\title{
Large Angle Washout Algorithms for Flight Simulators
}

\author{
by \\ Mikayla Micomonaco \\ A thesis submitted to the Faculty of Graduate and Postdoctoral Affairs in partial \\ fulfillment of the requirements for the degree of \\ Master of Applied Science \\ in \\ Aerospace Engineering \\ Department of Mechanical and Aerospace Engineering \\ Carleton University \\ Ottawa, Ontario
}

(c) 2019

Mikayla Micomonaco 


\section{Abstract}

This thesis presents modifications to classical washout algorithms to allow for large angular motion. This allows flight simulators with large angular motion envelopes to more effectively produce the motion cues for edge-of-envelope aircraft manoeuvres for

pilot training. Classical washout is commonly used for flight simulation motion cues, but was originally designed for small simulator angular motion. With the development of the Atlas motion base allowing unbounded rotation about any axis, there is a need for washout algorithms which can exploit this extended angular motion envelope.

Classical washout is implemented and the implementation is validated using numeric metrics, normalized Pearson correlation, integral of the error, and maximum error; and plots of the modelled vestibular response for the aircraft simulator pilot compared to previous reported results. This implementation is then used as a performance baseline for two proposed modifications to extend washout to larger angular motion envelopes. Large-angle washout uses quaternions and eliminates the small-angle approximations present in classical washout but continues to use highpass filters to impose restrictions on the simulator's angular motion during sustained angular velocity cues. Unrestricted angular washout removes the high-pass filters and allows for unbounded rotation about all axes.

Using the numerical metrics and vestibular responses to assess the performance of the algorithms, for manoeuvres with small angular motion cues, the original classical washout algorithm designed for small angles performs best, and unrestricted angular washout has comparable performance. For manoeuvres with large angular motion cues, unbounded angular velocity washout performs best. Large-angle washout outperforms the small-angle washout for some large angular motion cues, but is outperformed by unrestricted angular washout, and performs badly for manoeuvres with small angular motion. 


\section{Acknowledgments}

I would like to thank Professor Hayes, Professor Irani, and Professor Langlois for their guidance and support. I would also like to thank Karim Youssef for providing the X-Plane flight data for the test cases in Chapter 4. 


\section{Contents}

Abstract $\quad$ i

$\begin{array}{ll}\text { Acknowledgements } & \text { ii }\end{array}$

List of Tables $\quad$ iv

List of Figures $\quad$ V

Nomenclature $\quad$ vi

1 Introduction 1

1.1 Background . . . . . . . . . . . . . . . . 5

1.1.1 Washout Algorithms ................ 5

1.1.2 Washout Evaluation and Tuning . . . . . . . . . . . 7

1.1.3 Uses of Washout Algorithms . . . . . . . . . . . . . . . 8

1.1.4 Extensions to Classical Washout . . . . . . . . . . . . . 9

1.1.5 Vestibular System Modelling . . . . . . . . . . . . . . . 9

1.2 Mathematical Background . . . . . . . . . . . . . . . 11

1.3 Thesis Organization . . . . . . . . . . . . . . . . . 14

2 Washout Simulation $\quad 16$

2.1 Translational Acceleration and Specific Force . . . . . . . . . . . . . 17

2.2 Classical Washout . . . . . . . . . . . . . . . . . 18

2.2.1 Translational Channel ... . . . . . . . . . . . . . . . 19

2.2.2 Tilt Coordination . . . . . . . . . . . . . . . 20

2.2.3 Rotational Channel . . . . . . . . . . . . . . . . . . . . . 21

2.2.4 Washout Parameters .................. 22

2.3 Adaptive Washout . . . . . . . . . . . . . . . . . . . . . 23

2.4 Vestibular Response . . . . . . . . . . . . . . 25 
2.4 .1 Simulator Motion . . . . . . . . . . . . . . . . . 26

2.4 .2 Vestibular System . . . . . . . . . . . . . . . . . 27

2.5 Quantifying Washout Algorithm Performance . . . . . . . . 28

2.6 Washout Results . . . . . . . . . . . . . . . . . . . . . . . . 31

2.7 Summary . . . . . . . . . . . . . . . . . . . . 37

3 Classical Washout with Large Angles 39

3.1 Translational Channel . . . . . . . . . . . . . . . . . . . 40

3.2 Tilt Coordination . . . . . . . . . . . . . . . . . . . . 42

3.3 Rotational Channel . . . . . . . . . . . . . . . . . . . . . . . . 42

3.4 Vestibular Model . . . . . . . . . . . . . . . . . . . . . 44

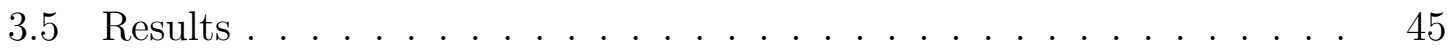

$3.5 .1 \quad$ M1: Turn Entries . . . . . . . . . . . . . . . . . 46

3.5.2 M2: Throttle Pulse . . . . . . . . . . . . . . . . . 49

3.5 .3 M3: Pushover/Pullup . . . . . . . . . . . . . . . . . 52

3.6 Angular Position Tracking . . . . . . . . . . . . . . . 55

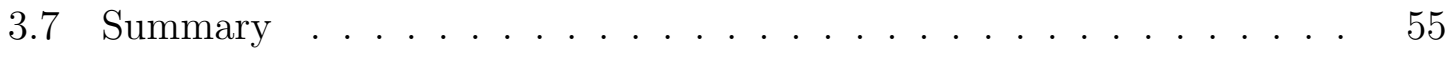

4 Case Studies $\quad 56$

4.1 Overview . . . . . . . . . . . . . . . . . . . . . . 57

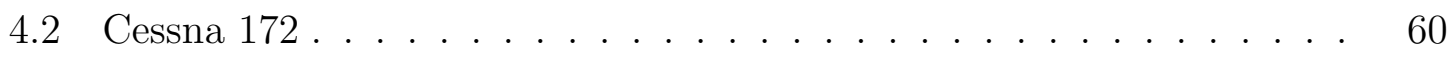

4.2 .1 Coordinated Turn . . . . . . . . . . . . . . . . . 60

4.2 .2 Cross-wind Landing . . . . . . . . . . . . . . . . . 65

4.2 .3 Single-axis Takeoff $\ldots \ldots \ldots \ldots 71$

$4.2 .4 \quad$ Spiral Dive . . . . . . . . . . . . . . . . 75

$4.2 .5 \quad$ Stall Recovery . . . . . . . . . . . . . . . . . 80

4.3 Other Aircraft . . . . . . . . . . . . . . . . . . . . . 85

$4.3 .1 \quad$ Boeing $737 \ldots \ldots \ldots \ldots \ldots \ldots$ 
$4.3 .2 \quad$ Columbia $400 \ldots \ldots \ldots \ldots \ldots$. . . . . . . . . . . 87

4.4 Summary of Large-angle Washout Results . . . . . . . . . . . 94

4.5 Unrestricted Angular Washout . . . . . . . . . . . . . . . 94

4.5.1 Columbia 400 Aerobatics . . . . . . . . . . . . . . . . 96

4.5.2 Cessna 172 Single-axis Takeoff . . . . . . . . . . . . . . 103

4.5.3 Cessna 172 Spiral Dive . . . . . . . . . . . . . . . . 105

4.6 Summary of Unrestricted Angular Washout _ . . . . . . . . 108

4.7 Conclusions . . . . . . . . . . . . . . . . . . . . . 108

$\begin{array}{lll}5 & \text { Conclusion } & 109\end{array}$

5.1 Objective 1: Modelling . . . . . . . . . . . . . . . . . 109

5.2 Objective 2: Extension of Classical Washout . . . . . . . . . 109

5.3 Objective 3: Performance Validation . . . . . . . . . . . 110

5.4 Future Work . . . . . . . . . . . . . . . . . . . 110

5.5 Publication $\ldots \ldots \ldots \ldots \ldots \ldots \ldots \ldots \ldots \ldots \ldots \ldots$

$\begin{array}{ll}\text { References } & 112\end{array}$

$\begin{array}{ll}\text { Appendix A Adaptive Washout } & 115\end{array}$

A.1 Translational Channel . . . . . . . . . . . . . . . 115

A.2 Rotational Channel . . . . . . . . . . . . . . . . 116

A.3 Surge/Pitch Adaptive Filters . . . . . . . . . . . . . . . . 116

A.4 Sway and Roll Adaptive Filters . . . . . . . . . . . . . . 117

A.5 Heave and Yaw Adaptive Filters . . . . . . . . . . . . 117

A.6 Washout Parameters . . . . . . . . . . . . . . . 118

$\begin{array}{lll}\text { Appendix B Small-angle Washout Test Cases } & 121\end{array}$

B.1 M1 . . . . . . . . . . . . . . . . . . 123

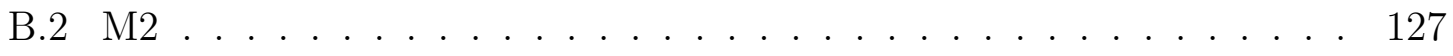




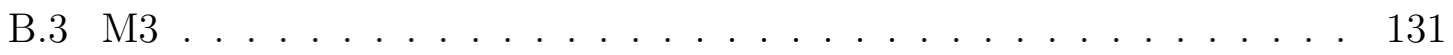

B.4 Cessna 172 Coordinated Turn . . . . . . . . . . . . . . 136

B.5 Cessna 172 Cross-wind Landing . . . . . . . . . . . . . . 140

B.6 Cessna 172 Single-axis Takeoff . . . . . . . . . . . . . . . . . . 144

B.7 Cessna 172 Spiral Dive . . . . . . . . . . . . . . . . . 148

B.8 Cessna 172 Stall Recovery . . . . . . . . . . . . . . . . . . 152

B.9 Boeing 737 Coordinated Turn . . . . . . . . . . . . 157

B.10 Boeing 737 Cross-wind Landing . . . . . . . . . . . . . 161

B.11 Boeing 737 Single-axis Takeoff . . . . . . . . . . . . . 165

B.12 Boeing 737 Spiral Dive . . . . . . . . . . . . . . . . . 169

B.13 Boeing 737 Stall Recovery . . . . . . . . . . . . . . . . 173

B.14 Columbia 400 Aerobatics . . . . . . . . . . . . . . . 178

B.15 Columbia 400 Coordinated Turn . . . . . . . . . . . . . 182

B.16 Columbia 400 Cross-wind Landing . . . . . . . . . . . . 186

B.17 Columbia 400 Single-axis Takeoff . . . . . . . . . . . . . . 190

B.18 Columbia 400 Spiral Dive . . . . . . . . . . . . . . . 194

B.19 Columbia 400 Stall Recovery . . . . . . . . . . . . . . . . 198

$\begin{array}{lll}\text { Appendix C Large-angle Washout Test Cases } & 202\end{array}$

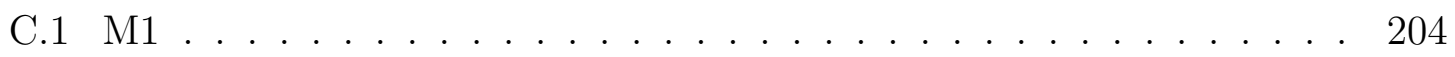

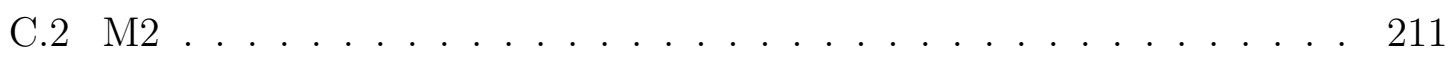

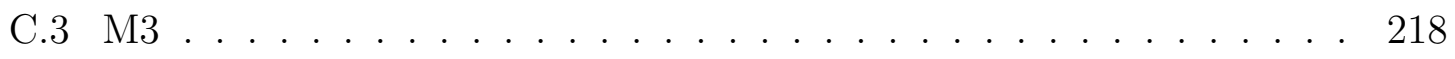

C.4 Cessna 172 Coordinated Turn . . . . . . . . . . . . . . 226

C.5 Cessna 172 Cross-wind Landing . . . . . . . . . . . . . 233

C.6 Cessna 172 Single-axis Takeoff . . . . . . . . . . . . . . . . 240

C.7 Cessna 172 Spiral Dive . . . . . . . . . . . . . . . . . 247

C.8 Cessna 172 Stall Recovery . . . . . . . . . . . . . . . . . . 254

C.9 Boeing 737 Coordinated Turn . . . . . . . . . . . . . . 262 
C.10 Boeing 737 Cross-wind Landing . . . . . . . . . . . . . . 269

C.11 Boeing 737 Single-axis Takeoff . . . . . . . . . . . . . 276

C.12 Boeing 737 Spiral Dive . . . . . . . . . . . . . . . . 283

C.13 Boeing 737 Stall Recovery . . . . . . . . . . . . . . . 290

C.14 Columbia 400 Aerobatics . . . . . . . . . . . . . . . . . . . 298

C.15 Columbia 400 Coordinated Turn . . . . . . . . . . . . . . 305

C.16 Columbia 400 Cross-wind Landing . . . . . . . . . . . . . . . 312

C.17 Columbia 400 Single-axis Takeoff . . . . . . . . . . . . . . 319

C.18 Columbia 400 Spiral Dive . . . . . . . . . . . . 326

C.19 Columbia 400 Stall Recovery . . . . . . . . . . . . . . . . 333

Appendix D Unrestricted Angular Washout Test Cases 340

D.1 Cessna 172 Coordinated Turn . . . . . . . . . . . . . . . . 342

D.2 Cessna 172 Cross-wind Landing . . . . . . . . . . . . . . . 349

D.3 Cessna 172 Single-axis Takeoff . . . . . . . . . . . . . . 356

D.4 Cessna 172 Spiral Dive . . . . . . . . . . . . . . . . . 363

D.5 Cessna 172 Stall Recovery . . . . . . . . . . . . . . . 370

D.6 Boeing 737 Coordinated Turn . . . . . . . . . . . . 378

D.7 Boeing 737 Cross-wind Landing . . . . . . . . . . . . 385

D.8 Boeing 737 Single-axis Takeoff . . . . . . . . . . . . . . . . . . . 392

D.9 Boeing 737 Spiral Dive . . . . . . . . . . . . . . . . . 399

D.10 Boeing 737 Stall Recovery . . . . . . . . . . . . . 406

D.11 Columbia 400 Aerobatics . . . . . . . . . . . . . . . . . . 414

D.12 Columbia 400 Coordinated Turn . . . . . . . . . . . . . . 421

D.13 Columbia 400 Cross-wind Landing . . . . . . . . . . . . 428

D.14 Columbia 400 Single-axis Takeoff _. . . . . . . . . . . 435

D.15 Columbia 400 Spiral Dive . . . . . . . . . . . . . . . 442

D.16 Columbia 400 Stall Recovery . . . . . . . . . . . . . . . . . 449 


\section{List of Tables}

1 Relationship between $\mathbf{a}_{\mathrm{AA}}$ and $\mathbf{f}_{\mathrm{AA}} \ldots \ldots \ldots \ldots$. . . . . . . 17

2 Classical washout parameter sets. . . . . . . . . . . . . . 22

$3 \quad$ M1 (turn entries) performance metrics for classical washout. . . . . . 37

$4 \quad$ M2 (throttle pulse) performance metrics for classical washout. . . . . 38

$5 \quad$ M3 (pushover/pullup) performance metrics for classical washout. . . . 38

6 Performance metrics for the turn entries manoeuvre (M1) for smalland large-angle washout (small-angle washout/large-angle washout). . 48

7 Performance metrics for the throttle pulse manoeuvre (M2) for smalland large-angle washout (small-angle washout/large-angle washout). . 51

8 Performance metrics for the pushover/pullup manoeuvre (M3) for smalland large-angle washout (small-angle washout/large-angle washout). . 54

$9 \quad$ X-Plane outputs. . . . . . . . . . . . . . . . 58

10 Equivalent axis for the washout algorithms and X-Plane. . . . . . . . 58

11 Ranked order of performance for each washout algorithm version for each manoeuvre. . . . . . . . . . . . . . . . . . . . 59

12 CW1 performance metrics for the coordinated turn manoeuvre by a Cessna 172 for small- and large-angle washout (small-angle washout/largeangle washout). . . . . . . . . . . . . . . . 64

13 CW1 performance metrics for the cross-wind landing manoeuvre by a Cessna 172 for small- and large-angle washout (small-angle washout/largeangle washout). . . . . . . . . . . . . . . . . . 70

14 CW1 performance metrics for the single-axis takeoff manoeuvre by a Cessna 172 for small- and large-angle washout (small-angle washout/largeangle washout). . . . . . . . . . . . . . . . . . 74 
15 CW1 performance metrics for the spiral dive manoeuvre by a Cessna 172 for small- and large-angle washout (small-angle washout/largeangle washout). . . . . . . . . . . . . . . . . 79

16 CW1 performance metrics for the stall recovery manoeuvre by a Cessna 172 for small- and large-angle washout (small-angle washout/largeangle washout). . . . . . . . . . . . . . . . . 84

17 CW1 performance metrics for manoeuvres with the Boeing 737 for small- and large-angle washout (small-angle washout/large-angle washout). 86

18 CW1 performance metrics for manoeuvres with the Columbia 400 for small- and large-angle washout (small-angle washout/large-angle

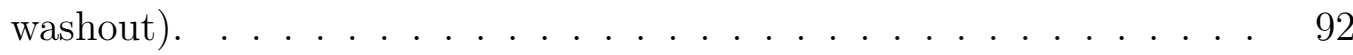

19 CW1 performance metrics for the aerobatics manoeuvre by a Columbia 400 for small- and large-angle washout (small-angle washout/largeangle washout). . . . . . . . . . . . . . . .

20 CW1 performance metrics for the aerobatics manoeuvre by a Columbia 400 for small-angle washout and unrestricted angular washout (smallangle washout/unrestricted angular washout). . . . . . . . . . . 102

21 CW1 performance metrics for the Cessna 172 for small-angle washout and unrestricted angular washout (small-angle washout/unrestricted angular washout). . . . . . . . . . . . . . . 107

22 Adaptive washout performance metrics. . . . . . . . . . . . . . 120 


\section{List of Figures}

1 Gough-Stewart Platform . . . . . . . . . . . . . . . . 2

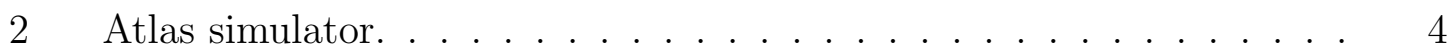

$\begin{array}{lll}3 & \text { Washout stages for a specific force cue in the surge degree of freedom. } & 7\end{array}$

4 Classical washout with Euler angles. . . . . . . . . . . . . 18

5 CW1 surge bode plot for the translational channel high pass filter and the tilt coordination low pass filter. . . . . . . . . . . . . . 23

6 Adaptive washout block diagram. . . . . . . . . . . . . . . . . 24

$7 \quad$ Vestibular response block diagram. . . . . . . . . . . . . . 25

8 Nonlinear relationship between the Pearson correlation and the normalized Pearson correlation. . . . . . . . . . . . . . . 30

9 Turn entries (M1) aircraft motion set points. . . . . . . . . . . . 32

10 Throttle pulse (M2) aircraft motion set points. . . . . . . . . . 33

11 Pullover/pushup (M3) aircraft motion set points. . . . . . . . . 34

12 CW1 M1 vestibular response from original report and current model. 35

13 Classical washout for large angles. . . . . . . . . . . . . . . 40

14 Translational channel for small-angle washout (top) and large-angle washout (bottom). . . . . . . . . . . . . . . . 41

15 Tilt coordination for small-angle washout (top) and large-angle washout

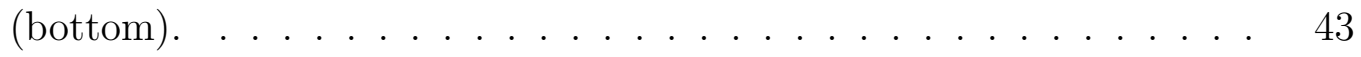

16 Rotational channel for small-angle washout (top) and large-angle washout

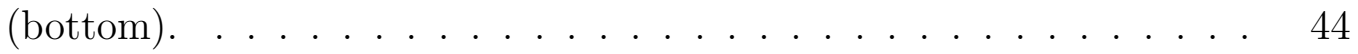

17 The CW1 vestibular response for M1, for small-angle washout and

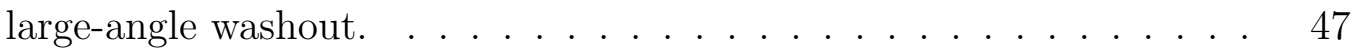

18 The CW1 vestibular response for M2, for small-angle washout and large-angle washout. . . . . . . . . . . . . . 50 
19 The CW1 vestibular response for M3, for small-angle washout and large-angle washout. . . . . . . . . . . . . . .

20 Aircraft motion at the pilot's head and the pilot's vestibular response for a Cessna 172 completing a coordinated turn. . . . . . . . . . . .

21 The vestibular response of the Cessna 172 aircraft pilot experiencing a coordinated turn manoeuvre, and the simulator pilot's vestibular response for small-angle washout and large-angle washout. . . . . . .

22 Aircraft motion at the pilot's head and the pilot's vestibular response for a Cessna 172 completing a cross-wind landing. . . . . . . . . . . .

23 The vestibular response of the Cessna 172 aircraft pilot experiencing a cross-wind landing manoeuvre, and the simulator pilot's vestibular response for small-angle washout and large-angle washout. . . . . . . 66

24 The vestibular response of the angular velocities of the Cessna 172 aircraft pilot at the end of a cross-wind landing manoeuvre, and the simulator pilot's vestibular response for small-angle washout and largeangle washout. . . . . . . . . . . . . . . .

25 Aircraft motion at the pilot's head for a Cessna 172 completing a singleaxis takeoff. . . . . . . . . . . . . . . . . . . . . 71

26 The vestibular response of the Cessna 172 aircraft pilot experiencing a single-axis takeoff manoeuvre, and the simulator pilot's vestibular response for small-angle washout and large-angle washout. . . . . .

27 Aircraft motion at the pilot's head for a Cessna 172 completing a spiral dive. . . . . . . . . . . . . . . . . . . 75

28 The vestibular response of the Cessna 172 aircraft pilot experiencing a spiral dive manoeuvre, and the simulator pilot's vestibular response for small-angle washout and large-angle washout. . . . . . . . . . . 76 
29 Aircraft motion at the pilot's head for a Cessna 172 completing a stall recovery. . . . . . . . . . . . . . . . . .

30 The vestibular response of the Cessna 172 aircraft pilot experiencing a stall recovery manoeuvre, and the simulator pilot's vestibular response for small-angle washout and large-angle washout. . . . . . . . . . .

31 Aircraft motion at the pilot's head for a Cessna 172 completing a stall recovery. . . . . . . . . . . . . . . . .

32 The vestibular response of the Columbia 400 aircraft pilot experiencing aerobatics, and the simulator pilot's vestibular response for small-angle washout and large-angle washout. . . . . . . . . . . . . . .

33 The aircraft's orientation in Euler angles for large-angle washout during aerobatics in the Columbia 400. . . . . . . . . . . . . . 91

34 The flight simulator's orientation in Euler angles for large-angle washout during aerobatics in the Columbia 400. . . . . . . . . . . . .

35 The flight simulator's orientation calculated as the integral of the angular velocity for unrestricted angular washout during aerobatics in the Columbia 400. . . . . . . . . . . . . . . .

36 The vestibular response of the Columbia 400 aircraft pilot experiencing aerobatics, and the simulator pilot's vestibular response for small-angle washout and unrestricted angular washout. . . . . . . . . . .

37 The vestibular response of the Columbia 400 aircraft pilot experiencing aerobatics, and the simulator pilot's vestibular response for unrestricted angular washout, and the components of gravity acting on the simulator. . . . . . . . . . . . . . . .

38 The vestibular response of the Cessna 172 aircraft pilot experiencing a single-axis takeoff, and the simulator pilot's vestibular response for small-angle washout and unrestricted angular washout. . . . . . . . 
39 The vestibular response of the Cessna 172 aircraft pilot experiencing a spiral dive, and the simulator pilot's vestibular response for small-angle washout and unrestricted angular washout. . . . . . . . . . . 106

40 Adaptive washout block diagram. . . . . . . . . . . . . . . 115

41 AW1 M1 vestibular response from original report and current model. 119 


\title{
Nomenclature
}

\author{
$\mathbf{a}_{\mathrm{AA}} \quad$ Aircraft translational acceleration in aircraft frame $\left(\mathrm{m} / \mathrm{s}^{2}\right)$ \\ $\mathbf{a}_{\mathrm{C}} \quad$ Aircraft translational acceleration in inertial frame $\left(\mathrm{m} / \mathrm{s}^{2}\right)$ \\ aps Translational acceleration at simulator pilot's head in simulator frame \\ $\left(\mathrm{m} / \mathrm{s}^{2}\right)$ \\ a Simulator translational acceleration in inertial frame $\left(\mathrm{m} / \mathrm{s}^{2}\right)$ \\ a Simulator translational acceleration in simulator frame $\left(\mathrm{m} / \mathrm{s}^{2}\right)$ \\ $\hat{f} \quad$ Specific force vestibular response in aircraft or simulator frame $\left(\mathrm{m} / \mathrm{s}^{2}\right)$ \\ $\mathbf{f}_{\mathrm{AA}} \quad$ Aircraft specific force in aircraft frame $\left(\mathrm{m} / \mathrm{s}^{2}\right)$ \\ $\mathbf{f}_{\mathrm{PS}} \quad$ Specific force at the simulator pilot's head, in simulator frame $\left(\mathrm{m} / \mathrm{s}^{2}\right)$ \\ $f_{x} \quad$ Specific force component along surge axis in simulator frame $\left(\mathrm{m} / \mathrm{s}^{2}\right)$ \\ $f_{y} \quad$ Specific force component along sway axis in simulator frame $\left(\mathrm{m} / \mathrm{s}^{2}\right)$ \\ $g \quad$ Acceleration due to gravity $\left(\mathrm{m} / \mathrm{s}^{2}\right)$ \\ g $_{\mathrm{AA}} \quad$ Gravity vector in aircraft frame $\left(\mathrm{m} / \mathrm{s}^{2}\right)$ \\ g $\quad$ Gravity vector in inertial frame $\left(\mathrm{m} / \mathrm{s}^{2}\right)$ \\ g $_{\mathrm{S}} \quad$ Gravity vector in simulator frame $\left(\mathrm{m} / \mathrm{s}^{2}\right)$ \\ $\mathbf{L}_{\mathrm{IS}} \quad$ Rotation matrix from simulator frame to inertial frame \\ $\mathbf{L}_{\mathrm{SI}} \quad$ Rotation matrix from inertial frame to simulator frame \\ NPC Normalized Pearson correlation \\ $p \quad$ Roll angular velocity ( $\mathrm{deg} / \mathrm{s})$ \\ $\mathbf{p}_{\mathbf{1}}, \mathbf{p}_{\mathbf{2}}, \mathbf{p}_{\mathbf{3}} \quad$ Adaptive filter parameters \\ PC Pearson correlation \\ $\dot{p}_{\mathrm{SS}} \quad$ Simulator roll angular acceleration $\left(\mathrm{deg} / \mathrm{s}^{2}\right)$ \\ $\dot{\mathbf{q}} \quad$ Quaternion rate \\ q Quaternion \\ $q \quad$ Pitch angular velocity (deg/s) \\ $\dot{q}_{\mathrm{SS}} \quad$ Simulator pitch angular acceleration $\left(\mathrm{deg} / \mathrm{s}^{2}\right)$




\begin{tabular}{|c|c|}
\hline$r$ & Roll angular velocity (deg/s) \\
\hline$\dot{r}_{\mathrm{SS}}$ & Simulator yaw angular acceleration $\left(\mathrm{deg} / \mathrm{s}^{2}\right)$ \\
\hline $\mathbf{R}_{\mathrm{SS}}$ & Position of pilot's head relative to simulator centre of gravity (m) \\
\hline $\mathbf{R}_{\mathrm{S}}$ & Matrix converting rate of change of Euler angles to angular velocities \\
\hline $\mathbf{T}_{\mathrm{S}}$ & Matrix converting angular velocities to rate of change of Euler angles \\
\hline$x$ & Surge $(\mathrm{m})$ \\
\hline$y$ & Sway $(\mathrm{m})$ \\
\hline$z$ & Heave $(\mathrm{m})$ \\
\hline$\dot{\boldsymbol{\beta}}_{\mathrm{C}}$ & Aircraft rate of change of Euler angles in inertial frame $(\mathrm{deg} / \mathrm{s})$ \\
\hline $\boldsymbol{\beta}_{\mathrm{S}}$ & Simulator Euler angle position set point in inertial frame (deg) \\
\hline$\dot{\boldsymbol{\beta}}_{\mathrm{S}}$ & Simulator rate of change of Euler angles set point in inertial frame $(\mathrm{deg} / \mathrm{s})$ \\
\hline$\Delta t$ & Timestep (s) \\
\hline$\hat{\omega}$ & Angular velocity vestibular response in aircraft or simulator frame (deg/s) \\
\hline$\omega_{\mathrm{AA}}$ & Aircraft angular velocity in aircraft frame (deg/s) \\
\hline $\boldsymbol{\omega}_{n}, \omega_{b}, \zeta$ & Transfer function coefficients \\
\hline$\dot{\boldsymbol{\omega}}_{\mathrm{SS}}$ & Simulator angular acceleration $\left(\mathrm{deg} / \mathrm{s}^{2}\right)$ \\
\hline $\boldsymbol{\omega}_{\mathrm{SS}}$ & Simulator angular velocity (deg/s) \\
\hline$\phi$ & Roll Euler angle (deg) \\
\hline$\psi$ & Yaw Euler angle (deg) \\
\hline$\theta$ & Pitch Euler angle (deg) \\
\hline
\end{tabular}




\section{Introduction}

Washout algorithms are commonly used for motion control in flight simulators. These algorithms are used to simulate a wider range of motion cues than would otherwise be available in the limited motion envelope of the flight simulator's hardware. For effective training, the simulator must recreate the pilot's perception of the translational specific force and angular velocity of the actual aircraft. Angular velocity simulation is limited to high-frequency cues by the hardware motion envelope; however, the specific force is simulated by a combination of translational acceleration and rotation. The translational accelerations are of a short duration, and simulate the high-frequency specific force cues usually associated with motion onset, while tilt coordination uses slow rotations of the simulator, below the perception threshold of the pilot, to move the gravity vector relative to the pilot to simulate the sustained specific force cues. The combination of translational and rotational motion allows for the simulation of sustained specific force that would not otherwise be possible.

Several different washout algorithms have been developed. The main three types of washout algorithms are classical washout, adaptive washout, and optimal control. In classical washout, high-pass filters are used to determine the high-frequency specific force and angular velocity cues, and low-pass filters are used for defining the cues representing the sustained translational acceleration. Adaptive washout, instead uses a set of cost functions and adaptive parameters to determine the translational and rotational set points for the simulator. Optimal control washout uses conventional optimal control techniques which have been tuned for the aircraft and manoeuvres intended to be simulated. Classical washout is most commonly used, since it is intuitive and easy to tune, although pilot testing has shown preference for the performance of adaptive washout. Optimal control has performed poorly during pilot testing [1-3].

Existing washout algorithms have been developed for simulators with restricted angular displacement. Figure 1 illustrates an example of a Gough-Stewart platform 


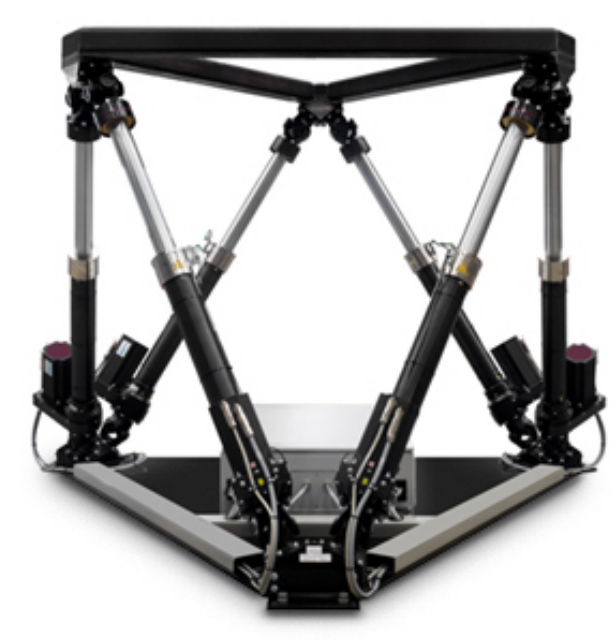

Figure 1: Gough-Stewart Platform [5].

that is the configuration most often used as the motion base for flight simulators. The geometry of the platform's legs restricts its angular displacement in all directions. Due to the restrictions of the simulators and for computational efficiency, washout algorithms are developed for a limited range of angular motion, resulting in these algorithms having several small-angle approximations, particularly in the implementation of tilt coordination. Tilt coordination is also designed with the assumption that the simulator's heave degree of freedom will remain closely aligned with the gravity vector, and not deviate from it by any large angle. For classical washout, to incorporate the angular position set point from tilt coordination and the angular velocity, the Euler angles of the two rotations are added, which is sufficiently accurate for the purposes of the aircraft simulator at small angles. Euler angles also can introduce singularities during large rotations of the simulator. There has been little progress in the development of washout algorithms since the 1980s [4], and therefore washout algorithms have not evolved to take advantage of advancements in simulator hardware.

As regulatory requirements for simulating edge-of-envelope aircraft motion are be- 
ing introduced, simulators with larger angular motion envelopes are being developed. One example of this type of simulator is the Atlas motion simulator developed by the Carleton University Simulator Project (CUSP), which has unbounded rotation about all axes. Figure 2 illustrates the simulator design. A Gough-Stewart motion base provides the translational motion, while three active mecanum wheels permit unbounded rotation in any direction for the rotational stage of the simulator [6-8]. To take advantage of the unbounded rotation, a series of changes to classical washout are required to accommodate large angles.

In this thesis, the necessary modifications to classical washout are made and the resulting simulator performance is evaluated. Small-angle approximations are removed, and the more computationally intensive unsimplified equations are used. Tilt coordination is allowed to act on all degrees of freedom, to ensure that the motion base is able to respond appropriately in all simulator orientations. Quaternions replace Euler angles for describing the orientation of the simulator, which eliminates the representational singularities present in existing classical washout. Finally, the high-pass filters are removed from the rotational channel to allow the simulator to rotate freely in response to the angular velocity cues.

In previous literature, classical and adaptive washout have been developed for small angles, and validated through simulations and experiments. The first objective of this thesis is to reproduce a model of classical washout and the vestibular response of the aircraft and simulator pilot. These algorithms will be implemented and tested, thereby allowing these algorithms to act as a baseline of performance.

The second objective of this thesis is to remove the small-angle restrictions and extend the range of angular motion for classical washout. Small-angle approximations and singularities will be removed, and the algorithm adapted so that it allows for a larger range of angular motion.

The final objective of this thesis is to demonstrate the performance of classical 


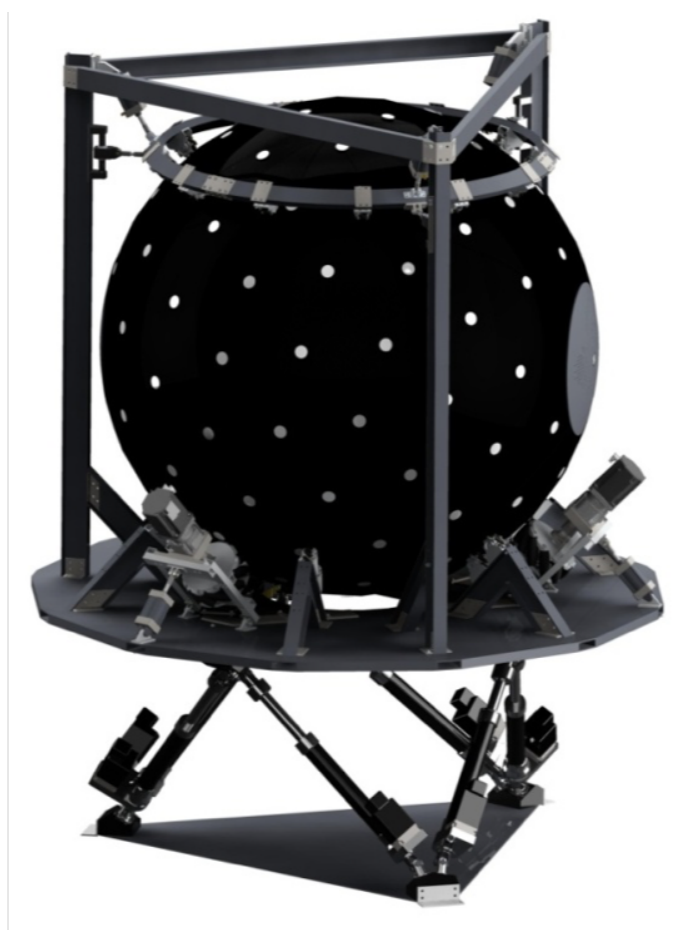

Figure 2: Atlas simulator.

washout at large angles in simulation. A case study of three different aircraft performing various manoeuvres is conducted and the performance of the algorithm against small-angle washout algorithms is assessed. In future work, adaptive washout can also be adapted for large angular motions.

The key contributions of this thesis include:

1. Creating a MATLAB Simulink model of the classical washout algorithm.

2. Extending classical washout to accommodate a larger range of angular motion.

3. Validating classical washout with the extended range through the use of computer simulation.

Additionally the work contained within this thesis has resulted in one conference publication: M. Micomonaco, M. J. D. Hayes, R. Irani, and R. Langlois, "Classical Washout Using Quaternions," in CCToMM Mechanisms, Machines, and Mechatron- 
ics $\left(M^{3}\right)$ Symposium, Montreal, Canada, May 16-17, 2019.

The following sections provide some background of washout algorithms and the quaternion math that will be used in this thesis.

\section{$1.1 \quad$ Background}

\subsubsection{Washout Algorithms}

There are three major types of washout algorithms: classical washout, adaptive washout, and optimal control [1]. Classical washout uses high- and low-pass filters, while adaptive washout and optimal control use adaptive and optimal controllers, respectively.

Reid and Nahon performed an extensive study of these washout algorithms [1-3]. They developed multiple benchmark cases for each algorithm type, and then subjected them to extensive pilot testing. Adaptive and classical washout performed well, with adaptive washout ranked best overall. As optimal control produced poor results, only adaptive washout and classical washout will be examined in this thesis.

Washout algorithms aim to simulate the translational specific force and angular velocity cues that would be experienced by an aircraft pilot while performing the same manoeuvre in a motion-enabled flight simulator, while remaining within the simulator hardware's motion envelope. They exploit the pilot's vestibular system by slowly tilting the simulator such that the gravity vector is interpreted as a sustained specific force cue. Some implementations of washout will also recentre the motion platform during sustained translational specific forces, to maximize the available translational motion envelope.

Classical washout accomplishes the simulation of translational specific force and angular velocity by using high-pass filters to restrict the translational and rotational motion of the simulator, and low-pass filters to tilt the simulator for the sustained specific force cues. Adaptive washout uses adaptive controllers and cost functions 
that work to match the specific force and angular velocity set points, while penalizing large values for the translational and rotational velocity and position.

Tilt coordination, which is the mechanism used to tilt the simulator so that the gravity vector is perceived as a sustained specific force cue is implemented for both algorithms for the surge and sway degrees of freedom. Washout was designed for small angular motions based on the limits of the motion actuation hardware available in the 1980s, and therefore as the gravity vector is closely aligned with the heave degree of freedom at all times, specific force cues in heave are unable to take advantage of tilt coordination. The tilt coordination limitation leads to poor performance in the heave degree of freedom compared to surge and sway, as it is limited to the acceleration that can be provided by the simulator's vertical motion, and this is often small relative to the magnitude of the cues.

An example of how tilt coordination works is illustrated in Figure 3, where there is a specific force cue forwards along the surge degree of freedom or the $x$-axis due to acceleration of the aircraft, as well as in the heave or $z$-axis due to gravity. To achieve these specific force cues, first the simulator accelerates forward, creating the specific force cue along the $x$-axis. As the simulator approaches the end of its motion envelope in 2, the simulator tilts, so that a component of the specific force cue in $x$ is coming from the simulator acceleration, and a component is coming from the gravity vector. In 3, the simulator has stopped accelerating, and the entirety of the sustained specific force cues are components of the gravity vector. When the surge specific force cue ends in 4 , the simulator returns to horizontal, and the simulator pilot experiences specific force only in heave.

Limited additional research has been completed since the Reid and Nahon study it and mostly focuses on tuning classical washout. Classical washout is still commonly used in the form proposed by Reid and Nahon due to its simplicity [4]. These washout algorithms can potentially be improved by eliminating the restrictions that limit them 

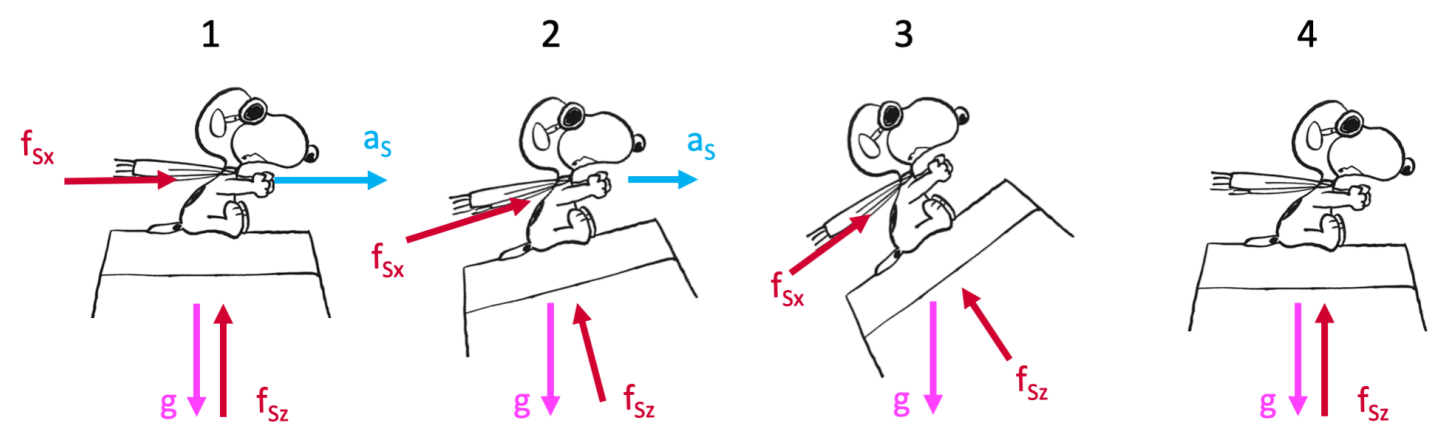

Figure 3: Washout stages for a specific force cue in the surge degree of freedom [10].

to small angles, to allow for better reproduction of angular velocity cues, and the ability to implement tilt coordination in heave.

\subsubsection{Washout Evaluation and Tuning}

The evaluation of washout algorithms in a simulation environment is challenging. The standard for evaluating the relative performance of different flight simulator motion algorithms is to conduct testing with aircraft pilots [3]. As pilot-in-the-loop tuning is impossible for a computer simulated environment, metrics can be used to attempt to quantify the performance of the algorithms, although pilot testing is still the preferred choice when available. The Casas et al. study in 2015 examined several metrics and compared them to the results from pilot testing, and concluded that the normalized Pearson correlation, which measures the degree the aircraft pilot's vestibular response and the simulator pilot's vestibular responses are positively correlated, is the best option for determining algorithm performance [4].

Tuning the simulator's transfer functions to improve the response of the system is another case where pilot evaluation is used. There are no automated or algorithmic ways of tuning a washout algorithm, and the best practice is to have an experienced 
pilot and someone familiar with washout tuning work together to tune the washout algorithm for the specified hardware. Grant and Reid outlined guidelines on how to address particular pilot concerns about the characteristics of the washout algorithm's response, and emphasized the inability to automate this process [11]. As with evaluation, completing tuning in a computer simulated environment is difficult, as it lacks the opportunity for pilot feedback.

There have been some methods proposed for automated tuning without the need for pilot-in-the-loop testing. Asadi et al. proposed using particle swarm optimization for tuning classical washout for a driving simulator, and showed that this increases motion fidelity and reduce incorrect motion cues, while also maximizing the use of the simulator's motion envelope [12]. Casas et al. performed similar work with particle swarm optimization for classical washout, and came to the same conclusions [13]. In addition to the particle swarm optimization, fuzzy logic and genetic algorithms with nonlinear filters have been successfully used to tune classical washout and are also found to reduce incorrect cues and maximize the use of the simulator's motion envelope $[14,15]$.

A limitation for all these tuning studies is that the results have not been evaluated with pilot-in-the-loop testing to compare their performance with manual tuning.

For the scope of this thesis, tuning will not be attempted in order to isolate the performance changes due to the modifications to classical washout's structure, rather than improvements in tuning algorithms.

\subsubsection{Uses of Washout Algorithms}

Classical washout is frequently used when studying motion simulators. Keshavarz et al. [16] use the classical washout algorithm proposed by Reid and Nahon to evaluate the impact motion, visual, and auditory cues have on experiencing motion sickness in driving simulators . Cleij et al. [17] used classical washout to evaluate how individ- 
uals perceive motion incongruities, or negative cues in driving simulators. Dalmeijer et al. [18] used classical washout when they were evaluating the objective testing methods for evaluating flight simulator performance for rotorcraft.

In these studies, classical washout is used without modifying it beyond tuning for the intended simulator and manoeuvres being studied.

\subsubsection{Extensions to Classical Washout}

The creation of the Desdemona simulator has inspired one extension of the washout algorithm. This simulator was developed with the goal of addressing the limitations in specific force that can be applied to the simulator pilot by conventional simulators, by adding a centrifuge component. To address the complications that the centrifuge introduces to the system, spherical washout using polar coordinates was developed [19]. While spherical washout is interesting, it does not have much bearing on the scope of this thesis, as the washout algorithms applicable in this thesis are intended for flight simulators that do not have the centrifuge, and therefore the polar coordinate system is unnecessary.

\subsubsection{Vestibular System Modelling}

As the primary goal of flight simulators is that the flight simulator pilot perceive the same motion as they would in an aircraft undergoing the same manoeuvre, a model of the pilot's vestibular system is used to determine how motion is perceived [3]. The vestibular system consists of two parts, the otolith that detects translational specific force, and the semi-circular canal that detects rotational motion.

There are many methods of modelling the components of the vestibular system. Meiry and Young [20] developed a model for the otolith system as a pair of transfer functions with a deadband between them to represent the threshold where the specific

force can be detected. A survey of vestibular models completed by Zacharaias [21] 
proposed reasonable threshold values for specific force detection. Reid and Nahon [1] used the Meiry and Young model with deadband values from the Zacharias report to model the pilot's specific force simulator response. However, for the second phase of Reid and Nahon's study [2] they no longer included the deadband when graphing the vestibular response of the simulator and aircraft pilots. A 2016 survey of otolith models completed by Asadi et al. [22] includes the Meiry and Young model used by Reid and Nahon. Asadi et al. [22] recommend the Telban and Cardullo model, which reduces the gain and phase lag impact for perceiving lower-frequency specific force compared to Meiry and Young, and chooses the deadband values used by Reid and Nahon. However, to maintain consistency for comparing the results of this thesis with the study conducted by Reid and Nahon, the Meiry and Young model will be used, and is discussed in Chapter 3.

Young and Oman [23] developed a model for the semi-circular canal that detects angular acceleration as transfer functions. Zacharaias [21] presents an adaption of this model for angular velocity, and as with the otolith model, proposed the detection threshold deadbands. Reid and Nahon [2] use the angular velocity model. However, once again in their second phase of the study omit the deadbands when graphing the vestibular response. Asadi et al. [24] conducted a survey of semicircular canal vestibular models in 2017, which concluded that the Young and Oman model used by Reid and Nahon, as well as the Telban and Cardullo model, were both reasonable models for the semicircular canal experiencing normal head movement; however, the Telban and Cardullo model was the most acceptable. As with the otolith system model, to maintain consistency with the Reid and Nahon report results, this thesis will use the Young and Oman model, which is discussed in Chapter 3.

The following section provides the mathematical foundation for the development of the large-angle washout algorithm. 


\subsection{Mathematical Background}

As part of this thesis, quaternions will be used as they are a useful replacement for Euler angles in classical washout, as quaternions can be used to represent rotations in three-dimensional space, and do not have the representational singularities present with Euler angles.

Quaternions are of the form

$$
q=q_{0}+q_{1} \mathbf{i}+q_{2} \mathbf{j}+q_{3} \mathbf{k}
$$

where $q_{0}, q_{1}, q_{2}, q_{3}$ are real and $\mathbf{i}, \mathbf{j}, \mathbf{k}$ are three imaginary numbers defined as

$$
\mathrm{ijk}=\mathbf{i}^{2}=\mathbf{j}^{2}=\mathbf{k}^{2}=-1,
$$

where these are quaternion products and not dot products, and

$$
\mathbf{i} \neq \mathbf{j} \neq \mathbf{k}
$$

The quaternion can also be represented as

$$
\mathbf{q}=\left[\begin{array}{l}
q_{0} \\
\mathbf{q}_{v}
\end{array}\right]=\left[\begin{array}{l}
q_{0} \\
q_{1} \\
q_{2} \\
q_{3}
\end{array}\right]
$$

where $q_{0}$ is the scalar or real part and $\mathbf{q}_{v}$ is the vector or imaginary part [25]. 
For unit quaternions these are

$$
\left[\begin{array}{l}
q_{0} \\
q_{1} \\
q_{2} \\
q_{3}
\end{array}\right]=\left[\begin{array}{c}
\cos \theta / 2 \\
k_{x} \sin \theta / 2 \\
k_{y} \sin \theta / 2 \\
k_{z} \sin \theta / 2
\end{array}\right],
$$

where $\mathbf{k}$ is a unit vector in the direction of the equivalent rotation axis and $\theta$ is the equivalent rotation angle about $\mathbf{k}$.

Given the rotation matrix

$$
{ }^{0} \mathbf{R}_{1}=\left[\begin{array}{ccc}
r_{11} & r_{12} & r_{13} \\
r_{21} & r_{22} & r_{23} \\
r_{31} & r_{32} & r_{33}
\end{array}\right]
$$

then

$$
\theta=\cos ^{-1}\left[\frac{r_{11}+r_{22}+r_{33}-1}{2}\right]
$$

and when $\theta=0$ or $\pi$ (or computationally close) the code then jumps to

$$
\mathbf{q}=\left[\begin{array}{l}
1 \\
0 \\
0 \\
0
\end{array}\right]
$$


for $\theta=0$ and

$$
\mathbf{q}=\left[\begin{array}{l}
0 \\
1 \\
1 \\
1
\end{array}\right]
$$

for $\theta=\pi$.

In terms of unit quaternions

$$
{ }^{0} \mathbf{R}_{1}=\left[\begin{array}{ccc}
1-2 q_{2}^{2}-2 q_{3}^{2} & 2\left(q_{1} q_{2}-q_{0} q_{3}\right) & 2\left(q_{1} q_{3}+q_{0} q_{2}\right) \\
2\left(q_{1} q_{2}+q_{0} q_{3}\right) & 1-2 q_{1}^{2}-2 q_{3}^{2} & 2\left(q_{2} q_{3}-q_{0} q_{1}\right) \\
2\left(q_{1} q_{3}-q_{0} q_{2}\right) & 2\left(q_{2} q_{3}+q_{0} q_{1}\right) & 1-2 q_{1}^{2}-2 q_{2}^{2}
\end{array}\right]
$$

Given ${ }^{0} \mathbf{R}_{1}$ then

$$
\begin{aligned}
q_{0}=\frac{1}{2}\left(1+r_{11}+\right. & \left.r_{22}+r_{33}\right)^{\frac{1}{2}} \\
q_{1} & =\frac{r_{32}-r_{23}}{4 q_{0}} \\
q_{2} & =\frac{r_{13}-r_{31}}{4 q_{0}} \\
q_{3} & =\frac{r_{21}-r_{12}}{4 q_{0}} .
\end{aligned}
$$

The quaternion product can be written in vector form

$$
\mathbf{p} \otimes \mathbf{q}=\left[\begin{array}{c}
p_{0} q_{0}-p_{1} q_{1}-p_{2} q_{2}-p_{3} q_{3} \\
p_{0} q_{1}+p_{1} q_{0}+p_{2} q_{3}-p_{3} q_{2} \\
p_{0} q_{2}-p_{1} q_{3}+p_{2} q_{0}+p_{3} q_{1} \\
p_{0} q_{3}+p_{1} q_{2}-p_{2} q_{1}+p_{3} q_{0}
\end{array}\right]
$$

It is important to note that the quaternion product is not commutative [25]. 
A quaternion can be used to rotate a vector with the equation

$$
r(\mathbf{v})=\mathbf{q} \otimes \mathbf{v} \otimes \mathbf{q}^{*}
$$

where $\mathbf{q}^{*}$ is the conjugate of the quaternion defined as

$$
\mathbf{q}^{*}=\left[\begin{array}{c}
q_{0} \\
-\mathbf{q}_{v}
\end{array}\right]
$$

Using the inverse quaternion, defined as

$$
\mathbf{q}^{-1}=\frac{\mathbf{q}^{*}}{\|\mathbf{q}\|^{2}}
$$

in Equation 16 will rotate the vector by the same measure but in the opposite direction as the rotation with $\mathbf{q}$.

Quaternion integration can be accomplished by knowing the angular rate, $\boldsymbol{\omega}$, and the previous angular position, $\mathbf{q}_{n-1}$, with the equation

$$
\mathbf{q}_{n}=\mathbf{q}_{n-1} \otimes\left[\begin{array}{c}
\cos (\|\boldsymbol{\omega}\| \Delta t / 2) \\
\frac{\boldsymbol{\omega}}{\|\boldsymbol{\omega}\|} \sin (\|\boldsymbol{\omega}\| \Delta t / 2)
\end{array}\right]
$$

where $\Delta t$ is the timestep [25].

\subsection{Thesis Organization}

This thesis consists of 5 chapters. This chapter gives the background of the problem and is a review of the existing literature relating to washout algorithms for flight simulators and the mathematical background for quaternion mathematics. Chapter 2 discusses coding the existing washout algorithms in MATLAB Simulink and compares their performance with each other an with a conventional implementation of 
these algorithms. Chapter 3 describes the modifications made to classical washout to accommodate large angular motion. In Chapter 4, case studies involving three aircraft are performed to demonstrate the performance of large-angle classical washout, with additional modifications to improve performance. Finally, Chapter 5 presents the conclusions of this thesis. 


\section{Washout Simulation}

Washout algorithms allow for flight simulators to reproduce the sensation of aircraft flight in the limited motion envelope of a flight simulator [1]. The simulator attempts to reproduce the sensations of the specific force and angular velocity that would be present in the aircraft, while restricting large translational and rotational displacements to prevent the simulator from exceeding its hardware limits.

The simulator represents high-frequency translational accelerations and angular velocities directly, although they may be scaled to remain within the simulator motion envelope. Following high-frequency translational accelerations, the platform may be recentred to allow for future motion. To reproduce sustained low-frequency translational acceleration for surge and sway motions, the simulator is slowly rotated to align the gravity vector with the direction of the specific force associated with the sustained translational acceleration. By maintaining the rotation rate to align the simulator with the gravity vector below $3 \mathrm{deg} / \mathrm{s}$ the pilot does not sense the rotation and instead interprets the acceleration from the gravity vector as the sustained translational acceleration of the aircraft [1].

There are three primary reference frames used in washout. The aircraft frame is fixed to the aircraft at the location of the pilot. The simulator frame is fixed to the simulator and aligned with the simulator pilot, and the inertial frame is the world frame. There are six degrees of freedom, surge: $x$, sway $y$, heave $z$, roll $\phi$, pitch $\theta$, and yaw $\psi$.

The key to good flight simulation is to match the vestibular response of the aircraft

pilot and simulator pilot [1]. The vestibular response is the pilot's perception of motion from their vestibular system, and accounts for their psychological perception thresholds of motion.

Reid and Nahon conducted an in-depth study of washout algorithms and how pilots evaluated their relative performance [1-3]. The three algorithm types studied 
were classical washout, adaptive washout, and optimal control. Testing proved that classical and adaptive washout performed better than optimal control.

To have a baseline to evaluate the performance of adaptions to the washout algorithms, first the original versions of classical washout were implemented in simulation as a benchmark. The performance if the implementations was evaluated against the reported performance in the original Reid and Nahon study [2] to validate the development.

\subsection{Translational Acceleration and Specific Force}

The vector $\mathbf{a}_{\mathrm{AA}}$ is the translational acceleration of the aircraft in the aircraft frame. Washout algorithms use the input of specific force $\mathbf{f}_{\mathrm{AA}}$ which is related to gravity by

$$
\mathbf{f}_{\mathrm{AA}}=\mathbf{a}_{\mathrm{AA}}-\mathbf{g}_{\mathrm{AA}}
$$

where $\mathbf{g}_{\mathrm{AA}}$ is the gravity vector in the aircraft frame, and the positive $\mathrm{z}$ axis is down. Table 1 lists some examples of the relationship between $\mathbf{a}_{\mathrm{AA}}$ and $\mathbf{f}_{\mathrm{AA}}$. In the first case, the aircraft is at rest with no acceleration, and there is a specific force applied to the pilot of $1 g$ up. In the freefall case, the acceleration is $1 g$ down, and the specific force applied to the pilot is $0 \mathrm{~m} / \mathrm{s}^{2}$. In the third case, the aircraft is rising with an acceleration of $1 g$ up, resulting in a specific force of $2 g$ up.

Table 1: Relationship between $\mathbf{a}_{\mathrm{AA}}$ and $\mathbf{f}_{\mathrm{AA}}$.

\begin{tabular}{ccccc} 
& Rest & $1 \mathrm{~g}$ Down (Freefall) & $1 \mathrm{~g}$ Up \\
\hline \hline $\mathbf{a}_{\mathrm{AA}}$ & {$\left[\begin{array}{lll}0 & 0 & 0\end{array}\right]^{\mathrm{T}}$} & {$\left[\begin{array}{lll}0 & 0 & g\end{array}\right]^{\mathrm{T}}$} & {$\left[\begin{array}{lll}0 & 0 & -g\end{array}\right]^{\mathrm{T}}$} \\
$\mathbf{g}_{\mathrm{AA}}$ & {$\left[\begin{array}{lll}0 & 0 & g\end{array}\right]^{\mathrm{T}}$} & {$\left[\begin{array}{lll}0 & 0 & g\end{array}\right]^{\mathrm{T}}$} & {$\left[\begin{array}{lll}0 & 0 & g\end{array}\right]^{\mathrm{T}}$} \\
$\mathbf{f}_{\mathrm{AA}}$ & {$\left[\begin{array}{lll}0 & 0 & -g\end{array}\right]^{\mathrm{T}}$} & {$\left[\begin{array}{lll}0 & 0 & 0\end{array}\right]^{\mathrm{T}}$} & {$\left[\begin{array}{lll}0 & 0 & -2 g\end{array}\right]^{\mathrm{T}}$}
\end{tabular}




\subsection{Classical Washout}

Figure 4 illustrates the block diagram of classical washout, where the translational, tilt coordination, and rotational channels are indicated. The algorithm takes in inputs of the specific force and angular velocity experienced by the aircraft pilot, and produces set points of the translational and angular positions for the motion simulator.

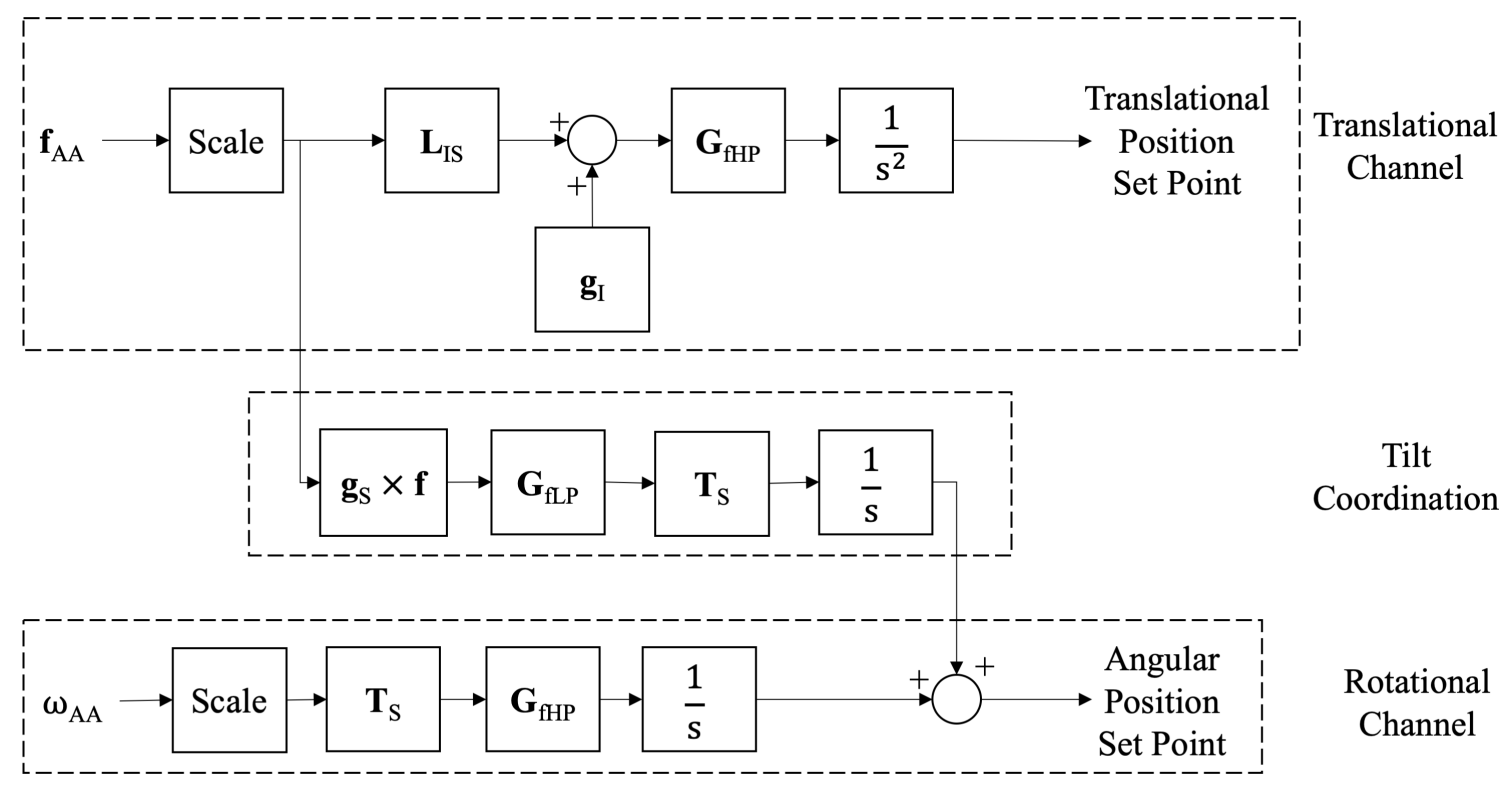

Figure 4: Classical washout with Euler angles.

Classical washout was implemented with two different versions, using high-order or low-order filters. The high-order filters act to recentre the motion simulator during sustained translational accelerations, allowing for the full motion envelope to be available in all directions. The low-order filters do not recentre the simulator, which reduces the available motion envelope but also reduces the negative motion cues experienced by the pilot due to the simulator motion. 


\subsubsection{Translational Channel}

The translational channel's input is the specific force experienced by the pilot of the aircraft being simulated, in the aircraft frame. First, the specific force may be optionally scaled, to assist in ensuring that the simulator motion set point remains within the hardware limits of the motion simulator. It is then converted into the inertial frame using the rotation matrix $\mathbf{L}_{\mathrm{IS}}$

$$
\mathbf{L}_{\mathrm{IS}}=\left[\begin{array}{ccc}
\cos \theta \cos \psi & \sin \phi \sin \theta \cos \psi & \cos \phi \sin \theta \cos \psi \\
& -\cos \phi \cos \psi & +\sin \phi \sin \psi \\
\cos \theta \sin \psi & \sin \phi \sin \theta \sin \psi & \cos \phi \sin \theta \sin \psi \\
& +\cos \phi \cos \psi & -\sin \phi \cos \psi \\
-\sin \theta & \sin \phi \cos \theta & \cos \phi \cos \theta
\end{array}\right]
$$

where $\phi, \theta$, and $\psi$ are the roll, pitch, and yaw Euler angles consistent with the $Z Y X$ rotation sequence based on the current orientation of the simulator [1]. Gravity $\mathbf{g}_{\mathrm{I}}$ is added in the inertial frame to convert the specific force to acceleration, and the result is then passed through a high-pass filter $\mathbf{G}_{\mathrm{fHP}}$ of the form

$$
\mathbf{G}_{\mathrm{fHP}}=\frac{\mathrm{s}^{2}}{\left(\mathrm{~s}+\omega_{n}\right)^{2}},
$$

for the low-order case, or the form

$$
\mathbf{G}_{\mathrm{fHP}}=\frac{\mathrm{s}^{3}}{\left(\mathrm{~s}^{2}+2 \zeta \omega_{n} \mathrm{~s}\right)\left(\mathrm{s}+\omega_{b}\right)},
$$

for the high-order case, where $\omega_{n}, \zeta$, and $\omega_{b}$ are chosen based on the desired washout algorithm performance characteristics. 
The output of the high-pass filter is then integrated twice to produce a position set point.

\subsubsection{Tilt Coordination}

While the translational channel simulates the high frequency specific forces, tilt coordination acts to simulate the low frequency sustained specific forces. To simulate sustained specific forces, tilt coordination aligns the gravity vector with the direction of the specific force. Reid and Nahon discuss two methods of tilt coordination [2].

One method, as illustrated in Figure 4, involves taking the cross product of $\mathbf{g}_{\mathrm{S}}$, the gravity vector in the simulator frame, and the scaled specific force $\mathbf{f}$. This is then multiplied by a scaling factor such that the result is an angular velocity which will align the gravity vector with the specific force set point without overshoot. As the simulator's rotation rate must be below the threshold that the pilot will detect, it is passed through a low-pass filter of the form

$$
\mathbf{G}_{\mathrm{fHP}}=\frac{\left(2 \omega_{n}\right)^{2}}{\mathrm{~s}^{2}+4 \omega_{n} \mathrm{~s}+\left(2 \omega_{n}\right)^{2}},
$$

for the low-order case, or with the addition of a $\zeta$ term, the form

$$
\mathbf{G}_{\mathrm{fHP}}=\frac{\left(2 \omega_{n}\right)^{2}}{\mathrm{~s}^{2}+4 \zeta \omega_{n} \mathrm{~s}+\left(2 \omega_{n}\right)^{2}},
$$

for the high-order case, with the same values for $\omega_{n}$ as those in the high-pass filter in the translational channel [2]. A rate limiter can also be added after the integrator if required to ensure the rotation produced by tilt coordination is below the pilot's perception threshold. The filtered angular velocity is converted to Euler angular rates 
using

$$
\mathbf{T}_{S}=\left[\begin{array}{ccc}
1 & \sin \phi \tan \theta & \cos \phi \tan \theta \\
0 & \cos \phi & -\sin \phi \\
0 & \sin \phi \sec \theta & \cos \phi \sec \theta
\end{array}\right]
$$

based on the current orientation of the simulator, and then integrated to update the Euler angles [1].

An alternative tilt coordination method relies on small-angle simplifications. Using small angles, the tilt coordination channel illustrated in Figure 4 can instead be modelled by removing the cross product and $\mathbf{T}_{\mathbf{s}}$, and inserting the equation

$$
\left[\begin{array}{l}
\phi \\
\theta \\
\psi
\end{array}\right]=\left[\begin{array}{c}
\frac{-f_{y}}{g} \\
\frac{f_{x}}{g} \\
0
\end{array}\right]
$$

between $\mathbf{G}_{\mathrm{fHP}}$ and the integrator. A rate limiter of $3 \mathrm{deg} / \mathrm{s}$ is placed after the integrator.

\subsubsection{Rotational Channel}

Finally, to simulate the angular velocity experienced by the aircraft pilot, the rotational channel takes the input of angular velocity in the aircraft frame. It is scaled, if required, and then converted to Euler angular rates using Equation 26. The Euler angular rates are then passed through a high-pass filter of the form

$$
\mathbf{G}_{\mathrm{fHP}}=\frac{\mathrm{s}}{\mathrm{s}+\omega_{n}}
$$


for the low-order case, or the form

$$
\mathbf{G}_{\mathrm{fHP}}=\frac{\mathrm{s}^{2}}{\left(\mathrm{~s}+\omega_{n}\right)^{2}},
$$

for the high-order case, where $\omega_{n}$ chosen based on the desired performance characteristics. The output of $\mathbf{G}_{\mathrm{fHP}}$ is integrated to produce angular position, and then added to the Euler angles produced from the tilt coordination. The addition of Euler angles from the tilt coordination and rotational channels is not strictly mathematically correct, but is an acceptable approximation for small angles [1]. The sum of the Euler angles is the angular position set point.

\subsubsection{Washout Parameters}

Reid and Nahon developed three classical washout parameter sets, with different simulator response characteristics [2]. Table 2 lists each parameter set and the order of the filters which were used. The parameters were chosen by observing the algorithm's response in simulation, and also in limited testing with pilots. The use of highorder filters allows for the simulator to recentre itself during sustained translational accelerations.

For the CW1 case in the surge degree of freedom, the parameters result in the

Table 2: Classical washout parameter sets.

\begin{tabular}{cccccc} 
Parameter Set & Degree of Freedom & Order & $\omega_{n}[\mathrm{deg} / \mathrm{s}]$ & $\zeta$ & $\omega_{b}[\mathrm{deg} / \mathrm{s}]$ \\
\hline \hline \multirow{2}{*}{ CW1 } & Surge & Low & 1.5 & - & - \\
& Sway, Heave & Low & 3.5 & - & - \\
& Roll, Pitch, Yaw & Low & 0.5 & - & - \\
\hline \multirow{2}{*}{ CW2 } & Surge & Low & 2.5 & - & - \\
& Sway, Heave & Low & 4 & - & - \\
& Roll, Pitch, Yaw & Low & 1 & - & - \\
\hline \multirow{2}{*}{ CW3 } & Surge, Sway, Heave & High & 3.1 & 1.4 & 0.2 \\
& Roll, Pitch, Yaw & High & 1.0 & - & -
\end{tabular}


translational channel high pass filter and tilt coordination low pass filter having a cutoff frequency of $0.31 \mathrm{~Hz}$ for the low pass filter and $0.37 \mathrm{~Hz}$ for the high pass filter. The Bode plot in Figure 5, illustrates how the filters in the two channels are able to isolate the high frequency and low frequency components of the signal, due to the similar cutoff frequencies and has unity DC gain.

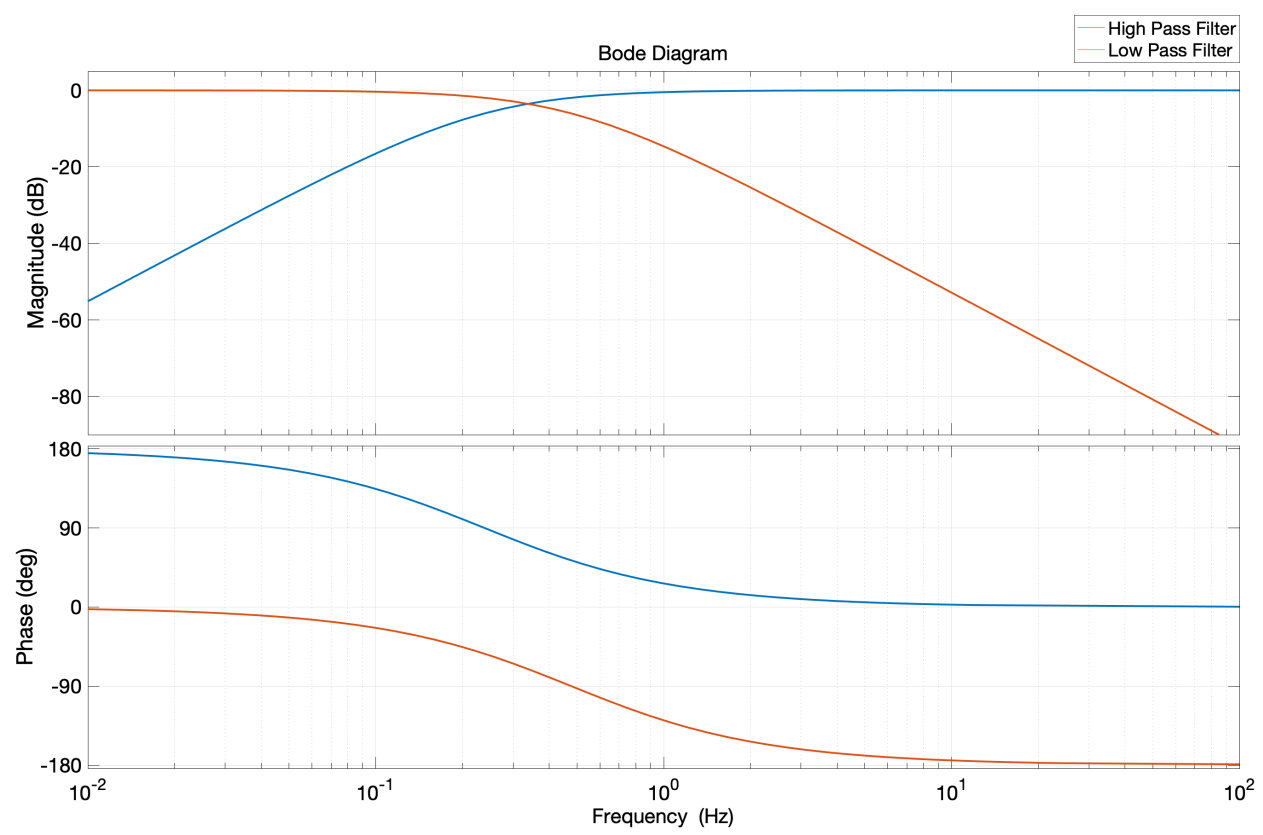

Figure 5: CW1 surge bode plot for the translational channel high pass filter and the tilt coordination low pass filter.

\subsection{Adaptive Washout}

Adaptive washout uses the same principles as classical washout, with translational acceleration and tilt coordination simulating the specific force cues, and rotation simulating the angular velocity cues. Figure 6 illustrates the block diagram for adaptive washout. The adaptive filter for the translational channel has one adaptive parameter, and attempts to match the translational acceleration input to the filter while penalizing large translational displacement and velocity to keep the simulator inside 
the motion envelope. The adaptive filter for the rotational channel has two adaptive parameters, one for tilt coordination and one for the rotational velocity set point. The tilt coordination is to not exceed the pilot's perception threshold, and the filter acts to match the angular velocity and tilt coordination set points while penalizing large angular displacement and velocity. Each adaptive parameter is updated by a cost function. Appendix A discusses the implementation of adaptive washout in greater detail.

Adaptive washout has been shown to have good performance compared to other implementations of washout [3]. However, it is less commonly used than classical washout because of its complexity. Due to this complexity, classical washout was selected for adaption to the larger angular motion envelope, and modifications to adaptive washout are beyond the scope of this thesis.

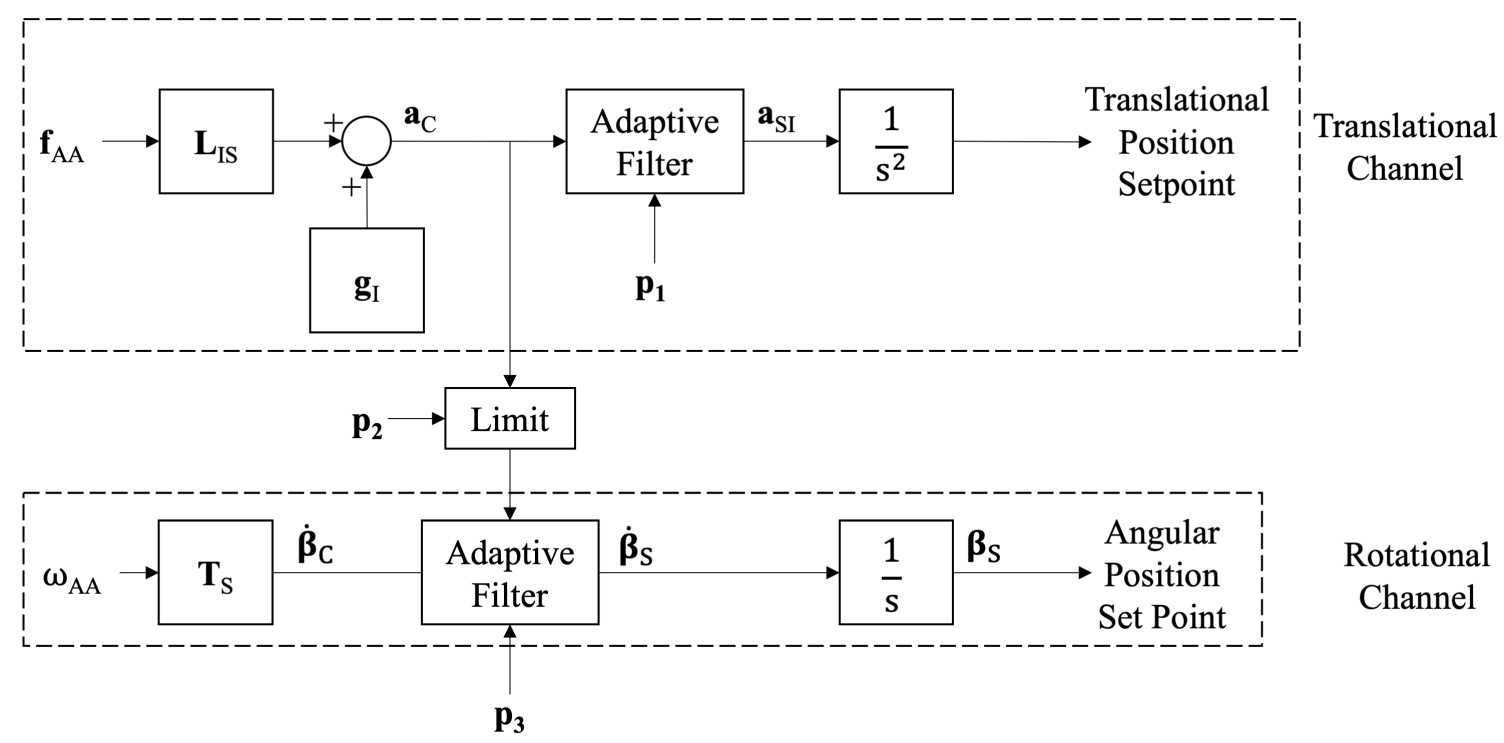

Figure 6: Adaptive washout block diagram. 


\section{$2.4 \quad$ Vestibular Response}

The pilot's vestibular system allows the pilot to perceive changes in specific force and angular velocity. To accurately simulate aircraft motion, the simulator needs to reproduce similar vestibular responses in the simulator pilot as would have been experienced by the aircraft pilot. Figure 7 illustrates the block diagram to determine the motion at the pilot's head and the pilot's vestibular response to that motion. The simulator's translational acceleration in the inertial frame $\mathbf{a}_{\mathrm{SI}}$ and angular position expressed in Euler angles $\boldsymbol{\beta}_{\mathrm{S}}$ are the inputs. The specific force at the pilot's head $\mathbf{f}_{\mathrm{PS}}$ and the angular velocity of the simulator $\boldsymbol{\omega}_{\mathrm{SS}}$ are calculated, and input into the vestibular response model, which calculates the vestibular response to the specific force $\hat{\mathbf{f}}$ and angular velocity $\hat{\boldsymbol{\omega}}$.

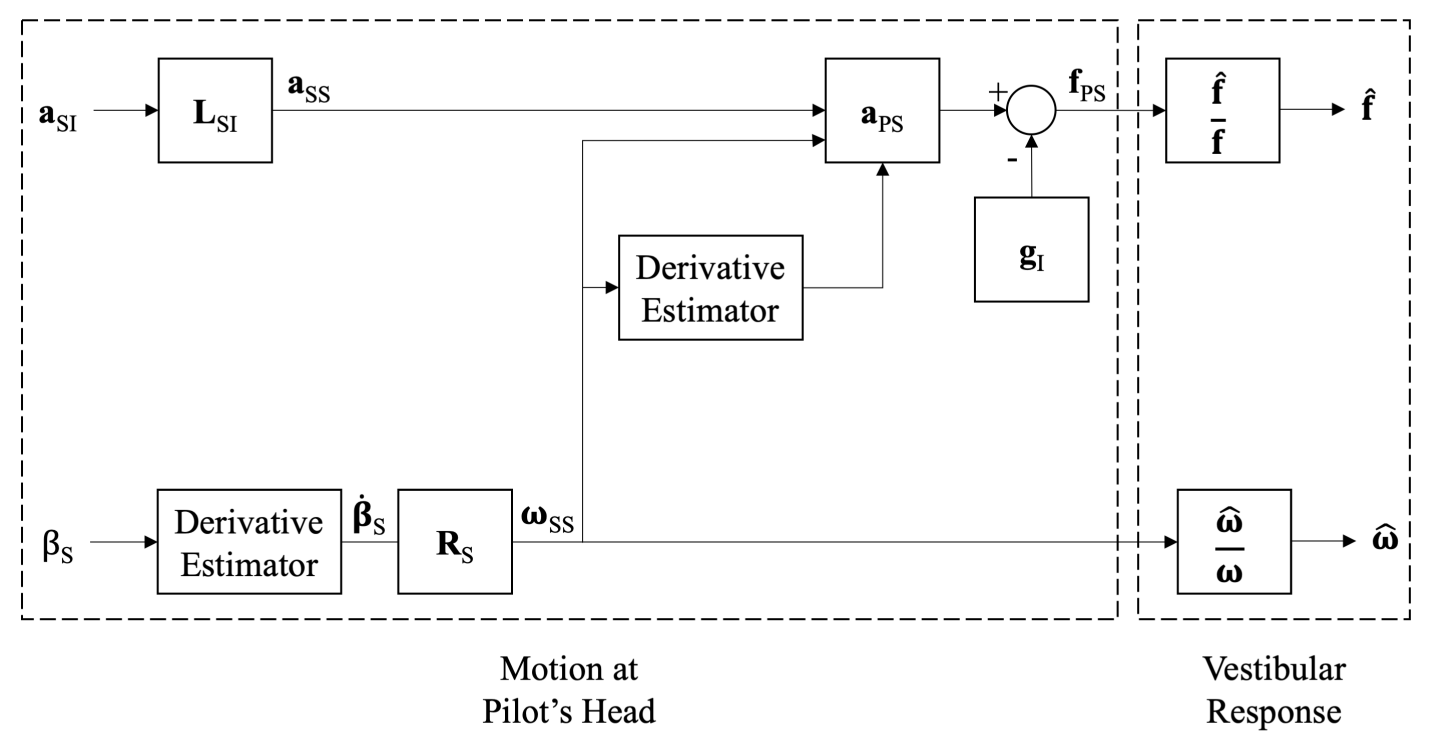

Figure 7: Vestibular response block diagram. 


\subsubsection{Simulator Motion}

The inputs to the vestibular system are the specific force and angular velocity experienced by the simulator pilot's head. For a physical simulator, the translational acceleration in the inertial frame, $\mathbf{a}_{\text {SI }}$, and the angular position expressed using Euler angles $\boldsymbol{\beta}_{\mathrm{S}}=\left[\begin{array}{lll}\phi & \theta & \psi\end{array}\right]^{\mathrm{T}}$ are measured. For the simulation, it is assumed that the simulator has perfect response to the given set point.

To calculate the specific force applied to the simulator pilot $\mathbf{f}_{\mathrm{S}}$ the acceleration is first converted into the simulator frame using the rotation matrix

$$
\mathbf{L}_{\mathrm{SI}}=\left[\begin{array}{ccc}
\cos \theta \cos \psi & \cos \theta \sin \psi & -\sin \theta \\
\sin \phi \sin \theta \cos \psi & \sin \phi \sin \theta \sin \psi & \sin \phi \cos \theta \\
-\cos \phi \cos \psi & +\cos \phi \cos \psi & \\
\cos \phi \sin \theta \cos \psi & \cos \phi \sin \theta \sin \psi & \cos \phi \cos \theta \\
+\sin \phi \sin \psi & -\sin \phi \cos \psi &
\end{array}\right],
$$

the inverse of Equation 21, to give the simulator acceleration in the simulator frame $\mathbf{a}_{\mathrm{SS}}$.

The equation

$$
\mathbf{G}_{\mathrm{est}}=\frac{\omega_{2} \mathrm{~s}}{\mathrm{~s}+\omega_{2}}
$$

where $\omega_{2}=12.5 \mathrm{rad} / \mathrm{s}$ is used to estimate a derivative. It is first used to estimate the rate of change for the Euler angles. The rate of change of Euler angles of the simulator is then converted into angular velocities, $\boldsymbol{\omega}_{\mathrm{SS}}=\left[\begin{array}{lll}p_{\mathrm{SS}} & q_{\mathrm{SS}} & r_{\mathrm{SS}}\end{array}\right]^{\mathrm{T}}$, using 
the matrix

$$
\mathbf{R}_{\mathrm{S}}=\left[\begin{array}{ccc}
1 & 0 & -\sin \theta \\
0 & \cos \phi & \sin \phi \cos \theta \\
0 & -\sin \phi & \cos \phi \cos \theta
\end{array}\right]
$$

The angular acceleration, $\dot{\boldsymbol{\omega}}_{\mathrm{SS}}=\left[\begin{array}{lll}\dot{p}_{\mathrm{SS}} & \dot{q}_{\mathrm{SS}} & \dot{r}_{\mathrm{SS}}\end{array}\right]^{\mathrm{T}}$, is estimated with the derivative estimator given in Equation 31.

The acceleration at the aircraft pilot's head is calculated using

$$
\mathbf{a}_{\mathrm{PS}}=\mathbf{a}_{\mathrm{SS}}+\left[\begin{array}{ccc}
-\left(q_{\mathrm{SS}}^{2}+r_{\mathrm{SS}}^{2}\right) & p_{\mathrm{SS}} q_{\mathrm{SS}}-\dot{r}_{\mathrm{SS}} & p_{\mathrm{SS}} r_{\mathrm{SS}}+\dot{q}_{\mathrm{SS}} \\
p_{\mathrm{SS}} q_{\mathrm{SS}}+\dot{r}_{\mathrm{SS}} & -\left(p_{\mathrm{SS}}^{2}+r_{\mathrm{SS}}^{2}\right) & q_{\mathrm{SS}} r_{\mathrm{SS}}-\dot{p}_{\mathrm{SS}} \\
p_{\mathrm{SS}} r_{\mathrm{SS}}-\dot{q}_{\mathrm{SS}} & q_{\mathrm{SS}} r_{\mathrm{SS}}+\dot{p}_{\mathrm{SS}} & -\left(p_{\mathrm{SS}}^{2}+q_{\mathrm{SS}}^{2}\right)
\end{array}\right] \mathbf{R}_{\mathrm{SS}}
$$

where the vector $\mathbf{R}_{\mathrm{SS}}$ is the displacement of the pilot's head from the origin of the simulator reference frame [1].

For the Atlas simulator, $\mathbf{R}_{\mathrm{SS}}=\left[\begin{array}{lll}0 & 0 & 0\end{array}\right]^{\mathrm{T}}$, as the pilot's head is positioned at the geometric centre of the simulator cockpit.

With the resulting acceleration, the specific force at the pilot's head, $\mathbf{f}_{\mathrm{PS}}$ is calculated using Equation 20.

\subsubsection{Vestibular System}

The primary organs in the human body for sensing specific force and rotational motion are the otolith and the semicircular canals, respectively.

The otolith response to specific force $\hat{\mathbf{f}}$ can be modelled as the transfer function

$$
\frac{\hat{f}_{i}}{f_{i}}=\frac{K\left(\tau_{\mathrm{a}} \mathrm{s}+1\right)}{\left(\tau_{\mathrm{L}} \mathrm{s}+1\right)\left(\tau_{\mathrm{S}} \mathrm{s}+1\right)}
$$

with typical parameter values of $\tau_{\mathrm{L}}=5.33 \mathrm{~s}, \tau_{\mathrm{S}}=0.66 \mathrm{~s}, \tau_{\mathrm{a}}=13.2 \mathrm{~s}$, and $K=0.4[1]$. 
The semicircular canal response to angular velocity $\hat{\boldsymbol{\omega}}$ can be modelled as the transfer function

$$
\frac{\hat{\omega}_{i}}{\omega_{i}}=\frac{T_{\mathrm{L}} T_{\mathrm{a}} \mathrm{s}^{2}}{\left(T_{\mathrm{L}} \mathrm{S}+1\right)\left(T_{\mathrm{S}} \mathrm{S}+1\right)\left(T_{\mathrm{a}} \mathrm{s}+1\right)},
$$

with typical parameter values of $T_{\mathrm{L}}=10.2 \mathrm{~s}, T_{\mathrm{S}}=0.1 \mathrm{~s}$, and $T_{\mathrm{a}}=30.0 \mathrm{~s}[1]$.

\subsection{Quantifying Washout Algorithm Performance}

Typically, the relative performance of washout algorithms is assessed by qualitative pilot evaluations. As these algorithms are currently a MATLAB Simulink simulation, three quantitative metrics, the normalized Pearson correlation, the integral of the error, and the maximum error, as well as qualitatively assessing the resulting graphs, are used to evaluate the relative performance of the algorithms.

As the goal of this section of the thesis is to evaluate how well the original washout algorithms were reproduced, the metrics will assess the difference between the original results reported by Reid and Nahon [2] and the new simulation, instead of comparing them to the aircraft motion set points.

The Pearson correlation (PC) evaluates how closely two signals are correlated and is calculated by,

$$
\mathrm{PC}(x, y)=\frac{\sum_{i=1}^{n}\left(x_{i}-\bar{x}\right)\left(y_{i}-\bar{y}\right)}{\sqrt{\sum_{i=1}^{n}\left(x_{i}-\bar{x}\right)^{2}} \sqrt{\sum_{i=1}^{n}\left(y_{i}-\bar{y}\right)^{2}}},
$$

where $x$ and $y$ represent the signals being correlated, $\bar{x}$ and $\bar{y}$ are the mean of $x$ and $y$ respectively, and $n$ is the number of samples. The Pearson correlation is a linear metric of the correlation of the two signals, where a value of 1 indicates the signals are completely correlated, 0 indicates they are uncorrelated, and -1 indicates they are negatively correlated.

An alternate metric proposed by Casas et al. after conducting a study comparing 
statistical evaluations of washout algorithm performance and pilot evaluations is the normalized Pearson correlation (NPC) and is calculated by,

$$
\operatorname{NPC}(x, y)=\frac{K}{1+\operatorname{PC}(x, y)}+1-\frac{K}{2},
$$

where $K=1$. Figure 8 illustrates the relationship between the Pearson correlation and the normalized Pearson correlation, where positively-correlated signals give a value of normalized Pearson correlation value of 1 , uncorrelated signals a value of 1.5, and negatively-correlated signals a value of $+\infty$. The normalized Pearson correlation is a non-linear metric which penalizes negative cues, where the simulator is providing a cue in the opposite direction to the actual aircraft motion, as this has significant negative impact on the aircraft pilot [4]. Limitations of this metric include that when the signal is close to constant, noise tends to overwhelm the correlation, and the signals will appear to be uncorrelated even if they are very similarly shaped.

The second metric compares the area between two signals, by taking the integral of the absolute value of the difference between the curves, calculated by,

$$
\operatorname{IntError}(x, y)=\int|x-y| \mathrm{d} t .
$$

Typically, the error metrics would be used to compare the aircraft pilot and simulator pilot's vestibular responses. However, since the goal is to determine how well the original algorithms have been reproduced, the simulator pilot's vestibular response will be evaluated against pilot's vestibular response results from the original report. The results for the normalized Pearson correlation, integral of the error, and maximum error for each degree of freedom for each algorithm version and manoeuvre are listed in tables. To eliminate the error caused by the transient responses, only the final 20 seconds of each manoeuvre were used. 


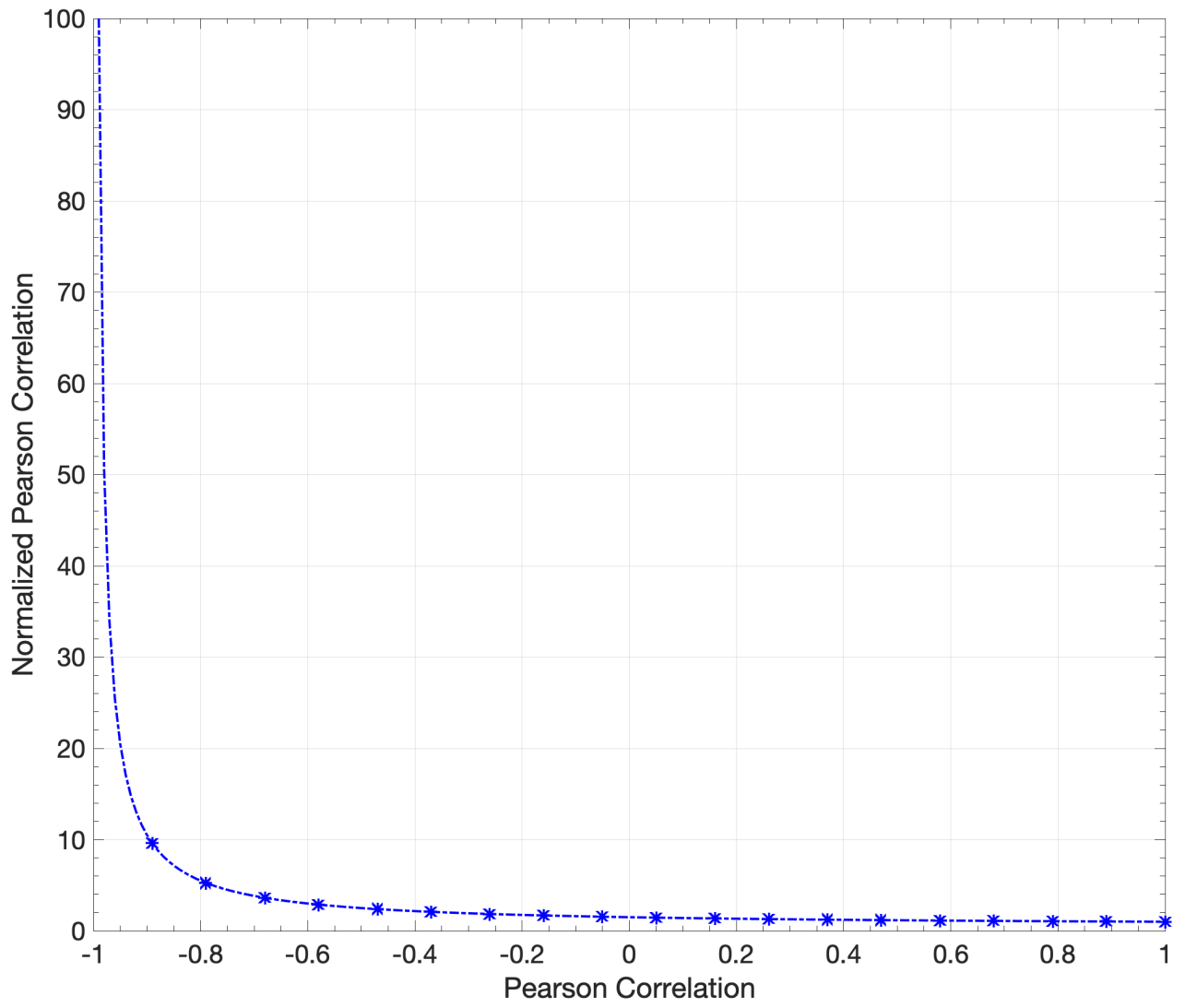

Figure 8: Nonlinear relationship between the Pearson correlation and the normalized Pearson correlation. 


\subsection{Washout Results}

For each algorithm type and parameter set, Reid and Nahon tested them for three manoeuvres. Figure 9 illustrates the turn entries (M1) manoeuvre has the aircraft specific force and angular velocity at the pilot's head for all six degrees of freedom. For M1, the dominant motion cue is the changing roll rate. Figure 10 illustrates the throttle pulse (M2) manoeuvre, which has a dominant motion cue of the change in the surge specific force, as well as small oscillations in the roll rate. Finally, Figure 11 illustrates the pushover/pullup (M3) manoeuvre, which has large changes in the heave specific force, as well as oscillations in the roll rate.

The sets of transfer function coefficients CW1, CW2, and CW3, listed in Table 2, were run for all three manoeuvres, and the vestibular response results were plotted. CW1 and CW2 are different coefficients for the low-order case, while the CW3 coefficients are for the high-order case. Figure 12 illustrates the response for the CW1 low-order algorithm for the M1 turn entries case. The grey line is the vestibular response of the aircraft pilot experiencing the manoeuvre. Blue line is the vestibular response for the simulator pilot reported in the original Reid and Nahon reports for the given manoeuvre and parameter set, and the black line is the simulator pilot's vestibular response for the algorithm implemented in MATLAB Simulink. For a successful implementation of the algorithm, the simulated results should be close to the results from the original report. The sway, heave, and pitch degrees of freedom initially diverge from the results from the original report, although all converge within 20 seconds [2].

For many degrees of freedom there are unwanted transient responses. The transient response is due to the differences in the simulation environment. The original testing had the simulator run level to allow the transient responses to dissipate be- 

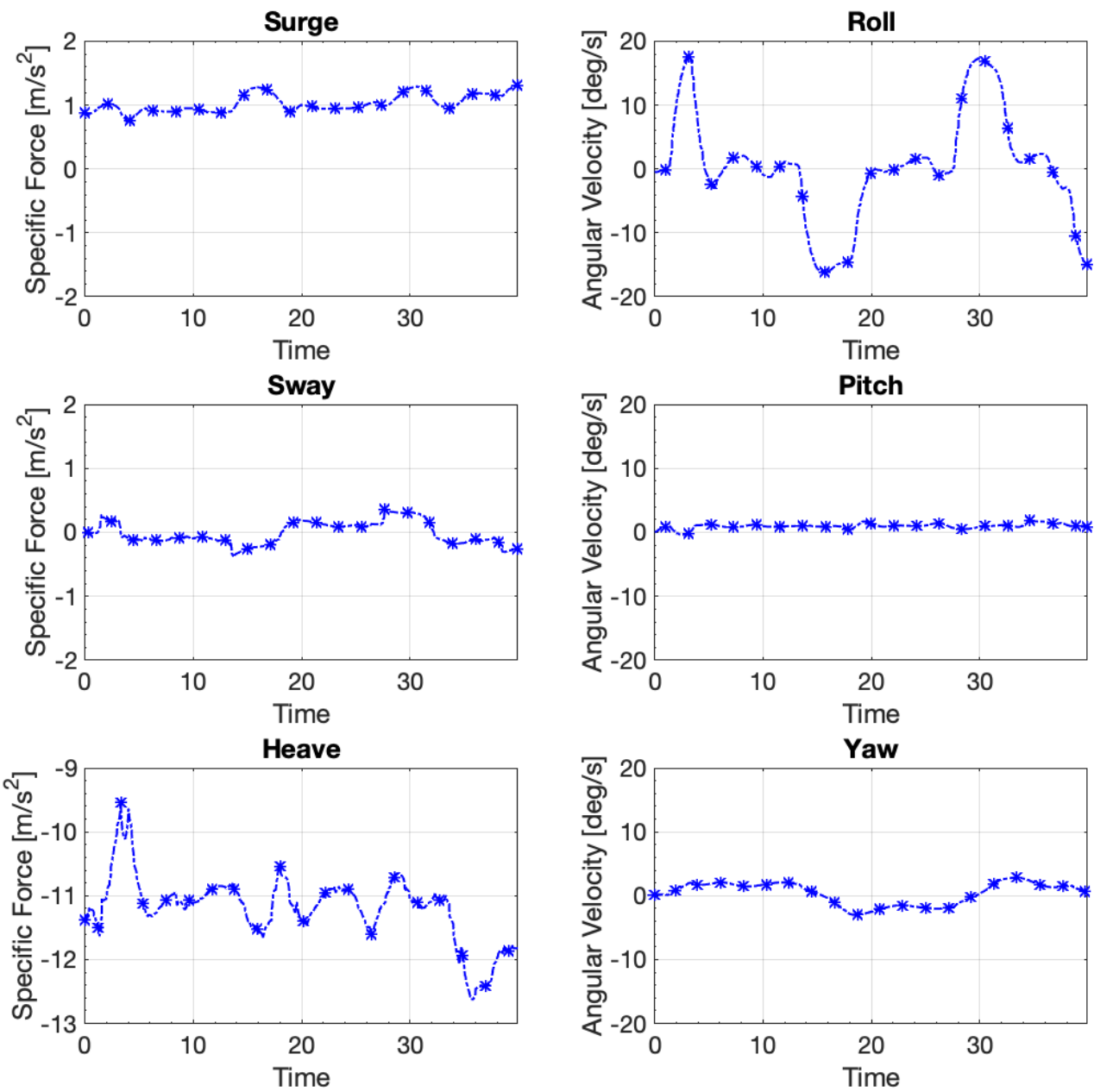

Figure 9: Turn entries (M1) aircraft motion set points. 

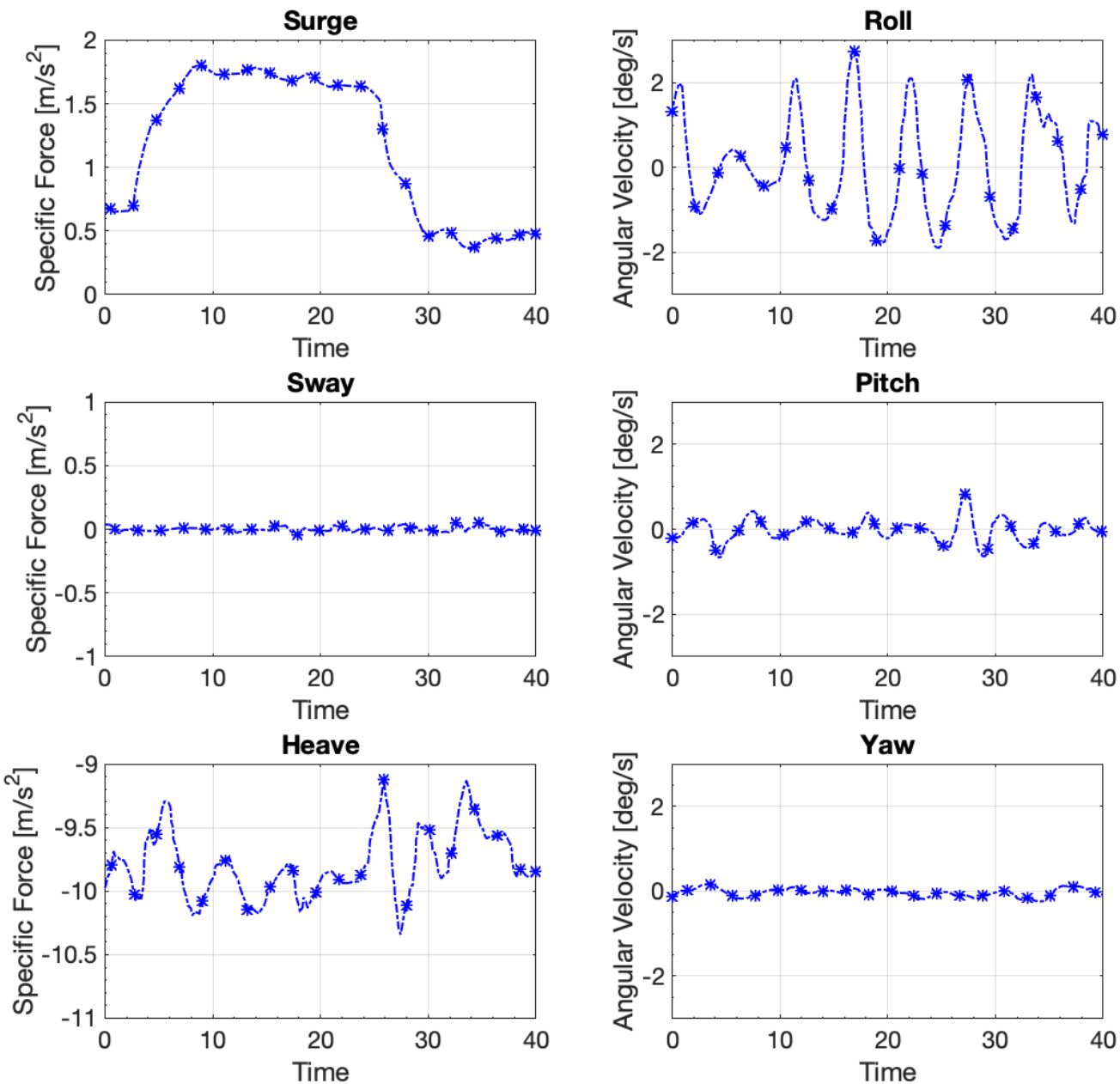

Figure 10: Throttle pulse (M2) aircraft motion set points. 

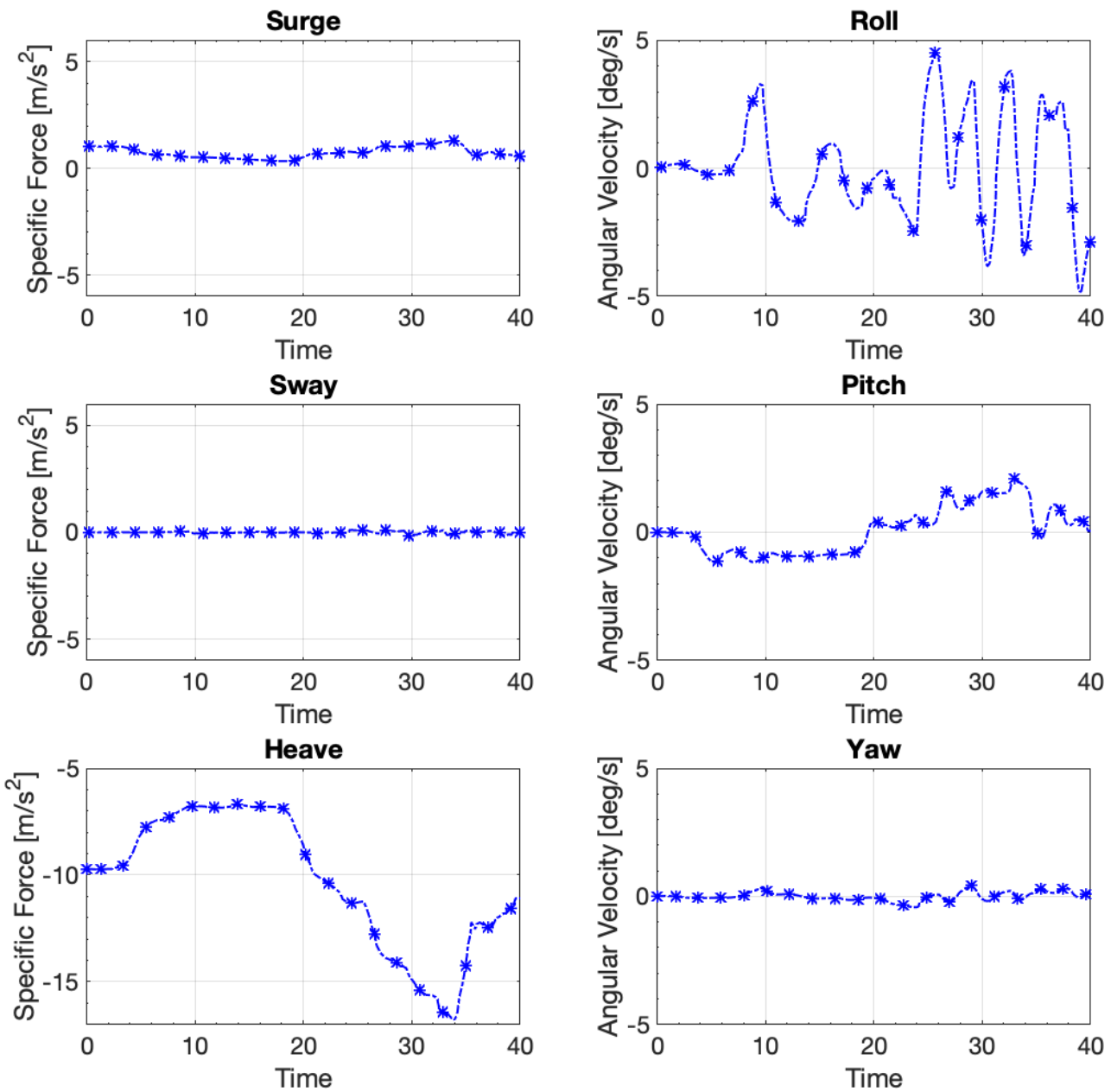

Figure 11: Pullover/pushup (M3) aircraft motion set points. 

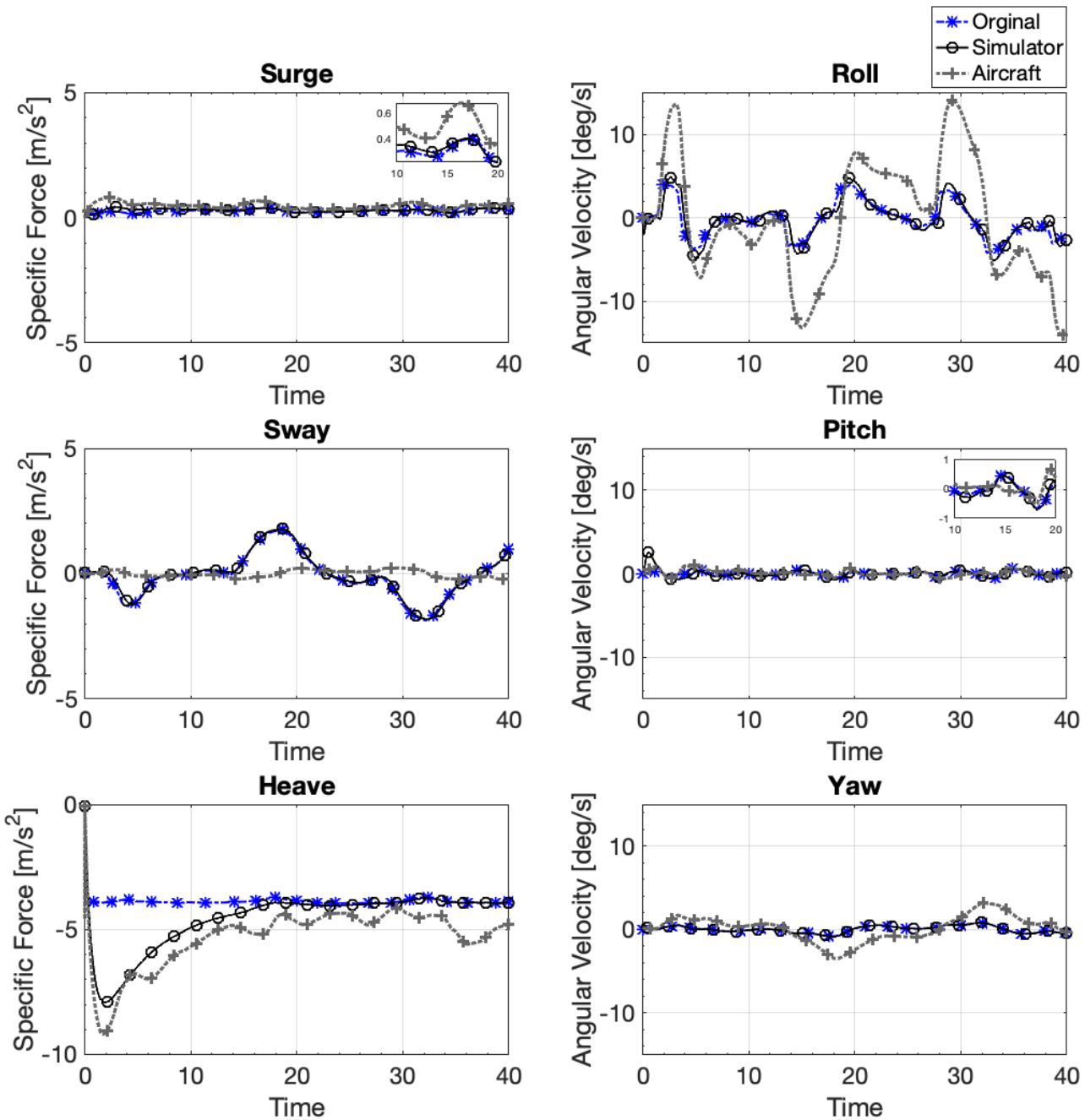

Figure 12: CW1 M1 vestibular response from original report and current model. 
fore the manoeuvres were executed. As those data were not included in the original report [2], it is impossible to recreate them such that the simulator would have the same translational and rotational positions, velocities, and accelerations at the start of the manoeuvre. As these initial values are important for reproducing the simulator response, the simulation begins at the start of the manoeuvre, with the initial conditions for the simulator indicated by the report. However, the transient response cannot be eliminated.

Table 3 lists the three performance metrics for the M1 manoeuvre for classical washout using each of the three parameter sets. For all the degrees of freedom for all the classical washout coefficient sets, the normalized Pearson correlation is close to one, with the exception of the heave degree of freedom for CW3. Examining the vestibular response in Figure 12 for the heave degree of freedom for CW1, it shows that the vestibular response for both the original and reproduced washout algorithms deviate very little during the final 20 seconds which are used to determine the performance metrics. The normalized Pearson correlation does not perform well for unvarying signals, as small deviations result in the two signals appearing uncorrelated. Examining the vestibular response shows that after the transient is completed, the two algorithms produce similar results, and the small values in the corresponding integral of the error and maximum error metrics support this conclusion.

Table 4 lists the performance metrics for the M2 manoeuvre, and Table 5 lists the performance metrics for the M3 manoeuvre for classical washout for each parameter set. The results are similar to the M1 case, with small integral of the error and maximum errors, and the normalized Pearson correlations being close to 1, with the exception of some of the heave degrees of freedom due to the nature of the vestibular response.

Appendix B includes additional graphs for each of the manoeuvres discussed in this section. 
Table 3: M1 (turn entries) performance metrics for classical washout.

\begin{tabular}{ccccc} 
Case & $\begin{array}{c}\text { Degree of } \\
\text { Freedom }\end{array}$ & $\begin{array}{c}\text { Normalized Pearson } \\
\text { Correlation }\end{array}$ & $\begin{array}{c}\text { Integral of Error } \\
{\left[\mathrm{m} / \mathrm{s}^{2}\right] \text { or }[\mathrm{deg} / \mathrm{s}]}\end{array}$ & $\begin{array}{c}\text { Maximum Error } \\
{\left[\mathrm{m} / \mathrm{s}^{\mathrm{s}}\right] \text { or }[\mathrm{deg} / \mathrm{s}]}\end{array}$ \\
\hline \hline \multirow{6}{*}{ CW1 M1 } & Surge & 1.00 & 0.16 & 0.02 \\
& Sway & 1.00 & 1.16 & 0.18 \\
& Heave & 1.04 & 0.94 & 0.13 \\
& Roll & 1.01 & 9.38 & 2.91 \\
& Pitch & 1.00 & 0.75 & 0.17 \\
& Yaw & 1.00 & 0.30 & 0.06 \\
\hline \multirow{6}{*}{ CW2 M1 } & Surge & 1.01 & 0.19 & 0.03 \\
& Sway & 1.01 & 1.27 & 0.26 \\
& Heave & 1.13 & 0.88 & 0.15 \\
& Roll & 1.06 & 12.41 & 3.77 \\
& Pitch & 1.01 & 1.19 & 0.29 \\
& Yaw & 1.00 & 0.55 & 0.10 \\
\hline \multirow{6}{*}{ CW3 M1 } & Surge & 1.02 & 0.37 & 0.05 \\
& Sway & 1.01 & 0.94 & 0.17 \\
& Heave & 1.40 & 0.87 & 0.16 \\
& Roll & 1.04 & 7.50 & 2.02 \\
& Pitch & 1.03 & 2.07 & 0.47 \\
& Yaw & 1.00 & 0.19 & 0.04
\end{tabular}

\subsection{Summary}

In this chapter, classical washout was examined in detail, and implemented in MATLAB Simulink. The vestibular responses for the implemented algorithms were compared against the results reported by Reid and Nahon, and they correlate closely for the final 20 seconds of each manoeuvre, once the impact of the transients and the incomplete information about the initial conditions of the manoeuvre have settled. The close correlations indicate that the implemented algorithms are a good reproduction of the original work and can be used as a baseline for assessing the performance of the modifications made to classical washout in the following chapters. 
Table 4: M2 (throttle pulse) performance metrics for classical washout.

\begin{tabular}{ccccc} 
Case & $\begin{array}{c}\text { Degree of } \\
\text { Freedom }\end{array}$ & $\begin{array}{c}\text { Normalized Pearson } \\
\text { Correlation }\end{array}$ & $\begin{array}{c}\text { Integral of Error } \\
{\left[\mathrm{m} / \mathrm{s}^{2}\right] \text { or }[\mathrm{deg} / \mathrm{s}]}\end{array}$ & $\begin{array}{c}\text { Maximum Error } \\
{\left[\mathrm{m} / \mathrm{s}^{2}\right] \text { or }[\mathrm{deg} / \mathrm{s}]}\end{array}$ \\
\hline \hline \multirow{6}{*}{ CW1 M2 } & Surge & 1.00 & 0.26 & 0.05 \\
& Sway & 1.01 & 0.29 & 0.05 \\
& Heave & 1.62 & 0.81 & 0.15 \\
& Roll & 1.03 & 4.24 & 0.83 \\
& Pitch & 1.01 & 1.59 & 0.44 \\
& Yaw & 1.01 & 0.16 & 0.04 \\
\hline & Surge & 1.00 & 1.11 & 0.02 \\
& Sway & 1.02 & 0.39 & 0.06 \\
CW2 M2 2 & Heave & 1.82 & 0.81 & 0.15 \\
& Roll & 1.05 & 4.40 & 0.83 \\
& Pitch & 1.03 & 1.94 & 0.70 \\
& Yaw & 1.01 & 0.09 & 0.02 \\
\hline \multirow{6}{*}{ CW3 M2 2} & 0.58 & 0.11 \\
& Surge & 1.01 & 0.42 & 0.06 \\
& Sway & 1.02 & 0.79 & 0.16 \\
& Heave & 2.08 & 2.75 & 0.48 \\
& Roll & 1.02 & 2.34 & 0.65 \\
& Pitch & 1.05 & 0.06 & 0.02
\end{tabular}

Table 5: M3 (pushover/pullup) performance metrics for classical washout.

\begin{tabular}{|c|c|c|c|c|}
\hline Case & $\begin{array}{l}\text { Degree of } \\
\text { Freedom }\end{array}$ & $\begin{array}{l}\text { Normalized Pearson } \\
\text { Correlation }\end{array}$ & $\begin{array}{l}\text { Integral of Error } \\
{\left[\mathrm{m} / \mathrm{s}^{2}\right] \text { or }[\mathrm{deg} / \mathrm{s}]}\end{array}$ & $\begin{array}{l}\text { Maximum Error } \\
{\left[\mathrm{m} / \mathrm{s}^{2}\right] \text { or }[\mathrm{deg} / \mathrm{s}]}\end{array}$ \\
\hline \multirow{6}{*}{ CW1 M3 } & Surge & 1.00 & 0.18 & 0.05 \\
\hline & Sway & 1.00 & 0.39 & 0.09 \\
\hline & Heave & 1.09 & 0.84 & 0.15 \\
\hline & Roll & 1.04 & 8.30 & 1.81 \\
\hline & Pitch & 1.00 & 1.62 & 0.35 \\
\hline & Yaw & 1.04 & 0.41 & 0.35 \\
\hline \multirow{6}{*}{ CW2 M3 } & Surge & 1.00 & 0.20 & 0.07 \\
\hline & Sway & 1.03 & 0.68 & 0.11 \\
\hline & Heave & 1.15 & 0.80 & 0.15 \\
\hline & Roll & 1.13 & 10.92 & 1.84 \\
\hline & Pitch & 1.01 & 1.73 & 0.41 \\
\hline & Yaw & 1.01 & 0.29 & 0.07 \\
\hline \multirow{6}{*}{ CW3 M3 } & Surge & 1.02 & 0.50 & 0.13 \\
\hline & Sway & 1.02 & 0.73 & 0.10 \\
\hline & Heave & 1.33 & 0.93 & 0.15 \\
\hline & Roll & 1.03 & 5.23 & 1.19 \\
\hline & Pitch & 1.01 & 2.33 & 0.54 \\
\hline & Yaw & 1.01 & 0.19 & 0.06 \\
\hline
\end{tabular}




\section{Classical Washout with Large Angles}

Washout algorithms were designed for small angular motions; therefore, problems arise as the range of angular displacement increases. For this thesis, classical washout was adapted to develop large-angle washout, as classical washout is widely used and is less complex to adapt for large angles than other algorithms such as adaptive washout.

There are several limitations that the small-angle classical washout experiences when used for large angular motions. Euler angles, regardless of the sequence used, will always have a singularity when the middle angle of the sequence reaches $\frac{\pi}{2} \pm i \pi$, where $i$ is any positive integer. Furthermore, the addition of the Euler angles from the tilt coordination and rotational channels to produce the angular position set point, which was an acceptable approximation at small angles [1], will cause significant error with larger angular displacements.

Large-angle washout uses a similar form to the small-angle classical washout block diagram, which was illustrated in Figure 4. The Euler angles are replaced with unit quaternions, which are a singularity free representation of orientation, where

$$
\mathbf{q}=\left[\begin{array}{l}
q_{0} \\
\mathbf{q}_{v}
\end{array}\right]=\left[\begin{array}{c}
q_{0} \\
q_{1} \\
q_{2} \\
q_{3}
\end{array}\right]=\left[\begin{array}{c}
\cos \theta / 2 \\
k_{x} \sin \theta / 2 \\
k_{y} \sin \theta / 2 \\
k_{z} \sin \theta / 2
\end{array}\right],
$$

and the rotation can be viewed as a single rotation of angle $\theta$ about the axis $\mathbf{k}=$ $\left[\begin{array}{lll}k_{x} & k_{y} & k_{z}\end{array}\right]$ and the unit quaternion must also satisfy the condition

$$
q_{0}^{2}+q_{1}^{2}+q_{2}^{2}+q_{3}^{3}=1 .
$$

This representation of orientation is considered singularity-free, as rotations of $\pi$ are treated as a special case, and the unit quaternion can be viewed as points on the 
surface of a unit 4D hypersphere, where distinct points represent distinct orientations [26]. Therefore, unit quaternions are used to replace Euler angles in classical washout to eliminate representational singularities at large angles.

The small-angle approximations are removed to improve the accuracy of the washout algorithm when the simulator is at large angles. Figure 13 illustrates the block diagram for large-angle washout with quaternions, which includes the translational, tilt coordination, and rotational channels. The following section discuss the changes made to each channel for large-angle washout.

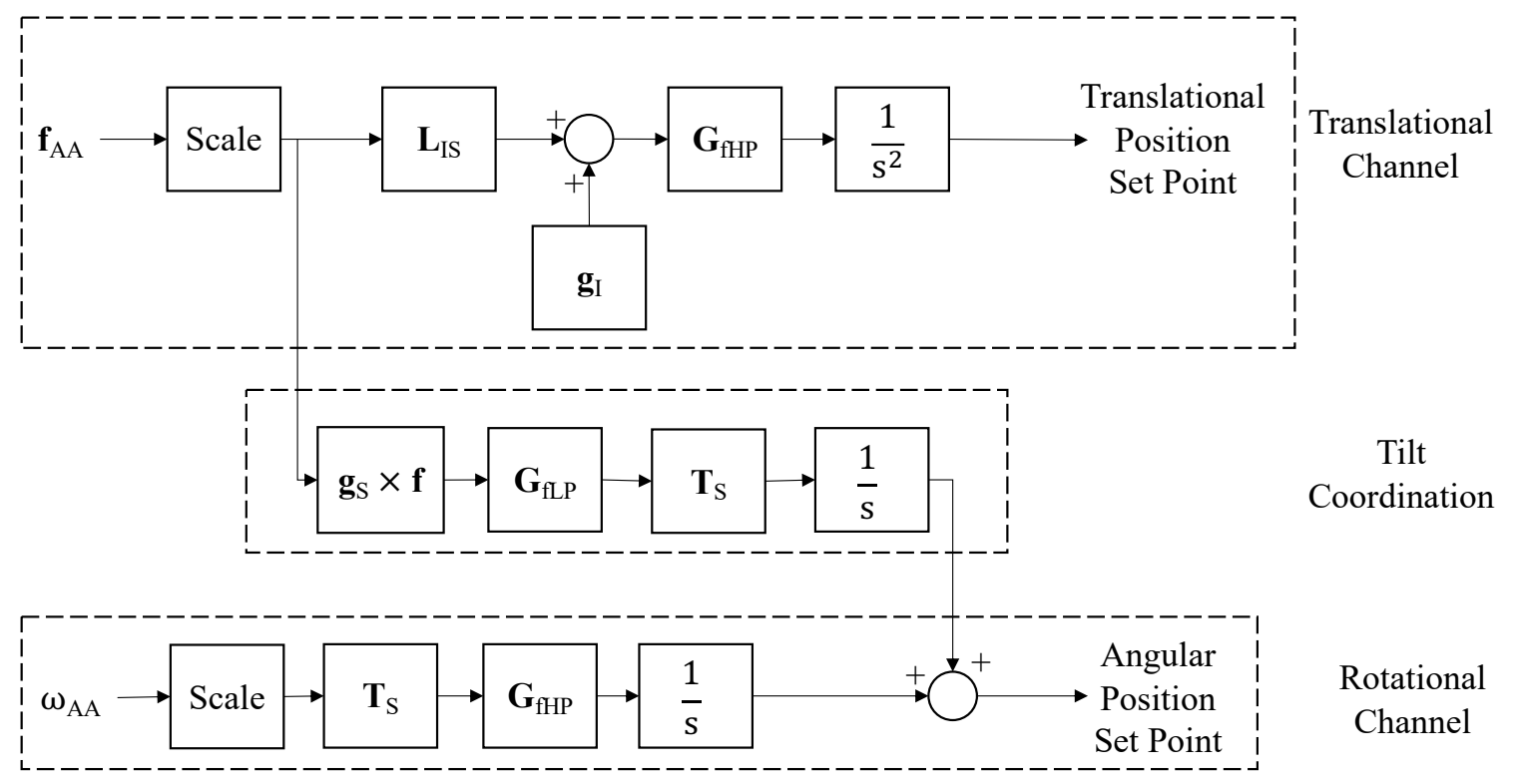

Figure 13: Classical washout for large angles.

\subsection{Translational Channel}

For large-angle washout, the only modification that needs to be made to the translational channel is the conversion from the aircraft frame and the inertial frame. Figure 14 illustrates how small-angle washout used the rotation matrix, $\mathbf{L}_{\mathrm{IS}}$ from 
Equation 21, which uses Euler angles and how the rotation matrix is replaced with a quaternion rotation,

$$
r(\mathbf{f})=(-\mathbf{q}) \otimes \mathbf{f} \otimes\left(-\mathbf{q}^{*}\right)
$$

where $\mathbf{f}$ is the scaled specific force, $\mathbf{q}$ is the orientation of the simulator, and $\mathbf{q}^{*}$ is the associated conjugate quaternion, which is similar to complex conjugate numbers,

$$
\mathbf{q}^{*}=\left[\begin{array}{c}
q_{0} \\
-\mathbf{q}_{v}
\end{array}\right] \text {. }
$$

\section{Small-angle Washout}

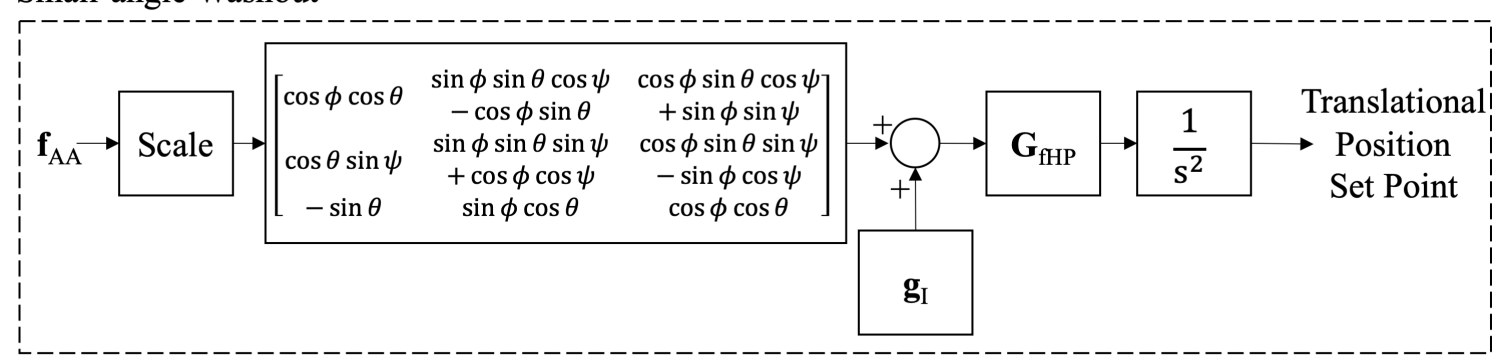

Large-angle Washout

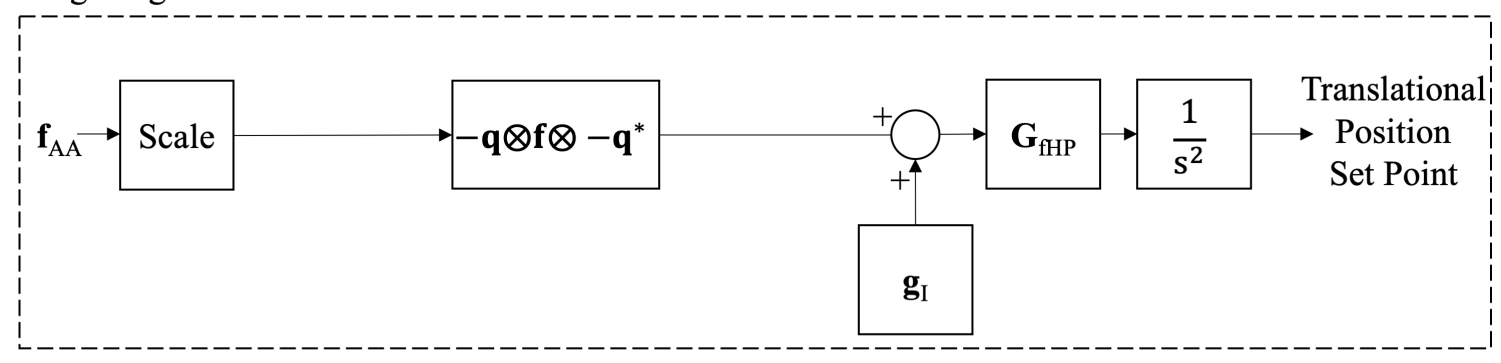

Figure 14: Translational channel for small-angle washout (top) and large-angle washout (bottom). 


\subsection{Tilt Coordination}

The next channel that requires modification is the tilt coordination channel. The small-angle approximation version of tilt coordination from Equation 27 cannot be used, as the small-angle approximations no longer hold.

Figure 15 illustrates that the tilt coordination channel for small-angle washout and for large-angle washout to compare the changes. First, the gravity vector in the simulator frame, $\mathbf{g}_{\mathrm{S}}$ was calculated by using the rotation matrix $\mathbf{L}_{\mathrm{SI}}$ from Equation 30, using the Euler angles of the simulator orientation. The Euler angle rotation matrix is replaced by a quaternion rotation similar to Equation 41 from the translational channel.

The second change is that instead of converting the angular velocity output from the low-pass filter to rate of change of Euler angles and then integrating to produce Euler angles, the angular velocity is directly output from the tilt coordination channel.

The third change is moving the low-pass filter to before the cross product, which is the location it is found in the small-angle simplification version of tilt coordination. Filtering the specific force instead of the angular velocity reduces unwanted oscillations in the tilt coordination velocity output.

\subsection{Rotational Channel}

The last channel to be modified is the rotational channel. Figure 16 illustrates the small-angle washout rotational channel as well as the large-angle washout rotational channel.

First, the elimination of Euler angles results in the high-pass filtering occurring in angular velocity rather than rate of change of Euler angles. Filtering in angular velocity has been shown to have a small negative impact on the flight simulator's

performance [2]. However, it also allows the addition of the outputs of the tilt co- 
Small-angle Washout

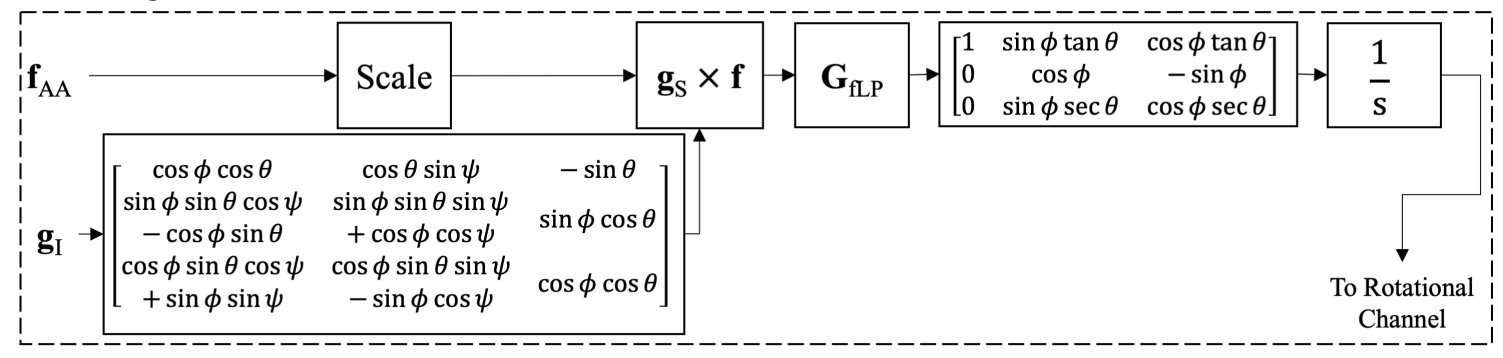

Large-angle Washout

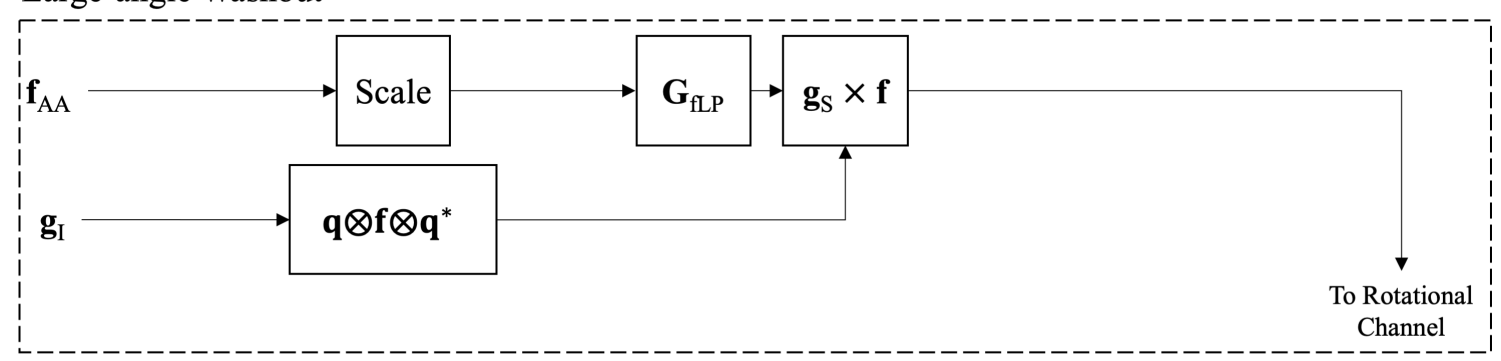

Figure 15: Tilt coordination for small-angle washout (top) and large-angle washout (bottom).

ordination and rotational channel to occur in angular velocities, removing the error introduced by the addition of Euler angles.

Next, as the channel is using angular velocities instead of converting to rate of change of Euler angles, the matrix $\mathbf{T}_{\mathrm{S}}$ is removed. After filtering, the angular velocity set point from tilt coordination is added to the rotational channel. To convert the angular velocity to an angular position set point expressed as a quaternion, a quaternion integrator is added, which is calculated using

$$
\mathbf{q}_{n}=\mathbf{q}_{n-1} \otimes\left[\begin{array}{c}
\cos (\|\omega\| \Delta t / 2) \\
\frac{\omega}{\|\omega\|} \sin (\|\omega\| \Delta t / 2)
\end{array}\right]
$$

where $\Delta t$ is the timestep and $\mathbf{q}_{n-1}$ is the previous angular position set point, to produce an angular position set point. 
Small-angle Washout

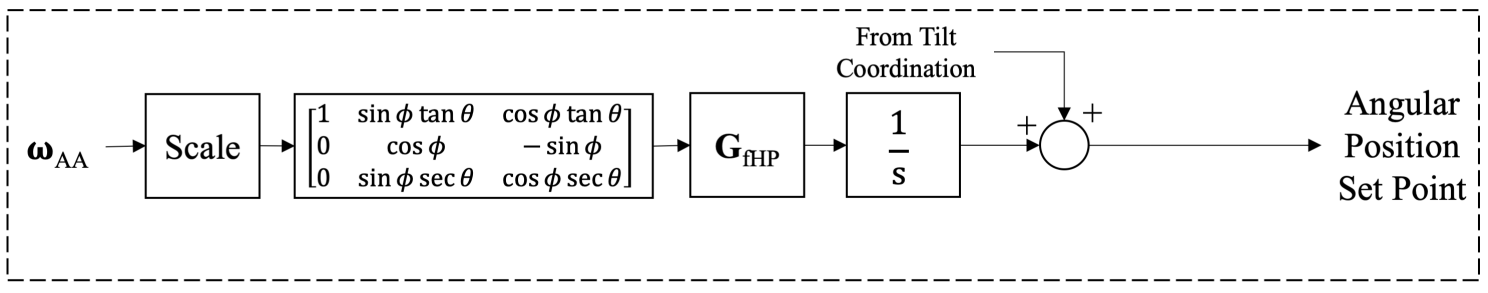

Large-angle Washout

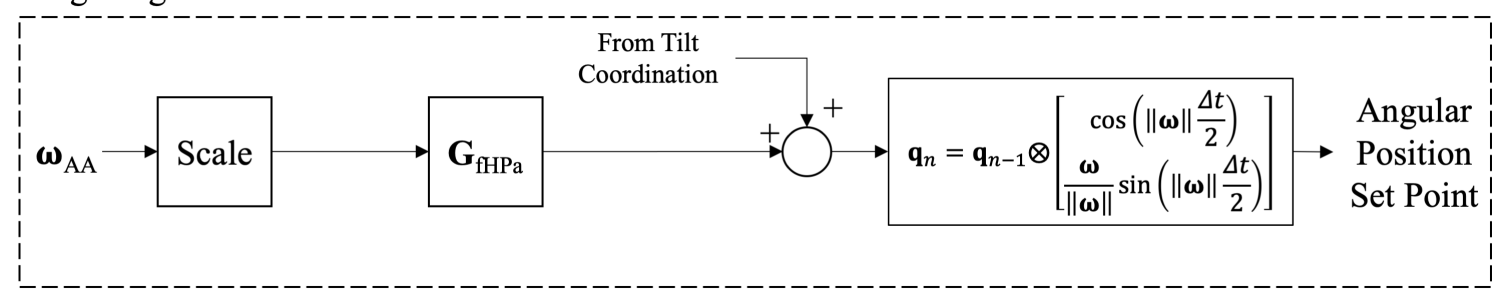

Figure 16: Rotational channel for small-angle washout (top) and large-angle washout (bottom).

\subsection{Vestibular Model}

Finally, the model of the pilot's vestibular system must be modified for large angles, by modifying the calculation of the motion at the pilot's head. For the translational acceleration, the rotation matrix $\mathbf{L}_{\mathbf{S I}}$ is replaced by a quaternion rotation. For the angles, the angular position is read as a quaternion, and the angular motion of the pilot's head $\boldsymbol{\omega}_{\mathrm{SS}}$ is calculated as

$$
\boldsymbol{\omega}_{\mathrm{SS}}=2 \mathbf{q}^{*} \otimes \dot{\mathbf{q}}
$$

where $\dot{\mathbf{q}}$, the quaternion rate, is given by

$$
\dot{\mathbf{q}}=\frac{\mathbf{q}_{n}-\mathbf{q}_{n-1}}{\Delta t} .
$$

The transfer functions used to model the pilot's vestibular system do not require any modifications. 


\subsection{Results}

The manoeuvres from Chapter 3, turn entries, throttle pulse, and pushover/pullup, are again used to assess the performance of the adapted algorithm. These are all small-angle manoeuvres, but it is important to ensure that there is still acceptable performance of the large-angle washout algorithm in these ranges. The next chapter will evaluate larger-angle manoeuvres. To evaluate the impact of the performance, each manoeuvre in this chapter is run for both small-angle washout and large-angle washout for the three parameter sets listed in Table 2, and there is a comparison of their performance metrics and a qualitative evaluation of the graphed responses. To evaluate the performance of each algorithm, the normalized Person correlation, integral of the error, and maximum error metrics are calculated to compare the aircraft pilot's response and the simulator pilot's response for each algorithm. For evaluation, only the last 20 seconds of the manoeuvre are used when calculating the performance metrics to eliminate the influence of the transients associated with imperfect initial conditions, as discussed in the previous chapter.

Each metric used can only give an indication of the performance of the response, as the primary method of evaluating flight simulators in practice is to have pilots perform subjective evaluation. The numeric metrics are best used to compare the relative results of two washout algorithms performing the same manoeuvre, with the metrics calculated for the vestibular response for the simulator pilot against the vestibular response for the aircraft pilot. The normalized Pearson correlation gives an indication of how well the shape of the simulator pilot's vestibular response curve matches the aircraft pilot's response, and the integral of the error and maximum error metrics give an indication of the distance between the curves. If all the numbers are smaller, it suggests that that algorithm gives a better response, and if the metrics are all larger it is likely worse. In many cases, one metric will improve while another worsens, which requires subjective evaluation of the responses. The following sections 
compare the new large-angle classical washout to the small-angle classical washout for the M1 turn entries, M2 throttle pulse, and M3 pullover/pushup manoeuvres for each of the classical washout parameter sets listed in Table 2. Appendix C includes additional graphs for the test cases in this chapter.

\subsubsection{M1: Turn Entries}

For the M1 turn entries manoeuvre, the dominant motion cue is the changing roll rate. For the roll degree of freedom, the large-angle washout performs slightly better for both performance metrics. The vestibular responses for small-angle washout pilot, large-angle washout pilot and the aircraft pilot are plotted in Figure 17 for each of the six degrees of freedom, and the normalized Pearson correlation, integral of the error, and maximum error, as well as the difference for each metric for small-angle washout and large-angle washout are listed in Table 6. A positive number in the difference column indicates that large-angle washout performed better for the given metric.

The CW1 parameter set sway vestibular response shows an improvement to the normalized Pearson correlation metric; however, the maximum error is the same,

and the integral of the error is worse. Examining the vestibular response shows that both the small-angle washout and large-angle washout vestibular responses have substantial unwanted specific force cues. These unwanted cues are due to the roll angular velocity set point, and cannot be eliminated by the algorithm.

For the CW1 parameter set roll response, all the performance metrics in Table 6 perform slightly worse for large-angle washout compared to small-angle washout. Examining the vestibular response in Figure 17 shows that the small-angle washout and large-angle washout responses are similar, but have differences at the points where there is peak perceived angular velocities. Small-angle washout may produce a better response based on the metrics.

The CW2 and CW3 parameter sets have similar performance for M1 as CW1. 
Overall, the vestibular response for large-angle washout produces a closer representation of the aircraft pilot's vestibular response than small-angle washout.
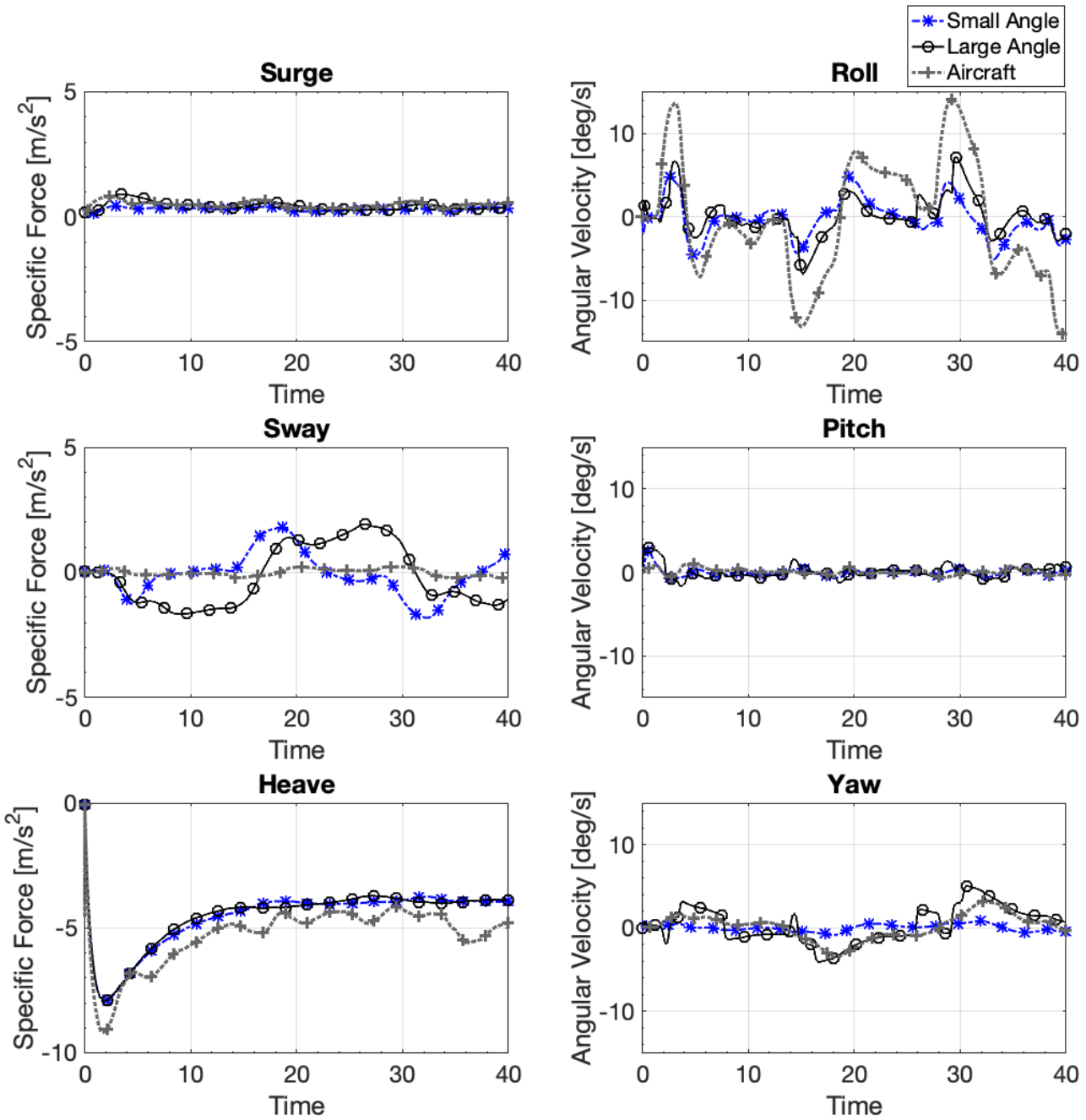

Figure 17: The CW1 vestibular response for M1, for small-angle washout and largeangle washout. 


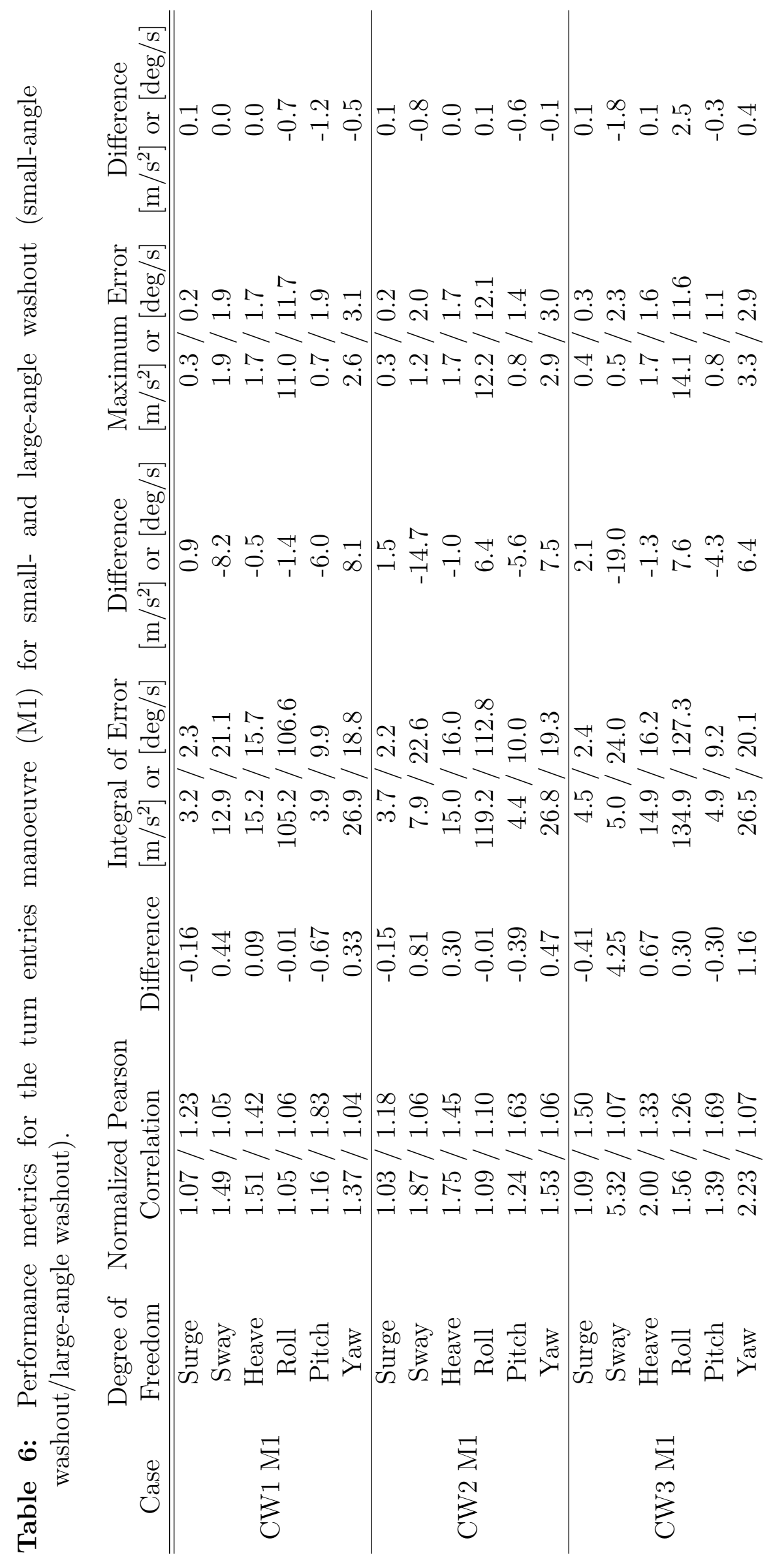




\subsubsection{M2: Throttle Pulse}

For the M2 throttle pulse manoeuvre, the main motion cue is the change of specific force in the surge degree of freedom, with some small oscillations in roll. Figure 18 illustrates the vestibular motion for the washout algorithms and the aircraft pilots for each of the six degrees of freedom, and Table 7 lists the performance metrics for each degree of freedom for each parameter set.

For the surge degree of freedom for CW1, the normalized Pearson correlation and maximum error is the same for both washout algorithms, and small-angle washout has a slightly better integral of the error. Examining the vestibular response shows that both washout algorithms follow the aircraft vestibular response closely.

The CW1 pitch degree of freedom response shows that large-angle washout performed worse for all metrics. Examining the plot shows that there is a large negative cue spike at 25 seconds, while the vestibular responses are close in the remainder of the time period simulated. The negative cue is present in both the small-angle washout and large-angle washout cases, and corresponds with the change in the surge set point, which will have an impact on the angular velocity due to tilt coordination. The negative cue is more pronounced in the large-angle washout case, as there is a larger change in specific force, which accounts for the decline in the metrics.

For roll for CW1, large-angle washout performed worse for all metrics in Table 7. Examining the vestibular response in Figure 18 shows that small-angle washout arrives at the angular velocity peaks more quickly than large-angle washout, which is resulting in a better correlation with the aircraft pilot's vestibular response.

The CW2 and CW3 parameter sets also produce similar results. Overall the motion cues are reproduced similarly by both algorithms, but small-angle washout has slightly better performance. 

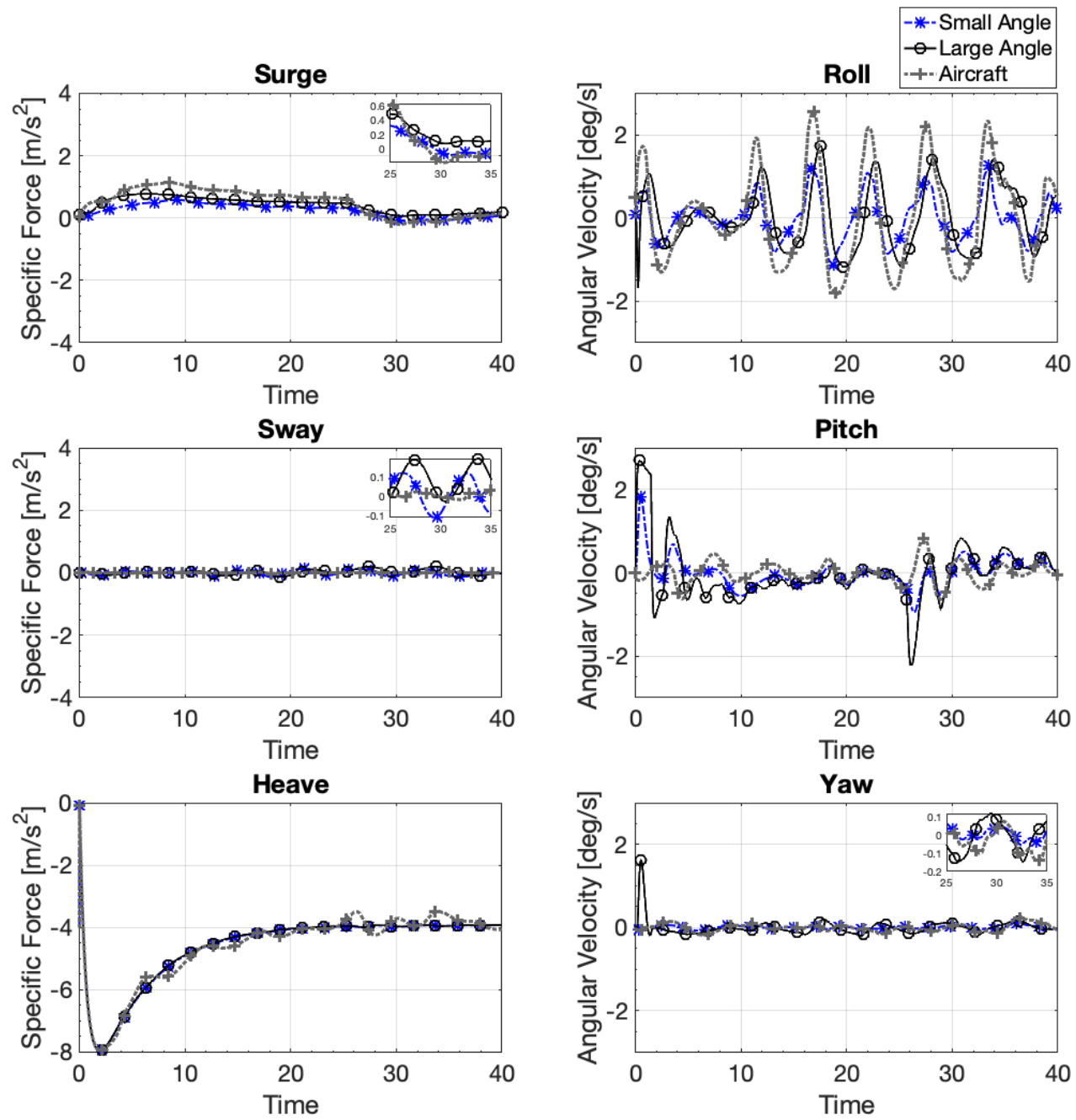

Figure 18: The CW1 vestibular response for M2, for small-angle washout and largeangle washout. 


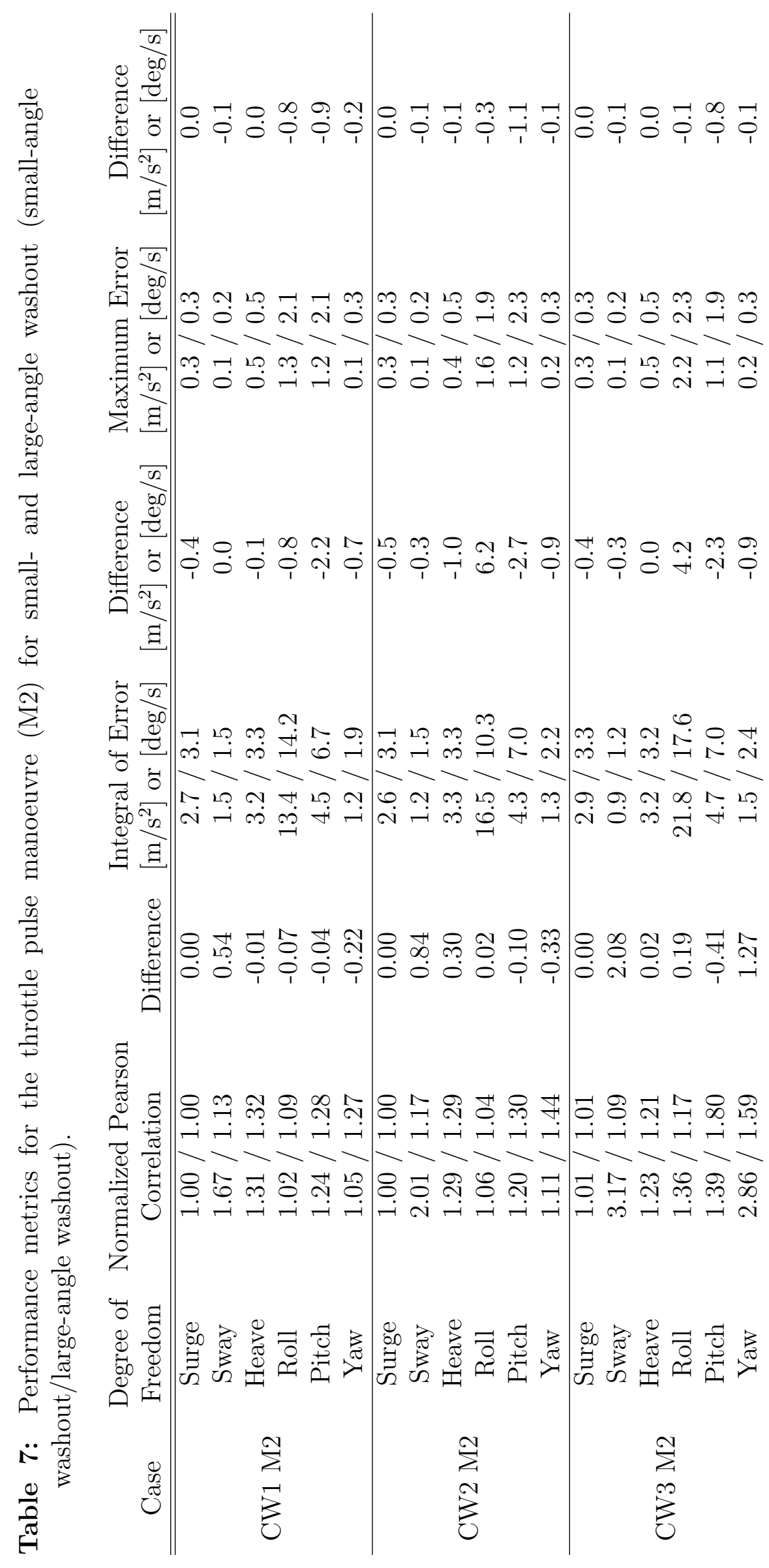




\subsubsection{M3: Pushover/Pullup}

For the M3 manoeuvre, the main motion cue is the large changes in the heave specific force, as well as oscillations in the roll degree of freedom. The performance metrics for each parameter set and degree of freedom are listed in Table 8 and the vestibular response is illustrated in Figure 19.

For the CW1 heave response, the normalized Pearson correlation shows that largeangle washout has improved performance, while the integral of the error and the maximum error metrics are similar for the two washout algorithms. Examining the vestibular response shows that both washout algorithms have very similar response and both fail to follow the vestibular response for the aircraft pilot in the heave degree of freedom, due to tilt coordination's limitations when the axis is aligned with the gravity vector.

For the CW1 roll response, the performance metrics in Table 8 show that smallangle washout performs better than large-angle washout for all the metrics. Examining the vestibular response in Figure 17 shows that the two algorithms have similar responses, but that small-angle washout reacts more quickly to the changes in the aircraft pilot's vestibular response.

The responses for the CW2 and CW3 parameter sets are similar. Overall, the two algorithm types perform similarly, but small-angle washout has better performance metrics for the majority of the degrees of freedom. 



Figure 19: The CW1 vestibular response for M3, for small-angle washout and largeangle washout. 


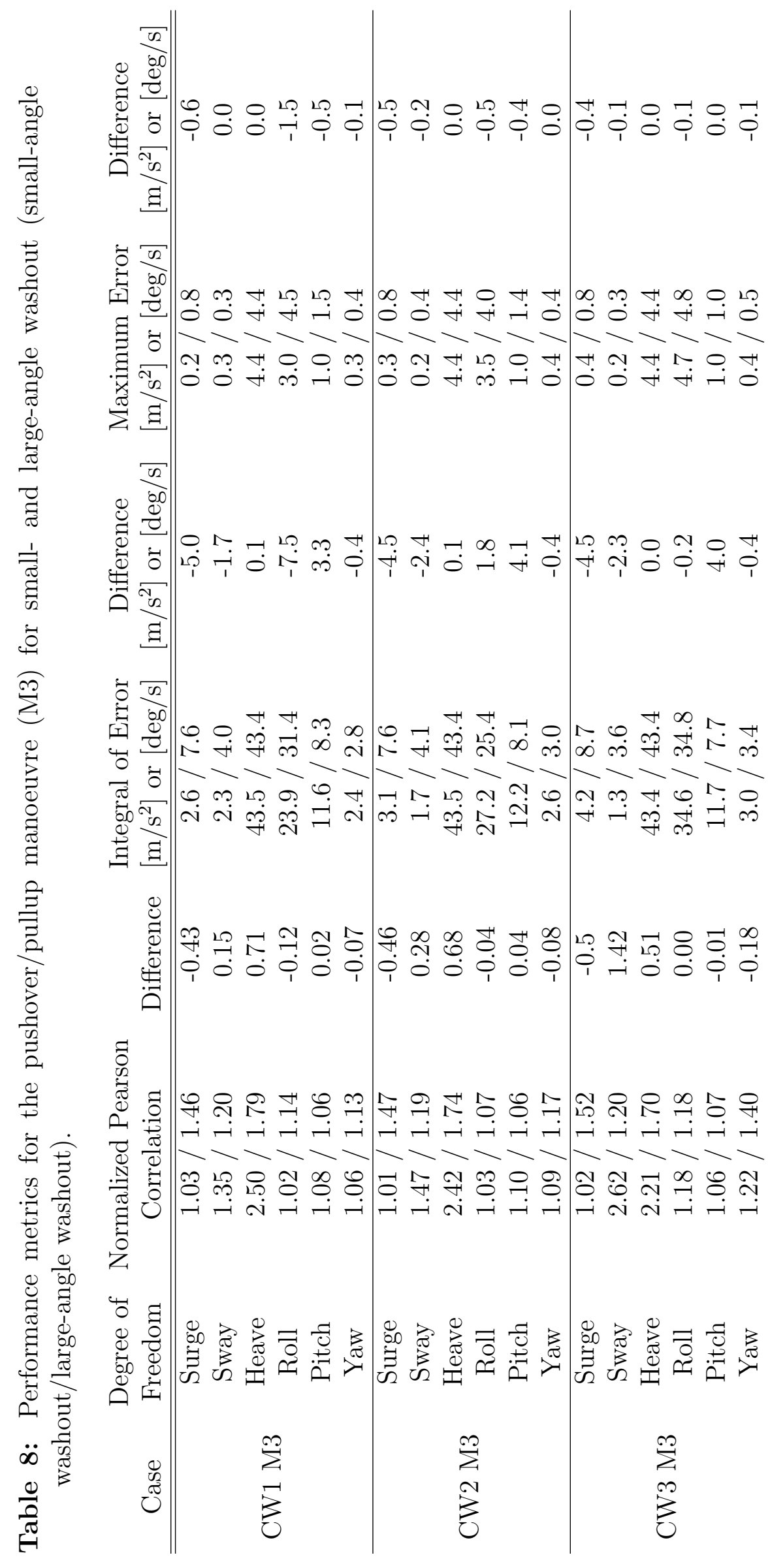




\subsection{Angular Position Tracking}

Washout algorithms use the angular velocity of the aircraft as their set point, and high-pass filters to restrict the range of angular motion. However, with the ability to use a larger motion envelope, it was possible that other methods may produce better vestibular responses for the simulator pilots.

One method that was considered was controlling based on the aircraft's angular position as well as angular velocity, to assist with reproducing the specific force impacts of the aircraft's z axis not being aligned with the gravity vector. However, it was determined that for many manoeuvres, such as a coordinated turn, where the aircraft rolls while turning at such a rate that the specific force is pointing down relative to the pilot, the orientation of the aircraft was not the dominant factor in determining the specific force. Therefore, this control method was not pursued further.

\subsection{Summary}

For the manoeuvres examined in this section, small-angle washout tends to perform better than large-angle washout for the majority of the degrees of freedom for the manoeuvres. The worse performance for large-angle washout is expected, as there was an expected performance reduction due to filtering the angular velocity instead of the rates of change of Euler angles for the rotational channel, and the manoeuvres in this chapter do not have large angular velocity cues, which would benefit from eliminating the small-angle approximations that are present in small-angle washout. The following chapter will examine manoeuvres with larger angular motion. 


\section{Case Studies}

To further compare the performance of large-angle washout and small-angle washout, a series of manoeuvres is completed using three aircraft with different flight characteristics: the Cessna 172, the Boeing 737, and the Columbia 400. These aircraft give examples of the performance of the algorithms for a small fixed-wing aircraft, a commercial passenger jet, and an acrobatic aircraft. The flight simulation program X-Plane, which uses precise models of different aircraft to calculate the forces reacting to the motion and the geometry of the control surfaces, was used to gather data on the manoeuvres for each aircraft. For each aircraft, the following manoeuvres were performed: a coordinated turn, a cross-wind landing, a single-axis takeoff, a spiral dive, and a stall recovery. For the Columbia 400 aerobatic aircraft, an additional aerobatic manoeuvre with large angular velocities in roll and pitch was also performed. Additional graphs for the large-angle washout test cases are provided in Appendix C.

The X-Plane flight simulation software outputs do not directly match the required information for the washout algorithm inputs. In particular, X-Plane does not provide outputs for the specific force experienced by the pilot, so before the data can be used by the washout algorithms it must be converted into a usable form.

Table 9 lists the following X-Plane outputs: the aircraft's translational acceleration at the aircraft's centre of gravity, the angular velocity, the angular acceleration, the location of the pilot's head relative to the centre of gravity, and the aircraft's orientation. Once the outputs have been collected, they are converted to match the washout requirements. Table 10 lists which axes in X-Plane are equivalent to the conventions used for washout. For the translational acceleration, the aircraft frame for X-Plane uses a different convention than is used in washout, so the translational acceleration must be converted to the washout frame. The angular velocity and orientation variables do not require conversion, as X-Plane uses a different reference frame for these variables, which aligns with the aircraft frame used in the washout 
algorithm.

Once all the data is in the washout algorithm's aircraft reference frame, the specific force must be calculated. Using Equation 33 and the converted outputs from X-Plane, the acceleration at the pilot's head $\mathbf{a}_{\mathrm{AA}}$ is calculated. Then the gravity in the aircraft frame $\mathbf{g}_{\mathrm{AA}}$ is calculated with the known orientation of the aircraft. Equation 20 is then used to give the specific force at the aircraft pilot's head in the aircraft frame $\mathrm{f}_{\mathrm{AA}}$.

As the Atlas simulator is designed such that the simulator pilot's head is at the centre of gravity of the simulator, this configuration will be the assumed for the flight simulator when calculating the simulator pilot's vestibular response.

In the previous chapters, versions of each algorithm with the transfer function coefficients defined by CW1, CW2, CW3, in the Reid and Nahon reports have been used when evaluating the washout algorithm's performance $[1,2]$. As the three versions produce similar results, and the focus of this chapter is to compare the results between small-angle washout and large-angle washout, a single set of the coefficients CW1 will be used.

\section{$4.1 \quad$ Overview}

The following sections of this chapter discuss the performance of small-angle washout and large-angle washout for a variety of manoeuvres. Unrestricted angular washout is also introduced to address the performance limitations of large-angle washout.

As the primary concern for assessing the algorithm performance is avoiding negative cues to the pilot, it is preferable to have a scaled cue then an incorrect cue. The normalized Pearson correlation gives a measurement of the correlation of the simulator pilot's vestibular response with the aircraft pilot's vestibular response, but 
Table 9: X-Plane outputs.

\begin{tabular}{|c|c|c|}
\hline Output & Variable Name & Units \\
\hline $\begin{array}{l}\text { Translational acceleration } \\
\text { at aircraft centre } \\
\text { of gravity }\end{array}$ & $\begin{array}{l}\text { sim/flightmodel/position/local_ax } \\
\text { sim/flightmodel/position/local_ay } \\
\text { sim/flightmodel/position/local_az }\end{array}$ & $\mathrm{m} / \mathrm{s}^{2}$ \\
\hline Angular velocity & $\begin{array}{l}\text { sim/flightmodel/position/Prad } \\
\text { sim/flightmodel/position/Qrad } \\
\text { sim/flightmodel/position/Rrad }\end{array}$ & $\mathrm{rad} / \mathrm{s}$ \\
\hline Angular acceleration & $\begin{array}{l}\text { sim/flightmodel/position/P_dot } \\
\text { sim/flightmodel/position/Q_dot } \\
\text { sim/flightmodel/position/R_dot }\end{array}$ & $\operatorname{deg} / \mathrm{s}^{2}$ \\
\hline Aircraft orientation & sim/flightmodel/position/q & quaternion \\
\hline $\begin{array}{l}\text { Location of pilot's head } \\
\text { relative to aircraft } \\
\text { centre of gravity }\end{array}$ & $\begin{array}{l}\text { sim/aircraft/acf_peX } \\
\text { sim/aircraft/acf_peY } \\
\text { sim/aircraft/acf_peZ }\end{array}$ & $\mathrm{m}$ \\
\hline
\end{tabular}

Table 10: Equivalent axis for the washout algorithms and X-Plane.

\begin{tabular}{cc} 
Washout axis & X-Plane axis \\
\hline \hline$+x$ & $-z$ \\
$+y$ & $+x$ \\
$+z$ & $-y$
\end{tabular}


Table 11: Ranked order of performance for each washout algorithm version for each manoeuvre.

\begin{tabular}{llccc} 
Aircraft & Manoeuvre & $\begin{array}{c}\text { Small-Angle } \\
\text { Washout }\end{array}$ & $\begin{array}{c}\text { Large-Angle } \\
\text { Washout }\end{array}$ & $\begin{array}{c}\text { Unrestricted Angular } \\
\text { Washout }\end{array}$ \\
\hline \hline \multirow{5}{*}{ Cessna 172 } & Coordinated Turn & 1 & 3 & 2 \\
& Cross-wind Landing & 1 & 3 & 2 \\
& Single-axis Takeoff & 2 & 3 & 1 \\
& Spiral Dive & 3 & 2 & 1 \\
& Stall Recovery & 1 & 3 & 2 \\
\hline Columbia 400 & Acrobatics & 2 & 3 & 1
\end{tabular}

cannot be used in isolation, as there are cases such as when the response is flat, for which the normalized Pearson correlation is not informative. Also, different algorithm versions may perform better for different degrees of freedom in the same manoeuvre, so judgement is necessary to determine which cues have more impact on the pilot for a given manoeuvre. For example, if there is low correlation in a degree of freedom but there is also little motion in that degree of freedom, improved correlation in a degree of freedom with larger cues can be of more importance.

Table 11 summarizes a subjective comparison of the performance of the three washout algorithm versions. The algorithms are ranked in terms of relative performance for the Cessna 172 for the coordinated turn, cross-wind landing, single-axis takeoff, spiral dive, and stall recovery; and for the Columbia 400 for the aerobatics manoeuvre. Small-angle washout performs best for the manoeuvres with small angular velocity cues, and unrestricted angular washout performs best for the manoeuvres with large angular velocity cues. Large angle washout performs worst for all the manoeuvres except the spiral dive, and the unrestricted angular washout outperforms it for that manoeuvre.

The following sections provide the details of the simulations that lead to these observations. 


\subsection{Cessna 172}

The first set of manoeuvres for the test cases was performed using a Cessna 172 .

\subsubsection{Coordinated Turn}

For the coordinated turn manoeuvre, Figure 20 illustrates the motion of the aircraft at the pilot's head. Each individual time history plot represents a degree of freedom, for surge, sway, and heave for the translational specific forces, and roll, pitch, and sway for the angular velocities, respectively. The coordinated turn primarily involves changes in the specific force for the surge and sway degrees of freedom, as well as small changes in all of the angular velocities.

The vestibular responses for small-angle and large-angle washout are illustrated in Figure 21 for the six degrees of freedom; where the dotted grey line represents the vestibular motion experienced by the aircraft pilot for the manoeuvre, the blue line represents the vestibular response for a simulator pilot while using the smallangle washout algorithm, and the black line represents the vestibular response for the simulator pilot using large-angle washout.

For the coordinated turn, the significant degrees of freedom for the vestibular response are surge and sway. The heave response in Figure 21 is comparatively small, and all the angular velocities remain within the perception threshold during the manoeuvre.

Table 12 lists the normalized Pearson correlation, the integral of the error, and the maximum error for small-angle washout and large-angle washout (small-angle value/large-angle value), as well as the difference between the two washout algorithms for each metric, with a positive number indicating large-angle washout performed better.

For surge, the normalized Pearson correlation in Table 12 is the same for small- 

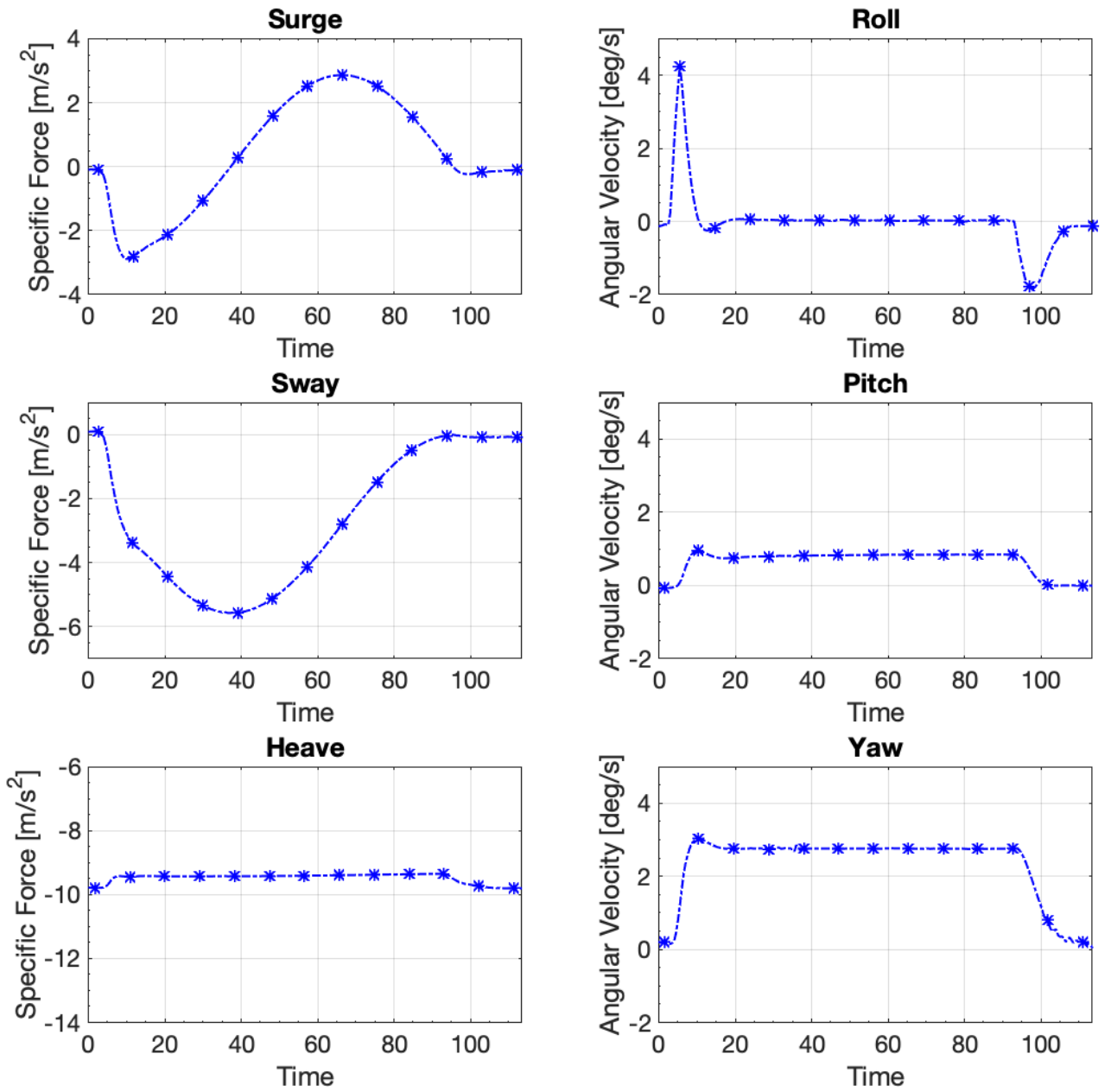

Figure 20: Aircraft motion at the pilot's head and the pilot's vestibular response for a Cessna 172 completing a coordinated turn. 

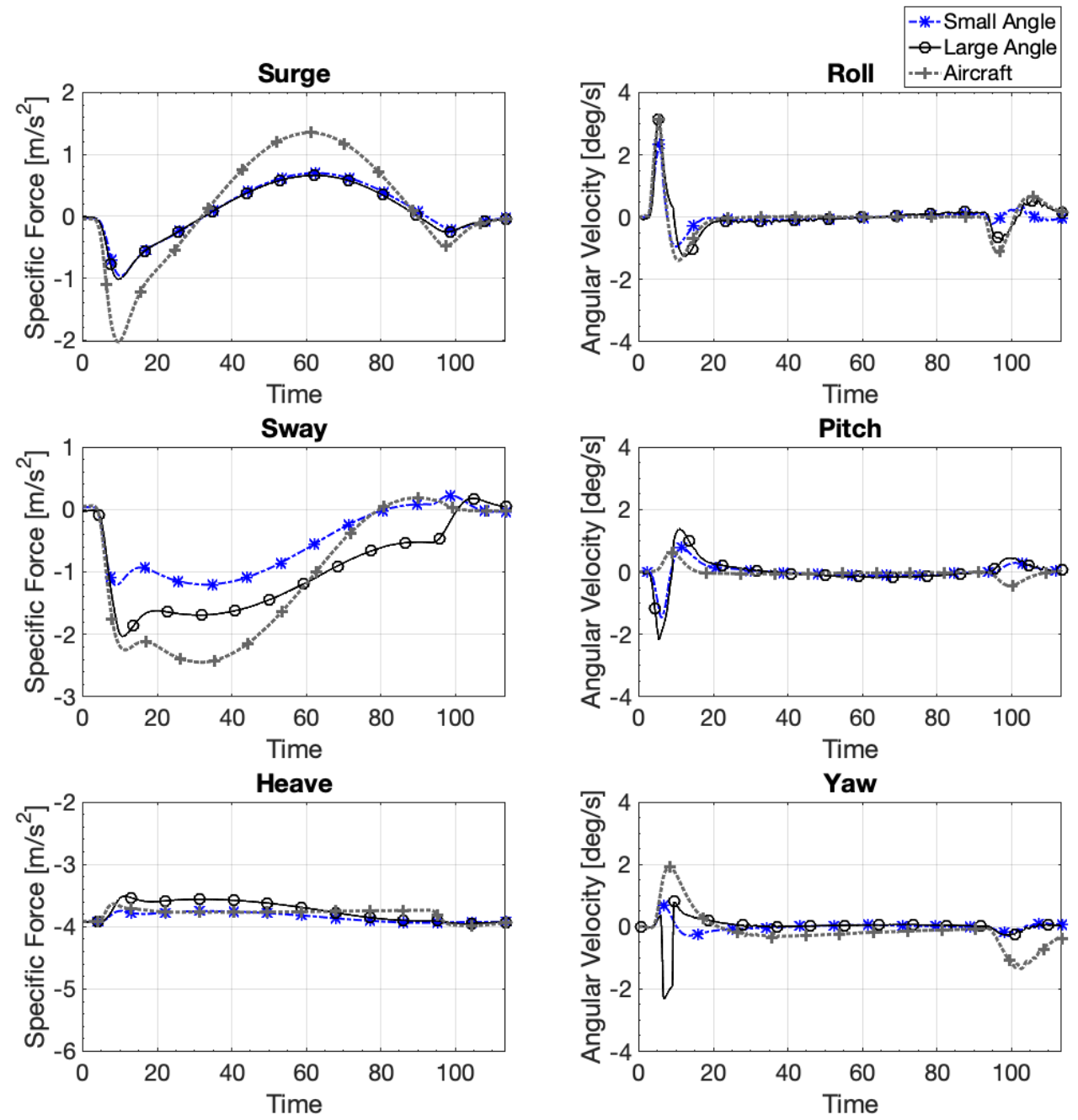

Figure 21: The vestibular response of the Cessna 172 aircraft pilot experiencing a coordinated turn manoeuvre, and the simulator pilot's vestibular response for small-angle washout and large-angle washout. 
angle washout and large-angle washout, with large-angle washout having a slightly worse integral of the error and a slightly better maximum error. Examining the vestibular response, small-angle washout and large-angle washout perform very similarly, as would be expected based on the performance metrics, and will likely perform equally well during pilot testing.

For sway, large-angle washout has a slightly worse normalized Pearson correlation in Table 12, and a substantial improvement in the integral of the error, with a slightly better maximum error. Examining the vestibular response in Figure 21 shows that the large-angle washout response is much closer to the aircraft's response at the beginning of the manoeuvre, but that small-angle washout performs better at the end of the manoeuvre. Pilot testing is needed to draw conclusions as to which behaviour is preferred.

While all the motion for yaw is within the vestibular response threshold, there is a noticeable difference in the response for the large-angle washout compared to the vestibular response for small-angle washout and the aircraft pilots. The difference is due to the impact of allowing tilt coordination to use the yaw degree of freedom, which is not present in small-angle washout. As the negative cue is well within the $3 \mathrm{deg} / \mathrm{s}$ threshold for the pilot's perception limits, it should not negatively impact the pilot.

Overall, the two washout algorithms have similar responses, and pilot testing will be needed to determine how the different sway responses impact the simulation's overall performance. 


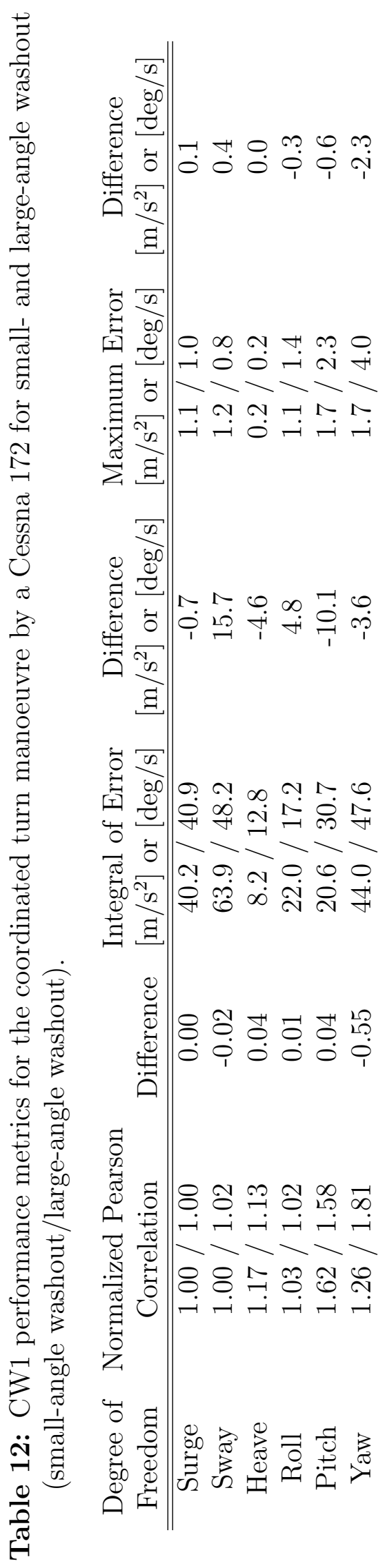




\subsubsection{Cross-wind Landing}

Figure 22 illustrates the six-degree-of-freedom motion for the cross-wind landing manoeuvre of the Cessna 172. The manoeuvre involves significant changes in heave and roll at the beginning of the manoeuvre, and oscillations in surge, sway, pitch, and yaw as the manoeuvre finishes.

The performance metrics for the cross-wind landing manoeuvre are listed in Table 13, and the six-degree-of-freedom vestibular response is illustrated in Figure 23.
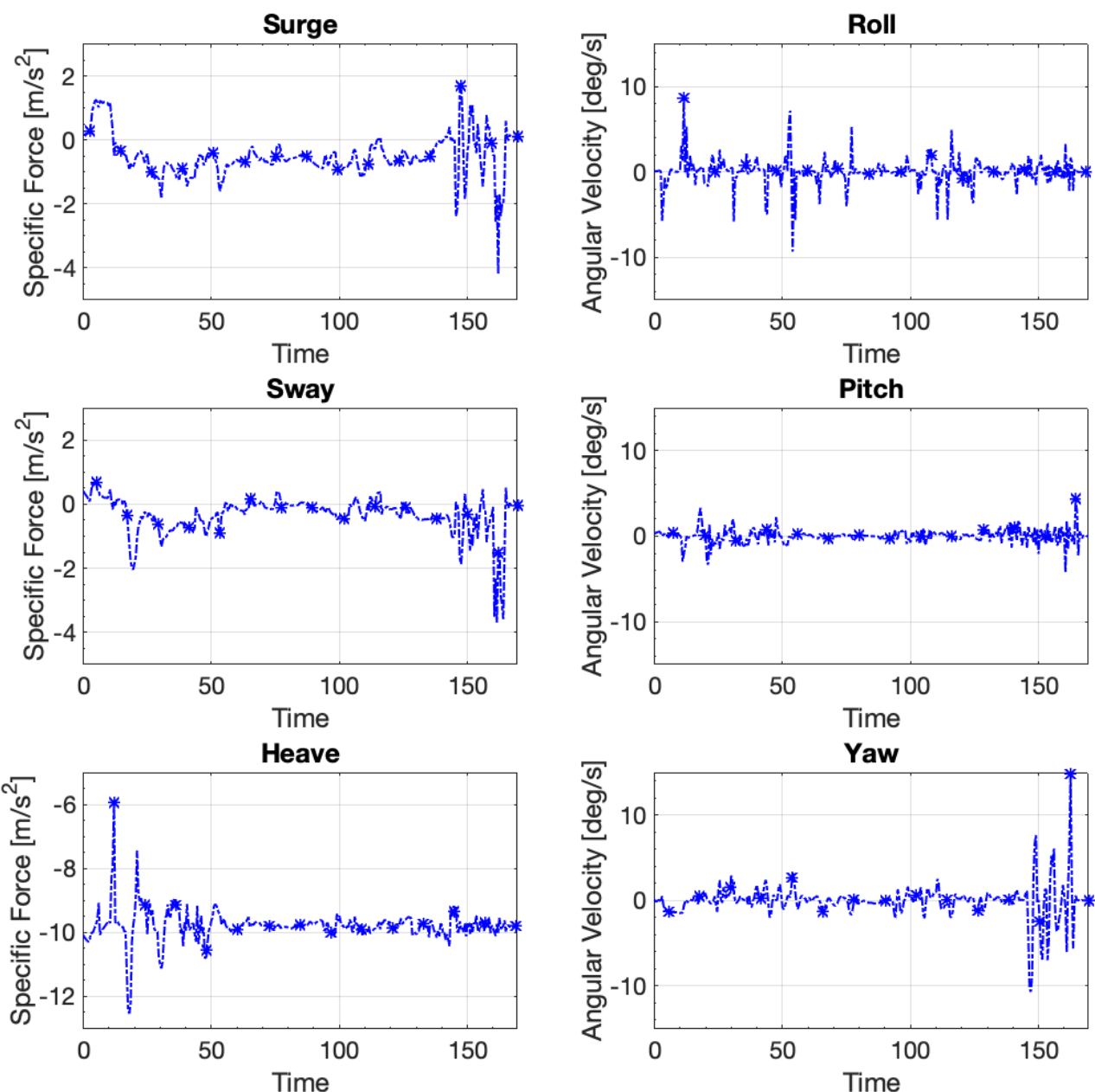

Figure 22: Aircraft motion at the pilot's head and the pilot's vestibular response for a Cessna 172 completing a cross-wind landing. 

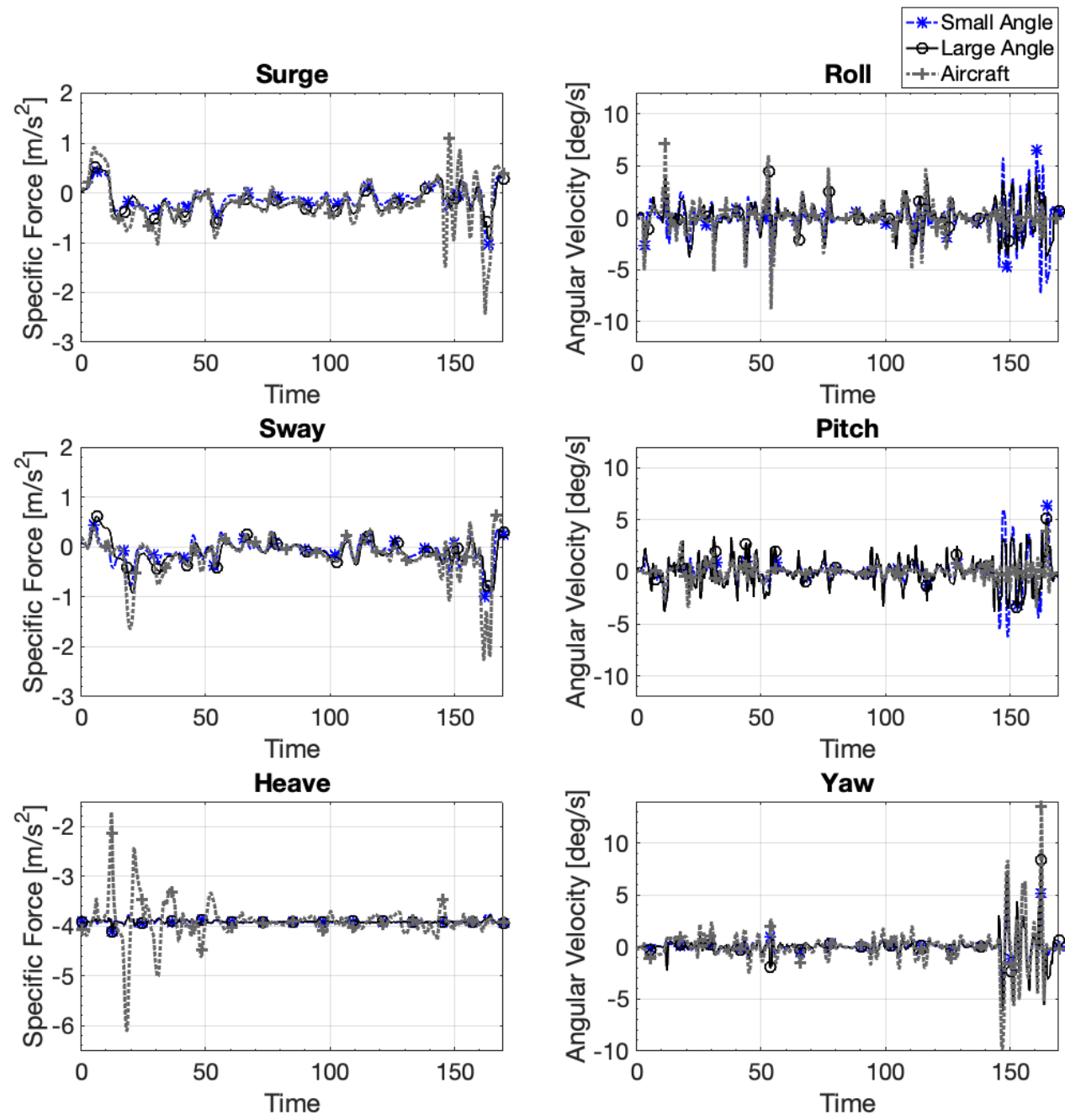

Figure 23: The vestibular response of the Cessna 172 aircraft pilot experiencing a cross-wind landing manoeuvre, and the simulator pilot's vestibular response for small-angle washout and large-angle washout.

For surge, the normalized Pearson correlation results are similar for both algorithms. The integral of the error is better for large-angle washout, and small-angle washout is slightly better for the maximum error. Examining the vestibular response shows that both washout algorithms perform similarly.

For sway in Table 13 the normalized Pearson correlation, integral of the error, and maximum error are better for small-angle washout. Examining the vestibular response in Figure 23 shows that the responses are similar, but particularly at the 
beginning the large-angle washout does not follow the aircraft response as closely as small-angle washout. These differences are minor, and pilot testing is required to determine the impact of the decline in response.

For heave, the normalized Pearson correlation is better for large-angle washout, with both washout algorithms having a similar integral of the error and the same maximum error. Examining the vestibular response shows that both washout algorithms fail to follow the large changes in specific force. The small changes in specific force in this degree of freedom is a known limitation of flight simulators of this type, as there is no way to add or remove specific force using methods like tilt coordination when the axis is aligned with the gravity vector.

For roll, small-angle washout performs better for the normalized Pearson correlation and the integral of the error, while large-angle washout has a slightly better maximum error. Examining the vestibular response in Figure 23, it shows that the small-angle washout and large-angle washout are similar until the final 30 seconds of the manoeuvre.

For the final 30 seconds of the manoeuvre there are significant angular velocity changes in all the rotational degrees of freedom. Figure 24 illustrates the changes the pilots' vestibular response for angular velocity for the final 30 seconds of the manoeuvre.

For roll, the aircraft pilot's vestibular response is small for the entirety of the manoeuvre; however, both large-angle washout and small-angle washout have significant cues that exceed the expected response. Small-angle washout has significantly larger and more unwanted cues compared to large-angle washout.

Examining the pitch response in Figure 24, the aircraft vestibular response cues remain small for almost the entire manoeuvre, with slightly larger cues in the final 10 seconds. Both small-angle washout and large-angle washout generate large negative cues, with small-angle washout having more and larger ones than large-angle washout. 

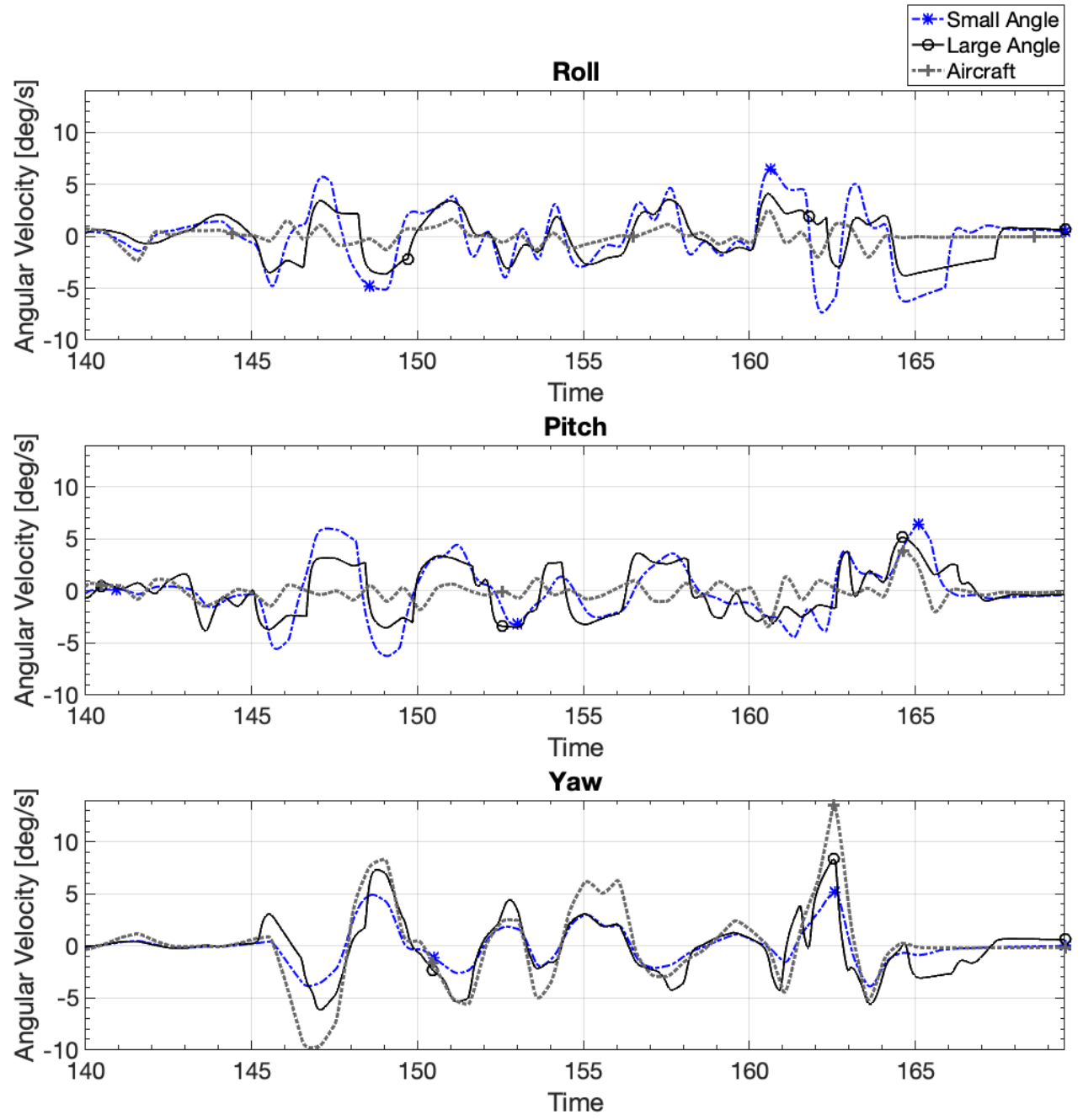

Figure 24: The vestibular response of the angular velocities of the Cessna 172 aircraft pilot at the end of a cross-wind landing manoeuvre, and the simulator pilot's vestibular response for small-angle washout and large-angle washout. 
For the yaw response in Figure 24, the aircraft vestibular motion has large cues multiple times during the final 30 seconds of the manoeuvre. Large-angle washout tends to follow the peaks of the aircraft response more closely; however, the response also has more negative cues than are present in the small-angle washout response.

Overall, small-angle washout performs better for the majority of the metrics. However, pilot testing may prove that some of the large-angle washout's behaviour for the rotational channels may be beneficial. 


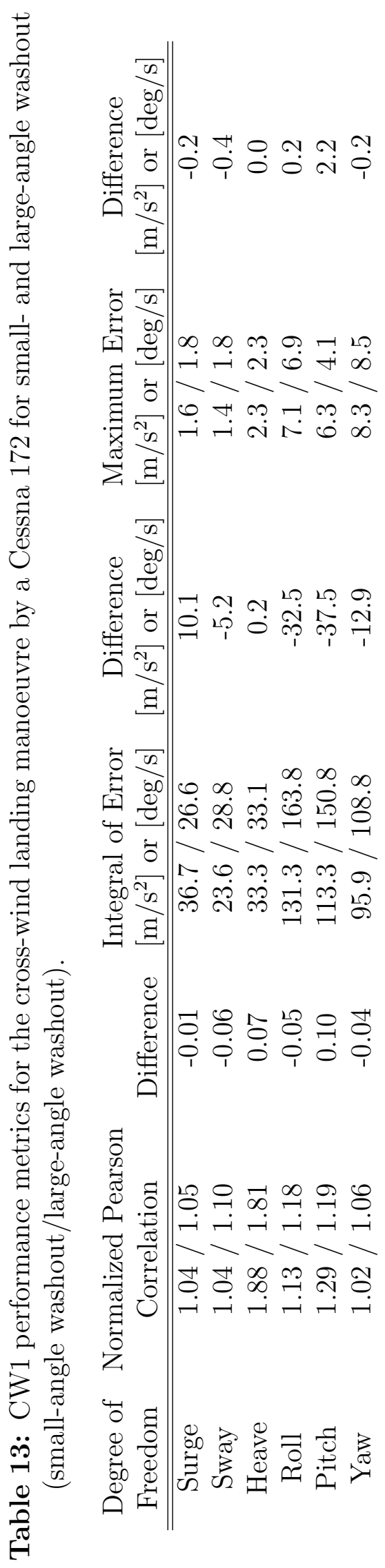




\subsubsection{Single-axis Takeoff}

The single-axis takeoff manoeuvre, illustrated in Figure 26 consists of abrupt specific forces in the surge and sway degrees of freedom, that gradually go back to zero, while the heave specific force oscillates. The angular velocities for all degrees of freedom fluctuate constantly throughout the manoeuvre, with roll having significant negative spikes in angular velocity. The performance metrics for the single-axis takeoff manoeuvre are listed in Table 14, and the vestibular response is illustrated in Figure 26.
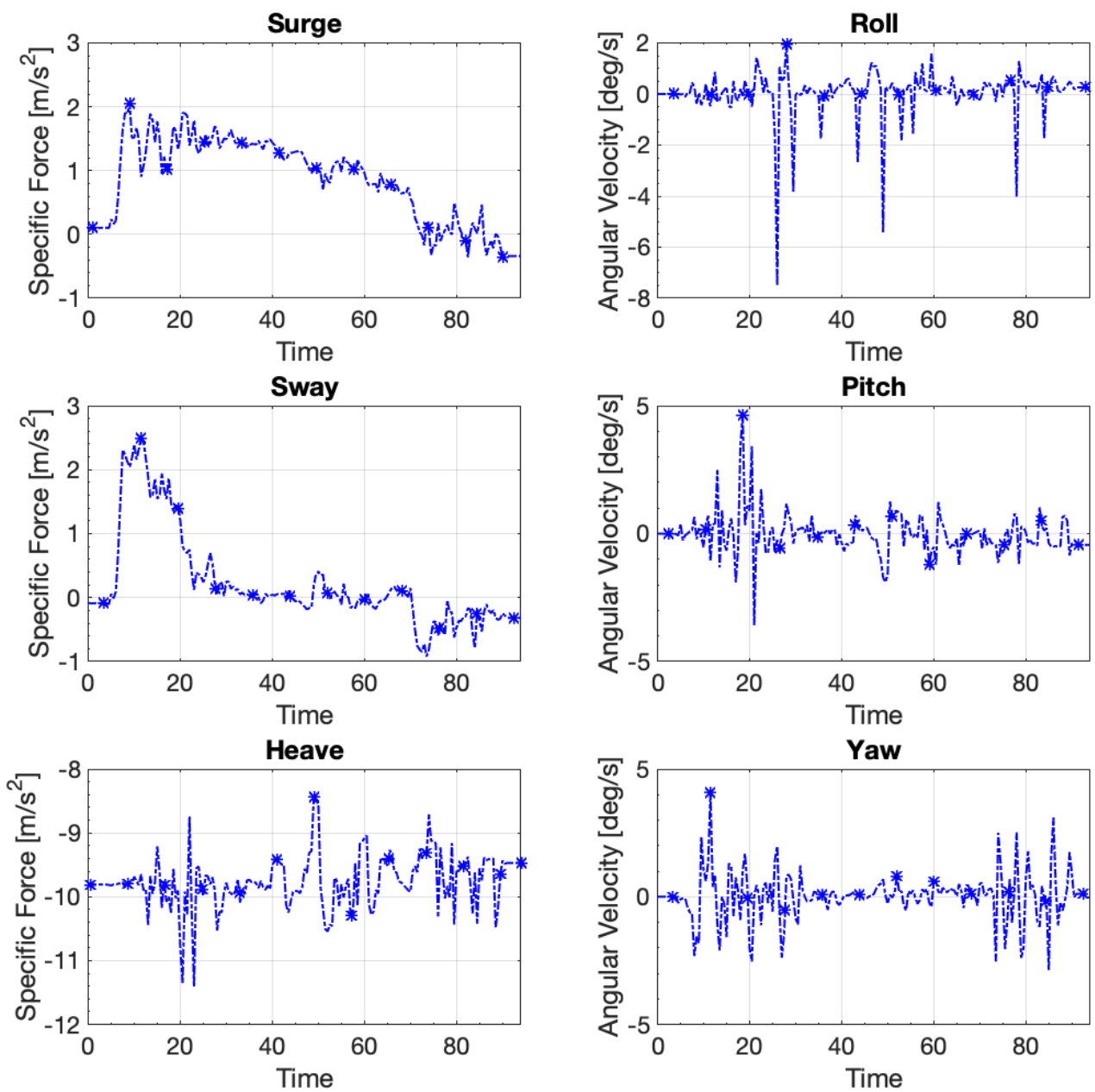

Figure 25: Aircraft motion at the pilot's head for a Cessna 172 completing a singleaxis takeoff. 

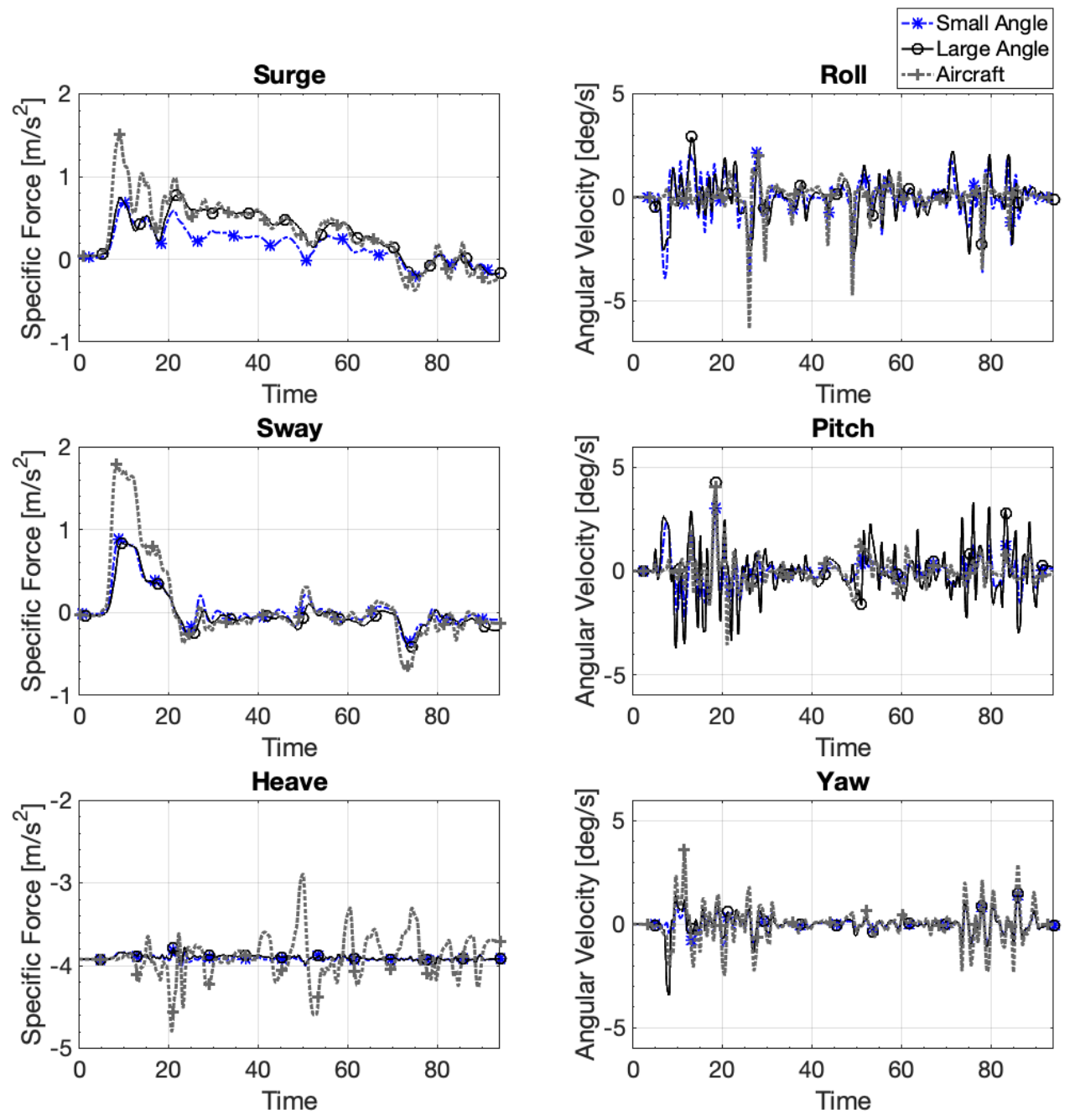

Figure 26: The vestibular response of the Cessna 172 aircraft pilot experiencing a single-axis takeoff manoeuvre, and the simulator pilot's vestibular response for small-angle washout and large-angle washout.

For surge, the normalized Pearson correlation is similar for the two washout algorithms, with the integral of the error better for large-angle washout and the maximum error better with small-angle washout. Examining the vestibular response in Figure 26 shows that the two washout algorithms perform similarly in the beginning and end of the manoeuvre, but that large-angle washout matches the aircraft pilot's vestibular response more closely during the middle part. It is likely that large-angle washout is an overall improvement in response, particularly as the metrics where it is performing 
worse are still very similar to small-angle washout.

For sway, small-angle washout performs better for all metrics listed in Table 14, but the differences are small. Examining the vestibular response in Figure 26 shows that both algorithm versions perform similarly. There are differences in how the algorithm versions respond to some of the smaller peaks in specific force later in the manoeuvre, which may impact the pilot's perception. However as these are small this will require pilot testing to assess.

For heave, as is expected, neither algorithm version follows the changes in specific force due to the limitations of the flight simulator.

For the angular velocity degrees of freedom, examining the vestibular response shows that for the majority of the manoeuvre there is minimal perceived motion. For the large spikes in the perceived angular velocity seen in the roll degree of freedom in Figure 26, both algorithm versions fail to fully reproduce them.

Overall, with the significant motion in the manoeuvre happening in surge and sway, the pilot's vestibular response for large-angle washout is closer to the aircraft pilot's vestibular response, and sway is similar for both algorithm types. Large-angle washout may provide improved performance overall for this manoeuvre; however, the majority of the metrics show that the response is similar or slightly worse than the small-angle washout response. 


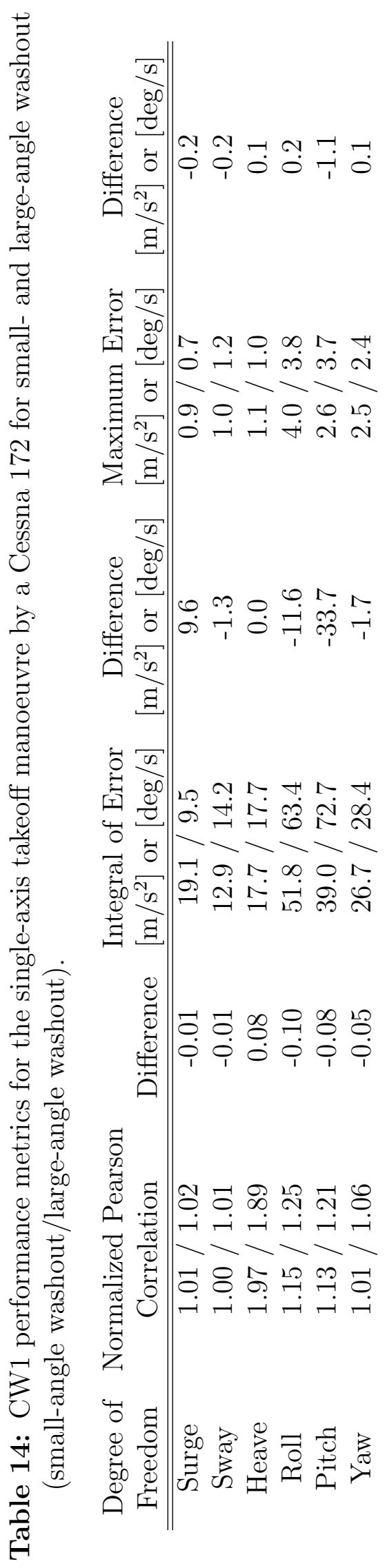




\subsubsection{Spiral Dive}

The spiral dive manoeuvre, illustrated in Figure 27, involves large changes in specific force or angular velocity for all degrees of freedom.

The performance metrics for the spiral dive manoeuvre are listed in Table 15, and the vestibular response is illustrated in Figure 28. This manoeuvre is an extreme case that requires specific forces and angular velocities beyond the abilities of the physical simulator this algorithm is designed for.
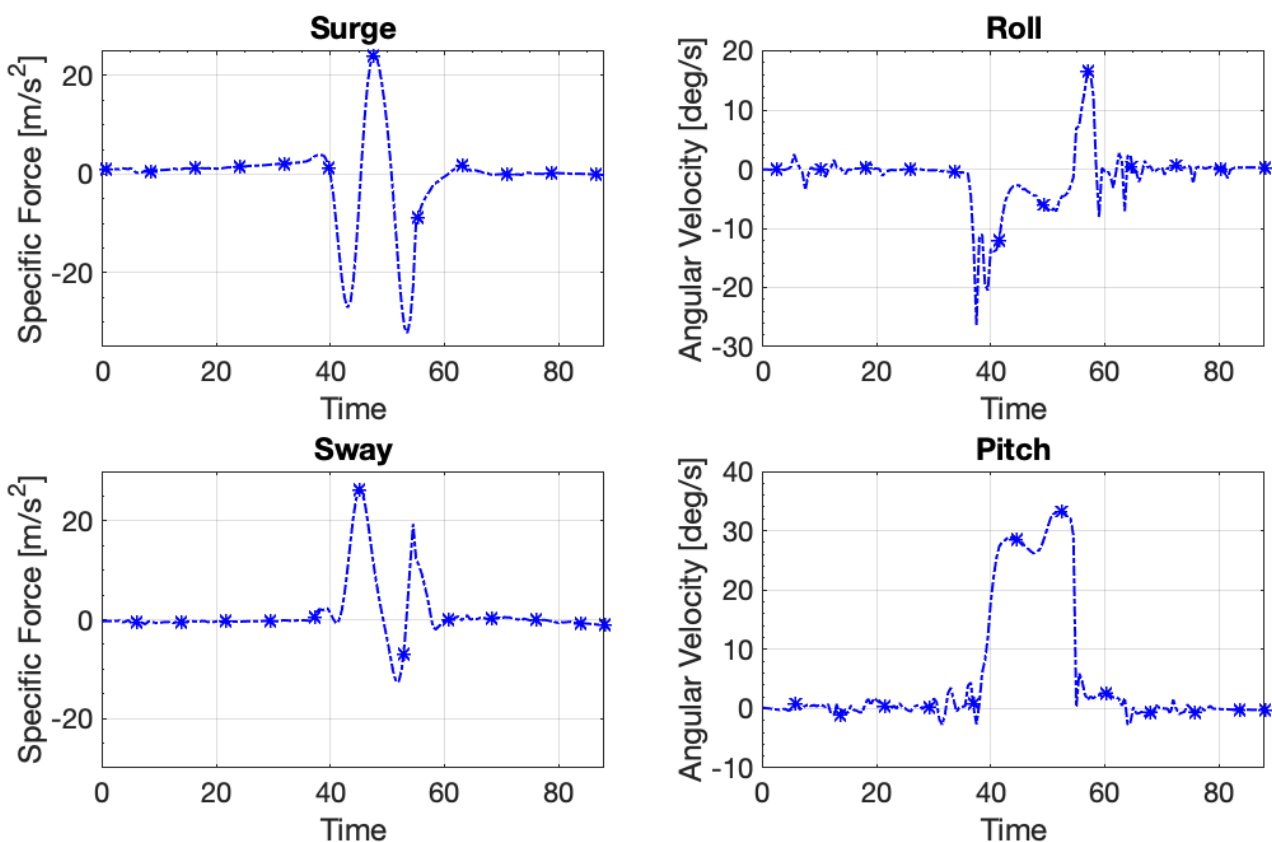

Heave
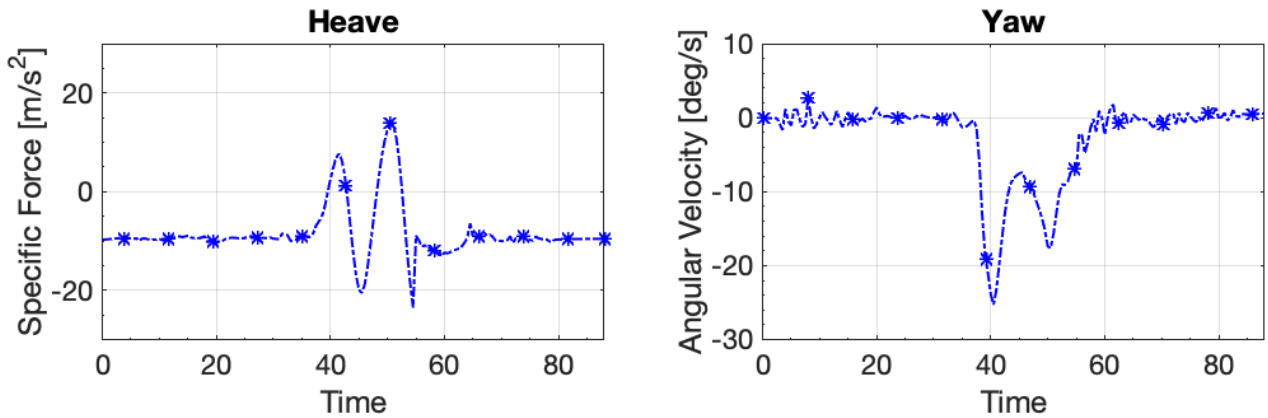

Figure 27: Aircraft motion at the pilot's head for a Cessna 172 completing a spiral dive. 

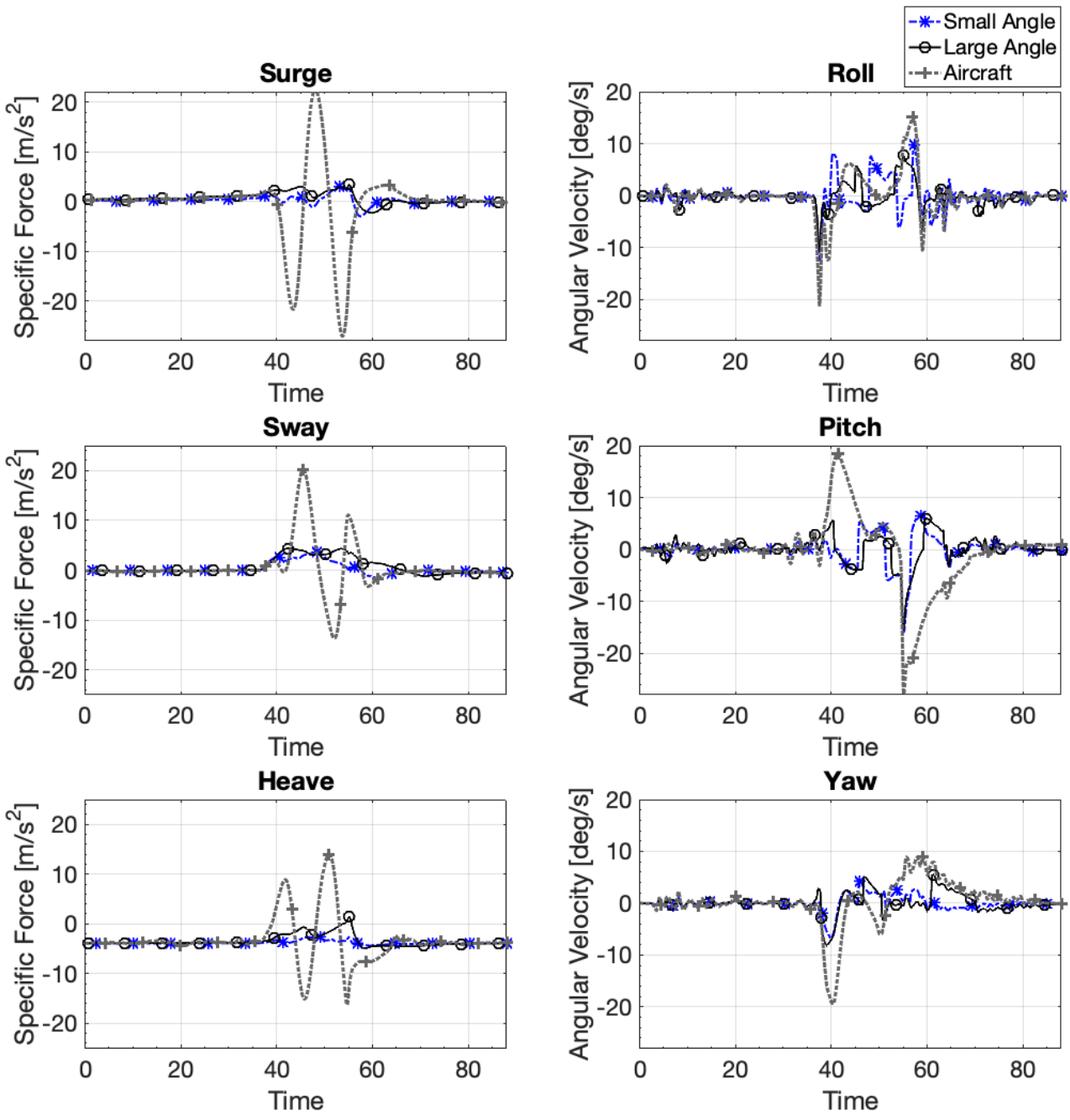

Figure 28: The vestibular response of the Cessna 172 aircraft pilot experiencing a spiral dive manoeuvre, and the simulator pilot's vestibular response for smallangle washout and large-angle washout.

For surge, in Table 15 large-angle washout has a large improvement in the normalized Pearson correlation, and both algorithms have similar results for the integral of the error, and the same maximum error. Examining the vestibular response, illustrated in Figure 28, shows that both washout versions fail to follow the changes in surge. This result is unsurprising, as the pilot is experiencing large specific forces exceeding $\pm 20 \mathrm{~m} / \mathrm{s}^{2}$, which is well beyond the specific force that can be provided by tilt coordination and gravity. 
For sway, the normalized Pearson correlation results in Table 15 are similar for both washout algorithms, with small-angle washout performing slightly better. The integral of the error shows that small-angle washout performed better, but the maximum error is very similar. Examining the vestibular response in Figure 28, there are similar characteristics to the response for surge, where the large changes in specific force experienced by the aircraft pilot are well beyond the limits of what can be provided by the simulator.

For heave, large-angle washout performs slightly worse for the normalized Pearson correlation and the integral of the error, but the maximum error is slightly smaller. Examining the vestibular response, the large-angle washout changes more than the small-angle washout, but this results in some negative cues.

For the roll response, there are large angular velocity cues. Large-angle washout shows considerable improvement for the normalized Pearson correlation and the integral of the error, as well as a smaller maximum error in Table 15. Examining the vestibular response in Figure 28, both small- and large-angle washout have difficulty following the aircraft pilot's vestibular cues, but large-angle washout does match the extremes of motion more closely, and with fewer negative cues.

Examining the pitch response, large-angle washout improves the normalized Pearson correlation and the maximum error, but has a slightly worse integral of the error compared to small-angle washout. Examining the vestibular response shows that both washout algorithms fail to reach the peaks of angular velocity experienced by the aircraft pilot, and both have similar unwanted negative cues. There may be slight improvements for large-angle washout, particularly around 40 seconds where the large-angle washout provides a positive cue that small-angle washout misses, but it will require pilot testing to determine the impact.

For the yaw response, the normalized Pearson correlation in Table 15 is very similar for small-angle washout and large-angle washout, and large-angle washout 
performs better for the integral of the error and the maximum error. Examining the vestibular response in Figure 28 shows that the large-angle washout matches the aircraft vestibular response better than the small-angle washout, but that both have negative cues and cannot match the peaks of the angular velocity.

Overall, neither algorithm reproduced the manoeuvre accurately due to the extreme nature of the manoeuvre and the limitations of the simulator; however, particularly in the roll degree of freedom there is potentially improved performance with large-angle washout. 


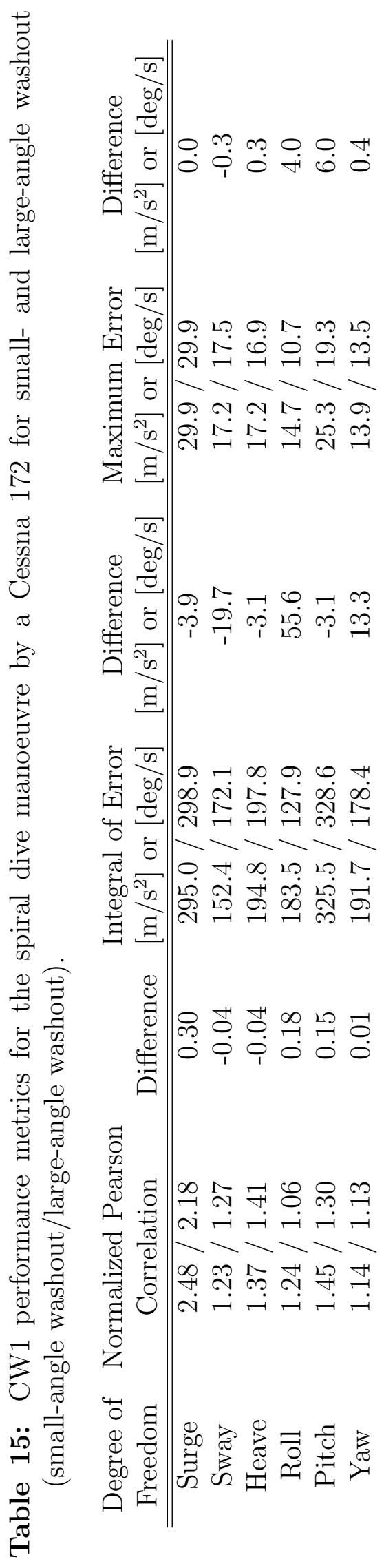




\subsubsection{Stall Recovery}

For stall recovery, the aircraft motion at the pilot's head is illustrated in Figure 29. This manoeuvre involves significant changes in specific force for surge, sway, and heave. The changes in angular velocity are primarily in roll and sway.
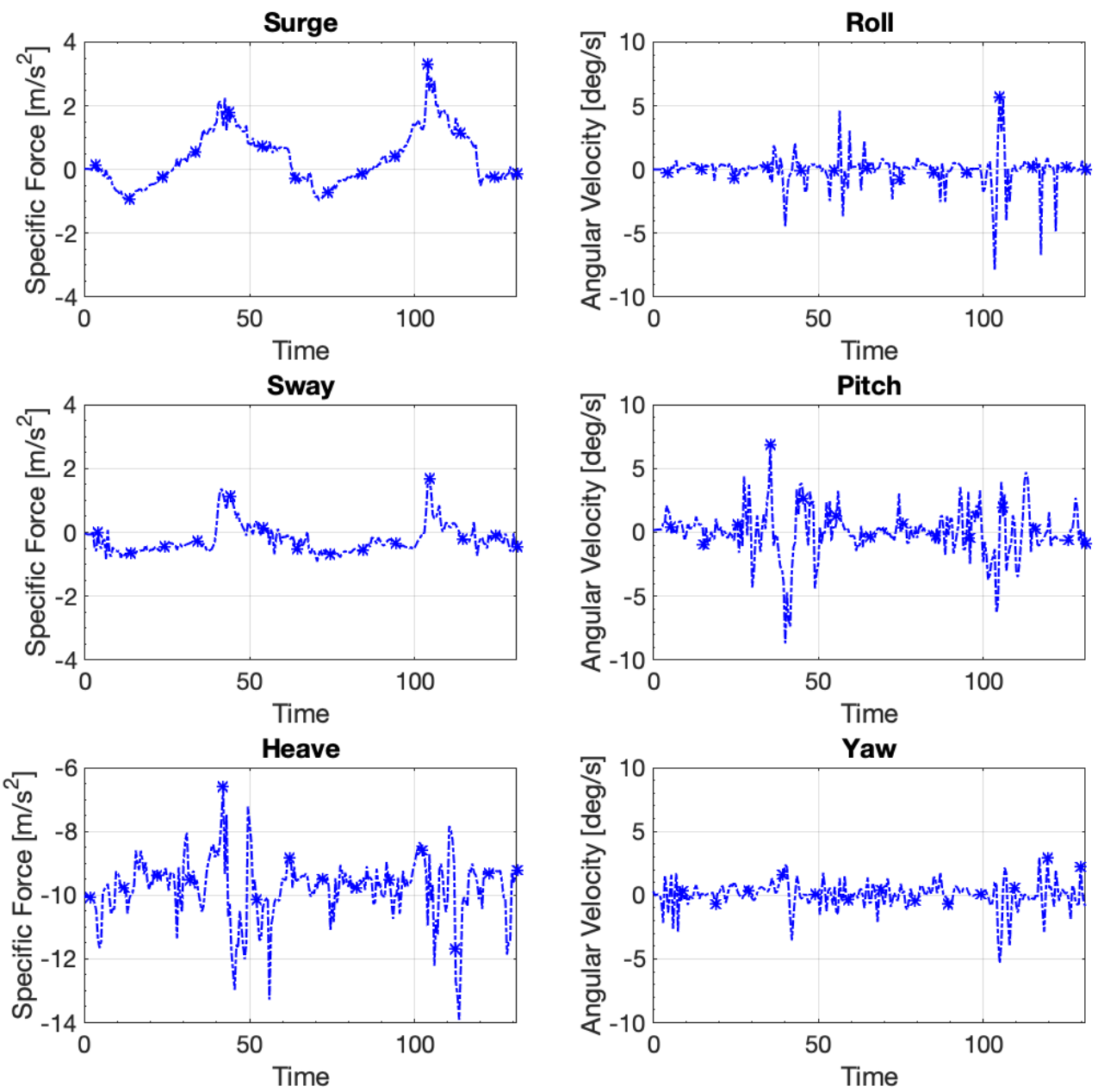

Figure 29: Aircraft motion at the pilot's head for a Cessna 172 completing a stall recovery.

The normalized Pearson correlation and error results are listed in Table 16, and the vestibular response is illustrated in Figure 30. 

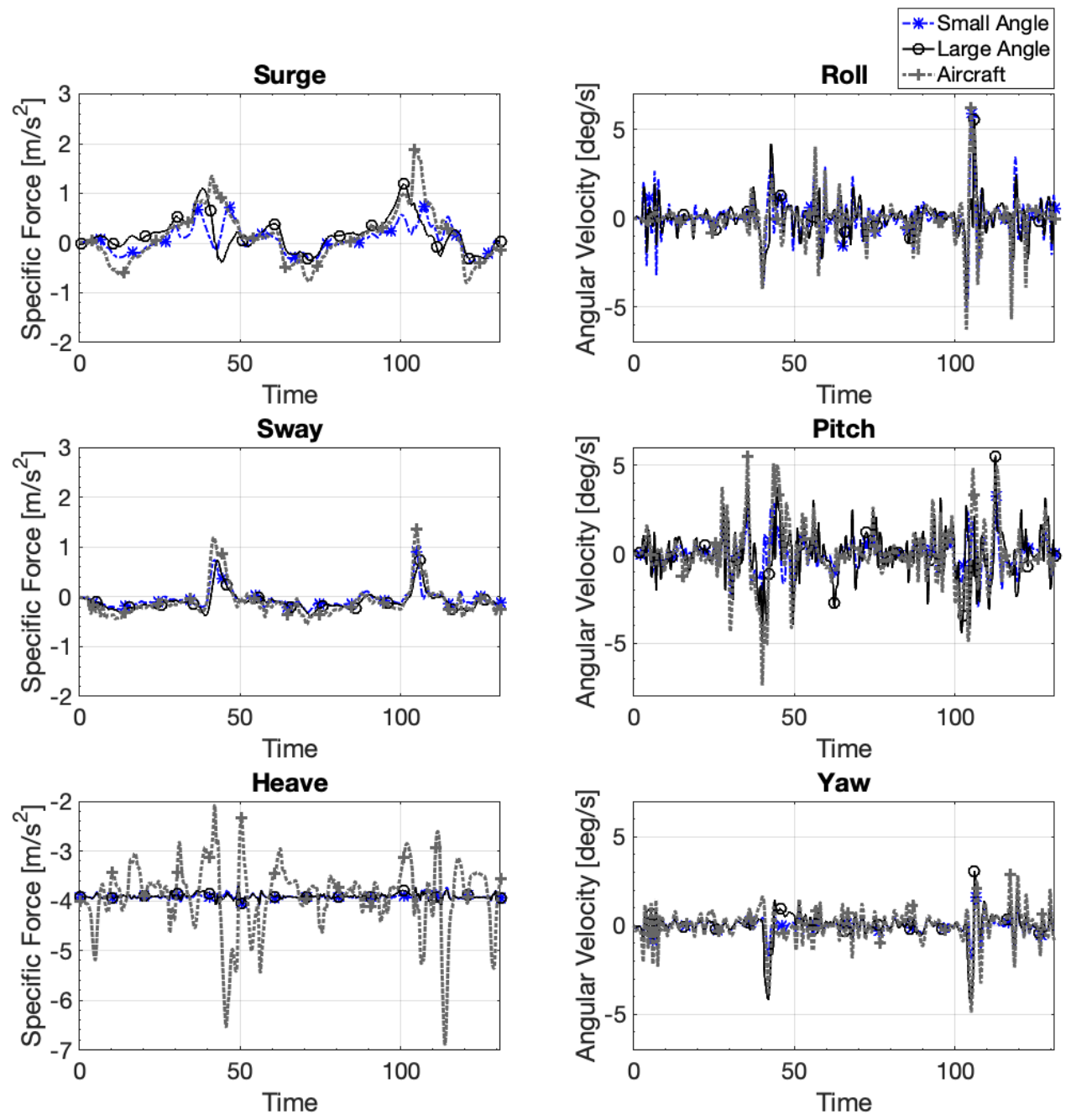

Figure 30: The vestibular response of the Cessna 172 aircraft pilot experiencing a stall recovery manoeuvre, and the simulator pilot's vestibular response for small-angle washout and large-angle washout. 
For the surge degree of freedom in Table 16, large-angle washout has worse performance for the normalized Pearson correlation and the integral of the error, but improves the maximum error. Examining the vestibular response in Figure 30, smallangle washout and large-angle washout each respond more closely to the aircraft response at different points of the manoeuvre, and both have negative cues. The relative performance of the two algorithms will also need to be assessed by pilot testing.

For sway, large-angle washout has a slightly worse normalized correlation and maximum error, and a better result for the integral of the error. Examining the vestibular response the responses for small-angle washout and large-angle washout are almost identical, and the pilot will likely not perceive any difference in performance.

For heave, as reflected in Table 16, large-angle washout offers a substantial improvement in the normalized Pearson correlation, and also a better result for the integral of the error. The maximum error is the same for both washout algorithms. Examining the vestibular response in Figure 30 shows that both washout algorithms fail to follow the changes in specific force, and while the change in normalized Pearson correlation might indicate that the pilot may prefer large-angle washout, it is difficult to draw conclusions based on the simulation.

For roll, large-angle washout performs worse for the normalized Pearson correlation, the integral of the error, and the maximum error. Examining the vestibular response shows that the majority of the manoeuvre has small angular velocity cues, and therefore should not impact the pilot's perception of the motion. For the angular velocity spikes, small-angle washout and large-angle washout appear to perform similarly.

For the pitch response, the normalized Pearson correlation in Table 16 is the same for small-angle washout and large-angle washout. Small-angle washout performs better for the integral of the error, while large-angle washout has a better maximum 
error. Examining the vestibular response in Figure 30, it appears that large-angle washout follows the aircraft vestibular response more closely for the large cues, so may result in an overall improvement. 


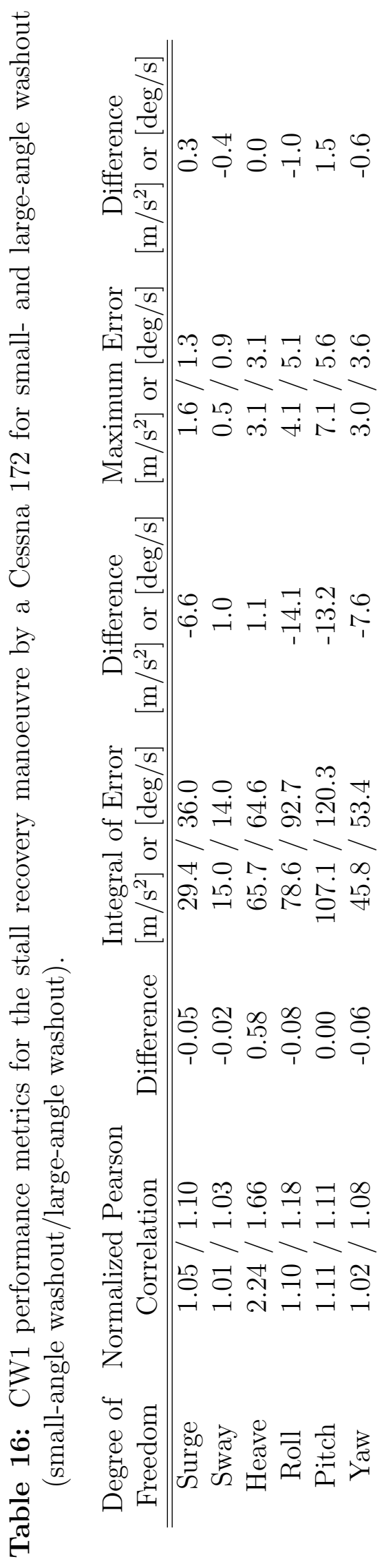


Overall, the two washout algorithms appear to perform similarly; however smallangle washout appears to have better performance in the majority of cases.

\subsection{Other Aircraft}

\subsubsection{Boeing 737}

The same manoeuvres; coordinated turn, cross-wind landing, single-axis takeoff, spiral dive, and stall recovery, were also completed in X-Plane using a Boeing 737 to examine the response of a large passenger jet. Table 17 lists the performance metrics for the manoeuvres using the CW1 coefficients and the graphs for these test cases are provided in Appendix C. For the majority of the metrics, small-angle washout outperforms large-angle washout. 


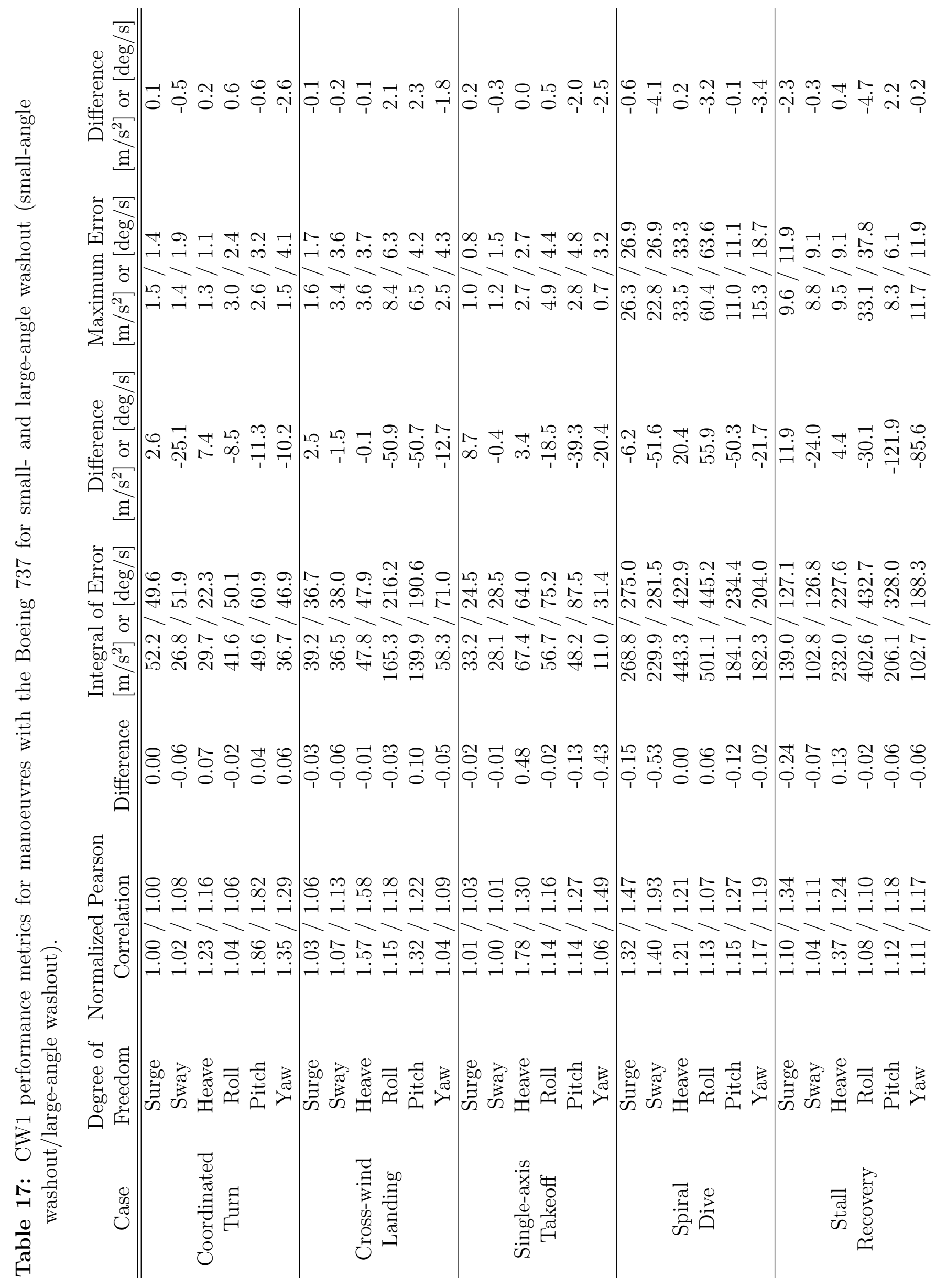




\subsubsection{Columbia 400}

The Columbia 400 aerobatic aircraft was also used to perform the same set of manoeuvres. The performance metrics for these manoeuvres are listed in Table 18. As with the Boeing 737, small-angle washout outperforms large-angle washout for the majority of the metrics.

In addition to these manoeuvres, an aerobatic manoeuvre was performed that would not be possible with the other aircraft. The aircraft motion at the pilot's head is illustrated in Figure 31 for all six degrees of freedom. The performance metrics are listed in Table 19, and the vestibular response is illustrated in Figure 32.

For all the degrees of freedom with the most significant changes, the simulator is unable to produce motions that can closely replicate the aircraft pilot's vestibular response, as the aerobatic manoeuvre is well outside the physical limitations of what the hardware could produce.

For surge, small-angle washout performs better for all the metrics in Table 19, although examining the vestibular response in Figure 32 shows that neither algorithm can follow the response, so it is difficult to determine if there is meaningful difference between the algorithm responses.

For the heave reponse, small-angle washout performs better for the normalized Pearson correlation and the integral of the error, and large-angle washout performs better for the maximum error. Once again, however, both algorithms do not follow the aircraft pilot's response.

For roll, large-angle washout performs better for the normalized Pearson correlation, the integral of the error, and the maximum error as listed in Table 19. Examining the vestibular response in Figure 32 shows that while the simulator is largely unable to follow the peaks of the angular velocity, large-angle washout captures the beginning of some peaks more accurately than small-angle washout, which may lead to 

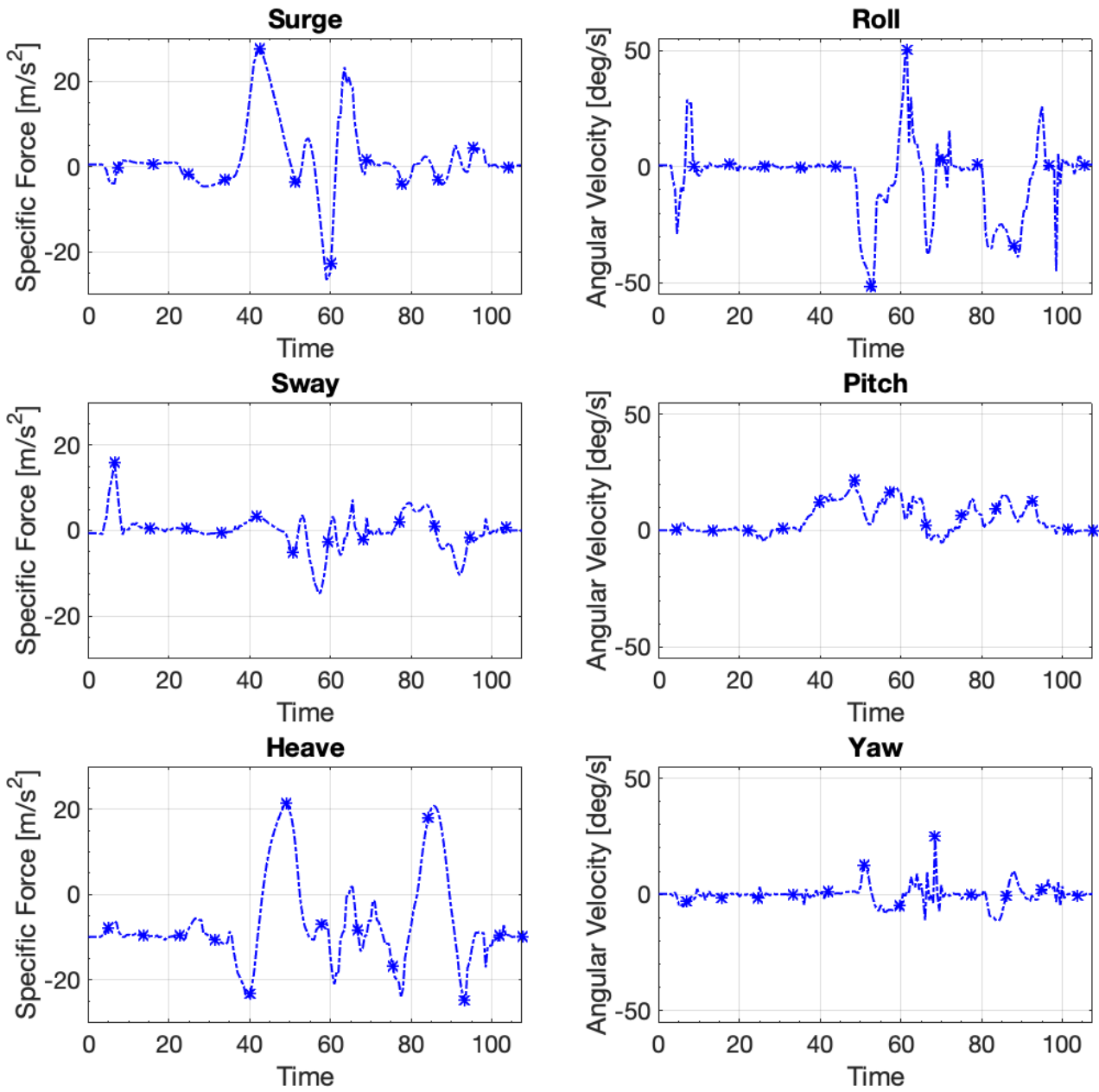

Figure 31: Aircraft motion at the pilot's head for a Cessna 172 completing a stall recovery. 

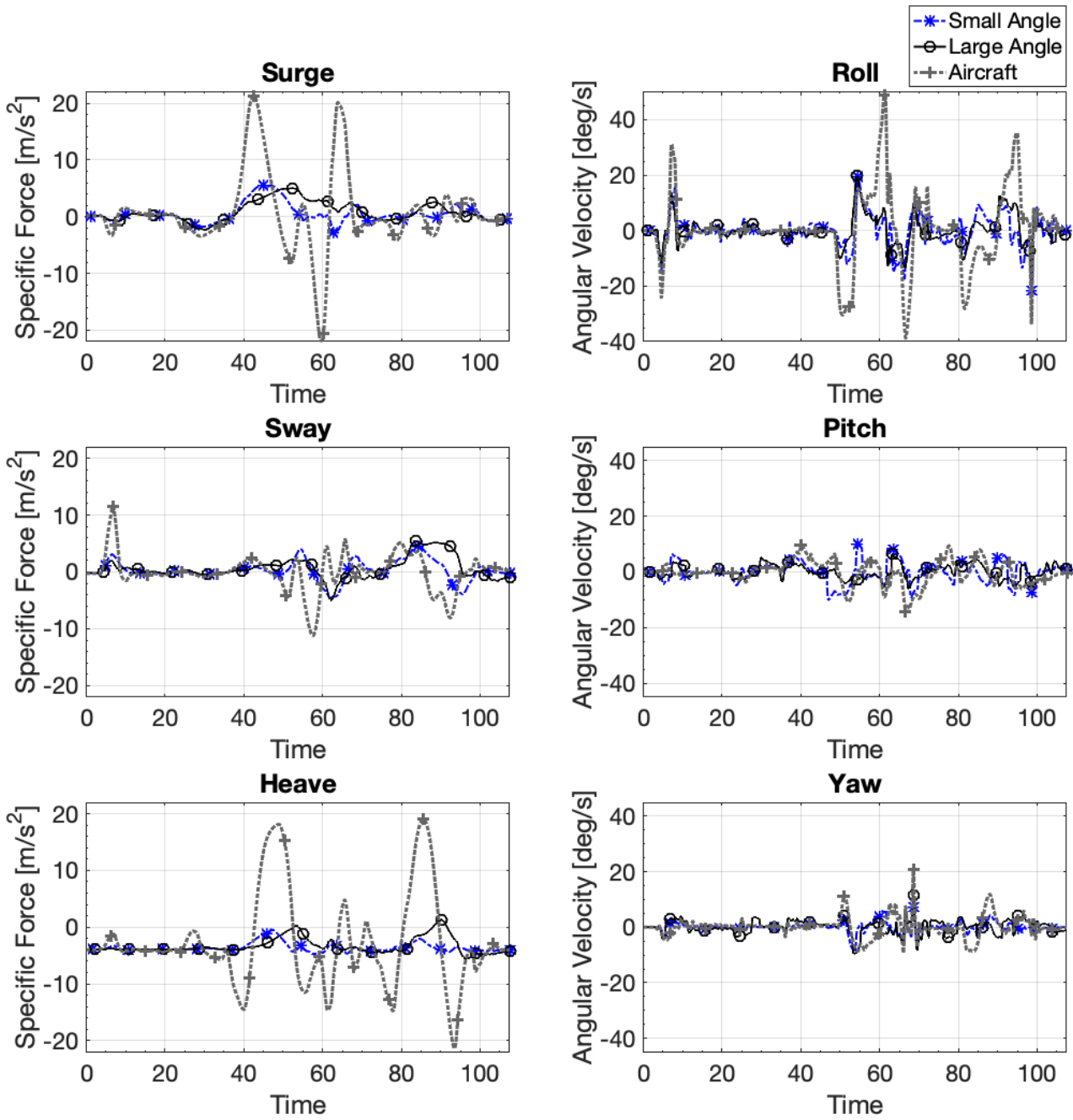

Figure 32: The vestibular response of the Columbia 400 aircraft pilot experiencing aerobatics, and the simulator pilot's vestibular response for small-angle washout and large-angle washout. 
some improvement.

Figure 33 illustrates the Euler angles for the aircraft orientation during the aerobatics manoeuvre. It is notable that there is a discontinuity in the aircraft roll Euler angle from X-Plane 86 seconds into the manoeuvre, which is used to calculate the specific force acting on the pilot; however examining the vestibular response in Figure 32 illustrates that small-angle washout is not negatively impacted by the presence of this discontinuity, as there is no evidence of disruption in the response at that point in the vestibular response.

Overall, this manoeuvre is outside the bounds of the designed system, and neither algorithm version performs accurately. However, small-angle washout outperforms large-angle washout for the normalized Pearson correlation and the integral of the error. 

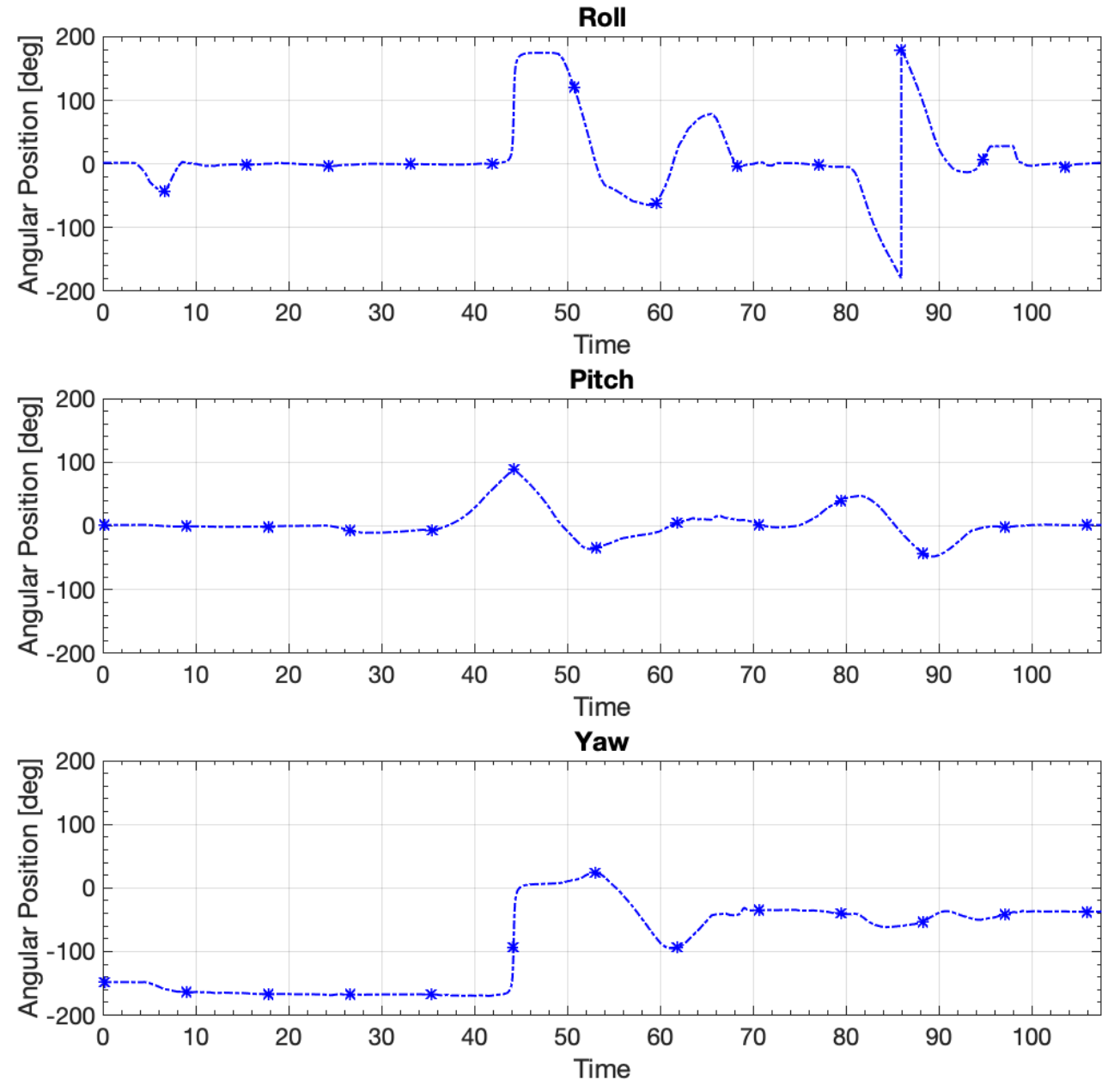

Figure 33: The aircraft's orientation in Euler angles for large-angle washout during aerobatics in the Columbia 400 . 


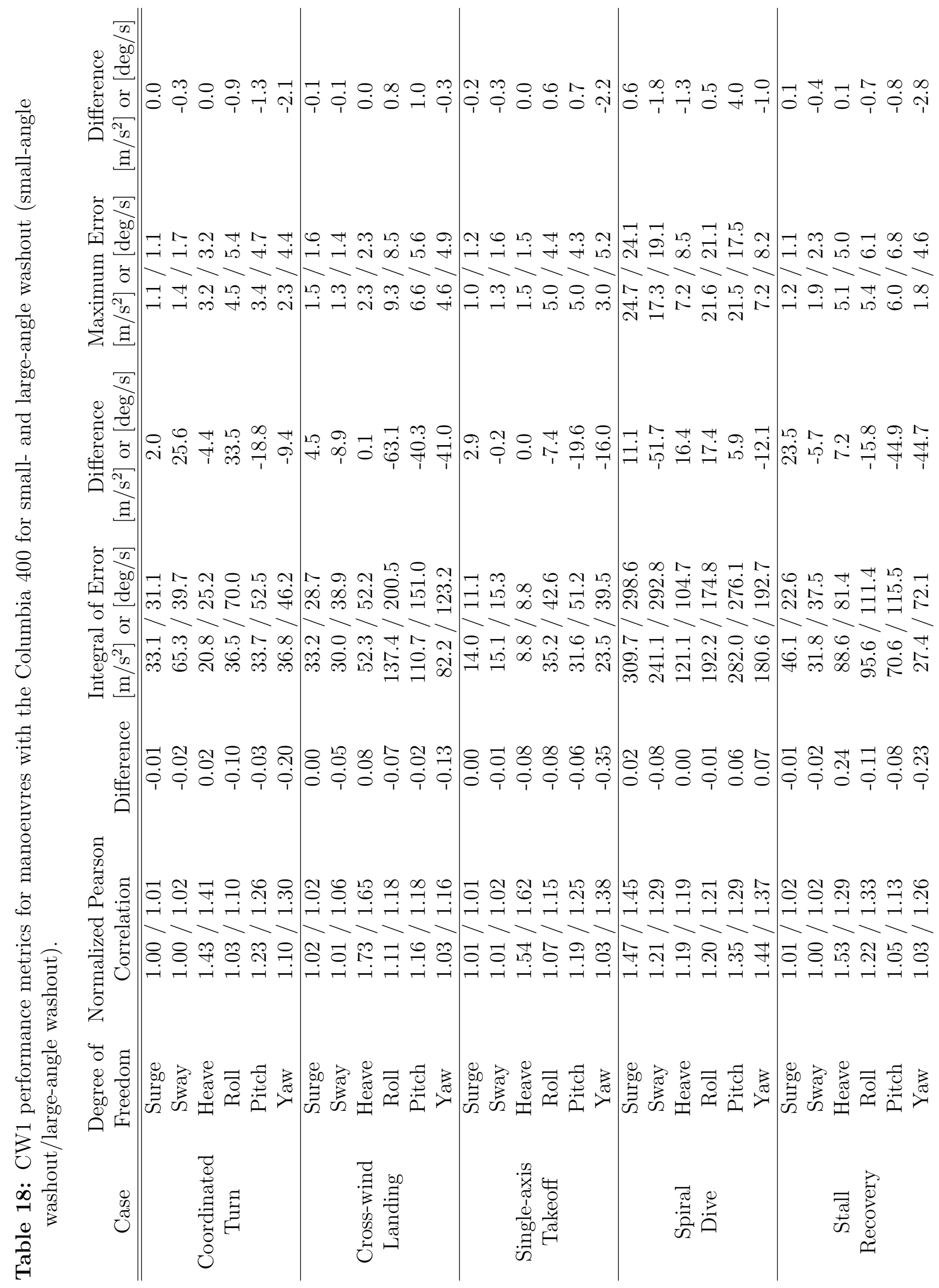




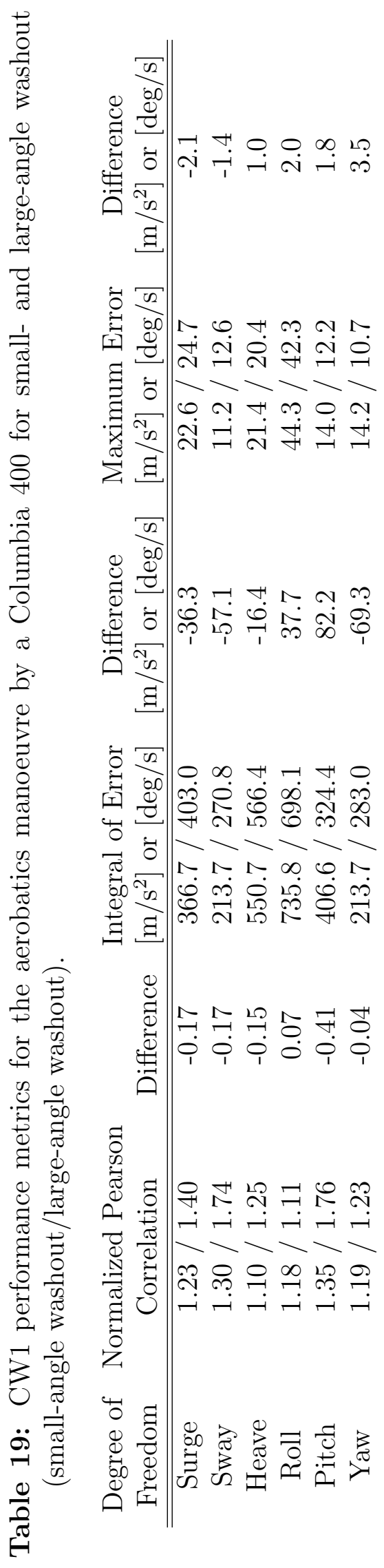




\subsection{Summary of Large-angle Washout Results}

In all the cases shown, large-angle washout can result in some improvement for some metrics, but overall does not present a compelling case for its use. The performance tradeoffs that were made for large-angle washout were intended to eliminate the problems that occur when the simulator was at sufficiently large angles; however the design of classical washout prevents the simulator from approaching the singularity point, even with manoeuvres such as the Columbia 400's aerobatics. The simulator's angular position in ZYX-Euler angles for the aerobatics, illustrated in Figure 34, demonstrates that despite the large angular motion present in the aircraft orientation during the manoeuvre, the simulator motion is restricted so that the Euler angles are continuous at all times, so the potential singularities do not impact the performance of small-angle washout.

There are other improvements in large-angle washout, such as eliminating the addition of Euler angles and the small-angle approximations which should reduce the error source in the algorithm. However, the test cases show that these changes often do not result in an overall improvement after the performance tradeoffs caused by filtering in angular velocity.

\subsection{Unrestricted Angular Washout}

As large-angle washout does not provide a substantial improvement in performance compared to small-angle washout, and to take advantage of simulators with large angular motion envelopes, an additional change to large-angle washout was made. The high-pass filters on the rotational channel exist to restrict the rotational motion of the simulator, so it does not exceed the hardware limits. Simulators such as Atlas do not have physical limits on the rotational motion, and therefore to take advantage of the full range of motion available, the $\omega_{n}$ values for the rotational high pass filters 

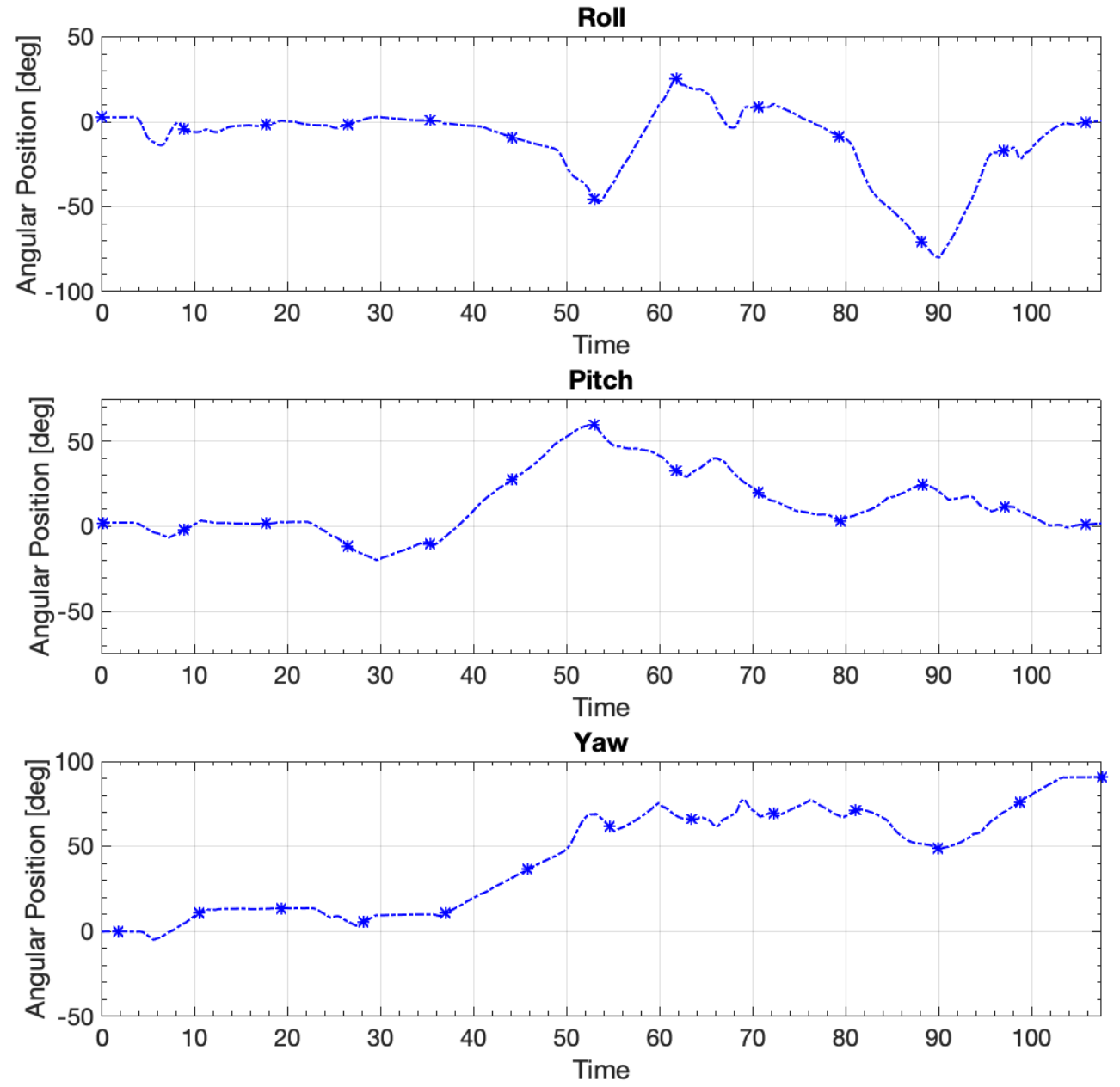

Figure 34: The flight simulator's orientation in Euler angles for large-angle washout during aerobatics in the Columbia 400. 
are set to zero, which effectively eliminates the high pass filter from the rotational channel. The limit for the angular velocity input to the rotational channel remains at $32.2 \mathrm{deg} / \mathrm{s}$ after scaling, to prevent washout from requesting velocities in excess of what can be produced by the hardware.

This solution is different than the angular position control considered in the previous chapter, as the washout algorithm continues to control based on the specific force and angular velocity, and is not trying to match the simulator's orientation with the aircraft's orientation.

The following sections discuss some of the manoeuvres covered in this chapter and additional graphs for the manoeuvres are included in Appendix D.

\subsubsection{Columbia 400 Aerobatics}

The Columbia 400 aircraft completing the aerobatics manoeuvre shows the impact of removing the rotational channel high-pass filters. Figure 35 shows the angular position for each rotational degree of freedom as integrals of the angular velocity (not Euler angles) which illustrates that the simulator completes more than a full rotation in roll and nearly a full rotation in pitch, which is much more angular motion than what was present with the high-pass filter in place. The extended angular motion allows the corresponding vestibular responses for angular velocity to achieve peaks that were not previously possible, and allows the tilt coordination to act on different degrees of freedom for different parts of the manoeuvre, as the simulator orientation changes.

The metrics for the manoeuvre with unrestricted angular washout, compared against small-angle washout and large-angle washout, are listed in Table 20. For

all the rotational degrees of freedom, the unrestricted angular washout improves the normalized Pearson correlation, the integral of the error, and the maximum error compared with small-angle washout. The results are mixed for the translational de- 

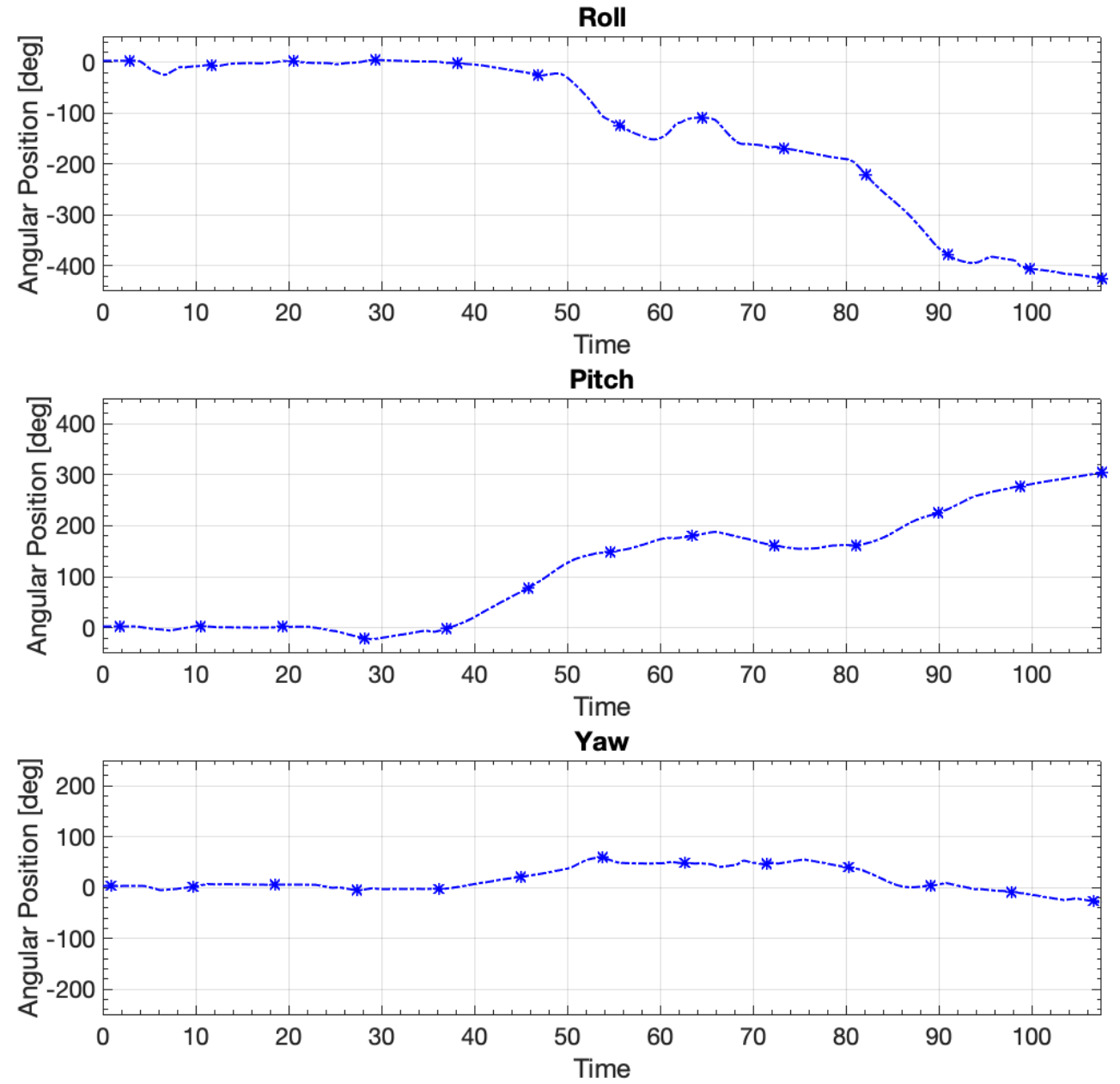

Figure 35: The flight simulator's orientation calculated as the integral of the angular velocity for unrestricted angular washout during aerobatics in the Columbia 400. 
grees of freedom, with surge and heave performing worse with unrestricted angular washout for all metrics, while sway has an improved normalized Pearson correlation and maximum error, with a worse result for the integral of the error.

The vestibular response for the aircraft pilot, small-angle washout, and unrestricted angular washout completing the aerobatics manoeuvre are illustrated in Figure 36. Particularly in roll, which has the most significant angular velocity cues, the unrestricted angular washout matches the angular velocity peaks more accurately than small-angle washout and does not have the negative cues that are present in the small-angle washout vestibular response.

Heave, despite performing worse for all metrics, shows that for some of the peaks, unrestricted angular washout is able to match some of the peaks much more accurately than small-angle washout. Capturing some of these peaks may be worth the overall reduction in the performance metrics.

Surge performs worse for all metrics, and examining the vestibular response shows that particularly in the second half of the manoeuvre the unrestricted angular washout pilot's vestibular response is not following the aircraft pilot's response. The performance reduction is likely due to the improved pitch performance, which may be dominating the response.

The relationship between the vestibular response for the simulator and the orientation of the gravity vector is illustrated in Figure 37. The first column of graphs shows the specific force vestibular response for the aircraft pilot and the simulator pilot for the unrestricted angular washout and the second column of graphs shows the component of the simulator's gravity vector along each axis during the manoeuvre. The components of the gravity vector are significant to understanding the specific force vestibular response, as when the gravity vector is aligned with a translational degree of freedom (its value is close to $\pm 9.81 \mathrm{~m} / \mathrm{s}^{2}$ ), the effect of tilt coordination is limited, as there is no way to increase the specific force applied to the pilot in those 

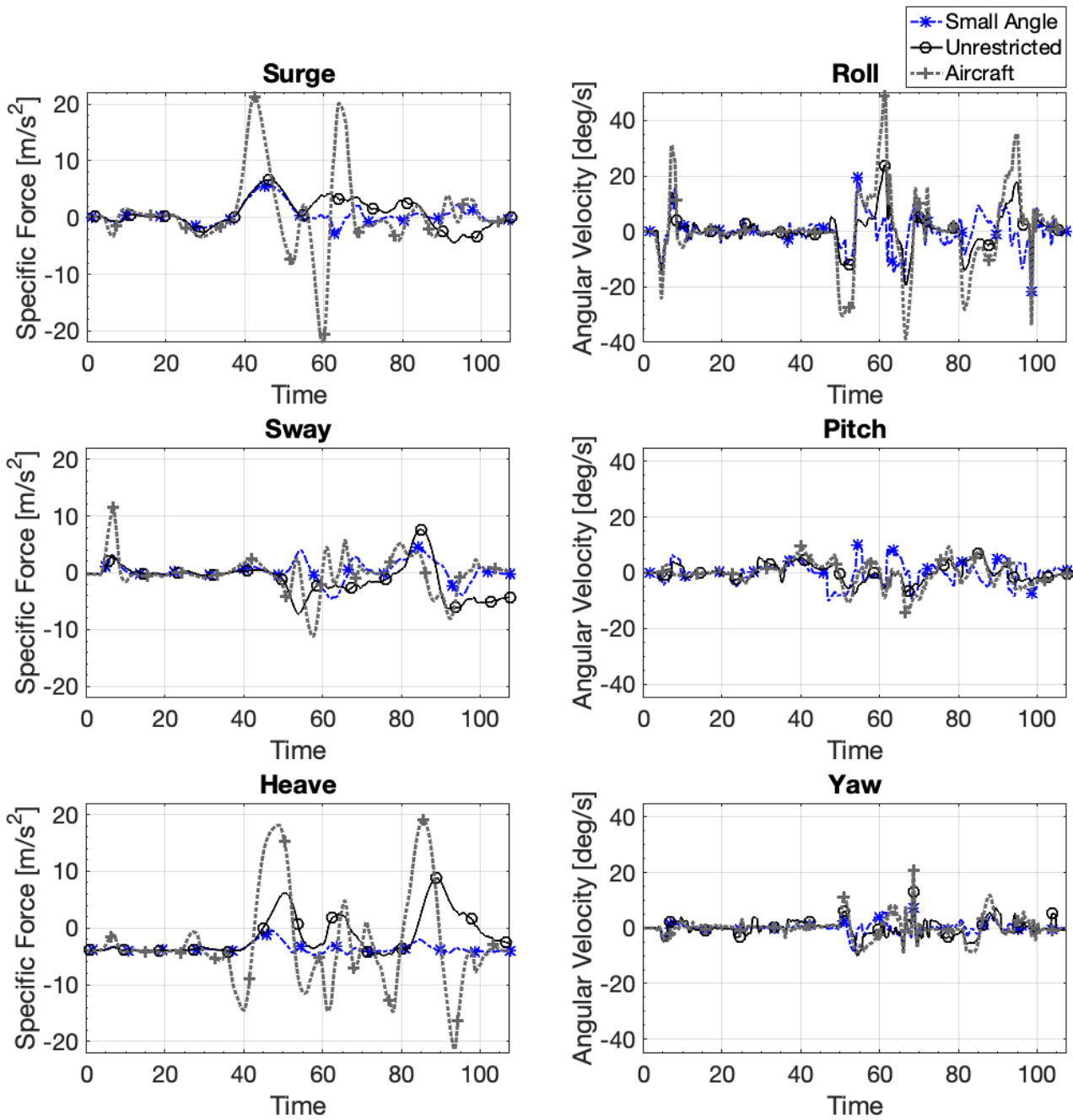

Figure 36: The vestibular response of the Columbia 400 aircraft pilot experiencing aerobatics, and the simulator pilot's vestibular response for small-angle washout and unrestricted angular washout. 
circumstances, as the maximum effect of gravity is already acting on that degree of freedom. Therefore, the simulator is limited to the translational acceleration, which is restricted due to the geometry of the simulator and the high-pass filters on the translational channel. In small-angle washout, the lack of tilt coordination is assumed to only impact the heave degree of freedom, as the small angular motion means that the gravity vector will be aligned with the heave axis, and only deviate from it by small angles. With the elimination of the effects of the high-pass filters, any translational degree of freedom can be aligned with the gravity vector during the course of the manoeuvre. It is important to remember that specific force is the force acting on the pilot from the simulator, and therefore the gravity component is in the opposite direction of the resulting specific force.

For surge, in Figure 36 at just over 40 seconds, the gravity component reaches maximum, which corresponds with the large peak in the specific force. While the gravity component is at its limit, the simulator is unable to match the peak of aircraft pilot's response, as it has reached its maximum specific force. For heave, the simulator does not follow the aircraft pilot's vestibular response for the first 40 seconds. During this time, the heave gravity component is maximized, as this axis is aligned with the gravity vector, which limits the range of specific force to what can be generated by the motion of the platform.

Overall, there is substantial improvement for the performance of the angular degrees of freedom. There is some reduction in performance for the translational degrees of freedom; however the response for both washout algorithms is not accurate as the manoeuvre being completed far exceeds the abilities of the simulator. 
Surge

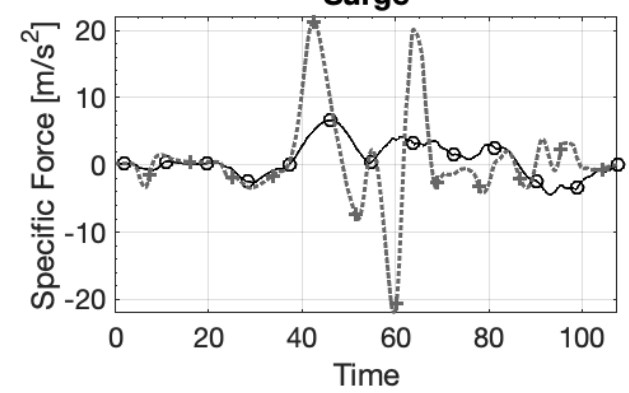

Sway

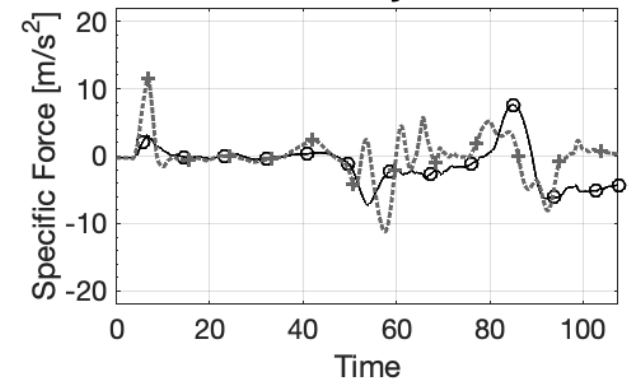

Heave

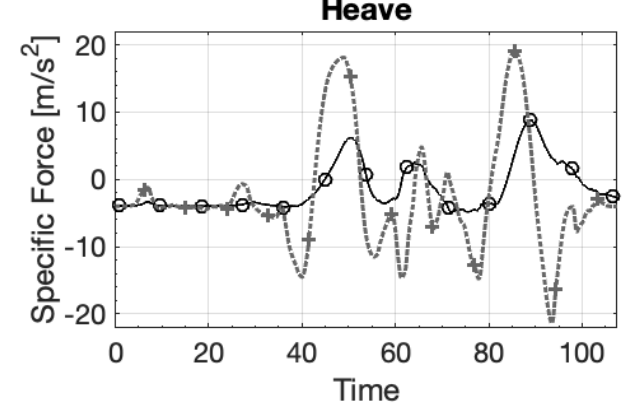

-Unrestricted

-+-Aircraft

Surge Gravity Component

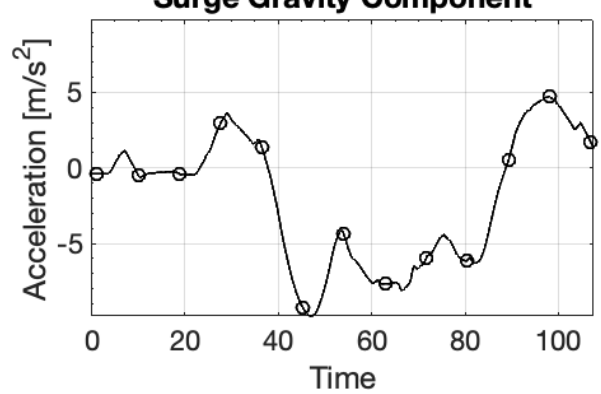

Sway Gravity Component
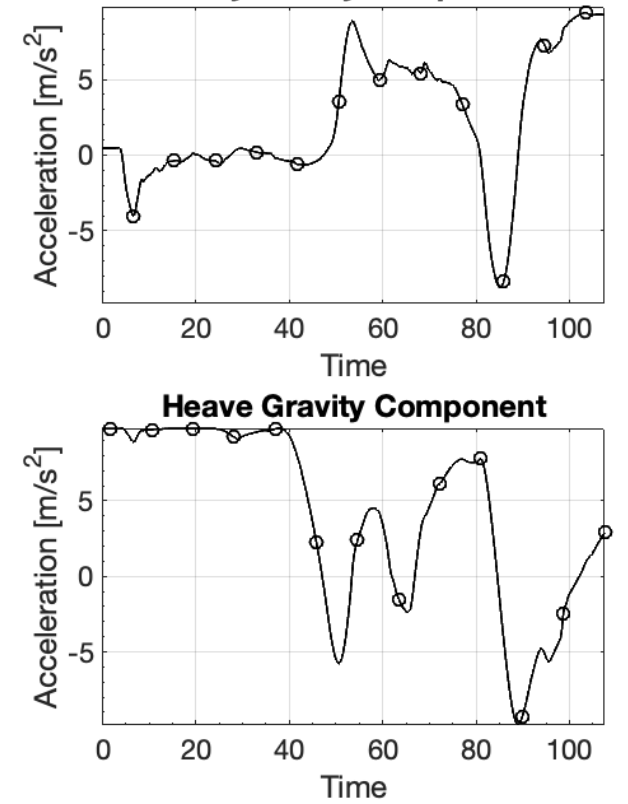

Figure 37: The vestibular response of the Columbia 400 aircraft pilot experiencing aerobatics, and the simulator pilot's vestibular response for unrestricted angular washout, and the components of gravity acting on the simulator. 


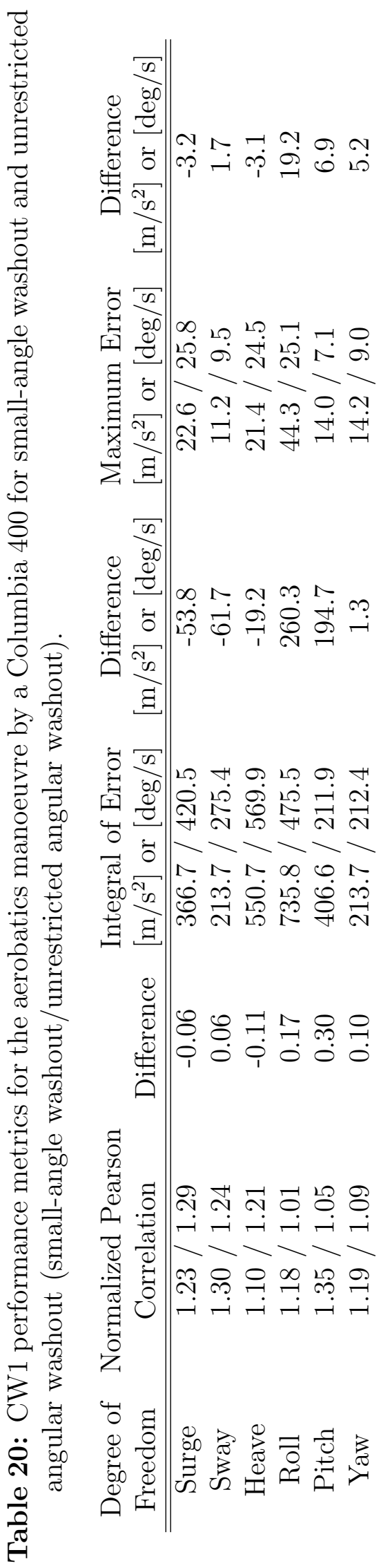




\subsubsection{Cessna 172 Single-axis Takeoff}

The manoeuvres with the Cessna 172 were completed with the unrestricted angular washout. The performance metrics for each manoeuvre are listed in Table 21.

The single-axis takeoff manoeuvre is an example of a manoeuvre with limited angular velocity and significant specific force values. For the unrestricted angular washout the normalized Pearson correlation is slightly worse for all degrees of freedom except heave. For the integral of the error, unrestricted angular washout improves the performance for surge and yaw, but otherwise the performance is similar or worse. For the maximum error, the values are similar for most degrees of freedom, with small-angle washout performing better for sway and pitch.

Examining the vestibular response illustrated in Figure 38 shows that the response for surge is similar for the two washout algorithms for the first 20 seconds, and then unrestricted angular washout is much closer to the aircraft pilot's vestibular response, despite the reduction in performance metrics. For all other degrees of freedom, the response for the two types of washout appear to be extremely similar.

Overall, the performance metrics indicate that for this small-angle manoeuvre, small-angle washout may be a better option, although the vestibular response indicates that this performance reduction may not be a major issue to the pilot's perception of the motion as the differences between the responses appear to be small. 

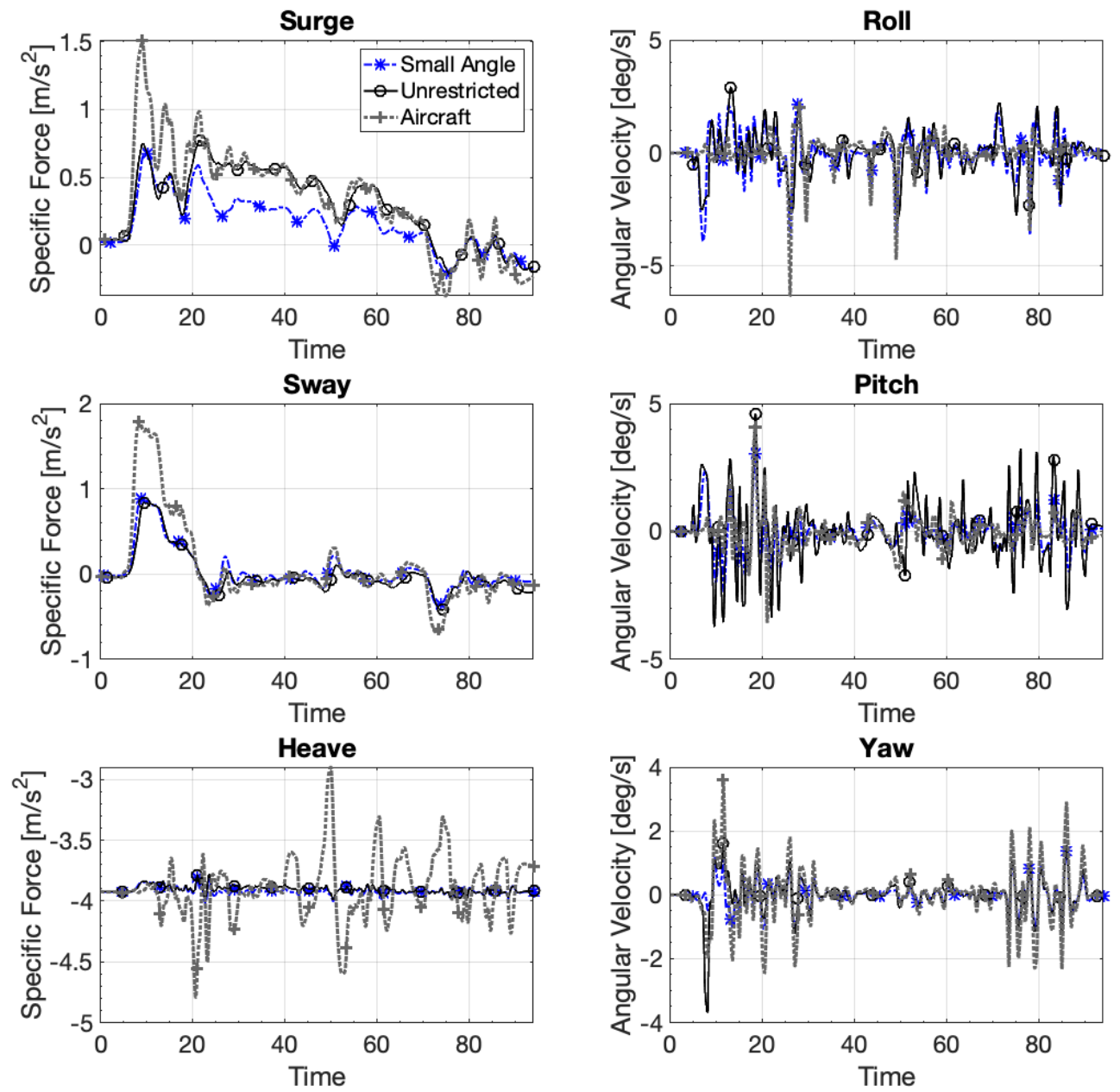

Figure 38: The vestibular response of the Cessna 172 aircraft pilot experiencing a single-axis takeoff, and the simulator pilot's vestibular response for small-angle washout and unrestricted angular washout. 


\subsubsection{Cessna 172 Spiral Dive}

The spiral dive is an example of another manoeuvre that has a large angular velocity component in the vestibular response. The performance metrics listed in Table 21 show that for all metrics the unrestricted angular washout is better for all degrees of freedom except sway.

Examining the vestibular response, illustrated in Figure 39, shows that for all the rotational degrees of freedom the unrestricted angular washout is significantly better at matching the peaks of the aircraft pilot's vestibular response, and does not have the negative cues associated with the small washout response.

For the translational degrees of freedom, neither washout algorithm is able to follow the specific force of the aircraft due to the limitations of the specific force that can be generated by the simulator. However the unrestricted angular washout response allows for larger peaks, particularly in heave.

Overall, the unrestricted angular washout response has a clear benefit, as the performance metrics are almost all improved, and there is a clear improvement in the response for the angular velocities. 

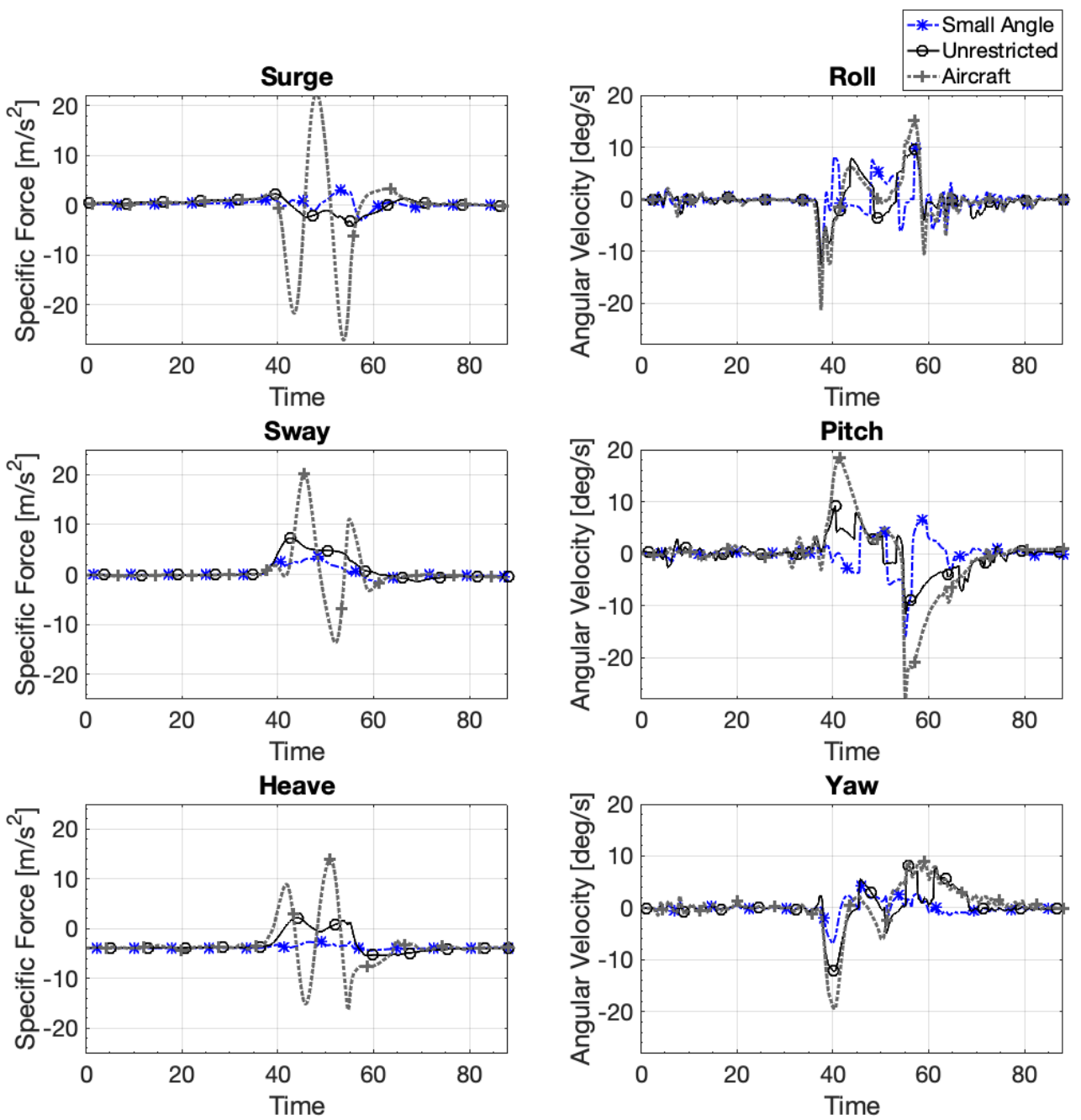

Figure 39: The vestibular response of the Cessna 172 aircraft pilot experiencing a spiral dive, and the simulator pilot's vestibular response for small-angle washout and unrestricted angular washout. 


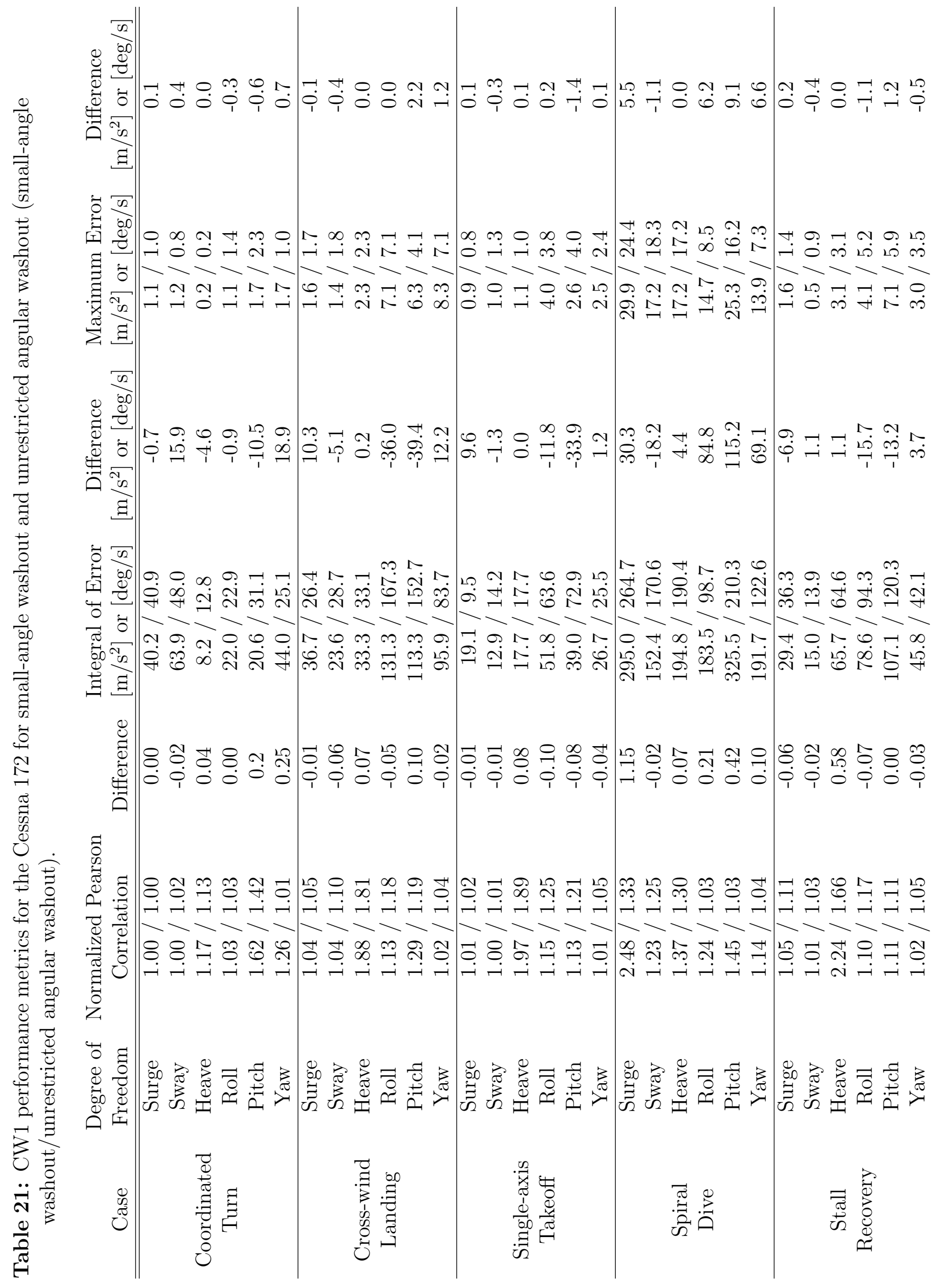




\subsection{Summary of Unrestricted Angular Washout}

For manoeuvres with large angular velocities, there is a clear benefit to the unrestricted angular washout, as it improves the performance for the rotational degrees of freedom, and usually without significant detriment to the translational degrees of freedom. For the manoeuvres with no significant angular velocity cues, the smallangle washout is likely the better option based on the performance metrics, although unrestricted angular washout does not seem to cause a large performance degradation. Further testing with pilots is necessary to confirm these results.

\subsection{Conclusions}

For aircraft manoeuvres with large angular motion, unrestricted angular washout has the best performance of the three washout algorithms examined in this chapter. While large-angle washout does have some performance benefits compared with smallangle washout for some manoeuvres with large angular motion, unrestricted angular washout outperforms large-angle washout for these manoeuvres, and for simulators with unrestricted angular motion envelopes, unrestricted angular washout should be used when simulating these large angular motion manoeuvres.

For aircraft manoeuvres with small angular motion, small-angle washout has the best performance of the three washout algorithms. However, unrestricted angular washout's performance is comparable to small-angle washout, and therefore it is also a reasonable choice for small-angle manoeuvres. Large-angle washout does not perform well for small angular manoeuvres compared to the other two washout algorithms, and should not be used for these manoeuvres. 


\section{Conclusion}

The objectives of this thesis were:

1. To code the models for classical washout, adaptive washout, and for the vestibular response of the aircraft and simulator pilots.

2. To remove the small angle restrictions and extend the range of angular motion for classical washout.

3. To demonstrate the performance of large-angle classical washout in simulation.

\subsection{Objective 1: Modelling}

In Chapter 2, implementation of the classical washout model and the model for the pilot vestibular response in MATLAB Simulink was described. The main contribution of this thesis related to the first objective is the creation of a MATLAB Simulink model of the classical washout algorithm. The model was developed to reproduce that described by Reid and Nahon $[1,2]$, and simulations were used to validate the performance of the model compared to the reported performance of their classical washout model by comparing the vestibular responses with numerical metrics and subjective observations of the plotted vestibular response. Creating this model allowed for a benchmark of performance for small-angle washout that was used in Chapters 3 and 4 as a benchmark for the expected performance from the washout algorithms.

\subsection{Objective 2: Extension of Classical Washout}

In Chapter 3, initial modifications for large-angle washout were described, and simulation results were used to compare the performance for small-angle manoeuvres. In Chapter 4, to improve performance, classical washout was extended to unbounded 
rotation by the removal of the high-pass filters for the rotational channel. The contribution of this thesis related to the second objective is extending classical washout to allow a larger angular range of motion. This washout model used quaternions instead of Euler angles and eliminated the small-angle approximations present in small-angle washout. Large-angle washout overall did not perform as well as small-angle washout for the manoeuvres tested in Chapter 3 and Chapter 4; however, unbounded angular velocity washout improves performance compared to small-angle washout for manoeuvres with large angular motion, and achieves similar performance at small angles.

\subsection{Objective 3: Performance Validation}

In Chapter 4, large-angle classical washout and unrestricted angular classical washout were simulated and compared against the performance of small-angle classical washout. The main contribution of this thesis related to the third objective is simulated validation of the performance of classical washout with an extended angular range of motion. Large-angle classical washout did not perform well compared to small-angle washout, and therefore was not a feasible replacement for that algorithm. The unrestricted angular washout performed well compared to small angle washout, particularly with manoeuvres that contain large angular velocity cues. Unrestricted angular washout shows clear advantages for use with manoeuvres that have large angular motion, and is comparable to small-angle washout in performance for small-angle cues.

\subsection{Future Work}

The following are suggested as potential areas of future work:

1. Hardware testing of unrestricted angular washout with pilot evaluation. All the tests conducted in this thesis were done with numerical metrics and subjective 
evaluation based on the graphed vestibular response. Pilot-in-the-loop testing will provide additional evaluation of the washout modifications.

2. Tuning the transfer function coefficients for improved washout performance. Chapter 1 discusses several tuning mechanisms for washout, including genetic algorithms, fuzzy logic, and particle swarm optimization that have been applied to small-angle classical washout. Unrestricted angular washout uses the transfer function coefficients that were reported by Reid and Nahon, and further improvements could be accomplished with additional tuning.

3. Development of an adaptive washout algorithm for large-angle washout. Adaptive washout is less commonly used than classical washout due to its complexity. However, it has good performance that could be improved with extending it to large angles.

\subsection{Publication}

The paper M. Micomonaco, M. J. D. Hayes, R. Irani, and R. Langlois, "Classical Washout Using Quaternions," in CCToMM Mechanisms, Machines, and Mechatronics $\left(M^{3}\right)$ Symposium, Montreal, Canada, May 16-17, 2019 was published based on the work in this thesis. 


\section{References}

[1] L. D. Reid and M. A. Nahon, "Flight simulation motion-base drive algorithms: Part 1 - developing and testing the equations," tech. rep., UTAIS, 1985.

[2] L. D. Reid and M. A. Nahon, "Flight simulation motion-base drive algorithms: Part 2 - selecting the system parameters," tech. rep., UTAIS, 1986.

[3] L. D. Reid and M. A. Nahon, "Flight simulation motion-base drive algorithms: Part 3 - pilot evaluations," tech. rep., UTAIS, 1986.

[4] S. Casas, I. Coma, J. V. Riera, and M. Fernández, "Motion-cuing algorithms: Characterization of users' perception," Human Factors, vol 57, pp 144-162, 2015.

[5] MOOG Inc., Moog Motion Base MB-E-6DOF/24/1.800KG: elektrische Bewegungsplattform für Traglasten bis zu 1.800 kg, 2018 (accessed May 3, 2019).

[6] M. J. D. Hayes and R. G. Langlois, "Atlas: A Novel Kinematic Architecture for Six DOF Motion Platforms," Transactions of the Canadian Society for Mechanical Engineering: Selected Papers of the $3^{\text {rd }}$ CCToMM Symposium on Machines, Mechanisms, and Mechatronics, vol. 29, no. 4, pp. 701-709, 2005.

[7] Z. Copeland, B. Jung, M. J. D. Hayes, and R. G. Langlois, "Full-scale Atlas Motion Platform: Structure, Actuation, and Control," International Journal of Mechanisms and Robotic Systems, vol. 3, no. 2/3, pp. 94-112, 2016.

[8] R. A. Irani, N. Schut, M. J. D. Hayes, and R. G. Langlois, "A Novel Flight Simulator Capable of Unbounded Rotation," Proceedings of the 2017 IEEE International Symposium on Systems Engineering (ISSE), Vienna, Austria, October 11-13, 2017. 
[9] M. Micomonaco, M. J. D. Hayes, R. Irani, and R. Langlois, "Classical Washout Using Quaternions," CCToMM Mechanisms, Machines, and Mechatronics ( $\left.M^{3}\right)$ Symposium, Montreal, Canada, May 16-17, 2019.

[10] C. M. Schultz, "World War I Flying Ace," 1965.

[11] P. R. Grant and L. D. Reid, "Protest: An expert system for tuning simulator washout filters," Journal of Aircraft, vol 34, pp 152-159, 1997.

[12] H. Asadi, A. Mohammadi, S. Mohamed, C. P. Lim, A. Khatami, A. Khosravi, and S. Nahavandi, "A Particle Swarm Optimization-based Washout Filter for Improving Simulator Motion Fidelity," IEEE International Conference on Systems, Man, and Cybernetics, Budapest, Hungary, October 9-12, 2016.

[13] S. Casas, C. Portalés, P. Morillo, and M. Fernández, "A particle swarm approach for tuning washout algorithms in vehicle simulators," Applied Soft Computing, 2018.

[14] H. Asadi, S. Mohamed, and S. Nahavandi, "Incorporating Human Perception With the Motion Washout Filter Using Fuzzy Logic Control," IEEE/ASME Transactions on Mechatronics, 2015.

[15] H. Asadi, S. Mohamed, D. R. Zadeh, and S. Nahavandi, "Optimization of nonlinear motion cueing algorithm based on genetic algorithm," Vehicle System Dynamics, 2015.

[16] B. Keshavarz, R. Ramkhalawansingh, B. Haycock, S. Shahab, and J. L. Campos, "Comparing simulator sickness in younger and older adults during simulated driving under different multisensory conditions," Transportation Research Part F: Traffic Psychology and Behaviour, 2018. 
[17] D. Cleij, J. Venrooij, P. Pretto, D. M. Pool, M. Mulder, and H. H. Bülthoff, "Continuous Subjective Rating of Perceived Motion Incongruence During Driving Simulation," IEEE Transactions on Human-Machine Systems, 2018.

[18] W. Dalmeijer, I. Miletovic, O. Stroosma, and M. Pavel, "Extending the Objective Motion Cuing Test to Measure Rotorcraft Simulator Motion Characteristics," 73rd Annual AHS International Forum and Technology Display, 2017.

[19] M. Wentink, W. Bles, R. Hosman, and M. Mayrhofer, "Design \& evaluation of spherical washout algorithm for Desdemona simulator," AIAA Modeling and Simulation Technologies Conference and Exhibit, San Francisco, California, August 15-18, 2005.

[20] L. R. Young and J. L. Meiry, "A revised dynamic otolith model," Aerospace Medicine, vol 39, pp 606-608, 1968.

[21] G. L. Zacharias, "Motion cue models for pilot-vehicle analysis," tech. rep., Aerospace Medical Research Laboratory, 1977.

[22] H. Asadi, S. Mohamed, C. P. Lim, and S. Nahavandi, "A review on otolith models in human perception," Behavioural Brain Research, 2016.

[23] L. R. Young and C. M. Oman, "Model for vestibular adaption to horizontal rotation," Aerospace Medicine, vol 40, pp 1076-1080, 1969.

[24] H. Asadi, S. Mohamed, C. P. Lim, S. Nahavandi, and E. Nalivaiko, "Semicircular canal modelling in human perception," Reviews in the Neurosciences, 2017.

[25] J. Solà, "Quaternion kinematics for the error-state kalman filter," 102017.

[26] J. Craig, Introduction to Robotics, Mechanics and Control. Upper Saddle River, New Jersey, USA: Pearson Prentice Hall, 3 ed., 2005. 


\section{A Adaptive Washout}

Adaptive washout works on the same principles as classical washout, with sustained specific force cues generated by a component of the gravity vector due to tilting the simulator. Figure 40 is the block diagram for adaptive washout. The diagram contains two channels, translational and rotational, but is missing the tilt coordination channel found in classical washout. The tilt coordination component of adaptive washout is part of the adaptive filter in the rotational channel.

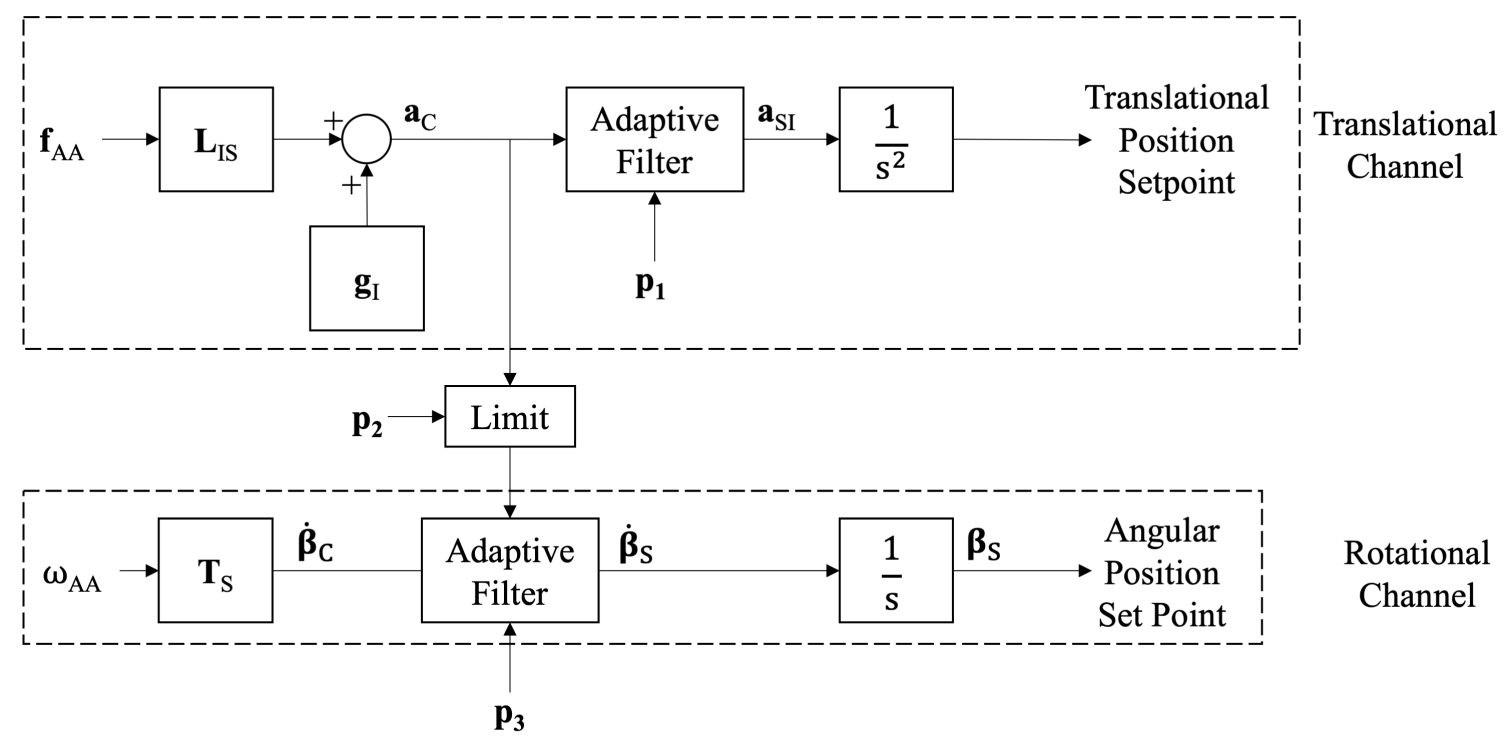

Figure 40: Adaptive washout block diagram.

\section{A.1 Translational Channel}

Adaptive washout's translational channel uses the aircraft's specific force in the aircraft frame $\mathbf{f}_{\mathrm{AA}}$ as the input. The rotation matrix $\mathbf{L}_{\mathrm{IS}}$ from Equation 21 in classical washout is used to convert the specific force to the inertial frame. The gravity vector is added to the specific force resulting in the translational acceleration of the aircraft in the inertial frame, $\mathbf{a}_{\mathrm{C}}$. 
The translational acceleration is then passed to the adaptive filter, along with the updated adaptive parameter $p_{1}$, to produce the translational acceleration set point for the simulator in the inertial frame $\mathbf{a}_{\mathrm{SI}}$. The resulting translational acceleration is then integrated twice to produce the translational position set point.

\section{A.2 Rotational Channel}

The rotational channel takes the input of the aircraft angular velocity $\boldsymbol{\omega}_{\mathrm{AA}}$ in the aircraft frame, where it is converted to rate of change of Euler angles $\dot{\boldsymbol{\beta}}_{\mathrm{C}}$.

The signals $\dot{\boldsymbol{\beta}}_{\mathrm{C}}$ and $\mathbf{a}_{\mathrm{C}}$, from the translational channel, are passed into the adaptive filter, along with the updated adaptive parameters $p_{2}$ and $p_{3}$. The output rate of change of Euler angles is then integrated to give the angular position set point in Euler angles $\boldsymbol{\beta}_{\mathrm{S}}=\left[\begin{array}{lll}\phi & \theta & \psi\end{array}\right]^{\mathrm{T}}$.

\section{A.3 Surge/Pitch Adaptive Filters}

The surge and pitch adaptive filters are related, as changes in pitch, $\theta$, impact the specific forces in the surge direction, $f_{\mathrm{Sx}}$.

The pair of equations for the adaptive filters are

$$
\ddot{S}_{\mathrm{Ix}}=p_{\mathrm{x} 1} a_{\mathrm{Cx}}-k_{\mathrm{x} 1} S_{\mathrm{Ix}}-k_{\mathrm{x} 2} \dot{S}_{\mathrm{Ix}}
$$

and

$$
\dot{\theta}_{\mathrm{S}}=\operatorname{LIM}\left(p_{\mathrm{x} 2} a_{\mathrm{Cx}}\right)+p_{\mathrm{x} 3} \dot{\theta}_{\mathrm{C}}
$$

where $p_{\mathrm{x} 1}, p_{\mathrm{x} 2}$, and $p_{\mathrm{x} 3}$ are the adaptive parameters. The constants $k_{\mathrm{x} 1}$ and $k_{\mathrm{x} 2}$ are chosen to reflect the desired simulator performance characteristics [1]. These filters have the effect of attempting to match the simulator translational acceleration $\ddot{S}_{\text {IX }}$ 
with the acceleration set point from the aircraft motion $a_{\mathrm{Cx}}$ and the simulator roll rate $\dot{\theta}_{\mathrm{S}}$ with the angular velocity set point from the aircraft motion, $\dot{\theta}_{\mathrm{C}}$ while restricting the translational and angular velocities and positions.

To achieve tracking the translational acceleration and angular velocity while restricting the translational and angular velocities and positions, the adaptive parameters are updated using the cost function

$$
\begin{aligned}
J_{\mathrm{x}}= & 0.5\left[\left(a_{\mathrm{Cx}}-\ddot{S}_{\mathrm{Ix}}\right)^{2}+W_{\mathrm{x} 1}\left(\dot{\theta}_{\mathrm{C}}-\dot{\theta}_{\mathrm{S}}\right)^{2}\right. \\
& +\rho_{\mathrm{x}}\left(W_{\mathrm{x} 2} \dot{S}_{\mathrm{Ix}}^{2}+W_{\mathrm{x} 3} S_{\mathrm{Ix}}^{2}+W_{\mathrm{x} 4} \dot{\theta}_{\mathrm{S}}^{2}+W_{\mathrm{x} 5} \theta_{\mathrm{S}}^{2}\right) \\
& \left.+W_{\mathrm{x} 6}\left(p_{\mathrm{x} 1}-p_{\mathrm{x} 10}\right)^{2}+W_{\mathrm{x} 7}\left(p_{\mathrm{x} 2}-p_{\mathrm{x} 20}\right)^{2}+W_{\mathrm{x} 8}\left(p_{\mathrm{x} 3}-p_{\mathrm{x} 30}\right)\right],
\end{aligned}
$$

where $W_{\mathrm{x} 1-8}, \rho_{\mathrm{x}}, p_{\mathrm{x} 10}, p_{\mathrm{x} 20}$, and $p_{\mathrm{x} 30}$ are constants [1]. The first row of the cost function minimizes the difference between the input and output translational acceleration and angular velocity for the adaptive filters. The second row of the equation minimizes the translational velocity, translational position, angular velocity, and angular position of the simulator, and the final row is to return the adaptive parameters to their initial values between manoeuvres.

\section{A.4 Sway and Roll Adaptive Filters}

The sway and roll adaptive filters are set up the same as the surge and pitch versions, using Equations 46 and 47 with $y$ instead of $x$ and $\phi$ instead of $\theta$.

\section{A.5 Heave and Yaw Adaptive Filters}

Unlike surge and sway, heave does not have a tilt coordination component to the adaptive filter, as at small angles the heave degree of freedom is already aligned with 
the gravity vector. The adaptive filter for heave is

$$
\ddot{S}_{\mathrm{Iz}}=p_{\mathrm{z}} a_{\mathrm{Cz}}-k_{\mathrm{z} 1} \int S_{\mathrm{Iz}}-k_{\mathrm{z} 2} S_{\mathrm{Iz}}-k_{\mathrm{z} 3} \dot{S}_{\mathrm{Iz}}
$$

with the corresponding cost function

$$
J_{\mathrm{z}}=0.5\left[\left(a_{\mathrm{Cz}}-\ddot{S}_{\mathrm{Iz}}\right)^{2}+\rho_{\mathrm{z}}\left(W_{\mathrm{z} 1} \dot{S}_{\mathrm{Iz}}^{2}+W_{\mathrm{z} 2} S_{\mathrm{Ix}}^{2}\right)+W_{\mathrm{x} 3}\left(p_{\mathrm{z}}-p_{\mathrm{z} 0}\right)^{2}\right]
$$

Finally, the adaptive filter for yaw is

$$
\dot{\psi}_{\mathrm{S}}=p_{\psi} \dot{\psi}_{\mathrm{C}}-k_{\psi 1} \int \psi_{\mathrm{S}}-k_{\psi 2} \psi_{\mathrm{S}}
$$

with the corresponding cost function

$$
J_{\psi}=0.5\left[\left(\dot{\psi}_{\mathrm{C}}-\dot{\psi}_{\mathrm{S}}\right)^{2}+\rho_{\psi}\left(W_{\psi 1} \dot{\psi}_{\mathrm{S}}^{2}+W_{\psi 2} \psi_{\mathrm{S}}^{2}\right)+W_{\psi 3}\left(p_{\psi}-p_{\psi 0}\right)^{2}\right]
$$

\section{A.6 Washout Parameters}

For testing, the M1 manoeuvre is used with the AW1 parameter set AW1 from the Reid and Nahon report. The performance metrics are listed in Table 22 for the final 20 seconds of the manoeuvre comparing the results of the implemented adaptive washout algorithm with the results reported by Reid and Nahon [2].

The normalized Pearson correlation is close to 1 for all the degrees of freedom, which indicates that the results are closely correlated. Heave has the worst results for the normalized Pearson correlation, but the small integral of error and maximum error, as well as examining the vestibular response in Figure 41, indicate that both the original report and the simulated heave values are closely correlated, and the normalized Pearson correlation result is due to the signals remaining steady during the final 20 seconds of the manoeuvre, therefore small changes have a large impact 
on the results. Roll has a large integral of error and maximum error compared to the other degrees of freedom. However examining the vestibular response shows that it is closely correlated for the majority of the response and only deviates in the final 5 seconds of the manoeuvre.

For further discussion on adaptive washout, see Reid and Nahon's technical reports "Flight Simulation Motion-Base Drive Algorithms: Part 1 - Developing and Testing the Equations" and "Part 2 - Selecting the System Parameters" [1,2].
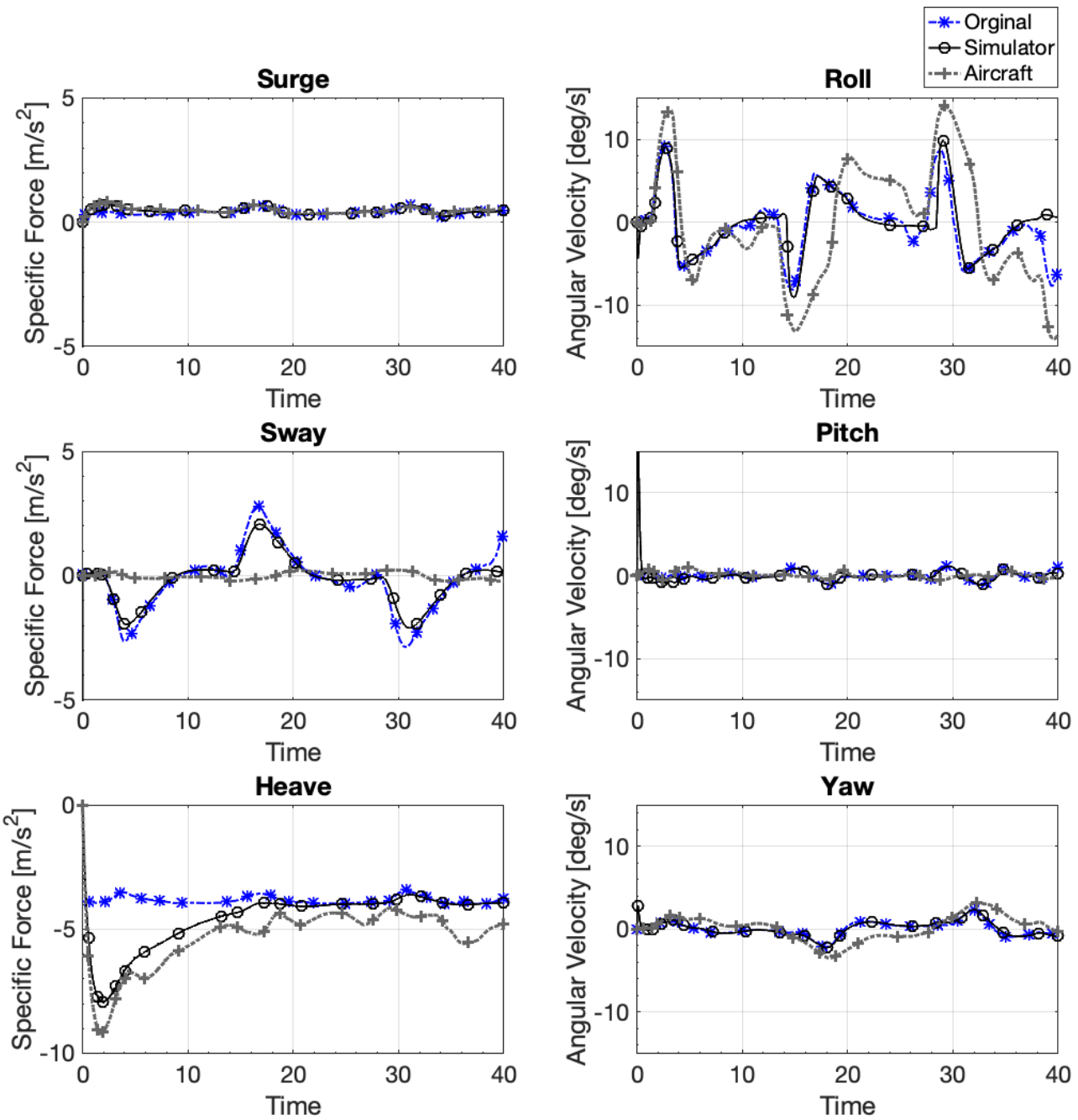

Figure 41: AW1 M1 vestibular response from original report and current model. 
Table 22: Adaptive washout performance metrics.

\begin{tabular}{ccccc} 
Case & $\begin{array}{c}\text { Degree of } \\
\text { Freedom }\end{array}$ & $\begin{array}{c}\text { Normalized Pearson } \\
\text { Correlation }\end{array}$ & Integral of Error & Maximum Error \\
\hline \hline \multirow{5}{*}{ AW1 M1 } & Surge & 1.00 & $0.36 \mathrm{~m} / \mathrm{s}^{2}$ & $0.04 \mathrm{~m} / \mathrm{s}^{2}$ \\
& Sway & 1.00 & $6.43 \mathrm{~m} / \mathrm{s}^{2}$ & $1.55 \mathrm{~m} / \mathrm{s}^{2}$ \\
& Heave & 1.08 & $2.55 \mathrm{~m} / \mathrm{s}^{2}$ & $0.44 \mathrm{~m} / \mathrm{s}^{2}$ \\
& Roll & 1.05 & $31.90 \mathrm{deg} / \mathrm{s}$ & $8.58 \mathrm{deg} / \mathrm{s}$ \\
& Pitch & 1.01 & $2.93 \mathrm{deg} / \mathrm{s}$ & $0.77 \mathrm{deg} / \mathrm{s}$ \\
& Yaw & 1.00 & $3.62 \mathrm{deg} / \mathrm{s}$ & $0.54 \mathrm{deg} / \mathrm{s}$
\end{tabular}




\section{B Small-angle Washout Test Cases}

This appendix includes graphs for the small-angle washout test cases, including the aircraft motion set points and the simulator motion. 


\section{B.1 M1}

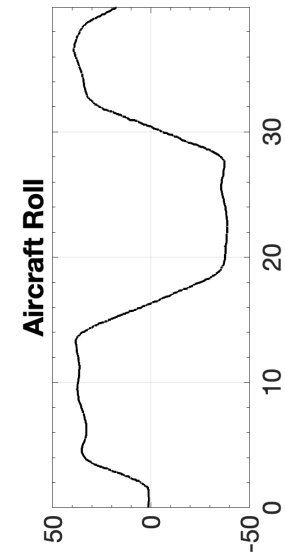

[бәр] uo!!!

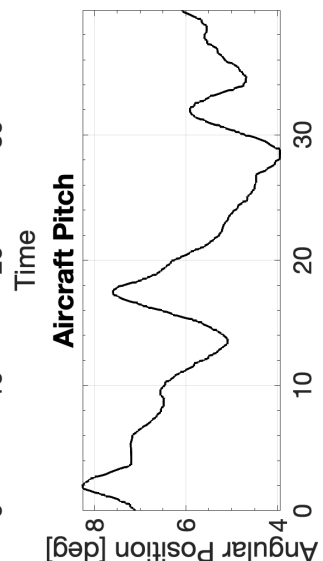

[6әр] uo!!! sod גe|n6u

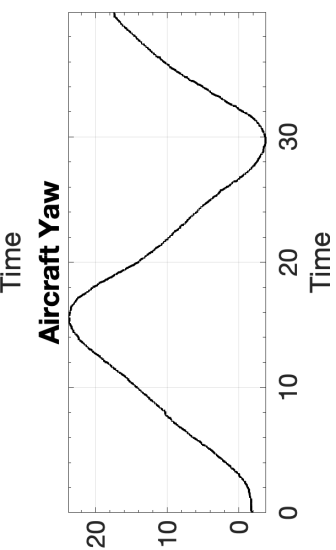

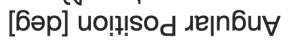

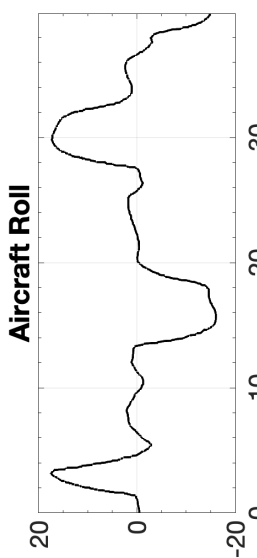

[s/бәр] หұ! оорл

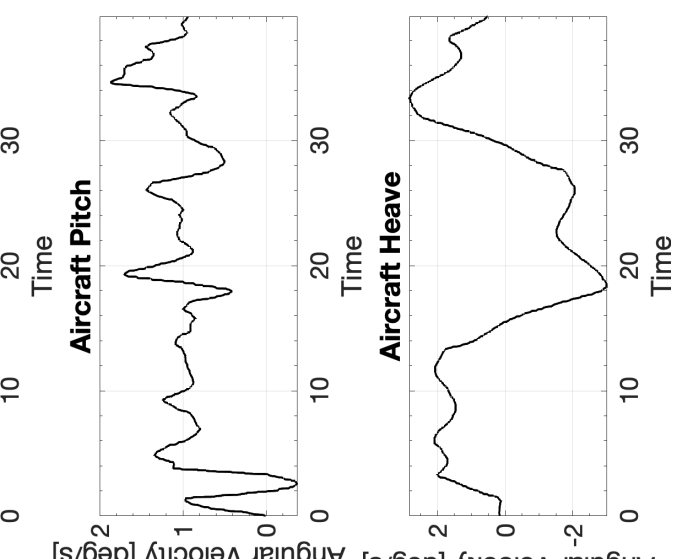

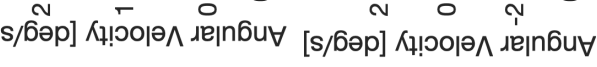

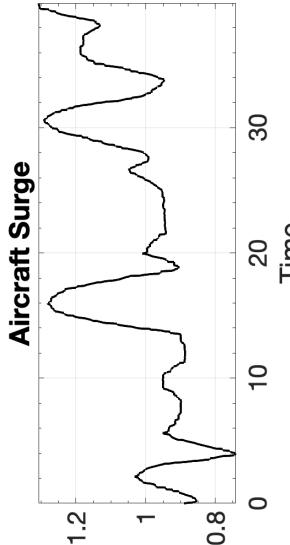

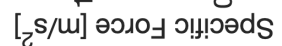

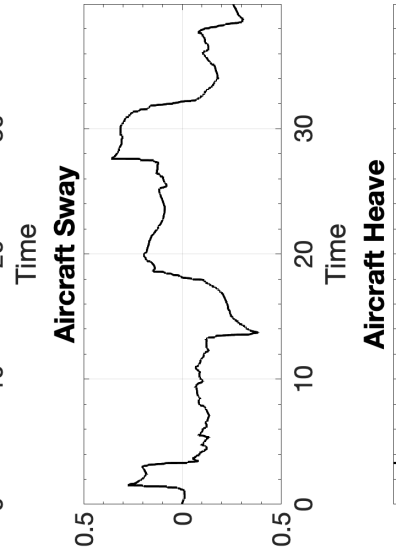

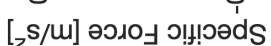

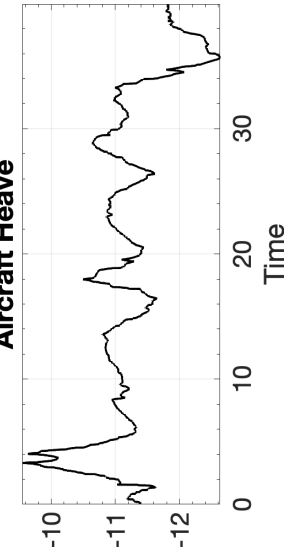

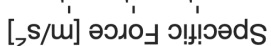
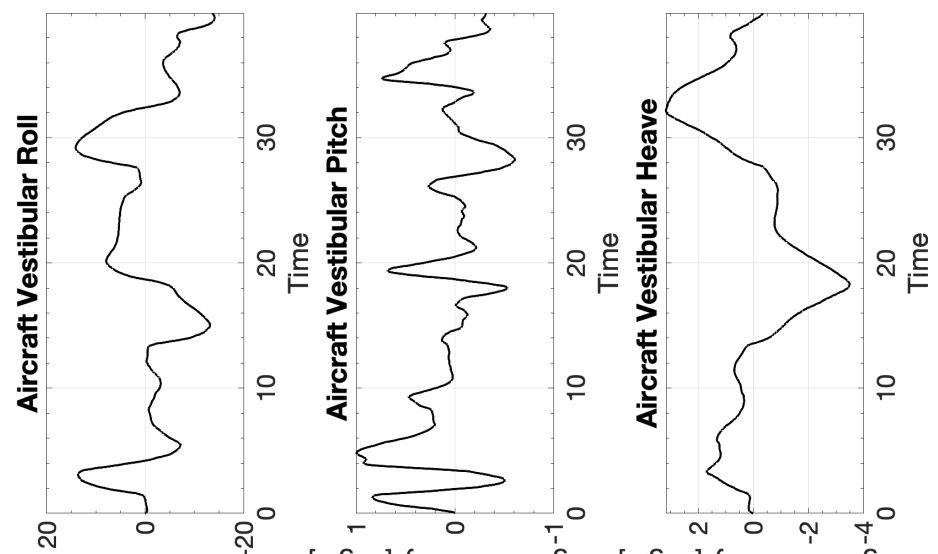

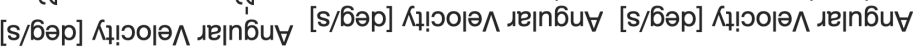

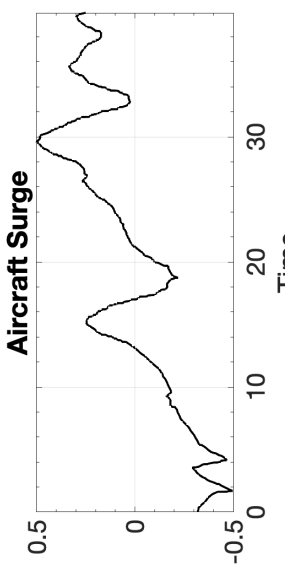

[s/u] uо!ฺвдә|әэว

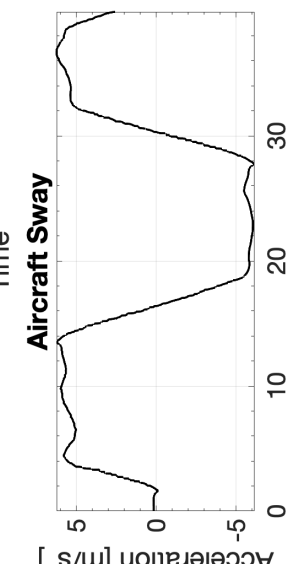

[

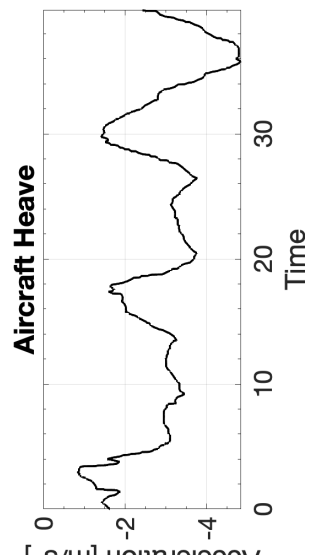

[

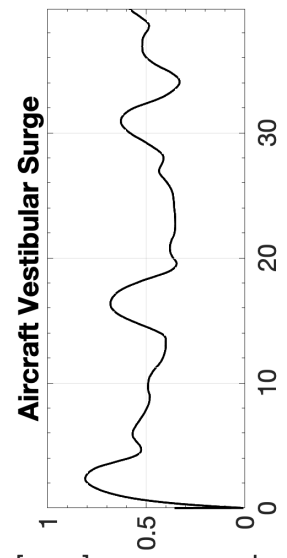

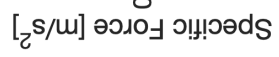

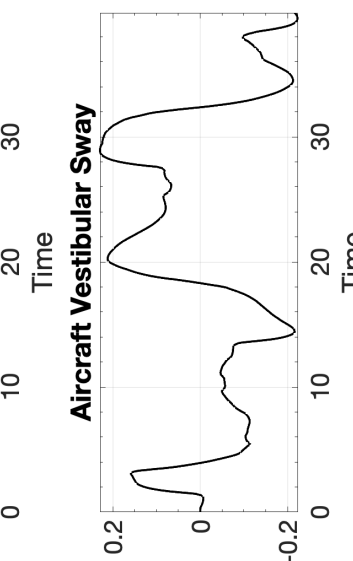

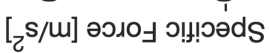

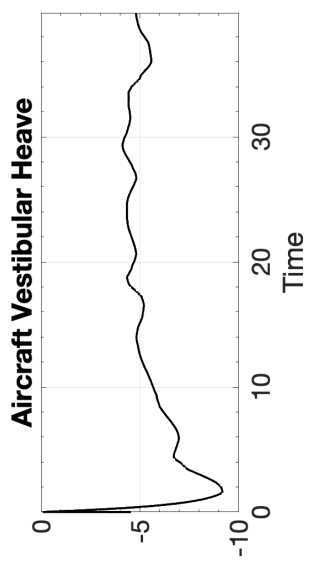

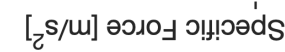




\section{B.1.1 CW1 M1}

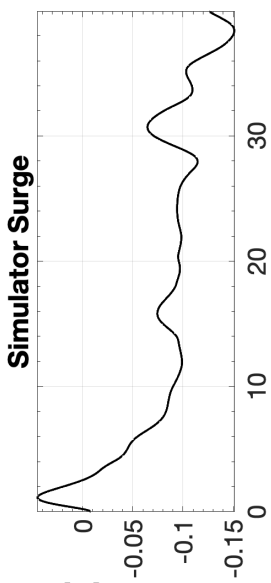

[u] uo!!!!sod

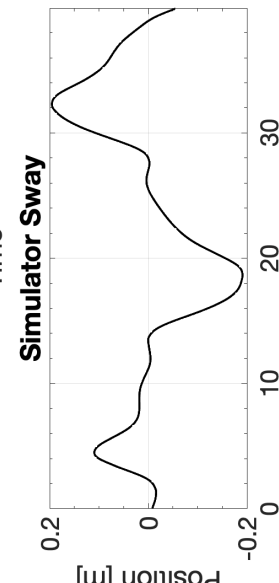

[w] uoln!sod

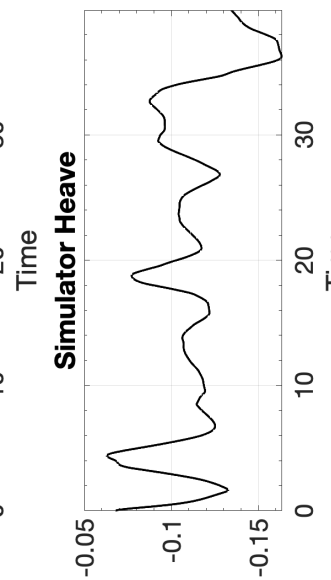

[m] uol!!sod

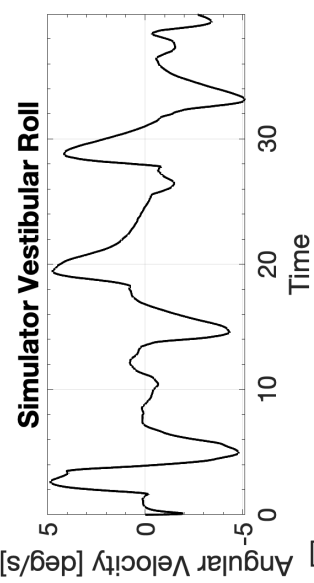

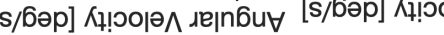
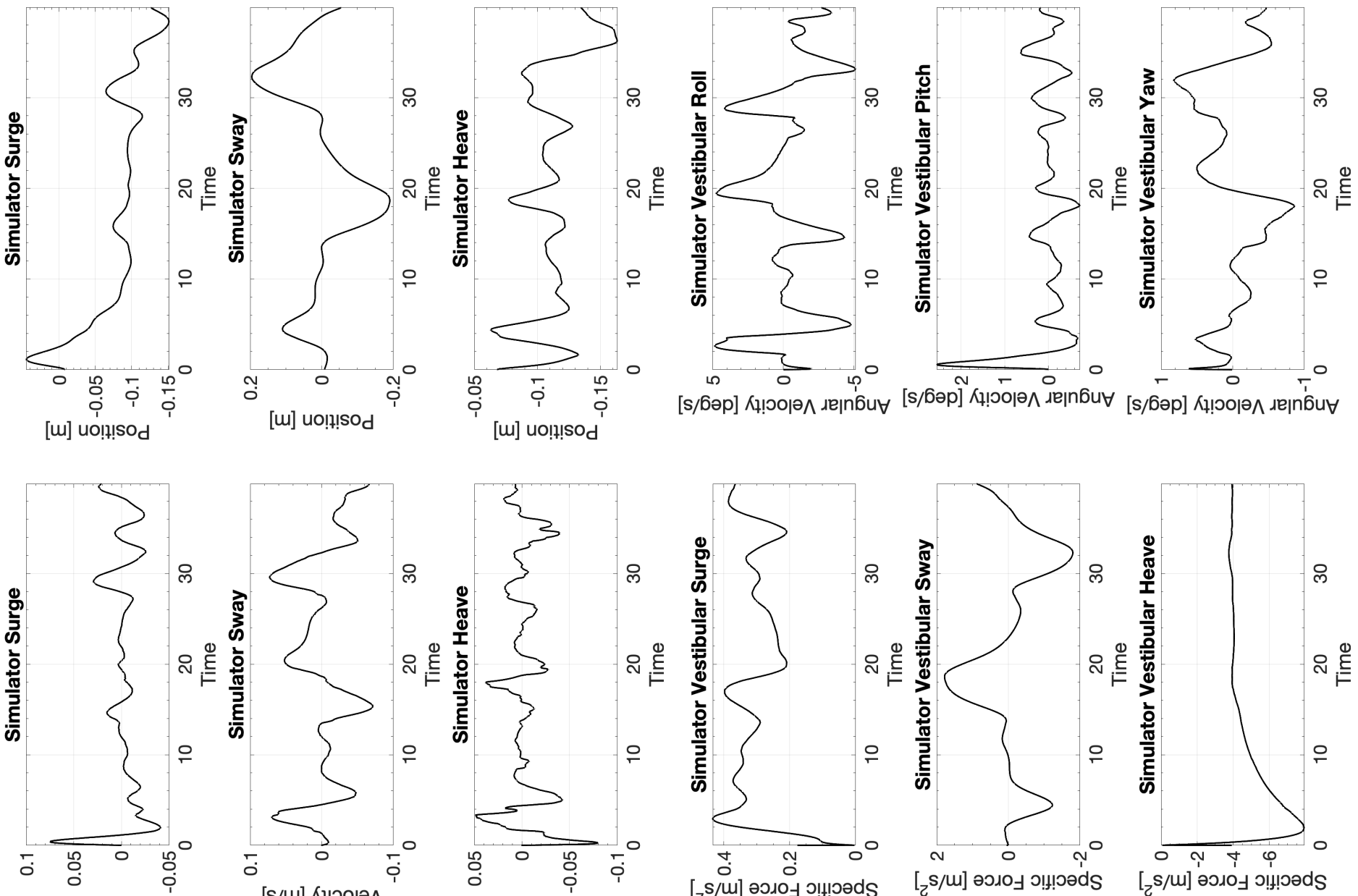

[s/u] К! ৩о夫 ^

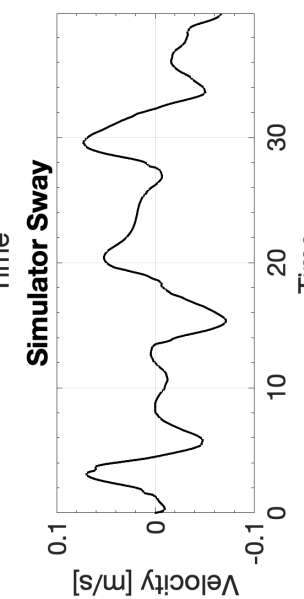

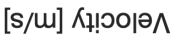
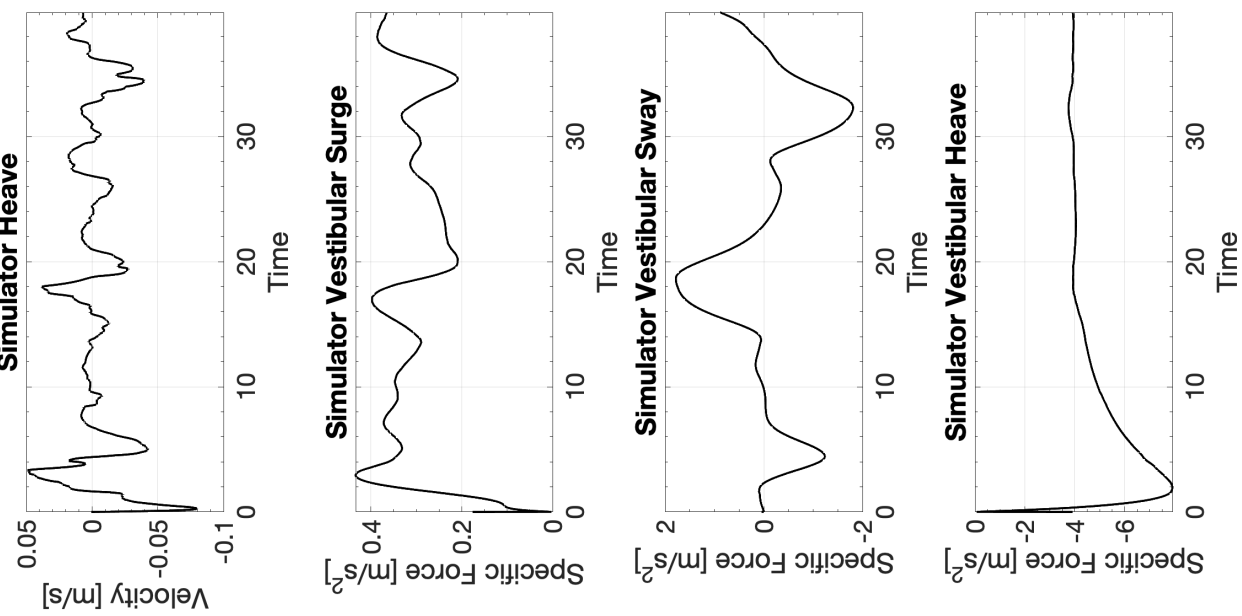

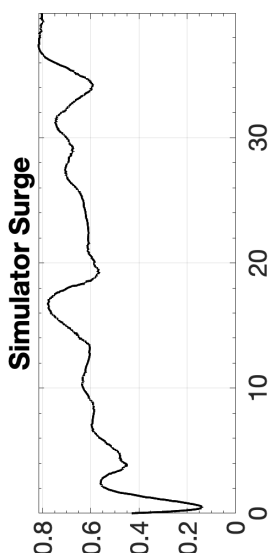

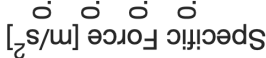
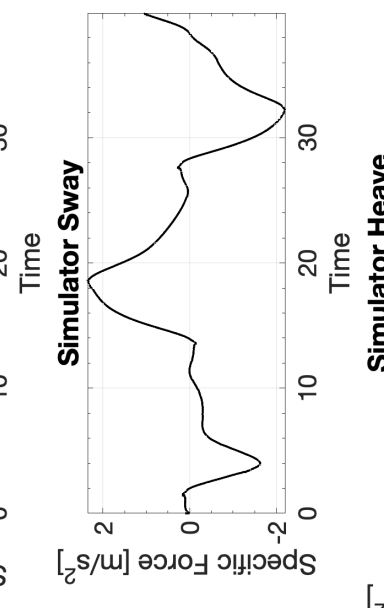

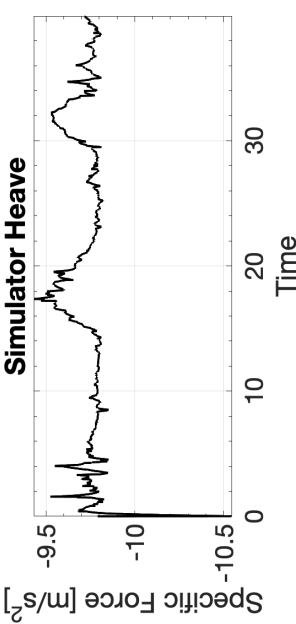

이
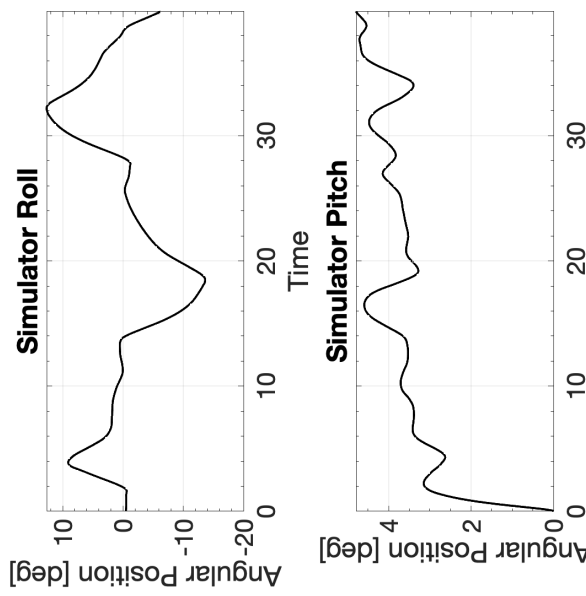

[6əp] uo!!!sod גe|n6u

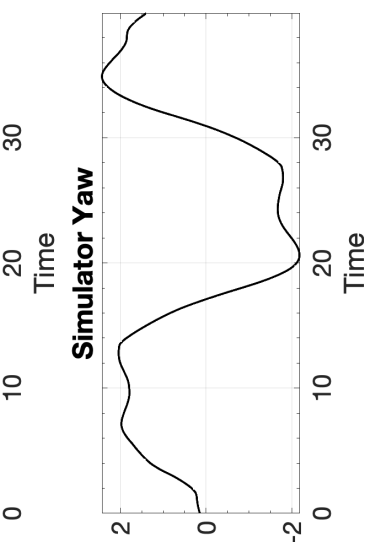

[бәр] uo!!!sod גeןn'́u

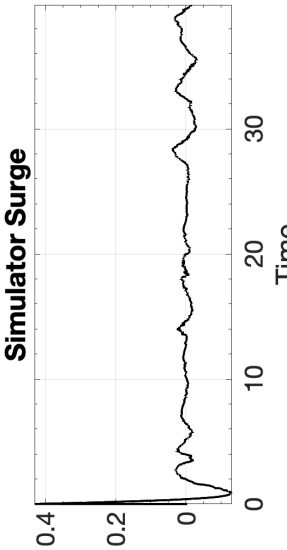

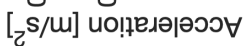

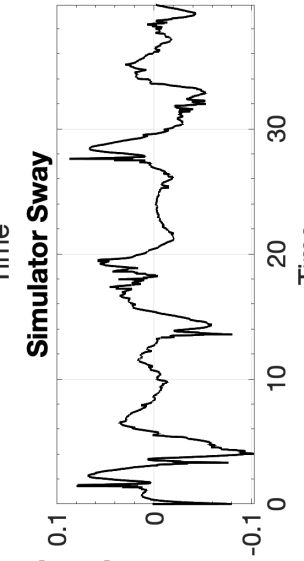

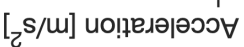

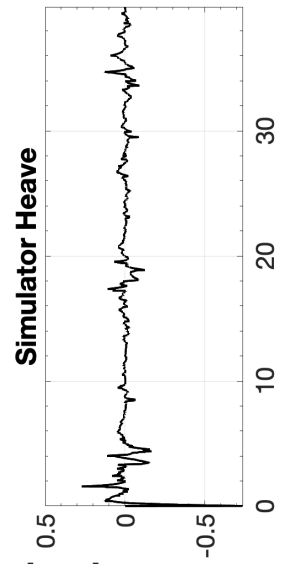

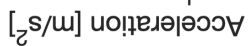

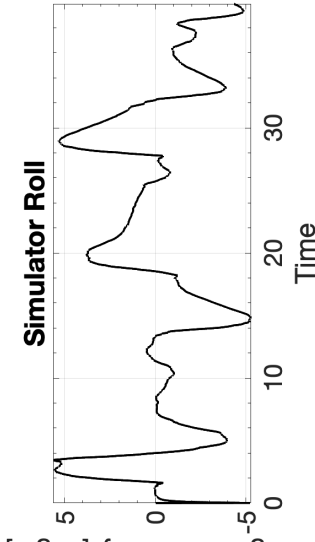

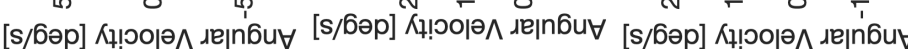




\section{B.1.2 CW2 M1}

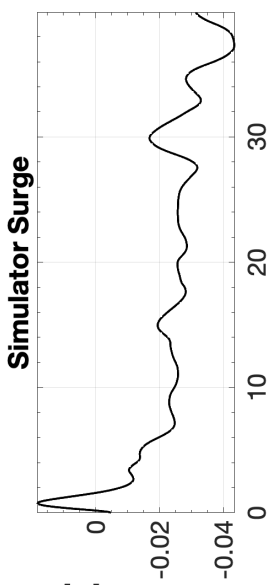

[u] uol!! sod

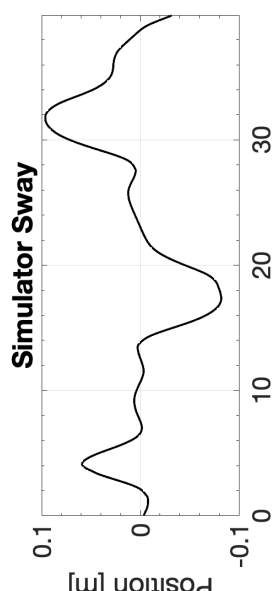

[w] uo!n! $\mathrm{sod}^{\mathrm{d}}$

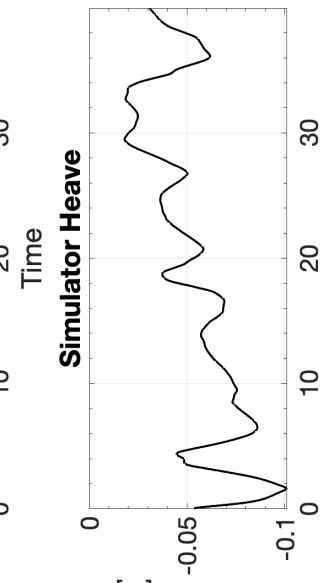

[u] uo!n!

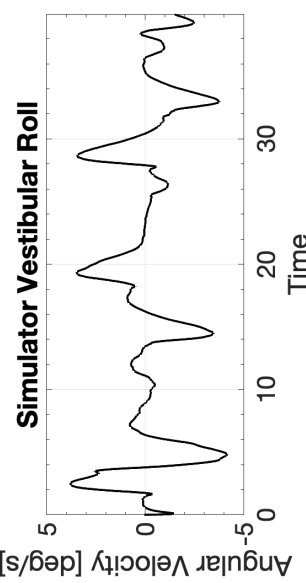

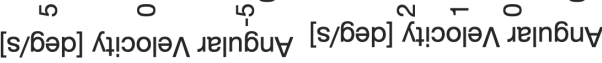

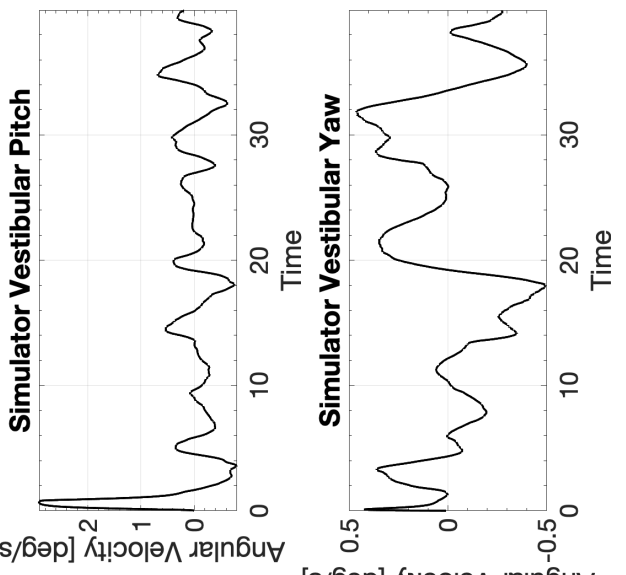

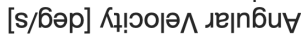

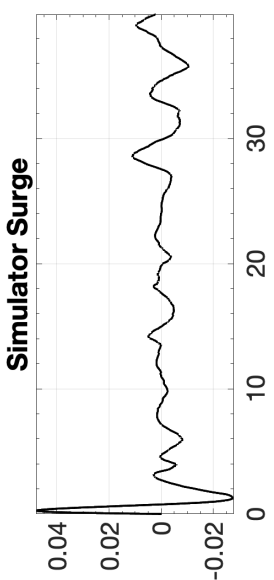

[s/u] К! ৩о夫

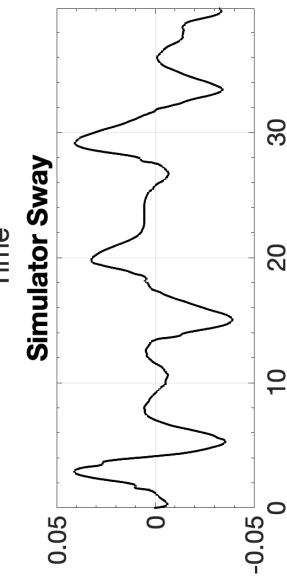

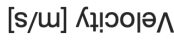

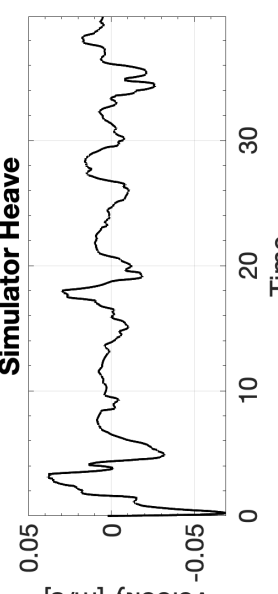

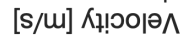
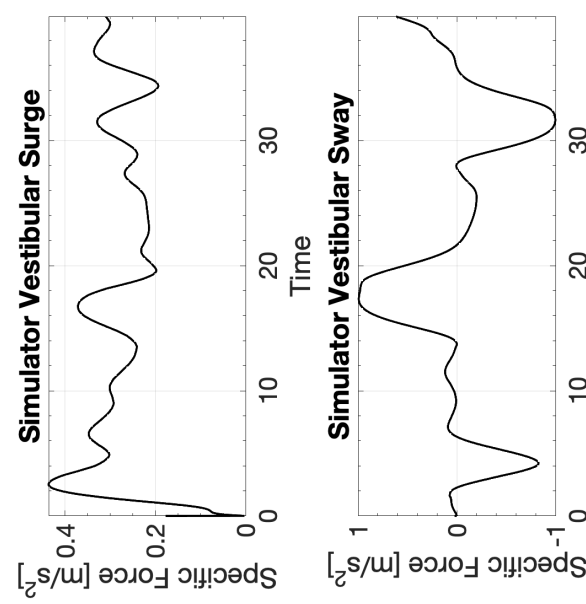

-2 o $T^{2}$

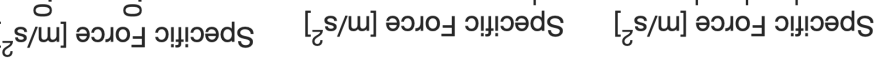

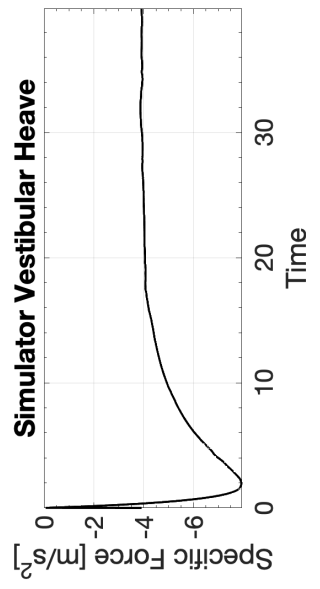

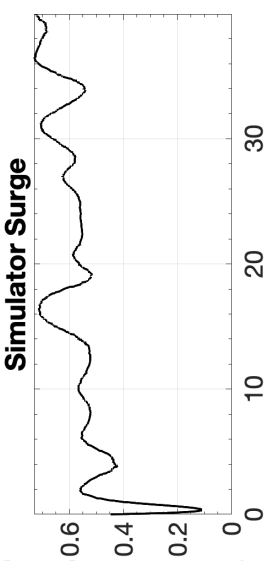

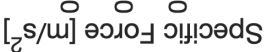

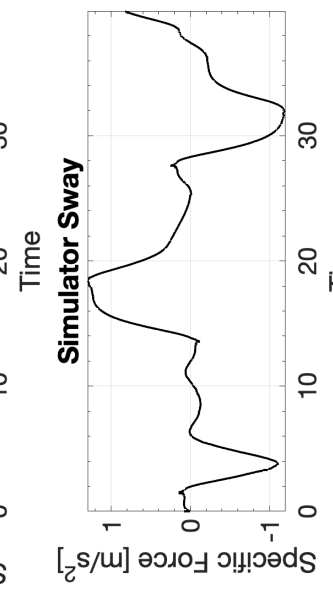

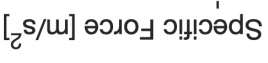

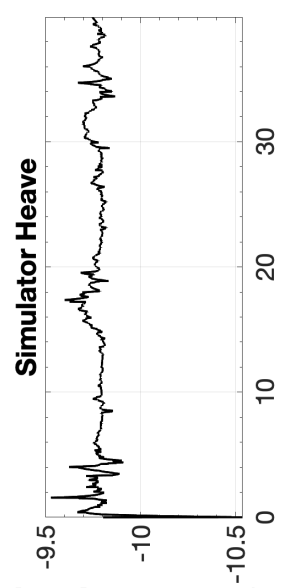

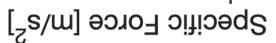

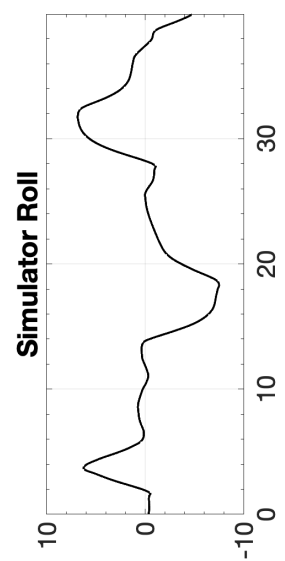

[6әр] uo!!!sod de|n6ư

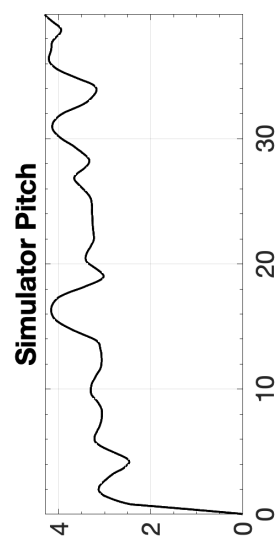

[бəp] uo!!!sod

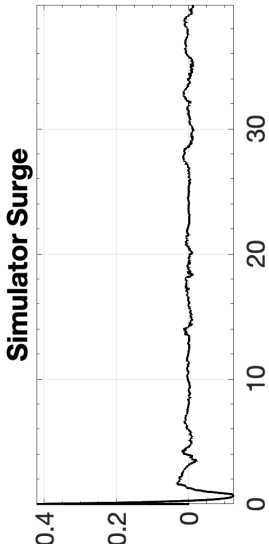

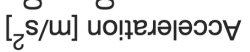

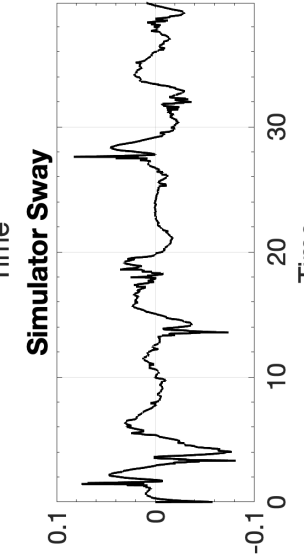

$[\mathrm{s} / \mathrm{L}]$ uо!ןедәәәэว

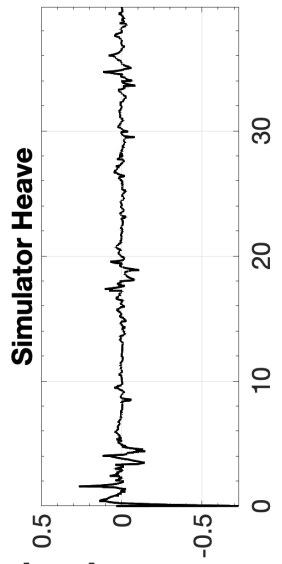

$\left[{ }_{2} \mathrm{~s} / \mathrm{M}\right]$ นо!ฺеләрәэว $\forall$

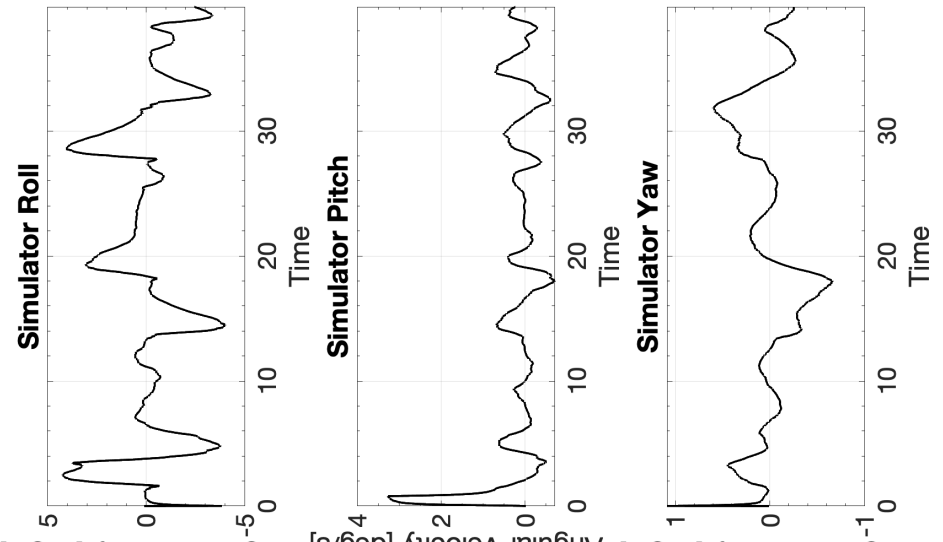

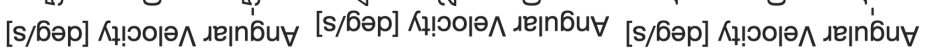




\section{B.1.3 CW3 M1}

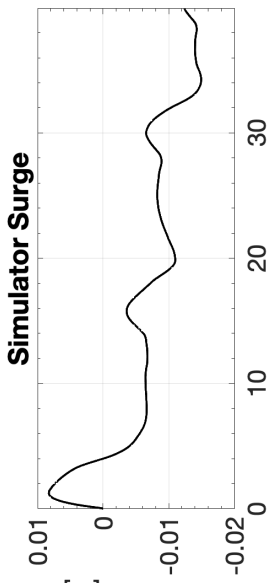

[w] uo!n!sod

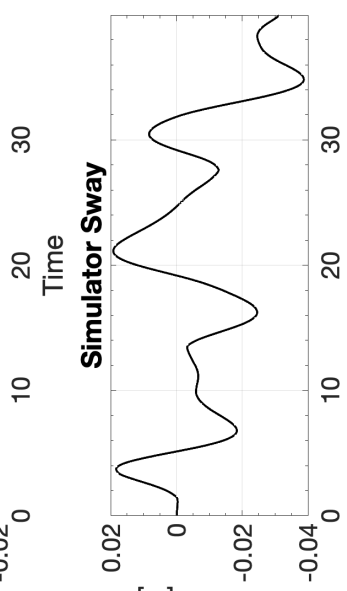

[u] uo!n!?sod

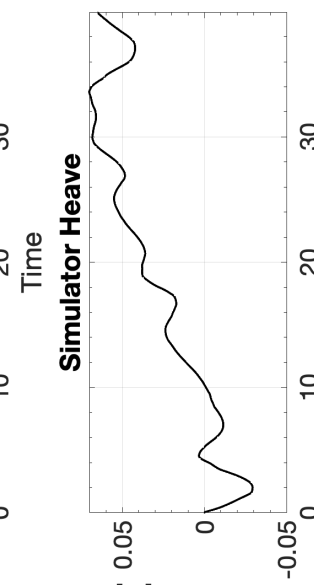

[w] uoli!nsod

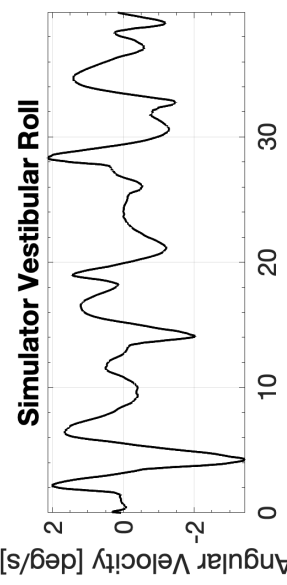

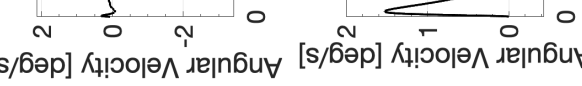

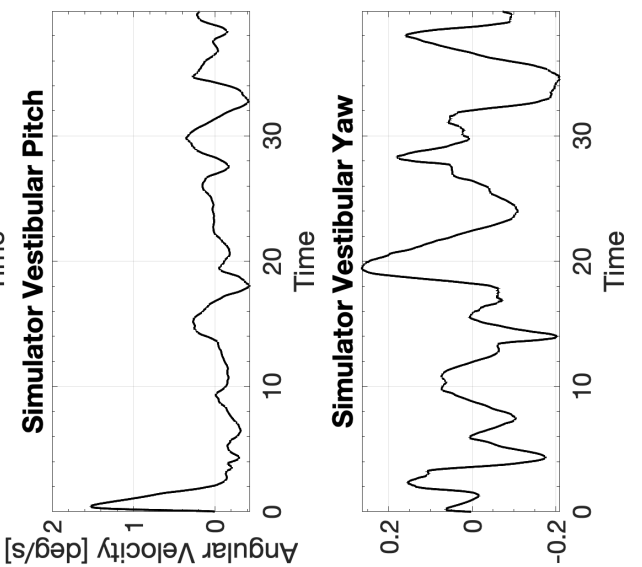

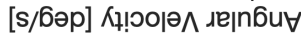

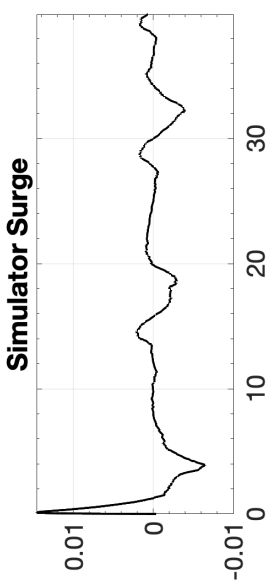

[s/u] Кц!юојә^

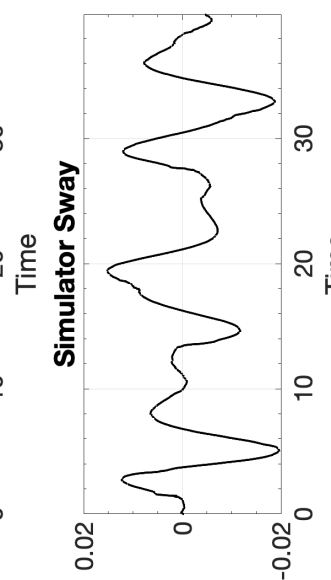

[s/u] Кұ!юо|ә^

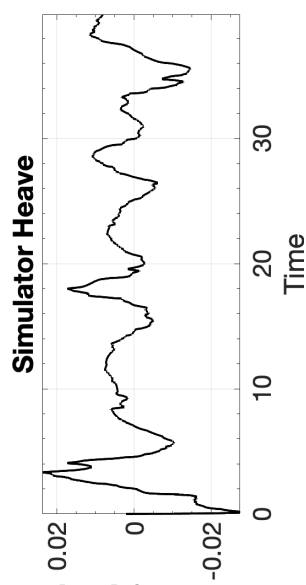

[s/m] К!о夫

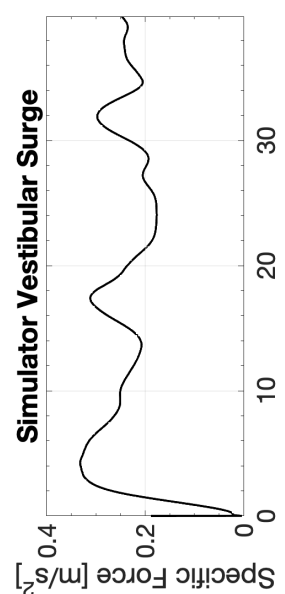

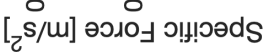

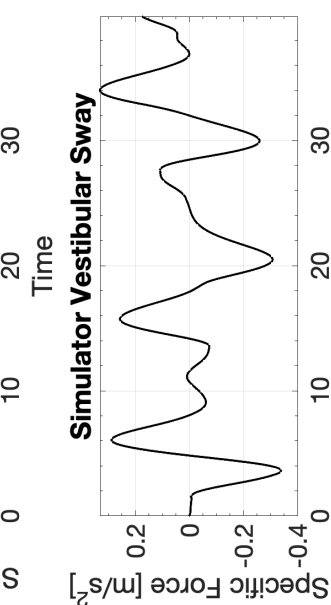

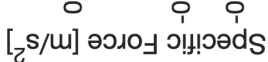
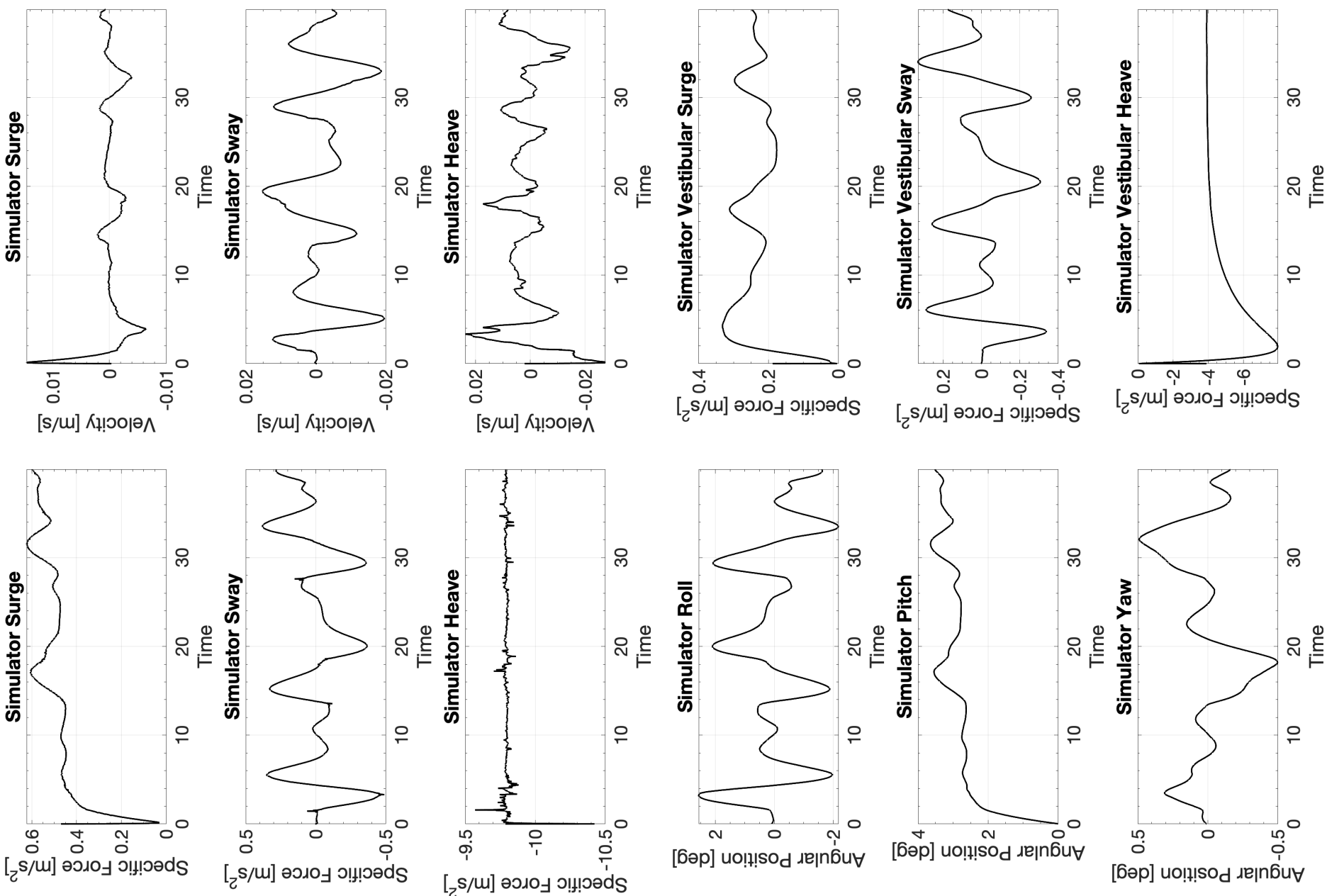

Bopilomend antion

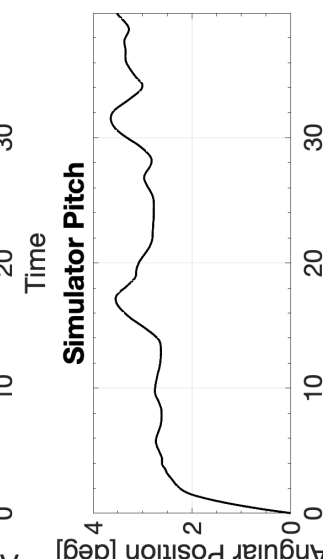

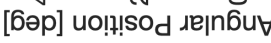

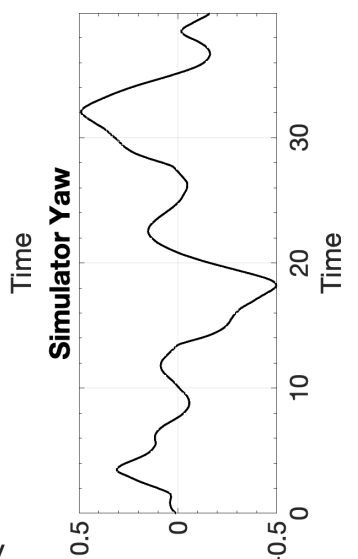

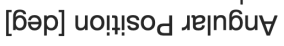

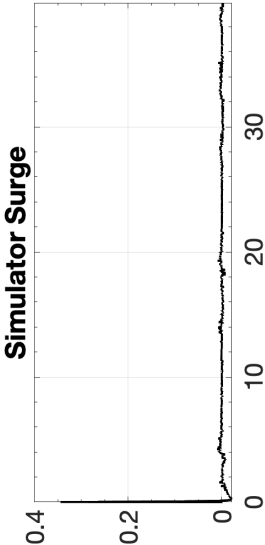

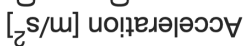

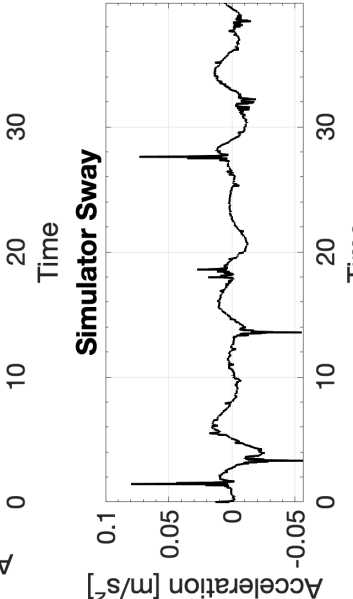

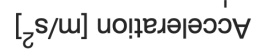

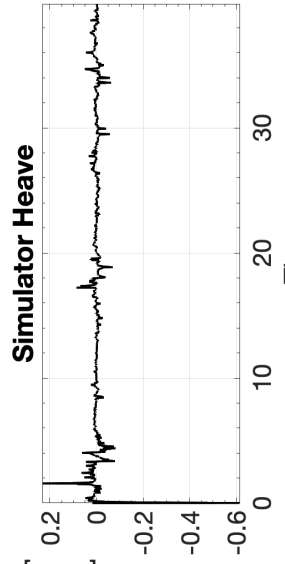

[zs/m] ио!ฺвдәәәэว

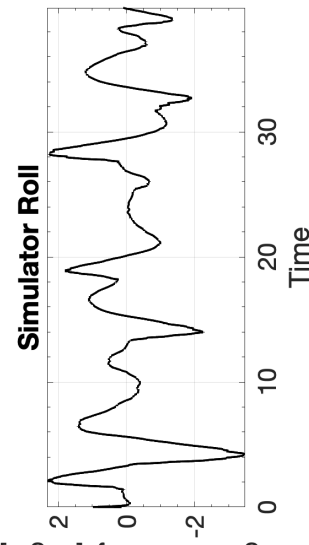

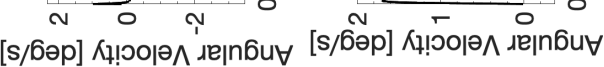
126 


\section{B.2 M2}
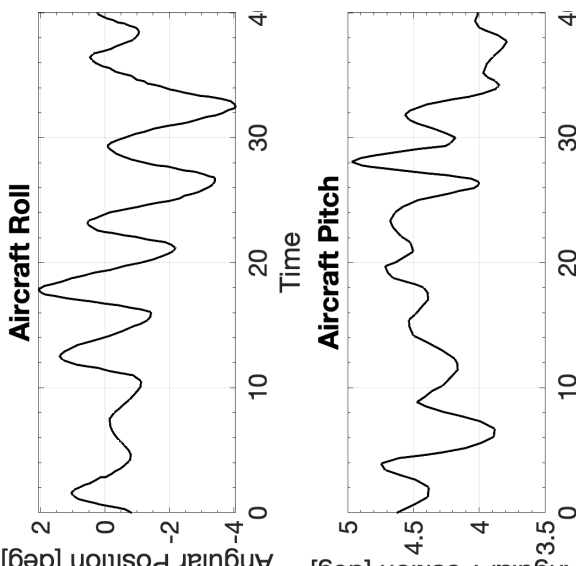

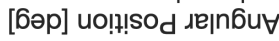

[6әр] uo!̣!sod גe|nติ
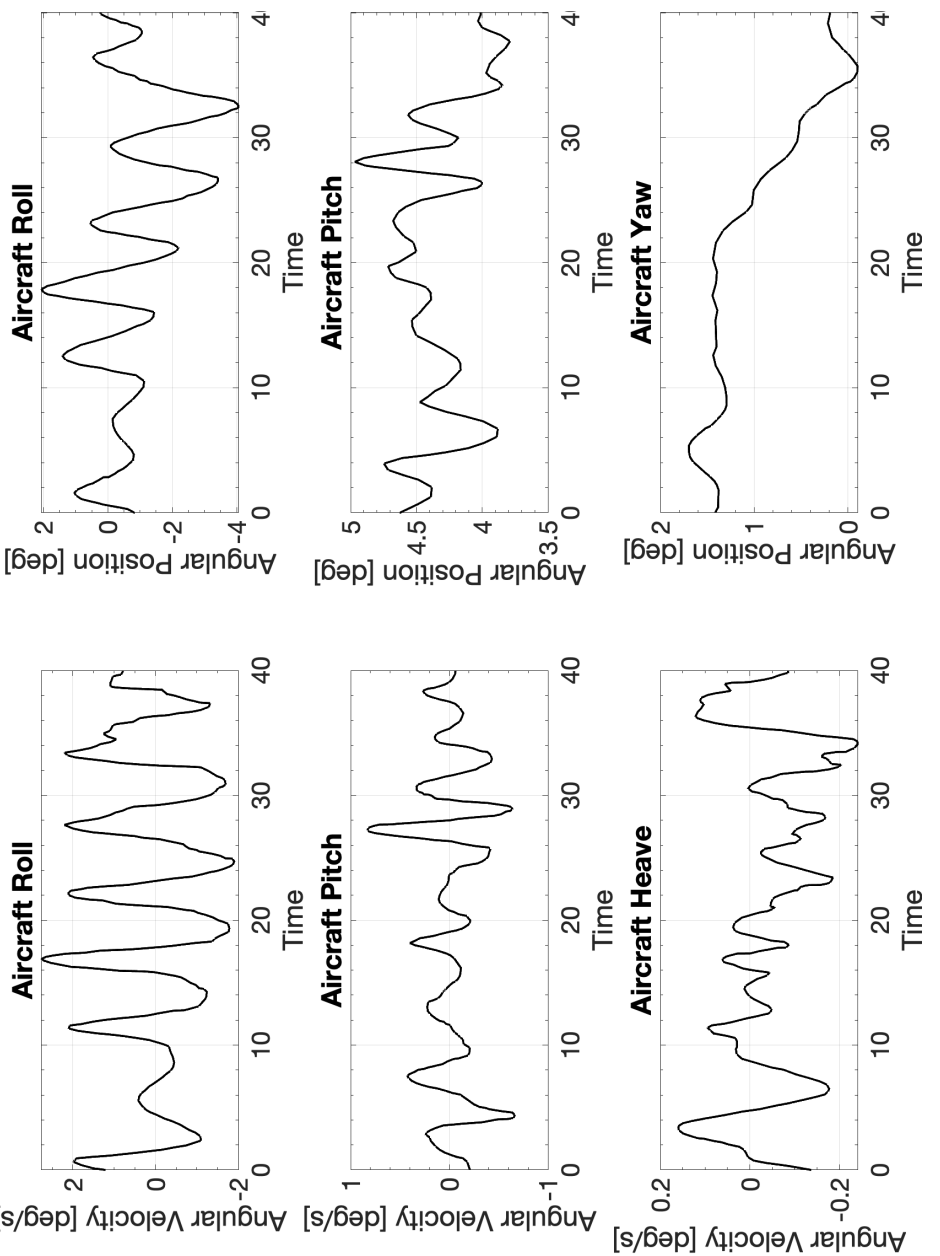

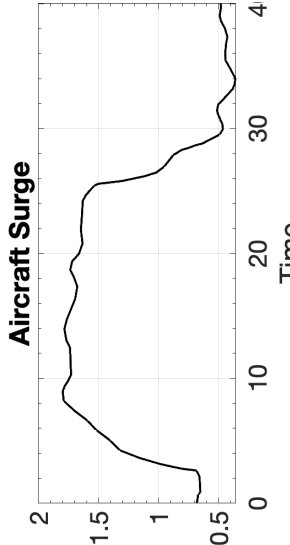

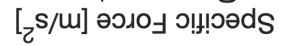

จ

음
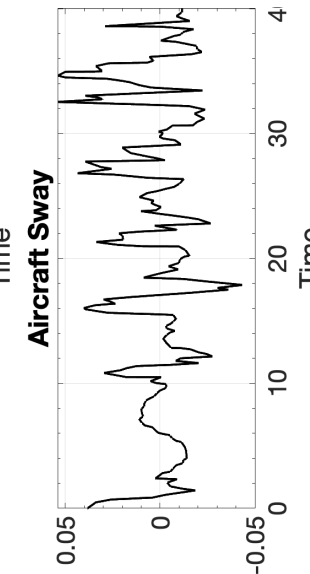

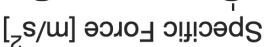
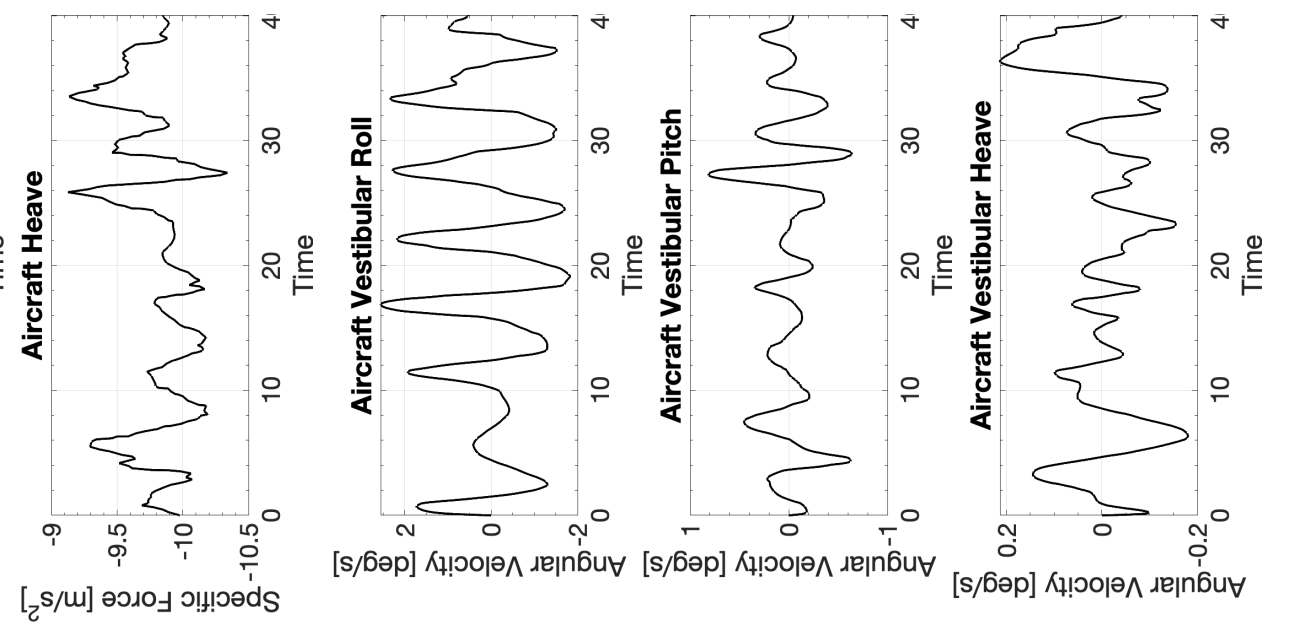

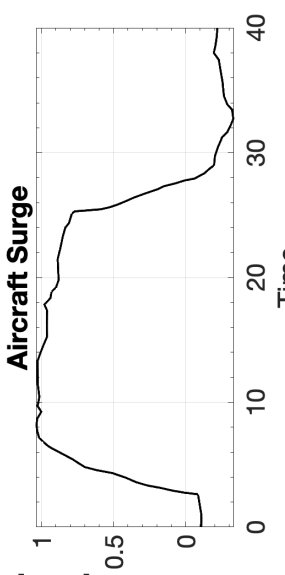

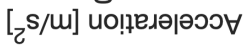

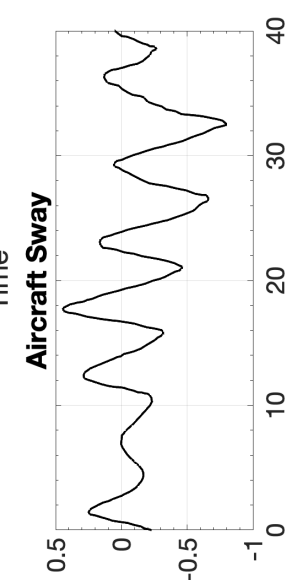

[zs/m] ио!ฺедәәәэว

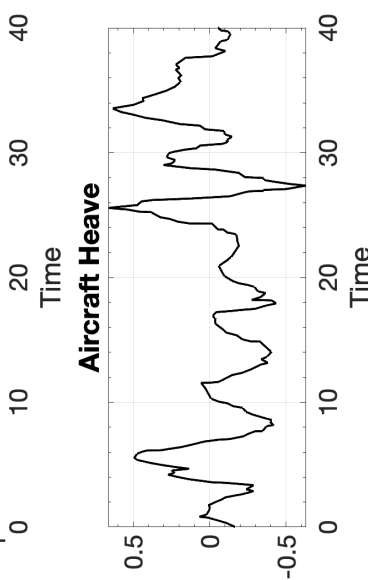

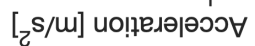

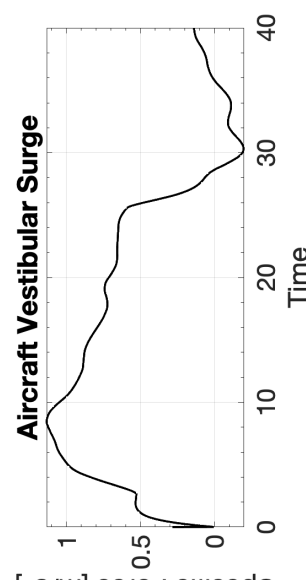

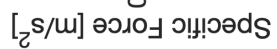

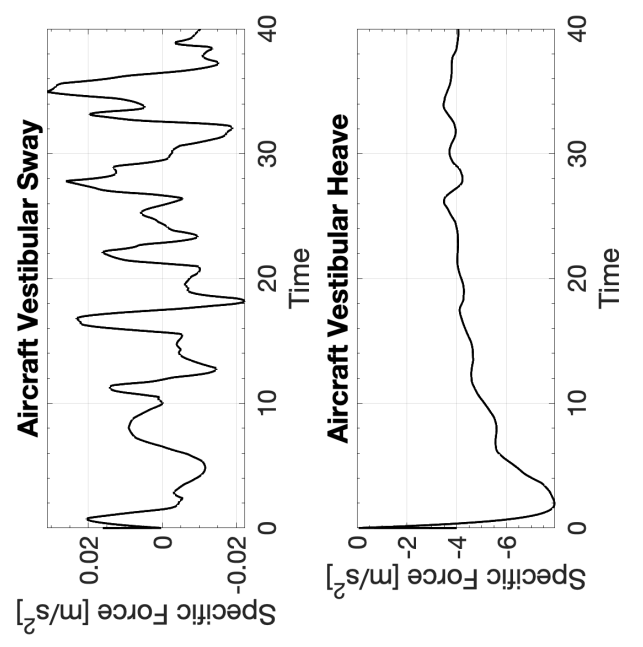




\section{B.2.1 CW1 M2}

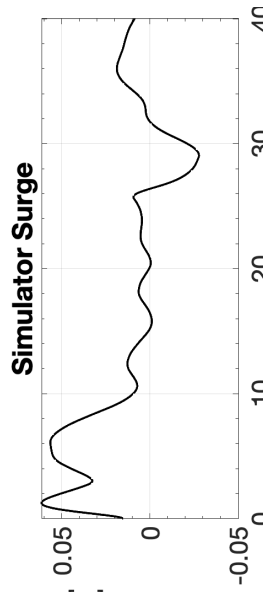

[u] uo!!! $\mathrm{sod}_{\mathrm{d}}$

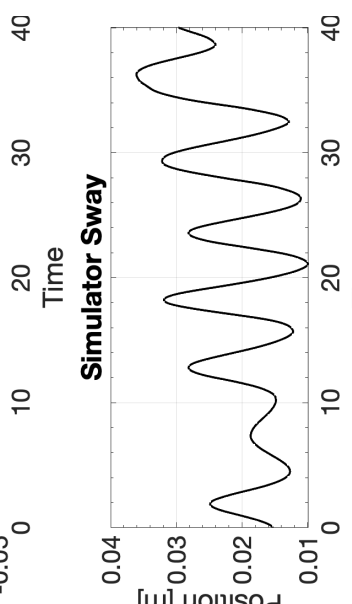

[w] uo!n!s $\mathrm{SOd}^{\mathrm{d}}$

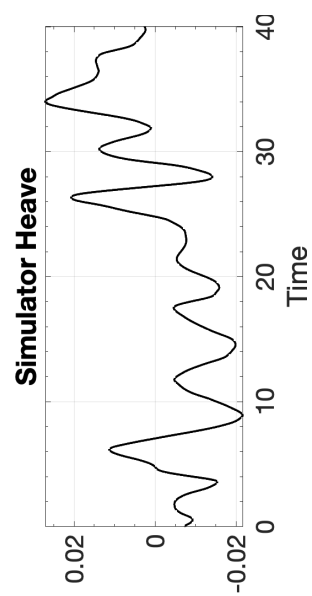

[u] uol!!!sod

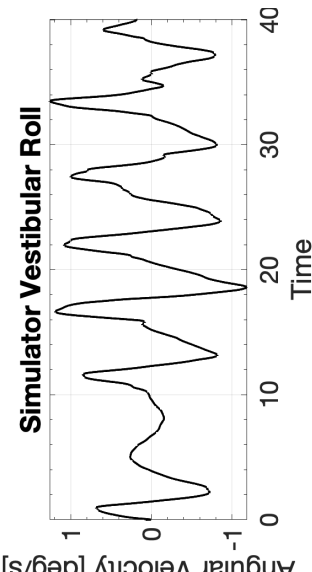

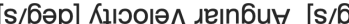

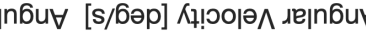

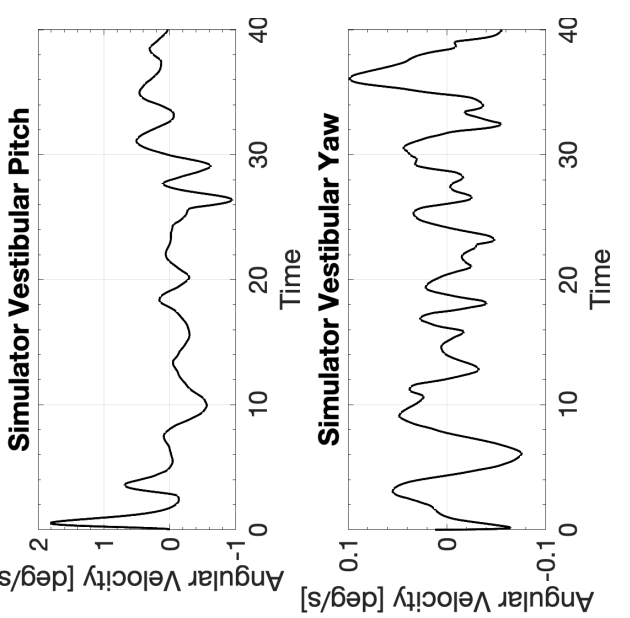

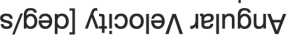

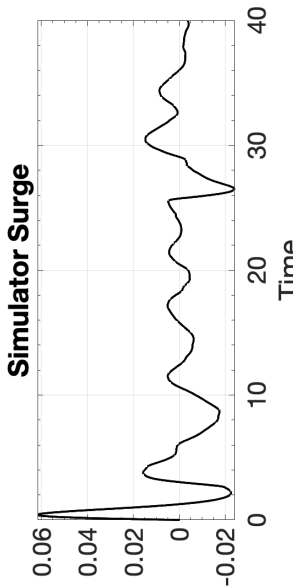

[s/u] К!ำю^

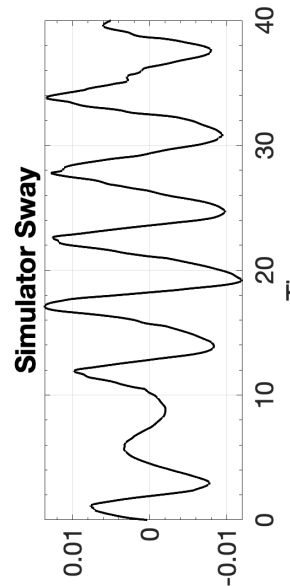

[s/m] К! Юо|ә^
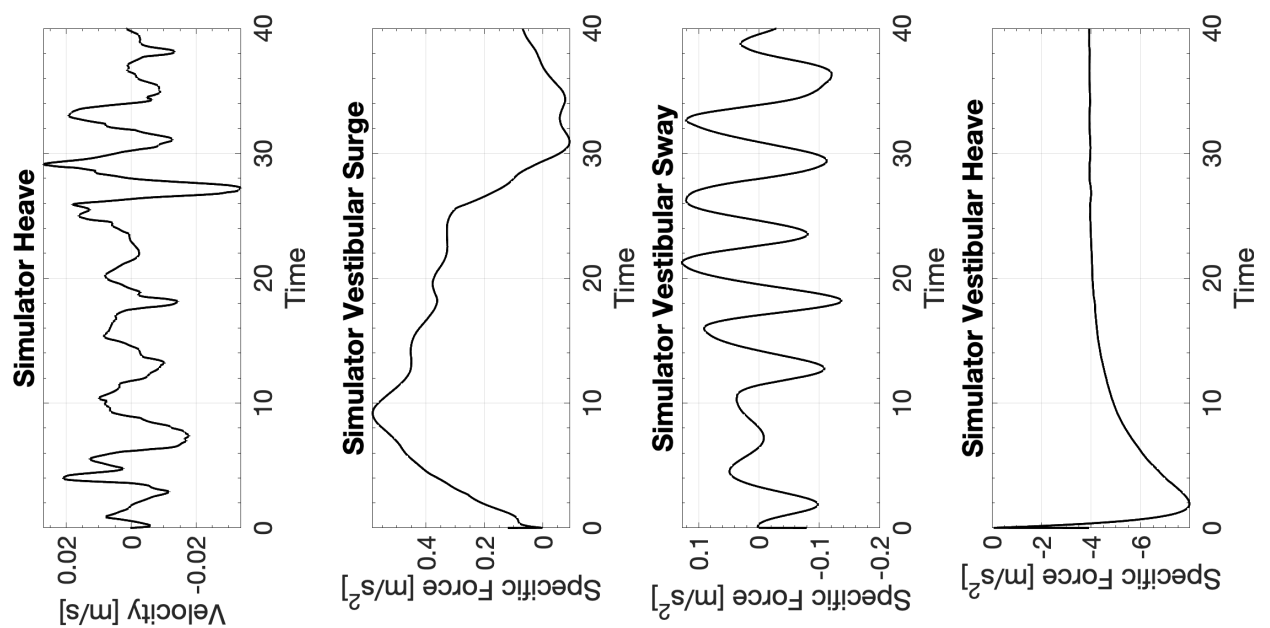

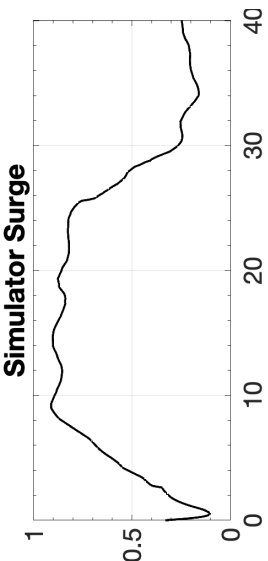

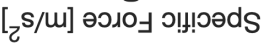

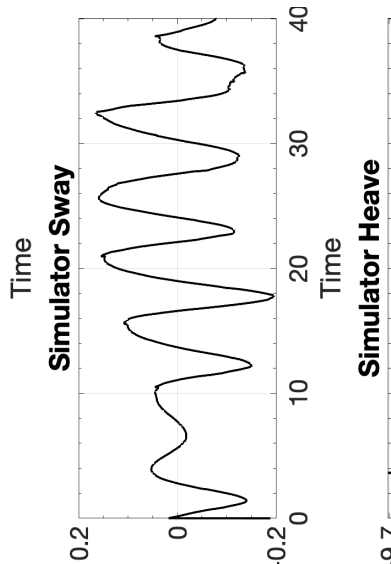

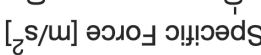

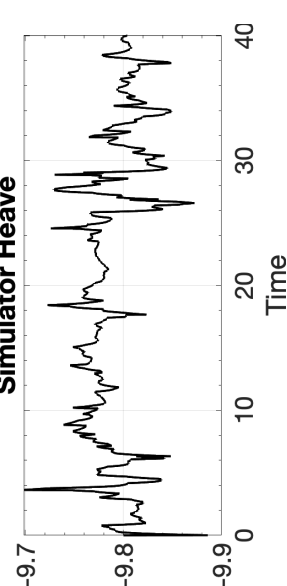

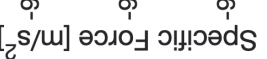

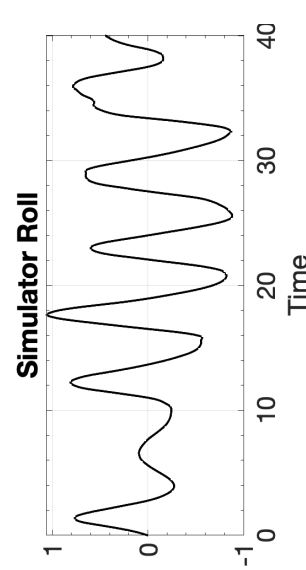

[бәр] uo!!! sod

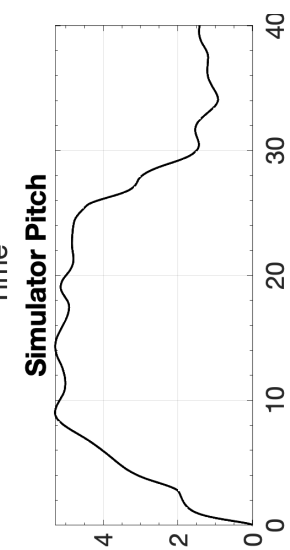

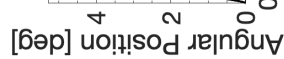

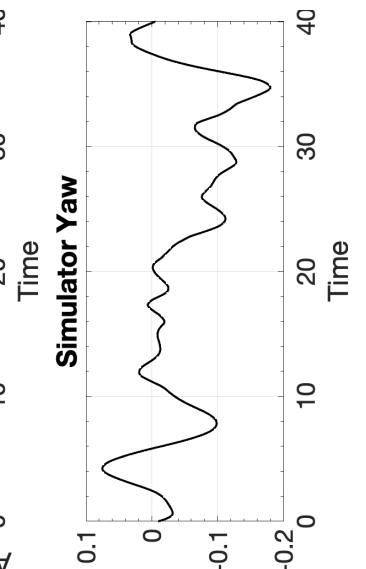

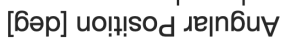

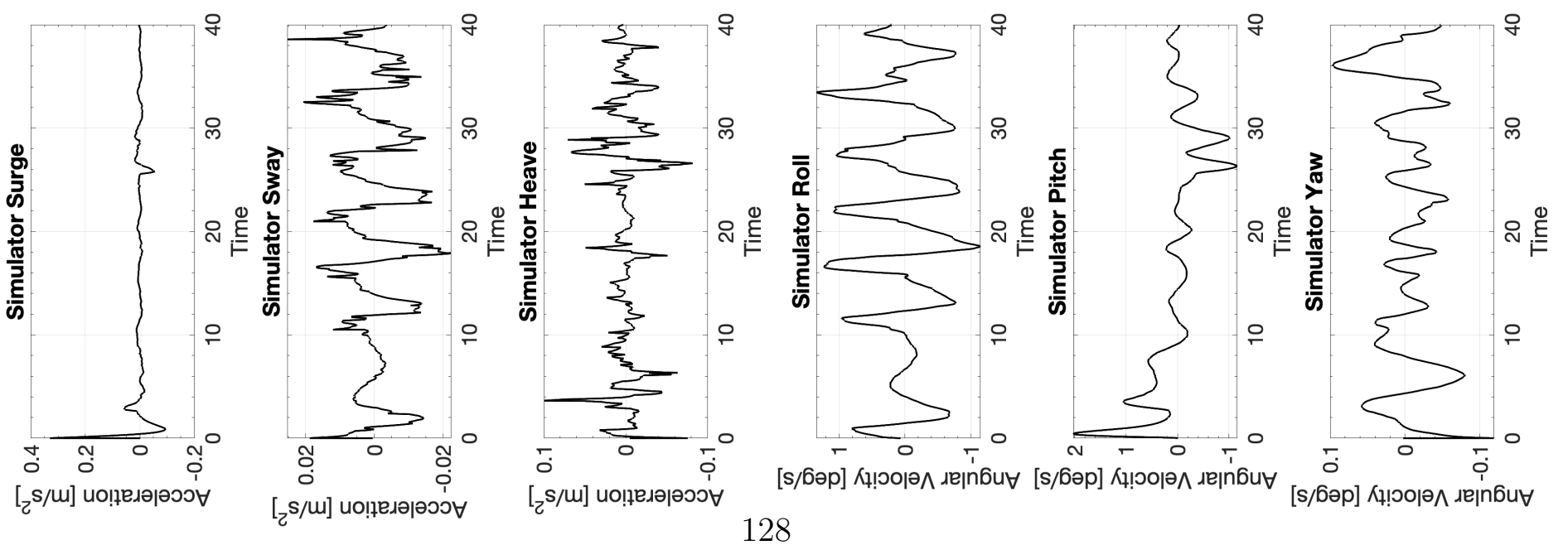




\section{B.2.2 CW2 M2}
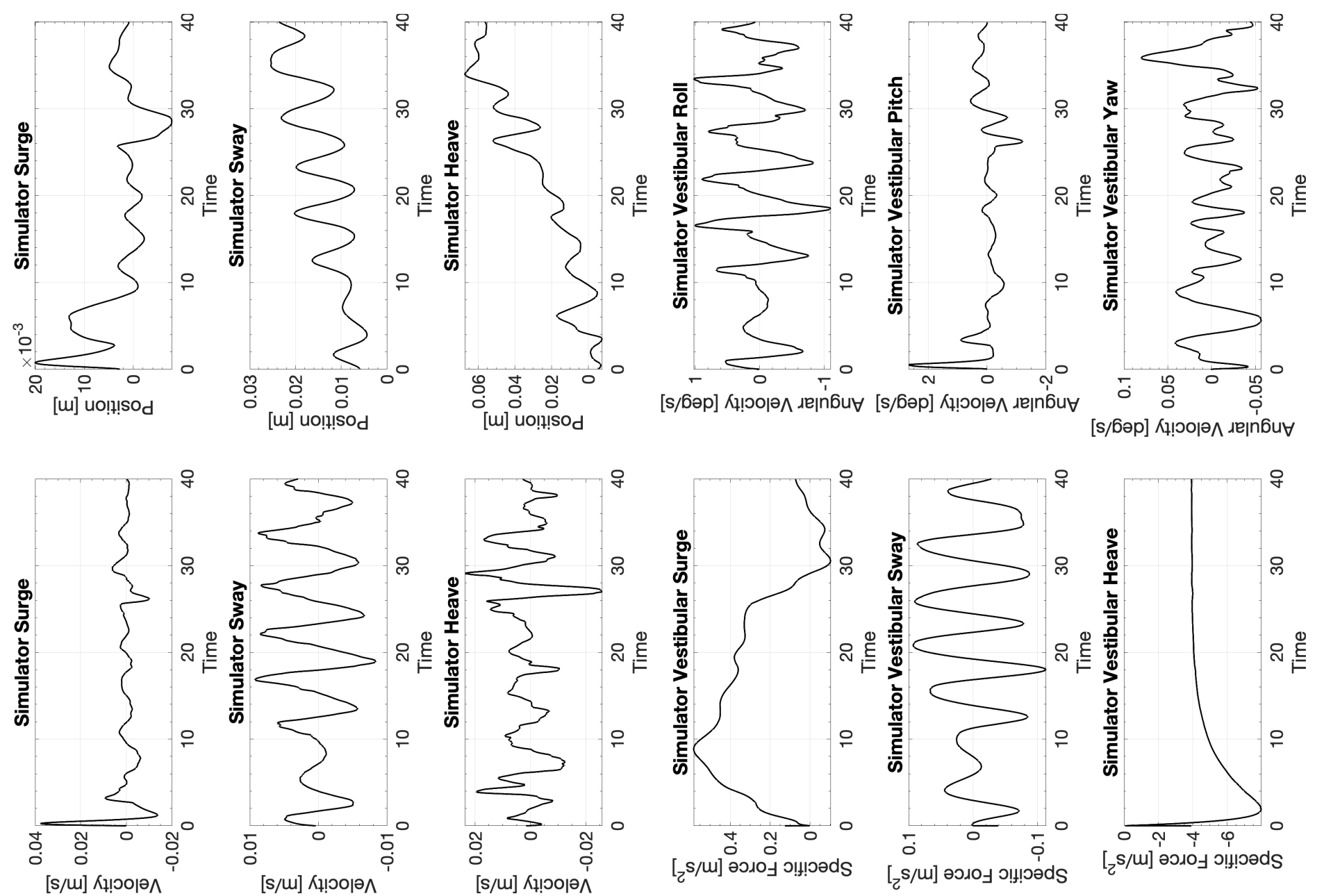

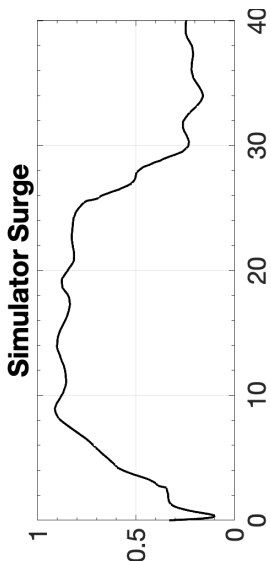

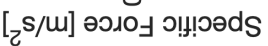

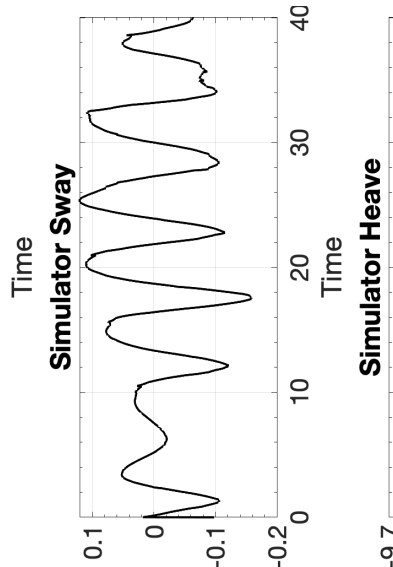

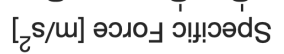

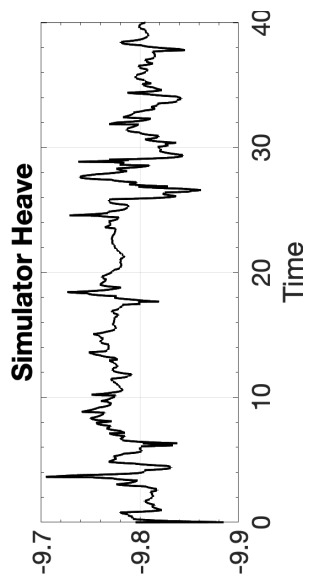

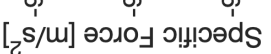

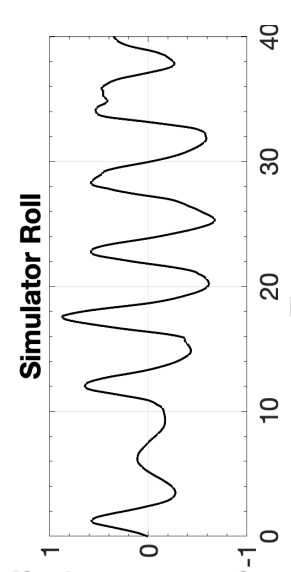

[6әр] uo!!?

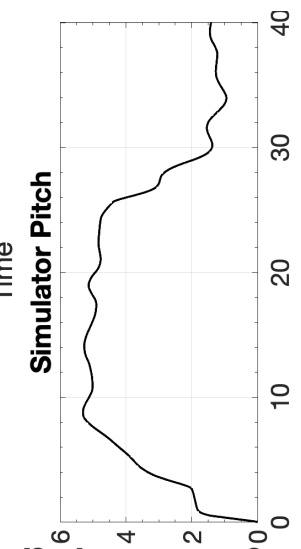

[ดว

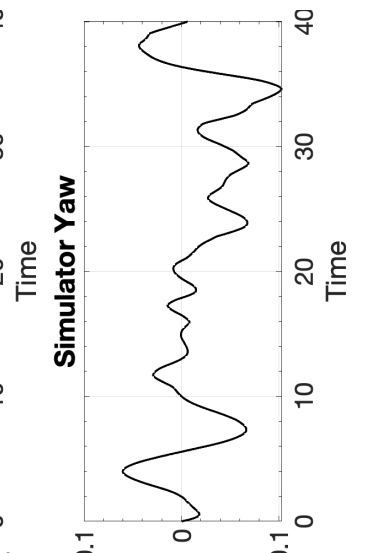

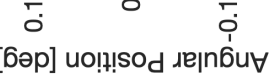

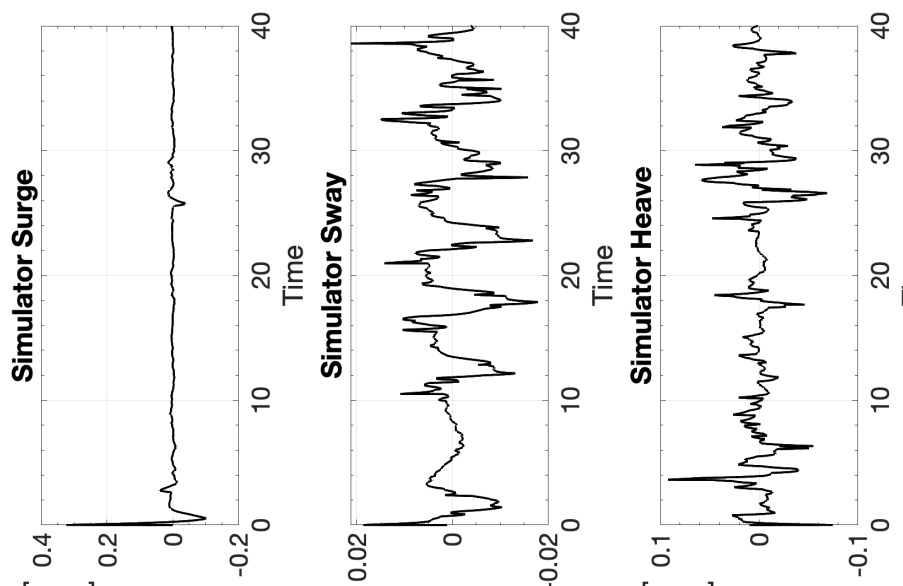

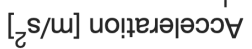

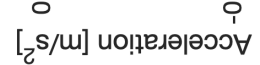

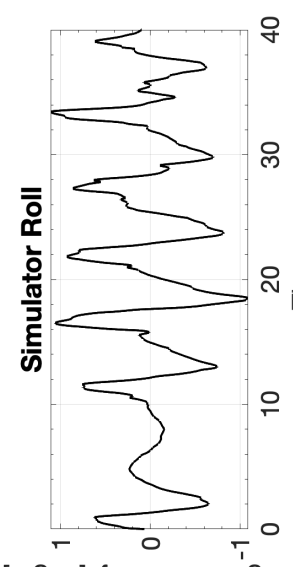

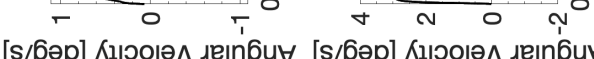

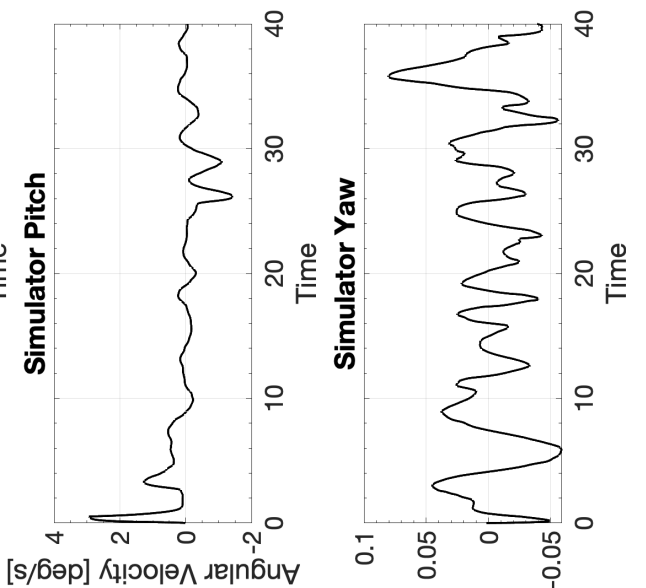

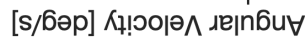




\section{B.2.3 CW3 M2}

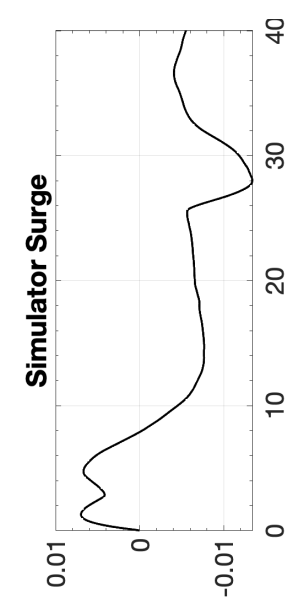

[u] uo!n!sod

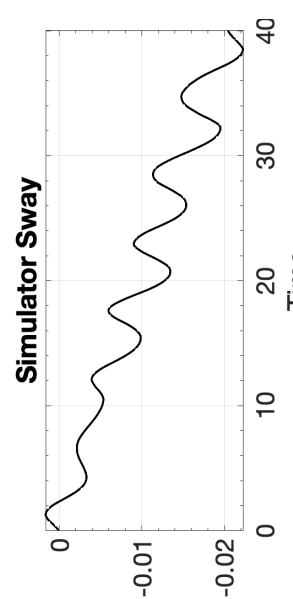

[u] uo!̣!!sod

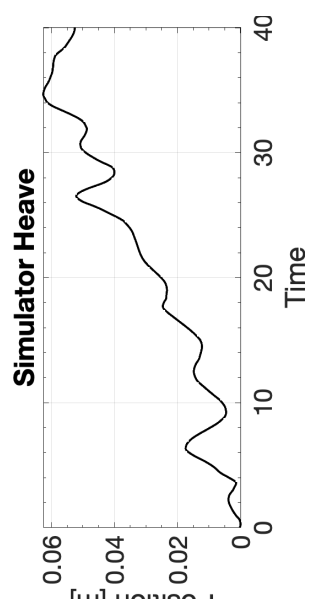

[u] uo!n!sod

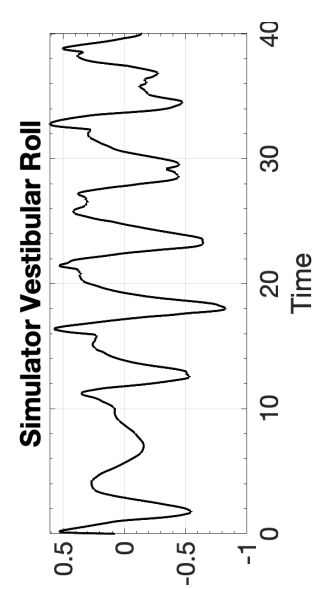

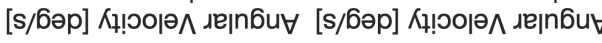

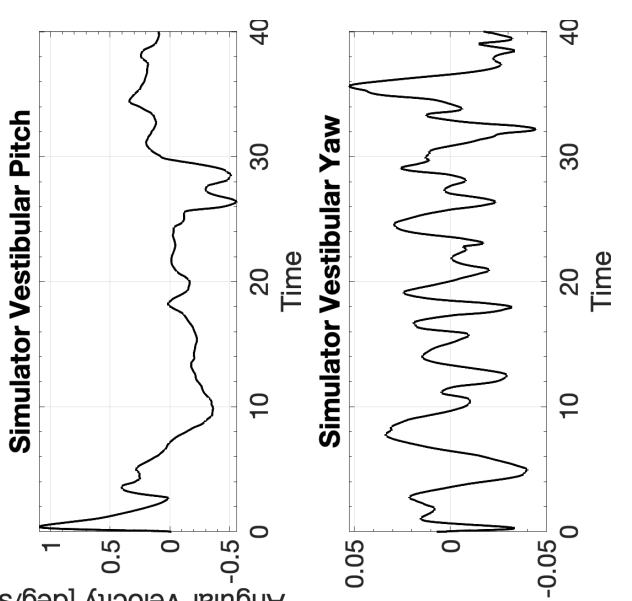

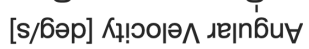

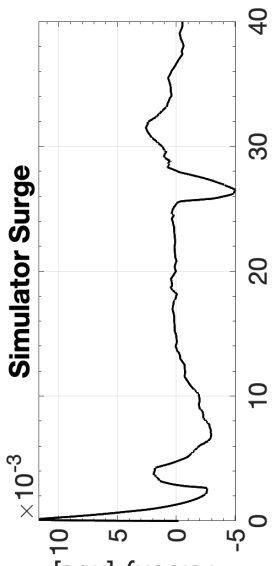

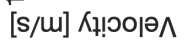

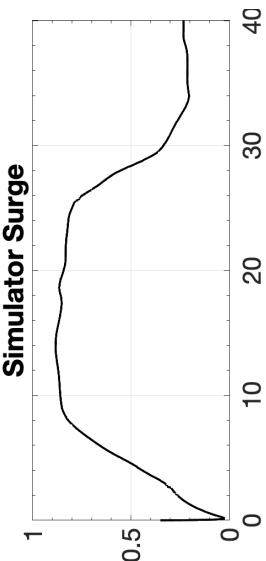

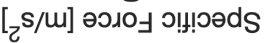

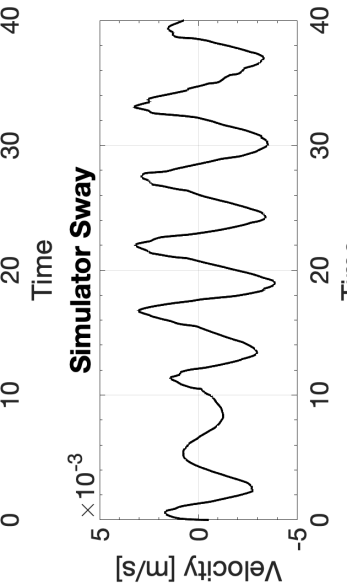

[s/m] Кㄲํㅇㅅ

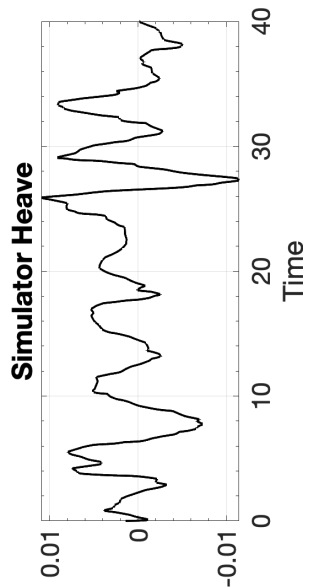

[s/m] Кำ잇

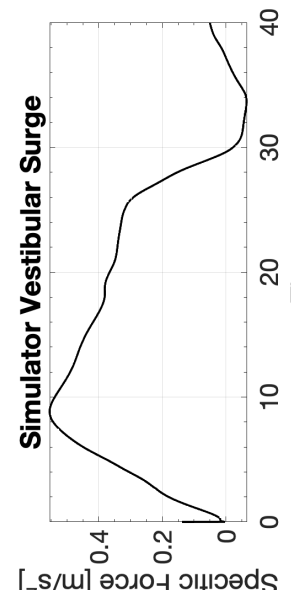

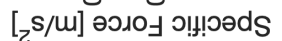

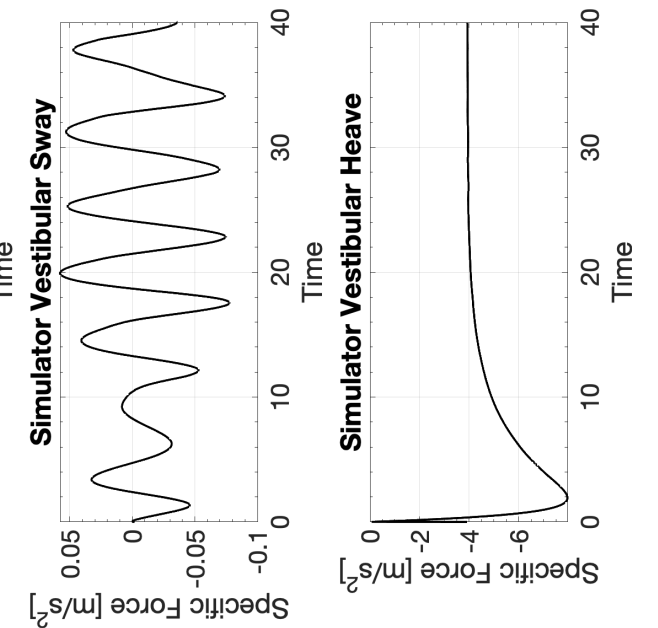

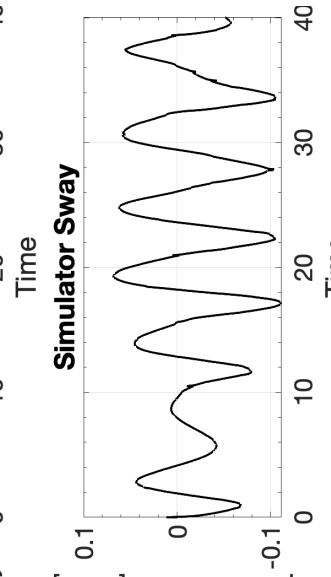

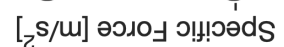

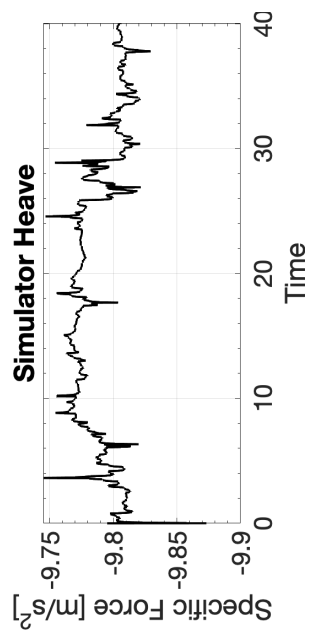

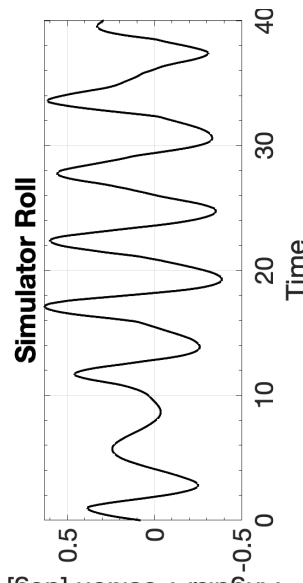

[бəp] uo!!!sod גe|nదินท

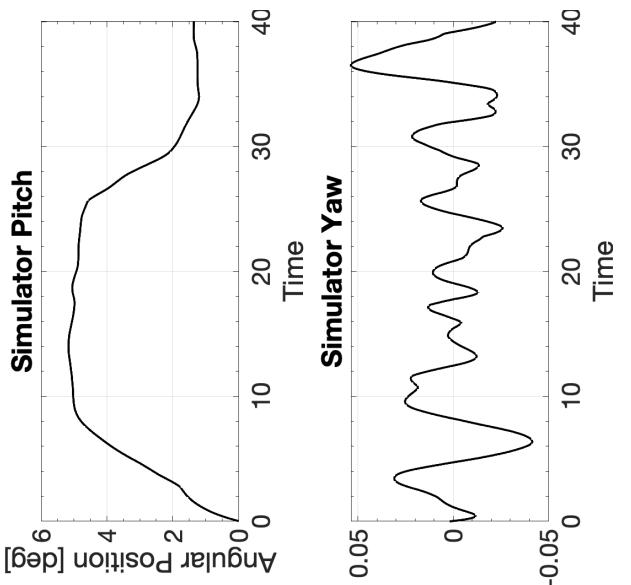

[6əp] uo!!!sod גe|nбü

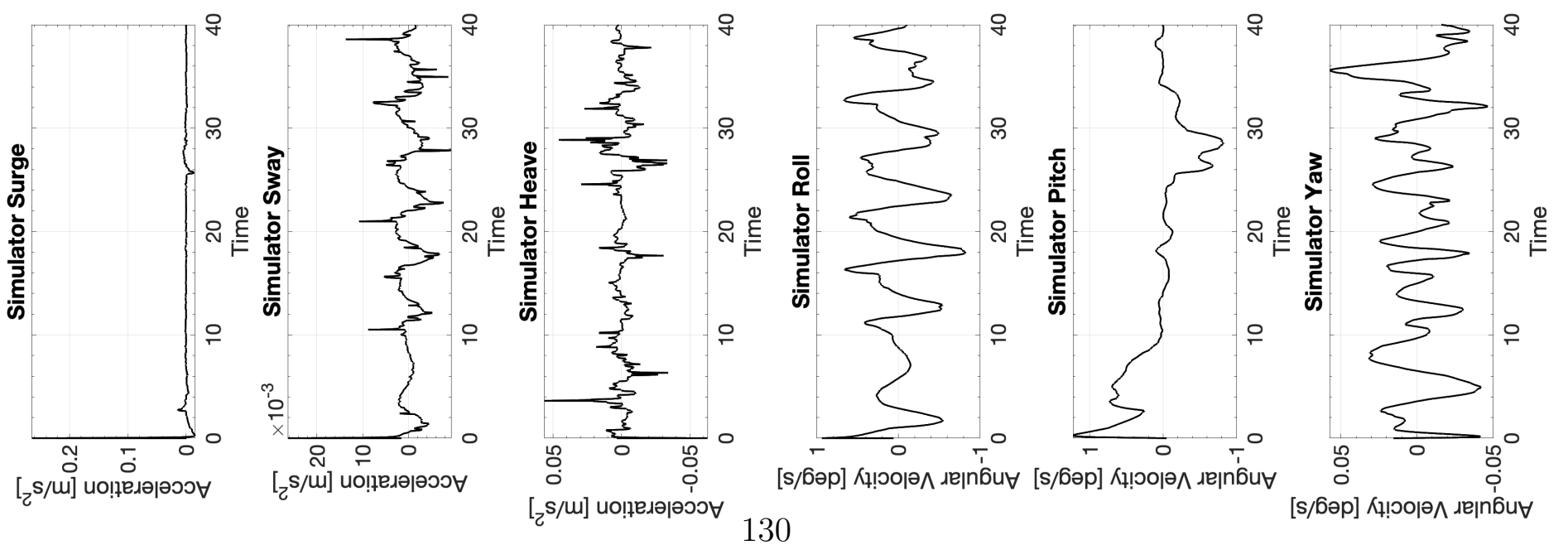




\section{B.3 M3}

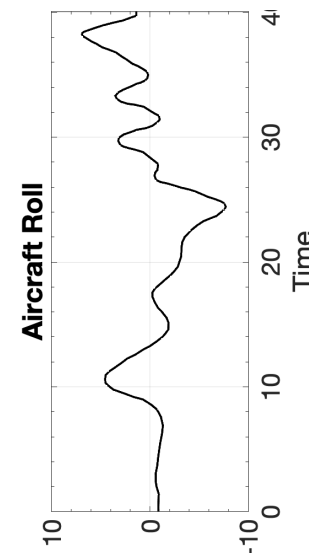

[бәр] uo!!̣!sod

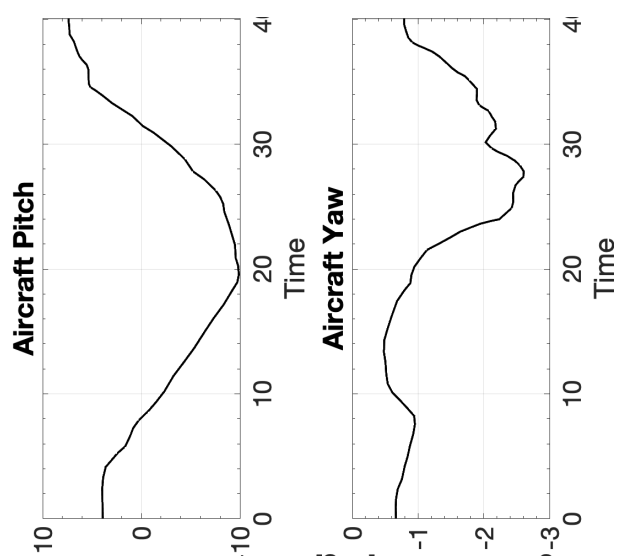

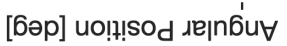

[бәр] uo!̣!sod גe|nбu

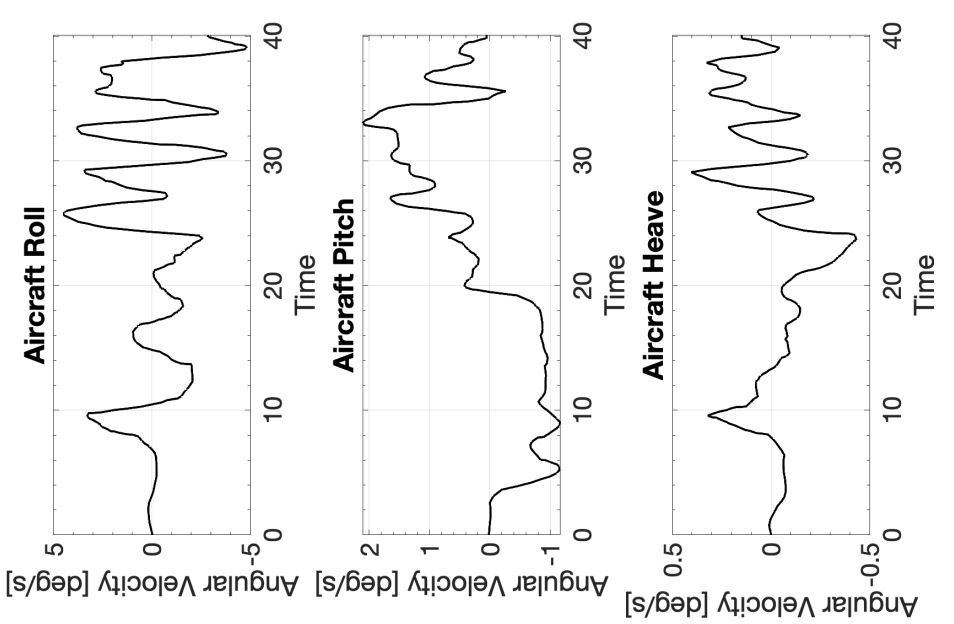

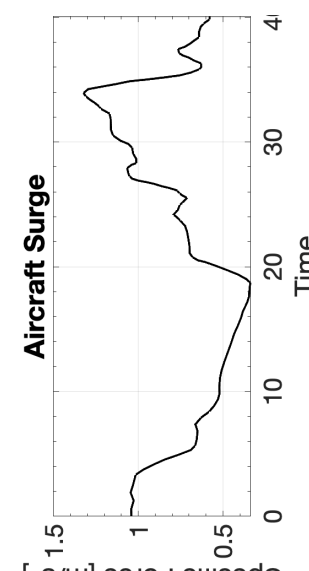

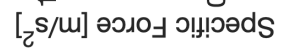

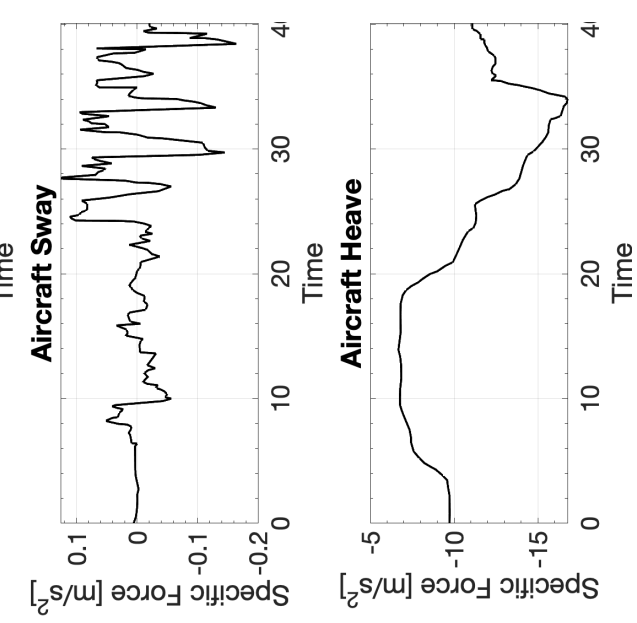

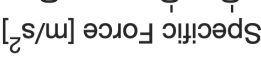

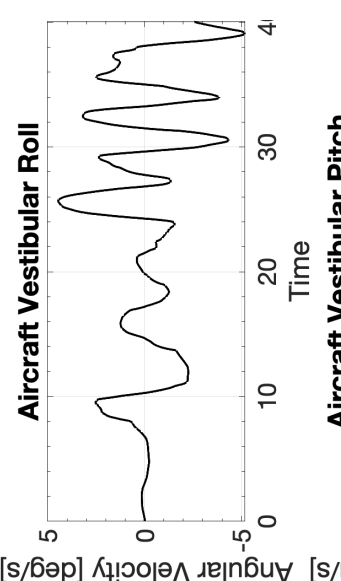

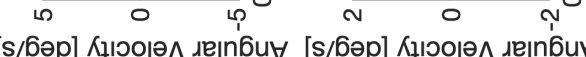

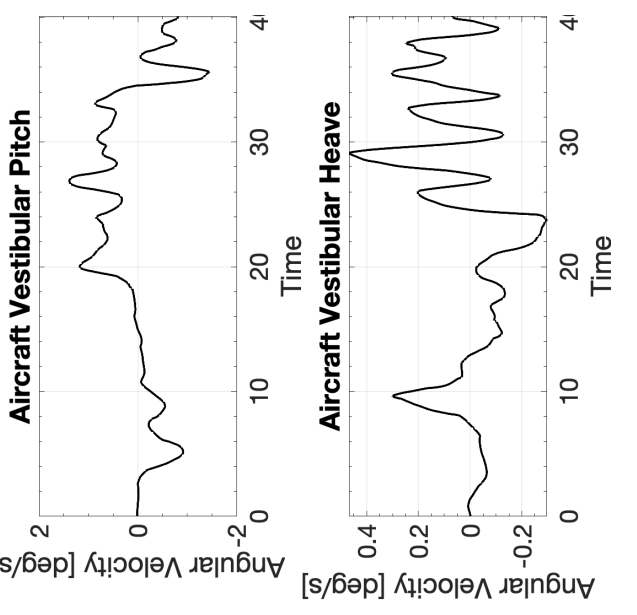

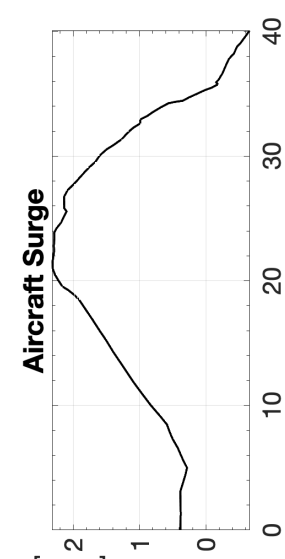

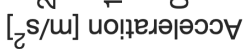

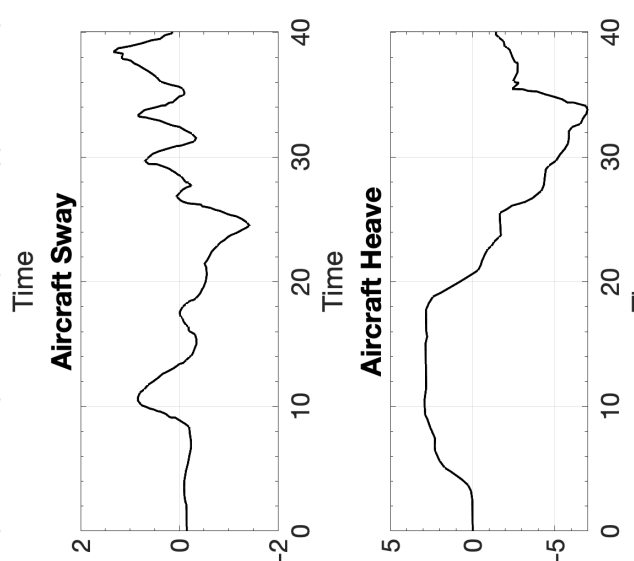

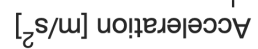

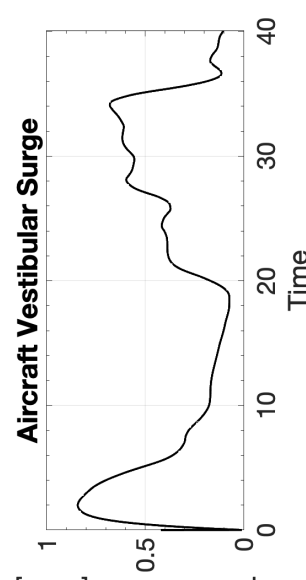

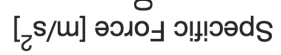

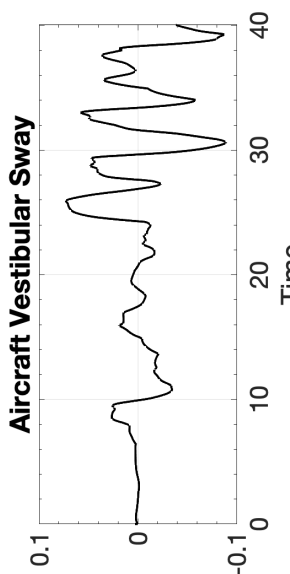

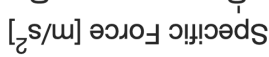

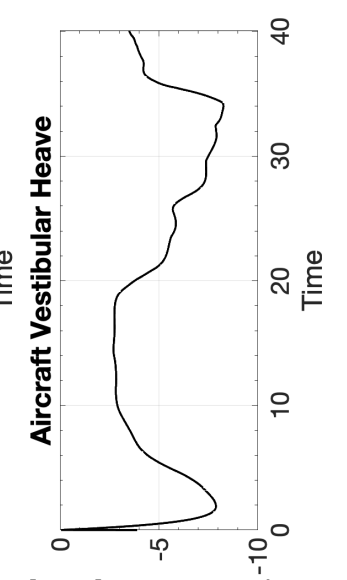

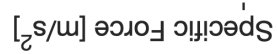




\section{B.3.1 CW1 M3}

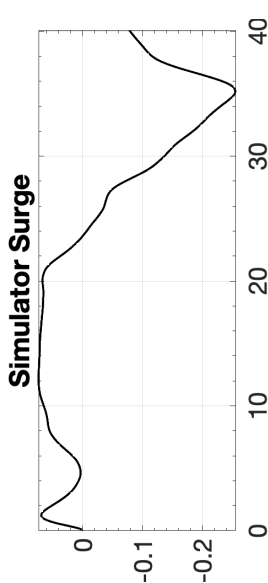

[w] uo!!!sod

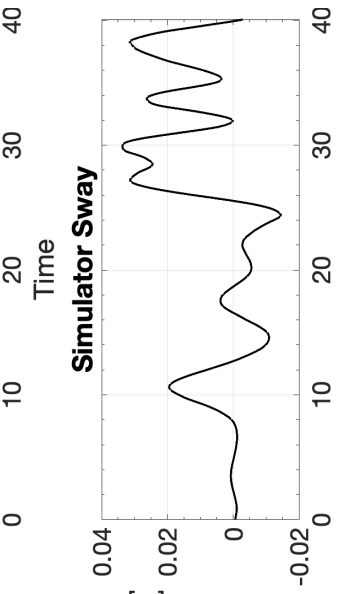

[w] uol!!!sod

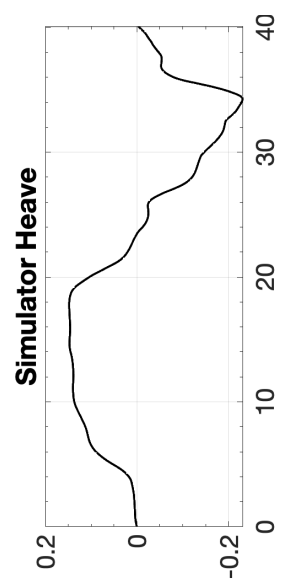

[w] uo!n!sod

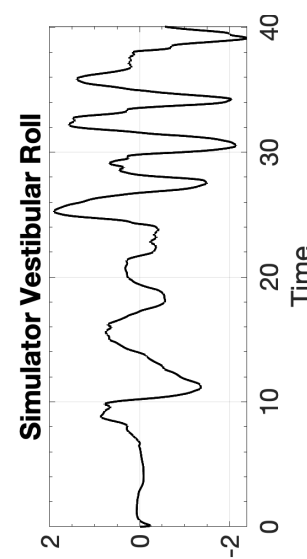

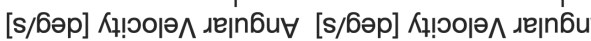

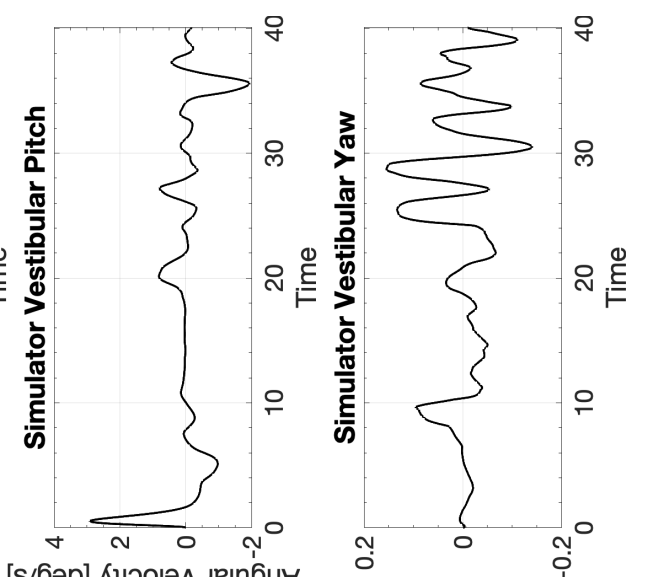

[s/6әр] Кұ!юојә^ «е|nбu

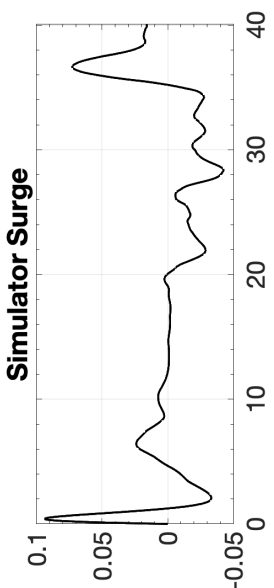

[s/u] Кұ! ৩оюә
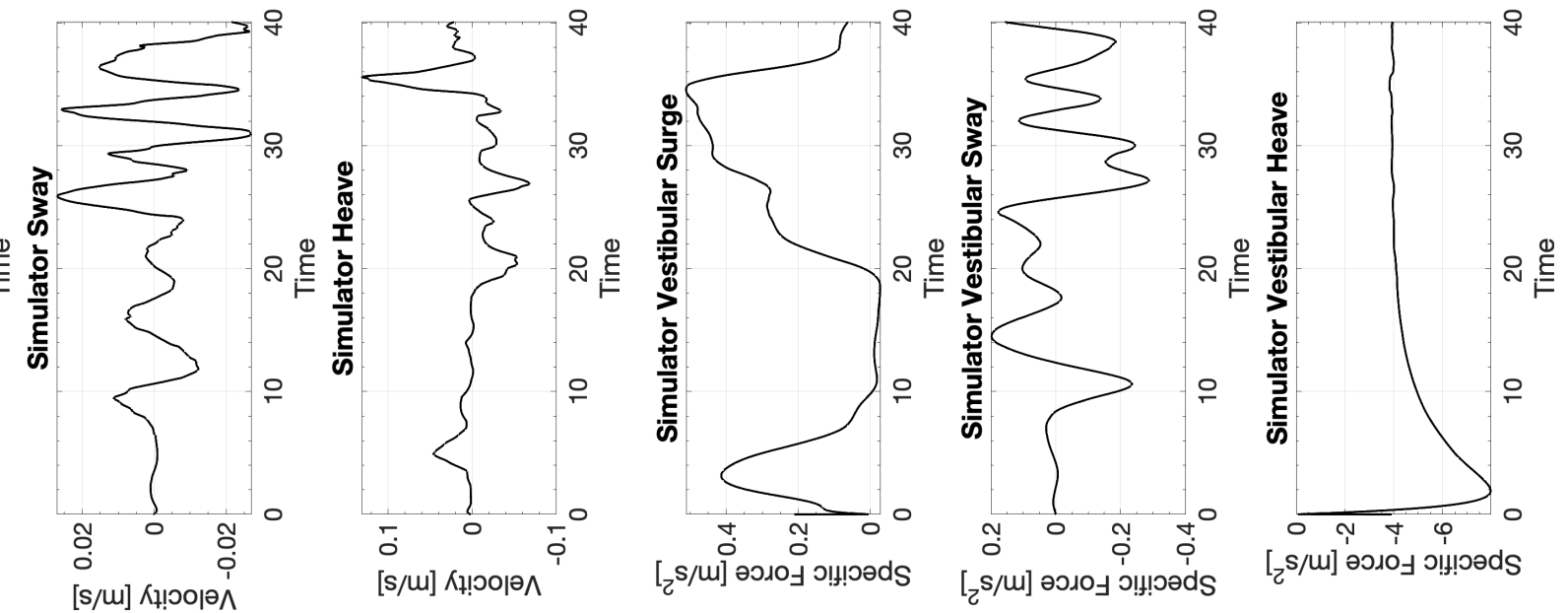

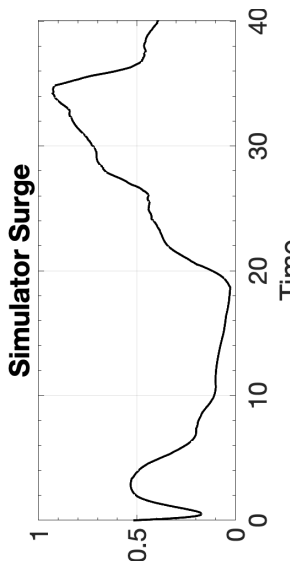

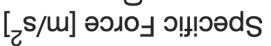

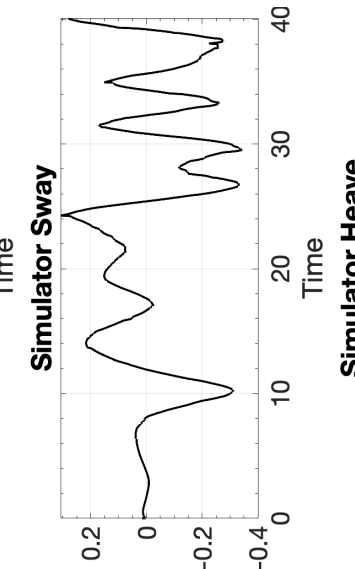

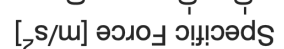

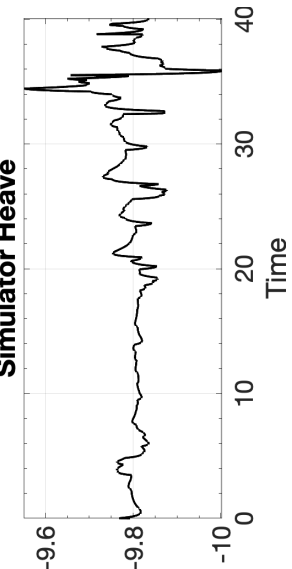

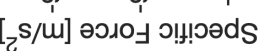

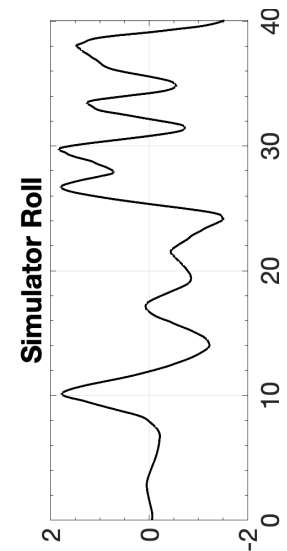

[бәр] uo!!!

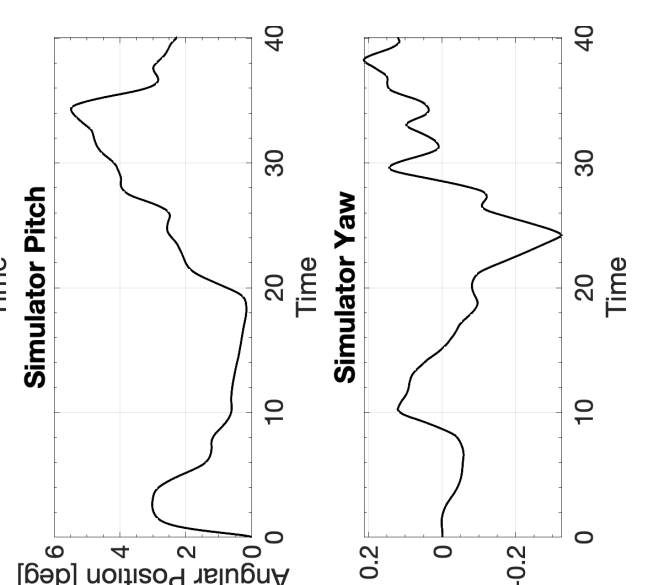

[бәр] uo!!!!sod

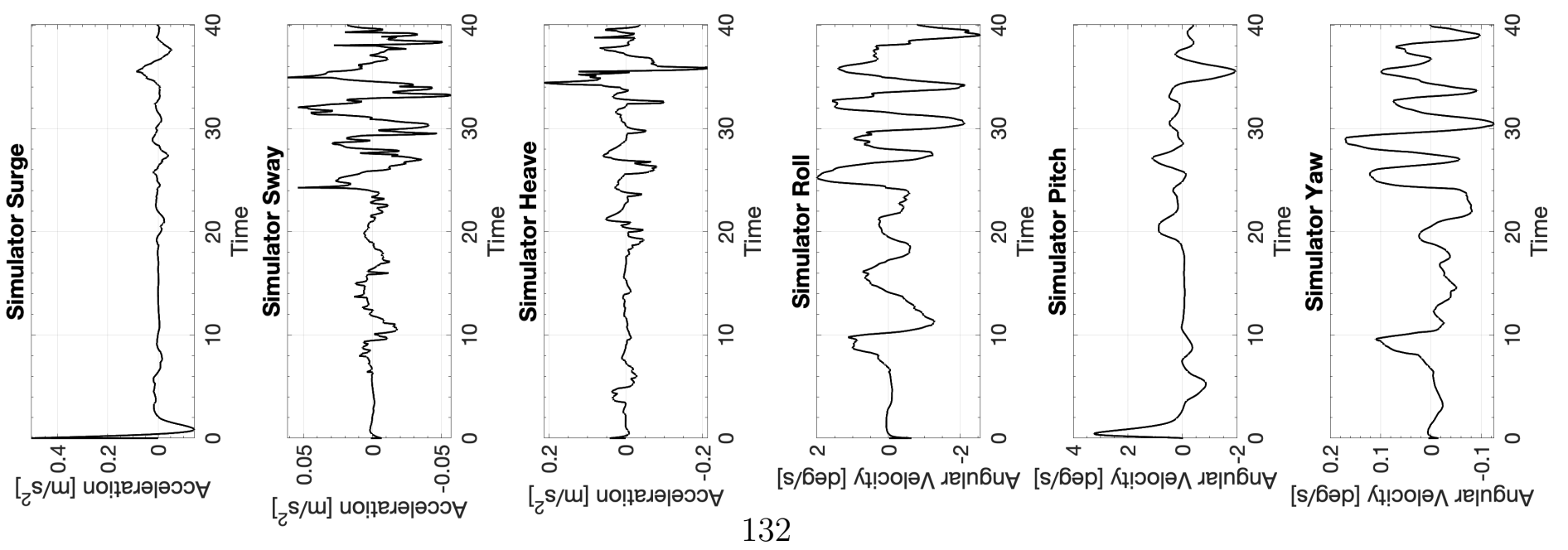




\section{B.3.2 CW2 M3}

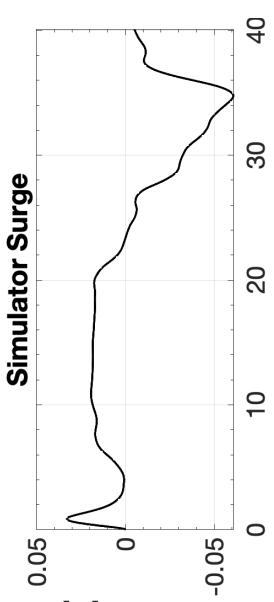

[u] uol!!

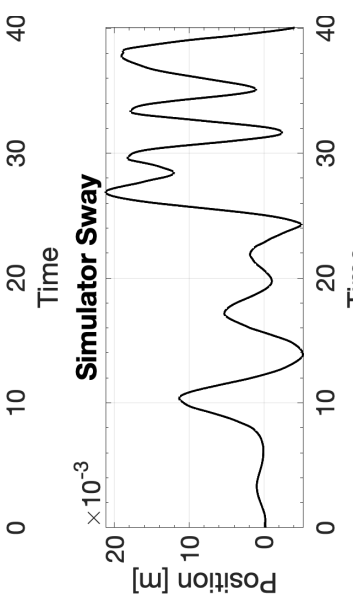

[u] uo!n! sod

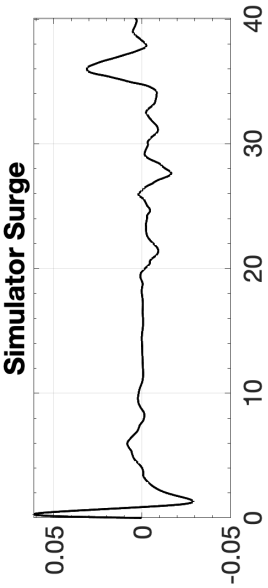

[s/u] Кц!юојә^

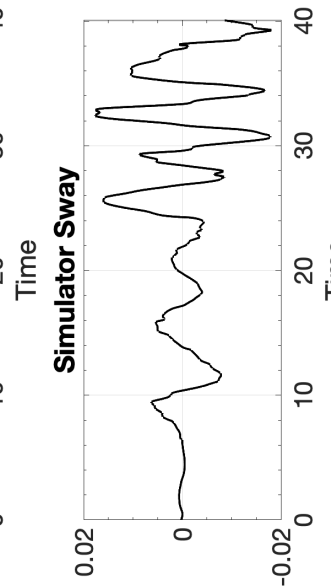

[s/m] К!оəㅅำ

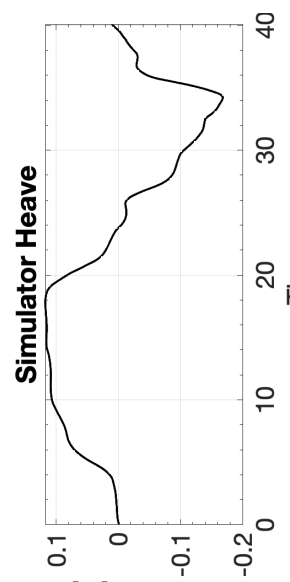

[w] uolt!sod

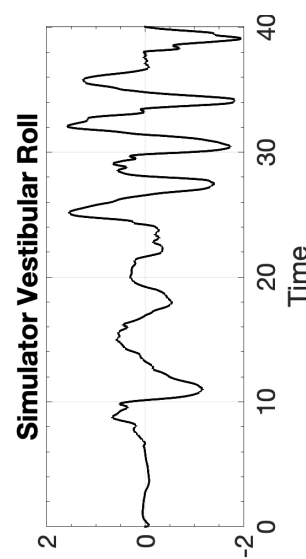

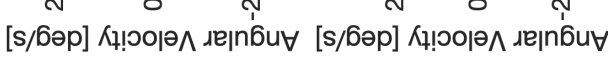

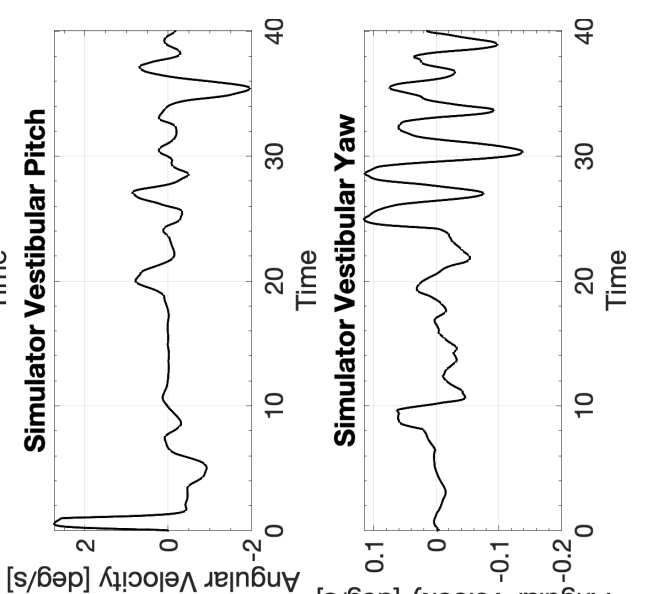

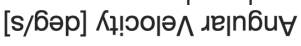

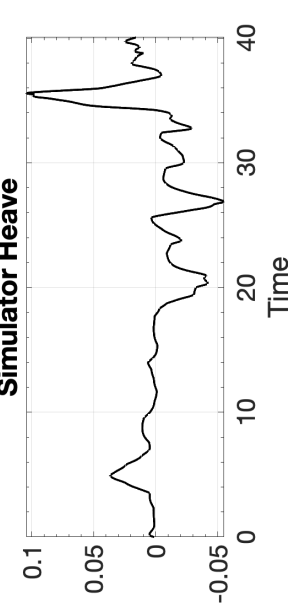

[s/m] К! ๖о|ә^
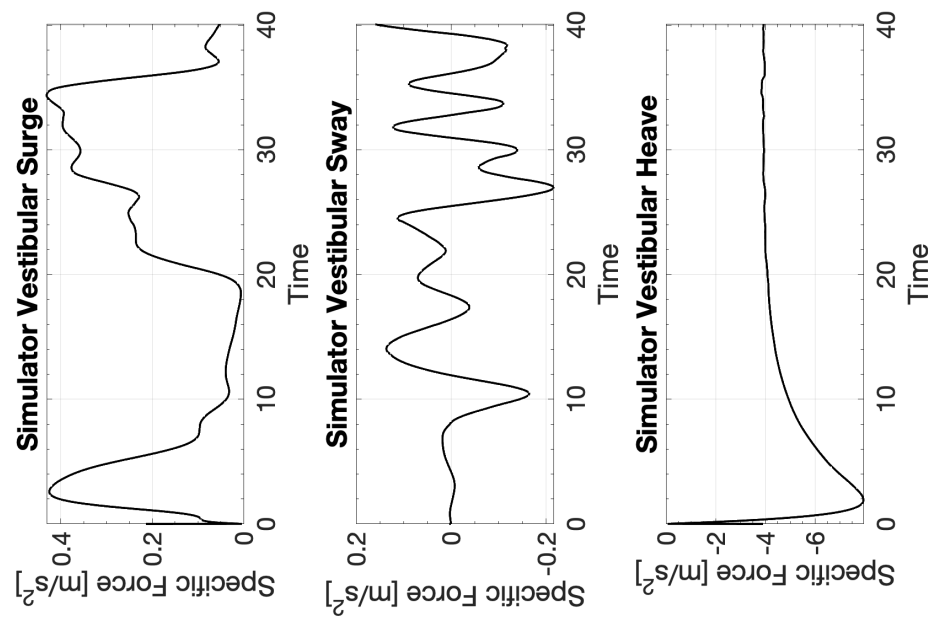

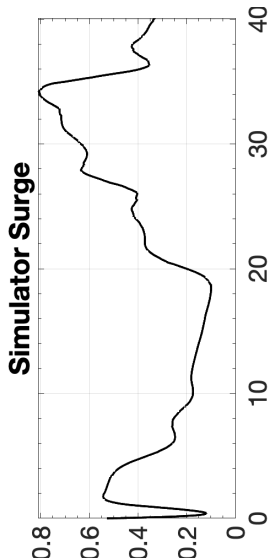

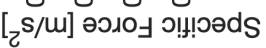

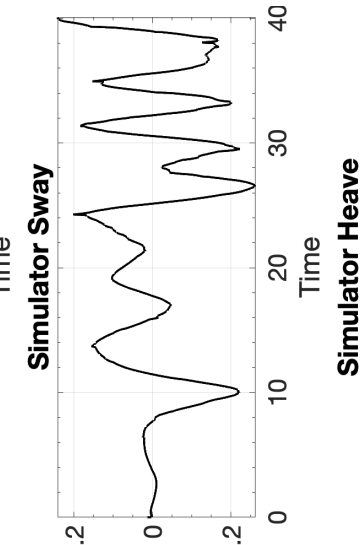

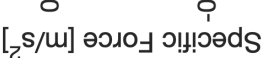

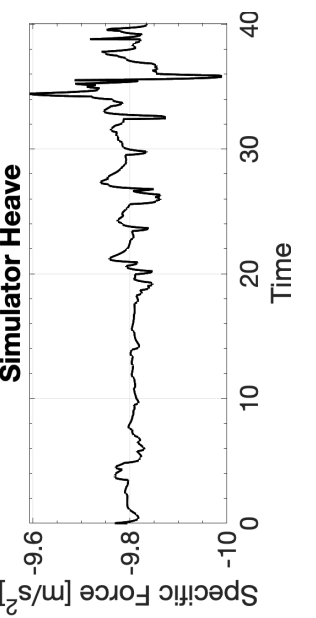

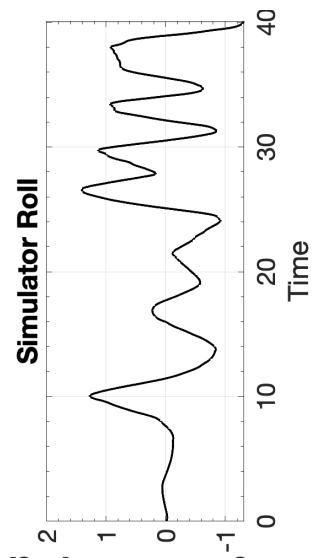

[бәр] uo!!!!sod גe|n்

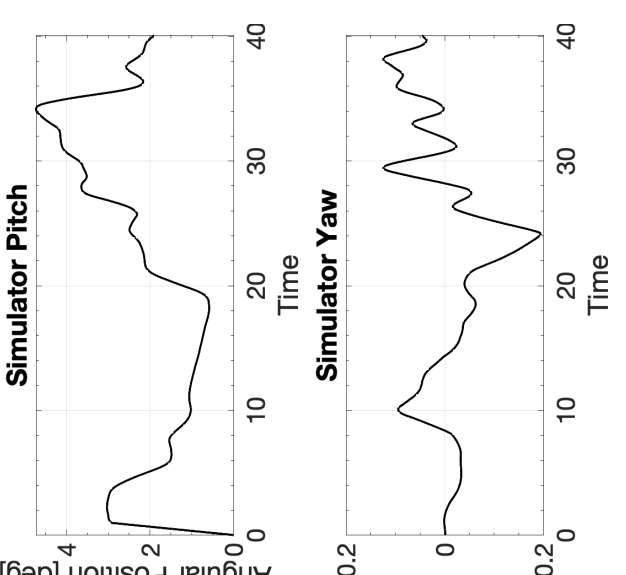

[6әp] uo!!!!sod גe|n6u

[6әр] uo!?!sod ле|n6̆

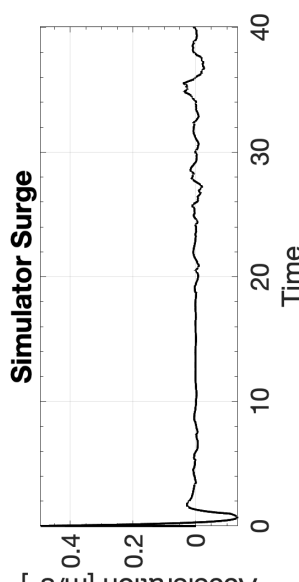

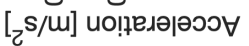

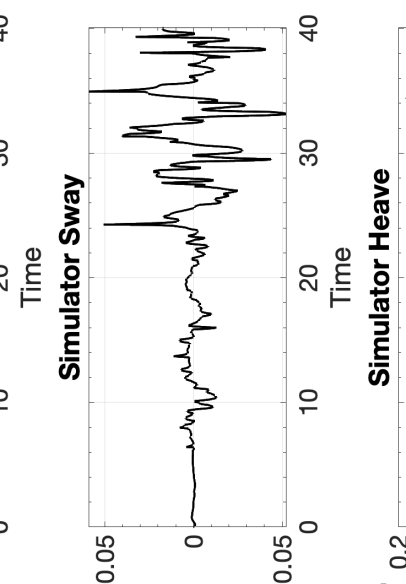

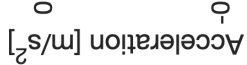

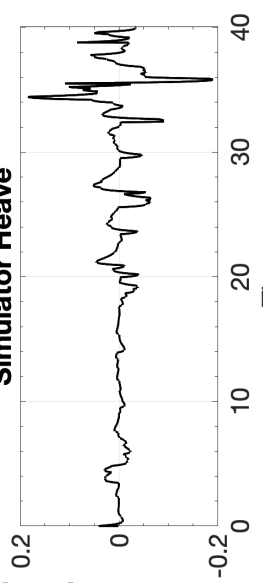

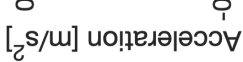

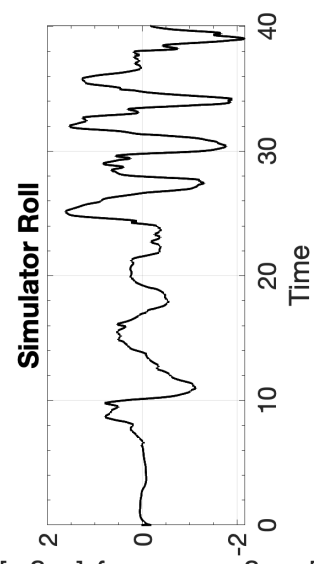

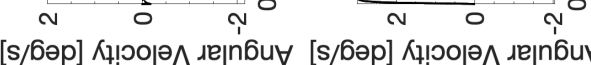

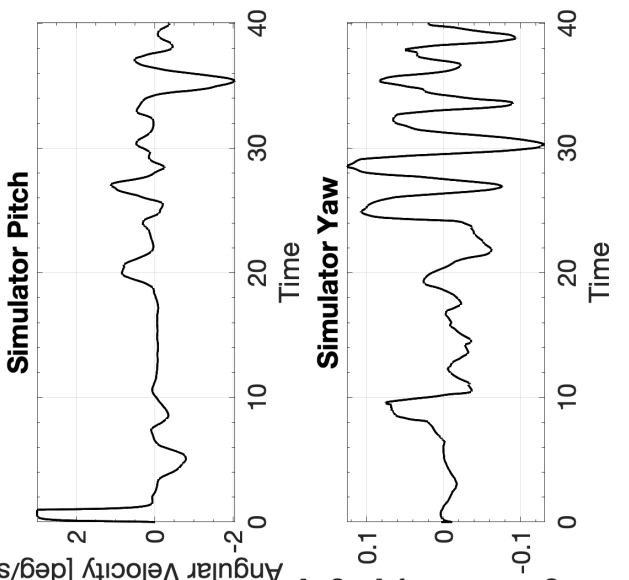

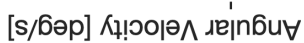




\section{B.3.3 CW3 M3}

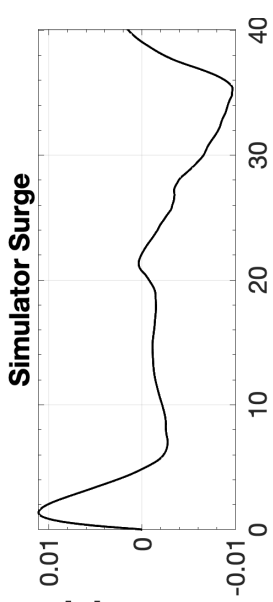

[u] uo!!!sod

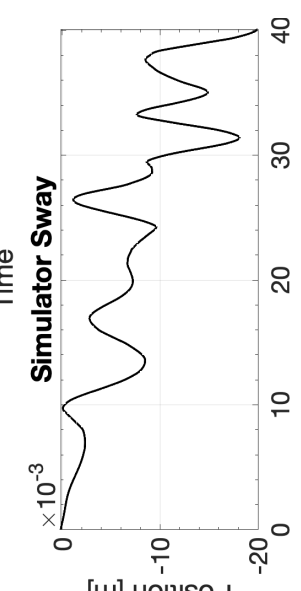

[w] uo!n!sod

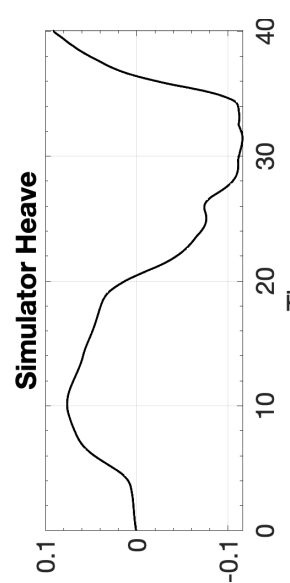

[u] uo!!!sod

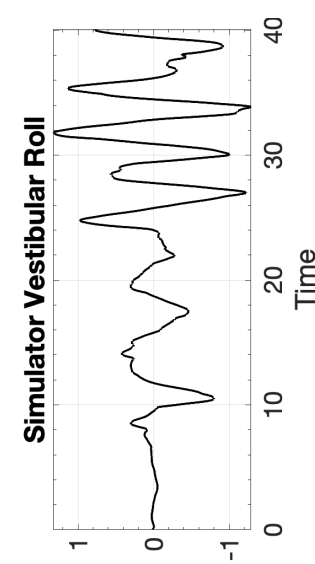

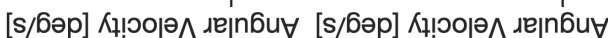

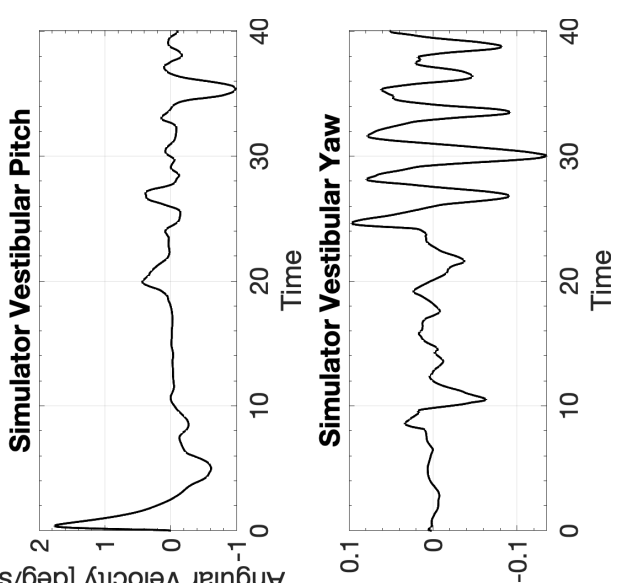

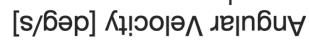

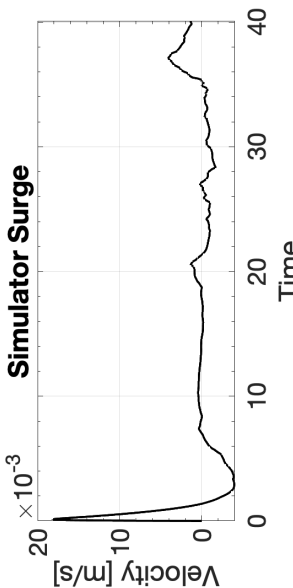

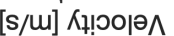

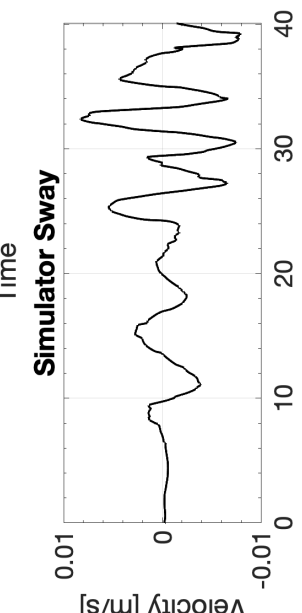

[s/m] ᄉұ!৩ою^

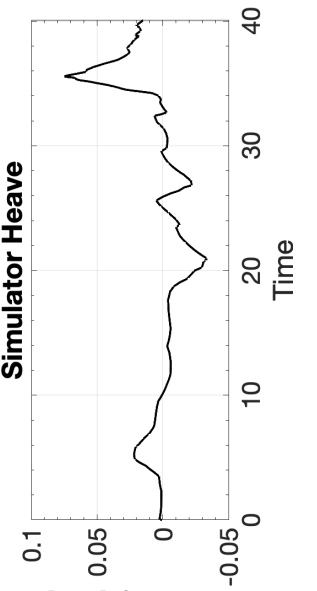

[s/m] К!ำю^
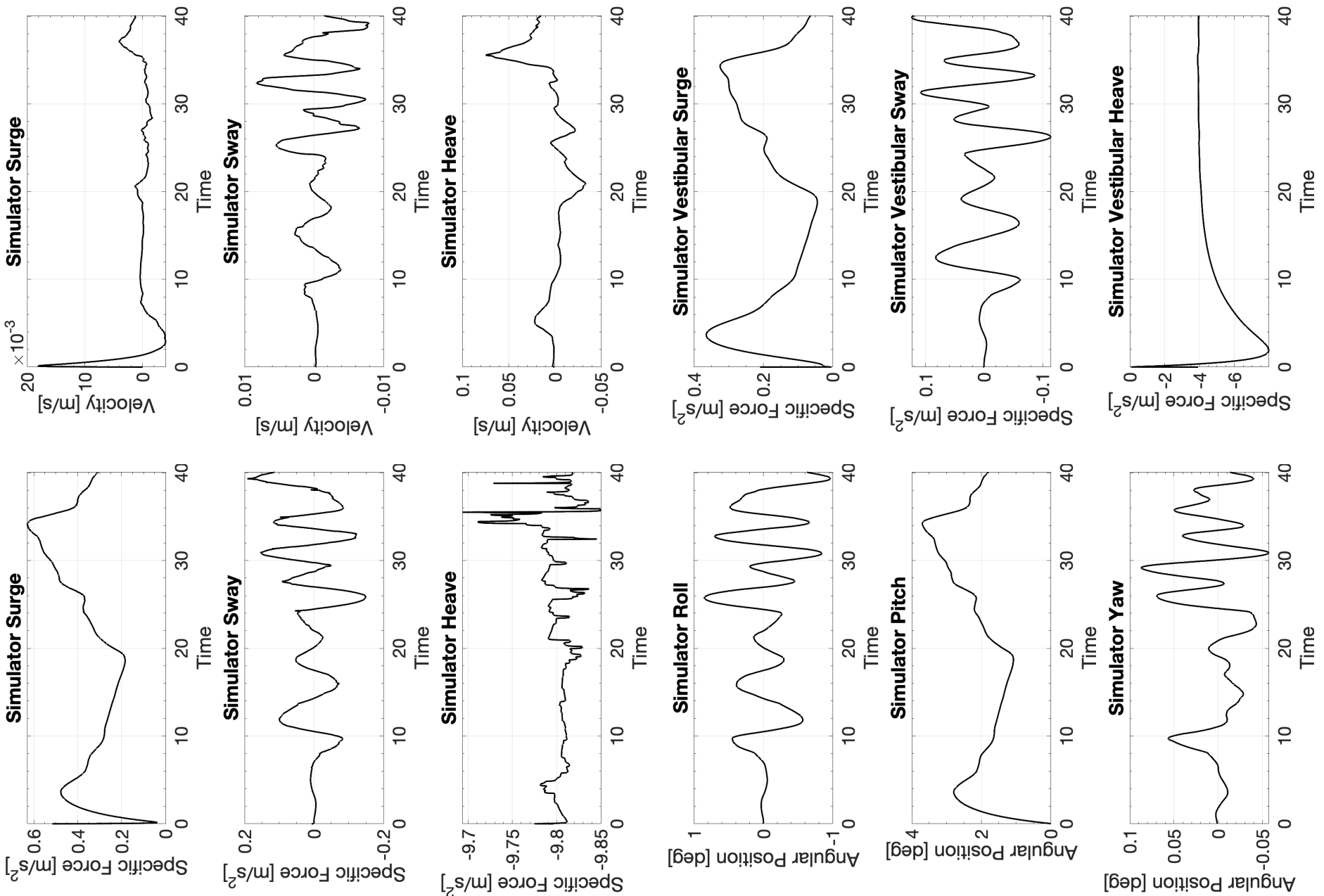

[бәр] uo!!!sod

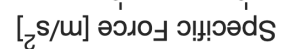

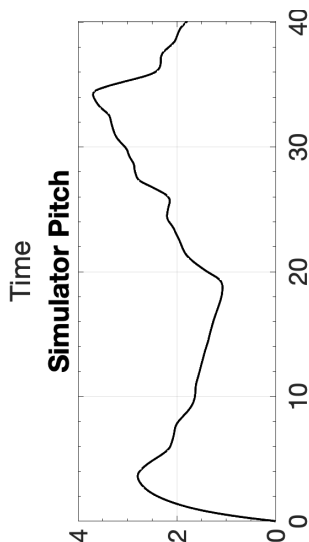

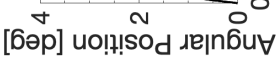

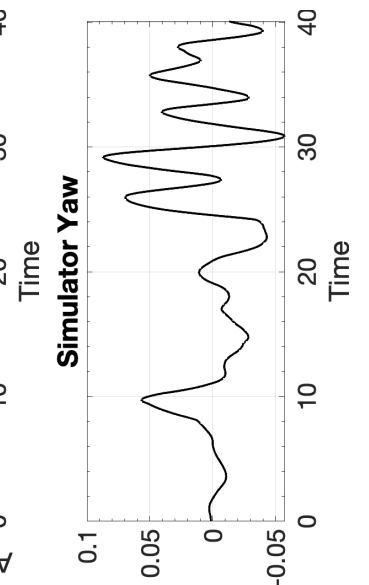

[6әр] uo!?! $\mathrm{sod}_{\mathrm{d}}$ גejn6u

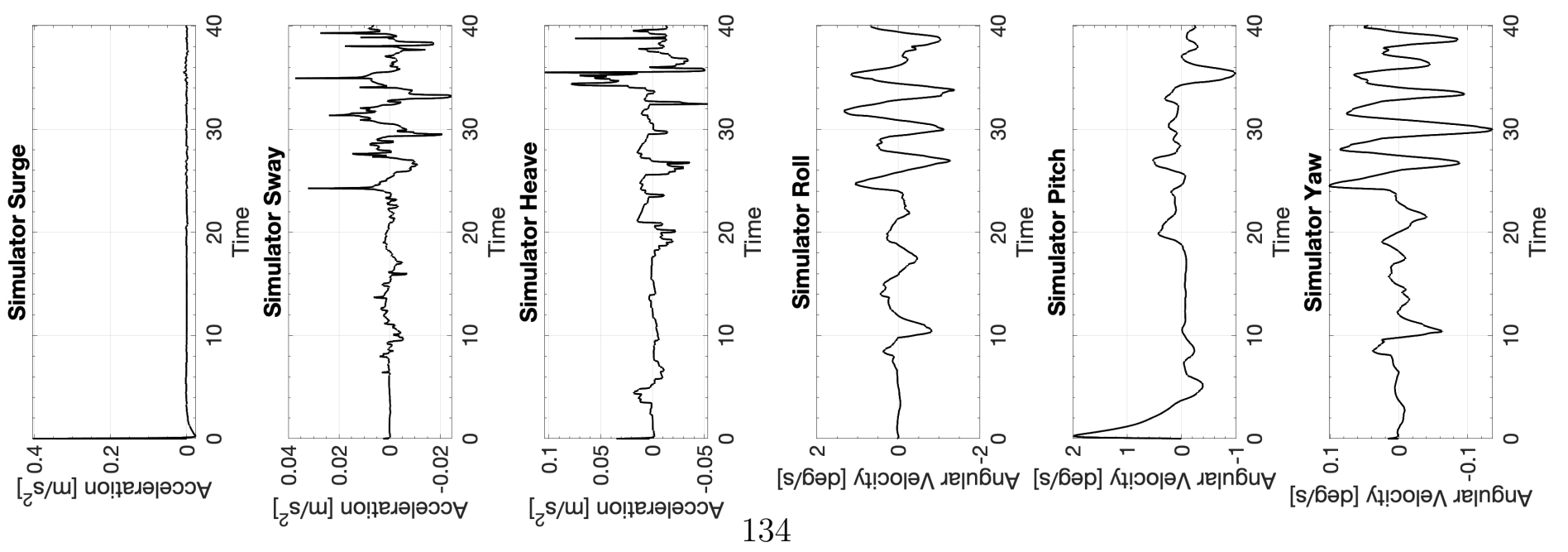




\section{B.4 Cessna 172 Coordinated Turn}
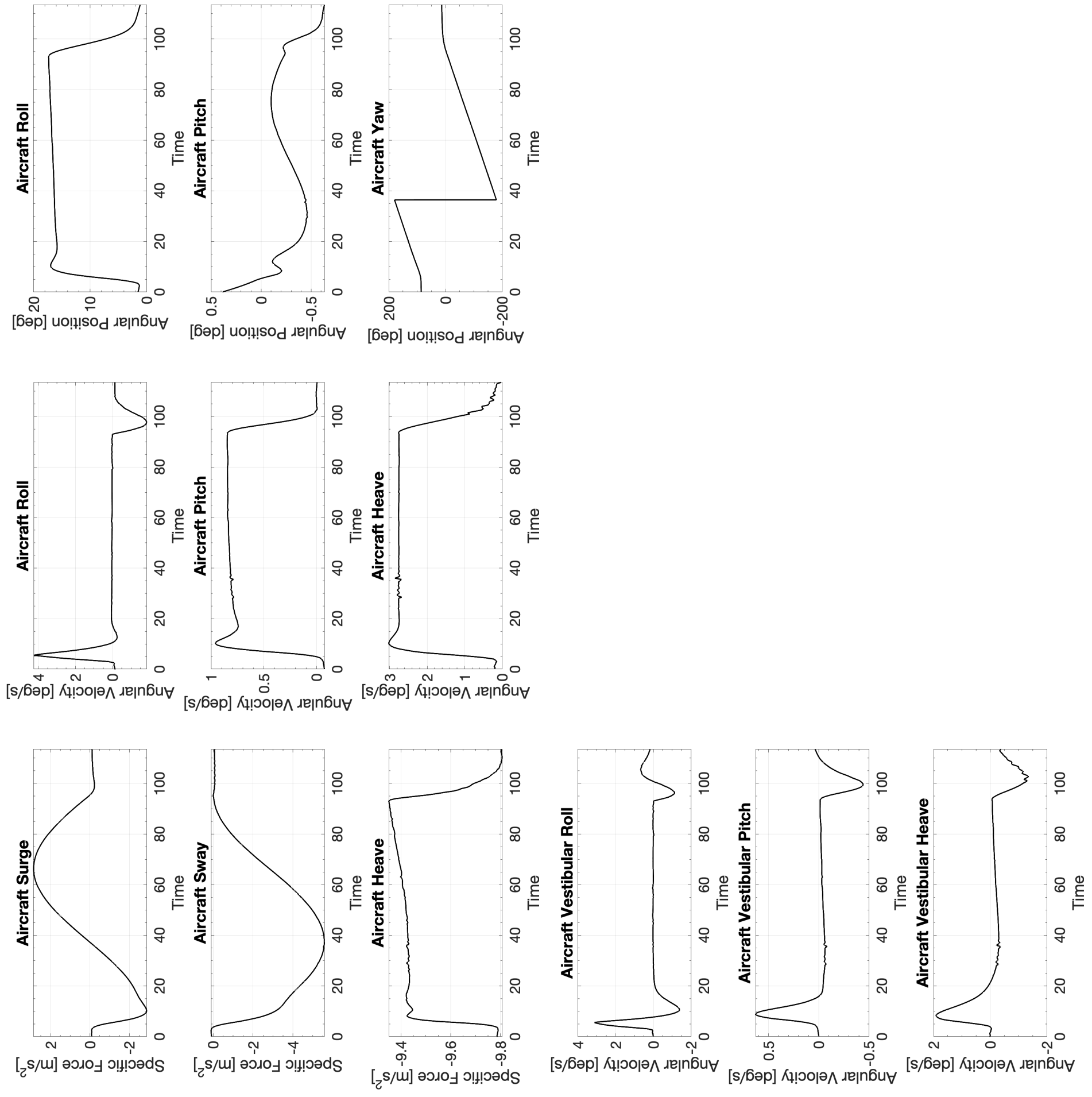

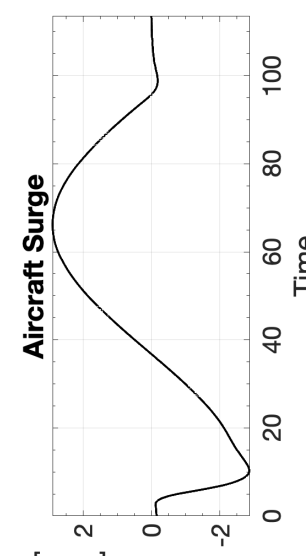

[z/m] ио!ฺвдә|әэว

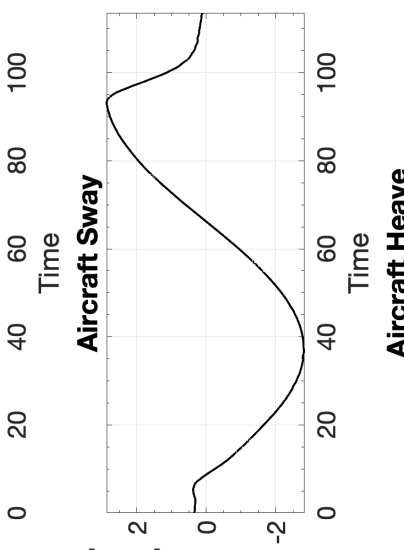

[s/m] ио!ฺеגә|әэว

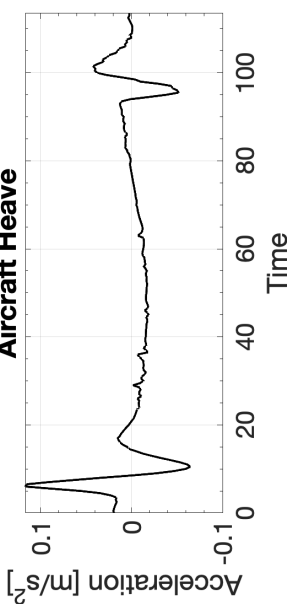

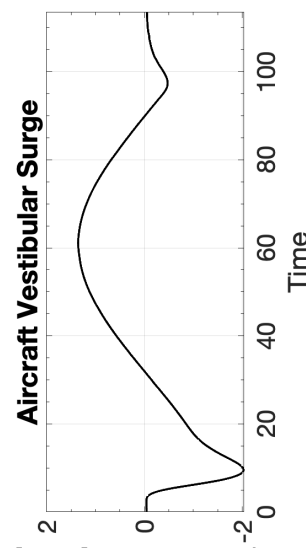

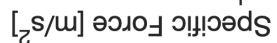




\section{B.4.1 CW1 Cessna 172 Coordinated Turn}

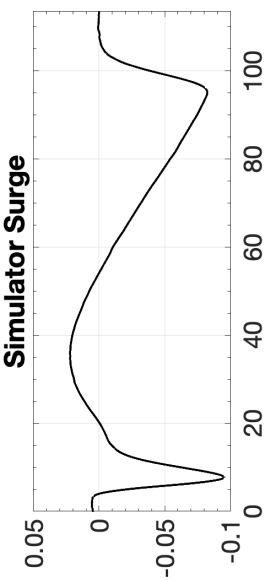

[w] uo!n! sod

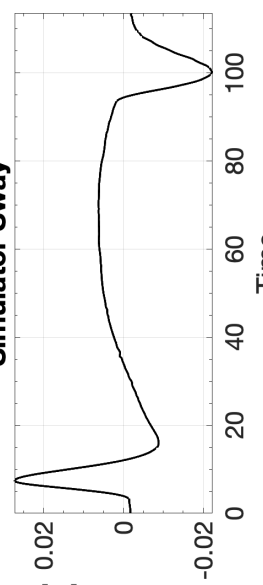

[u] uo!̣!!sod

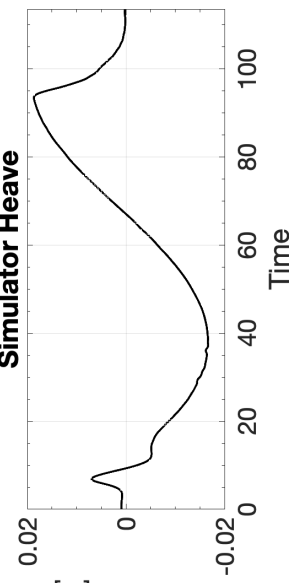

[w] uol!! $\mathrm{sod}^{\mathrm{d}}$

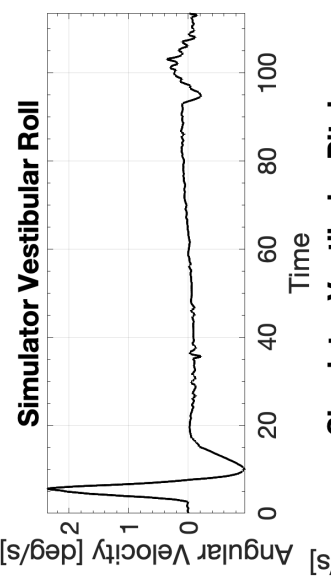

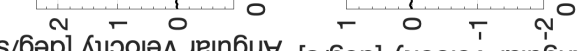

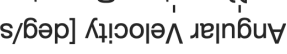

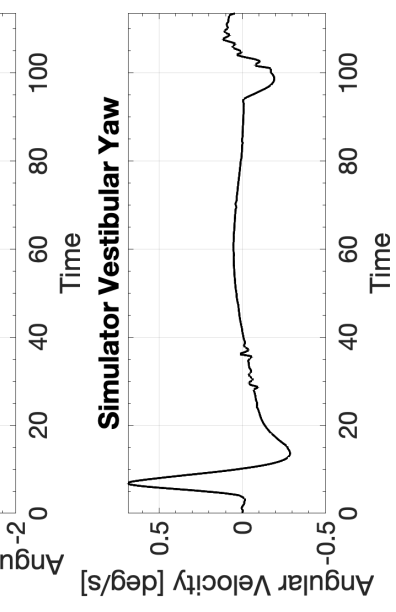

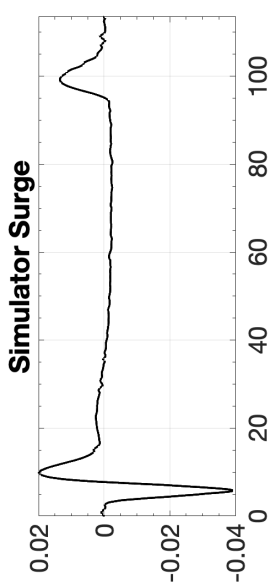

[s/u] ᄉ! ৩оюㅅ

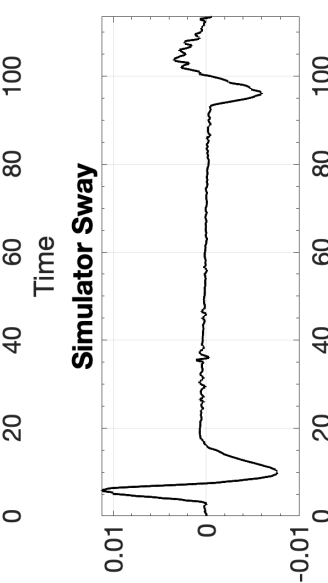

[s/u] Кц!юојә^

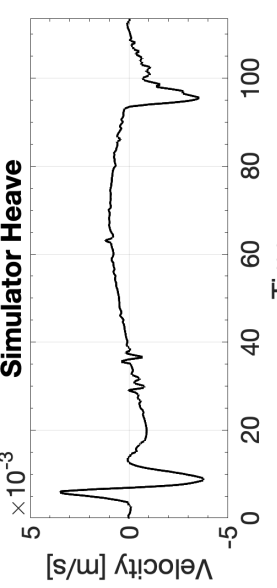

[s/u] К!ㅇำ

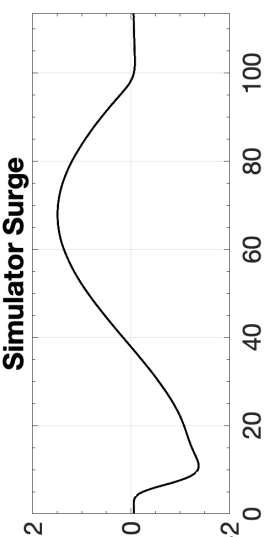

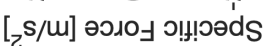

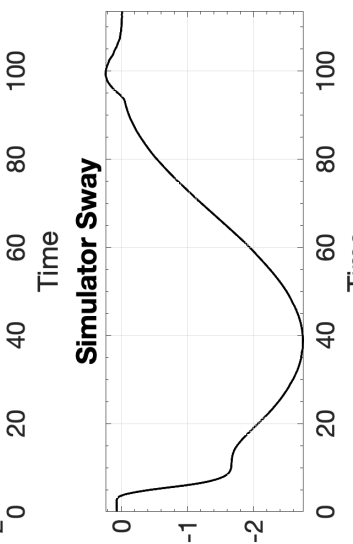

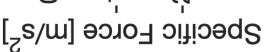

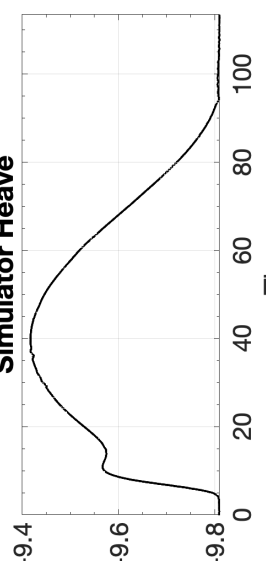

$\left[\begin{array}{c}2 \\ 1\end{array}\right.$

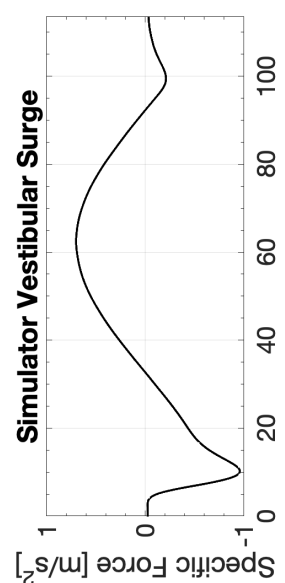

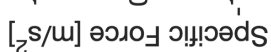

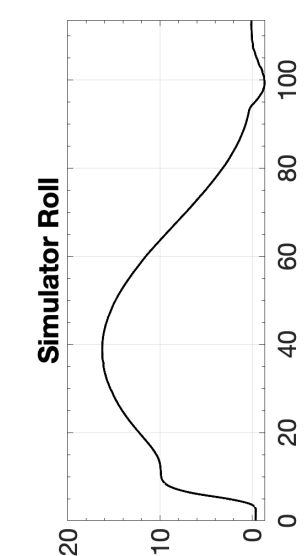

[6әр] uo!!!

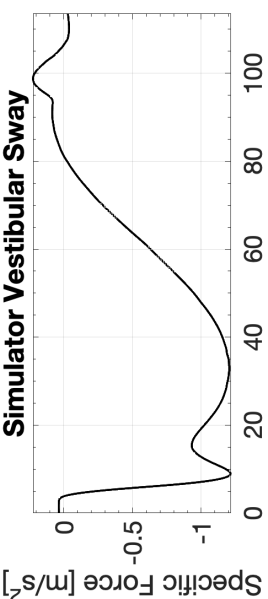

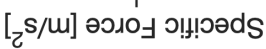

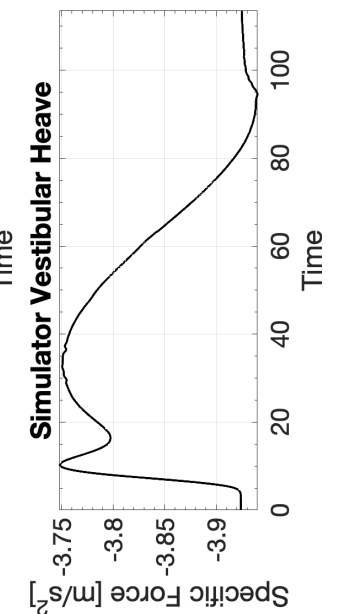

$8 \stackrel{\oplus !}{\xi}$

S

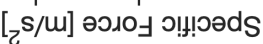

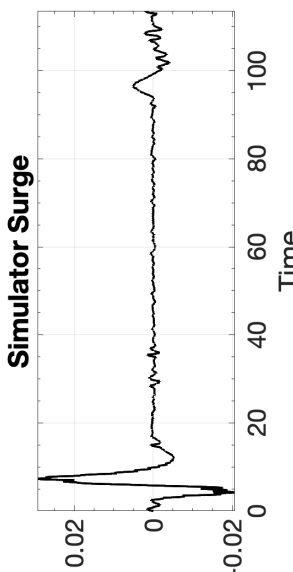

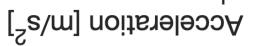

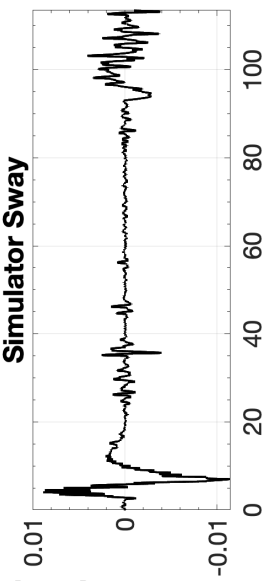

[zs/m] ио!ןедәәәэว

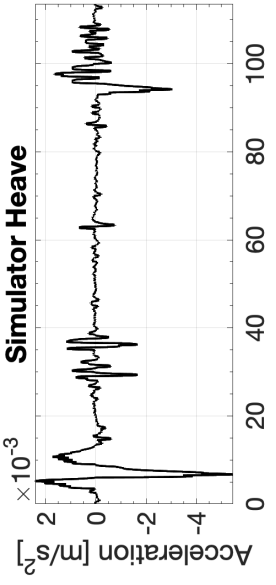

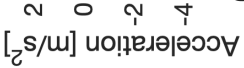

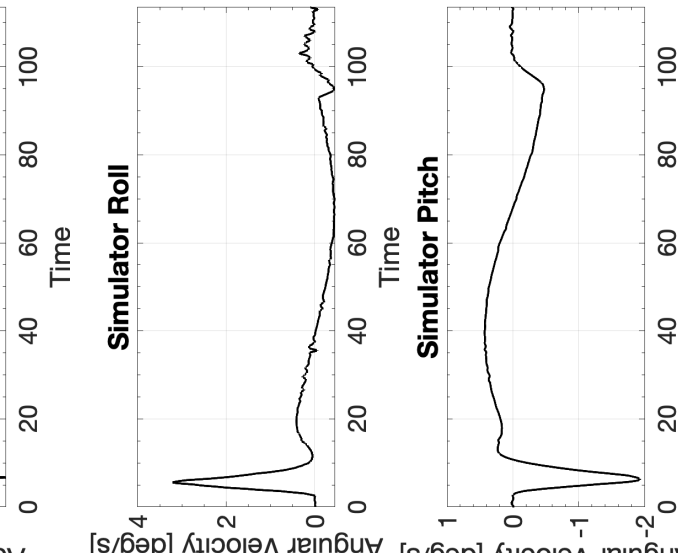

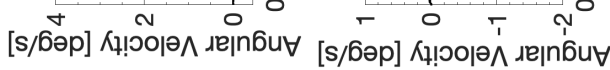

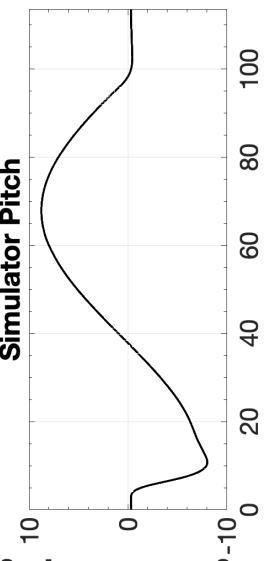

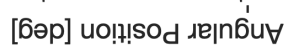

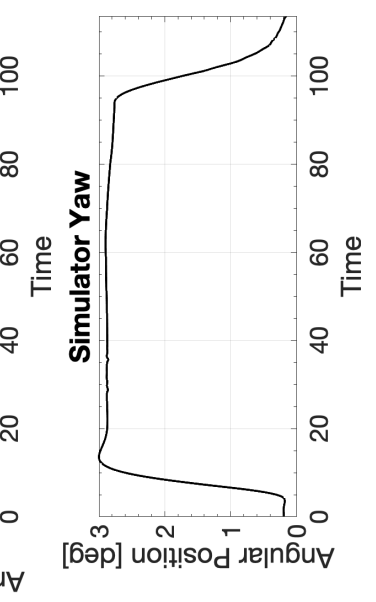




\section{B.4.2 CW2 Cessna 172 Coordinated Turn}

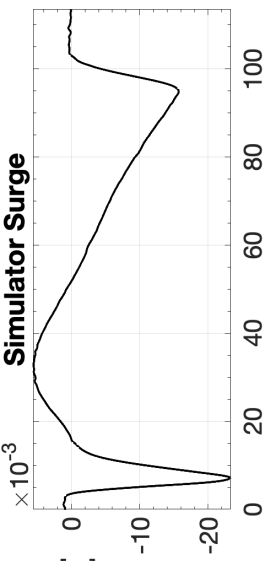

[u] uo!n! sod

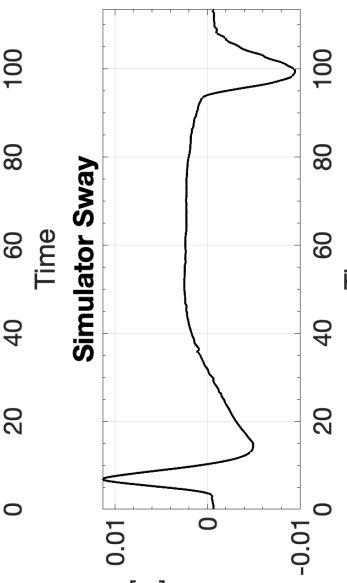

[u] uol!!? $\mathrm{SO}_{\mathrm{d}}$

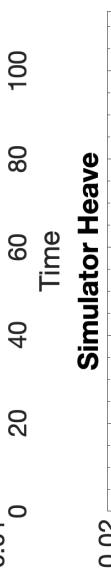

[w] uolp!ssod

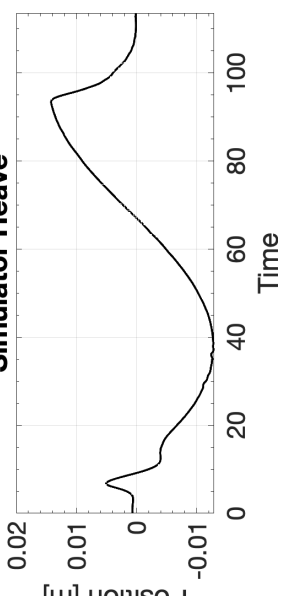

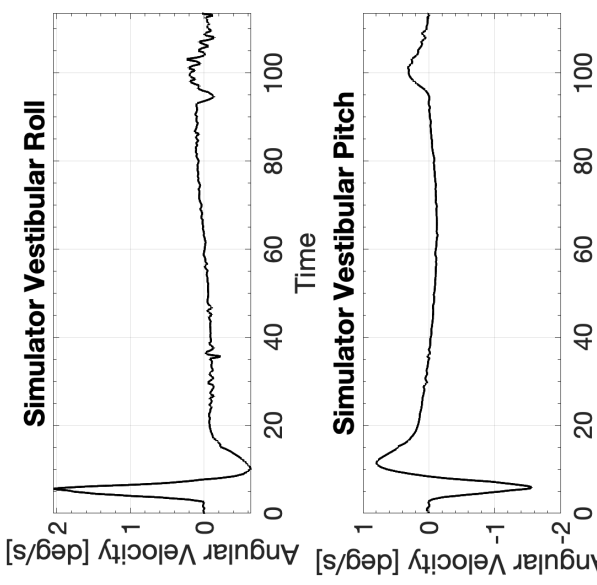

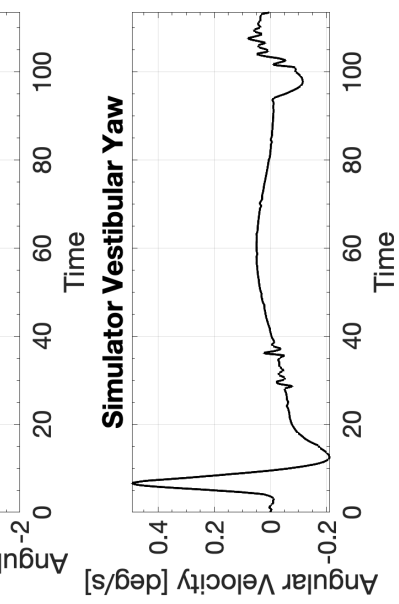

$\therefore \stackrel{0}{\stackrel{E}{F}}$

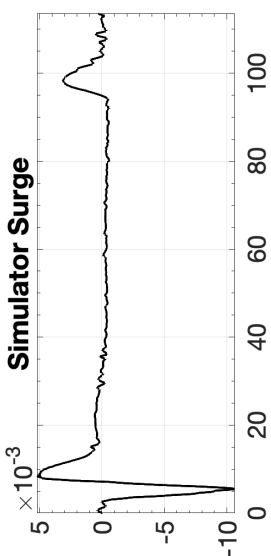

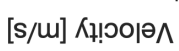

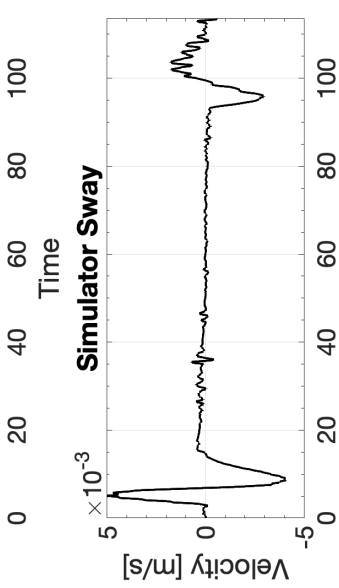

[s/u] К!๐ㅣㅅ

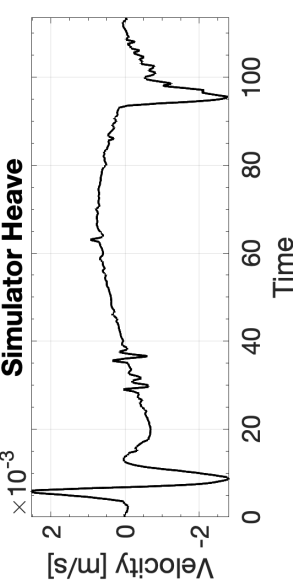

[s/m] Кұ!юојә'

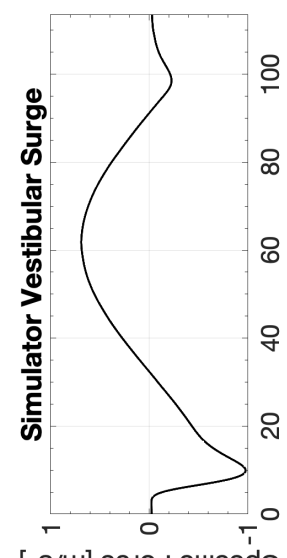

[

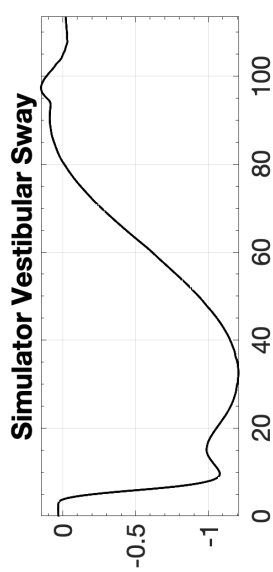

[

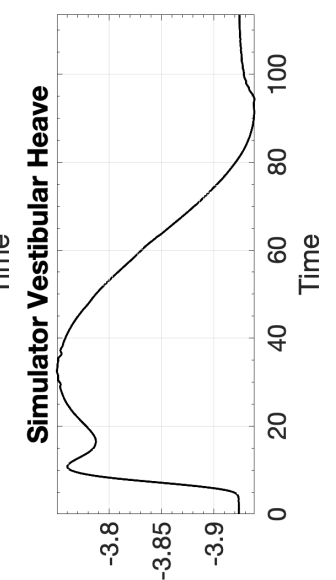

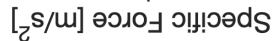

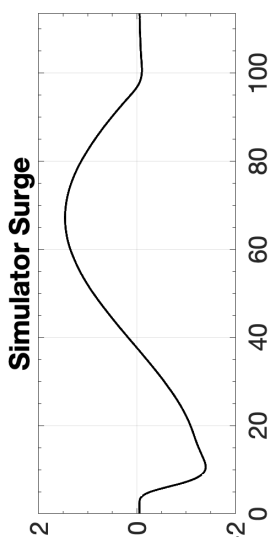

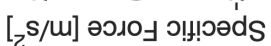

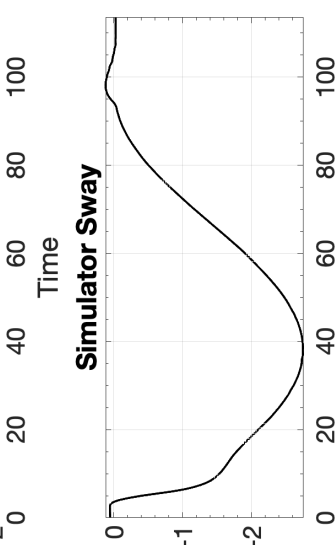

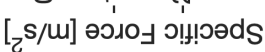

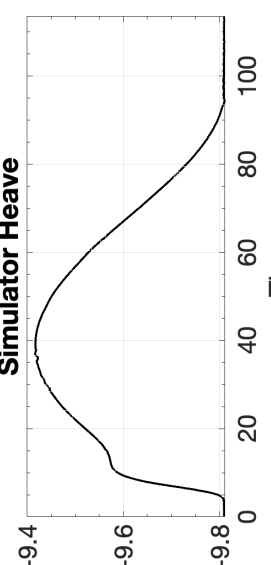

[

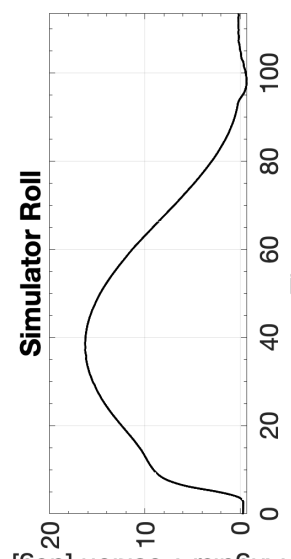

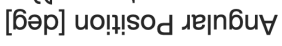

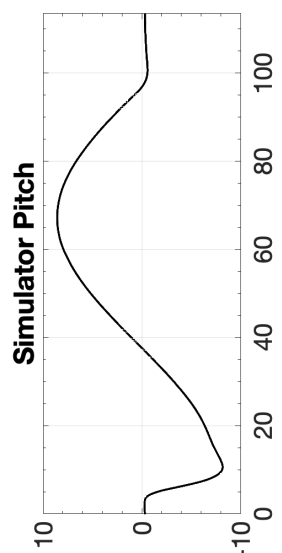

[6əp] uo!!!sod גe|nбuy

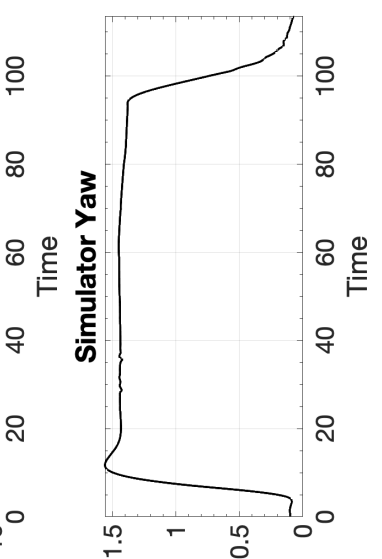

[6әр] uo!!!sod גejn6u

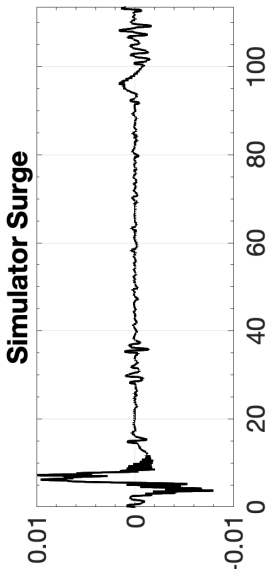

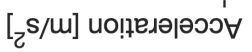

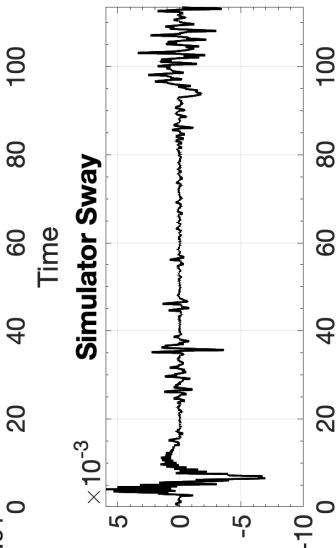

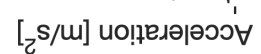

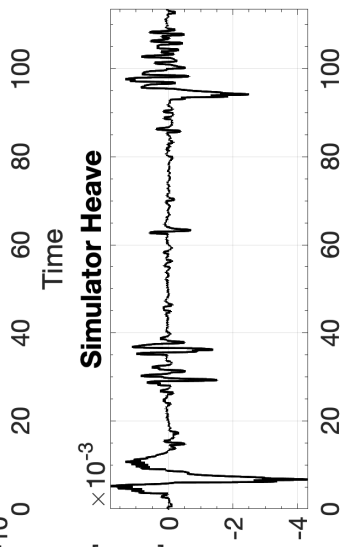

[s/m] ио!ฺอ
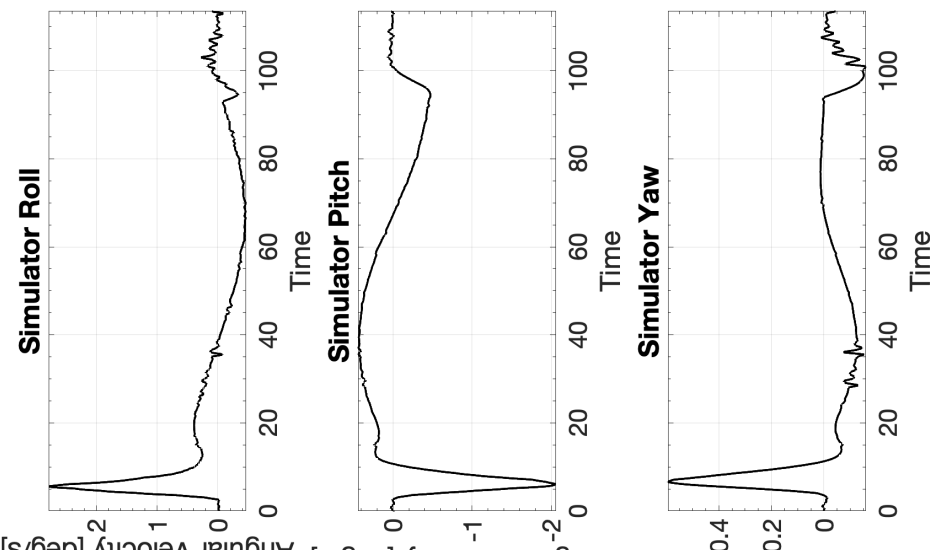

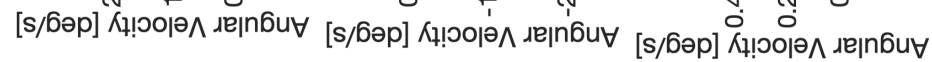




\section{B.4.3 CW3 Cessna 172 Coordinated Turn}

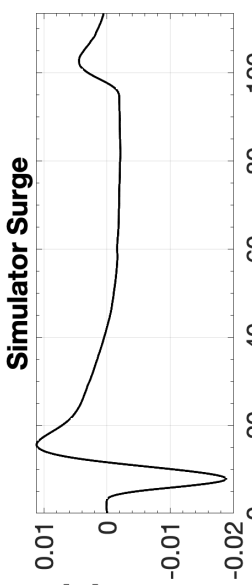

[u] uo!n!!sod

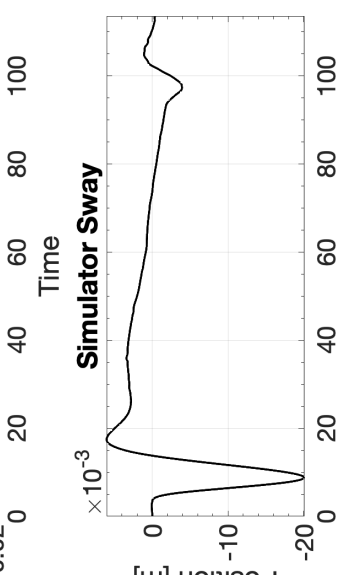

[w] uo!!! sod

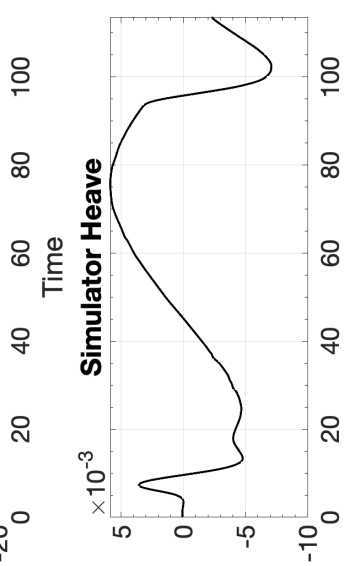

[u] uo!t!!sod

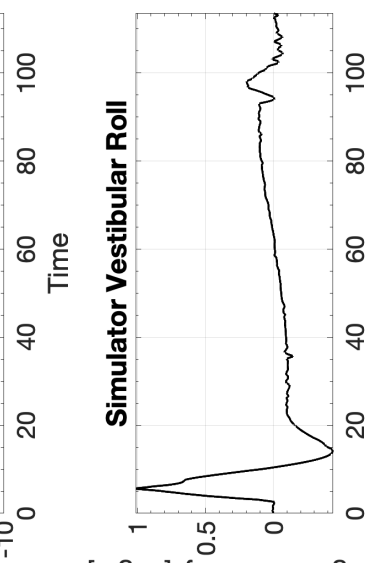

[s/6әр] Кำ
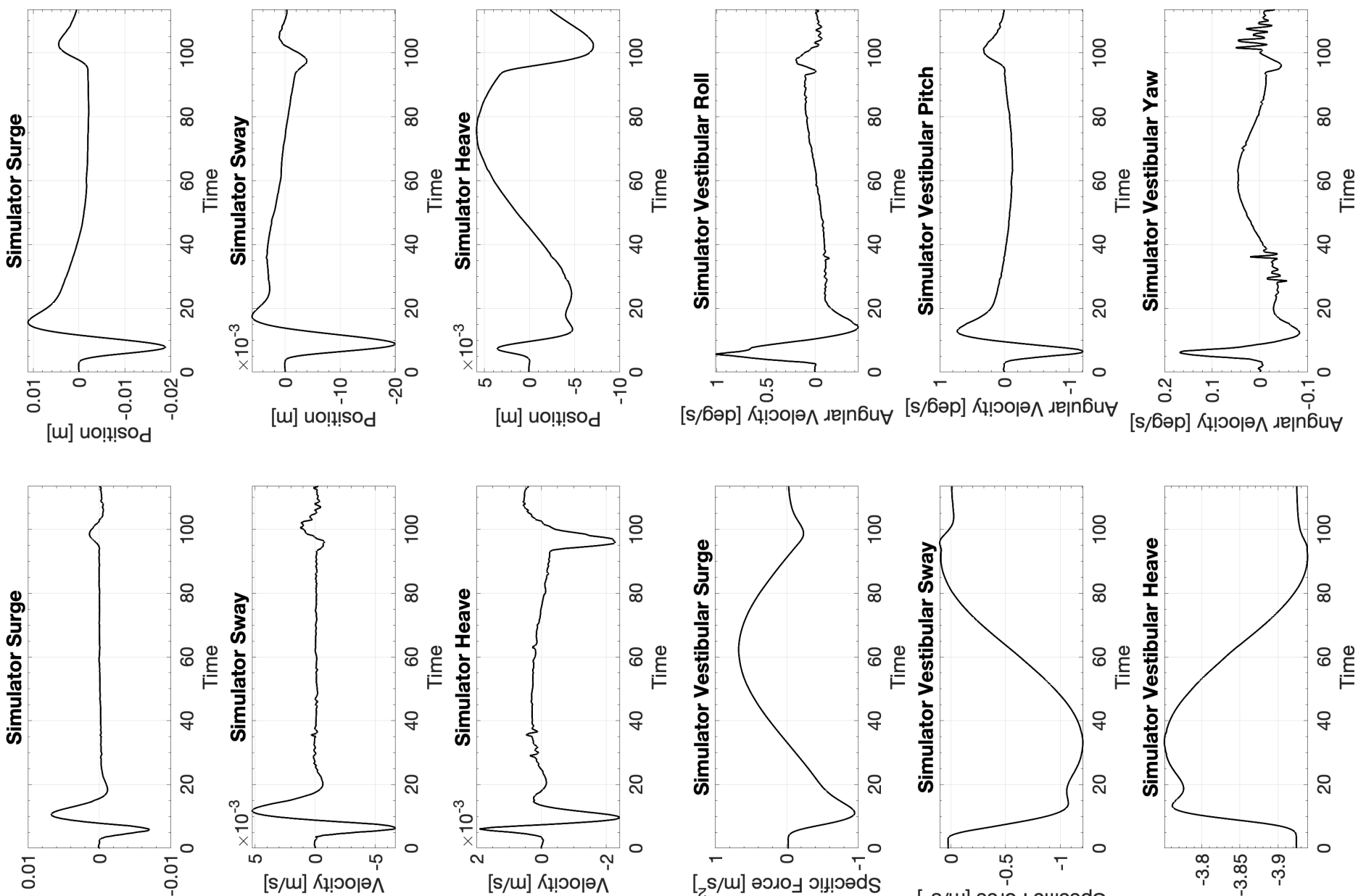

[s/u] Кц!юојә^
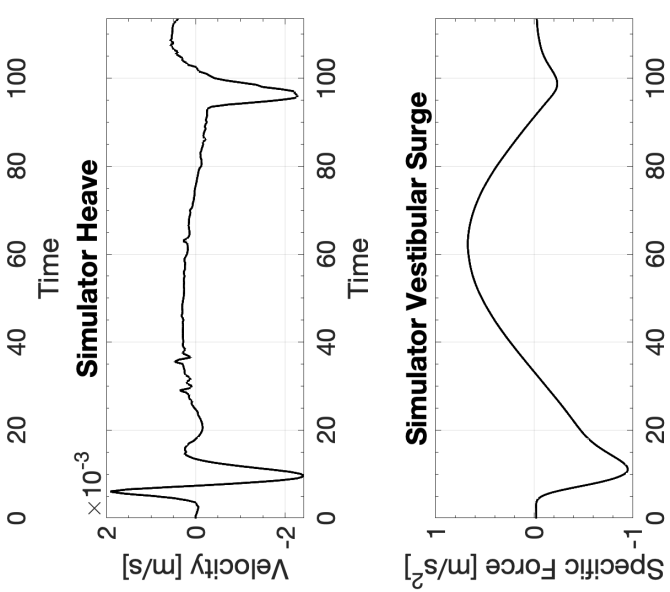

[
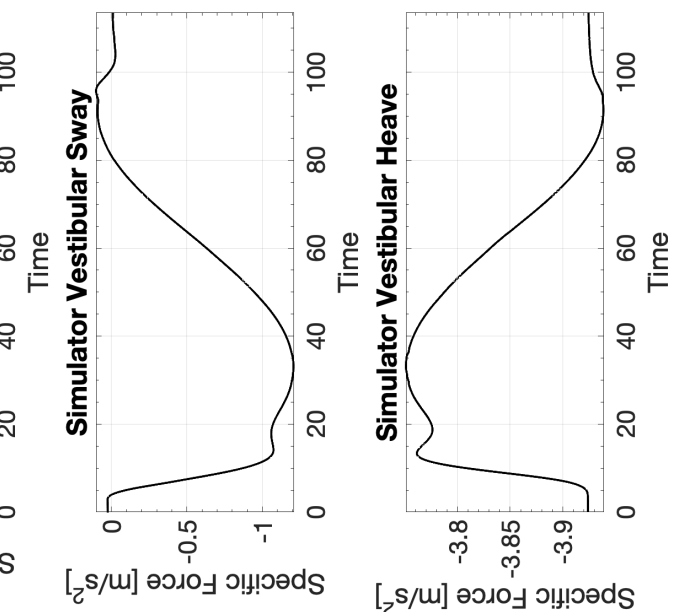

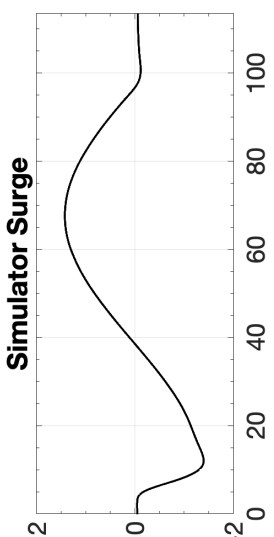

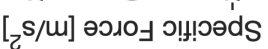

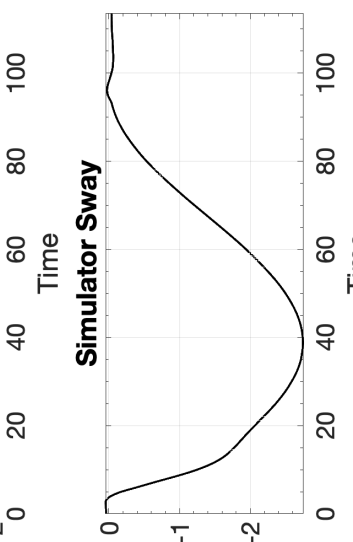

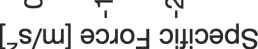

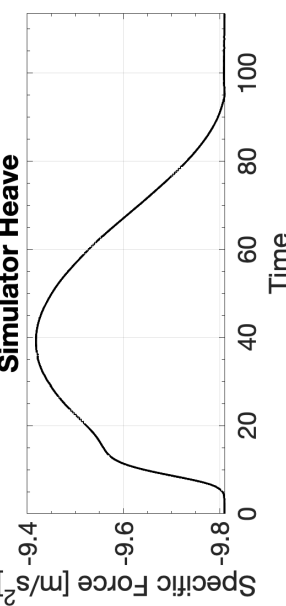

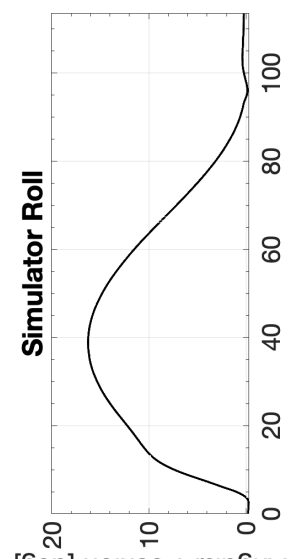

[бәр] uo!!!

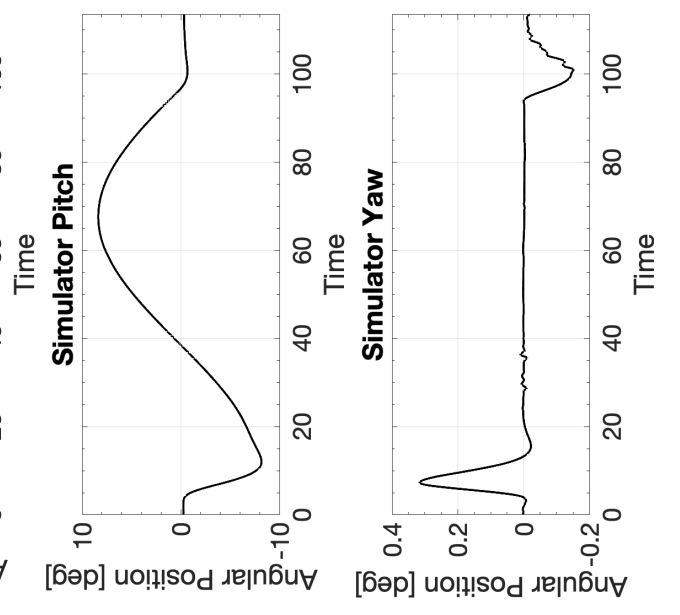

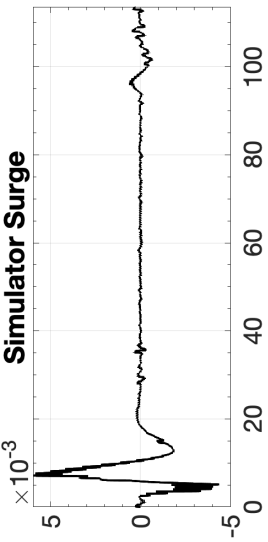

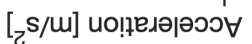

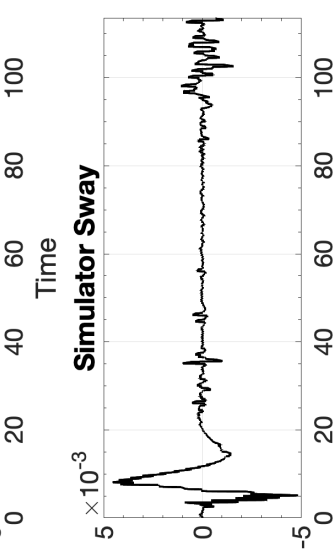

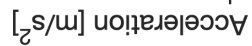

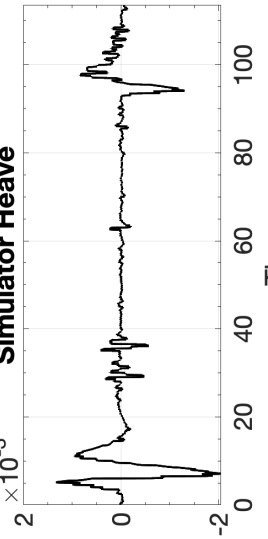

$[\mathrm{c} / \mathrm{m}]$ uо!ฺедәрәэว
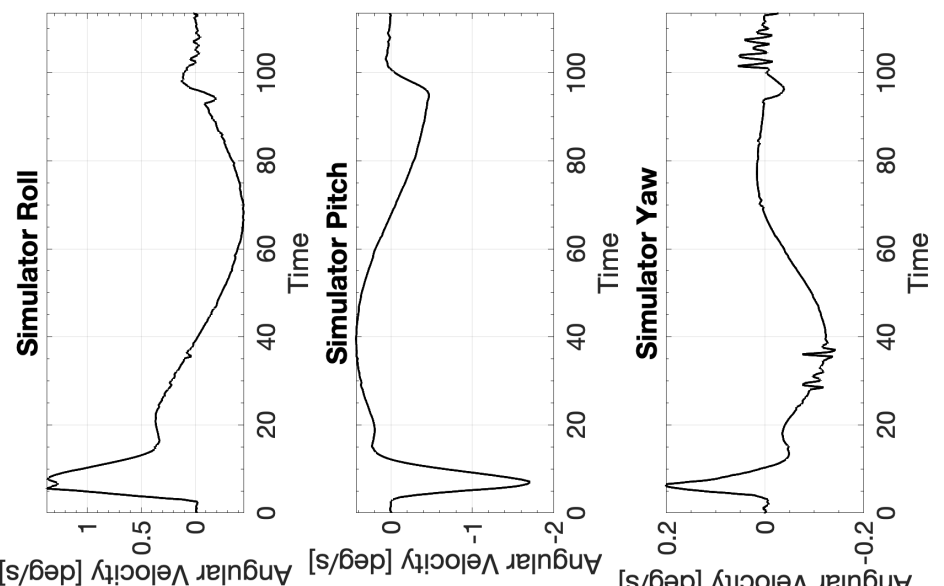


\section{B.5 Cessna 172 Cross-wind Landing}

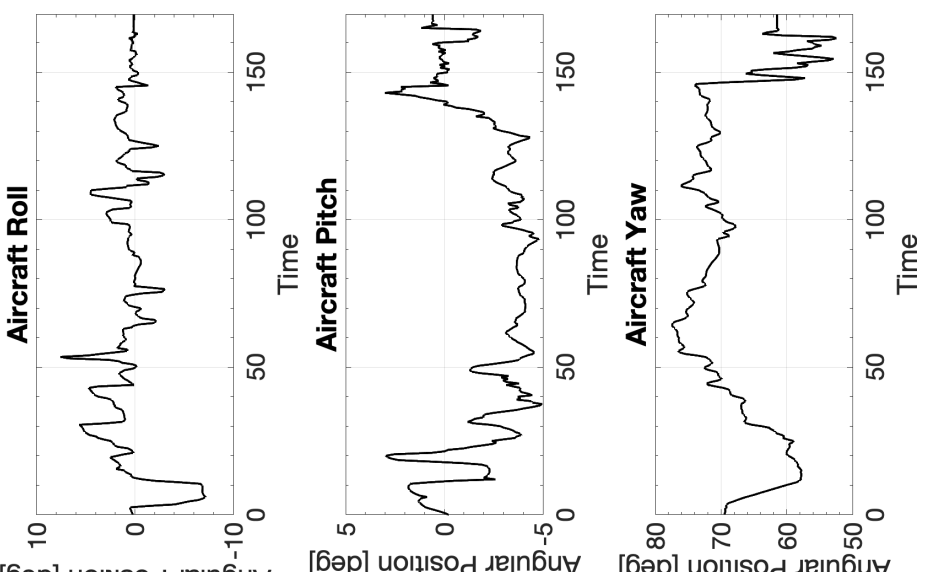

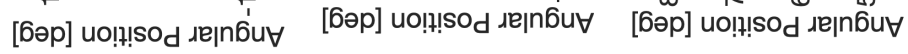

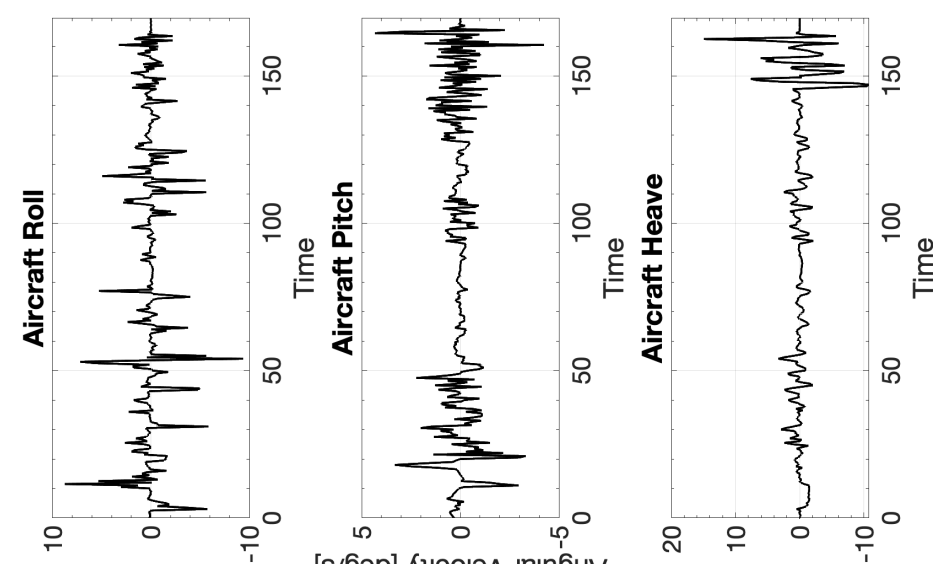

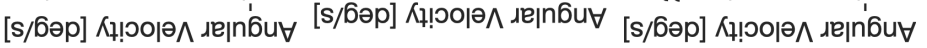

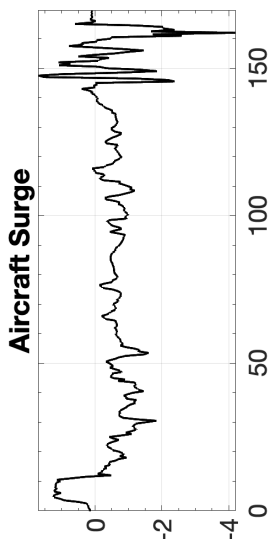

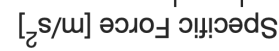

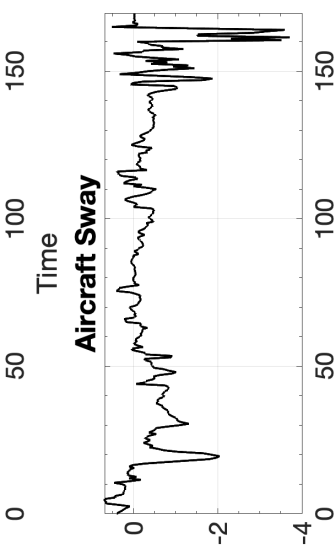

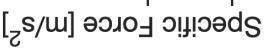

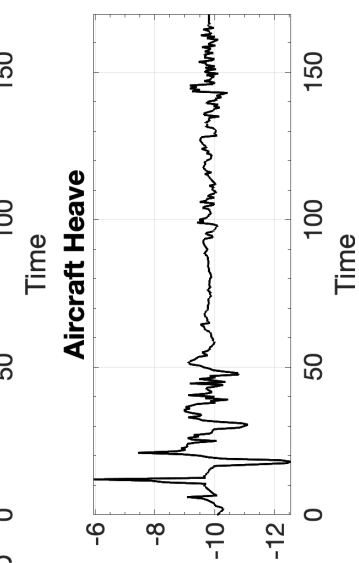

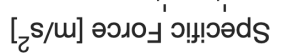

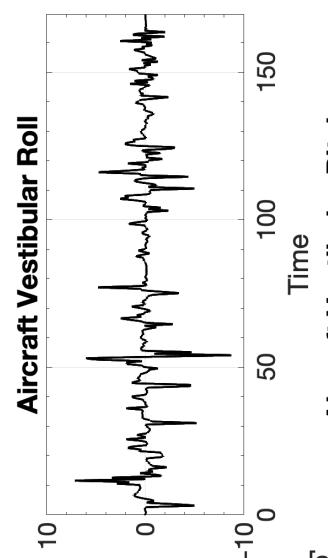

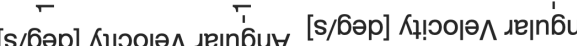

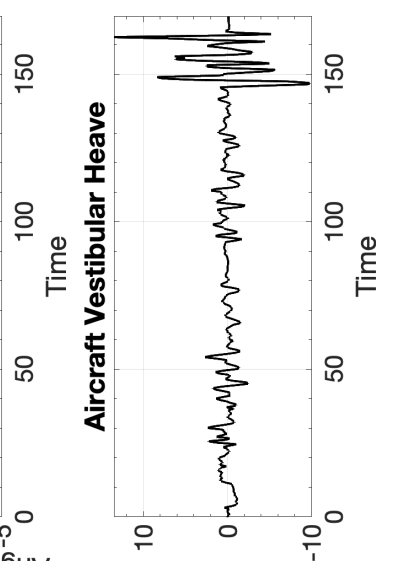

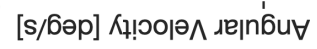

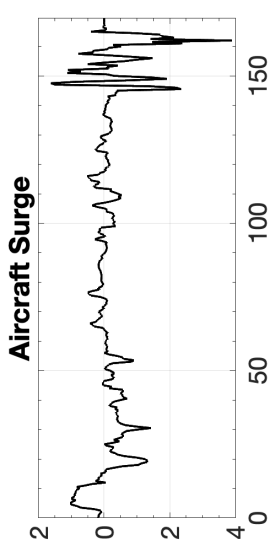

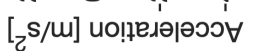

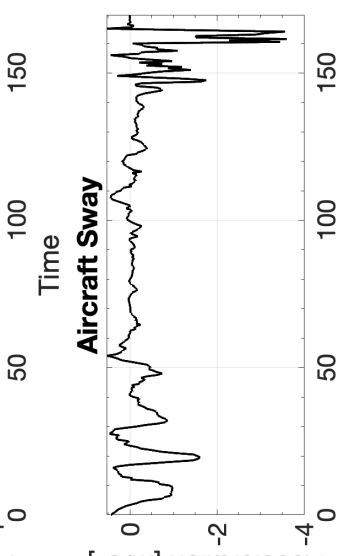

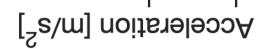

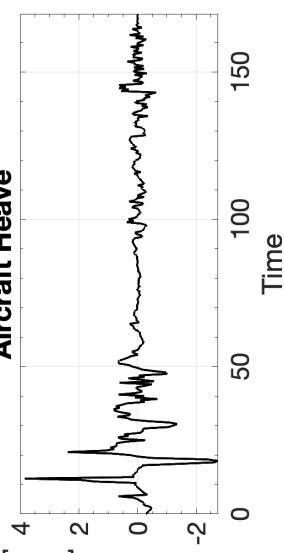

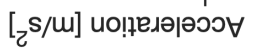

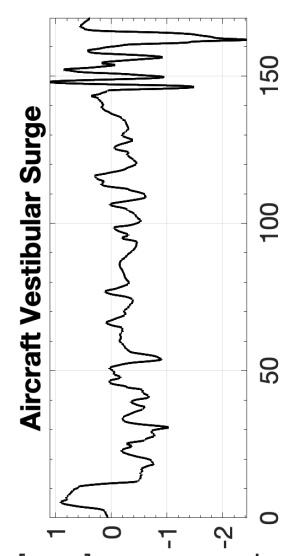

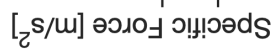

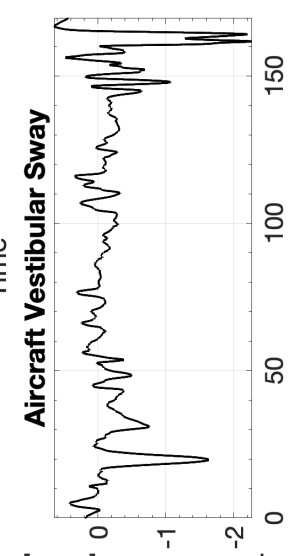

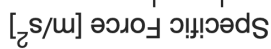

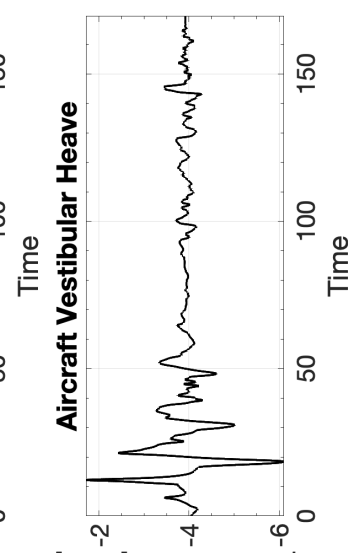

[ 


\section{B.5.1 CW1 Cessna 172 Cross-wind Landing}

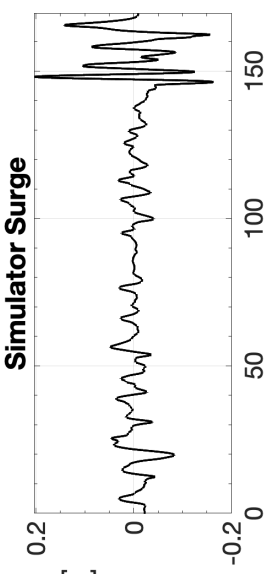

[u] uo!n! sod

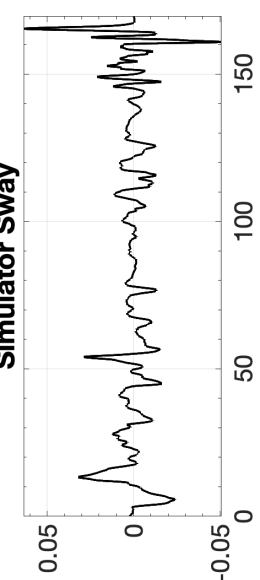

[w] uo!n! sod

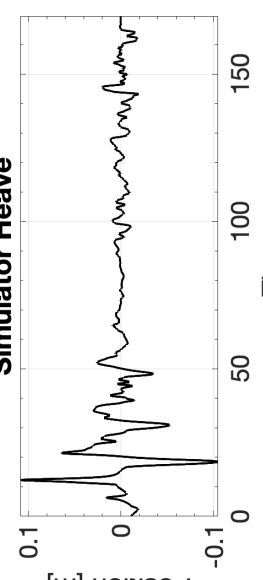

[w] uo!n!sod

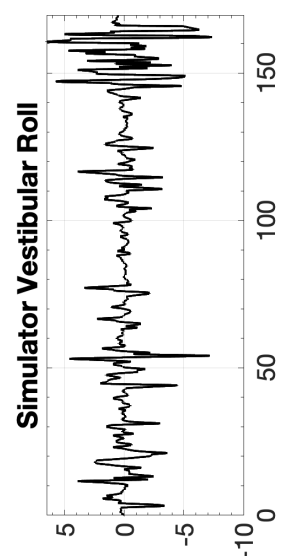

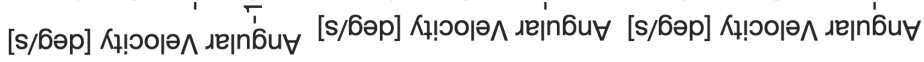
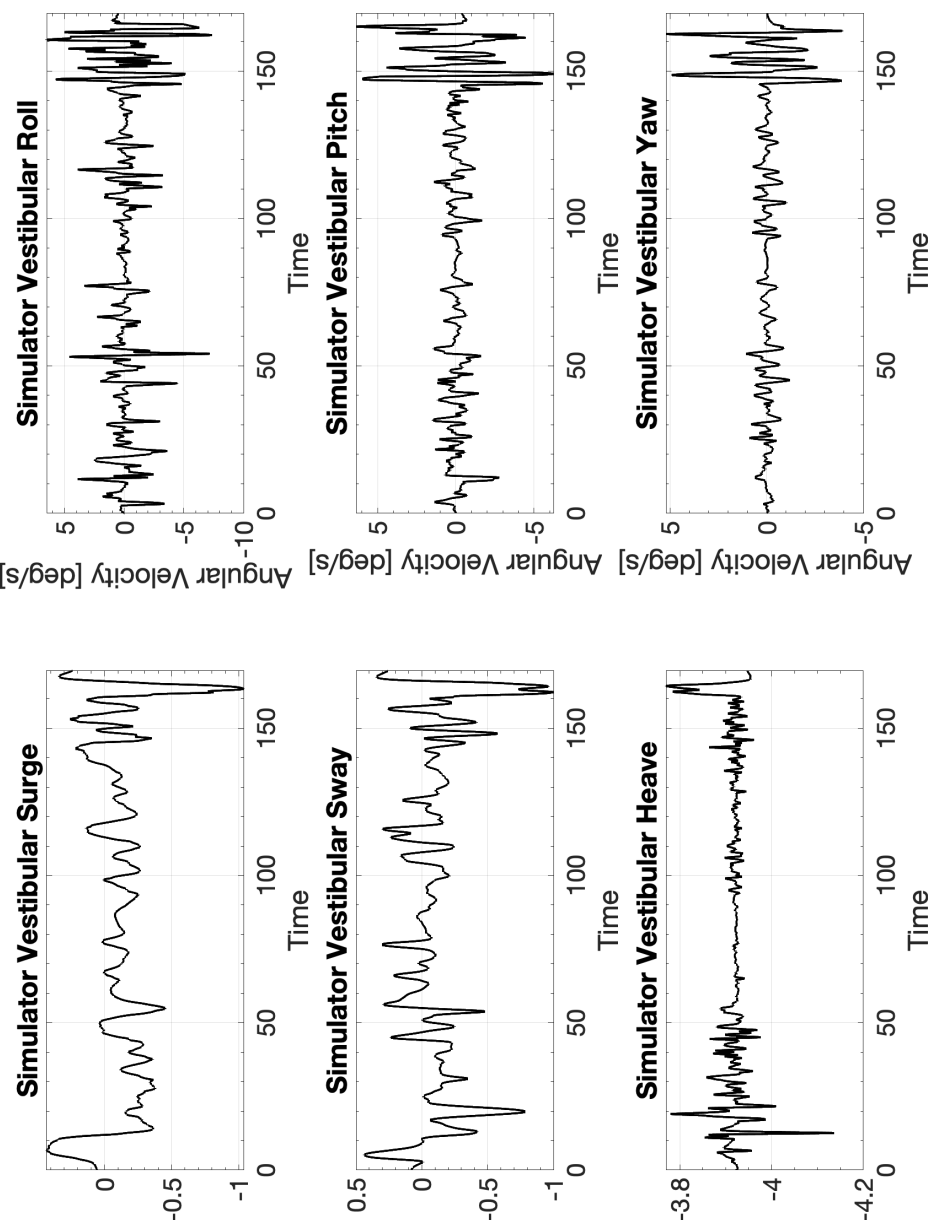

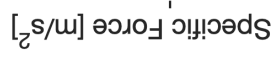

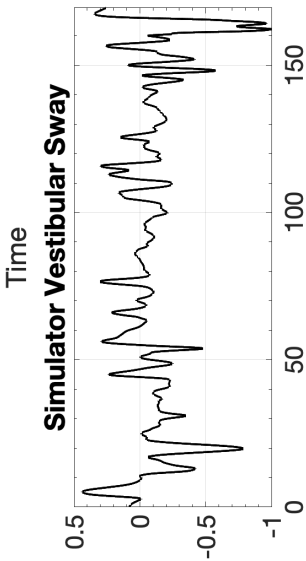

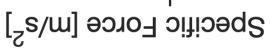

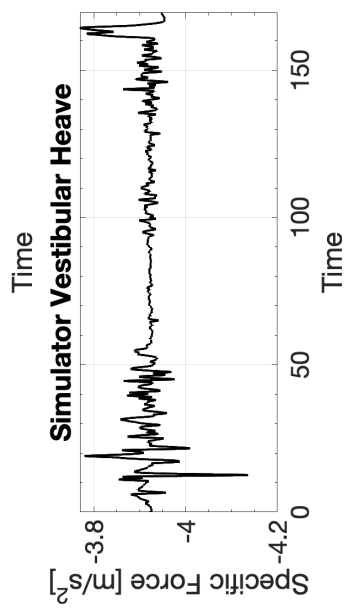

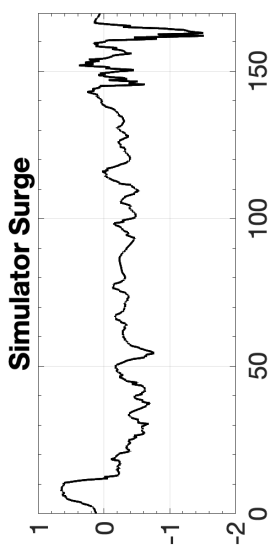
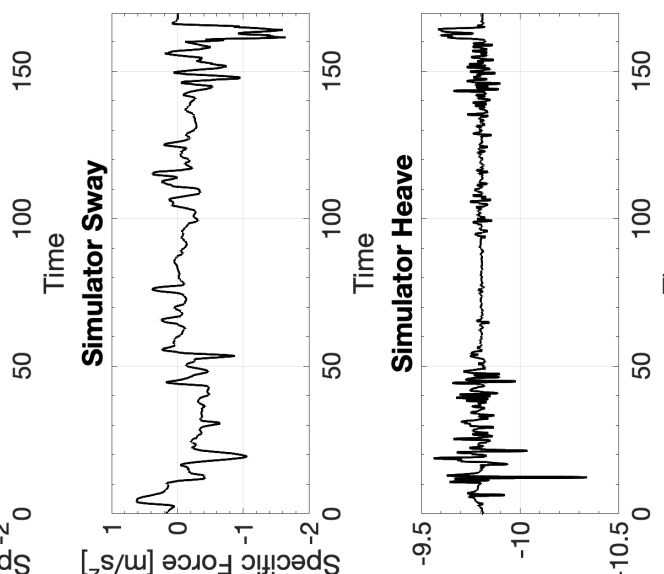

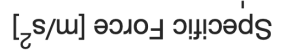

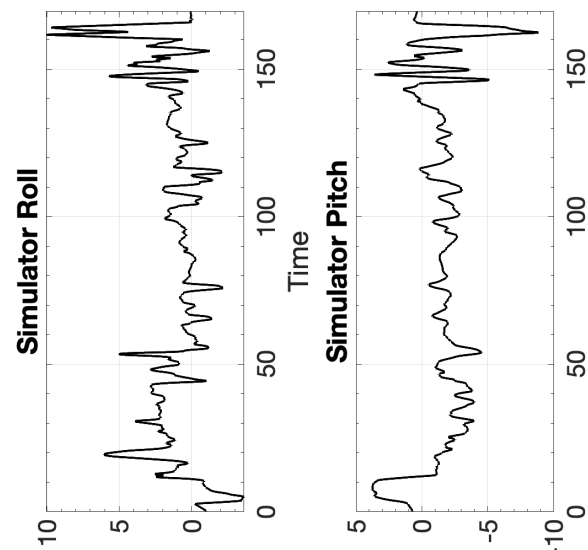

[бәр] uo!!!!sod גe|n6u

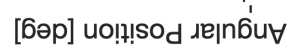

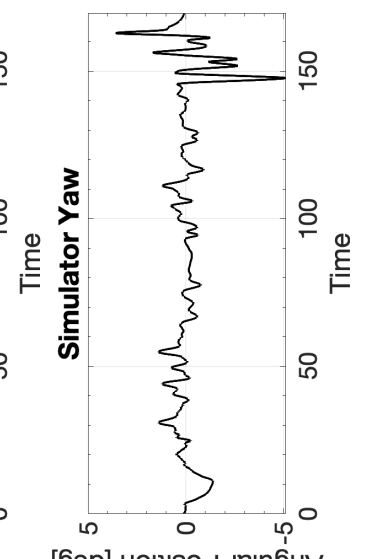

[бәр] uo!?!sod גejnбu

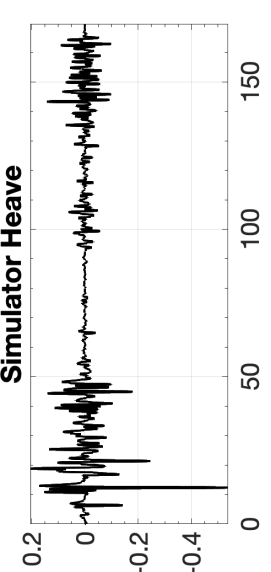

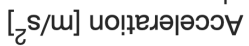

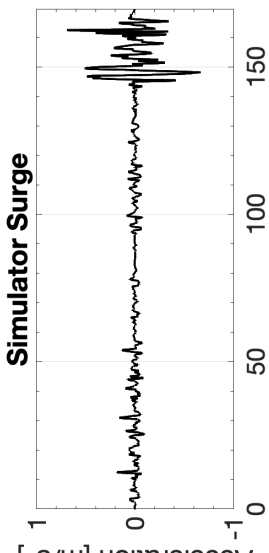

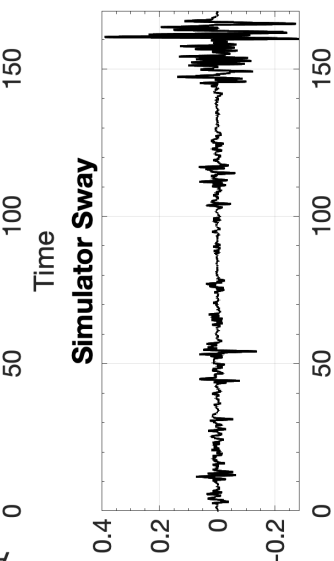

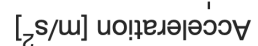
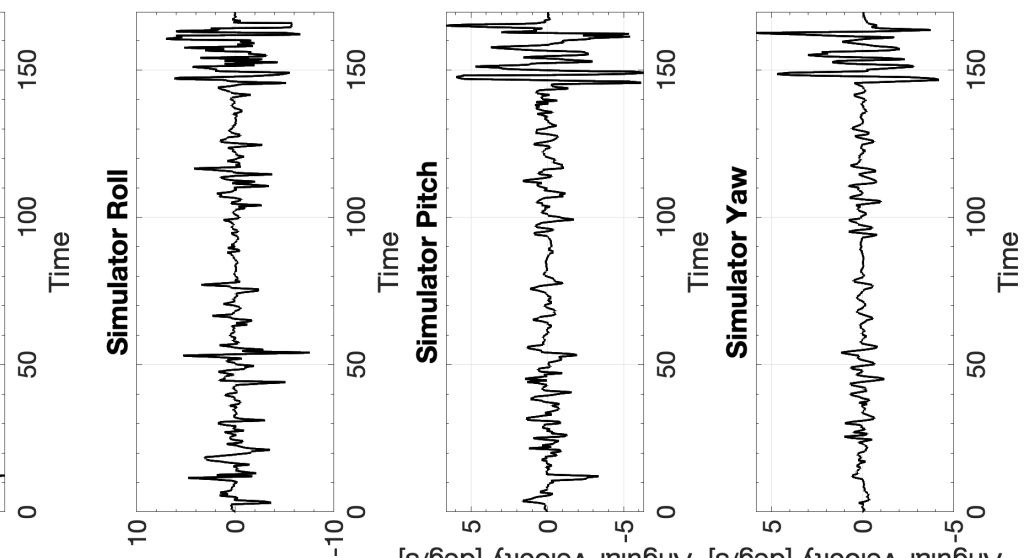


\section{B.5.2 CW2 Cessna 172 Cross-wind Landing}

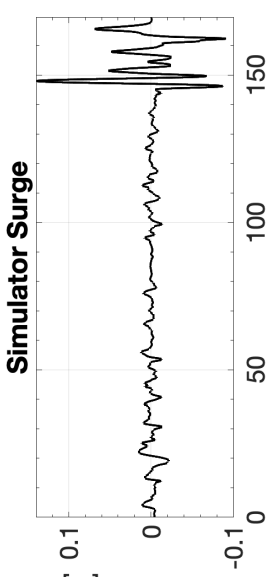

[m] uo!!!!sod

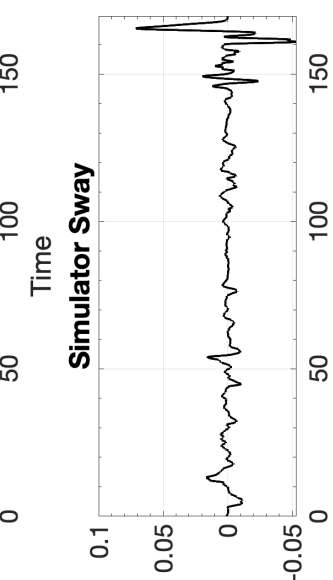

[u] uo!n!!sod

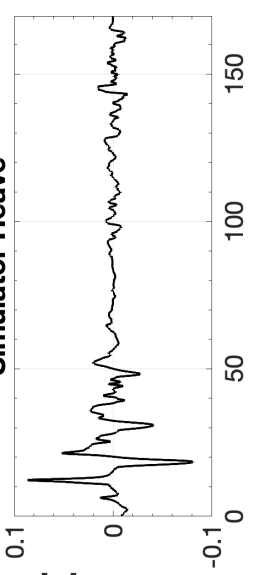

[w] uo!n!sod

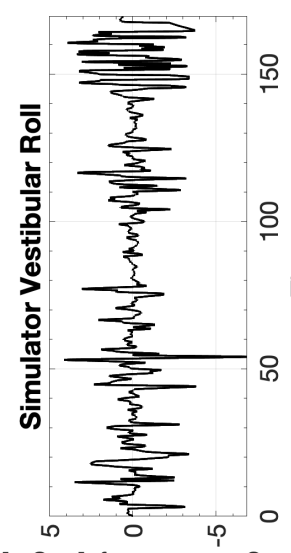

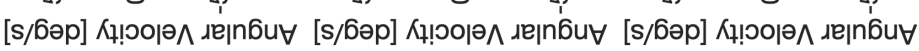
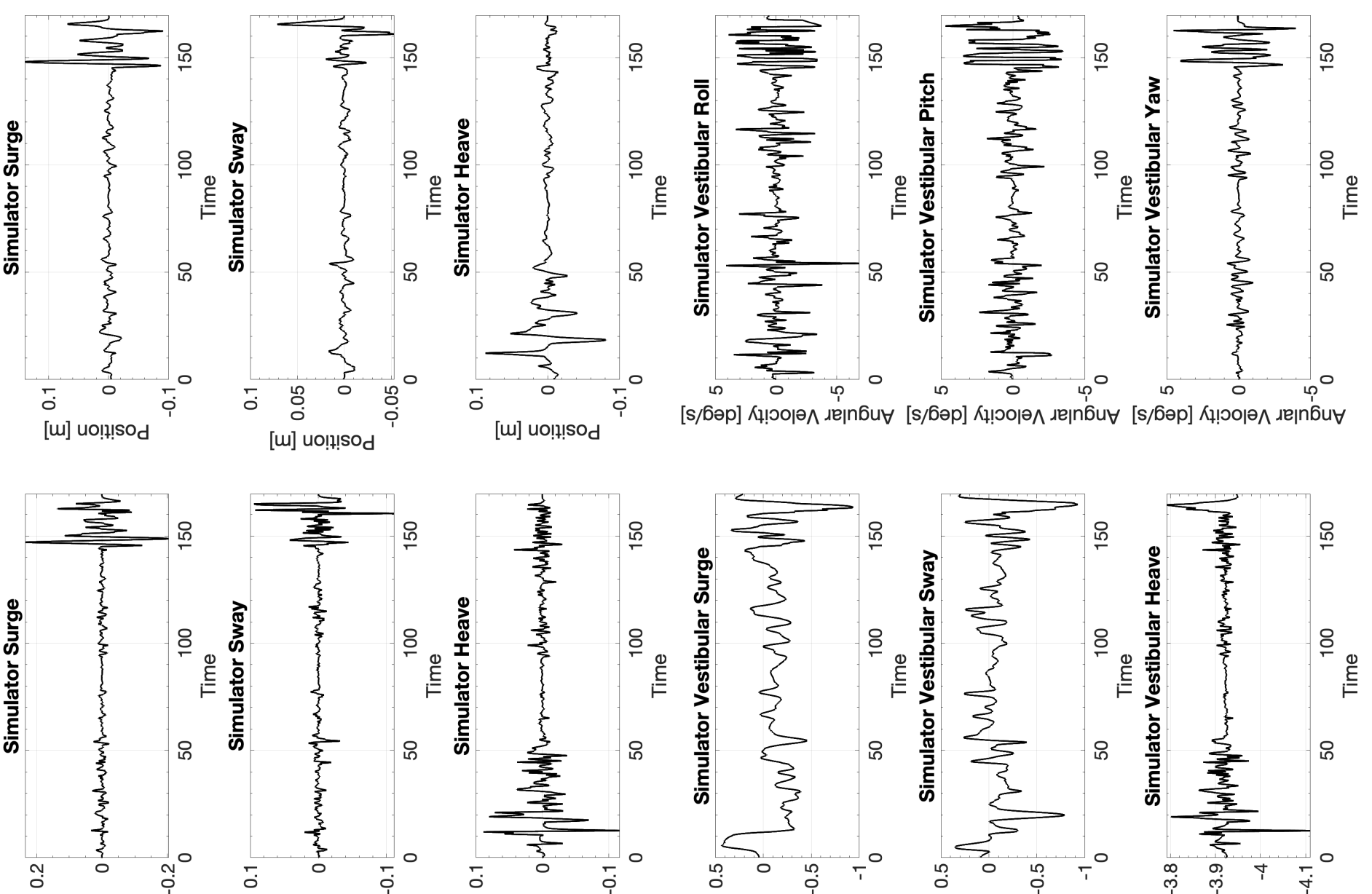

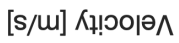

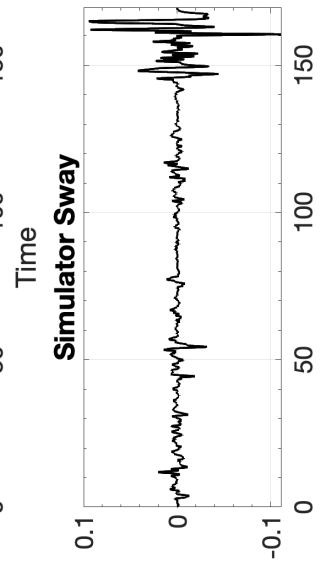

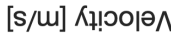

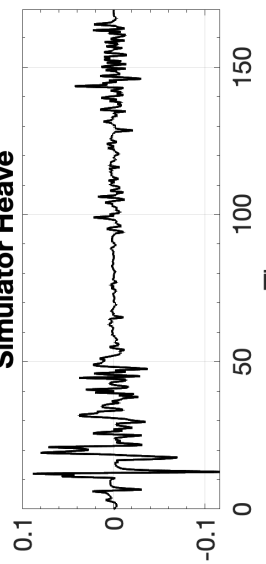

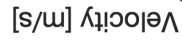

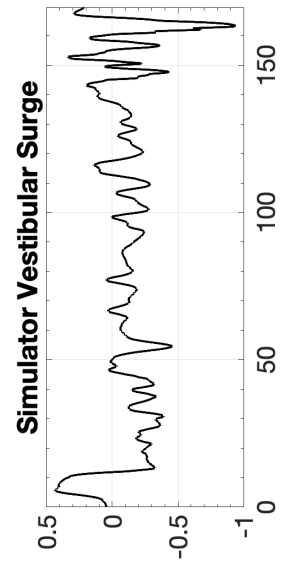

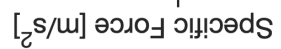

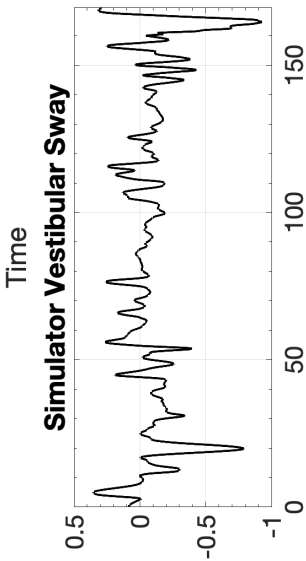

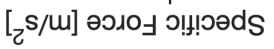

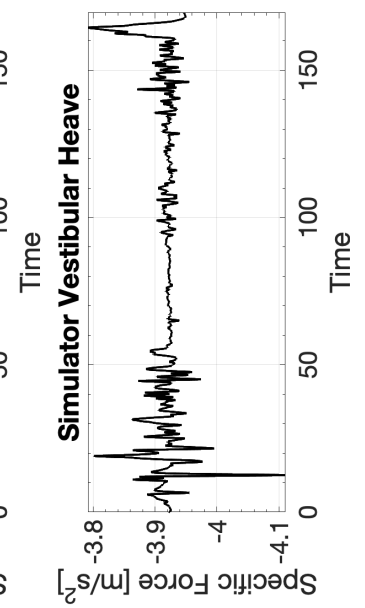

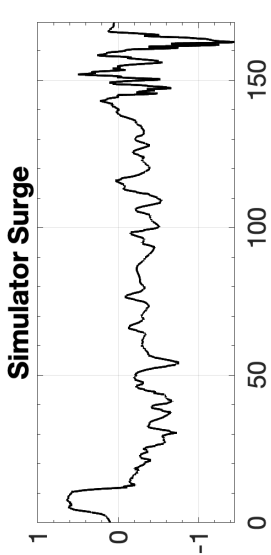

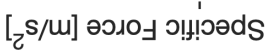

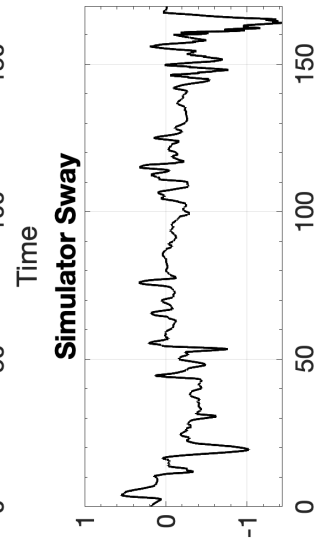

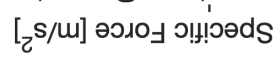

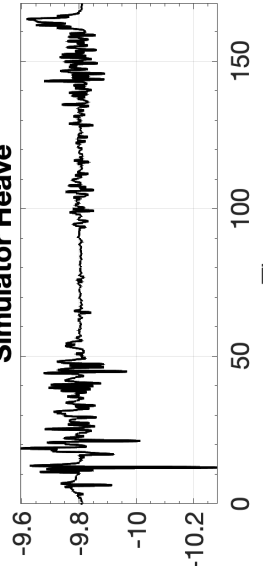

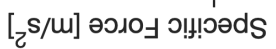

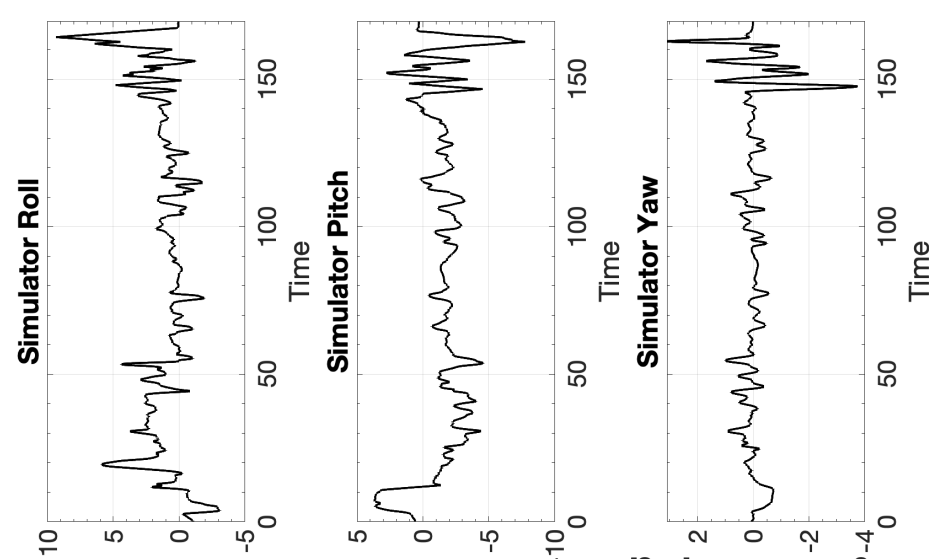

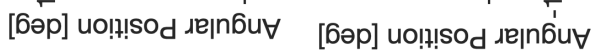

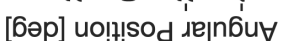

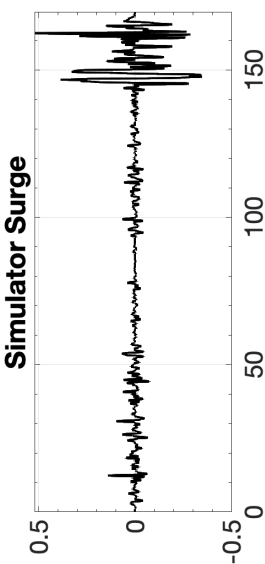

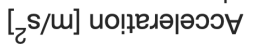

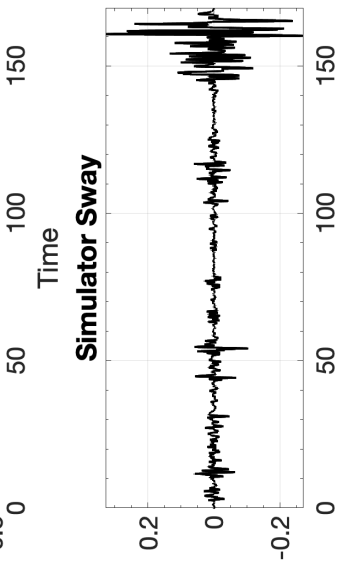

$[\mathrm{z} / \mathrm{s}]$ ио!ฺеләрәэว

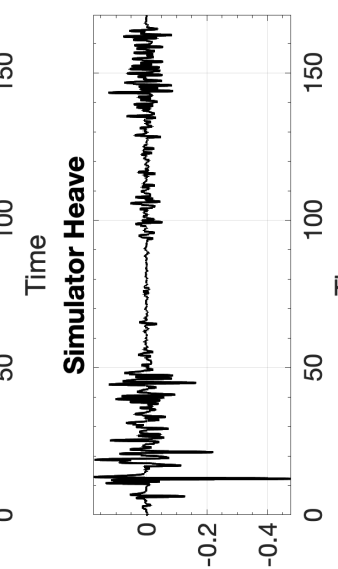

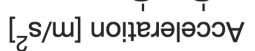
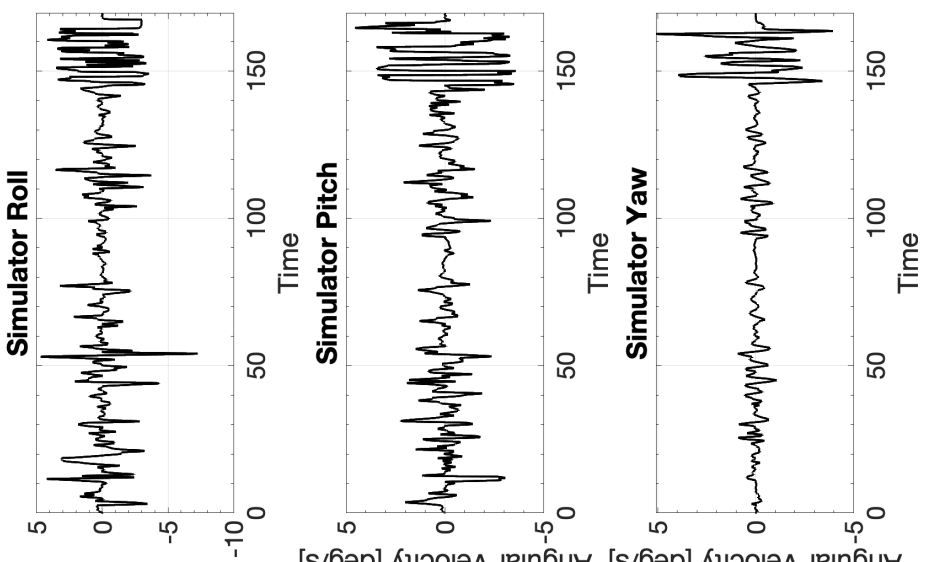

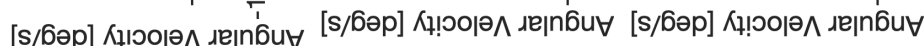




\section{B.5.3 CW3 Cessna 172 Cross-wind Landing}

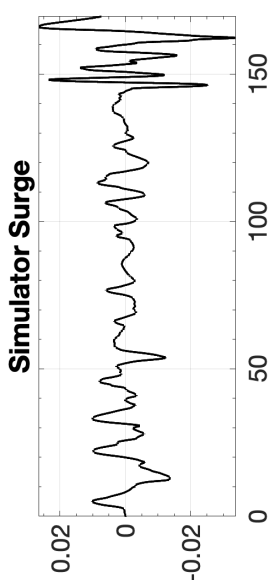

[w] no!!!sod

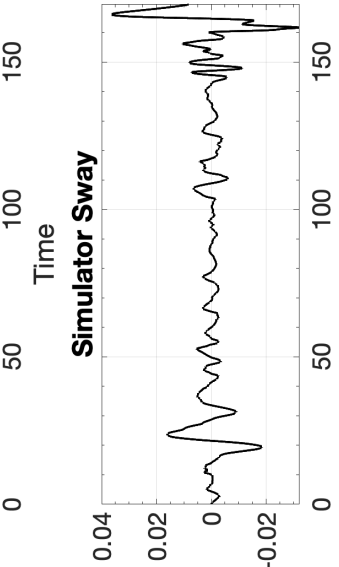

[w] uol!?!sod

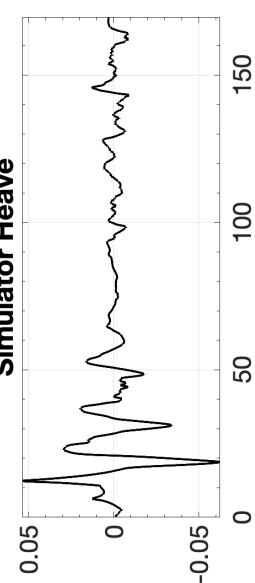

[u] uolp!nsod

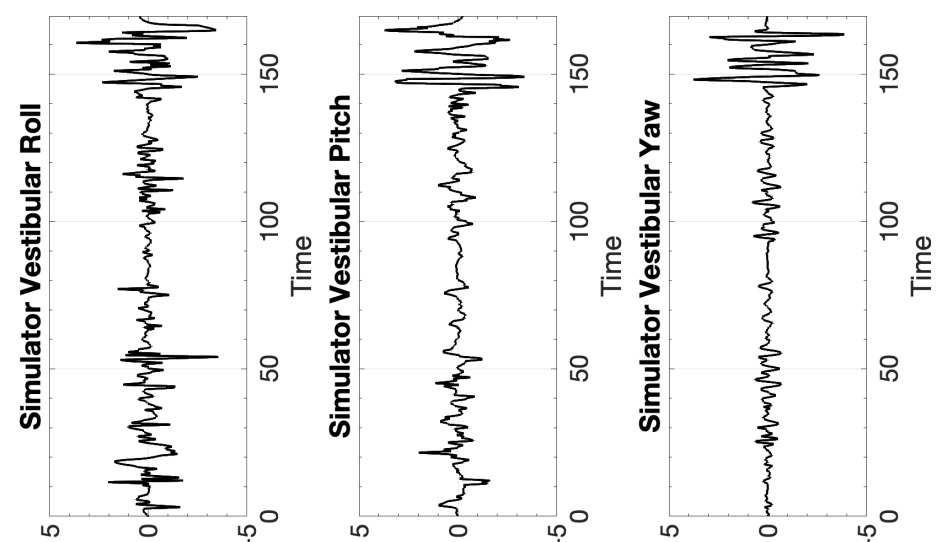

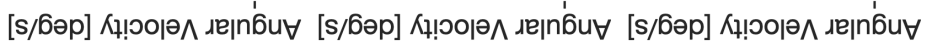

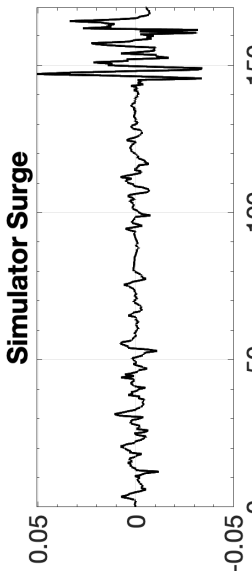

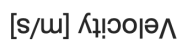

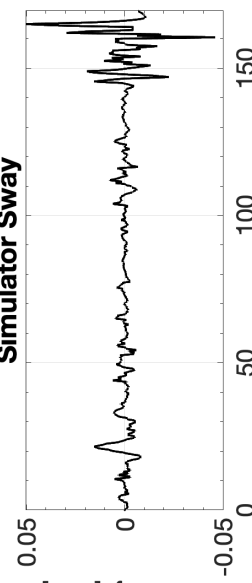

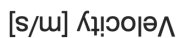

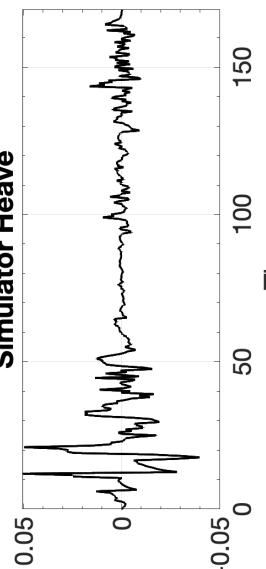

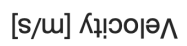

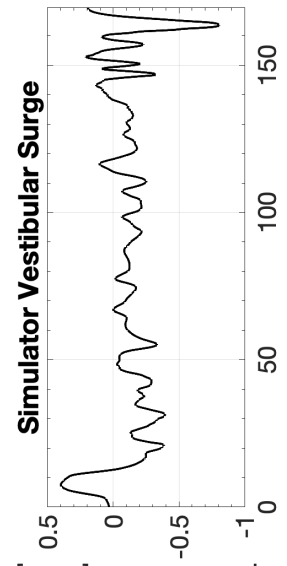

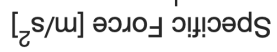

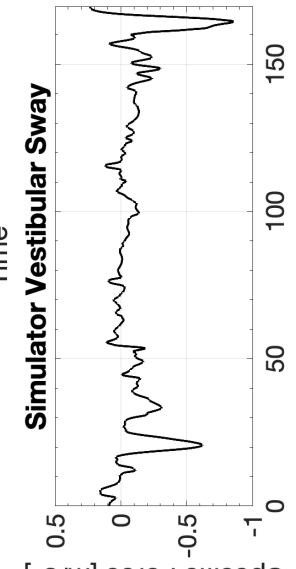

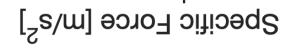

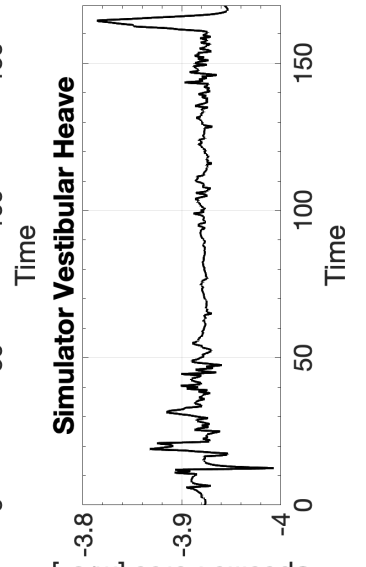

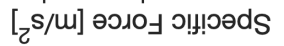

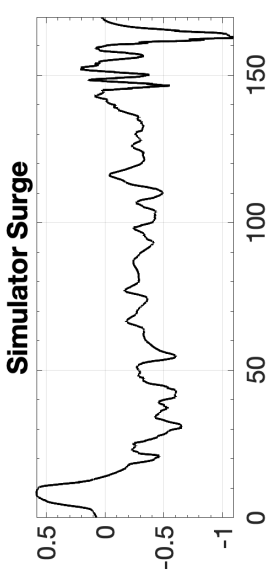

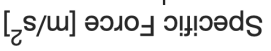

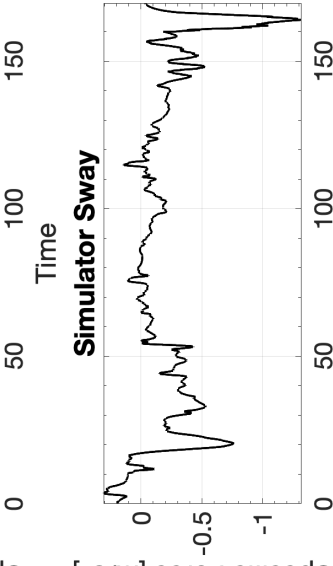

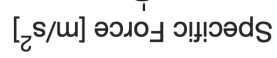

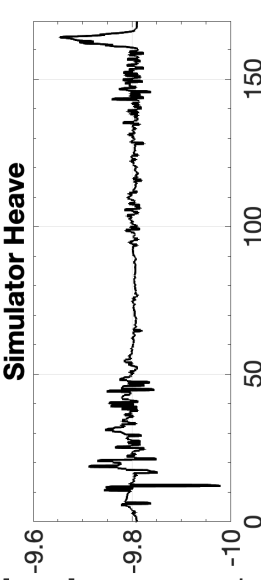

[

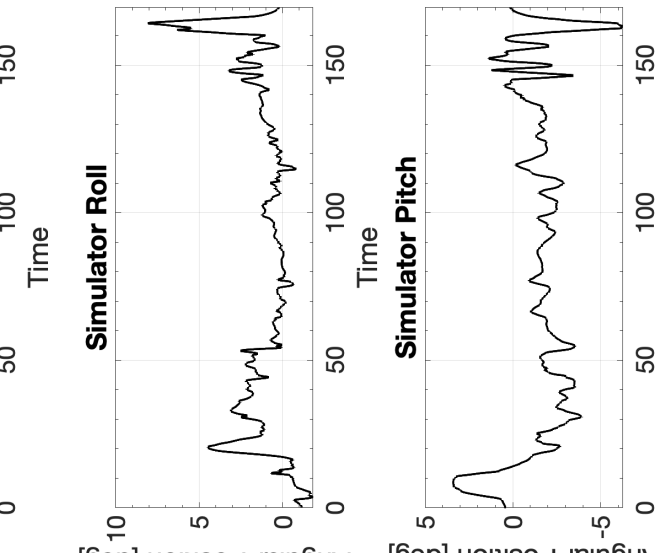

[6әр] uo!!!

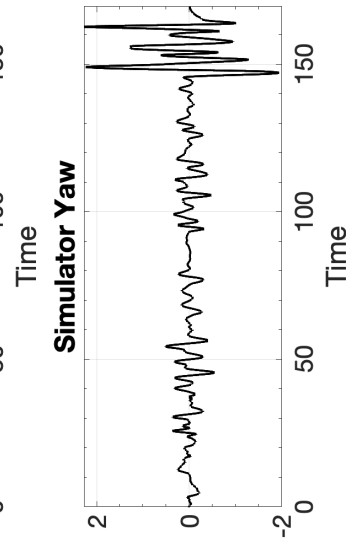

[6əp] uo!!!!sod גeןn6u

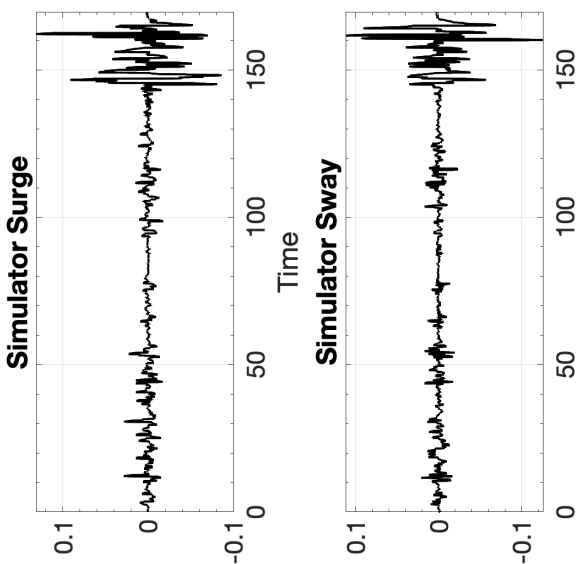

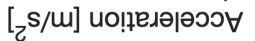

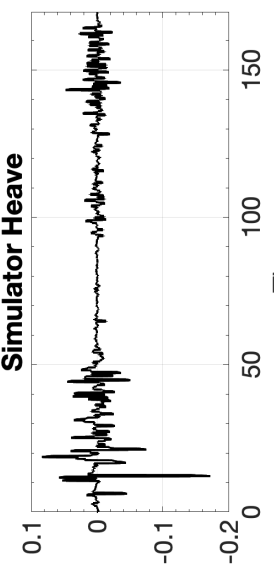

[zs/m] ио!ฺеләрәэว

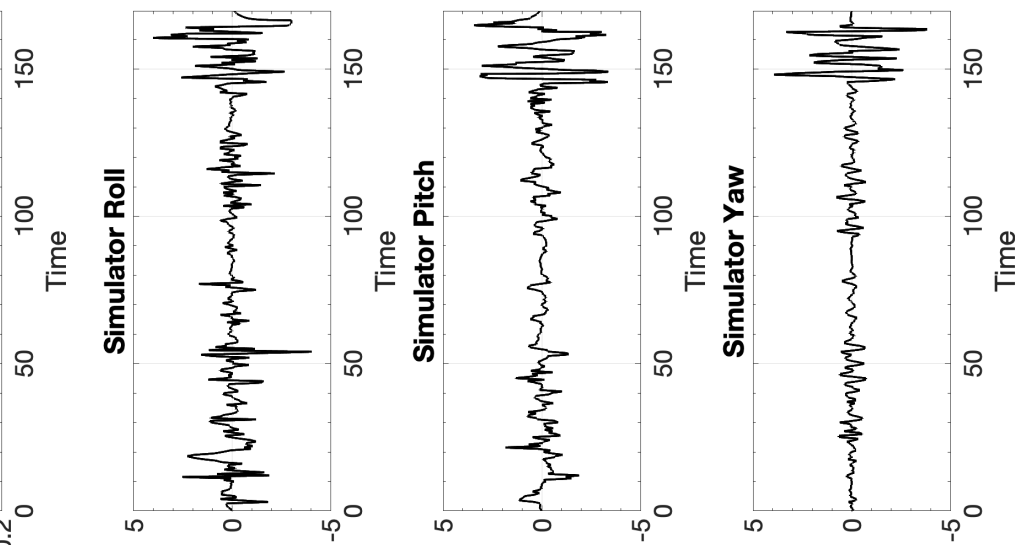

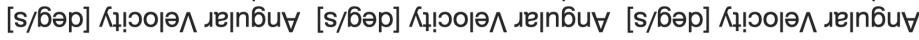




\section{B.6 Cessna 172 Single-axis Takeoff}
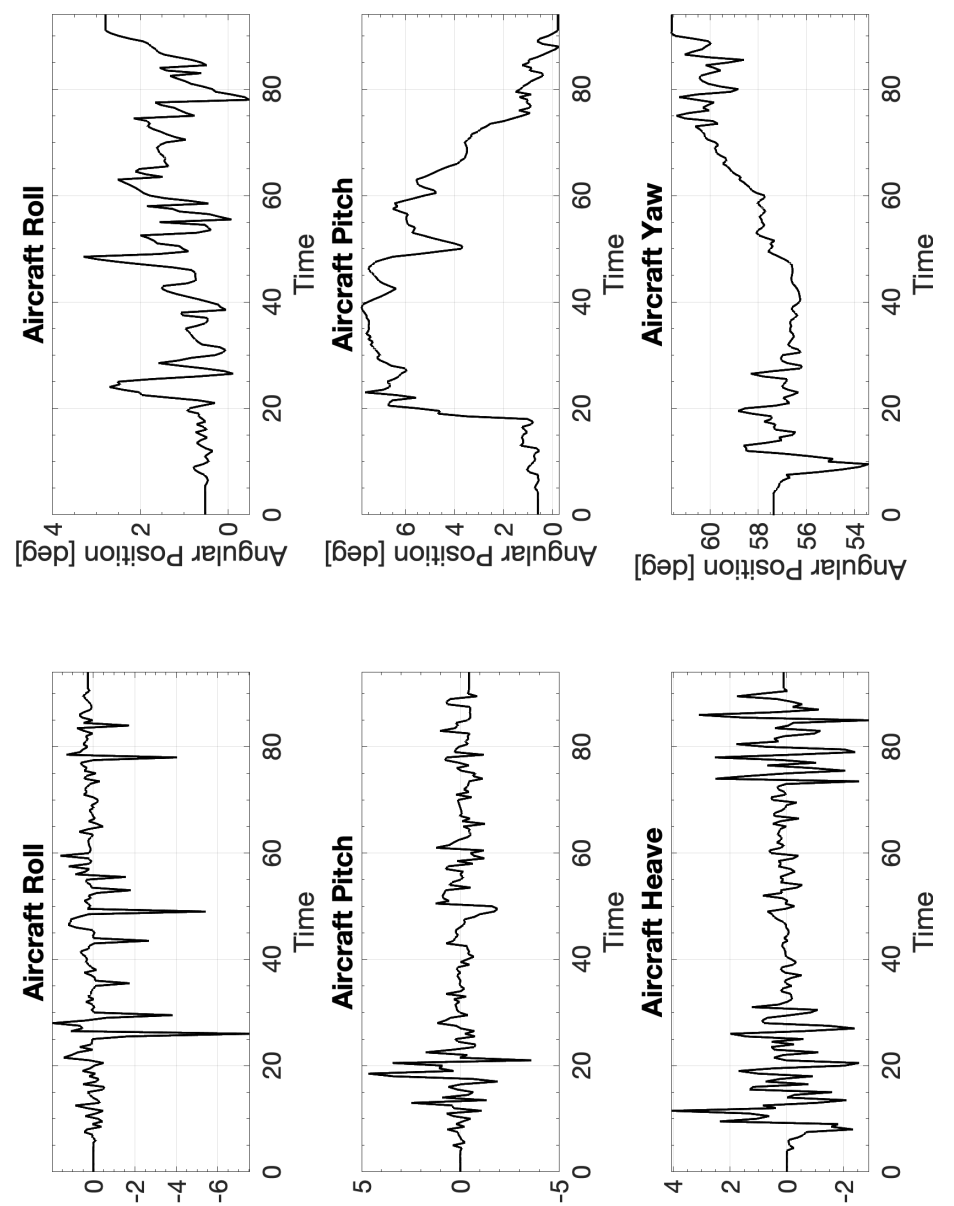

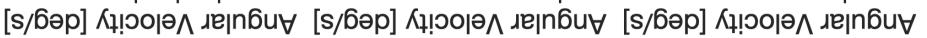

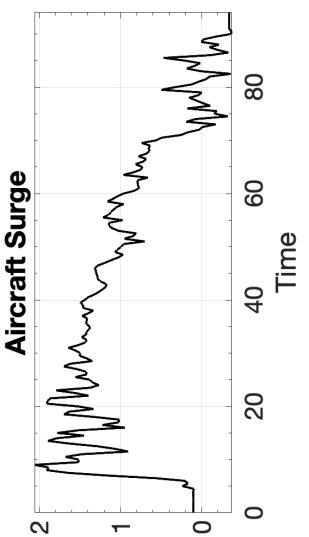

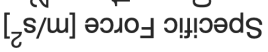
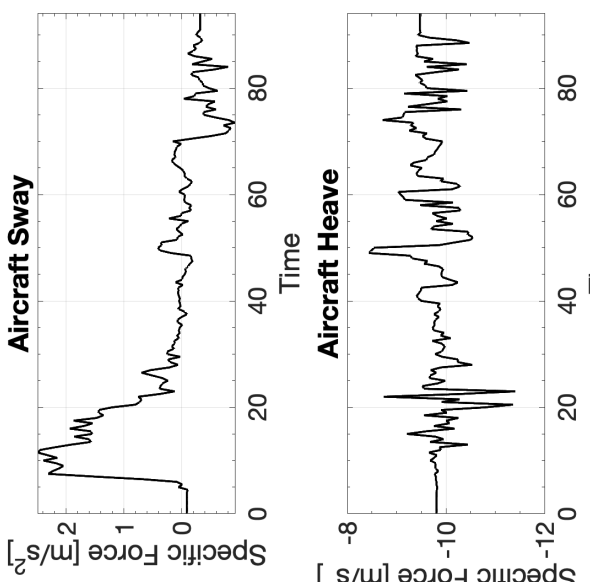

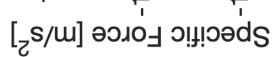

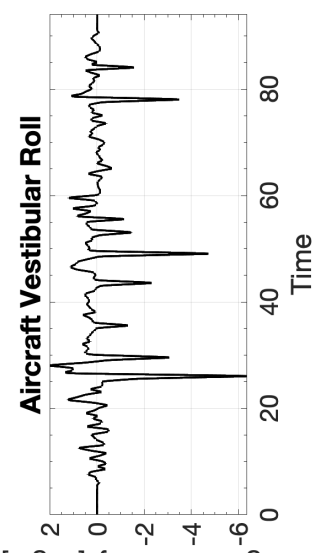

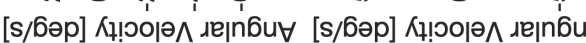

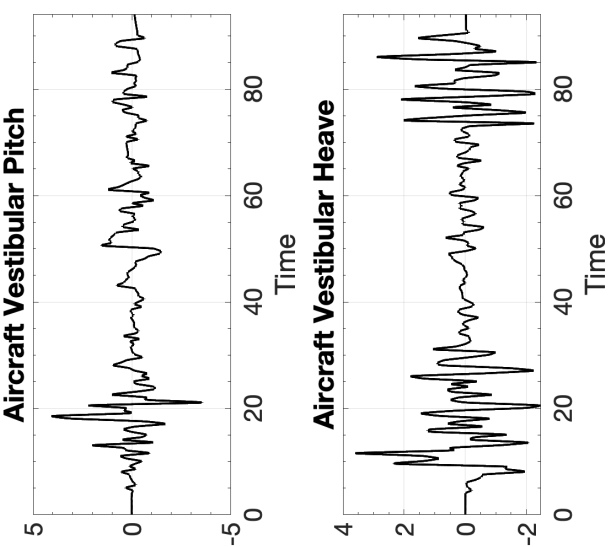

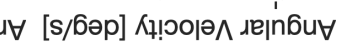

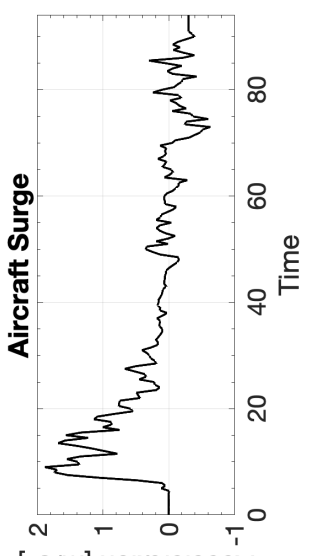

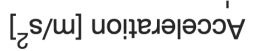

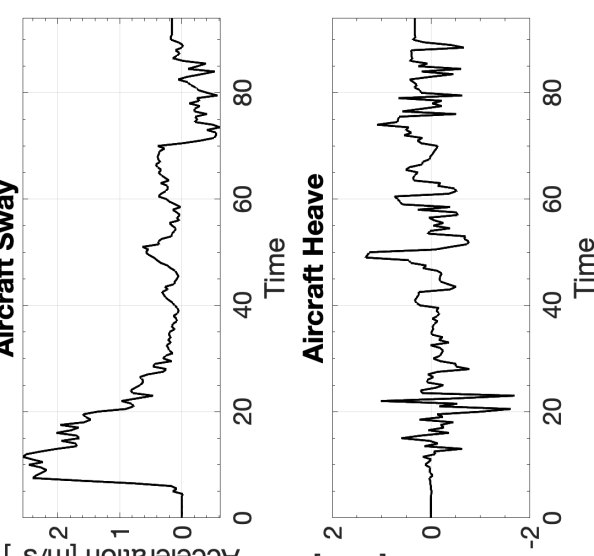

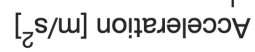

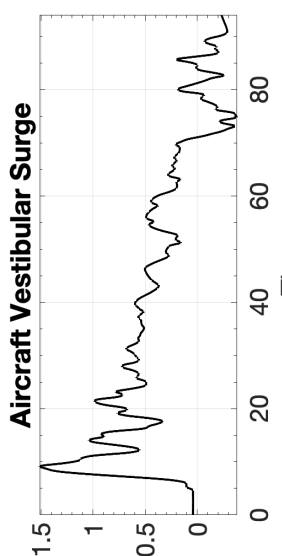

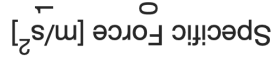

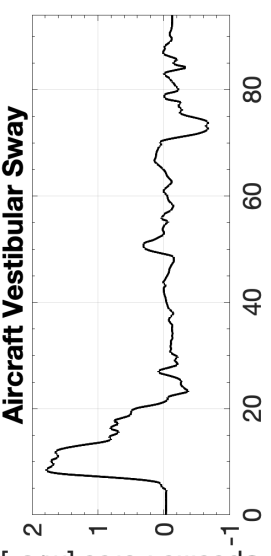

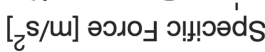

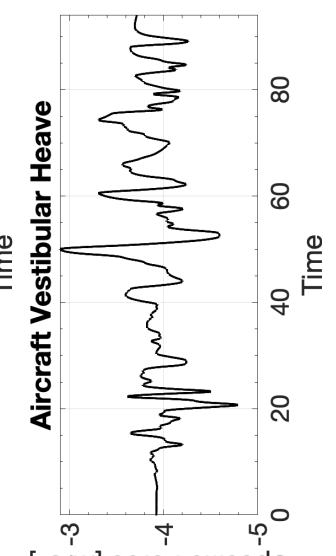

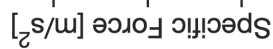




\section{B.6.1 CW1 Cessna 172 Single-axis Takeoff}

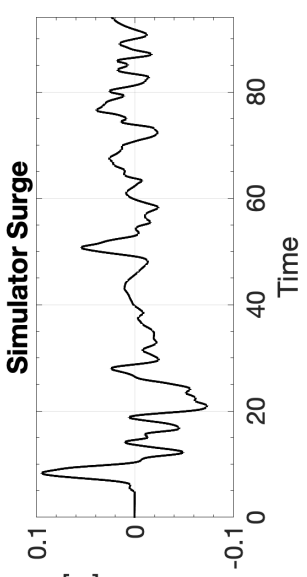

[u] uo!n! sod

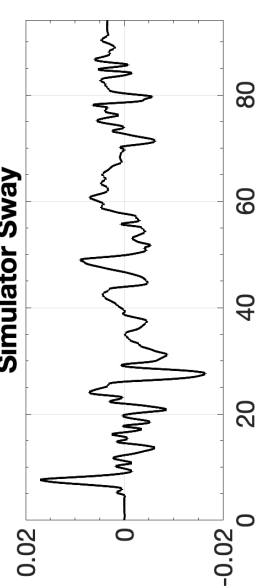

[u] uo!n!!sod

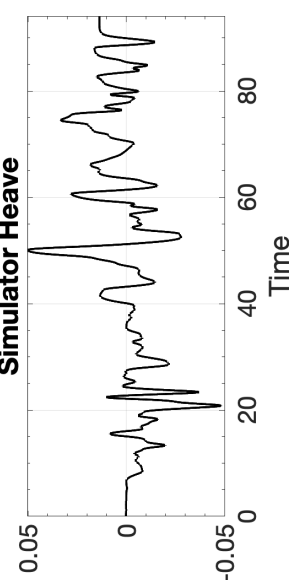

[w] uol!!sod

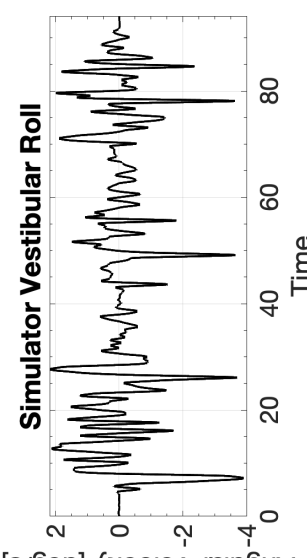

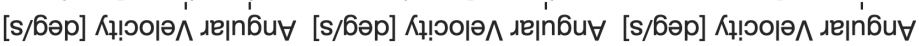

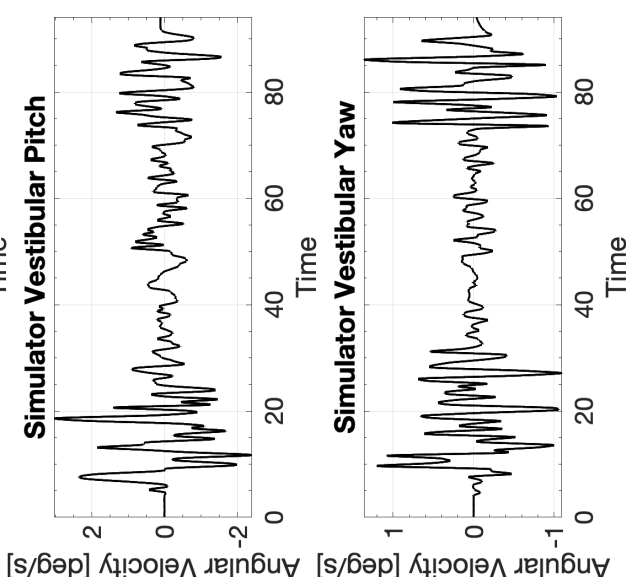

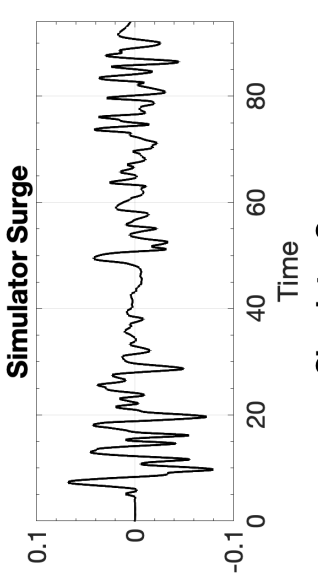

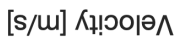

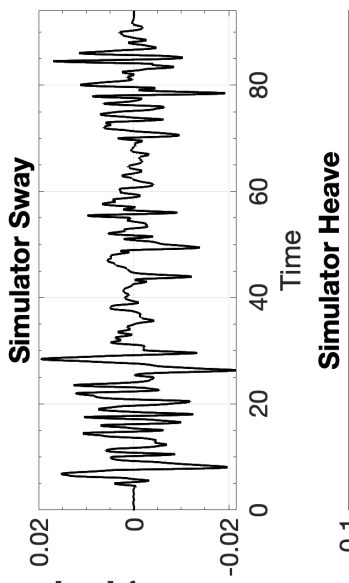

[s/m] Кұ!юор夫
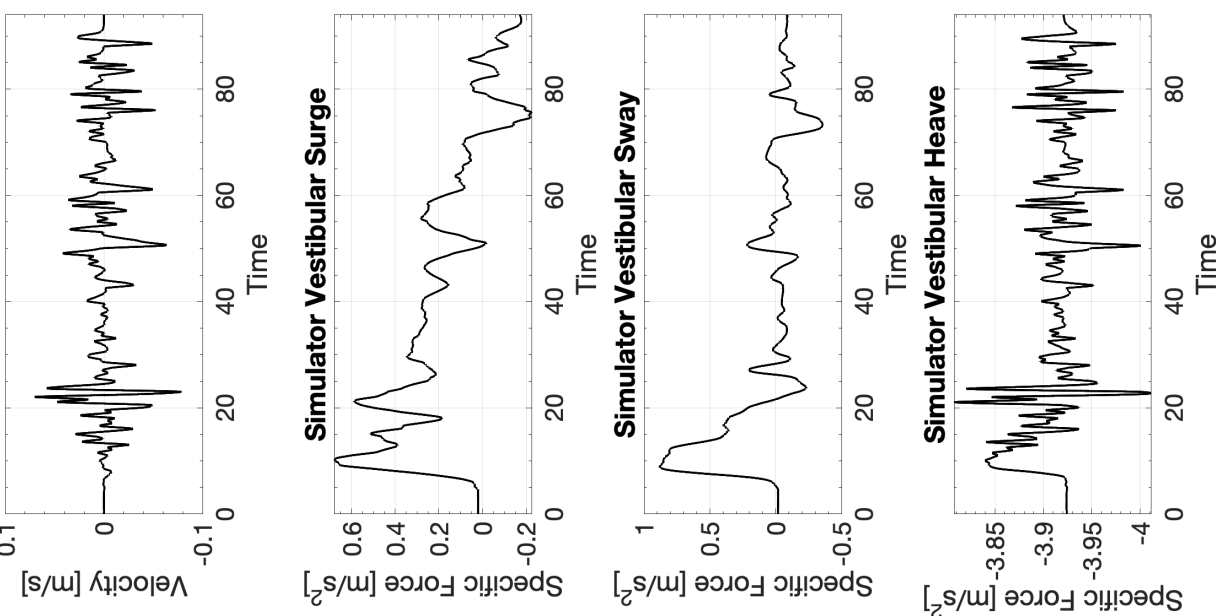

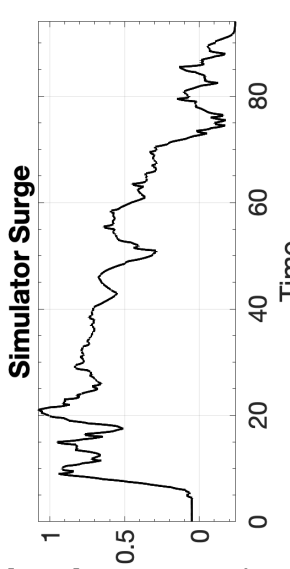

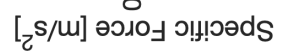

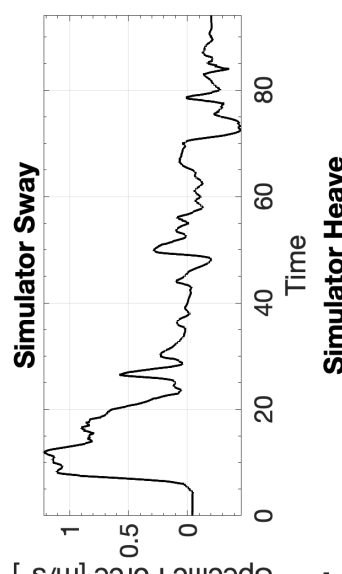

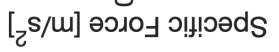

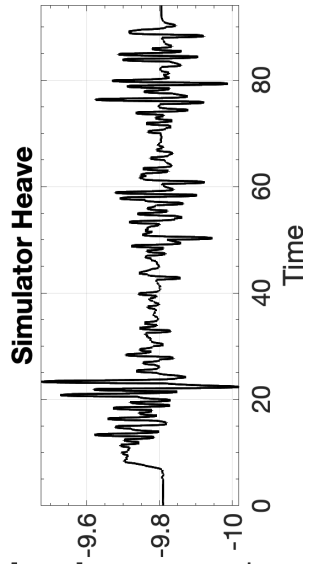

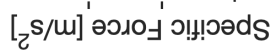

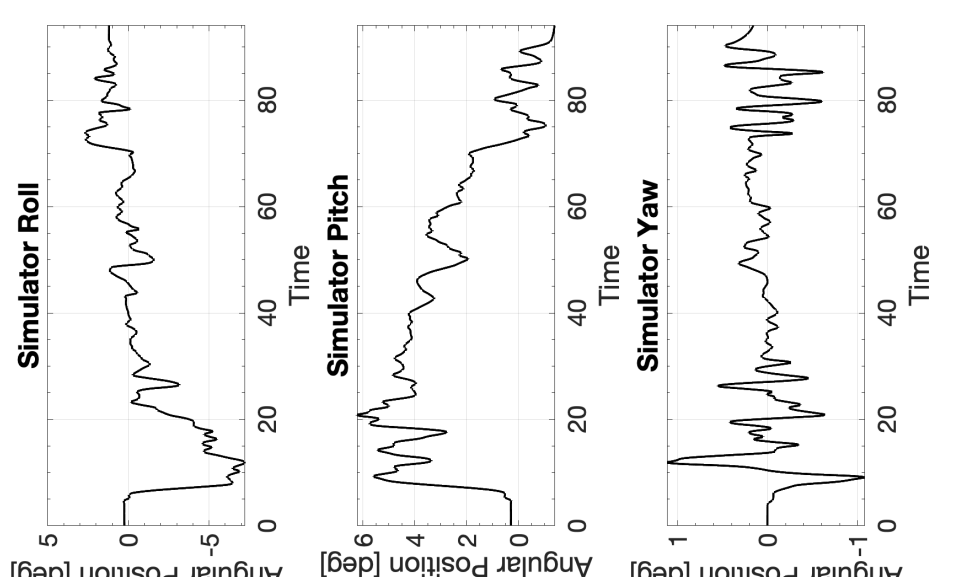

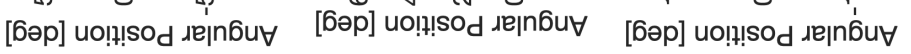

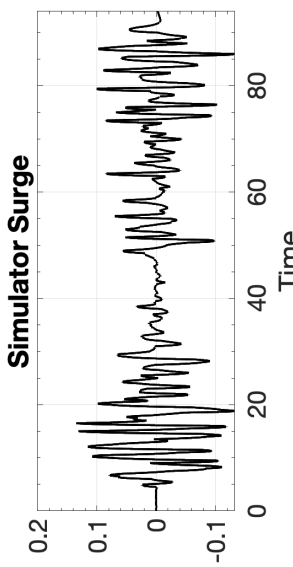

$[\mathrm{c} / \mathrm{L}] \mathrm{uo!̣}$

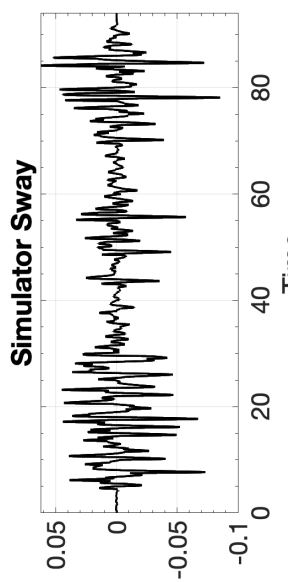

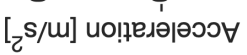

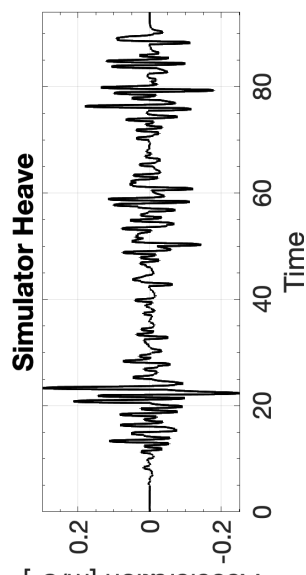

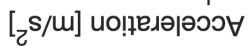
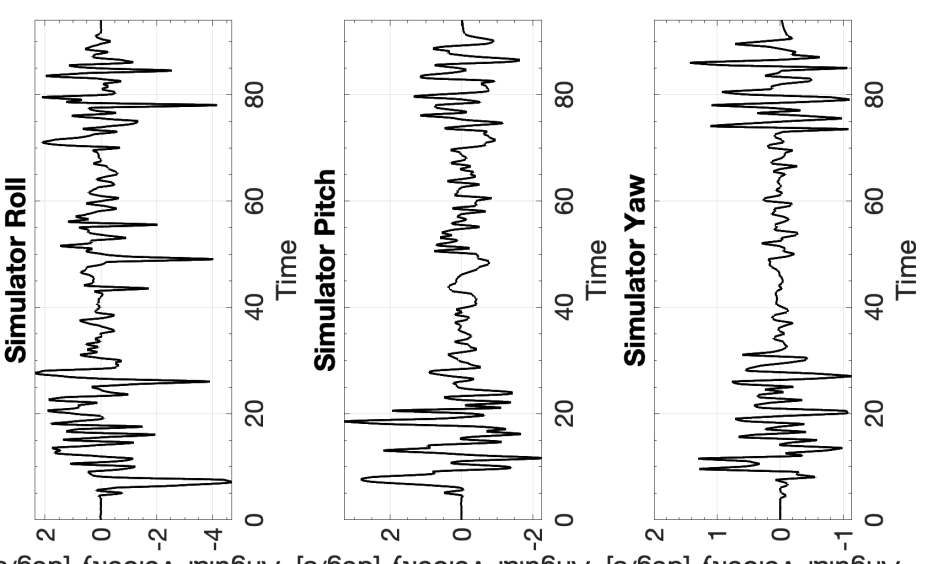

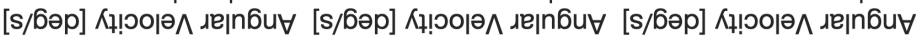




\section{B.6.2 CW2 Cessna 172 Single-axis Takeoff}

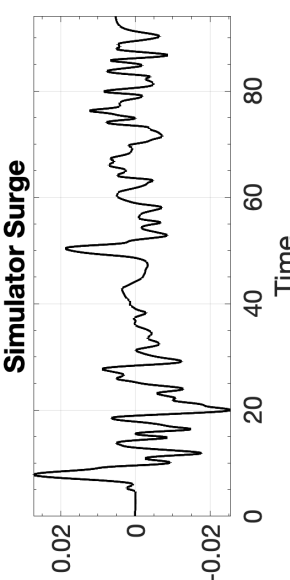

[w] uo!n!sod

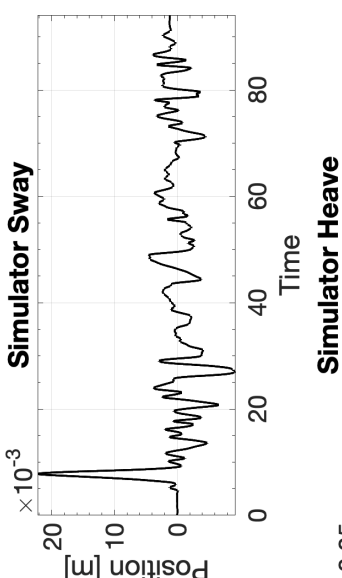

[w] uol!!sod

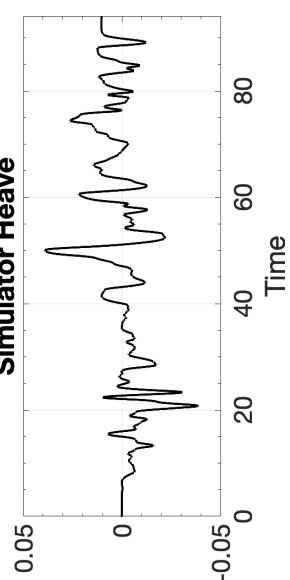

[w] uo!n!

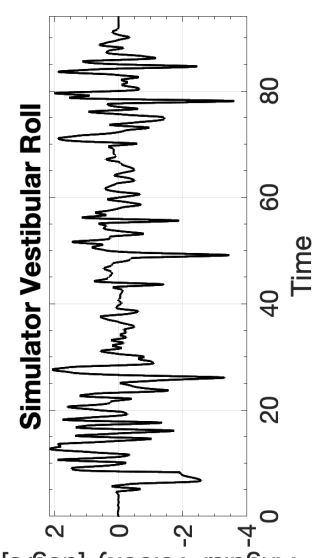

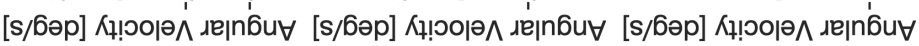
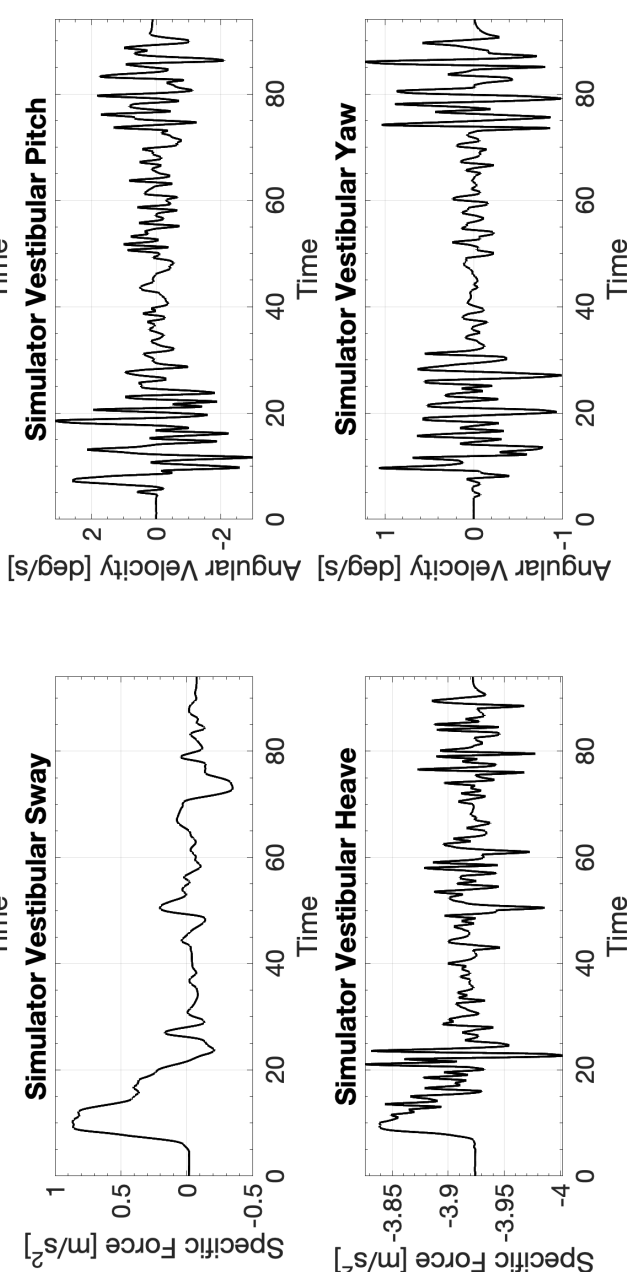

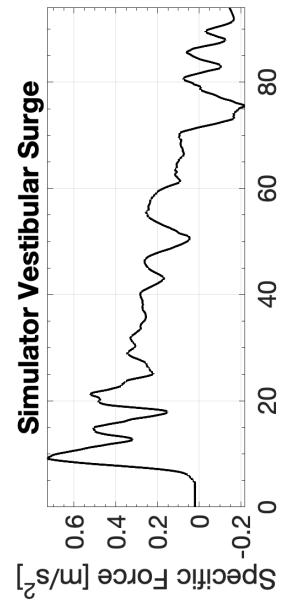

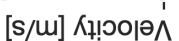

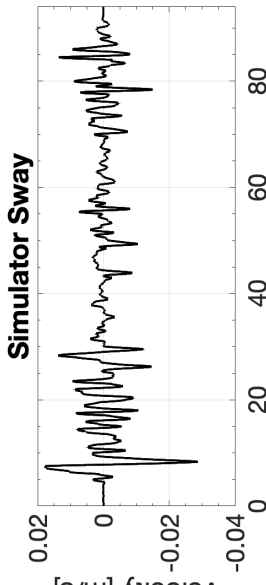

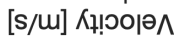
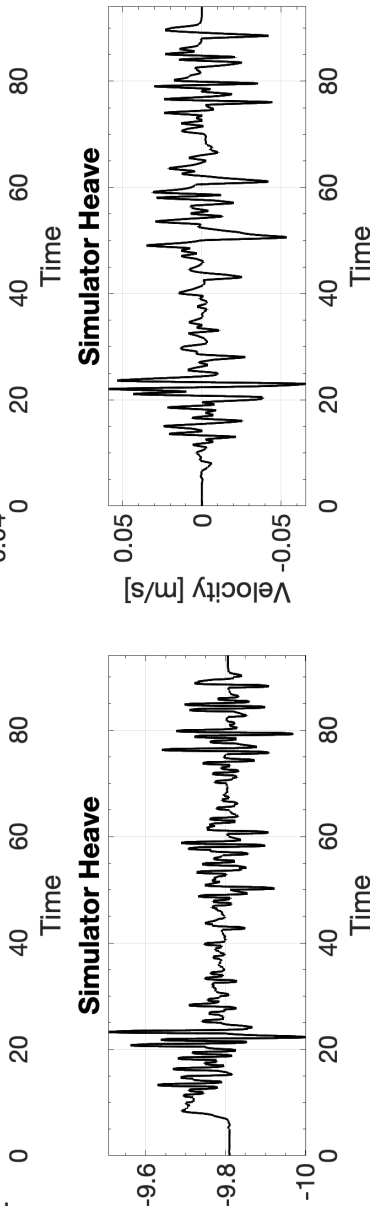

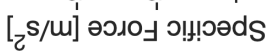

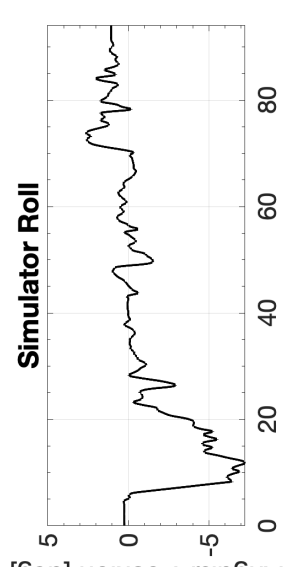

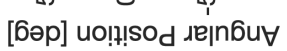

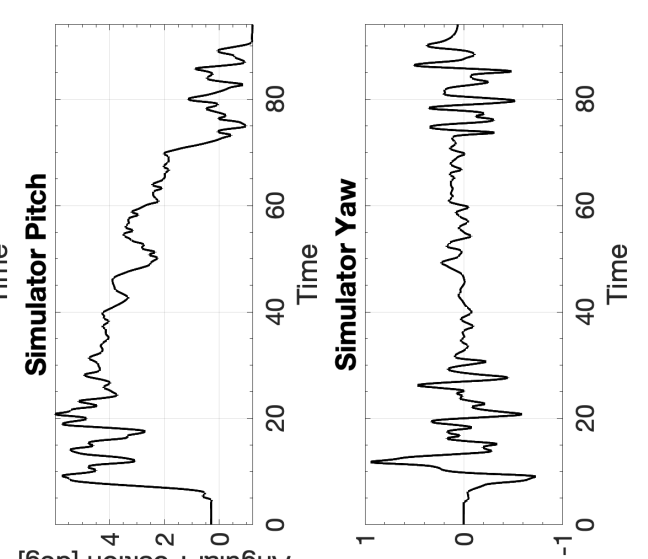

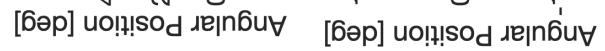

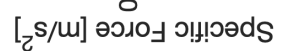

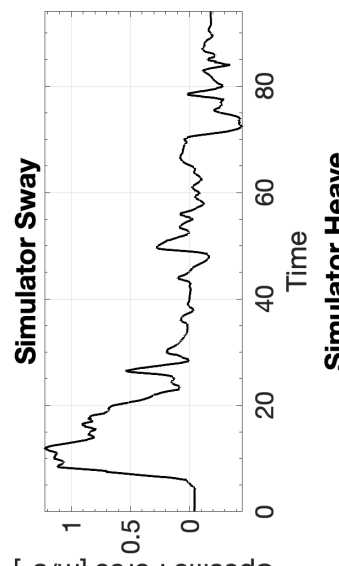

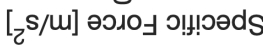

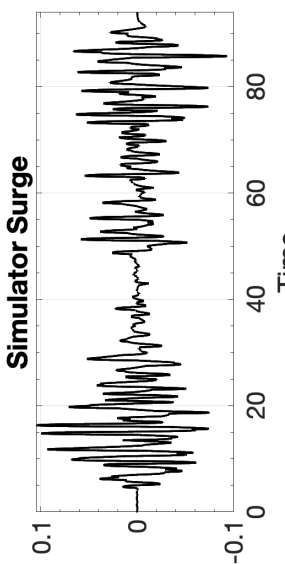

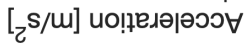

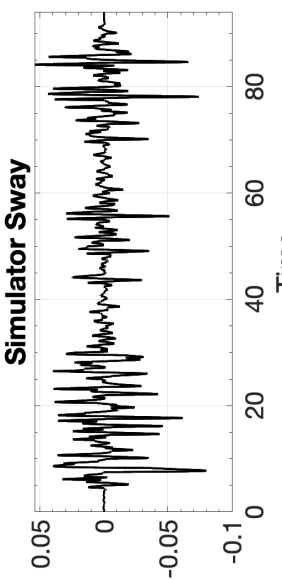

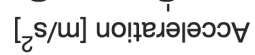

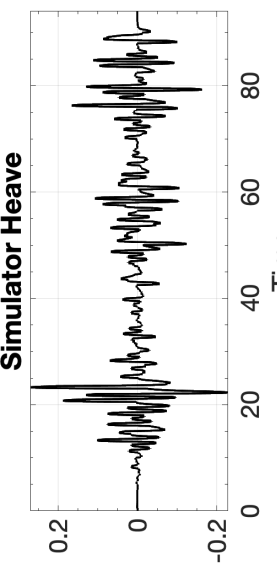

[s/m] uo!̣eגə|әэว
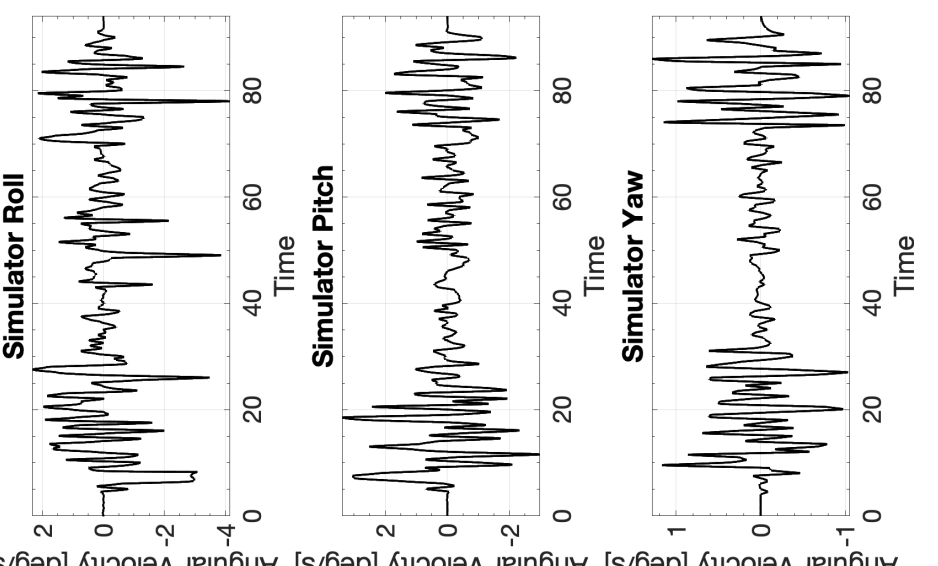

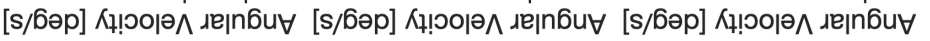




\section{B.6.3 CW3 Cessna 172 Single-axis Takeoff}

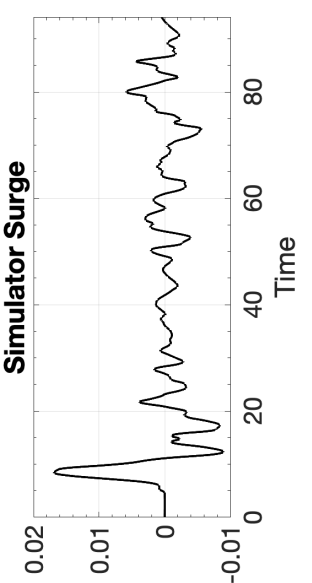

[w] uo!!!!sod

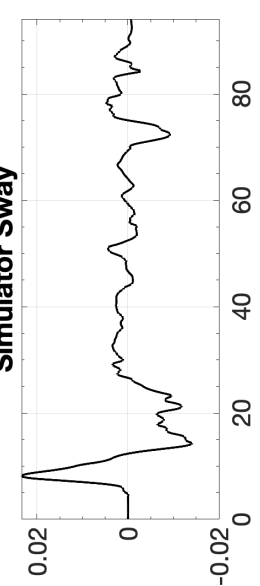

[u] uo!n! sod

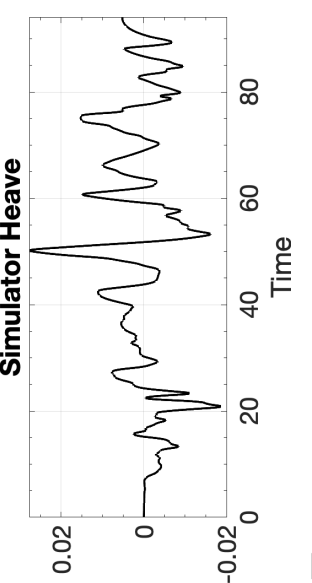

[u] uo!n!s $\mathrm{sod}^{\mathrm{d}}$

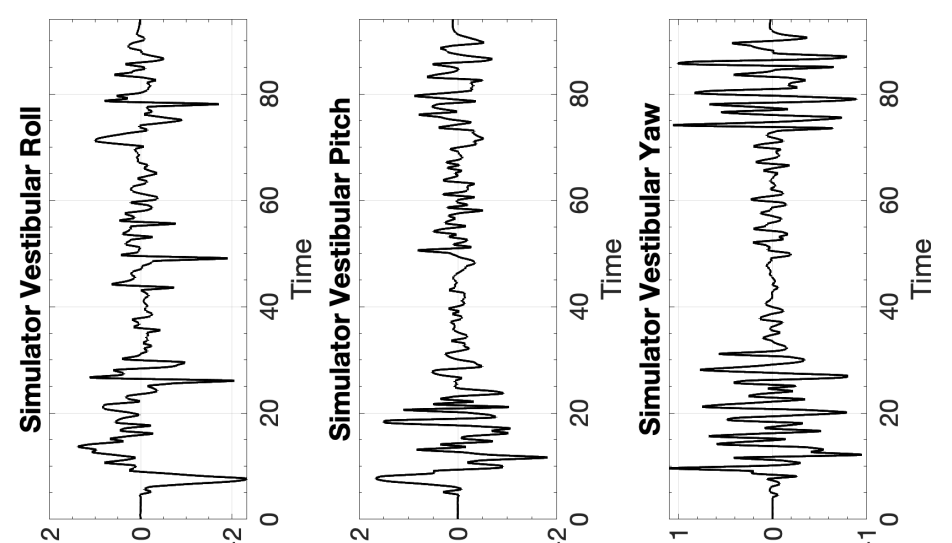

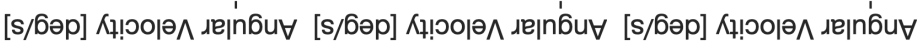

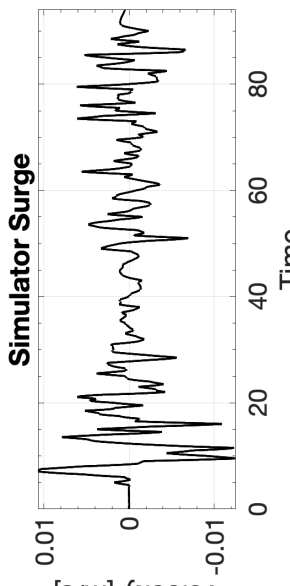

[s/m] Кұ!оојә

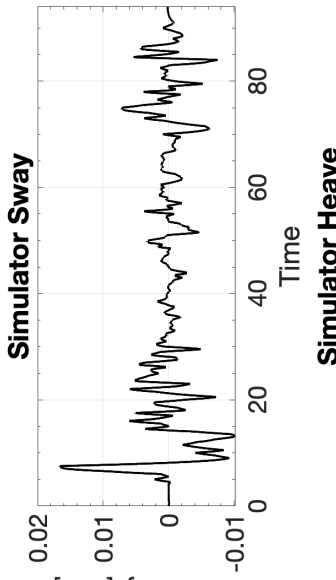

[s/u] Кұ!юор夫

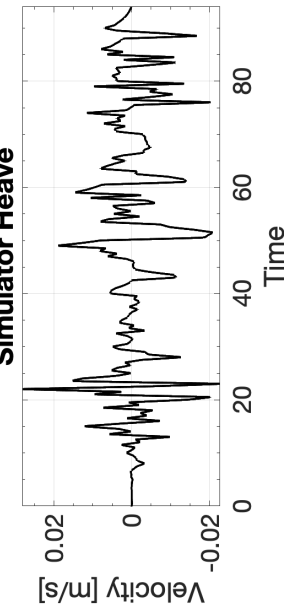

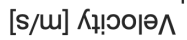

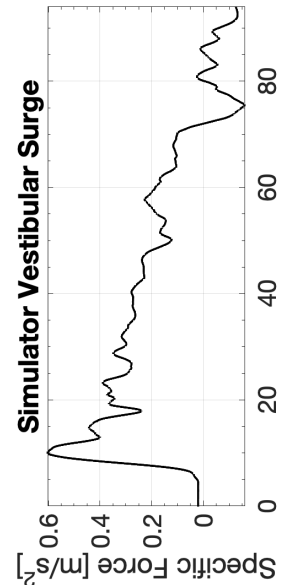

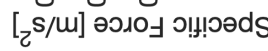
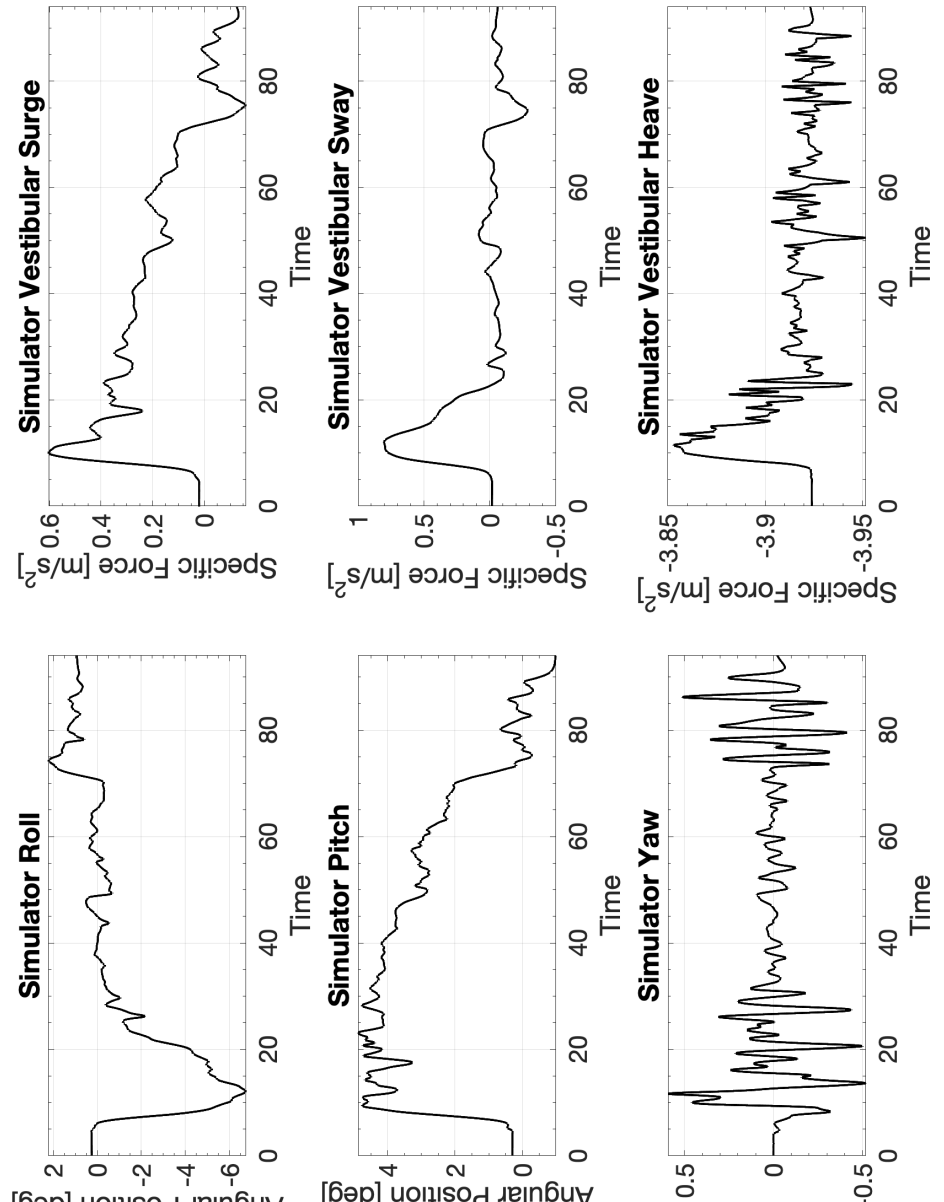

[бәр] uo!m!sod

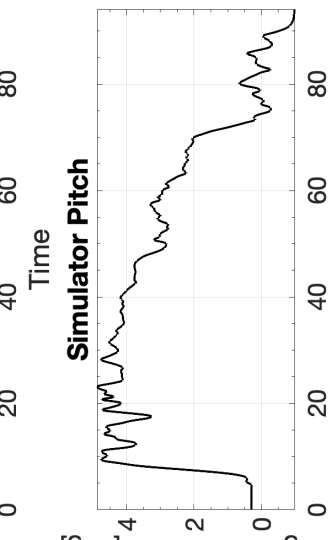

[6әр] uo!!!!sod

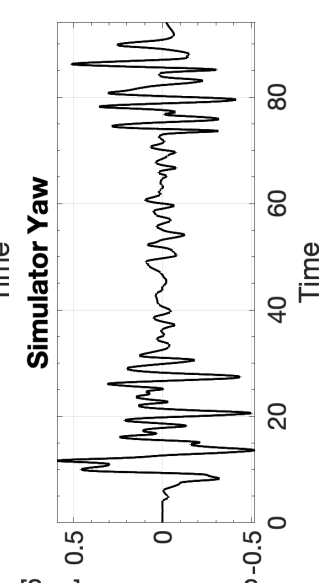

[6әр] uo!!!sod

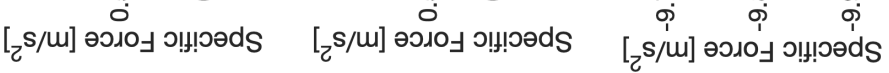

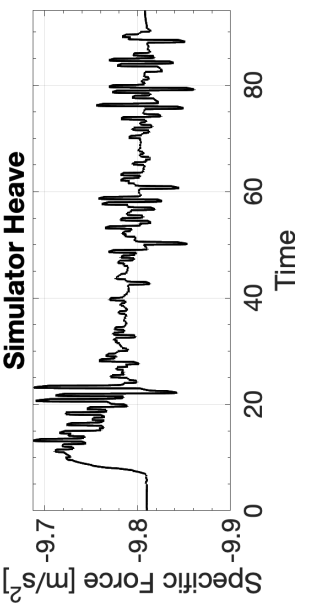

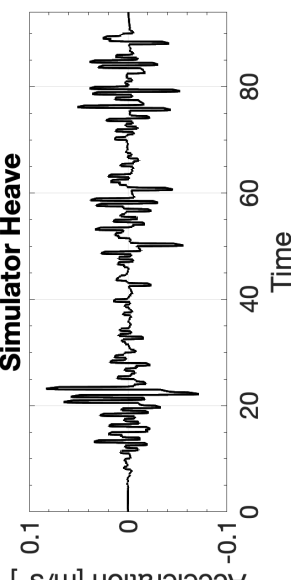

[z/m] ио!ฺелә|әэว

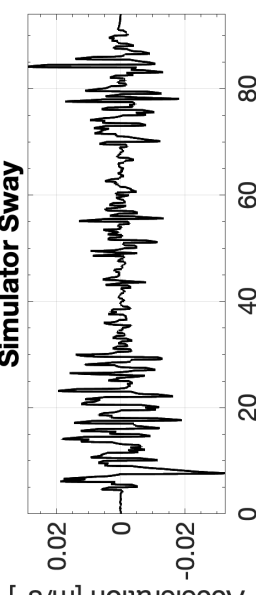

[z/m] ио!ฺвдәәәэว
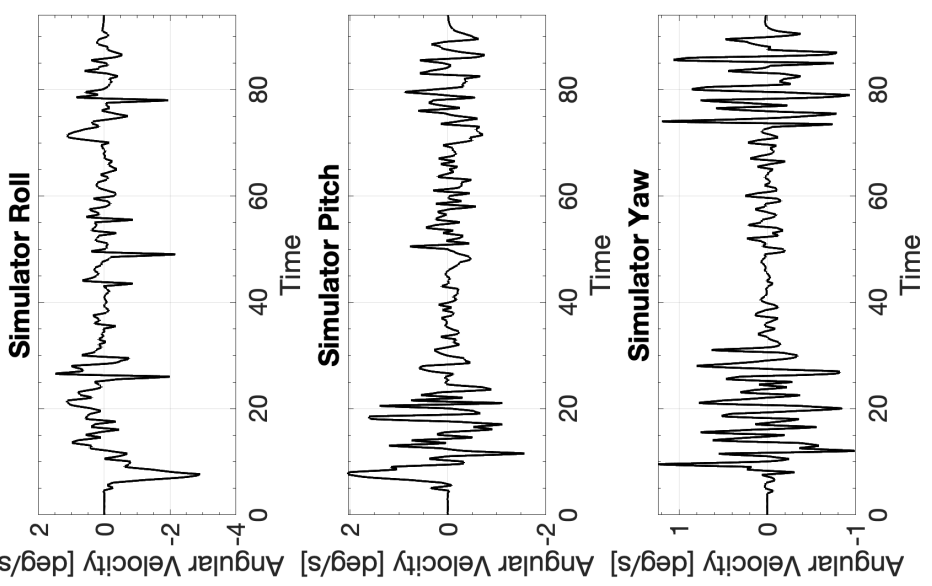

147 


\section{B.7 Cessna 172 Spiral Dive}

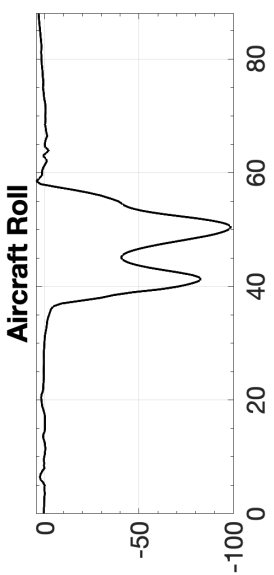

[6әр] uo!!!!sod

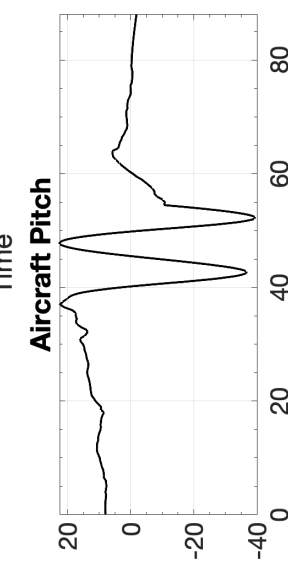

[бəр] uo!!]!sod גe|n6u

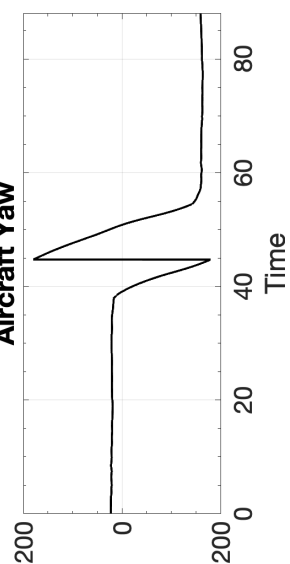

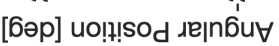

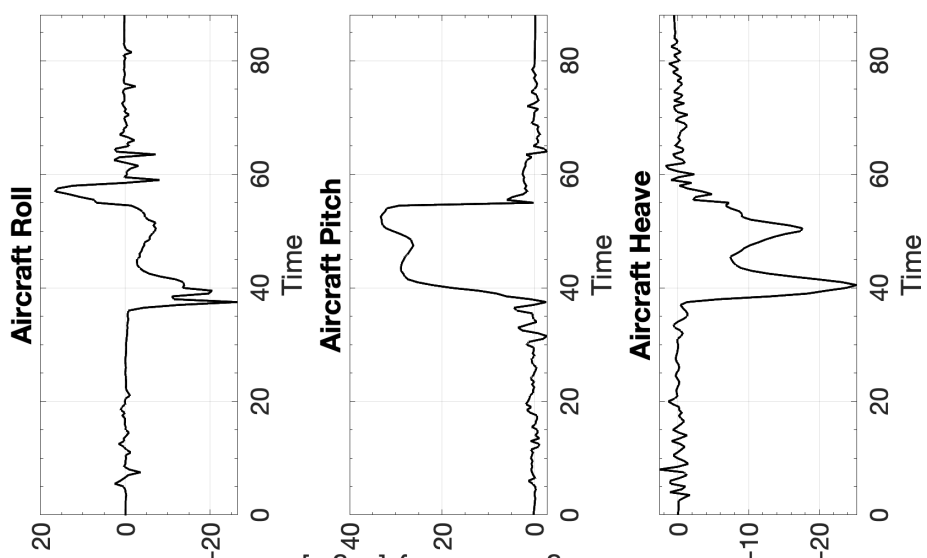

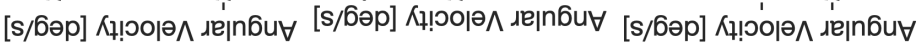
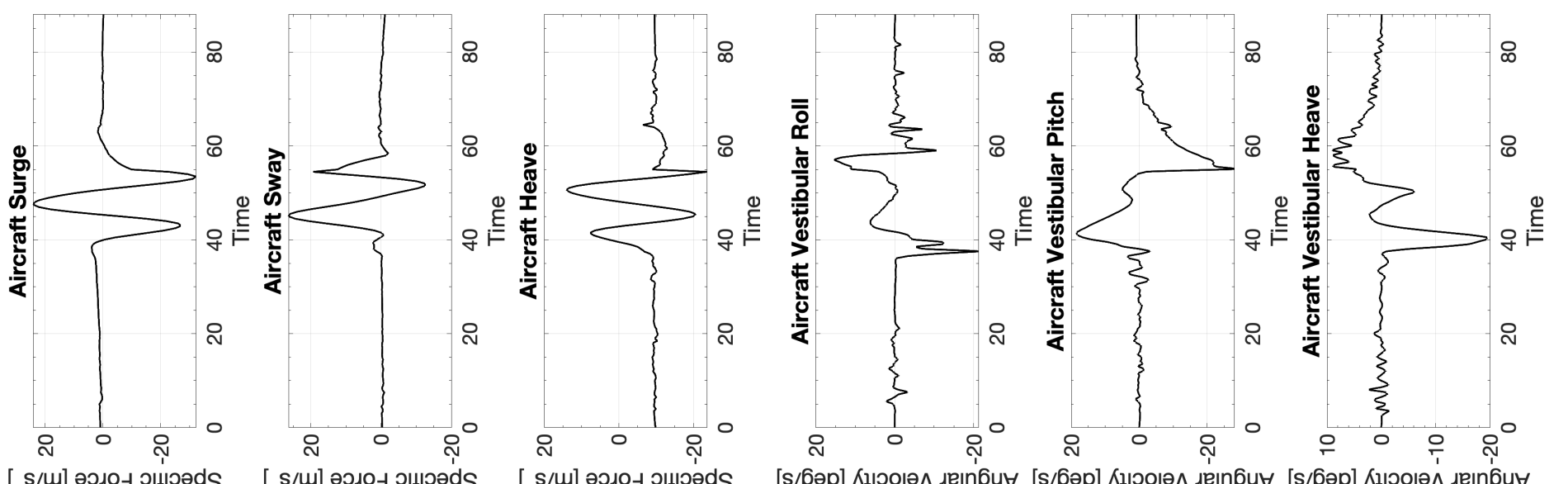

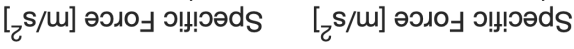

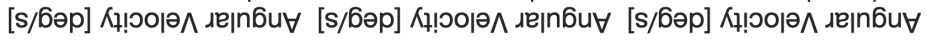

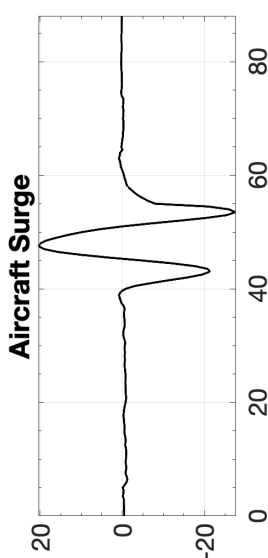

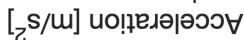

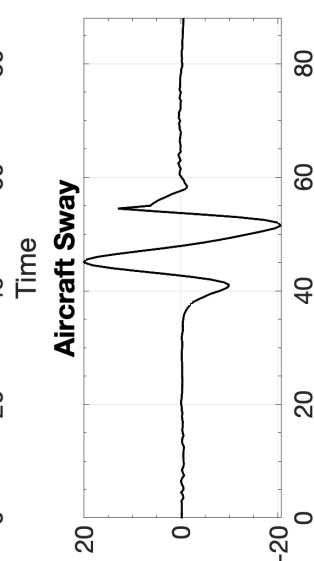

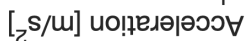

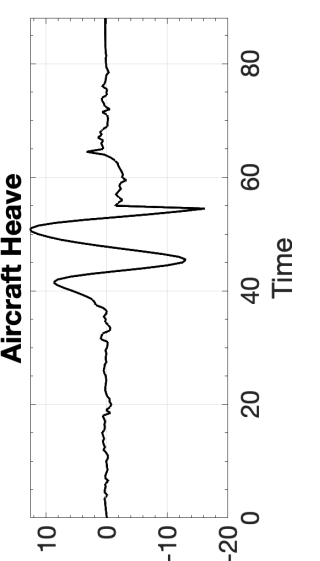

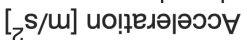
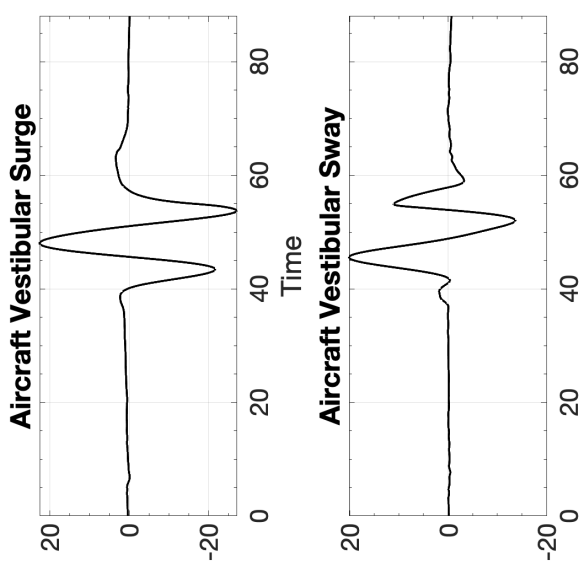

[ $\left.]_{2} \mathrm{~s} / \mathrm{m}\right]$ ә

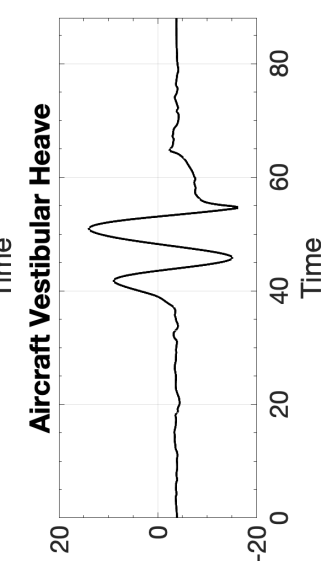

[zs/m] ә010๖ 0!!!อəds 


\section{B.7.1 CW1 Cessna 172 Spiral Dive}

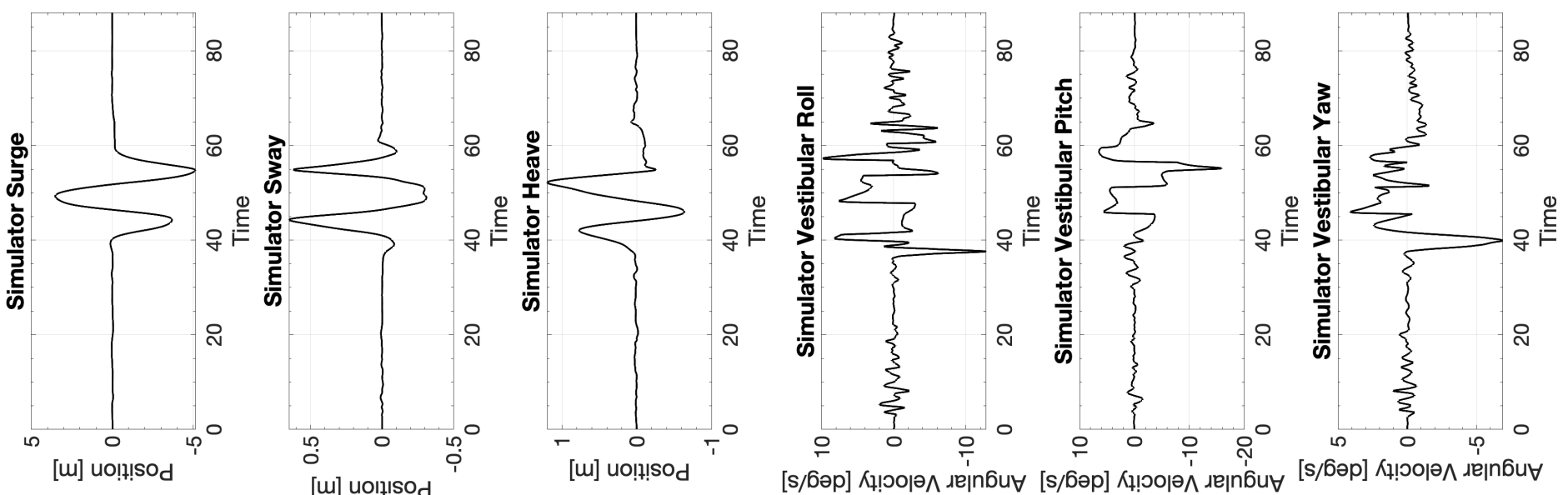

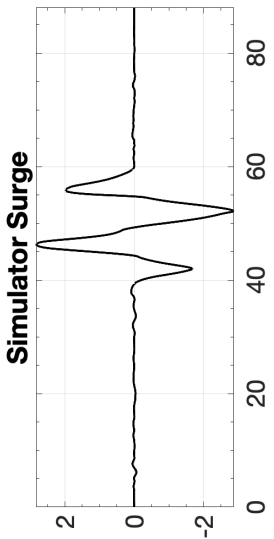

[s/u] Кџ!юојә^

[w] uolt!!sod

[m] uo!!!!sod

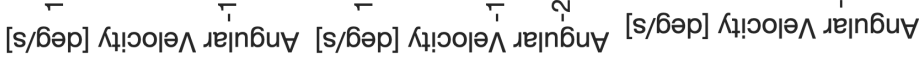
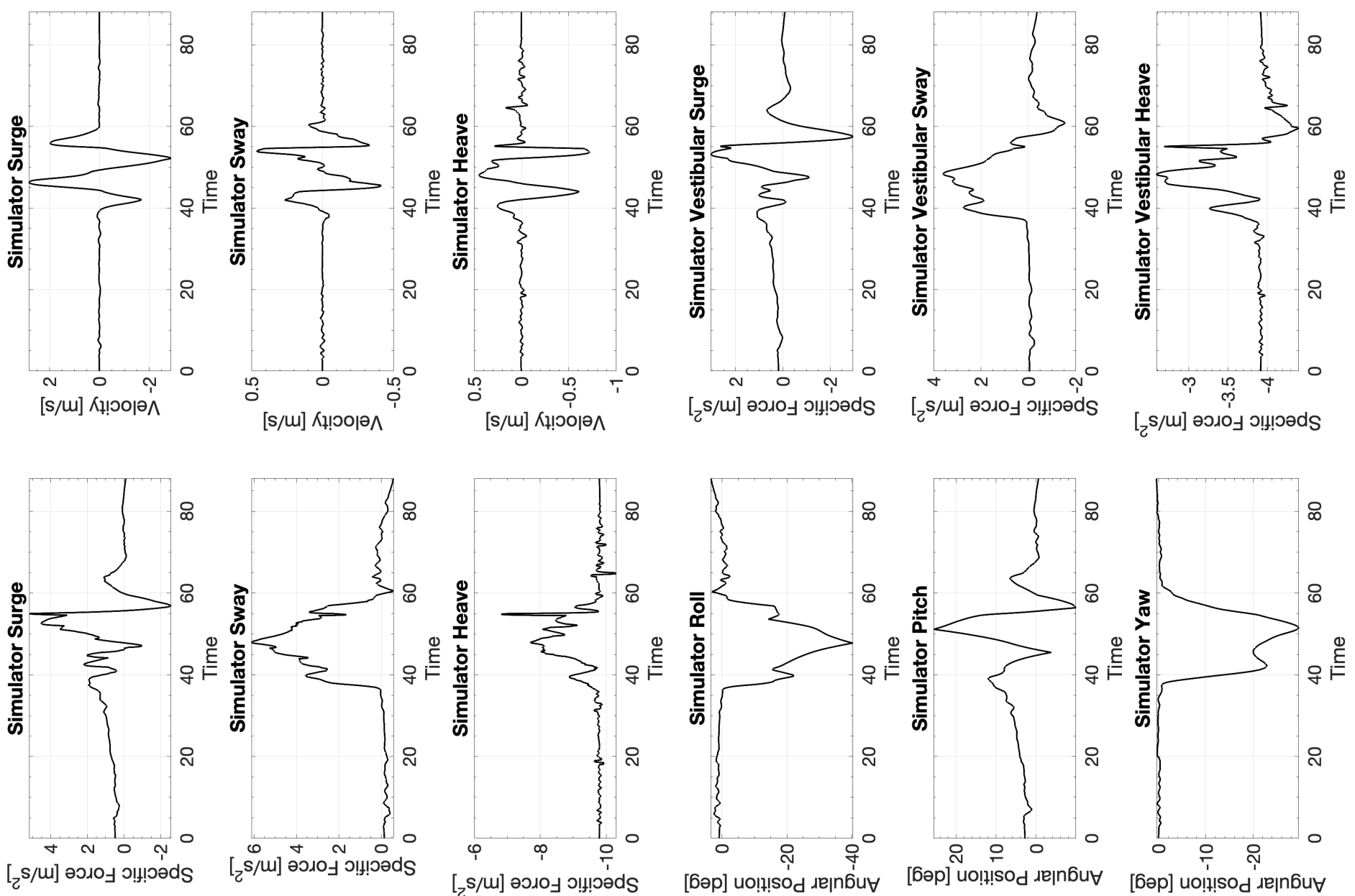

[6əp] uo!!!sod גe|n6u

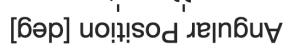
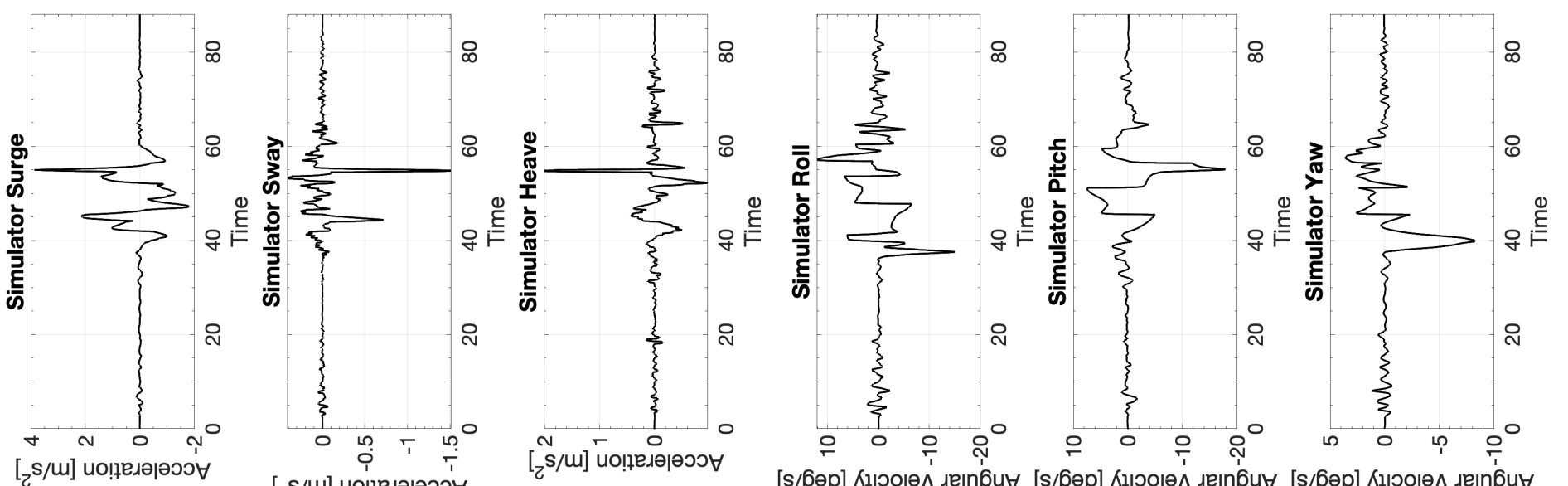


\section{B.7.2 CW2 Cessna 172 Spiral Dive}

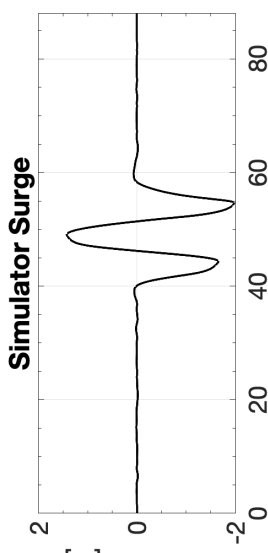

[u] uo!!!!sod

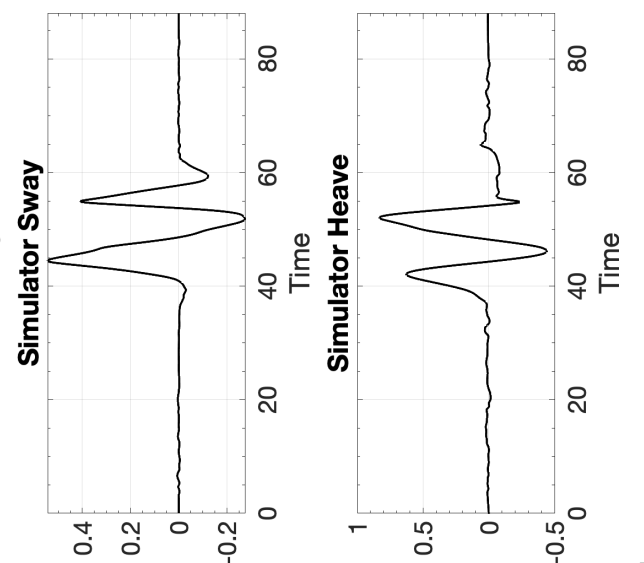

[u] uo!n! sod

[w] uolt!!sod

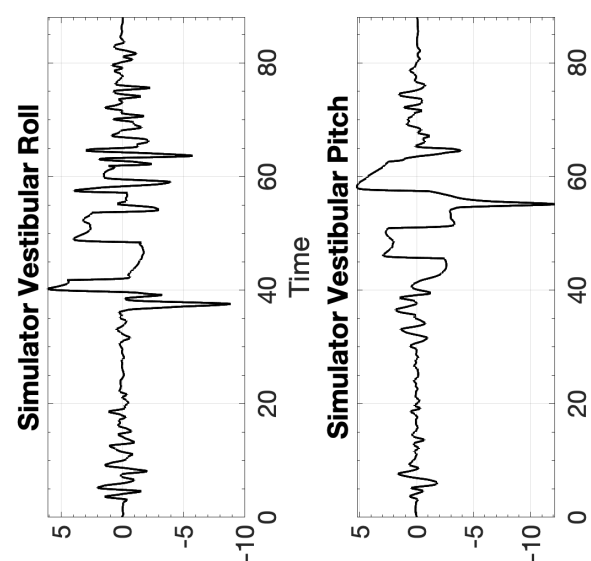

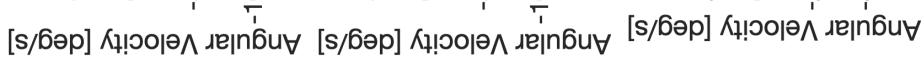

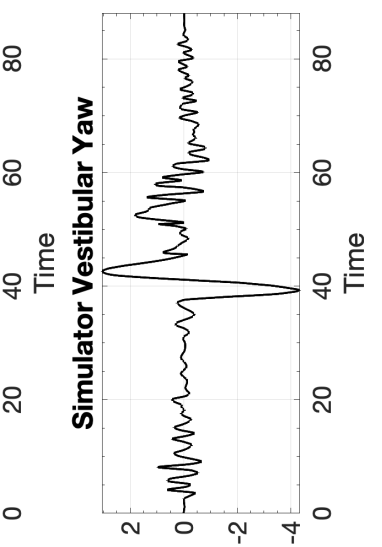

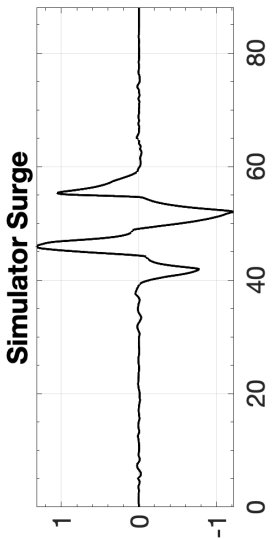

[s/m] Кџ!৩ојә^'
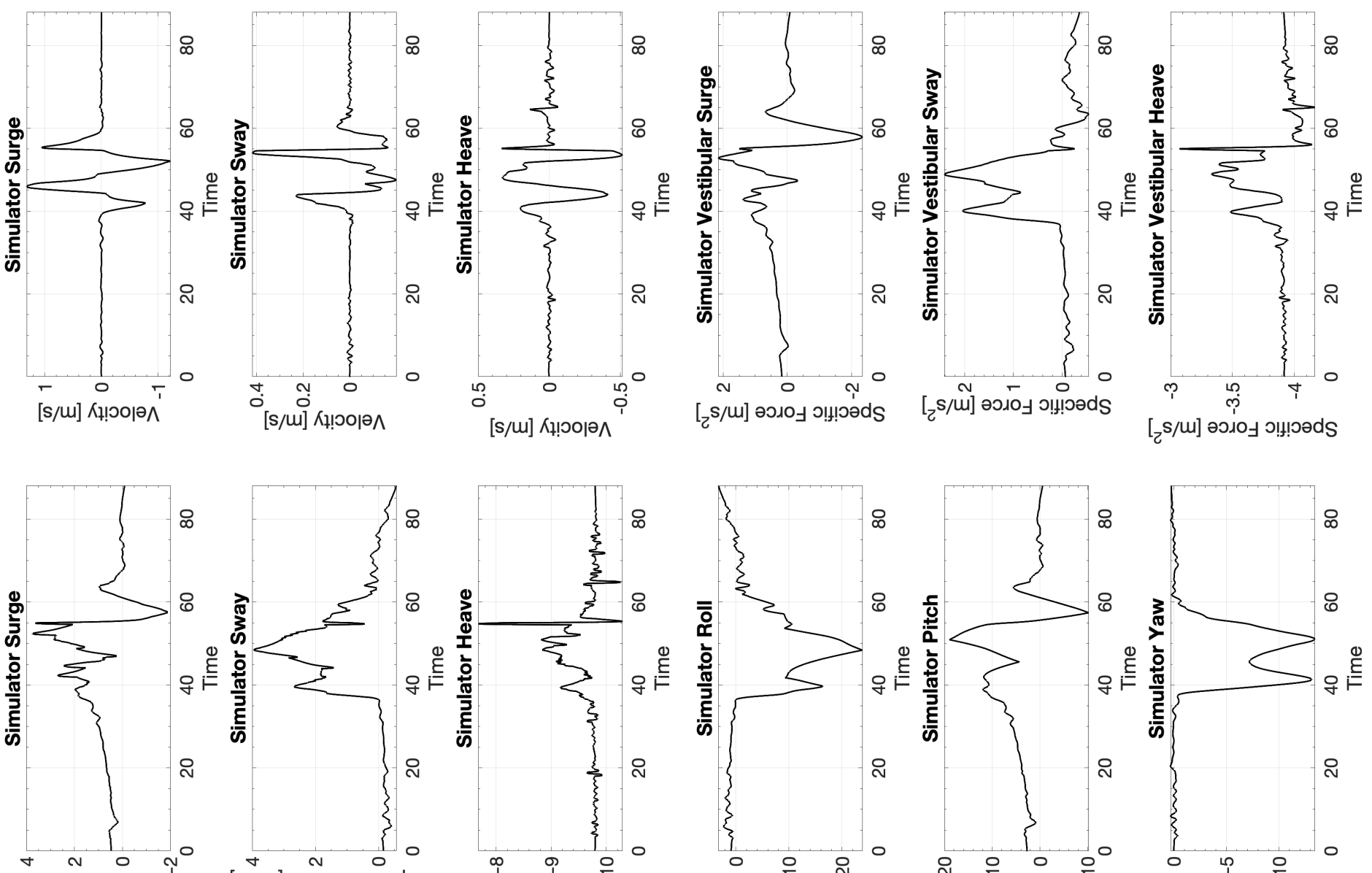

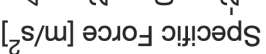

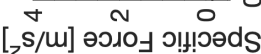

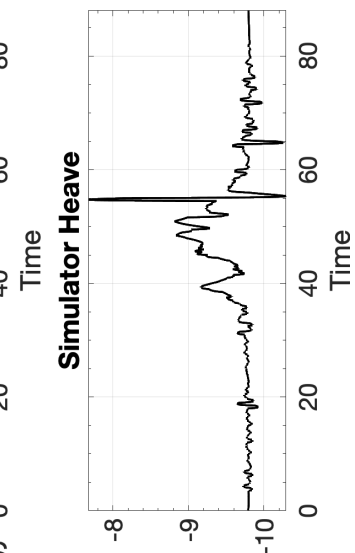

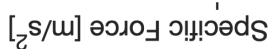
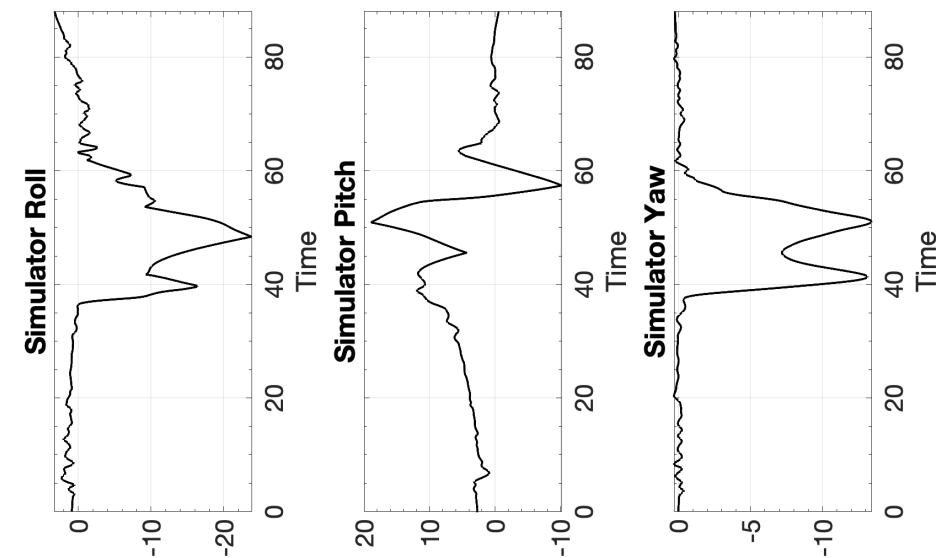

[бəp] uo!!!

[6әр] uo!!!

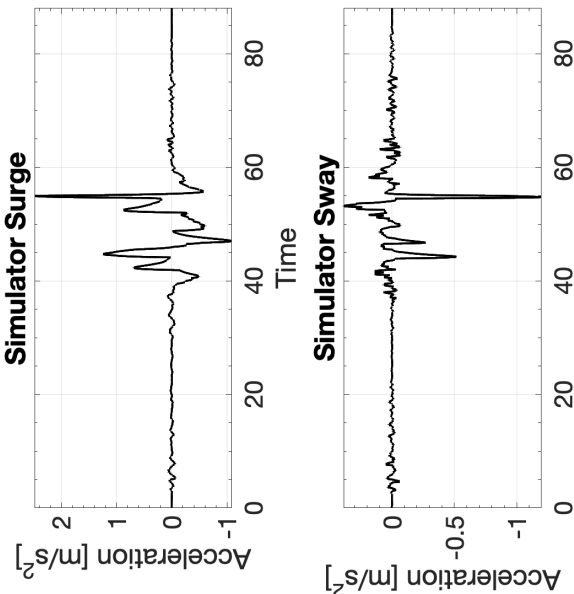

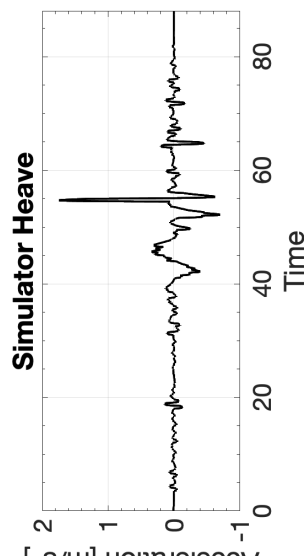

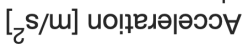
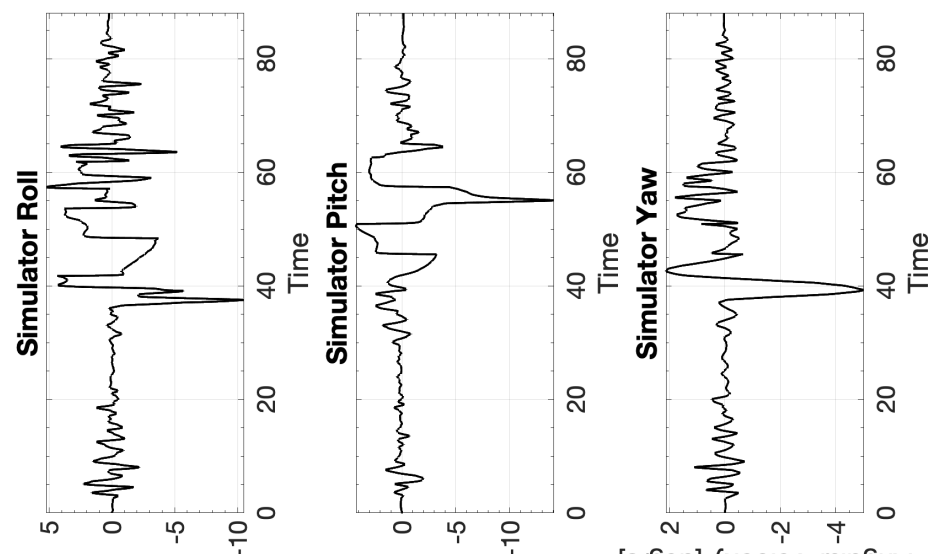

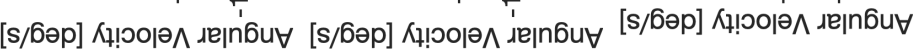




\section{B.7.3 CW3 Cessna 172 Spiral Dive}

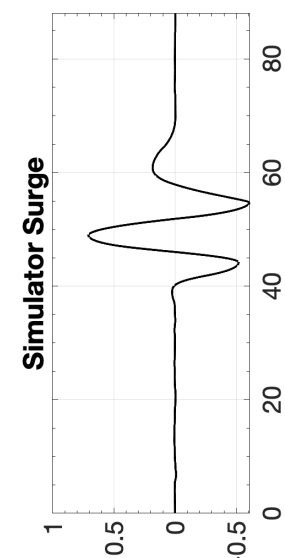

[w] uol!!sod

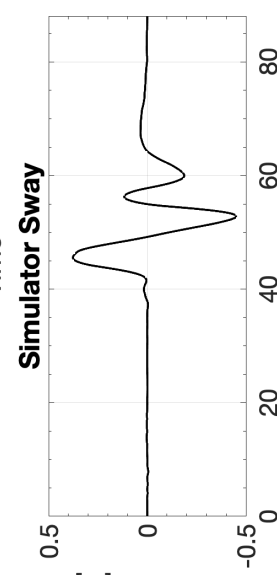

[m] uoli!nsod

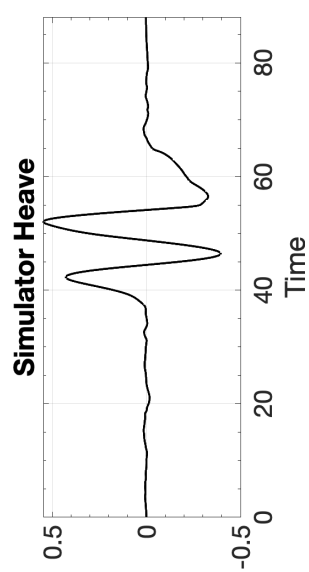

[u] uol!!!sod

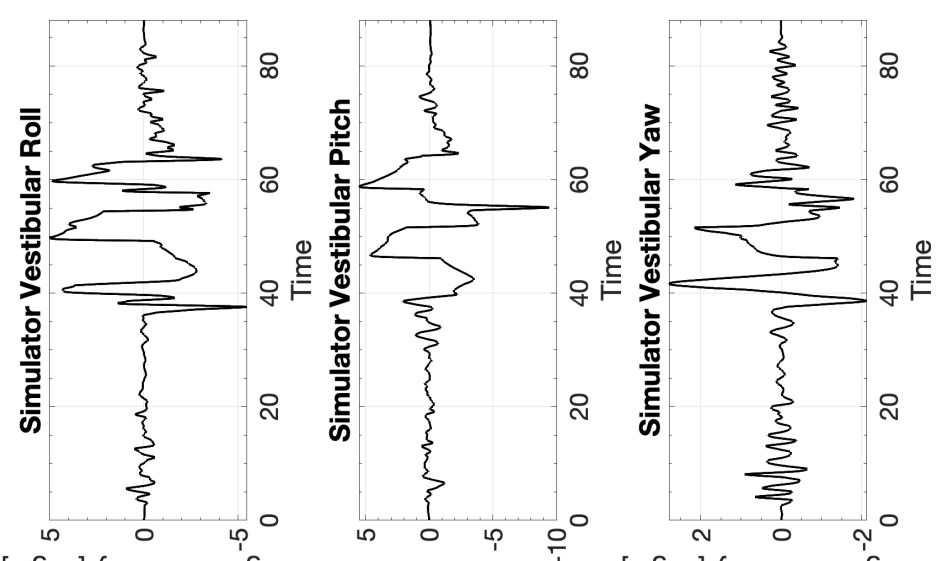

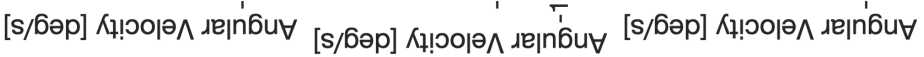
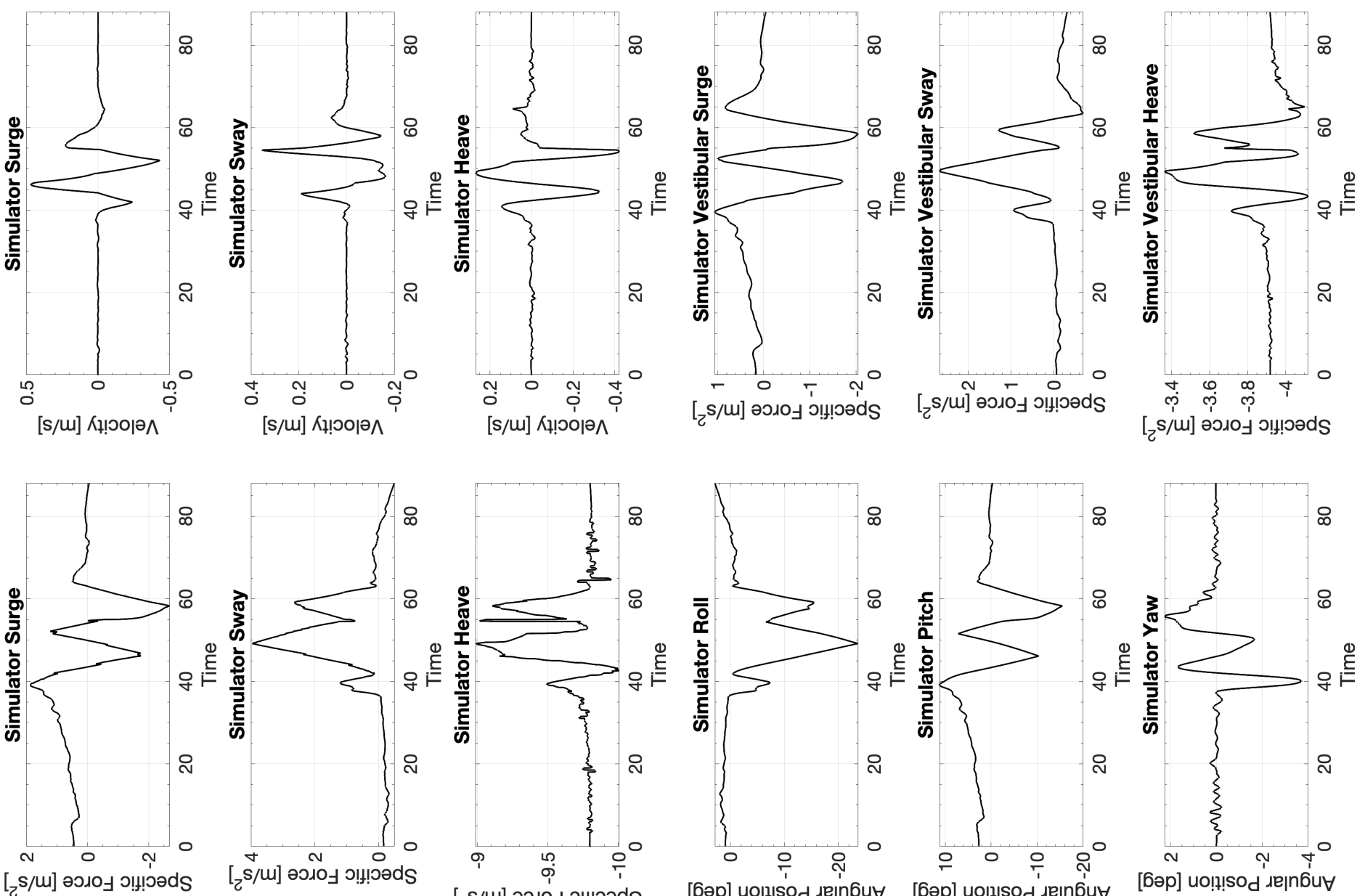

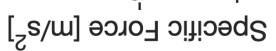

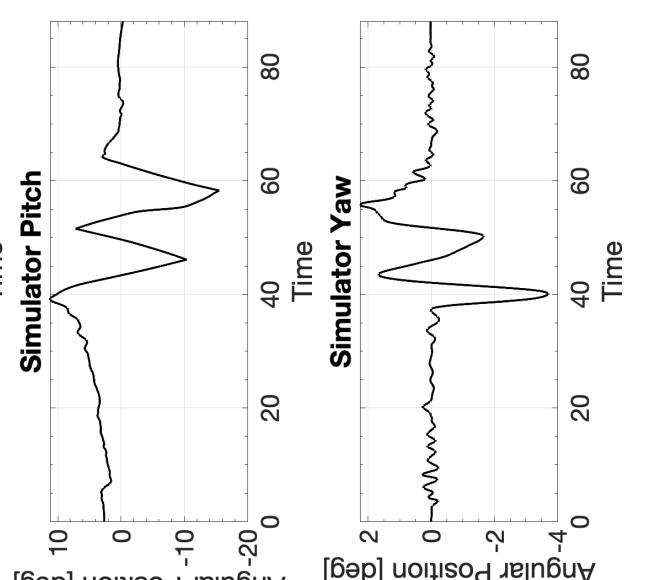

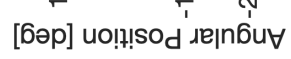

[6әр] uo!!!sod $\lambda$ ejn6u

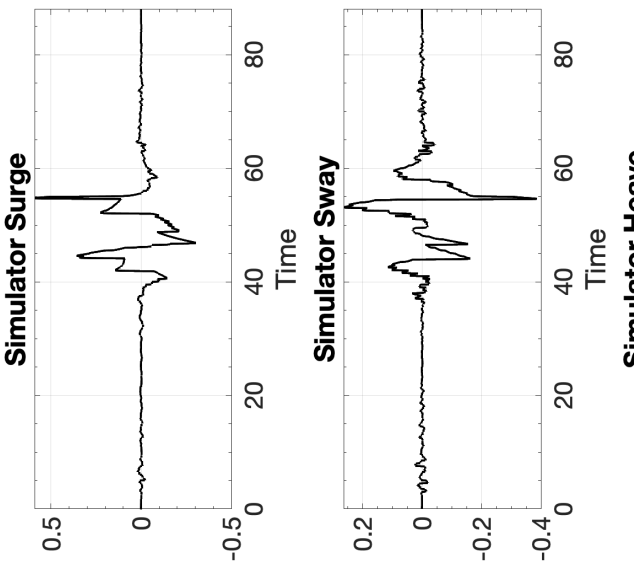

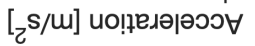

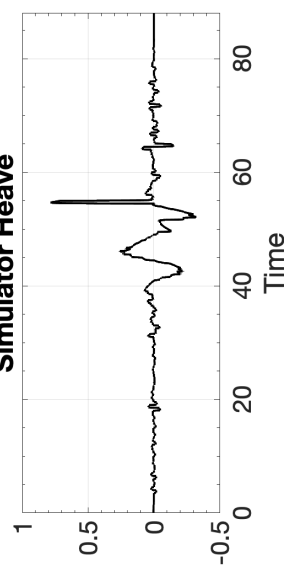

$[\mathrm{z} s / \mathrm{m}]$ ио!ฺедәәәоэ
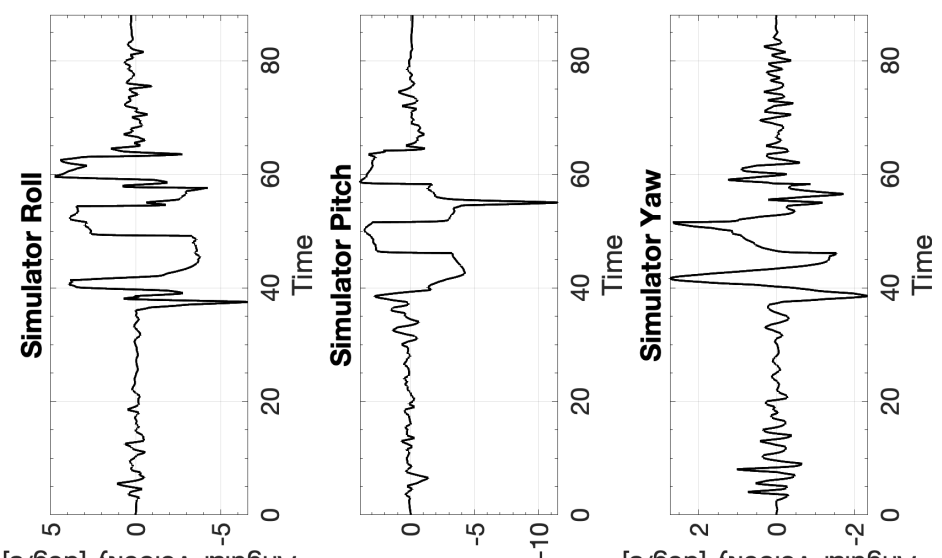

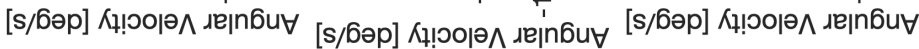




\section{B.8 Cessna 172 Stall Recovery}
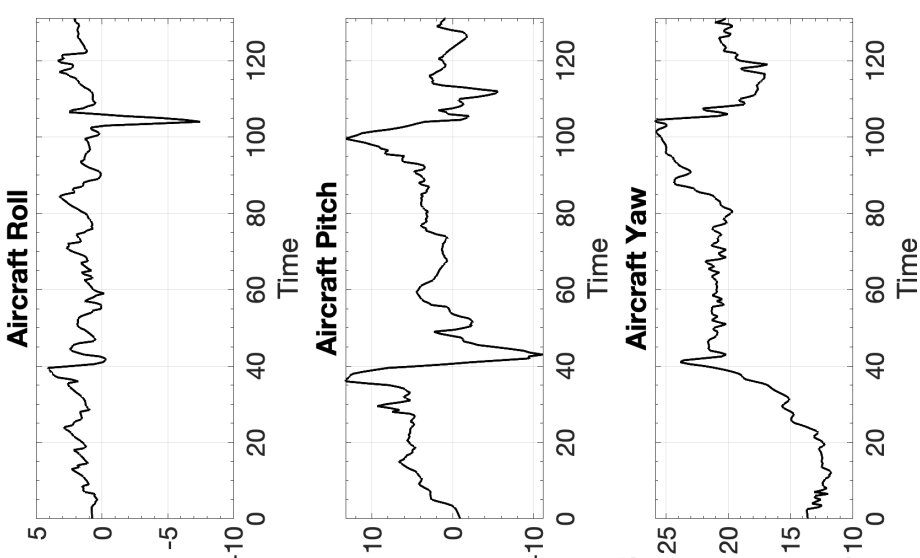

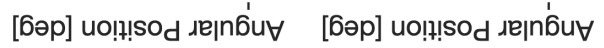

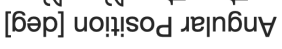

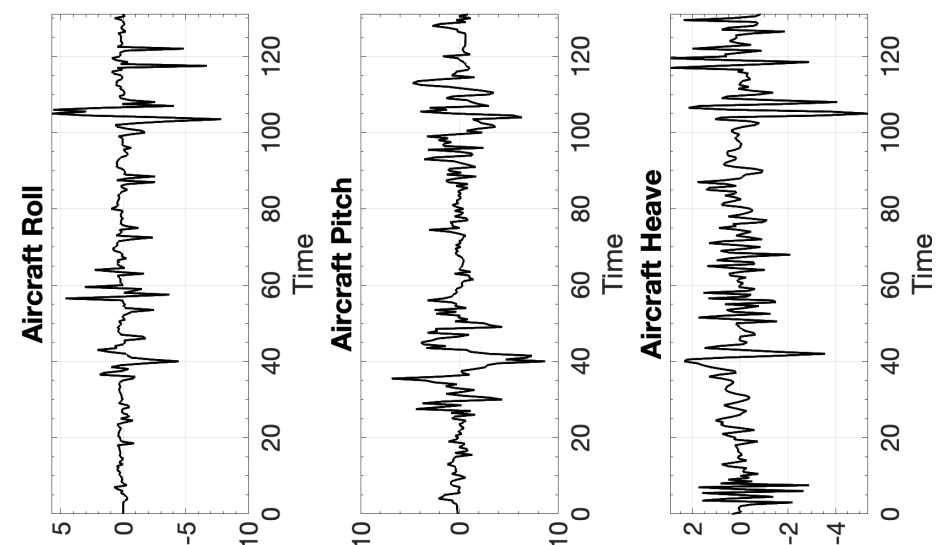

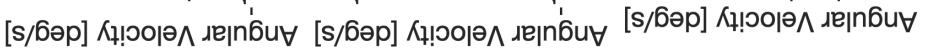

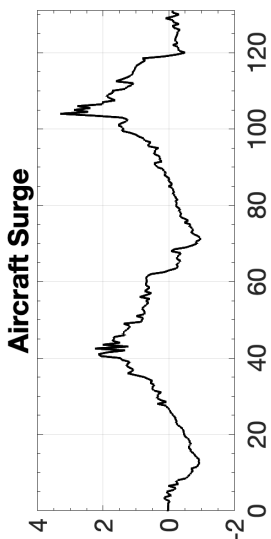

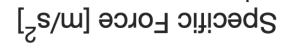

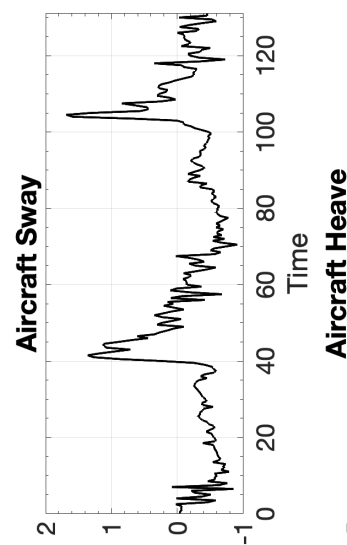

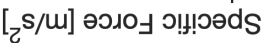

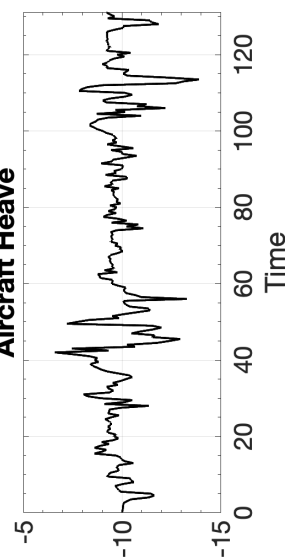

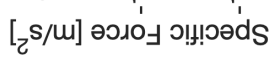

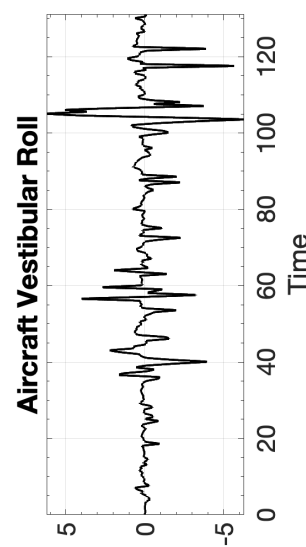

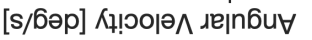
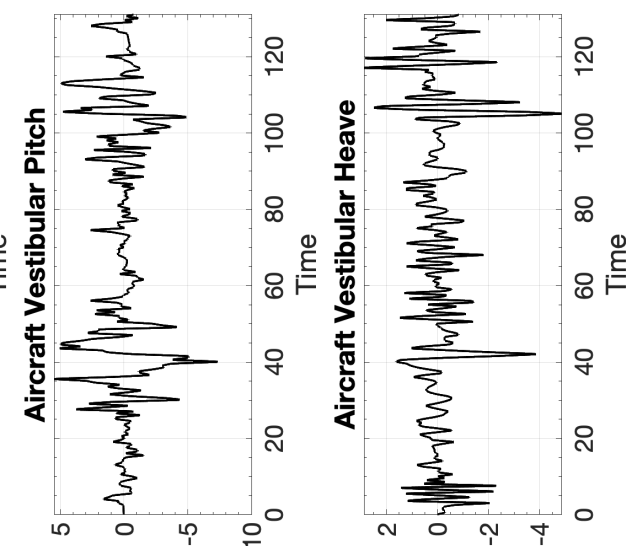

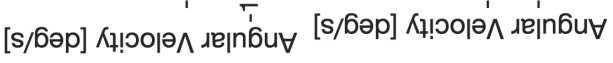

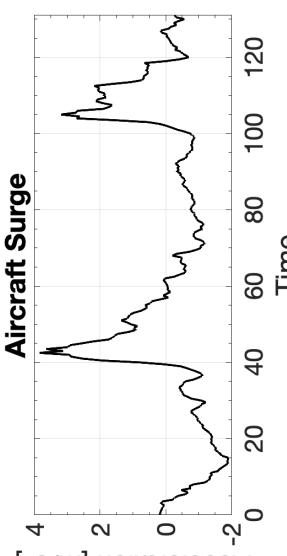

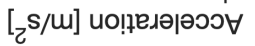

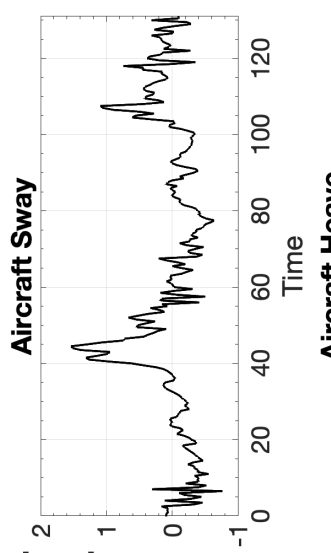

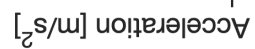

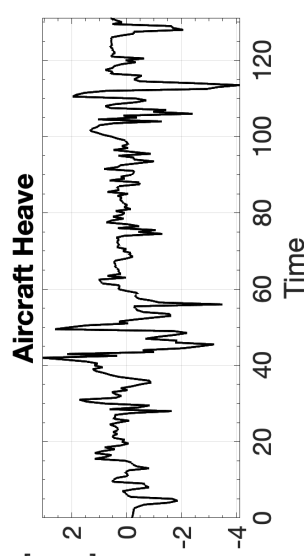

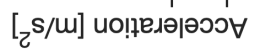

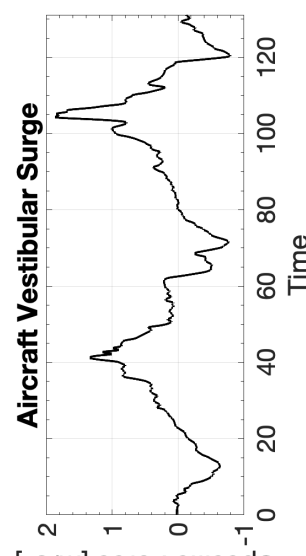

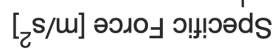

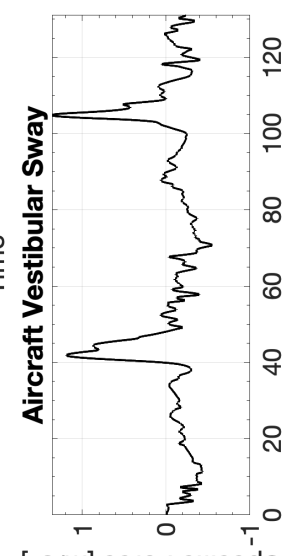

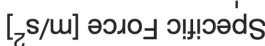

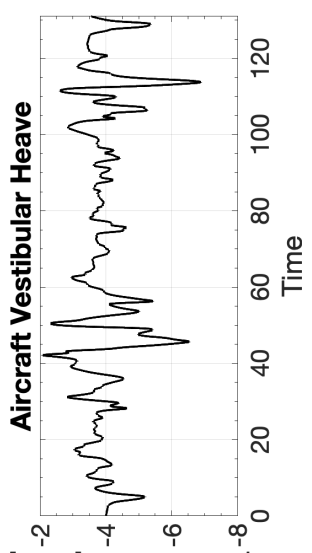

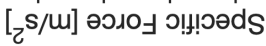




\section{B.8.1 CW1 Cessna 172 Stall Recovery}

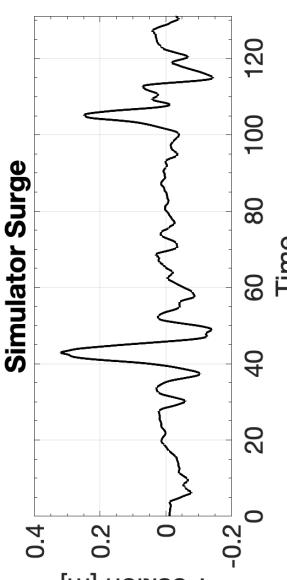

[u] uo!!!!sod

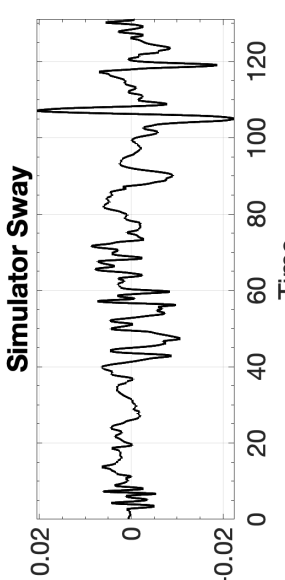

[u] uo!n!?sod

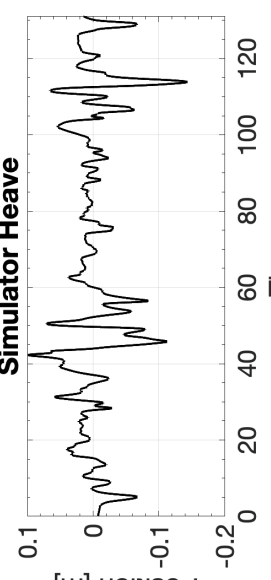

[u] uol!!!sod

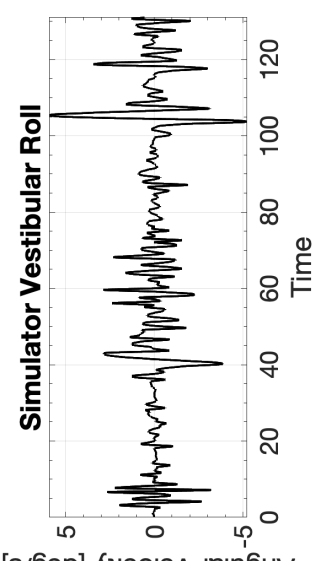

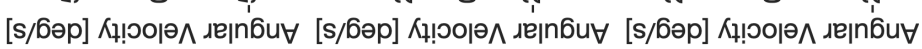


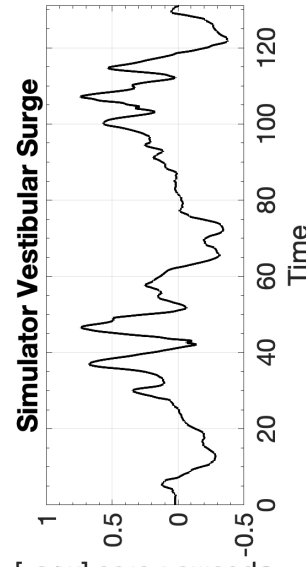

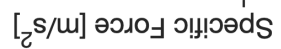

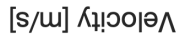

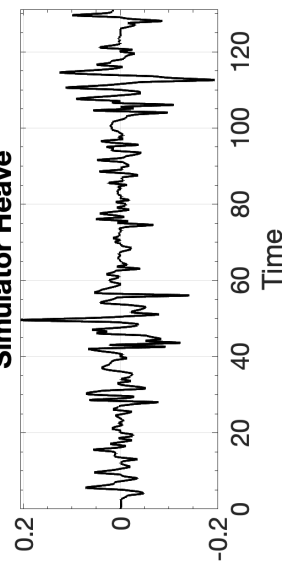

[s/u] ᄉұ!юำ

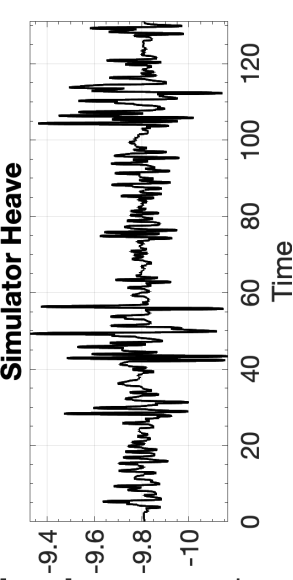

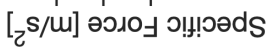

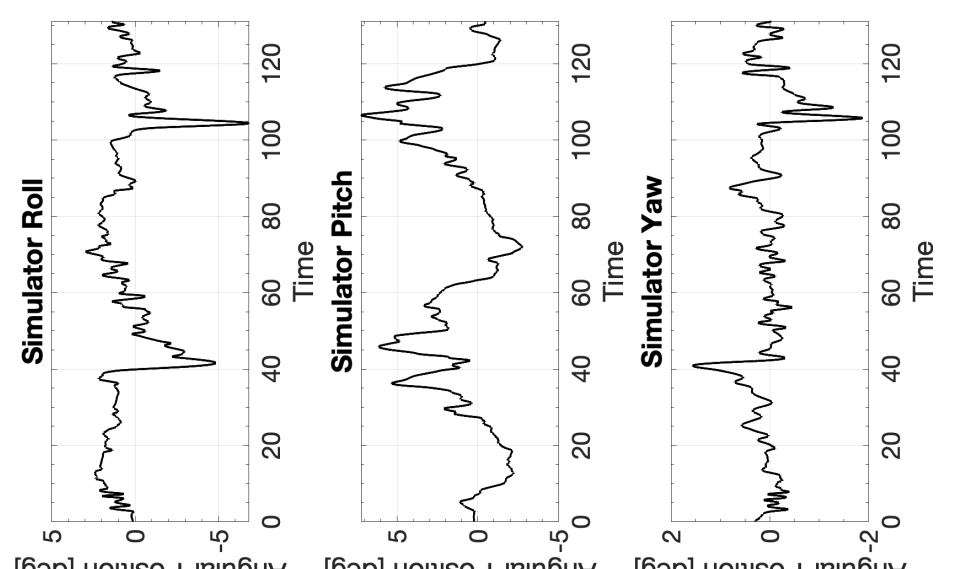

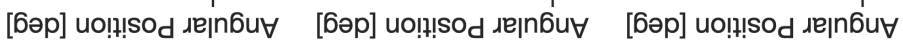

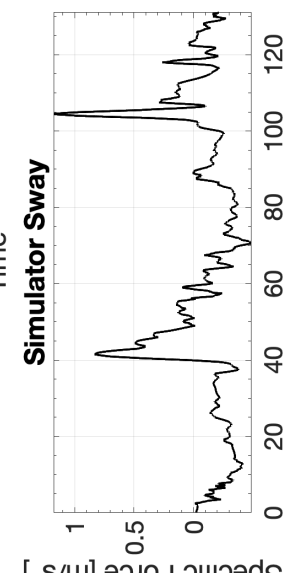

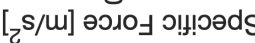

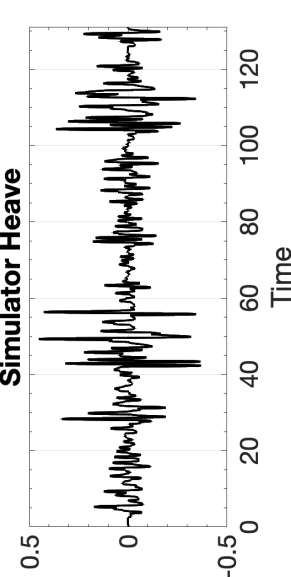

$[\mathrm{C} / \mathrm{L}]$ ио!ฺв

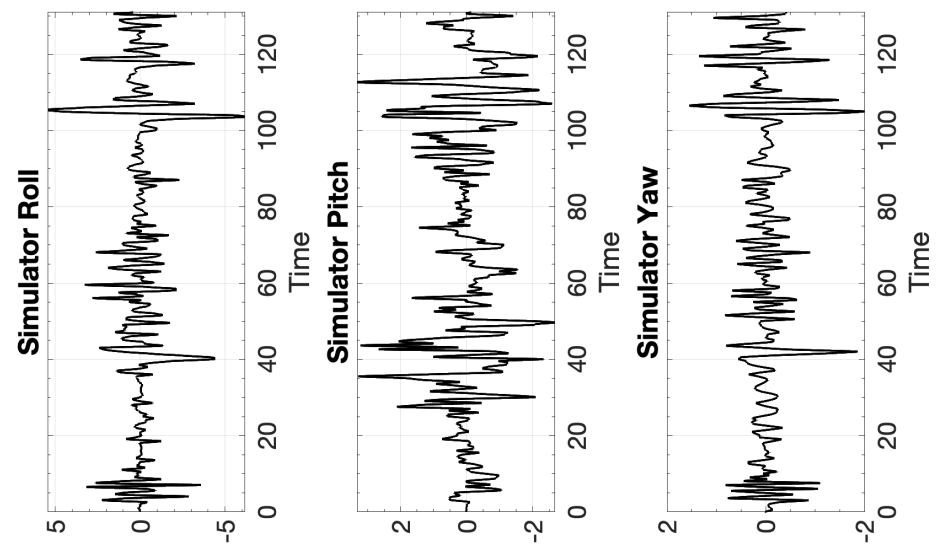

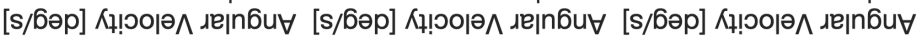




\section{B.8.2 CW2 Cessna 172 Stall Recovery}

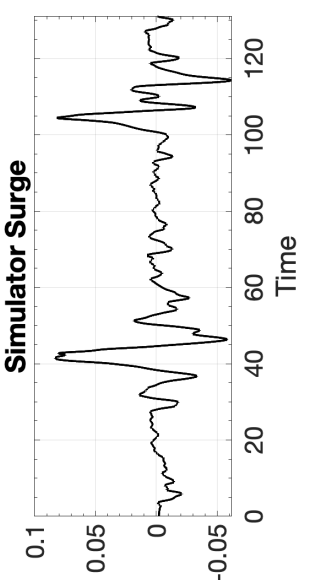

[w] uo!!!?sod

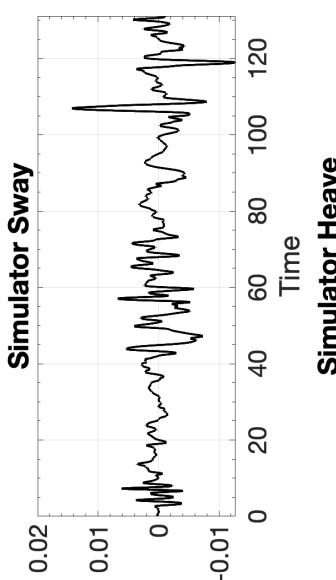

[w] uo!n!sod

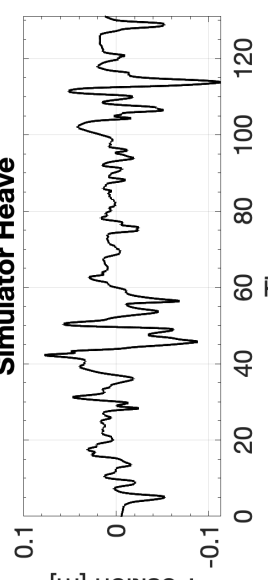

[w] uo!!!ssod

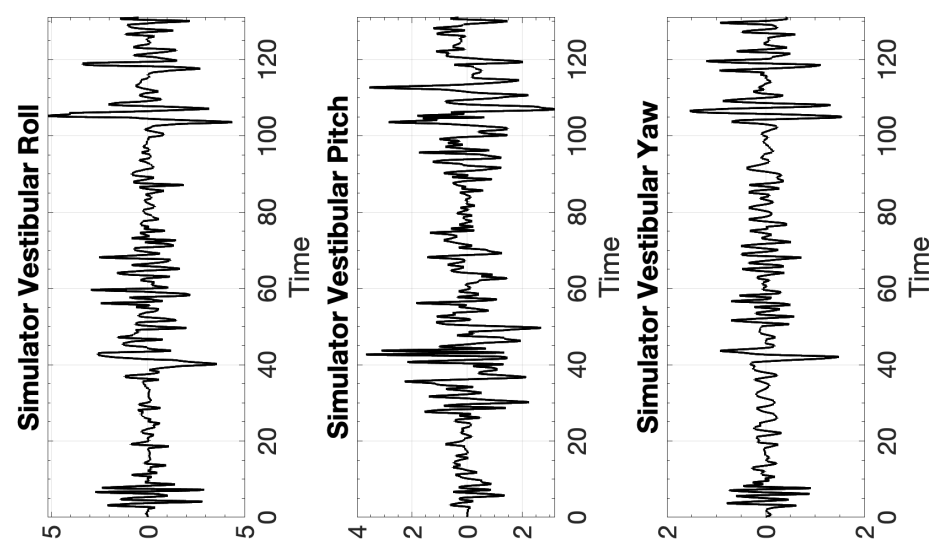

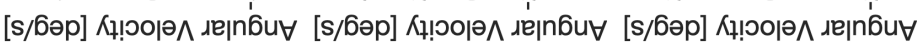

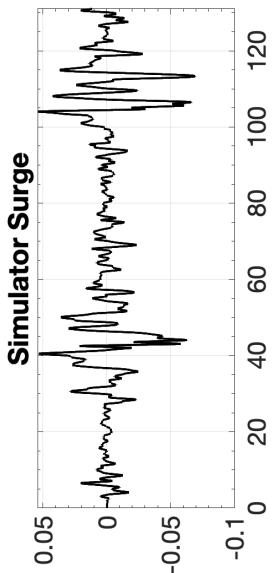

[s/m] К१!०o|ə^

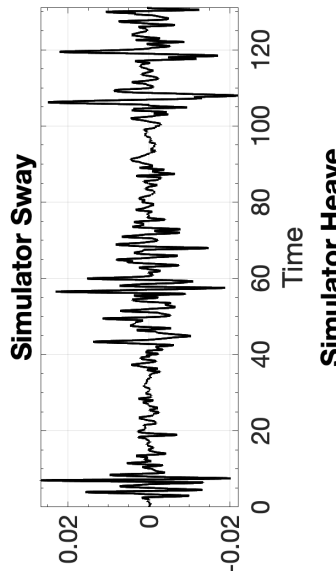

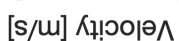

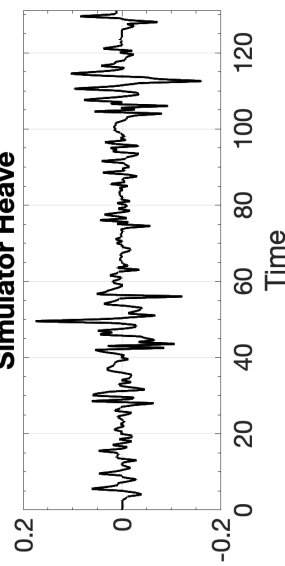

$[\mathrm{s} / \mathrm{M}]$ ᄉำ잇 $\Lambda$

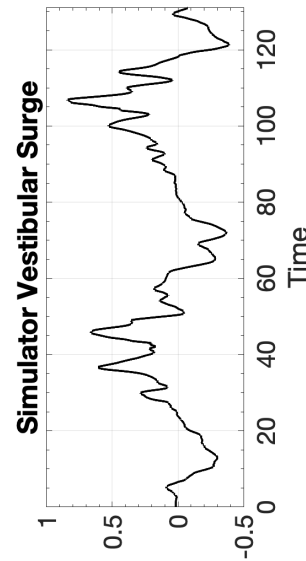

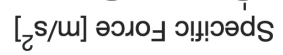

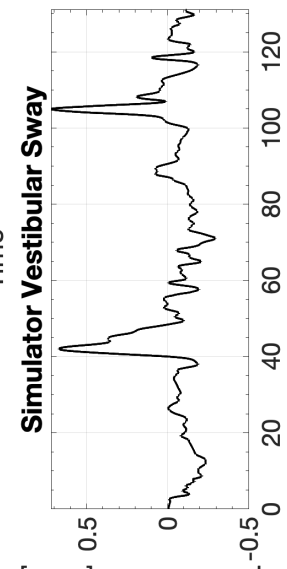

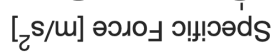

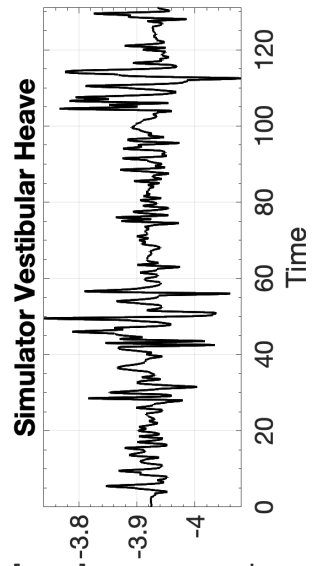

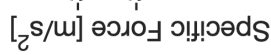

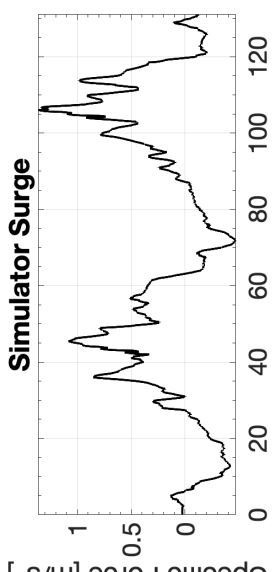

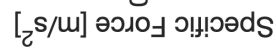

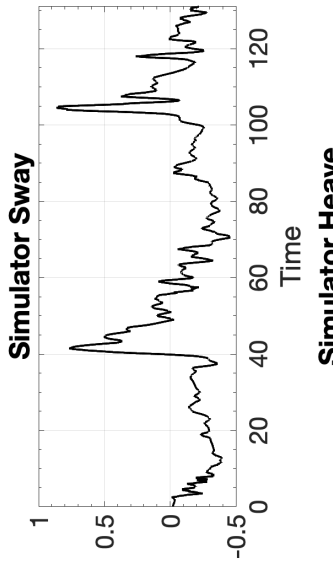

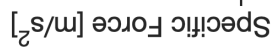

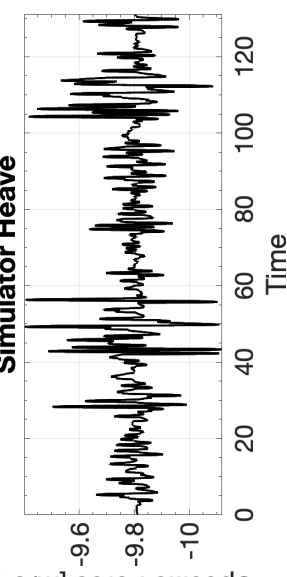

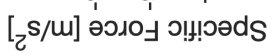

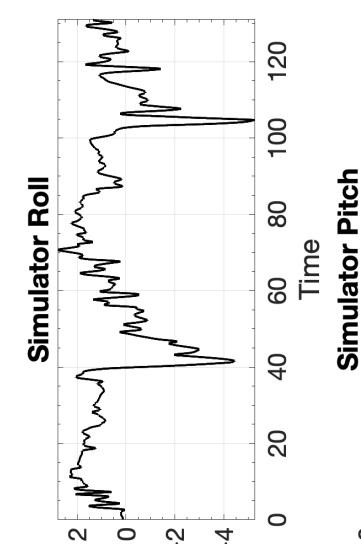

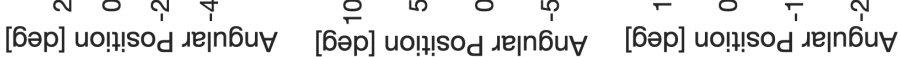
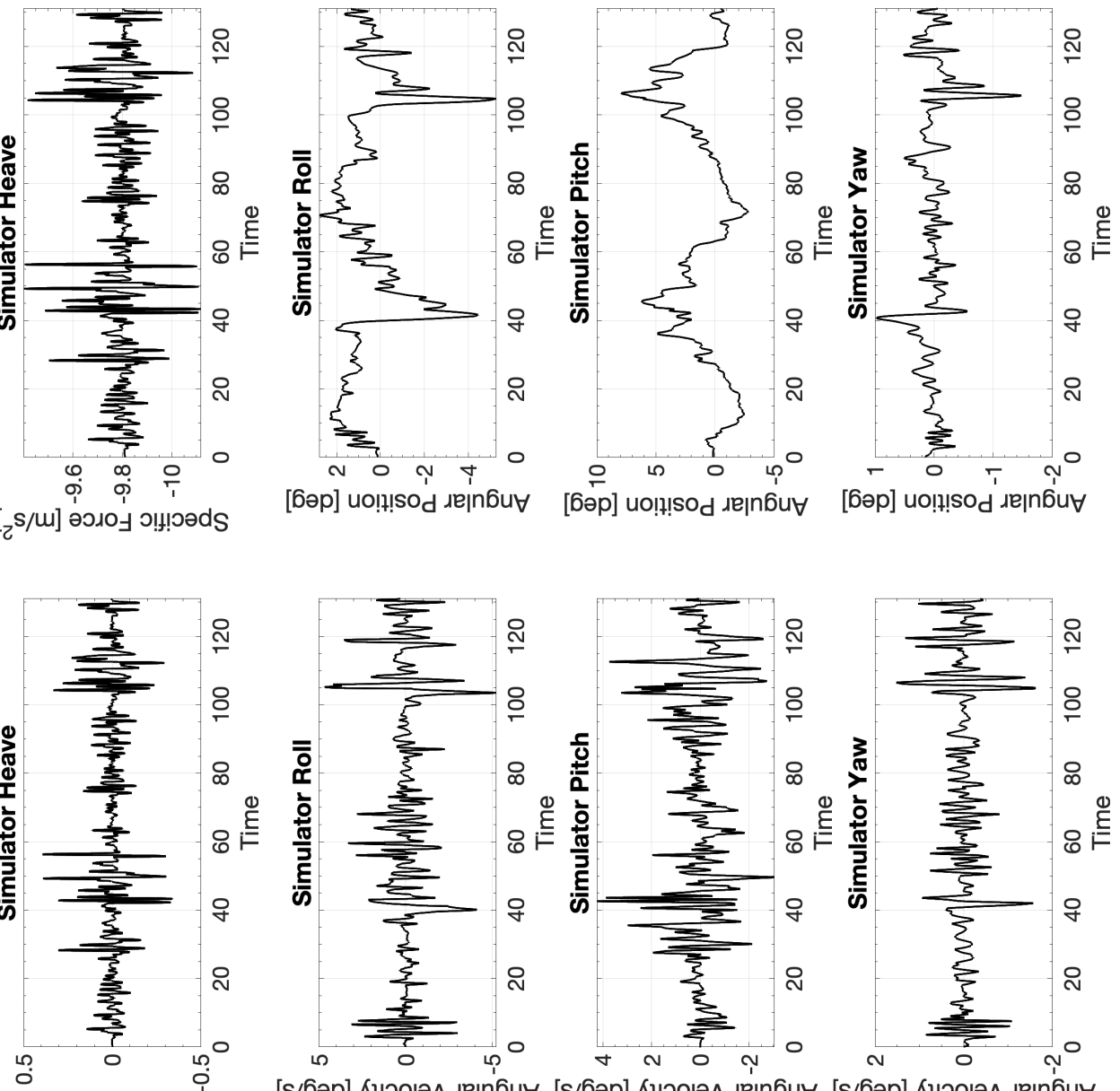

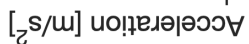

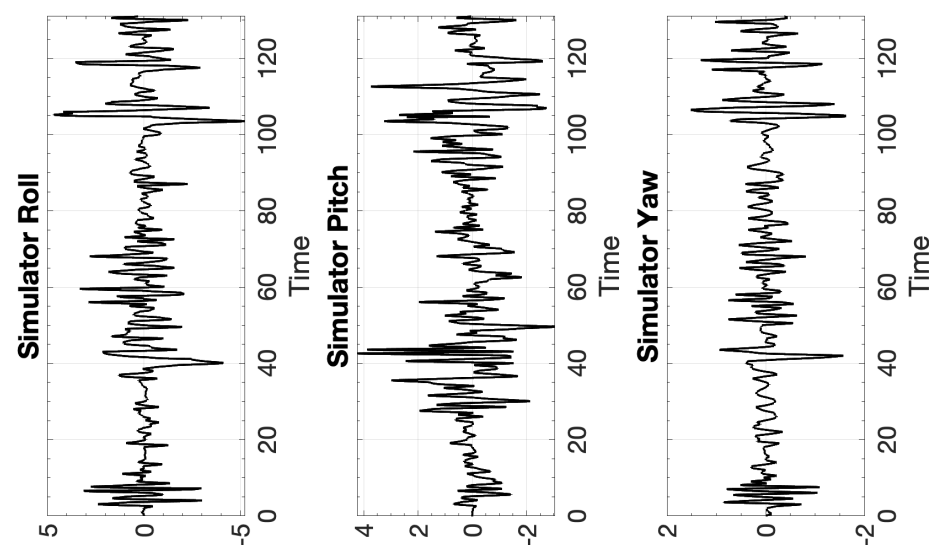

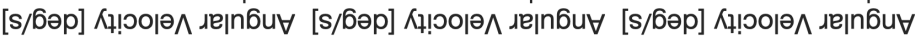

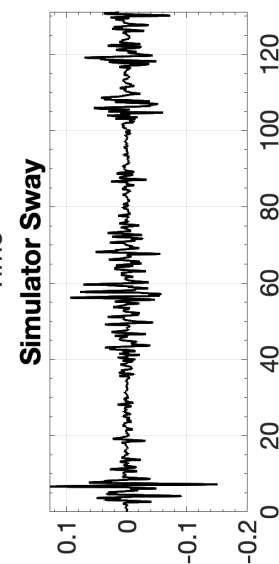

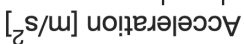

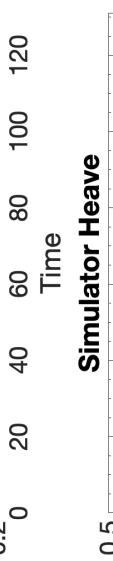

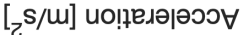

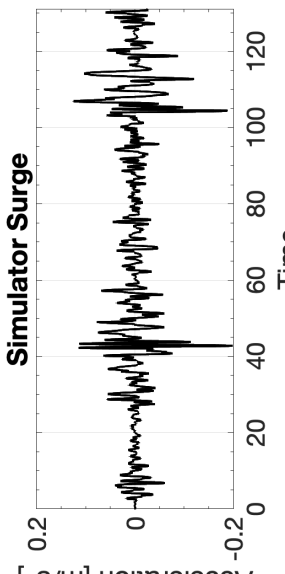




\section{B.8.3 CW3 Cessna 172 Stall Recovery}

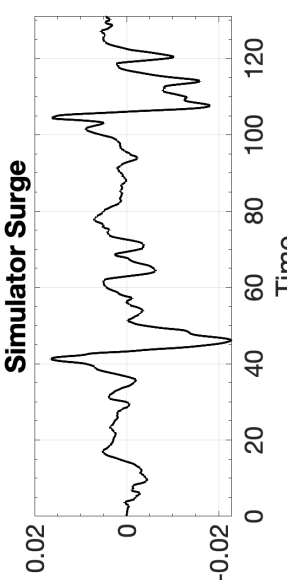

$[\mathrm{m}]$ uo!̣!!sod

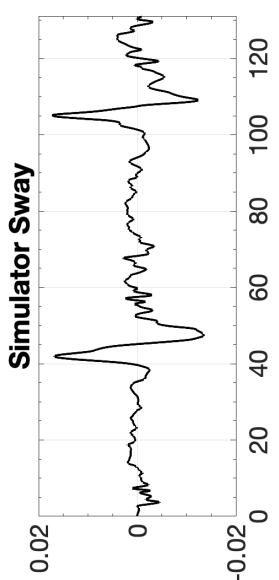

[u] uol!?!sod

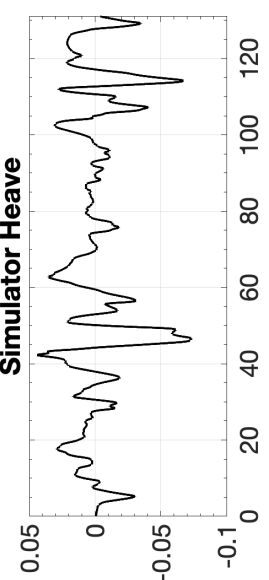

[w] uol!?!sod
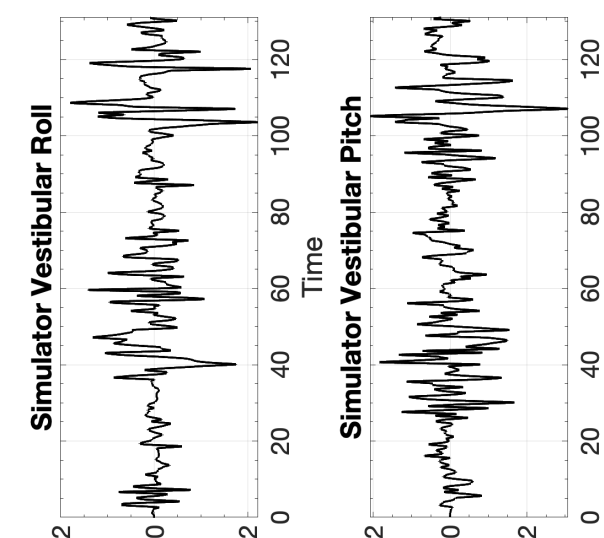

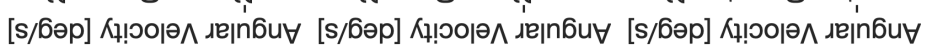
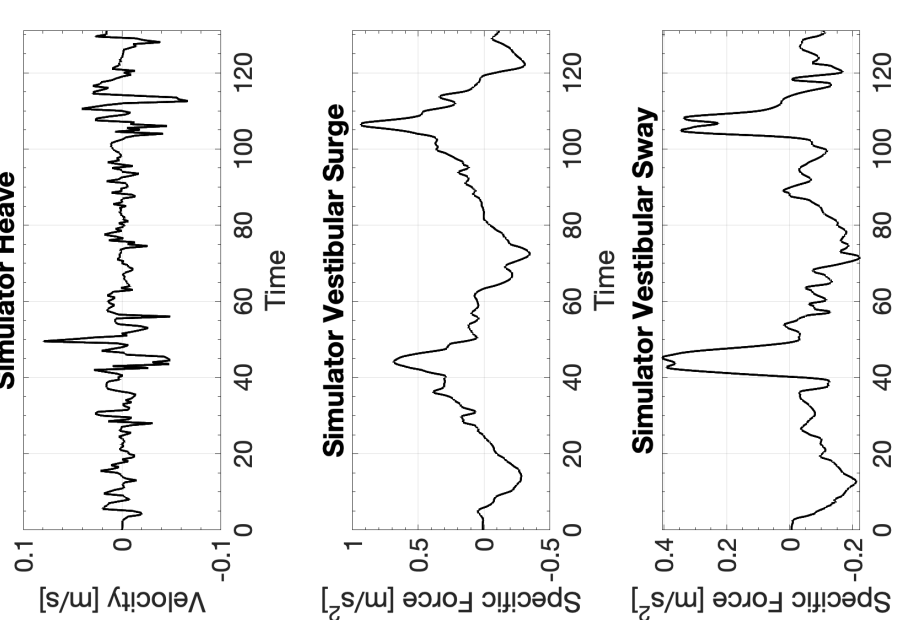

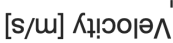

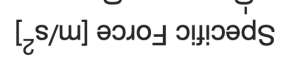

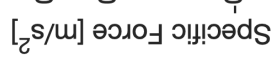
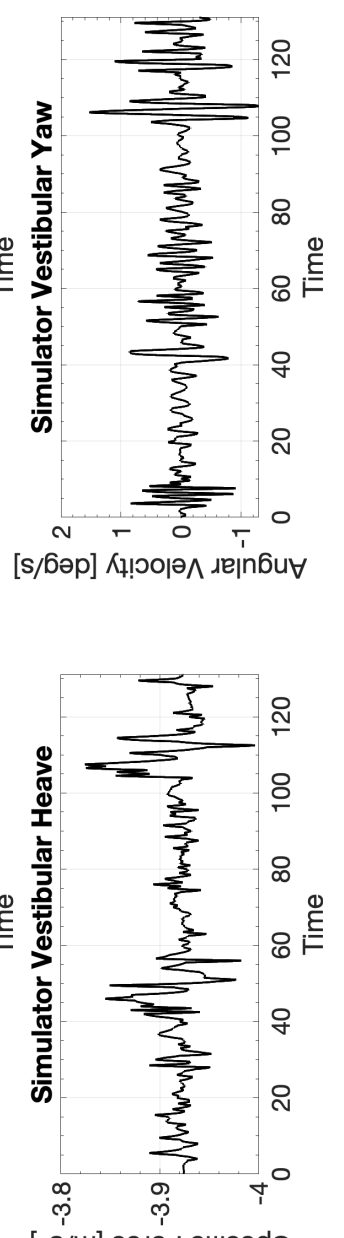

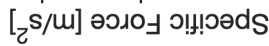

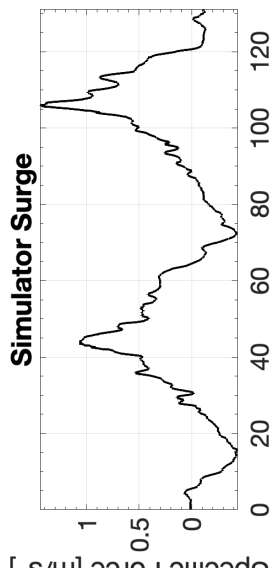

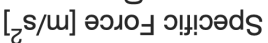

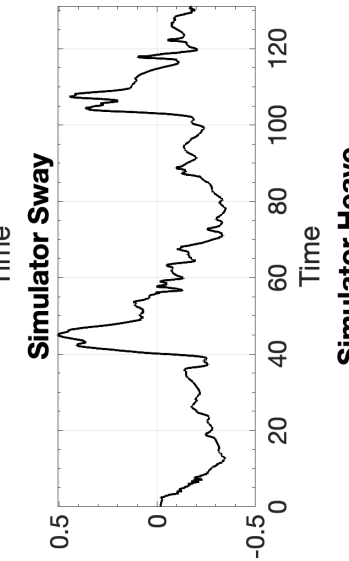

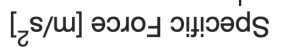

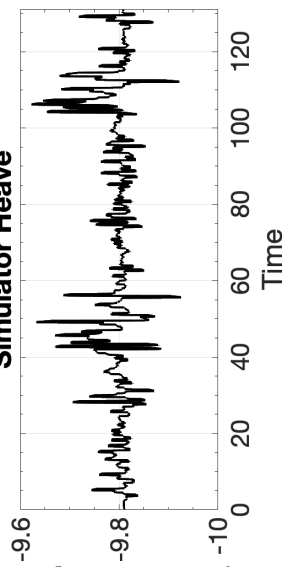

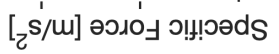

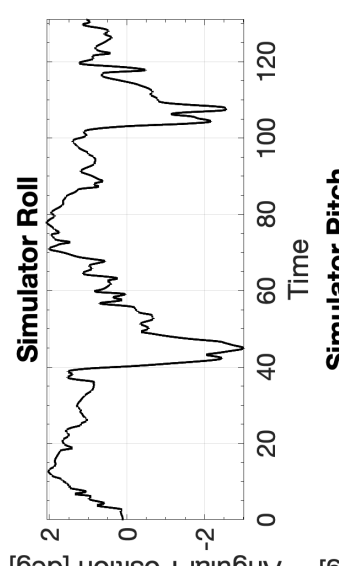

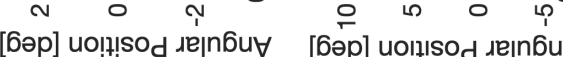

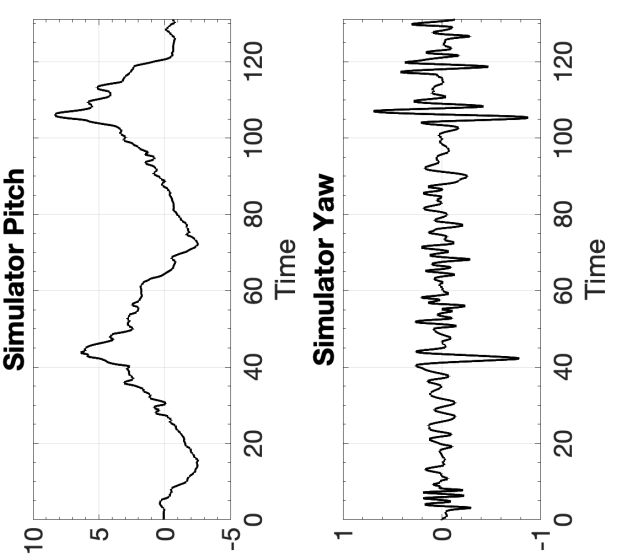

[6әр] uo!?!sod גeןn6u

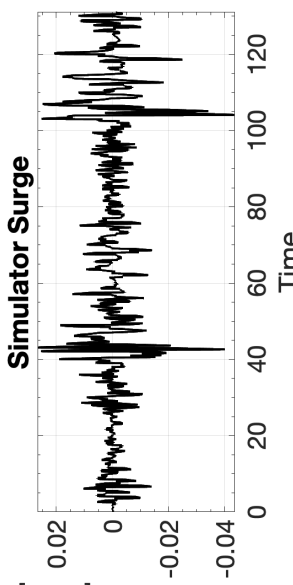

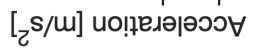

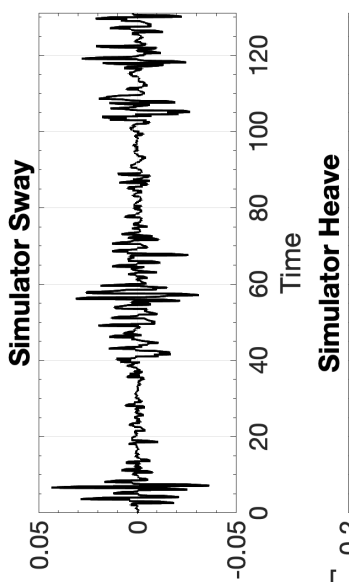

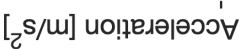

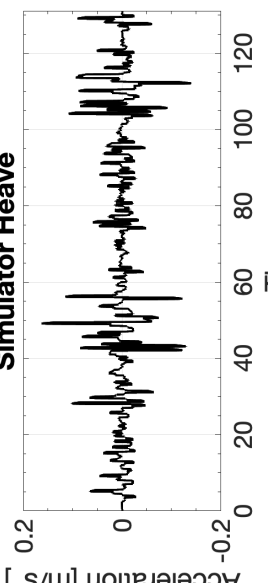

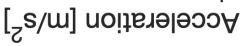

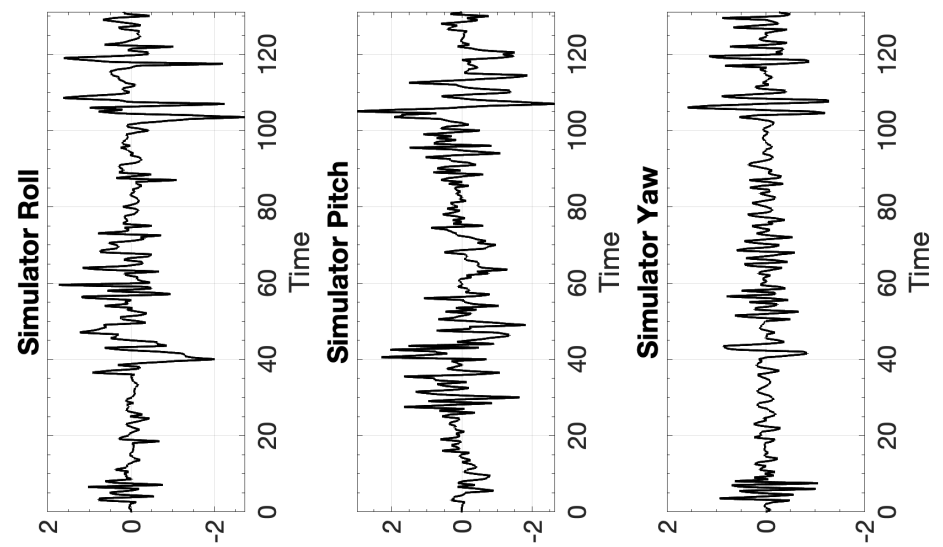

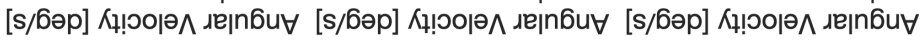




\section{B.9 Boeing 737 Coordinated Turn}
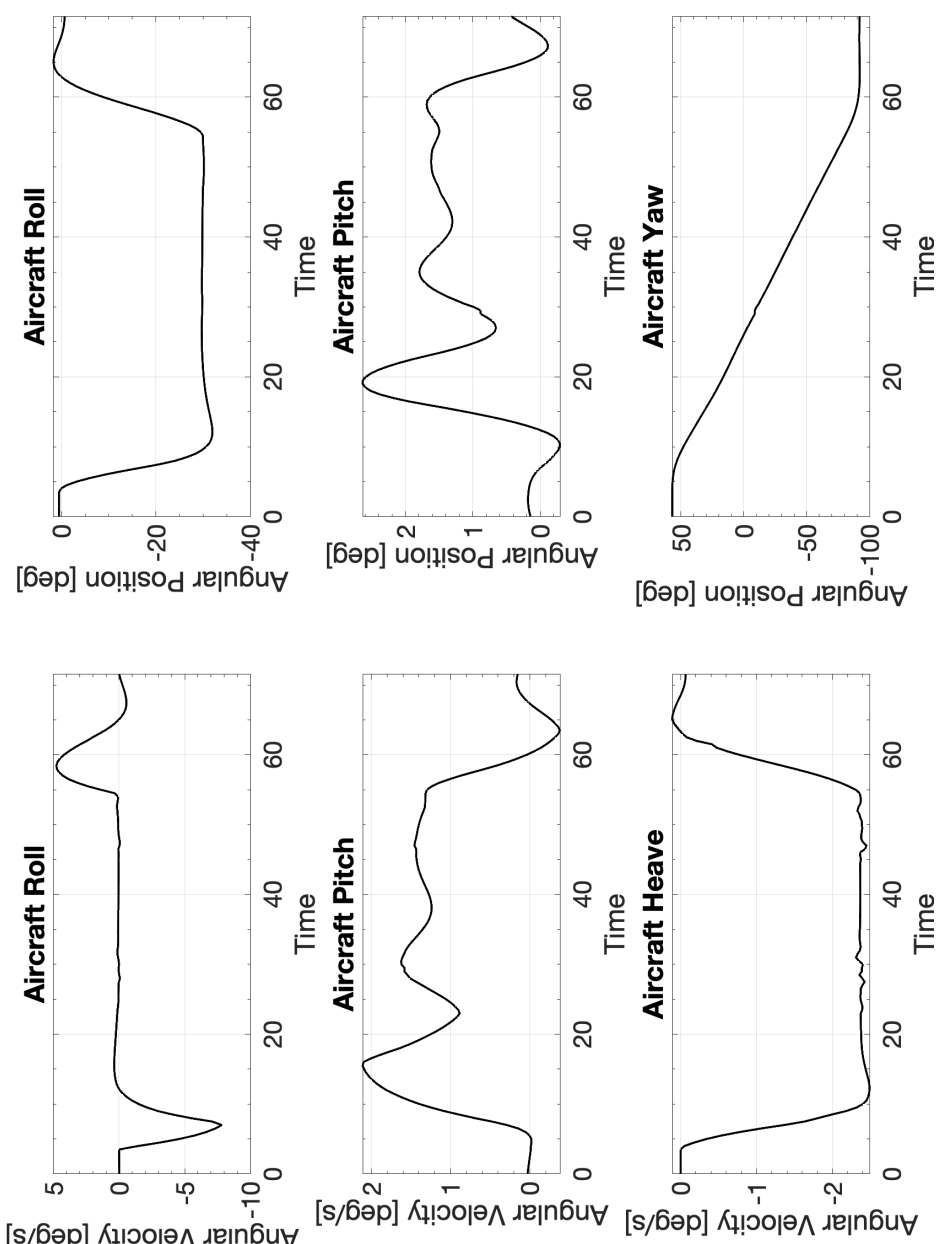

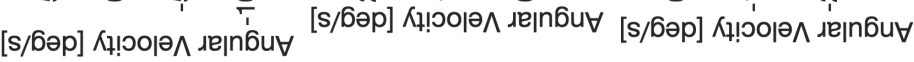

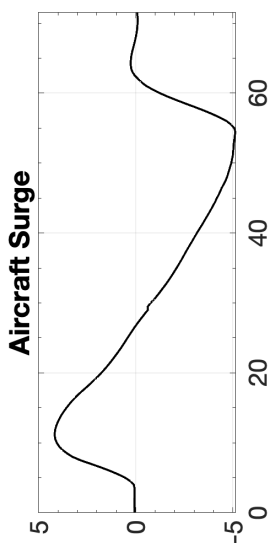

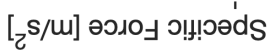

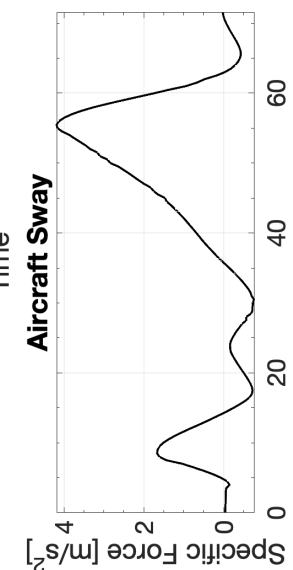

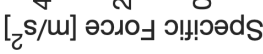

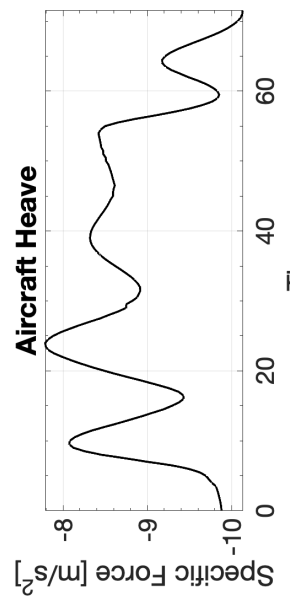

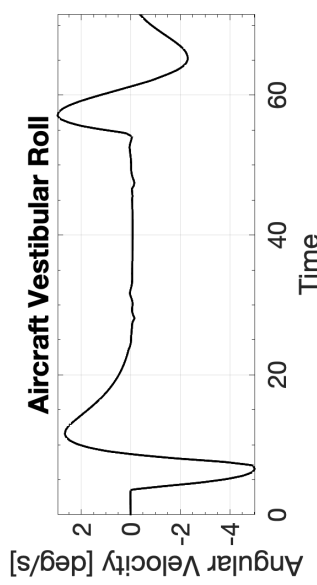

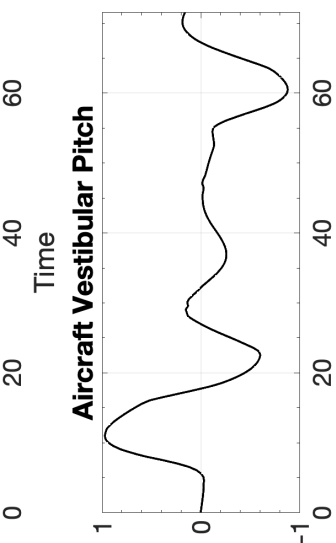

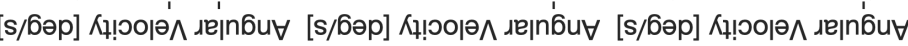

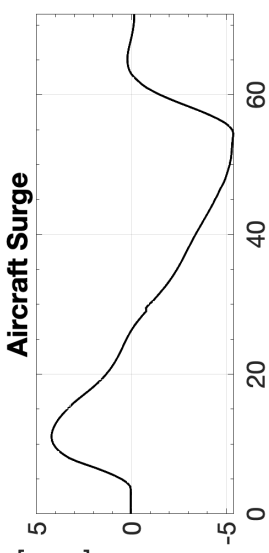

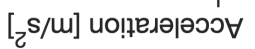

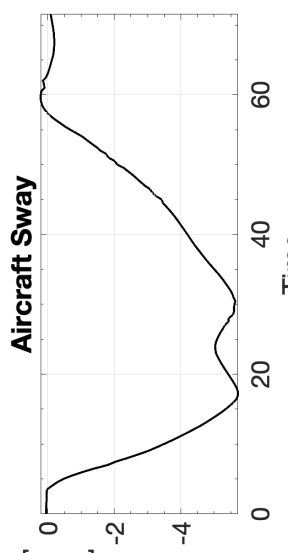

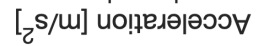

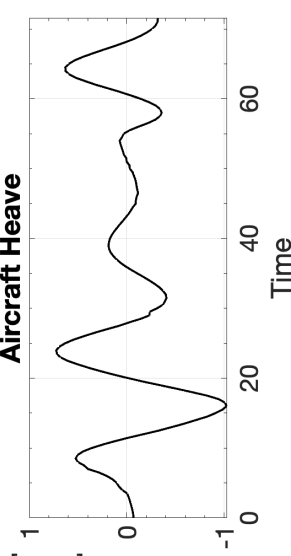

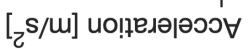

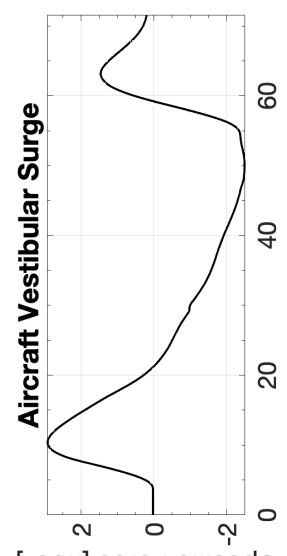

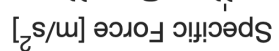

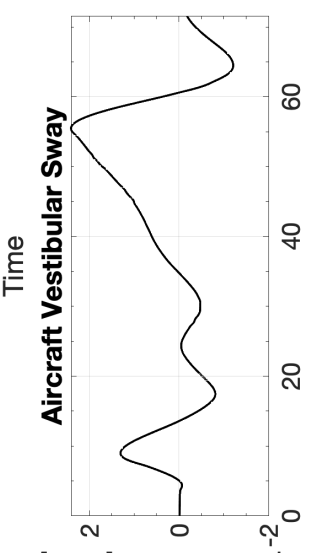

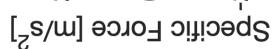

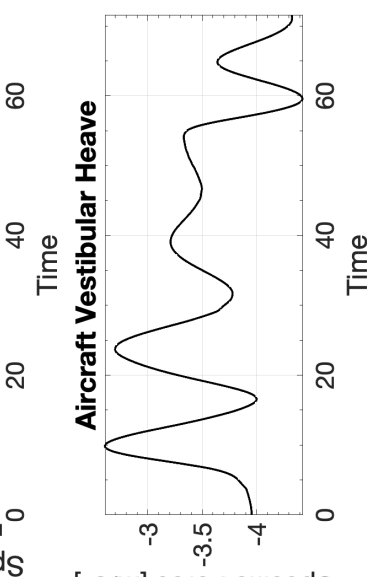

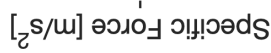




\section{B.9.1 CW1 Boeing 737 Coordinated Turn}

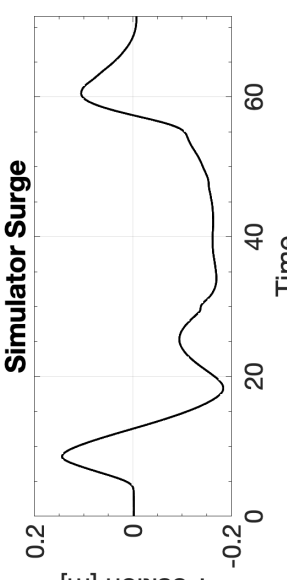

[u] uo!!!!sod

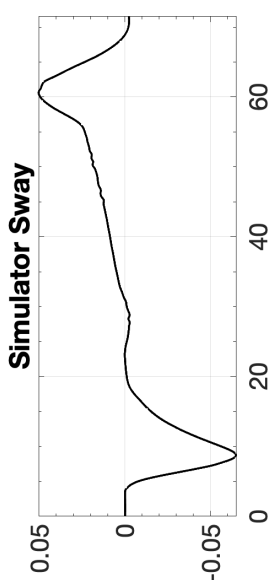

[u] uolt!sod

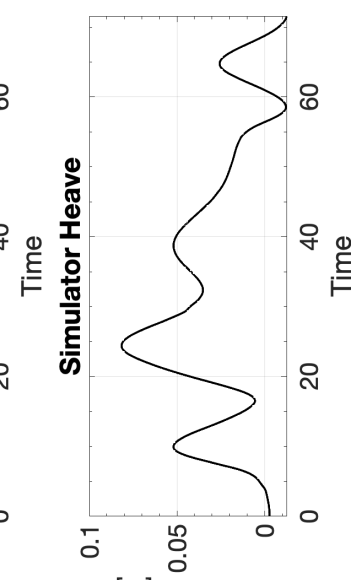

[w] uo!!! sod

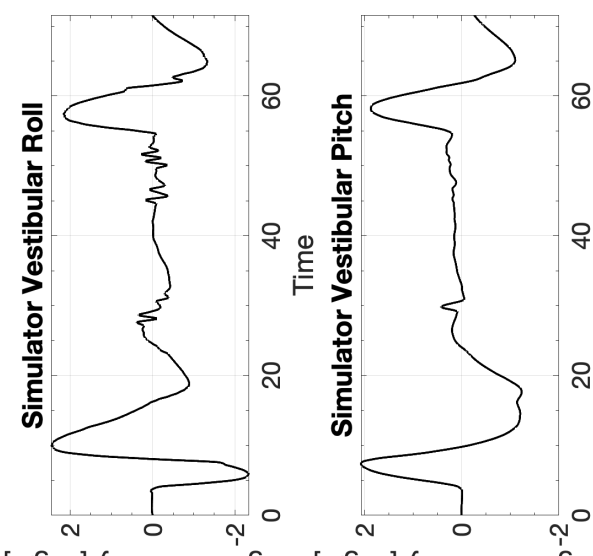

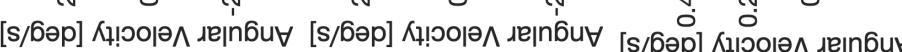

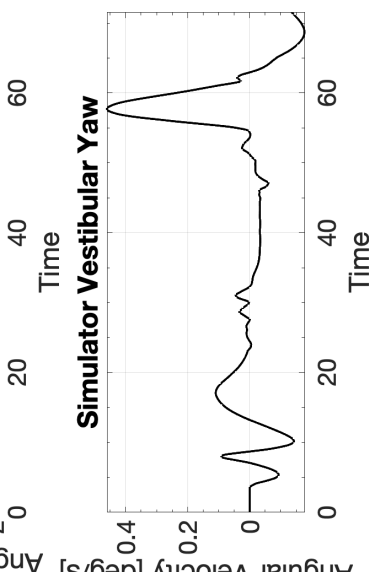

$\stackrel{q}{\stackrel{\oplus}{\xi}}$

ลิ

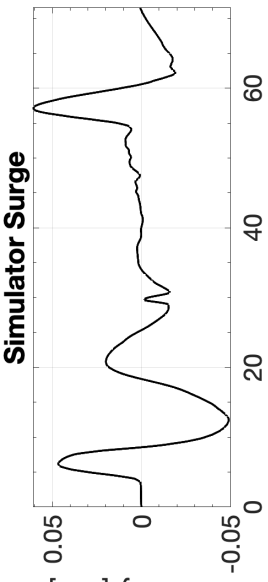

[s/m] К१! 잇

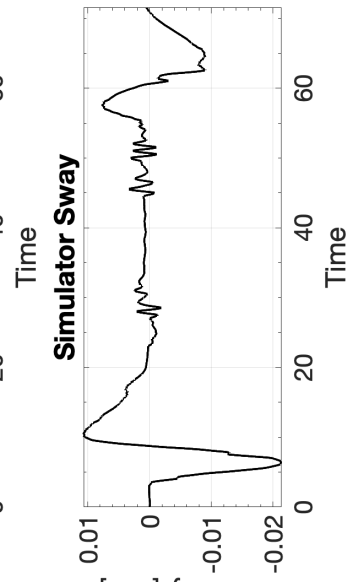

[s/m] Кұ! 잇

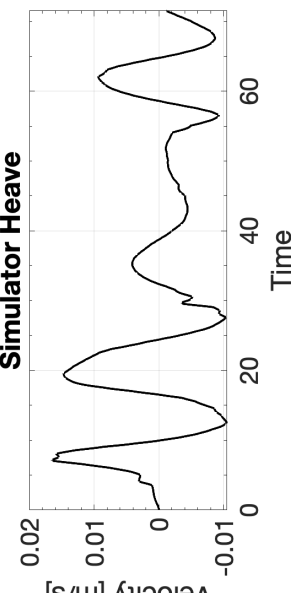

[s/m] Кұ!৩о|ә

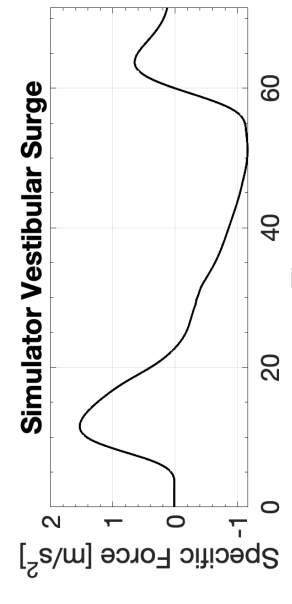

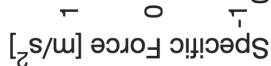

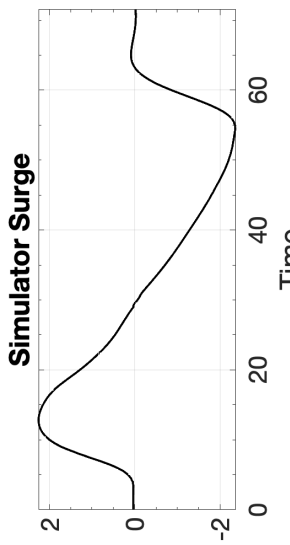

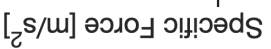

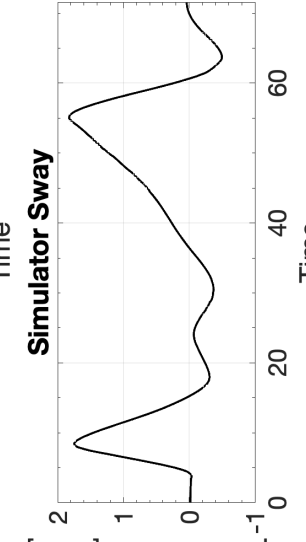

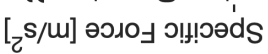

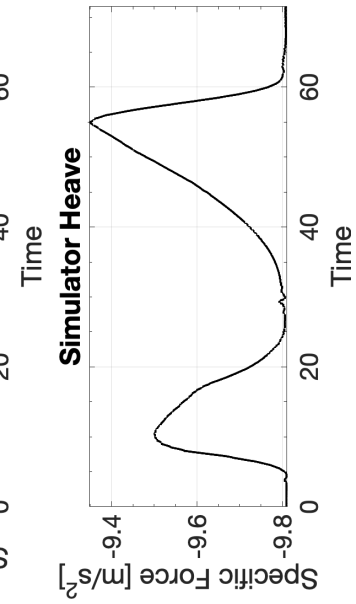

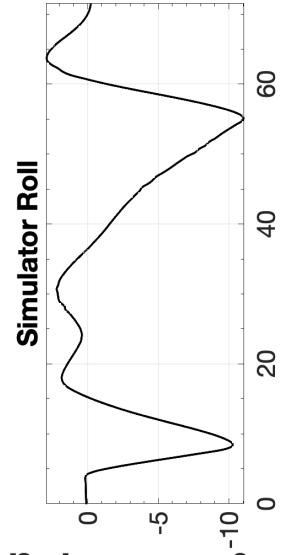

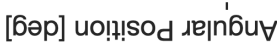
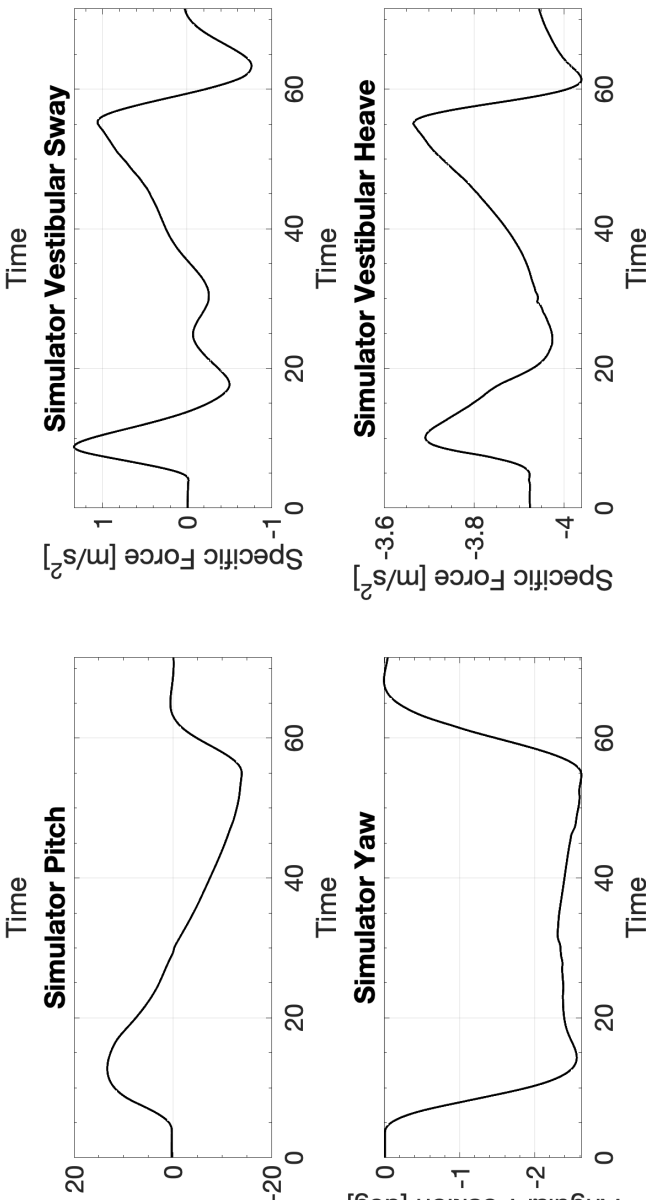

[6әp] uo!!!sod

[6әр] uo!̣!!sod jejn6u

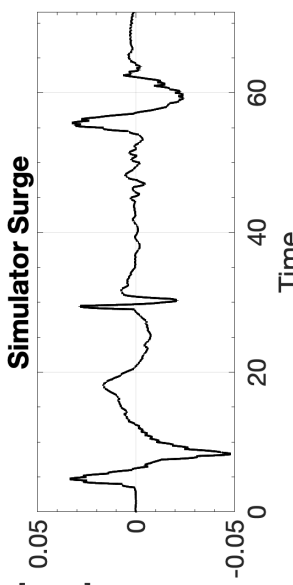

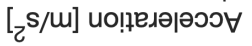

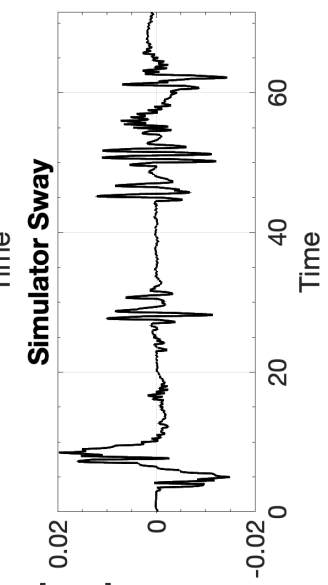

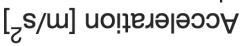

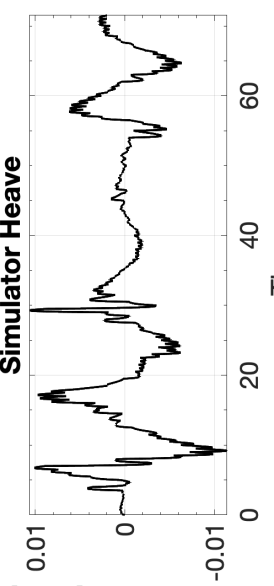

[z/m] uо!ฺелә|әээ $\forall$

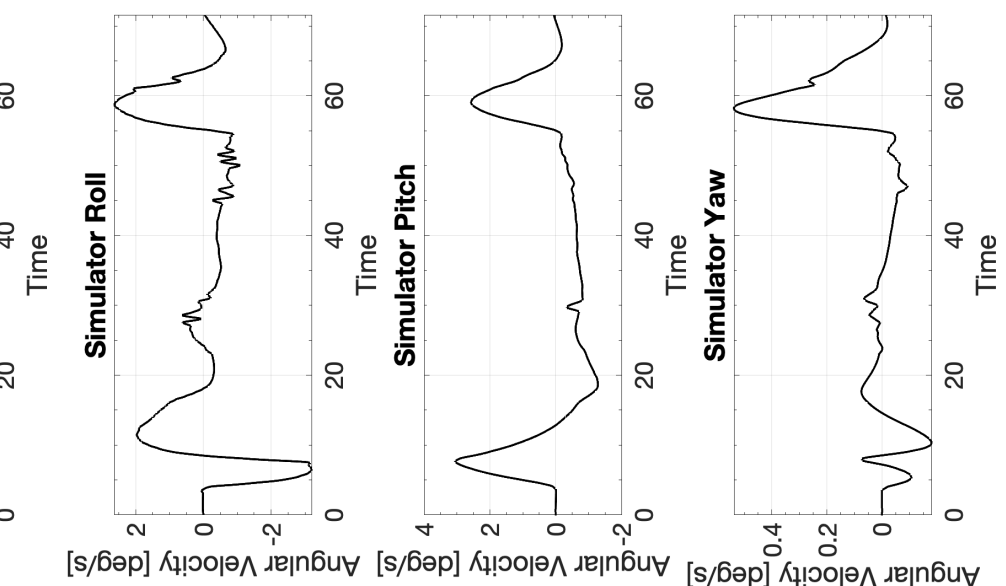

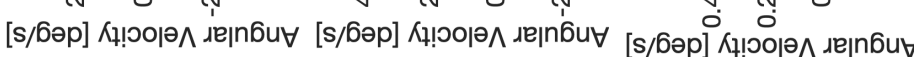




\section{B.9.2 CW2 Boeing 737 Coordinated Turn}

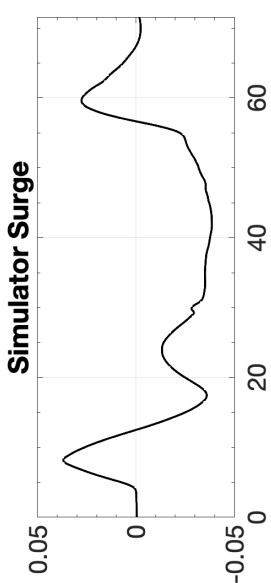

[w] uo!n!lsod

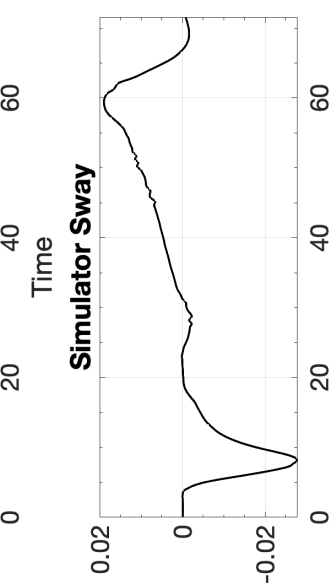

[w] uo!n! sod

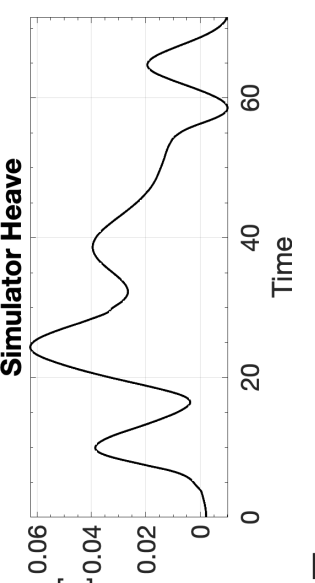

[w] uo!!?!sod

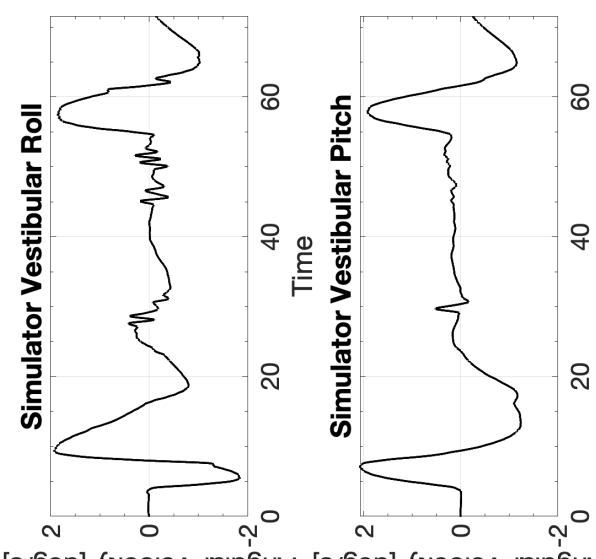

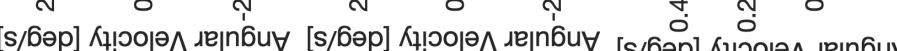

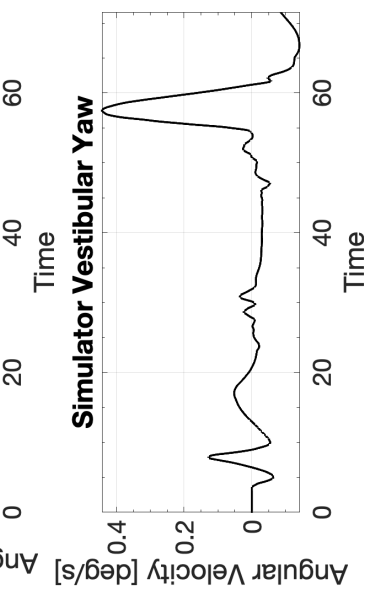

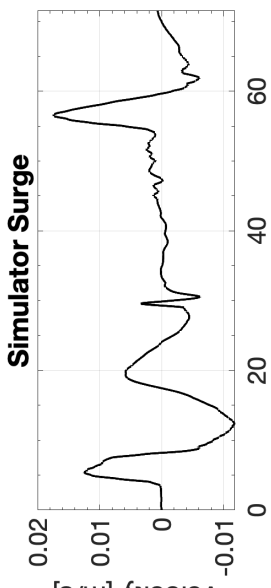

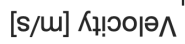

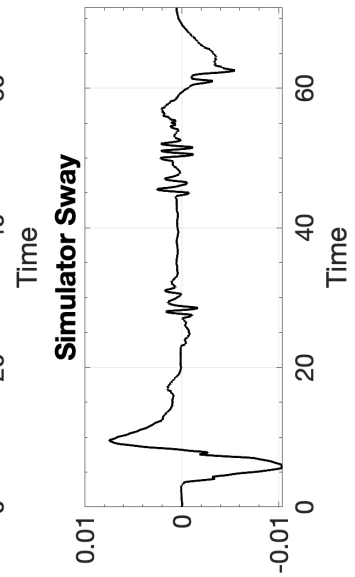

[s/m] Кұ!๐о|ә^

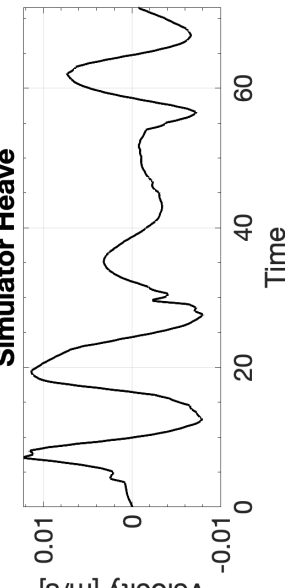

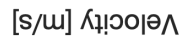

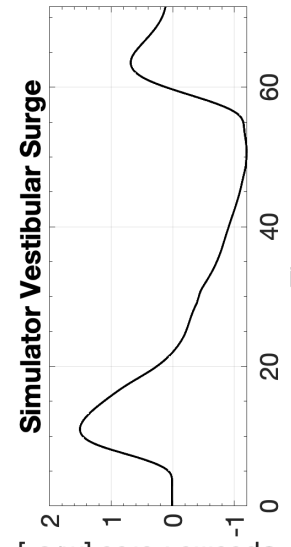

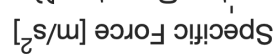

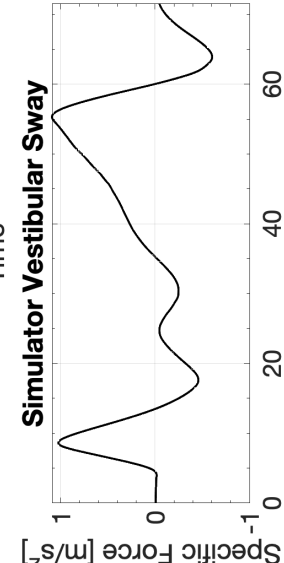

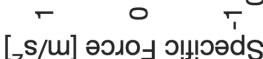


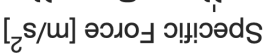

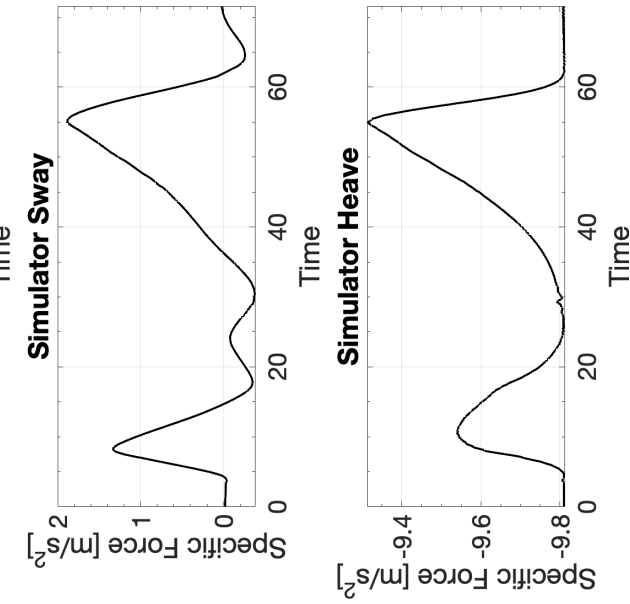

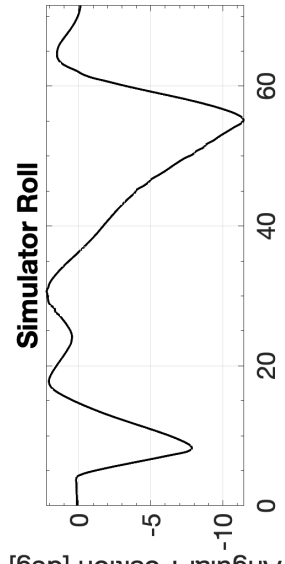

[бәр] uo!n!sod גeןn6u

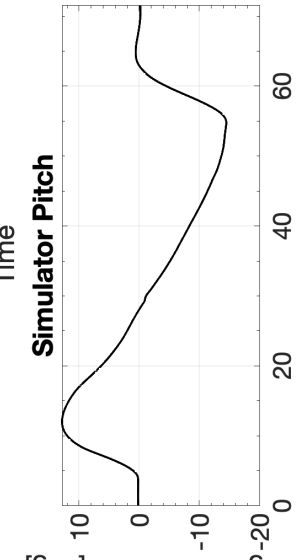

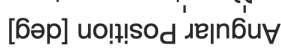

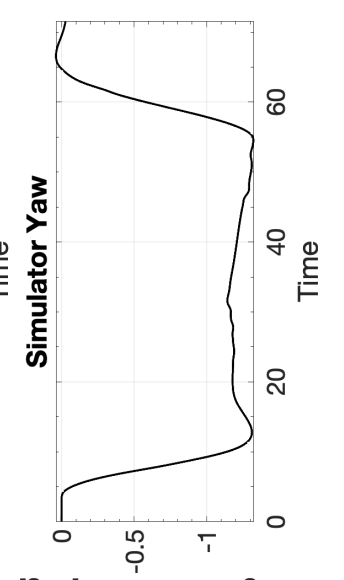

[6әр] uo!!!sod rejn6u

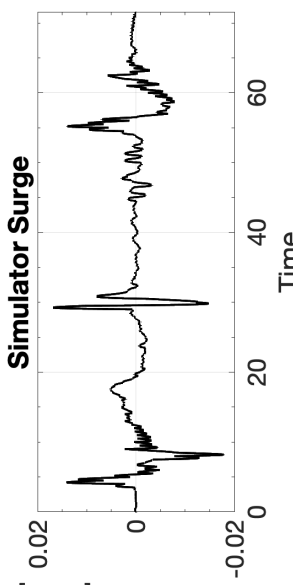

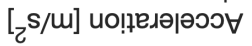

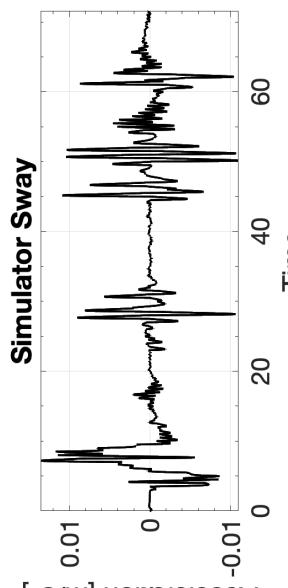

[z $\mathrm{s} / \mathrm{m}]$ ио!цедәрәоэ

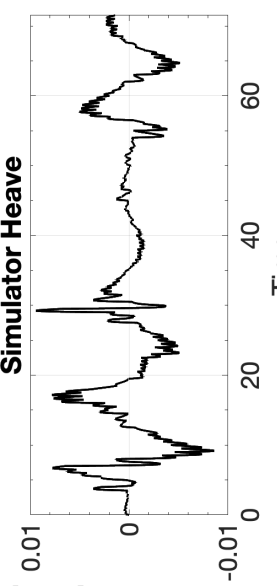

[zs/m] ио!редәәәээ

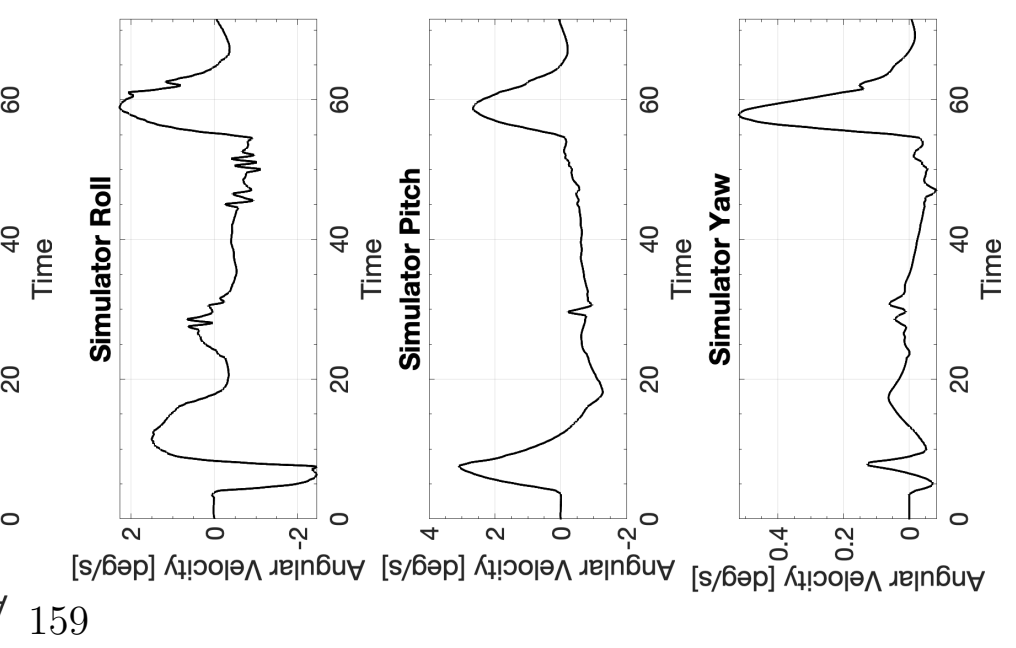




\section{B.9.3 CW3 Boeing 737 Coordinated Turn}

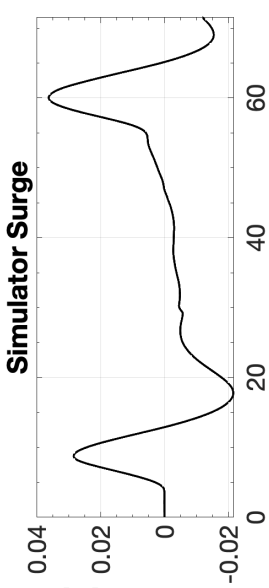

[u] uo!n! sod

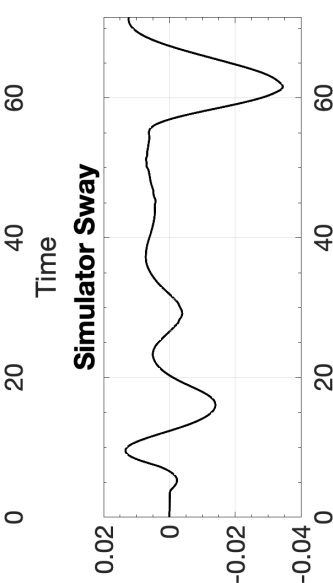

[w] uo!n!lsod

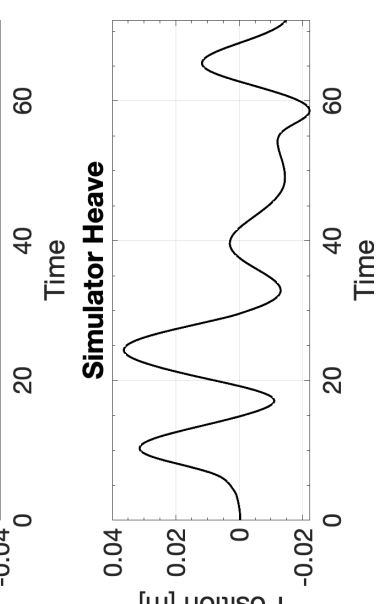

[w] uo!n!s $\mathrm{SO}_{\mathrm{d}}$

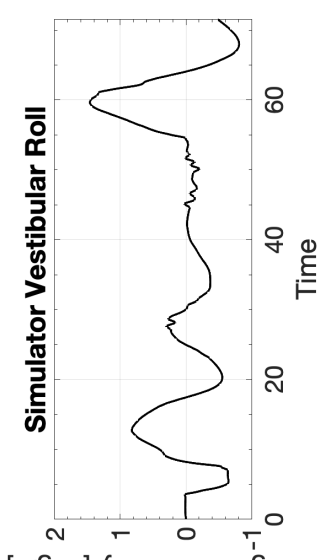

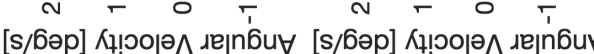

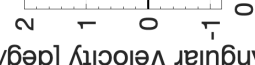

[s/бəp]

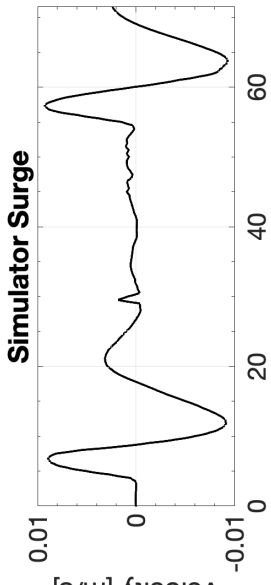

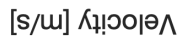

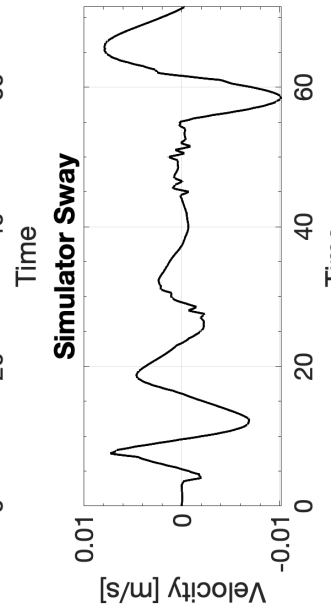

[s/m] Кұ!๐о|ә^

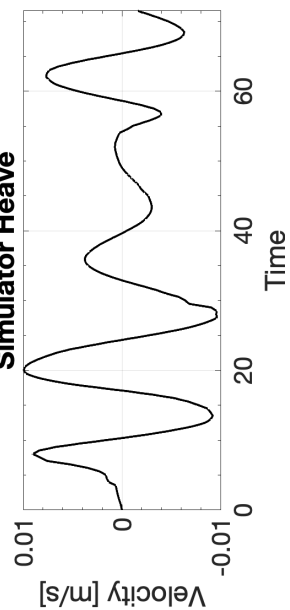

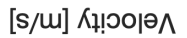
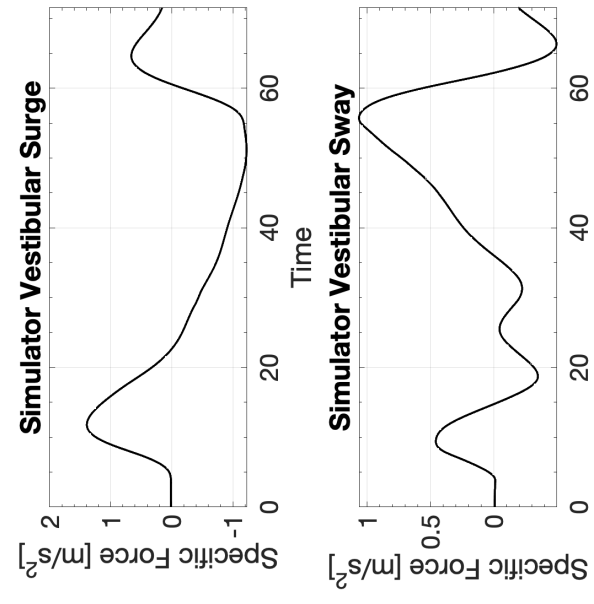

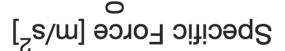

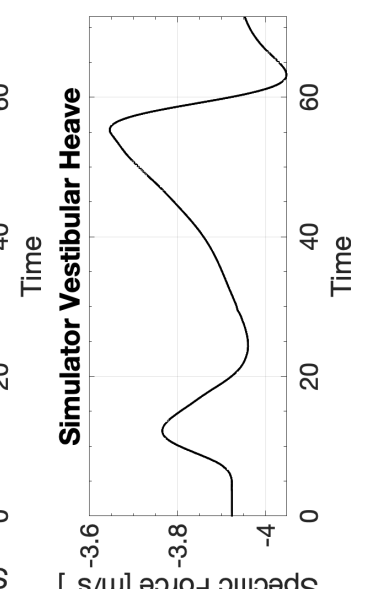

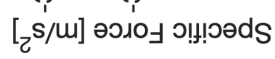
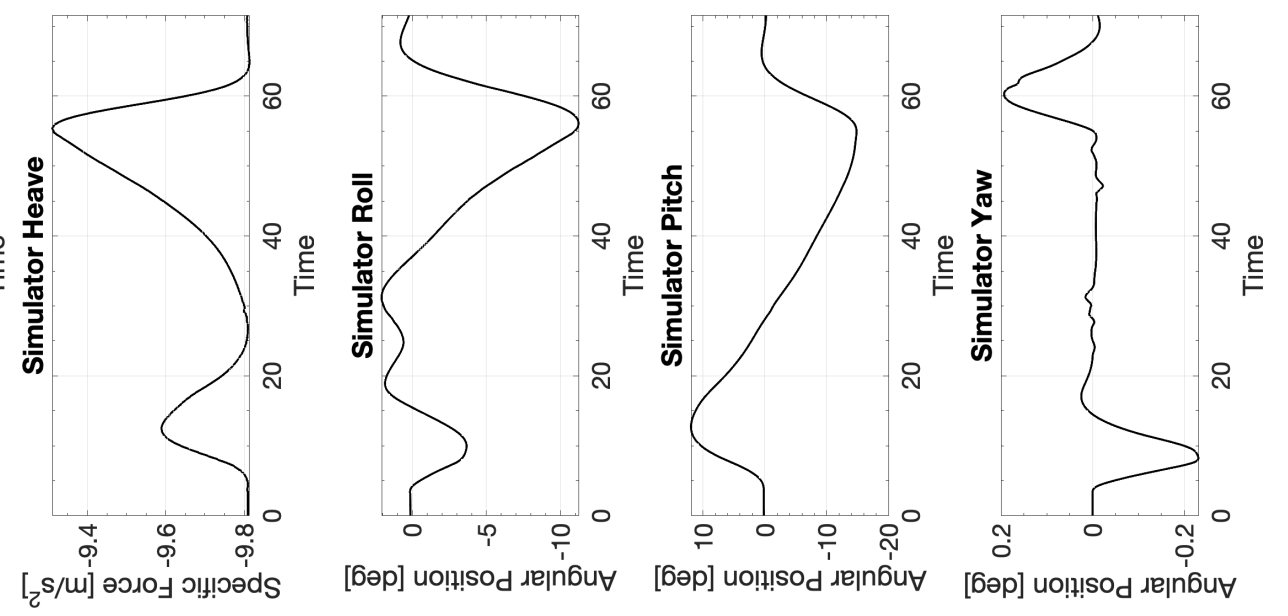

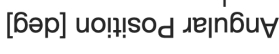

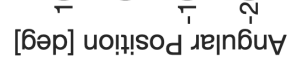

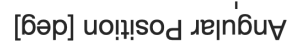

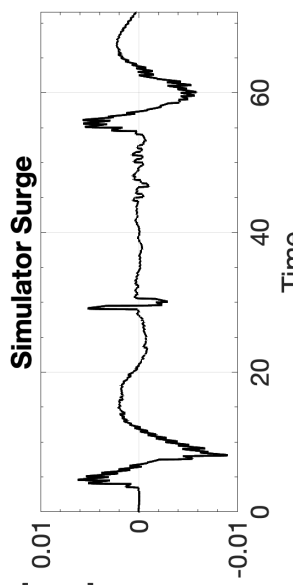

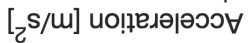

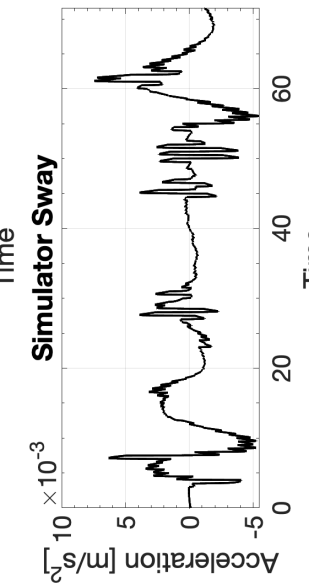

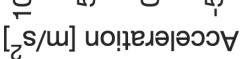

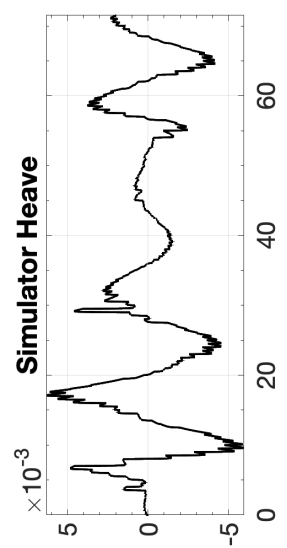

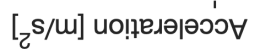

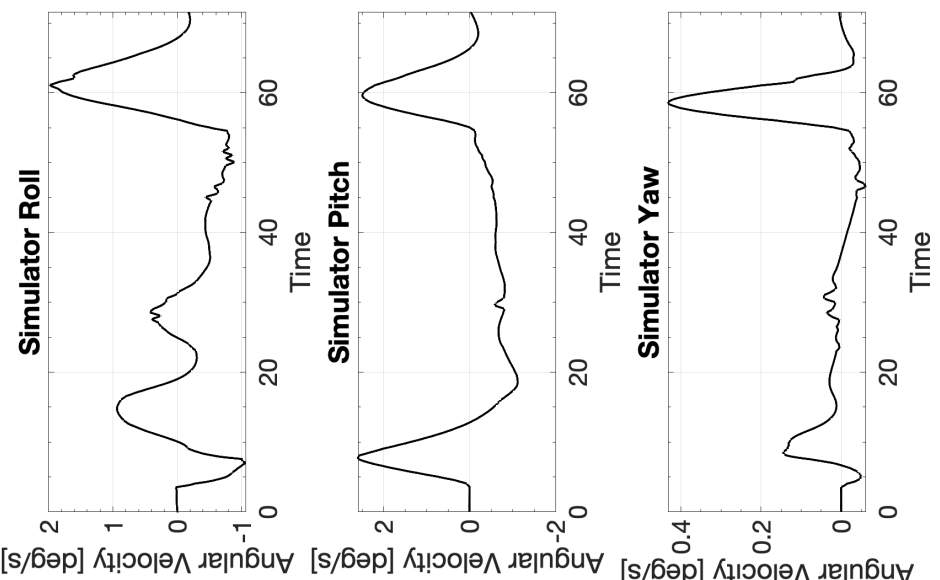

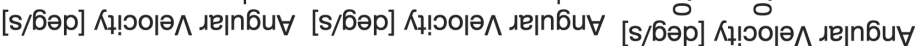


B.10 Boeing 737 Cross-wind Landing

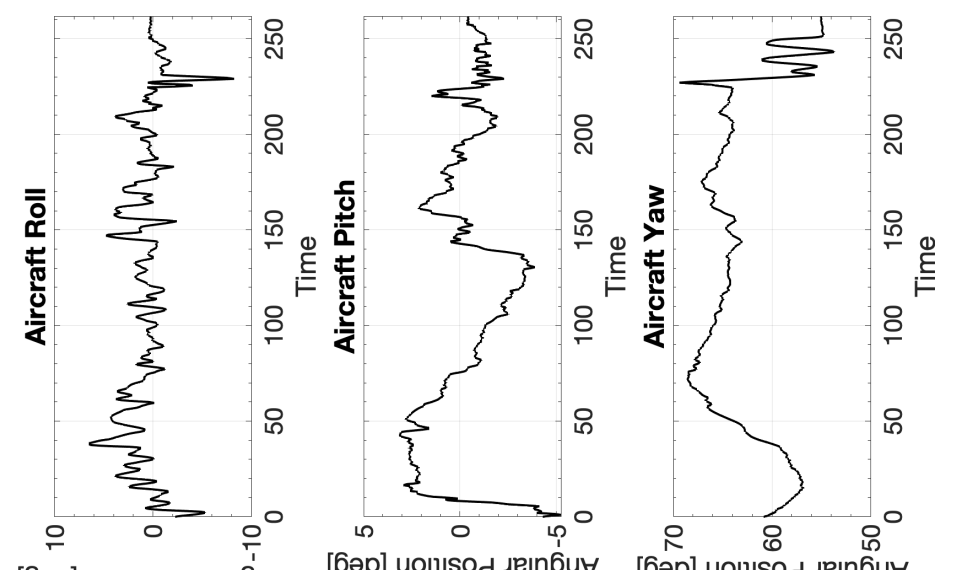

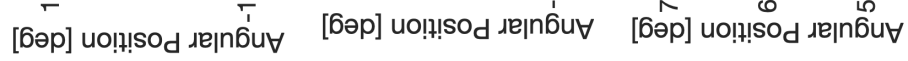

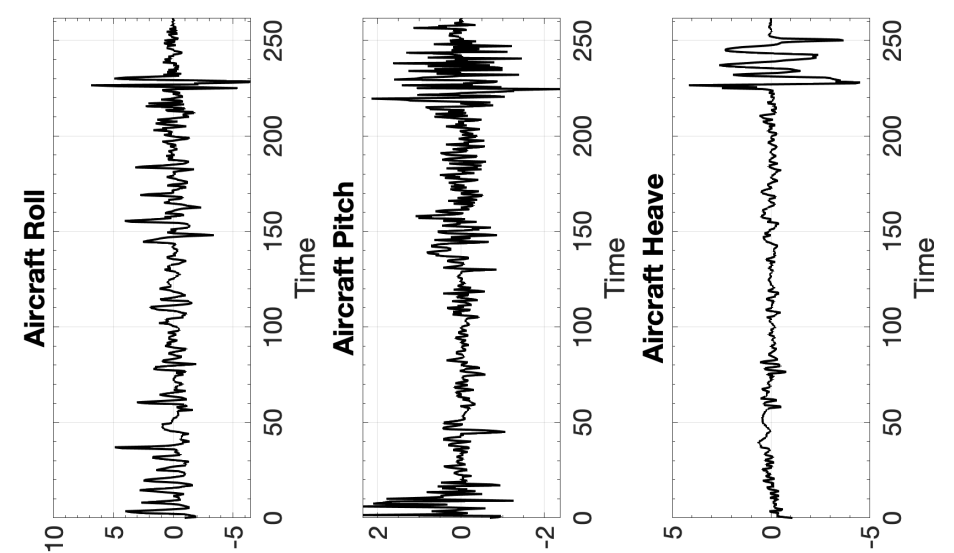

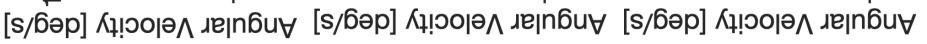

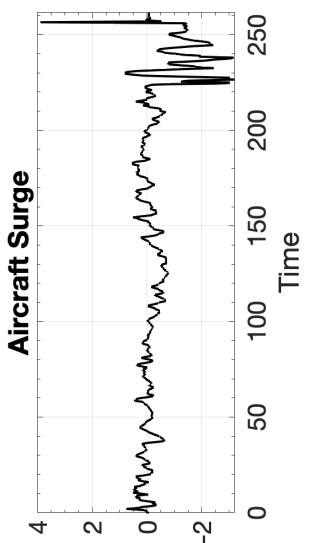

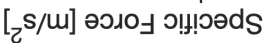

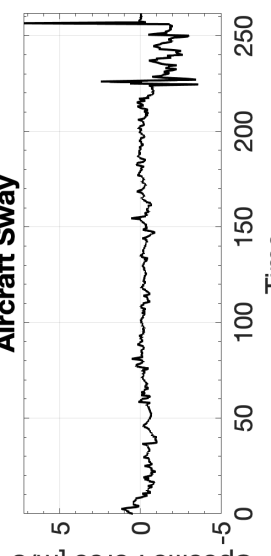

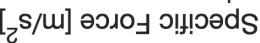

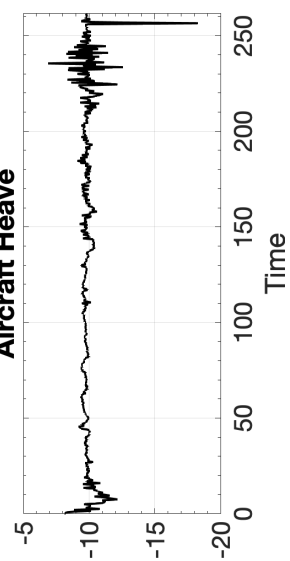

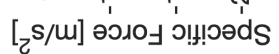

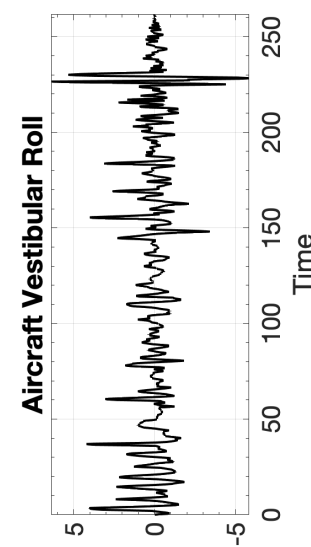

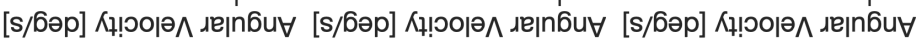

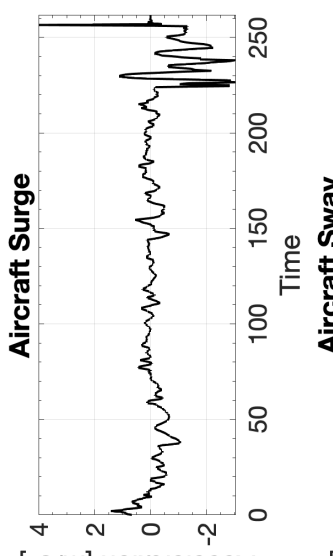

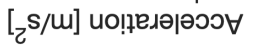

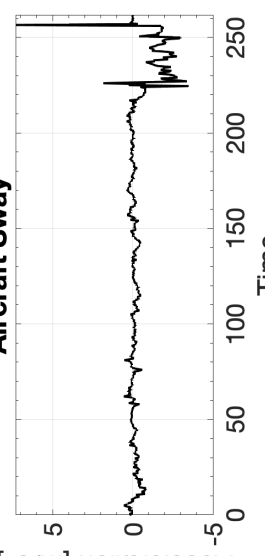

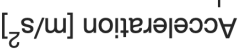

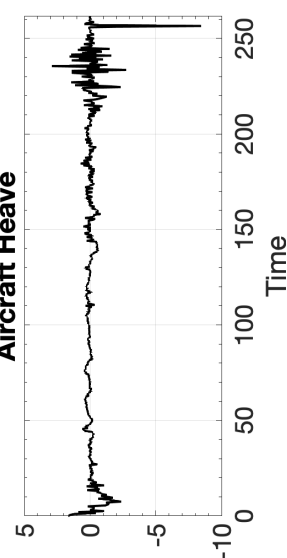

$[z s / m]$ uo!̣eләәәэว

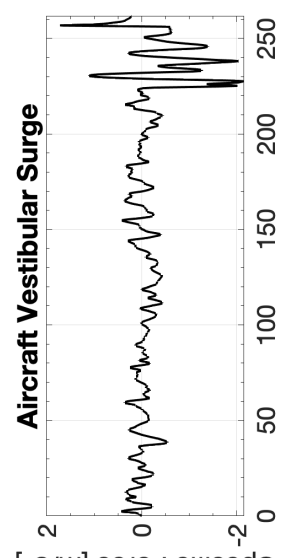

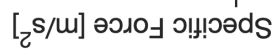
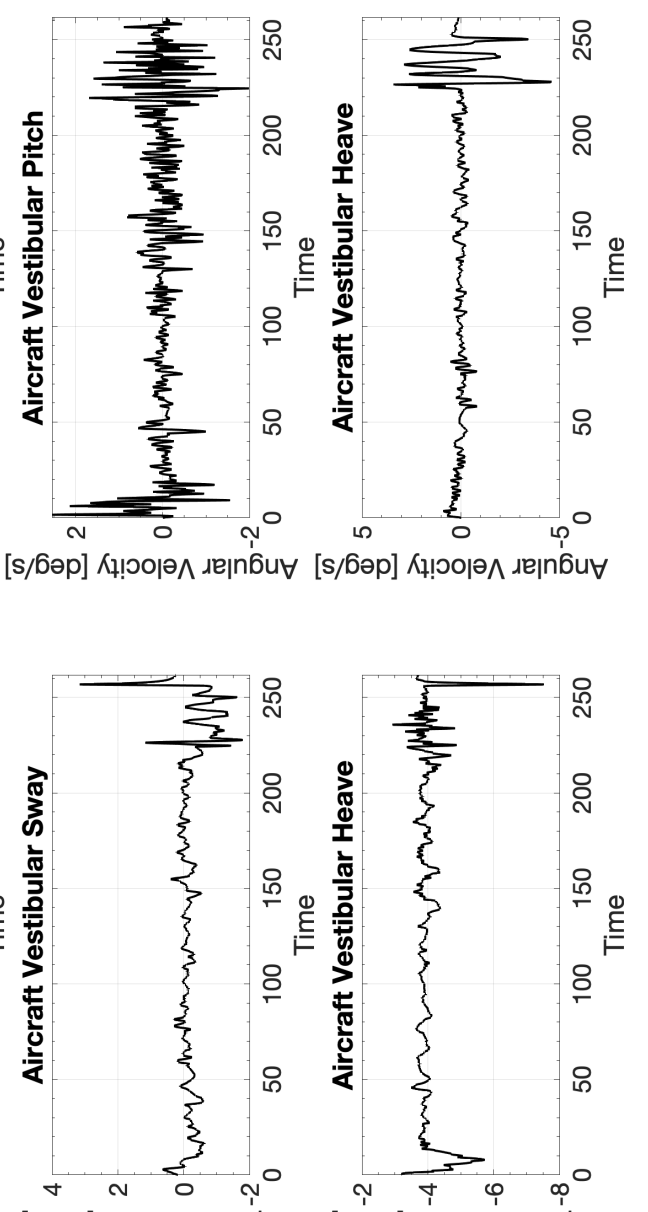

[ 


\section{B.10.1 CW1 Boeing 737 Cross-wind Landing}

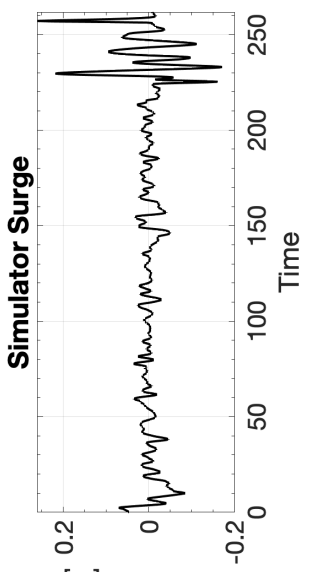

[u] uo!!! sod

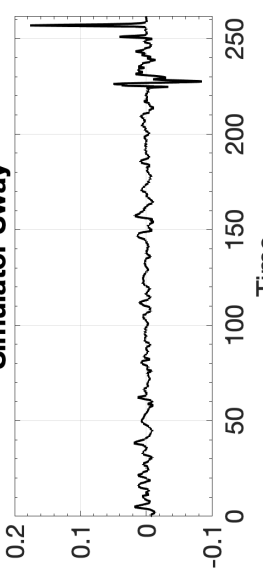

[w] uo!n!!sod

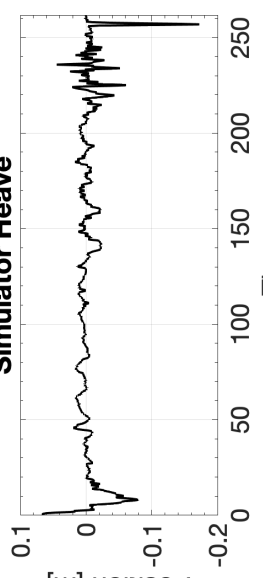

[u] uolt!!sod

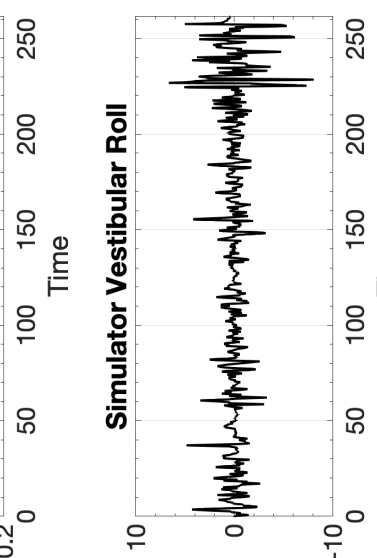

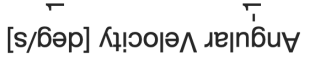

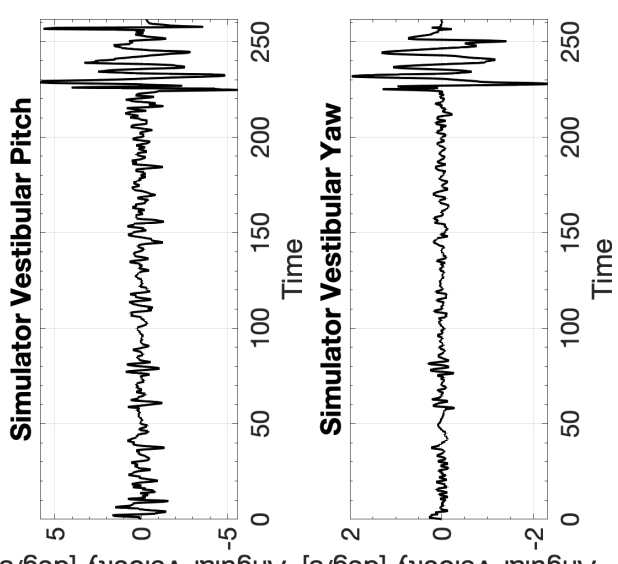

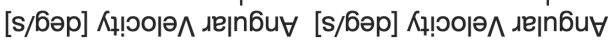

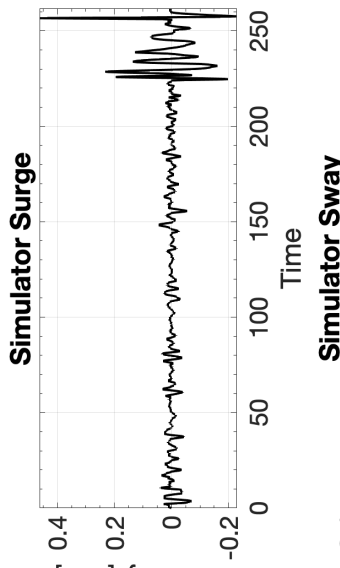

[s/u] К! ৩оюә $\Lambda$

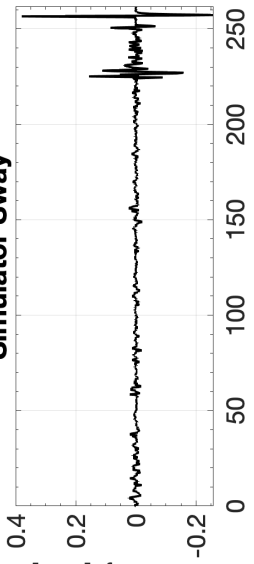

[s/m] К!ำю

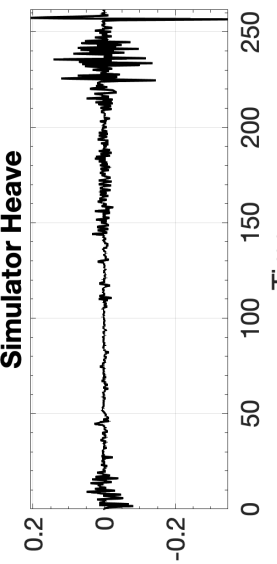

[s/m] К!ำю^

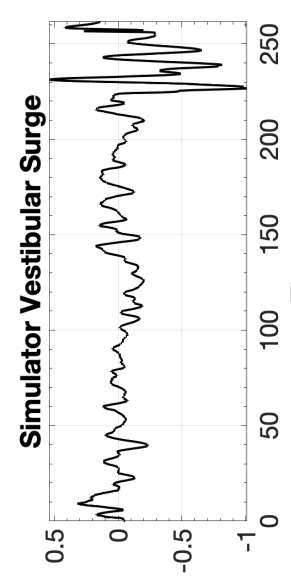

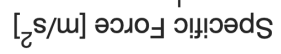

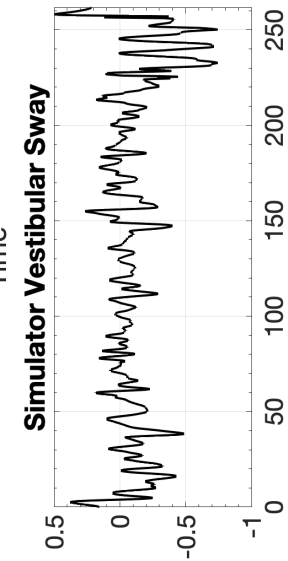

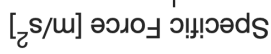
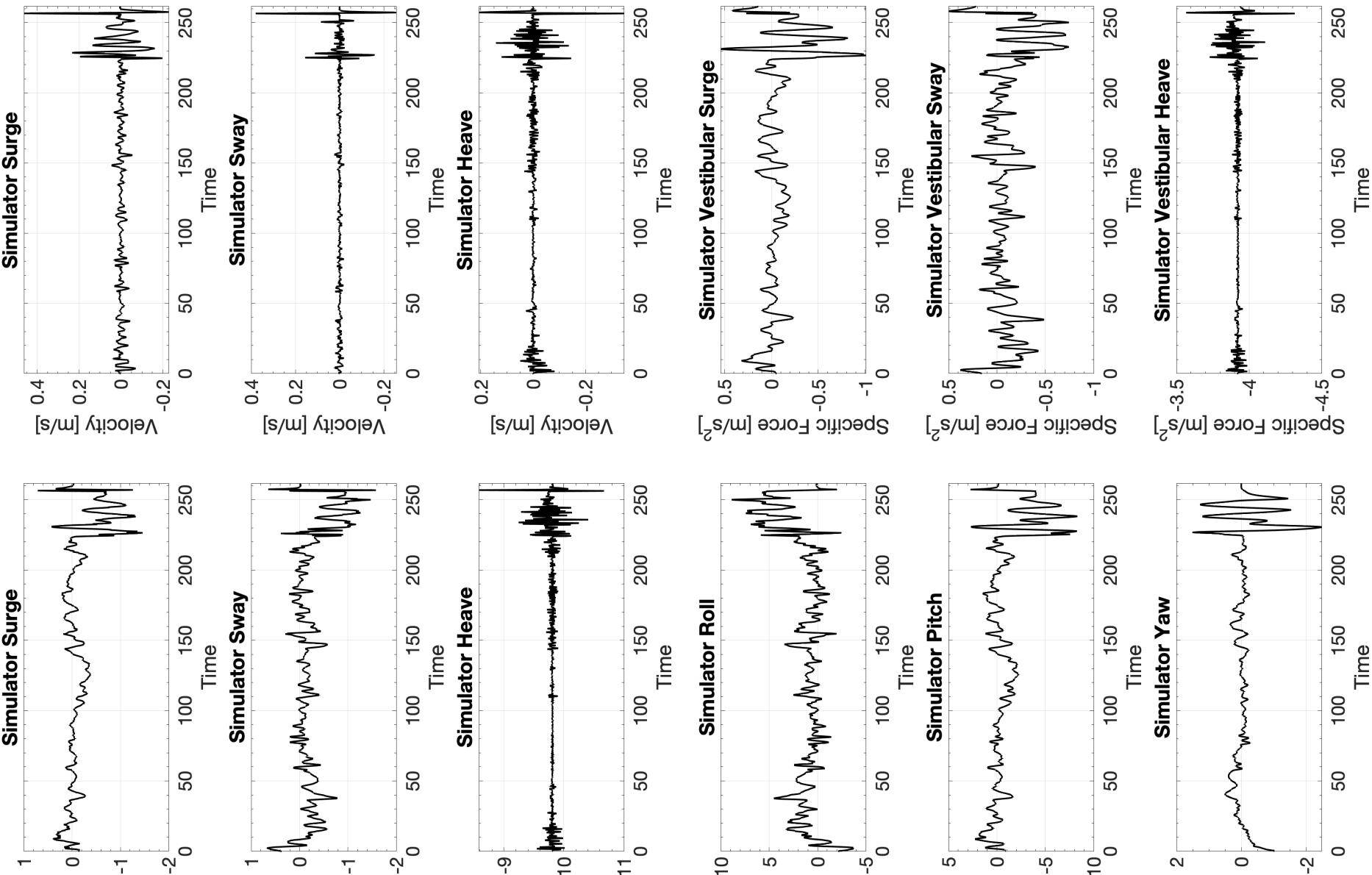

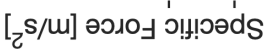

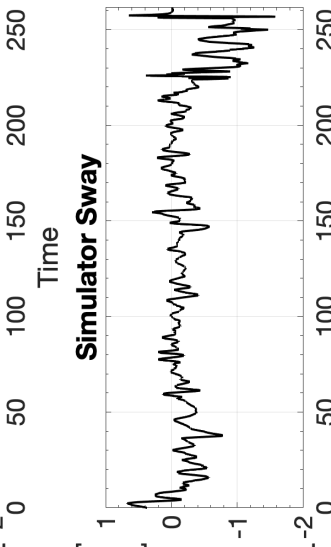

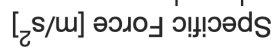

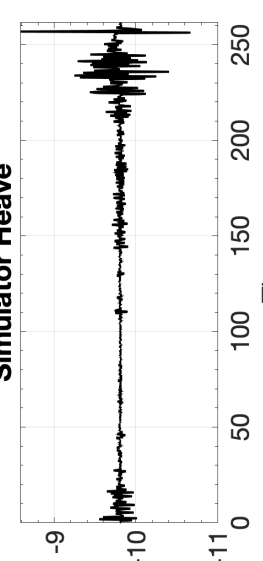

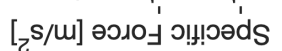

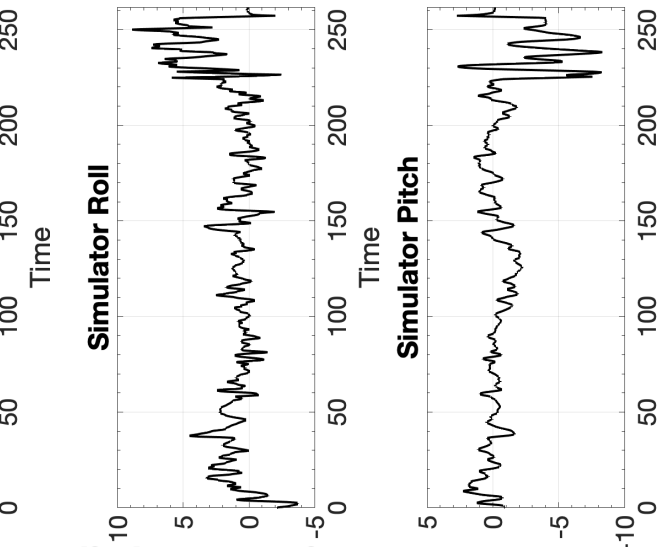

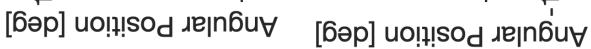

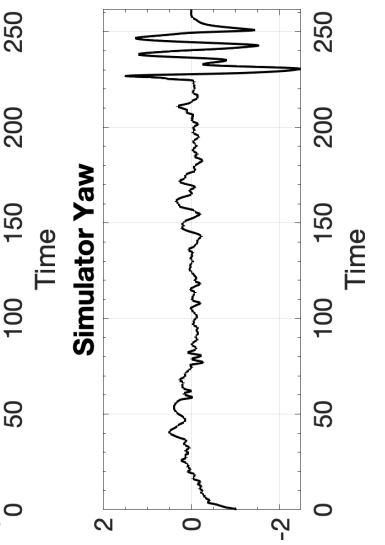

[6әр] uo!̣!!sod גejn6u

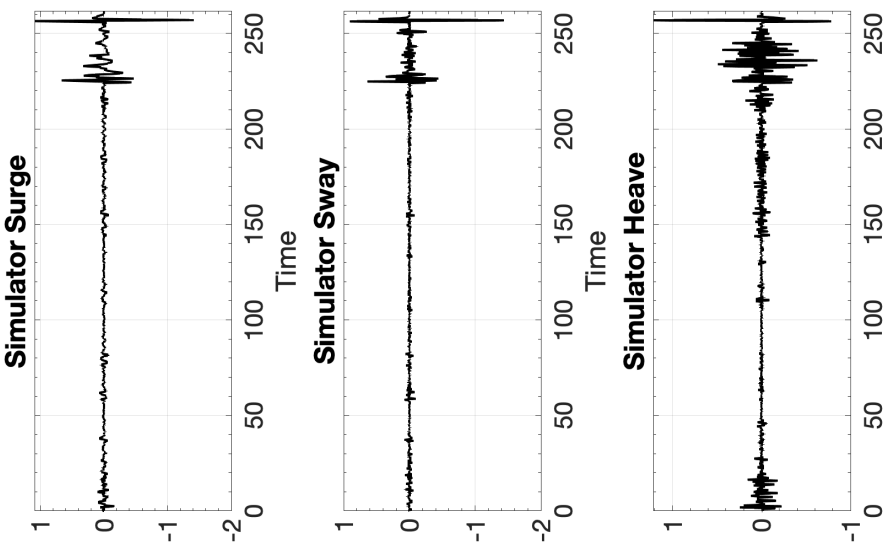

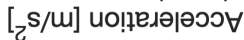

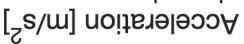

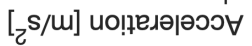

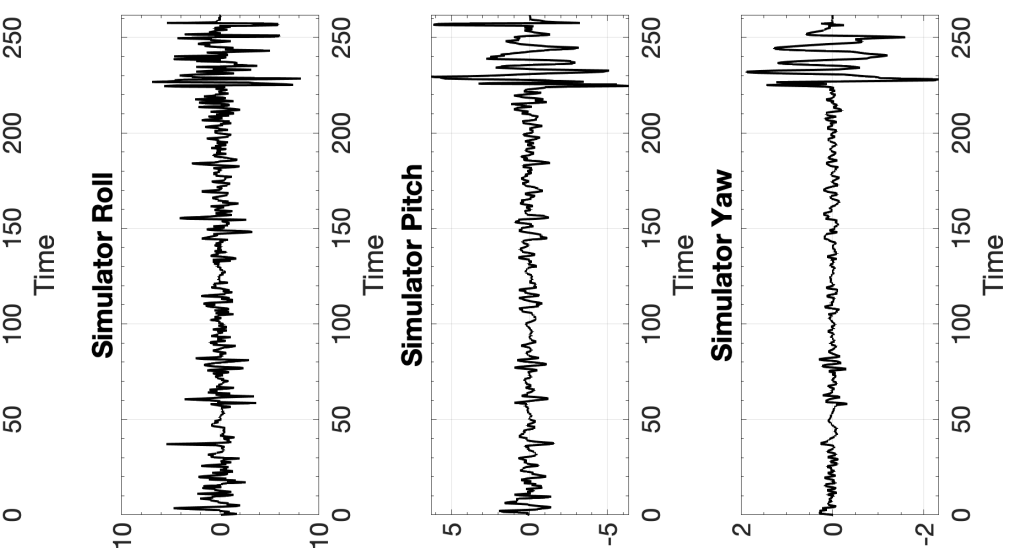

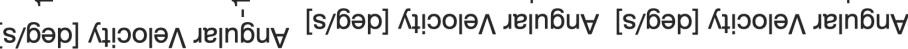




\section{B.10.2 CW2 Boeing 737 Cross-wind Landing}

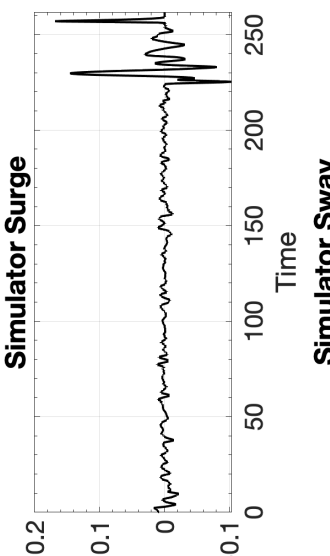

[m] uo!!!!sod

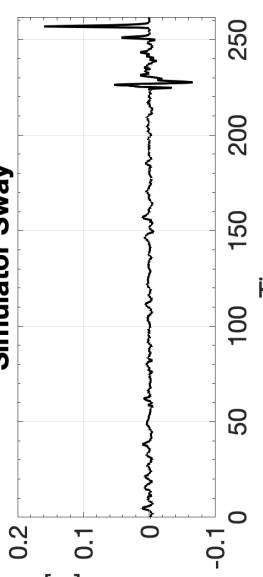

[w] uo!n!sod

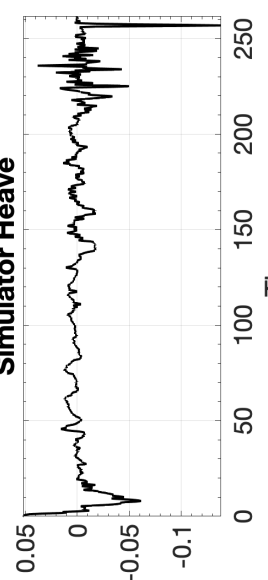

$[\mathrm{m}]$ uo!̣!?sod
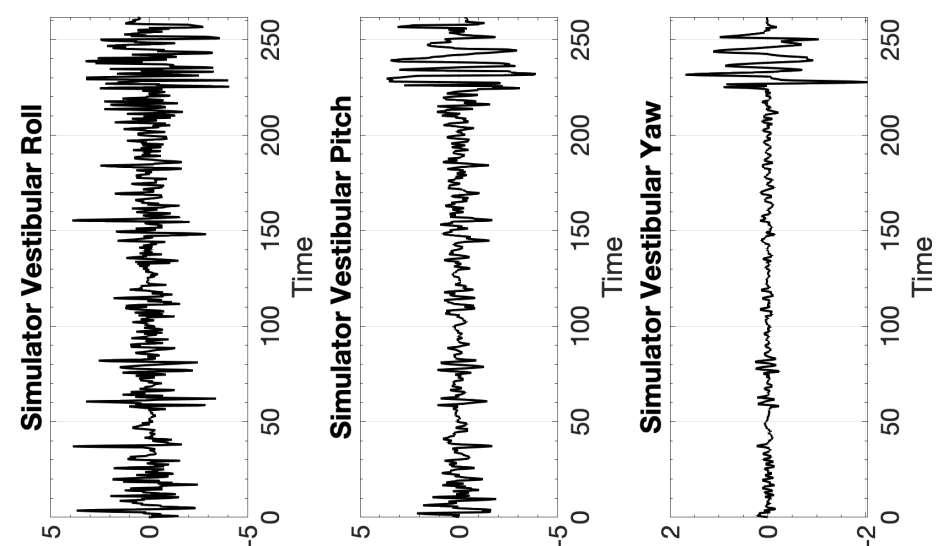

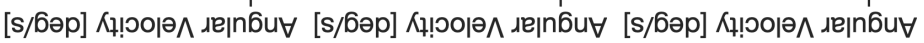

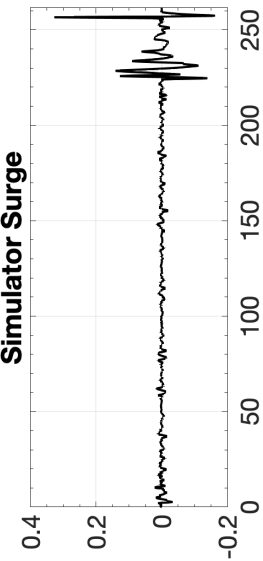

[s/u] К! ৩оำ

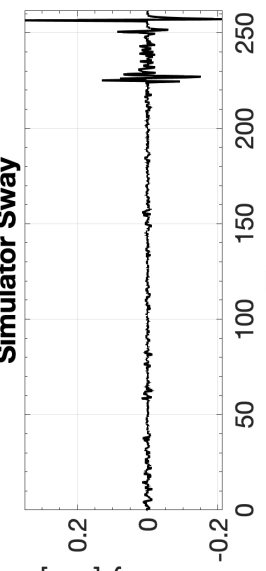

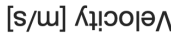

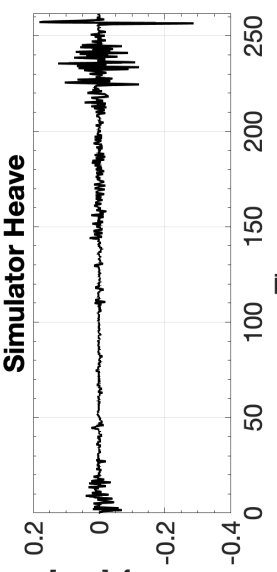

[s/m] К!ํㅣㅅ

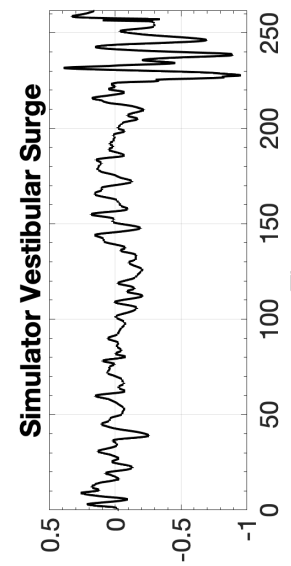

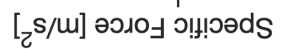

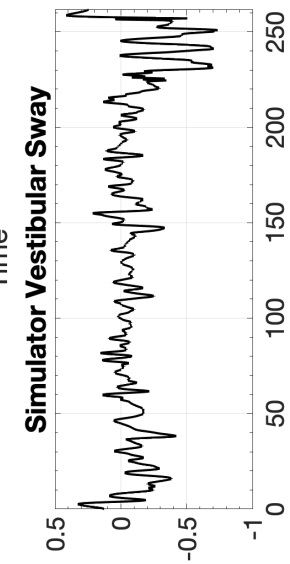

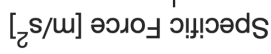

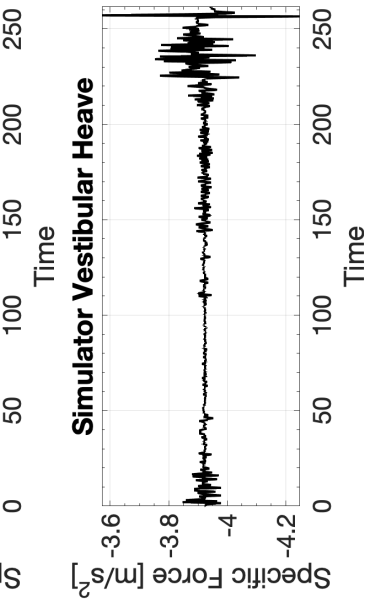

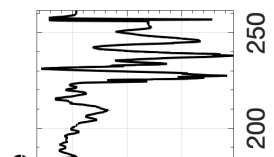

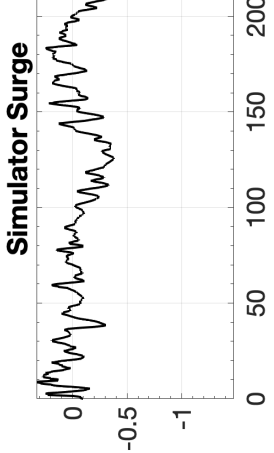

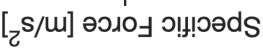
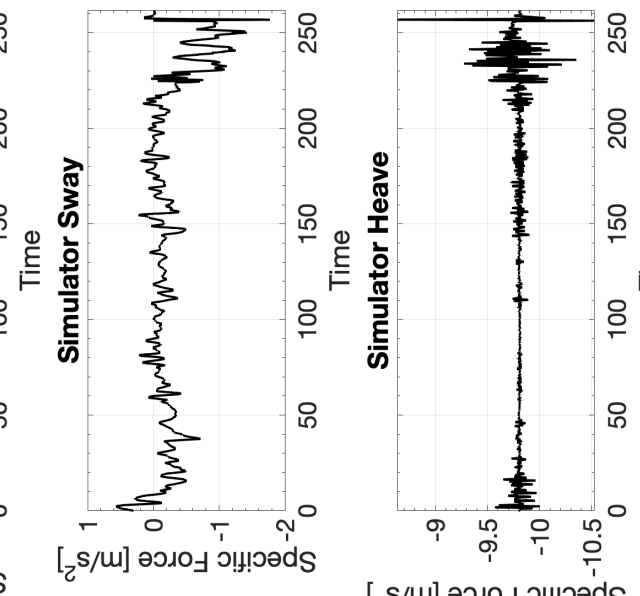

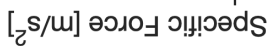
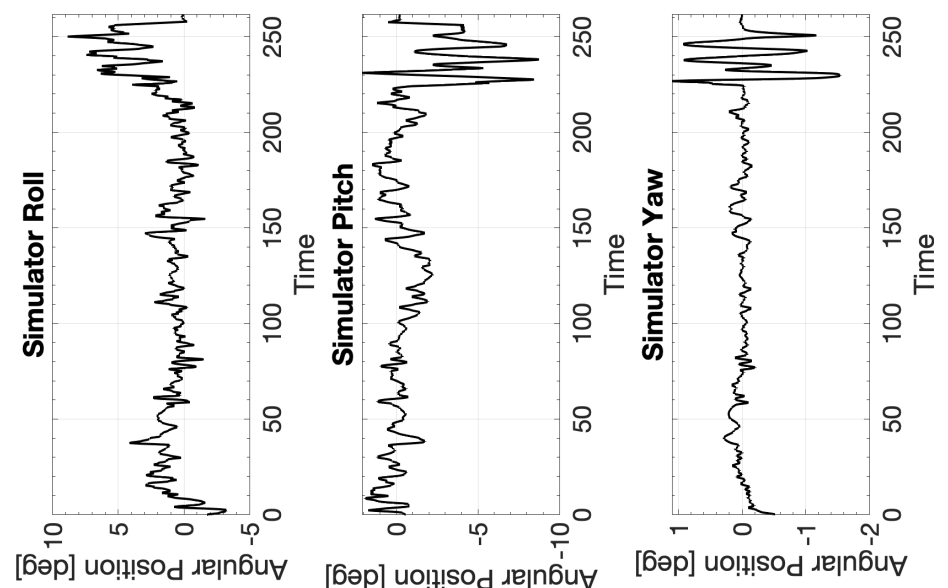

[6әр] uo!̣!

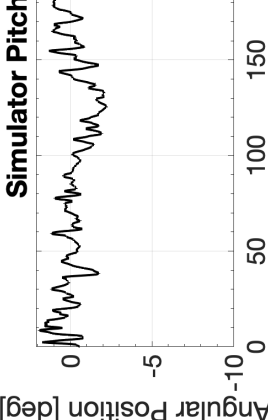

[6әр] uo!?! sod גejnбü
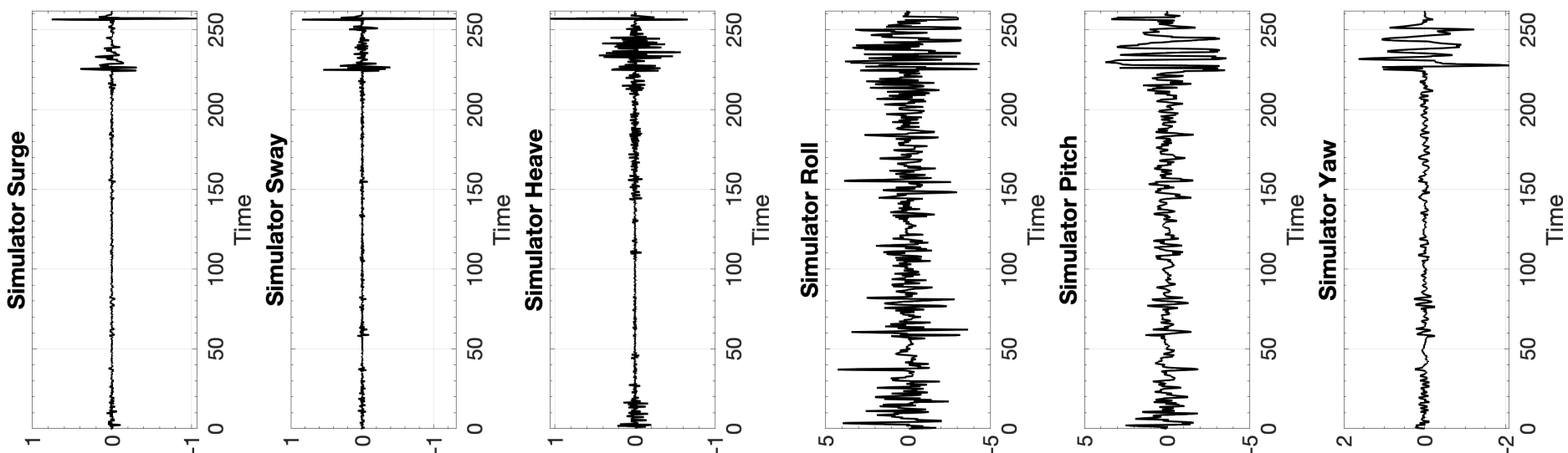

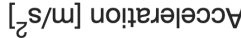

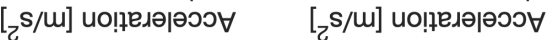

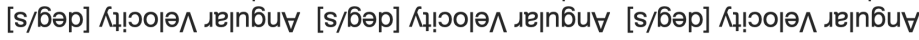




\section{B.10.3 CW3 Boeing 737 Cross-wind Landing}

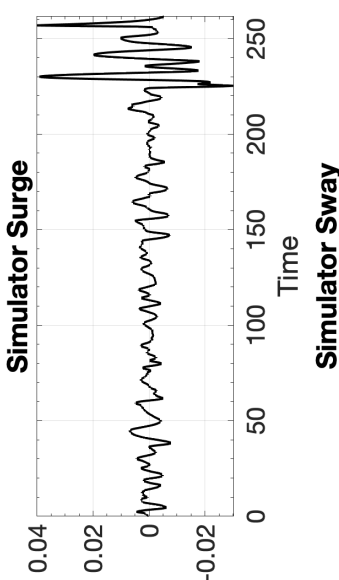

[u] uo!n!sod

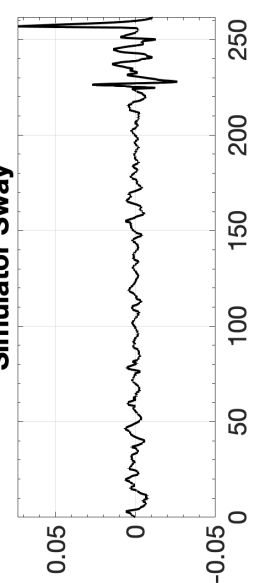

[u] uol!?!sod

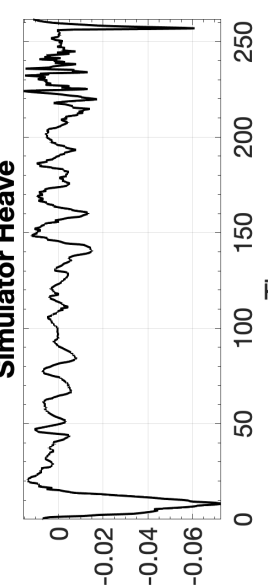

$[\mathrm{m}] \mathrm{uo!p!ls} \mathrm{SO}_{\mathrm{d}}$
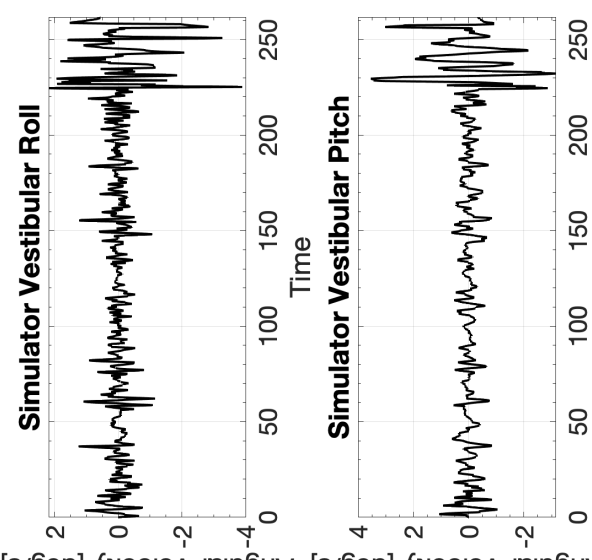

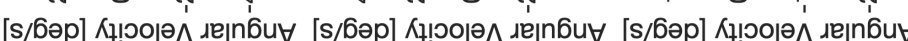

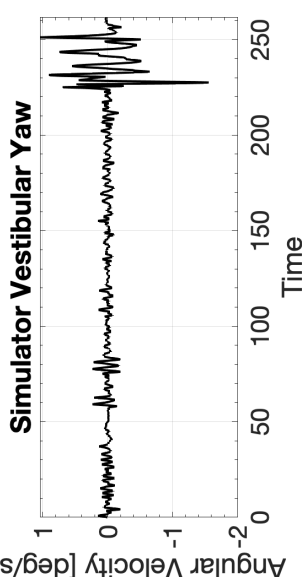

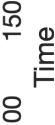

웅

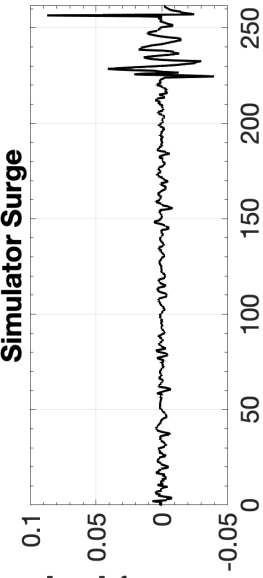

[s/u] Кұ! Ро|ә^

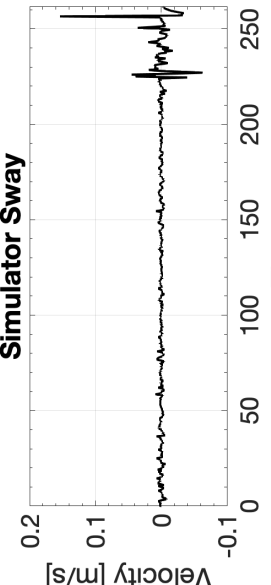

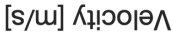

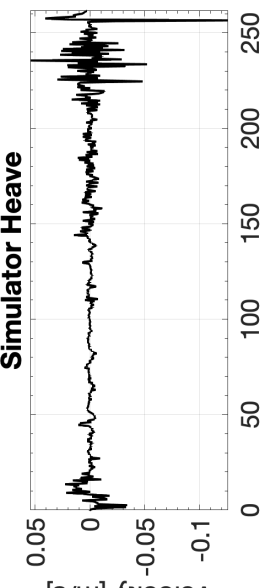

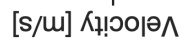

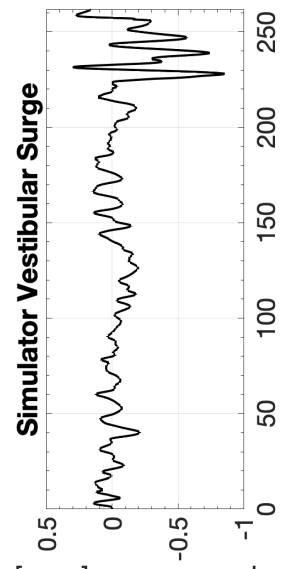

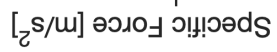
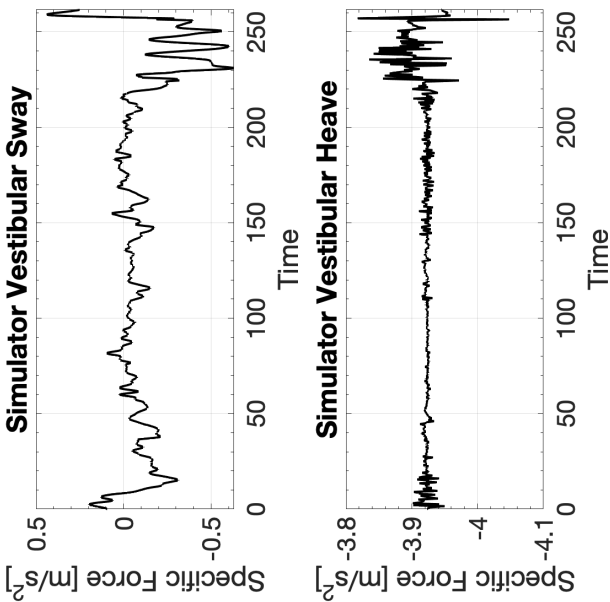

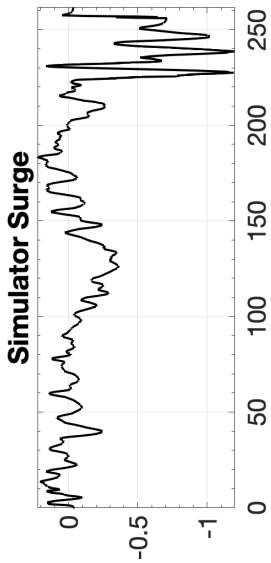

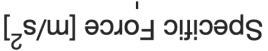

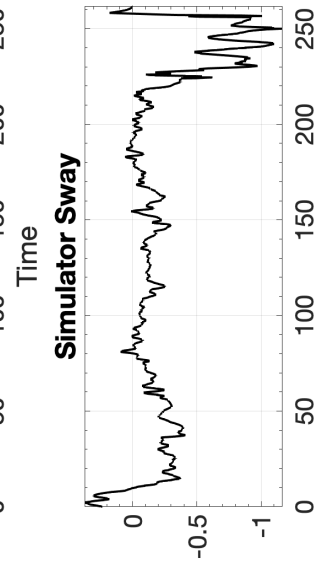

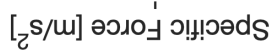

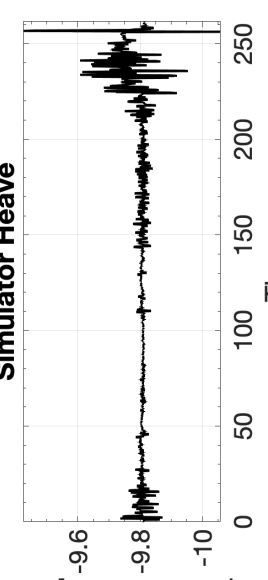

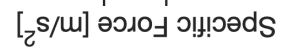

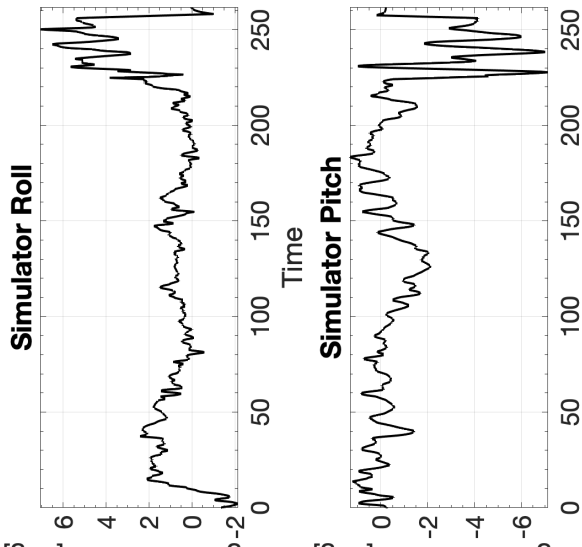

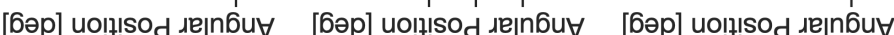

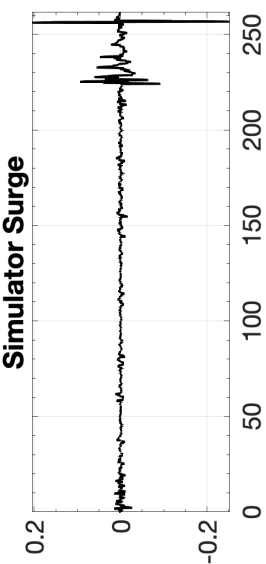

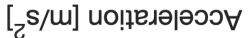

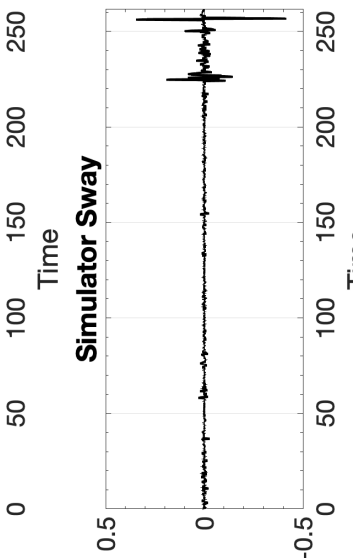

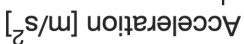

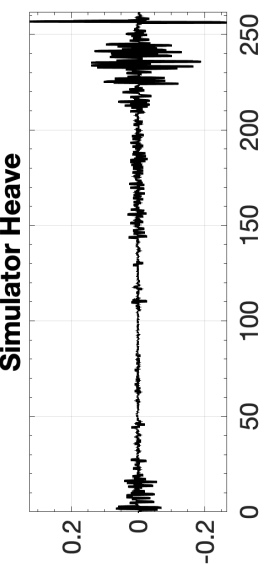

[ $\mathrm{s} / \mathrm{m}]$ ио!ฺедәрәэว
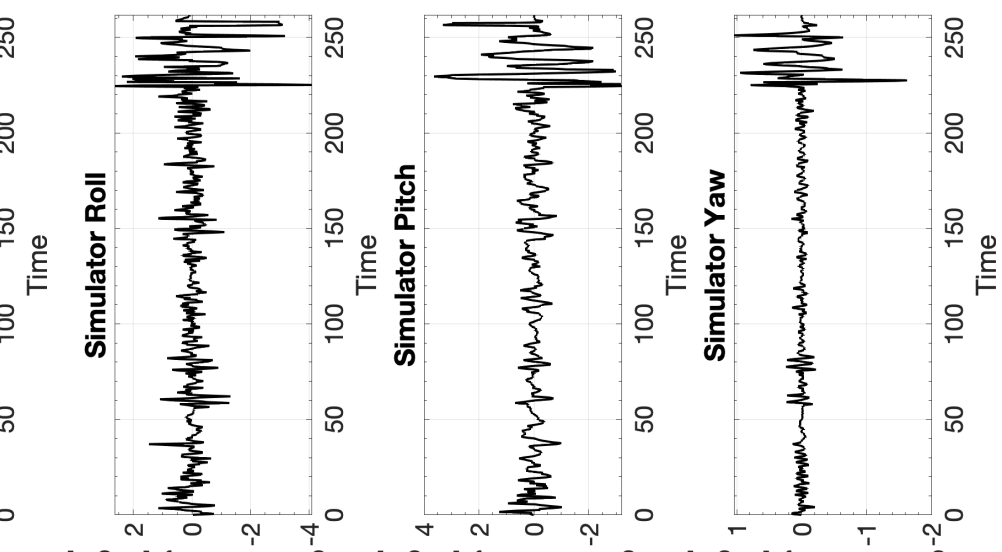

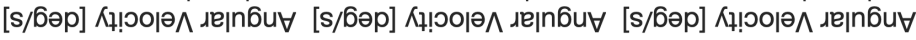




\section{B.11 Boeing 737 Single-axis Takeoff}
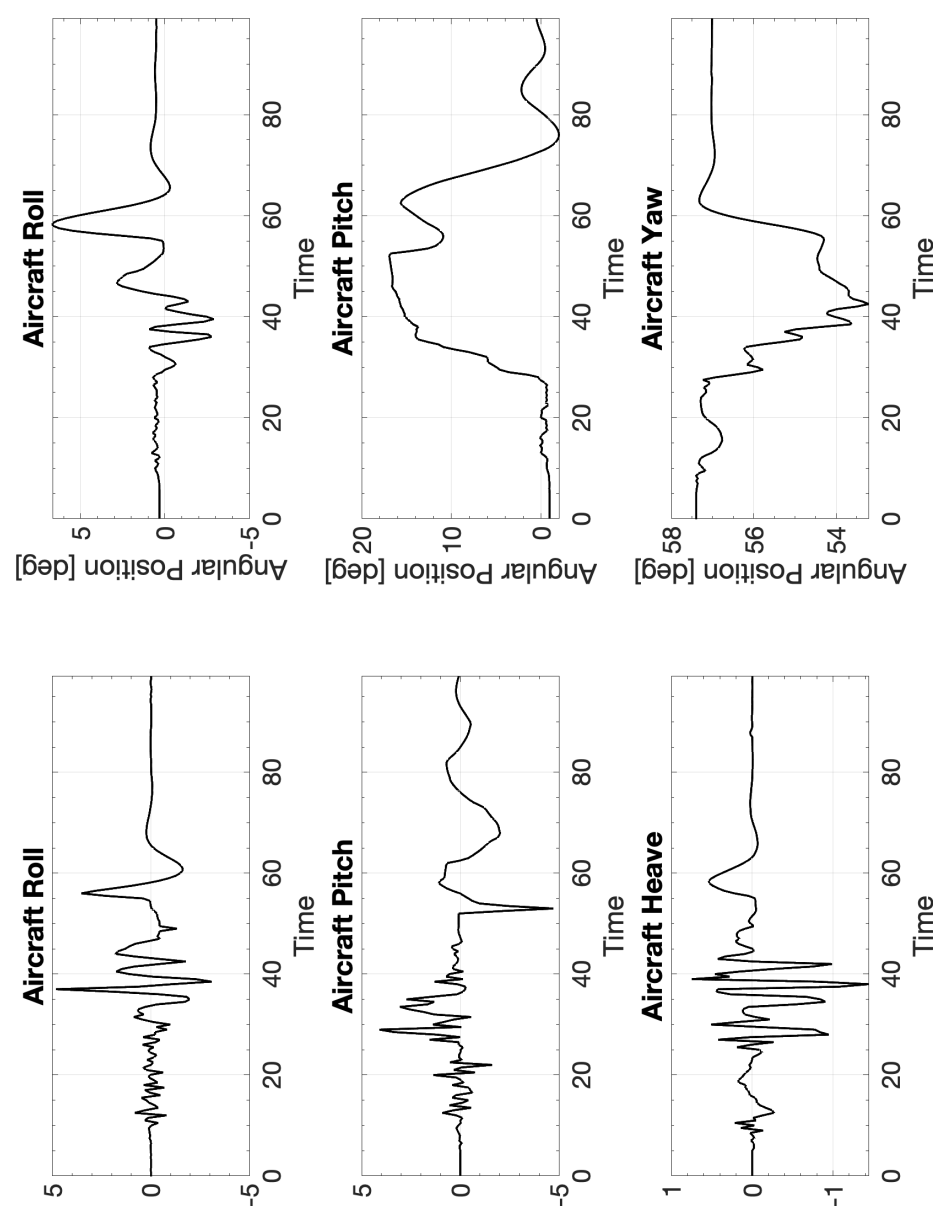

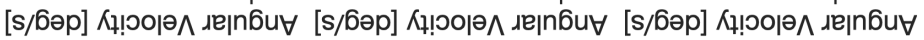

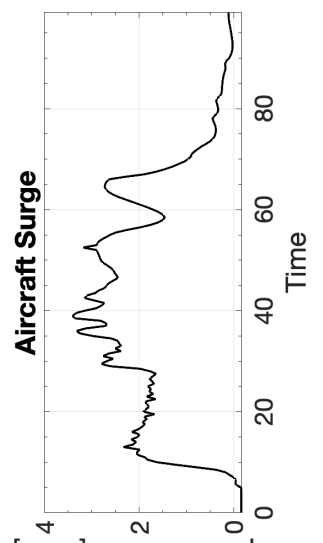

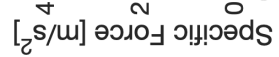

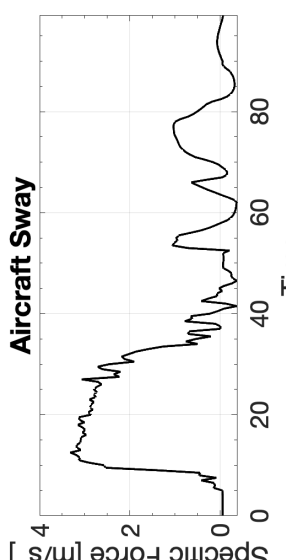

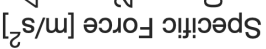

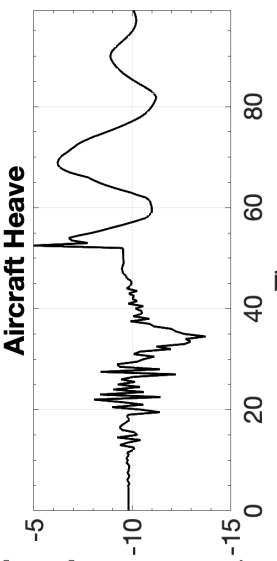

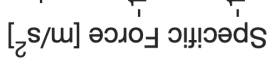

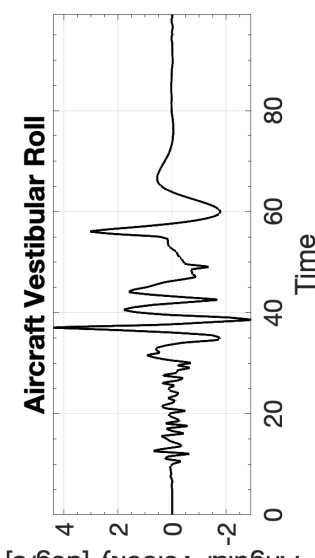

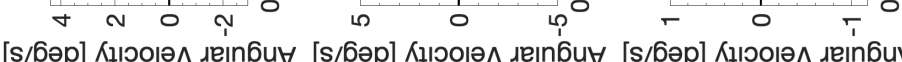

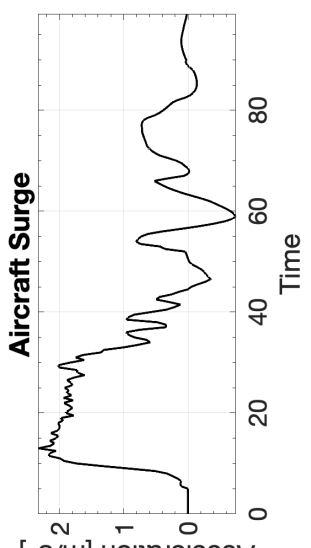

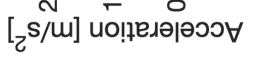

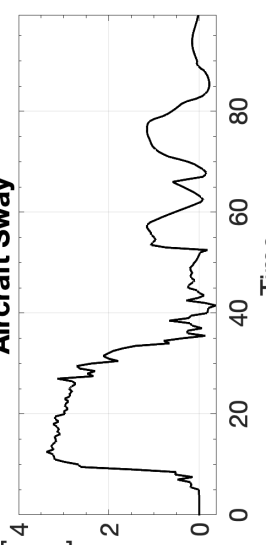

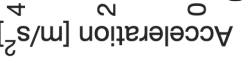

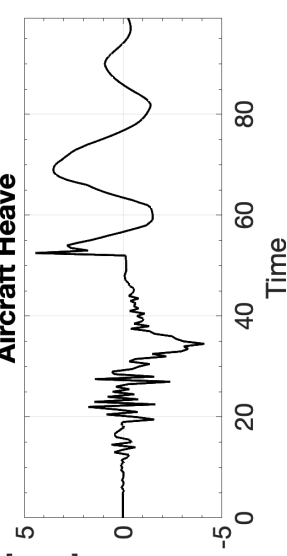

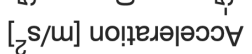

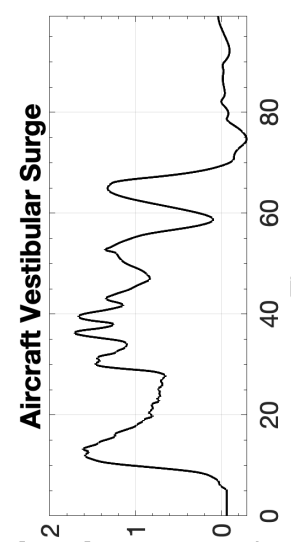

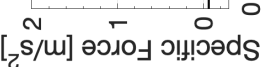
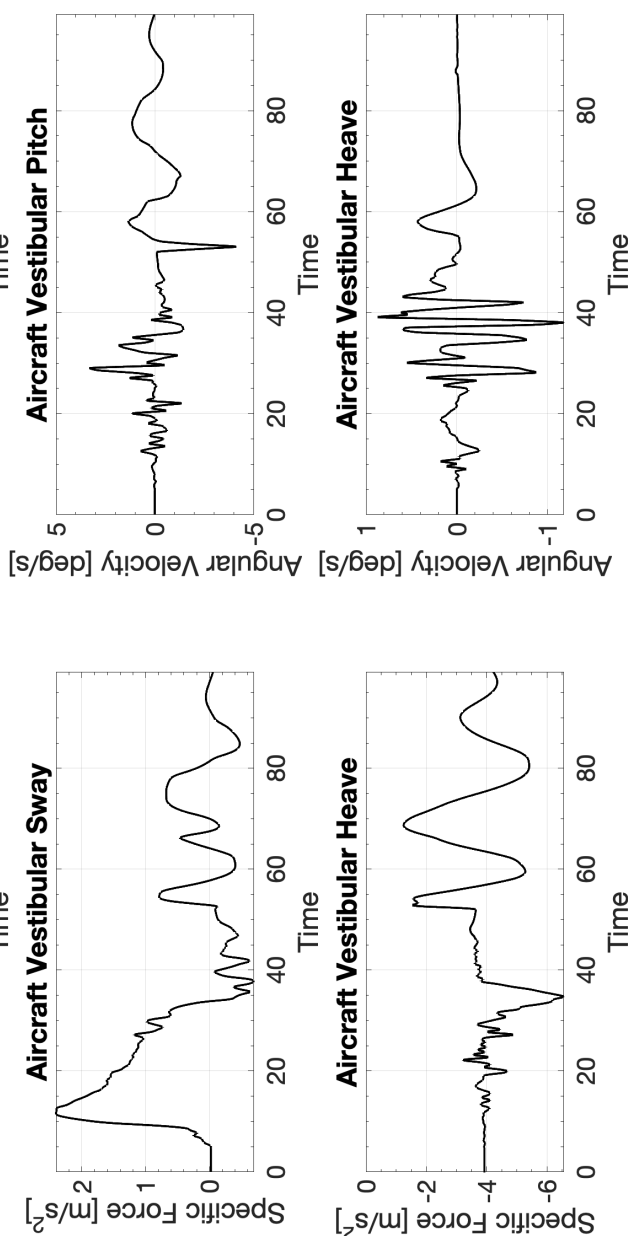


\section{B.11.1 CW1 Boeing 737 Single-axis Takeoff}

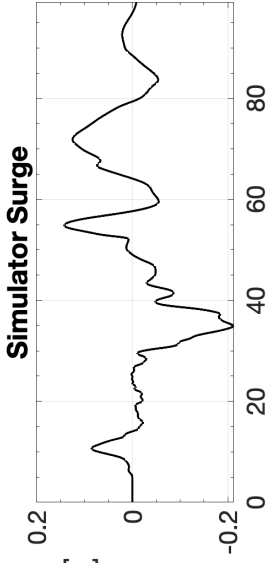

[u] uo!!!!sod

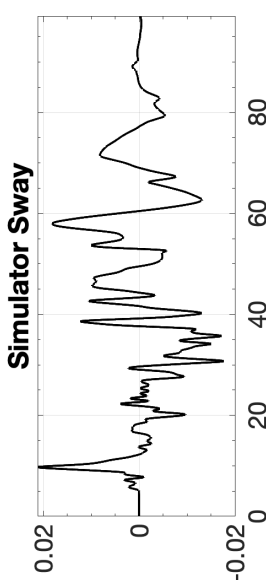

[u] uol!?!sod

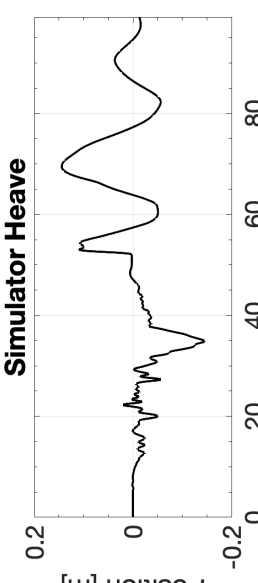

[w] uo!̣!sod

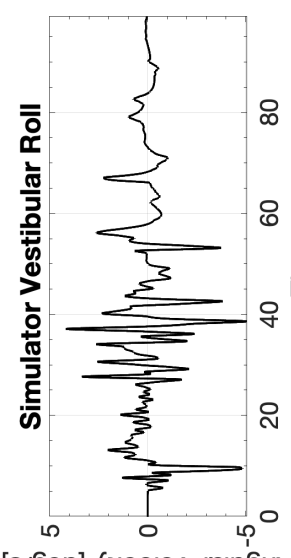

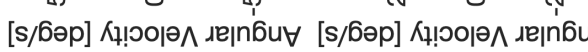

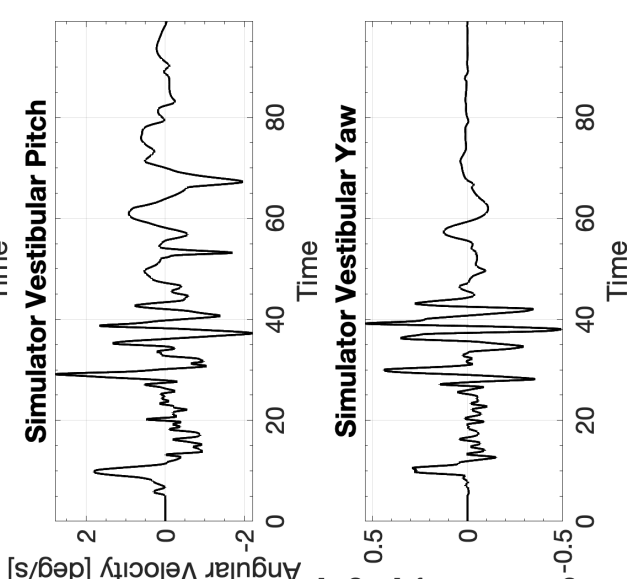

[s/6әр] Кұ!юорә

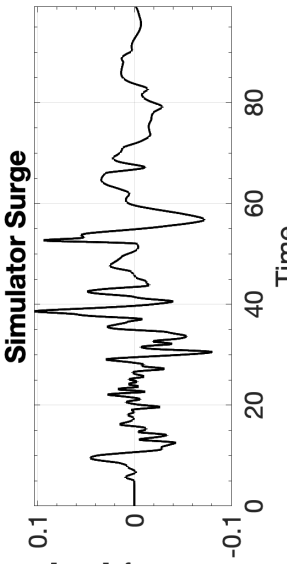

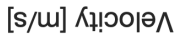

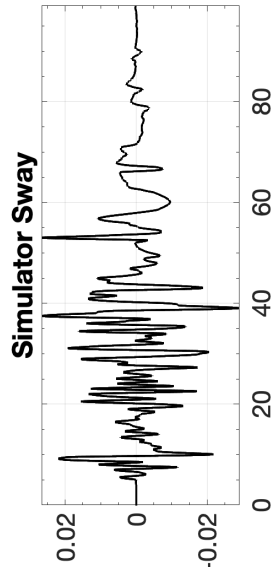

[s/u] Кㄲо잇

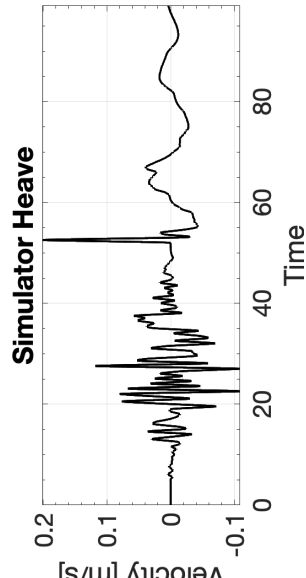

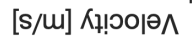

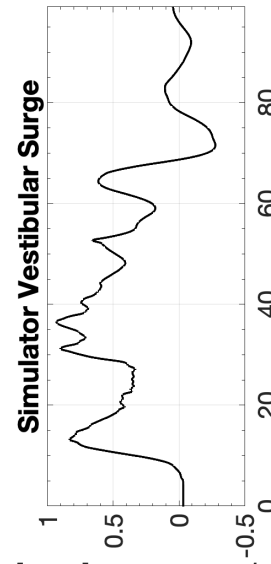

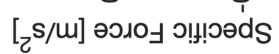

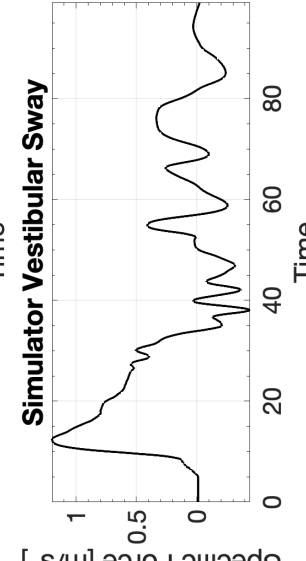

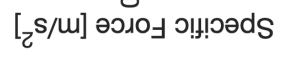

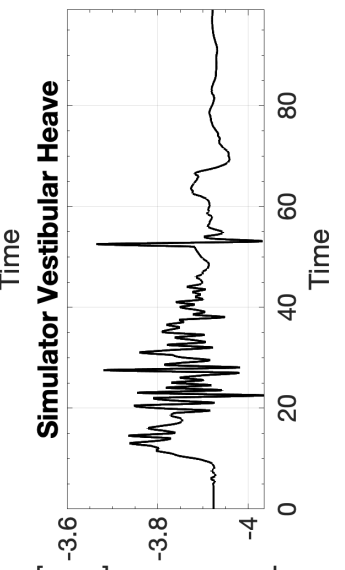

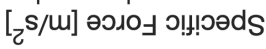

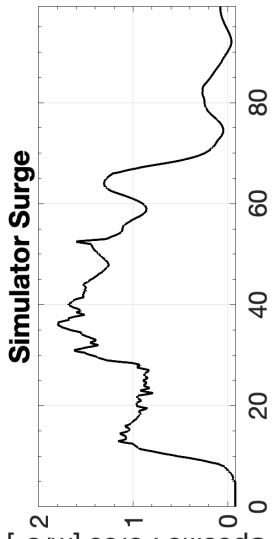

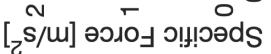

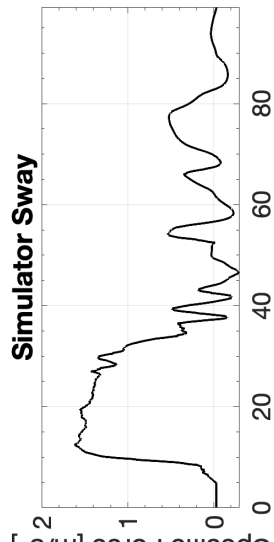

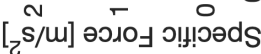

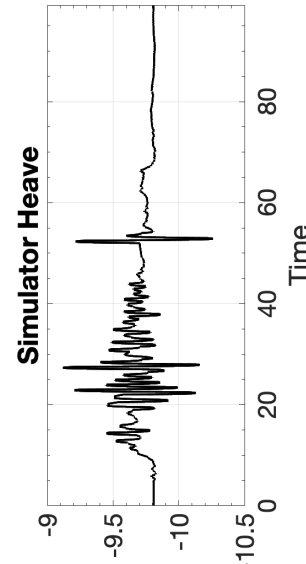

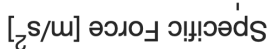

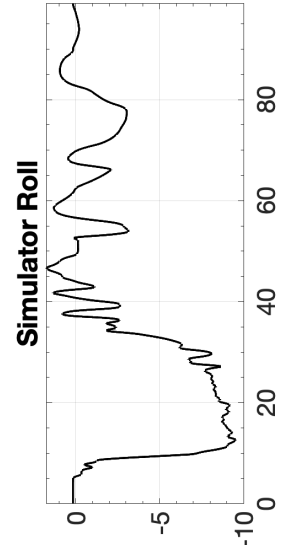

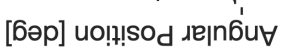

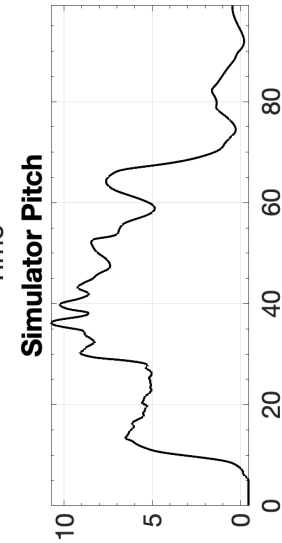

[6әр] uo!!!!sod גe|nбu

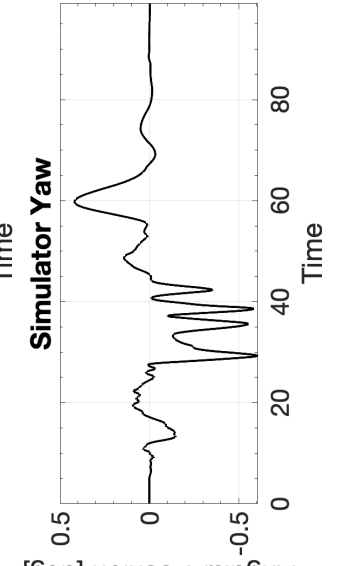

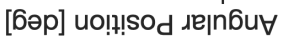

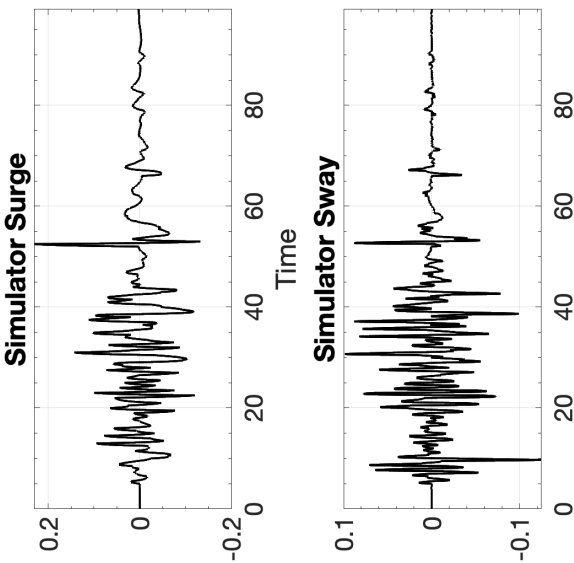

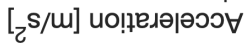

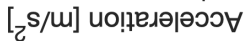

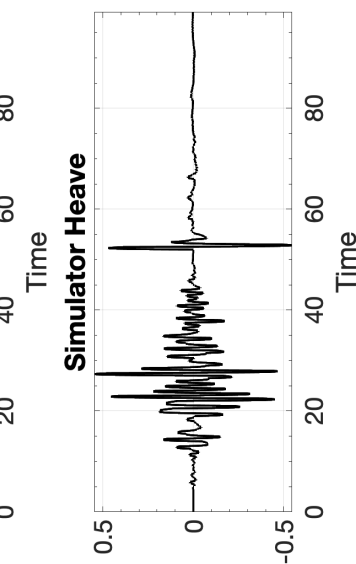

[z $\mathrm{s} / \mathrm{m}]$ ио!џедәәәэว $\forall$

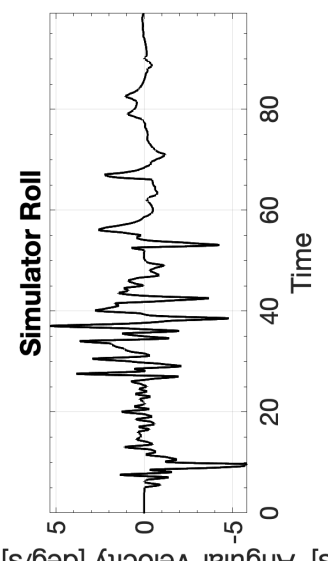

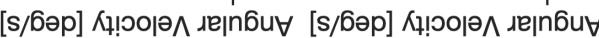

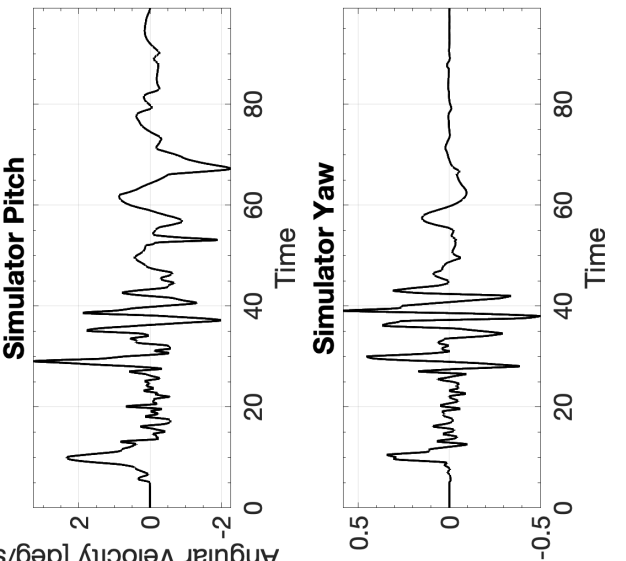

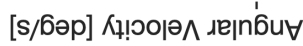




\section{B.11.2 CW2 Boeing 737 Single-axis Takeoff}

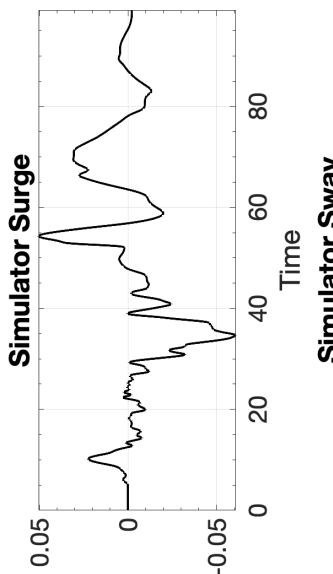

[w] uo!n!sod

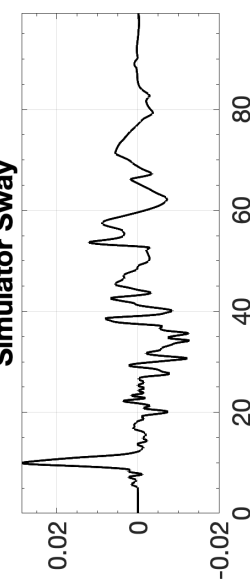

[w] uol!?!sod

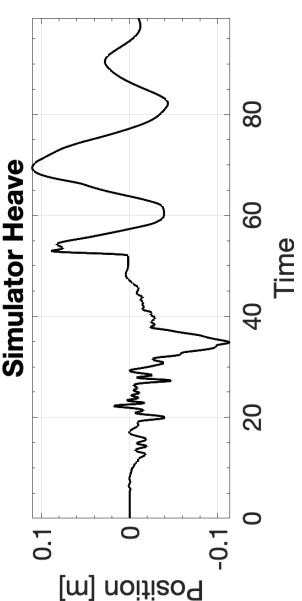

[w] uo!t!!sod

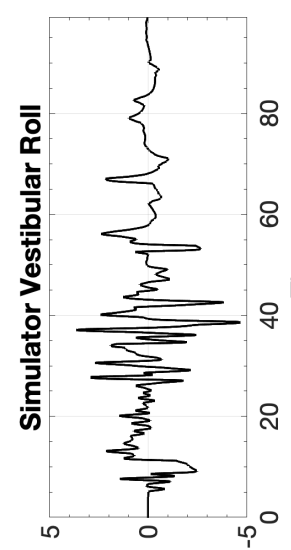

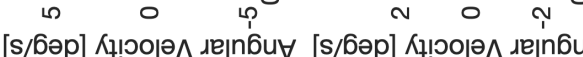

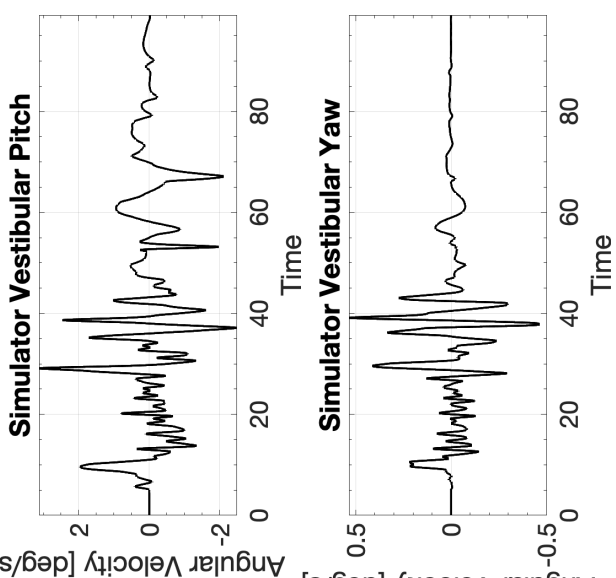

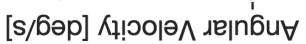

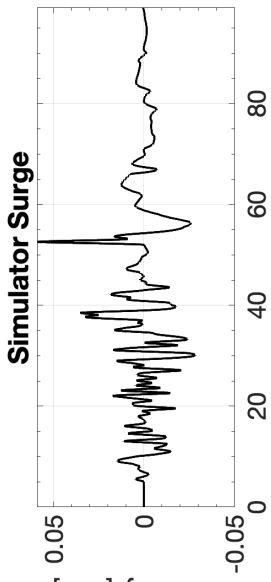

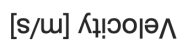

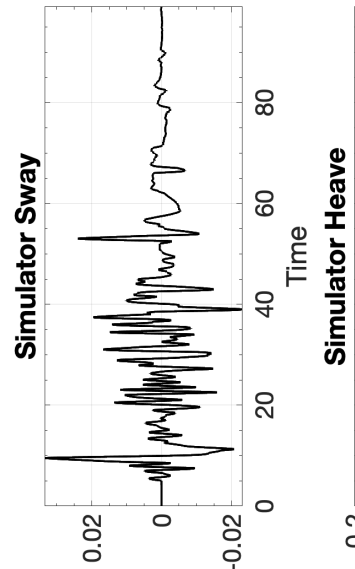

[s/u] К!ำю ^
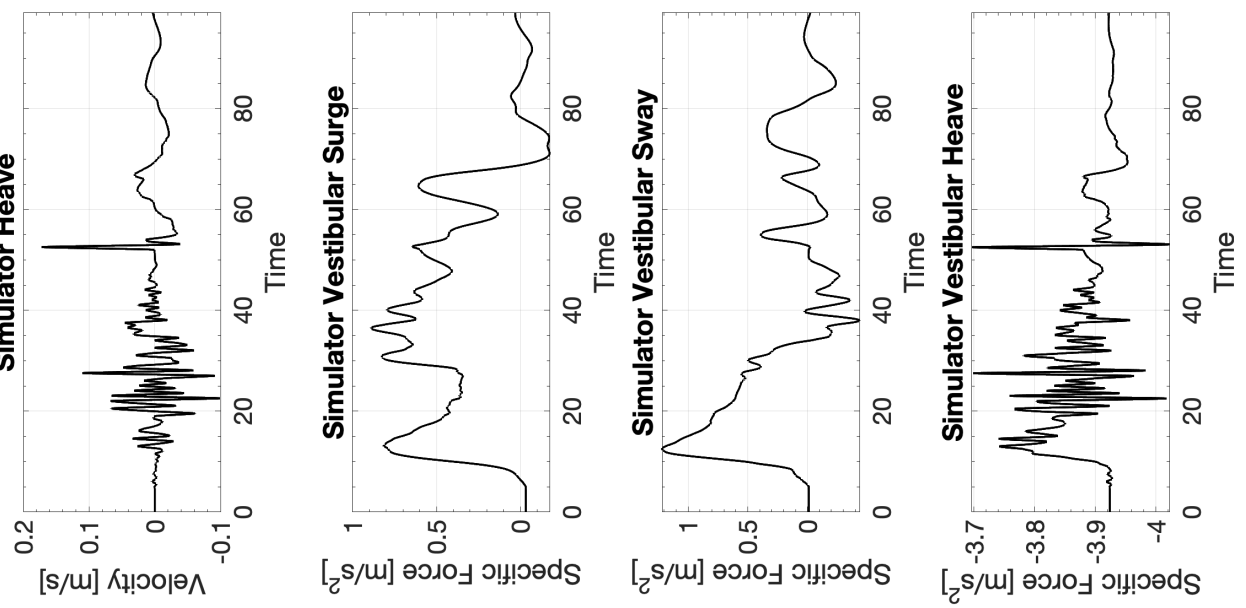

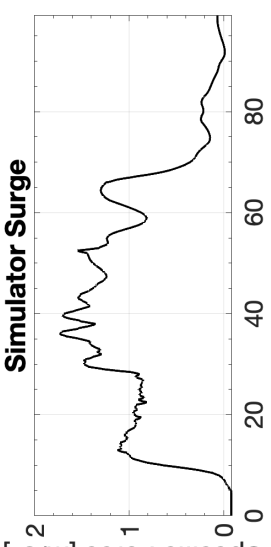

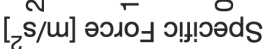

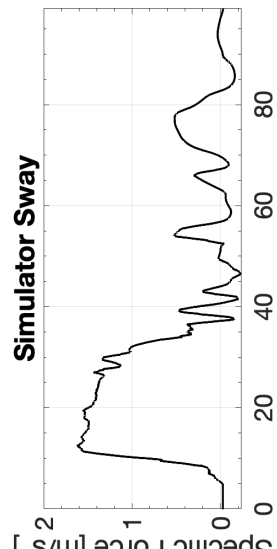

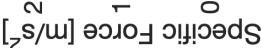

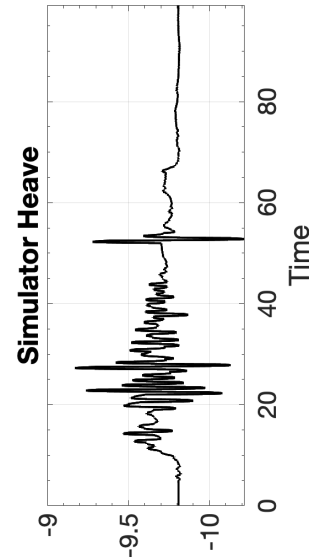

[

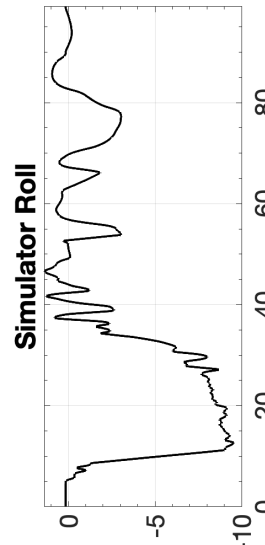

[бəp] uo!̣!!sod

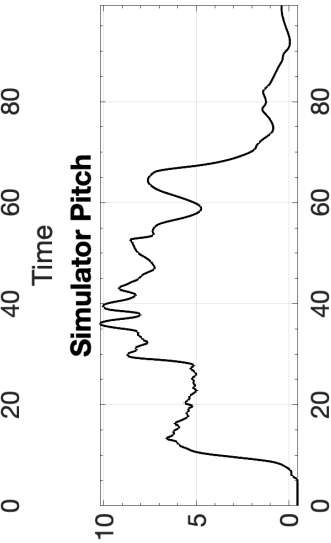

[бəp] uo!!! sod גe|n6u

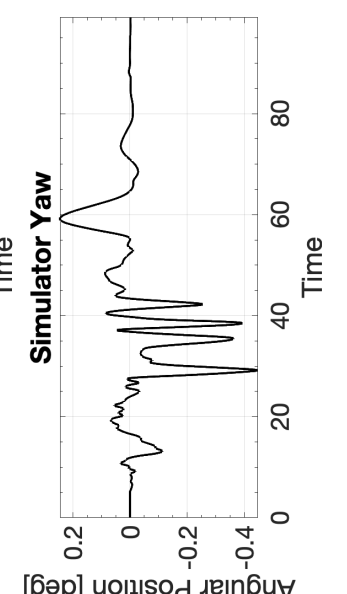

[6әр] uo!!!sod teןn6uy

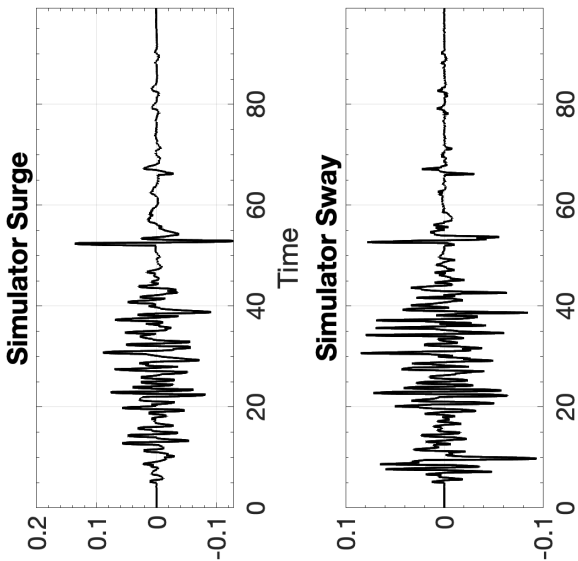

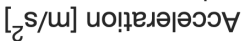

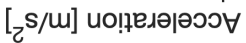

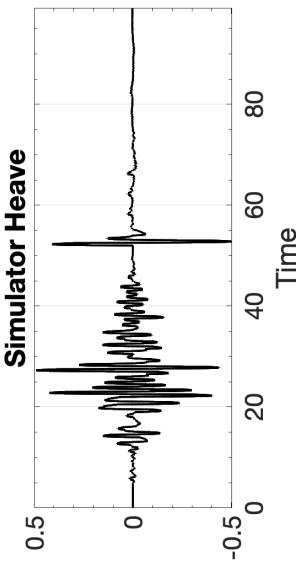

$[\mathrm{c} / \mathrm{m}]$ ио!ฺедә|әэว

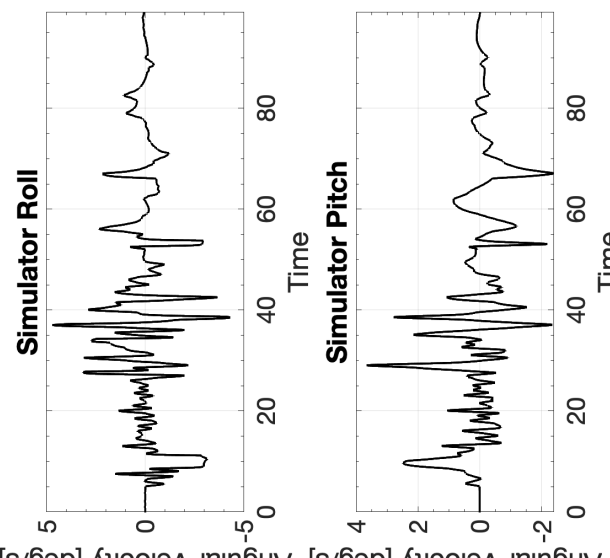

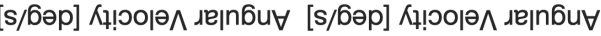
167

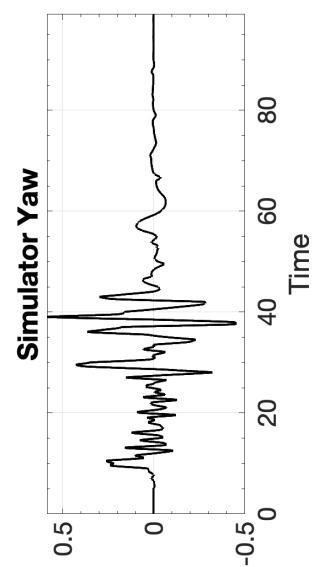

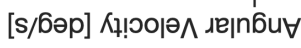




\section{B.11.3 CW3 Boeing 737 Single-axis Takeoff}

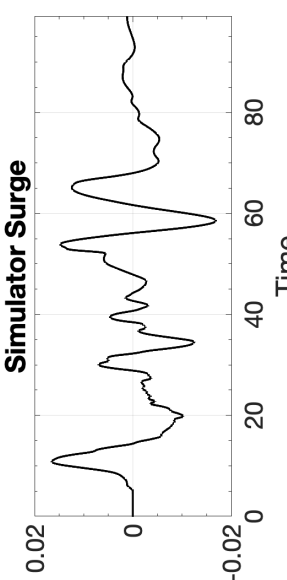

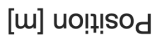

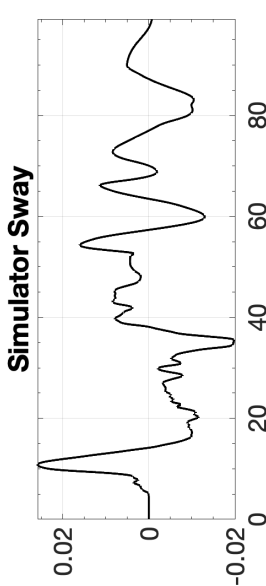

[w] no!n!sod

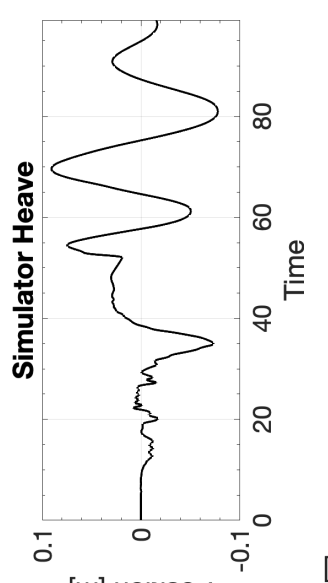

[u] uo!t!sod

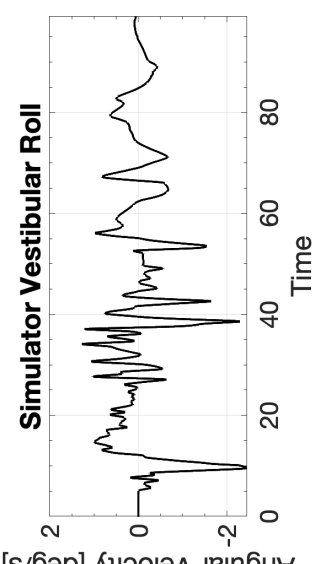

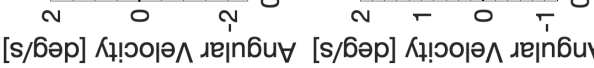

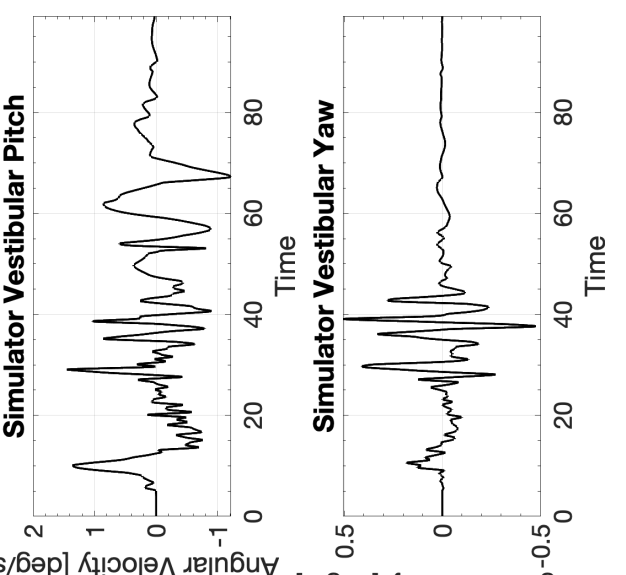

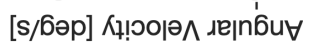

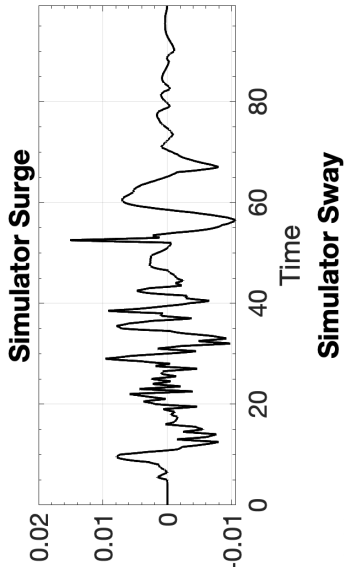

[s/u] Кұ!৩о|ә^

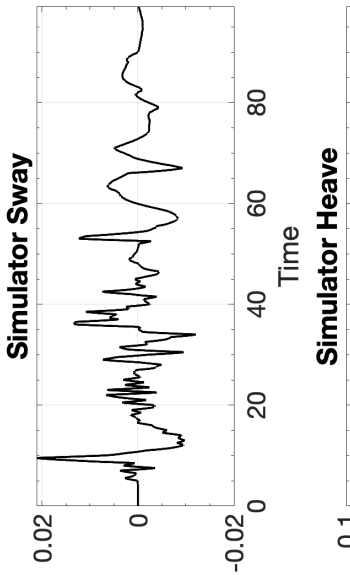

[s/m] К! юорә^

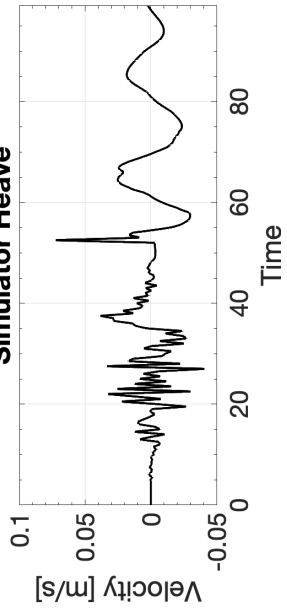

[s/m] К! оㅣㅅ

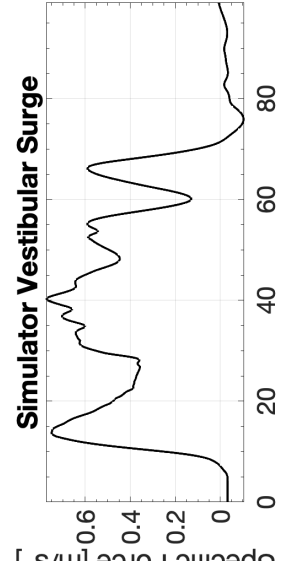

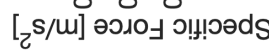

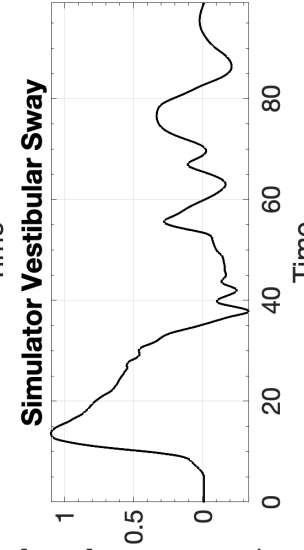

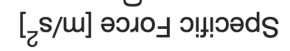

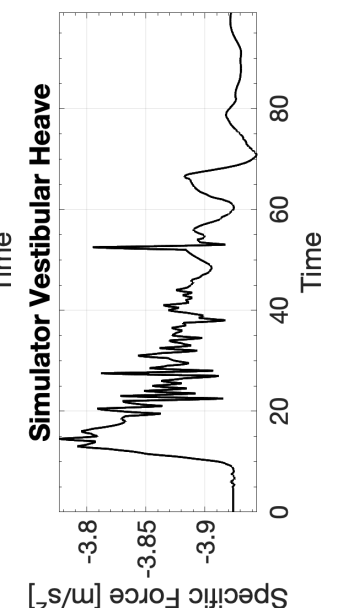

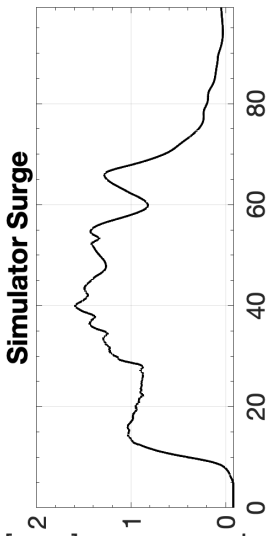

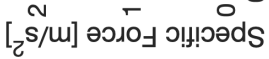

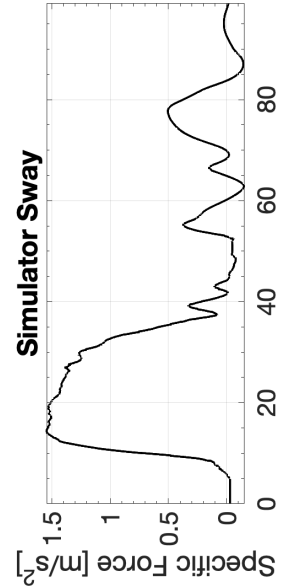

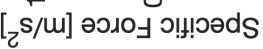

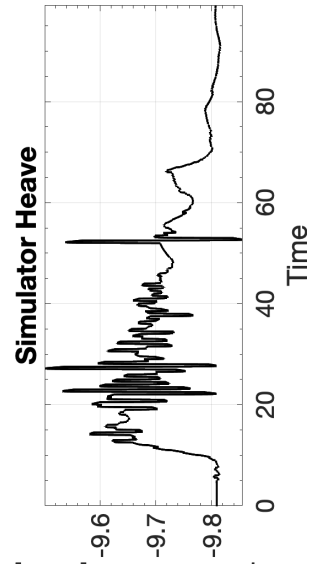

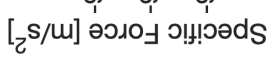

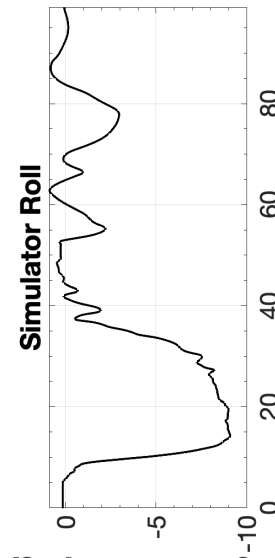

[бәр] uo!!!sod גe|nธ่นี

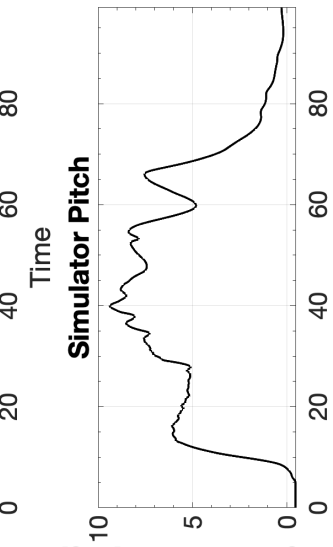

[6əp] uo!!!sod גe|nదu

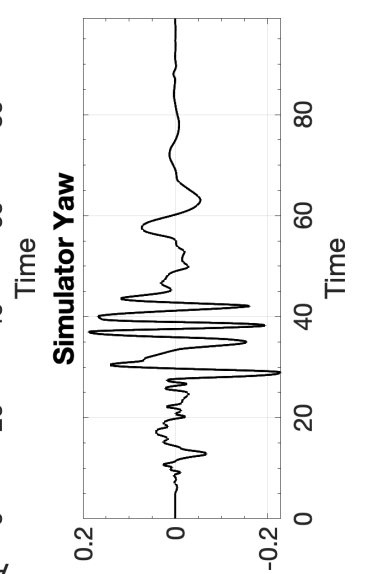

[6əр] uo!!!sod גe|n6u

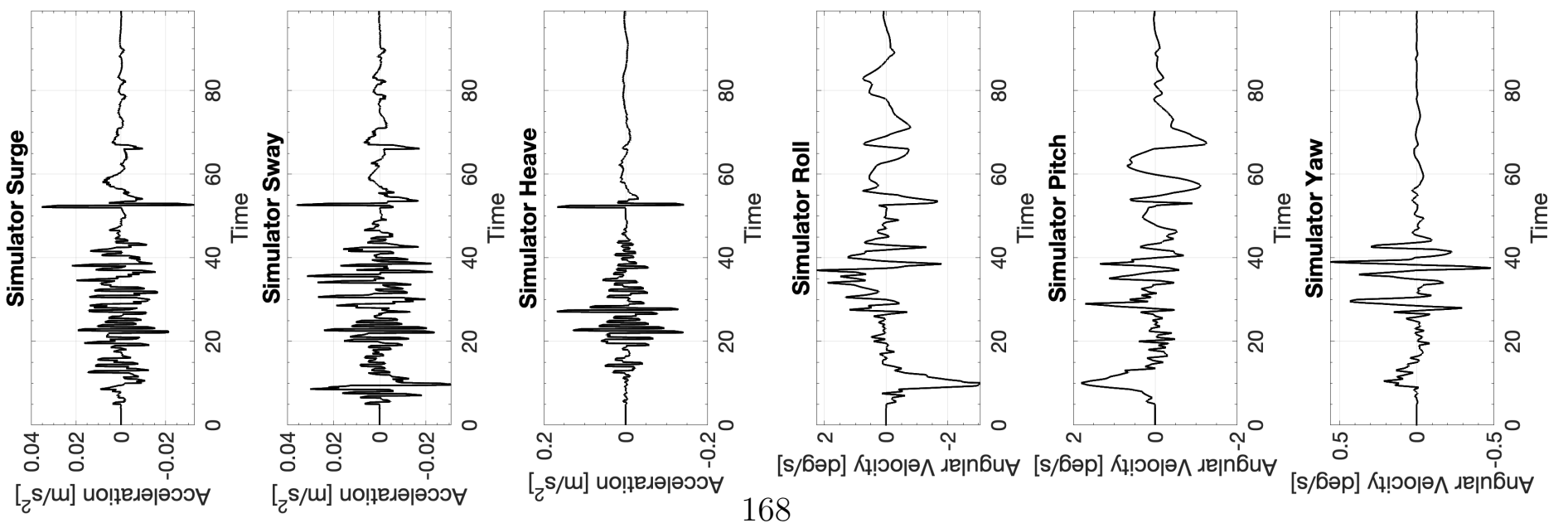




\section{B.12 Boeing 737 Spiral Dive}

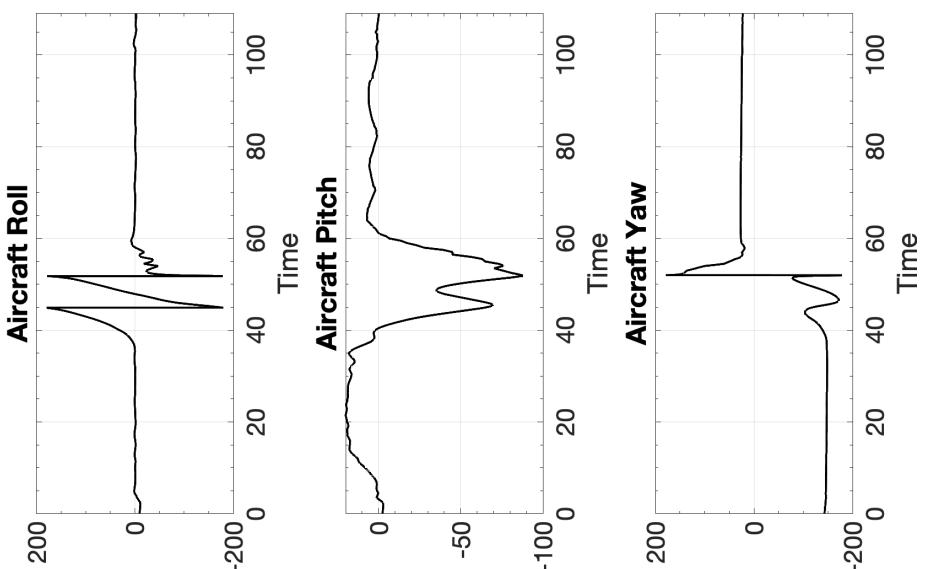

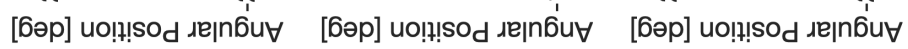

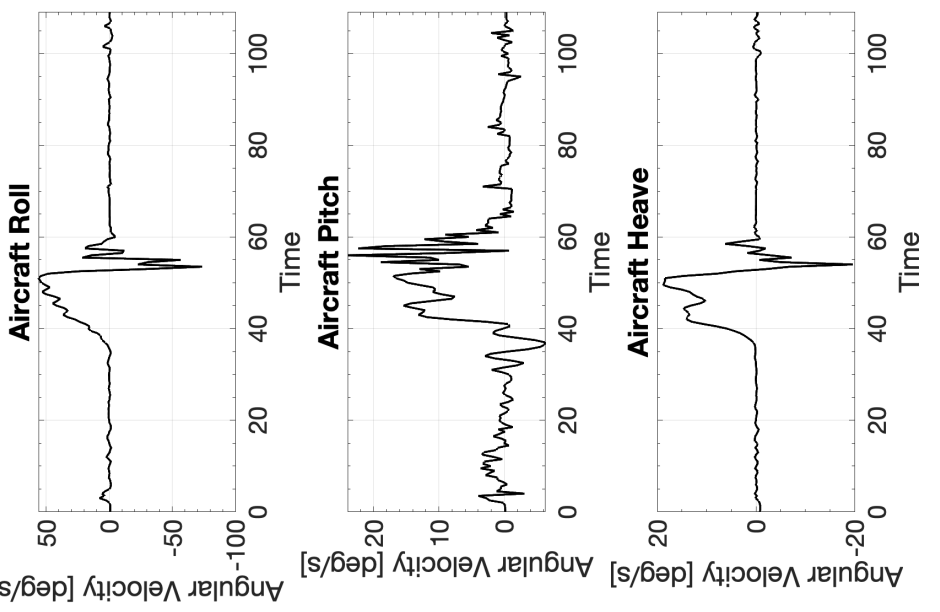

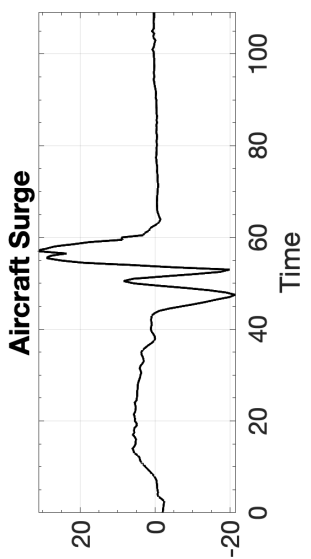

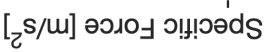

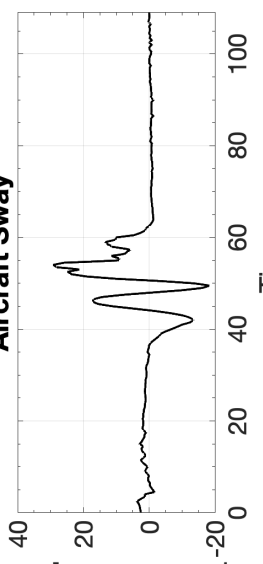

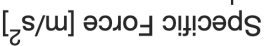

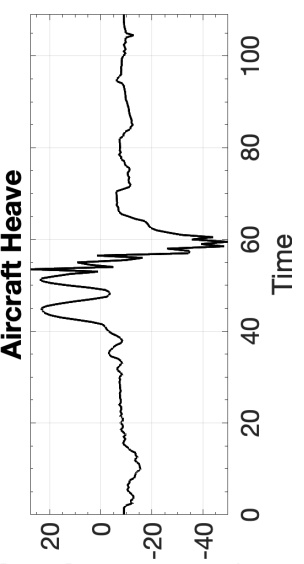

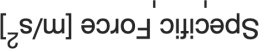

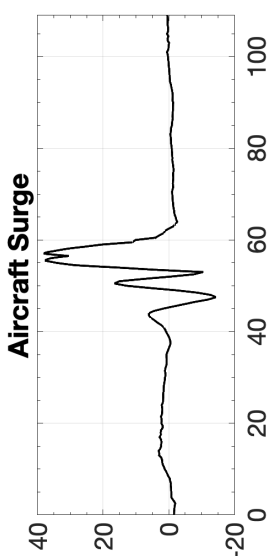

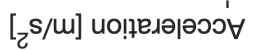

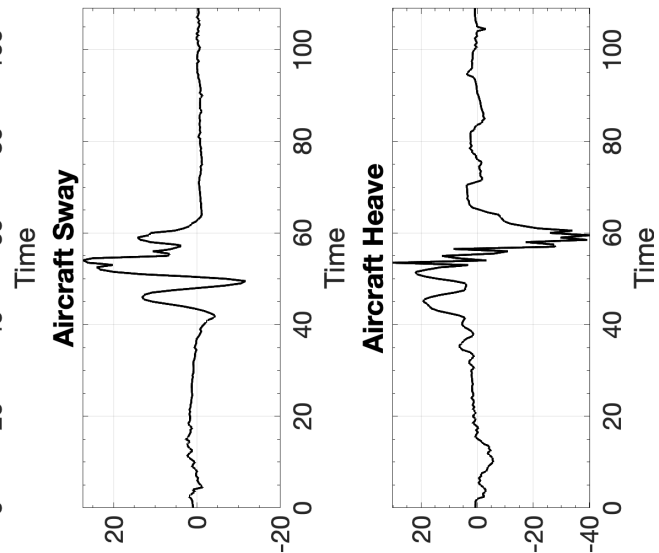

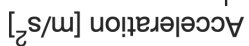

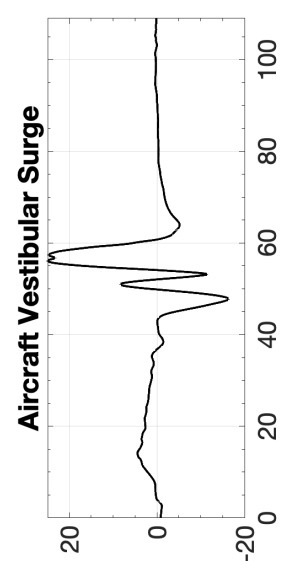

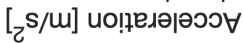

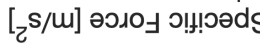

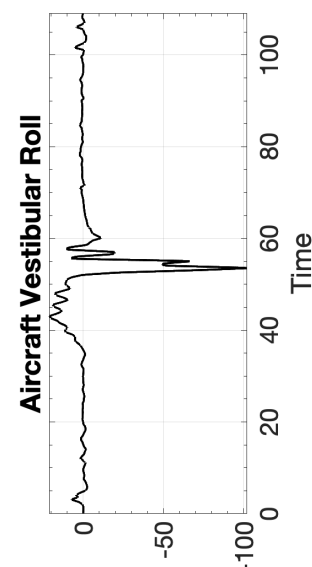

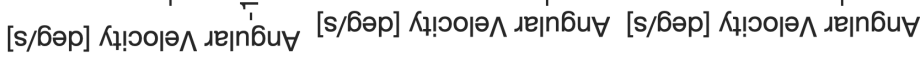
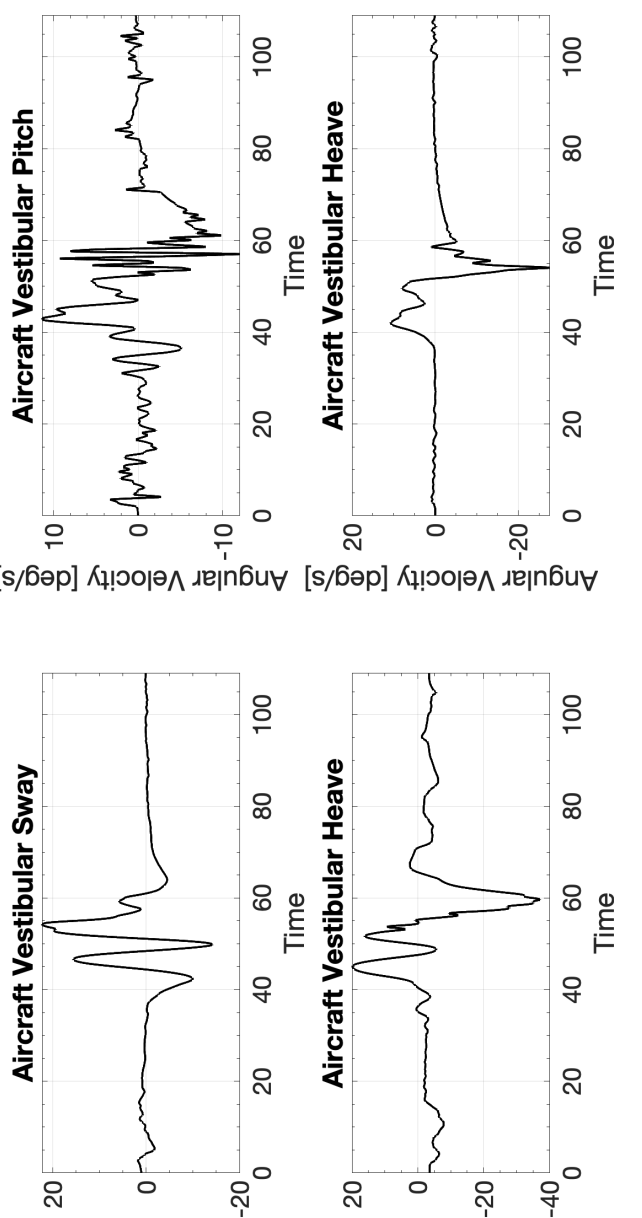

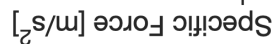

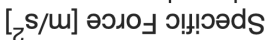




\section{B.12.1 CW1 Boeing 737 Spiral Dive}

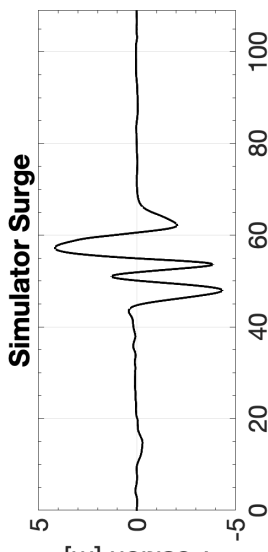

[u] uo!t!!sod

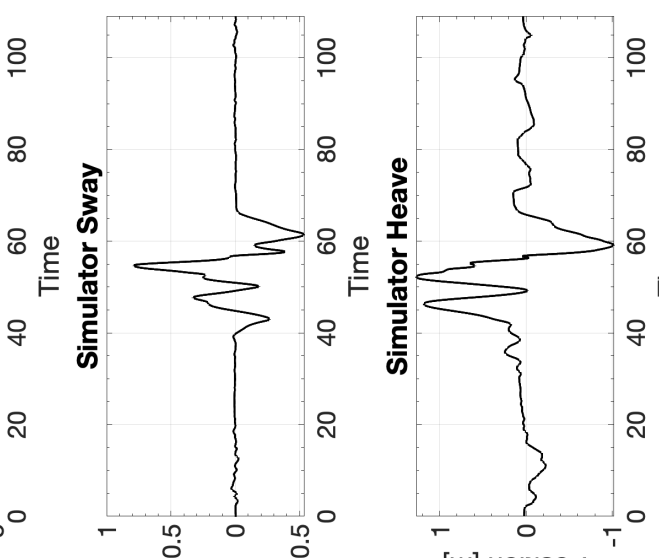

[u] uo!n!sod

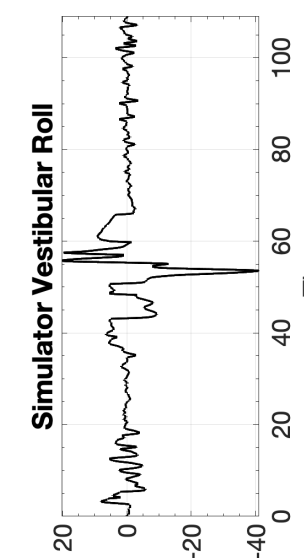

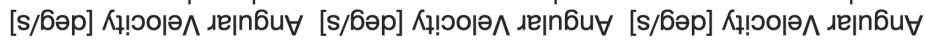

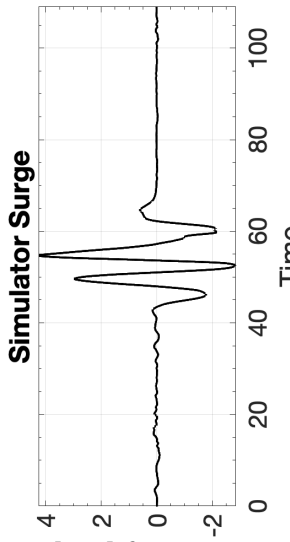

[s/u] Кұ!००|ә^
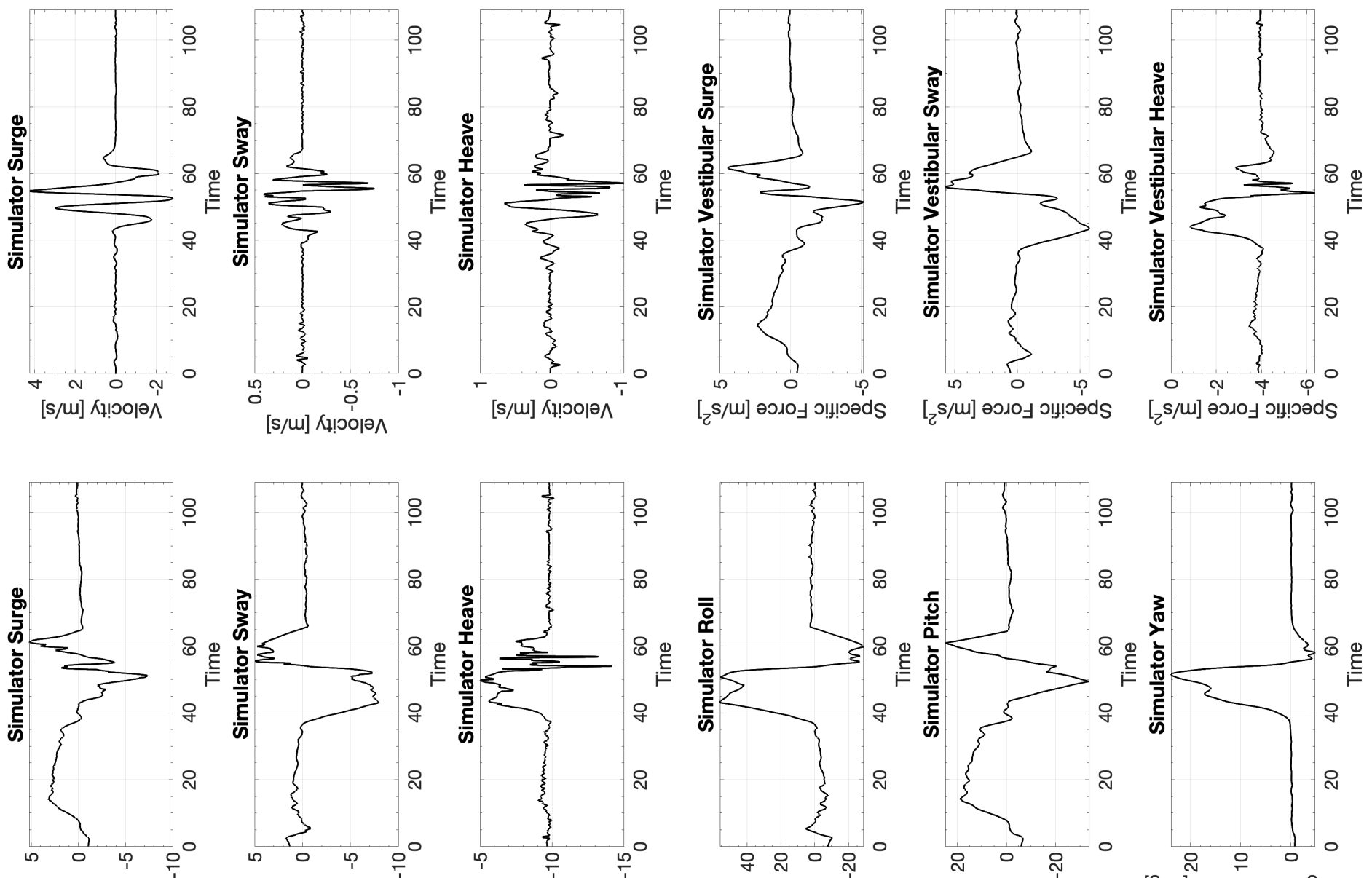

[z/m] ә

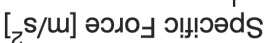

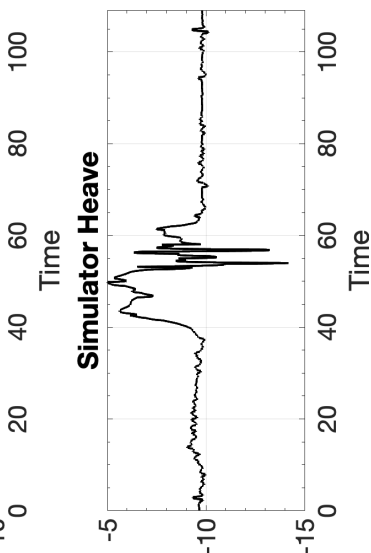

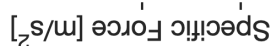
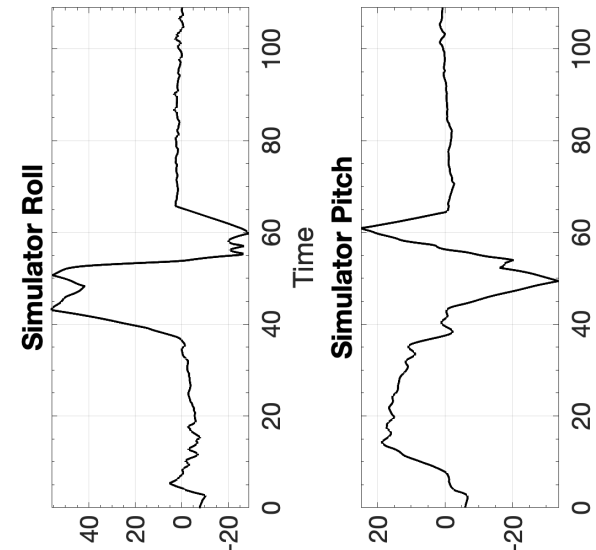

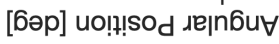

[6әр] uo!̣!sod גe|nбư

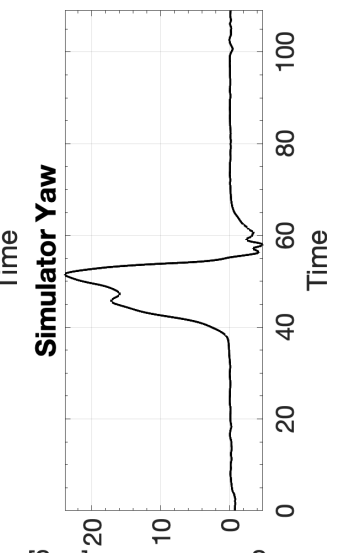

[бәр] uo!!!!sod גe|nбu

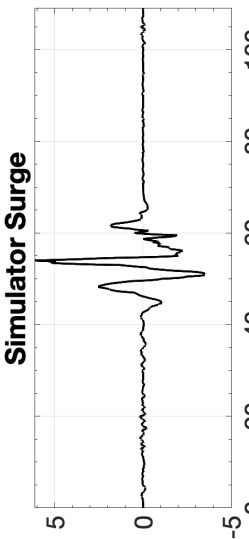

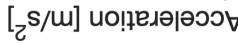

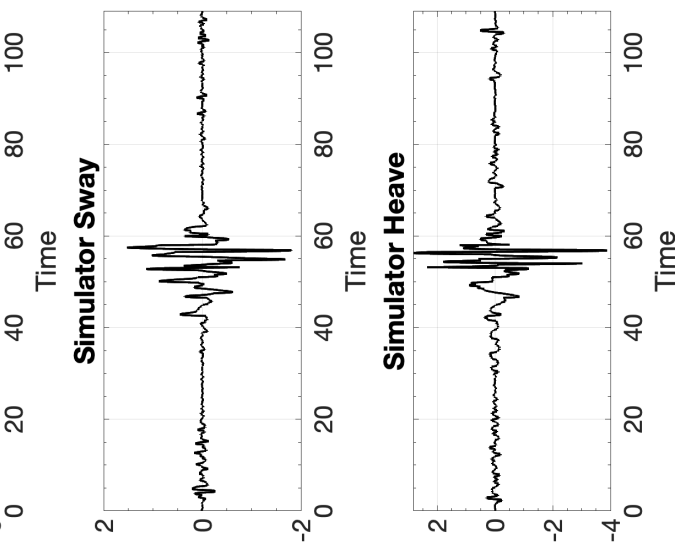

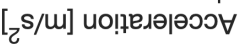

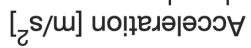

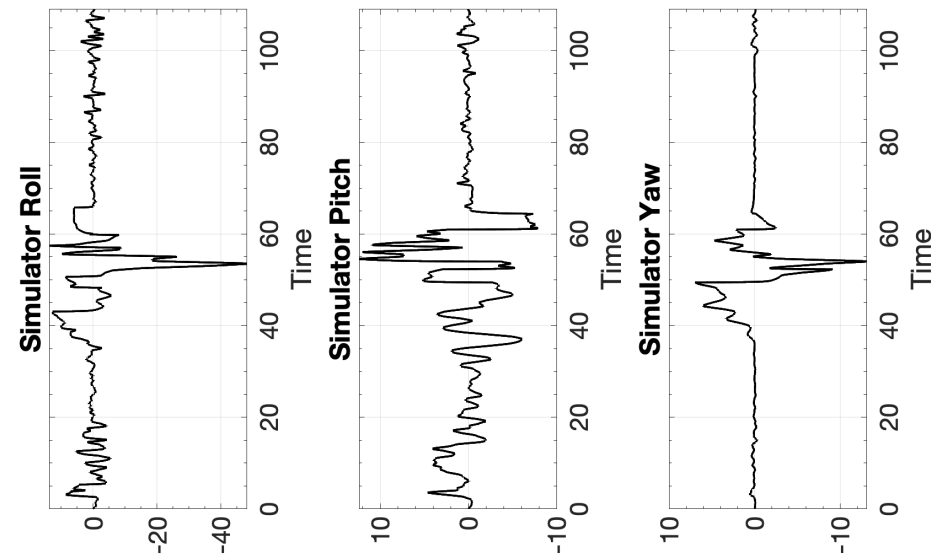

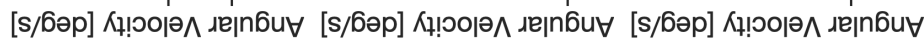




\section{B.12.2 CW2 Boeing 737 Spiral Dive}

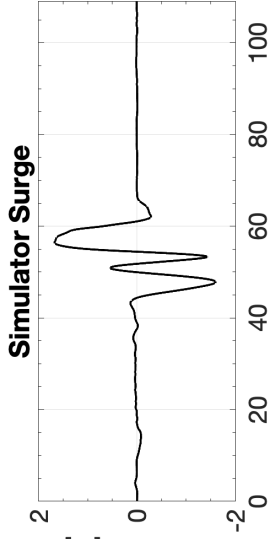

[w] uo!!!!sod

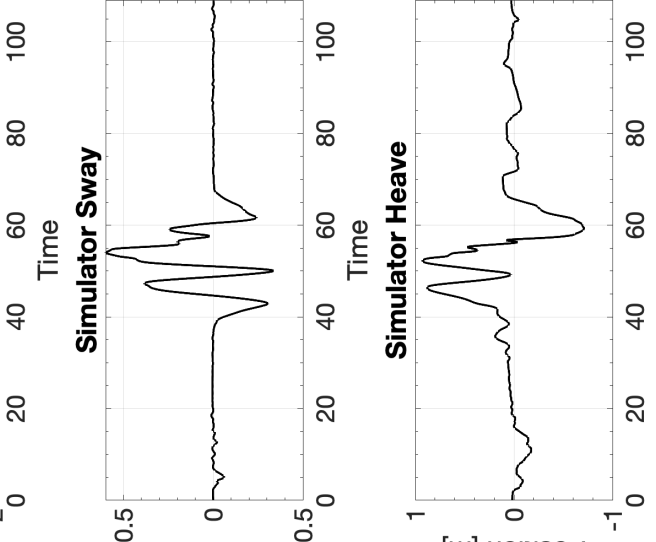

[w] uo!!!!sod

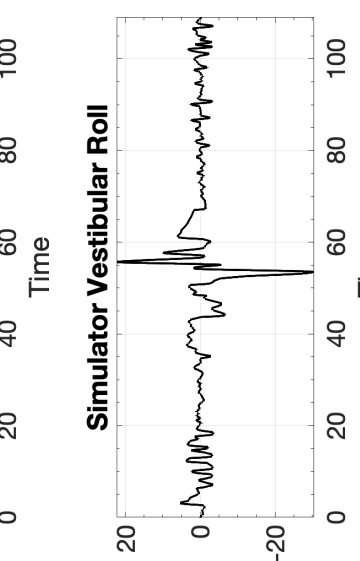

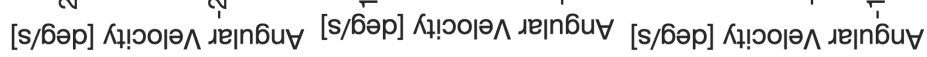

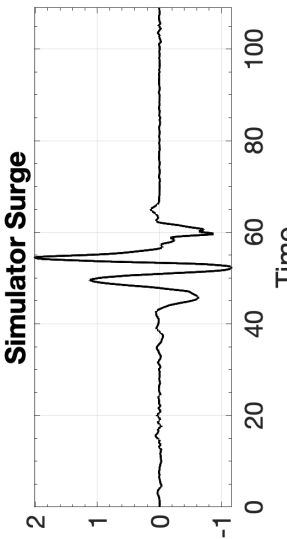

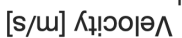

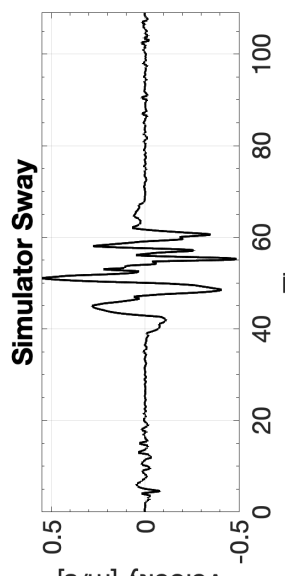

[s/u] Кł!วоㅋ^
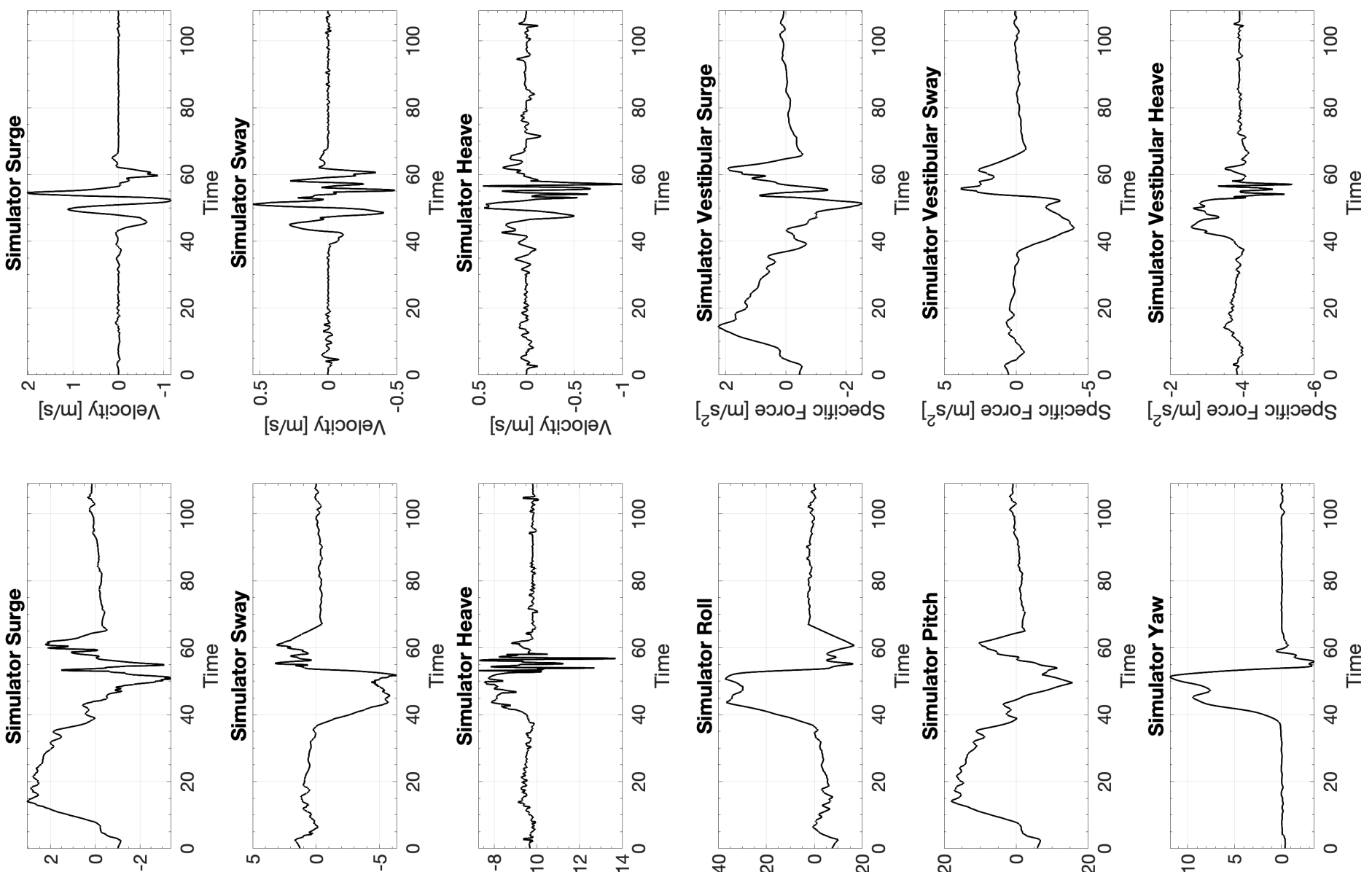

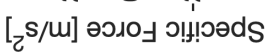
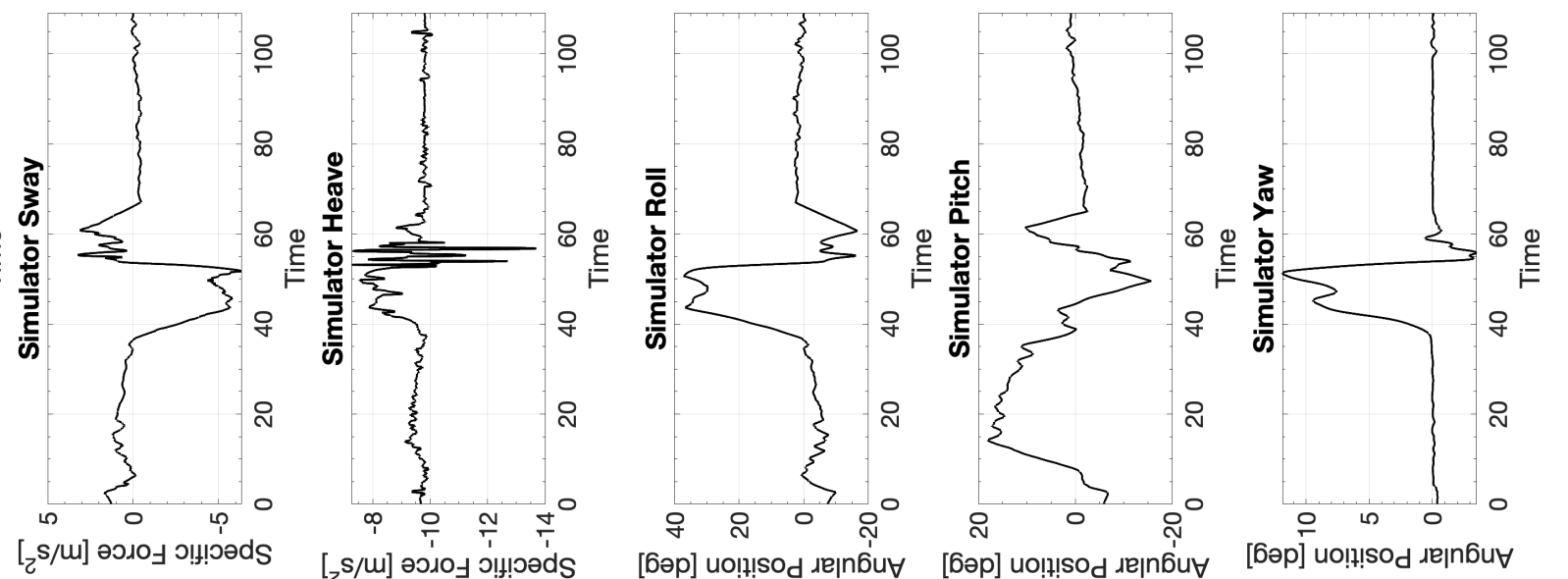

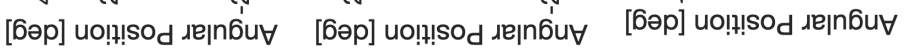

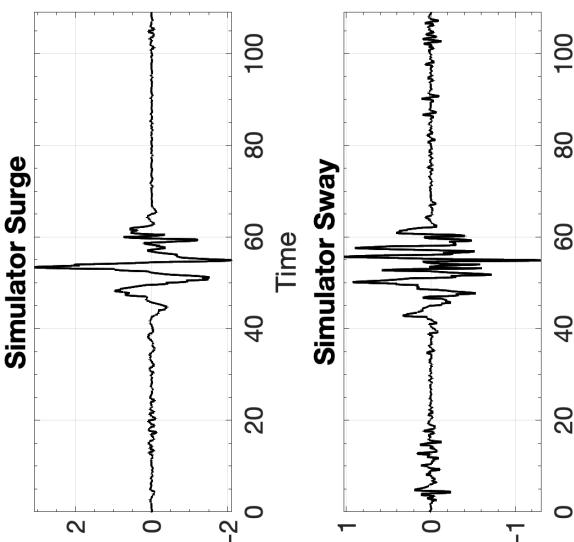

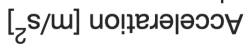

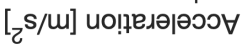

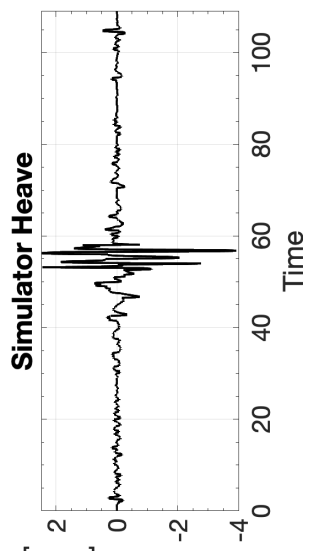

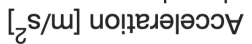

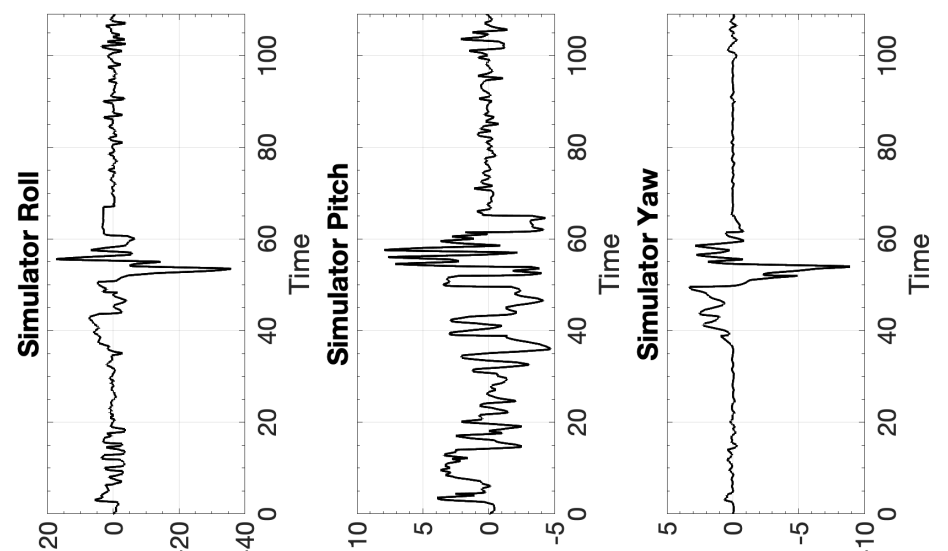

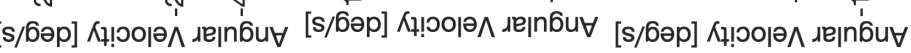




\section{B.12.3 CW3 Boeing 737 Spiral Dive}

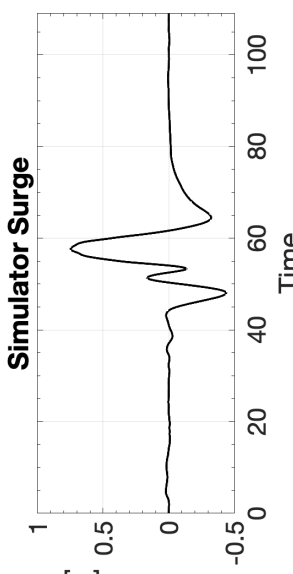

[m] uo!!!!sod

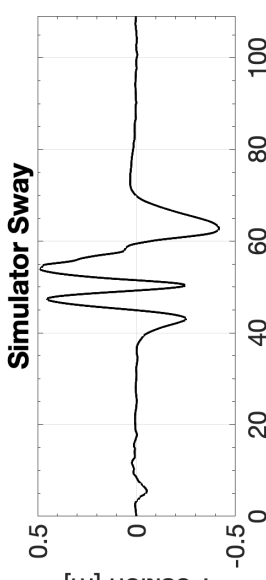

[w] uo!n!sod

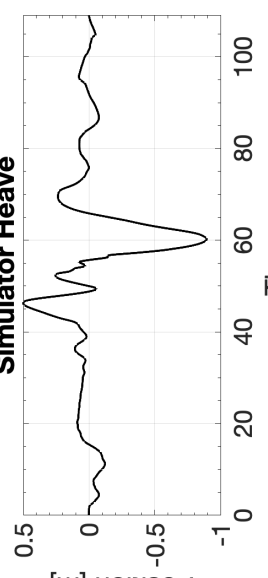

[u] uolt!!sod
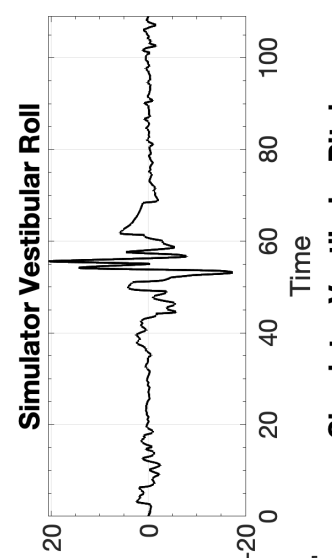

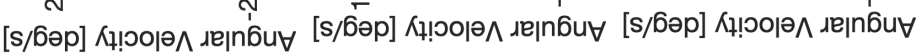

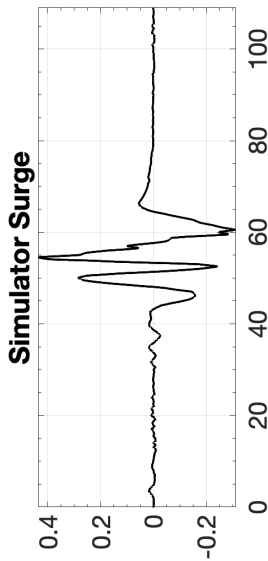

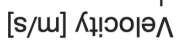

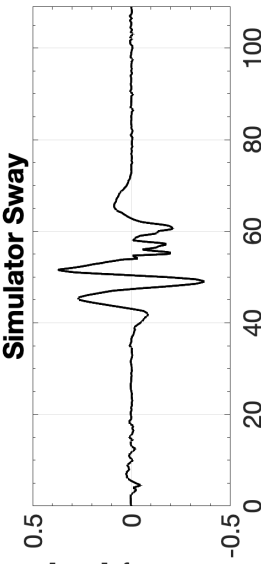

[s/m] К!ำю

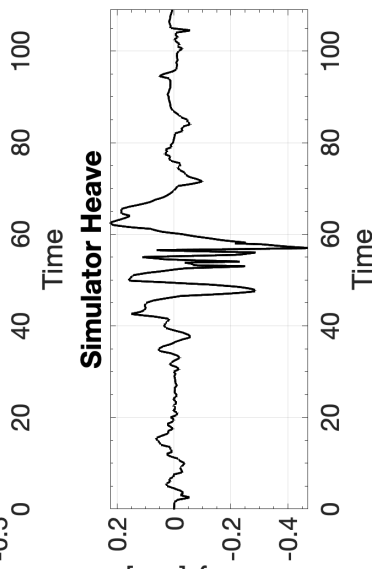

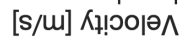
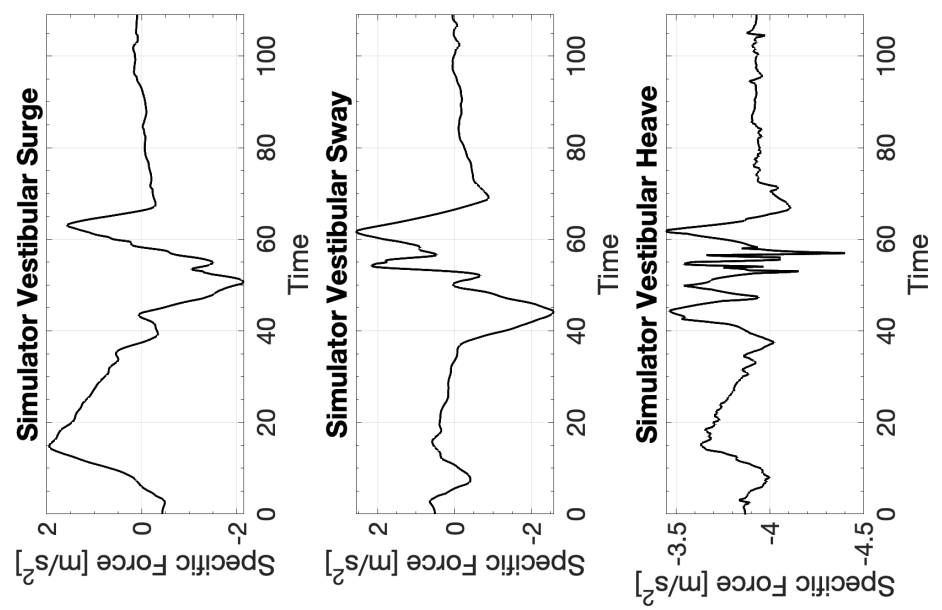
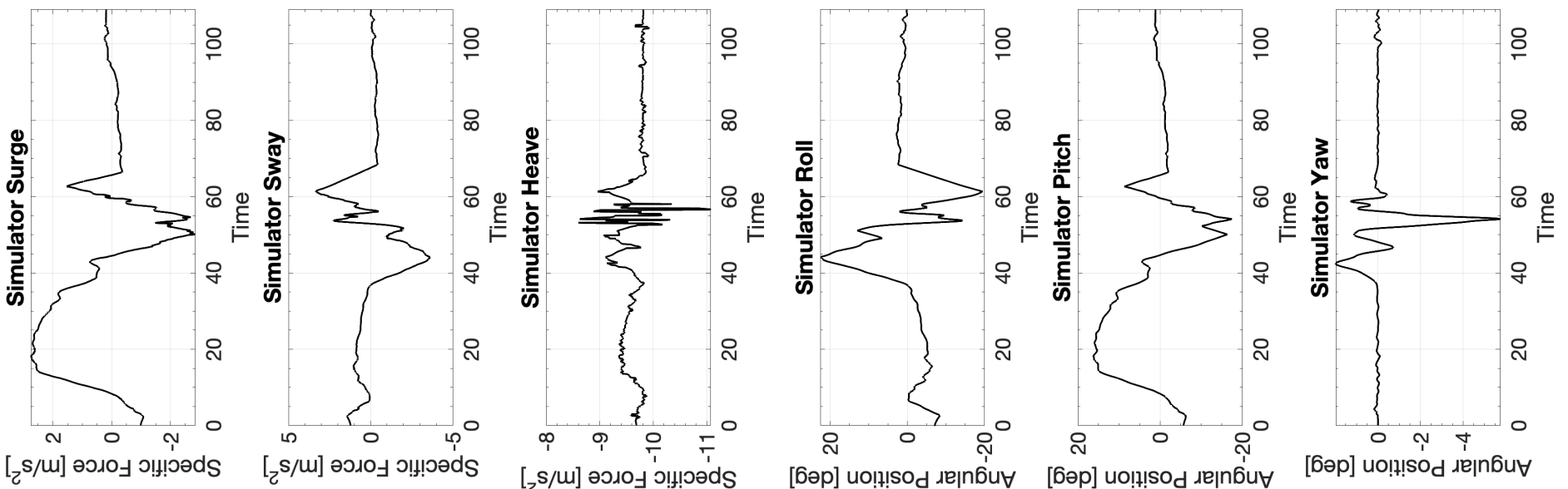

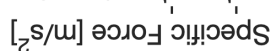

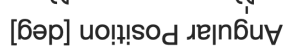

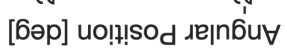

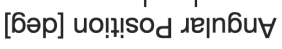

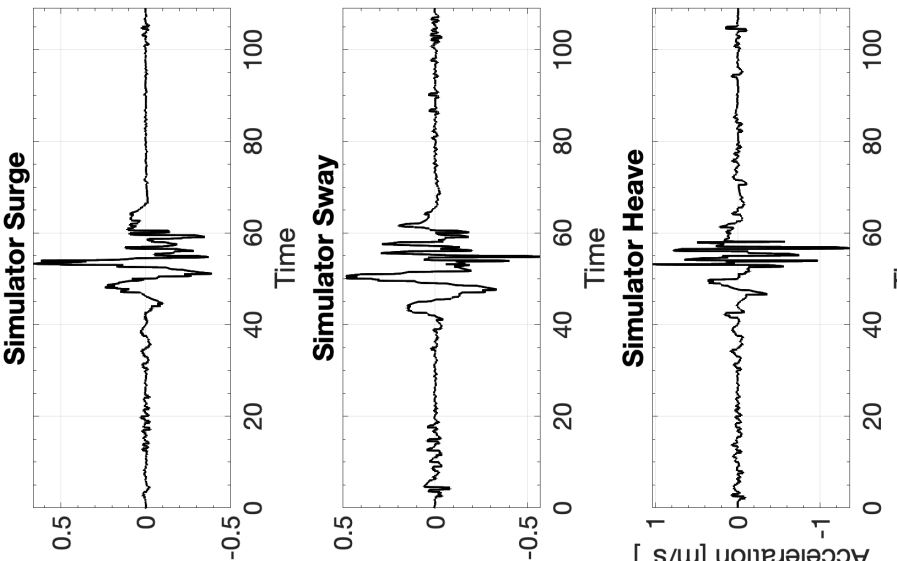

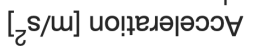

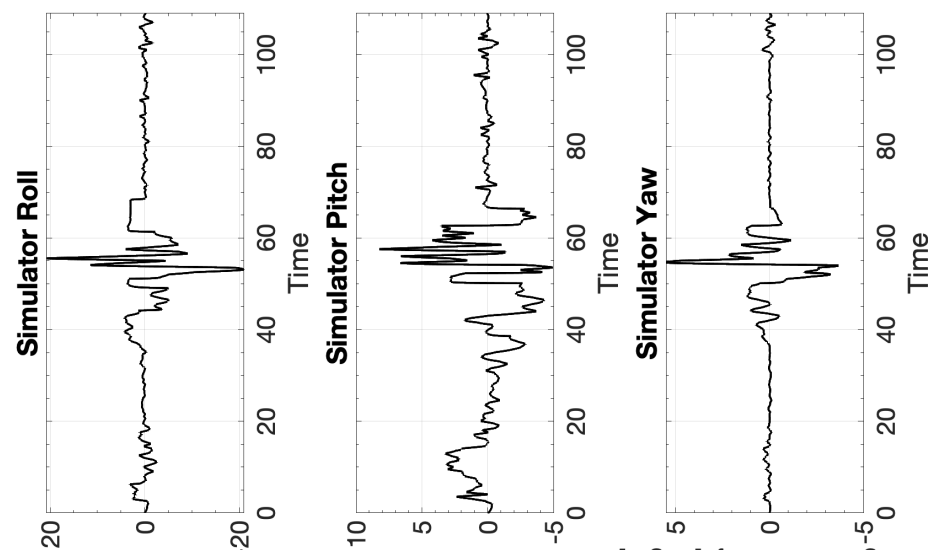

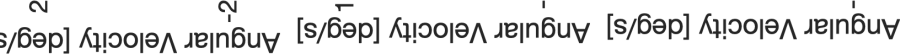




\section{B.13 Boeing 737 Stall Recovery}

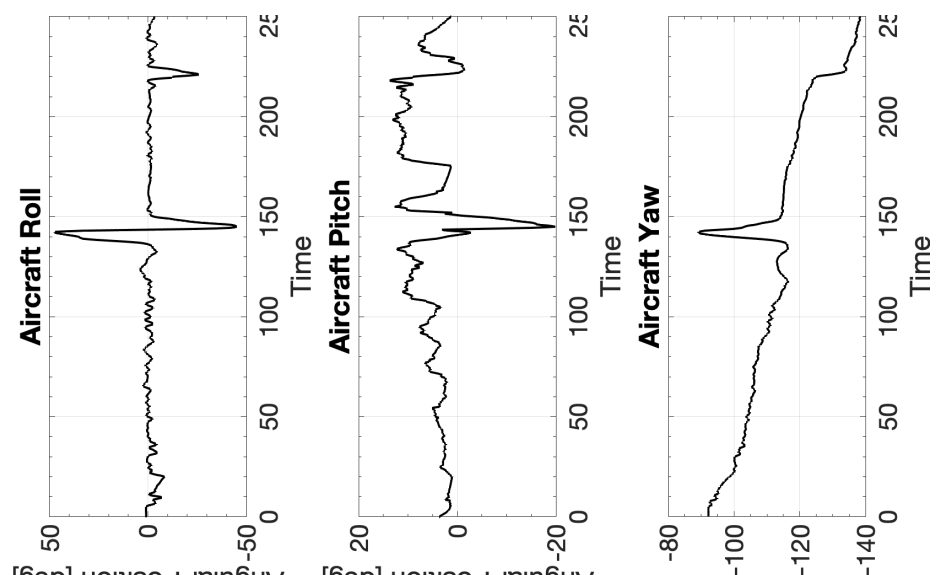

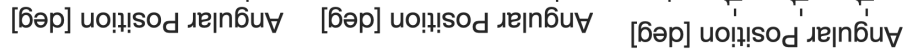

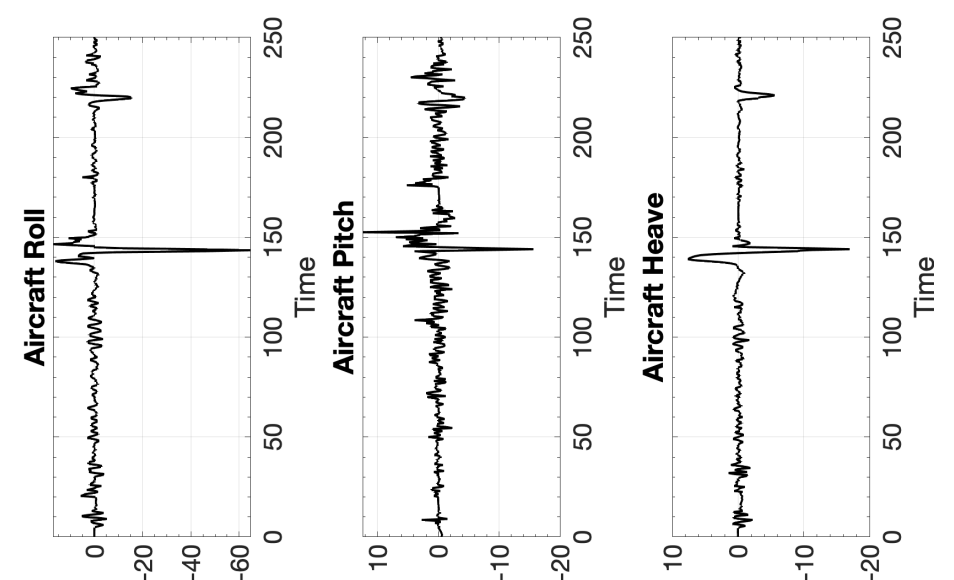

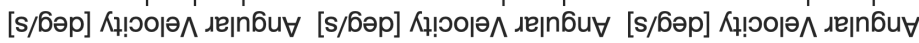
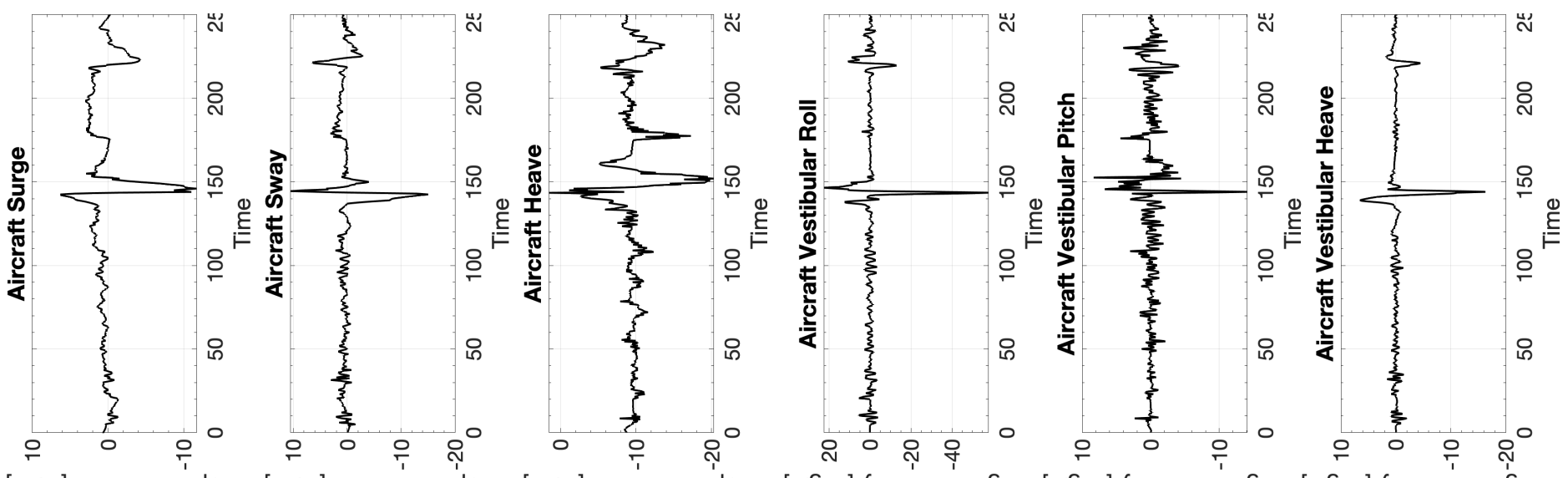

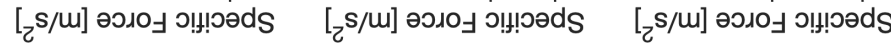

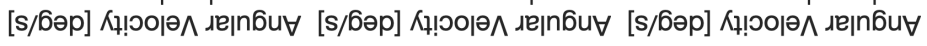

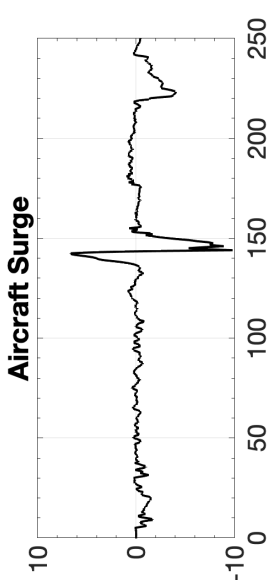

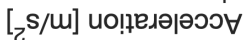

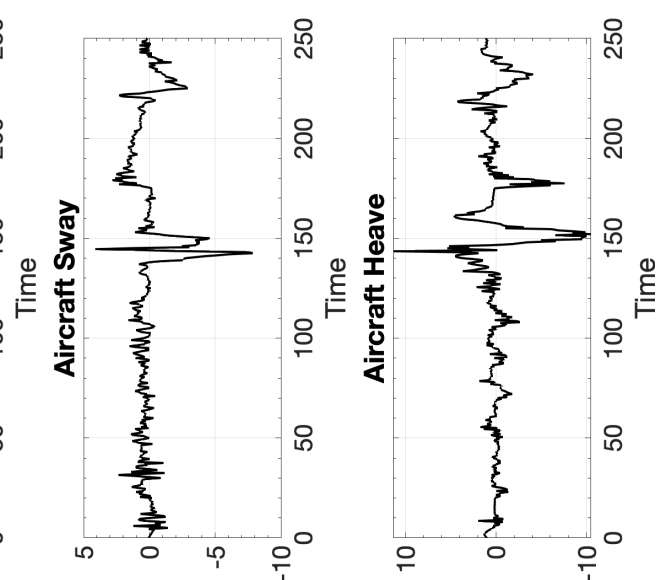

[zs/u] uo!̣eдәəәэว $\forall$

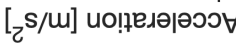
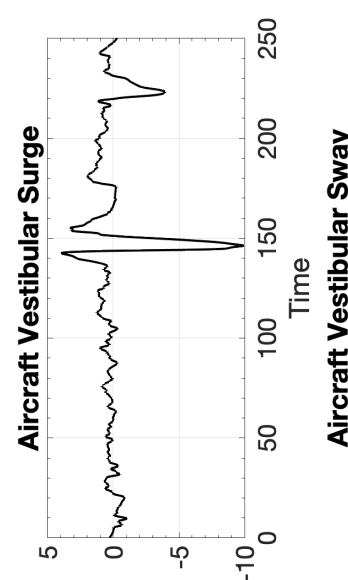

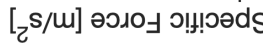
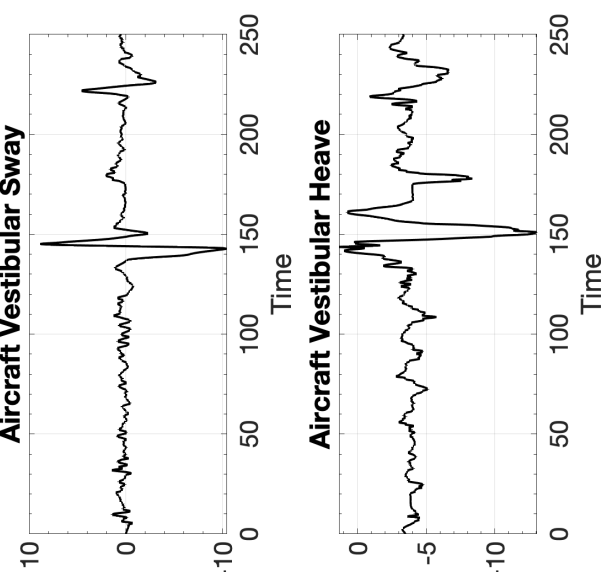

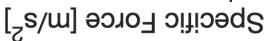

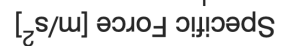




\section{B.13.1 CW1 Boeing 737 Stall Recovery}

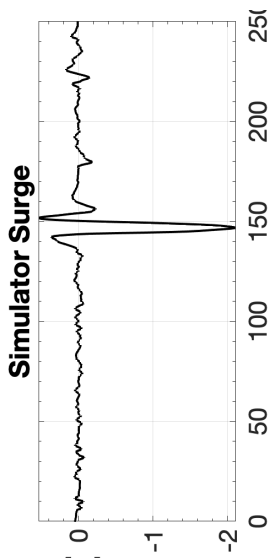

[u] uo!!!!sod

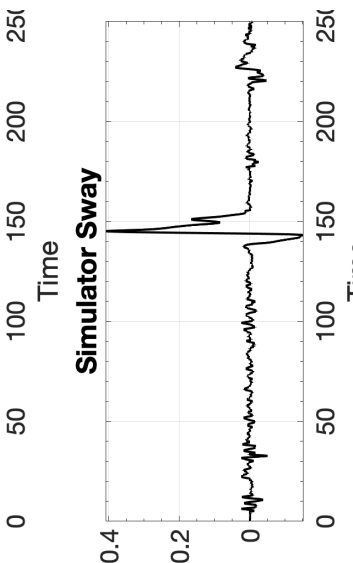

[u] uol!!!sod

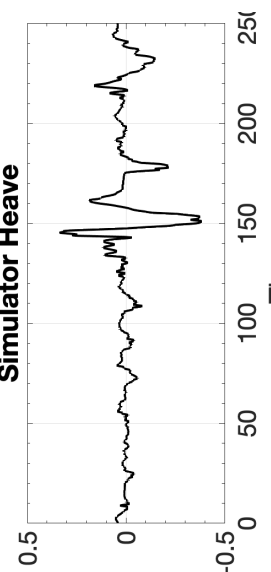

[w] uo!n!sod
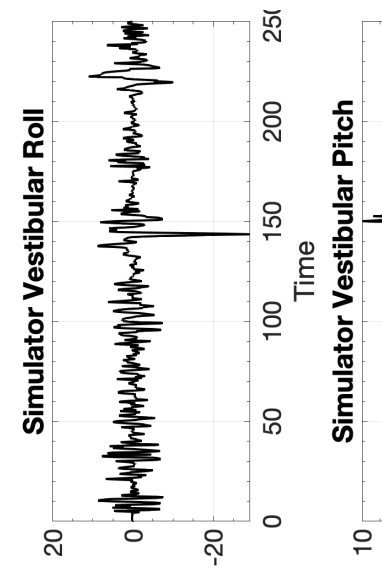

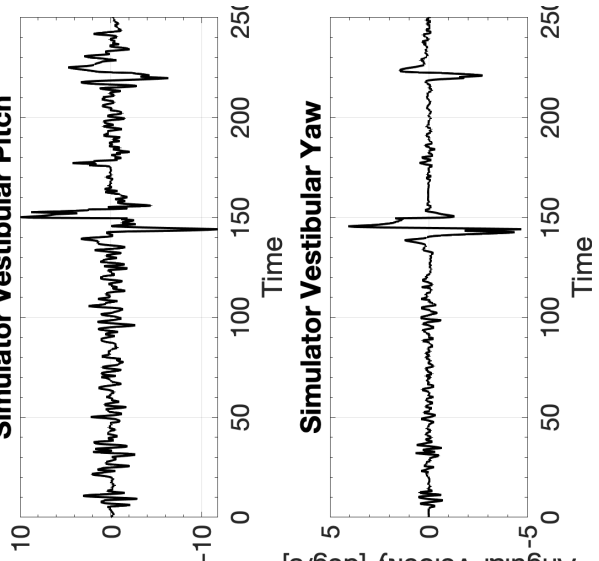

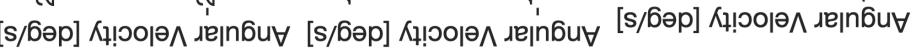

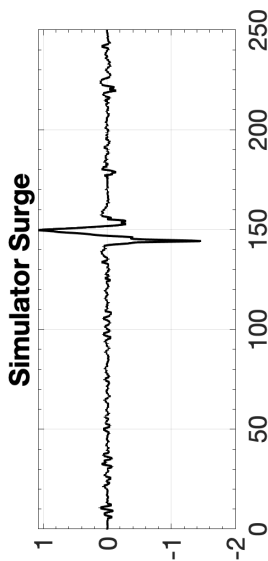

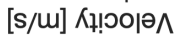

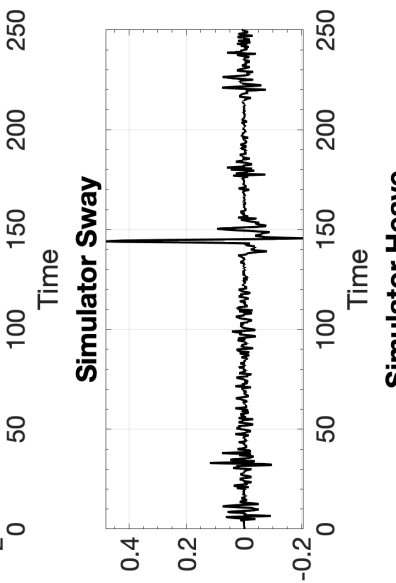

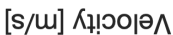

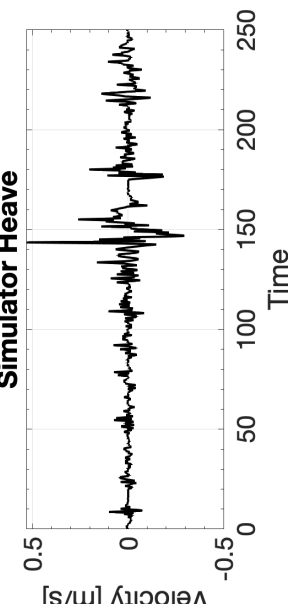

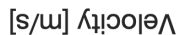
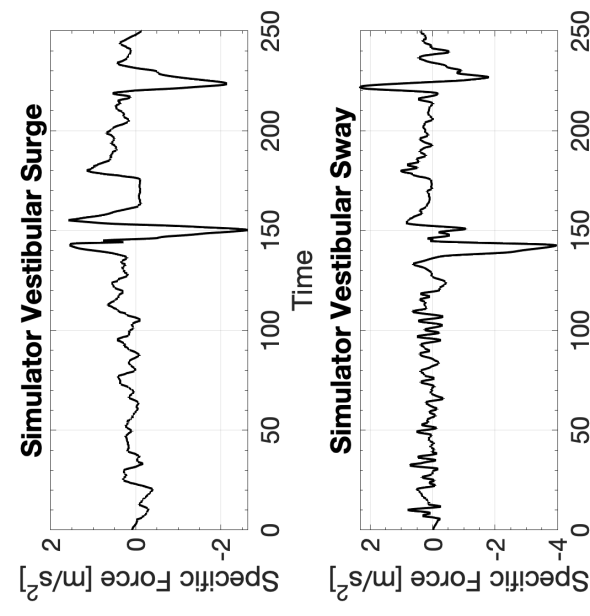

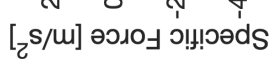
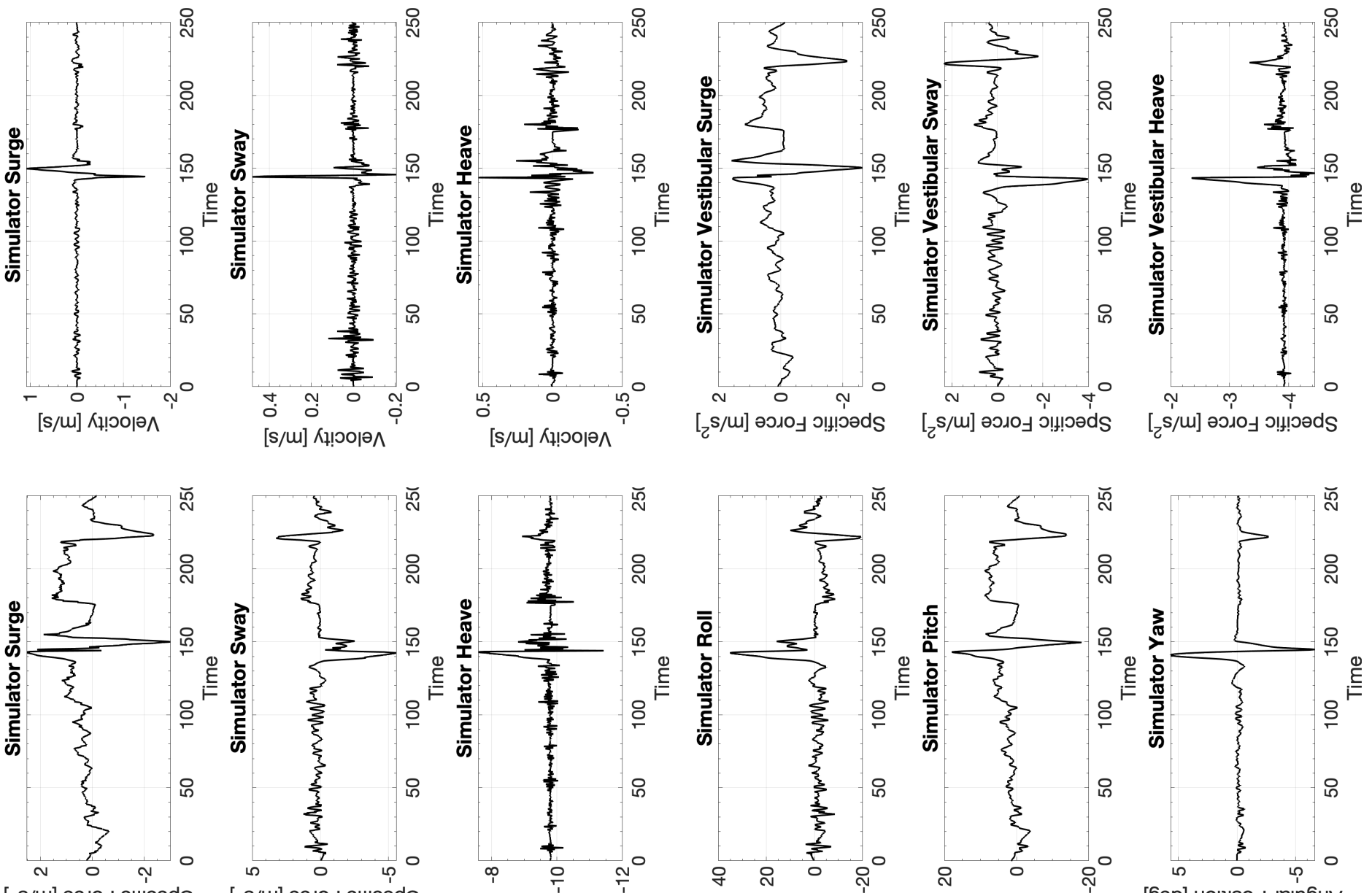

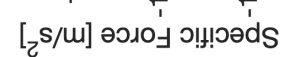
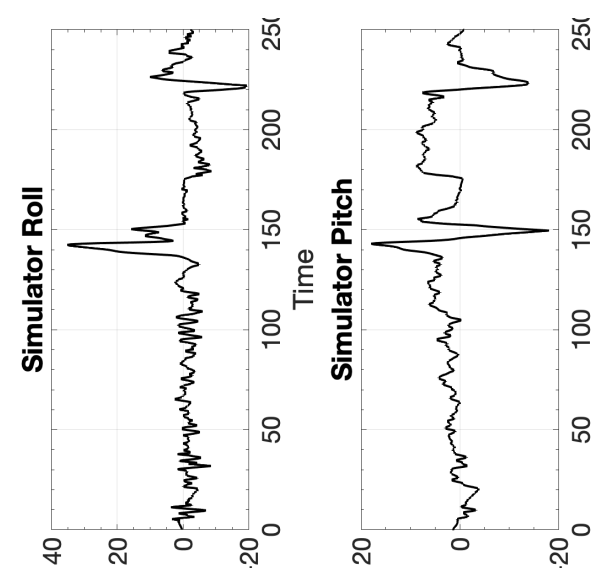

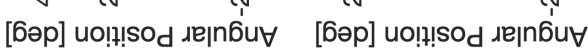

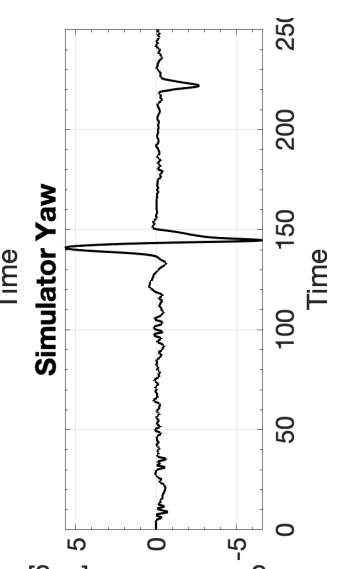

[бәр] uo!!!sod גeןn6u

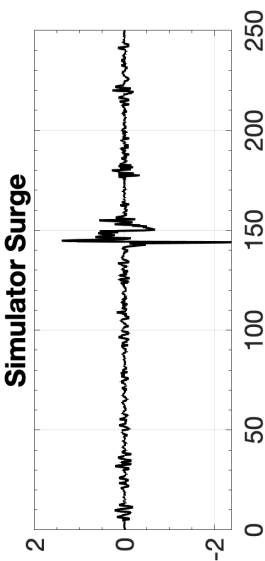

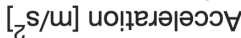

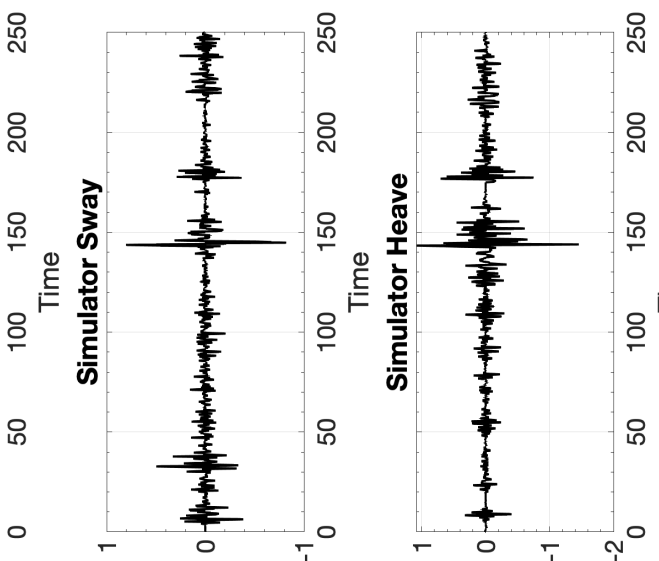

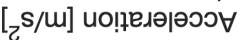

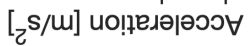
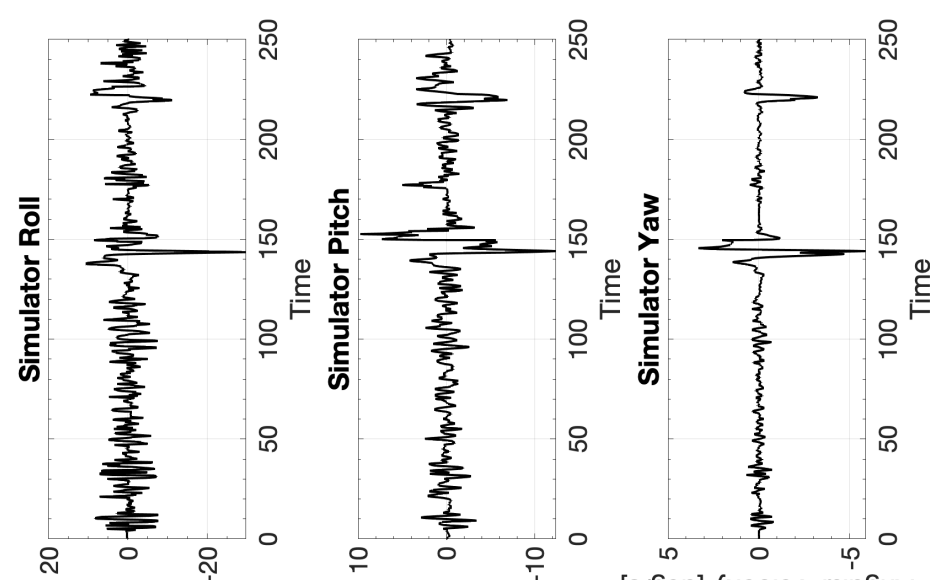

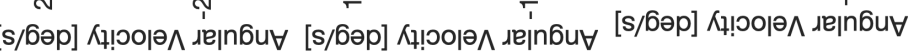




\section{B.13.2 CW2 Boeing 737 Stall Recovery}

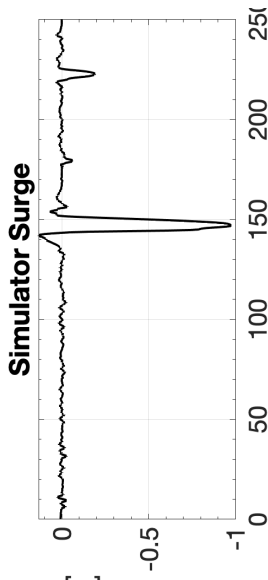

[w] uo!!!!sod

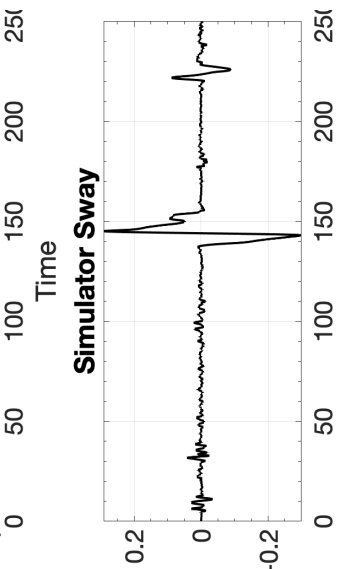

[w] uo!n!sod

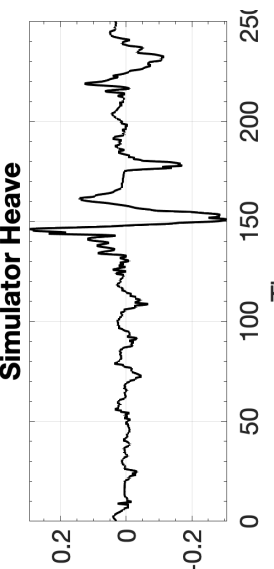

[u] uolp!sod

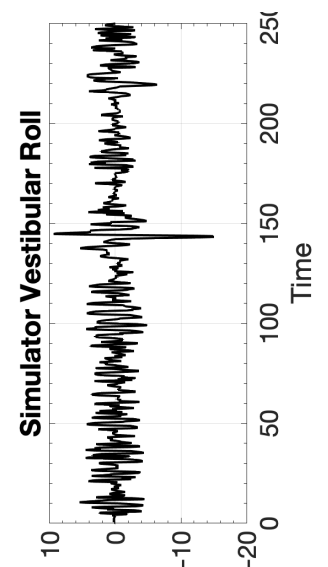

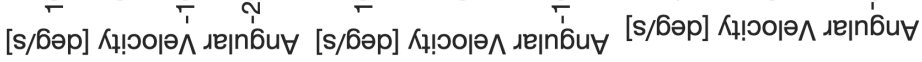
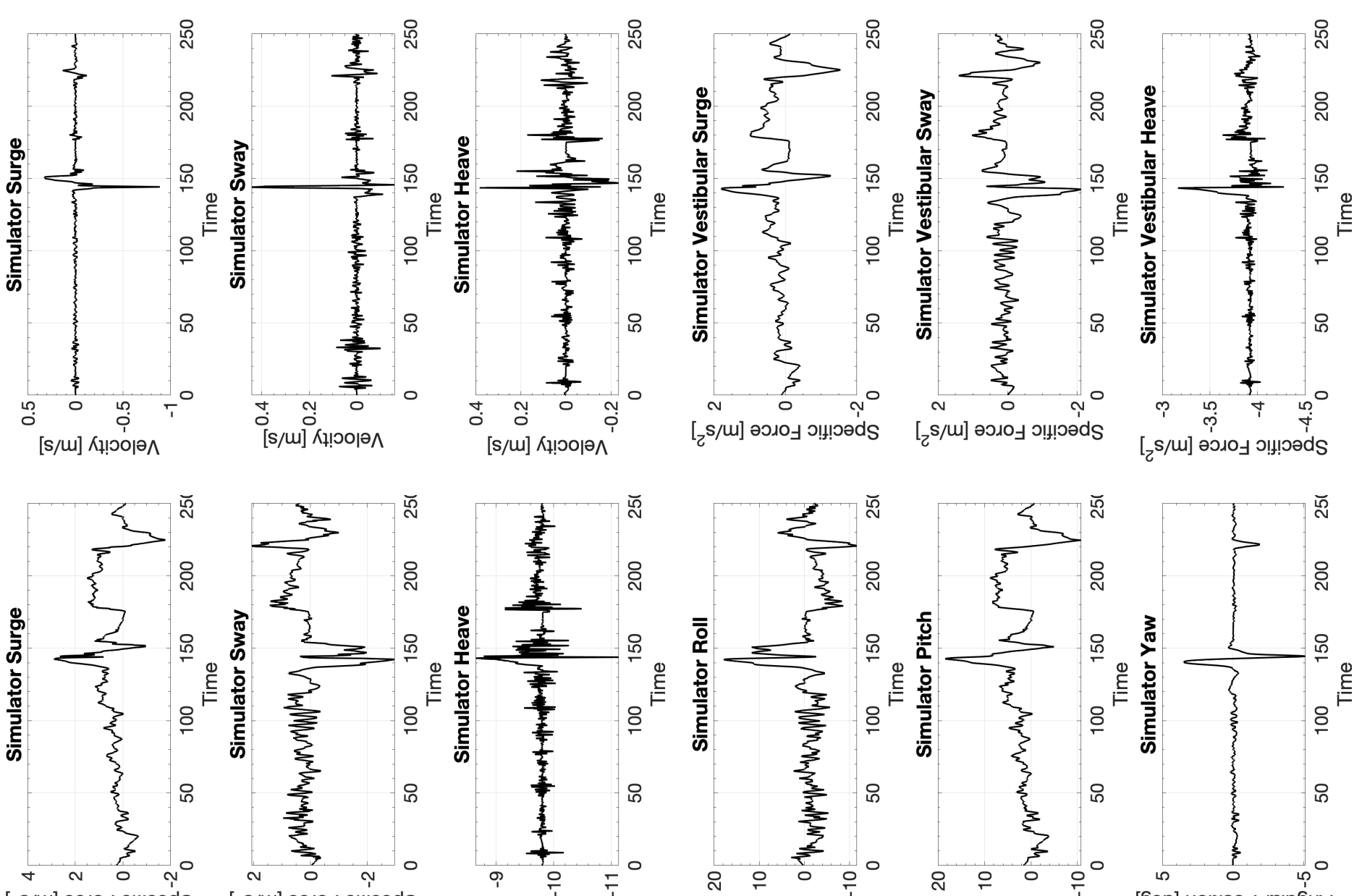

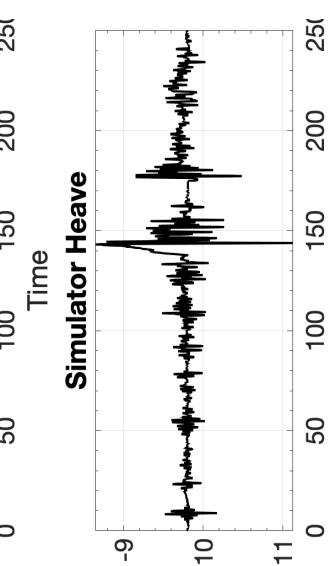

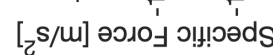

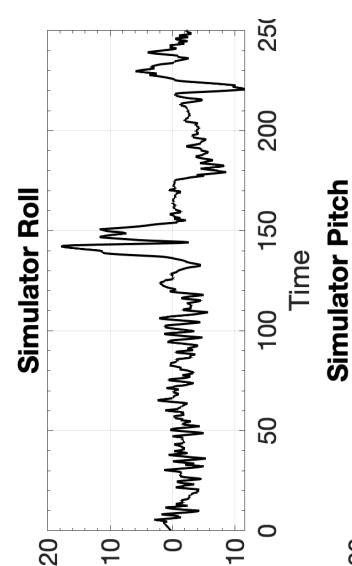

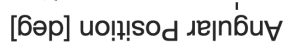
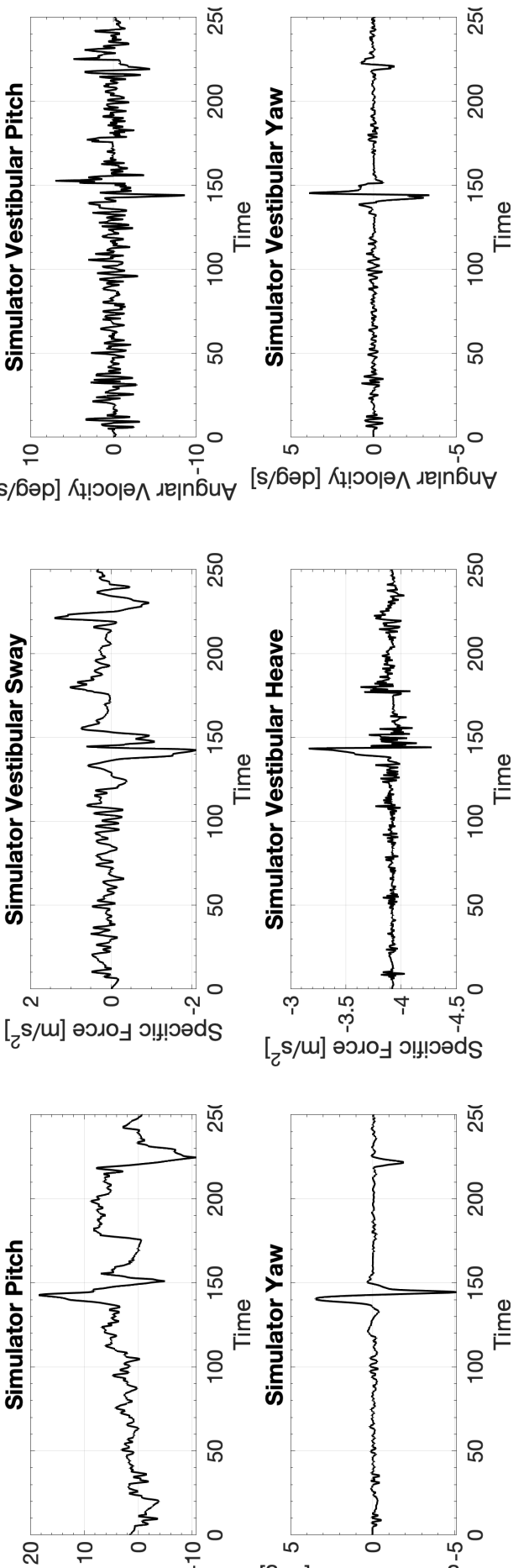

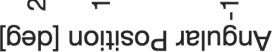

[бәр] uo!!!!sod גejnб์u

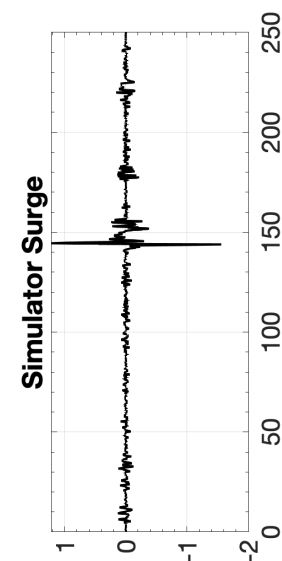

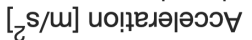

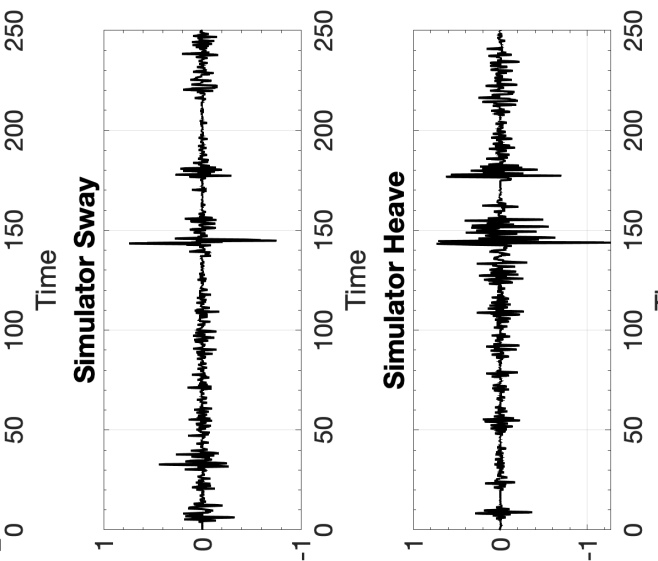

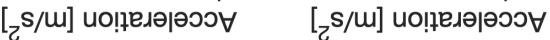
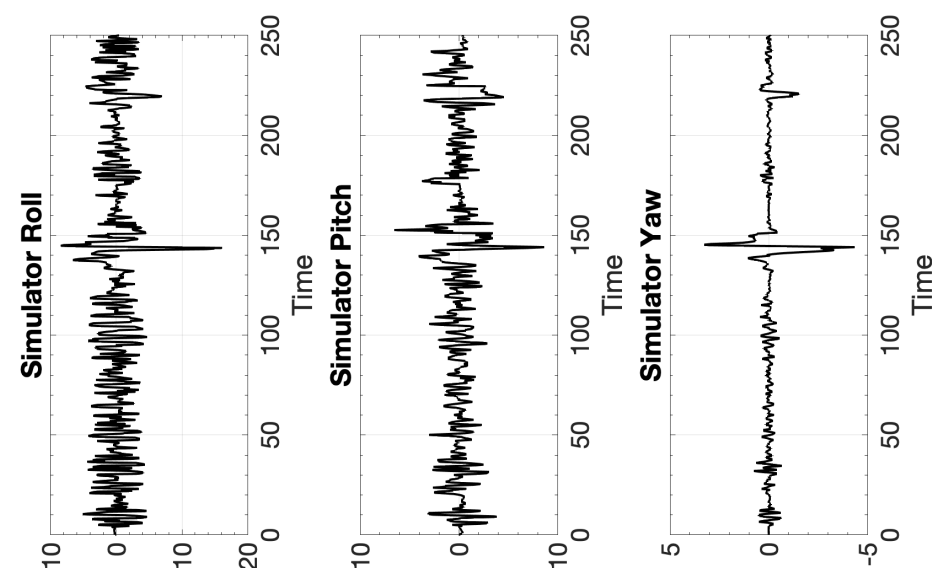

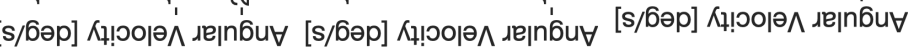




\section{B.13.3 CW3 Boeing 737 Stall Recovery}

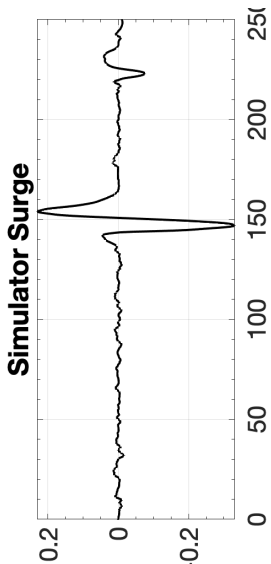

[u] uo!!!!sod

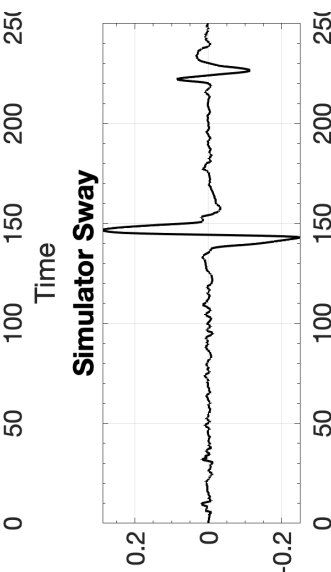

[w] uo!n!!sod

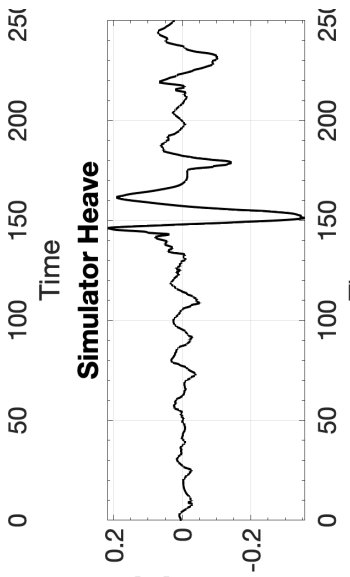

[u] uo!n!

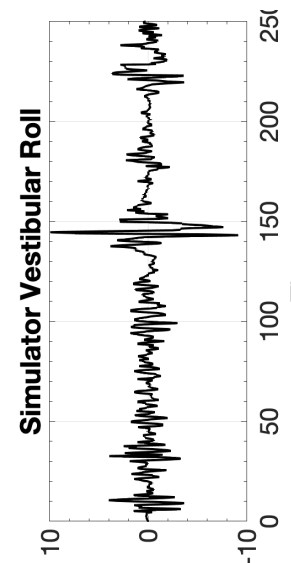

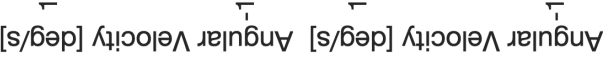

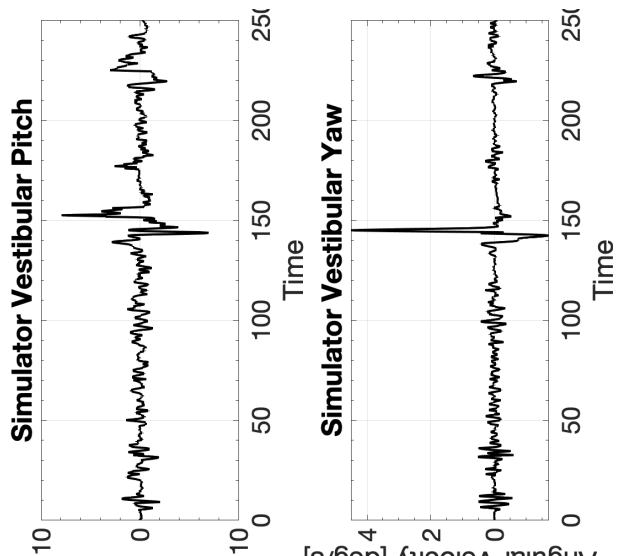

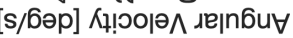

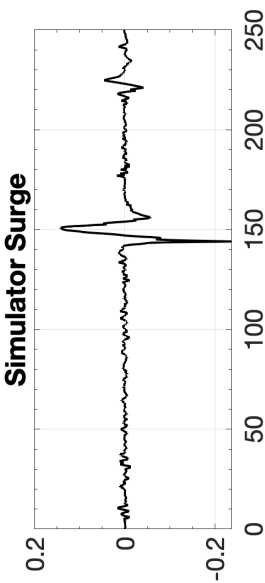

[s/m] Кł!००|ә^

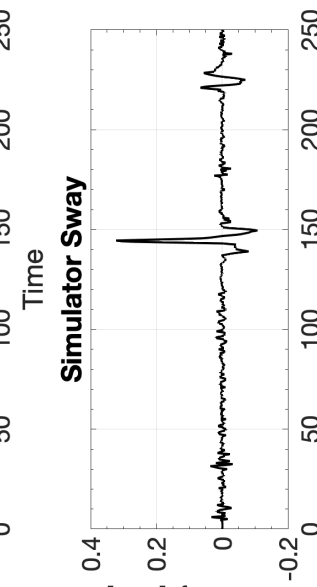

[s/m] Кұ!৩૦|ә

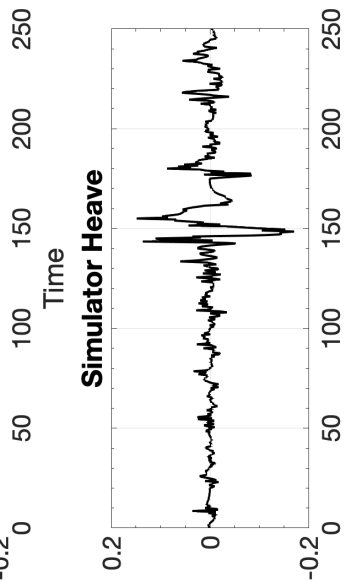

[s/m] Кł!ю૦ор^
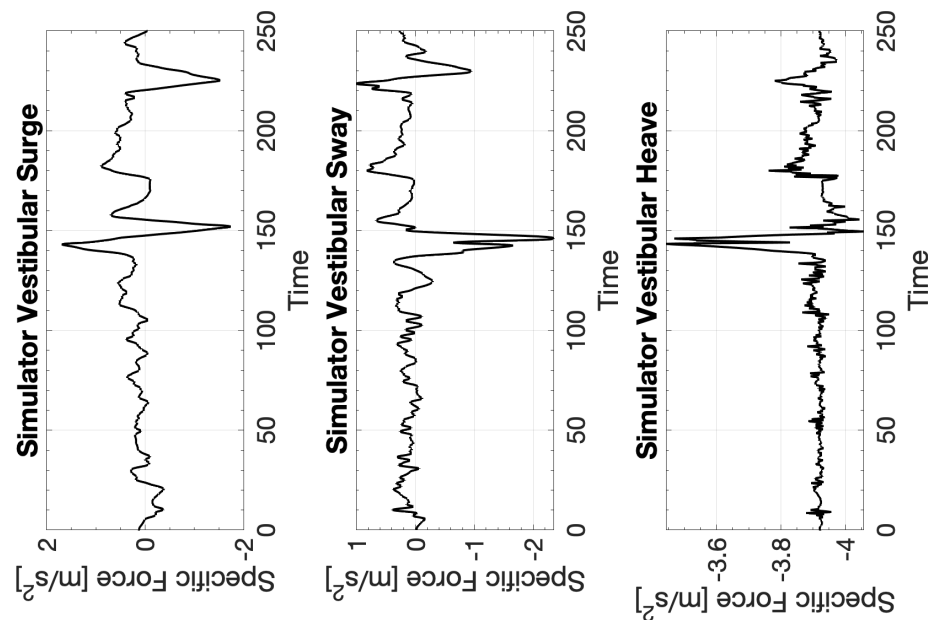

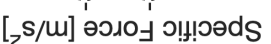

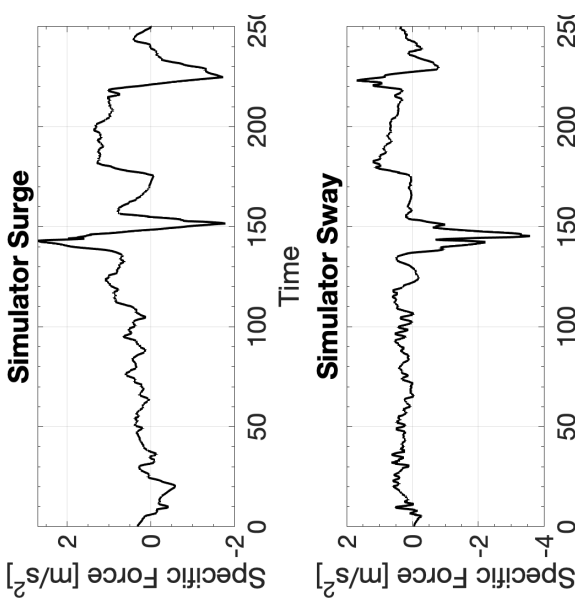

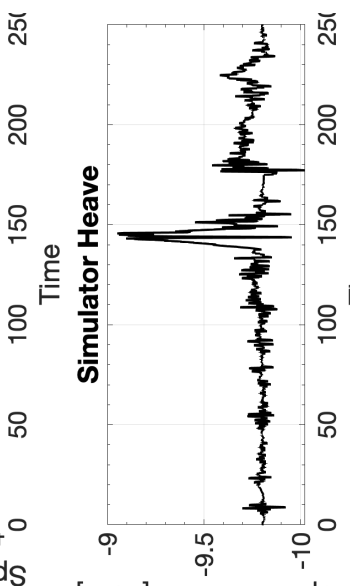

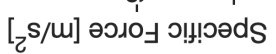

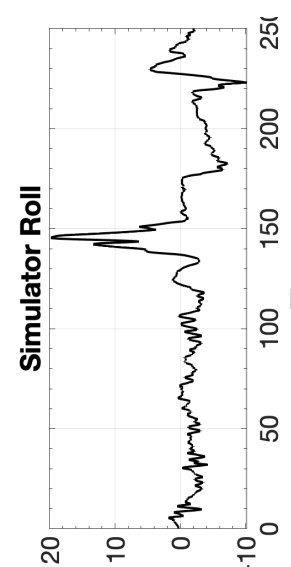

[бәр] uo!!! sod

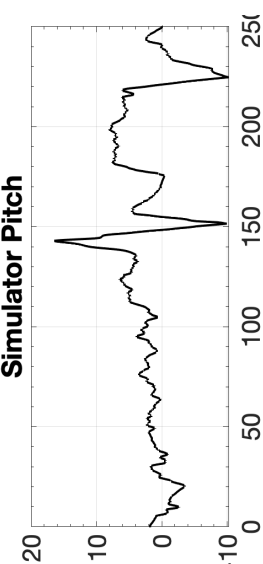

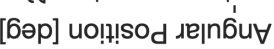

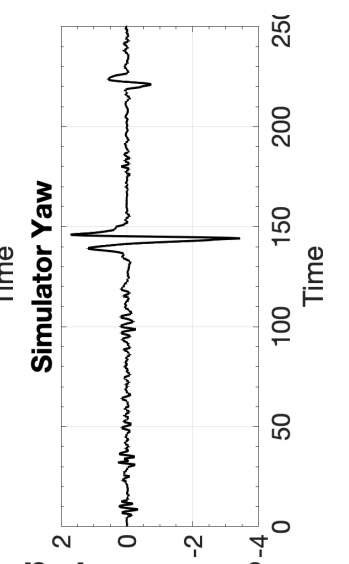

[бәр] uo!!!sod גeןn6u

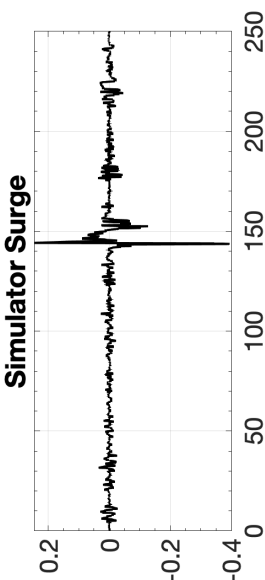

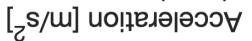

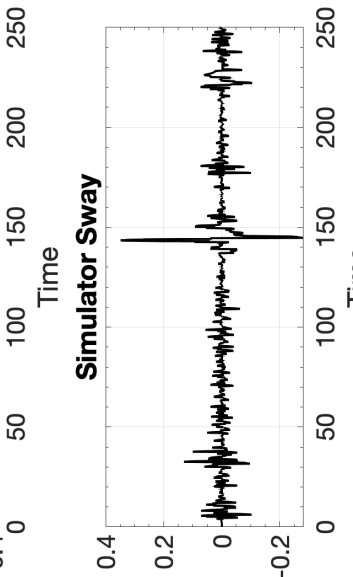

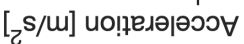

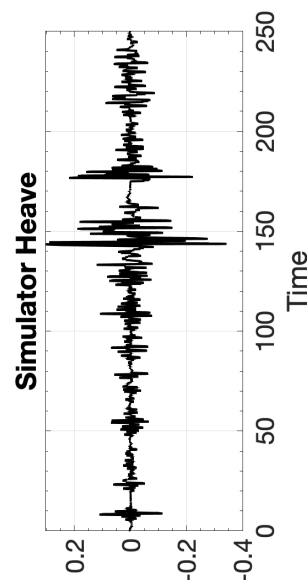

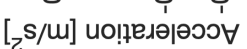
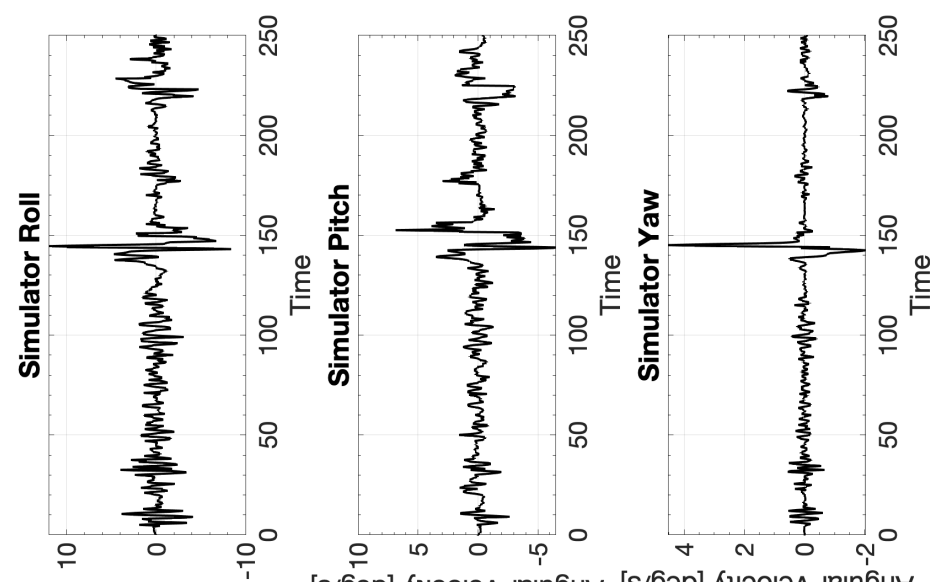


\section{B.14 Columbia 400 Aerobatics}

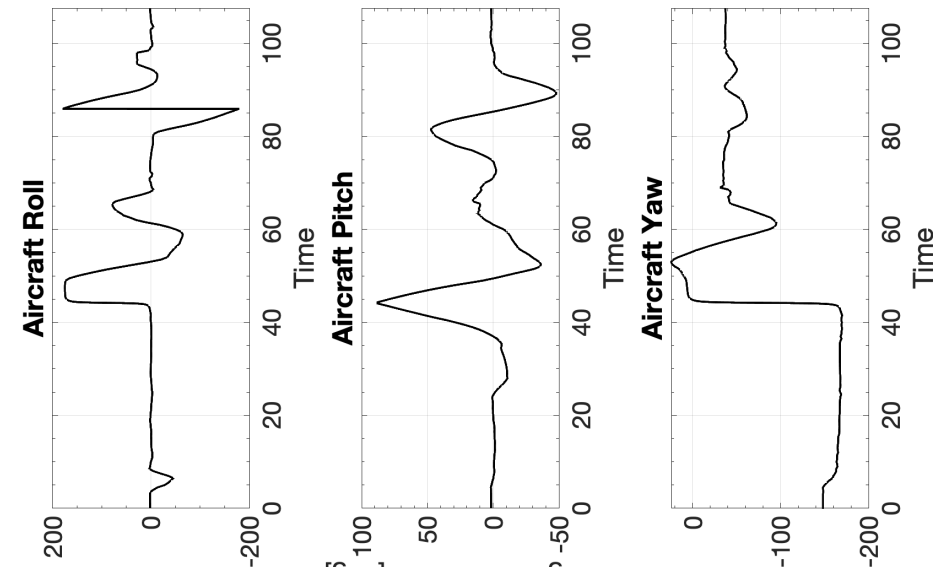

[6әp] uo!!!sod xe|n6́u

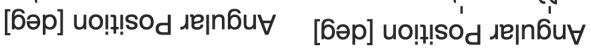

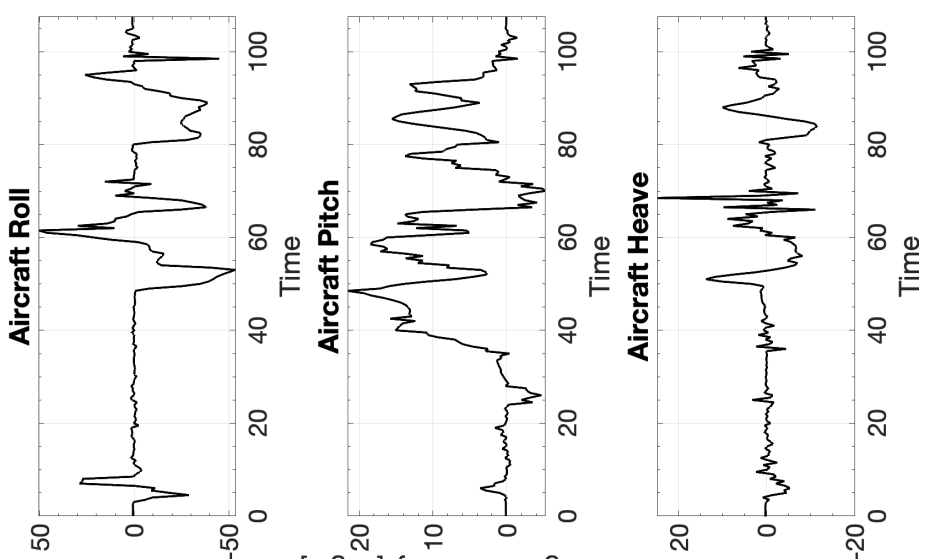

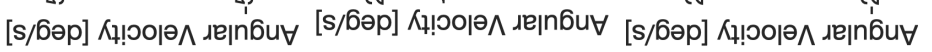

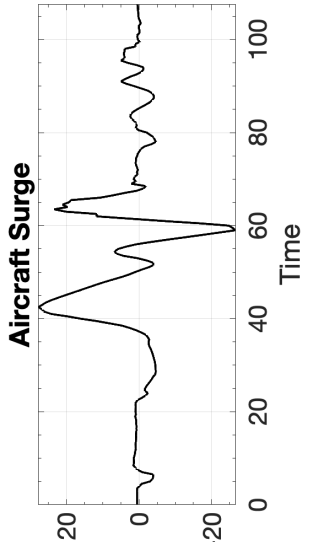

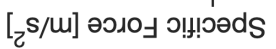

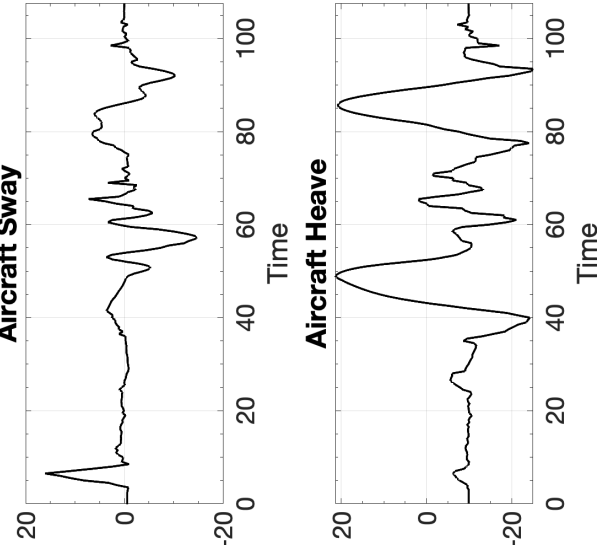

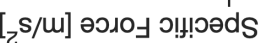
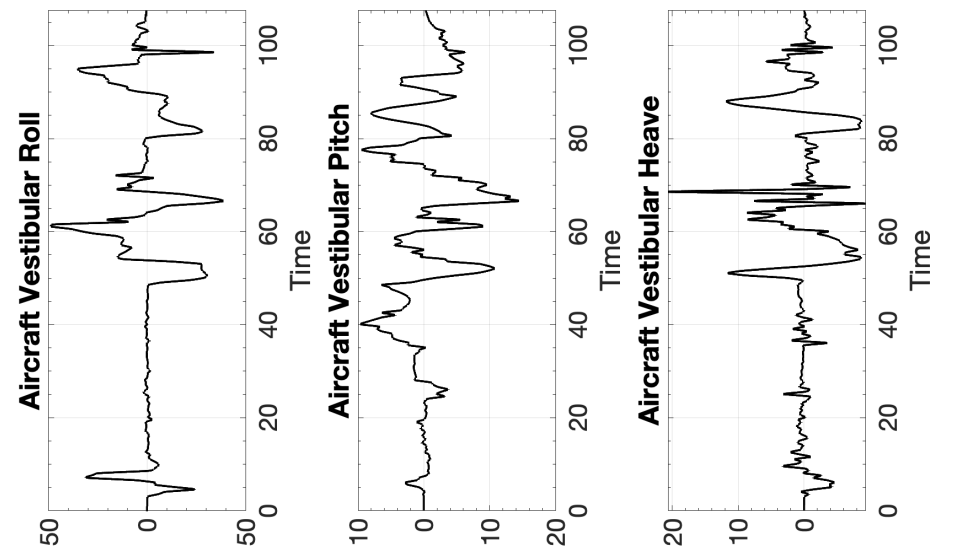

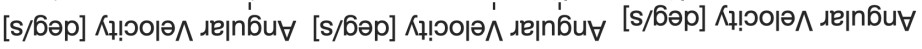

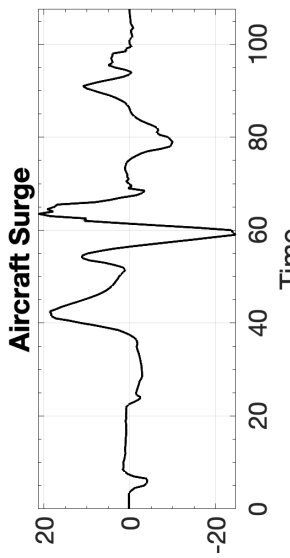

$\left[{ }^{2} \mathrm{~s} / \mathrm{m}\right]$ ио!ฺеләрәэว

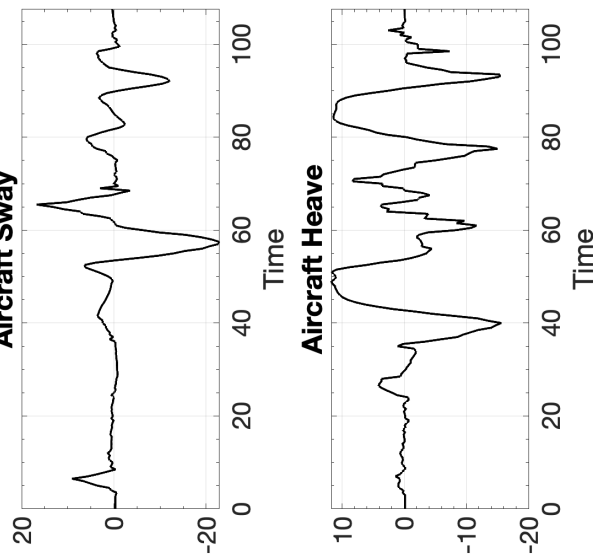

[z/m] ио!ฺвдәәәоэ

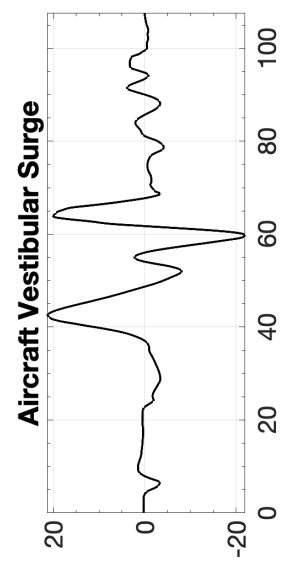

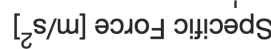

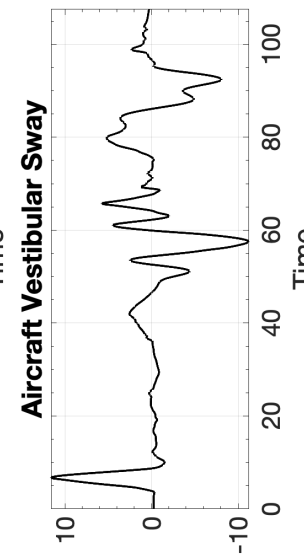

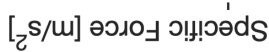

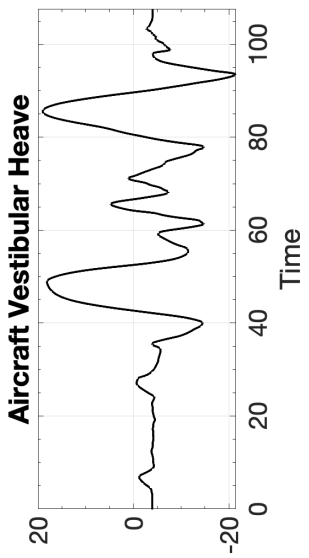

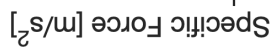




\section{B.14.1 CW1 Columbia 400 Aerobatics}

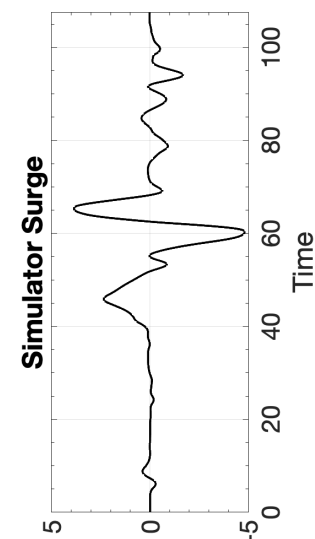

[u] uo!!! sod

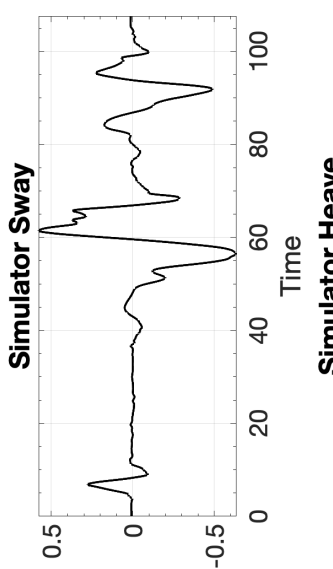

[m] uol!?!sod

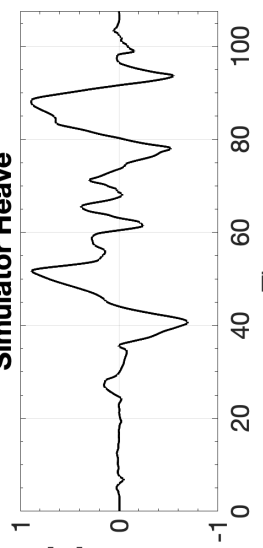

[w] uol!!sod
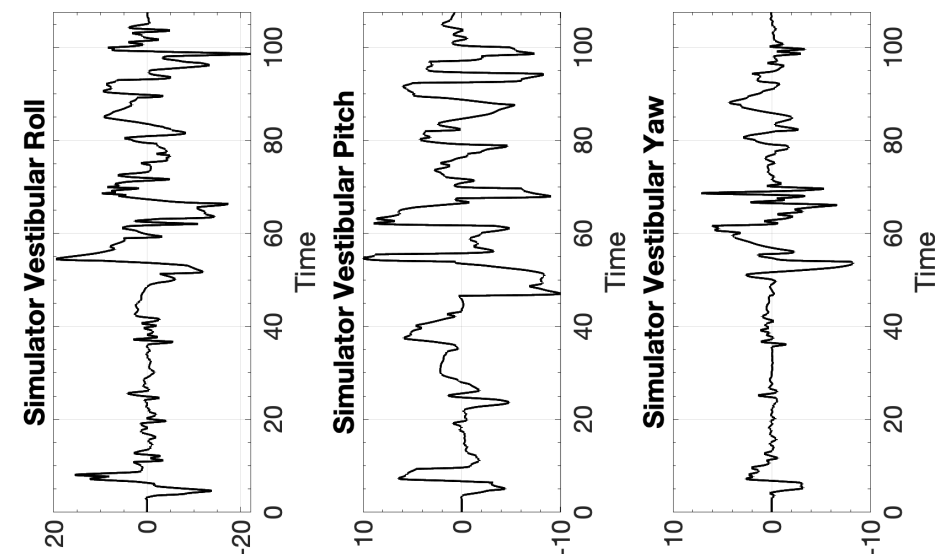

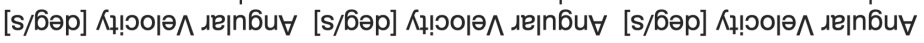

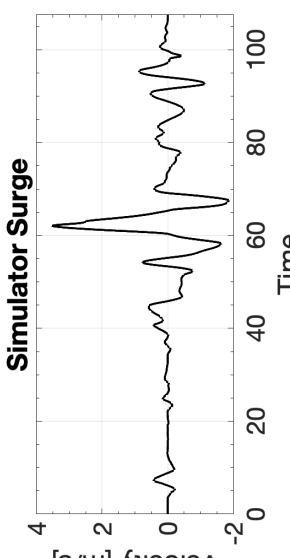

[s/u] Кұ!৩о|ә^
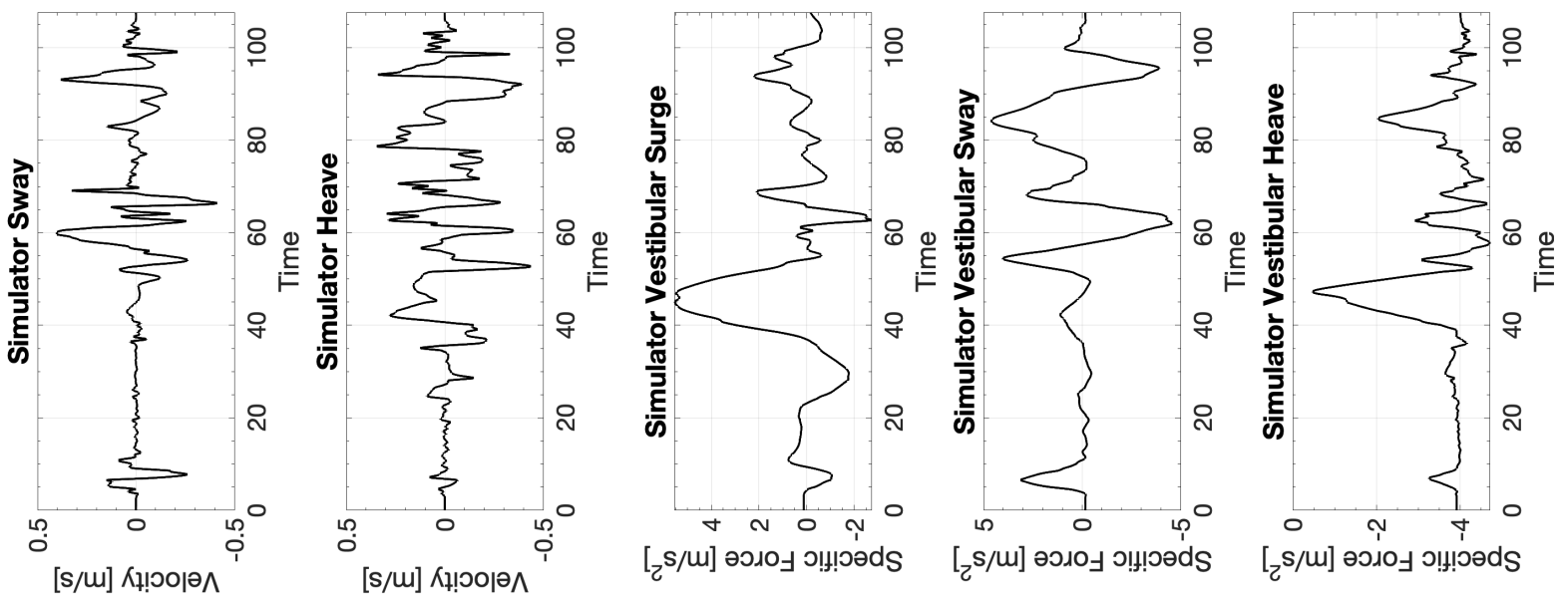

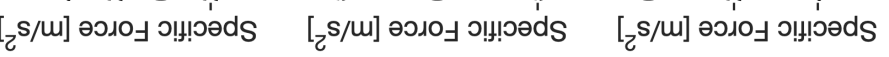

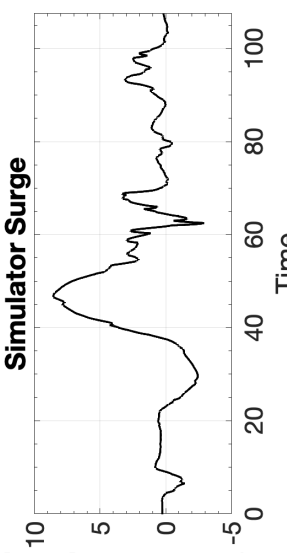

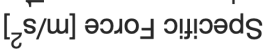

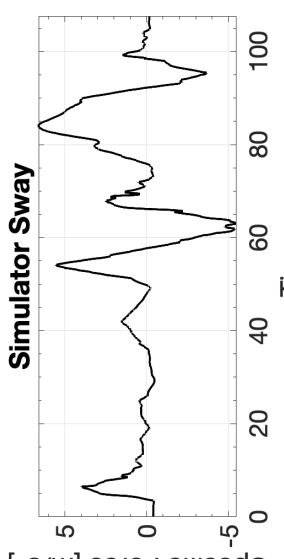

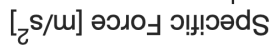

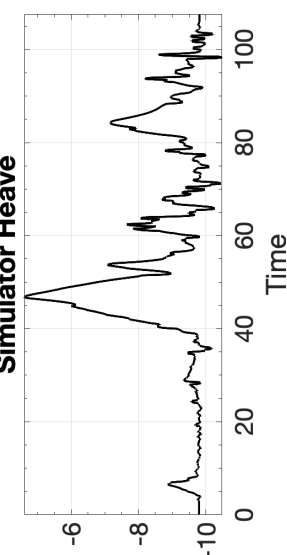

$[\mathrm{z} / \mathrm{s} / \mathrm{m}$ әอง

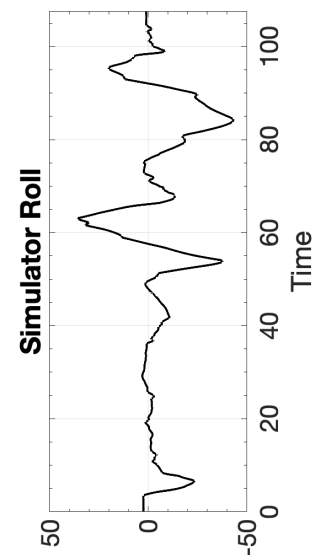

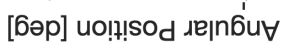

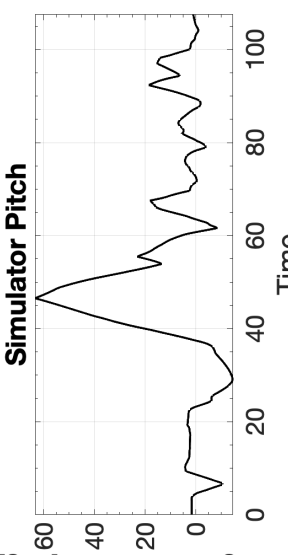

[6əp] uo!!! sod גeןn6u

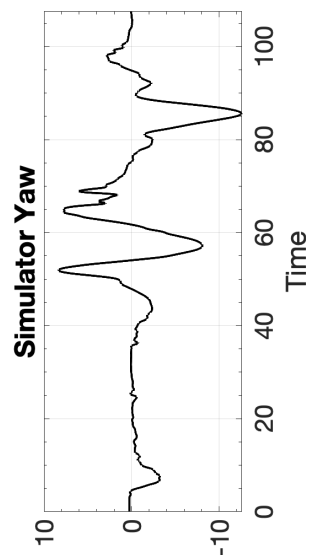

[6әр] uo!!!

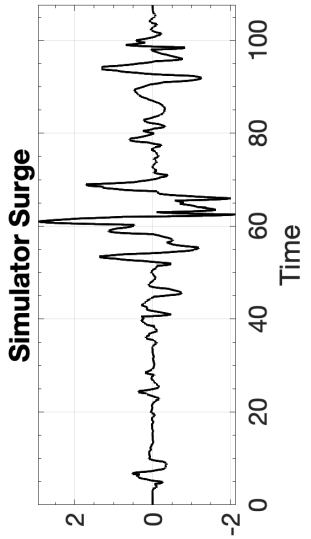

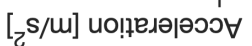

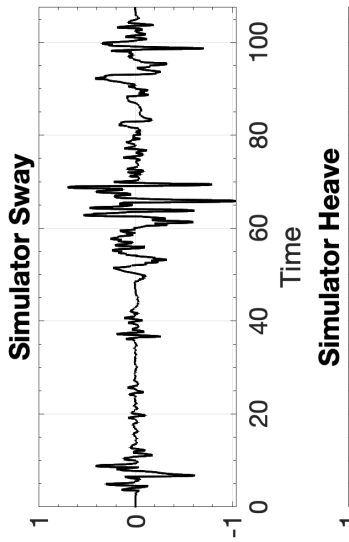

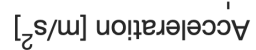

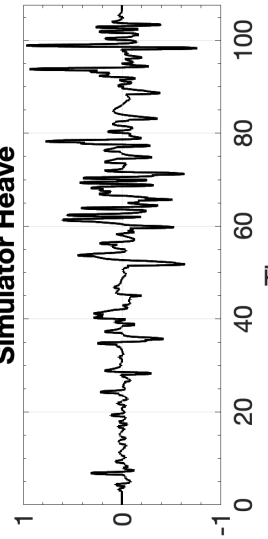

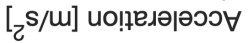

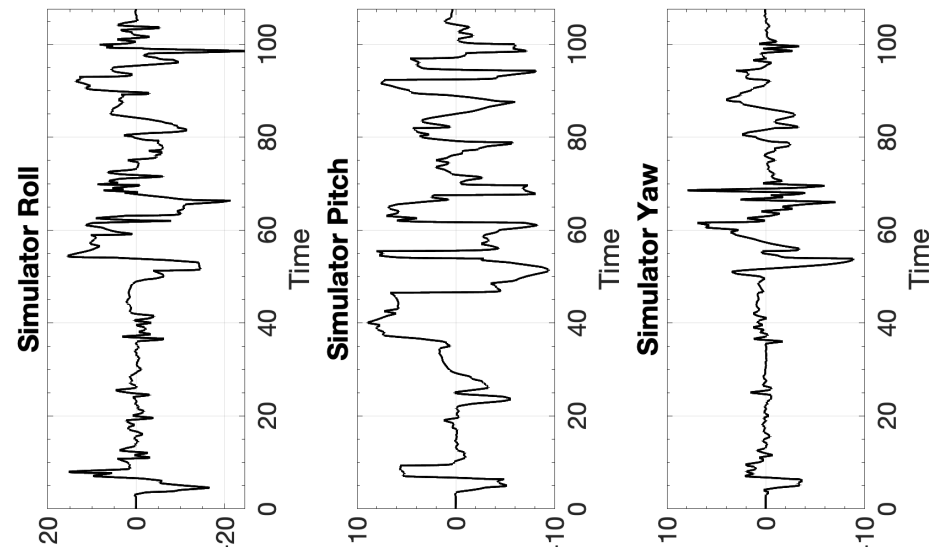

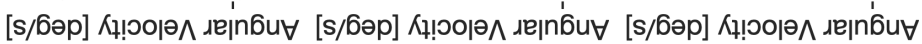




\section{B.14.2 CW2 Columbia 400 Aerobatics}

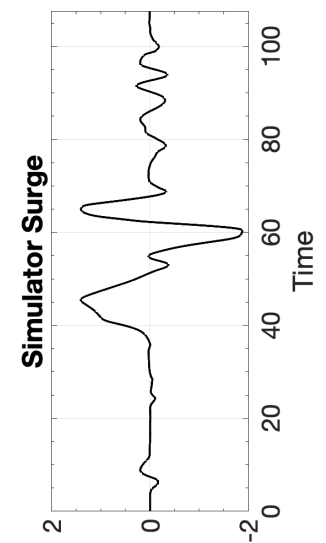

[w] uo!!!sod

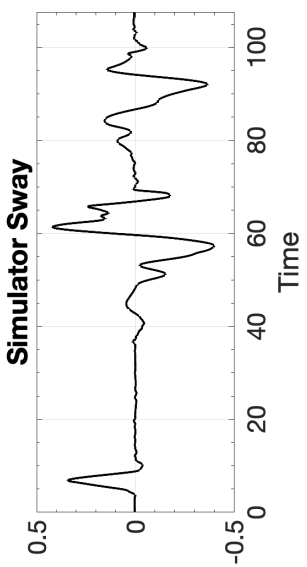

[w] uo!n!sod

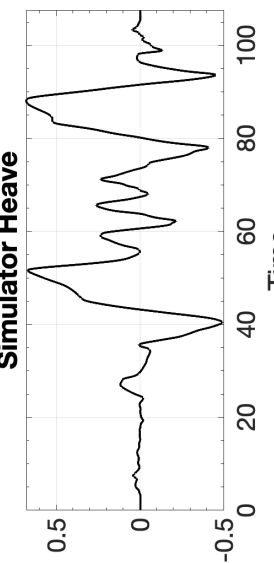

[w] uo!n!sod
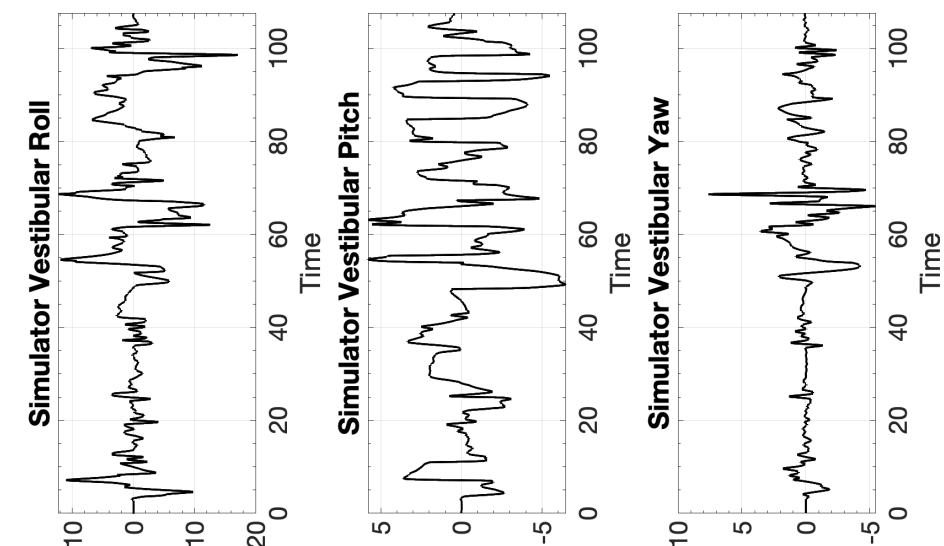

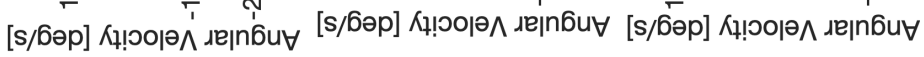

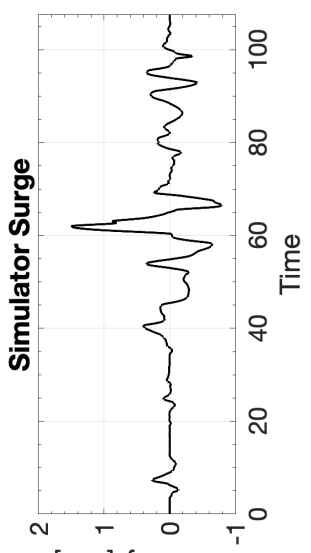

[s/u] Кұ!०о|ә

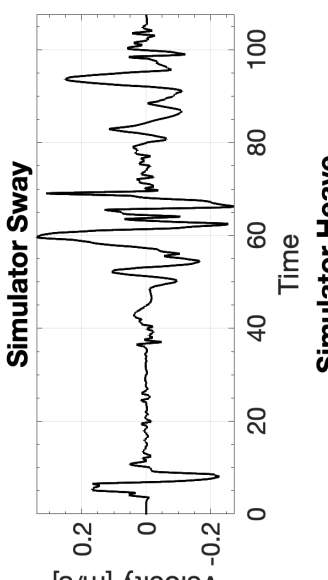

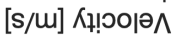

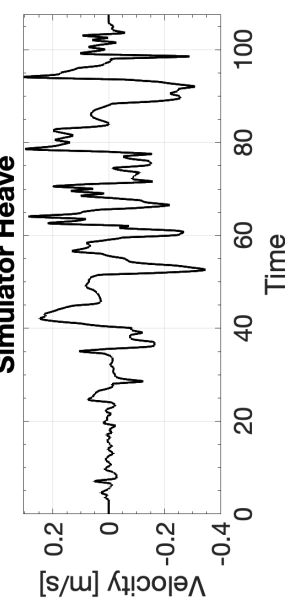

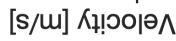
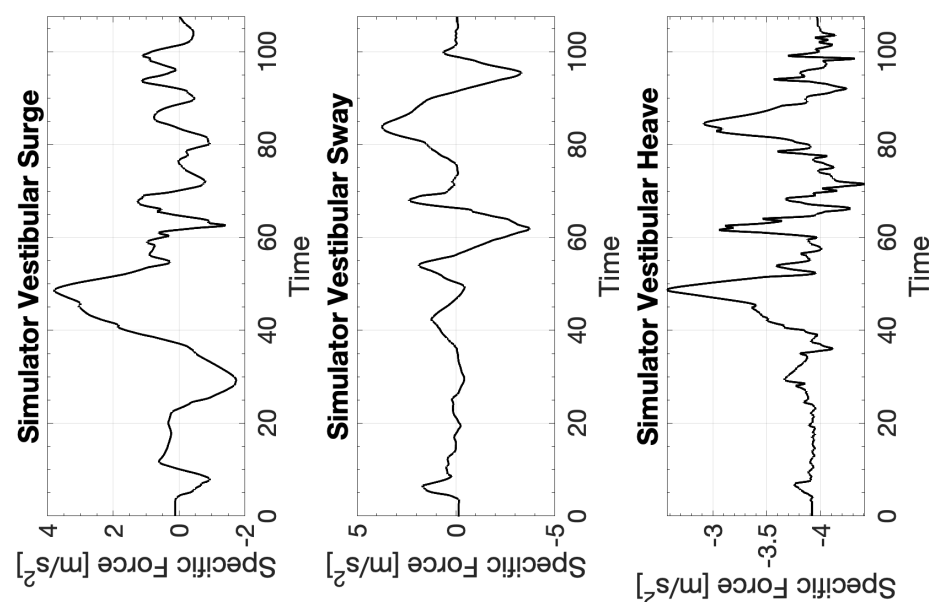

$[\mathrm{s} / \mathrm{s}]$ ә ә

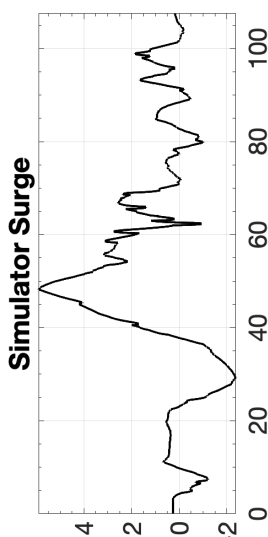

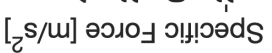

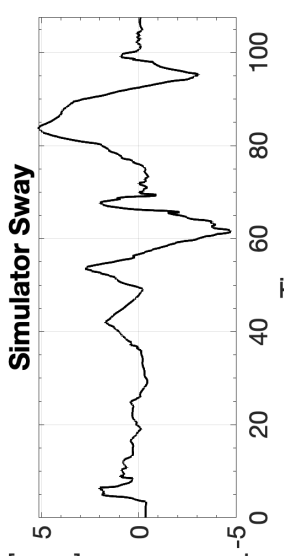

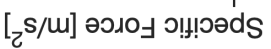

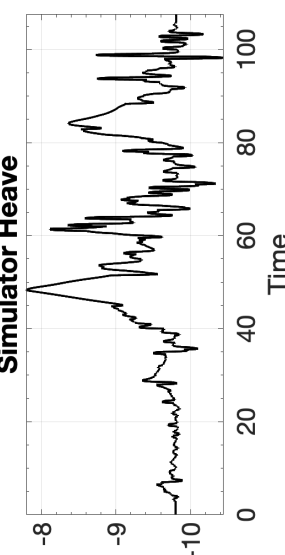

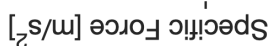

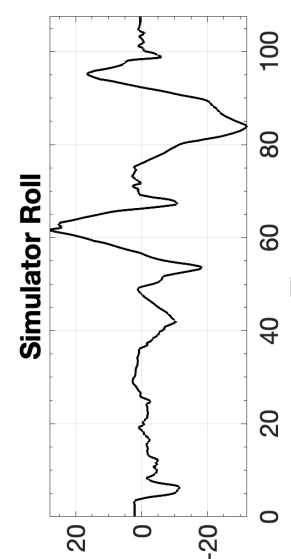

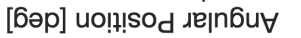
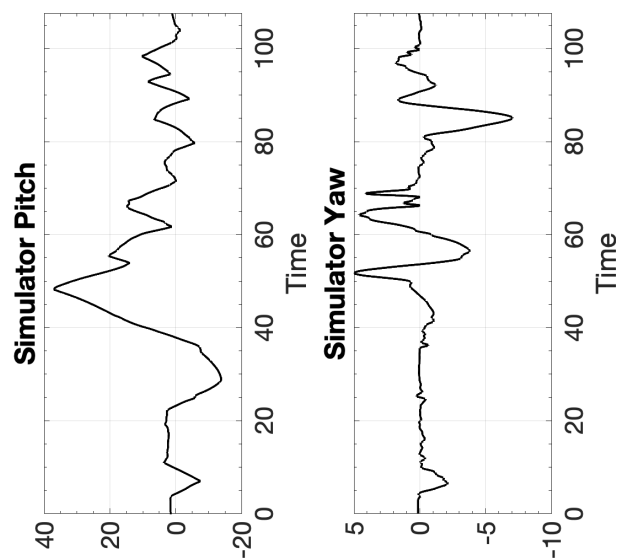

[бəр] uo!!!

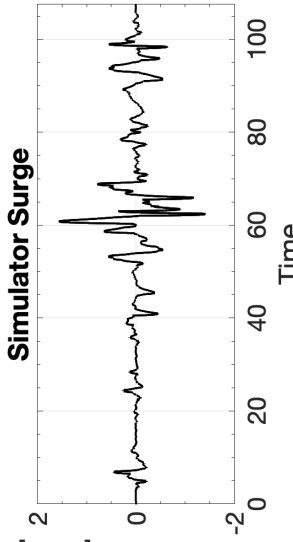

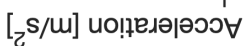

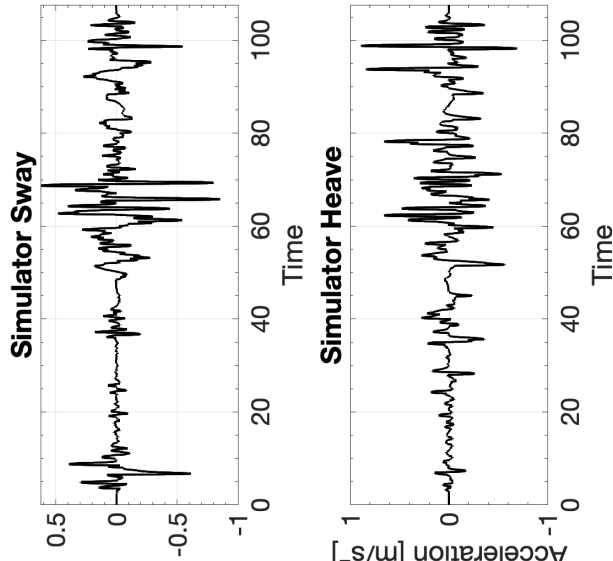

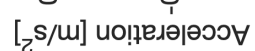
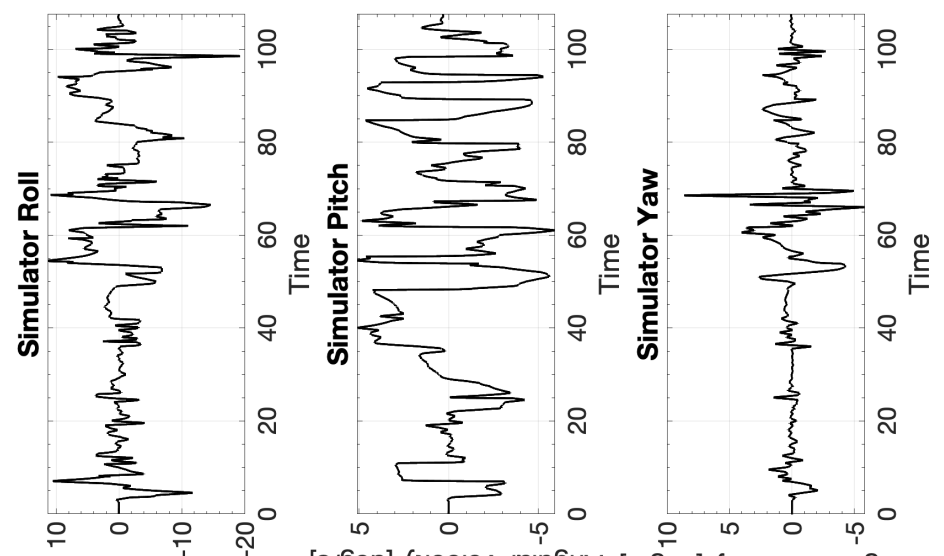

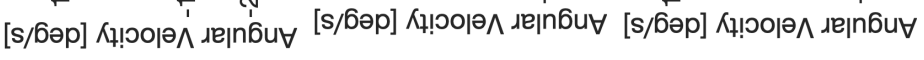




\section{B.14.3 CW3 Columbia 400 Aerobatics}

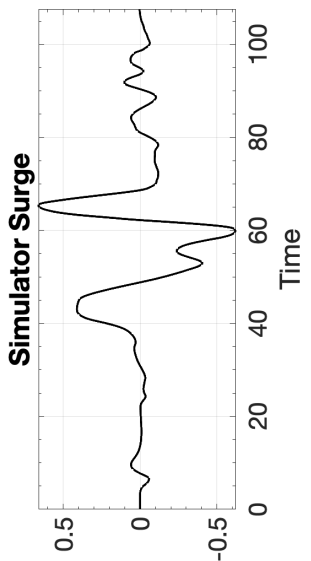

[w] uol!!sod

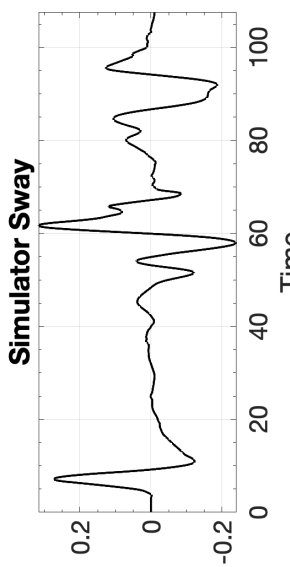

[w] uo!n!sod

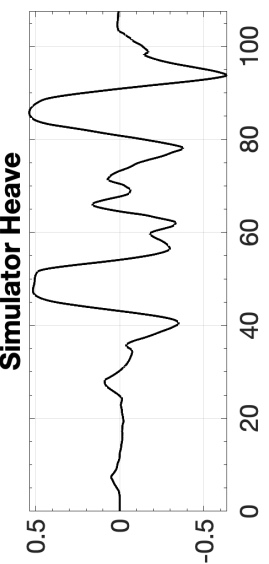

[w] uo!!!!sod
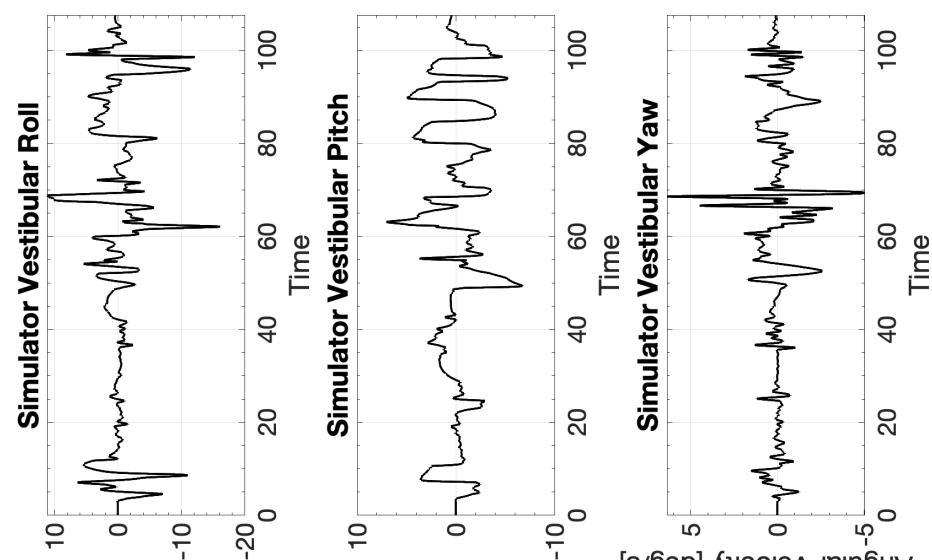

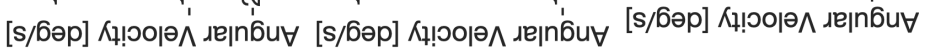

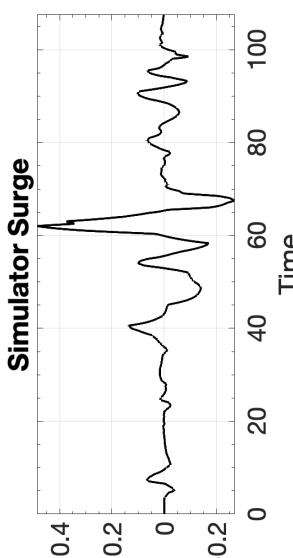

[s/u] Кұ!००|ә^

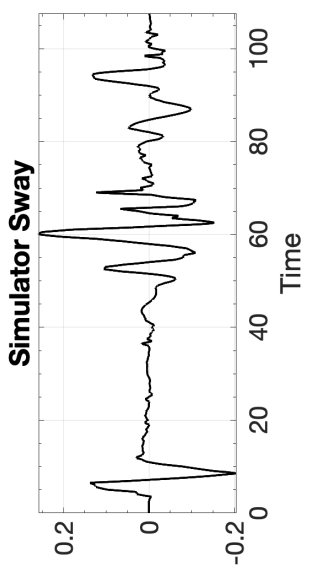

[s/u] Кұ!৩о|ә

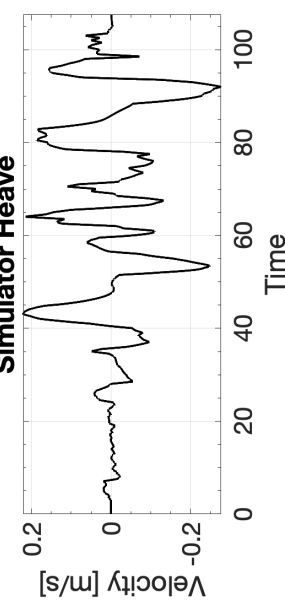

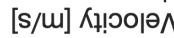

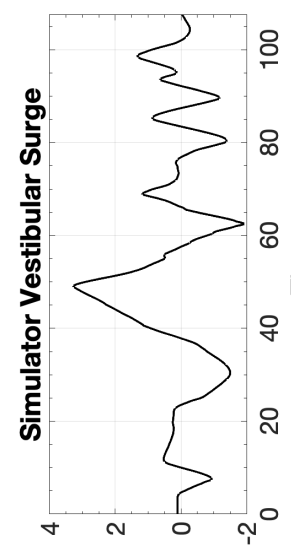

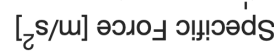

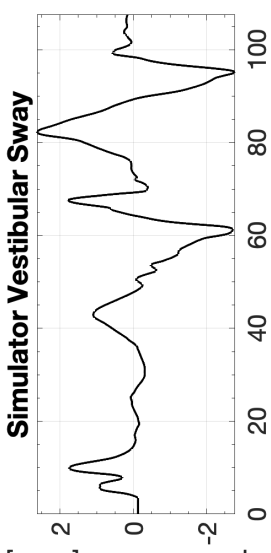

$[\mathrm{c} / \mathrm{s} / \mathrm{m}$ ә

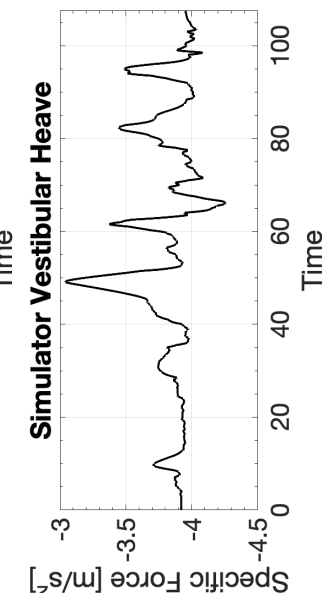

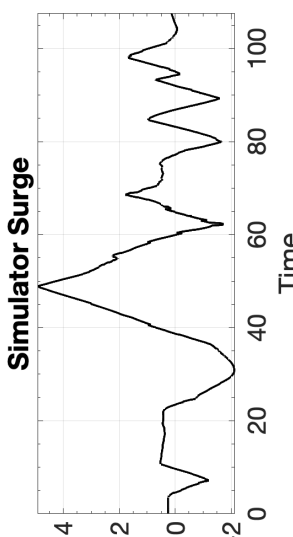

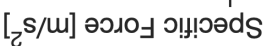

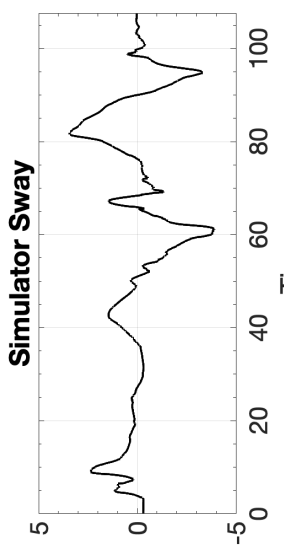

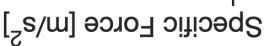

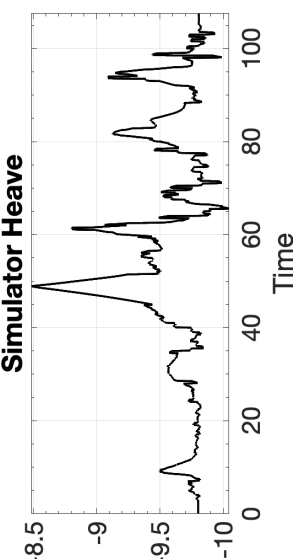

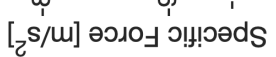

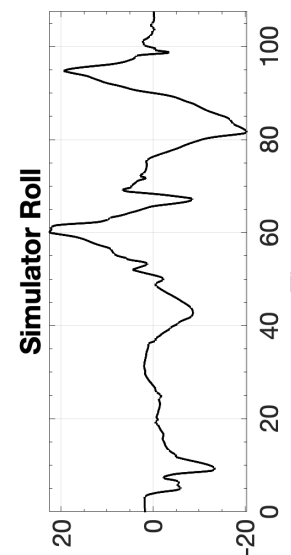

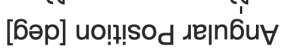

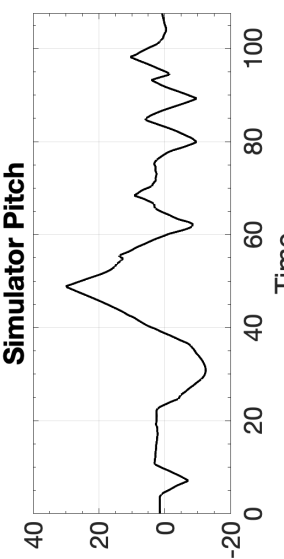

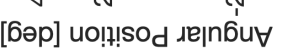

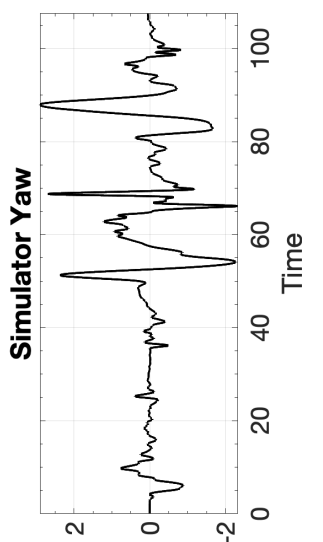

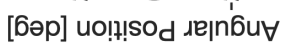

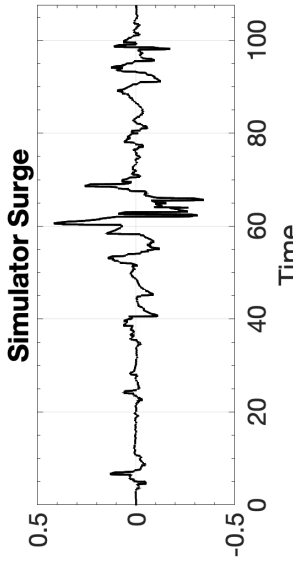

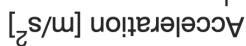

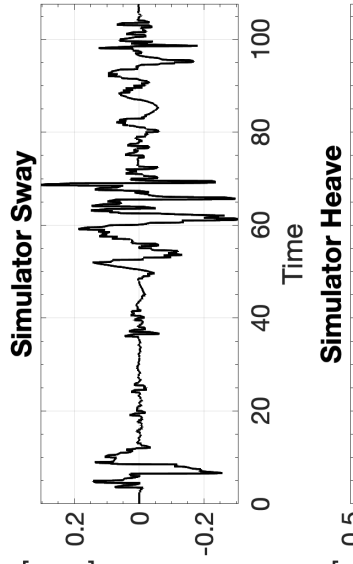

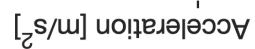

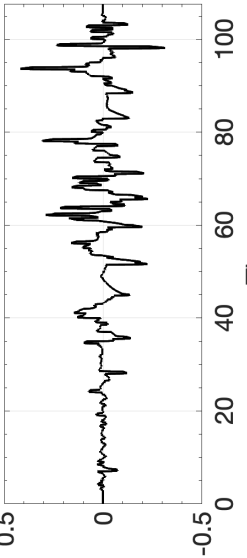

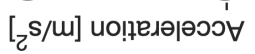
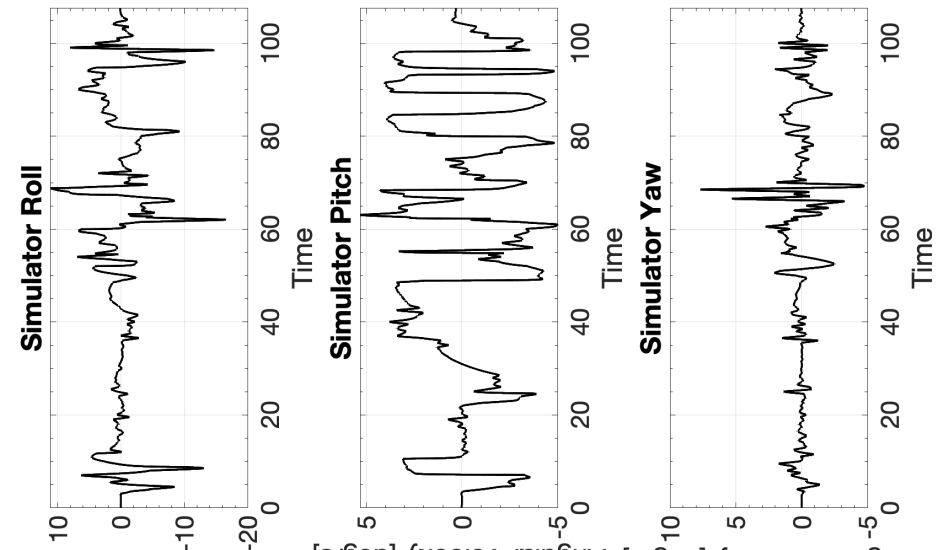

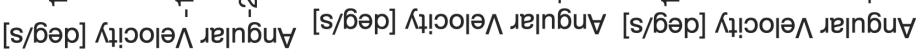




\section{B.15 Columbia 400 Coordinated Turn}
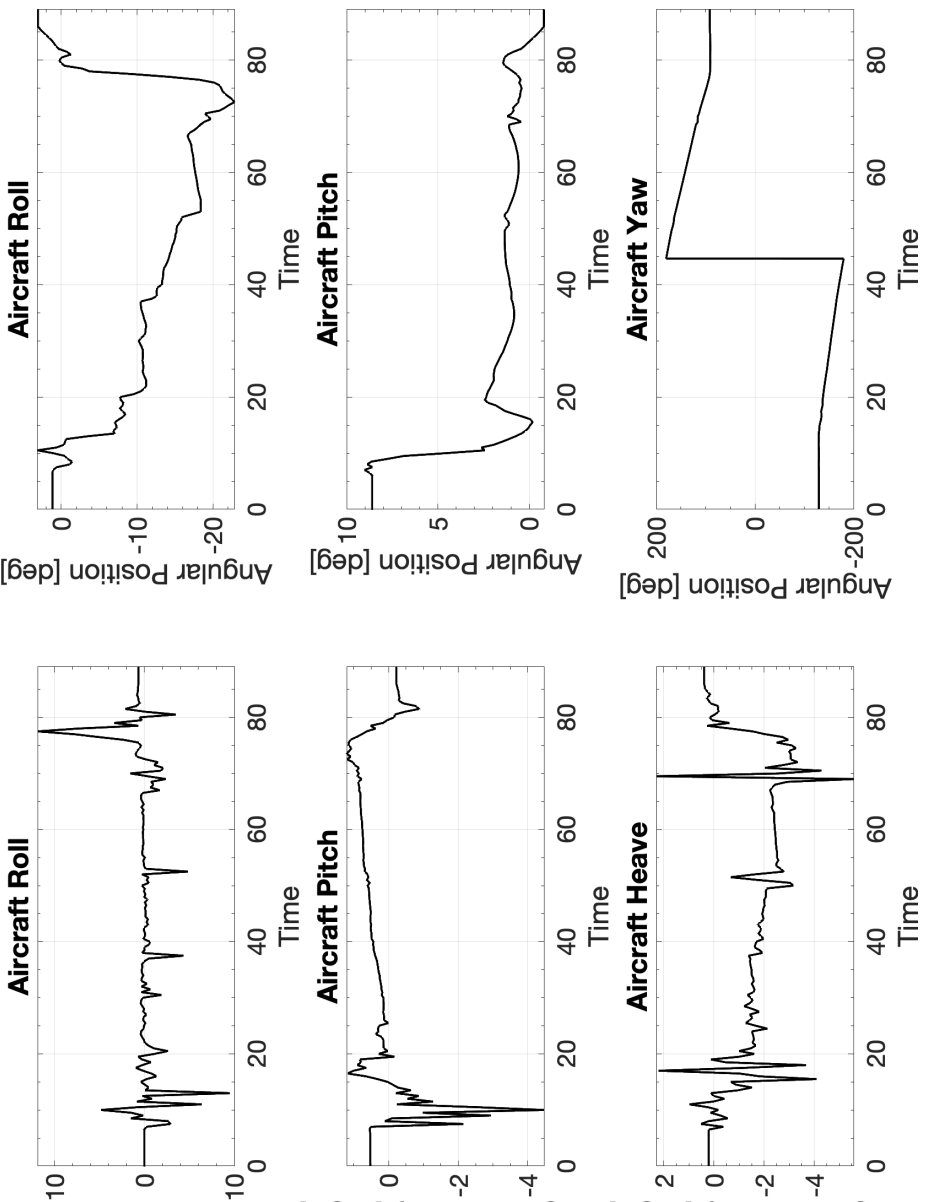

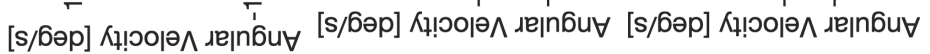
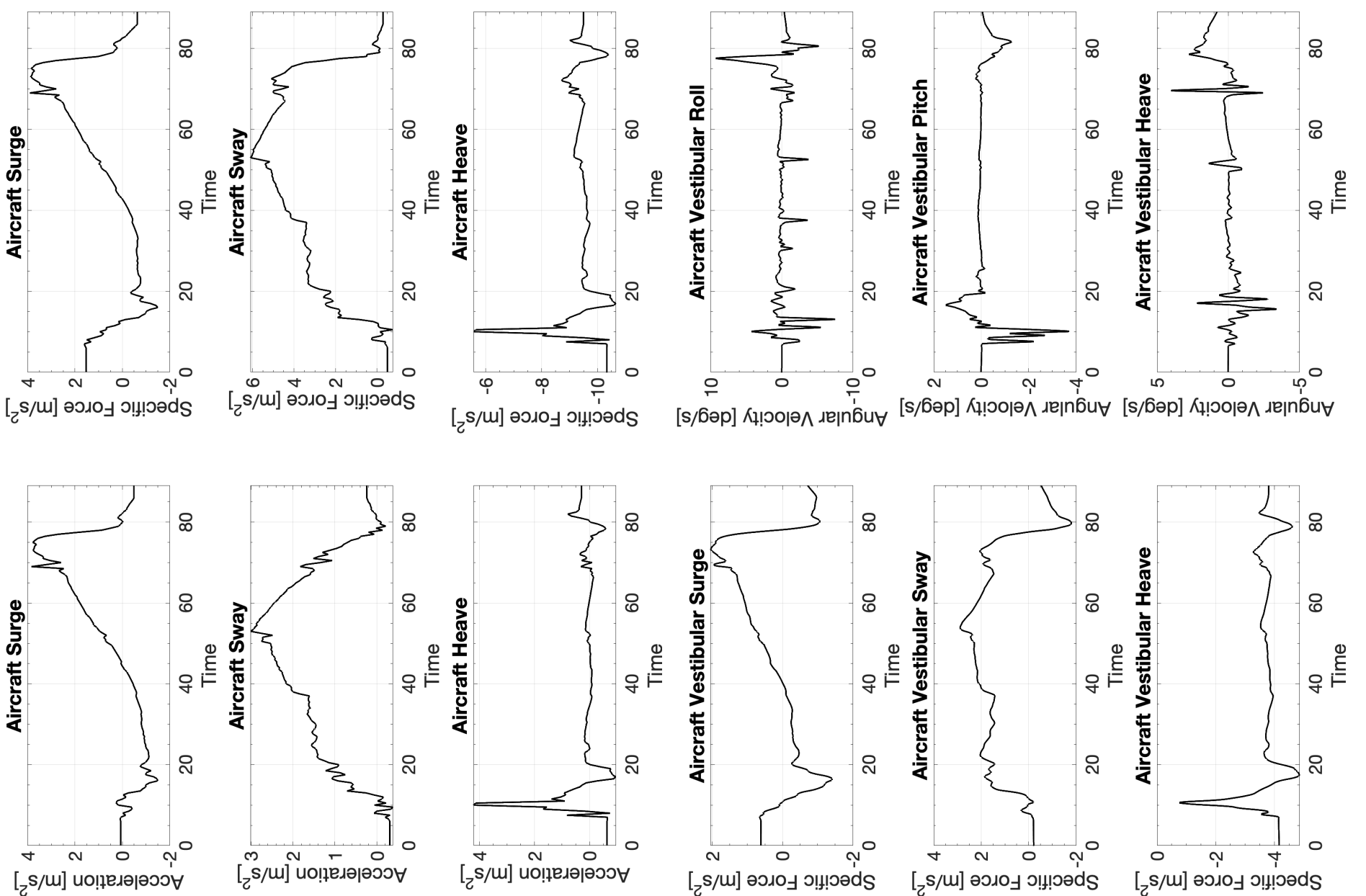

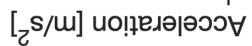

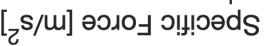

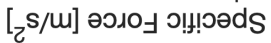

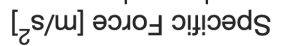




\section{B.15.1 CW1 Columbia 400 Coordinated Turn}

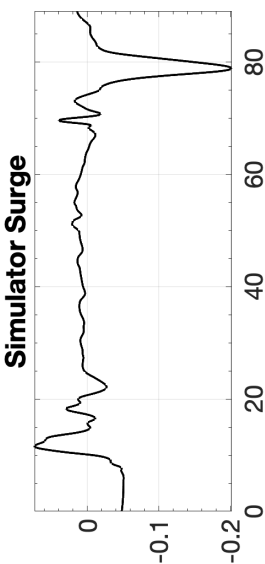

[u] uol!t!sod

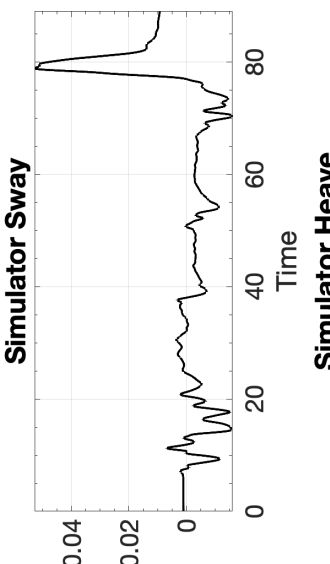

[u] uo!n!sod

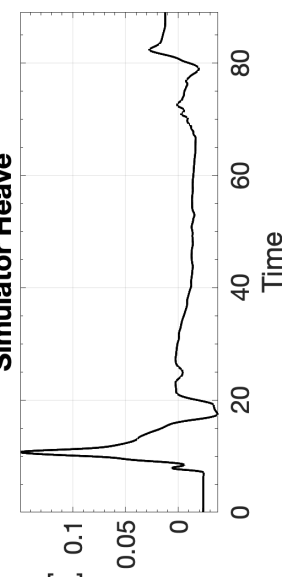

[u] uo!n!sod
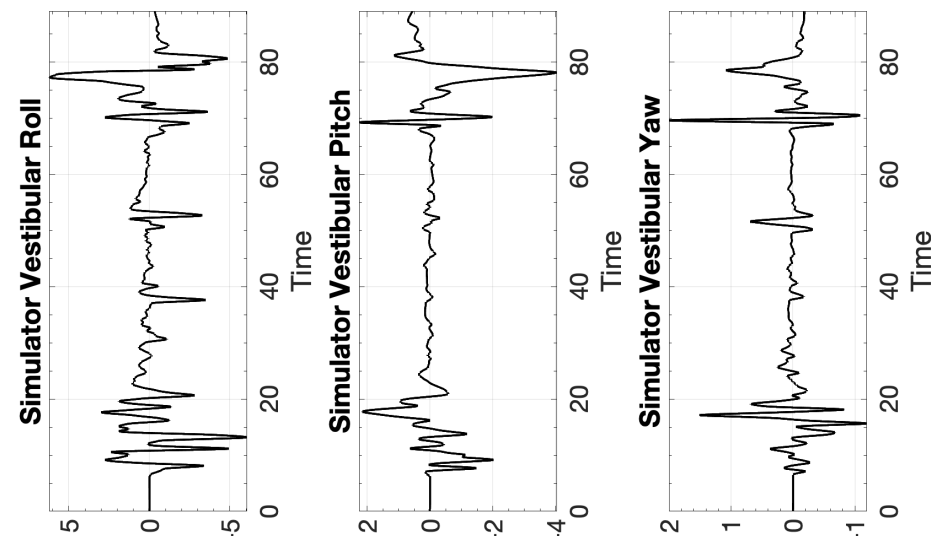

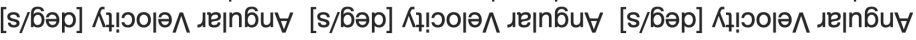

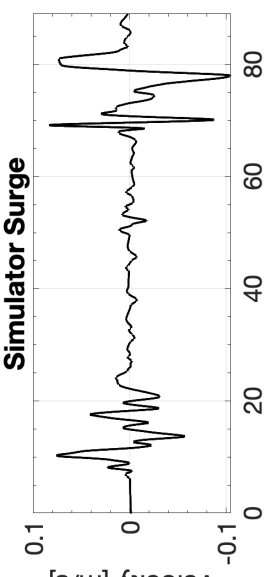

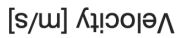

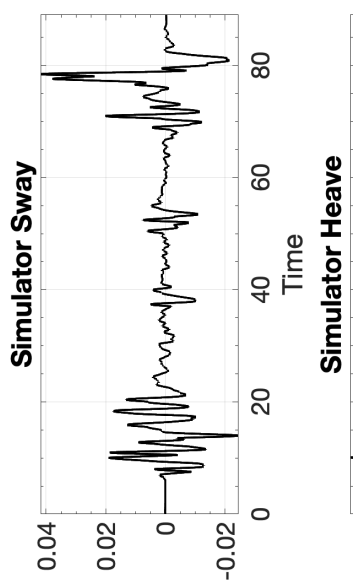

[s/m] Кұ!юор夫

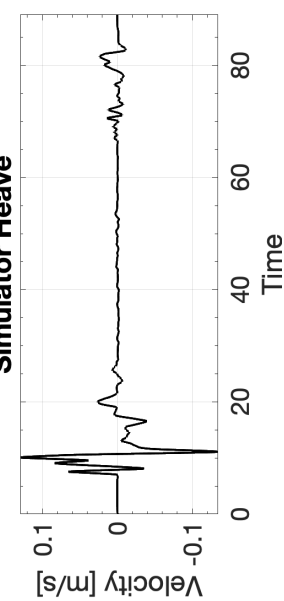

[s/u] Кұ!৩оюю

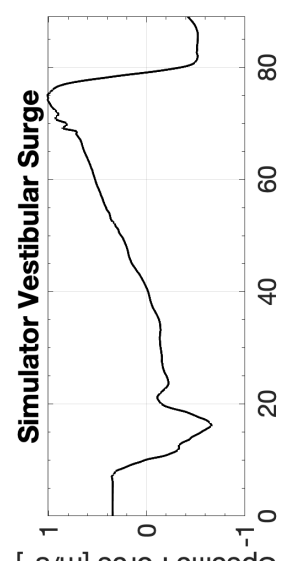

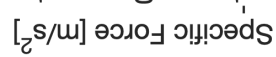

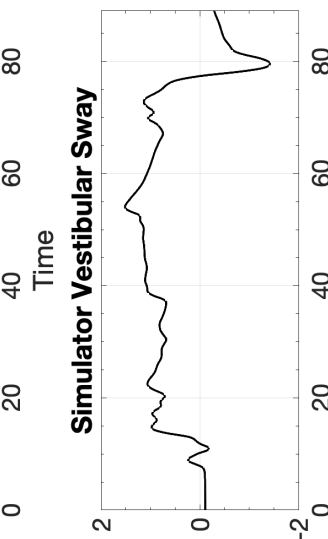

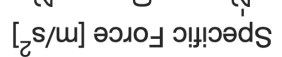

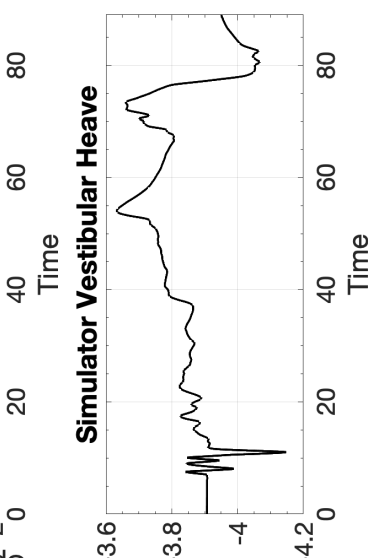

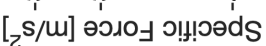

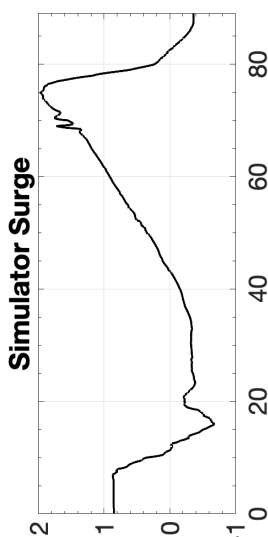

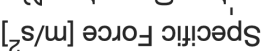

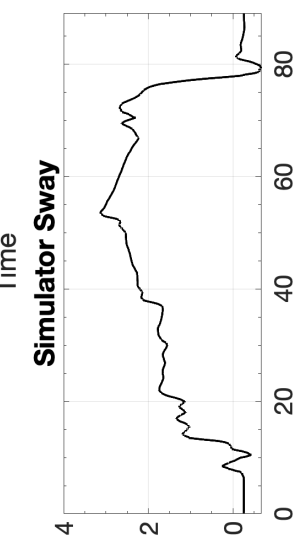

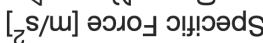

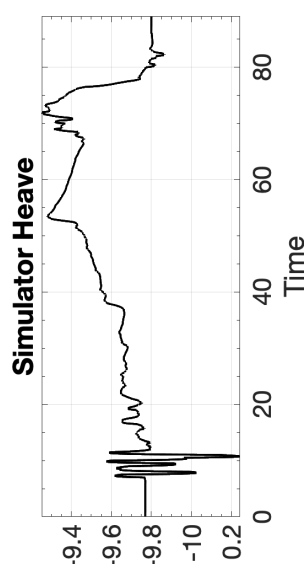

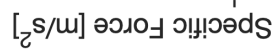

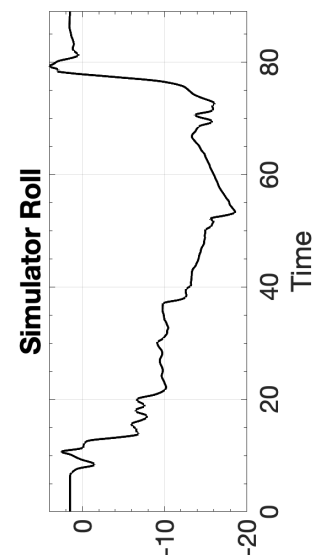

[бәр] uo!̣!so'd

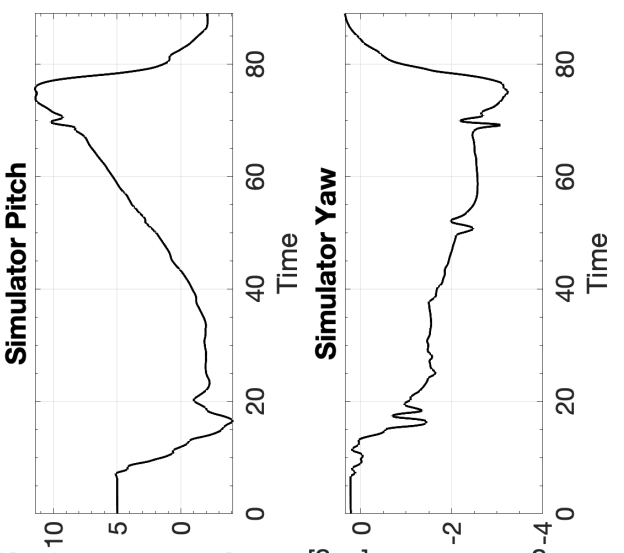

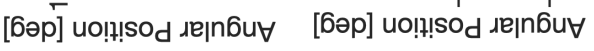

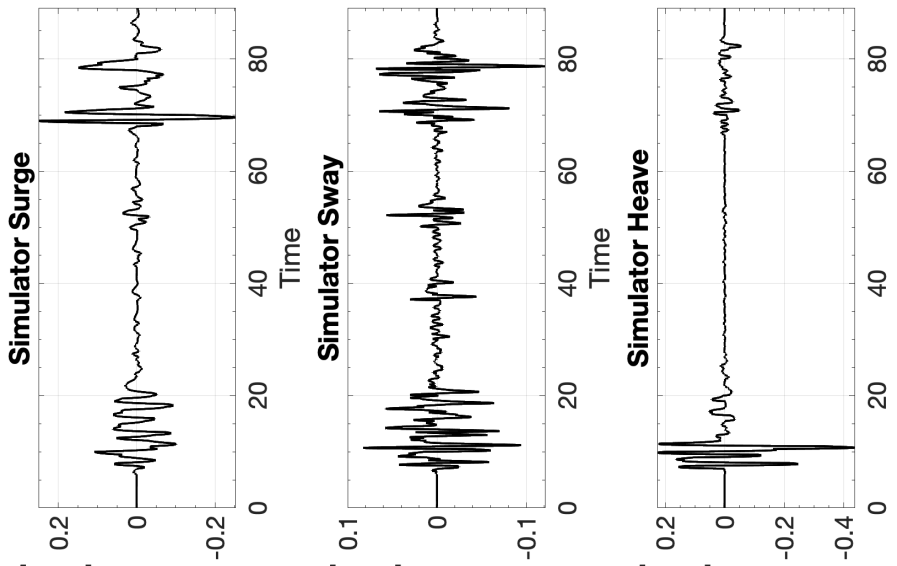

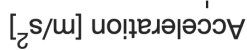

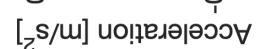
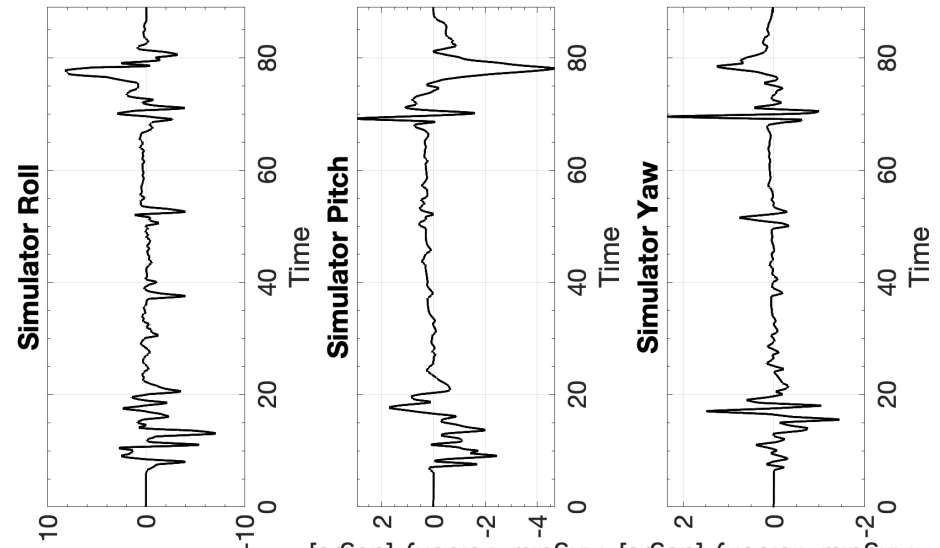

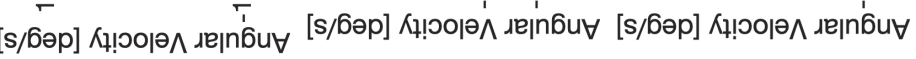




\section{B.15.2 CW2 Columbia 400 Coordinated Turn}

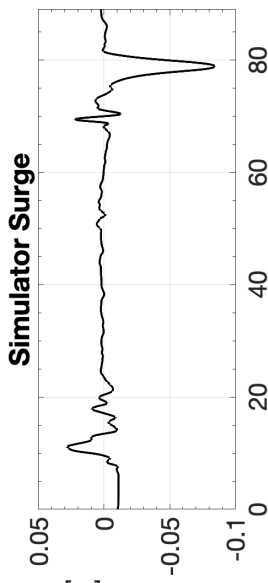

[w] uo!n! $\mathrm{SO}_{\mathrm{d}}$

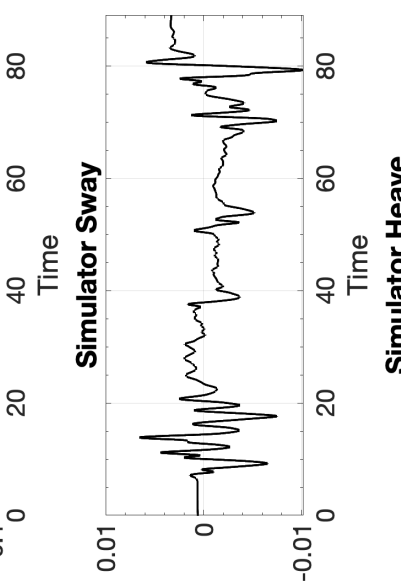

[u] uol!!!sod

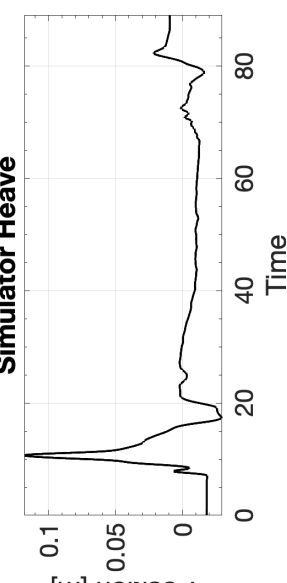

[w] uo!n!sod

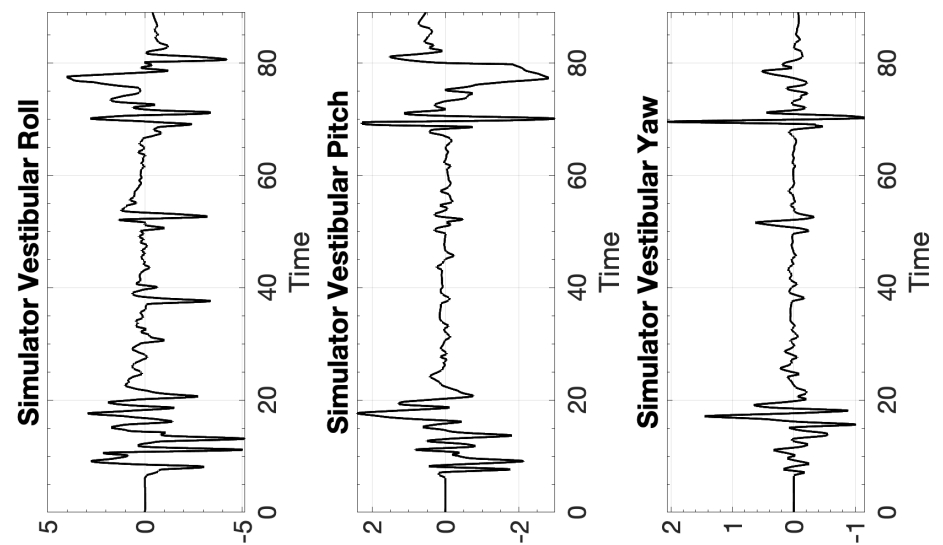

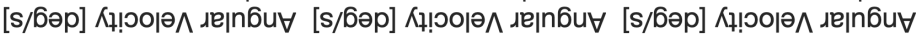

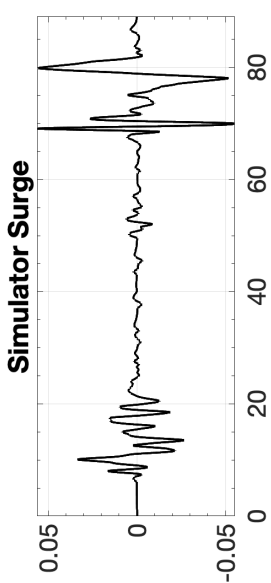

[s/u] ᄉң!юојә^

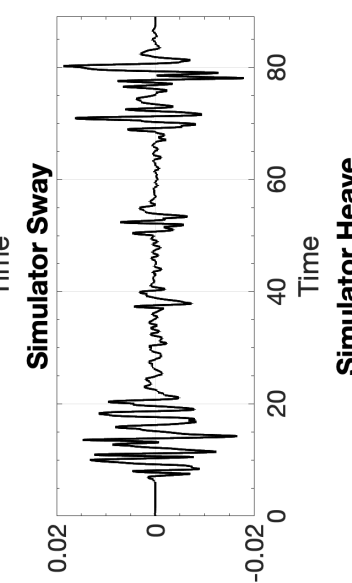

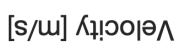

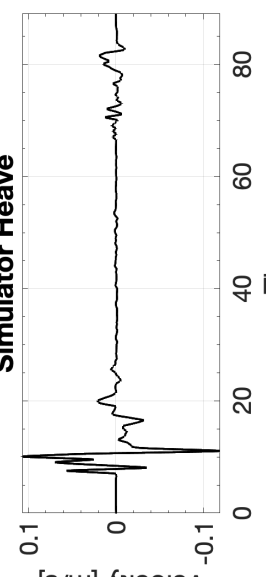

[s/u] Кұ!юою^

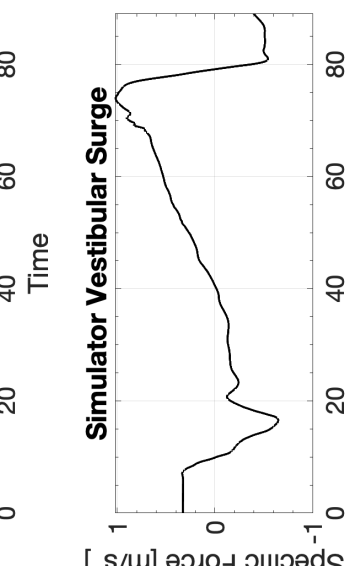

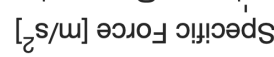

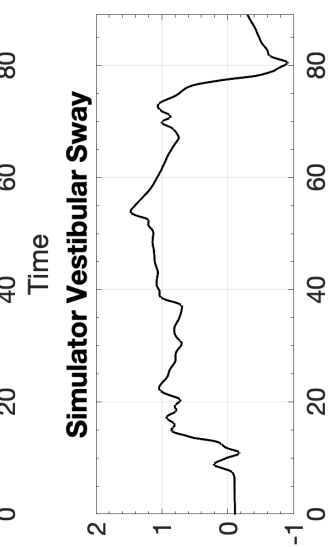

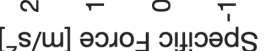

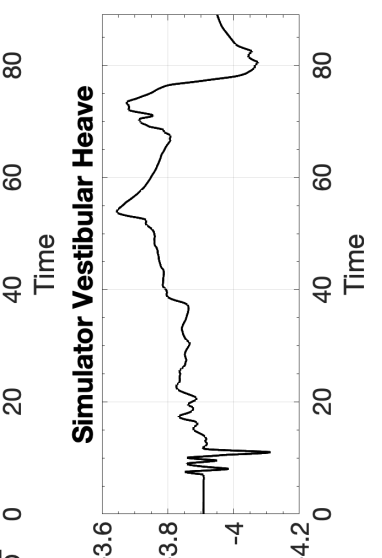

[

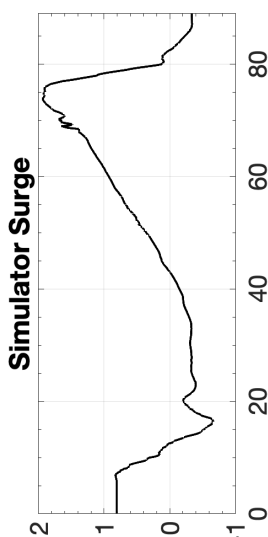

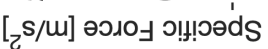

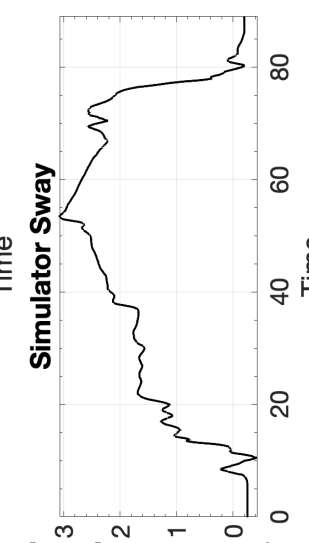

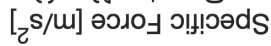
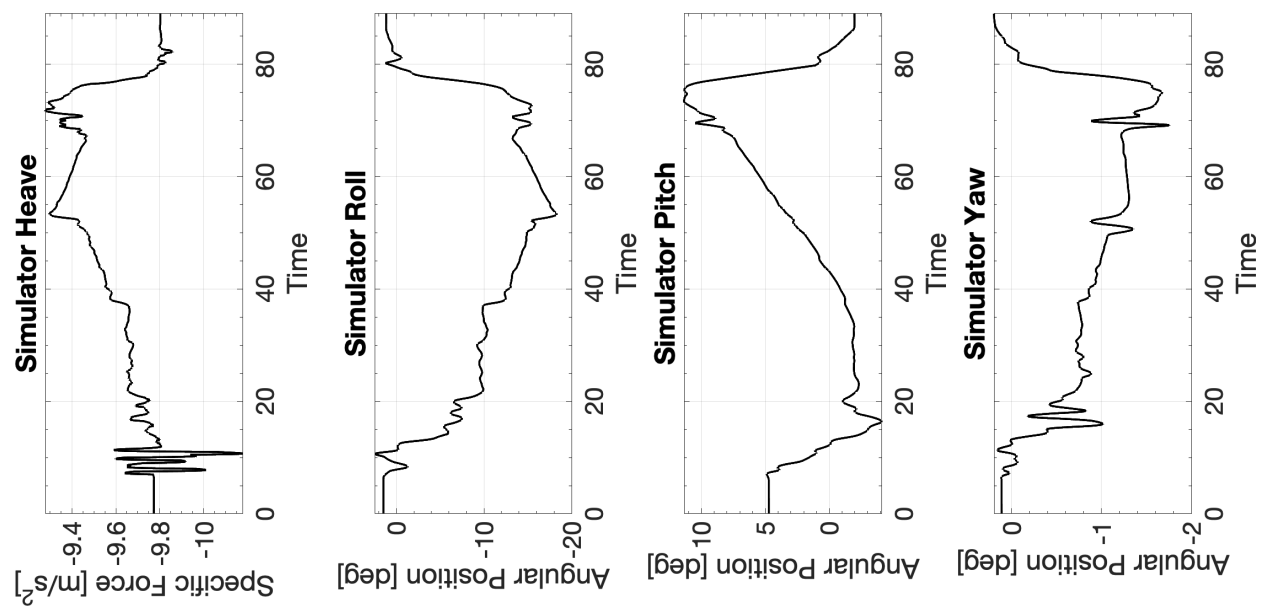

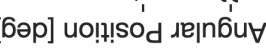

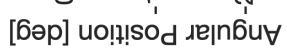

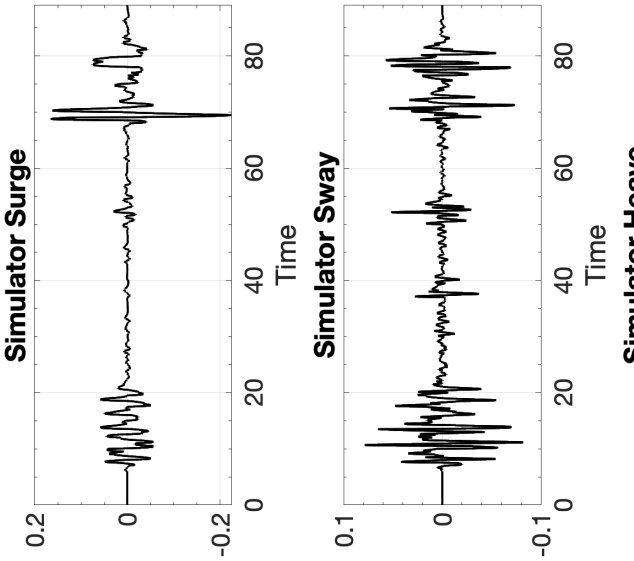

[z/m] иопฺеләрәээ

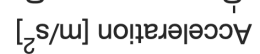

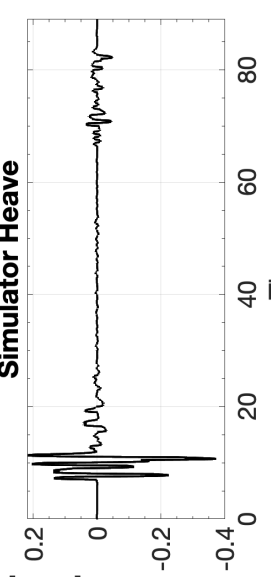

[z $s / m]$ uо!ฺедәәәоэ

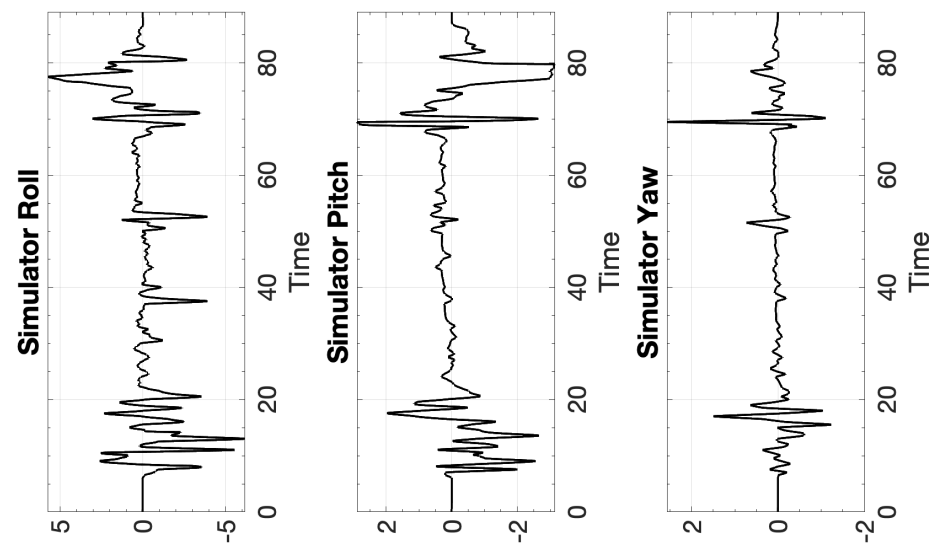

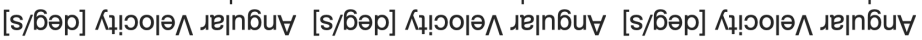




\section{B.15.3 CW3 Columbia 400 Coordinated Turn}

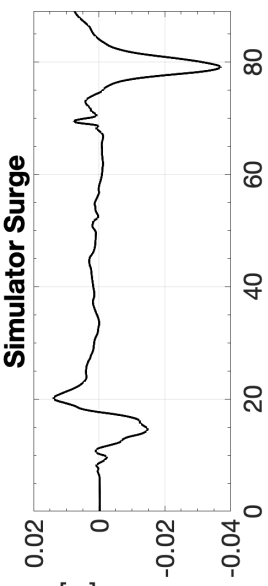

[u] uo!n!sod

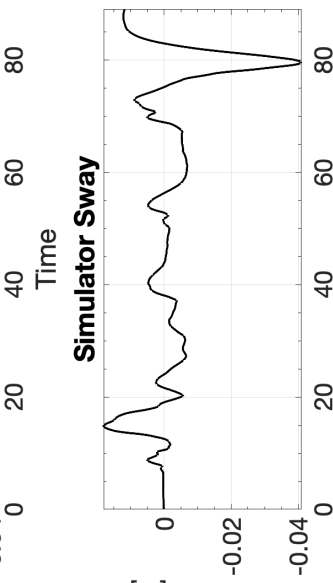

[u] uo!n!sod

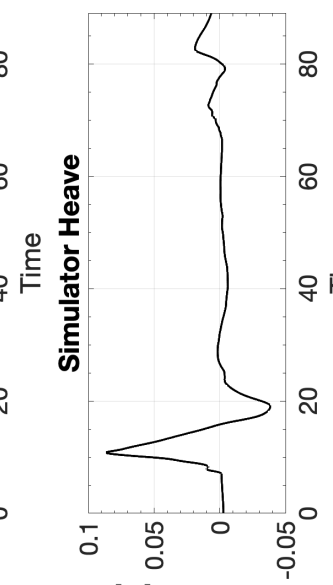

[w] uol!!sod

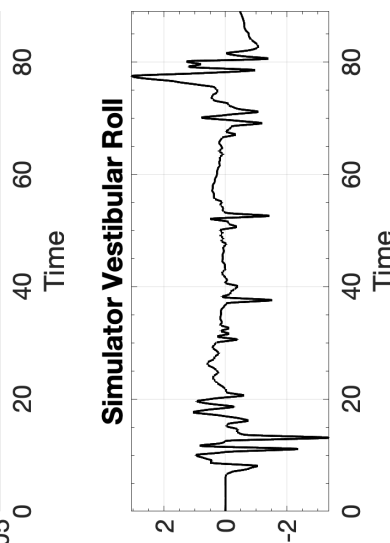

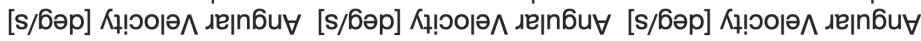
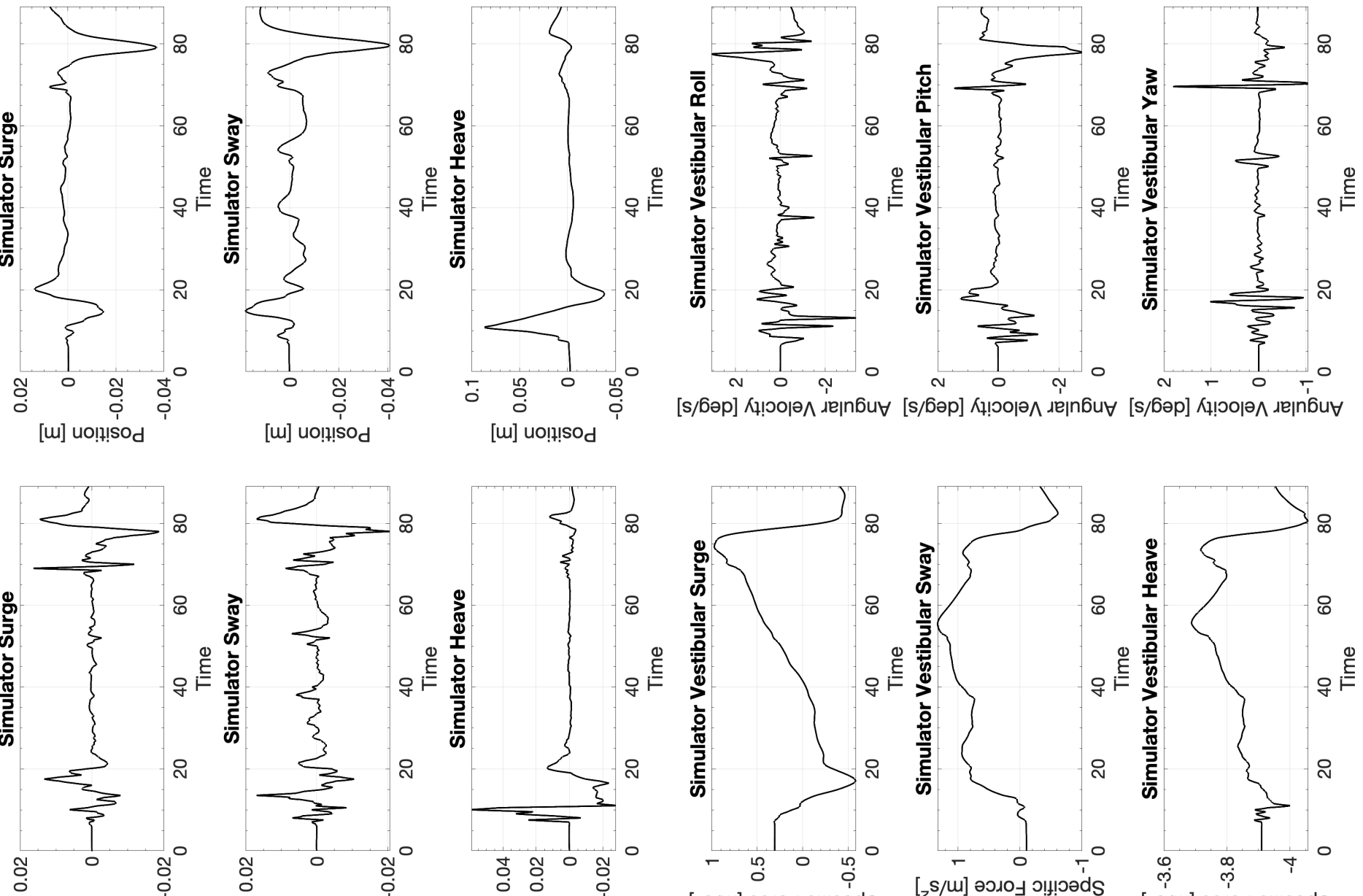

[s/u] Кц!оојә

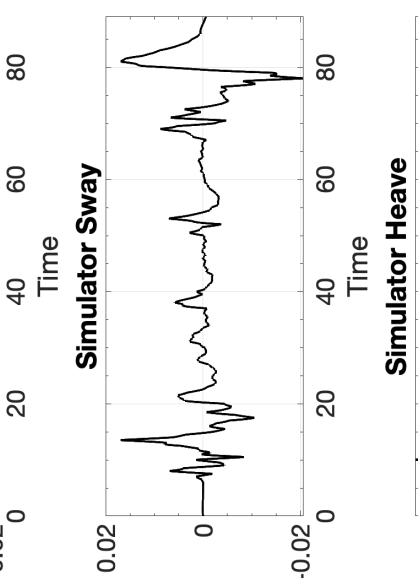

[s/m] Кџ!วо夫

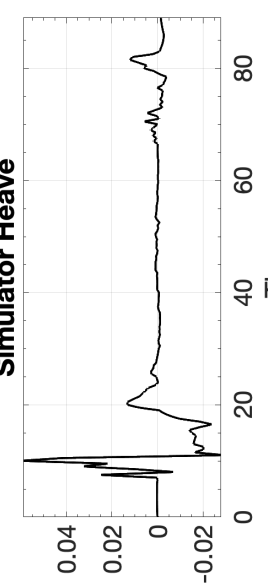

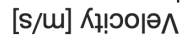

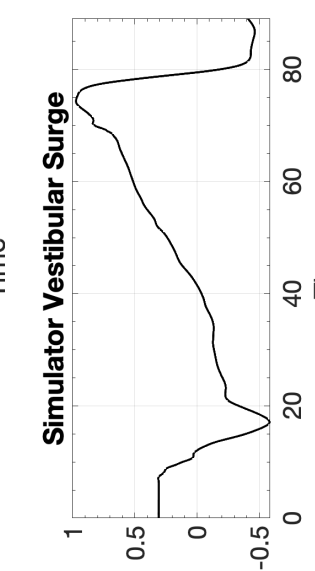

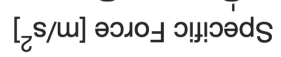

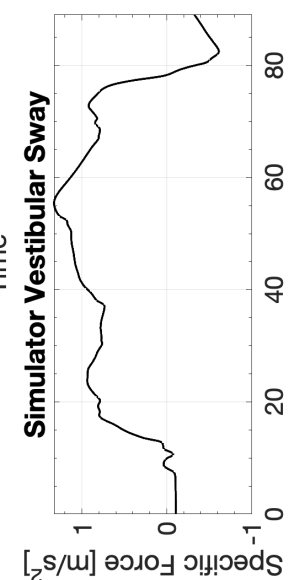

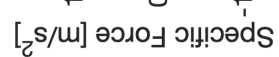

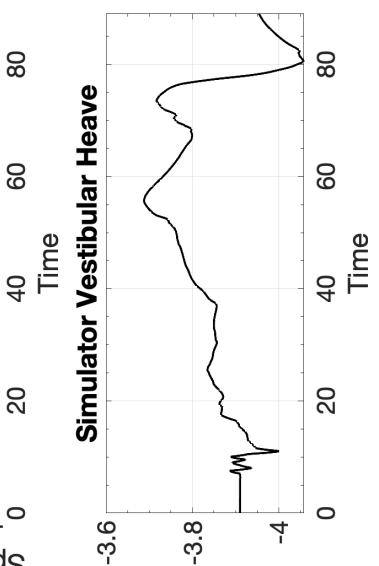

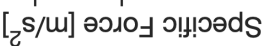

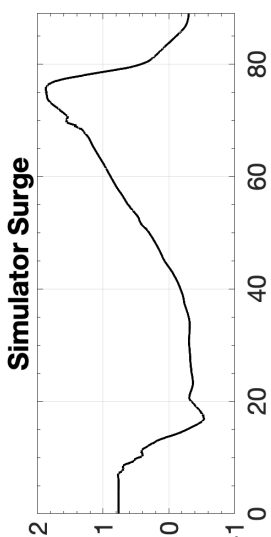

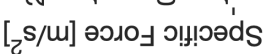

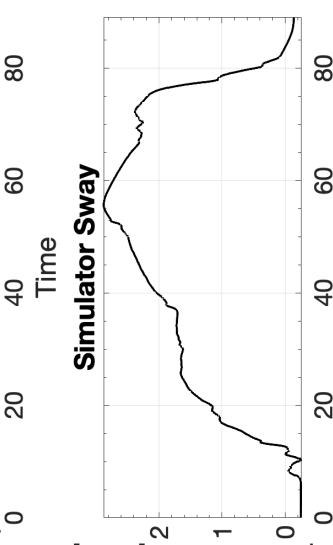

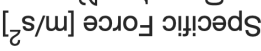

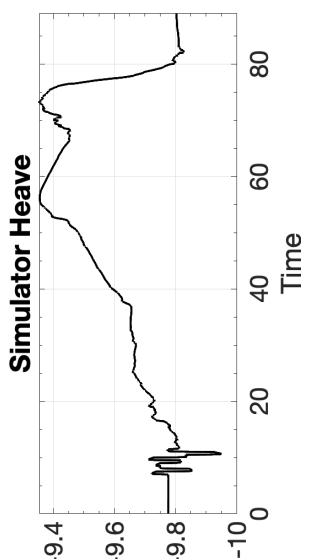

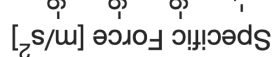

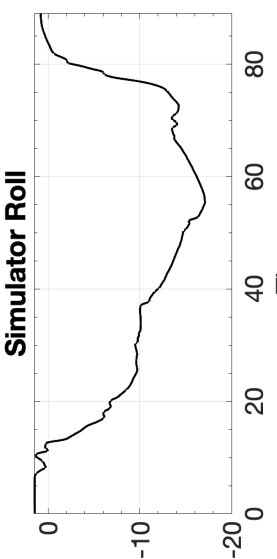

[бəp] uo!!!

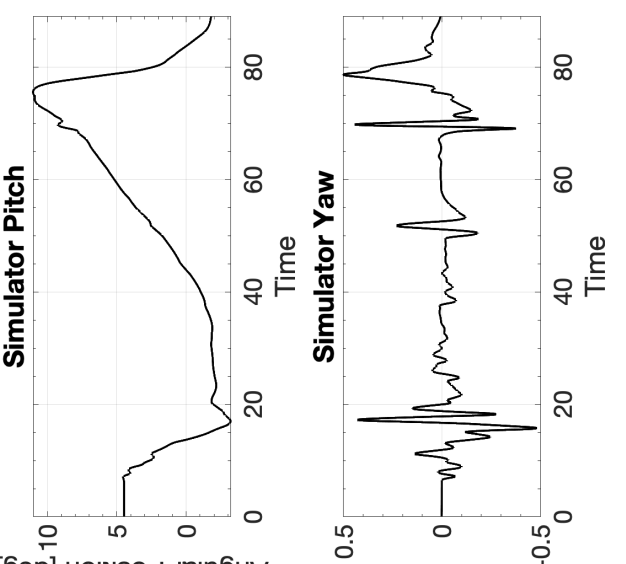

[6əp] uo!!!sod גejn6u

[бәр] uo!!!sod

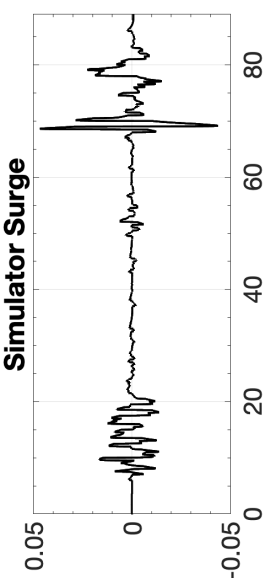

[zs/u] uо!ฺвләәәоэ

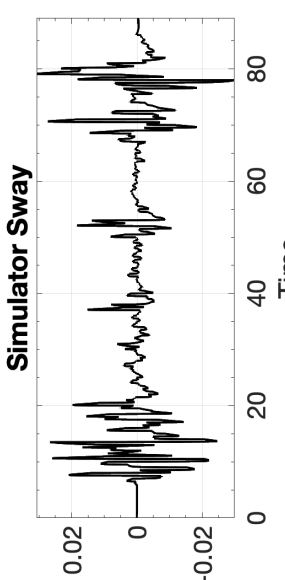

[z/m] ио!ฺедәәәэว

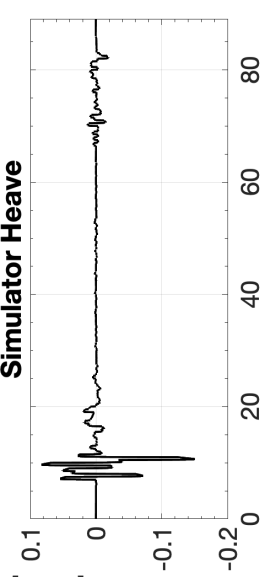

[z $s / m]$ uо!ฺедәәәэว

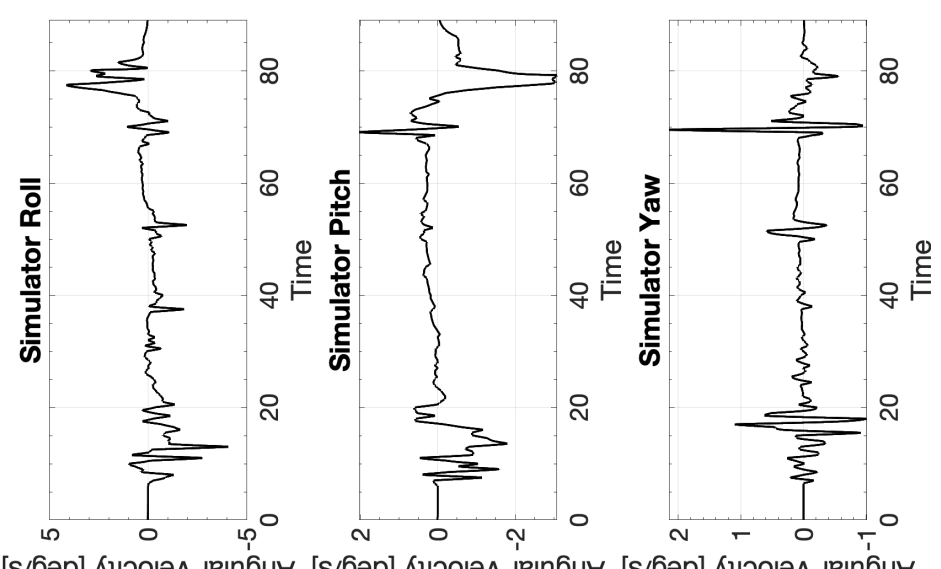

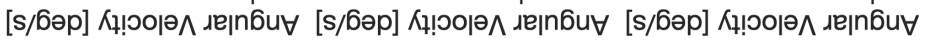




\section{B.16 Columbia 400 Cross-wind Landing}
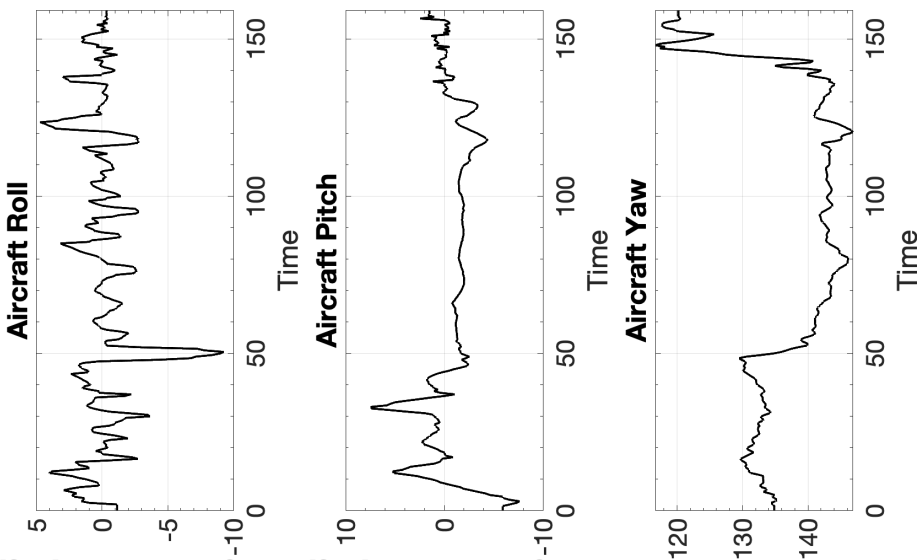

[6әр] uo!!!sod $\lambda$ גe|n6u

[бәр] uo!!!!sod גe|n6u

[бәр] uo!̣!!'sod dén6ü

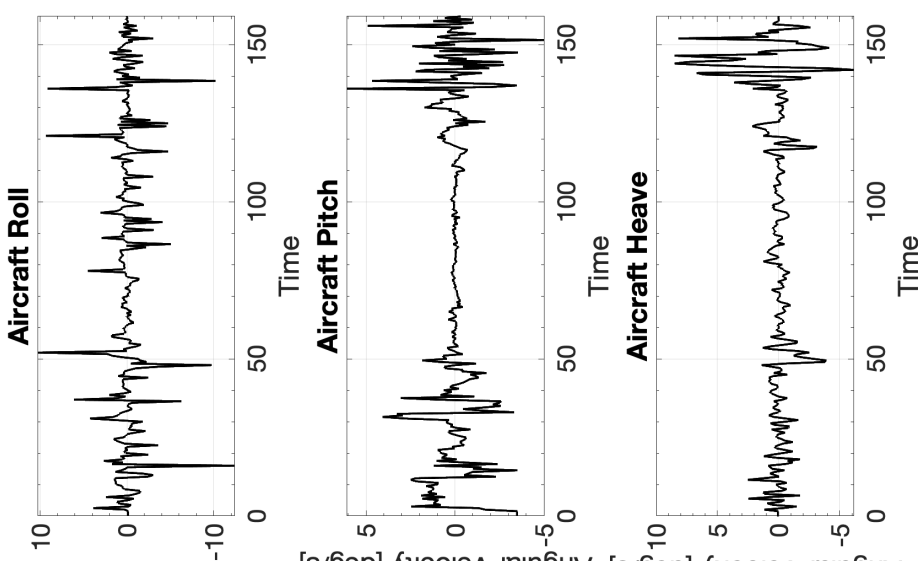

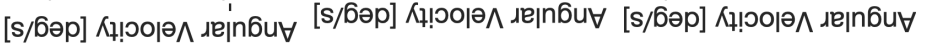

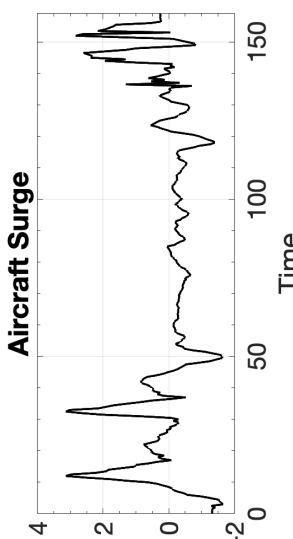

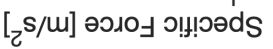

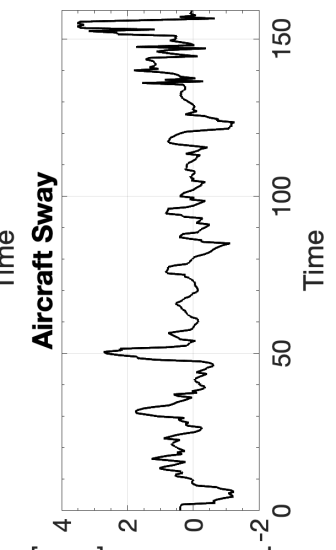

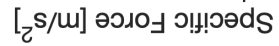
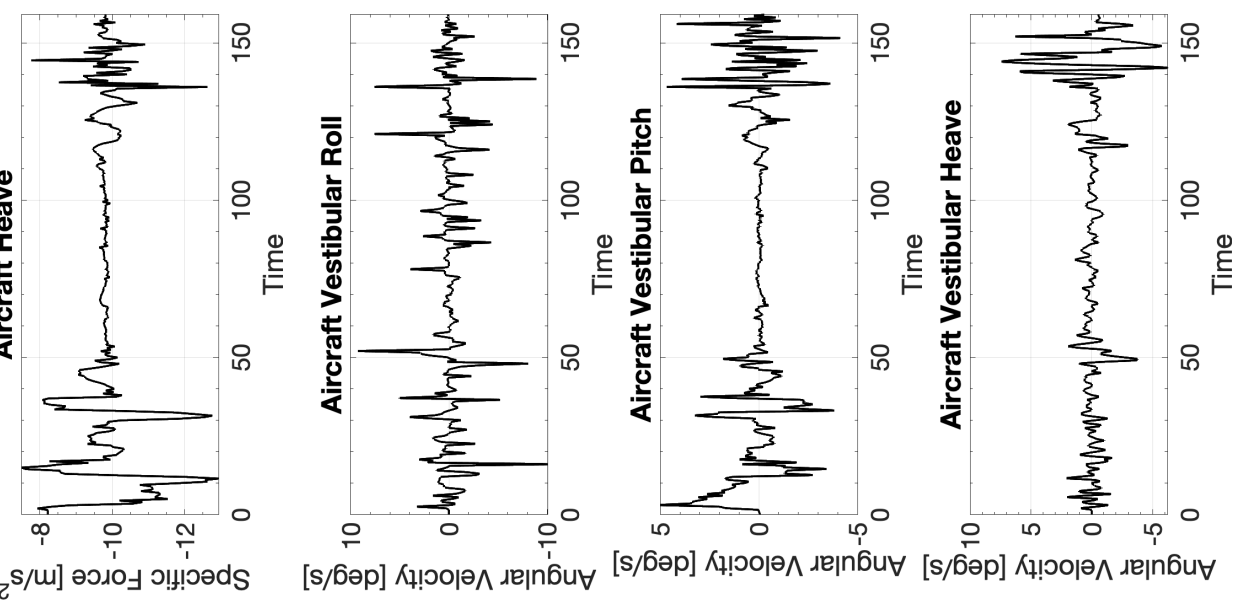

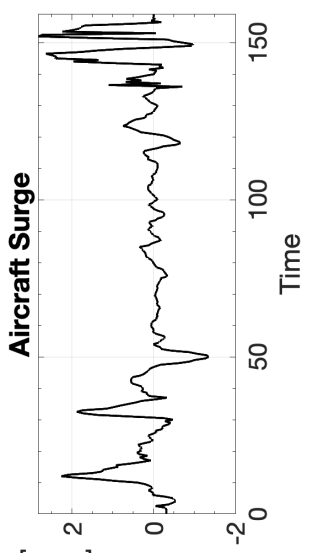

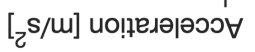

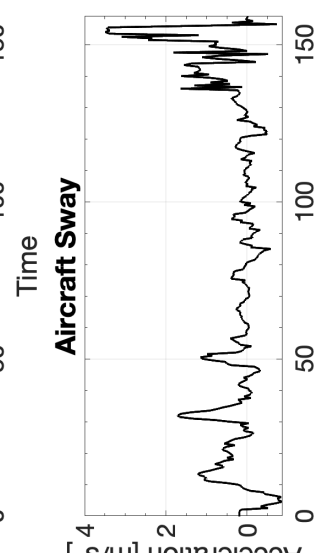

$[\mathrm{c} s / \mathrm{m}]$ นо!!ฺ

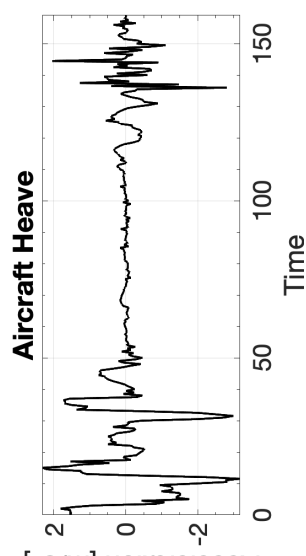

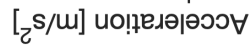

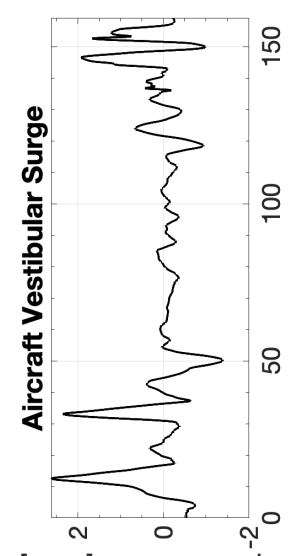

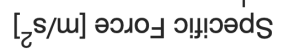

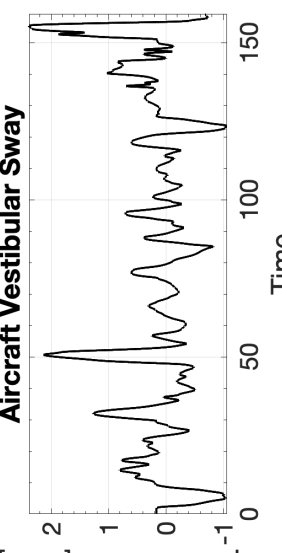

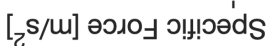

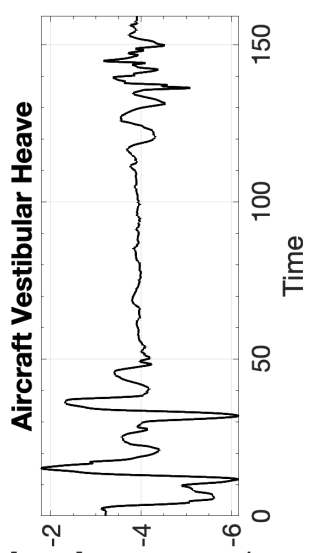

[ 


\section{B.16.1 CW1 Columbia 400 Cross-wind Landing}

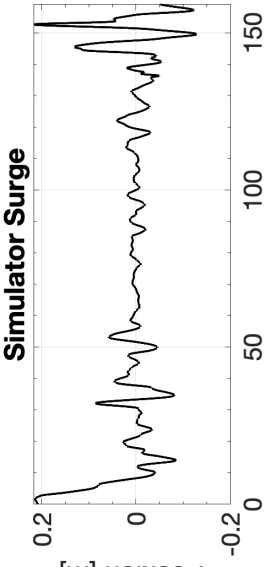

[m] uo!n!

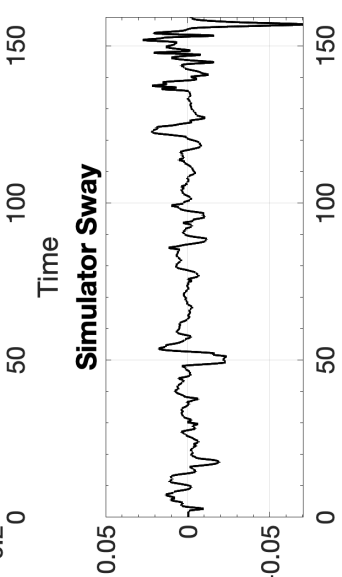

[w] uolp!sod

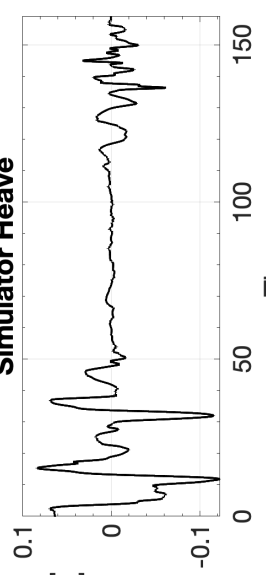

[w] uo!n!sod

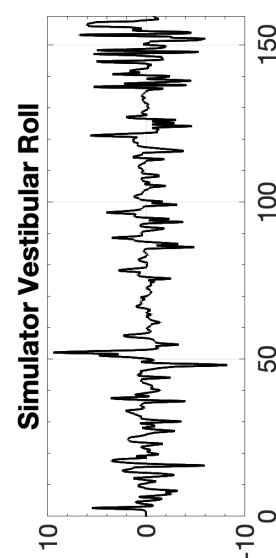

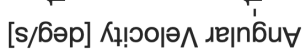

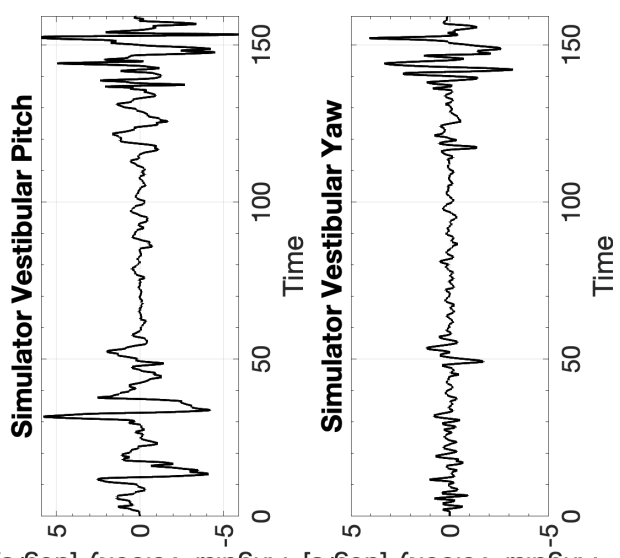

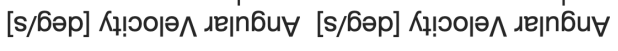

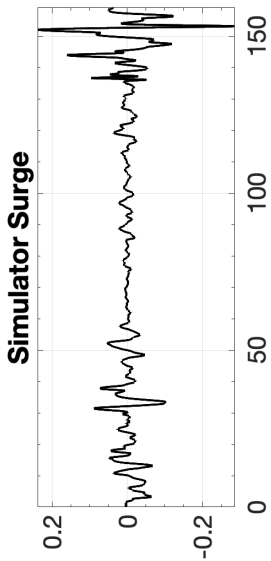

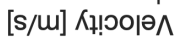

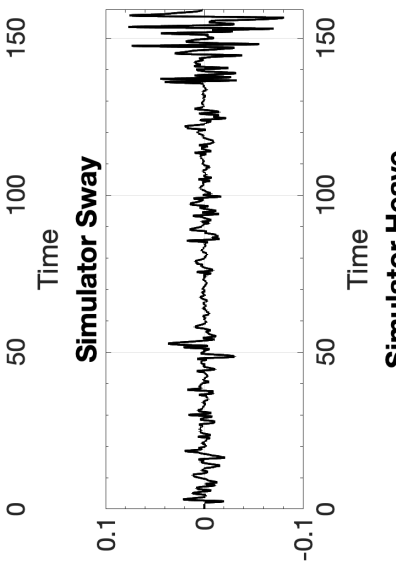

[s/u] К!ำ๐

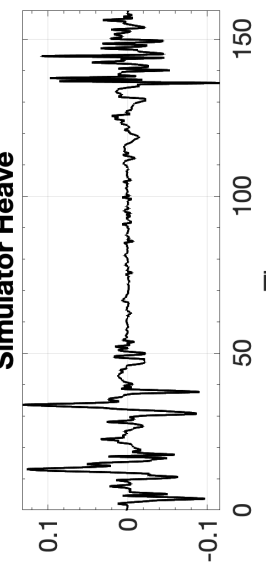

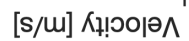

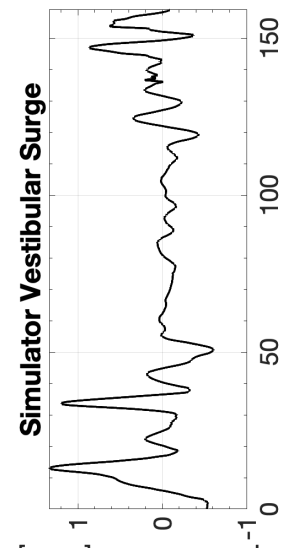

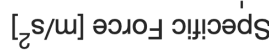

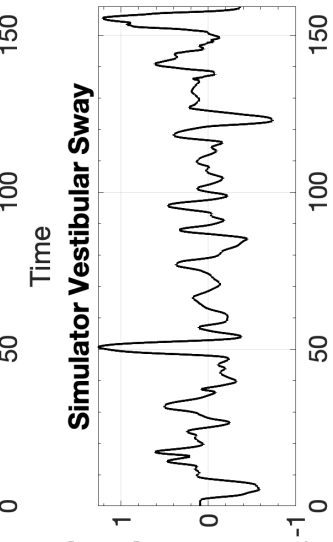

[zs/m] әวג0」 כ!!!วəd's

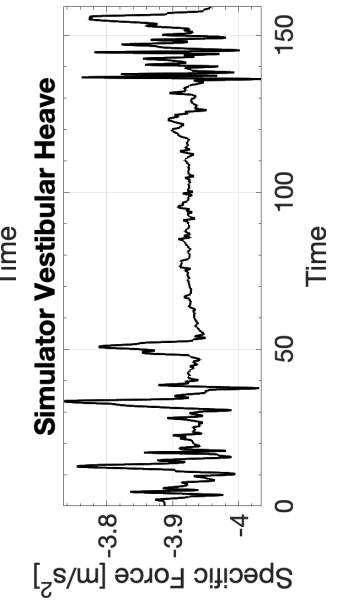

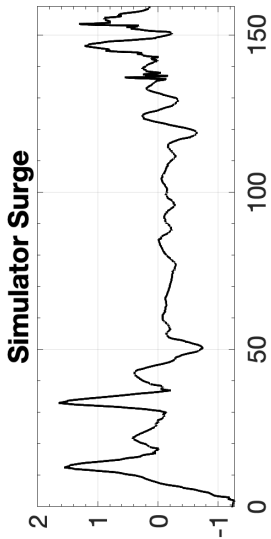

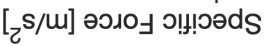

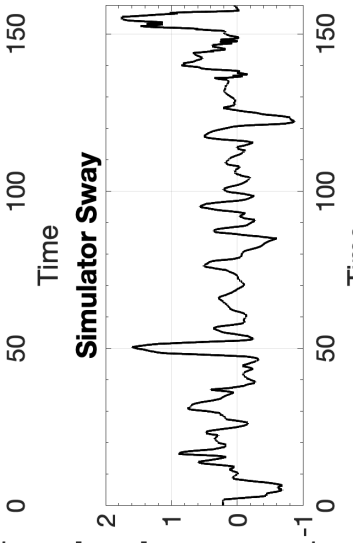

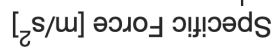

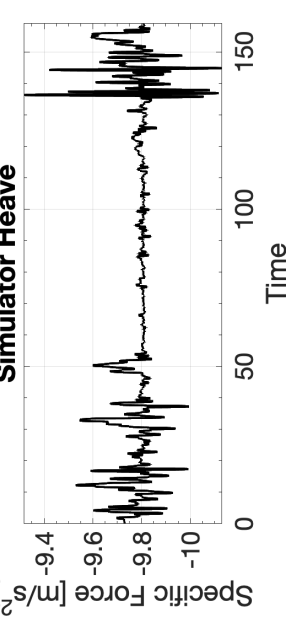

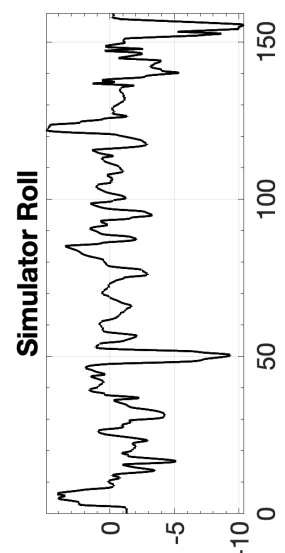

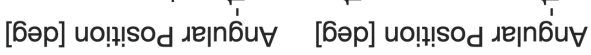

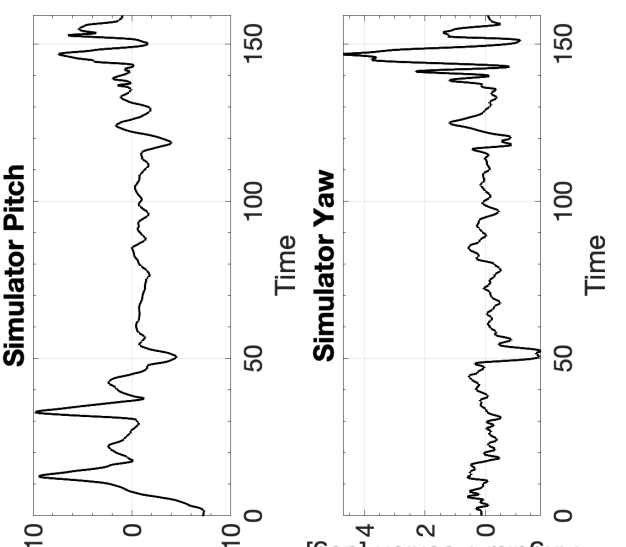

[6әр] uo!i!nsod גejn6ur

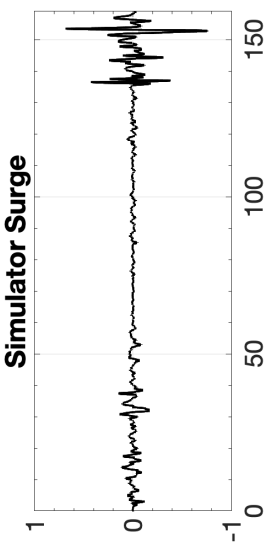

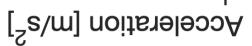

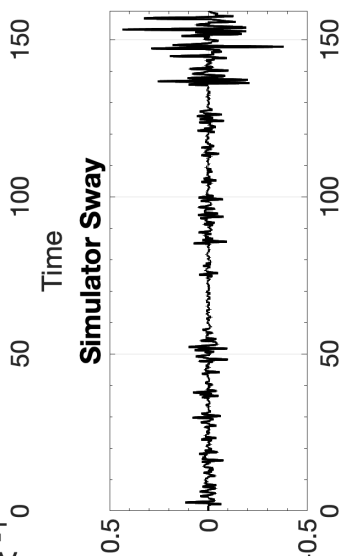

$[\mathrm{s} / \mathrm{m}]$ ио!ฺе

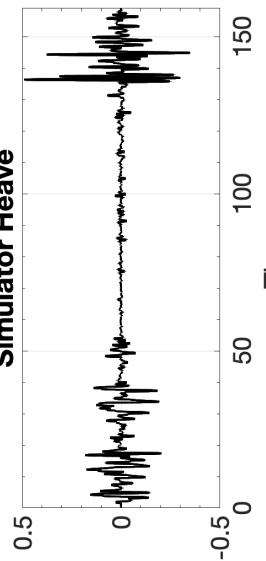

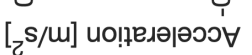

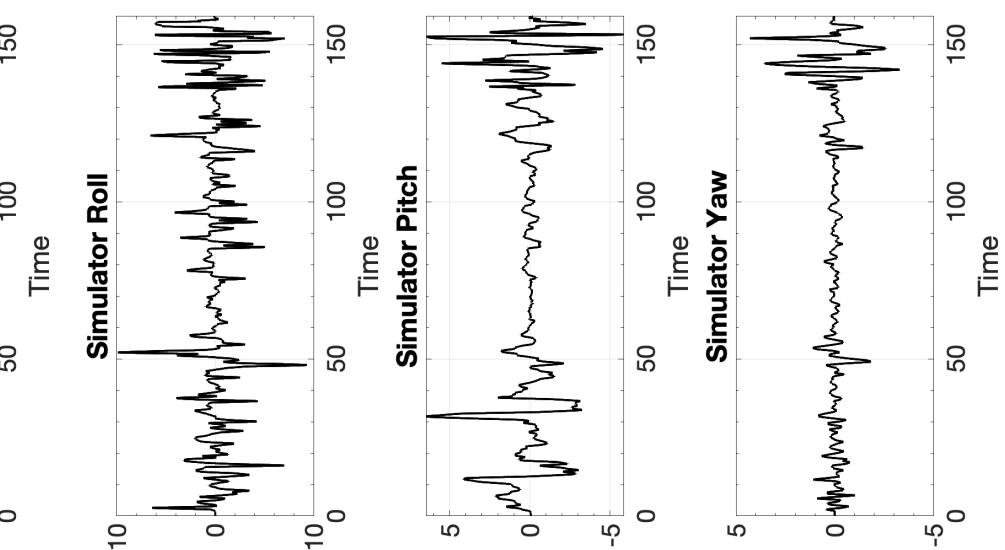

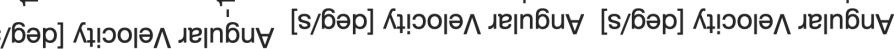




\section{B.16.2 CW2 Columbia 400 Cross-wind Landing}

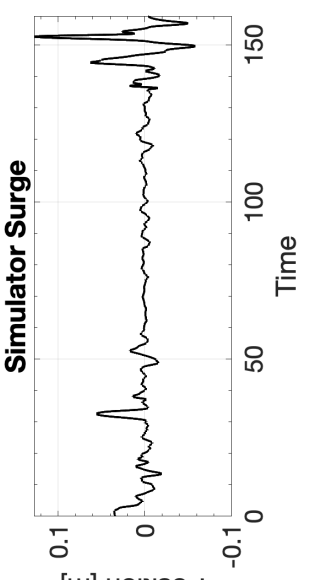

[m] uo!!!!sod

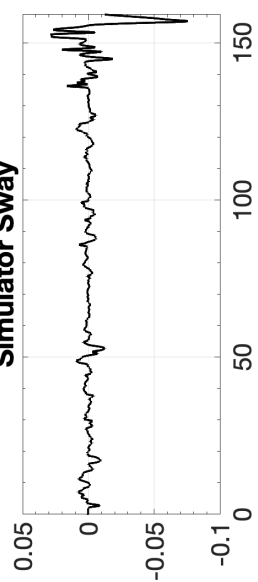

[u] uol!!sod

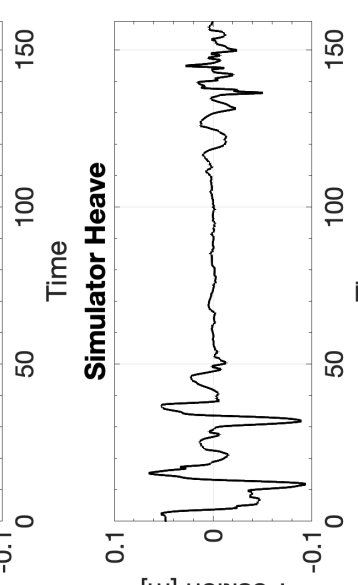

[w] uo!n!sod

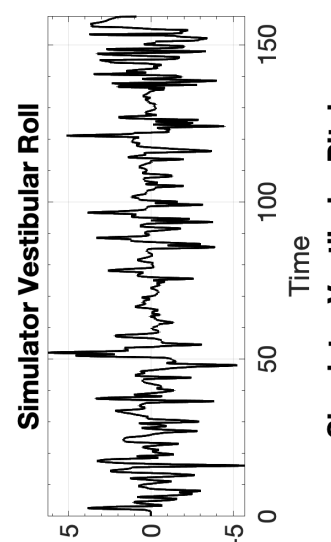

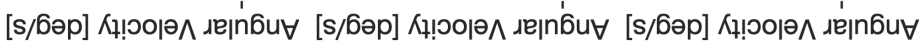
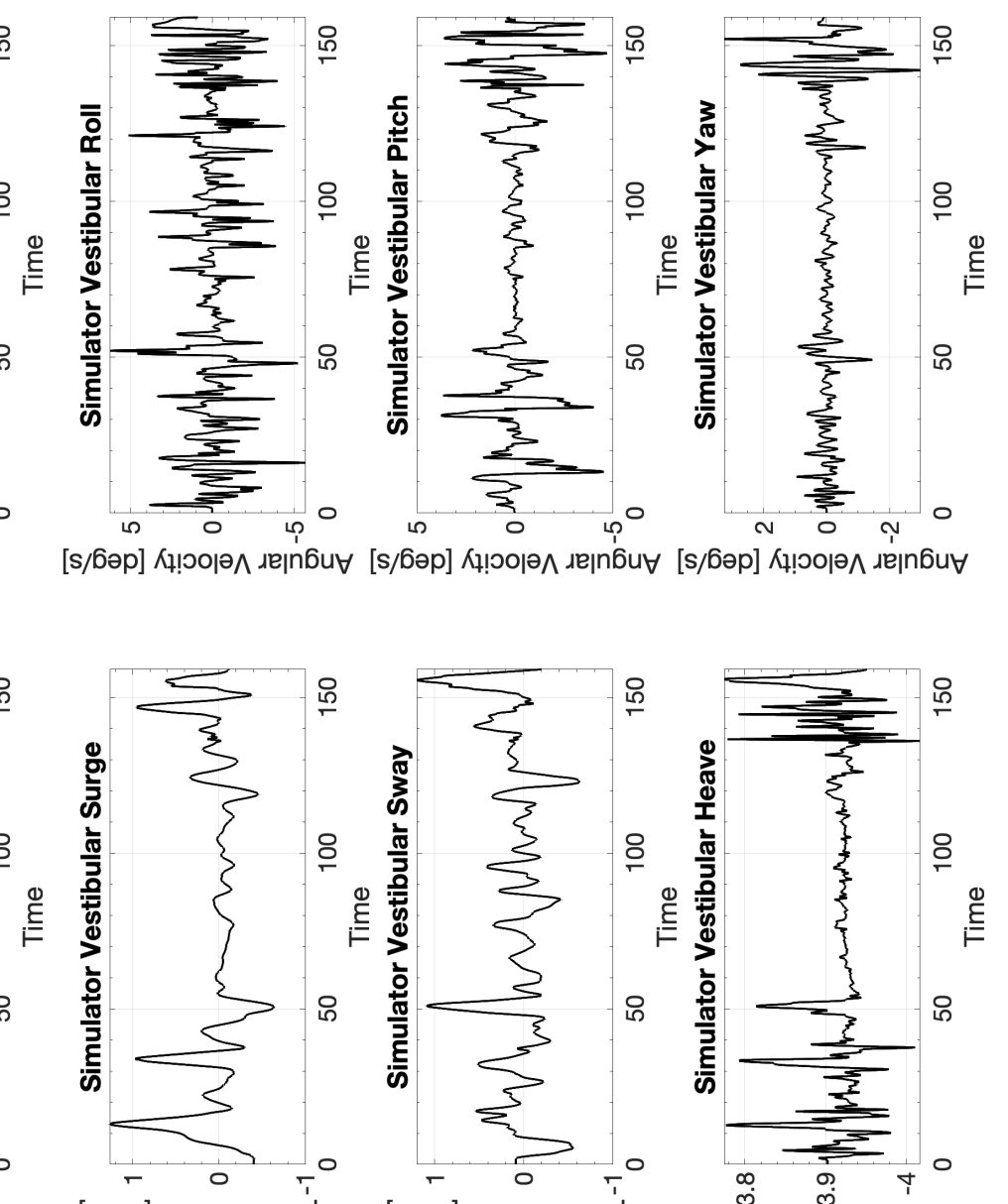

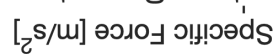

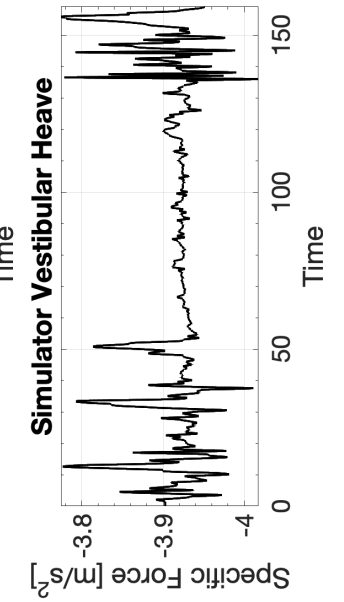

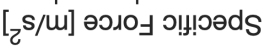

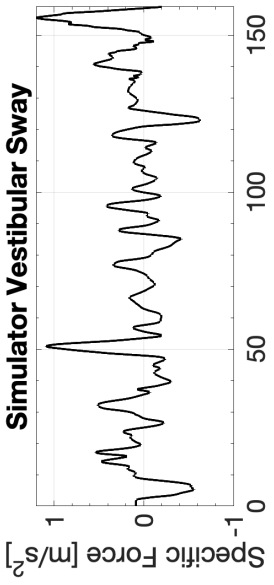

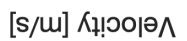

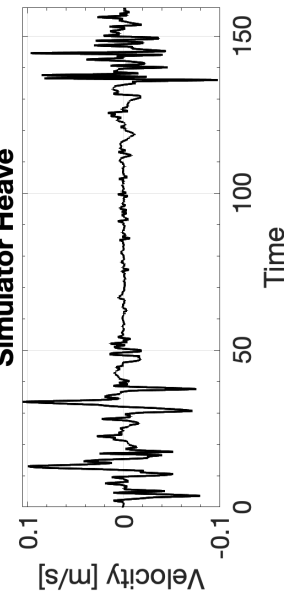

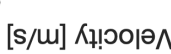

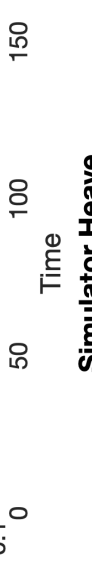

[s/u] Кұ!००|ә^

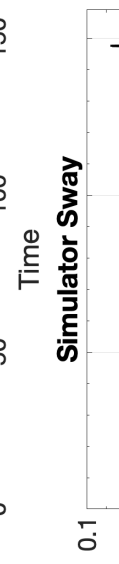

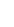

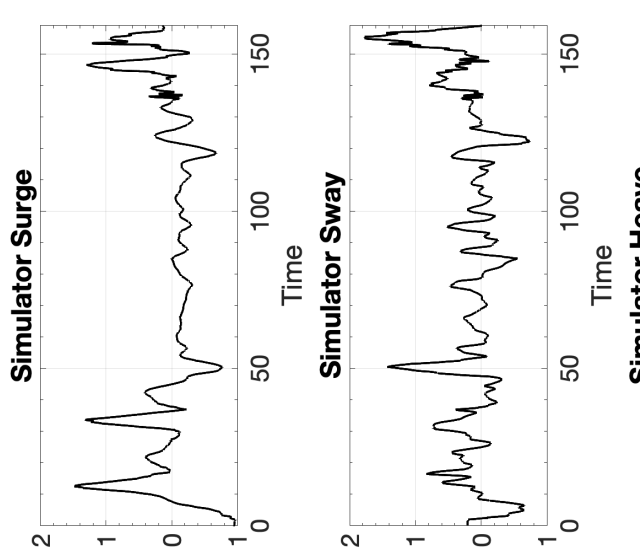

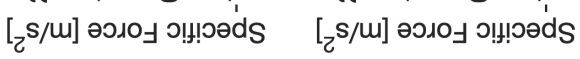

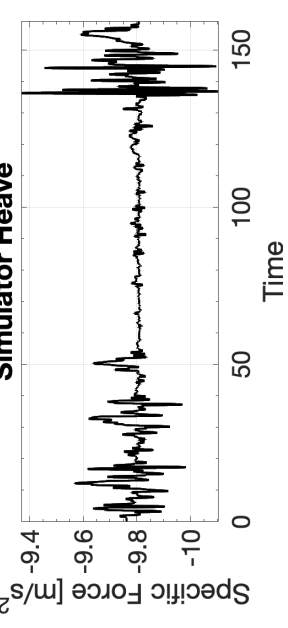

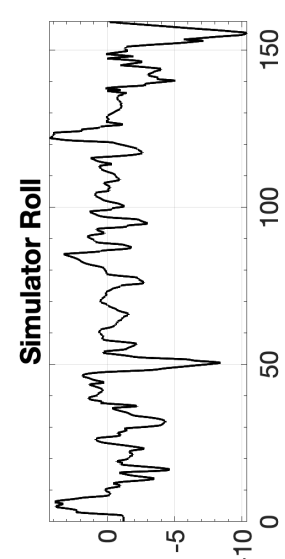

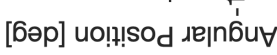

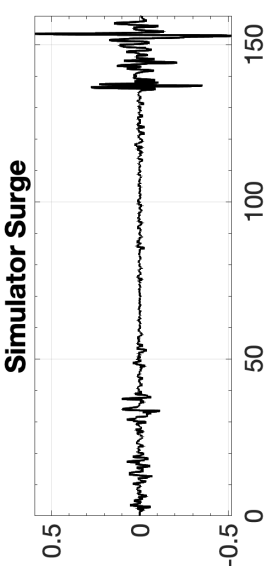

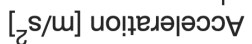

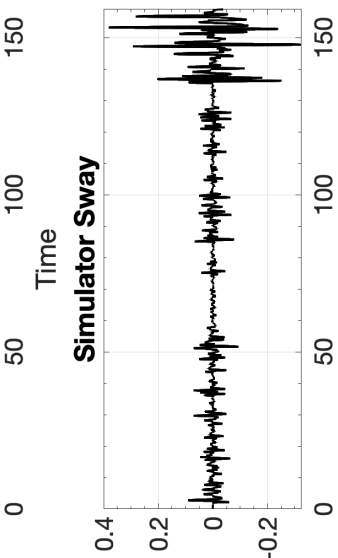

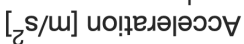

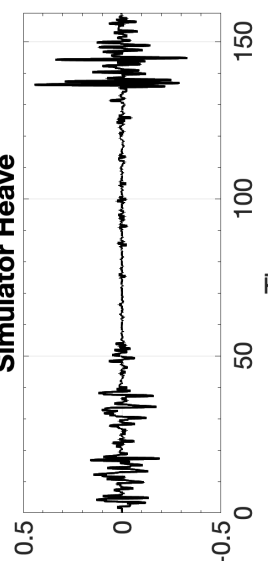

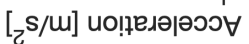
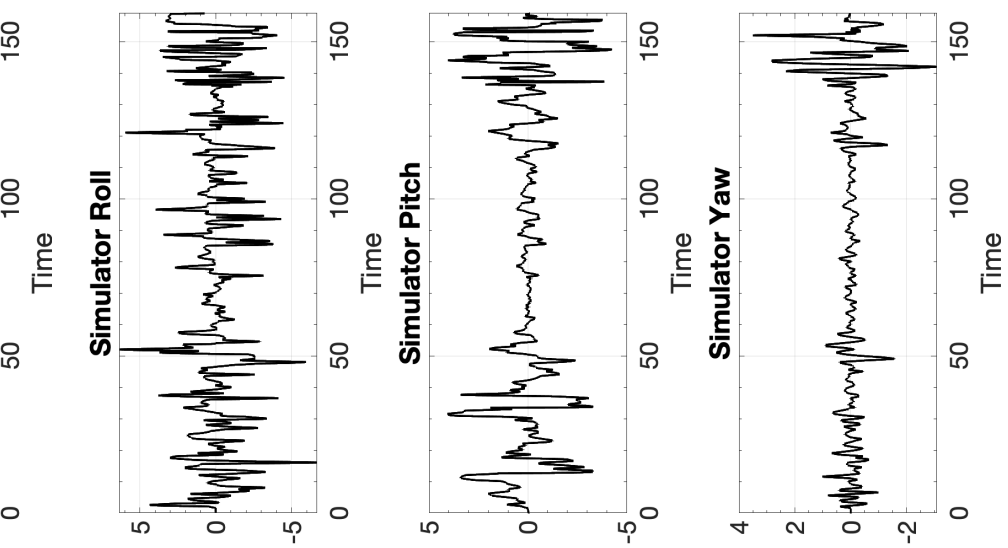

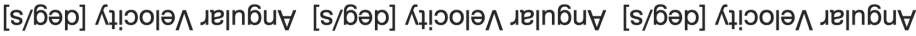




\section{B.16.3 CW3 Columbia 400 Cross-wind Landing}

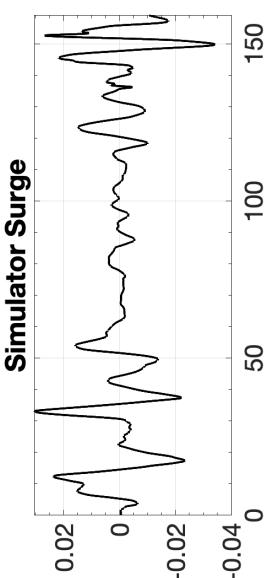

[u] uo!n! $\mathrm{sod}_{\mathrm{d}}$

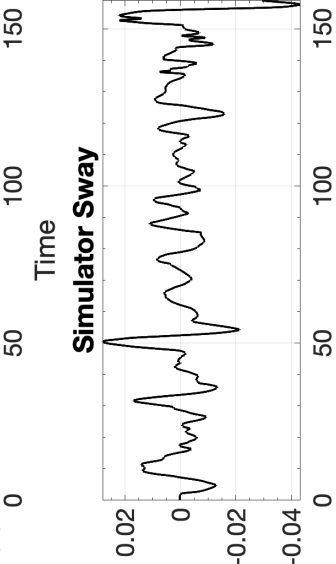

[w] uol!?!sod

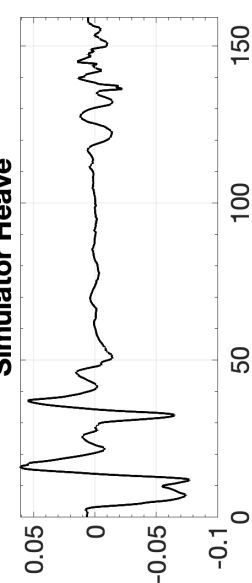

[w] uo!n! $\mathrm{sOd}_{\mathrm{d}}$
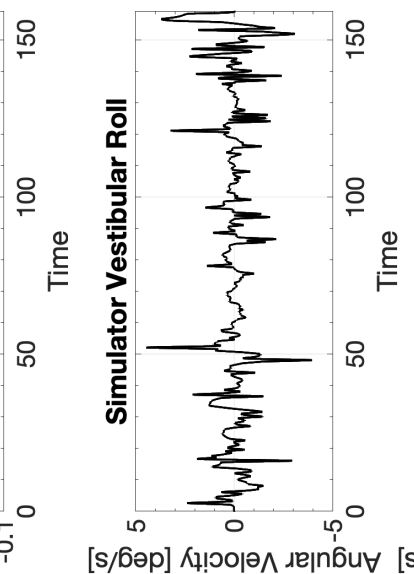

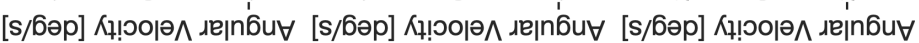

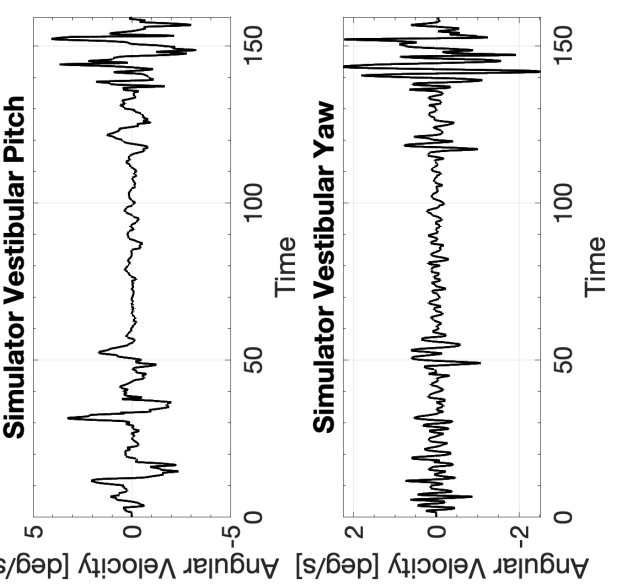

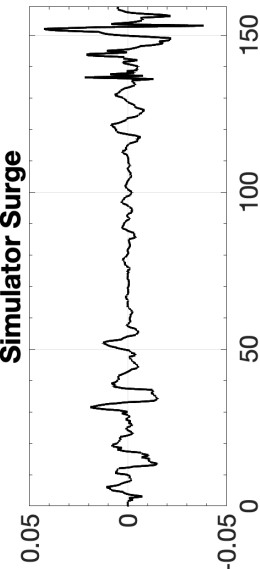

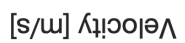

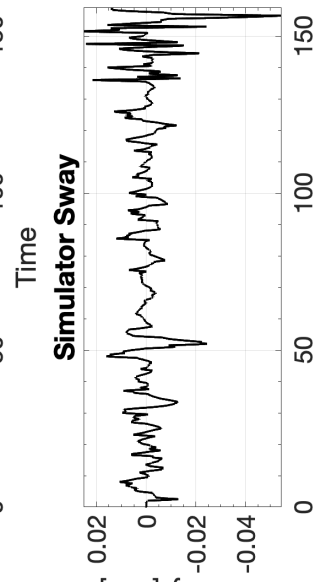

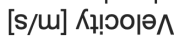

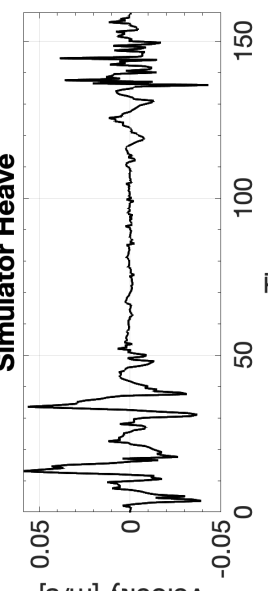

[s/m] Кұ! ৩оюә^

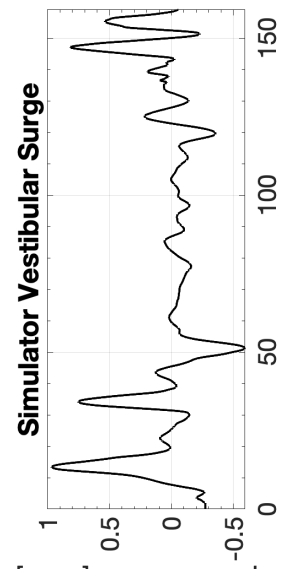

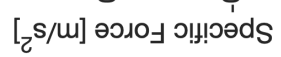

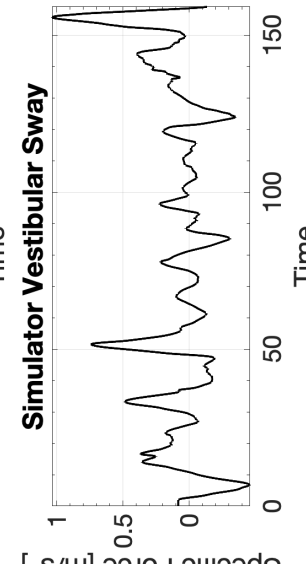

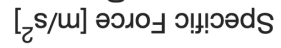

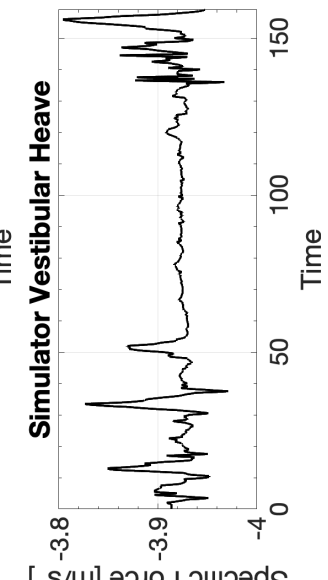

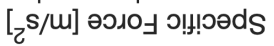

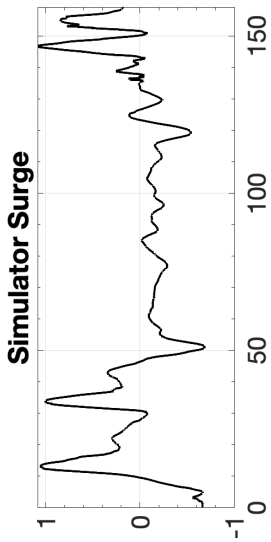

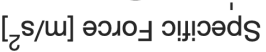

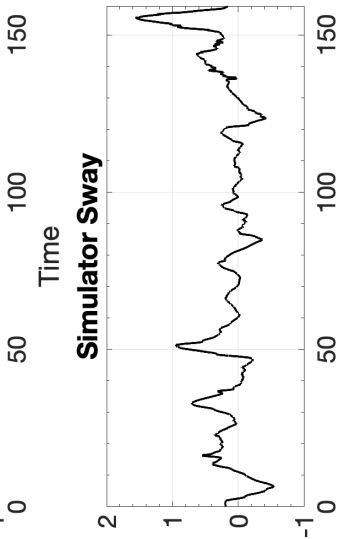

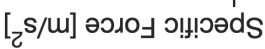

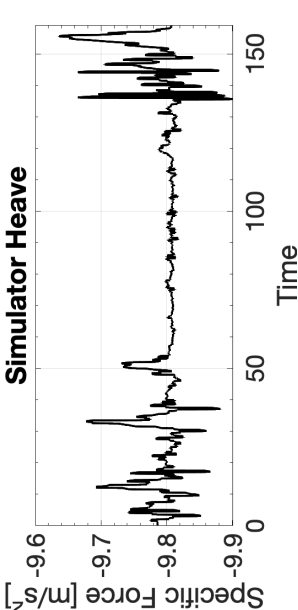

$\left[\begin{array}{c}1 \\ \text { L }\end{array}\right.$

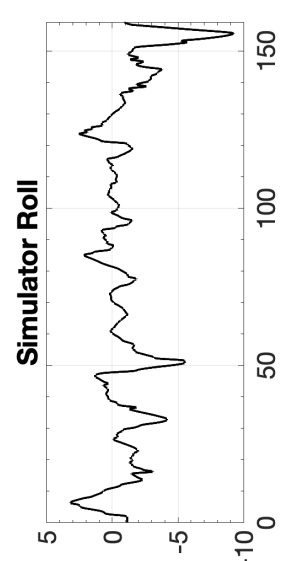

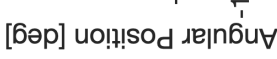

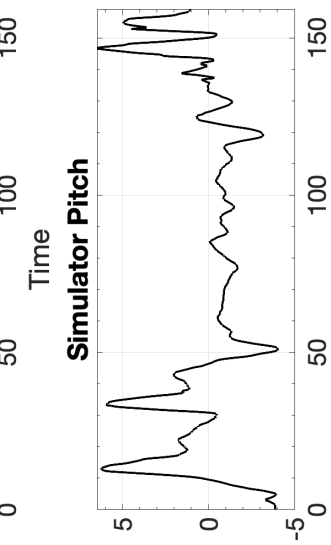

[бəр] uo!!!!sod גe|nбิu

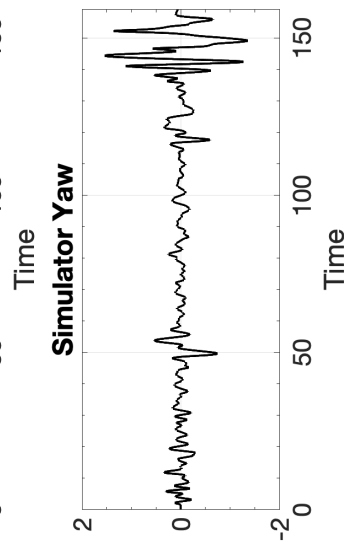

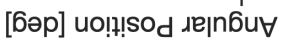

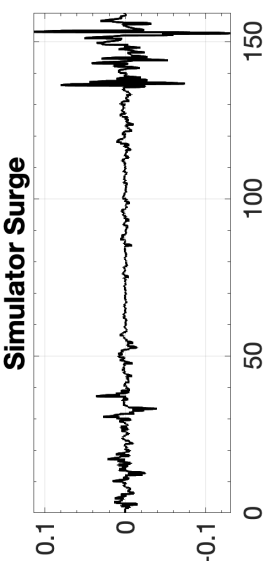

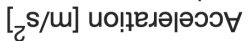

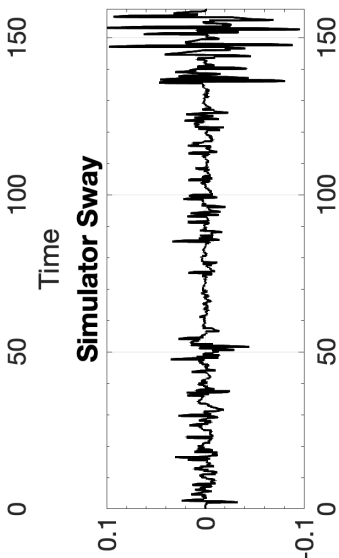

[s/m] ио!цедәәәоэн
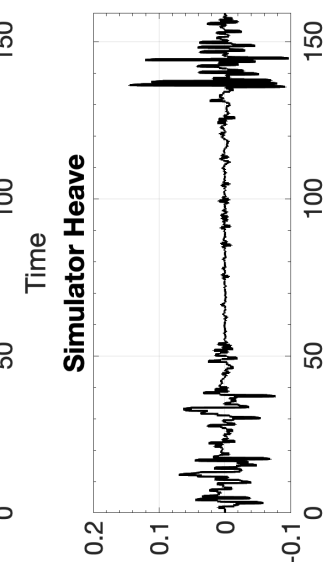

[s/m] ио!ฺедәәәэว
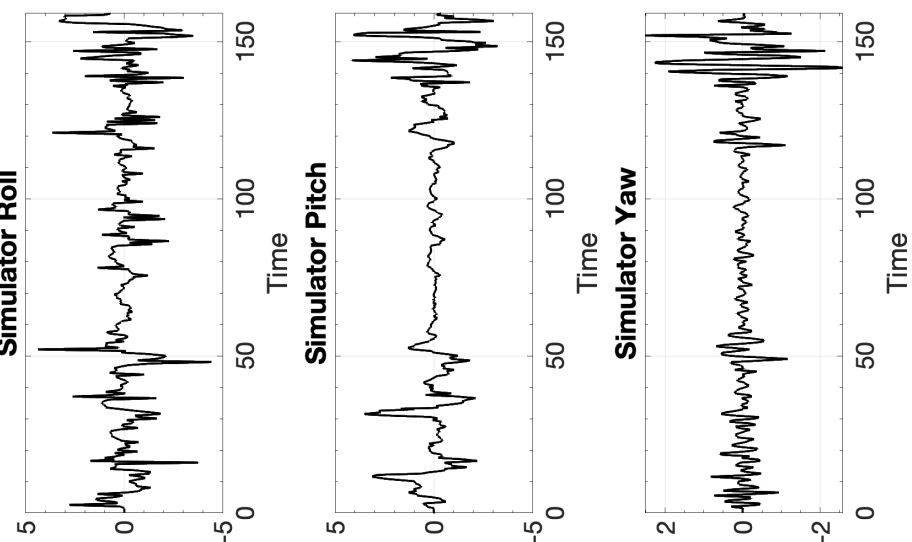

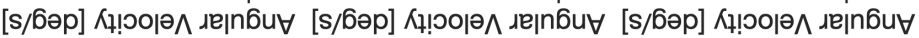




\section{B.17 Columbia 400 Single-axis Takeoff}
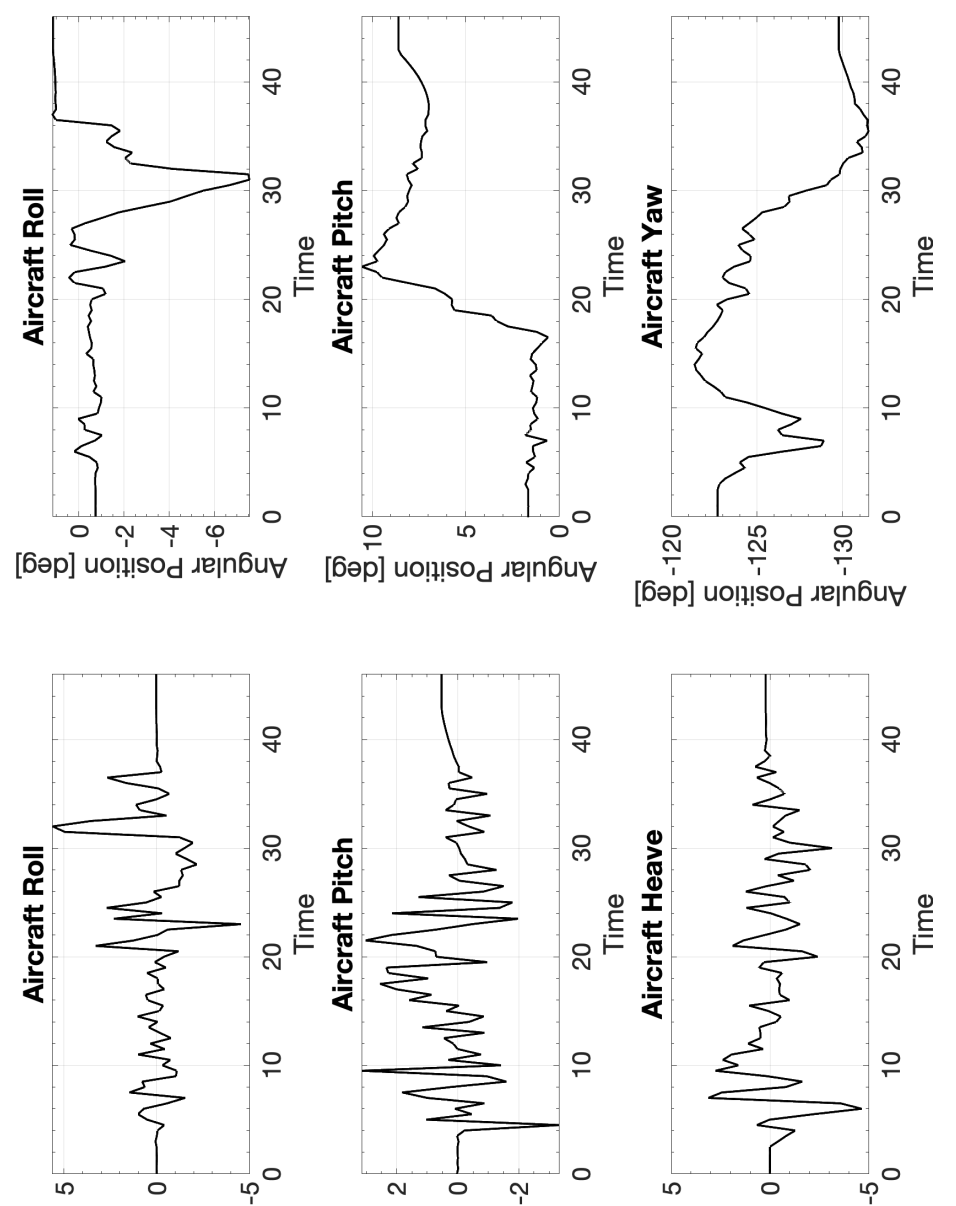

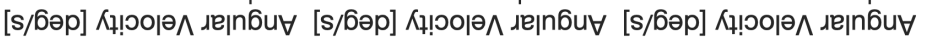

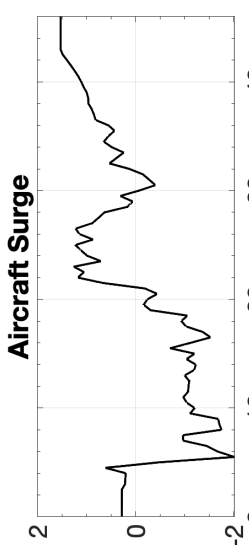

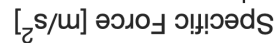

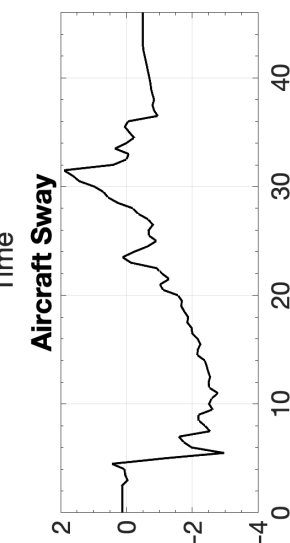

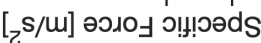

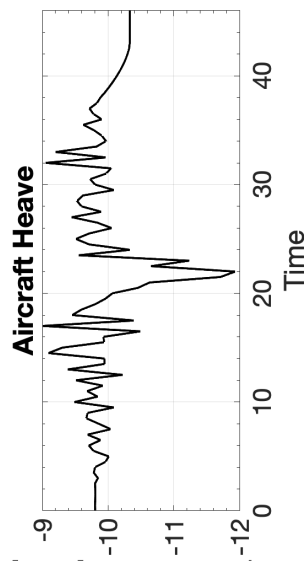

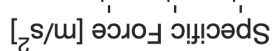

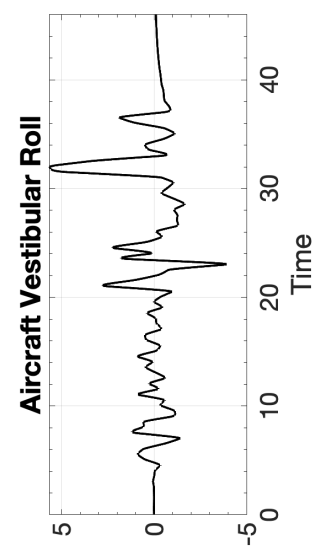

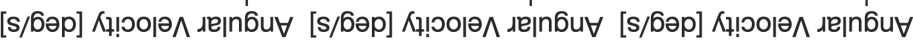

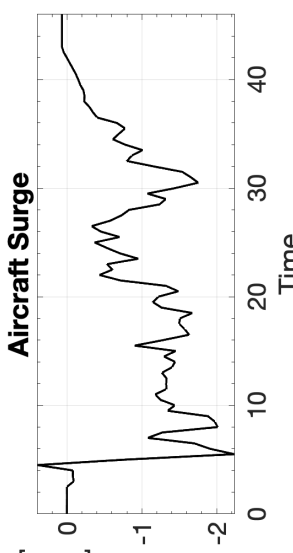

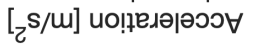

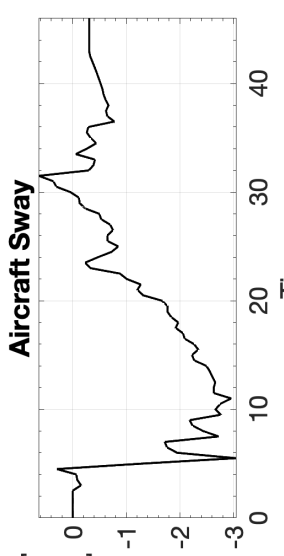

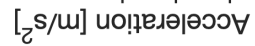

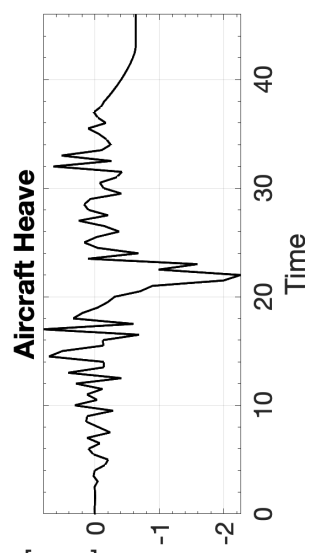

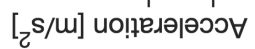

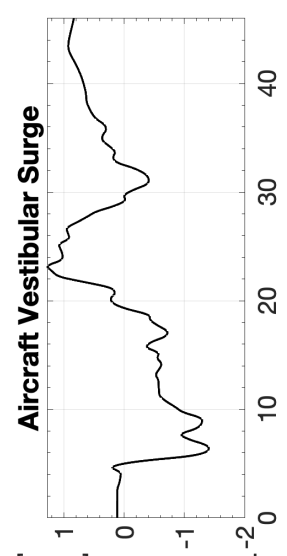

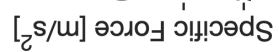
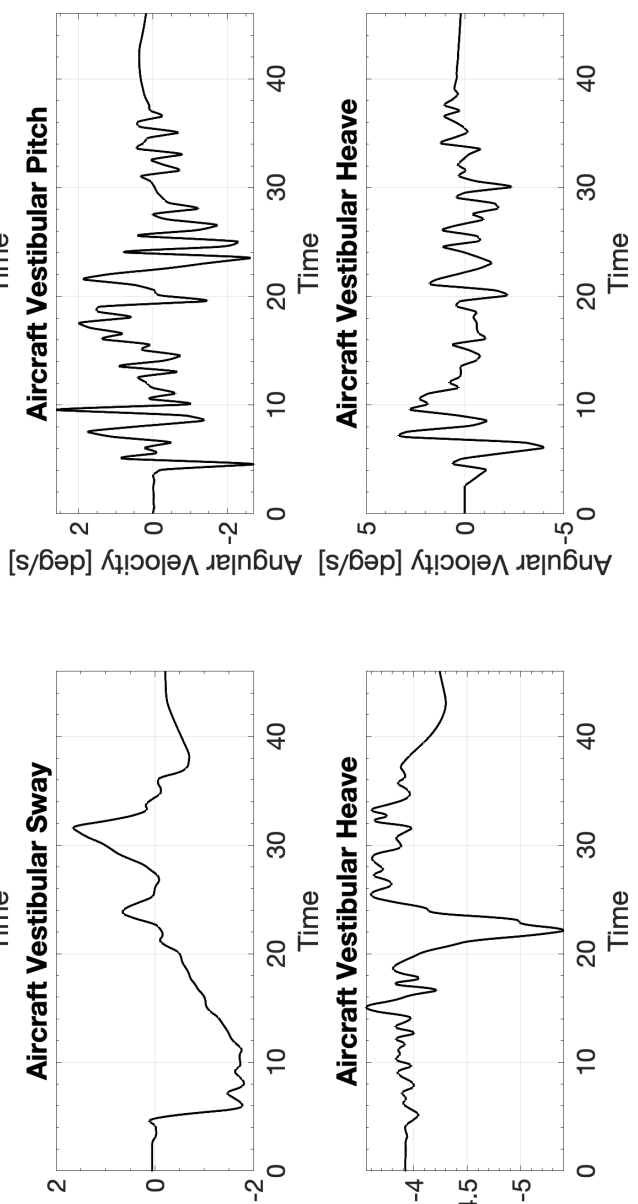

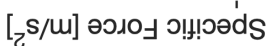

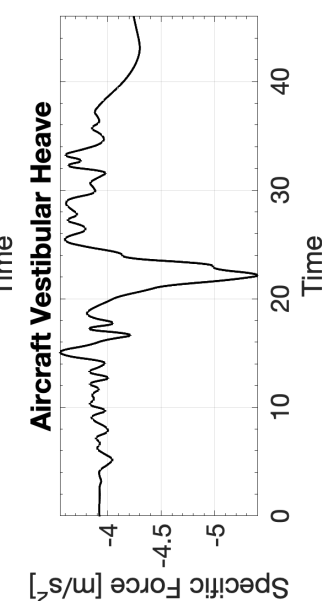

[ 


\section{B.17.1 CW1 Columbia 400 Single-axis Takeoff}

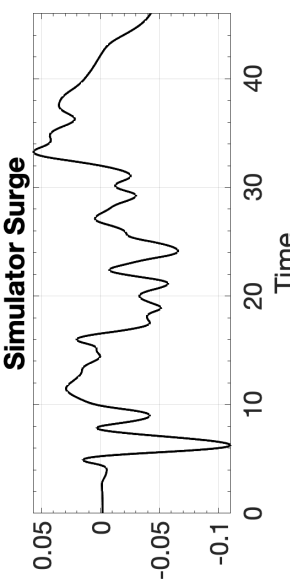

[w] uo!̣!!sod

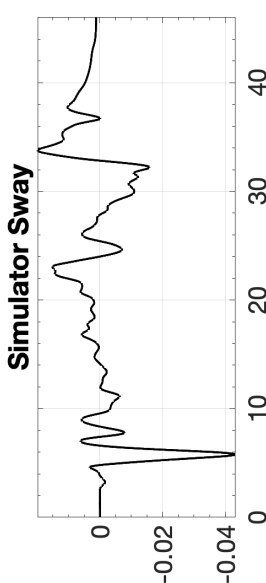

[u] uol!?!sod

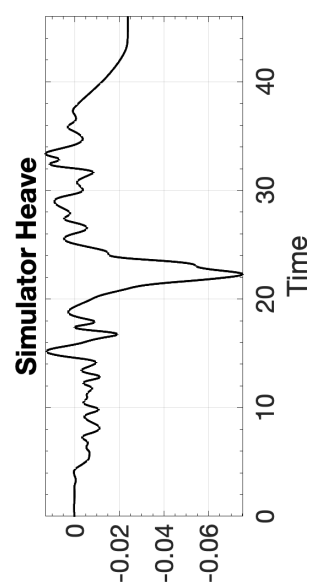

[w] uol!? sod

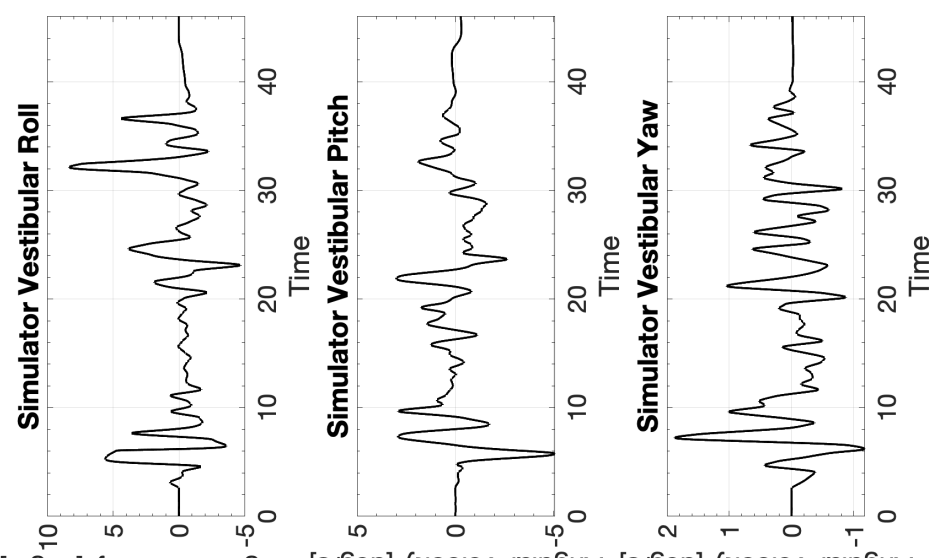

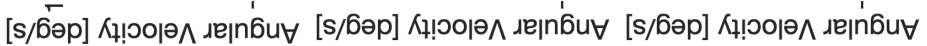

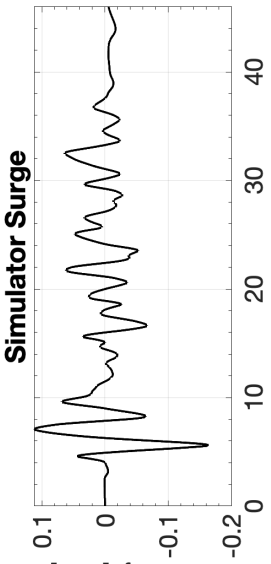

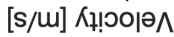

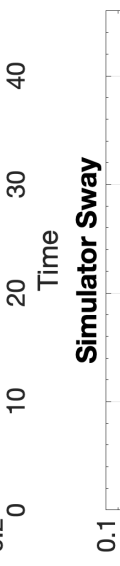

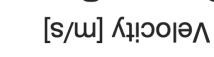

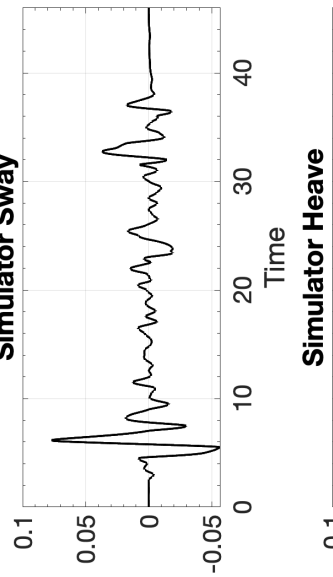

-

[s/m] Кұ!юорә

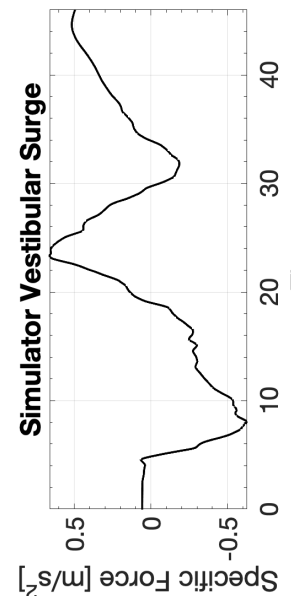

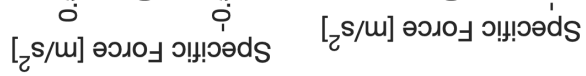
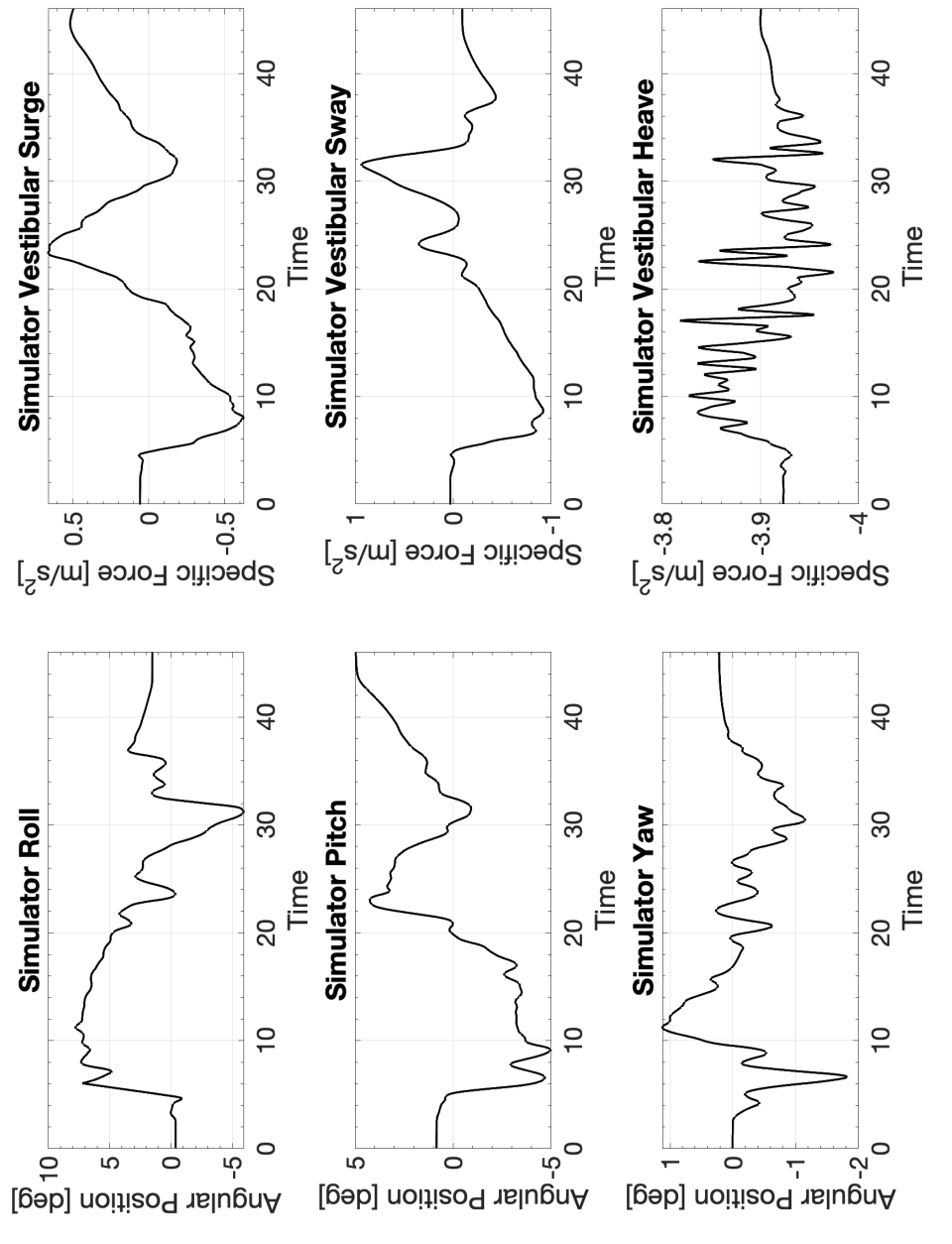

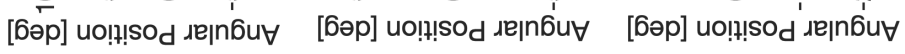

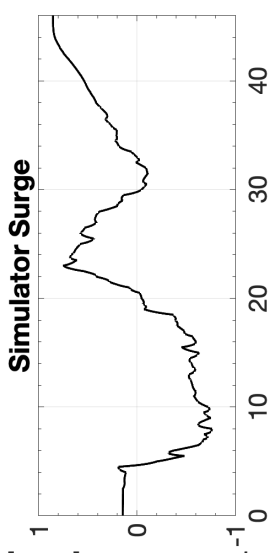

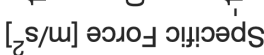

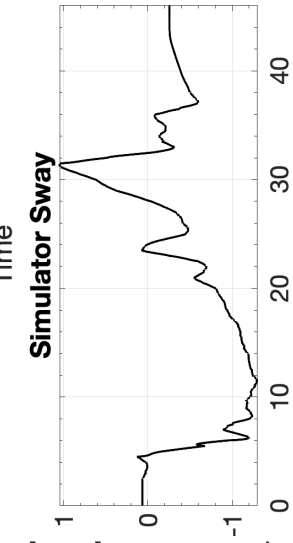

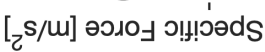

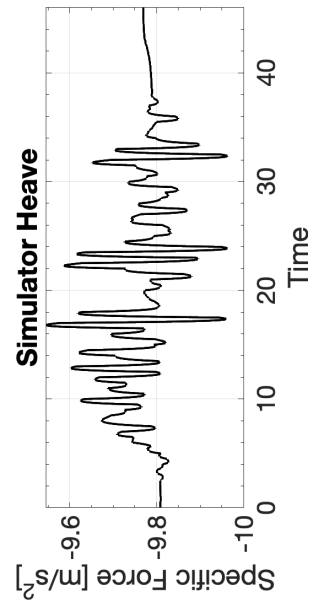

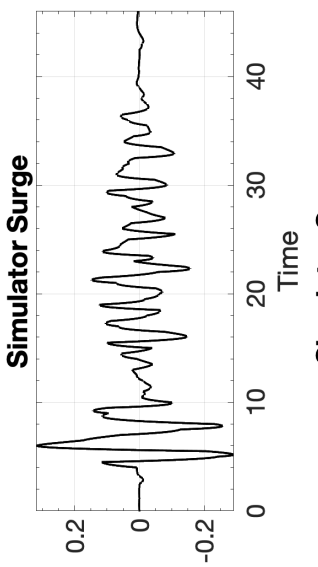

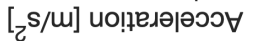

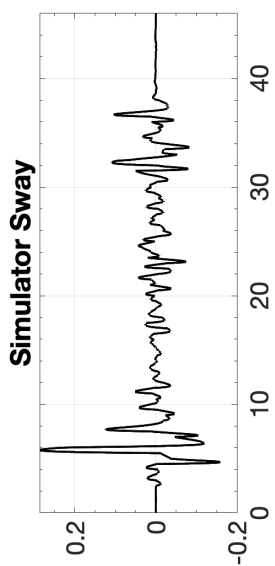

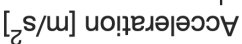

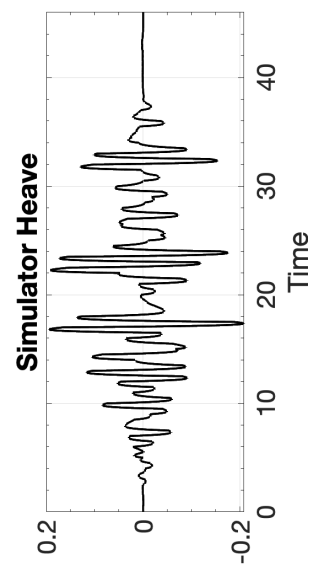

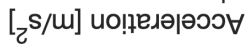

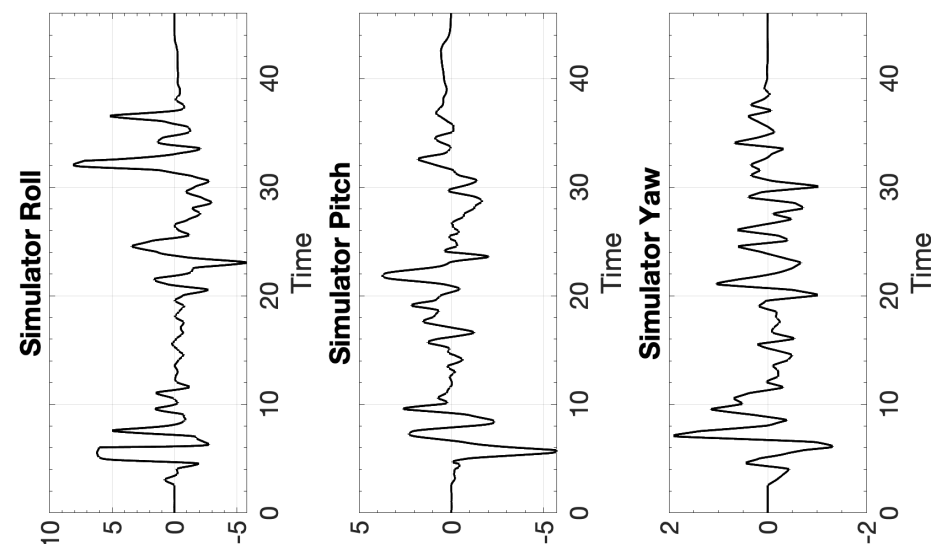

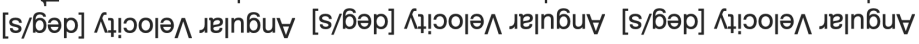




\section{B.17.2 CW2 Columbia 400 Single-axis Takeoff}

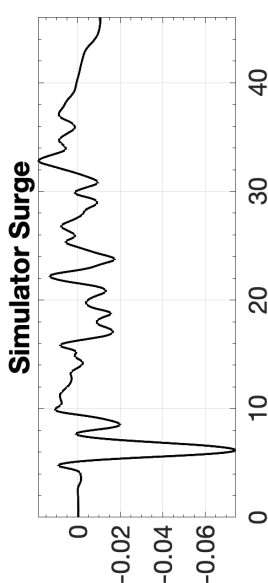

[u] uo!n! sod

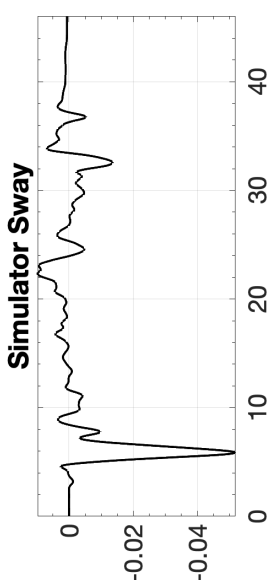

[u] uo!̣!!sod

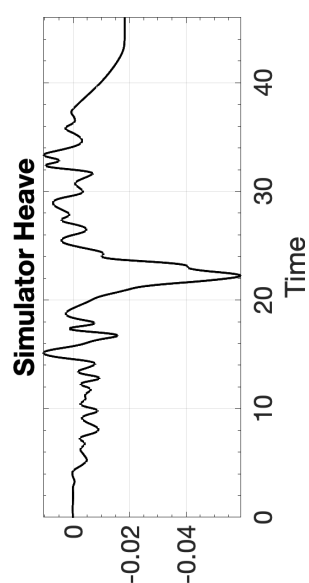

[w] uo!n!s sod

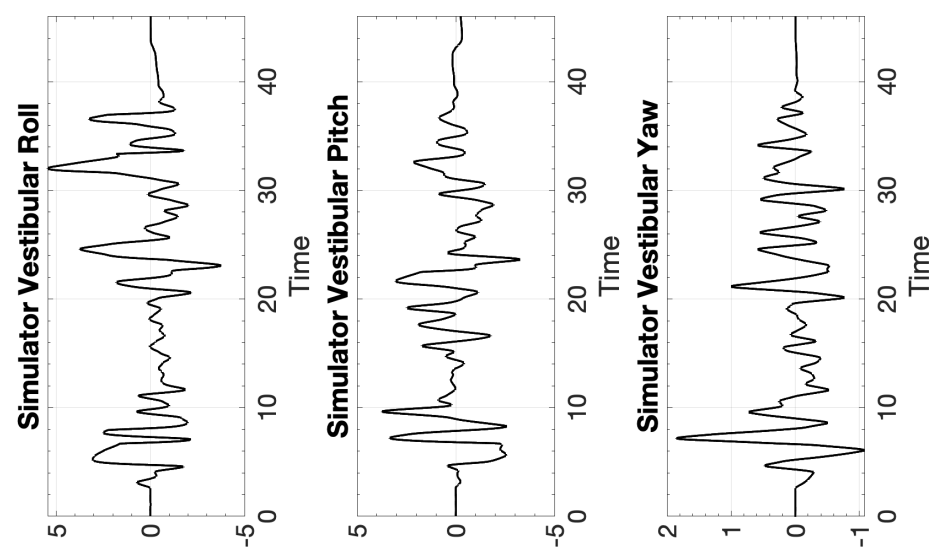

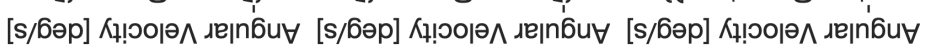

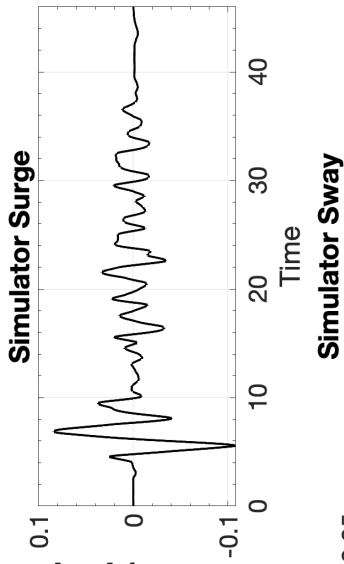

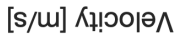

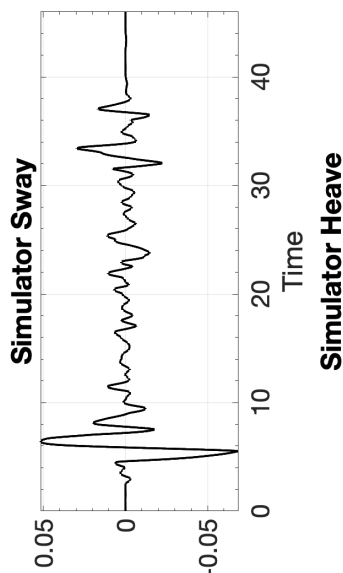

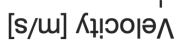

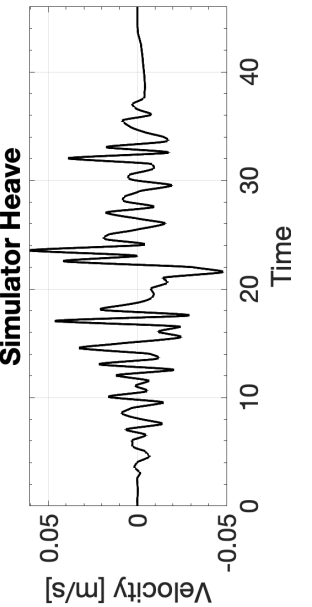

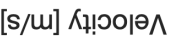

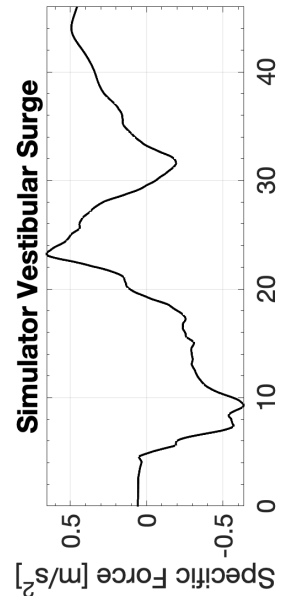

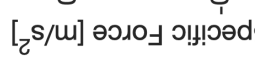
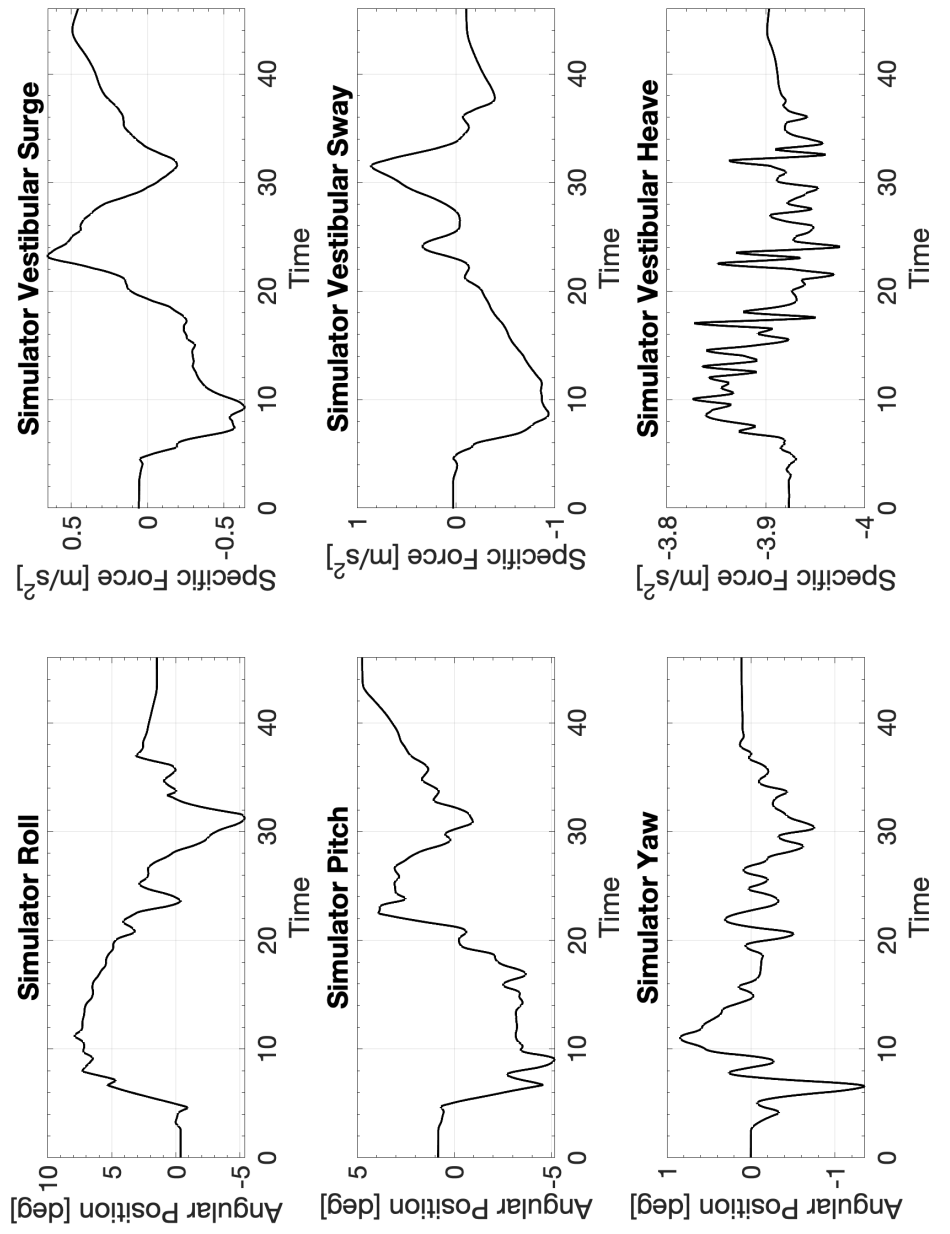

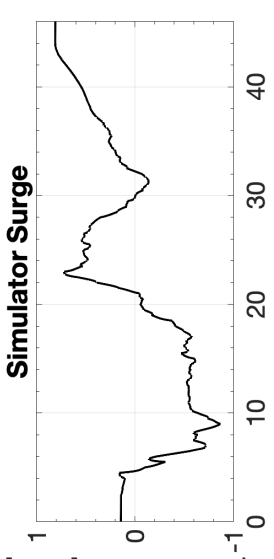

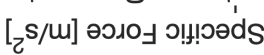

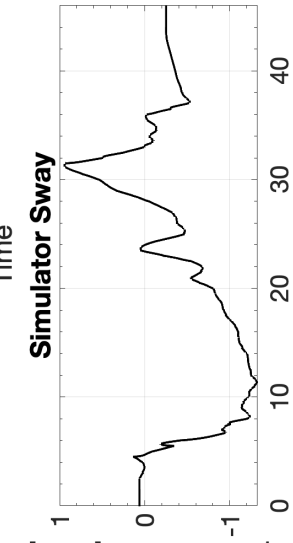

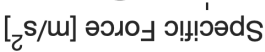

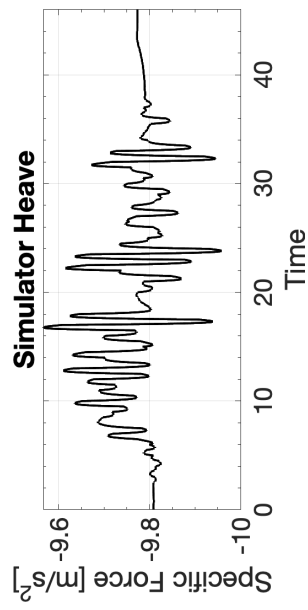

[бәр] uo!̣!

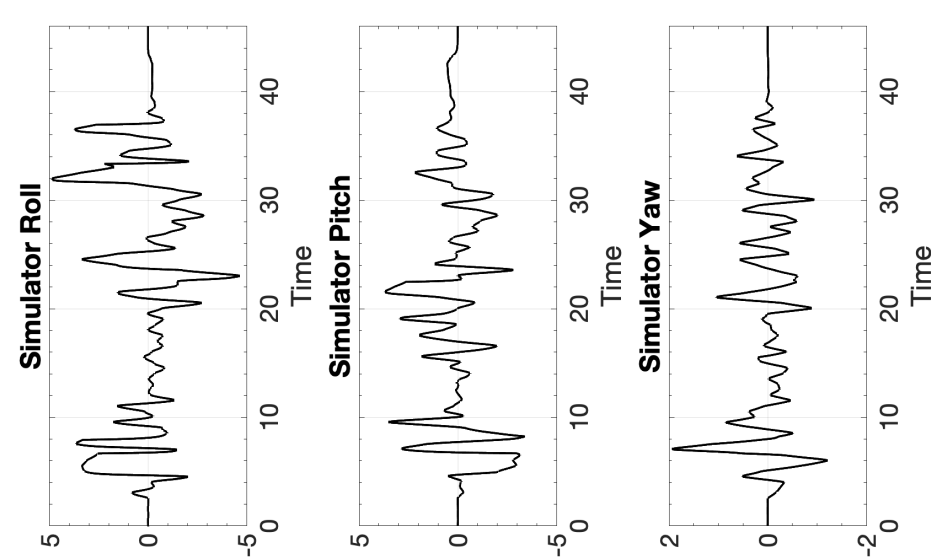

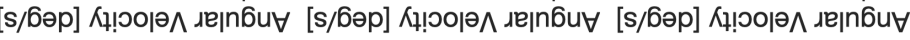

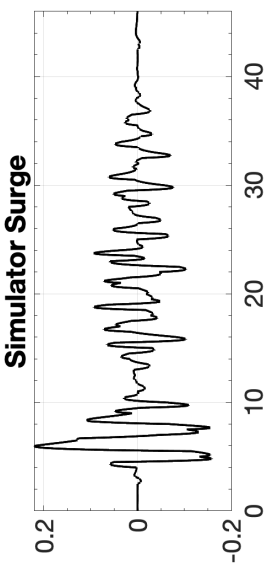

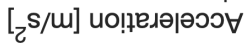

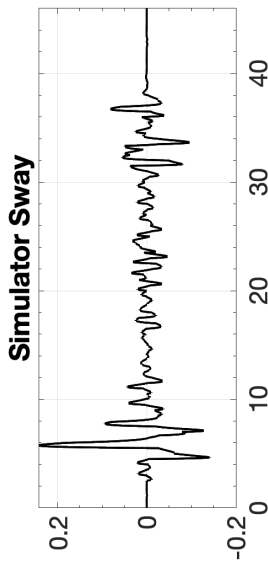

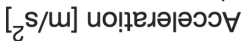

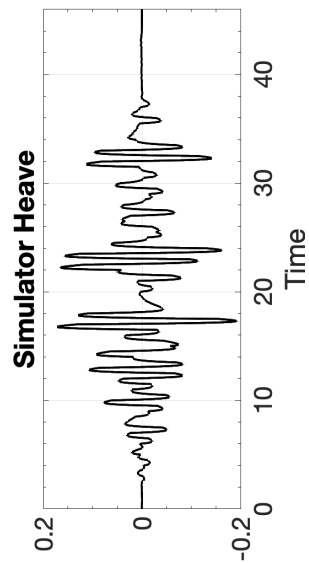

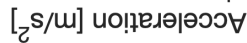




\section{B.17.3 CW3 Columbia 400 Single-axis Takeoff}

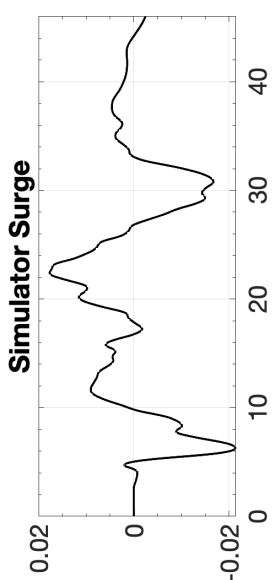

[w] uo!n! $\mathrm{sod}_{\mathrm{d}}$

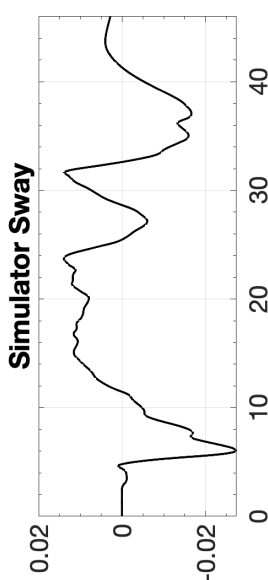

[u] uol!?!sod

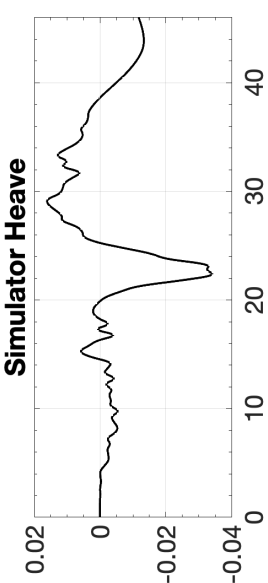

[u] uolp!!sod

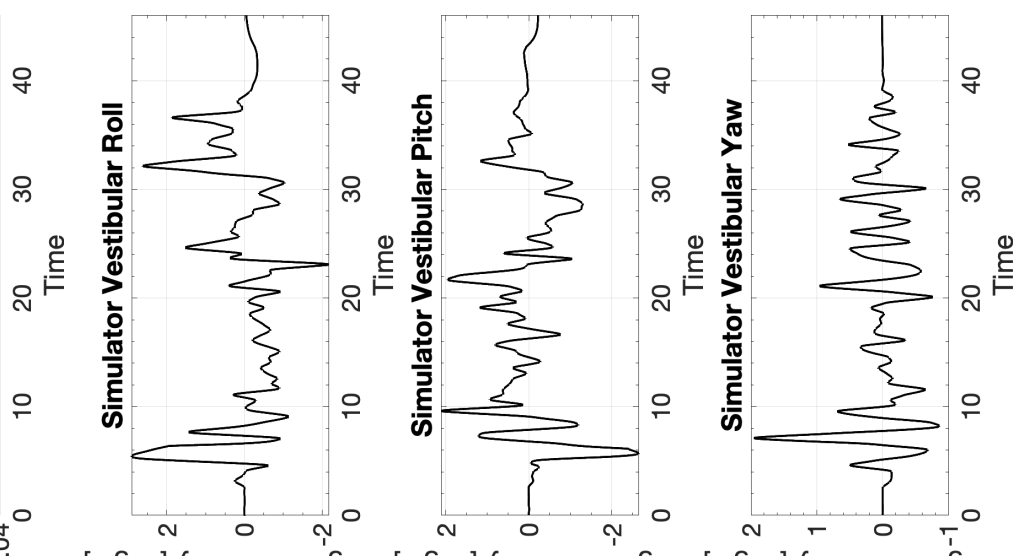

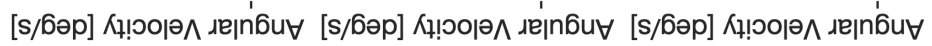

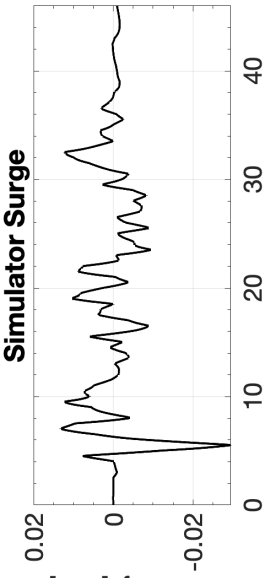

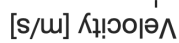

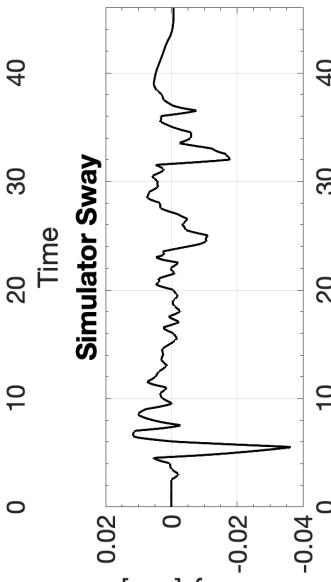

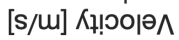

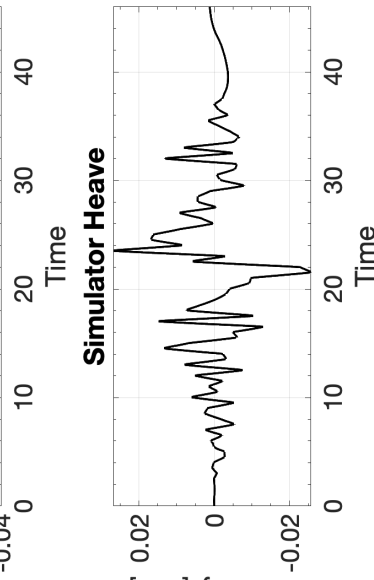

[s/m] Кұ!юорю

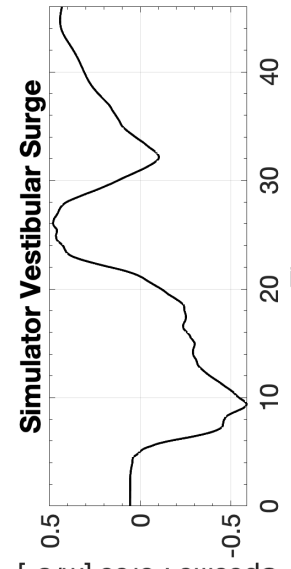

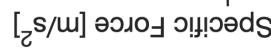
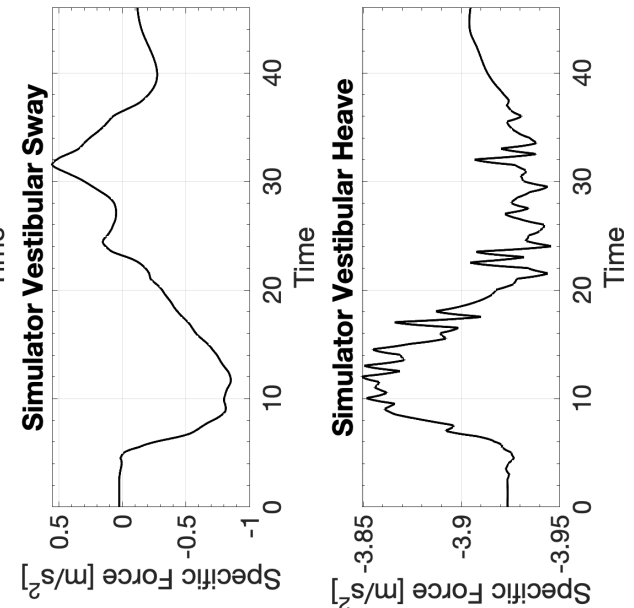

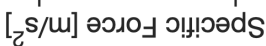

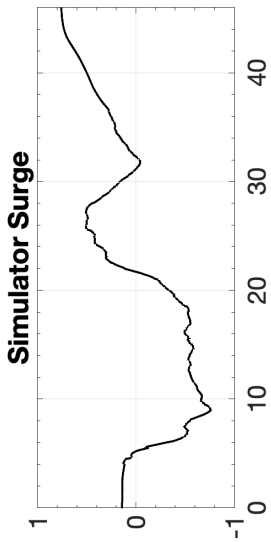

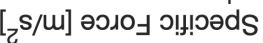
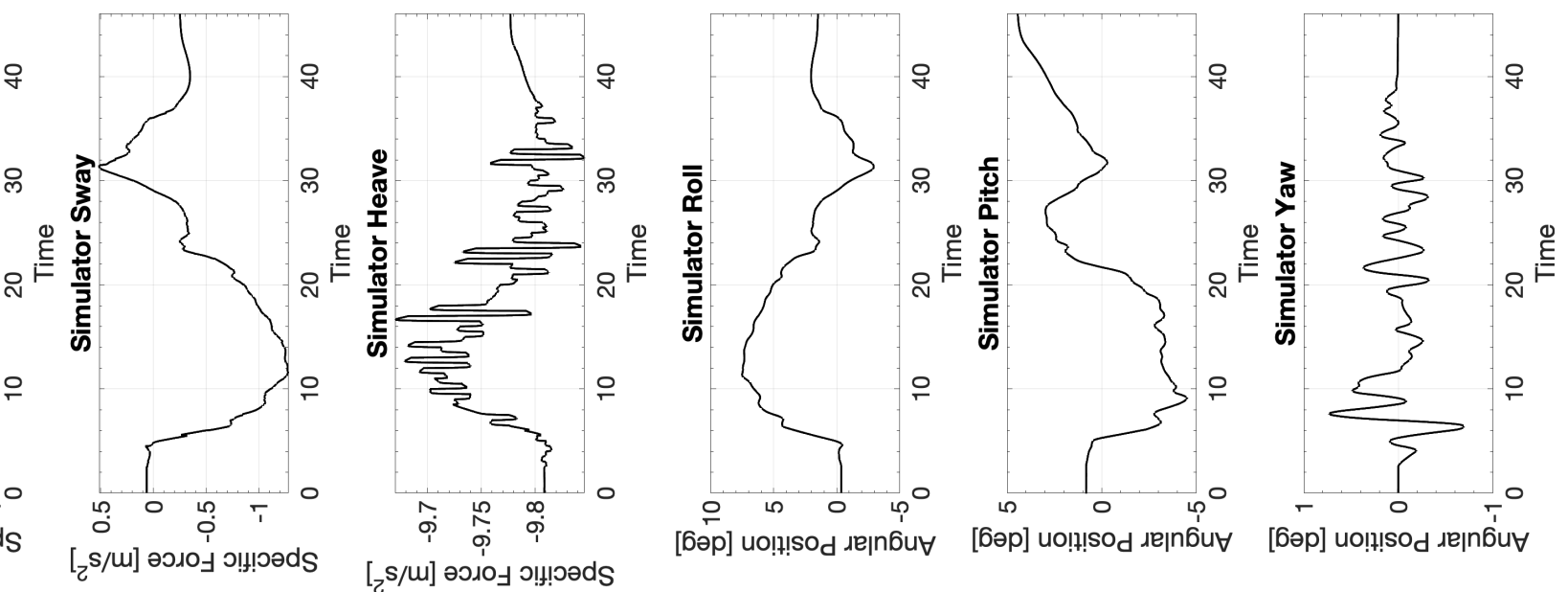

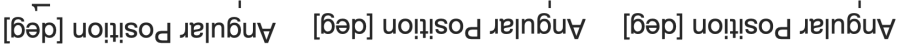

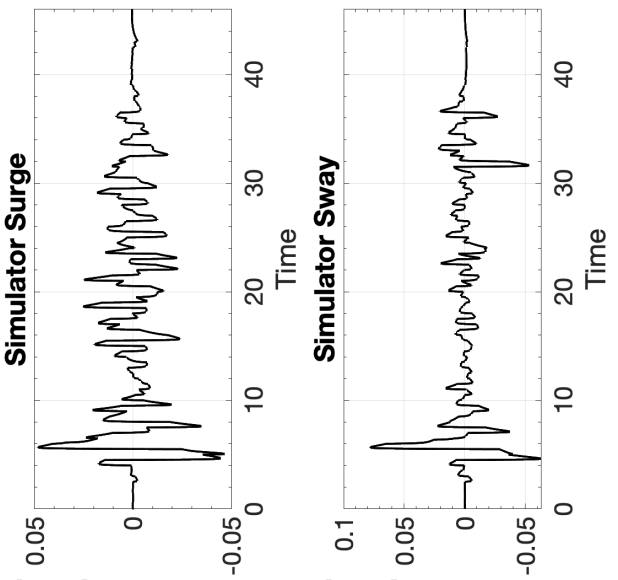

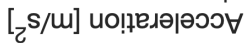

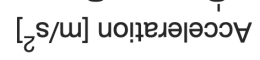
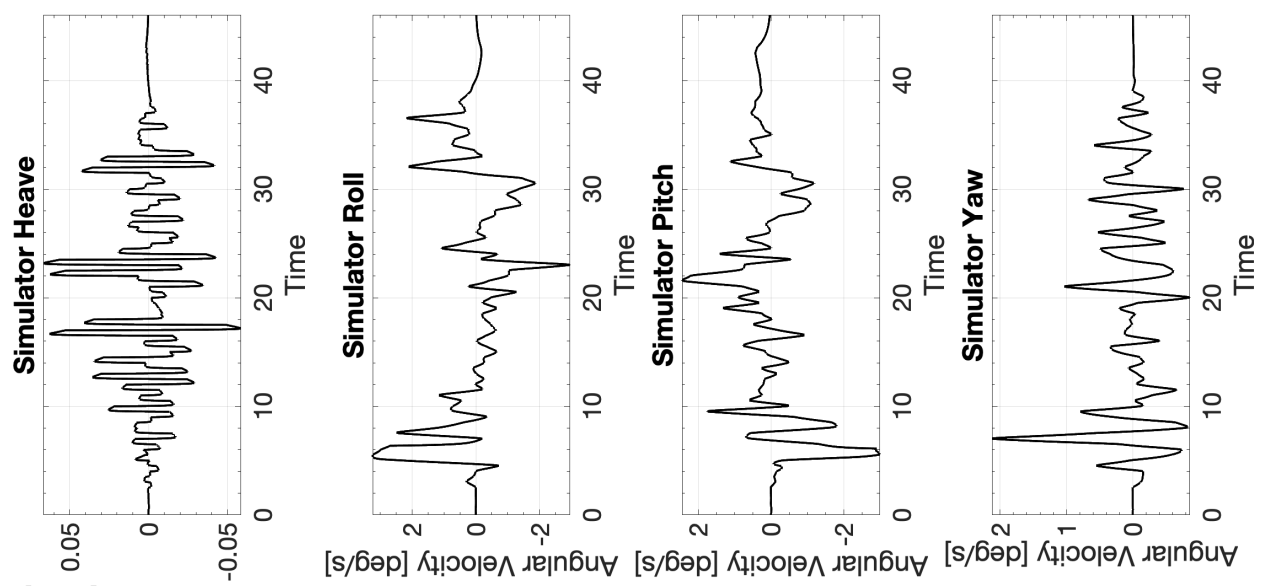

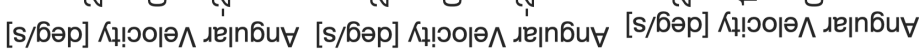




\section{B.18 Columbia 400 Spiral Dive}
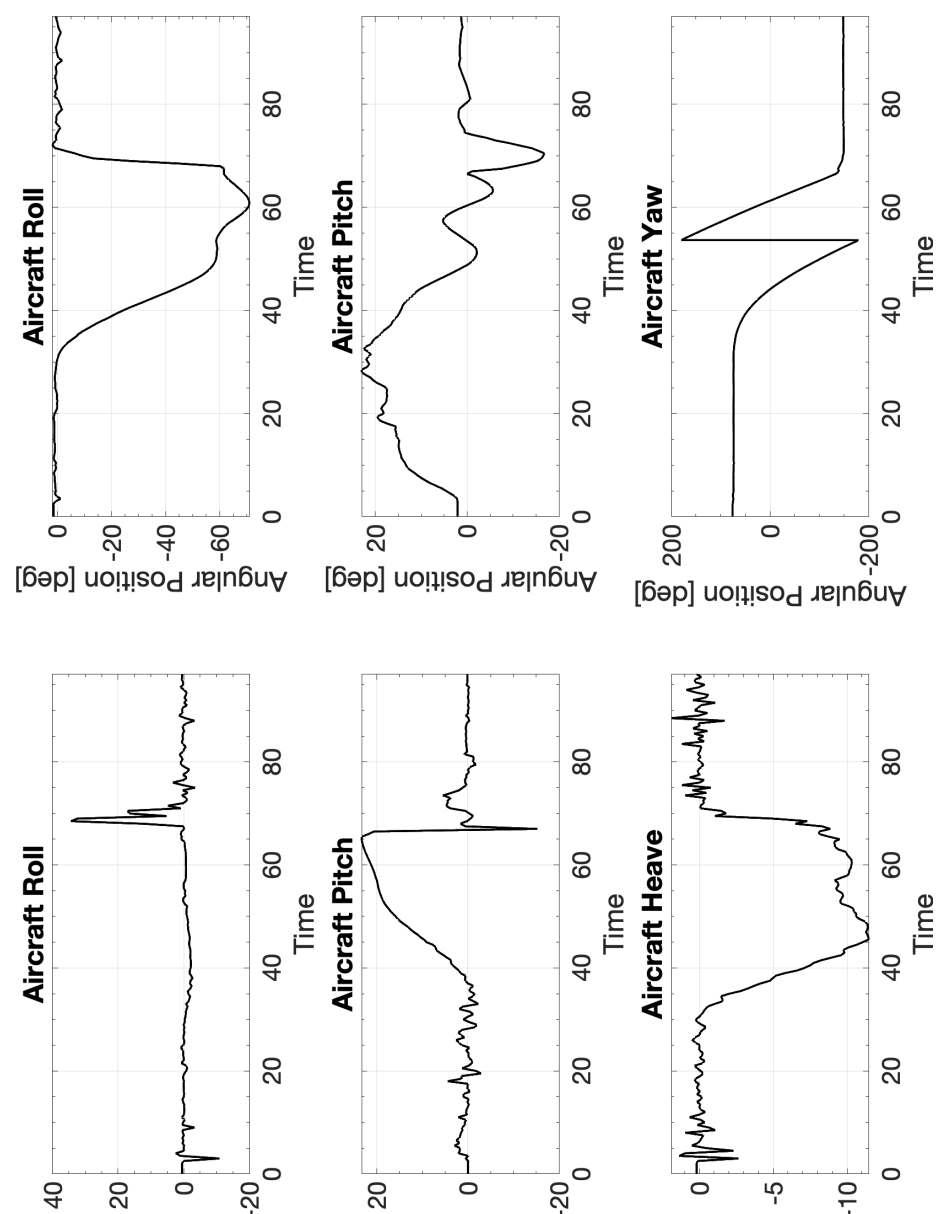

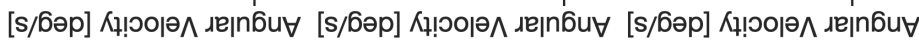

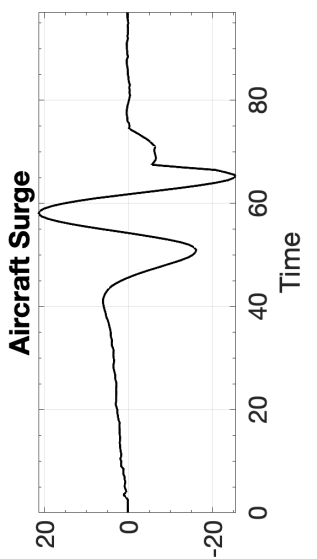

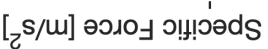

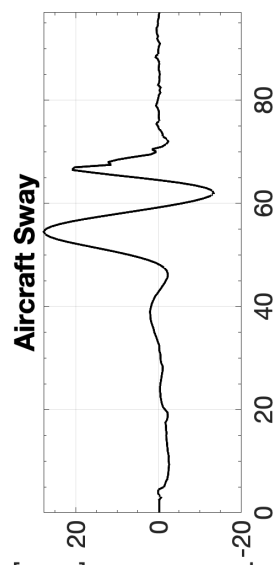

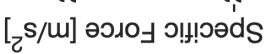

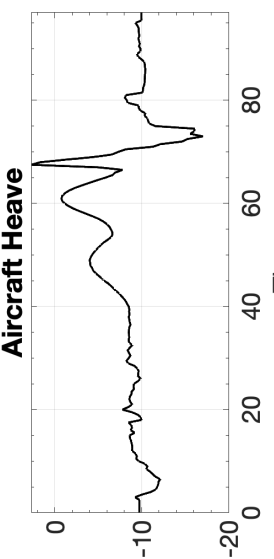

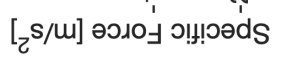
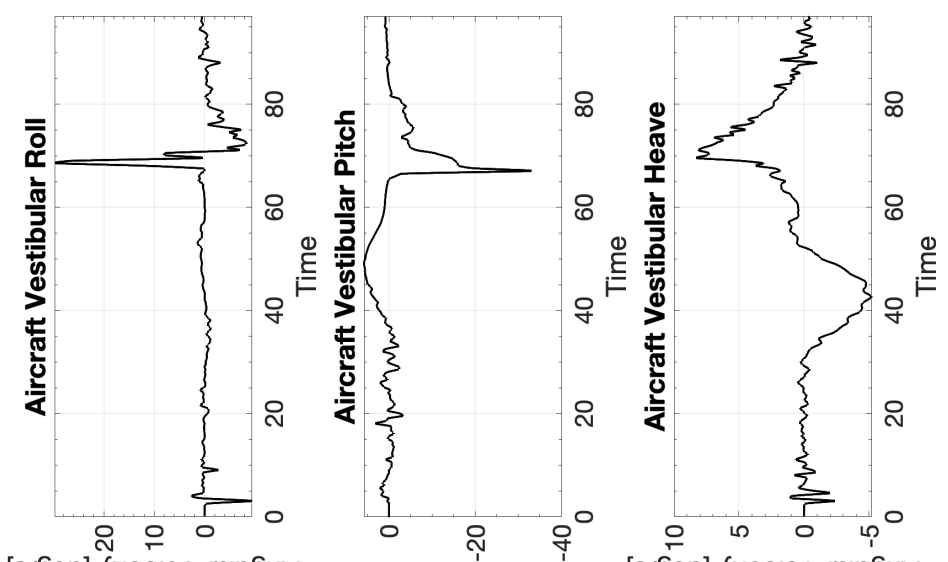

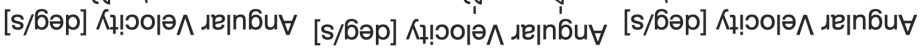

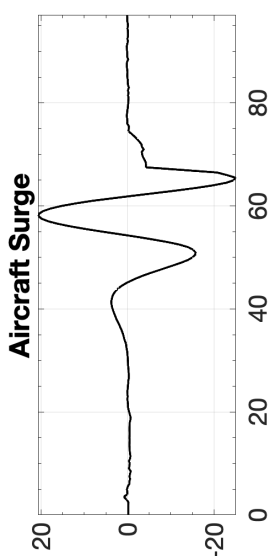

[zs/u] uо!ฺедәрәэ $\forall$

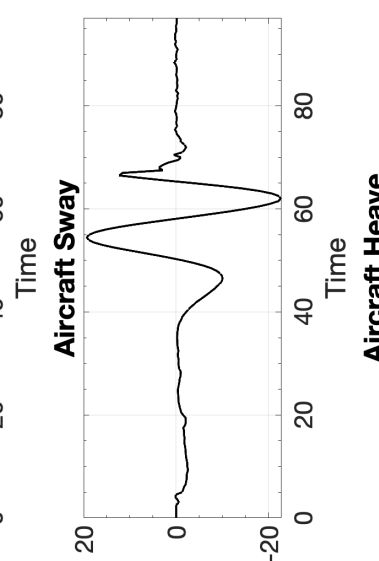

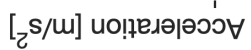

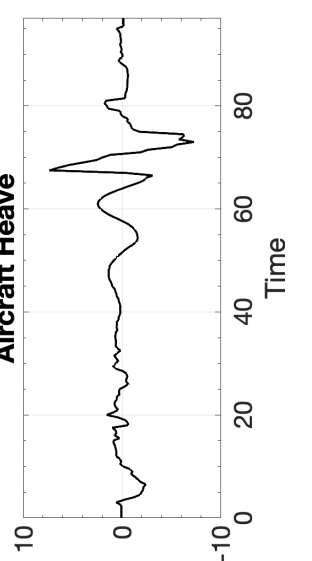

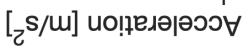

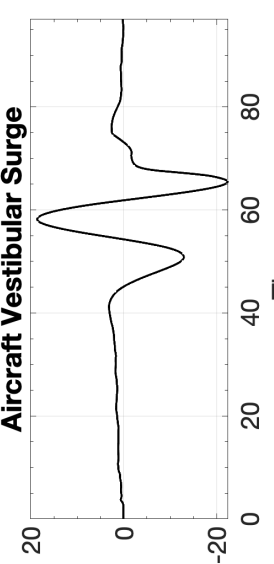

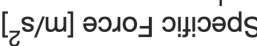

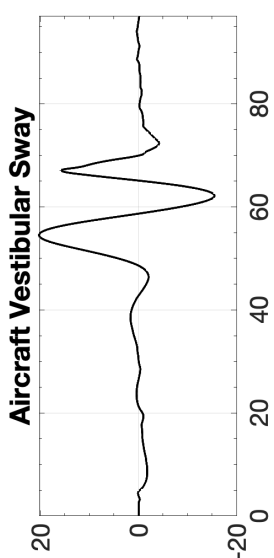

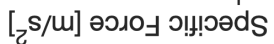

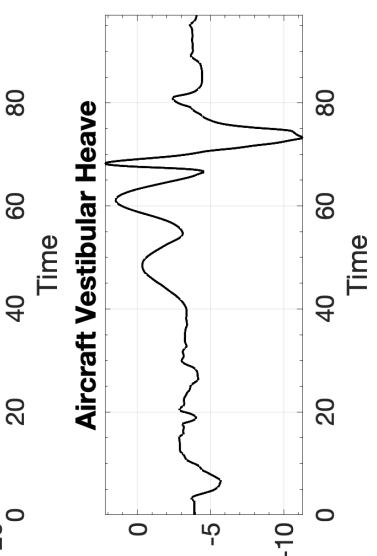

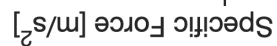




\section{B.18.1 CW1 Columbia 400 Spiral Dive}

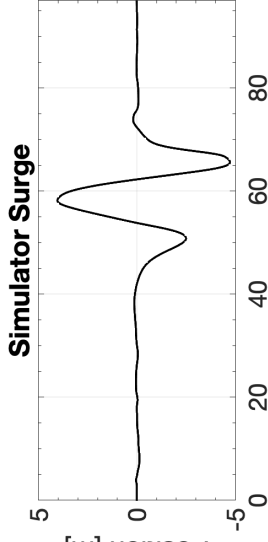

[u] uo!!!!sod

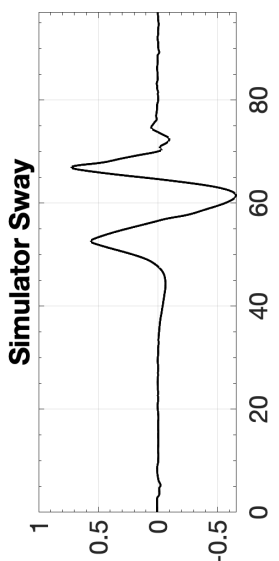

[w] uo!n!!sod

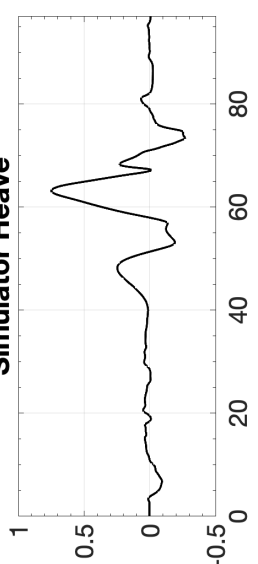

[u] uolp!!sod

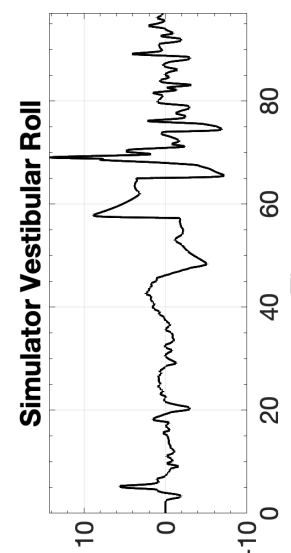

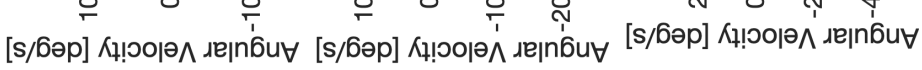

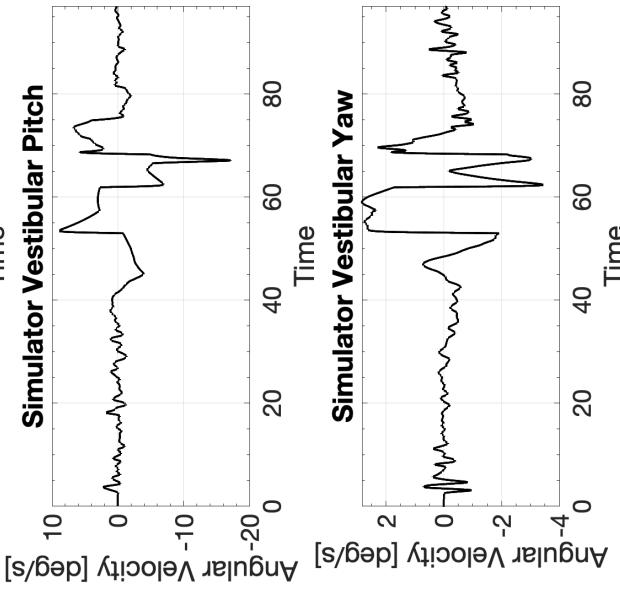

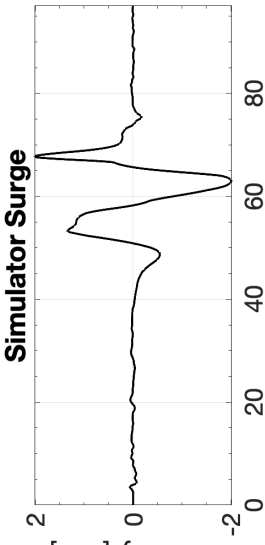

[s/u] Кł!००|ә^
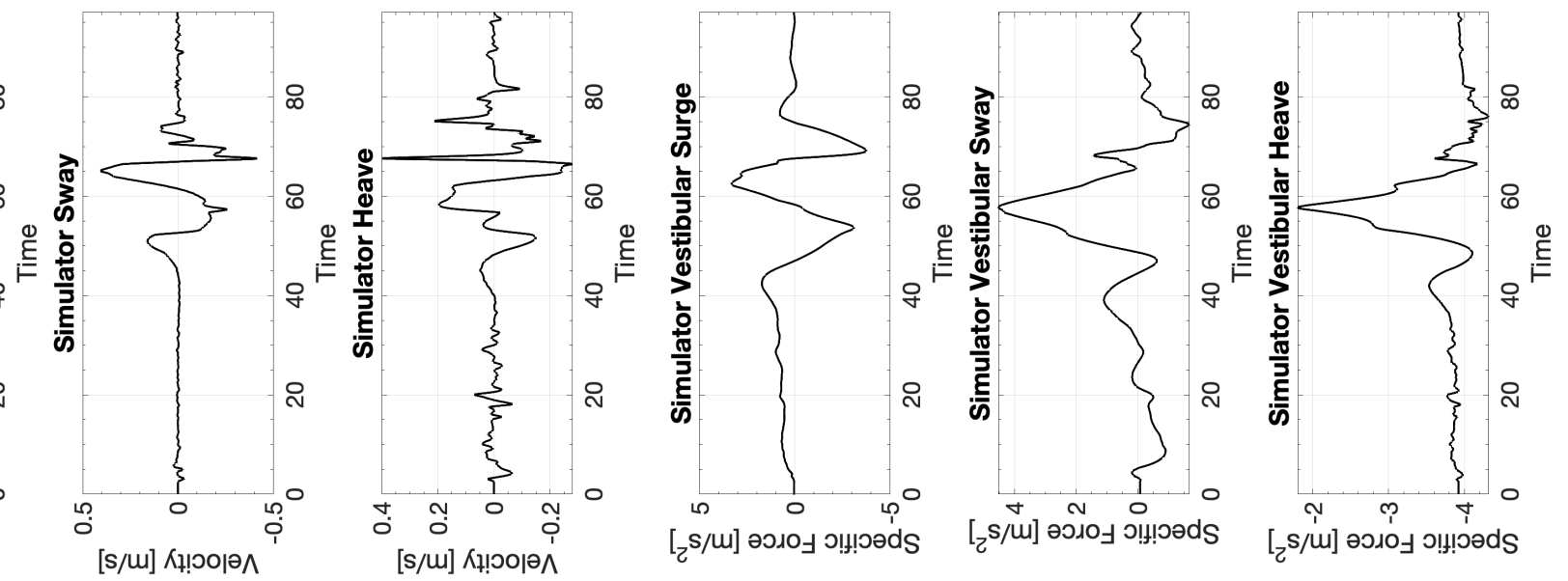

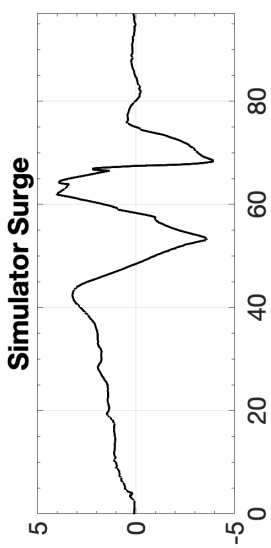

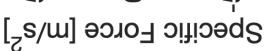

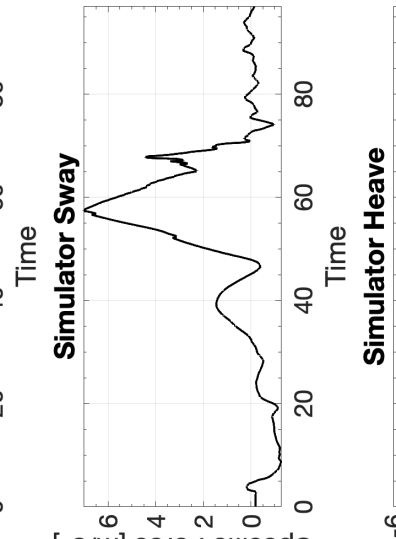

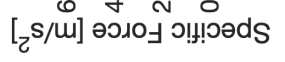

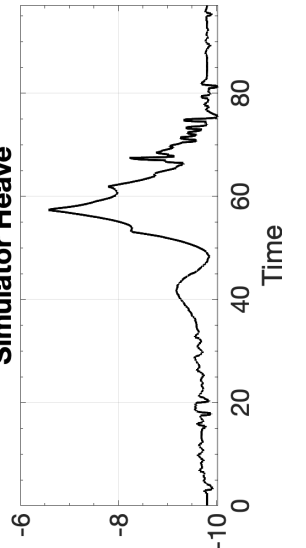

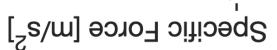

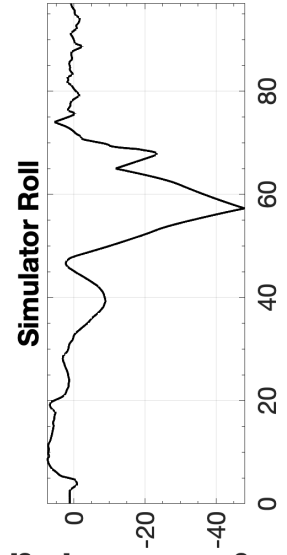

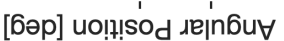

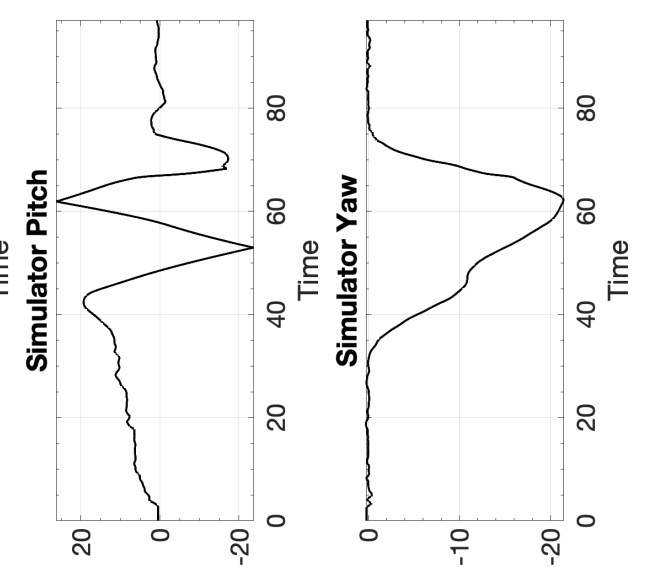

[6әр] uo!!!

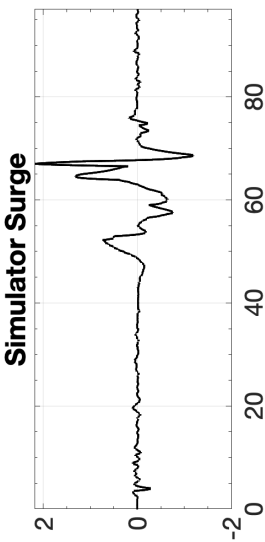

[zs/u] uо!ןелә|әэว

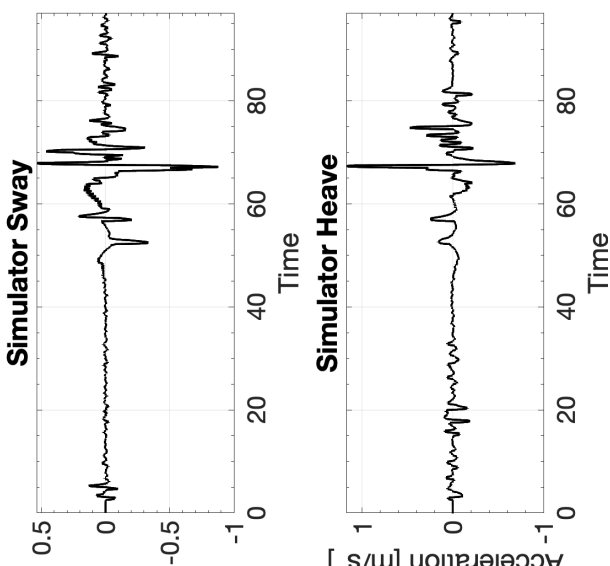

[zs/w] иопцедәәәоэн
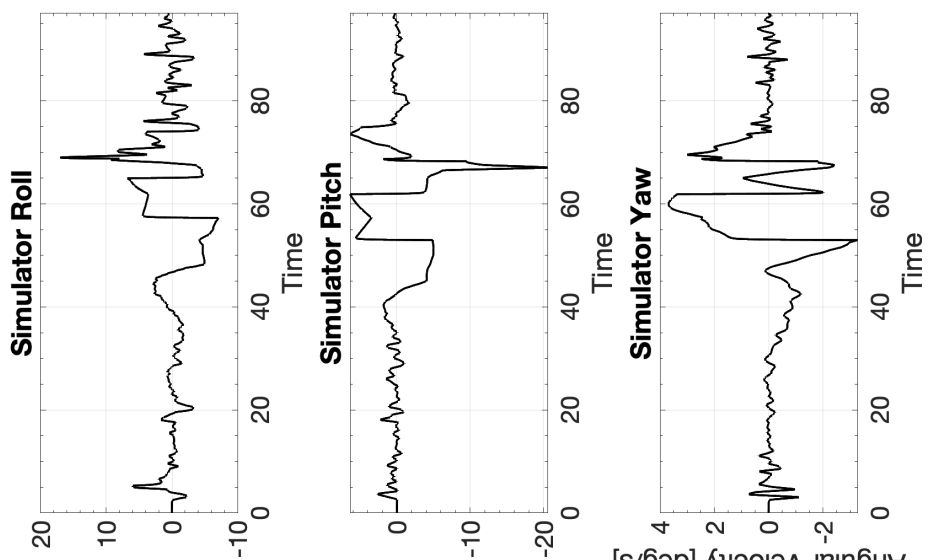


\section{B.18.2 CW2 Columbia 400 Spiral Dive}

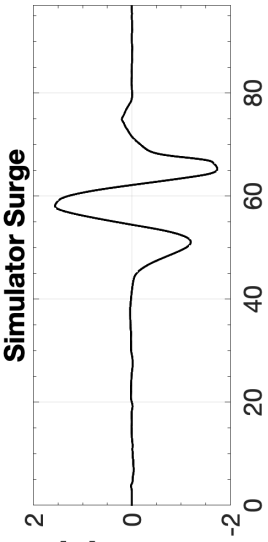

[u] uo!!!!sod

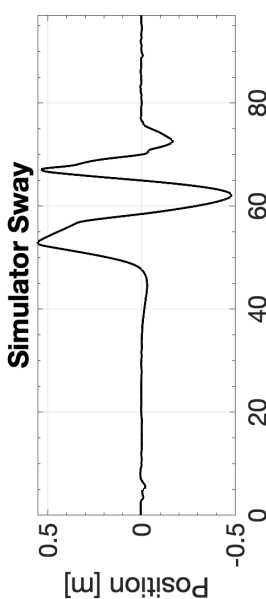

[m] uol!!!sod

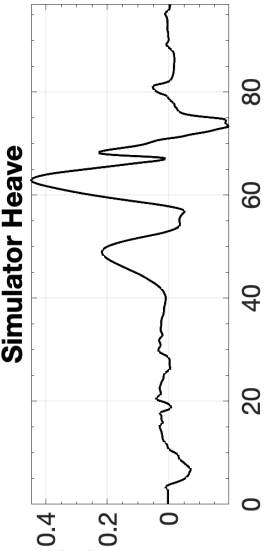

[u] uolt!nsod

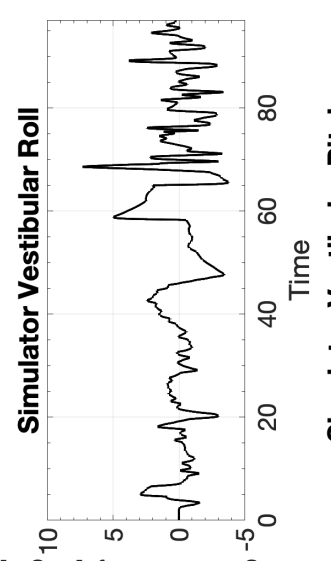

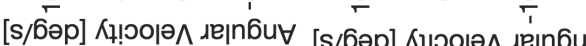

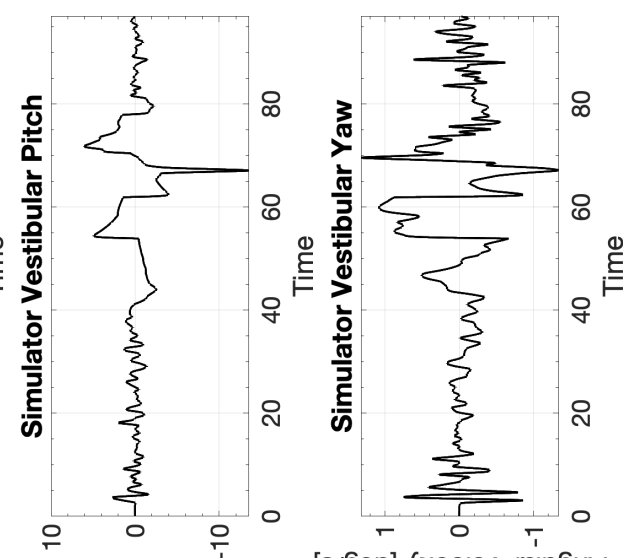

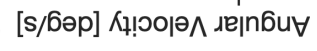

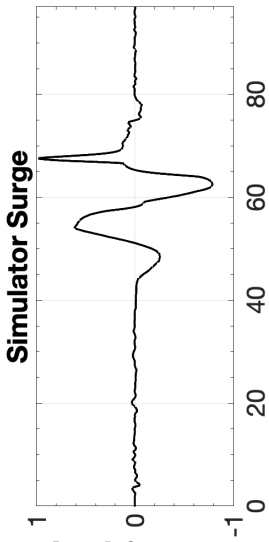

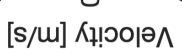

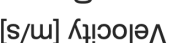

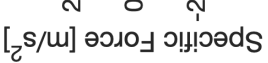

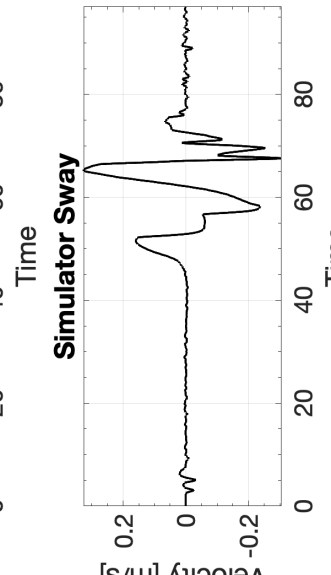

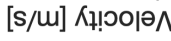

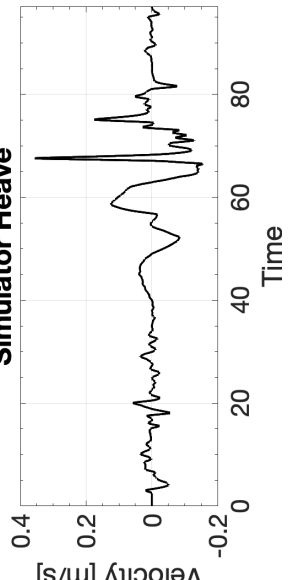

[s/u] К! ৩о।ә^
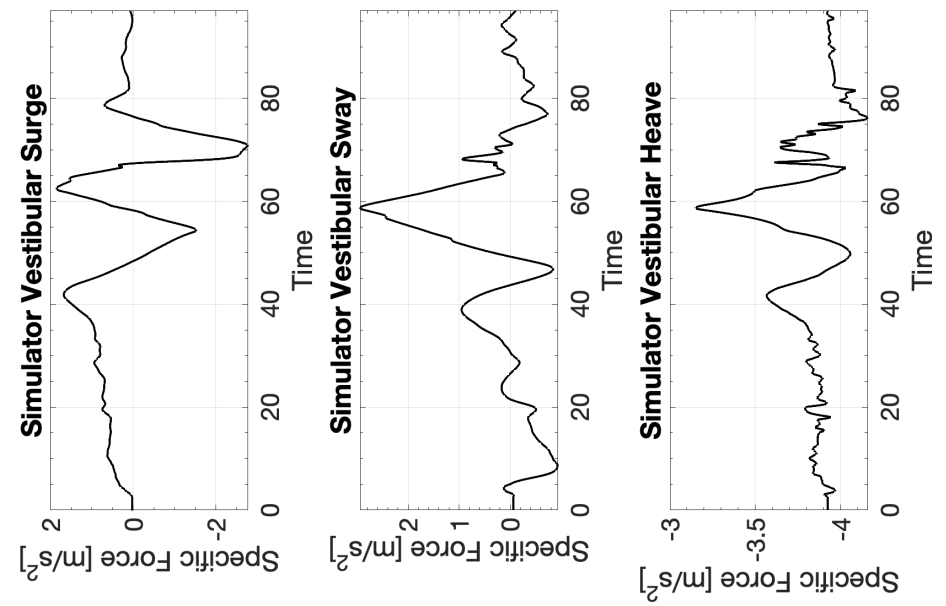

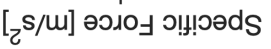

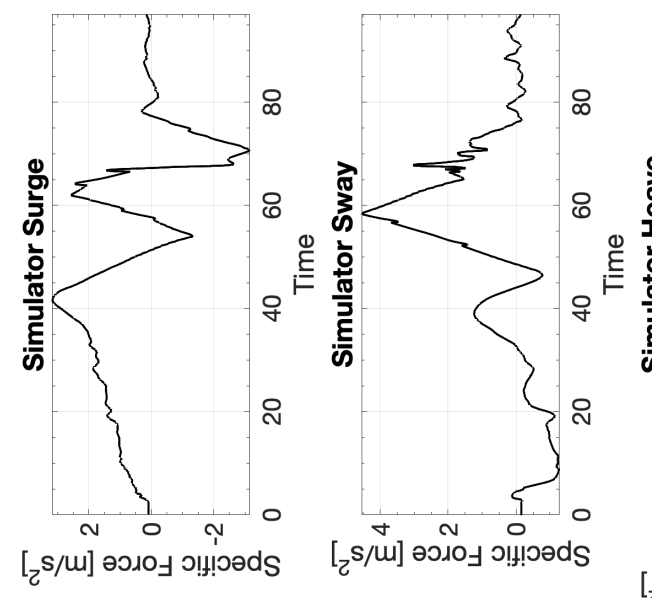

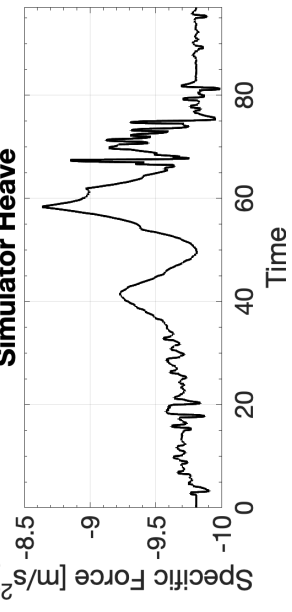
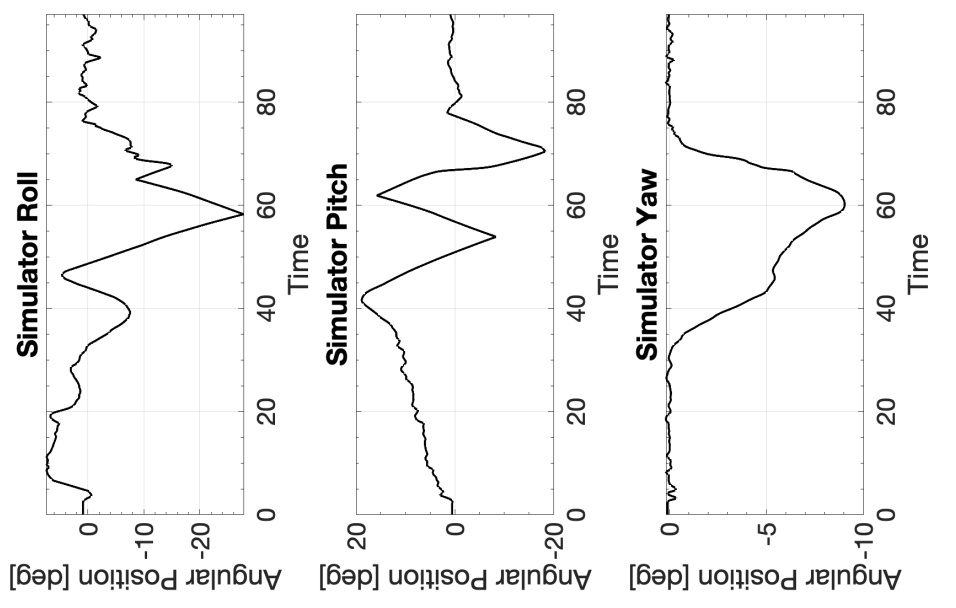

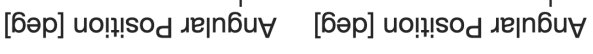

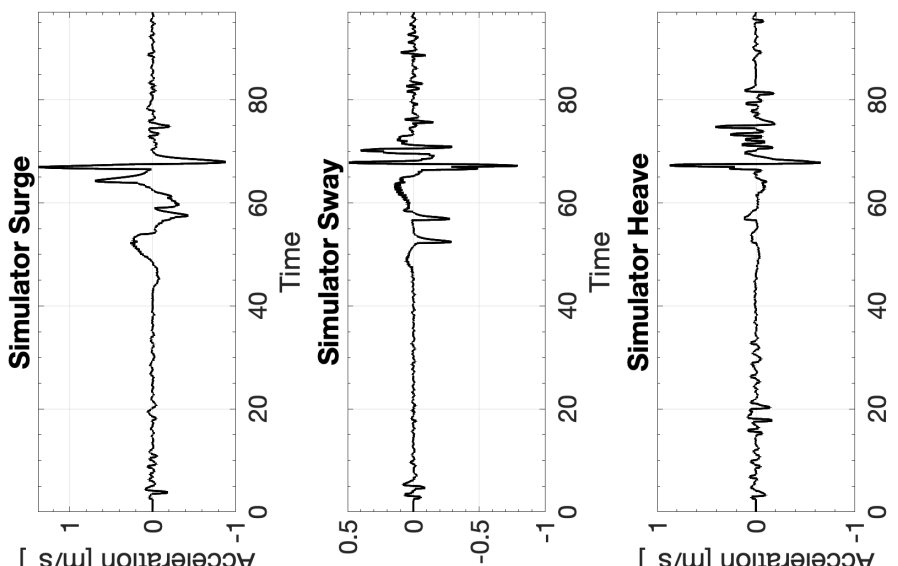

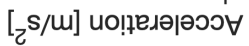

$[\mathrm{s} / \mathrm{L}]$ иопฺе
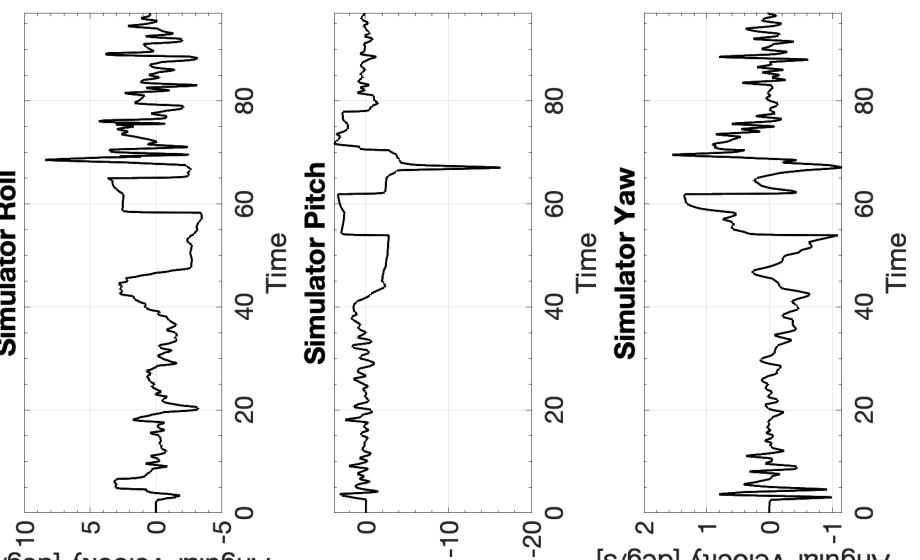


\section{B.18.3 CW3 Columbia 400 Spiral Dive}

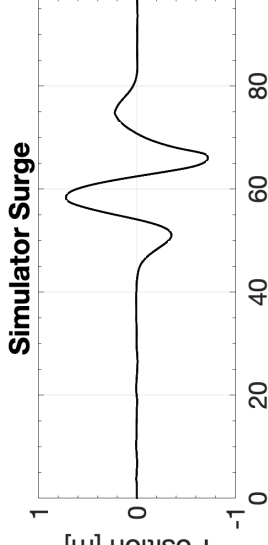

[u] uo!!!!sod

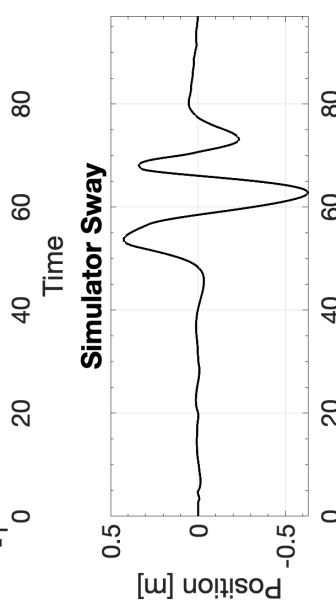

[u] uol!!!sod

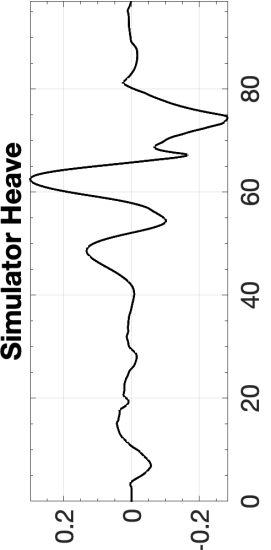

[w] uolp!ssod

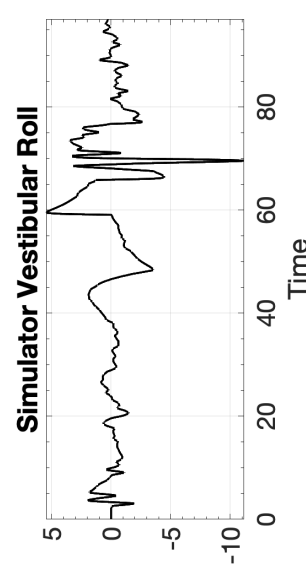

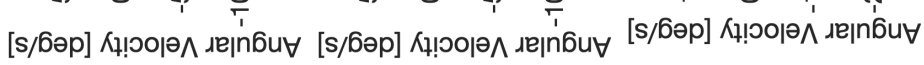

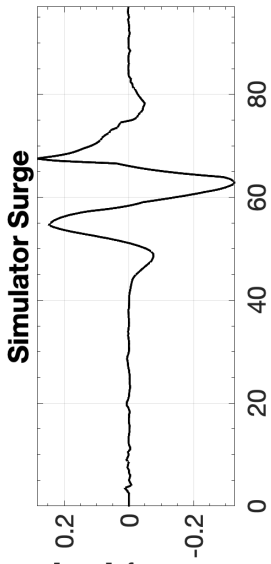

[s/m] Кұ!००|ә^

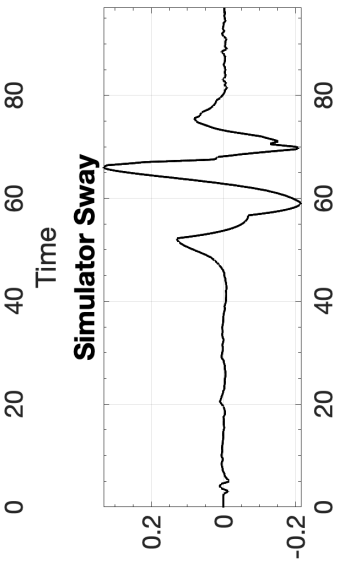

[s/m] Кұ!००|ә^

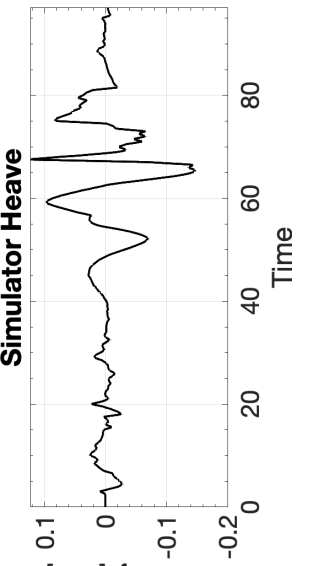

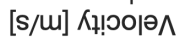
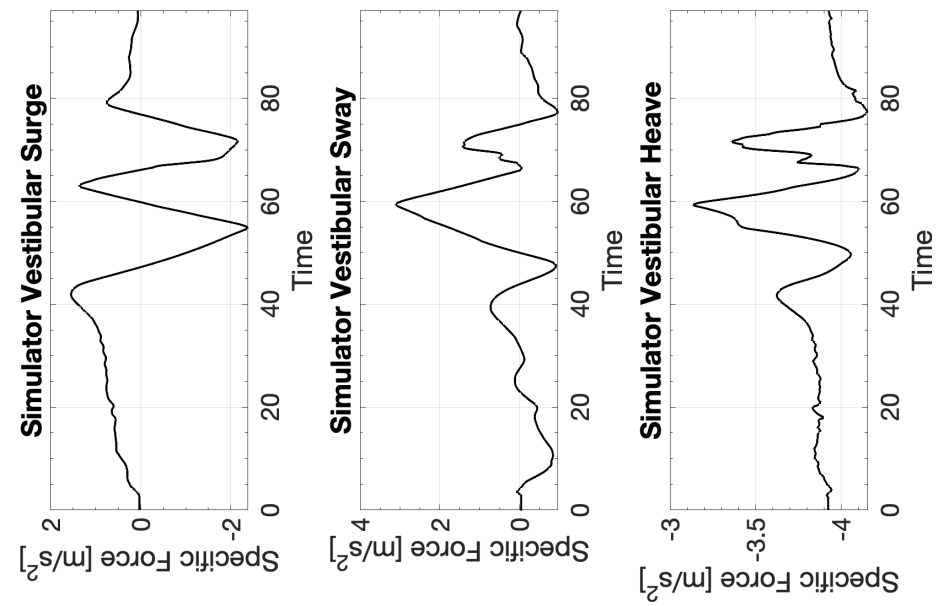

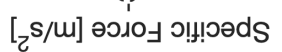

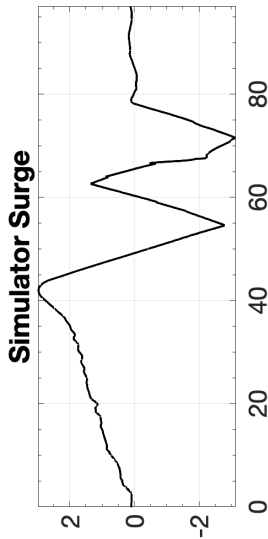

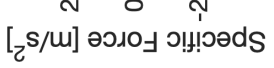

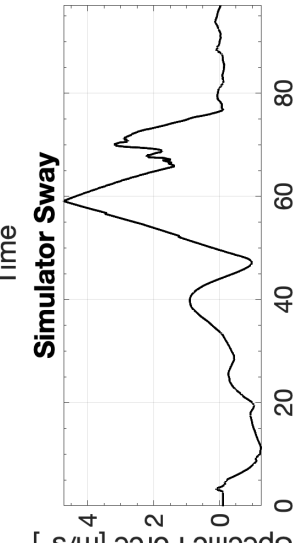

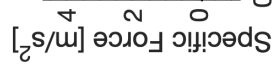
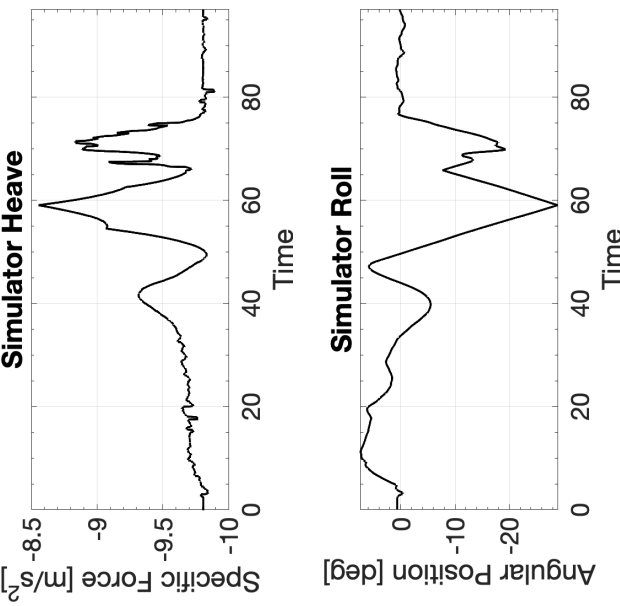

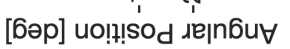

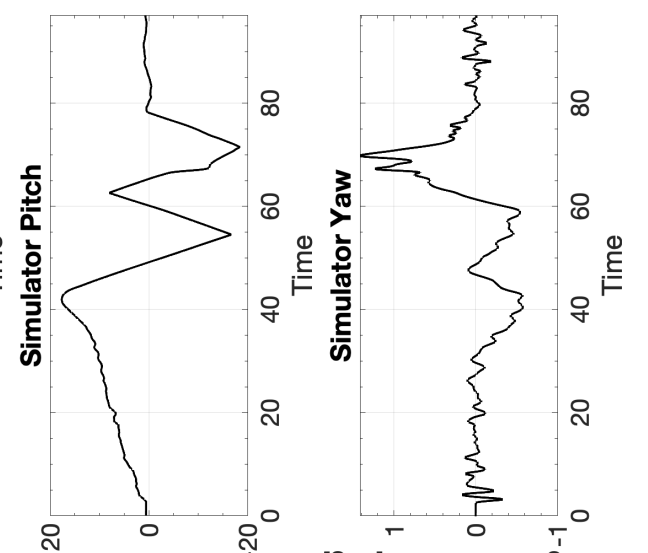

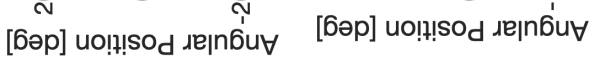

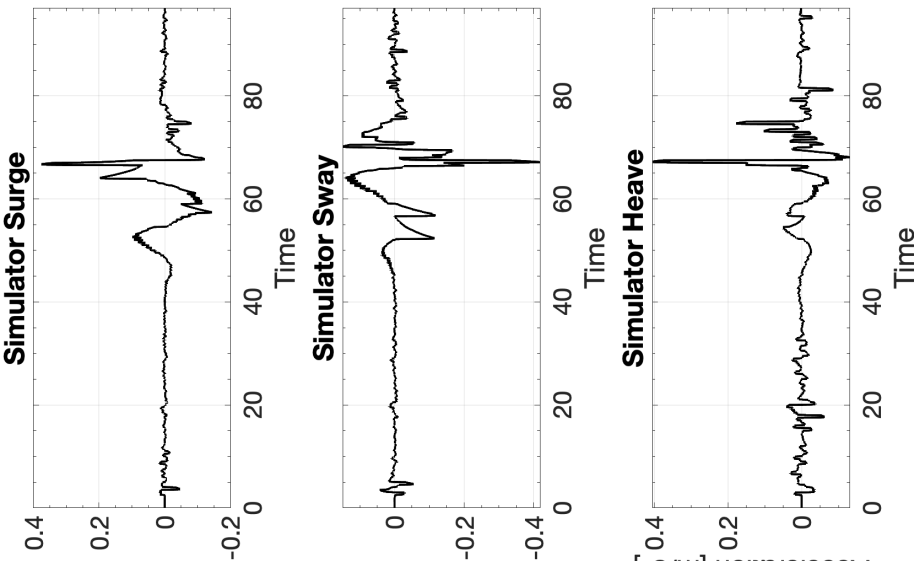

[zs/u] ио!ฺедә|әэว $\forall$

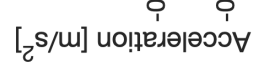
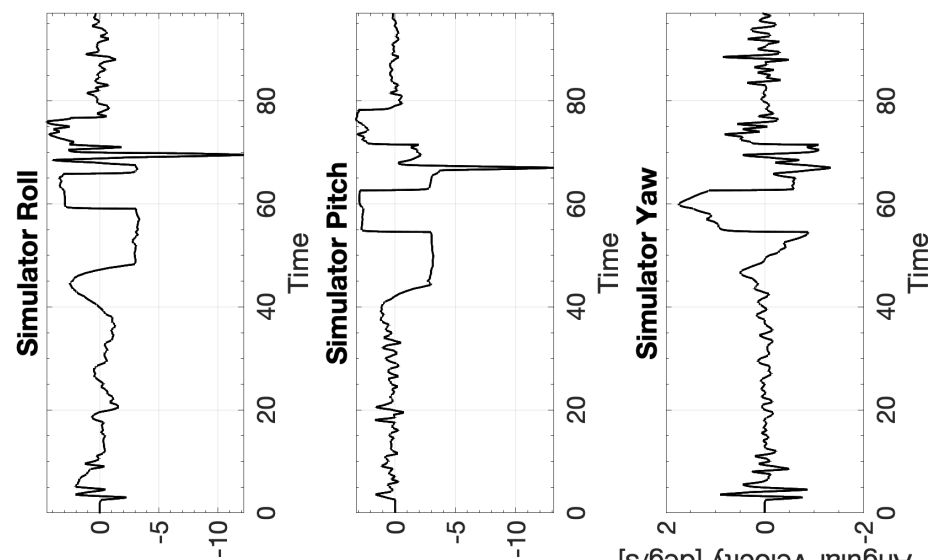


\section{B.19 Columbia 400 Stall Recovery}

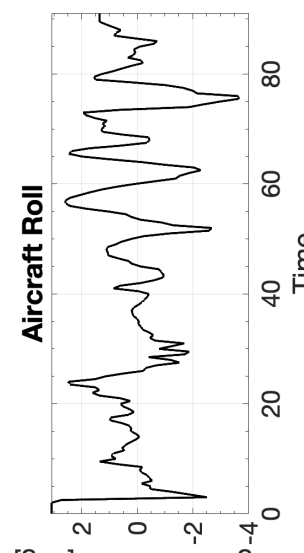

[6әр] uo!!!sod
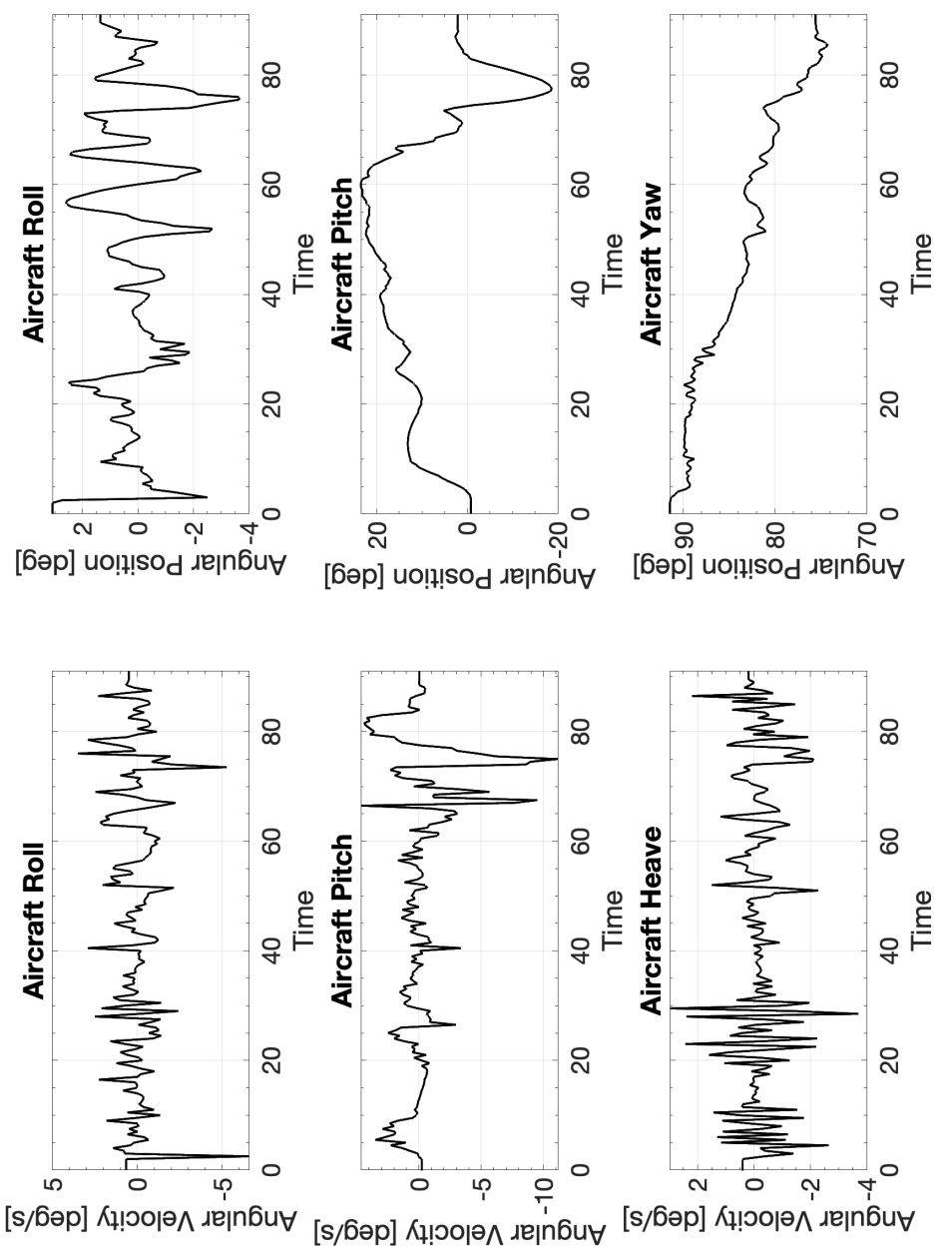

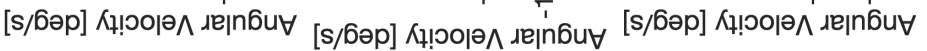

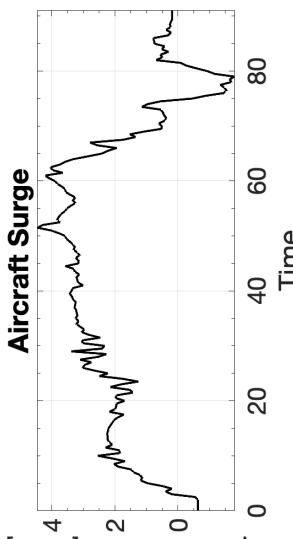

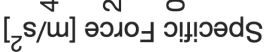

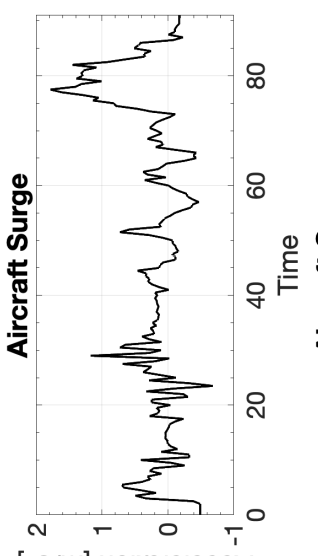

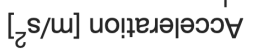
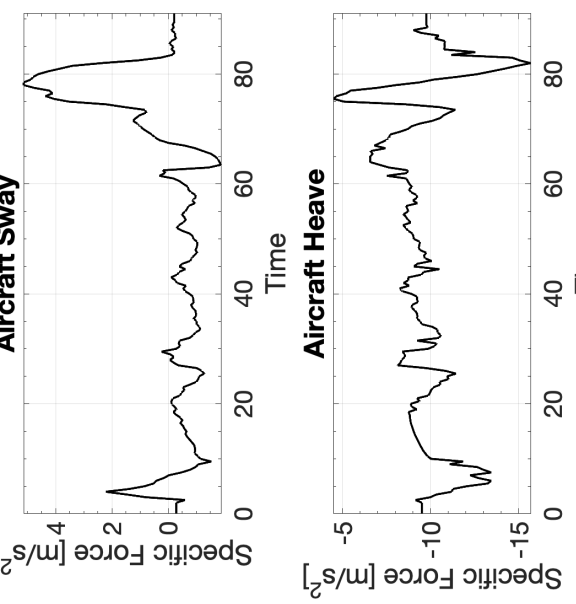

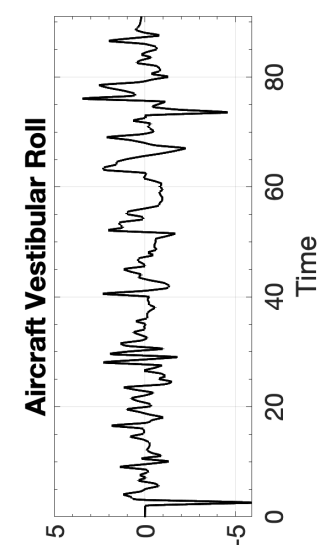

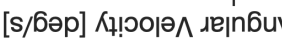

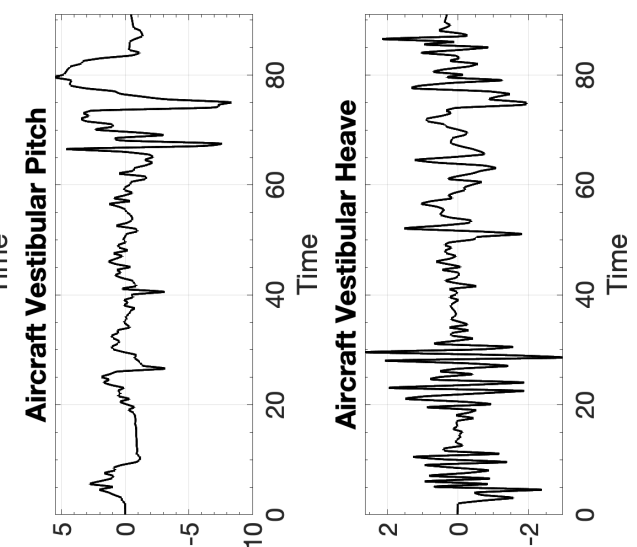

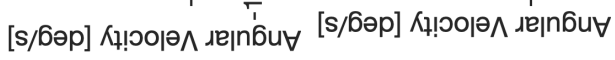

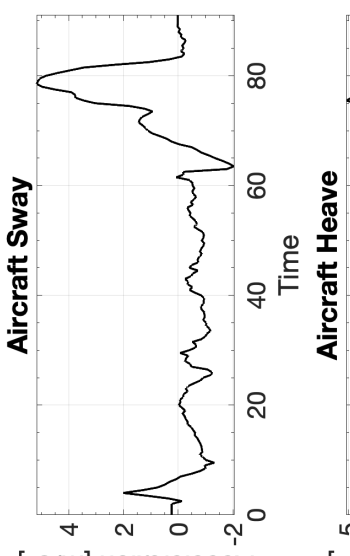

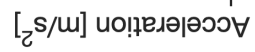

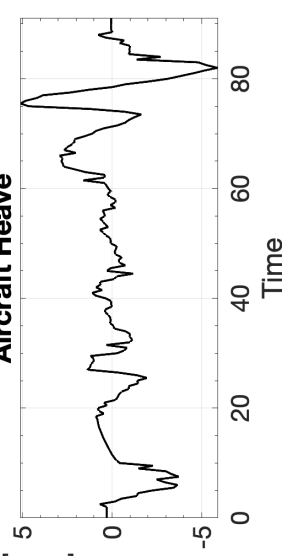

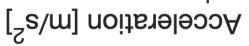

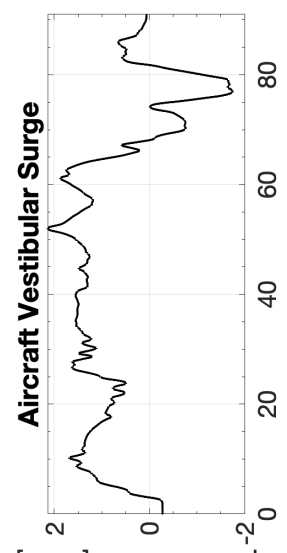

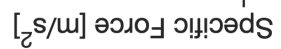

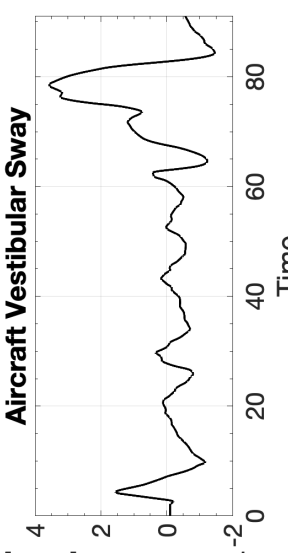

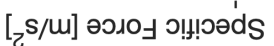

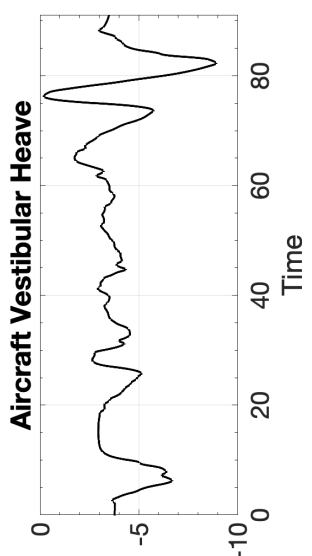

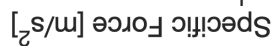




\section{B.19.1 CW1 Columbia 400 Stall Recovery}

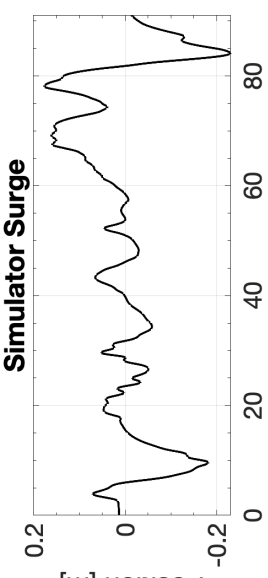

[u] uo!n! $\mathrm{Sod}^{\mathrm{d}}$

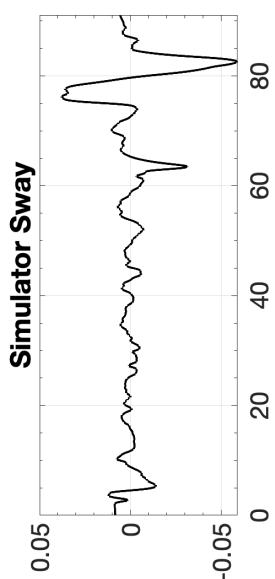

[u] uo!n! sod

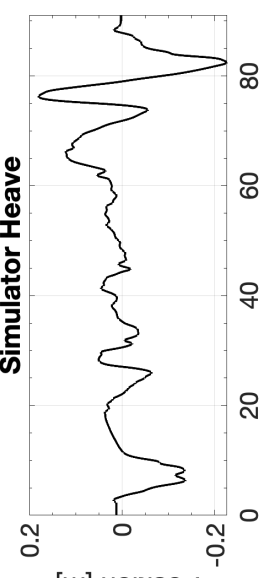

[w] uo!!!!sod

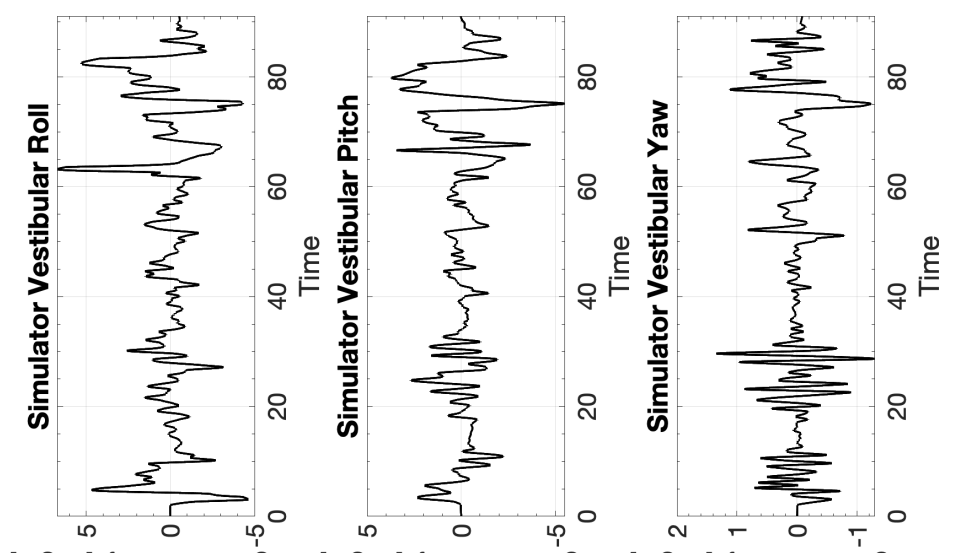

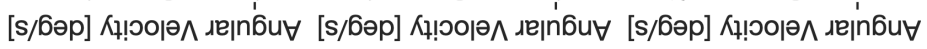

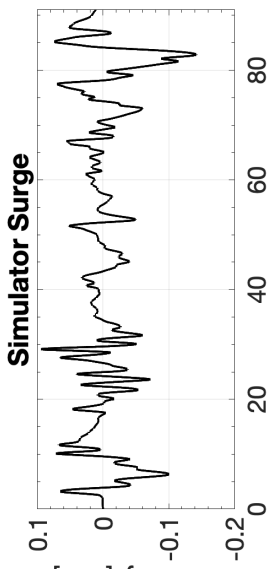

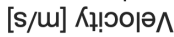

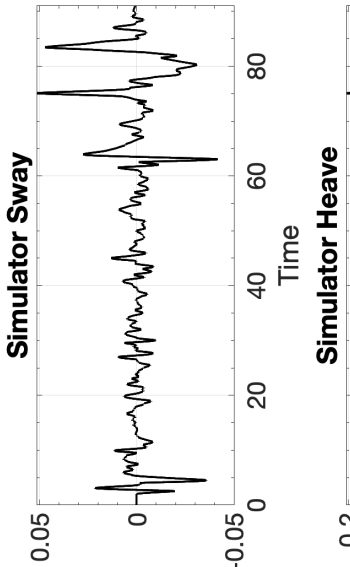

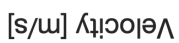

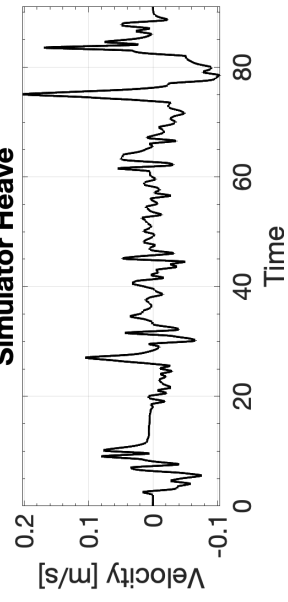

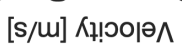

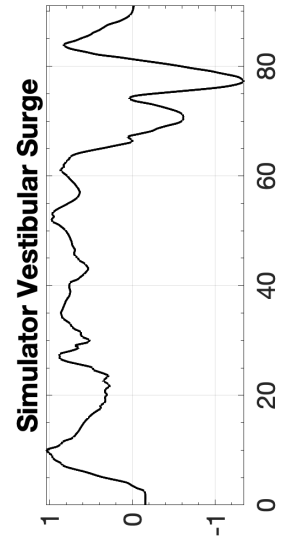

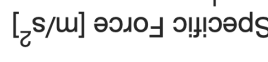
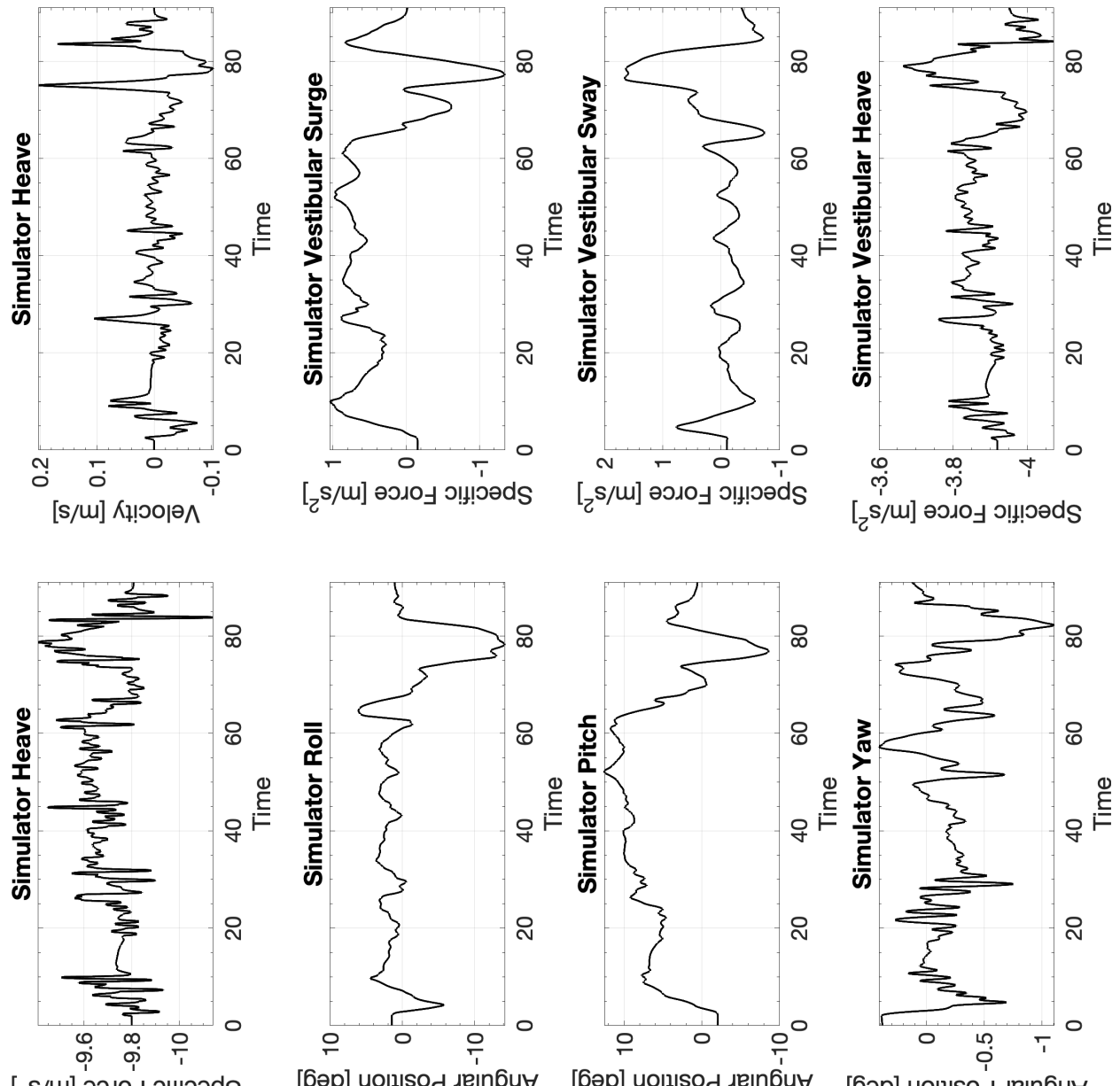

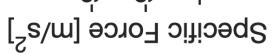

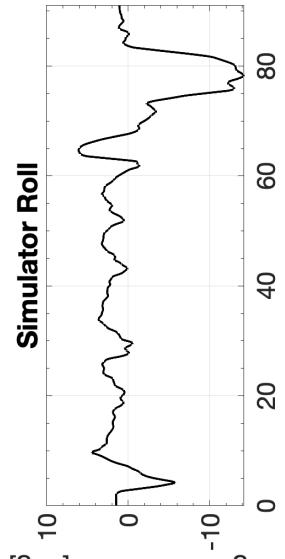

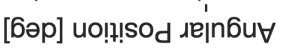

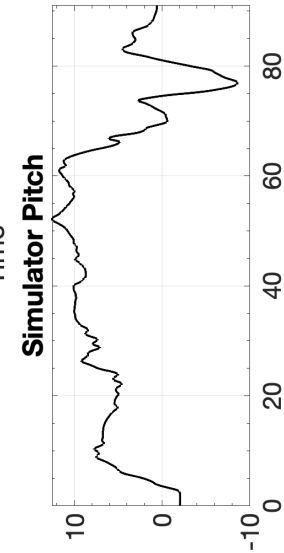

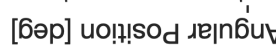

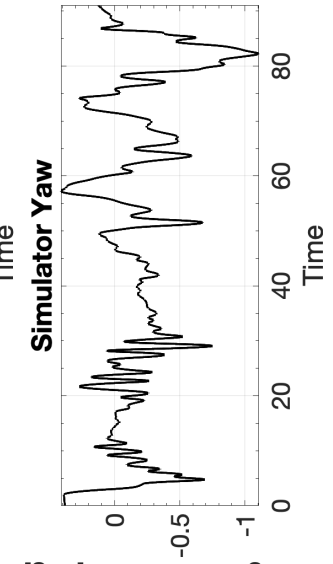

[6әр] uo!!!sod ıе|n6u

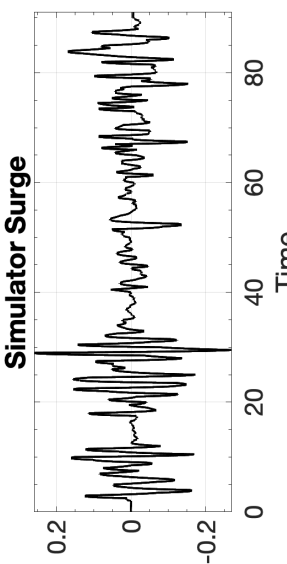

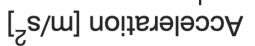

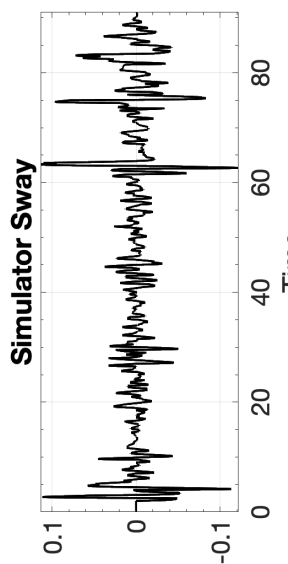

$[\mathrm{s} / \mathrm{s}]$ uо!ฺвдәәәээ

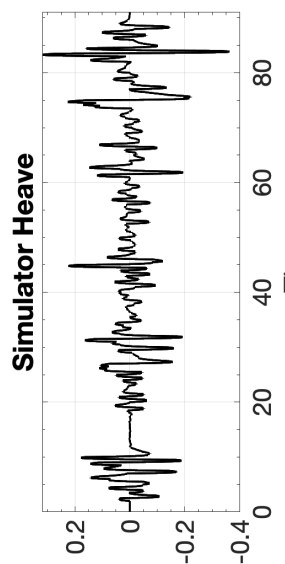

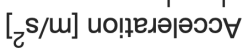

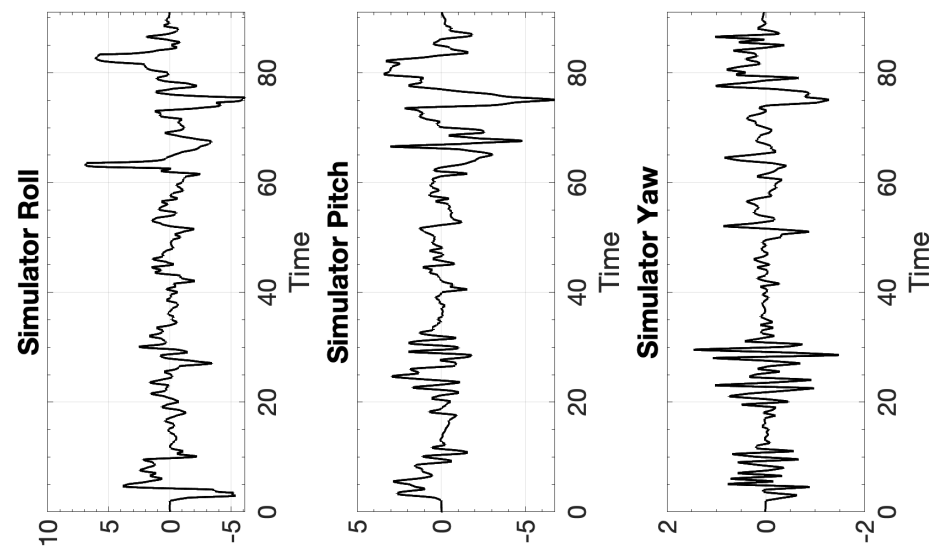

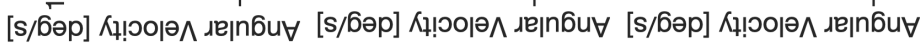




\section{B.19.2 CW2 Columbia 400 Stall Recovery}

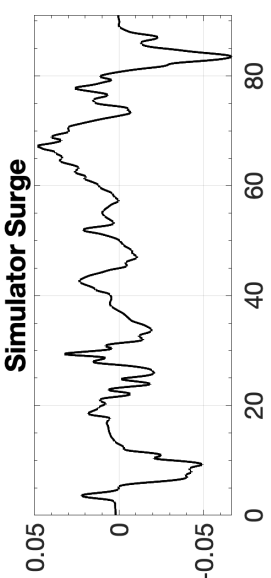

[w] uo!n!

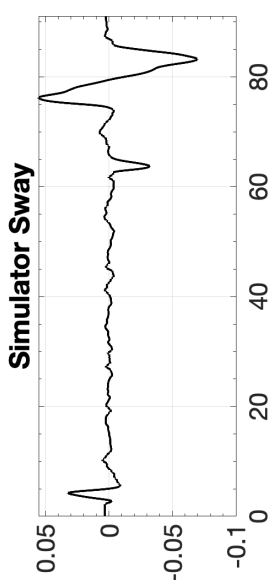

[u] uo!n!

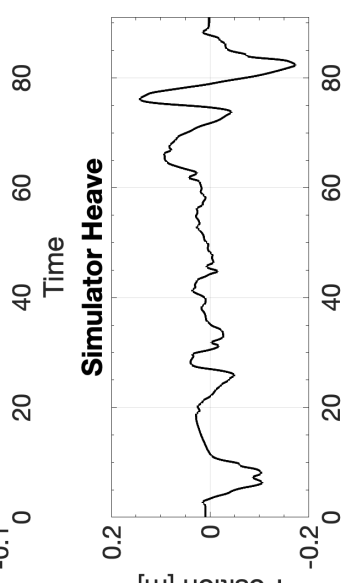

[u] uo!t!sod

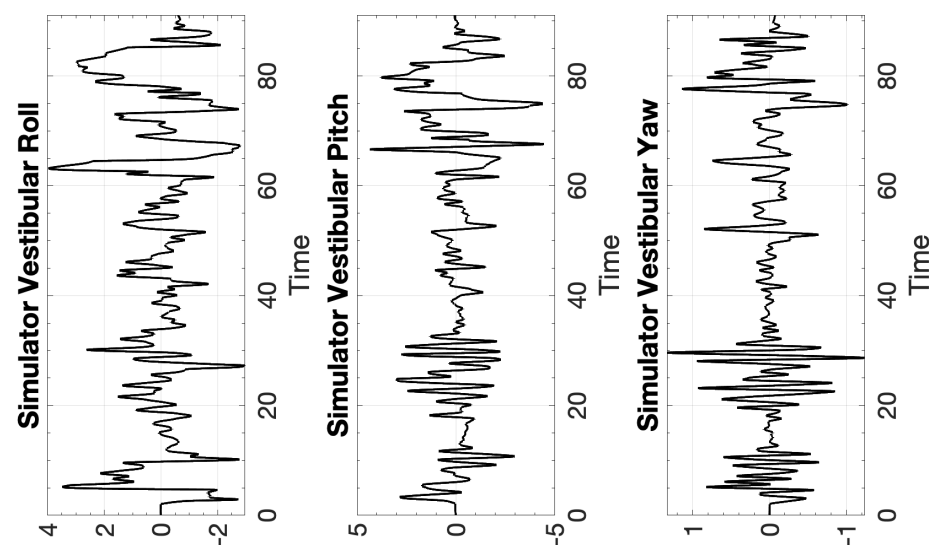

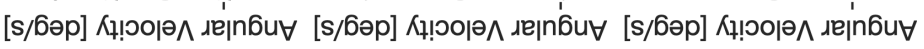

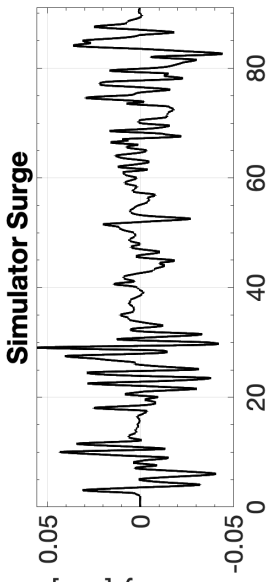

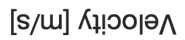

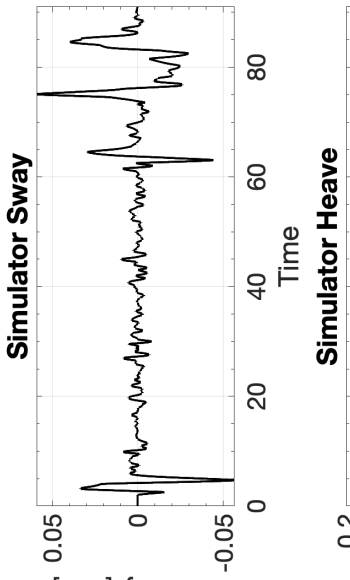

[s/m] Кџ!৩ою^

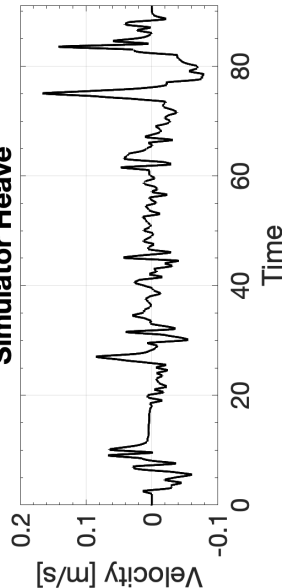

[s/m] Кұ!৩о夫

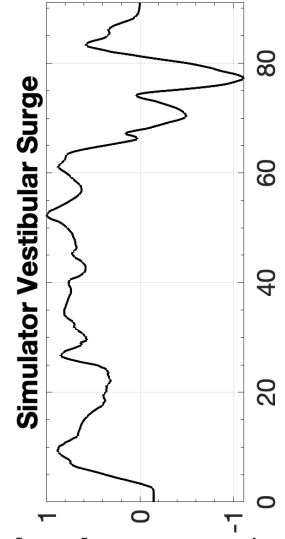

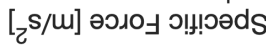

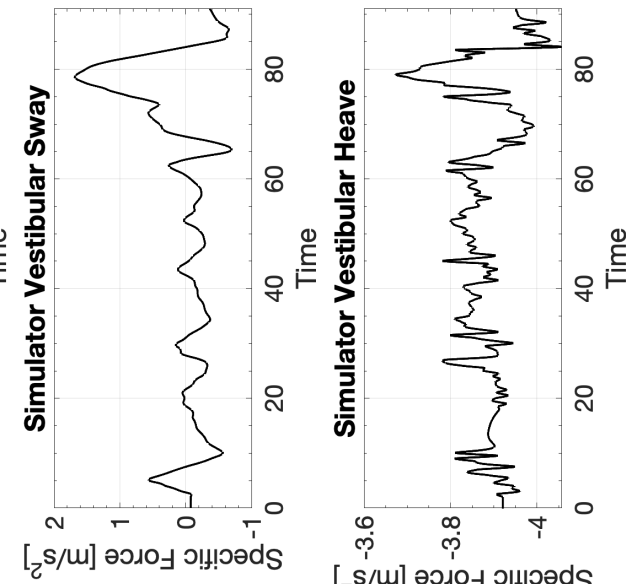

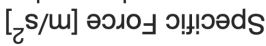

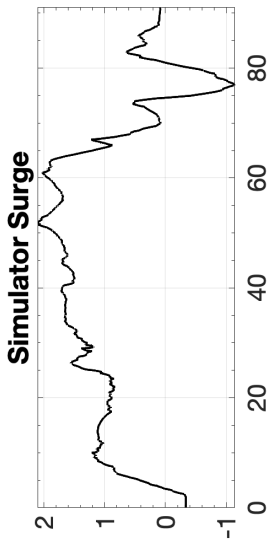

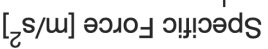

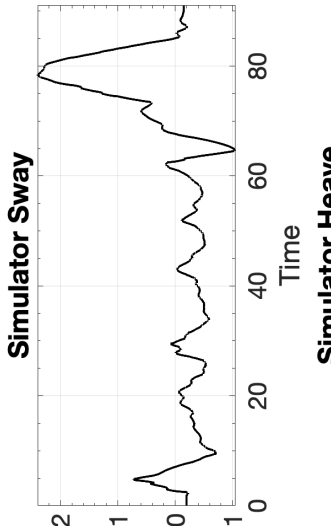

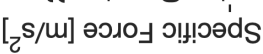

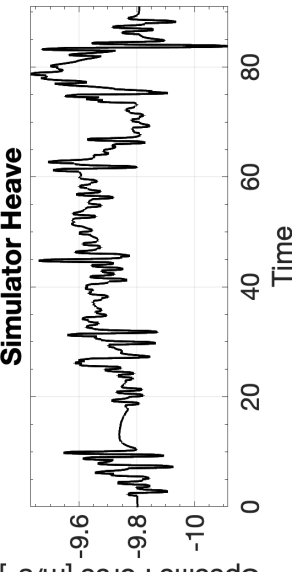

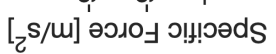

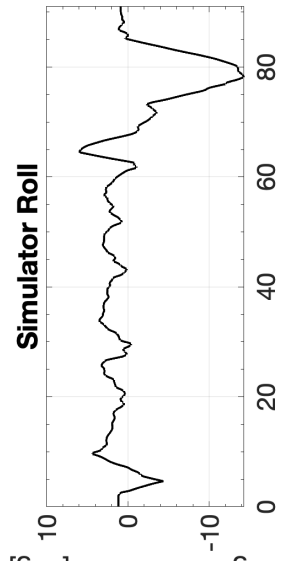

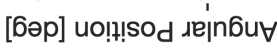

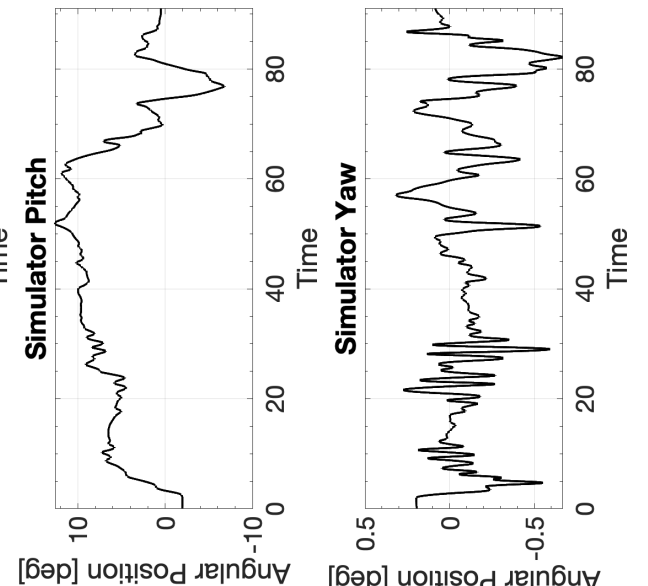

[6әp] uo!!!sod

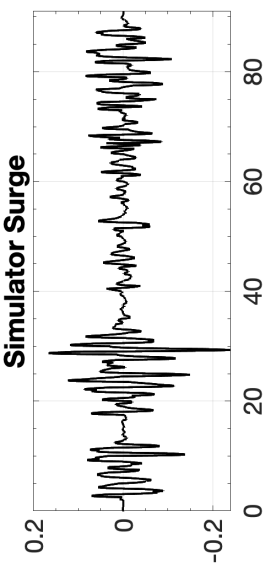

$[\mathrm{c} / \mathrm{L}] \mathrm{uo!̣}$

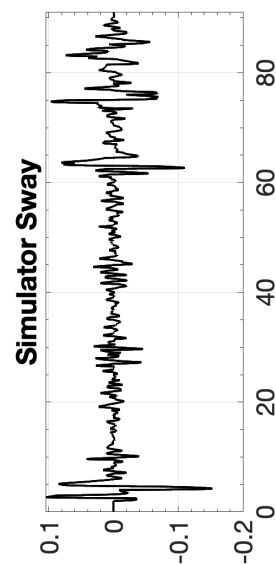

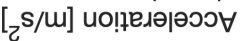

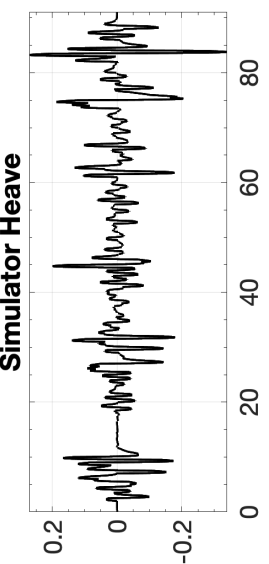

$[\mathrm{s} / \mathrm{m}]$ ио!ฺвдәәәэว

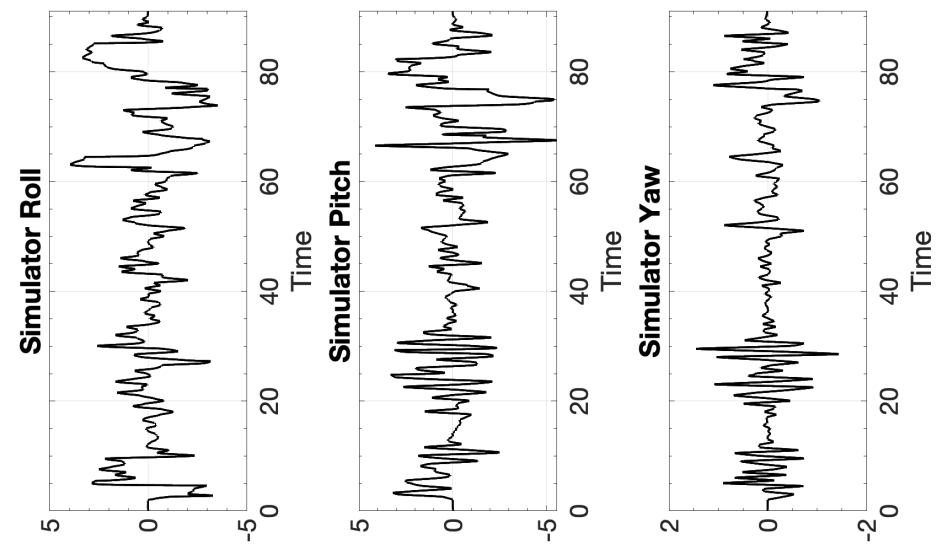

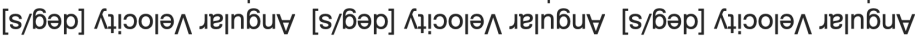




\section{B.19.3 CW3 Columbia 400 Stall Recovery}

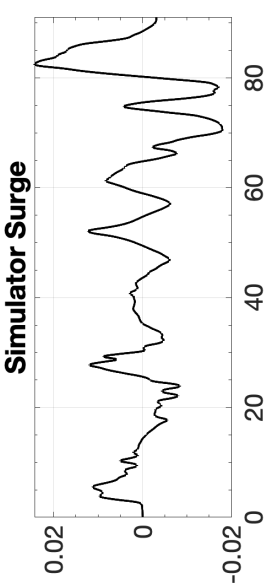

[u] uo!!!!sod

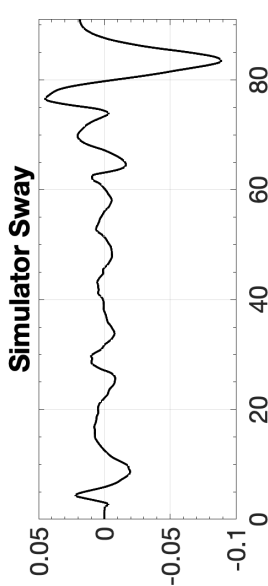

[u] uolp!!sod

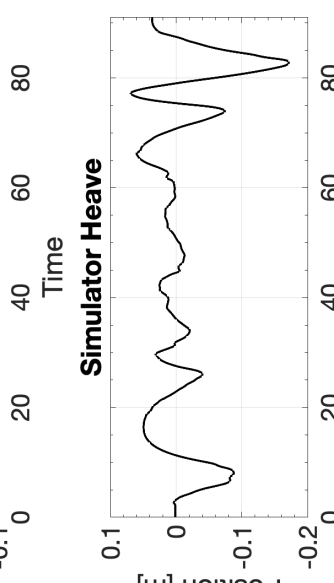

[u] uolt!!sod

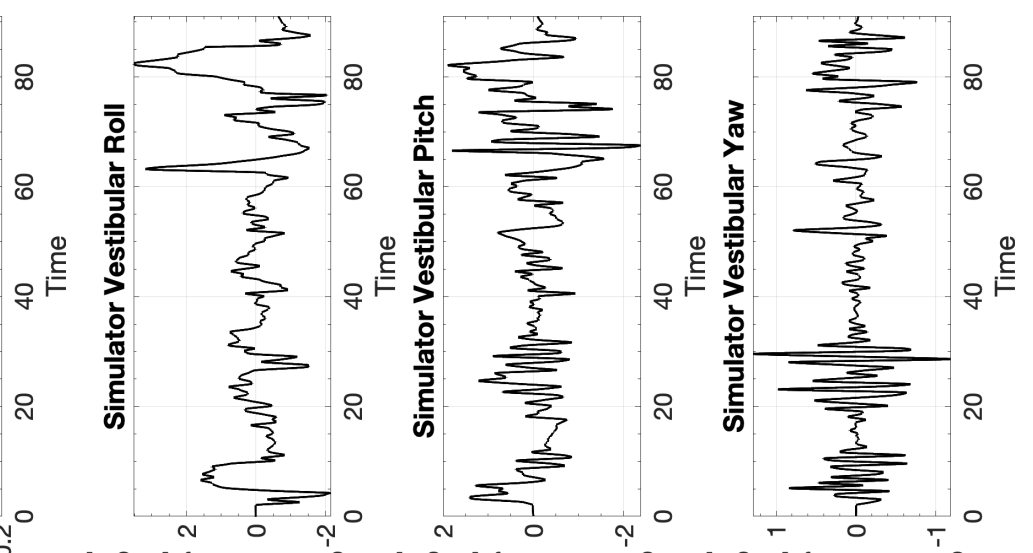

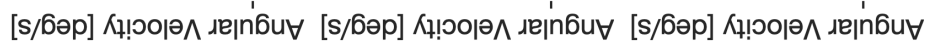

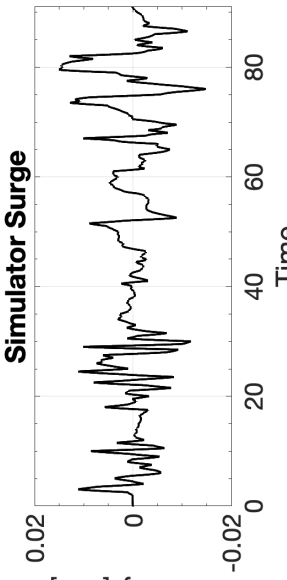

[s/m] Кұ!วо|ә^

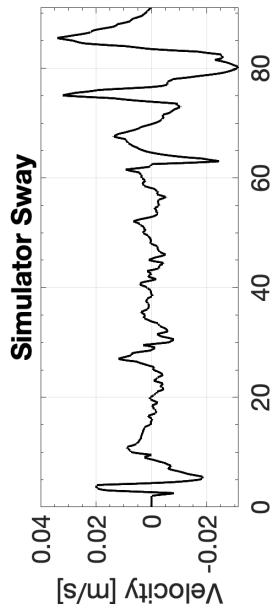

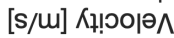

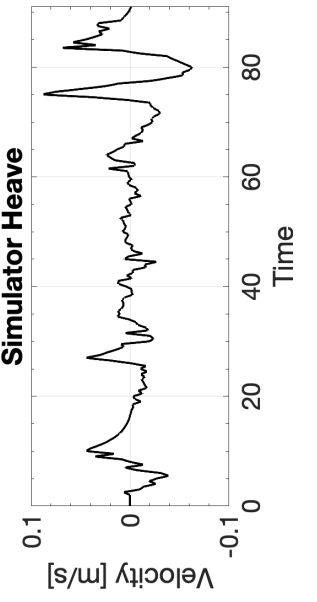

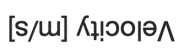
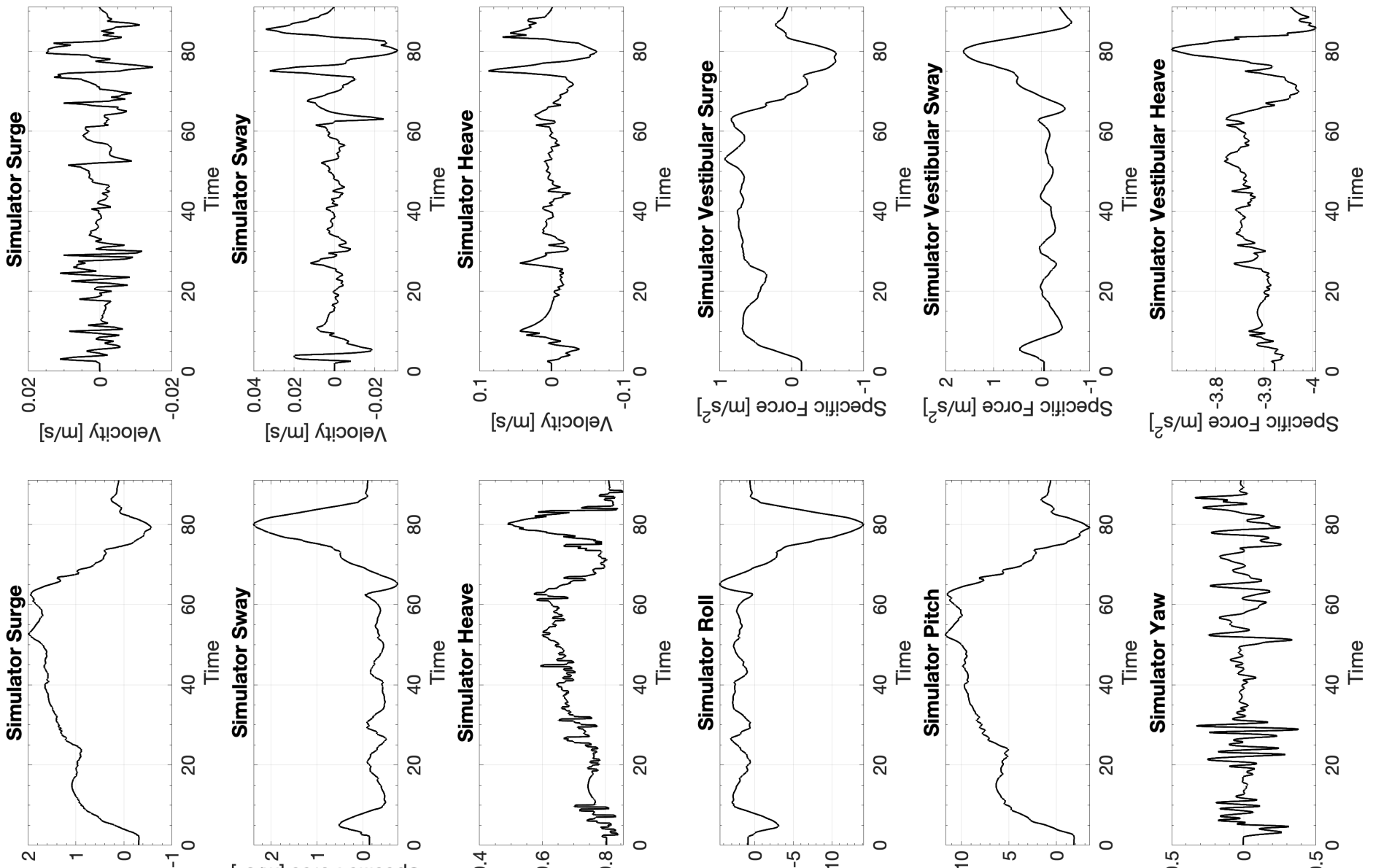

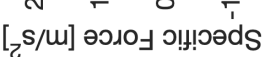

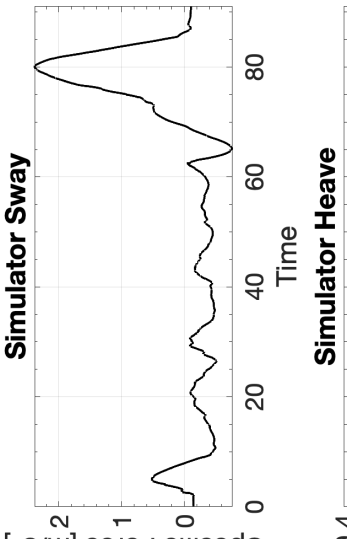

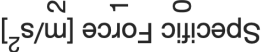
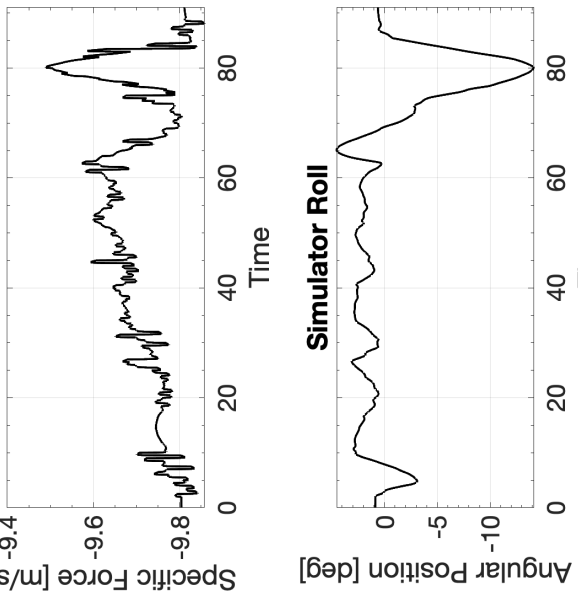

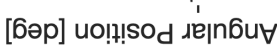

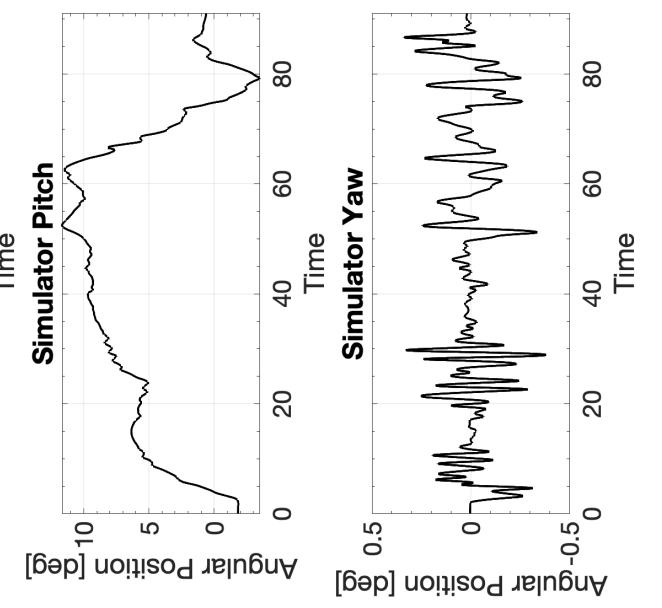

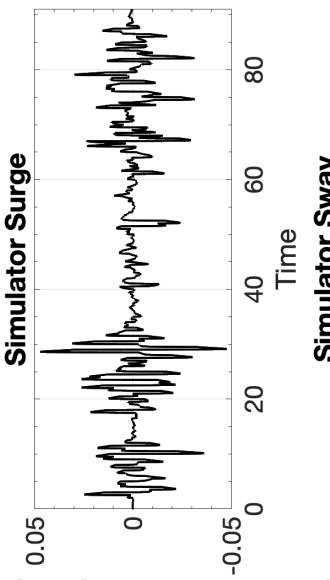

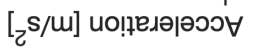

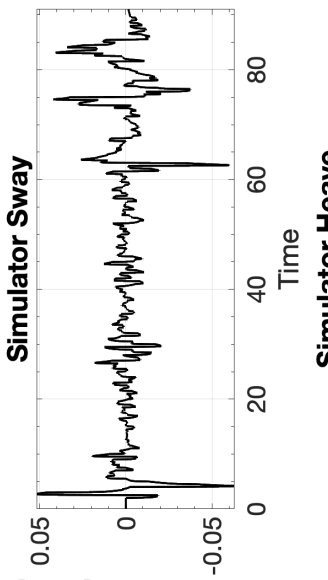

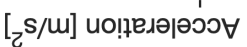

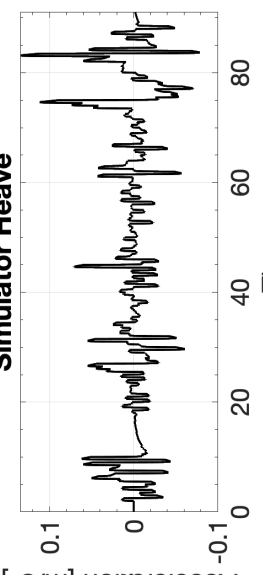

[z/m] ио!ฺеגә|әоэ甘

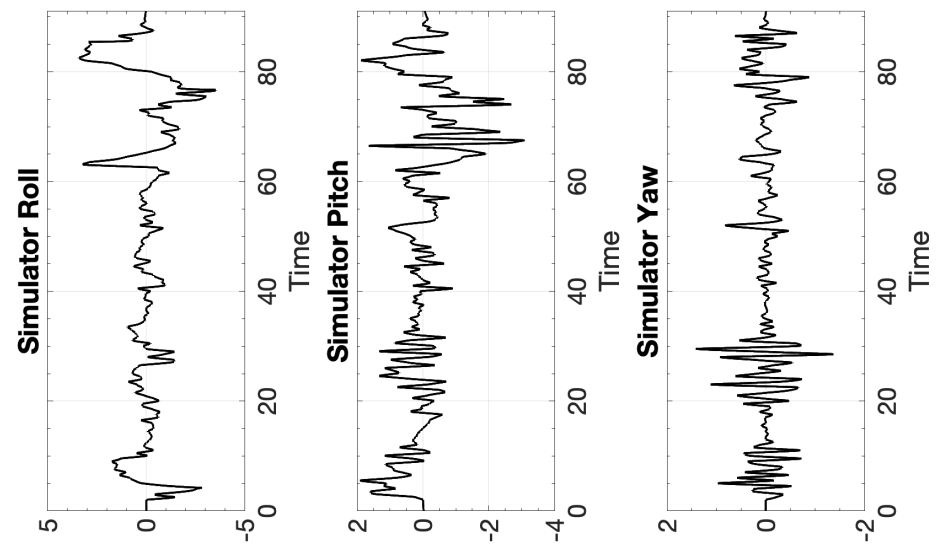

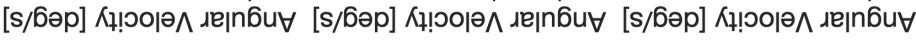




\section{Large-angle Washout Test Cases}

This appendix includes graphs for the large-angle washout test cases, including the aircraft motion set points and the simulator motion. 
C.1 M1
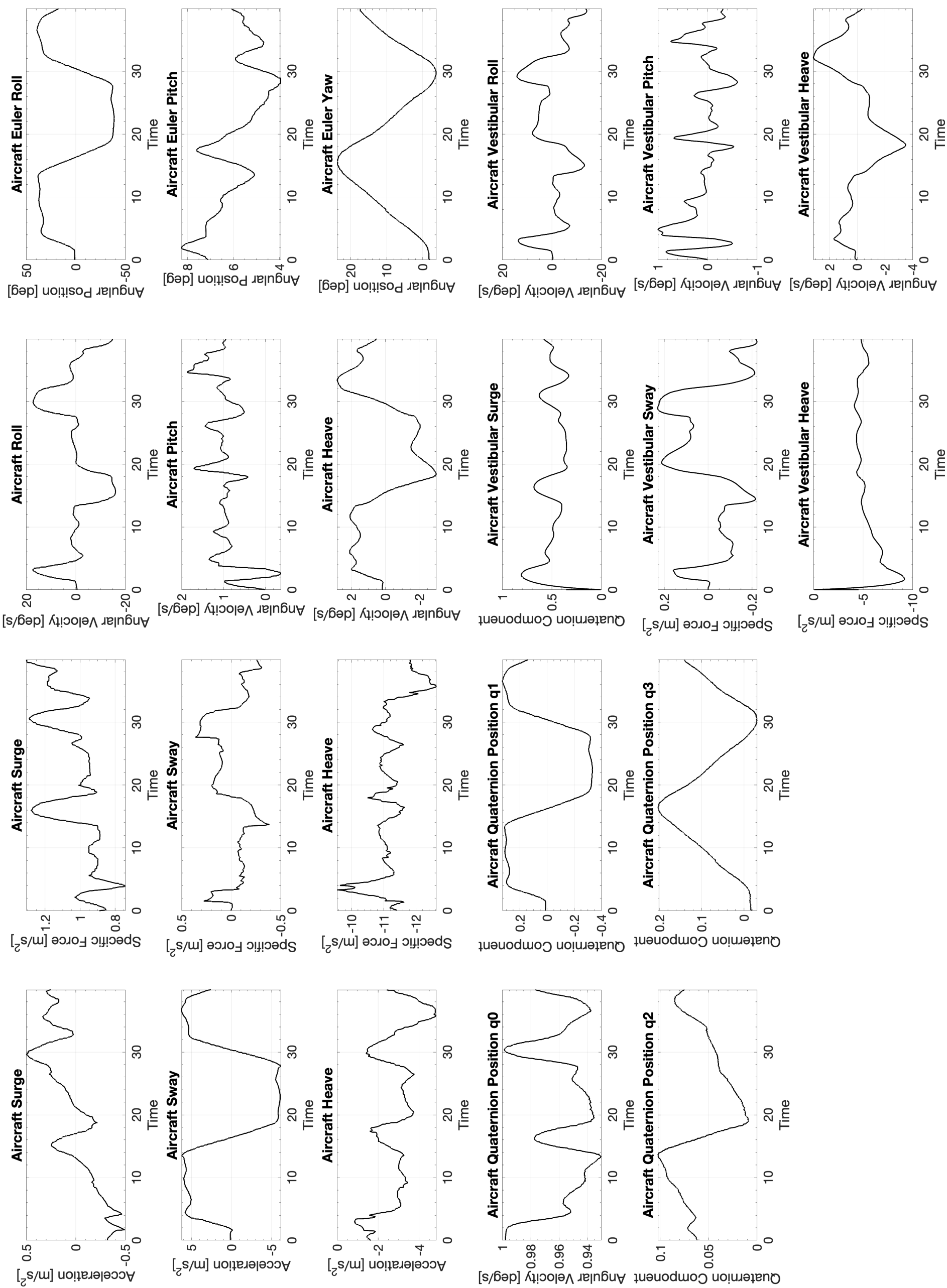

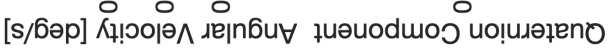
204 


\section{C.1.1 CW1 M1}
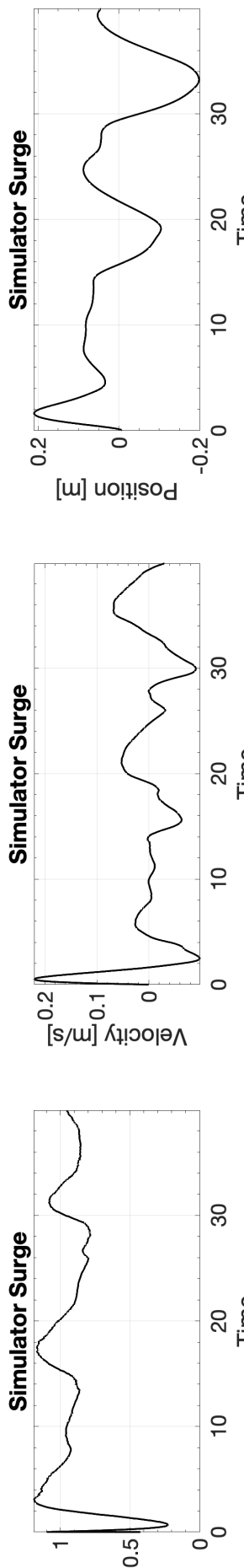

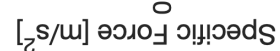

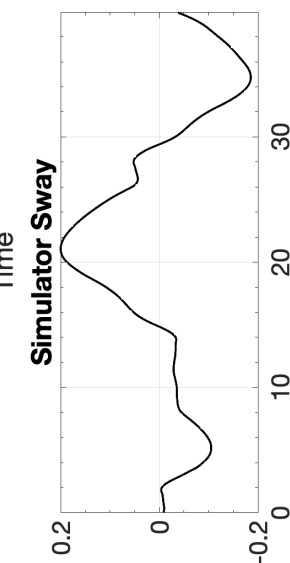

[w] uo!!!!sod

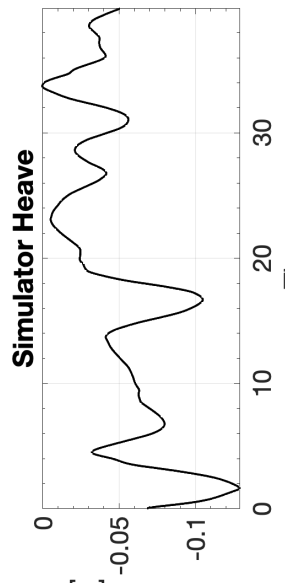

[u] uol?!sod

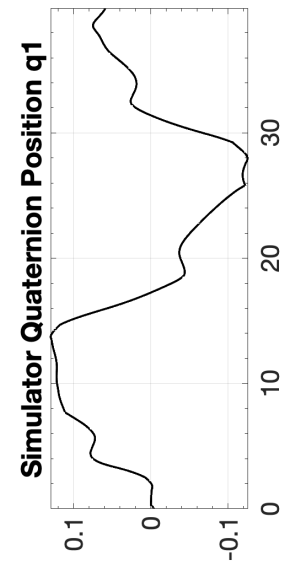

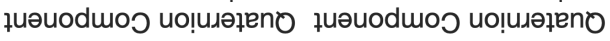

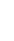

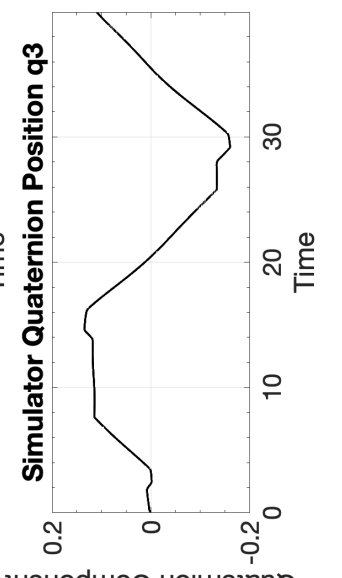

$\stackrel{\text { E }}{\underline{E}}$

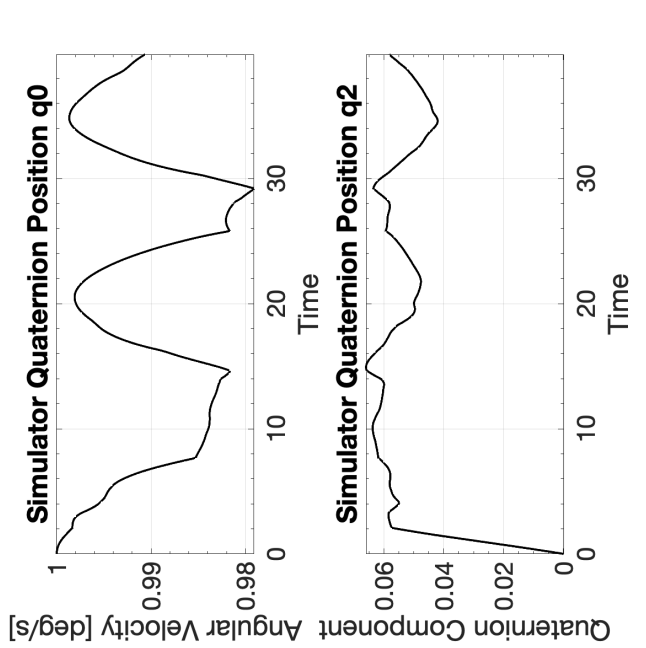

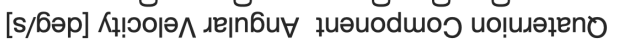

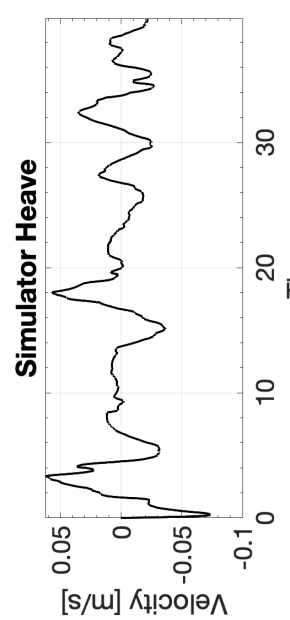

[s/u] Кџ!วоә

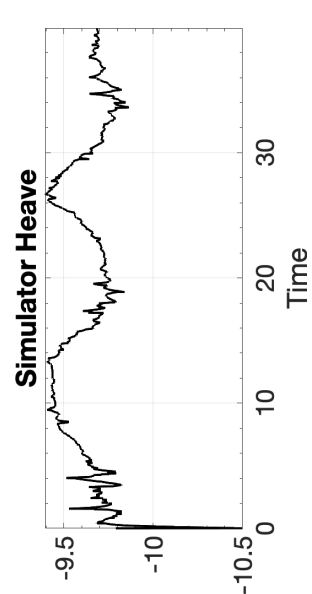

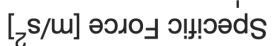
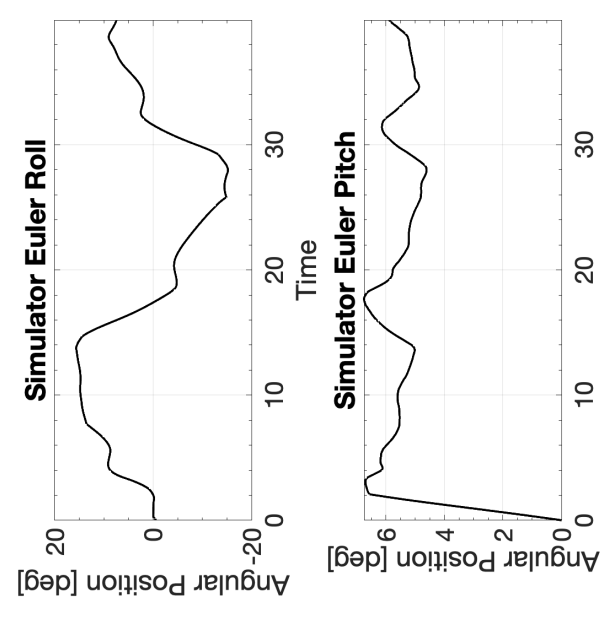

[6әр] uol]!sod גe|n6u

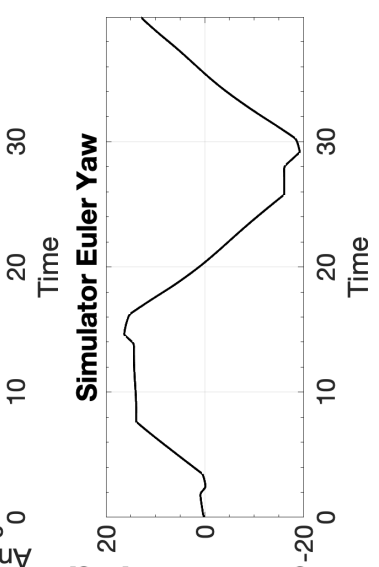

[6әр] uo!!! sod dejn6u

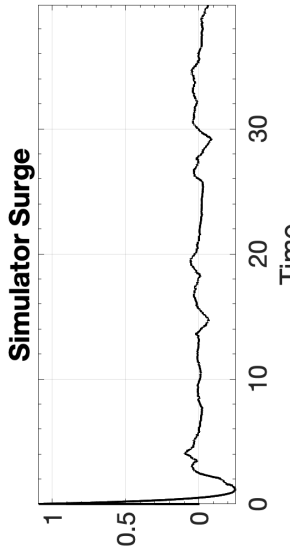

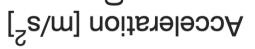

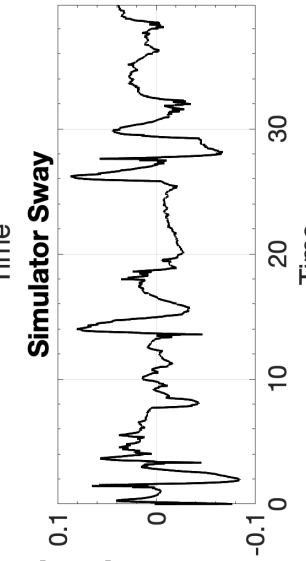

$[s / m]$ นо!ฺеләәәоэ

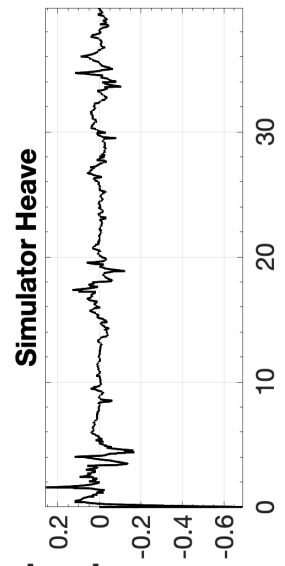

[z/m] ио!ฺвләрәээ

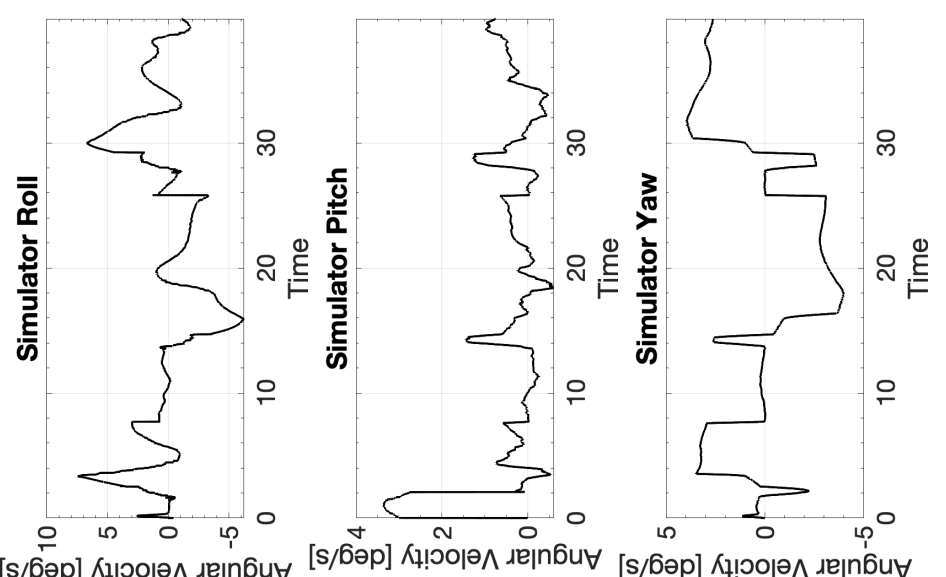



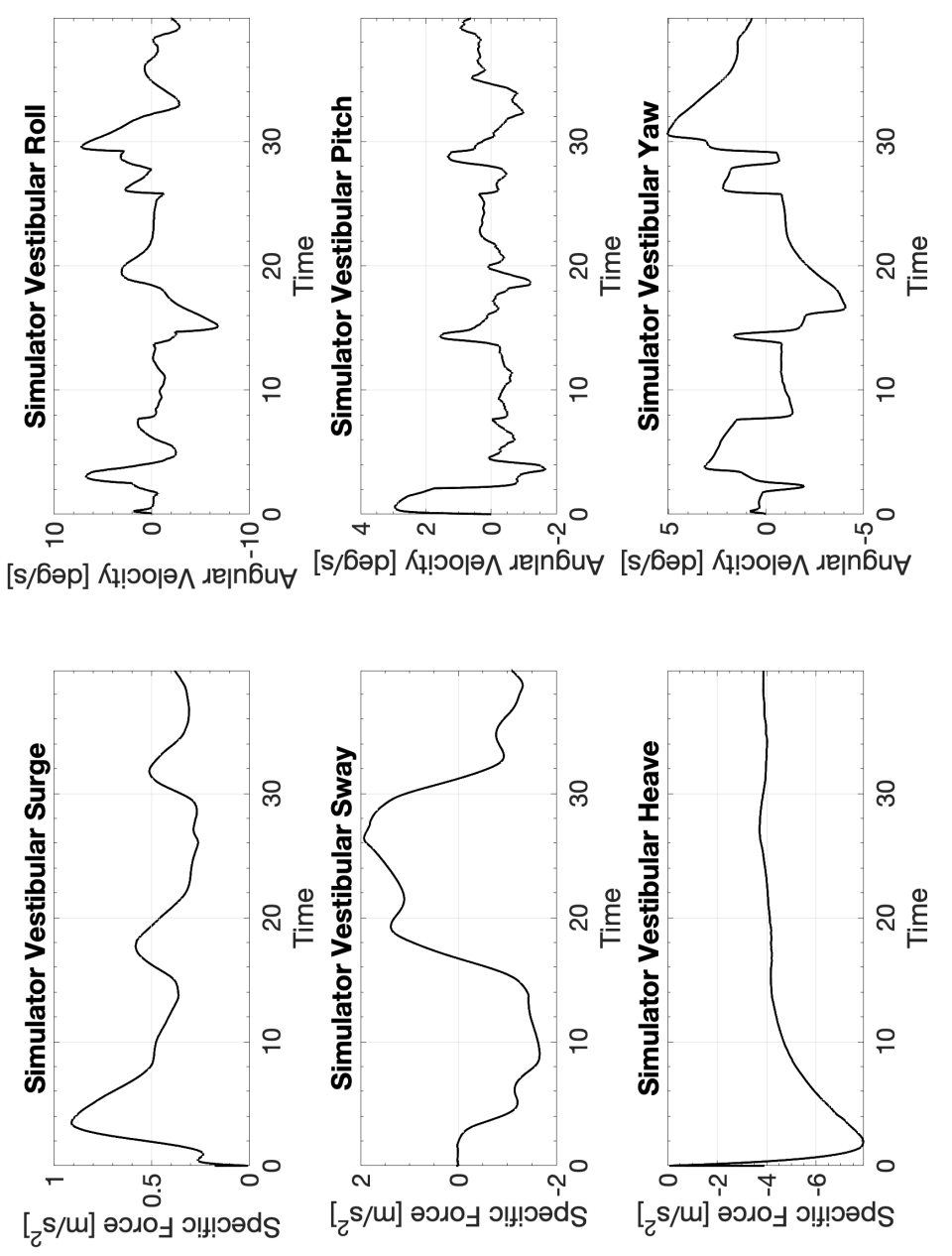


\section{C.1.2 CW2 M1}

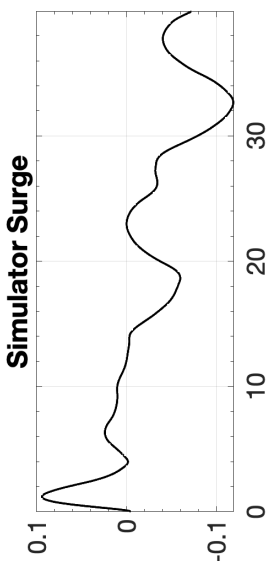

[w] uo!!!!sod

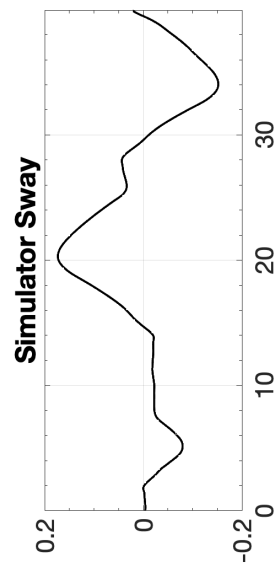

[w] uo!n!sod

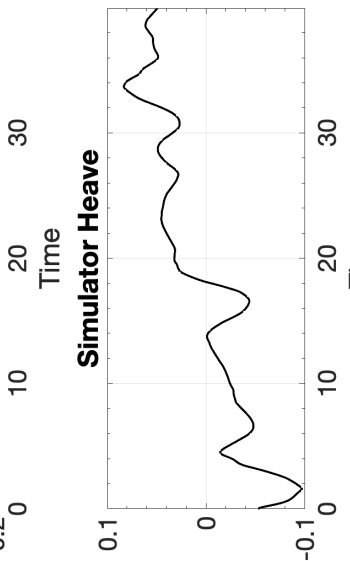

[w] uo!t!sod

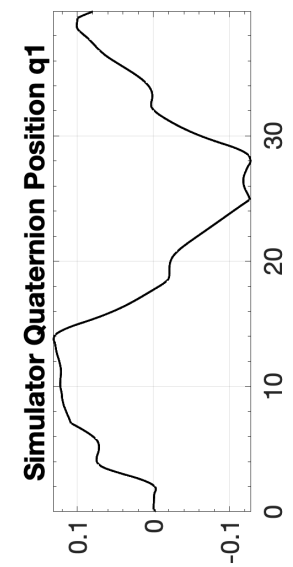

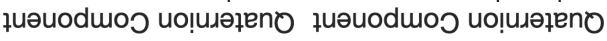

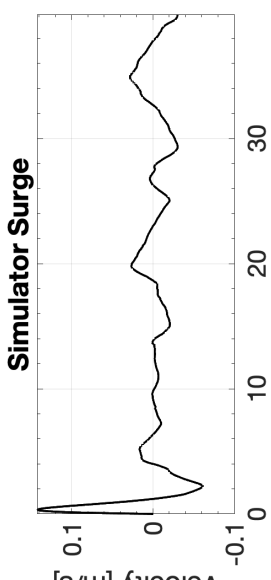

[s/m] К१!०орә^

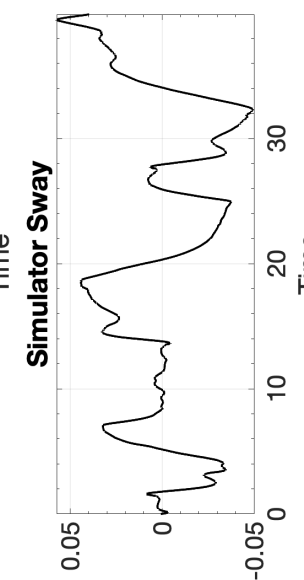

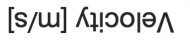

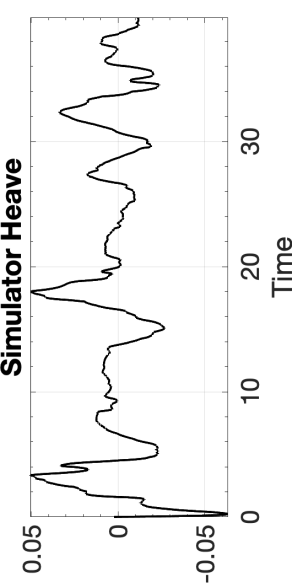

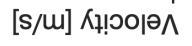

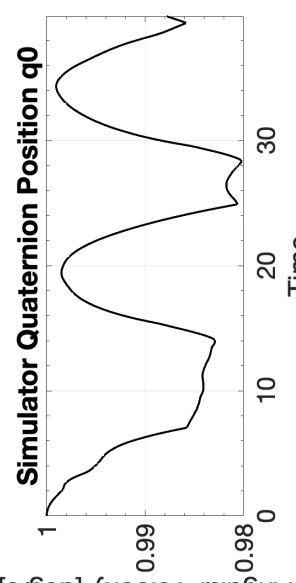

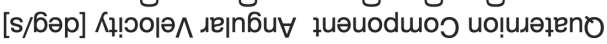

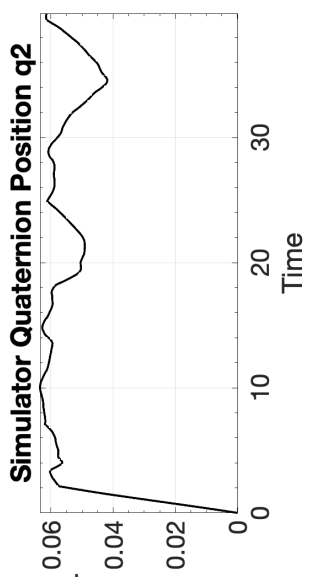

월

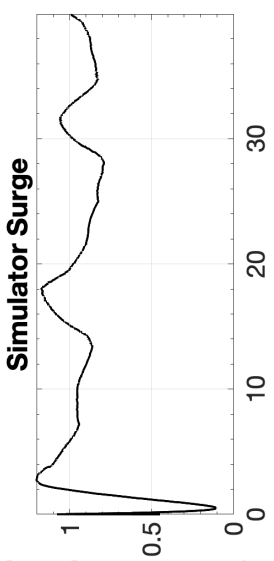

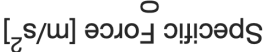

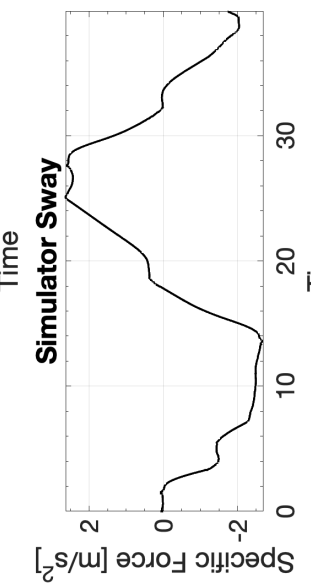

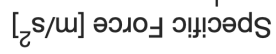

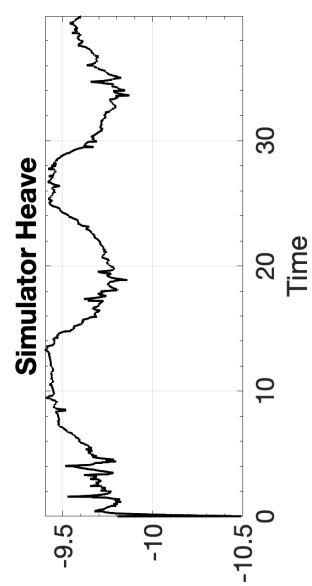

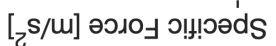
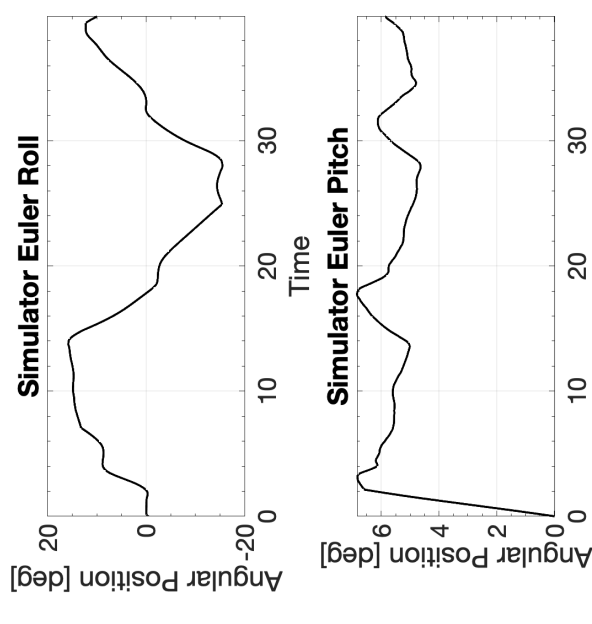

[6әp] uo!!! sod גe|n6u

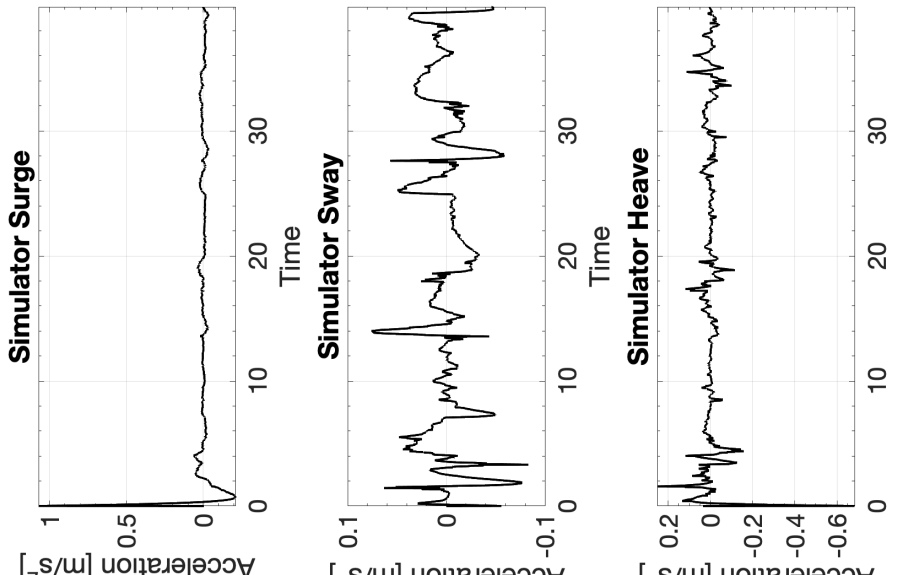

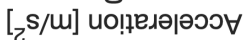

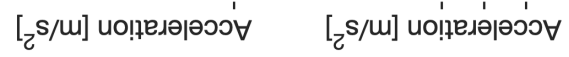

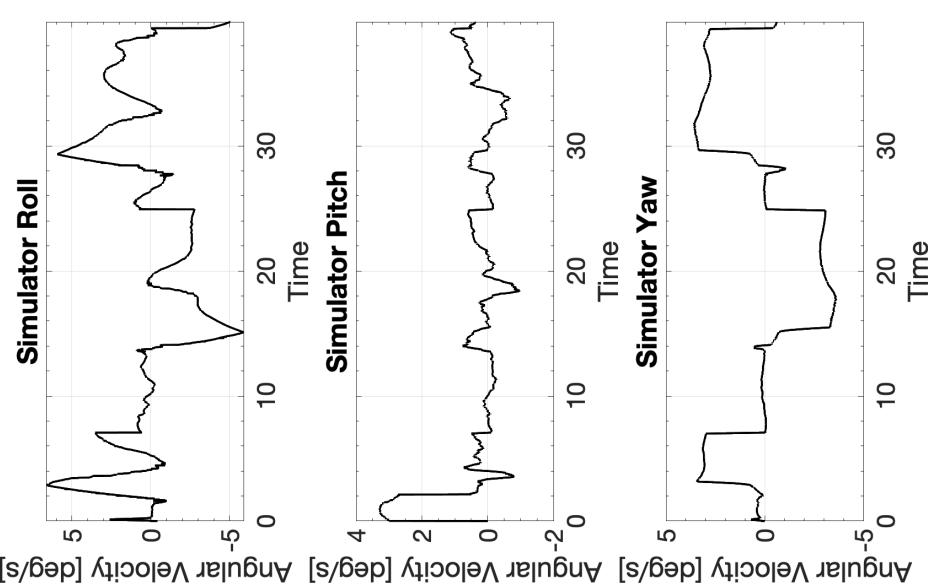

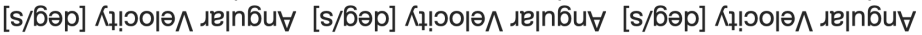



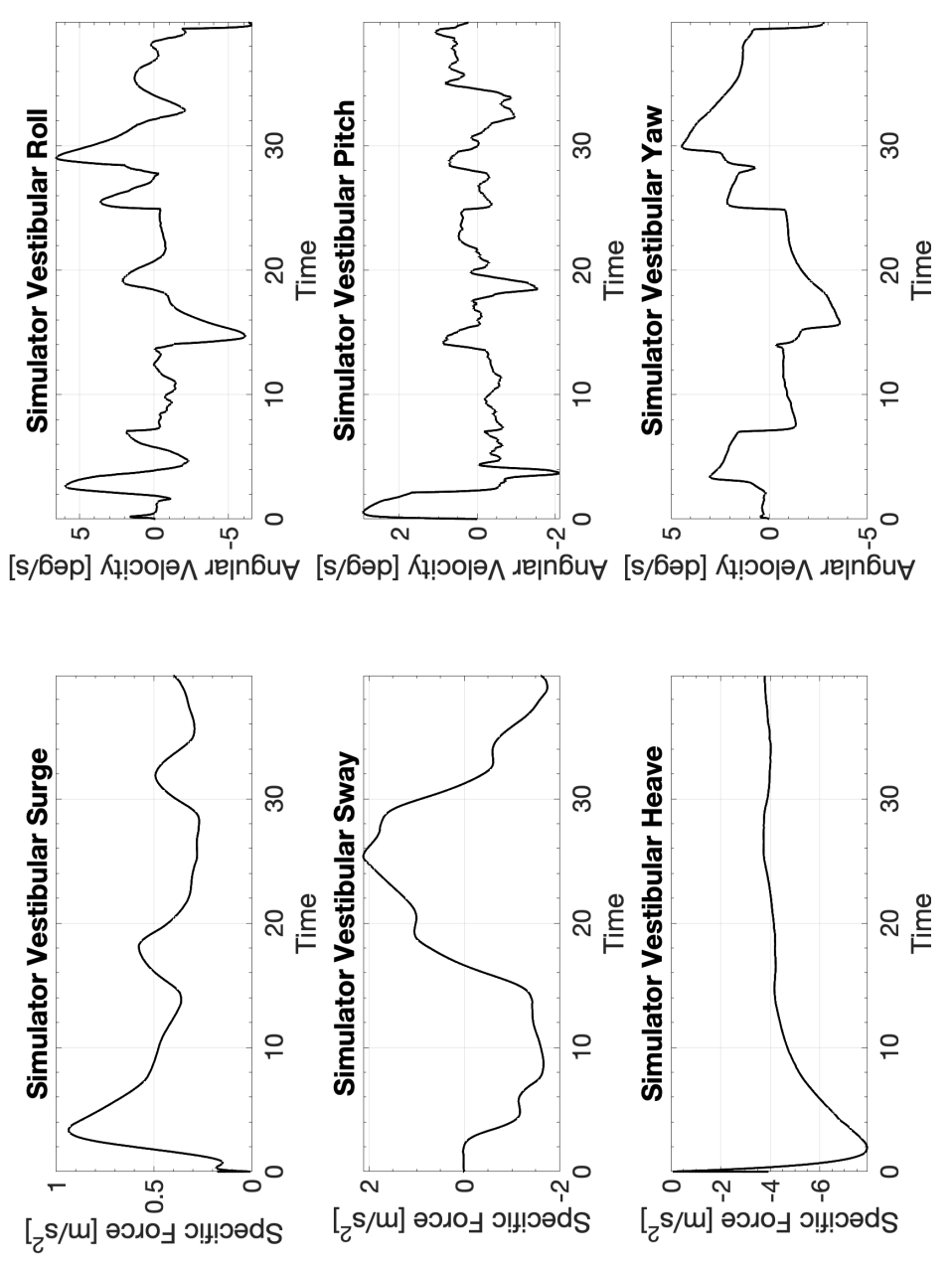


\section{C.1.3 CW3 M1}

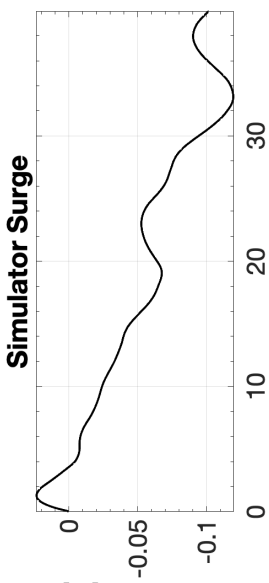

[u] uo!!!!sod

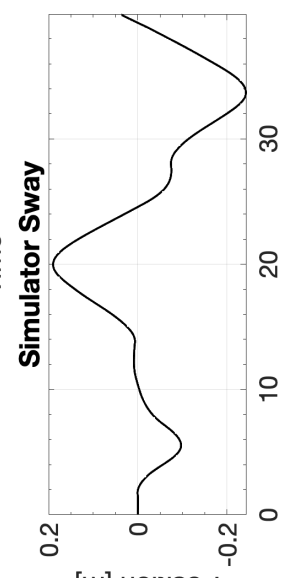

[w] uo!n!sod

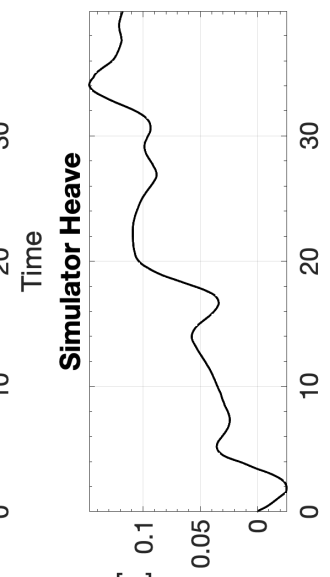

[w] uo!!!sod

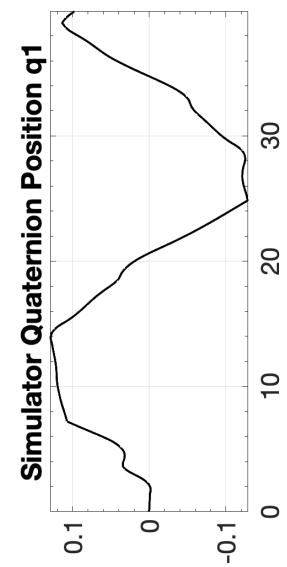

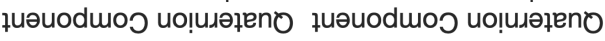

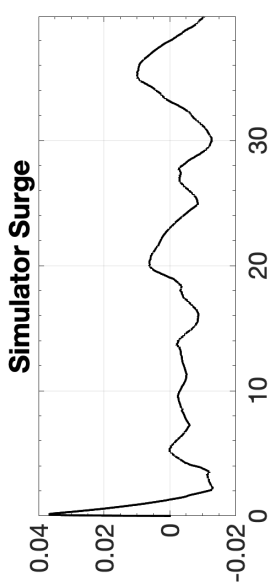

[s/u] Кц!оојә

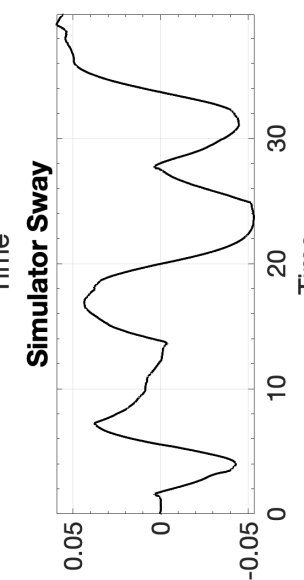

[s/u] Кұ!юо|ә^

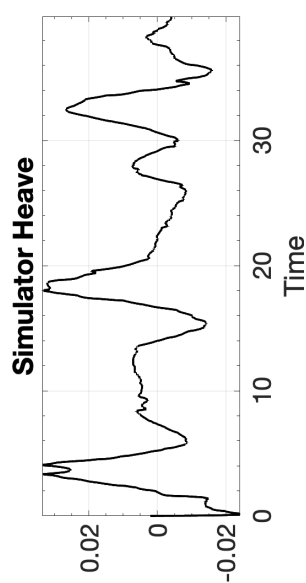

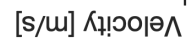

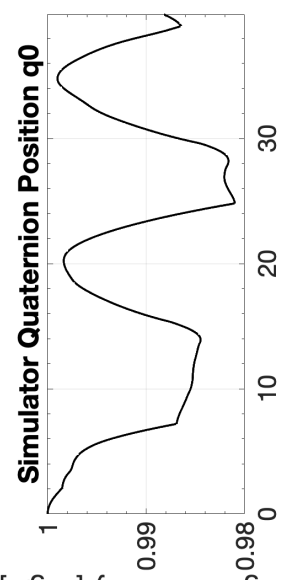

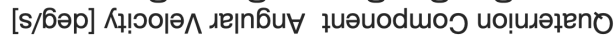

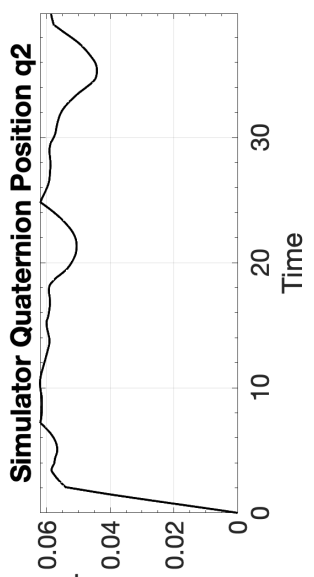

욜

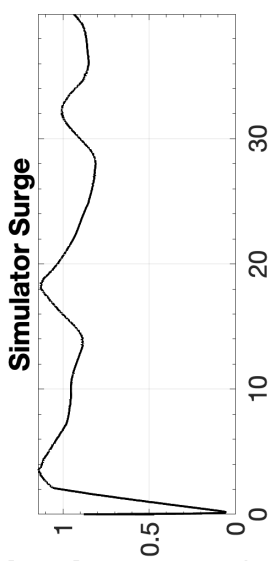

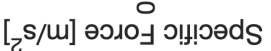

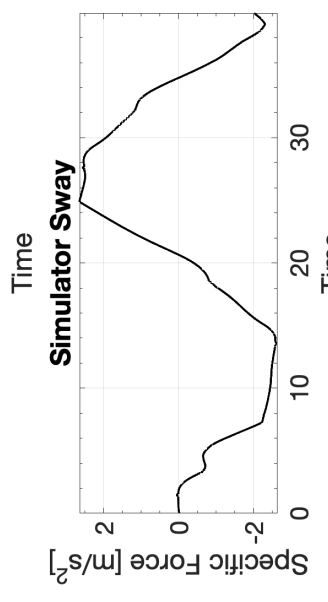

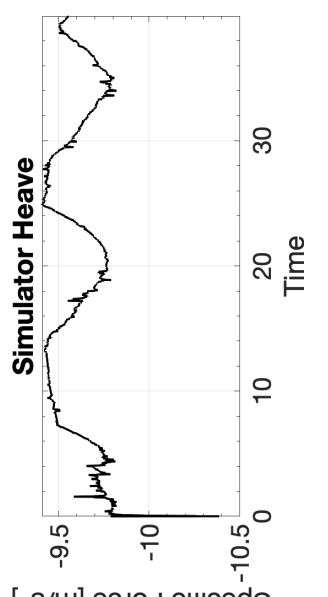

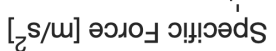
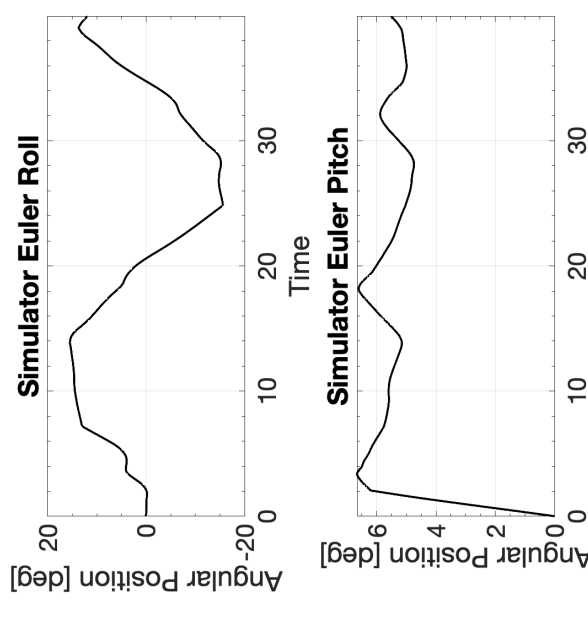

[6әp] uo!!! sod
จ $\stackrel{\oplus}{\stackrel{\oplus}{E}}$

우

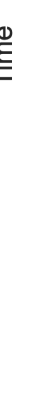

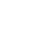

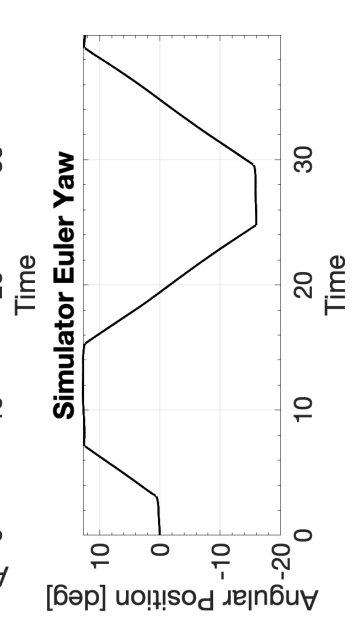

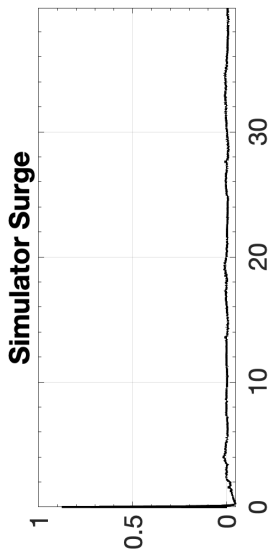

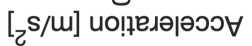

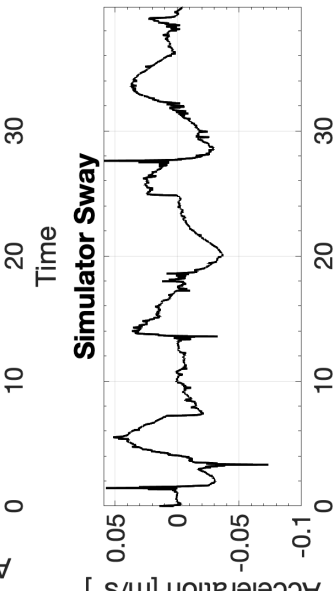

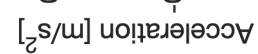

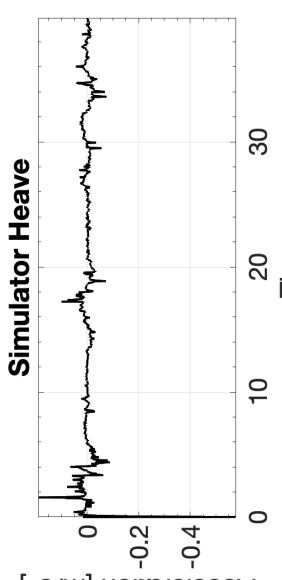

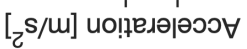

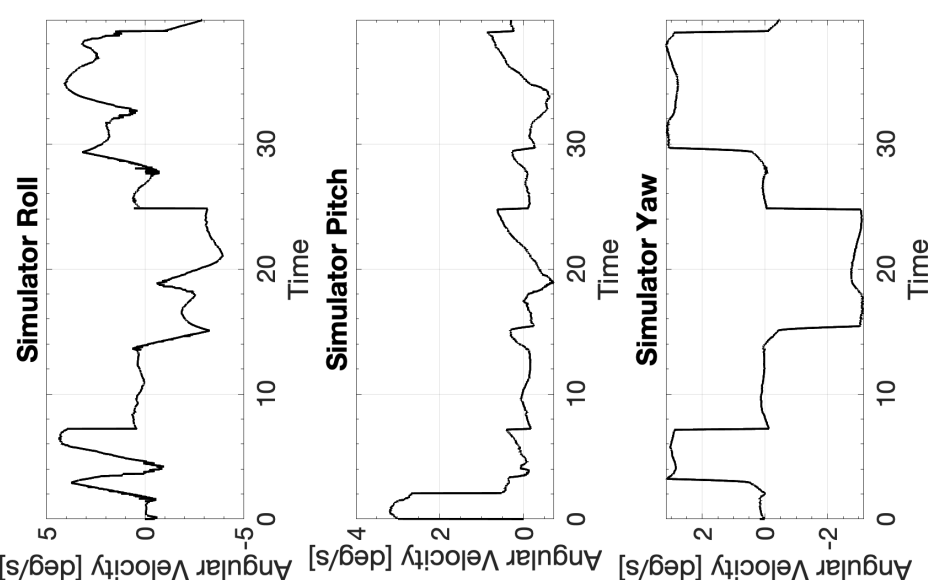

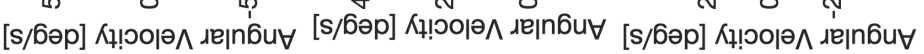




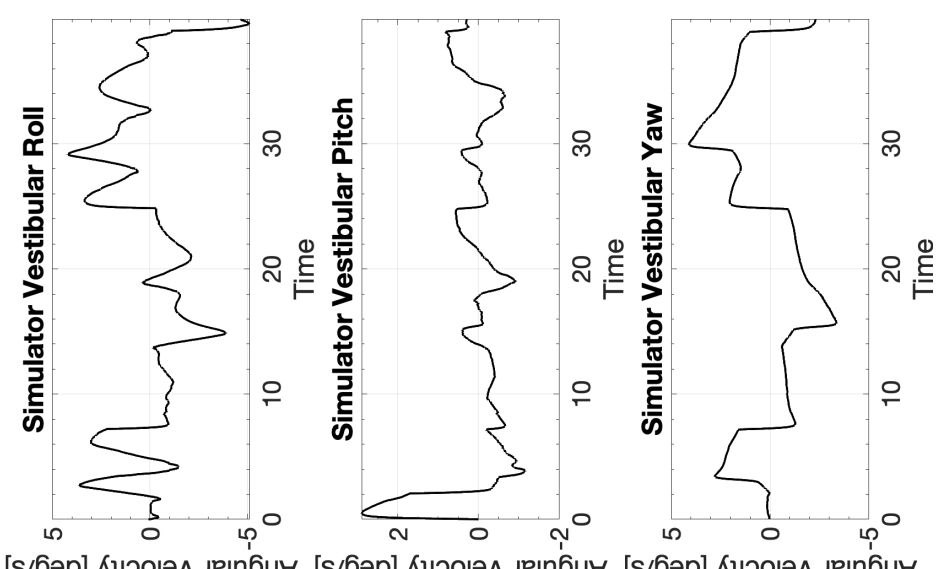

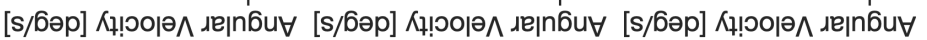

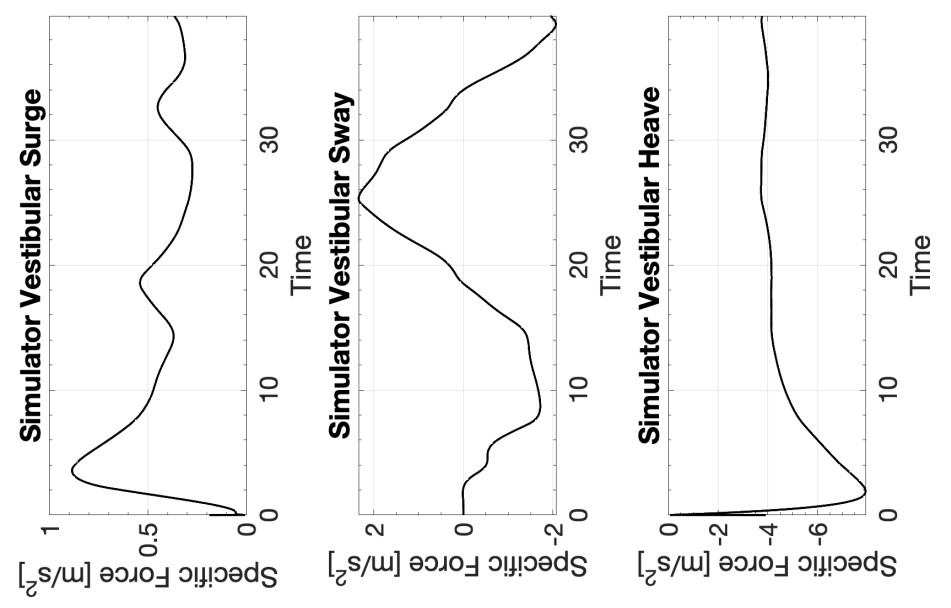




\section{C.2 M2}

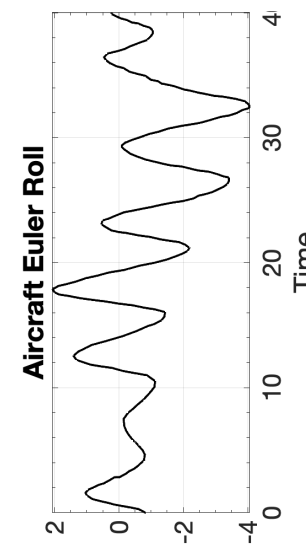

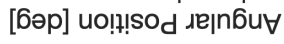

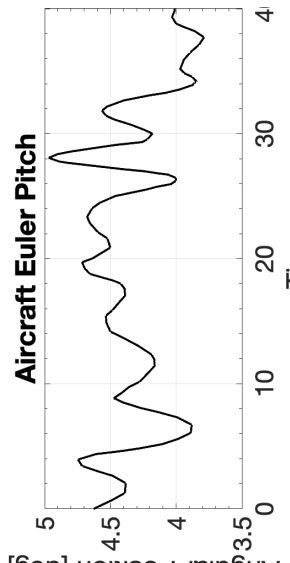

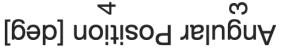

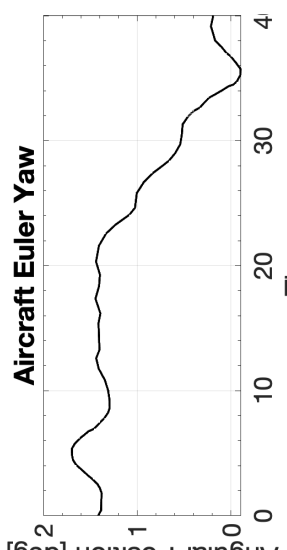

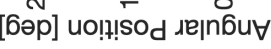

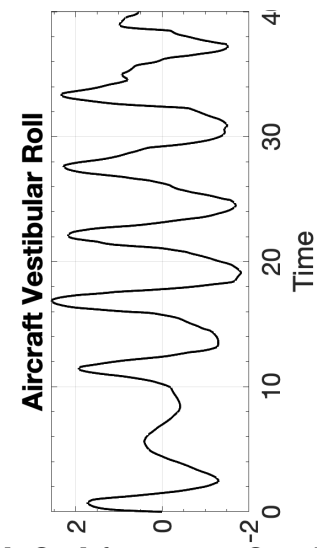

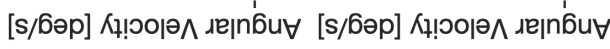

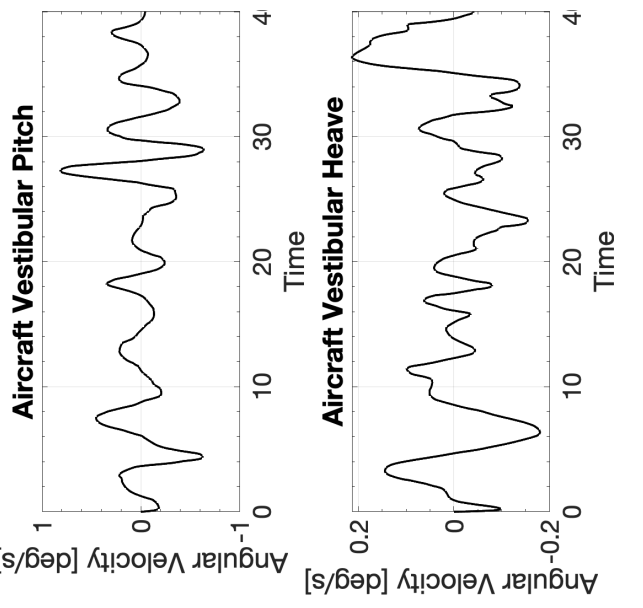

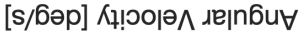

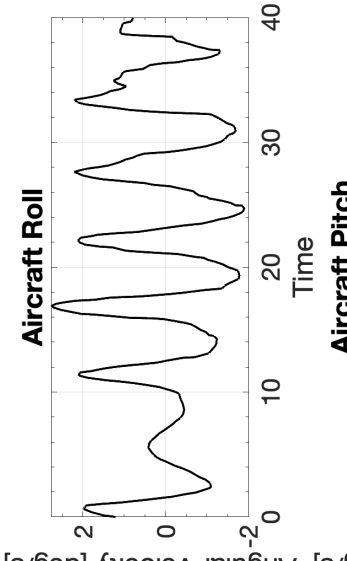

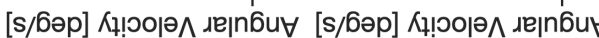

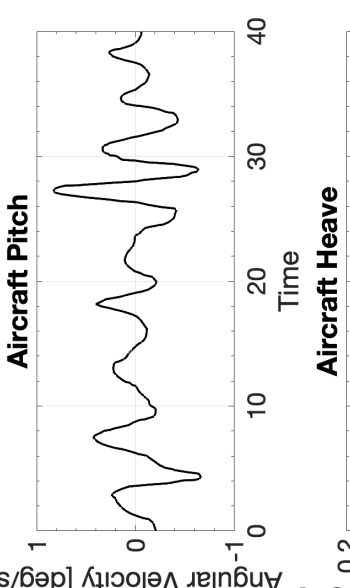

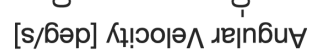

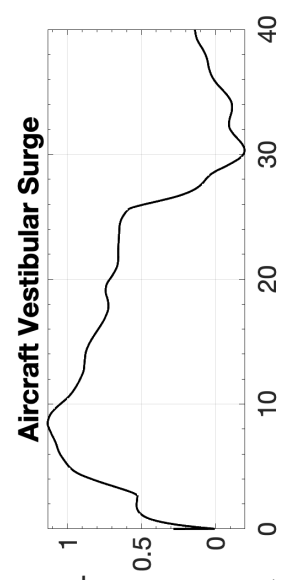

ұนəuodmoว uo!̣uəłeno
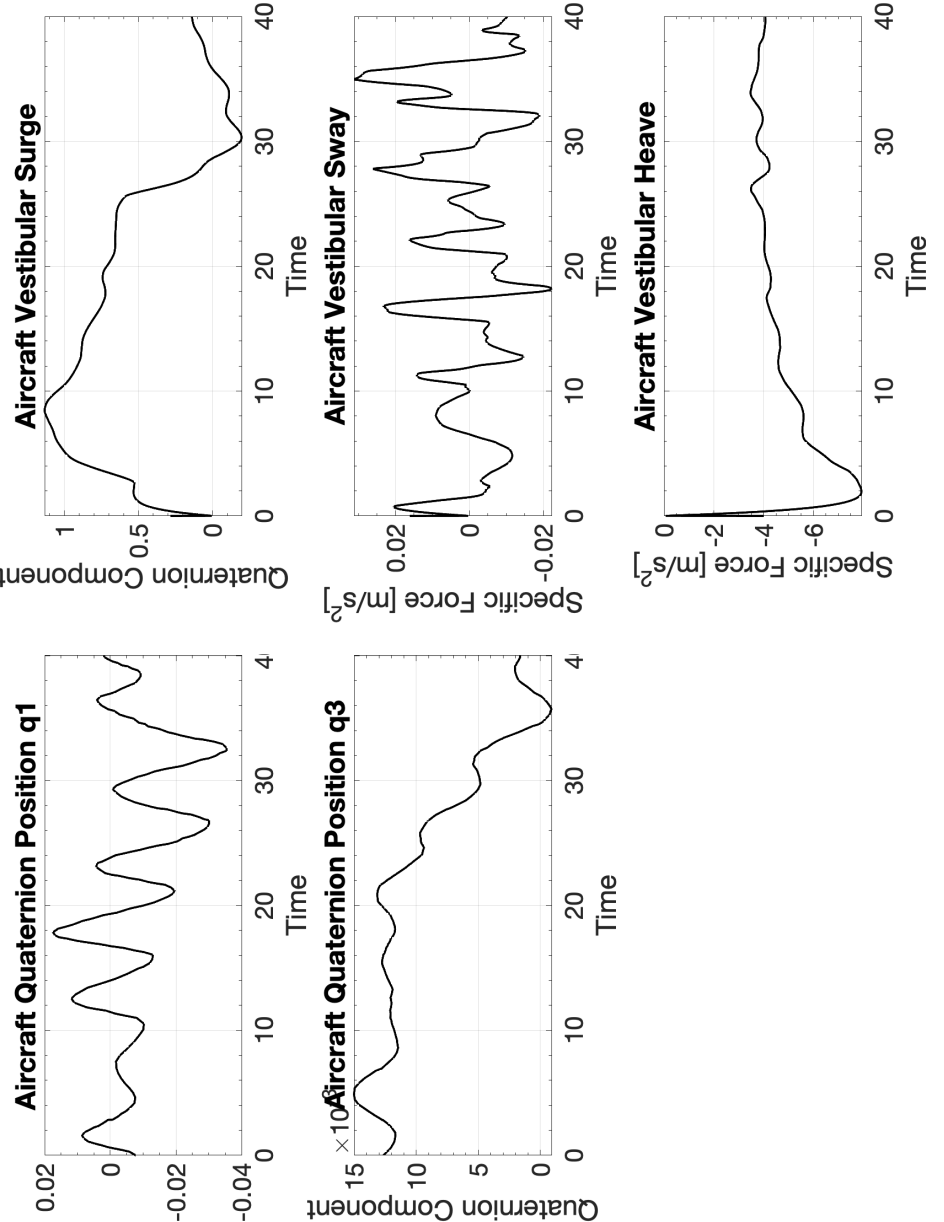

quәuodmo uo!̣uəteno

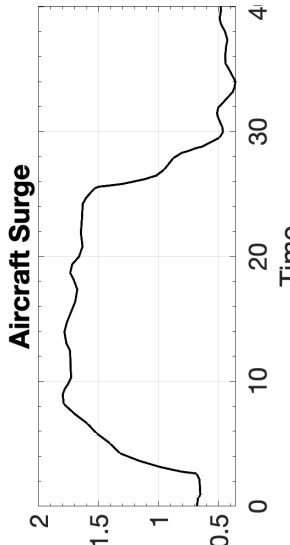

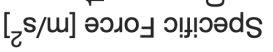

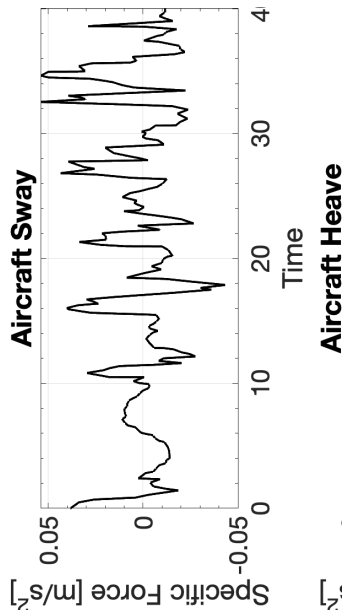

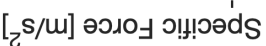

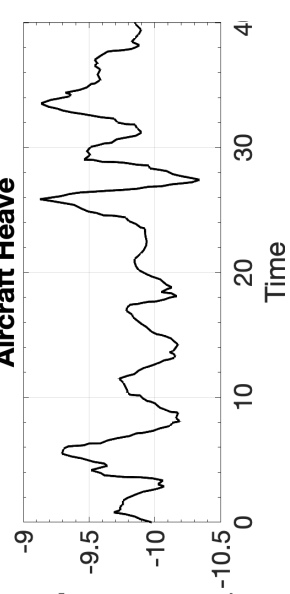

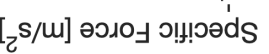

to

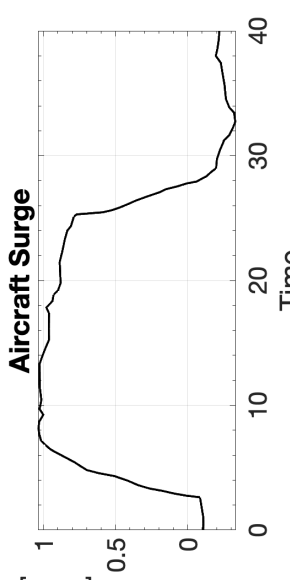

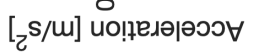

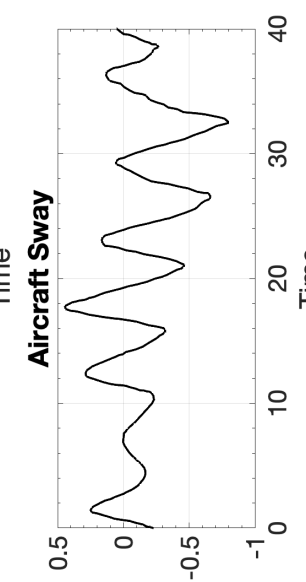

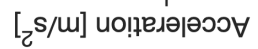

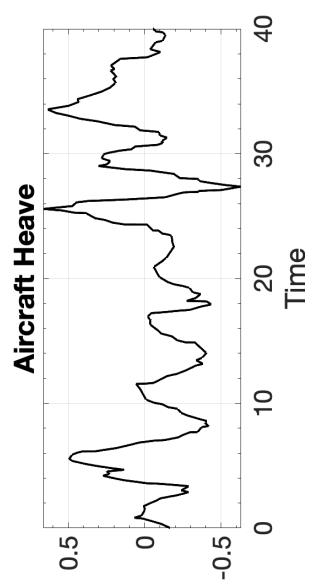

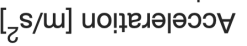
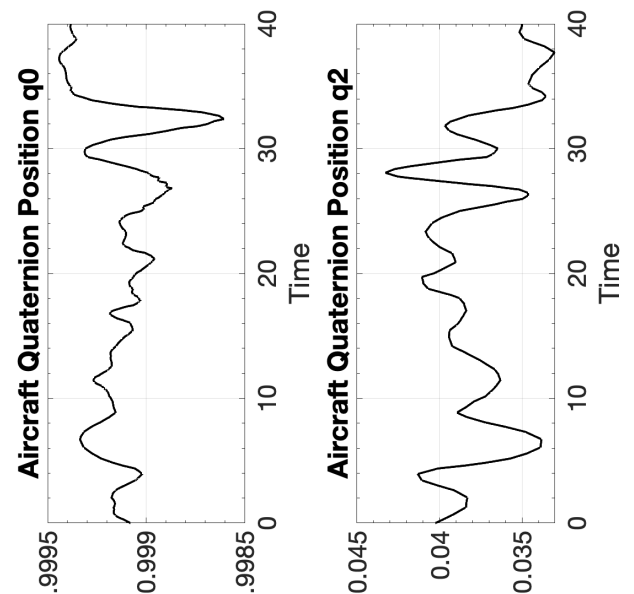


\section{C.2.1 CW1 M2}

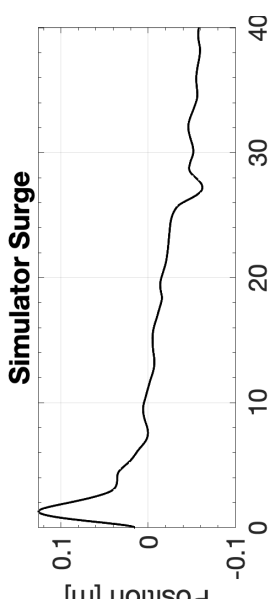

[u] uo!!!!sod

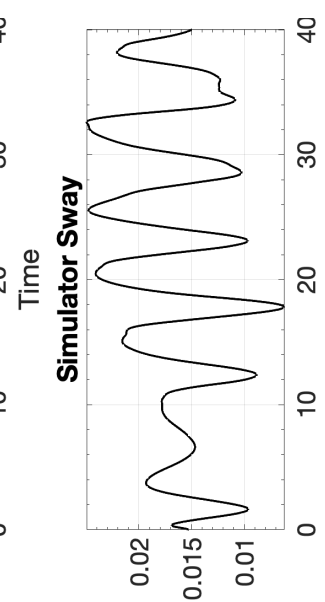

[u] uol!!sod

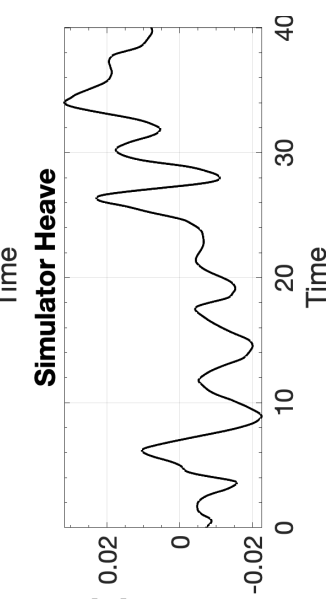

[u] uo!!! sod

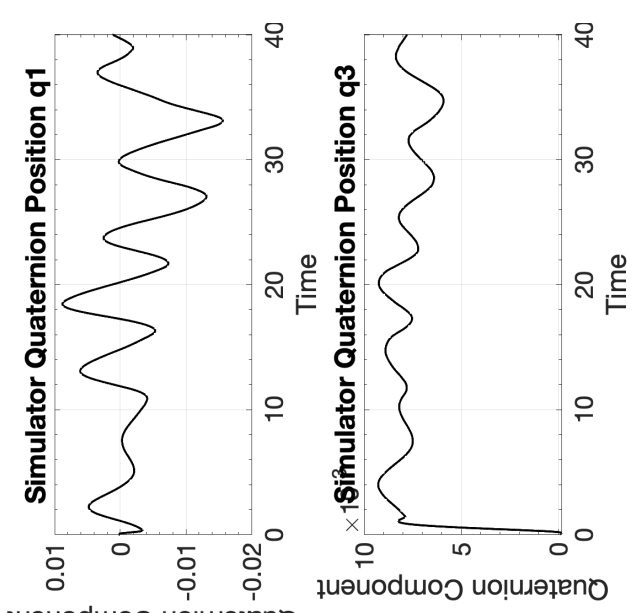

ұuəuodmos uo!udeleno

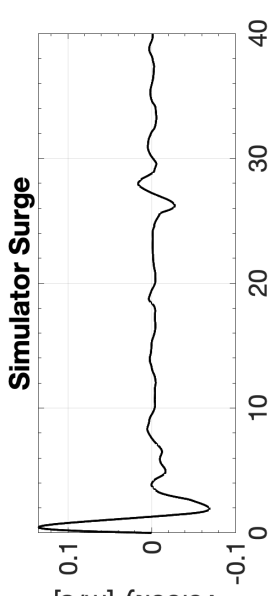

[s/m] К!оㅣㅅㅅ

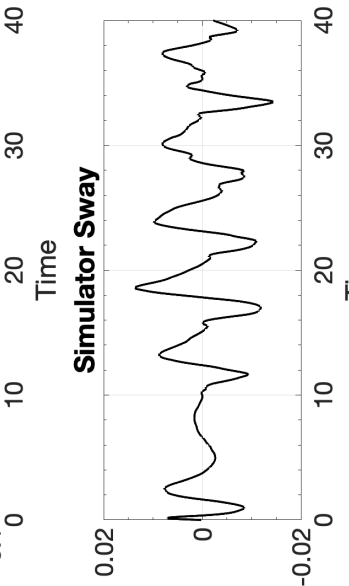

[s/m] К!이

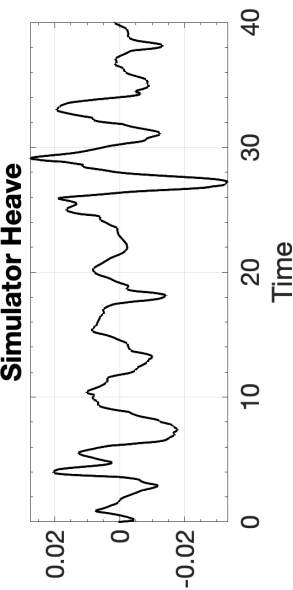

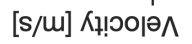
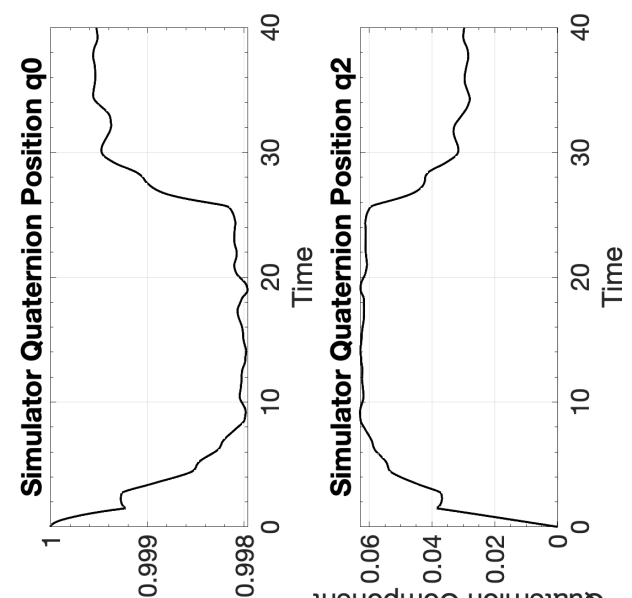

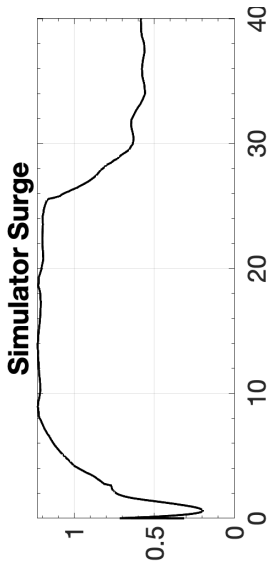

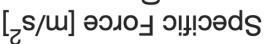

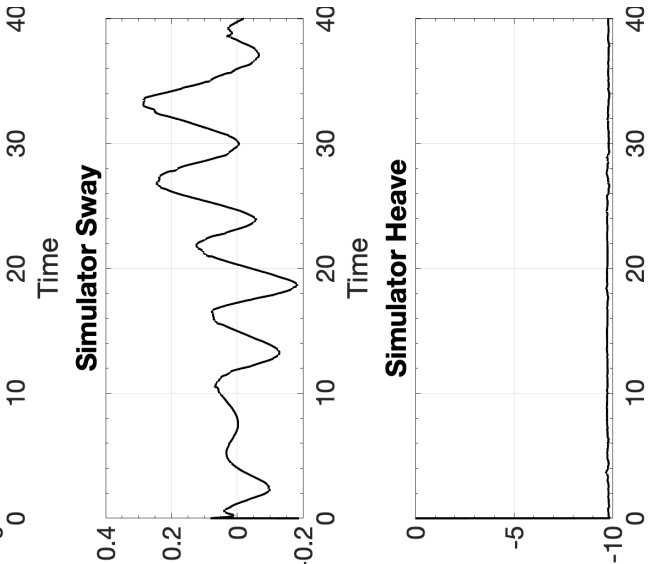

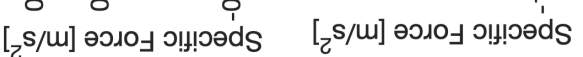

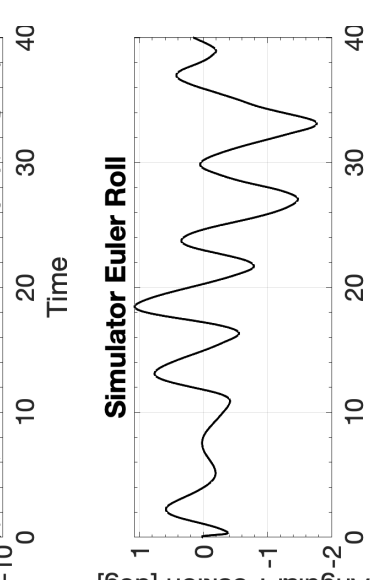

[6әр] uo!!!sod

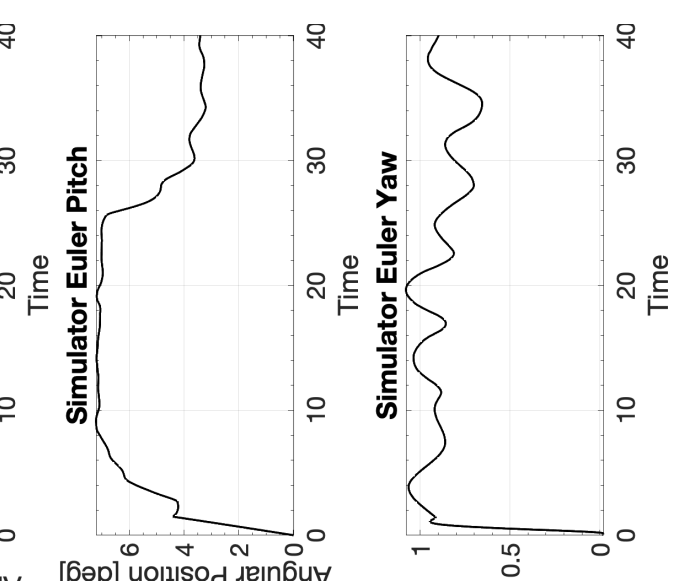

[6әр] uo!!!!sod גejn6u

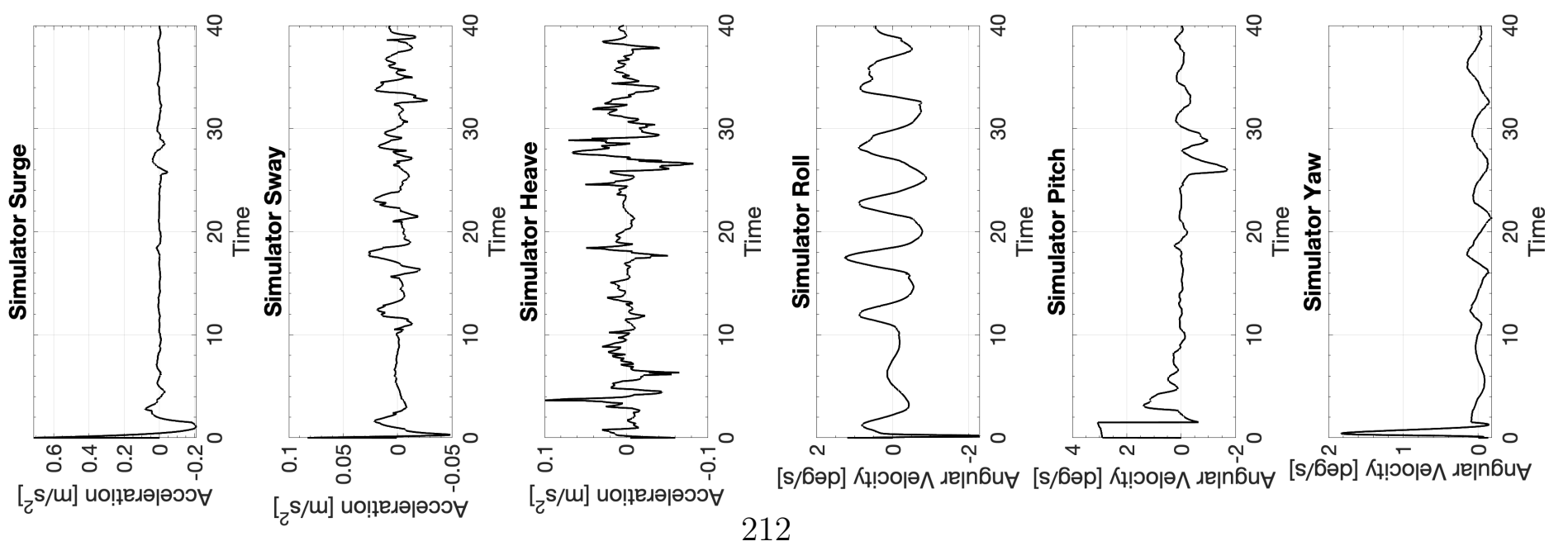



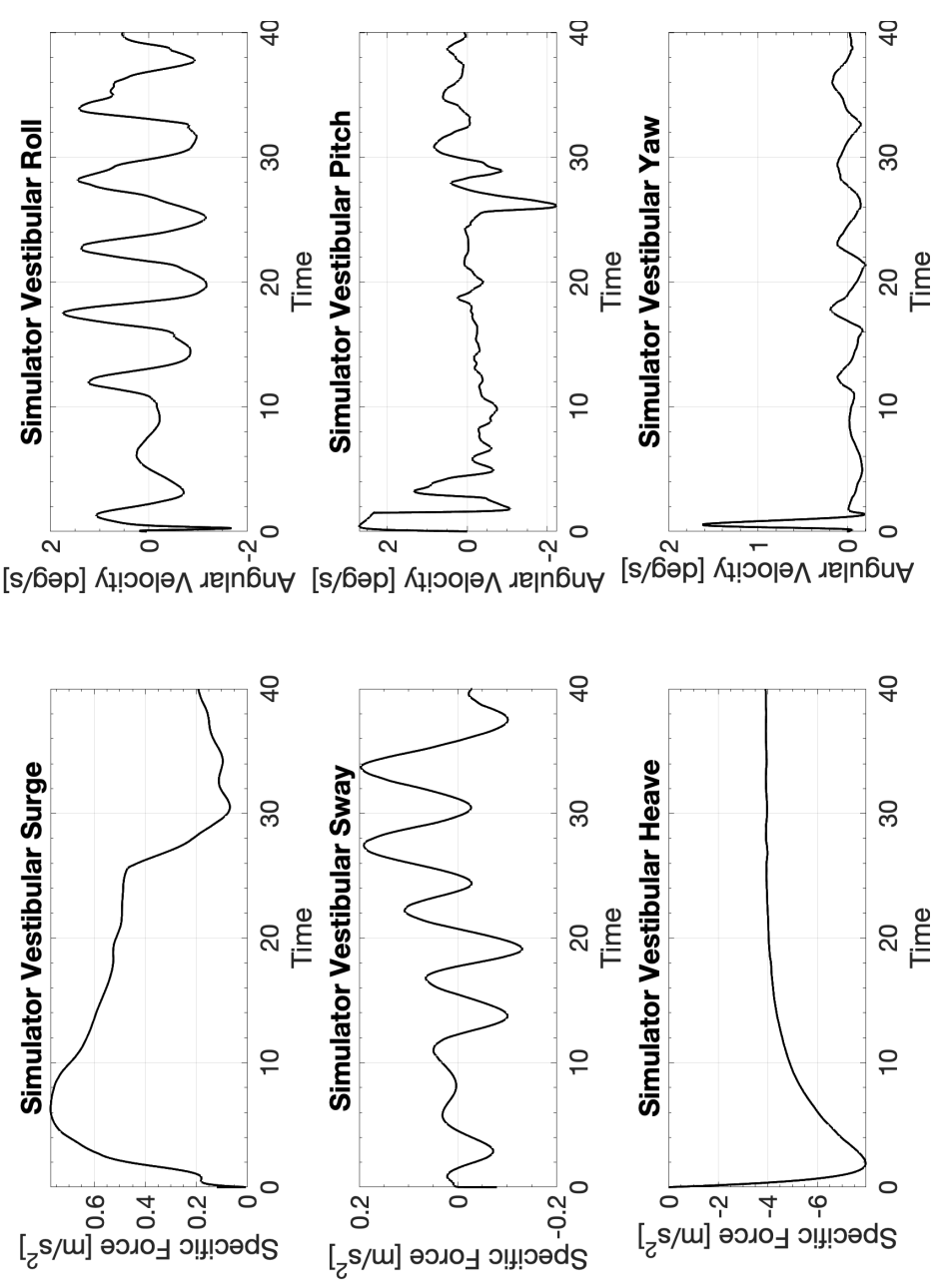


\section{C.2.2 CW2 M2}

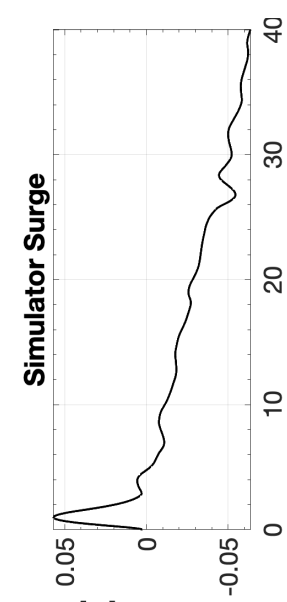

[w] uo!!!!sod

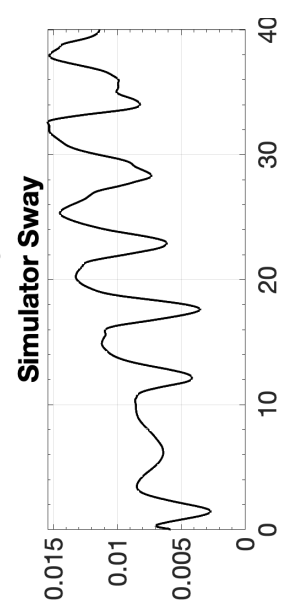

[u] uo!n!!sod

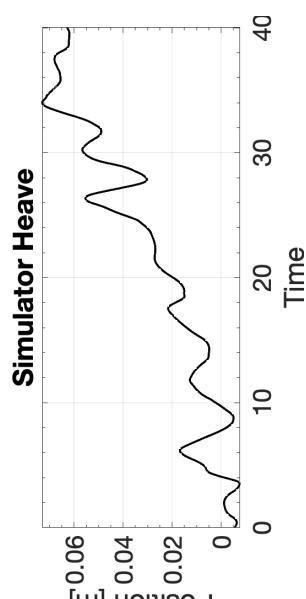

[u] uo!!!!sod

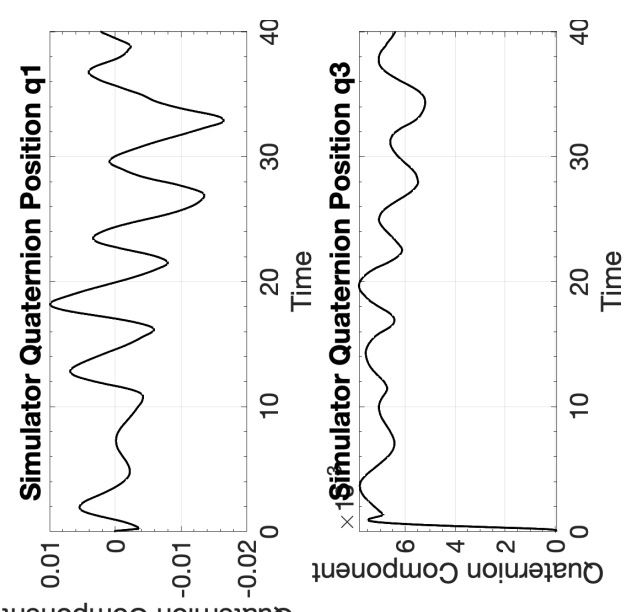

ұuәuodmos uo!udejeno

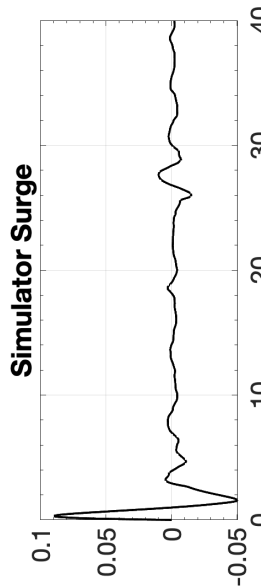

[s/m] К! ৩оə

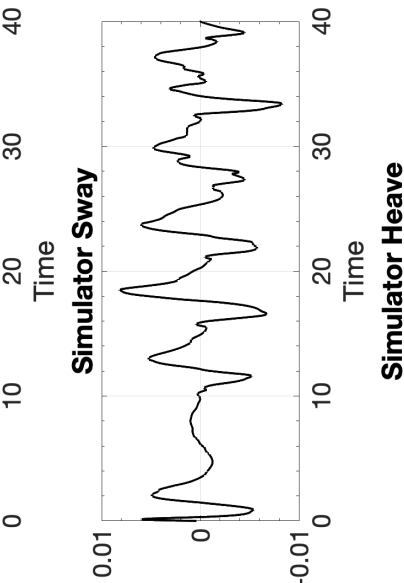

[s/u] К!잇

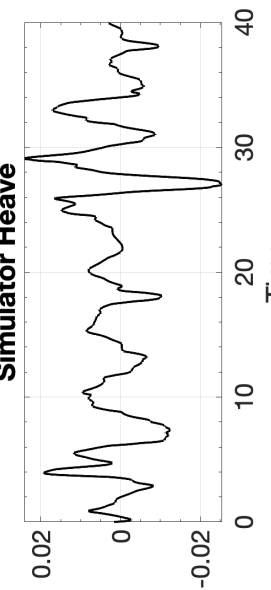

[s/u] Кұ! оә
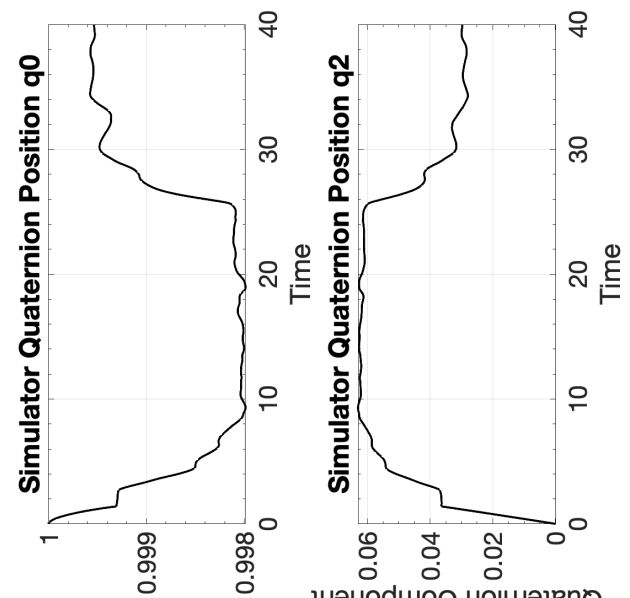

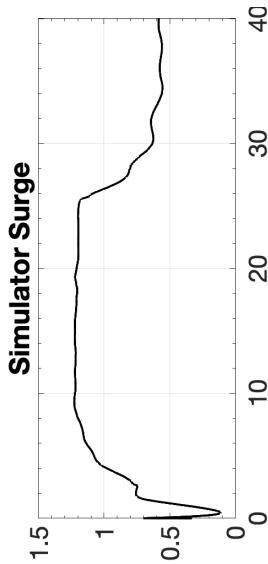

[ร

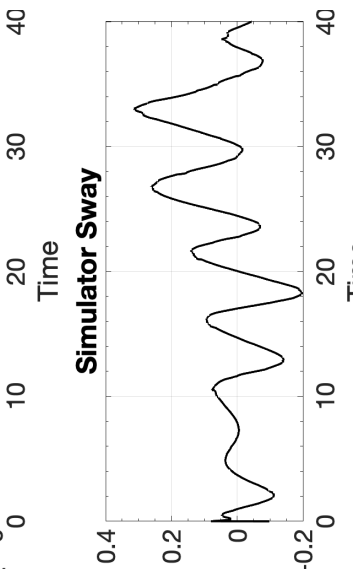

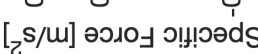

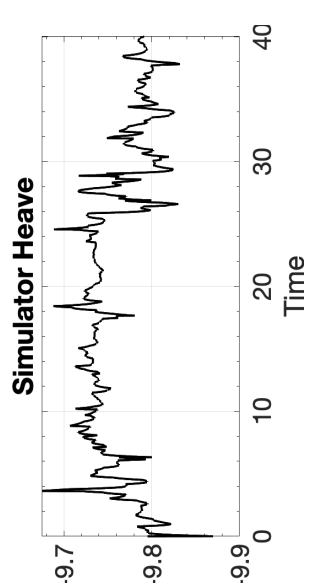

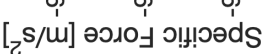

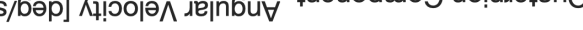

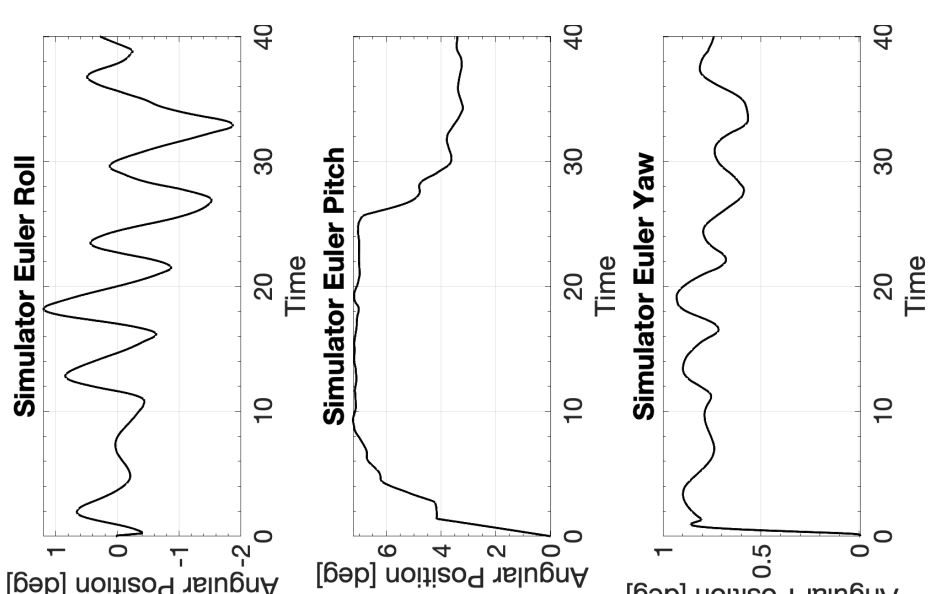

[бәр] uo!!!sod

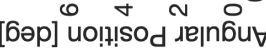

[6әр] uo!?!sod גeןn6u

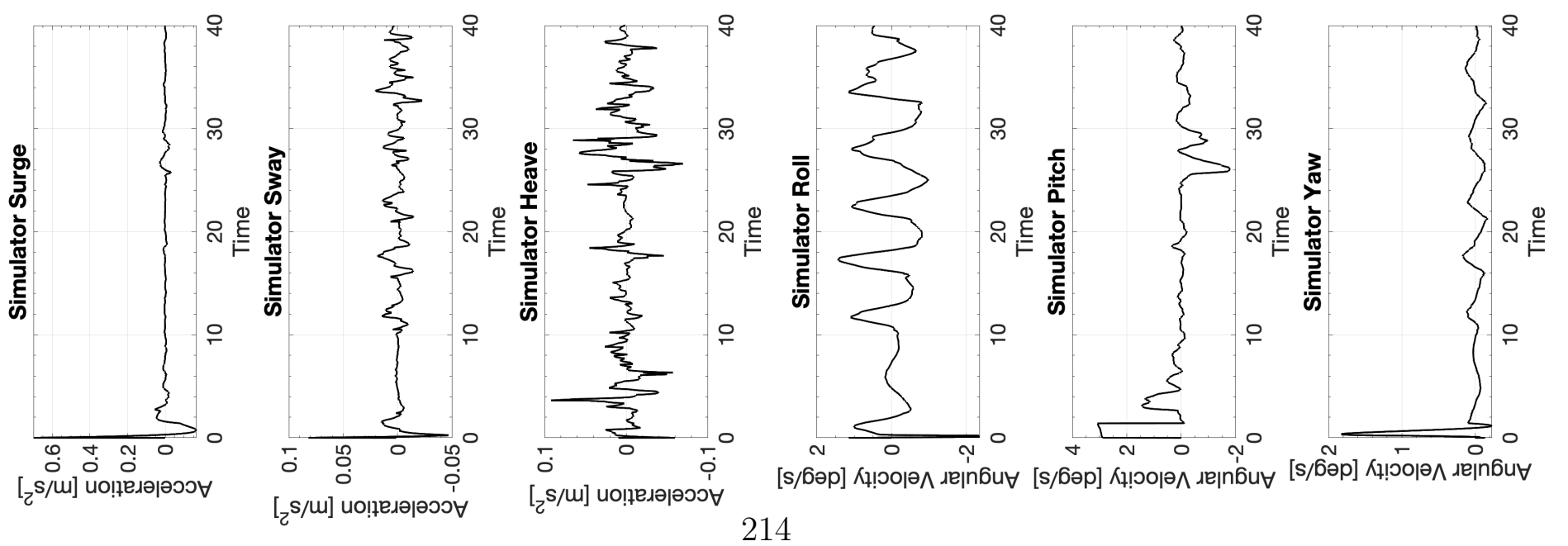




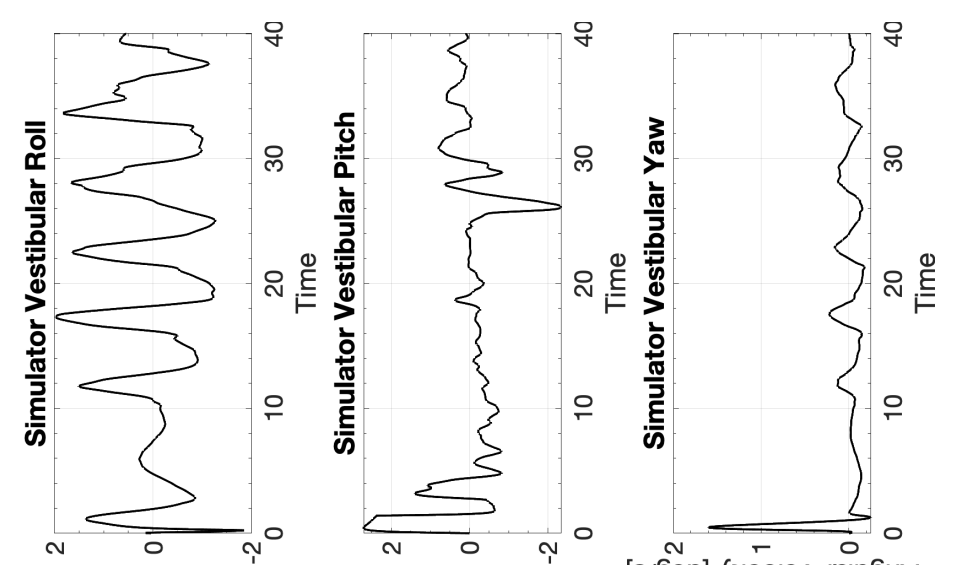

[s/бәр] кұ!юорә גе|пби

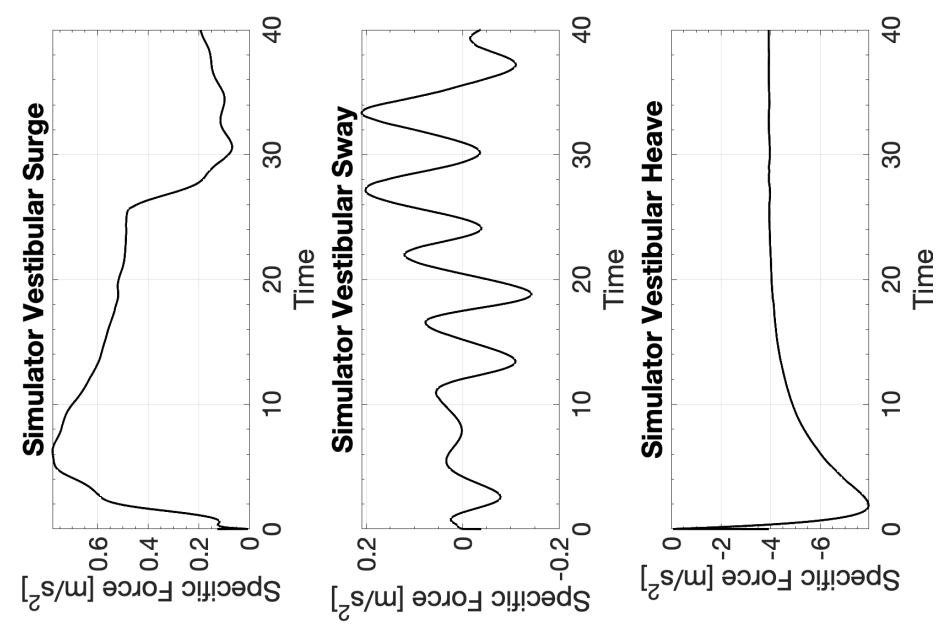




\section{C.2.3 CW3 M2}

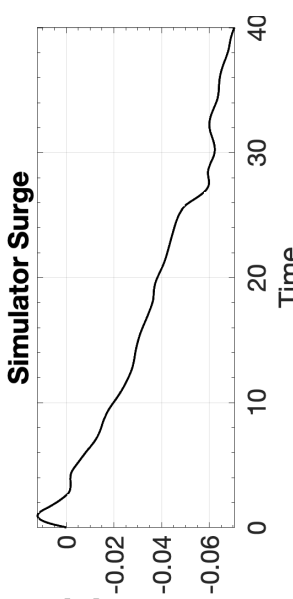

[u] uo!!! sod

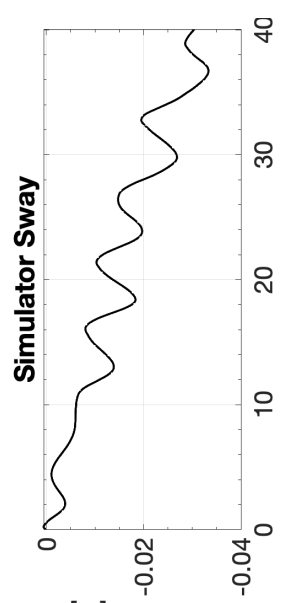

[w] uo!n! sod

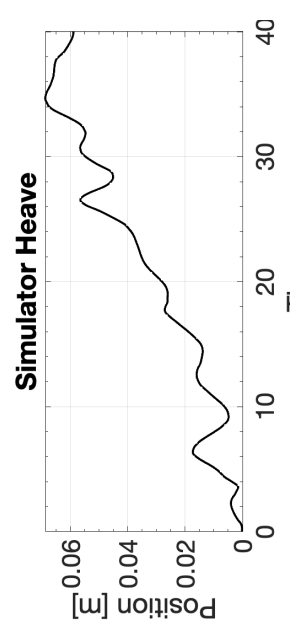

[w] uol!! sod

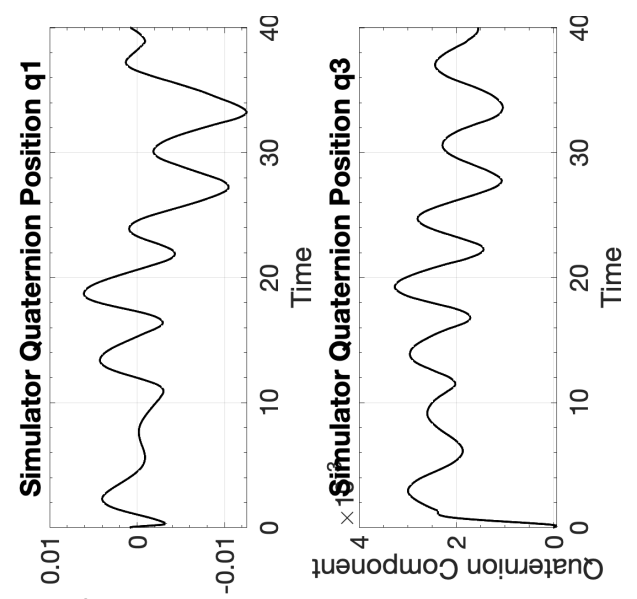

ұuəuodmo uo!̣uəłeno

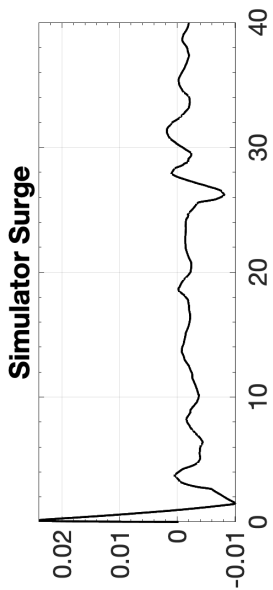

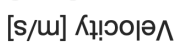

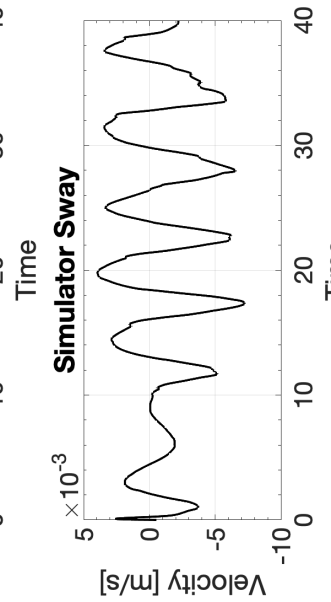

[s/u] К!ำю^

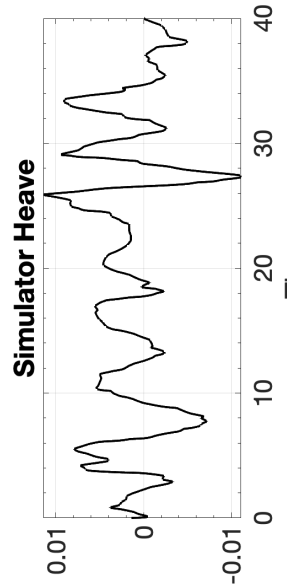

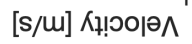

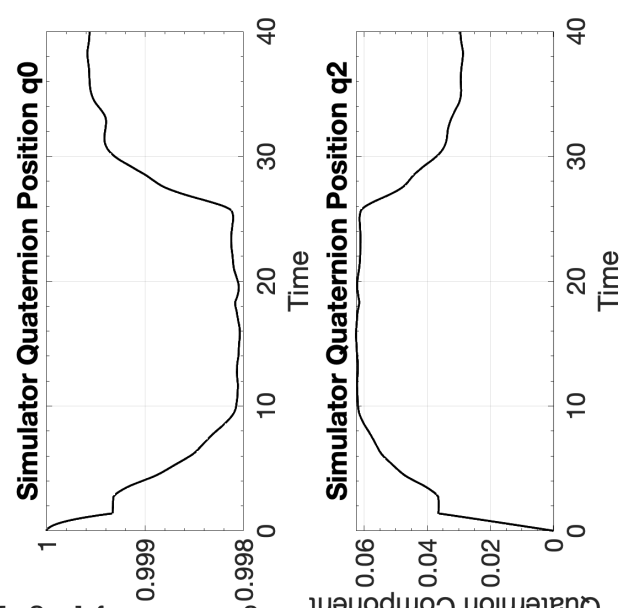

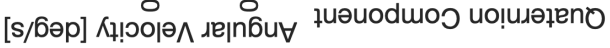

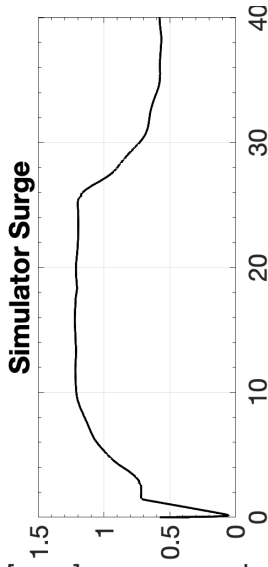

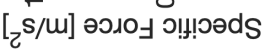

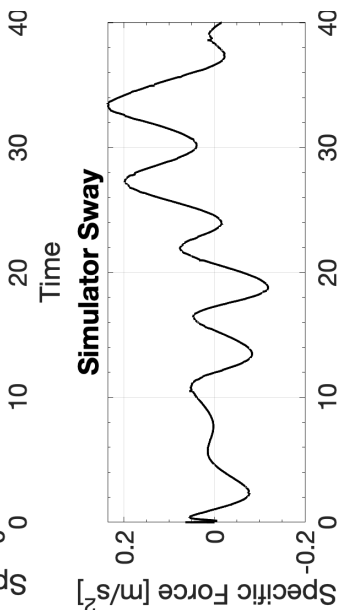

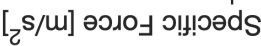

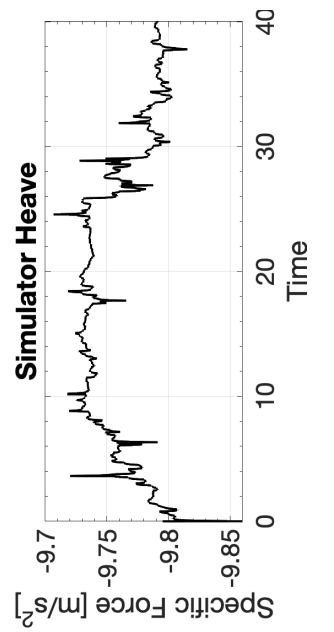

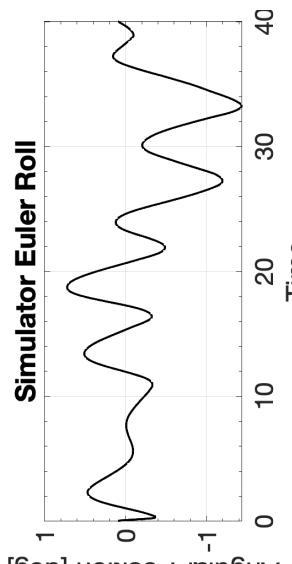

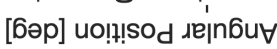

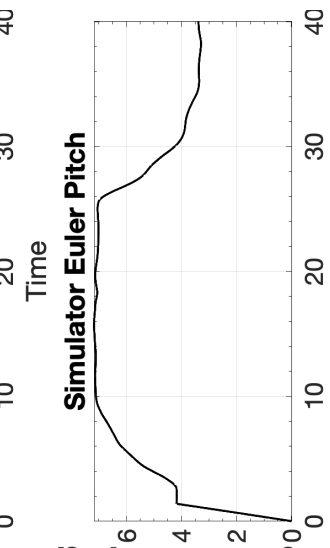

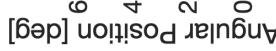

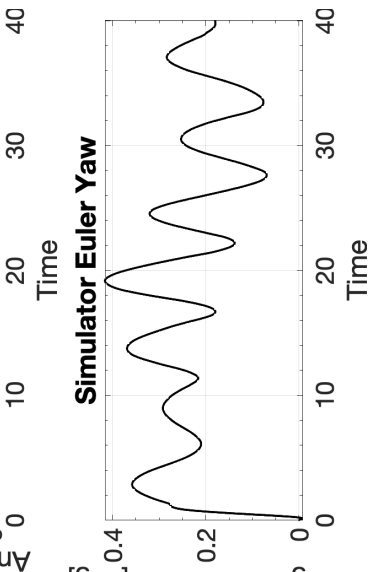

[6әр] uo!?! sod גejn6u

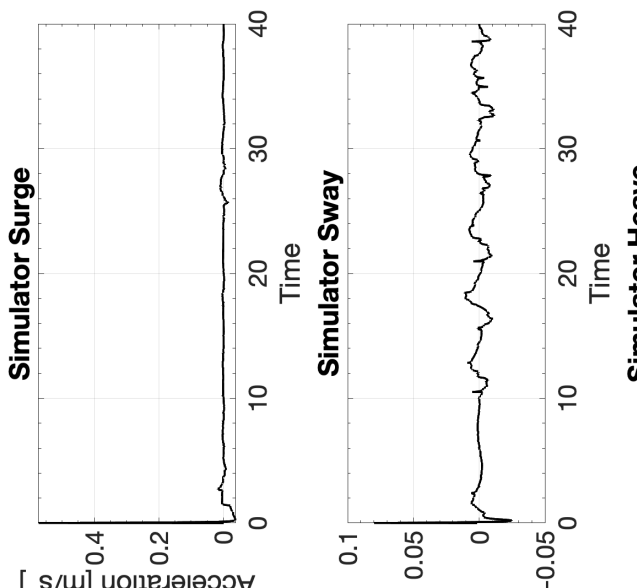

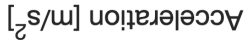

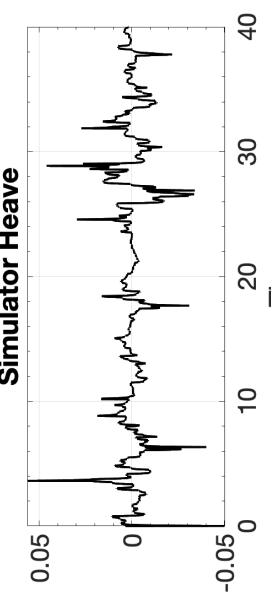

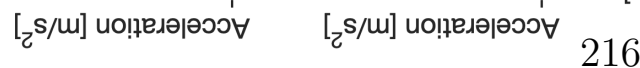

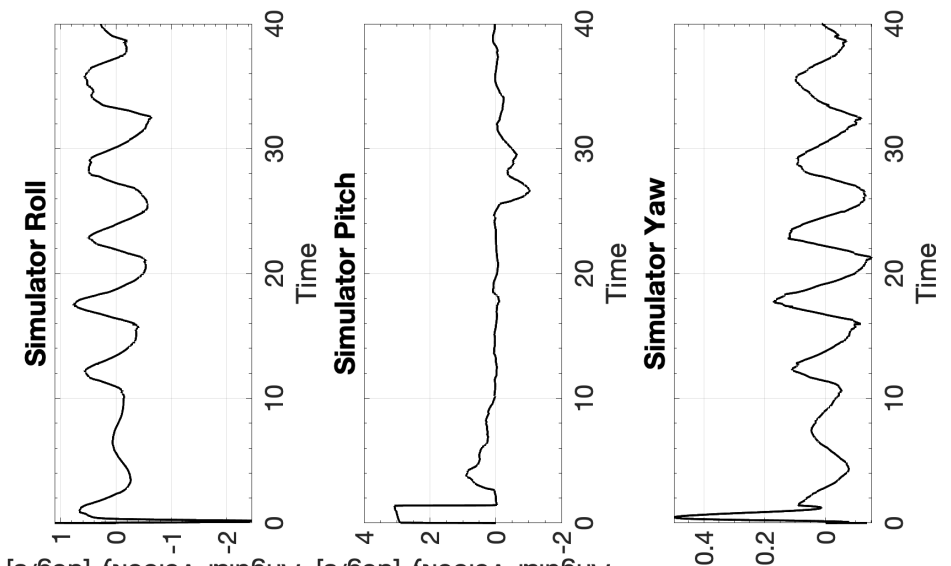

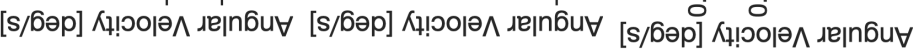



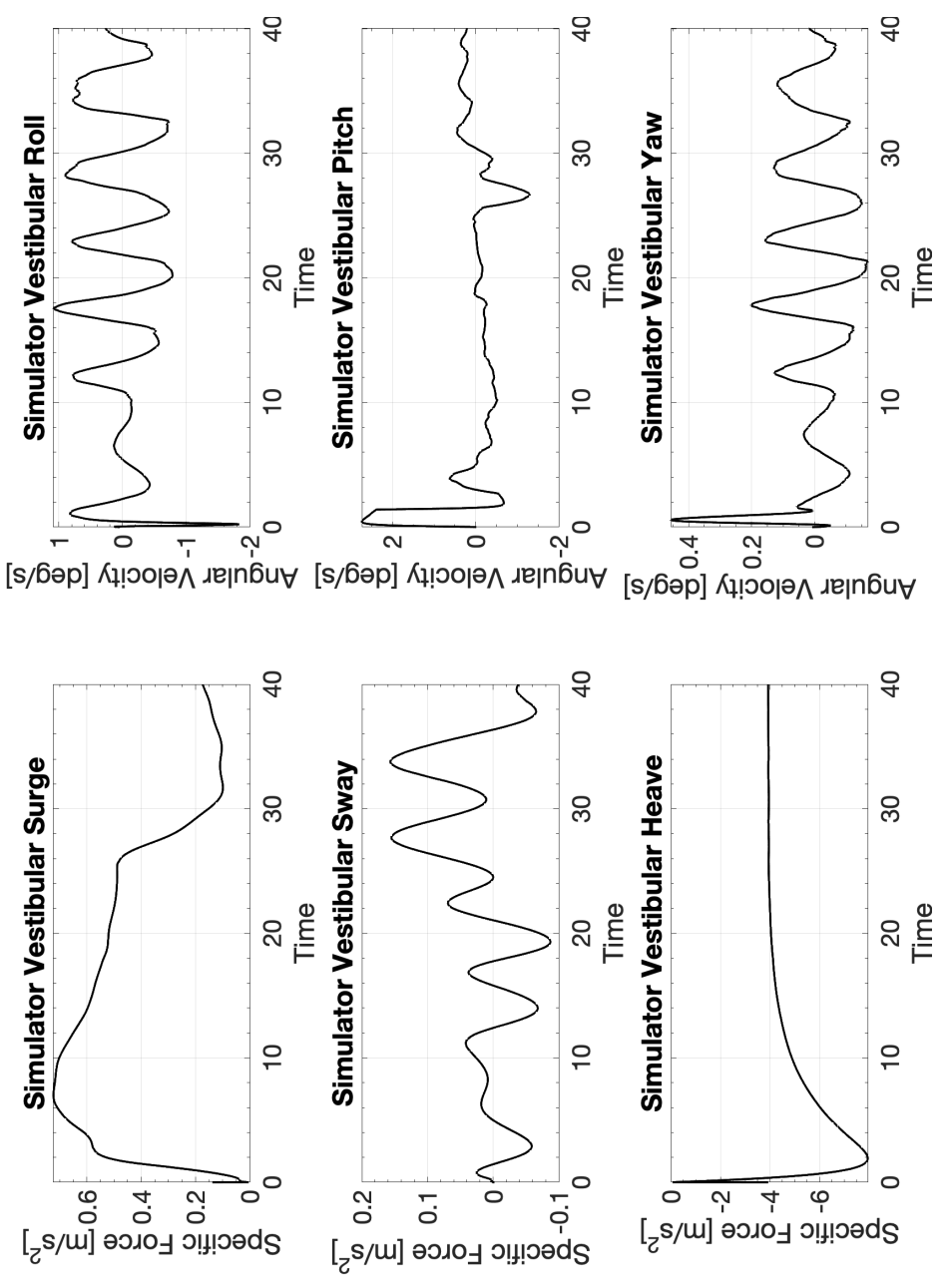


\section{C.3 M3}

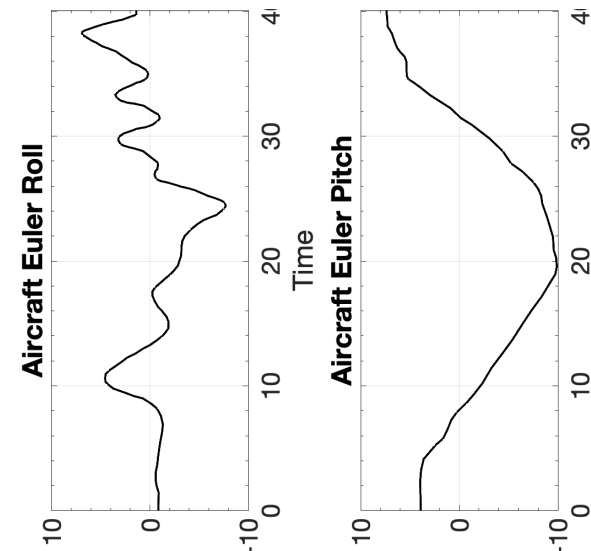

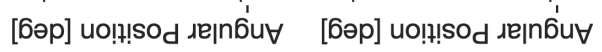

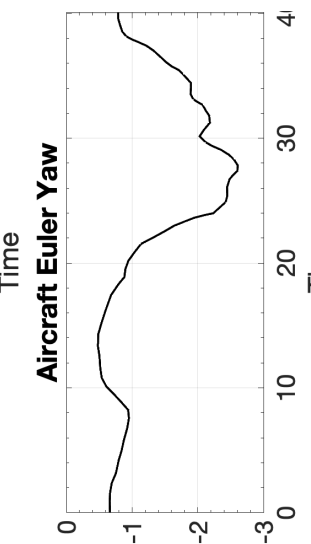

[бәр] uo!!!!sod גe|nбü

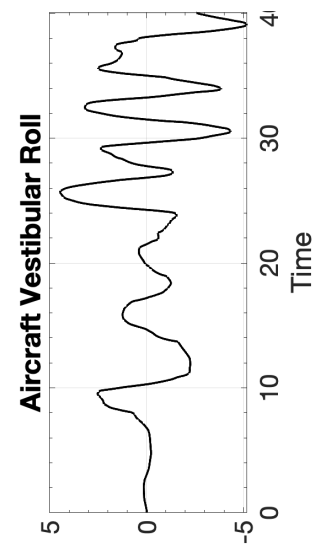

[2/6әp]

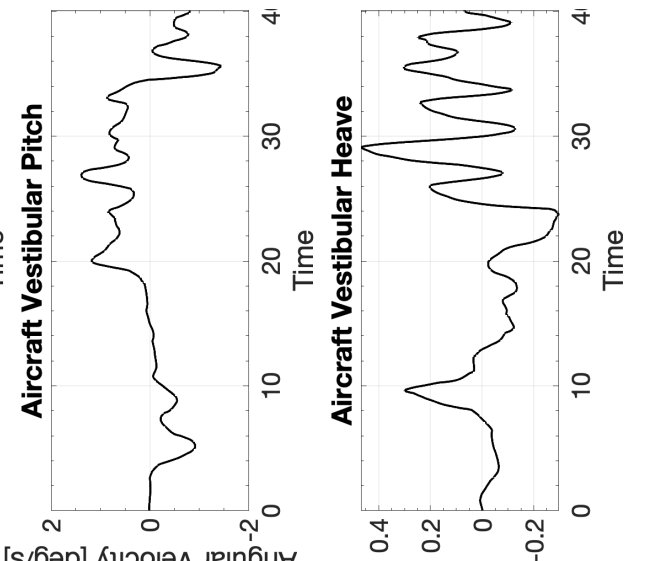

[s/6әр] Кұ!юорә
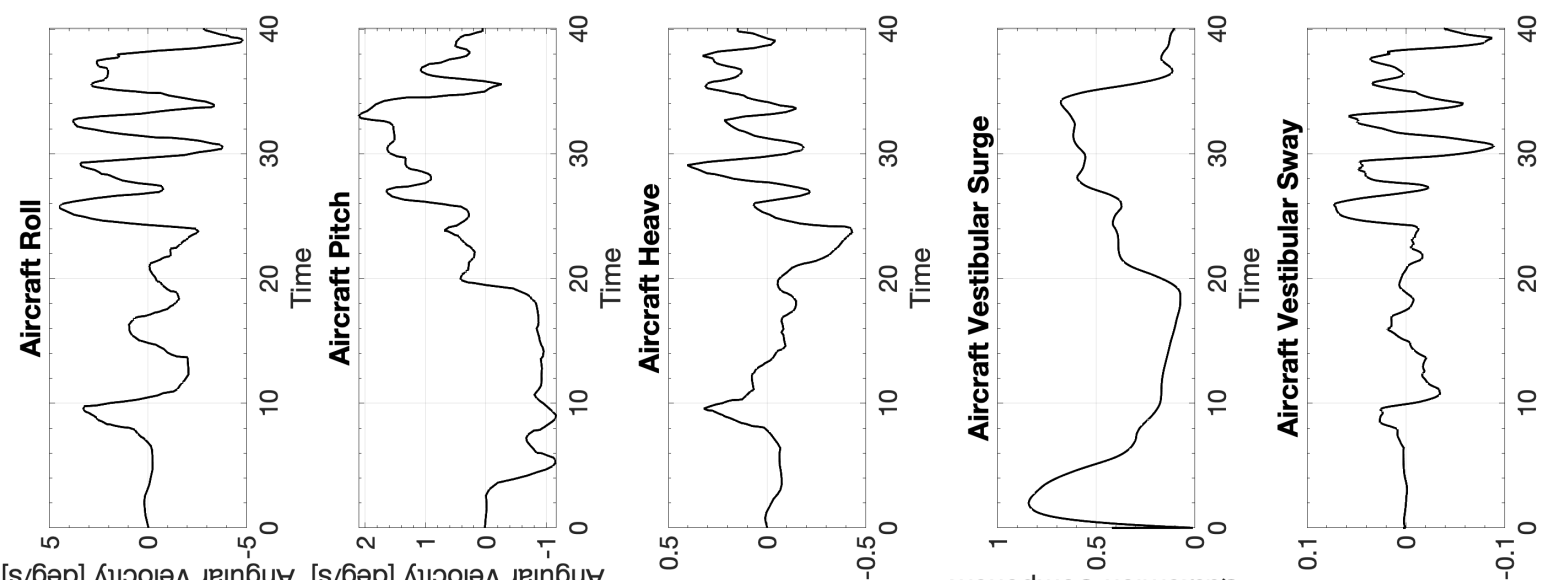

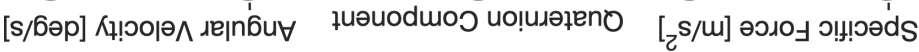

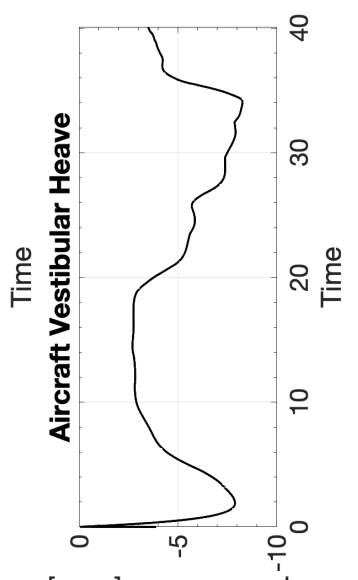

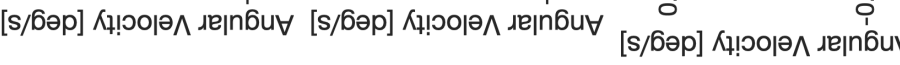

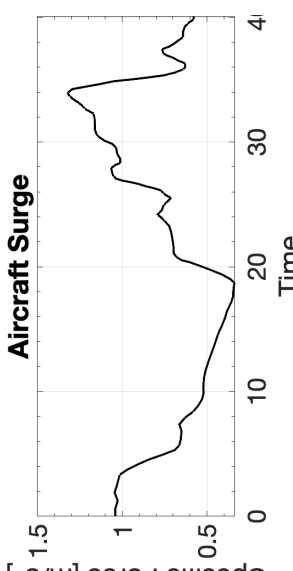

$[\bar{c} \mathrm{~s} / \mathrm{m}]$ ә

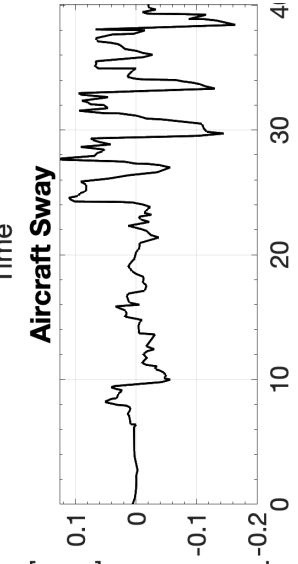

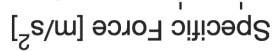

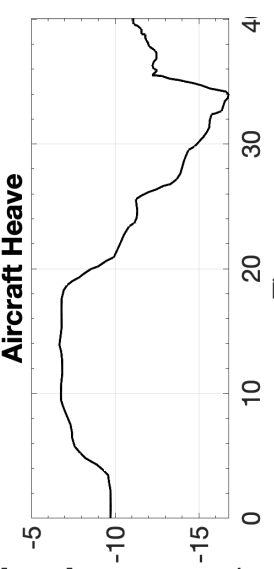

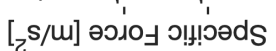

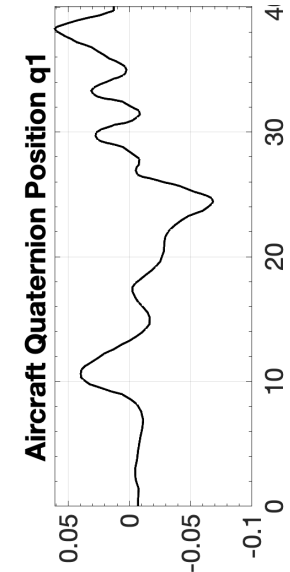

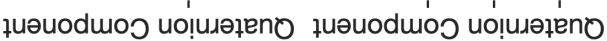

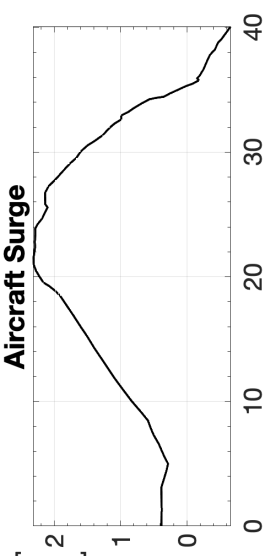

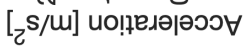

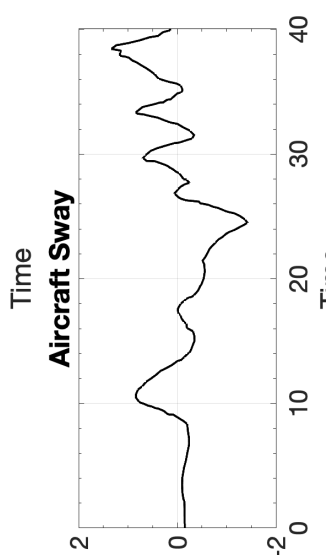

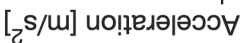

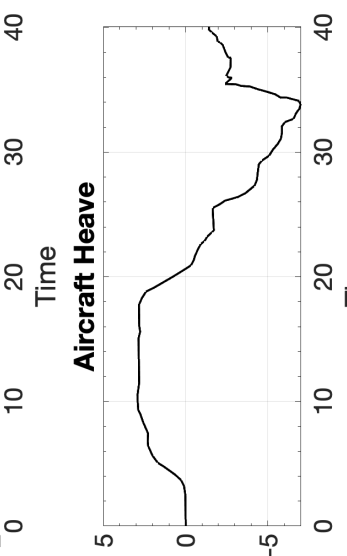

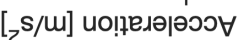
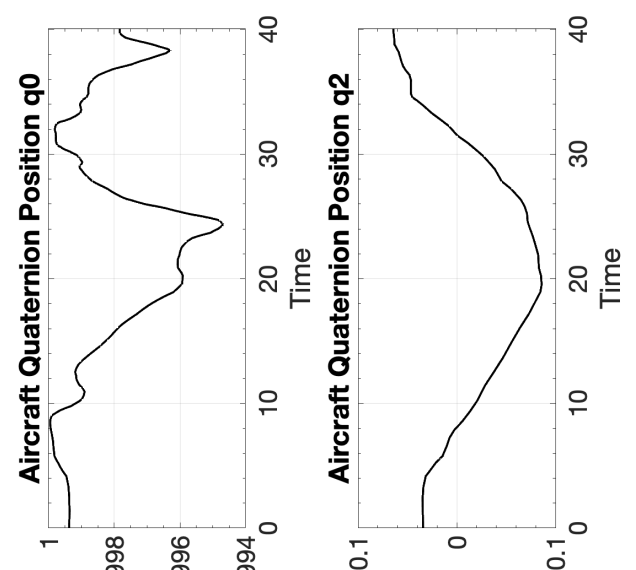

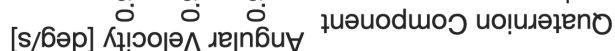




\section{C.3.1 CW1 M3}

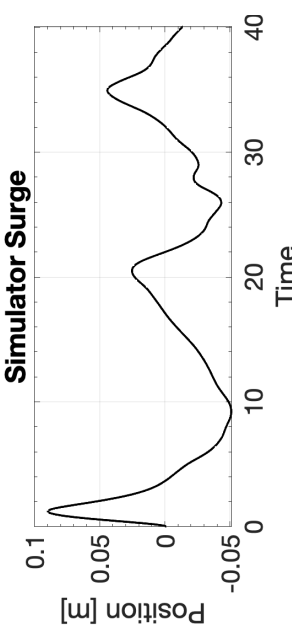

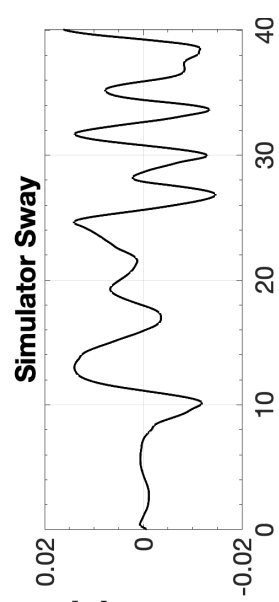

[u] uol!!!sod

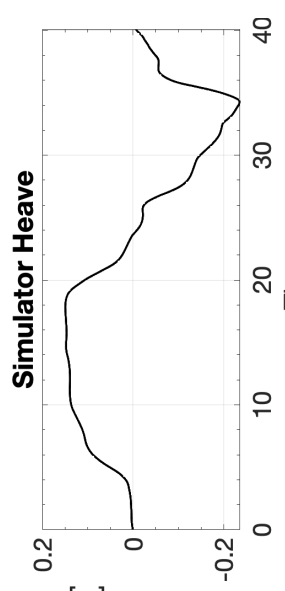

[w] uo!!!sod

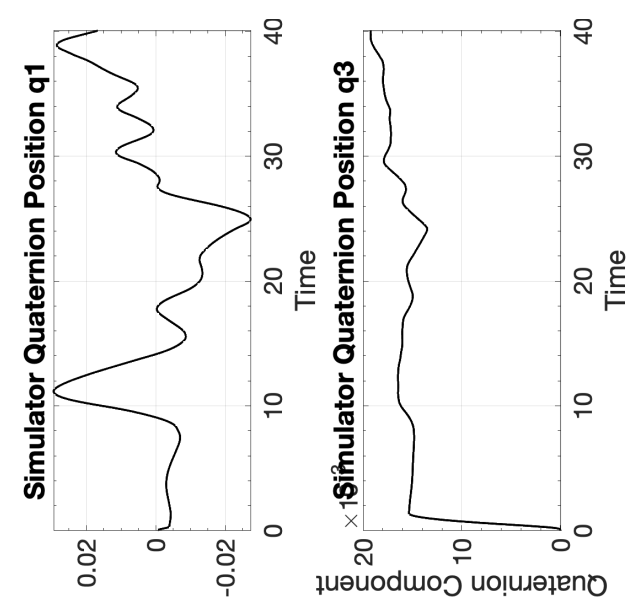

ұuәuodmos uo!̣uəłeno

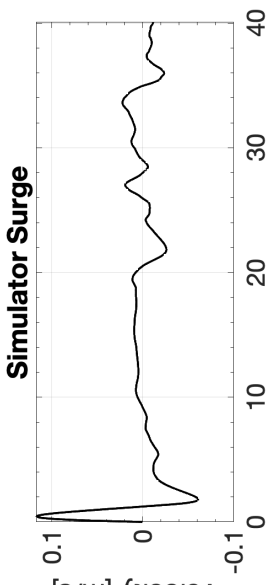

[s/m] Кџ! Оою๐

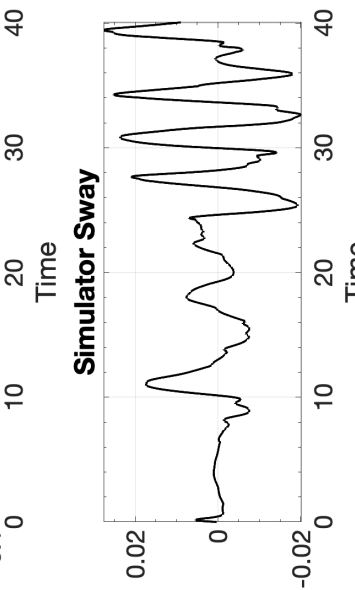

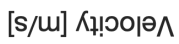

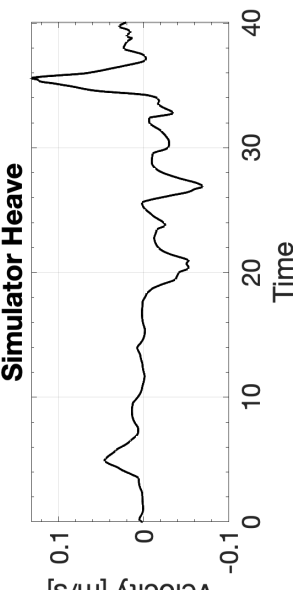

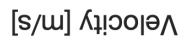
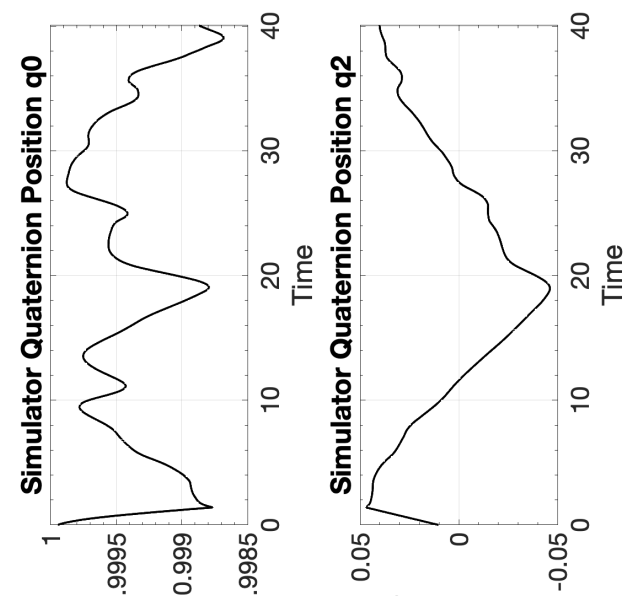

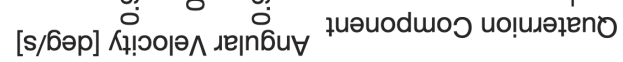

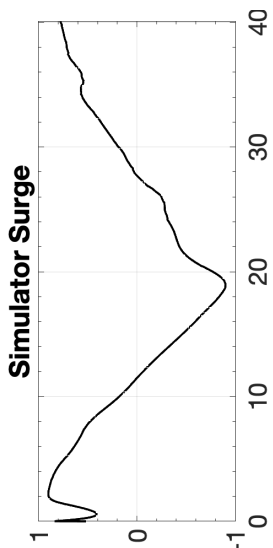

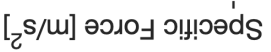
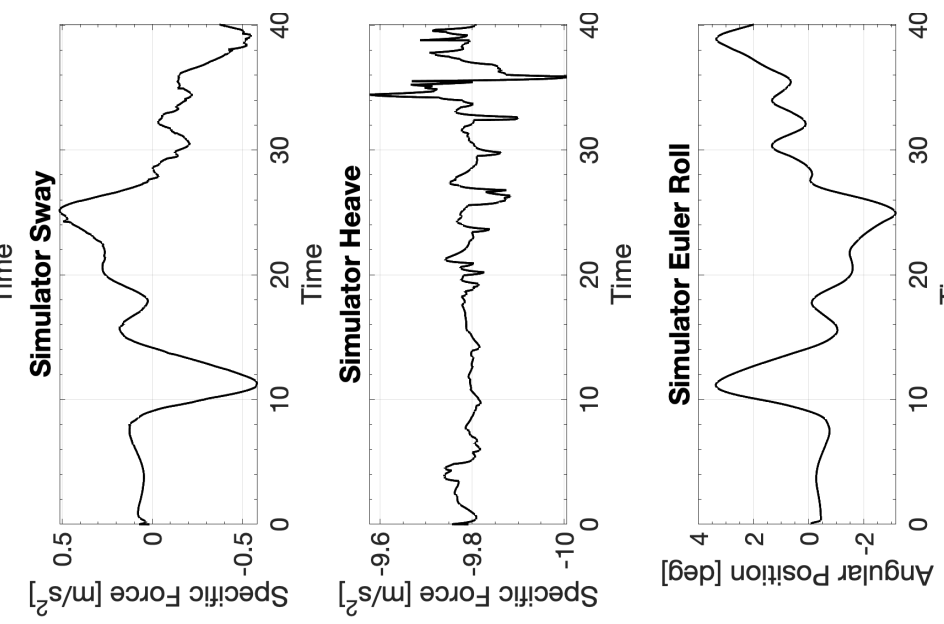

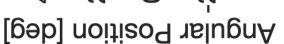

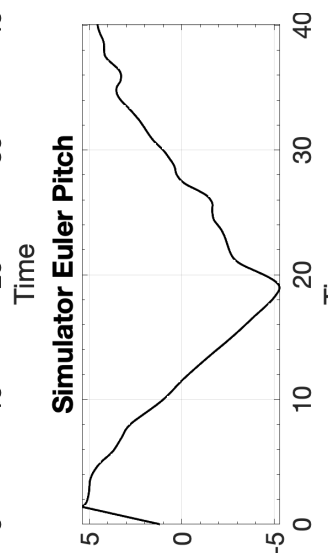

[6әр] uo!!!

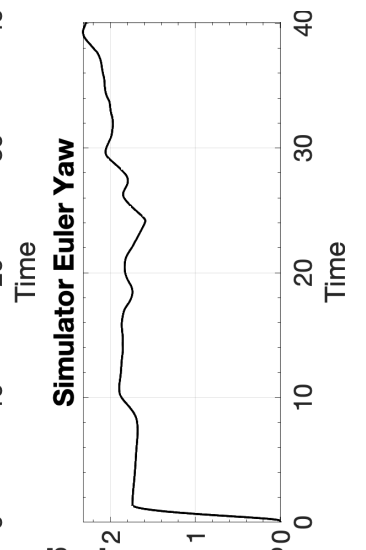

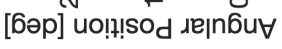

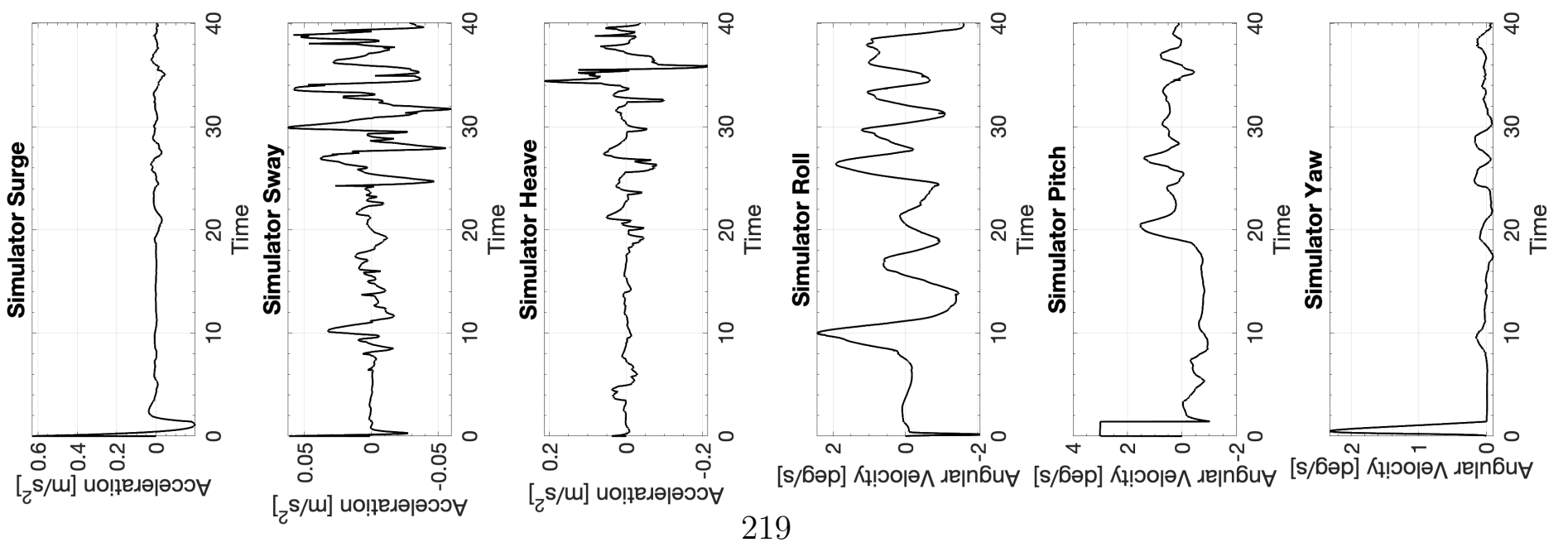




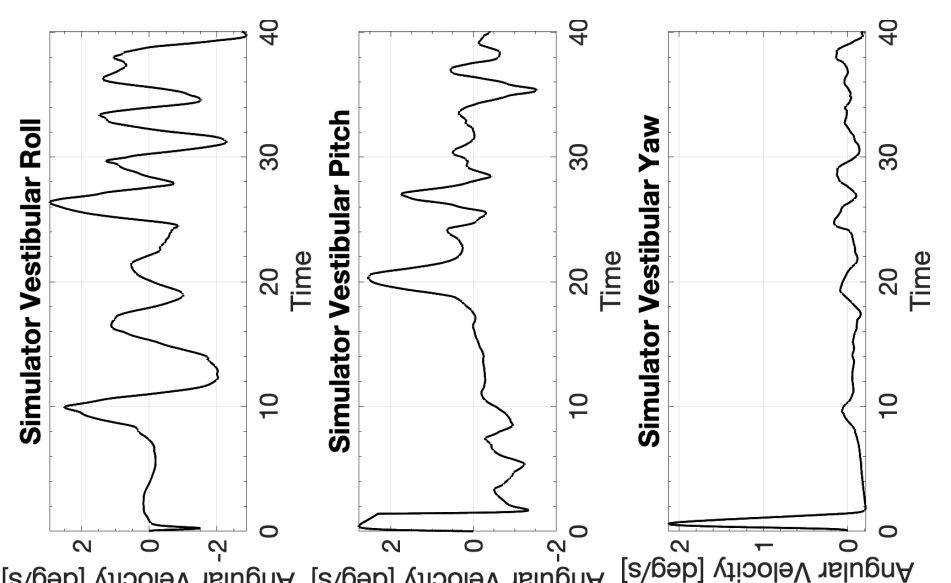

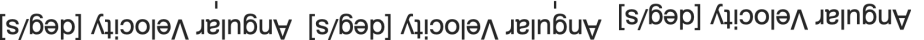

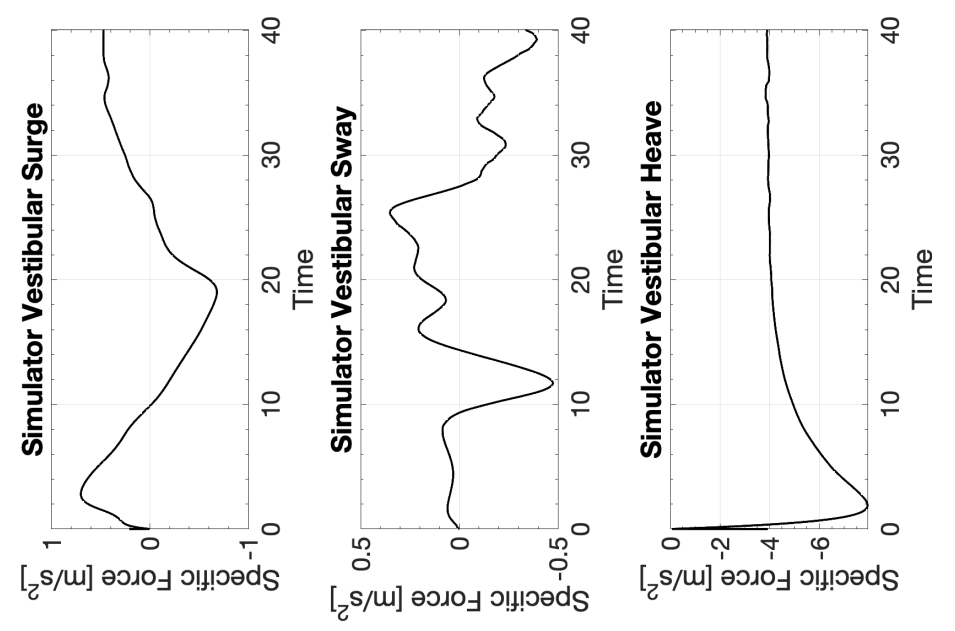




\section{C.3.2 CW2 M3}

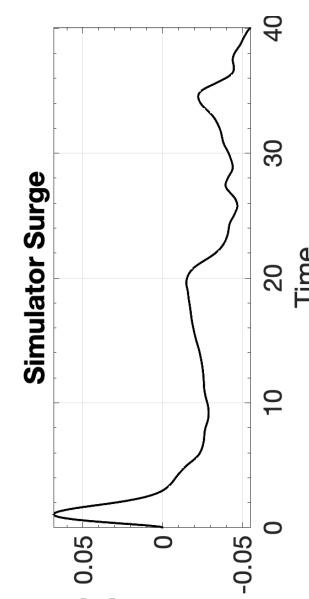

[u] uo!!!!sod

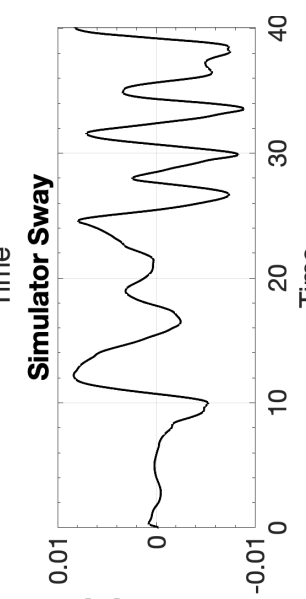

[u] uol!!!sod

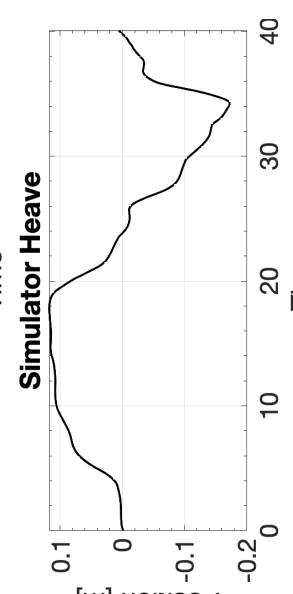

[w] uo!!!sod

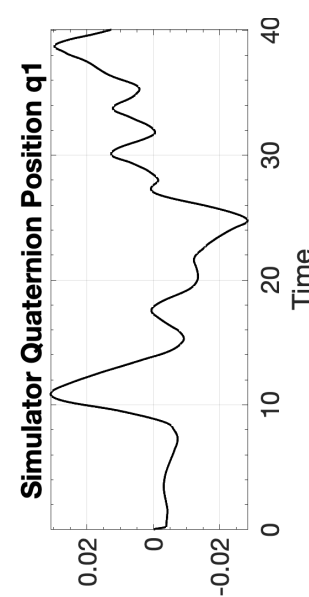

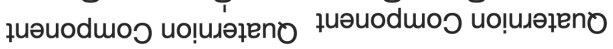

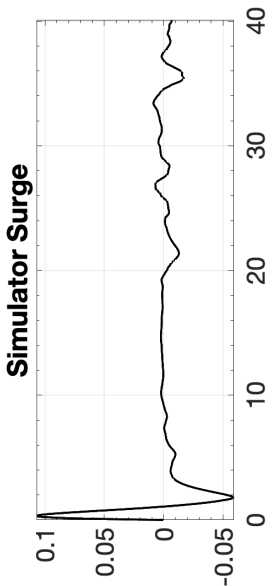

[s/m] К! ৩ою ^

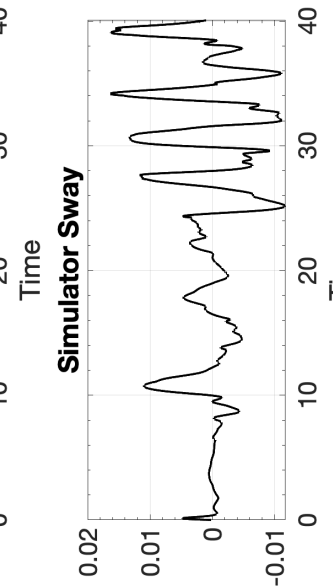

[s/m] Кц!юојә^

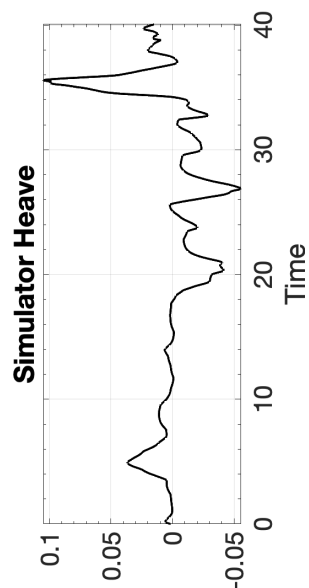

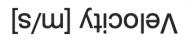

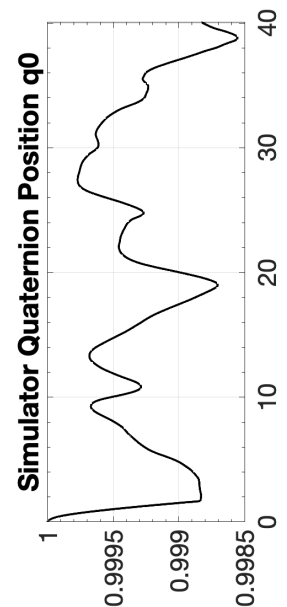

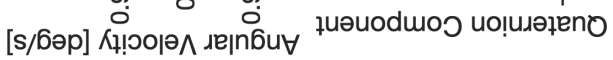

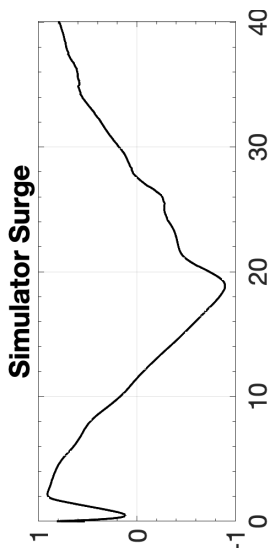

$[\mathrm{z} / \mathrm{L} / \mathrm{m}$ ә ә

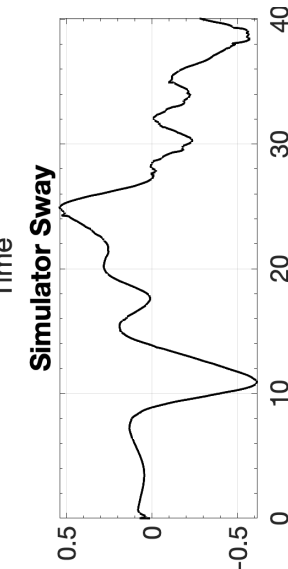

[s/m] ә010」 כ!!!
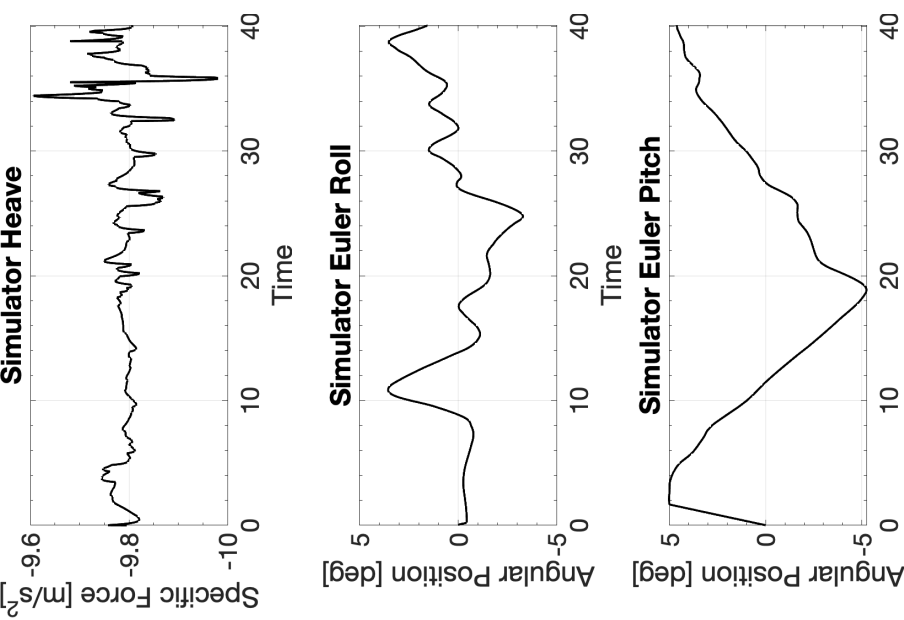

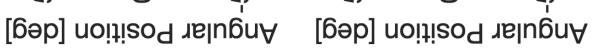

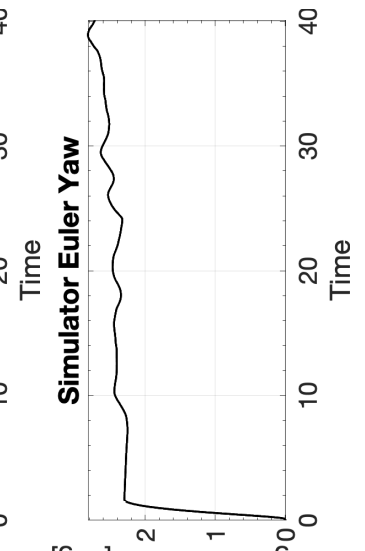

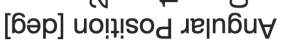

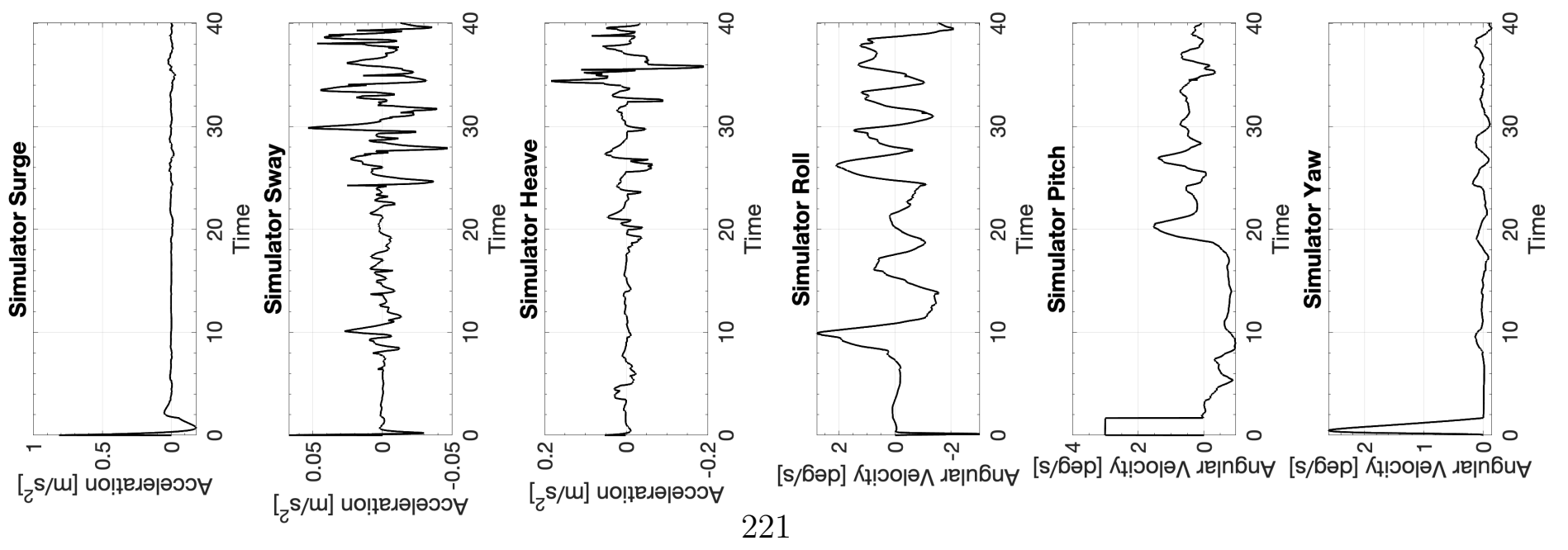



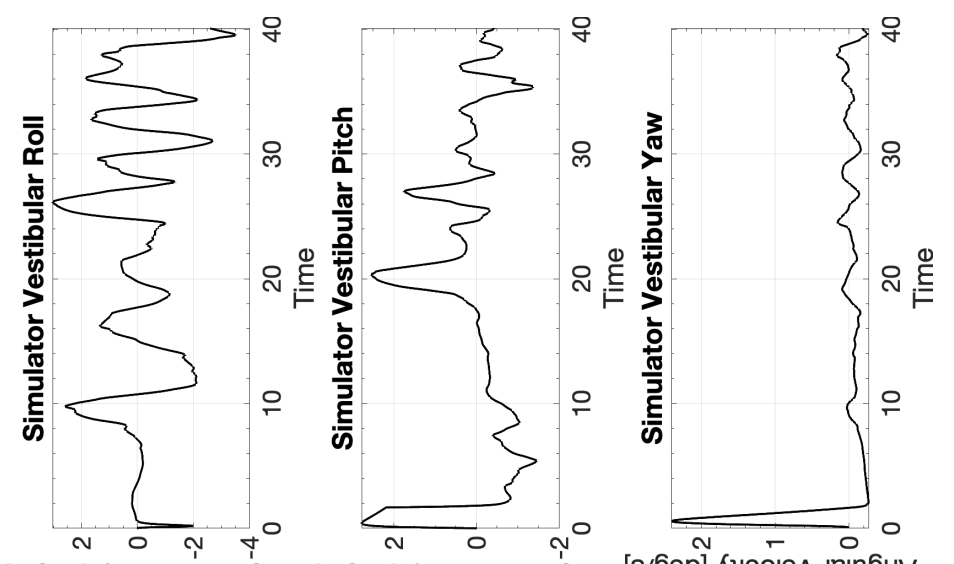

[s/бәр] Кұ!юорә $\wedge$ גе|nби

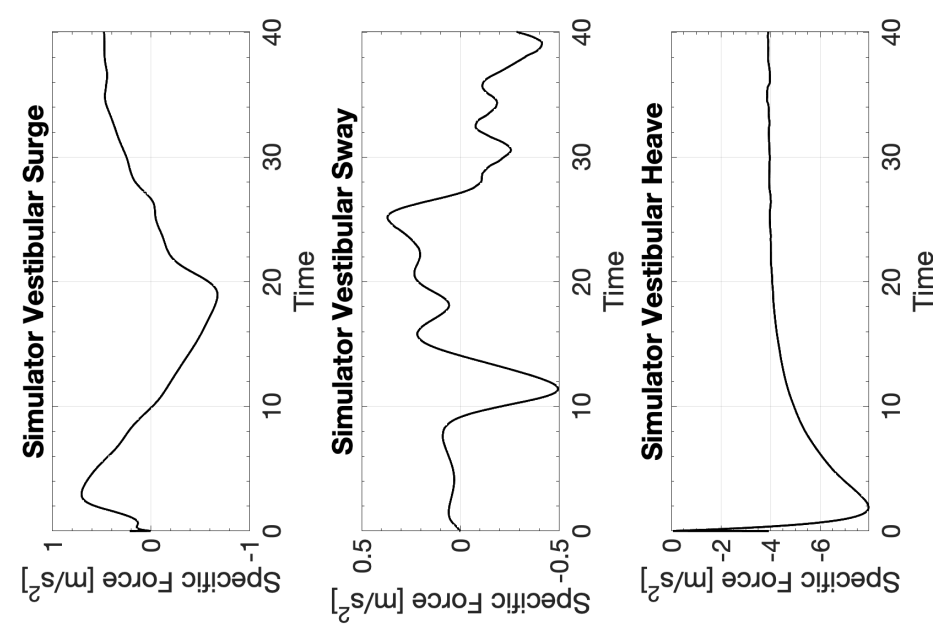




\section{C.3.3 CW3 M3}

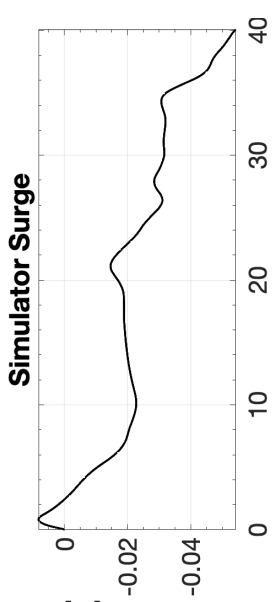

[w] uo!n!sod

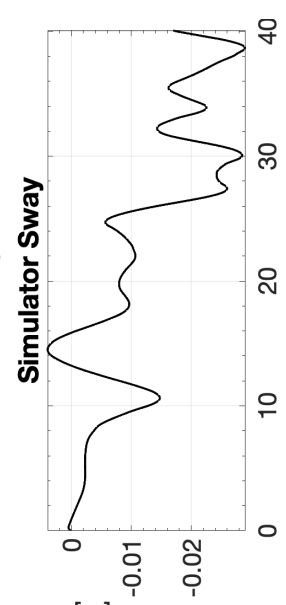

[u] uo!n!!sod

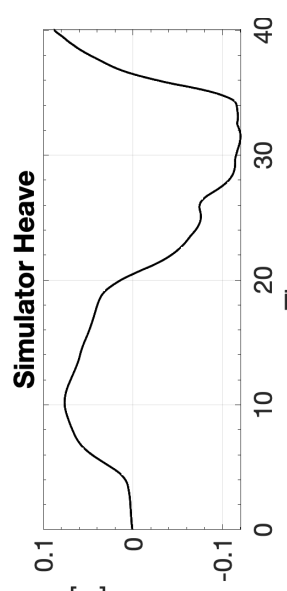

[w] uo!!!!sod

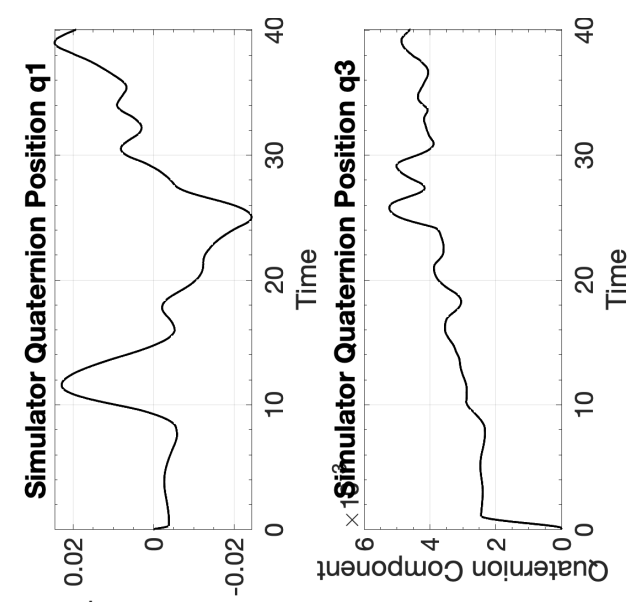

ұuəuodmo uo!̣uəłeno

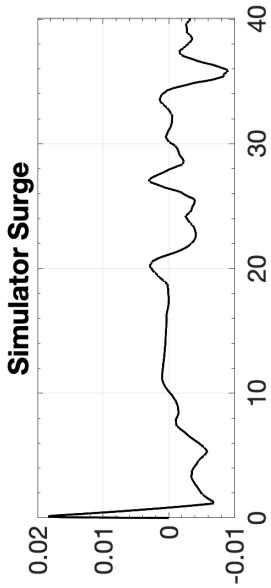

[s/u] Кц!юојә

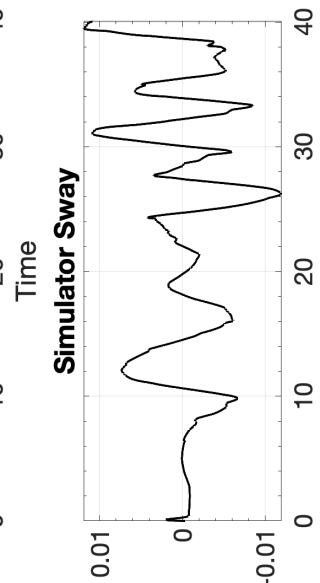

[s/m] К! ๖оəㅅ

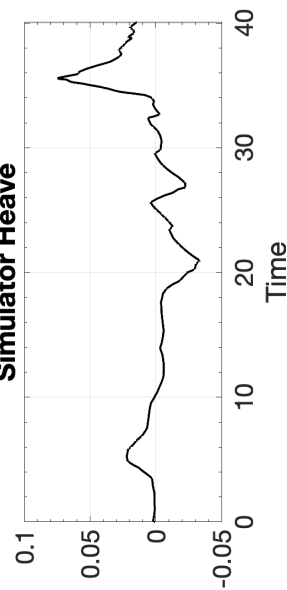

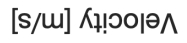

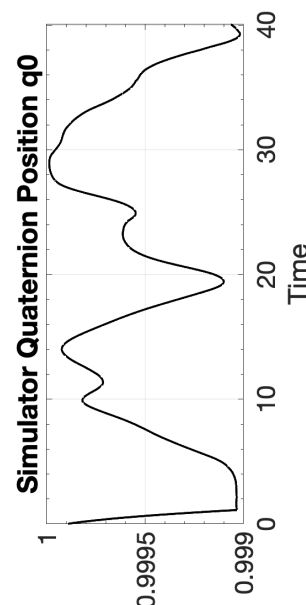

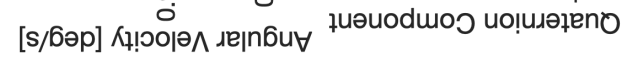

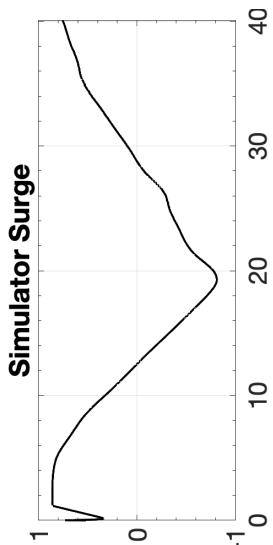

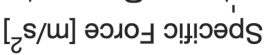
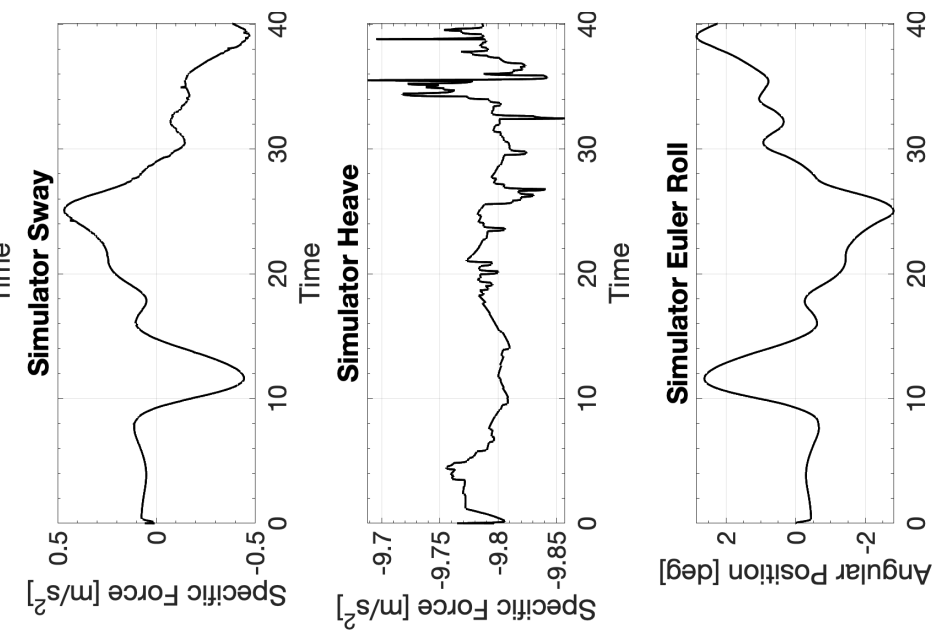

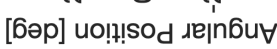

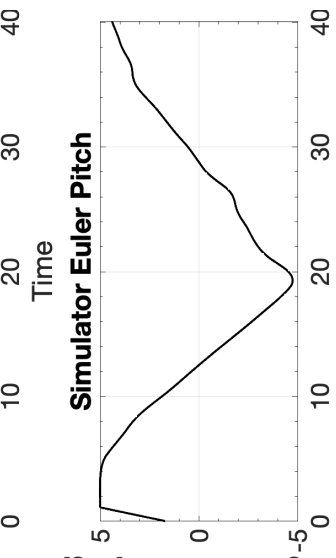

[6əp] uo!!!!sod גeןnదินี

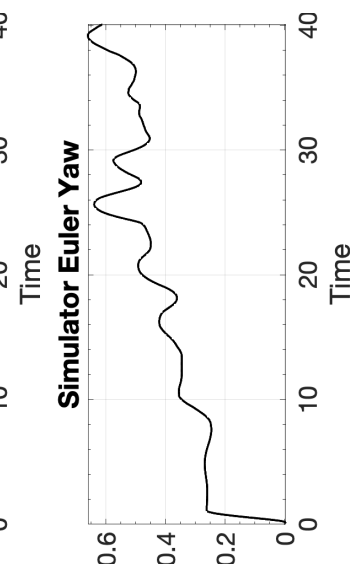

[6әр] uo!!!sod גejn6u
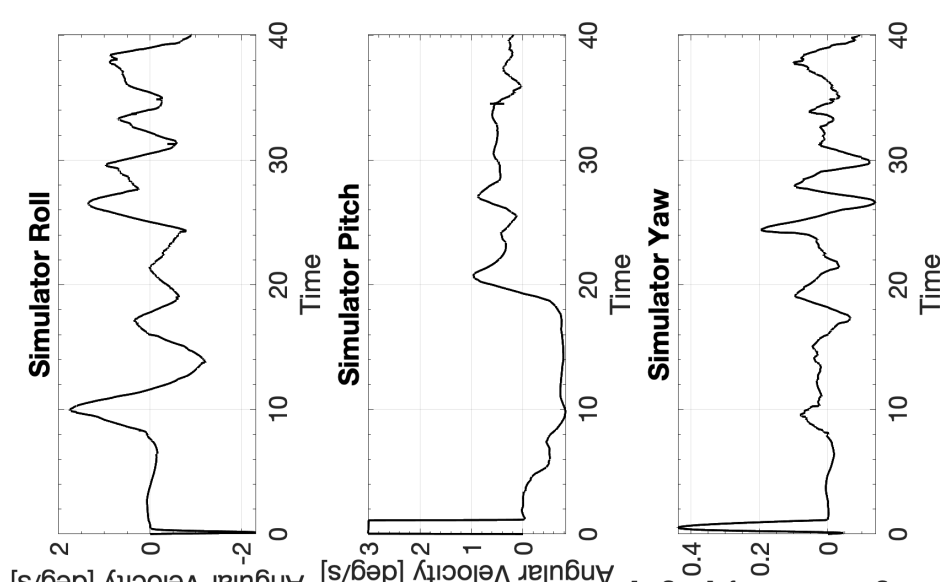

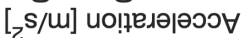

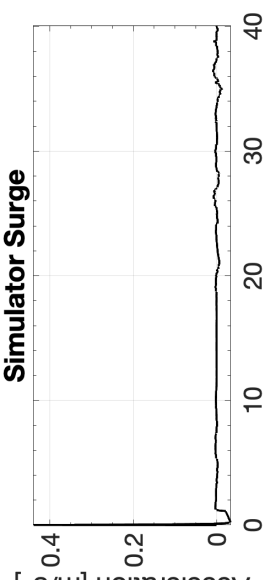

टे

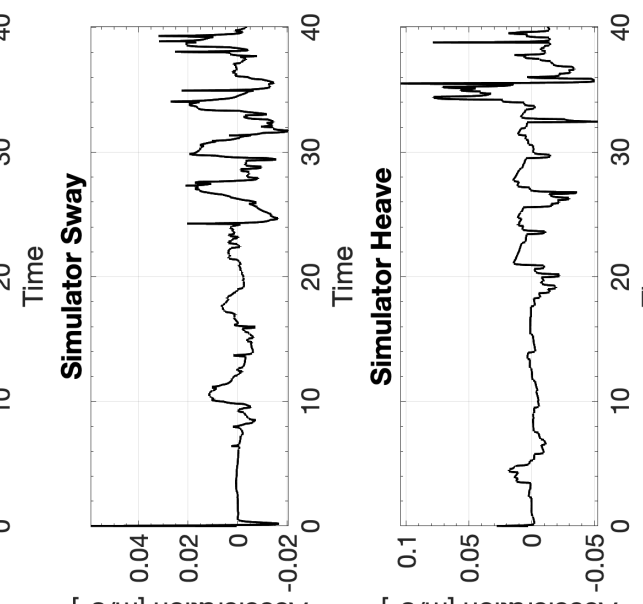

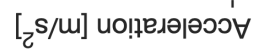

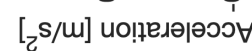

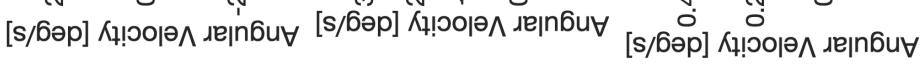
223 

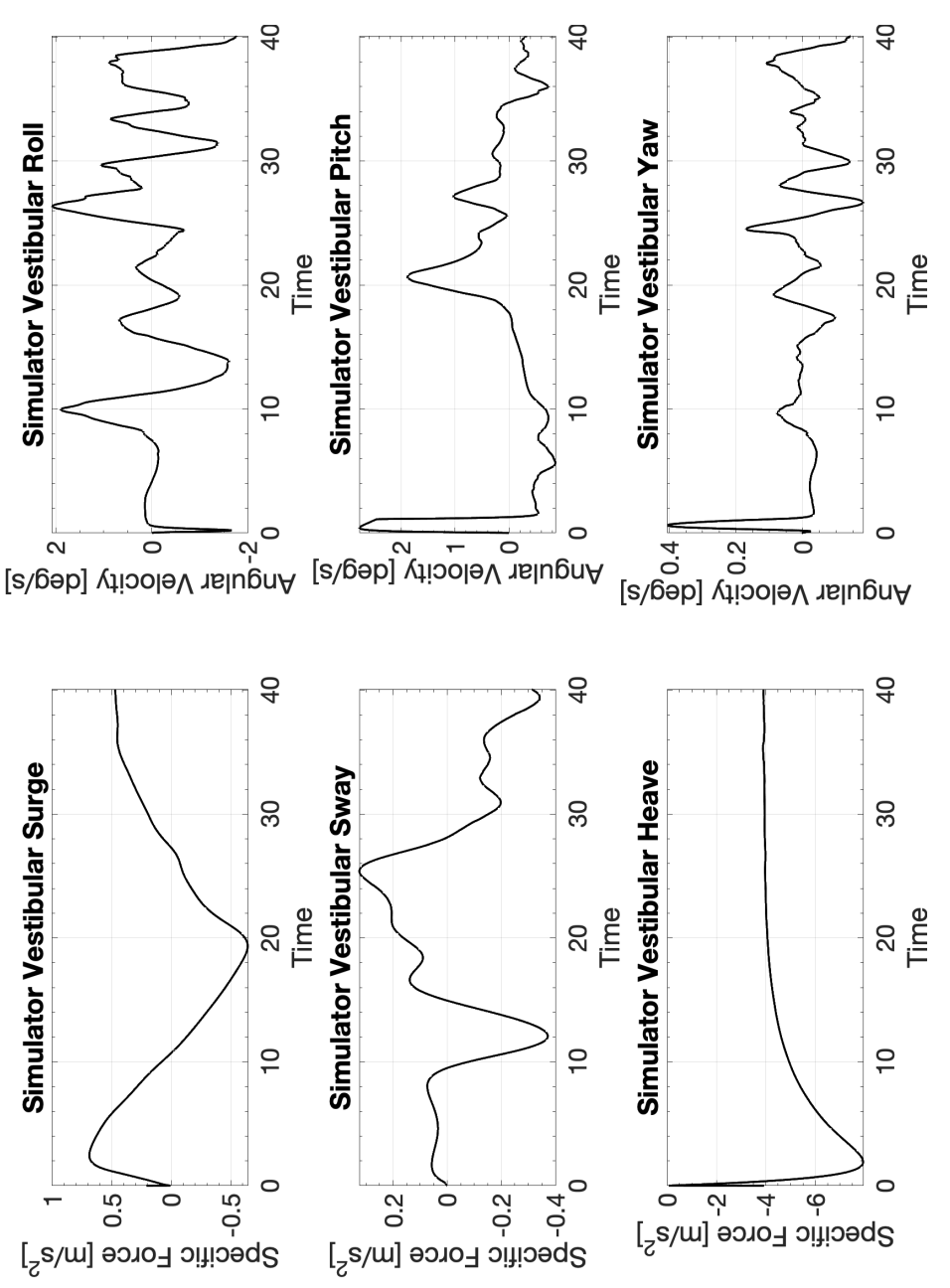


\section{C.4 Cessna 172 Coordinated Turn}

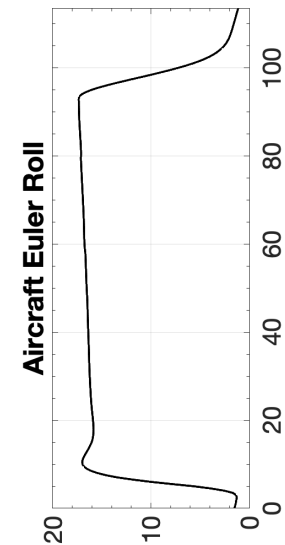

[6әp] uo!!!sod ле|n6u

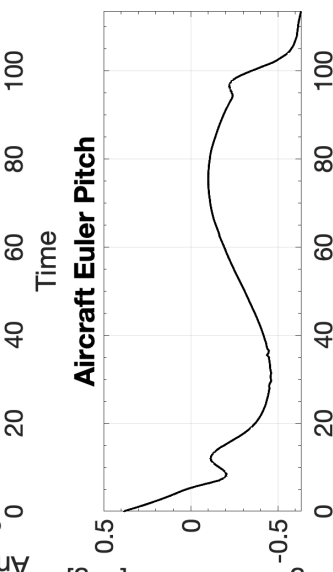

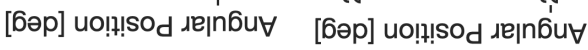

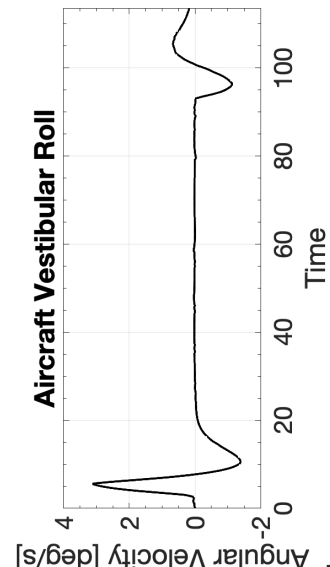

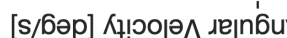
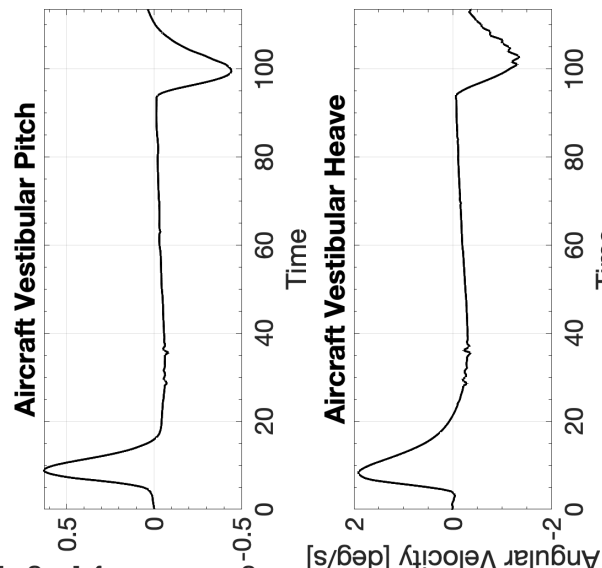

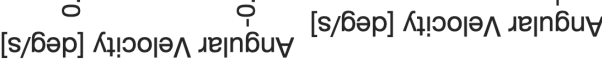

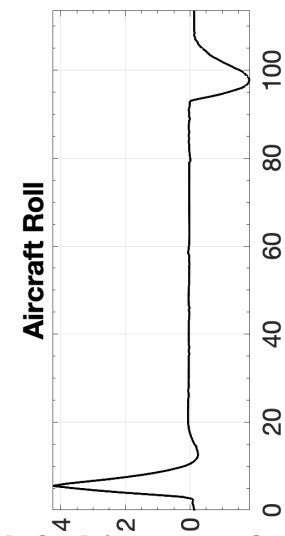

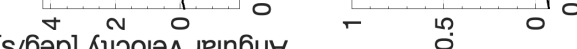

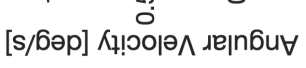

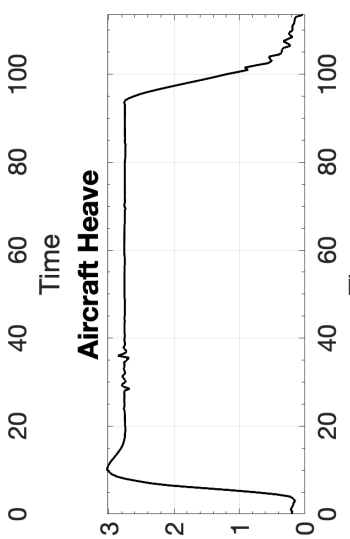

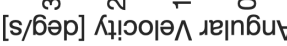

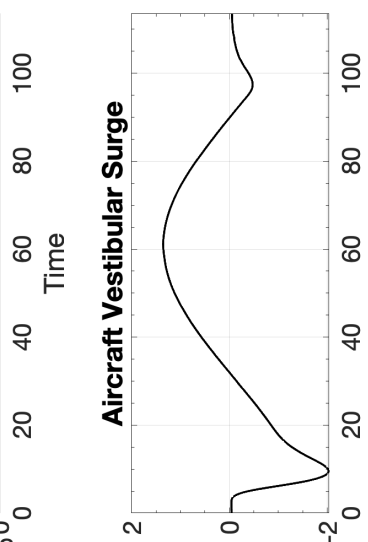

ұuәuodmos uọudəłeno

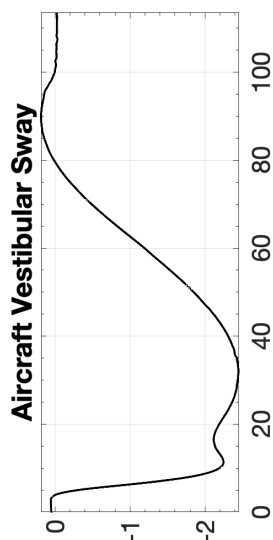

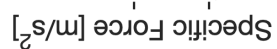
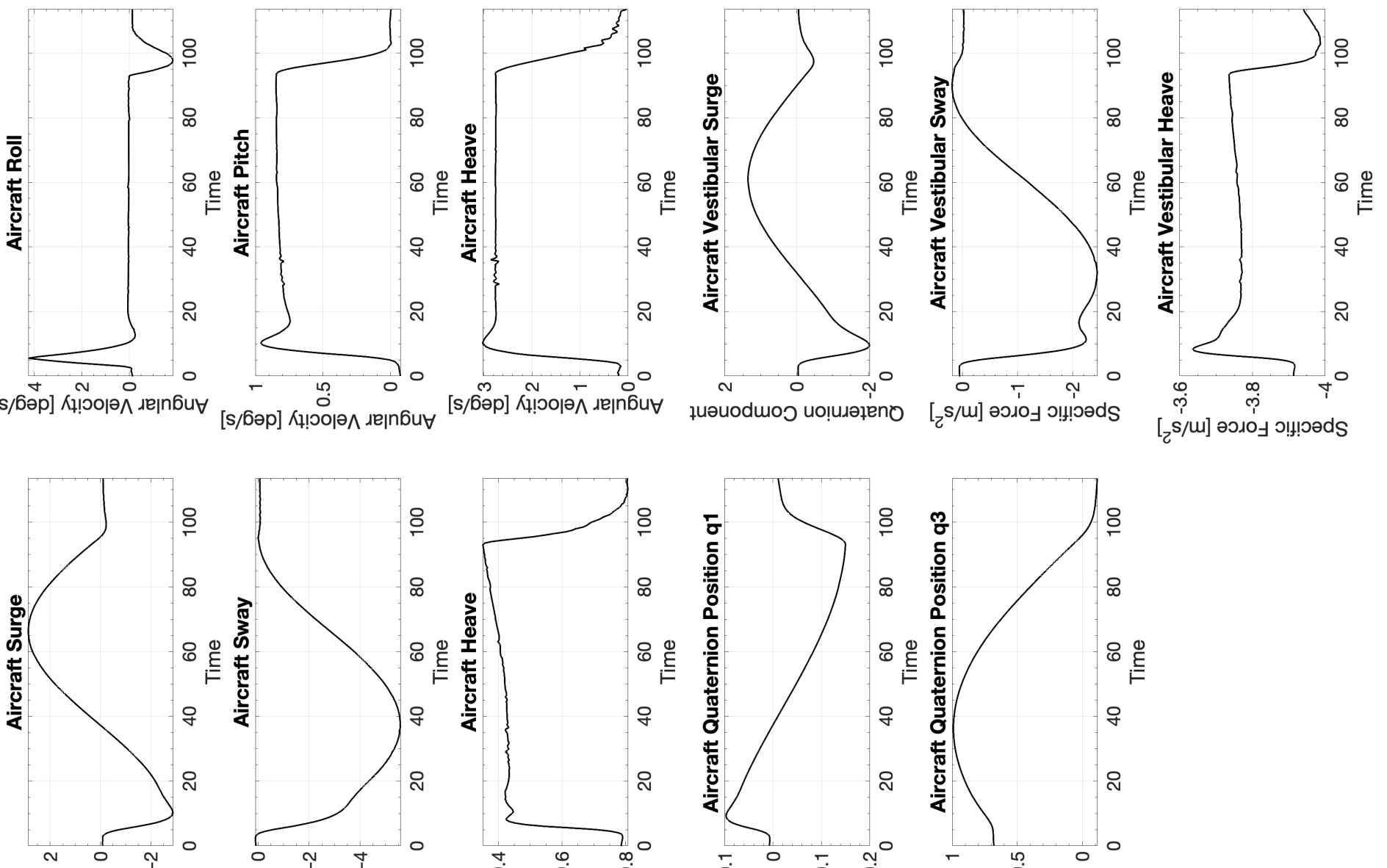

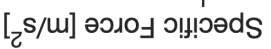

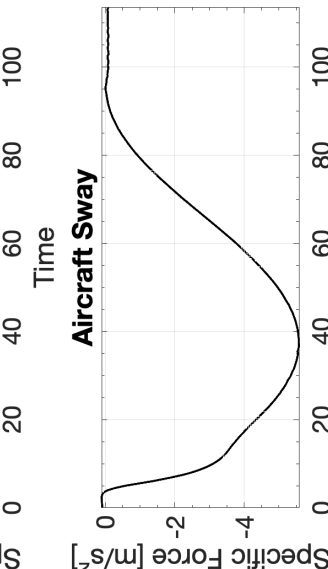

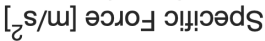
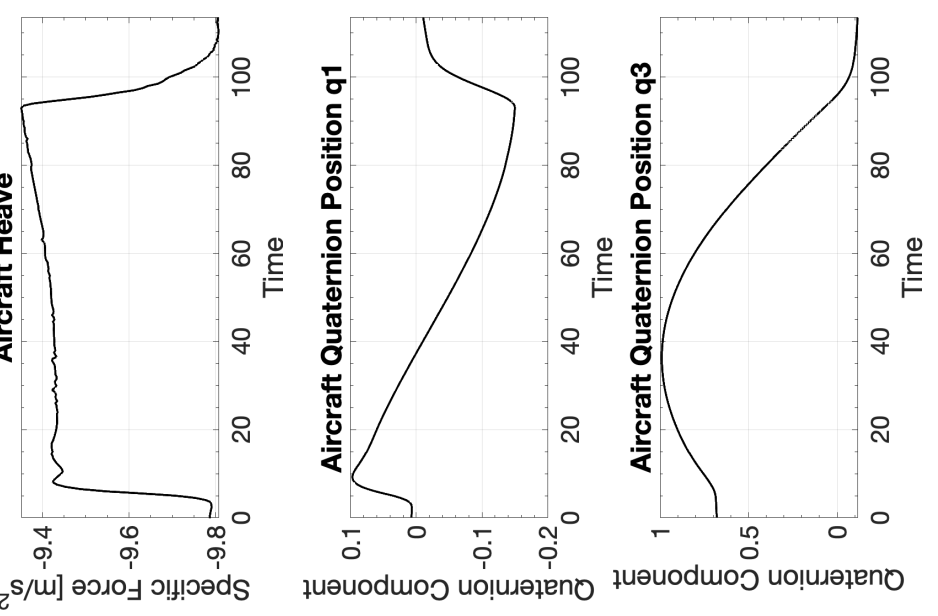

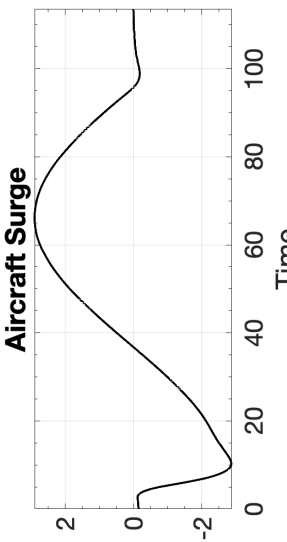

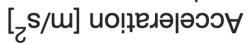

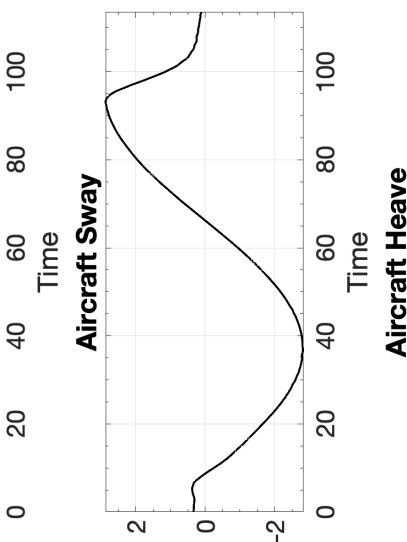

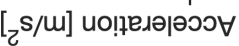

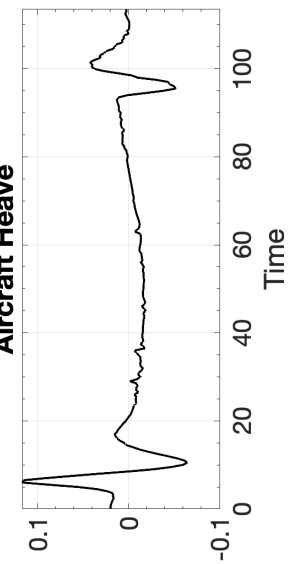

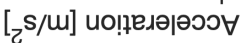

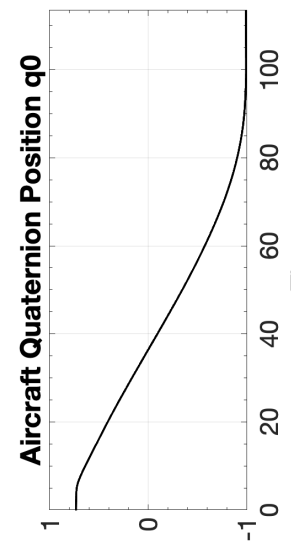

[s/6әр] Кұ!юо|ә^ גe|n6u

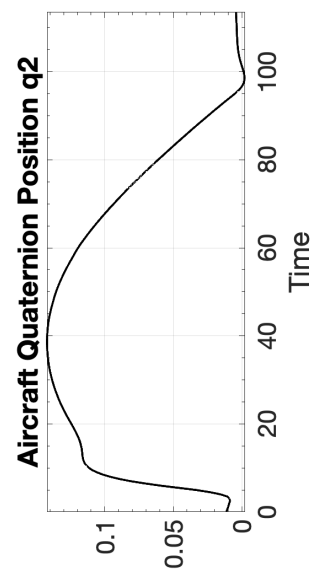

ұuəuodmo uo!̣uәəeno 


\section{C.4.1 CW1 Cessna 172 Coordinated Turn}


[s/u] К! ৩оำ

[s/m] Кұ!юојә^

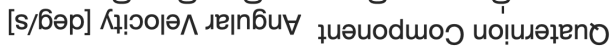

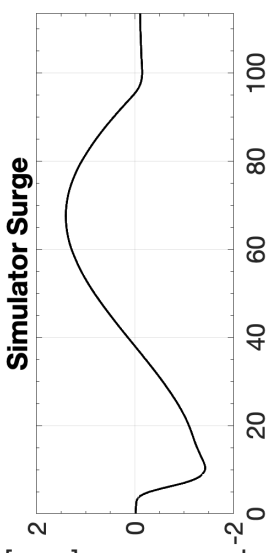

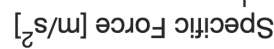

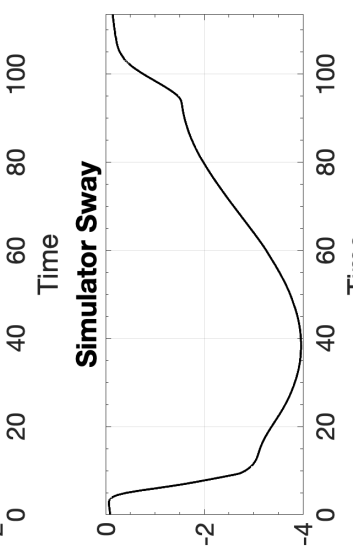

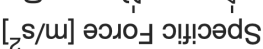

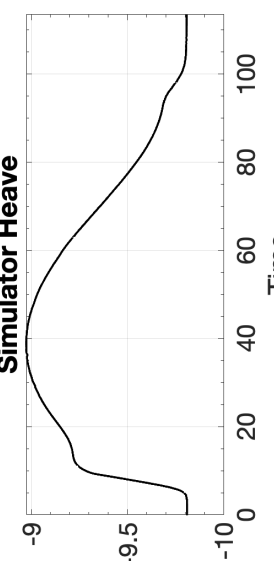

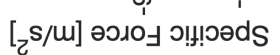

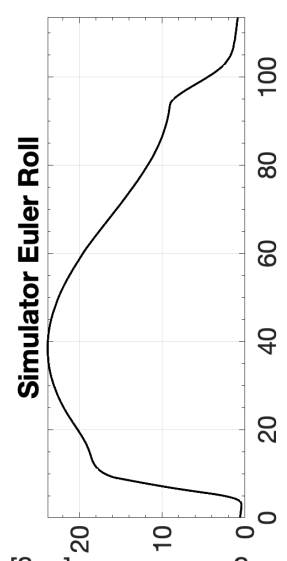

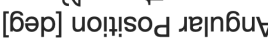

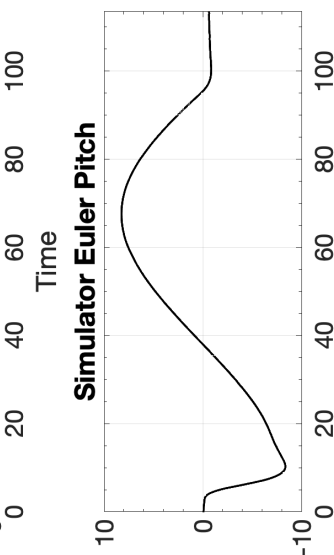

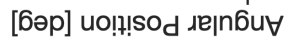

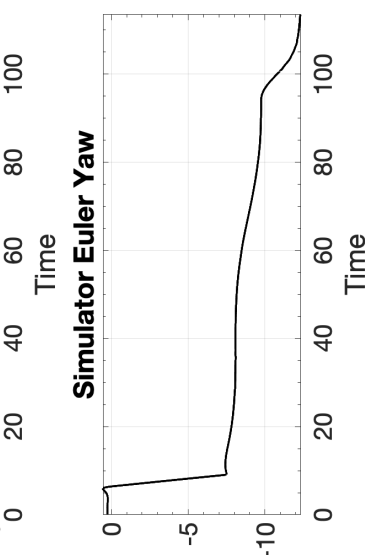

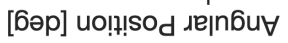

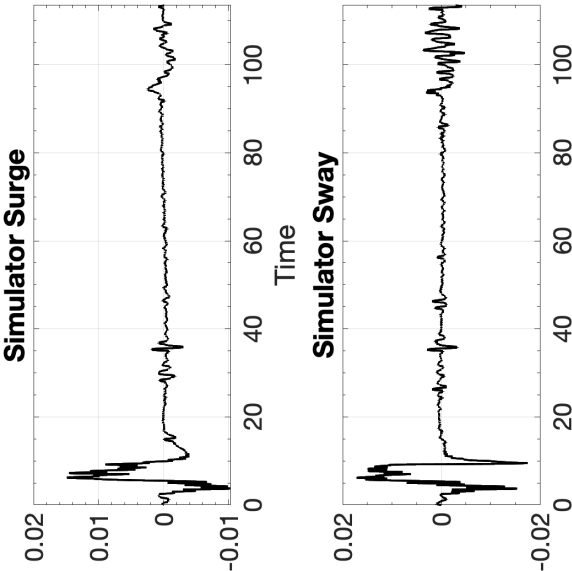

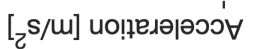

torsul
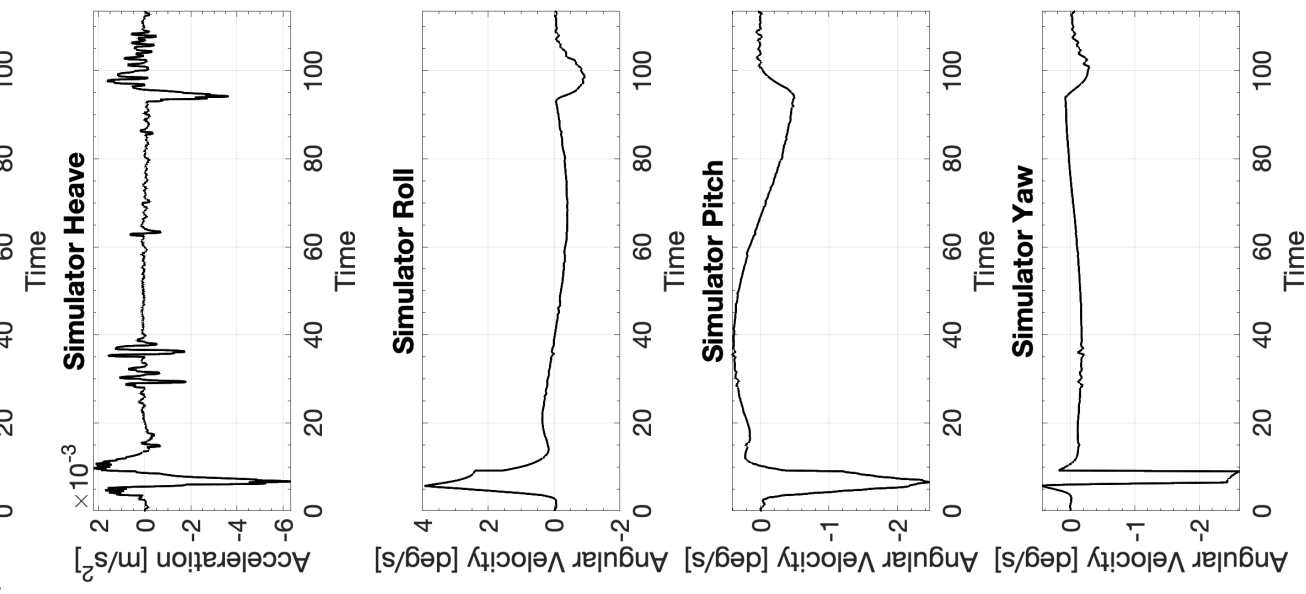

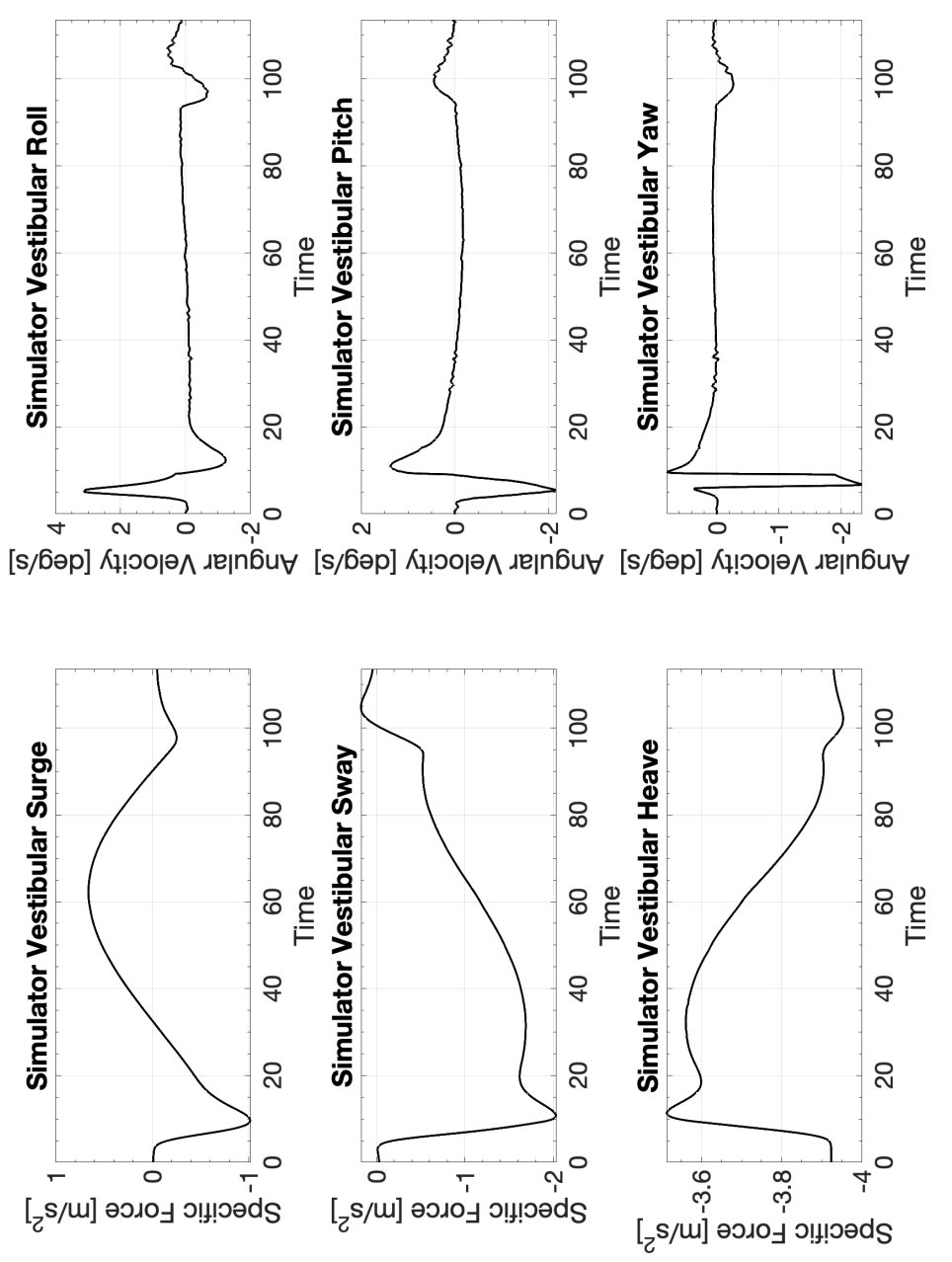


\section{C.4.2 CW2 Cessna 172 Coordinated Turn}

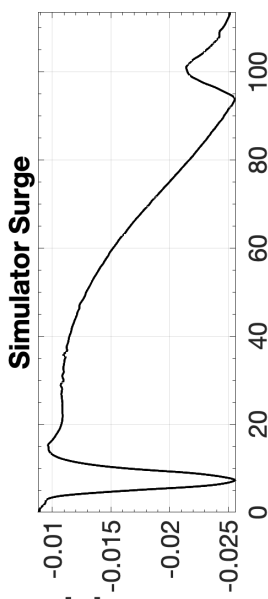

[w] uo!!!!sod

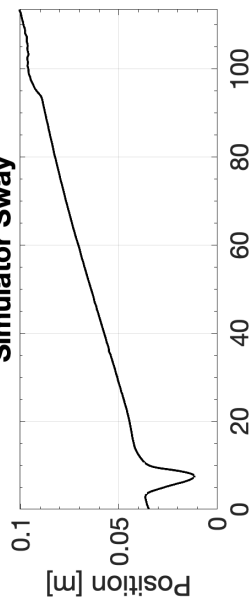

[w] uo!n!sod
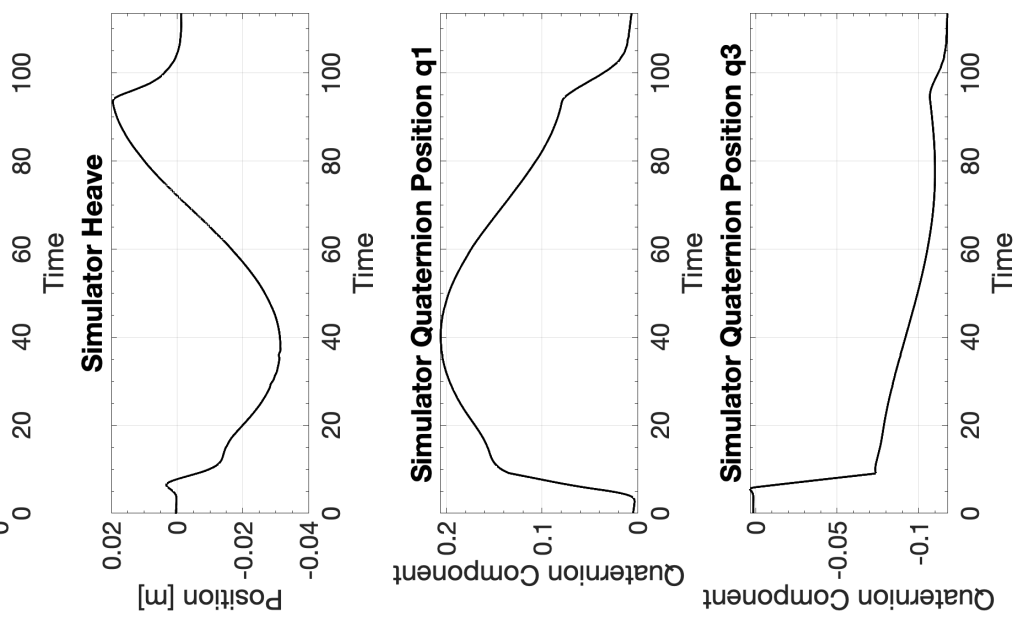

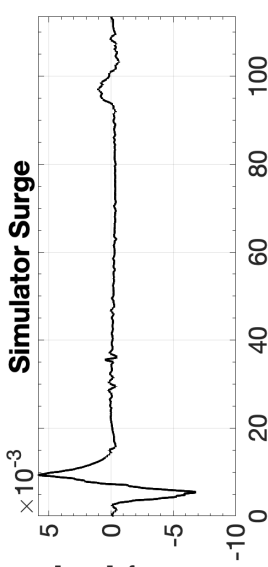

[s/u] К! ৩о夫 ^

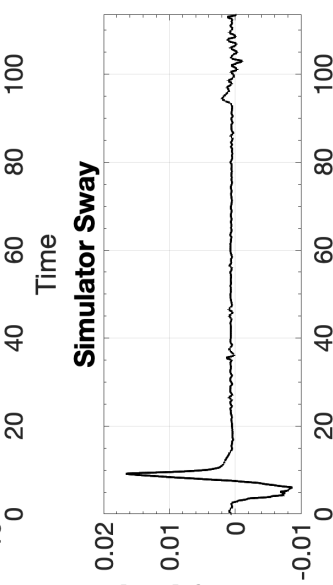

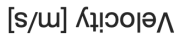

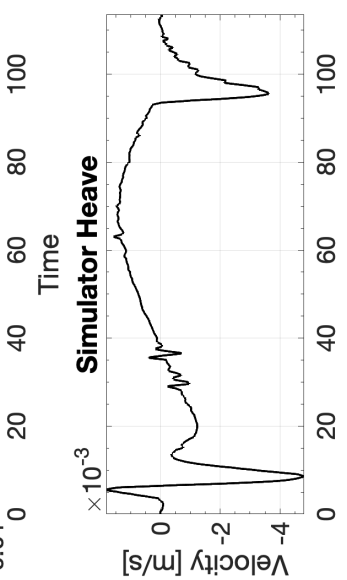

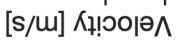
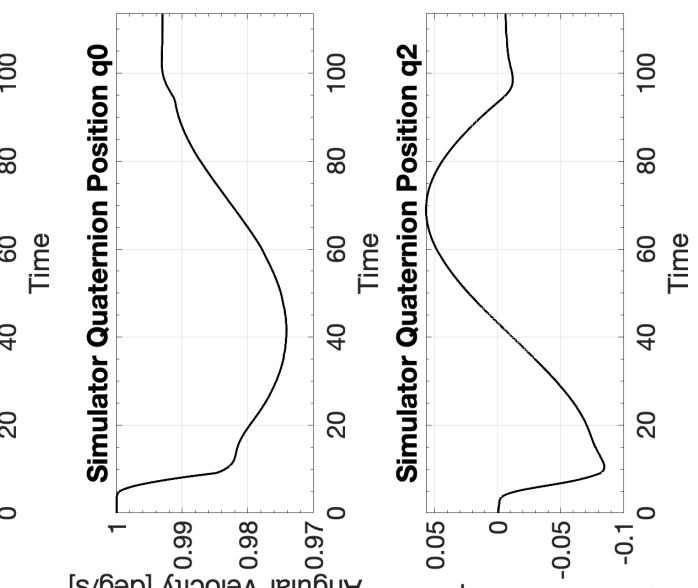

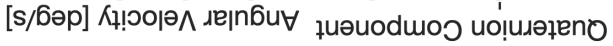

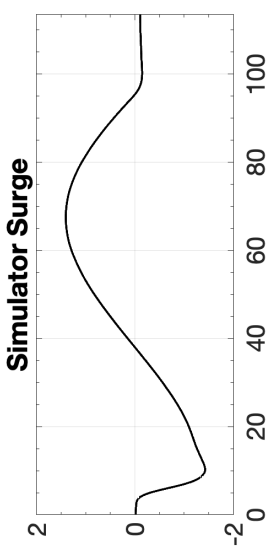

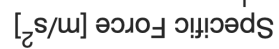

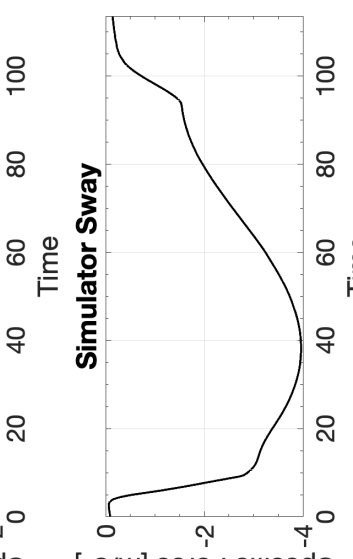

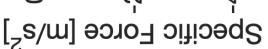

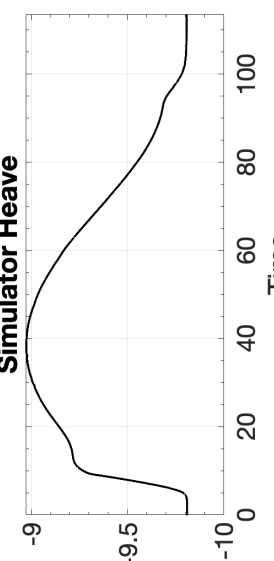

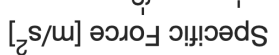

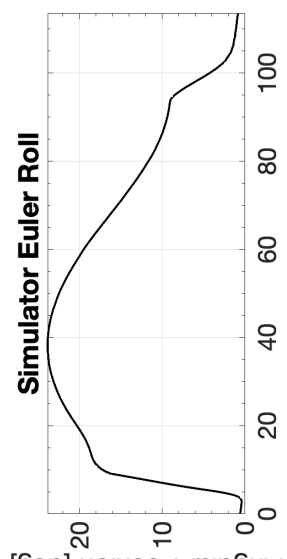

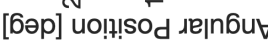

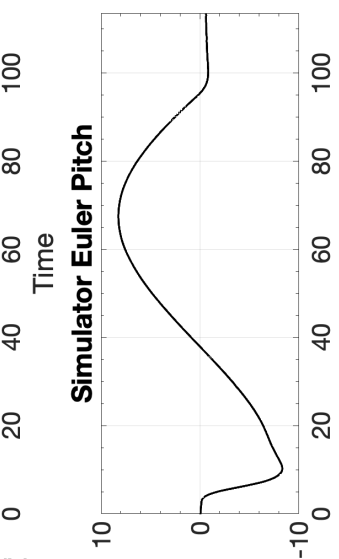

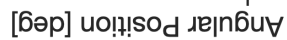

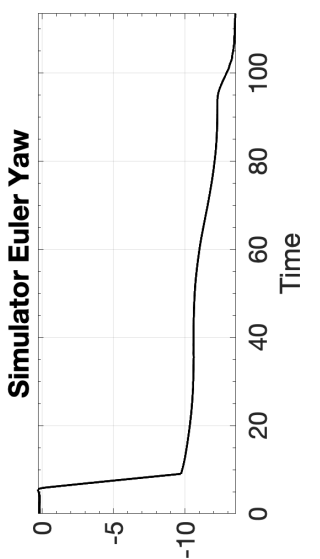

[6әр] uo!!!
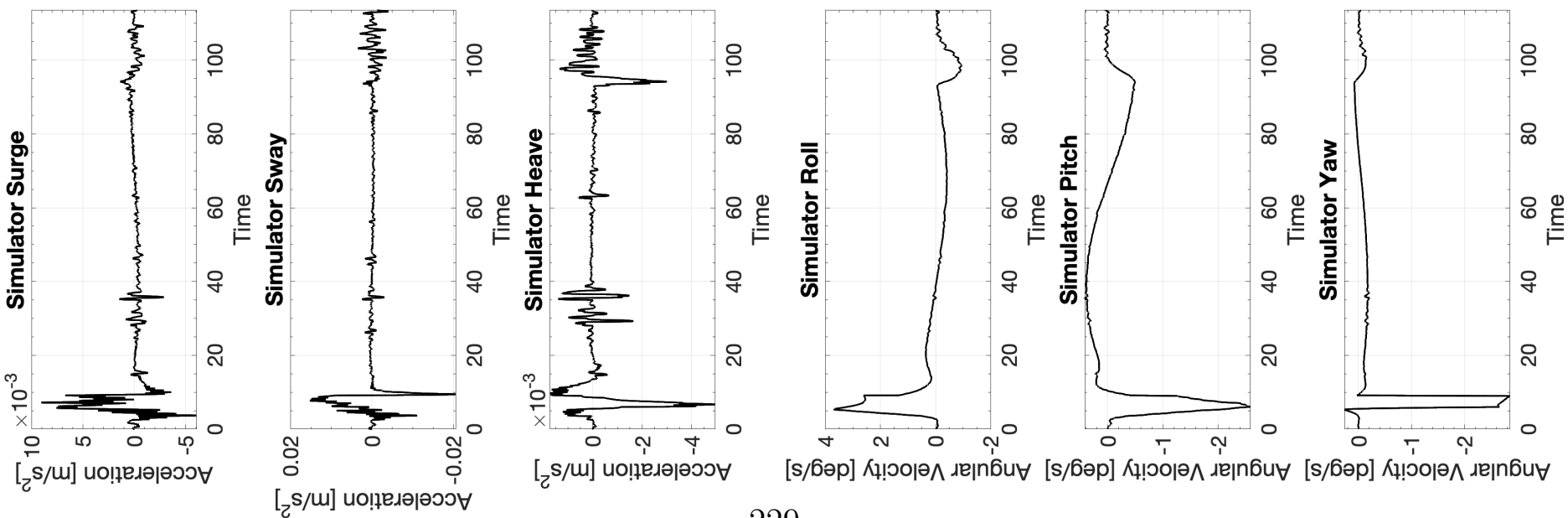

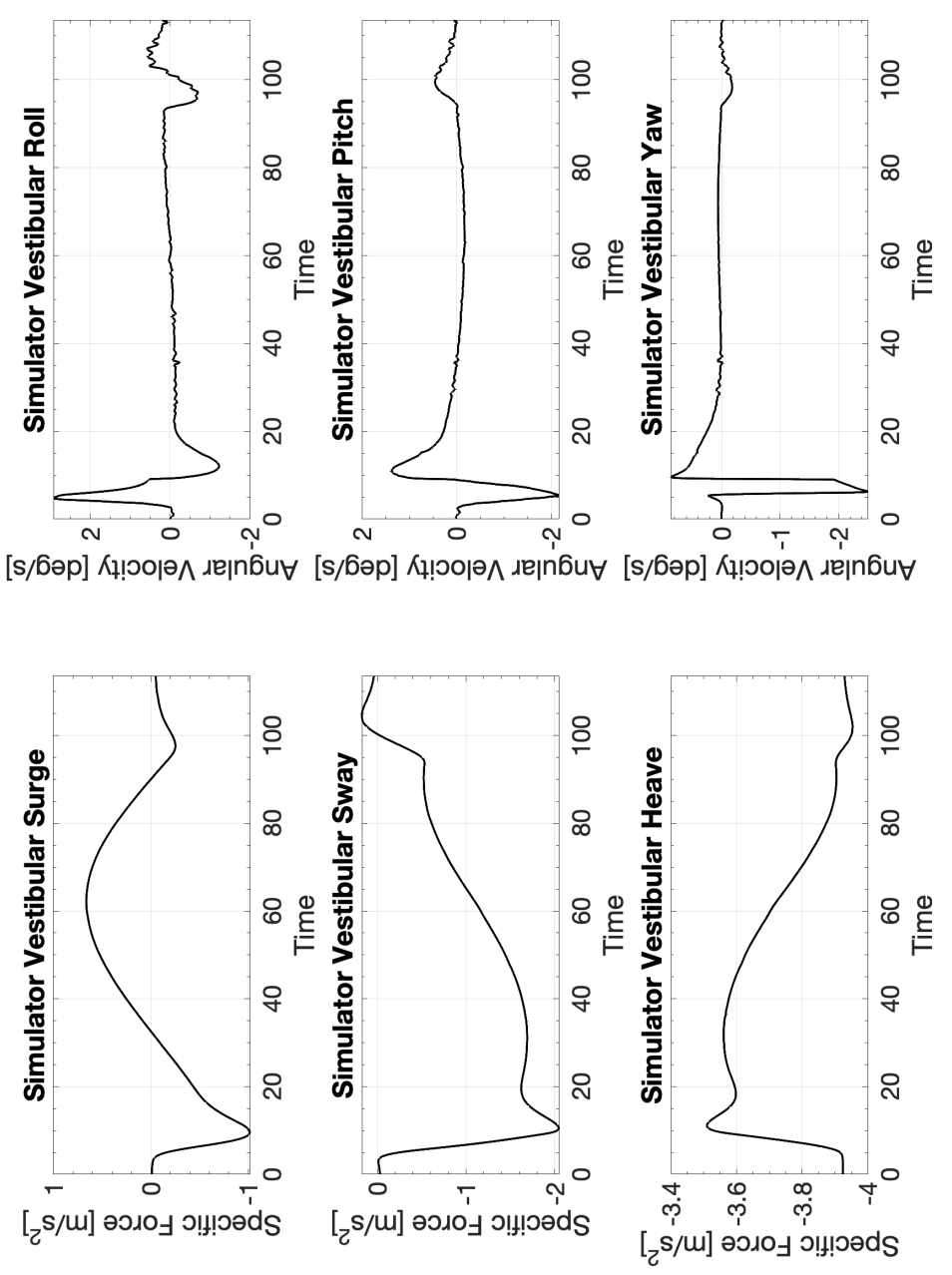


\section{C.4.3 CW3 Cessna 172 Coordinated Turn}

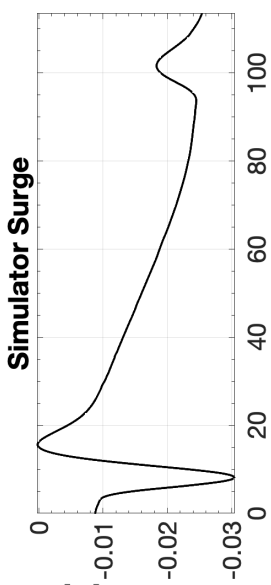

[m] uo!!!

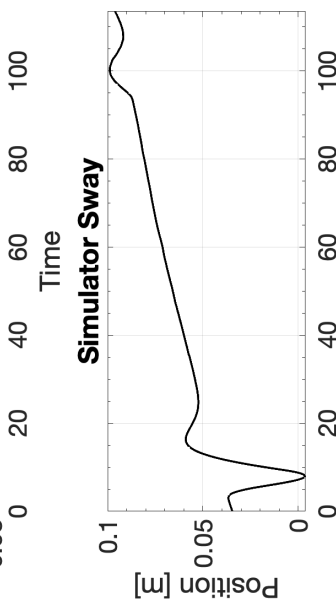

[u] uo!t!!sod

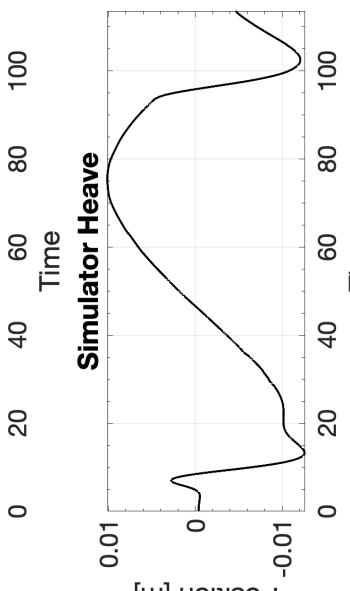

[w] uo!̣!!sod

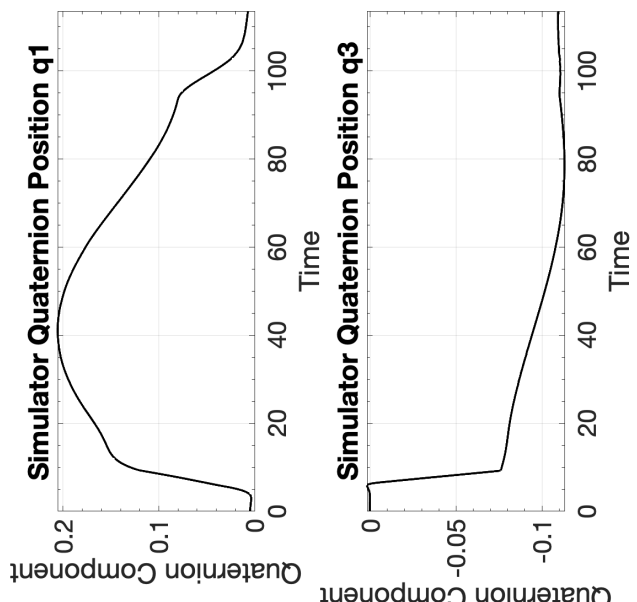

łuəuoduos uoluxəןeno

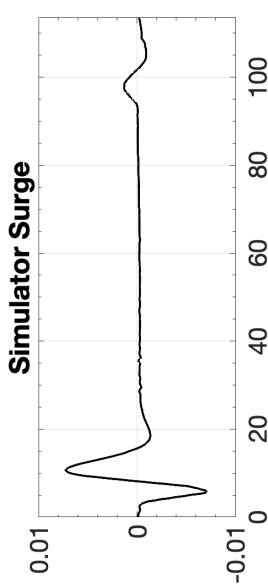

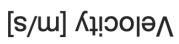

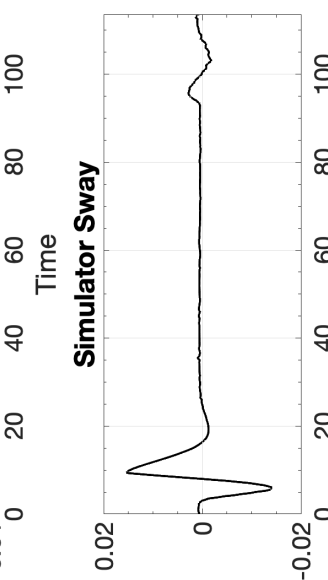

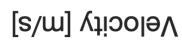

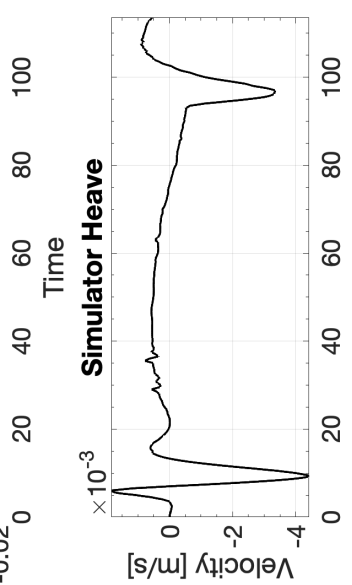

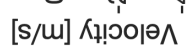

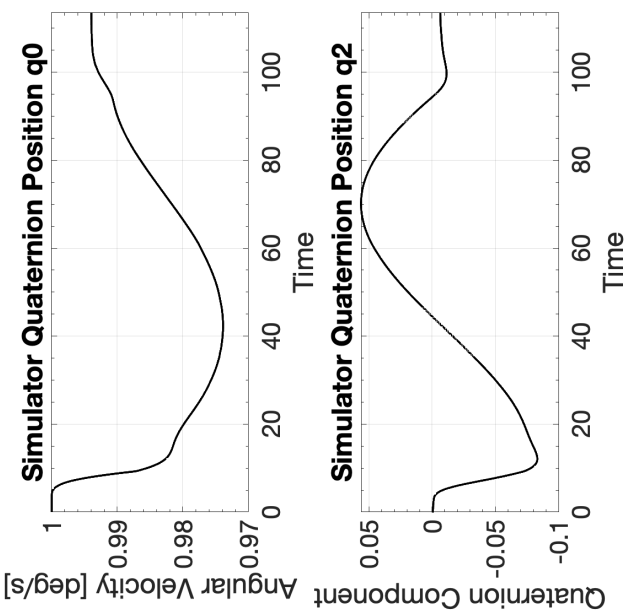

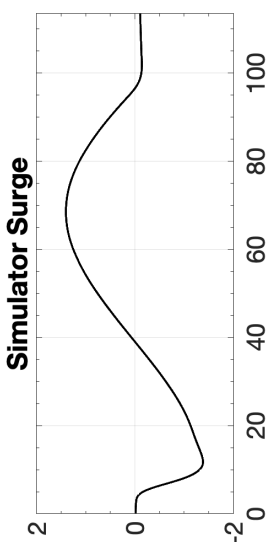

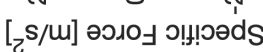

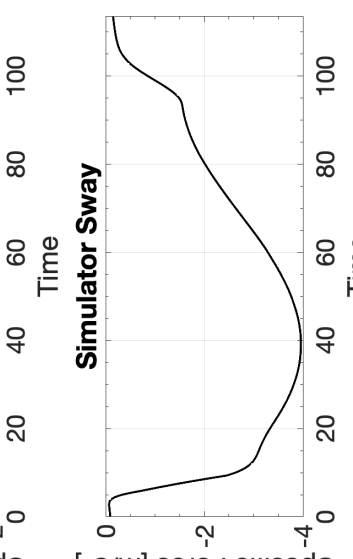

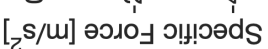

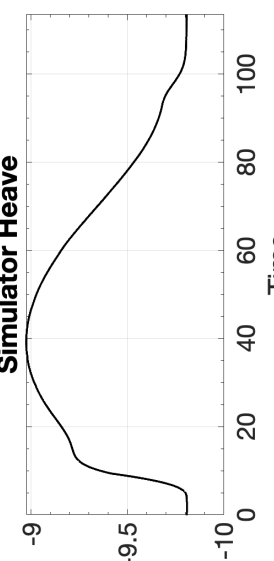

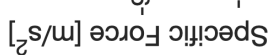

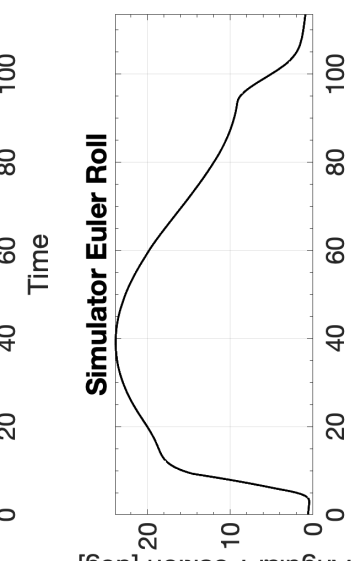

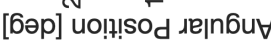

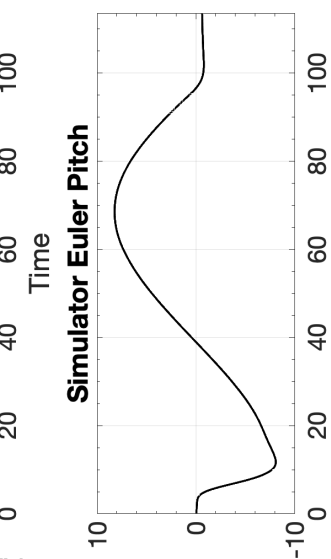

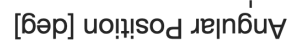

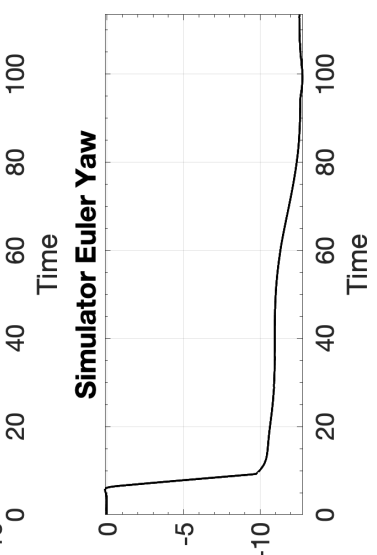

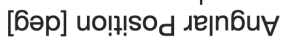

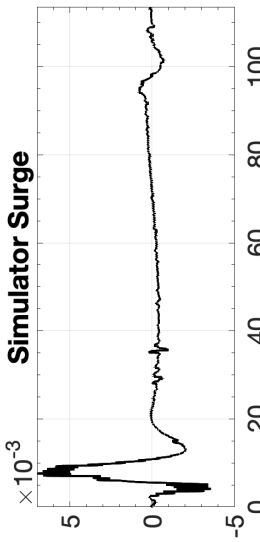

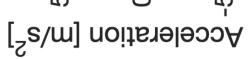

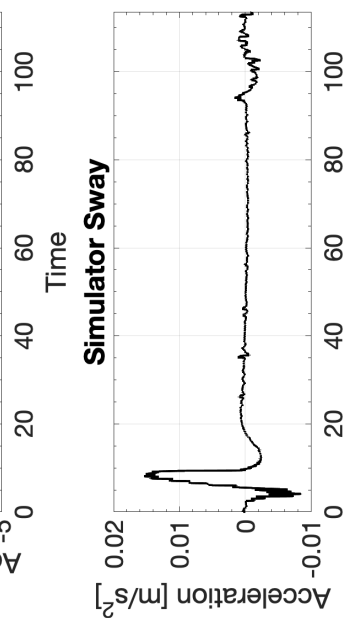

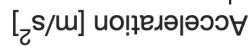

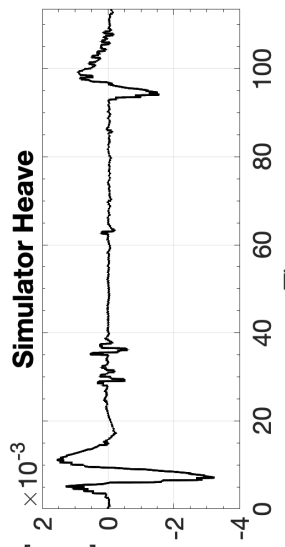

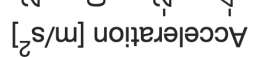
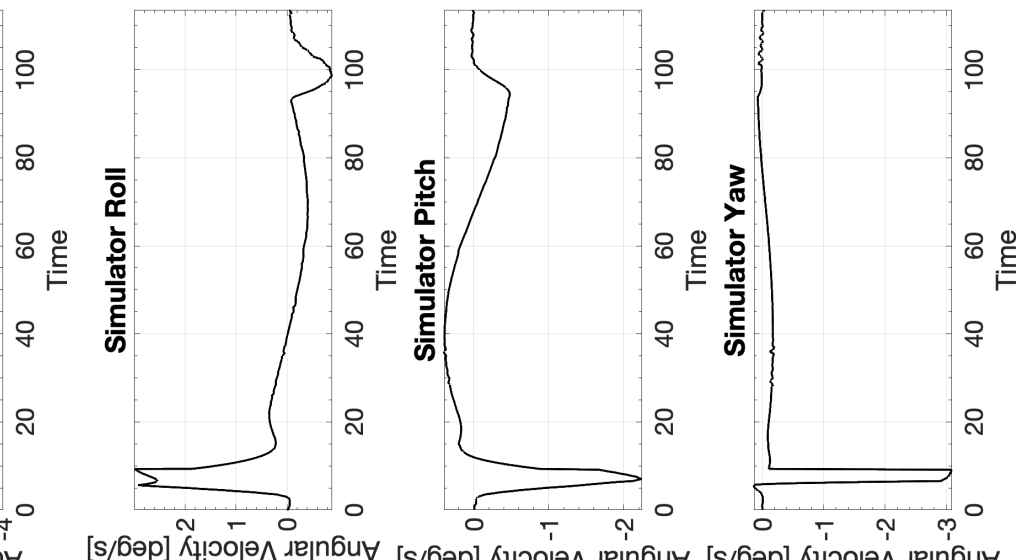

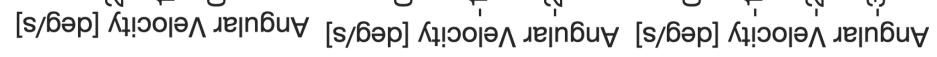



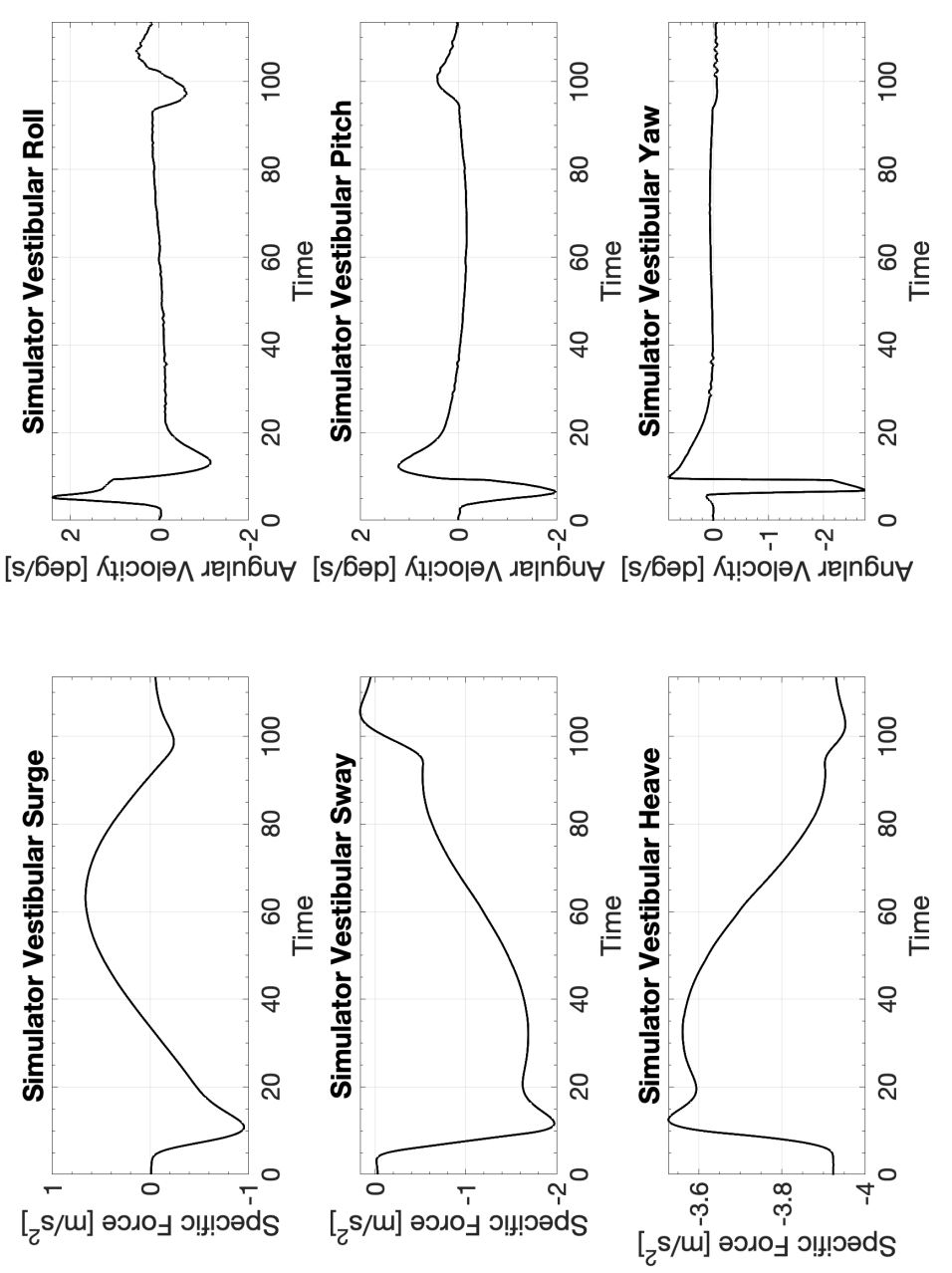


\section{C.5 Cessna 172 Cross-wind Landing}

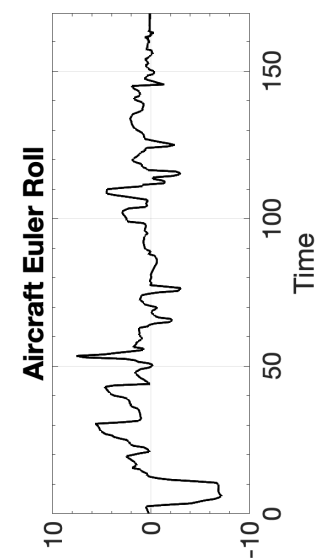

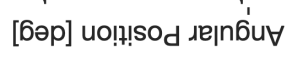

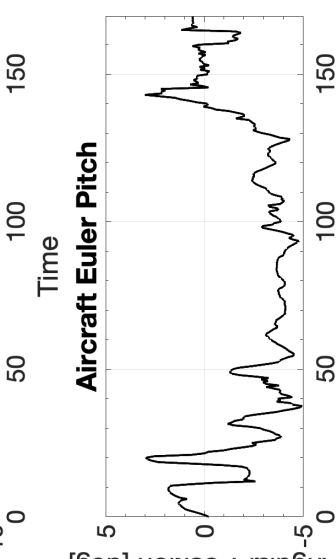

[6әр] uo!!!sod גe|n6u

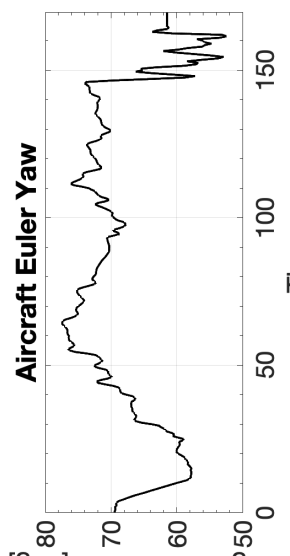

[6әр] uo!n!sod dejn6u
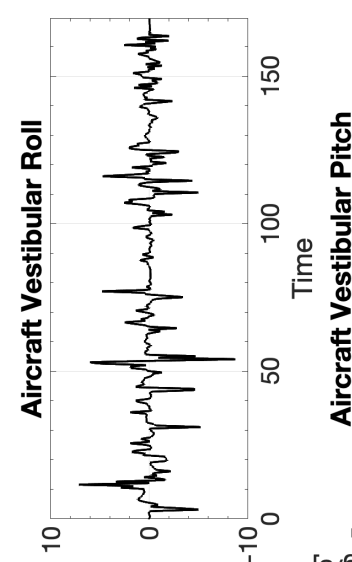

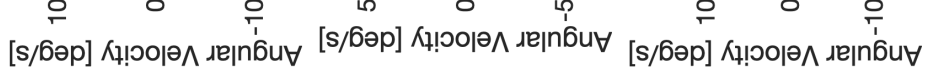

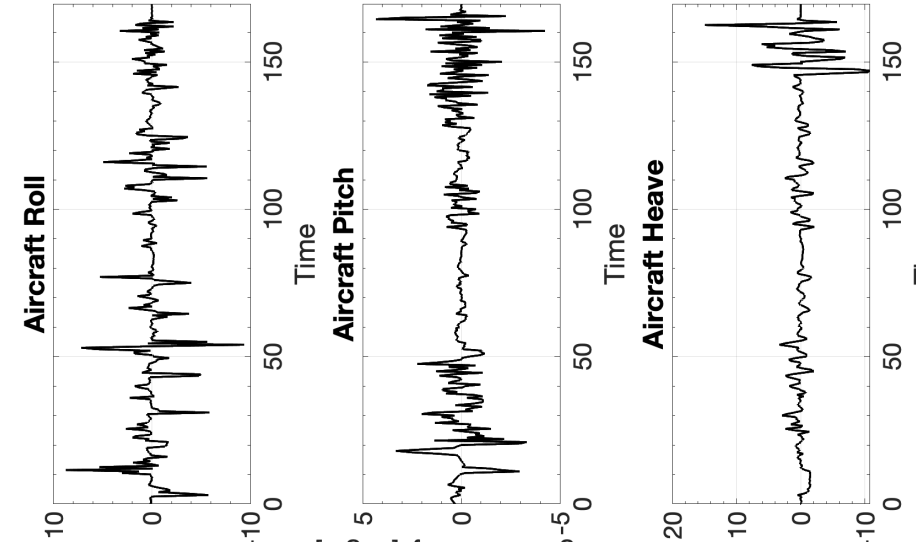

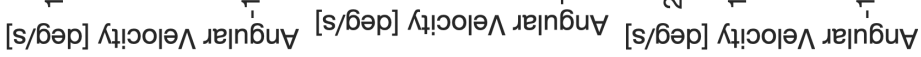
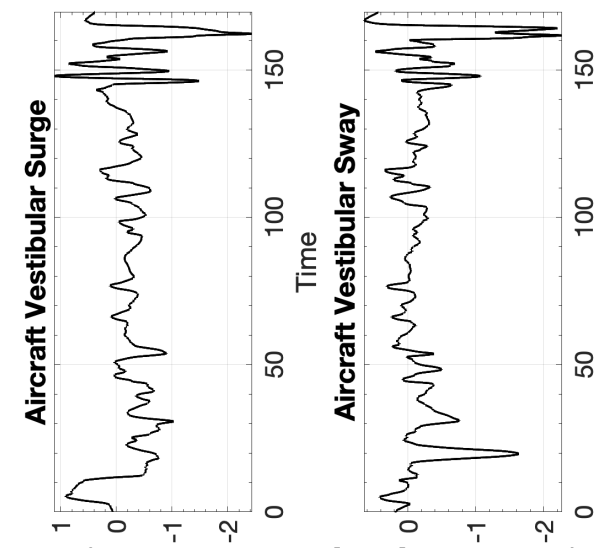

T/

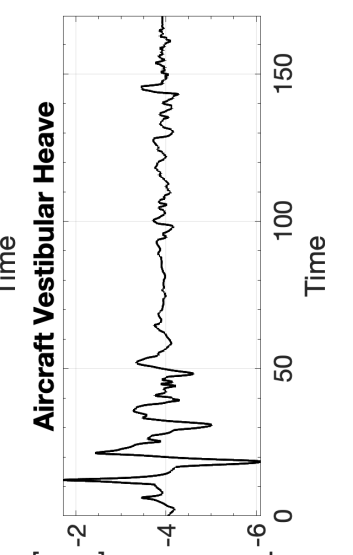

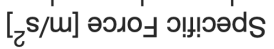

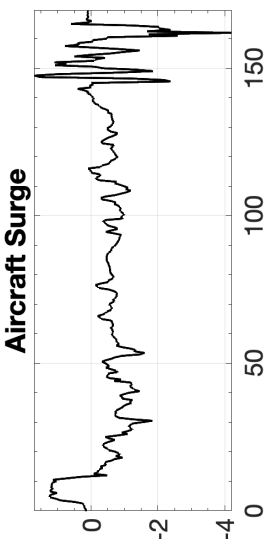

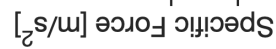

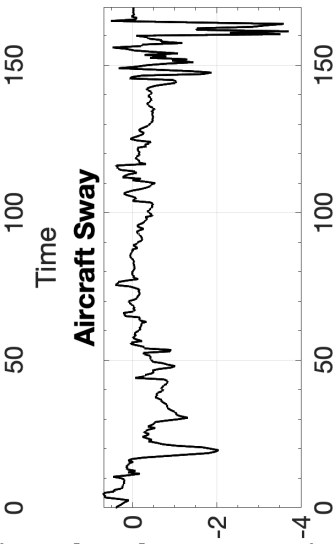

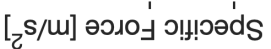

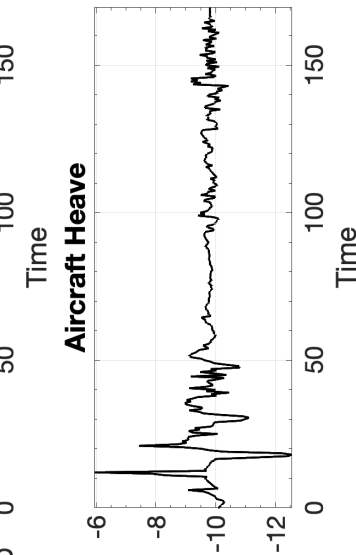

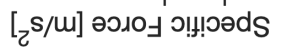

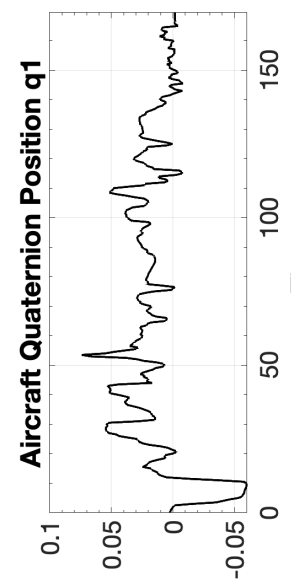

ұuəuodmos uo!̣uəłeno

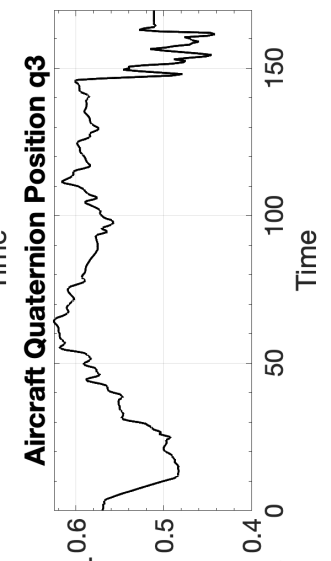

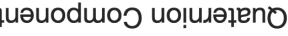

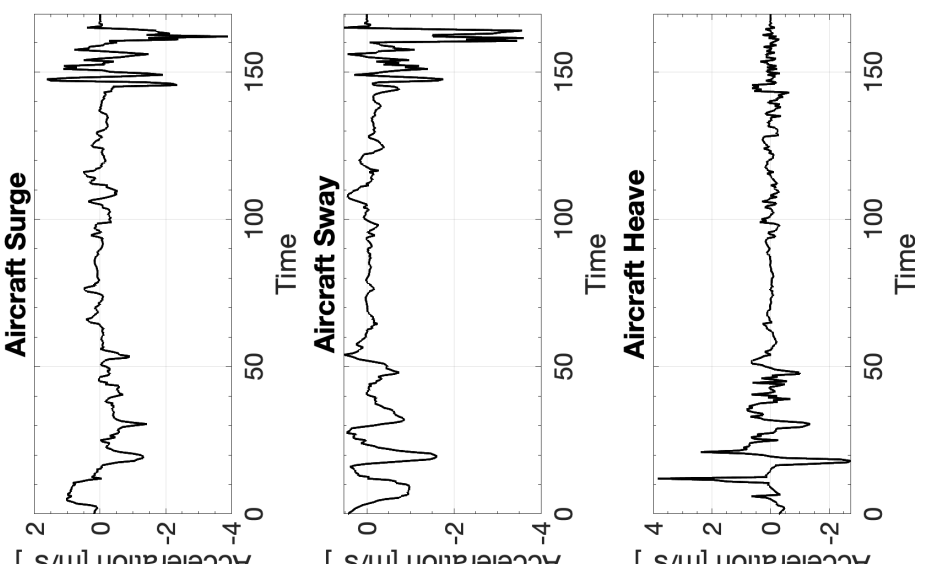

[z/m] ио!реләрәоэ

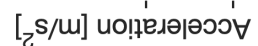

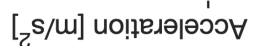
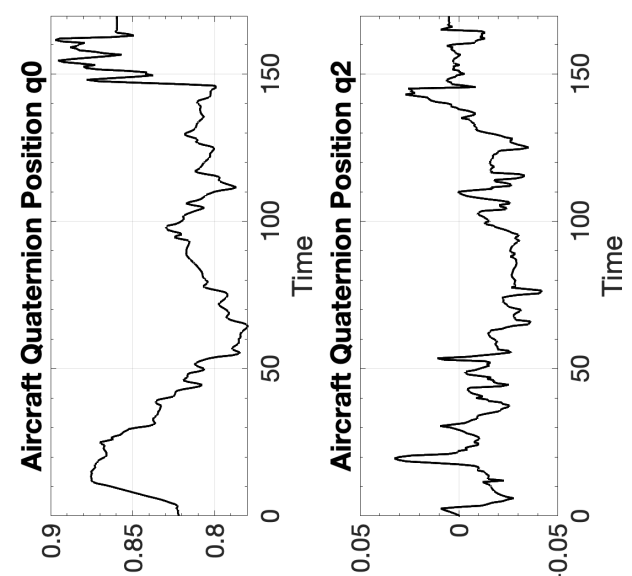


\section{C.5.1 CW1 Cessna 172 Cross-wind Landing}

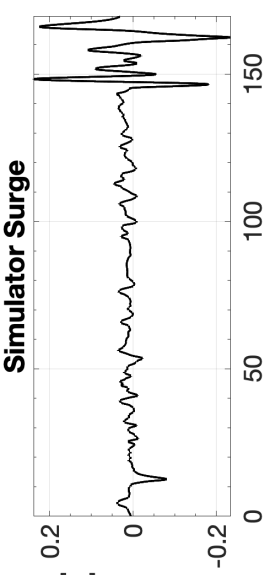

[u] uo!n! sod

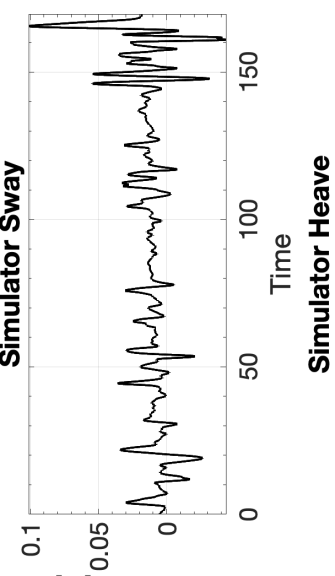

[w] uolp!sod

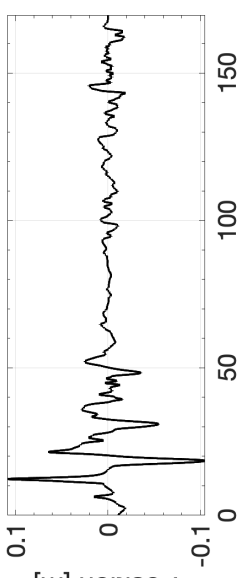

[w] uo!n!sod

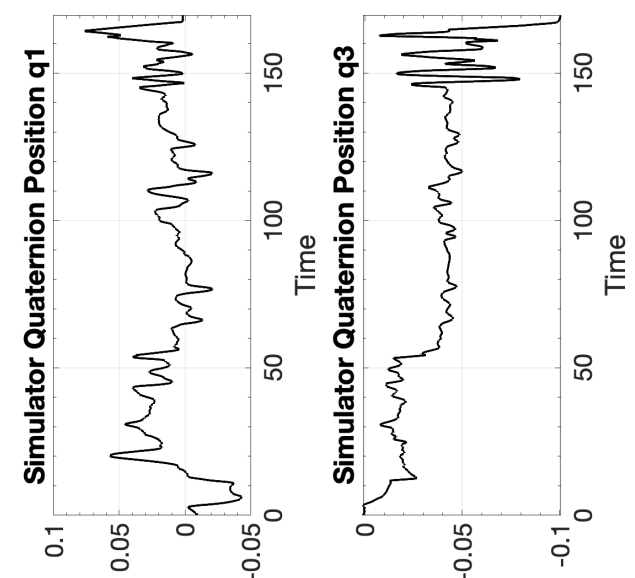

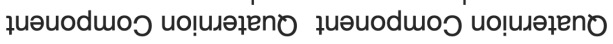

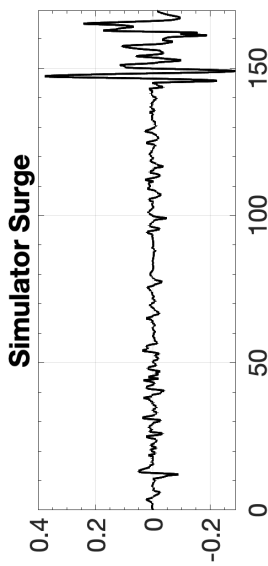

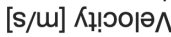

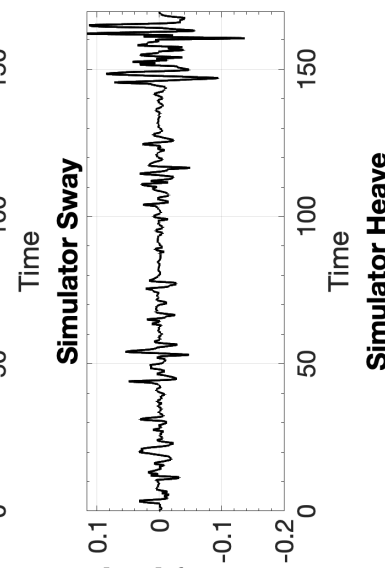

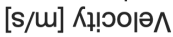

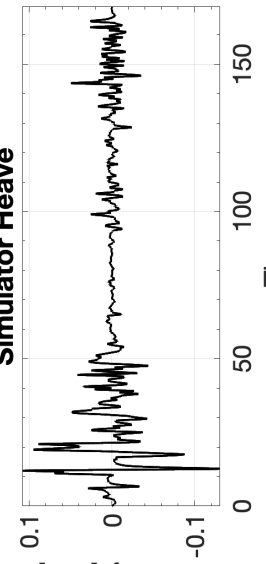

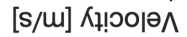

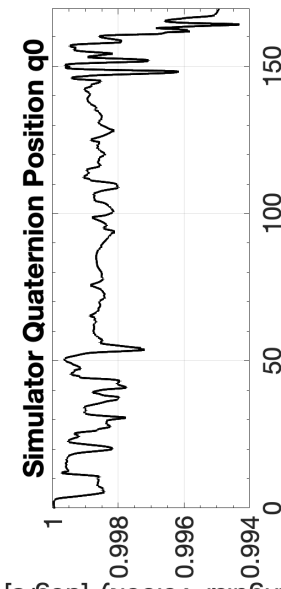

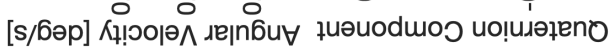

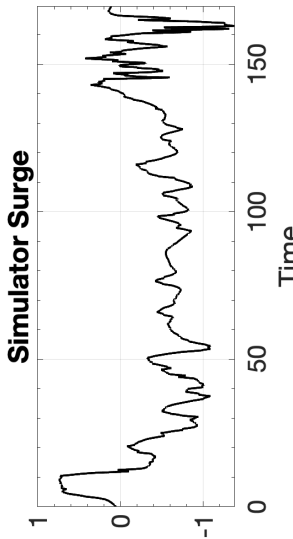

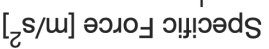

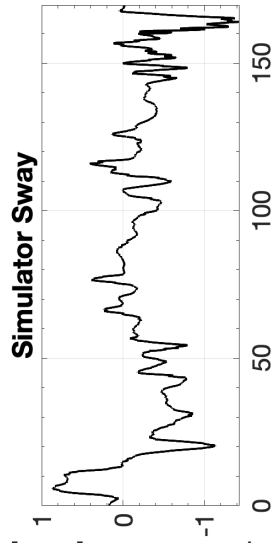

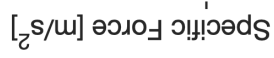

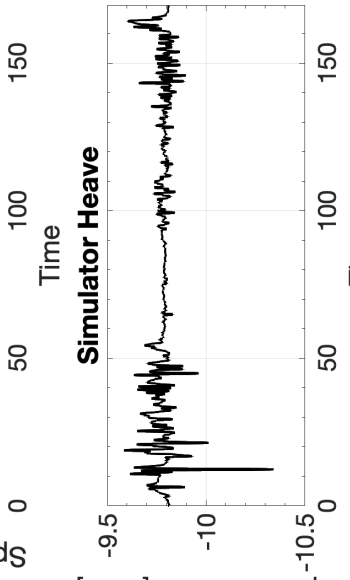

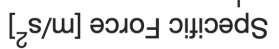

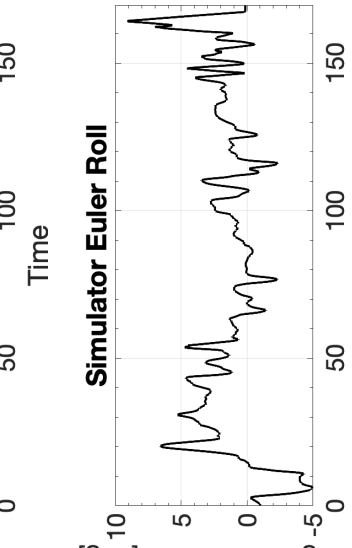

[6әр] uo!̣!!sod גeןnбü
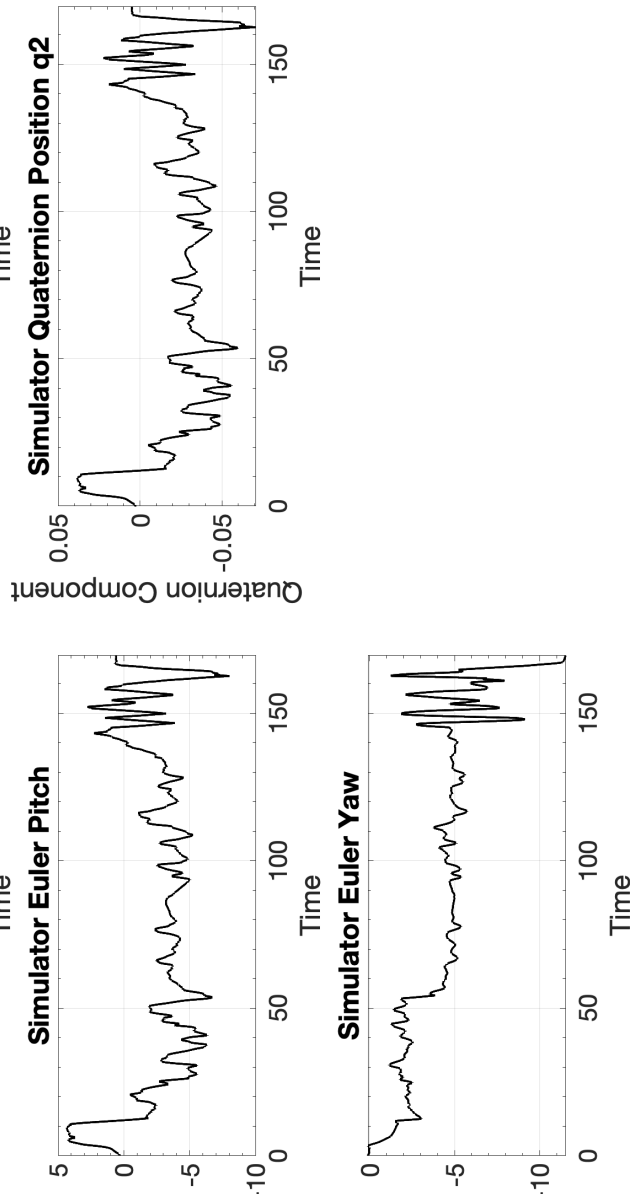

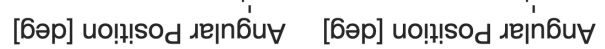

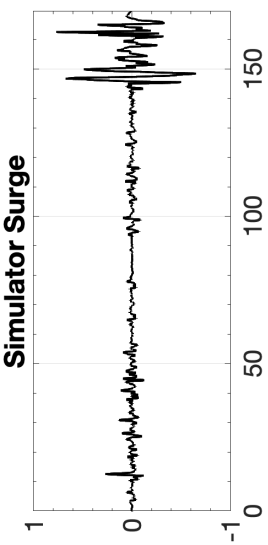

$[\mathrm{c} / \mathrm{L}]$ uо!ן

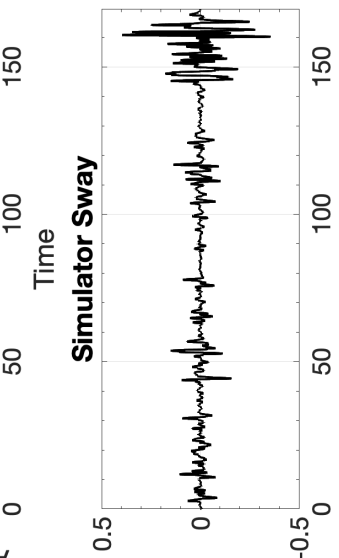

$[\mathrm{s} / \mathrm{m}]$ ио!ฺв

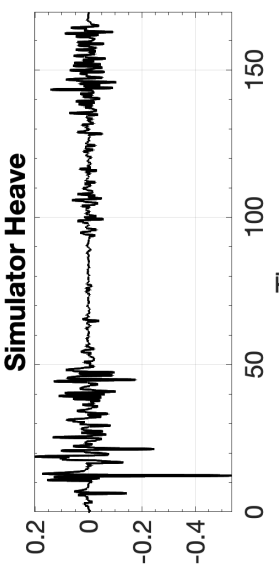

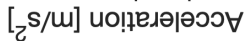

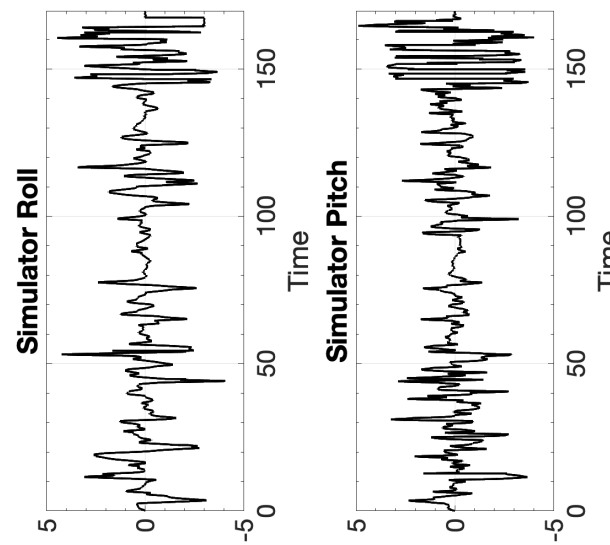

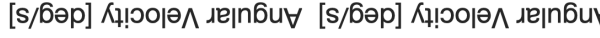

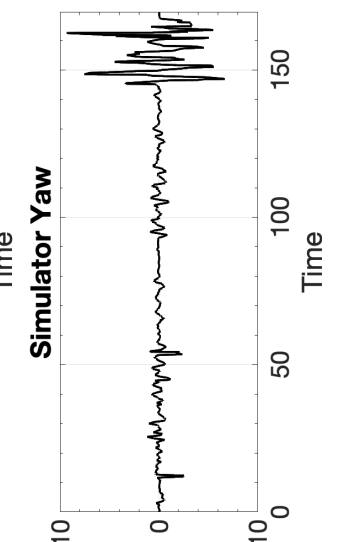

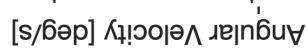




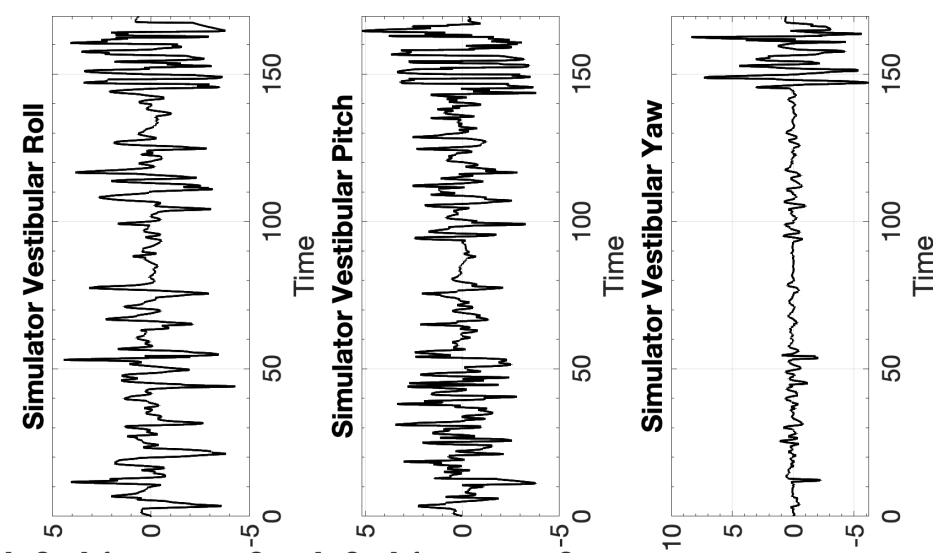

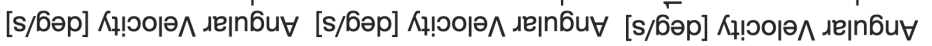

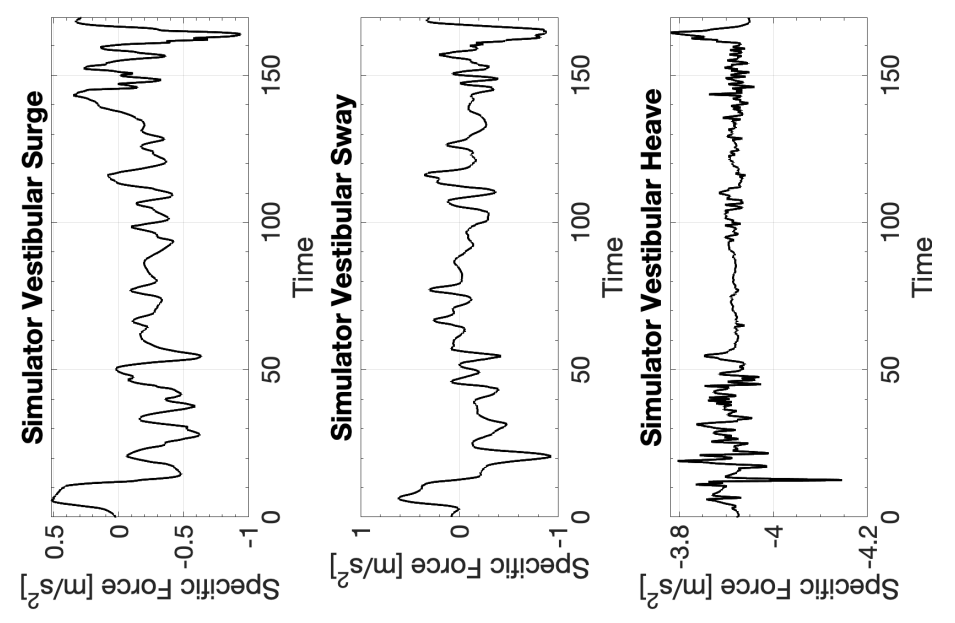




\section{C.5.2 CW2 Cessna 172 Cross-wind Landing}

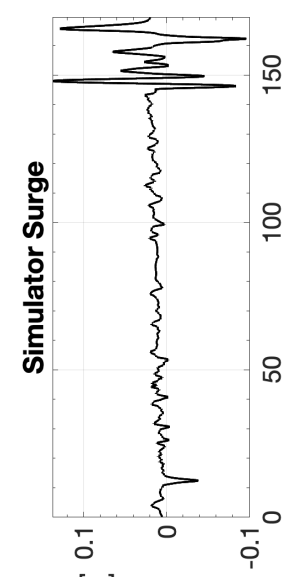

[w] uo!!!!sod

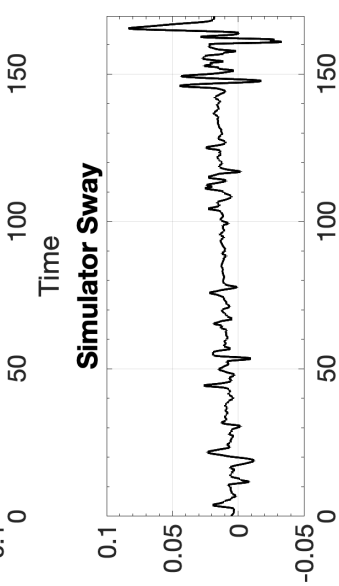

[w] uolp!sod

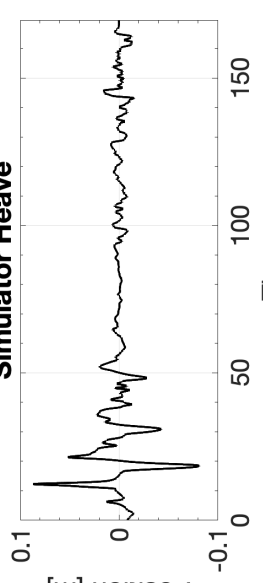

[u] uo!!!sod

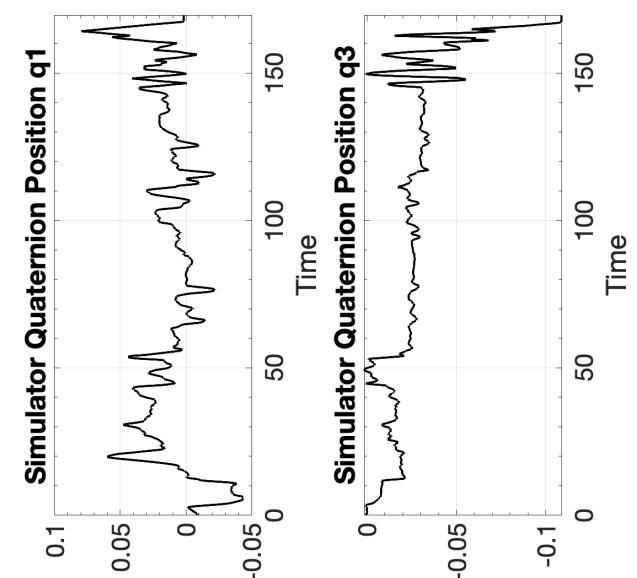

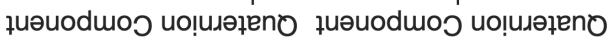

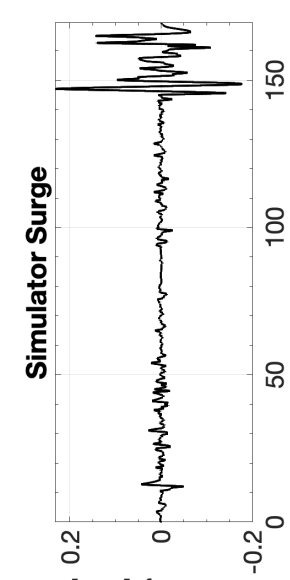

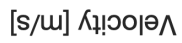

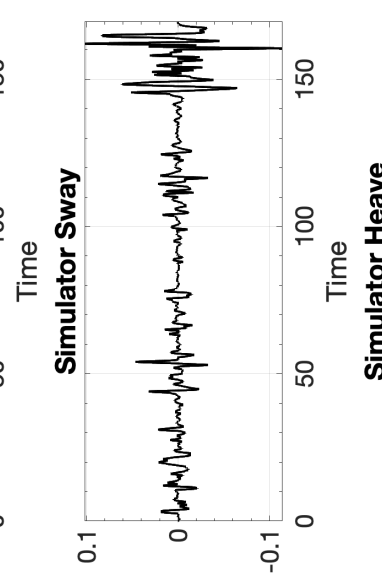

[s/m] Кұ!००|ә^

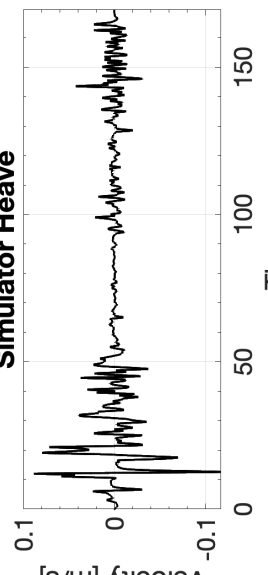

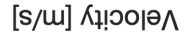
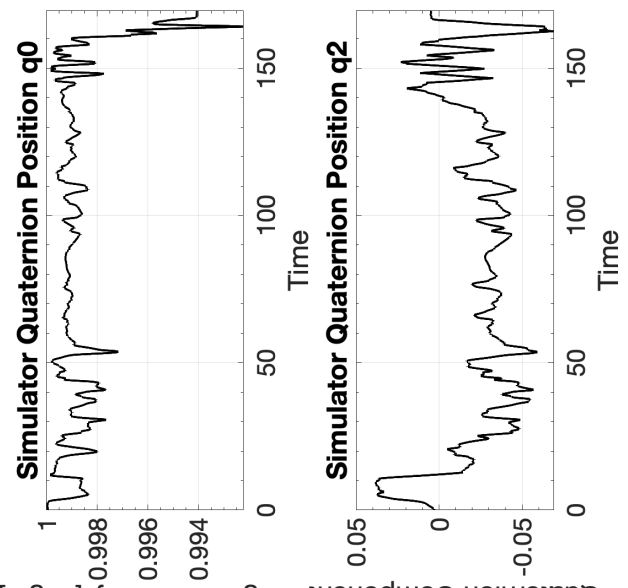

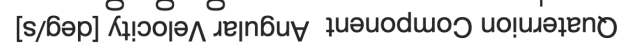

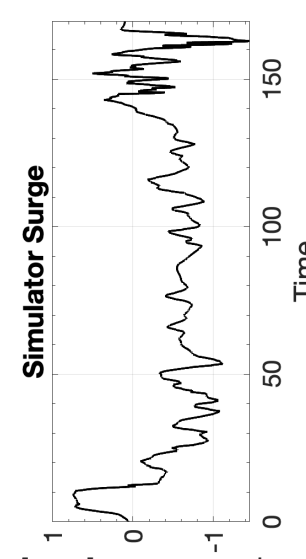

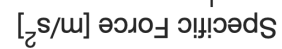

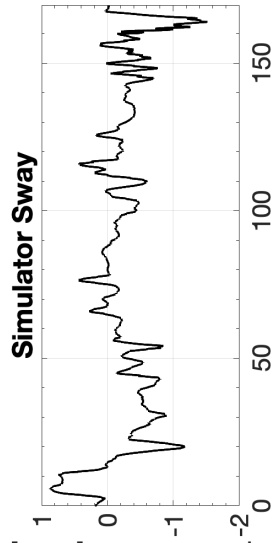

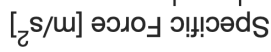

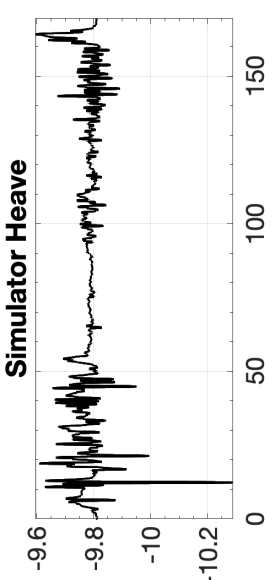

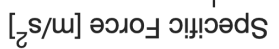

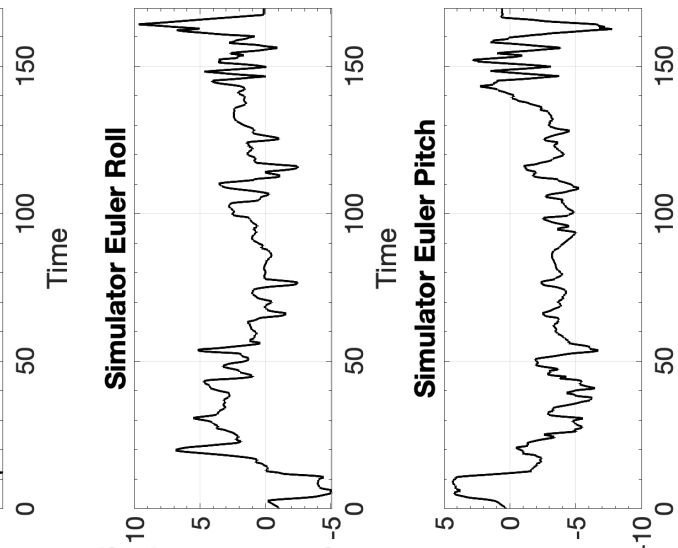

[бәр] uo!!!!sod גe|nбuY

[6әр] uo!!!sod xe|nб์uี

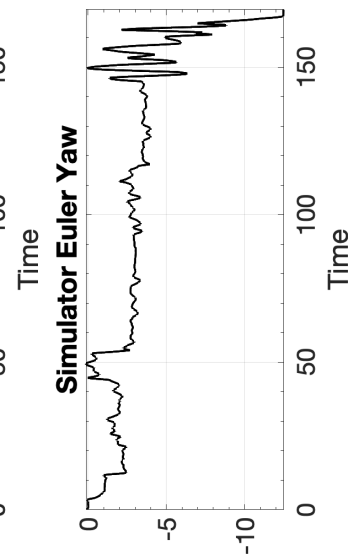

[6әр] uo!!!

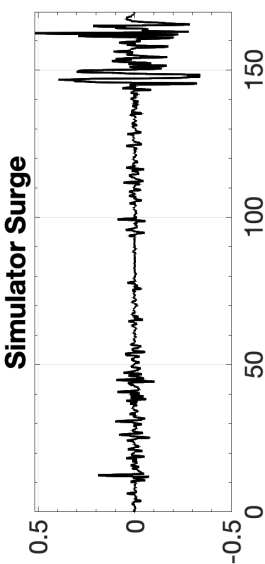

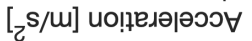

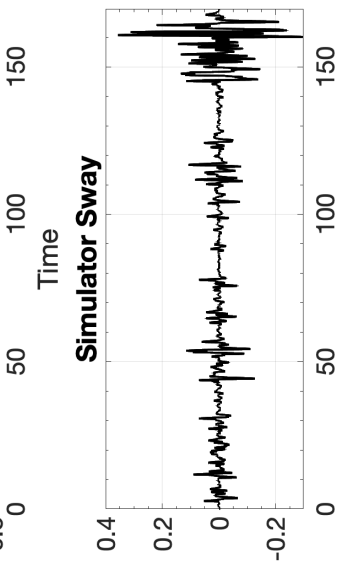

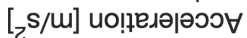

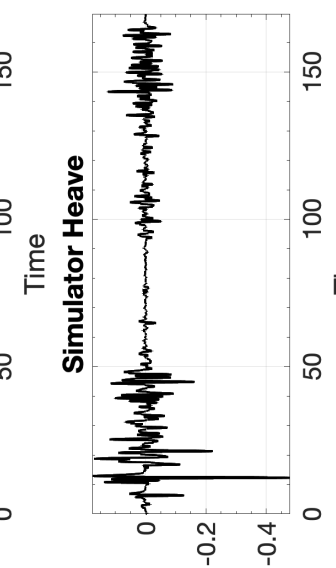

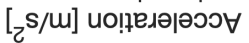

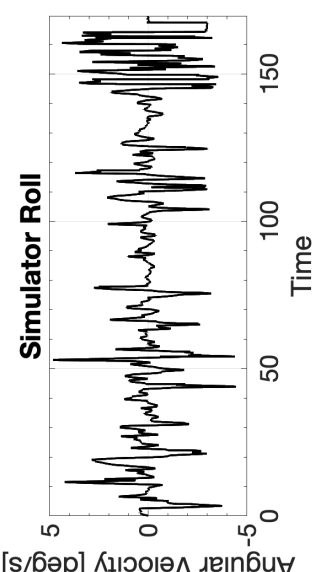

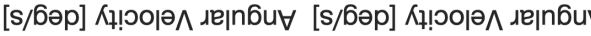

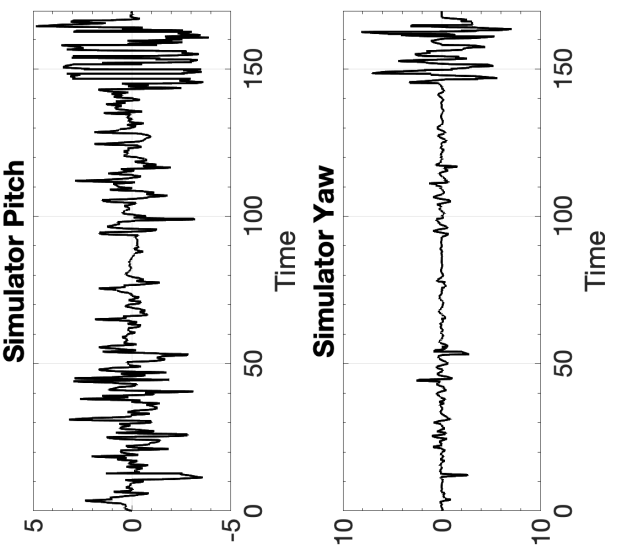

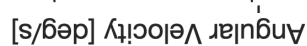



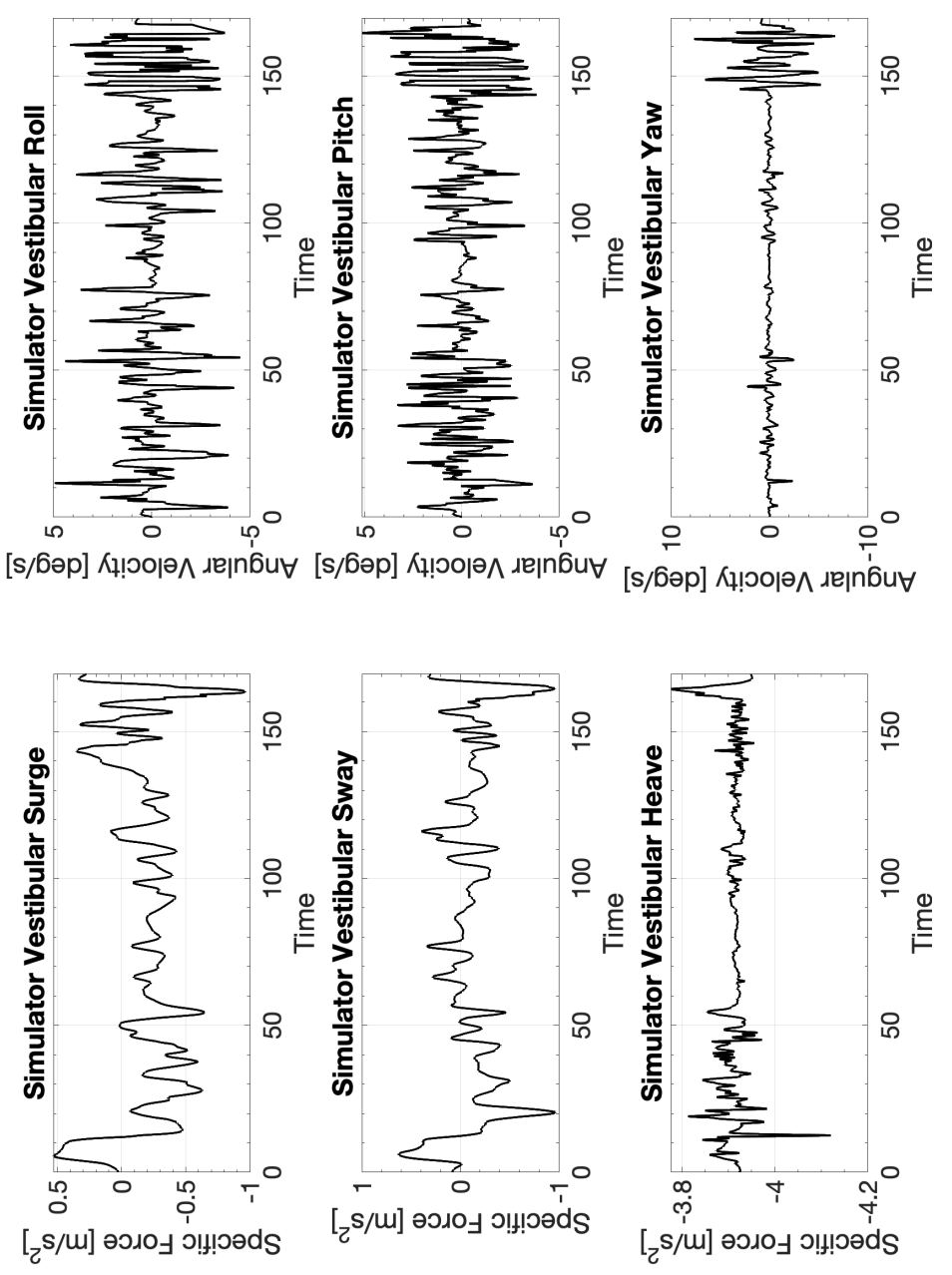


\section{C.5.3 CW3 Cessna 172 Cross-wind Landing}

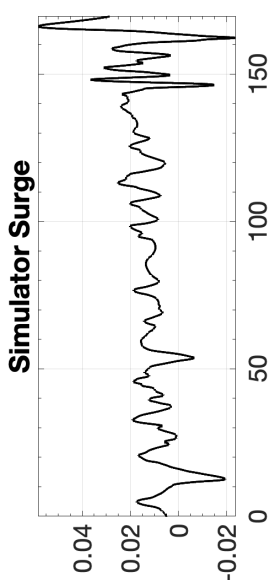

[w] uo!n!sod

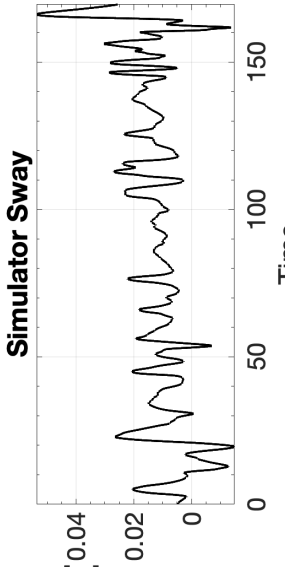

[u] uol!! sod

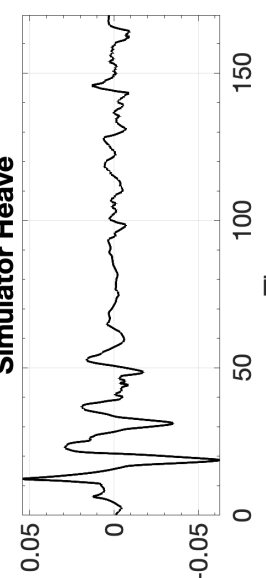

[u] uolp!nsod

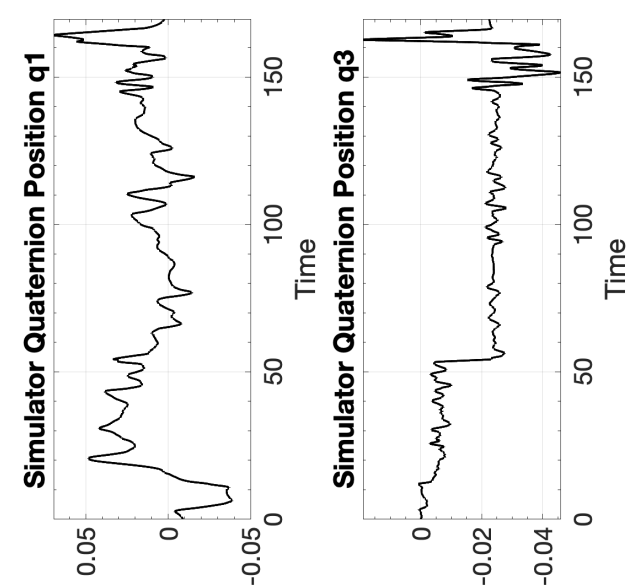

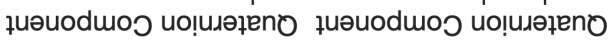

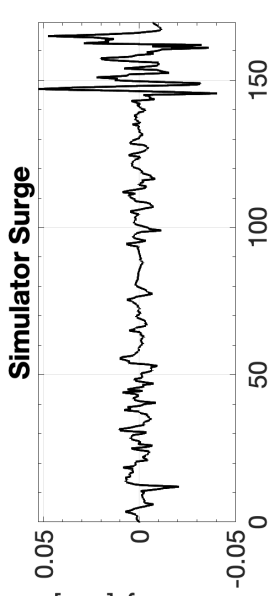

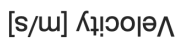

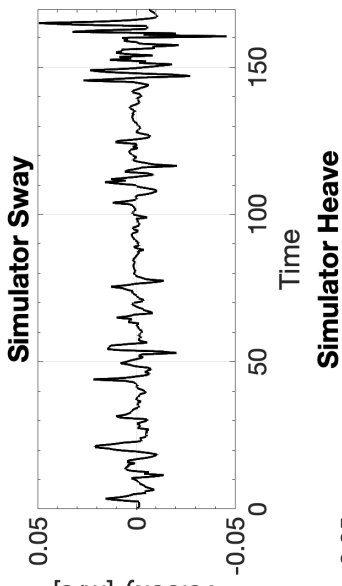

[s/m] Кł!эоюә

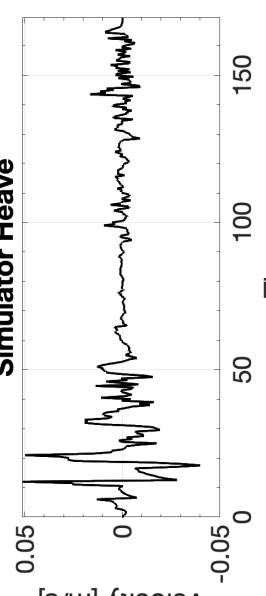

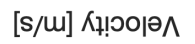

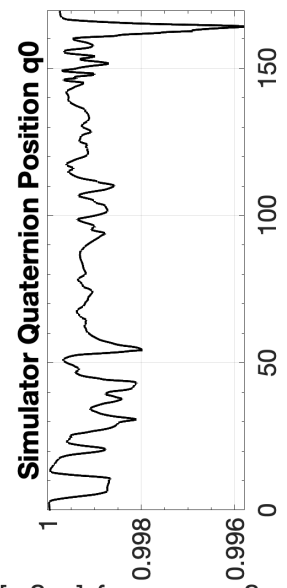

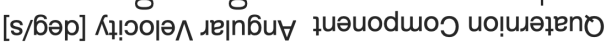

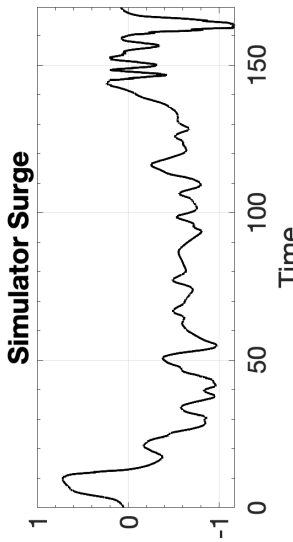

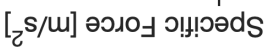

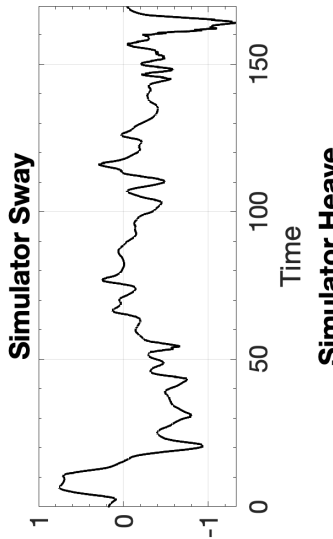

[

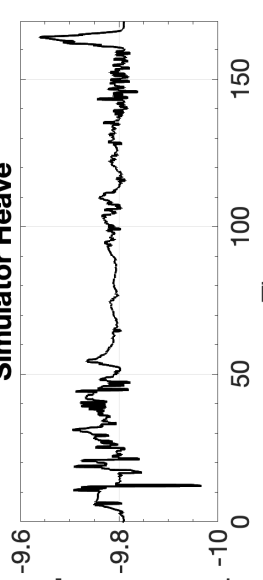

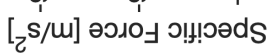

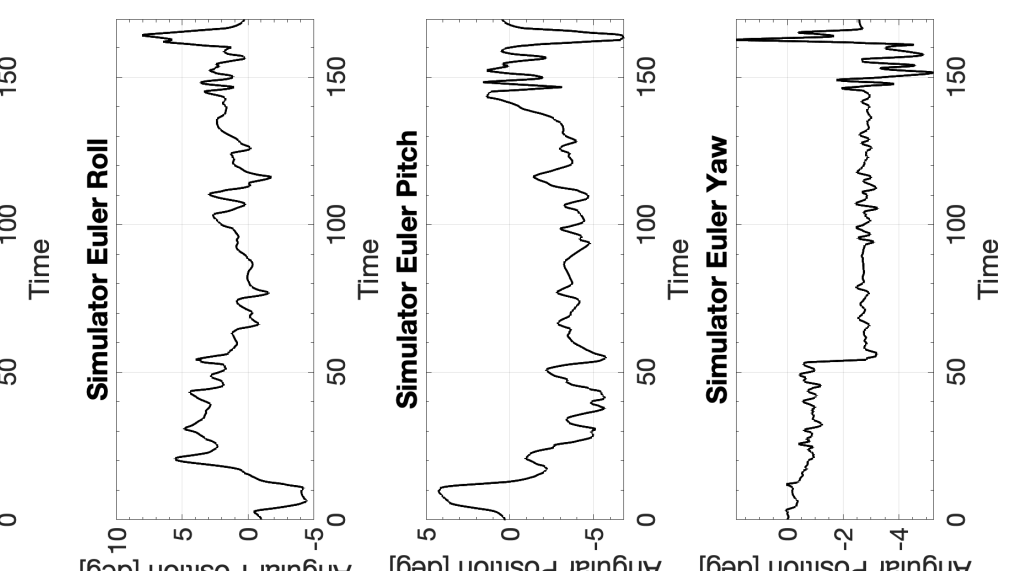

[6ә̄] uo!!!

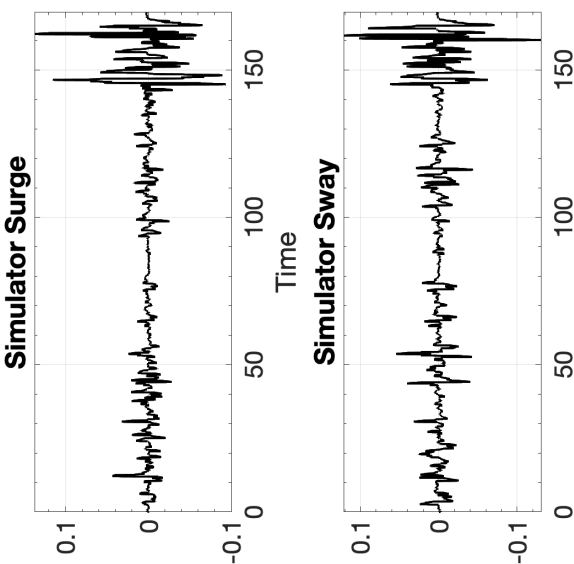

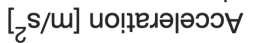

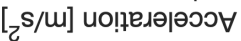

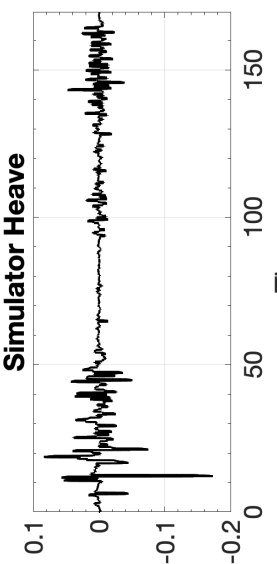

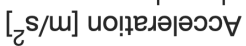
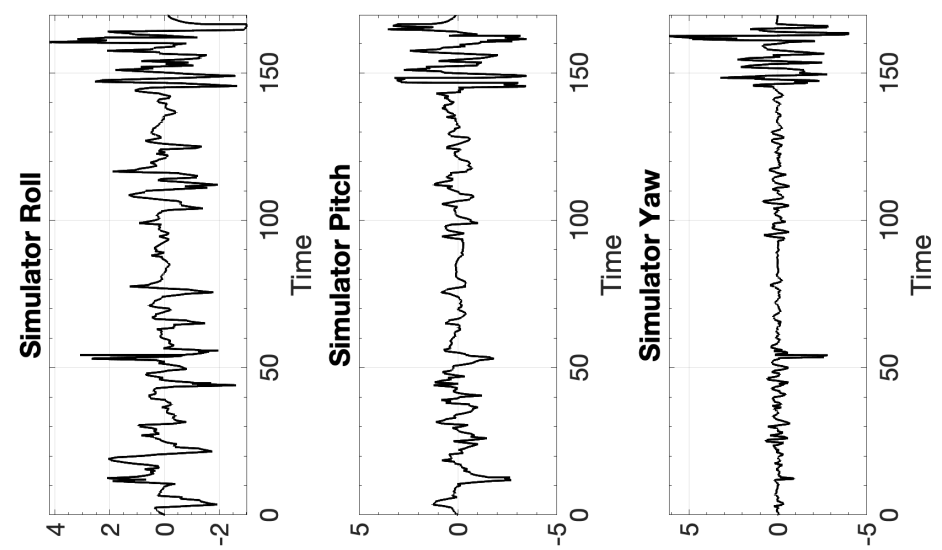

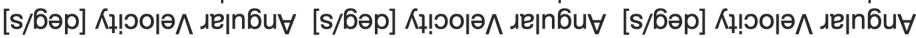




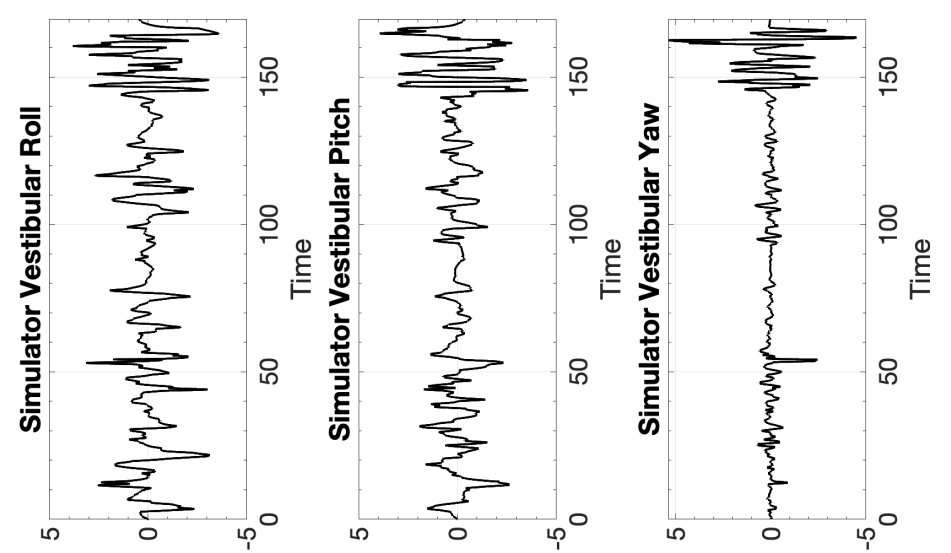

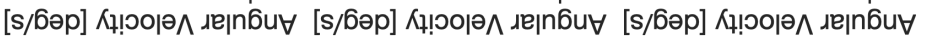

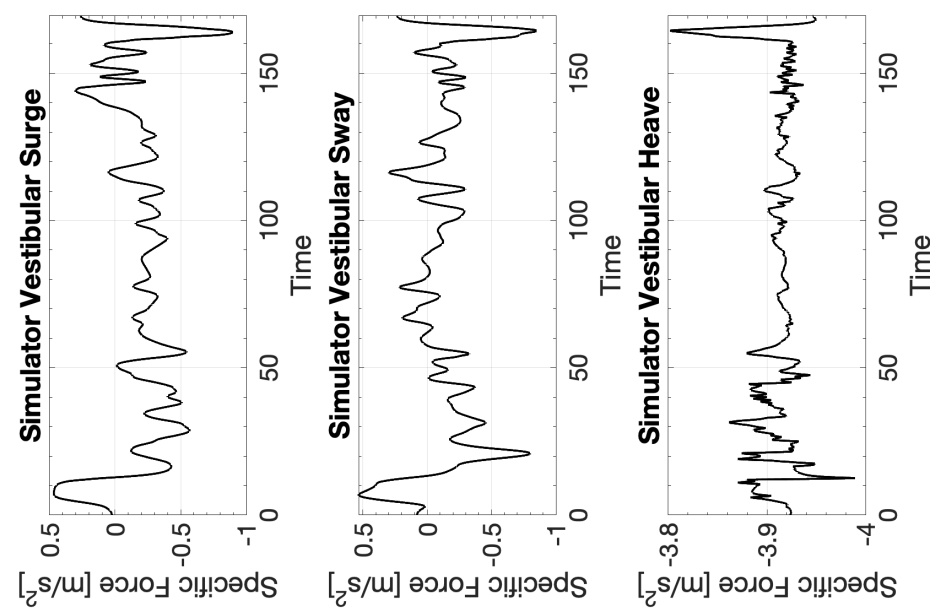




\section{C.6 Cessna 172 Single-axis Takeoff}

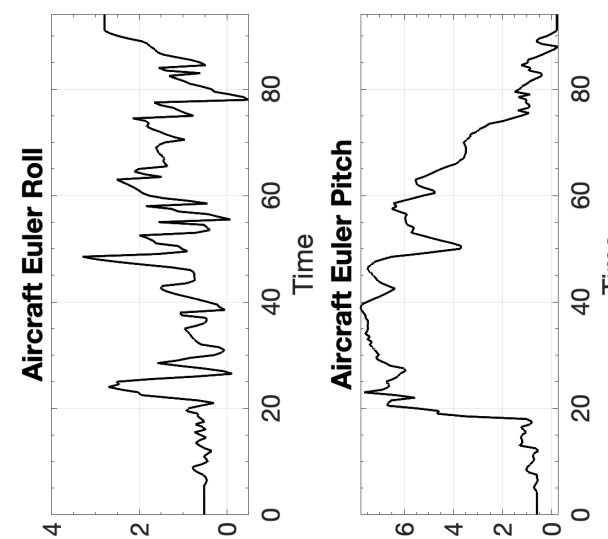

[6әр] uo!!!sod

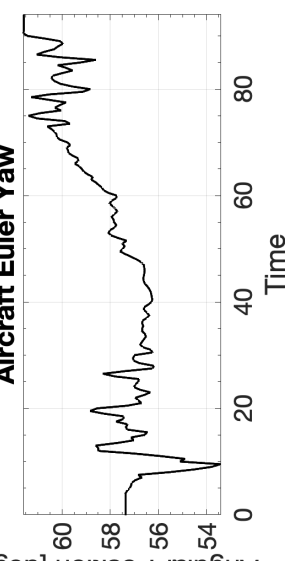

[6әр] uo!t!sog
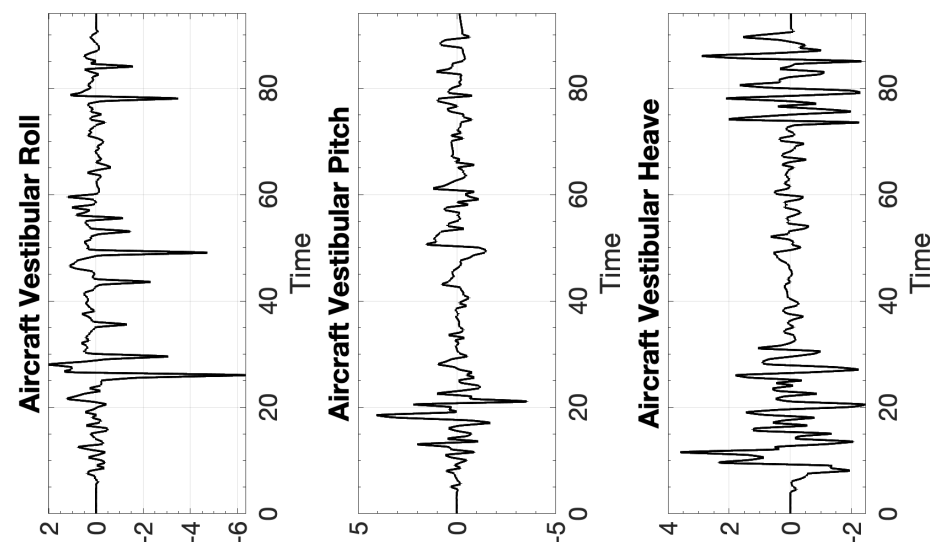

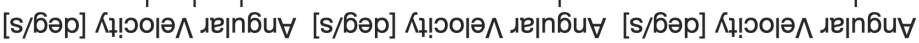

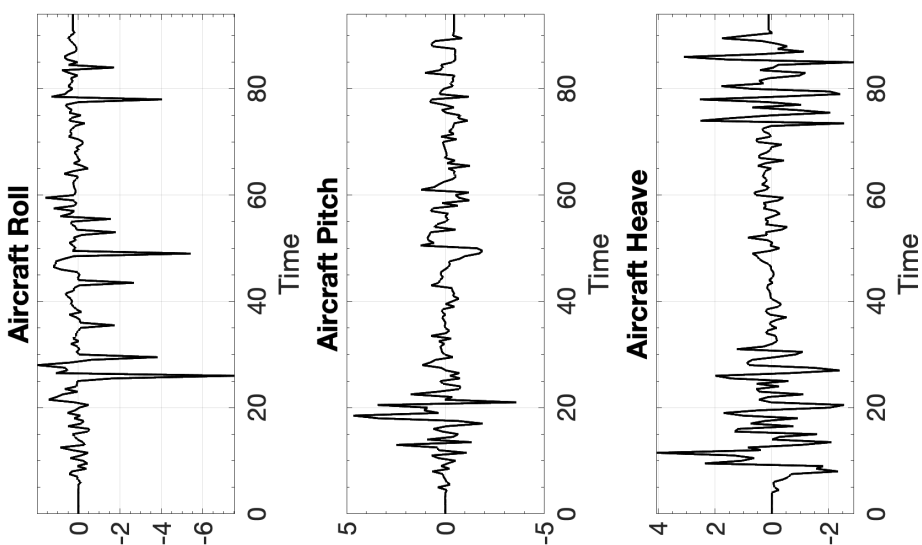

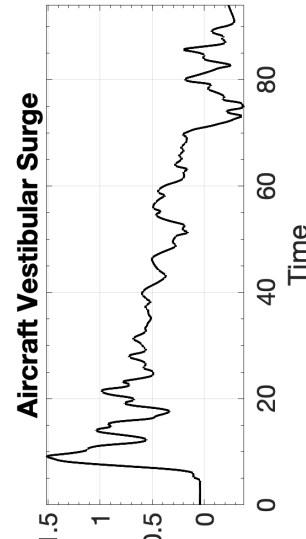
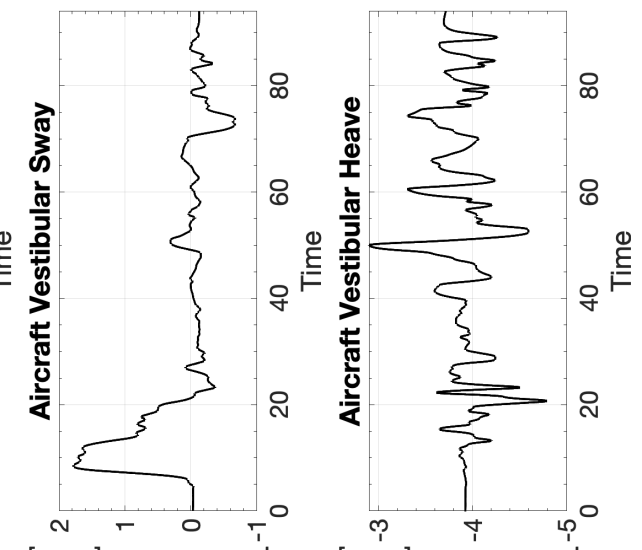

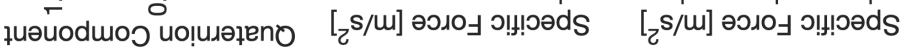

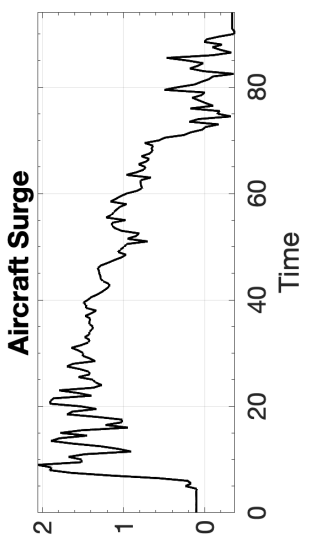

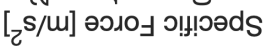
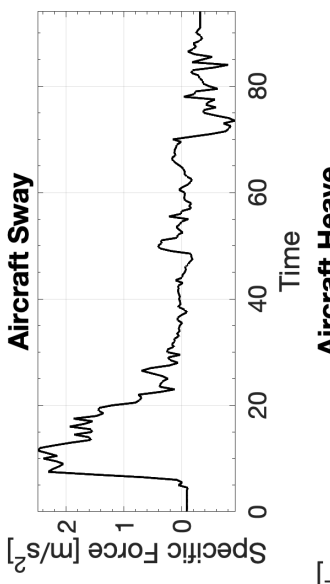

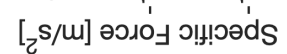
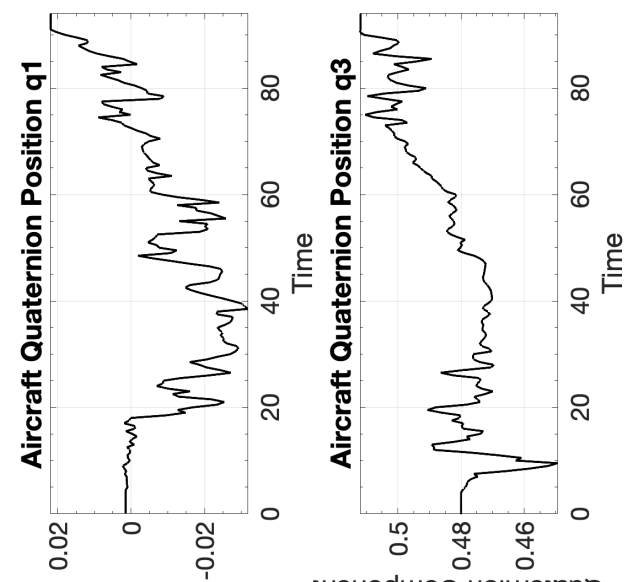

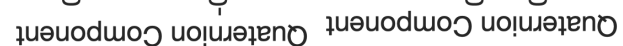

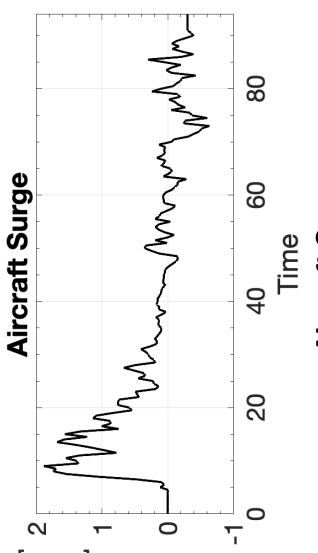

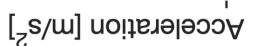

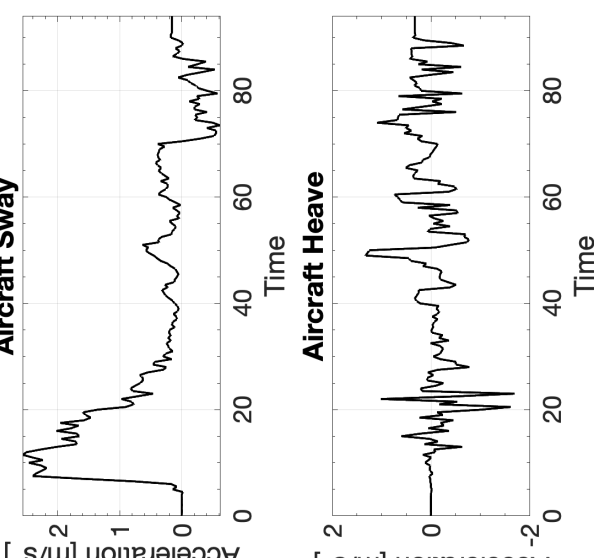

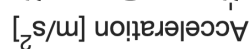

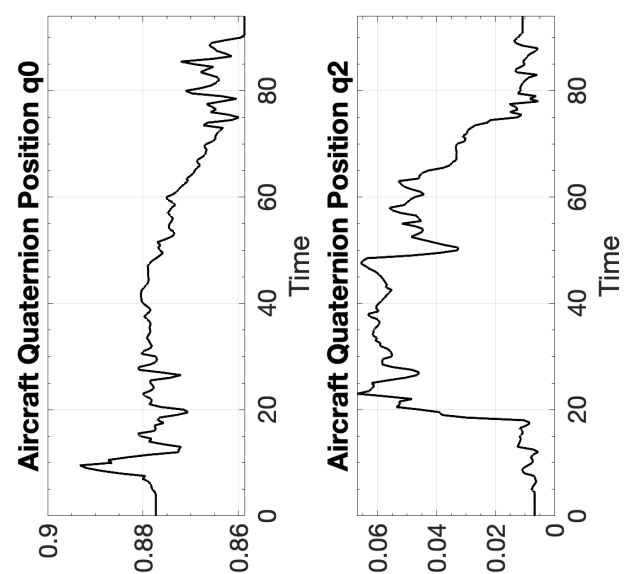

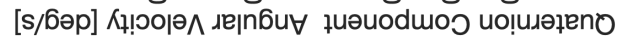




\section{C.6.1 CW1 Cessna 172 Single-axis Takeoff}

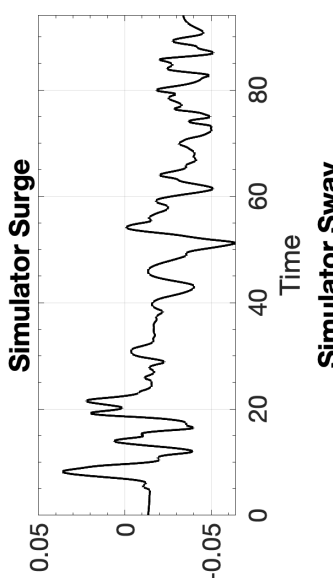

[w] uo!!!!sod

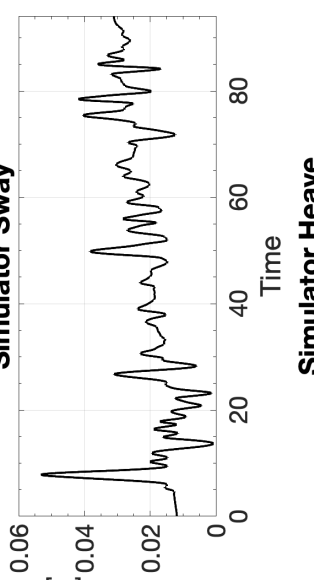

[w] uo!n! sod

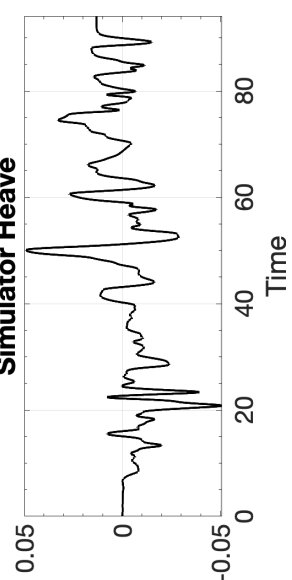

[u] uo!n!sod

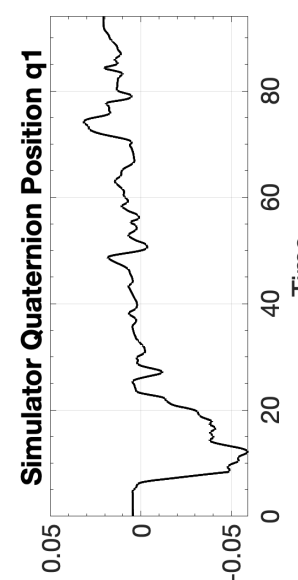

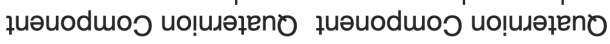

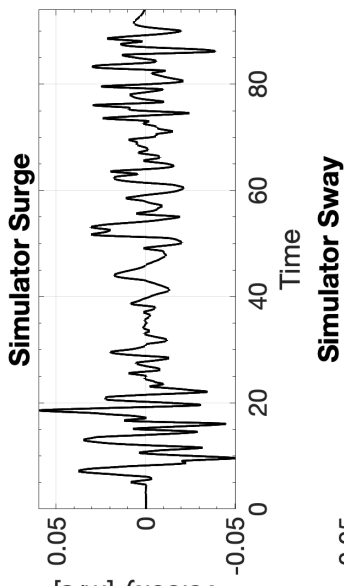

[s/m] Кұ!๐о|ә^

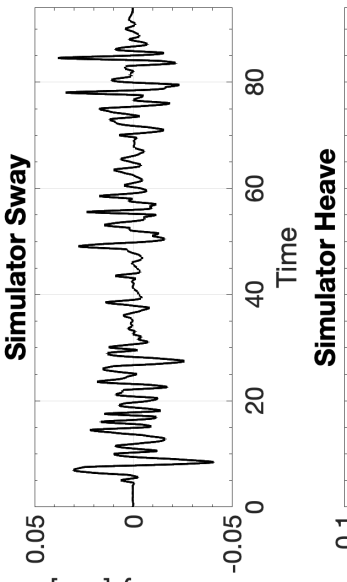

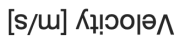

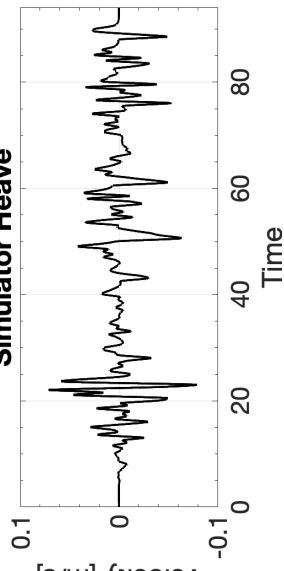

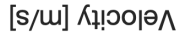

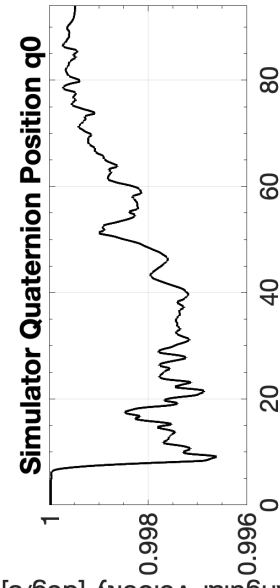

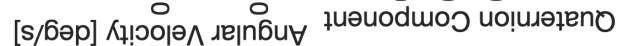

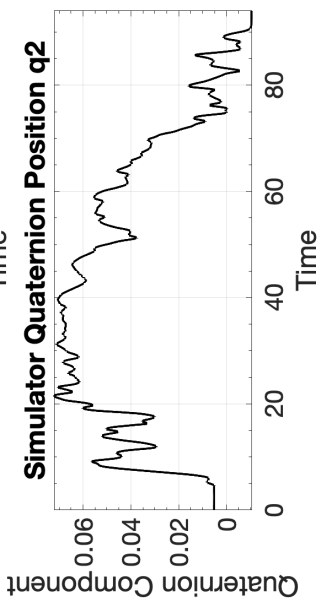

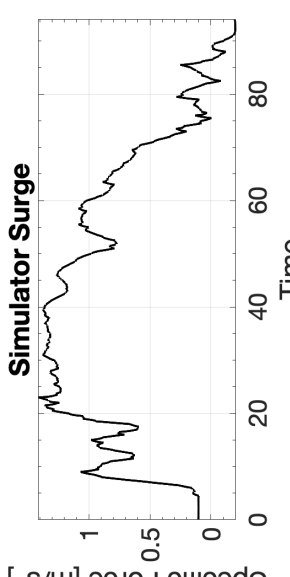

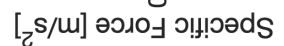

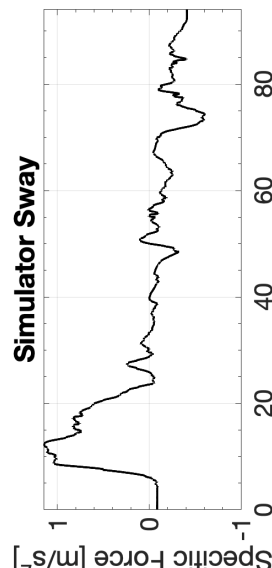

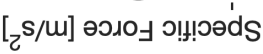


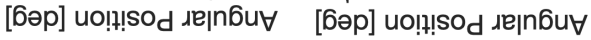

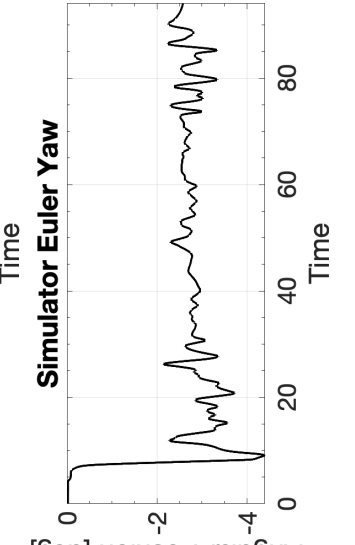

[6әр] uo!!! sod גeןn6u

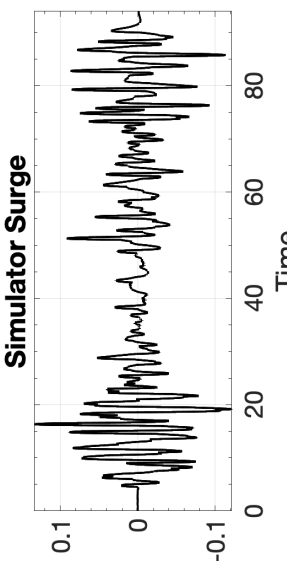

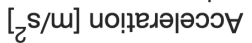

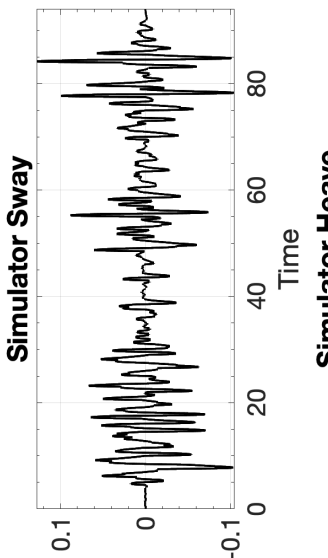

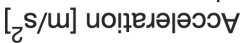

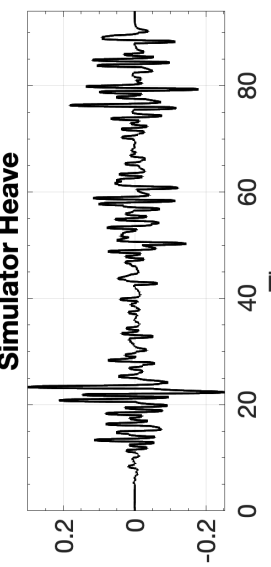

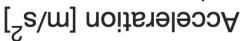

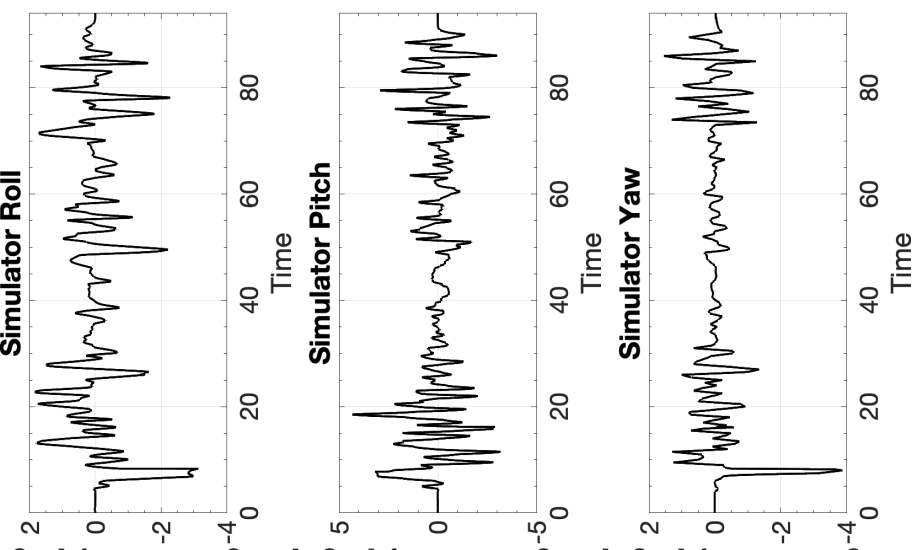

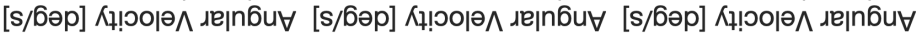




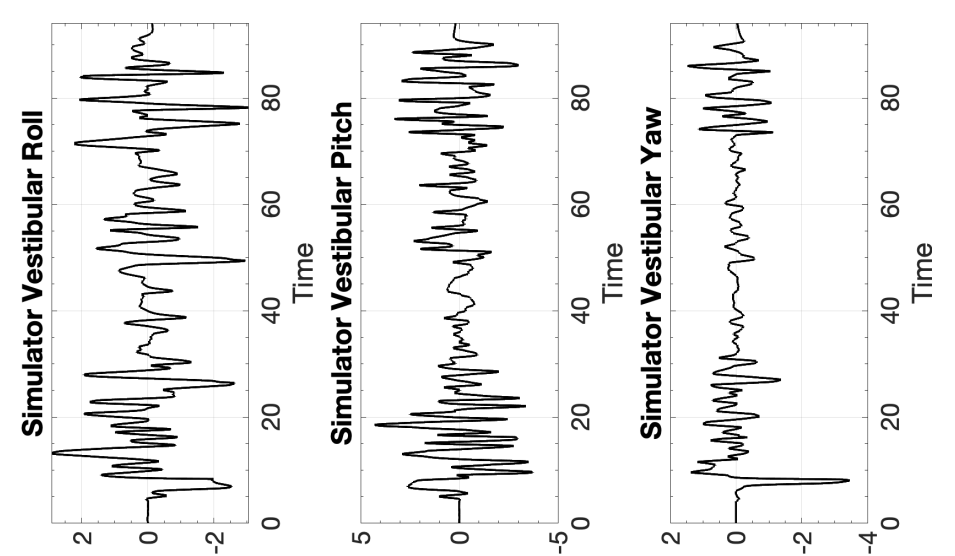

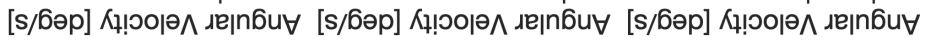

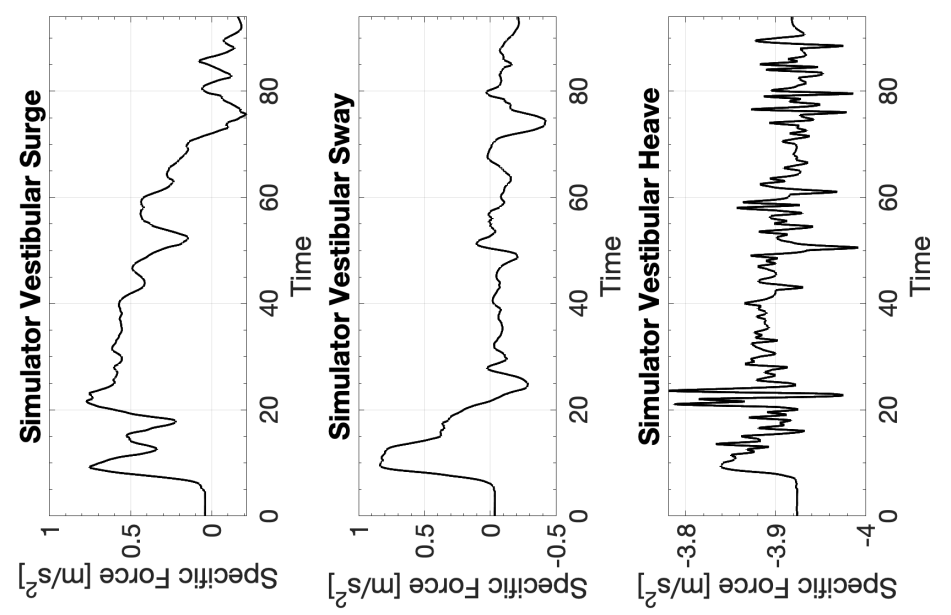




\section{C.6.2 CW2 Cessna 172 Single-axis Takeoff}

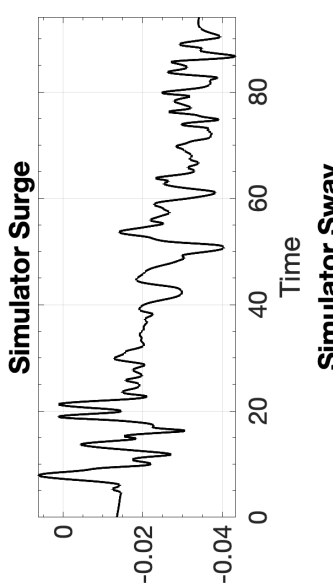

[w] uo!!!!sod

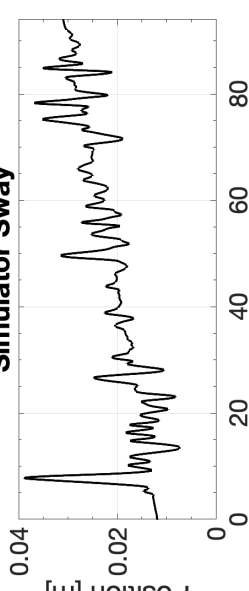

[w] uo!n!!sod

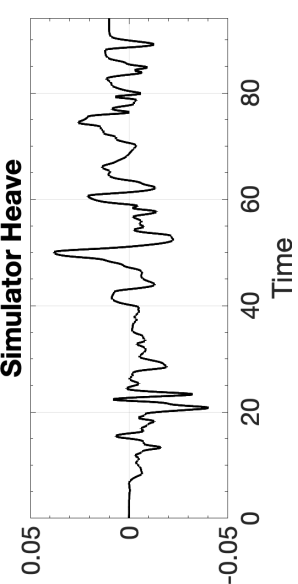

[u] uo!̣!!sod
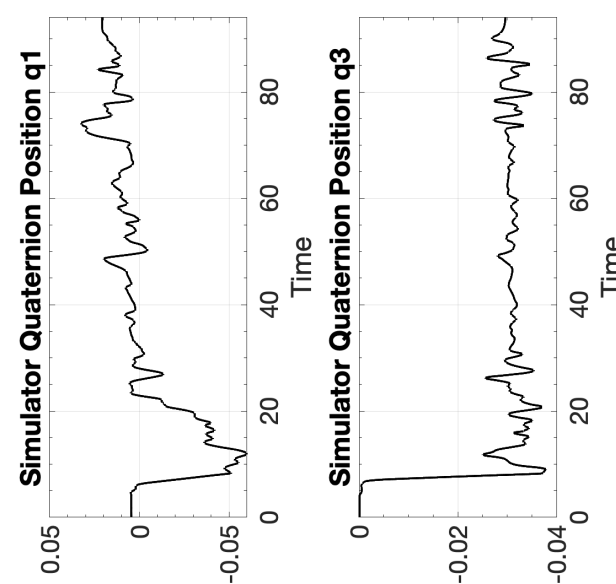

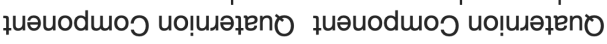

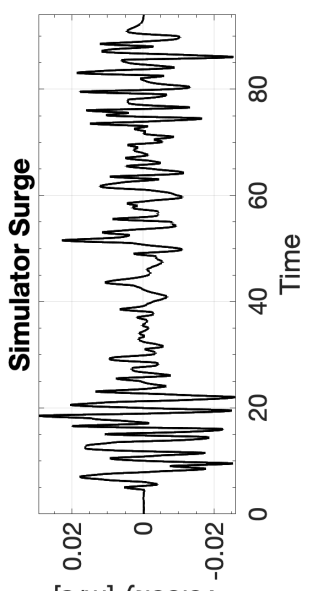

[s/u] Кұ!юорә

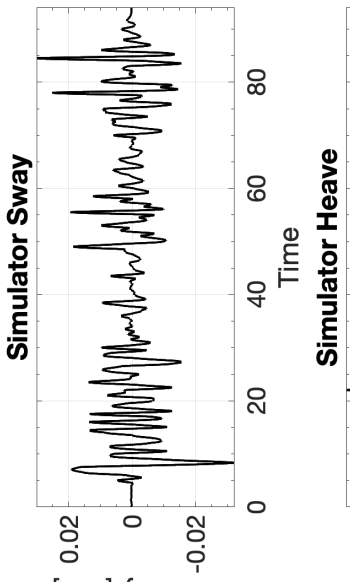

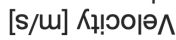

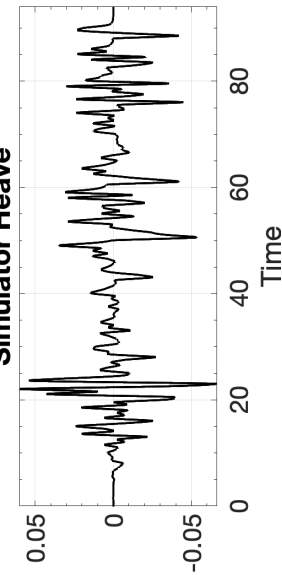

[s/u] Кұ!จојә

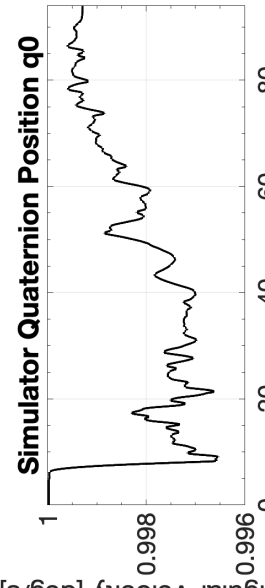

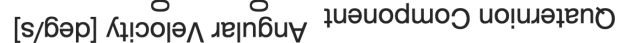

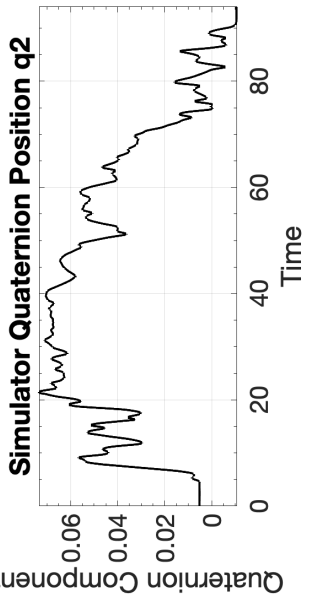

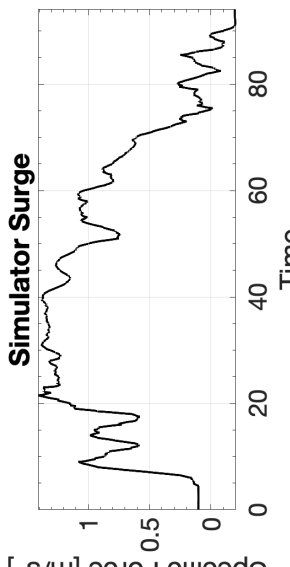

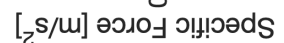

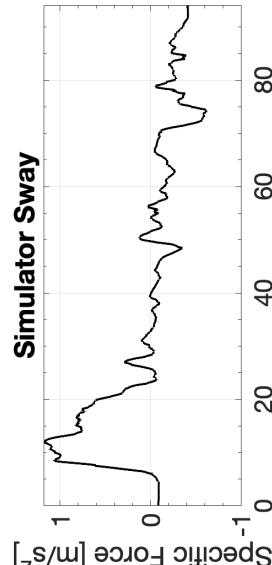

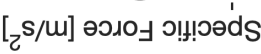
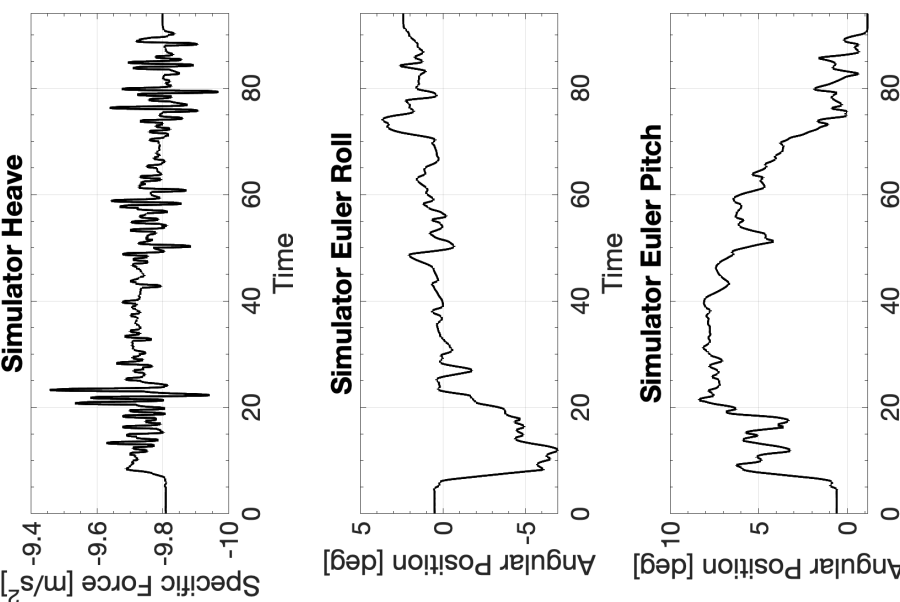

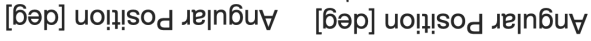

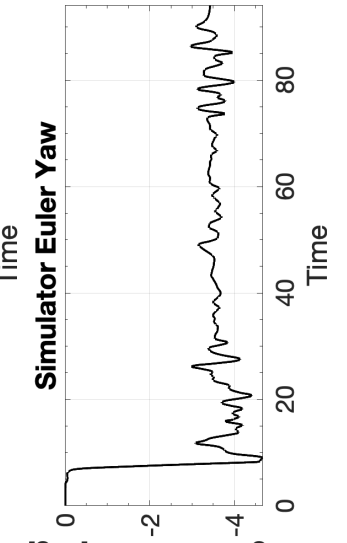

[6әр] uo!!! sod גeןn6u

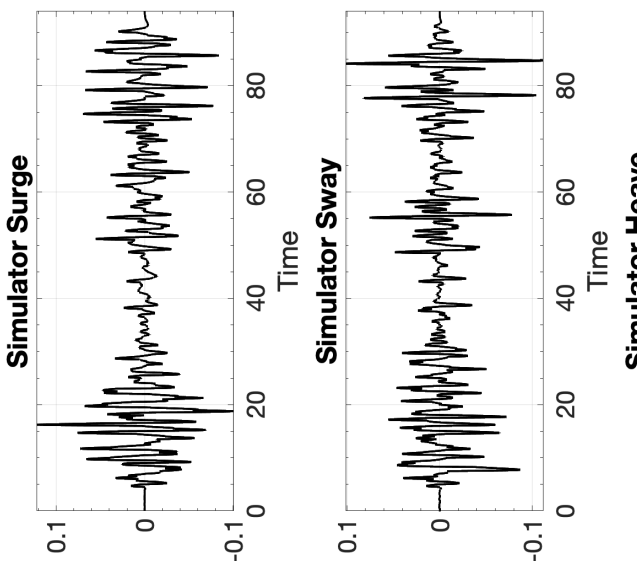

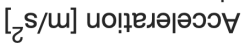

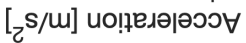

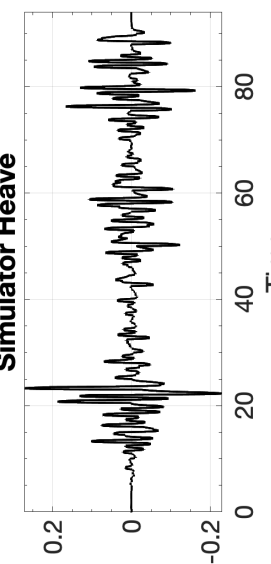

[ $\mathrm{s} / \mathrm{m}]$ ио!ฺедә|әэว
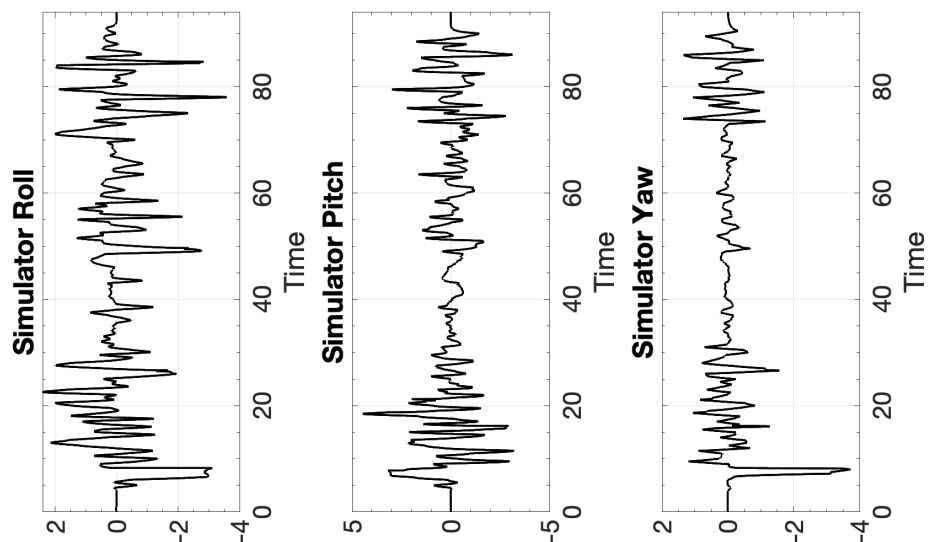

[s/6әр] Кұ! оорл גе|nби 


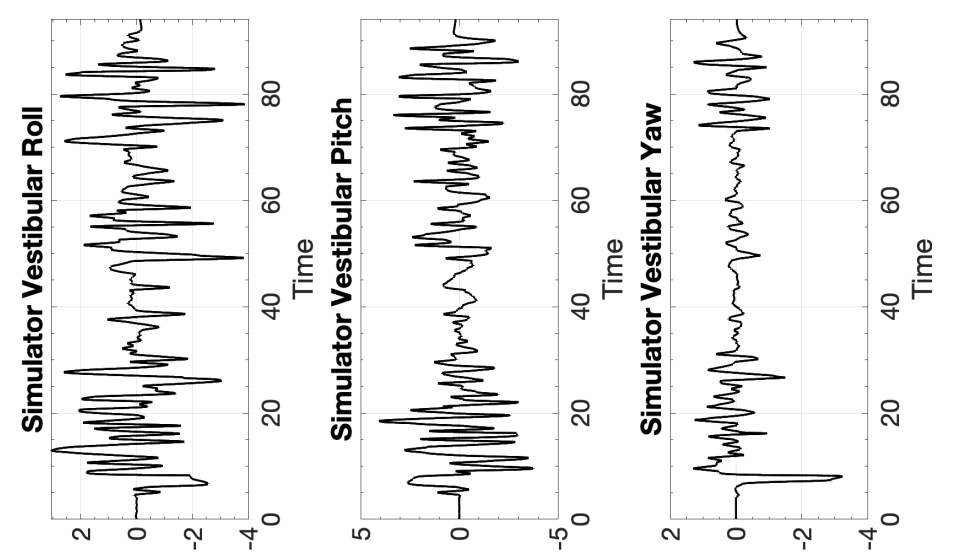

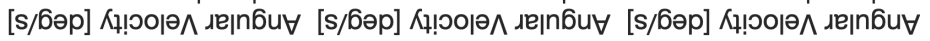

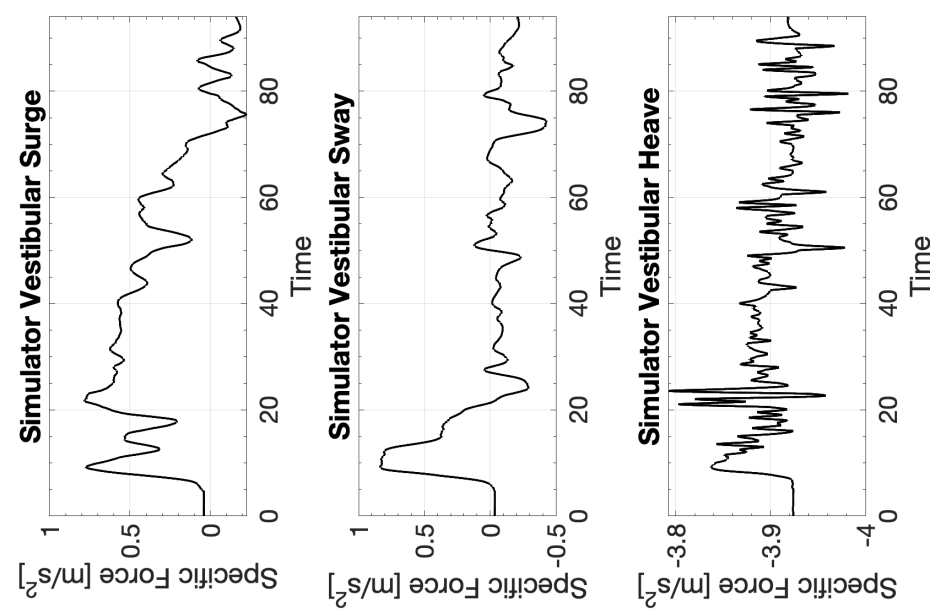




\section{C.6.3 CW3 Cessna 172 Single-axis Takeoff}

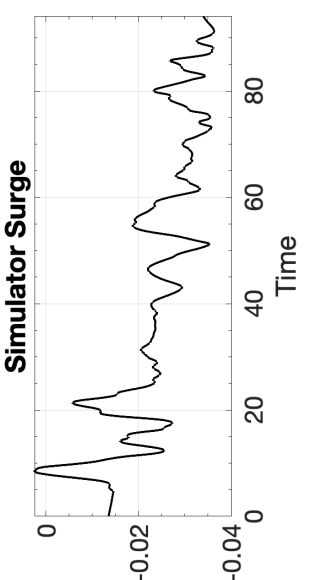

[u] uol!?!sod

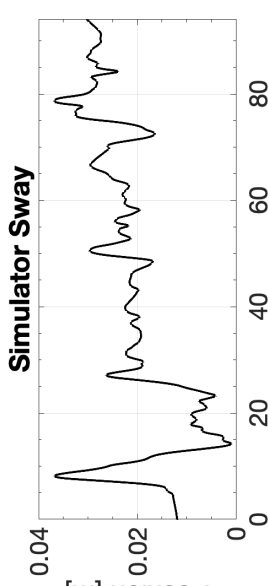

[w] uo!n! sod

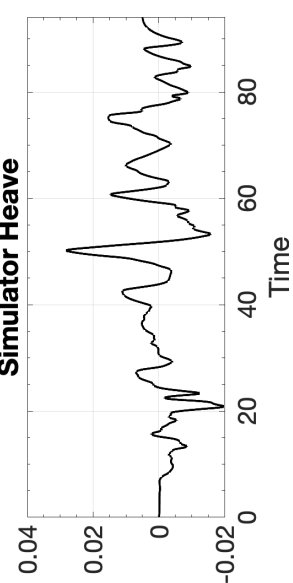

[u] uo!n!sod

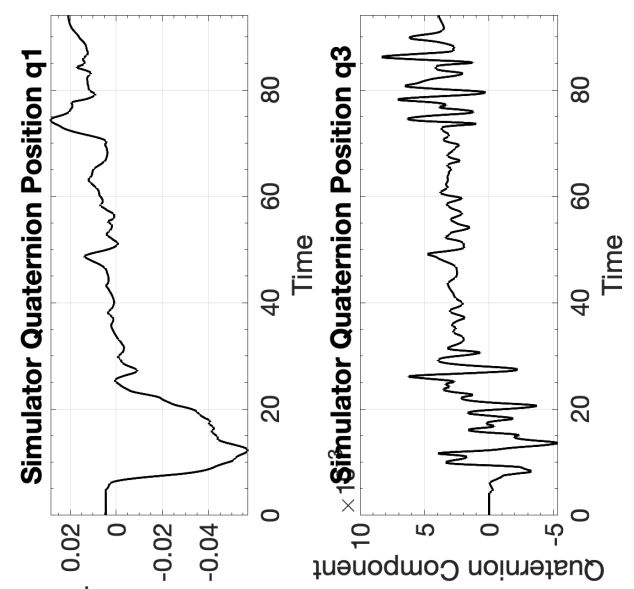

ұuəuodmos uọudəłeno

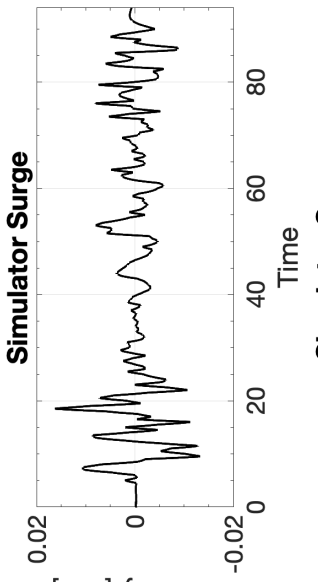

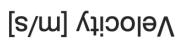

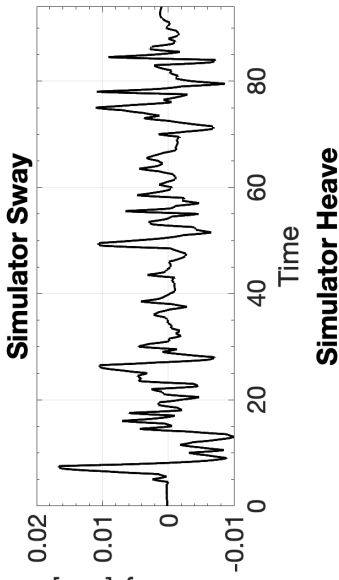

[s/u] Кұ!วо夫

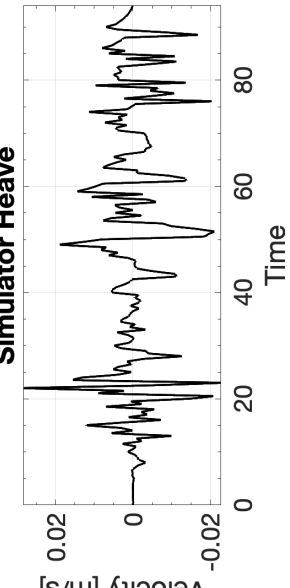

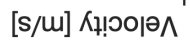

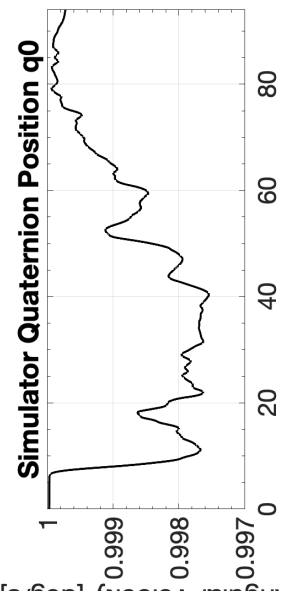

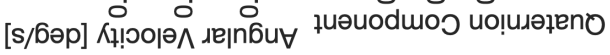

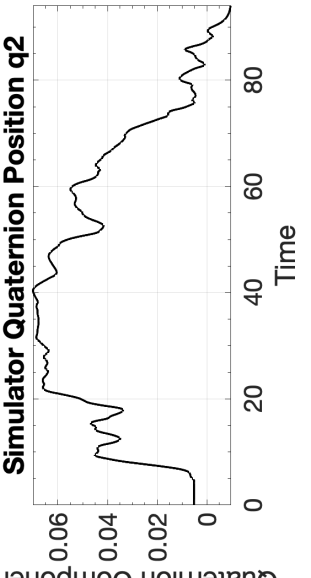

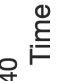
8

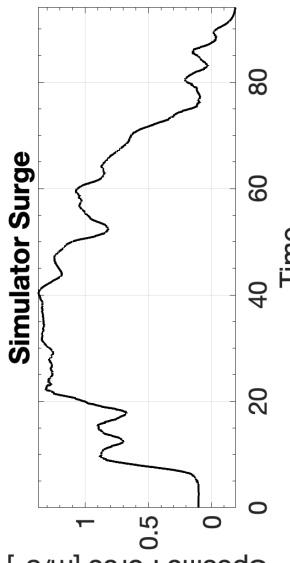

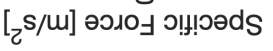

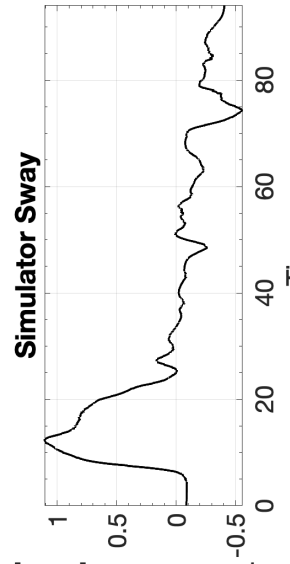

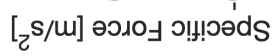

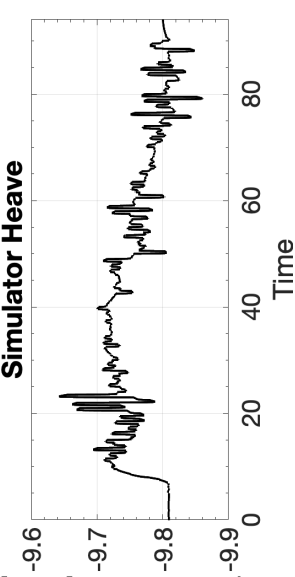

[

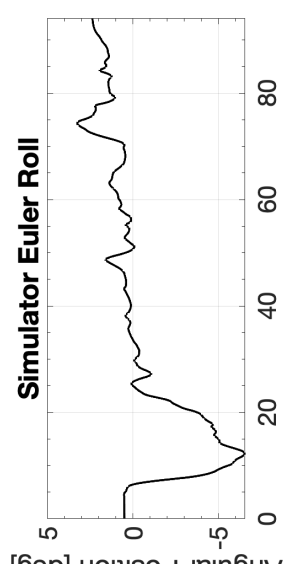

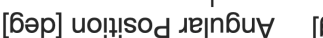
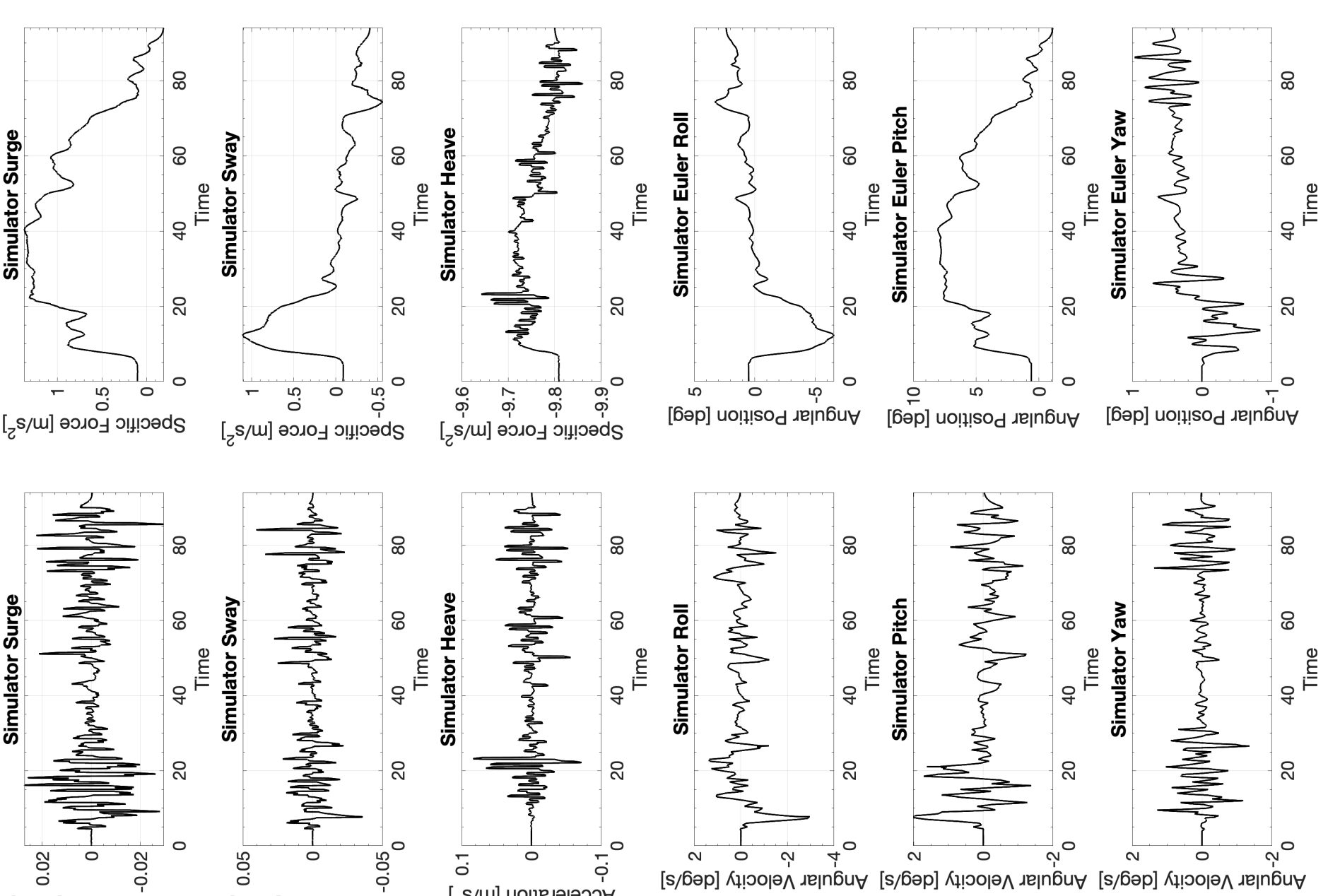

$\left[{ }_{2} \mathrm{~s} / \mathrm{M}\right]$ ио!ฺедәәәээ

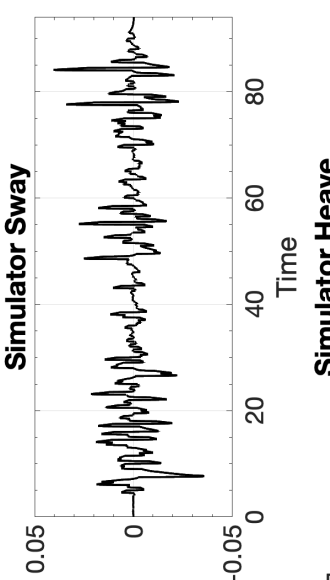

$\left[{ }^{2} s / m\right]$ uо!ฺеләәәэо

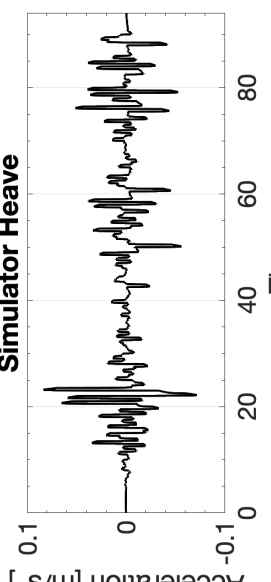

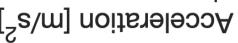

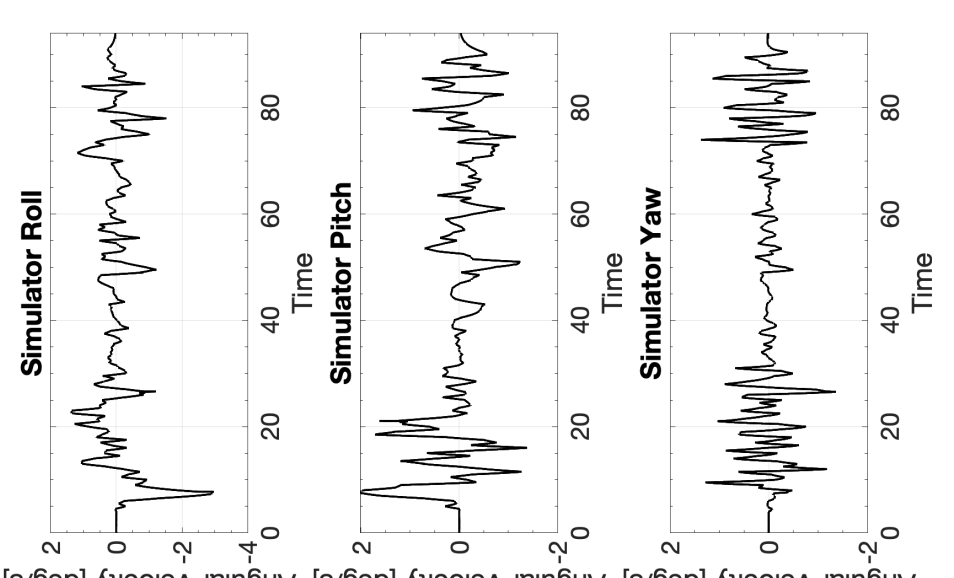

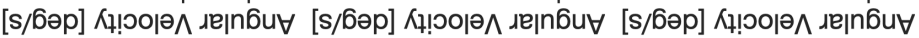



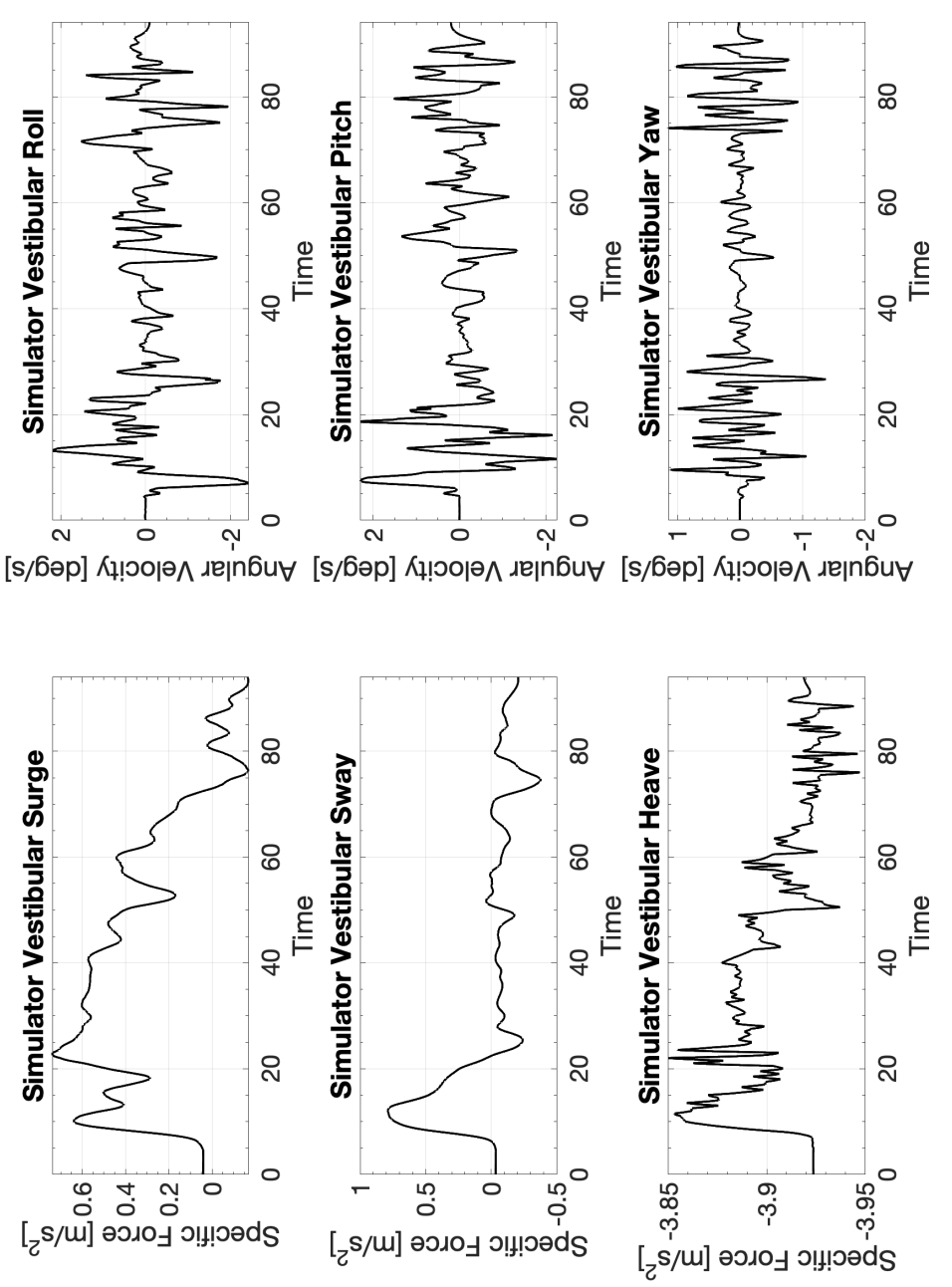


\section{C.7 Cessna 172 Spiral Dive}

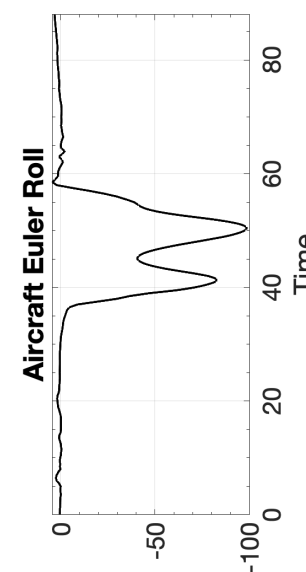

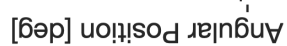

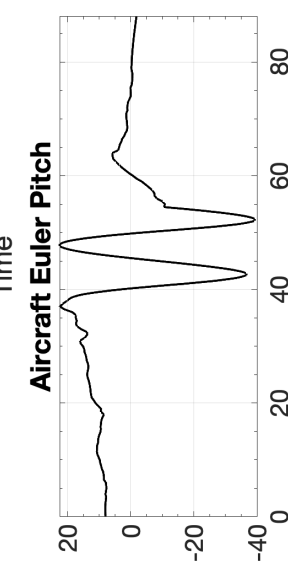

[6әр] uo!!!!sod גe|nбu

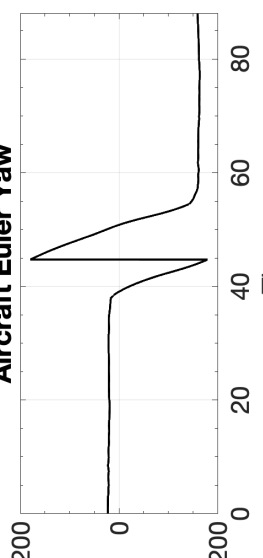

[6әр] uo!!!!sod de|nб̂́,

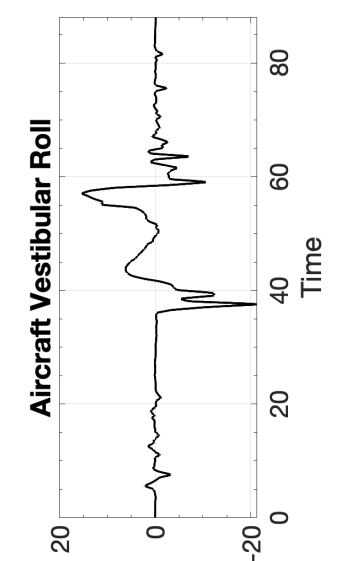

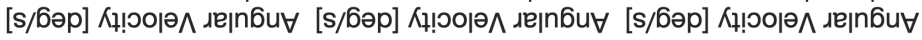
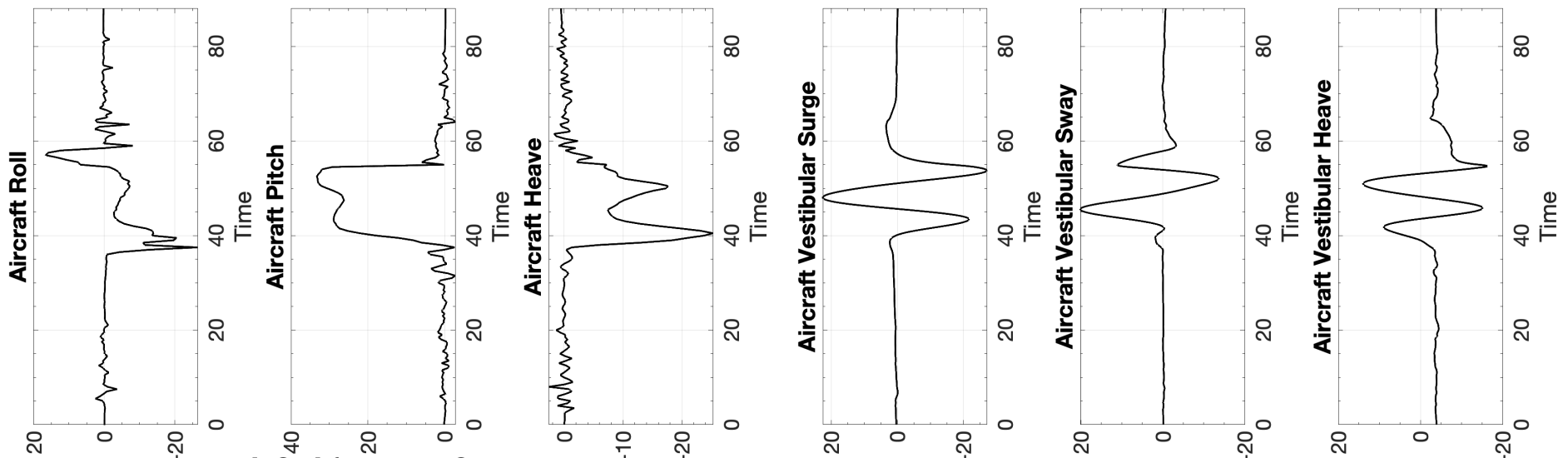

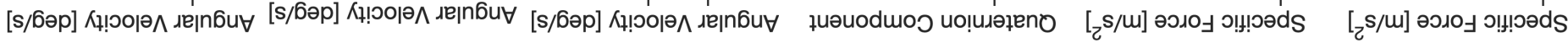
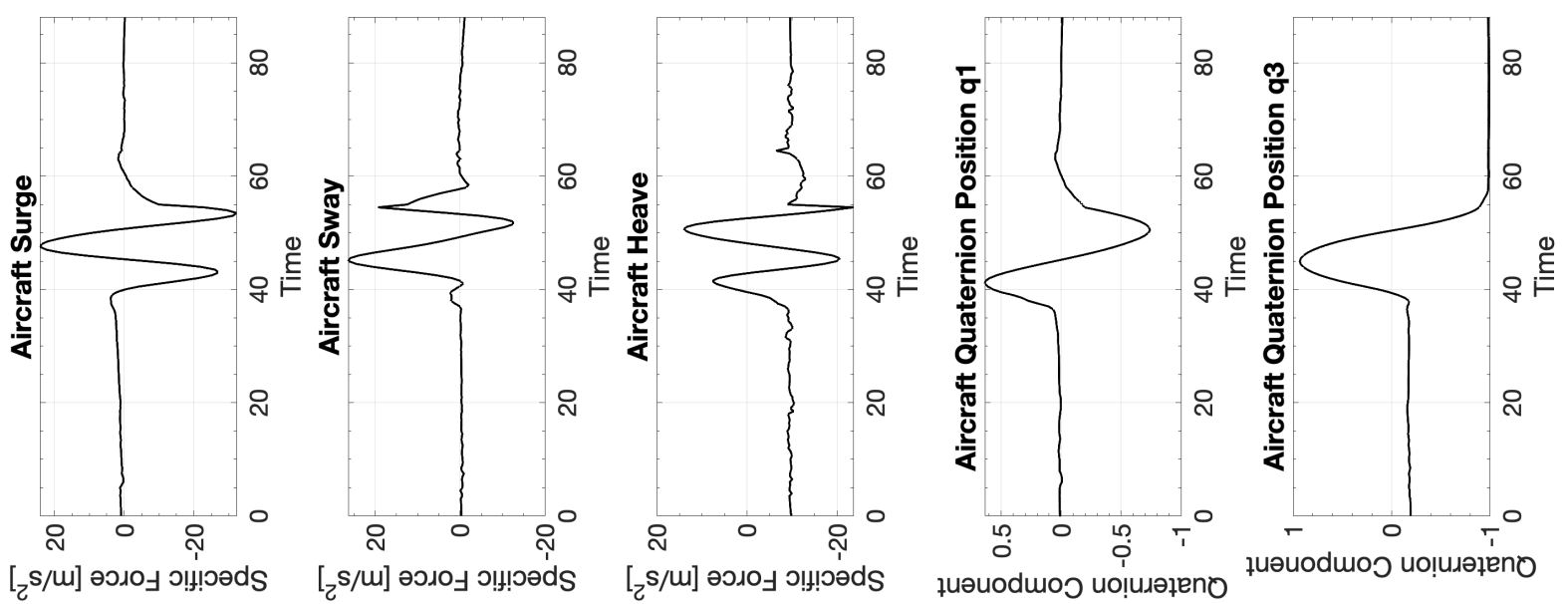

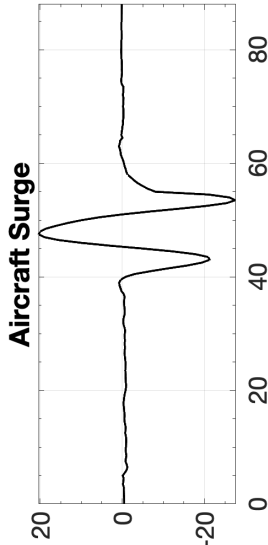

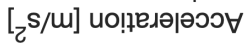

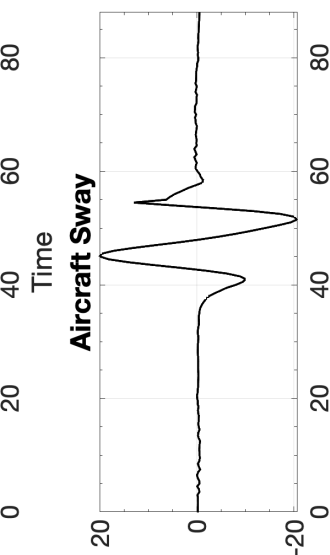

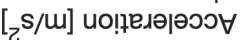

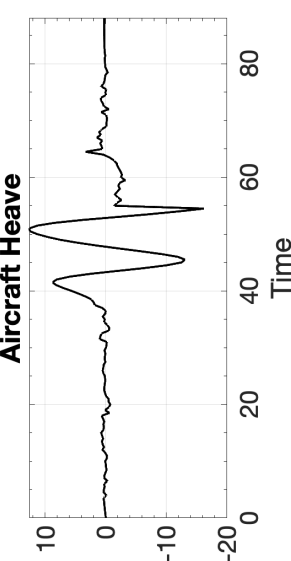

[ح $\mathrm{s} / \mathrm{m}]$ иопџедәрәоэ

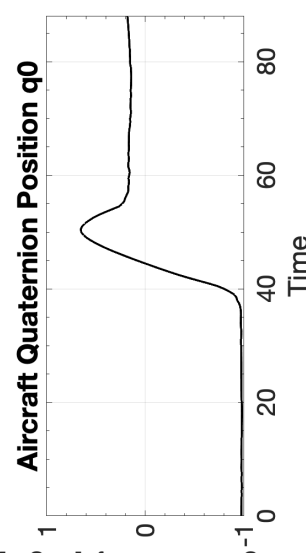

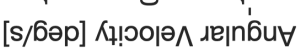

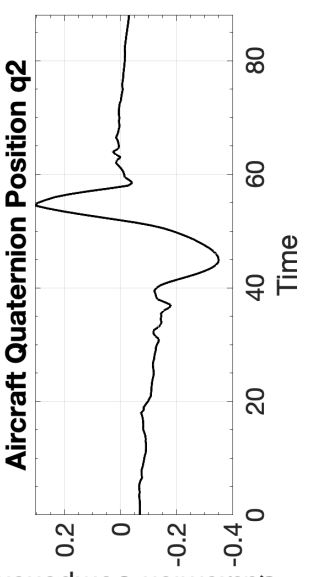

łuəuodmos uo!̣uәəteno 


\section{C.7.1 CW1 Cessna 172 Spiral Dive}
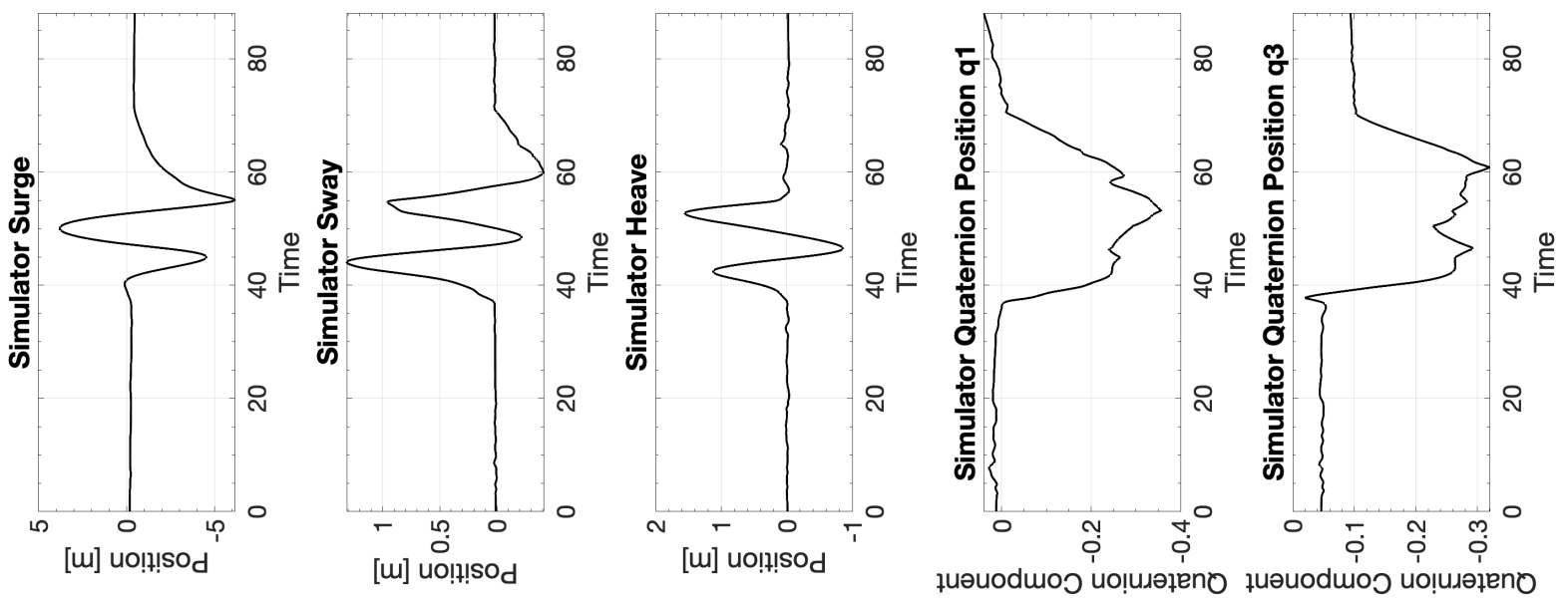

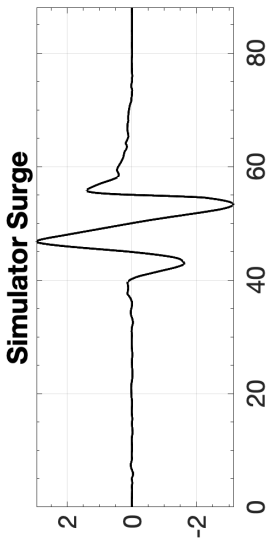

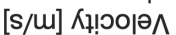
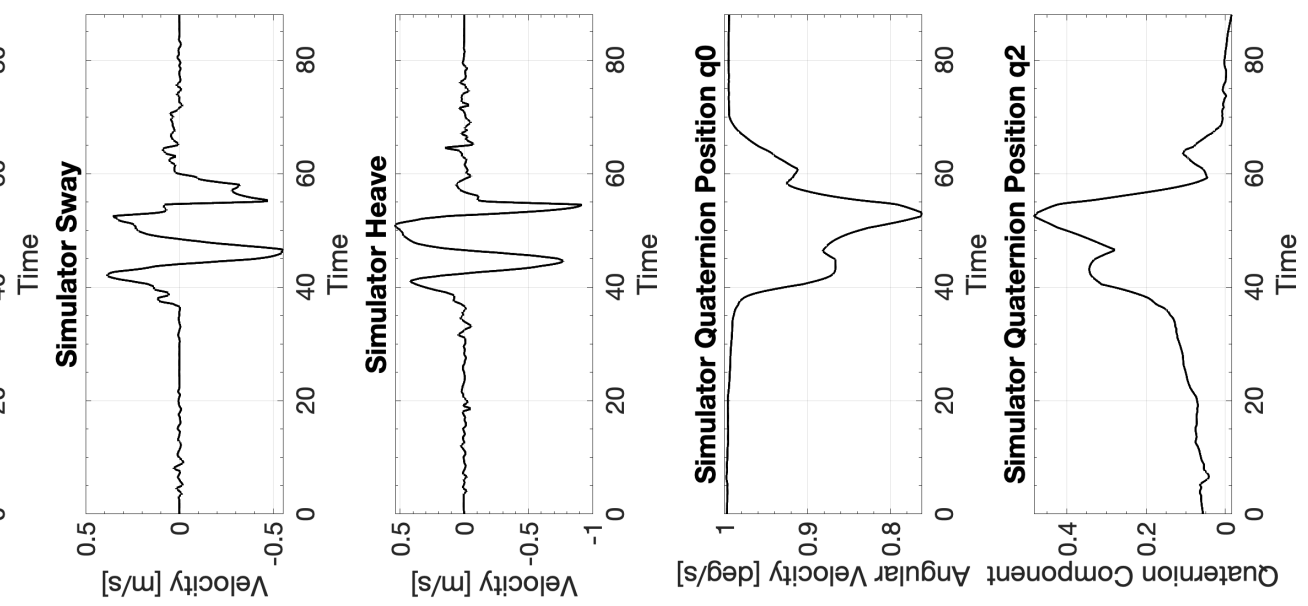

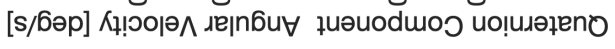

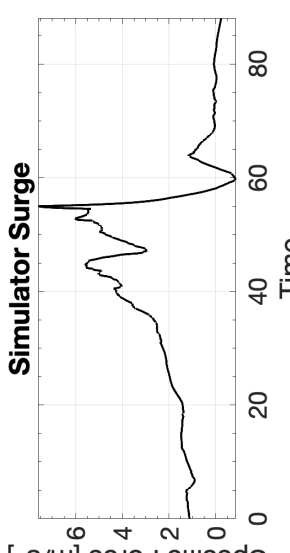

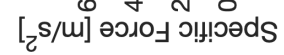
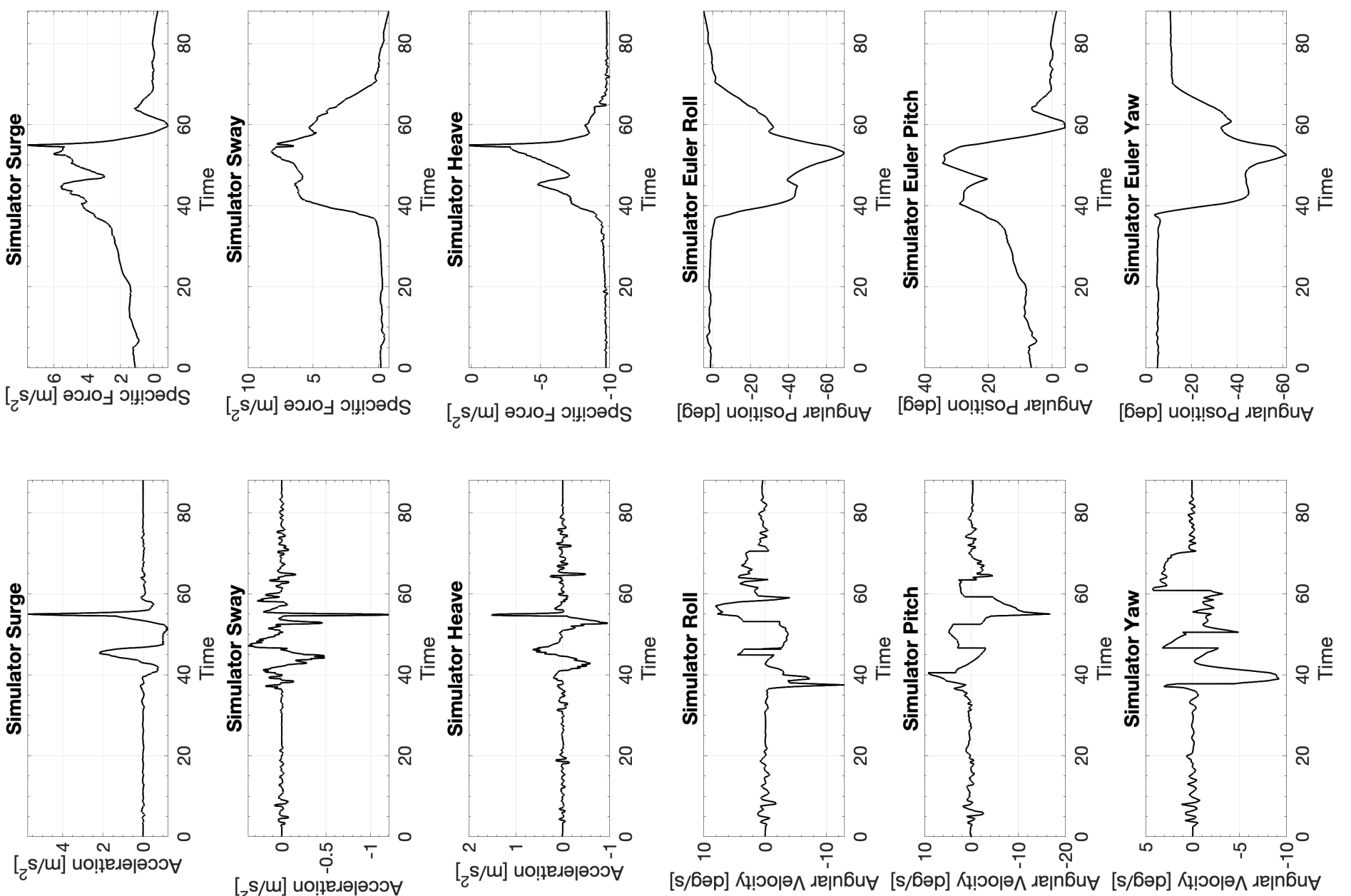

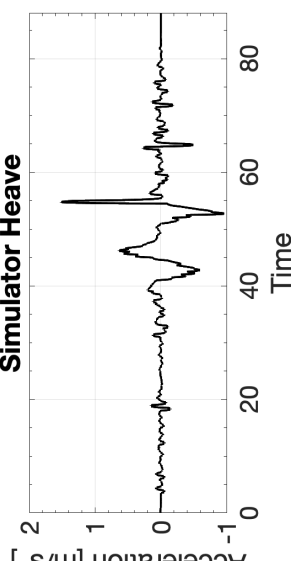

[zs/m] uо!ןеләрәэว

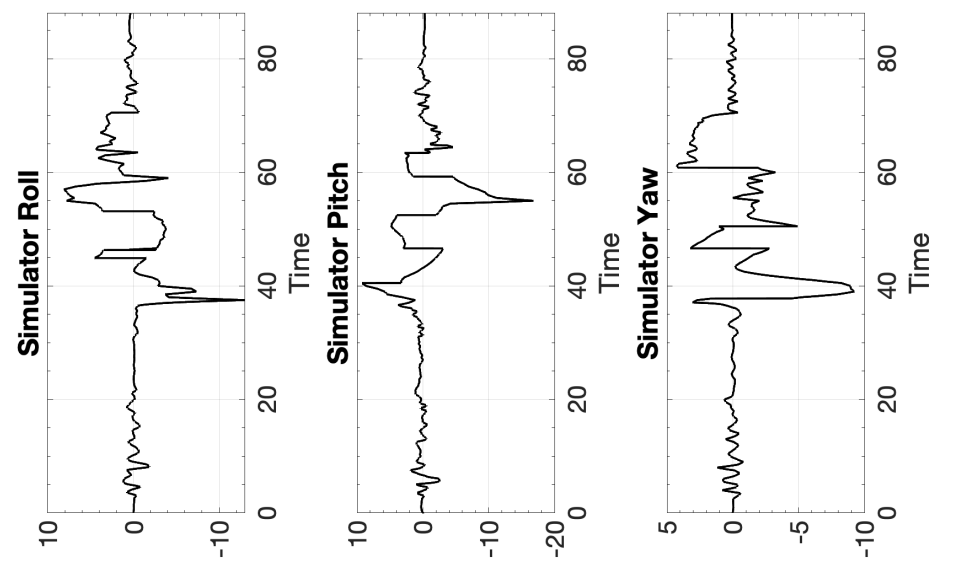

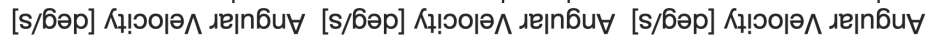




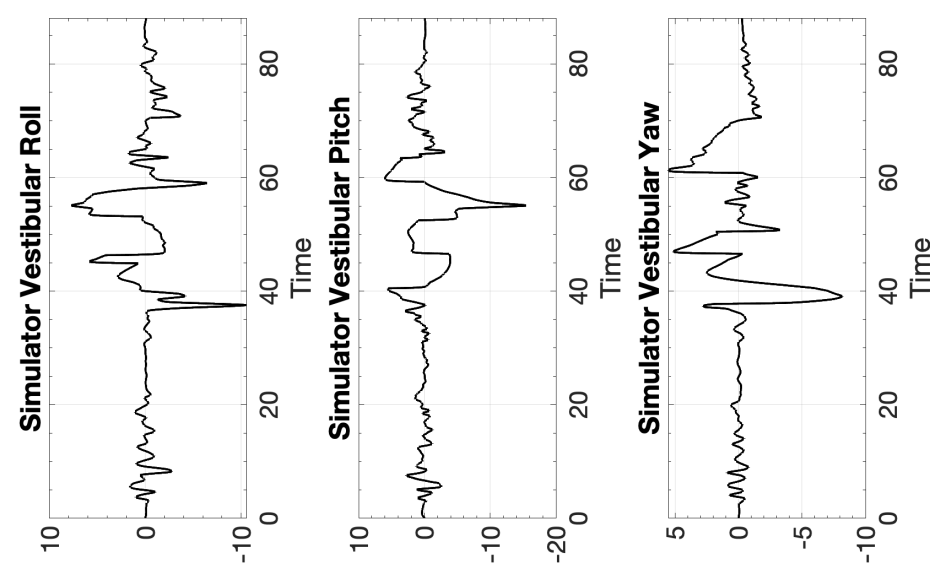

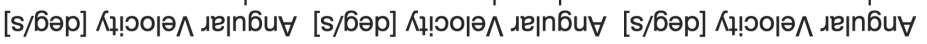

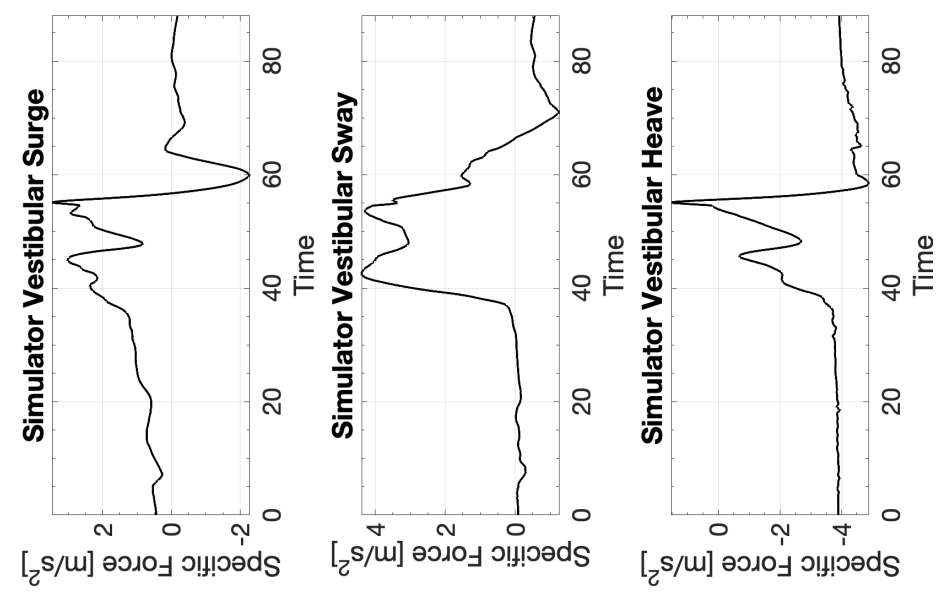




\section{C.7.2 CW2 Cessna 172 Spiral Dive}

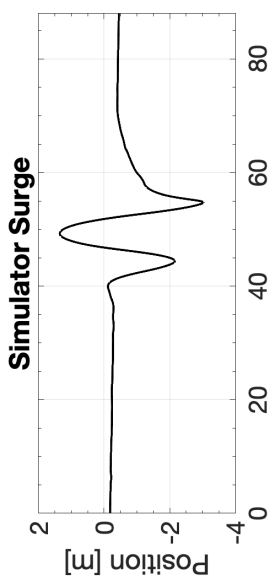

[w] uo!n! sod

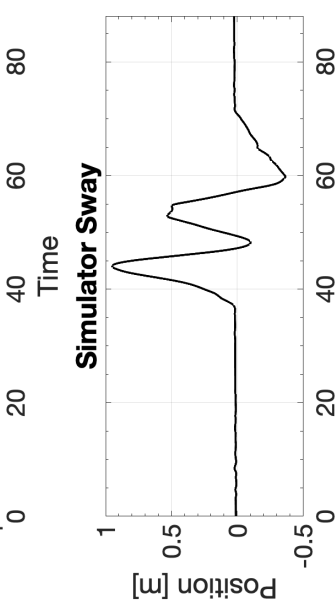

[m] uol!m!sod

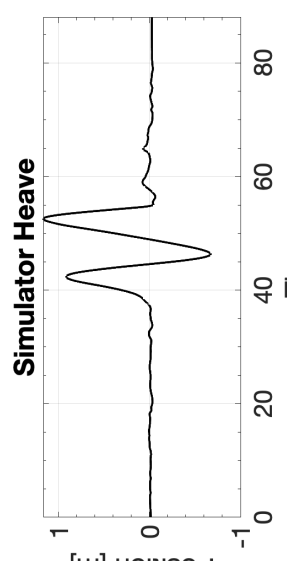

[u] uo!!! $\mathrm{sod}^{\mathrm{d}}$

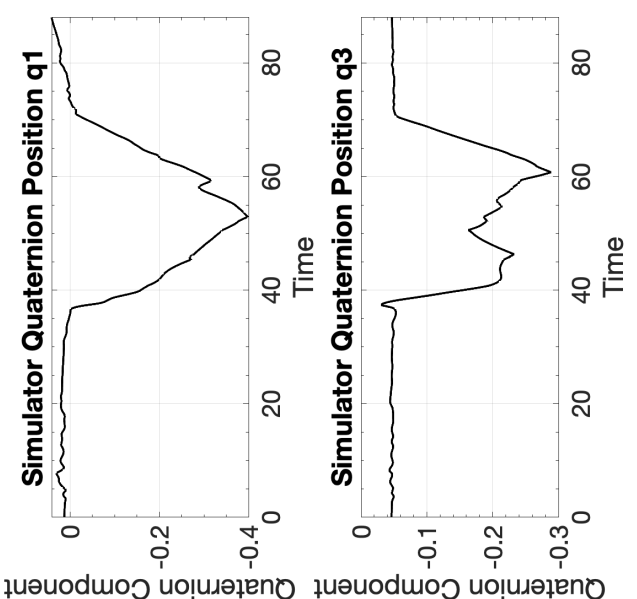

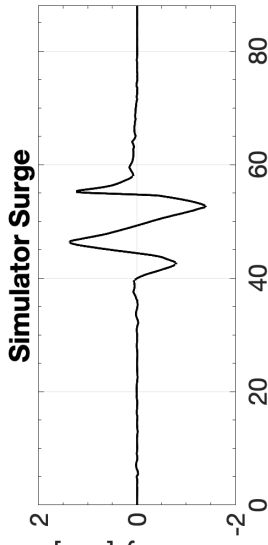

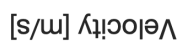

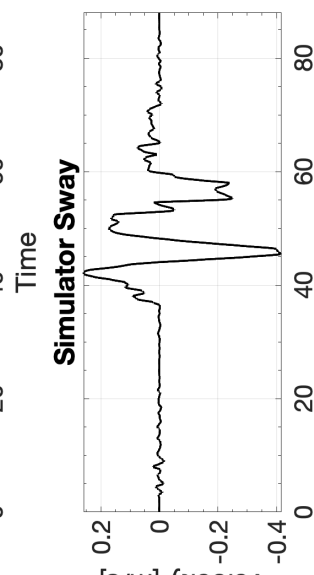

[s/u] Кұ!юำ^

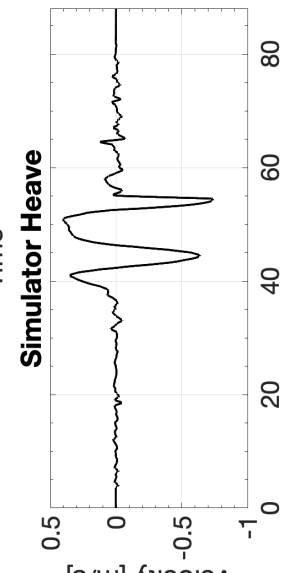

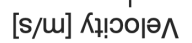

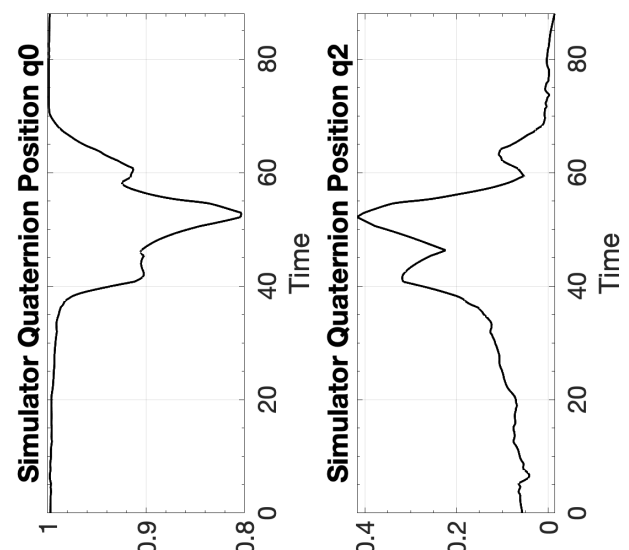

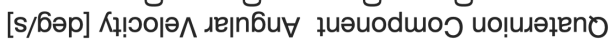

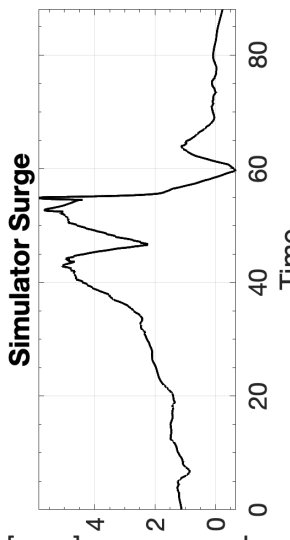

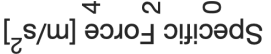

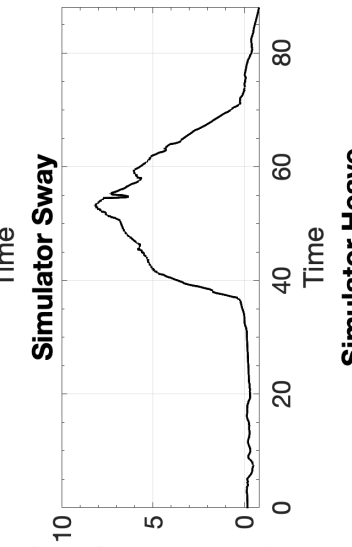

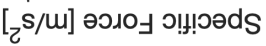

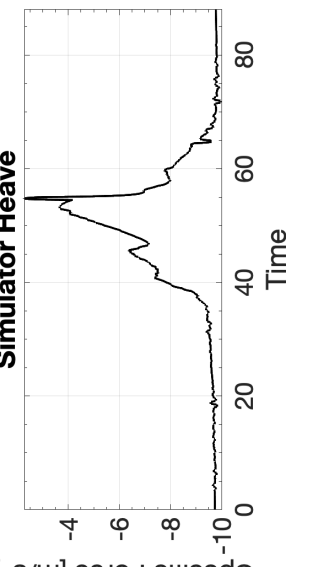

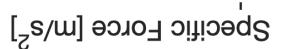
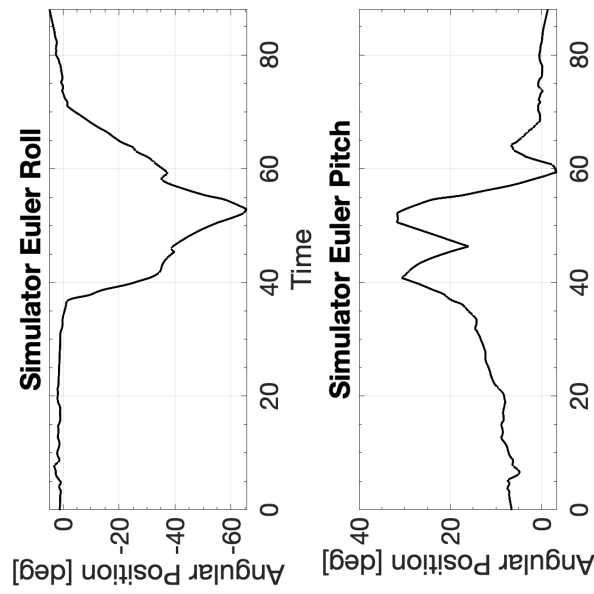

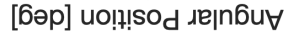

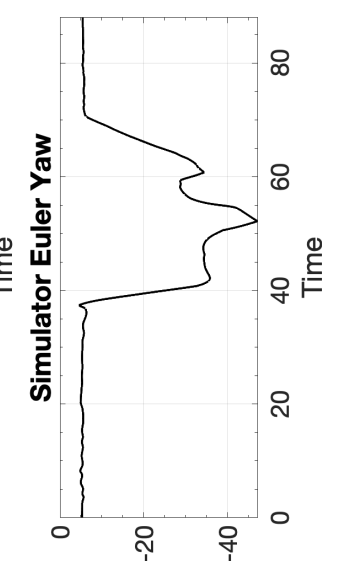

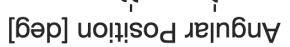

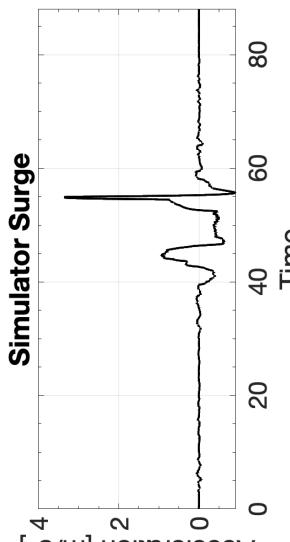

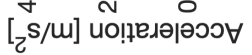

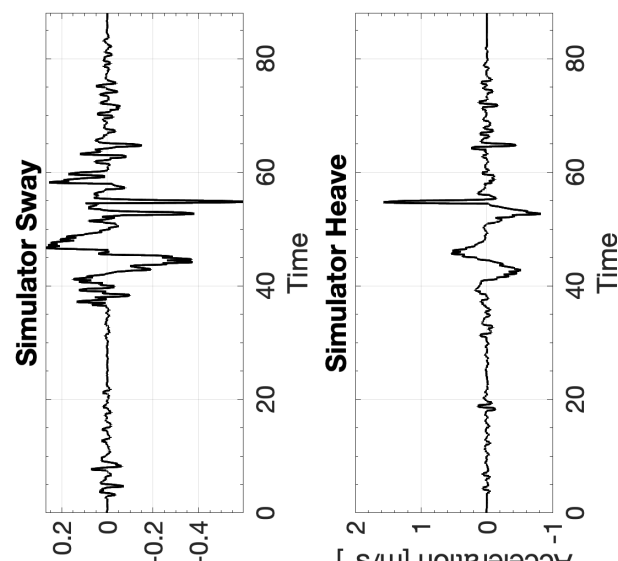

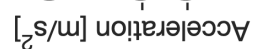
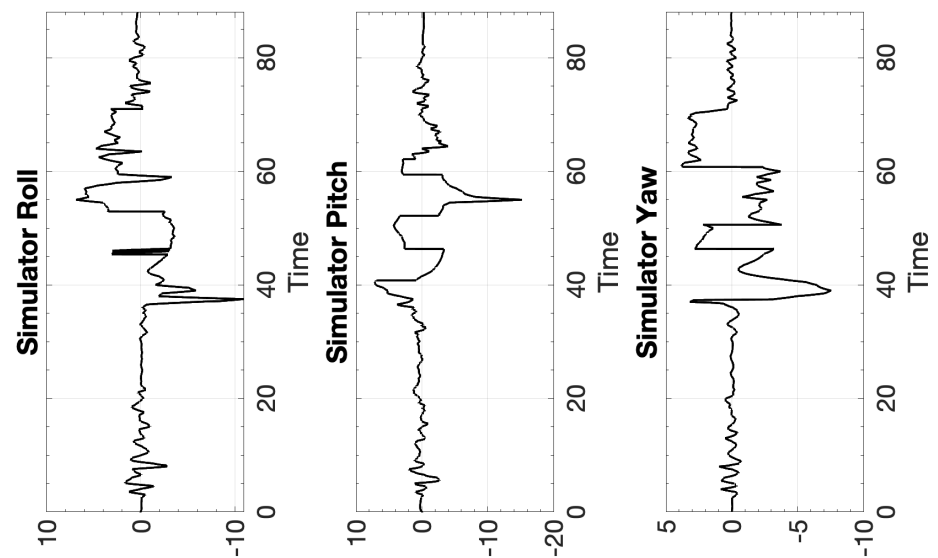

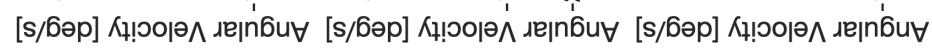




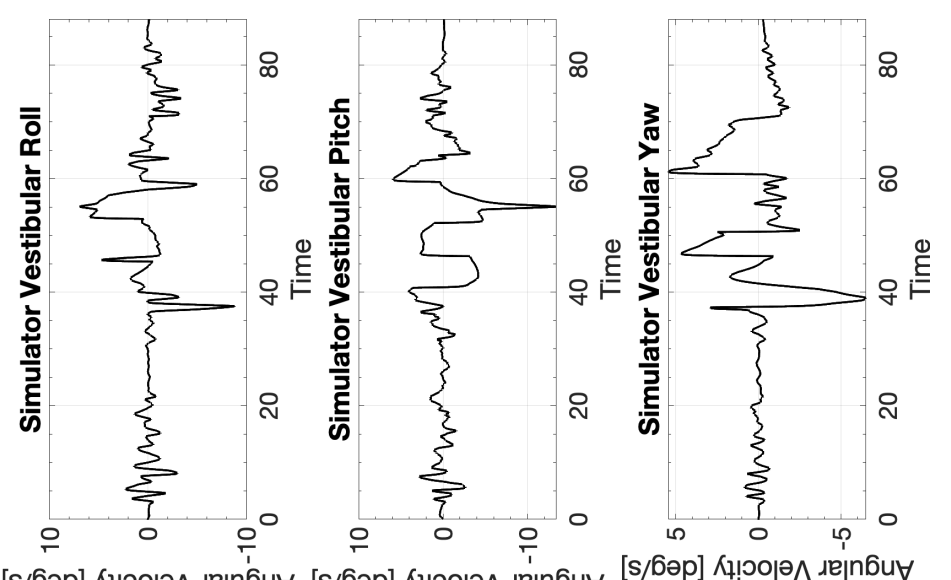

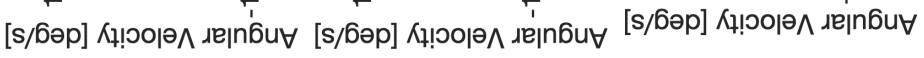

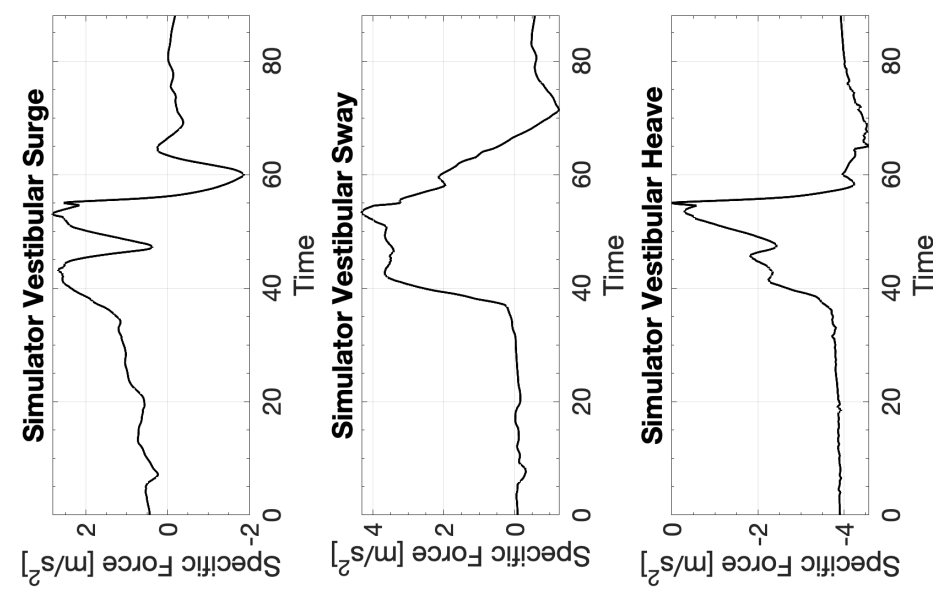




\section{C.7.3 CW3 Cessna 172 Spiral Dive}

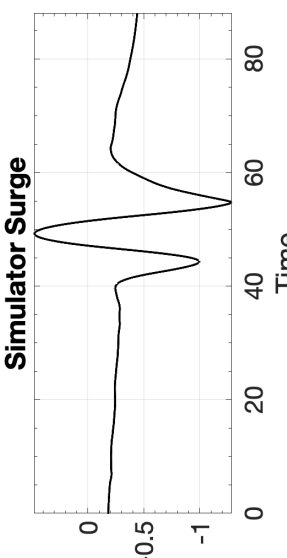

[w] uo!!!!sod

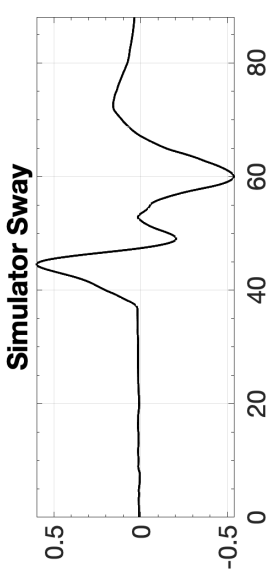

[m] uolin!sod

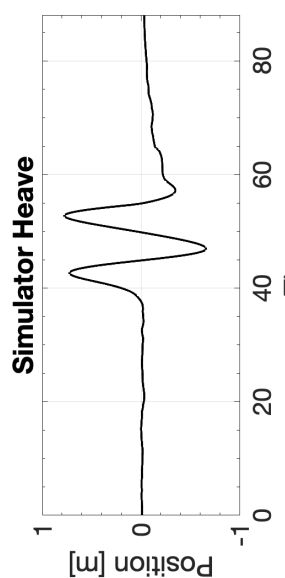

[w] uo!̣!!sod

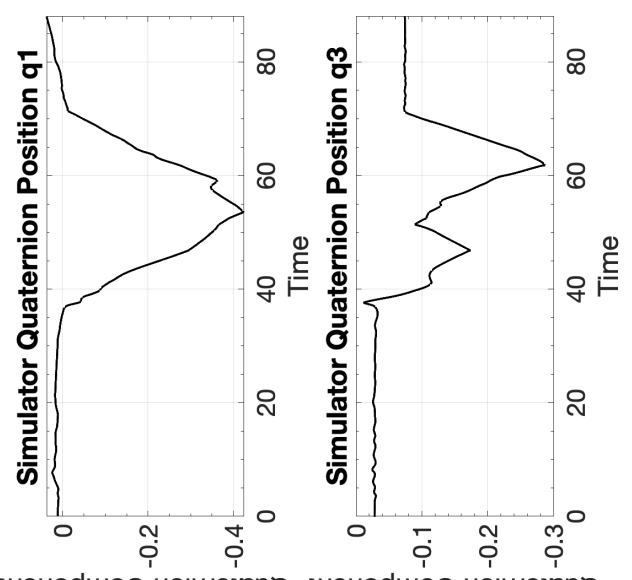

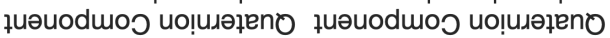

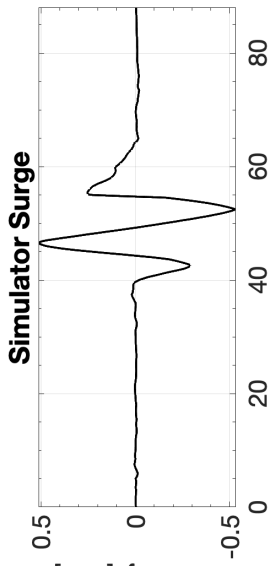

[s/u] К! ৩ою

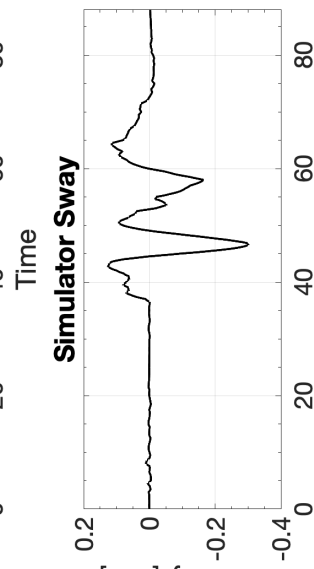

[s/u] Кұ!юо|ә

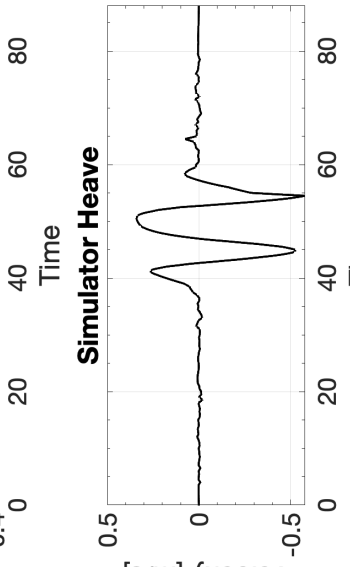

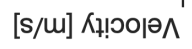

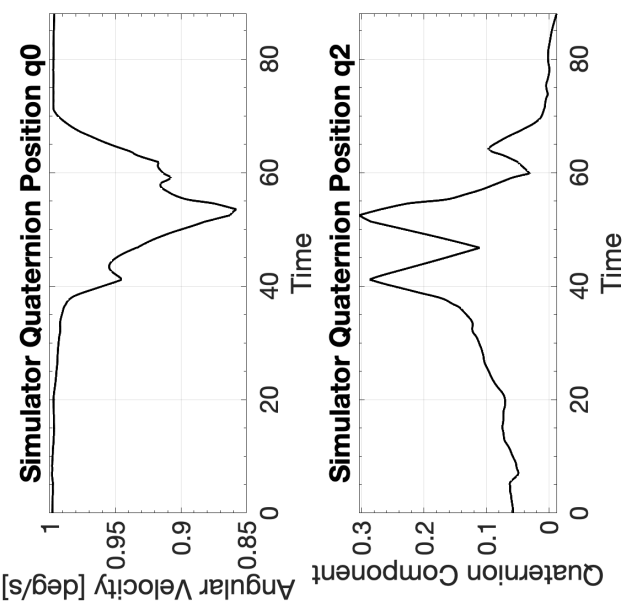

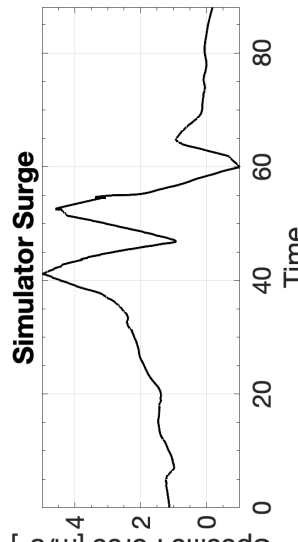

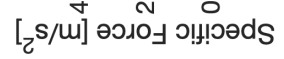
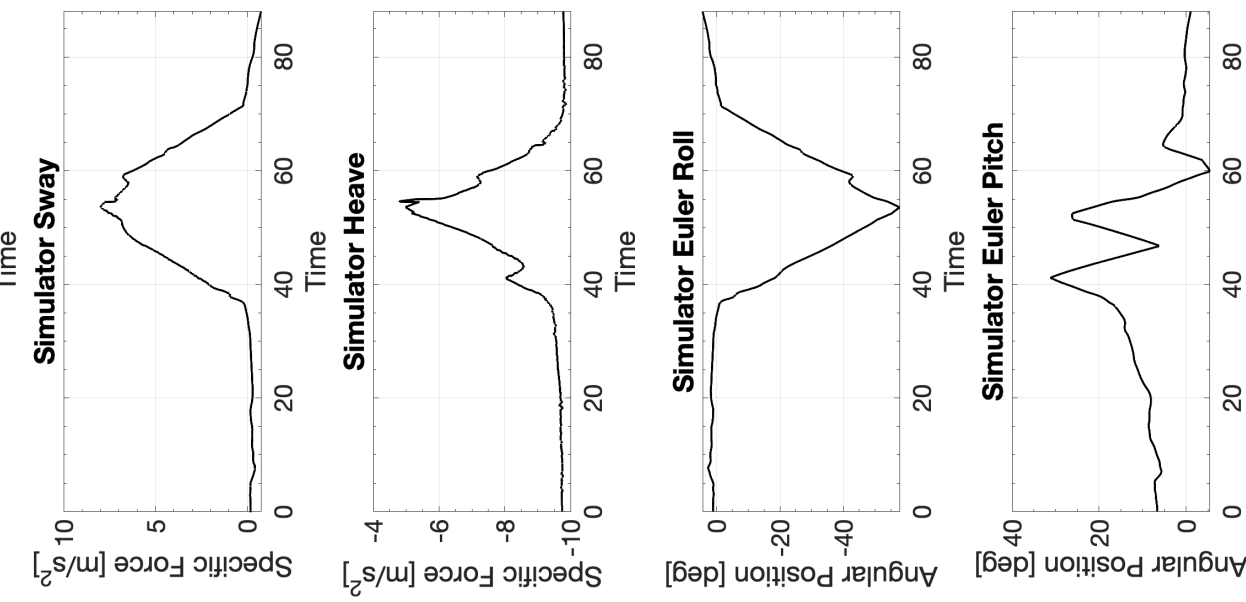

[6əp] uo!!!sod גe|n6u

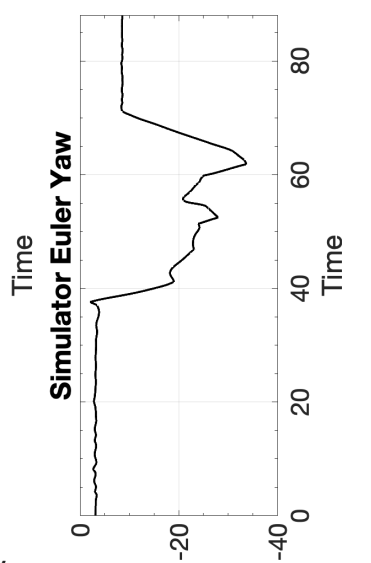

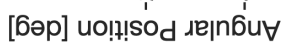

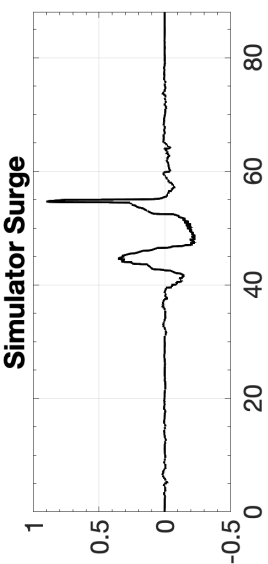

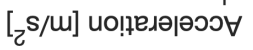

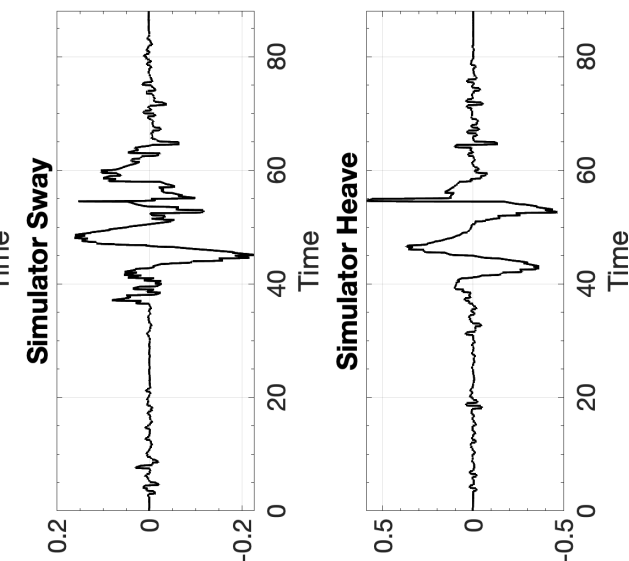

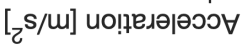

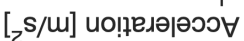
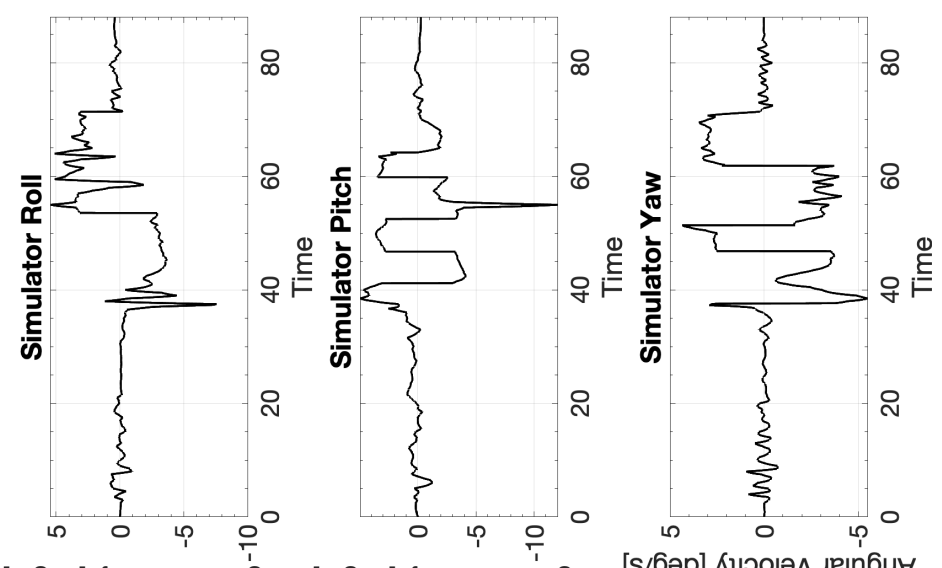

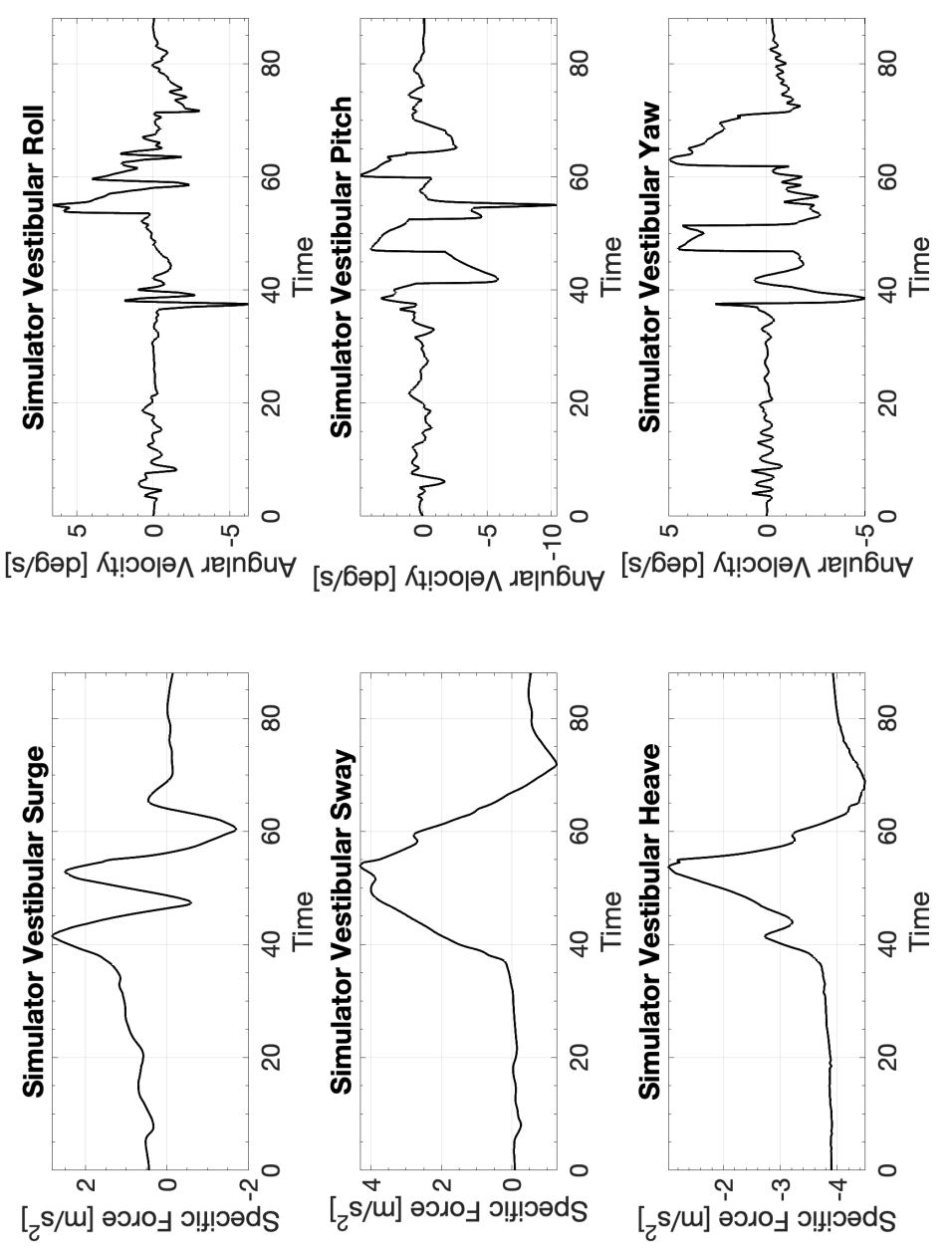


\section{C.8 Cessna 172 Stall Recovery}

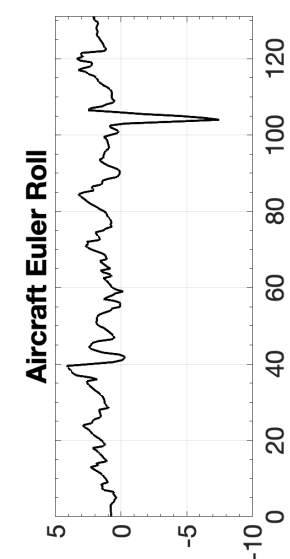

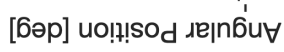

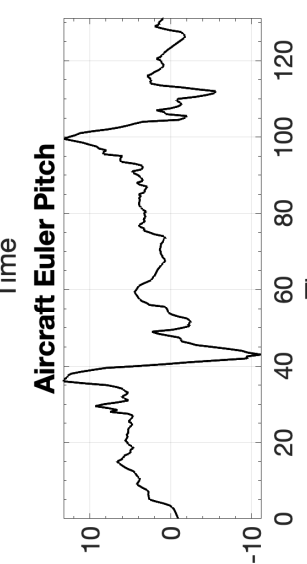

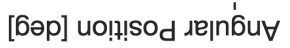

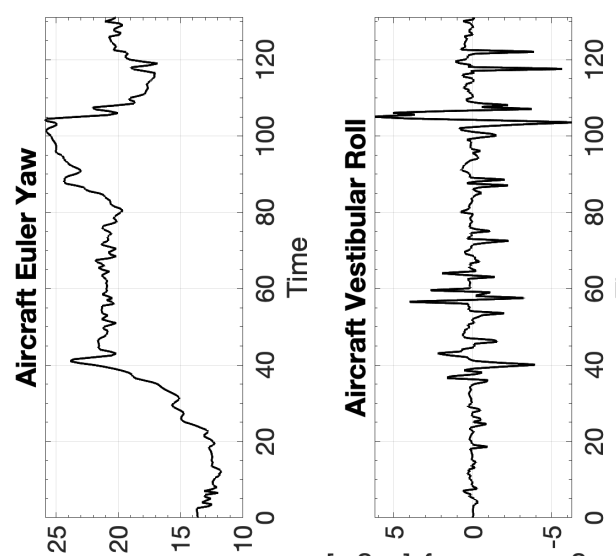

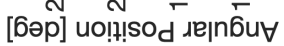
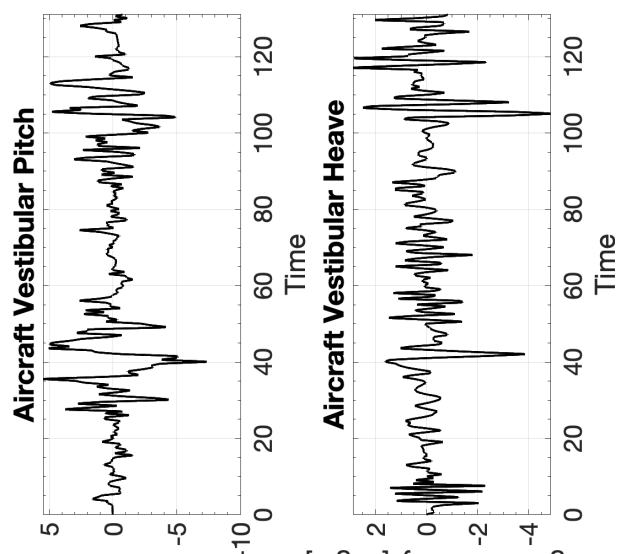

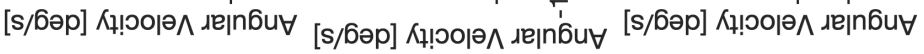
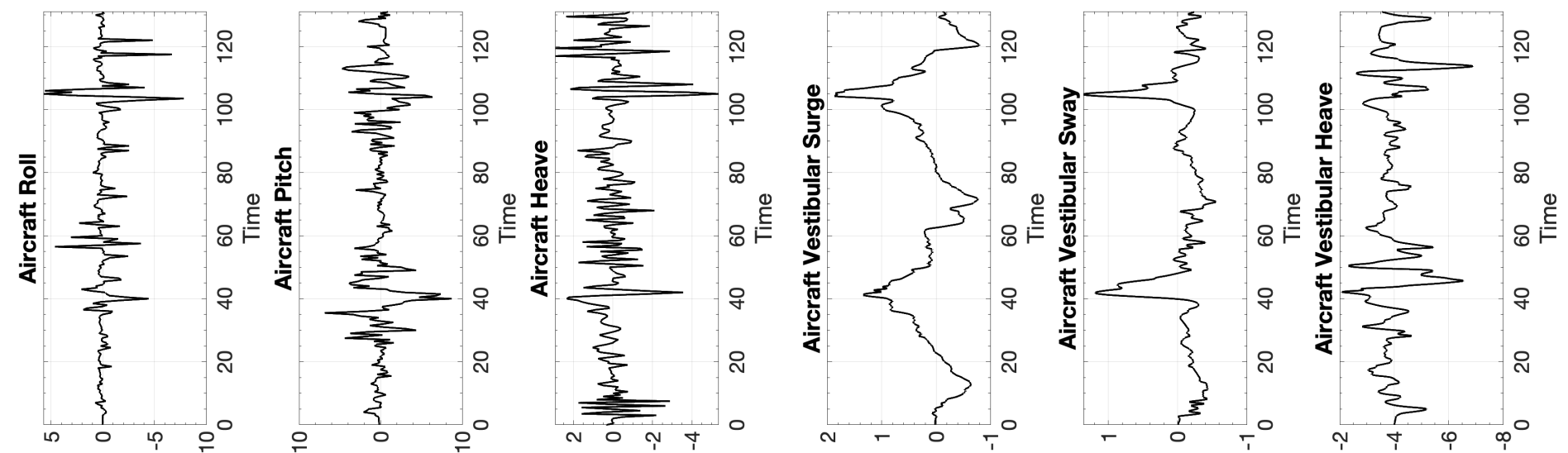

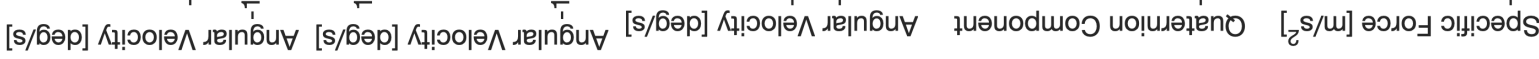

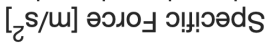

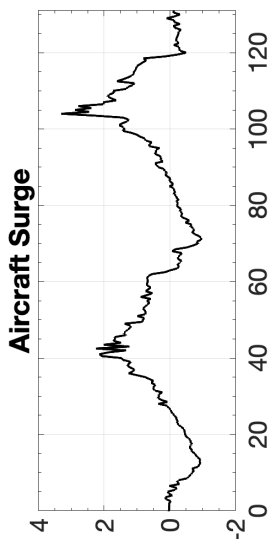

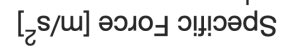

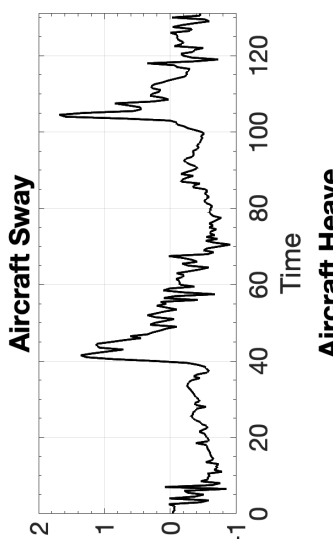

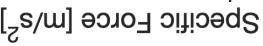

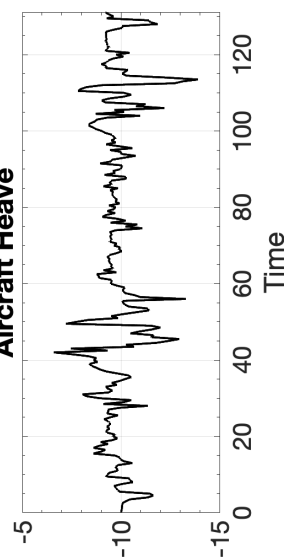

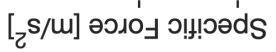
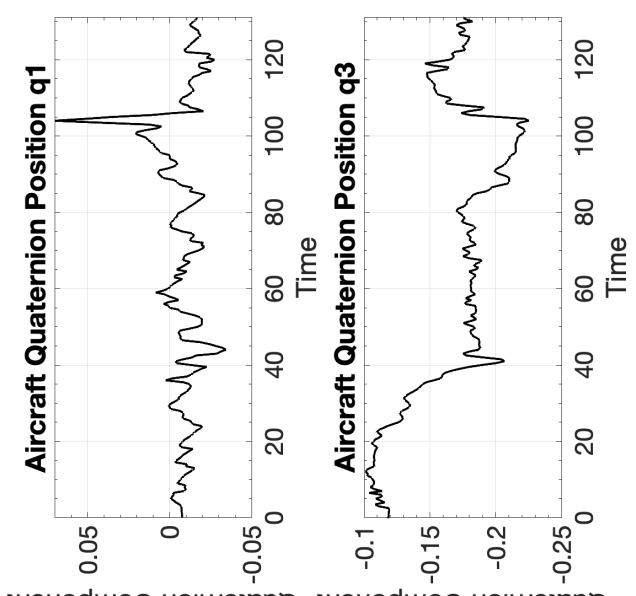

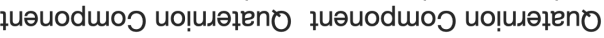

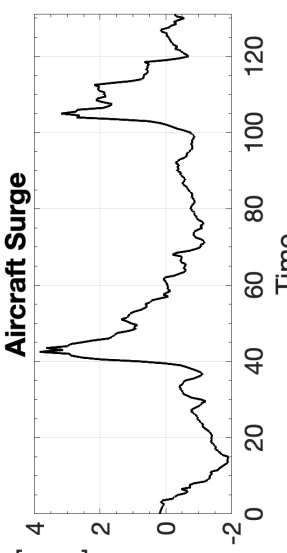

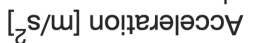

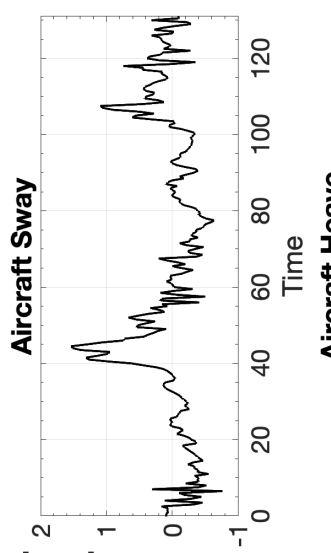

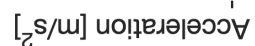

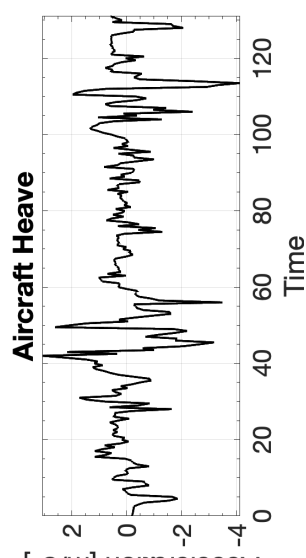

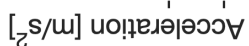
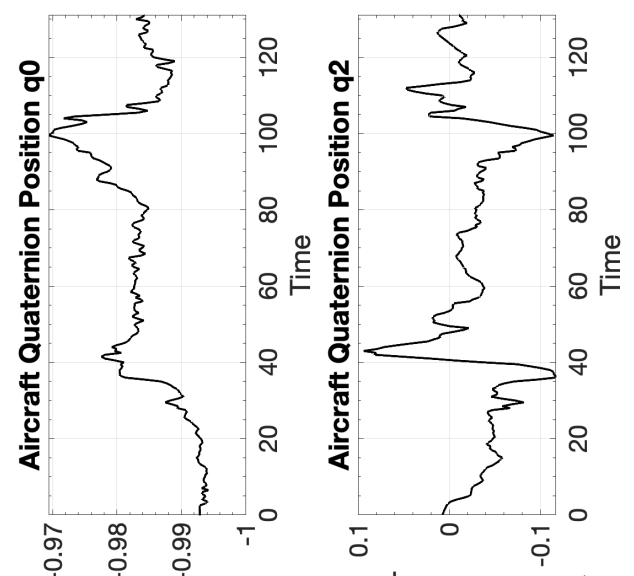

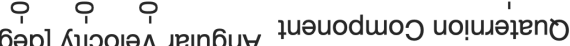




\section{C.8.1 CW1 Cessna 172 Stall Recovery}

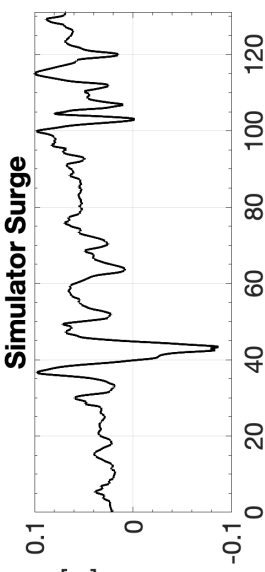

[u] uo!n! sod

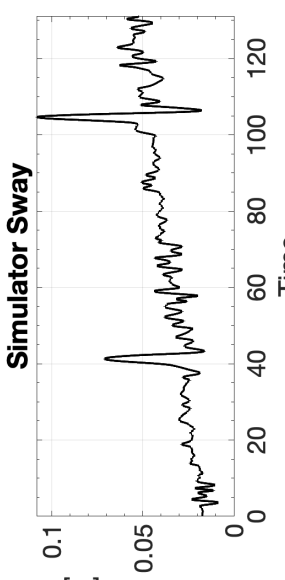

[w] uol!!sod

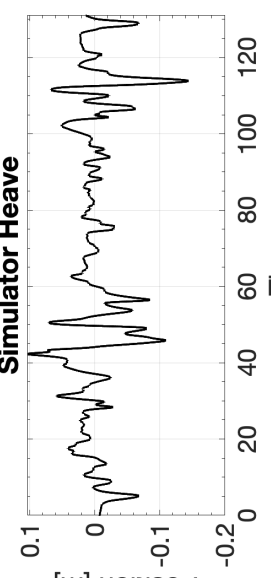

[w] uo!t!sod

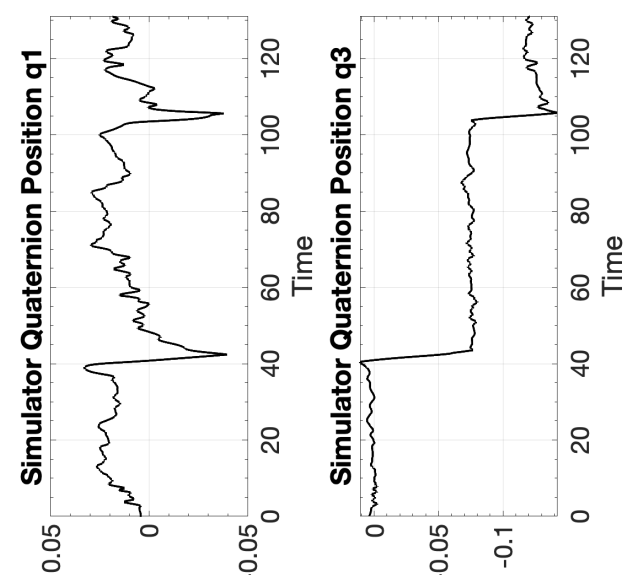

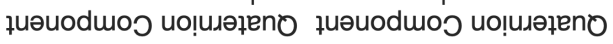

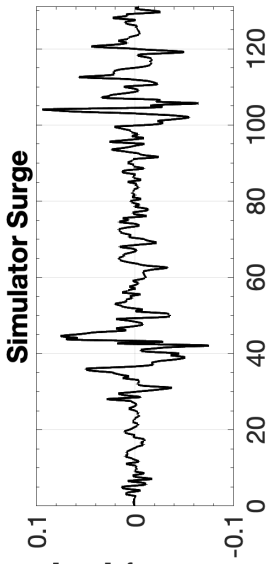

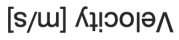

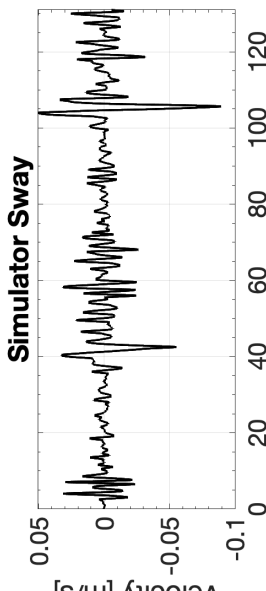

[s/m] Кұ!юор夫

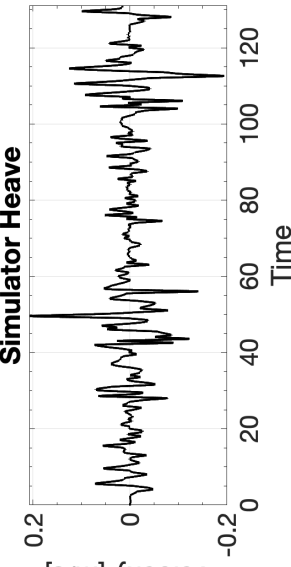

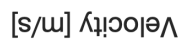

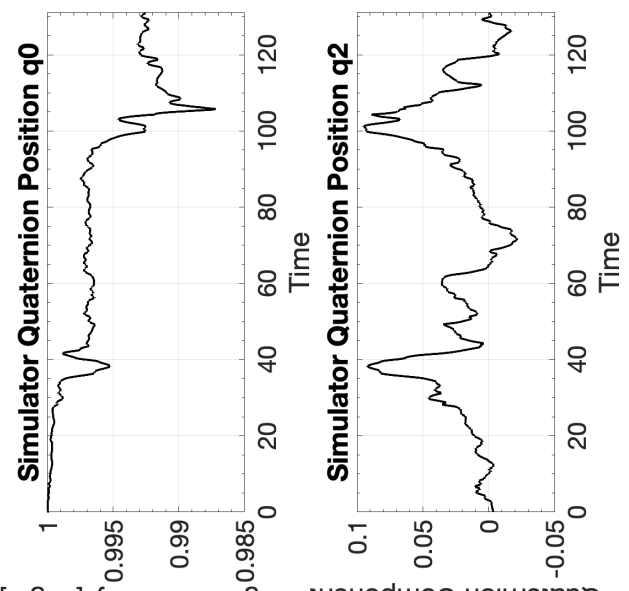

[s/6әр] К! юо

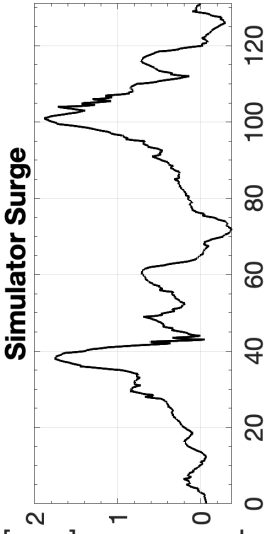

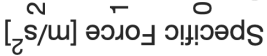

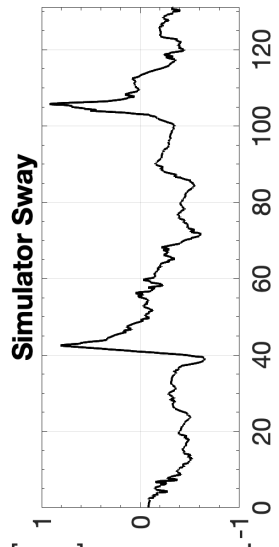

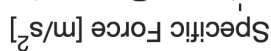
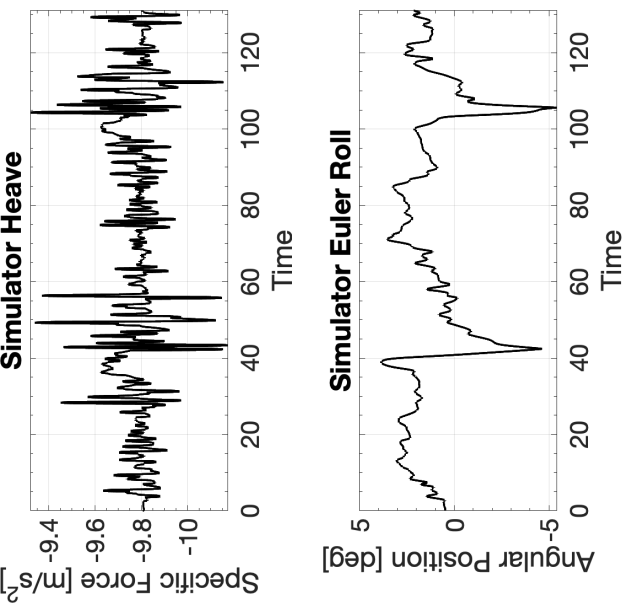

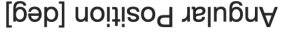

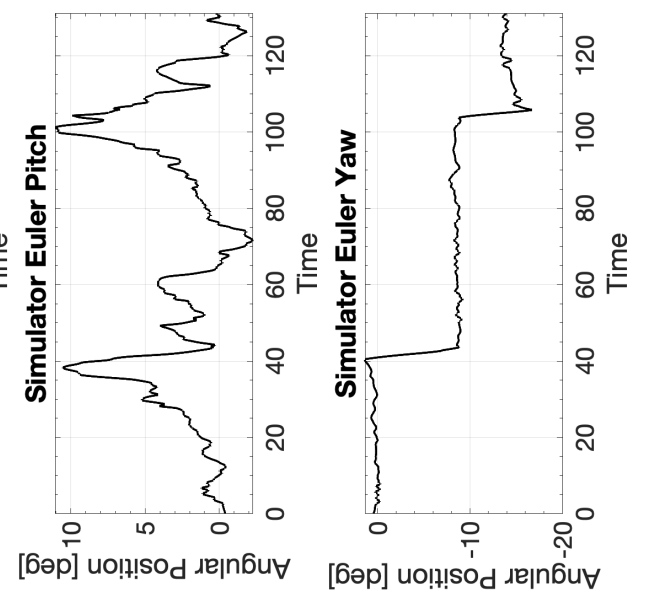

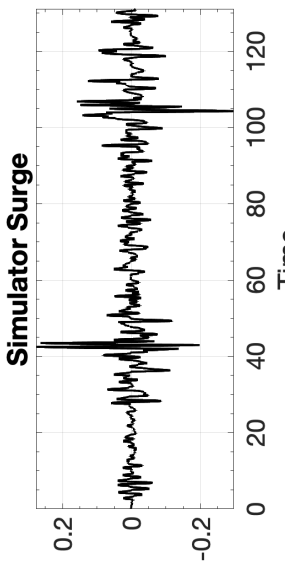

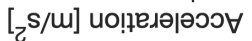

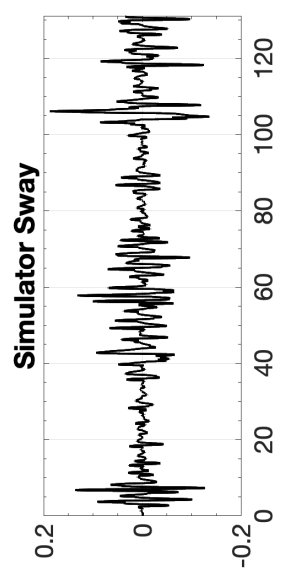

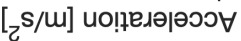

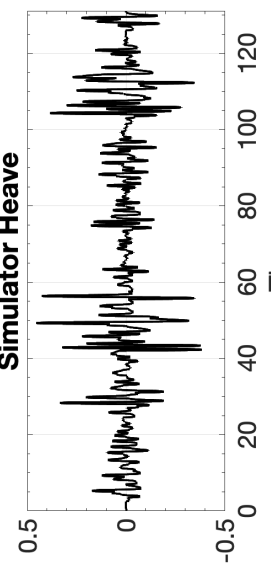

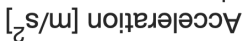

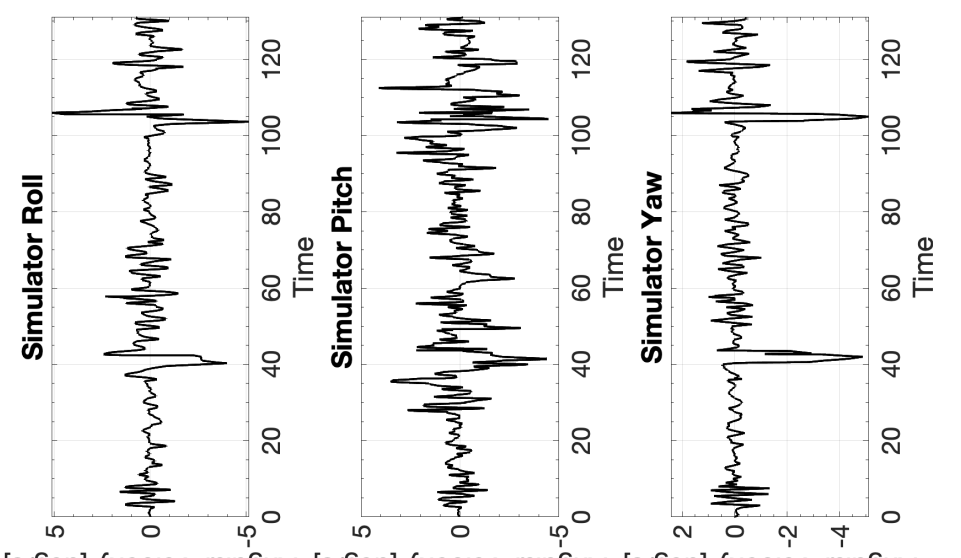

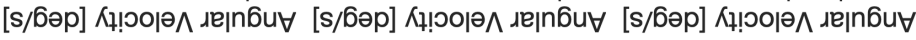




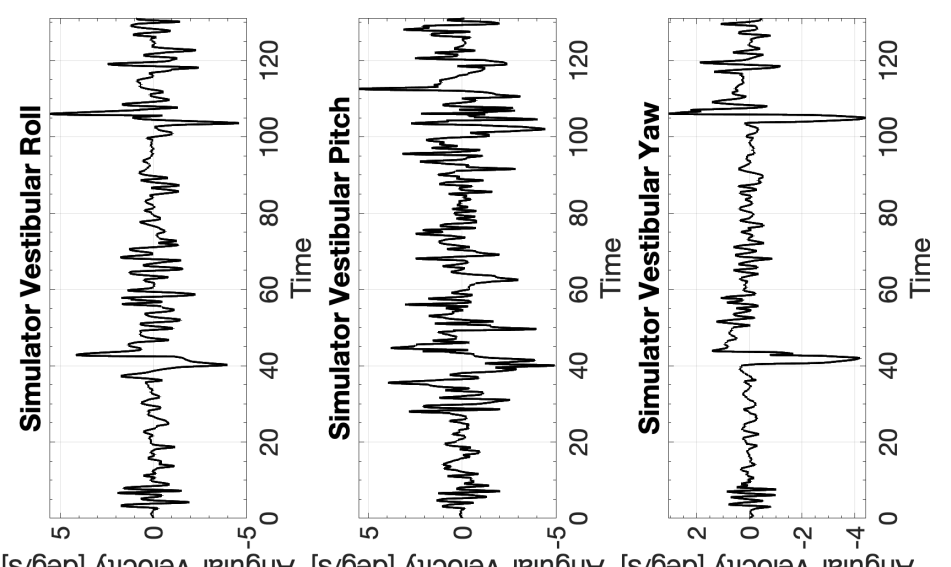

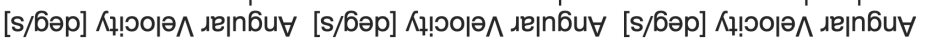

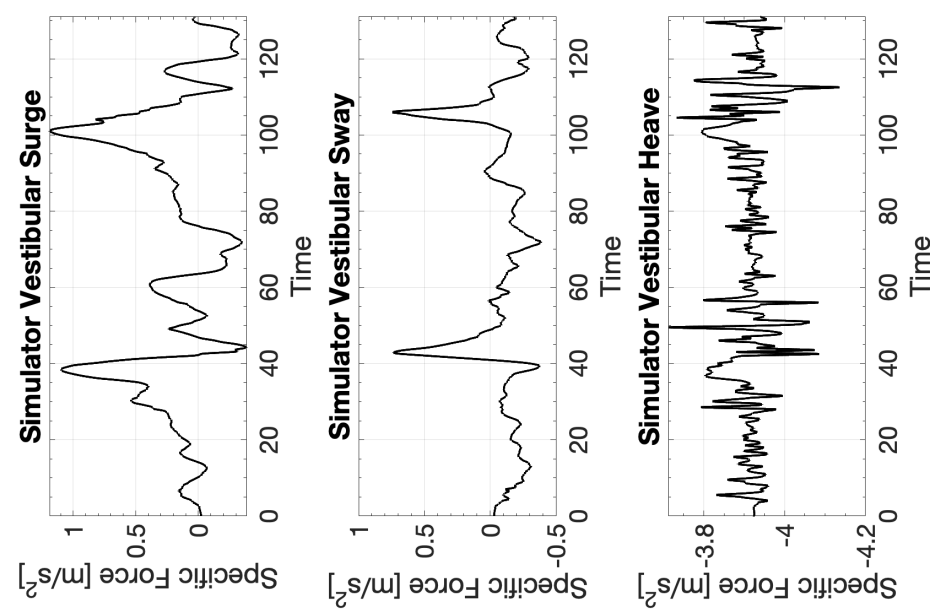




\section{C.8.2 CW2 Cessna 172 Stall Recovery}

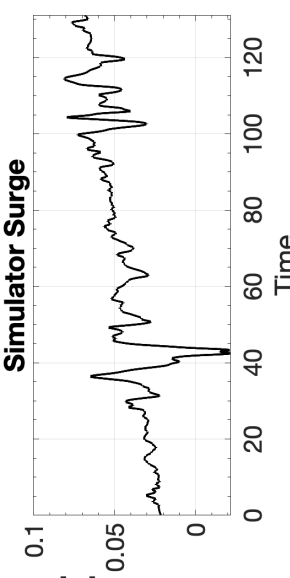

[u] uo!n! $\mathrm{sod}^{\mathrm{d}}$

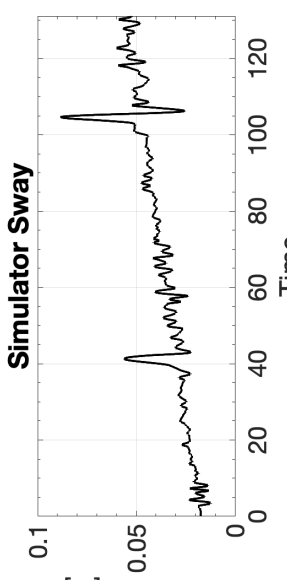

[w] uol!!sod

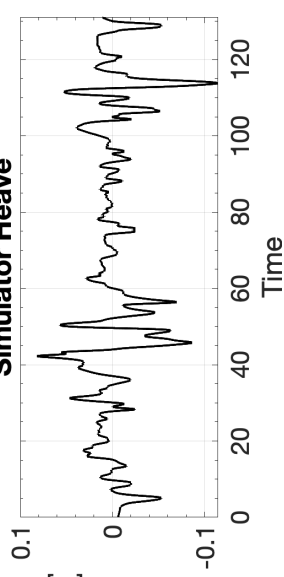

[w] uo!n!!sod

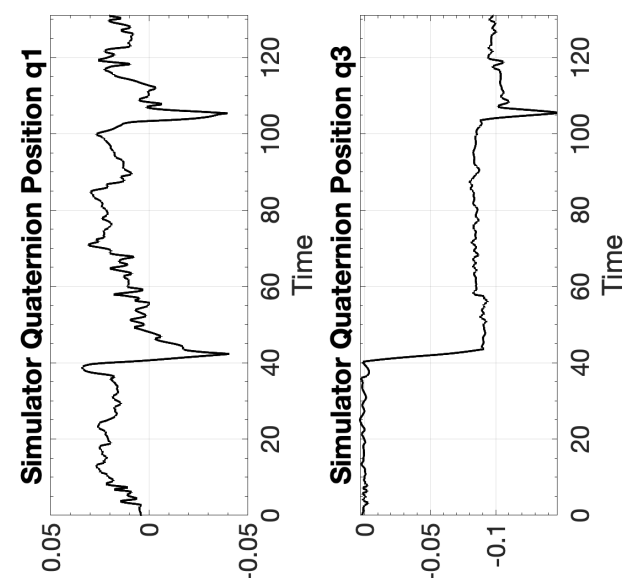

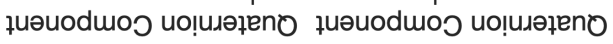

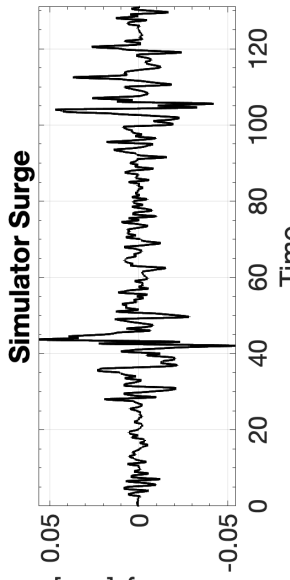

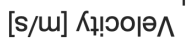

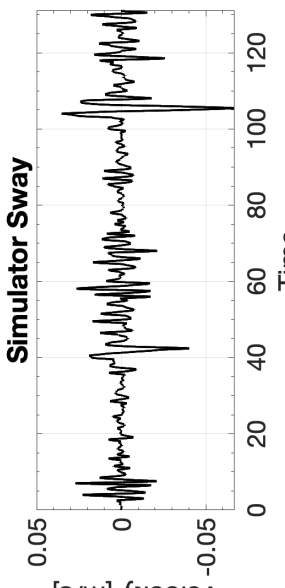

[s/m] Кұ!юою^

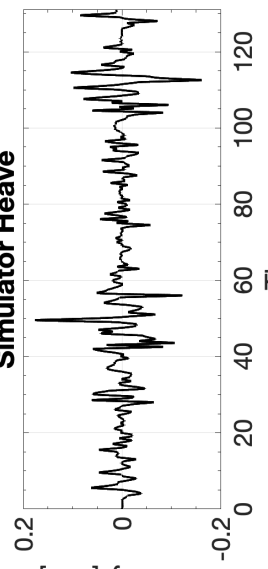

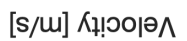

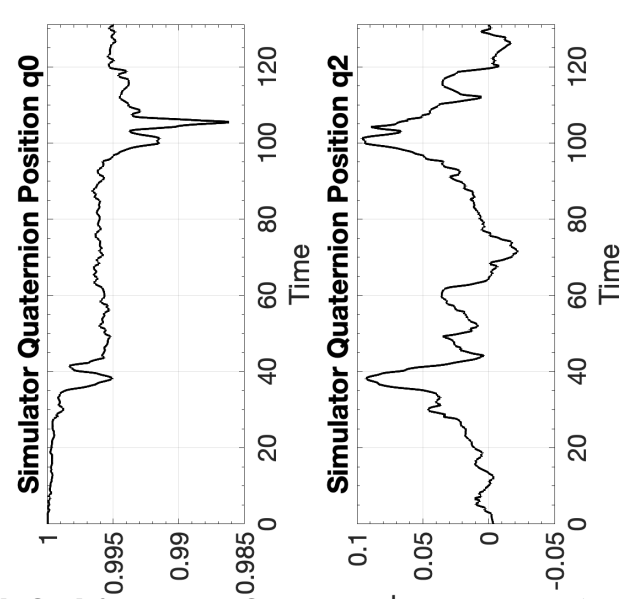

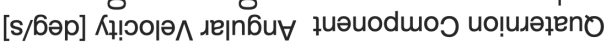

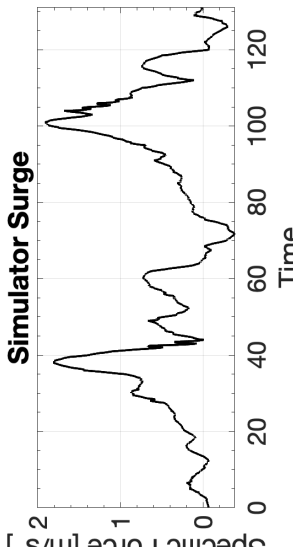

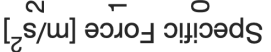

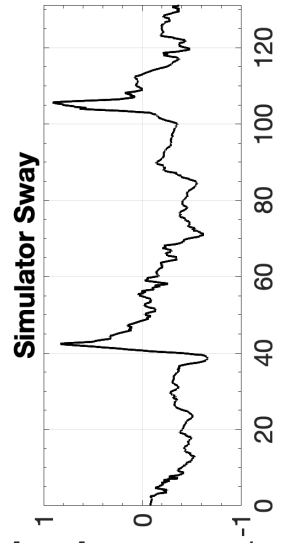

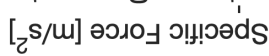
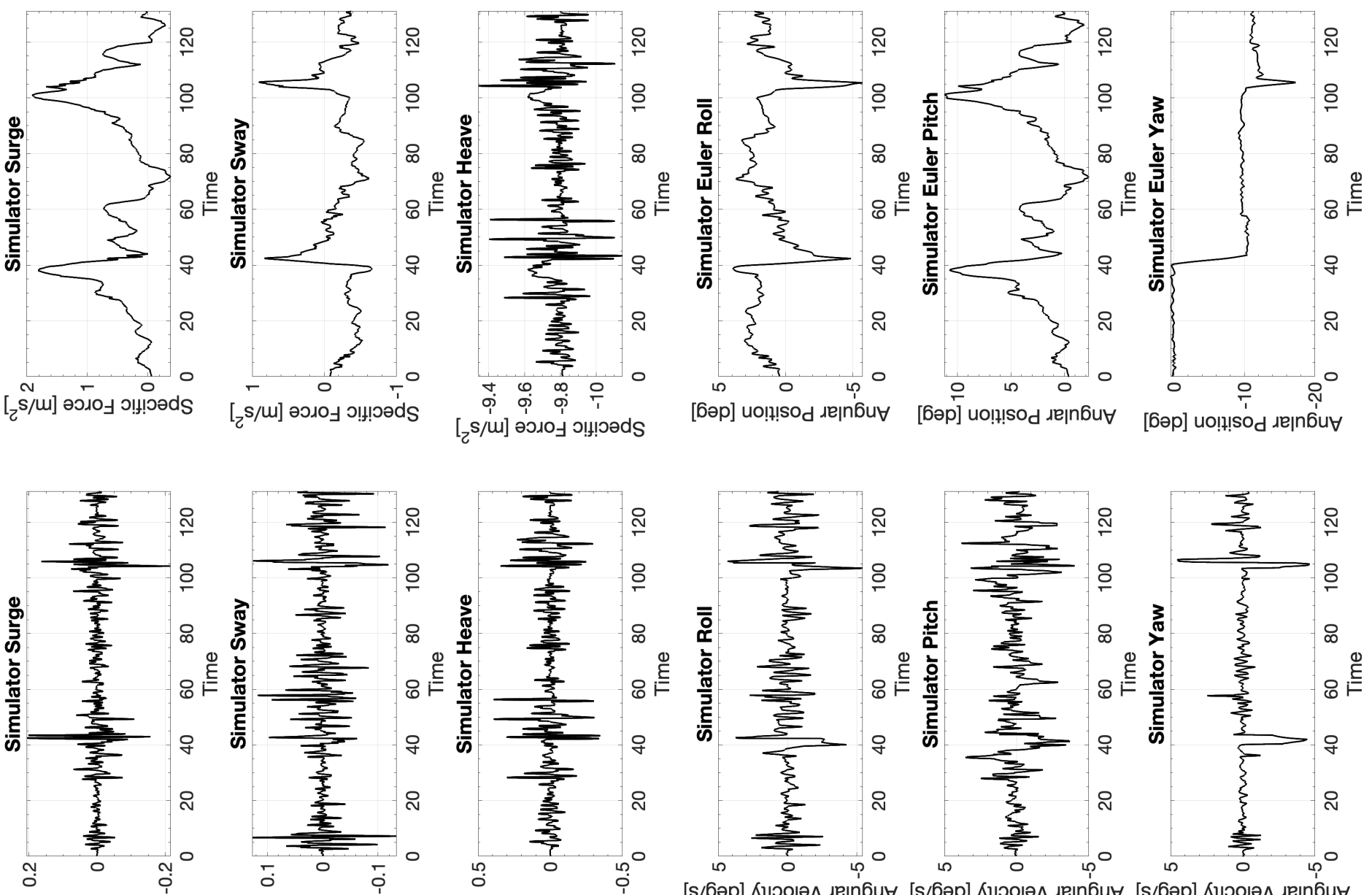

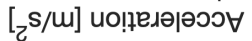

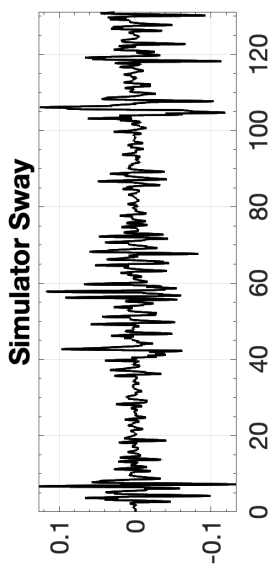

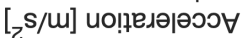

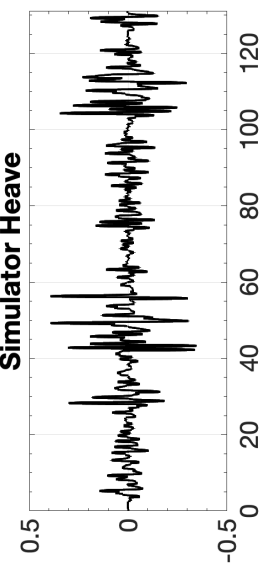

[zs/m] ио!ฺедәәәэว

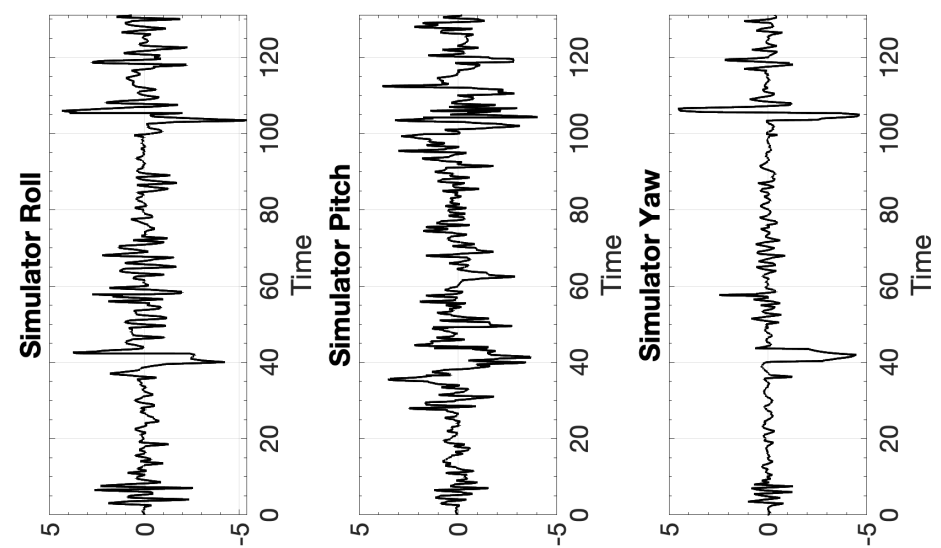

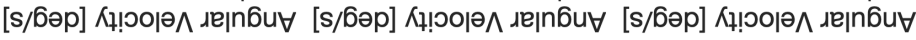




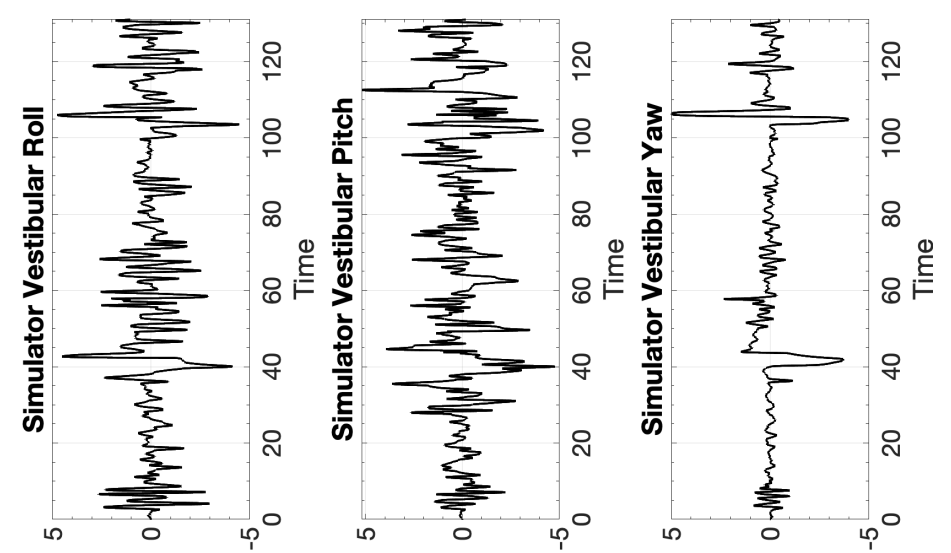

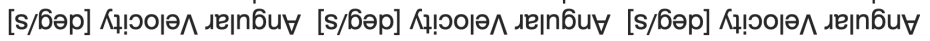

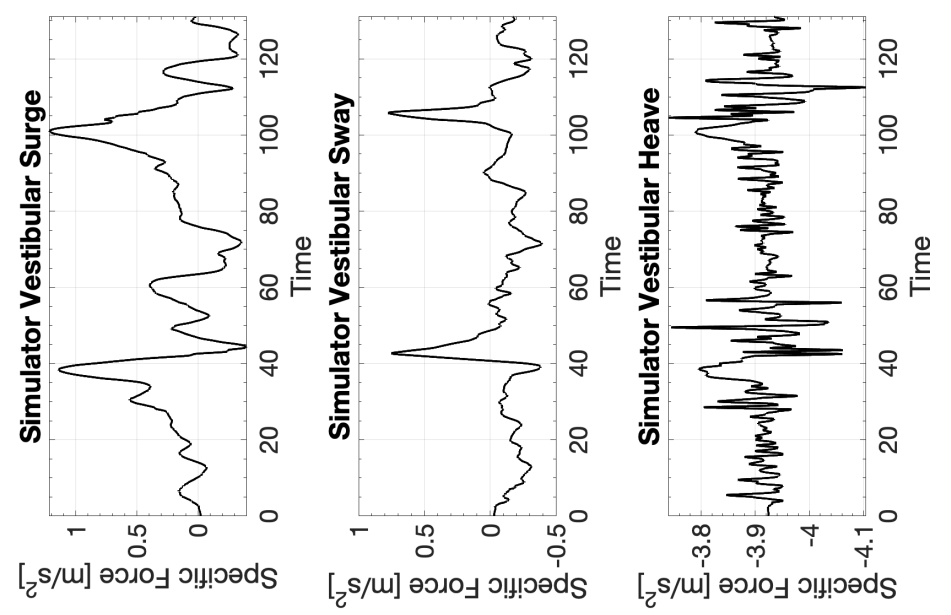




\section{C.8.3 CW3 Cessna 172 Stall Recovery}

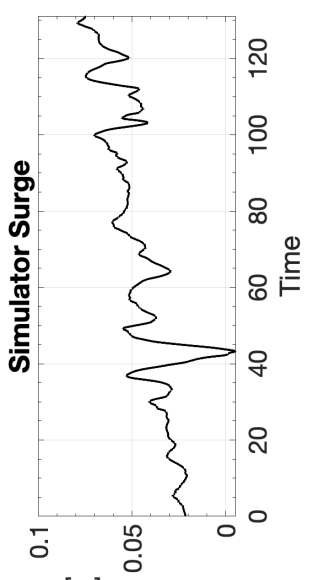

[w] uo!n!sod

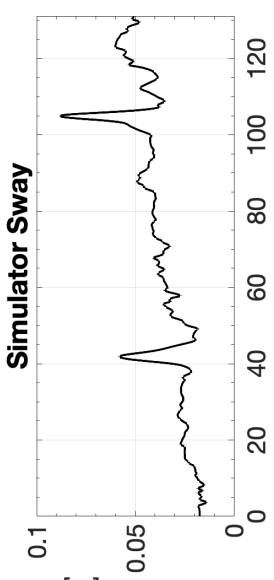

[u] uo!n!!sod

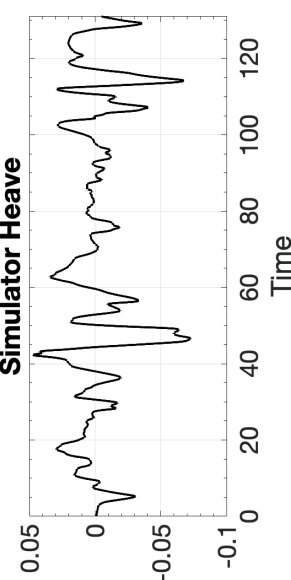

[u] uolt!!sod
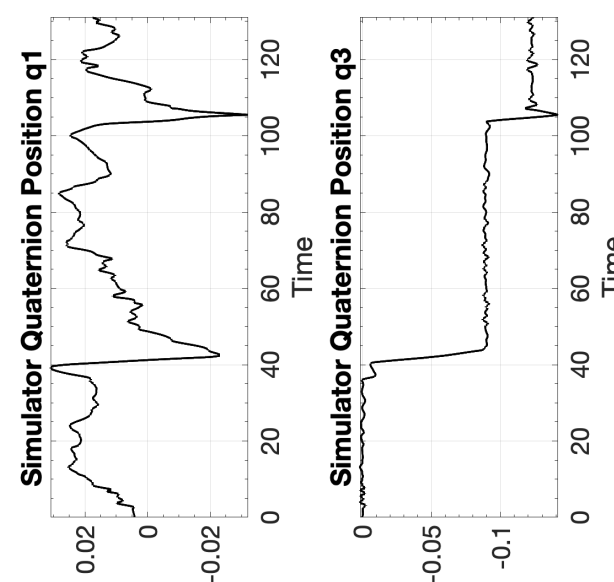

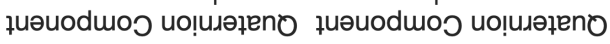

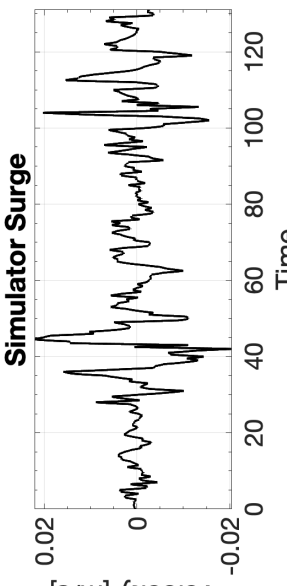

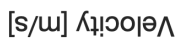

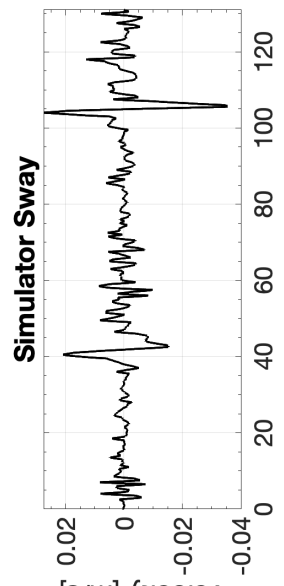

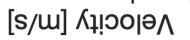

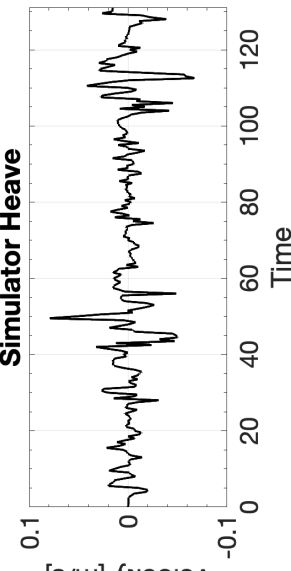

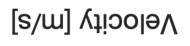

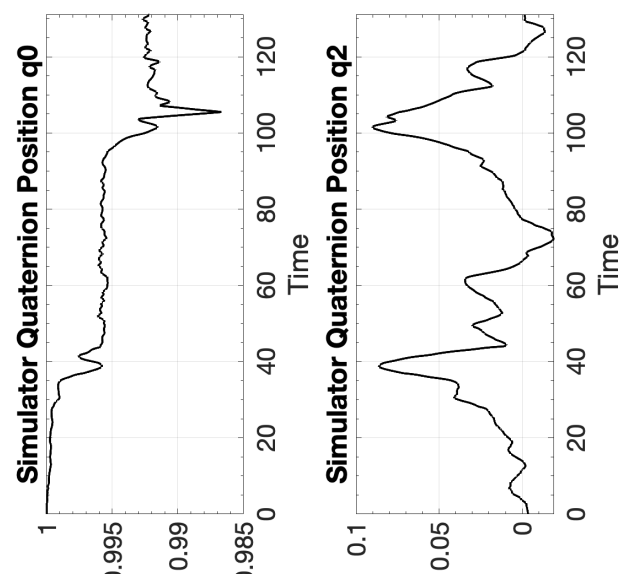

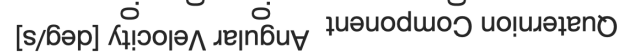

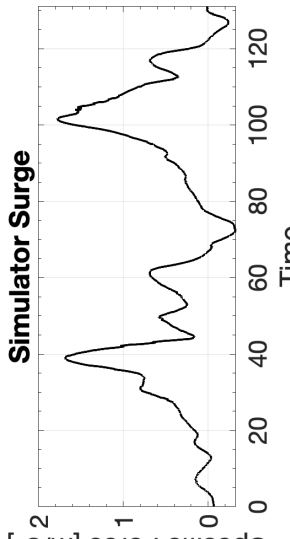

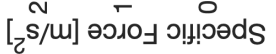
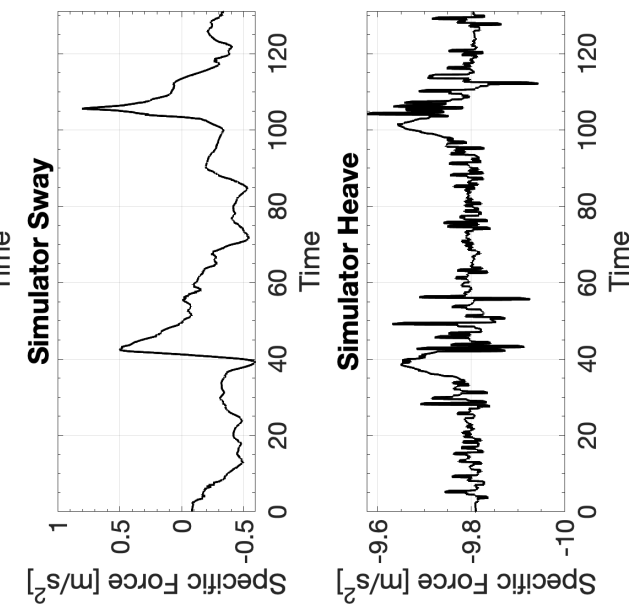

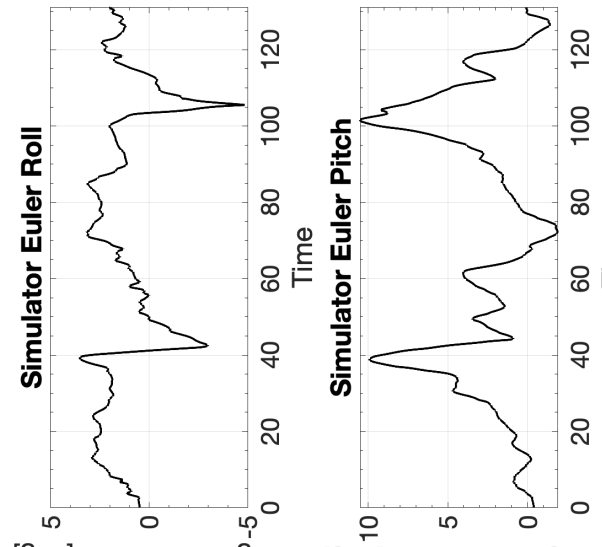

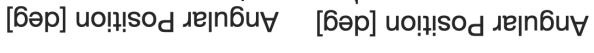

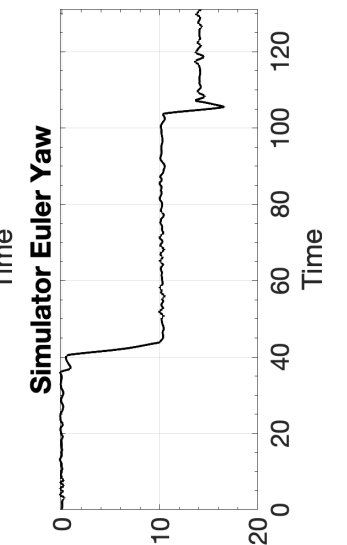

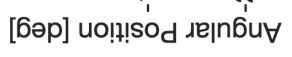

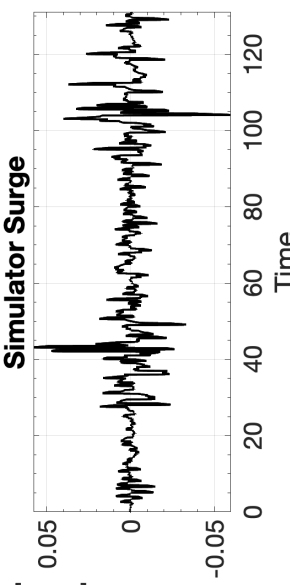

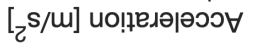

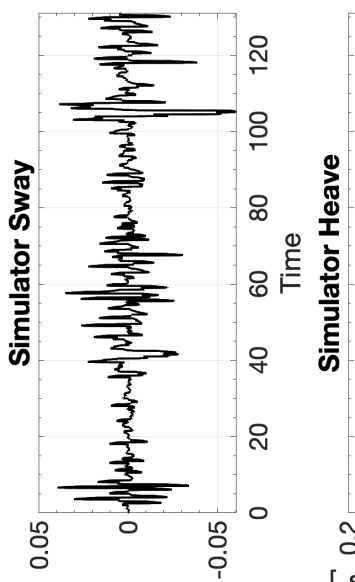

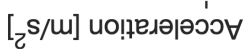

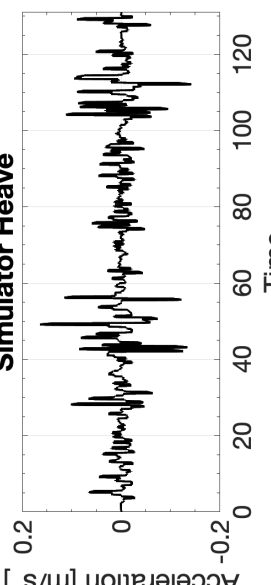

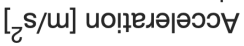

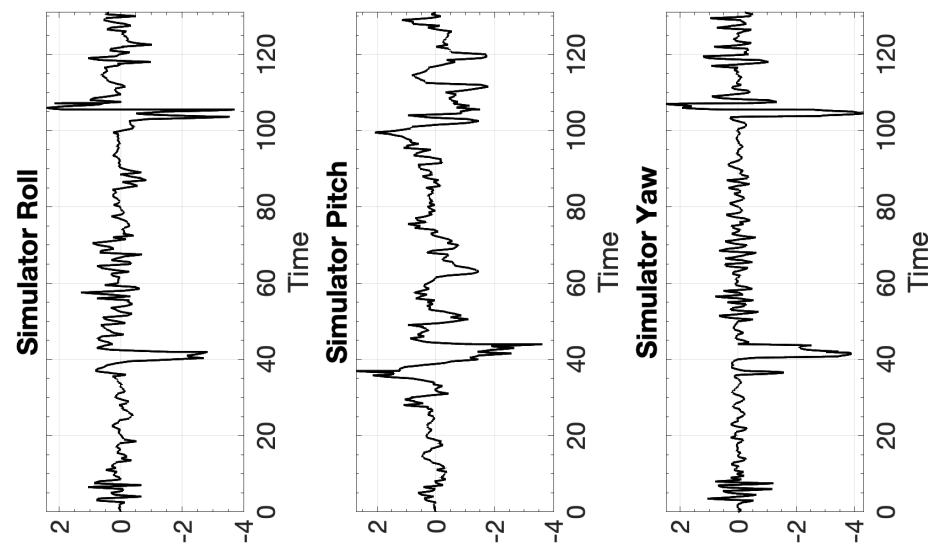

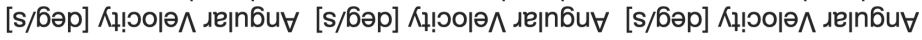




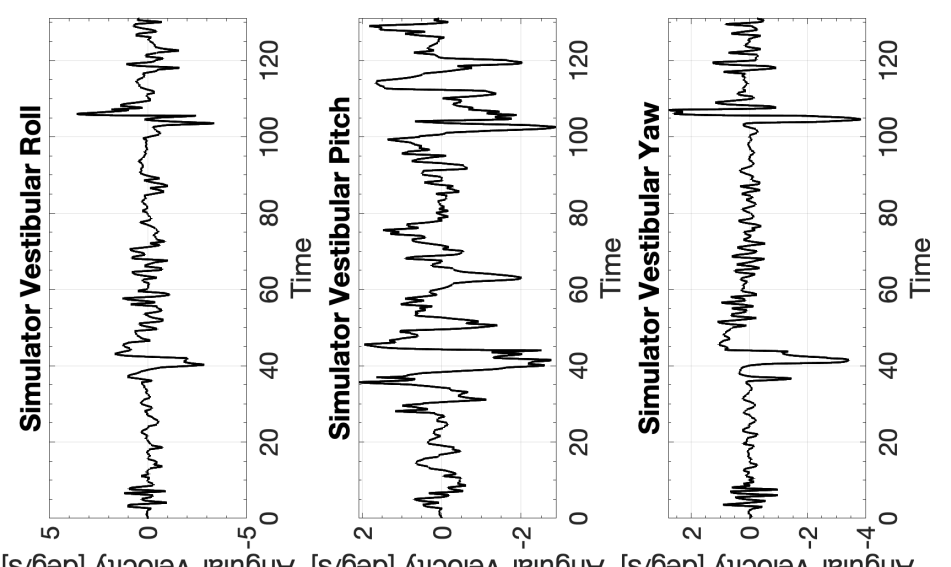

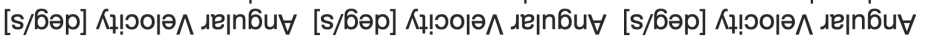

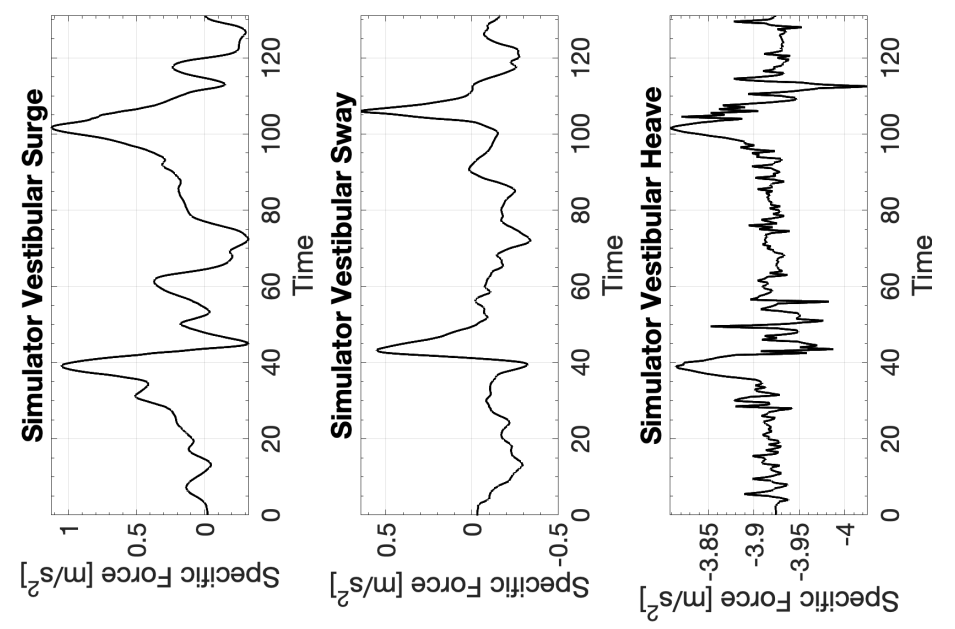




\section{C.9 Boeing 737 Coordinated Turn}

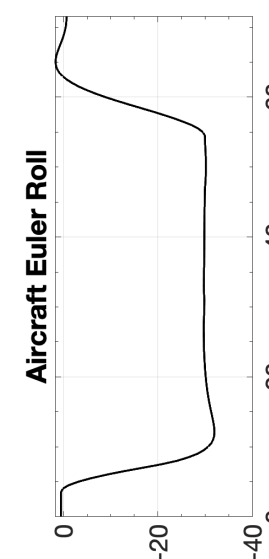

[6əp] uo!!!sod גe|n6u

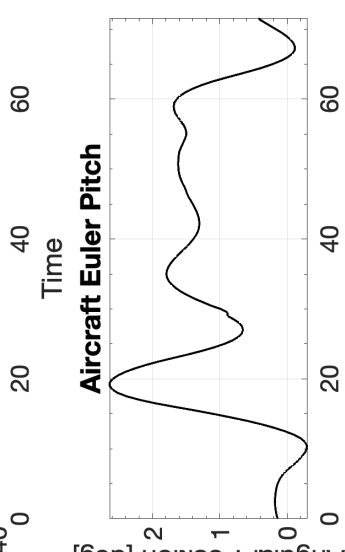

[бәр] uo!?!!sod

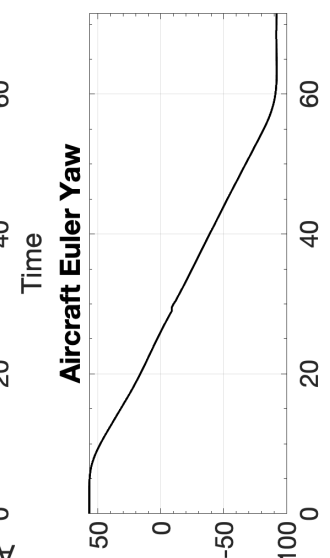

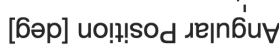

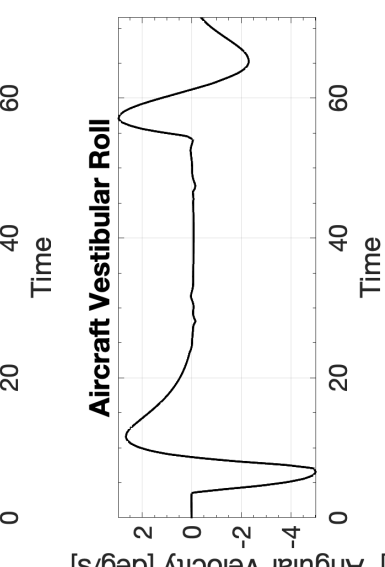

[s/6әр]

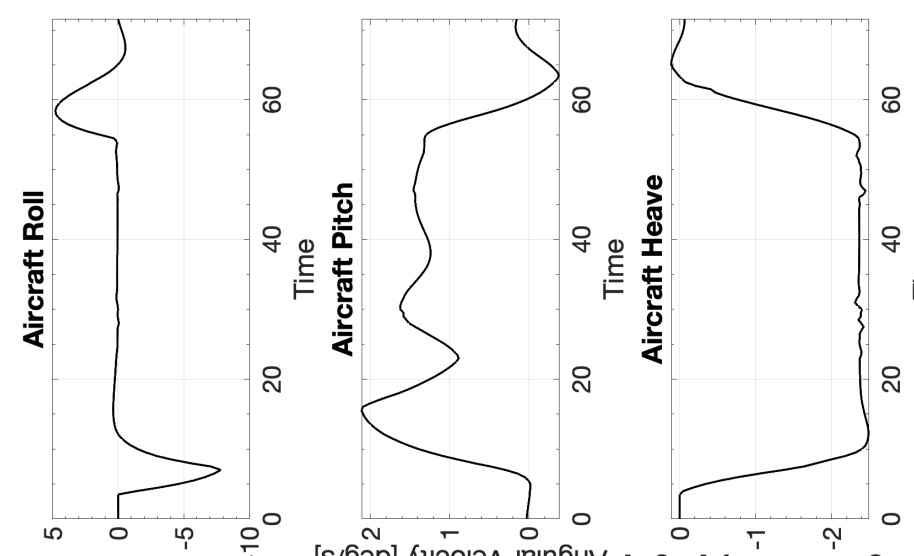

[s/бәр] Кұ! оорә

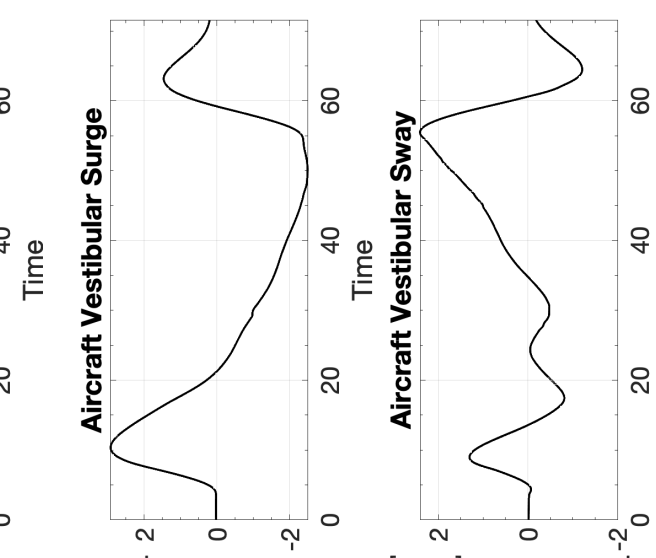

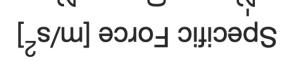

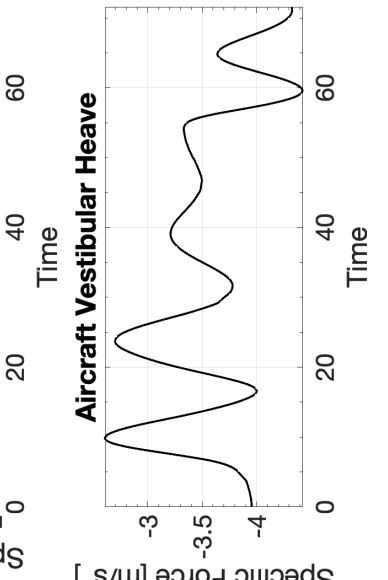

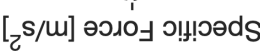

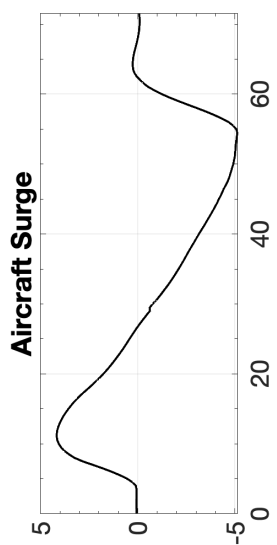

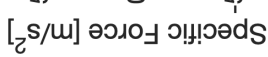

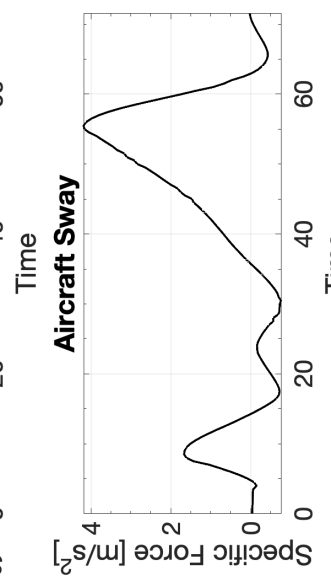

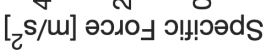

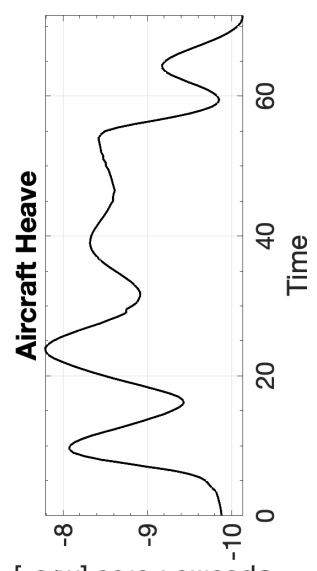

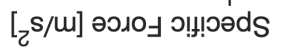

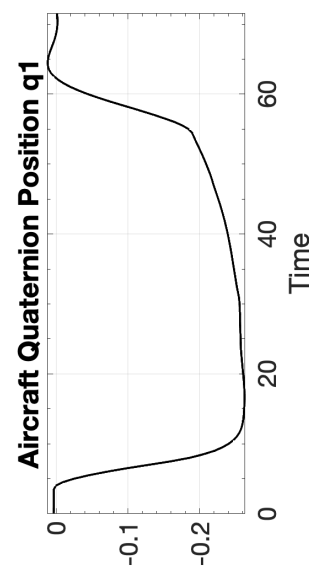

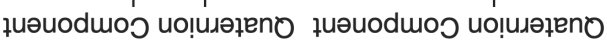

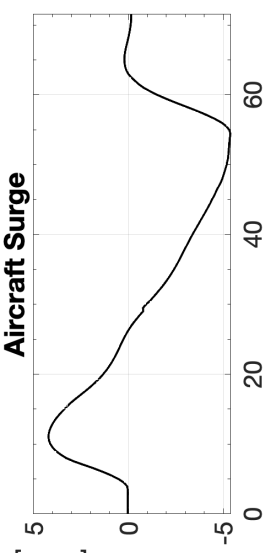

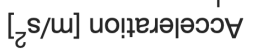

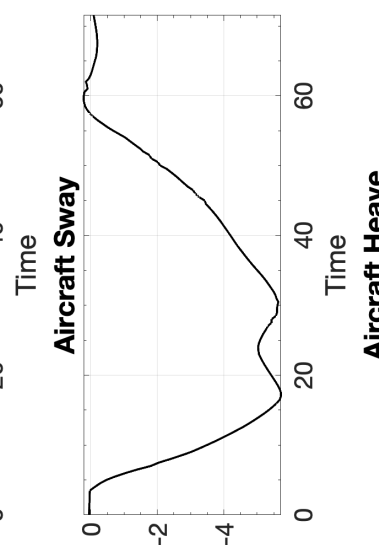

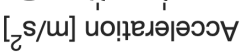

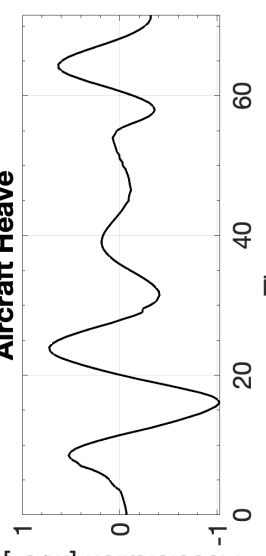

$[\mathrm{z} / \mathrm{m}]$ uо!ןеләрәоэ $\forall$

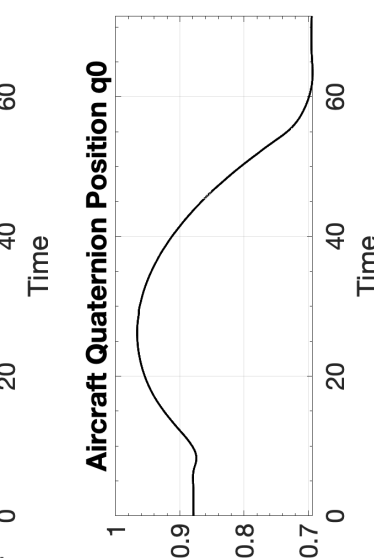

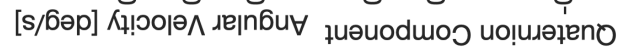
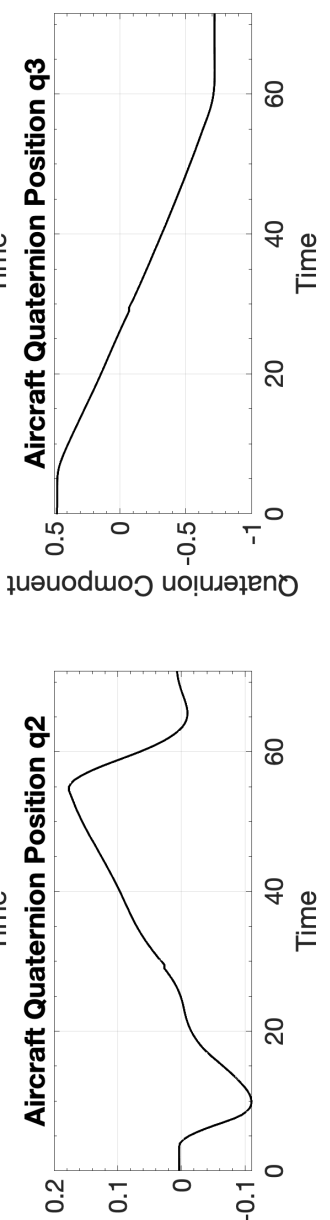


\section{C.9.1 CW1 Boeing 737 Coordinated Turn}

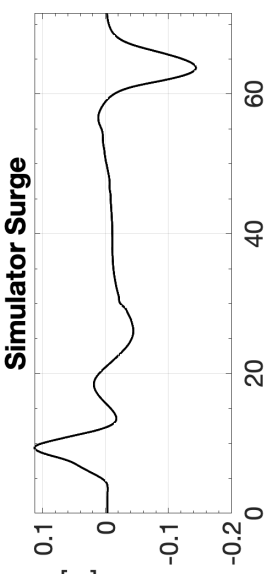

[u] uo!!!!sod

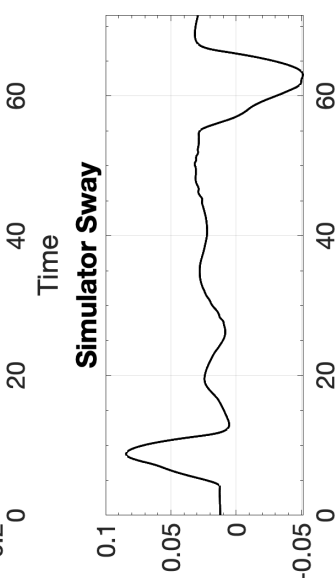

[u] uo!n! sod

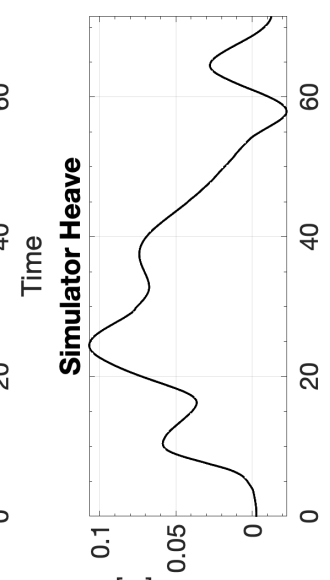

[w] uo!n!sod

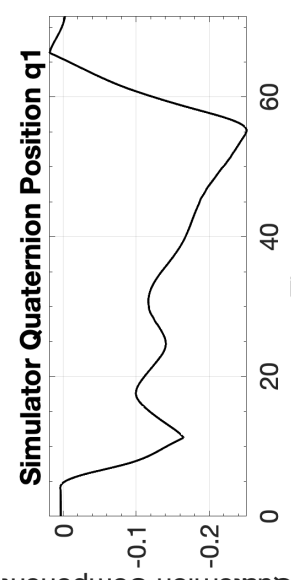

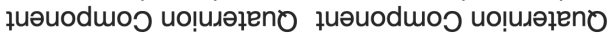
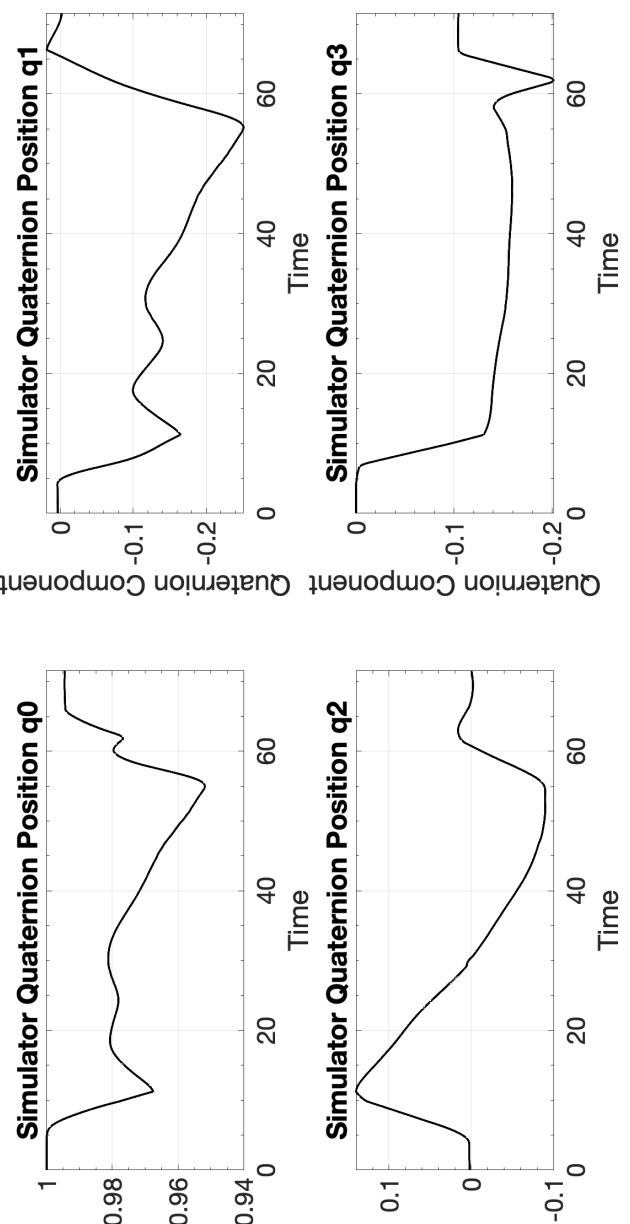

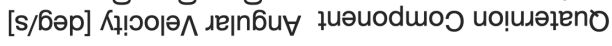

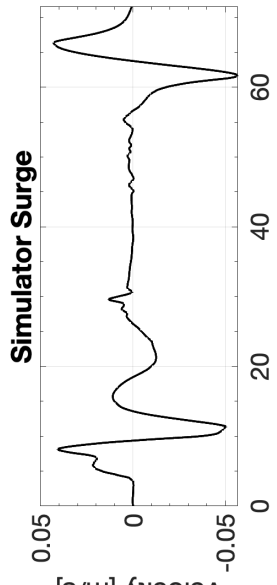

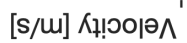

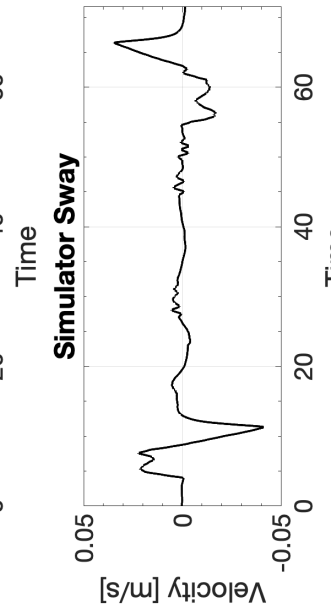

[s/m] Кұ!๐о|ә^

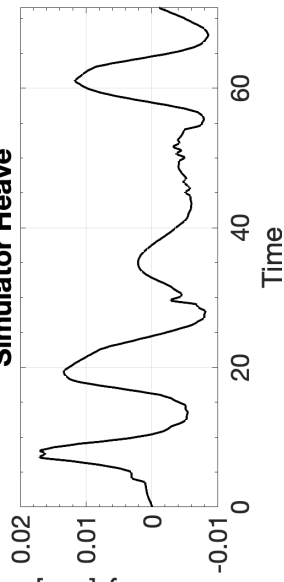

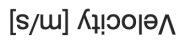

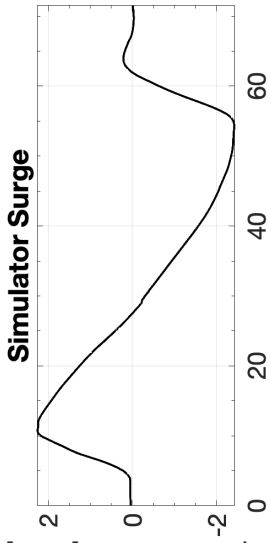

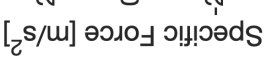

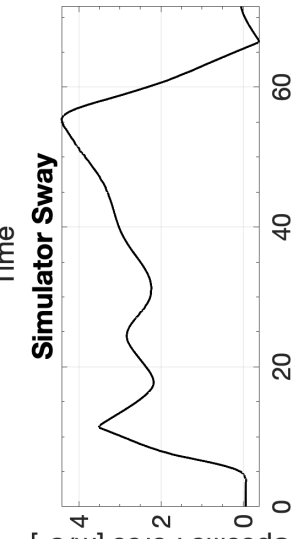

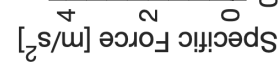
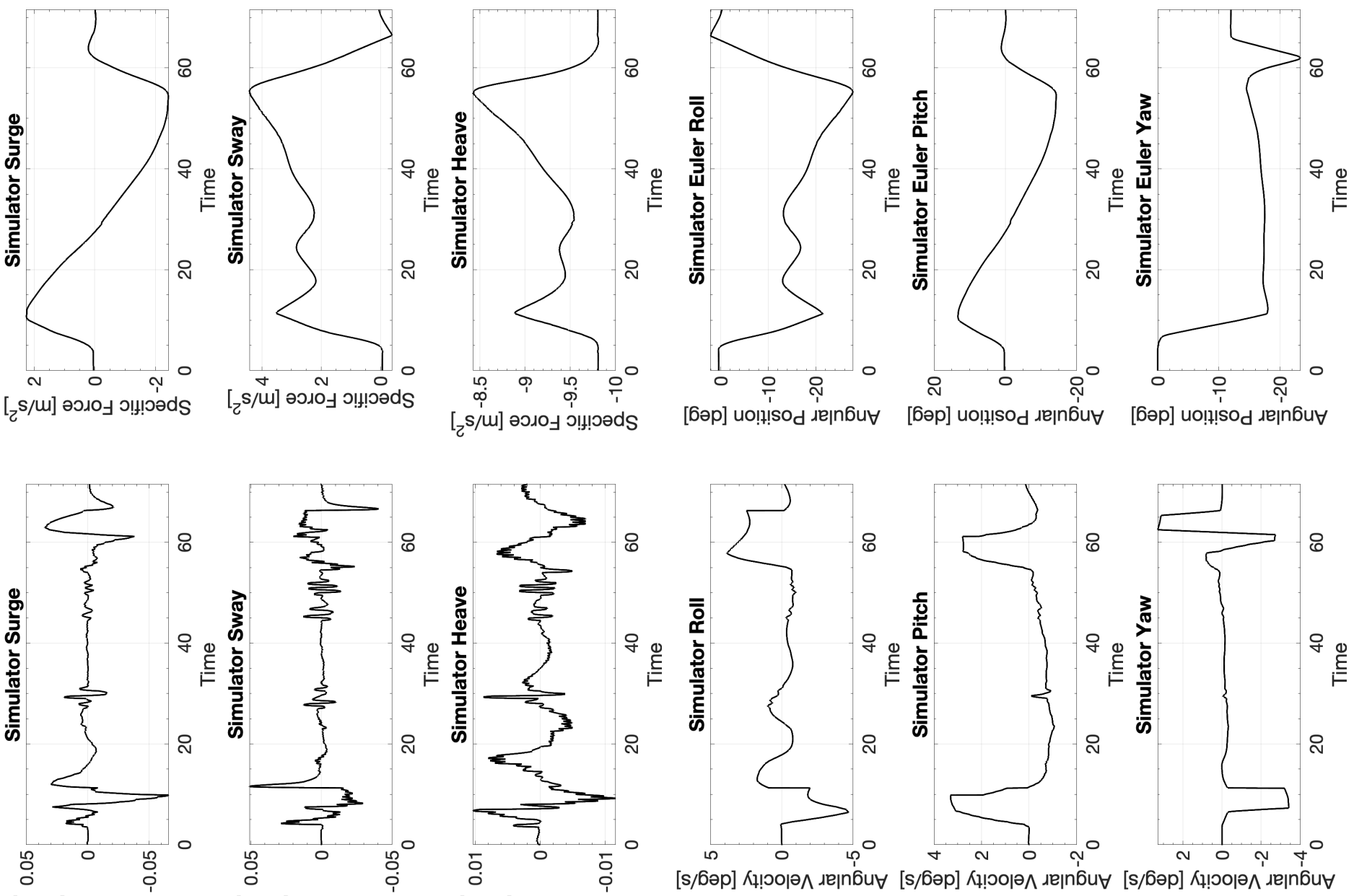

$\left[{ }_{2} \mathrm{~s} / \mathrm{m}\right]$ uо!ฺеләрәэ७

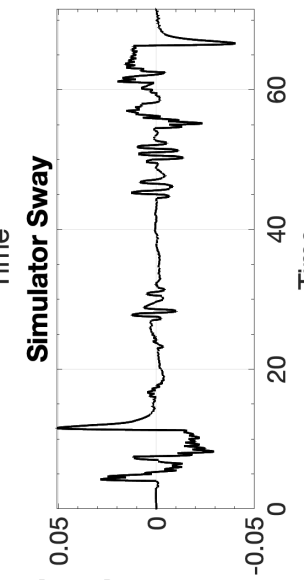

$\left[{ }_{2} s / m\right]$ uо!ฺеләәәэо

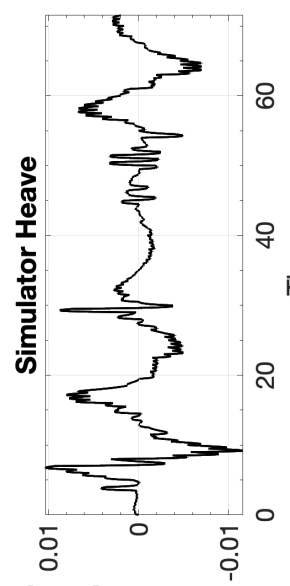

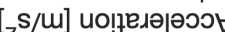

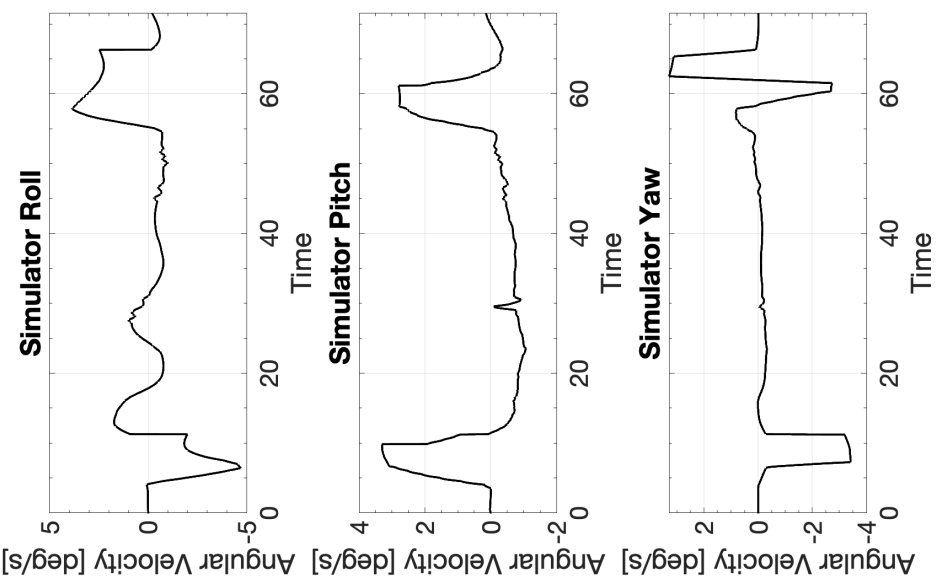

263 


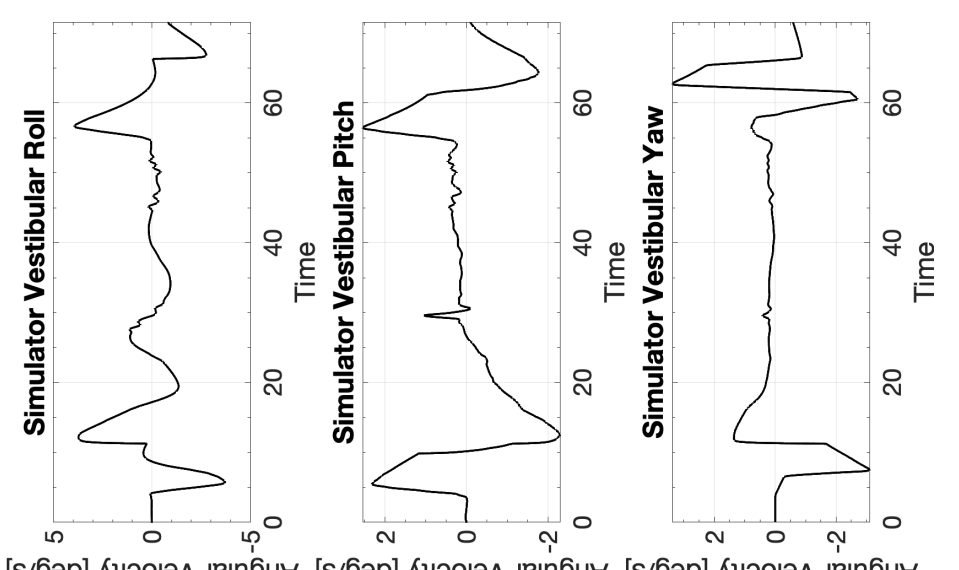

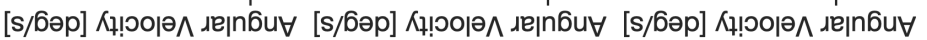

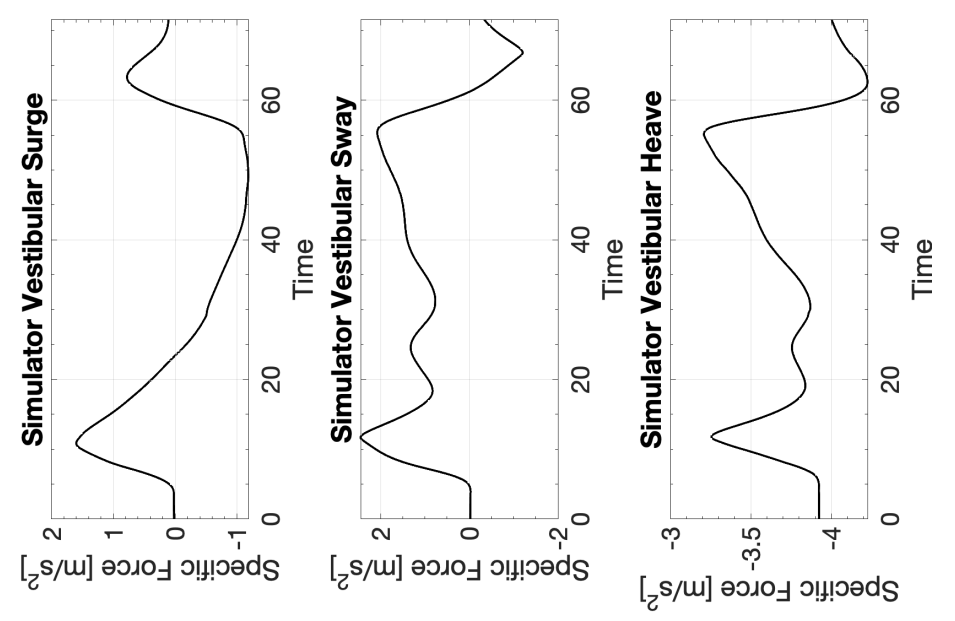




\section{C.9.2 CW2 Boeing 737 Coordinated Turn}

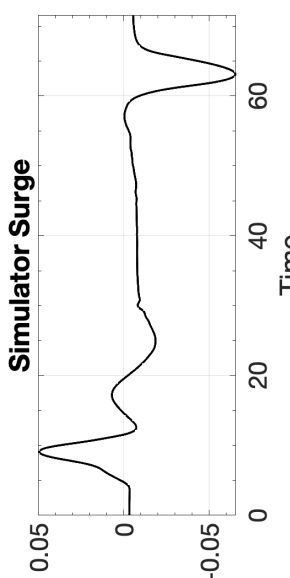

[w] uo!n!sod

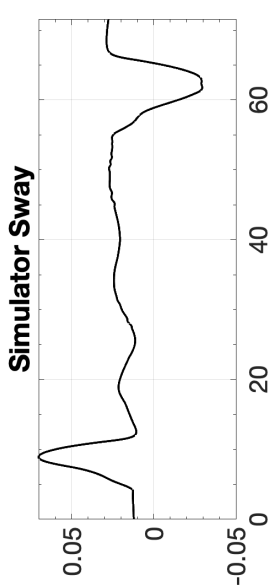

[w] uo!n! sod

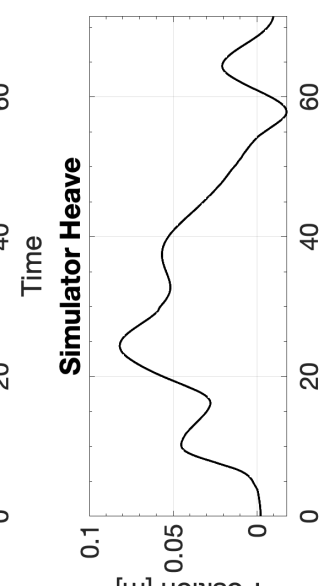

[w] uo!!!sod
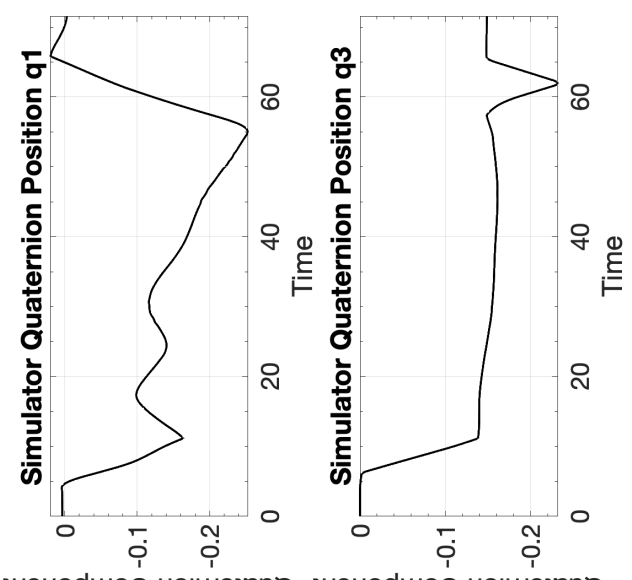

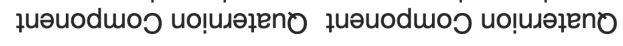

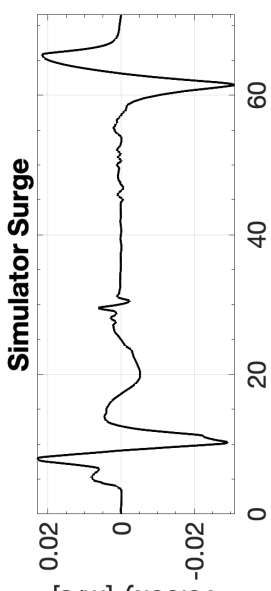

[s/m] Кұ! оорә

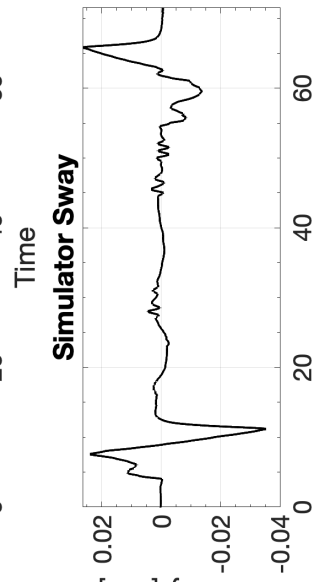

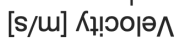

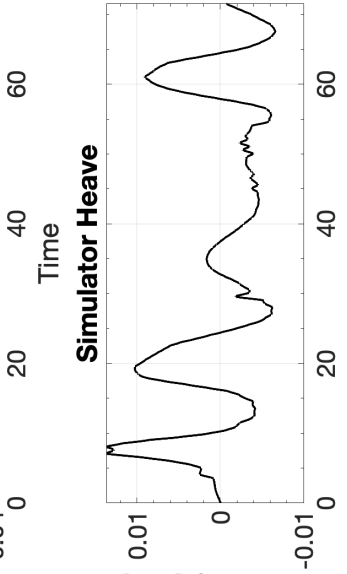

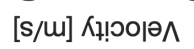

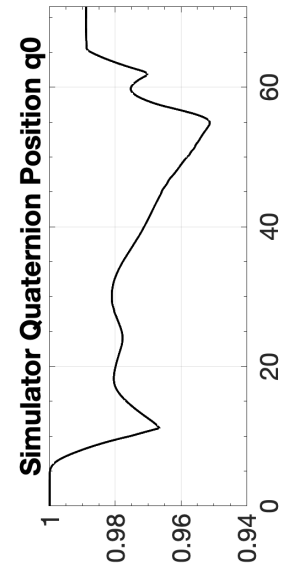

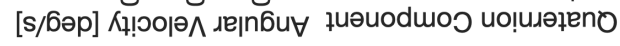

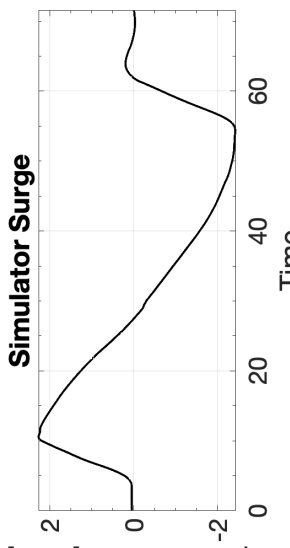

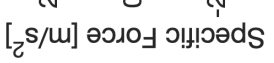

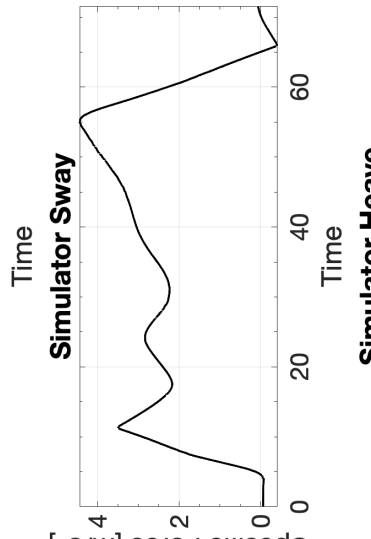

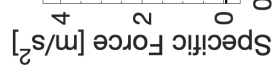
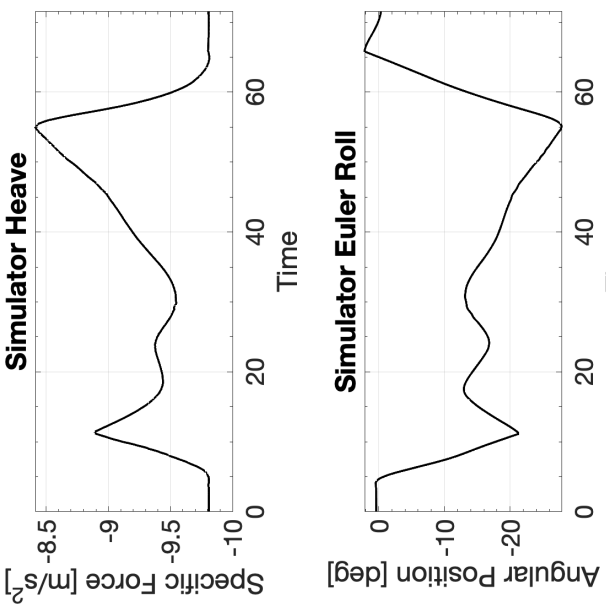
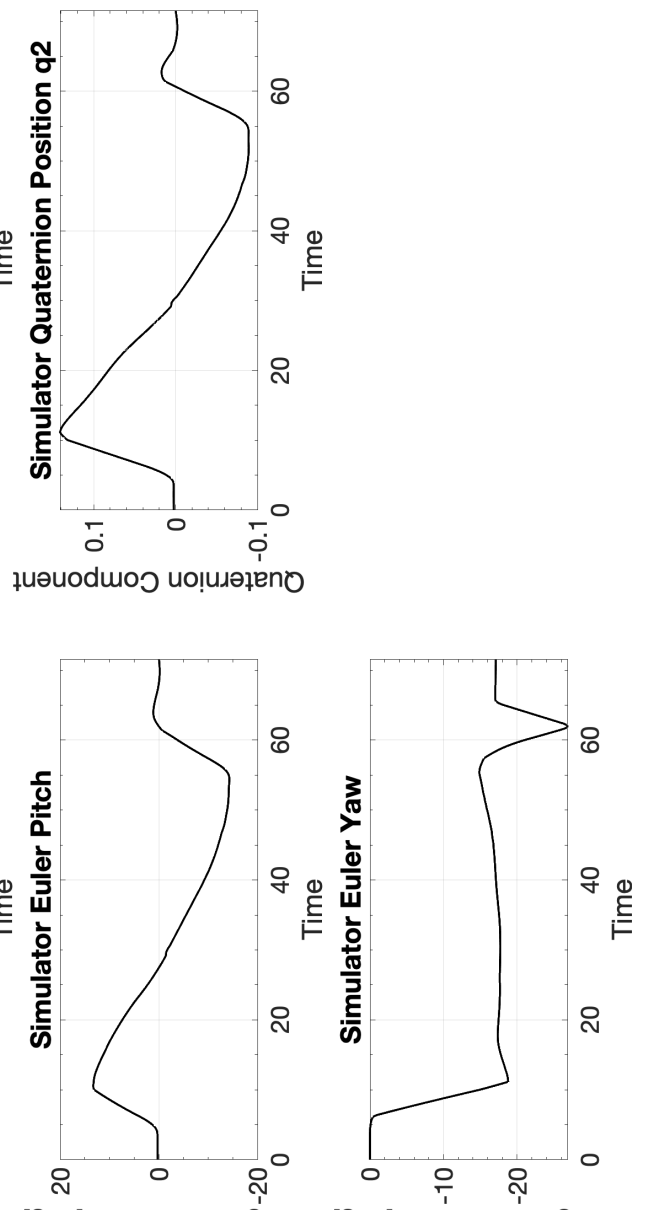

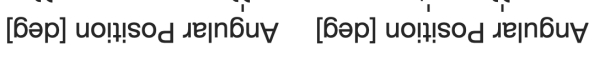

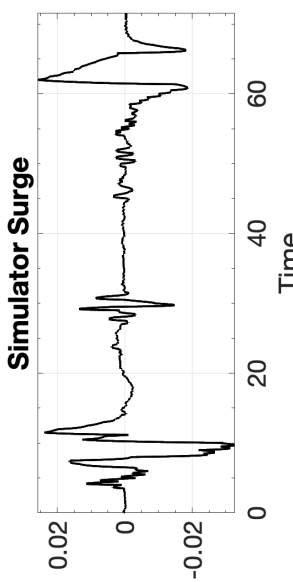

$[\mathrm{c} / \mathrm{M}]$ uо!ฺелә|әээ

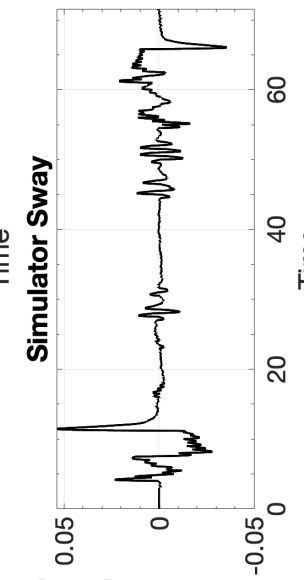

[

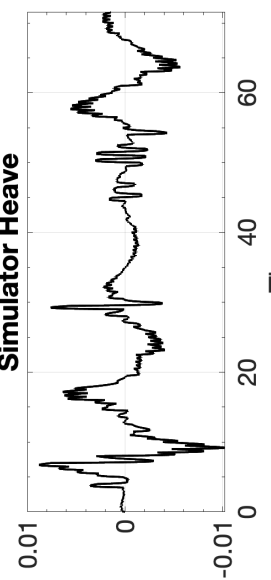

[s/m] иоำ

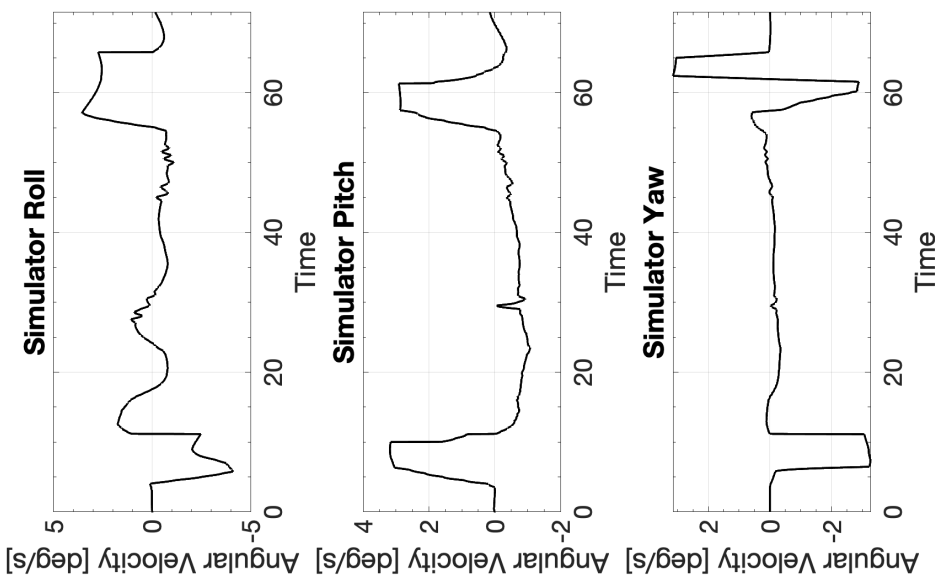

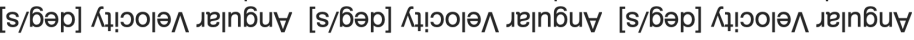




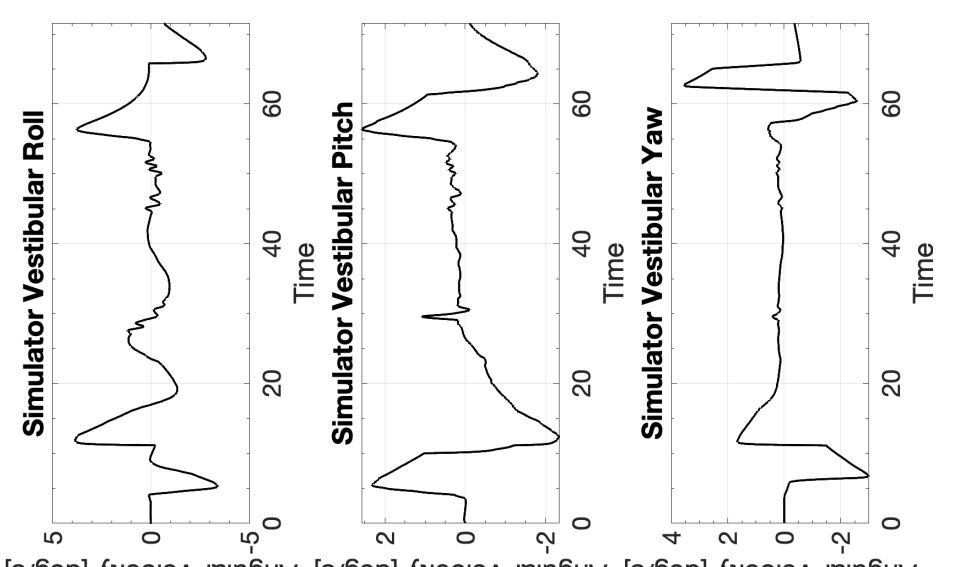

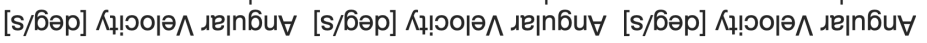

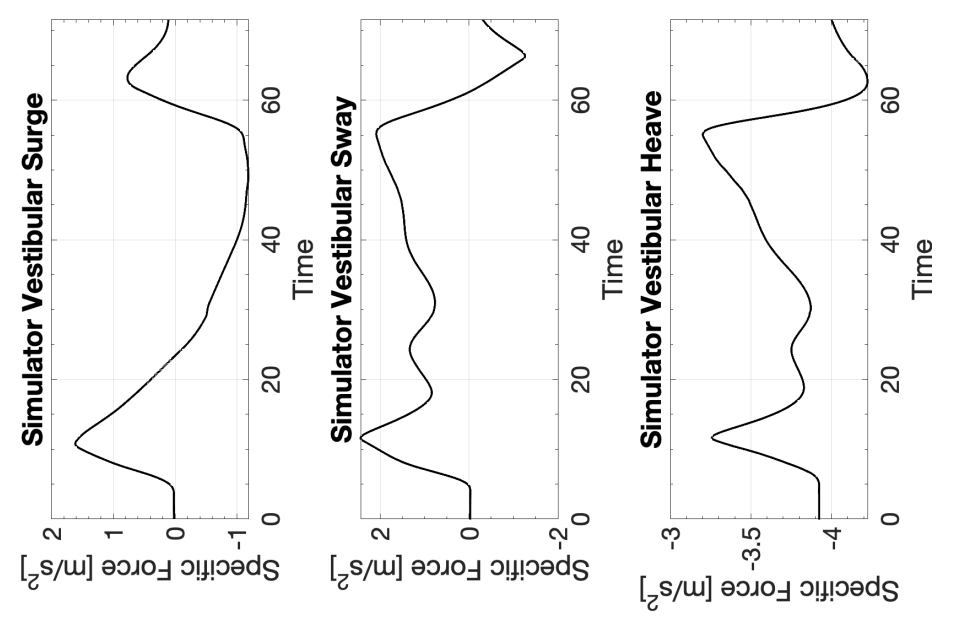




\section{C.9.3 CW3 Boeing 737 Coordinated Turn}

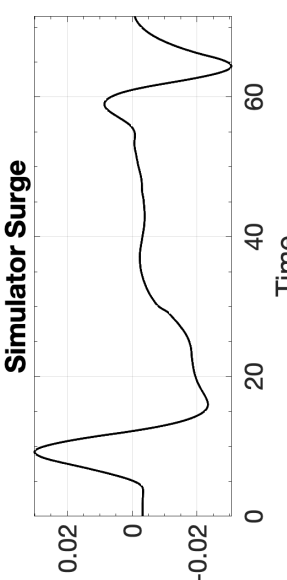

[w] uo!n!sod

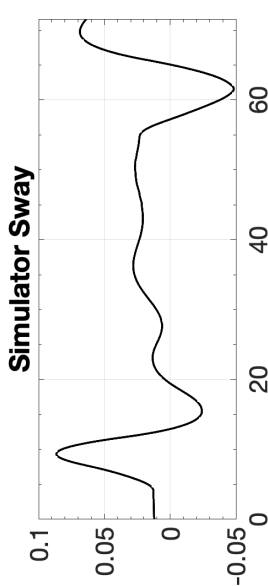

[w] uo!n! sod

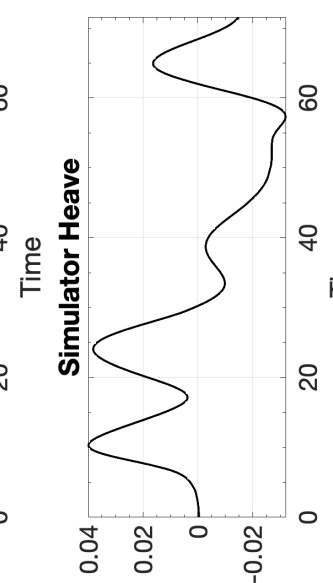

[w] uo!n!sod
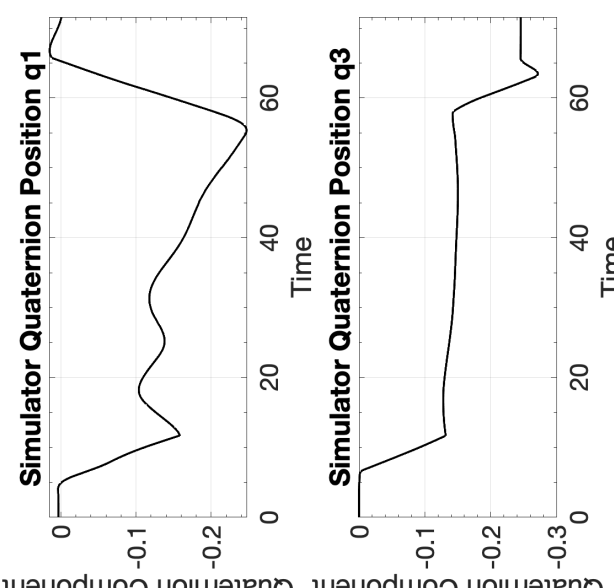

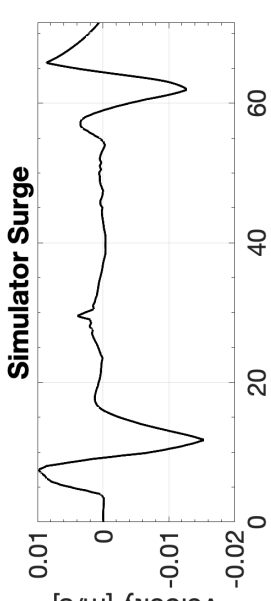

[s/m] Кұ!วо|ә^

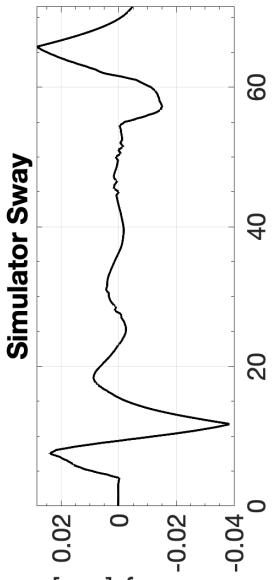

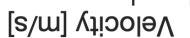

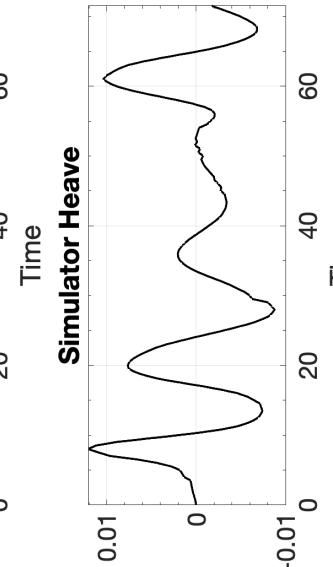

[s/m] ᄉұ!юоюю

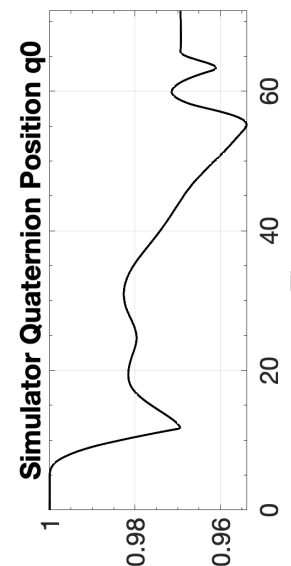

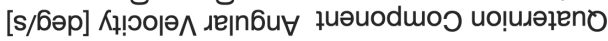

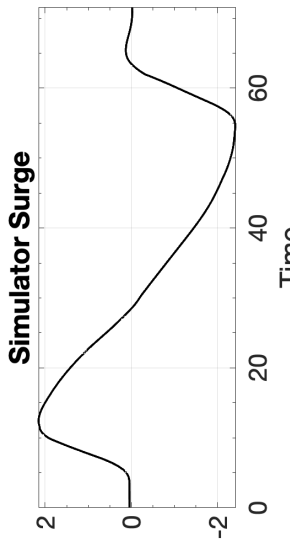

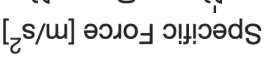

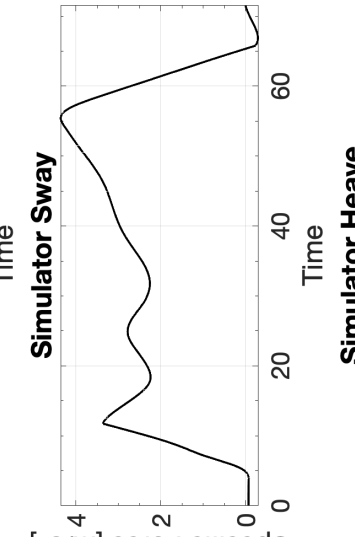

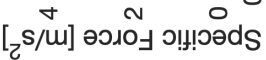

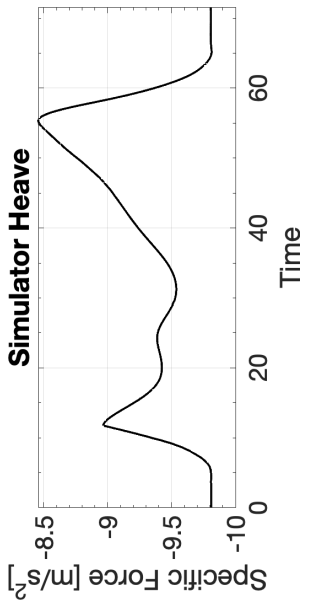

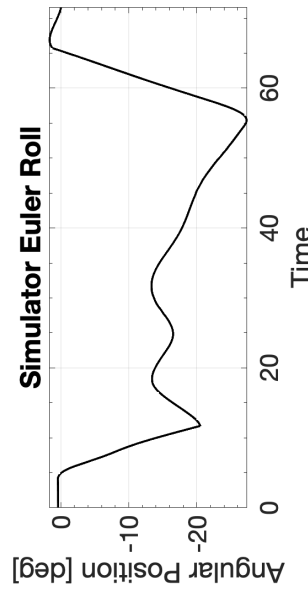

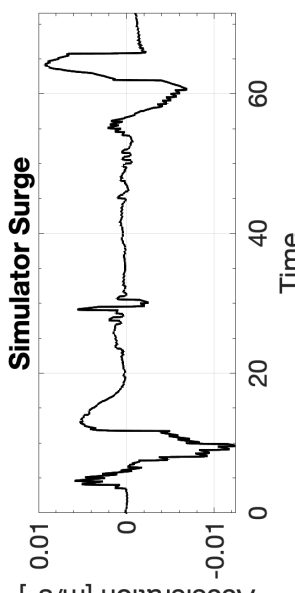

$[\mathrm{z} / \mathrm{L}]$ uo!̣อлә|әэว

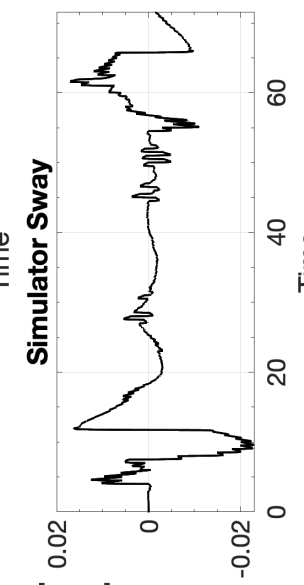

$[\mathrm{z} / \mathrm{s}]$ uо!ฺв

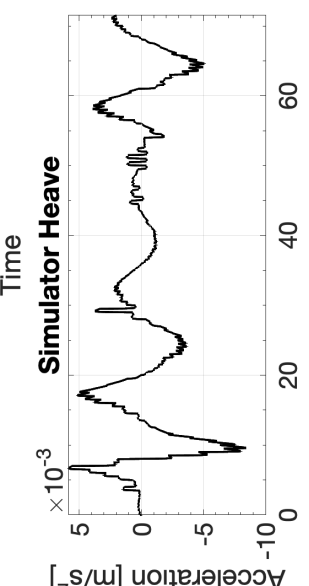

[zs/m] uо!̣едәәәоэ'

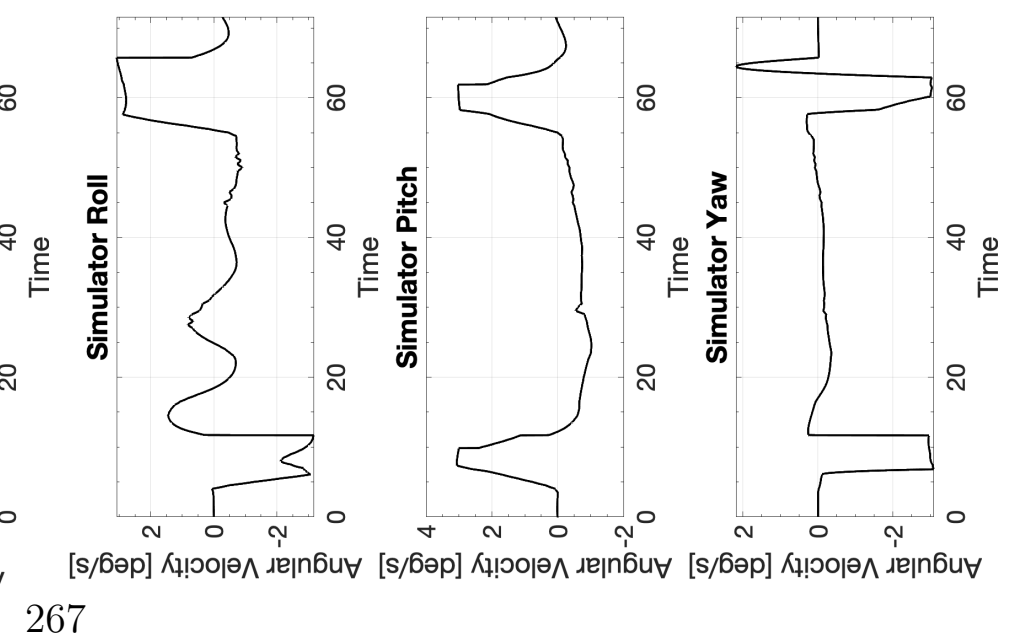

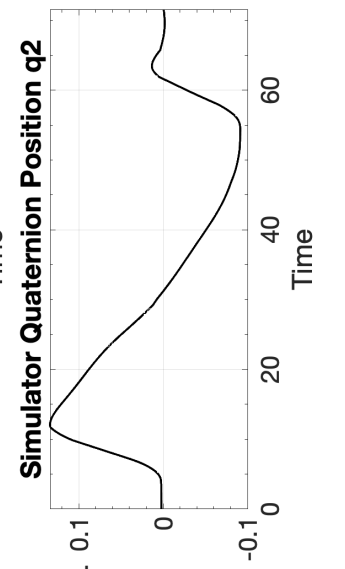

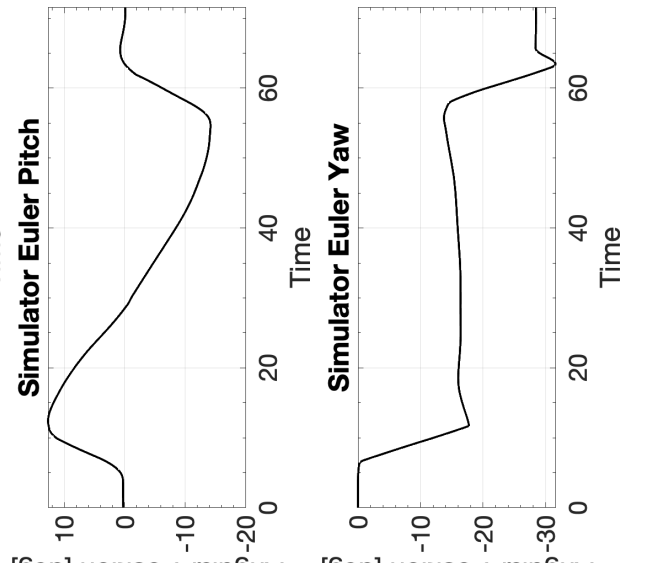

[бәр] uo!!! 


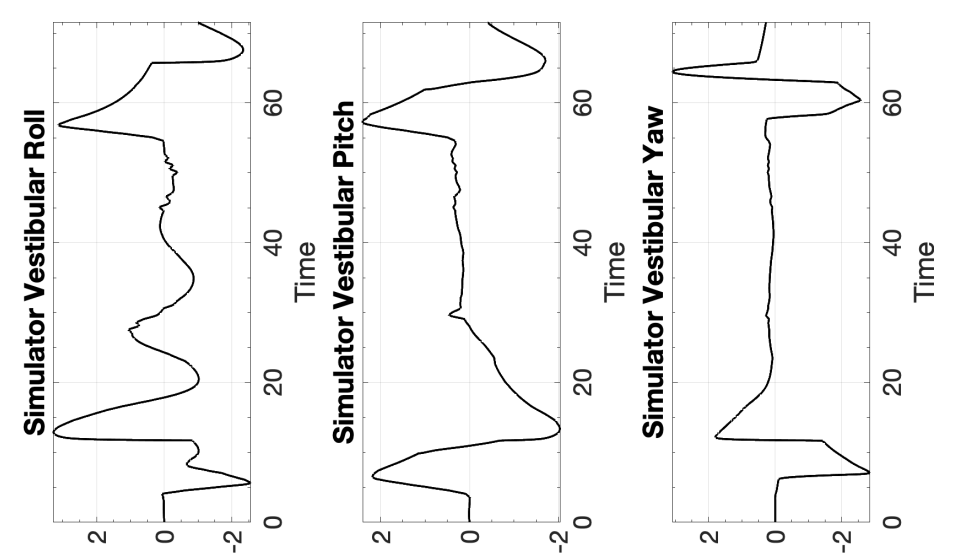

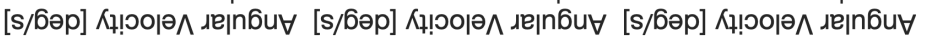

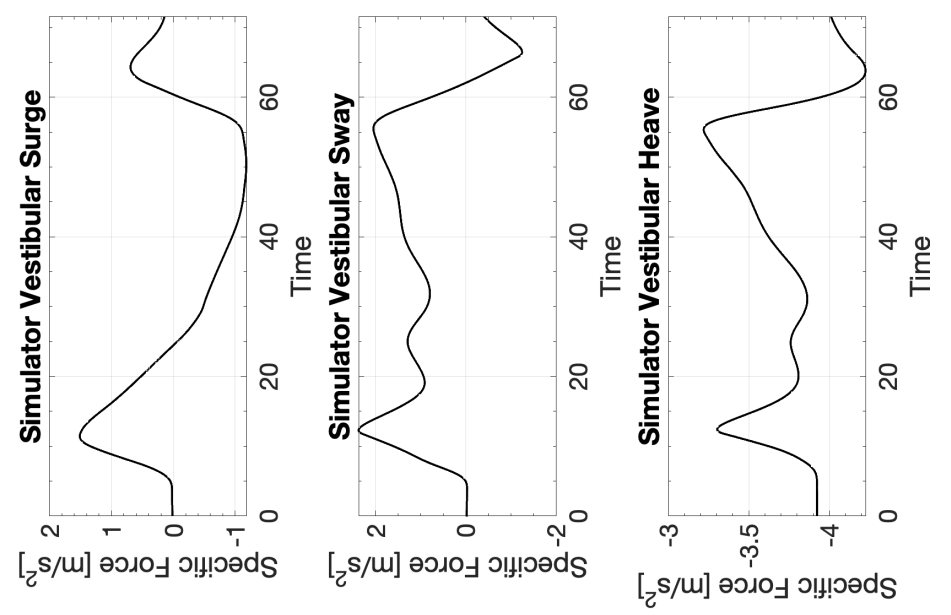


C.10 Boeing 737 Cross-wind Landing

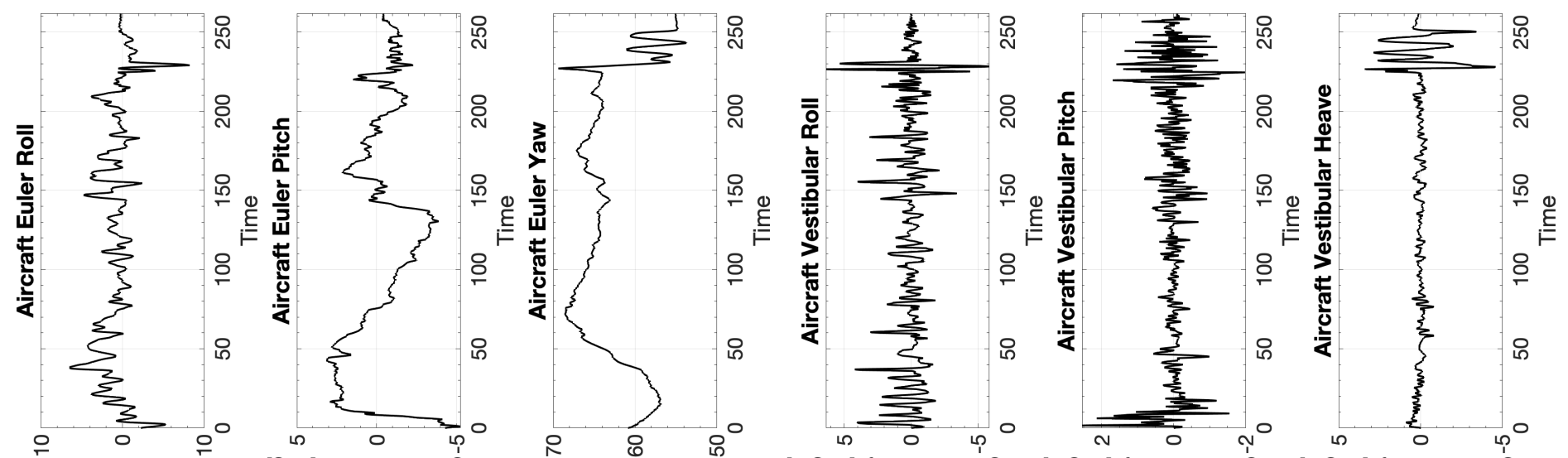

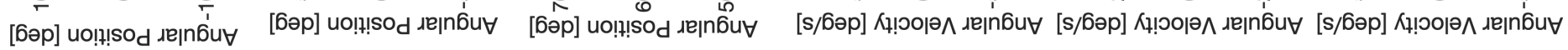
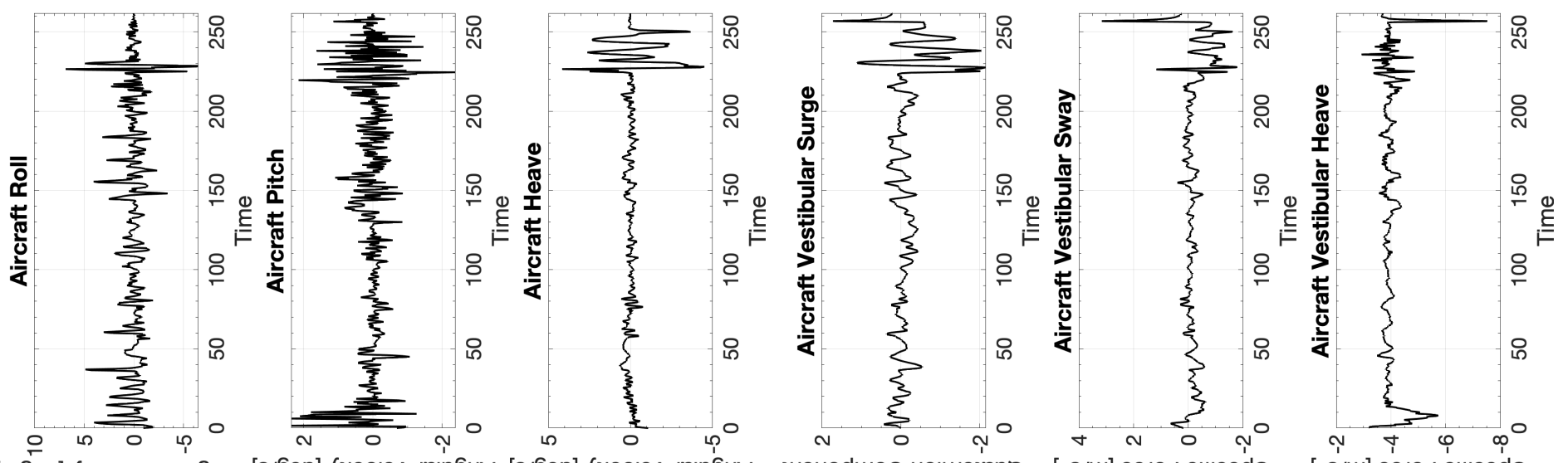

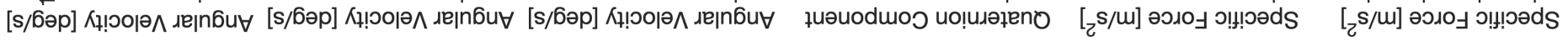

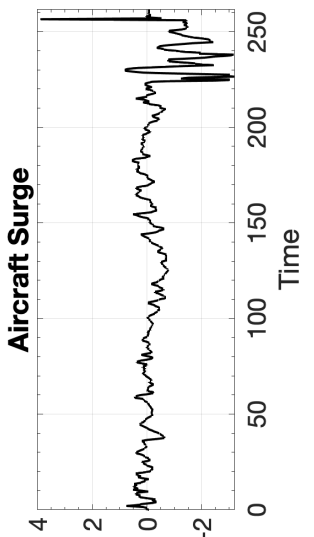

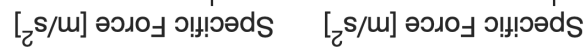
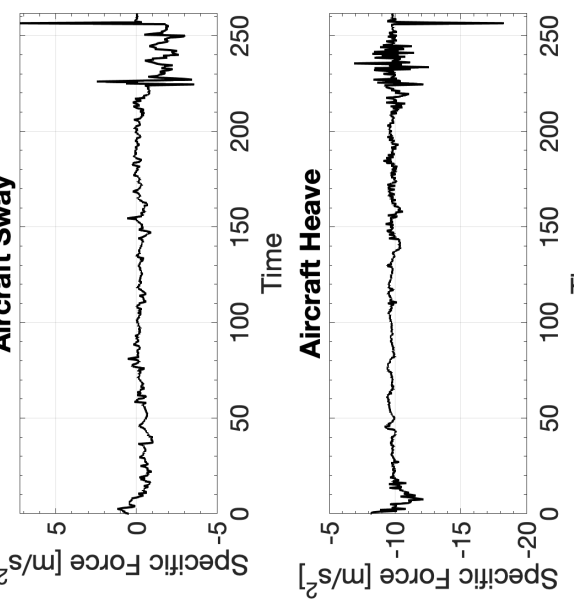

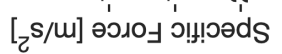

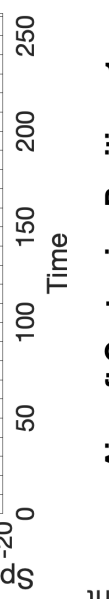

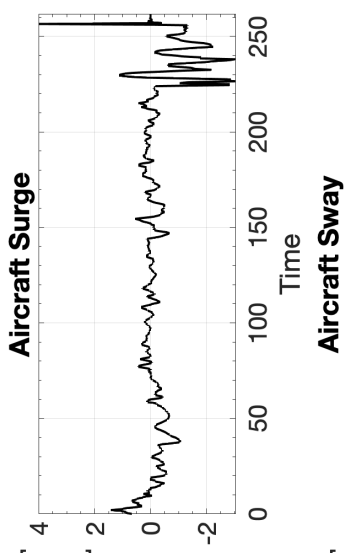

[zs/u] uо!ฺвлә|әээ

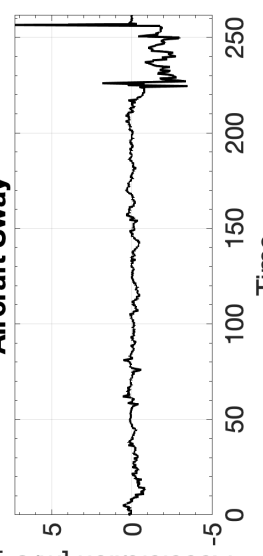

[z/m] ио!ฺвдәәәоว

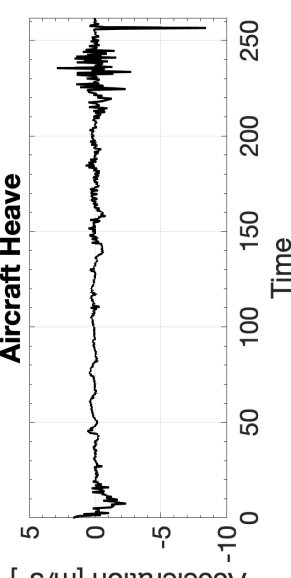

[zs/m] uо!ฺвдә|әоэ
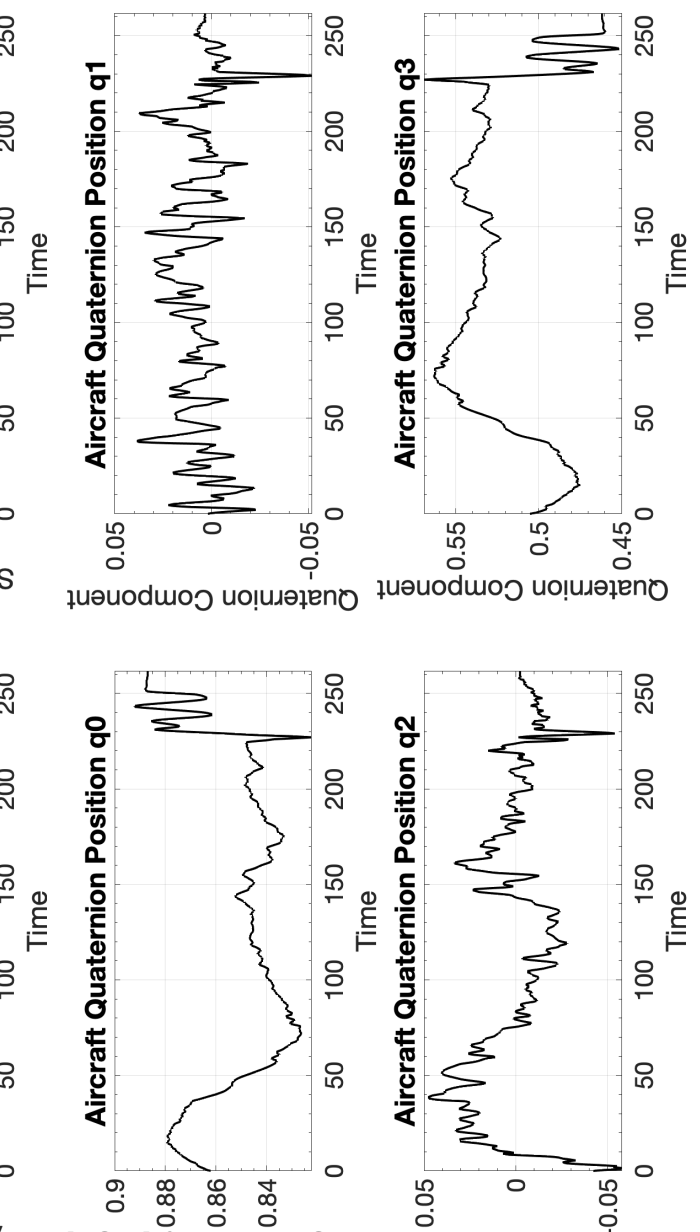


\section{C.10.1 CW1 Boeing 737 Cross-wind Landing}

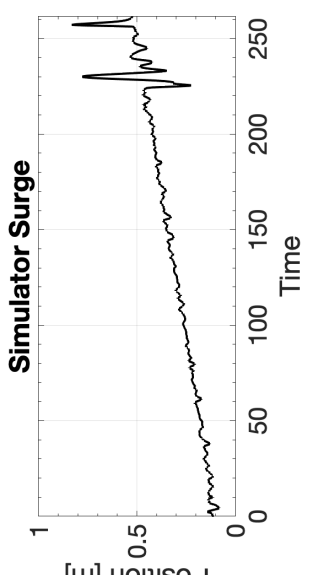

[u] uo!!!sod

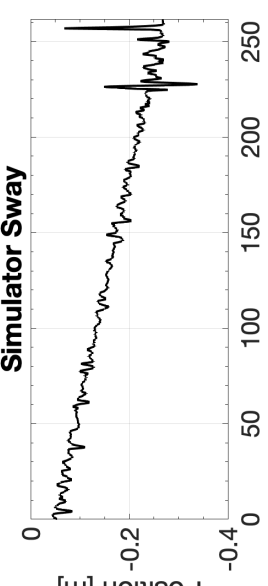

[w] uo!n!lsod

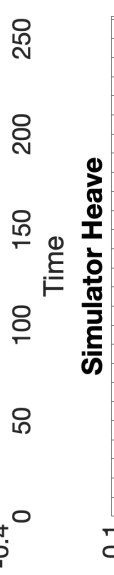

-

[w] uol!! sod
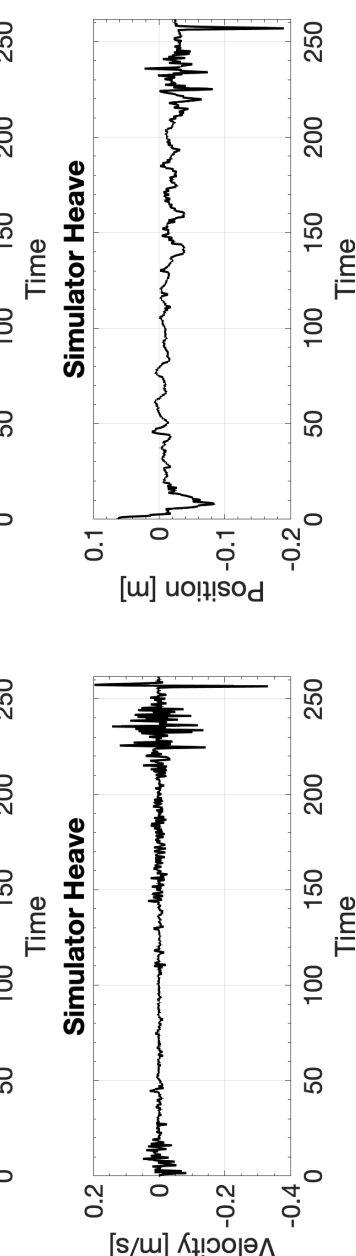

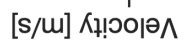
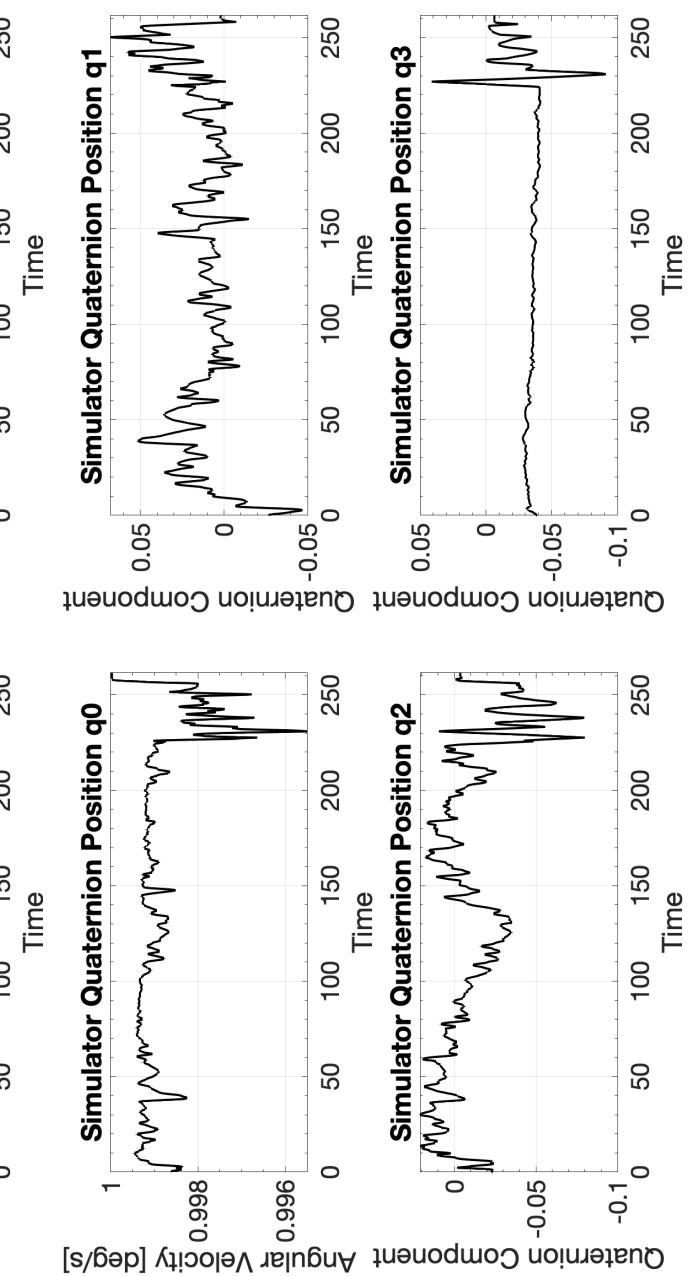

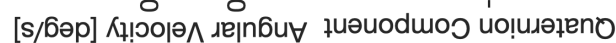

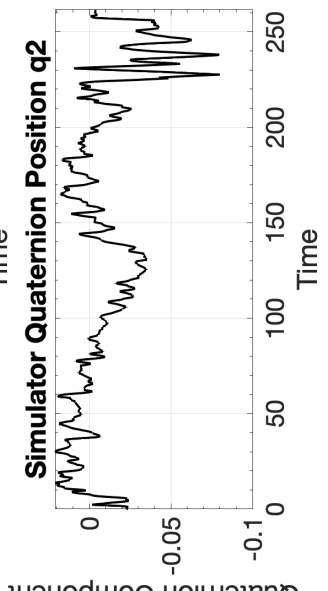

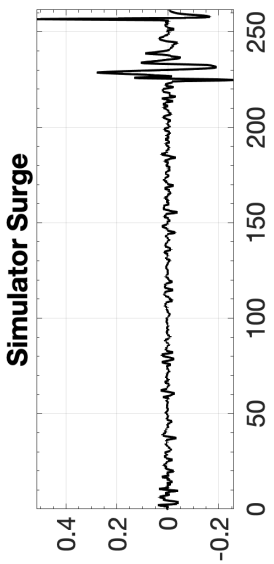

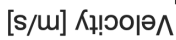

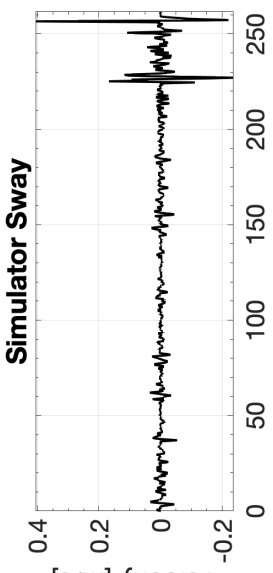

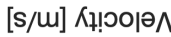
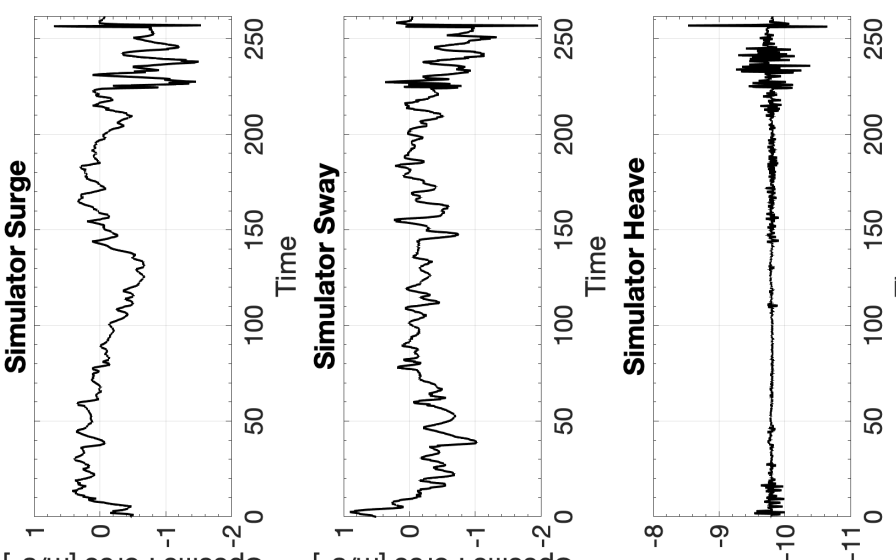

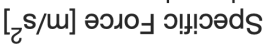

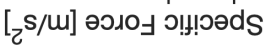

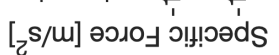
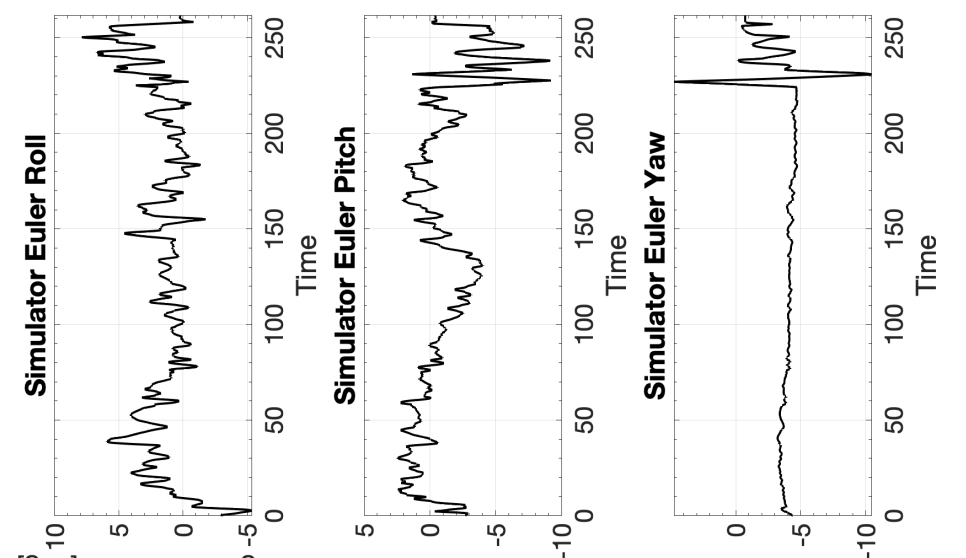

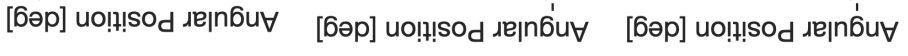

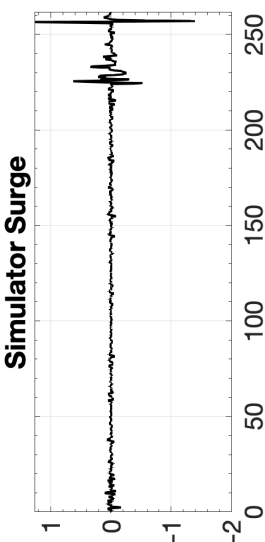

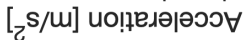

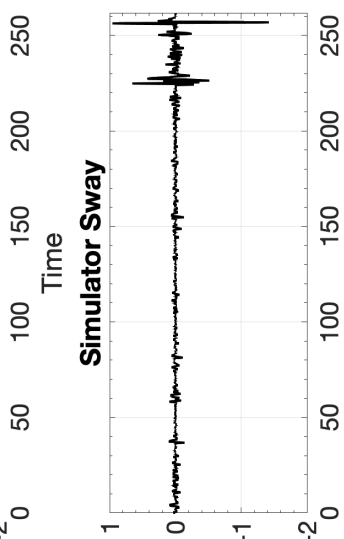

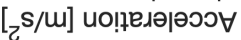

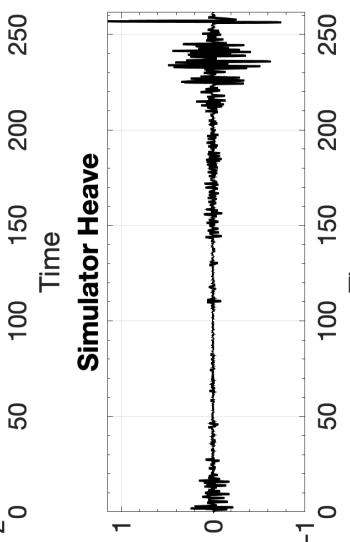

$[\mathrm{s} / \mathrm{m}]$ uо!ฺвдәәәэว

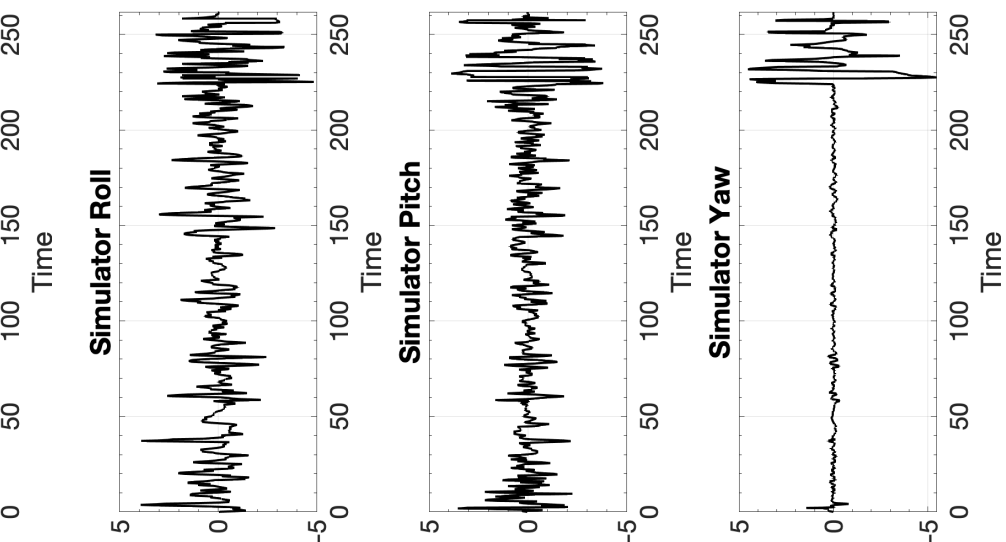

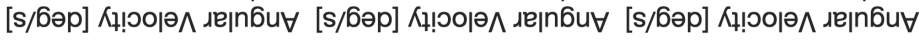




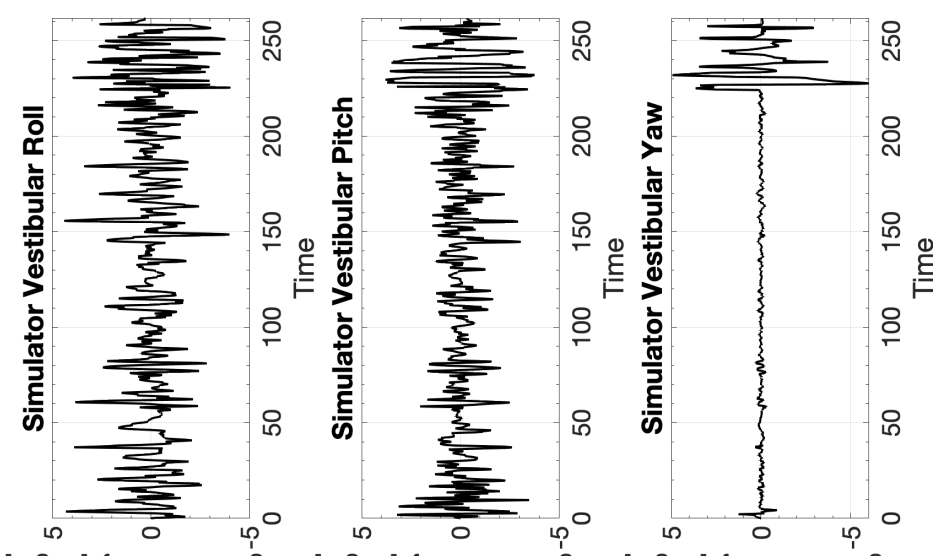

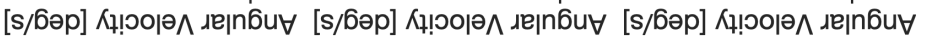

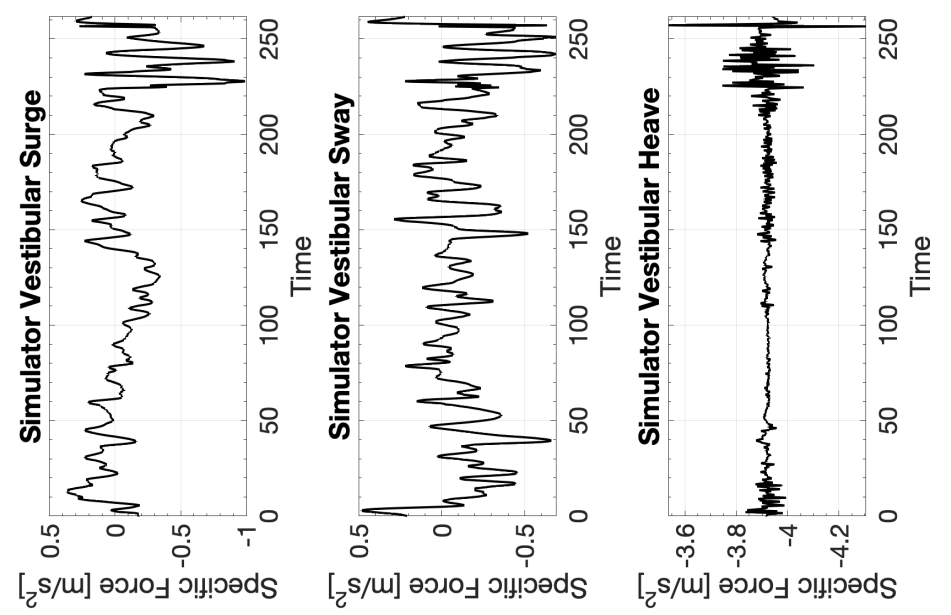




\section{C.10.2 CW2 Boeing 737 Cross-wind Landing}

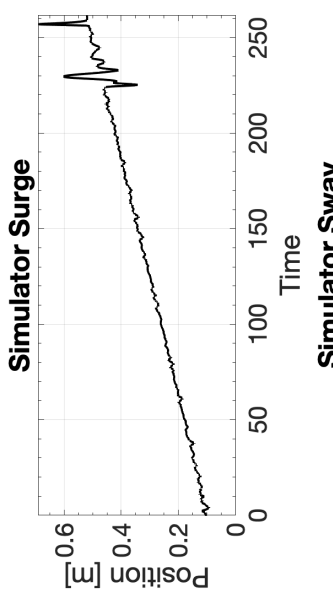

[w] uol!! sod

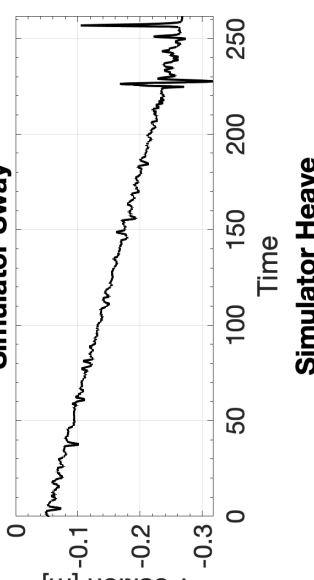

[m] uol!m!sod

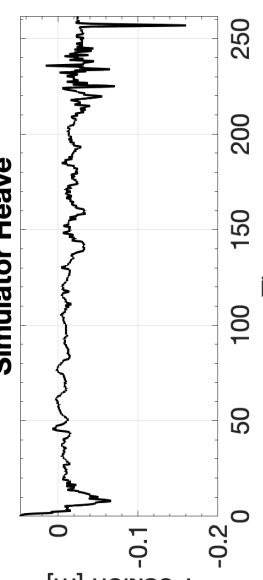

[m] uol!?!sod

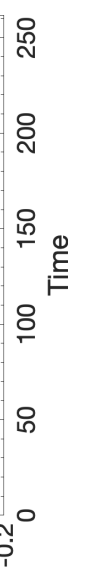

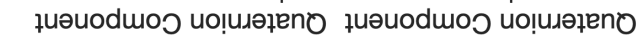
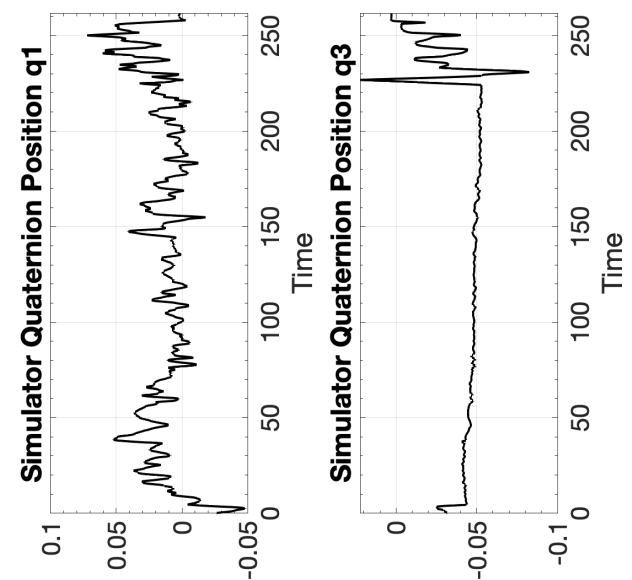

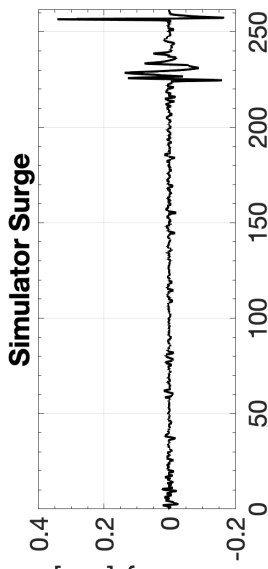

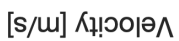

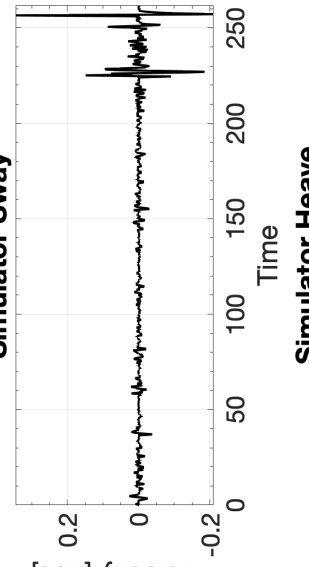

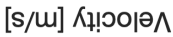

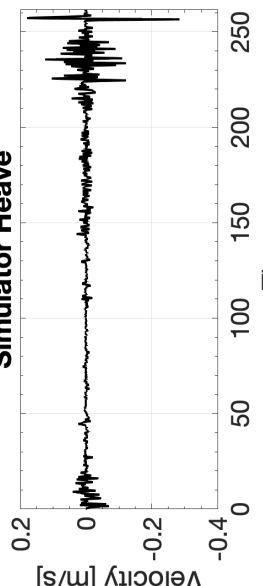

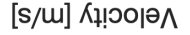

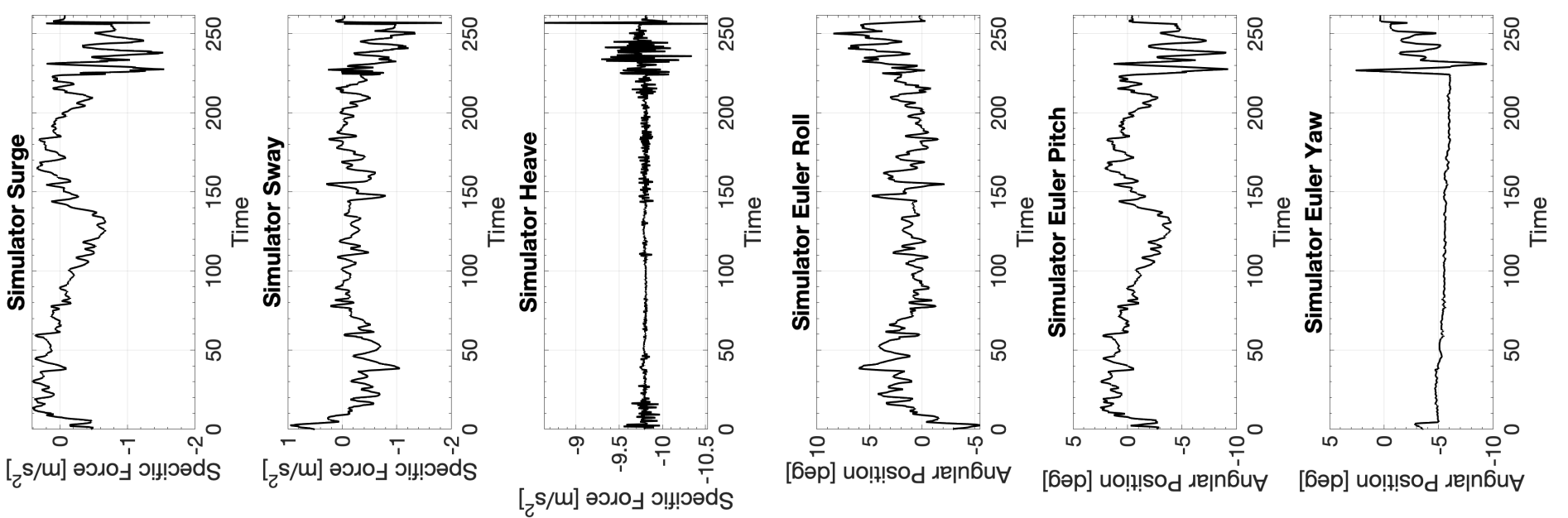

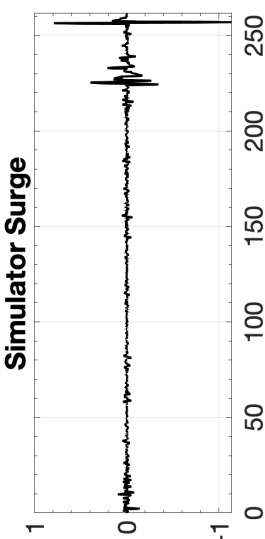

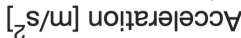

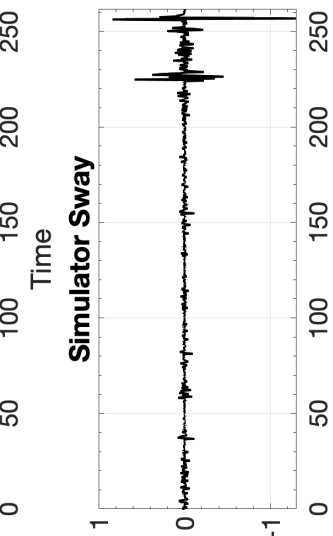

$[s / m]$ uо!̣едәәәэว

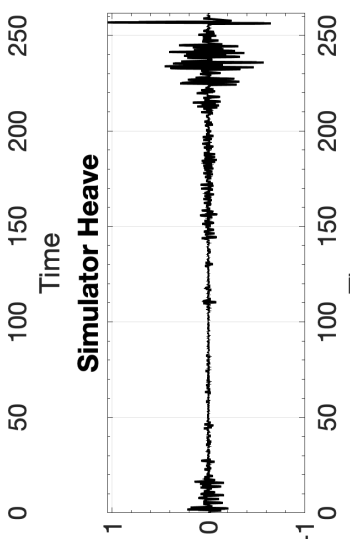

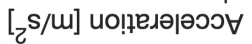

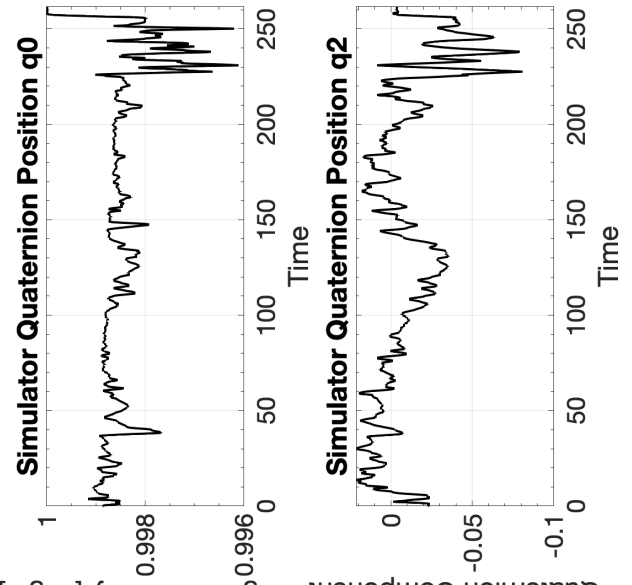

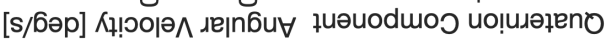

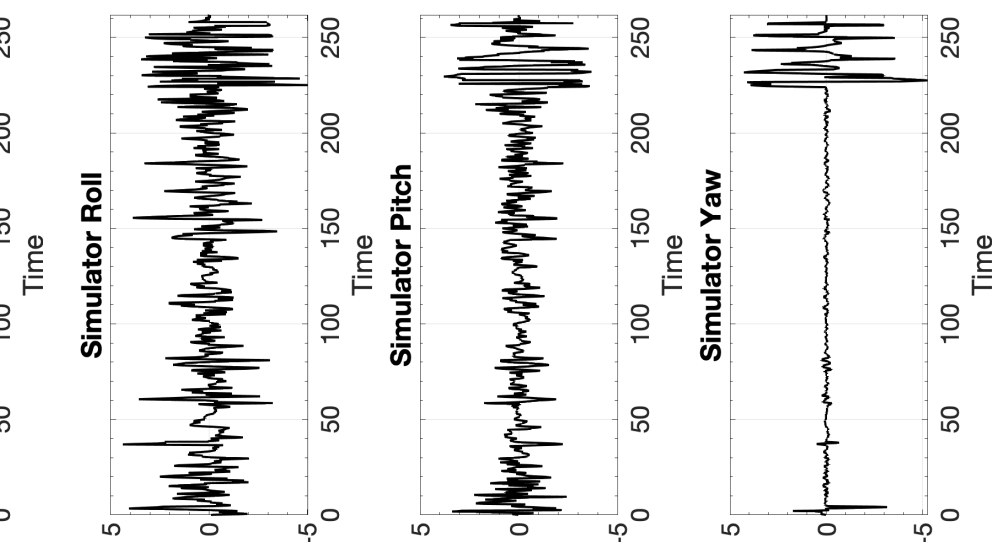

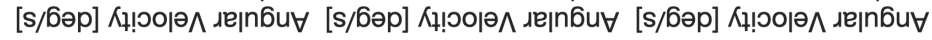




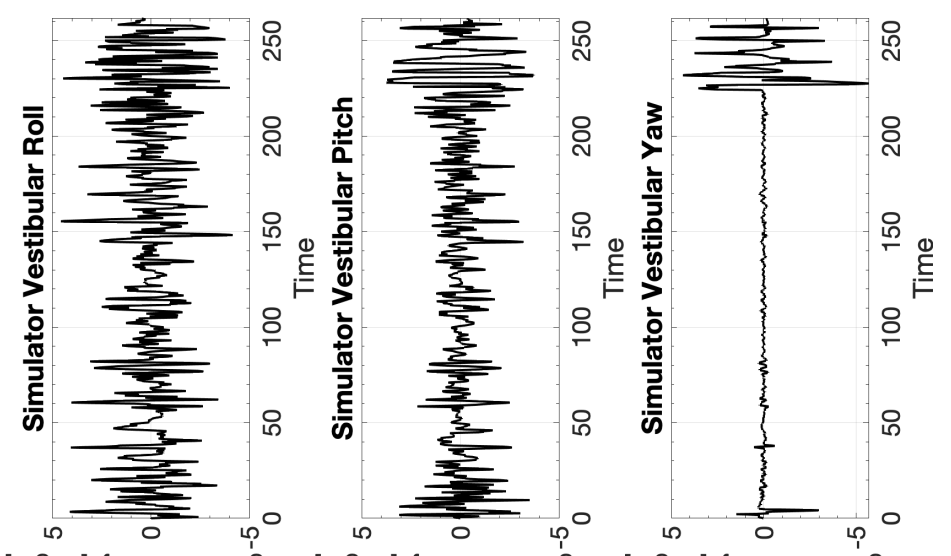

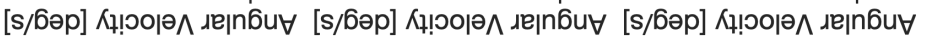

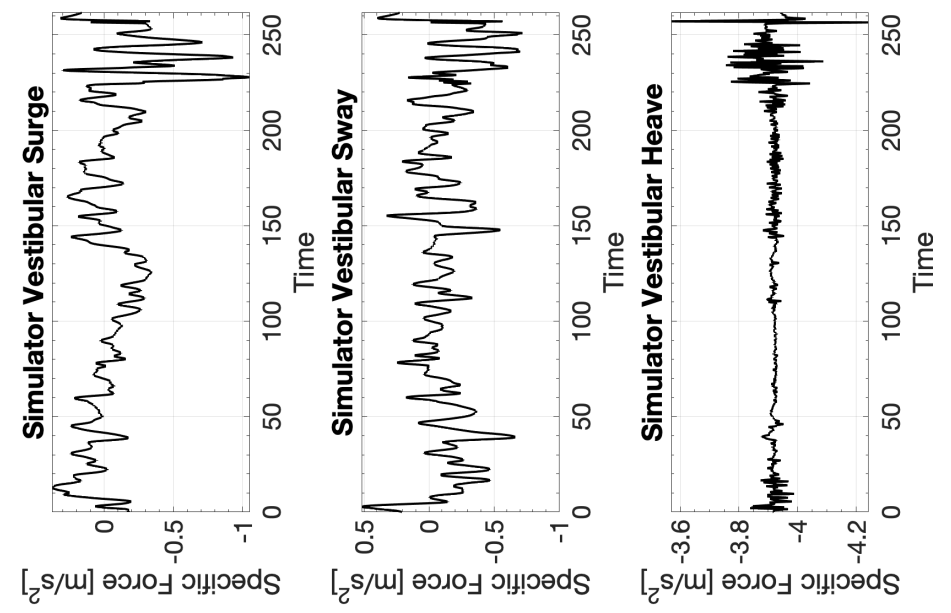




\section{C.10.3 CW3 Boeing 737 Cross-wind Landing}
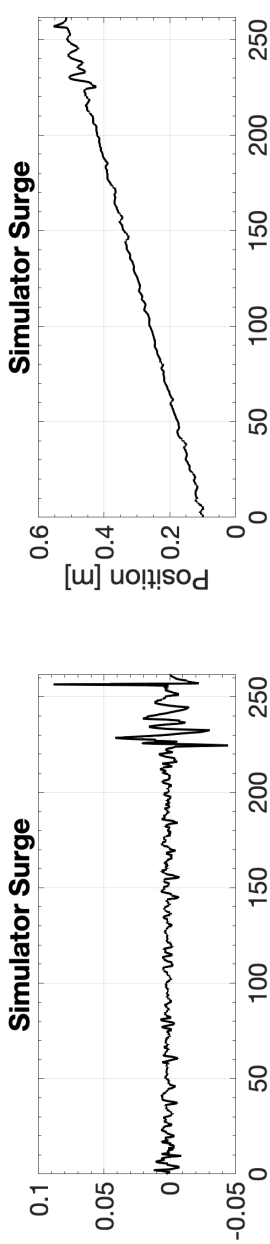

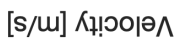

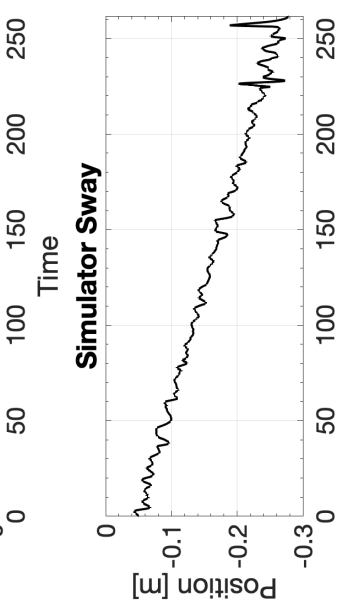

[m] uol!!!sod

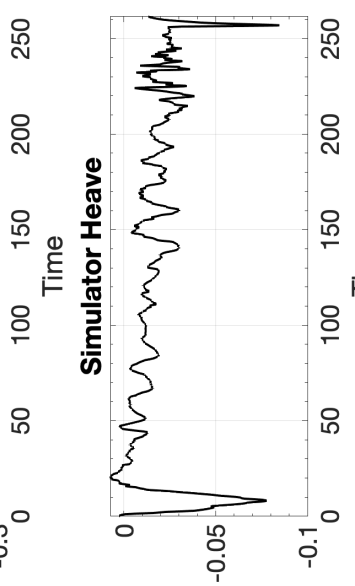

[w] uo!n!ssod

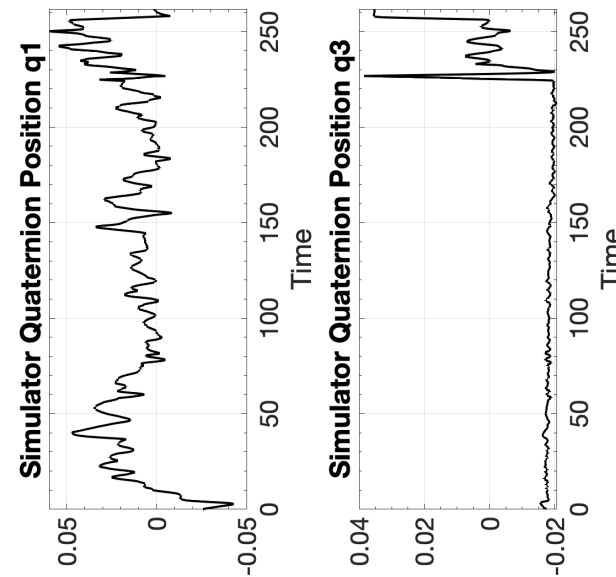

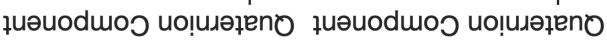

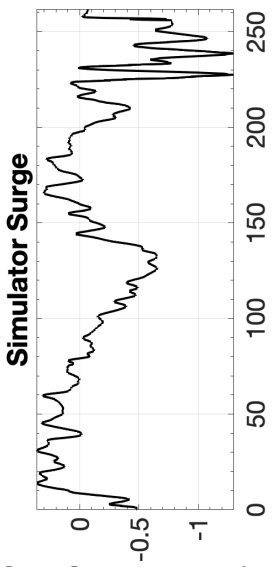

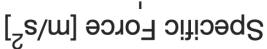

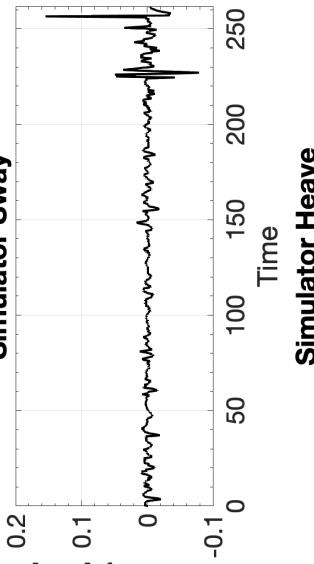

[s/m] К!ำ^

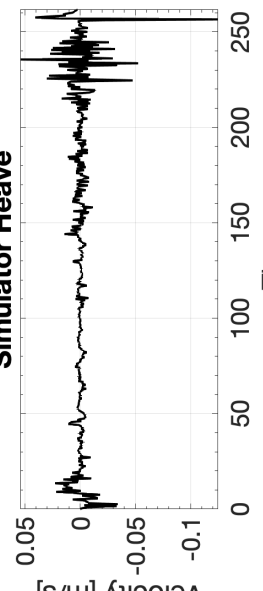

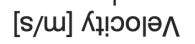

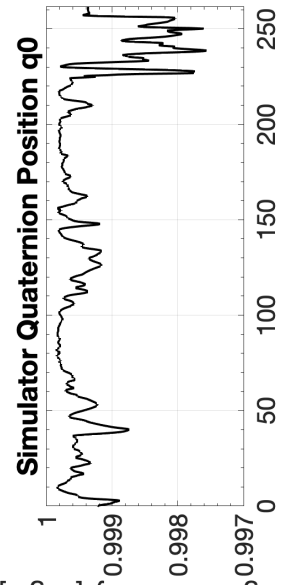

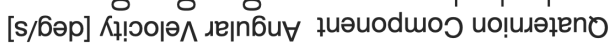

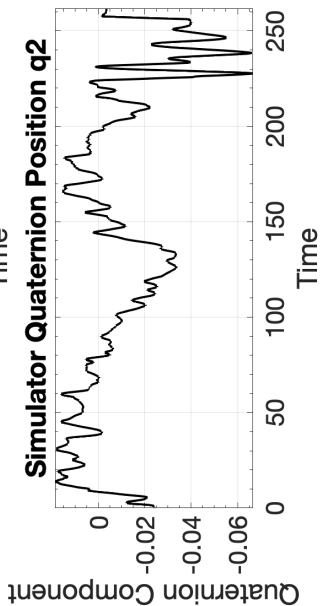

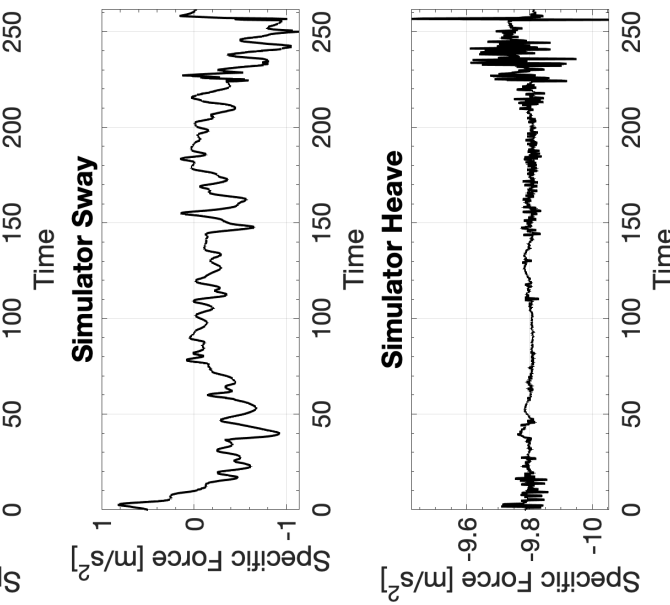
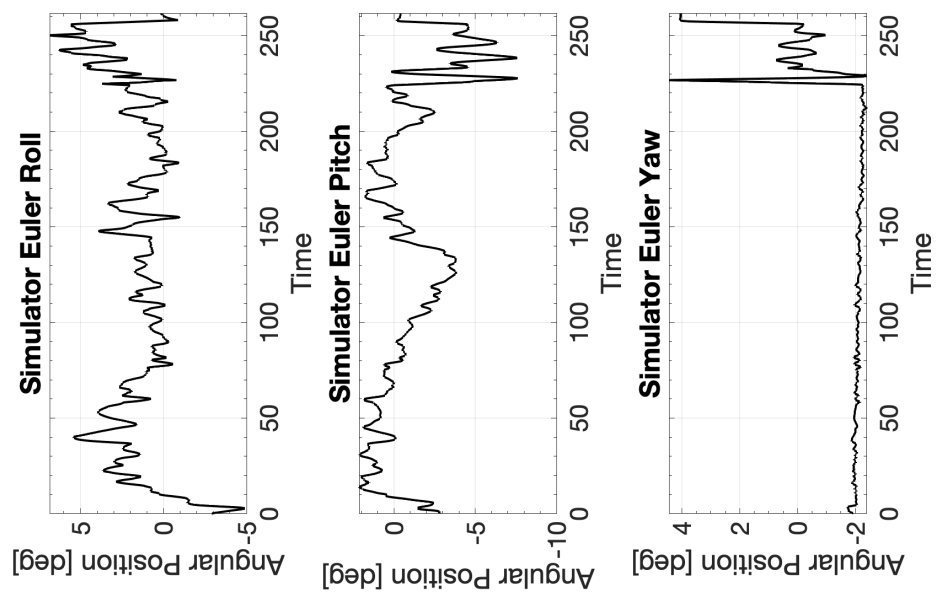

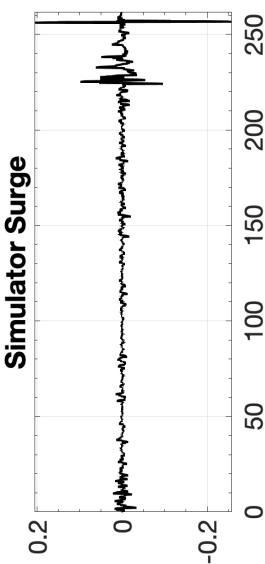

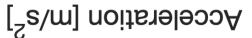

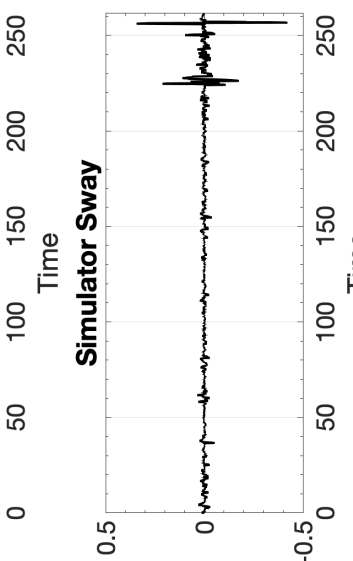

[zs/m] ио!ฺедәәәэว

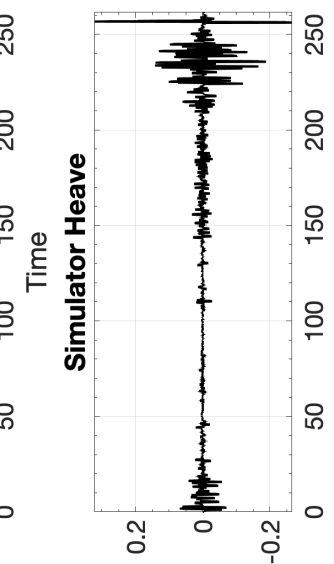

[s/m] ио!ฺедә|әэว
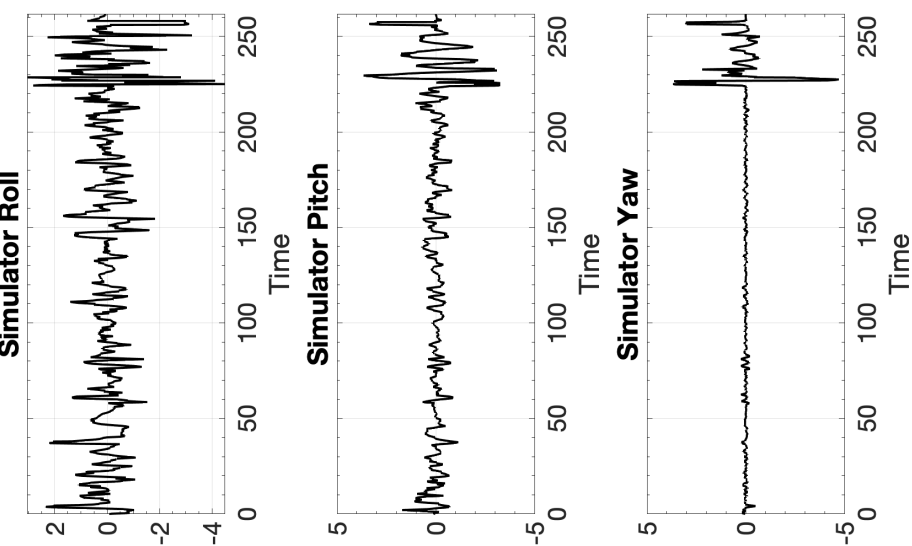

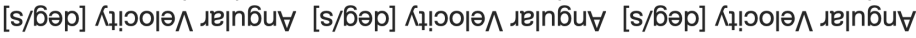




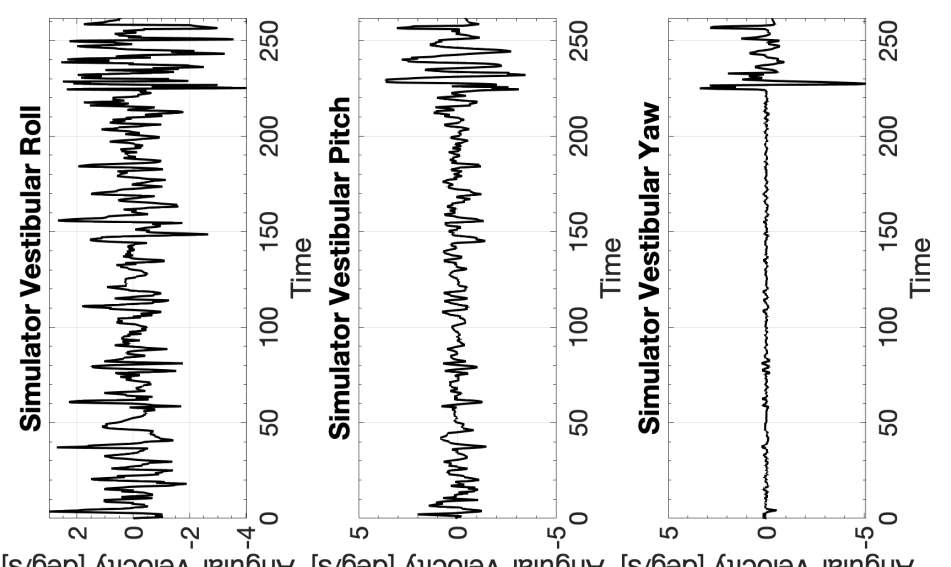

[s/бәр] Кұ!юорә גе|nби

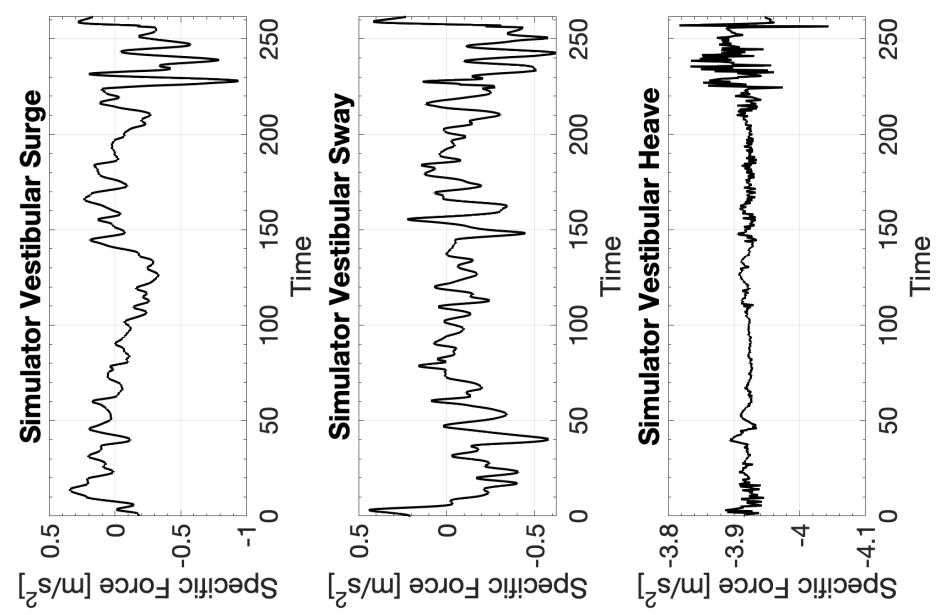




\section{C.11 Boeing 737 Single-axis Takeoff}

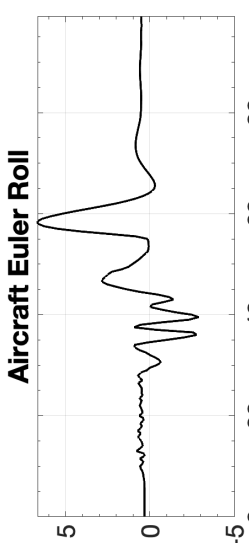

[6әр] uo!̣!!sod

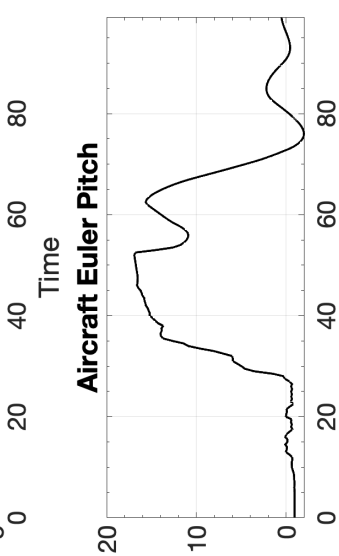

[6әр] uol]isod dejn6ut

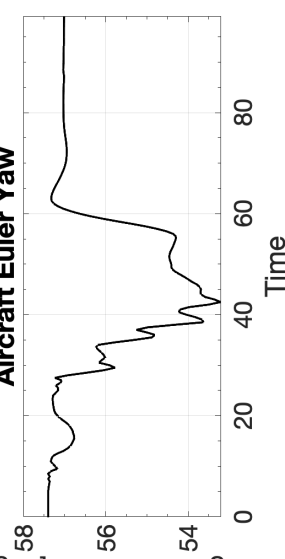

[6วp] uol]!sod גe|nదu

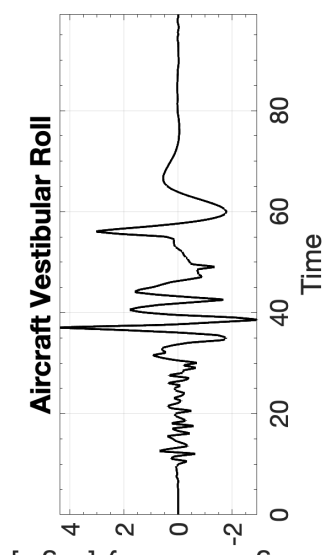

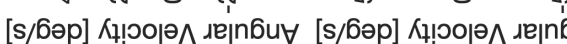

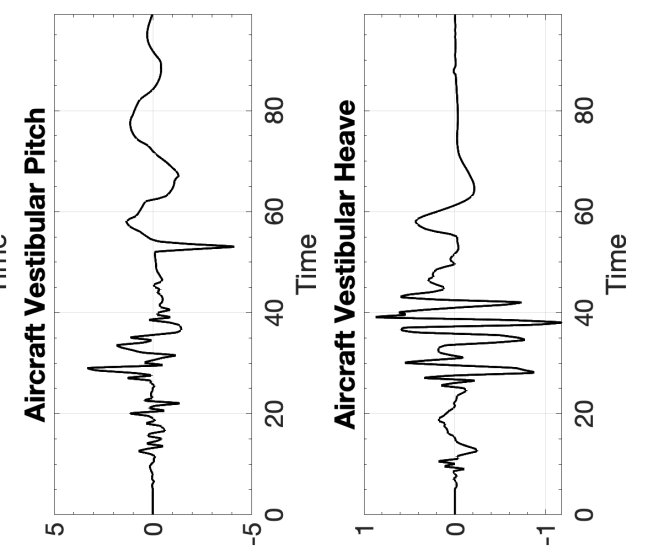

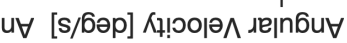

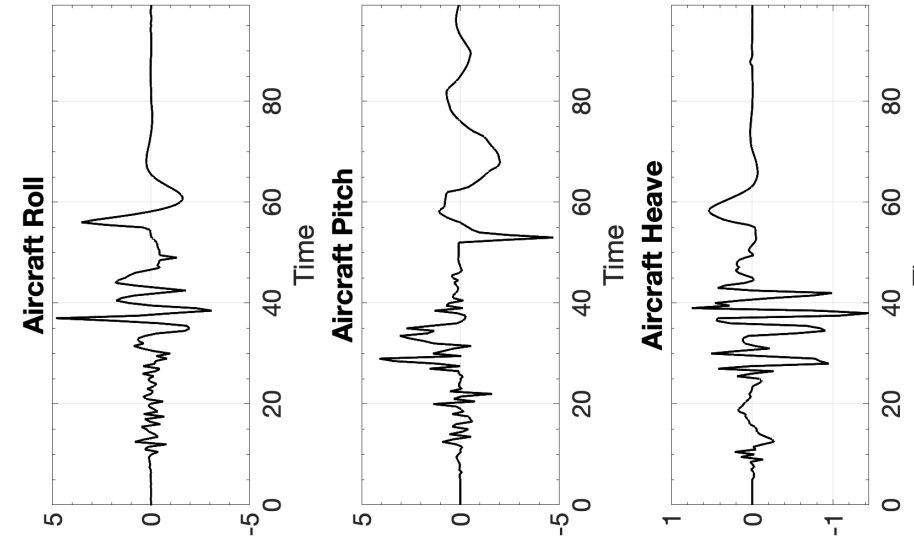

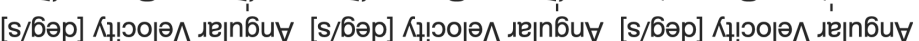

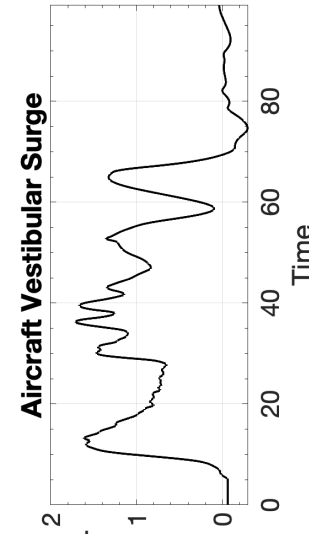

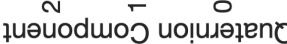

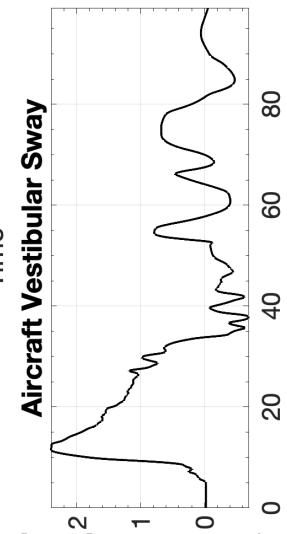

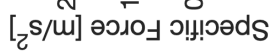

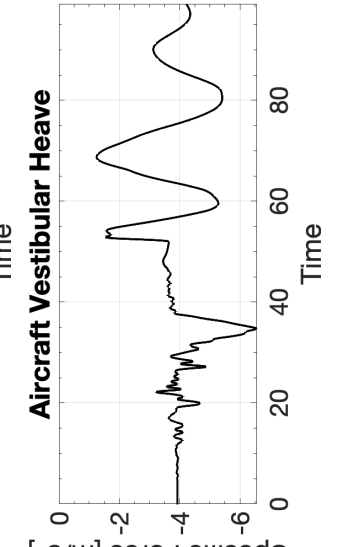

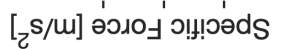

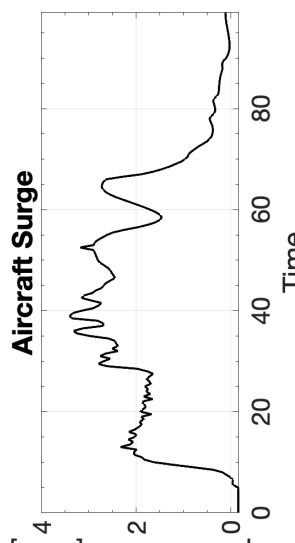

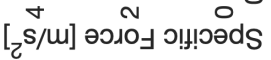

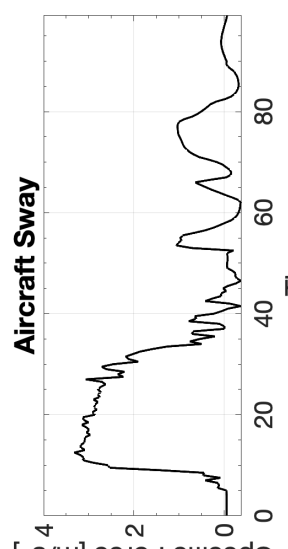

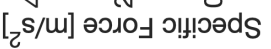

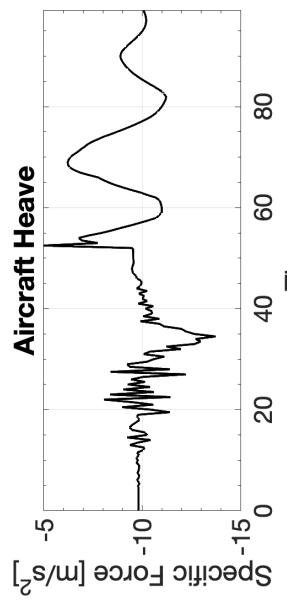

[

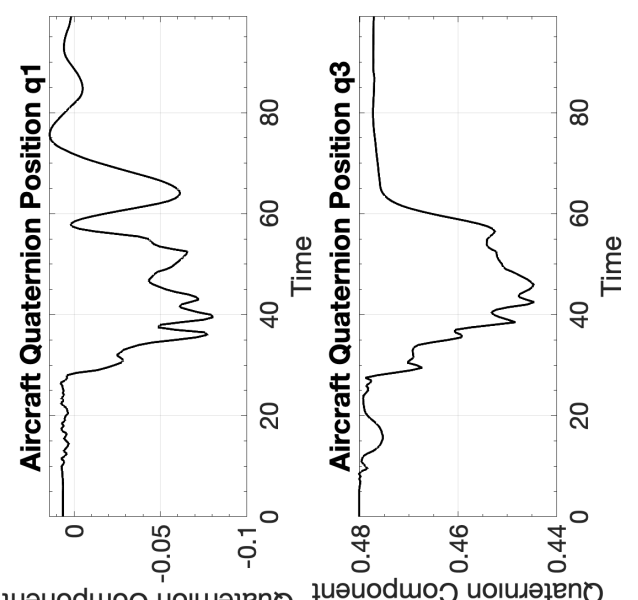

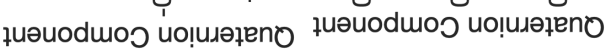

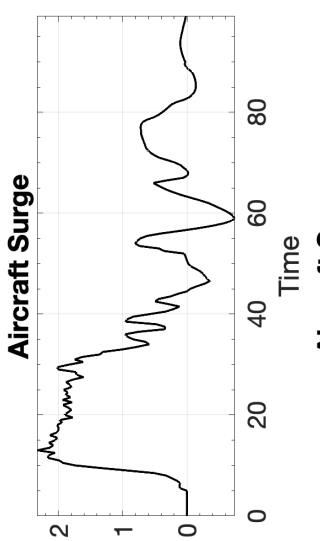

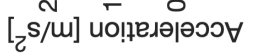

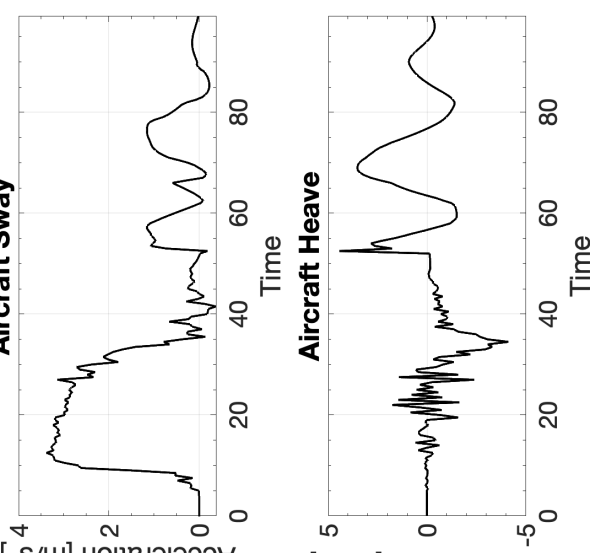

$\left[\begin{array}{c}2 \\ \text { [ }\end{array}\right.$

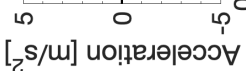

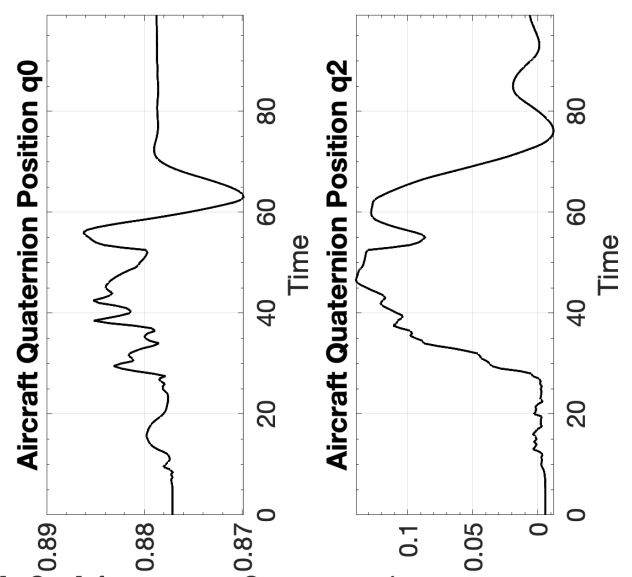

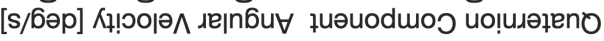




\section{C.11.1 CW1 Boeing 737 Single-axis Takeoff}

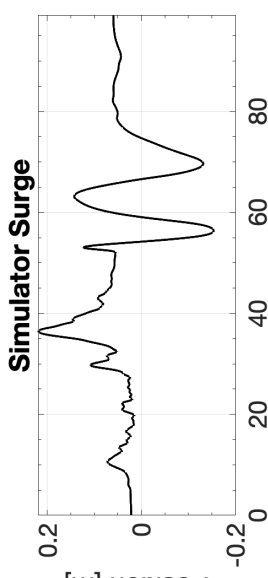

[w] uo!n!!sod

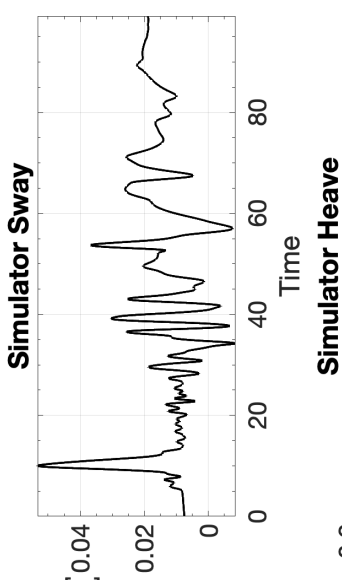

[u] uo!n!sod

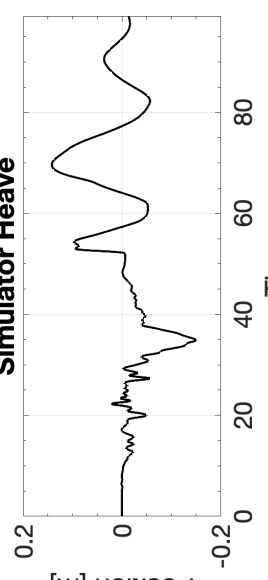

[w] uol!!!sod

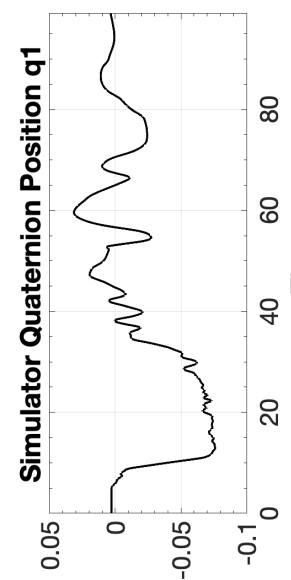

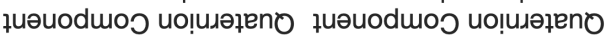

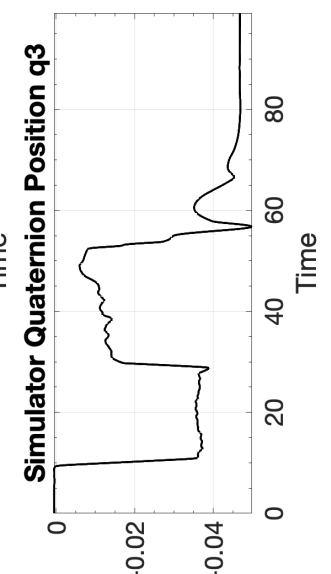

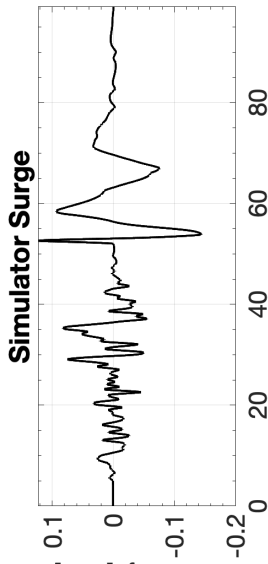

[s/m] К१!৩о|९

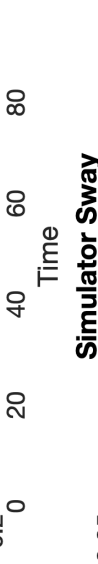

i.

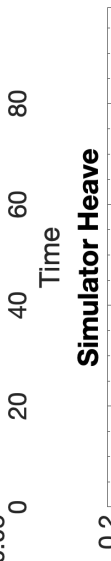

[s/m] ᄉ!ํㅇㅅ $\Lambda$
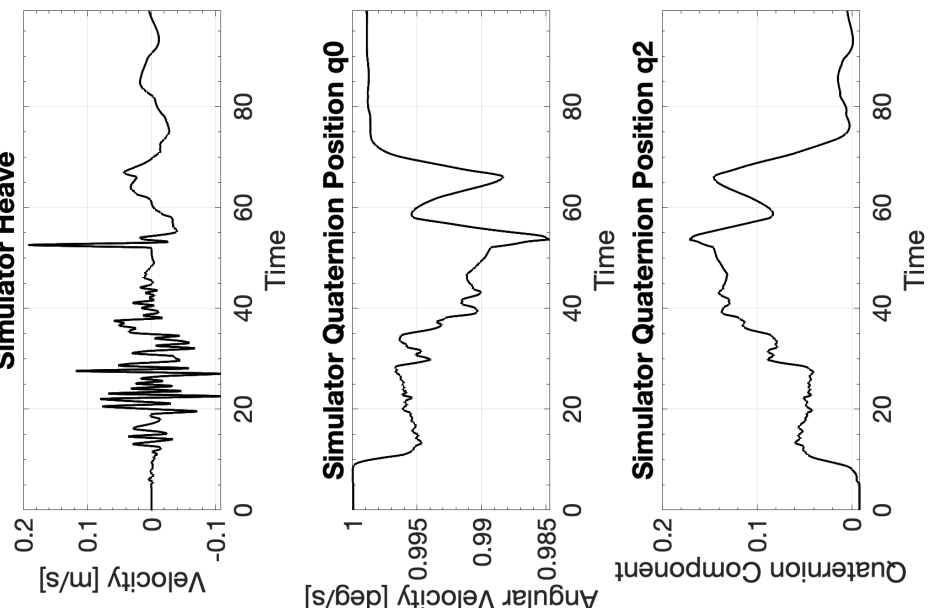

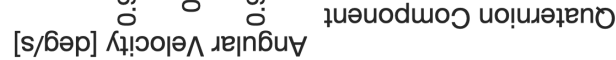

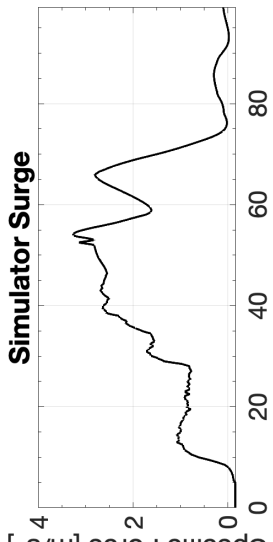

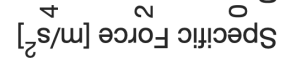

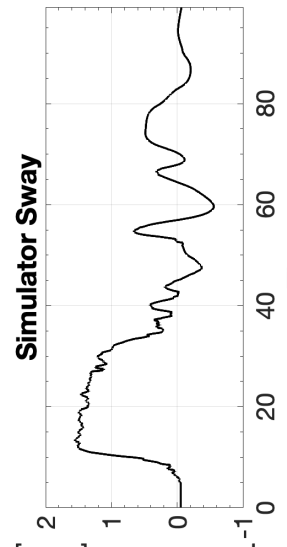

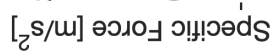

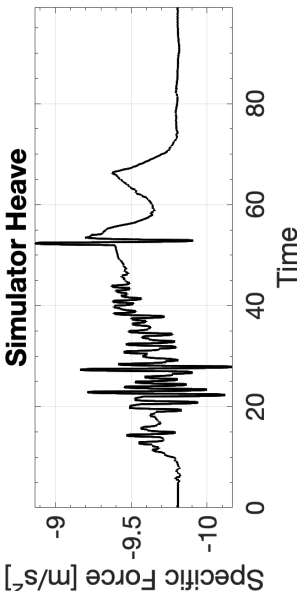

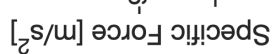

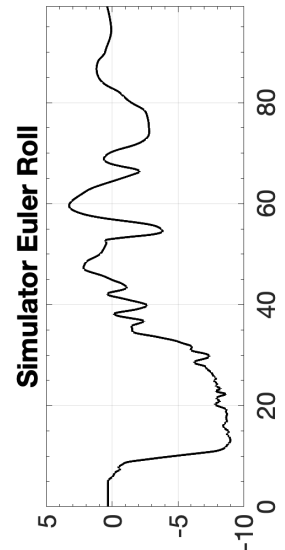

[бәр] uo!!!sod גe|nб́u

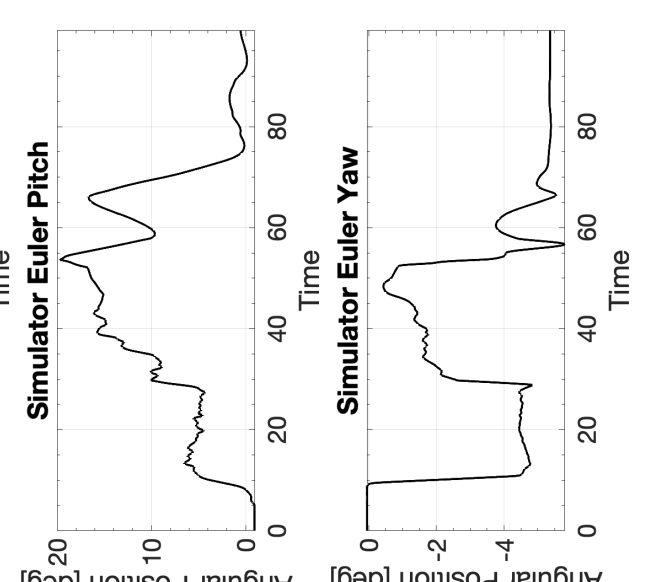

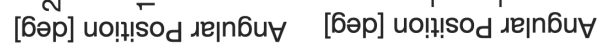

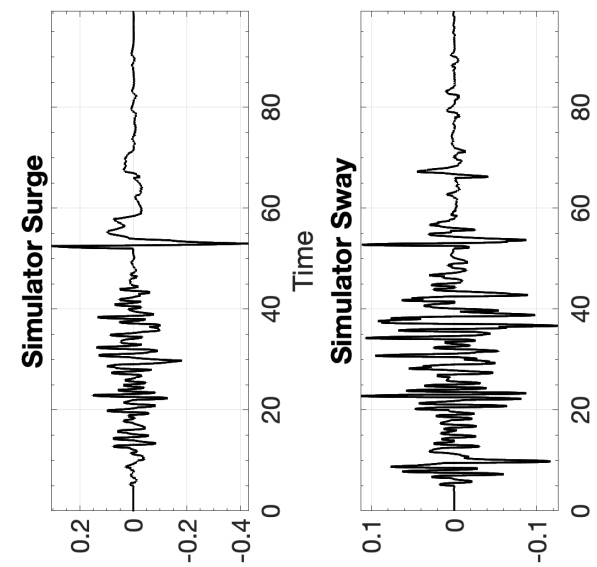

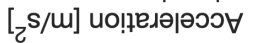

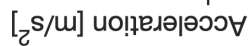

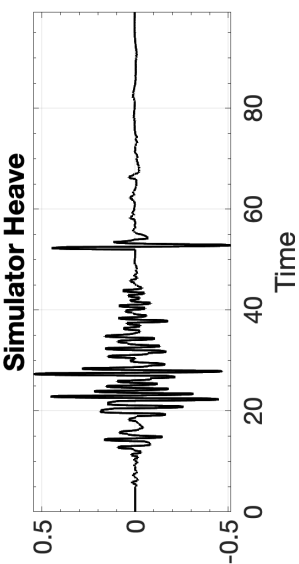

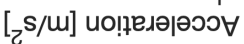

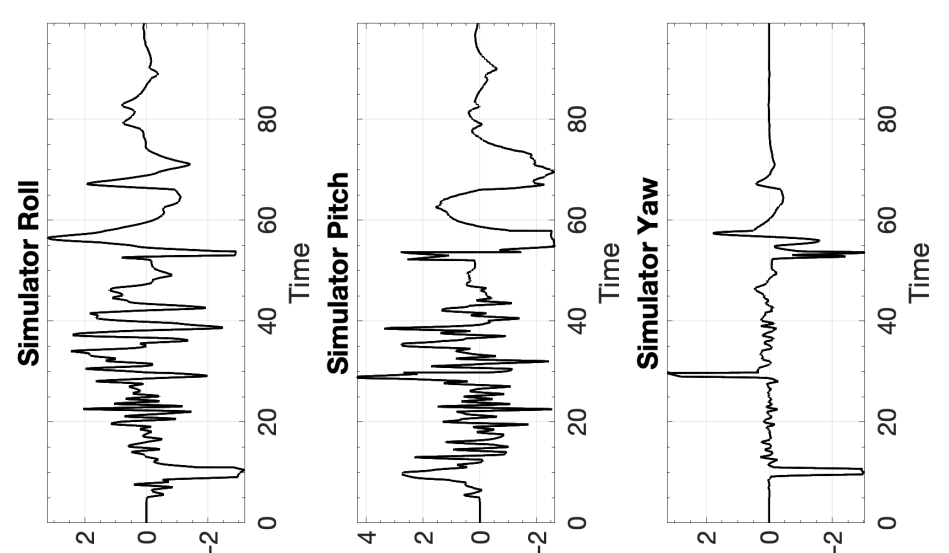

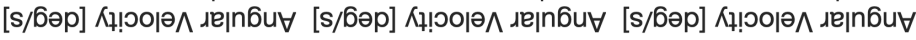




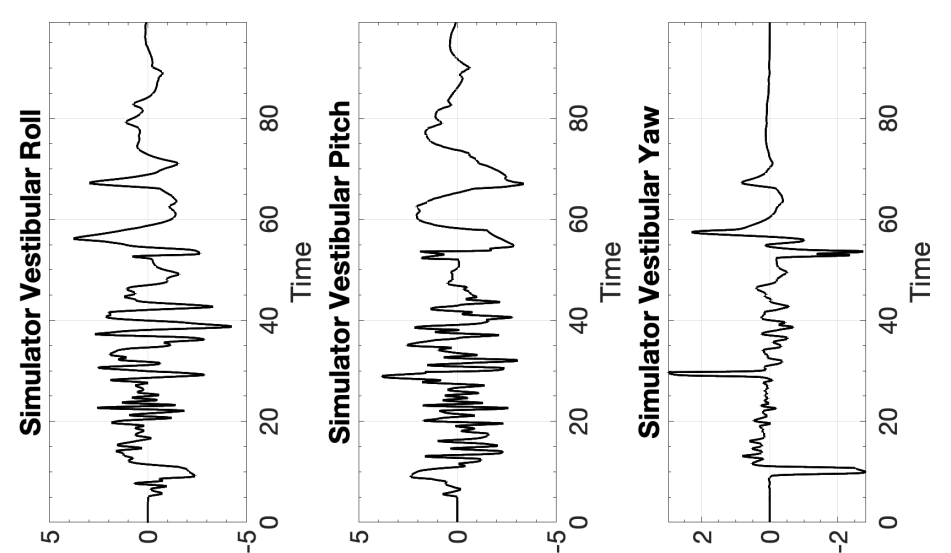

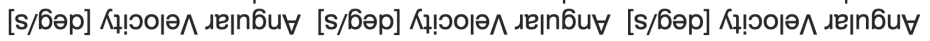

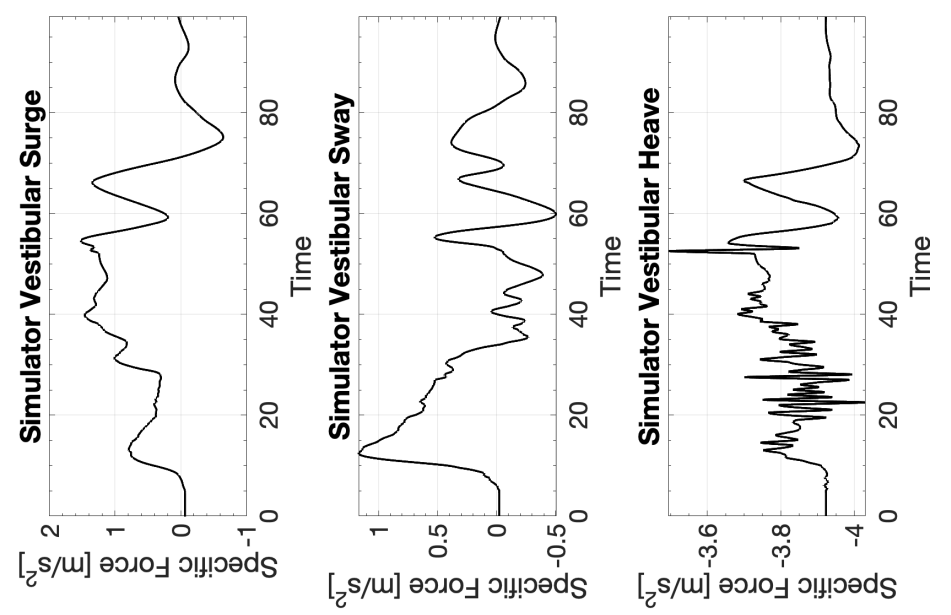




\section{C.11.2 CW2 Boeing 737 Single-axis Takeoff}

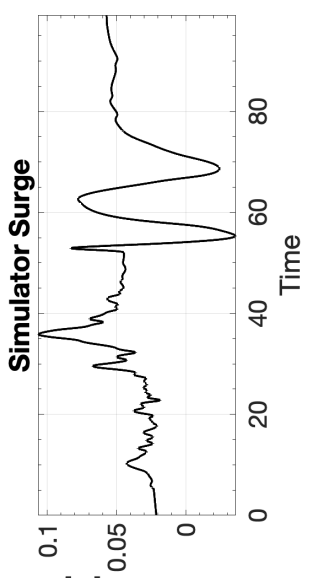

[u] uo!n! $\mathrm{sod}^{\mathrm{d}}$

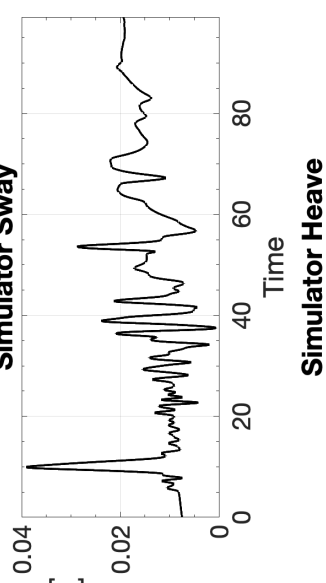

[w] uol!!sod

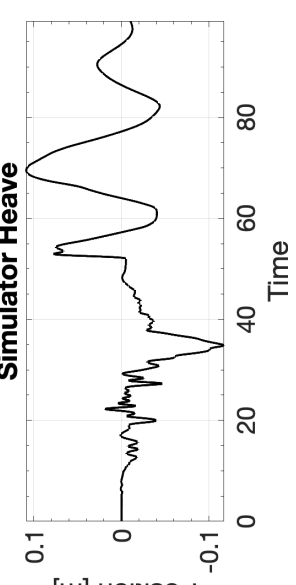

[w] uo!n!sod

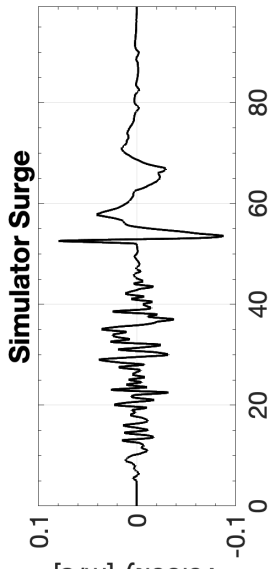

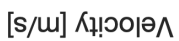

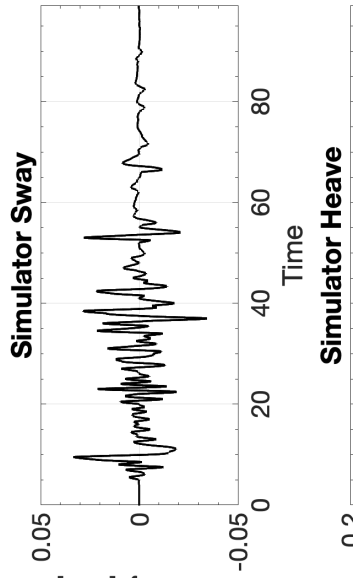

[s/u] Кџ!วо夫

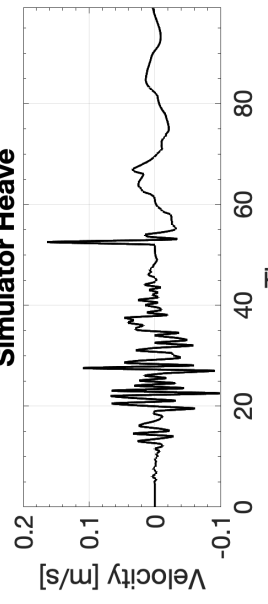

s/u] Кџ!юор夫

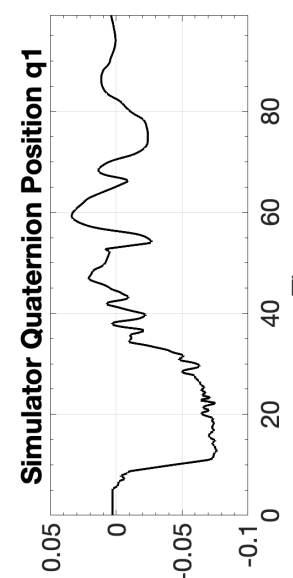

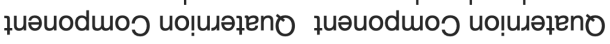

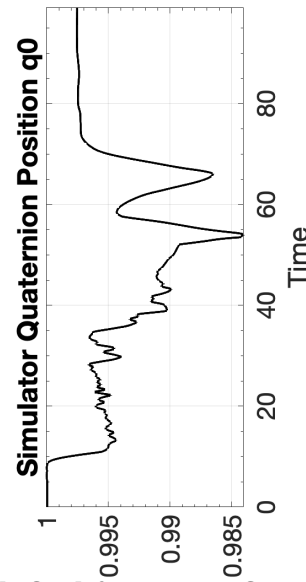

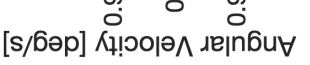
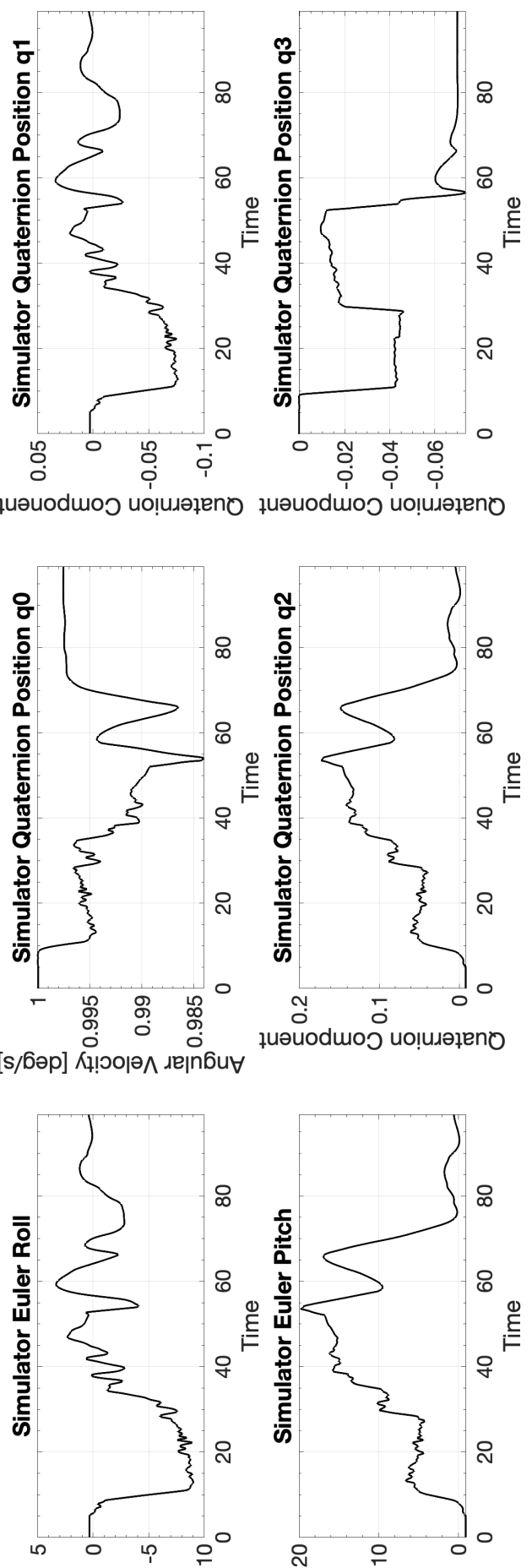

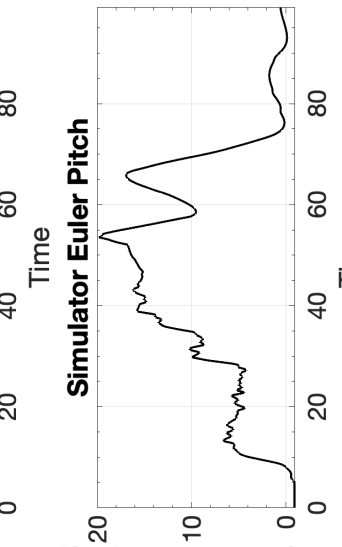

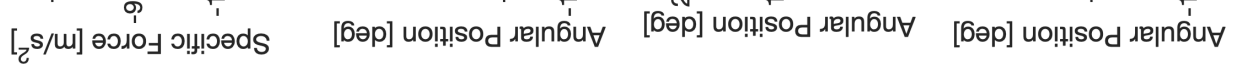

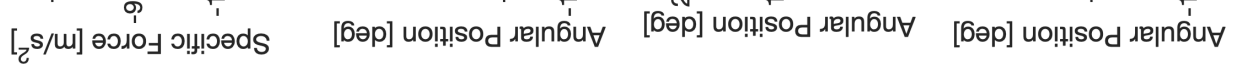

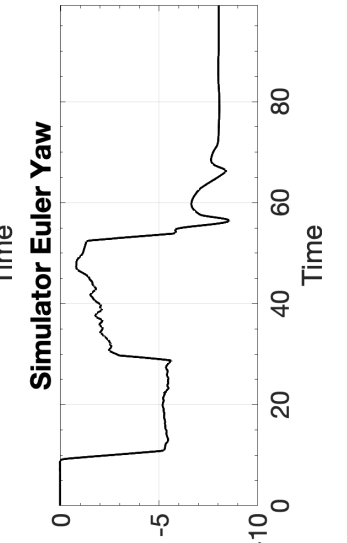

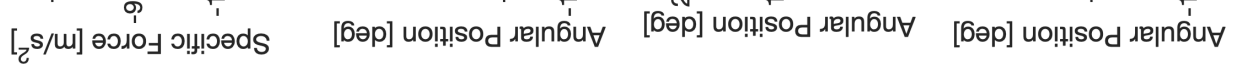

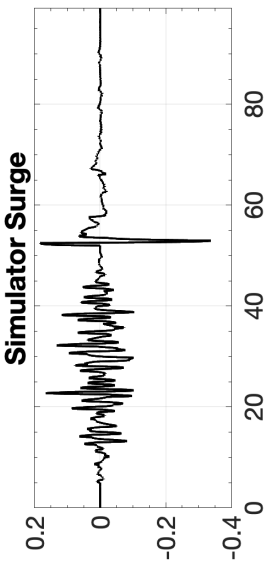

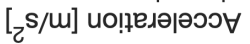

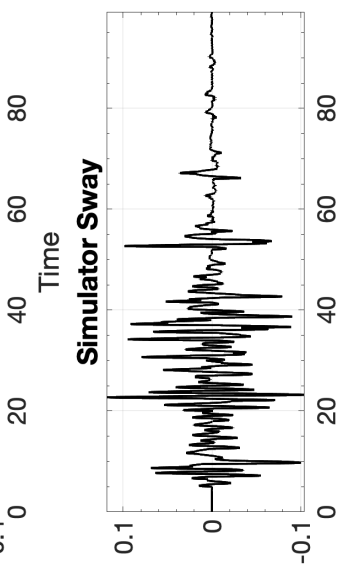

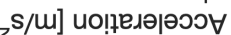

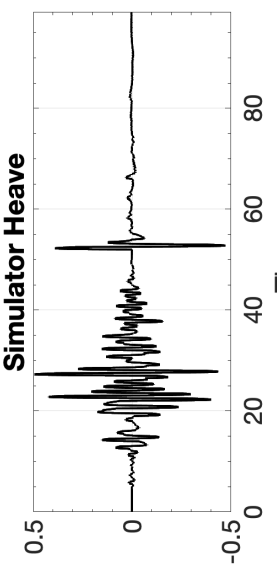

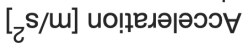

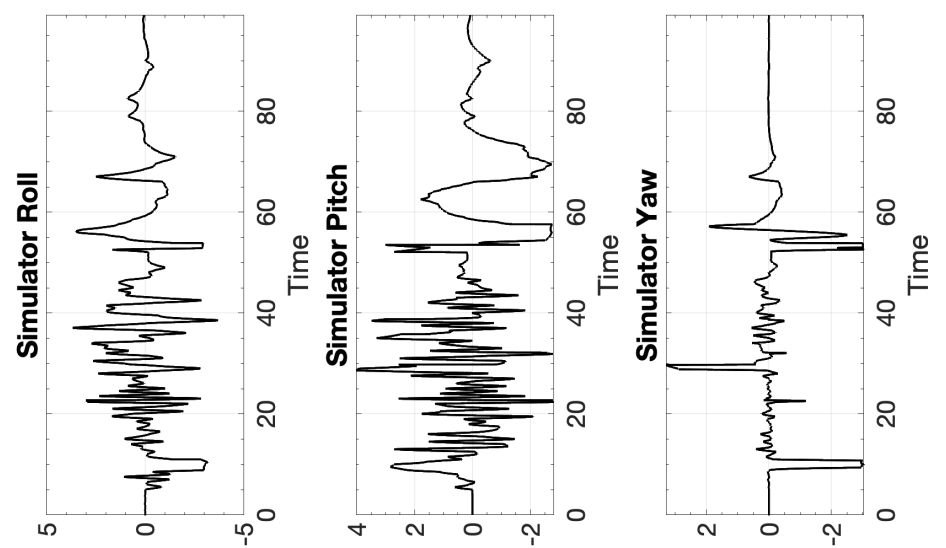

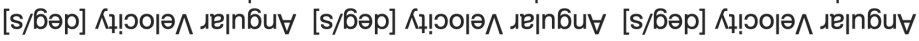




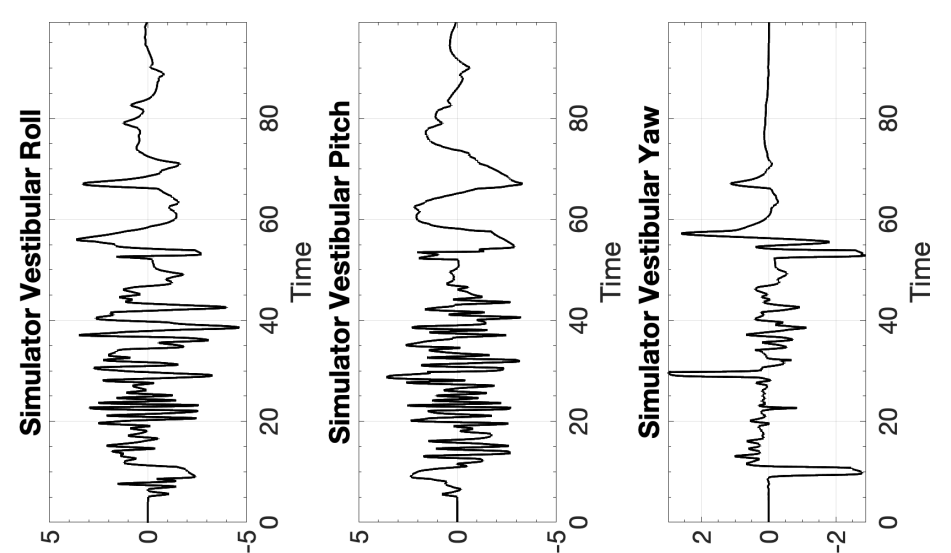

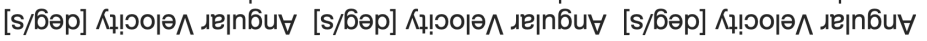

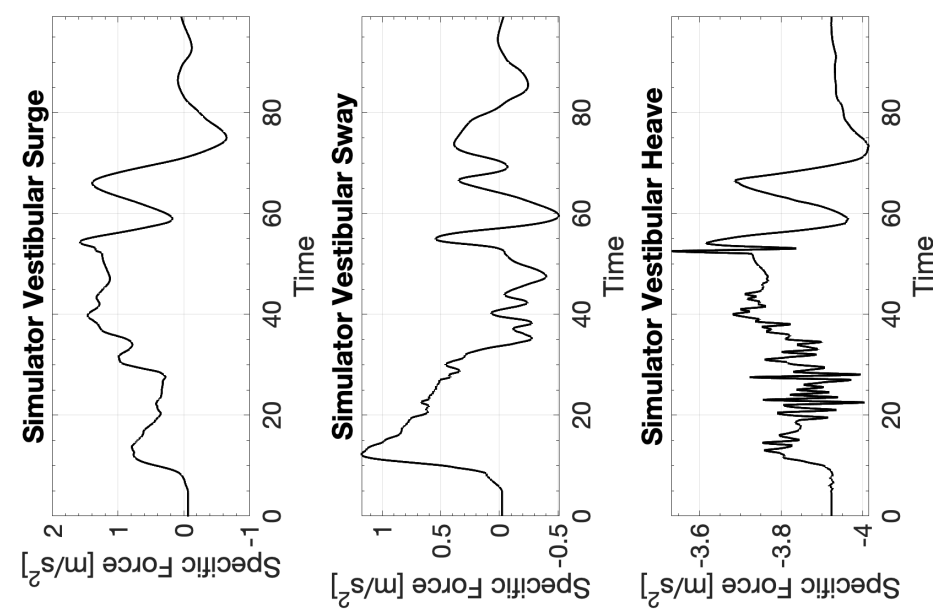




\section{C.11.3 CW3 Boeing 737 Single-axis Takeoff}

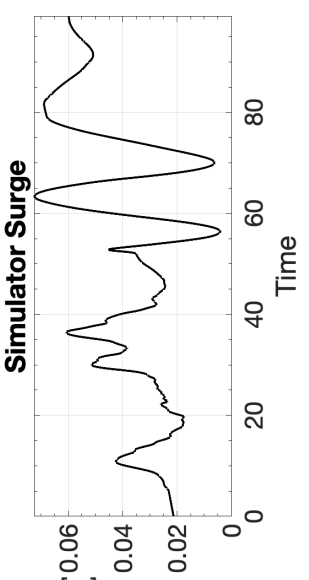

[u] uo!!!!sod

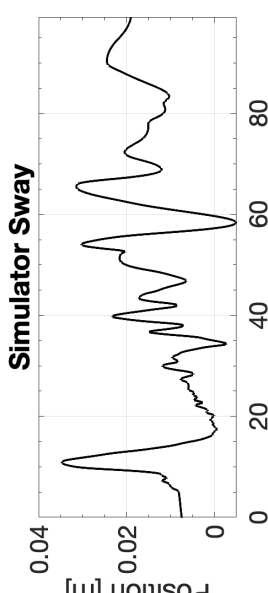

[w] uol!!sod

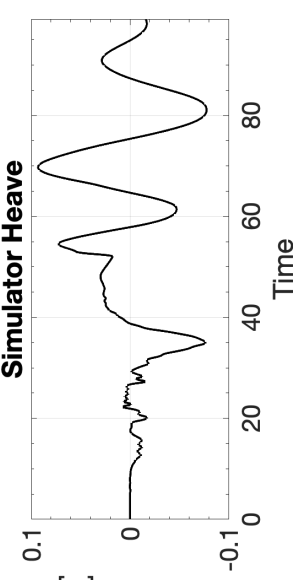

[u] uo!t!sod

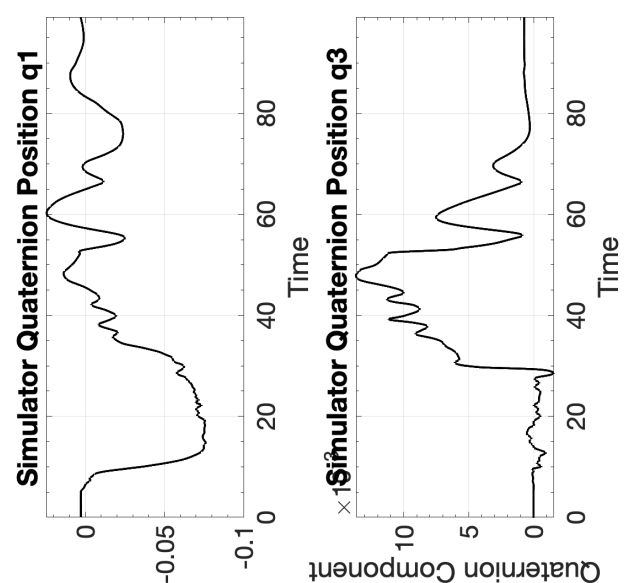

ұuəuodmos uọudəłeno

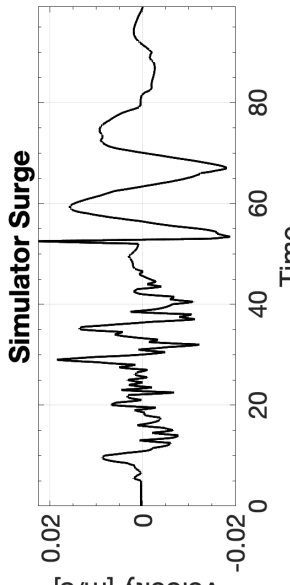

[s/u] Кұ!юор^

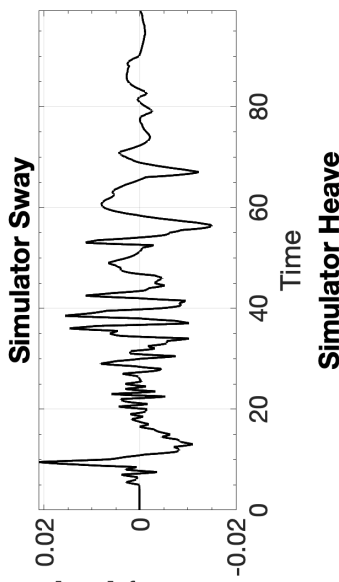

[s/m] К! юорә

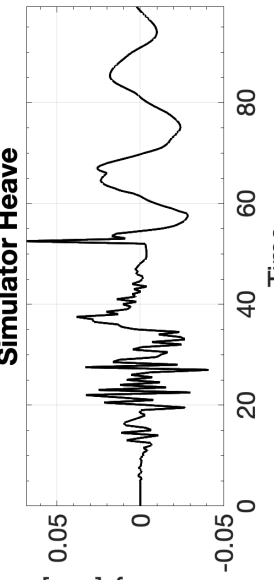

[s/m] К! Роюә^

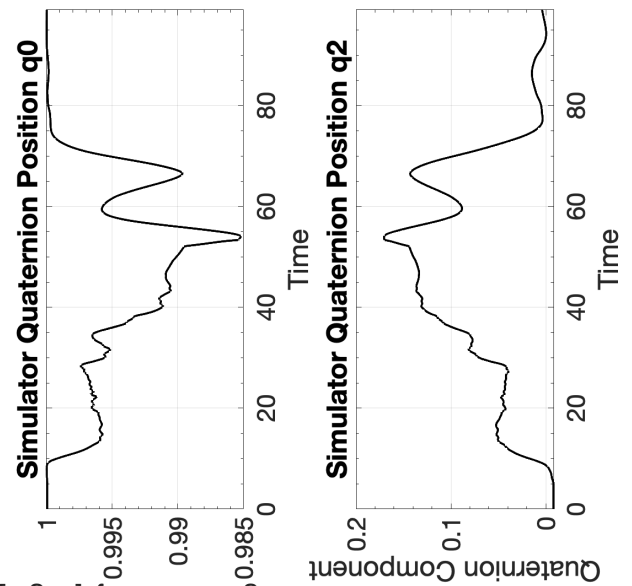

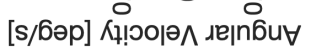

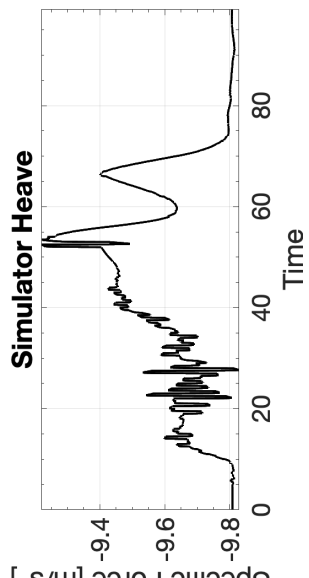

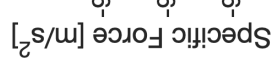

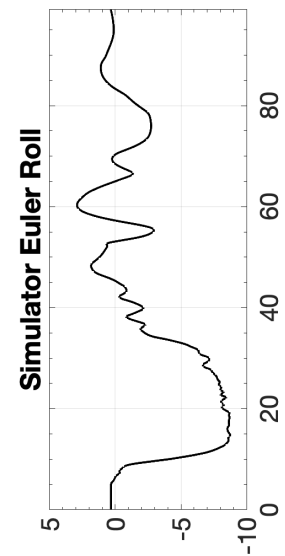

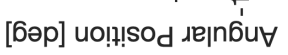

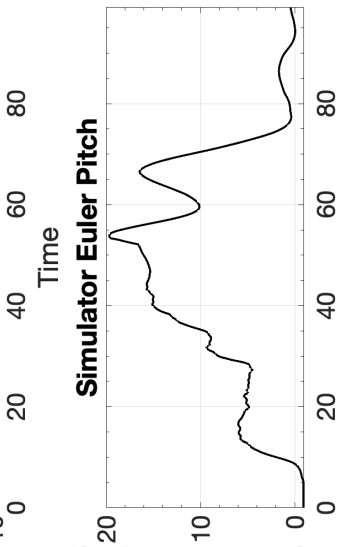

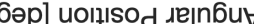

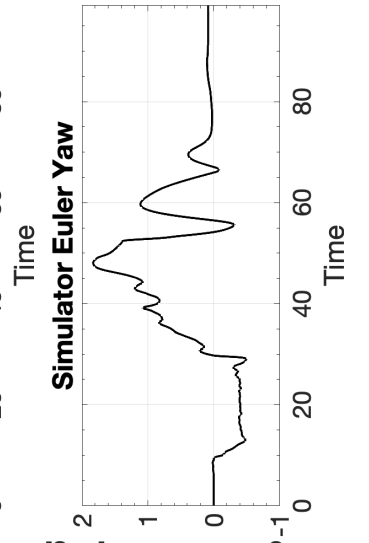

[6әр] uo!?!sod גeןn6u

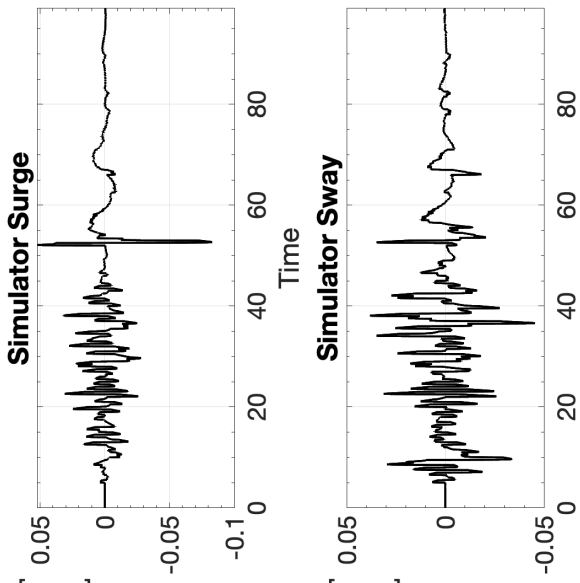

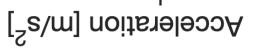

[z $s / m]$ uо!ฺеләәәэว

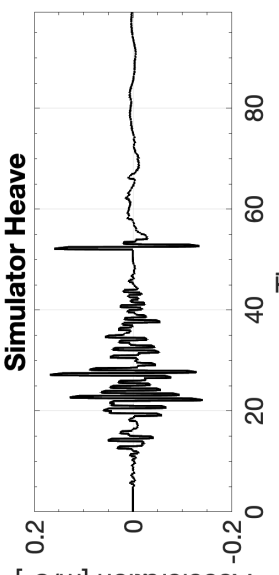

[S/m] ио!ฺедәрәээ

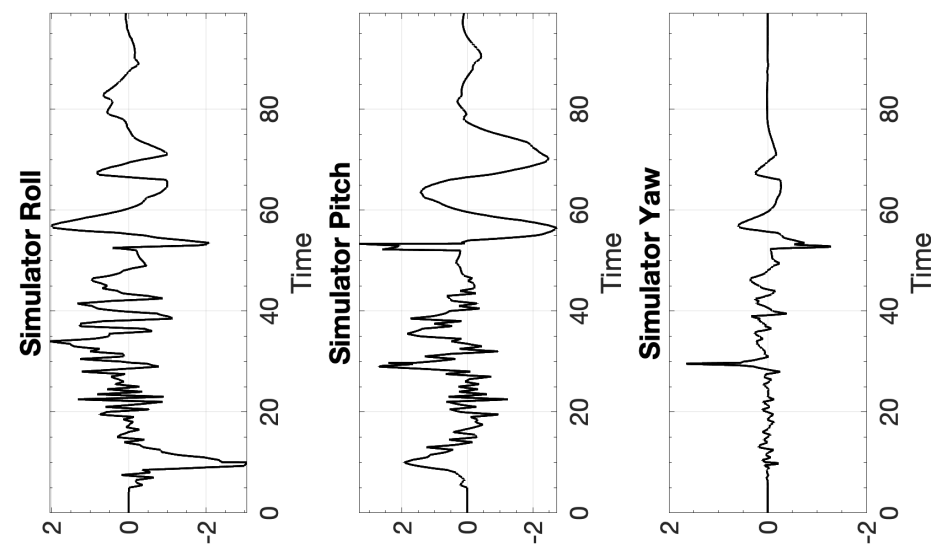

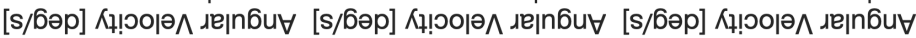



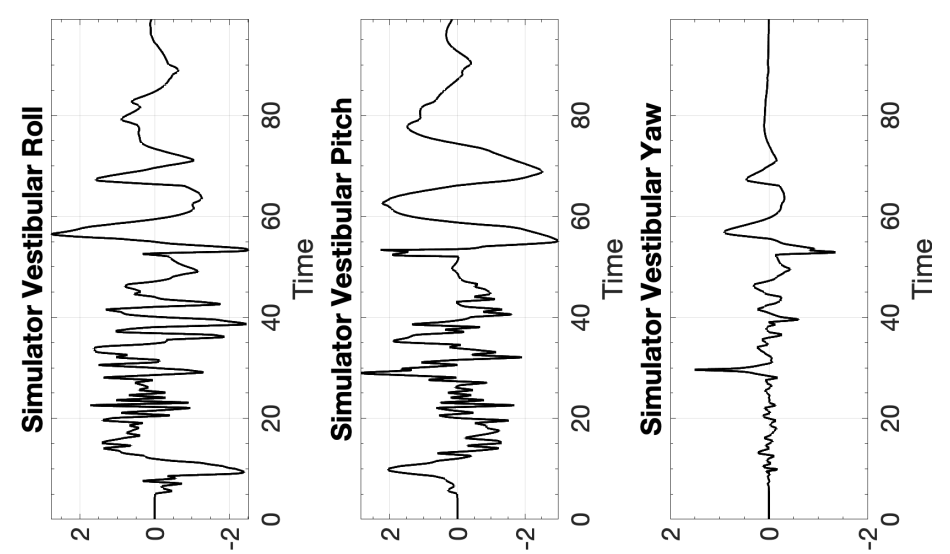

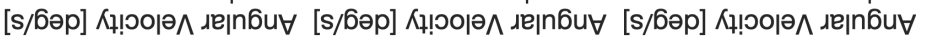

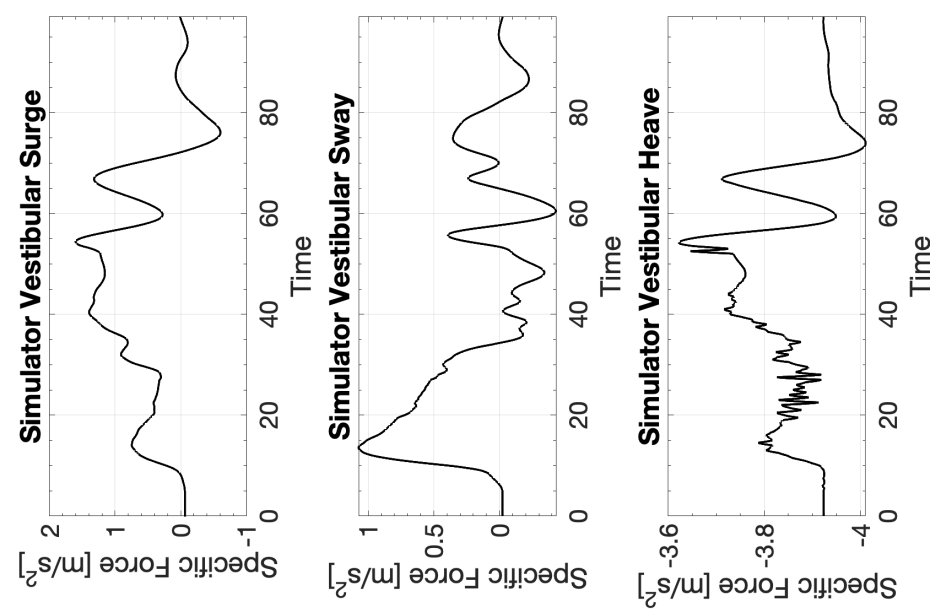




\section{C.12 Boeing 737 Spiral Dive}
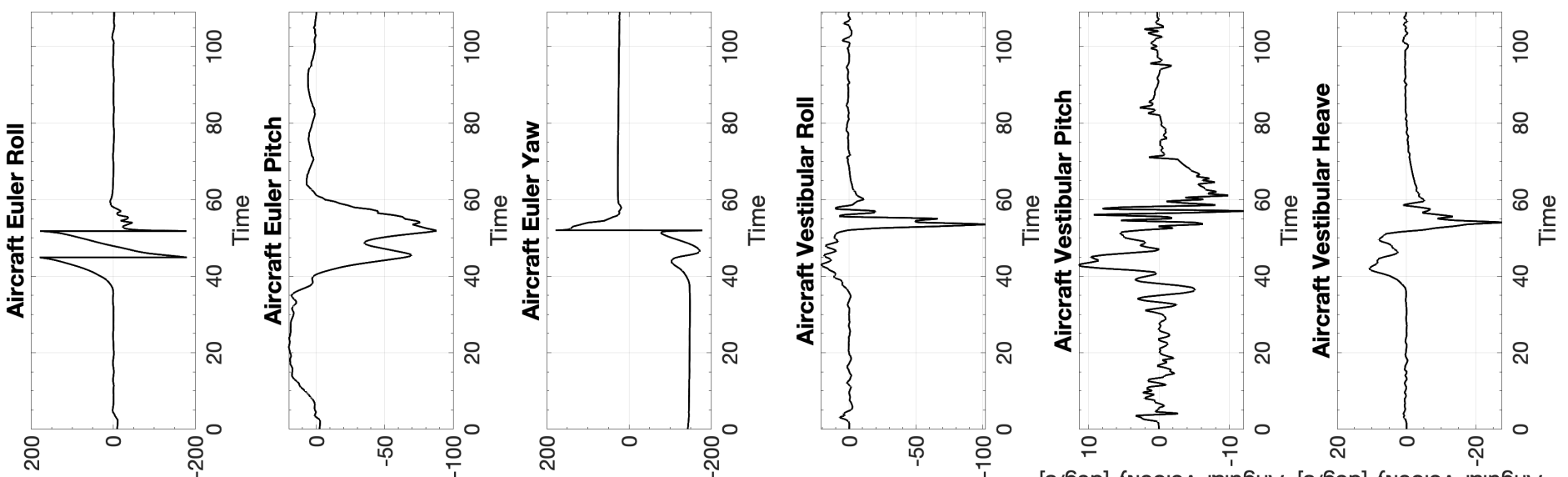

[6әр] uo!!!!sod גe|n6́u

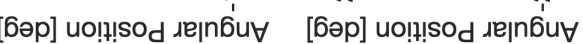

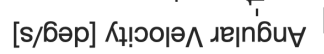

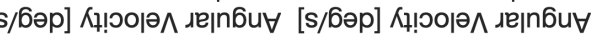
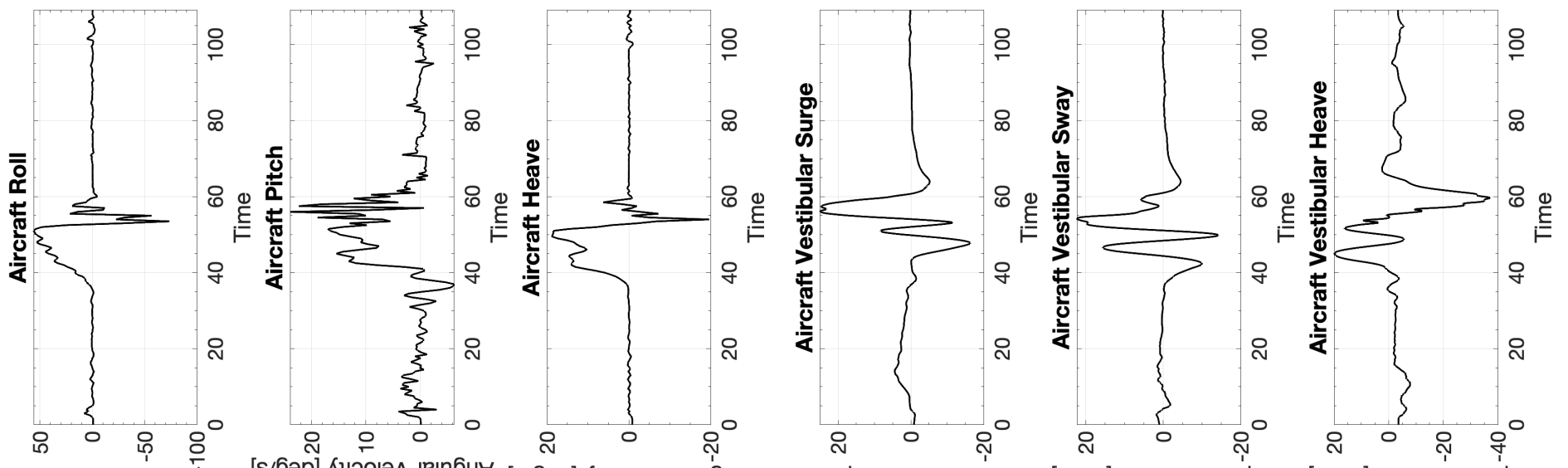

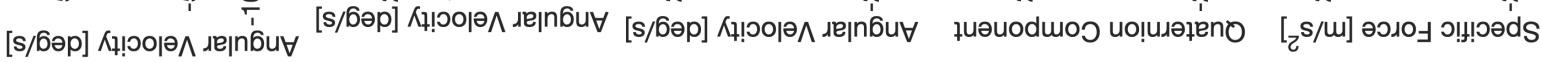

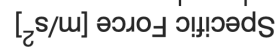

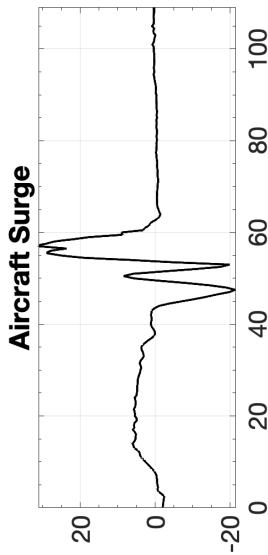

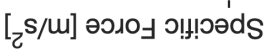

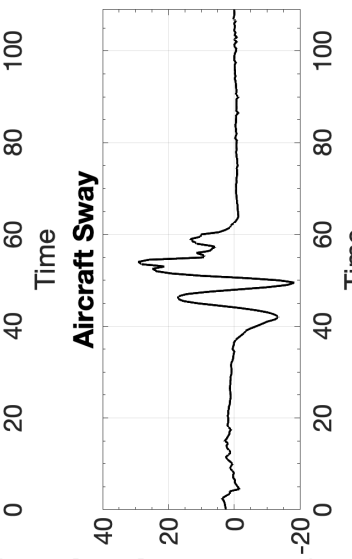

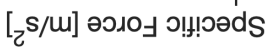

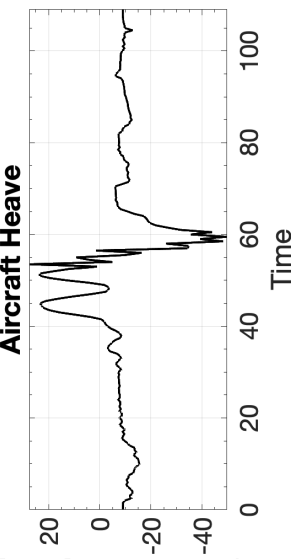

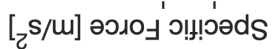

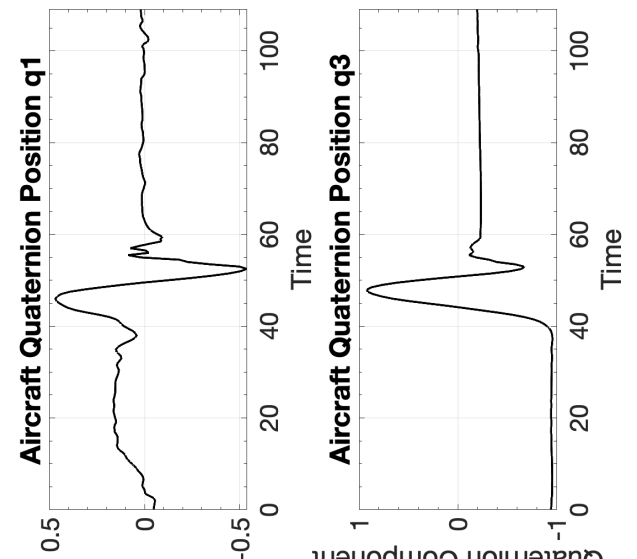

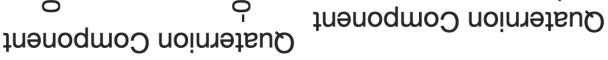

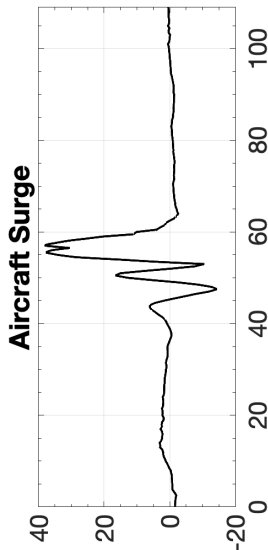

[zs/u] uо!ฺеләәәоэ $\forall$

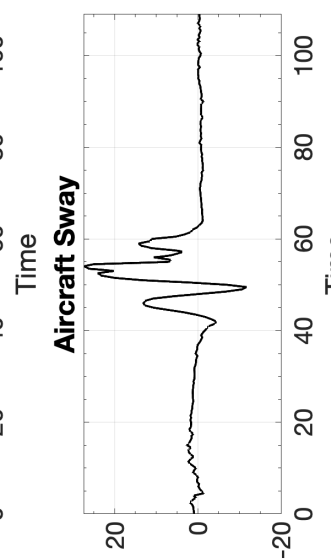

[s/m] ио!ฺедәрәоэ

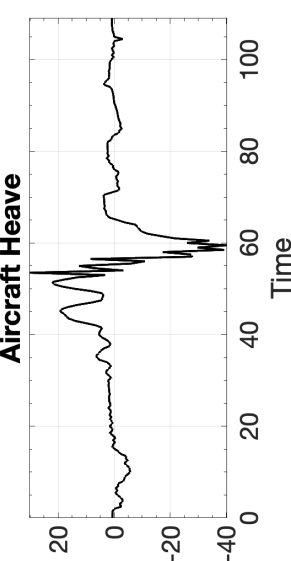

[s/m] uо!ฺвдәрәоэ

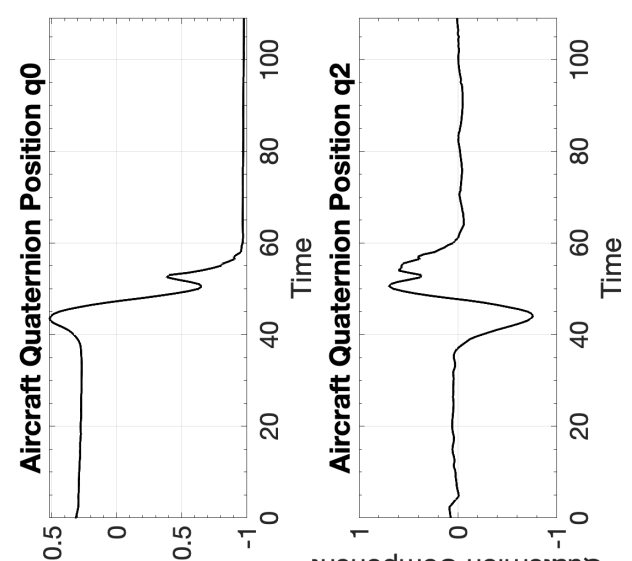

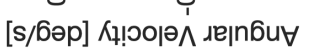

łuəuodmos uo!̣uəłeno 


\section{C.12.1 CW1 Boeing 737 Spiral Dive}

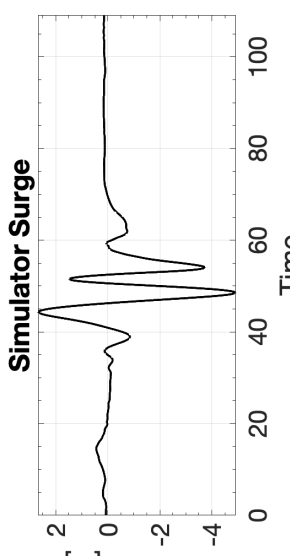

[w] uo!!!sod

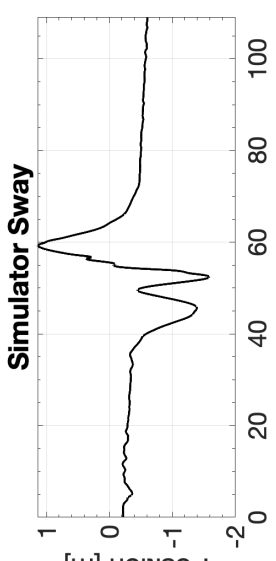

[u] uo!?! sod

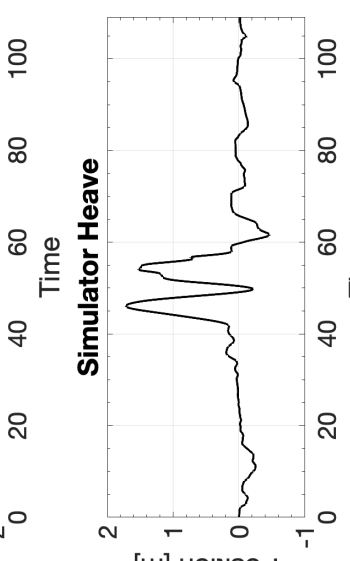

[w] uo!n! sod

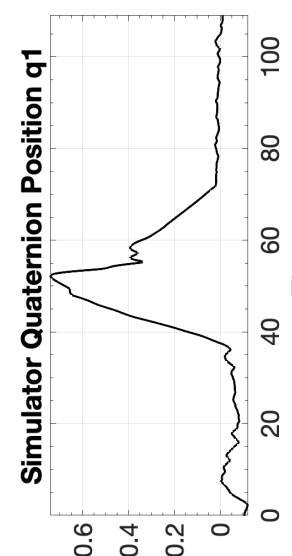

@ั

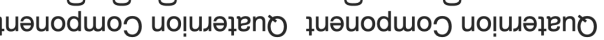

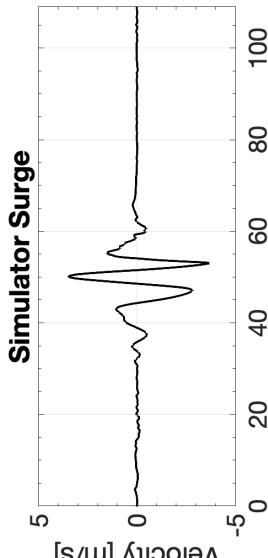

[s/u] Кұ! оо|ә
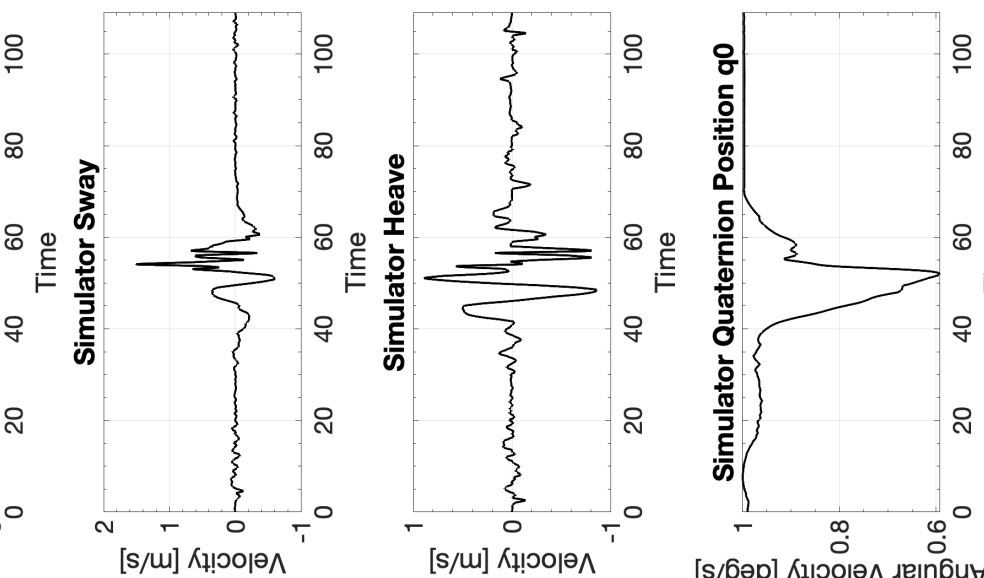

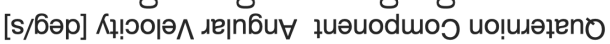

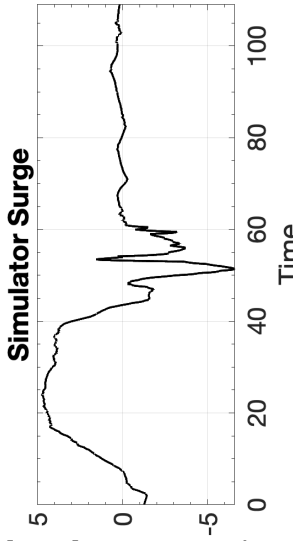

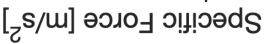

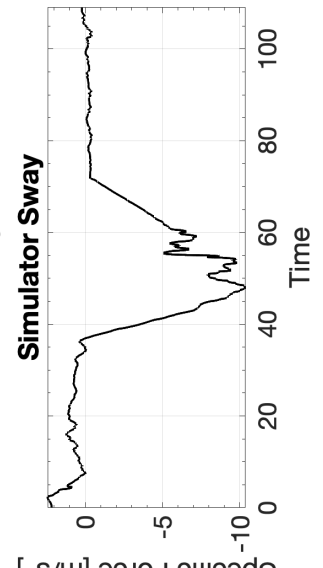

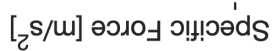

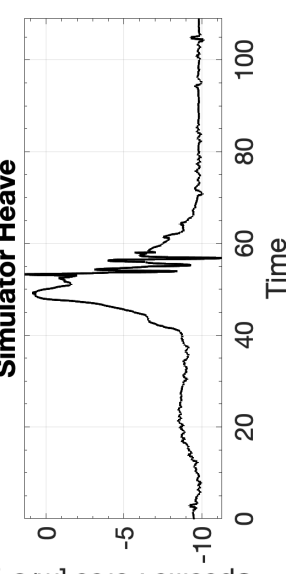

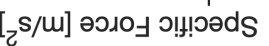

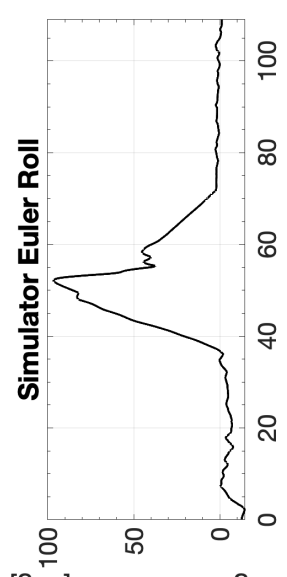

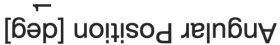
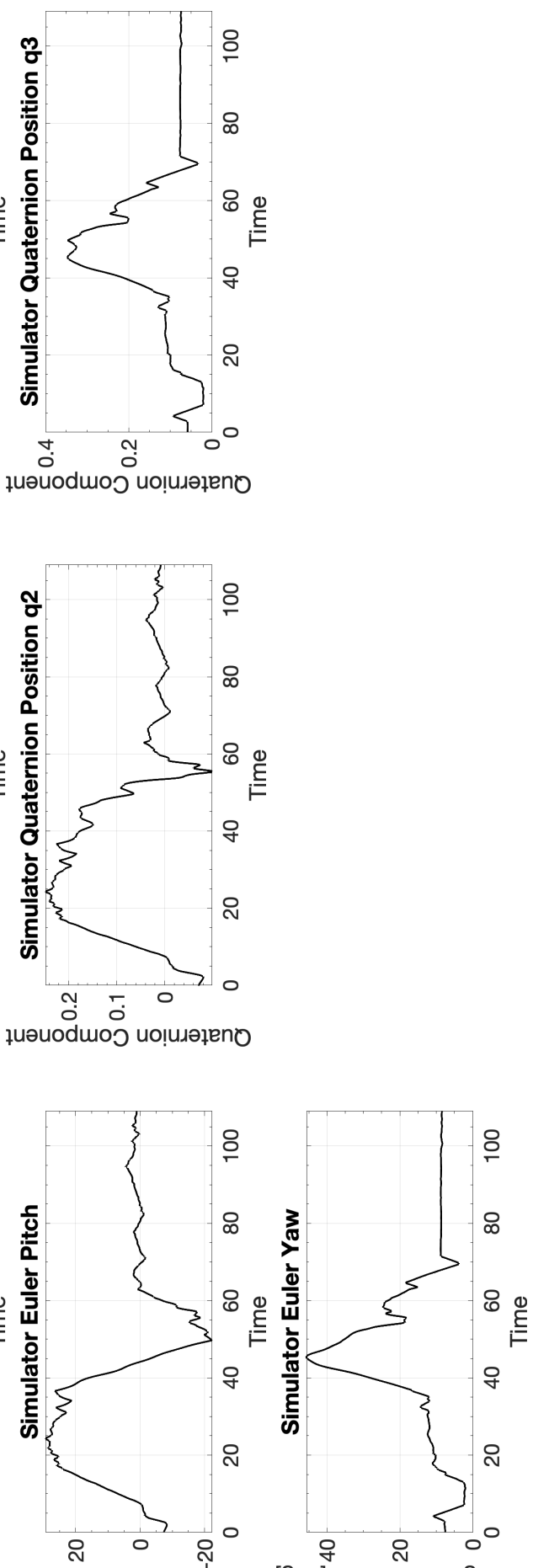

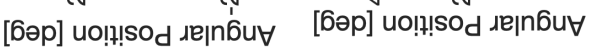

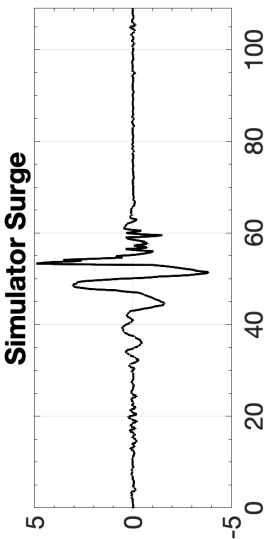

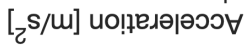

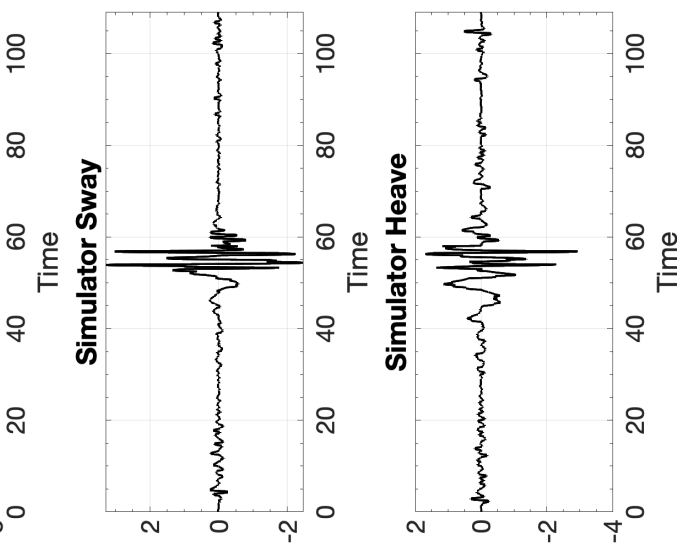

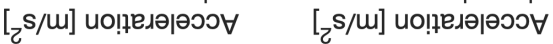

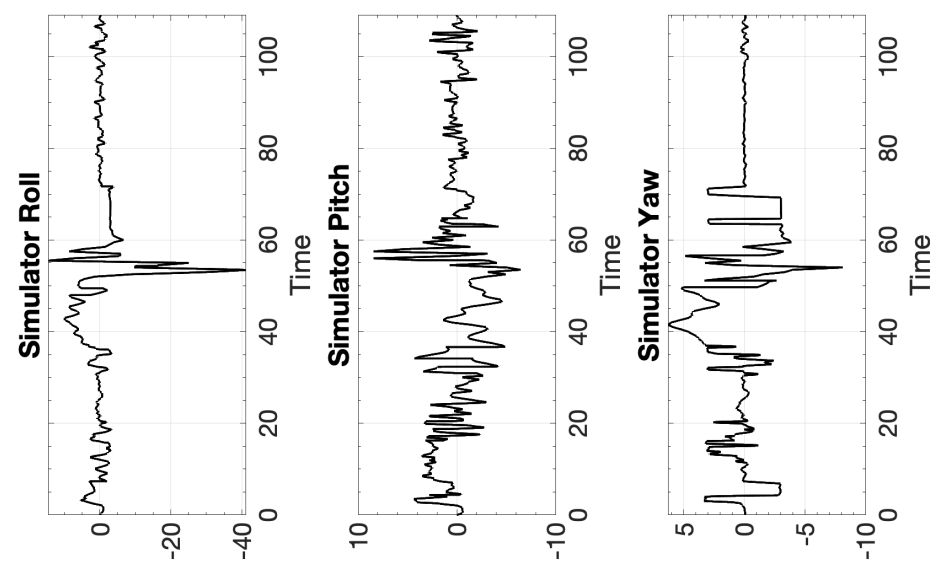

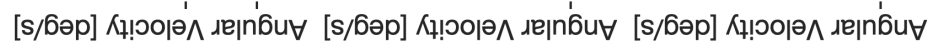



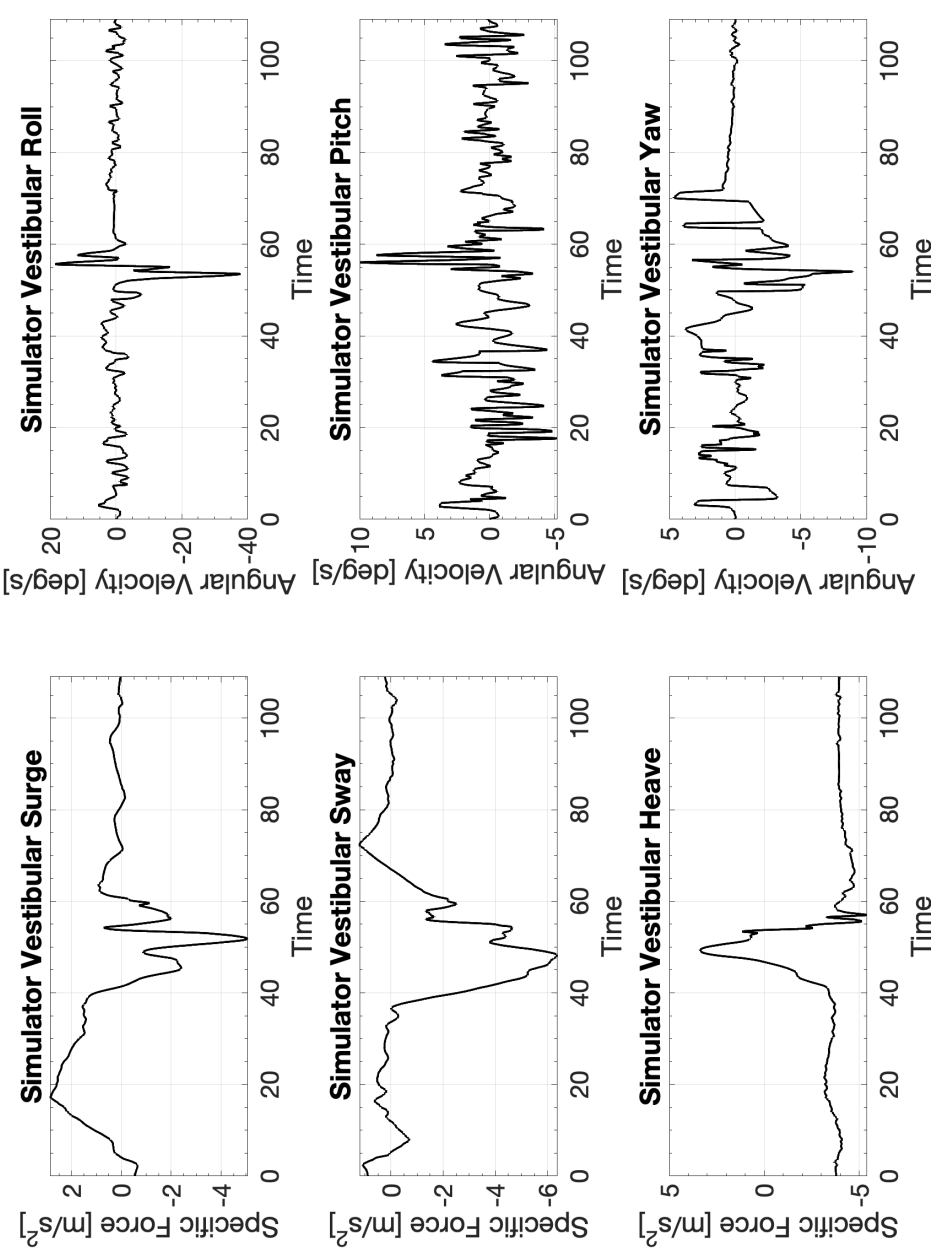


\section{C.12.2 CW2 Boeing 737 Spiral Dive}
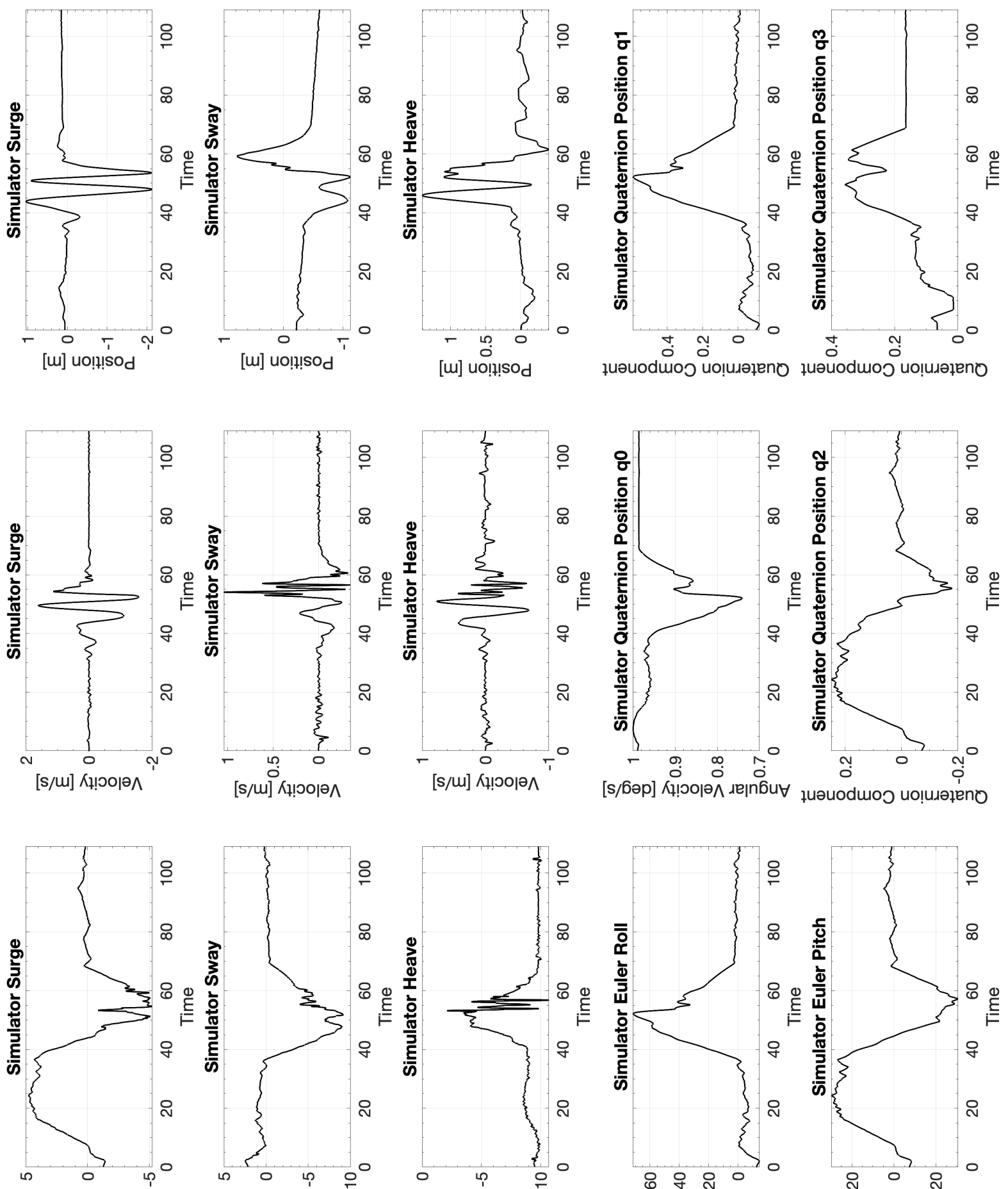

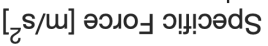
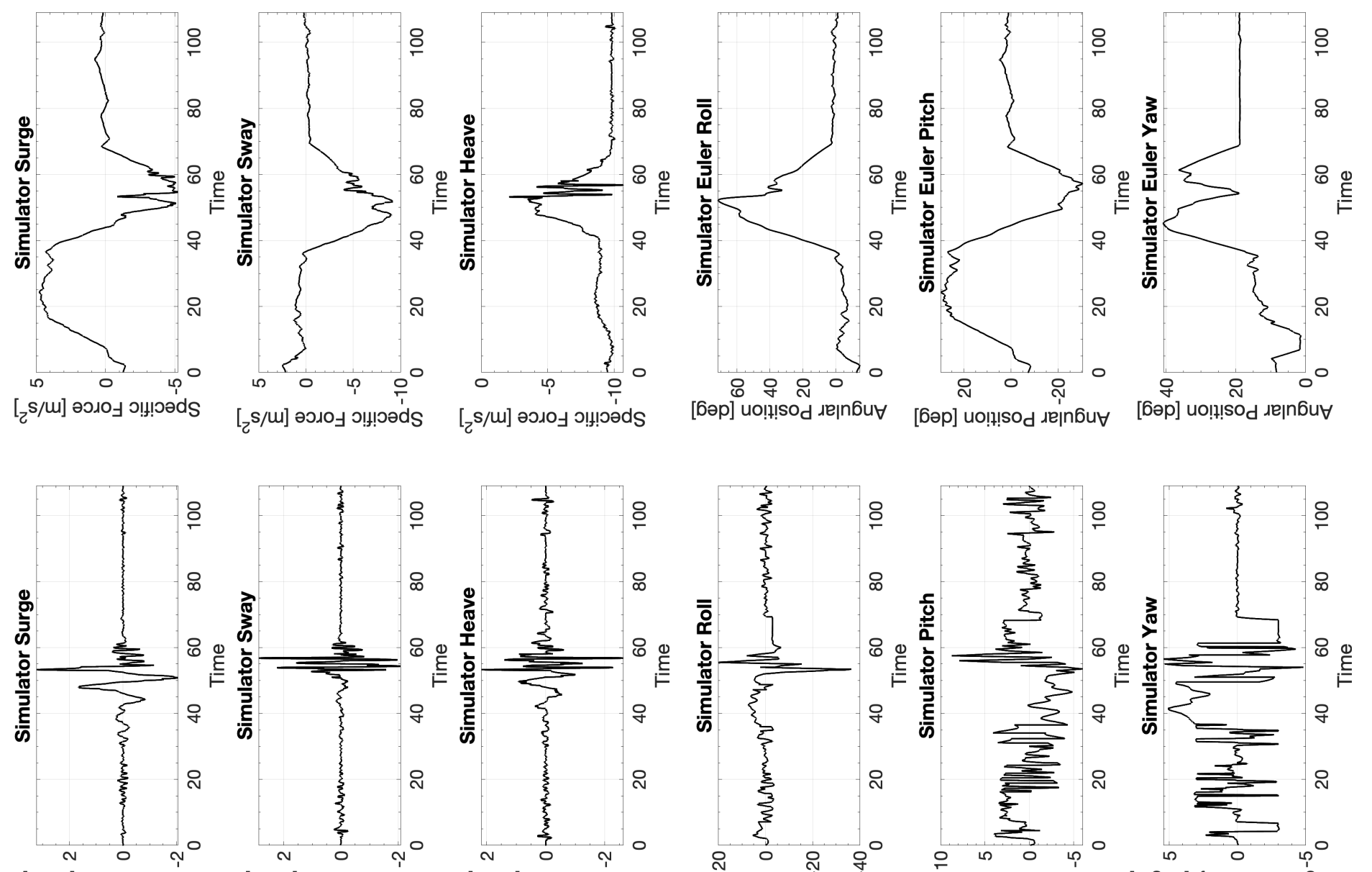

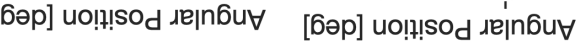

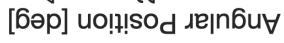

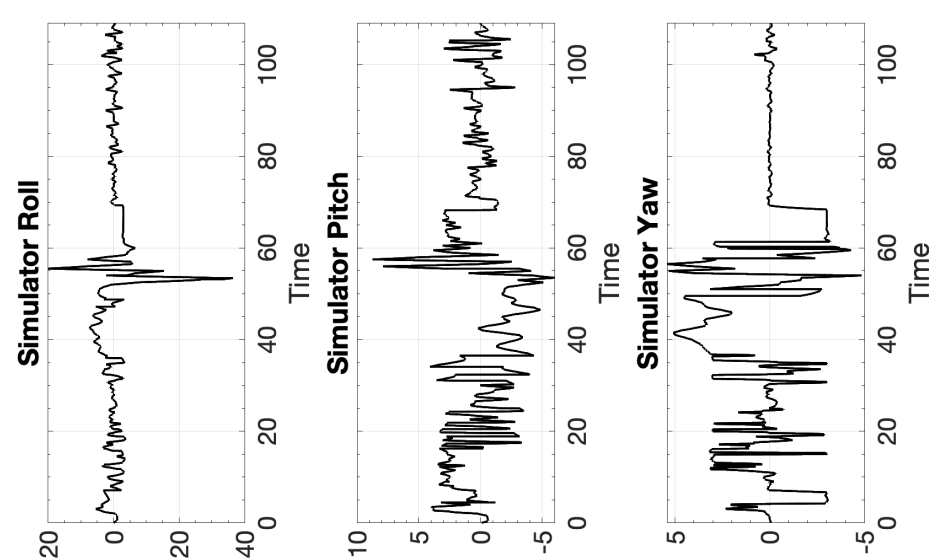



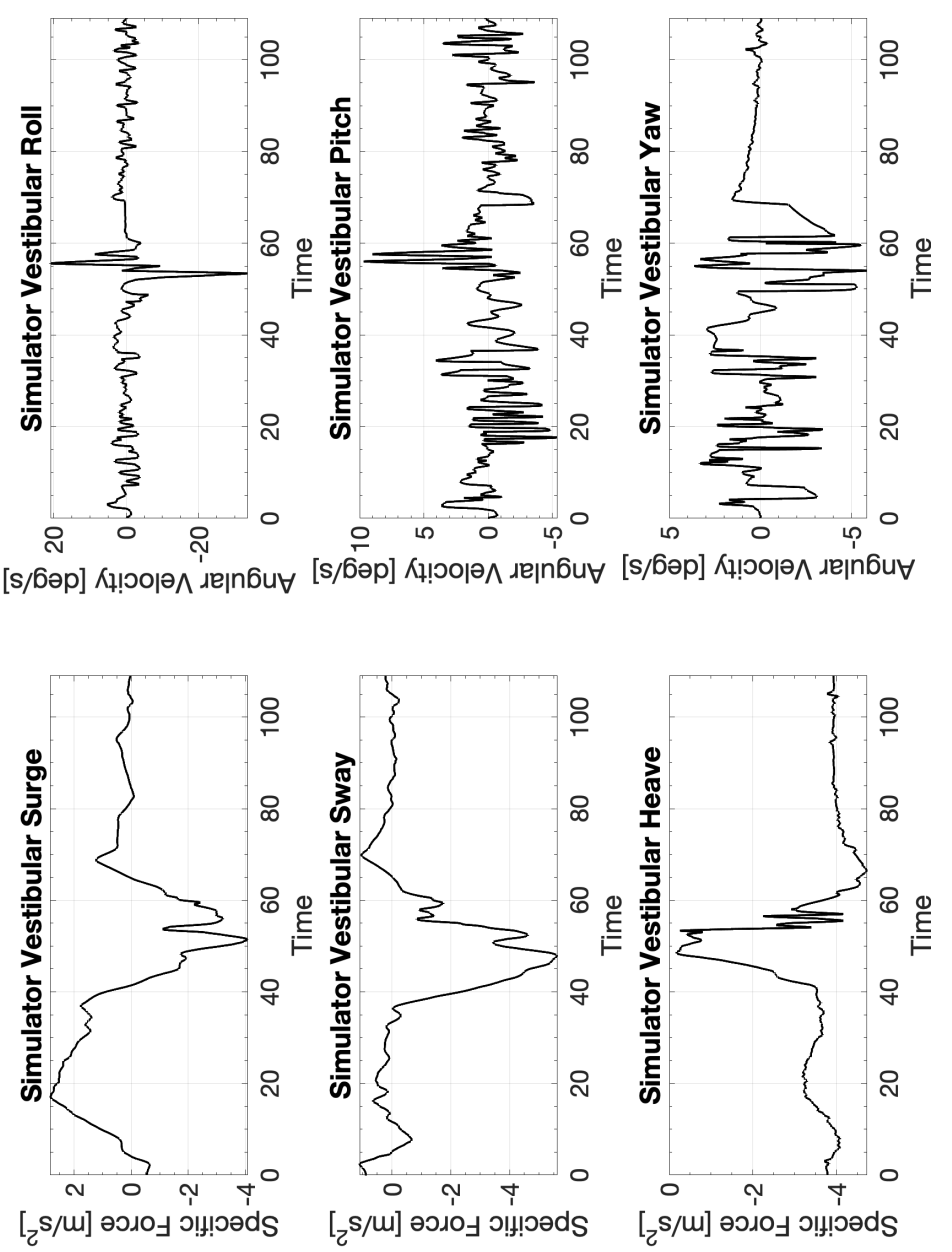


\section{C.12.3 CW3 Boeing 737 Spiral Dive}

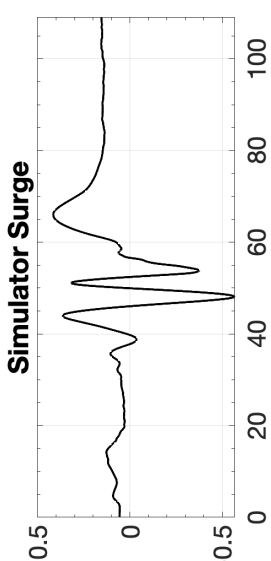

[m] uo!n!

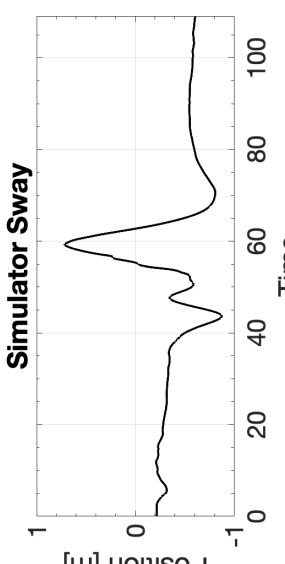

[w] uo!!!!sod

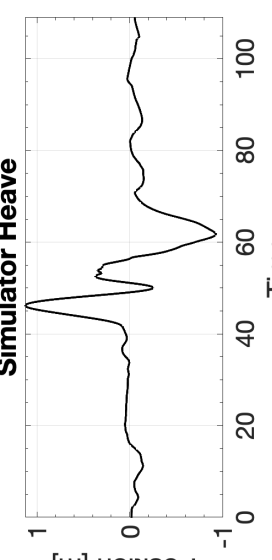

[u] uo!n!sod

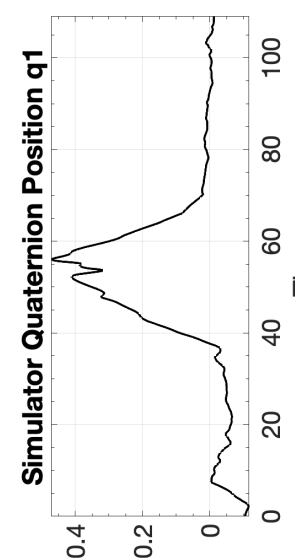

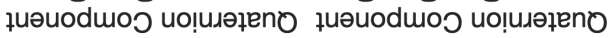

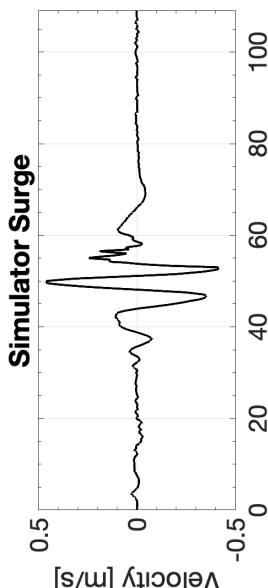

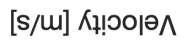
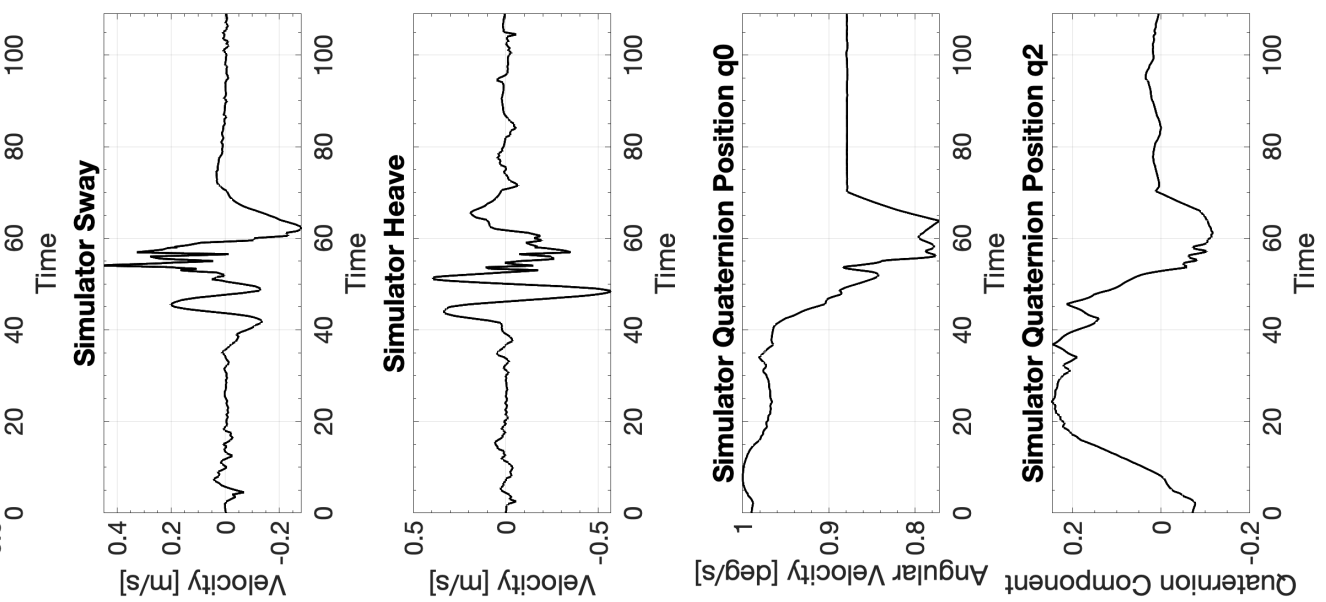

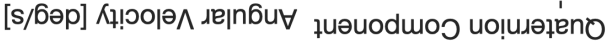

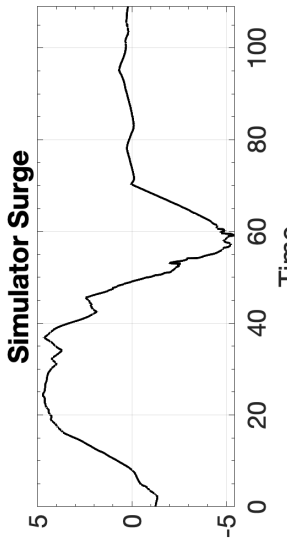

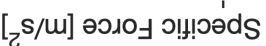

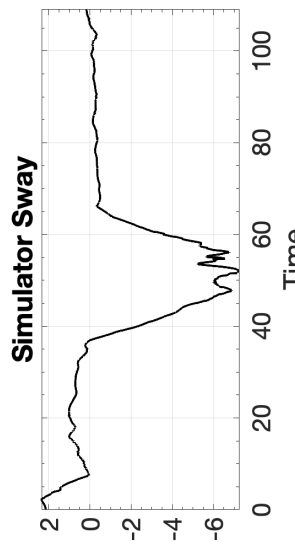

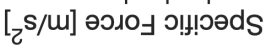

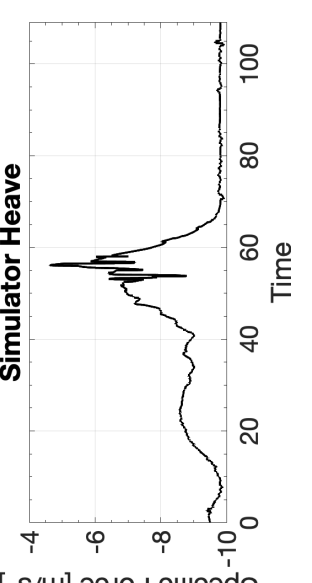

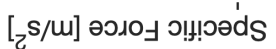

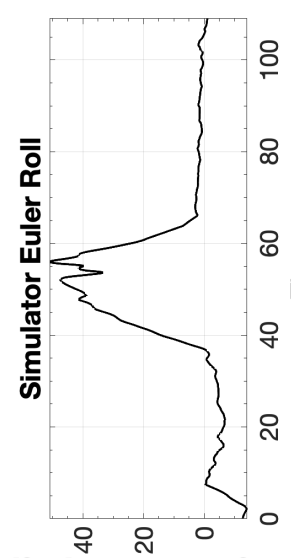

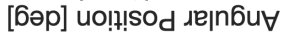

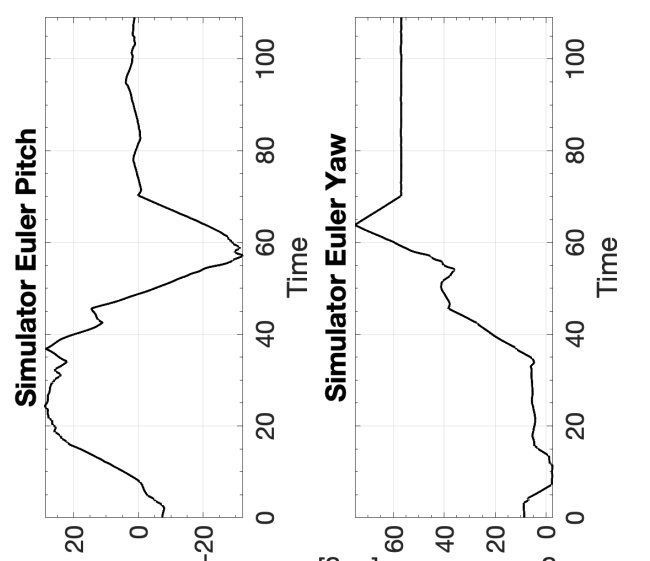

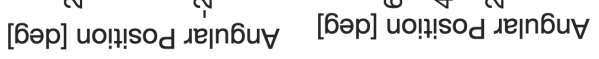

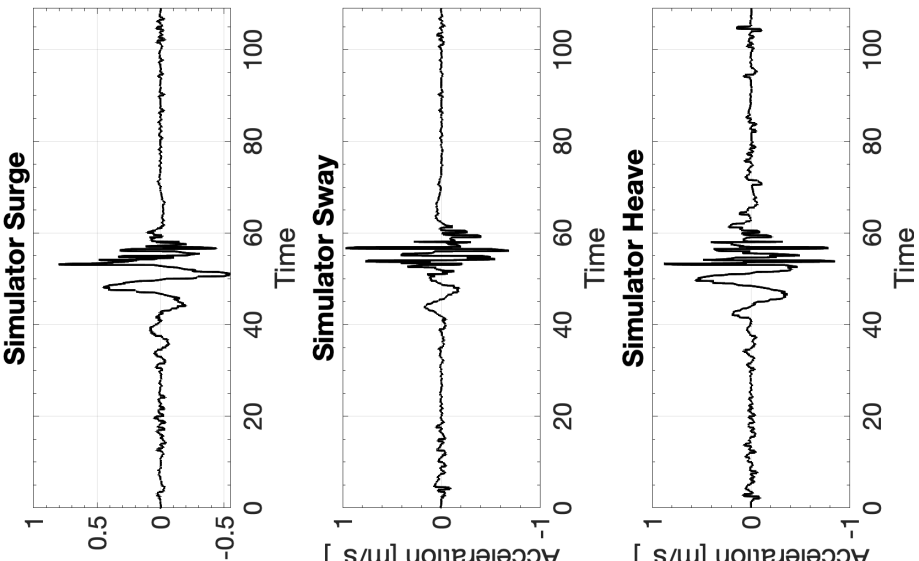

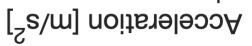

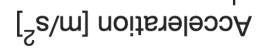

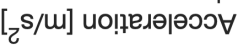

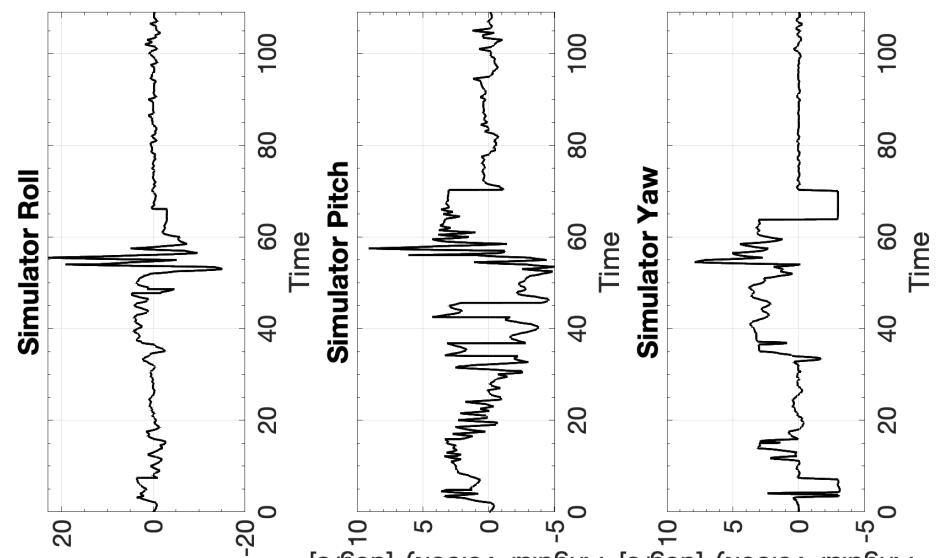

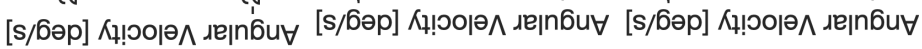




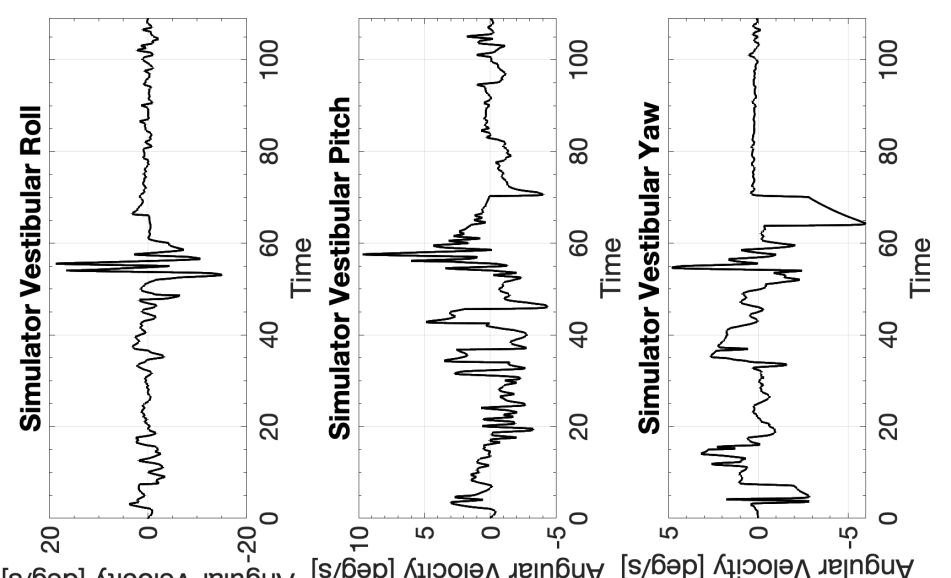

[s/бәр] Кұ! оорә

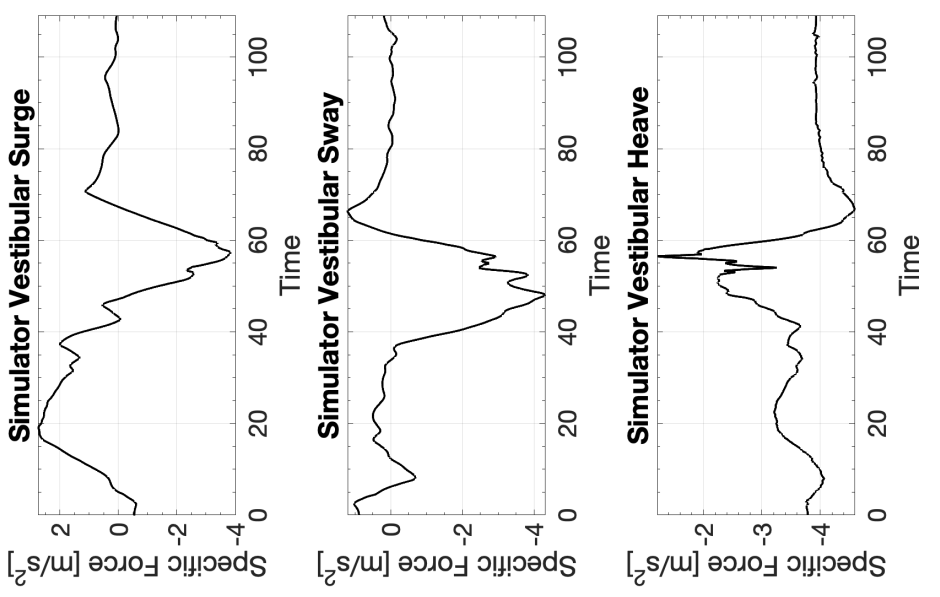




\section{C.13 Boeing 737 Stall Recovery}

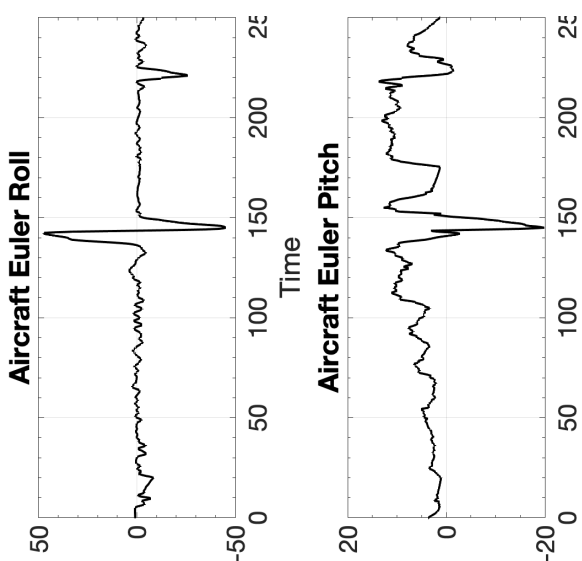

[6əp] uo!!!sod

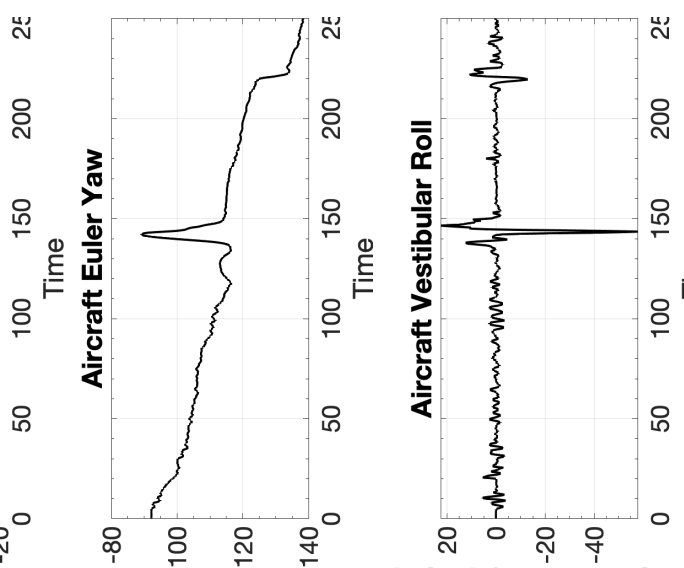

[6əp] uo!!! $\mathrm{SO}_{\mathrm{d}} \lambda \mathrm{e} \mid \mathrm{n} 6 \mathrm{u} \forall$

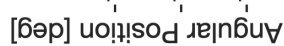
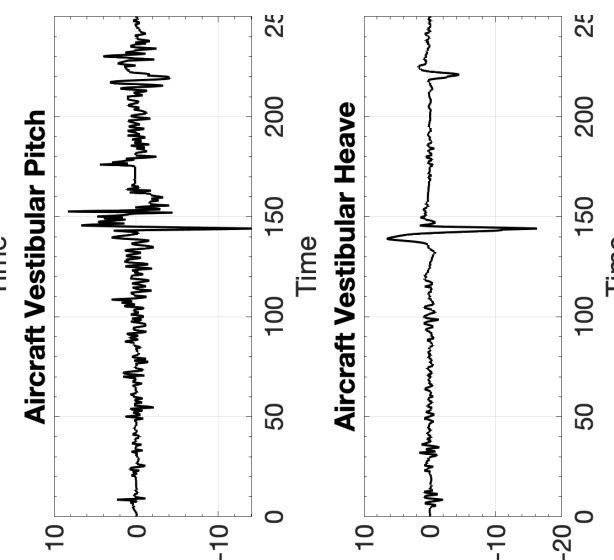

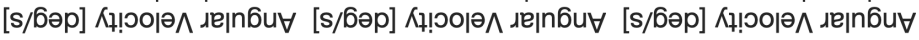
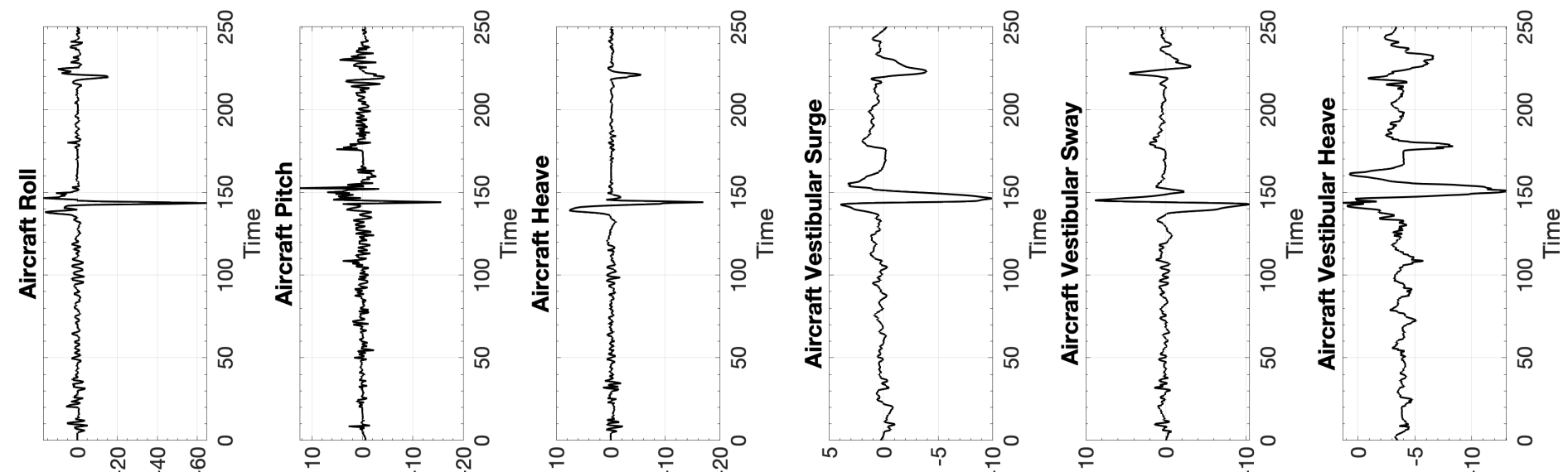

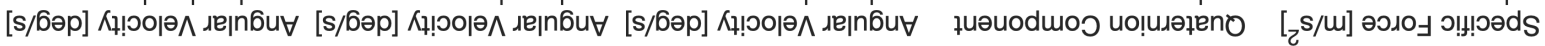

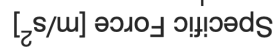

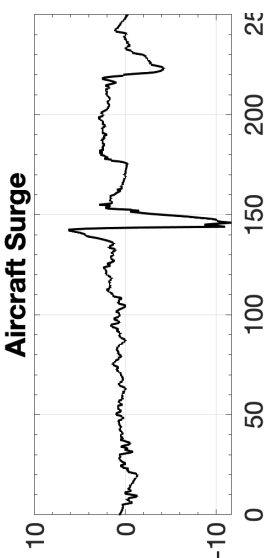

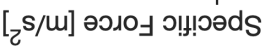

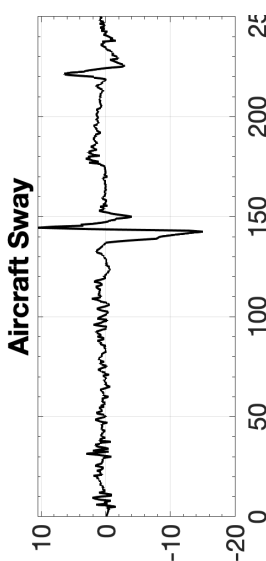

$[\mathrm{s} / \mathrm{m}]$ әองด

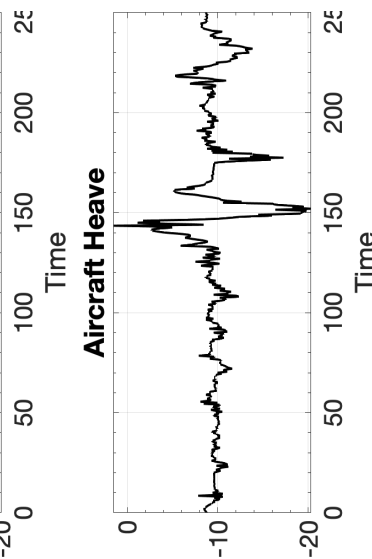

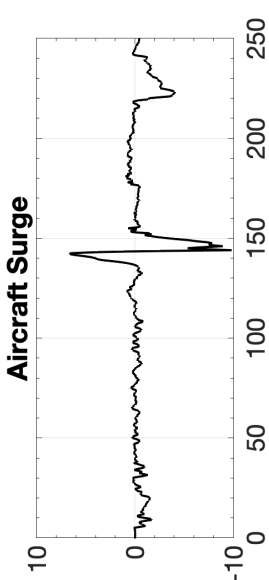

$\left[{ }_{2} \mathrm{~s} / \mathrm{m}\right]$ uо!̣อлә|әэว $\forall$

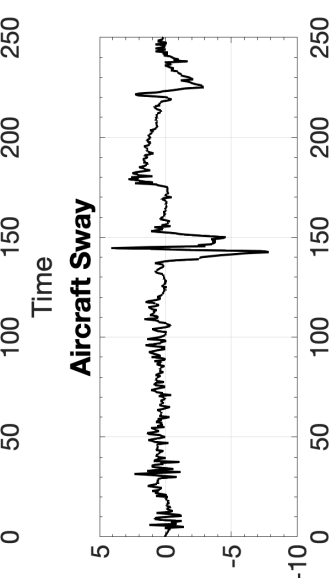

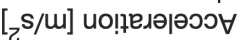

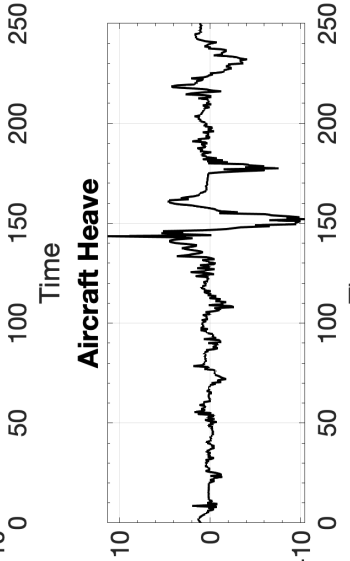

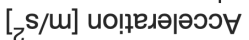

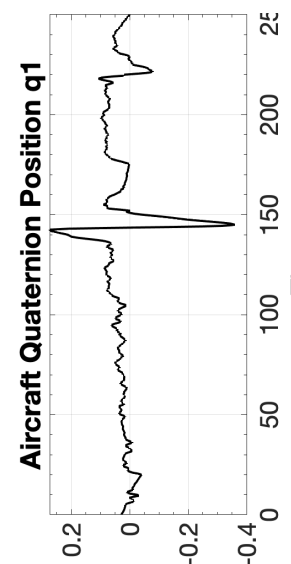

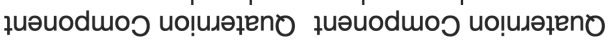

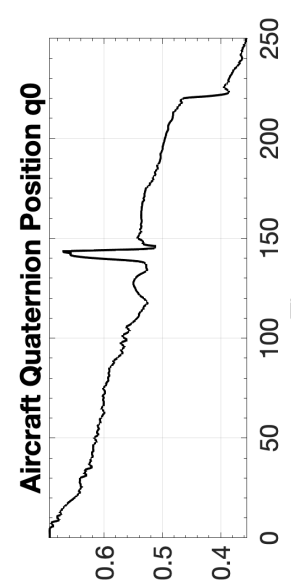

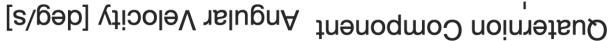
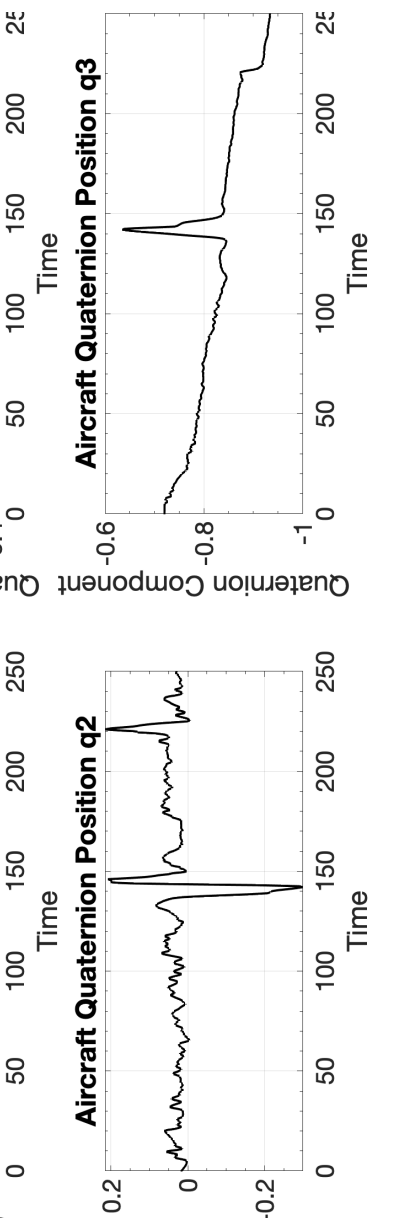


\section{C.13.1 CW1 Boeing 737 Stall Recovery}

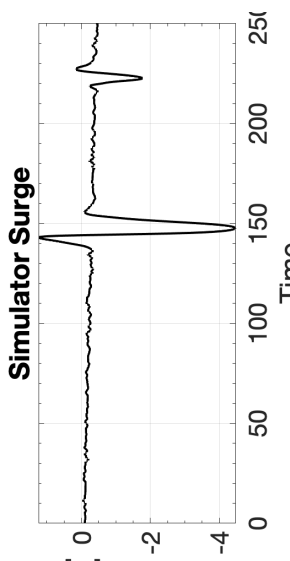

[w] uo!!!!sod

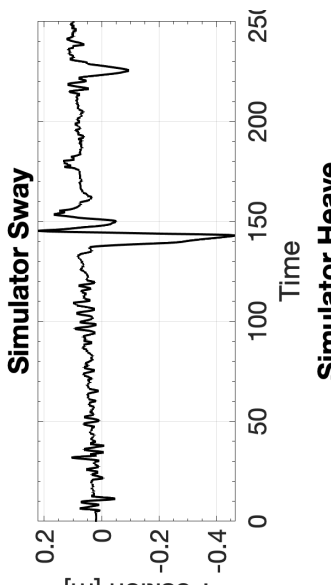

[m] uo!m!sod

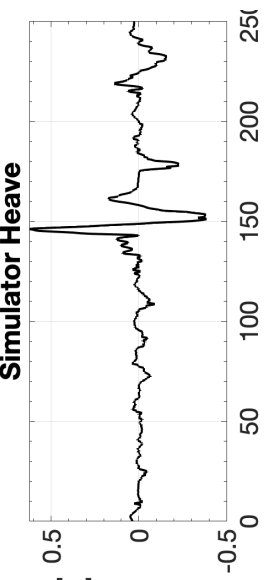

[w] uo!n!sod

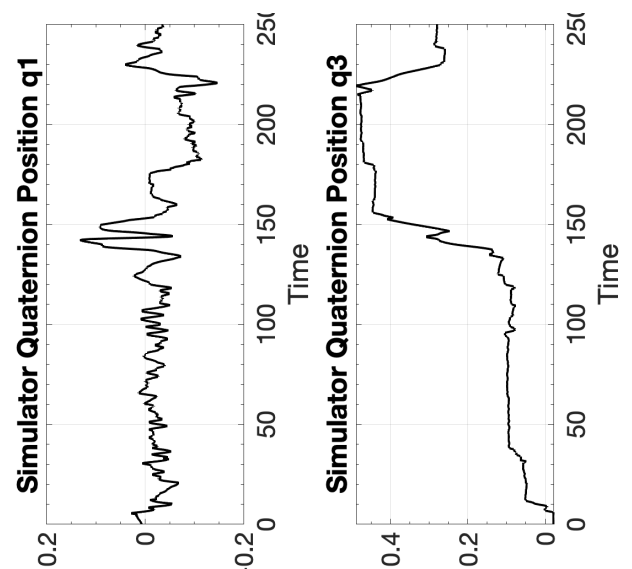

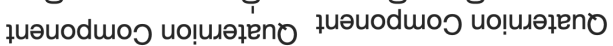

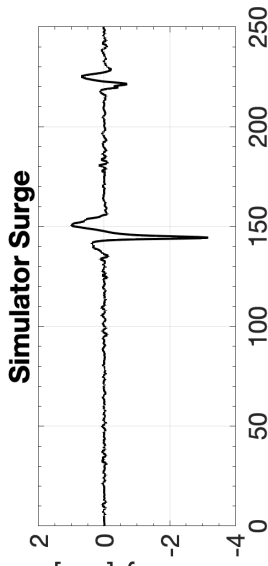

[s/u] Кұ!०оำ
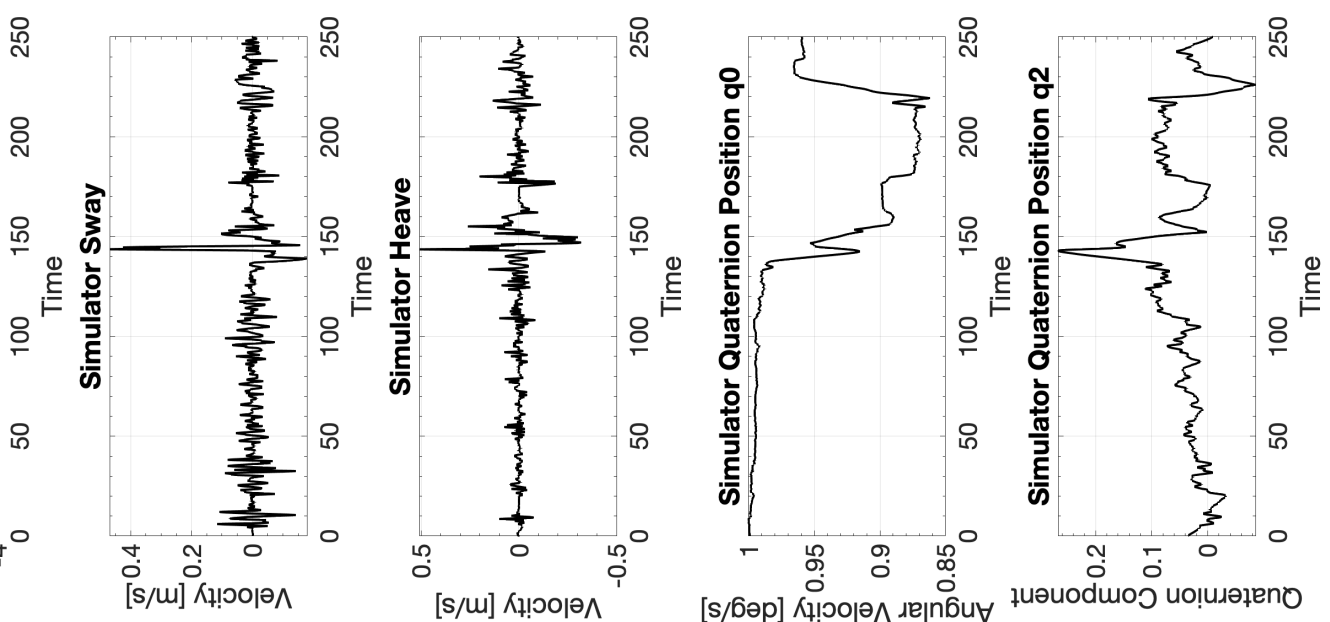

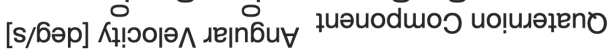

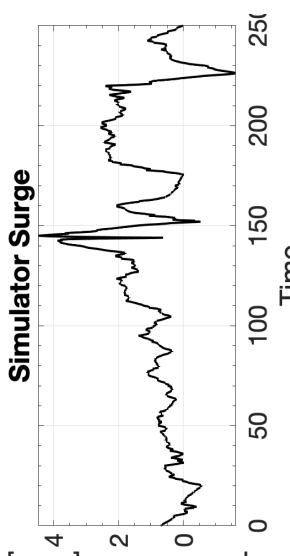

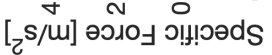
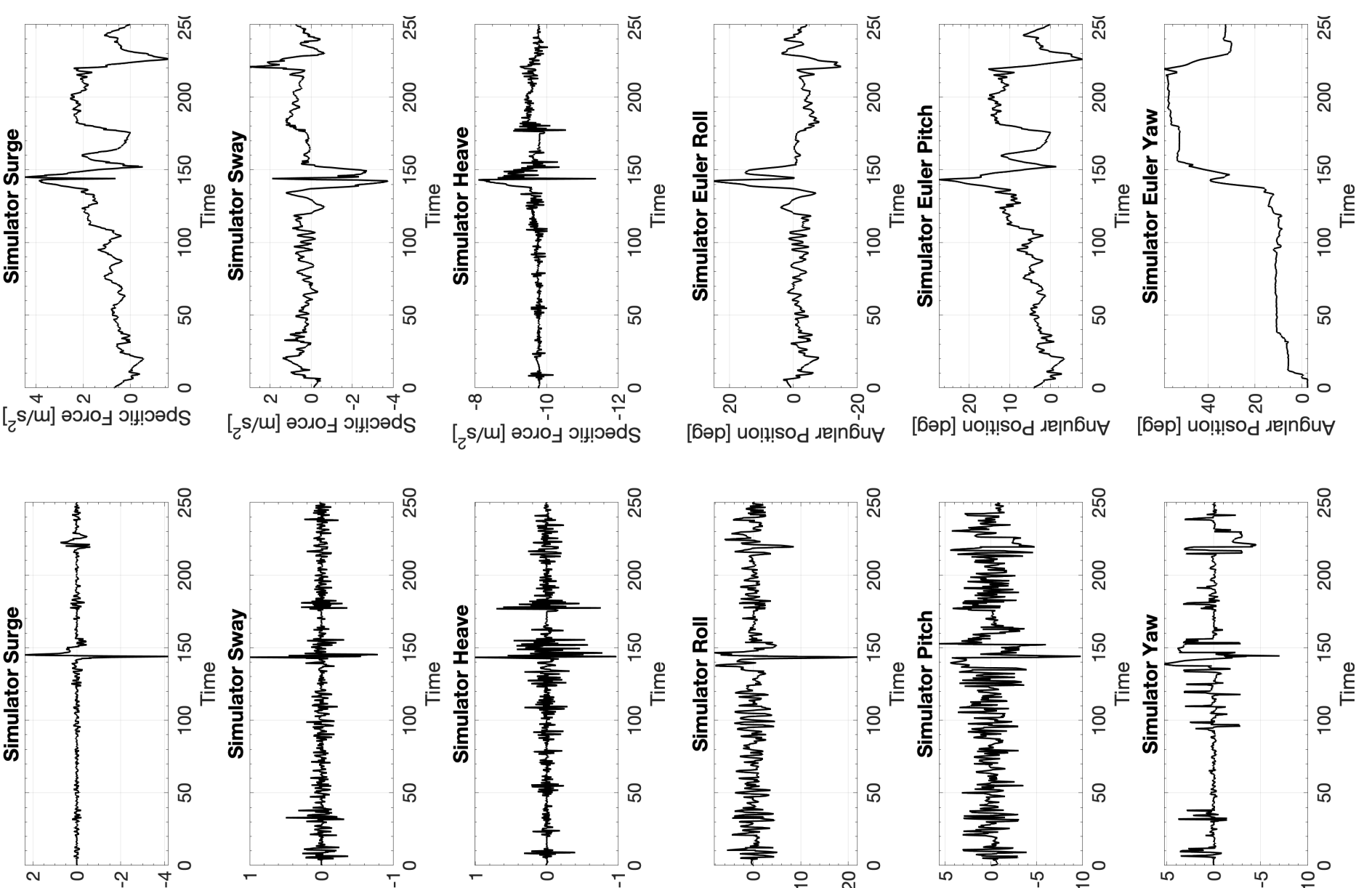

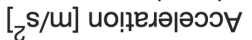

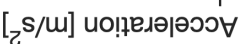

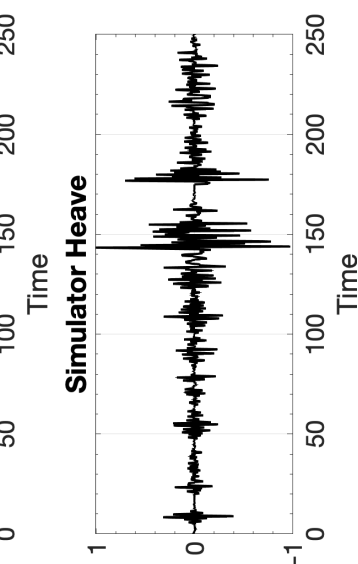

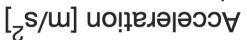
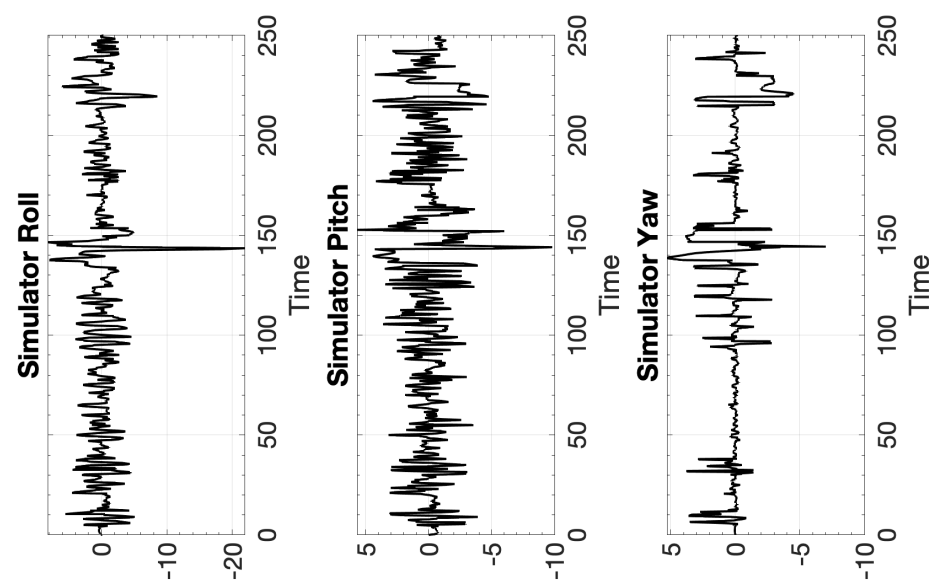

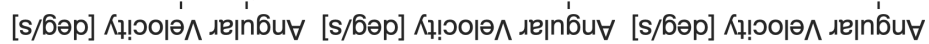



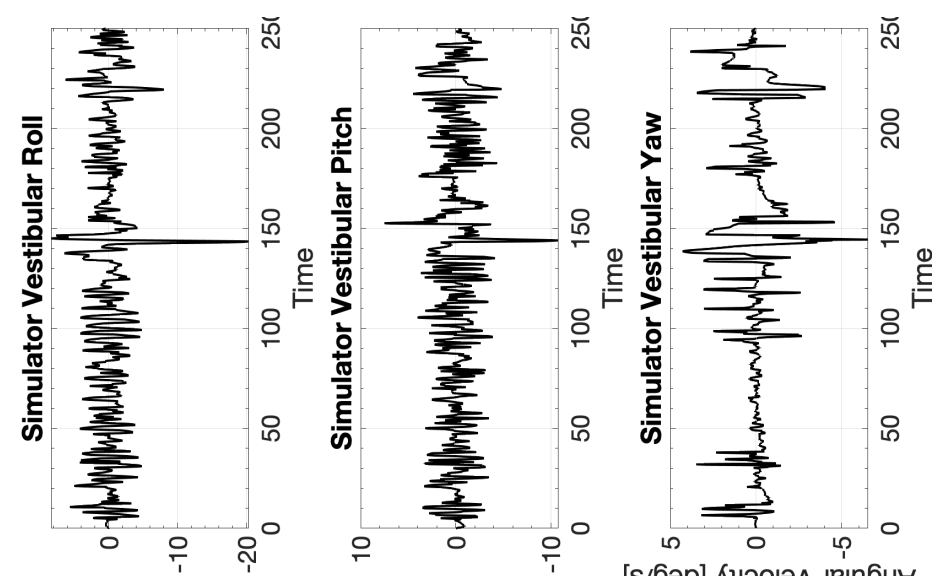

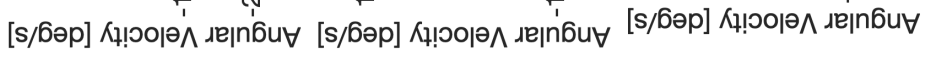

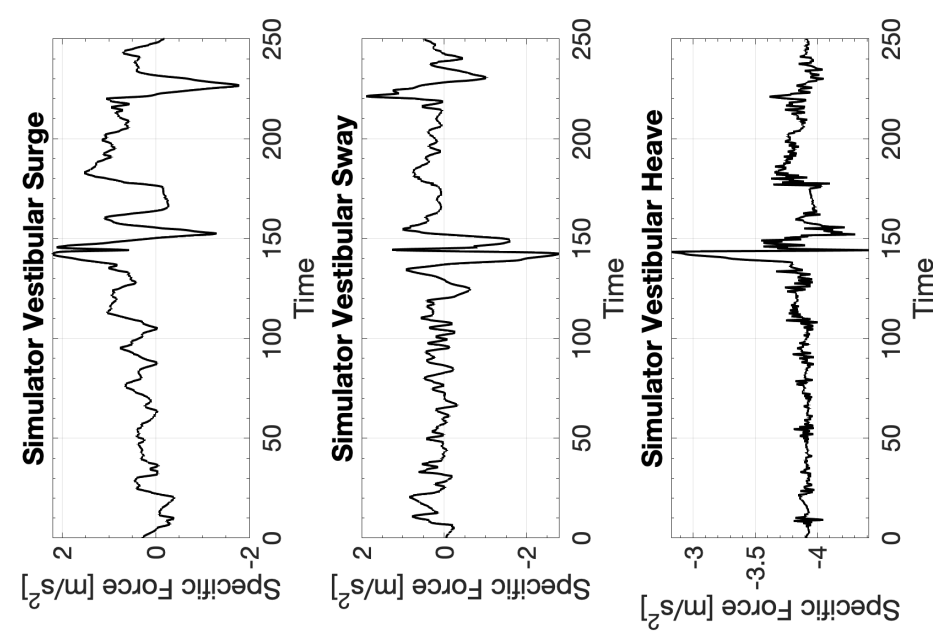




\section{C.13.2 CW2 Boeing 737 Stall Recovery}

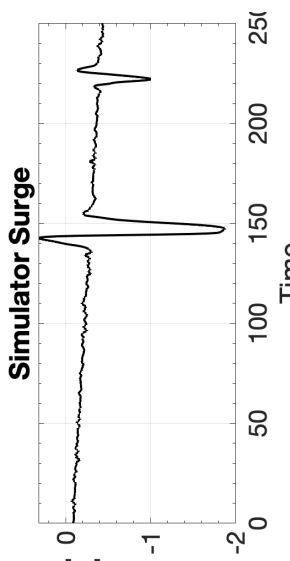

[w] uo!!!!sod

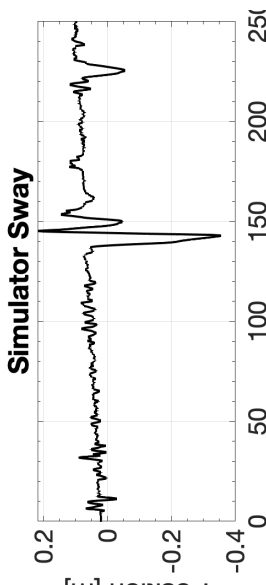

[u] uolp!sod

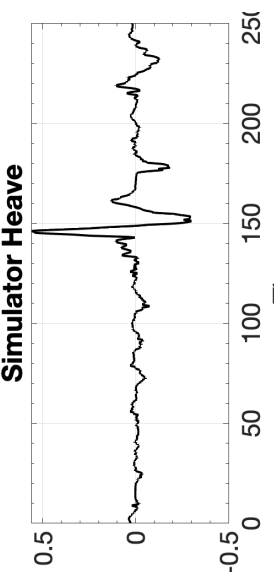

[w] uo!n!sod

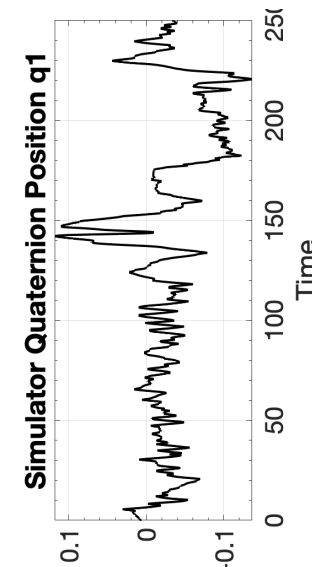

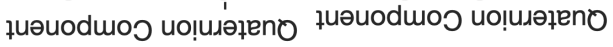

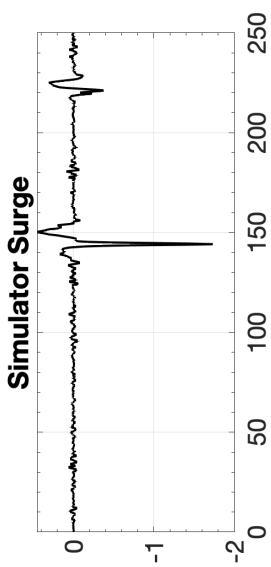

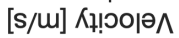
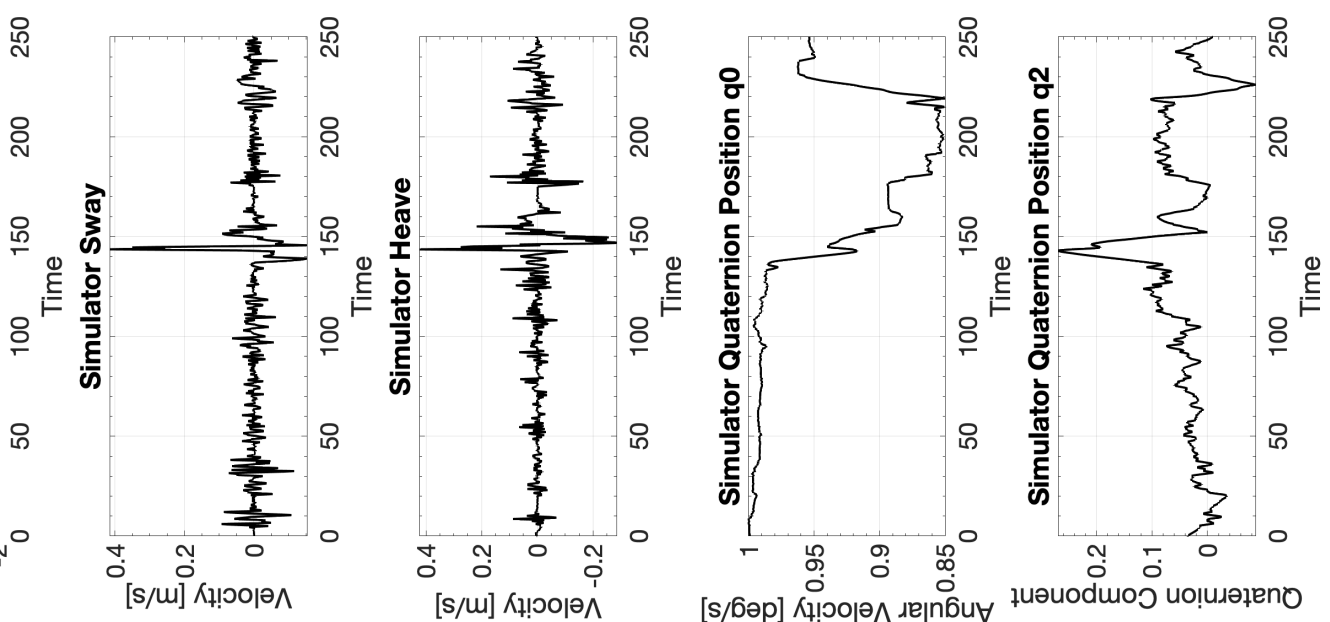

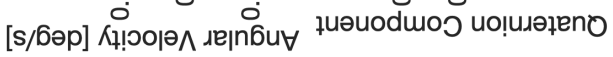
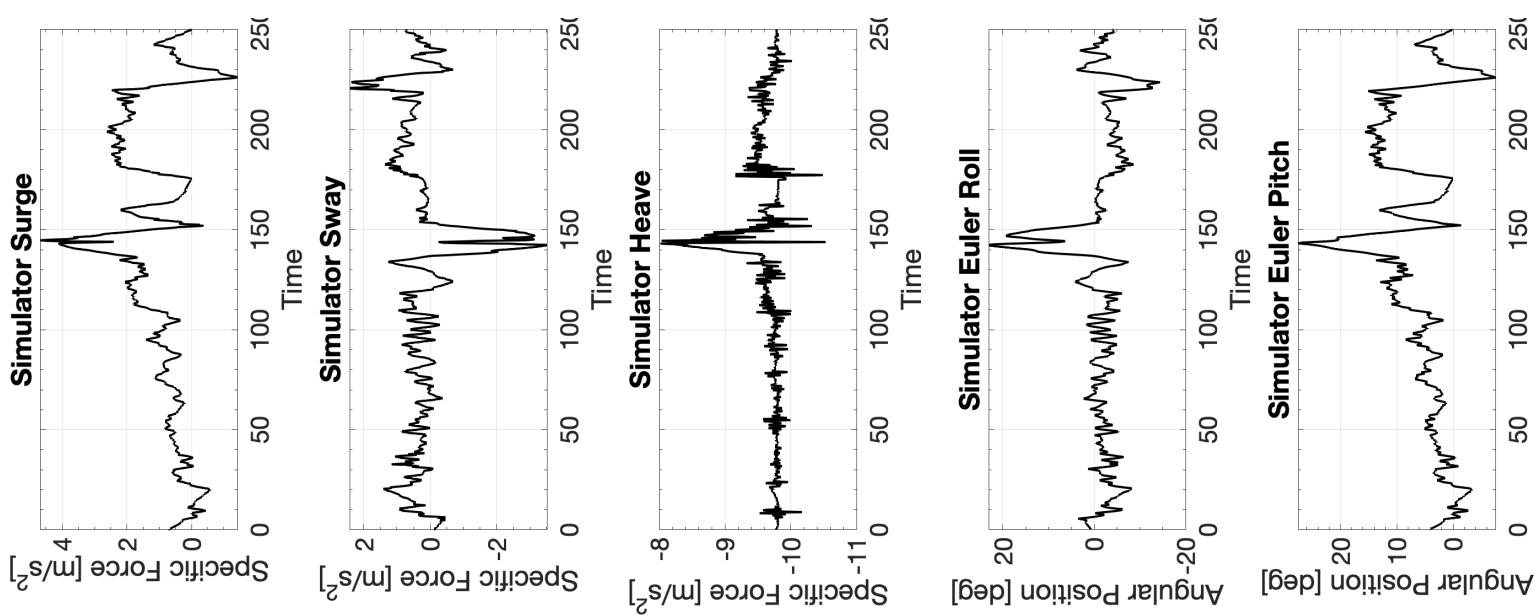

[6әр] uo!!!!sod גe|n6u
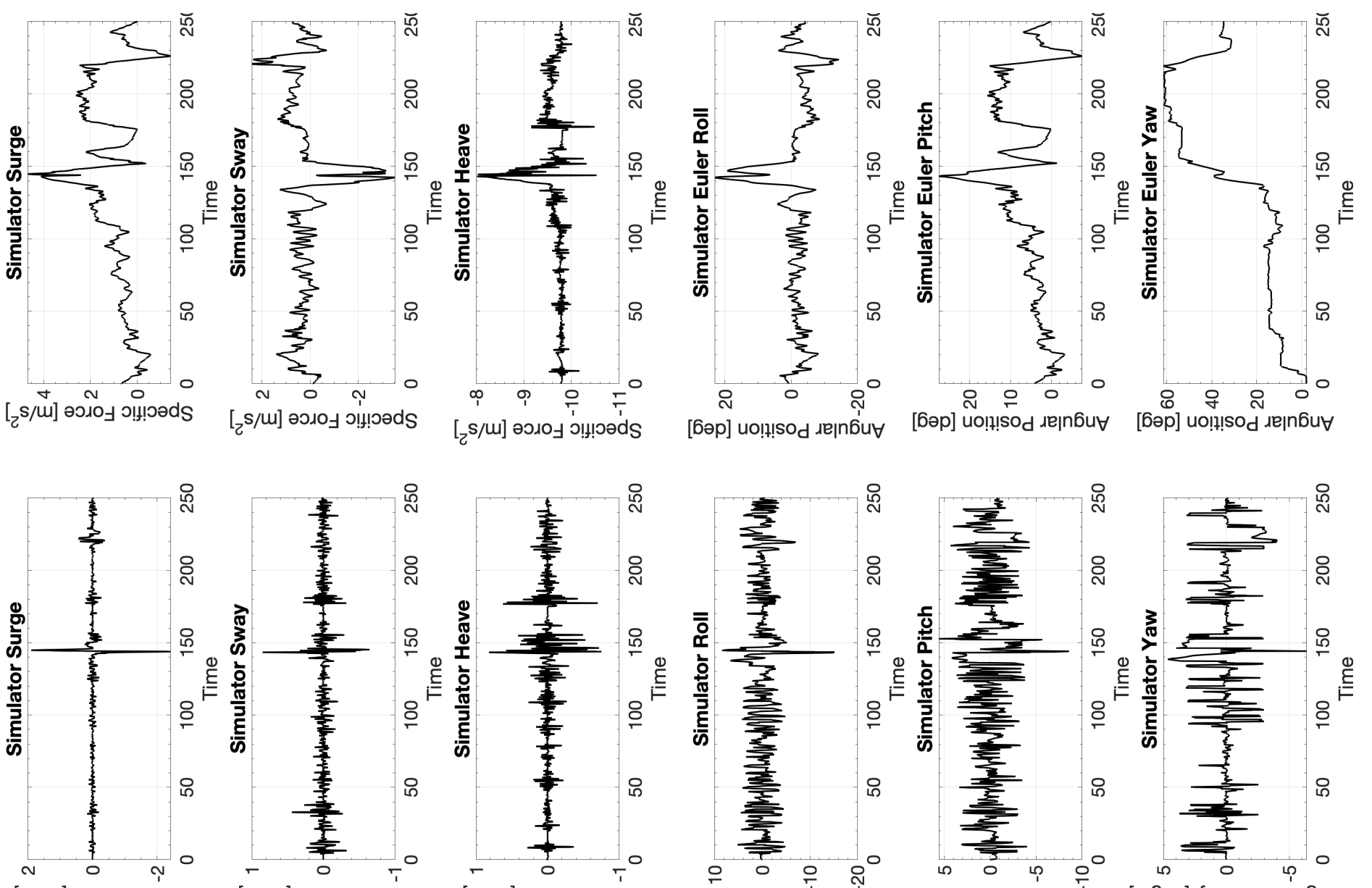

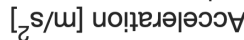

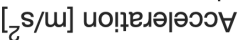

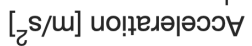



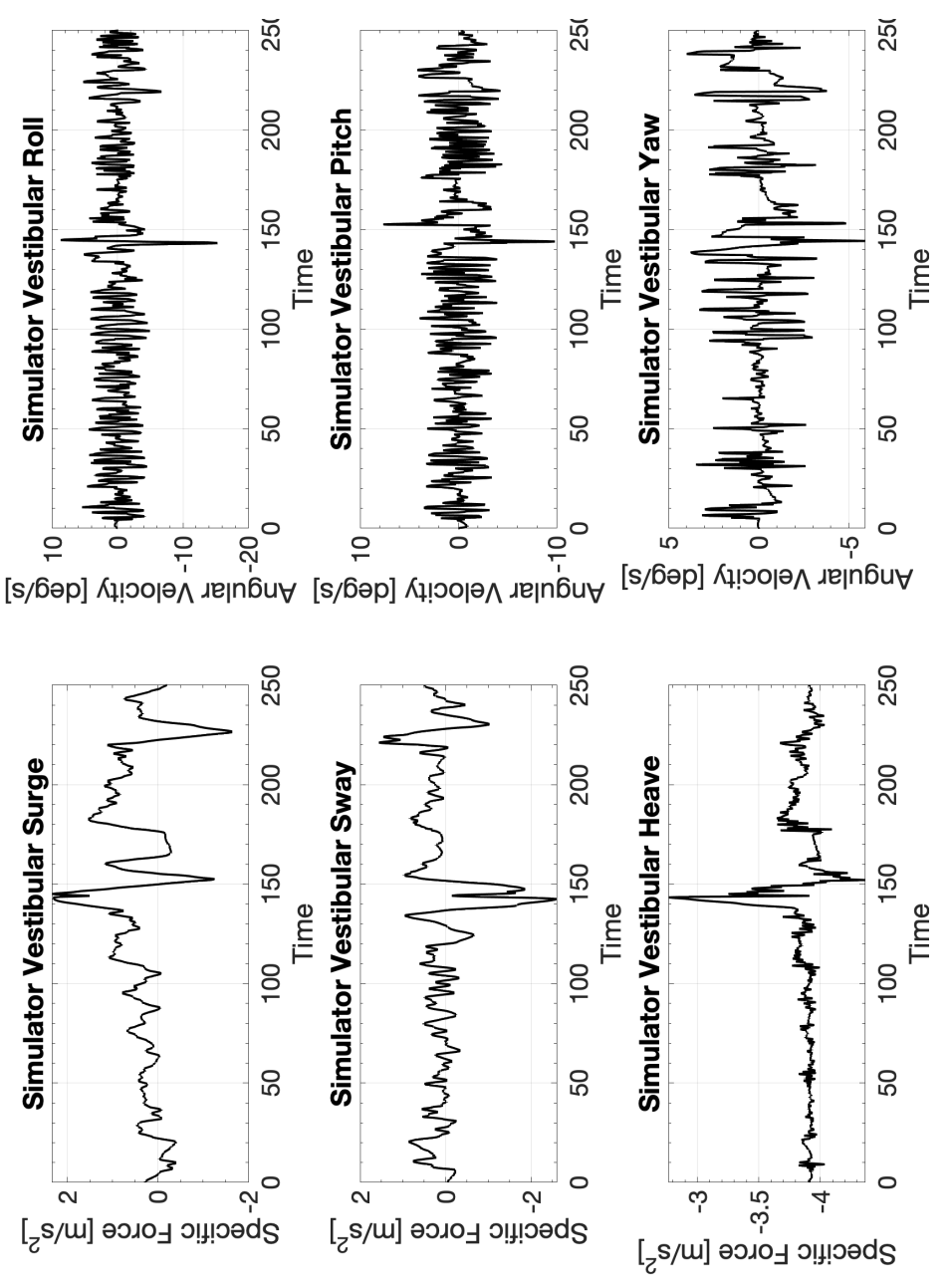


\section{C.13.3 CW3 Boeing 737 Stall Recovery}

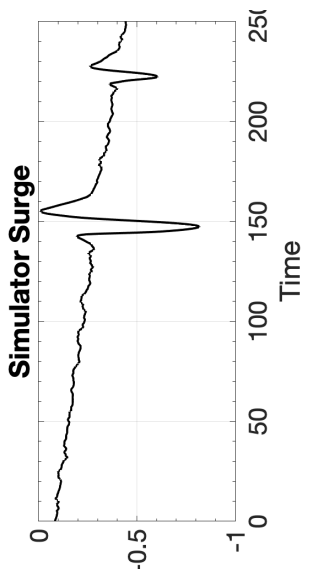

[u] uo!!!!sod

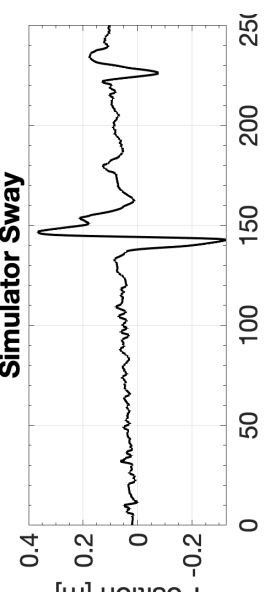

[u] uol!!!sod

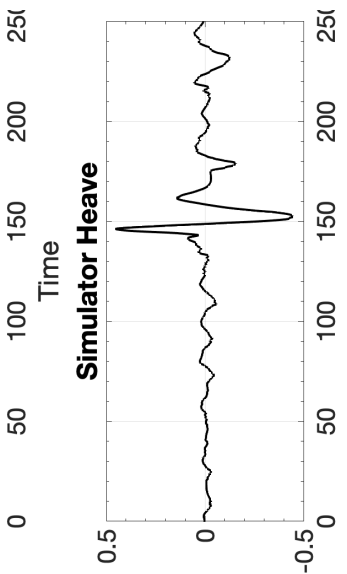

[w] uo!n!sod

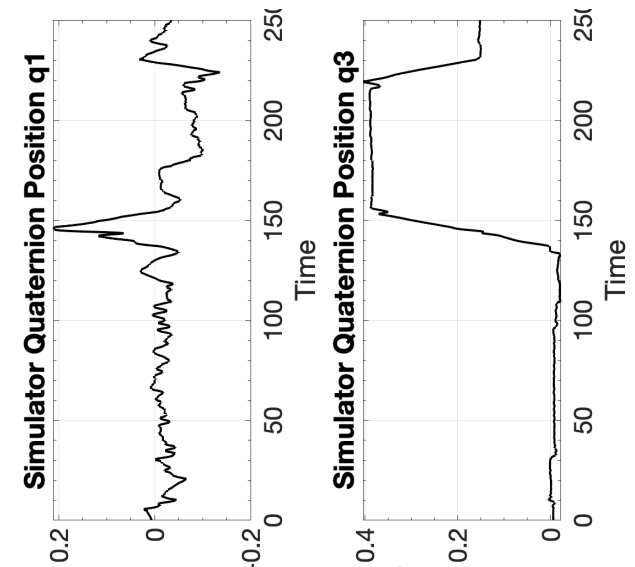

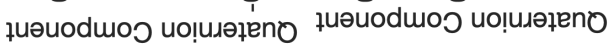

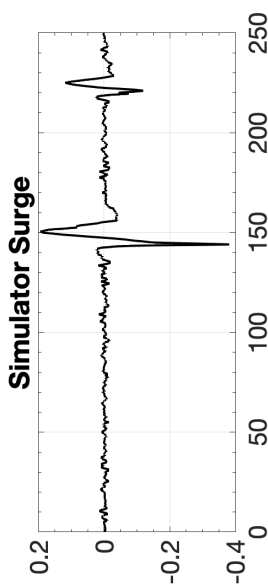

[s/u] Кұ!०орА

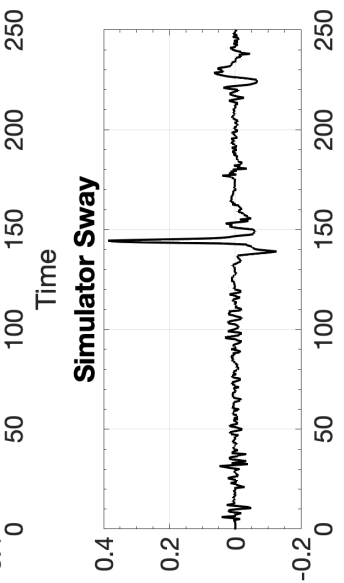

[s/u] Кұ!юор夫

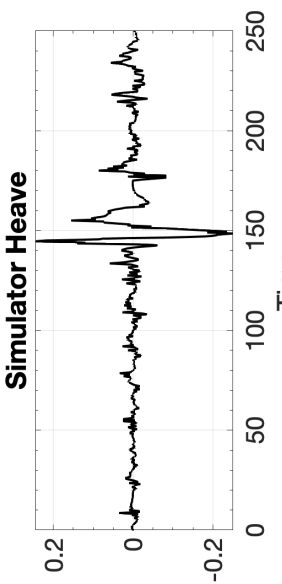

[s/m] Кұ!юор^
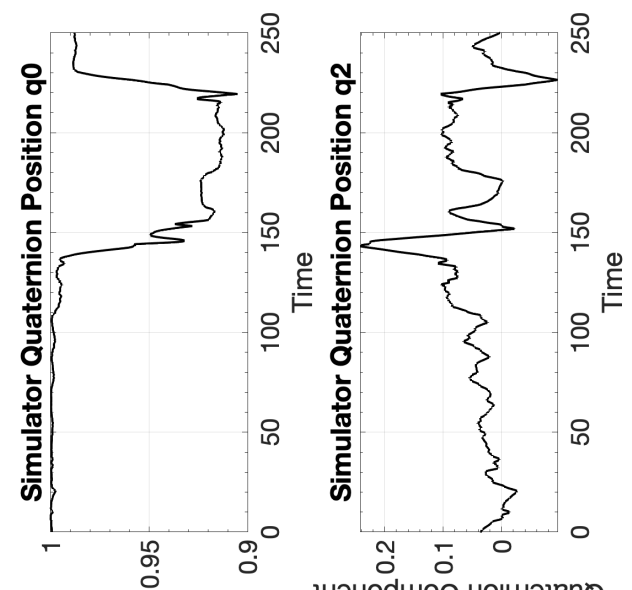

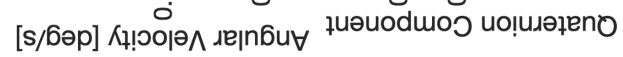

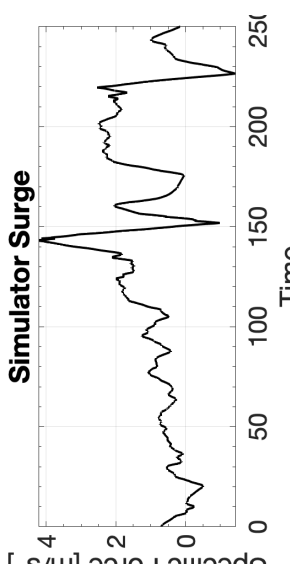

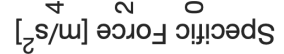

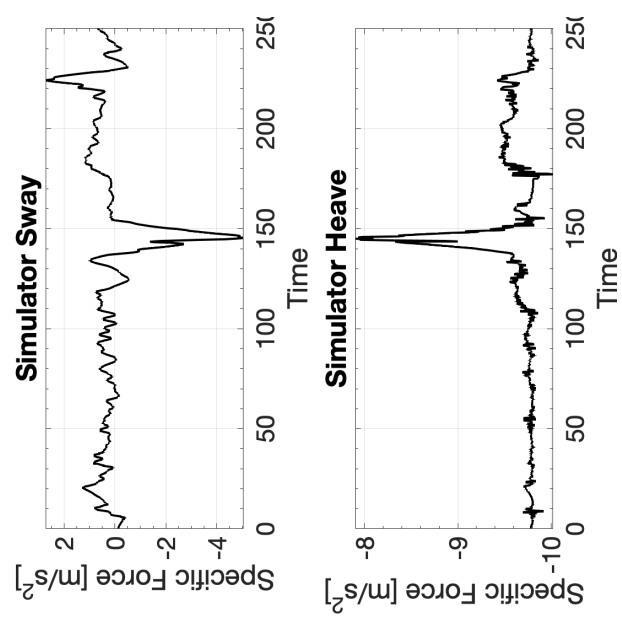

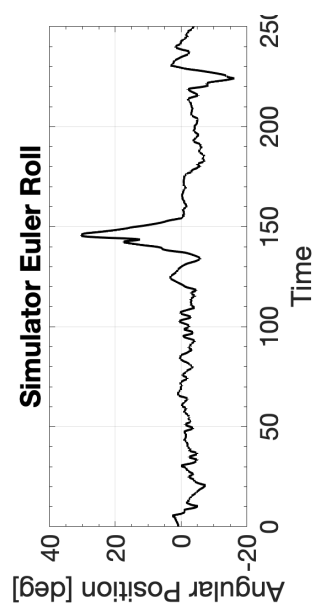

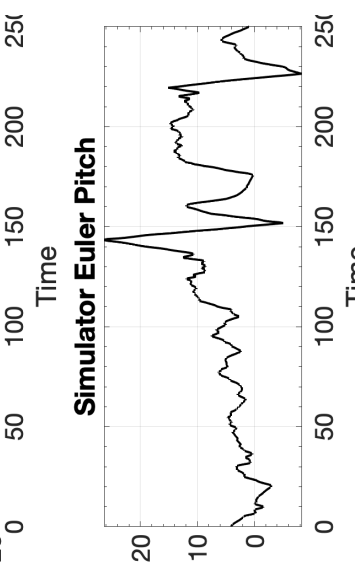

[бәр] uo!!!sod גe|n6u

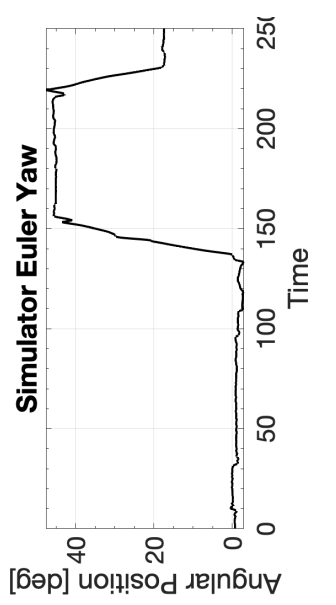

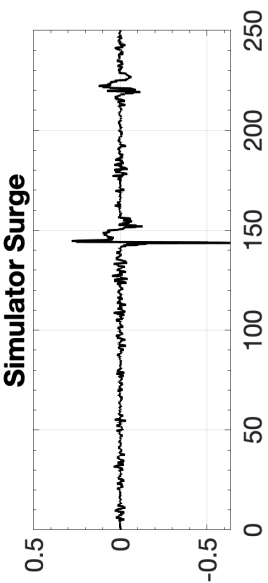

$[\mathrm{c} / \mathrm{L}] \mathrm{uo!̣}$

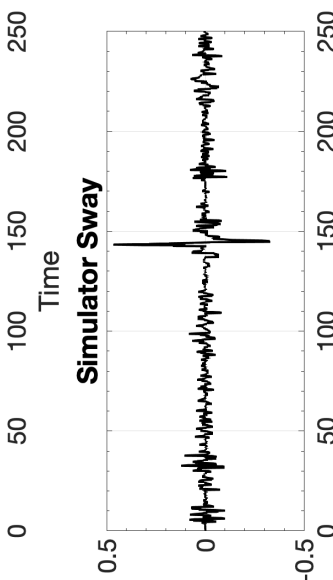

[zs/m] ио!ฺедәәәэว

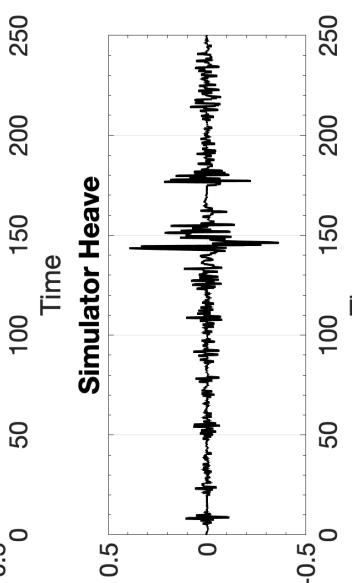

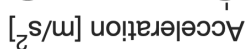

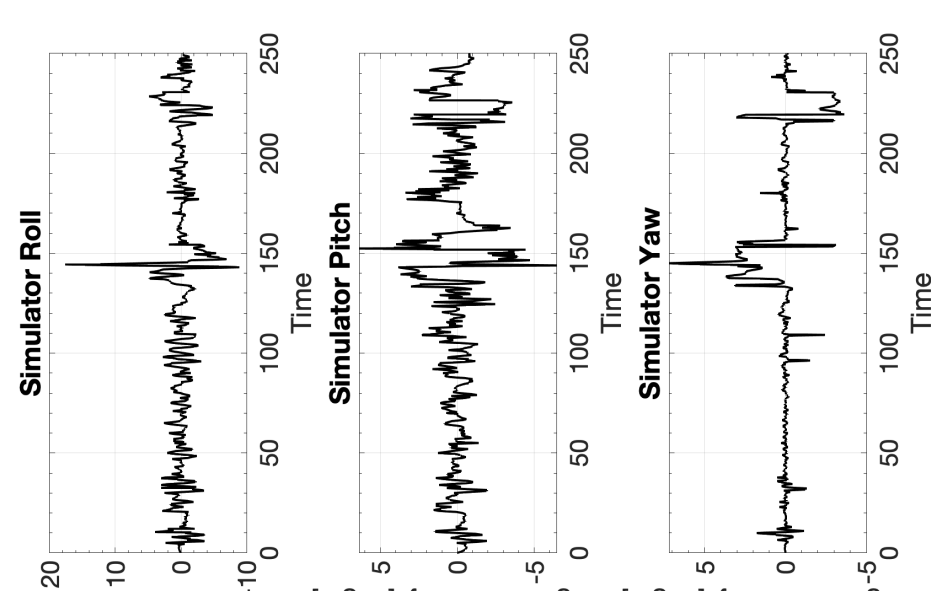

ก 


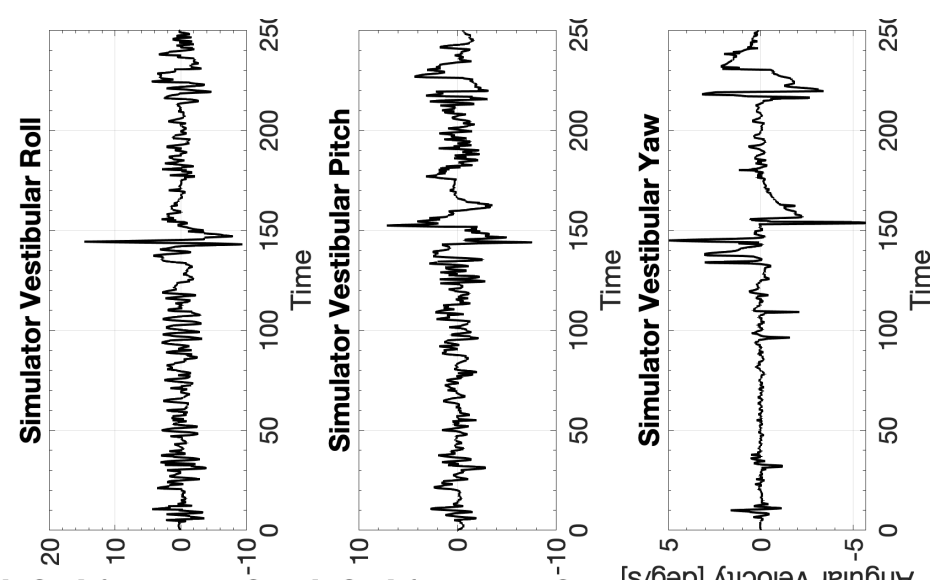

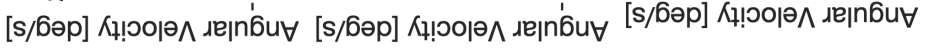

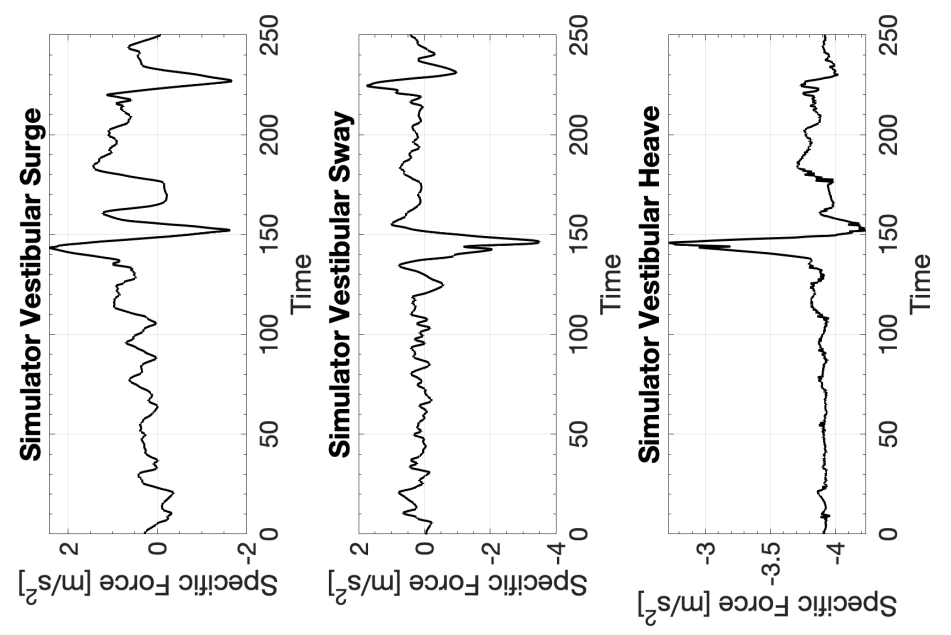




\section{C.14 Columbia 400 Aerobatics}

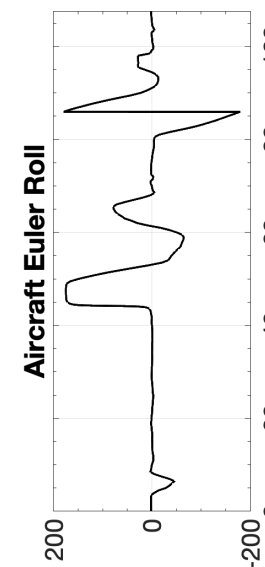

[6əp] uo!!!sod xe|n6́u

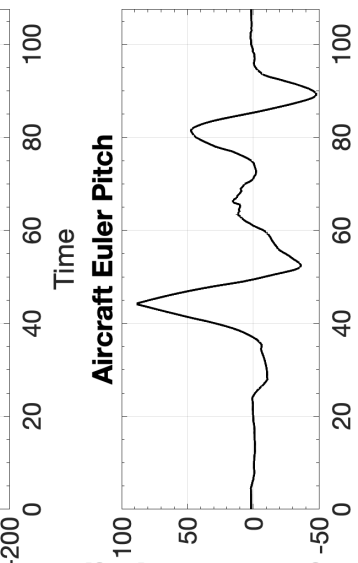

[6әр] uo!!!sod גejn6u

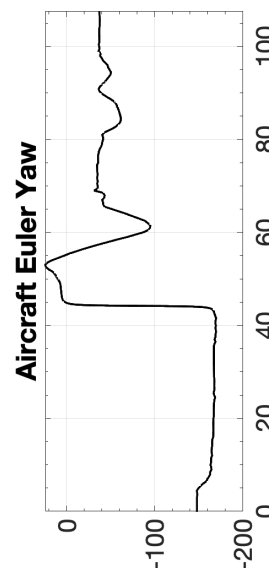

[бәр] uo!̣!so'd

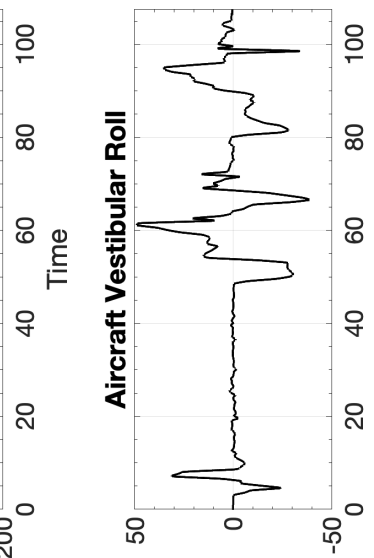

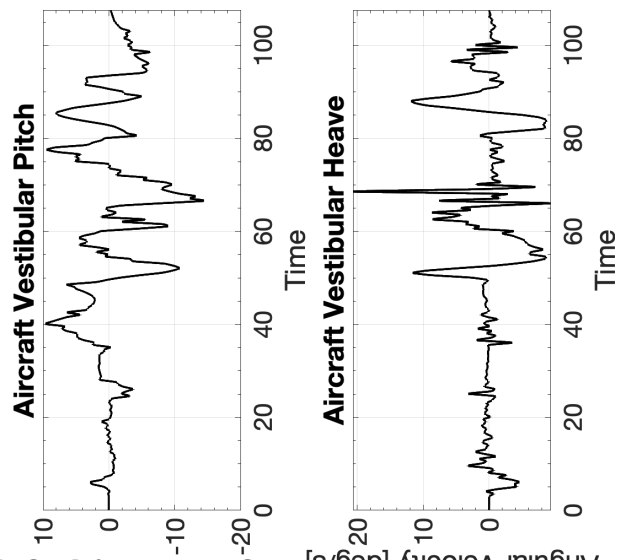

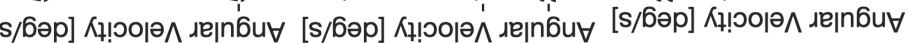

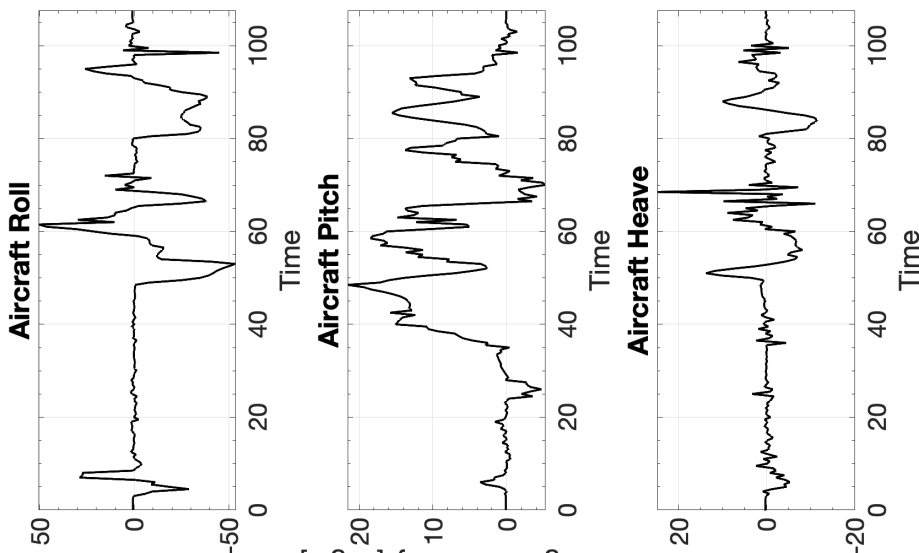

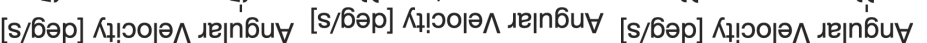

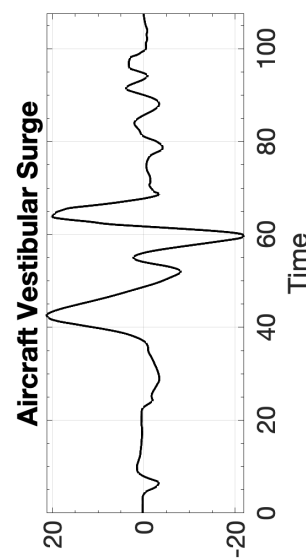

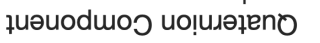

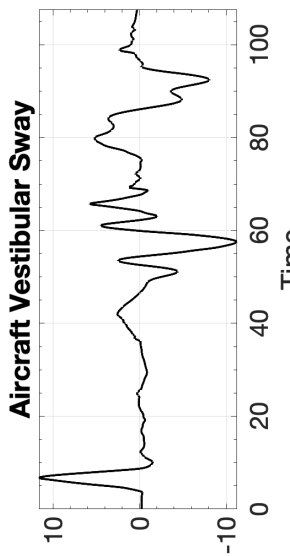

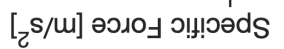

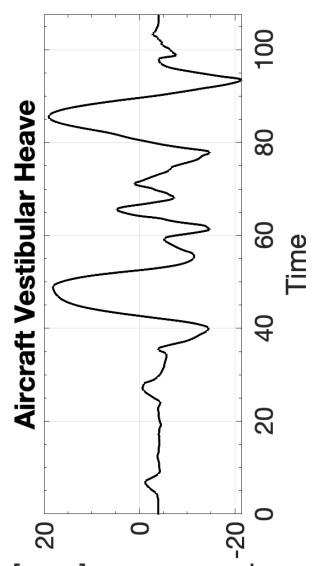

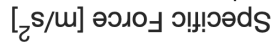

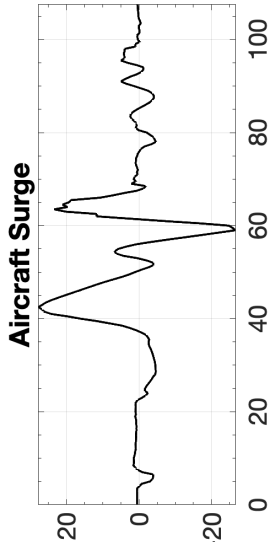

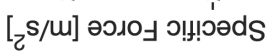

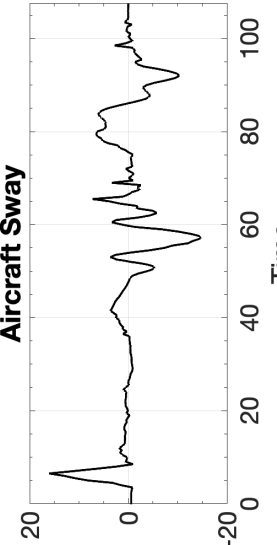

$[\mathrm{z} / \mathrm{s}]$ ә0ر0

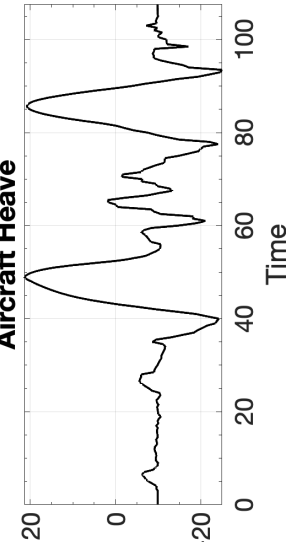

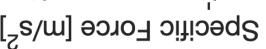
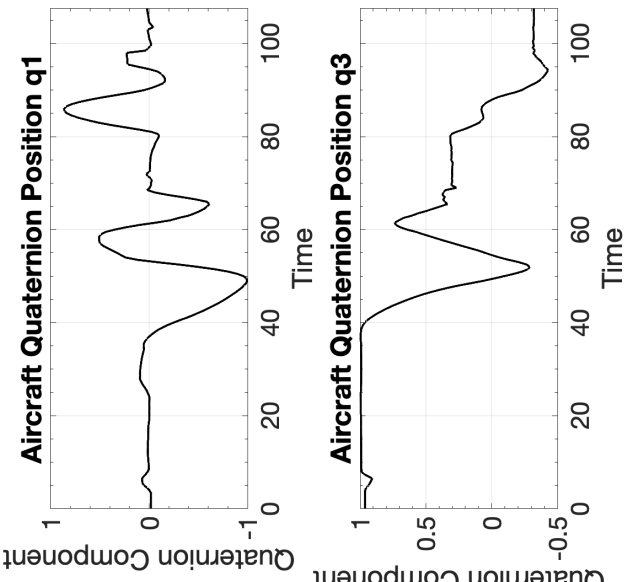

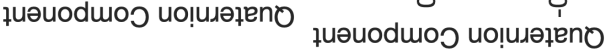

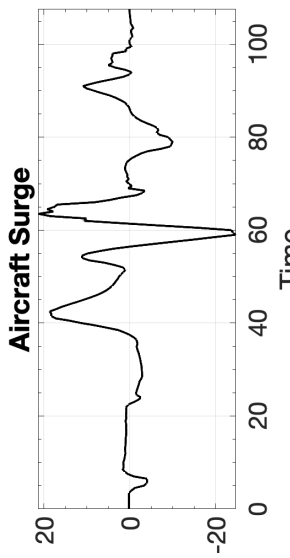

$\left[{ }^{2} \mathrm{~s} / \mathrm{m}\right]$ ио!ฺеләрәэว

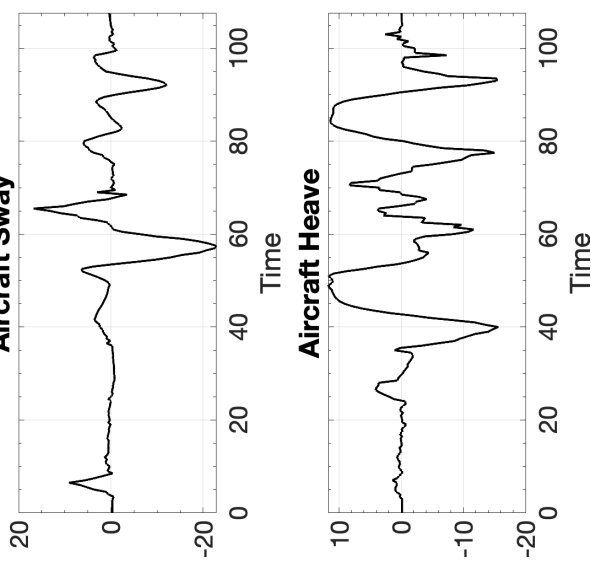

[zs/m] ио!ฺедәәәоэ

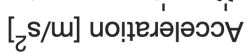

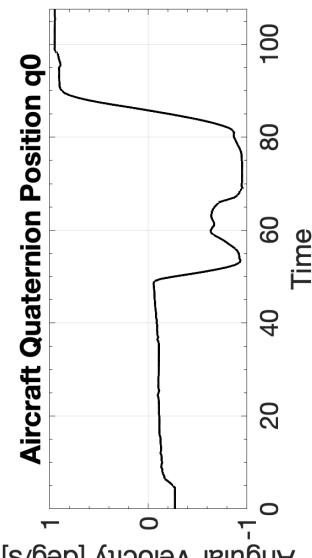

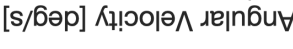

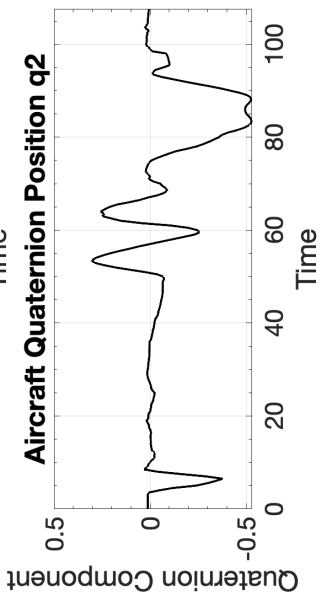




\section{C.14.1 CW1 Columbia 400 Aerobatics}

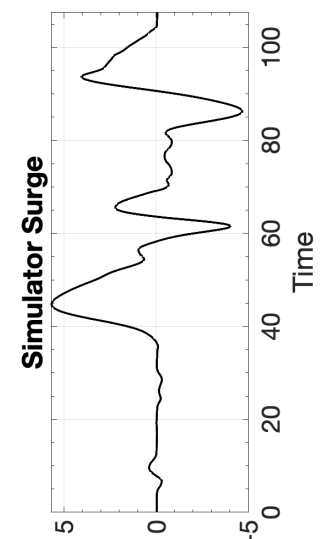

[u] uo!l!!sod

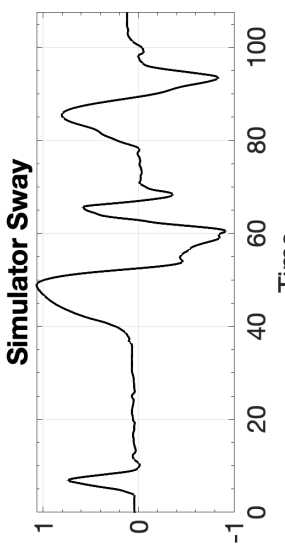

[u] uo!!!!sod

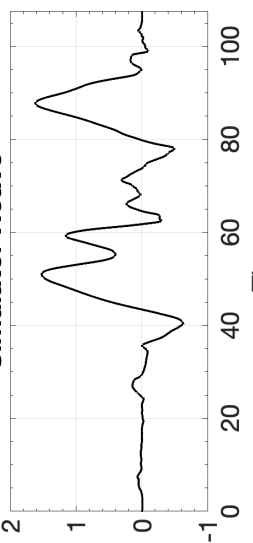

[w] uo!t!sod

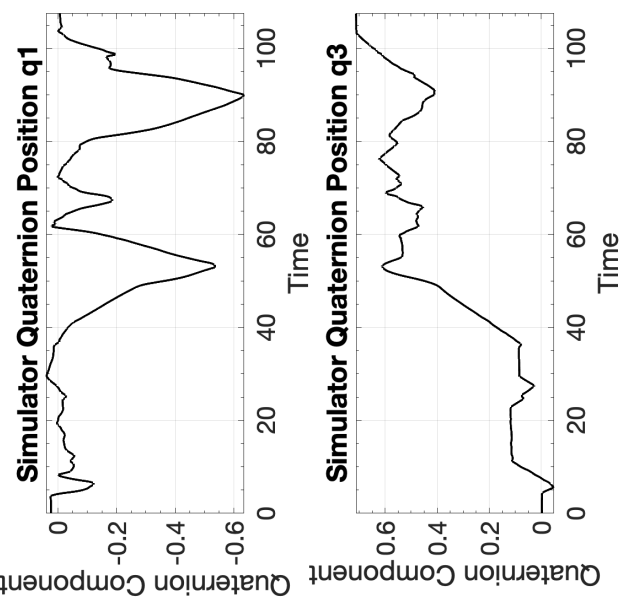

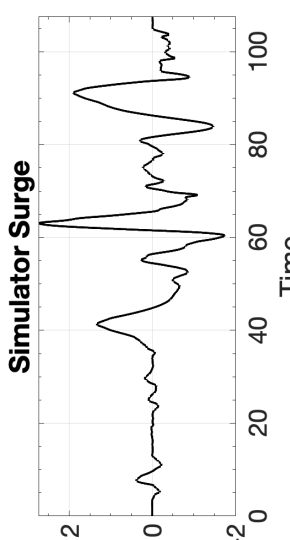

[s/u] Кц!৩о|ә

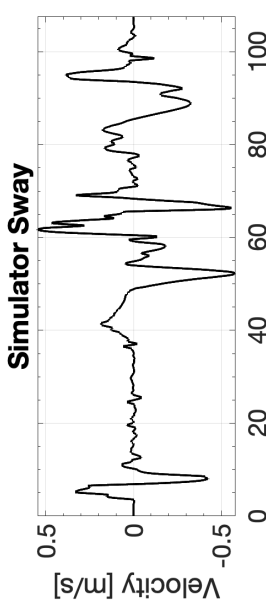

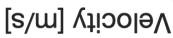

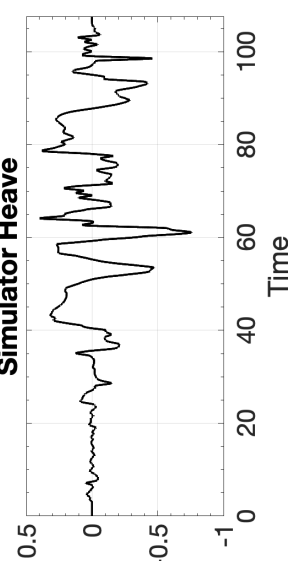

[s/m] Кł!юㅇㅅ

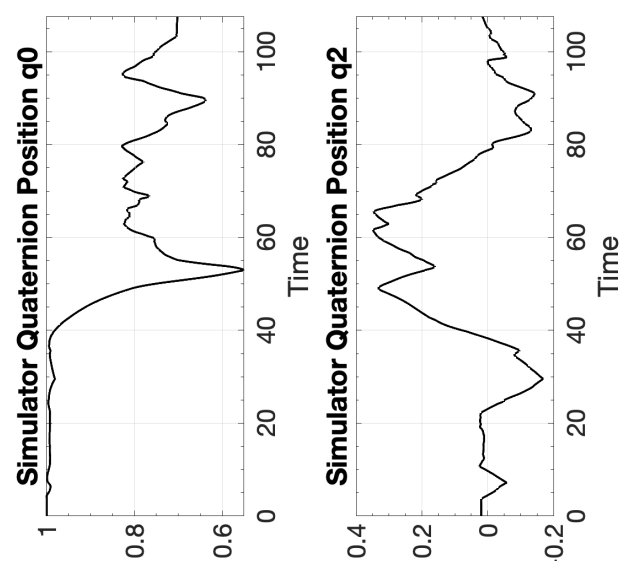

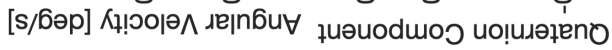

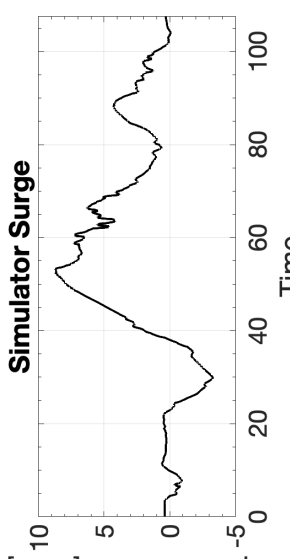

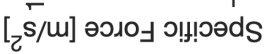

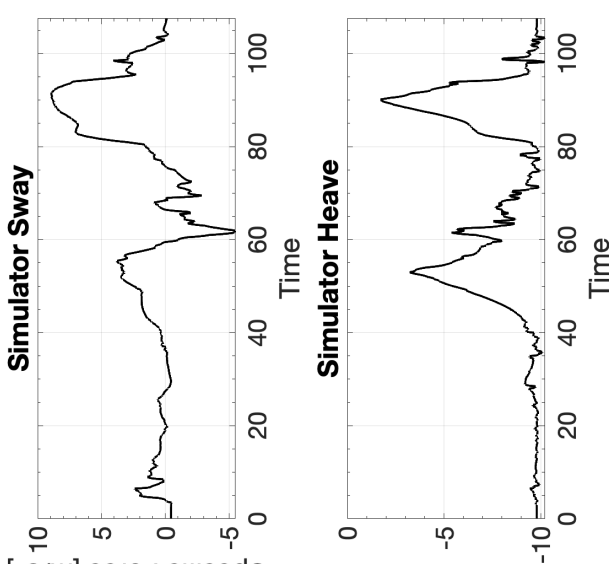

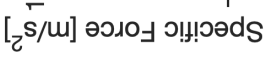

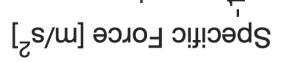

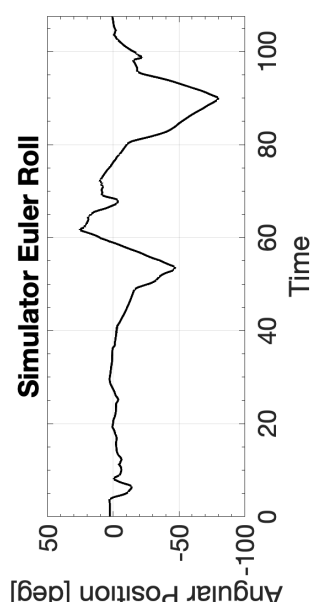

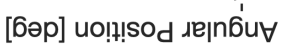

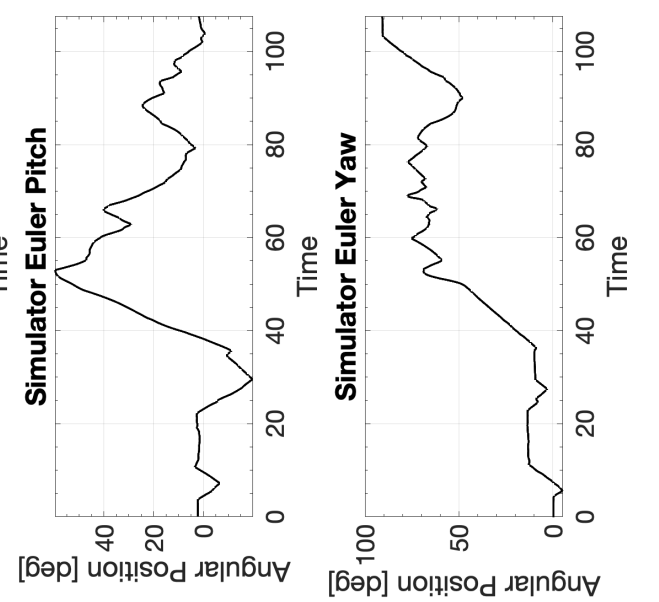

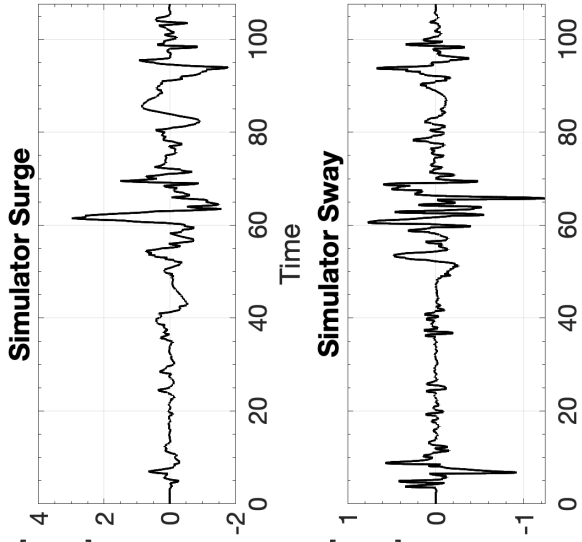

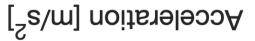

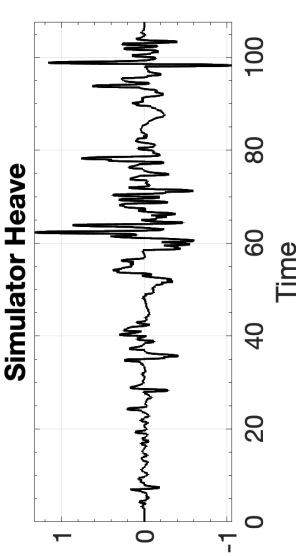

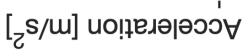

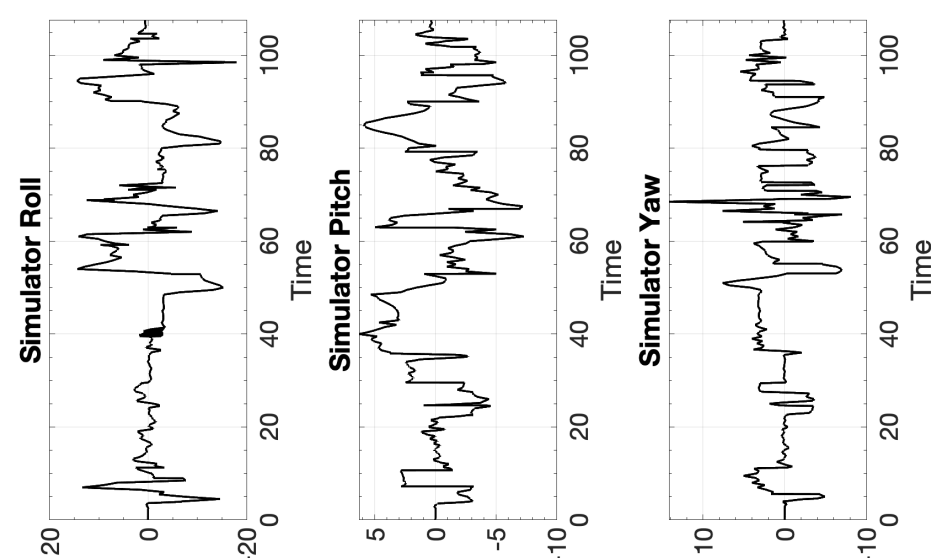

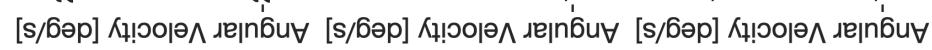



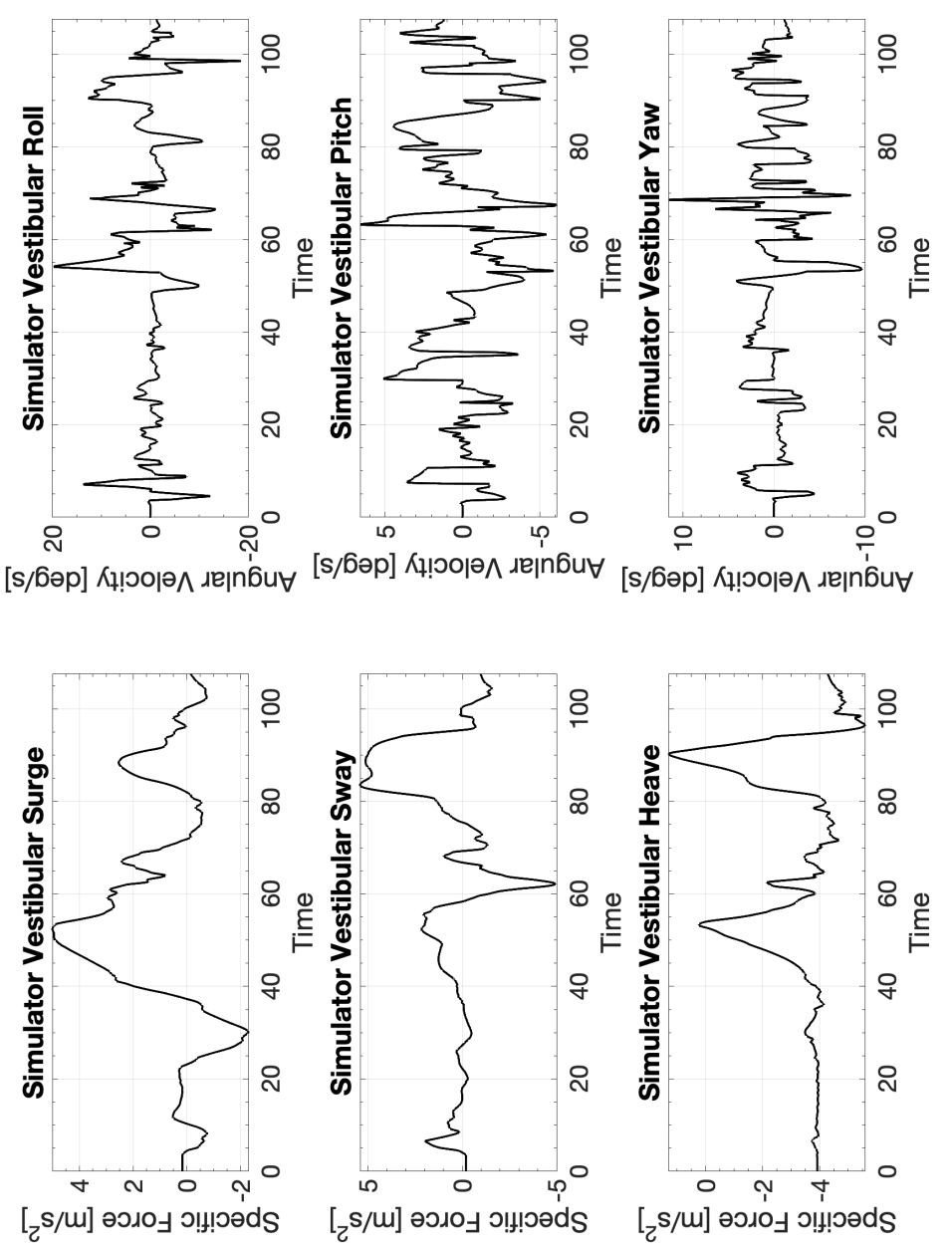


\section{C.14.2 CW2 Columbia 400 Aerobatics}

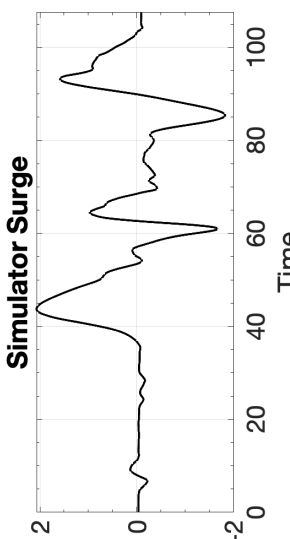

[u] uo!!!sod

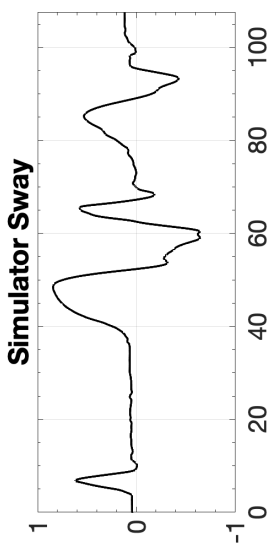

[w] uo!!!!sod

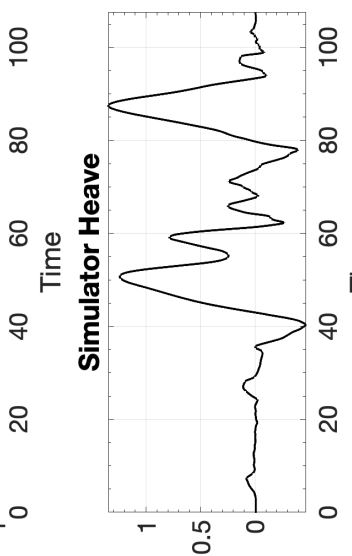

[w] uolt!sod

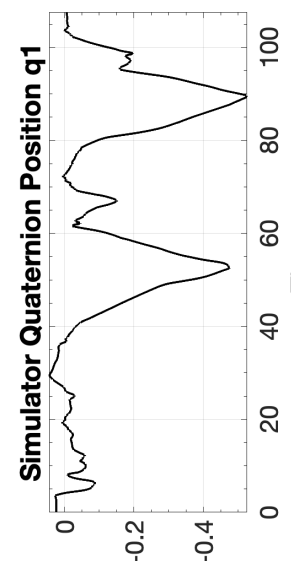

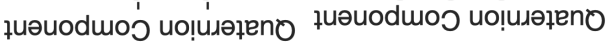

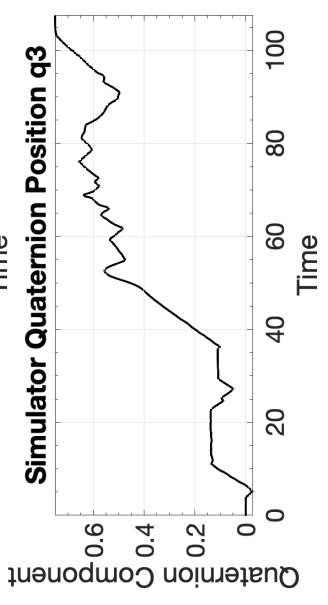

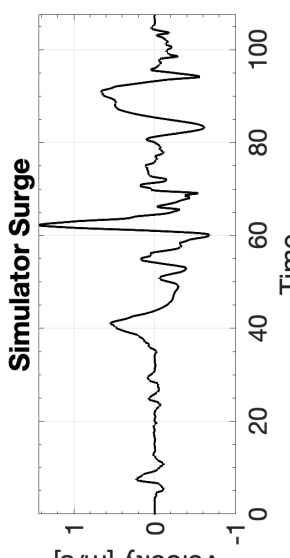

[s/m] Кำоำ

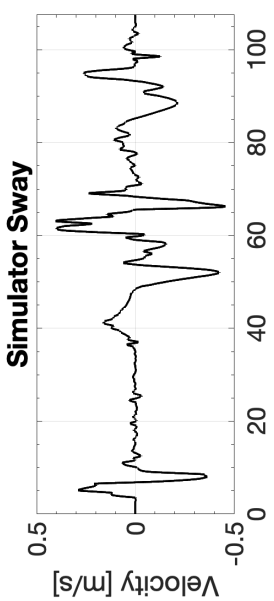

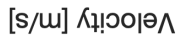

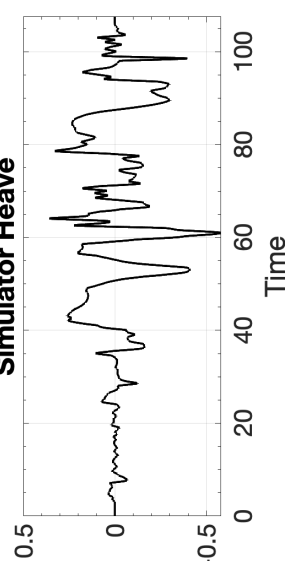

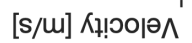
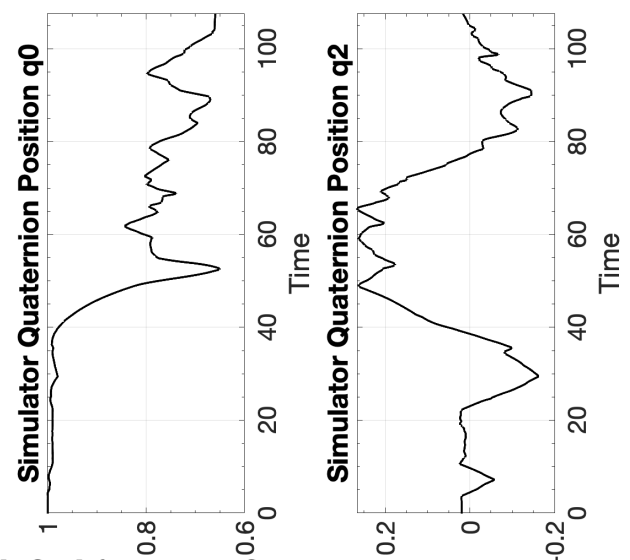

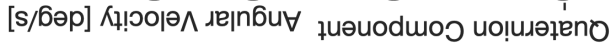

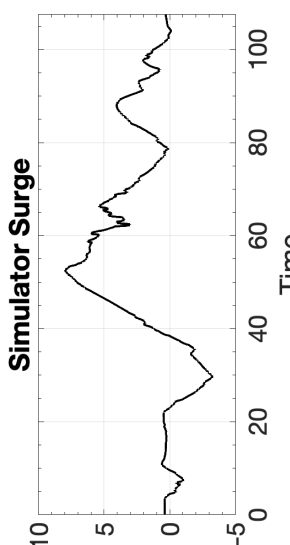

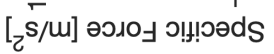

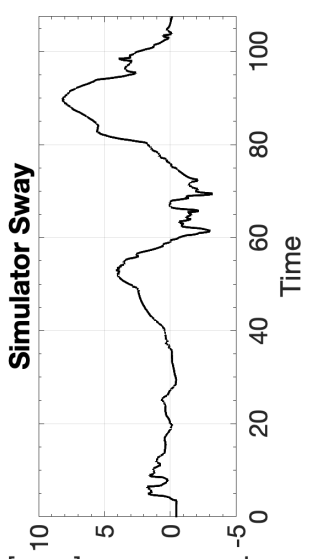

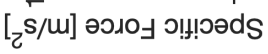

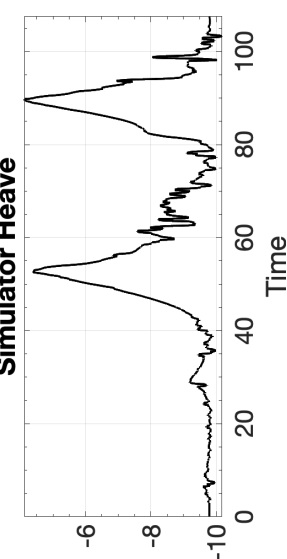

$[\mathrm{s} / \mathrm{s}]$ ә

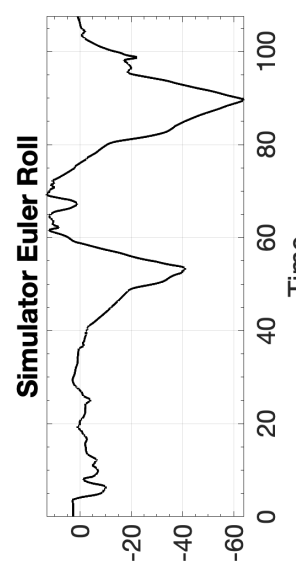

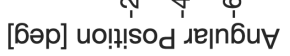

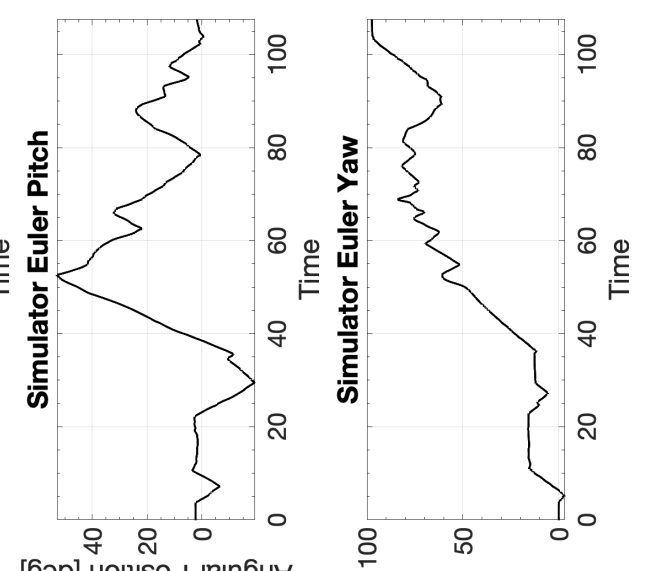

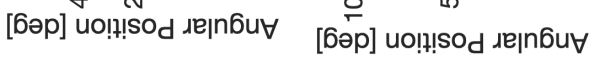

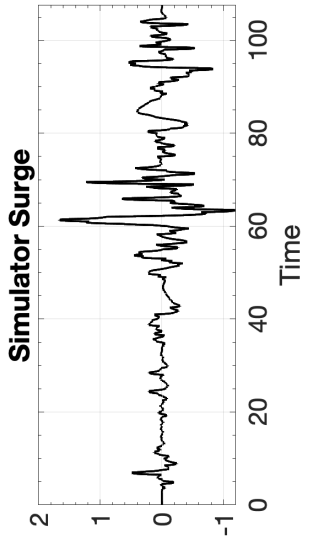

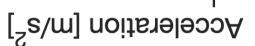

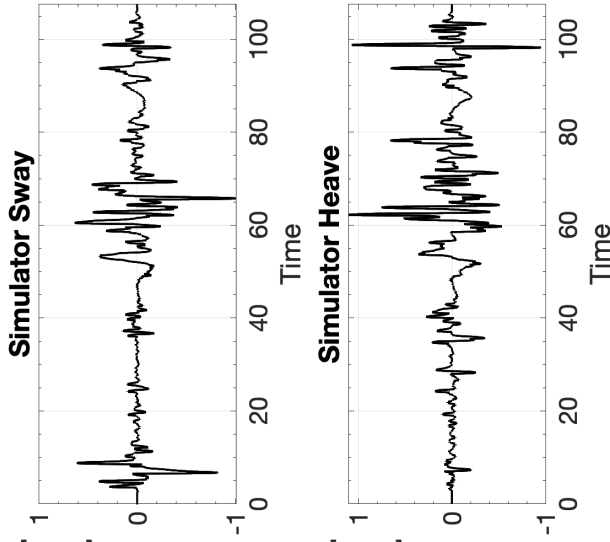

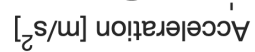

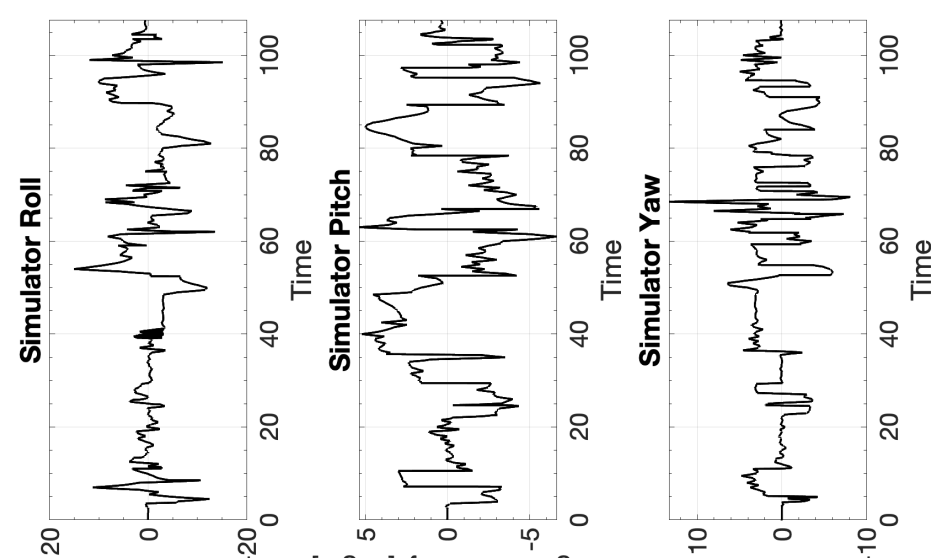

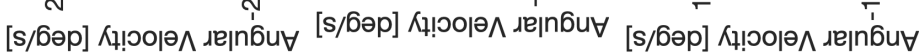



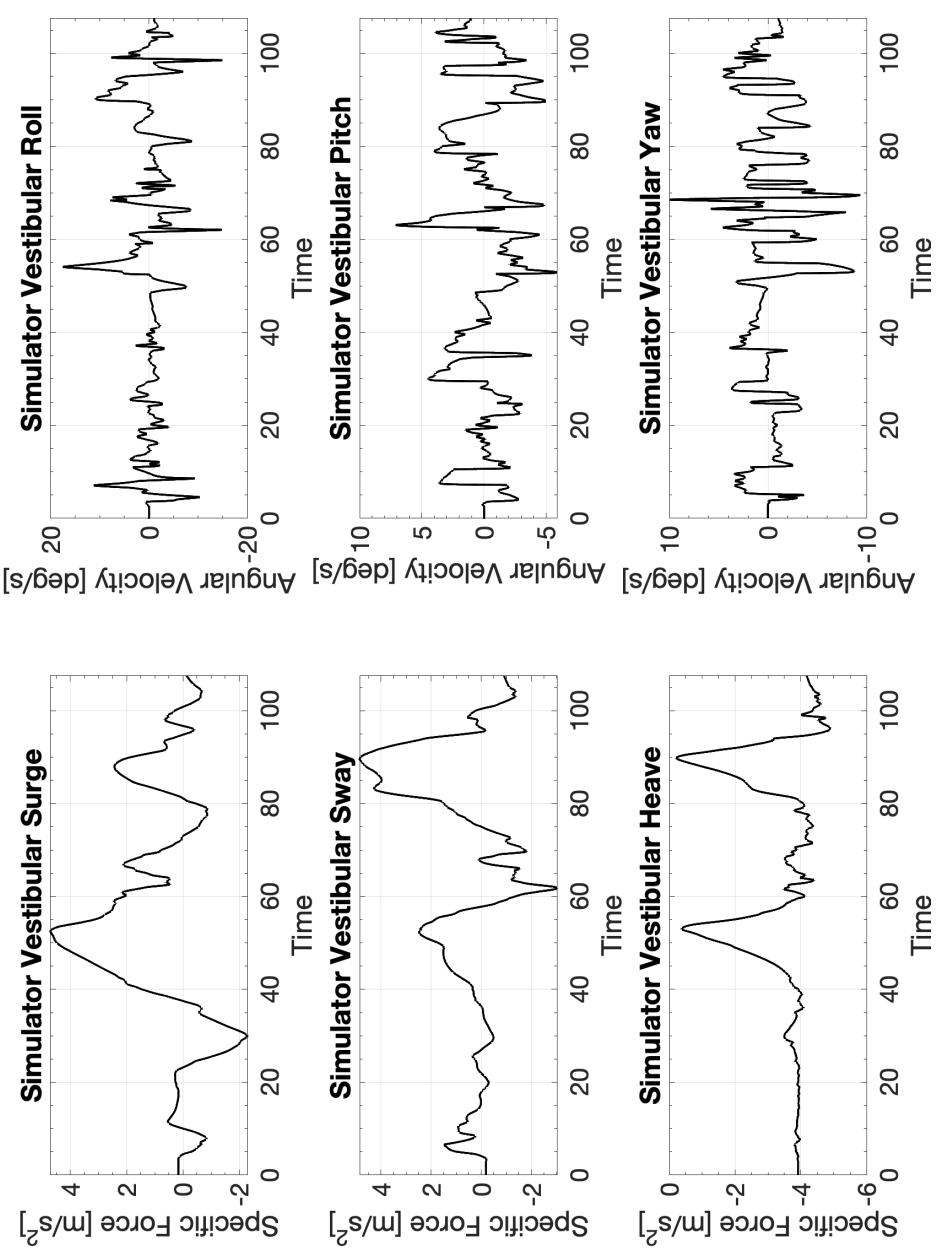


\section{C.14.3 CW3 Columbia 400 Aerobatics}

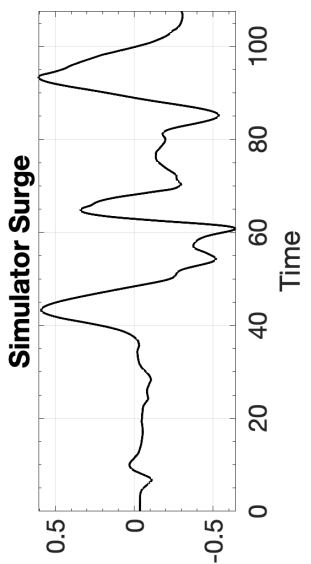

[u] uo!n!sod

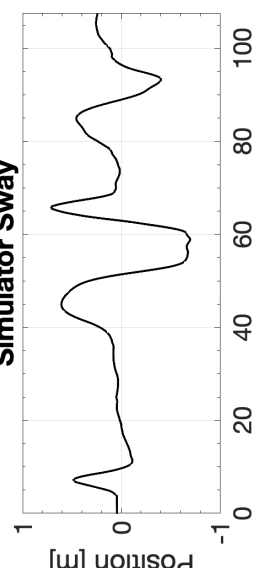

[w] uo!!!sod

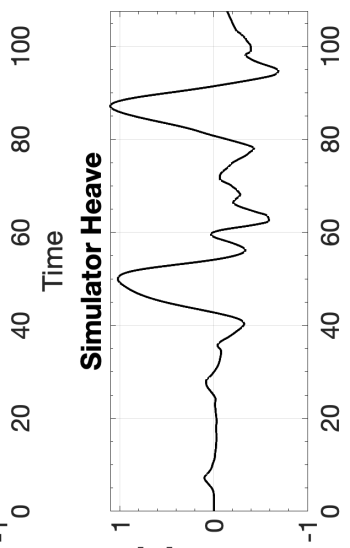

[w] uol!!sod

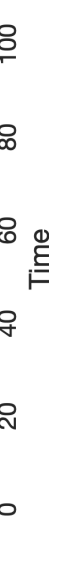

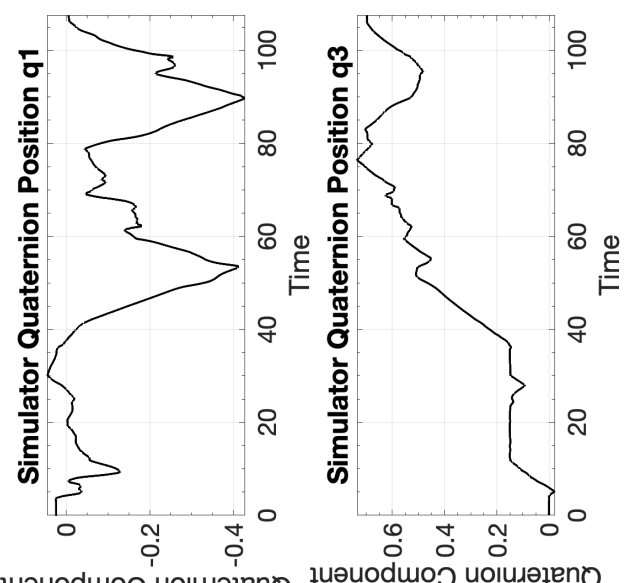

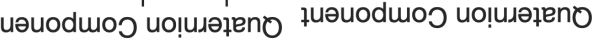

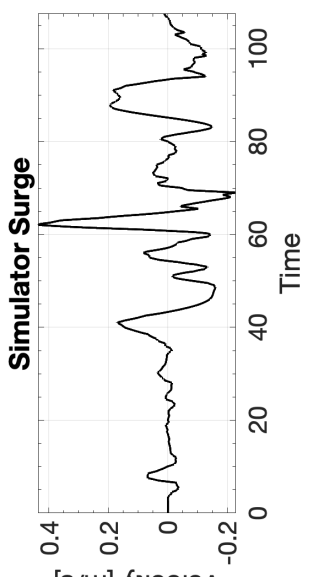

[s/u] Кұ! 이 ^

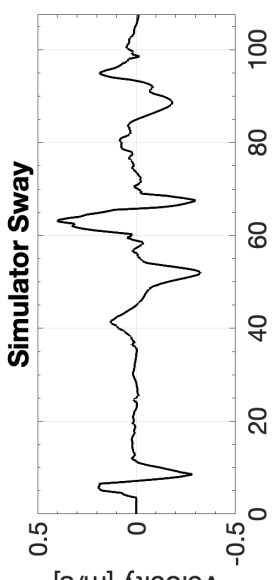

[s/u] Кұ!৩о|ә $\Lambda$

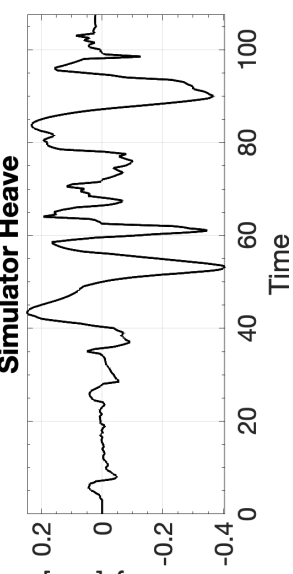

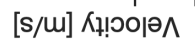

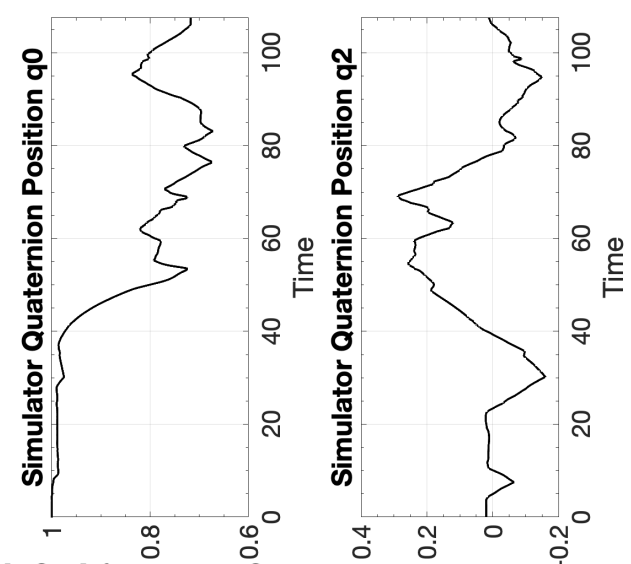

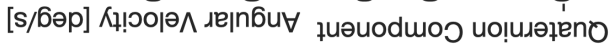

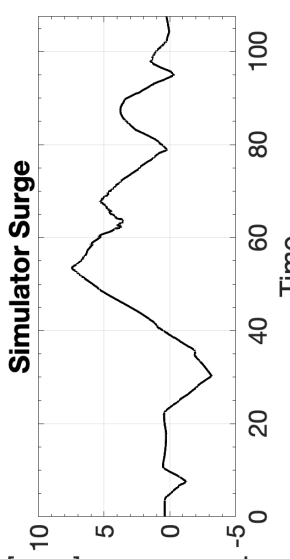

[ट

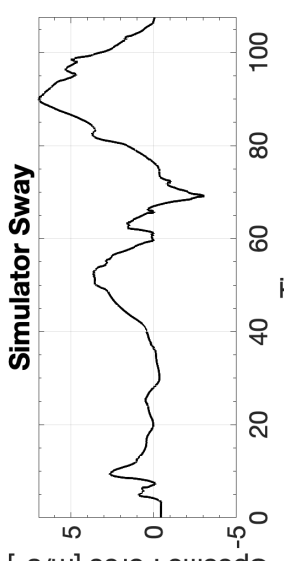

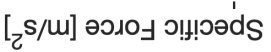

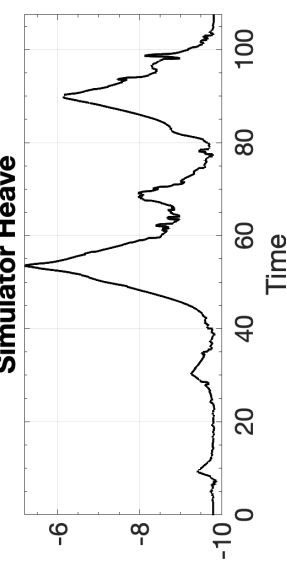

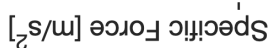

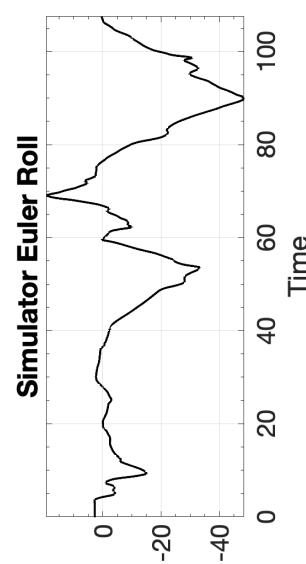

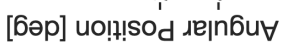

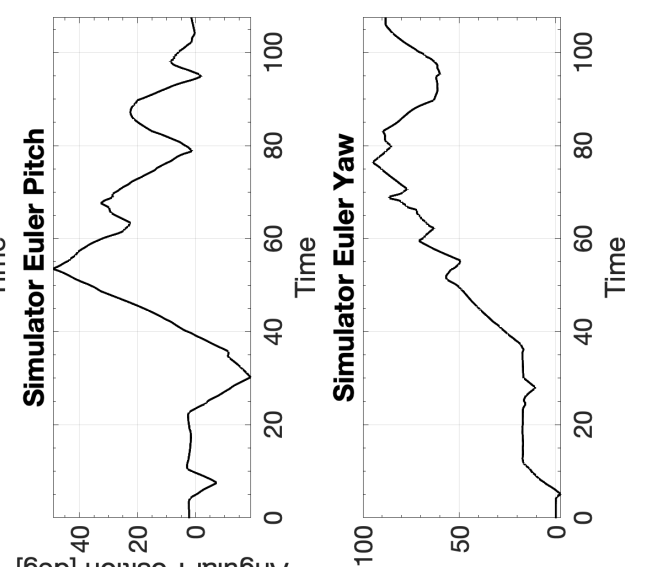

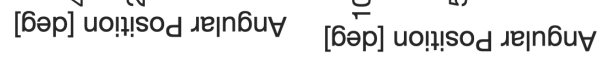

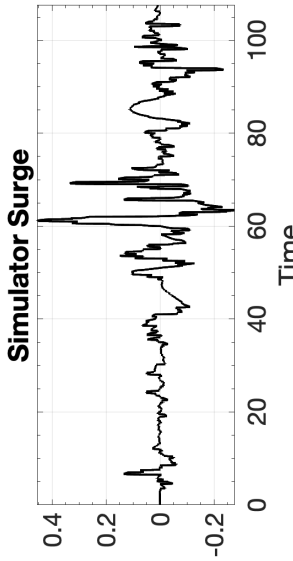

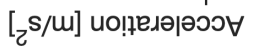

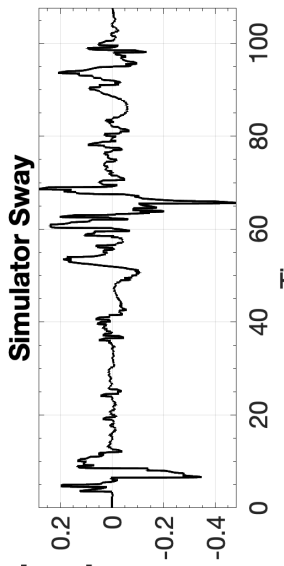

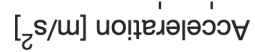

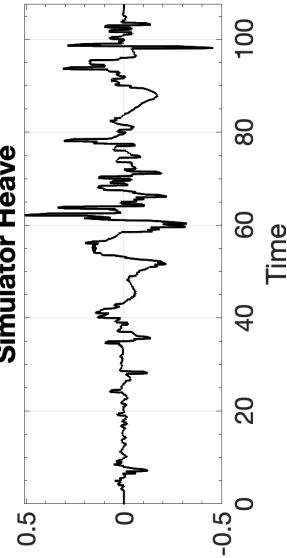

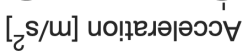

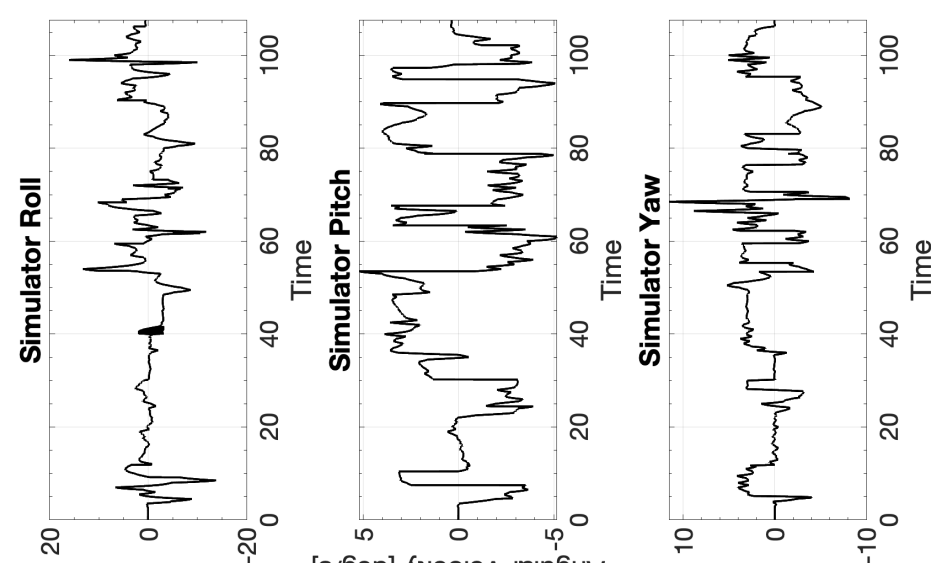

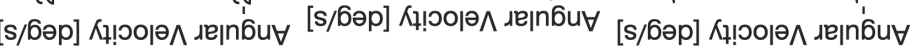



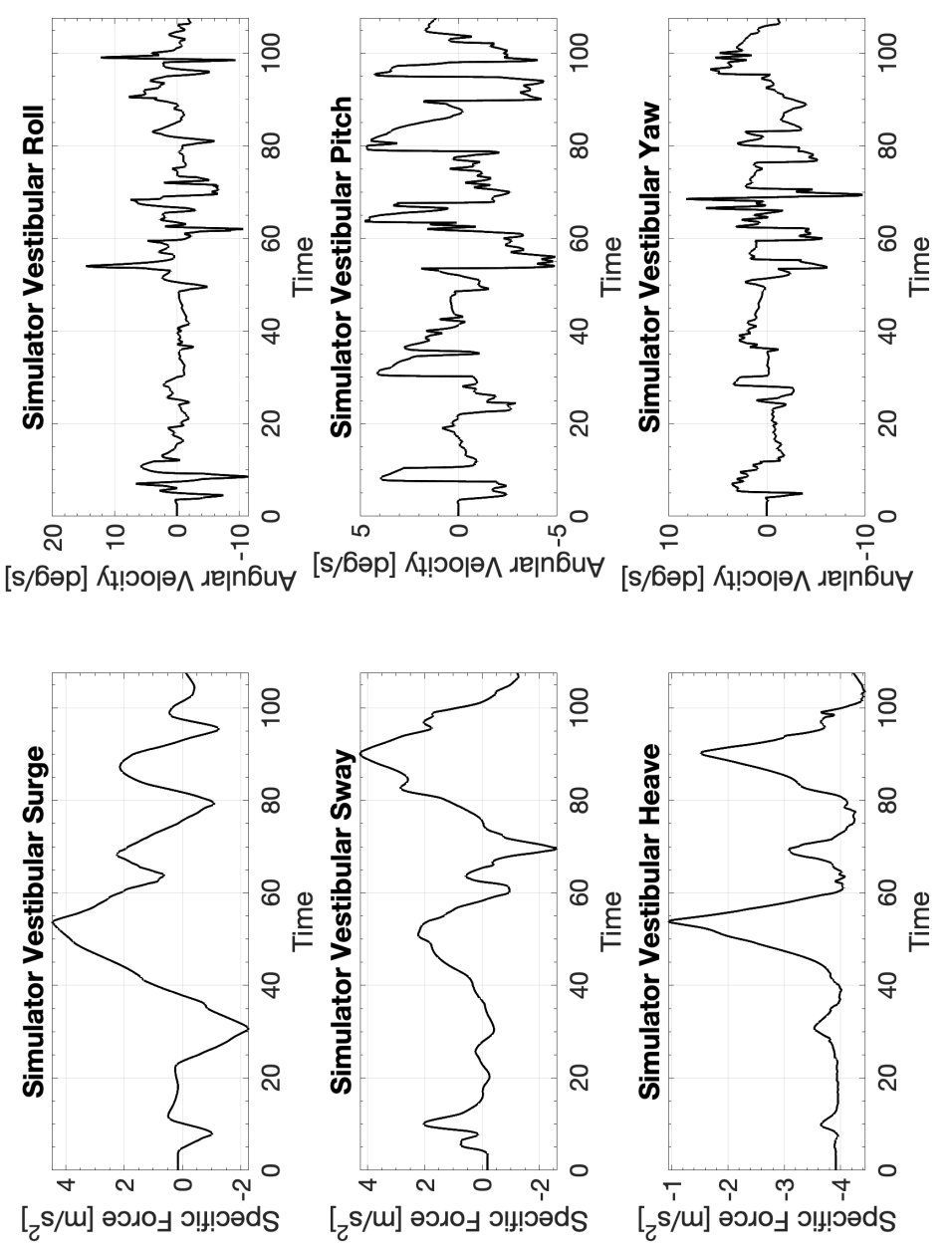


\section{C.15 Columbia 400 Coordinated Turn}

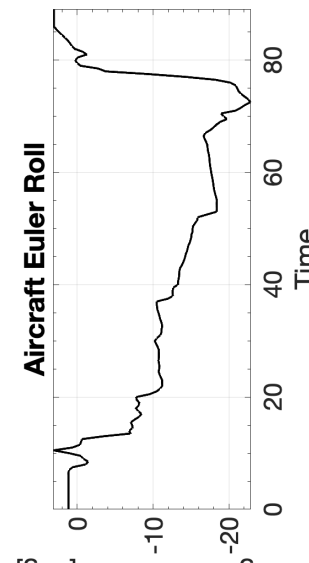

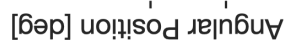

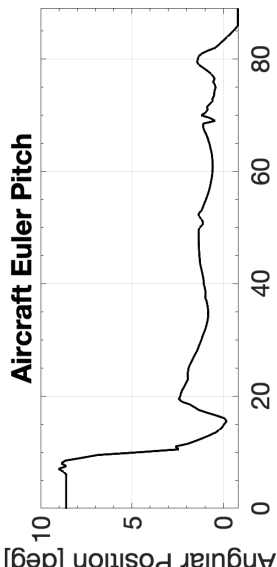

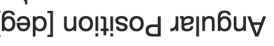

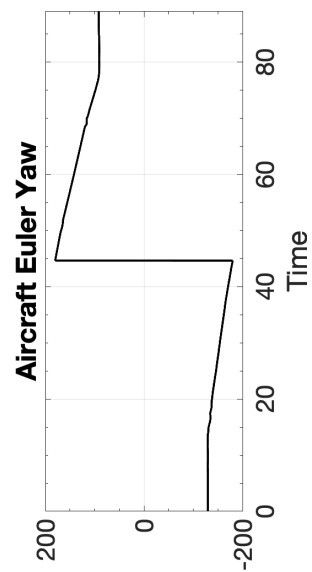

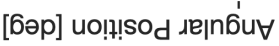
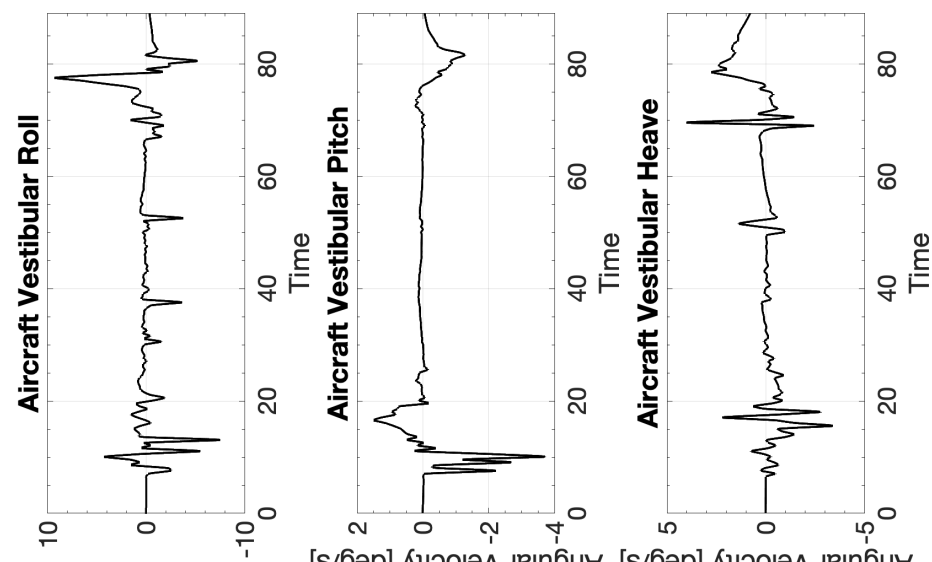

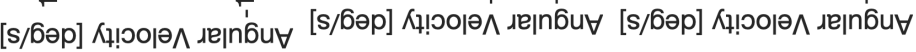

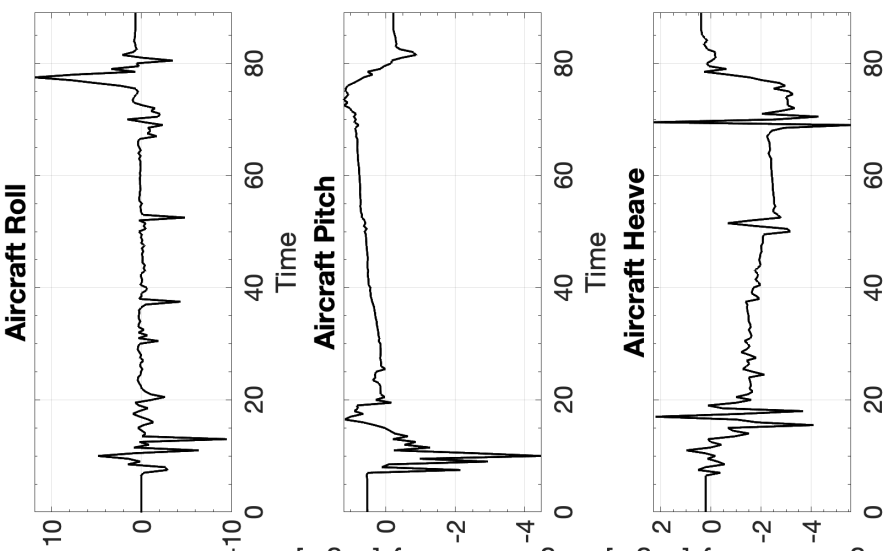

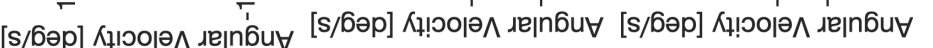

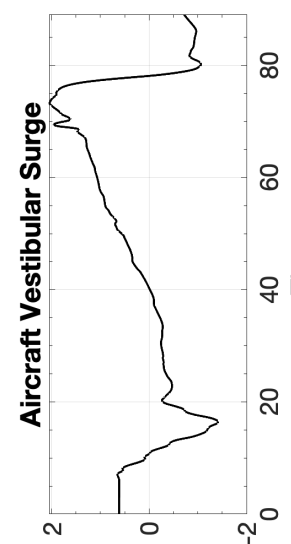

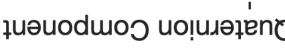

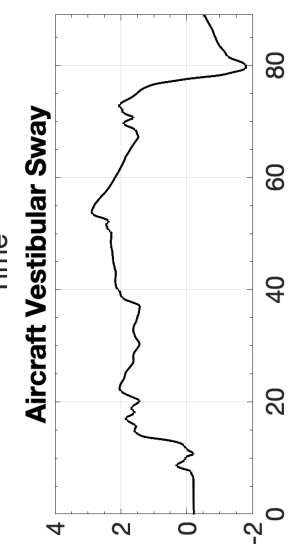

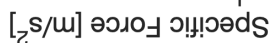

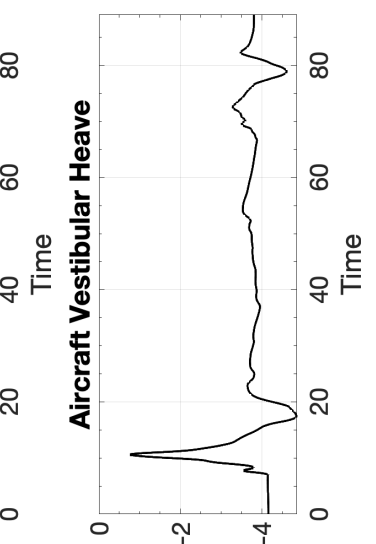

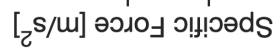

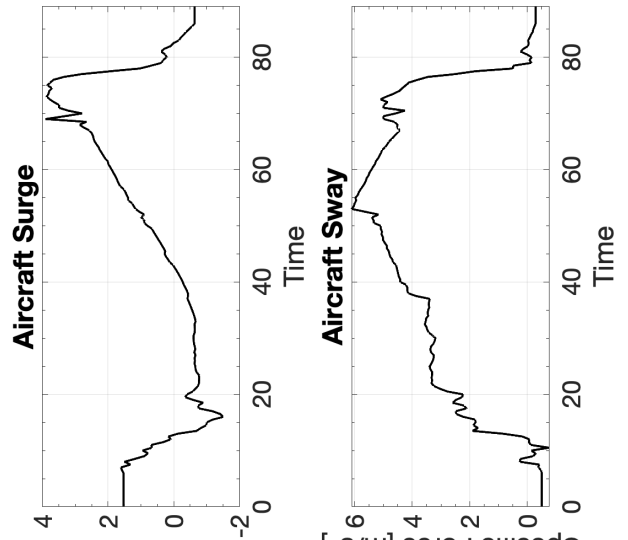

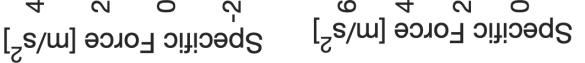
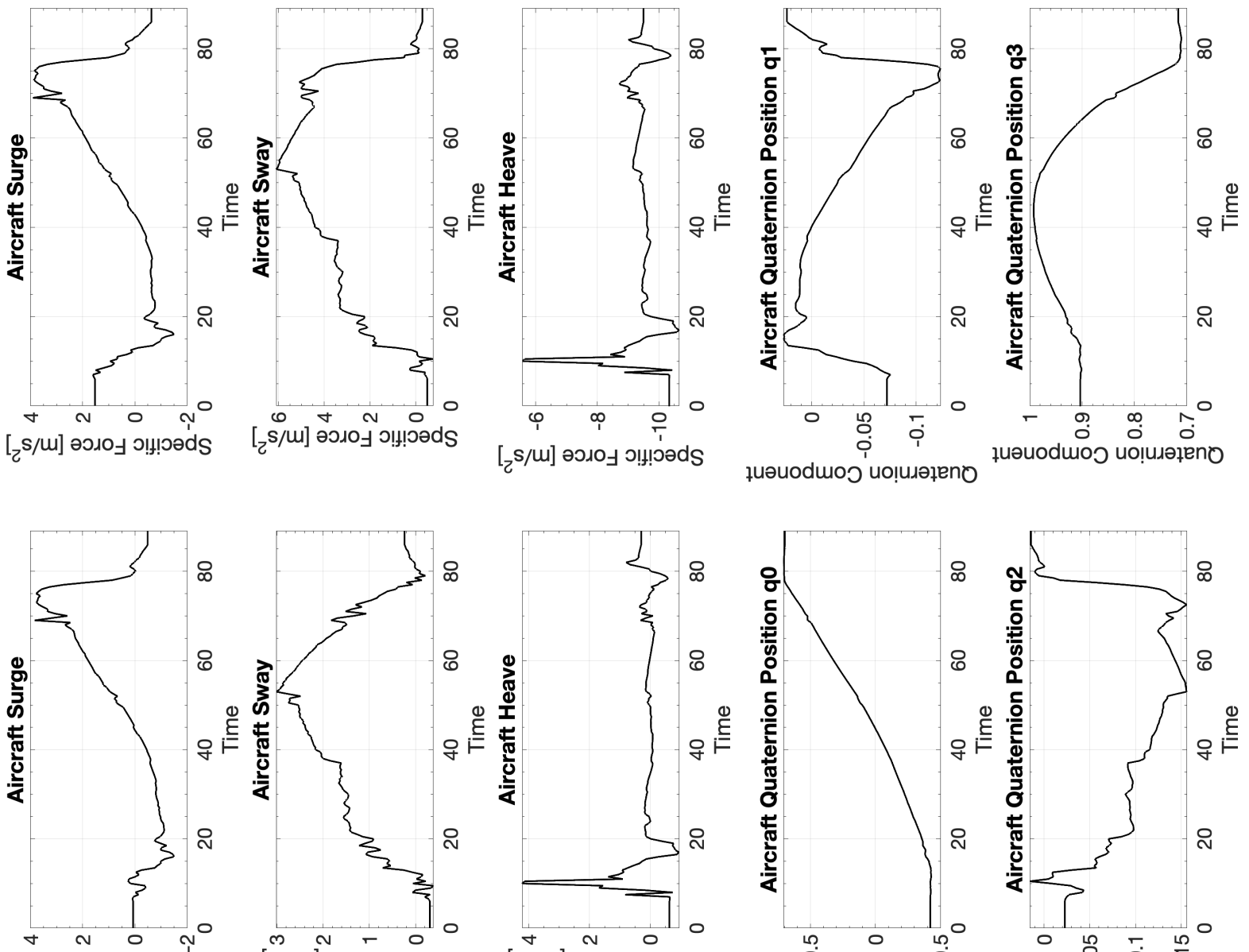

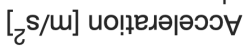

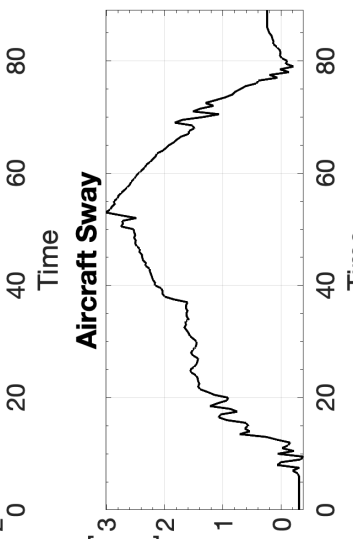

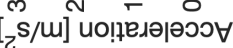

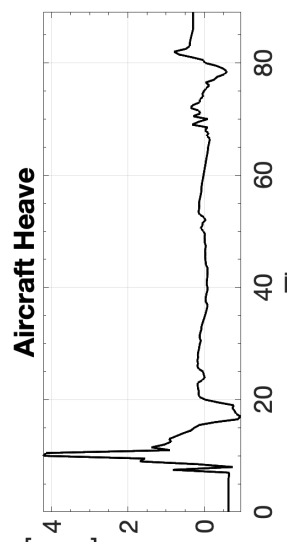

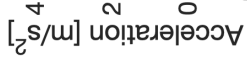

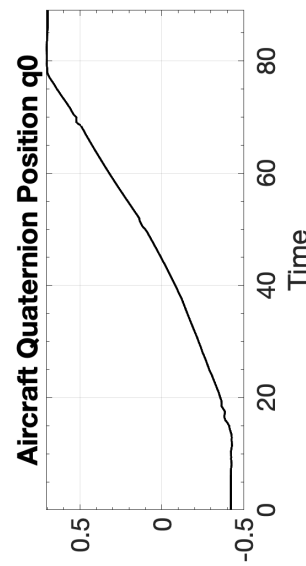

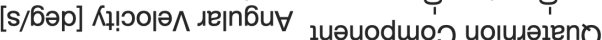

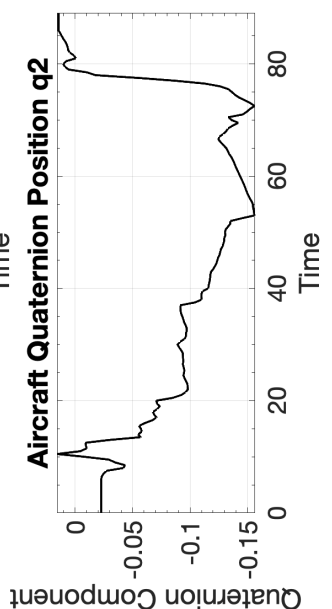




\section{C.15.1 CW1 Columbia 400 Coordinated Turn}
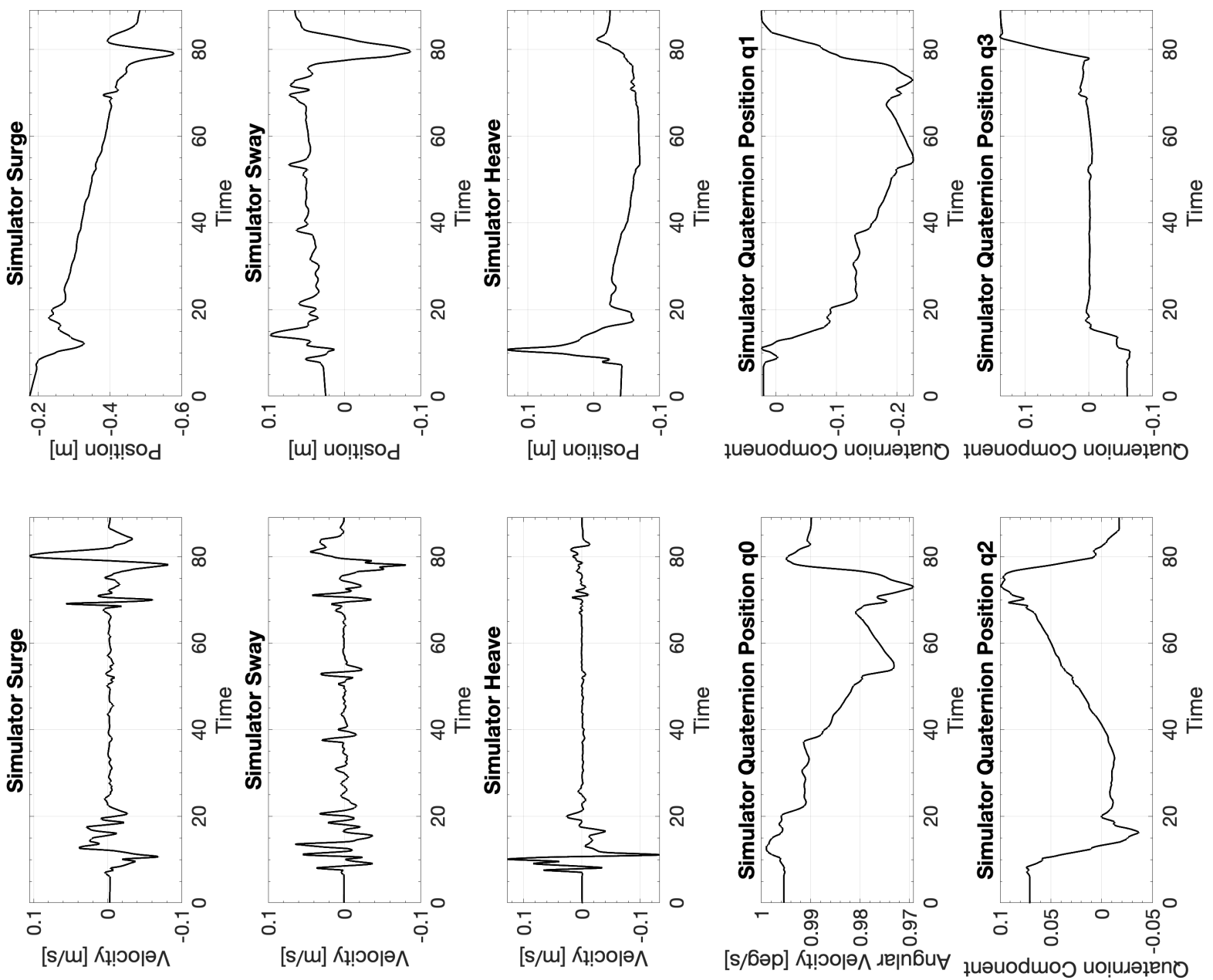

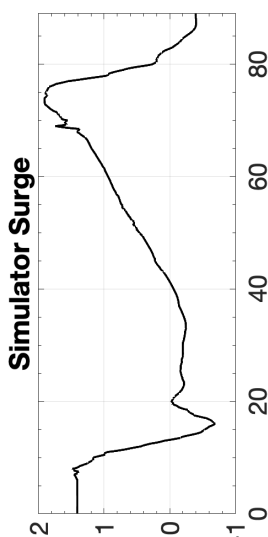

[z
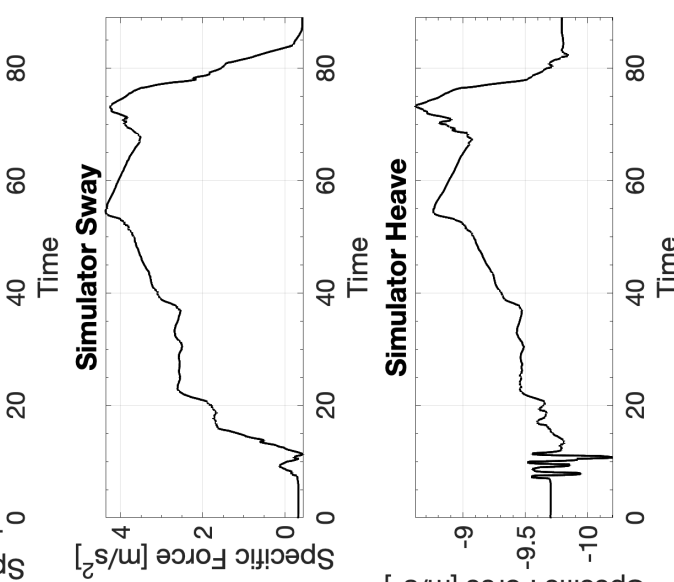

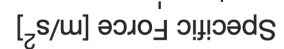

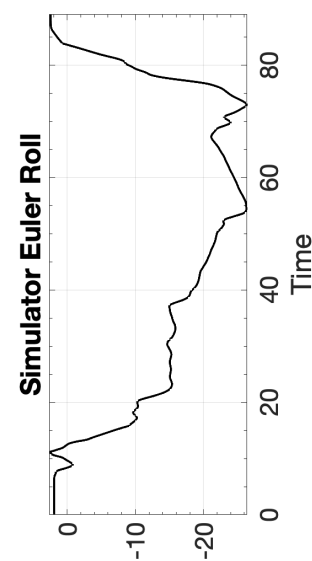

[бәр] uo!!!!sod גe|n6u

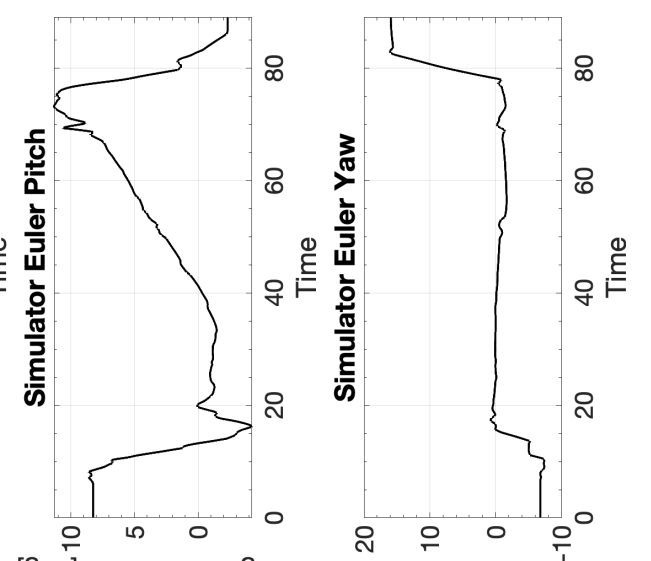

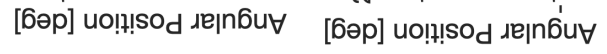

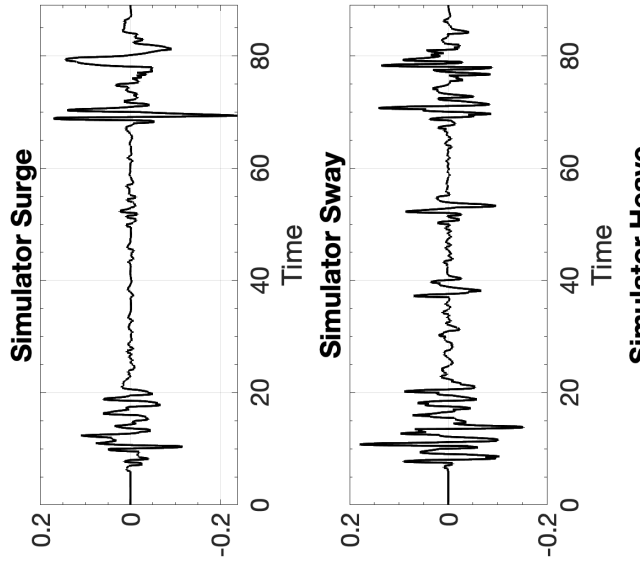

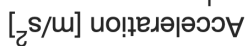

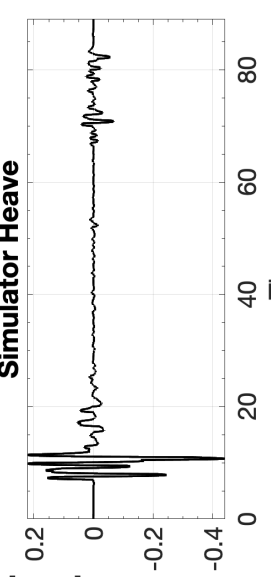

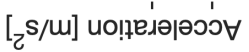

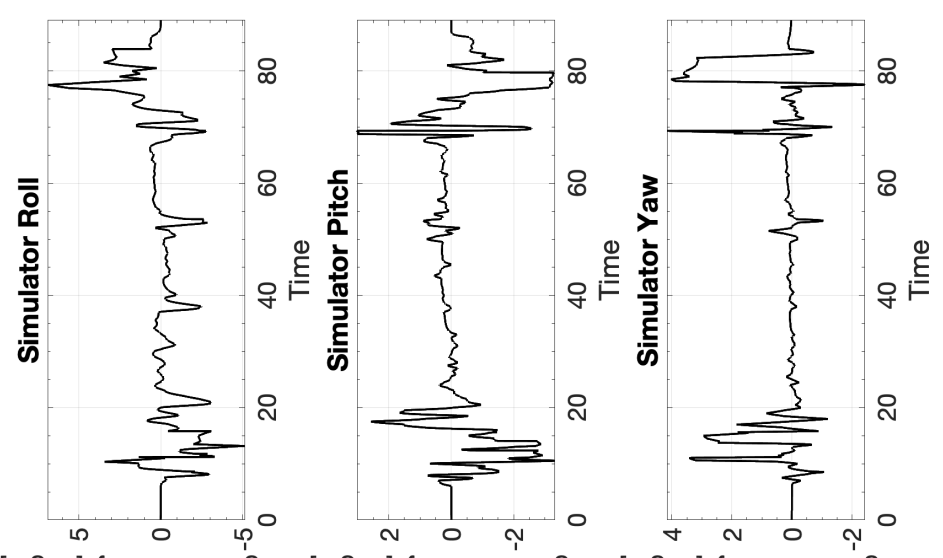

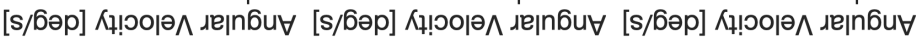




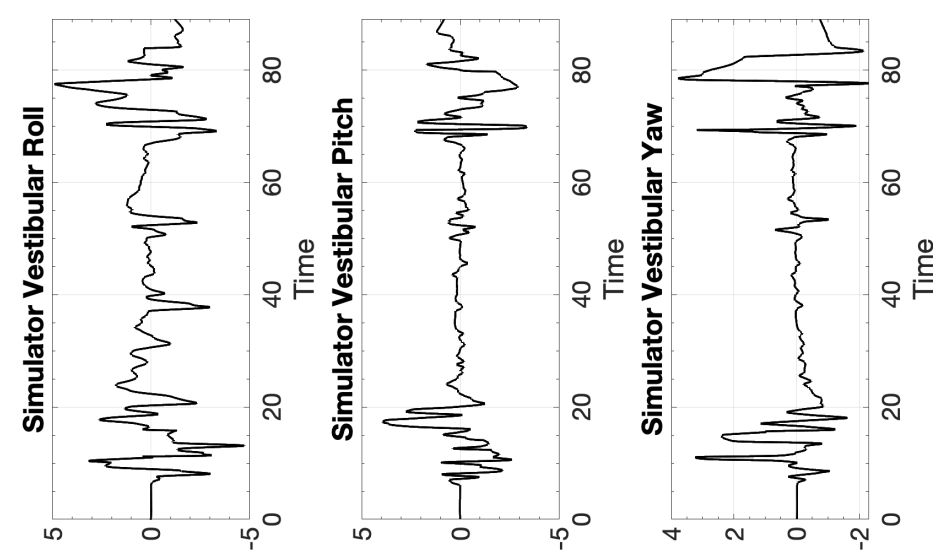

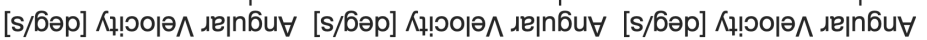

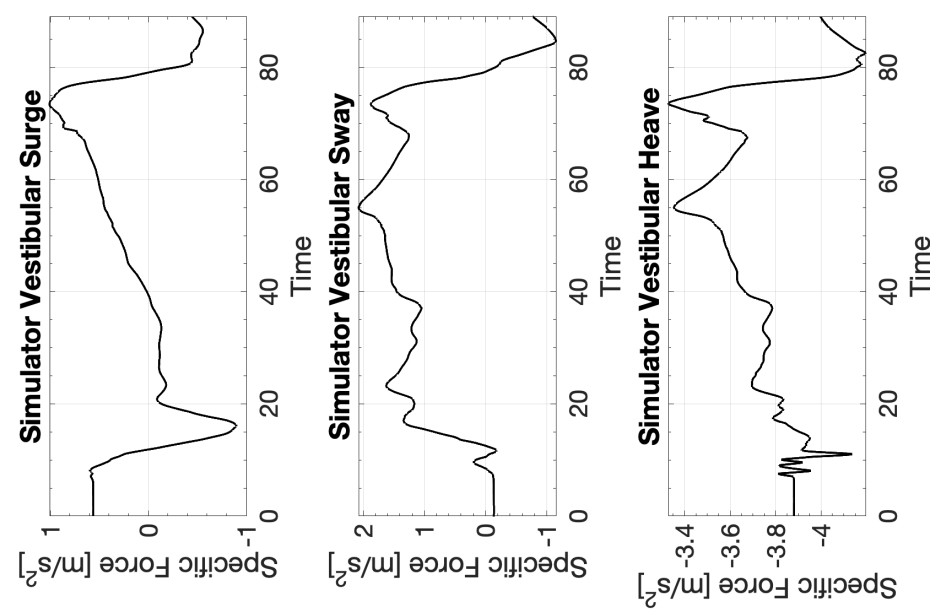




\section{C.15.2 CW2 Columbia 400 Coordinated Turn}
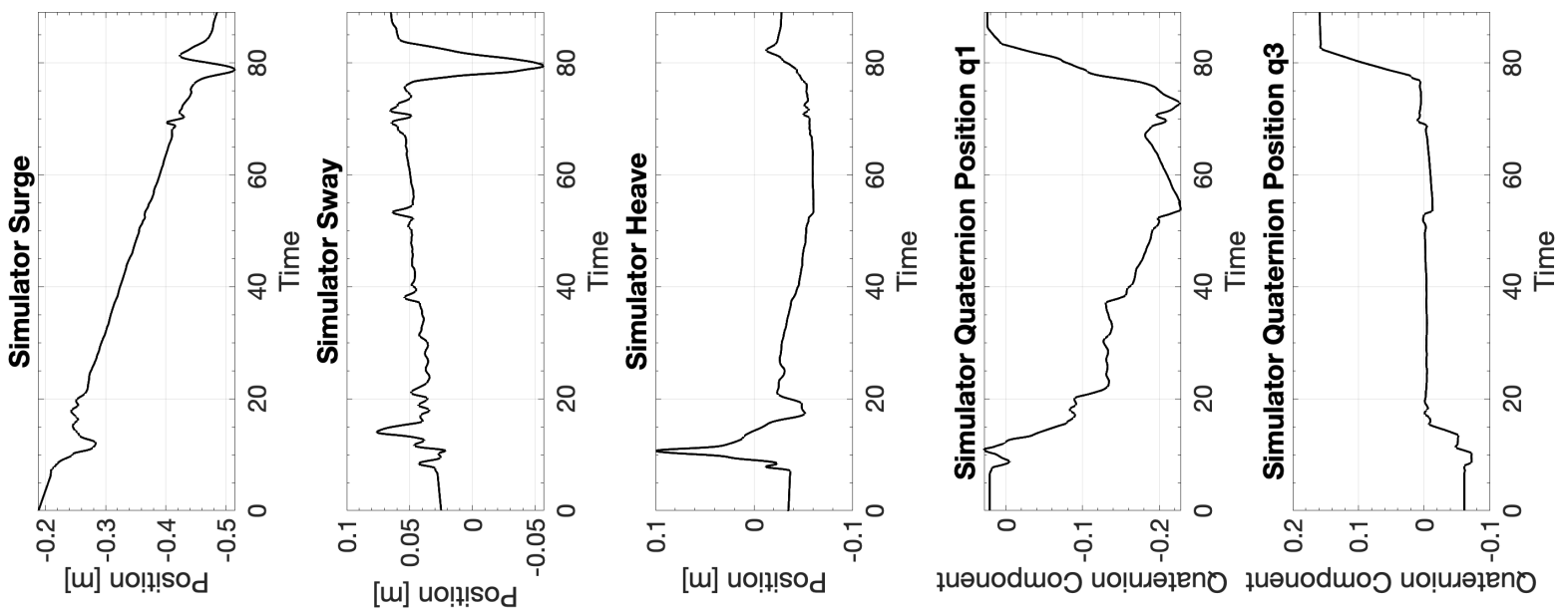

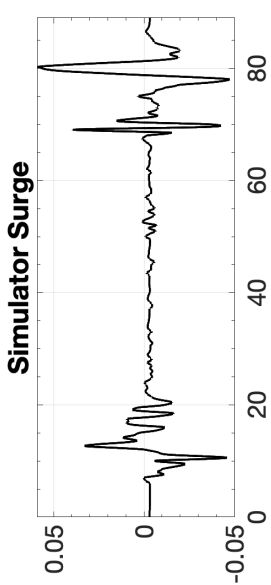

[s/u] Кұ!৩ојә^

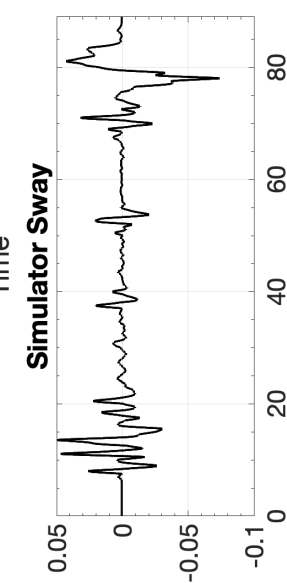

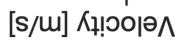

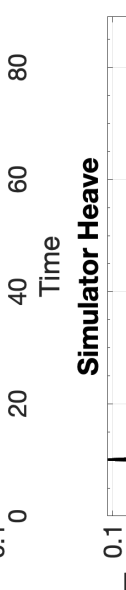

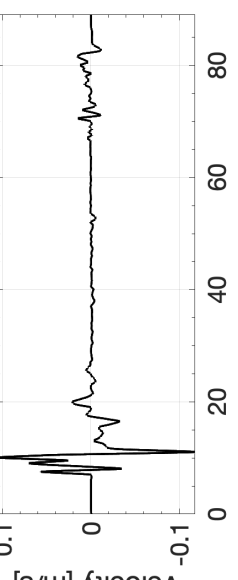

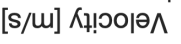

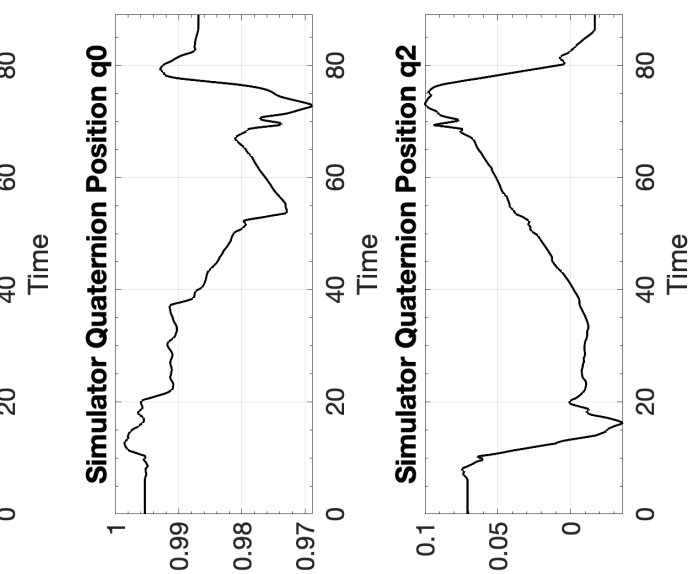

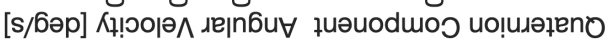

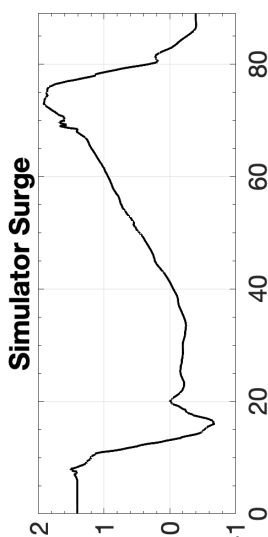

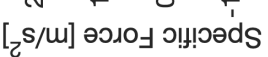

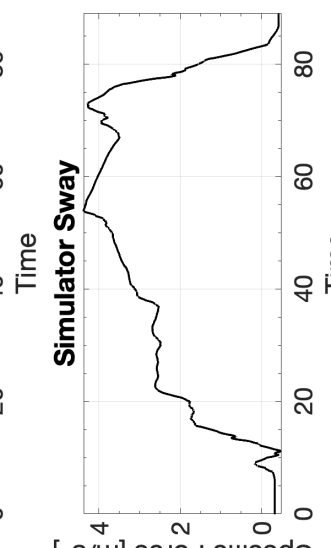

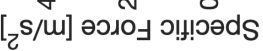

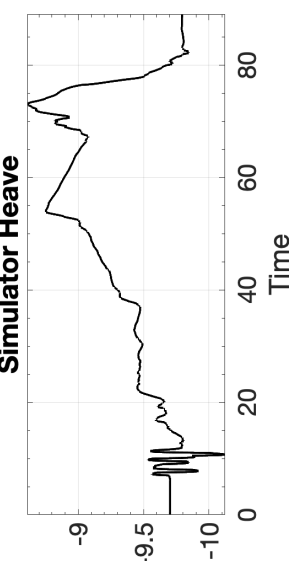

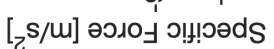

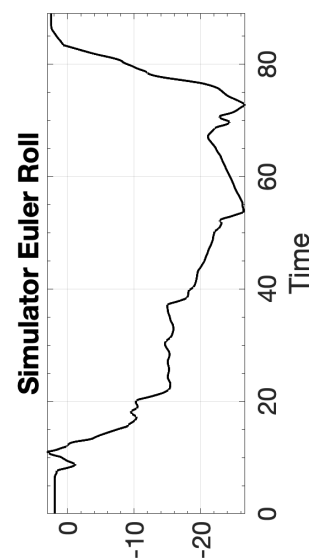

[бәр] uo!̣!'sod de|n6u

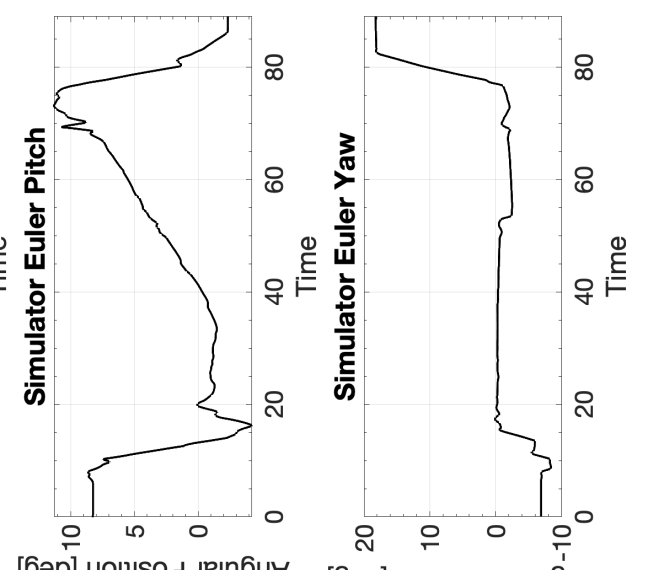

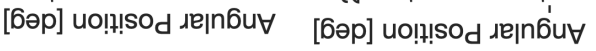

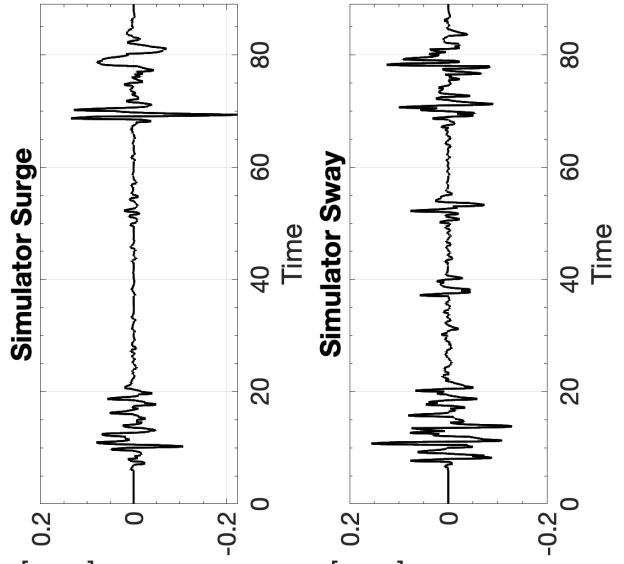

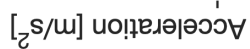

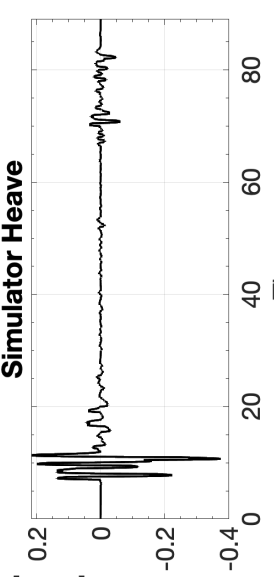

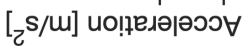

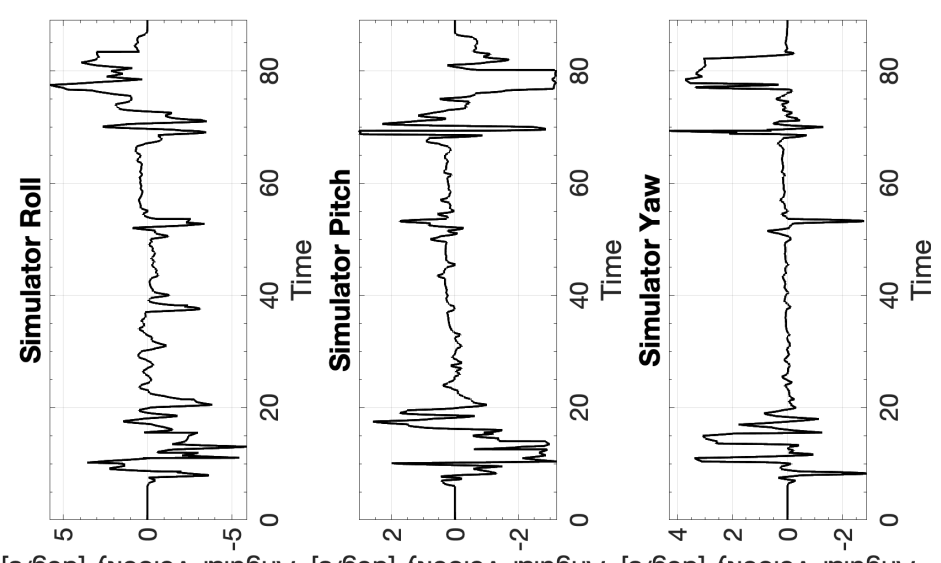

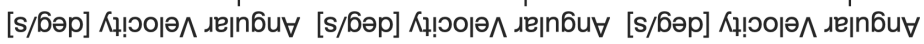




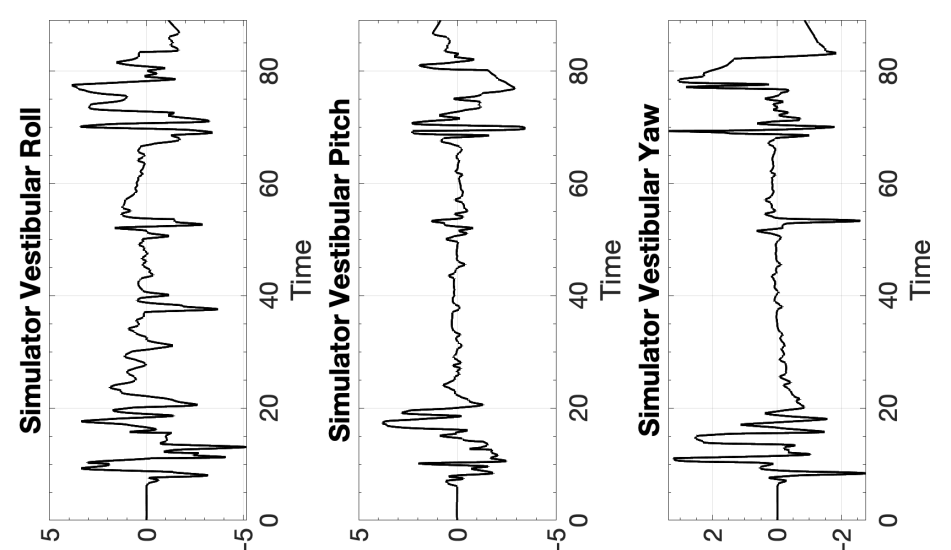

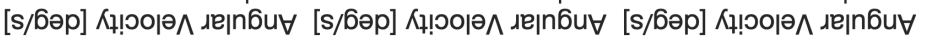

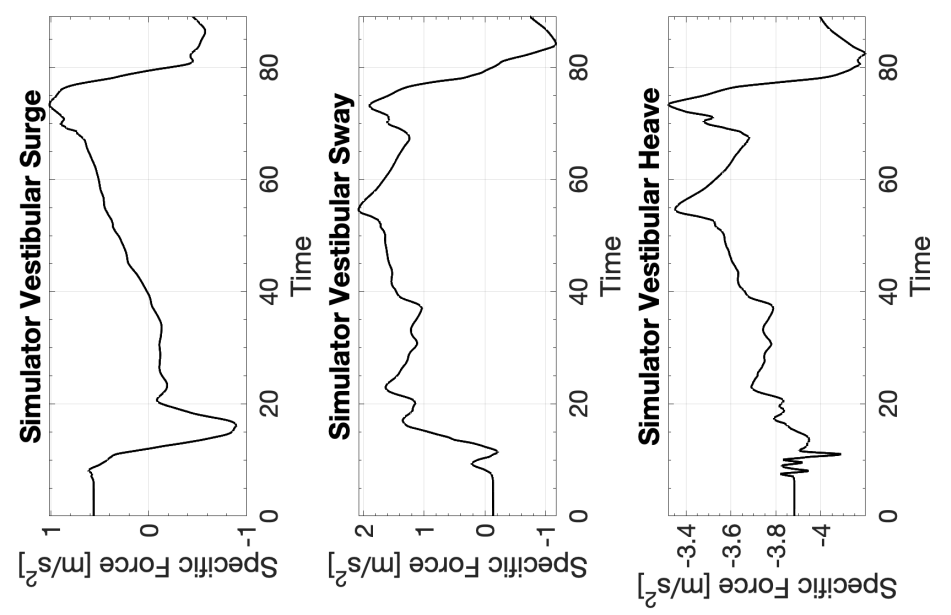




\section{C.15.3 CW3 Columbia 400 Coordinated Turn}
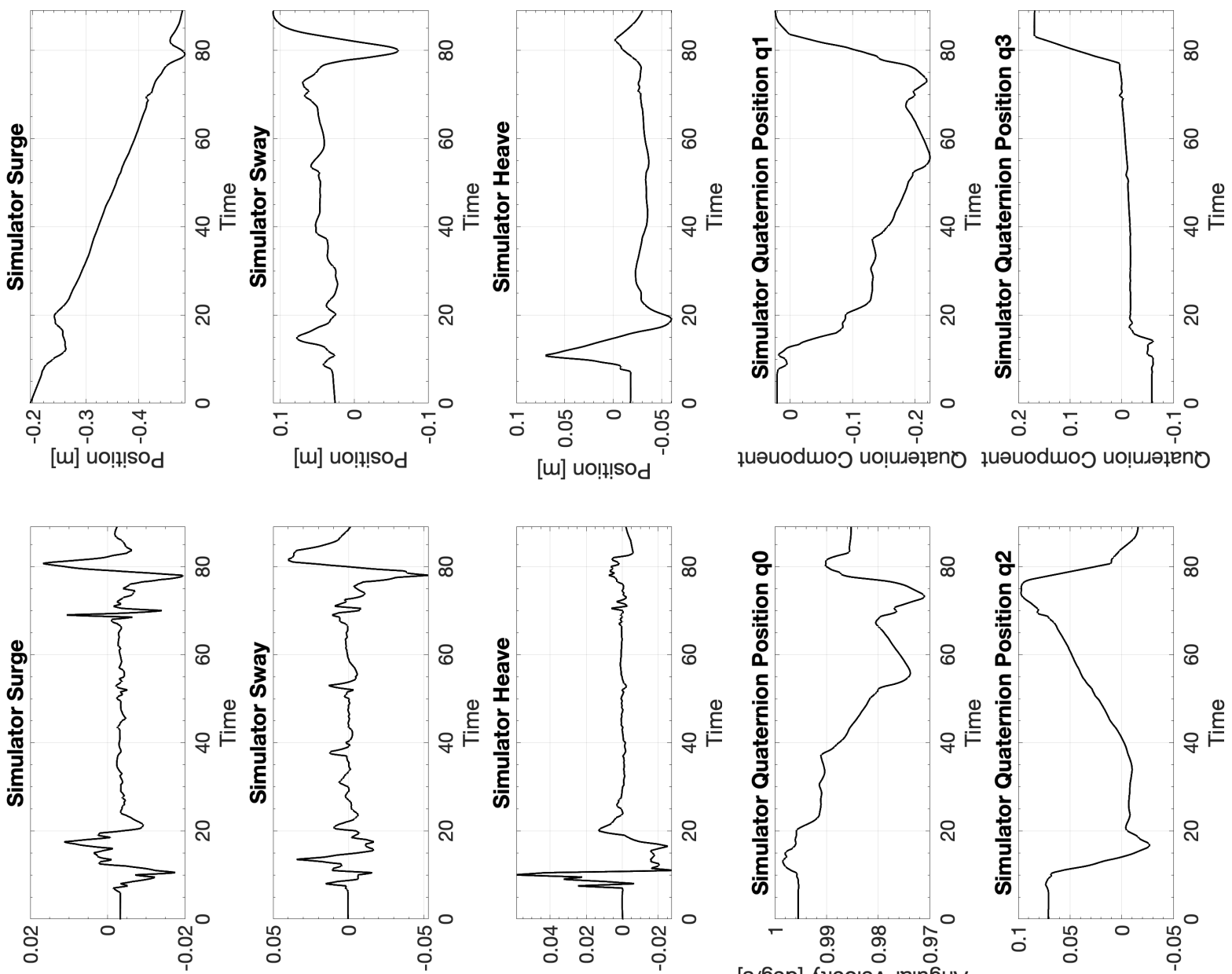

[u] uol!!sod

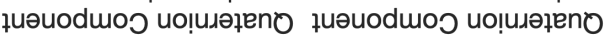

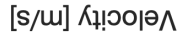

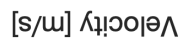
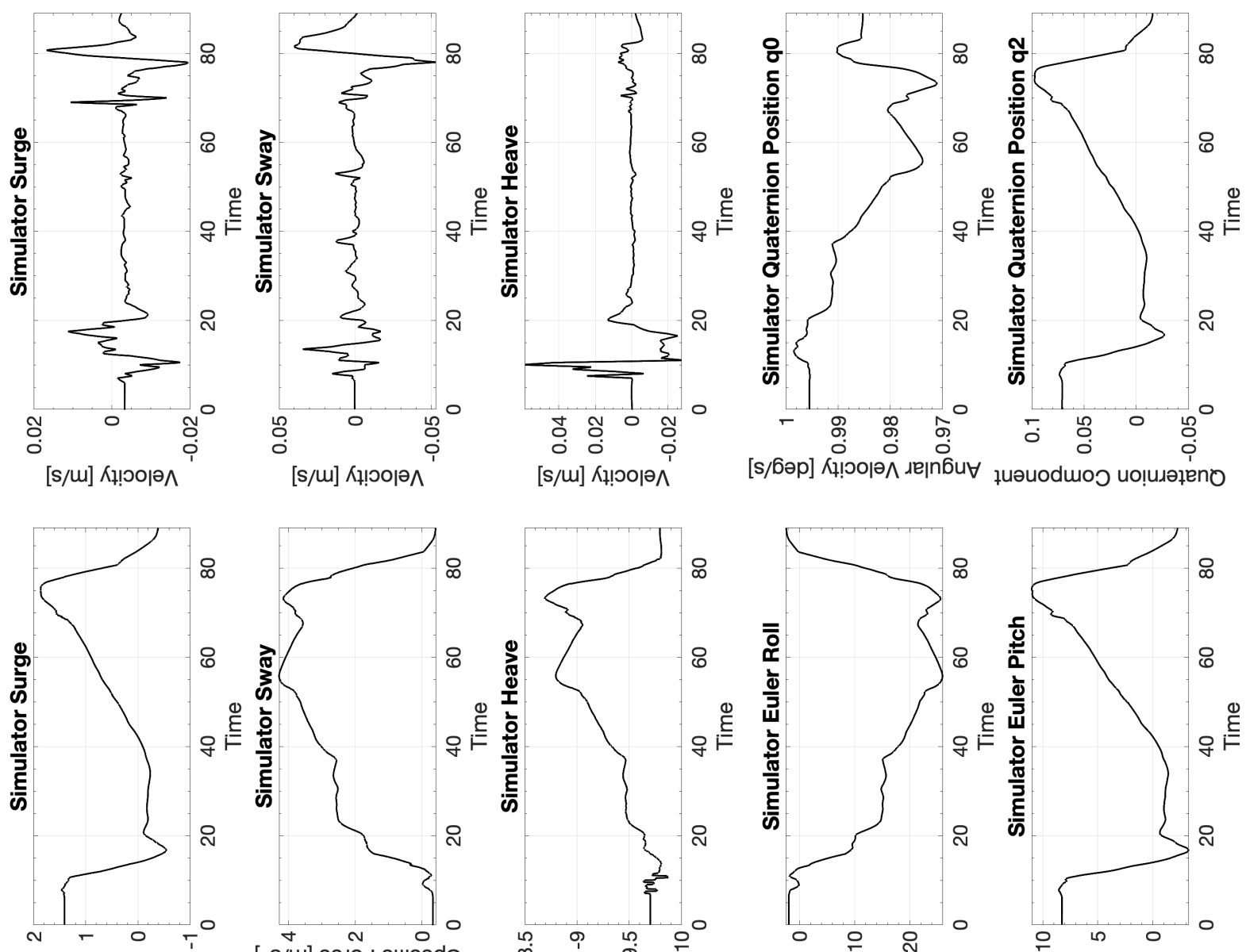

[s/m] ᄉำ잇

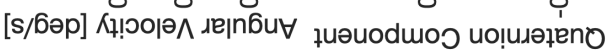

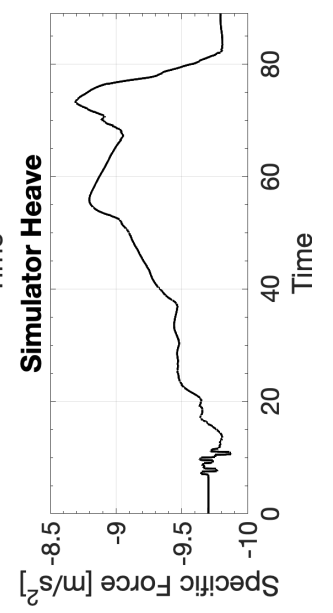

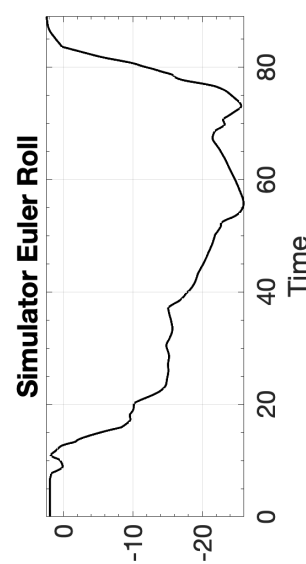

[бәр] uo!!!!sod נé|n6u

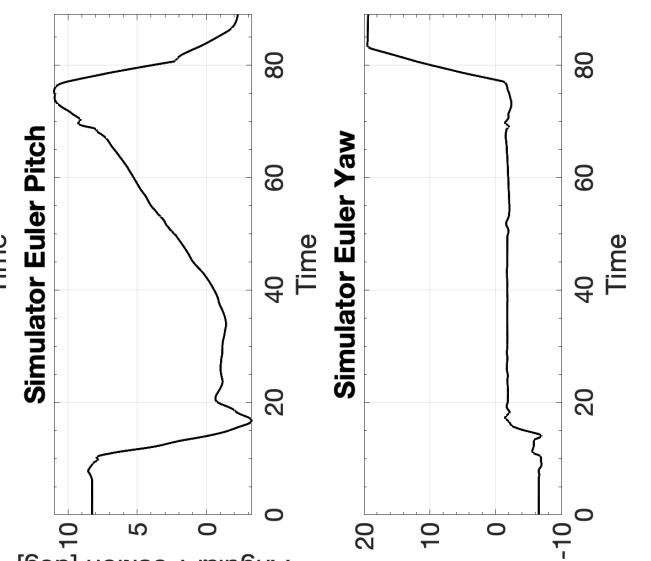

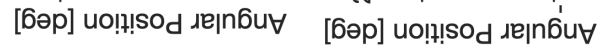

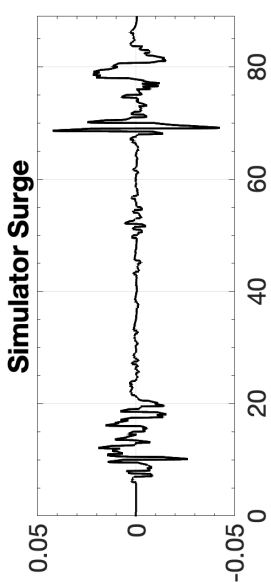

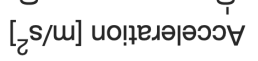

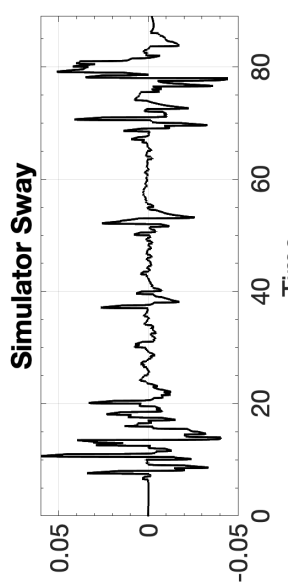

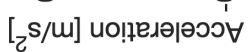

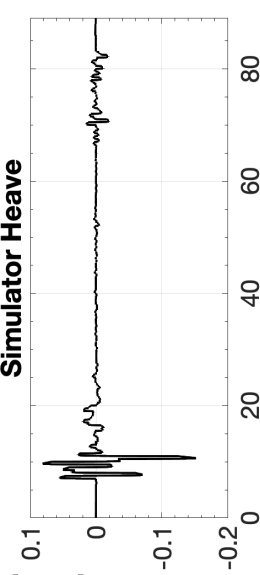

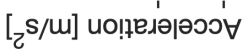

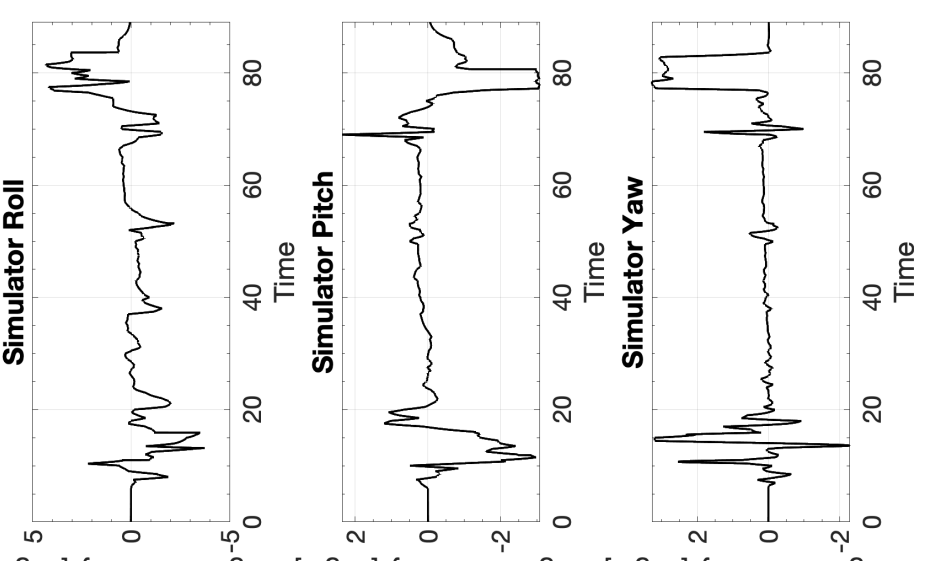

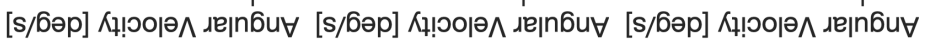



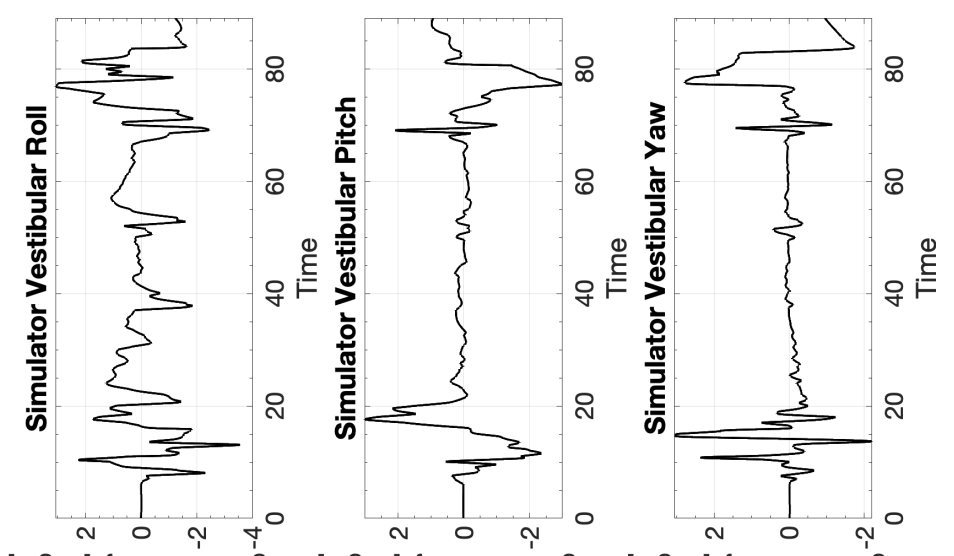

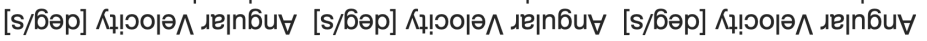

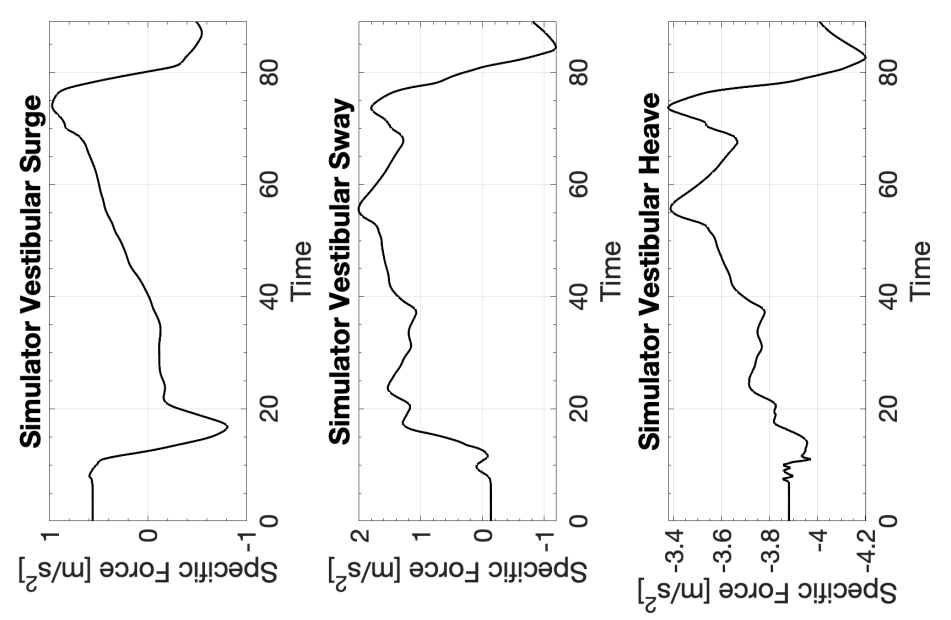




\section{C.16 Columbia 400 Cross-wind Landing}

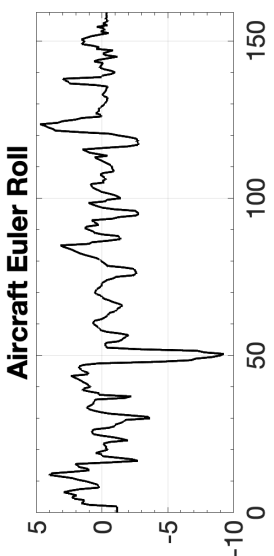

[6əp] uo!!!sod גe|n6u

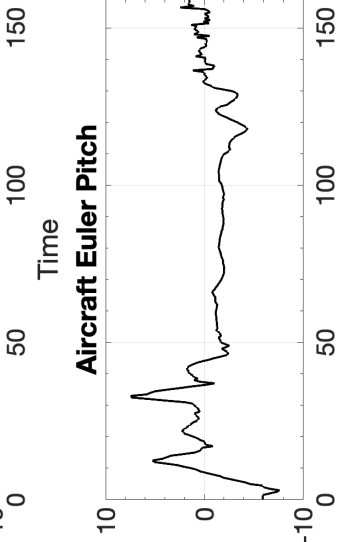

[6әр] uo!!! sod גeןn6u

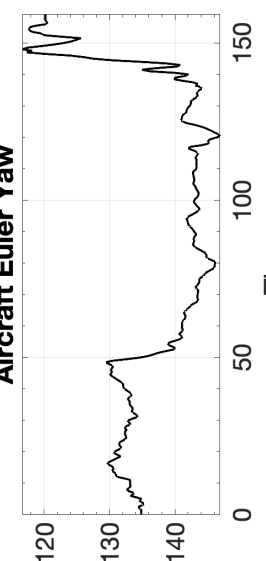

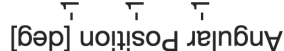
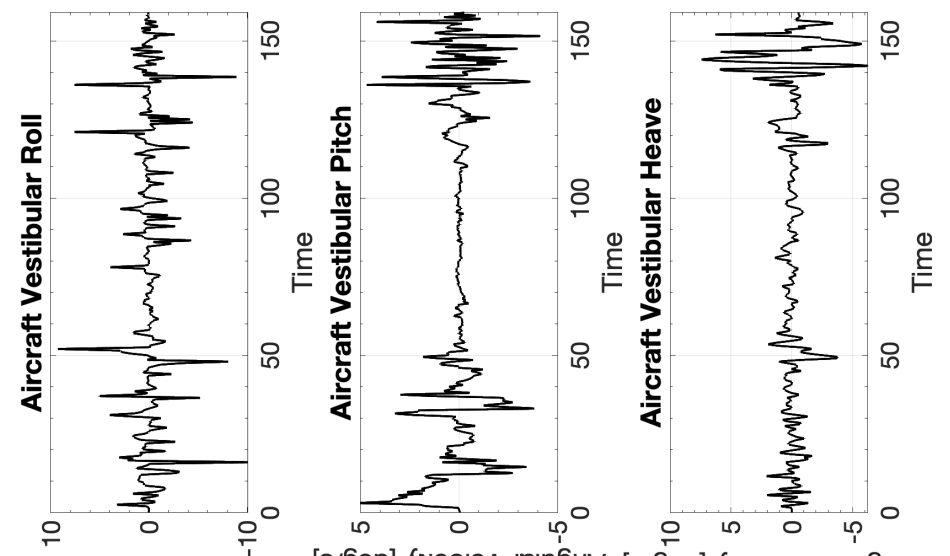

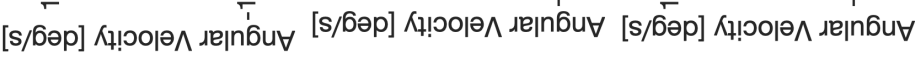

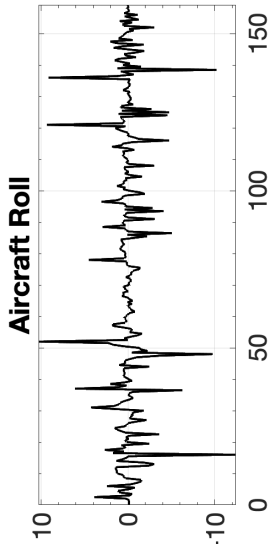

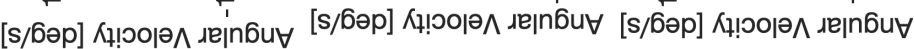
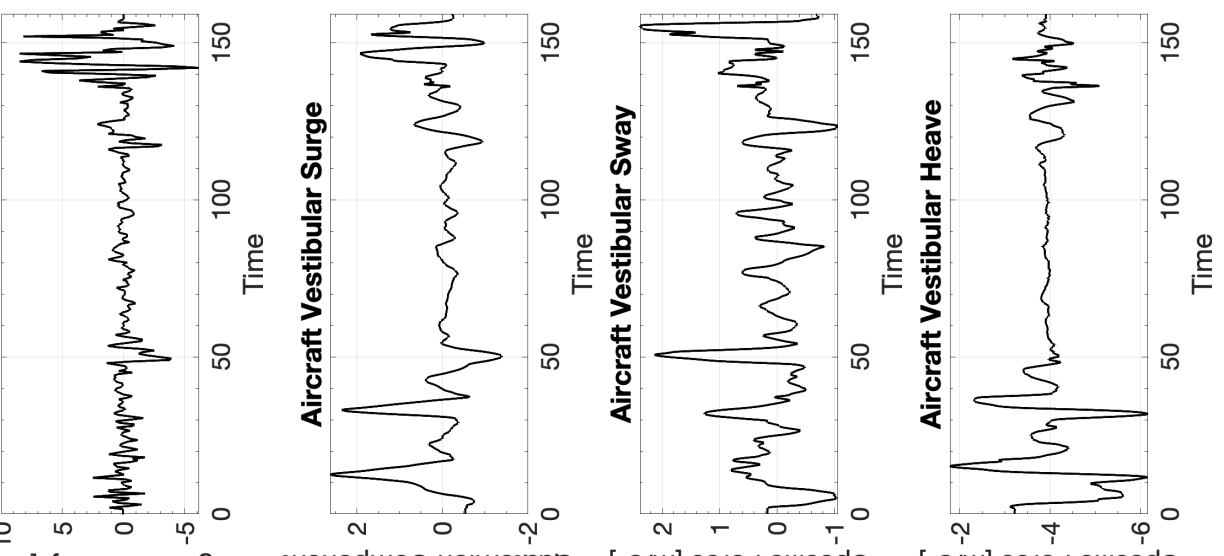

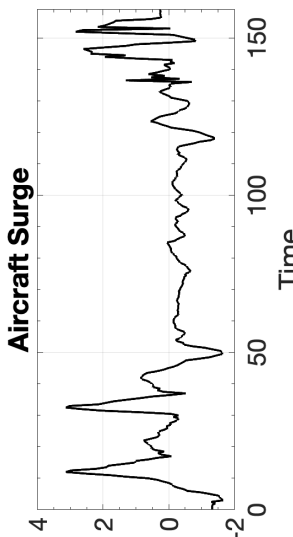

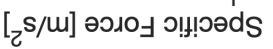

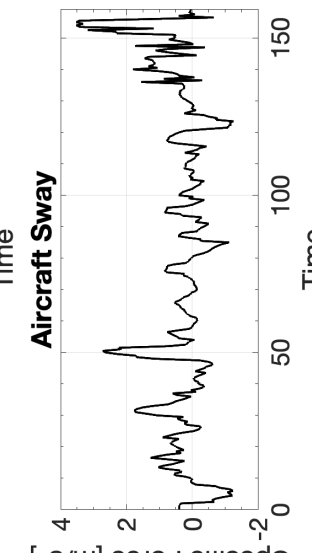

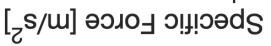

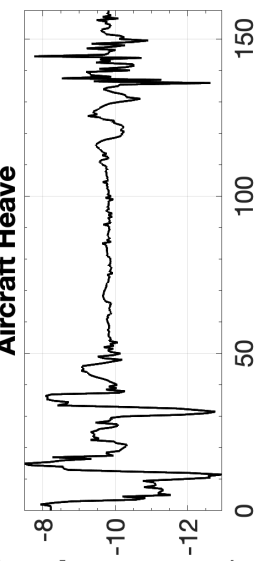

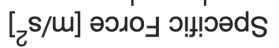

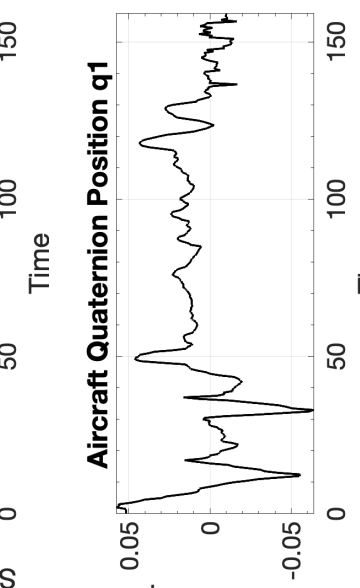

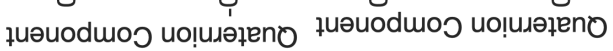

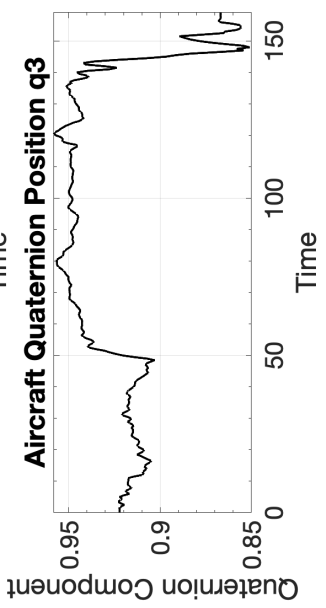

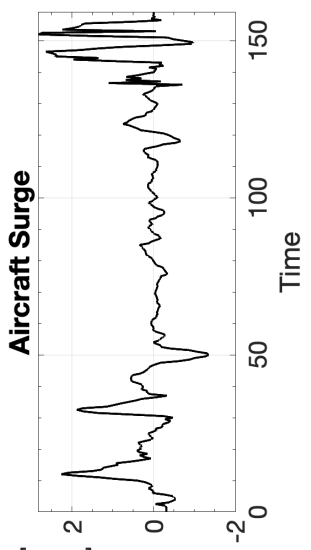

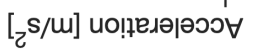

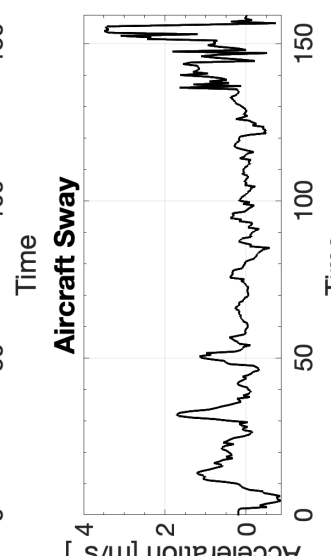

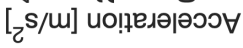

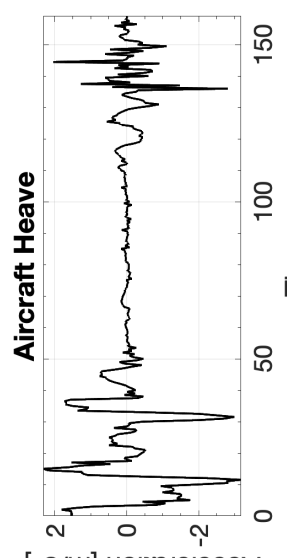

[zs/m] ио!ฺедә|әоว

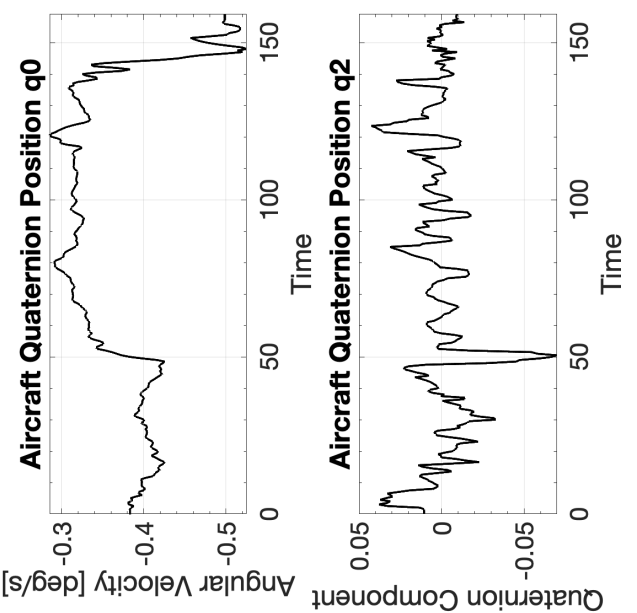




\section{C.16.1 CW1 Columbia 400 Cross-wind Landing}

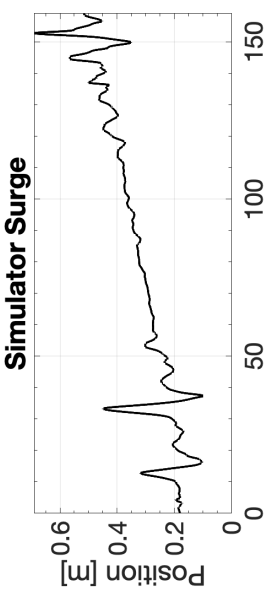

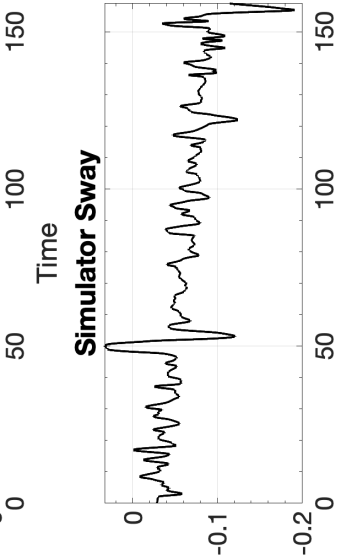

[w] uo!n!sod

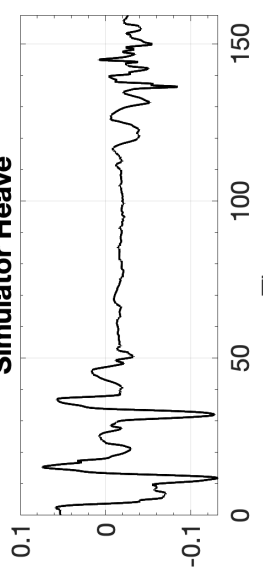

[w] uol!!sod

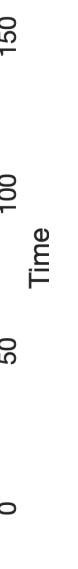

quәuodmos uọı

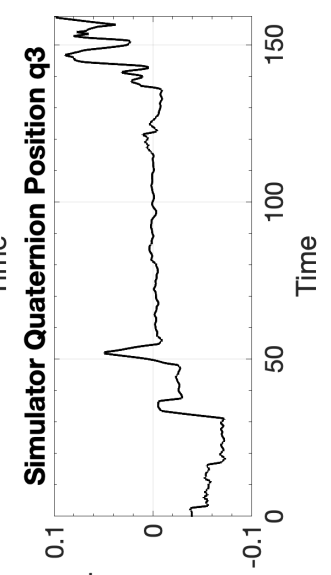

łuəuodmo uo!̣uәəeno

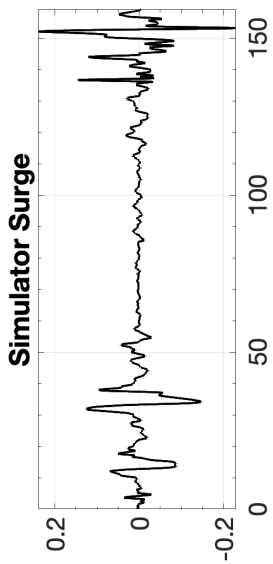

[s/u] К! ৩оюә

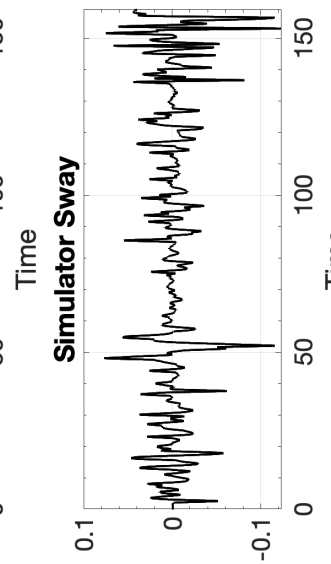

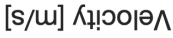

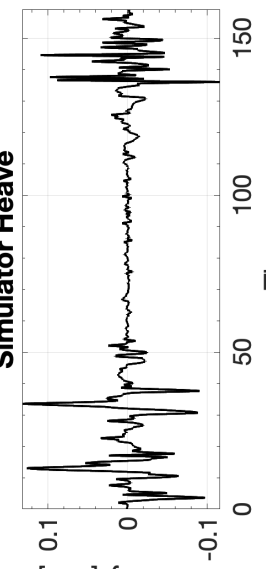

[s/u] Кұ!৩ојә^
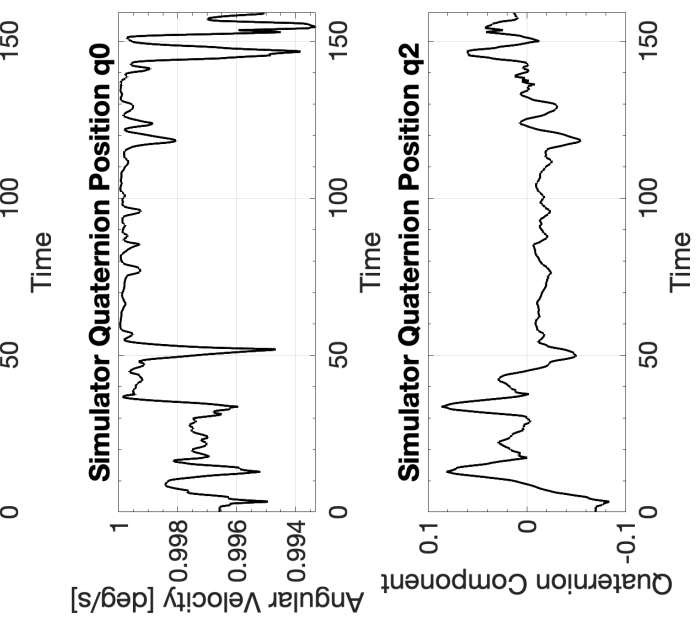

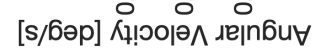

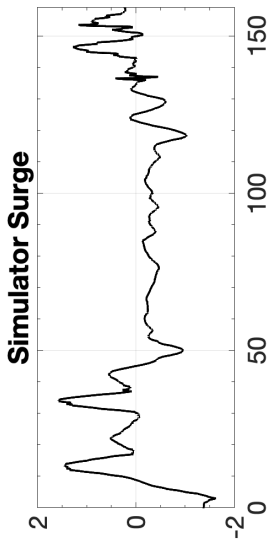

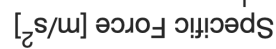

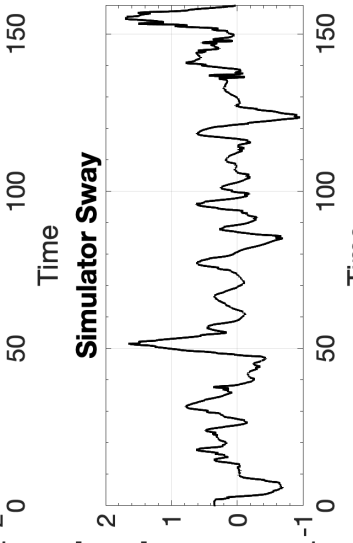

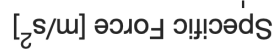

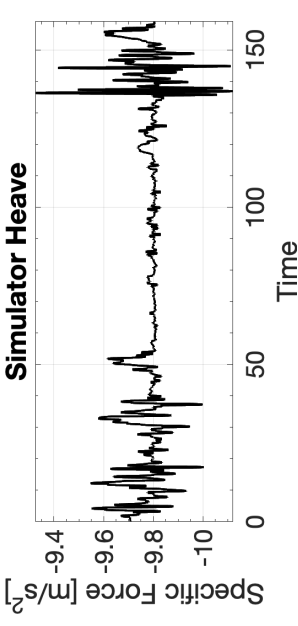

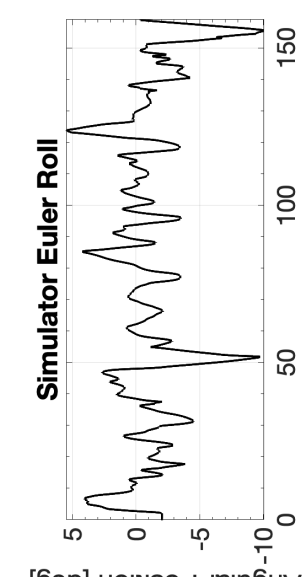

[бәр] uo!!!
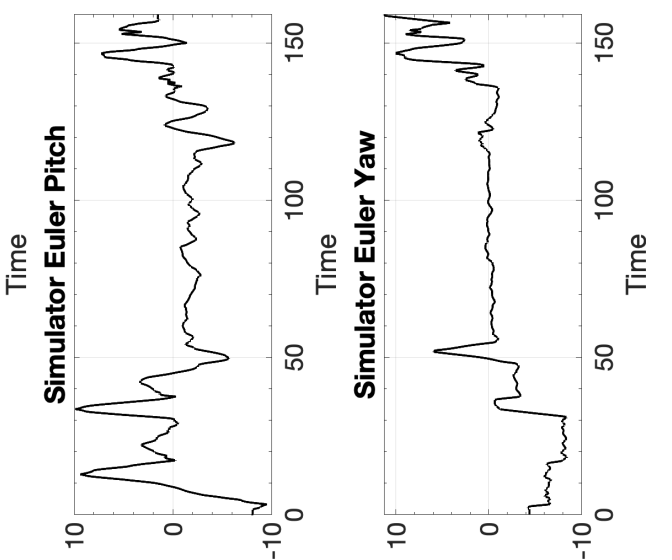

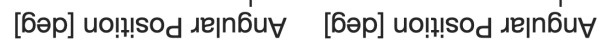

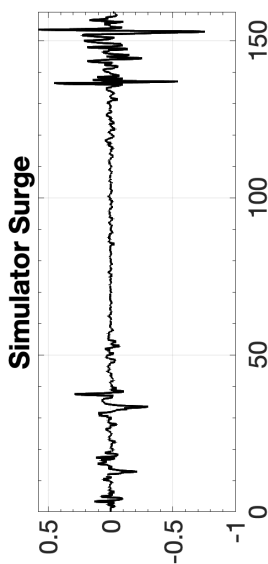

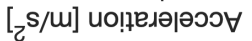

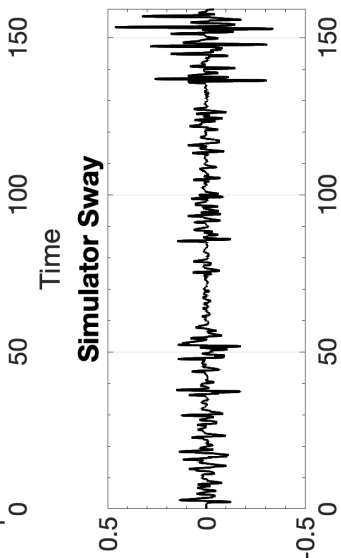

[zs/m] uо!!eגə|әэว

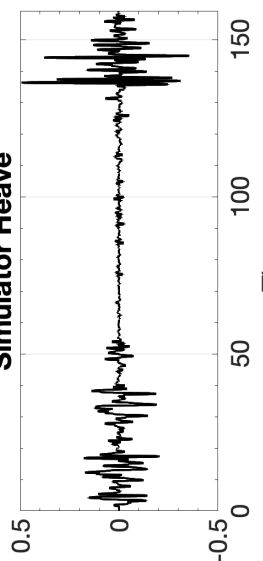

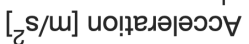

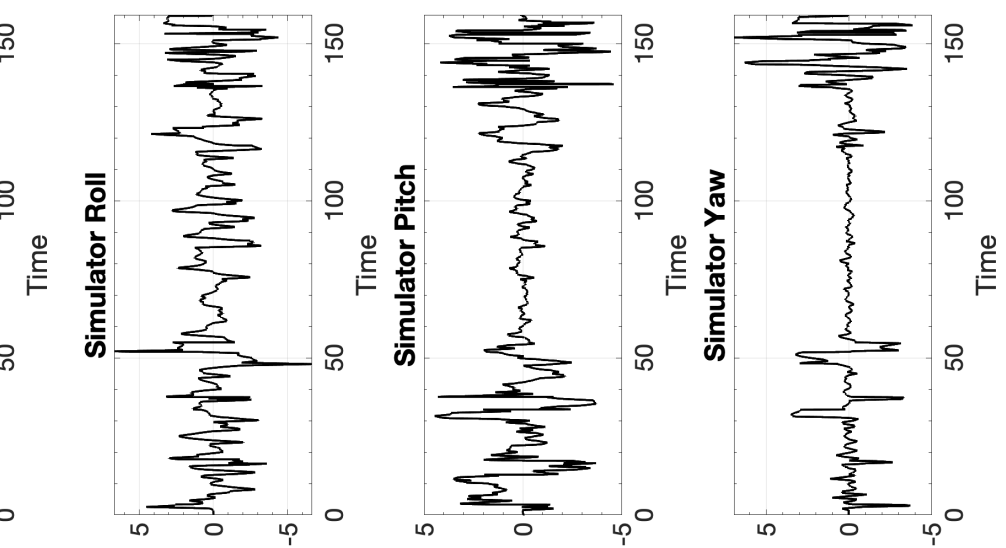

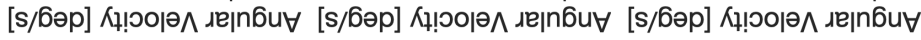



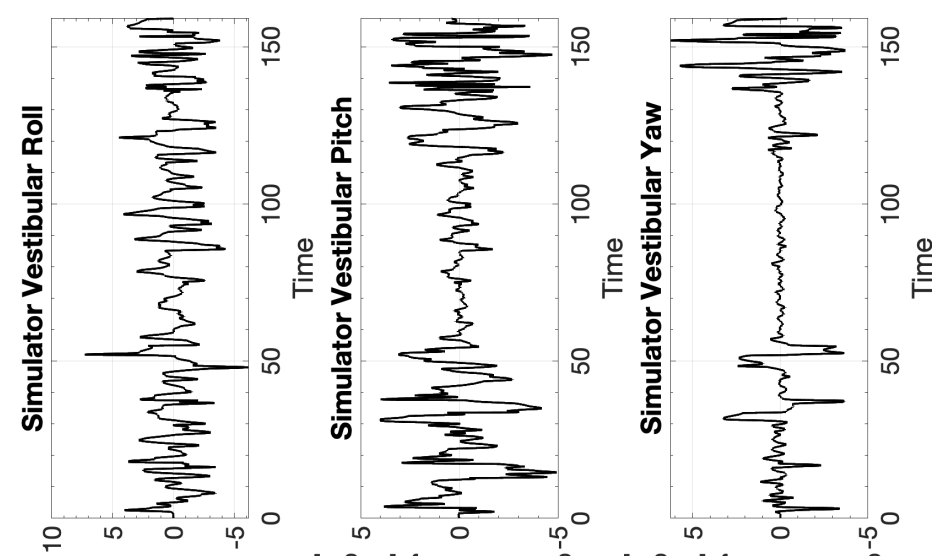

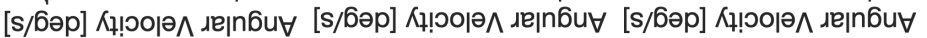
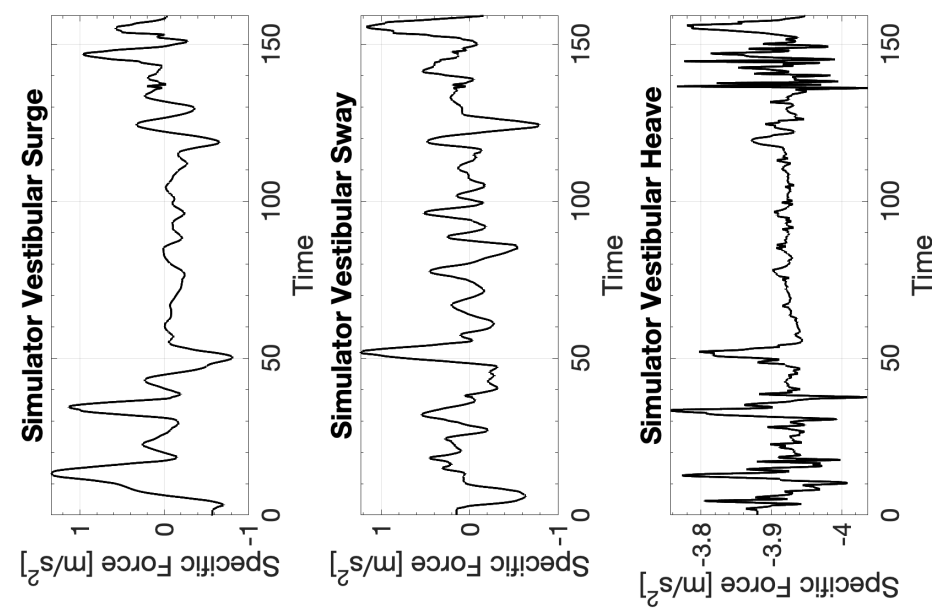

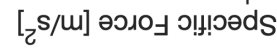

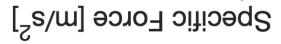

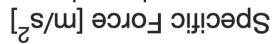




\section{C.16.2 CW2 Columbia 400 Cross-wind Landing}

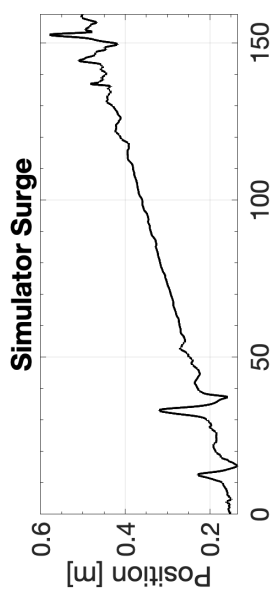

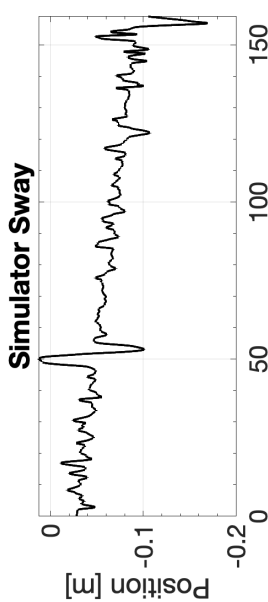

[m] uo!n!?sod

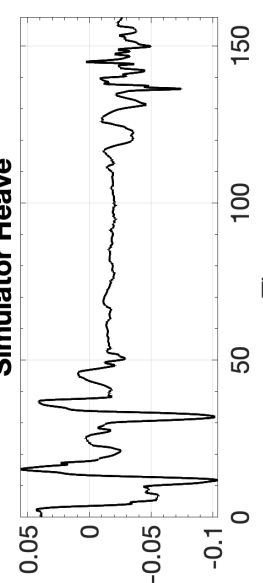

[u] uo!n!sod

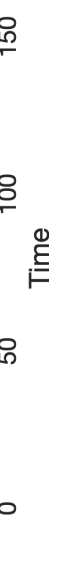

ұuəuodmos uọureteno

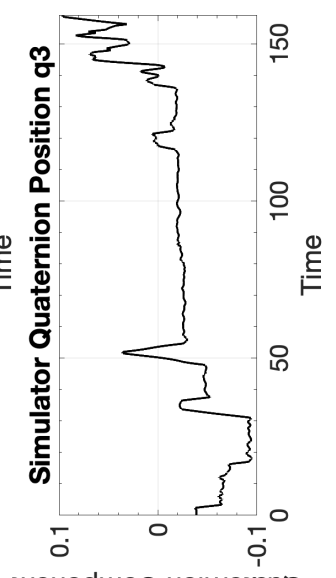

ұuəuoduo uo!̣uəteno

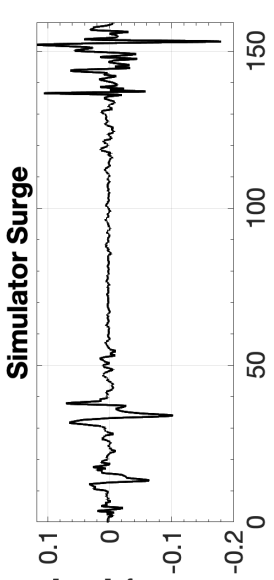

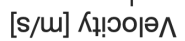

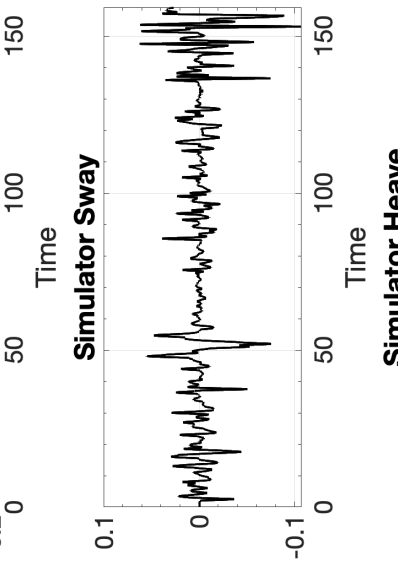

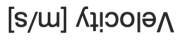

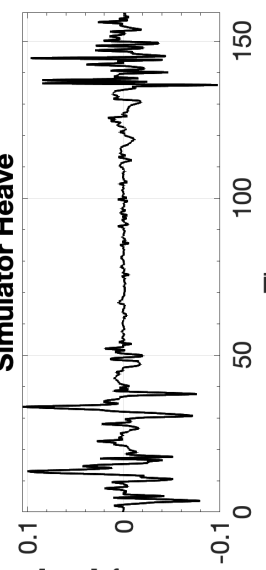

[s/u] Кұ!৩ою^

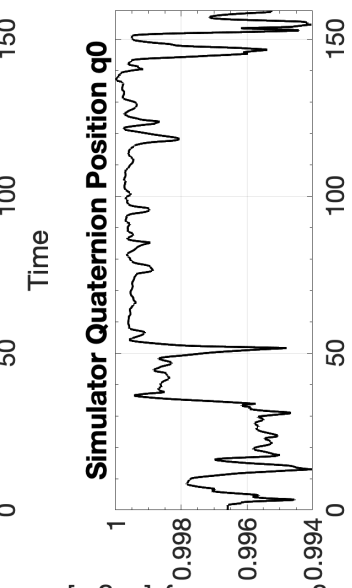

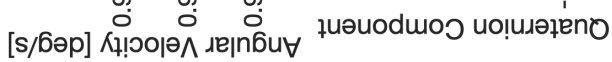

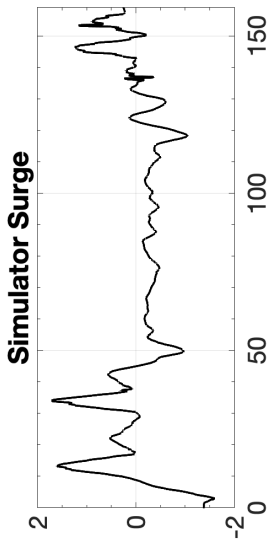

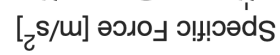

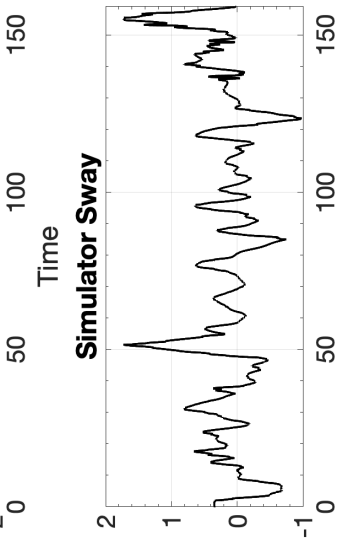

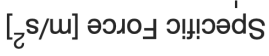

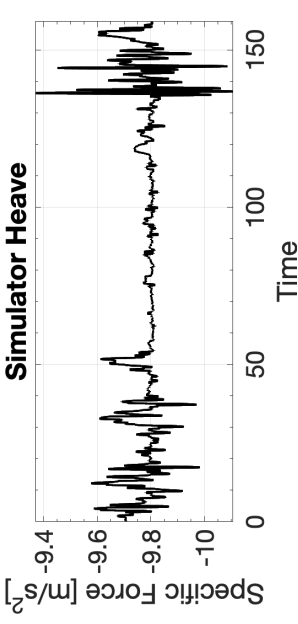

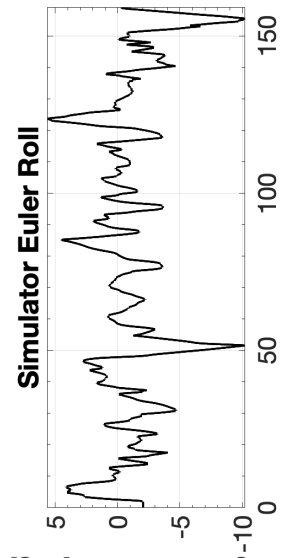

[бәр] uo!!!sod $\mu$ se|nб́u
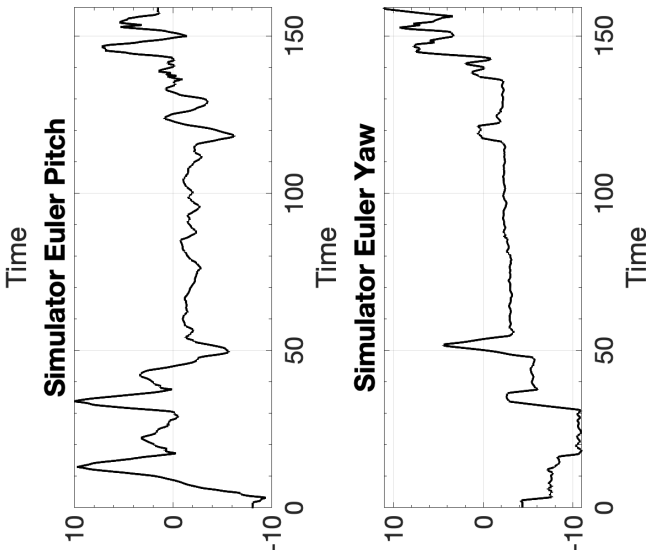

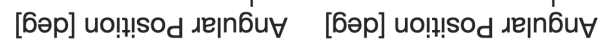

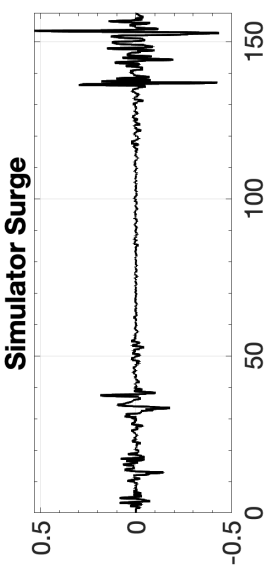

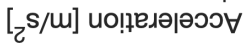

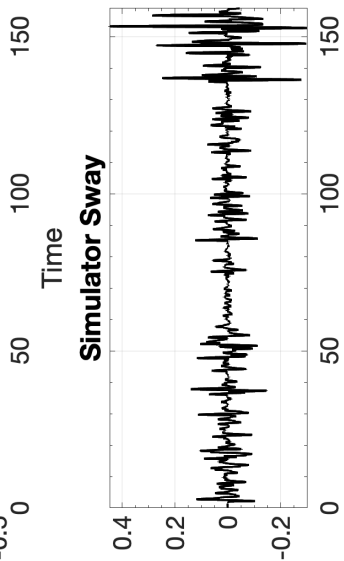

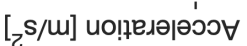

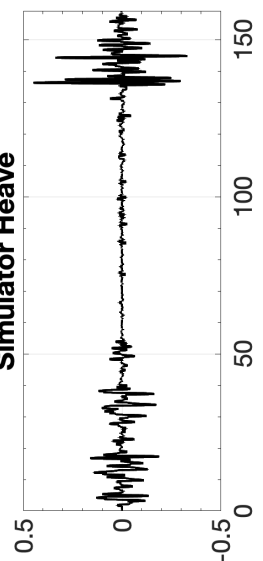

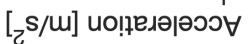
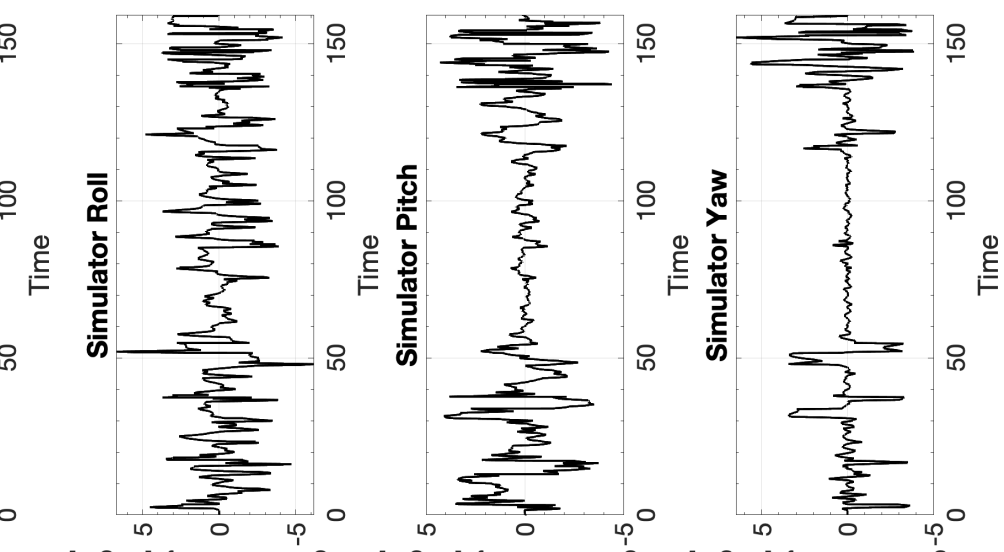

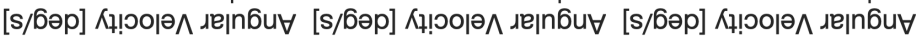



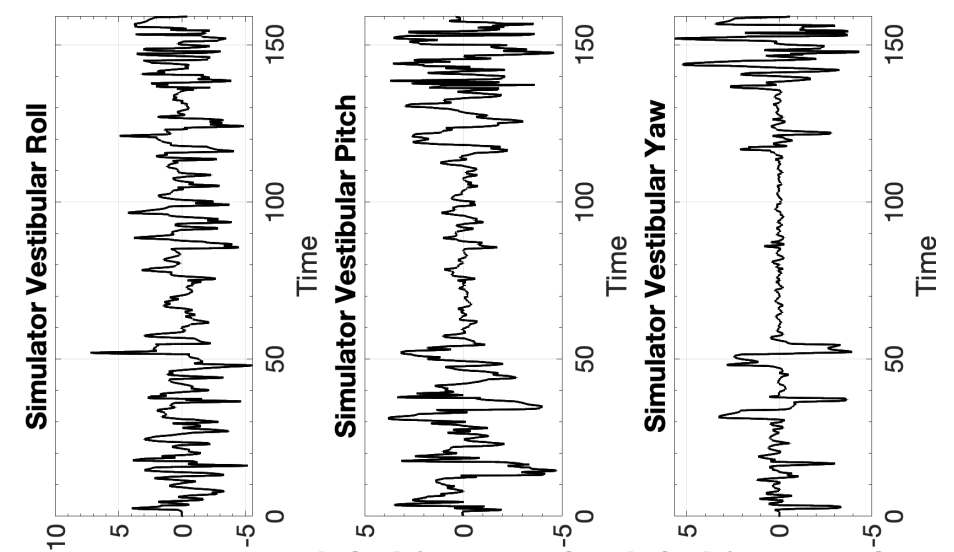

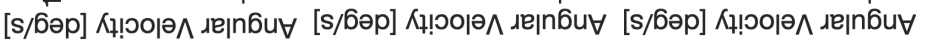
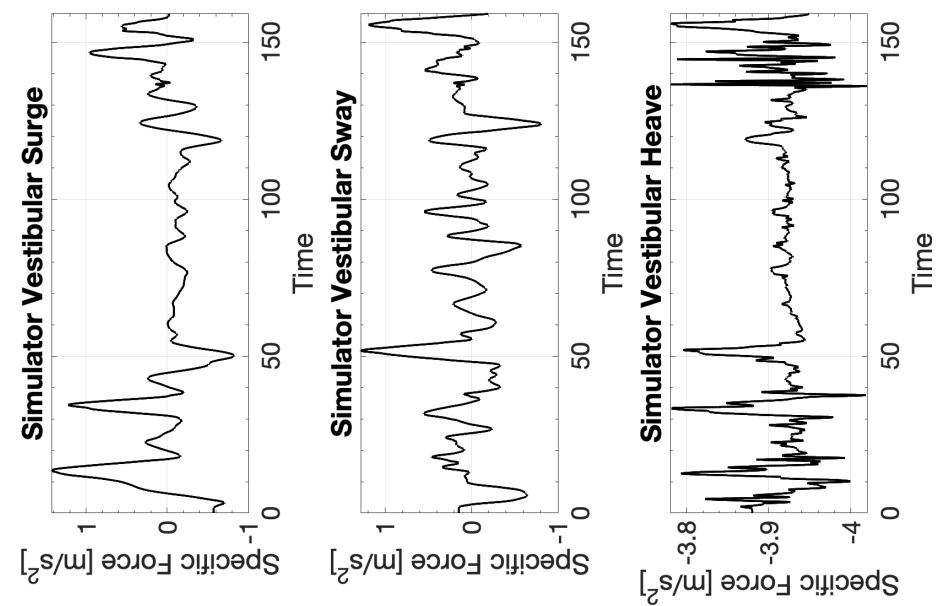

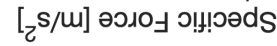

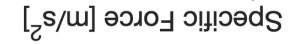

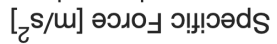




\section{C.16.3 CW3 Columbia 400 Cross-wind Landing}
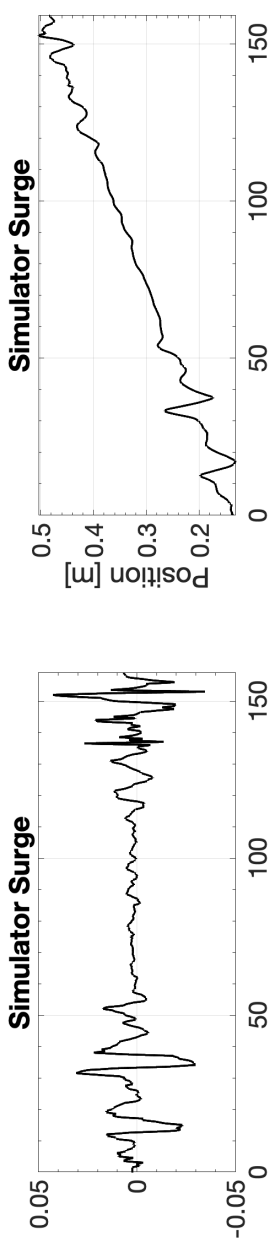

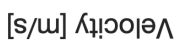

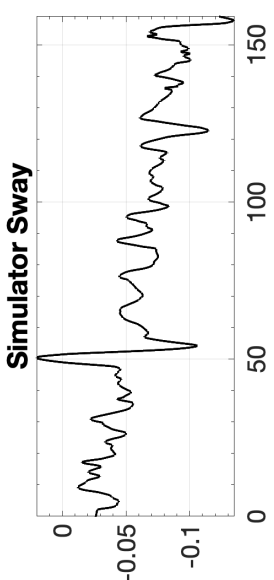

[w] uol!!!sod

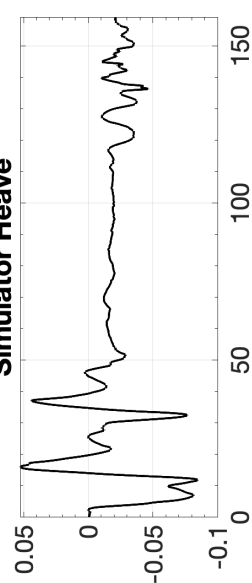

[w] uolı!!sod

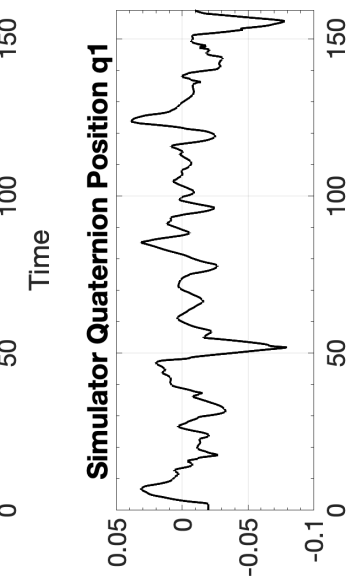

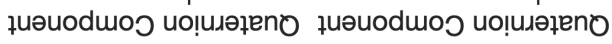
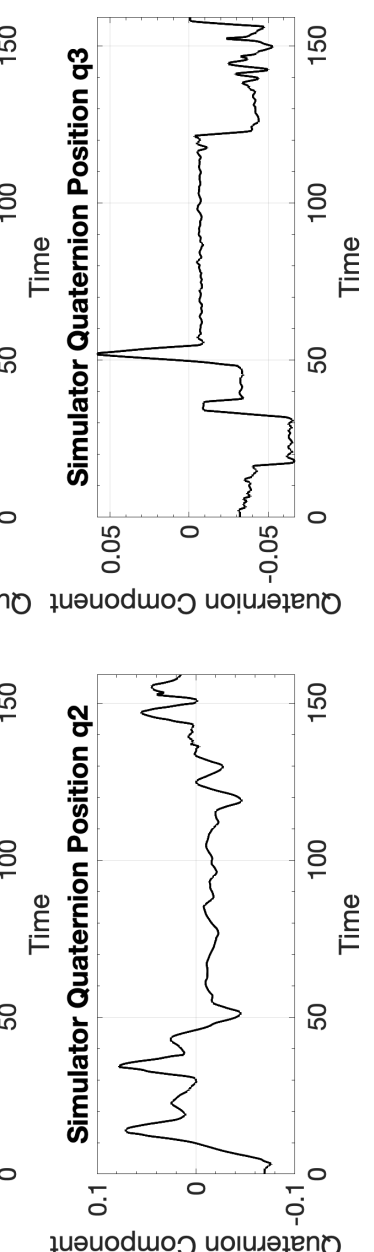

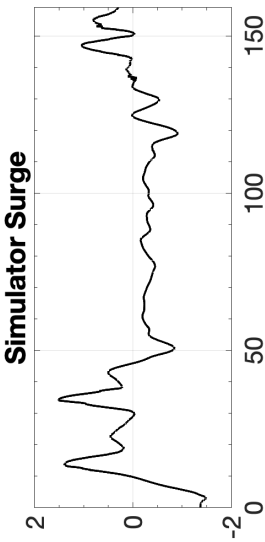

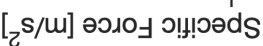

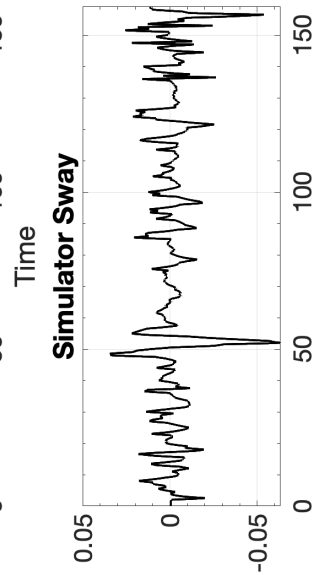

[s/m] Кұ!юорә

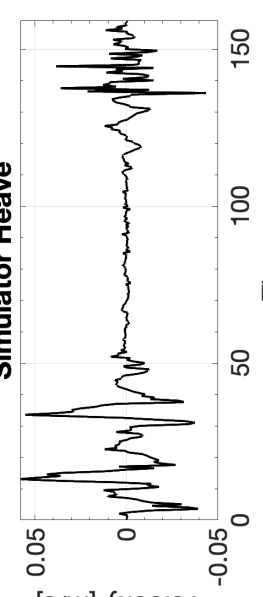

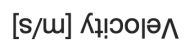

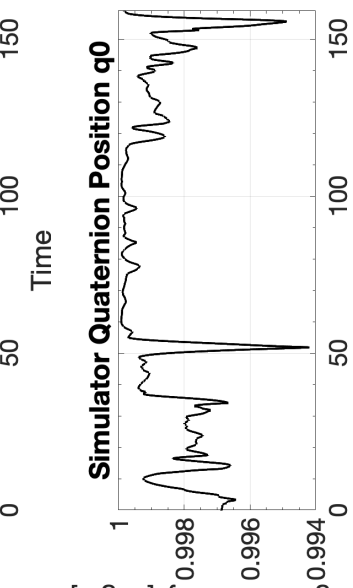

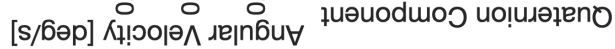

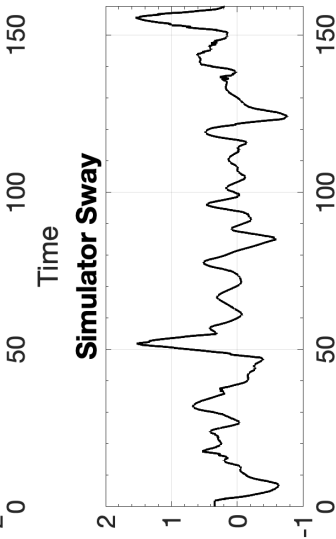

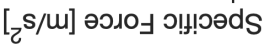

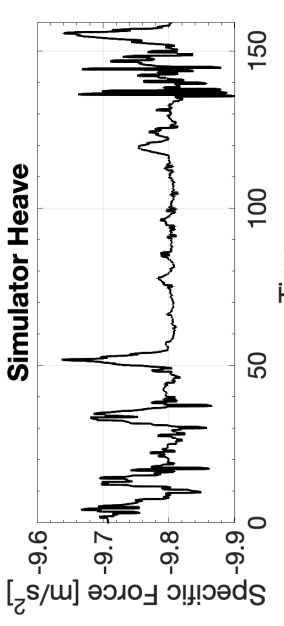

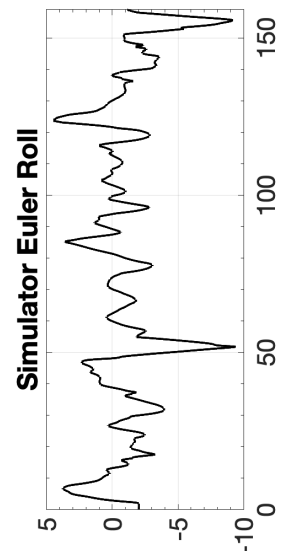

[бəp] uo!!! sod
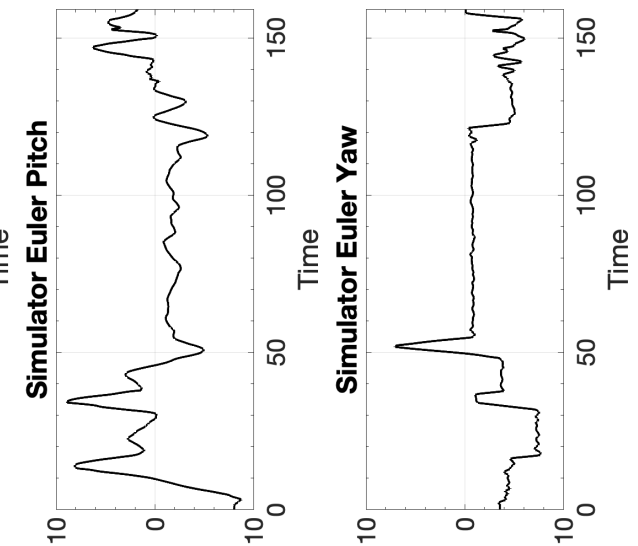

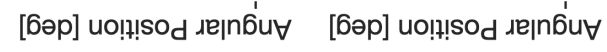

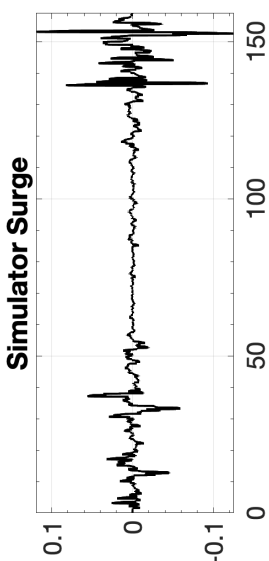

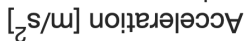

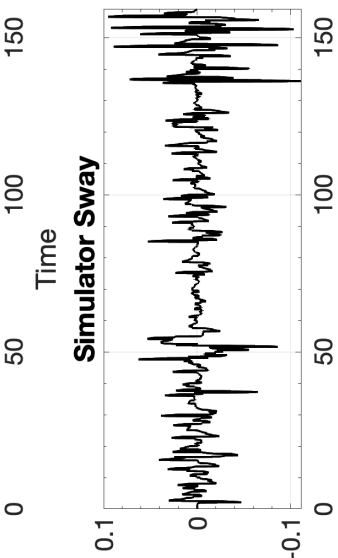

[zs/m] ио!ןеגә|әэว

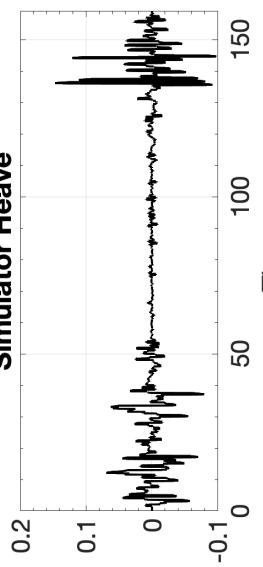

[zs/m] ио!ฺедәәәэว
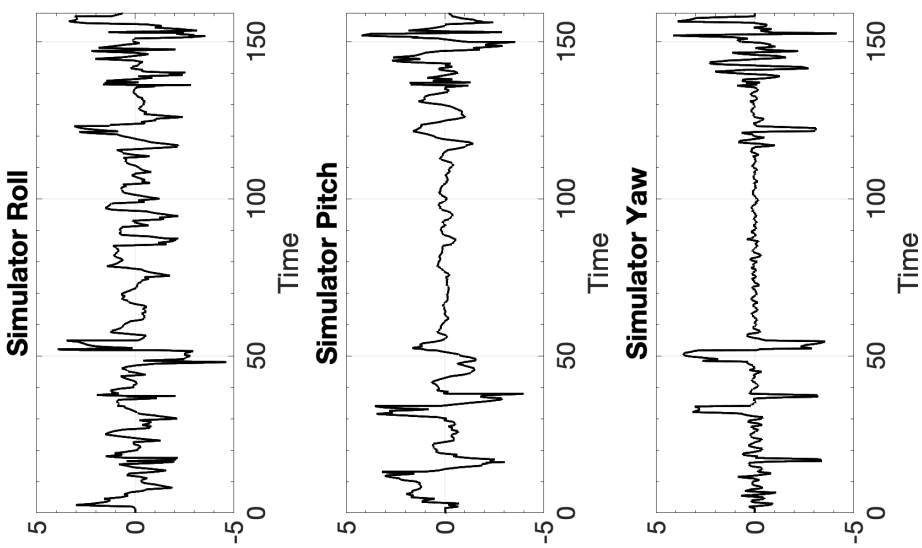

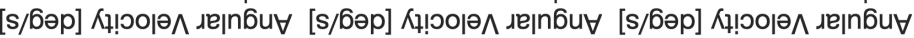




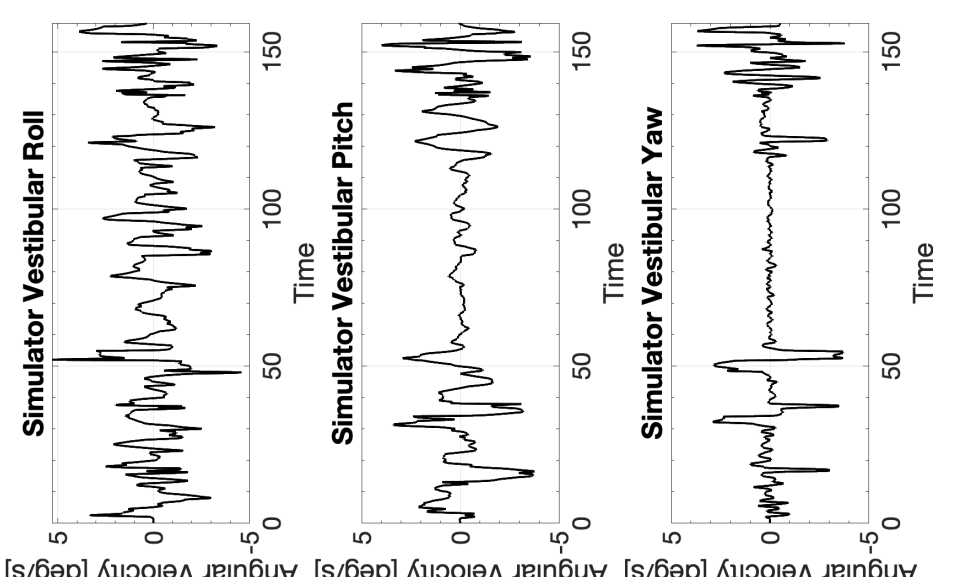

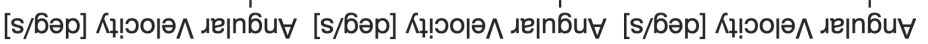

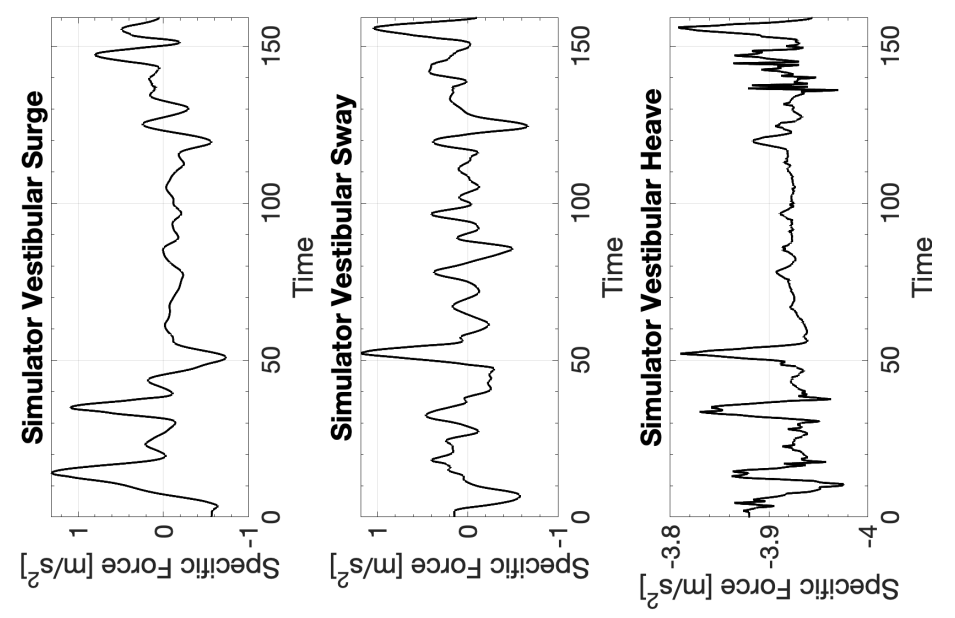




\section{C.17 Columbia 400 Single-axis Takeoff}

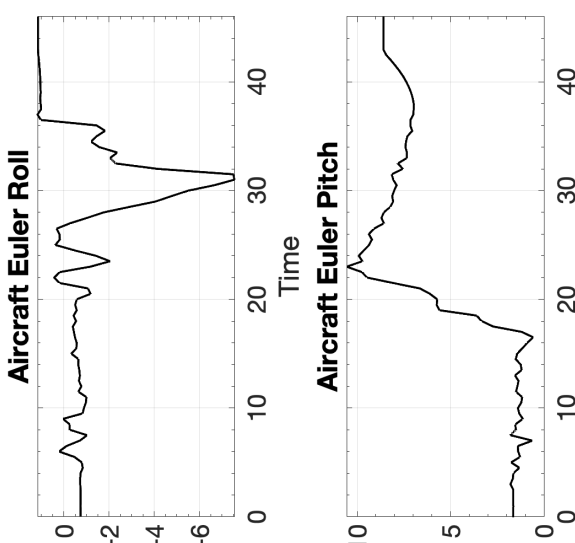

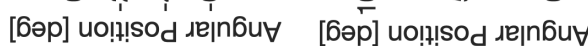

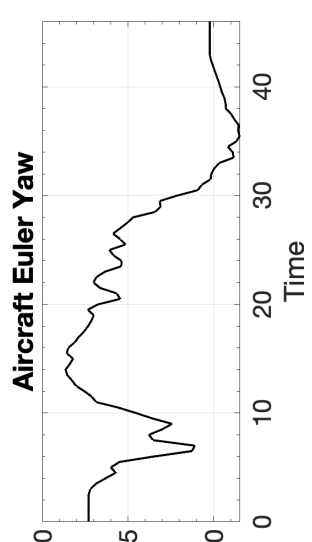

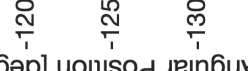

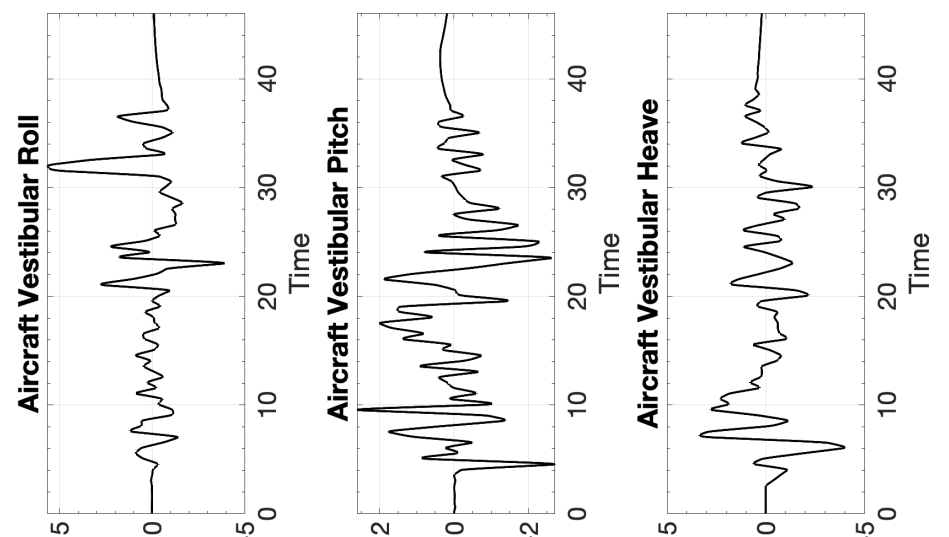

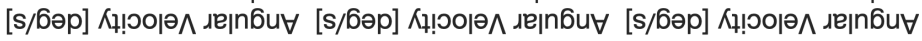

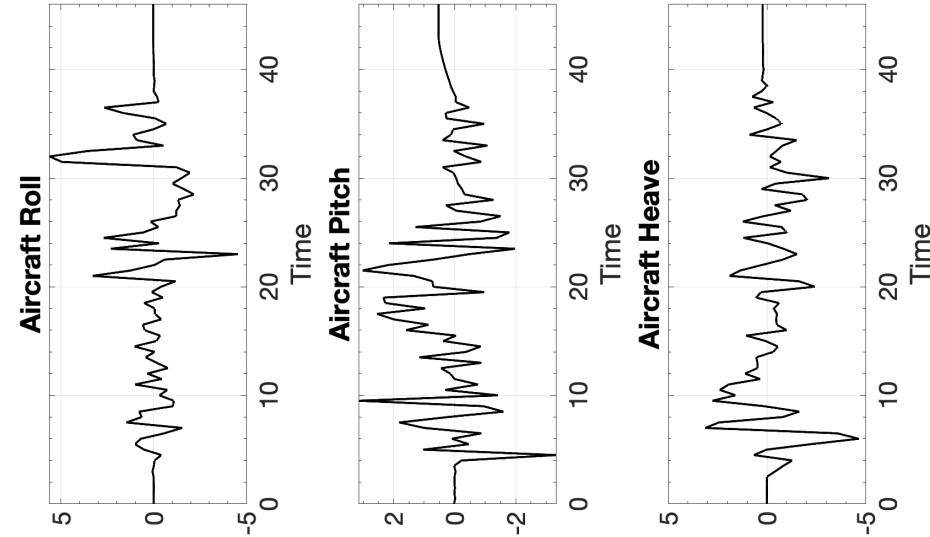

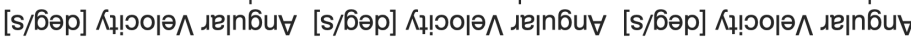

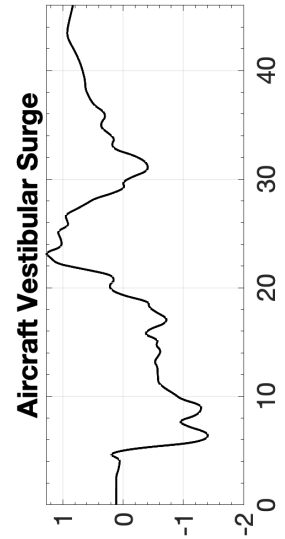

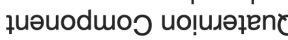

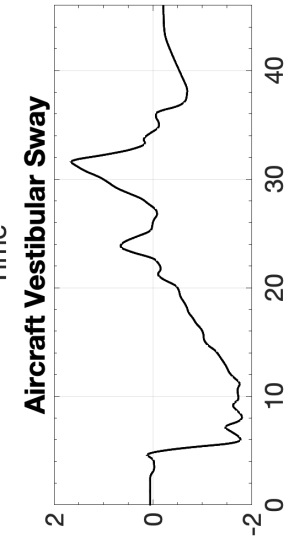

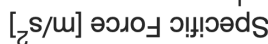

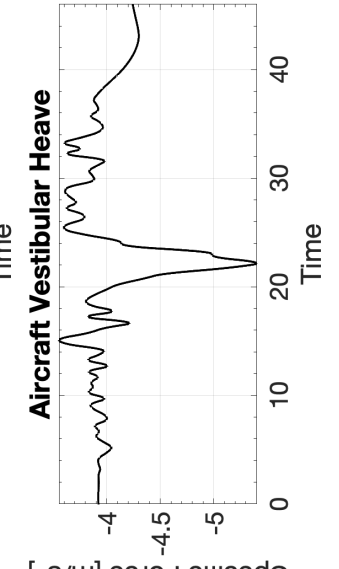

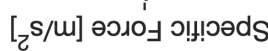

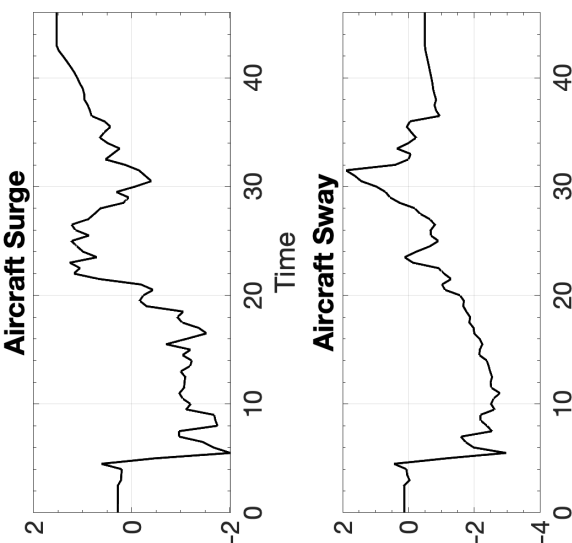

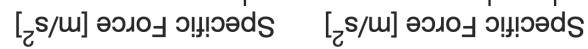

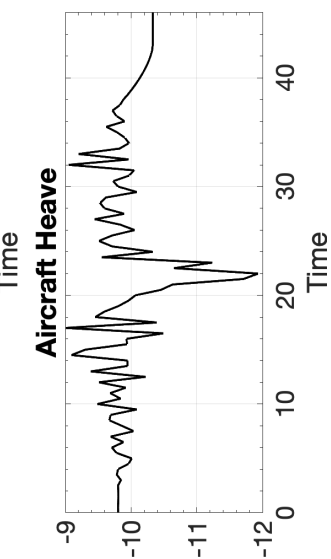

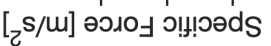

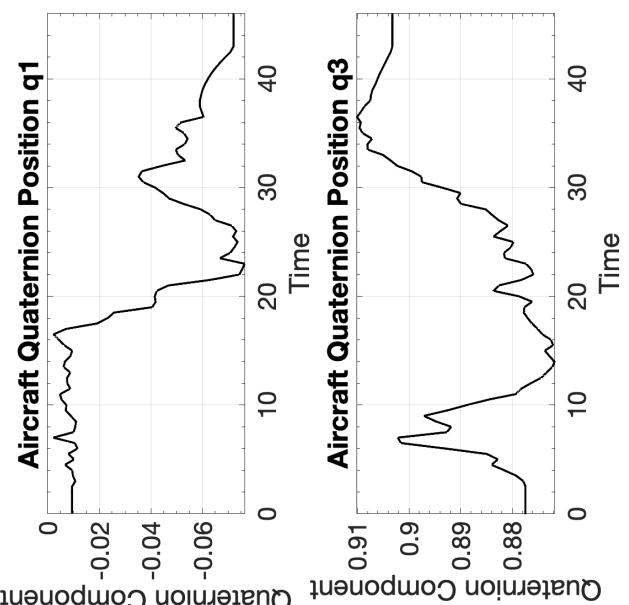

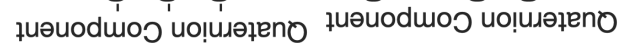

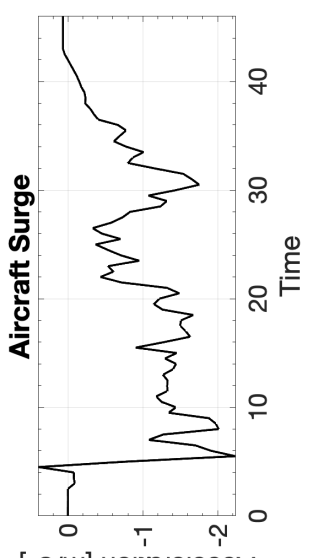

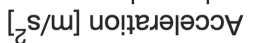

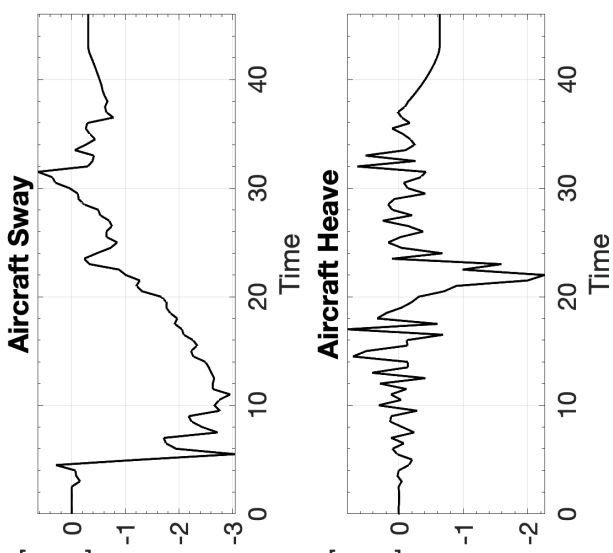

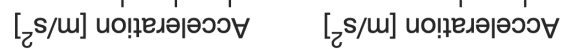

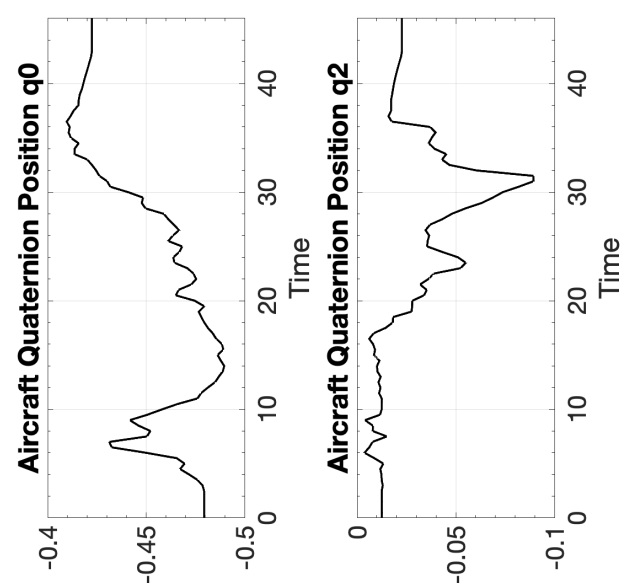

19 


\section{C.17.1 CW1 Columbia 400 Single-axis Takeoff}

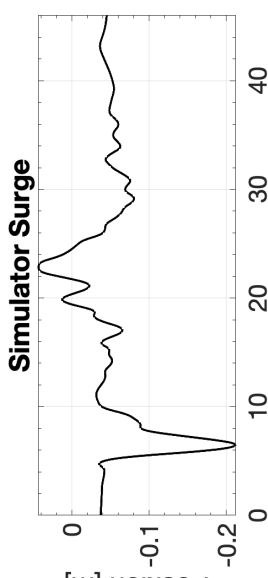

$[\mathrm{m}]$ uo!n!!sod

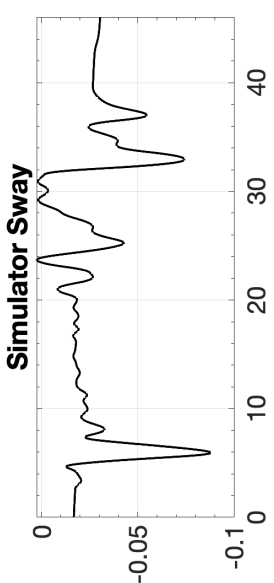

[u] uol!?!sod

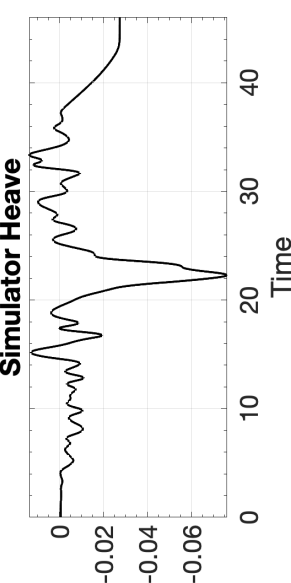

[w] uo!n!ssod

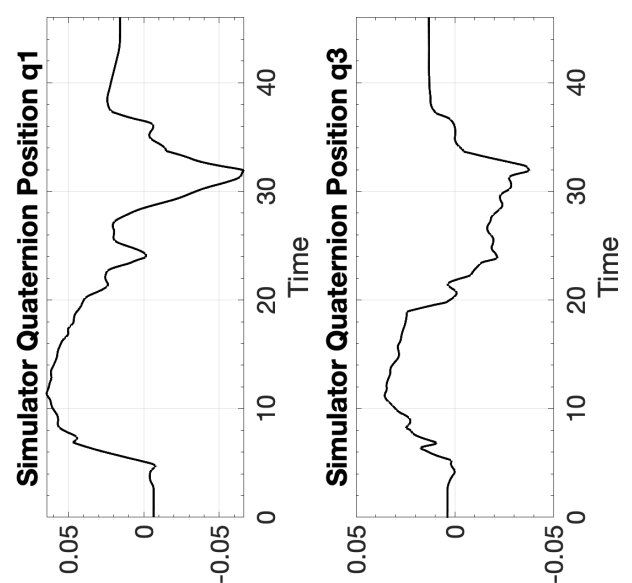

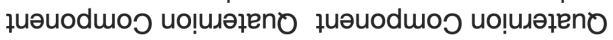

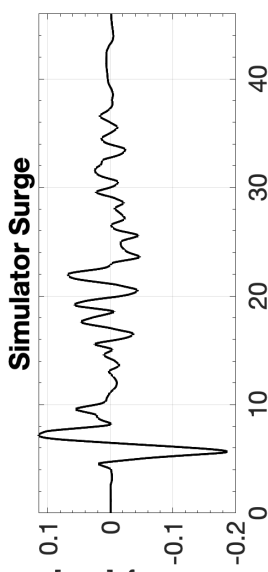

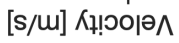

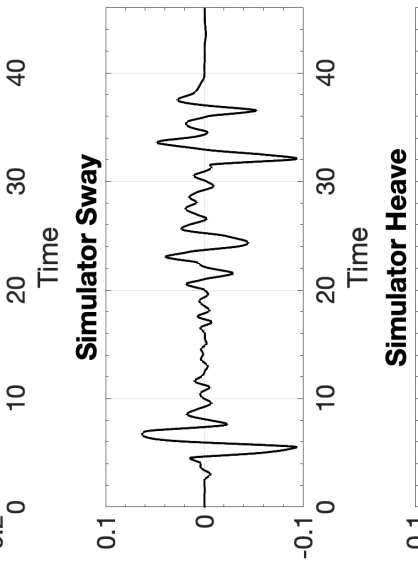

[s/u] Кұ!ю이

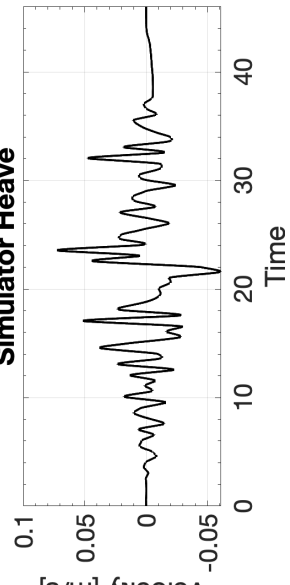

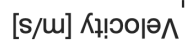

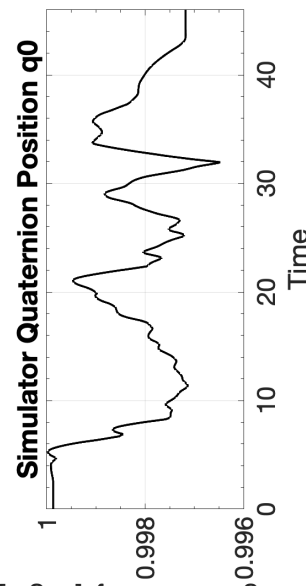

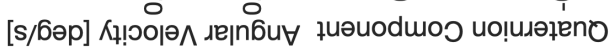

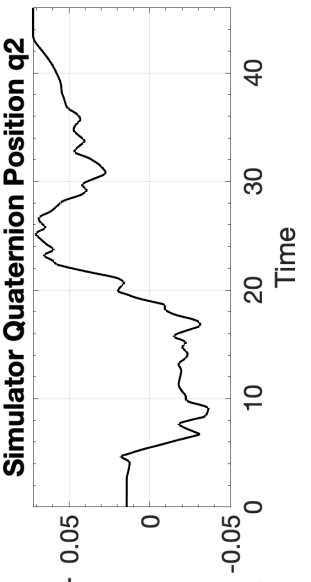

$\stackrel{\oplus}{\underline{F}}$

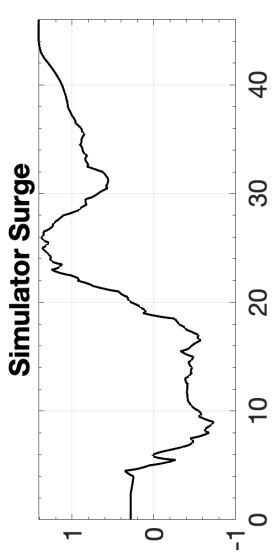

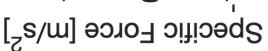

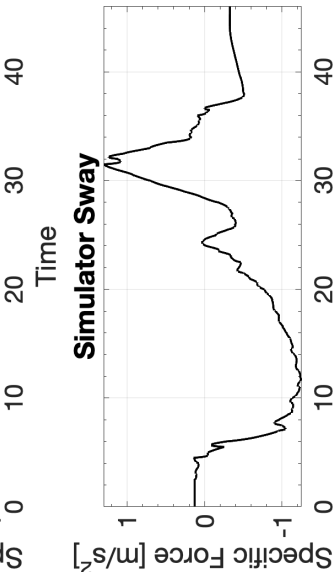

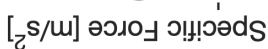

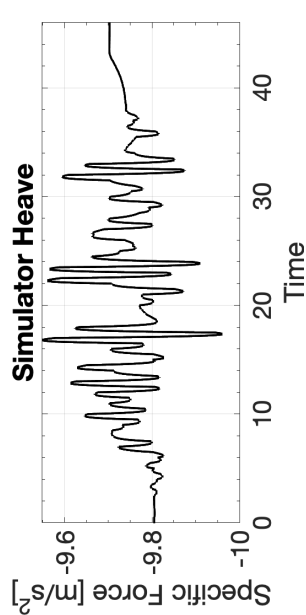

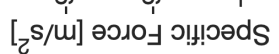

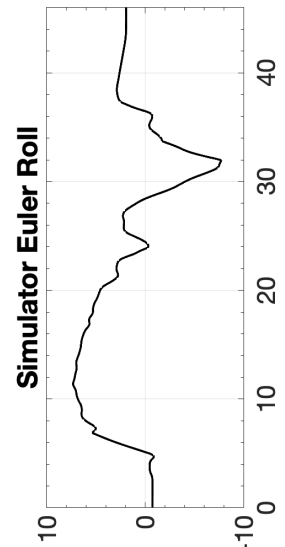

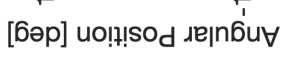

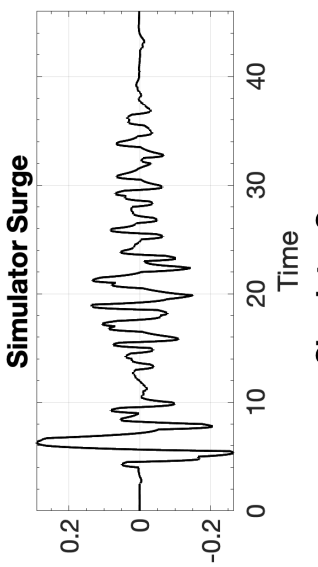

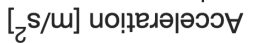

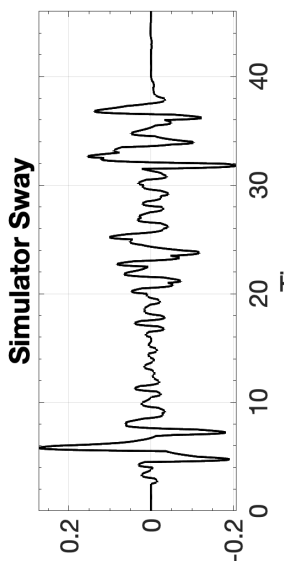

$[\mathrm{s} / \mathrm{m}]$ uо!̣еגә|әэว

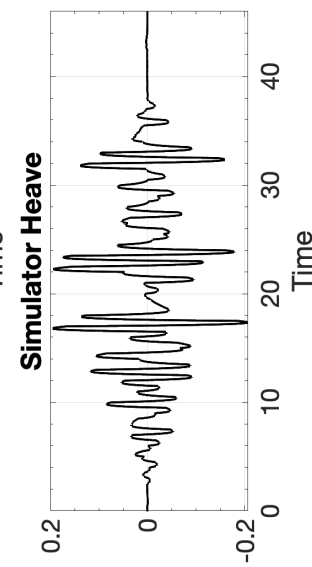

[z/m] ио!ฺедәәәоэ

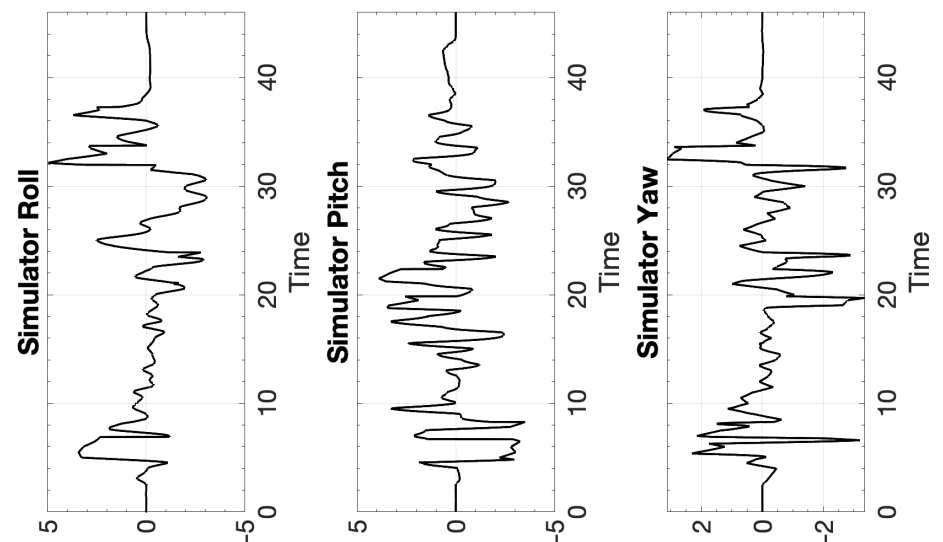

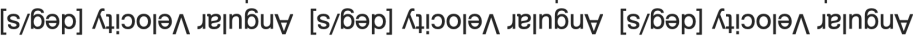




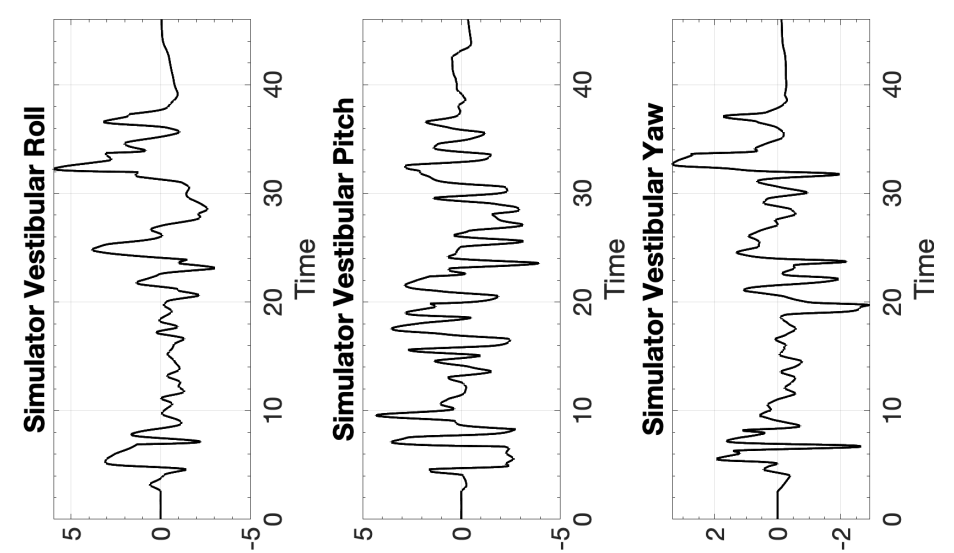

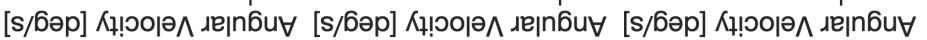

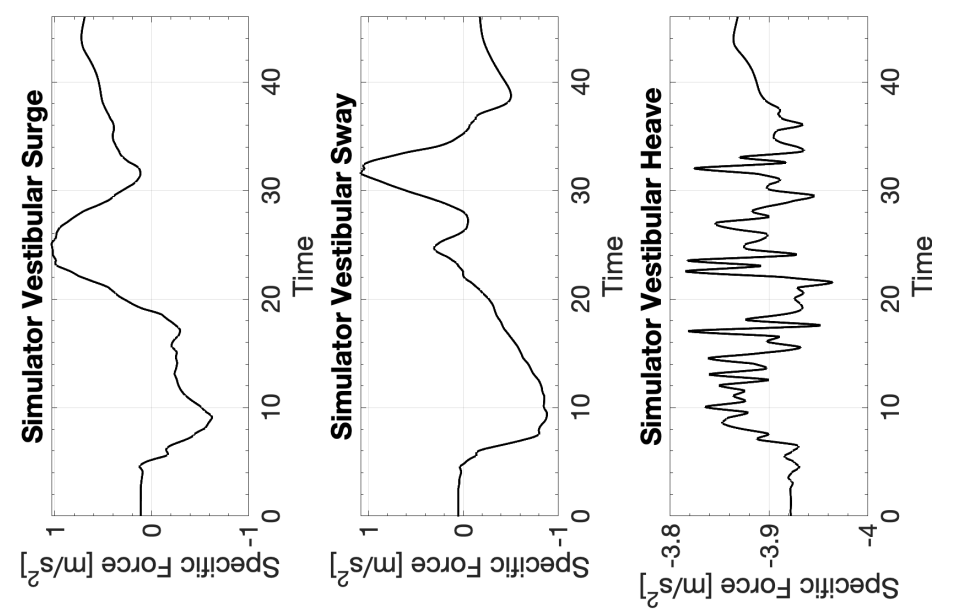




\section{C.17.2 CW2 Columbia 400 Single-axis Takeoff}

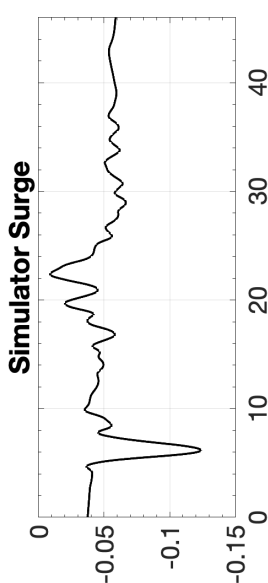

[w] uol!?!sod

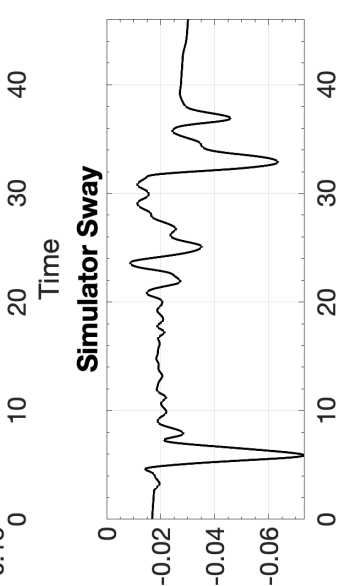

[u] uo!n! sod

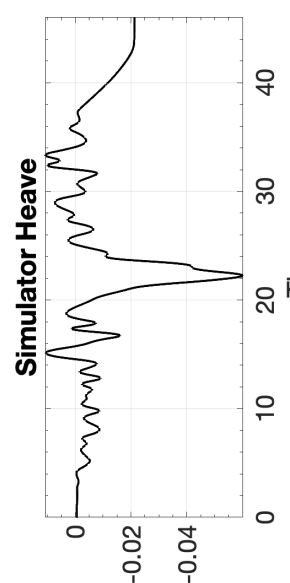

[w] uo!n!s $\mathrm{sOd}^{\mathrm{d}}$

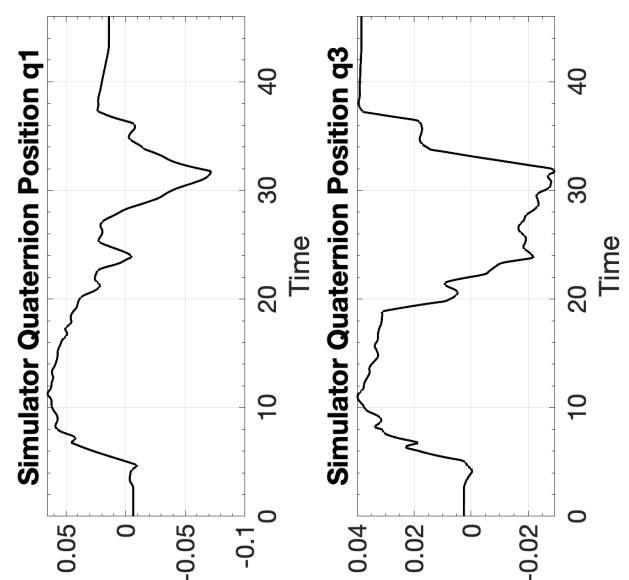

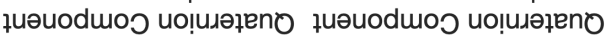
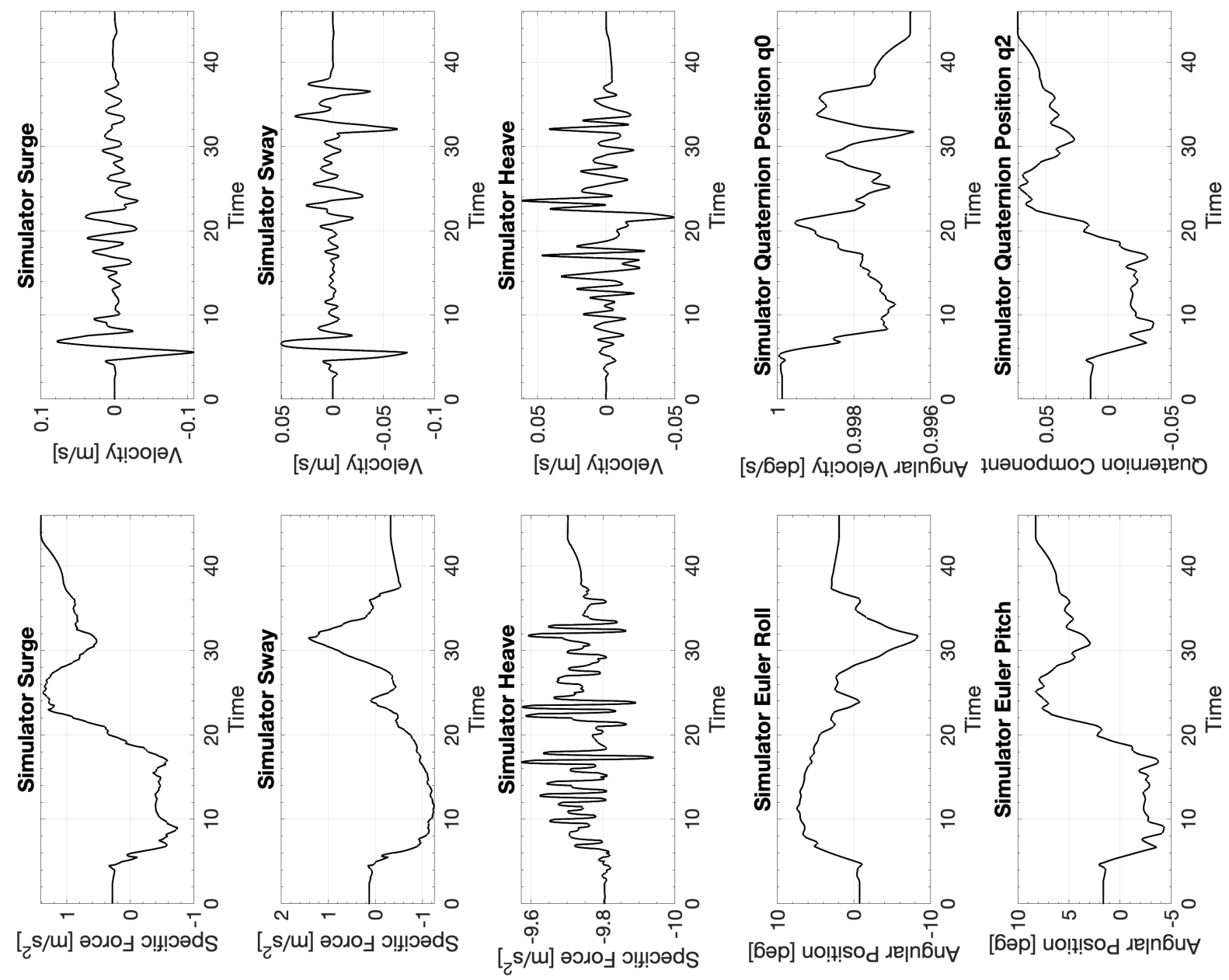

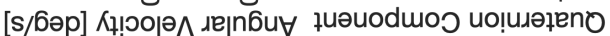

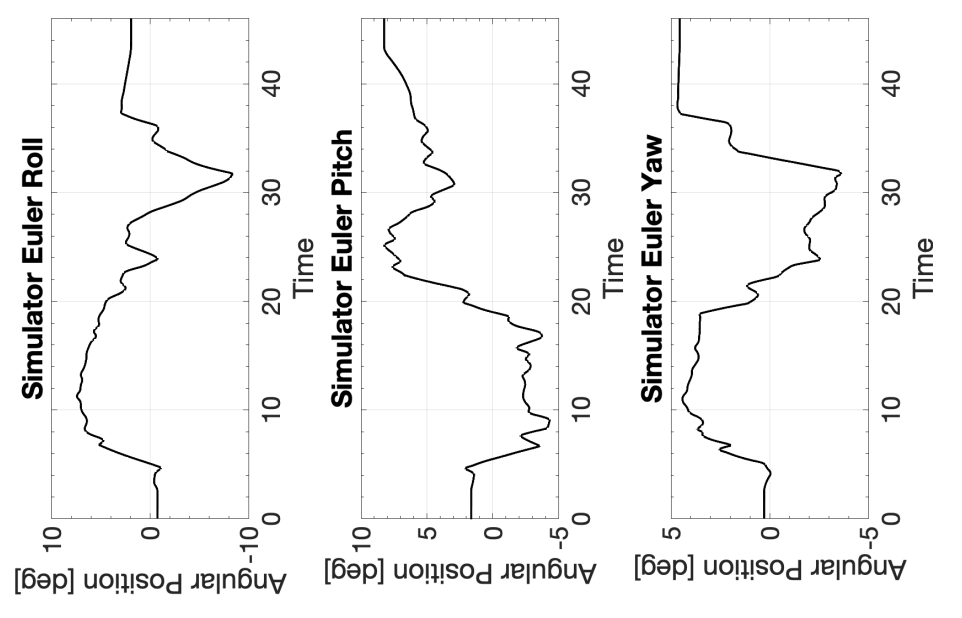

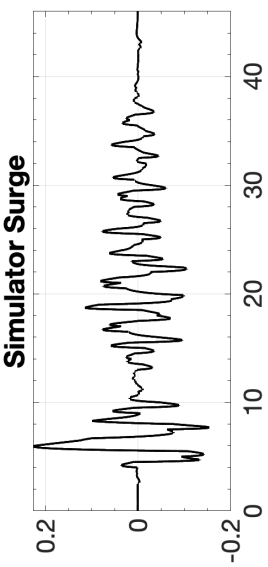

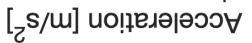

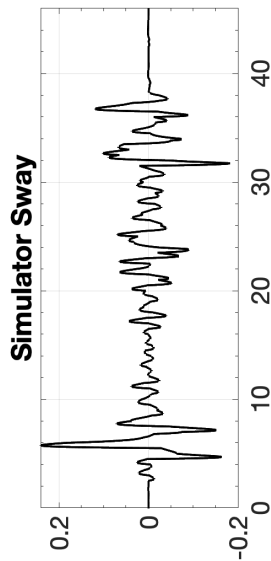

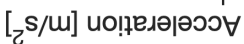

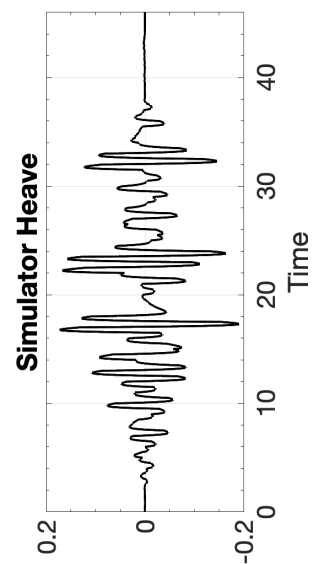

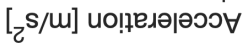

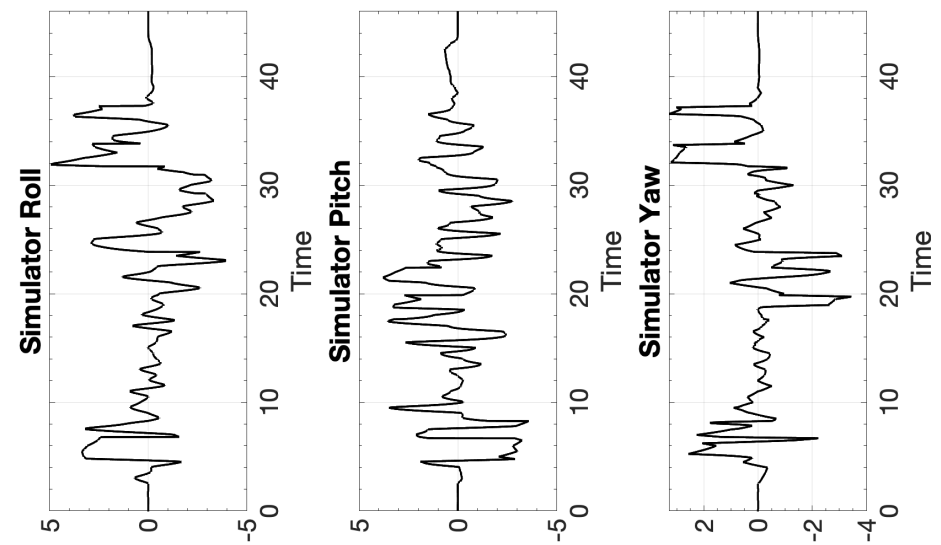

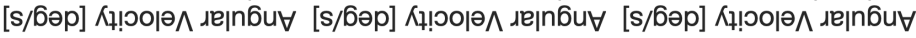




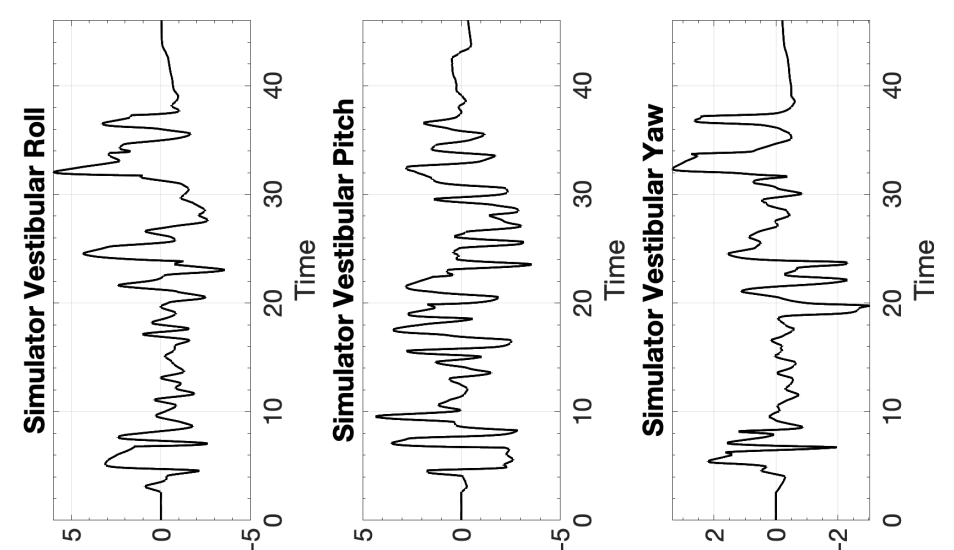

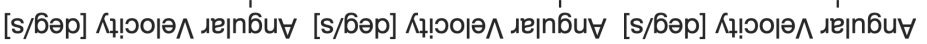

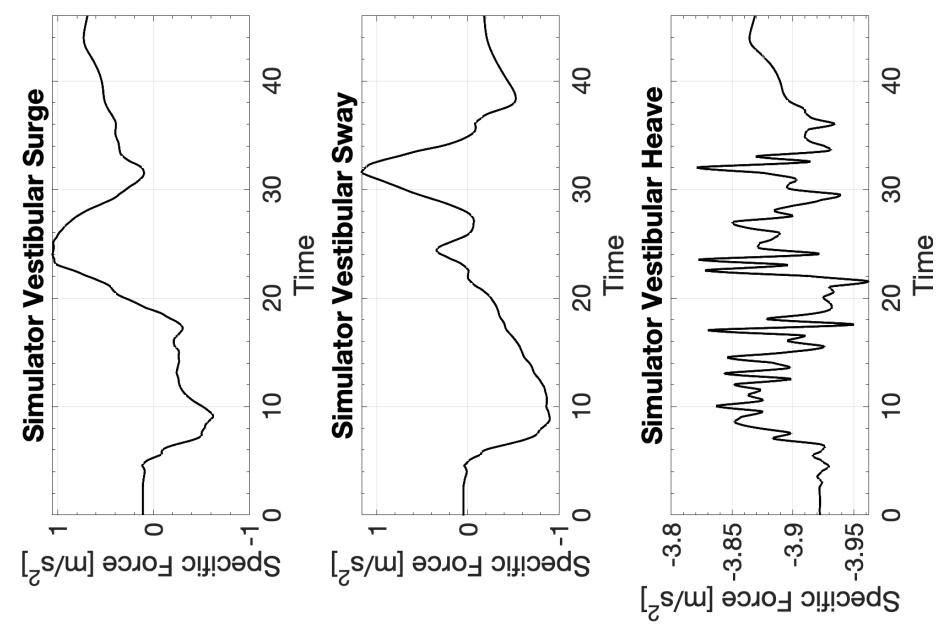




\section{C.17.3 CW3 Columbia 400 Single-axis Takeoff}

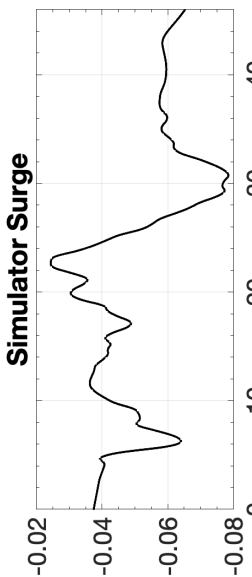

[u] uo!n! sod

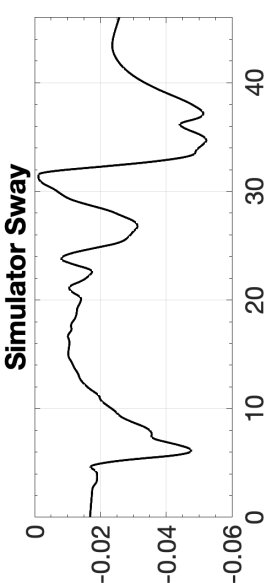

[u] uol!?!sod

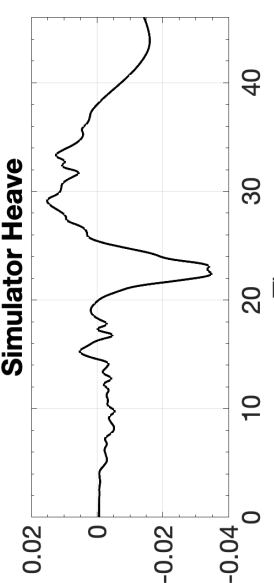

[w] uo!n! $\mathrm{sOd}^{\mathrm{d}}$

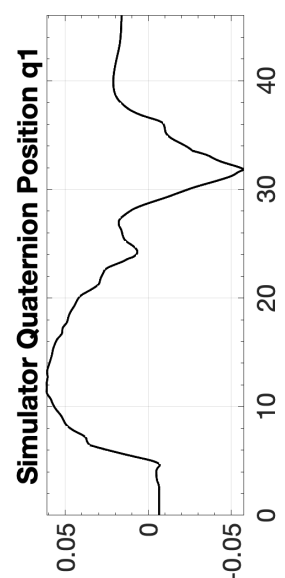

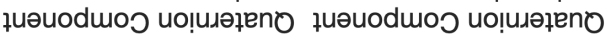

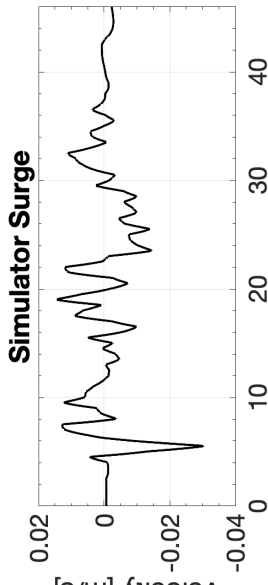

[s/m] ᄉұ! ○о|ә^

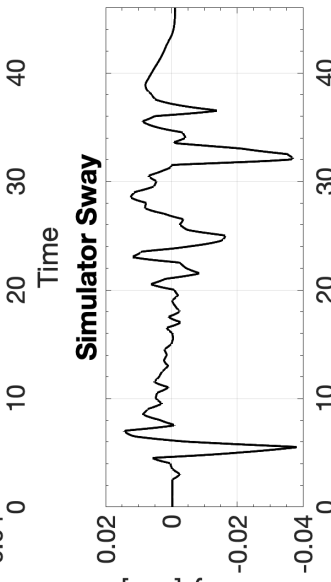

[s/u] Кұ!०о|ә

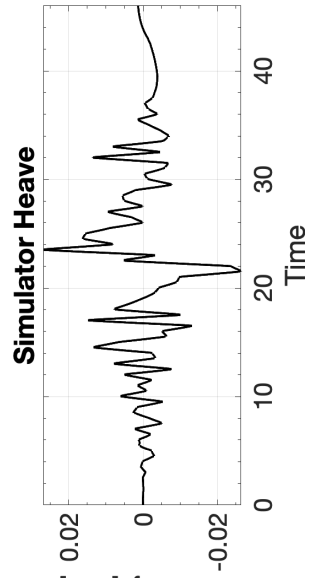

[s/m] Кұ!юори

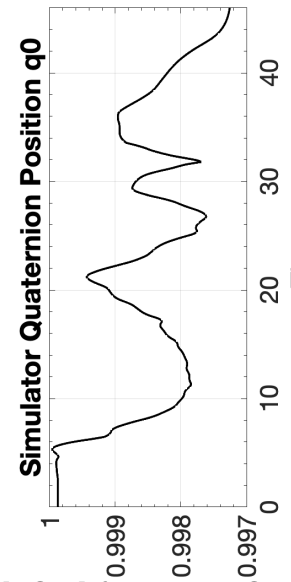

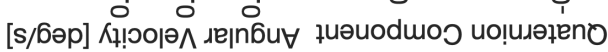

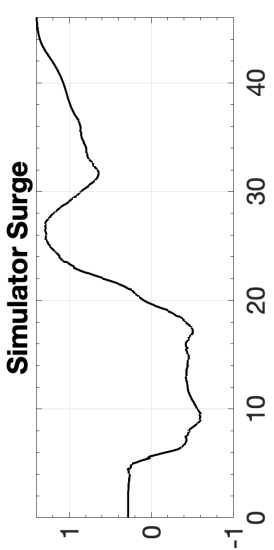

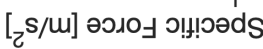

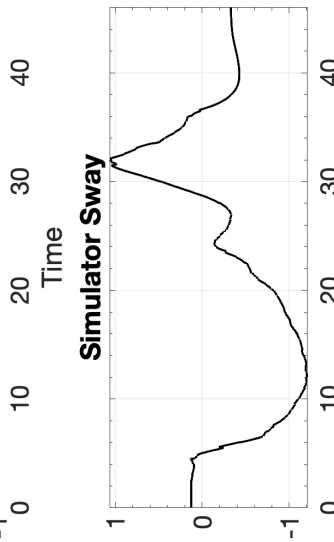

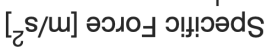

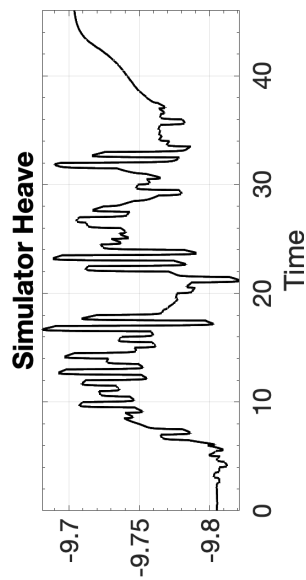

[

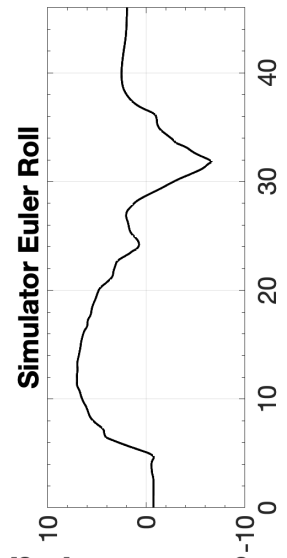

[бәр] uo!̣!!sod
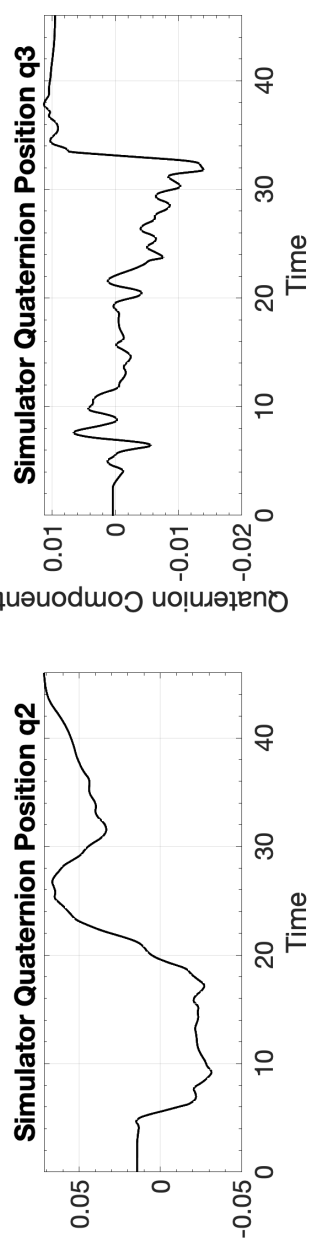
$\stackrel{\oplus}{\xi}$

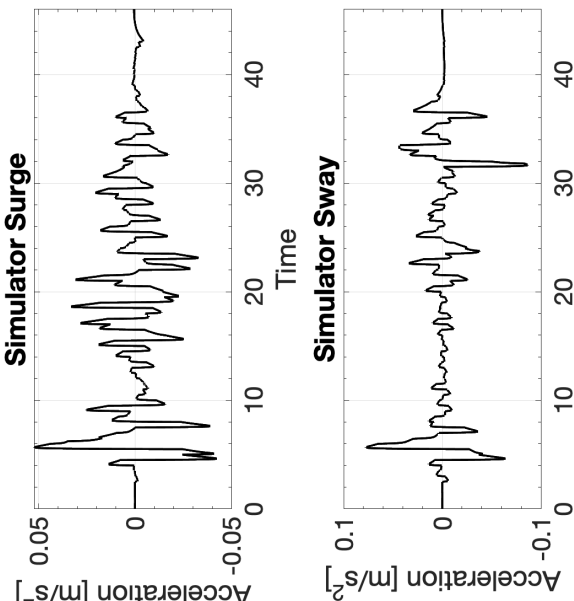

$[z$ s/u] uо!ฺеләәәэว $\forall$

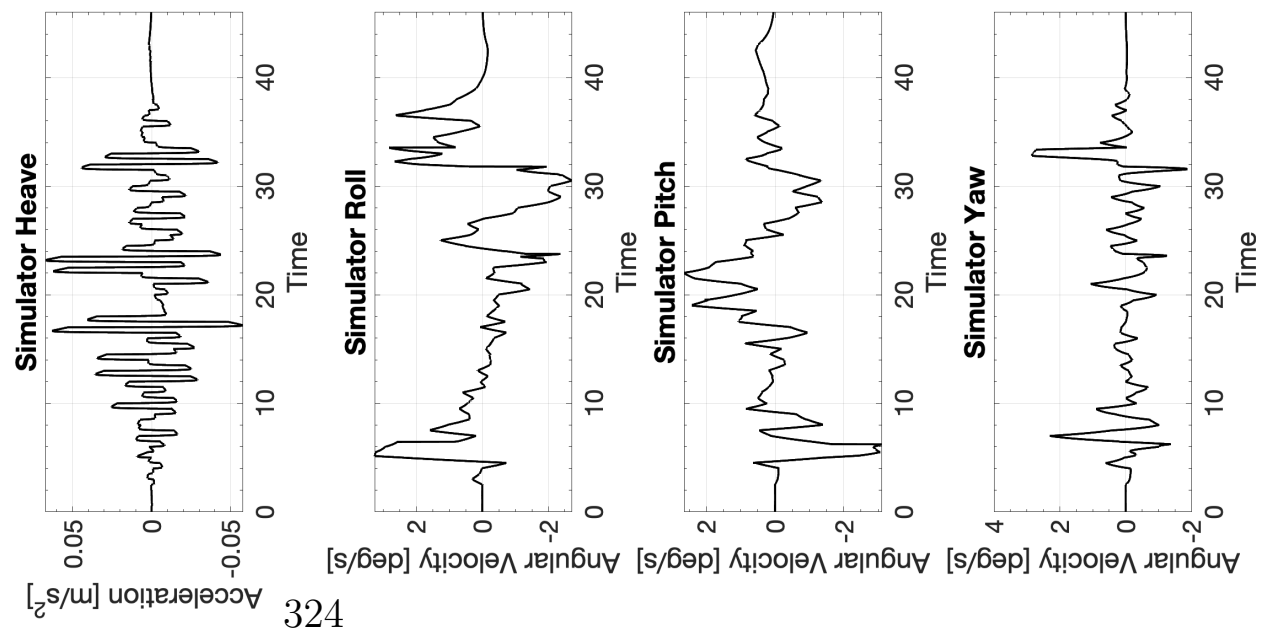



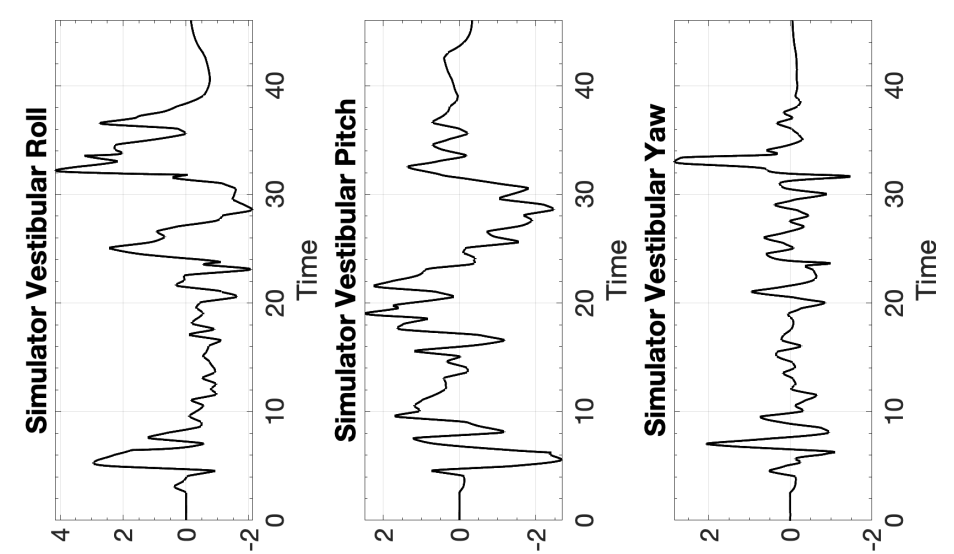

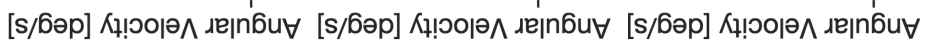

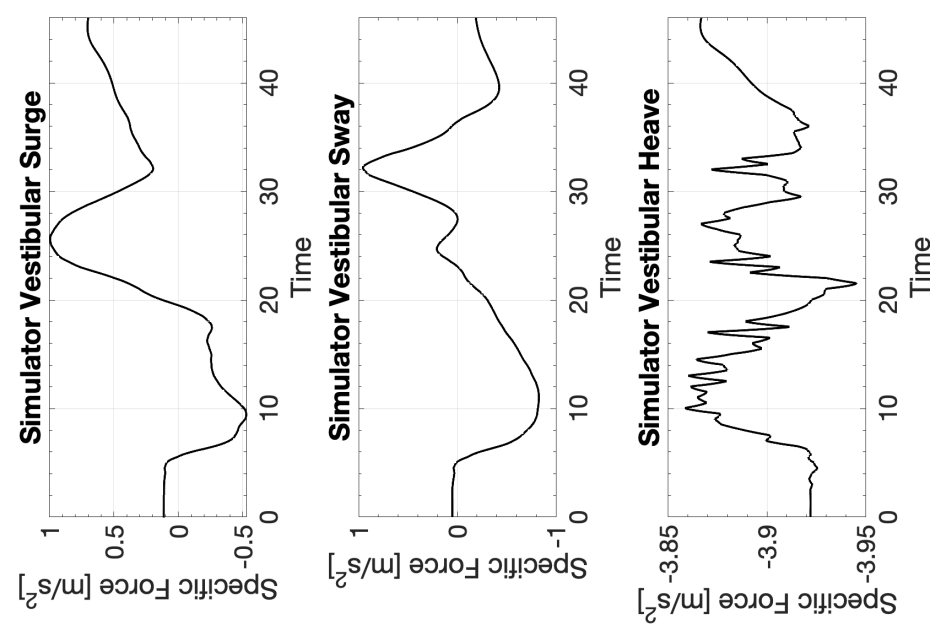




\section{C.18 Columbia 400 Spiral Dive}
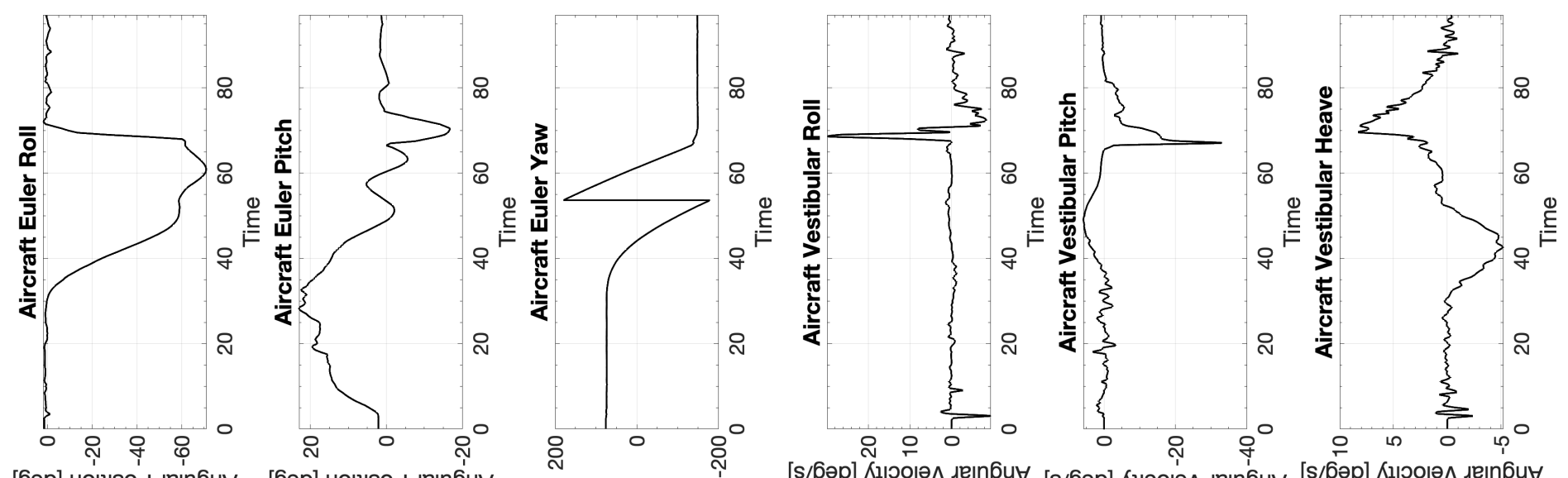

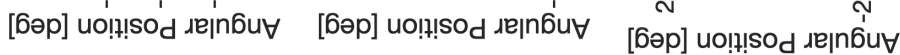
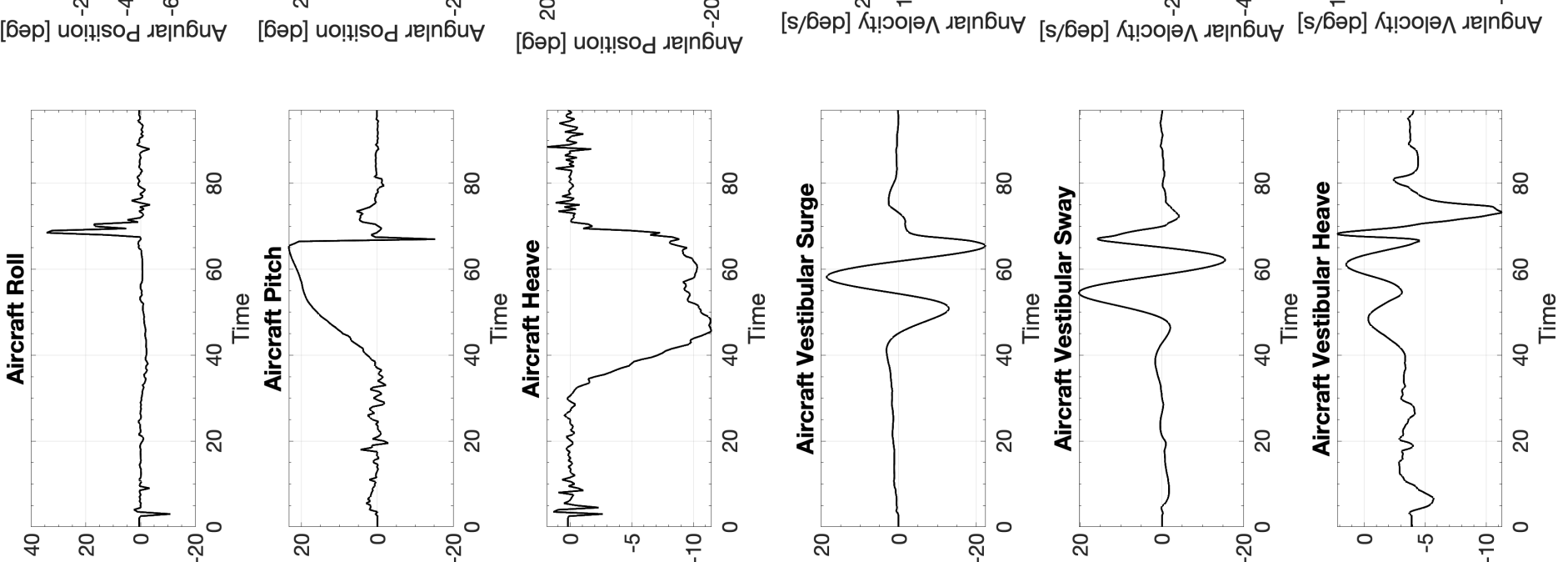

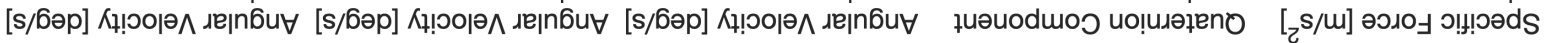

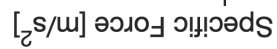
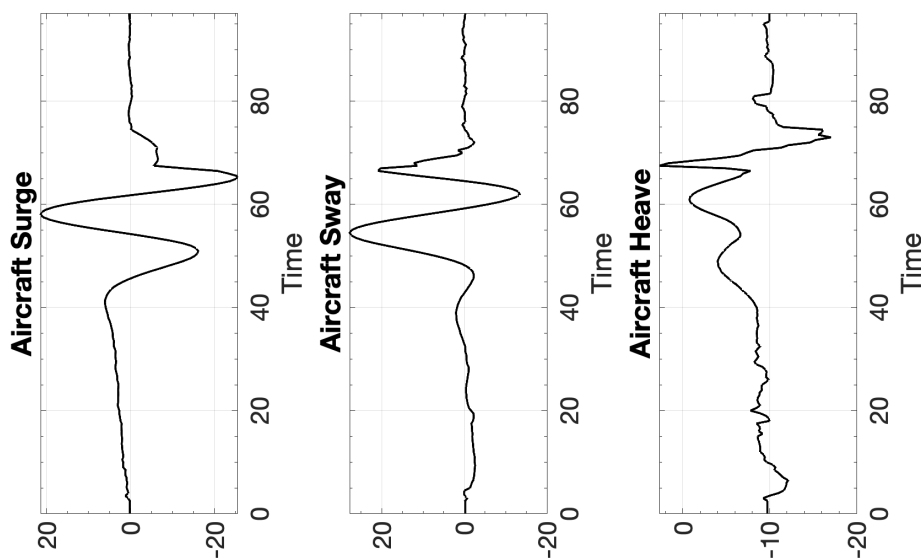

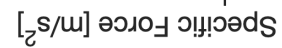

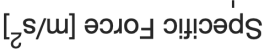

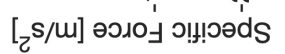

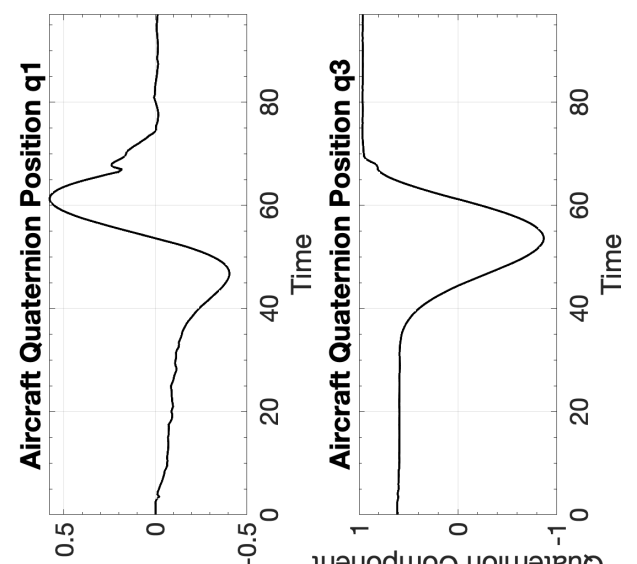

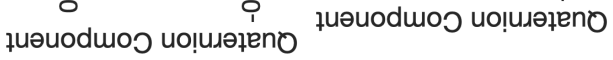

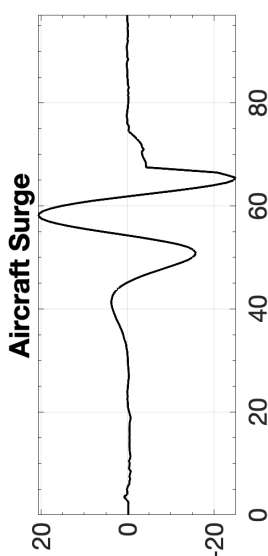

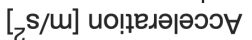

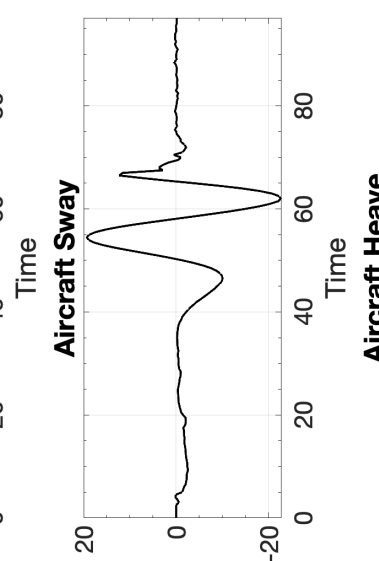

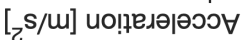

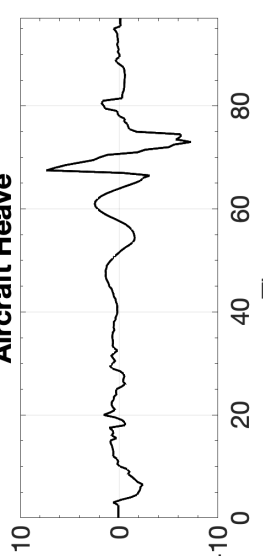

[zs/m] ио!ฺедәәәоэ

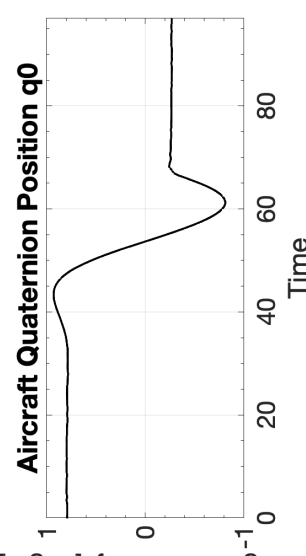

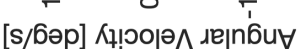

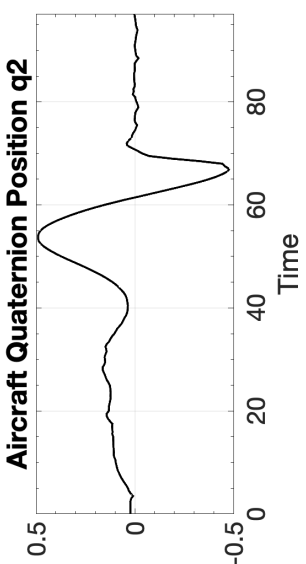

łuəuodmo uoḷuəfeno 


\section{C.18.1 CW1 Columbia 400 Spiral Dive}

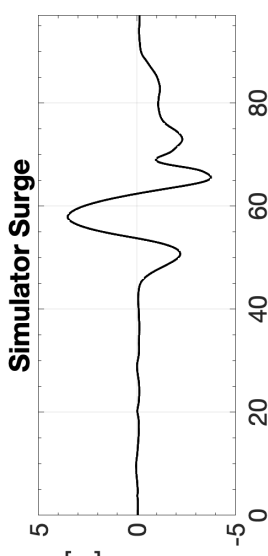

[u] uo!!!!sod

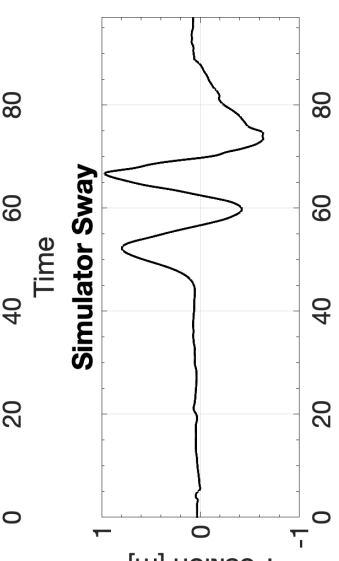

[u] uo!!!!sod

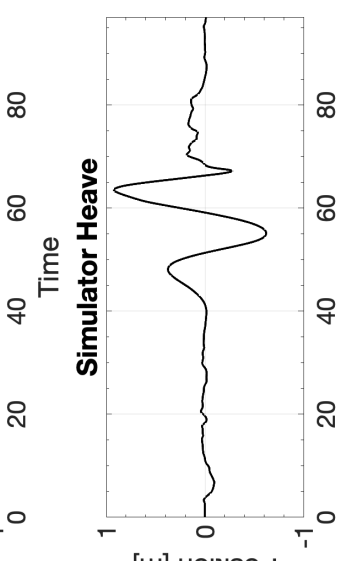

[w] uol!! sod

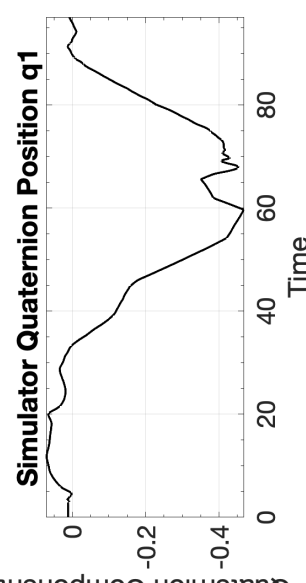

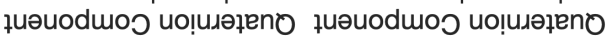

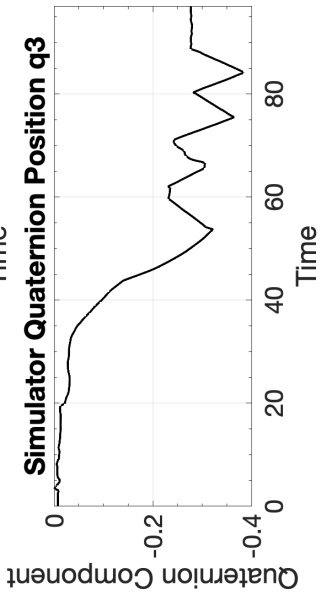

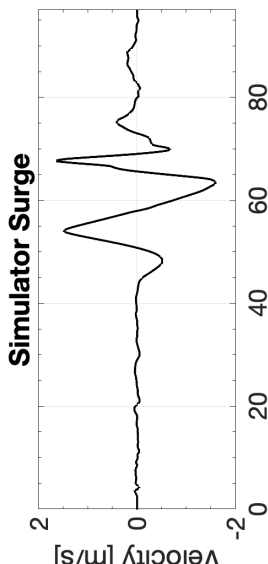

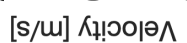

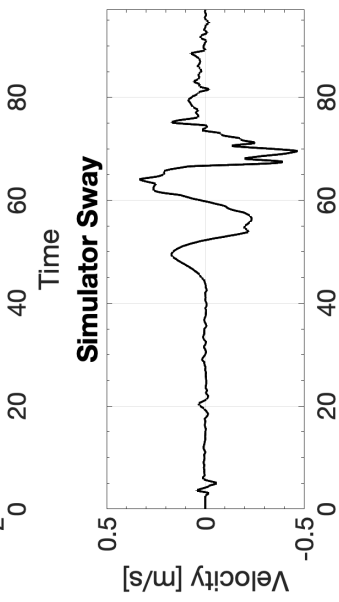

[s/u] Кұ!๐о|ю

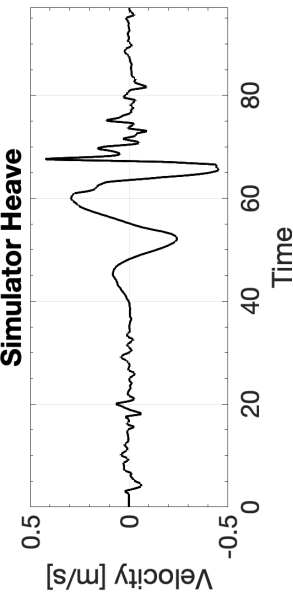

[s/m] Кұ! 이 ^

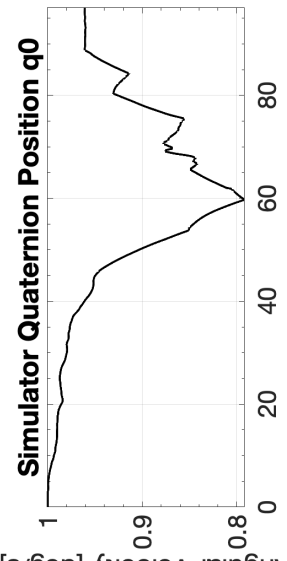

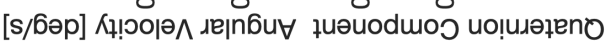

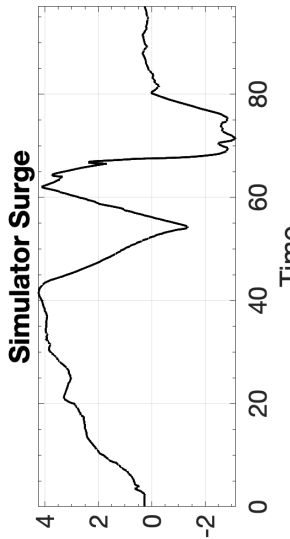

[z

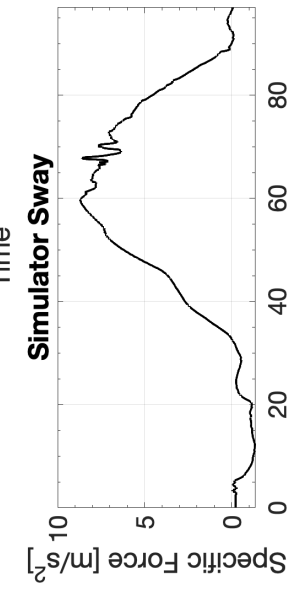

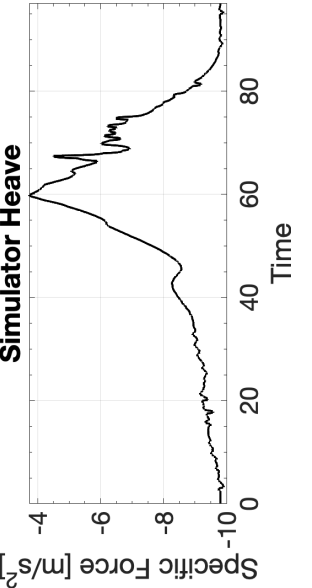

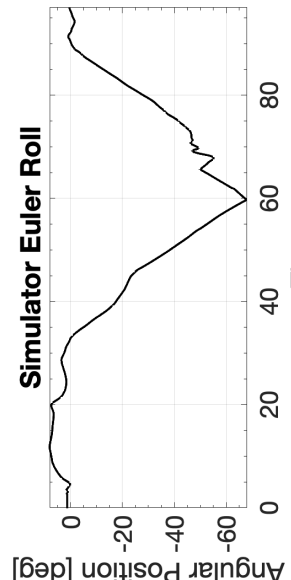

[бəp] uo!̣!!sod se|n'bu
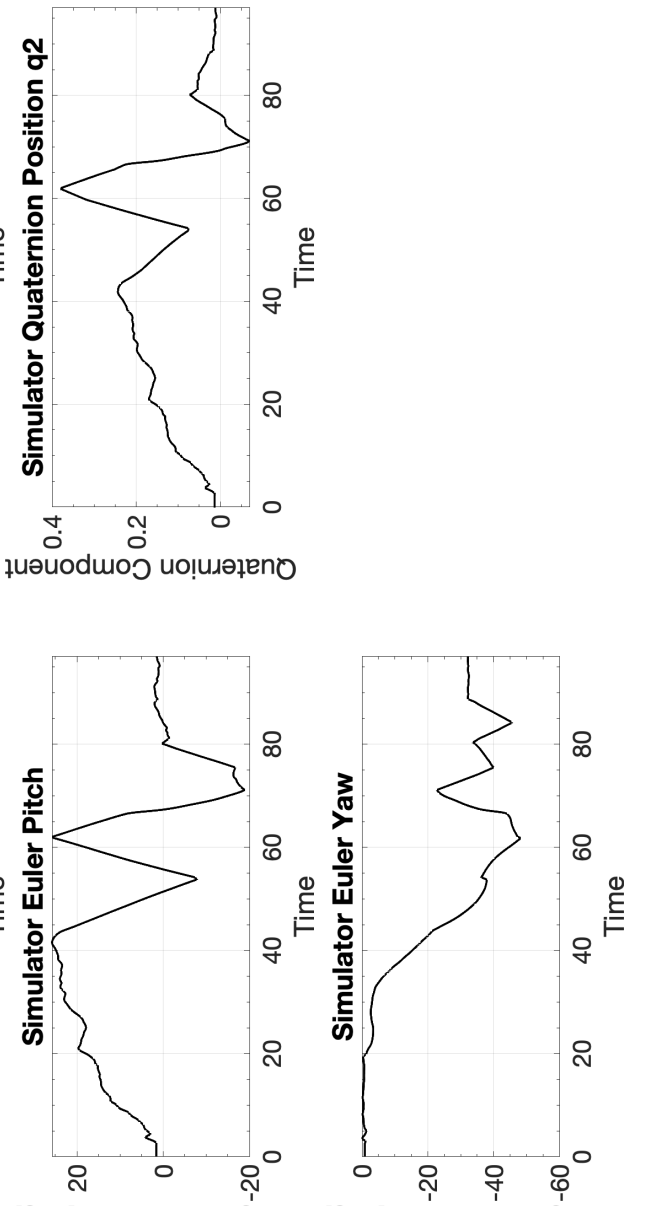

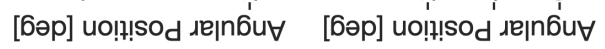

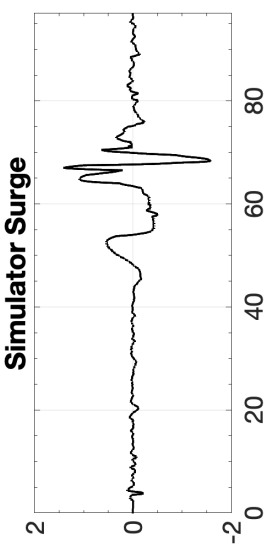

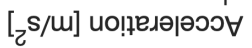

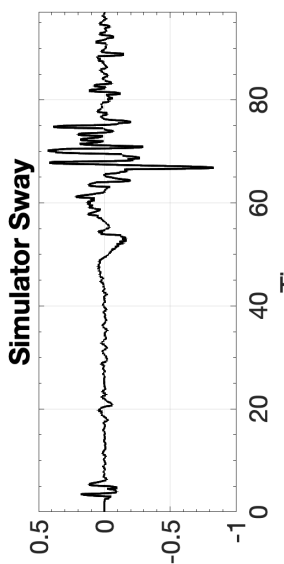

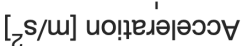

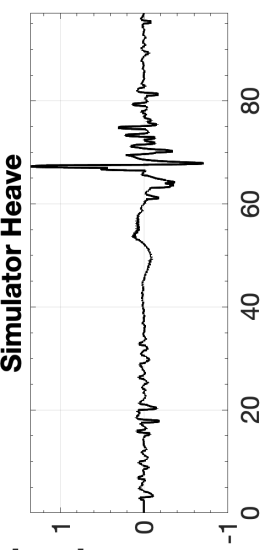

$[\mathrm{z} / \mathrm{m}]$ uо!ฺвдә|әэว
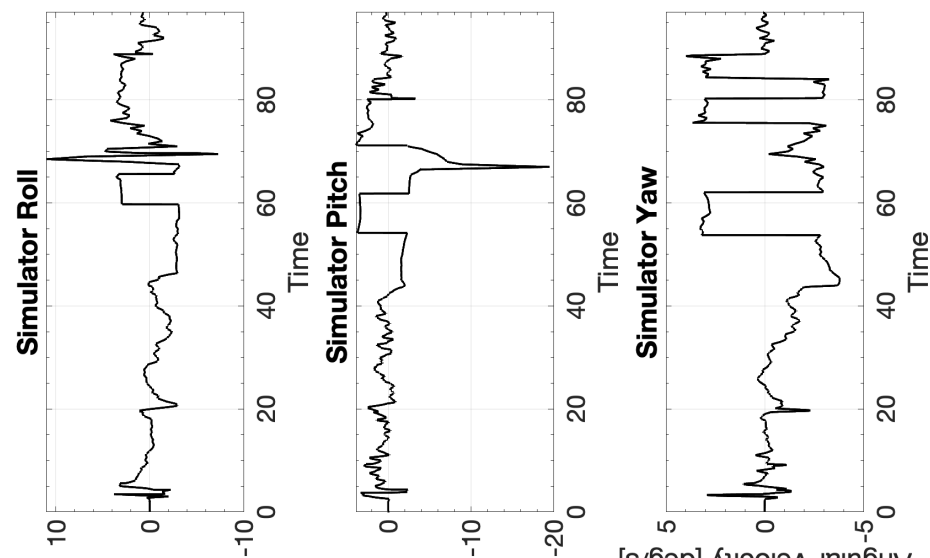

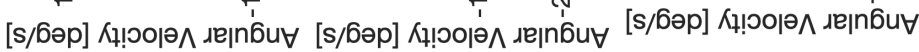



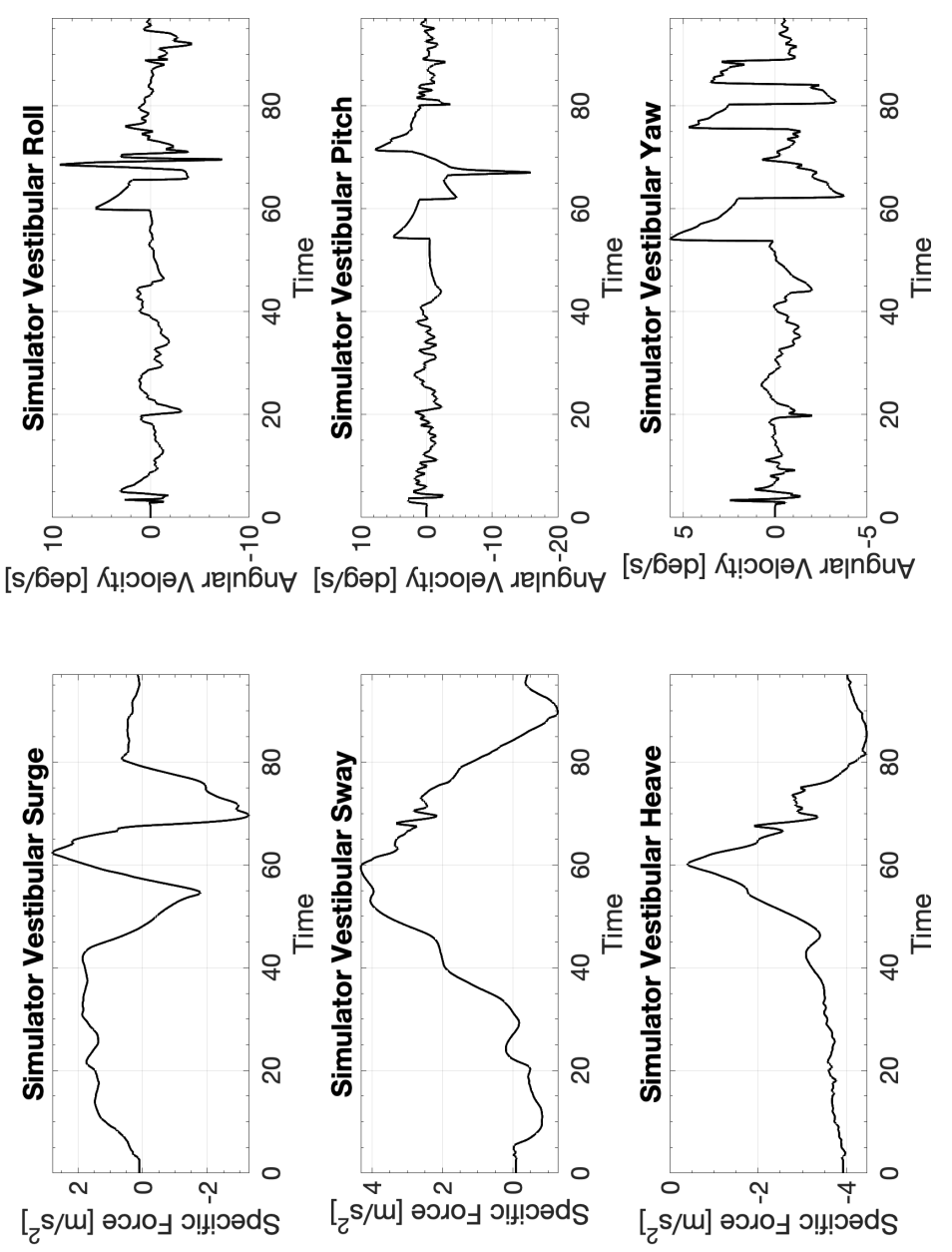


\section{C.18.2 CW2 Columbia 400 Spiral Dive}

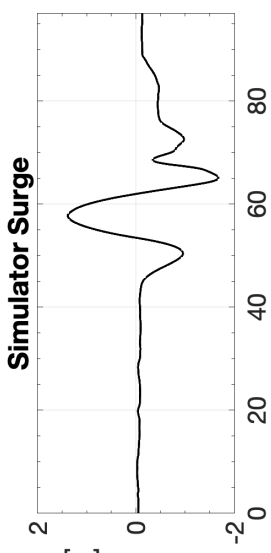

[u] uo!t!!sod

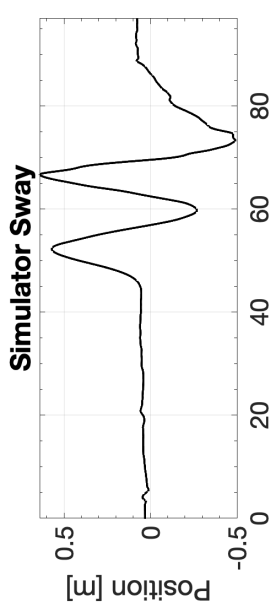

[w] uo!t!!sod

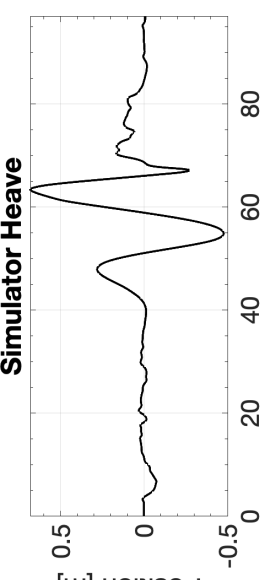

[w] uolt!!sod

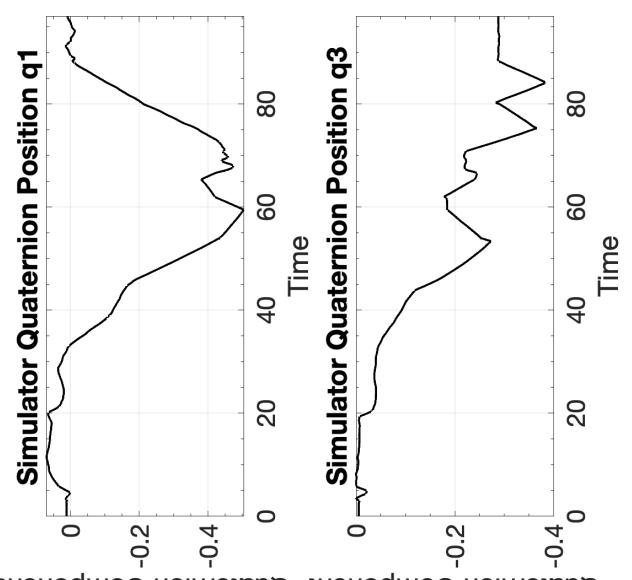

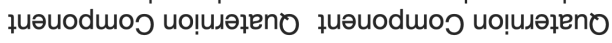

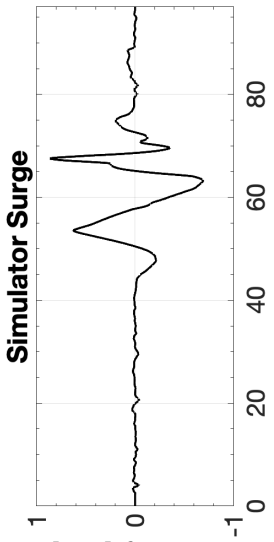

[s/u] Кц!००|ә
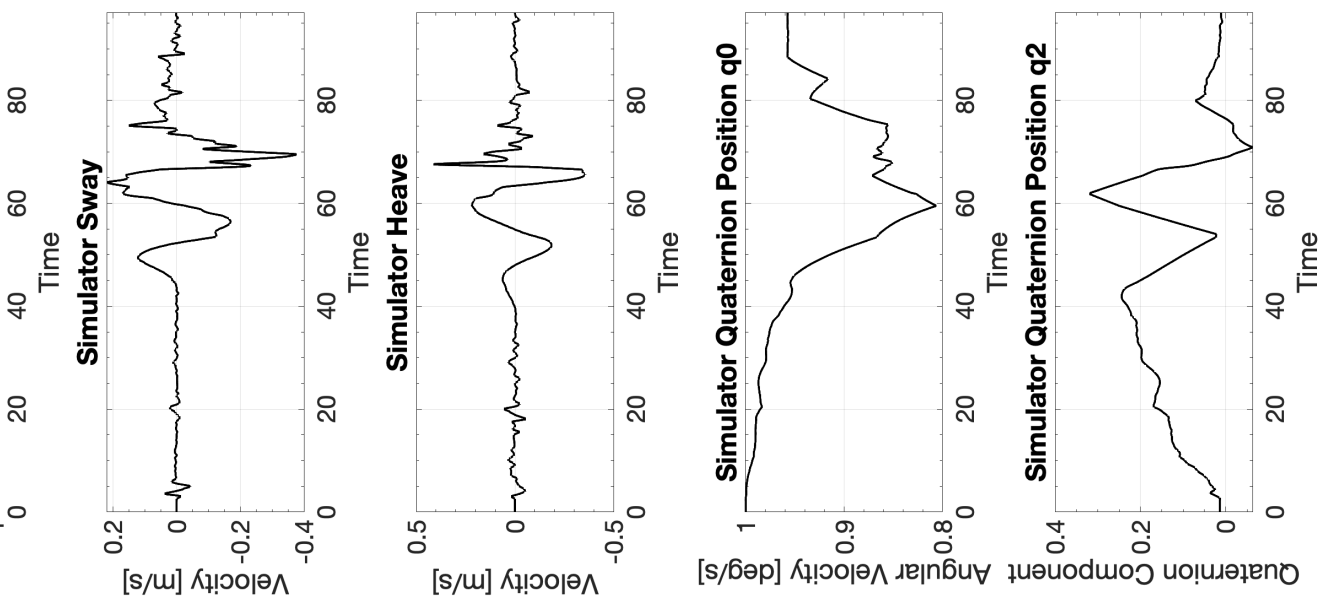

[s/u] Кұ!ю이

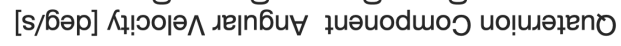

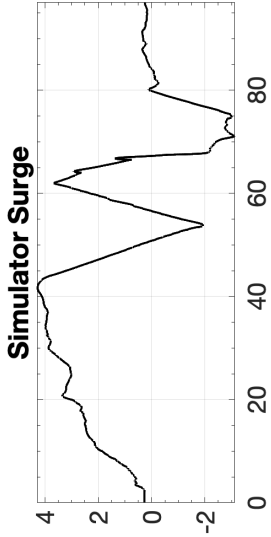

[ح

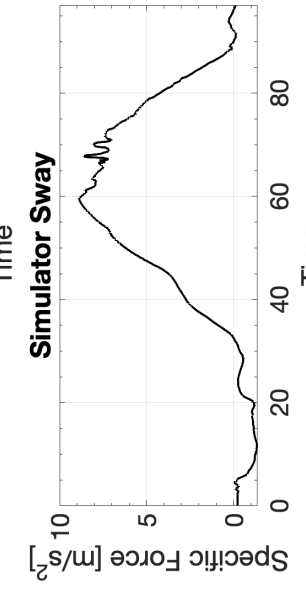

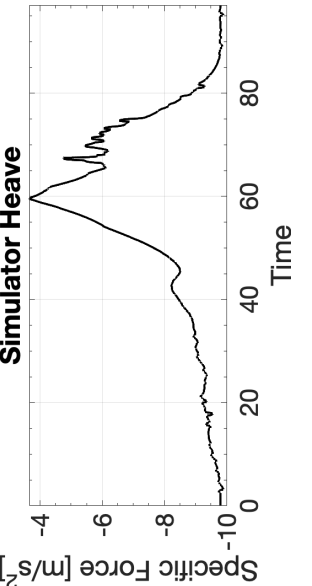

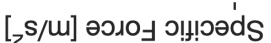
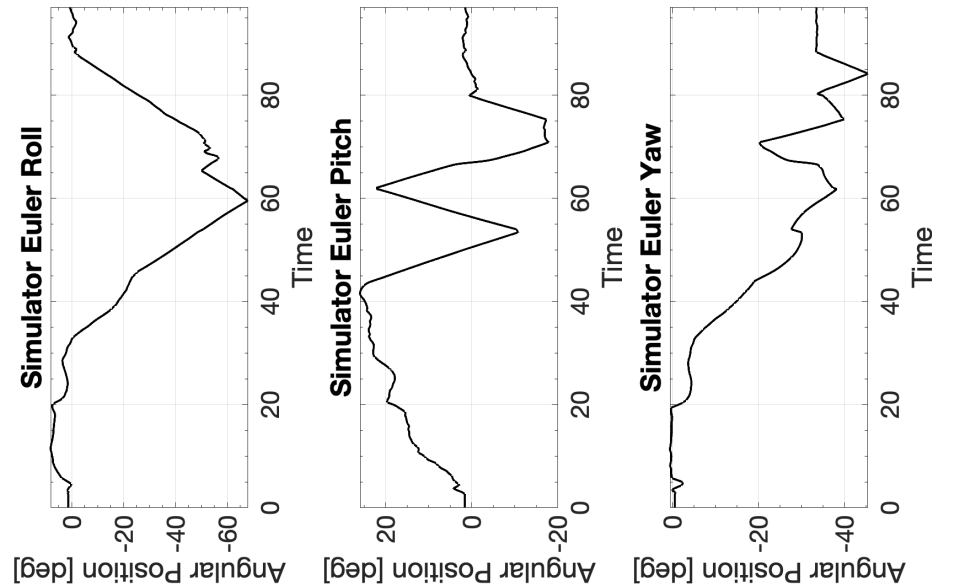

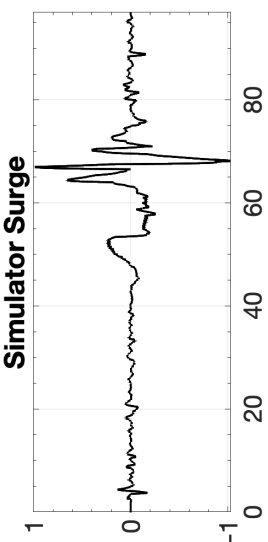

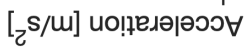

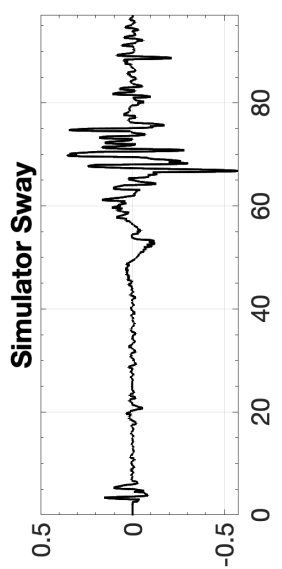

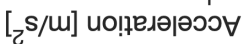

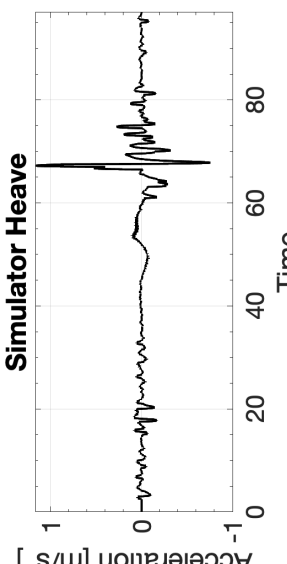

$[\mathrm{s} / \mathrm{m}]$ uо!ฺвдәәәэว
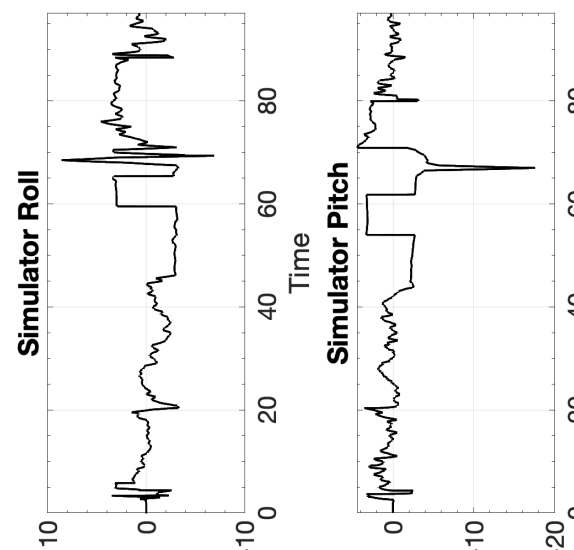

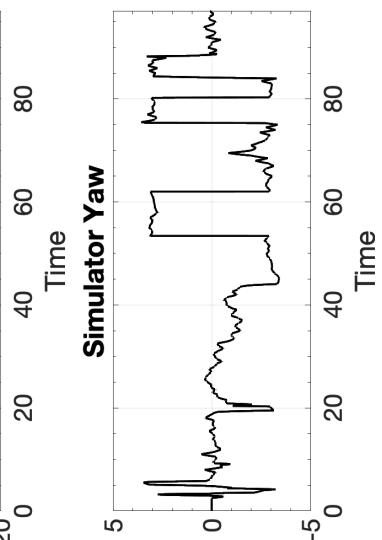

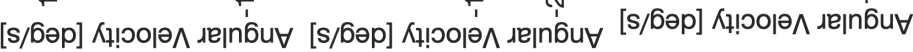



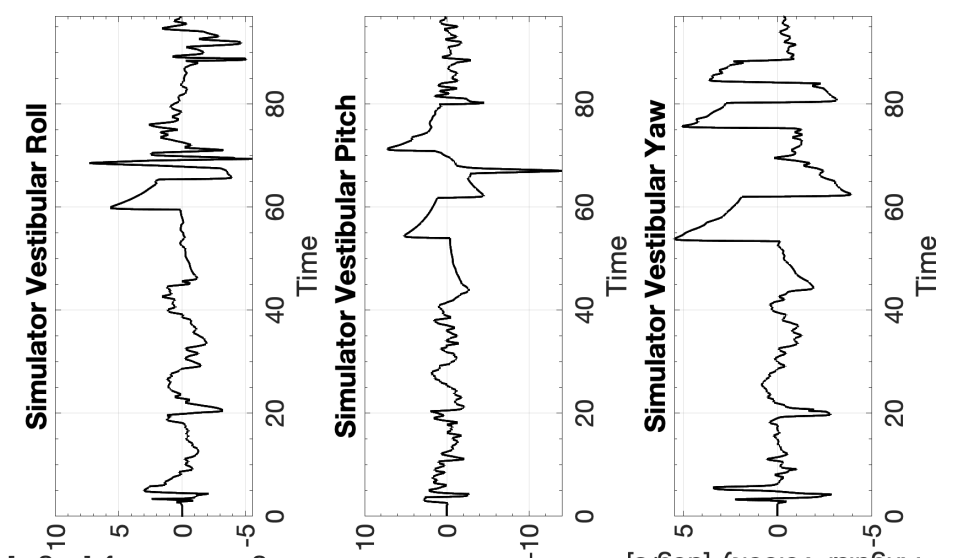

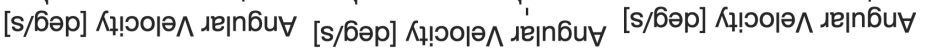

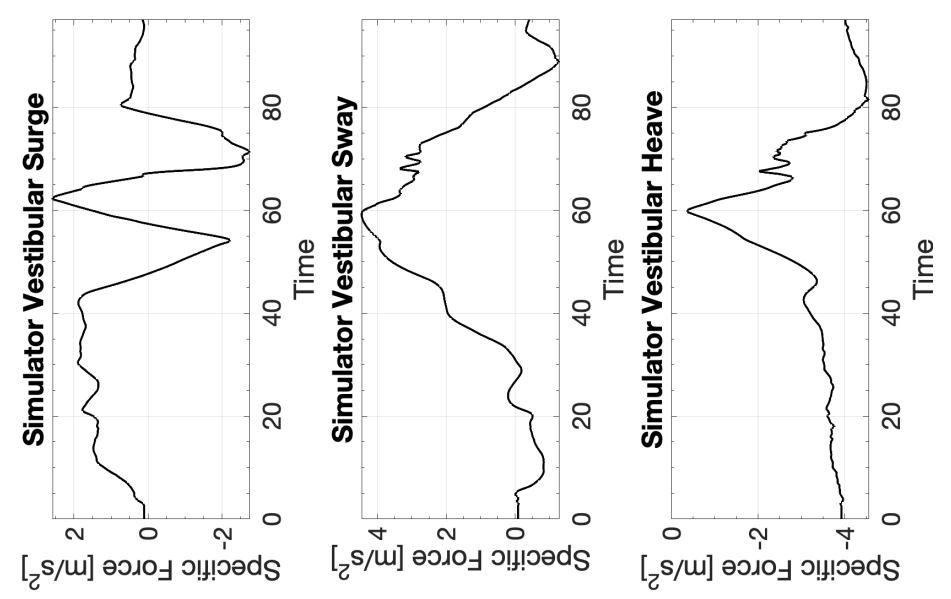




\section{C.18.3 CW3 Columbia 400 Spiral Dive}

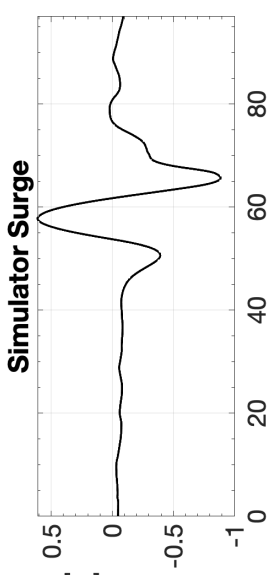

[w] uo!n!!sod

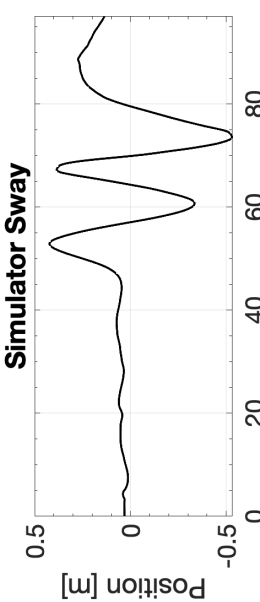

[w] uo!t!sod

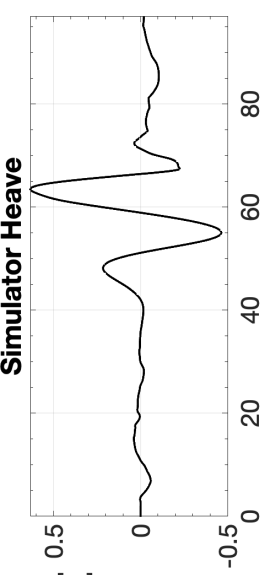

[u] uo!t!!sod

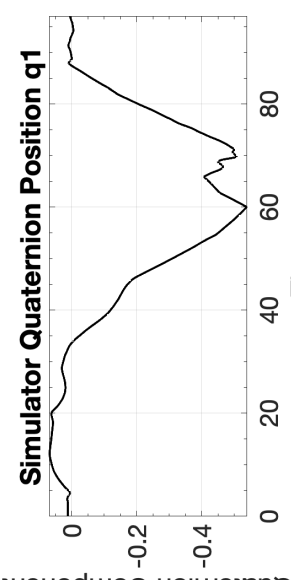

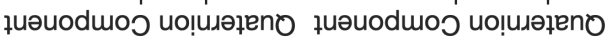

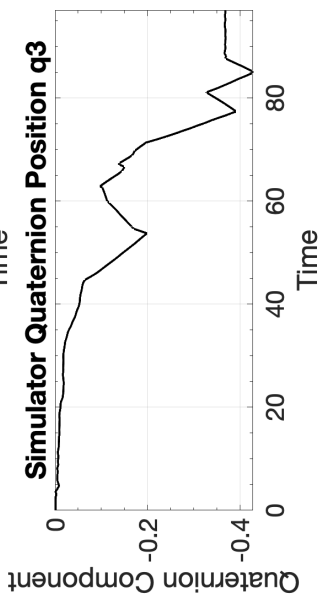

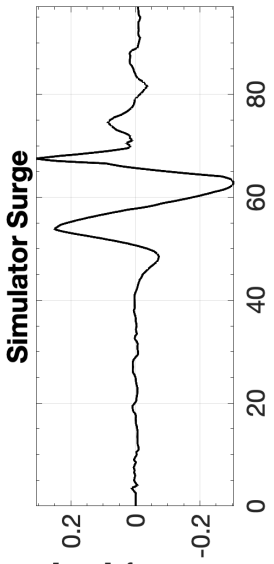

[s/m] К१!००|ә

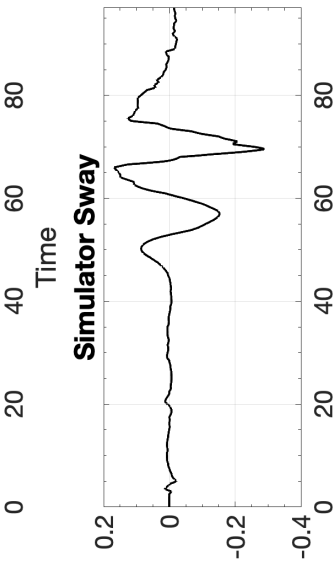

[s/u] Кұ!юо|ә

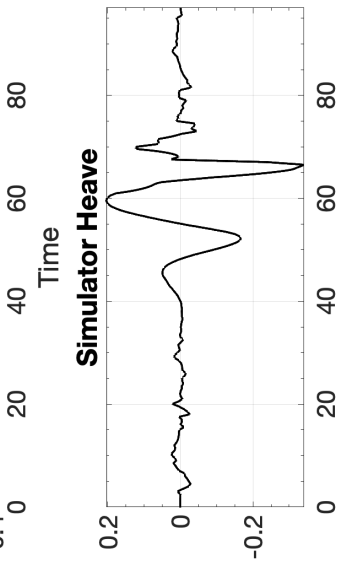

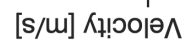

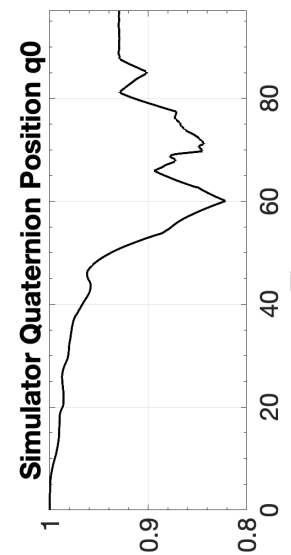

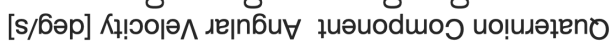

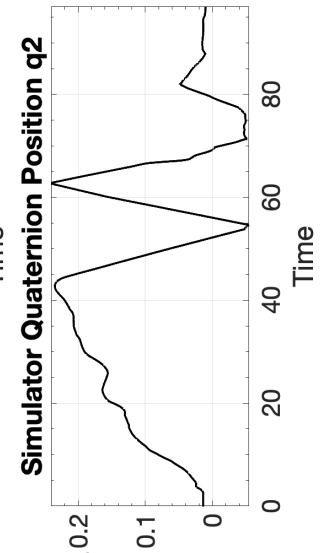

요

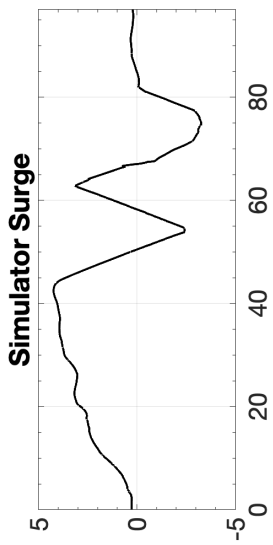

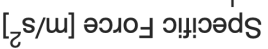

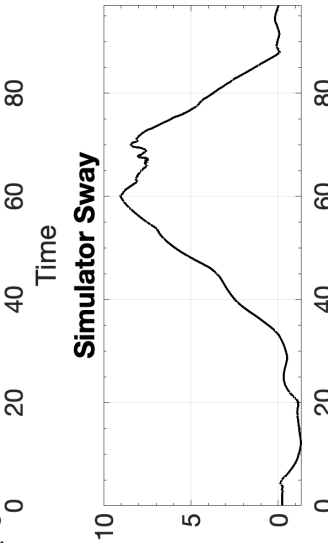

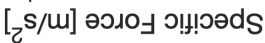

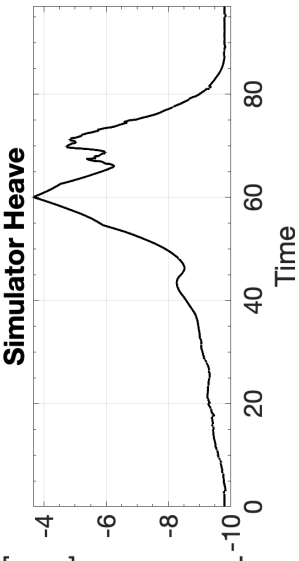

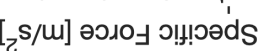

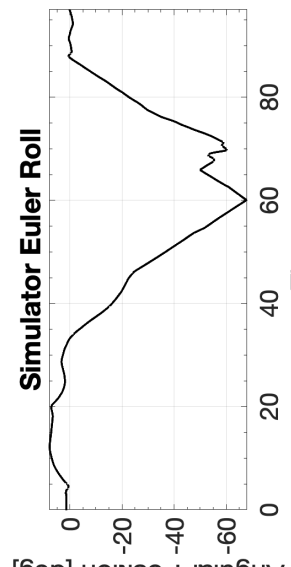

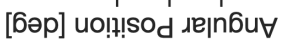

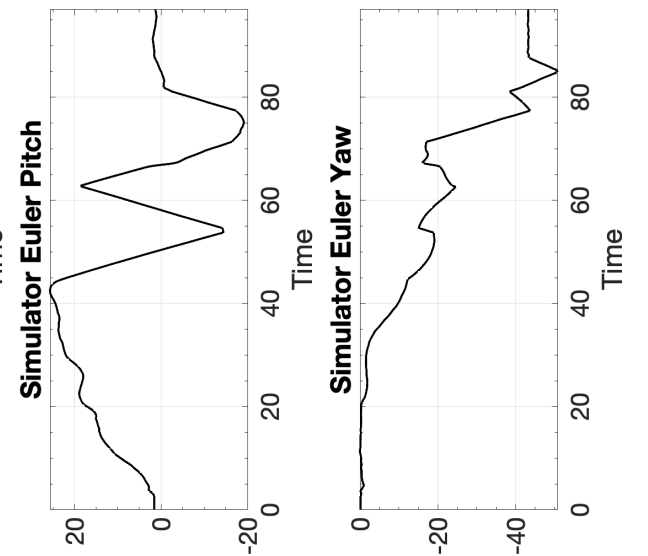

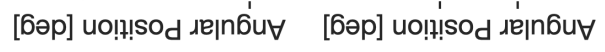

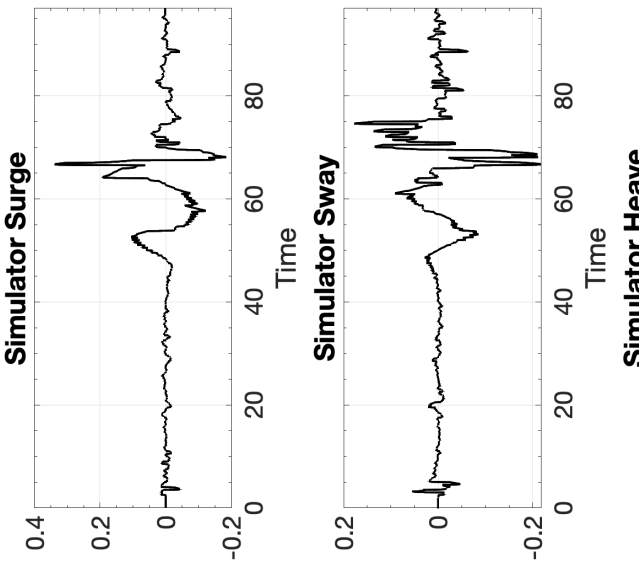

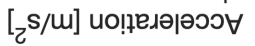

$\left[{ }_{2} \mathrm{~s} / \mathrm{M}\right]$ ио!ฺедәәәэว
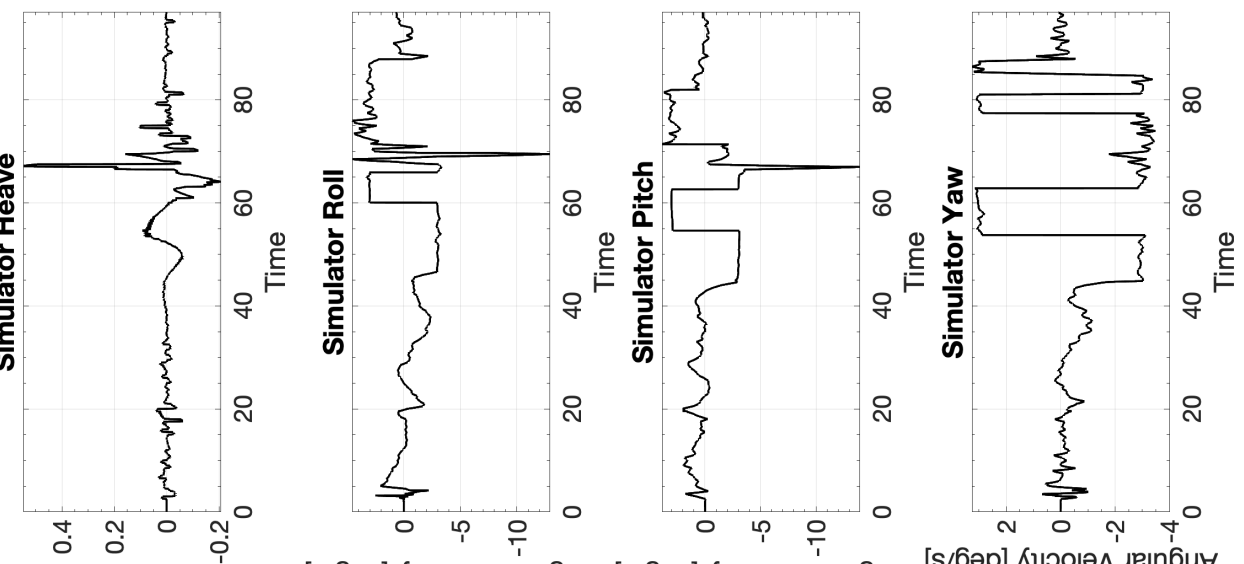

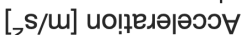



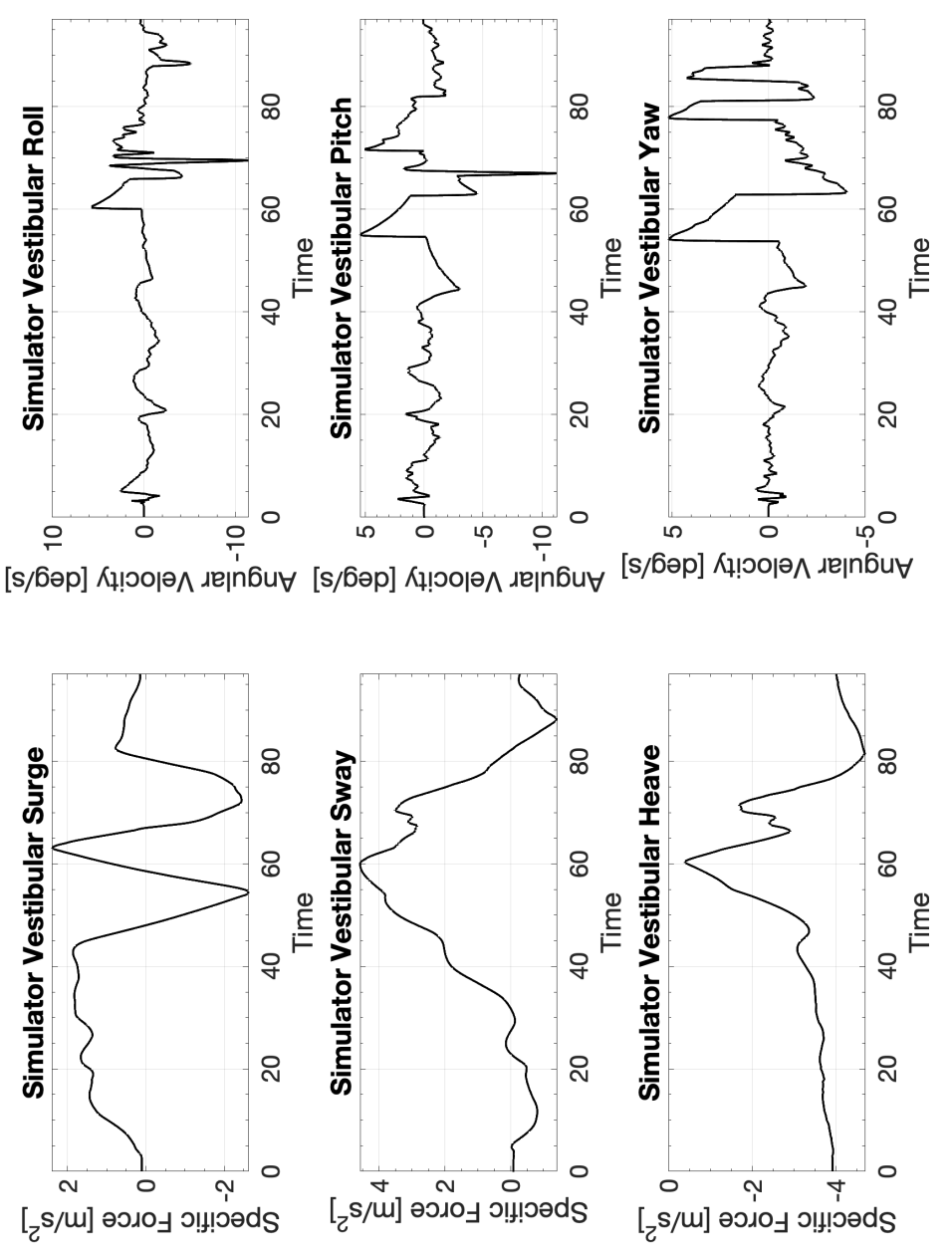


\section{C.19 Columbia 400 Stall Recovery}

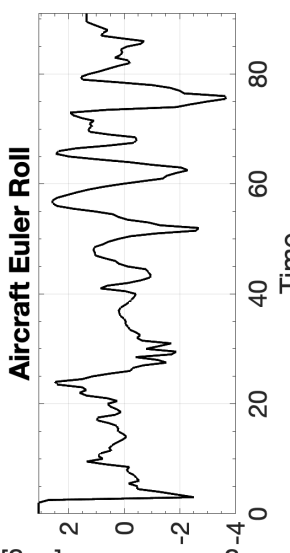

[6әр] uo!!!sod

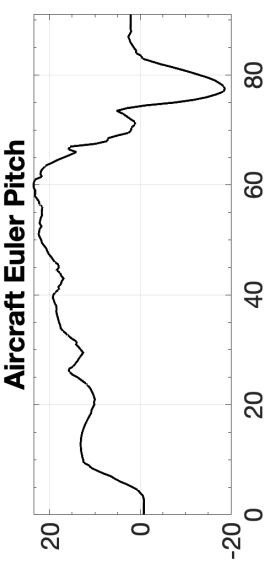

[6әр] uo!!!sod ıе|n6̆u

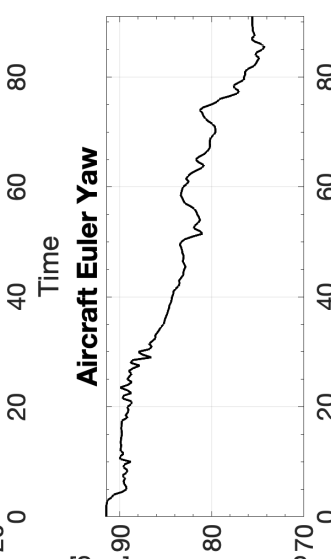

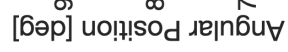

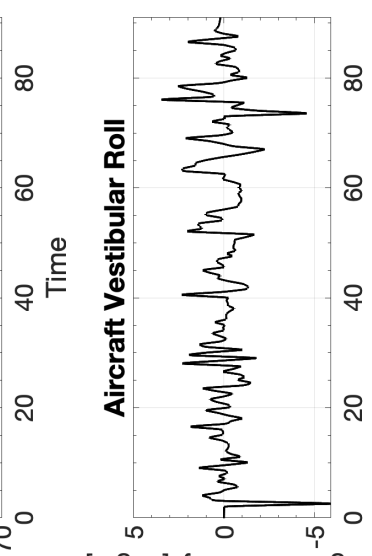

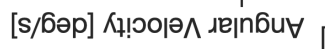

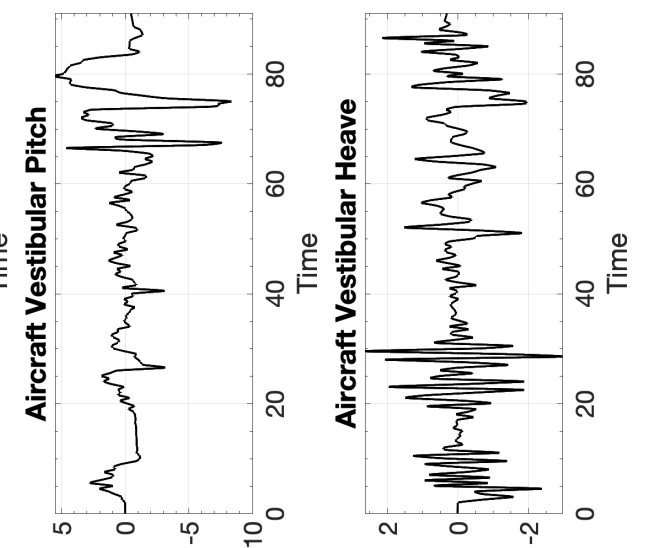

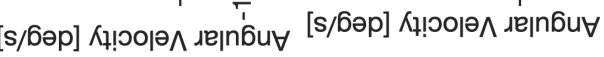
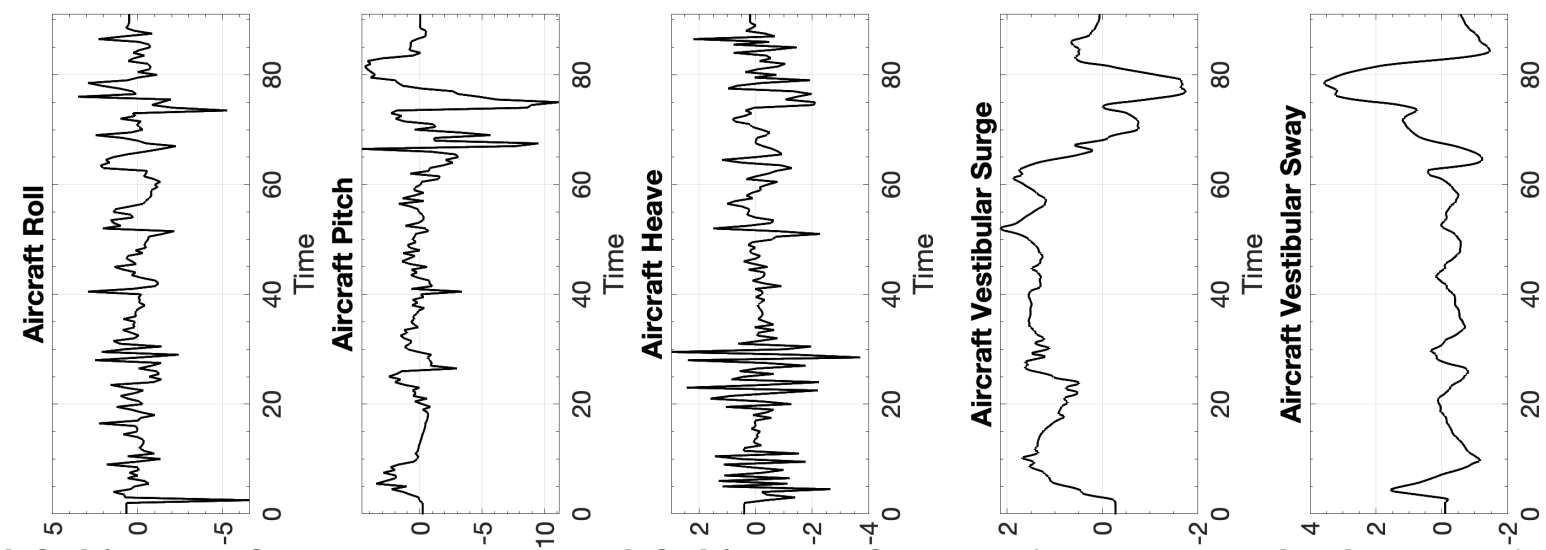

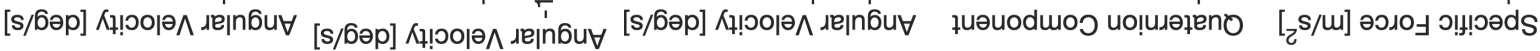

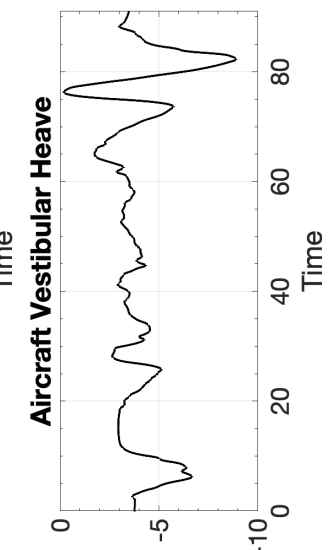

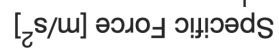

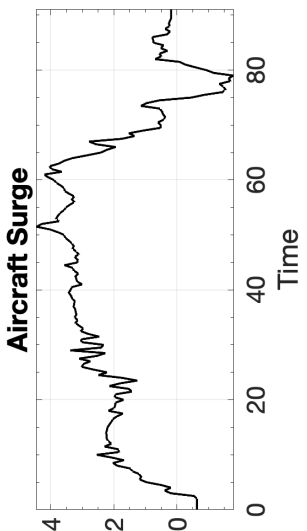

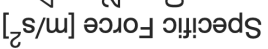

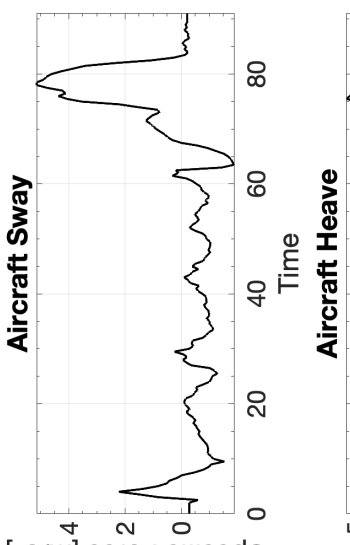

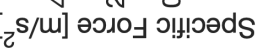

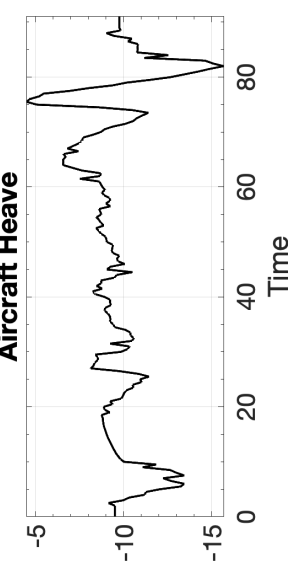

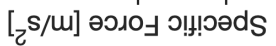

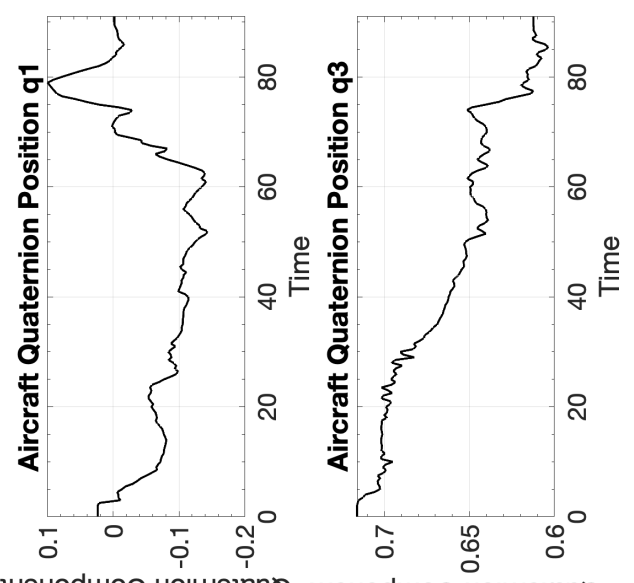

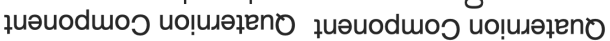

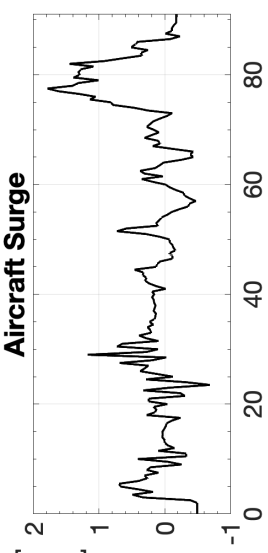

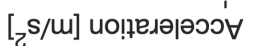

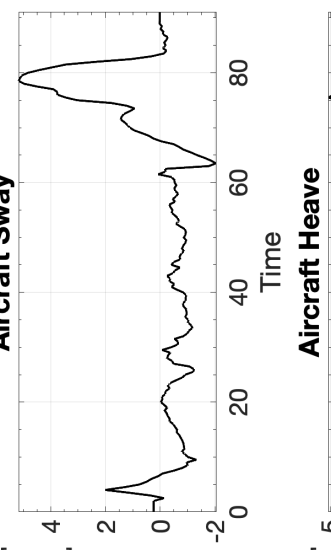

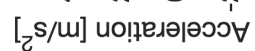

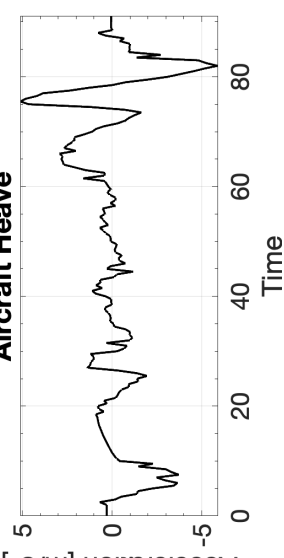

$[\mathrm{z} / \mathrm{s}]$ uо!ฺвләәәэว

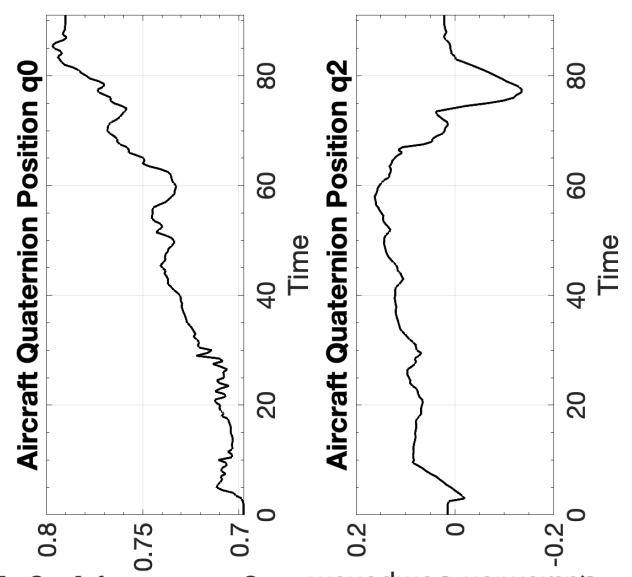

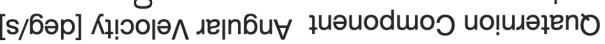




\section{C.19.1 CW1 Columbia 400 Stall Recovery}

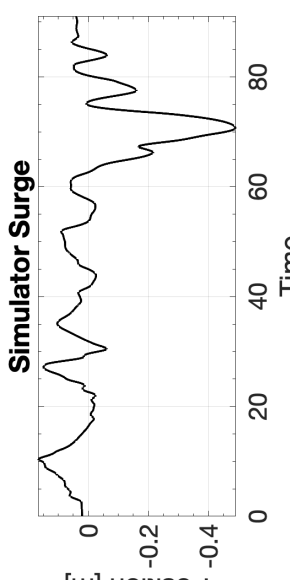

[u] uo!n! sod

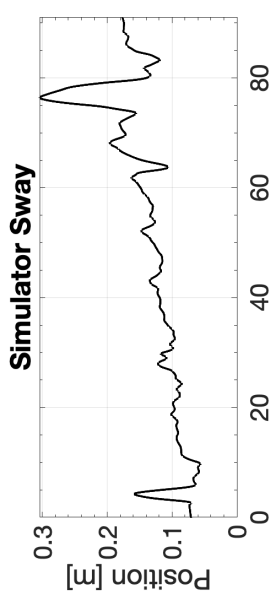

[u] uo!n!sod

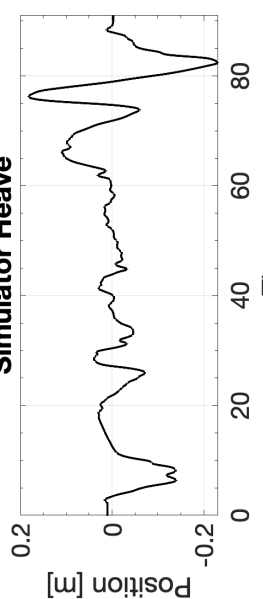

[m] uol!!sod

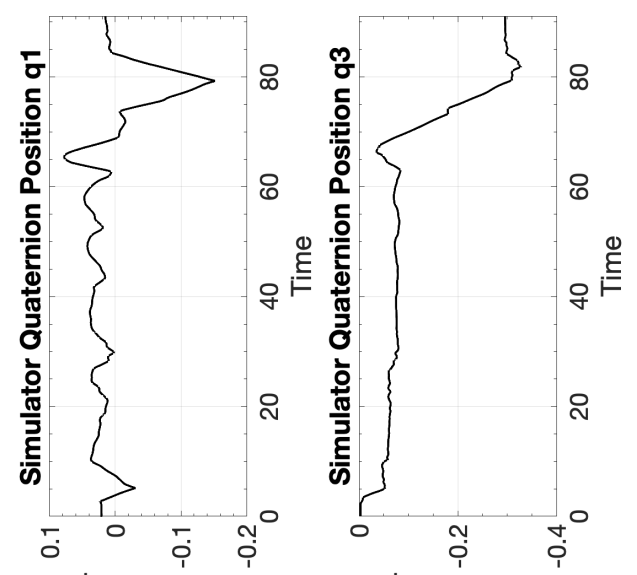

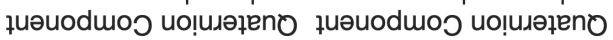

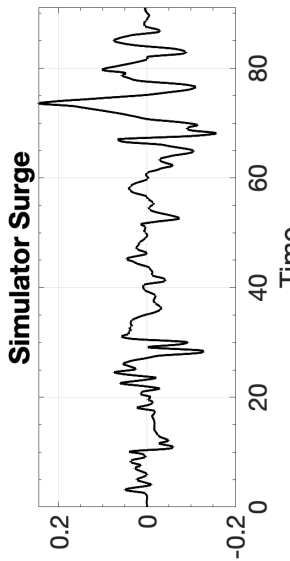

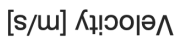

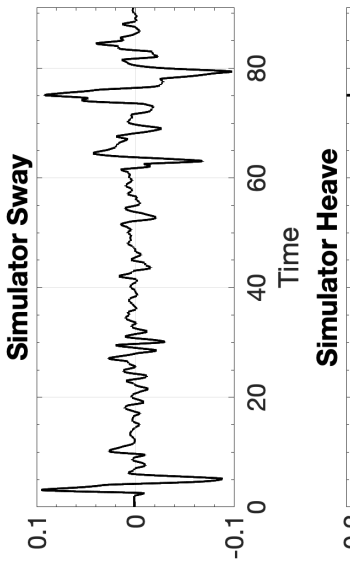

[s/u] Кұ!юо|ә

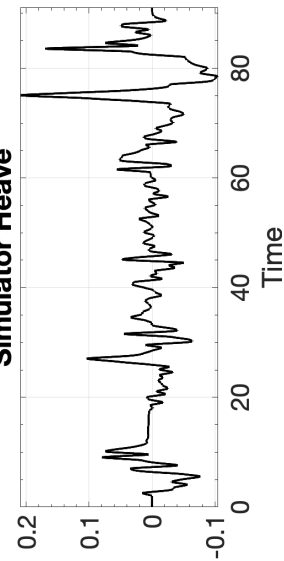

[s/u] Кұ!৩о|ә
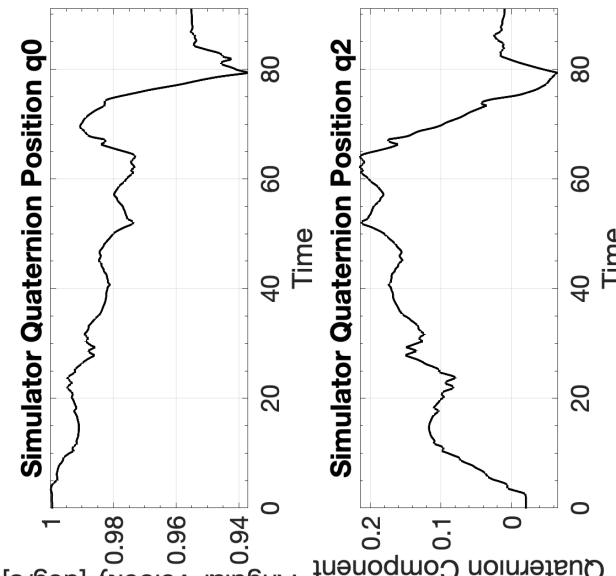

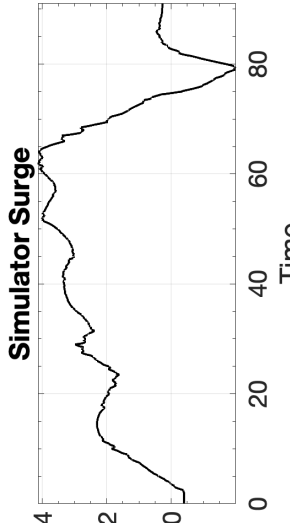

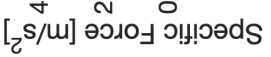
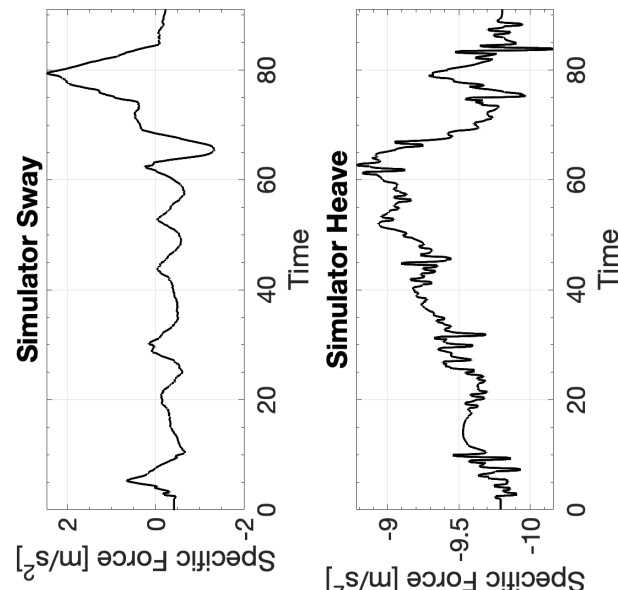

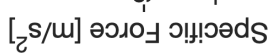

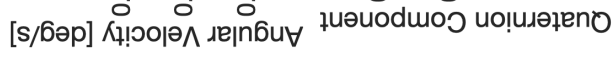

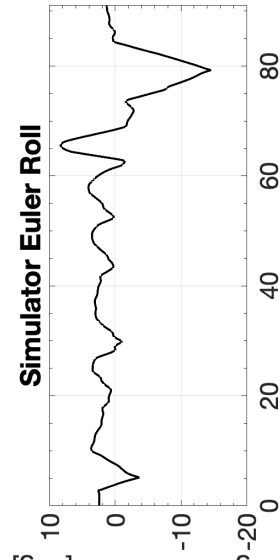

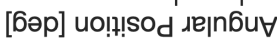

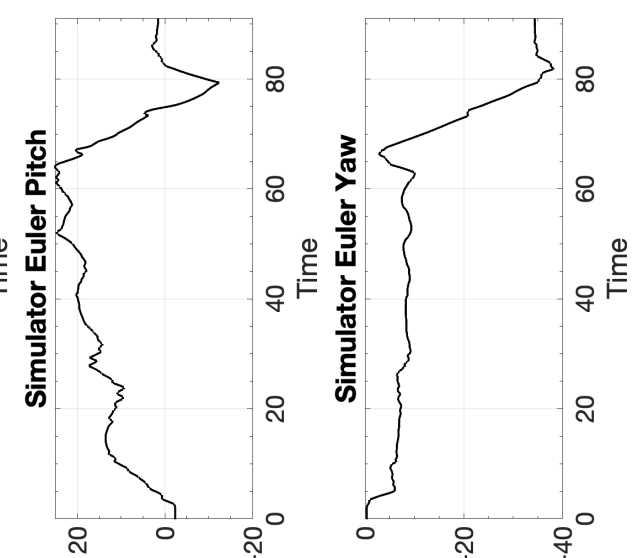

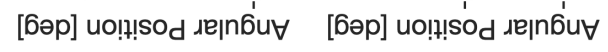

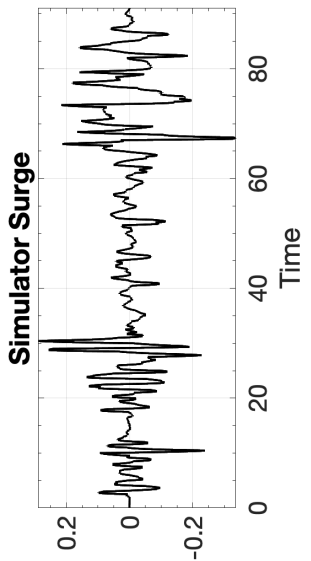

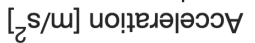

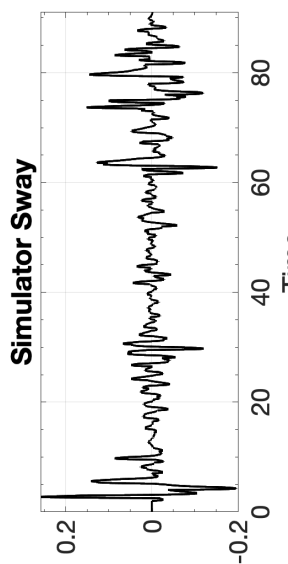

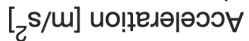

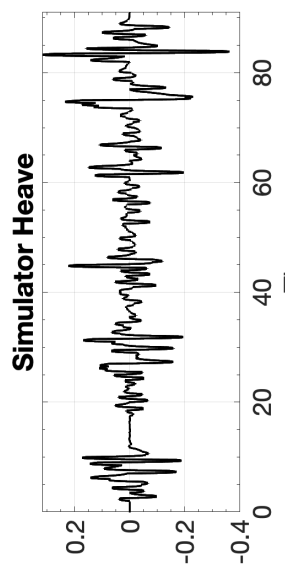

[s/m] ио!ฺедә|әэว

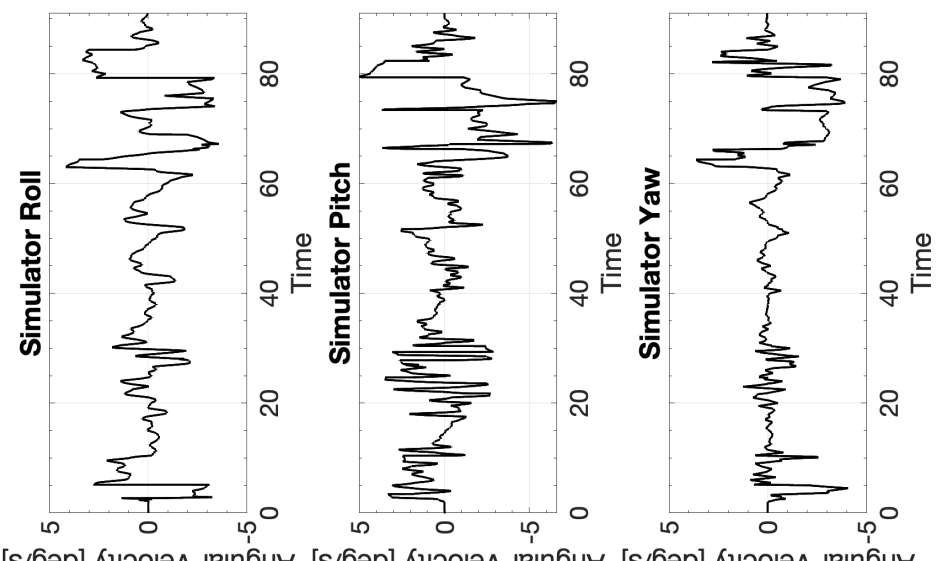

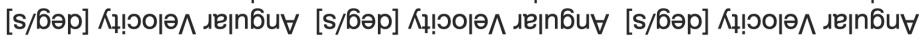




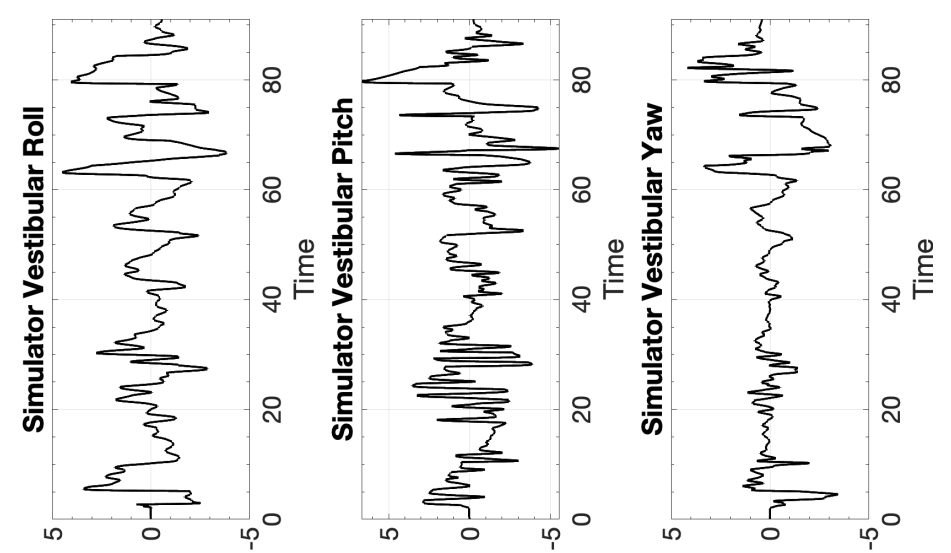

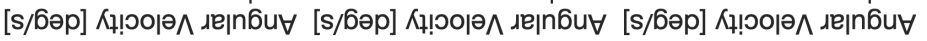

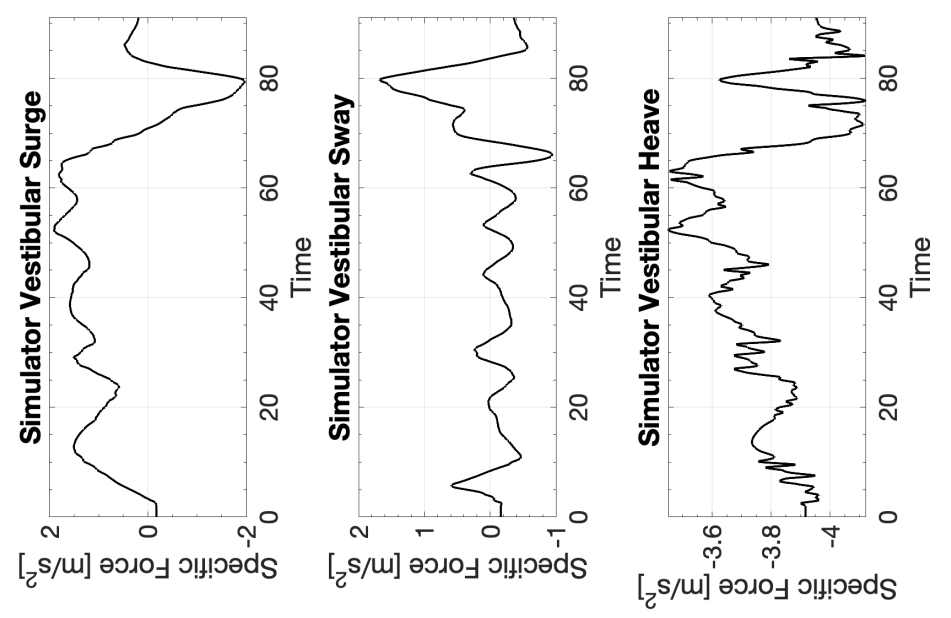




\section{C.19.2 CW2 Columbia 400 Stall Recovery}

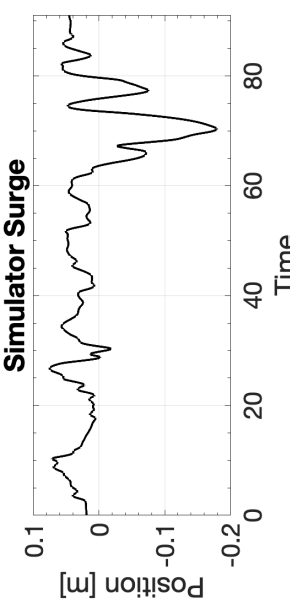

[u] uol!!!sod

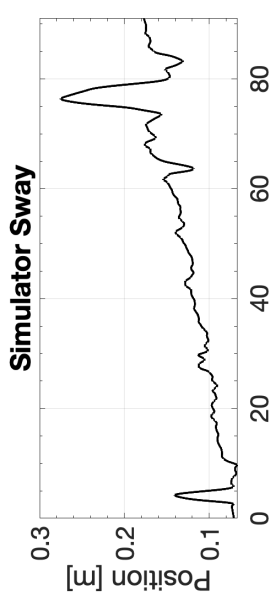

[u] uo!n!sod

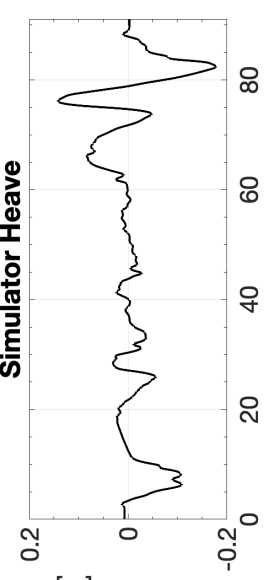

[m] uo!!!!sod
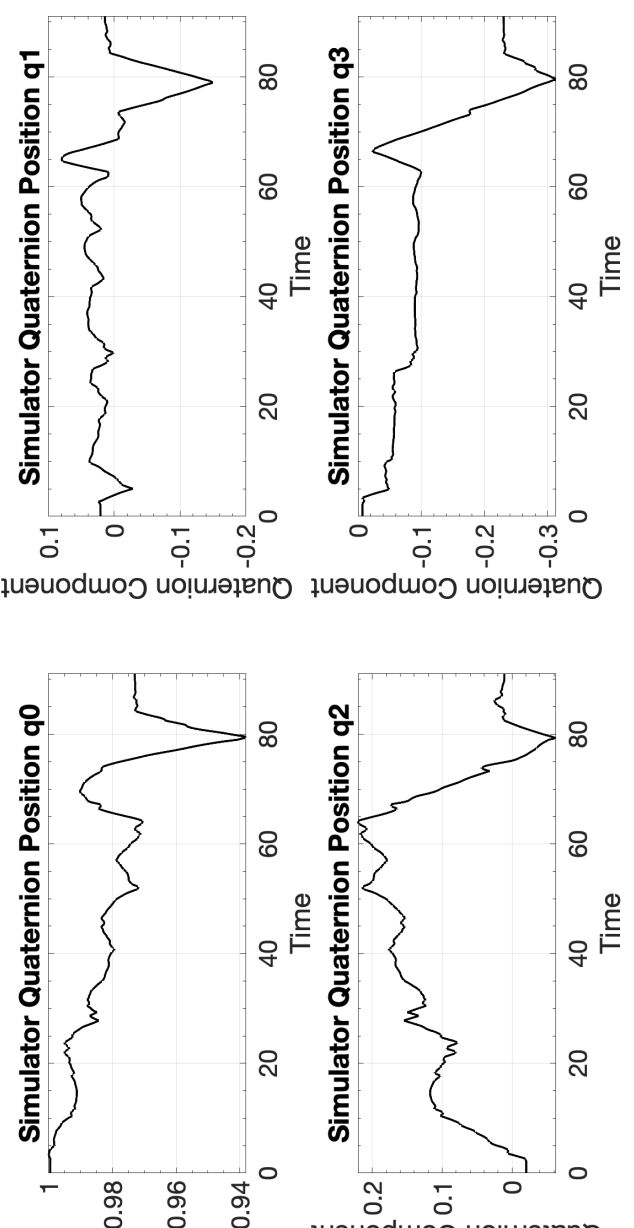

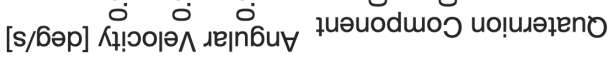

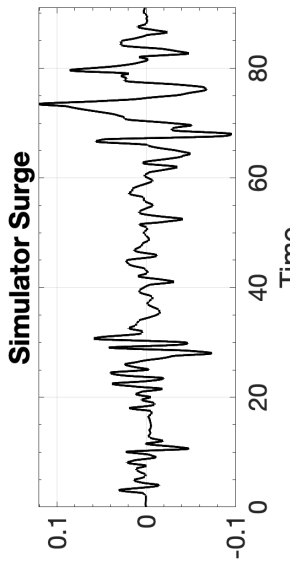

[s/u] Кұ! ৩орә $\Lambda$

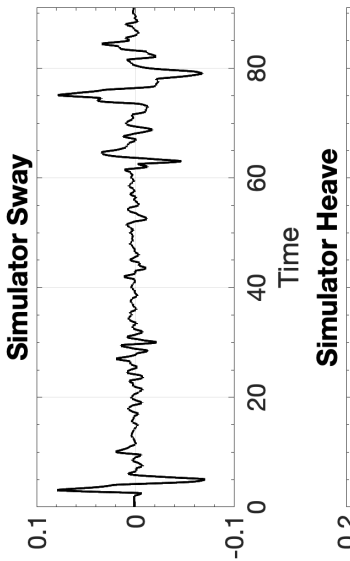

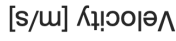

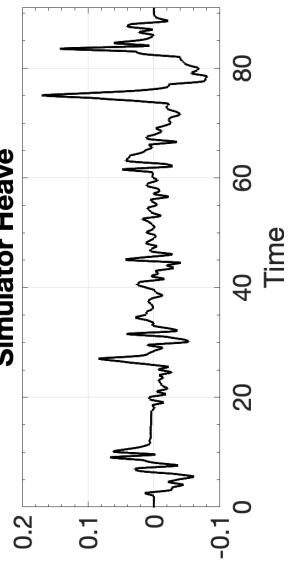

[s/u] Кұ!৩о|ә

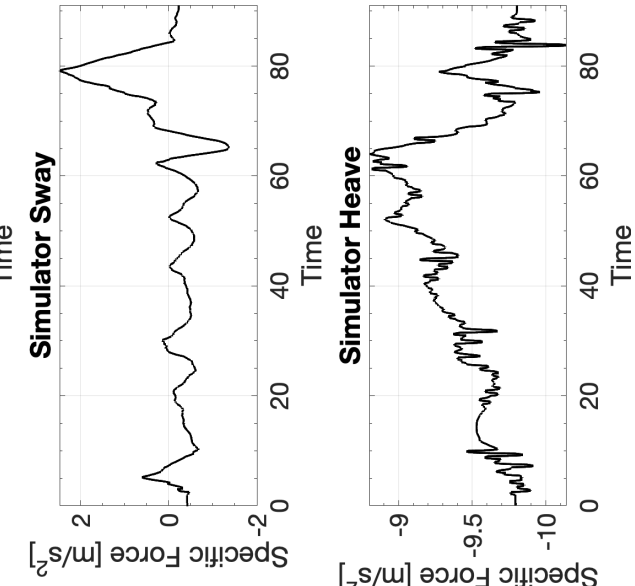

[

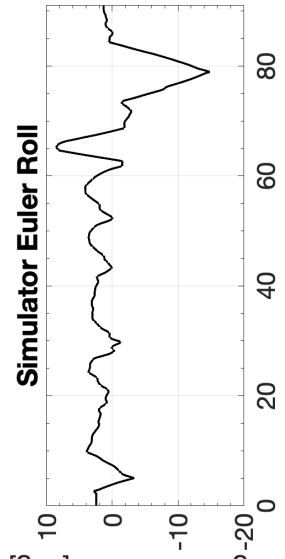

[бәр] uo!!! sod

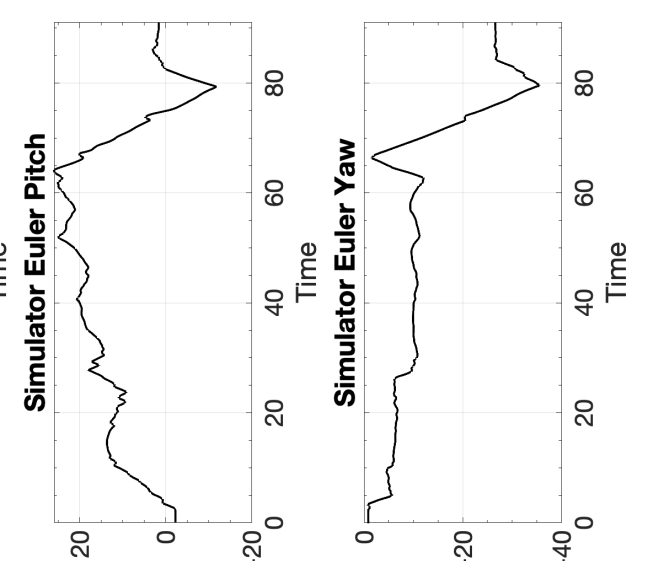

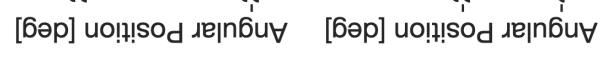

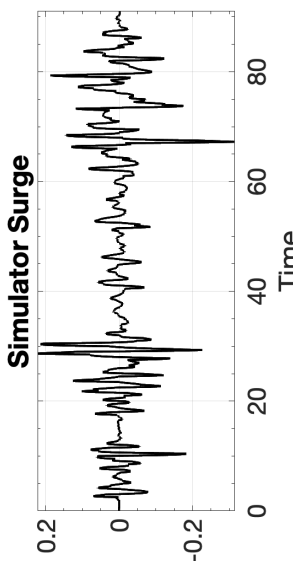

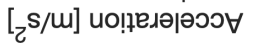

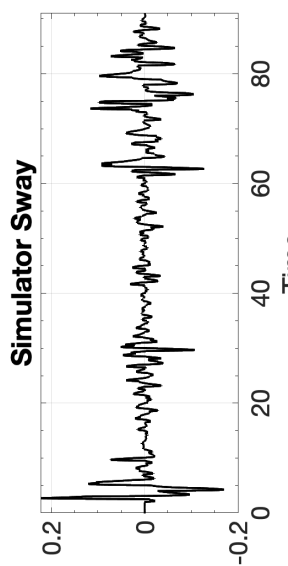

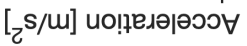

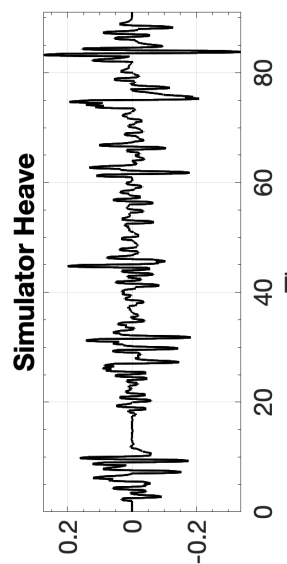

$[\mathrm{s} / \mathrm{m}]$ ио!ฺвләәәэว

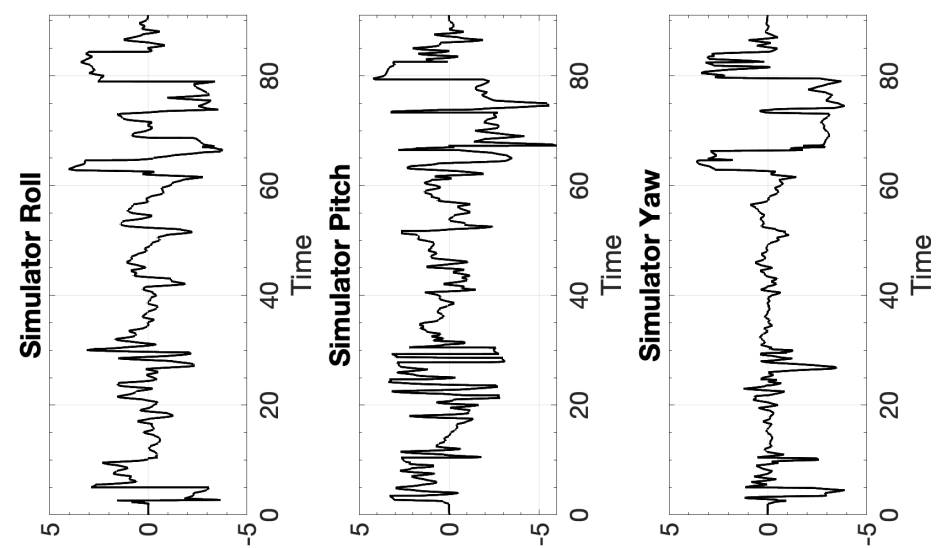

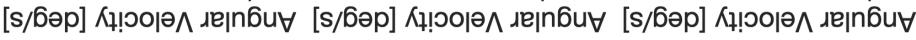




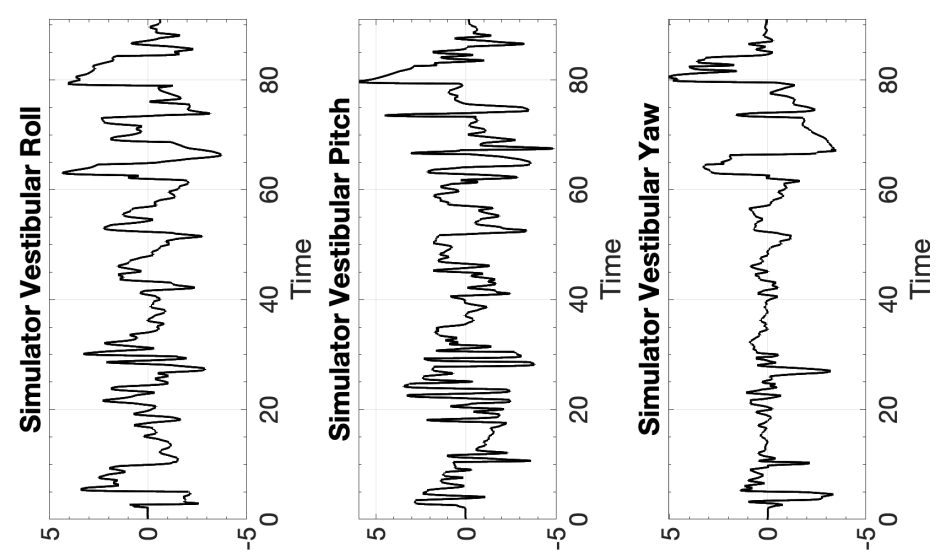

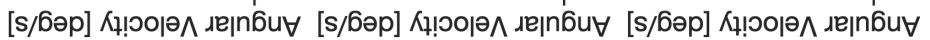

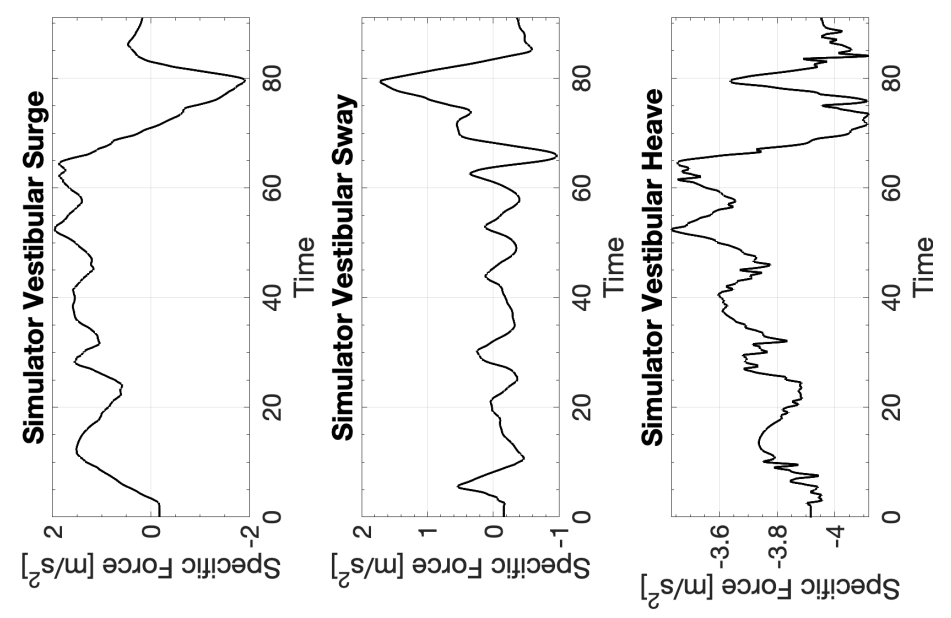




\section{C.19.3 CW3 Columbia 400 Stall Recovery}

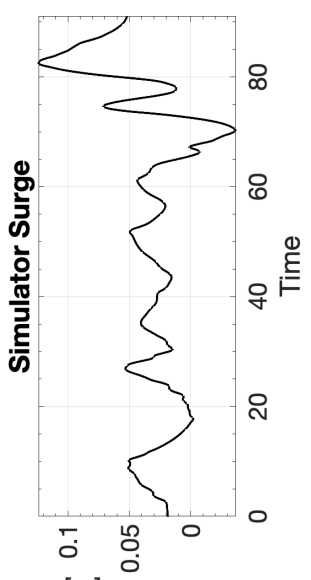

[u] uo!!!!sod

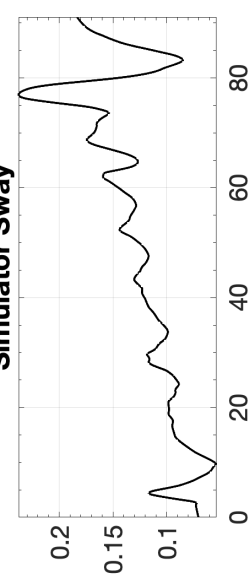

[w] uol!!sod

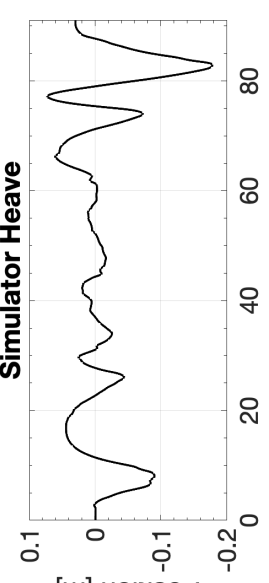

[w] uo!n!

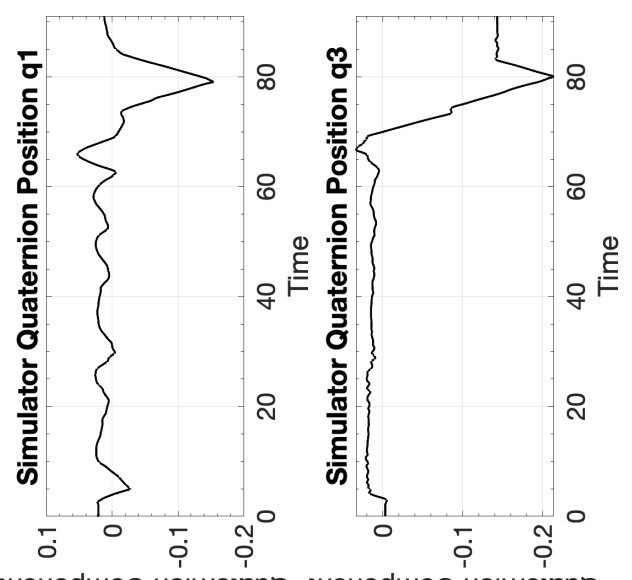

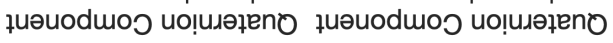

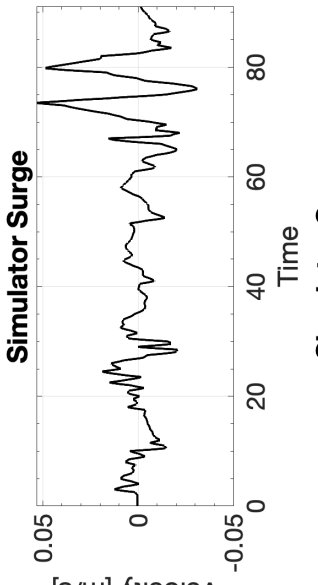

[s/u] Кұ!৩о|ә^

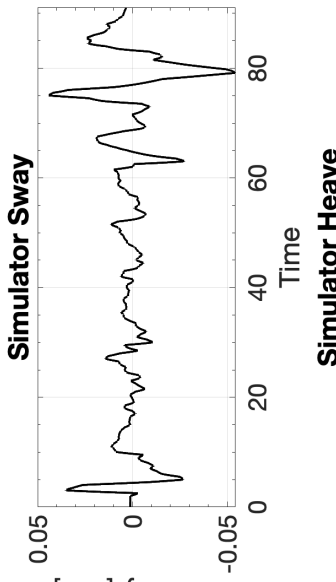

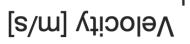
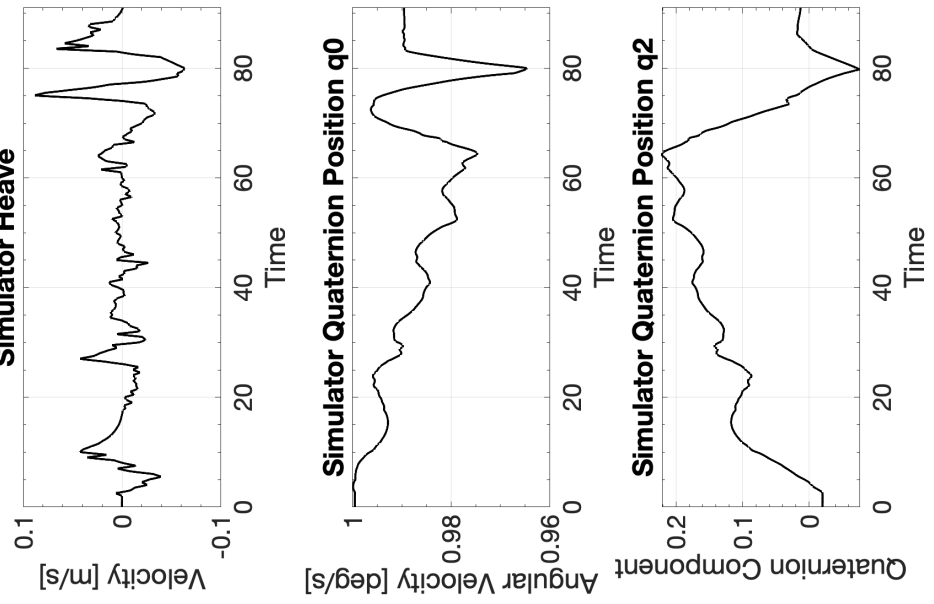

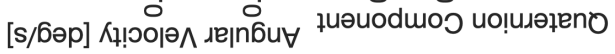

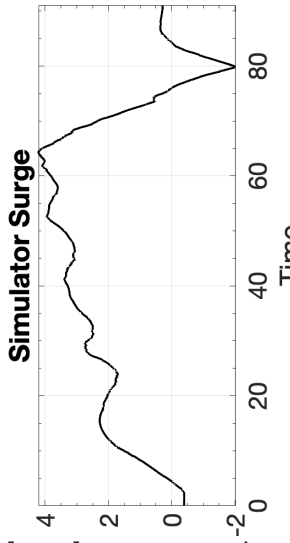

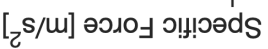

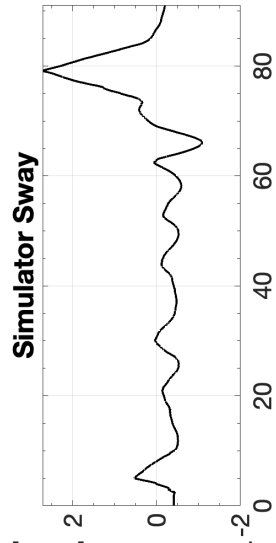

[z

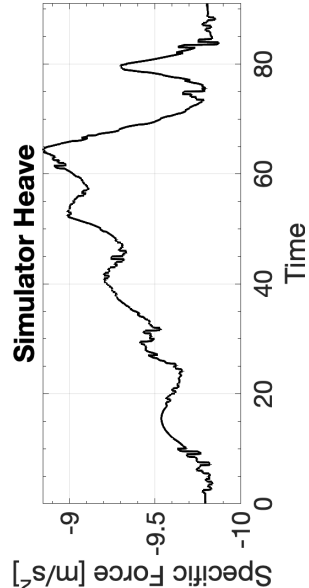

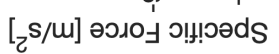

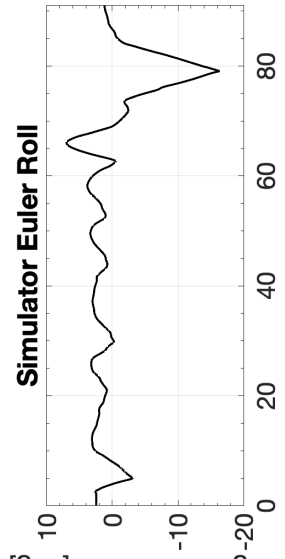

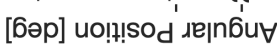

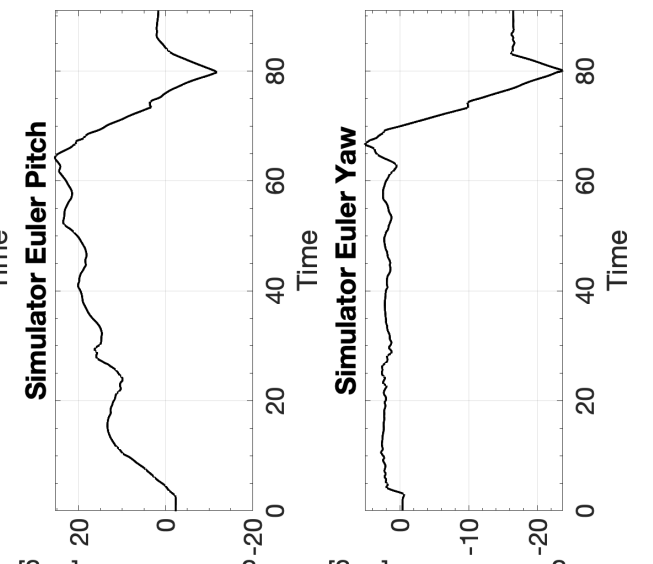

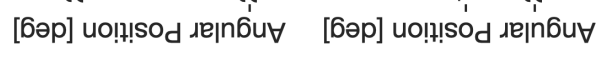

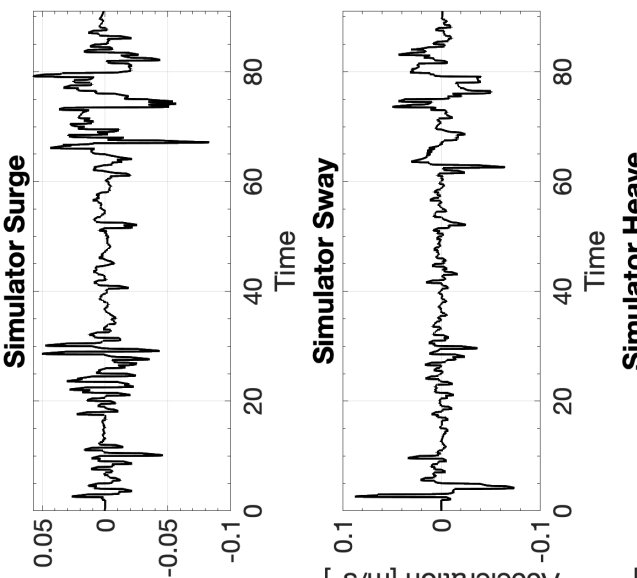

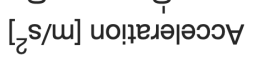

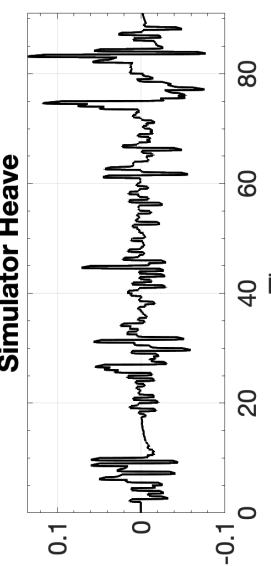

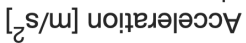

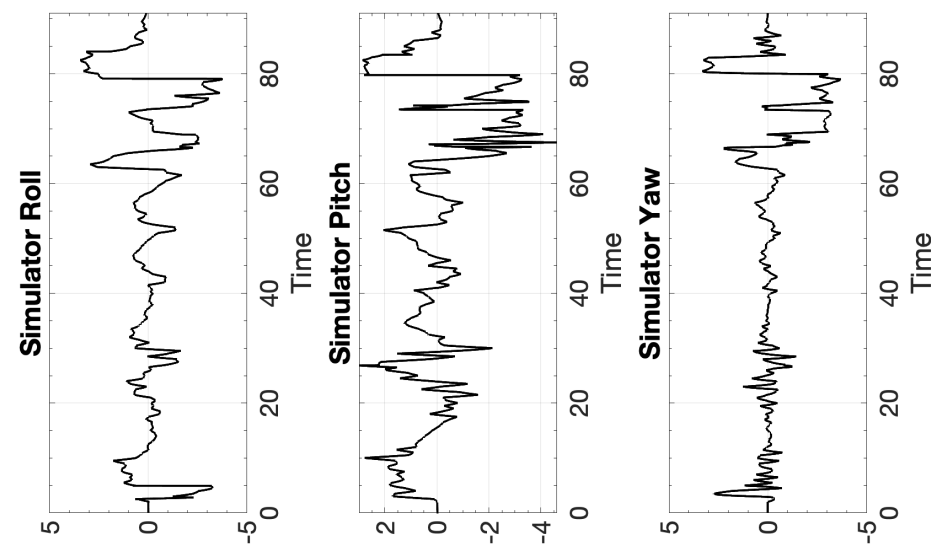

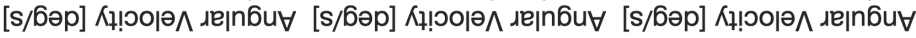




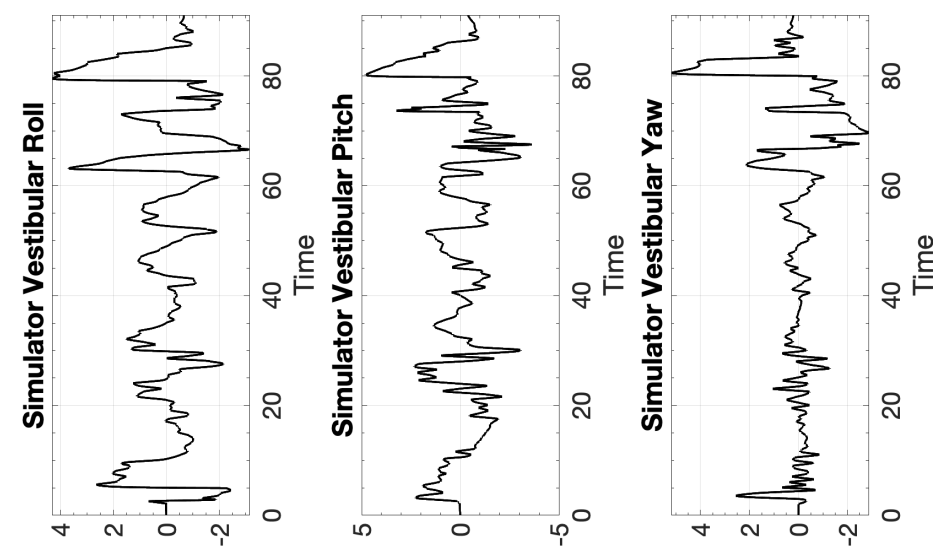

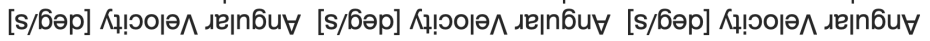

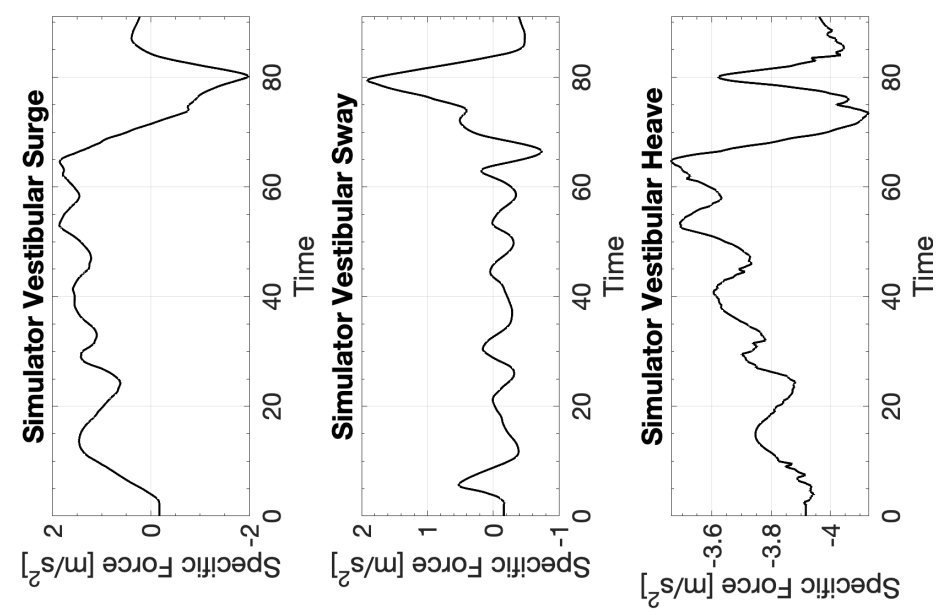




\section{Unrestricted Angular Washout Test Cases}

This appendix includes graphs for the unrestricted angular washout test cases, including the aircraft motion set points and the simulator motion. 


\section{D.1 Cessna 172 Coordinated Turn}

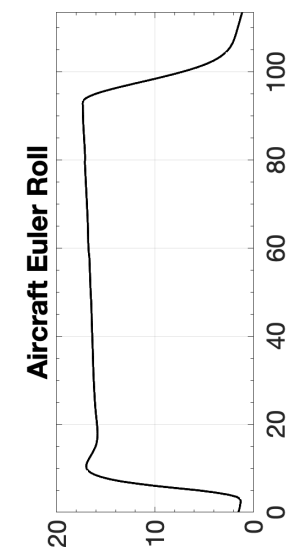

[6әp] uo!!!sod ле|n6u

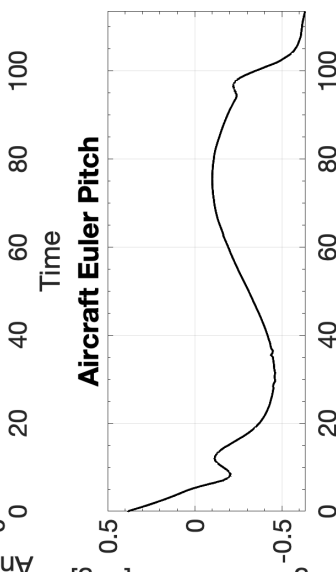

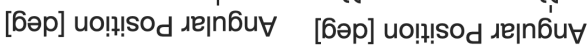

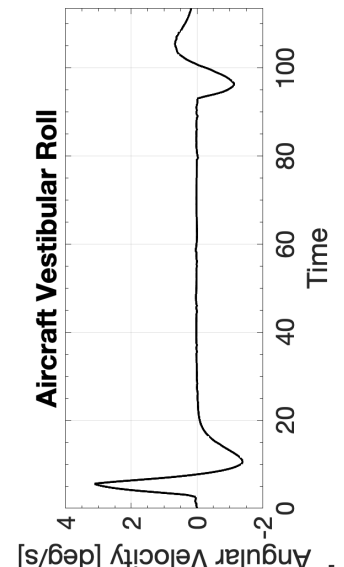

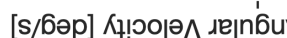
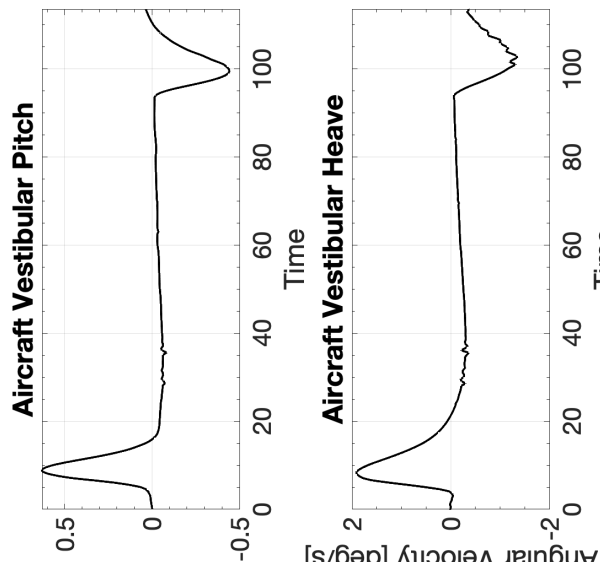

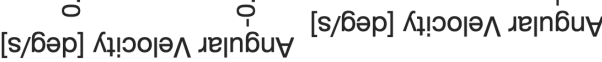

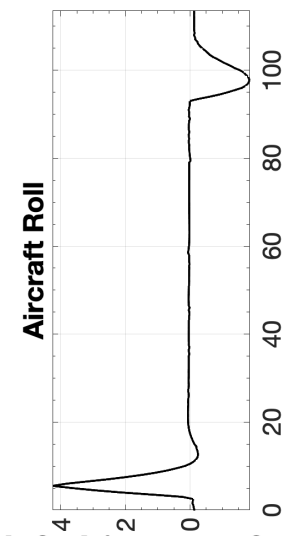

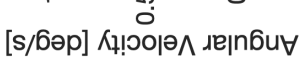

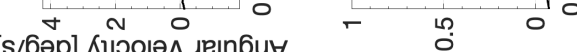

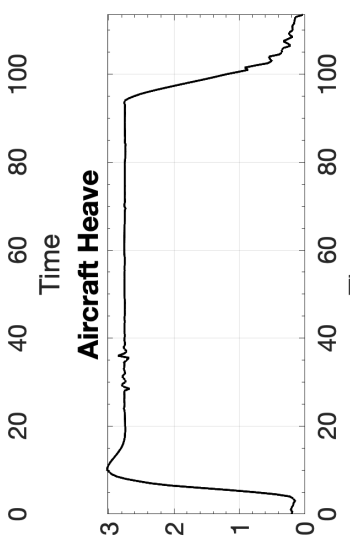

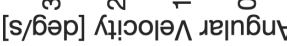

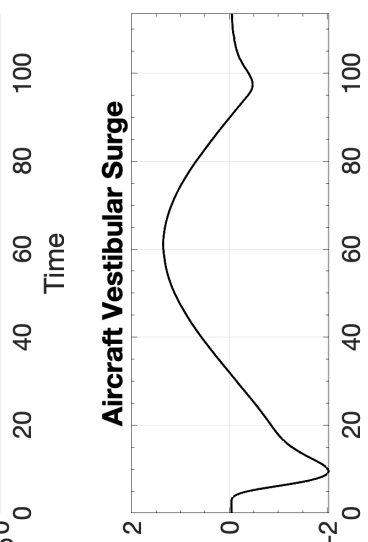

ұuәuodmos uọudəłeno

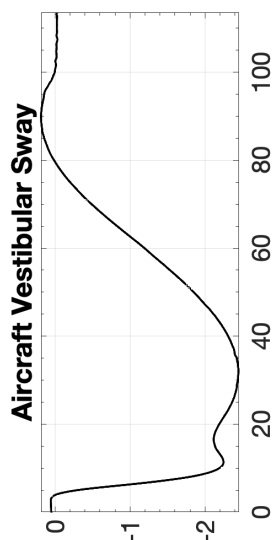

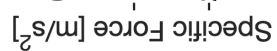
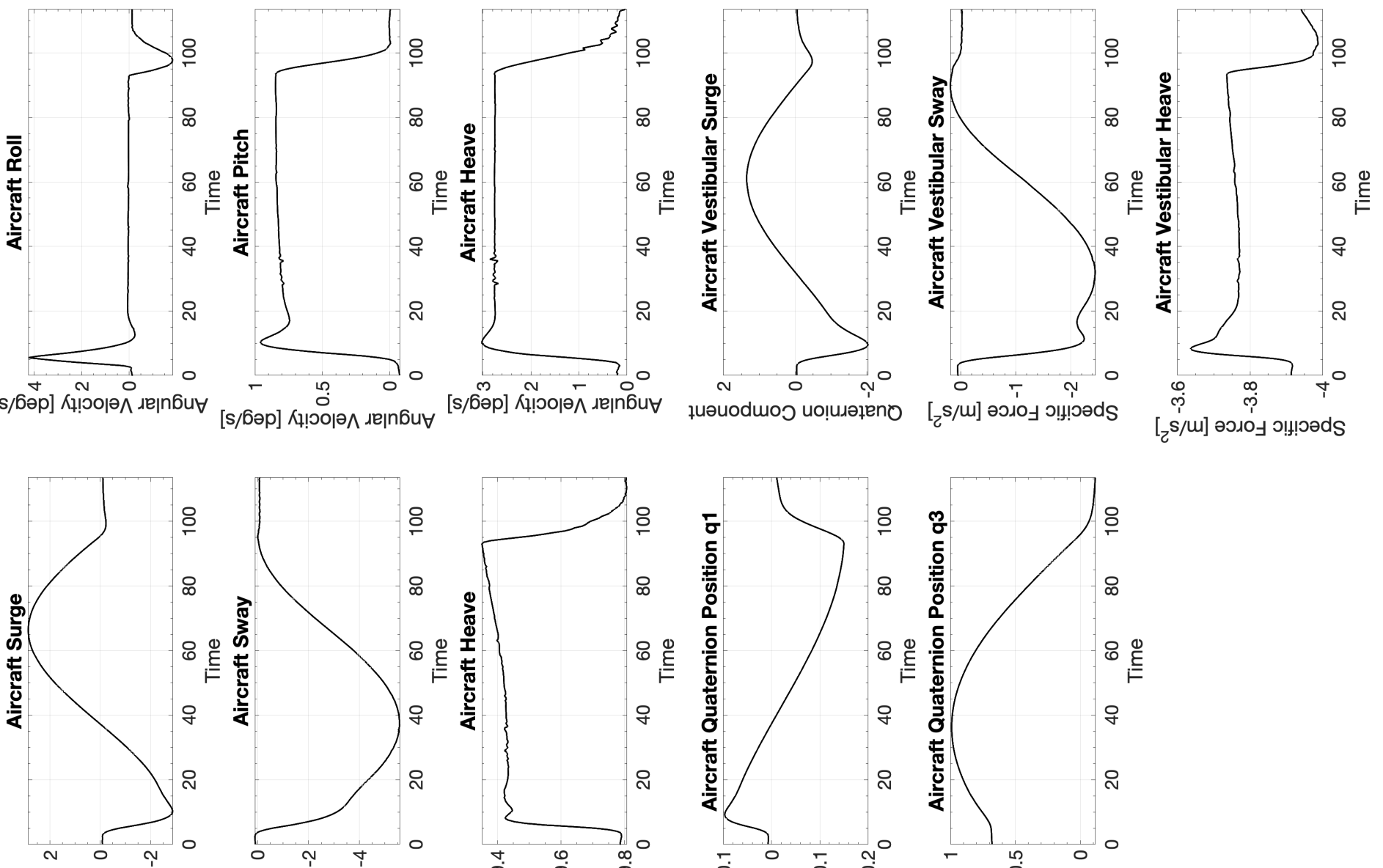

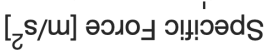

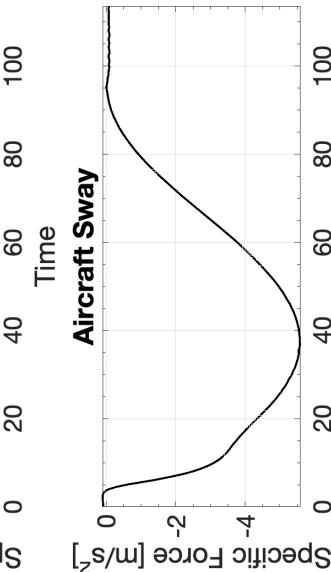

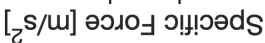
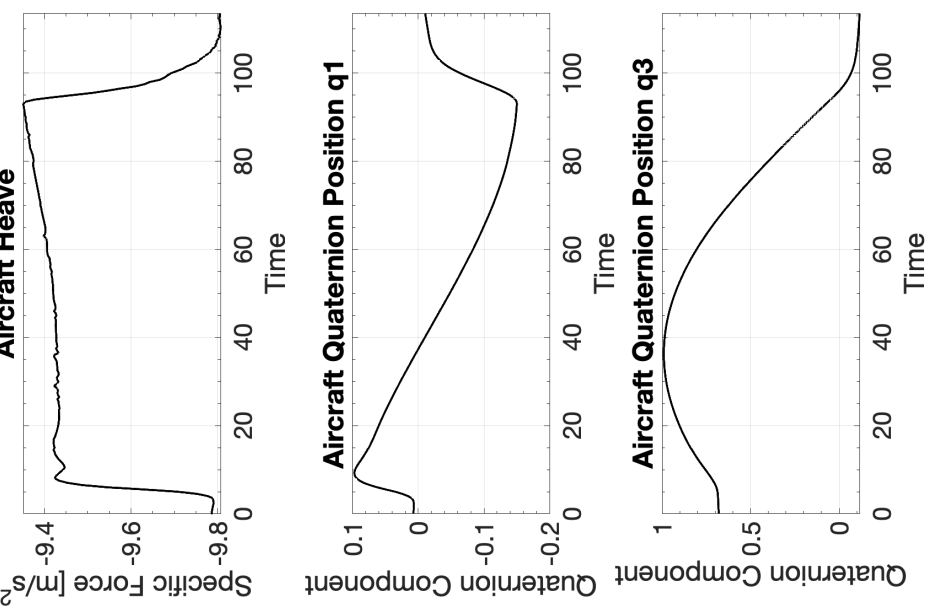

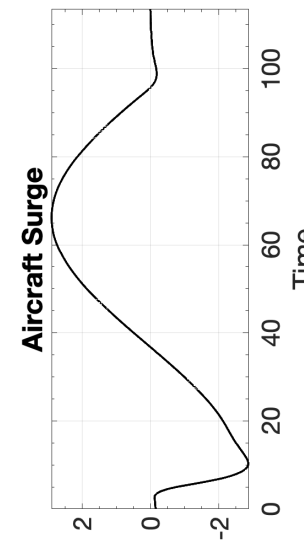

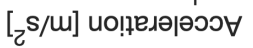

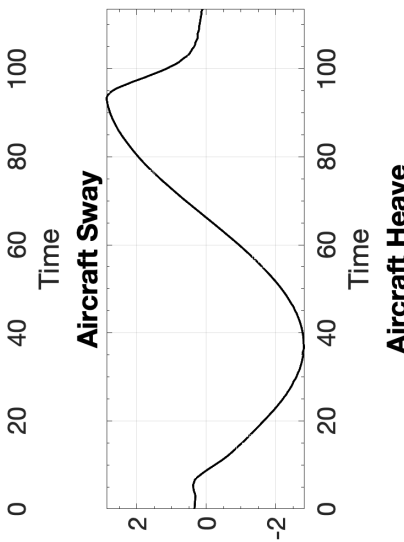

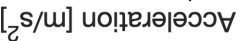

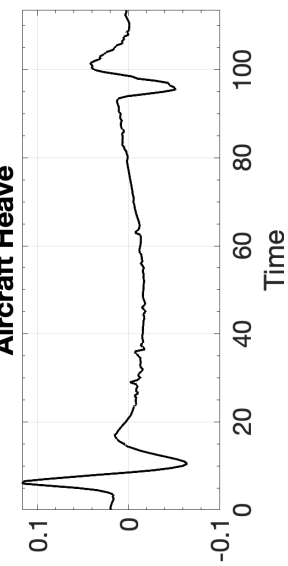

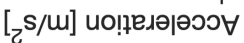

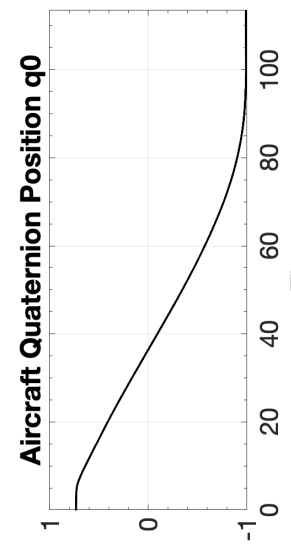

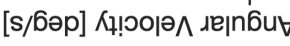

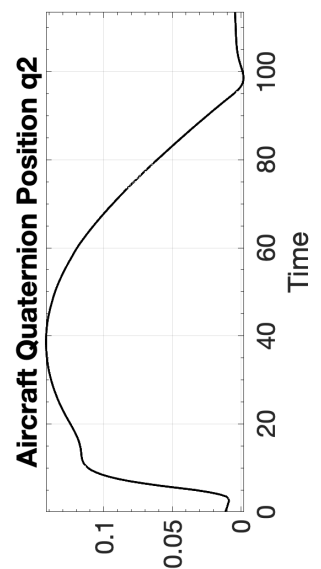

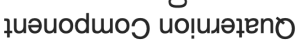




\section{D.1.1 CW1 Cessna 172 Coordinated Turn}

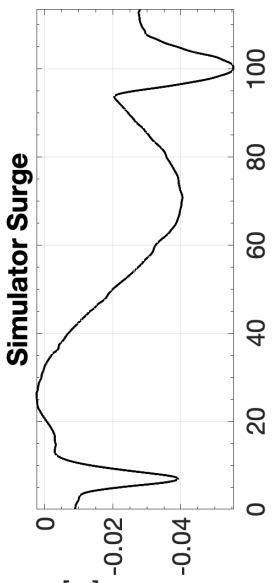

[w] uo!n!sod

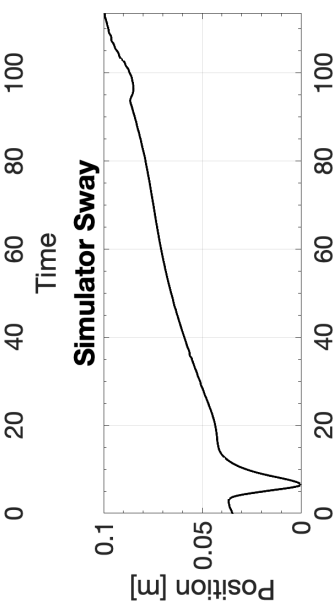

[u] uo!t!!sod

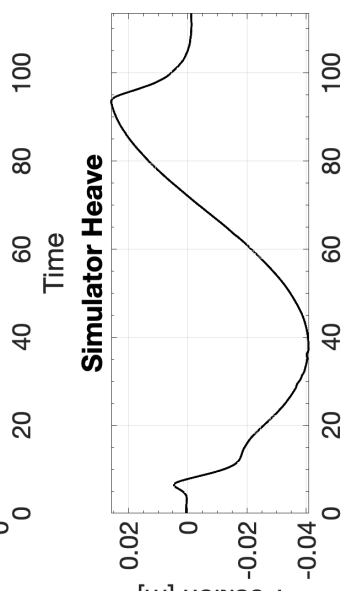

[w] uolt!!sod

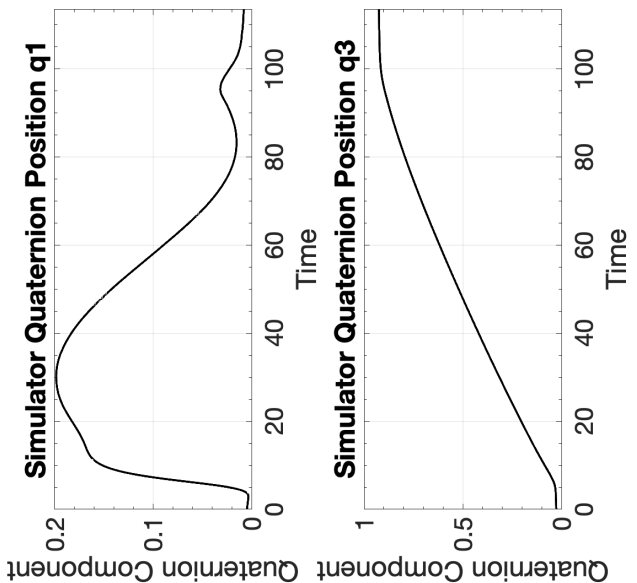

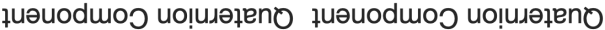

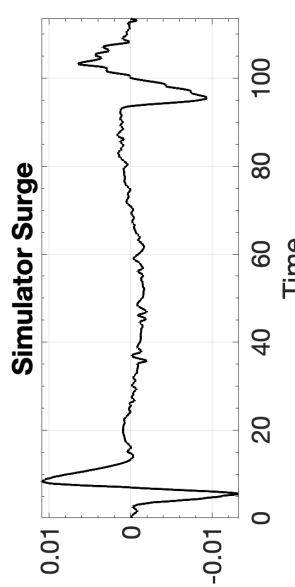

[s/u] Кұ!юор^

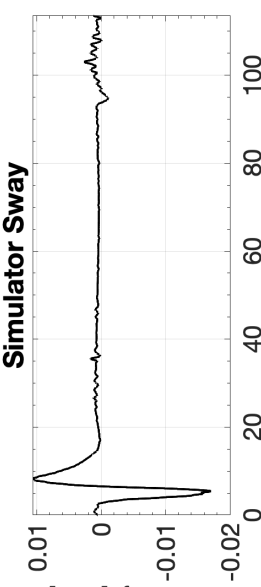

[s/u] Кұ!৩о夫

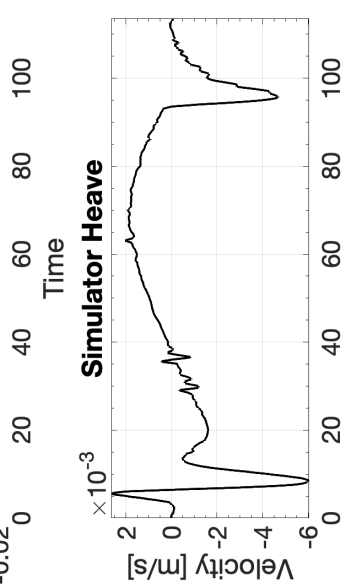

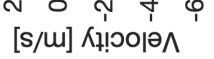

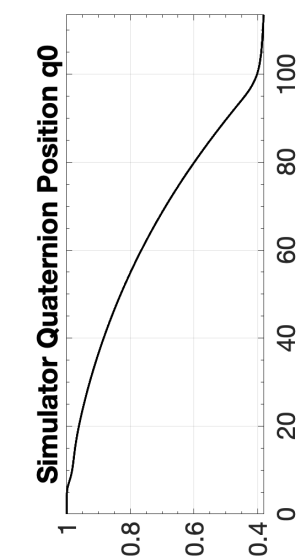

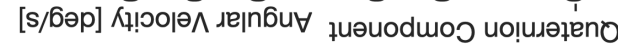

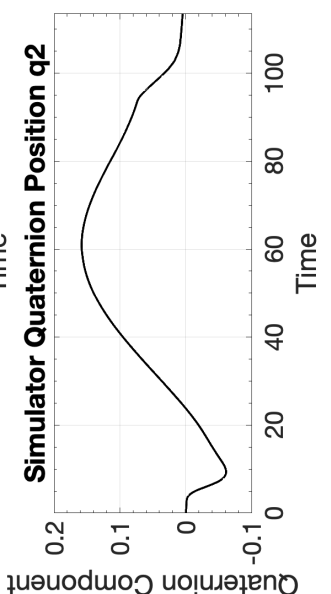

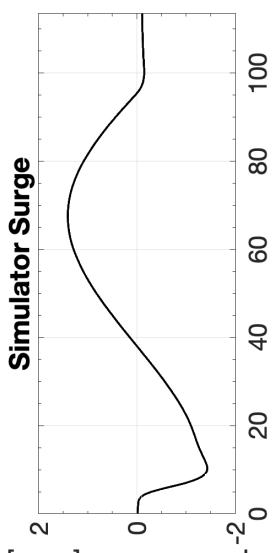

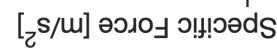

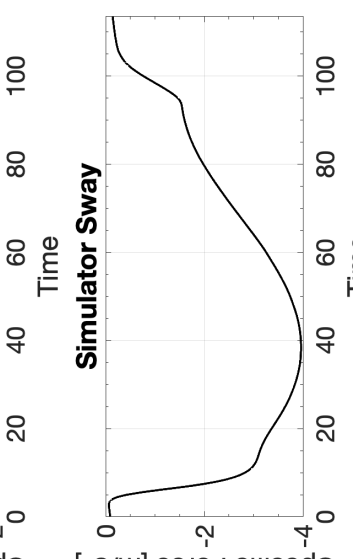

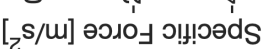

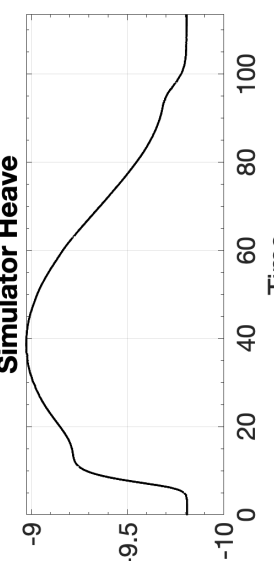

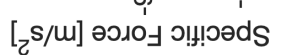

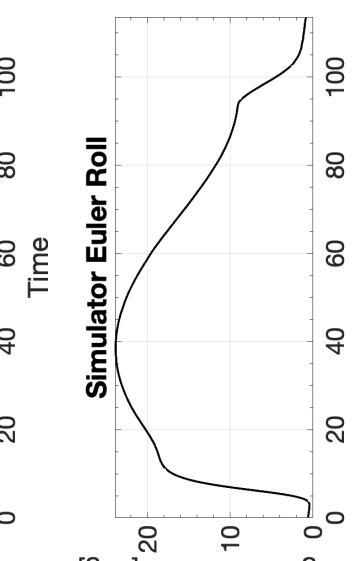

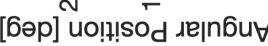

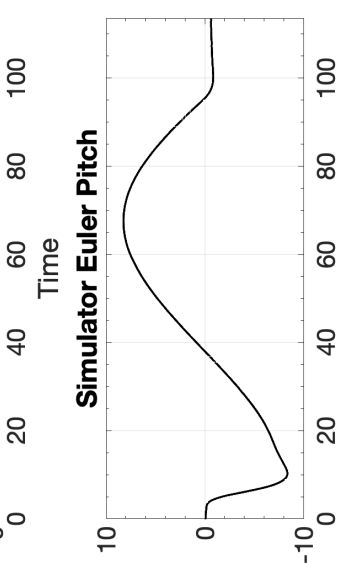

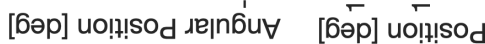

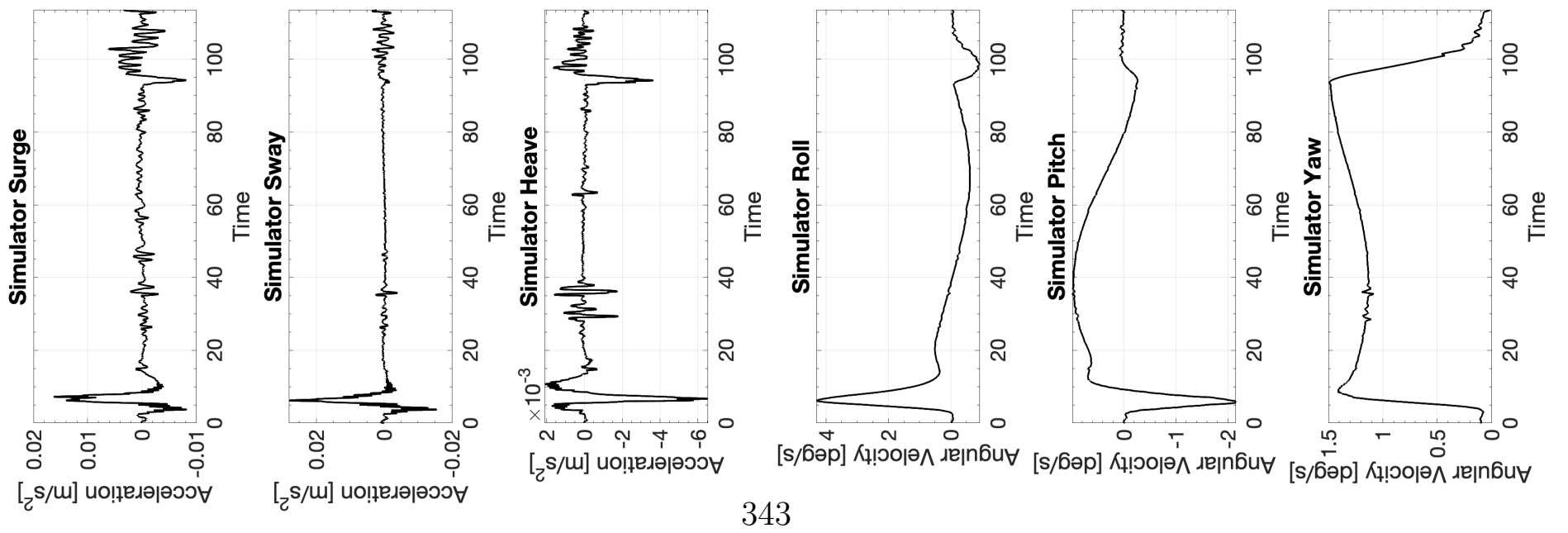




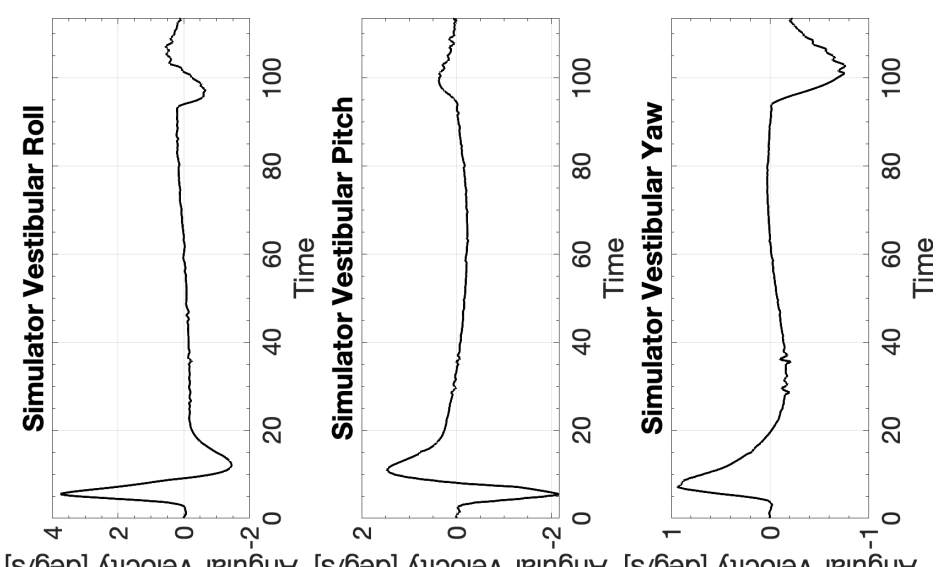

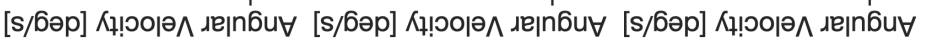

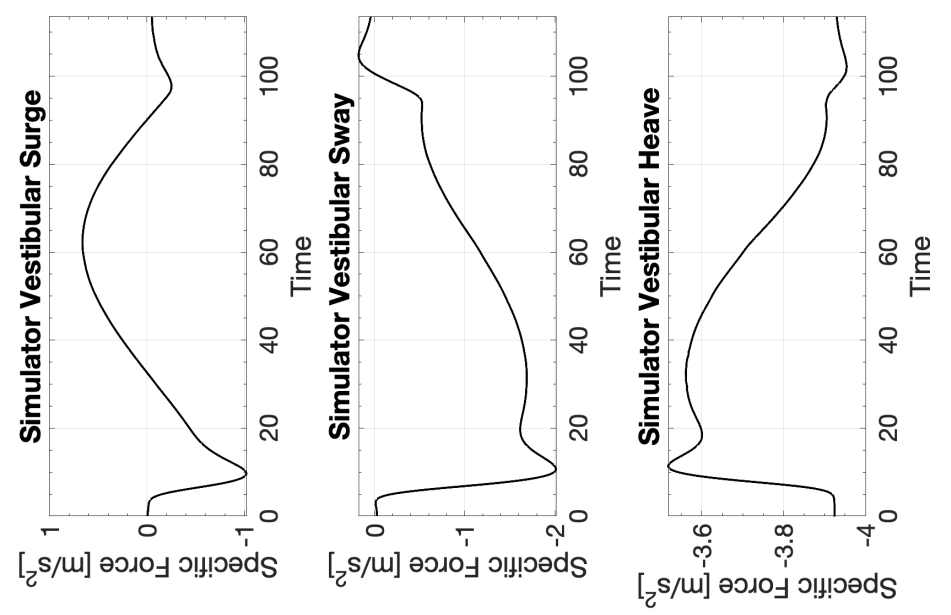




\section{D.1.2 CW2 Cessna 172 Coordinated Turn}
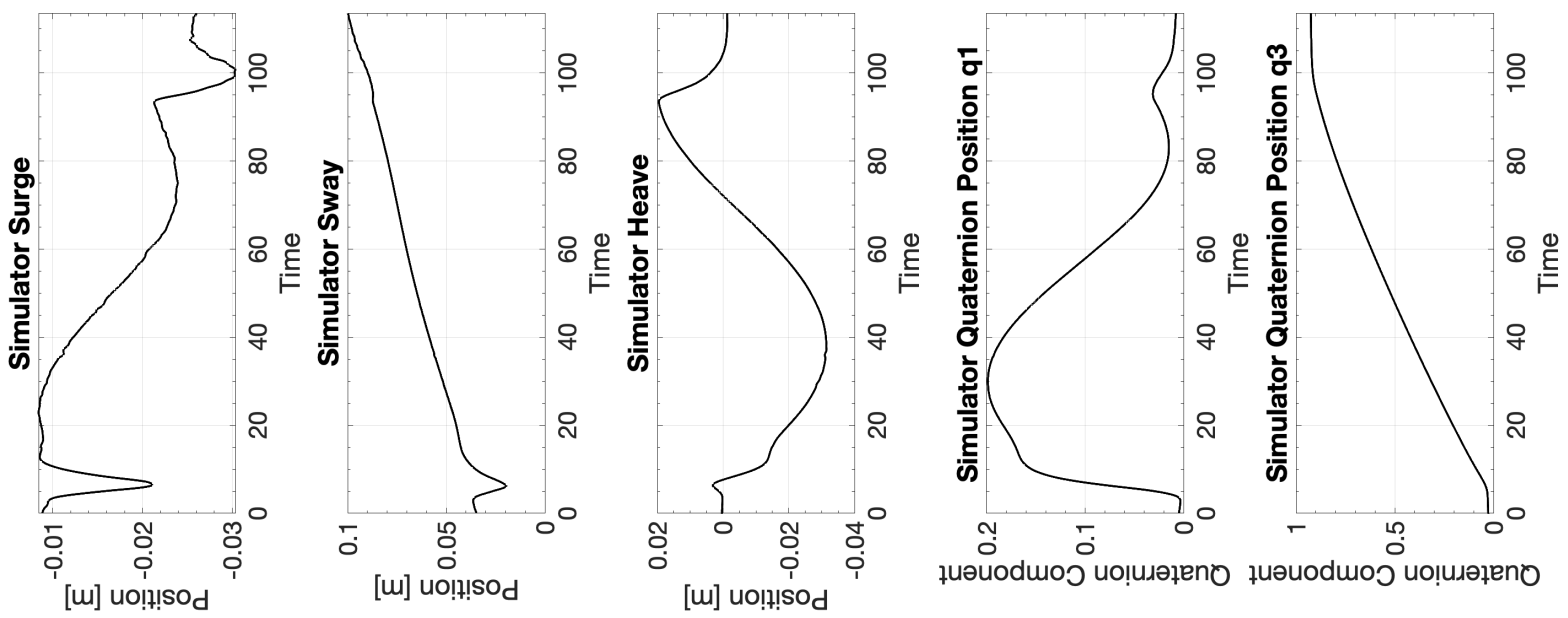

[w] uo!n! $\mathrm{SO}_{\mathrm{d}}$

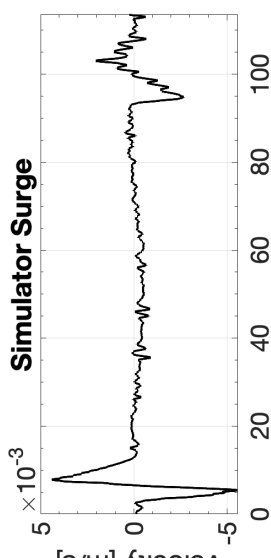

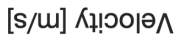

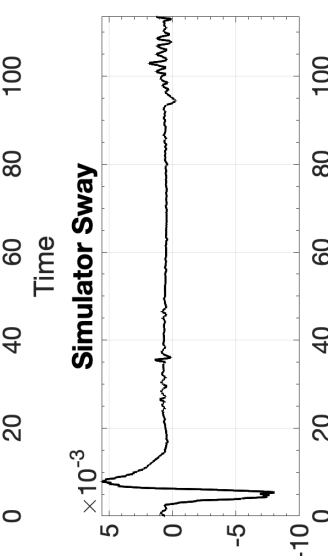

[s/u] Кł! Юо|ә

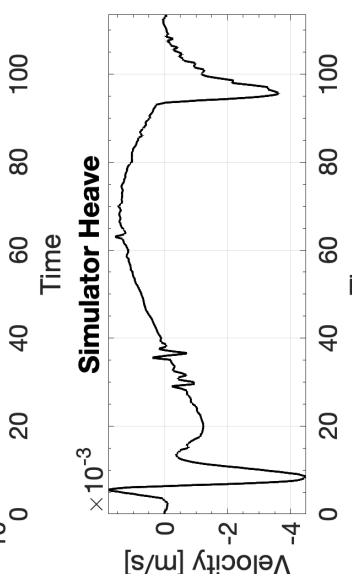

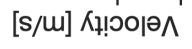
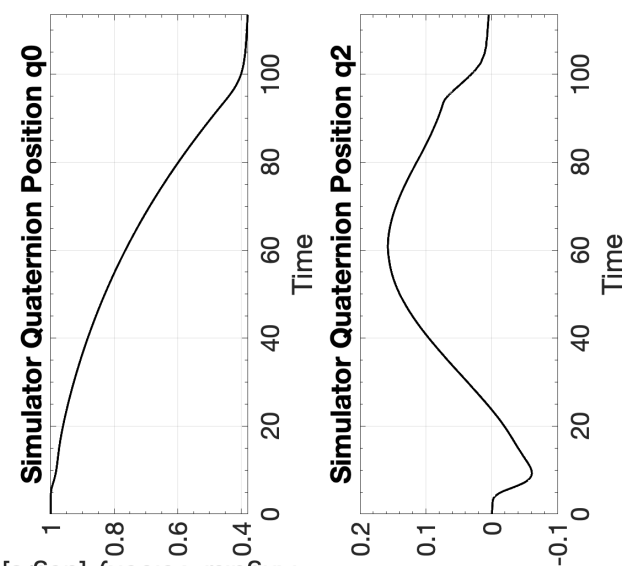

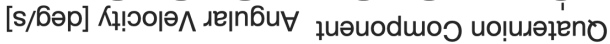

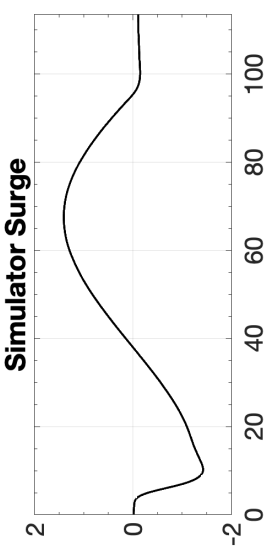

$\left[\begin{array}{l}5 \\ {[2 / m]}\end{array}\right.$

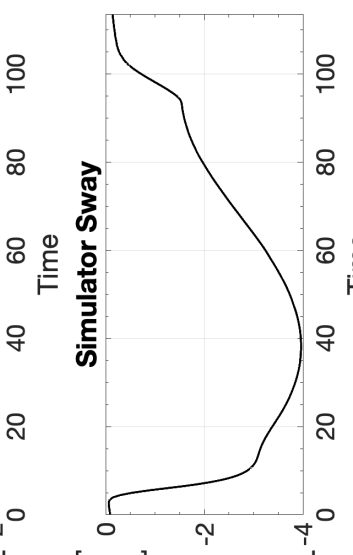

[

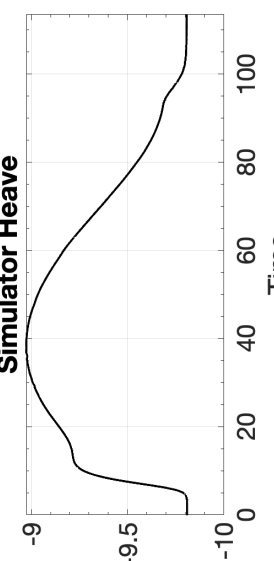

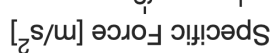

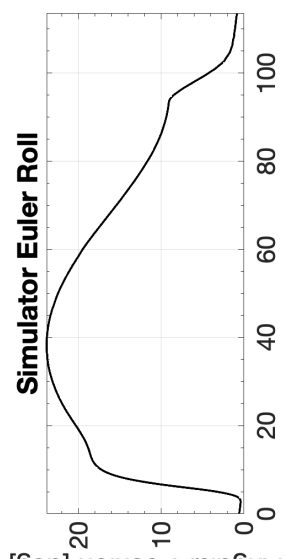

[бəp] uo!!!

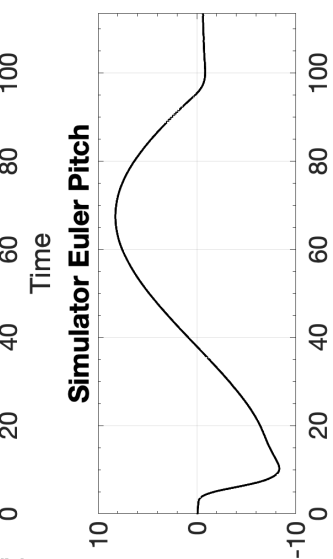

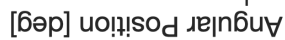

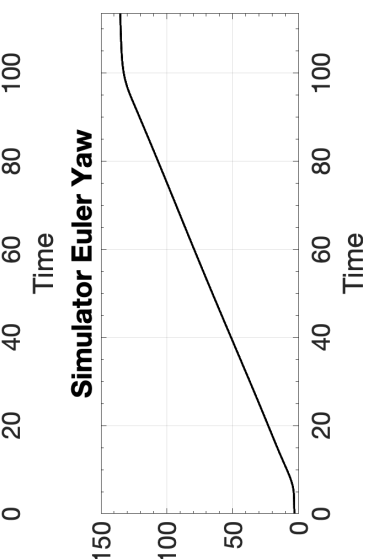

[6ә̄] uō!!!sod

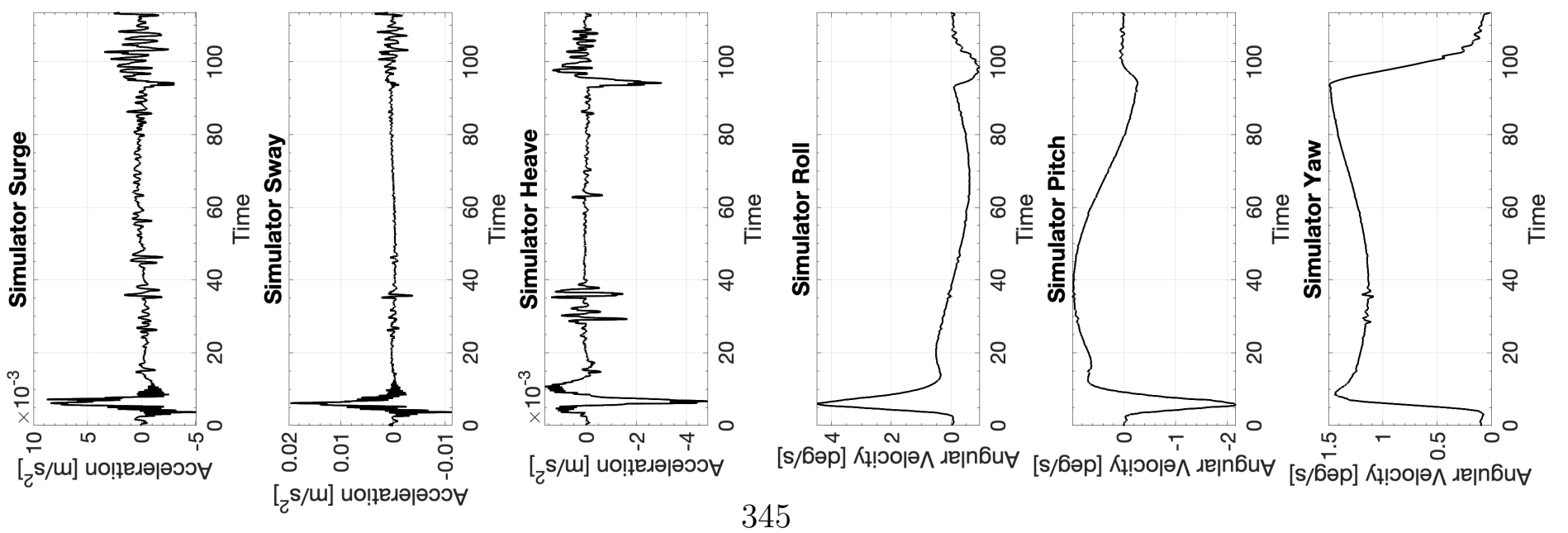




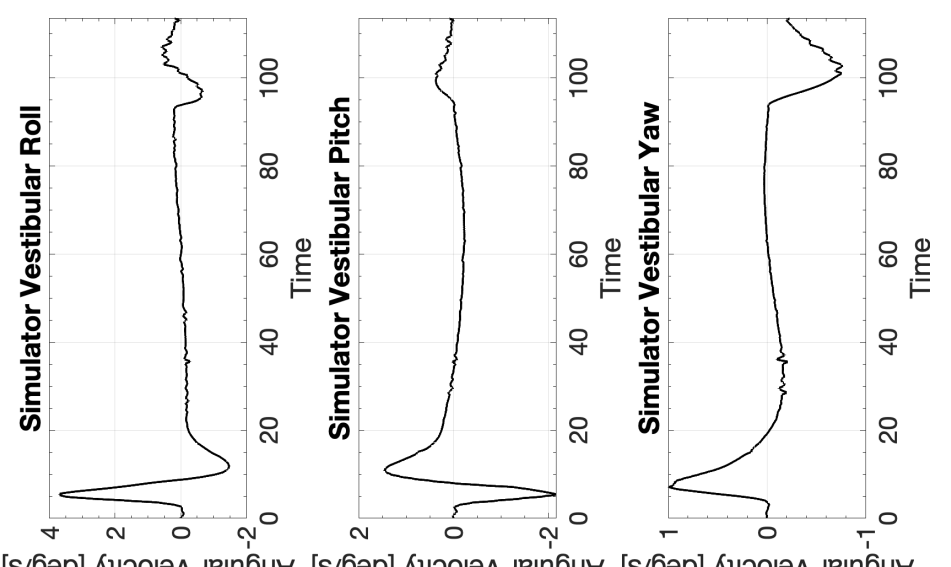

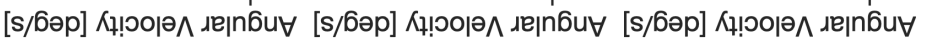

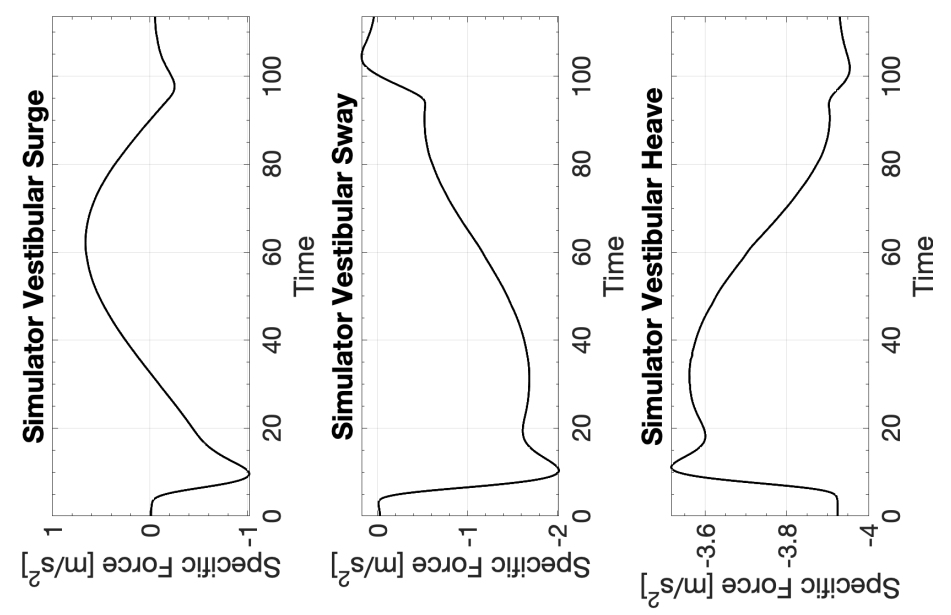




\section{D.1.3 CW3 Cessna 172 Coordinated Turn}
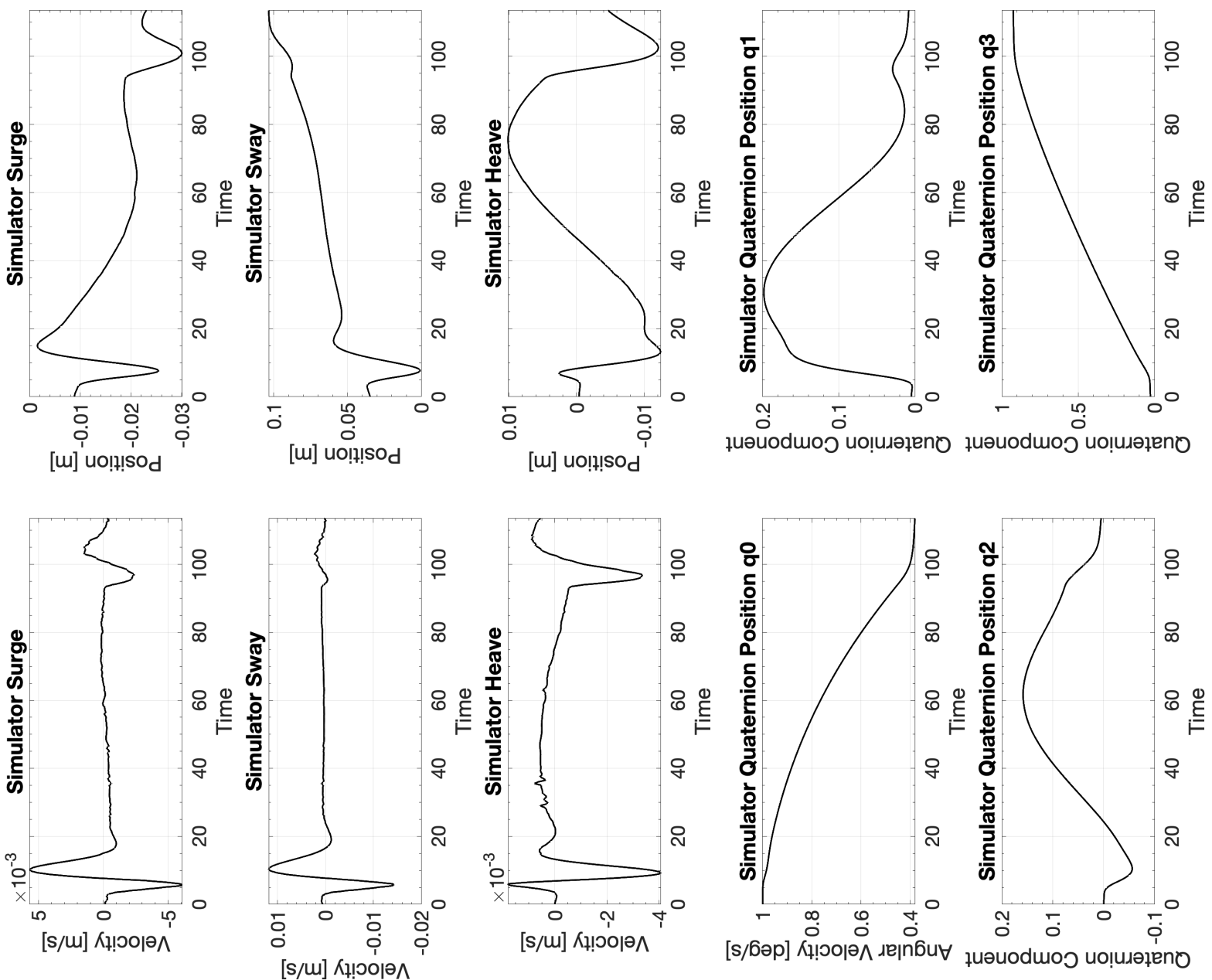

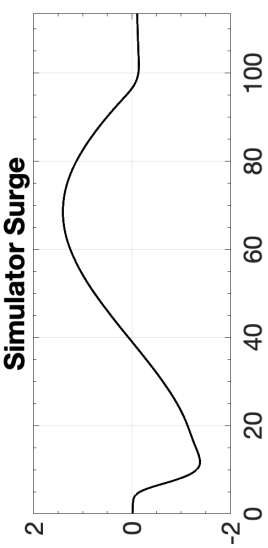

$\left[\begin{array}{l}2 \\ {[2 / m]}\end{array}\right.$

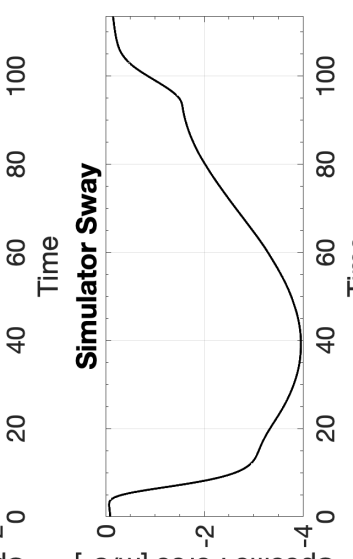

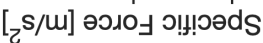

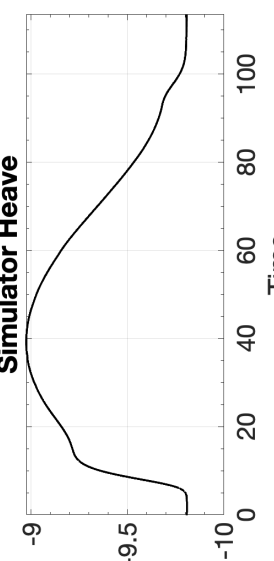

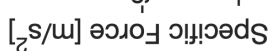

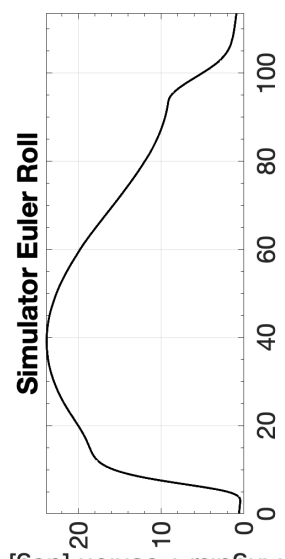

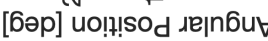

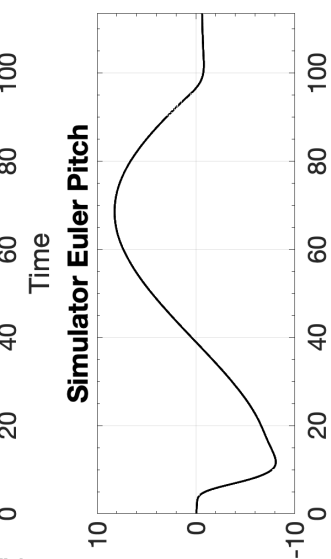

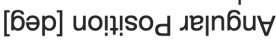

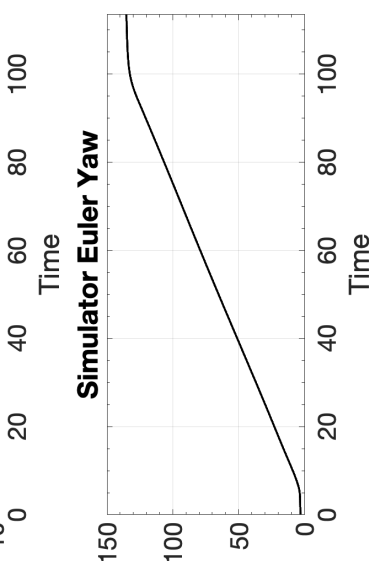

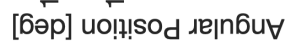

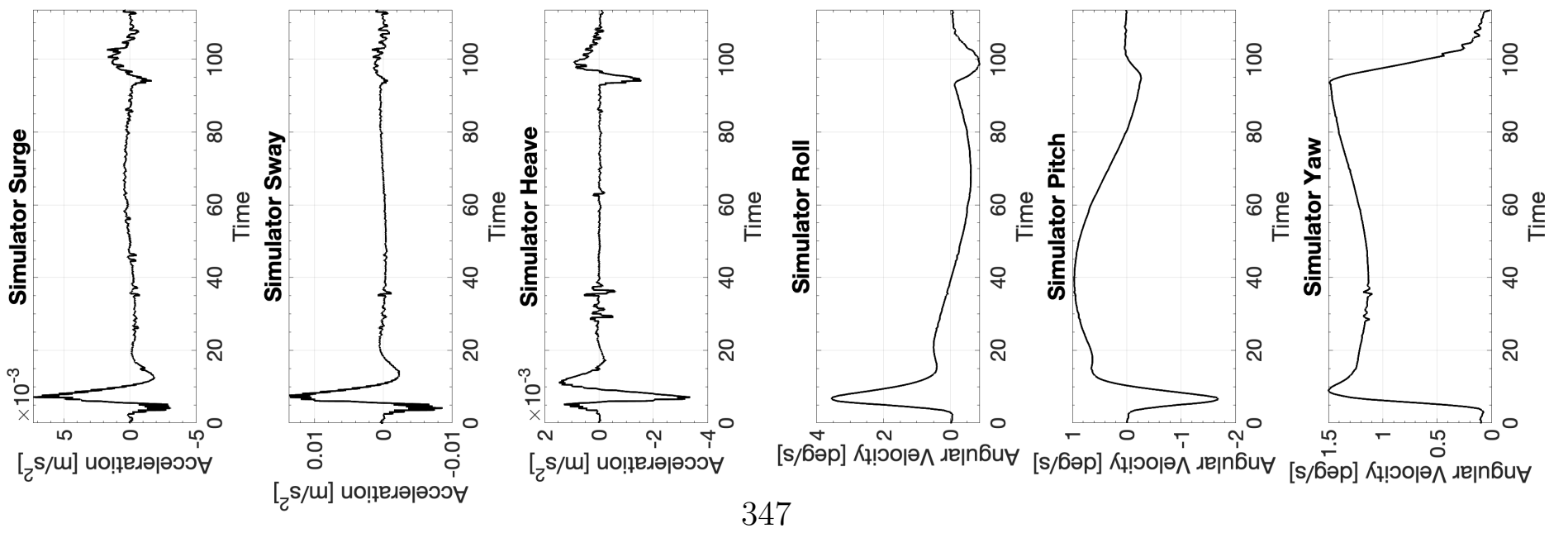




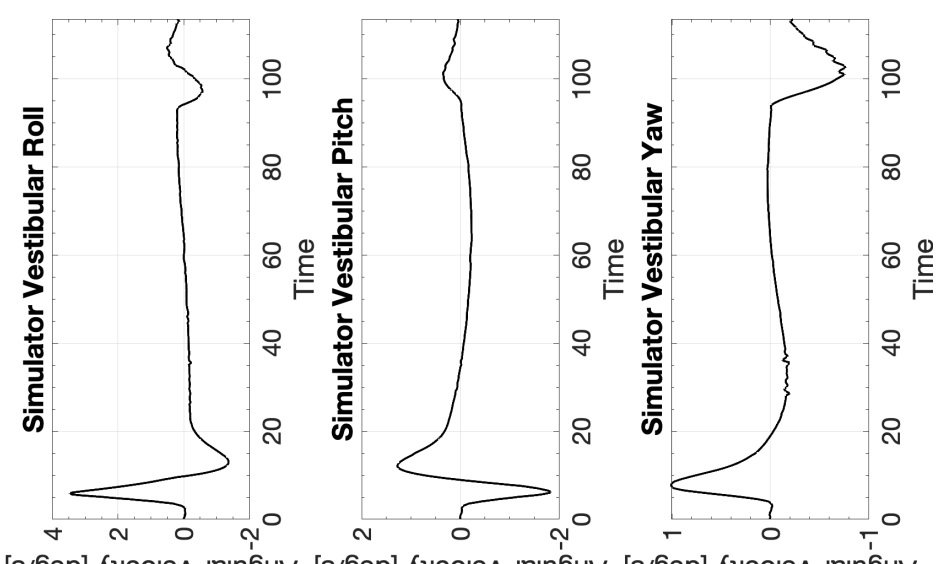

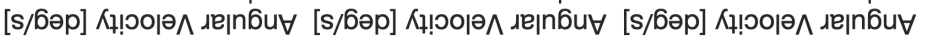

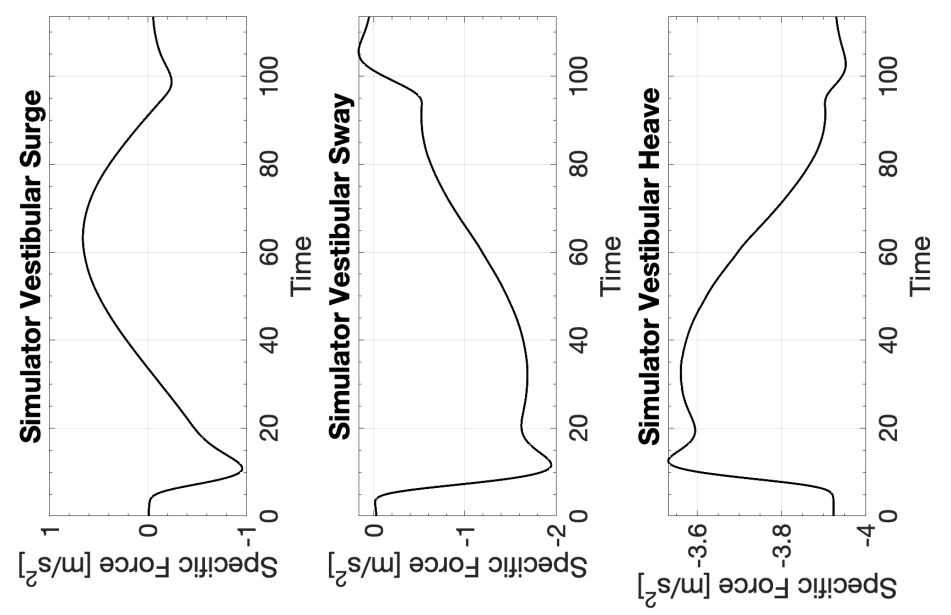




\section{D.2 Cessna 172 Cross-wind Landing}

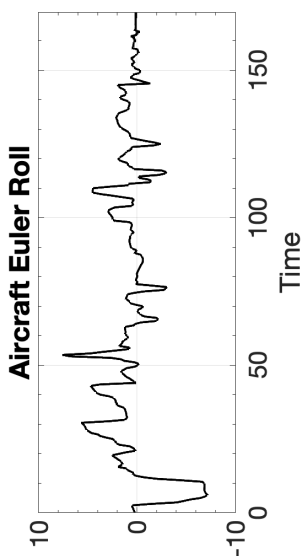

[бәр] uo!!!sod re|nбิ'

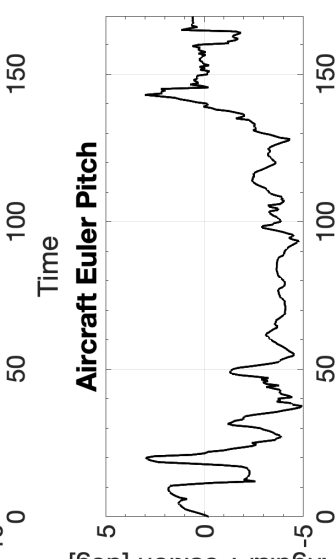

[6әр] uo!!!sod גe|n6u

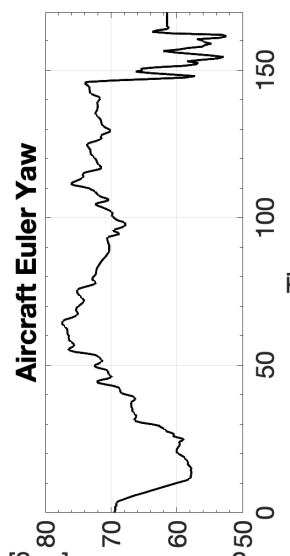

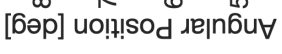
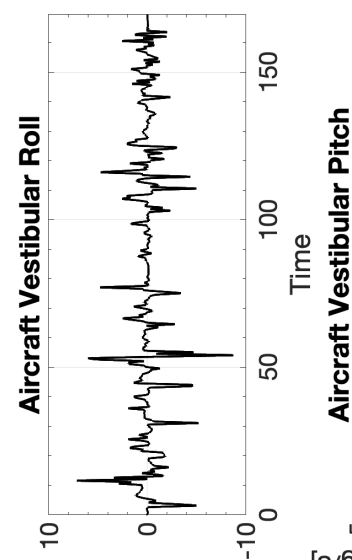

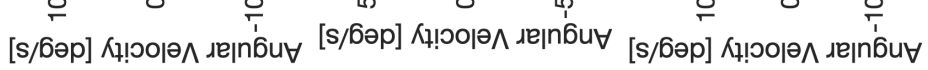

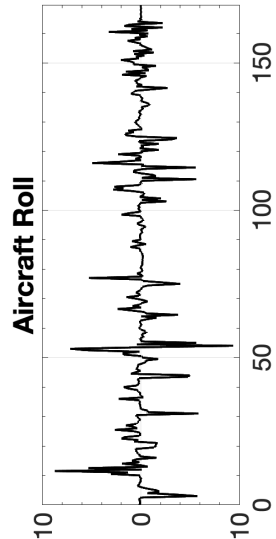

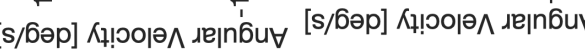
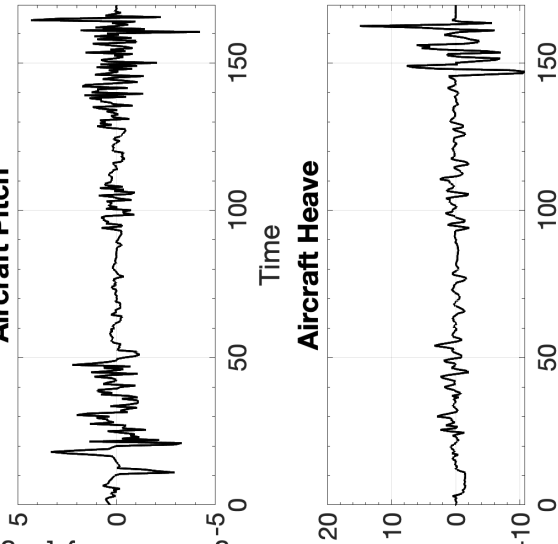

[s/6әр] К! อо
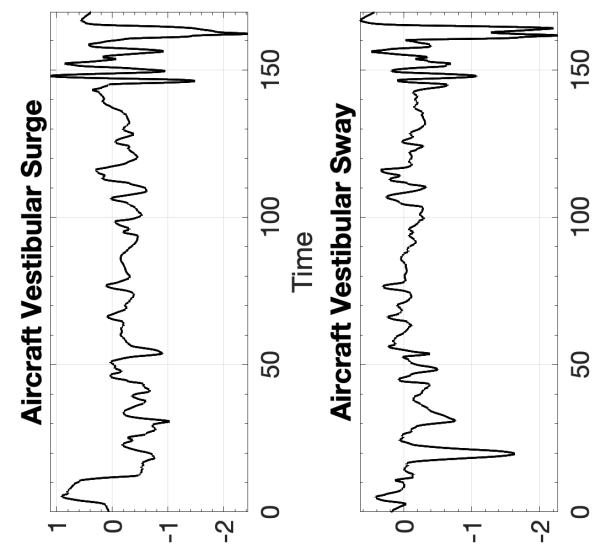

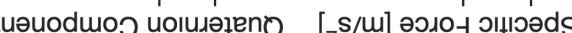

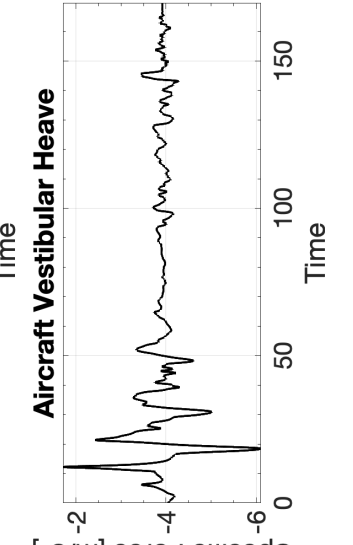

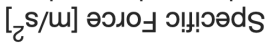

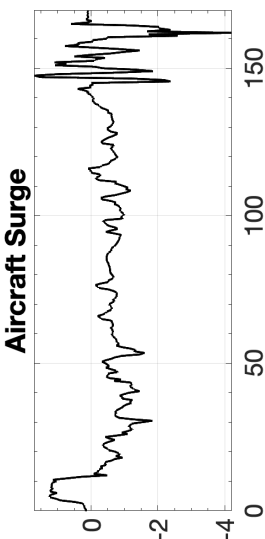

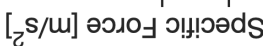

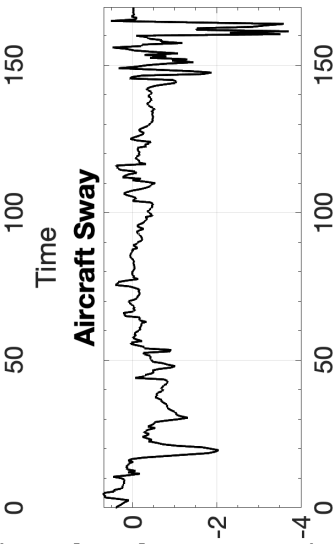

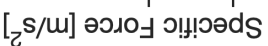

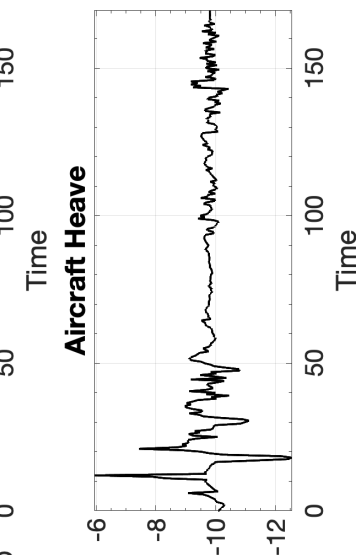

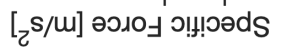

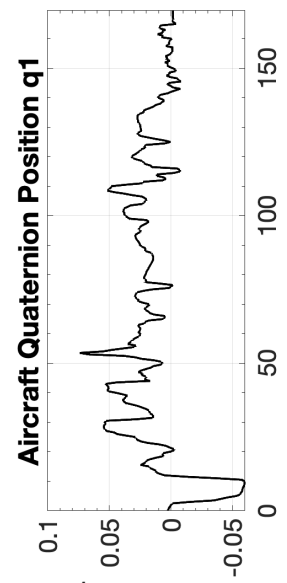

ұuәuodmo uo!̣uəieno

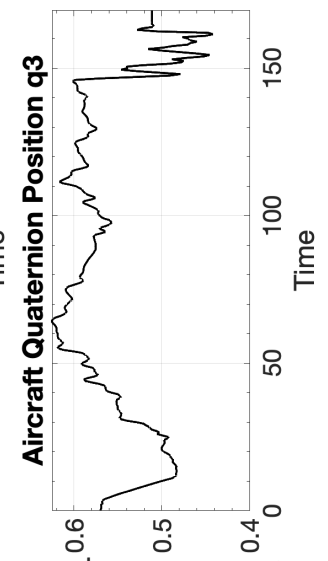

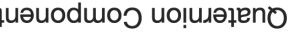

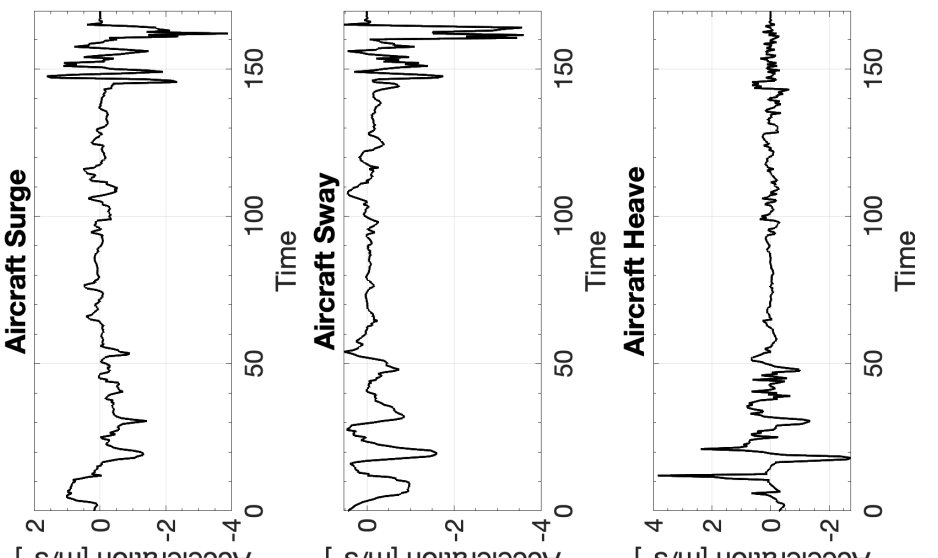

$[\mathrm{c} / \mathrm{L}]$ uо!ฺвләәәоэ

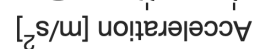

[zs/m] ио!ฺеләәәэว

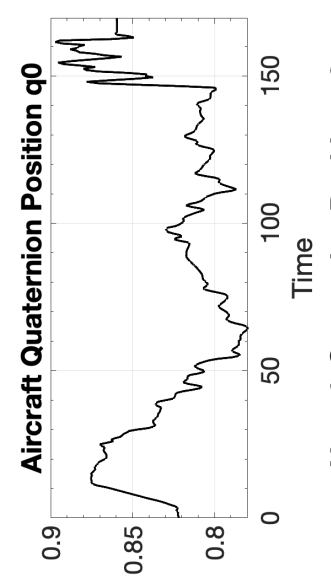

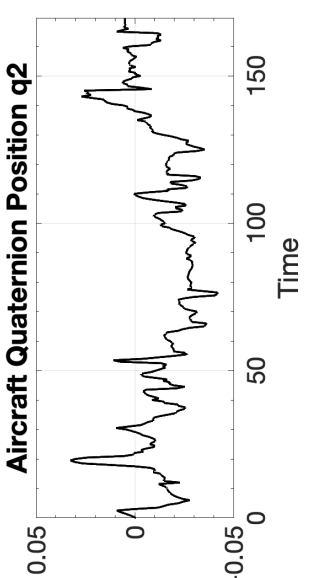

uəuoduos uo!̣uəténo 


\section{D.2.1 CW1 Cessna 172 Cross-wind Landing}

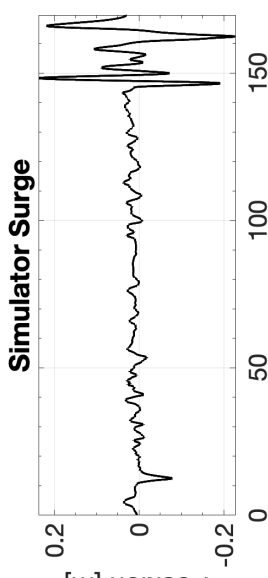

[m] uo!!!!sod

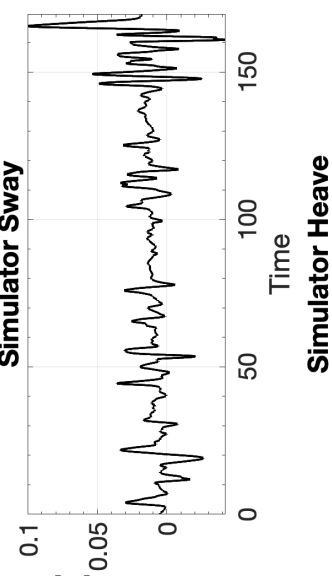

[w] uolp!sod

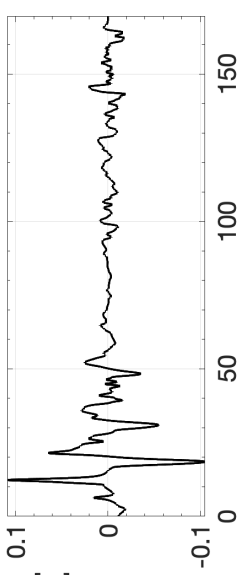

[w] uo!t!sod

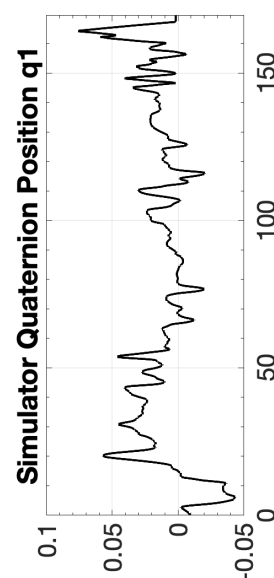

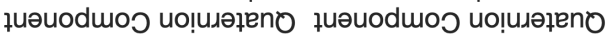
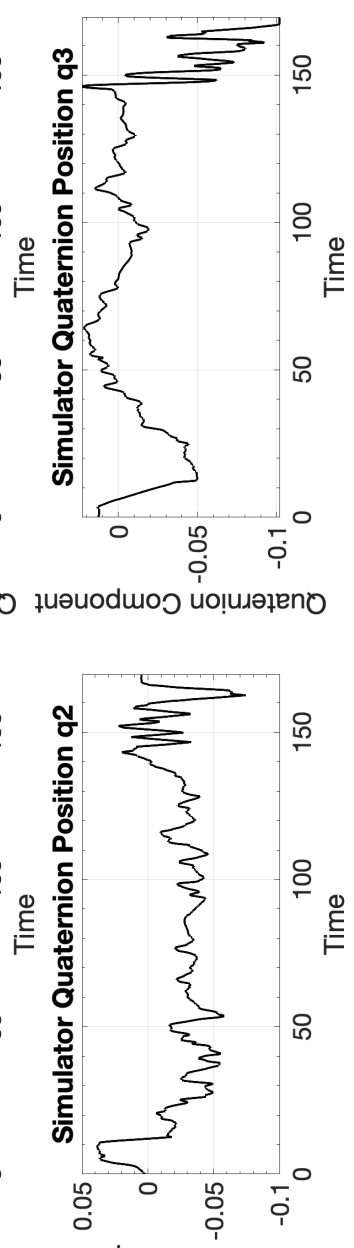

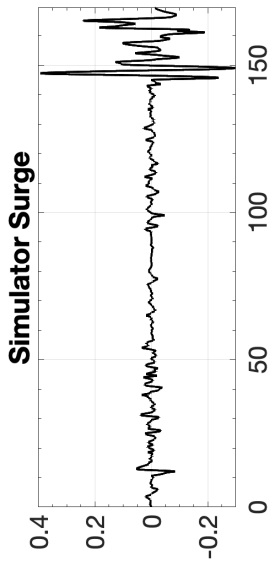

[s/u] Кұ!юор夫

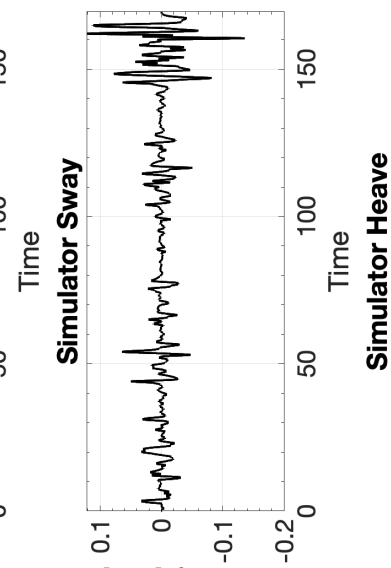

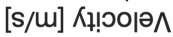

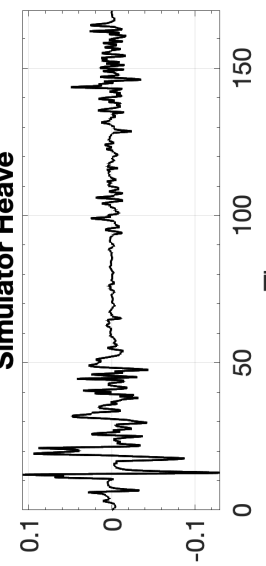

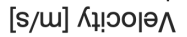

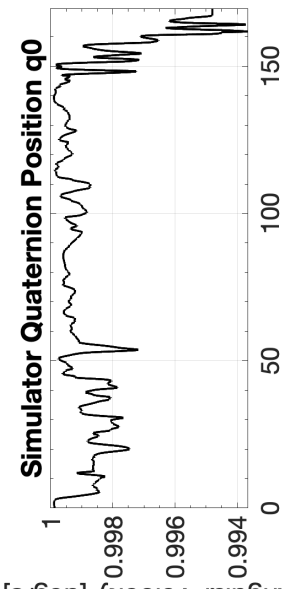

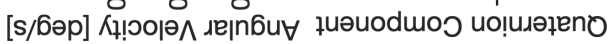

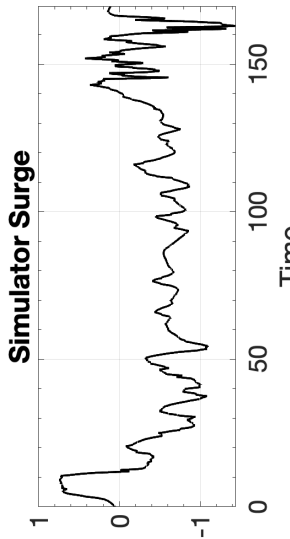

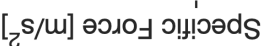

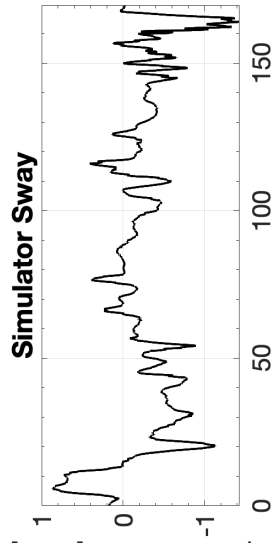

[

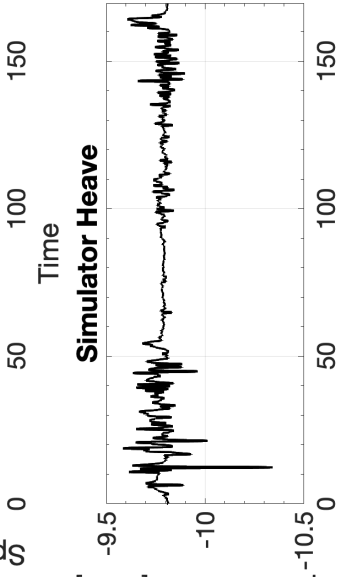

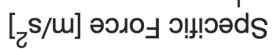

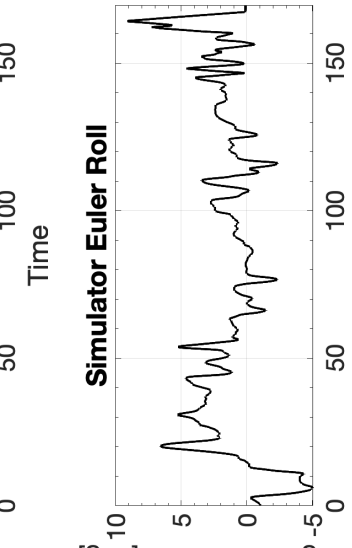

[6әр] uo!̣!!sod גeןnбü

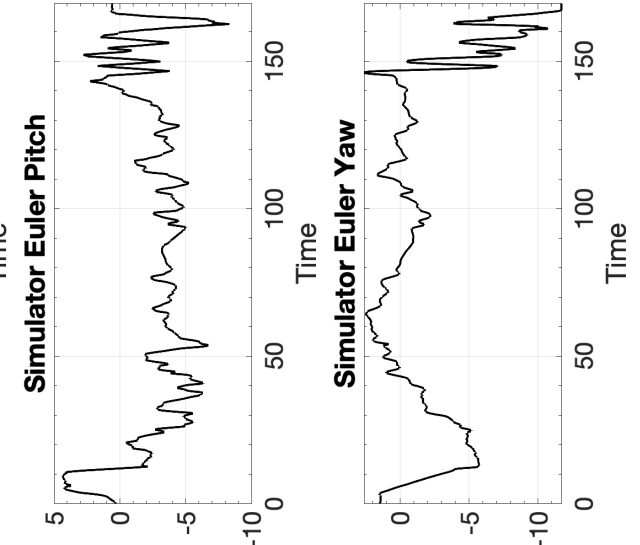

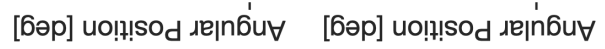

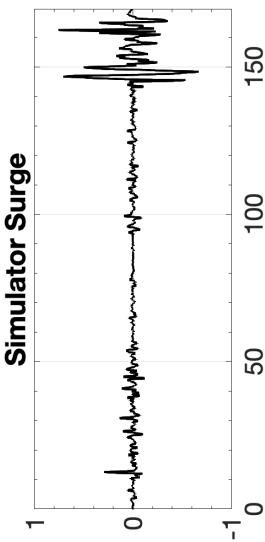

$[\mathrm{c} / \mathrm{L}]$ uо!ן

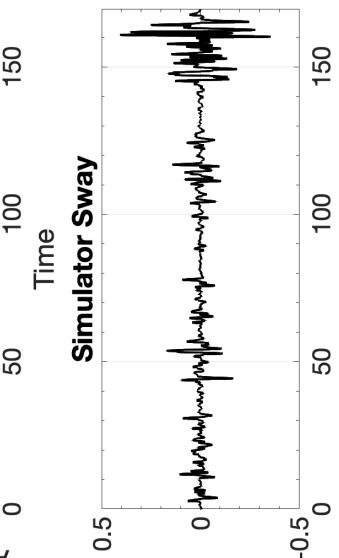

$[\mathrm{s} / \mathrm{m}]$ ио!ฺв

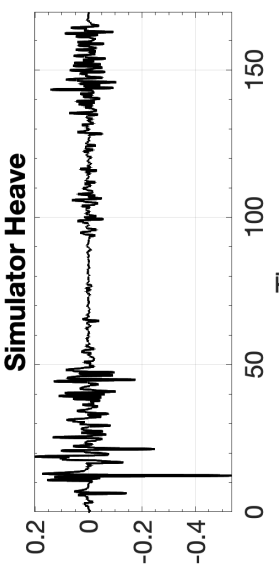

$[\mathrm{s} / \mathrm{m}]$ ио!ฺвләәәэว

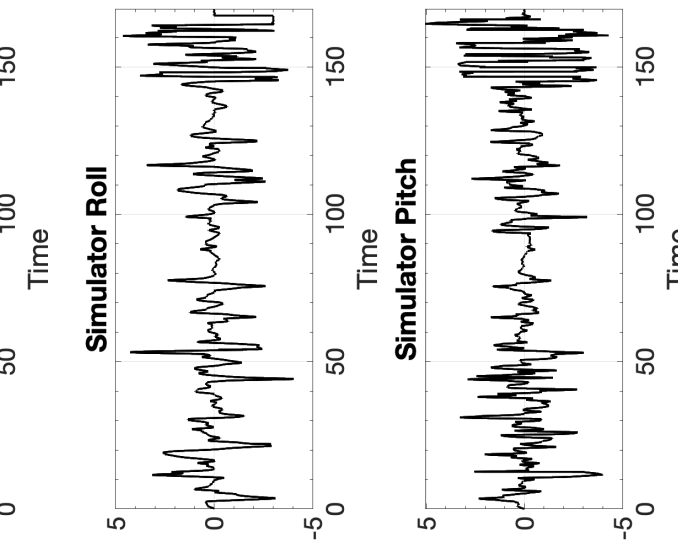

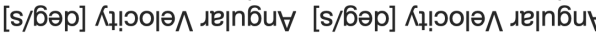

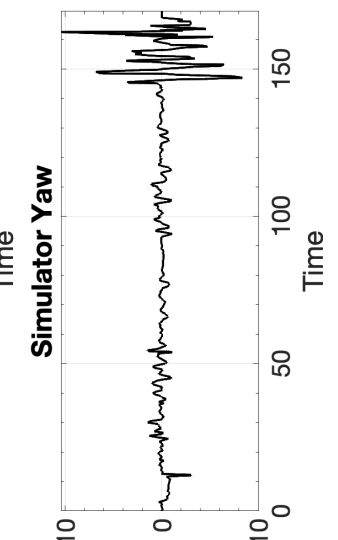

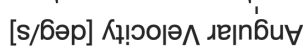



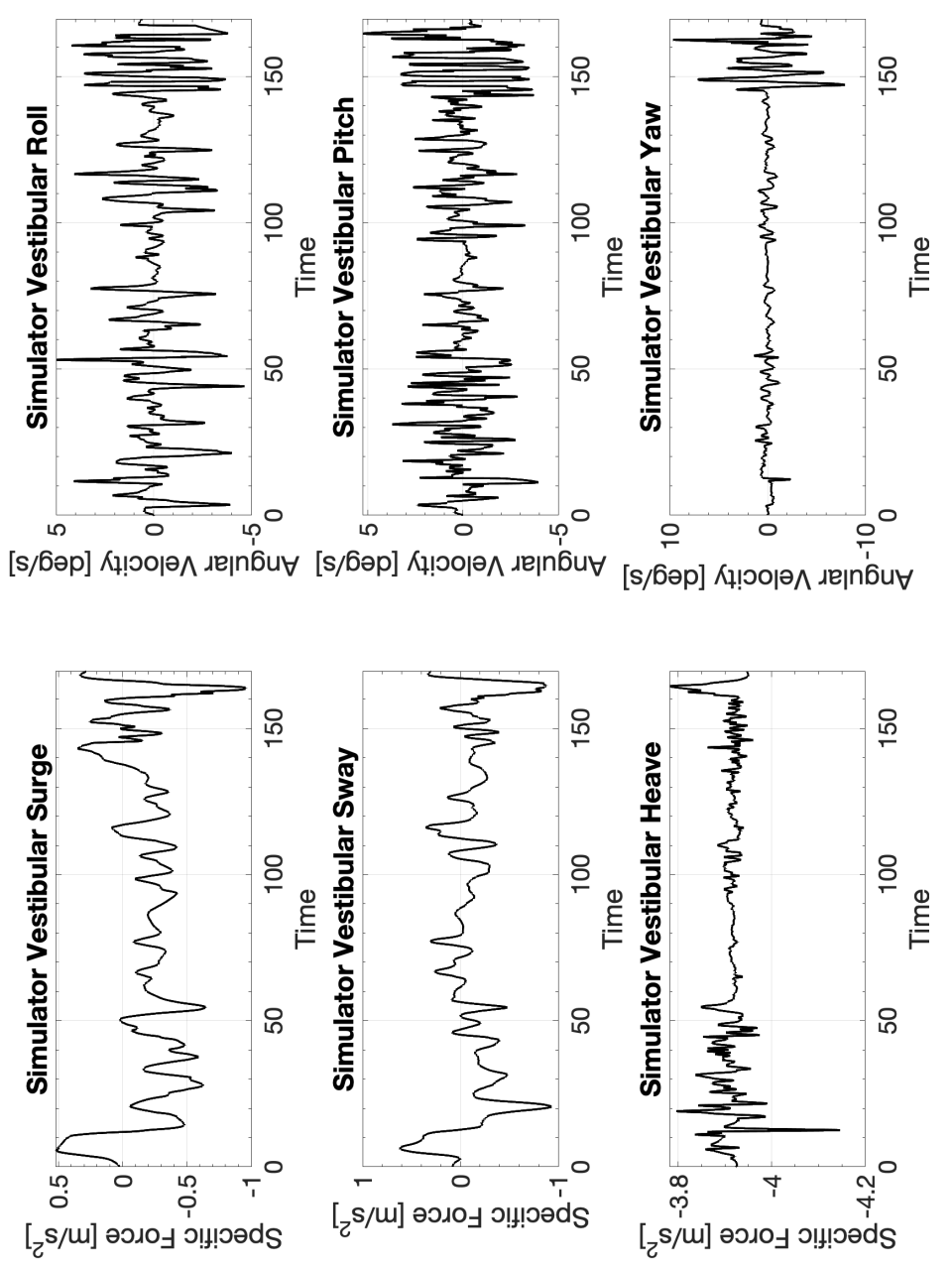


\section{D.2.2 CW2 Cessna 172 Cross-wind Landing}

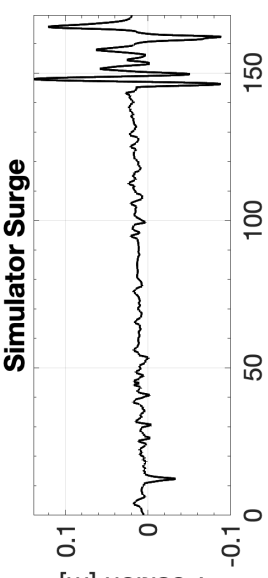

[m] uo!!!!sod

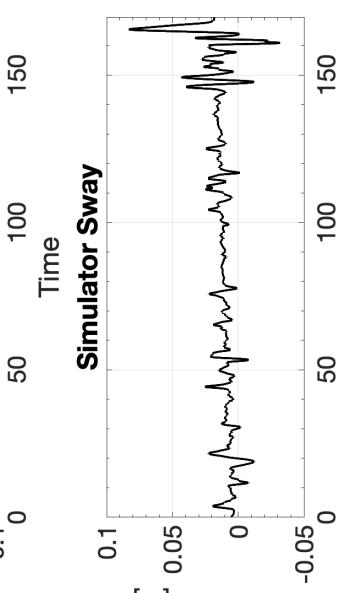

[w] uo!n! sod

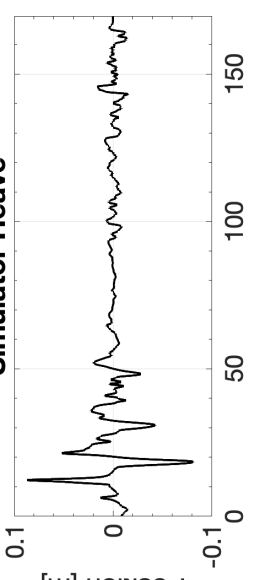

[u] uo!!!sod

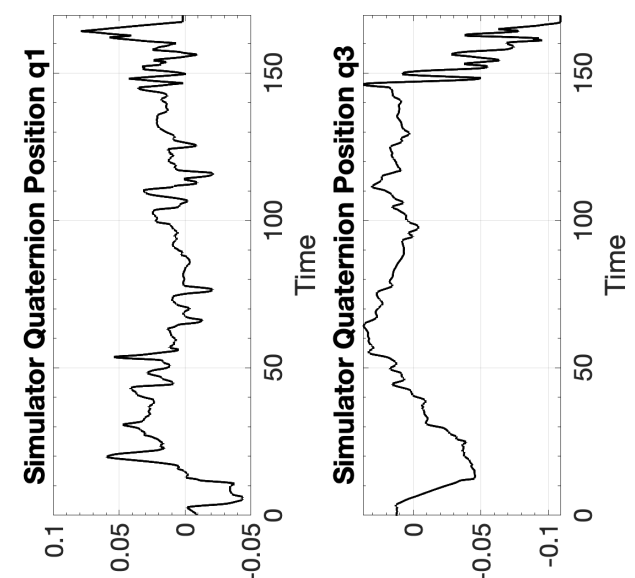

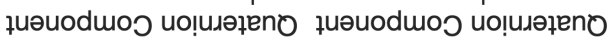

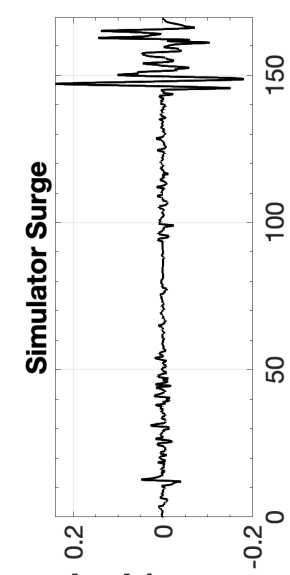

[s/m] Кұ!००|ә^

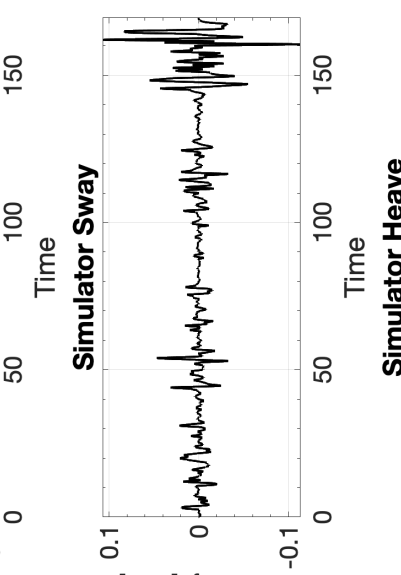

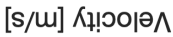

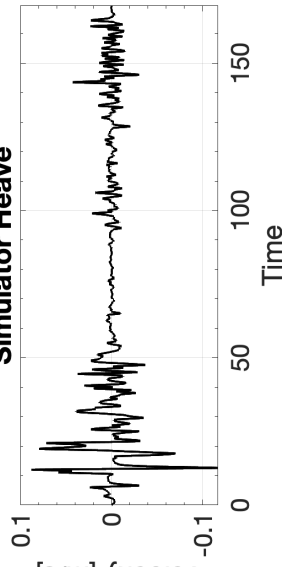

[s/m] К!

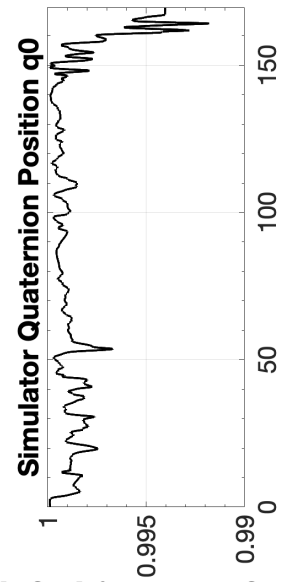

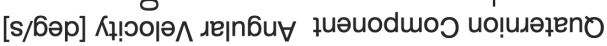

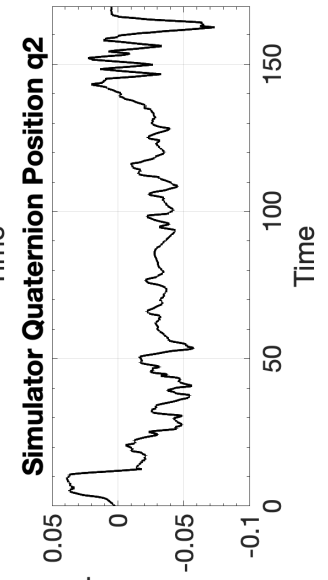

$\stackrel{\oplus}{\underline{E}}$

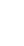

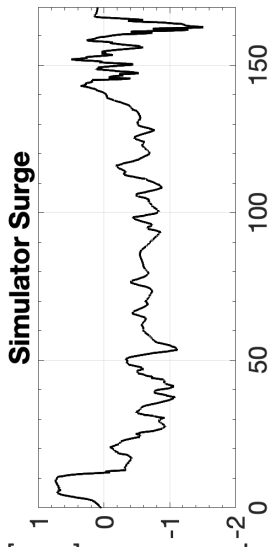

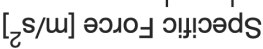

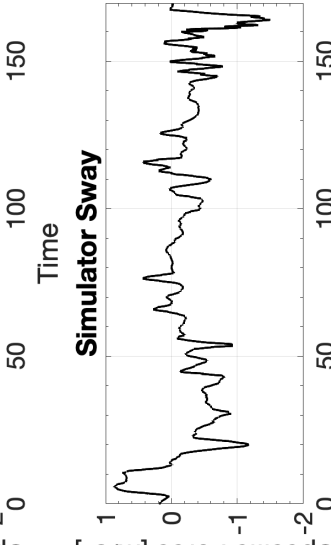

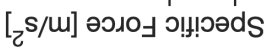

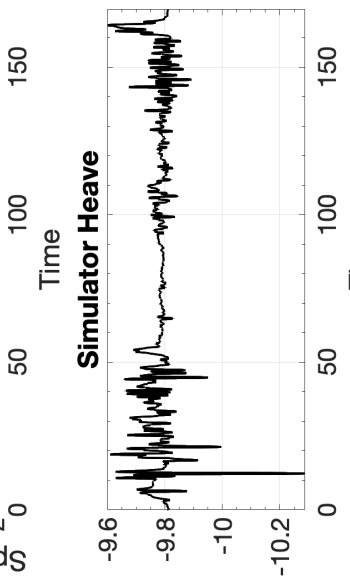

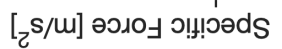

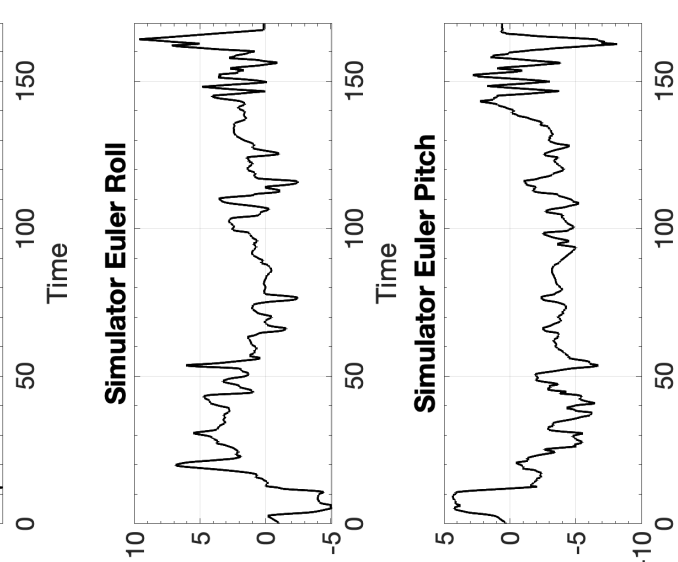

[бәр] uo!!!!sod גe|nбuY

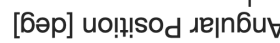

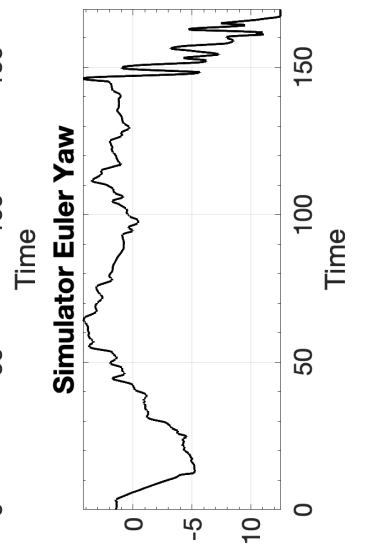

[6әр] uo!!!sod

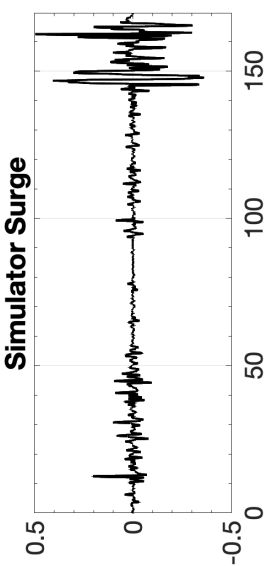

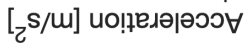

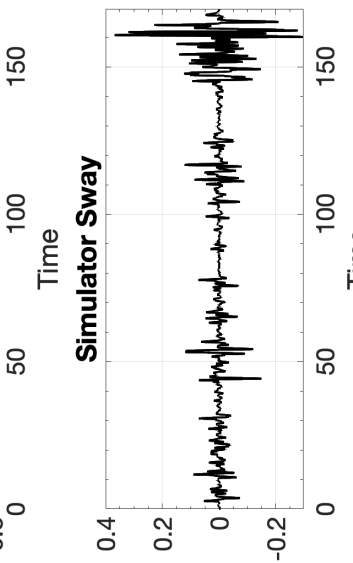

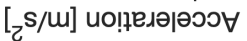

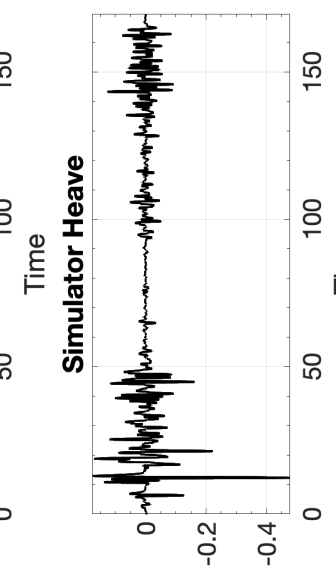

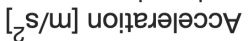

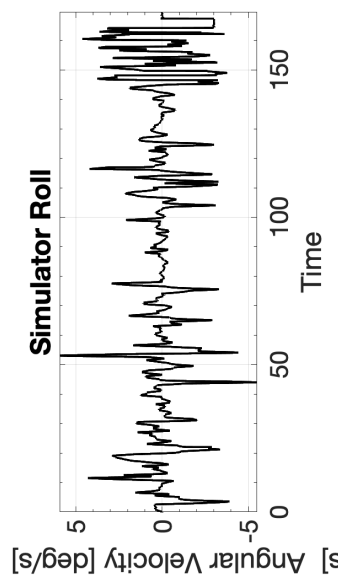

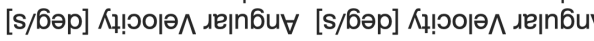
352 

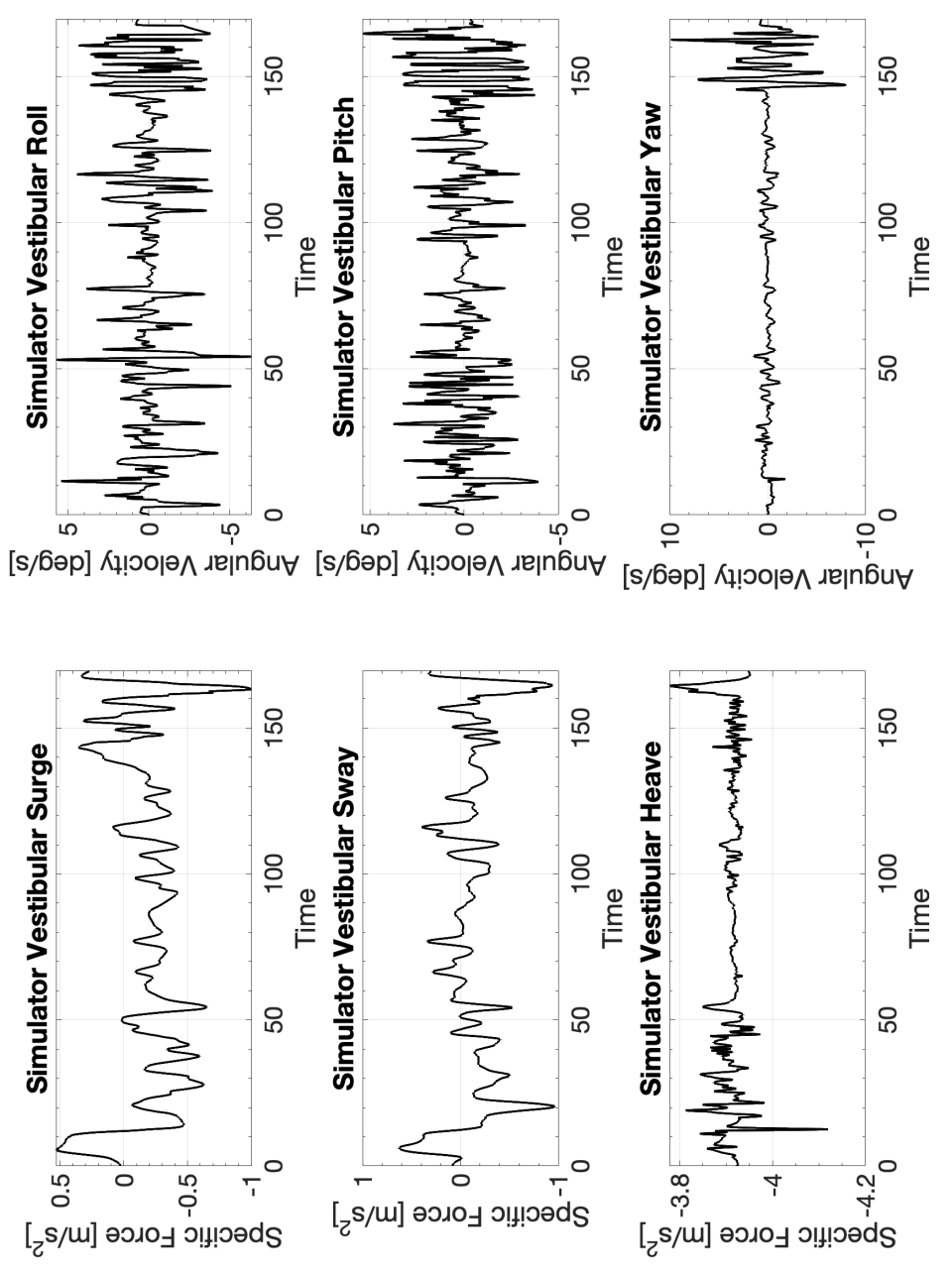


\section{D.2.3 CW3 Cessna 172 Cross-wind Landing}

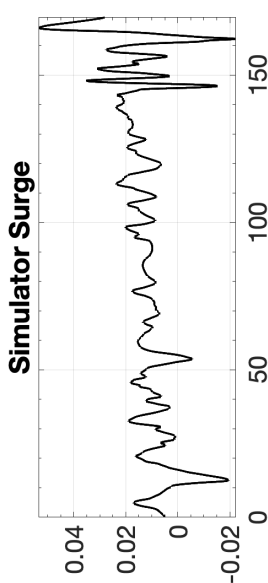

[w] uo!!!sod

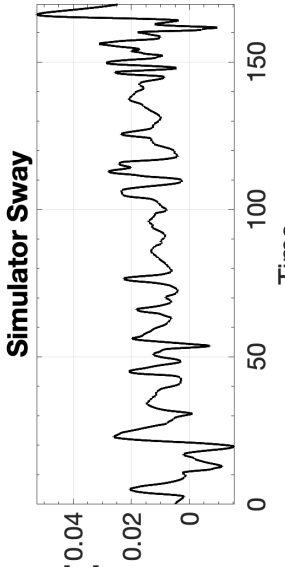

[u] uo!?!sod

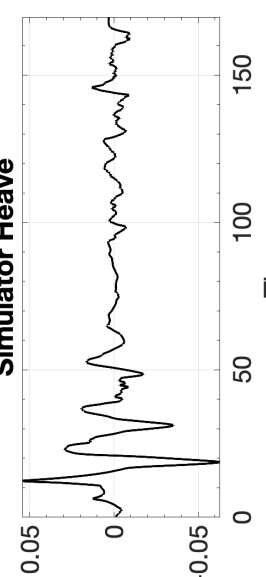

[m] uol!! sod

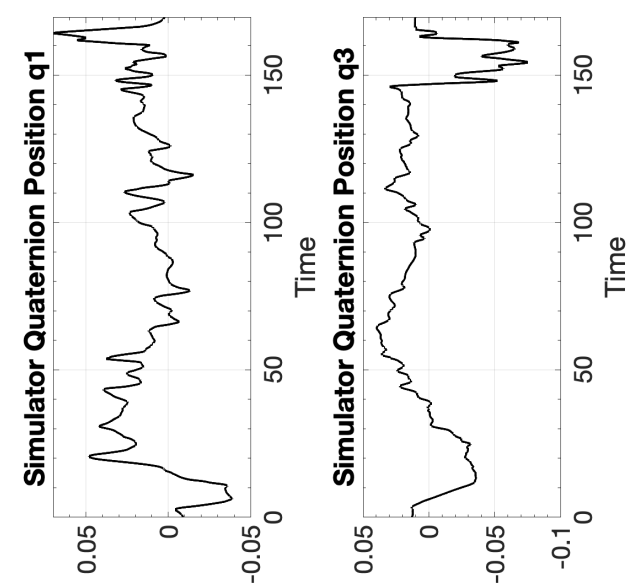

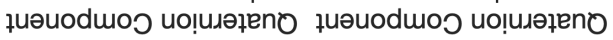

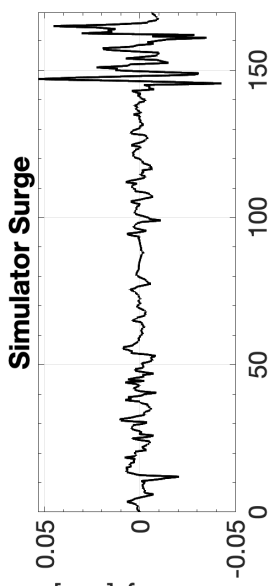

[s/u] Кұ!юо|ә

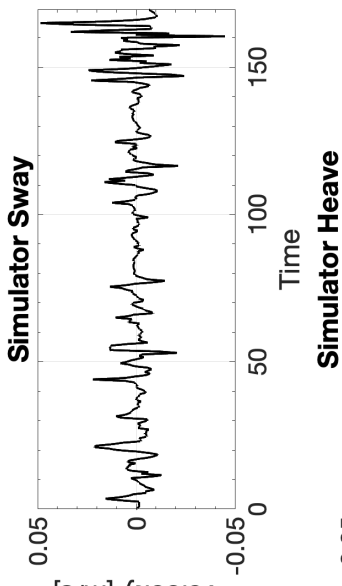

[s/u] ᄉұ!৩о夫 ^

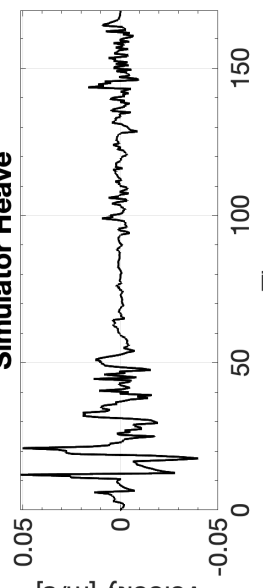

[s/u] Кұ! оə $\Lambda$

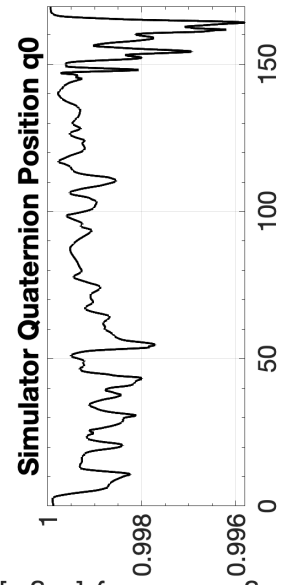

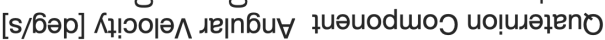

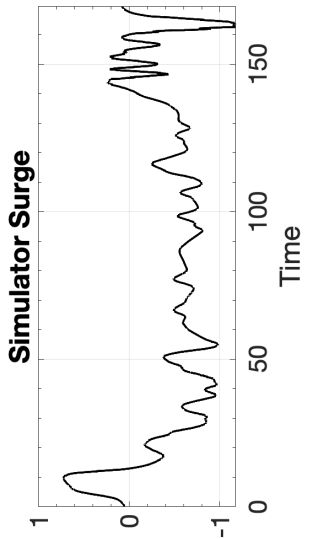

[

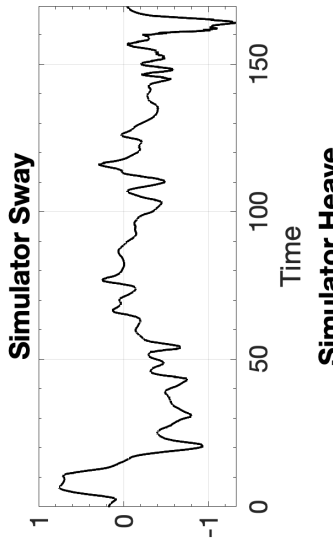

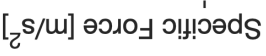

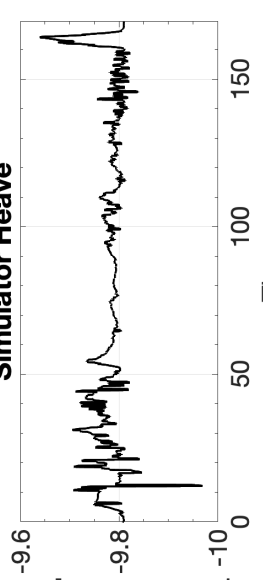

[

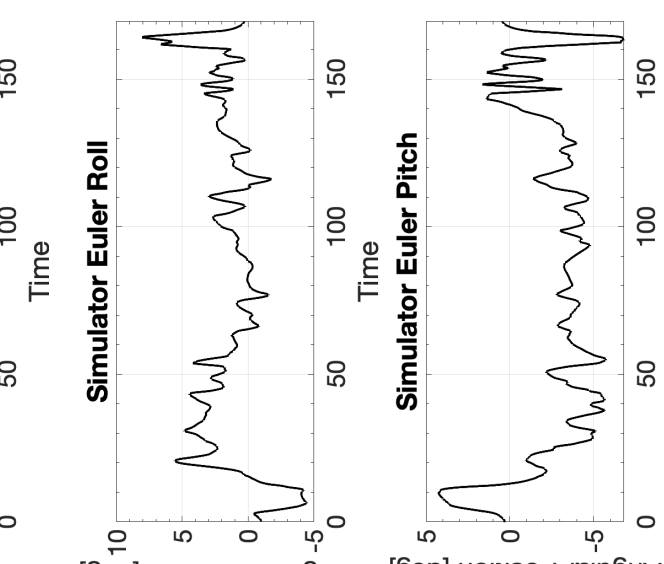

[бәр] uo!?!sod

[6әp] uolp!!sod d

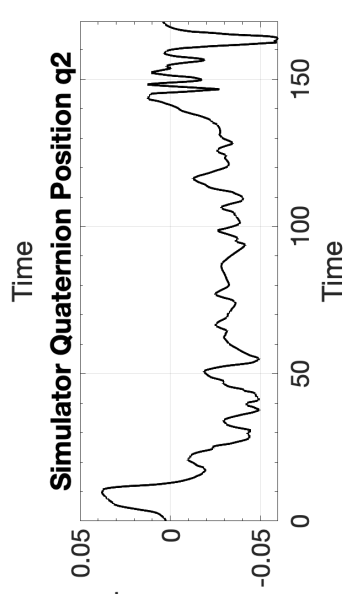

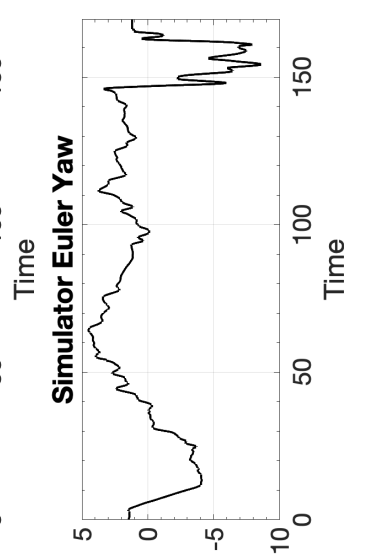

[6әр] uo!ı!sod

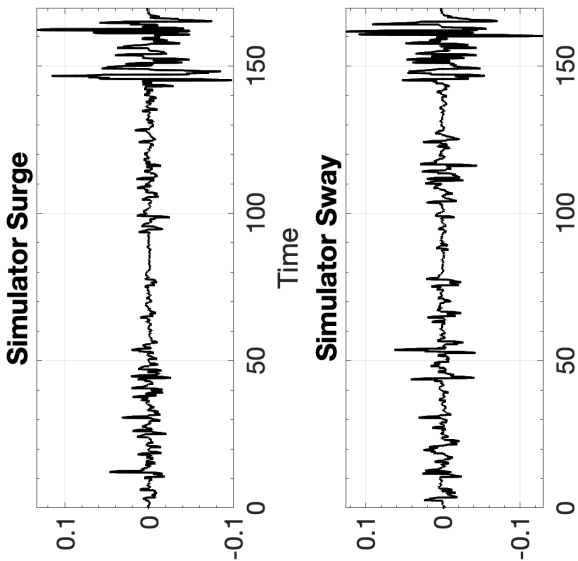

[z/m] ио!ฺеләрәэว

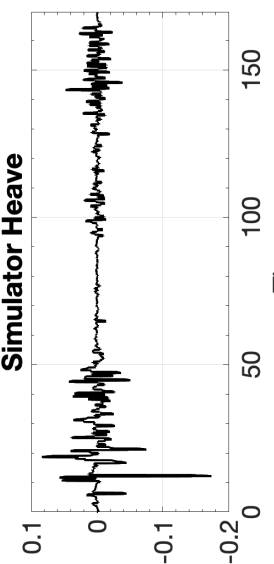

[s/m] ио!ฺедә|әэว
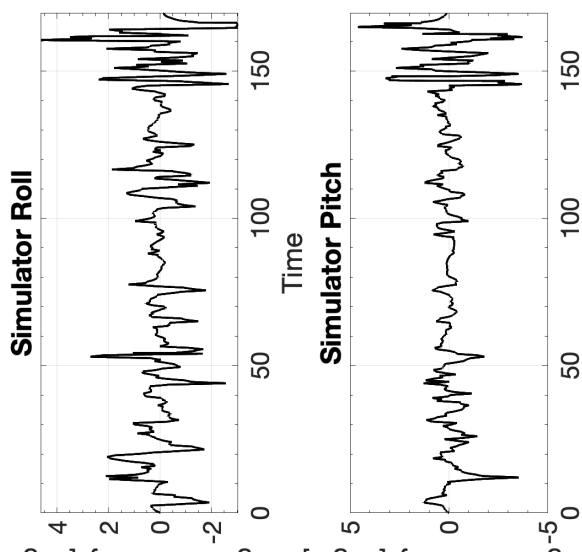

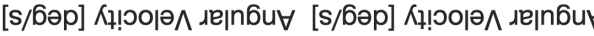
354 

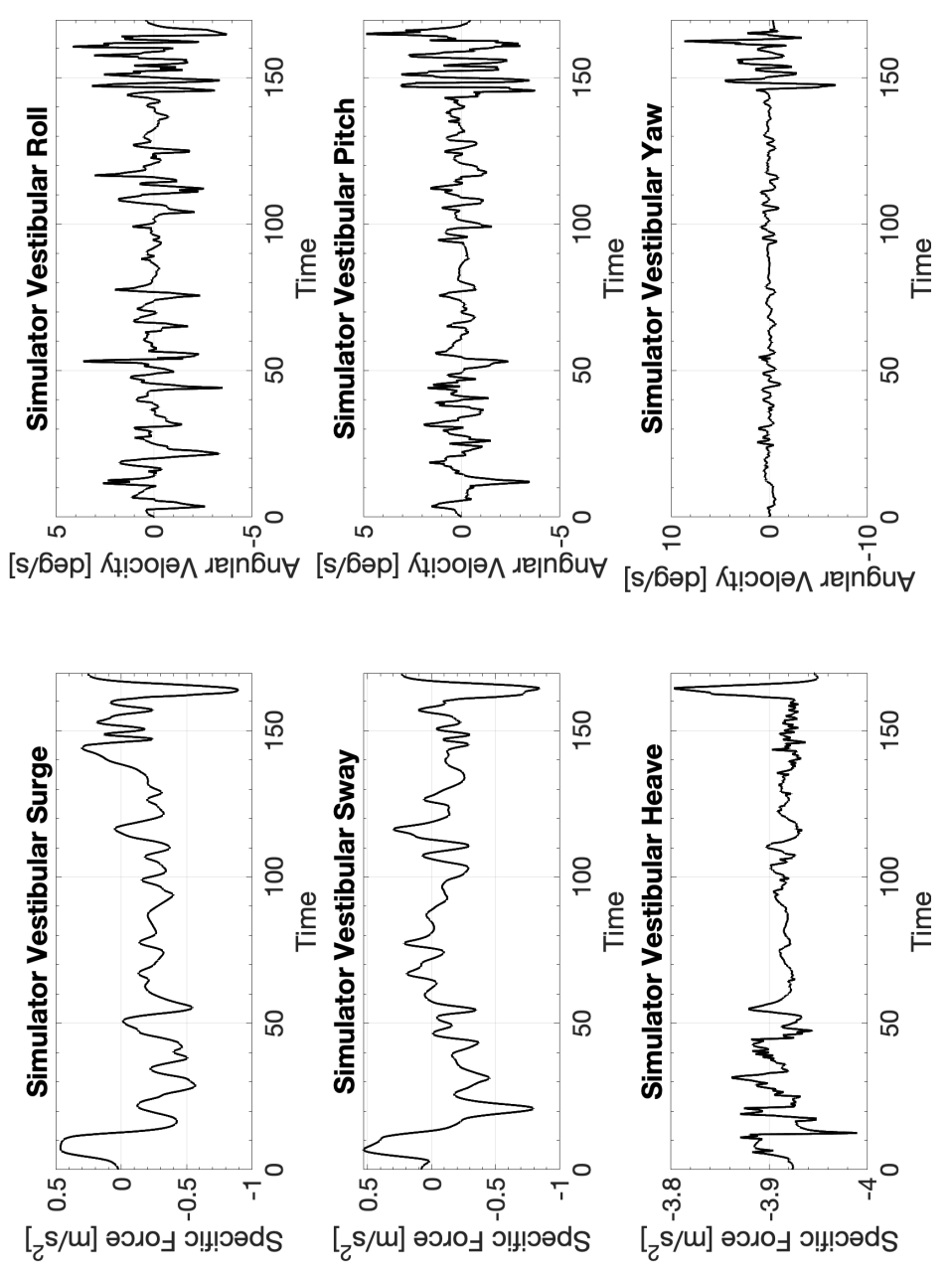


\section{D.3 Cessna 172 Single-axis Takeoff}

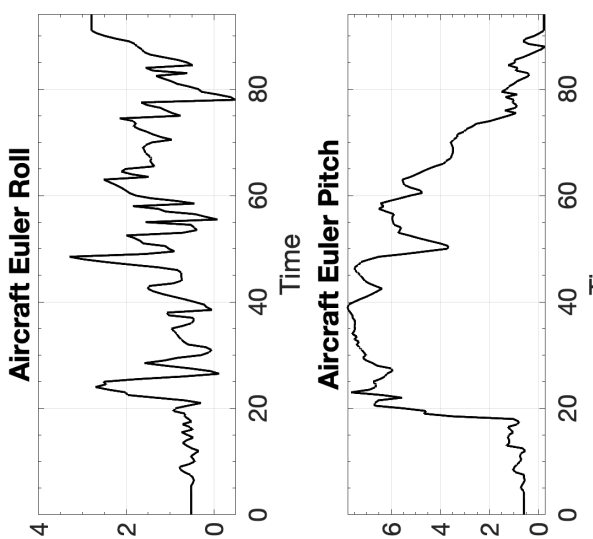

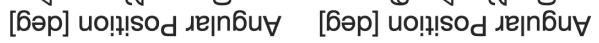

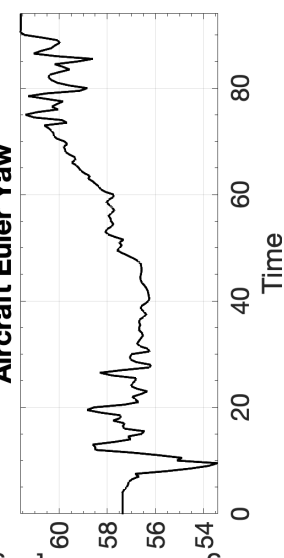

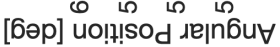
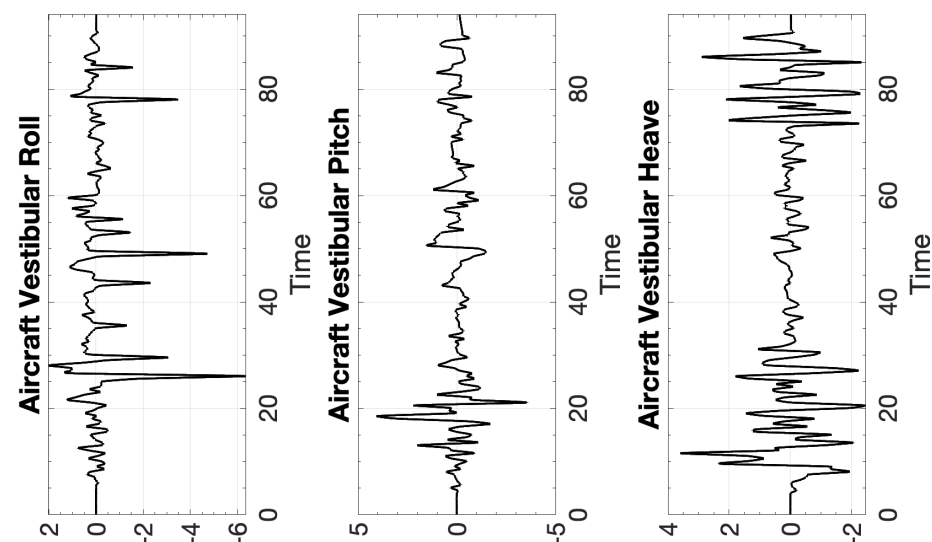

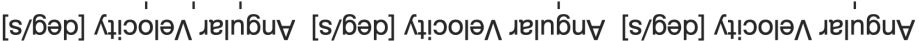

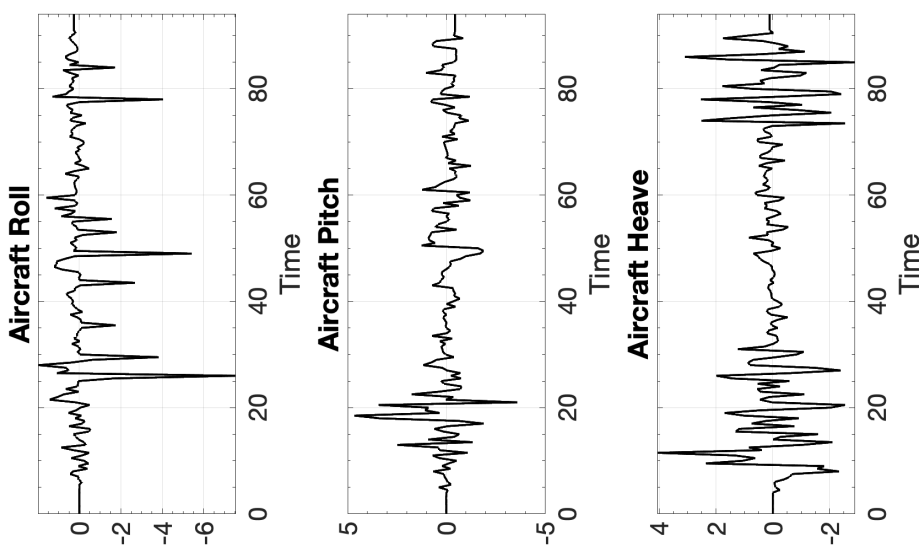

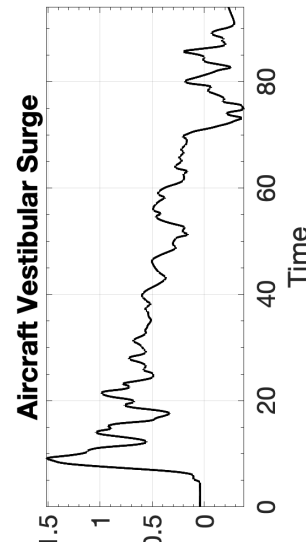
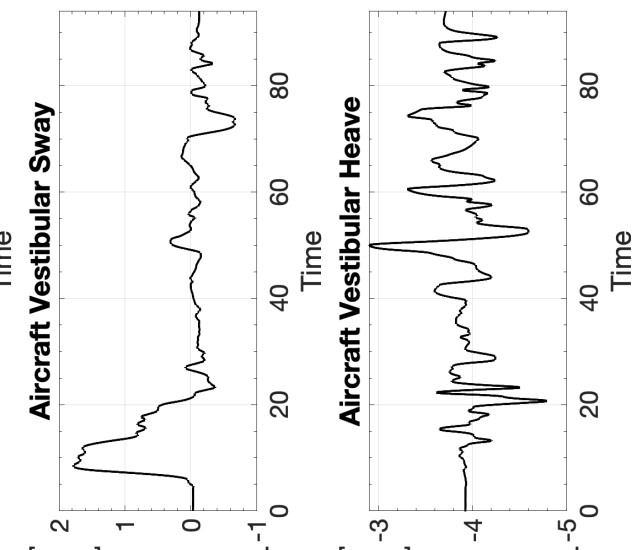

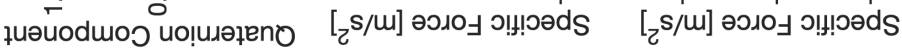

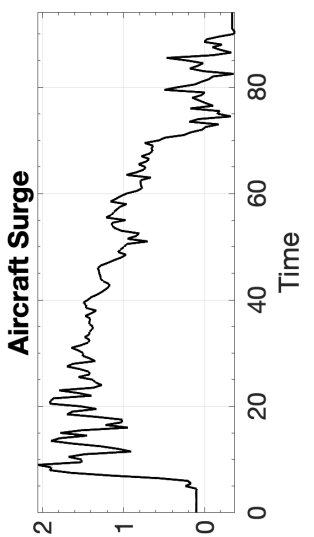

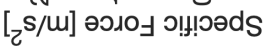
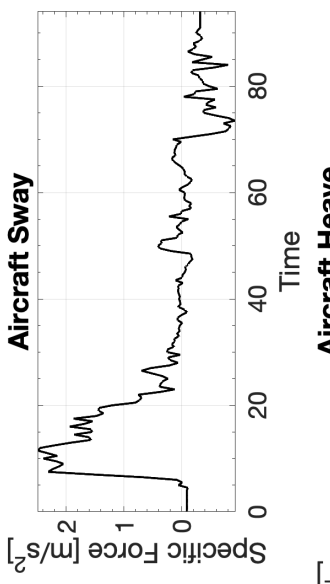

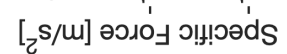
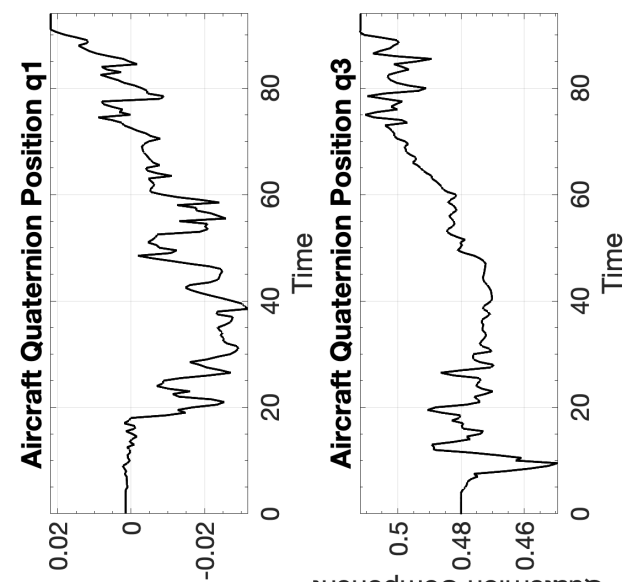

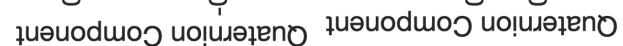

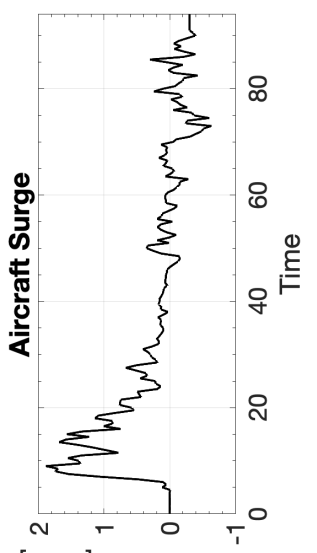

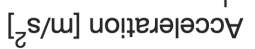

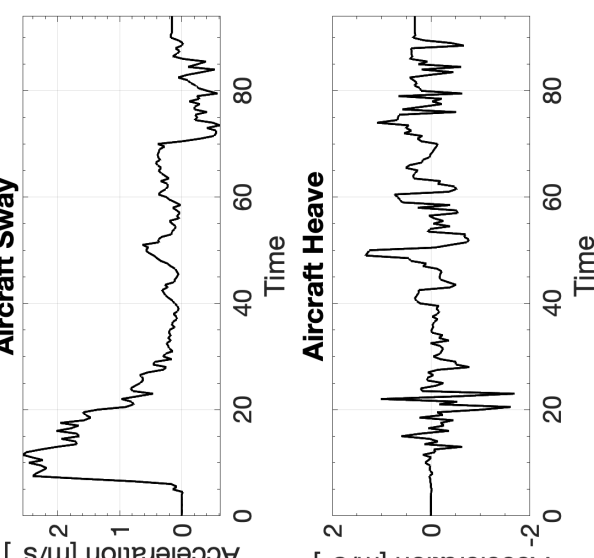

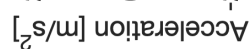

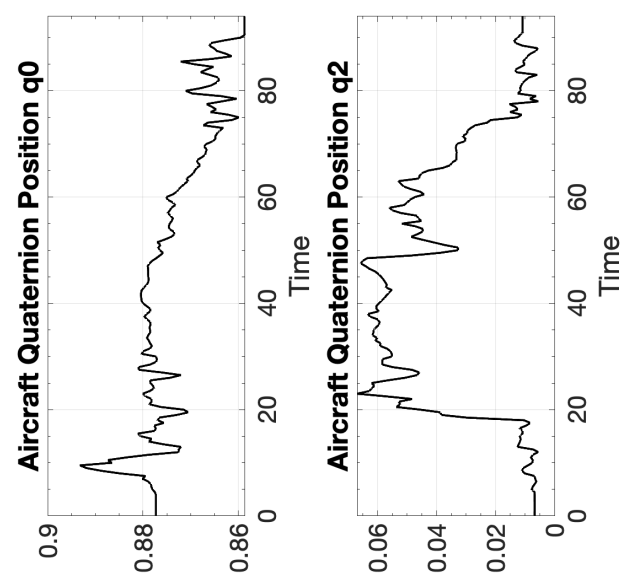

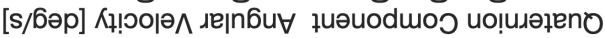




\section{D.3.1 CW1 Cessna 172 Single-axis Takeoff}

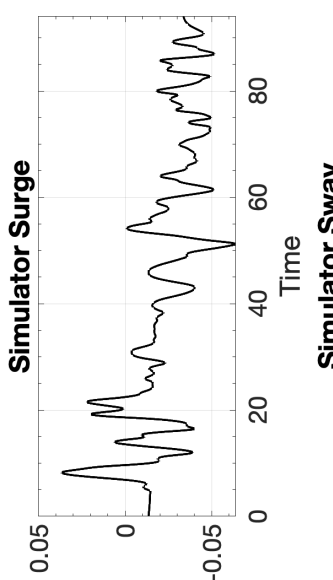

$[\mathrm{m}]$ uo!̣!!sod

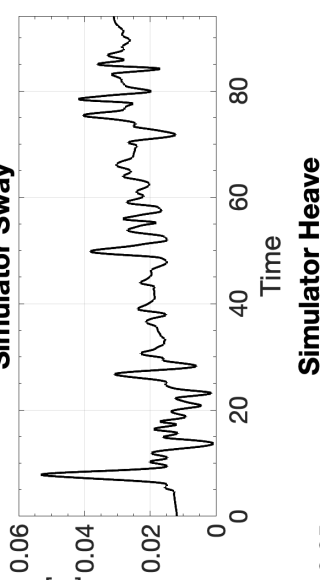

[w] uo!n! sod

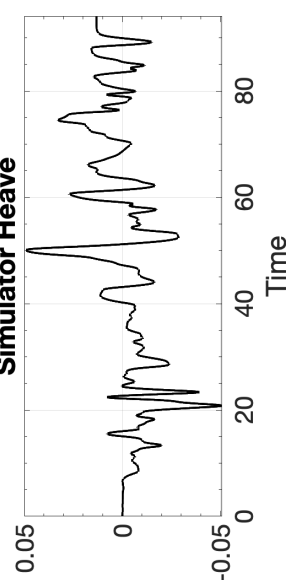

[u] uo!n!sod

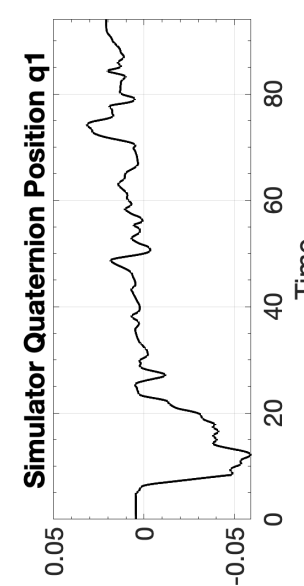

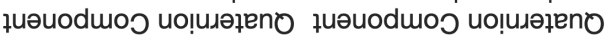

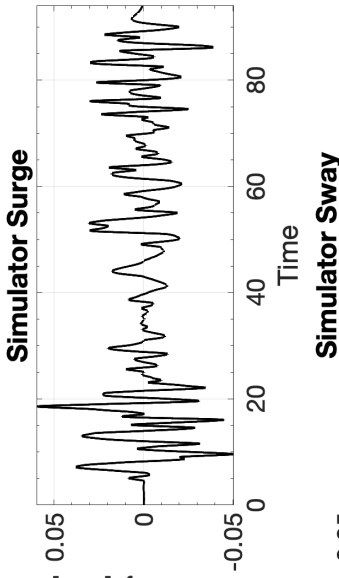

[s/m] Кұ!๐о|ә^

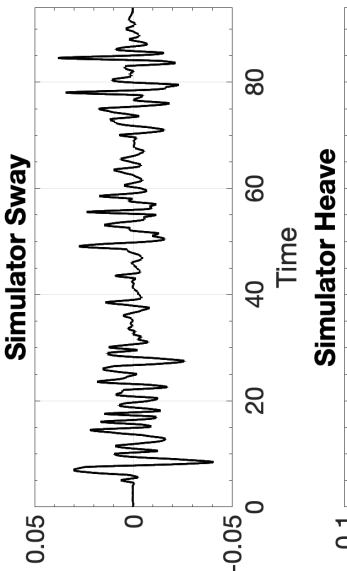

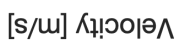

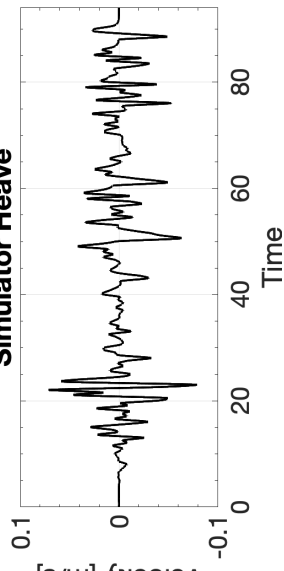

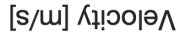

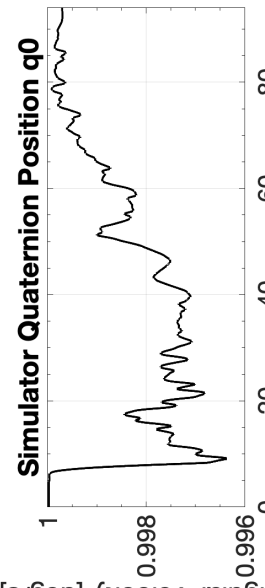

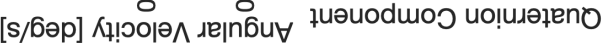

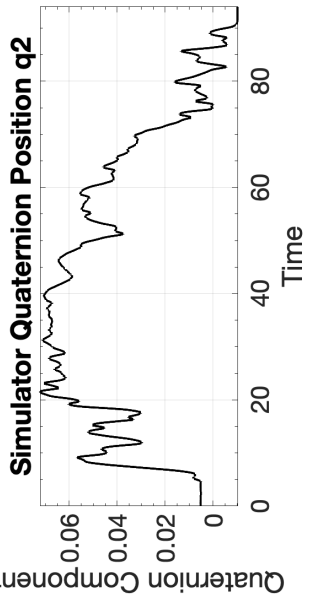

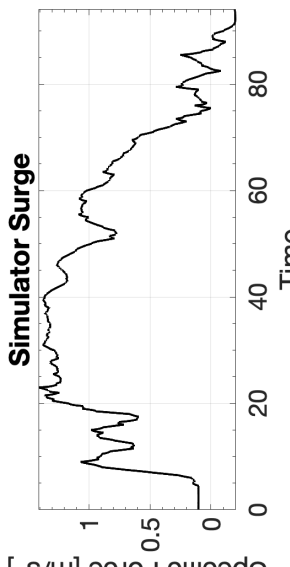

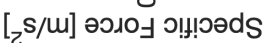

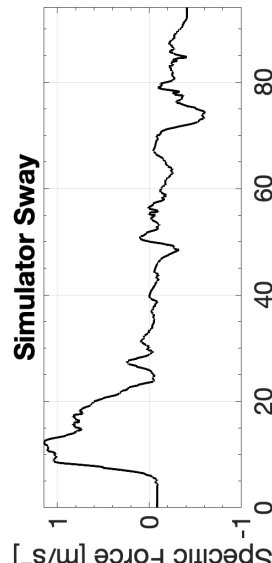

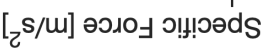
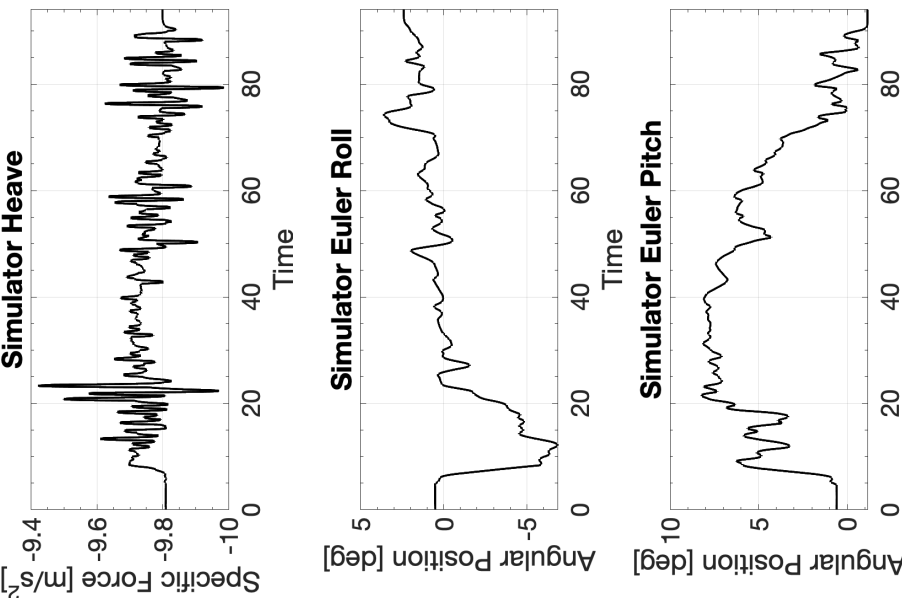

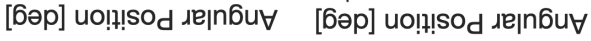

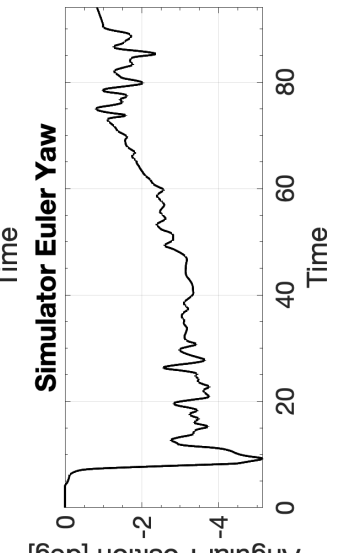

[6әр] uo!!!!sod גe|n6u

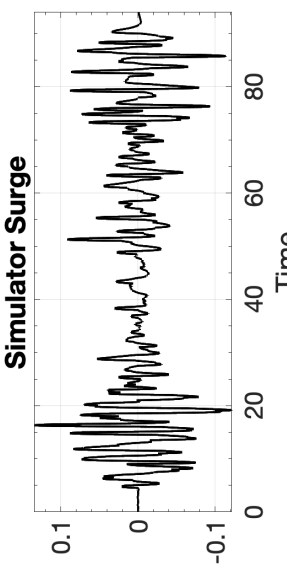

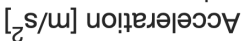

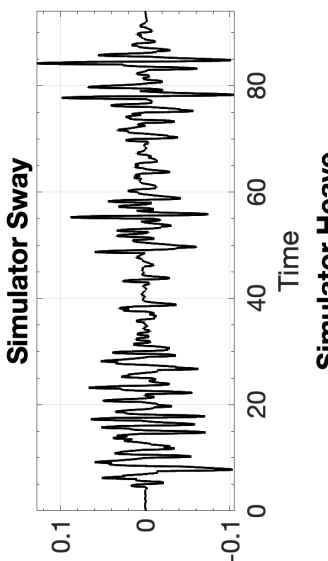

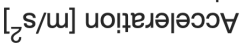

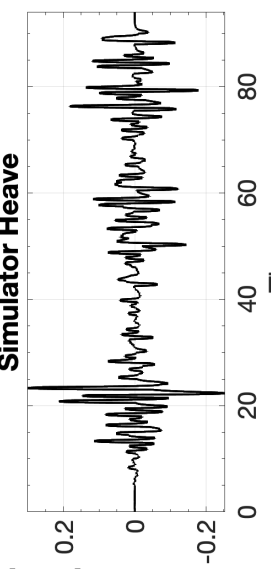

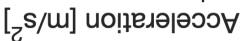

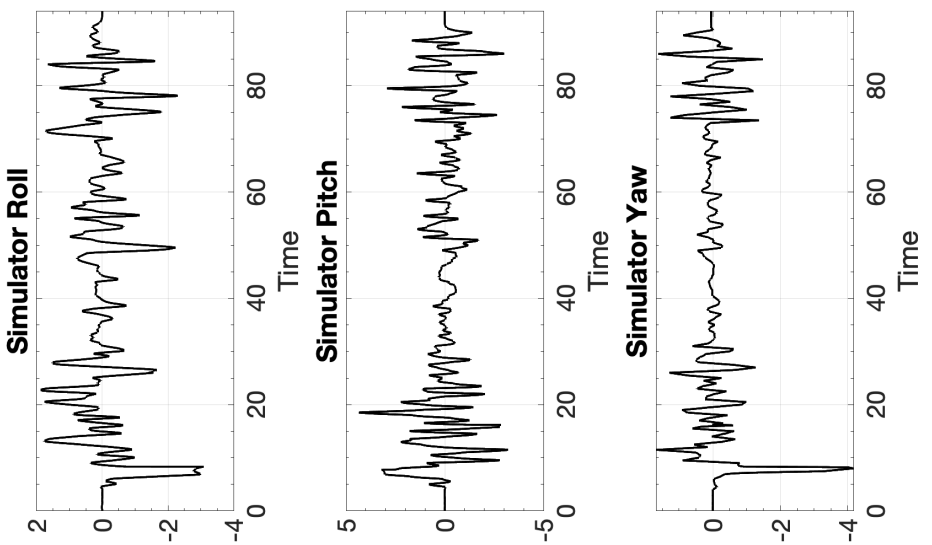

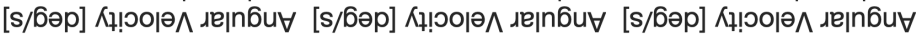




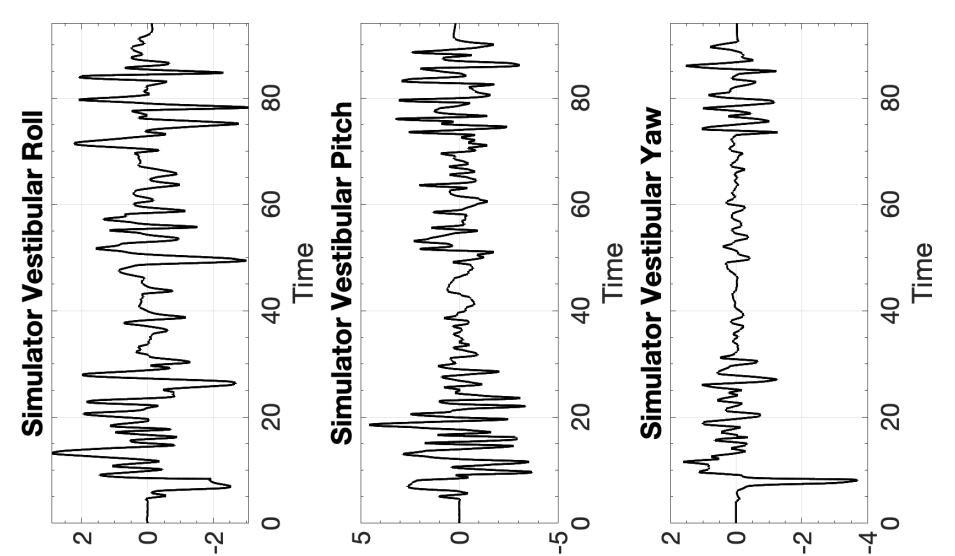

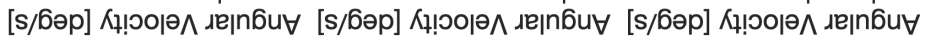

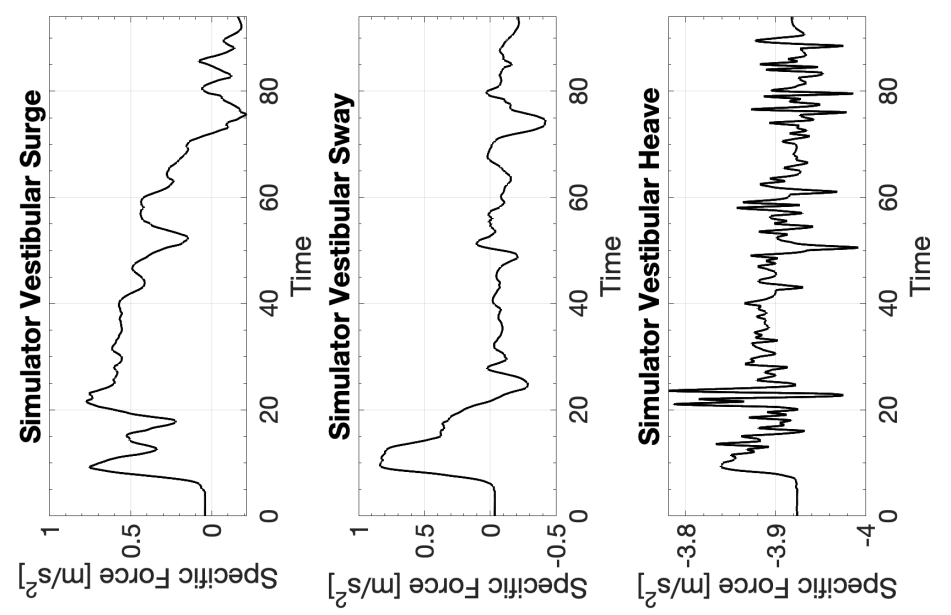




\section{D.3.2 CW2 Cessna 172 Single-axis Takeoff}

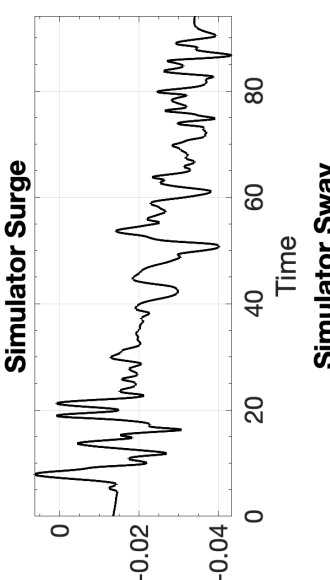

[w] uo!n!sod

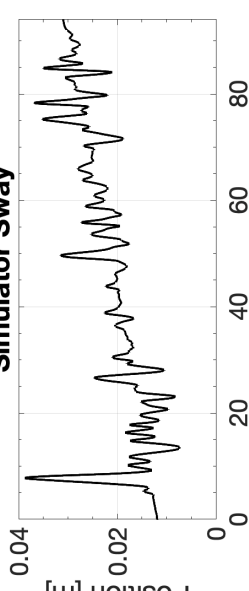

[w] uo!n! sod

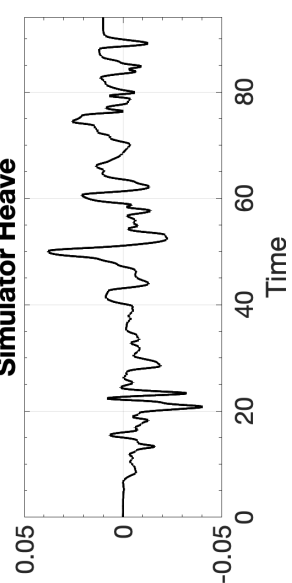

[u] uo!n! sod
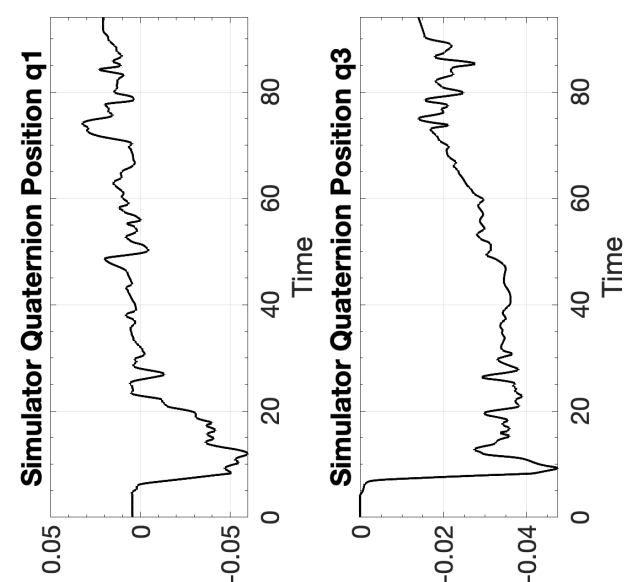

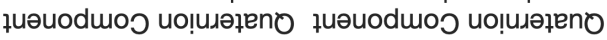

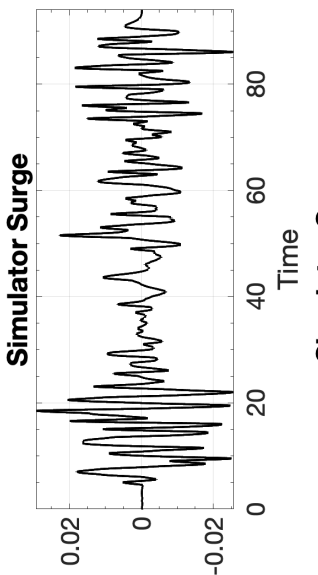

[s/m] Кұ!оојә

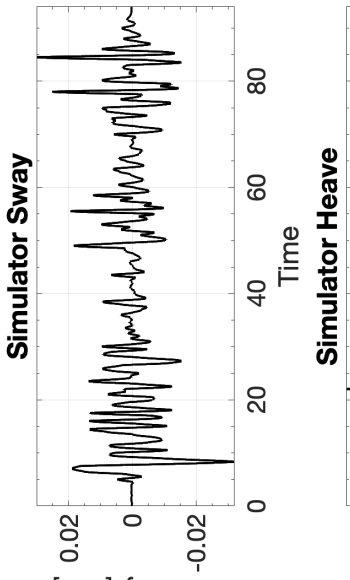

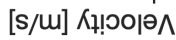

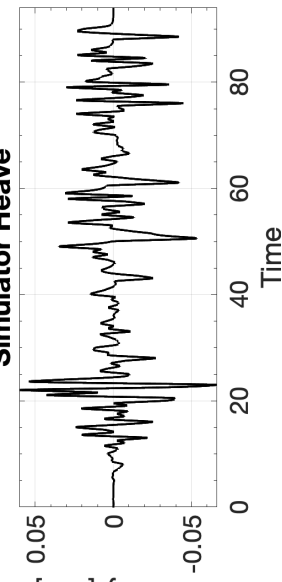

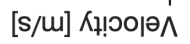

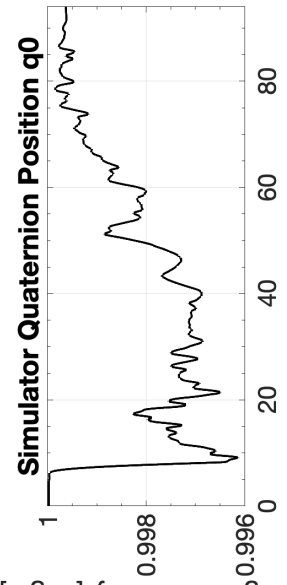

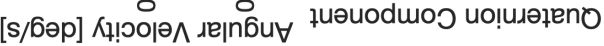

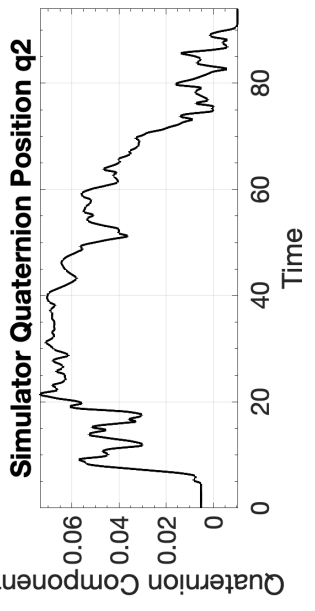

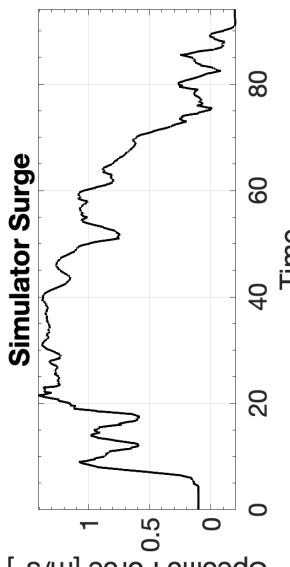

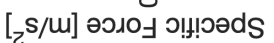

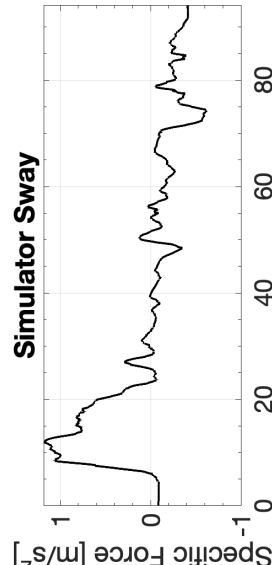

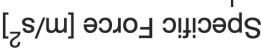
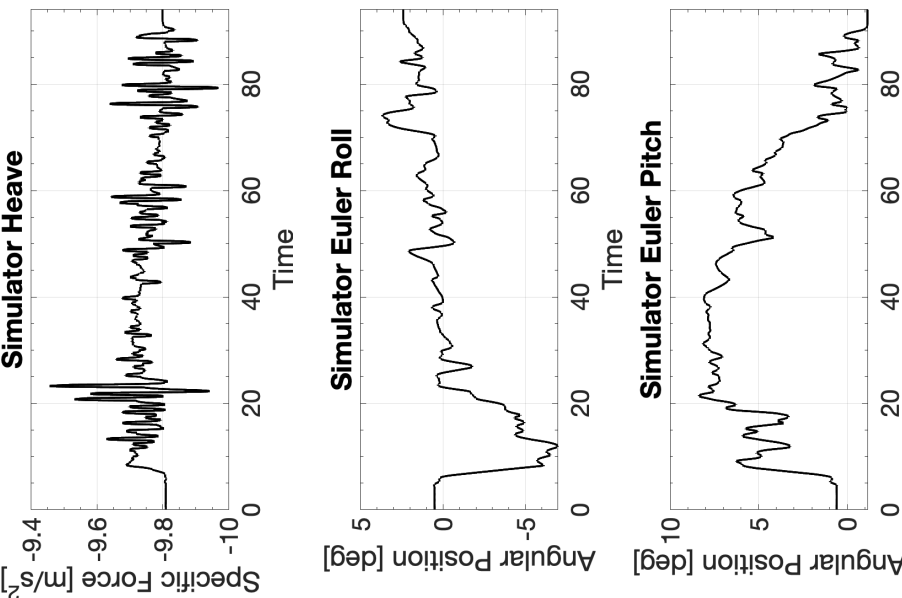

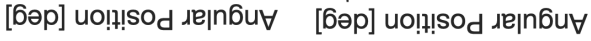

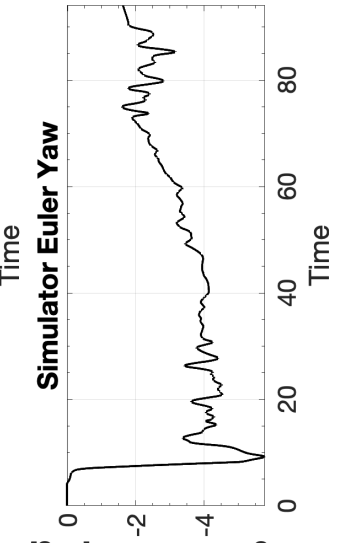

[6әр] uo!̣!sod גe|n6u

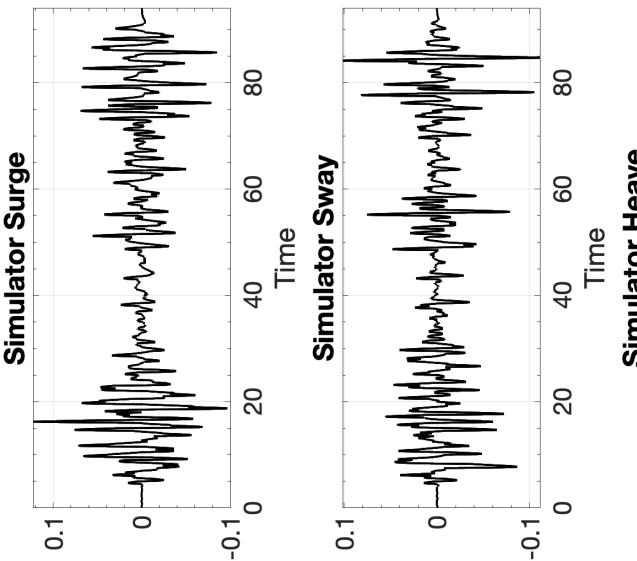

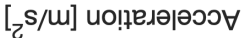

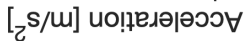

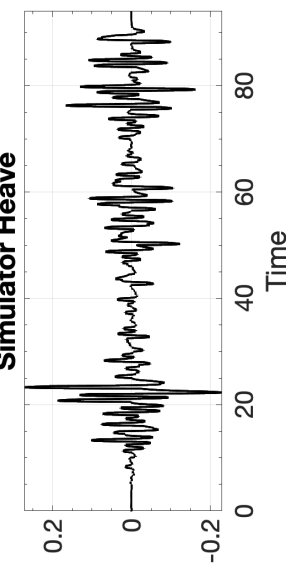

[ $\mathrm{s} / \mathrm{m}]$ uо!ฺвдәрәэว

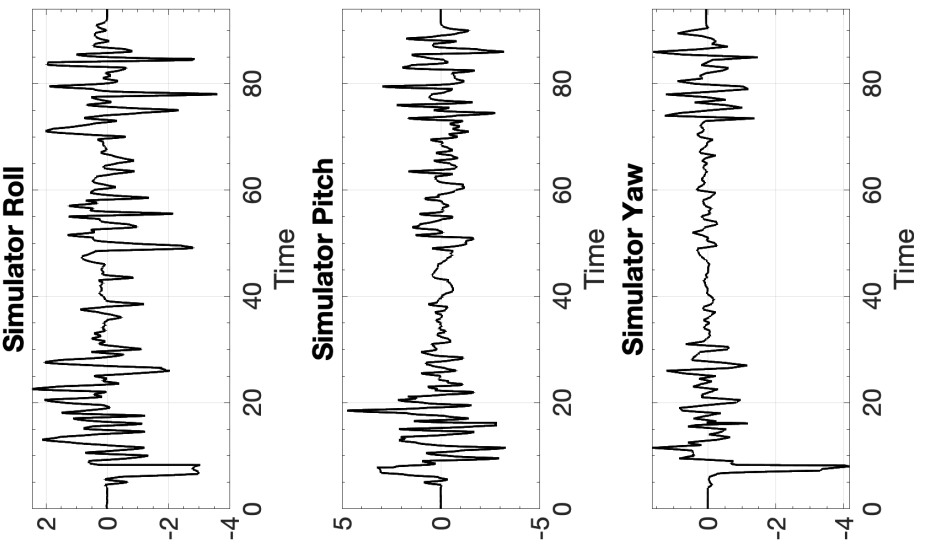

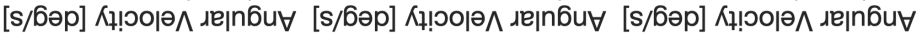




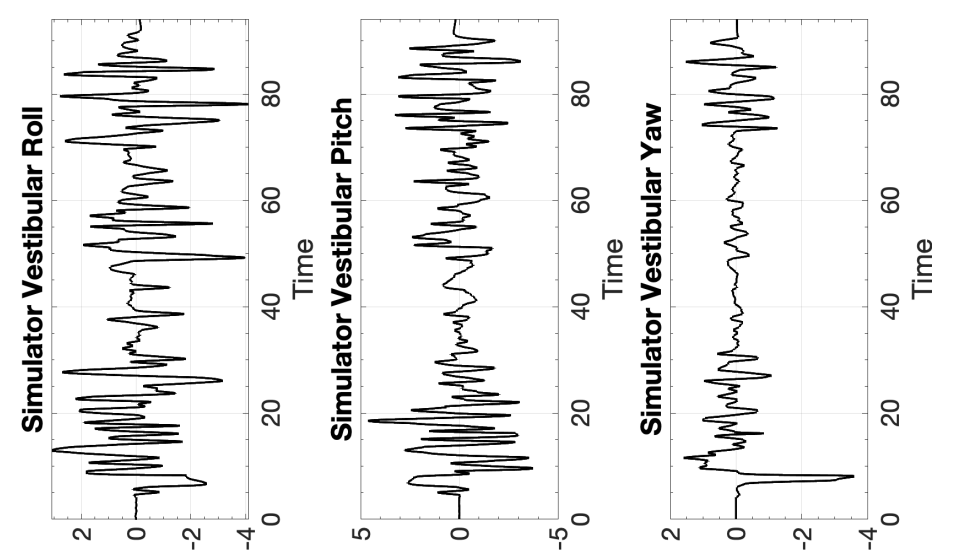

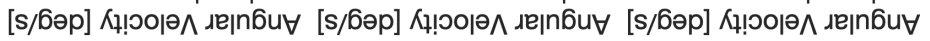

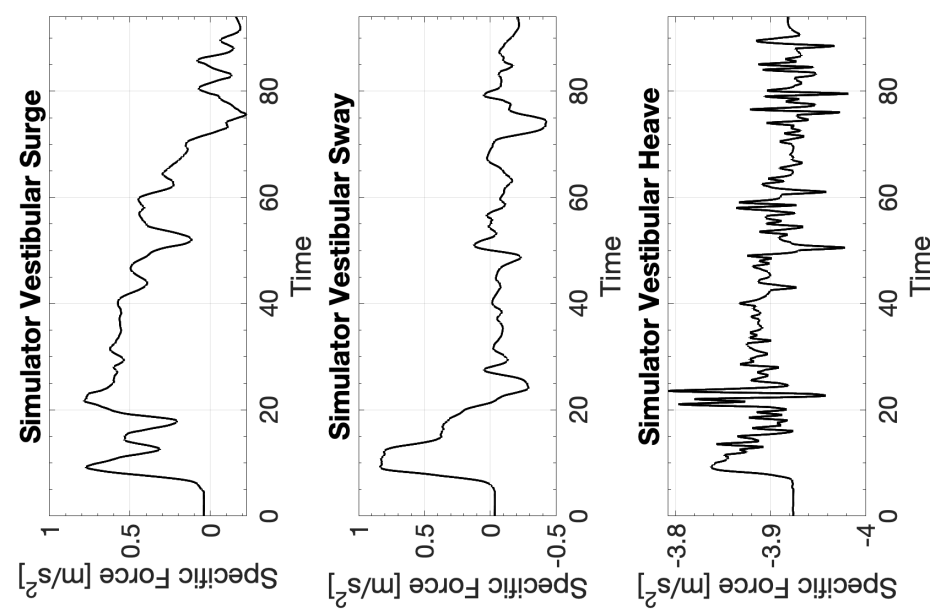




\section{D.3.3 CW3 Cessna 172 Single-axis Takeoff}

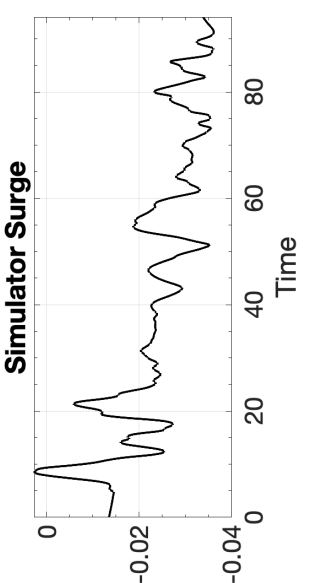

[w] uo!!!!sod

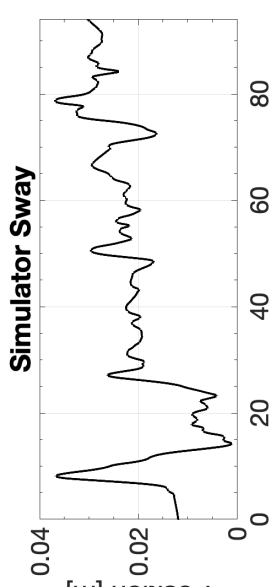

[w] uo!n! sod

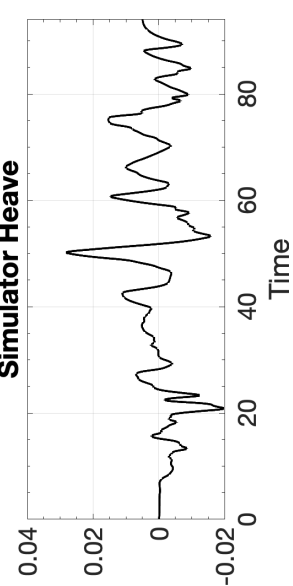

[u] uo!n!sod
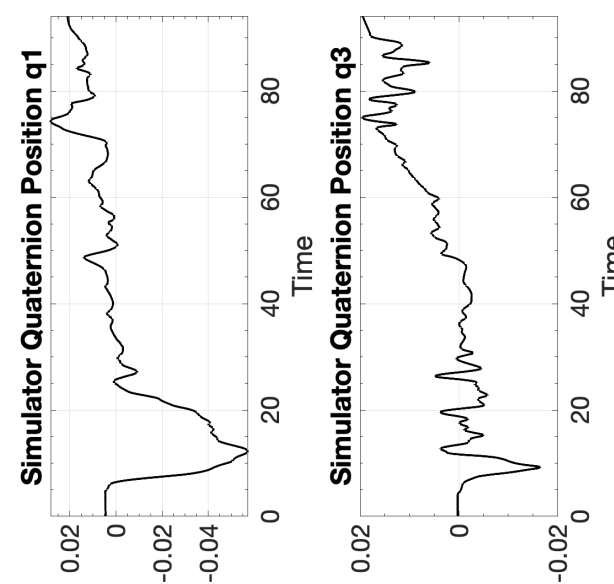

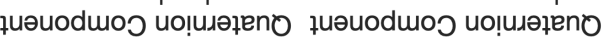

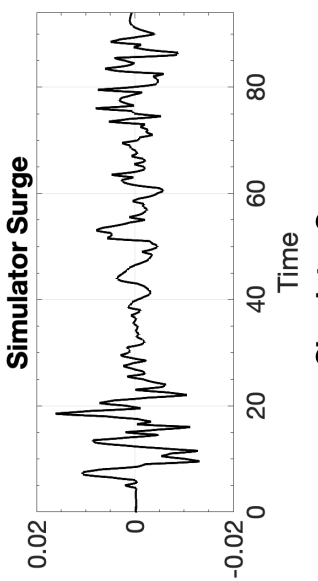

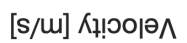

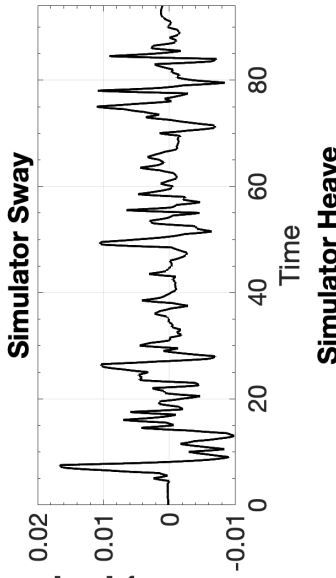

[s/m] Кұ!๐о|ә

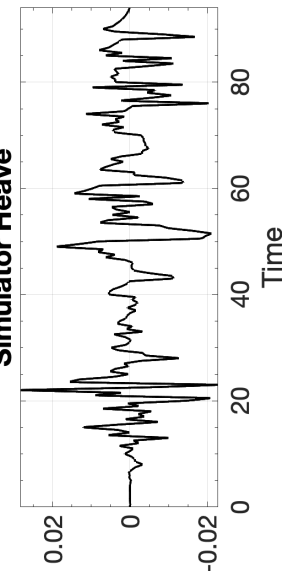

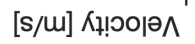

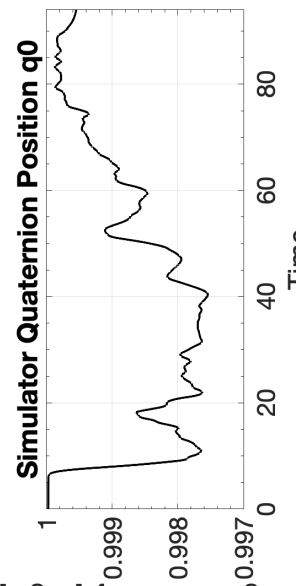

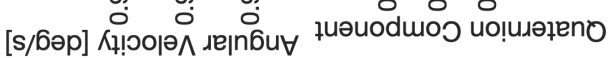

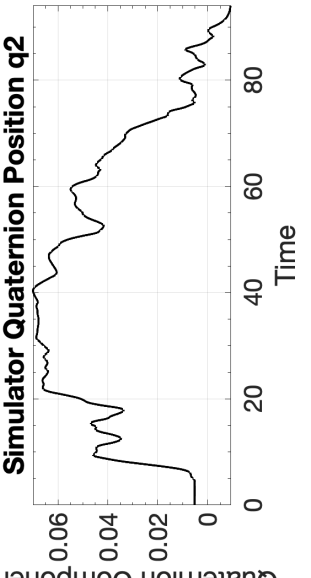
站

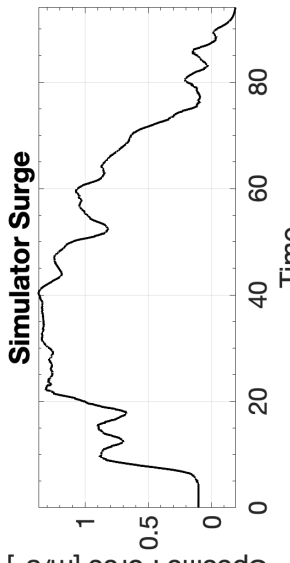

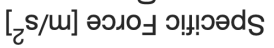

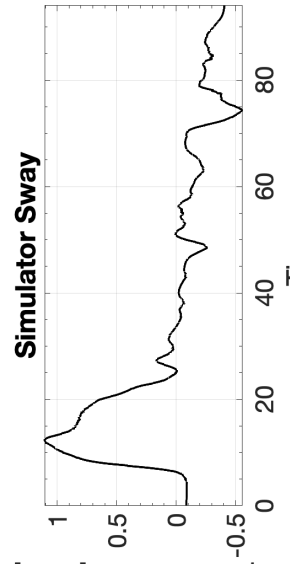

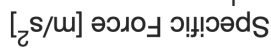

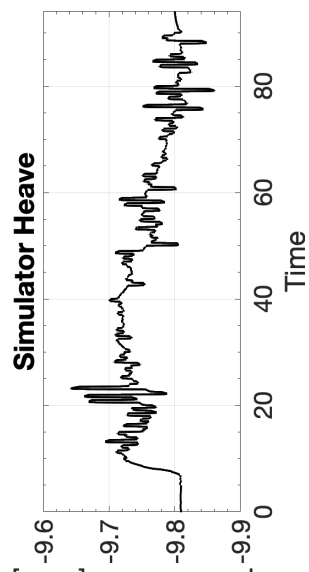

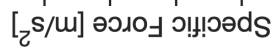

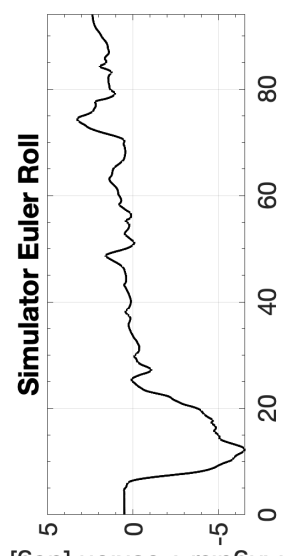

[бәр] uolissod

$\underset{q}{\stackrel{0}{F}}$

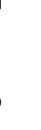
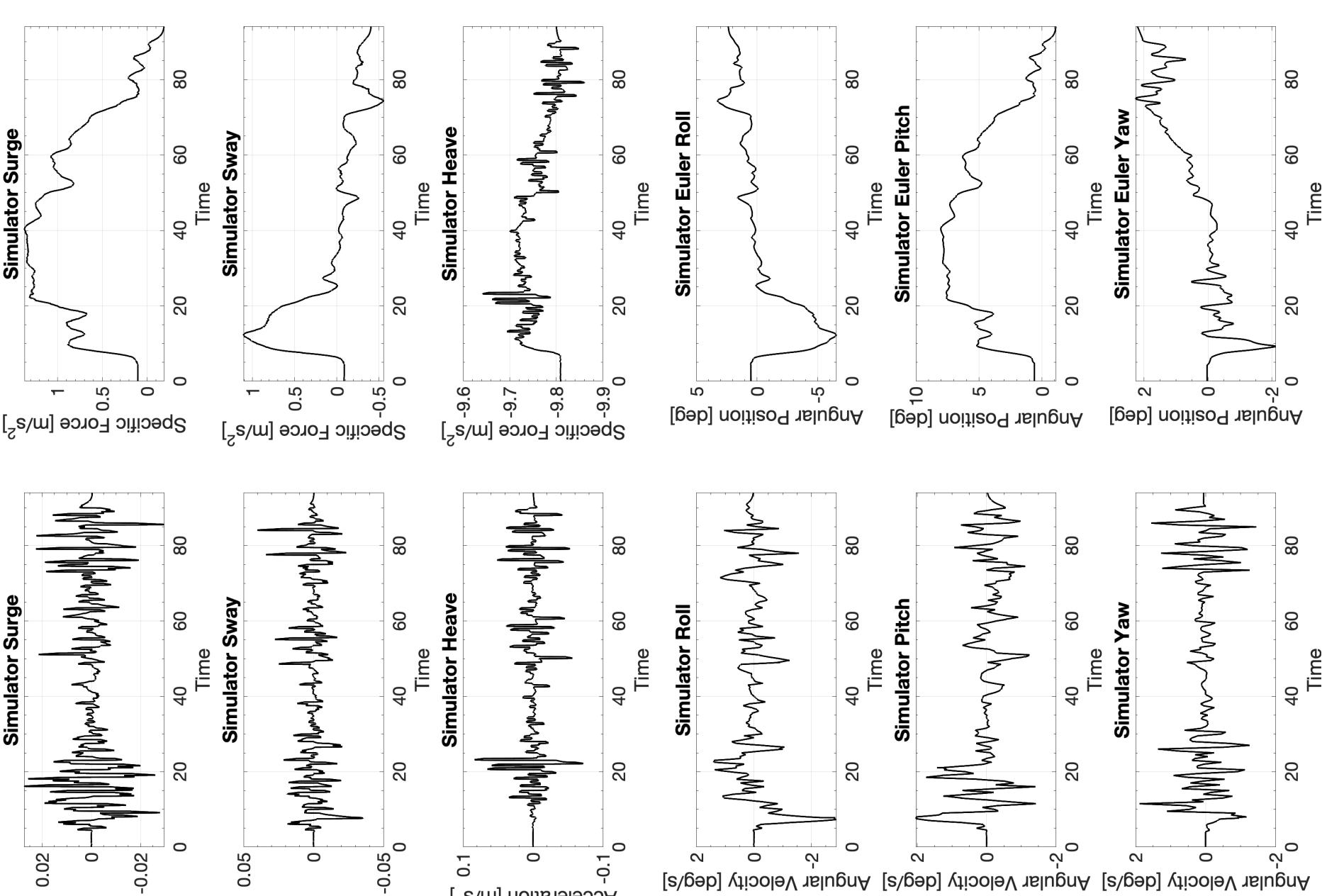

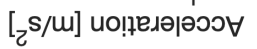

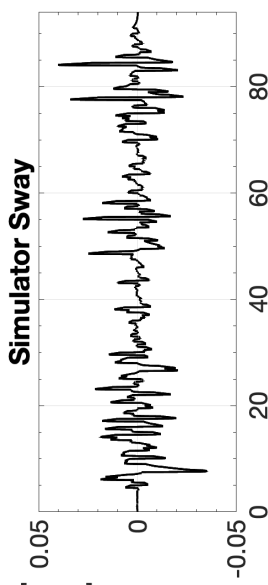

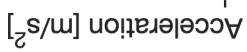

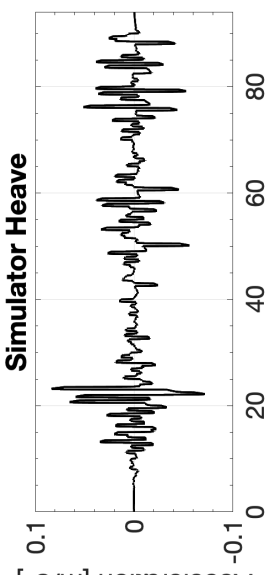

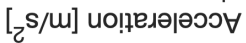

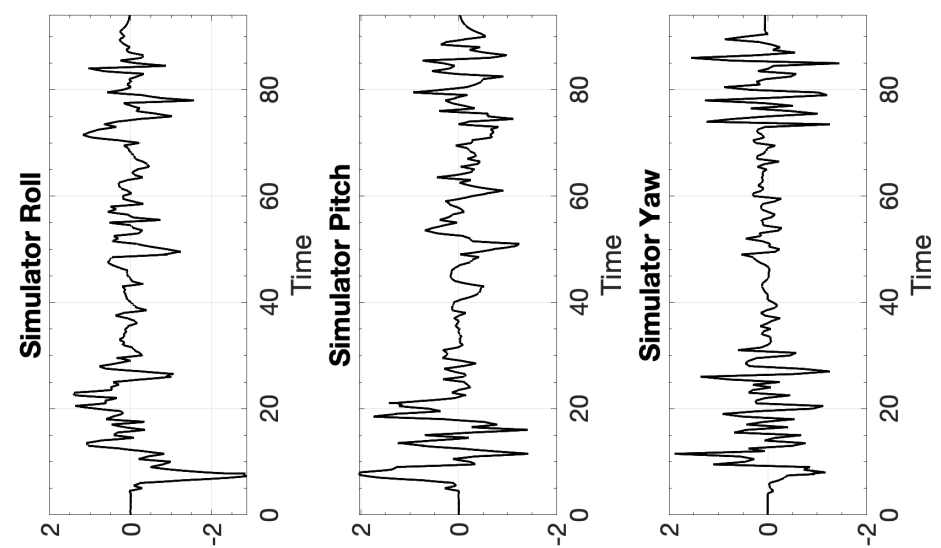

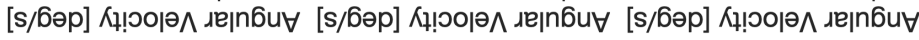



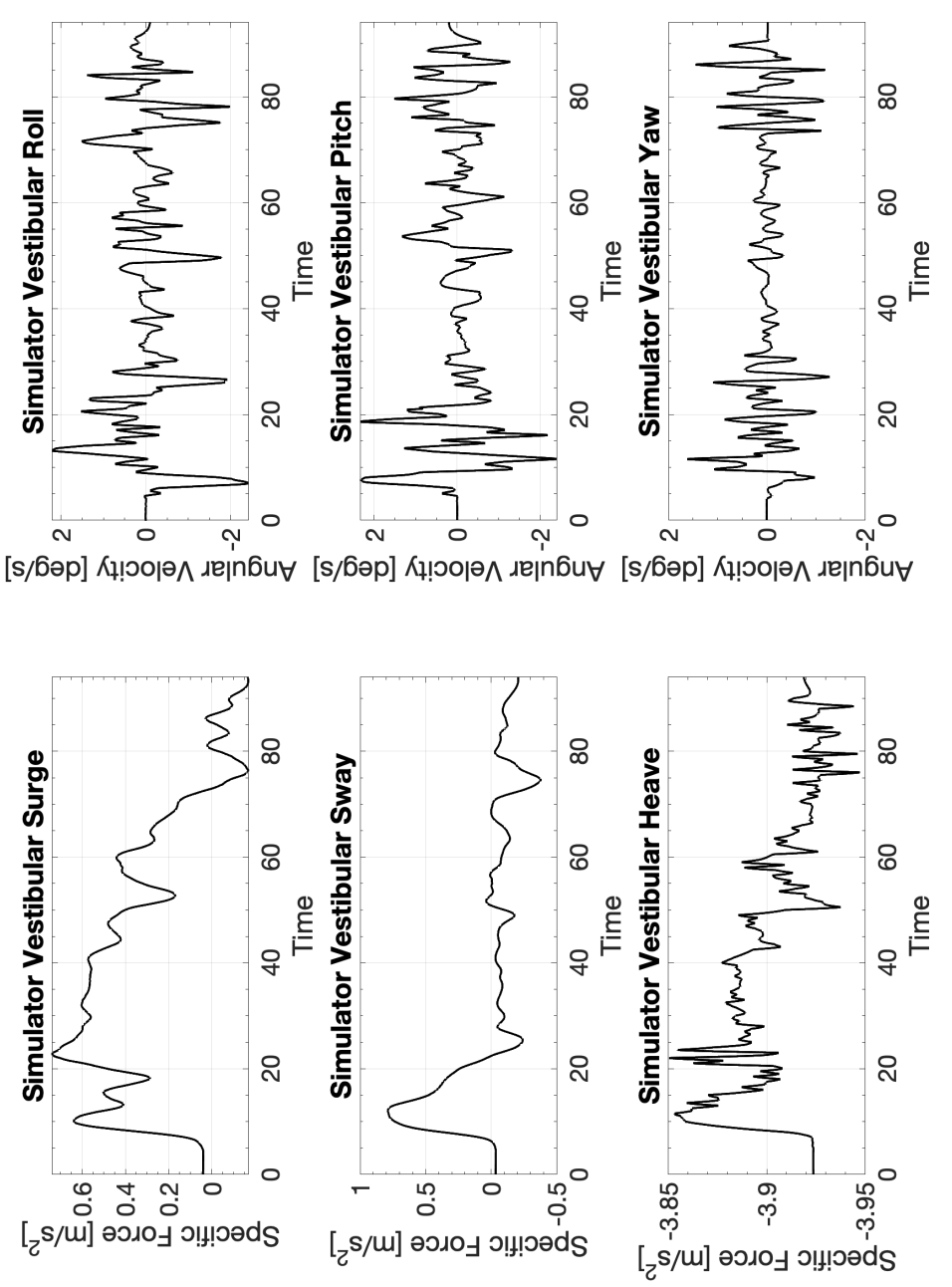


\section{D.4 Cessna 172 Spiral Dive}

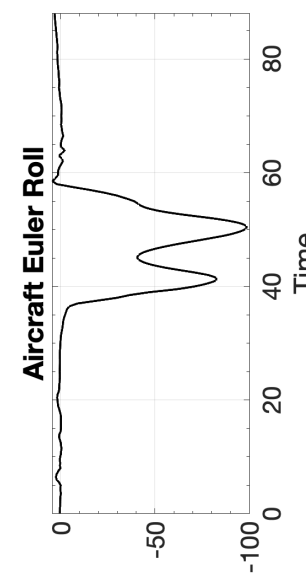

[6əp] uo!̣!sod גeןn6́ü

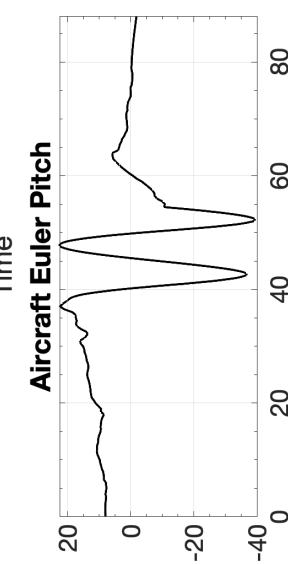

[6әр] uo!!!!sod גe|nбu

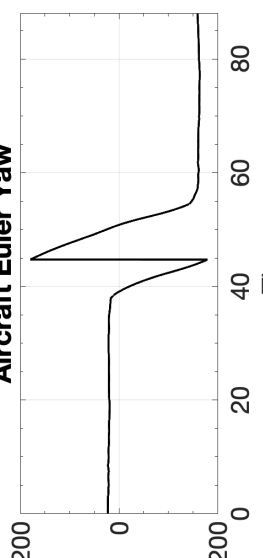

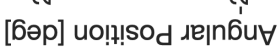

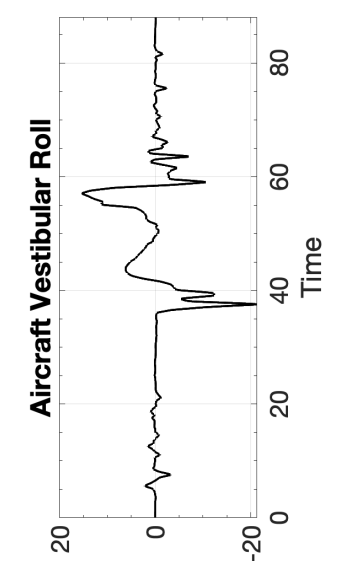

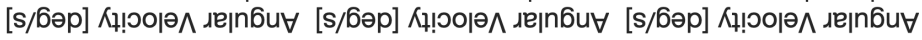
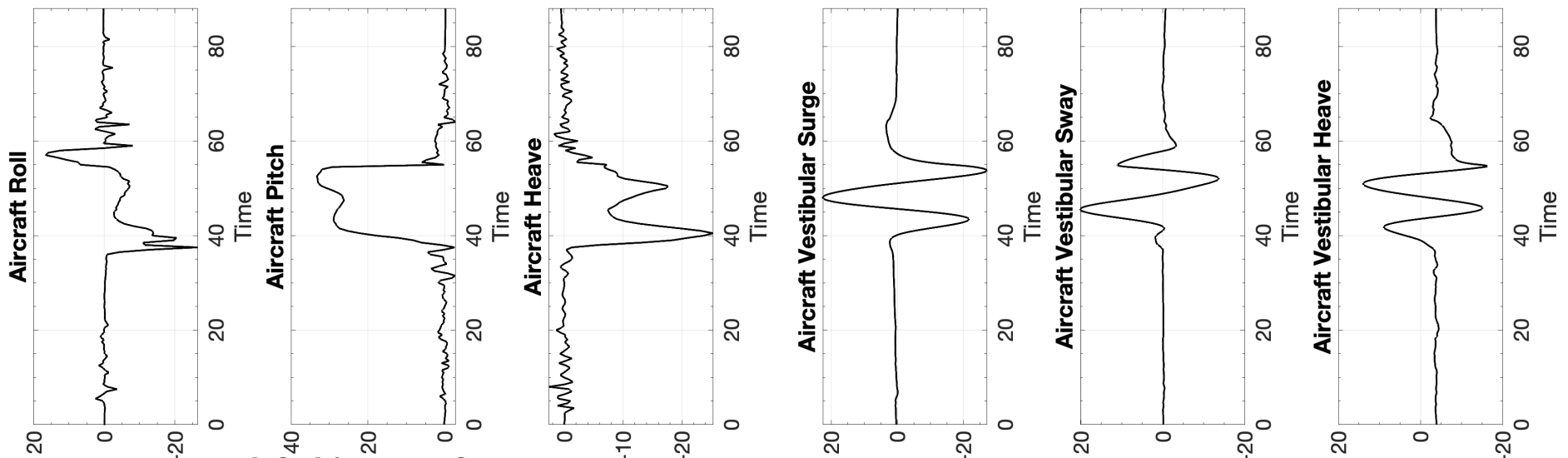

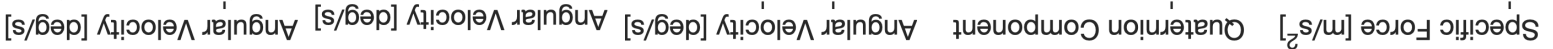

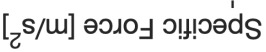
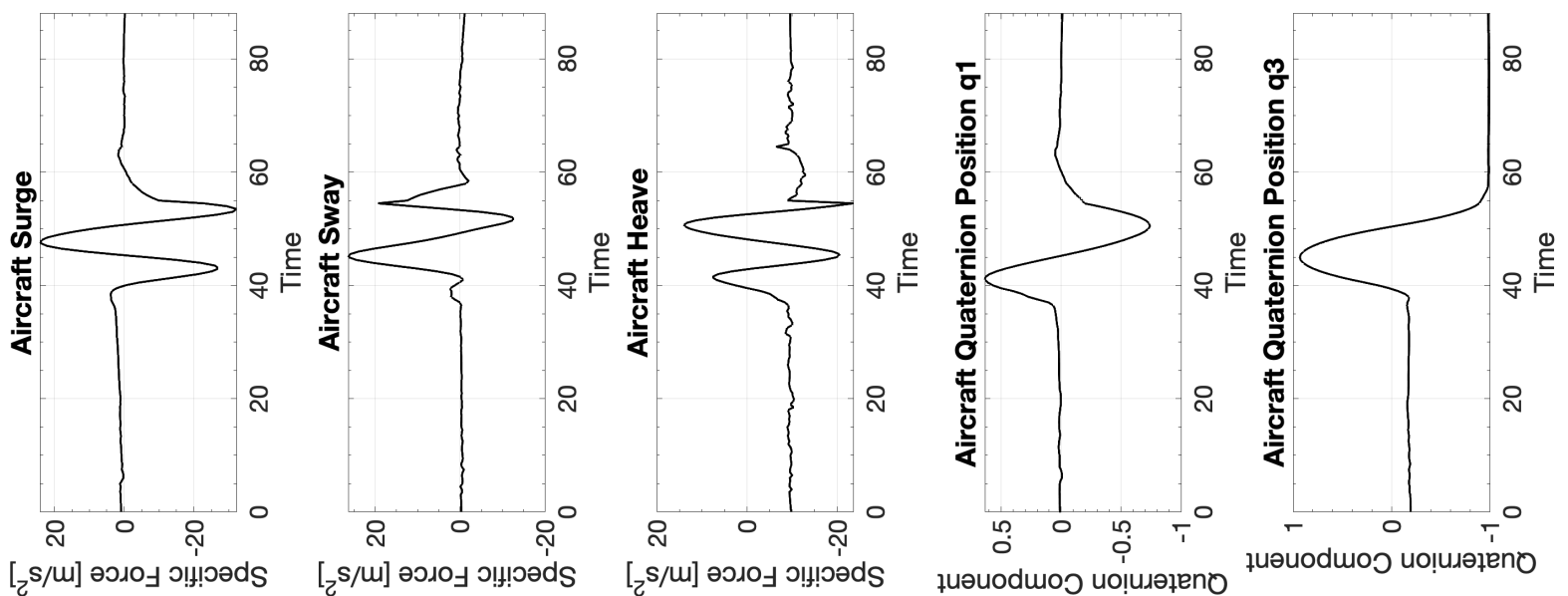

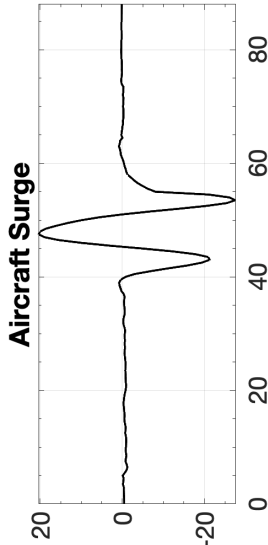

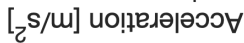

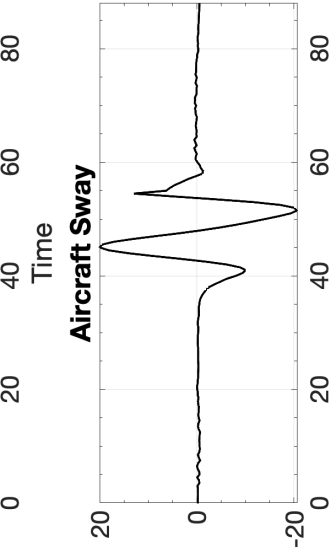

[z/m] ио!ฺеләрәоэ

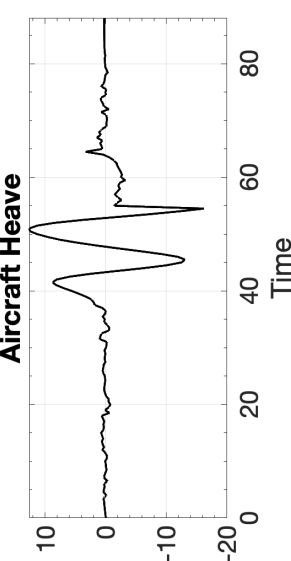

[ح $\mathrm{s} / \mathrm{m}]$ иопџедәрәоэ

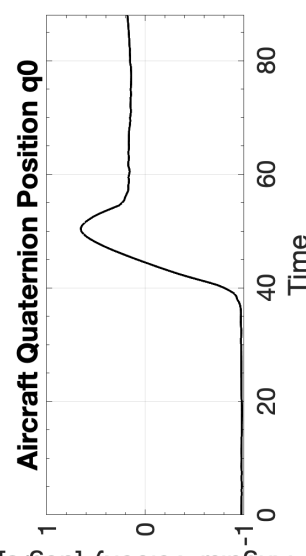

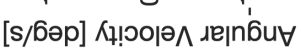

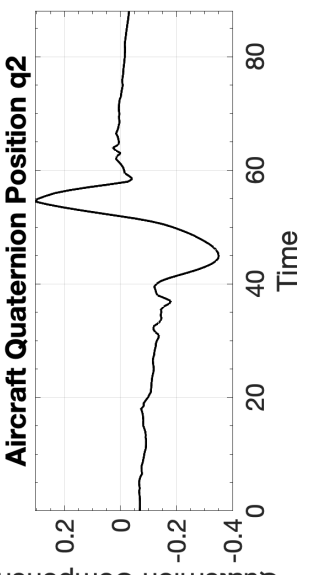

łuəuodmos uo!̣uәəteno 


\section{D.4.1 CW1 Cessna 172 Spiral Dive}
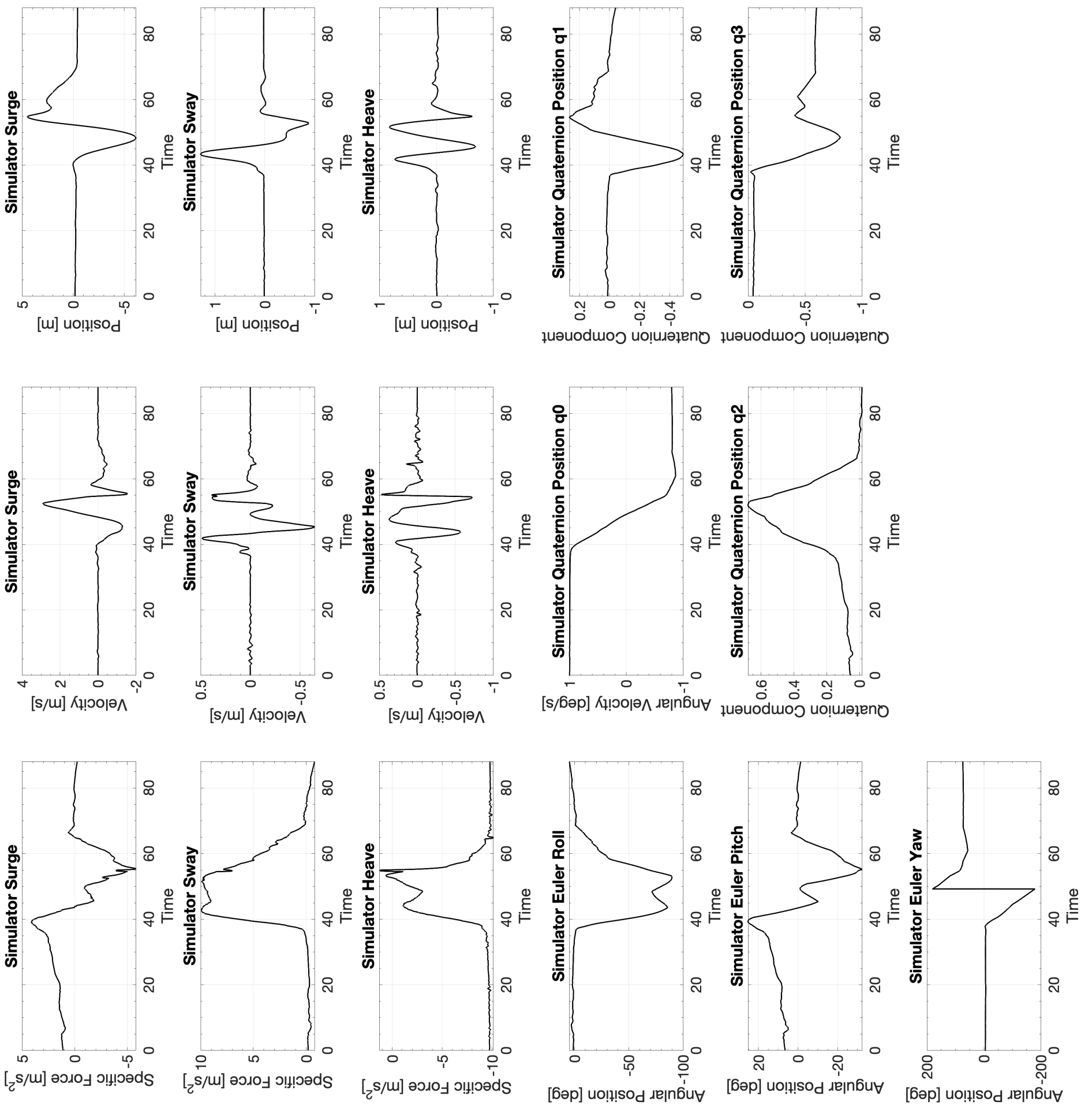

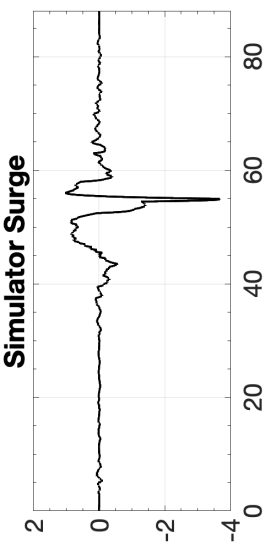

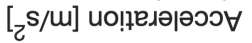

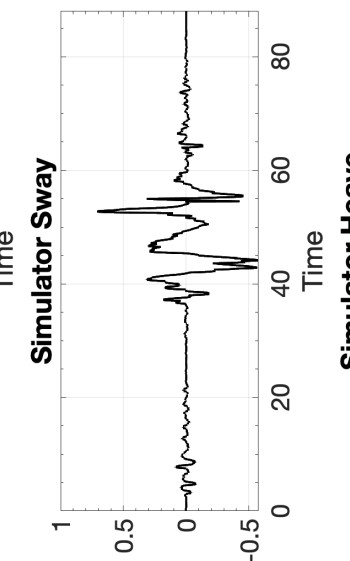

[
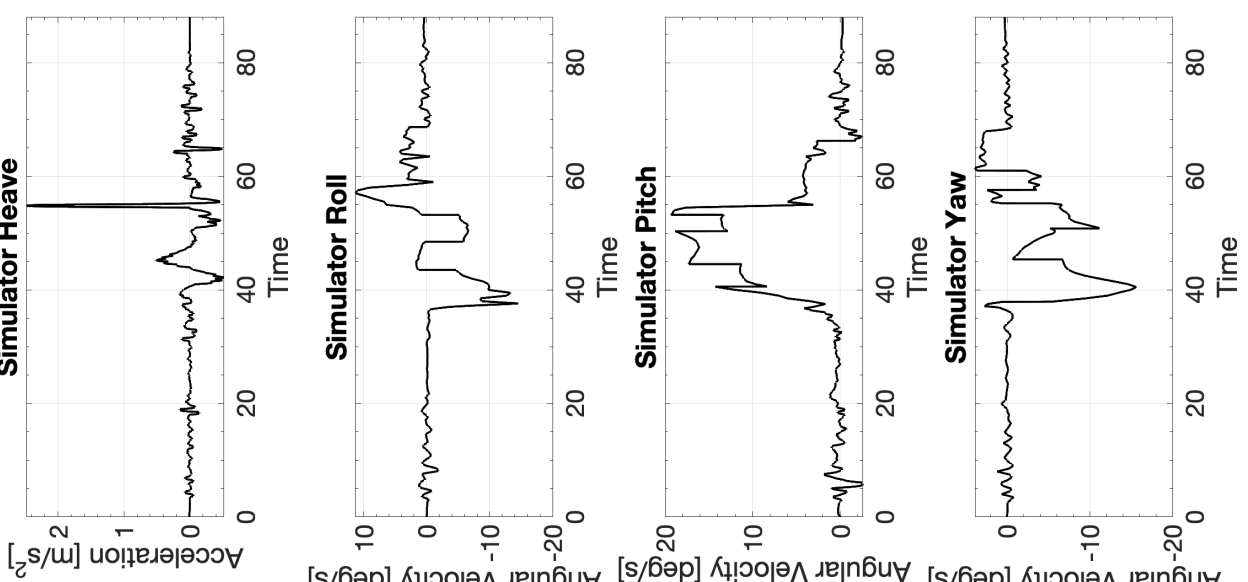


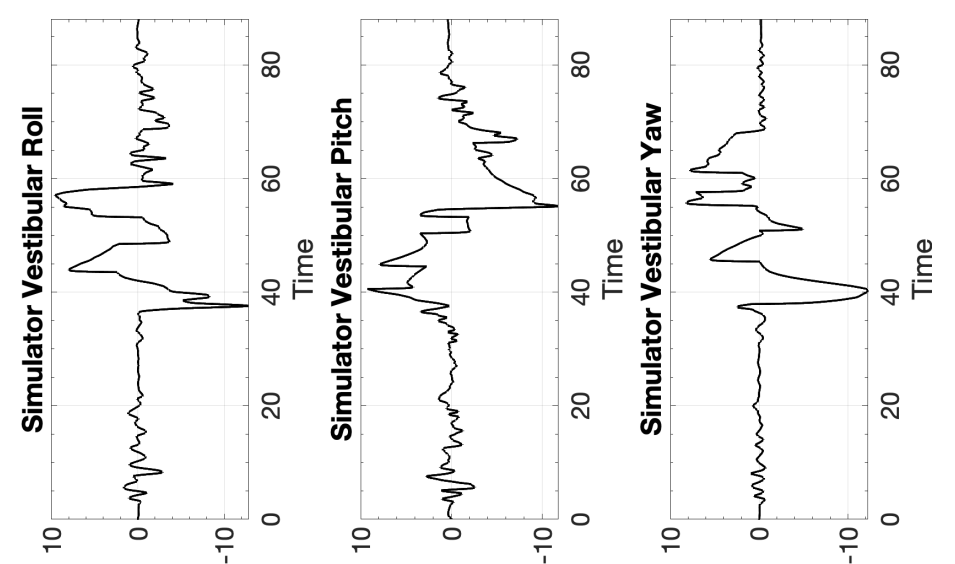

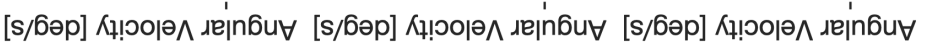

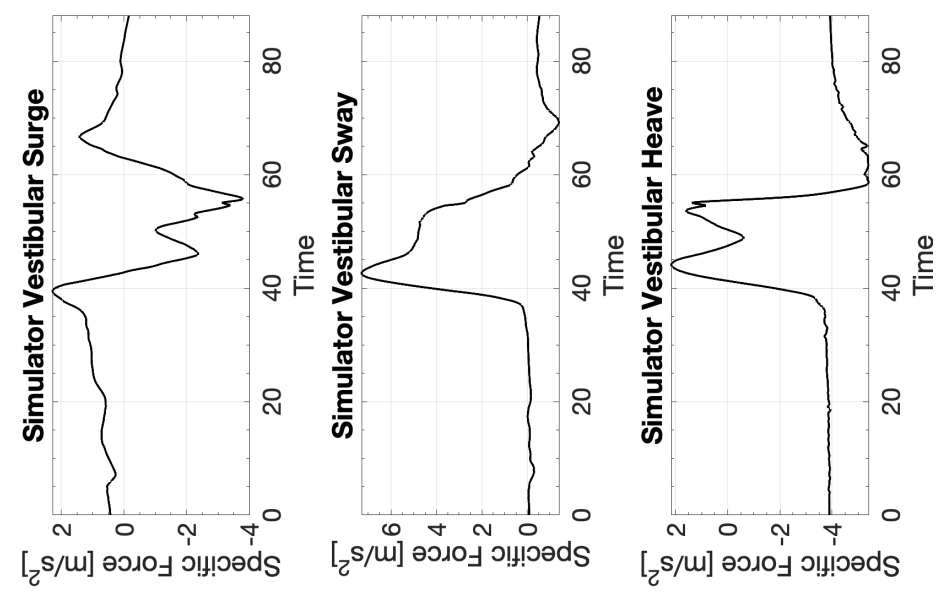




\section{D.4.2 CW2 Cessna 172 Spiral Dive}

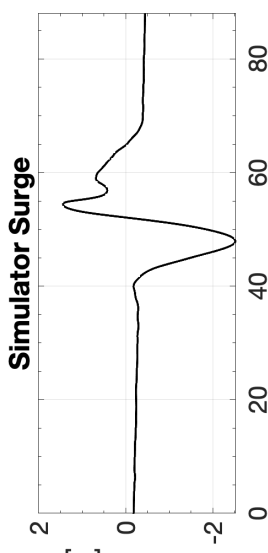

[u] uo!!!!sod

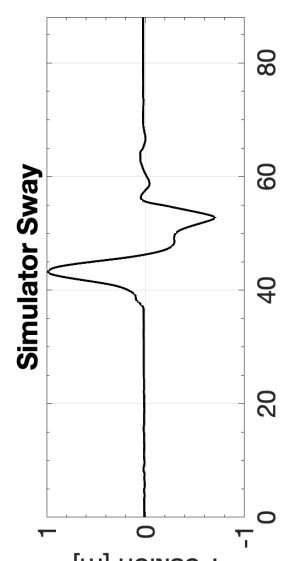

[u] uo!!!!sod

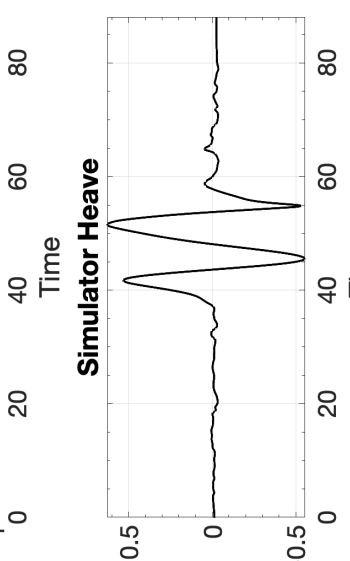

[u] uol!!!sod

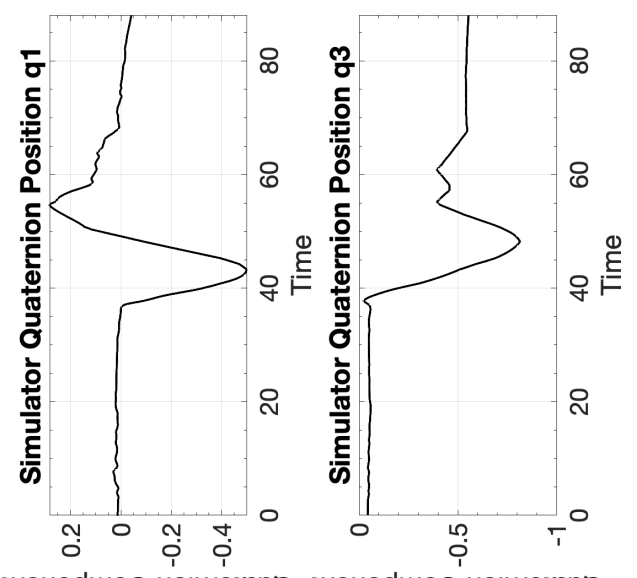

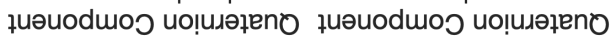

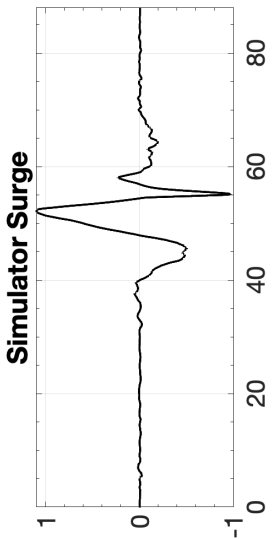

[s/u] Кџ!०о|ә^
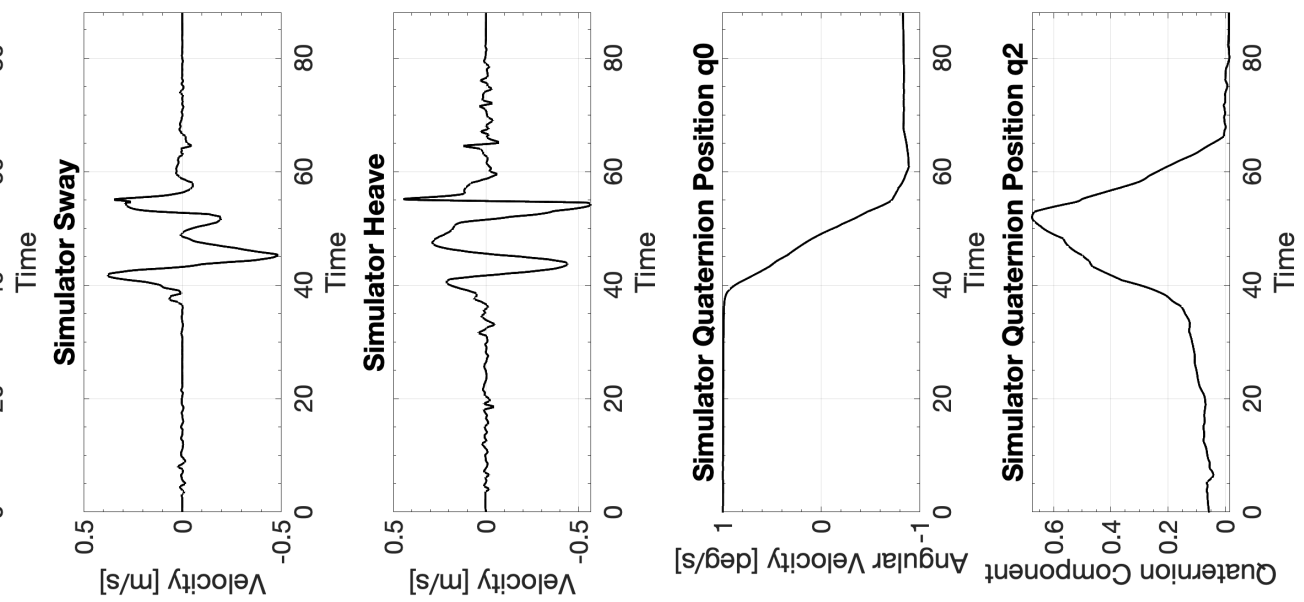

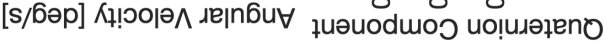

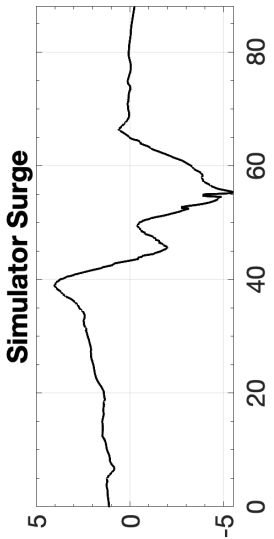

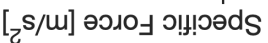

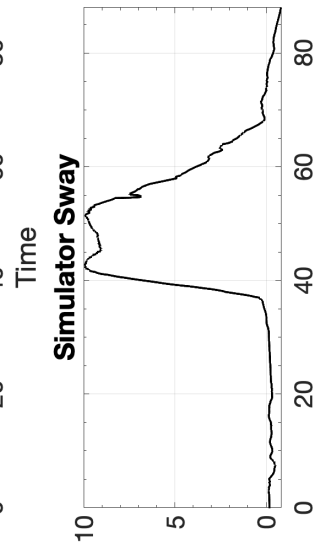

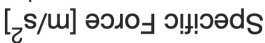

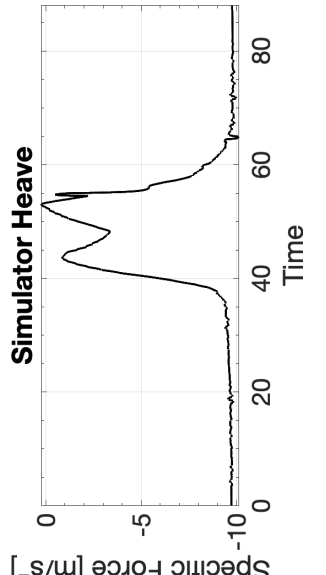

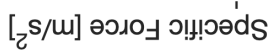

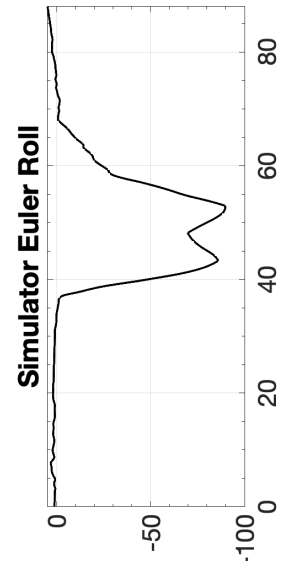

[бəp] uo!̣!sod גe|nб́u

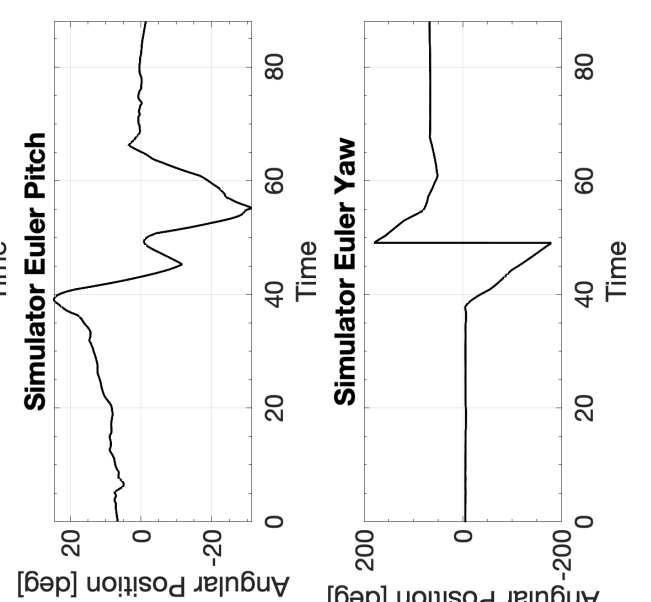

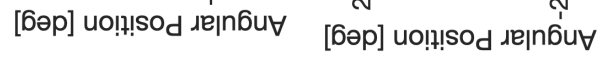

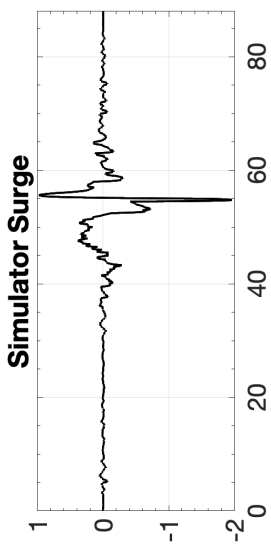

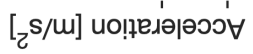

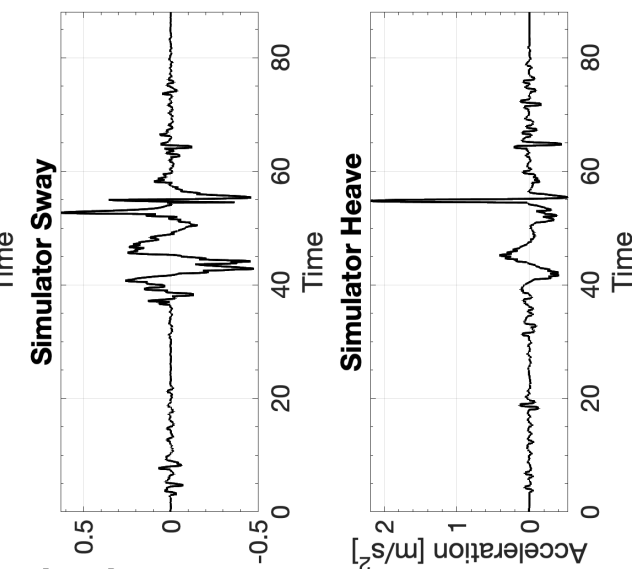

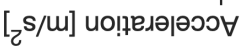
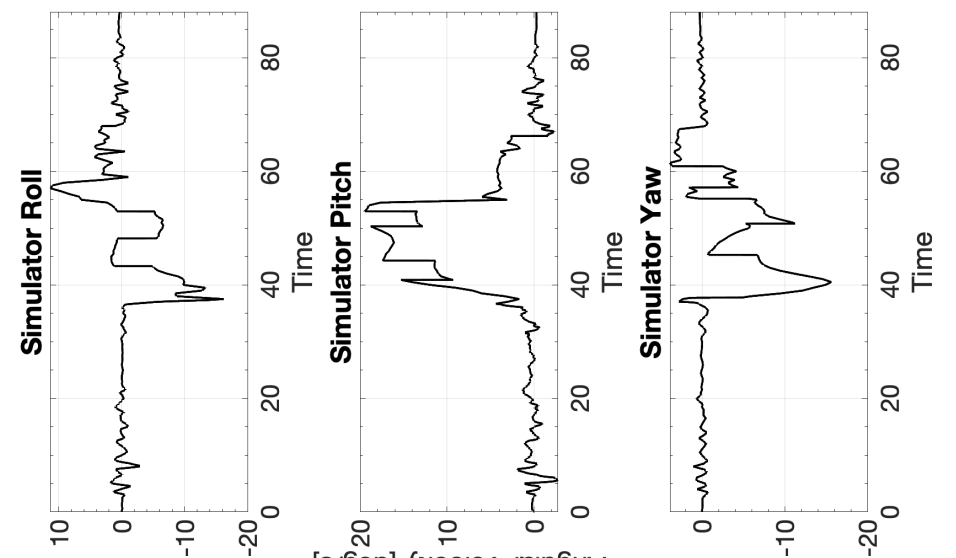


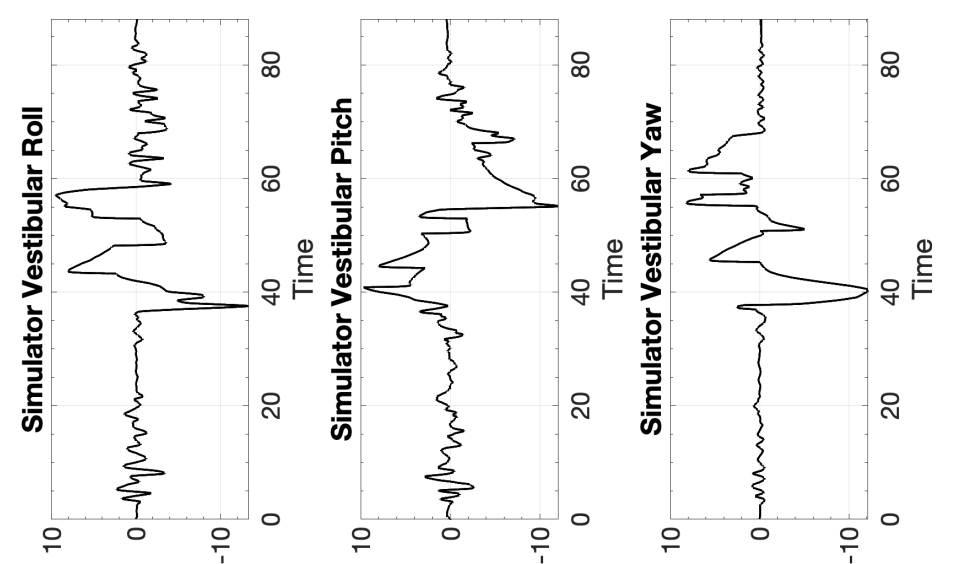

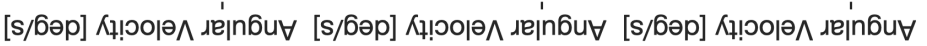

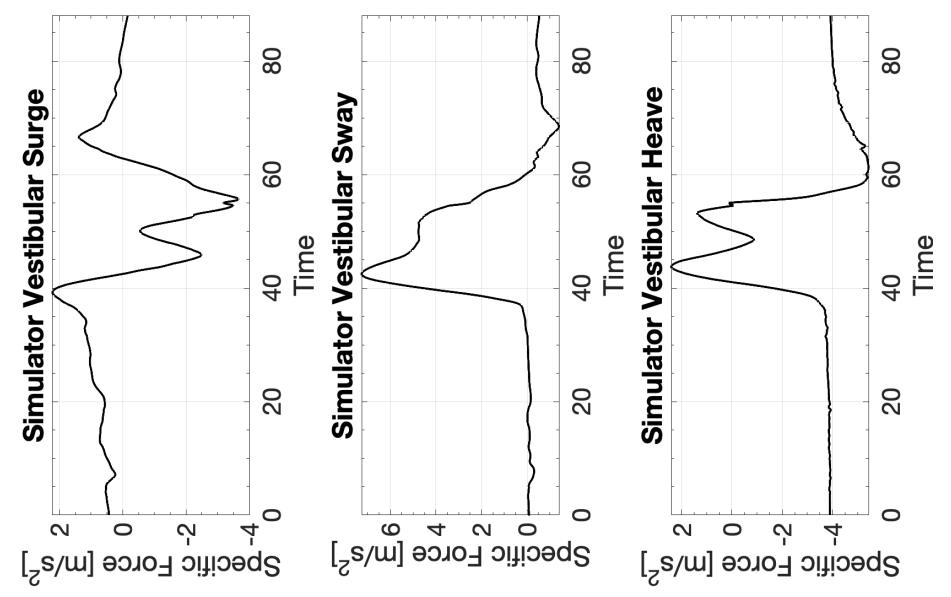




\section{D.4.3 CW3 Cessna 172 Spiral Dive}

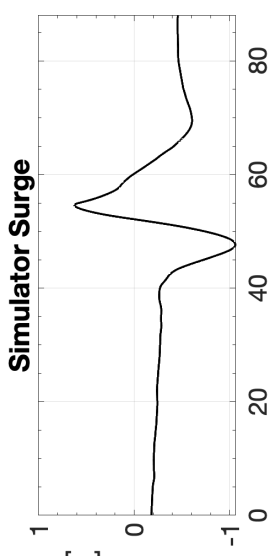

[u] uo!!!!sod

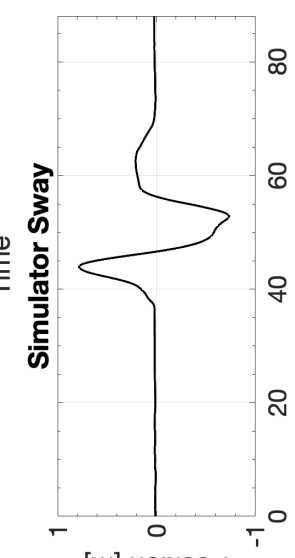

[u] uo!!!!sod

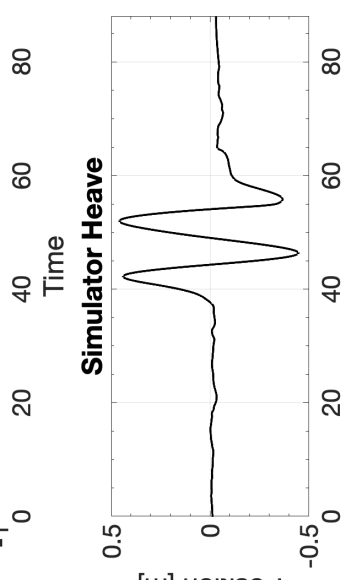

[w] uo!n!sod

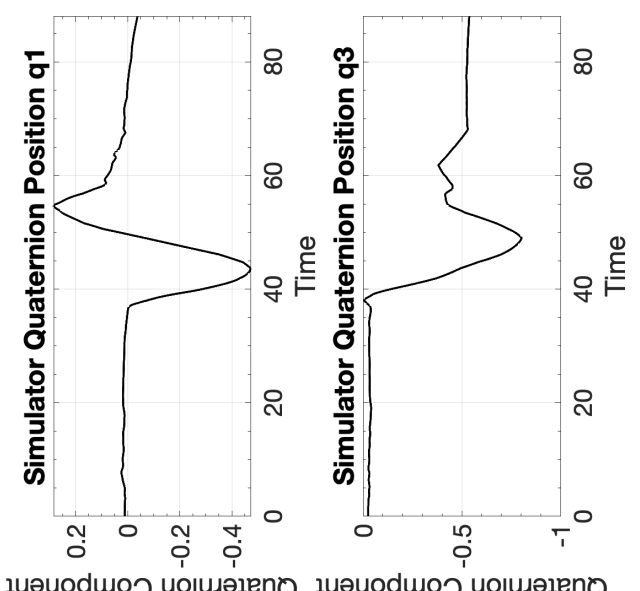

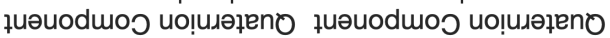

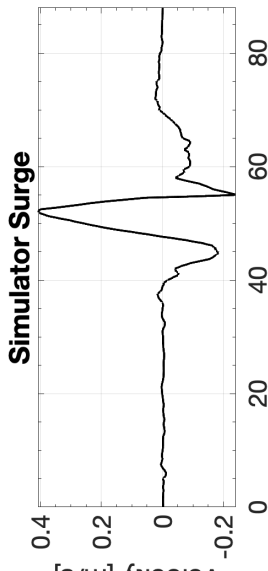

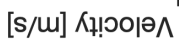

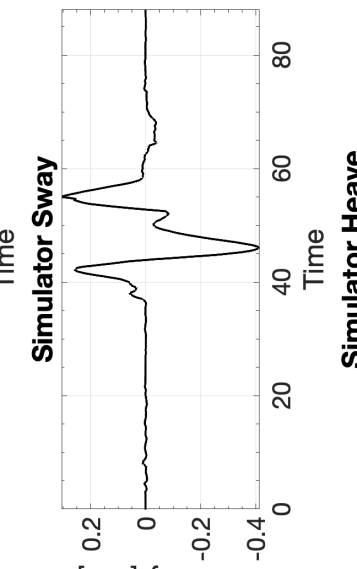

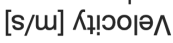

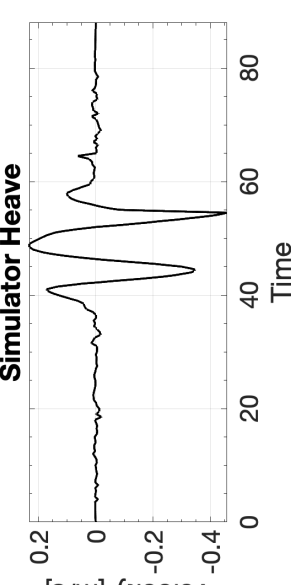

[s/m] Кұ! 잇

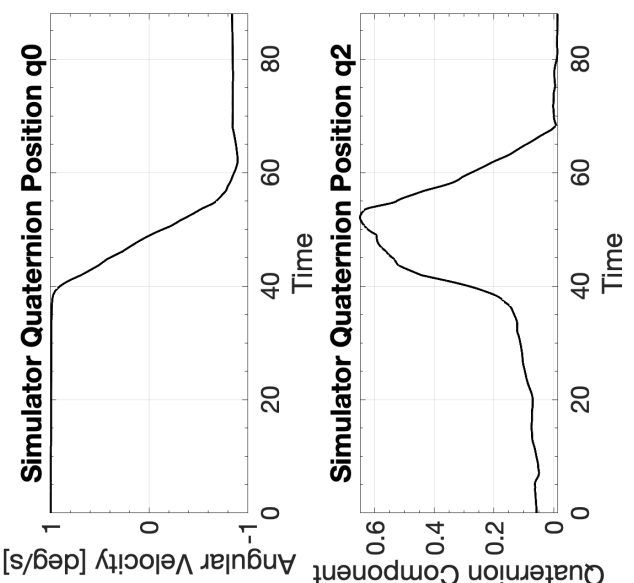

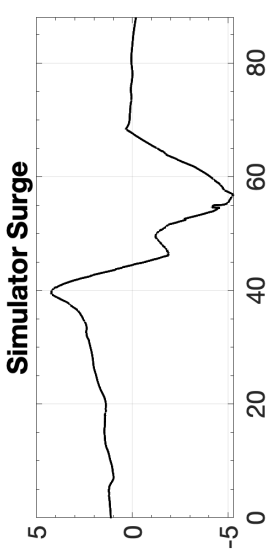

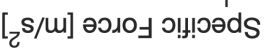

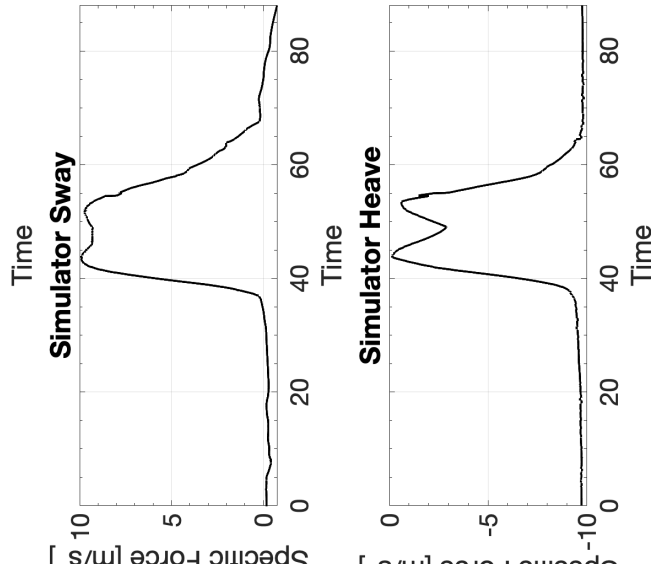

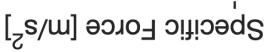

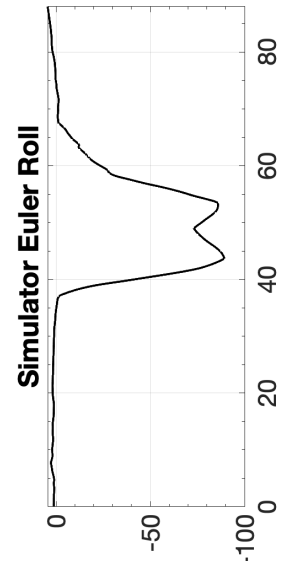

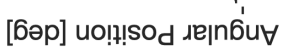

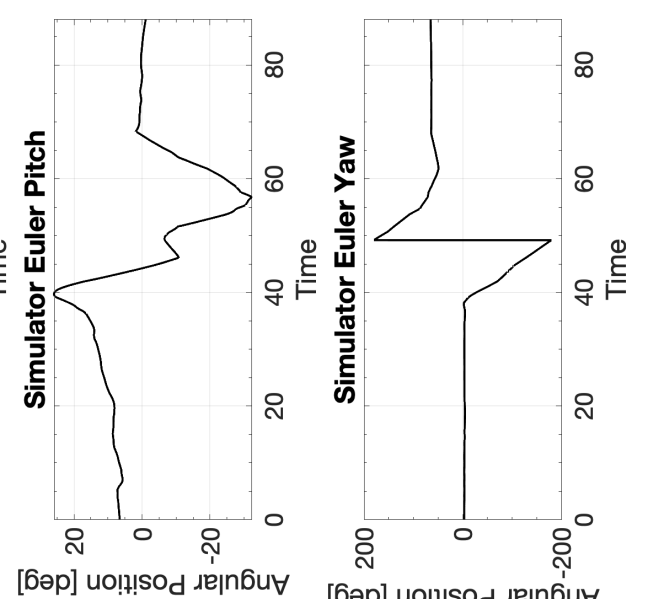

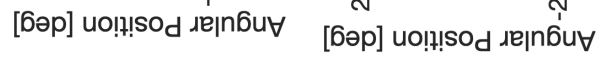

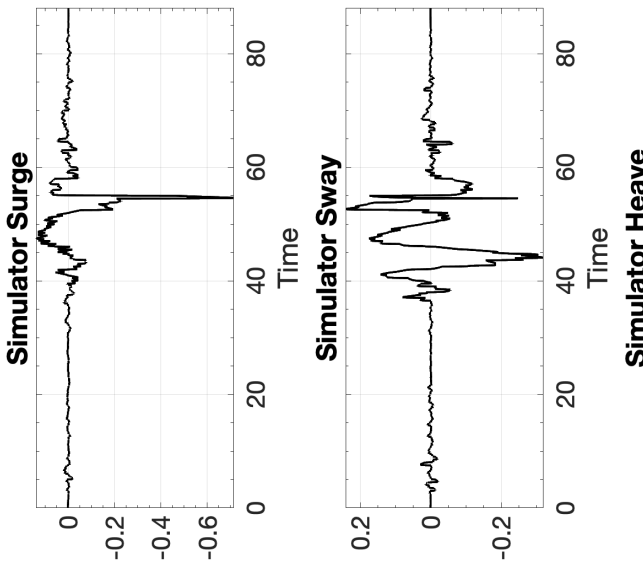

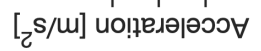

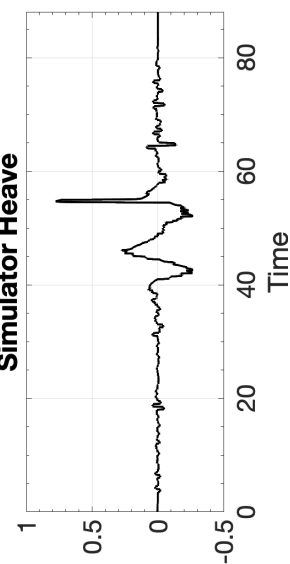

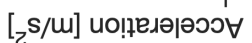

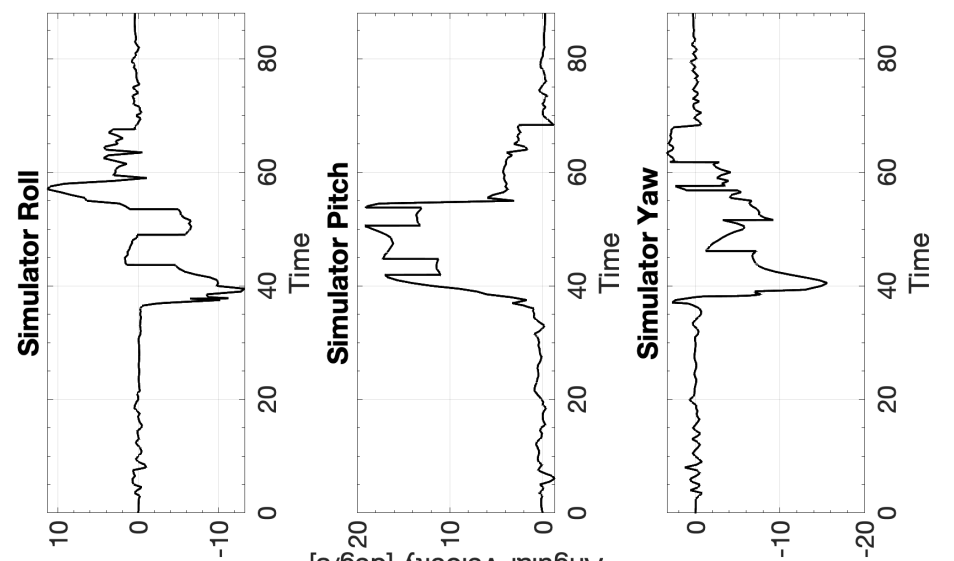

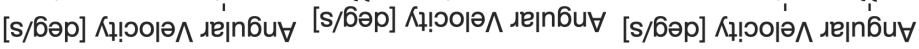




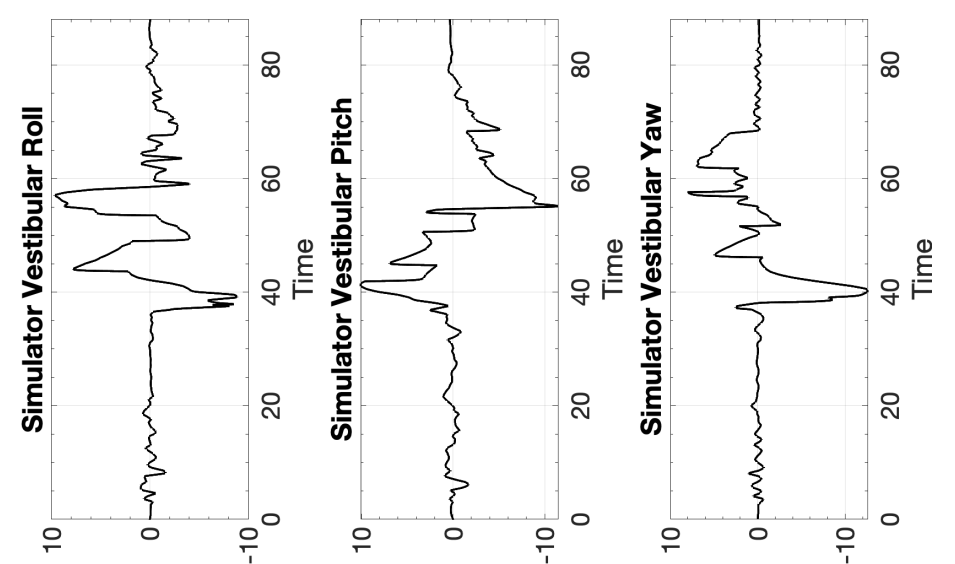

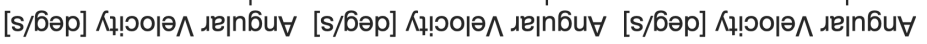

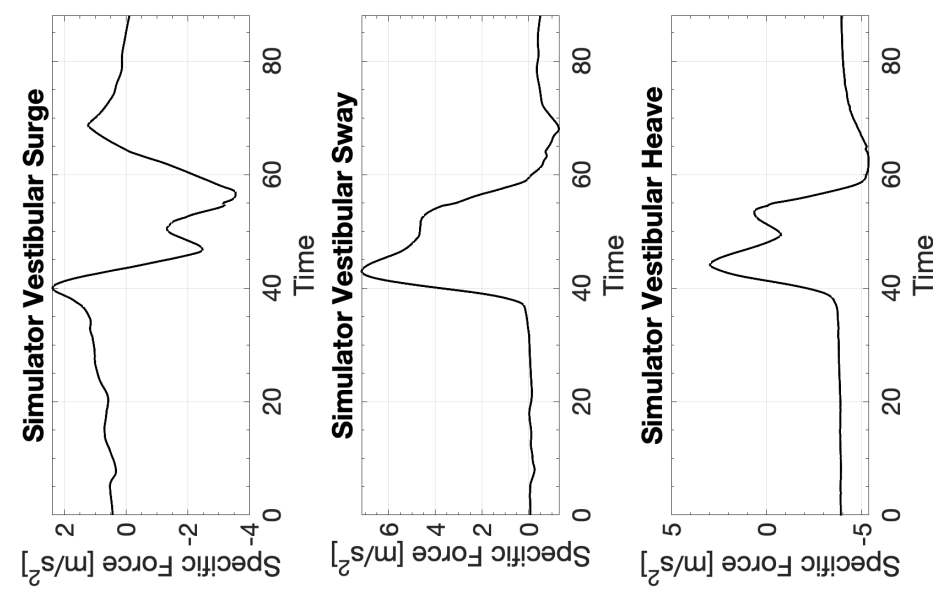




\section{D.5 Cessna 172 Stall Recovery}

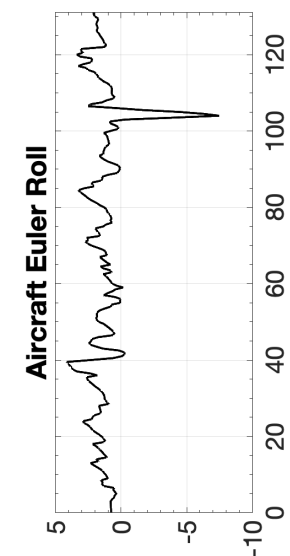

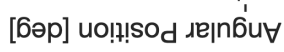

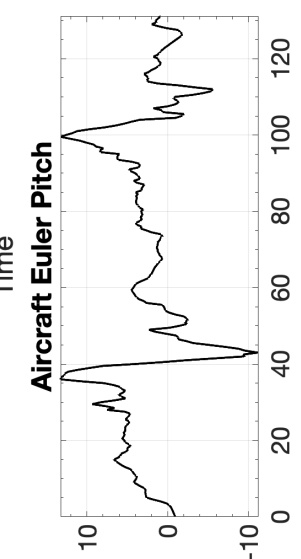

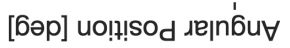

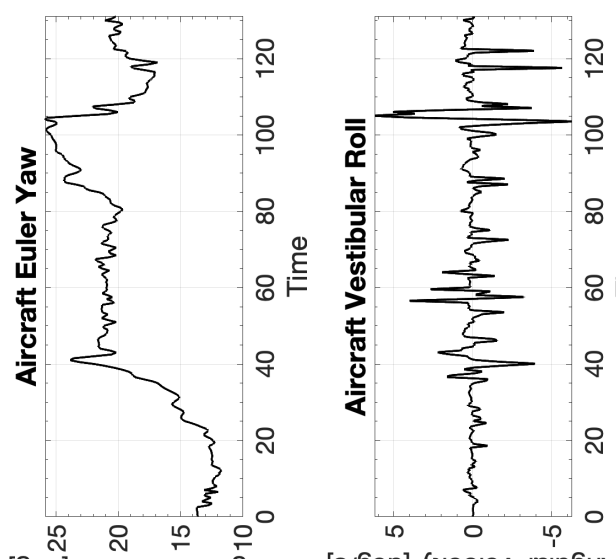

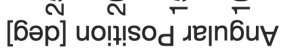
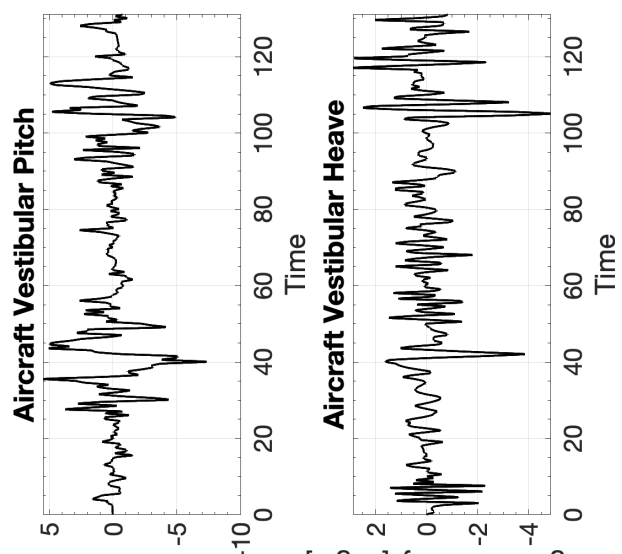

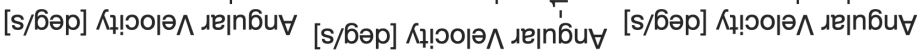
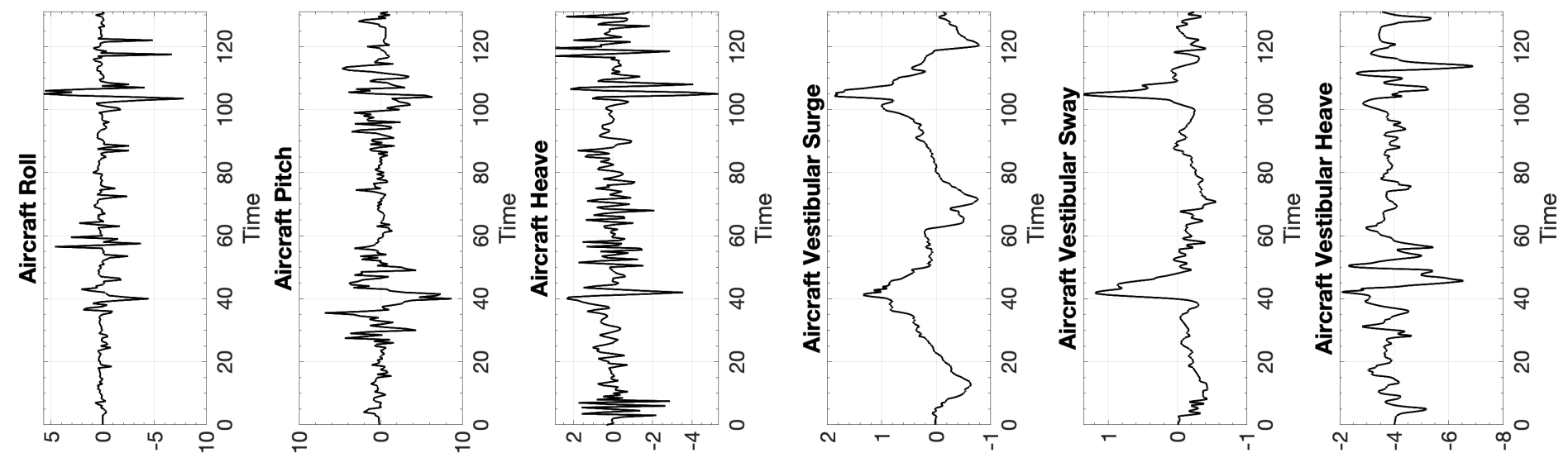

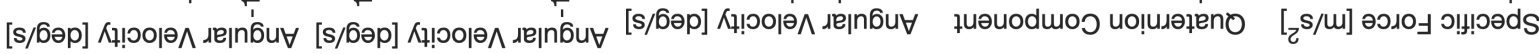

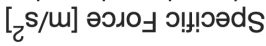

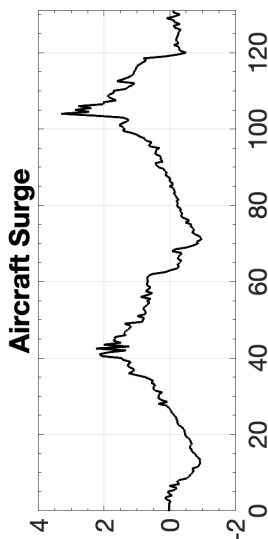

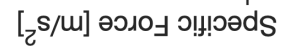

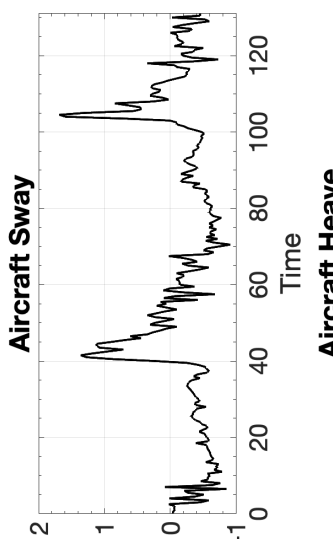

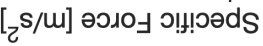

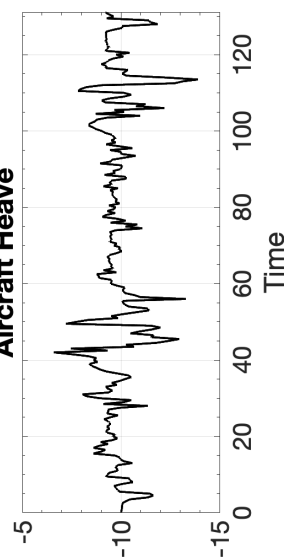

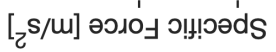
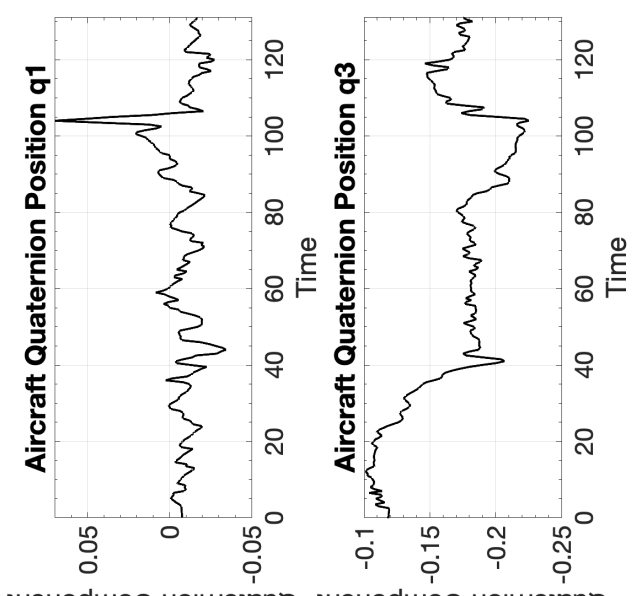

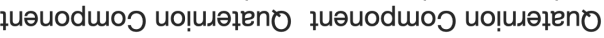

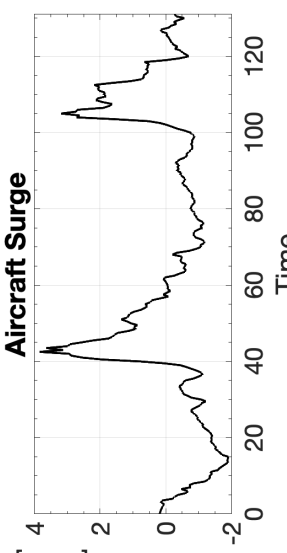

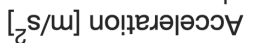

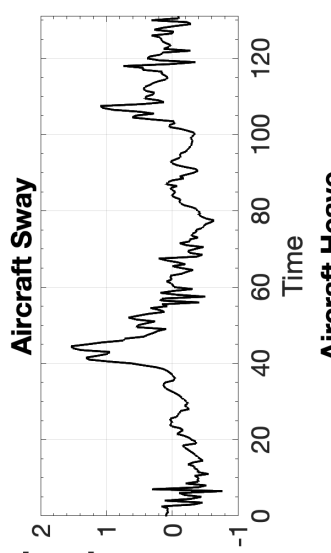

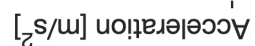

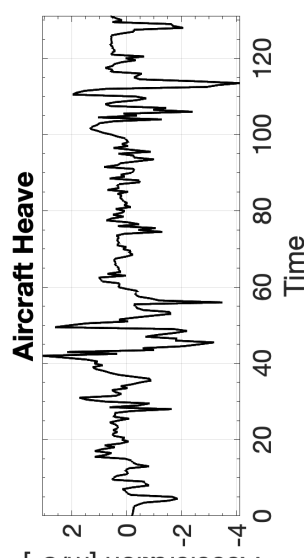

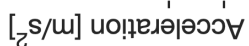
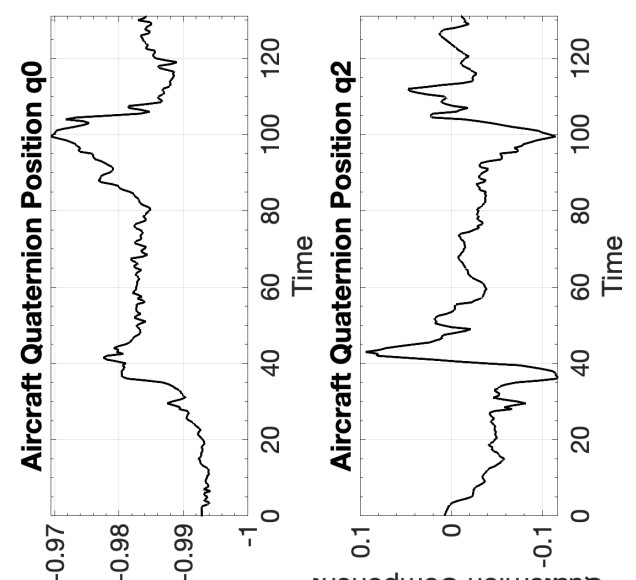

s/[ə்] 


\section{D.5.1 CW1 Cessna 172 Stall Recovery}

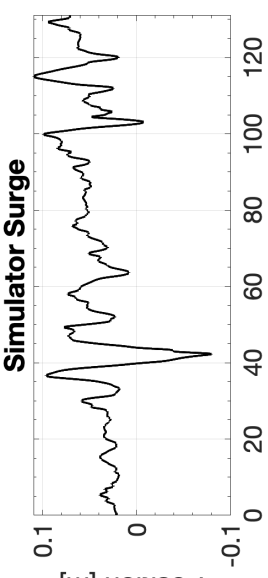

[w] uol!!sod

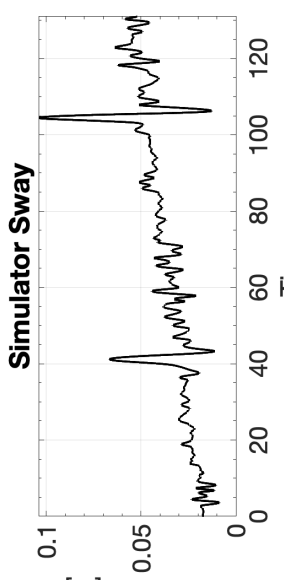

[w] uo!!!!sod

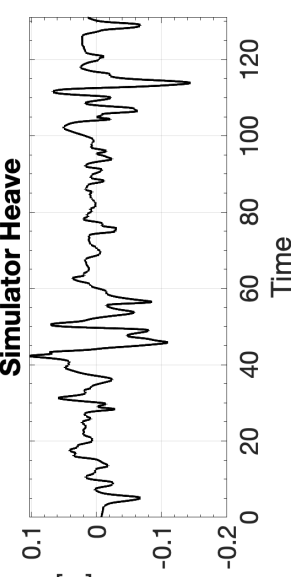

[w] uo!t!sod

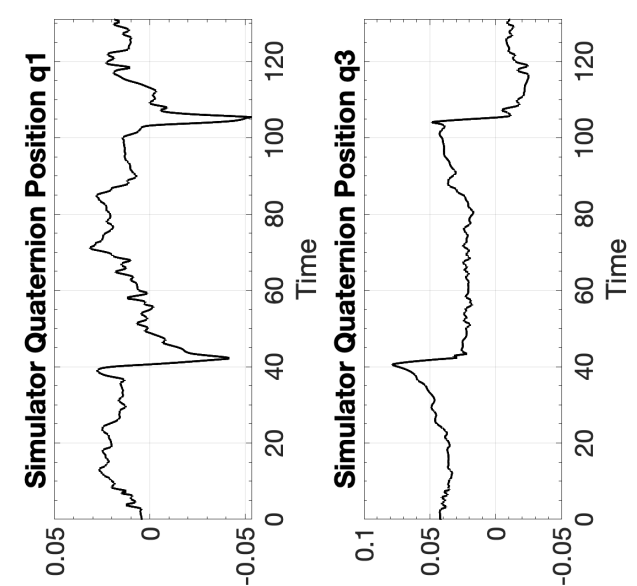

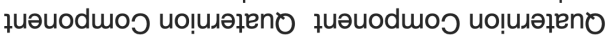

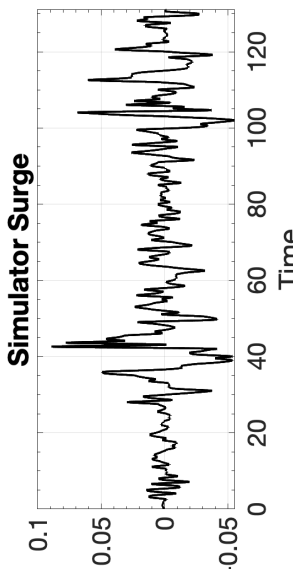

[s/u] Кұ! оㅣ $\Lambda$

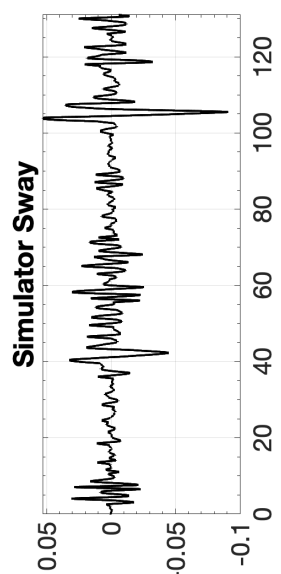

[s/m] Кұ!юор夫

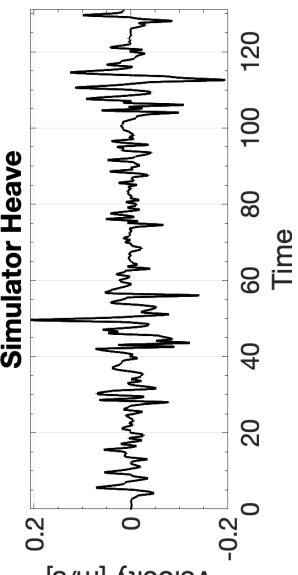

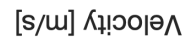

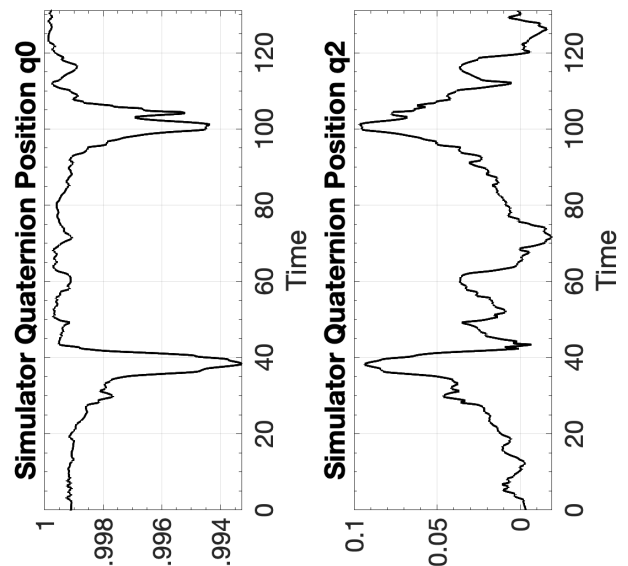

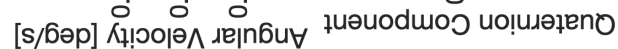

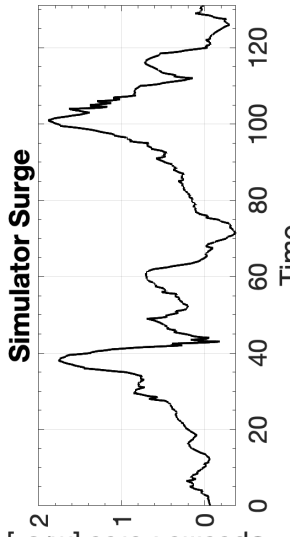

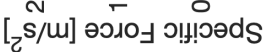

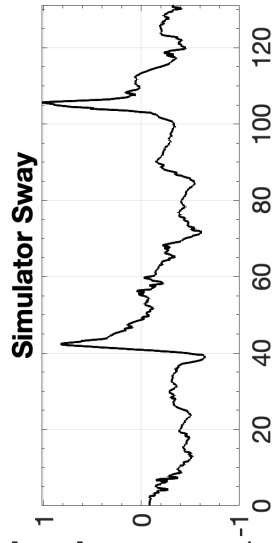

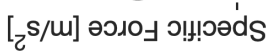
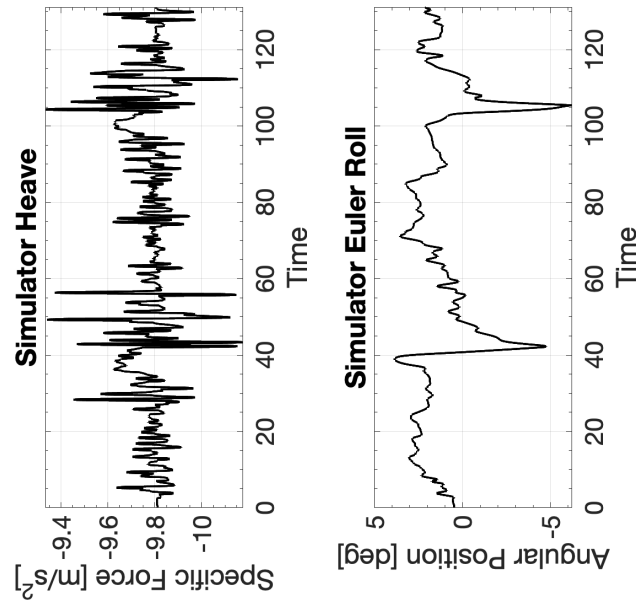

[6әр] uo!!!!sod de|n6ü

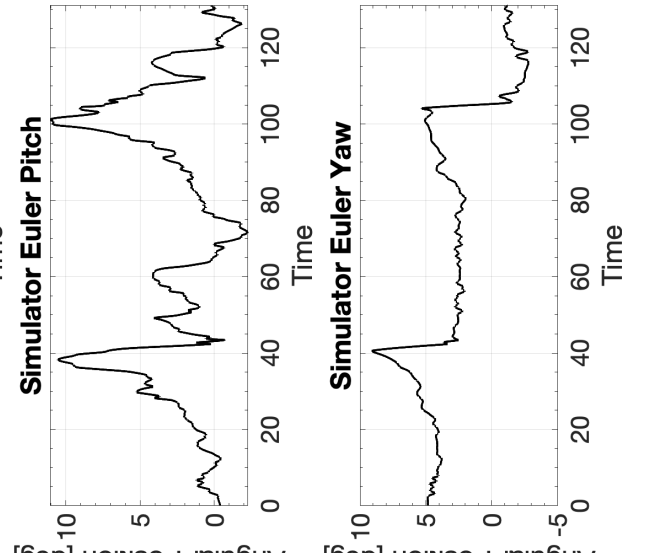

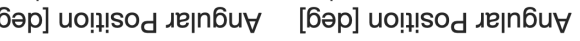

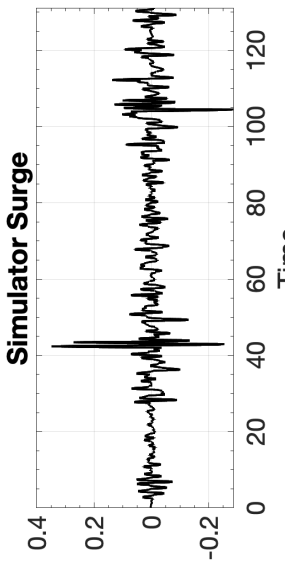

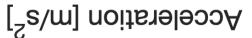

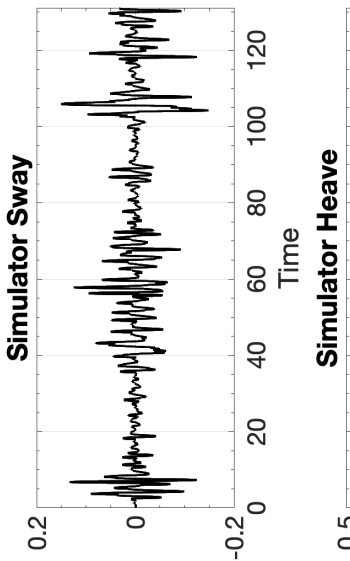

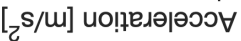

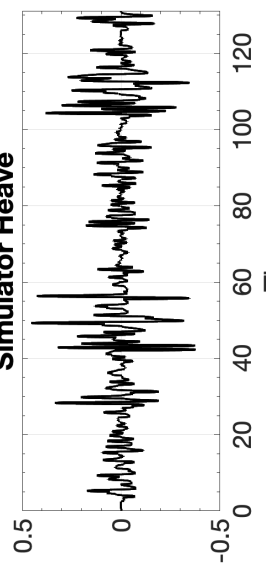

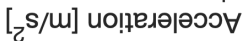

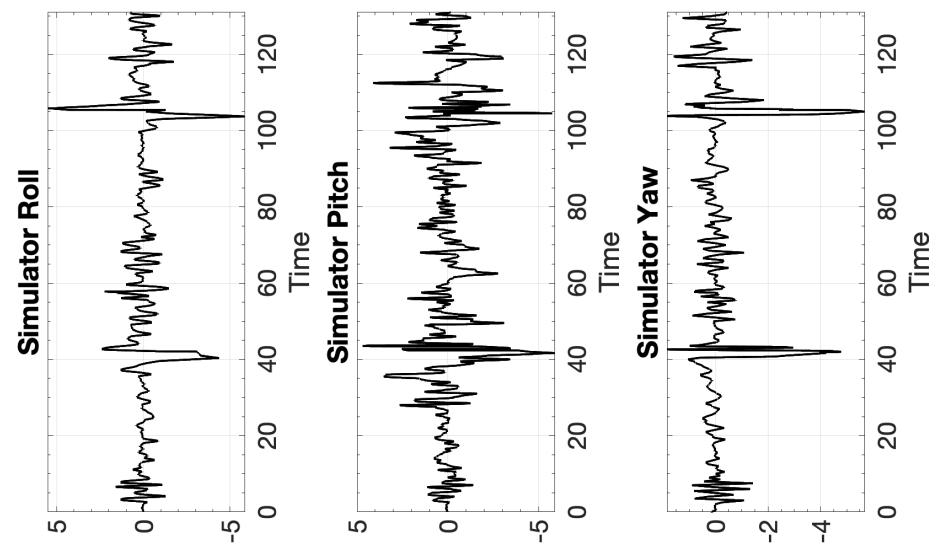

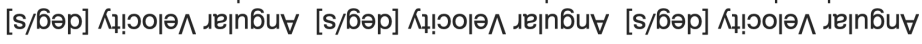




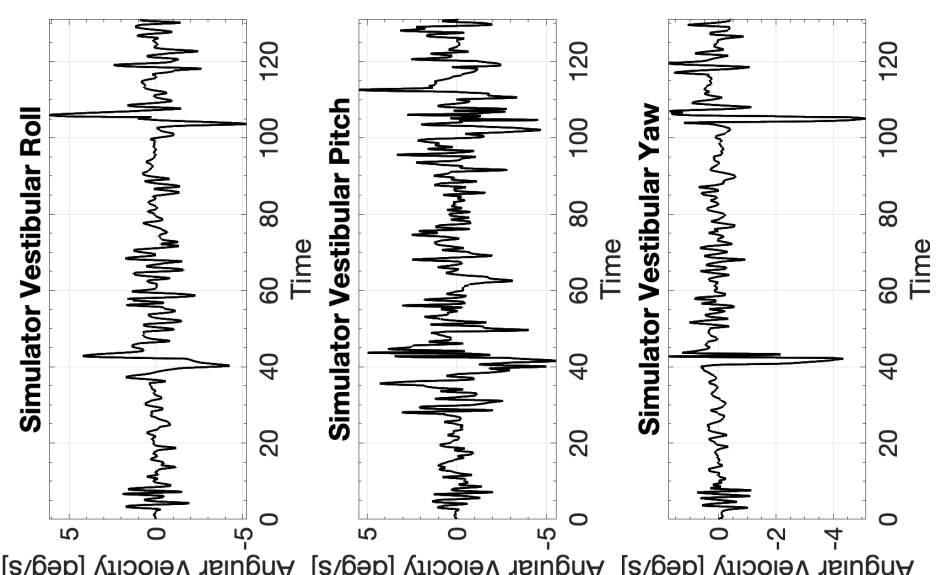

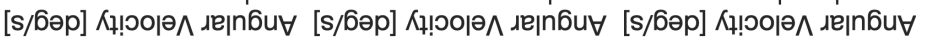

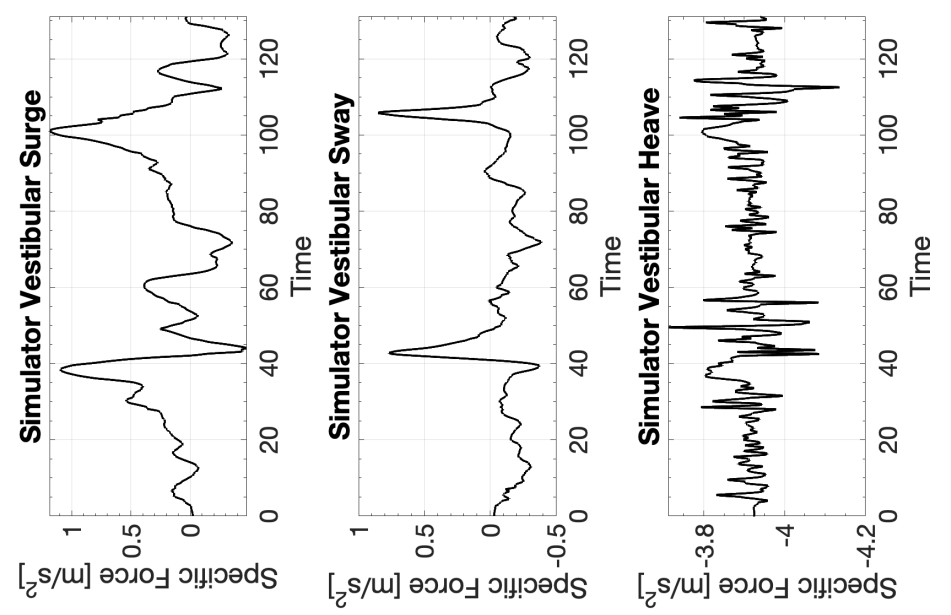




\section{D.5.2 CW2 Cessna 172 Stall Recovery}

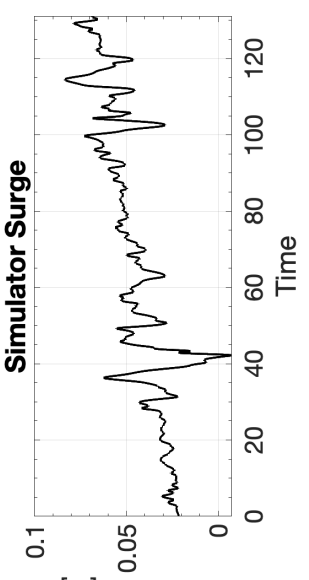

[u] uol!?!sod

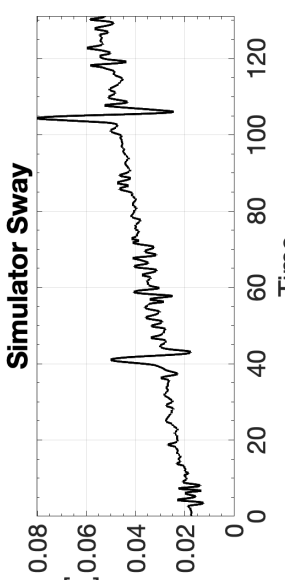

[u] uo!n!!sod

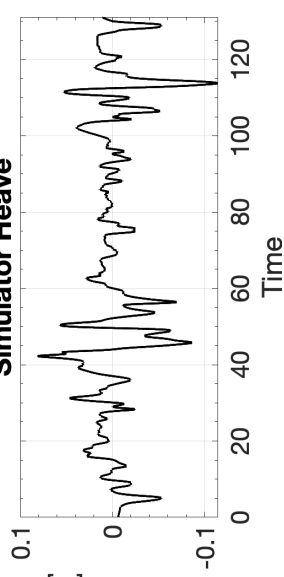

[w] uo!!!sod

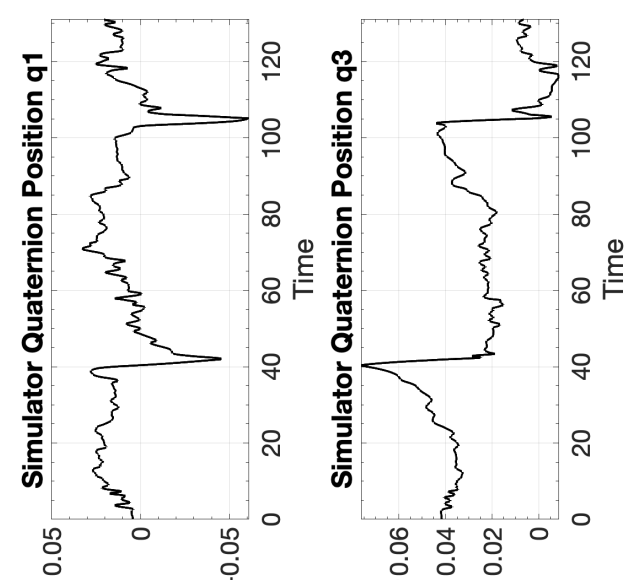

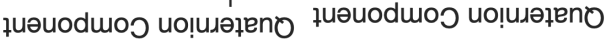

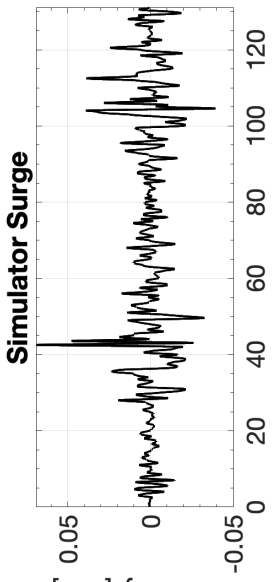

[s/u] Кұ!৩о|ә

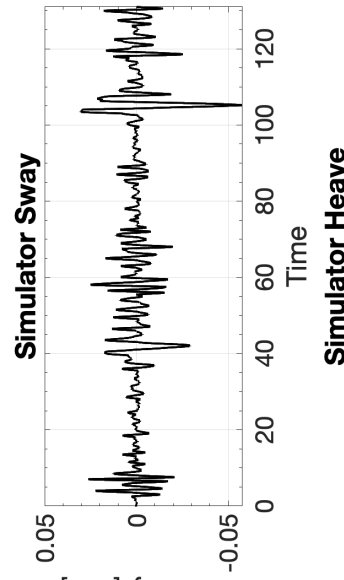

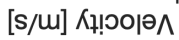

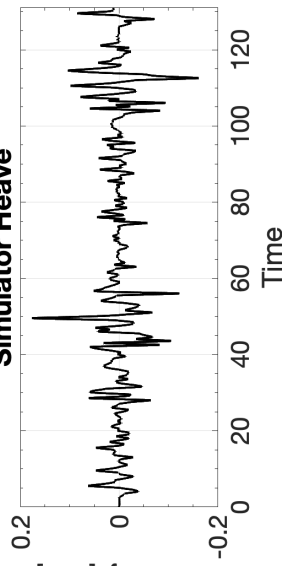

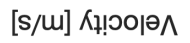

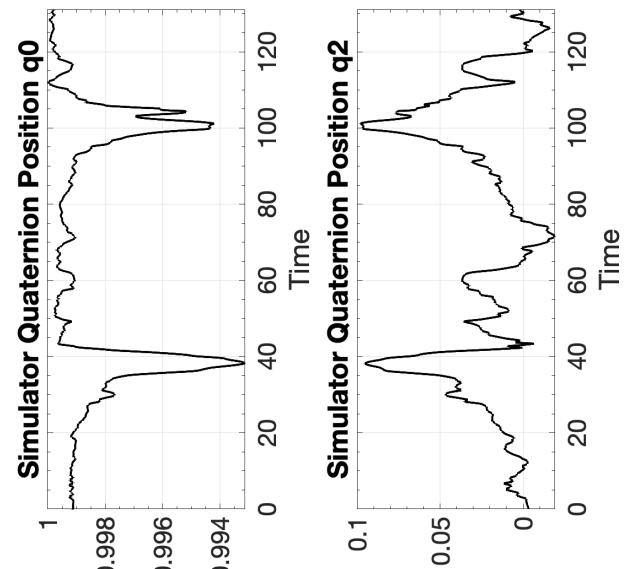

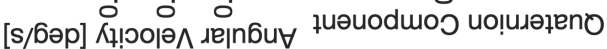

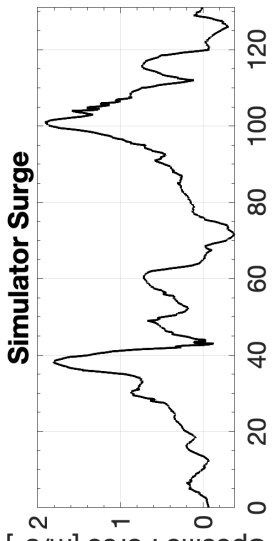

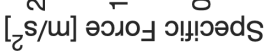

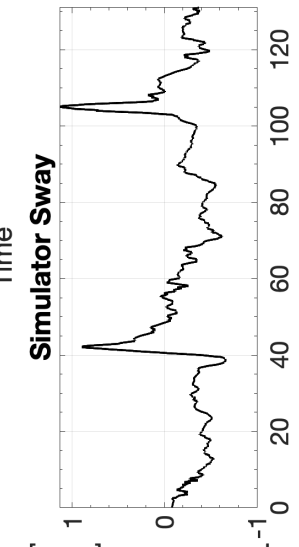

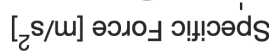
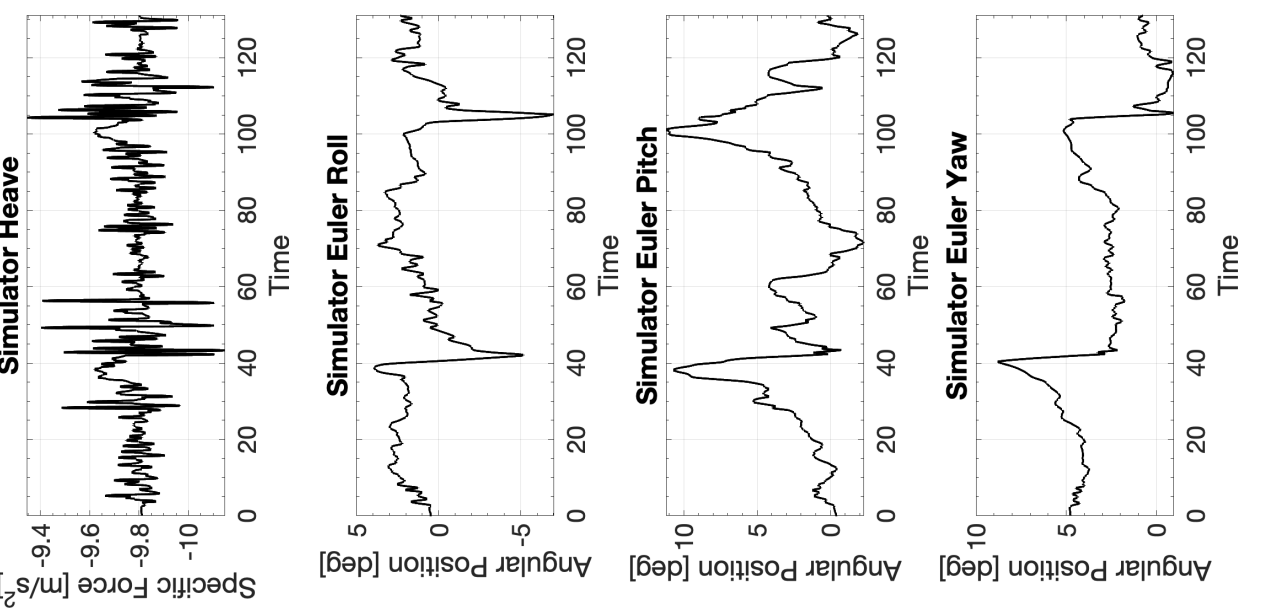

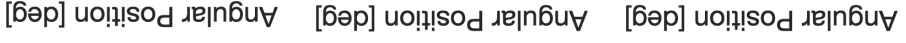

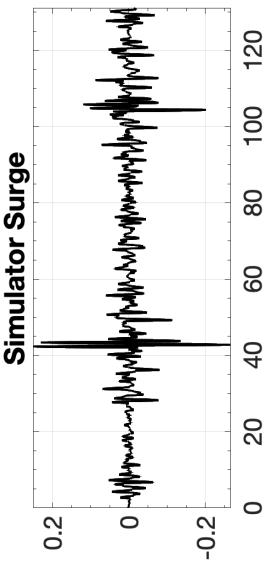

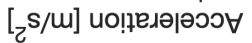

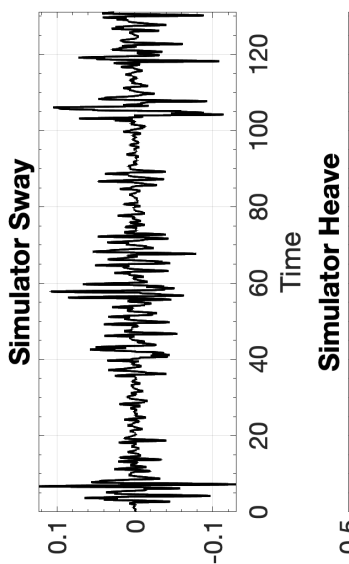

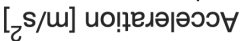

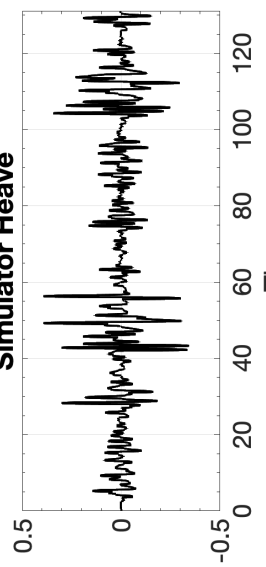

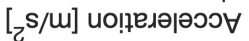

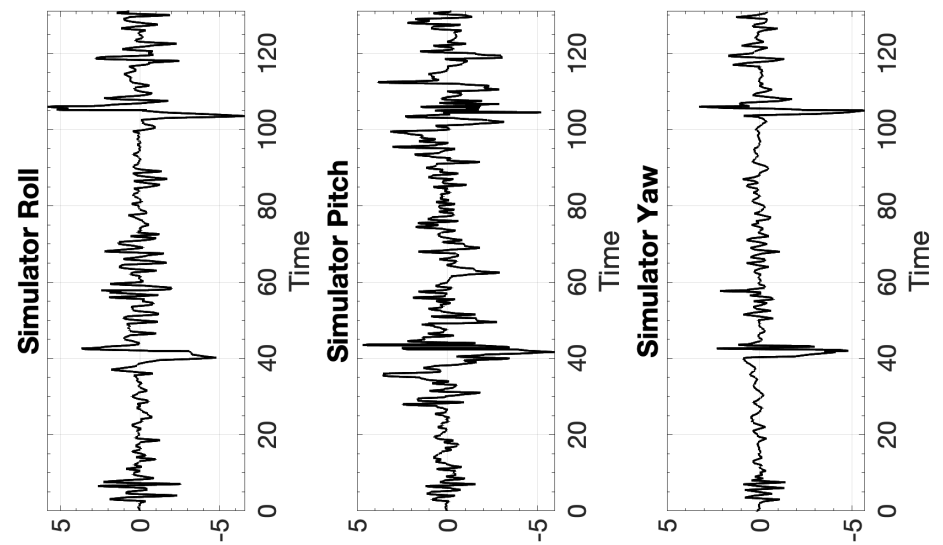

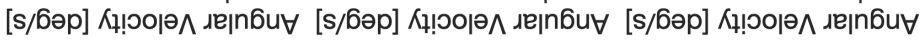



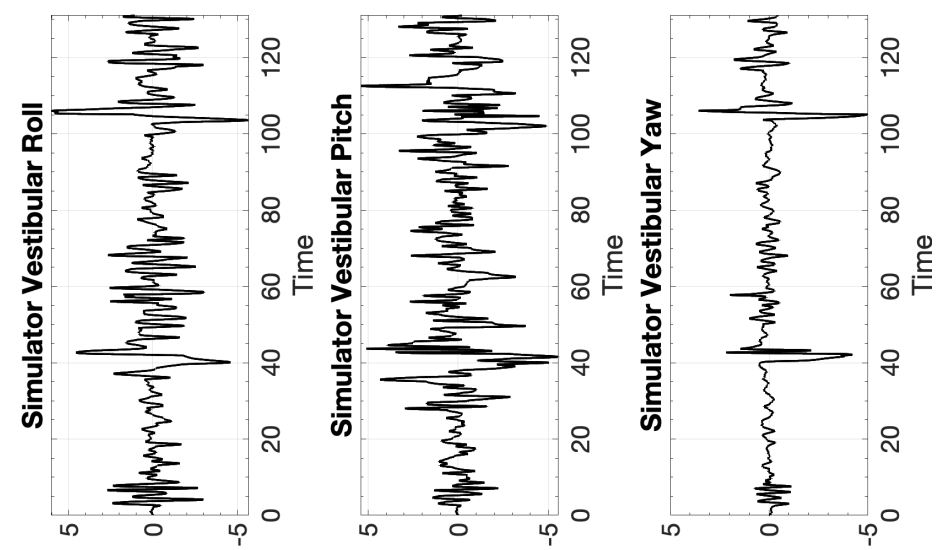

[s/бәр] Кұ!юою夫

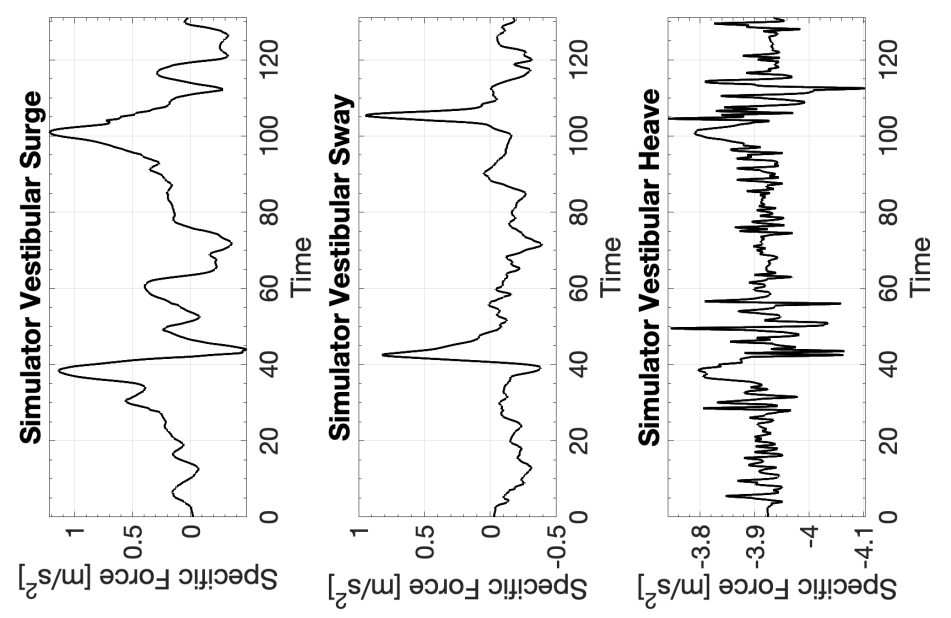




\section{D.5.3 CW3 Cessna 172 Stall Recovery}

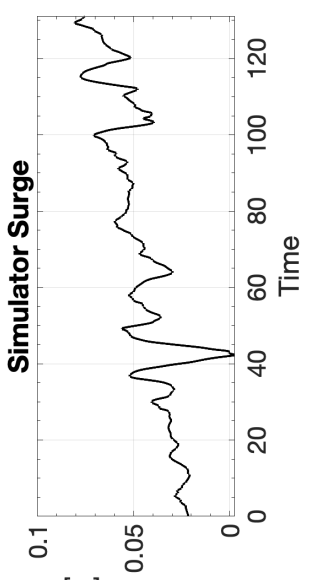

[w] uo!n!sod

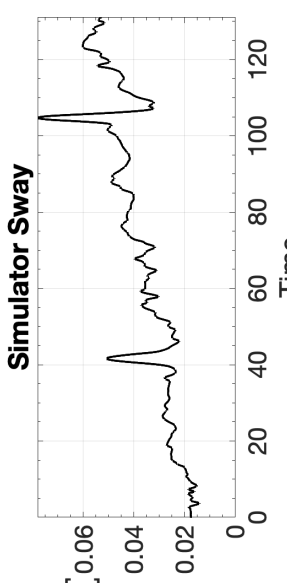

[w] uo!n! $\mathrm{SO}_{\mathrm{d}}$

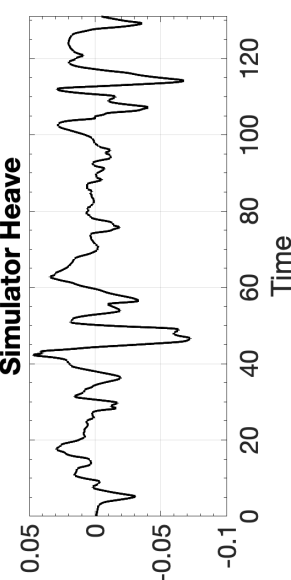

$[\mathrm{m}]$ uo!n! $\mathrm{sO}_{\mathrm{d}}$

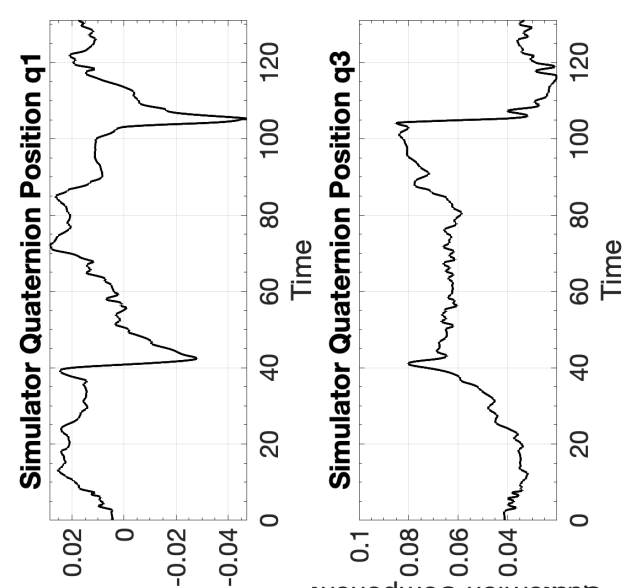

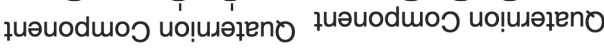

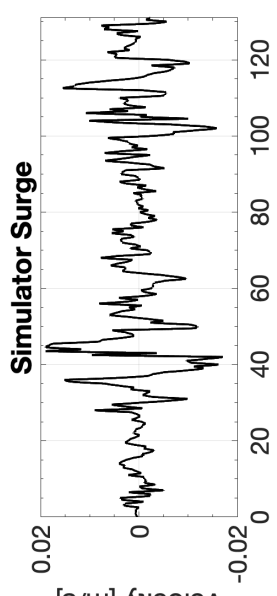

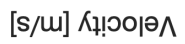

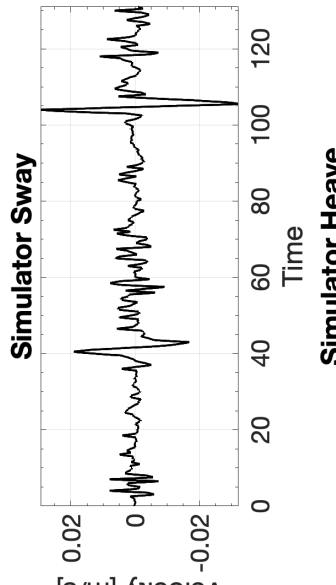

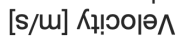

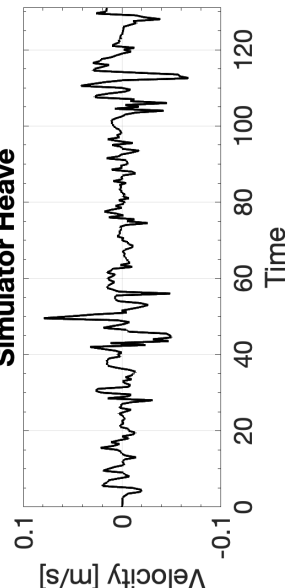

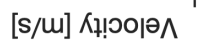

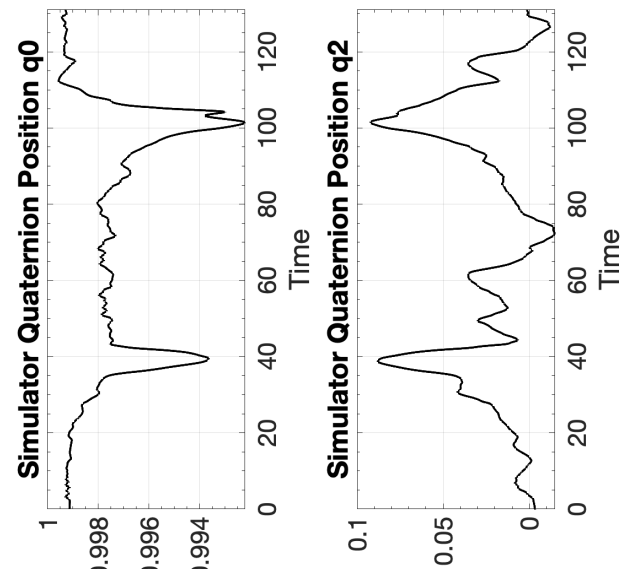

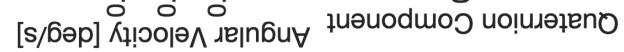

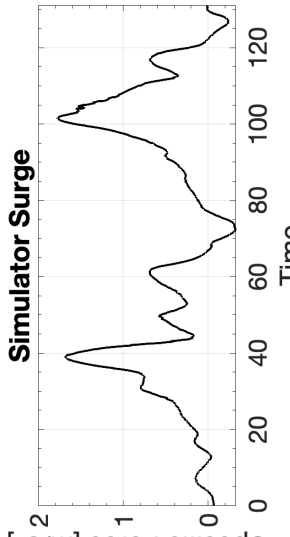

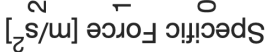
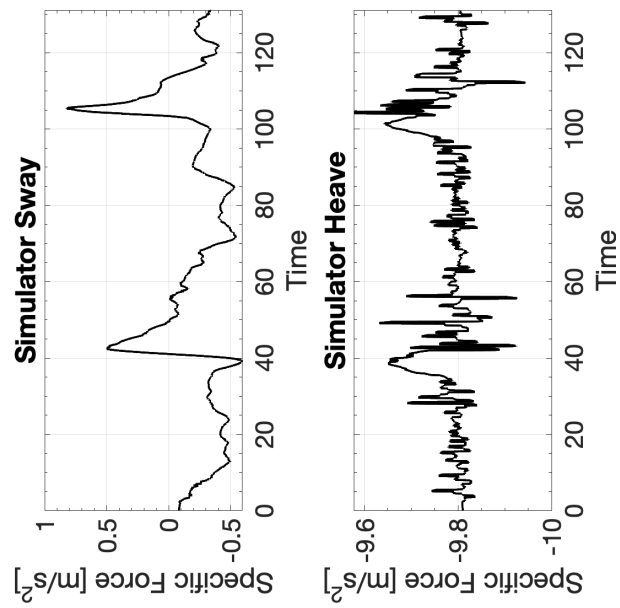

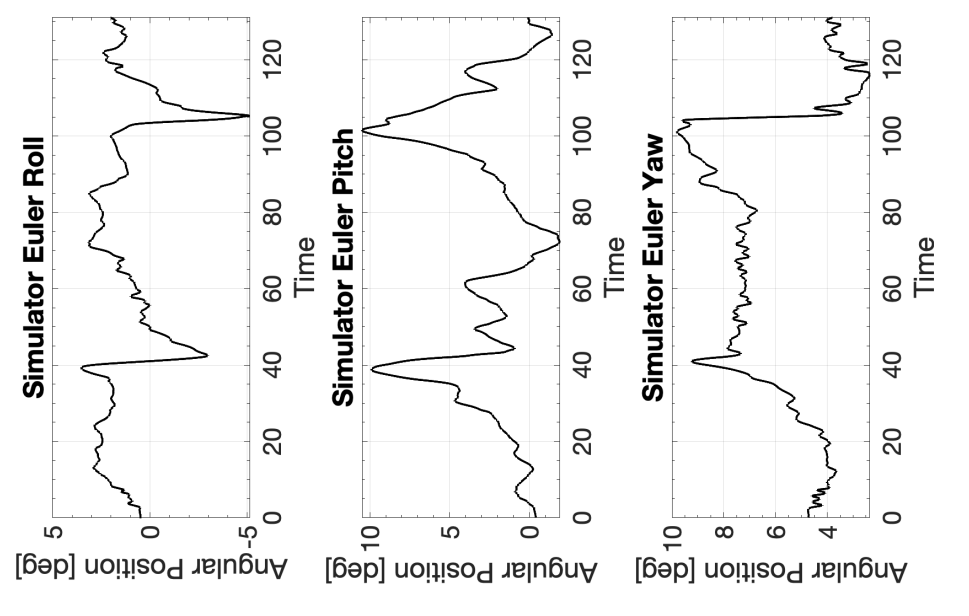

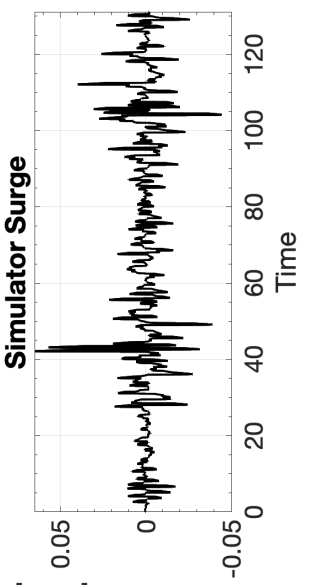

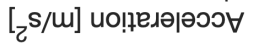

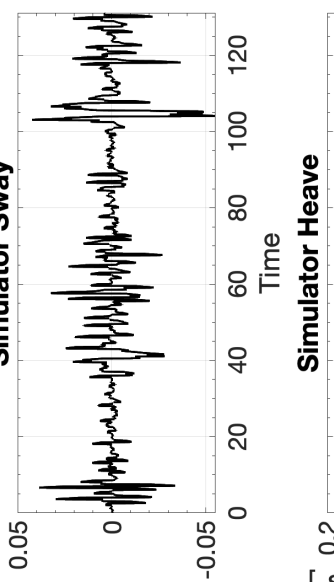

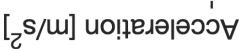

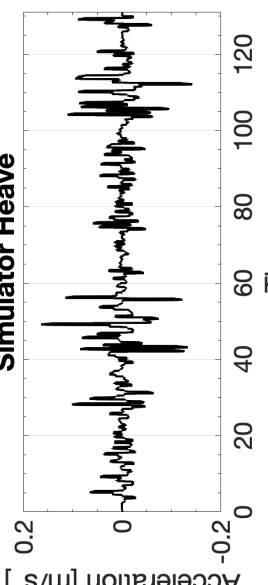

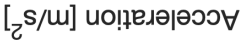
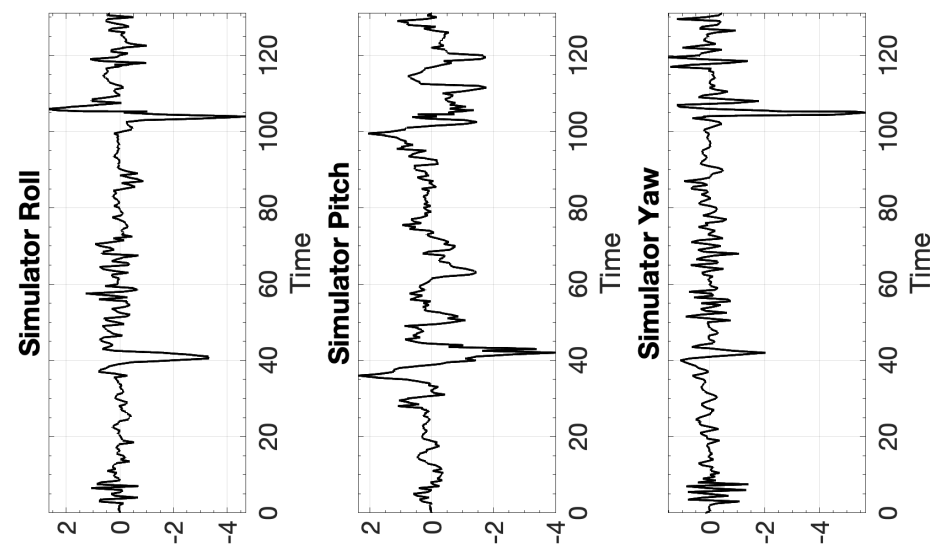

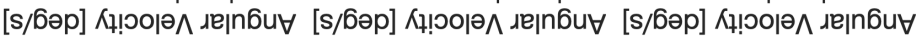



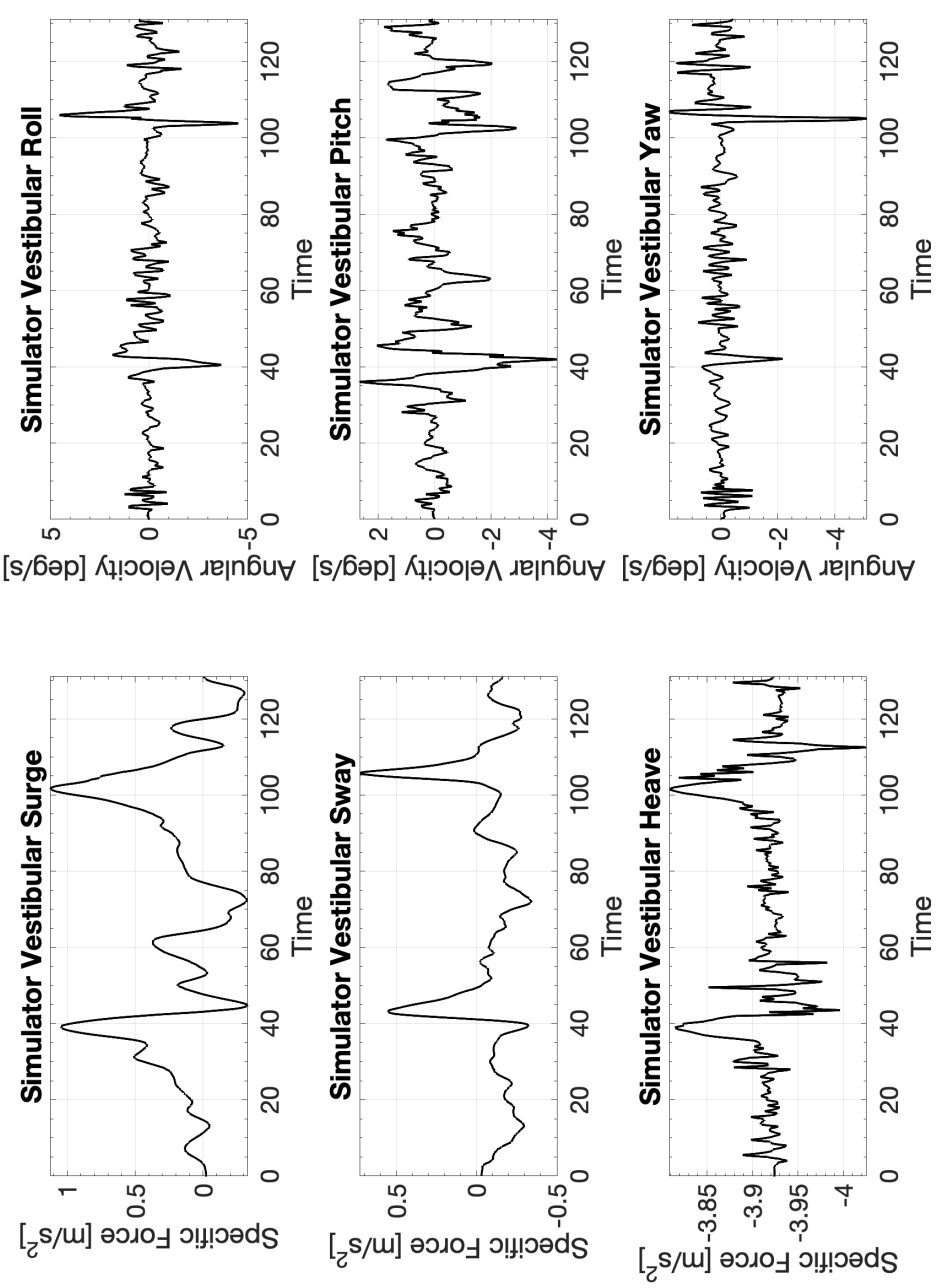


\section{D.6 Boeing 737 Coordinated Turn}

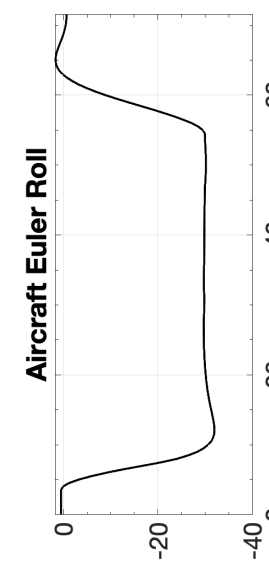

[6əp] uo!!!sod גe|n6ü

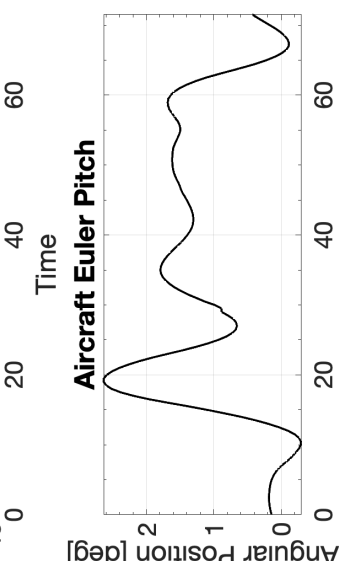

[6әр] uolı!sod גe|n6ur

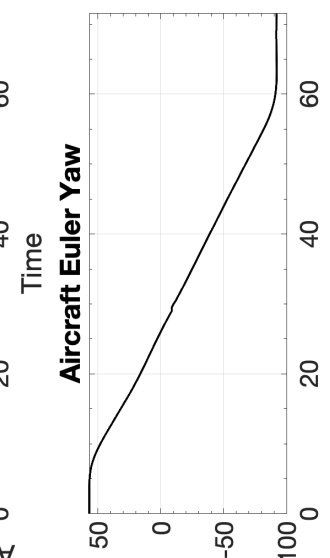

[бәp] uo!̣!!sod גe|nб์u $\forall$

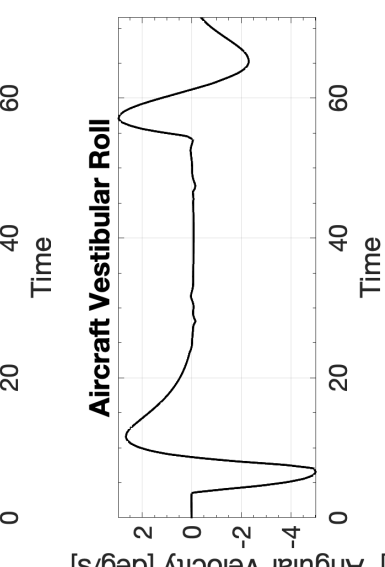

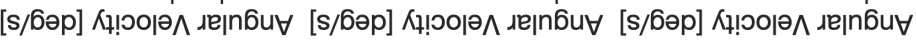

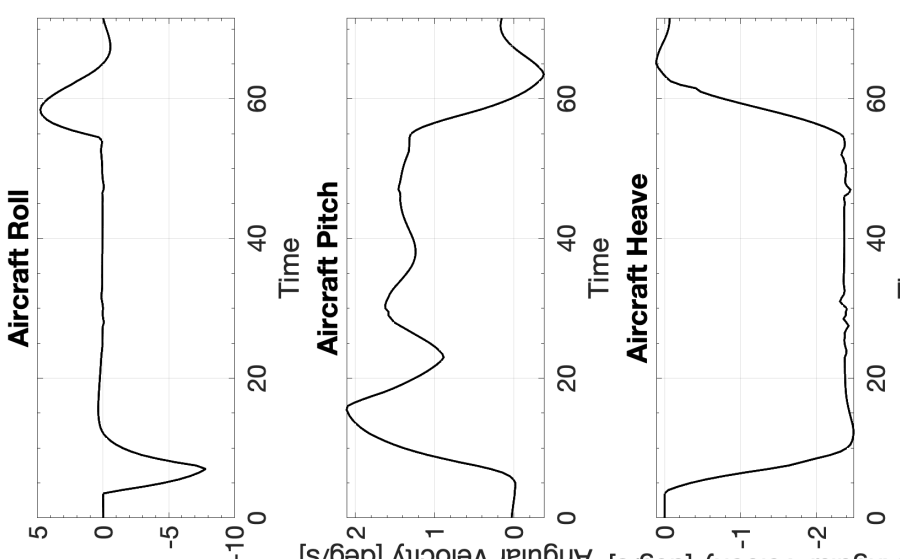

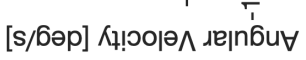

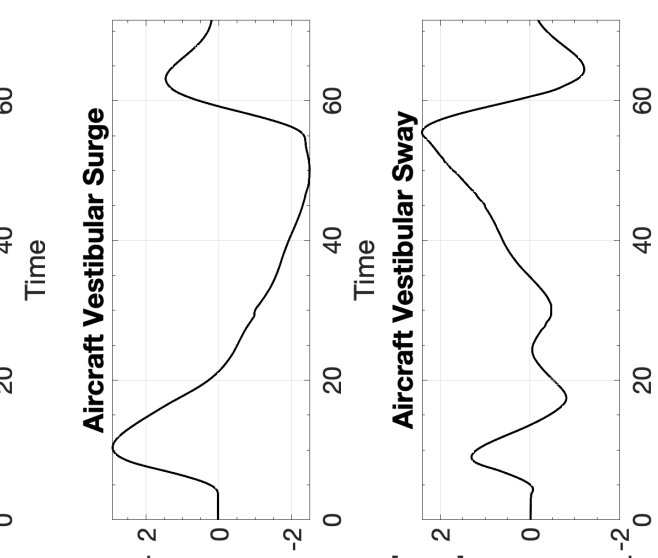

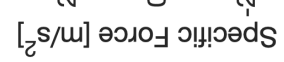

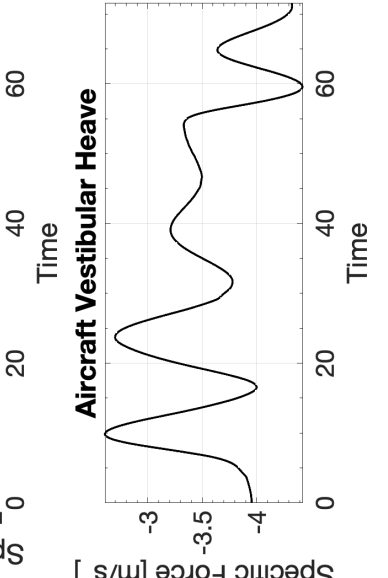

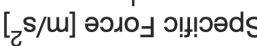

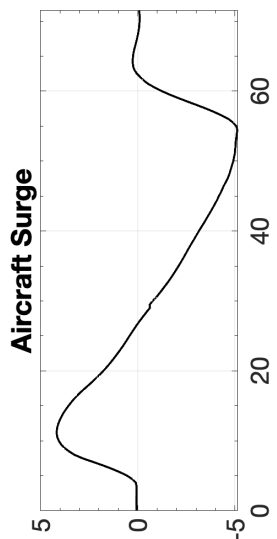

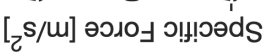

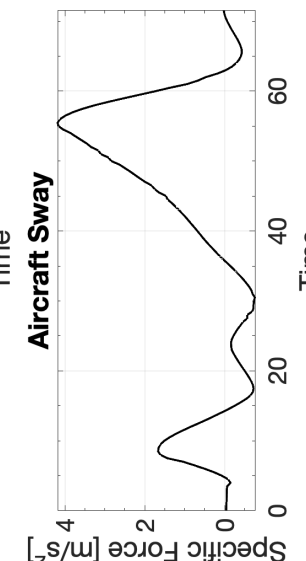

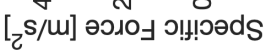

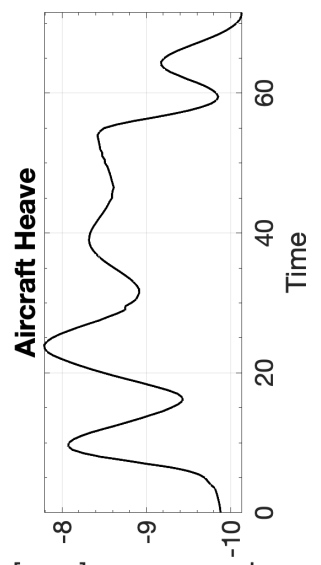

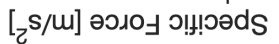

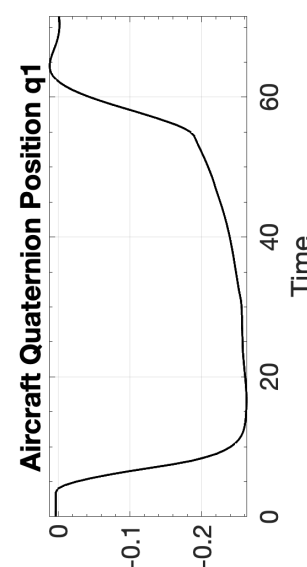

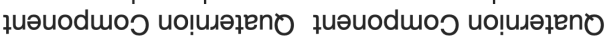

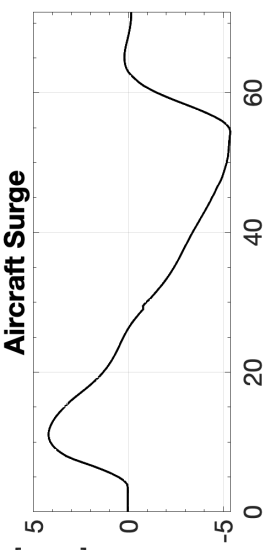

$[\mathrm{c} s / \mathrm{m}]$ uо!ฺвләрәээ

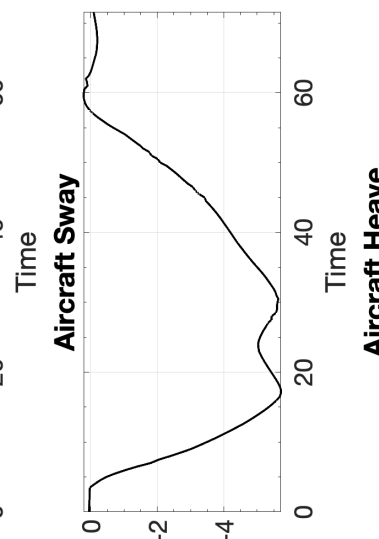

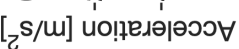

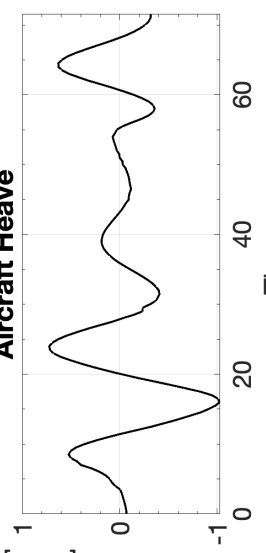

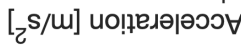

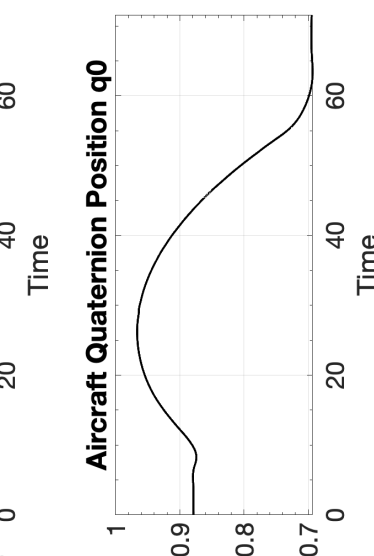

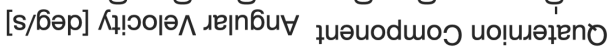
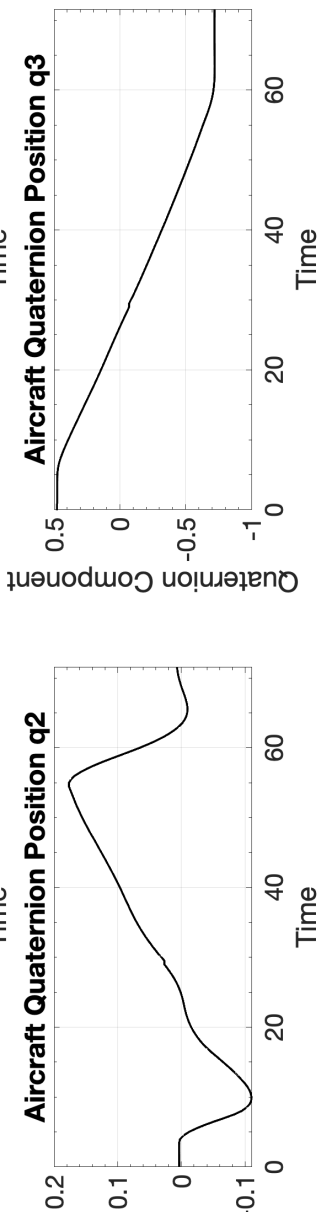


\section{D.6.1 CW1 Boeing 737 Coordinated Turn}

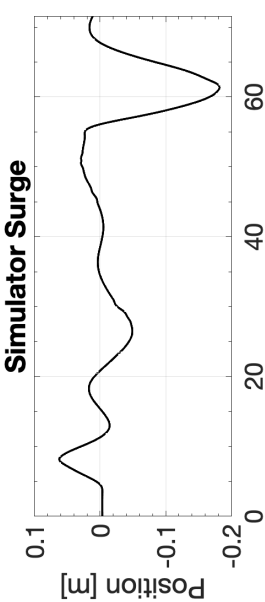

[m] uol!!!sod

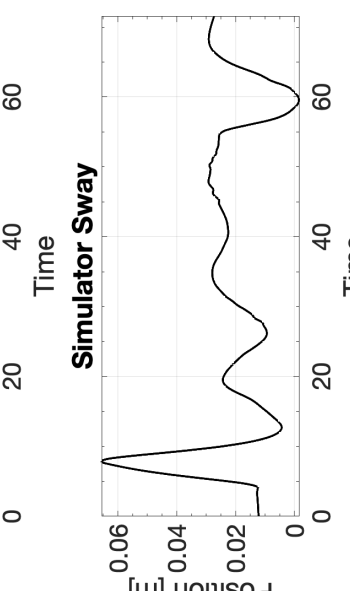

[u] uol!!!sod

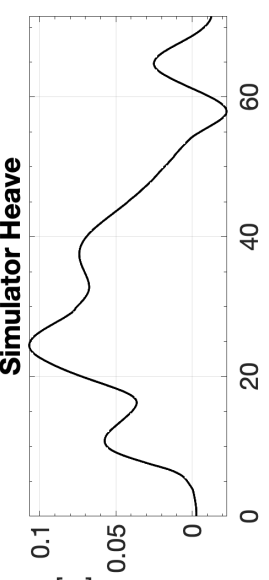

[w] uol!!sod

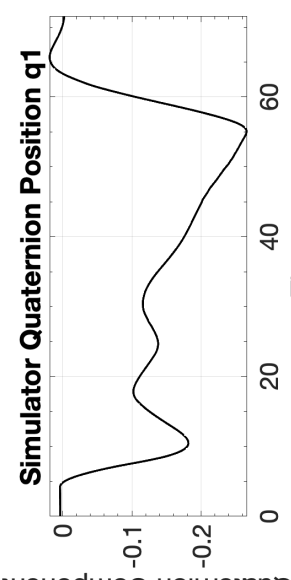

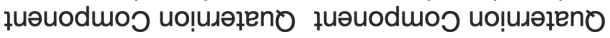

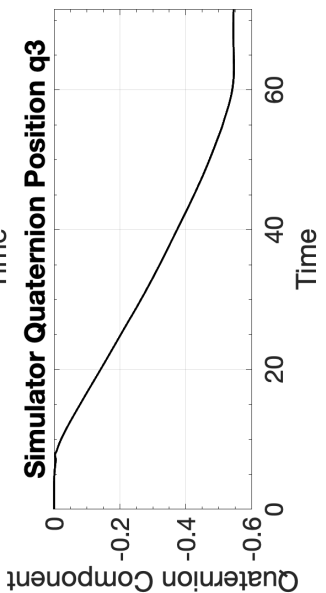

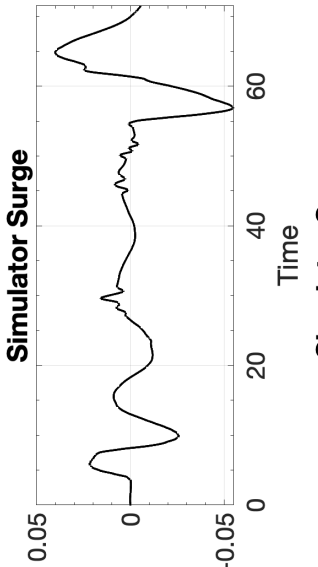

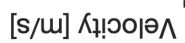

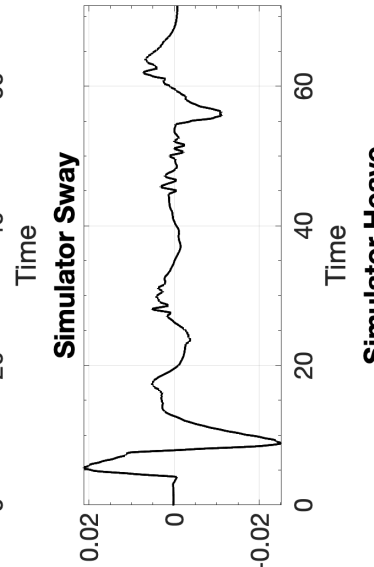

[s/m] Кұ!юою^

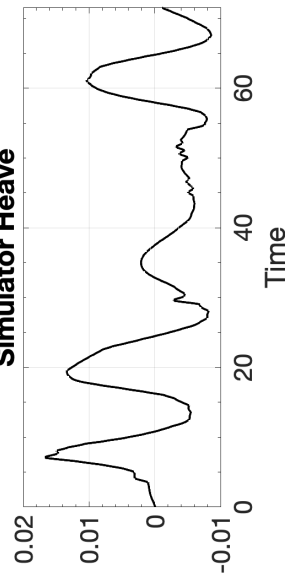

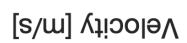
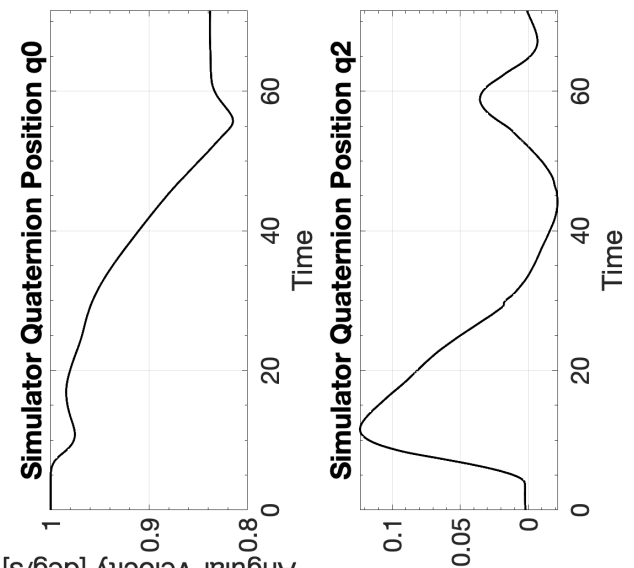

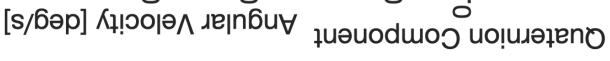

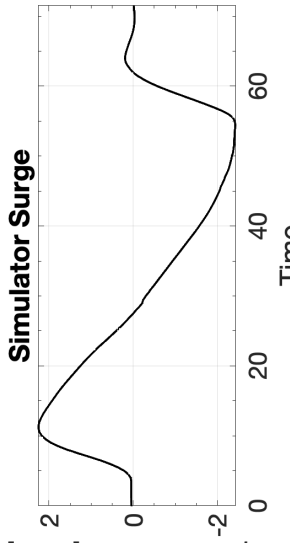

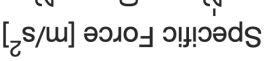

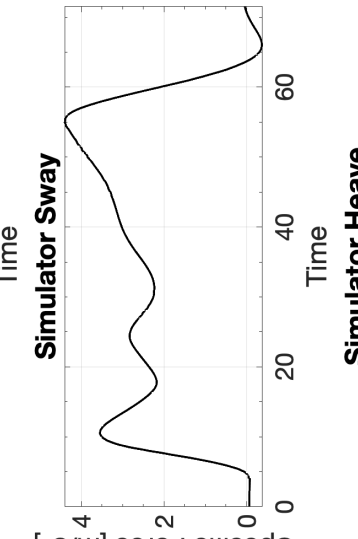

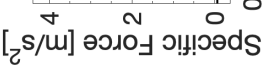
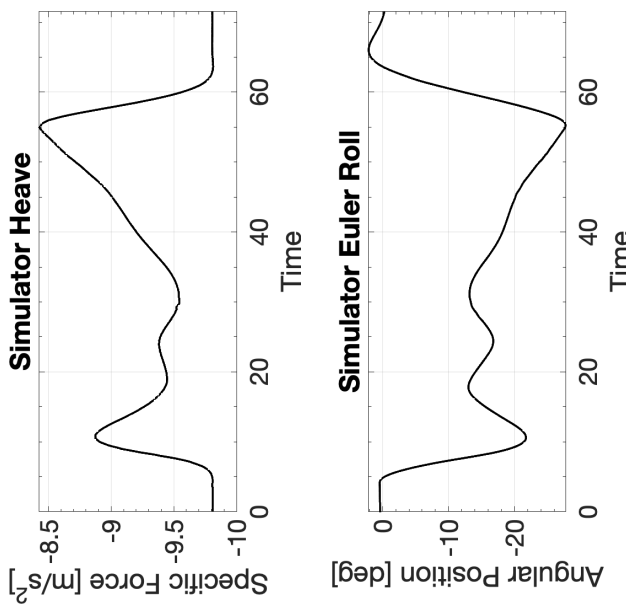

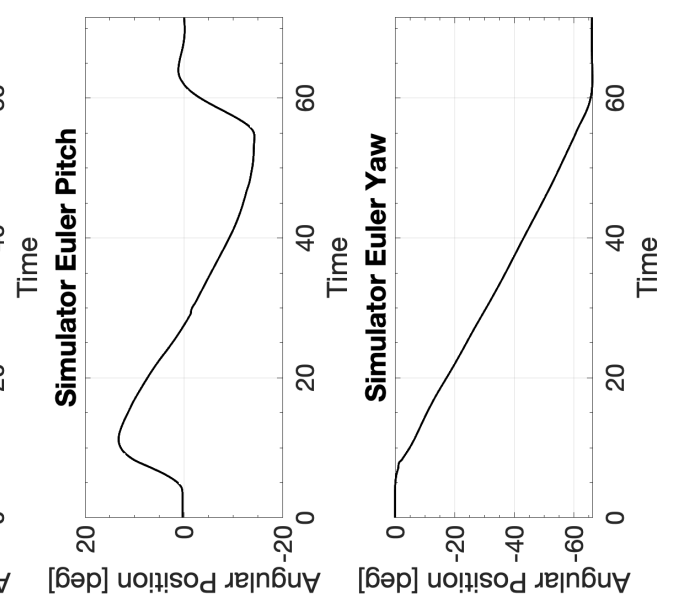

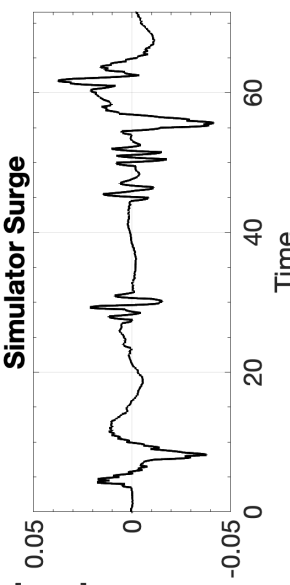

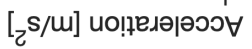

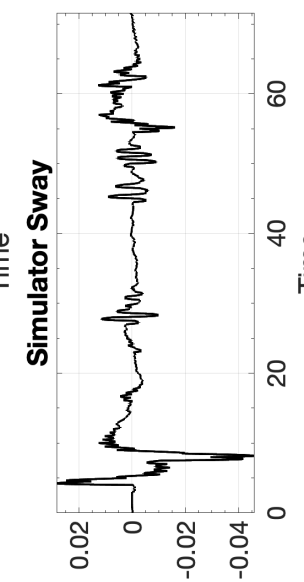

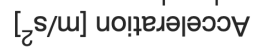

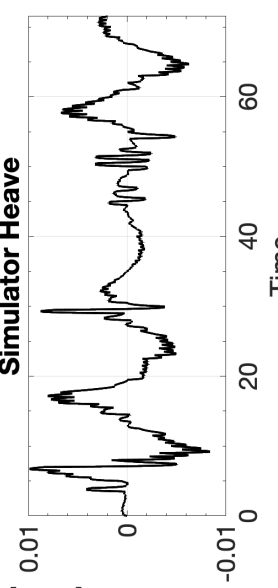

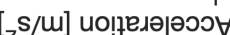

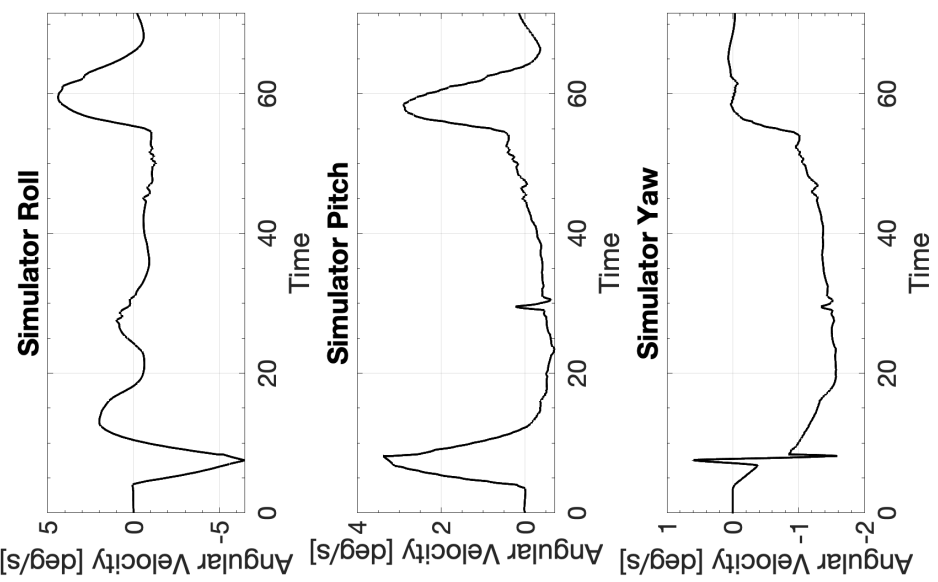

379 

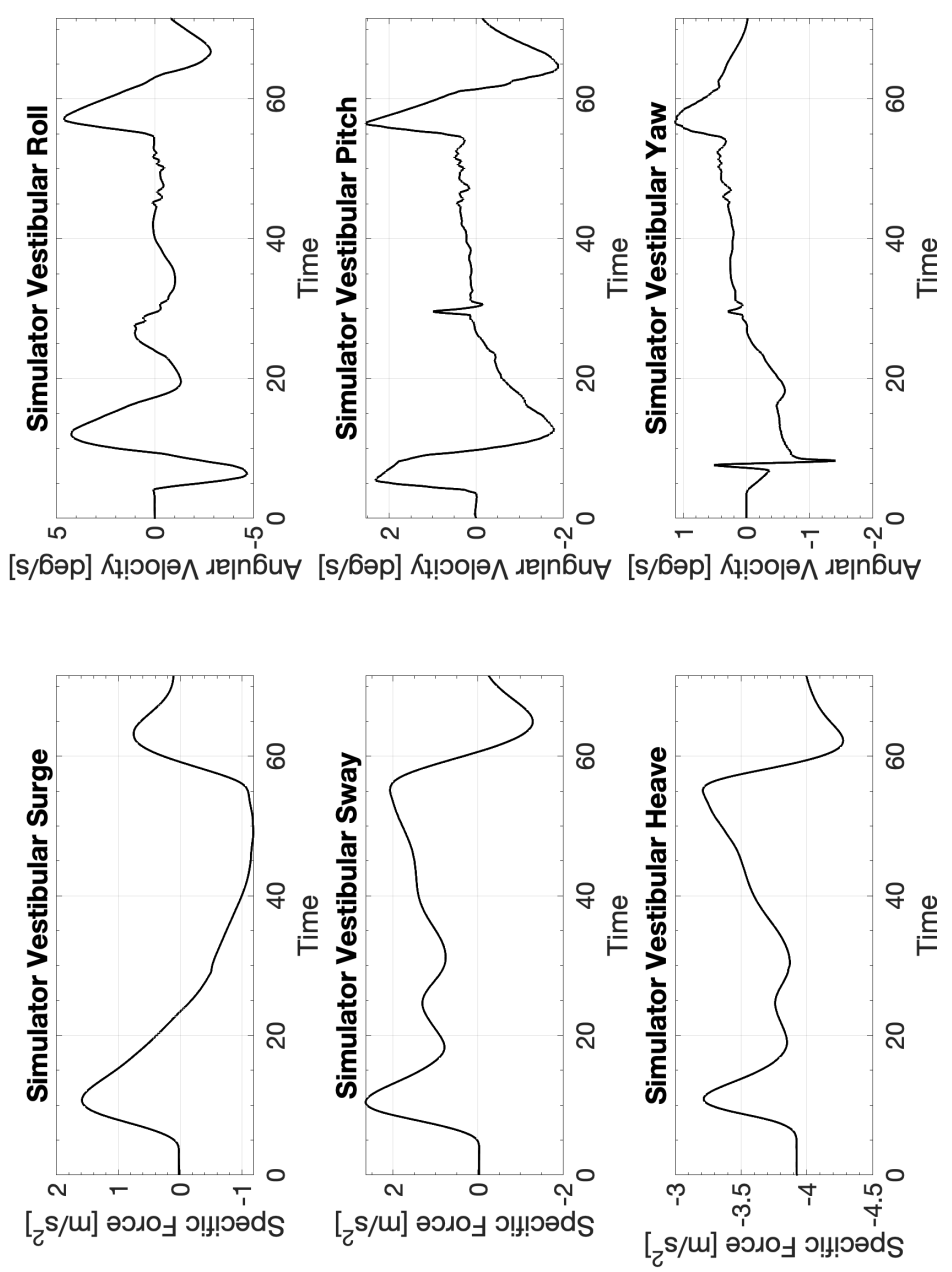

380 


\section{D.6.2 CW2 Boeing 737 Coordinated Turn}

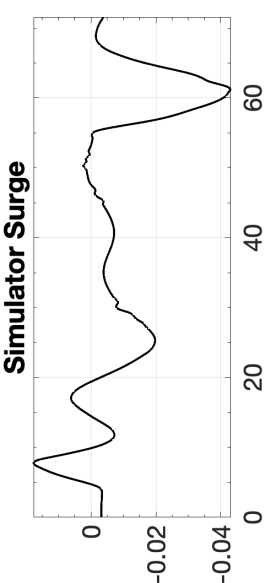

[w] uo!n!sod

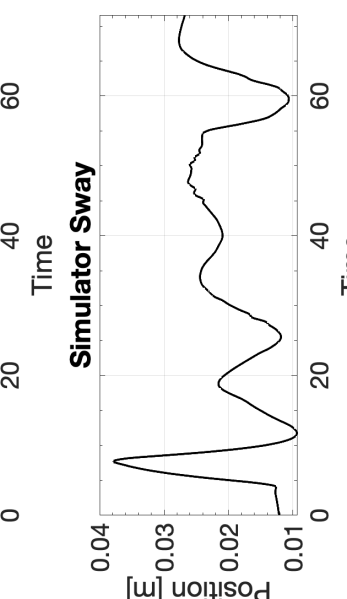

[w] uo!!!!sod

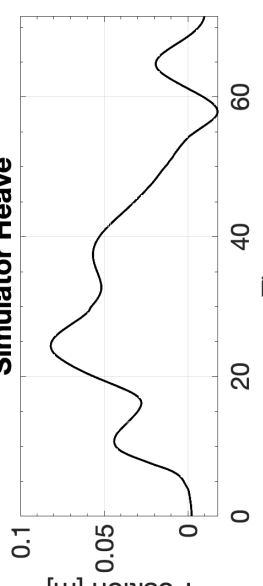

[w] uo!n!sod
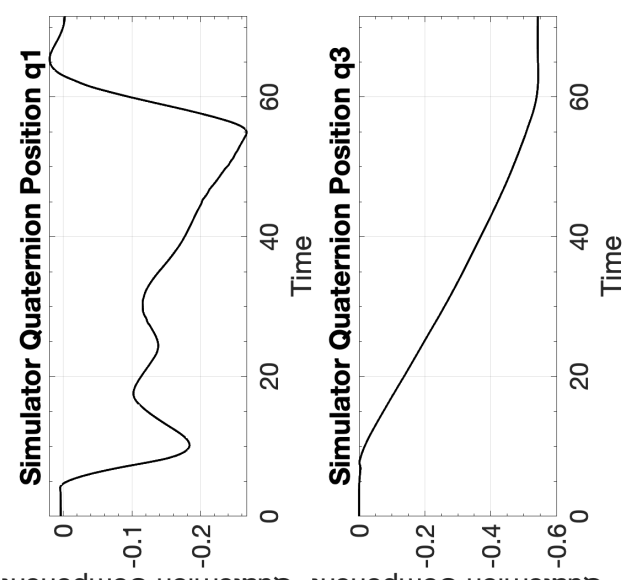

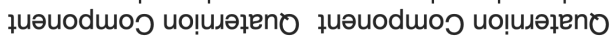

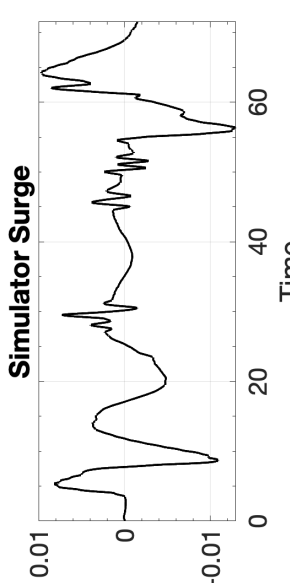

[s/u] Кұ!ројә^

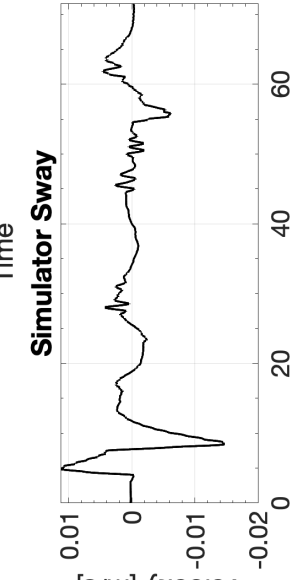

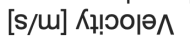

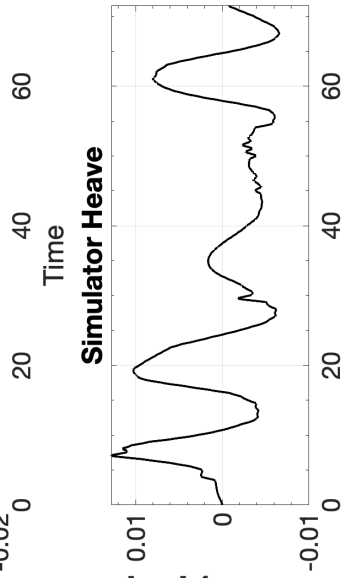

[s/m] ᄉұ! ७о|ә^
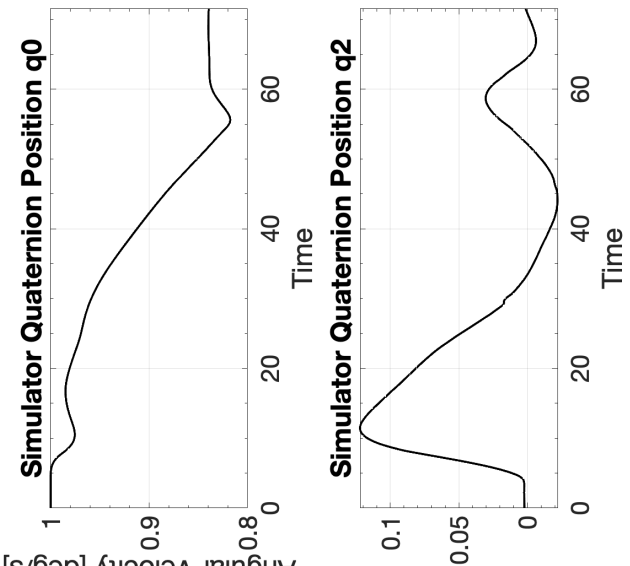

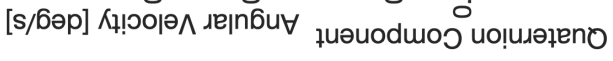

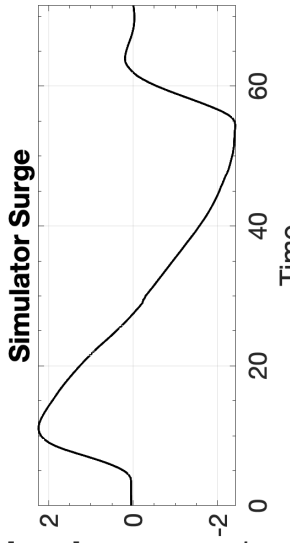

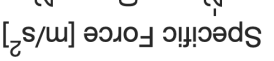

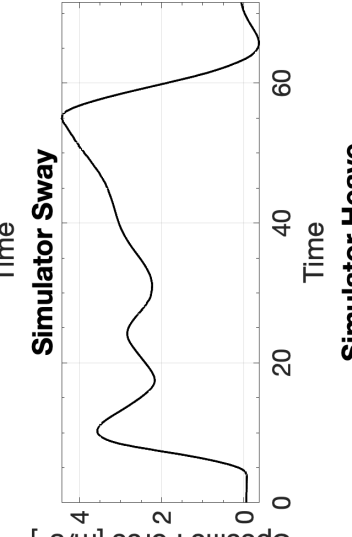

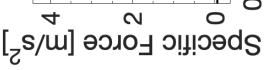
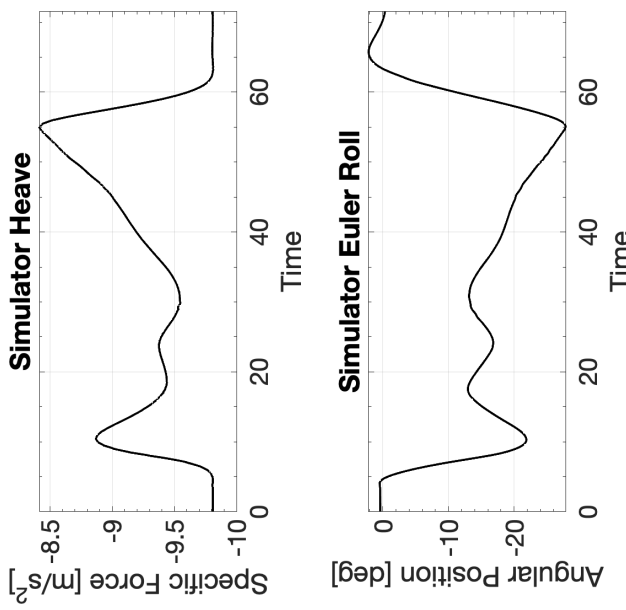
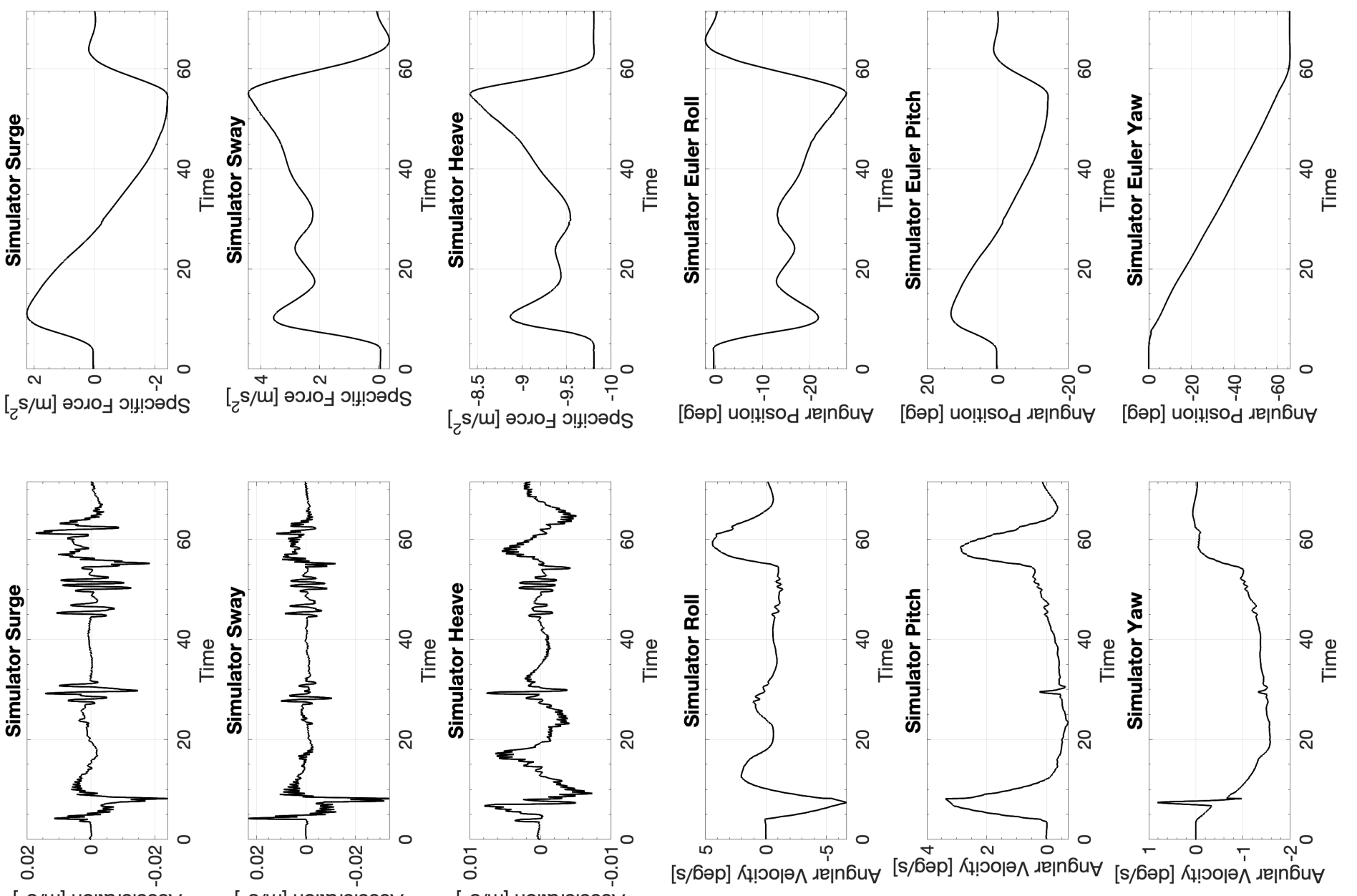

$\left[{ }_{2} \mathrm{~s} / \mathrm{M}\right]$ ио!ฺеләрәэว

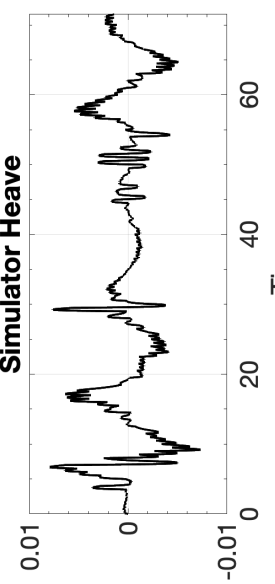

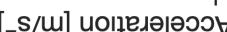

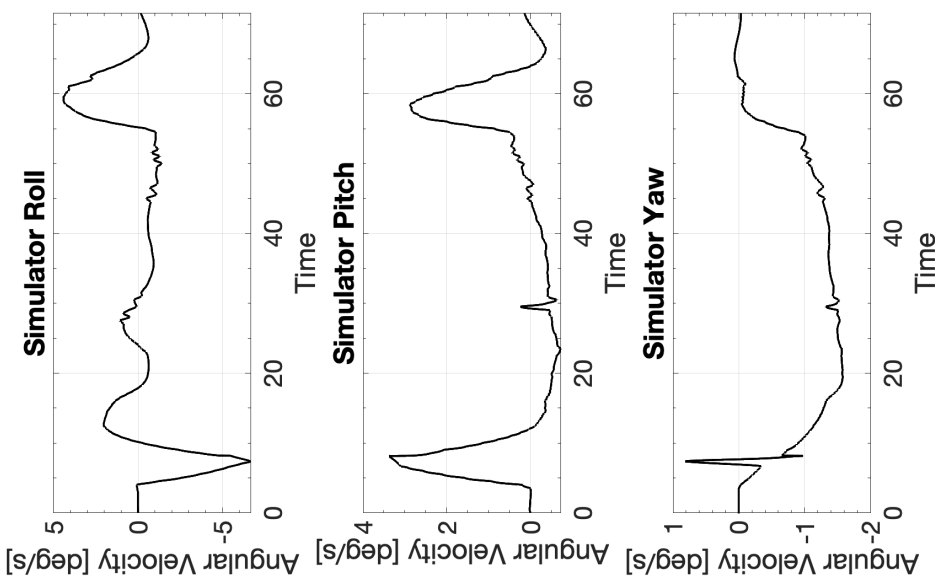

381 


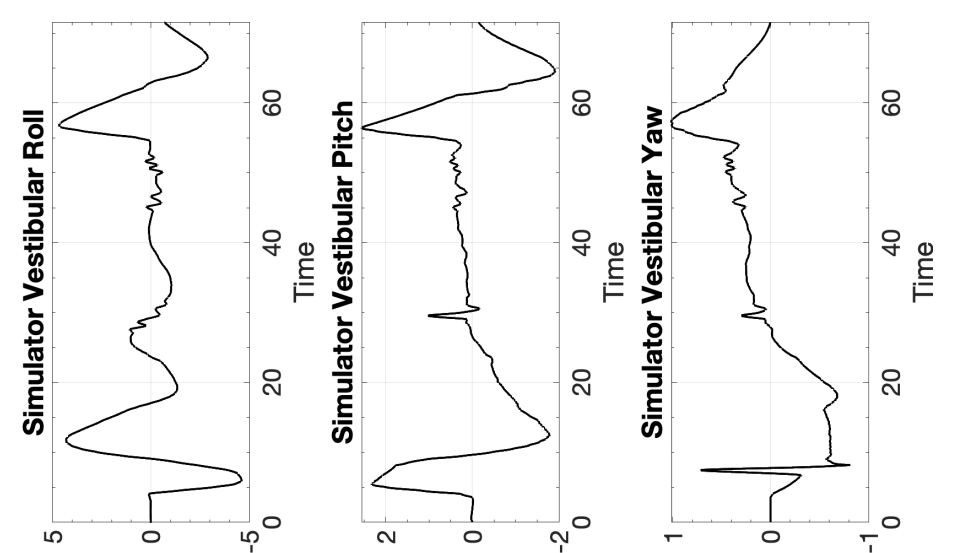

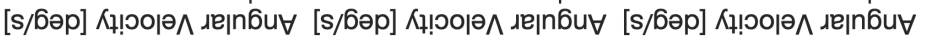

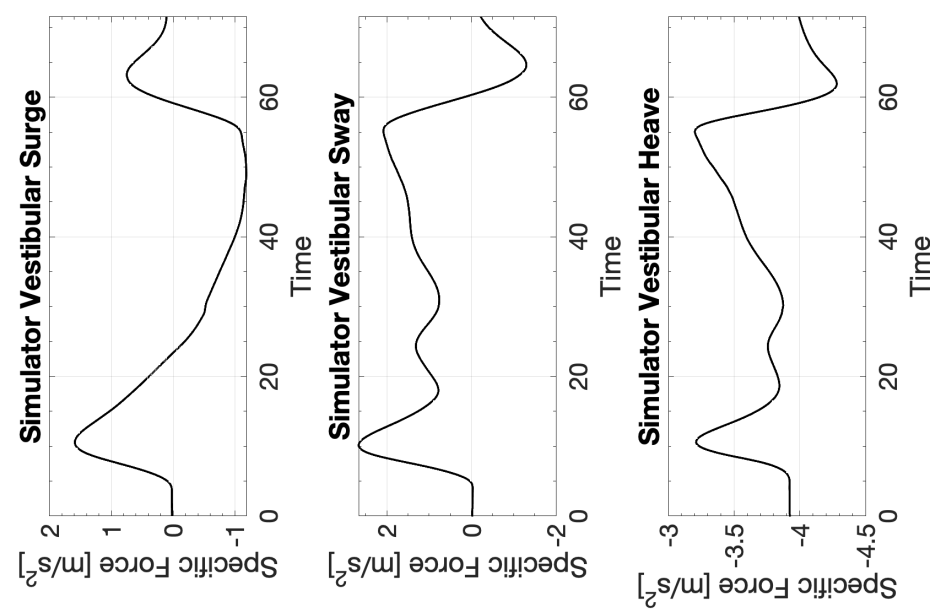




\section{D.6.3 CW3 Boeing 737 Coordinated Turn}

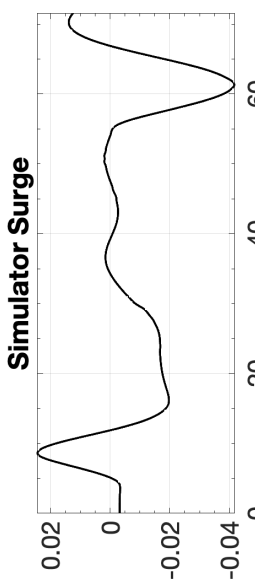

[w] uo!n!sod

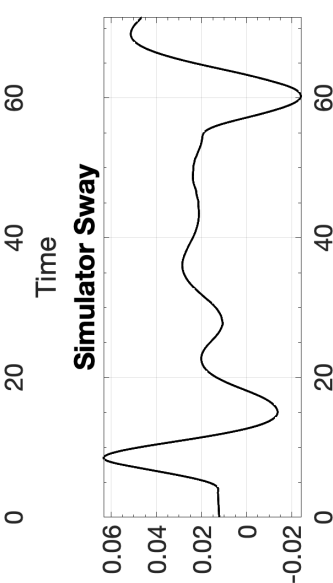

[u] uo!n! sod

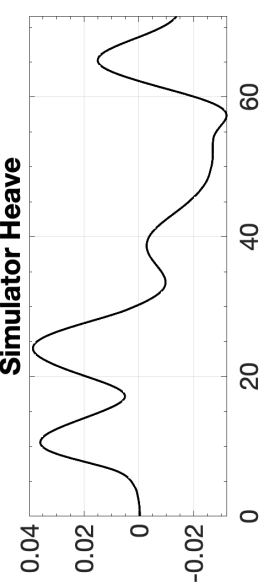

[w] uolt!sod
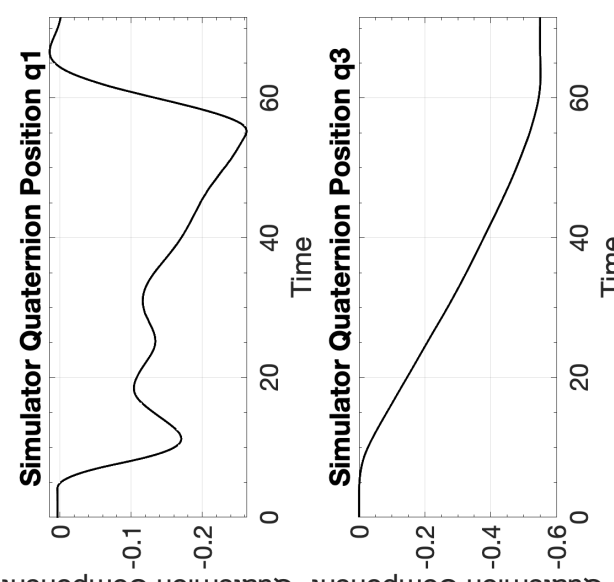

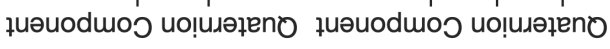

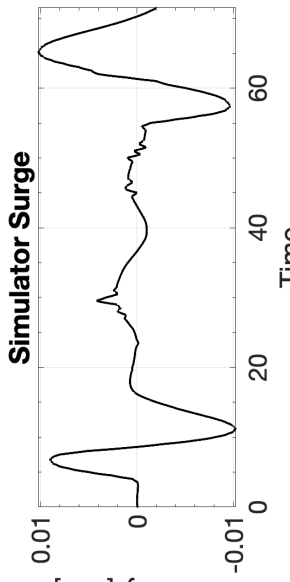

[s/m] ᄉұ! ৩оюә^

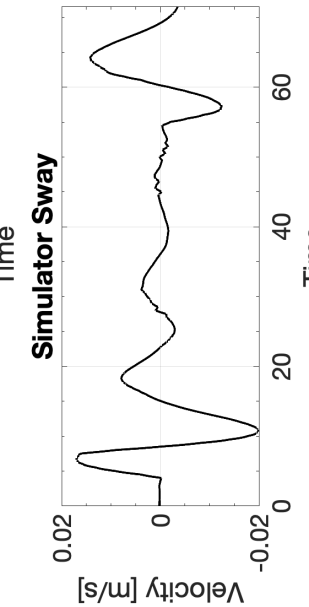

[s/m] Кұ!๐о|ә^

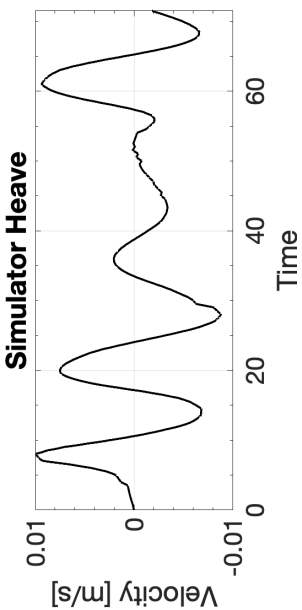

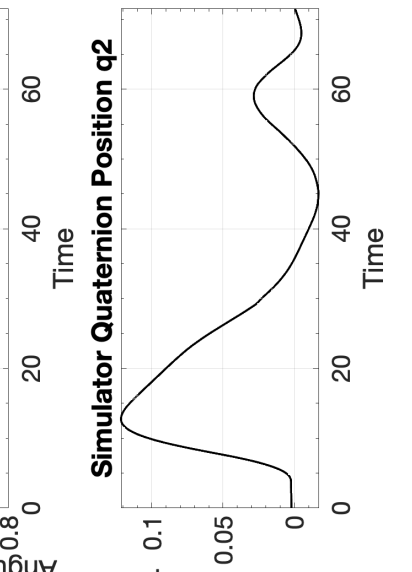

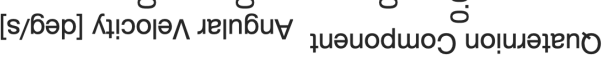

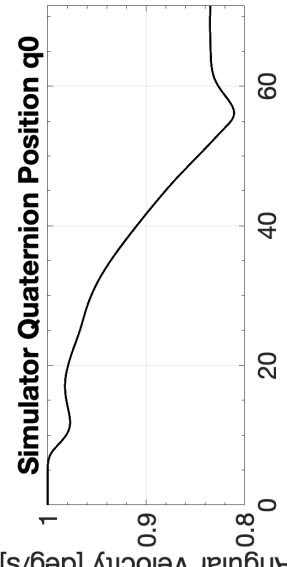

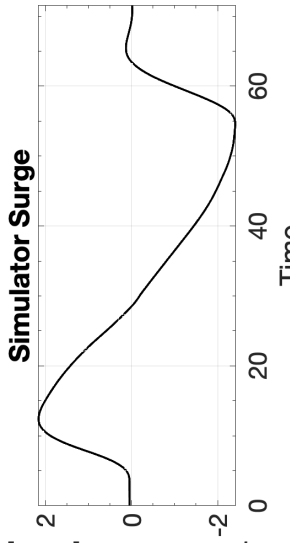

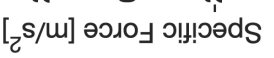

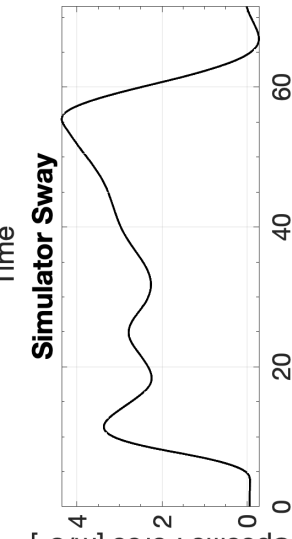

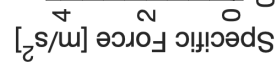

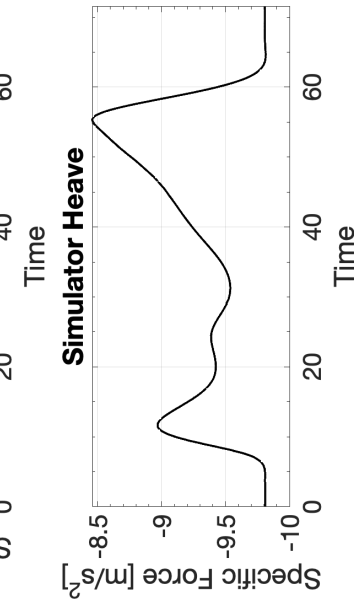

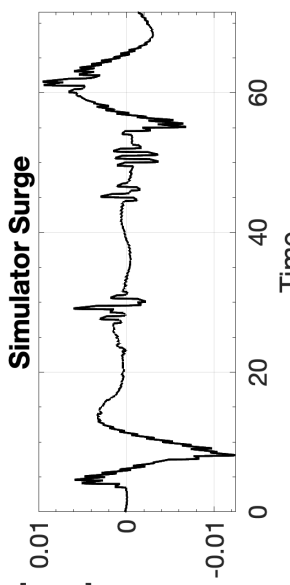

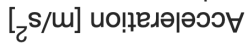

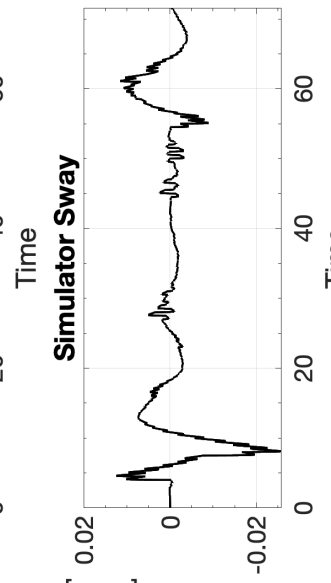

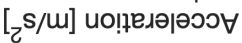

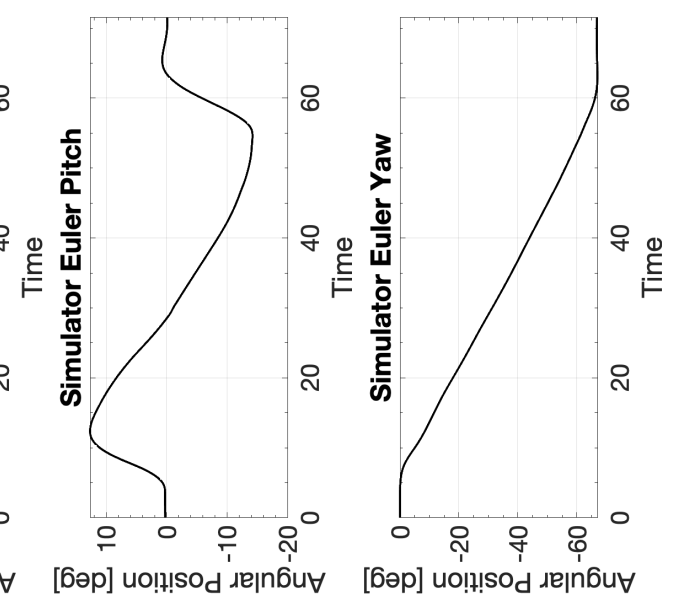

[бәр] uo!!!

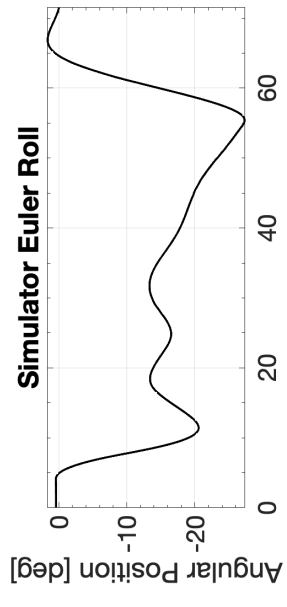

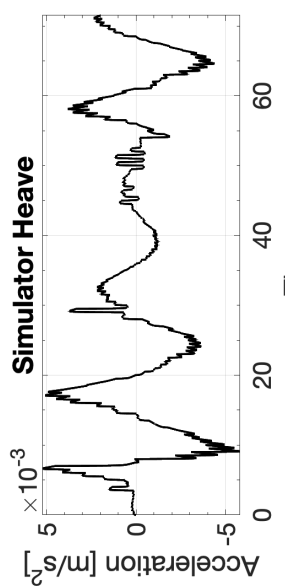

$[\mathrm{c} / \mathrm{m}]$ ио!ฺвдәәәэว

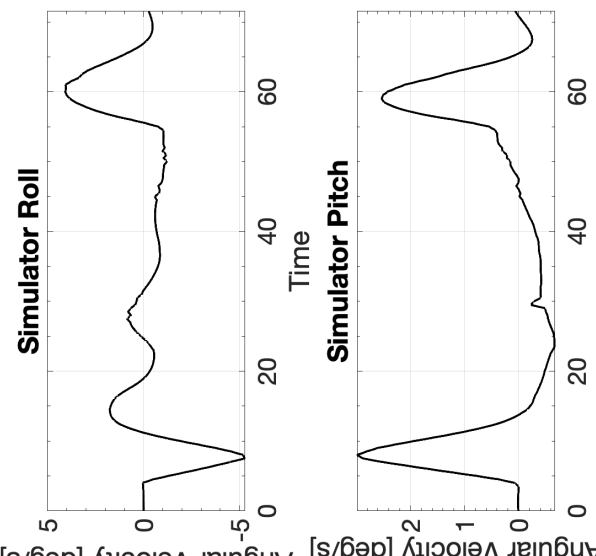

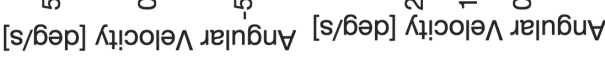

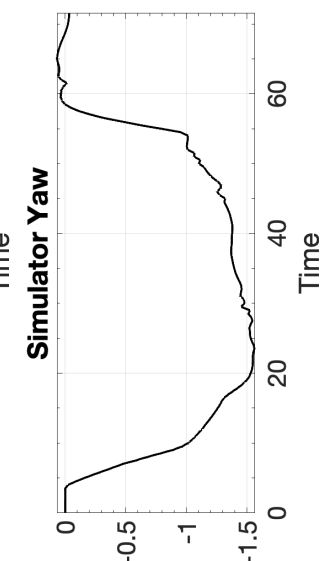

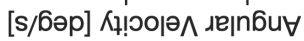




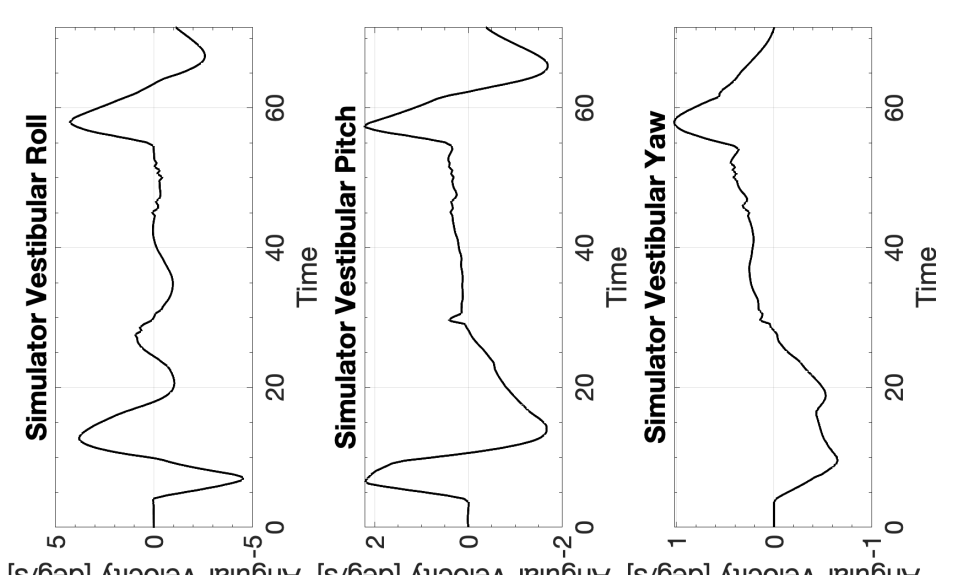

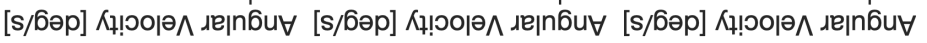

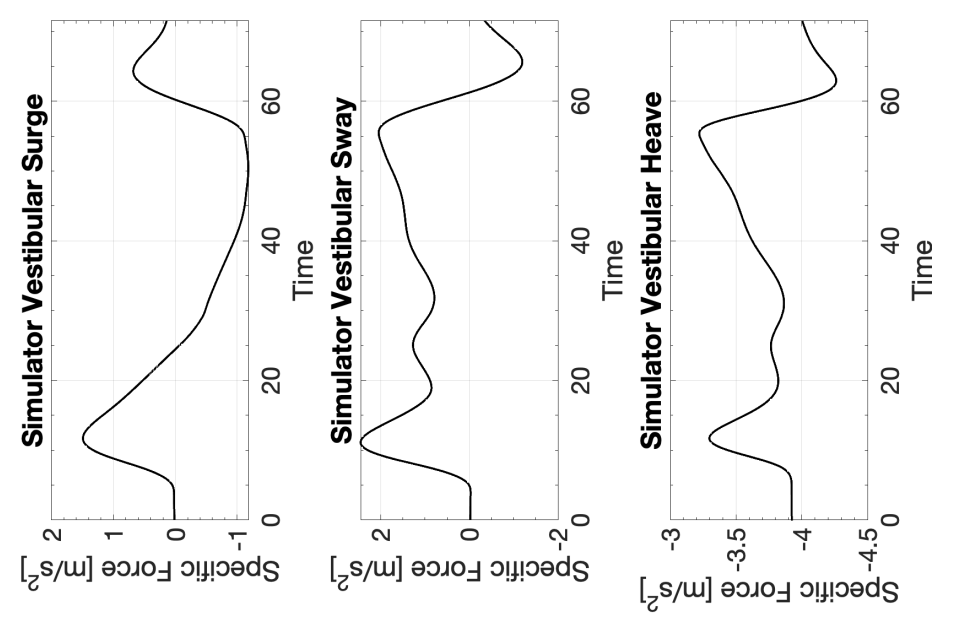


D.7 Boeing 737 Cross-wind Landing

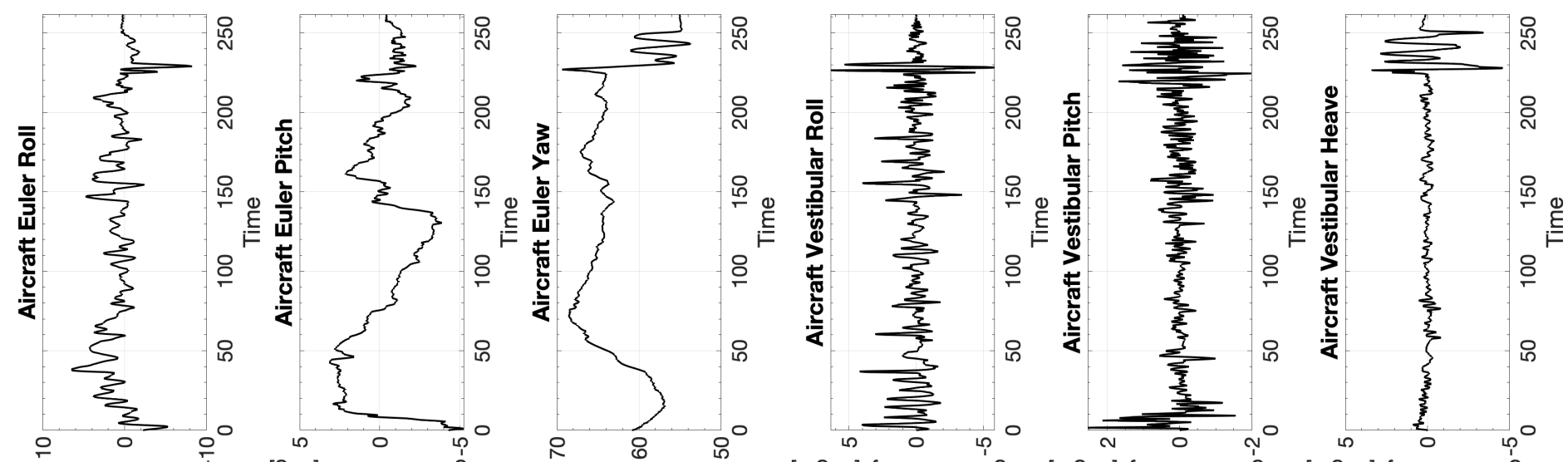

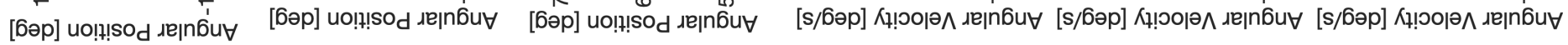
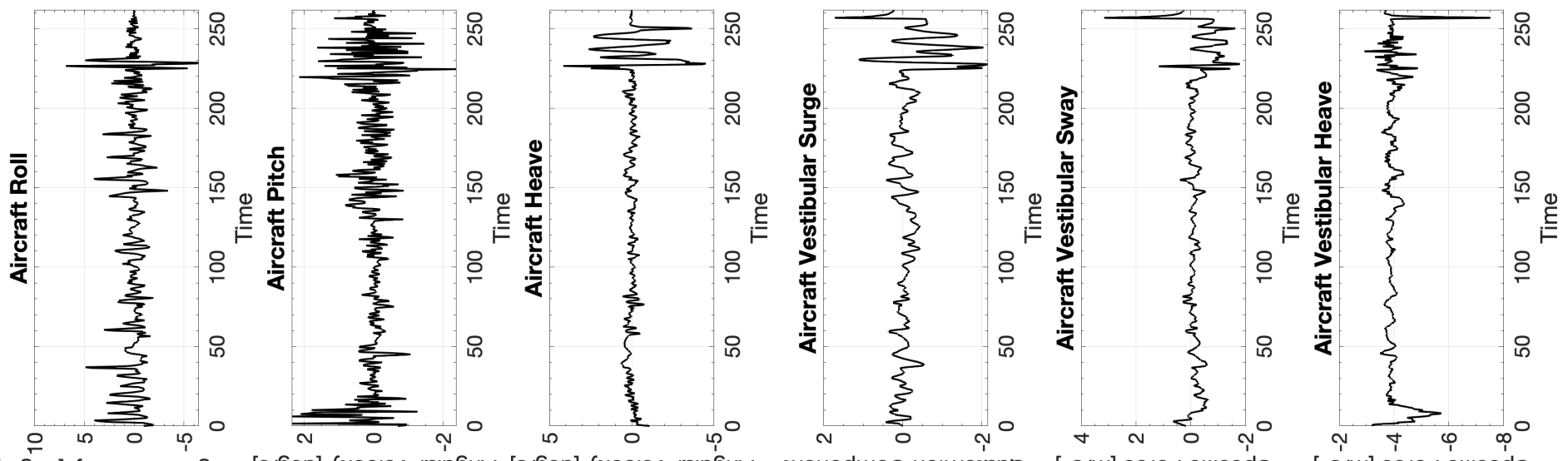

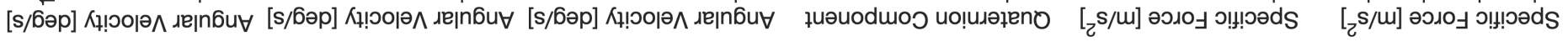

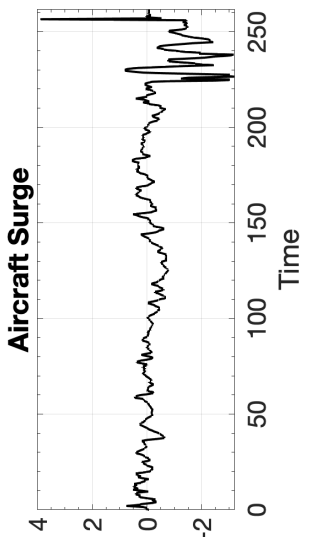

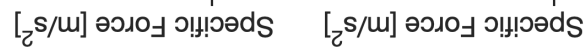
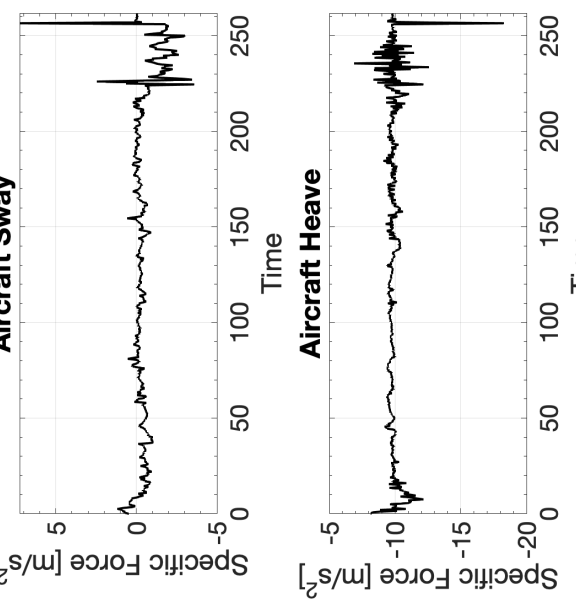

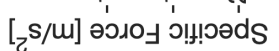

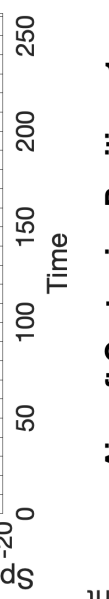

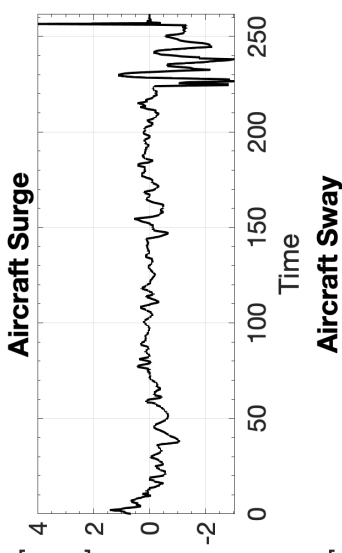

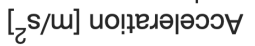

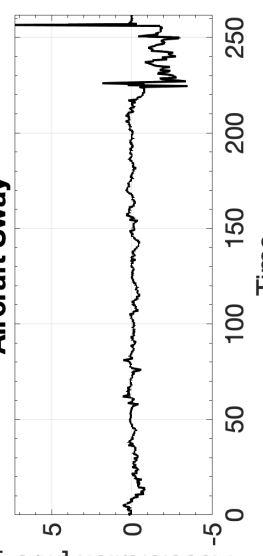

$[\mathrm{z} / \mathrm{s}]$ ио!ฺвдәәәоว

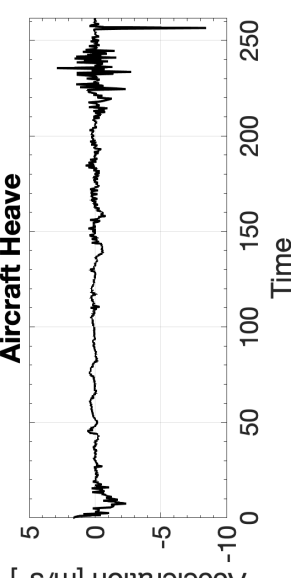

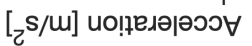
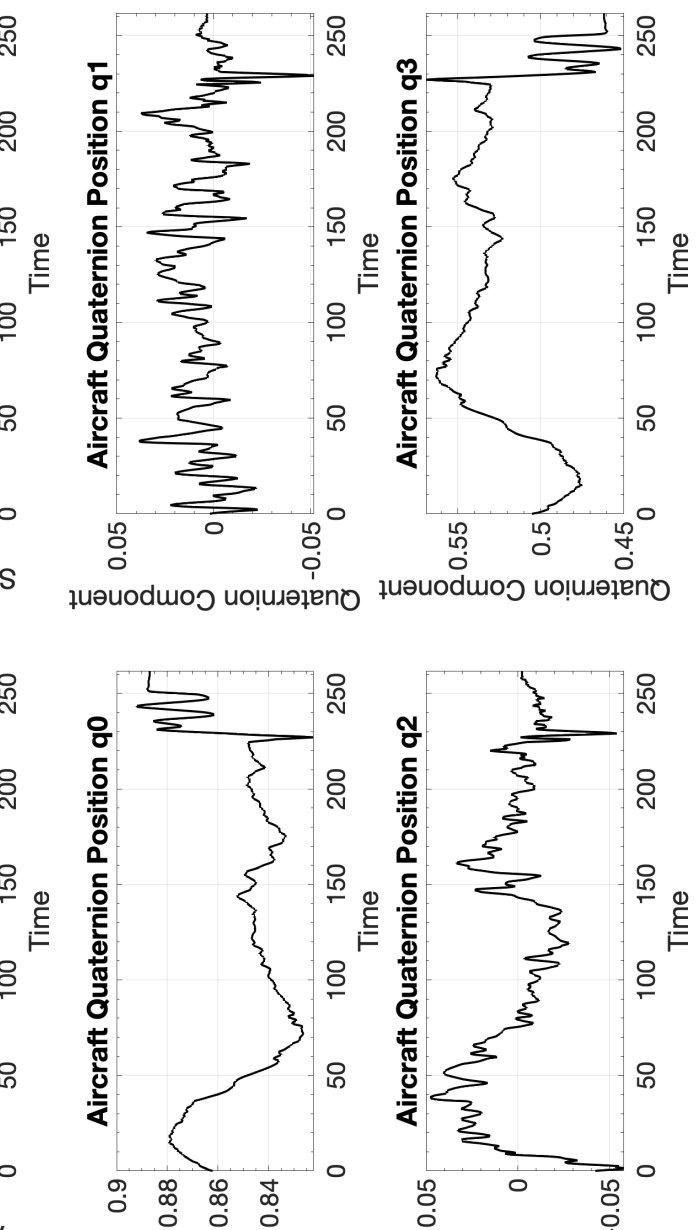

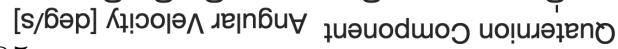




\section{D.7.1 CW1 Boeing 737 Cross-wind Landing}

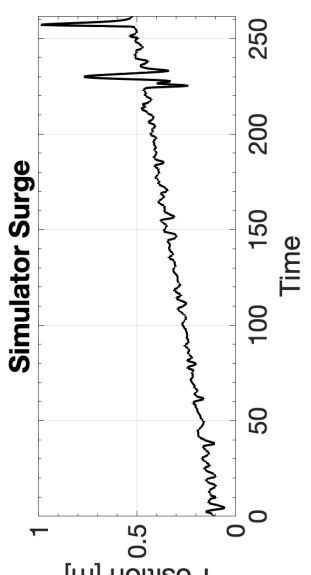

[u] uo!!!sod

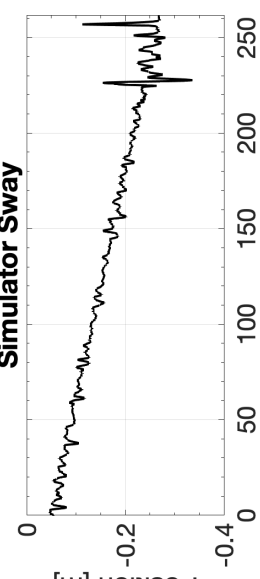

[u] uo!m!nsod

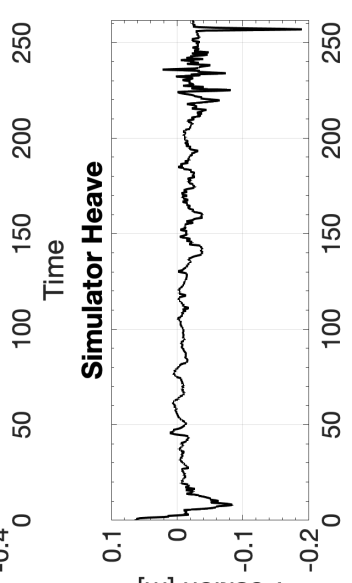

[w] uol!!!sod
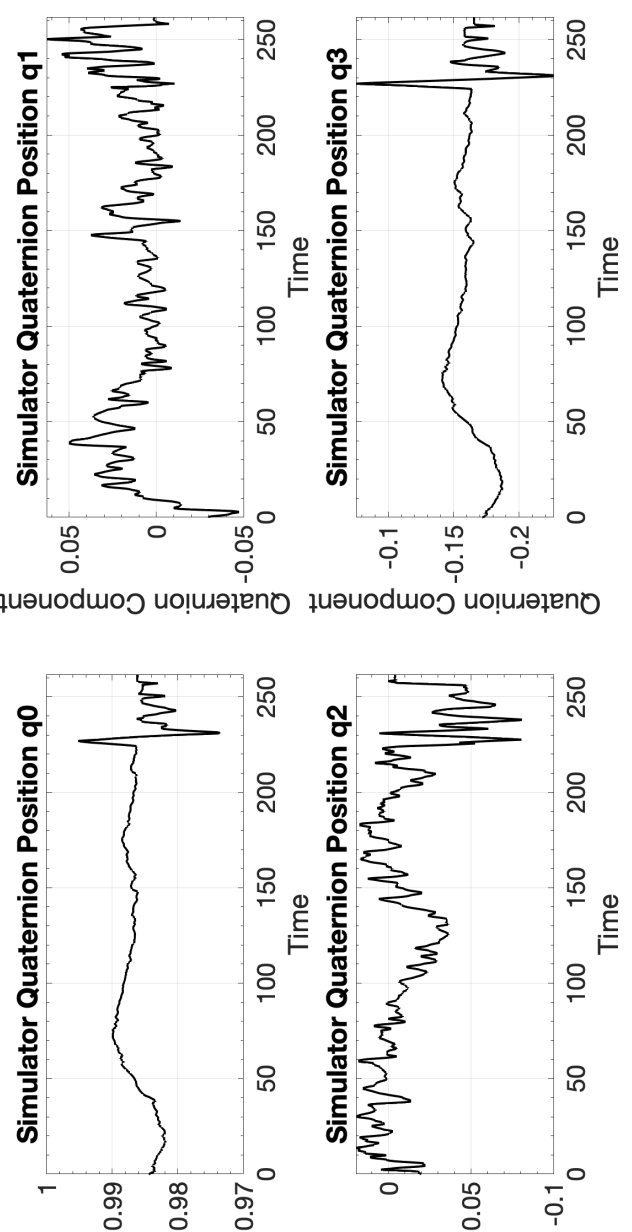

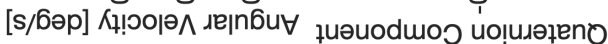

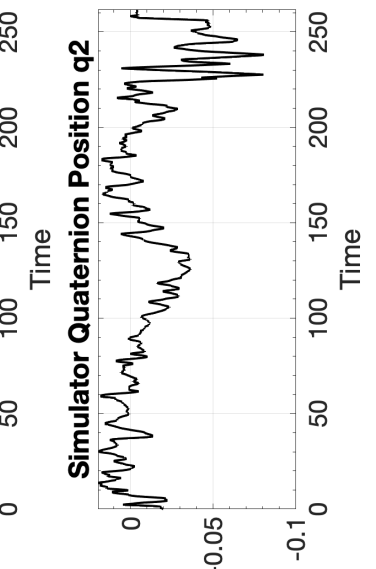

오

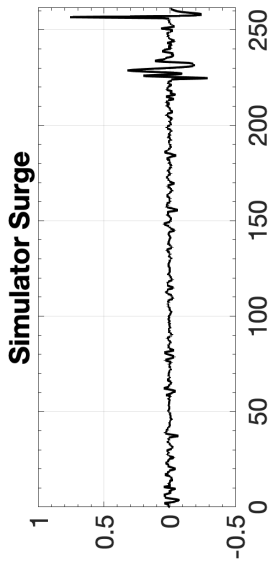

[s/m] Кł!००|ә^

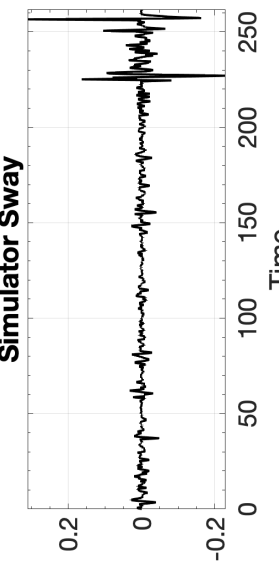

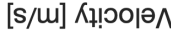

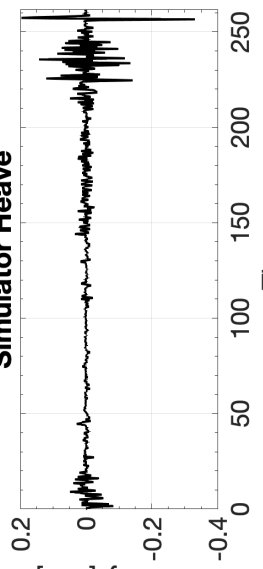

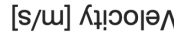

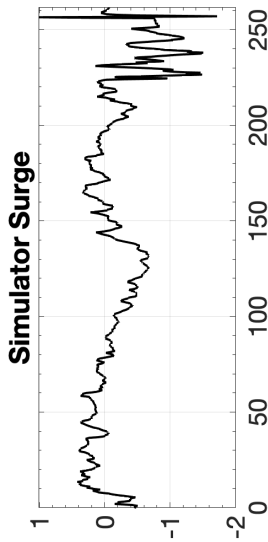

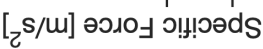

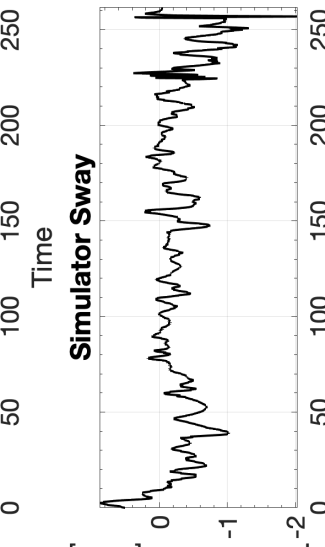

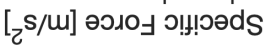

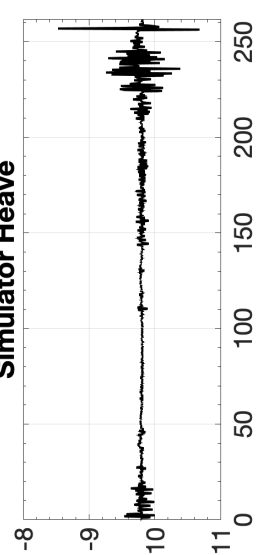

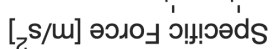

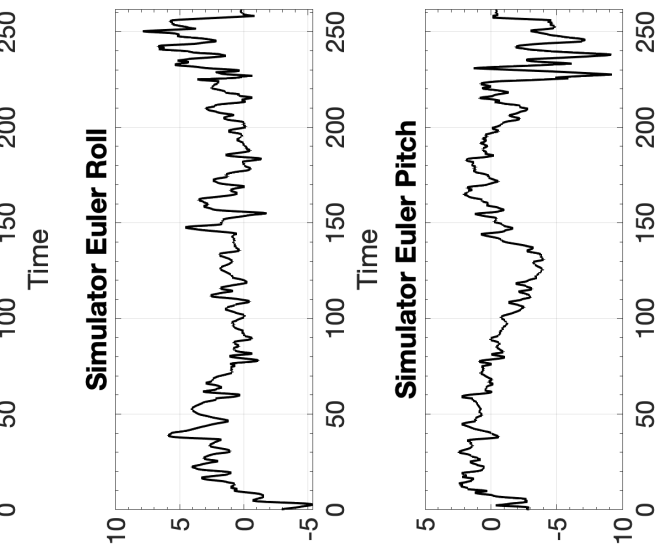

[бәр] uo!!!!sod גe|nбu

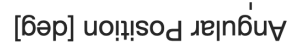

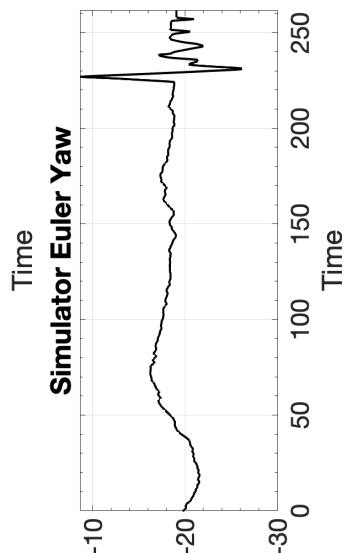

[бәр] uo!!!

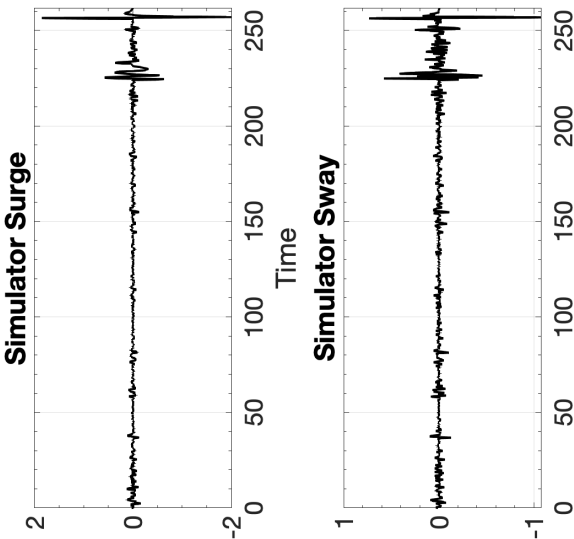

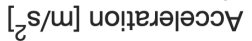

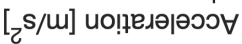

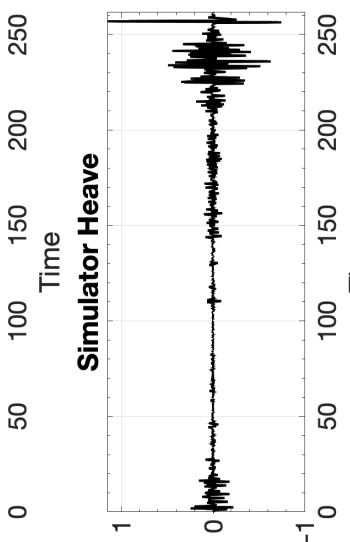

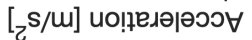

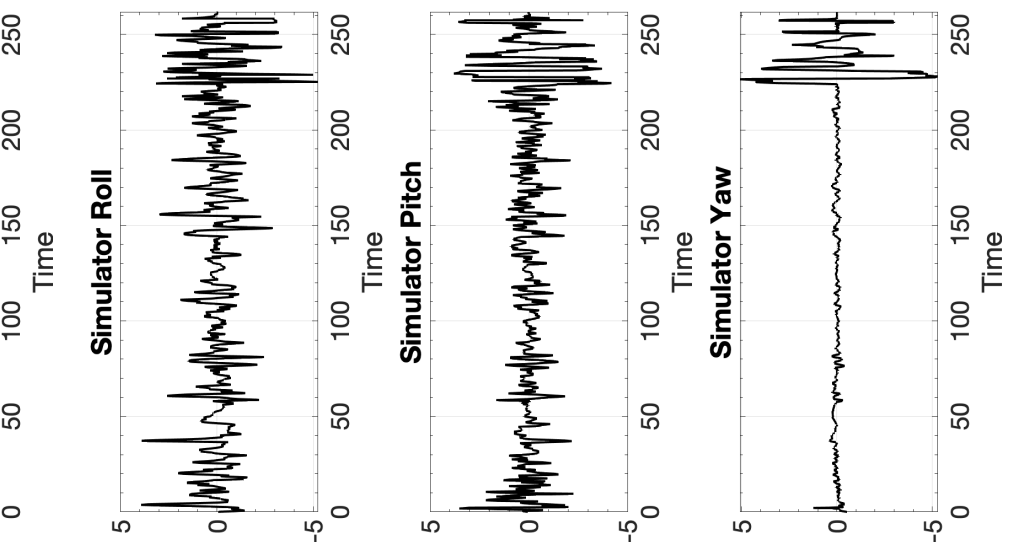

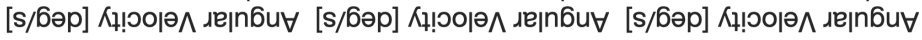




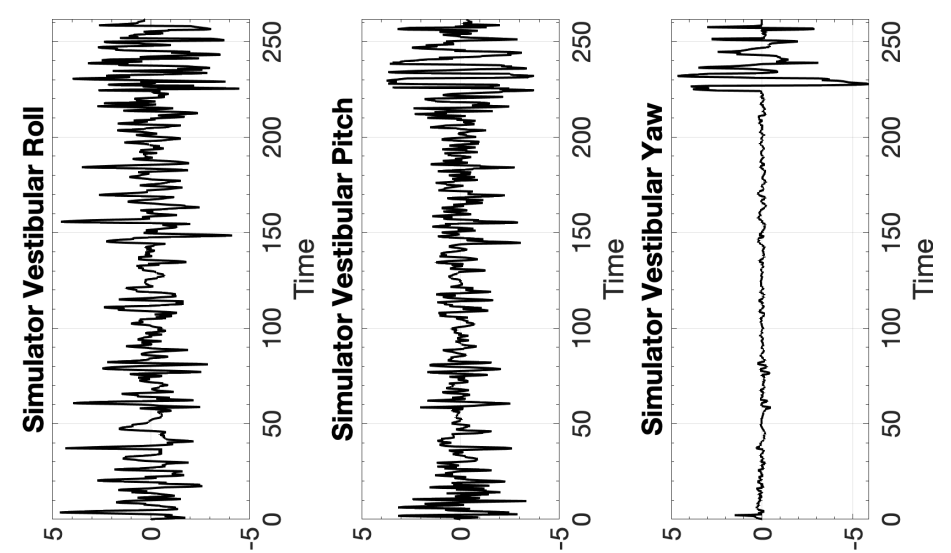

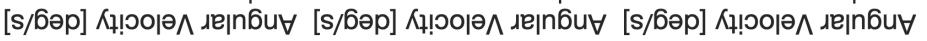
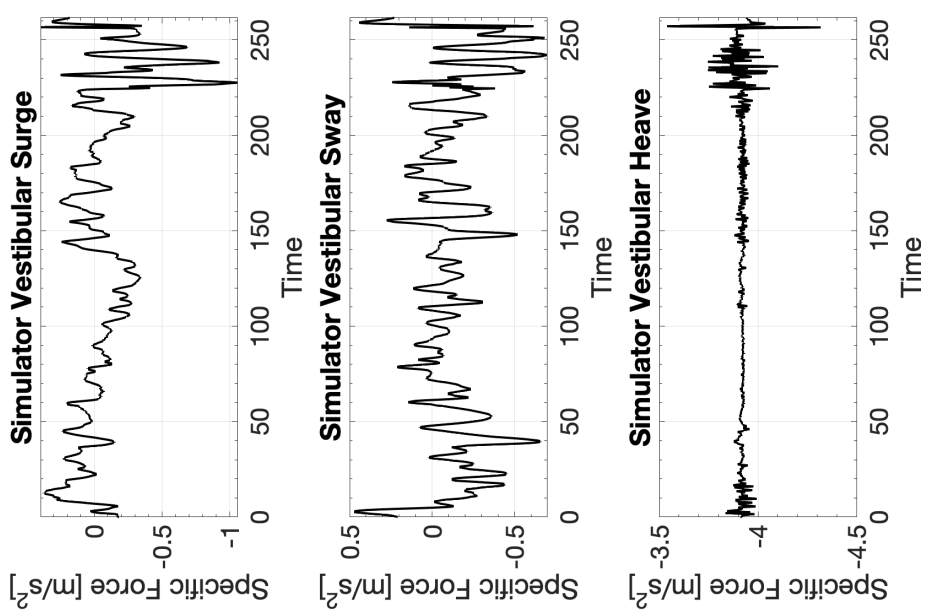

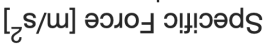




\section{D.7.2 CW2 Boeing 737 Cross-wind Landing}

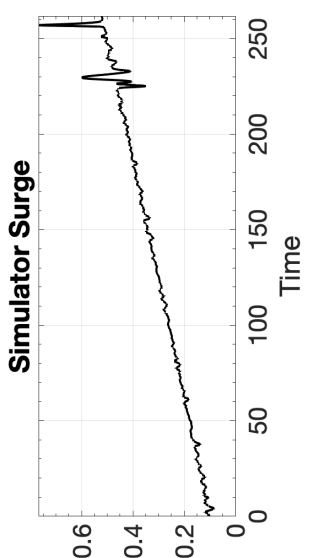

[u] uo!n!sod

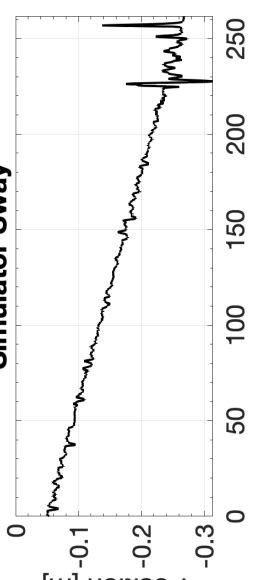

[m] uol!m!sod

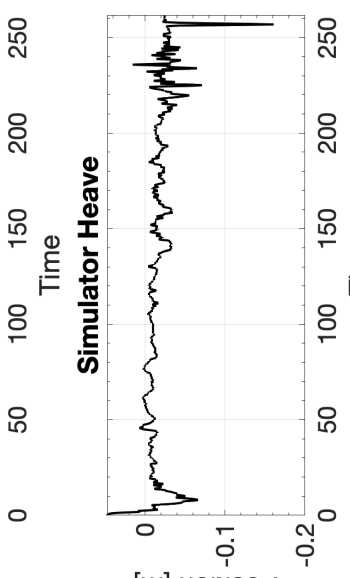

[m] uol!!!sod

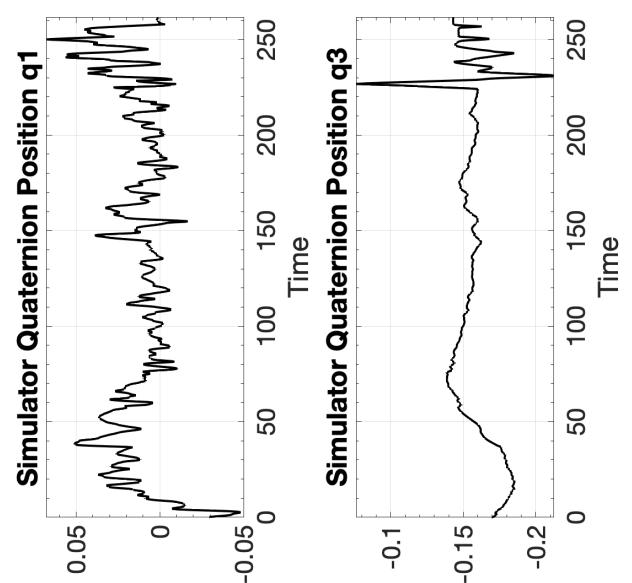

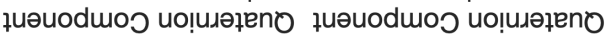

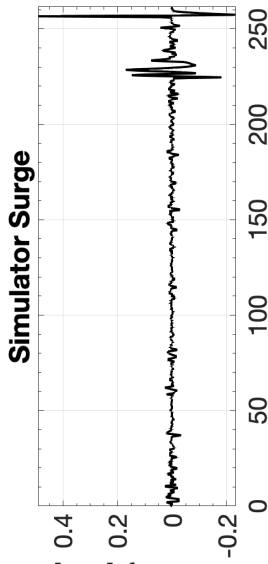

[s/m] Кł!००|ә^

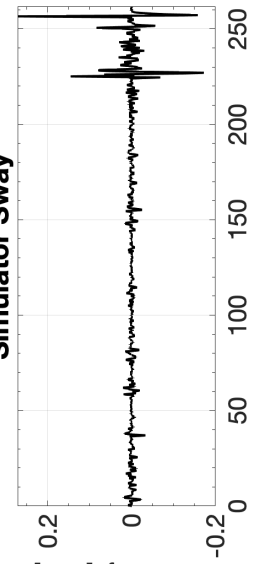

[s/m] Кұ!৩૦|ә

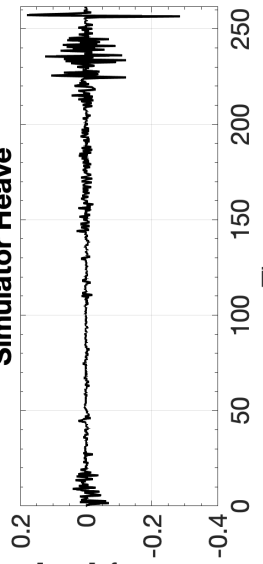

[s/m] К१!००|ә^

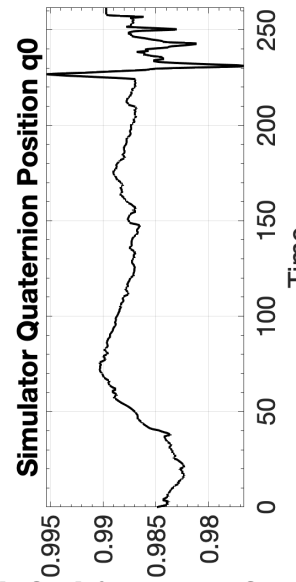

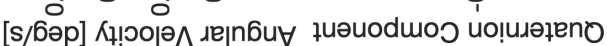

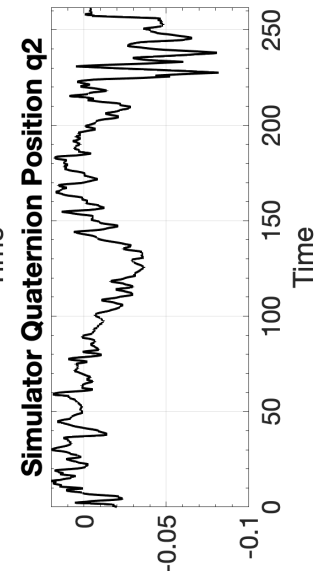

$\stackrel{8}{\circ}$
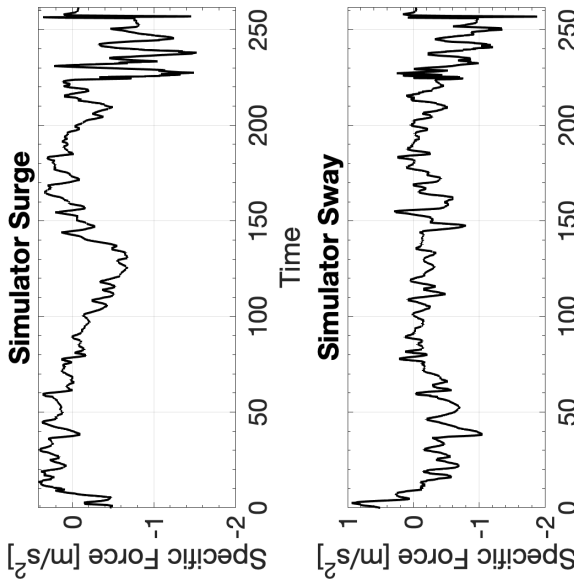

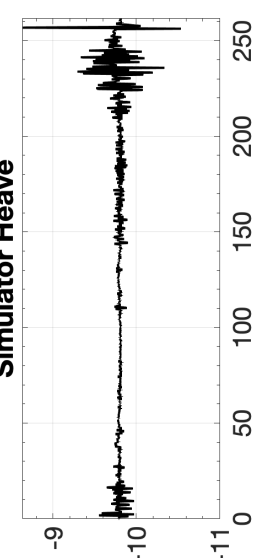

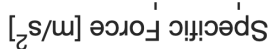

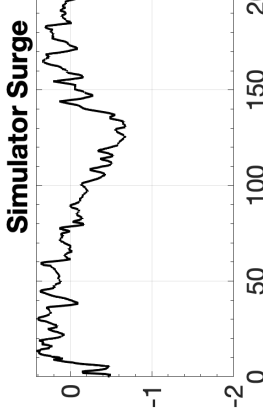

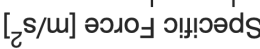

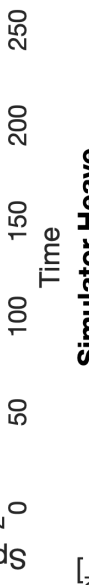

[бәр] uo!!! sod גeןn6u

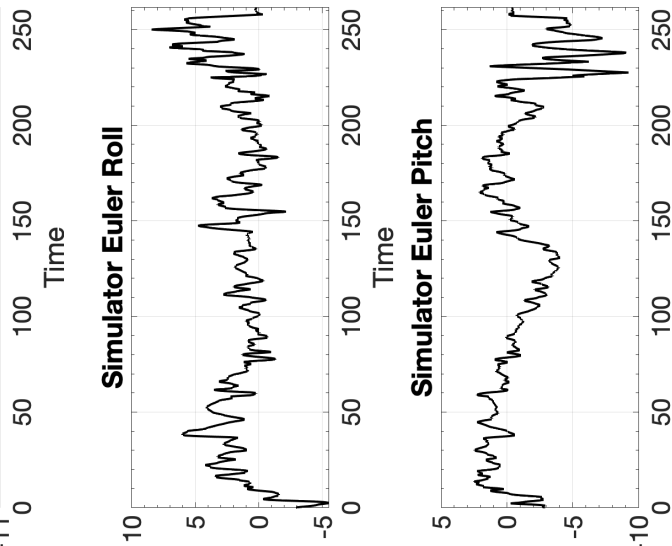

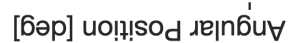

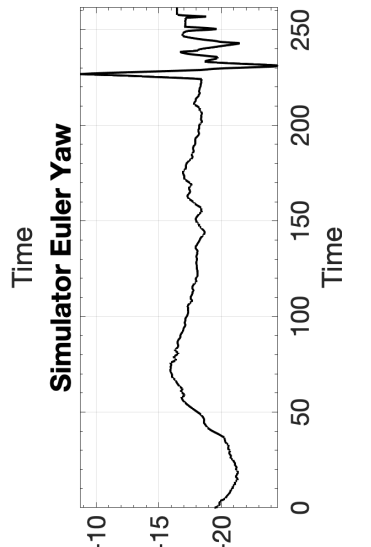

[6әр] uo!!!!sod de|n6u

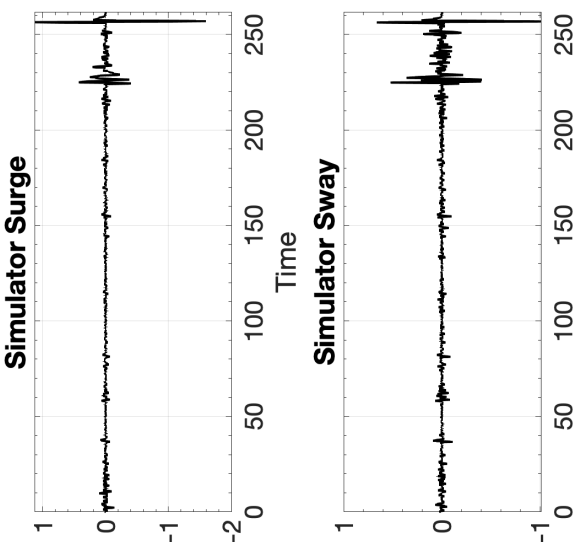

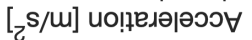

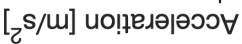

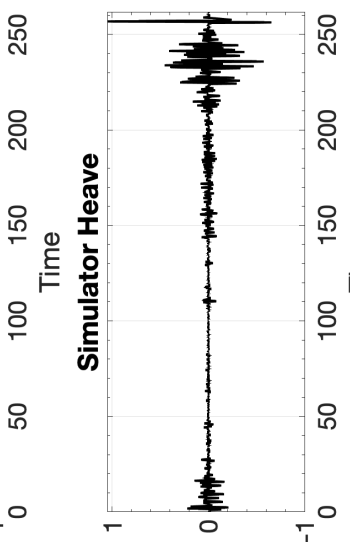

$[\mathrm{c} / \mathrm{m}]$ uо!ฺвләәәэว $\forall$

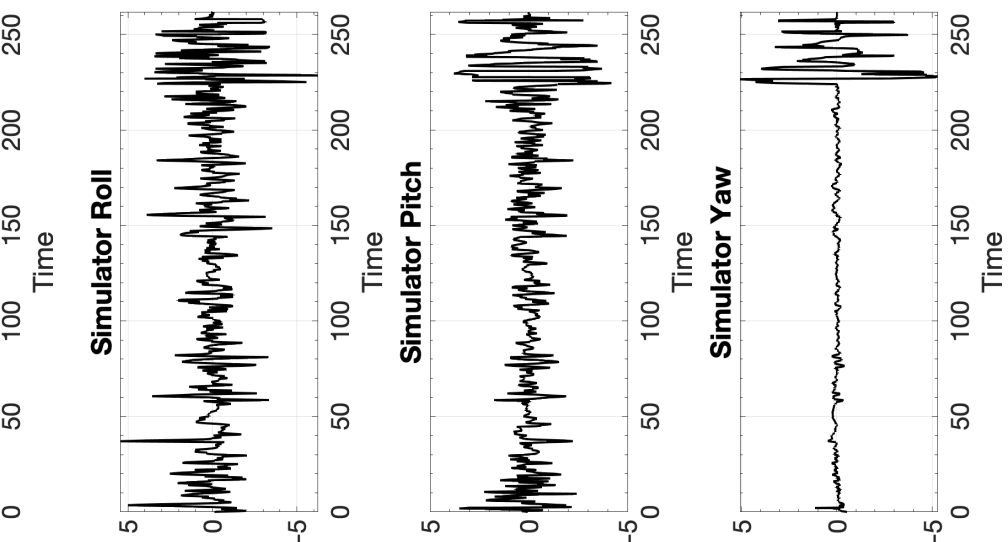

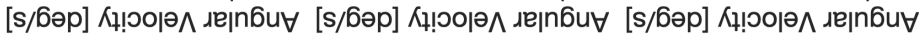




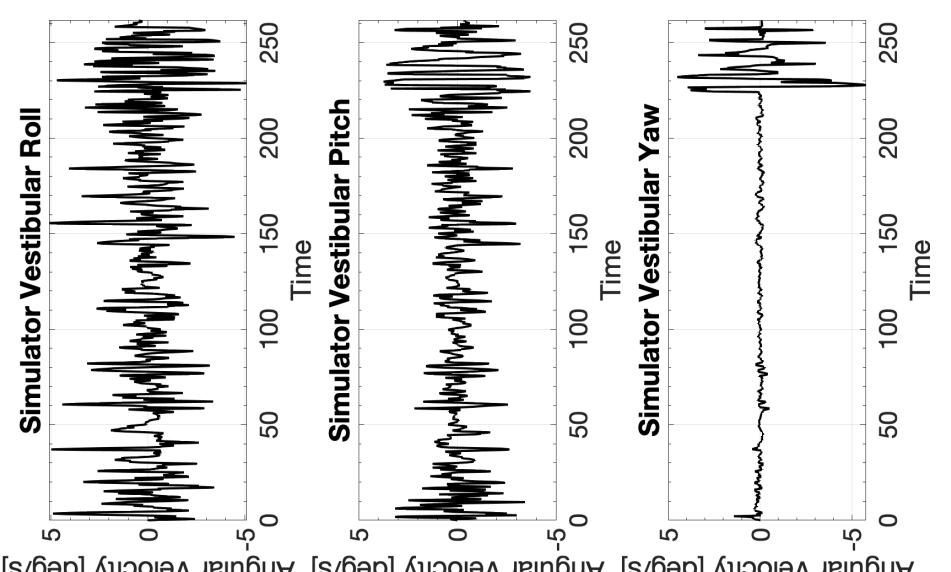

[s/бәр] Кұ!юорл

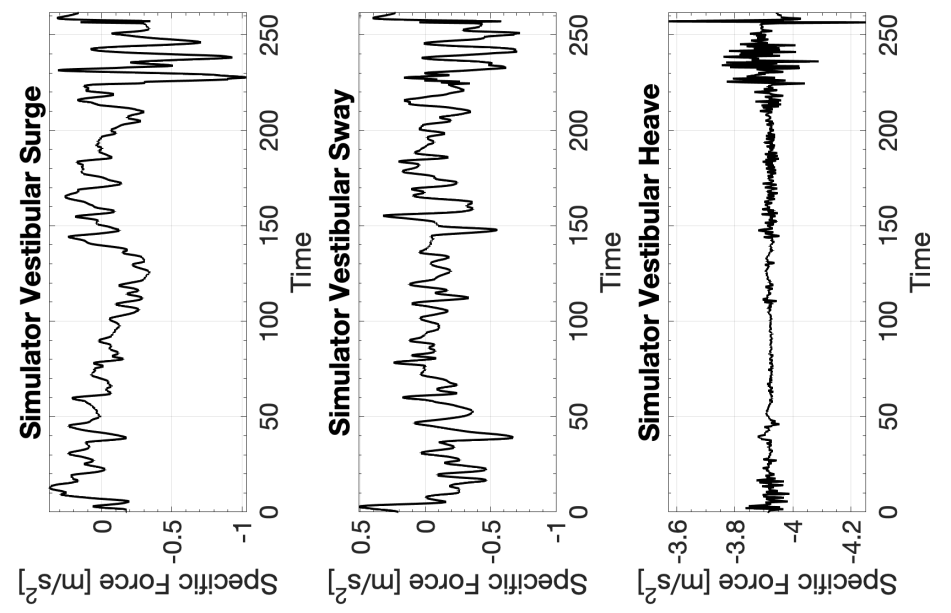




\section{D.7.3 CW3 Boeing 737 Cross-wind Landing}
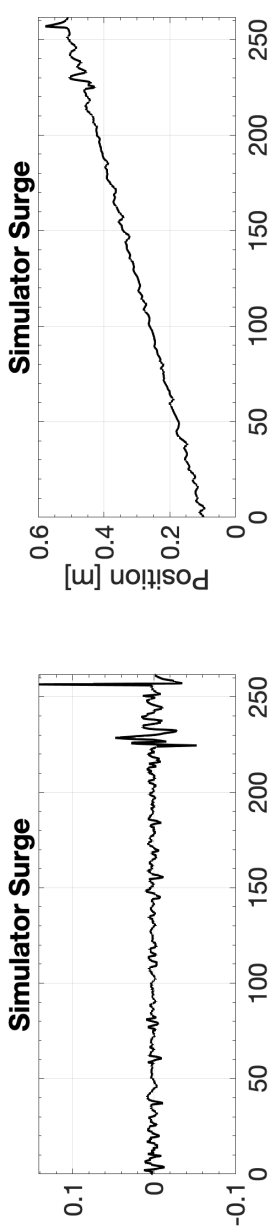

[s/m] Кұ!०о|ә

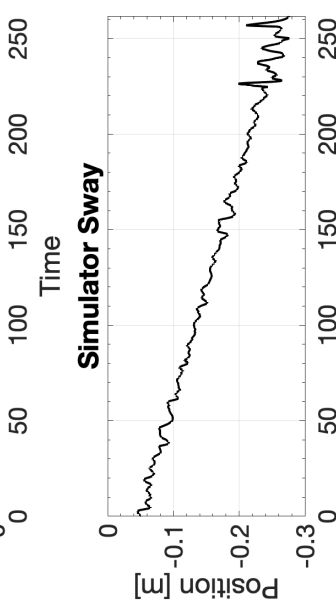

[w] uo!n!?sod

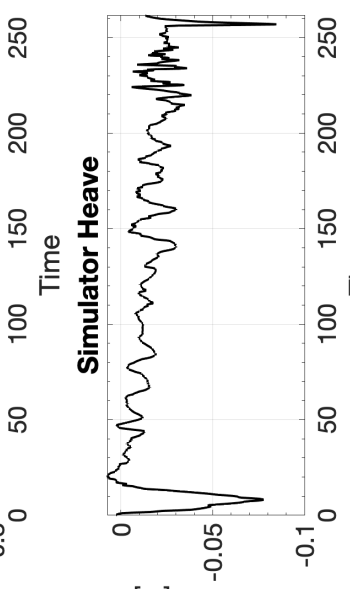

[w] uo!n!ssod

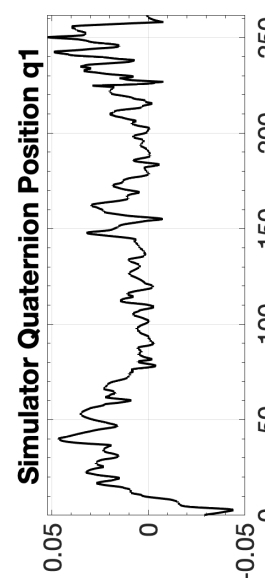

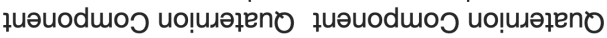
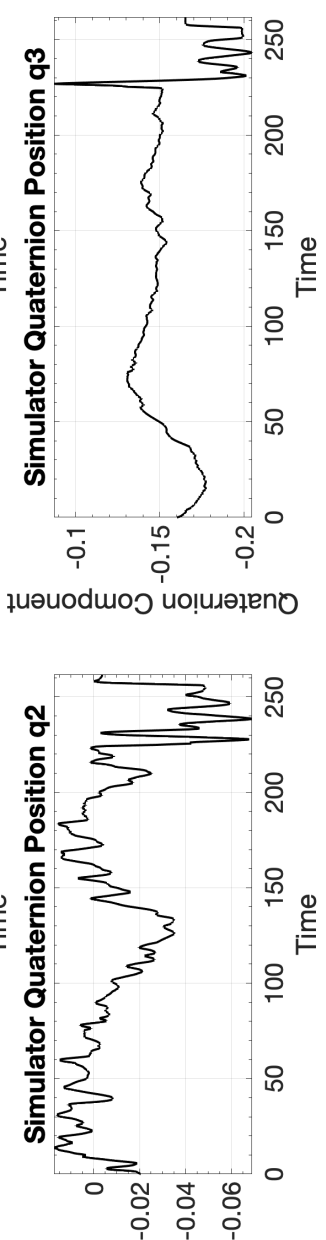

$[\mathrm{s} / \mathrm{m}]$ К!ํ이

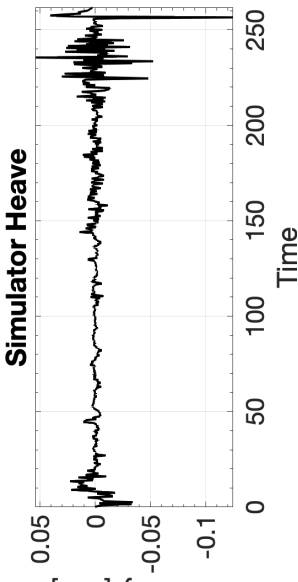

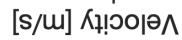

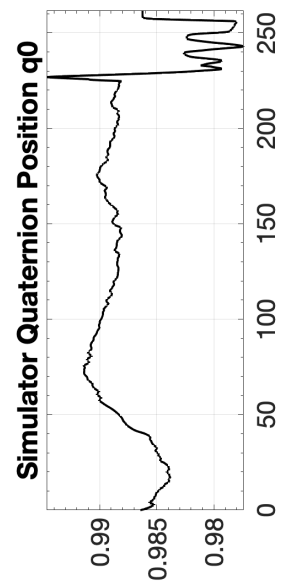

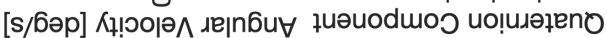

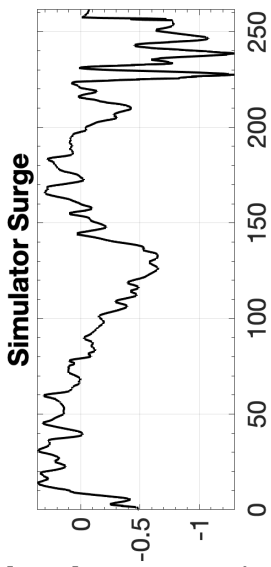

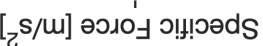
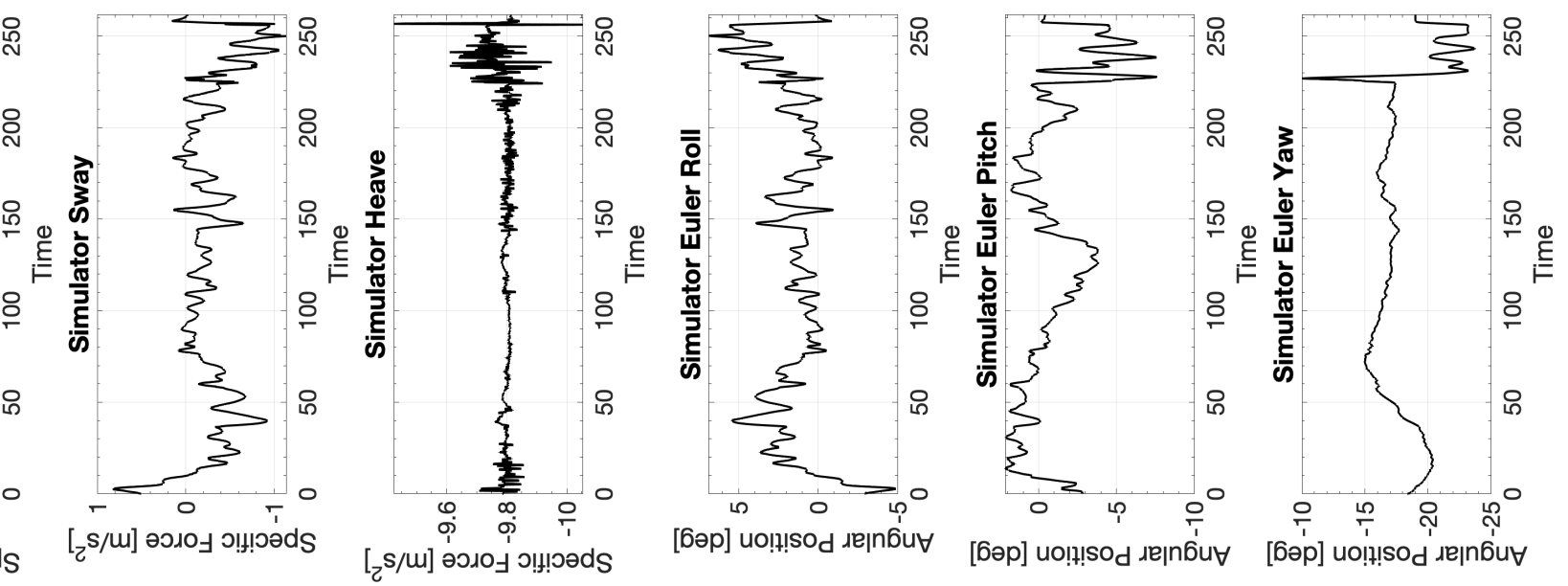

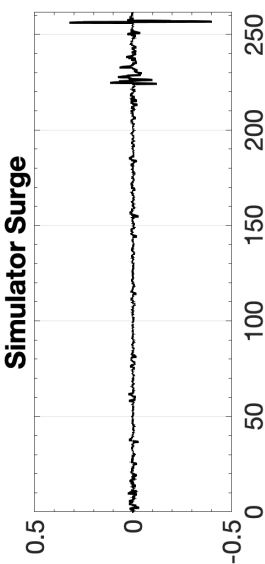

$[\mathrm{c} / \mathrm{L}] \mathrm{uo!̣}$

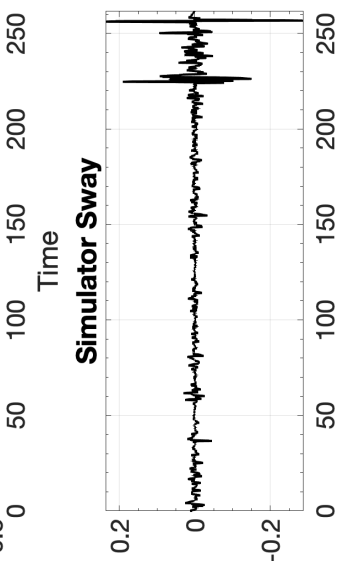

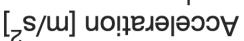

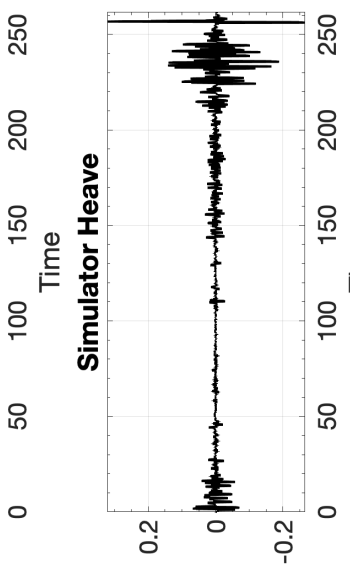

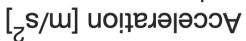

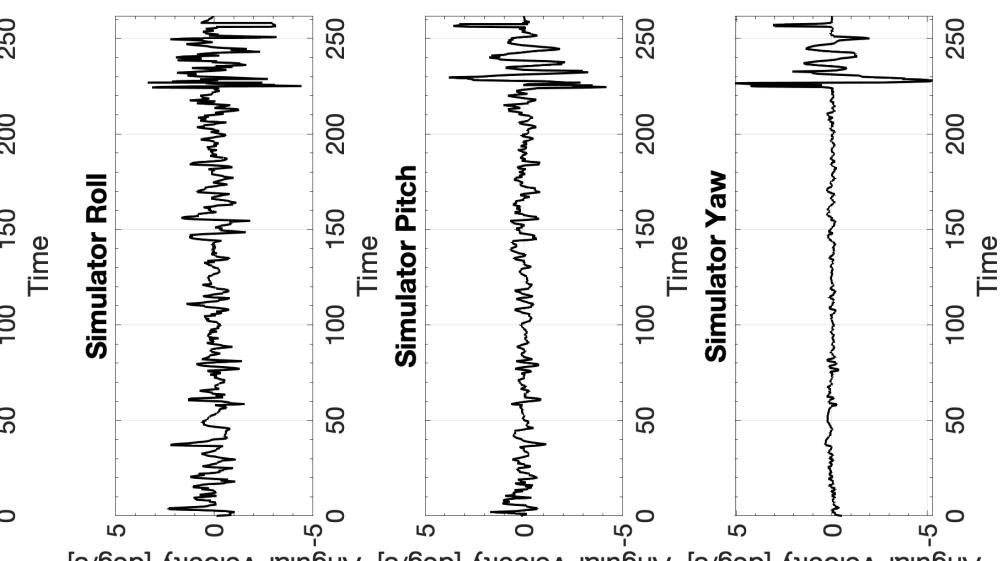

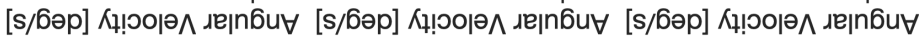




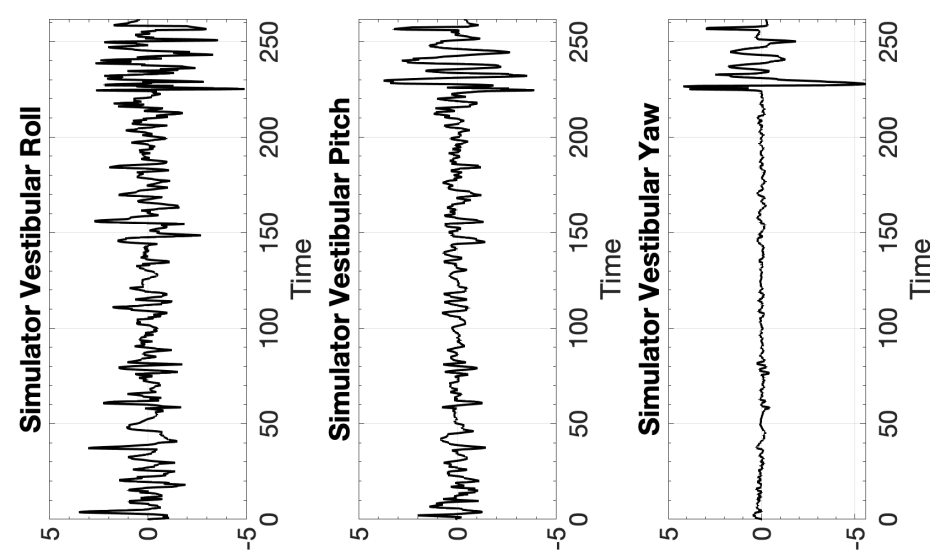

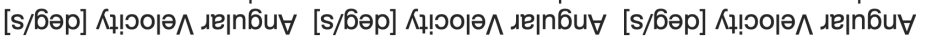

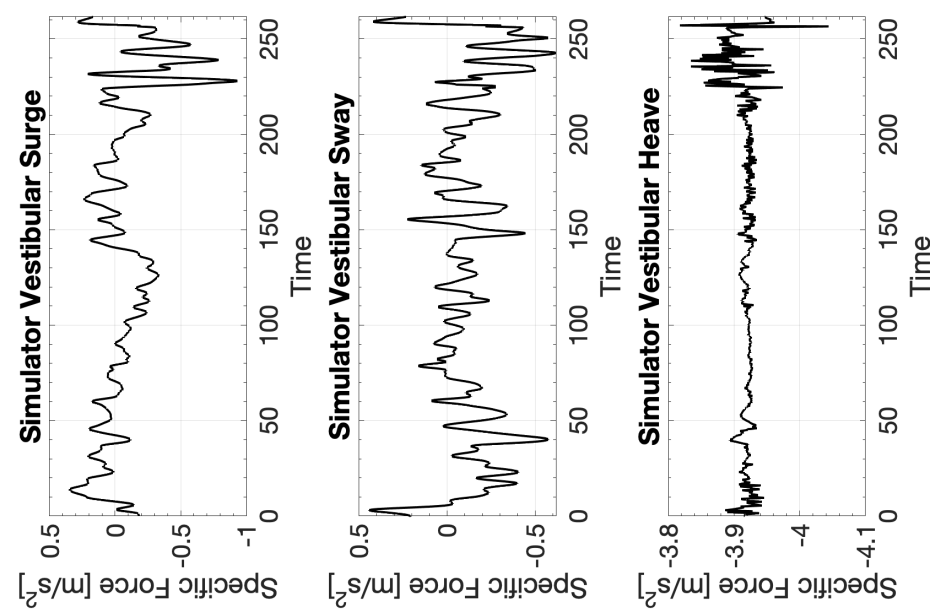


D.8 Boeing 737 Single-axis Takeoff

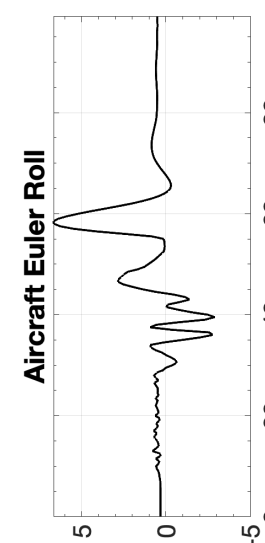

[бəp] uo!̣!sod

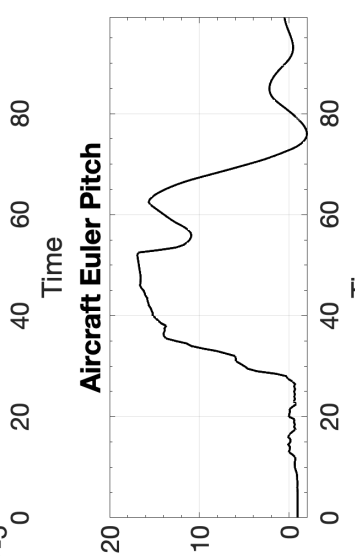

[6әр] uoliısod גe|n6u

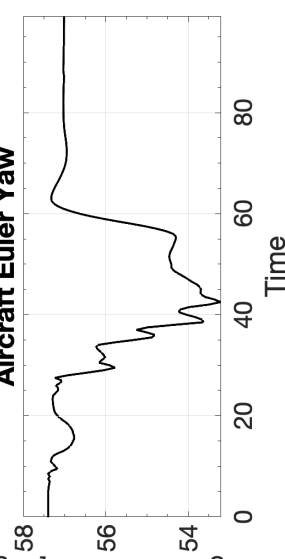

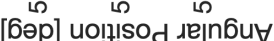

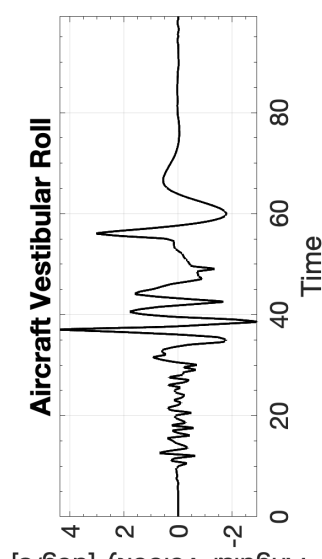

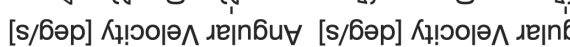

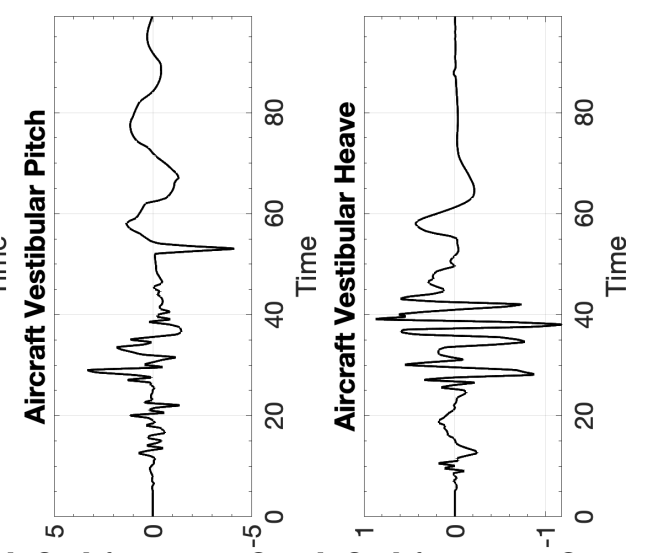

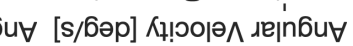

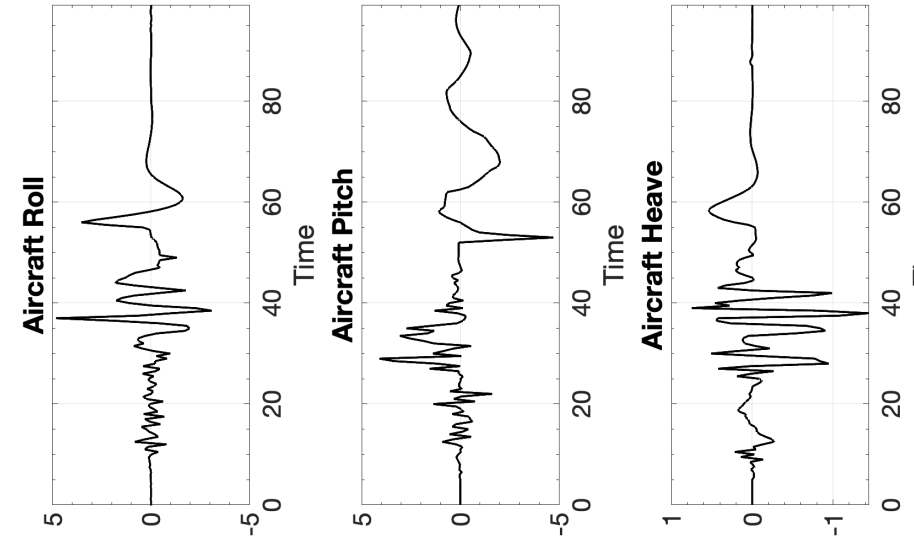

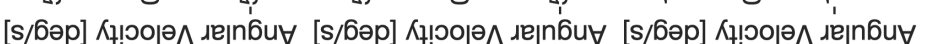

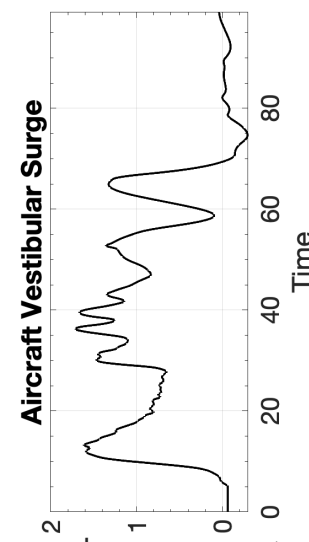

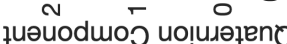

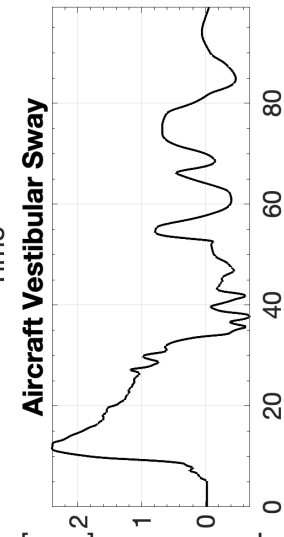

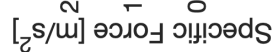

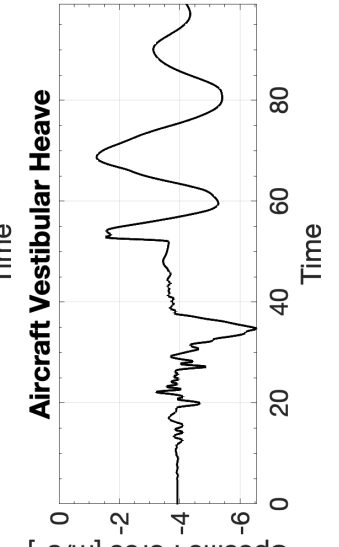

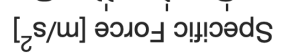

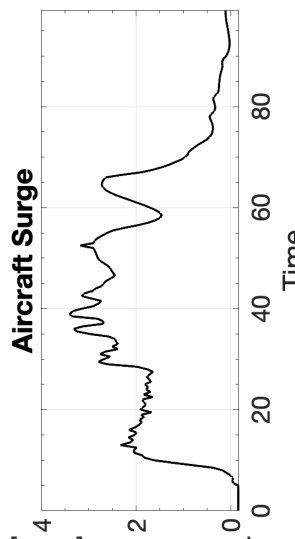

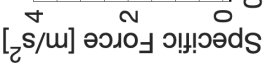

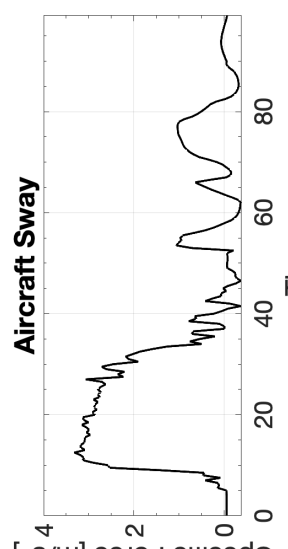

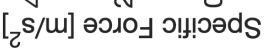

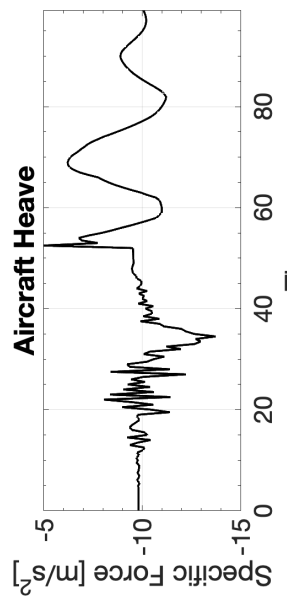

[

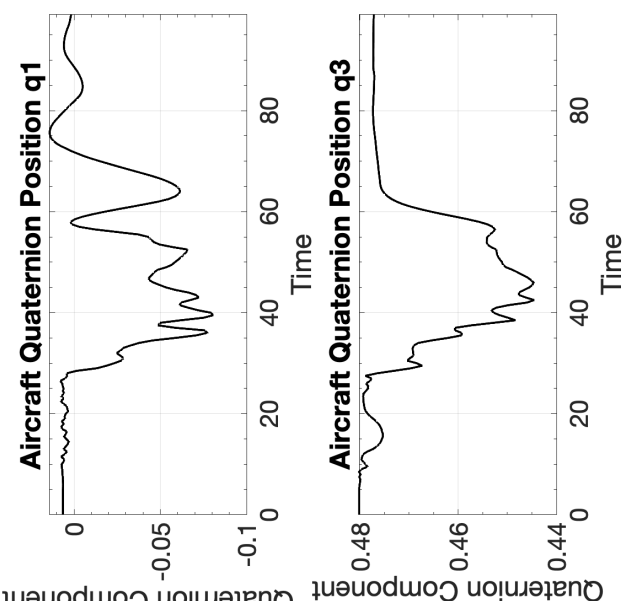

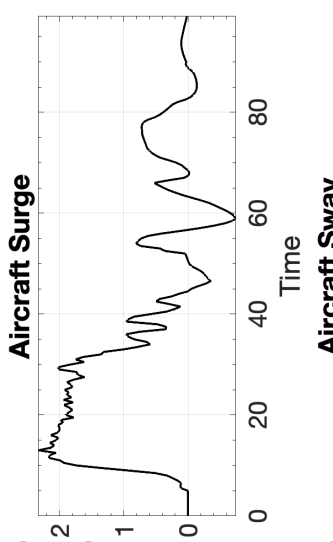

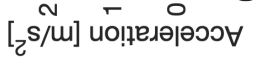

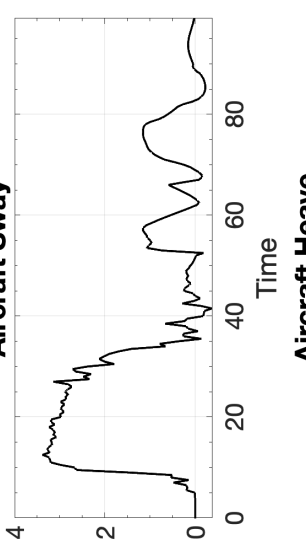

[ั]

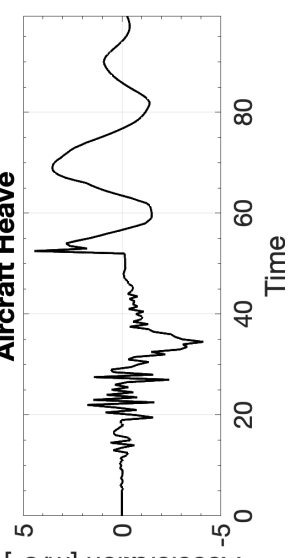

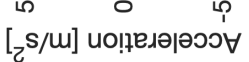

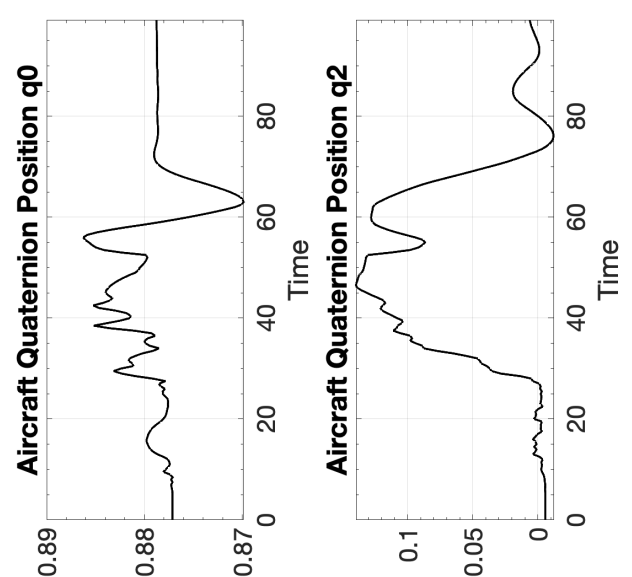

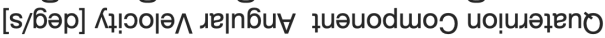




\section{D.8.1 CW1 Boeing 737 Single-axis Takeoff}

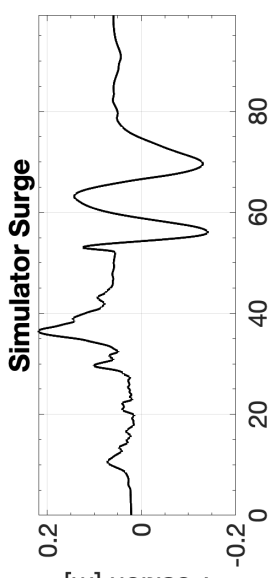

$[\mathrm{m}]$ uo!n!!sod

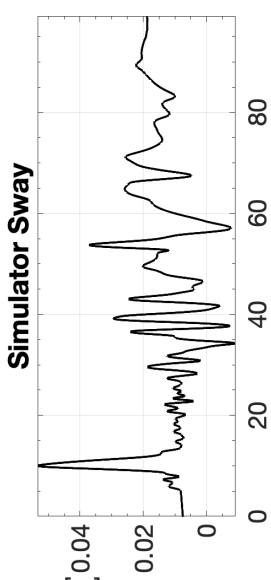

[u] uol!!!sod

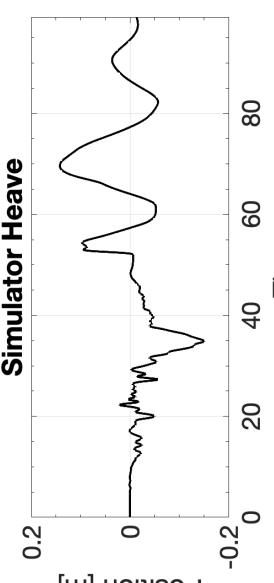

[u] uo!n!sod

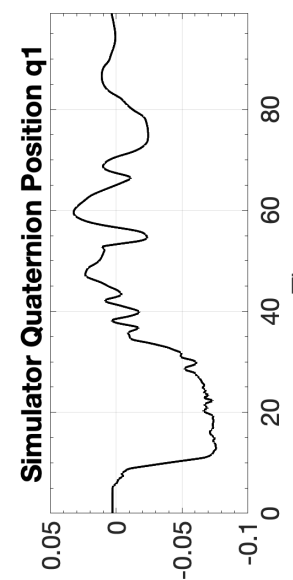

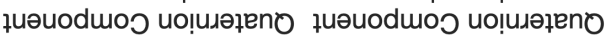

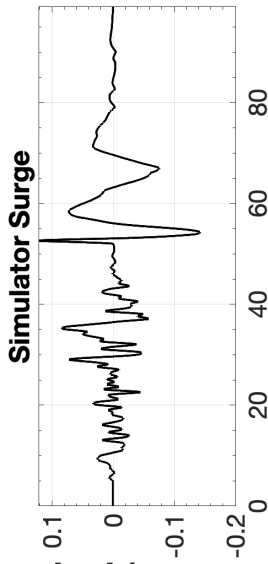

[s/m] К१!৩о|९

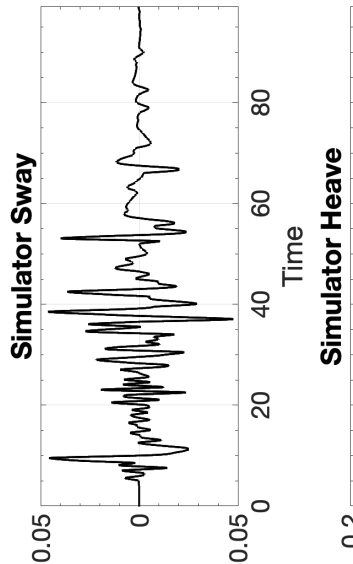

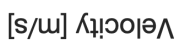

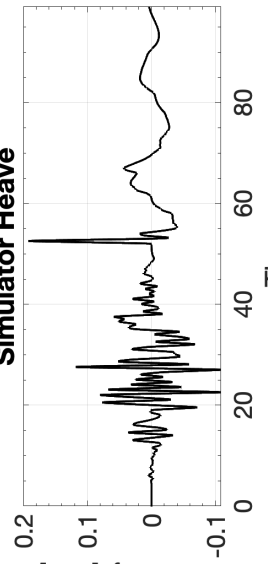

[s/u] Кұ!৩о|ә
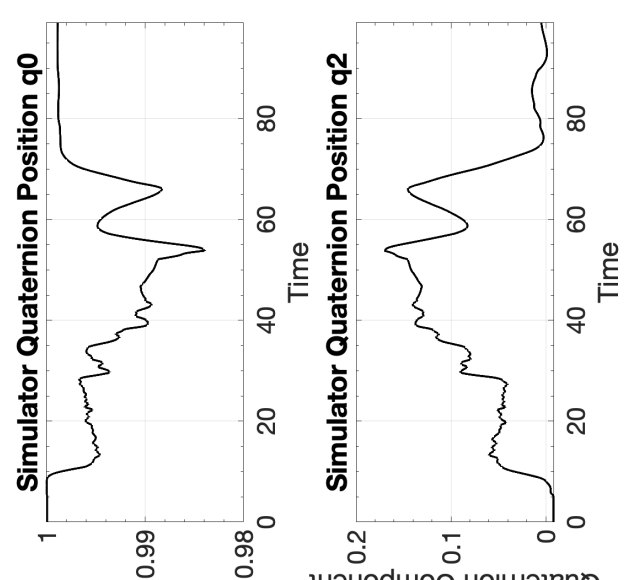

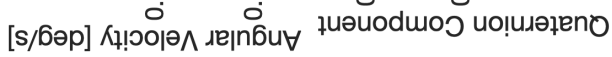

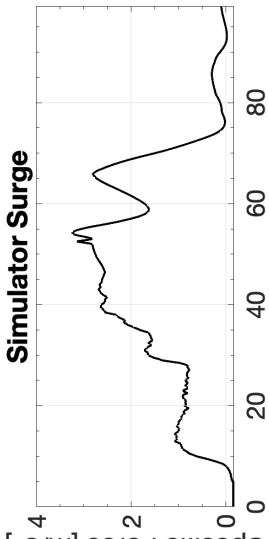

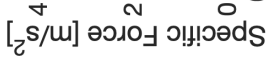

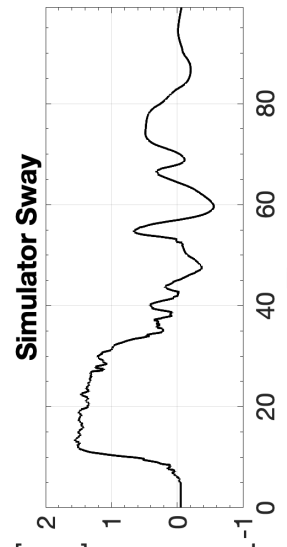

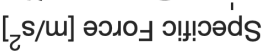

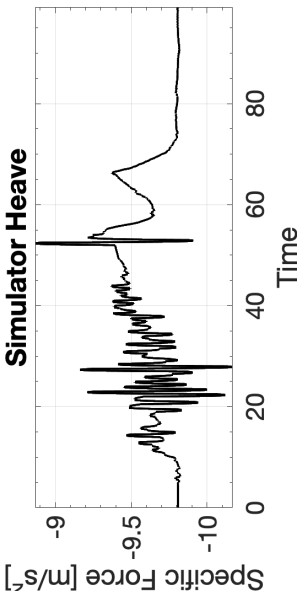

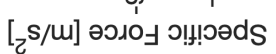

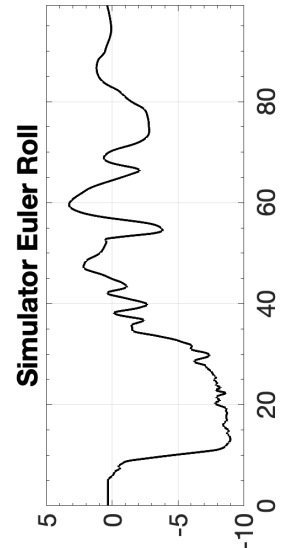

[бәр] uo!!!!sod
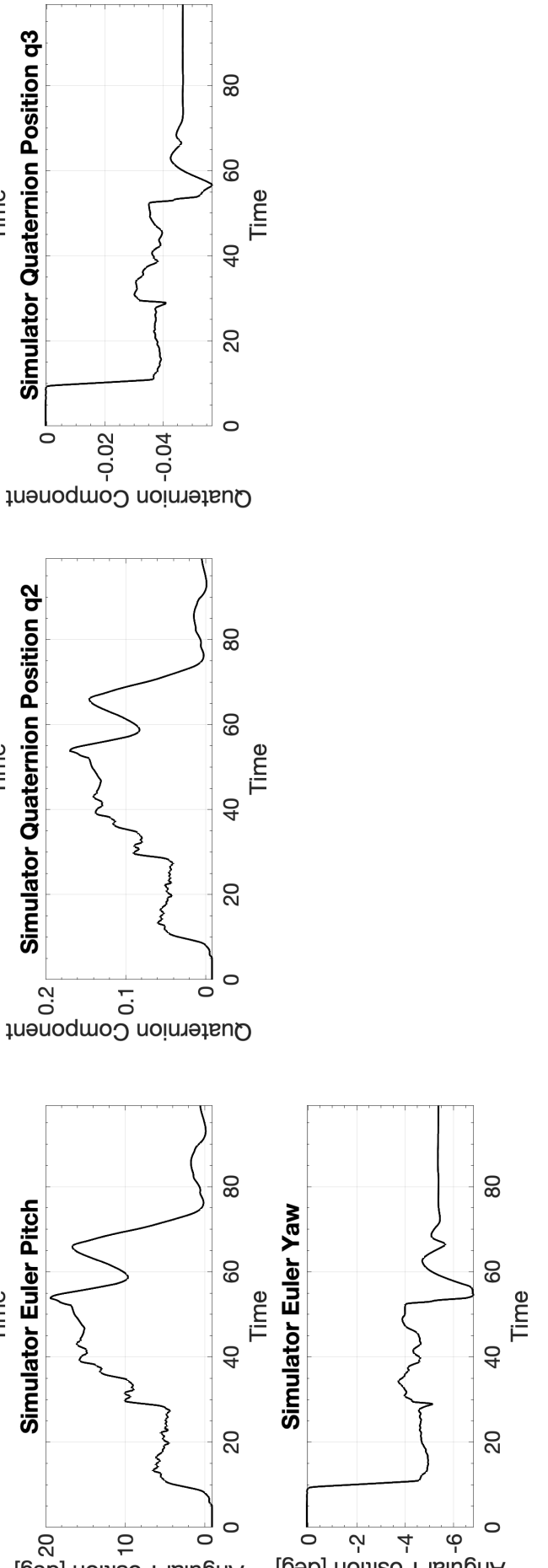

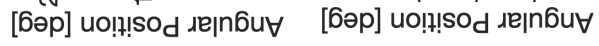

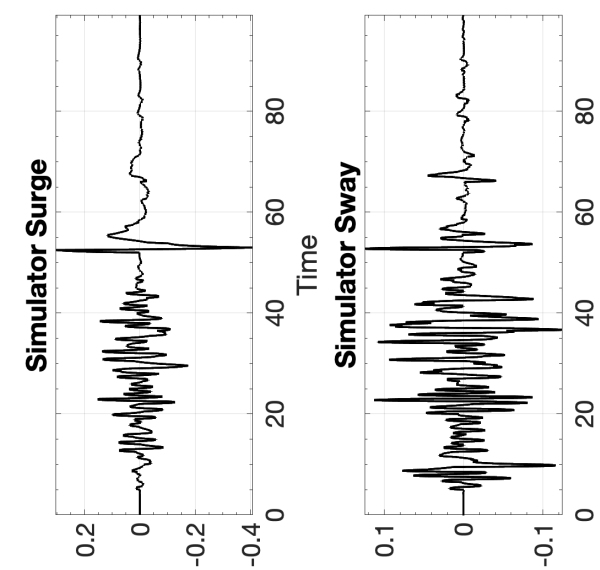

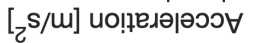

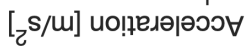

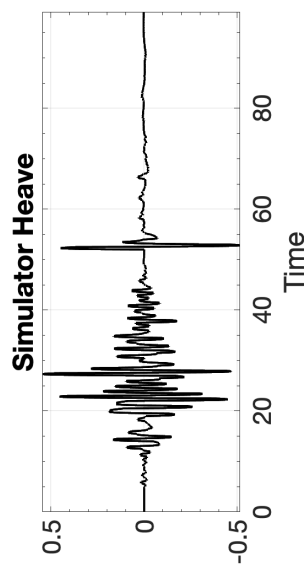

$[\mathrm{s} / \mathrm{m}]$ ио!ฺвдәәәэว $\forall$

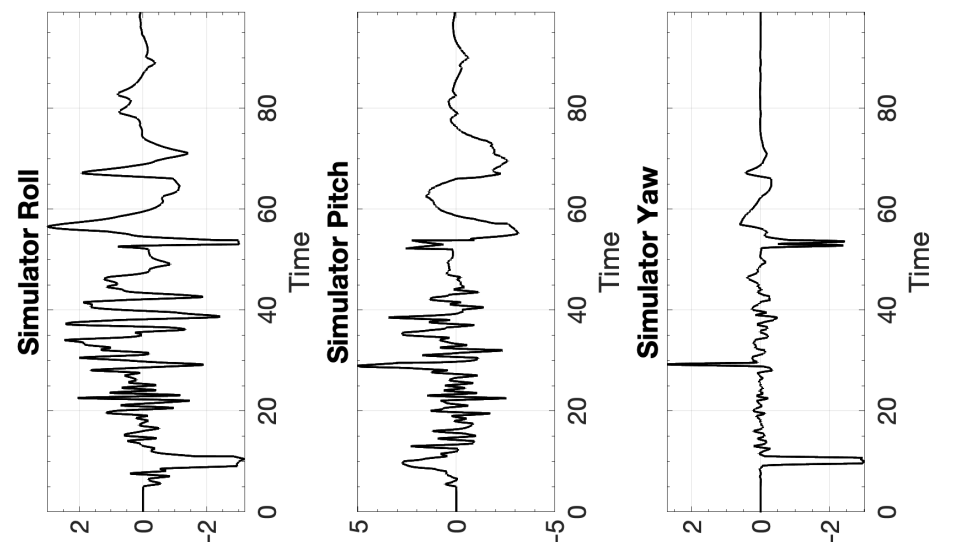

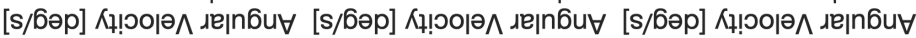




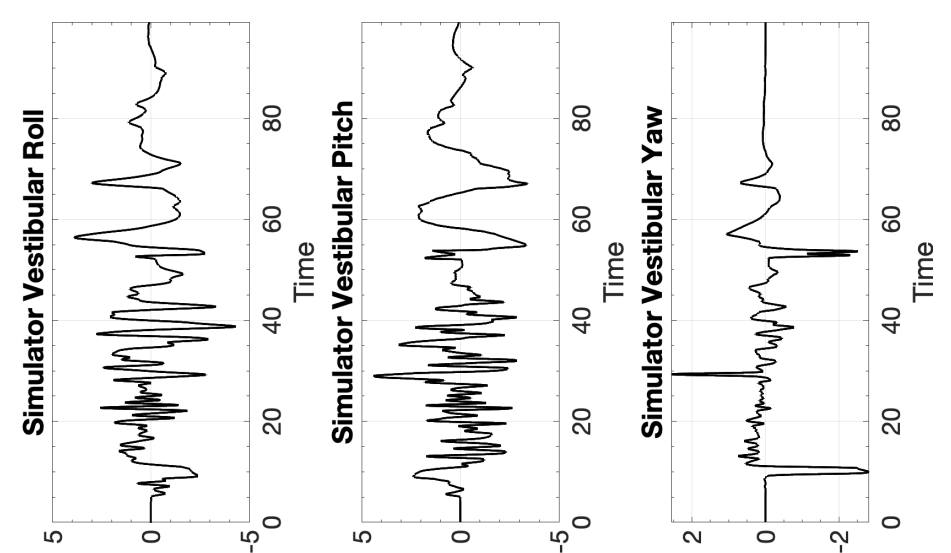

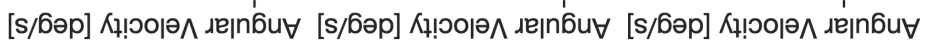

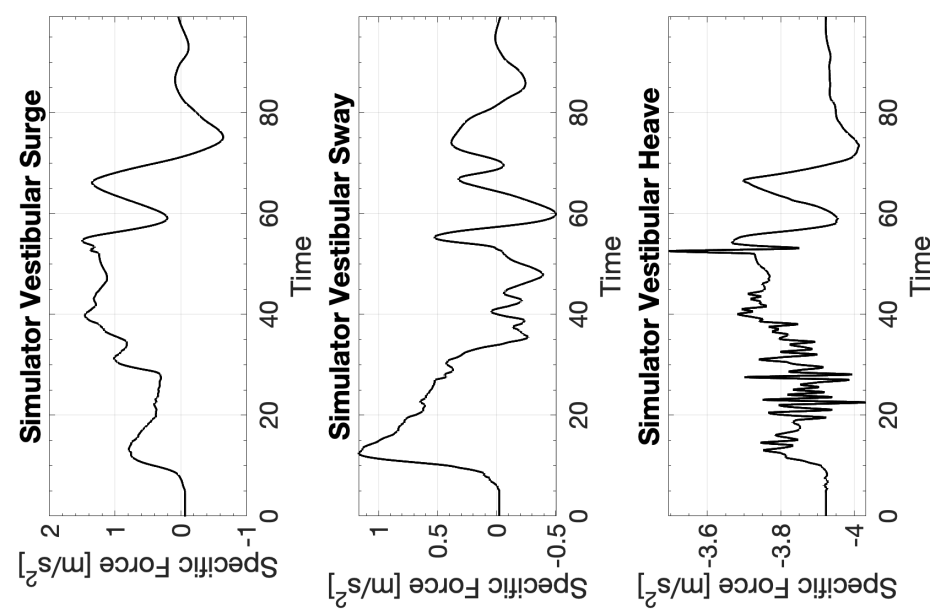




\section{D.8.2 CW2 Boeing 737 Single-axis Takeoff}

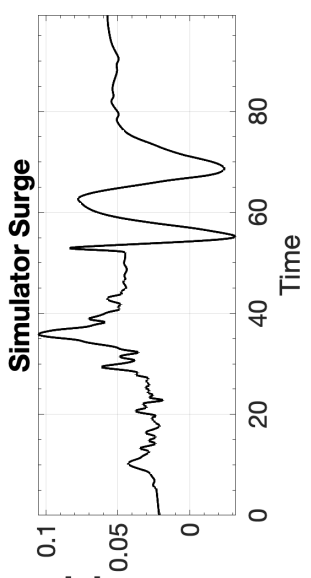

[u] uo!n!!sod

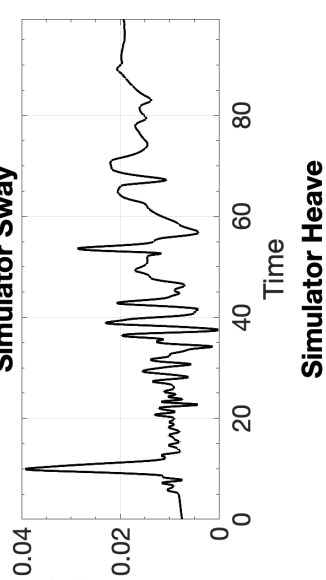

[w] uol!!sod

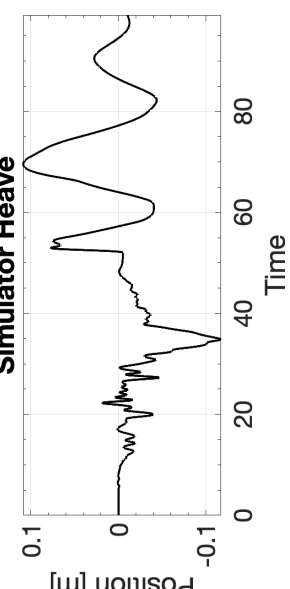

[m] uoli!nsod

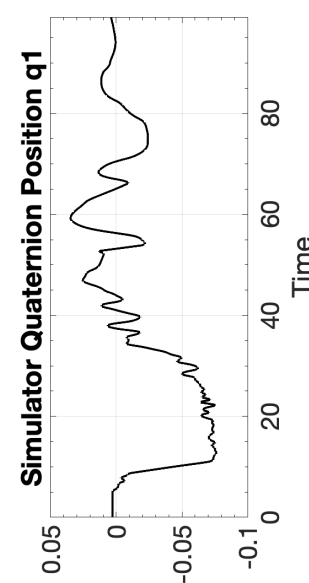

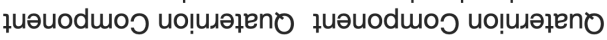

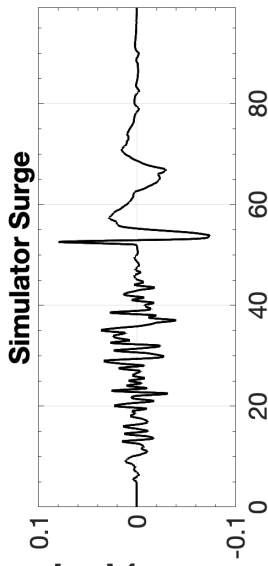

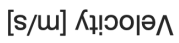

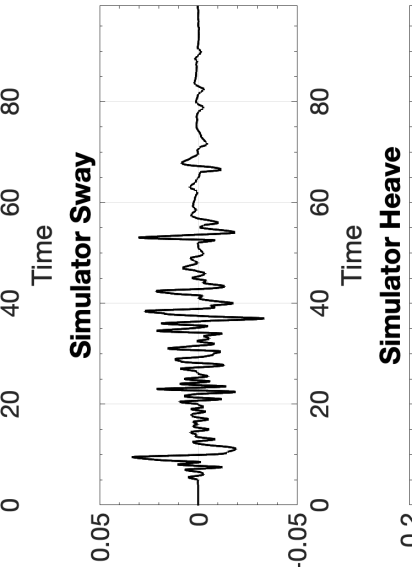

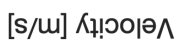

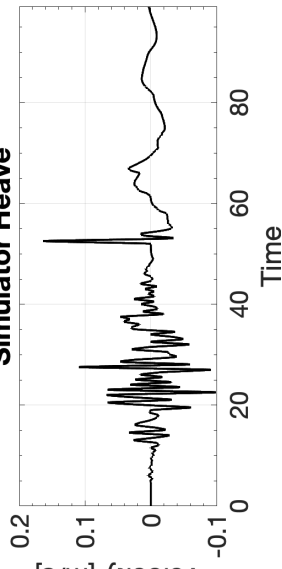

[s/u] К!๐ю ^
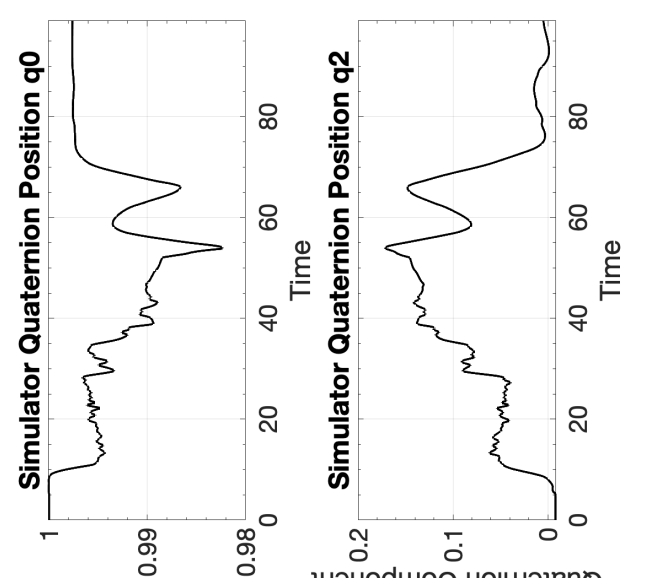

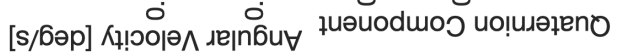

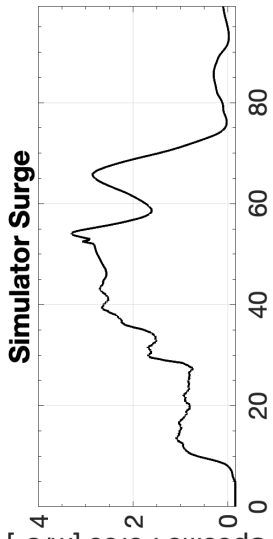

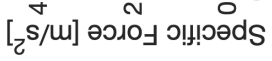

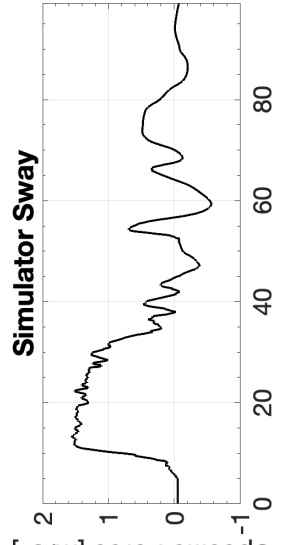

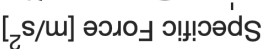

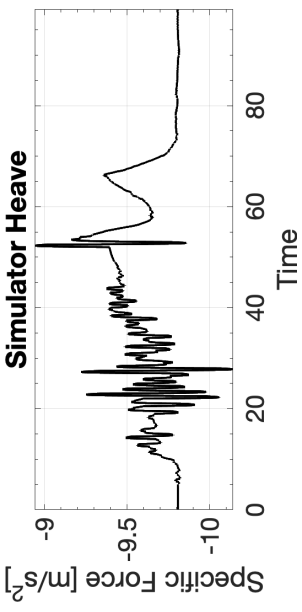

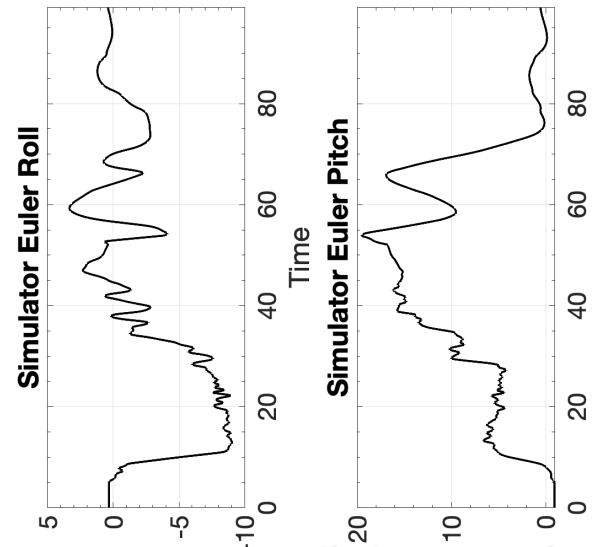

[бəp] uo!!!

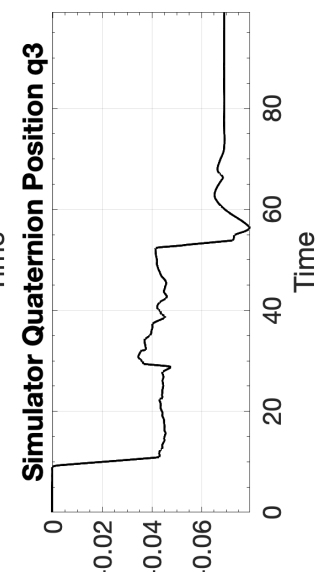




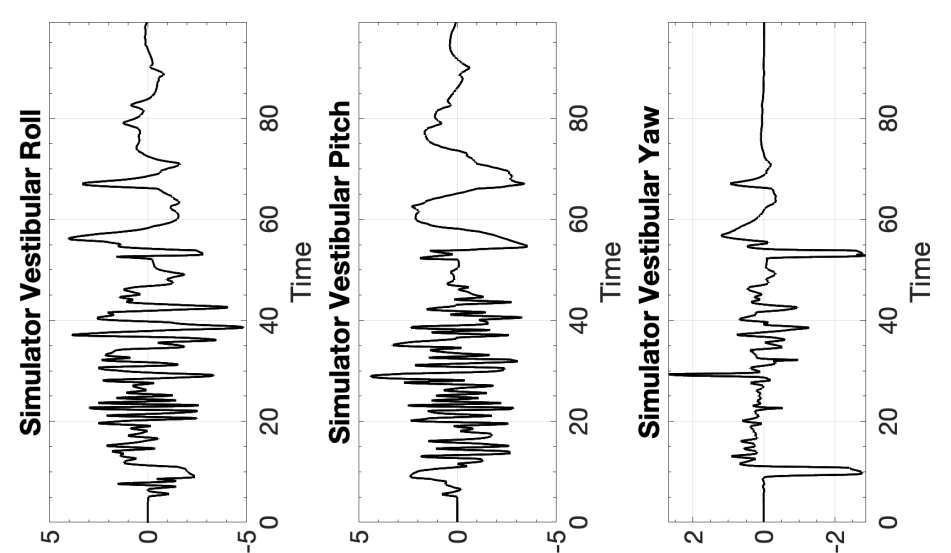

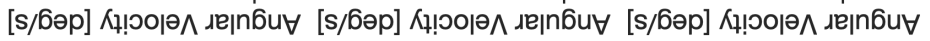

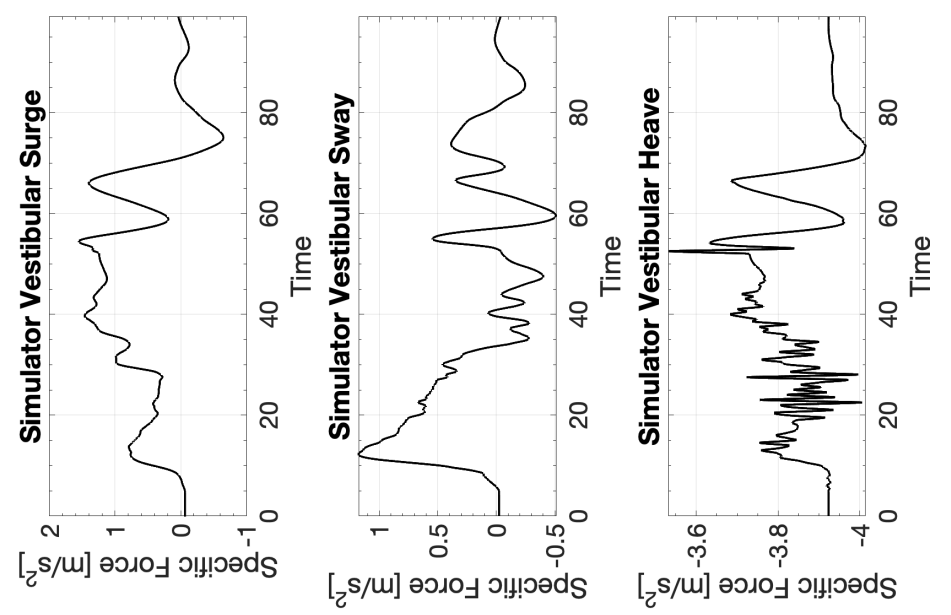




\section{D.8.3 CW3 Boeing 737 Single-axis Takeoff}

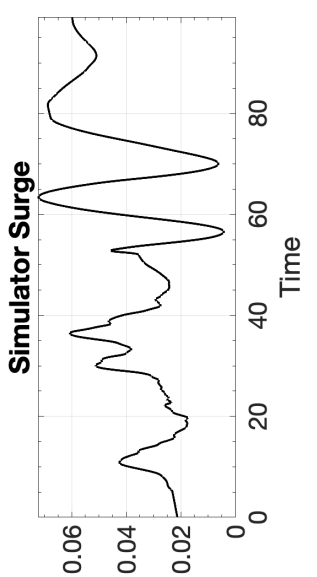

[u] uo!n! sod

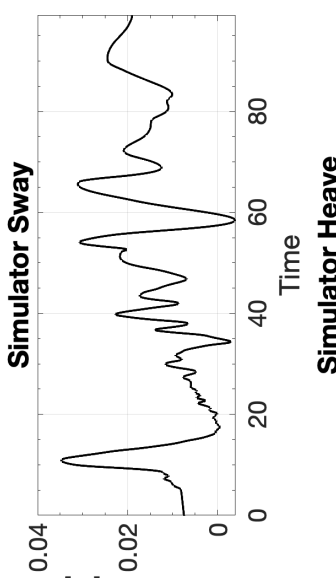

[w] uol!!sod

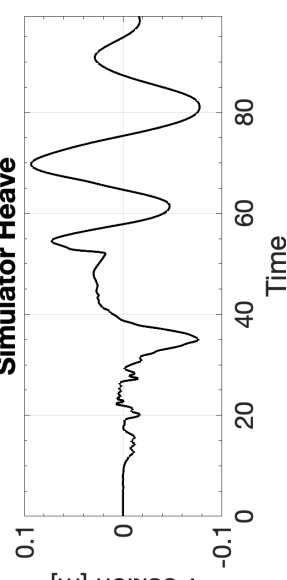

[u] uo!t!sod

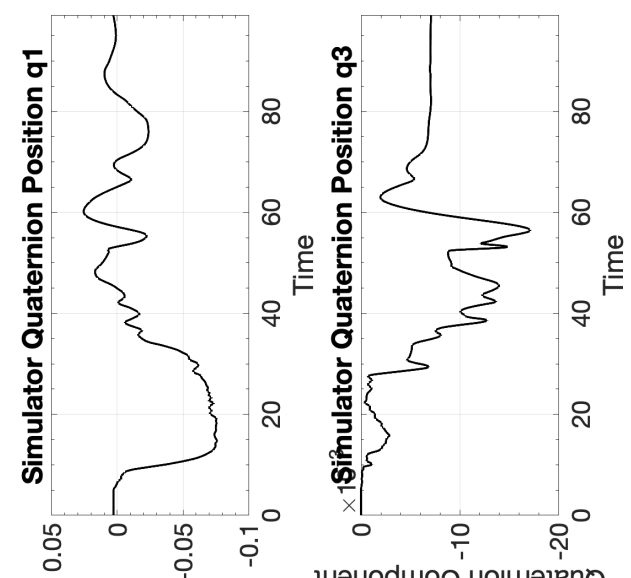

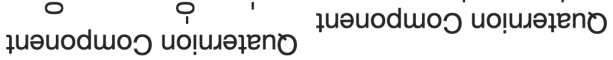

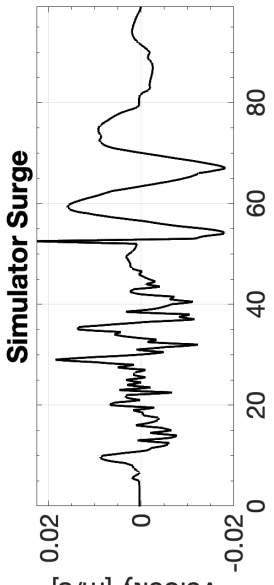

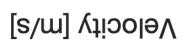

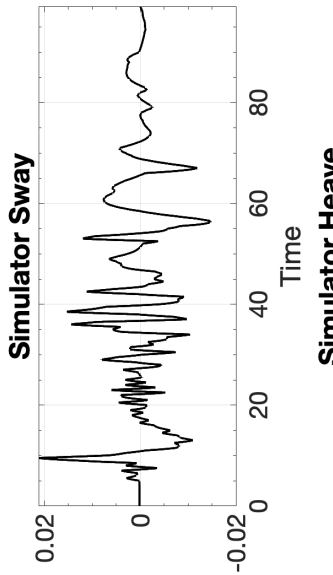

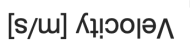

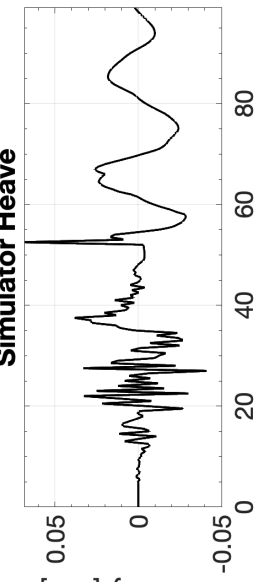

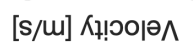

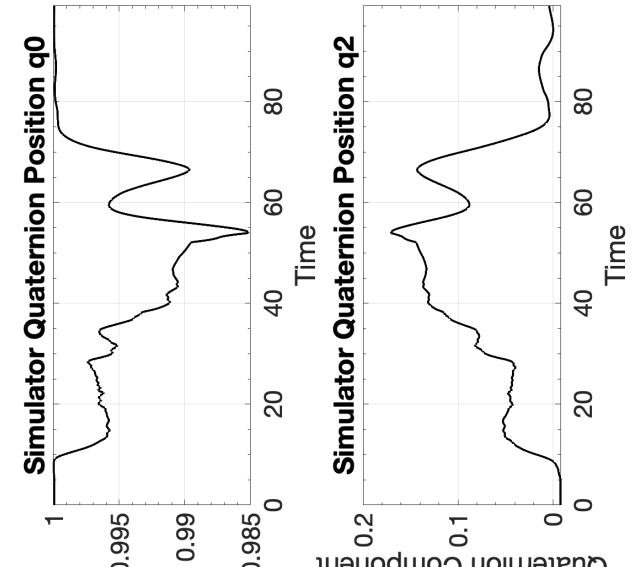

[s/6әр] Кำоорл

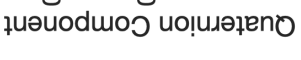

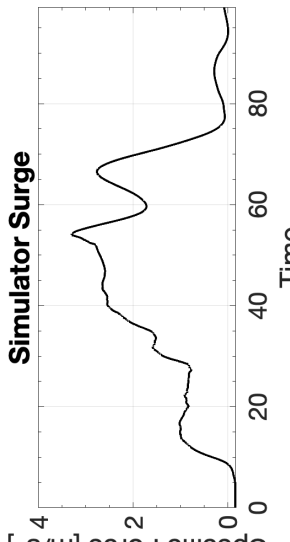

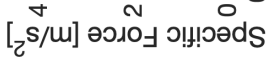

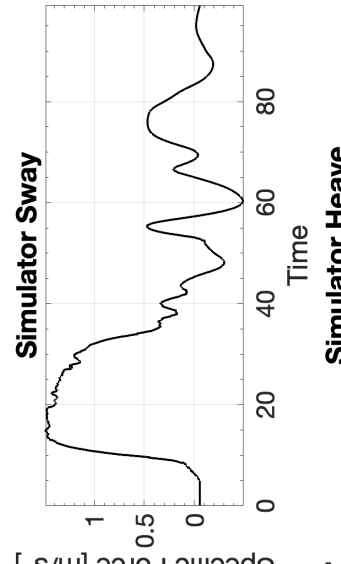

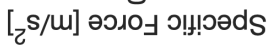
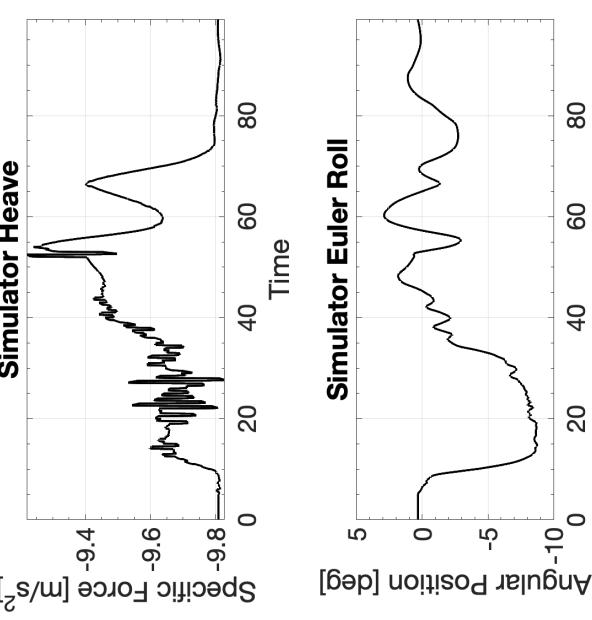

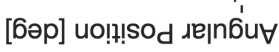

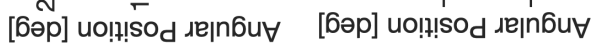
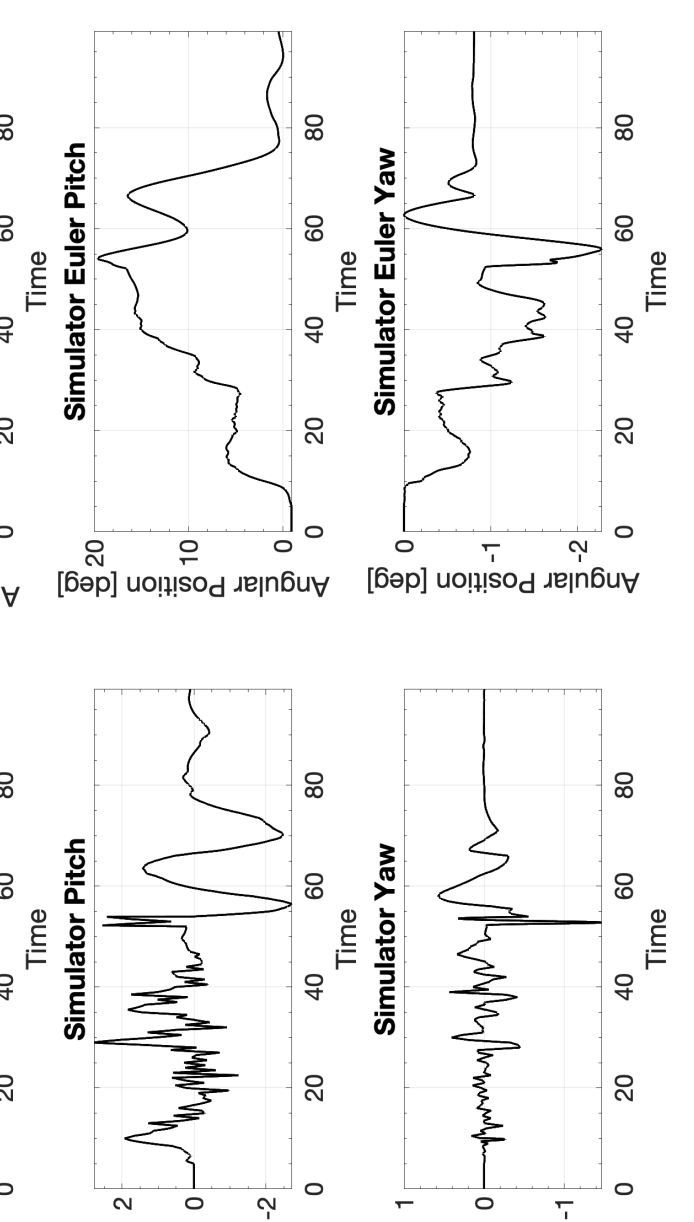

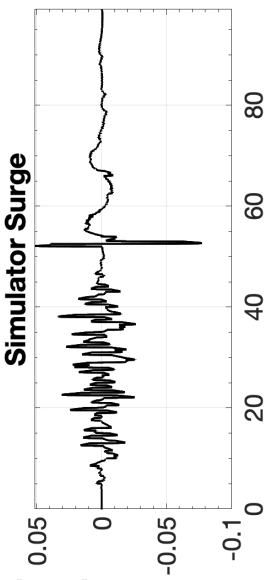

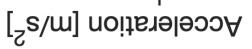

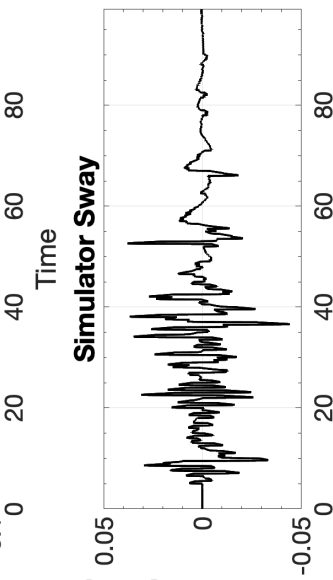

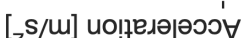

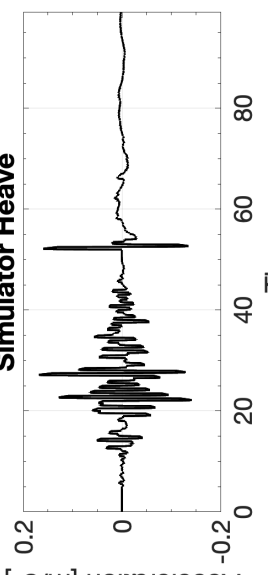

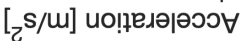

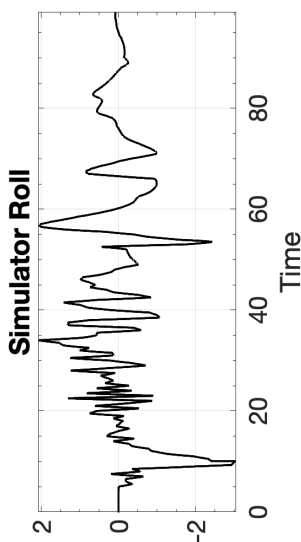

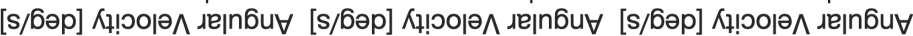



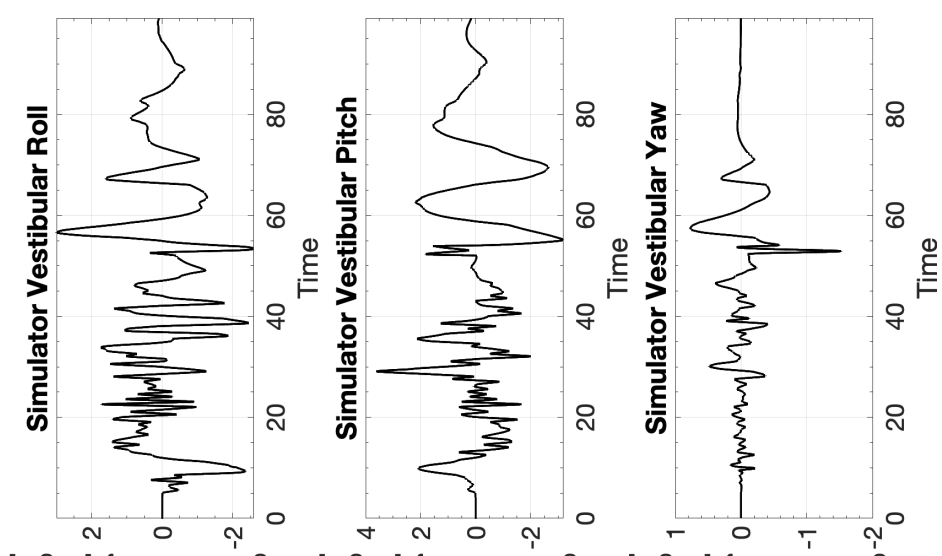

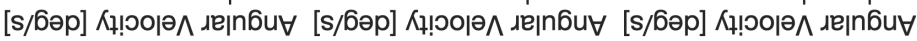

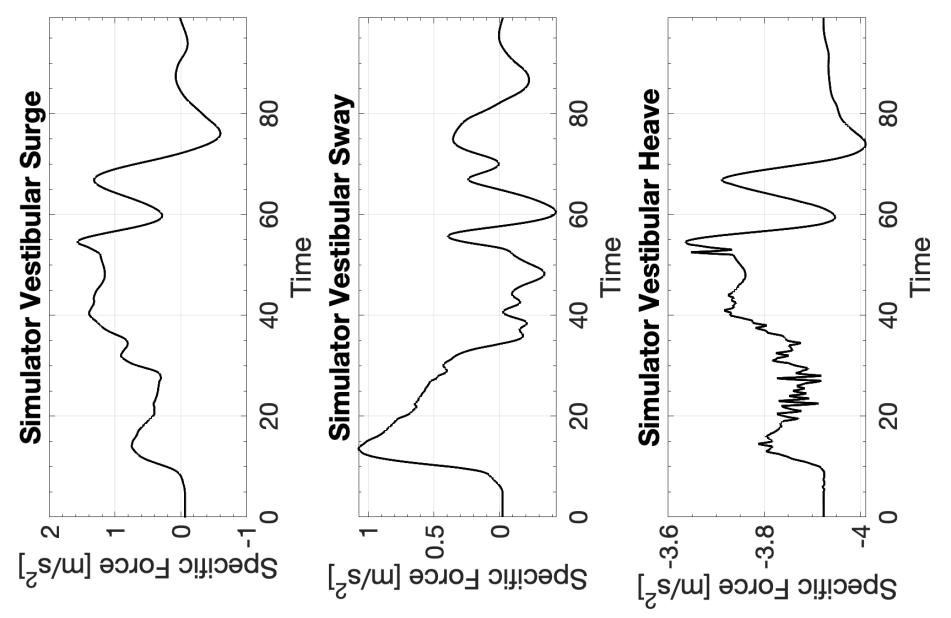




\section{D.9 Boeing 737 Spiral Dive}
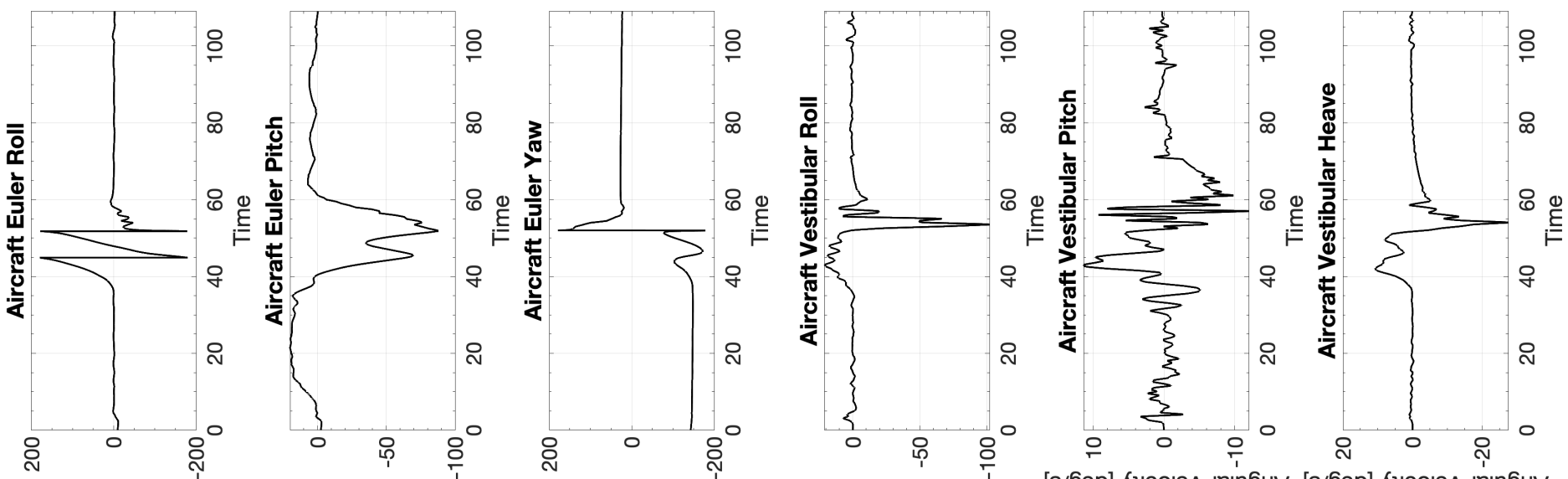

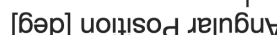

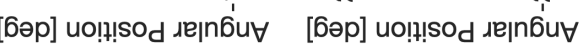

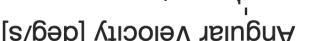

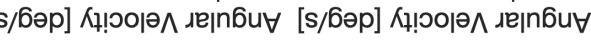
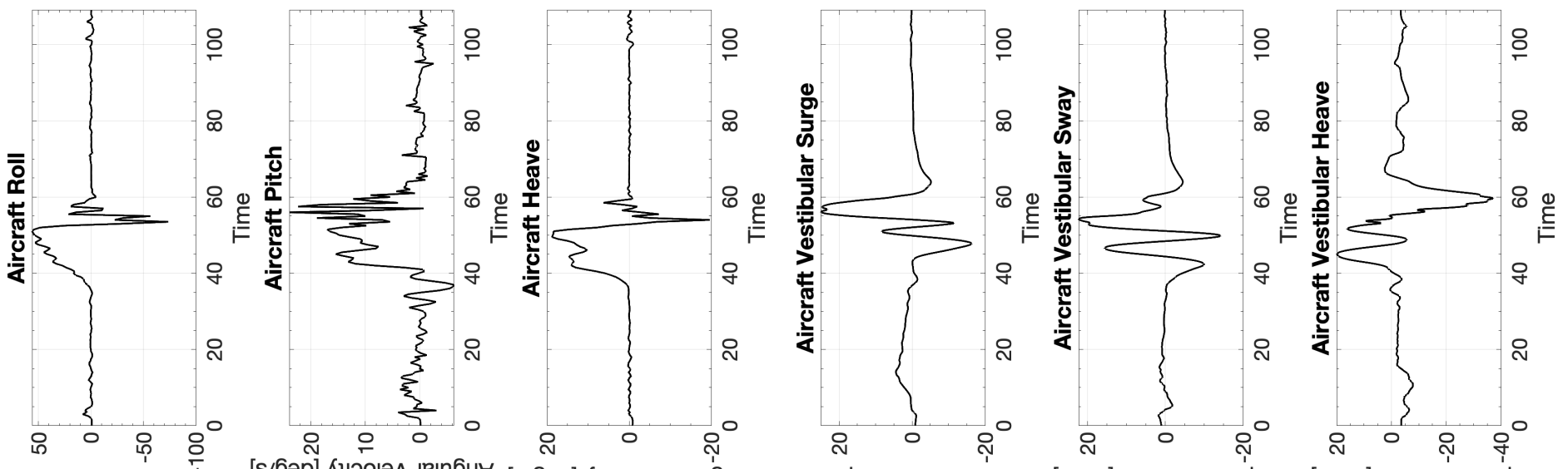

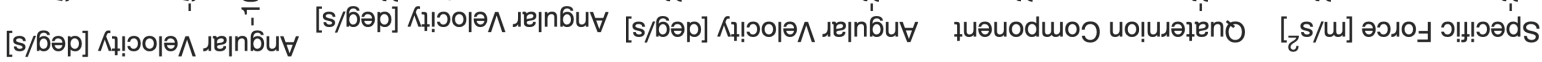

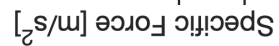

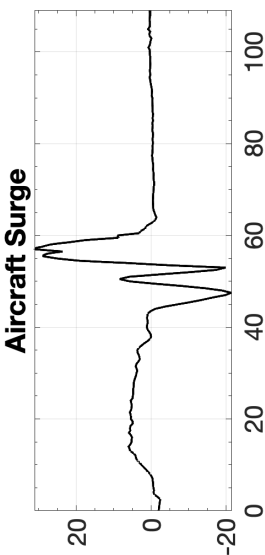

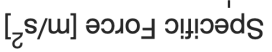

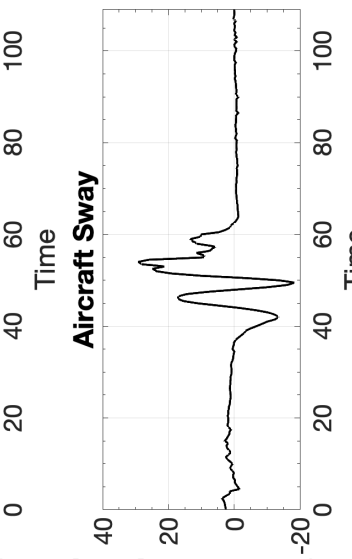

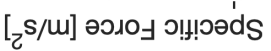

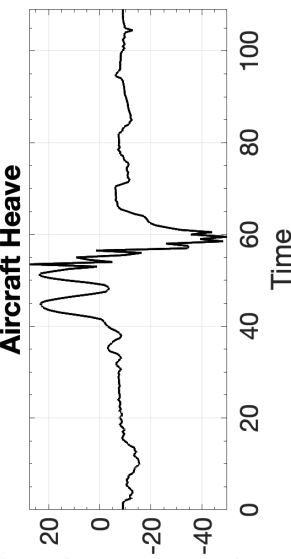

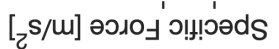

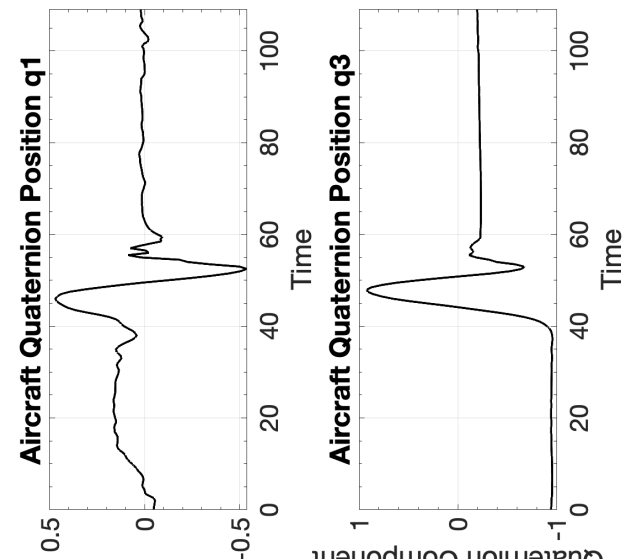

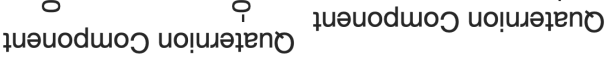

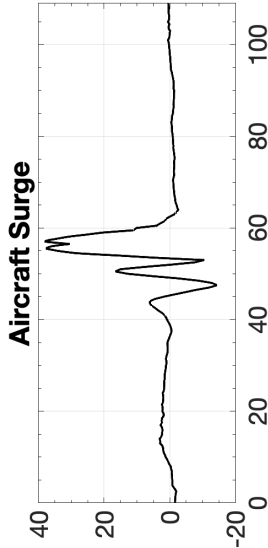

$[z$ S/u] uо!ฺеләәәэว $\forall$

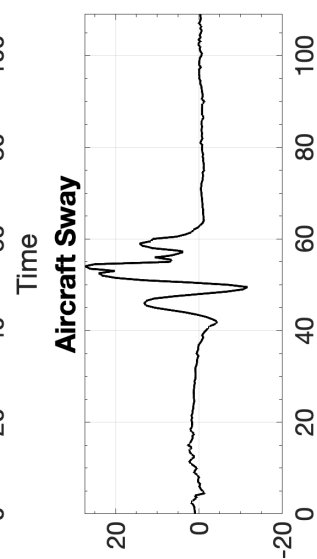

[s/m] ио!ฺедәрәоэ

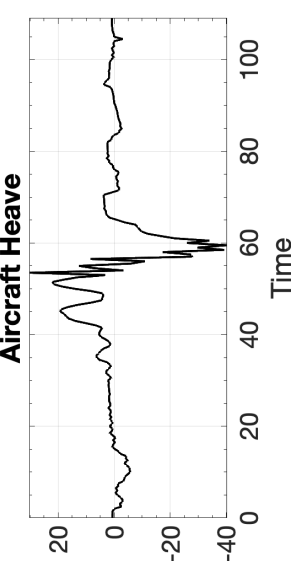

[s/m] uо!ฺвдәрәоэ

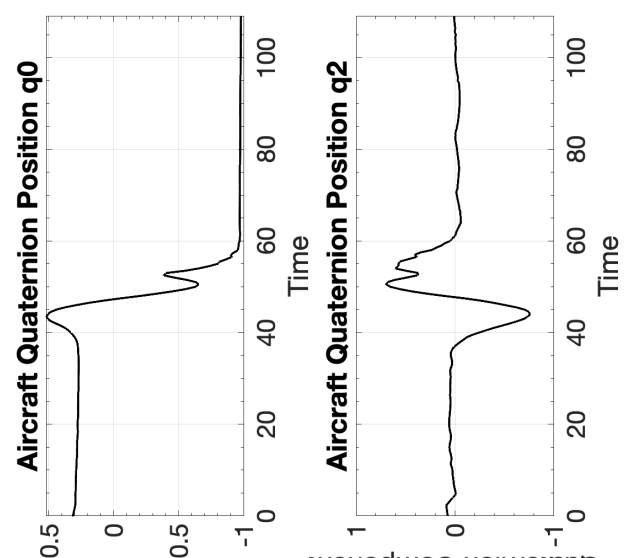

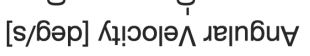

ұนəuoduos uo!̣uəłeno 


\section{D.9.1 CW1 Boeing 737 Spiral Dive}

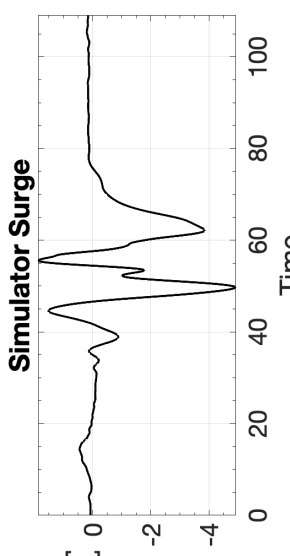

[u] uol!!!sod

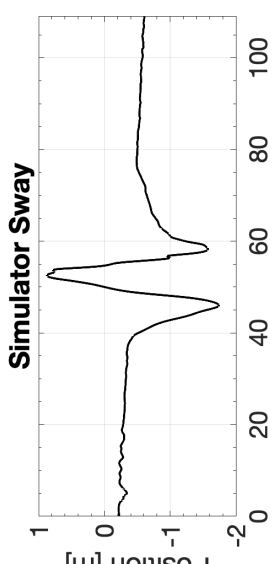

[u] uo!n!sod

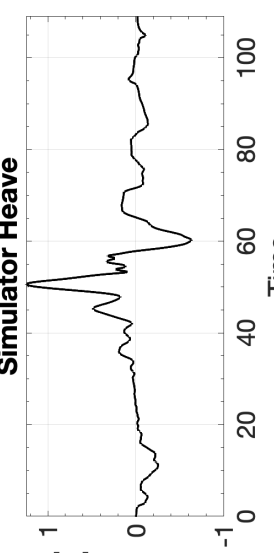

[u] uolt!nsod

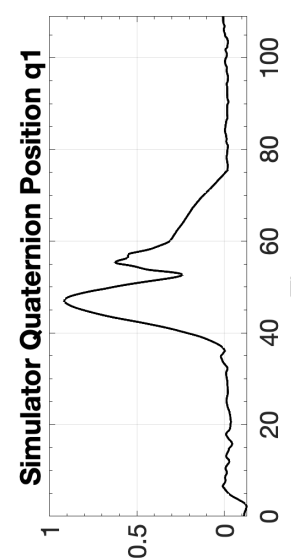

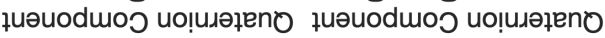

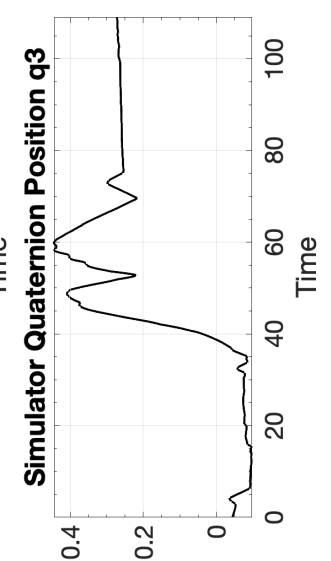

$\stackrel{\oplus}{\text { F }}$

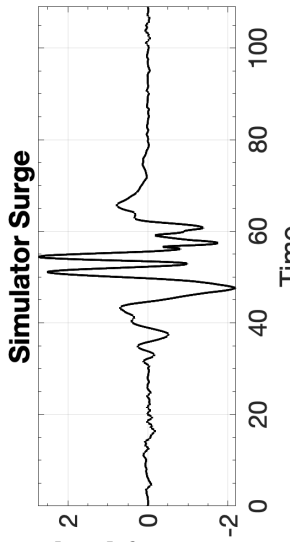

[s/u] Кұ!०о|ә^

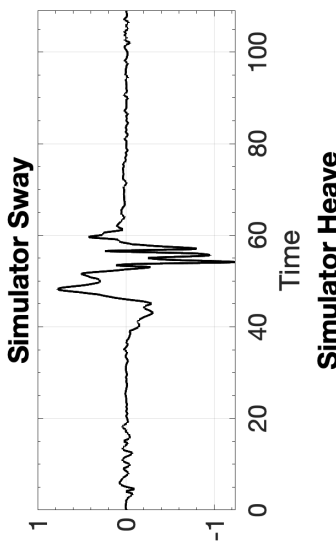

[s/m] Кұ!юорә

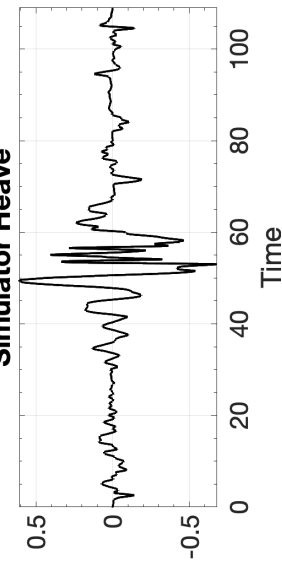

[s/m] Кł!ฺо夫

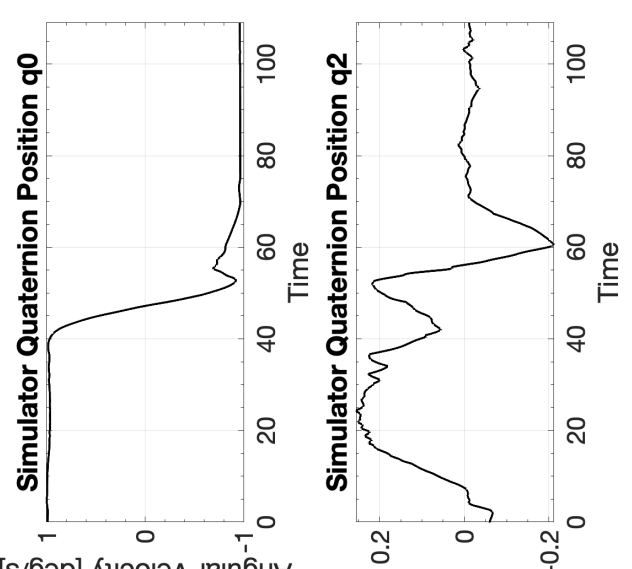

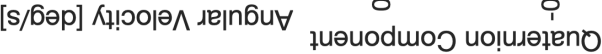

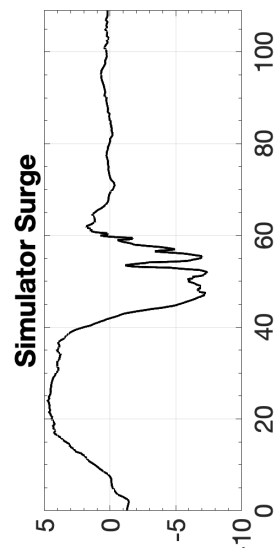

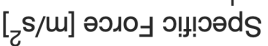

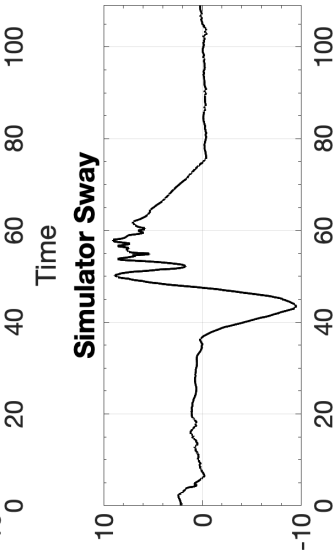

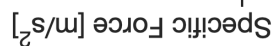

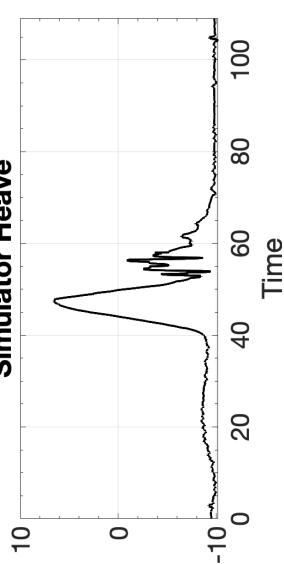

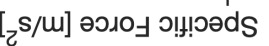

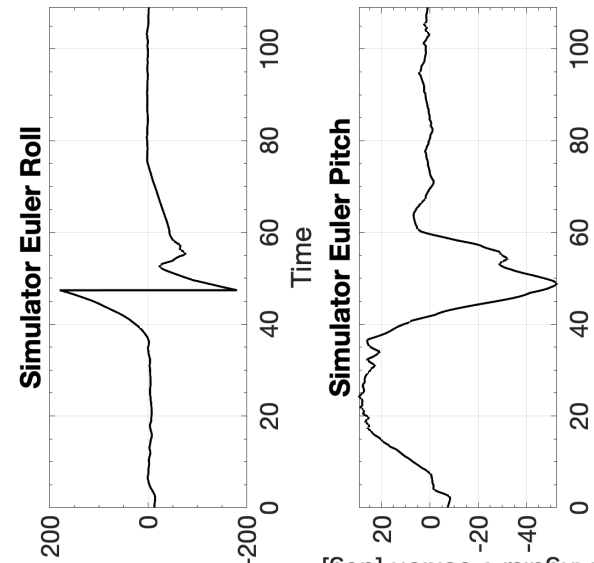

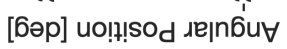

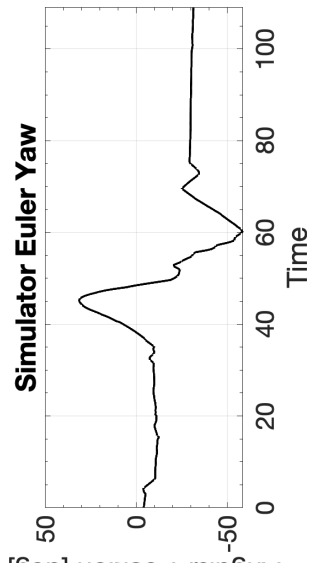

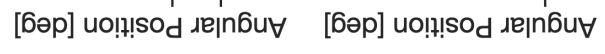

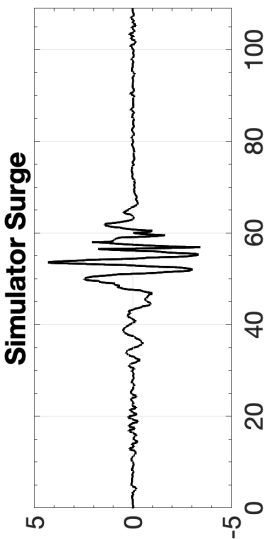

$[\mathrm{z} / \mathrm{s}]$ uо!ฺеגә|әэว $\forall$

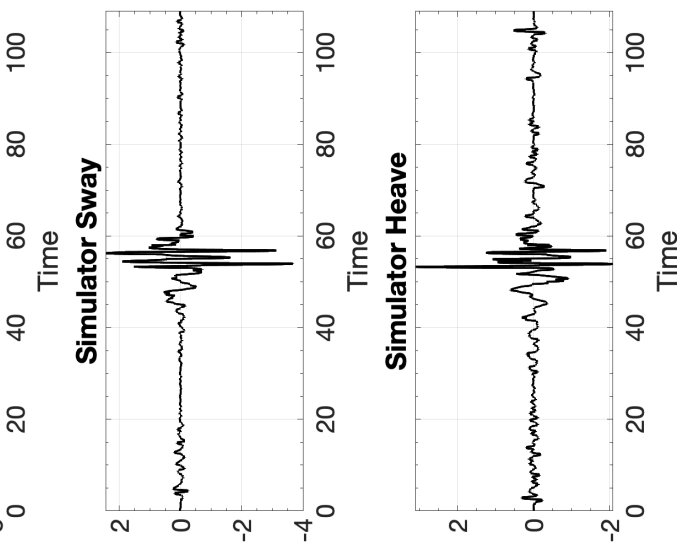

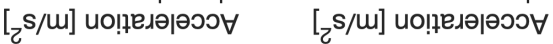

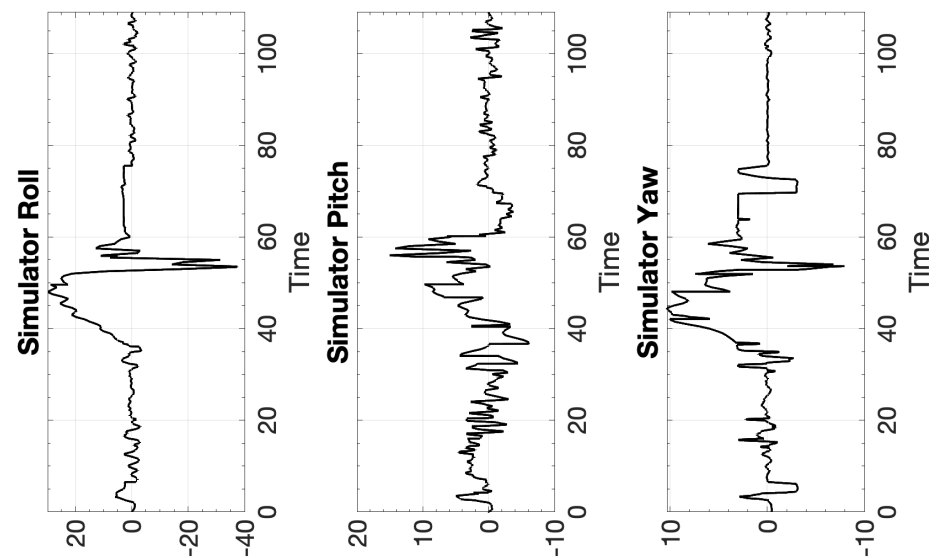

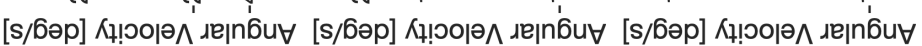




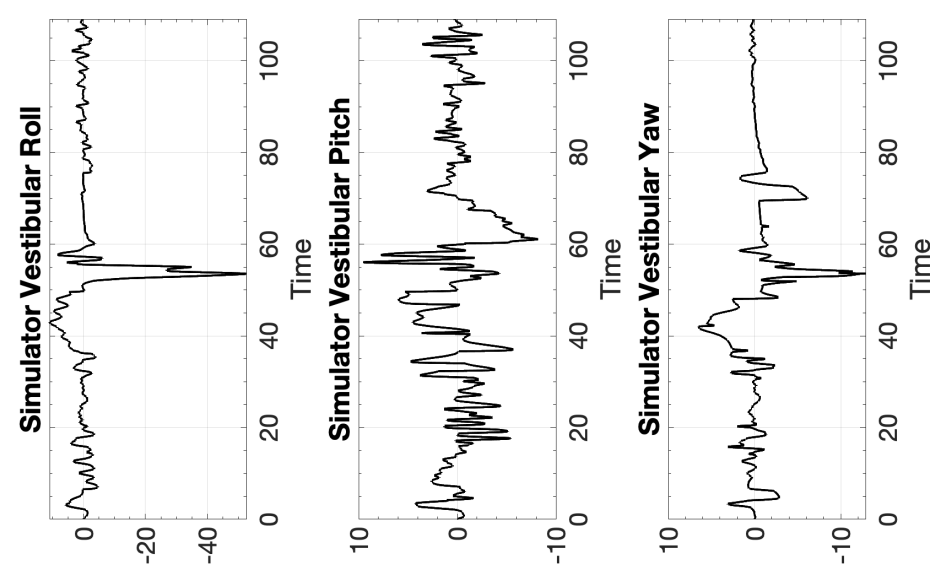

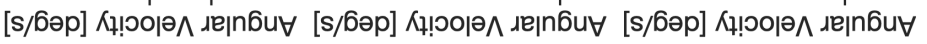

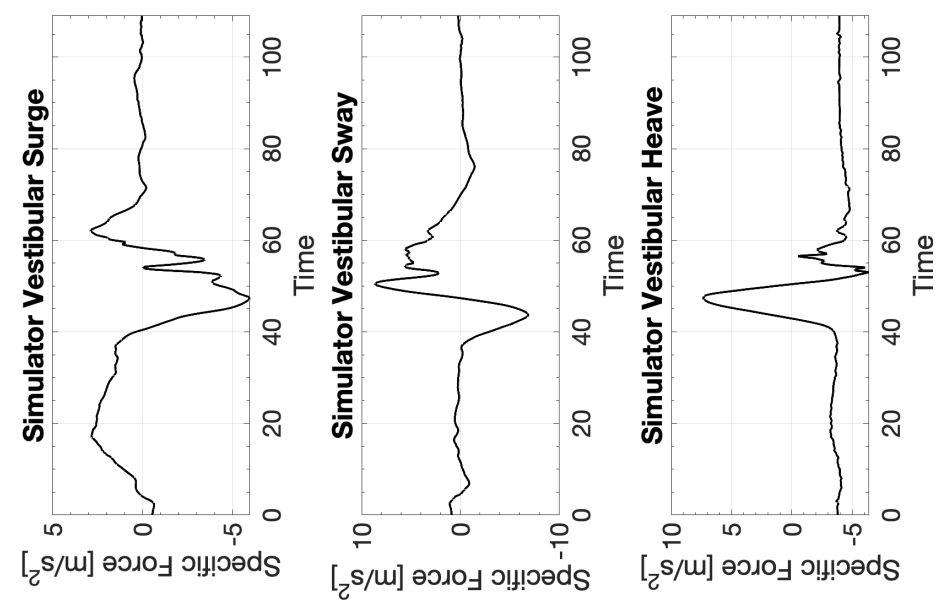




\section{D.9.2 CW2 Boeing 737 Spiral Dive}

[w] uo!!!!sod

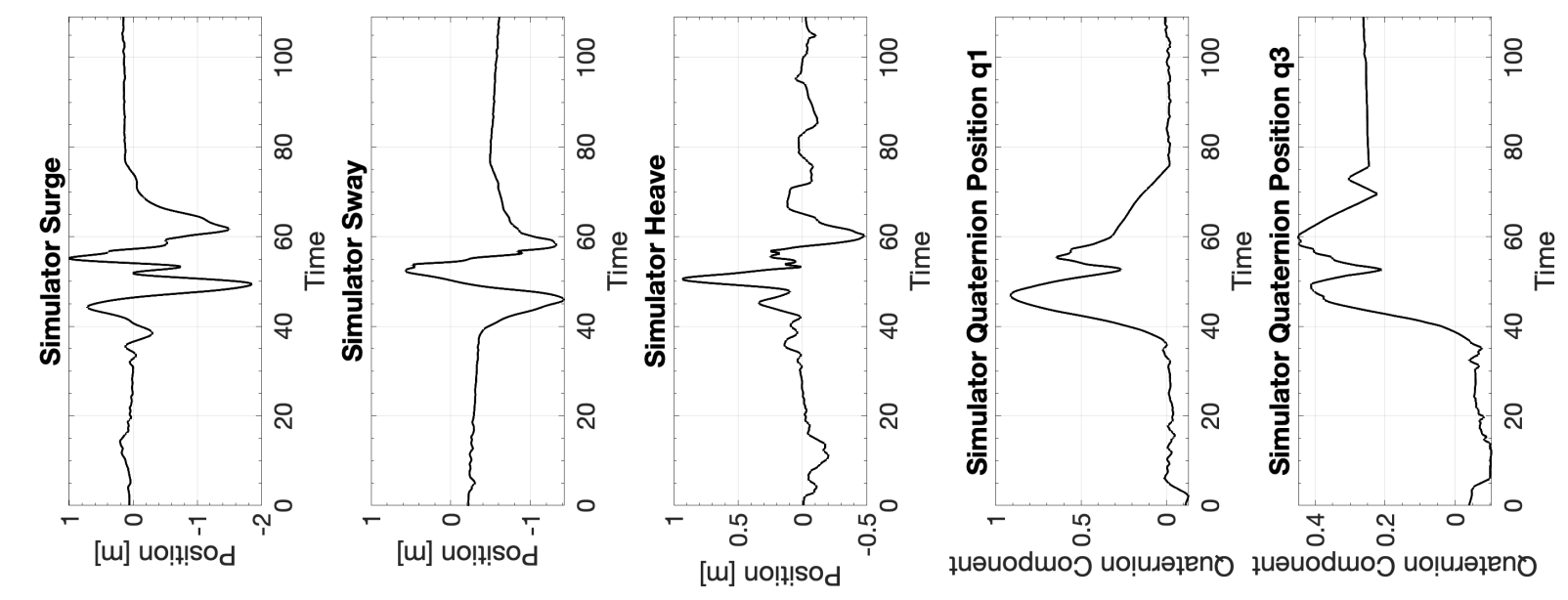

[u] uol!!!sod

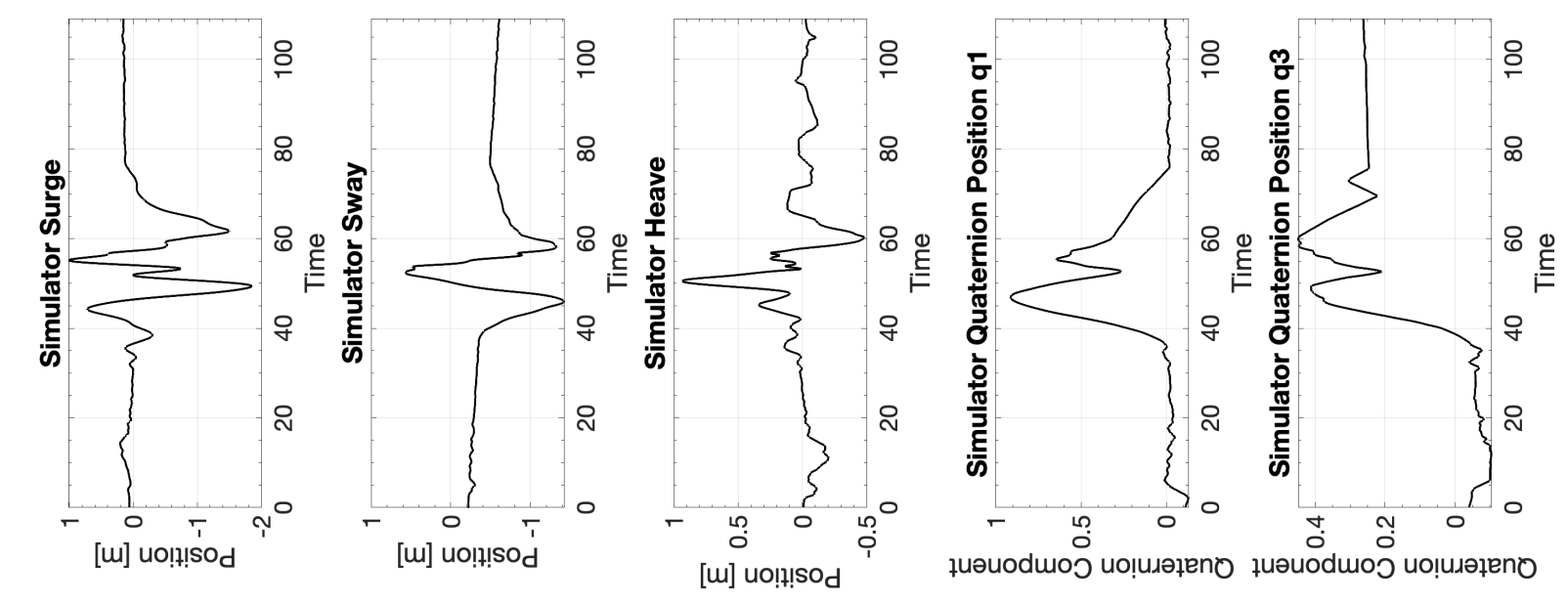

[w] uolitsod

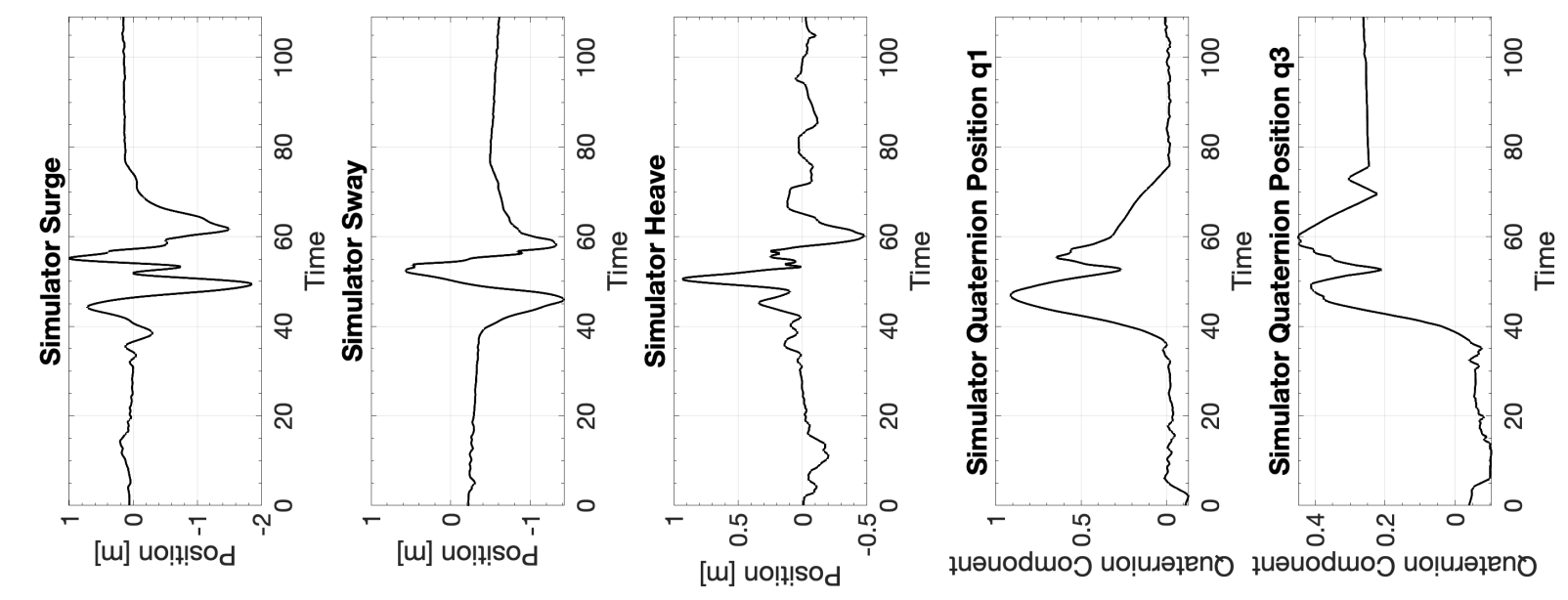

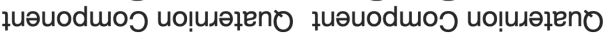

$\stackrel{\oplus}{\xi}$

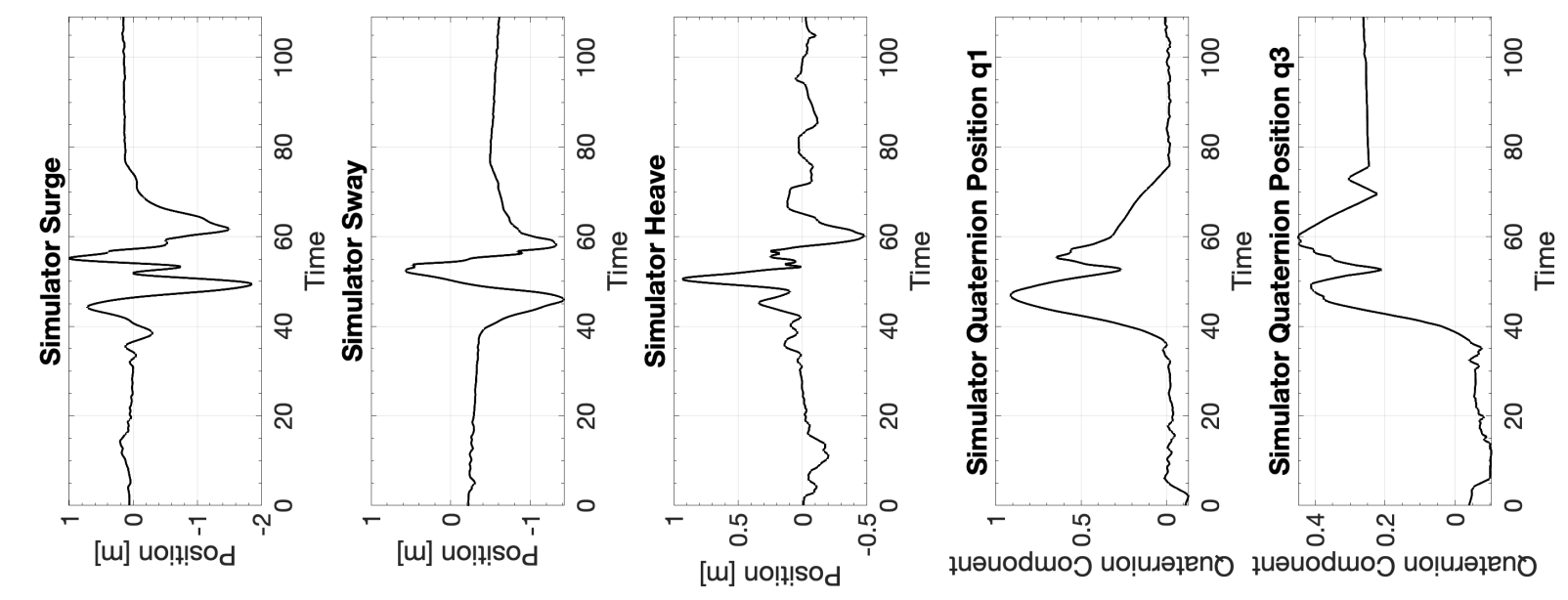

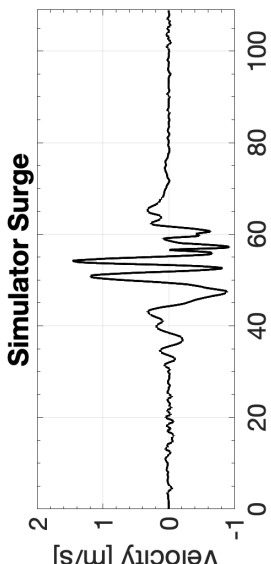

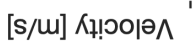
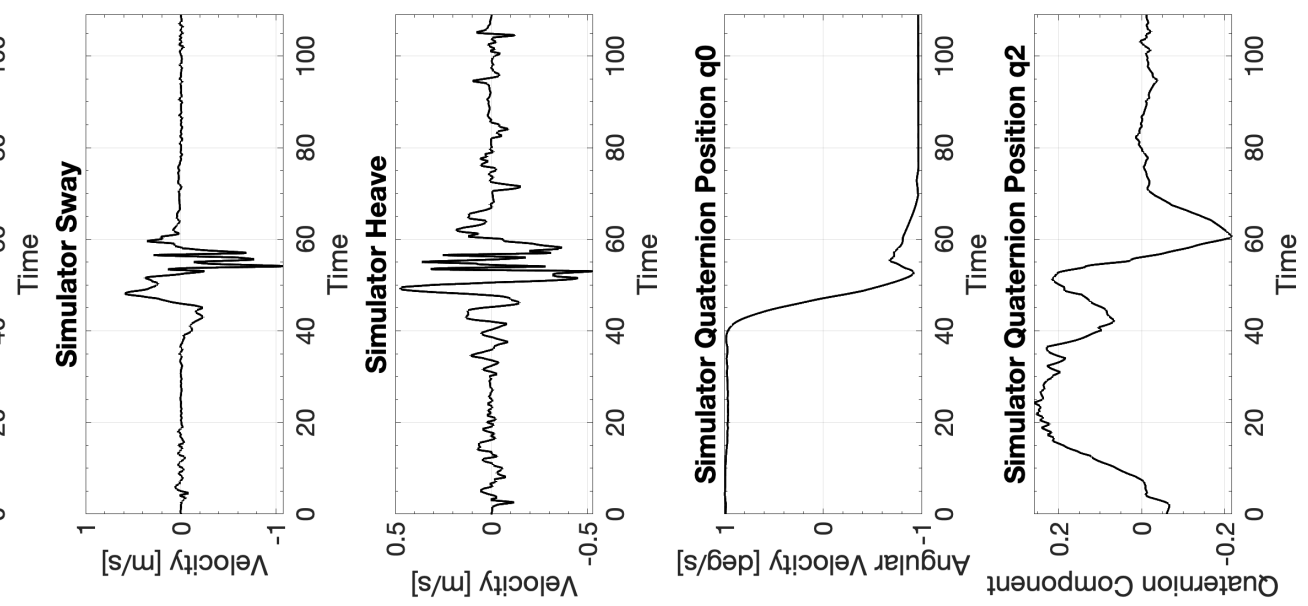

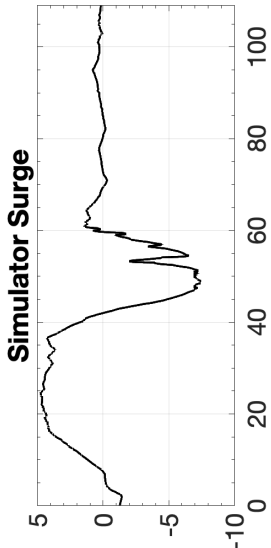

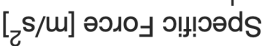

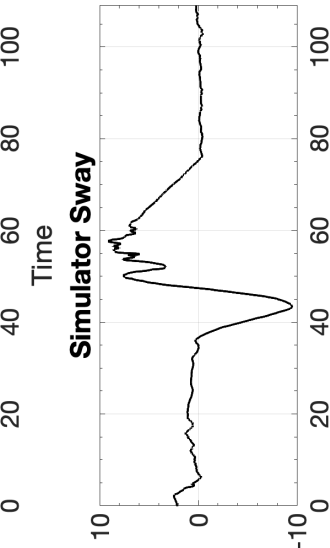

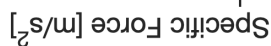

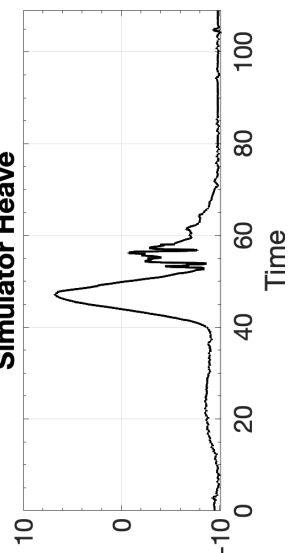

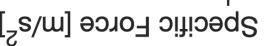

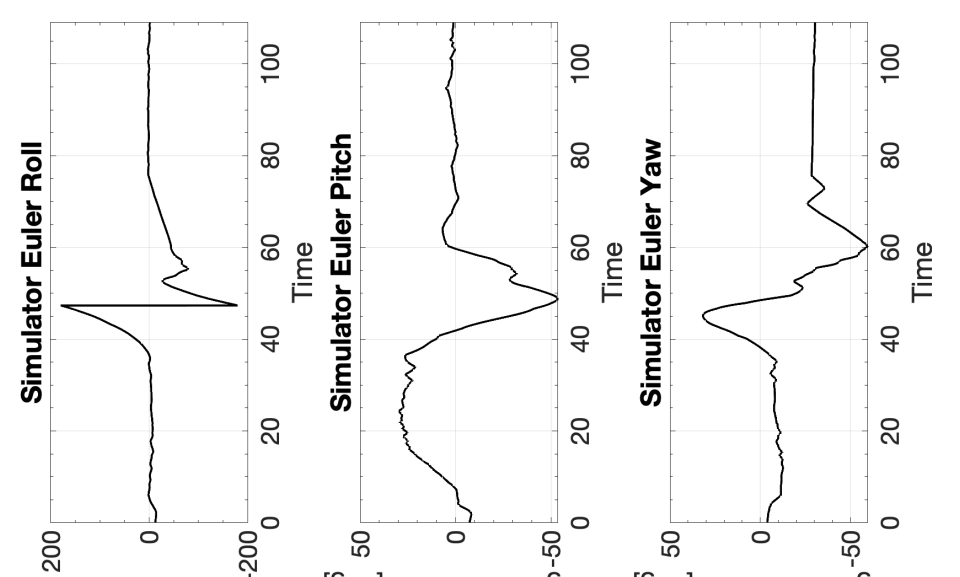

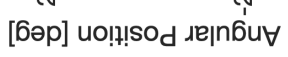
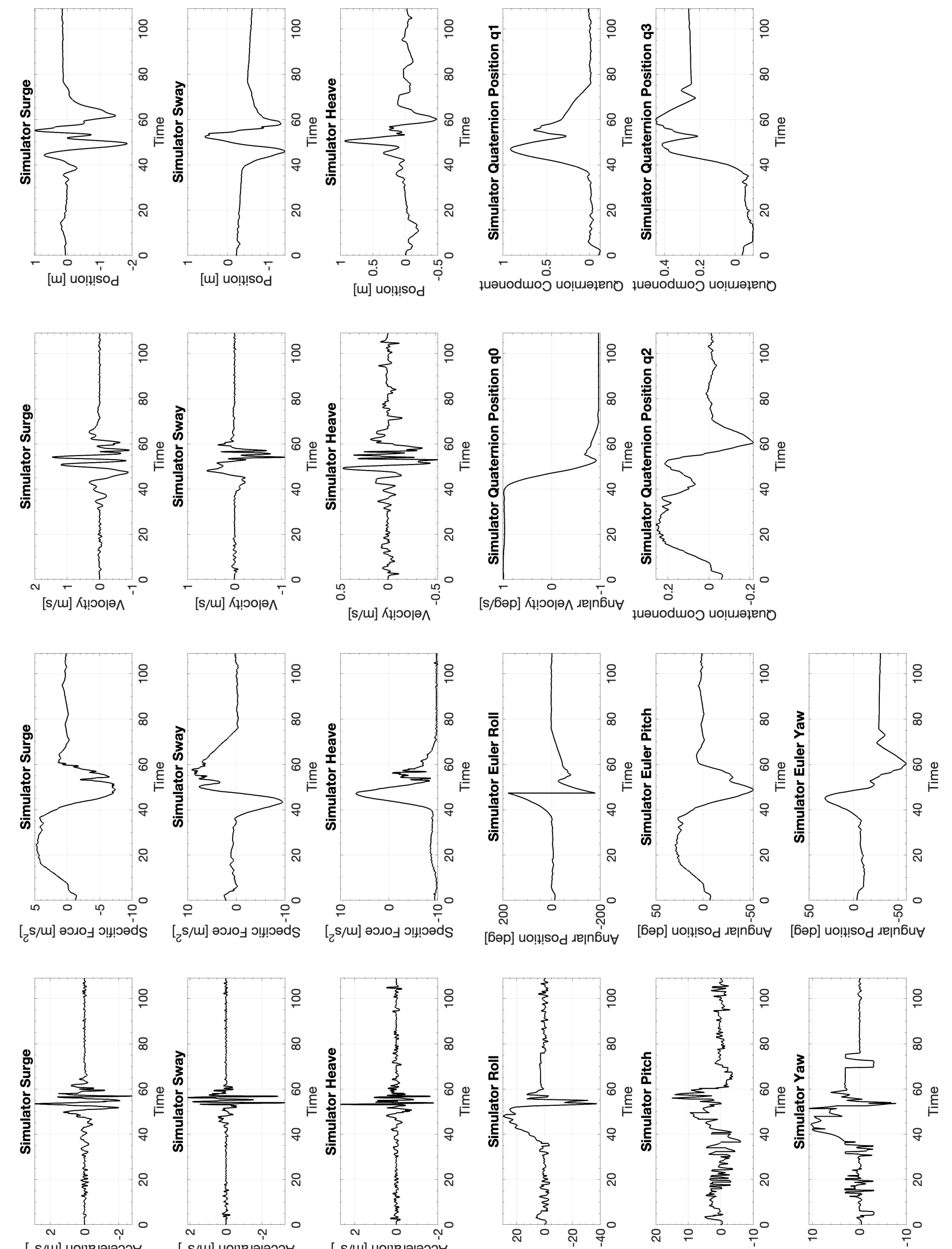

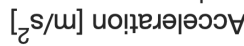

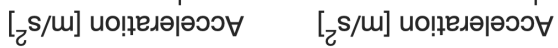

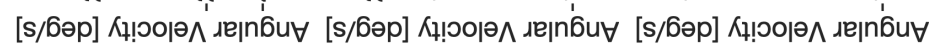




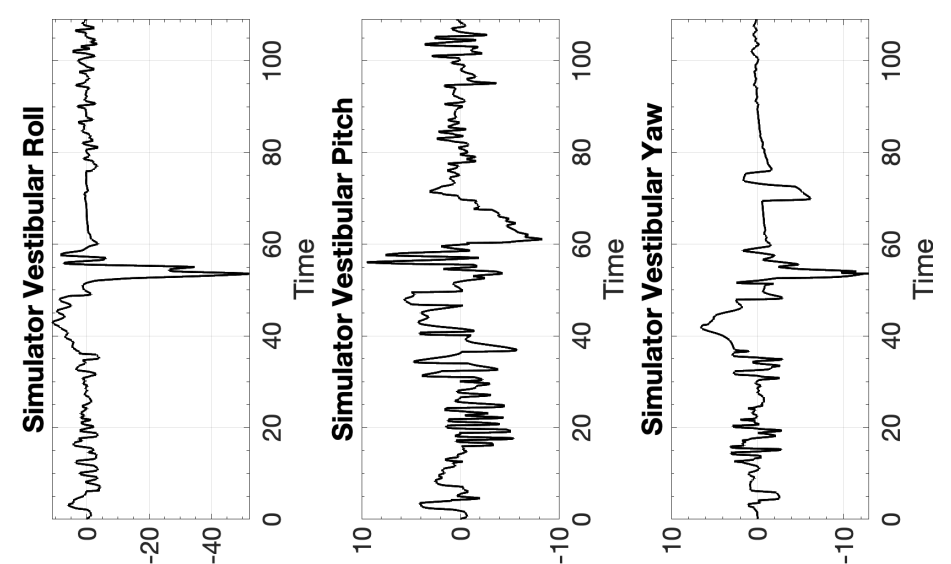

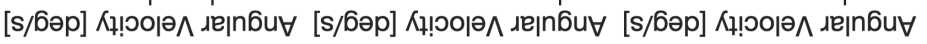

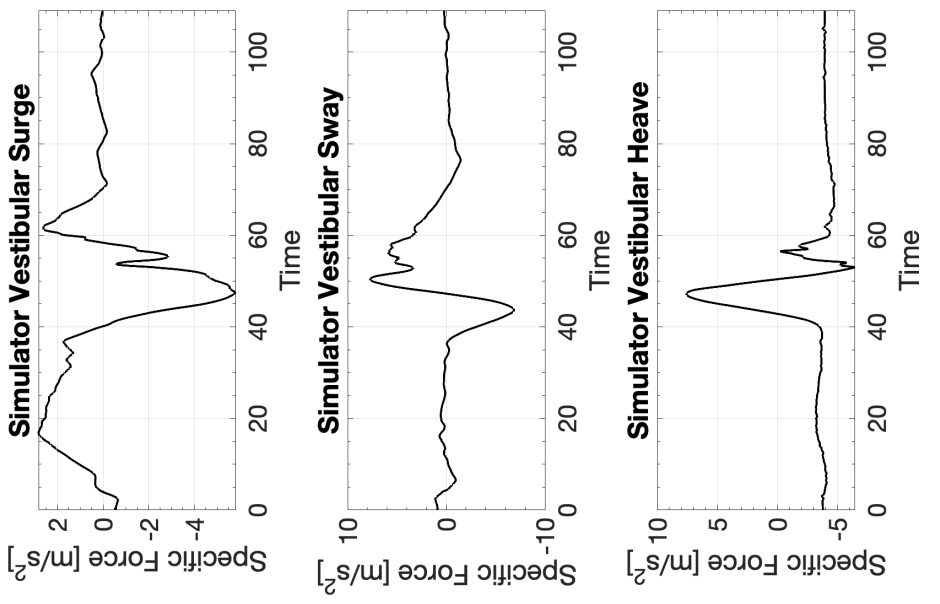




\section{D.9.3 CW3 Boeing 737 Spiral Dive}

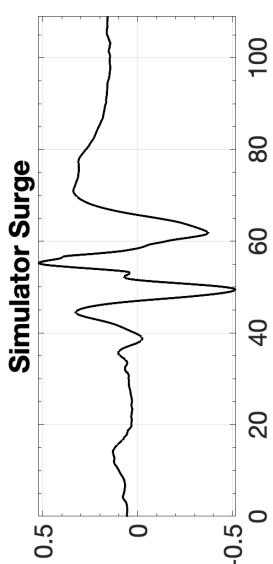

[u] uo!n! sod

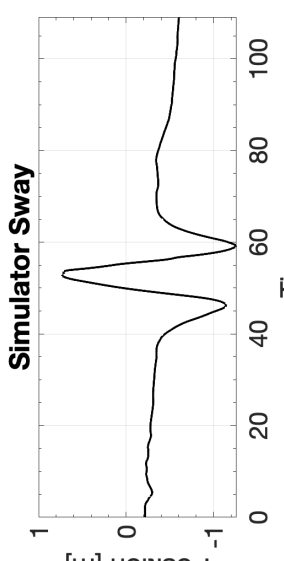

[w] uo!!!!sod

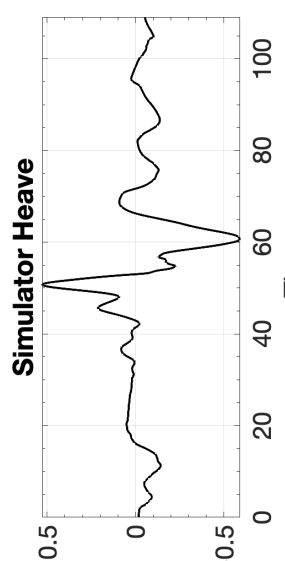

[u] uo!n!sod

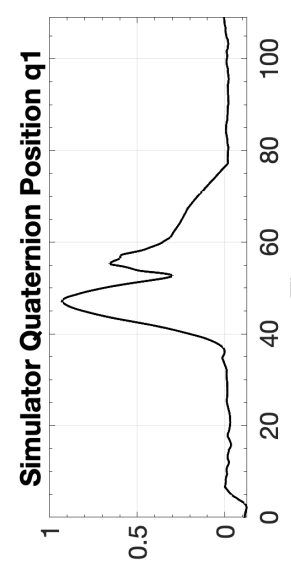

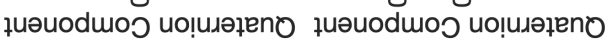
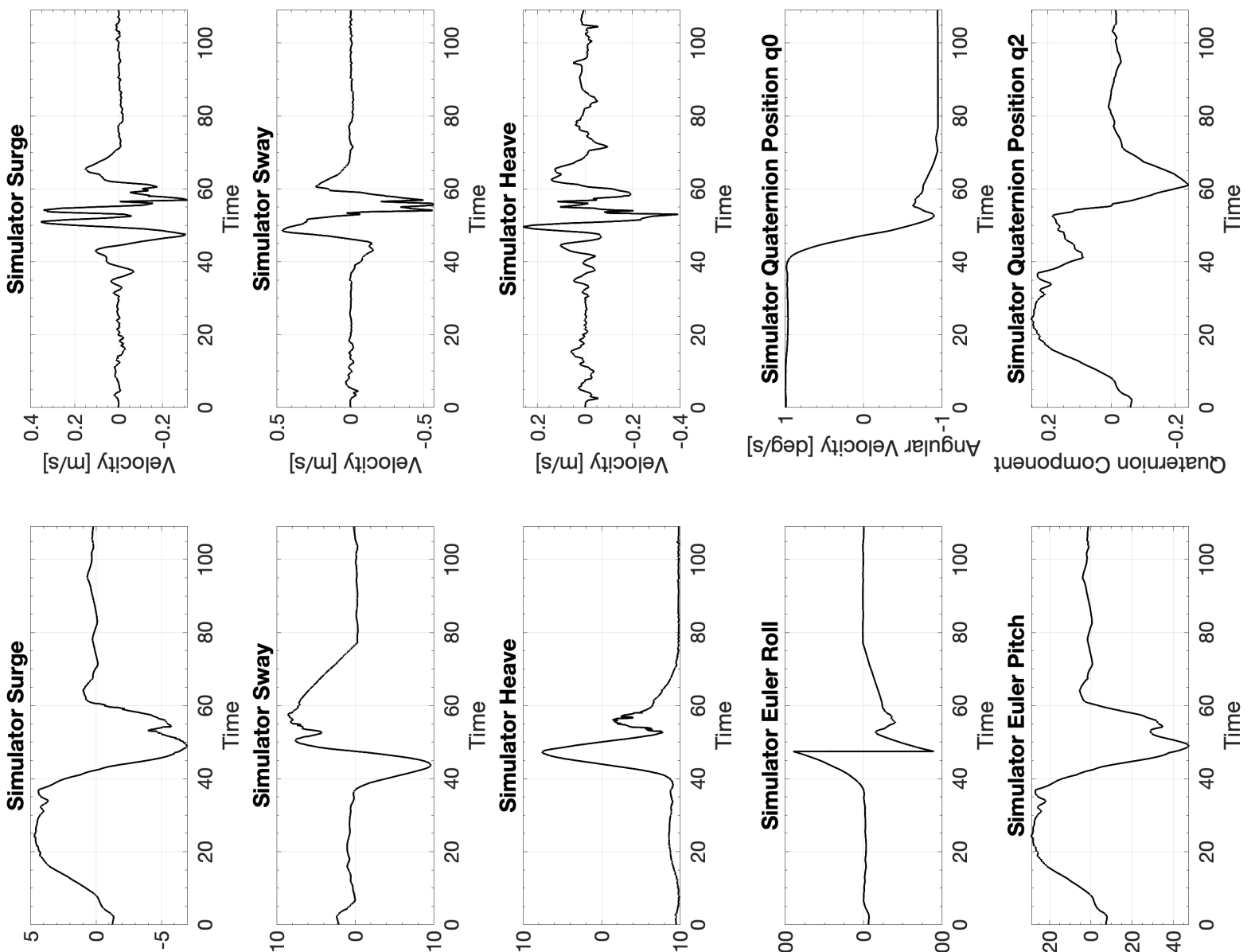

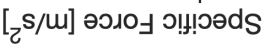
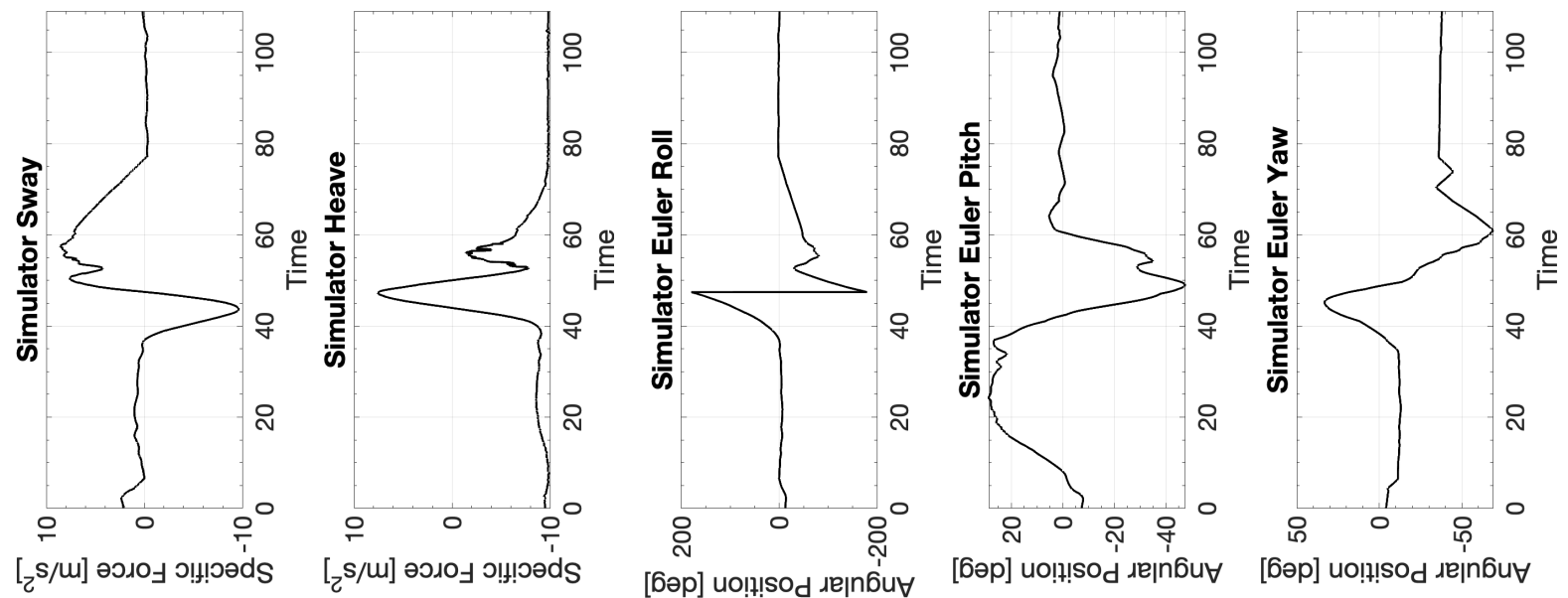

[бәр] uo!̣!sod גe|n6́u

[6әр] uo!!!sod גe|nбü [6әр] uo!!!sod גe|n6u

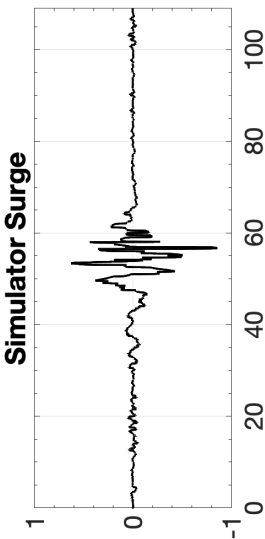

$[\mathrm{z} / \mathrm{s} / \mathrm{u}]$ uо!ฺеגә|әэว $\forall$

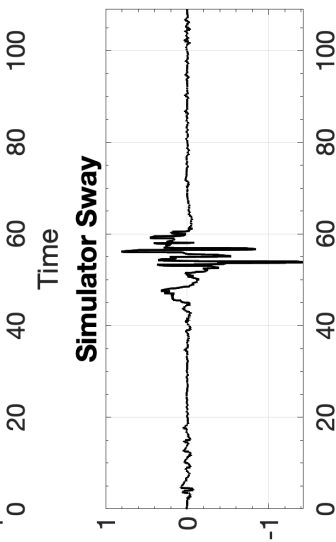

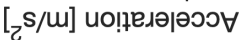

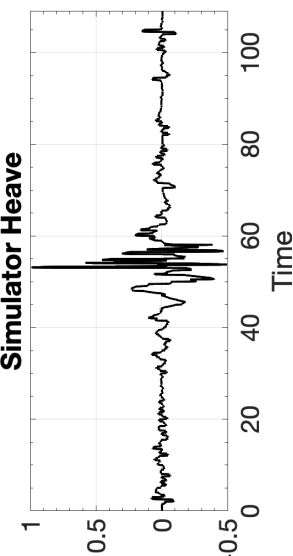

[z $s / m]$ ио!ฺедәрәээ

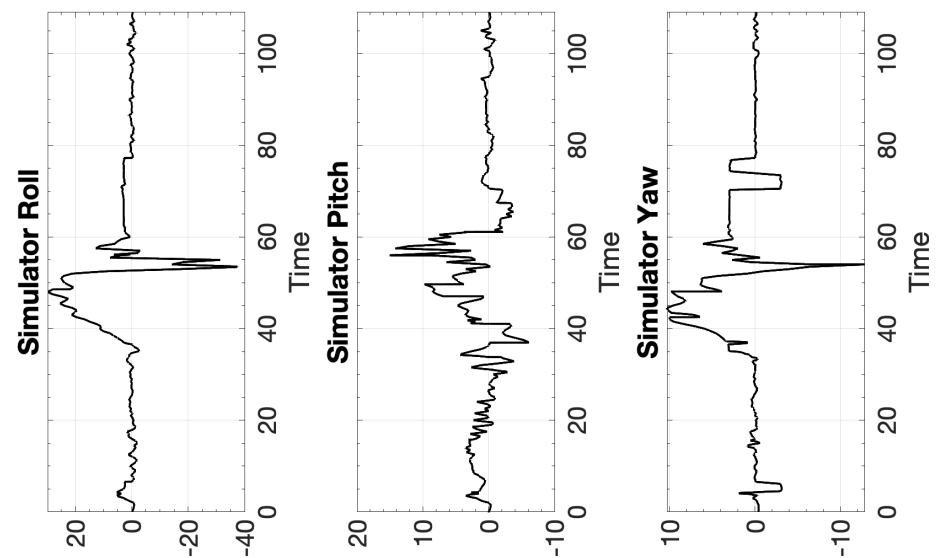




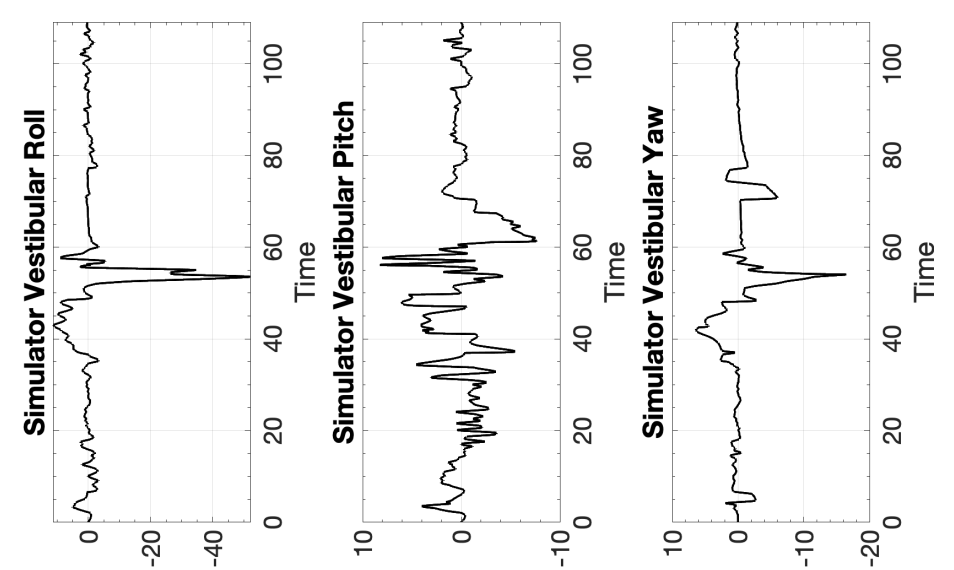

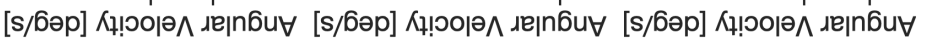

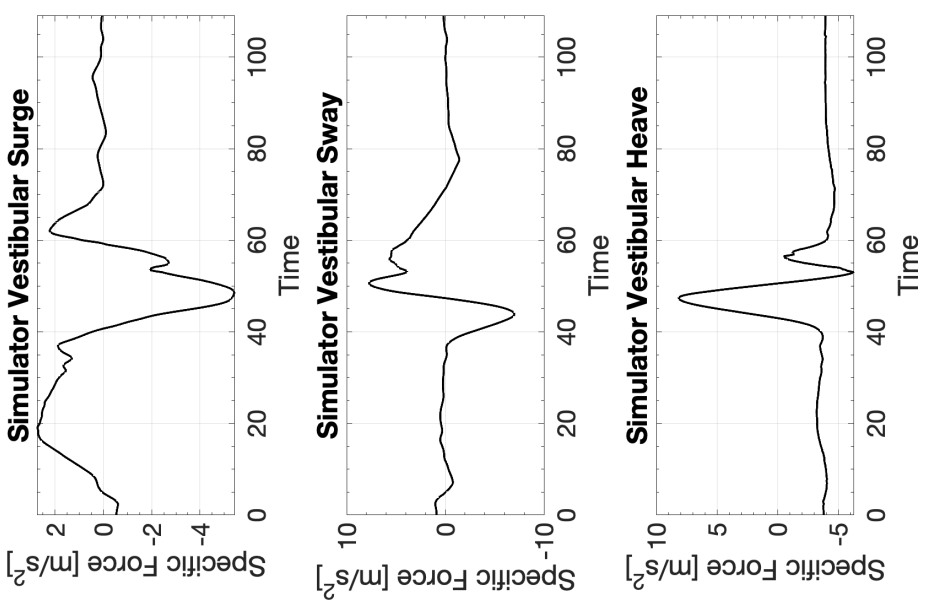




\section{D.10 Boeing 737 Stall Recovery}

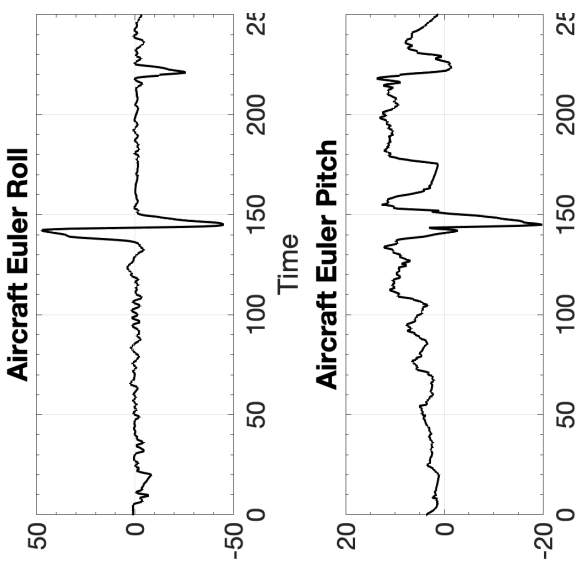

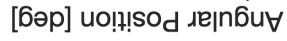

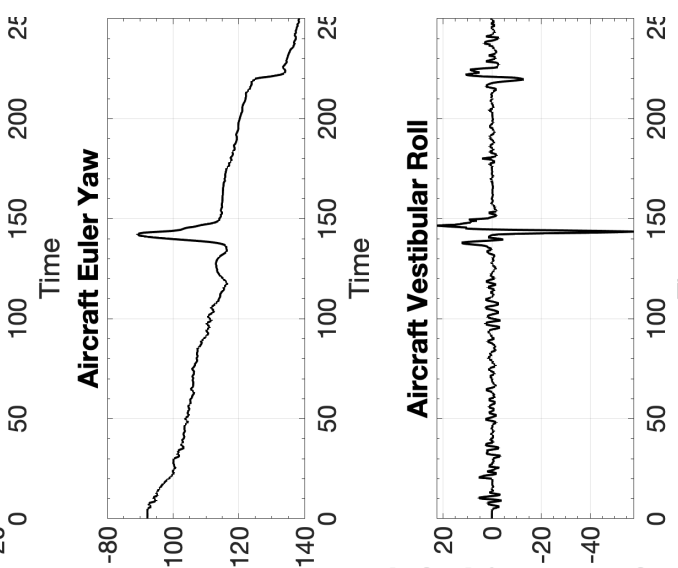

[6əp] uo!!! $\mathrm{SO}_{\mathrm{d}} \lambda \mathrm{e} \mid \mathrm{n} 6 \mathrm{u} \forall$

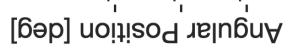
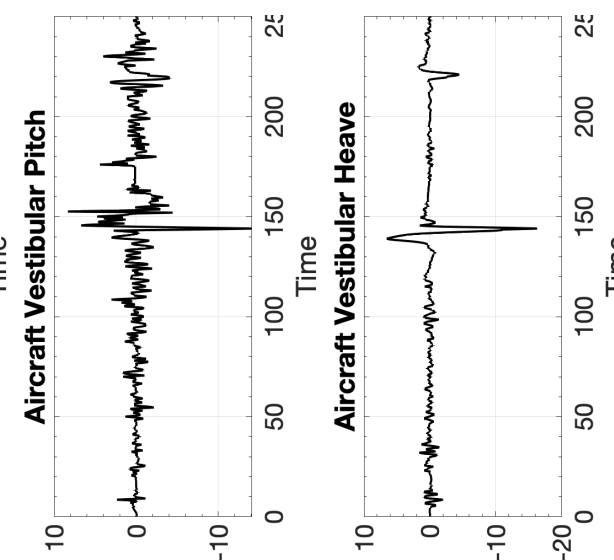

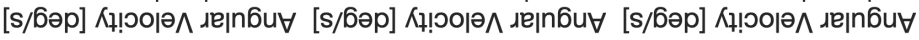
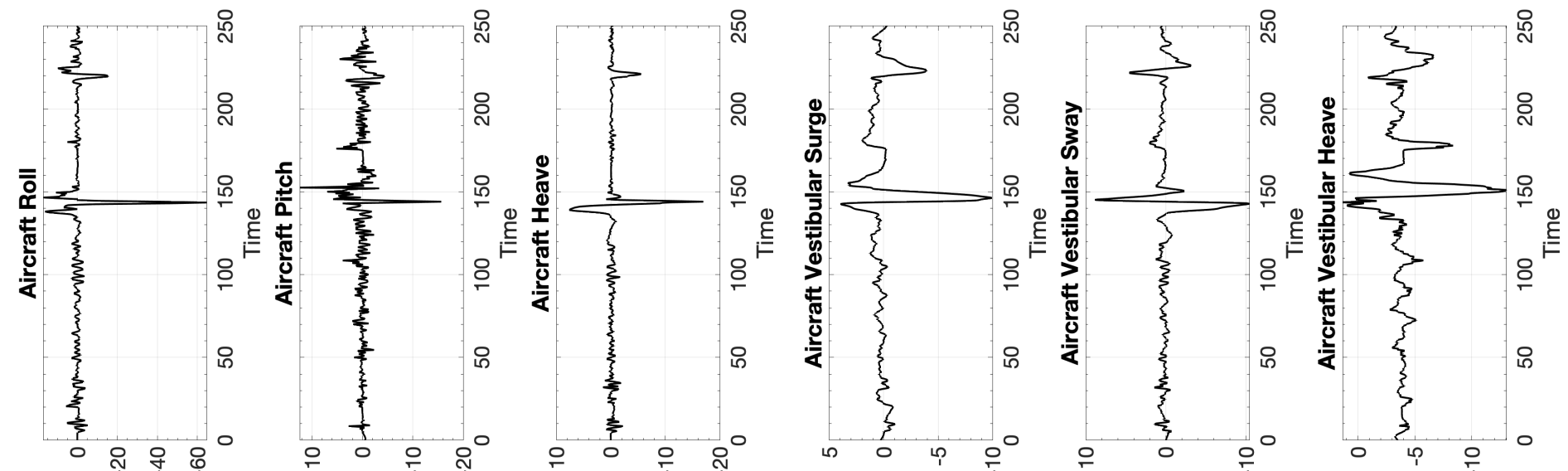

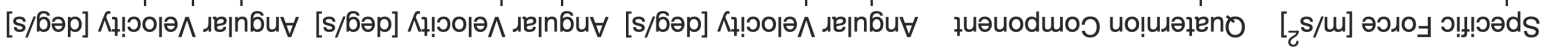

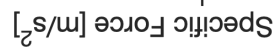

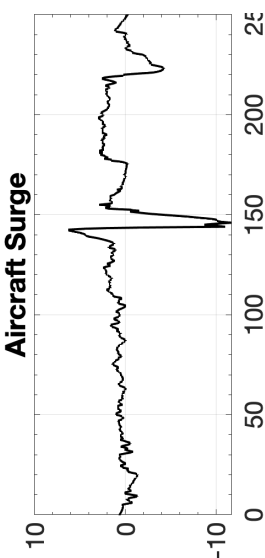

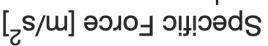

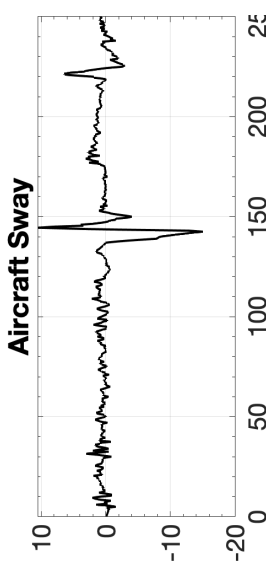

[s/u] әכر०

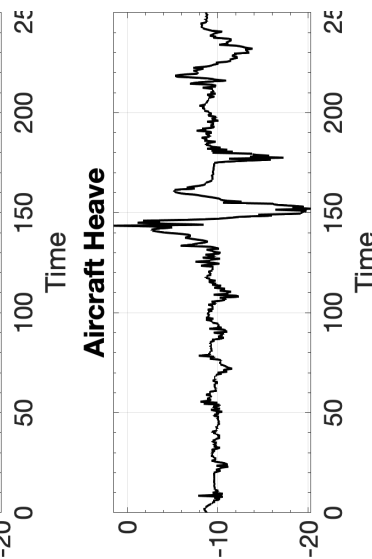

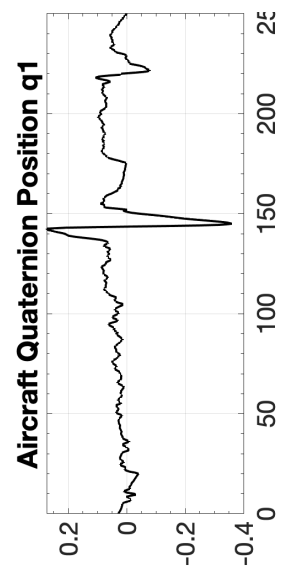
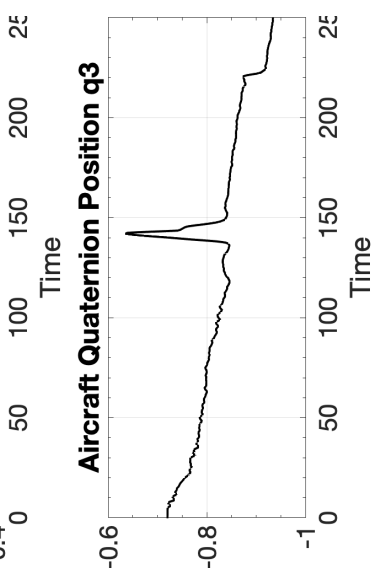

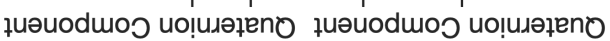

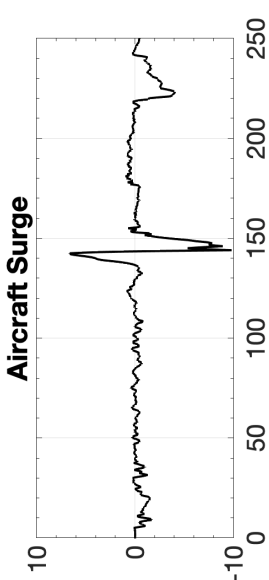

$\left[{ }_{2} \mathrm{~s} / \mathrm{m}\right]$ uо!̣อлә|әэว $\forall$

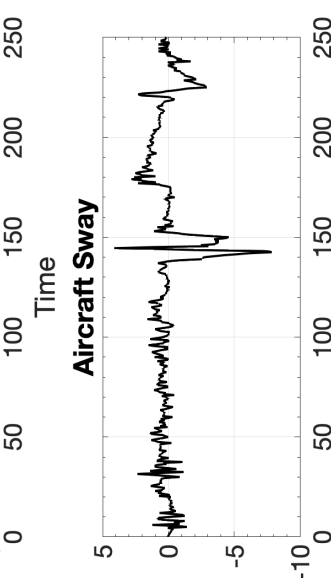

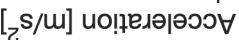

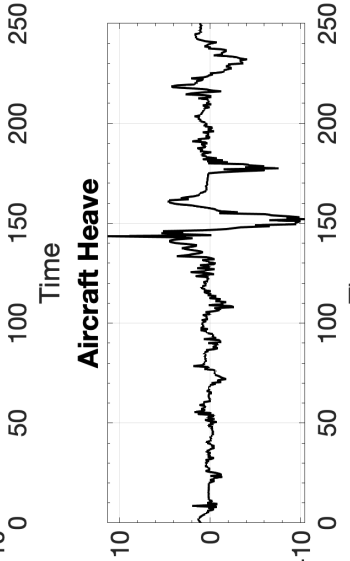

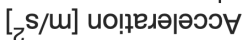
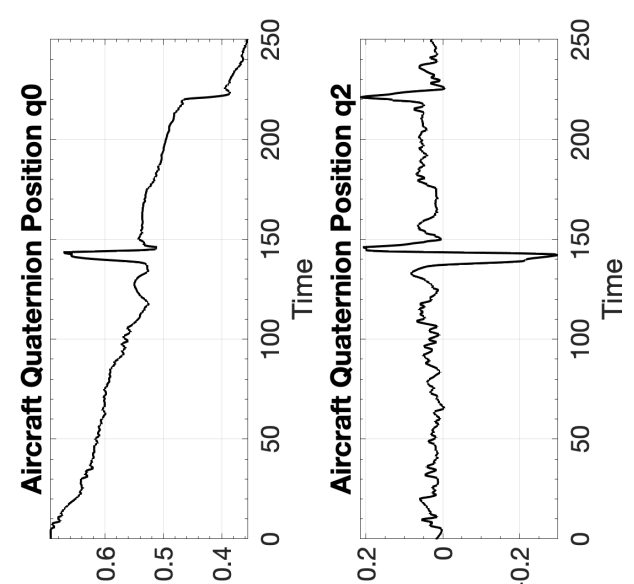

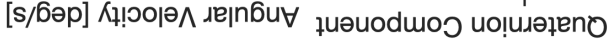




\section{D.10.1 CW1 Boeing 737 Stall Recovery}

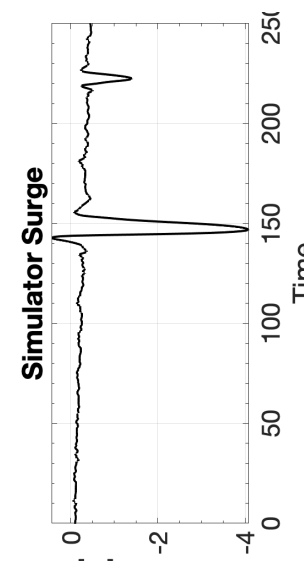

[u] uo!!!!sod

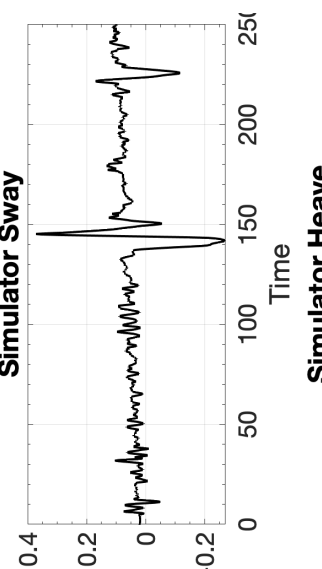

[w] uo!n! sod

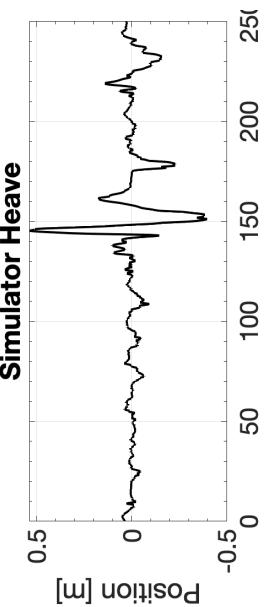

[w] uol!!!sod

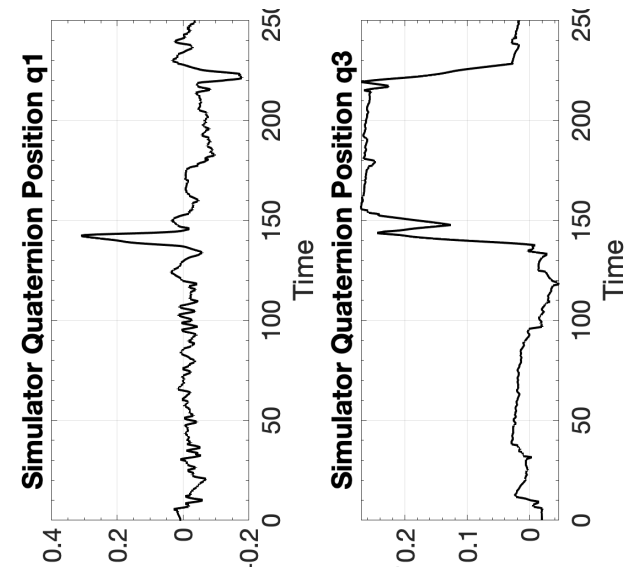

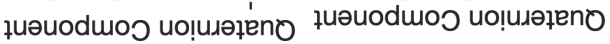

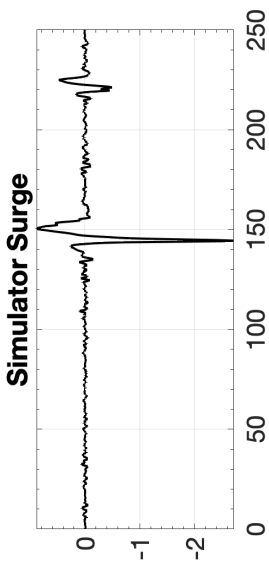

[s/u] К! ৩о夫

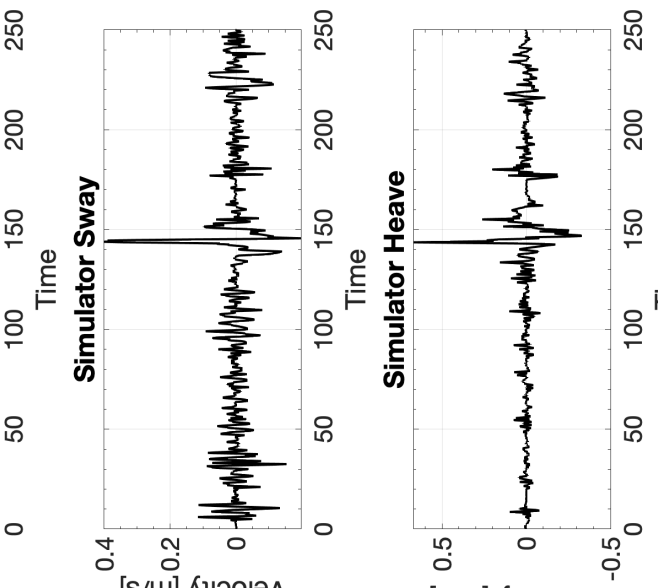

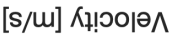
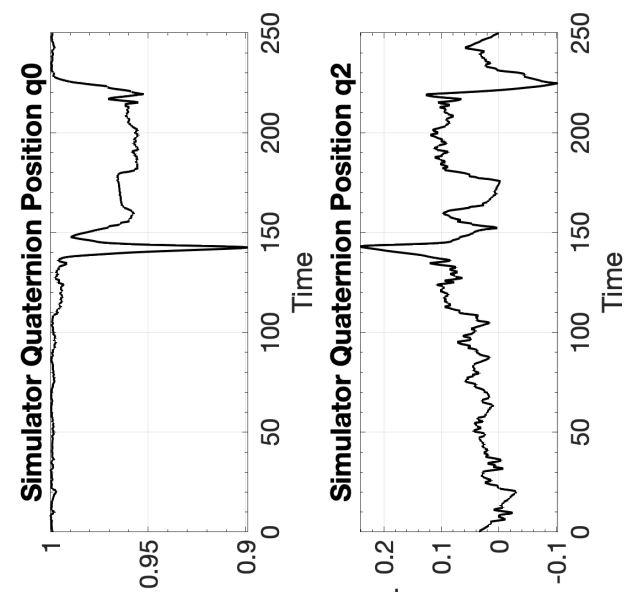

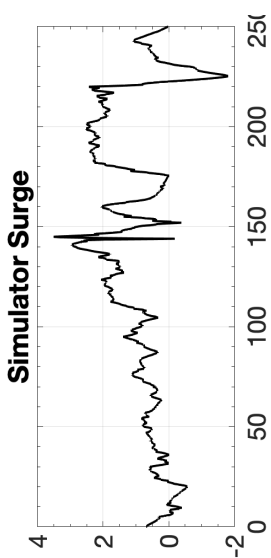

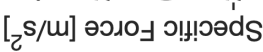
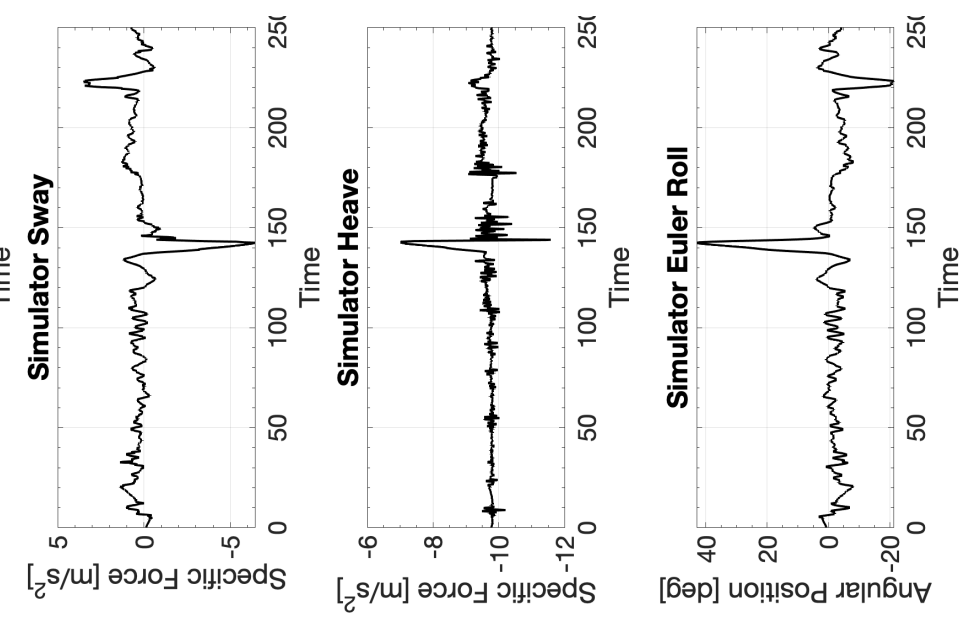

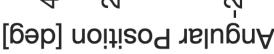

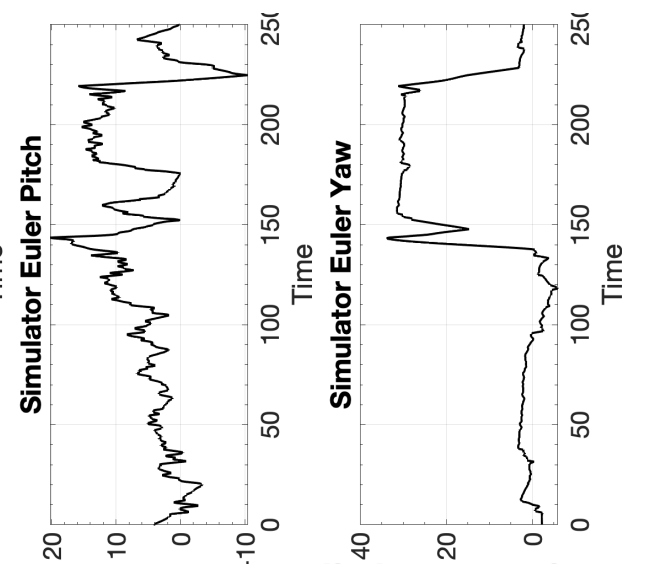

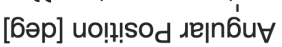

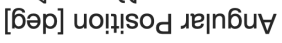

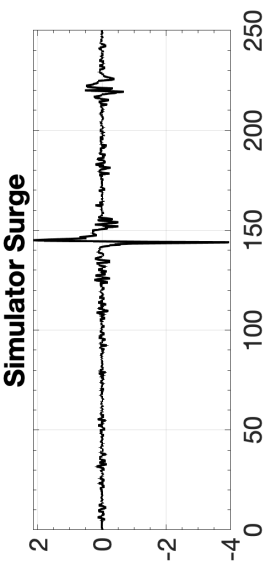

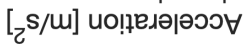

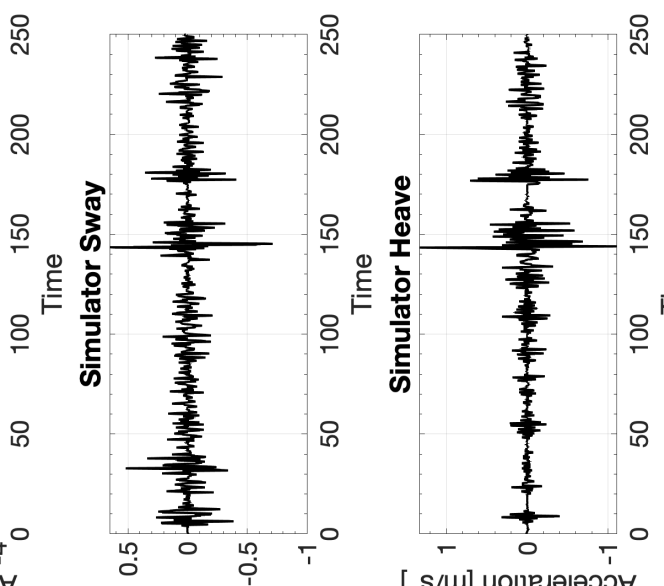

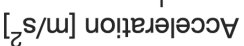
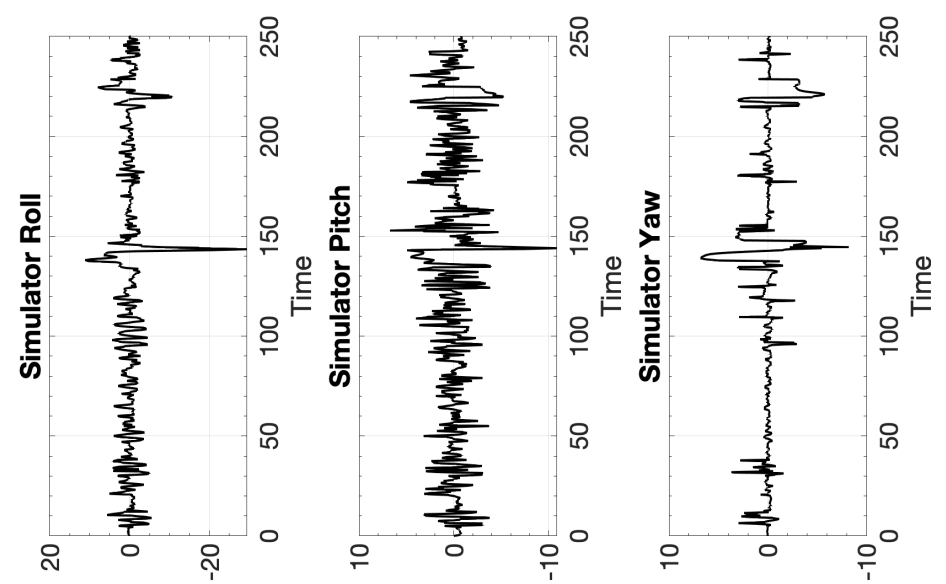

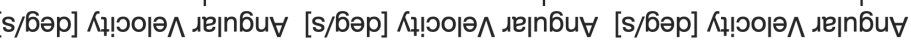




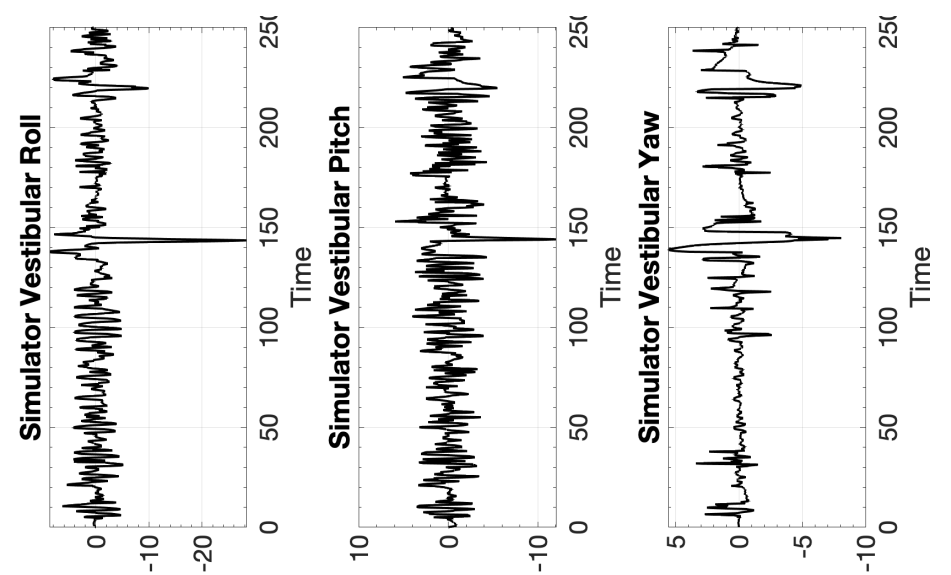

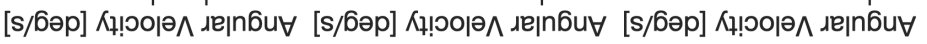

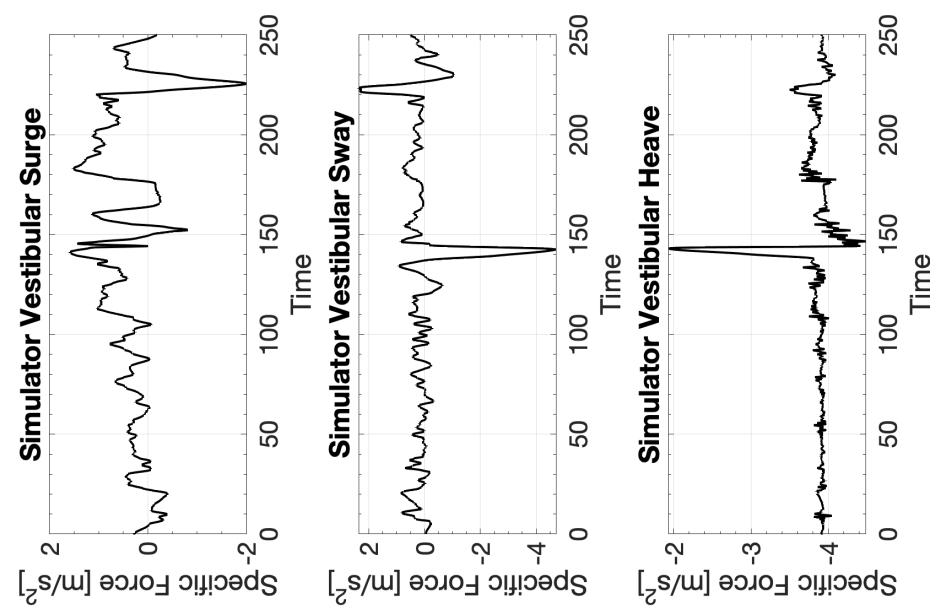




\section{D.10.2 CW2 Boeing 737 Stall Recovery}

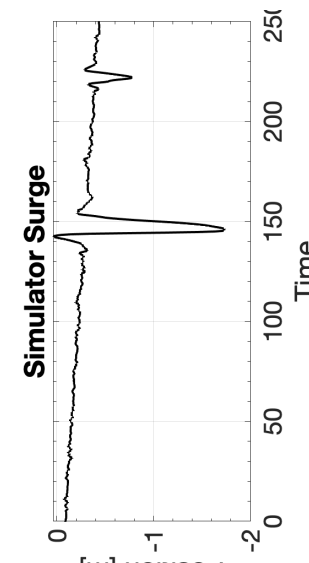

[u] uo!n!!sod

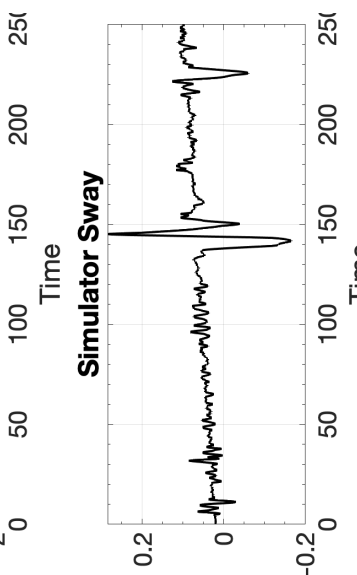

[u] uol!!sod

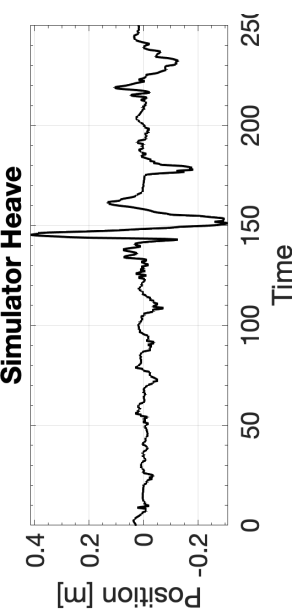

[w] uol!! sod

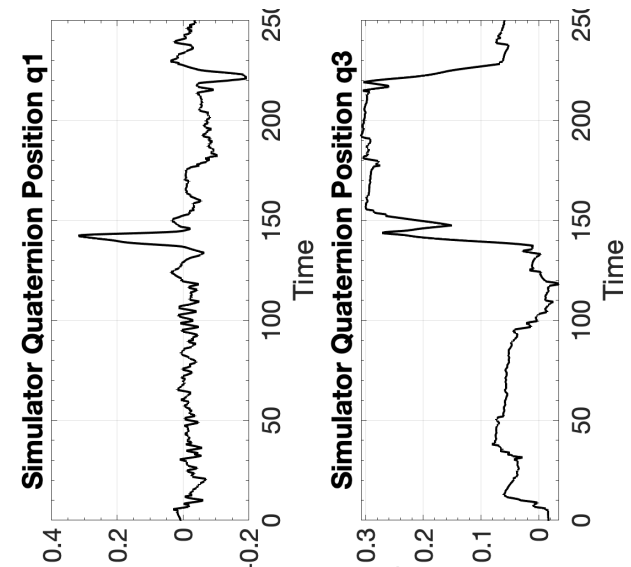

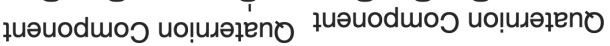

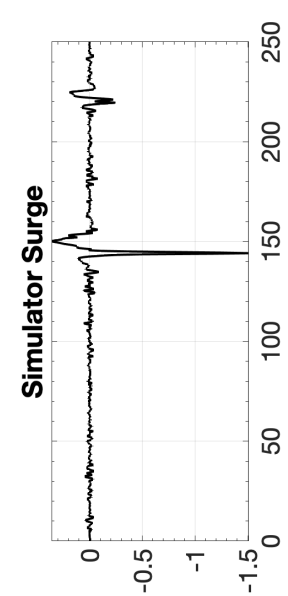

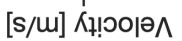

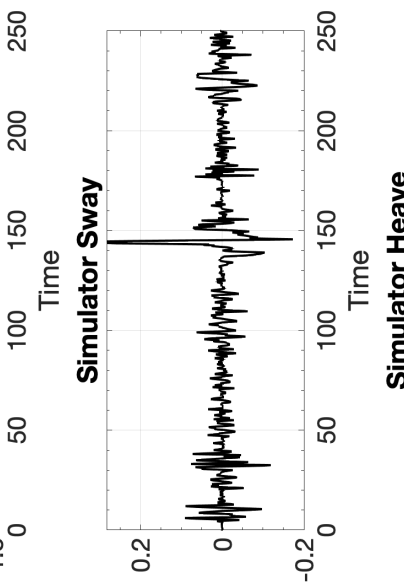

[s/m] Кұ!०о|ә^

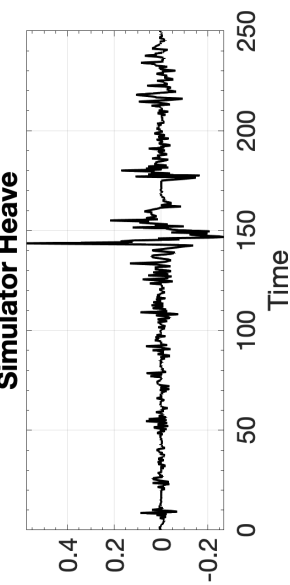

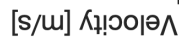

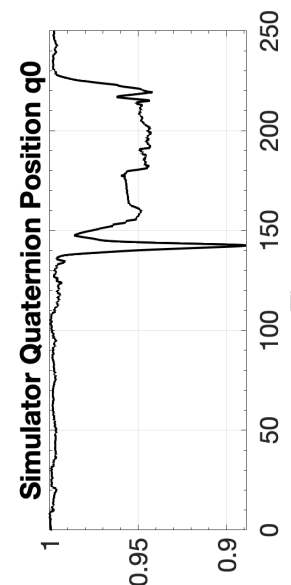

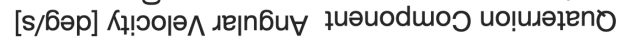

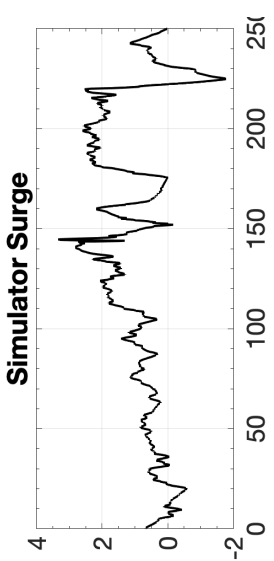

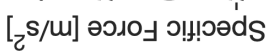

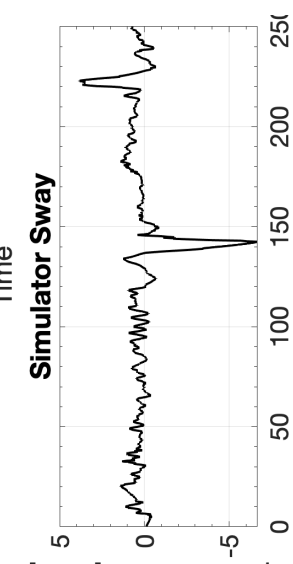

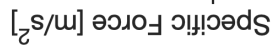
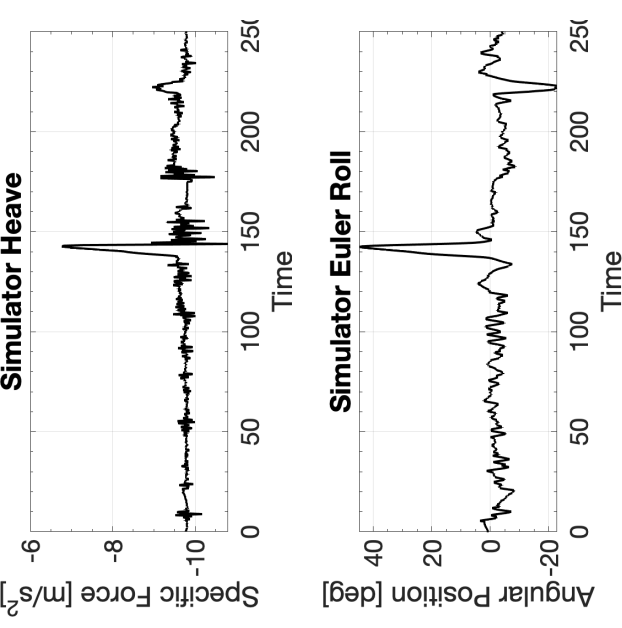

[бəp] uo!!!!sod גe|nโิu
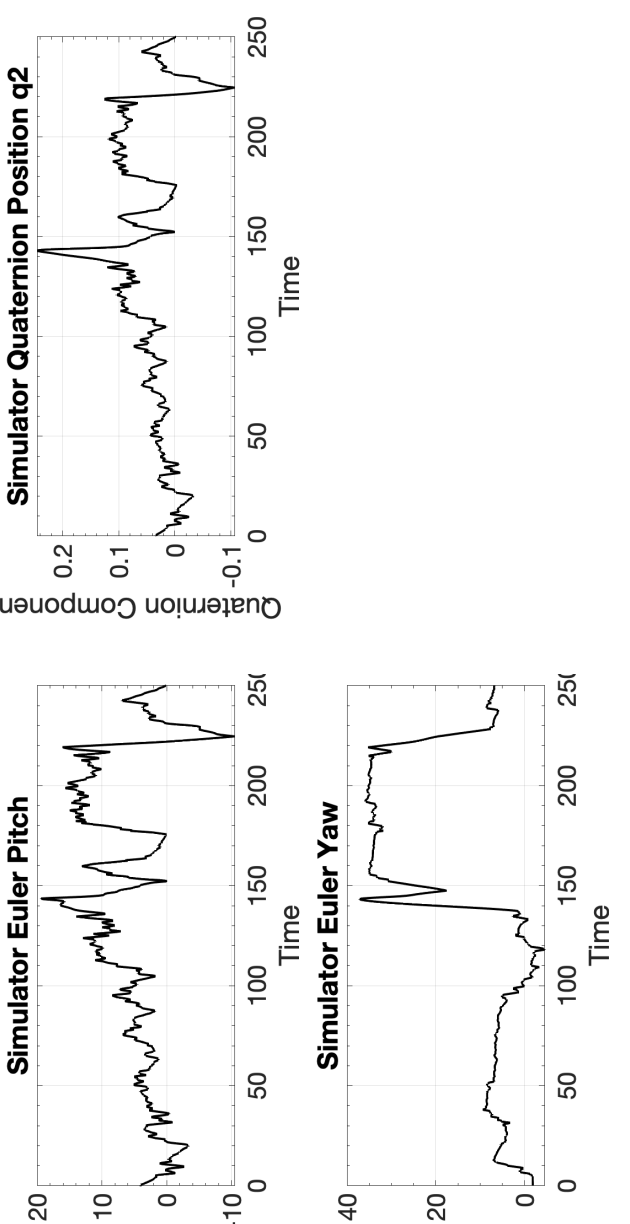

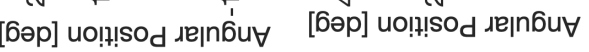

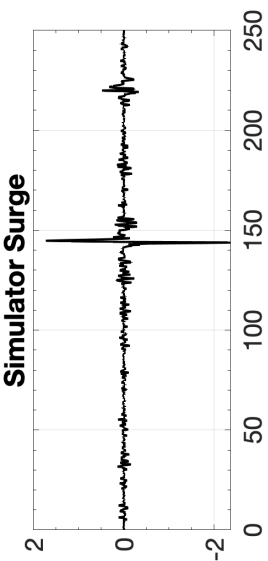

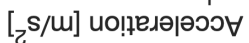

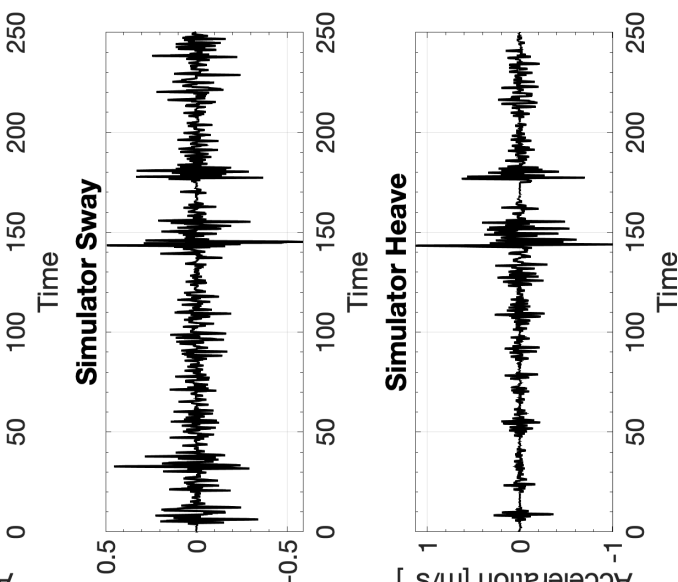

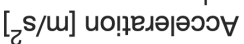
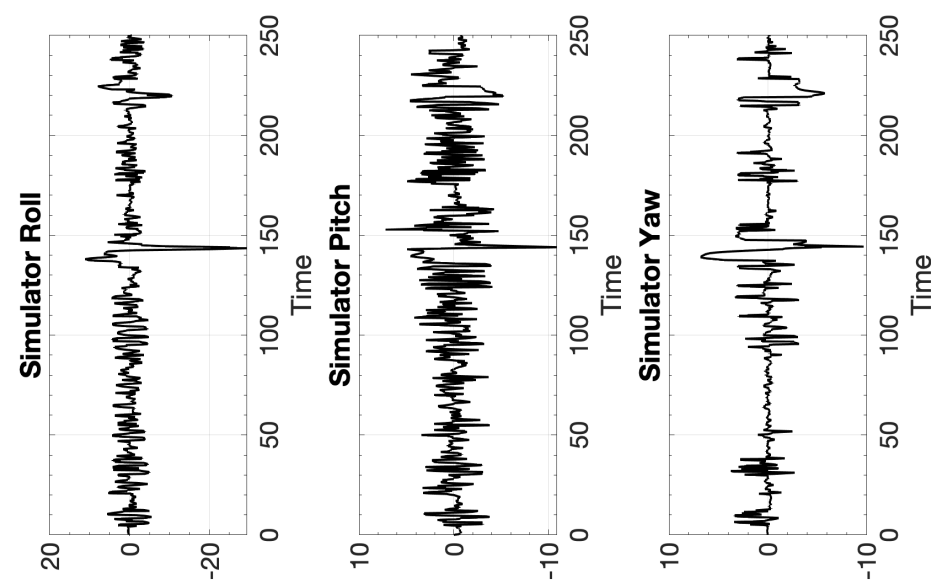

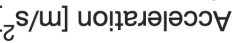




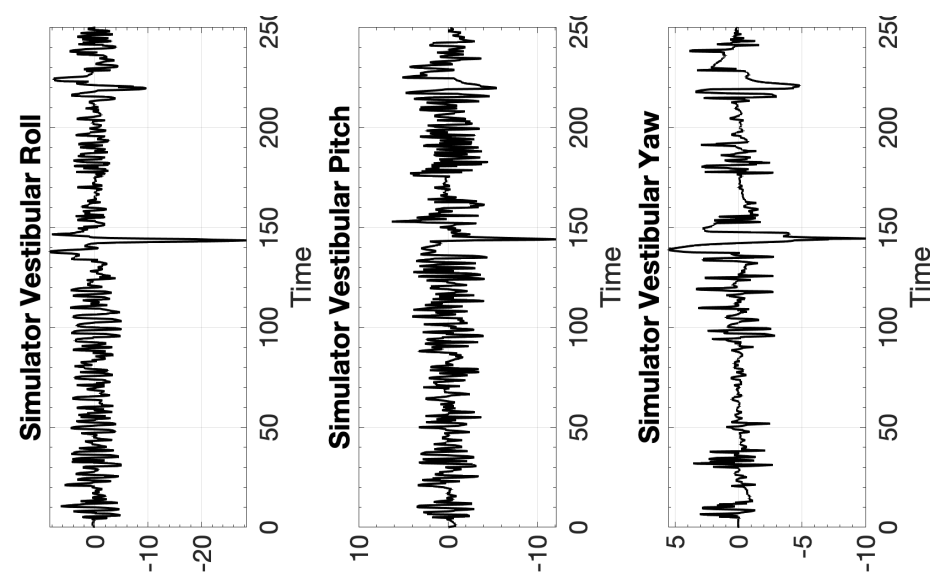

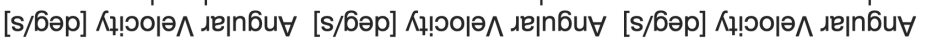

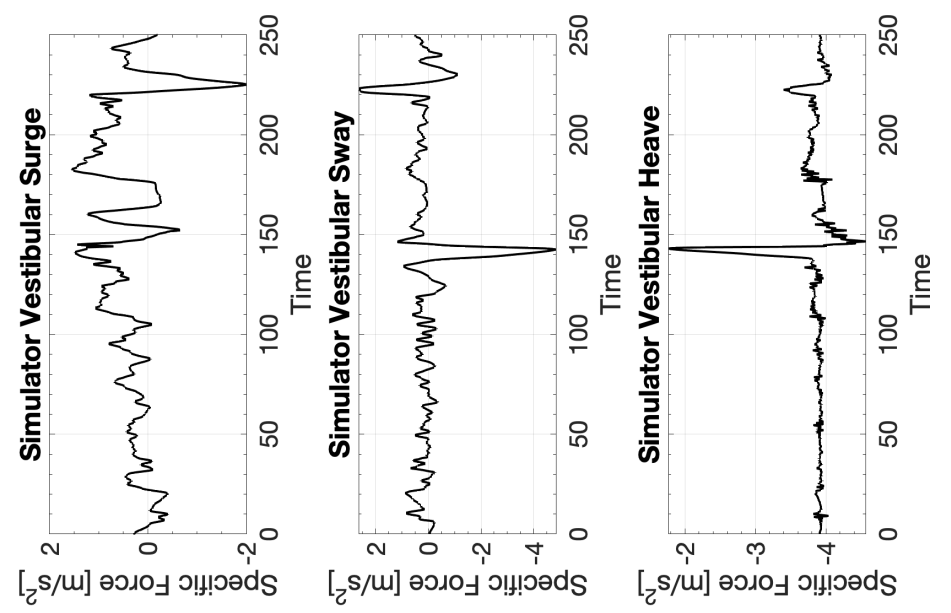




\section{D.10.3 CW3 Boeing 737 Stall Recovery}
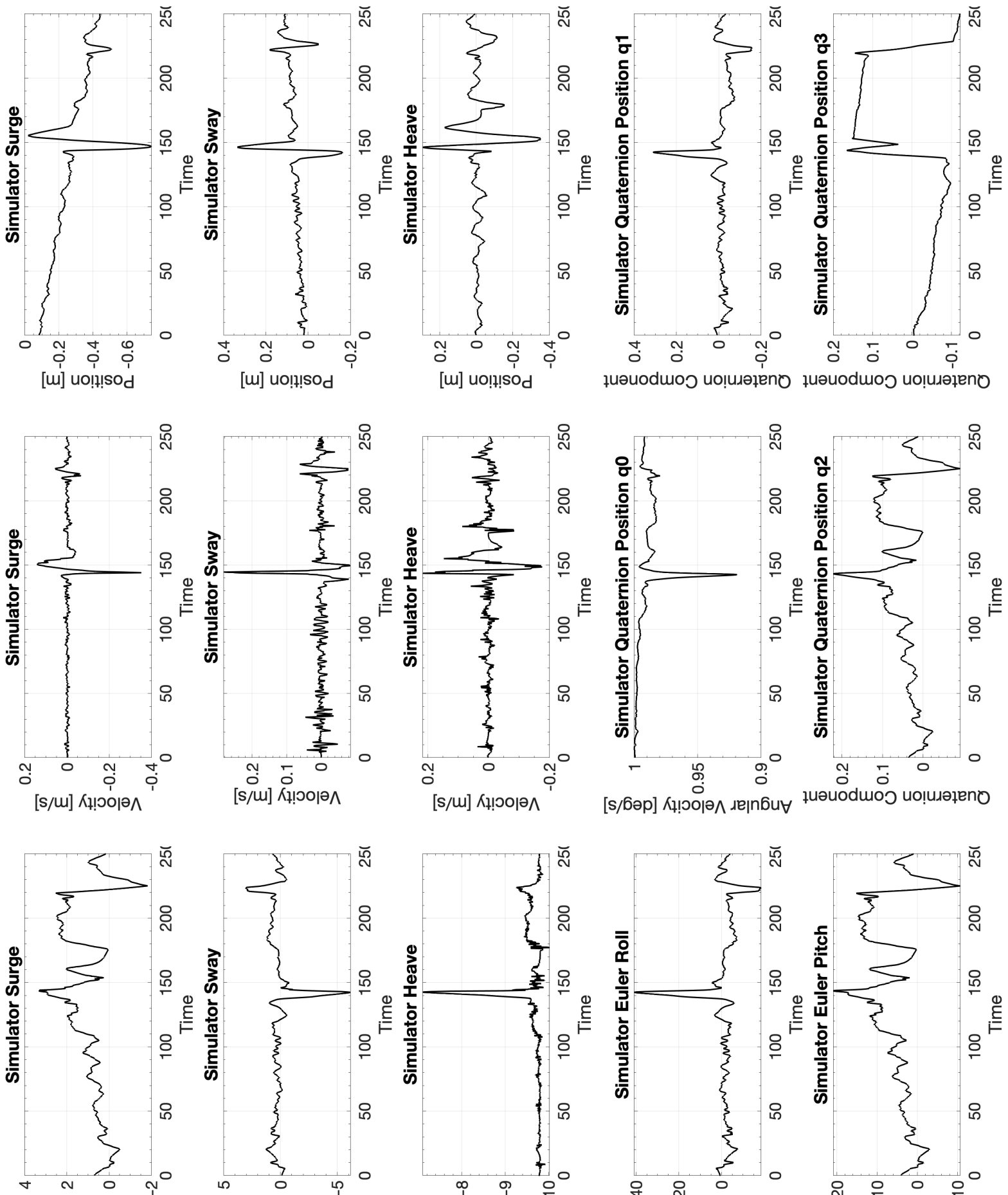

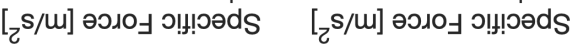
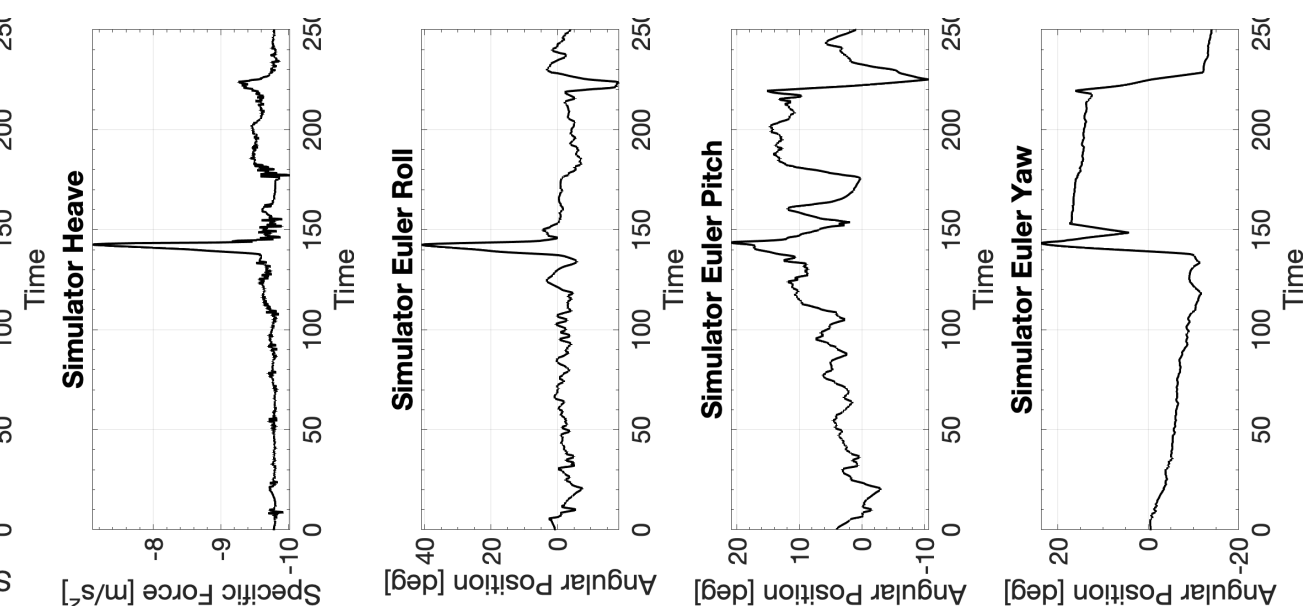

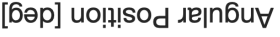

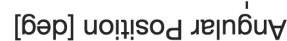

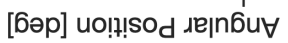

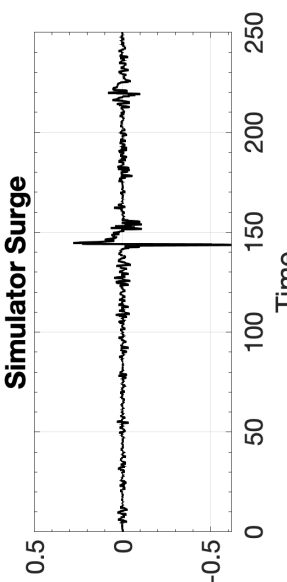

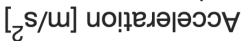

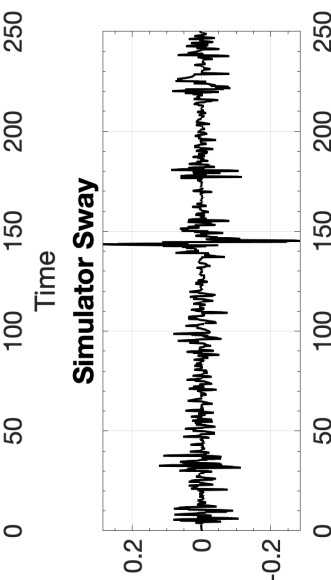

[zs/m] ио!ฺедәәәэว

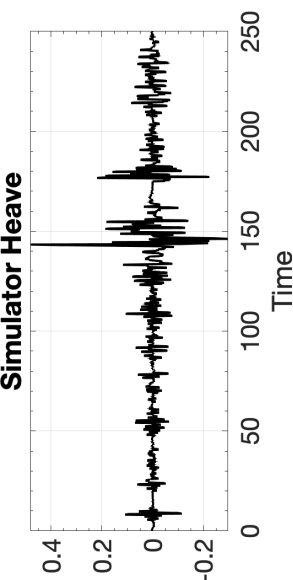

[zs/m] ио!ฺеләрәวо $\forall$
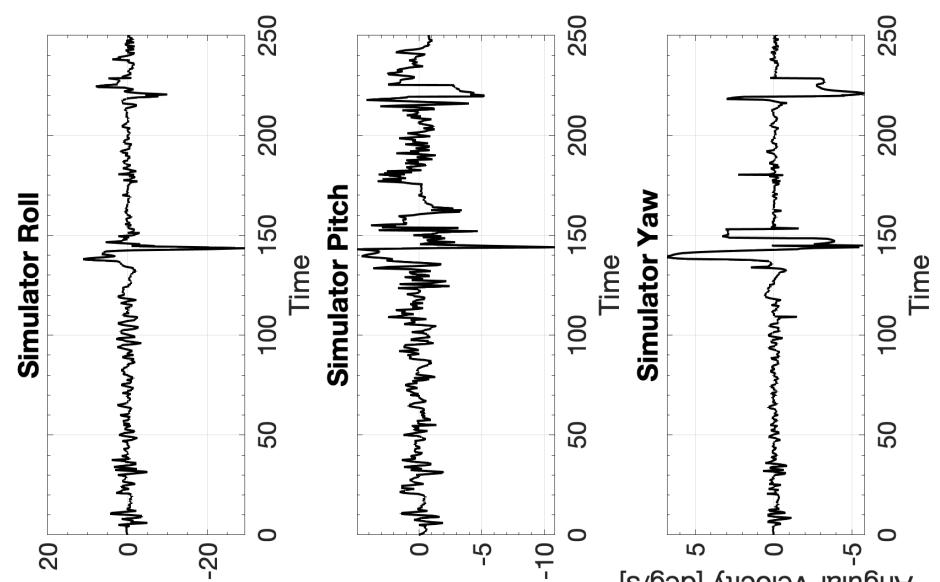
411 


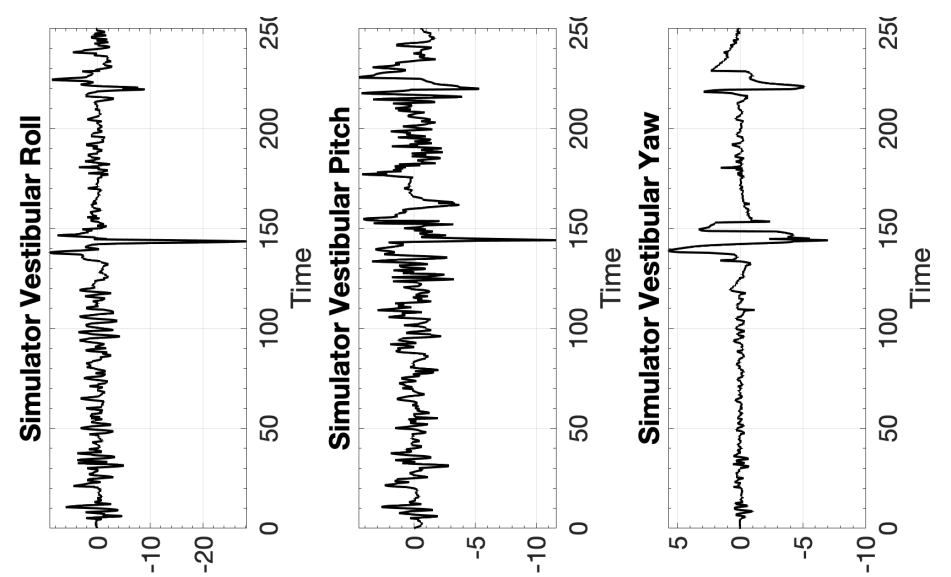

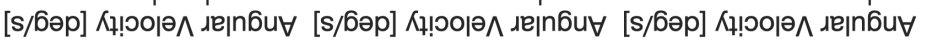

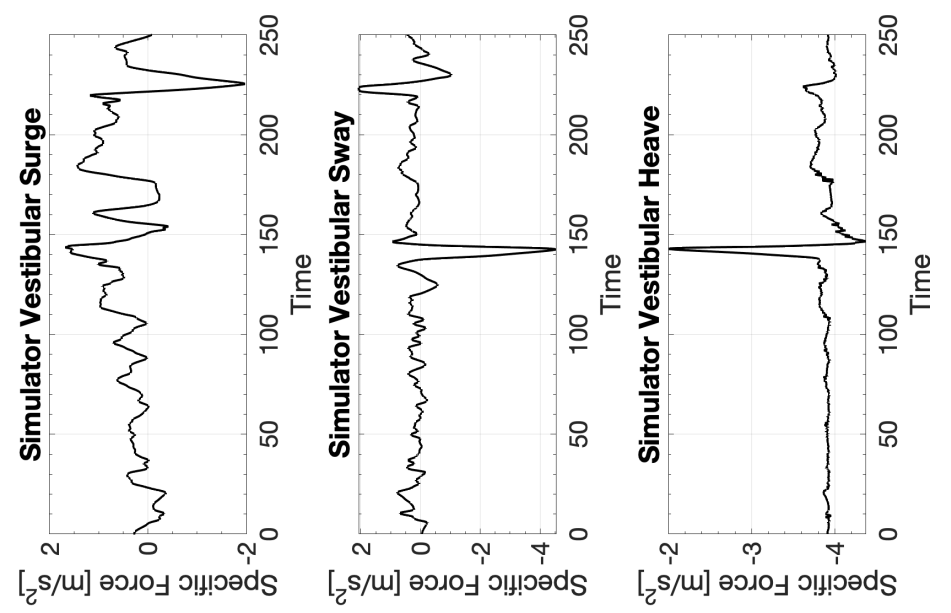




\section{D.11 Columbia 400 Aerobatics}

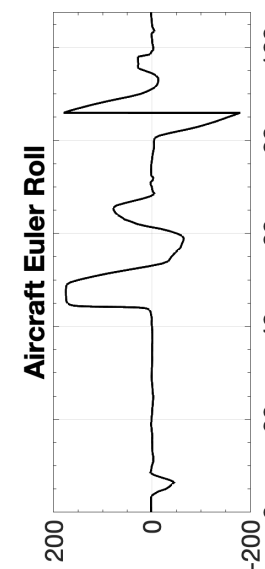

[6əp] uo!!!sod גe|n6́ü

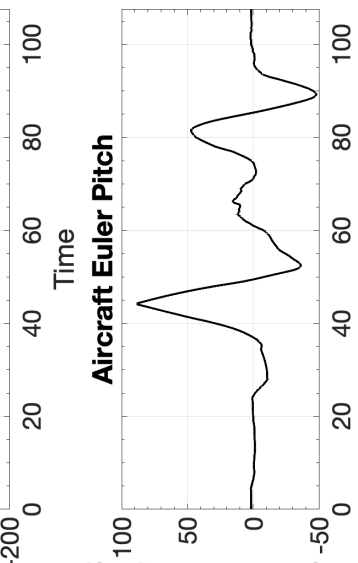

[6әр] uo!!!sod גejn6u

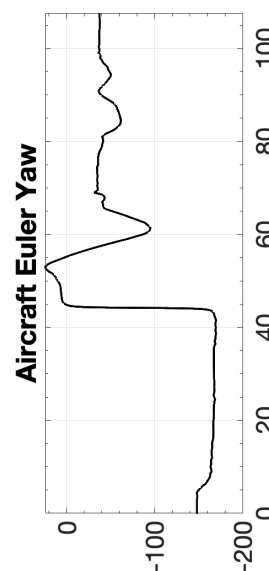

[бəp] uo!̣!so'd

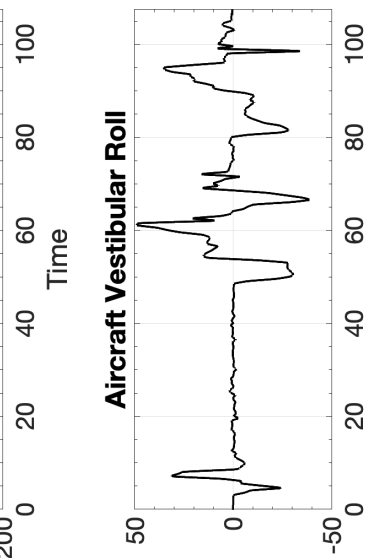

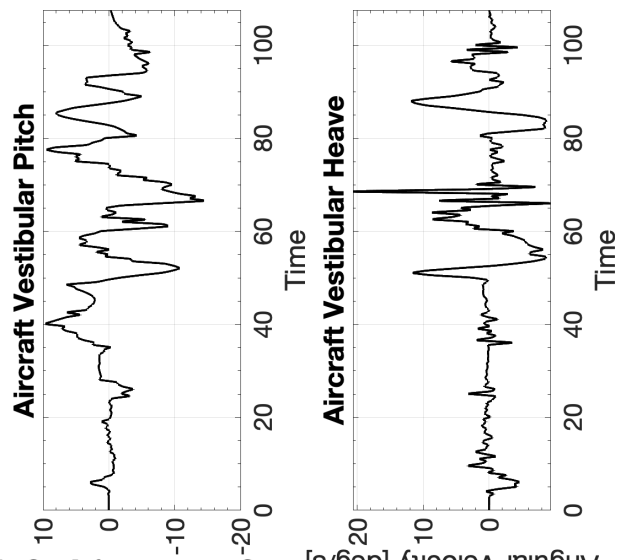

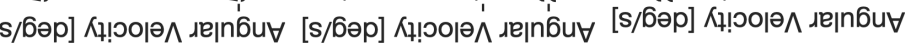

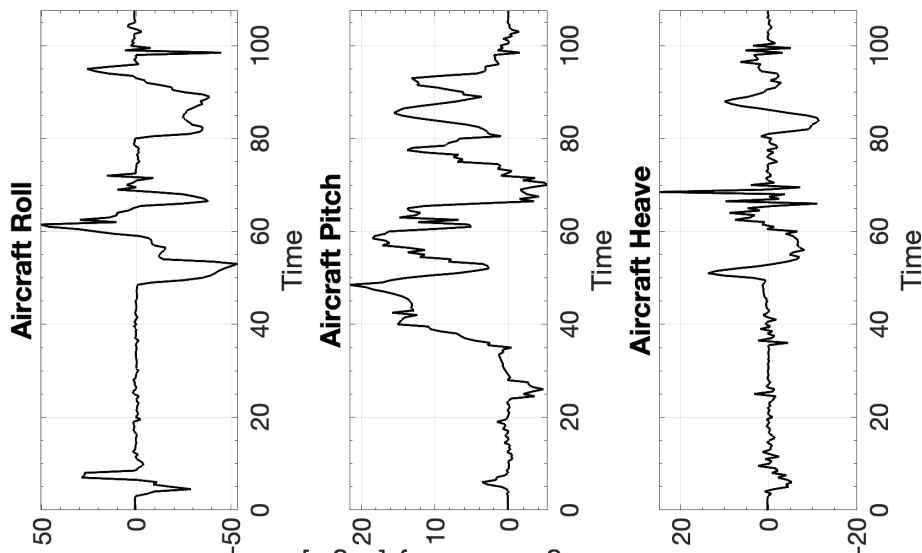

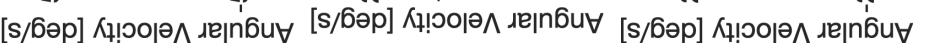

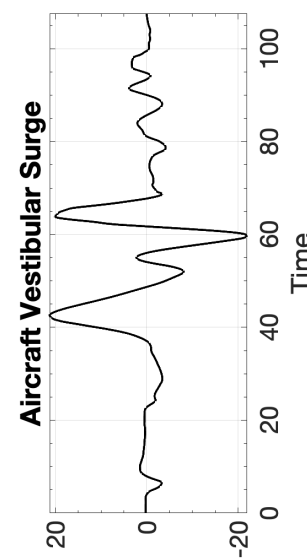

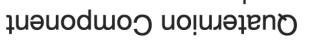

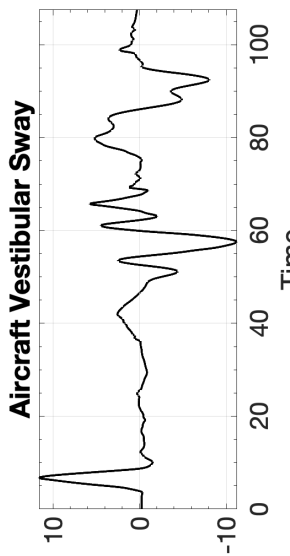

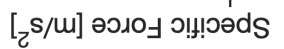

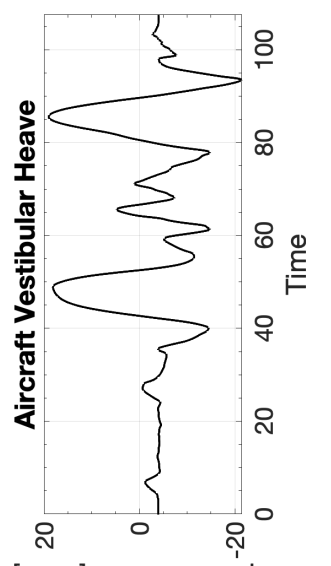

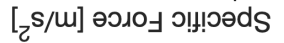

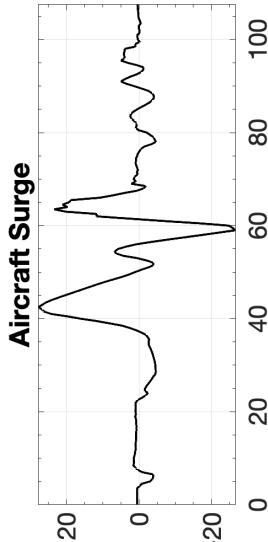

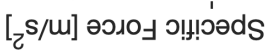

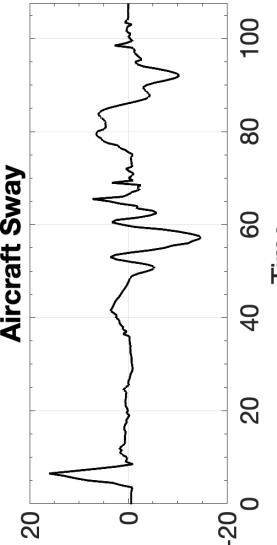

$[\mathrm{z} / \mathrm{s}]$ ә0ر0

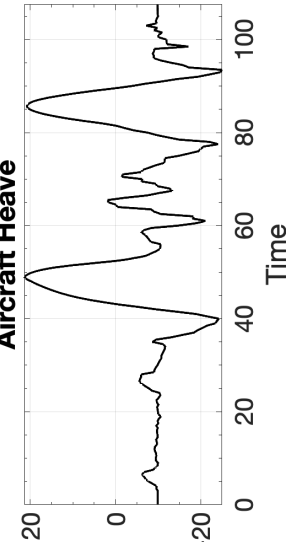

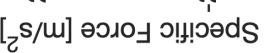
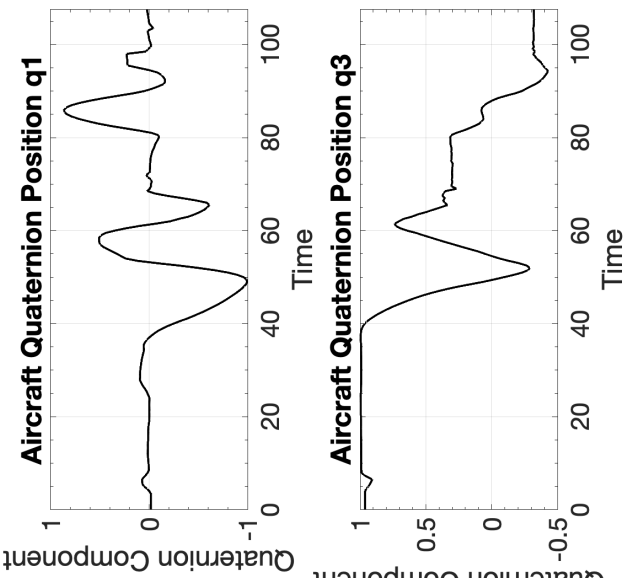

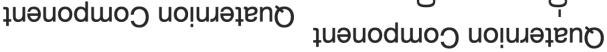

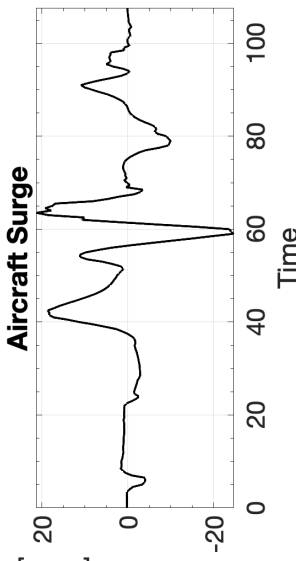

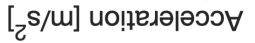

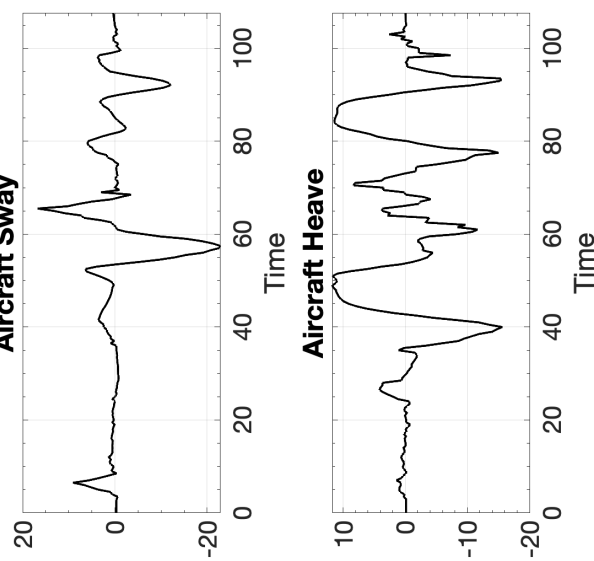

[zs/m] ио!ฺедәәәоэ

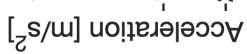

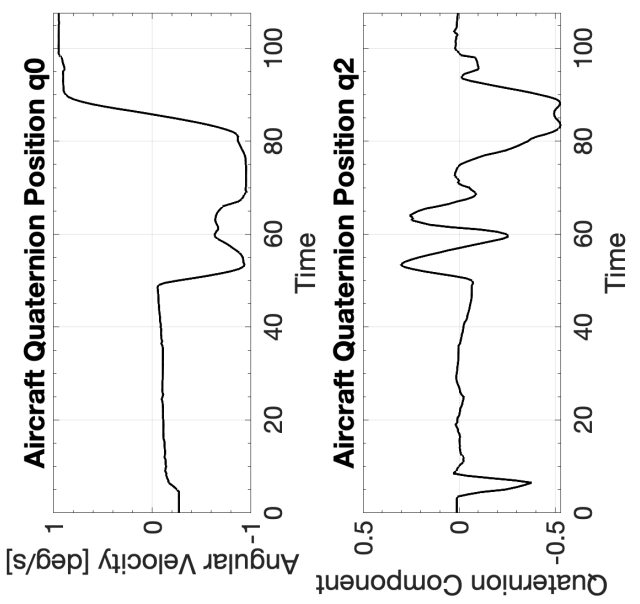




\section{D.11.1 CW1 Columbia 400 Aerobatics}

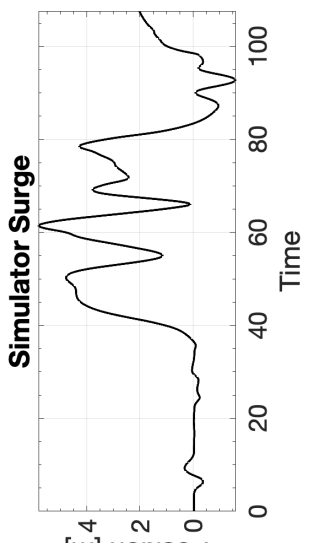

[w] uo!!!sod

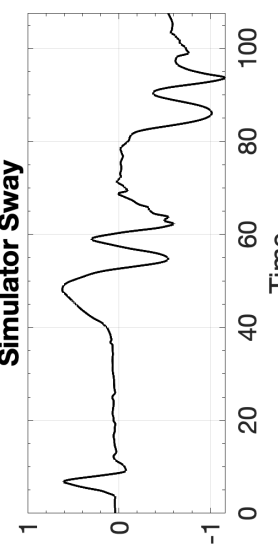

[u] uo!!! sod

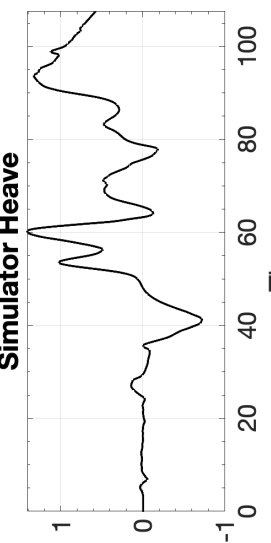

[w] uolt!sod

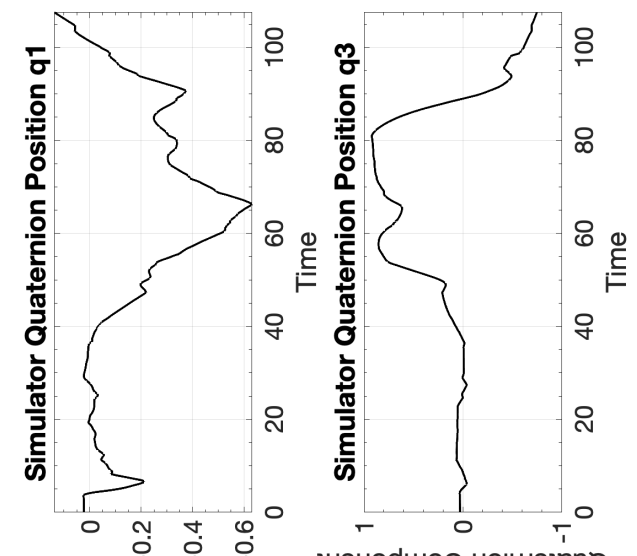

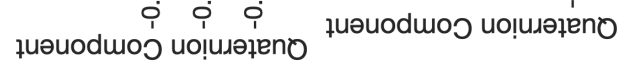

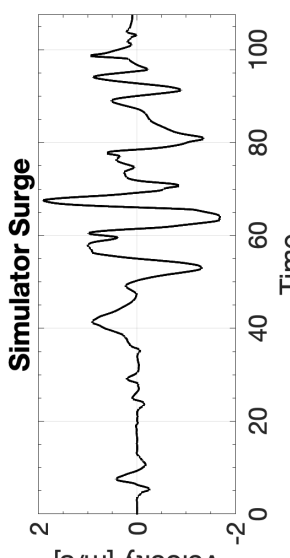

[s/u] Кұ!৩о|ә

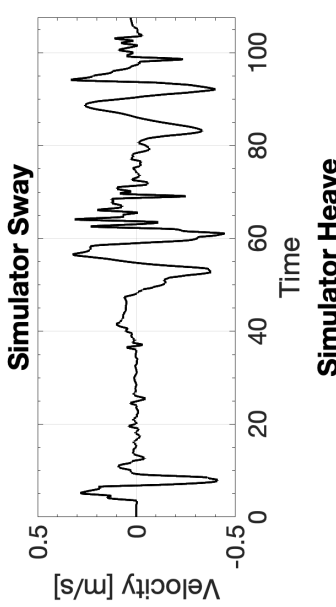

[s/u] Кł!৩о|ә^

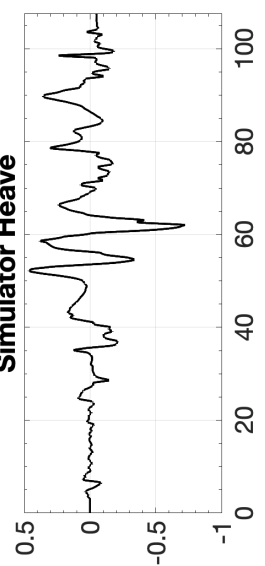

[s/m] Кł!วоำ

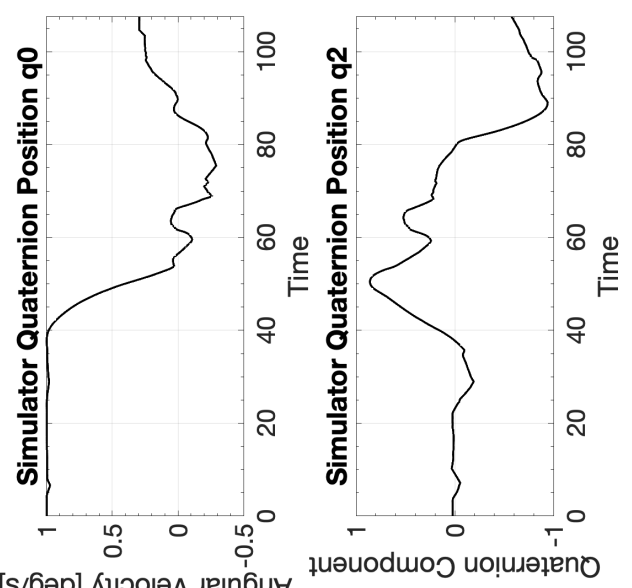

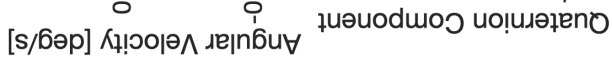

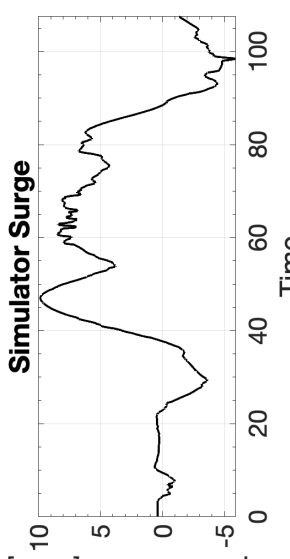

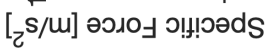

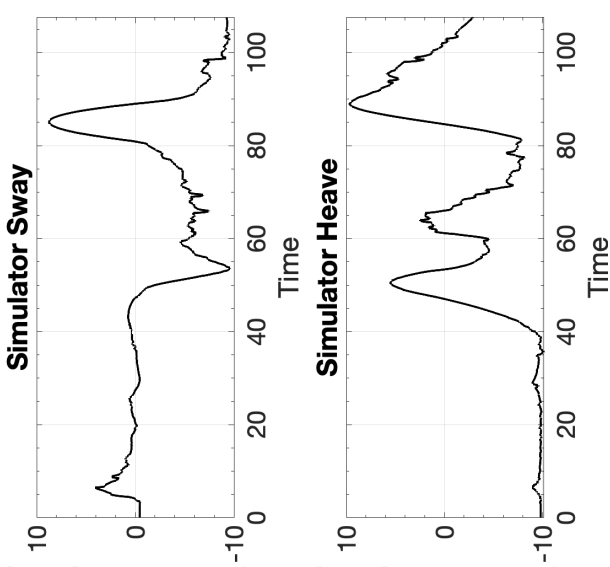

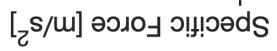

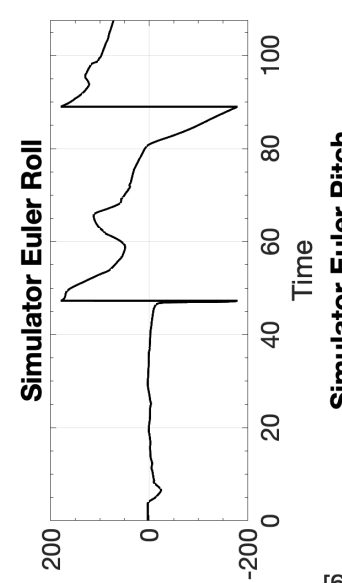

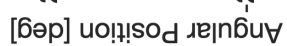

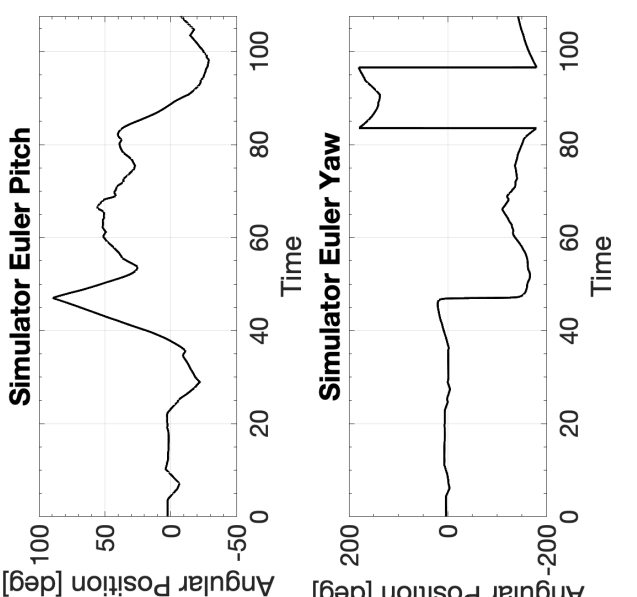

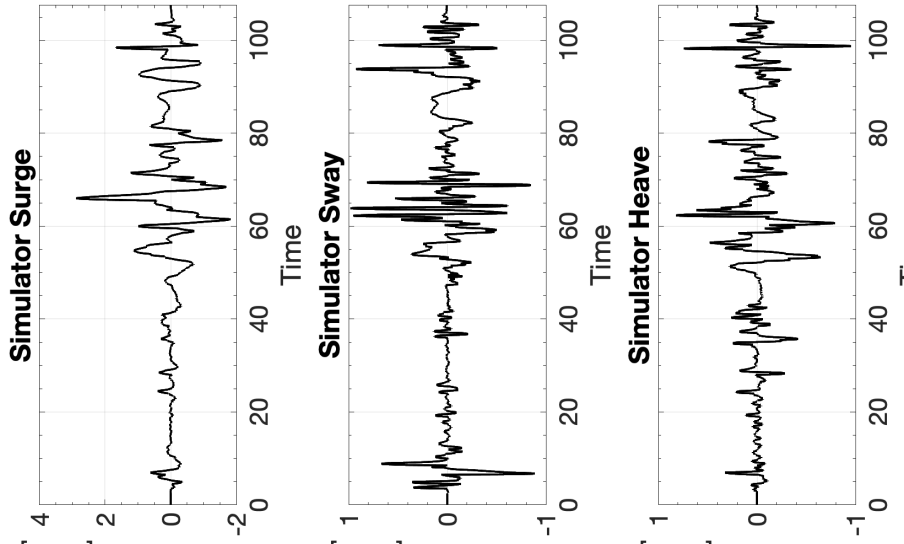

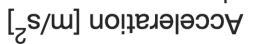

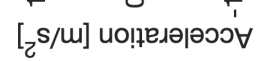
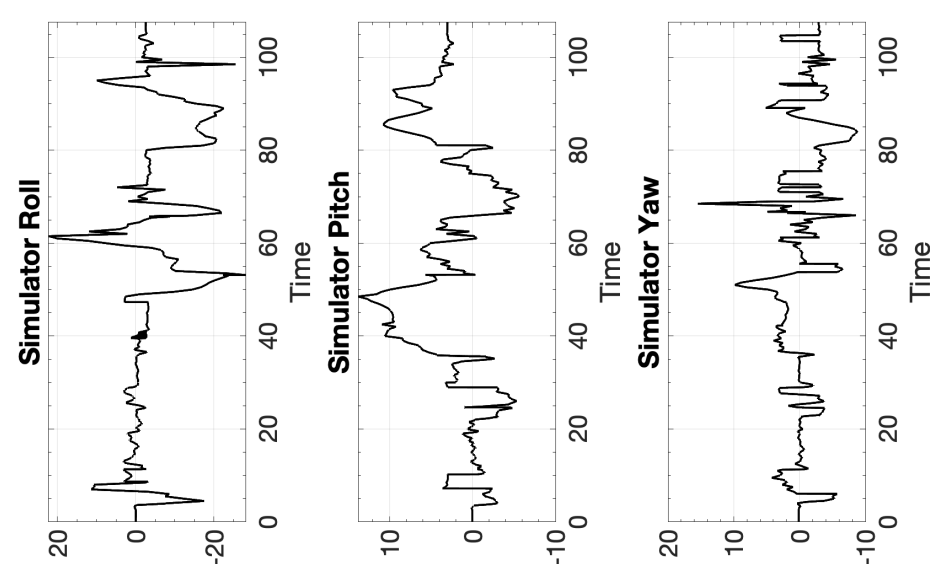

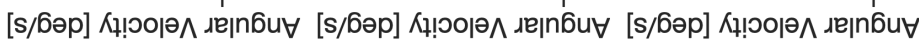




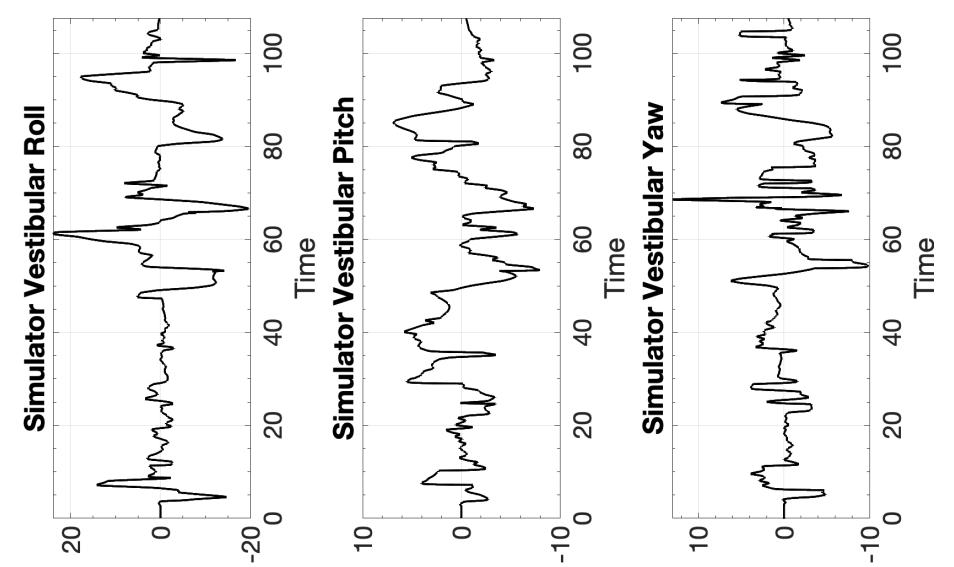

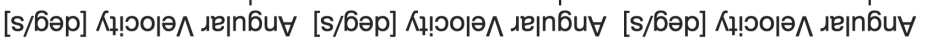

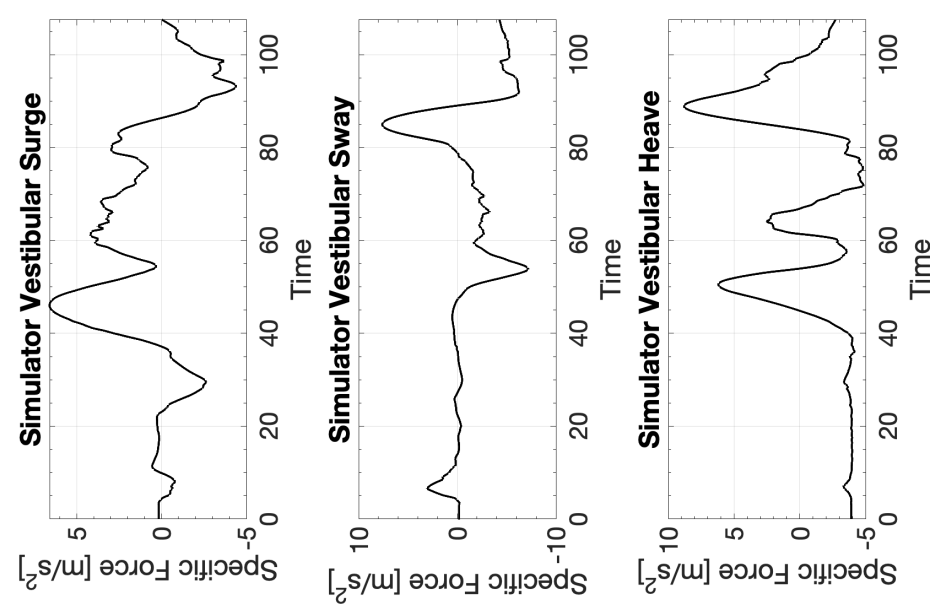




\section{D.11.2 CW2 Columbia 400 Aerobatics}

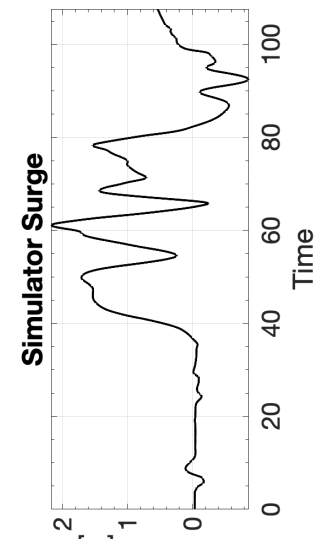

[w] uol!!!sod

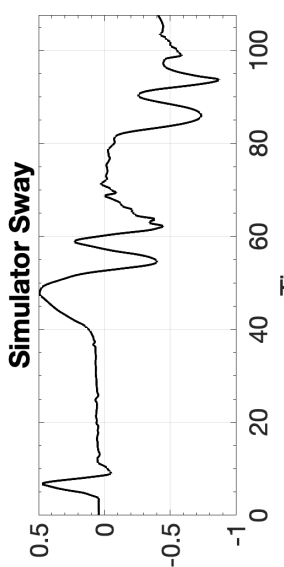

[m] uo!̣!sod

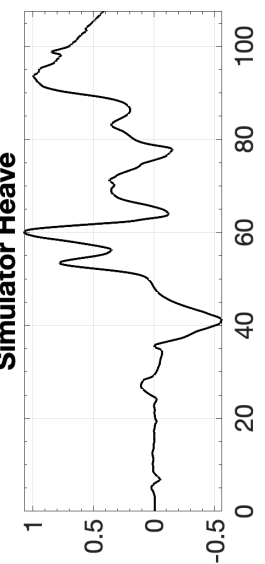

[m] uol!!!sod

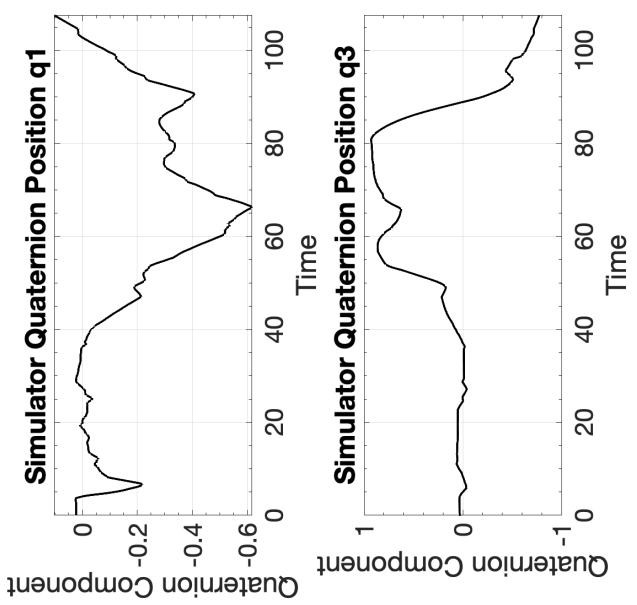

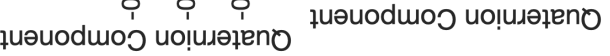

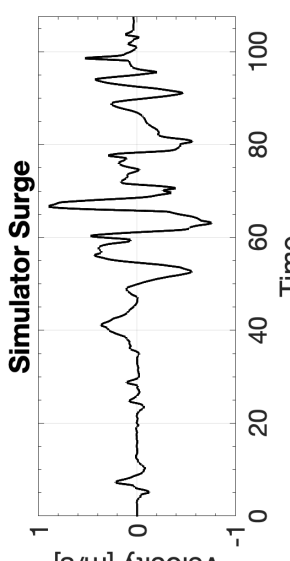

$[\mathrm{s} / \mathrm{M}] \Lambda_{\uparrow}$

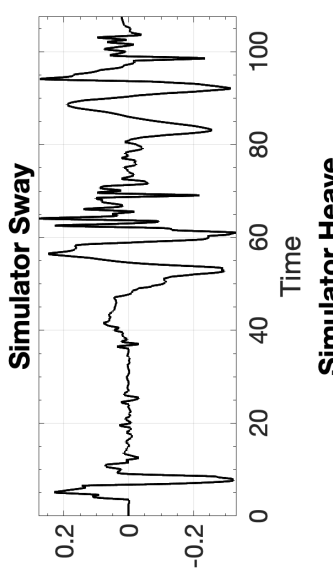

[s/m] ᄉ!잇

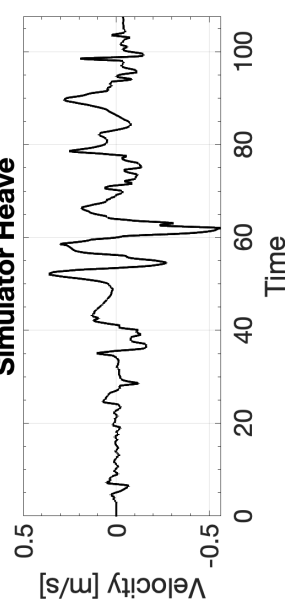

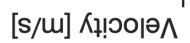

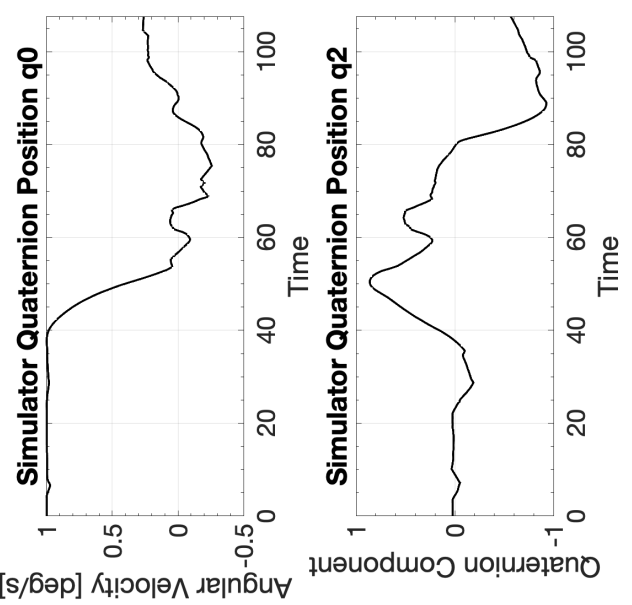

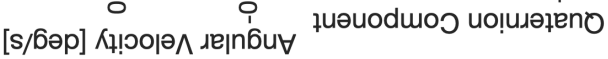

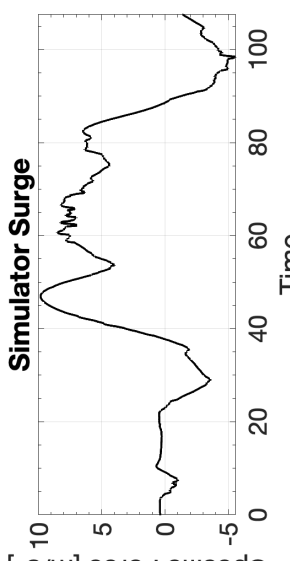

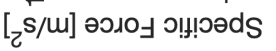

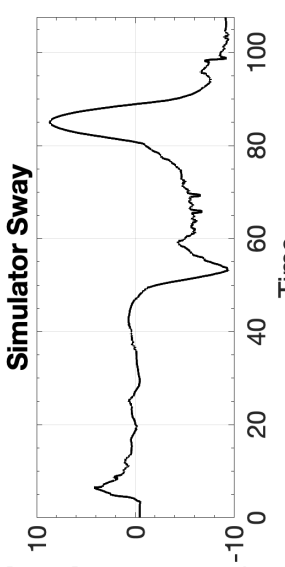

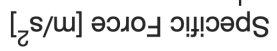

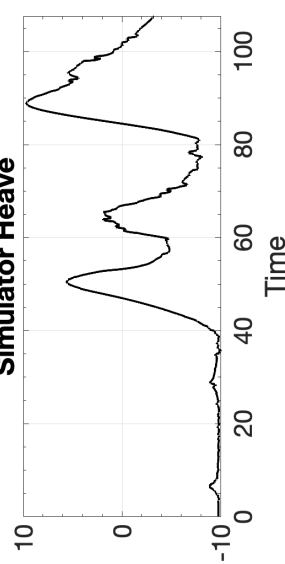

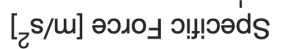

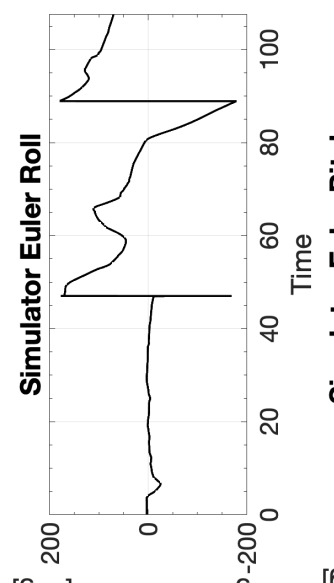

[6əp] uo!!!!sod גe|nదินที

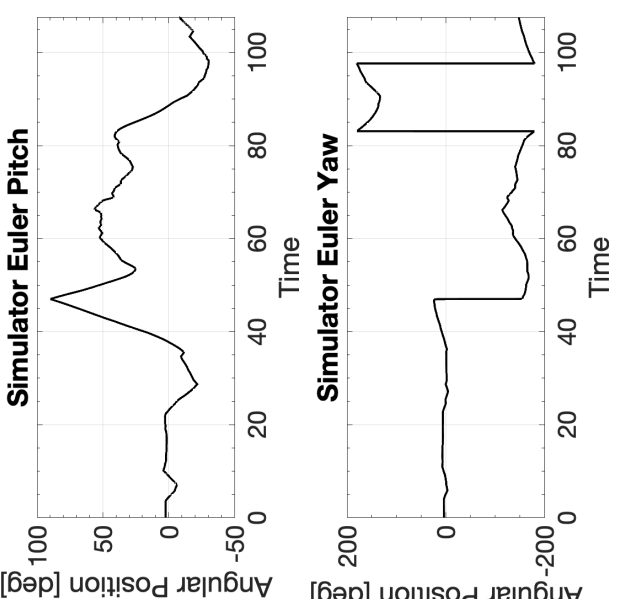

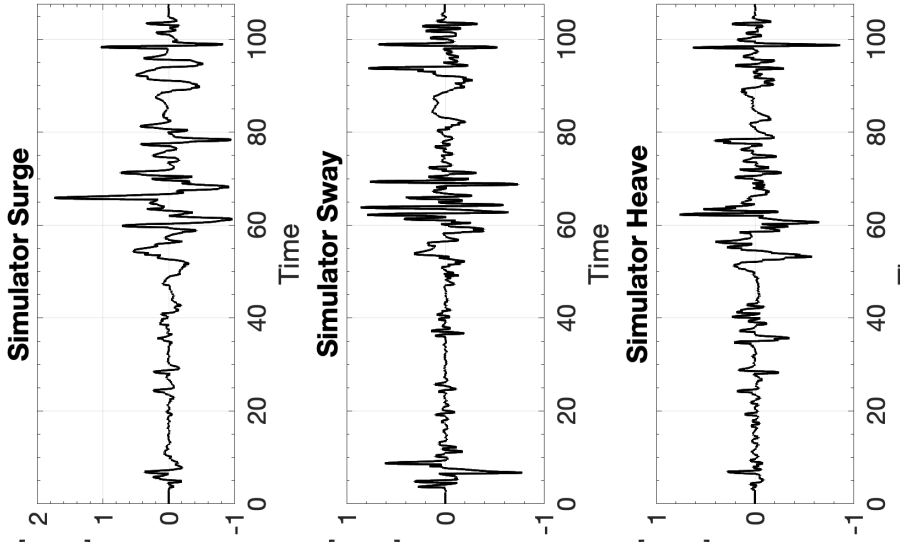

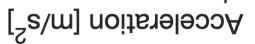

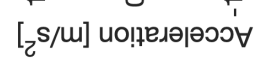
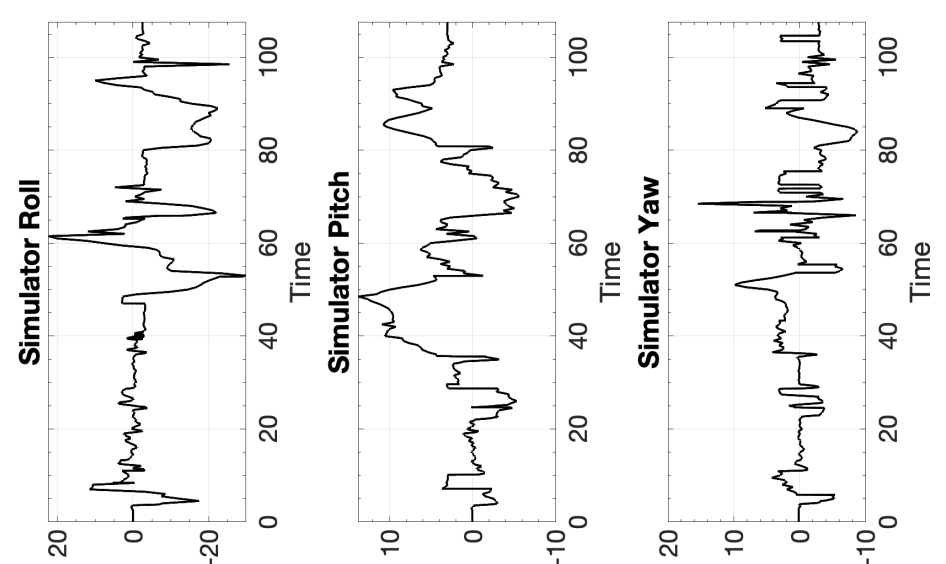

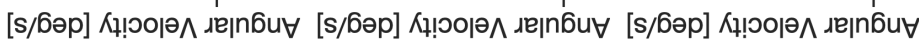




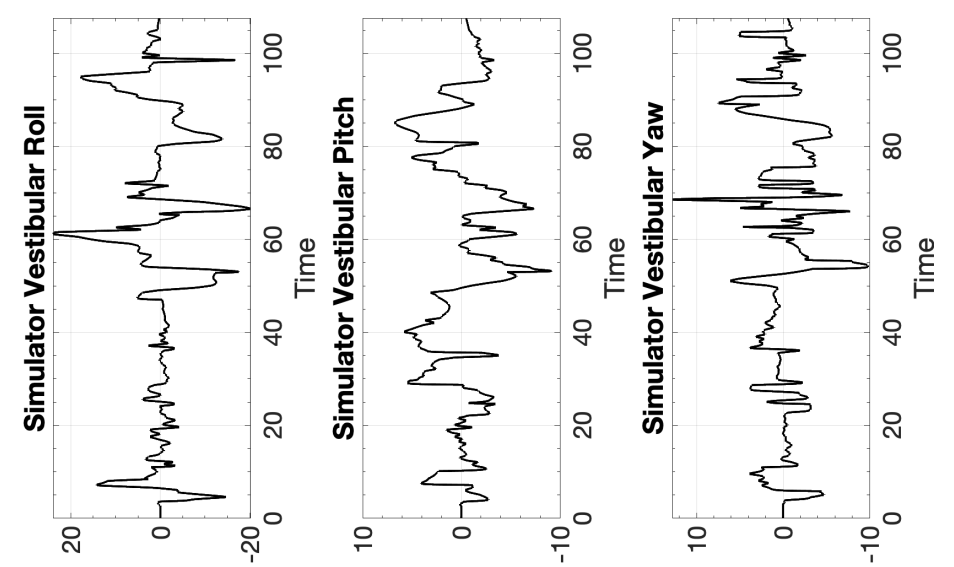

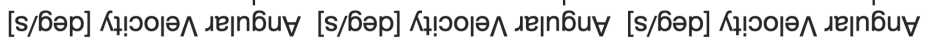

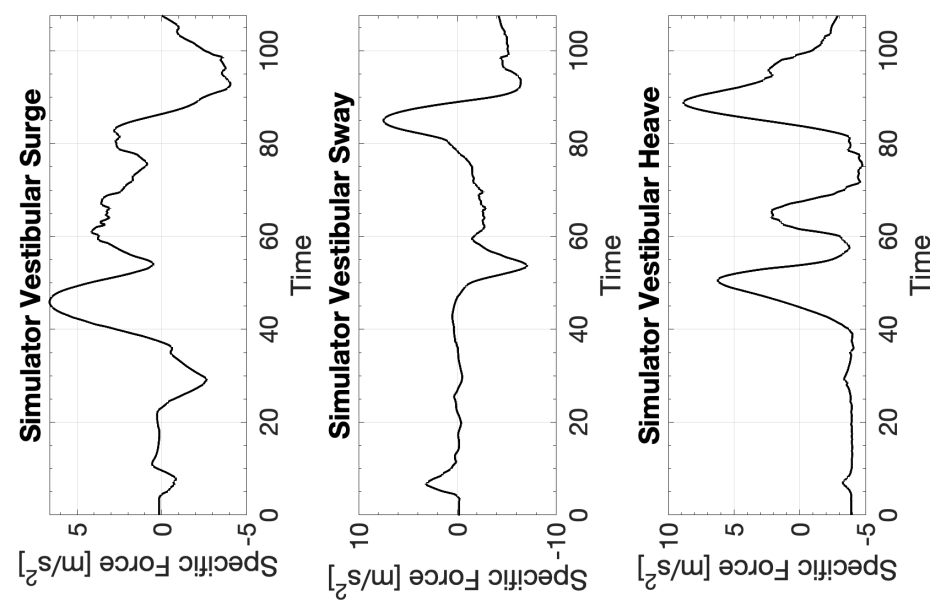




\section{D.11.3 CW3 Columbia 400 Aerobatics}

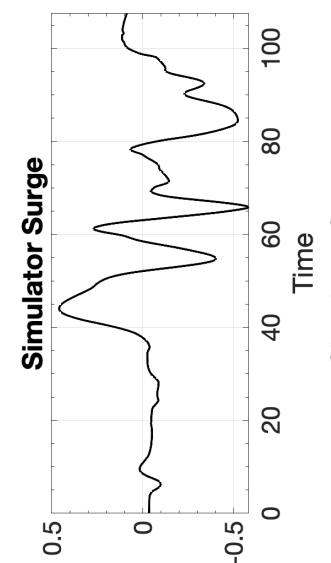

[w] uo!!!!sod

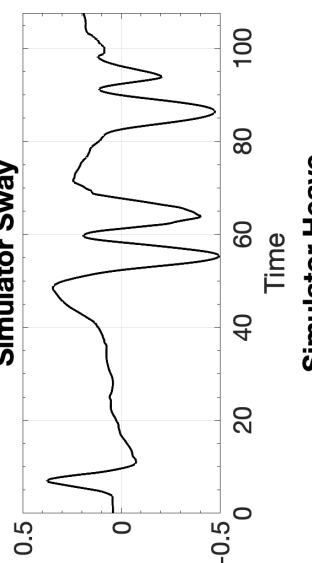

[m] uo!m!sod

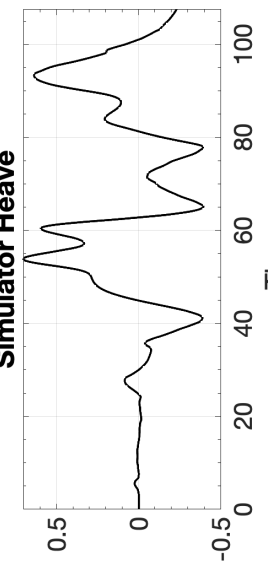

[w] uo!n!sod

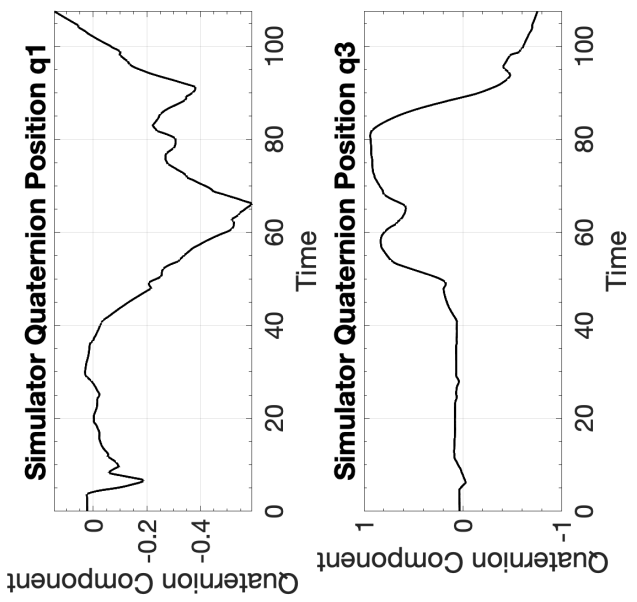

ұuəuodmoj no!̣uəteno fịurjeno

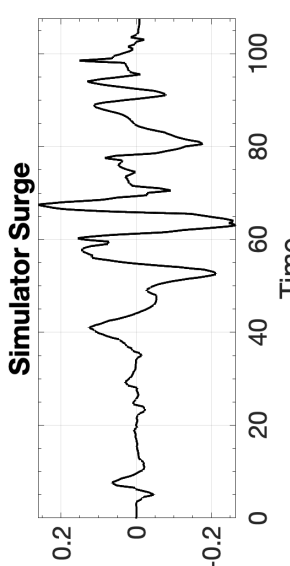

[s/u] Кц!৩оюю

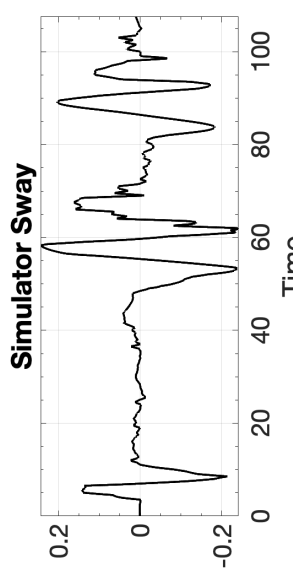

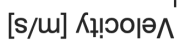

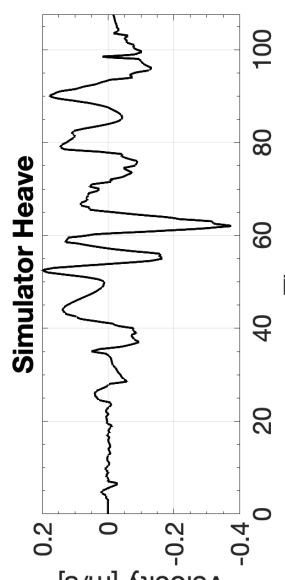

[s/m] Кұ!юำ

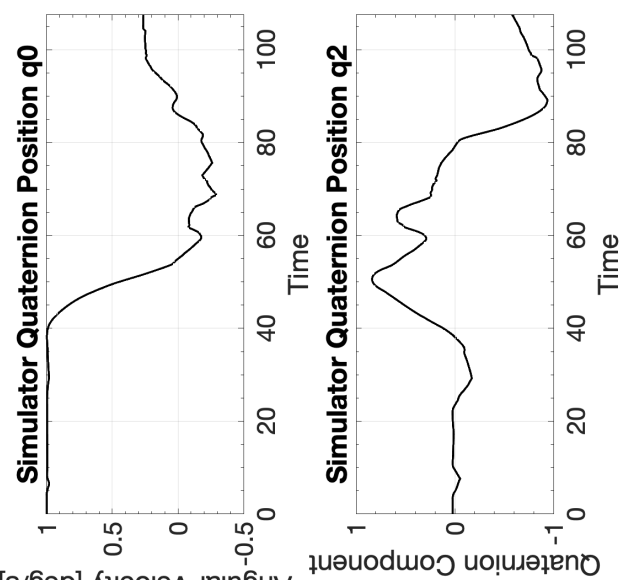

[s/6әр] К!

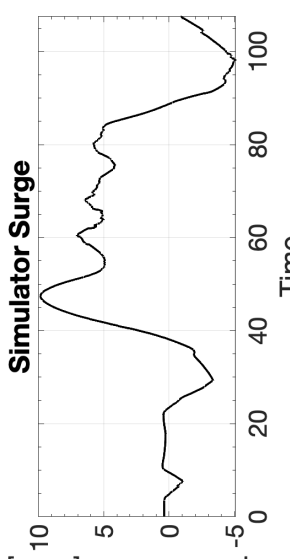

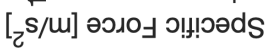

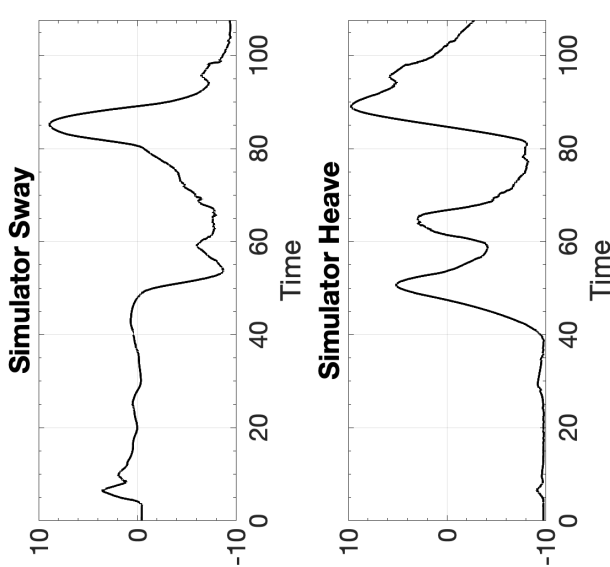

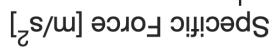

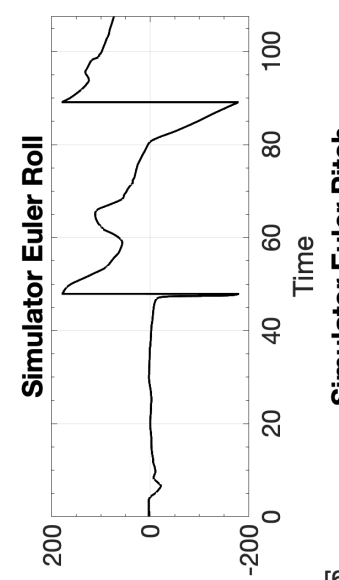

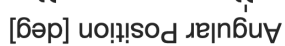

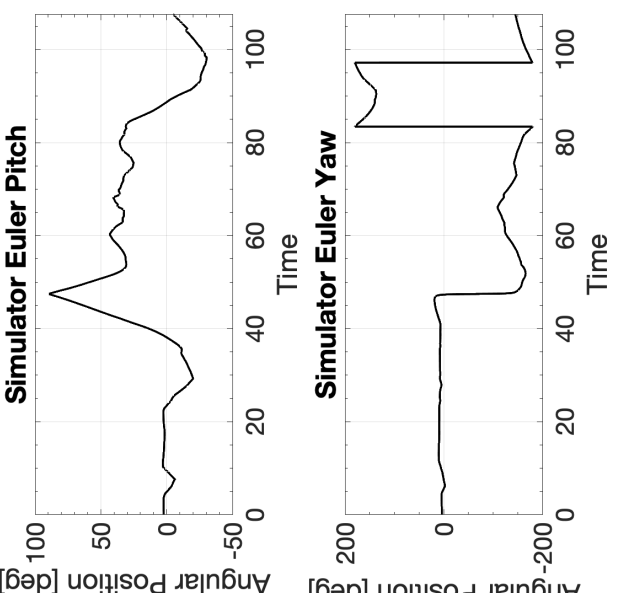

[6әр] uo!!!sod ле|n6u

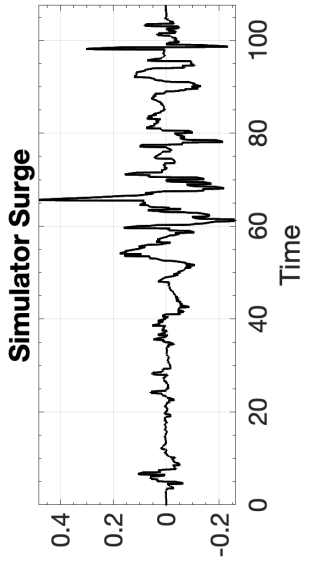

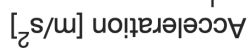

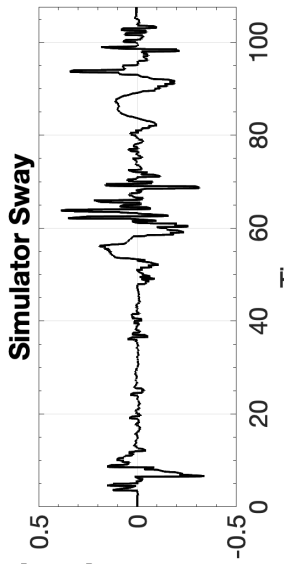

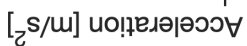

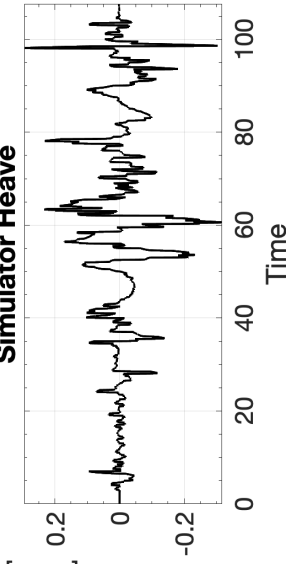

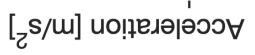

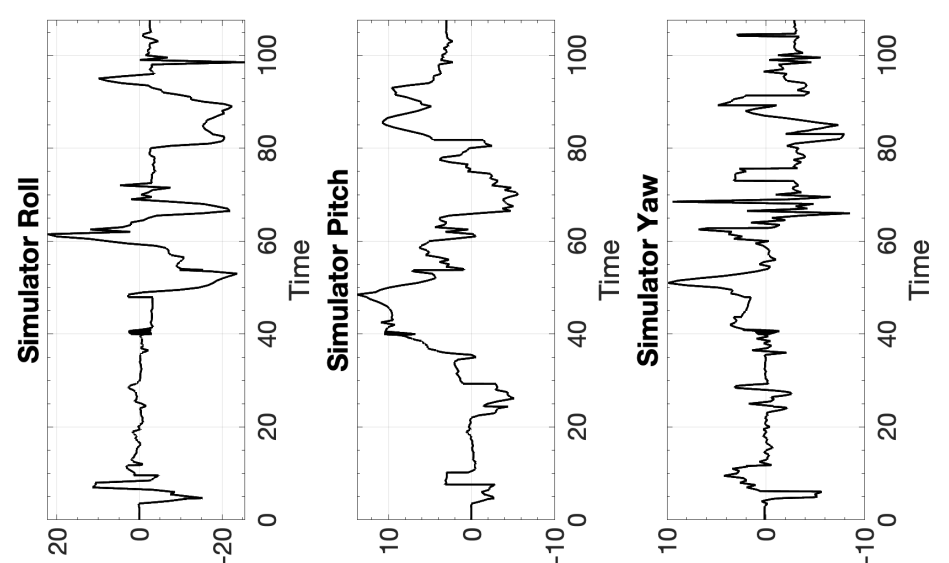

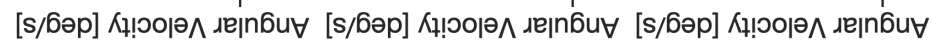




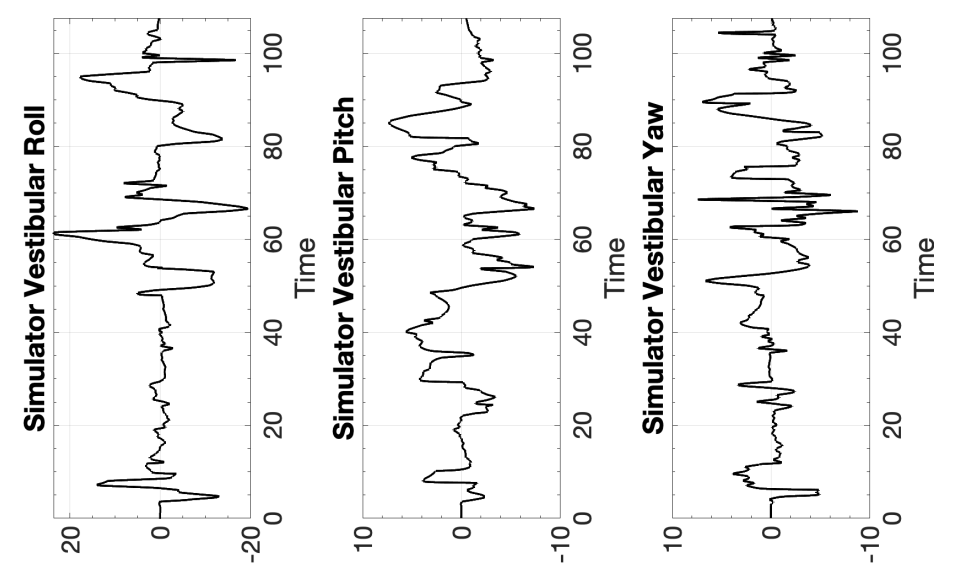

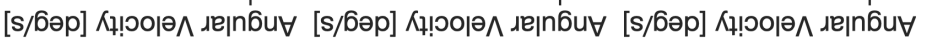

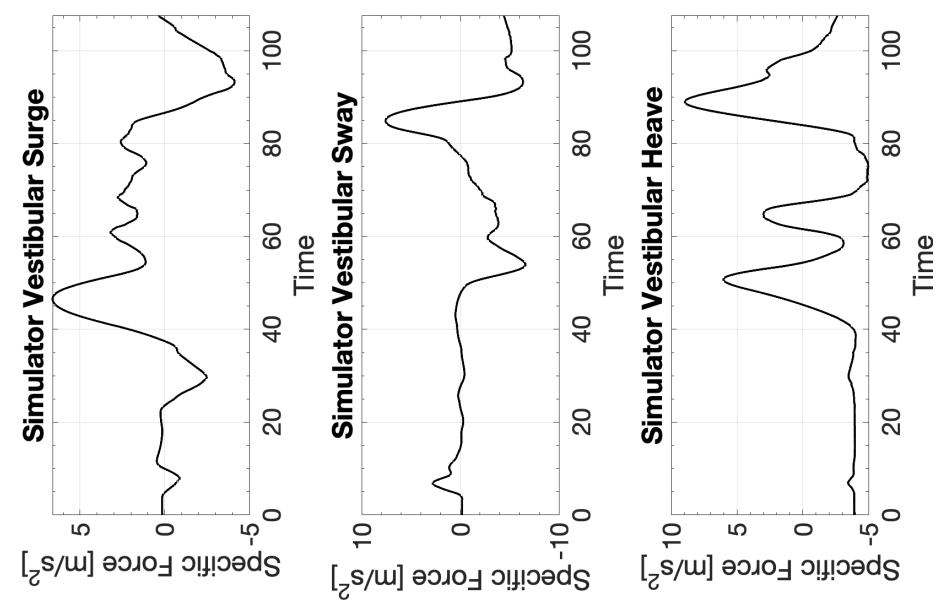




\section{D.12 Columbia 400 Coordinated Turn}

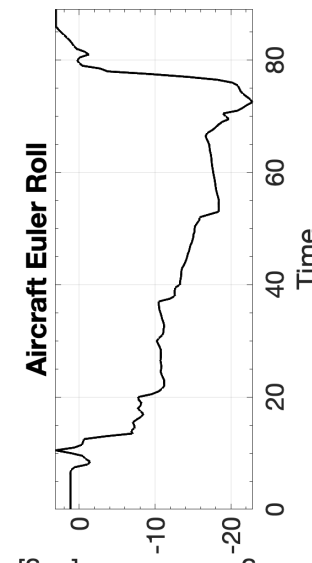

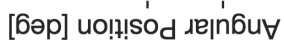

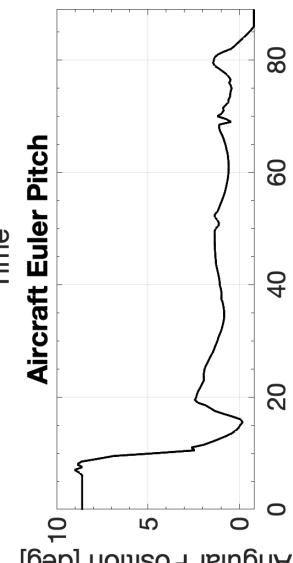

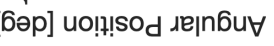

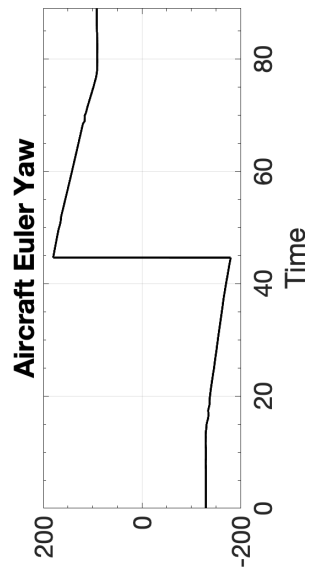

[6әр] uo!!!sod de|n6́u
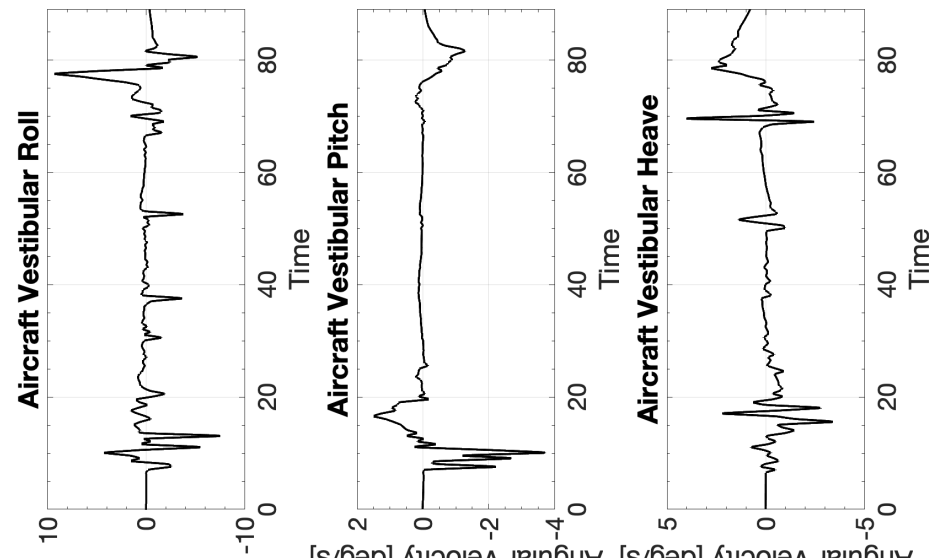

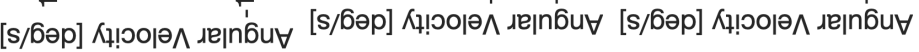

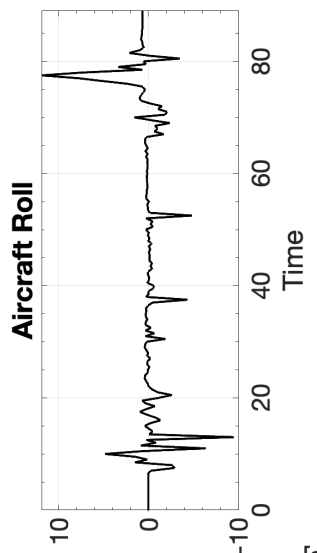

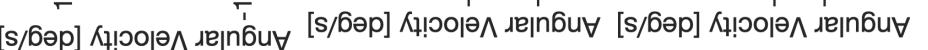
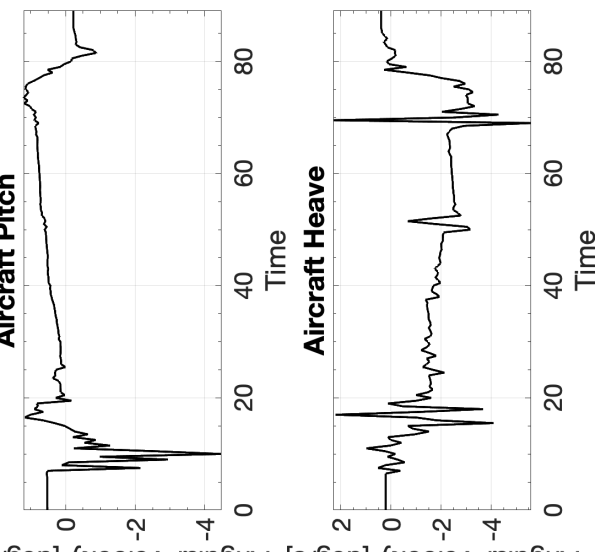

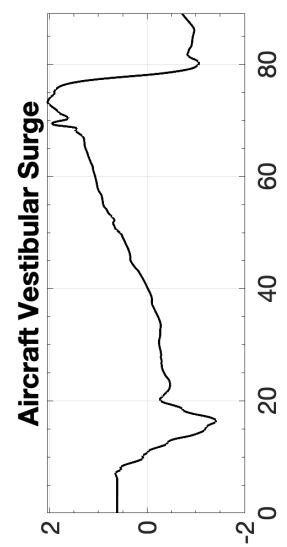

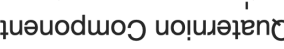

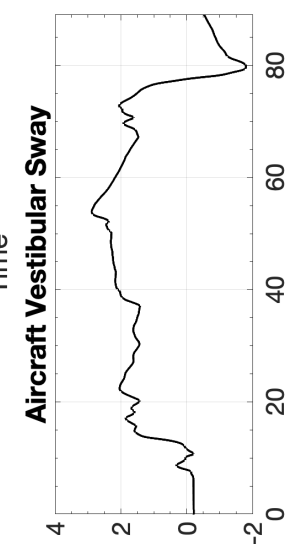

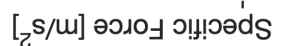

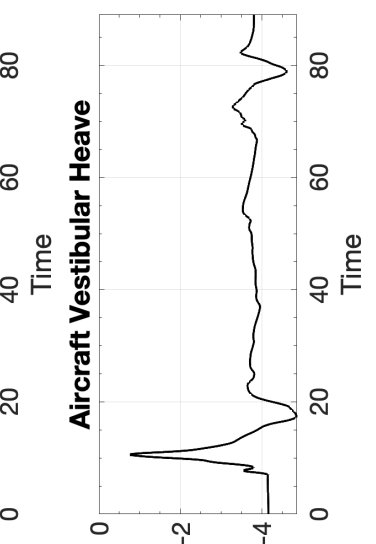

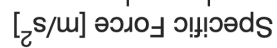

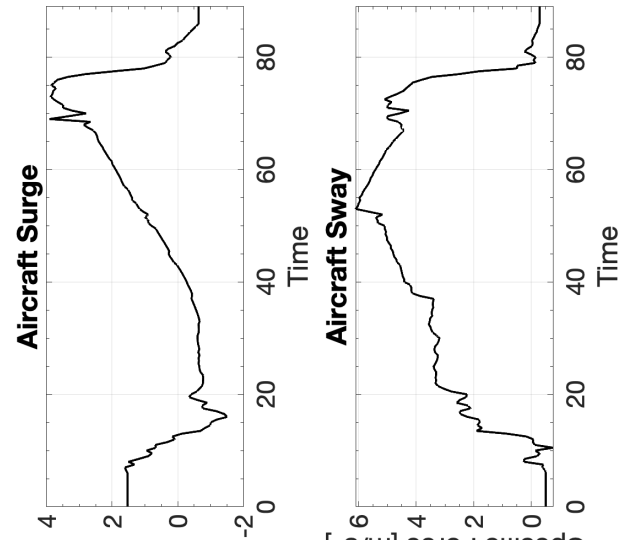

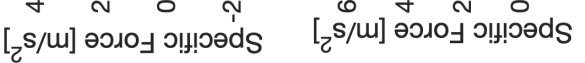
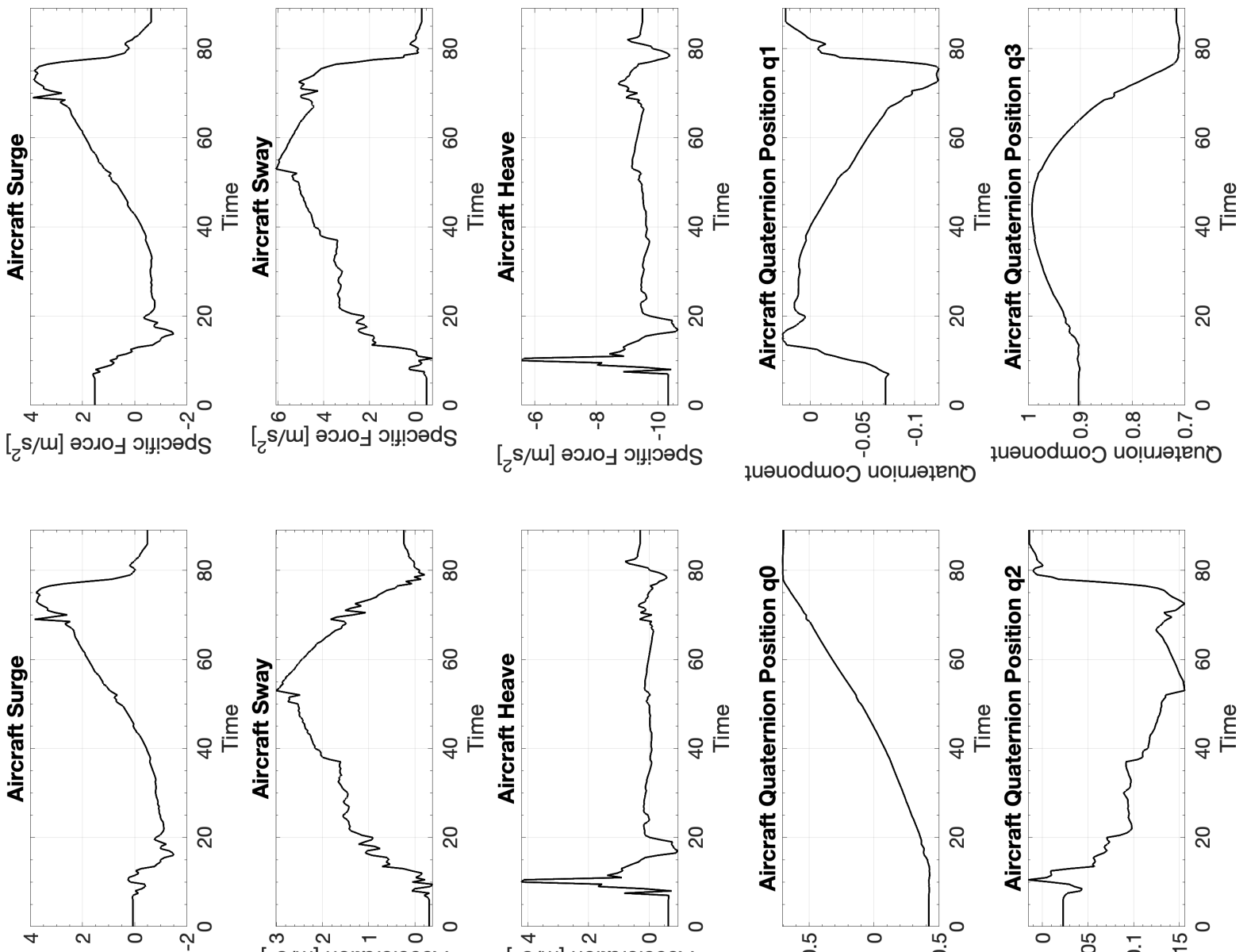

$[\mathrm{c} / \mathrm{m}] \mathrm{uo!̣}$

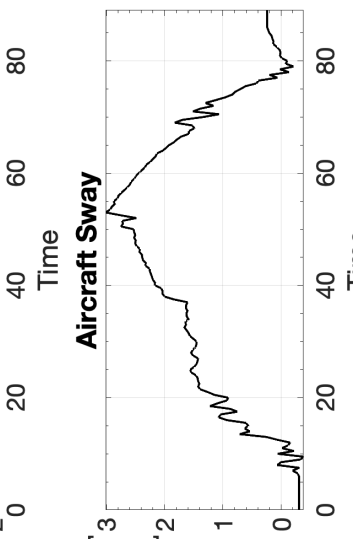

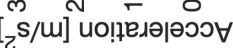

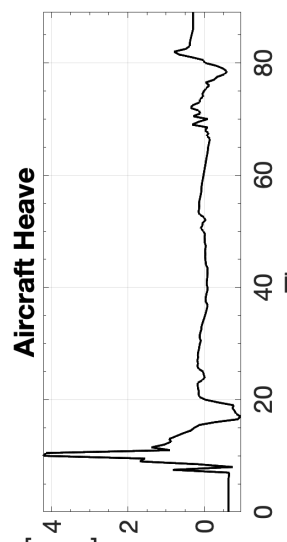

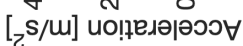

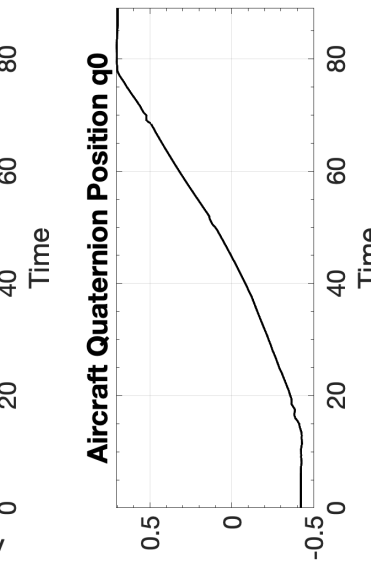

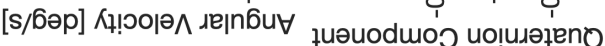

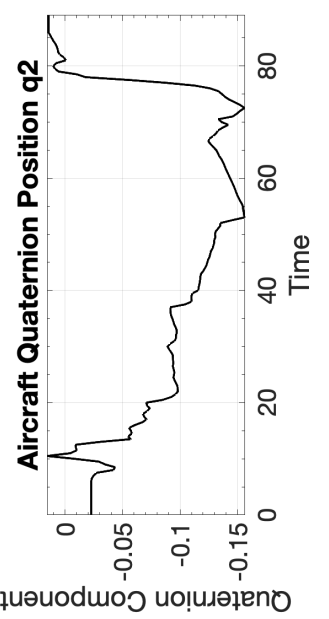




\section{D.12.1 CW1 Columbia 400 Coordinated Turn}

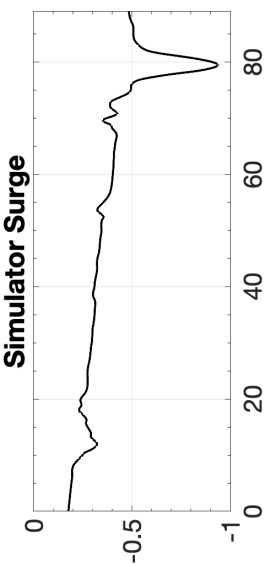

[w] uo!!!!sod

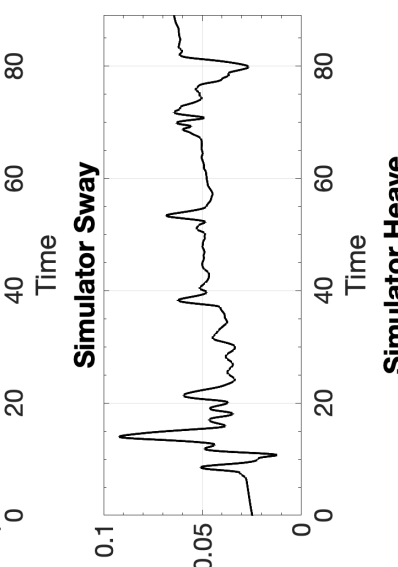

[u] uo!n!sod

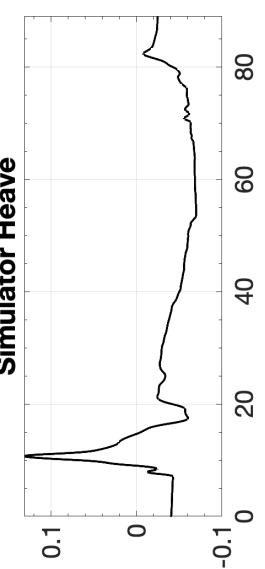

[m] uol!! sod

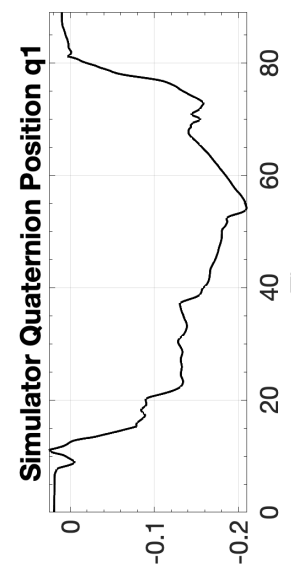

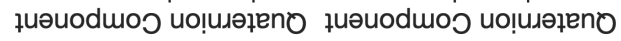

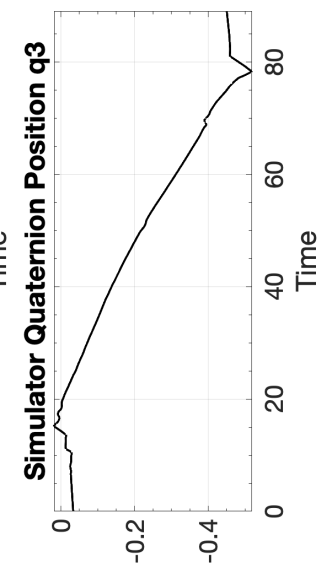

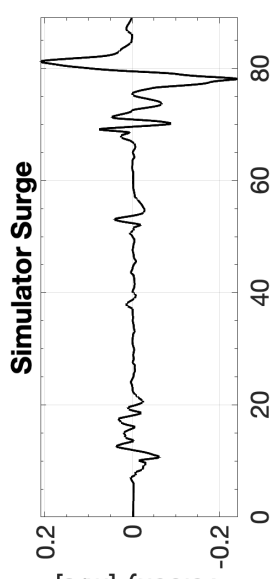

[s/m] Кұ!৩ою^

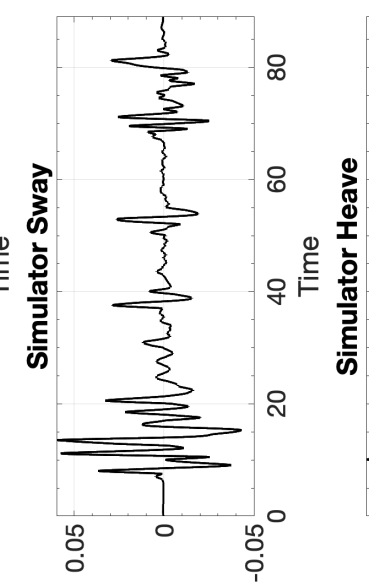

[s/m] Кџ!วо夫

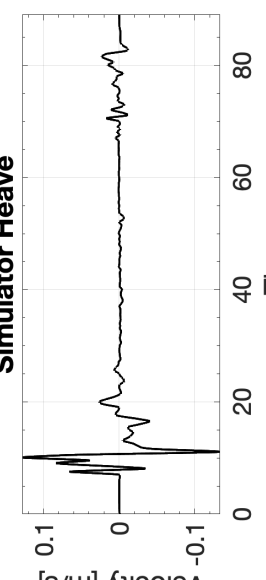

[s/u] К!ำю^
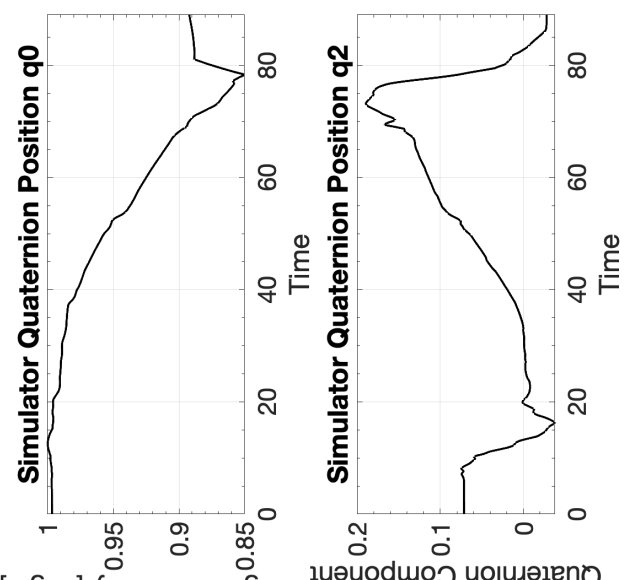

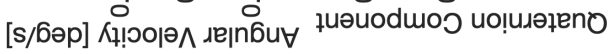

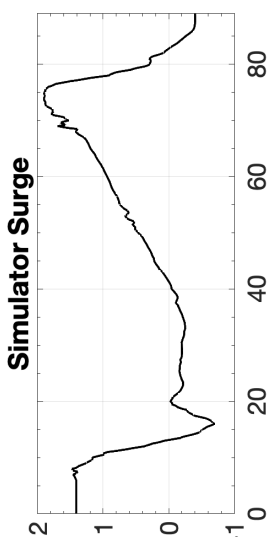

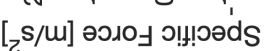

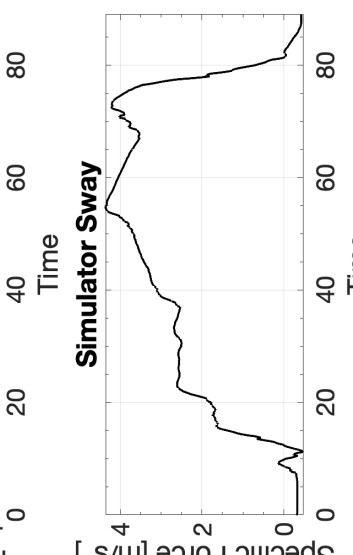

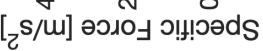

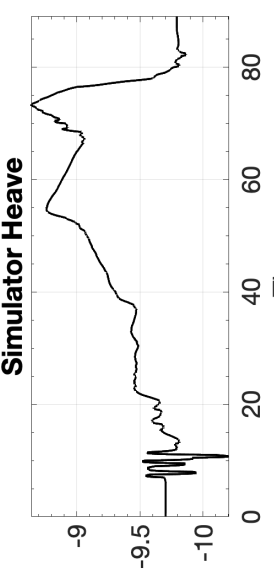

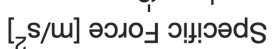

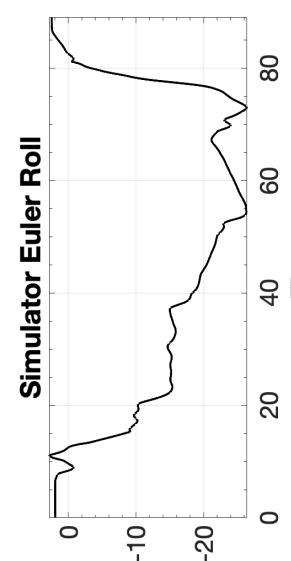

[6әр] uo!!!'sod גeןn6ur

gi

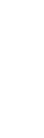




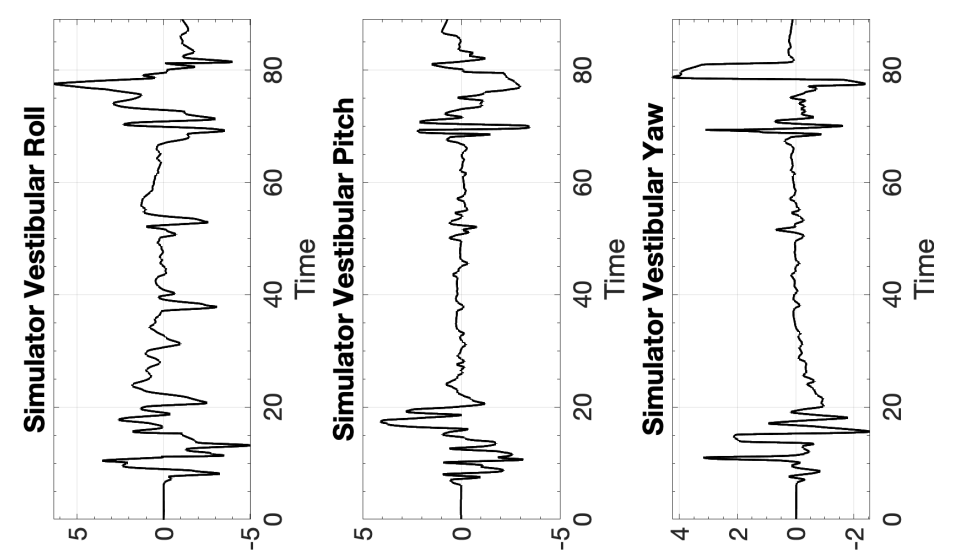

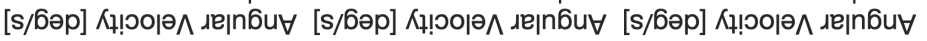

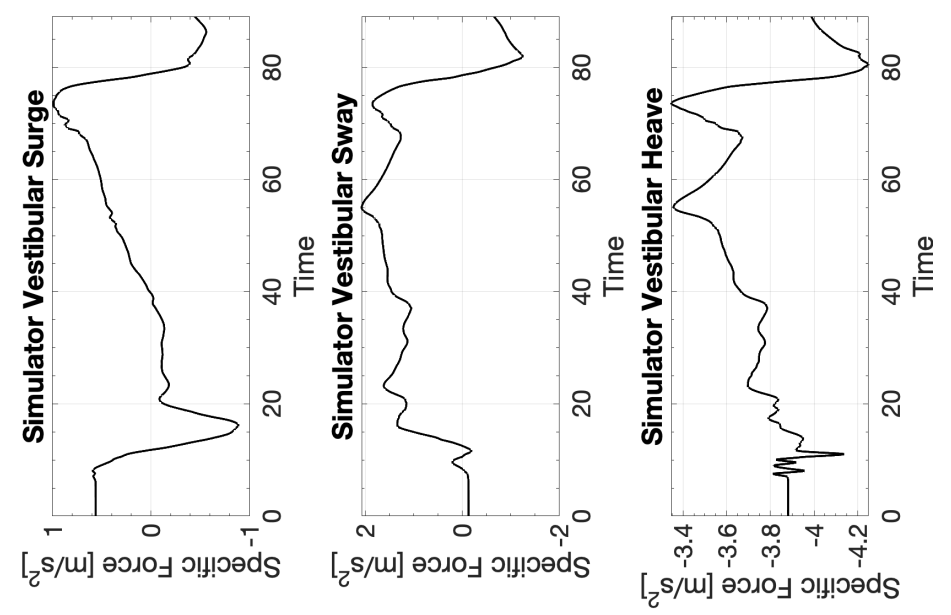




\section{D.12.2 CW2 Columbia 400 Coordinated Turn}
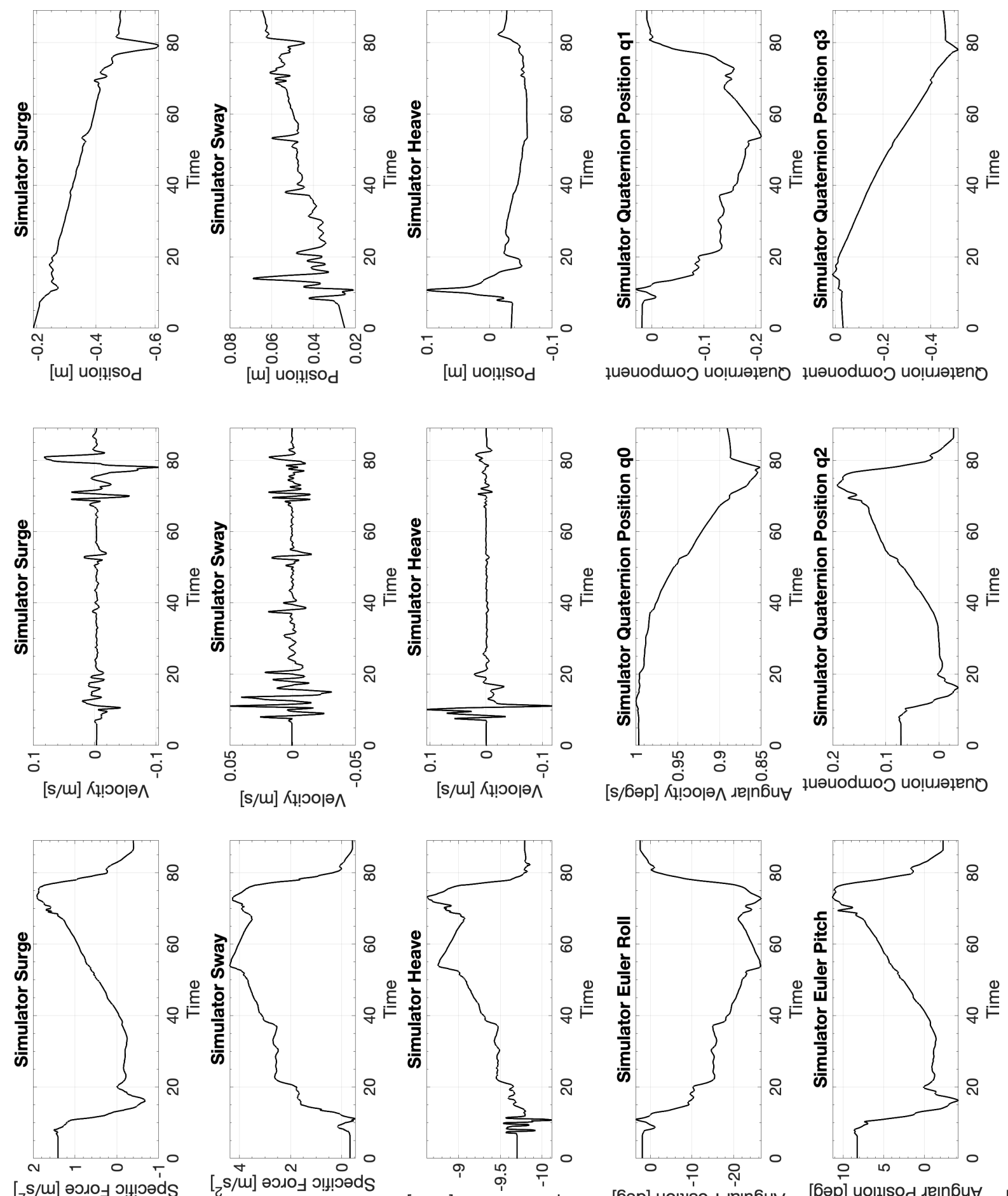

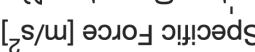

$\left[\begin{array}{l}5 \\ {[}\end{array}\right.$

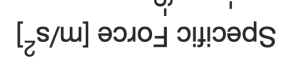

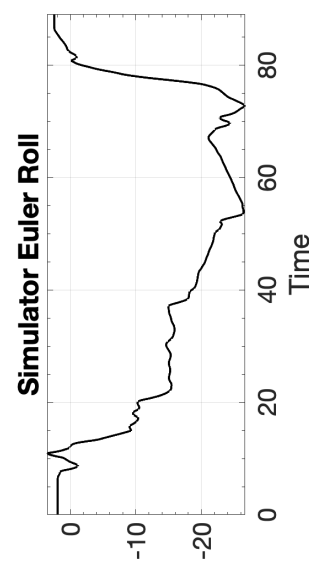

[6әр] uo!!!!sod

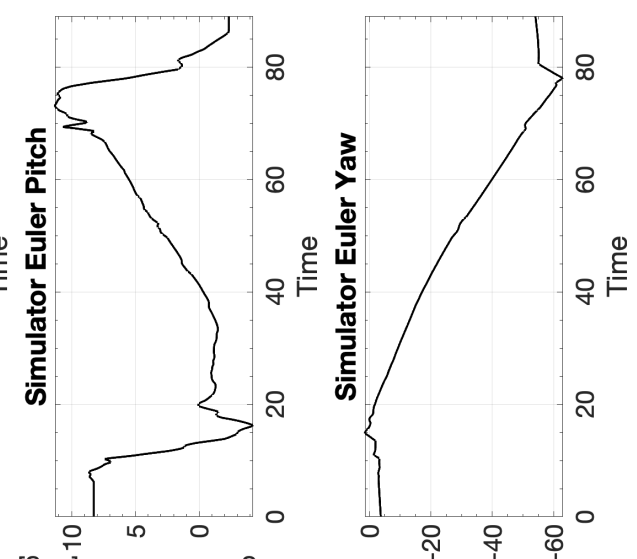

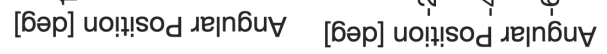

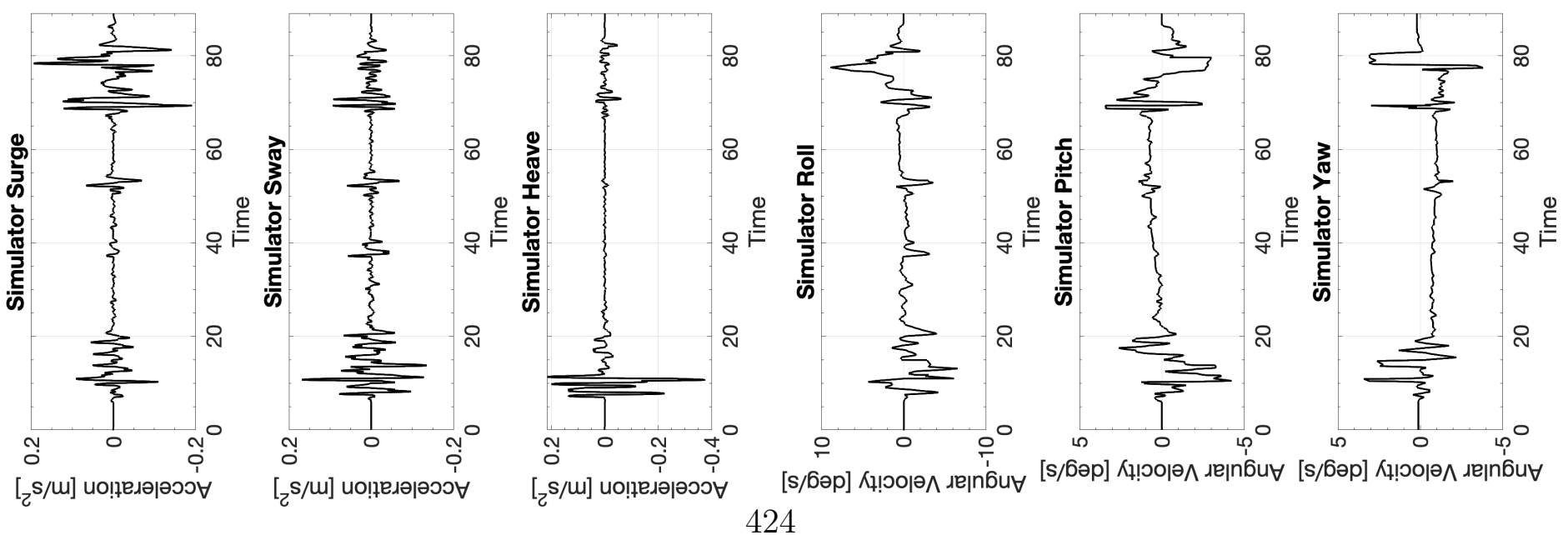



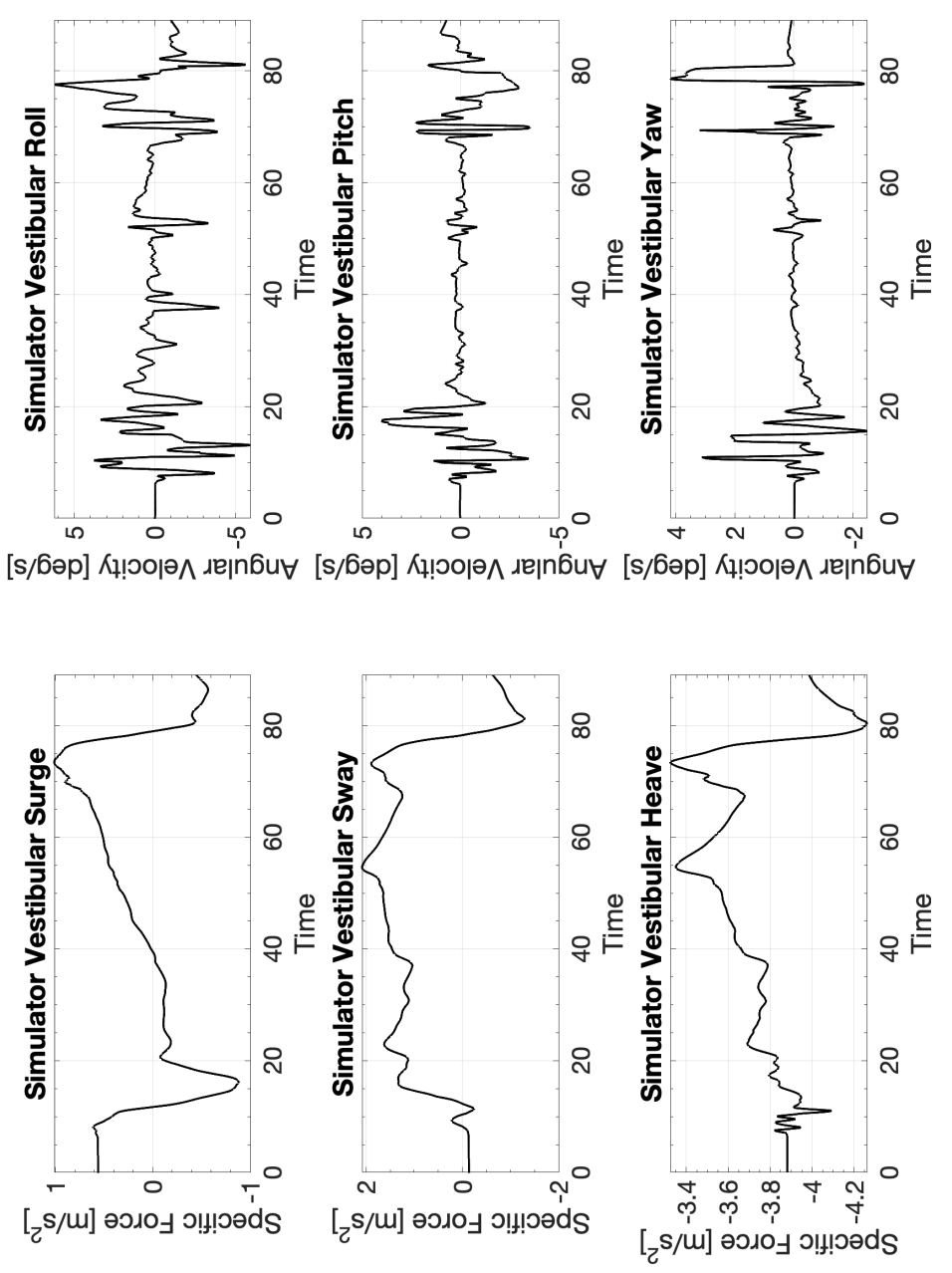


\section{D.12.3 CW3 Columbia 400 Coordinated Turn}
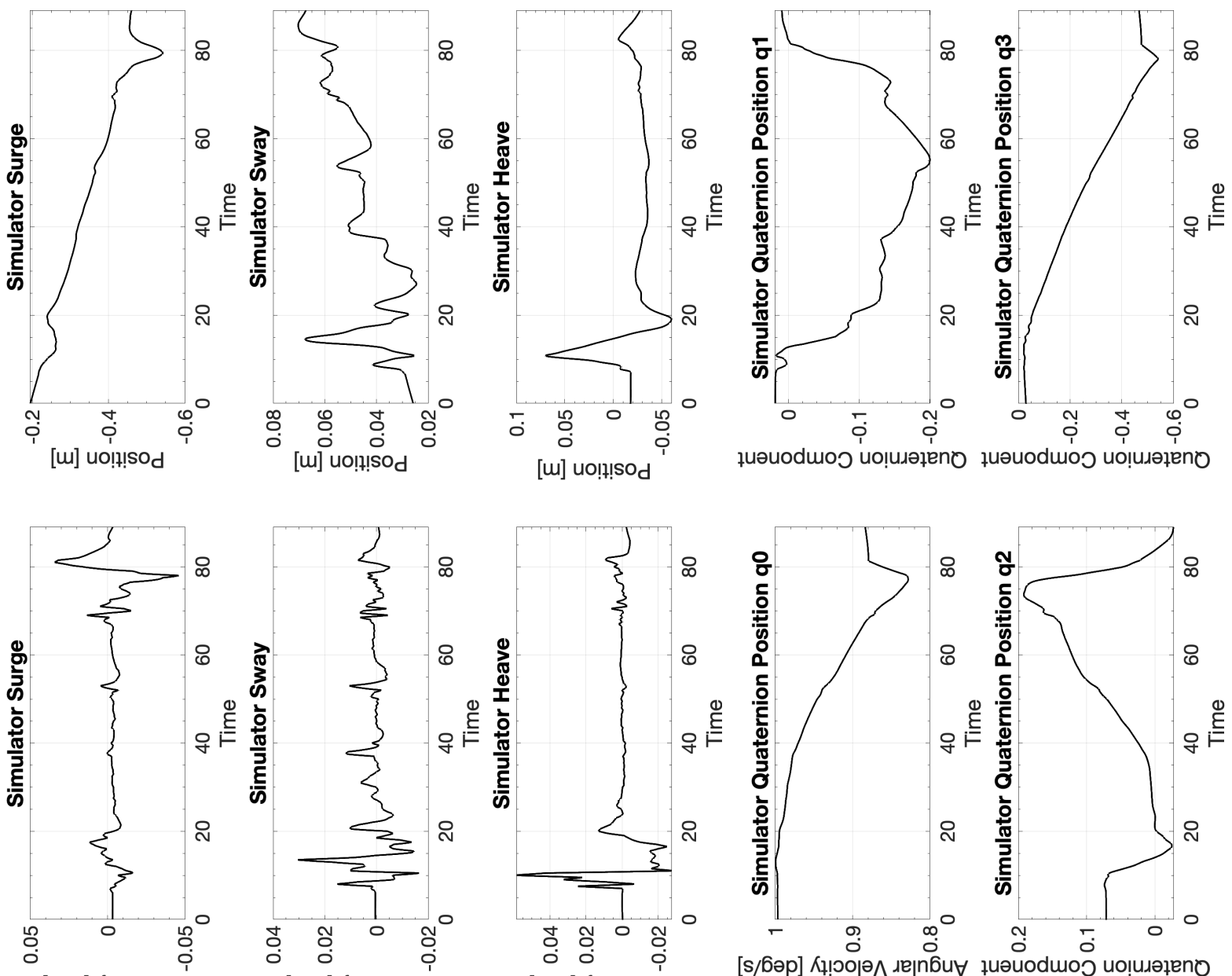

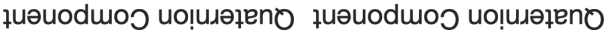

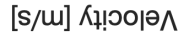

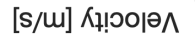

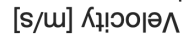

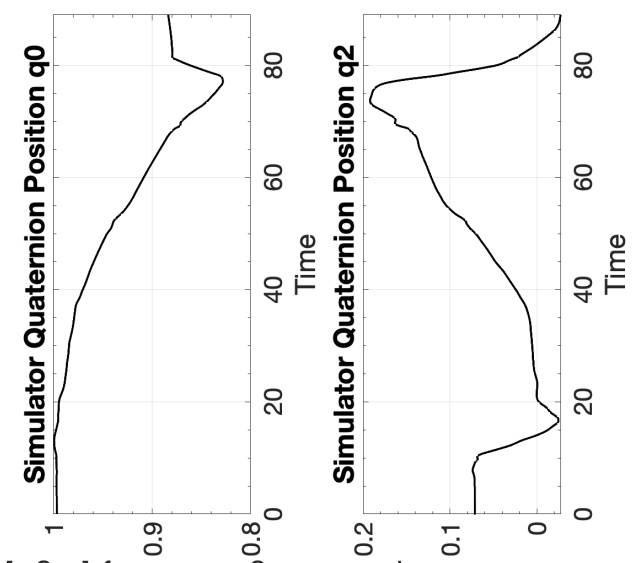

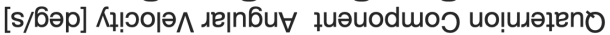

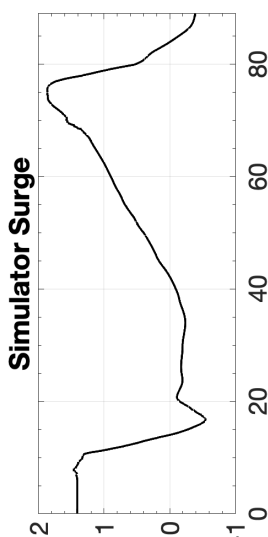

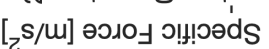

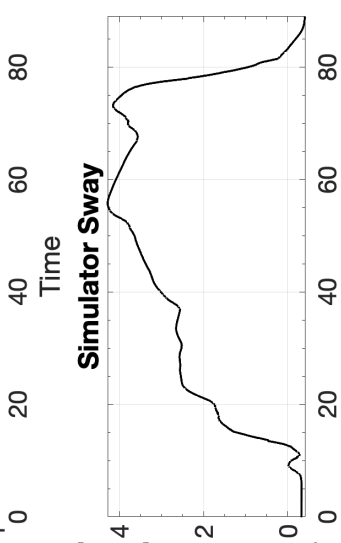

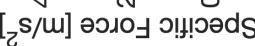

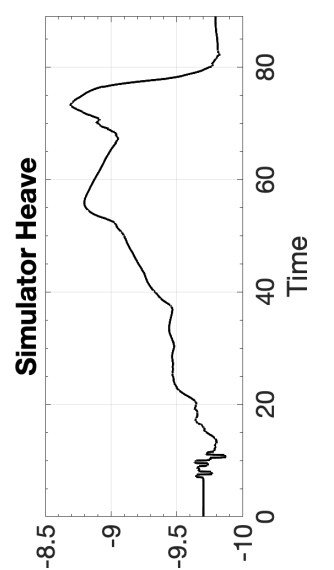

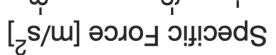

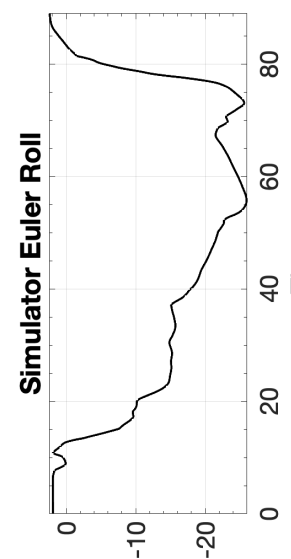

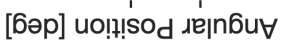

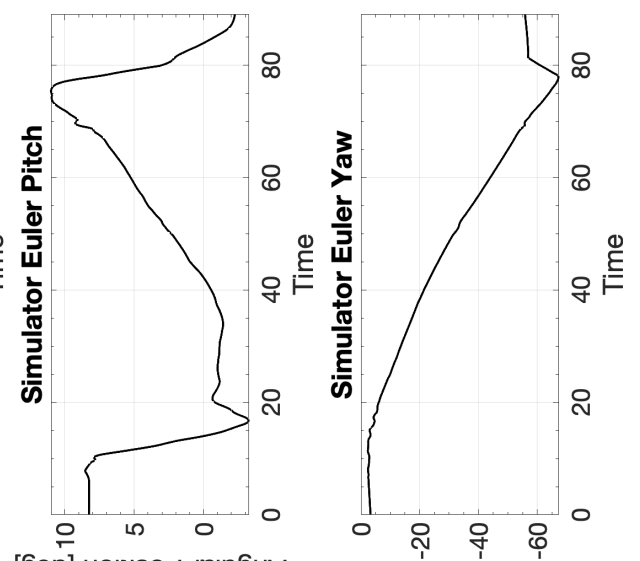

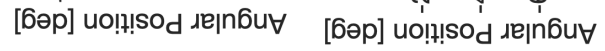

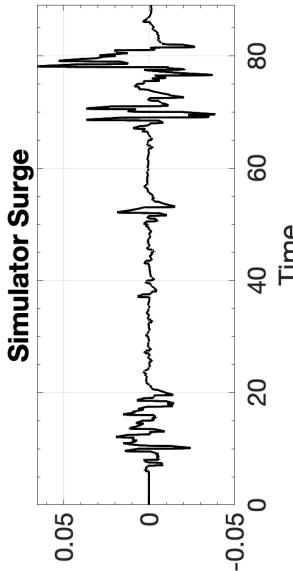

$\left[{ }^{2} \mathrm{~s} / \mathrm{M}\right]$ uо!ฺยләәәоэ

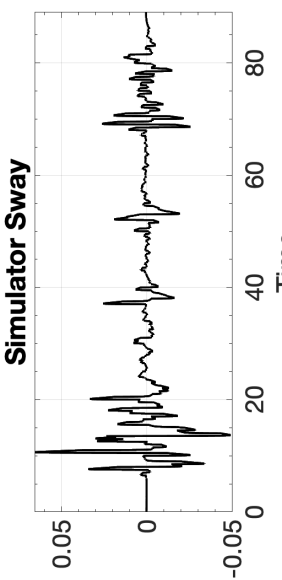

$[z s / m]$ นо!ฺеләәәэว

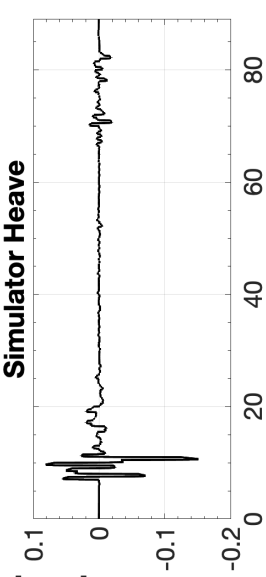

[z $s / m]$ uо!ฺедәәәэว

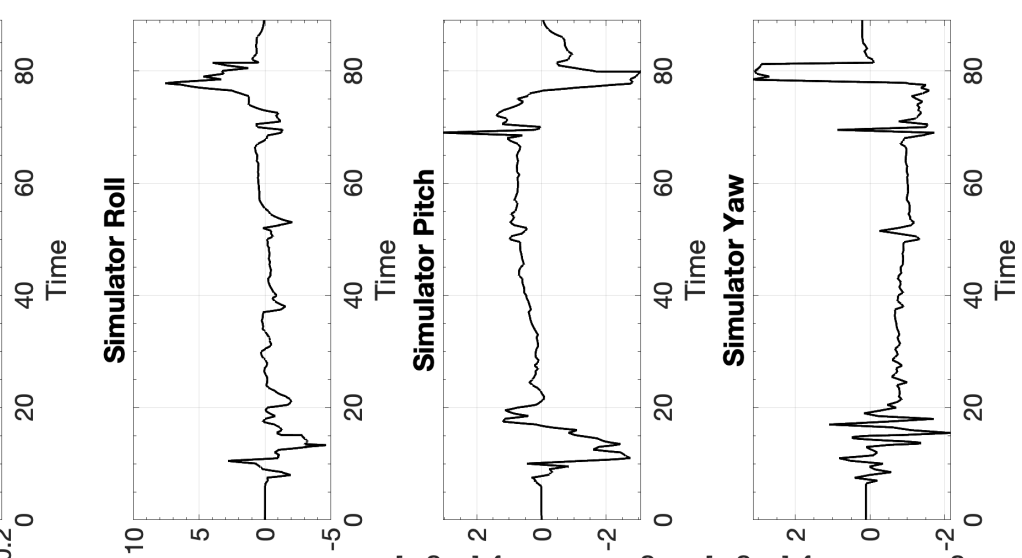

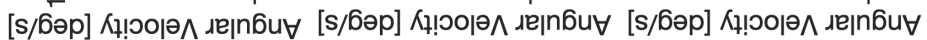



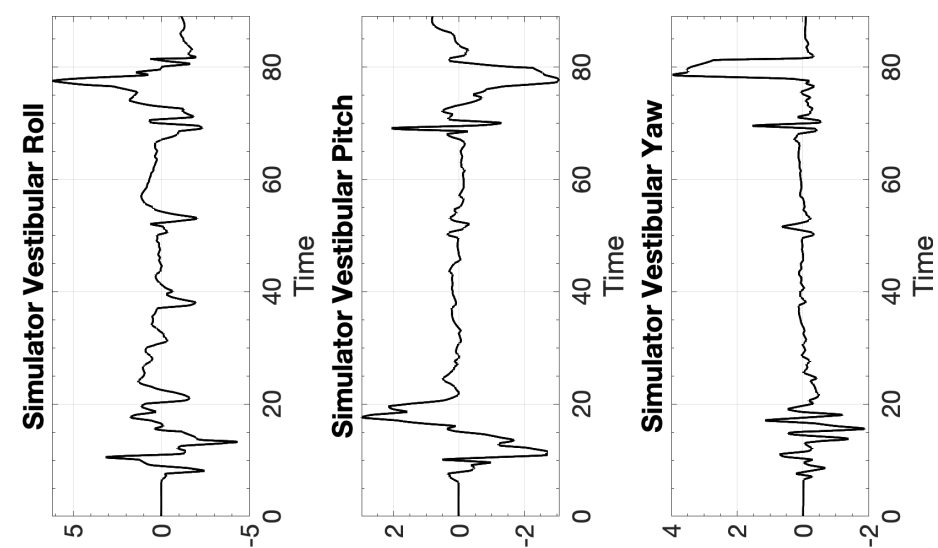

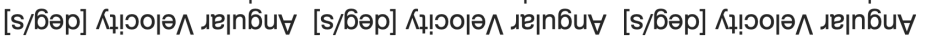

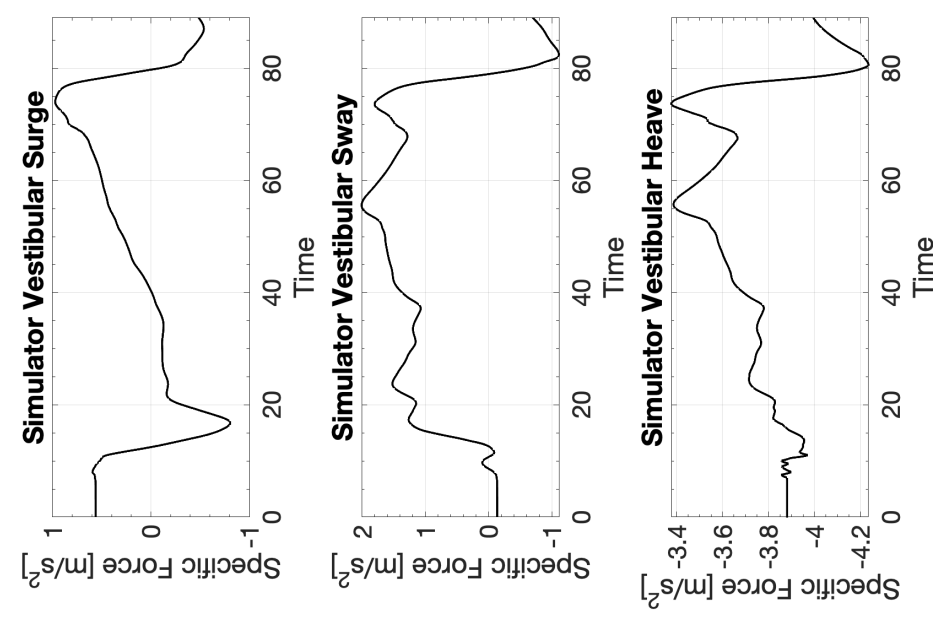




\section{D.13 Columbia 400 Cross-wind Landing}

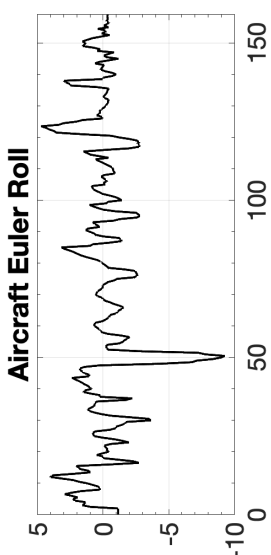

[6əp] uo!!!sod גe|n6u

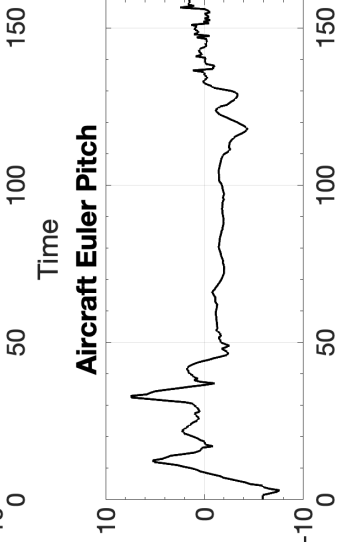

[6әр] uo!!!!sod גe|n6u

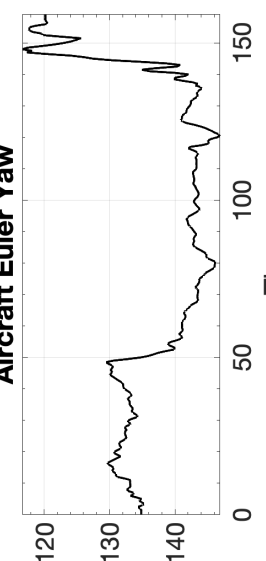

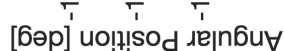
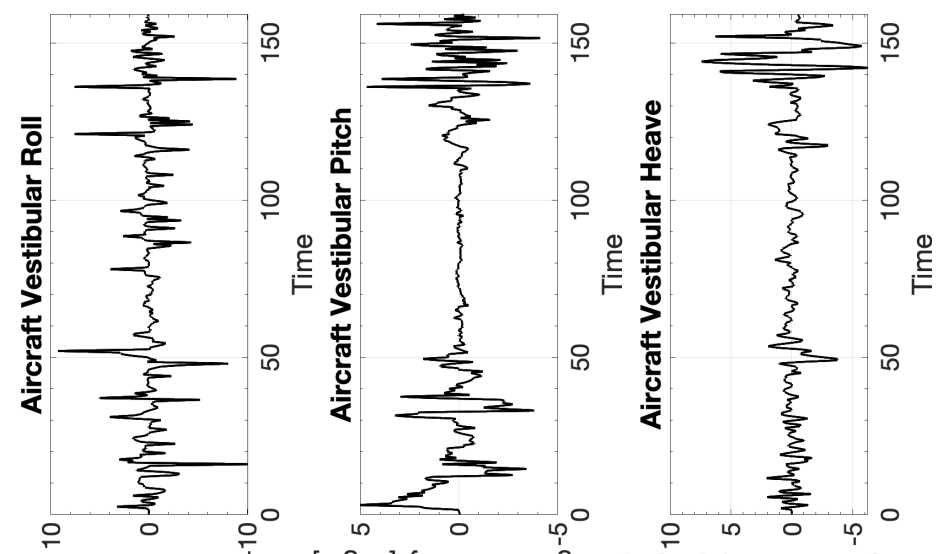

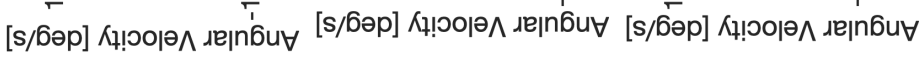

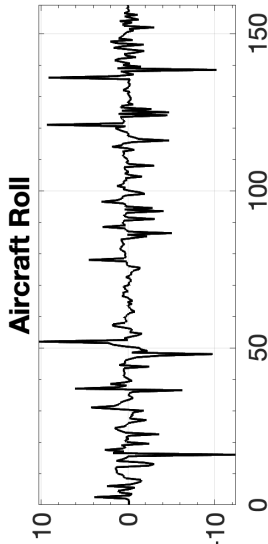

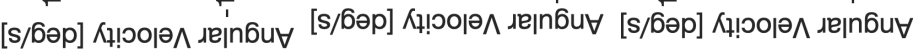
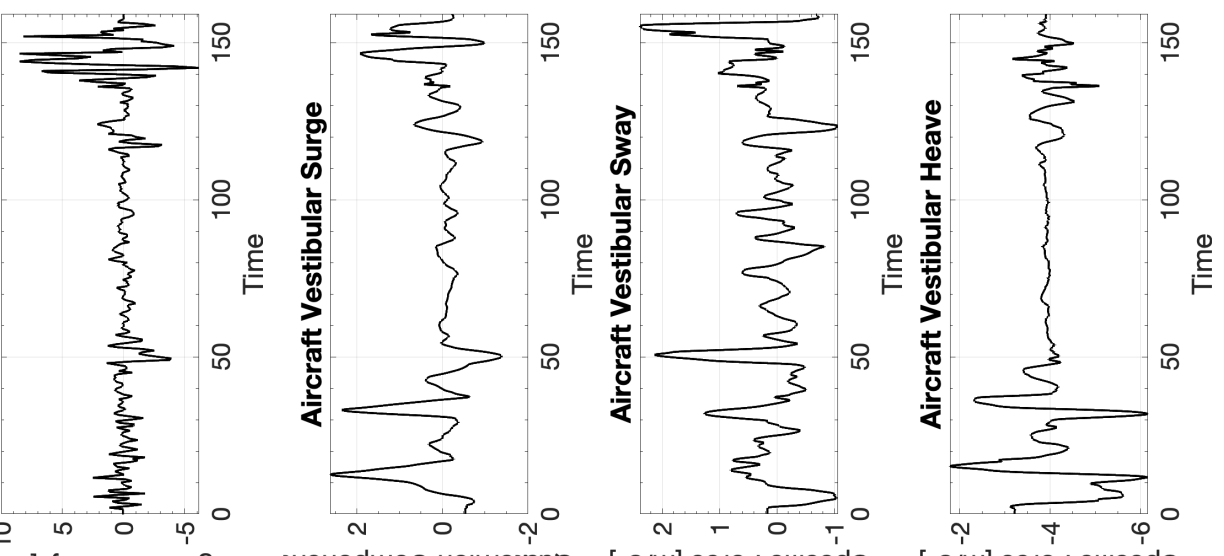

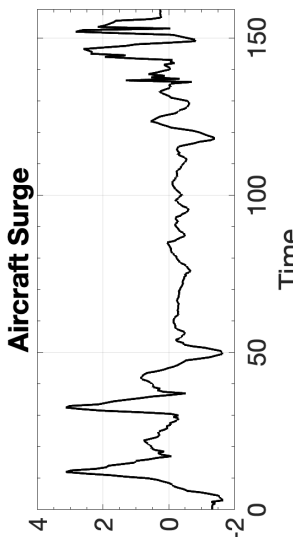

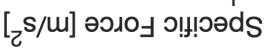

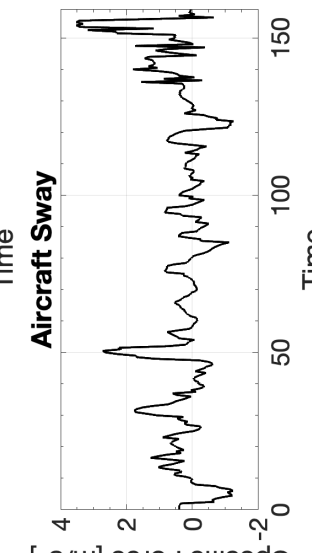

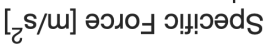

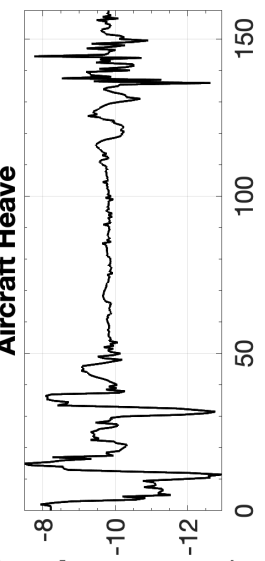

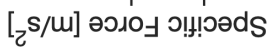

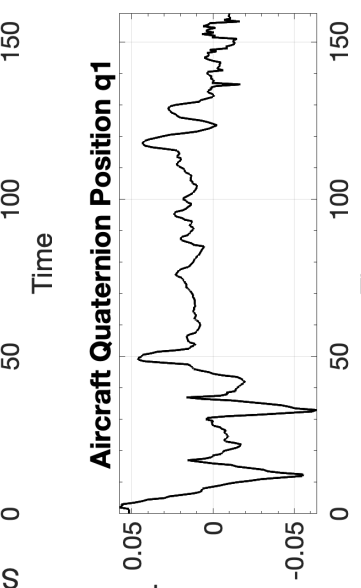

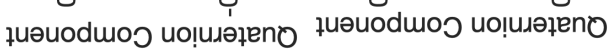

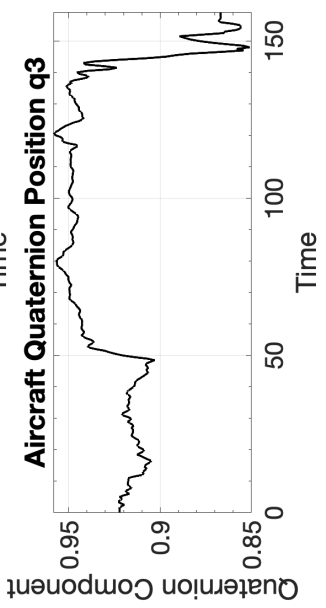

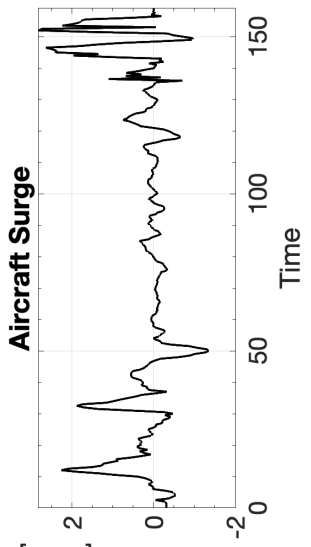

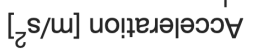

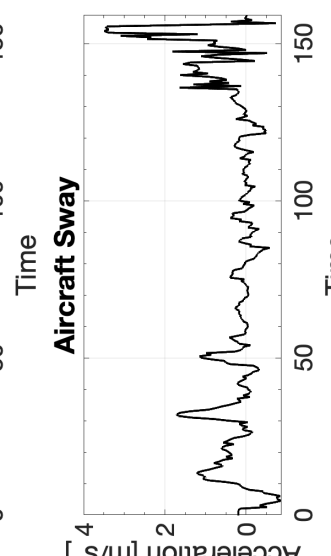

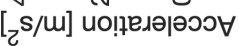

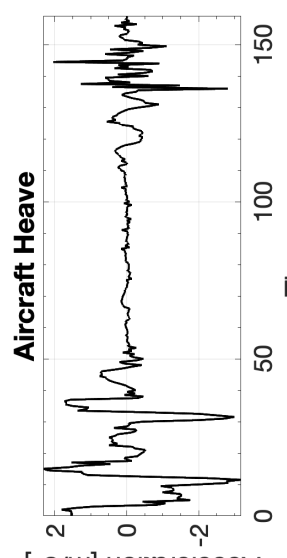

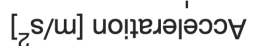

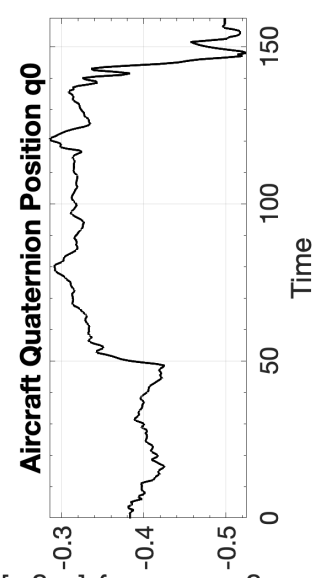

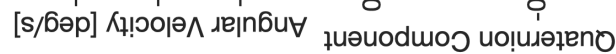




\section{D.13.1 CW1 Columbia 400 Cross-wind Landing}
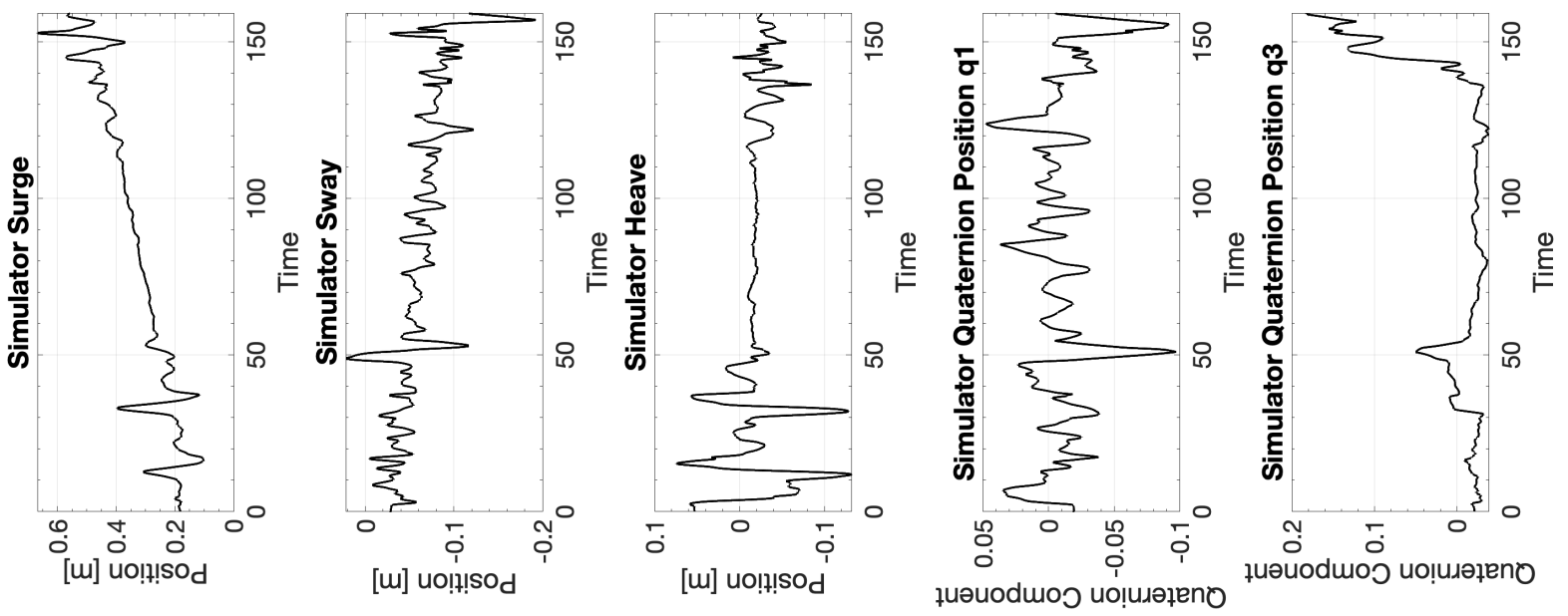

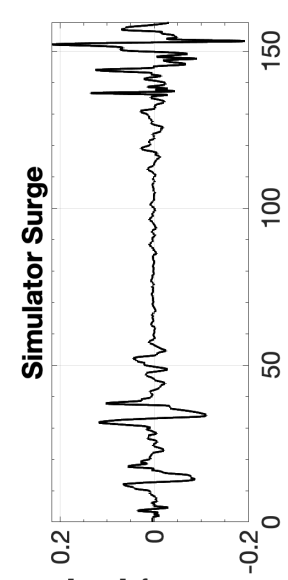

[s/u] К! ৩о夫 ^

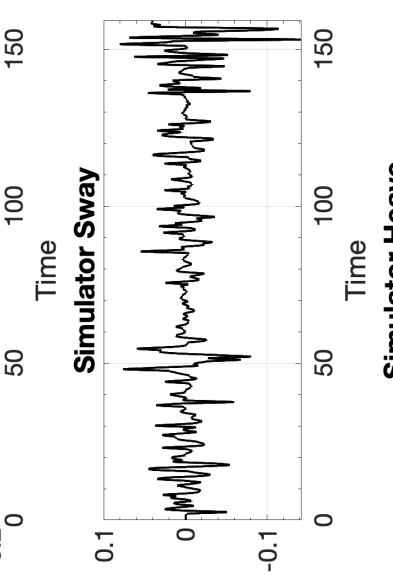

[s/u] Кұ!ю૦Ю^

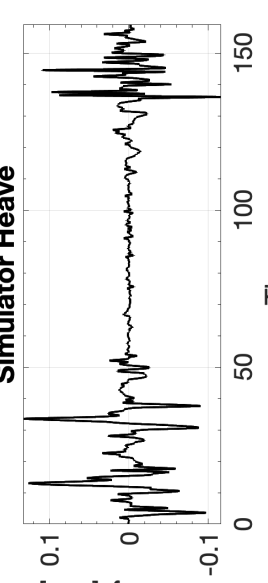

[s/u] Кұ!৩ојә^

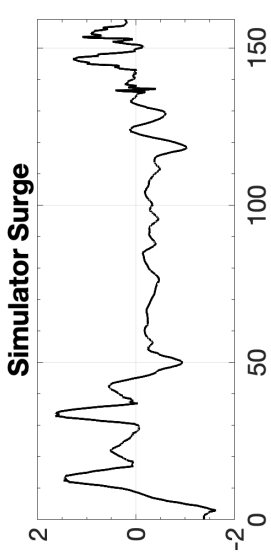

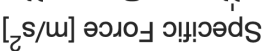

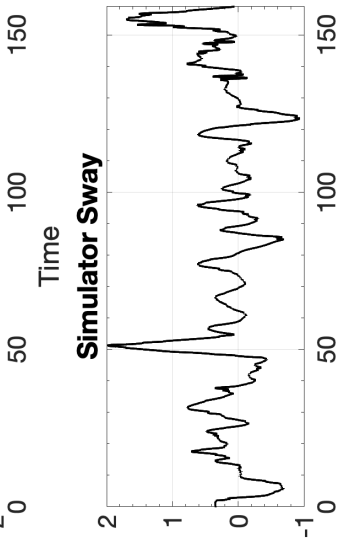

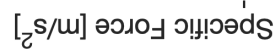

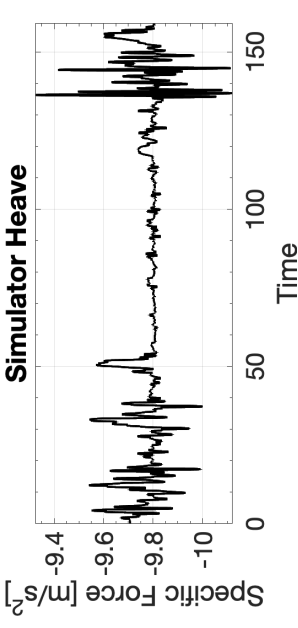

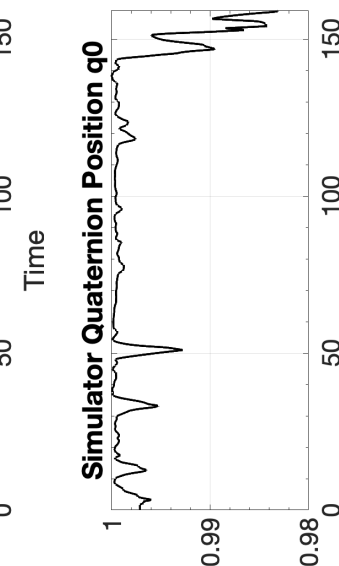

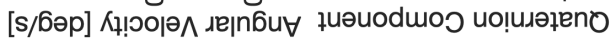

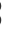
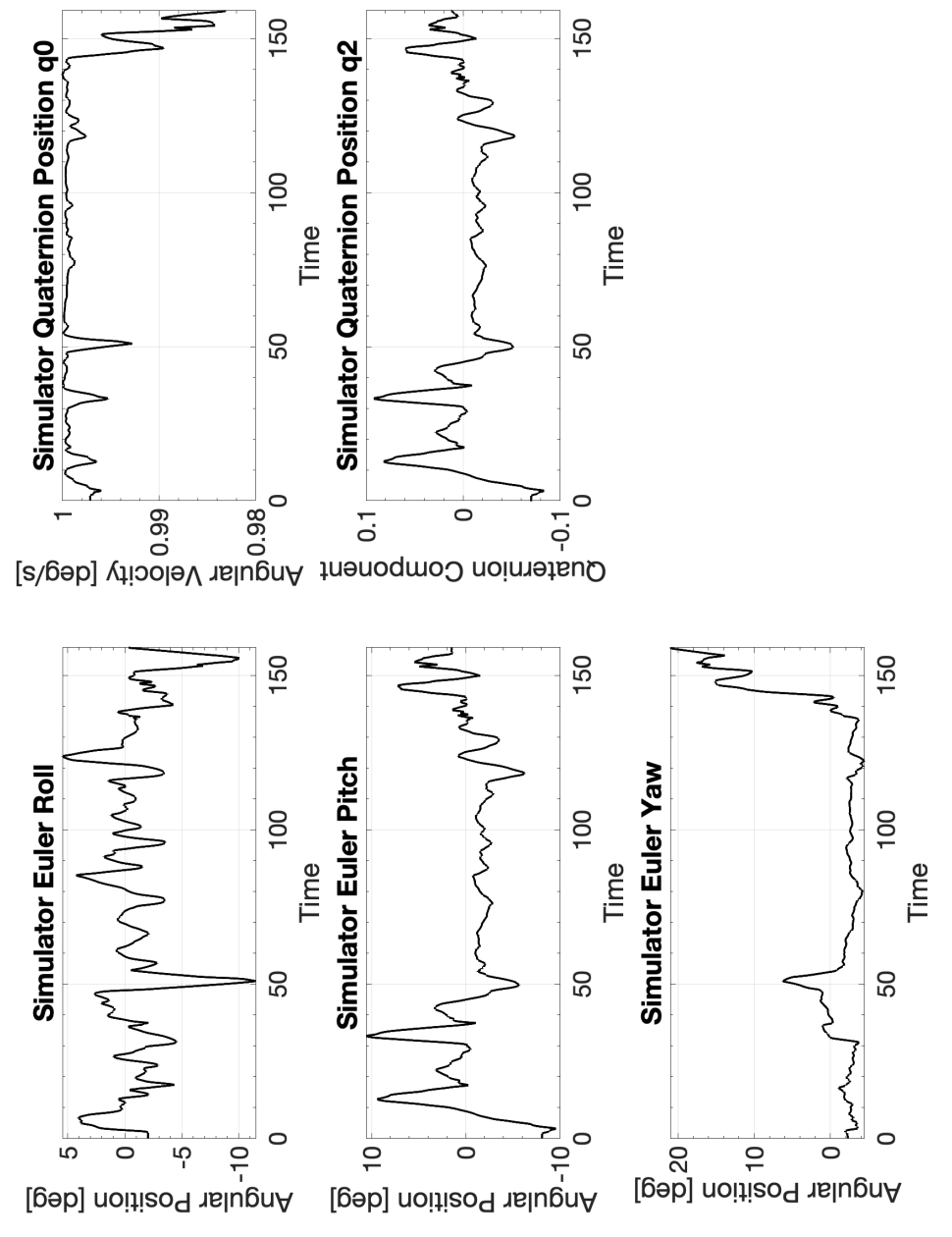

$\stackrel{\oplus}{\underline{E}}$

[6әр] uo!!!sod ıejn6u

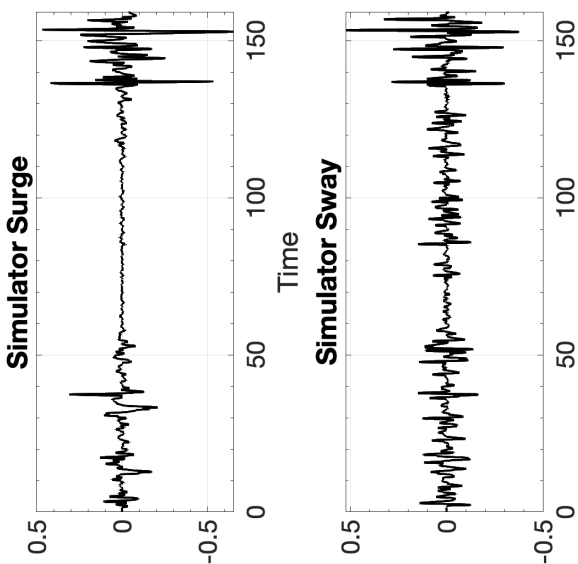

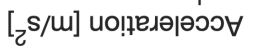

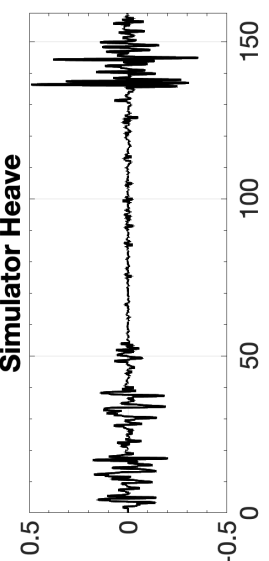

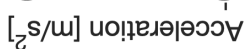

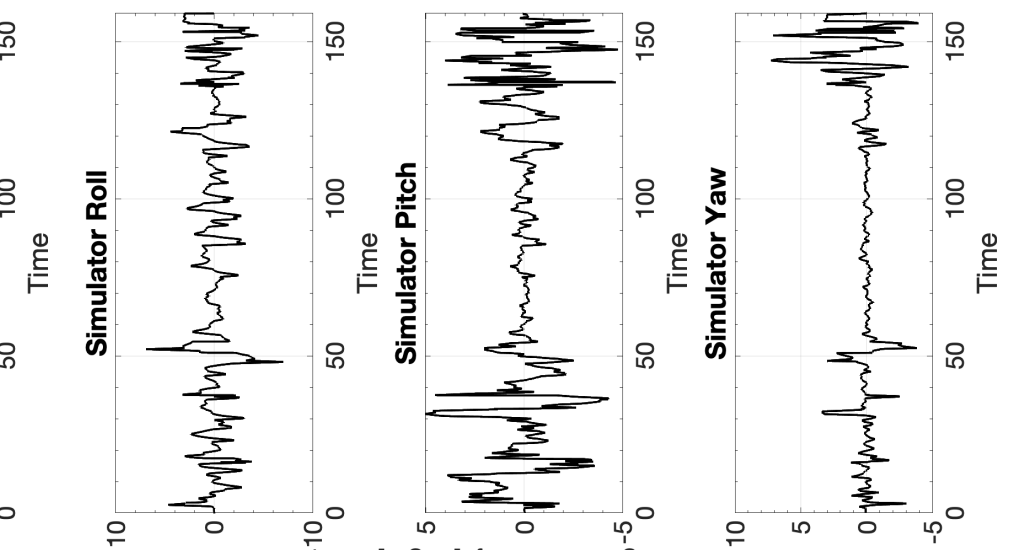

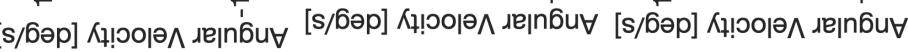



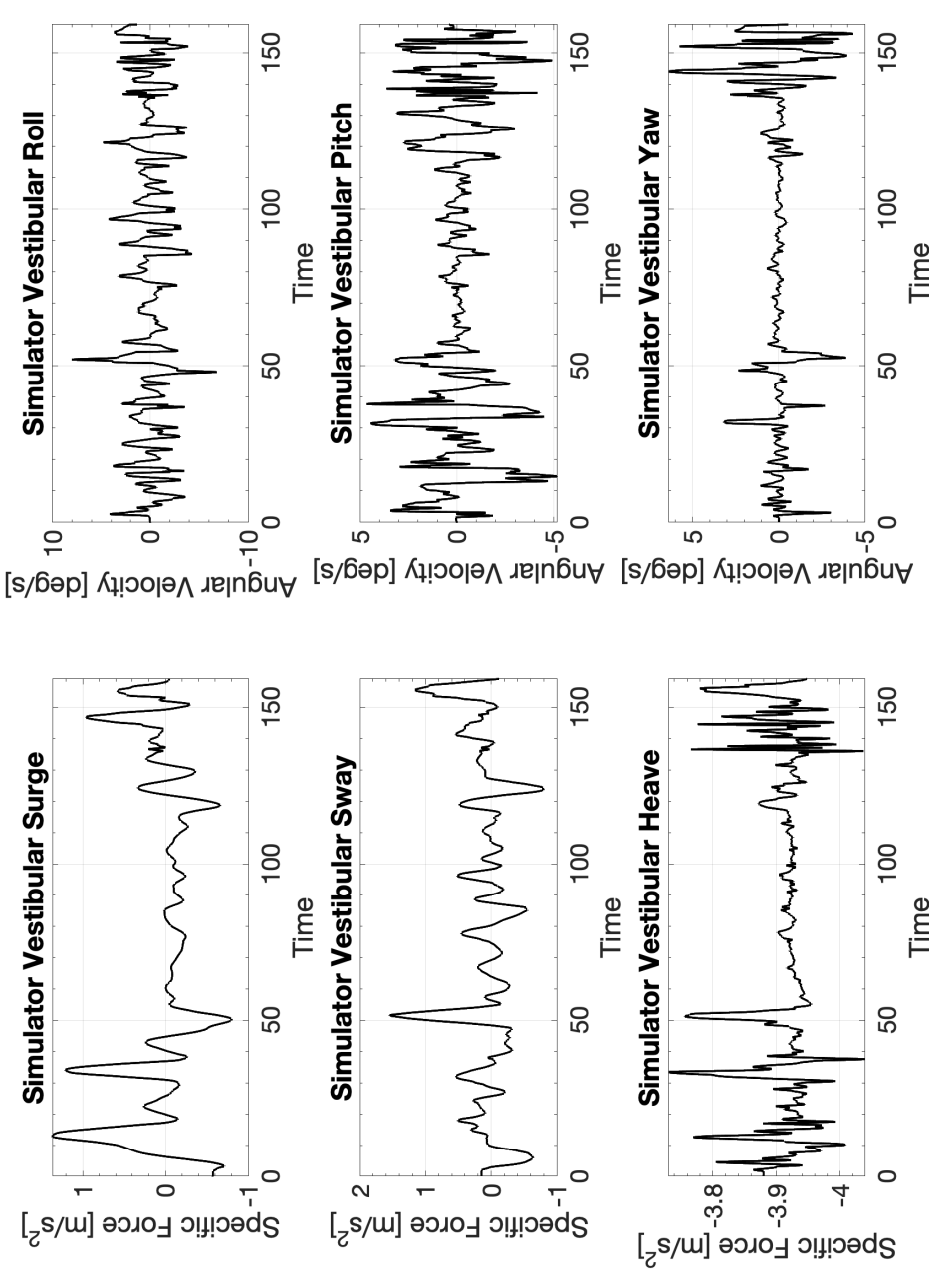


\section{D.13.2 CW2 Columbia 400 Cross-wind Landing}
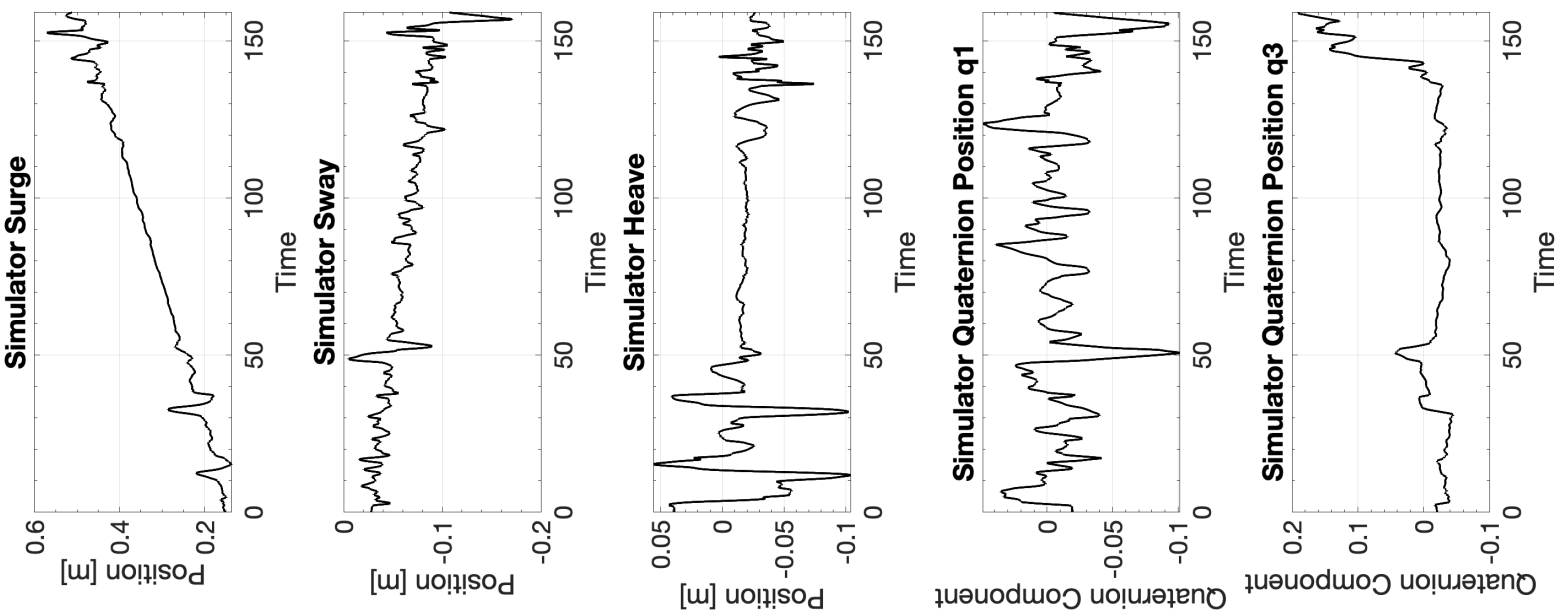

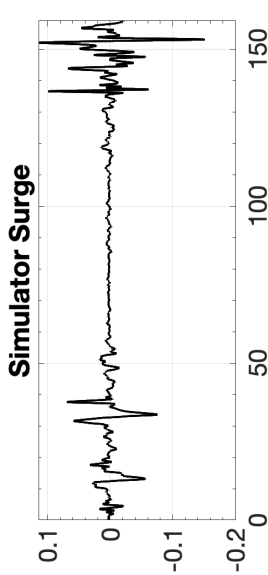

[s/m] К१!৩о|९

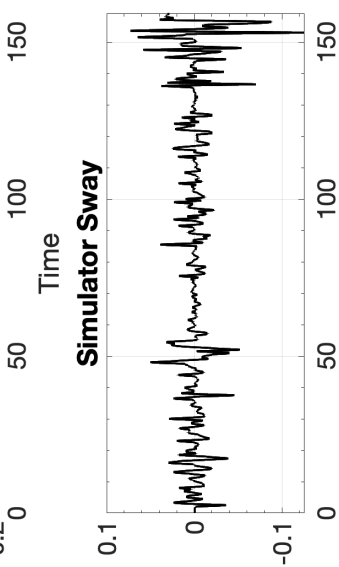

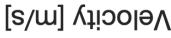

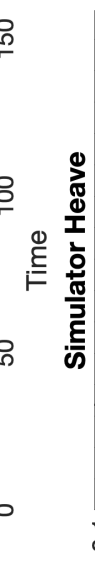

[s/u] Кұ! Рорә

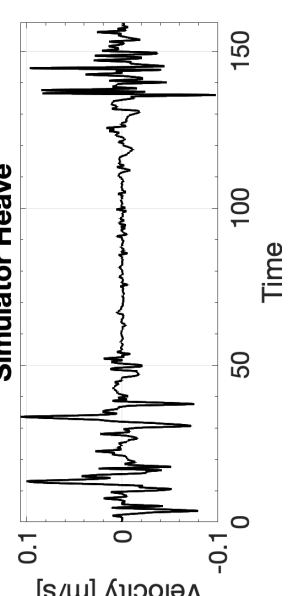

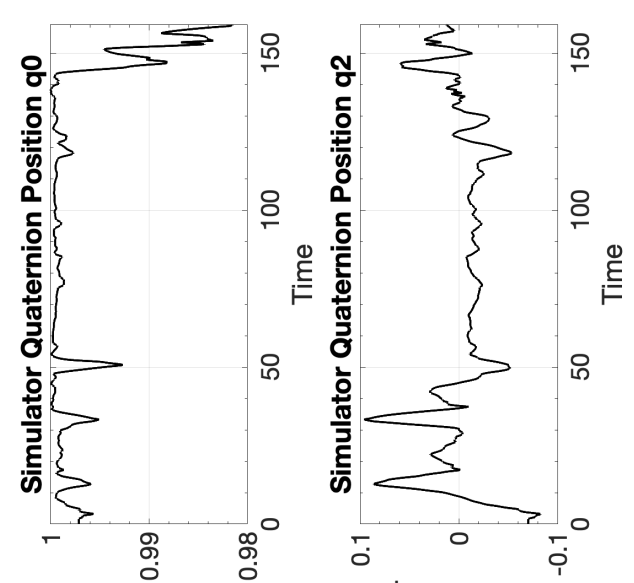

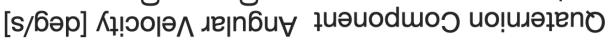

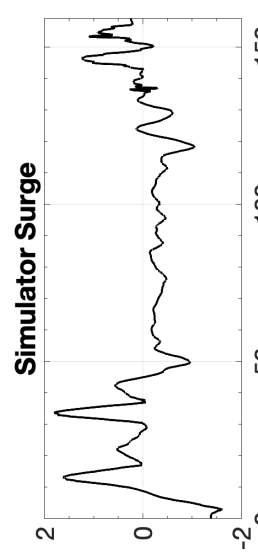

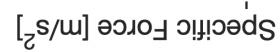

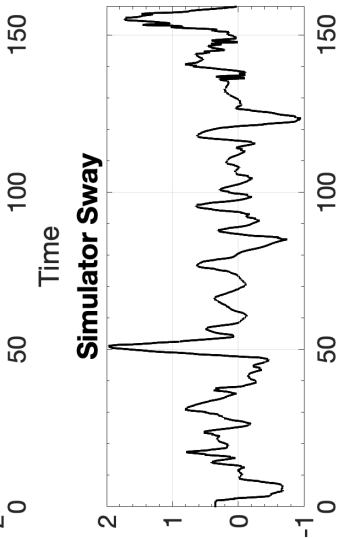

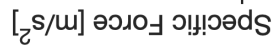

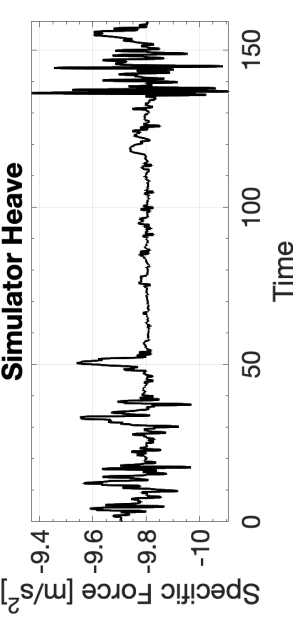

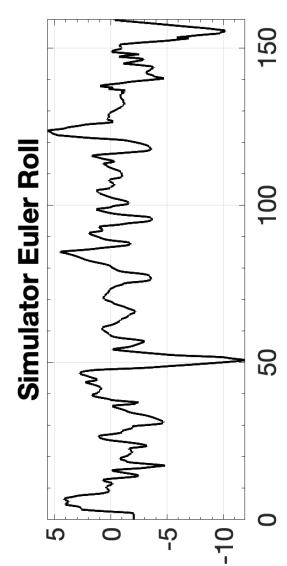

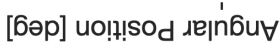

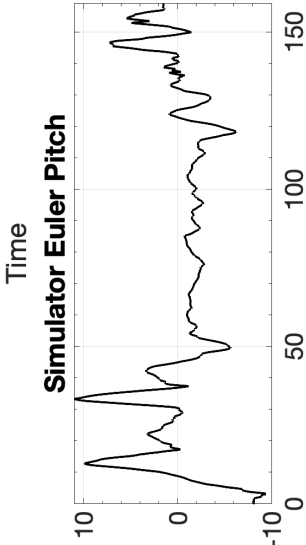

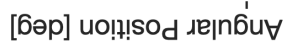

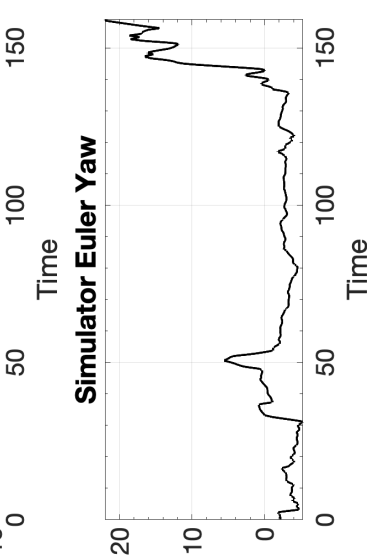

[бәр] uo!!!sod ıejn6u

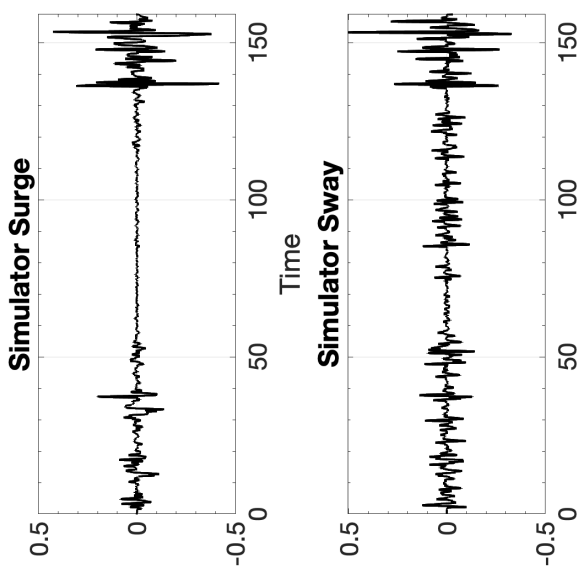

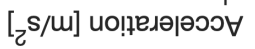

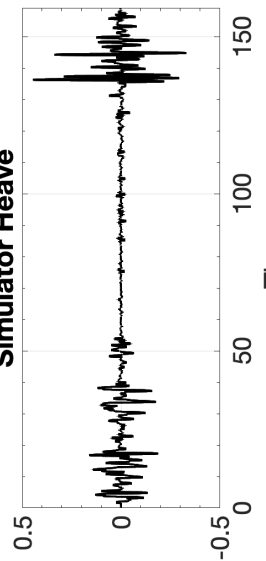

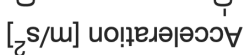

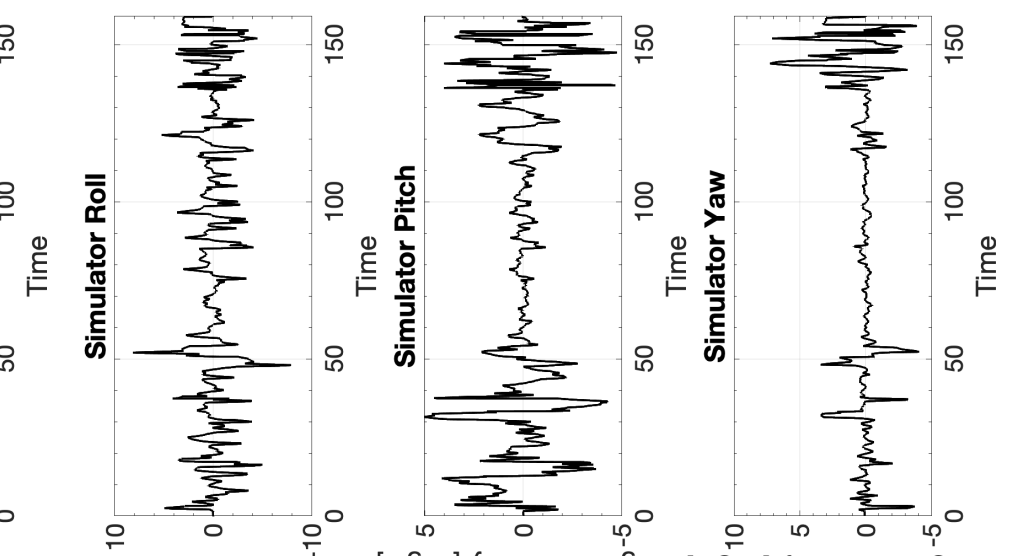

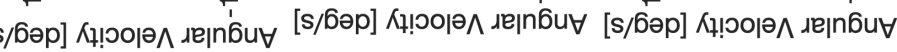




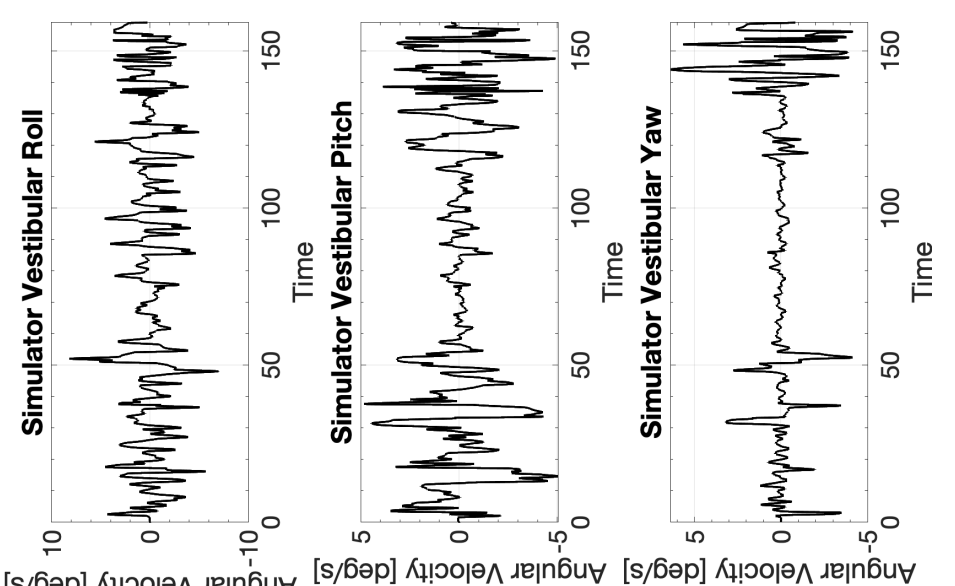

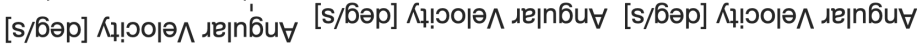

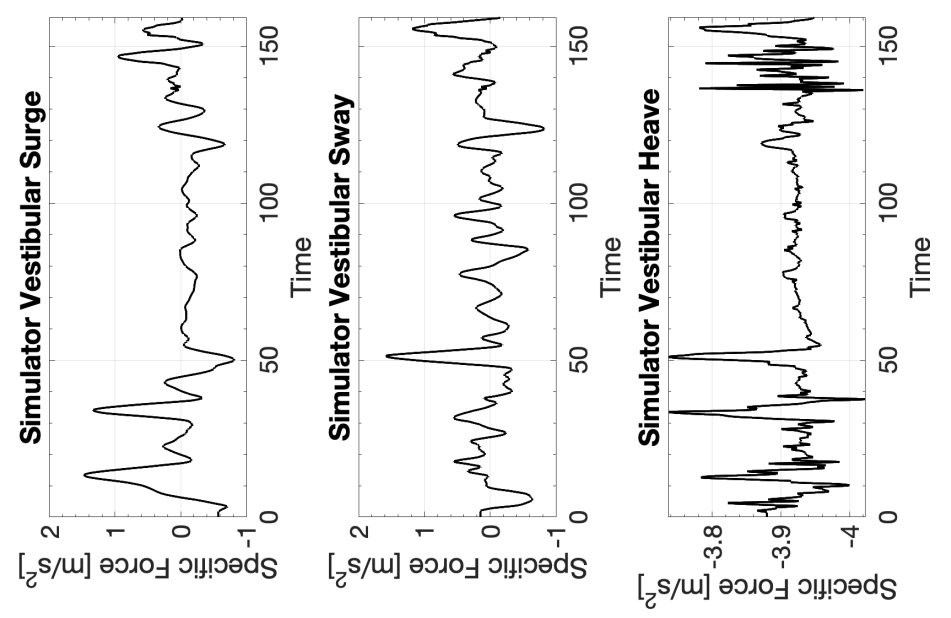




\section{D.13.3 CW3 Columbia 400 Cross-wind Landing}
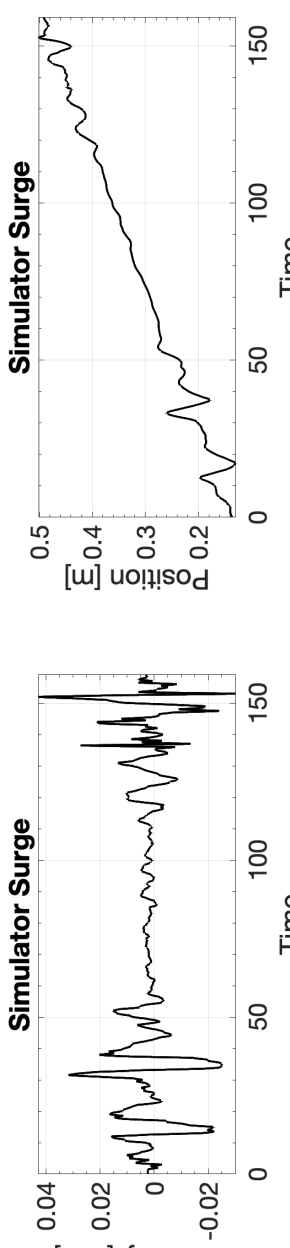

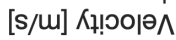

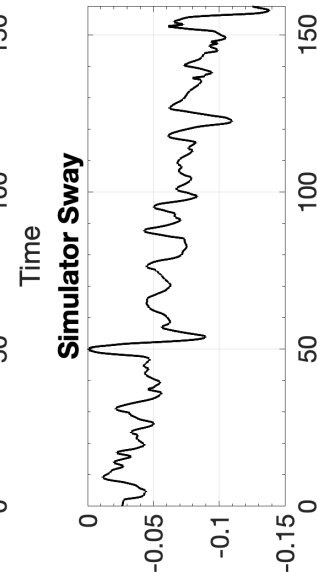

[u] uol!?!sod

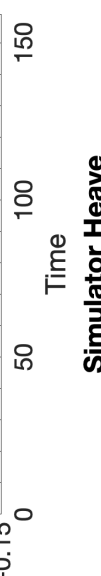

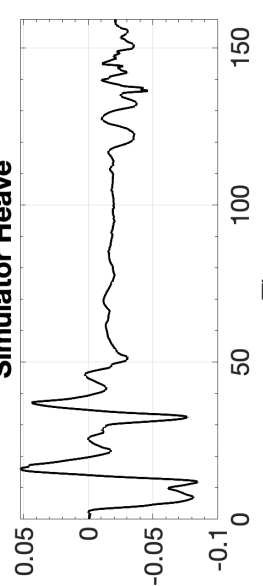

[w] uol!!sod

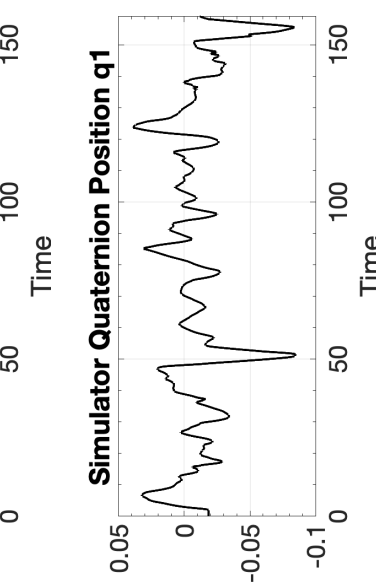

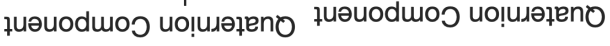

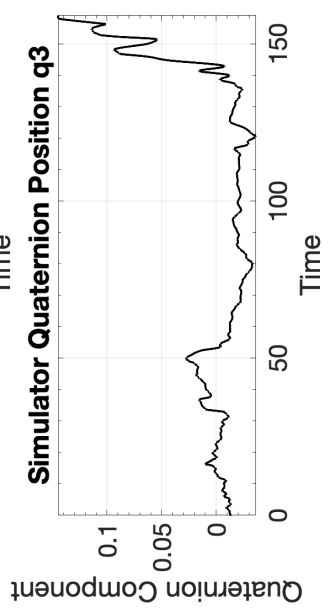

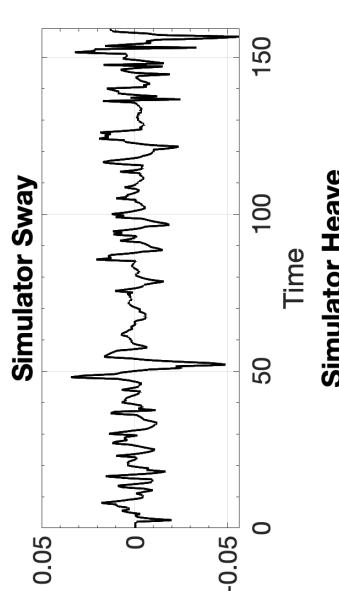

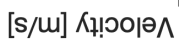
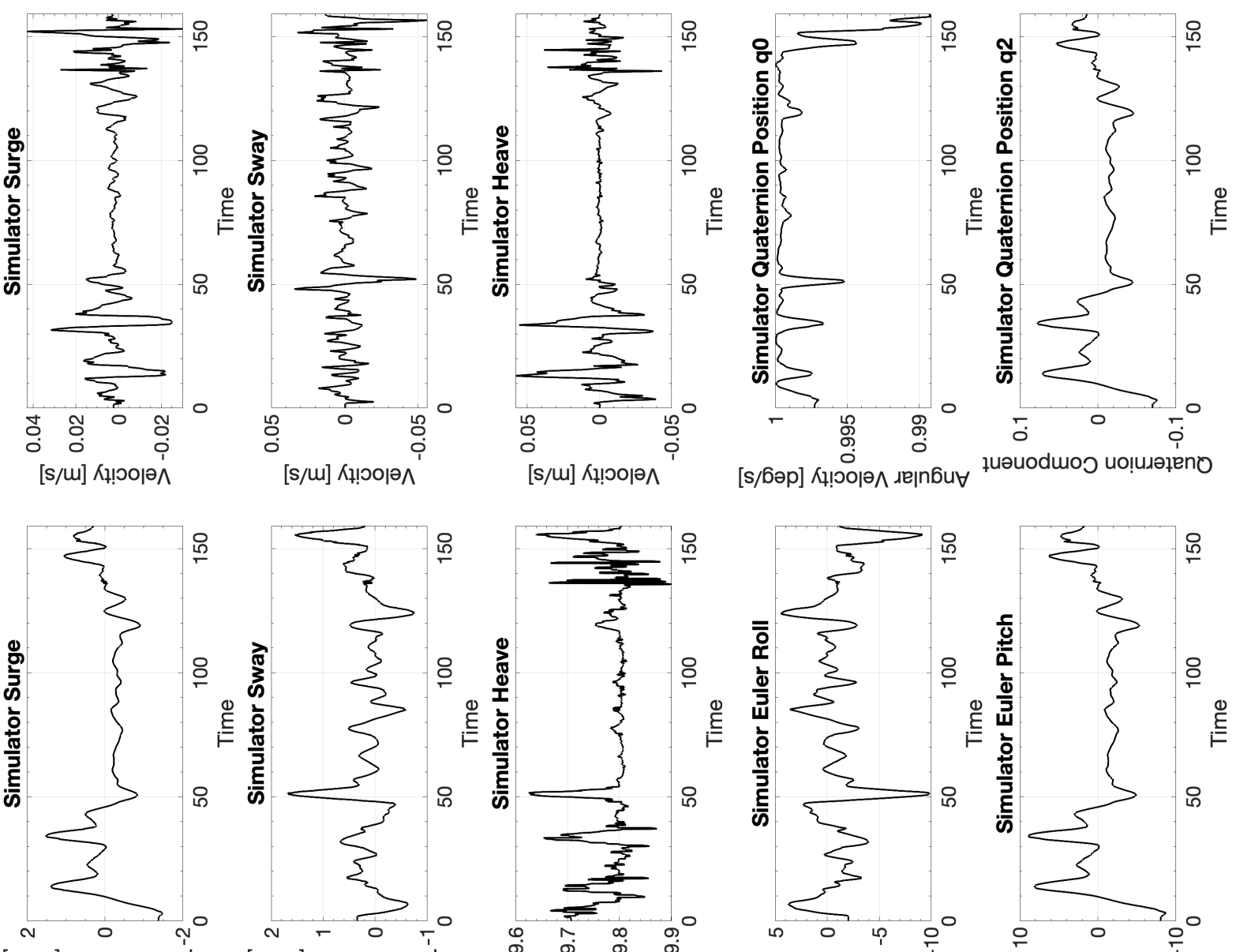

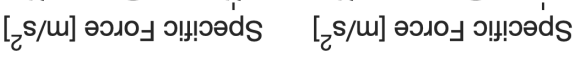
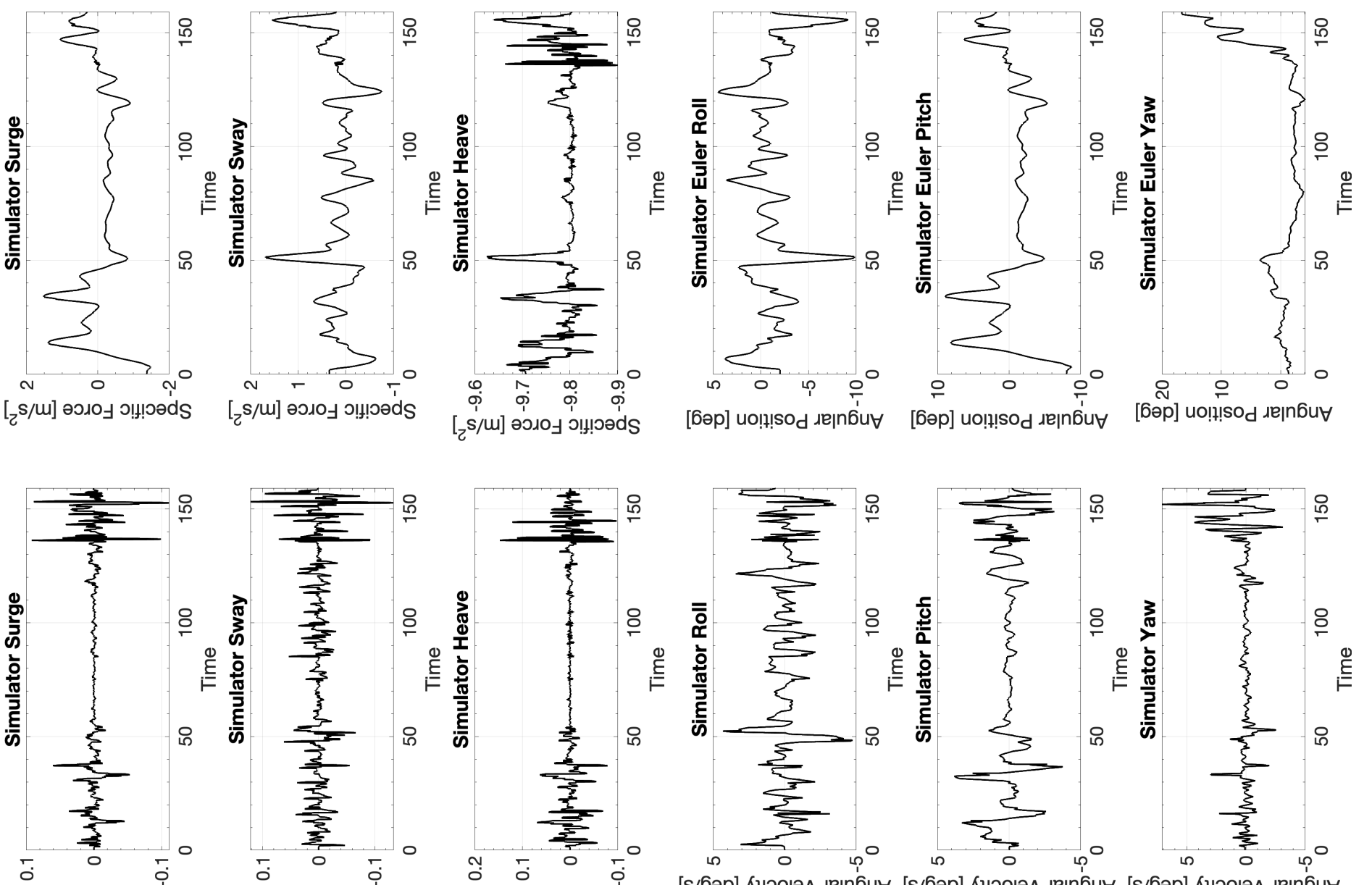

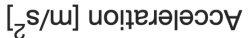

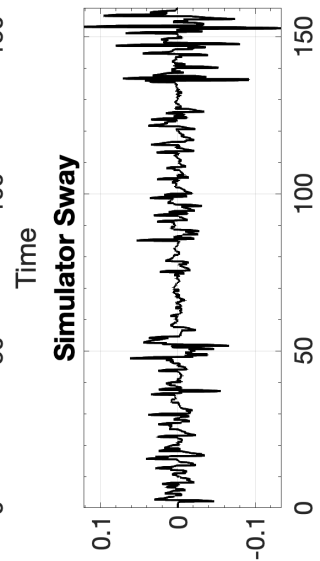

[s/m] ио!̣едә|әэว
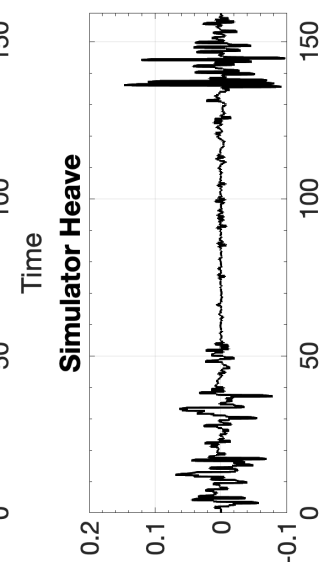

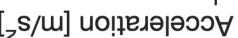

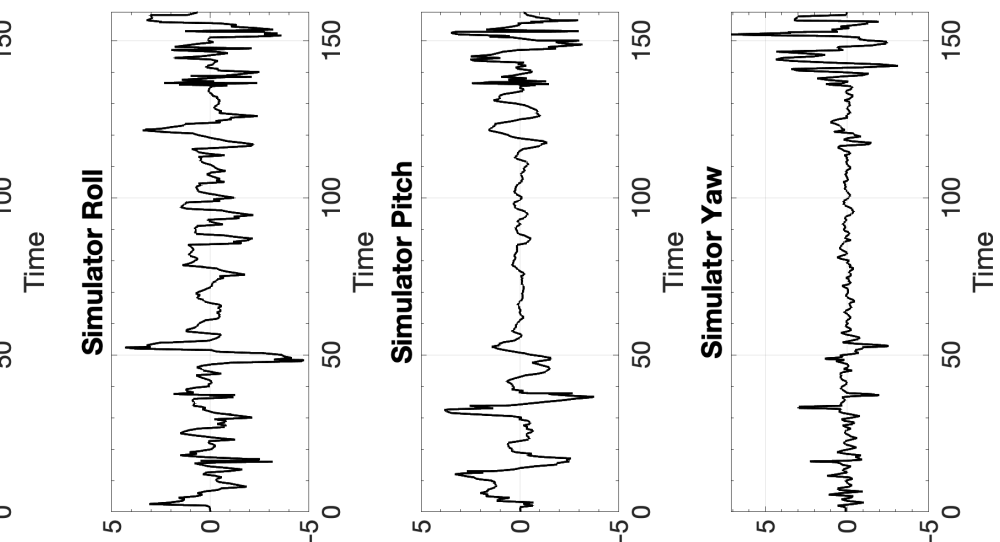

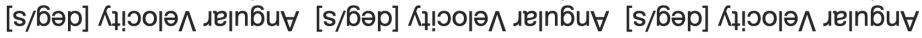



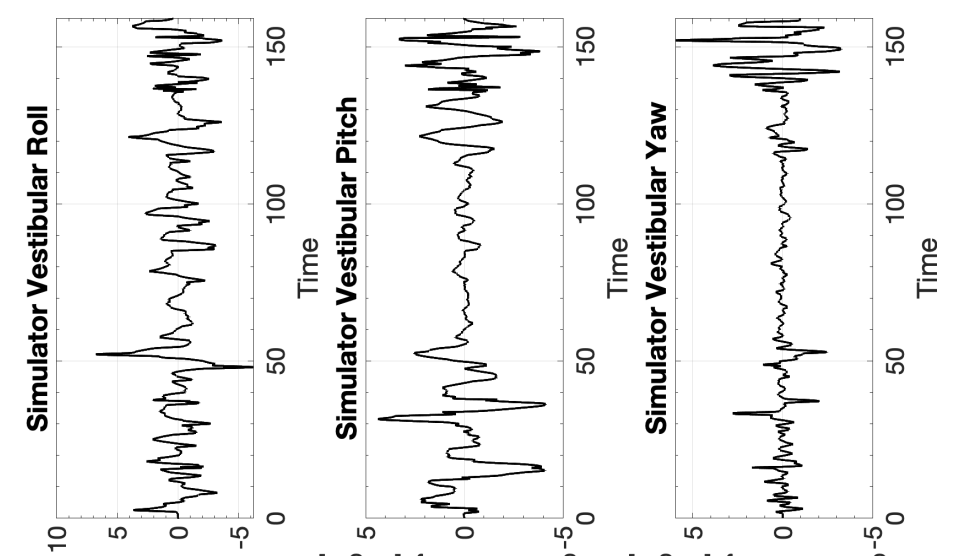

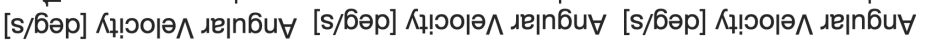
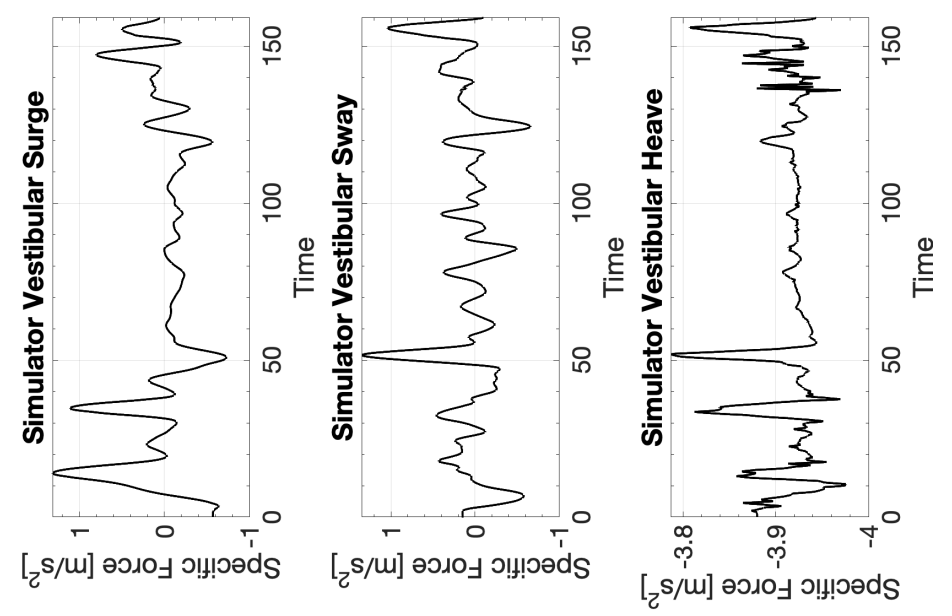

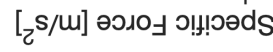




\section{D.14 Columbia 400 Single-axis Takeoff}

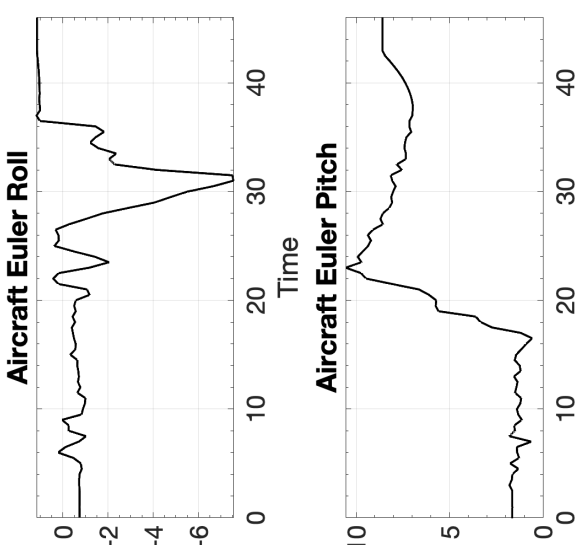

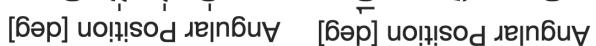

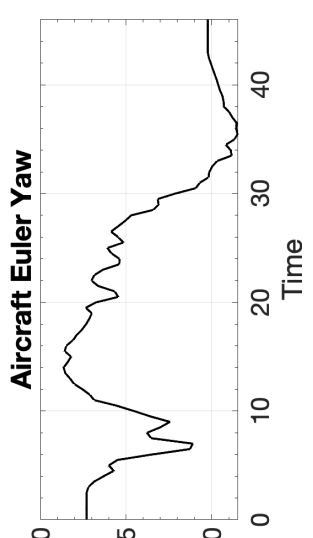

[ั)

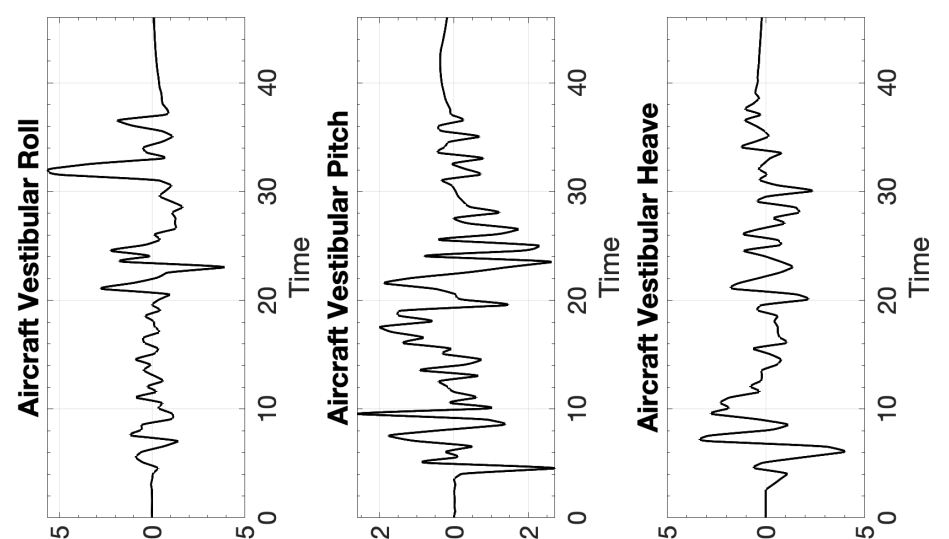

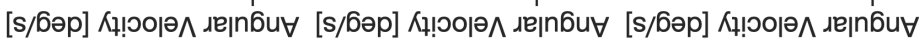

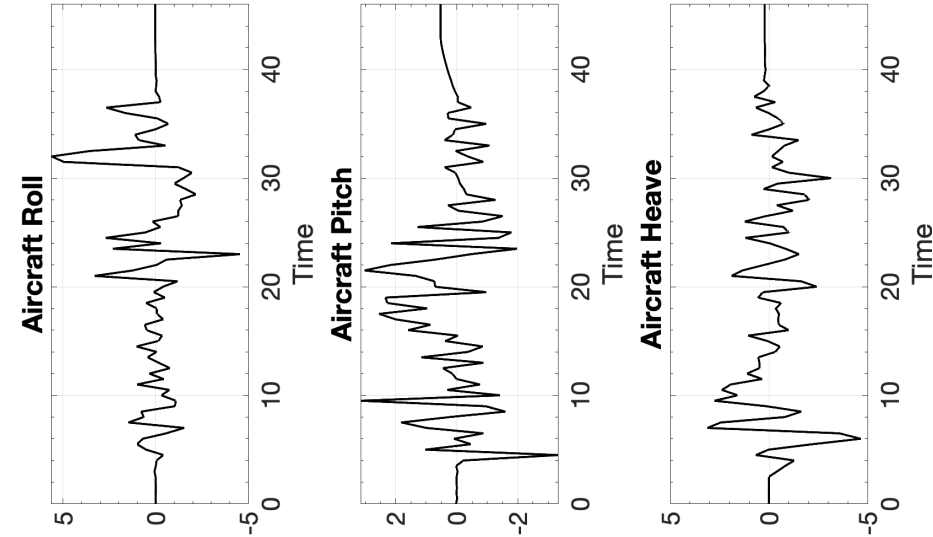

[s/6әр] Кұ!юорл

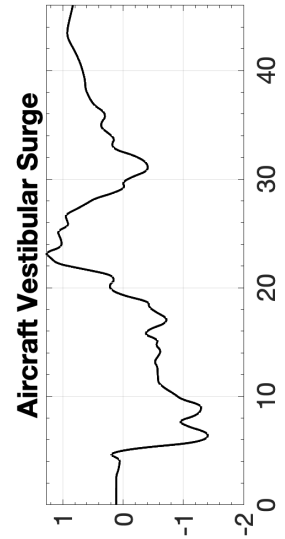

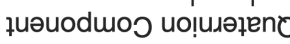

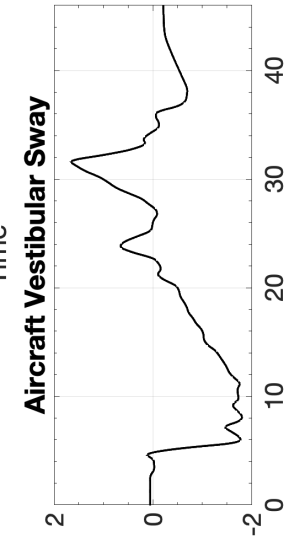

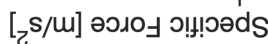

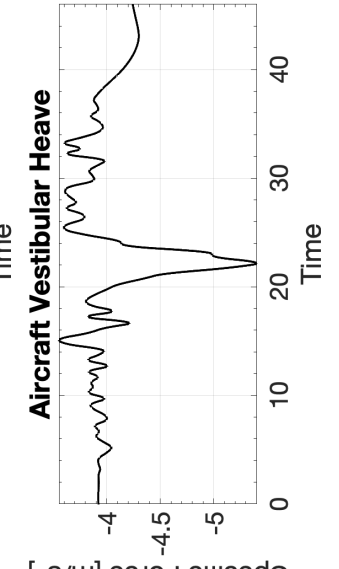

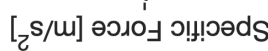

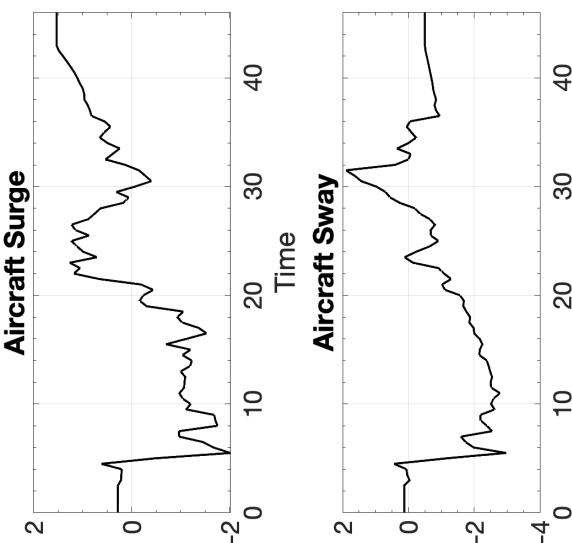

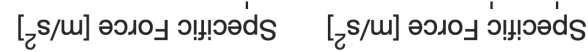

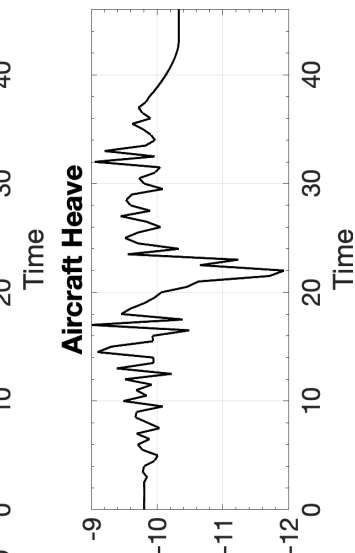

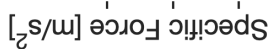

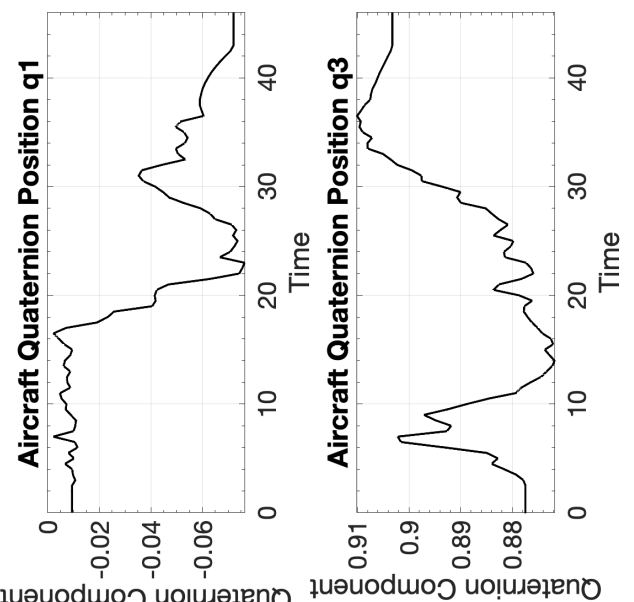

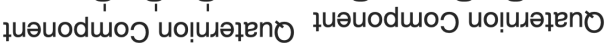

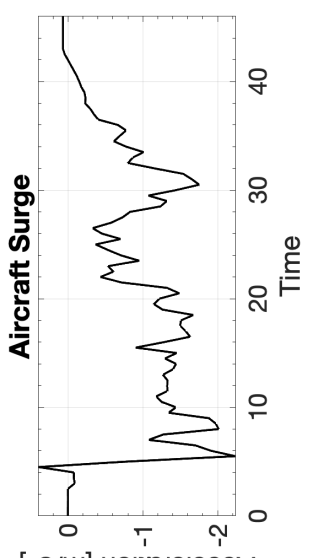

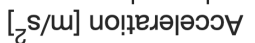

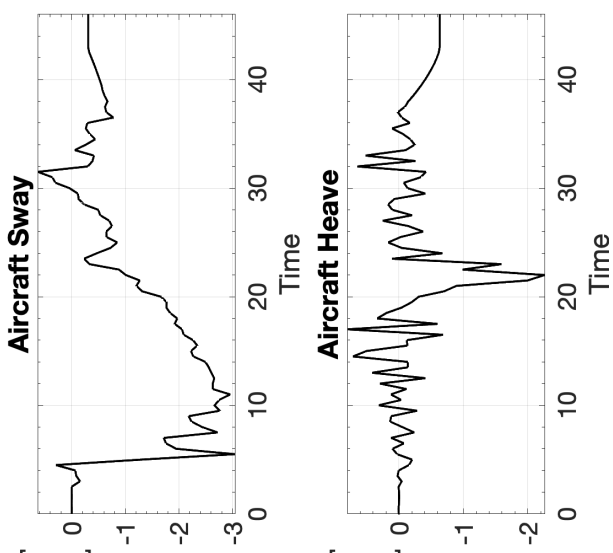

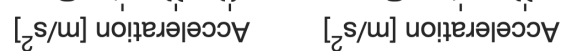

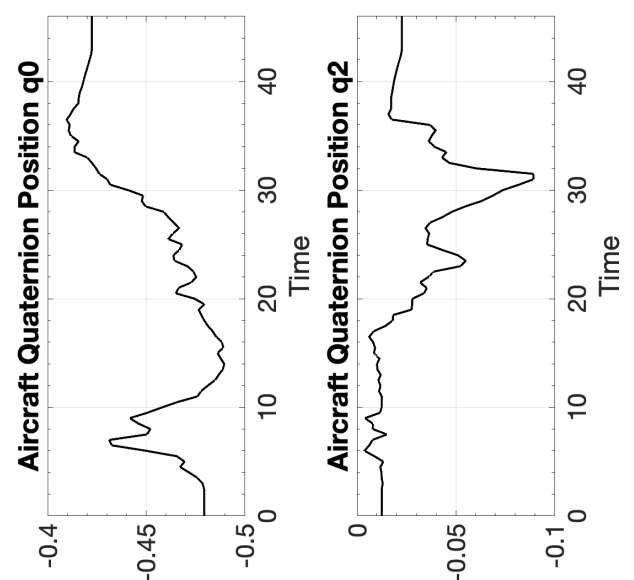

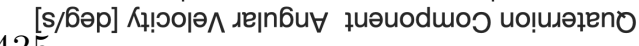




\section{D.14.1 CW1 Columbia 400 Single-axis Takeoff}

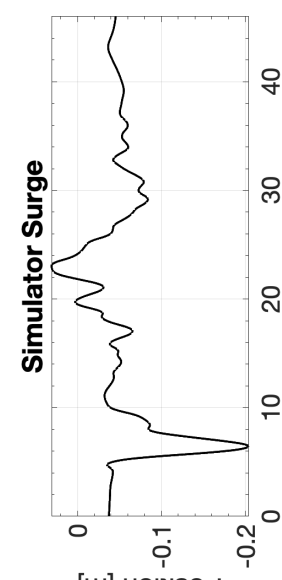

[u] uol!!!sod

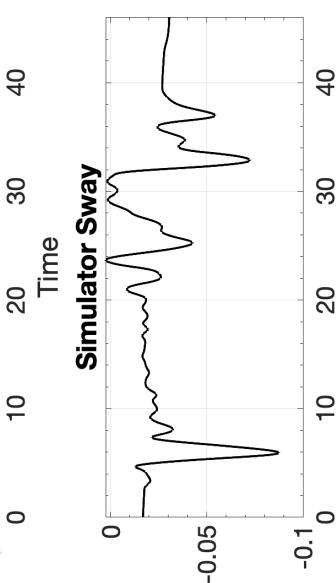

[u] uo!n!!sod

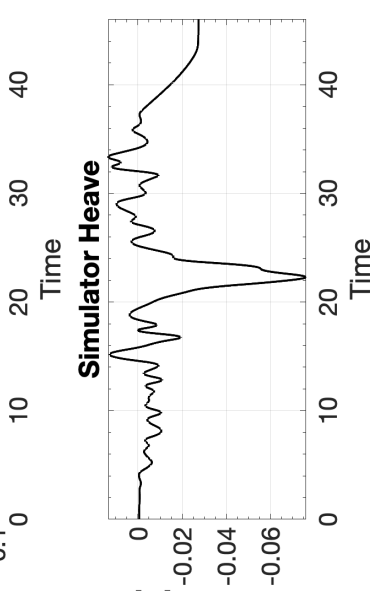

[w] uolt!nod

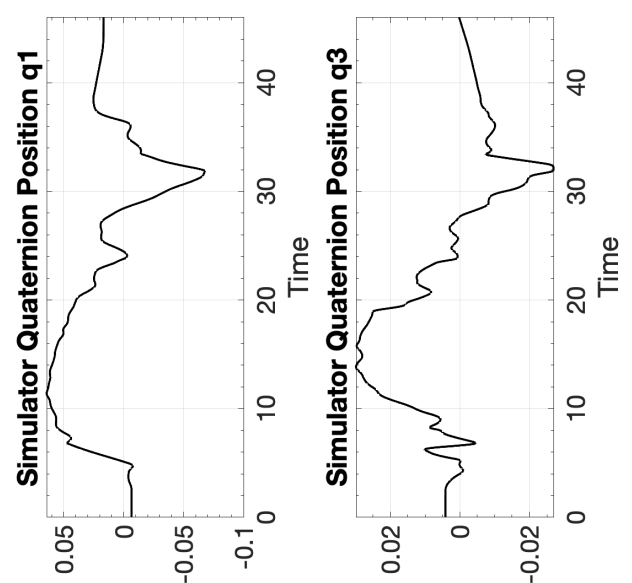

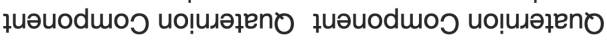

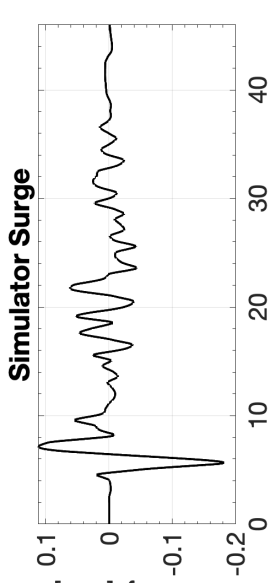

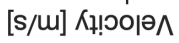

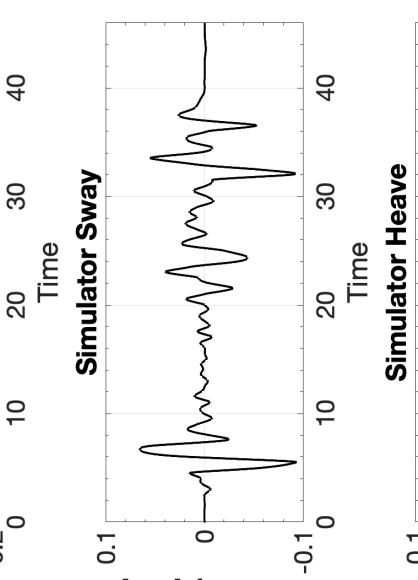

[s/u] Кұ!ю이

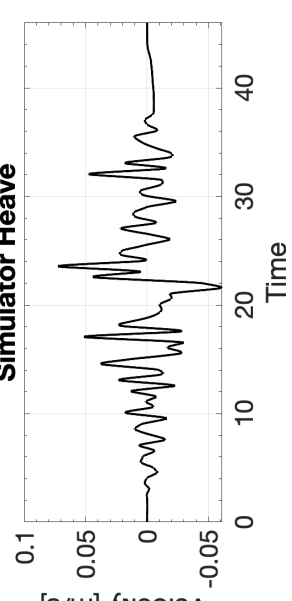

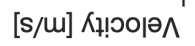

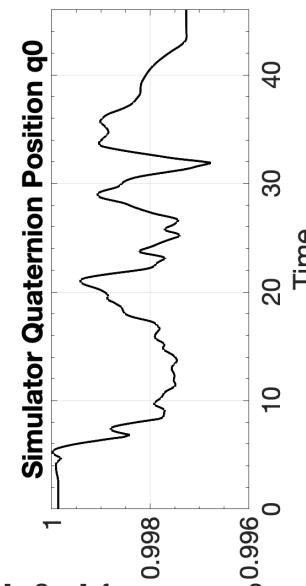

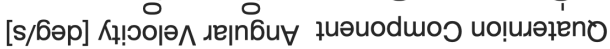

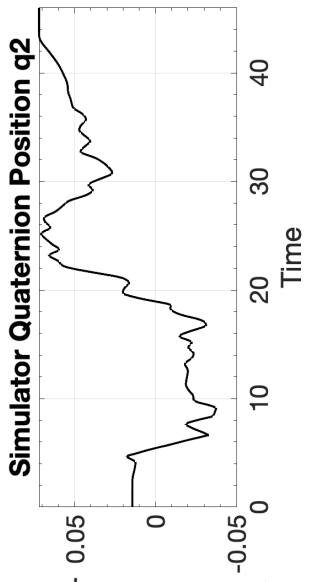

$\stackrel{\oplus}{\underline{F}}$

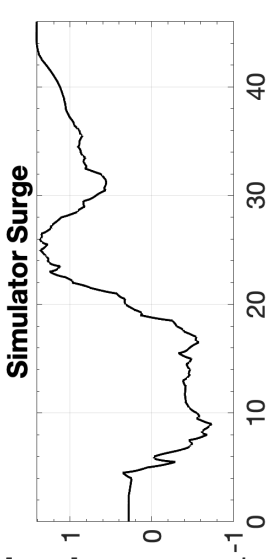

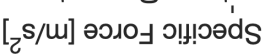
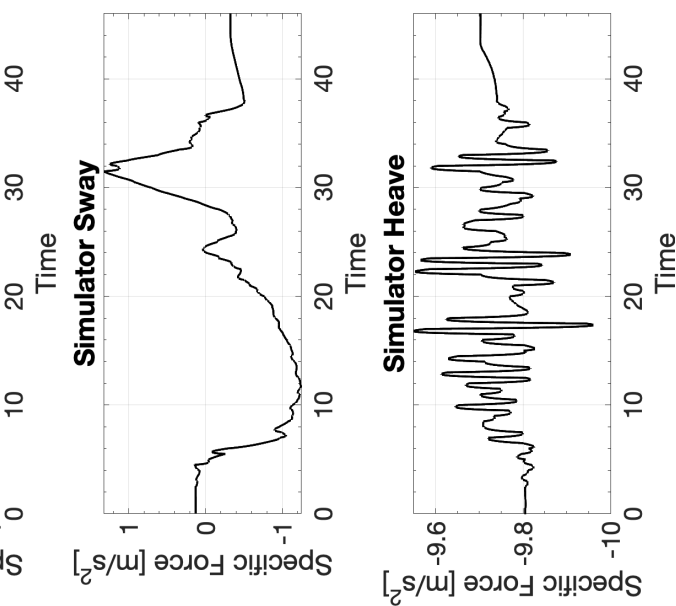

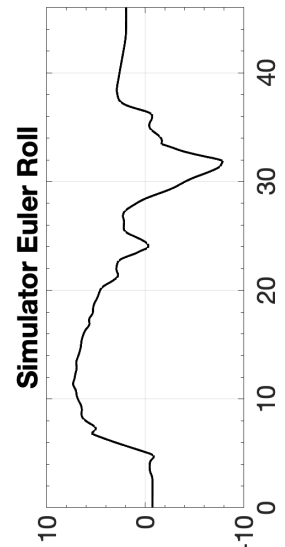

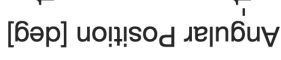

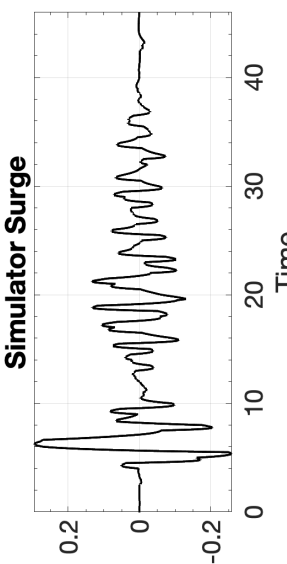

$[\mathrm{c} / \mathrm{L}] \mathrm{uo!̣}$

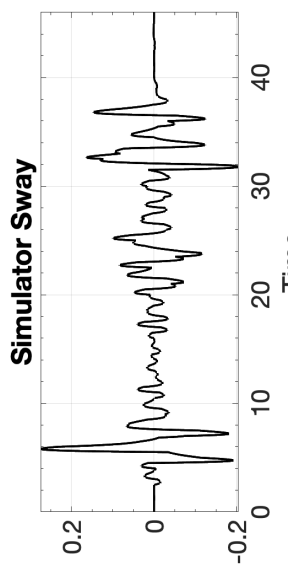

$[\mathrm{s} / \mathrm{m}]$ uо!̣еגә|әэว

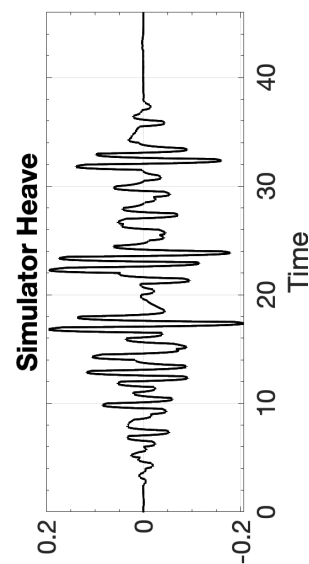

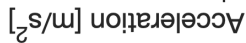

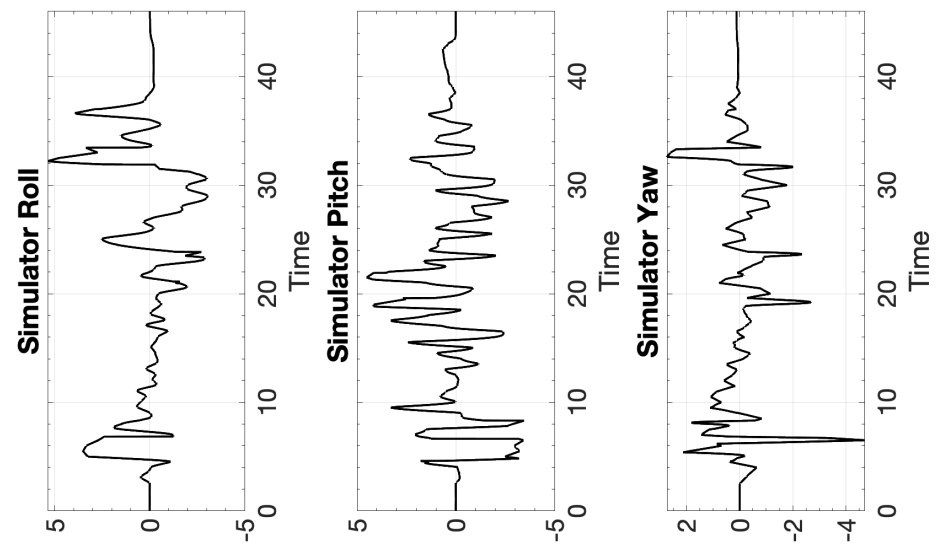

[s/6әр] Кұ!оорә גе|nби 


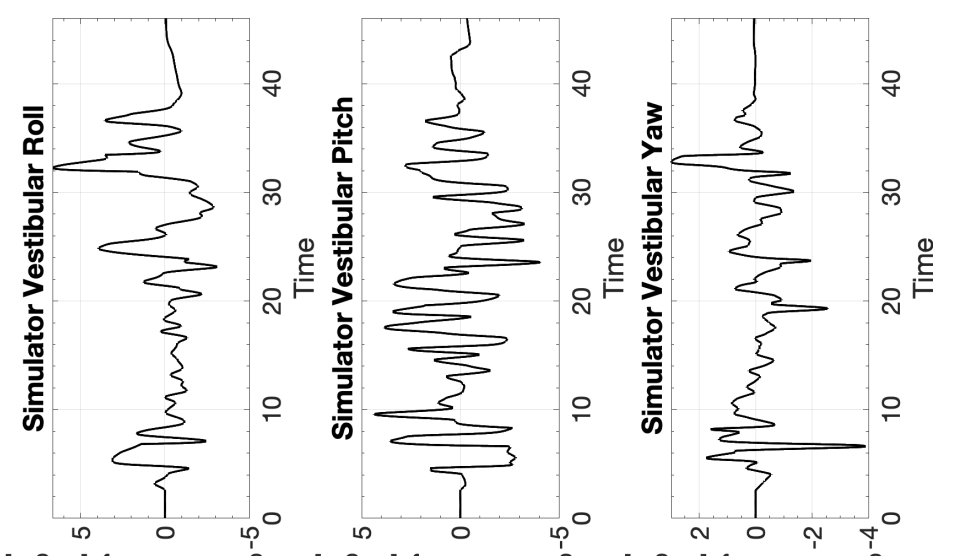

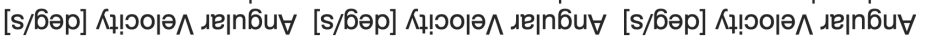

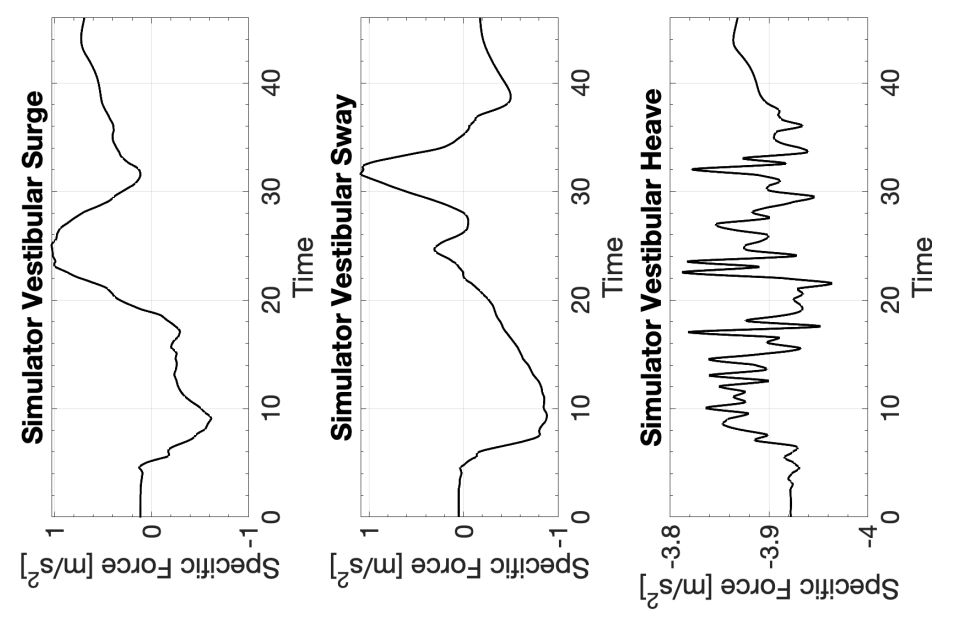




\section{D.14.2 CW2 Columbia 400 Single-axis Takeoff}

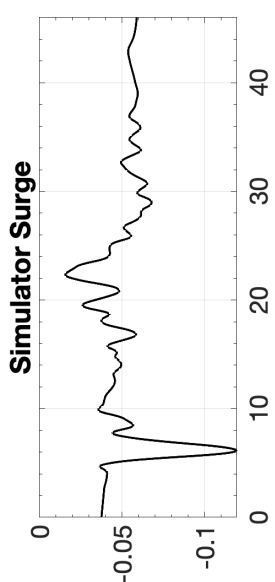

$[\mathrm{m}]$ uo!n!lsod

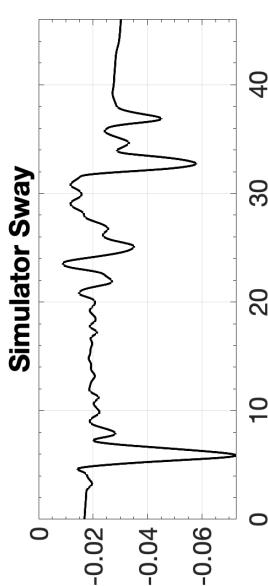

[w] uol!?!sod

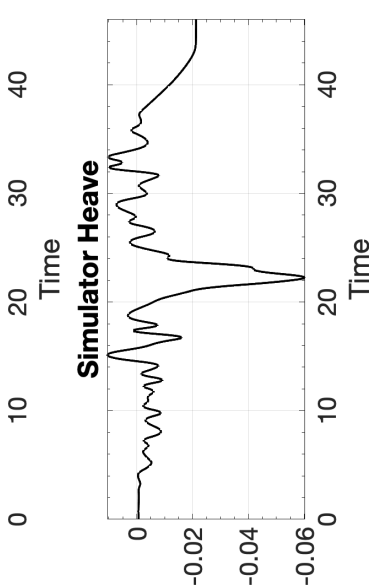

[w] uo!n!ssod
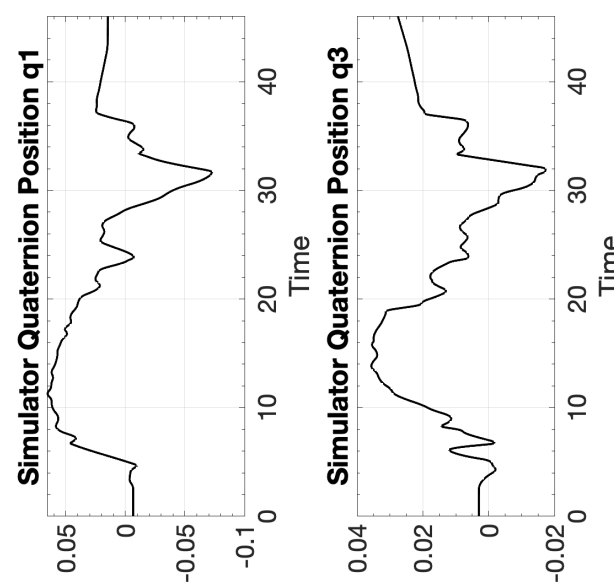

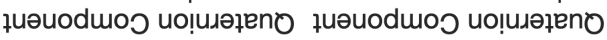

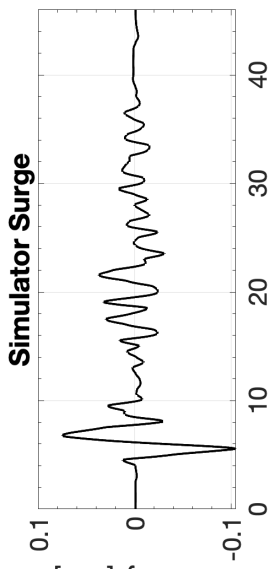

$[\mathrm{s} / \mathrm{m}]$ ᄉ!

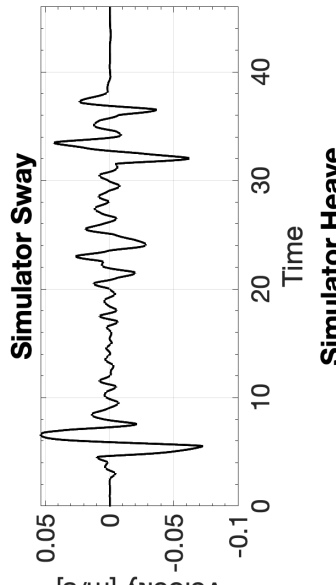

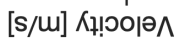

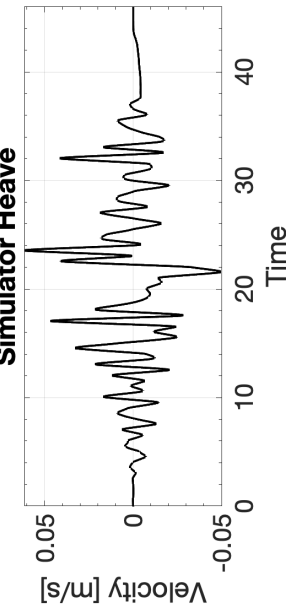

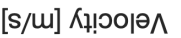

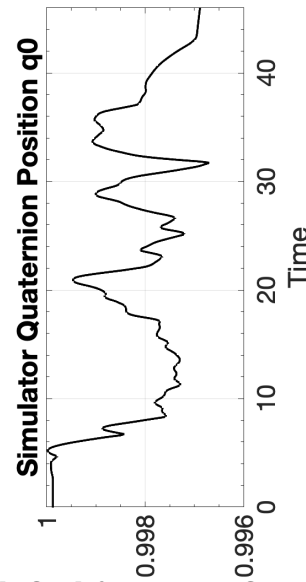

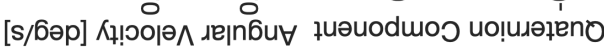

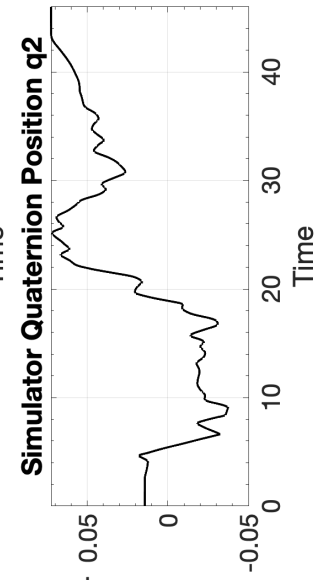

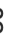

兽

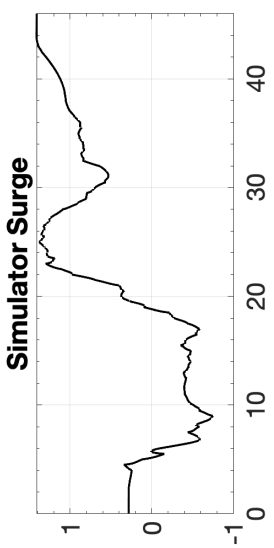

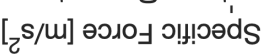

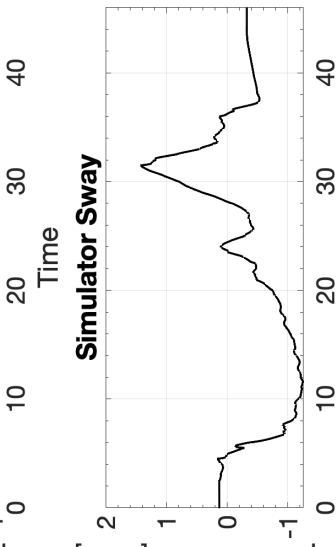

[

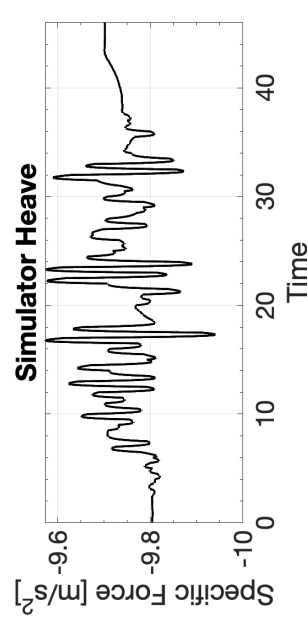

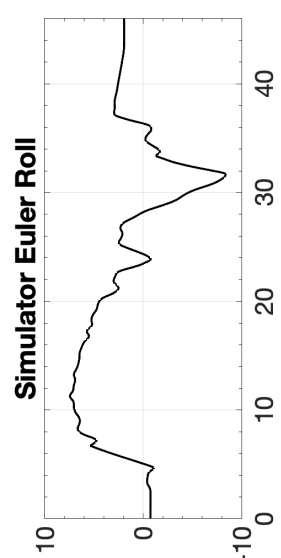

[6әр] uo!n!sod

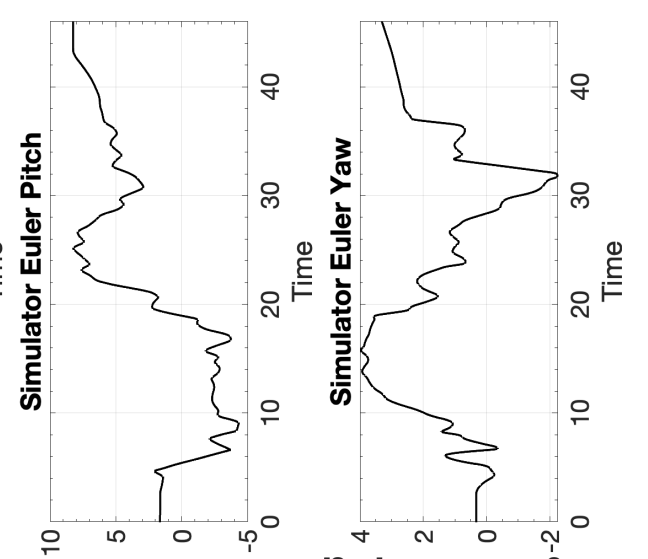

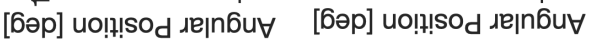

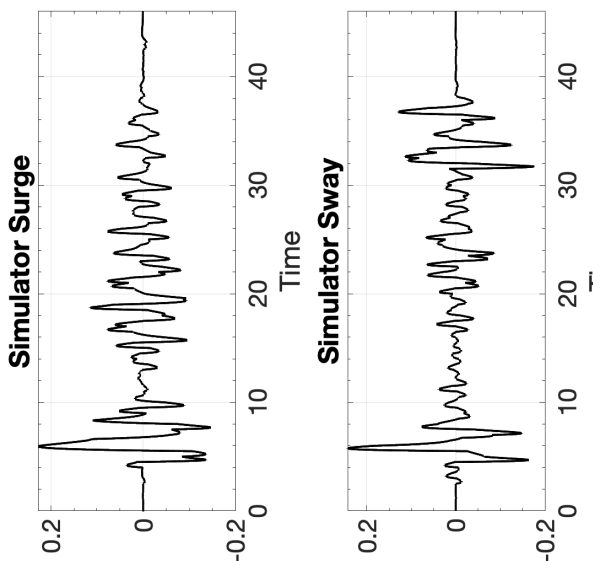

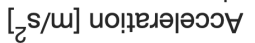

$[\mathrm{z} / \mathrm{m}]$ ио!ฺвдә|әэว

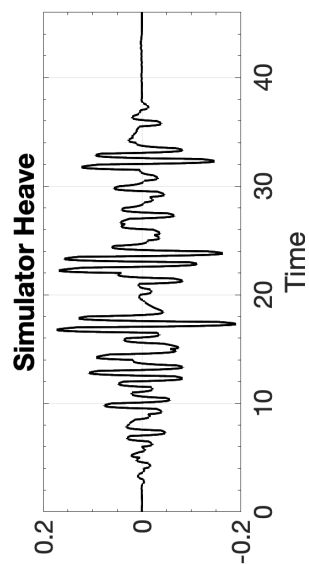

[z/m] ио!ฺеләрәэว
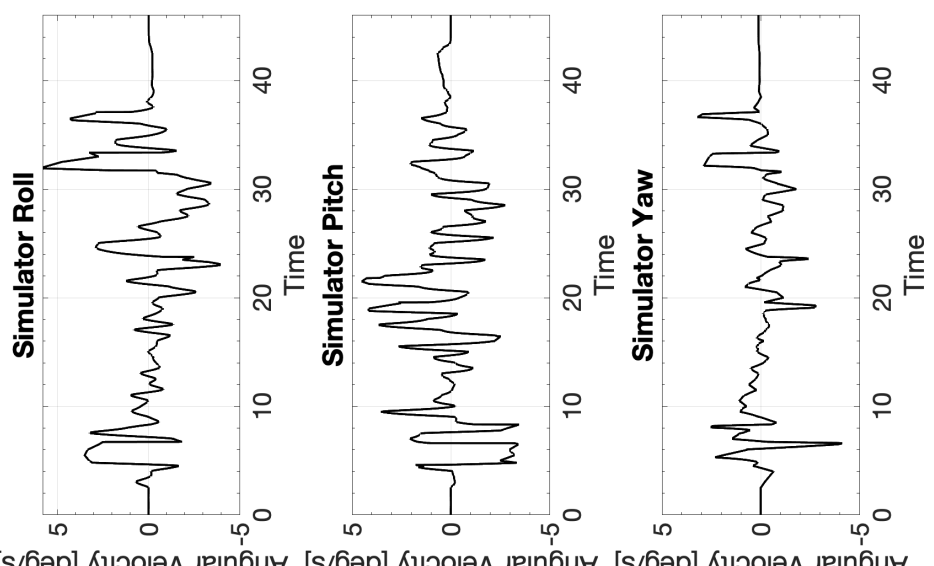

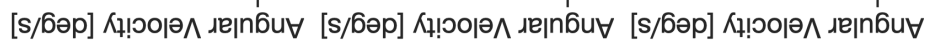




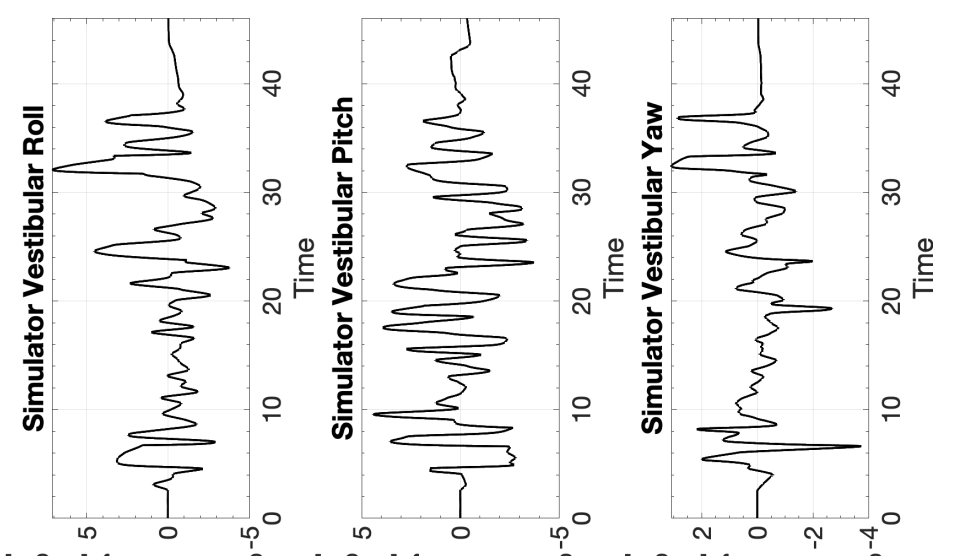

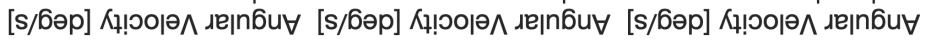

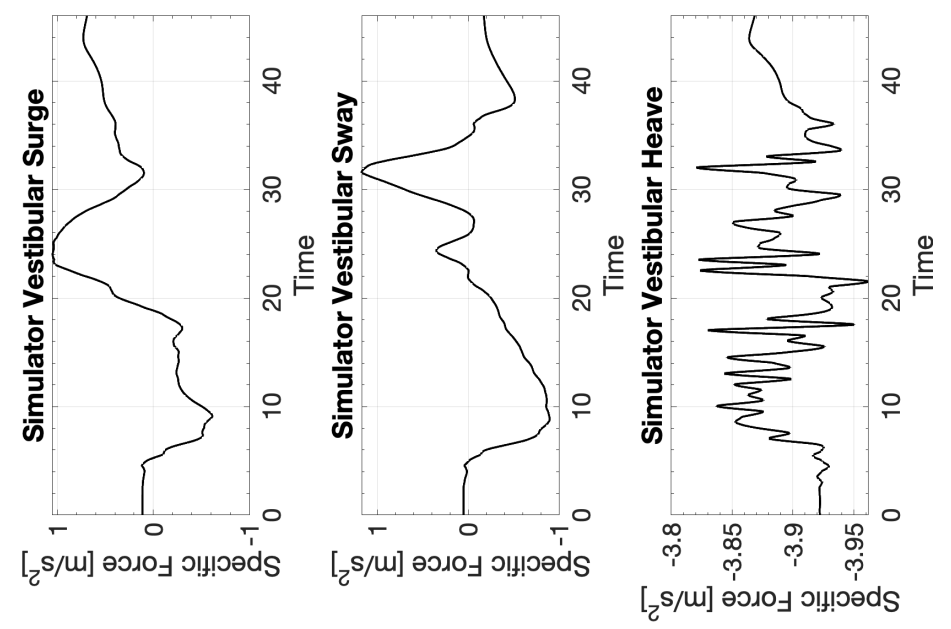




\section{D.14.3 CW3 Columbia 400 Single-axis Takeoff}
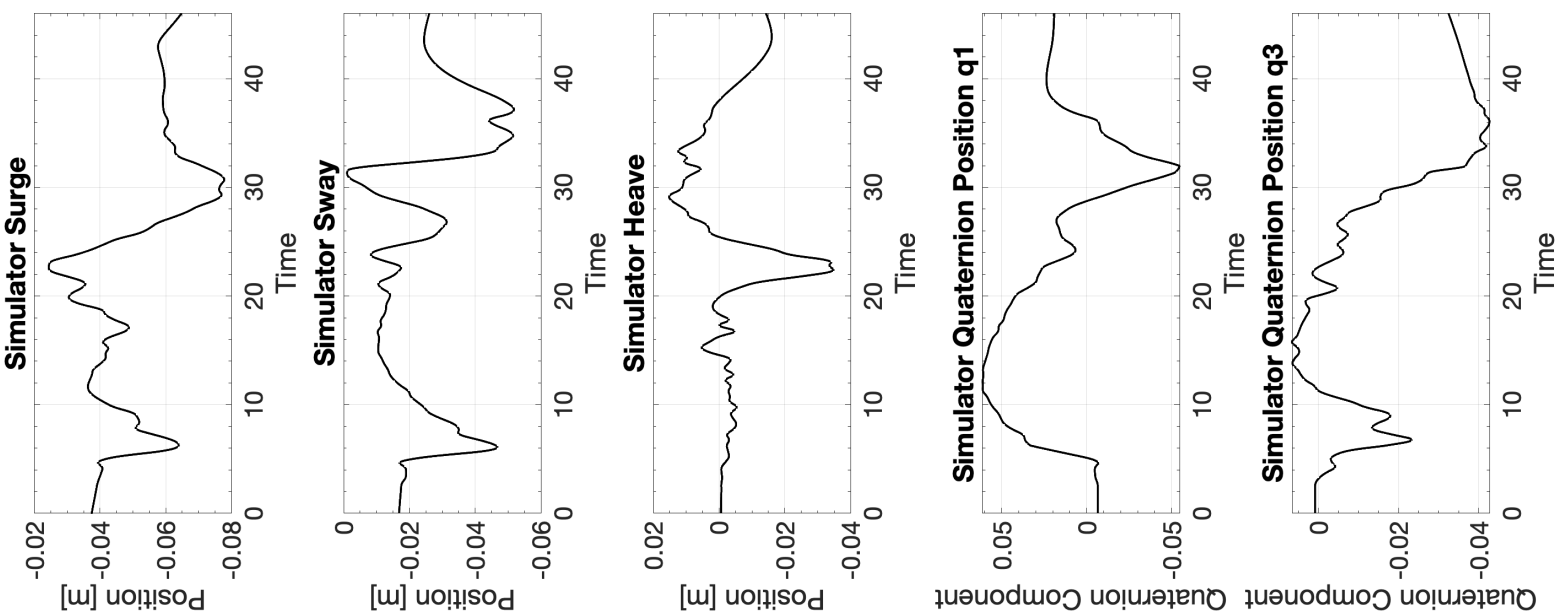

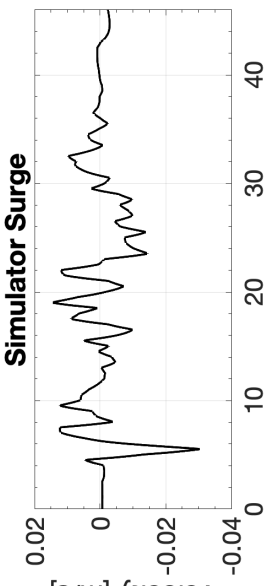

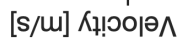

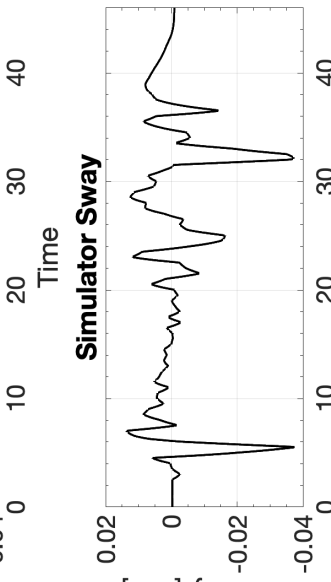

$[\mathrm{s} / \mathrm{m}]$ ㅅ!잇

$[w]$ uo!!!

ұuәuodmo

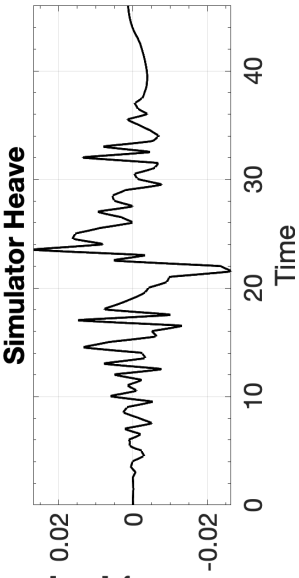

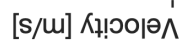
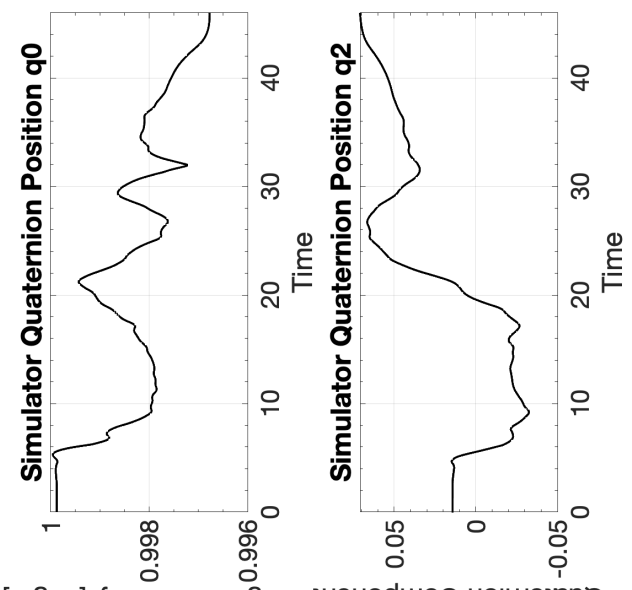

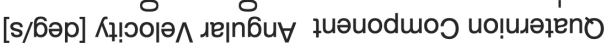

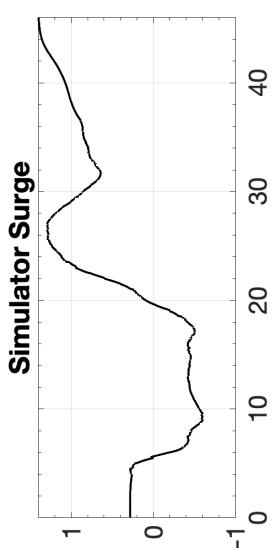

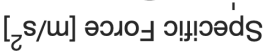

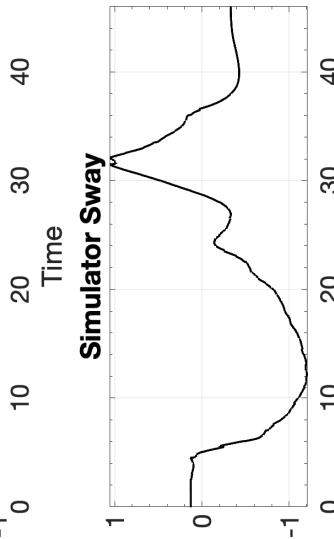

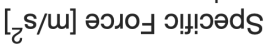

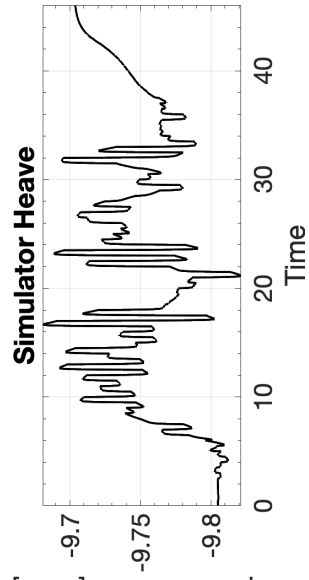

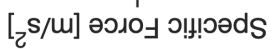

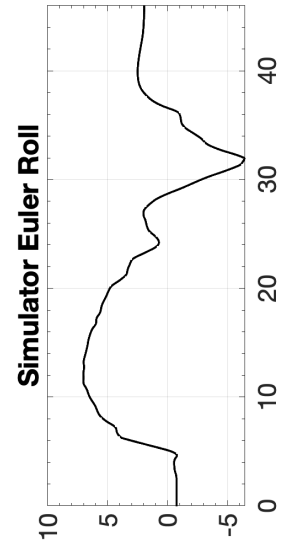

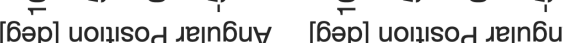

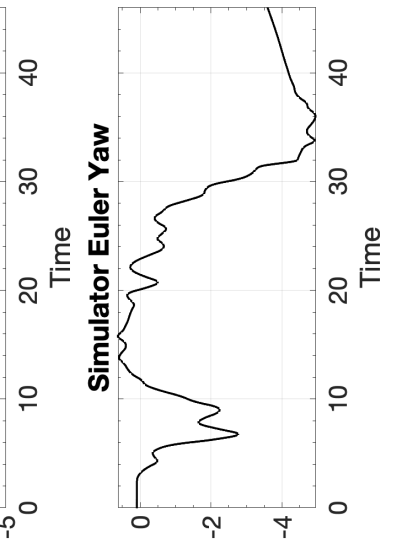

[бəp] uo!!!!sod גejn6u

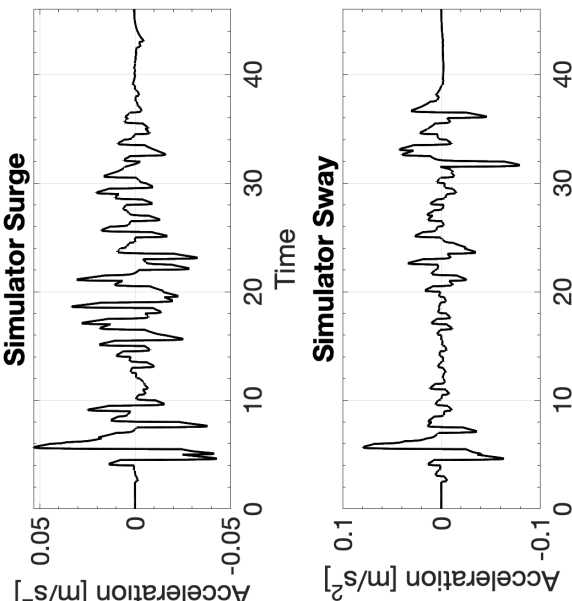

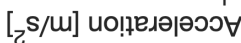

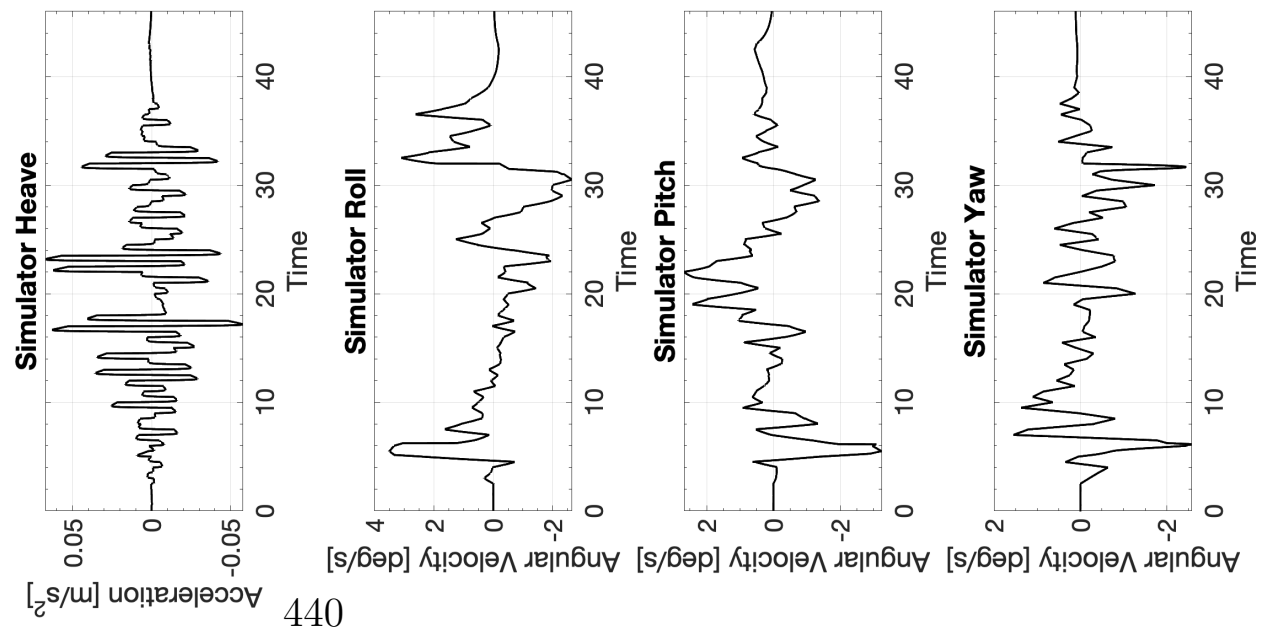




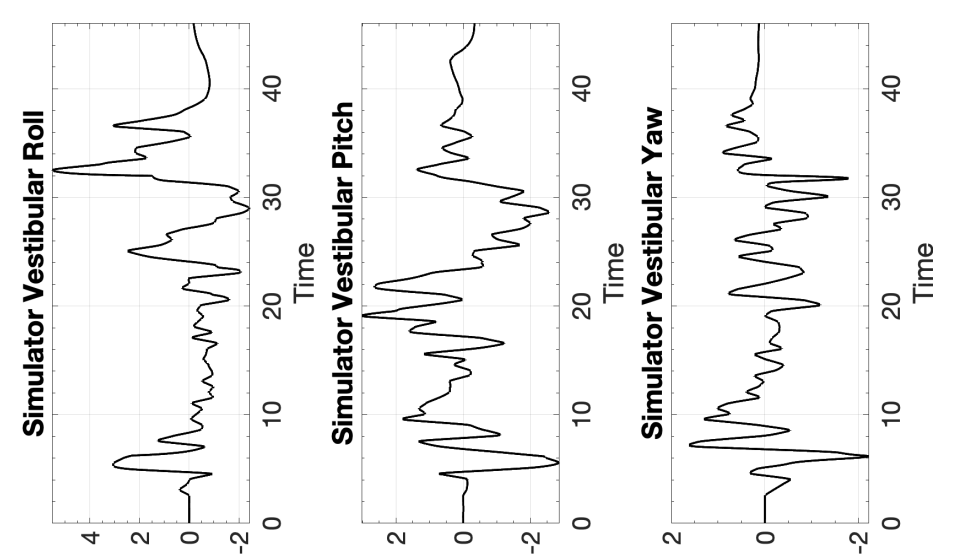

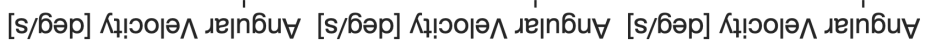

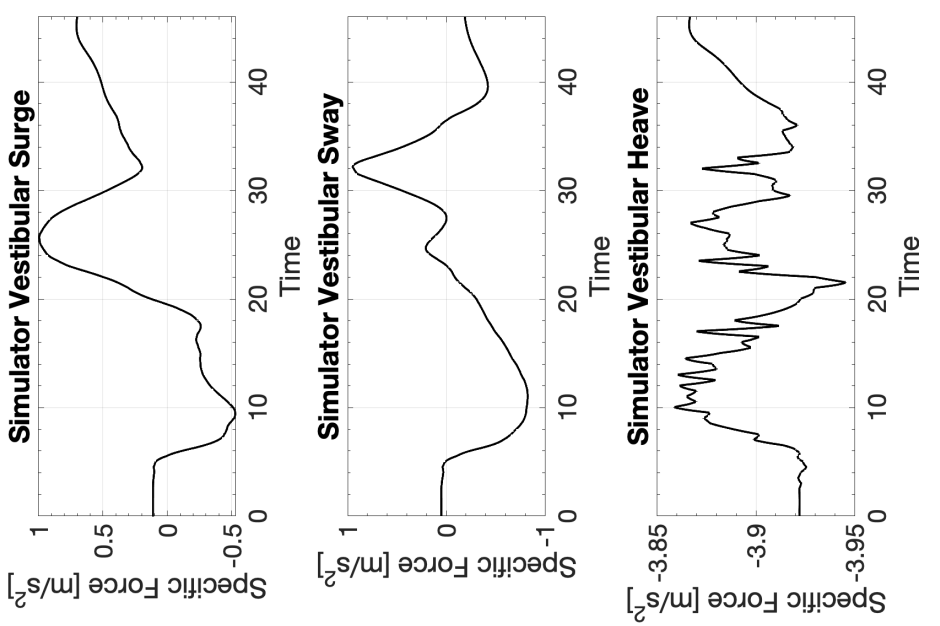




\section{D.15 Columbia 400 Spiral Dive}

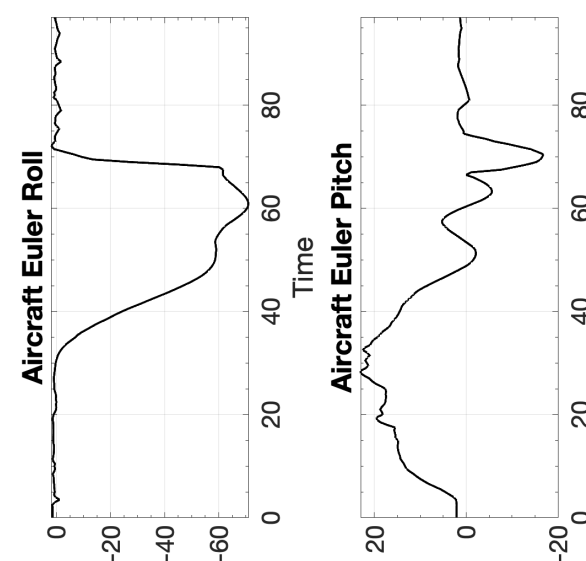

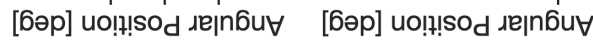

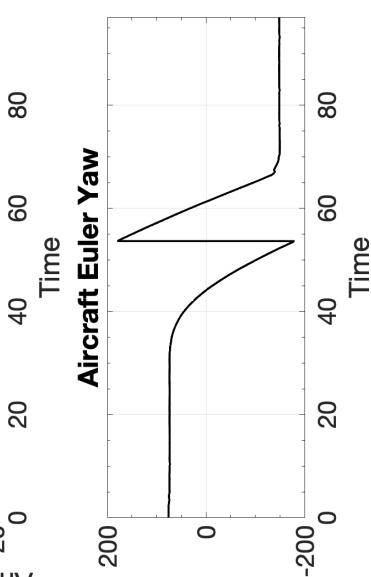

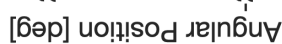
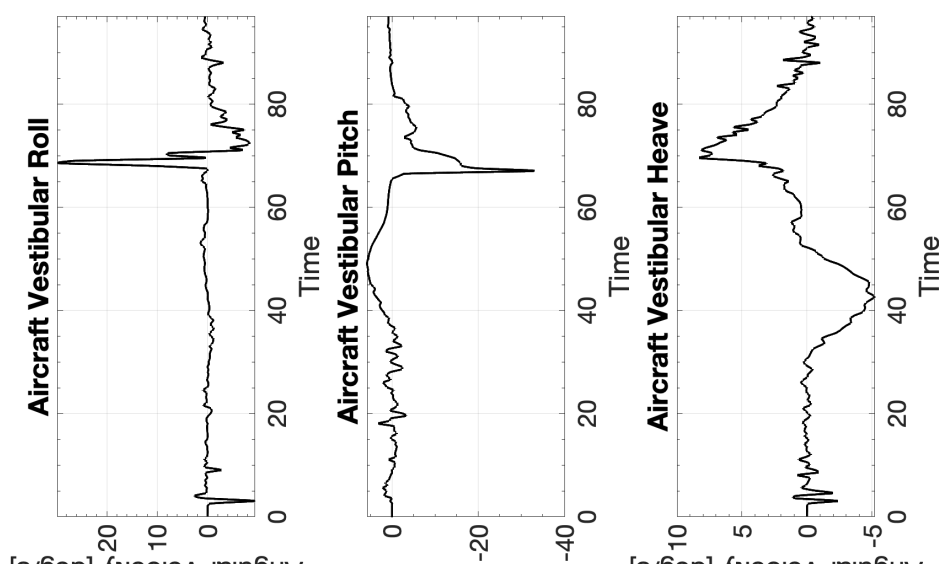

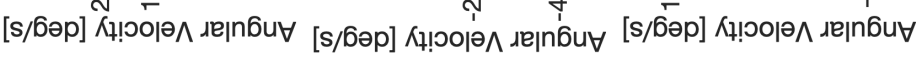

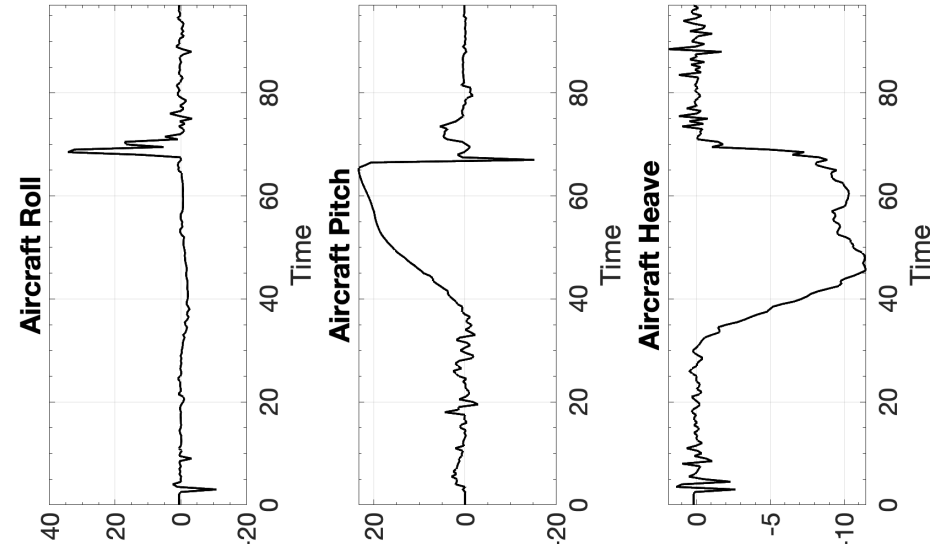

[s/бәр] Кұ! оорә

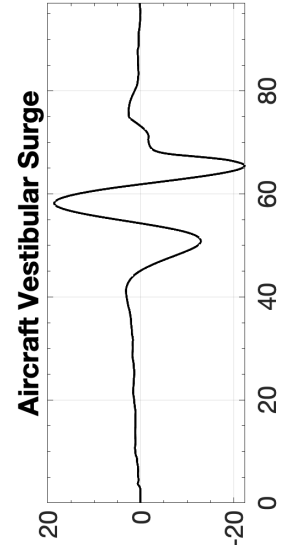

ұuәuodmoว uo!̣uəłeno

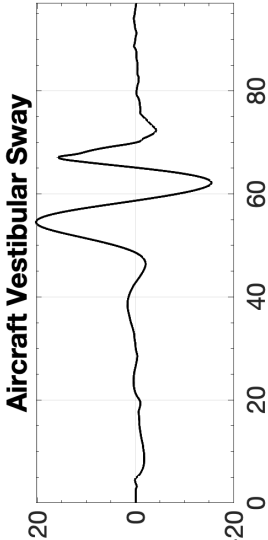

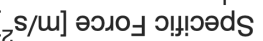

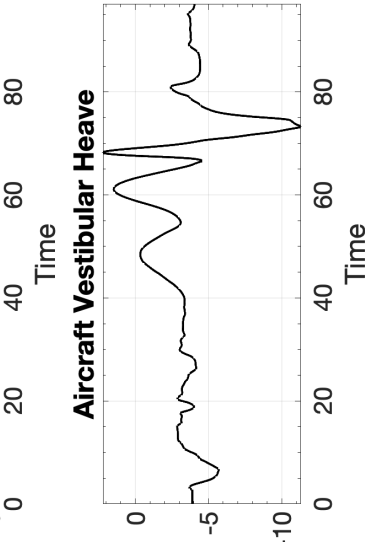

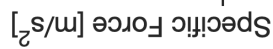

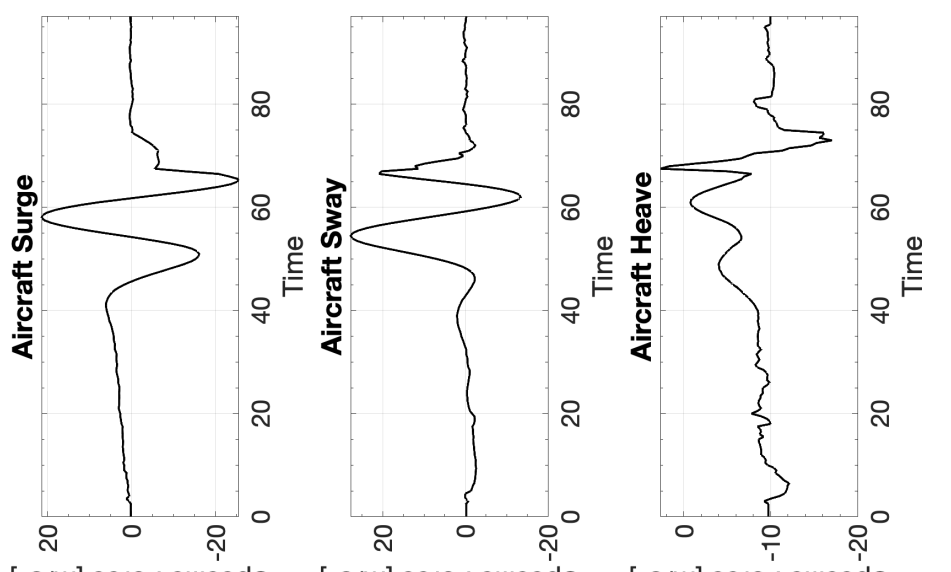

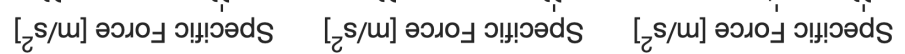

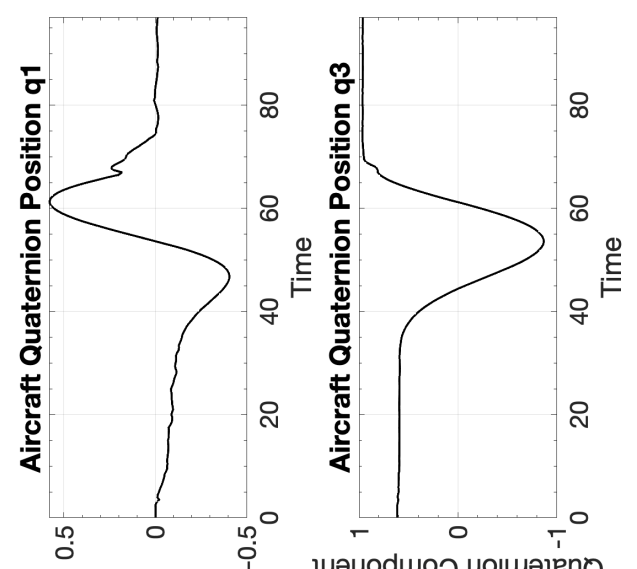

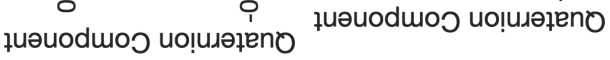

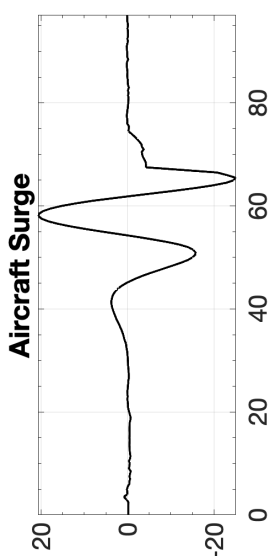

[zs/u] uo!ןелә|әэว

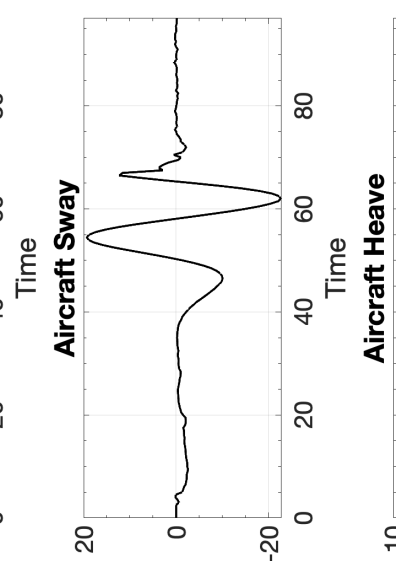

[zs/m] ио!ฺедәәәоэ

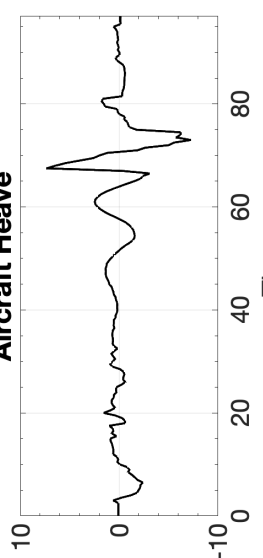

[zs/m] ио!једәрәоэ

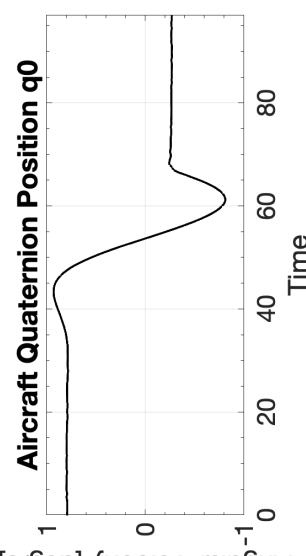

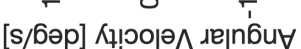

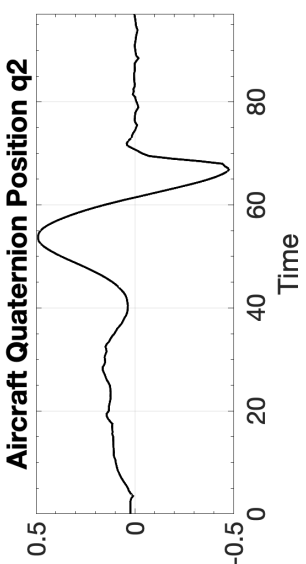

łuəuodmo uo!̣uəfeno 


\section{D.15.1 CW1 Columbia 400 Spiral Dive}

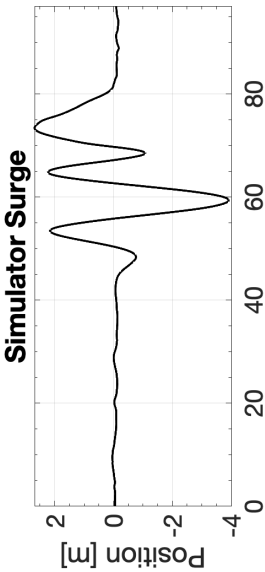

[w] uo!n!sod

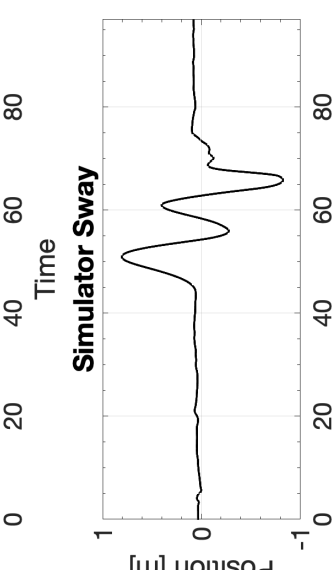

[w] uo!!!!sod

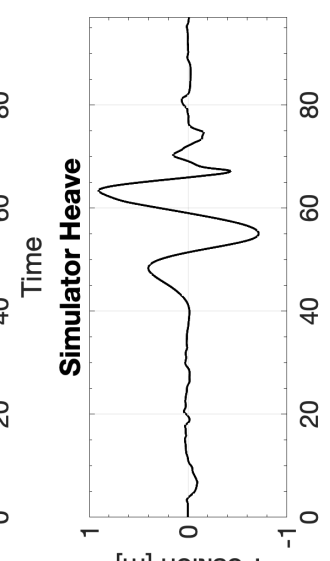

[w] uol!! sod

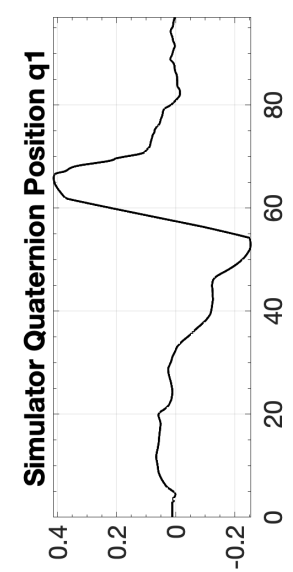

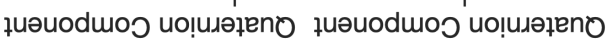

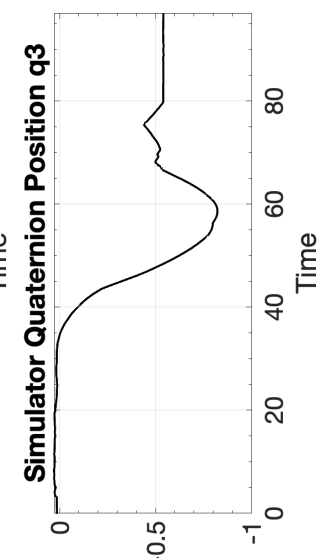

$\stackrel{\text { E }}{i=}$

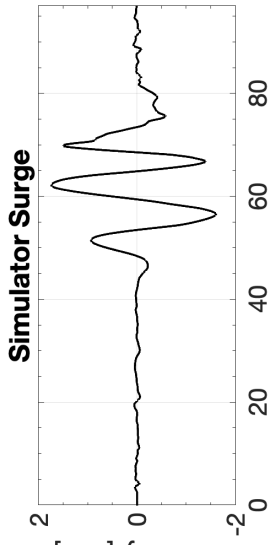

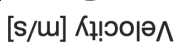

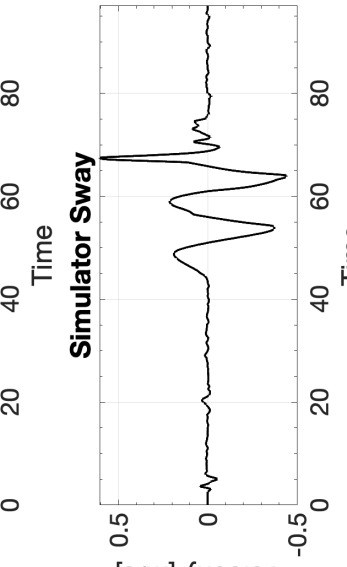

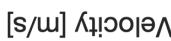

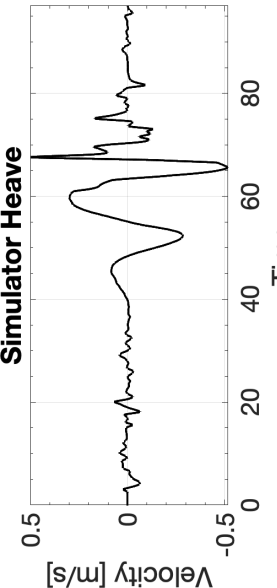

[s/m] Кұ! 잇

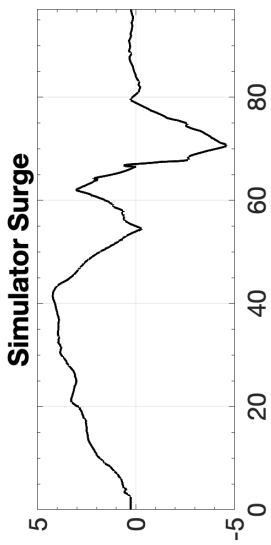

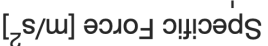

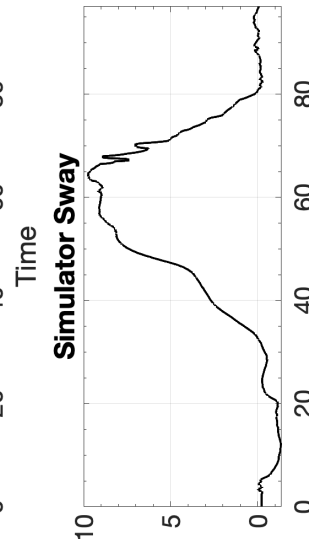

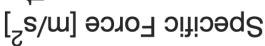

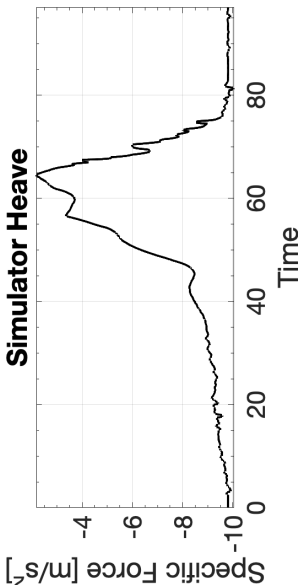

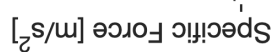

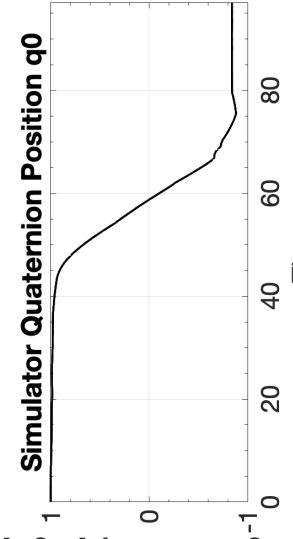

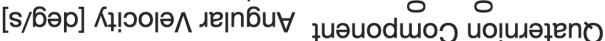

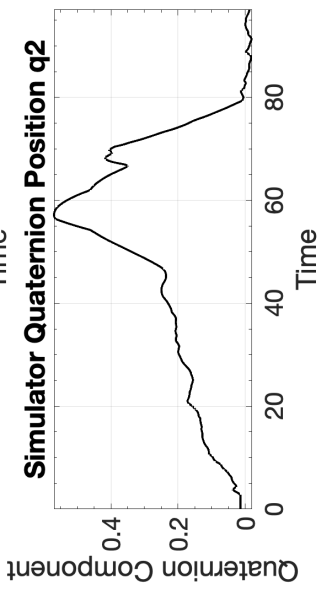

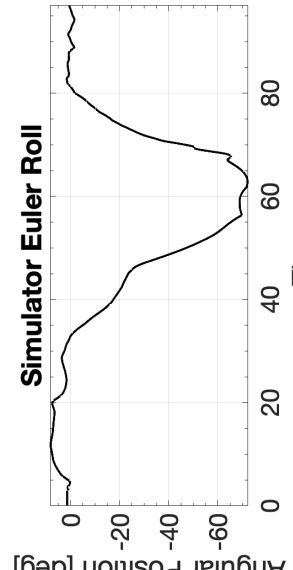

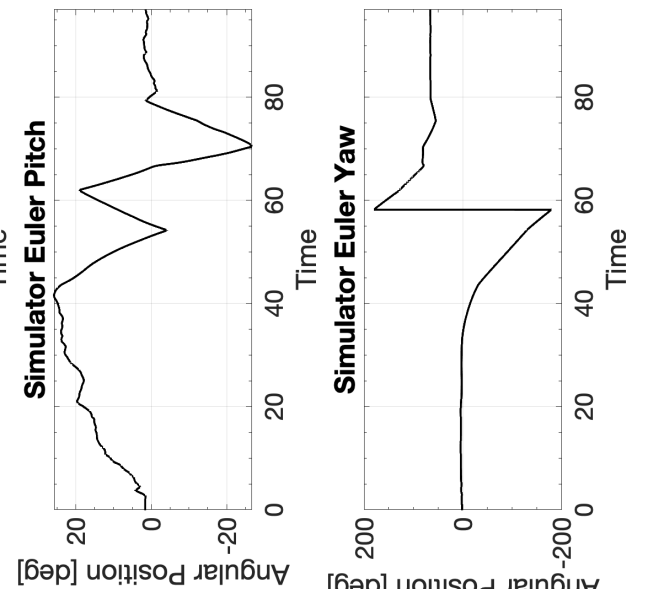

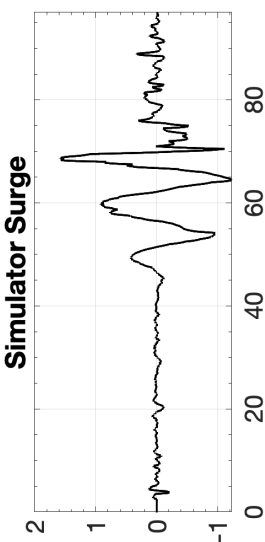

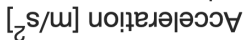

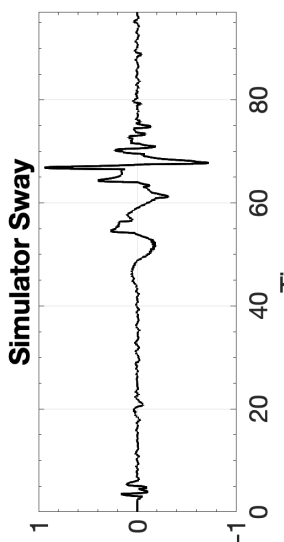

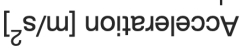

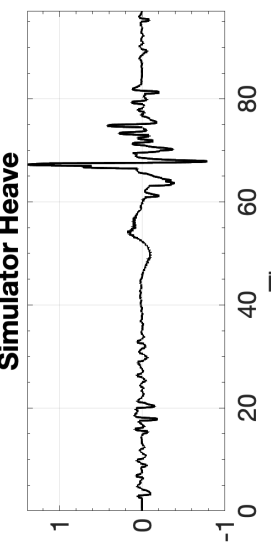

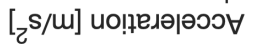

[6әp] uo!!!sod גe|nбü 

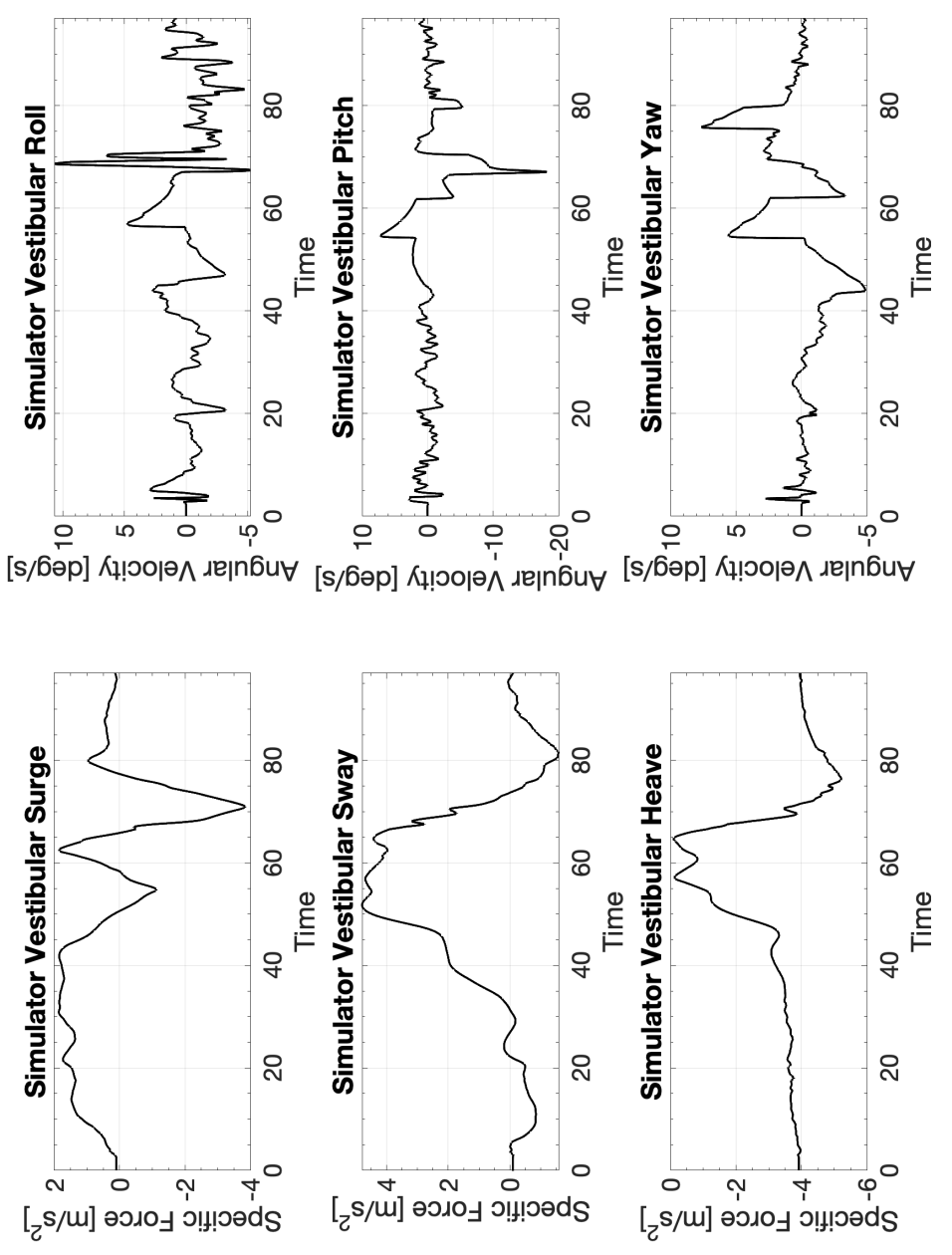


\section{D.15.2 CW2 Columbia 400 Spiral Dive}

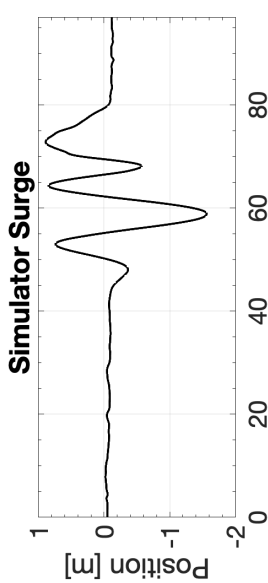

[w] uo!̣!!sod

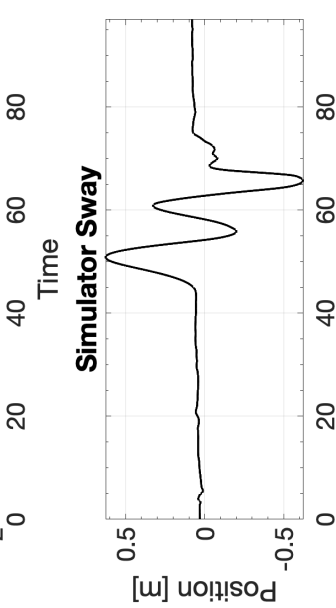

[m] uol!m!sod

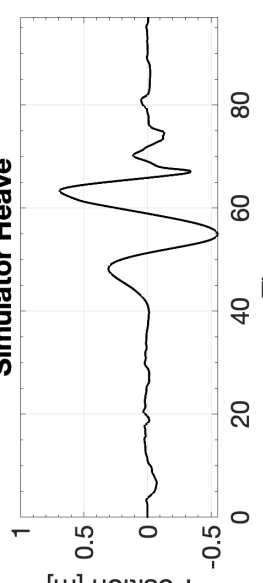

[u] uo!t!!sod

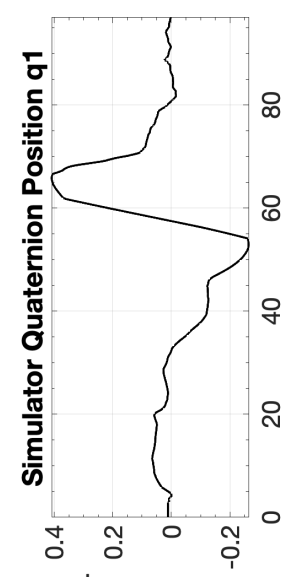

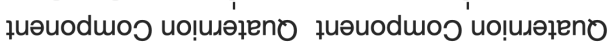

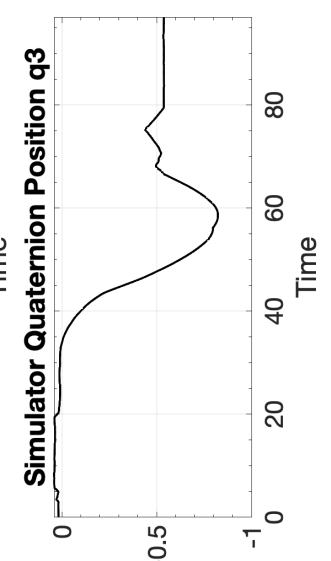

$\stackrel{\text { E }}{i=}$

요

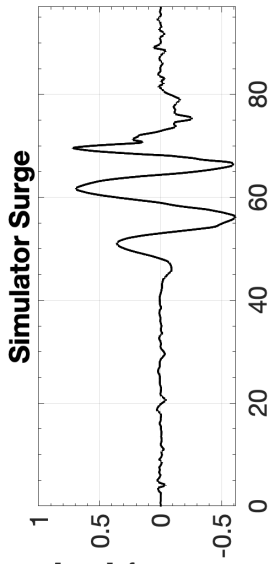

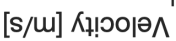

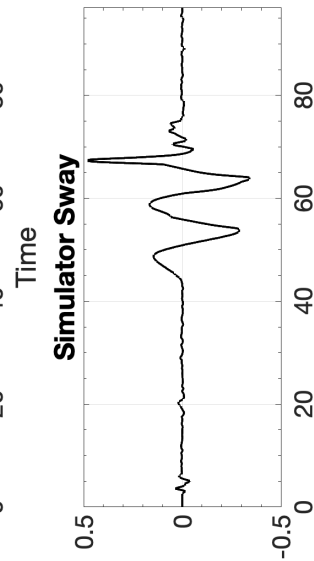

[s/u] К!ำю^

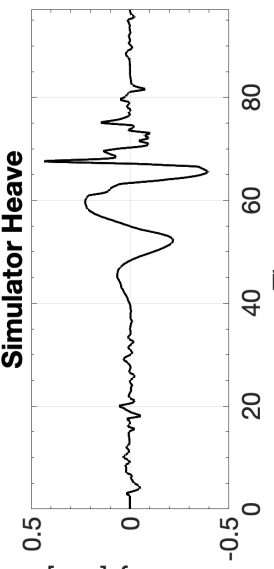

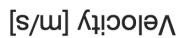

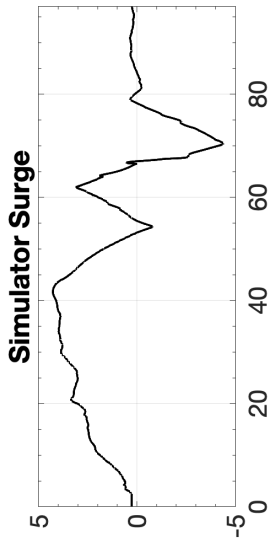

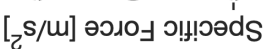

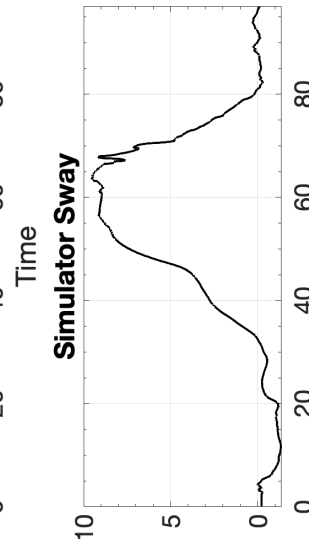

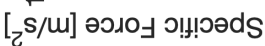

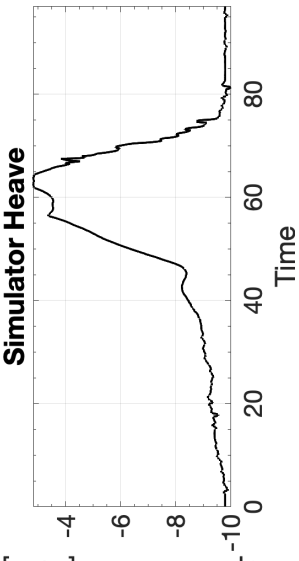

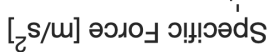

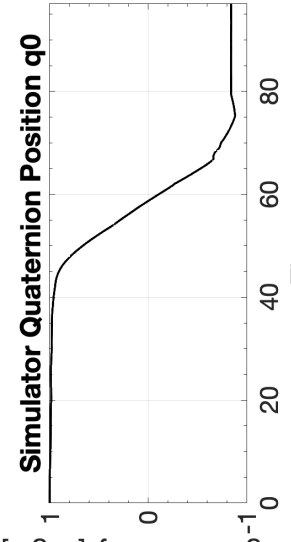

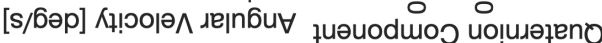

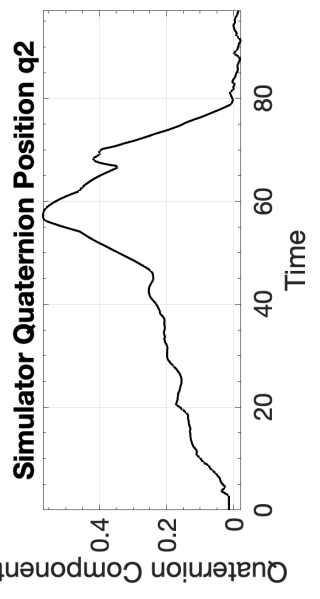

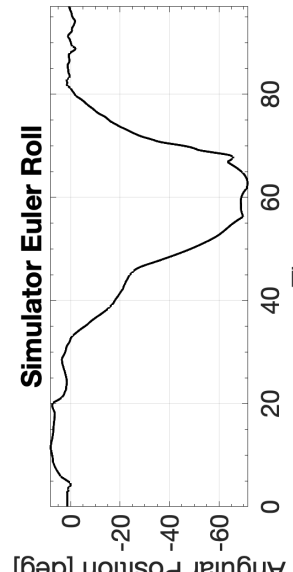

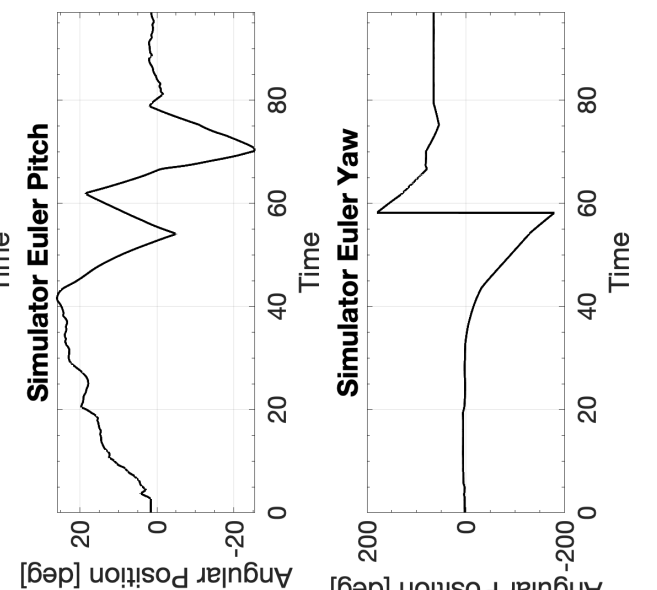

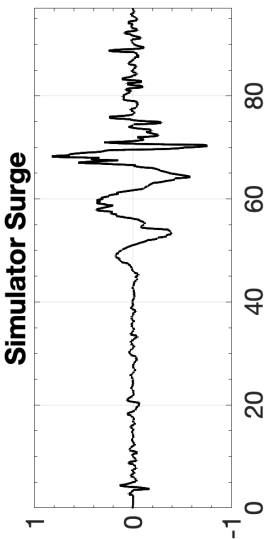

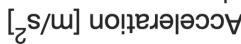

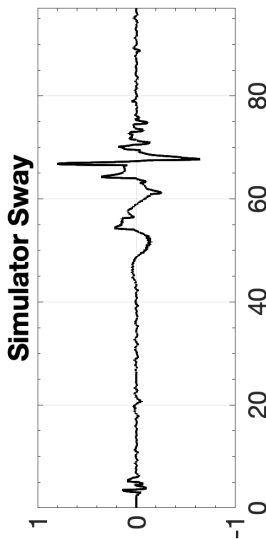

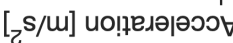

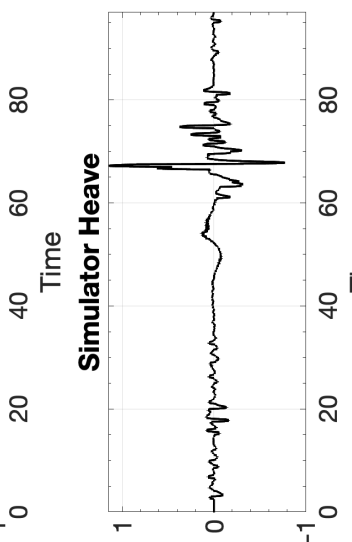

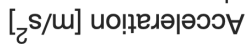
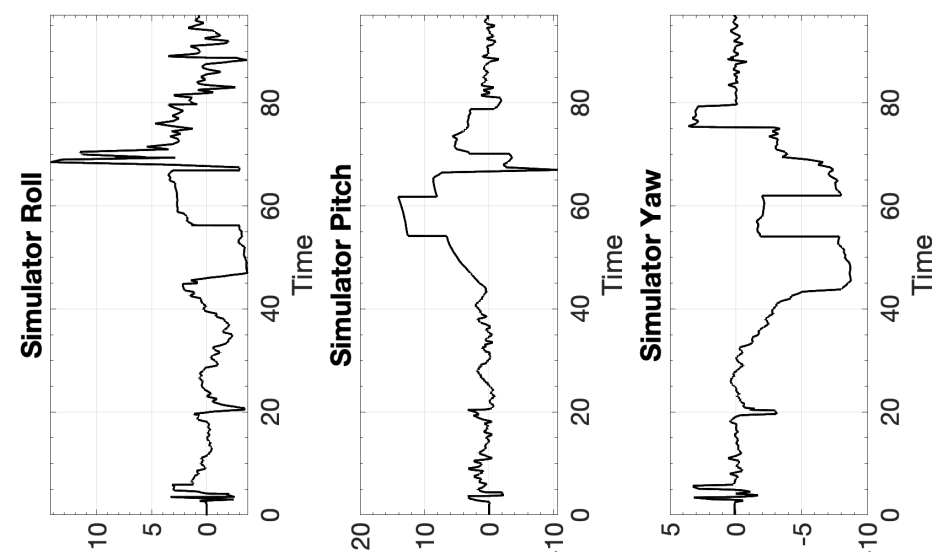

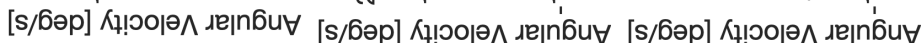



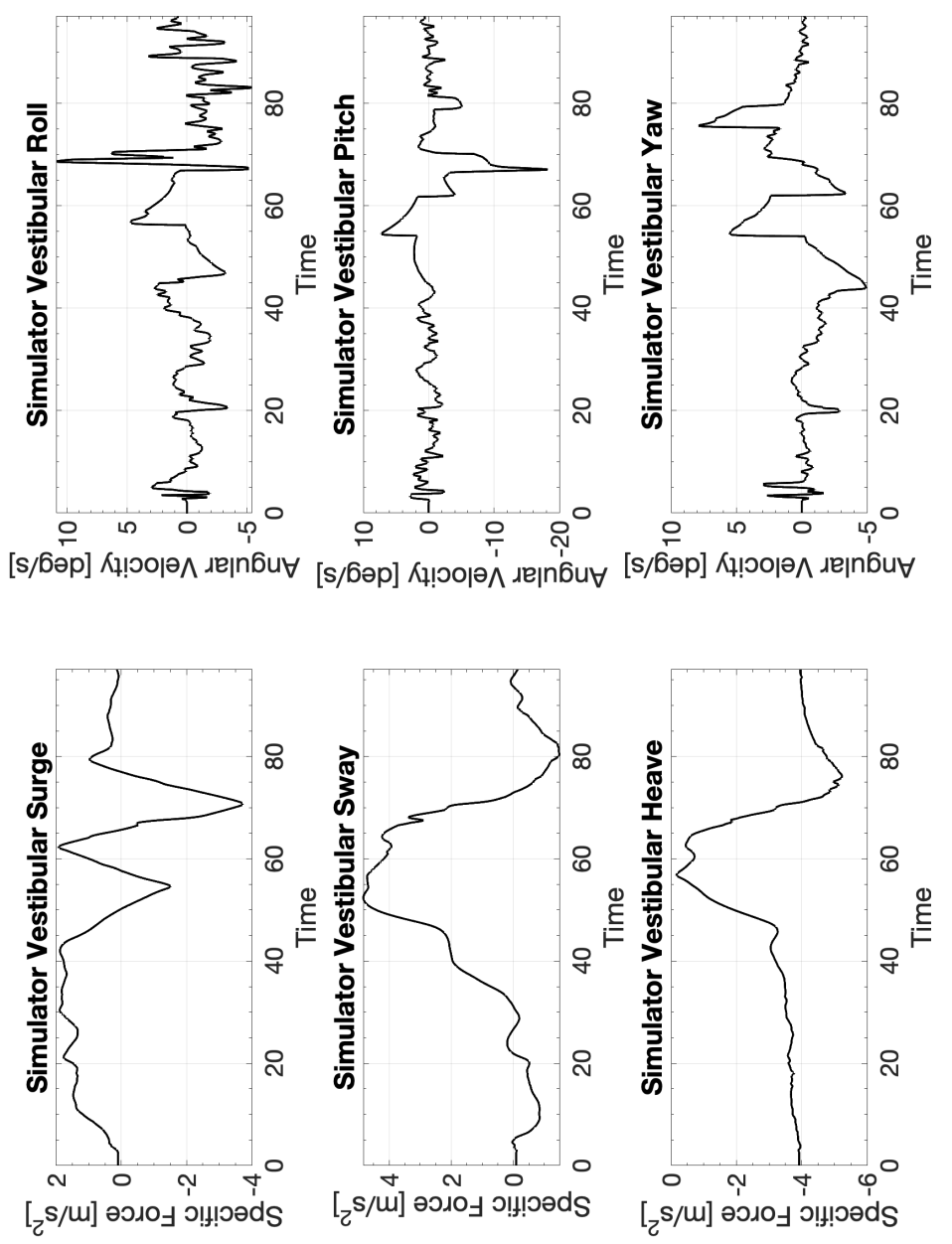


\section{D.15.3 CW3 Columbia 400 Spiral Dive}

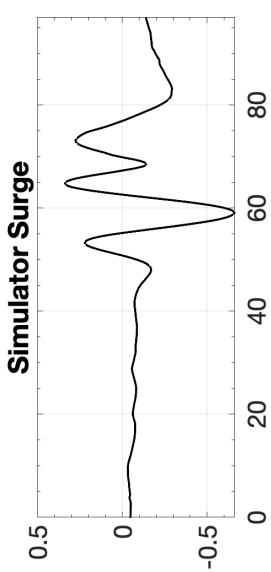

[w] uo! t!!sod

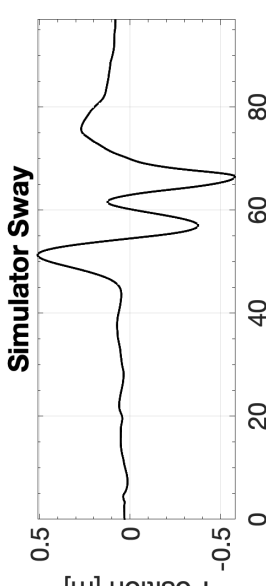

[w] uolt!!sod

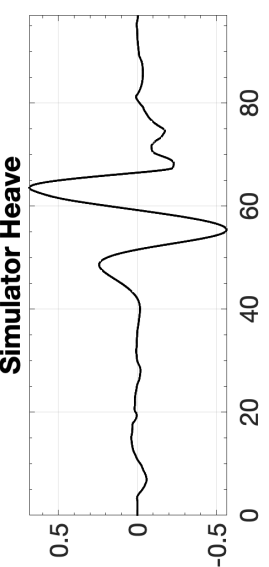

[u] uol!!sod

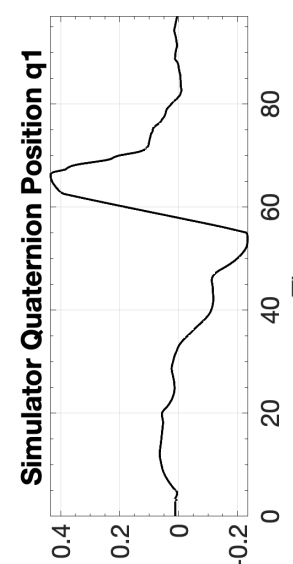

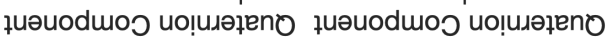

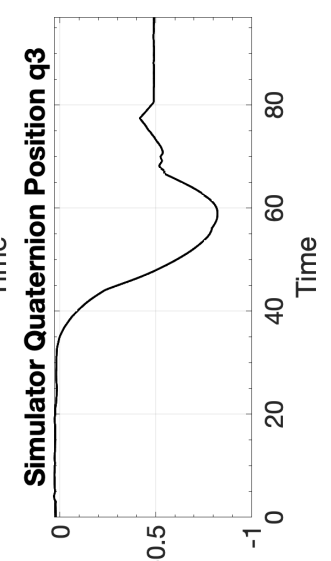

$\stackrel{\varrho}{E}$

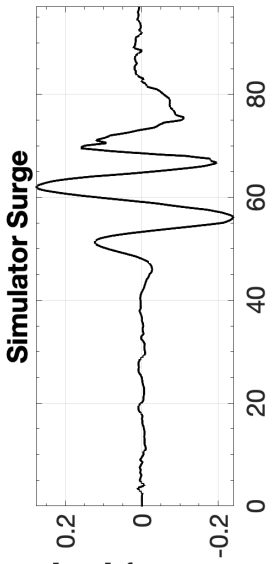

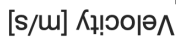

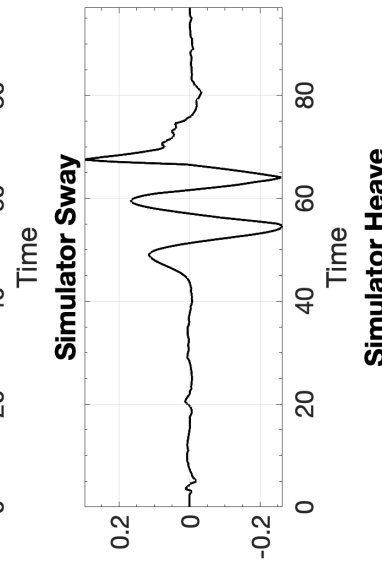

[s/u] Кł!วо夫

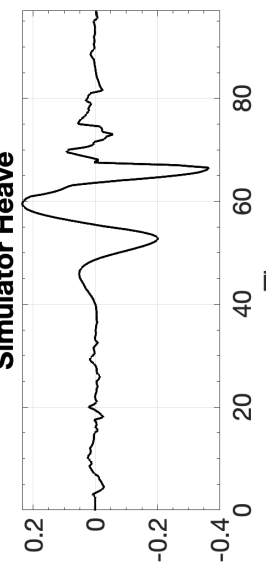

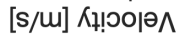

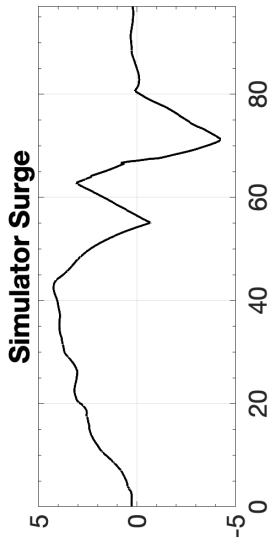

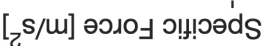

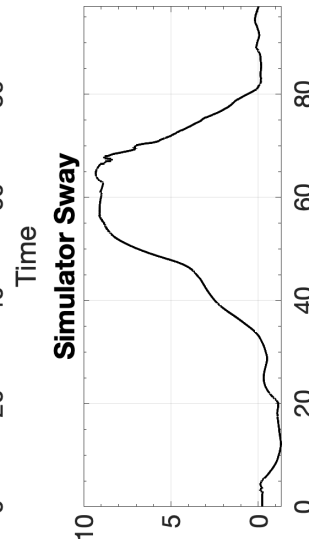

[ح

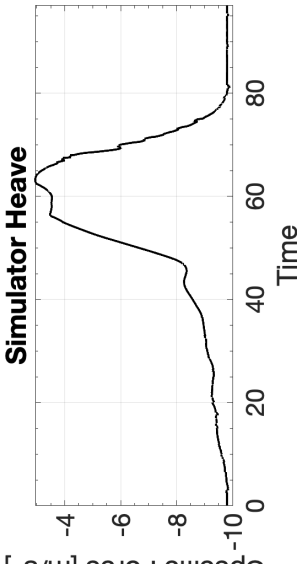

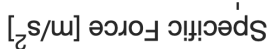

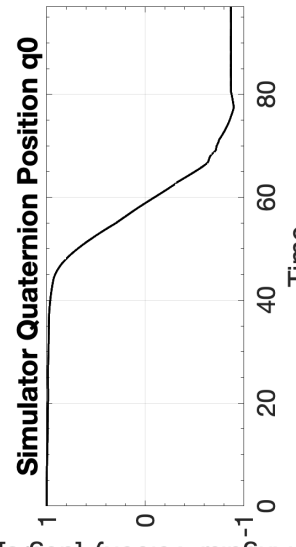

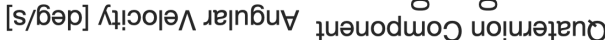

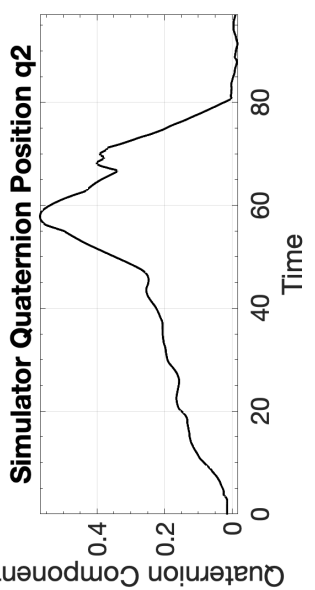

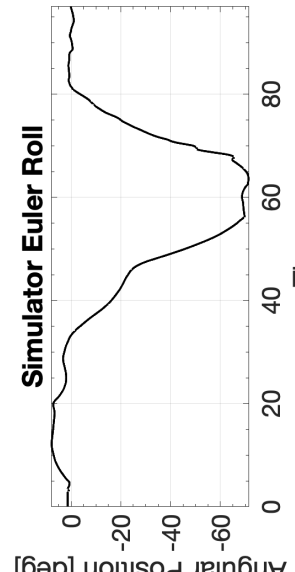

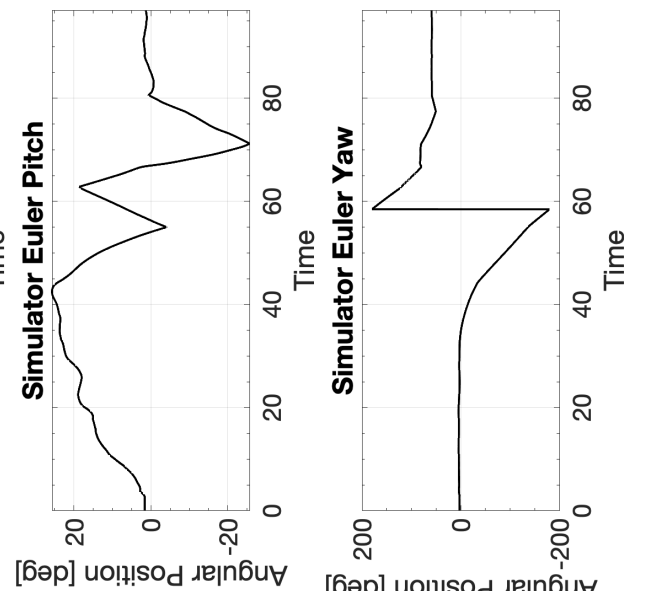

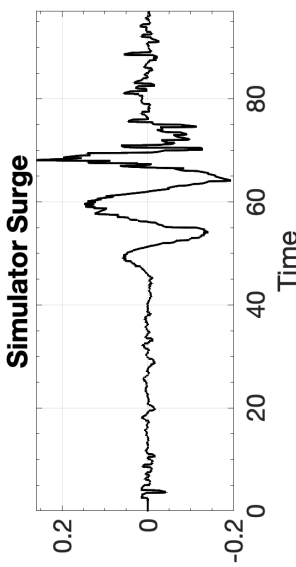

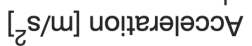

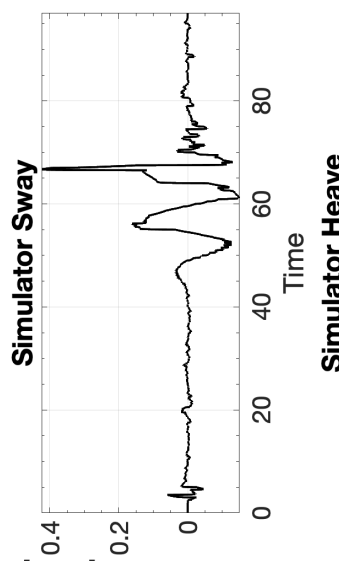

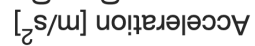

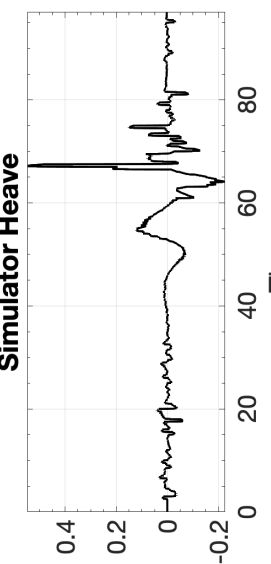

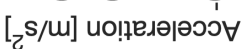

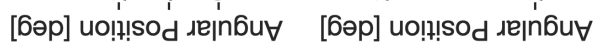

[6əp] uo!!!!sod גe|n6u

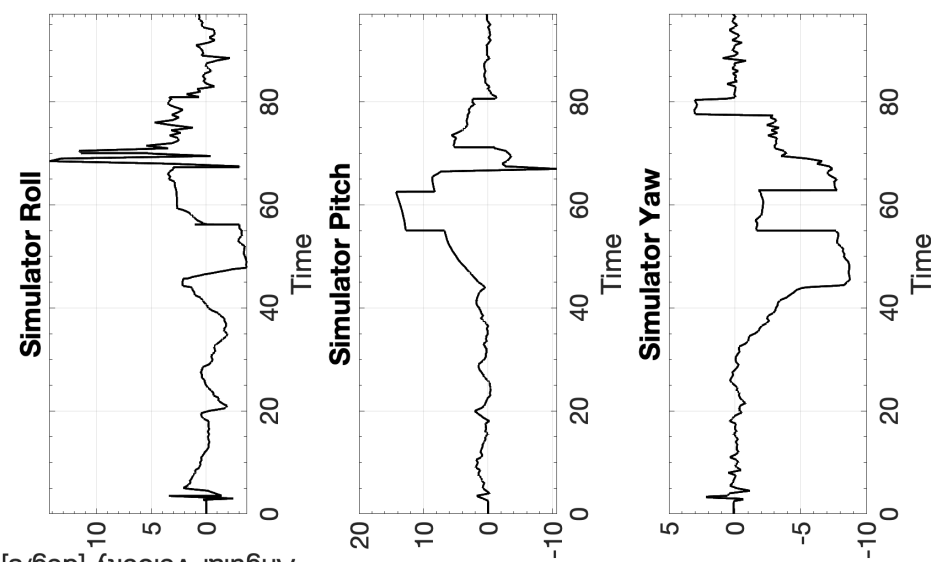

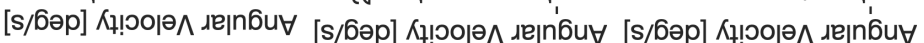



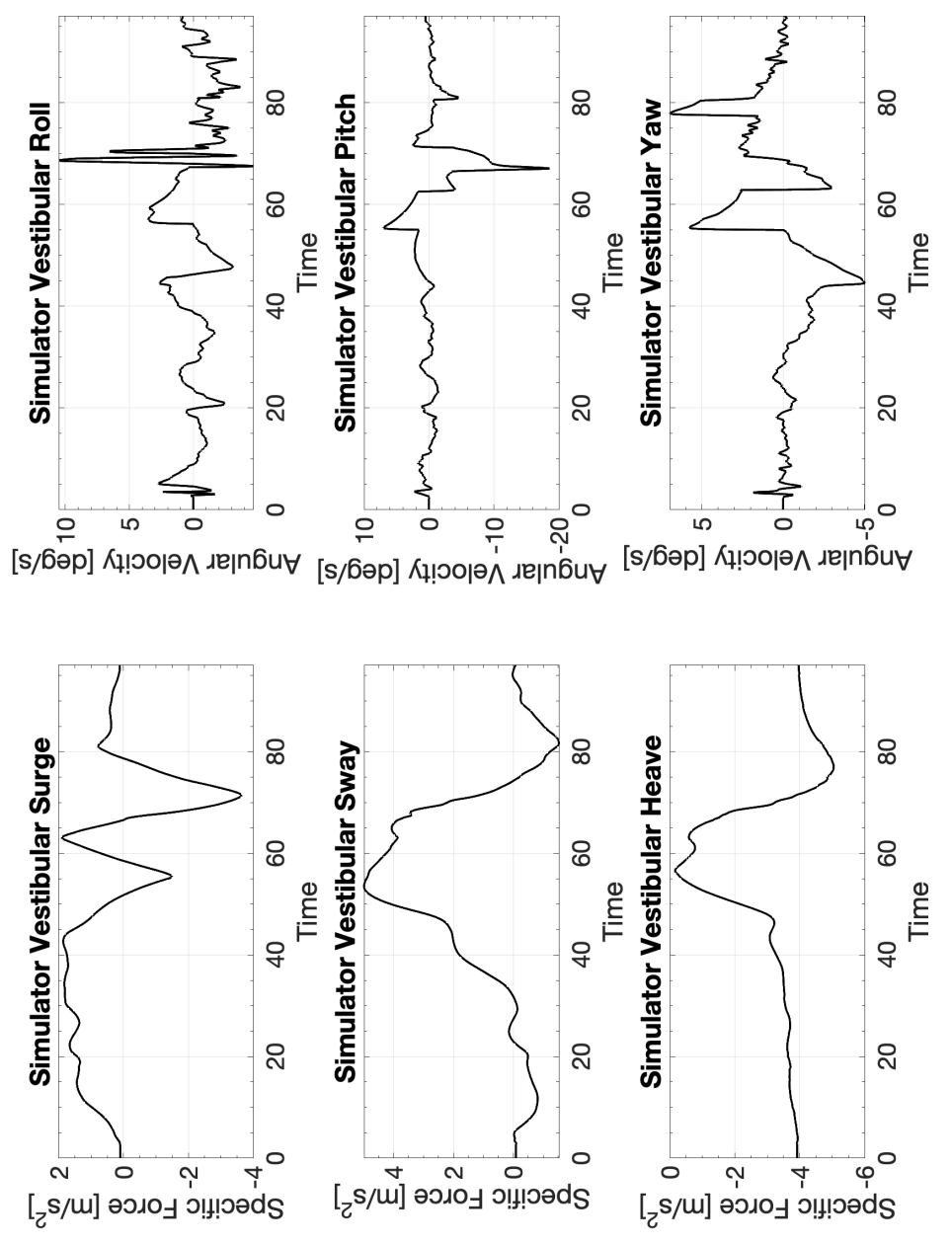


\section{D.16 Columbia 400 Stall Recovery}

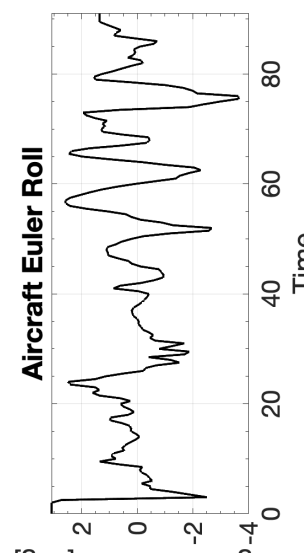

[6әр] uo!!!sod

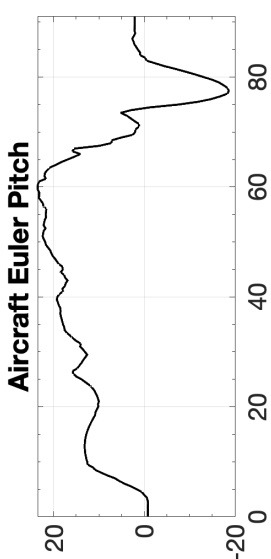

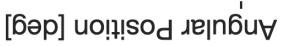

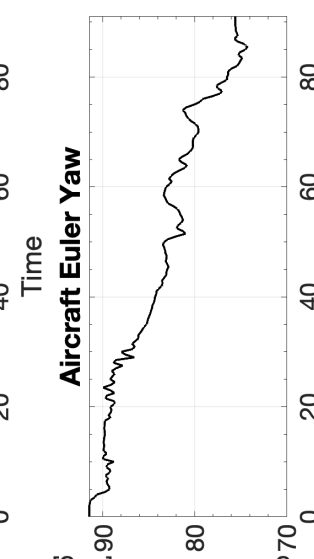

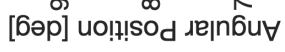

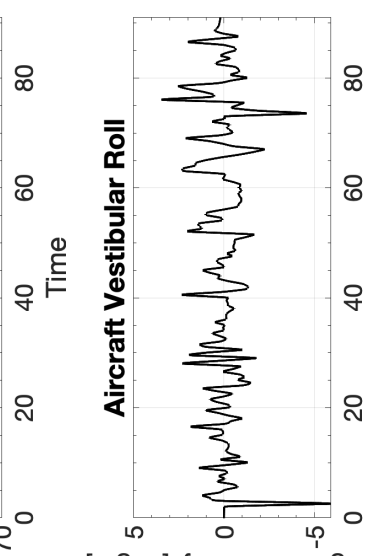

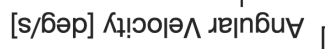

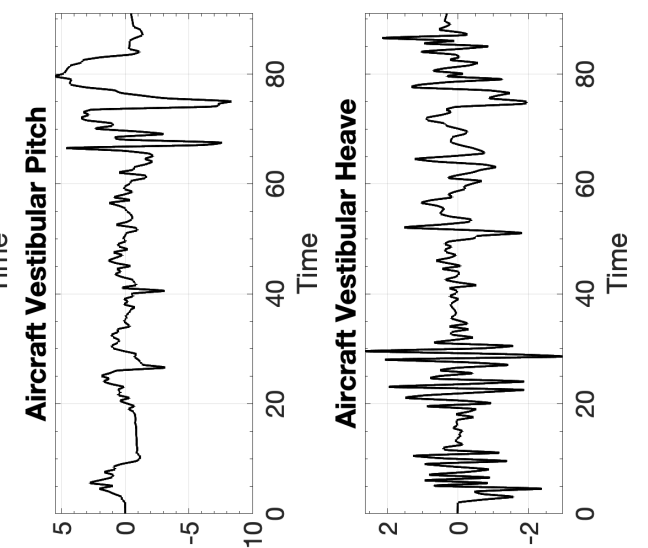

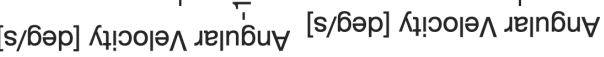
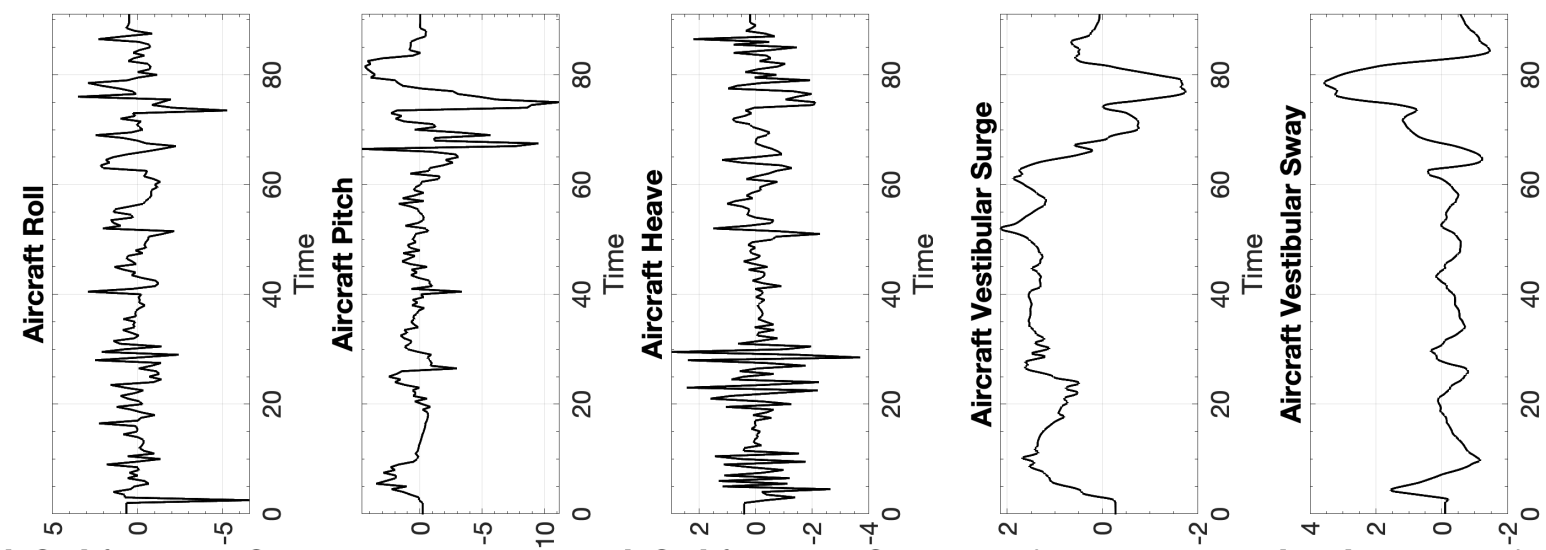

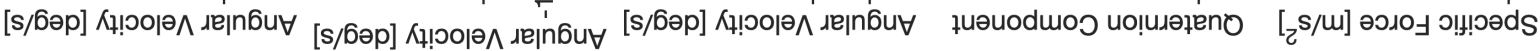

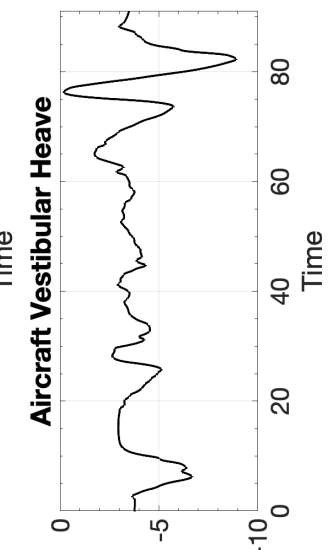

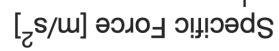

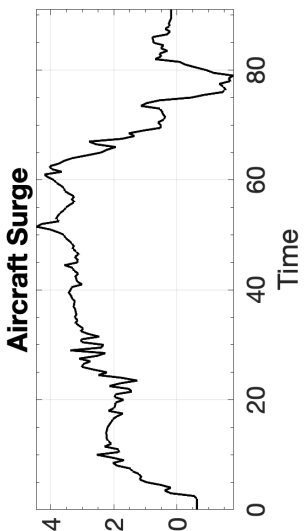

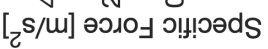

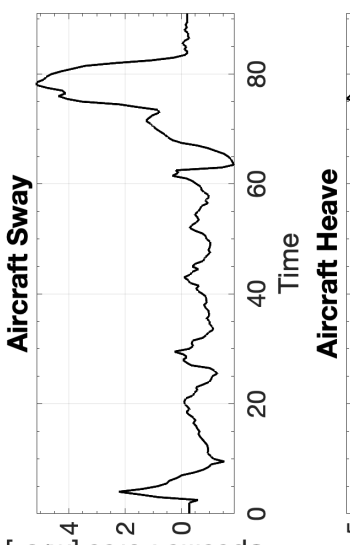

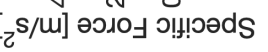

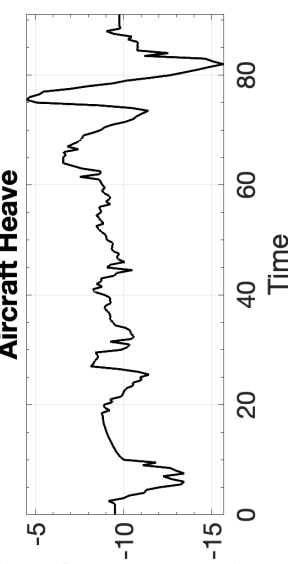

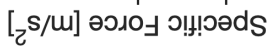

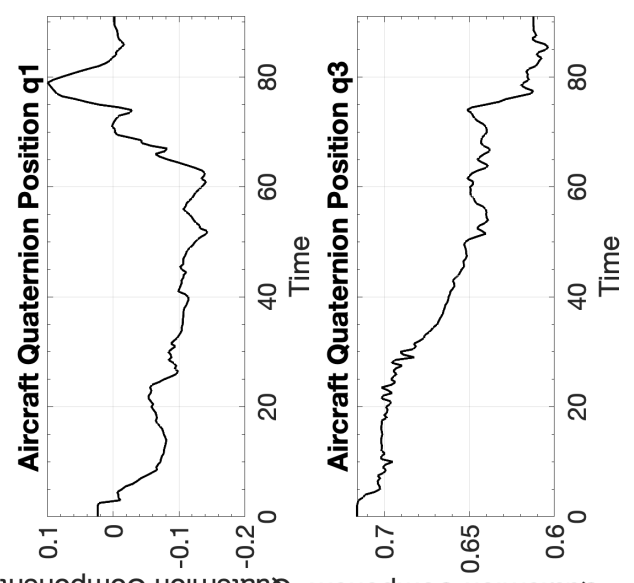

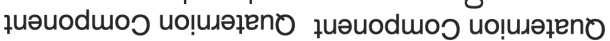

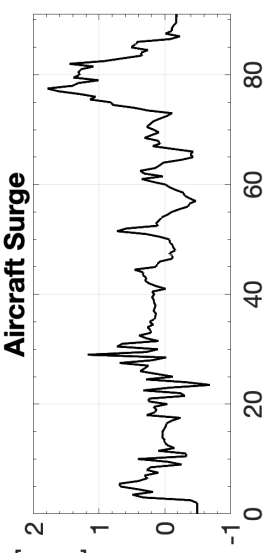

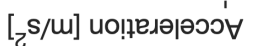

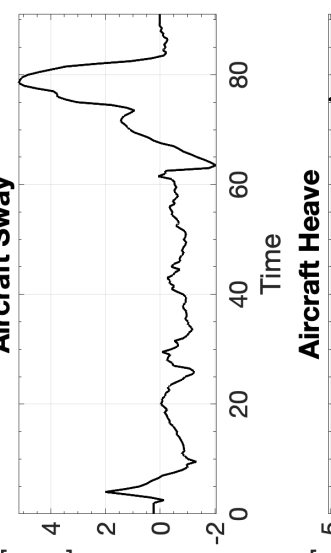

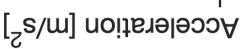

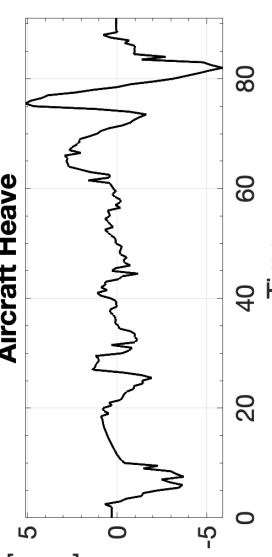

[z/m] uо!ฺеләрәэว

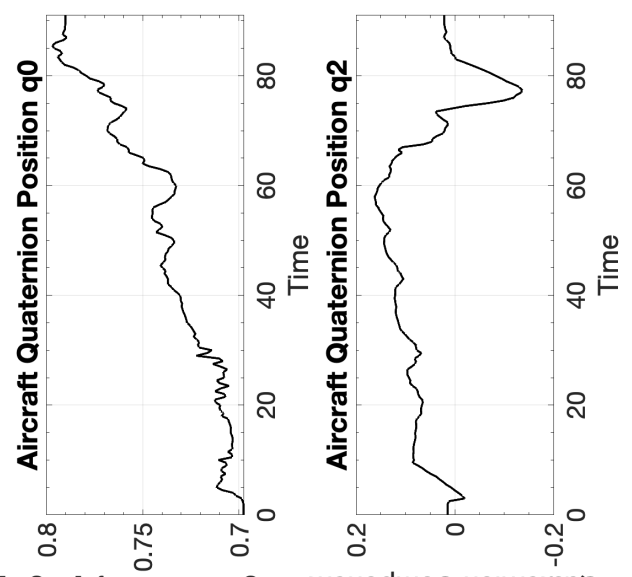

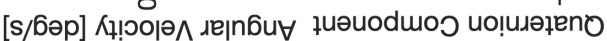




\section{D.16.1 CW1 Columbia 400 Stall Recovery}

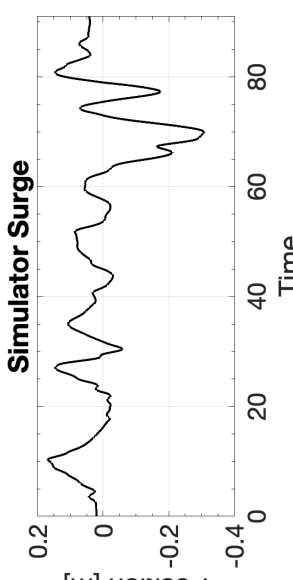

[u] uo!n!sod

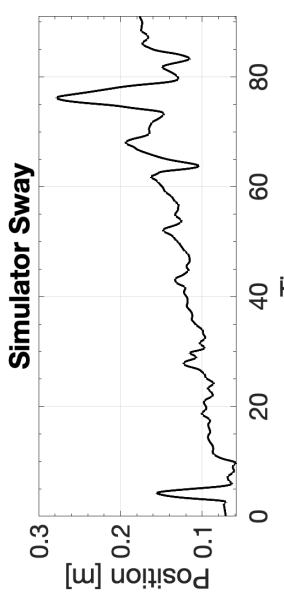

[w] uo!!!!sod

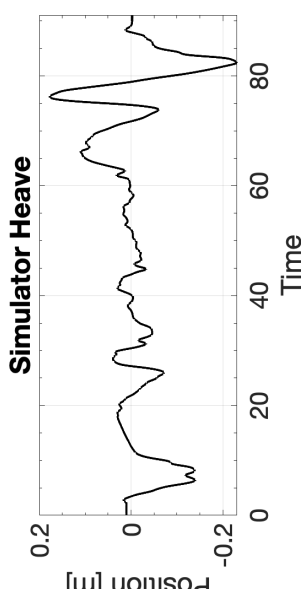

[w] uo!n!sod
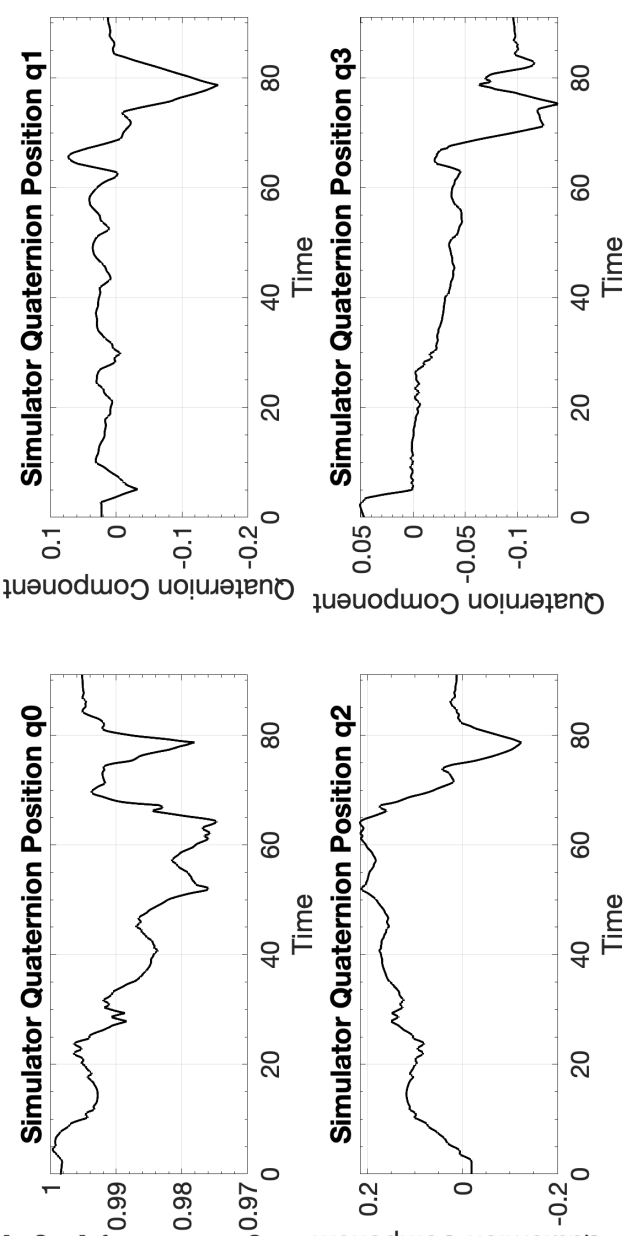

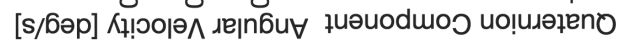

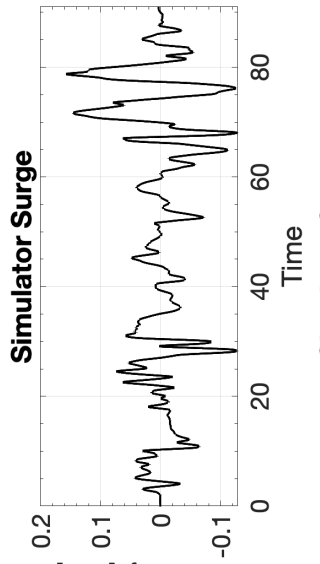

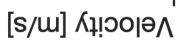

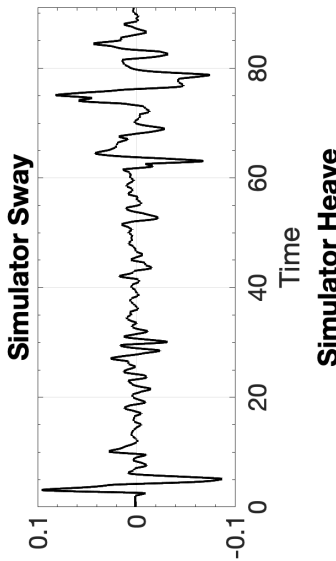

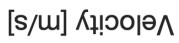

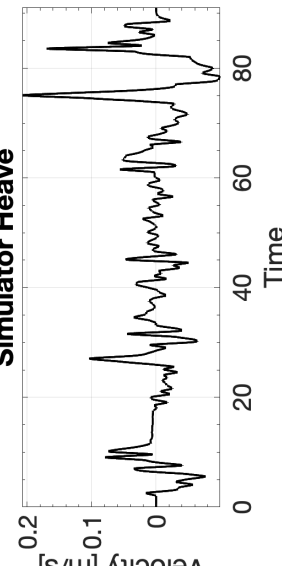

[s/u] К! ๖이

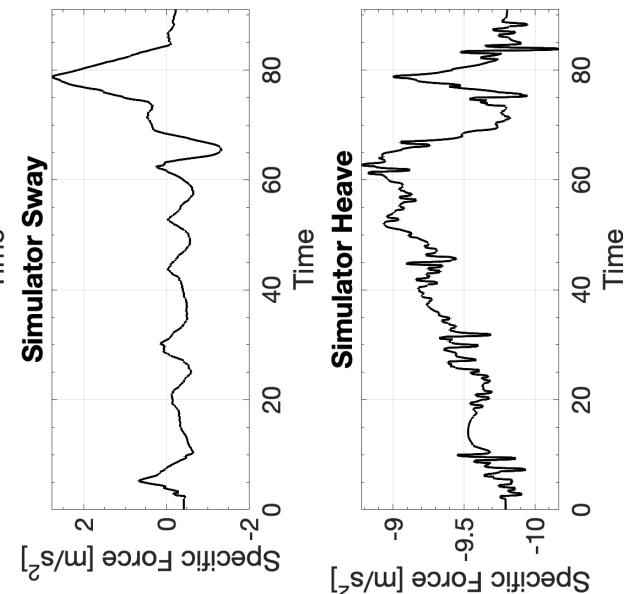

[

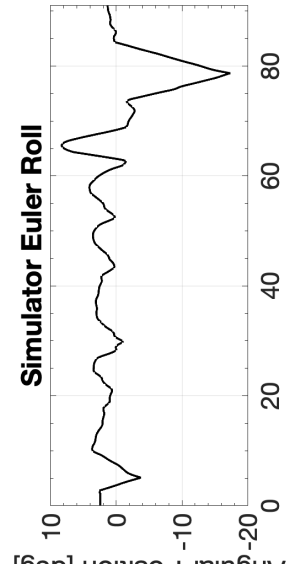

[бəp] uo!!!sod $\lambda$ גe|nб́u

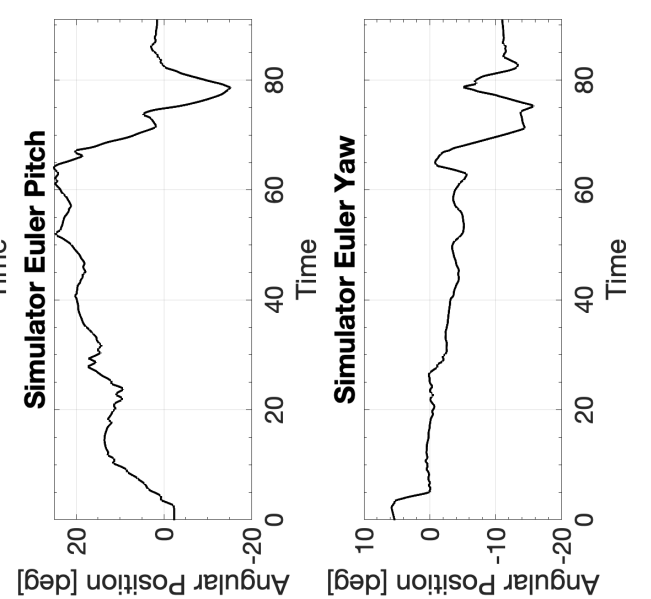

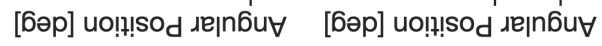

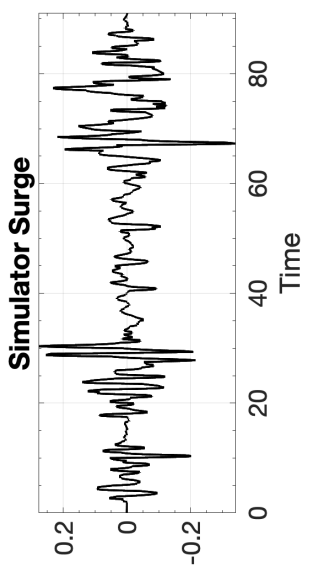

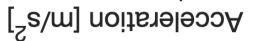

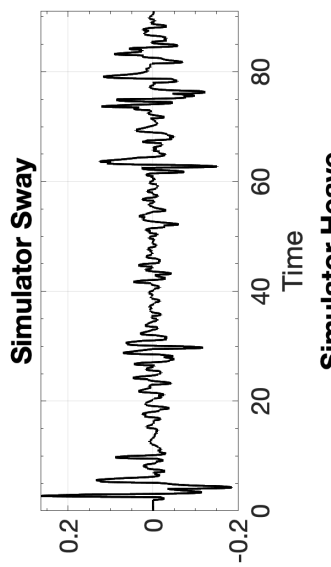

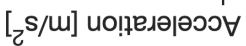

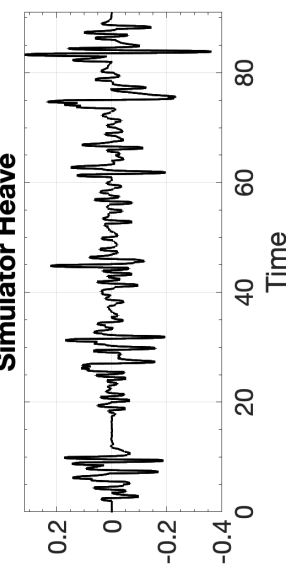

[ $\mathrm{s} / \mathrm{m}]$ ио!ฺедә|әэว

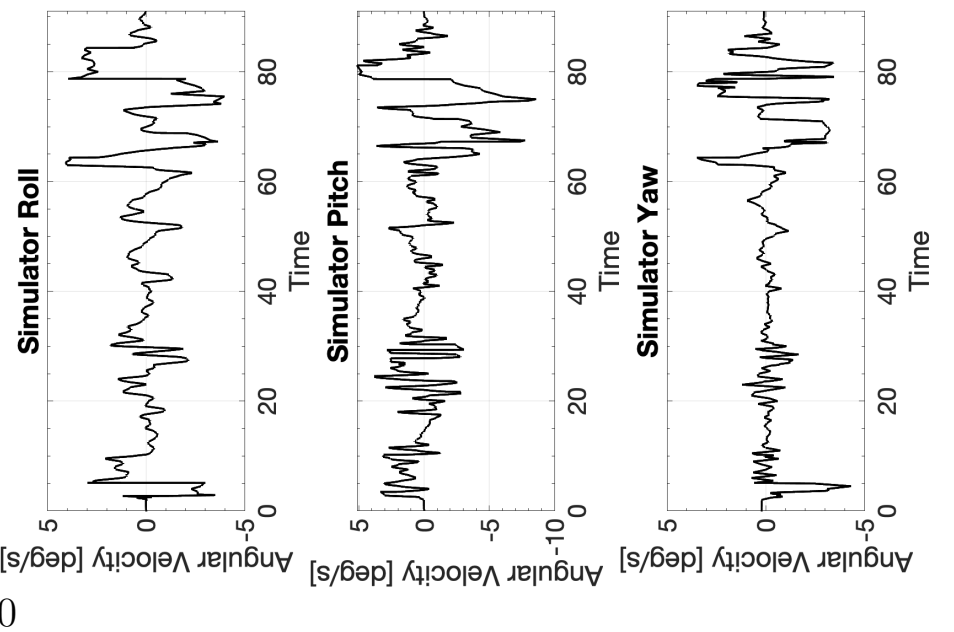



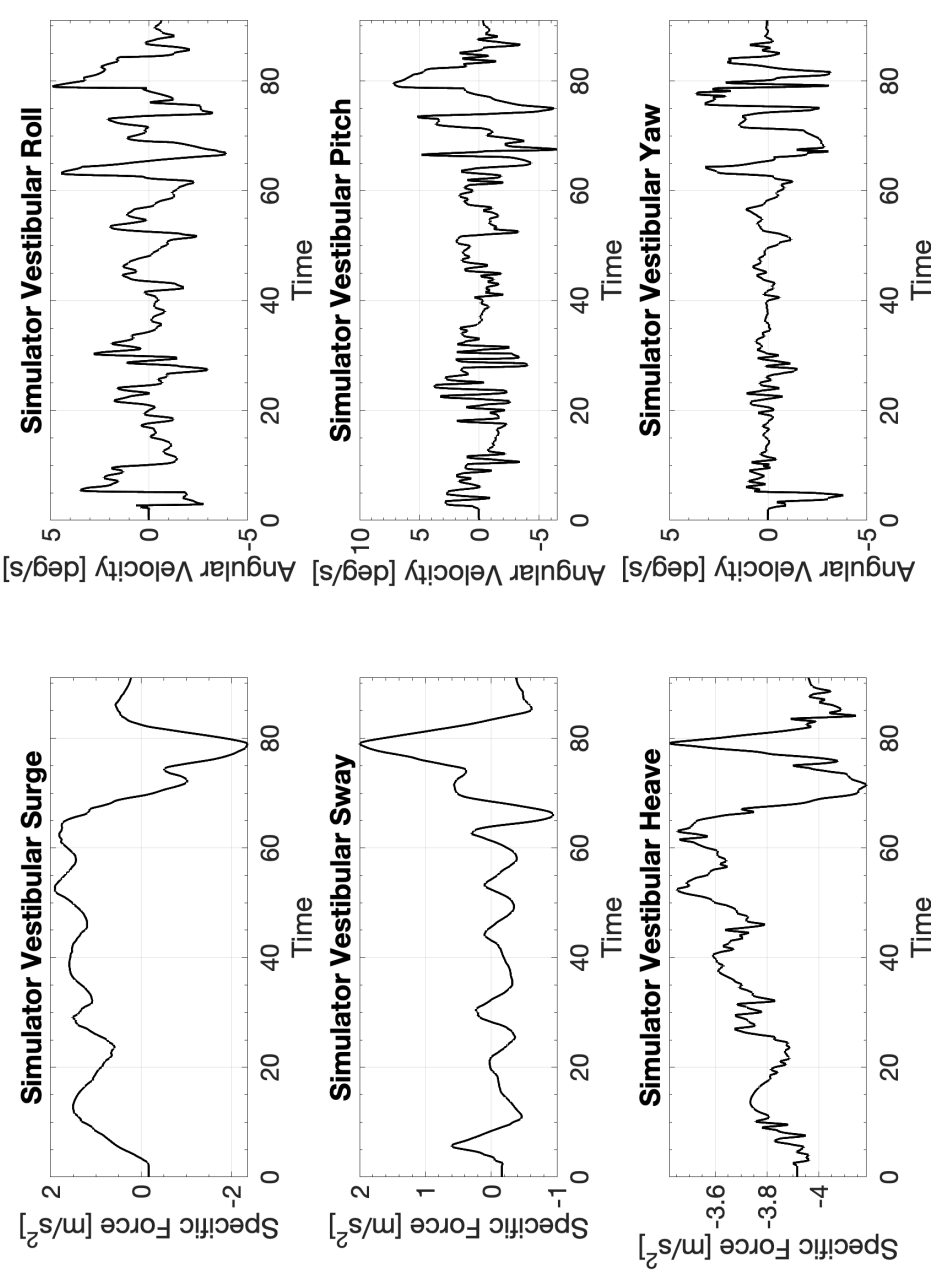


\section{D.16.2 CW2 Columbia 400 Stall Recovery}

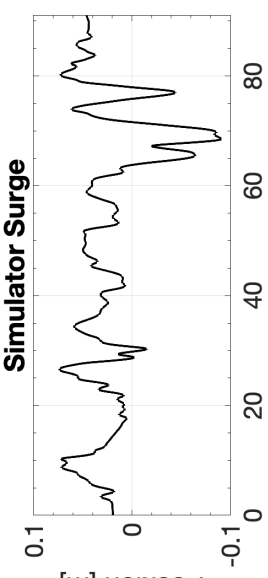

[w] uol!!sod

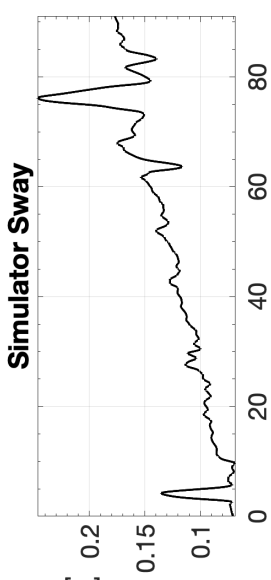

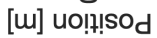

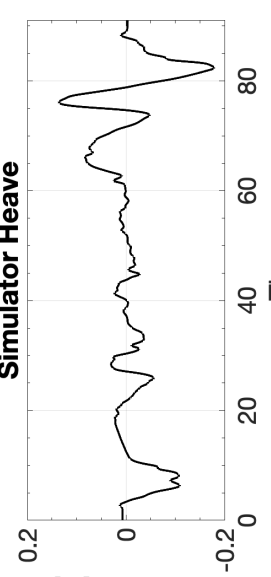

[w] uol!!sod

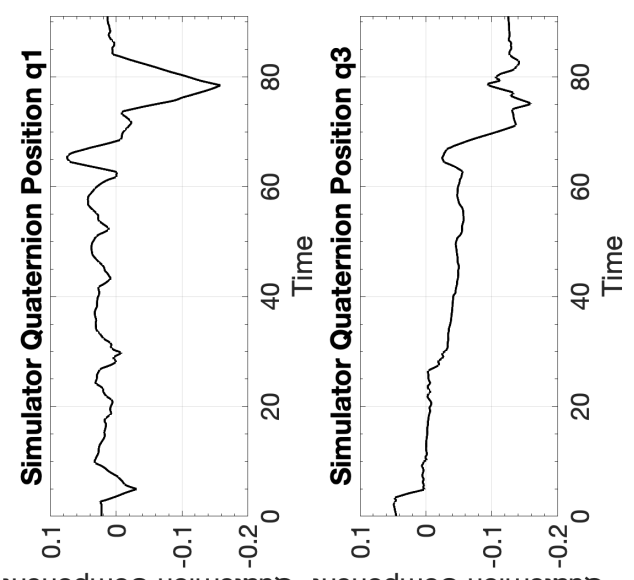

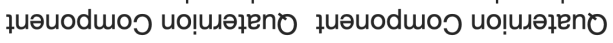
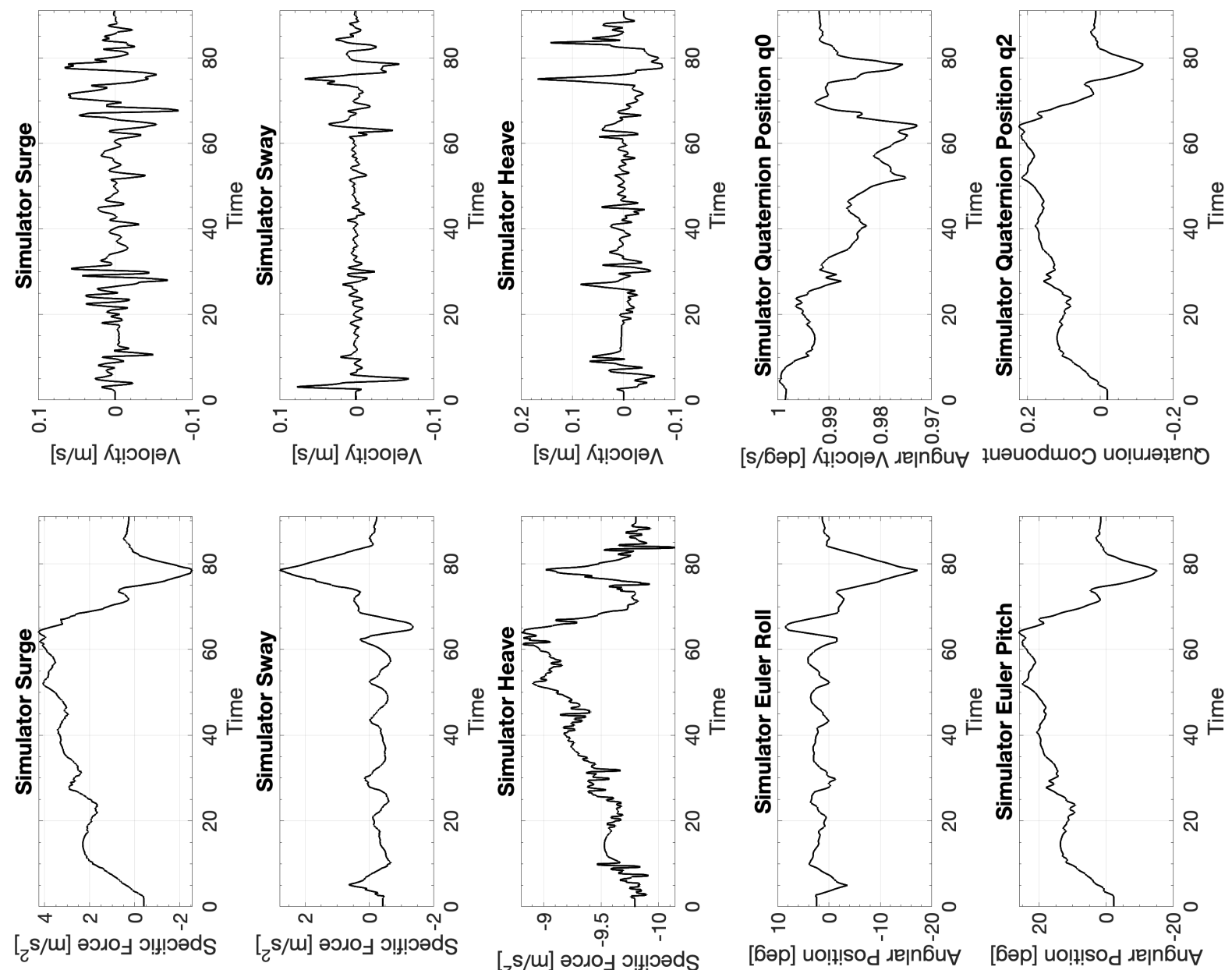

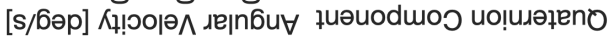

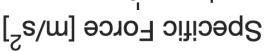

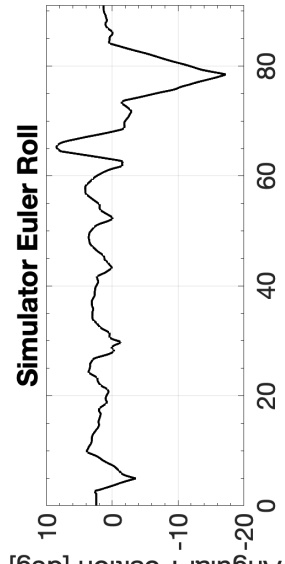

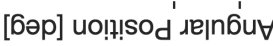

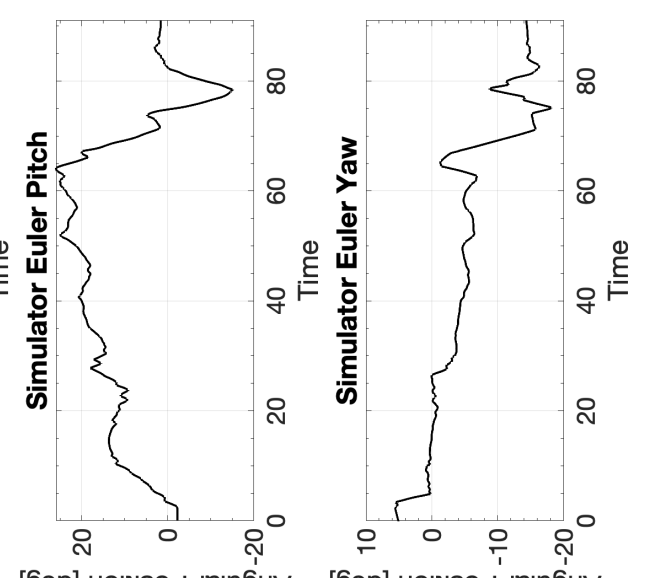

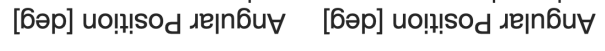

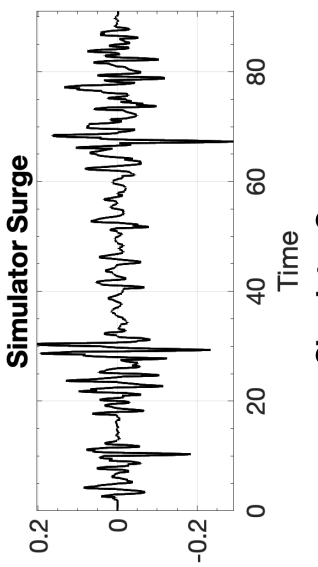

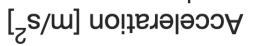

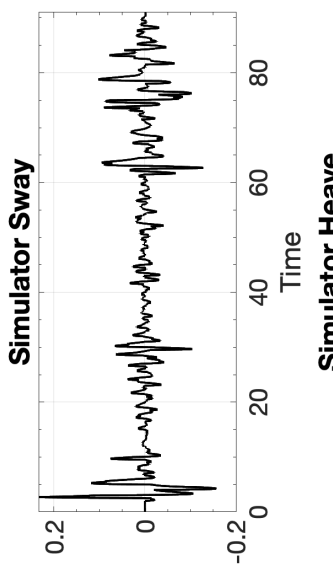

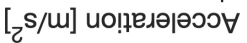

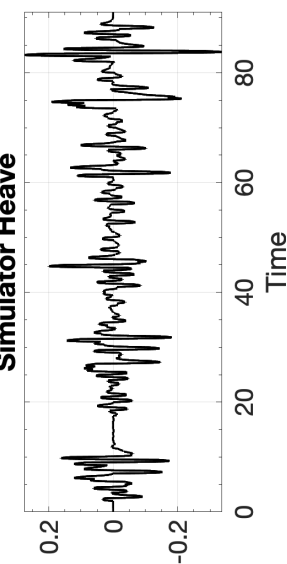

$[\mathrm{s} / \mathrm{s}]$ ио!ฺщелә|әэว

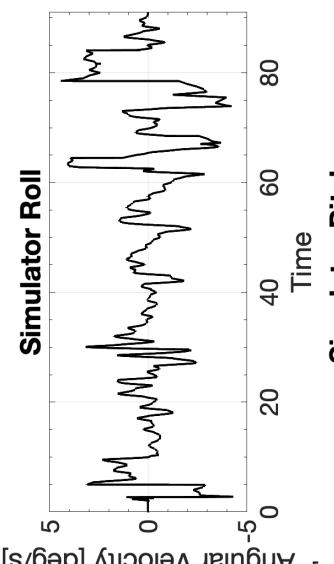

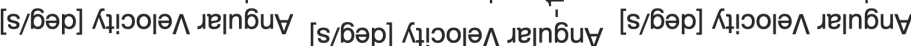

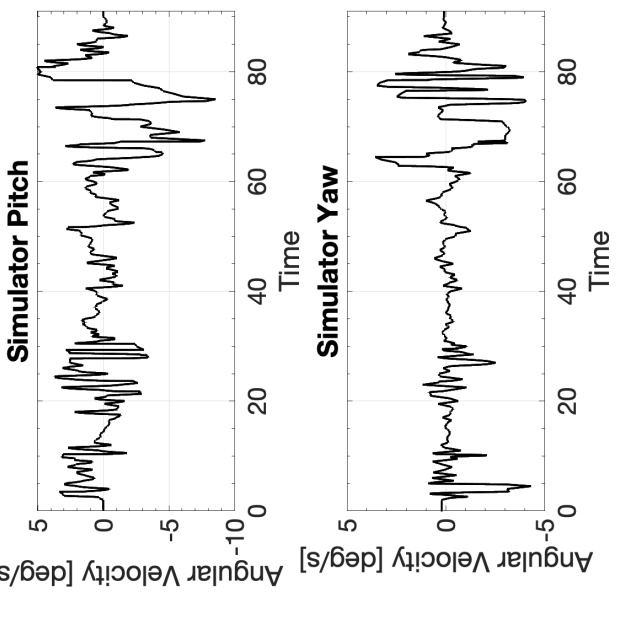



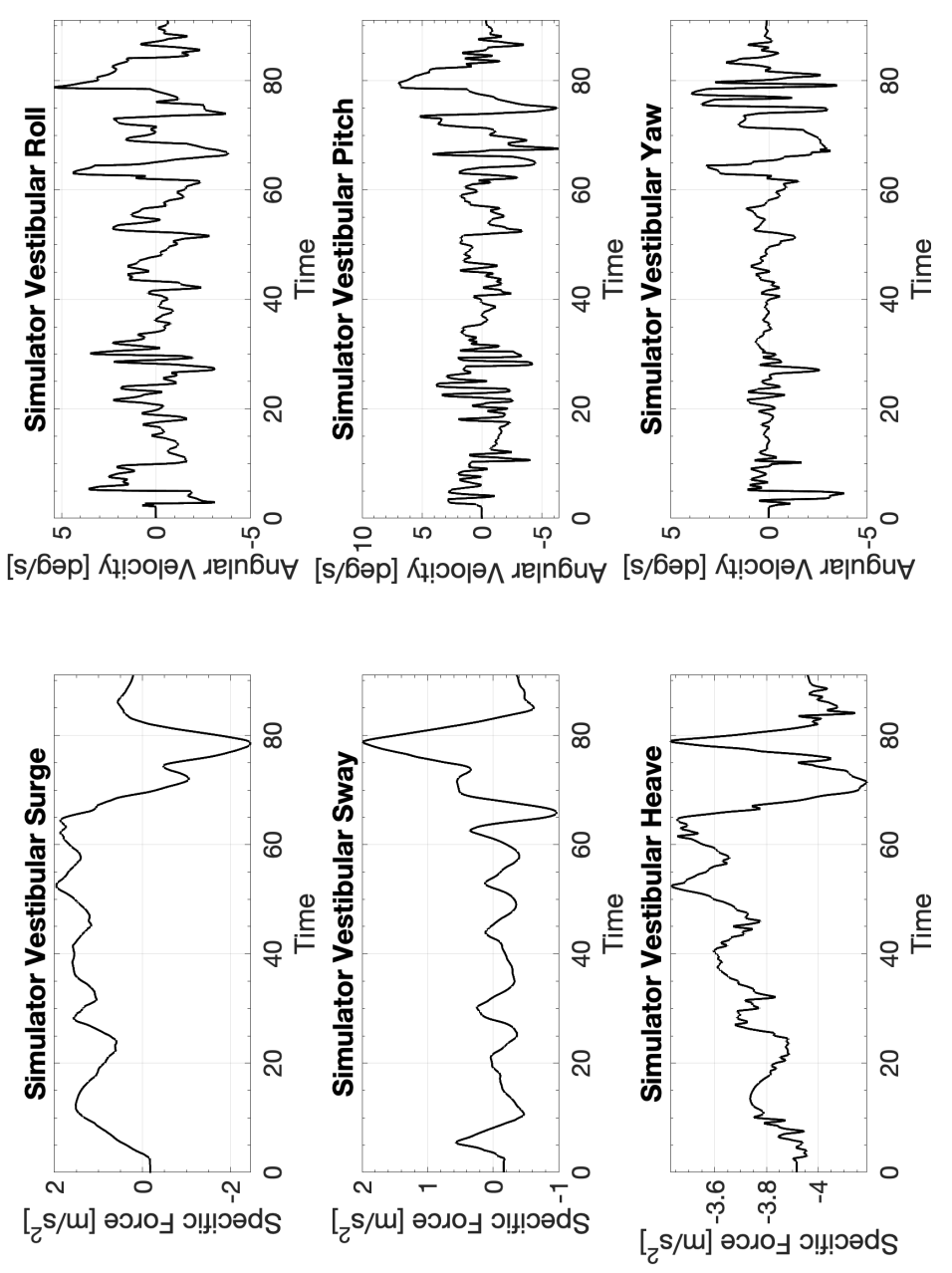


\section{D.16.3 CW3 Columbia 400 Stall Recovery}

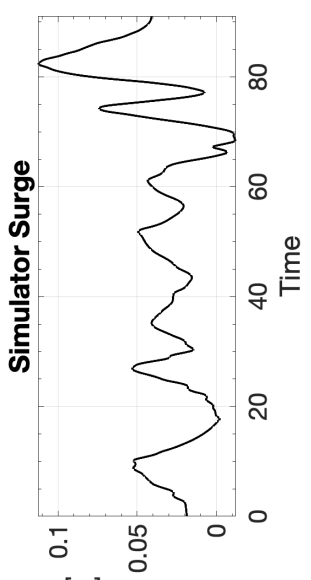

[w] uo!!!!sod

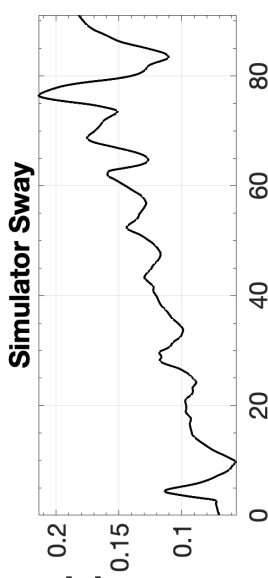

[w] uo!!!sod

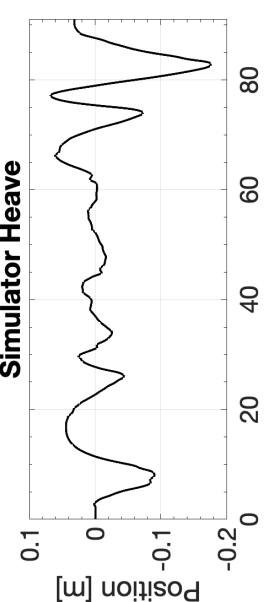

[u] uo!t!sod

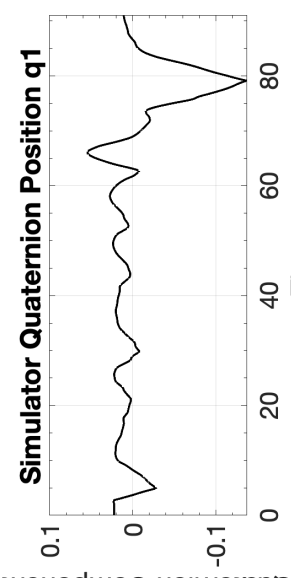

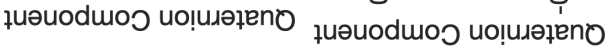

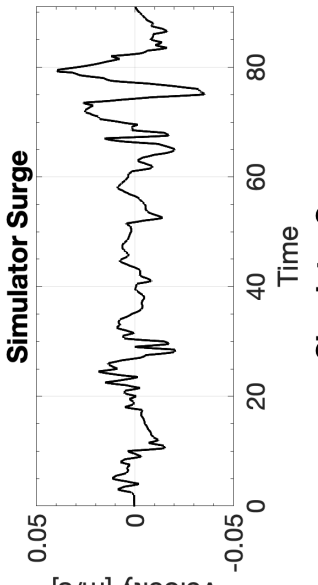

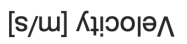

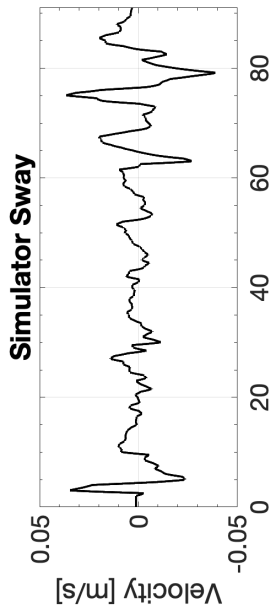

[s/m] Кұ!๐о|ә

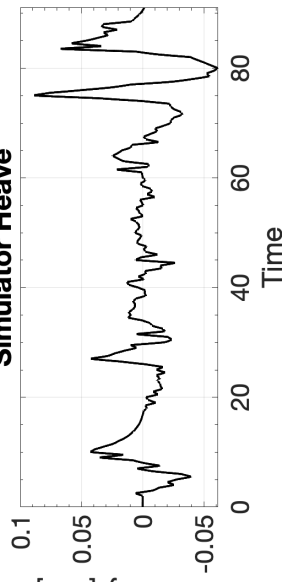

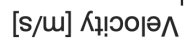
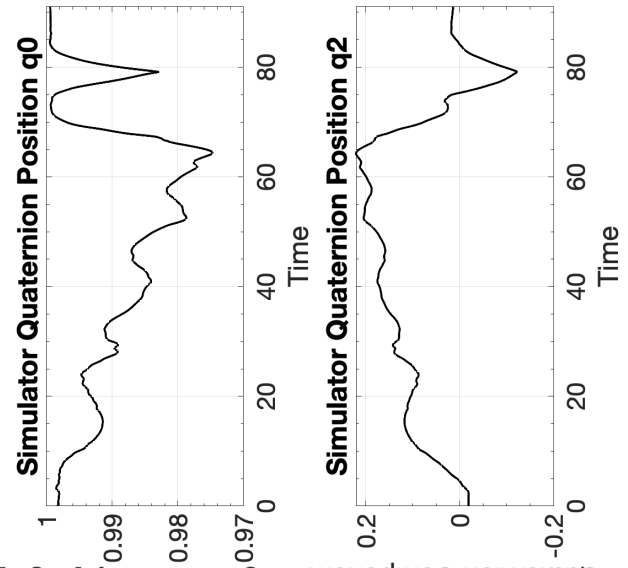

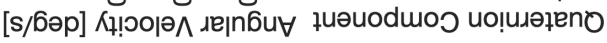

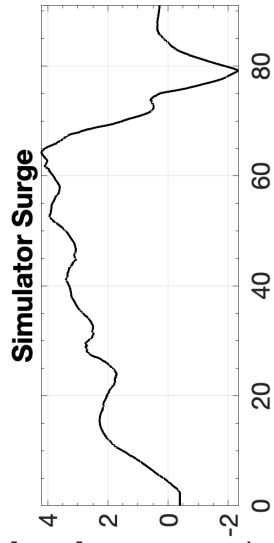

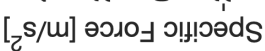
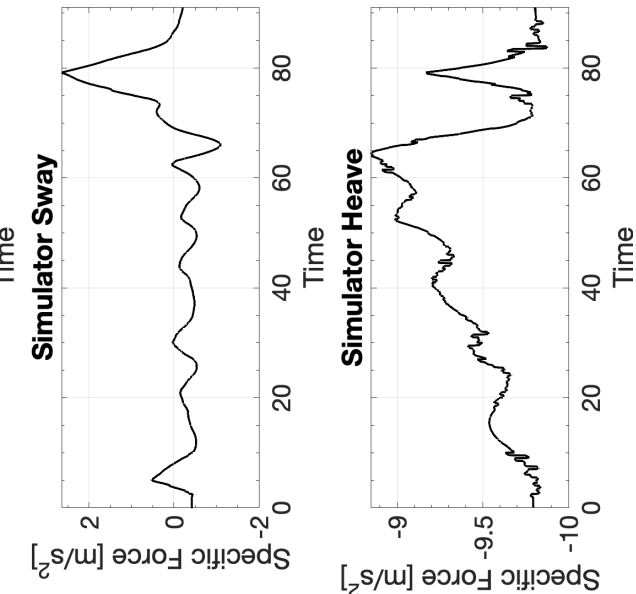

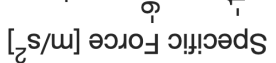

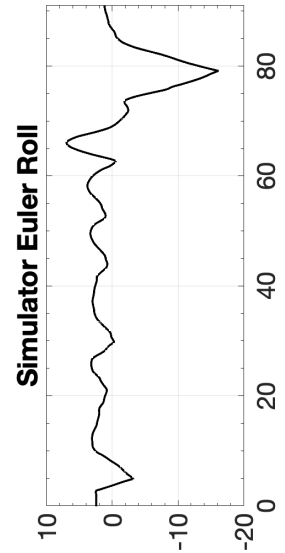

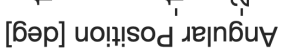

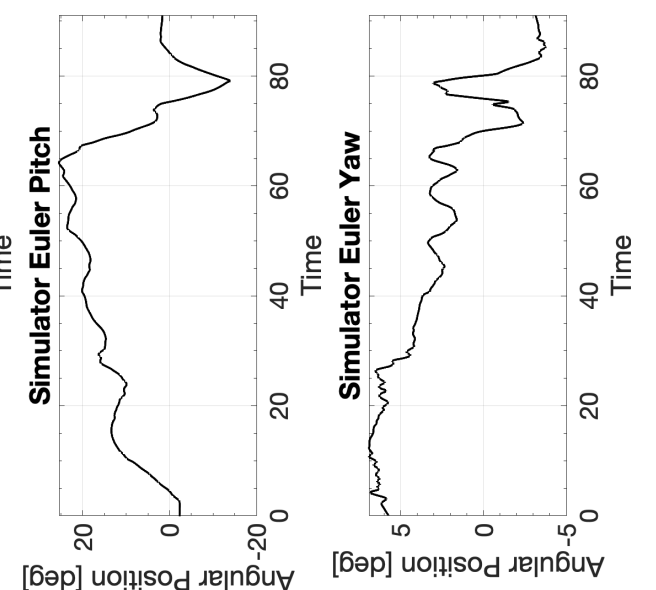

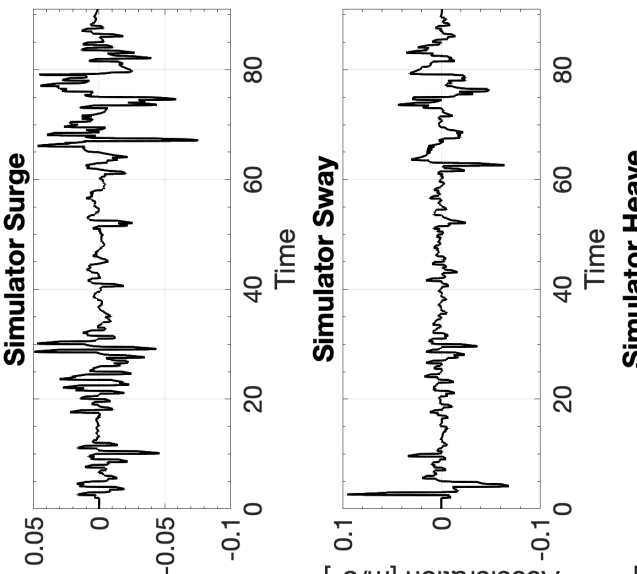

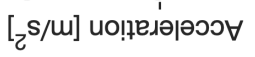

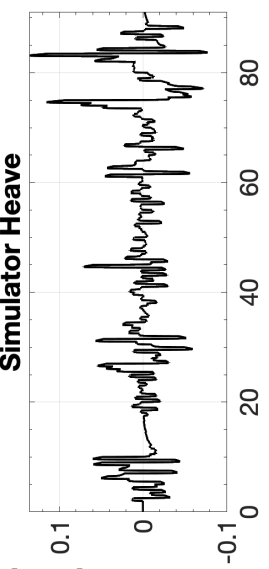

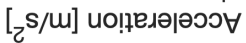

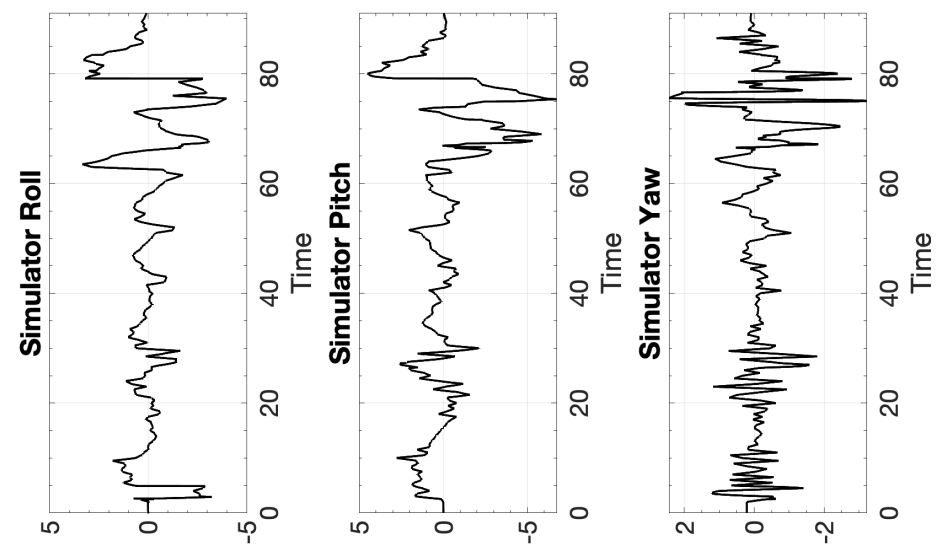

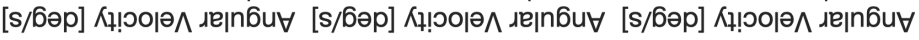




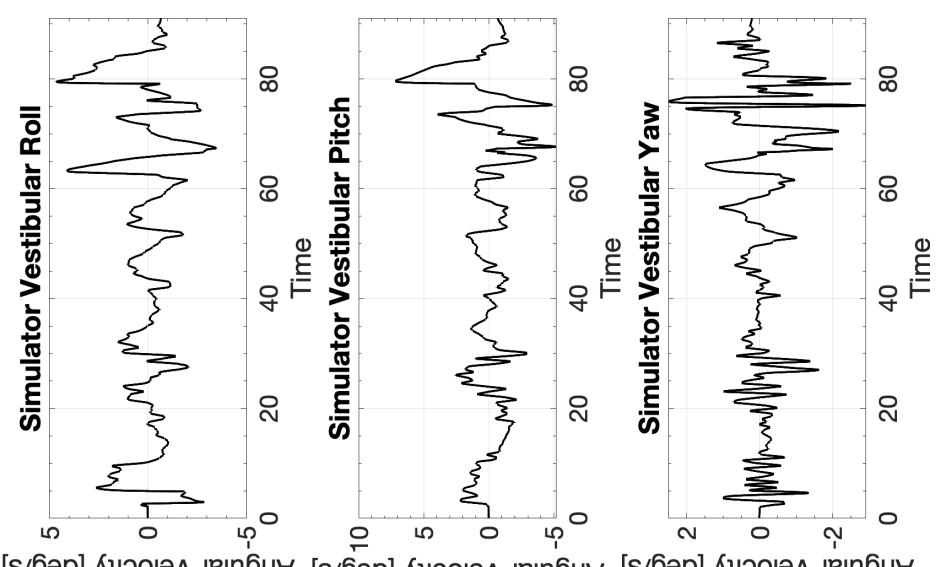

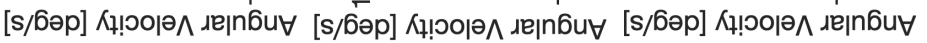

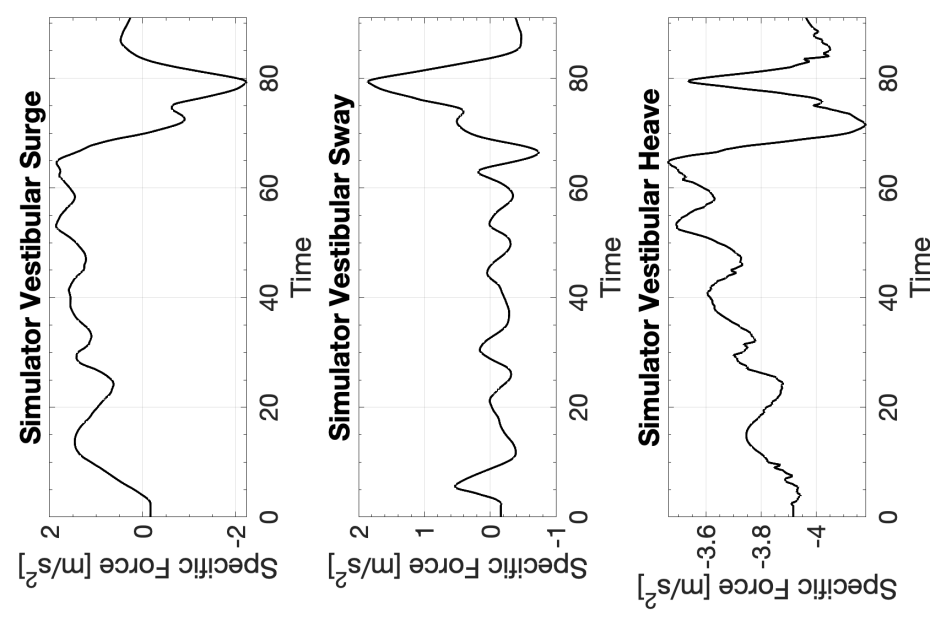

